\title{
Abstracts from the 2019 Annual Meeting of the Society of General Internal Medicine
}

\section{ABSTRACTS OF SUBMISSIONS ACCEPTED FOR PRESENTATION}

\section{SGIM 2019 Abstracts}

\section{'REACH-IN': USING THE INPATIENT ENCOUNTER TO EX- PAND ACCESS TO PRIMARY CARE-BASED TREATMENT FOR OPIOID USE DISORDER}

Trevor G. Lee; Michael Herscher; Reema Navalurkar; Leeza Hirt; Matthew Fine; Dillan Villavisanis; Benjamin Shuham; Jeffrey Weiss; Stacey Rubin; Linda Wang. Icahn School of Medicine at Mount Sinai, New York, NY. (Control ID \#3186383)

BACKGROUND: Maintenance opioid agonist therapy (OAT) with buprenorphine reduces mortality and is a well-established treatment for opioid use disorder (OUD). Despite the availability of effective therapy, approximately $80 \%$ of persons with OUD do not receive it. Patients with OUD represent an estimated $4-11 \%$ of hospitalized patients and are increasingly admitted for opioid-related complications. The inpatient encounter represents an opportunity to begin OAT and coordinate continued treatment in the primary care (PC) setting. Prior research has demonstrated that in-hospital buprenorphine initiation with linkage to outpatient care is associated with increased engagement in care and decreased opioid use.

METHODS: REACH-IN is a quality-improvement program to expand access to OAT by identifying hospitalized adult patients with OUD, initiating buprenorphine, and facilitating the transition to outpatient care at REACH, a PC-based program that offers buprenorphine. Based at a large, urban hospital, REACH-IN identifies eligible patients in two ways: 1) a daily electronic report of inpatient or emergency department encounters that documented opioid use, and 2) direct referrals from providers. Eligible patients were evaluated, , started on buprenorphine if appropriate, and titrated to a stable dose. All evaluated patients were offered a facilitated transition of care to REACH post-discharge. This involved meeting with REACH staff for a "warm" hand-off prior to discharge, receiving care coordination from a patient navigator, and being scheduled for a buprenorphine intake appointment within one week.

RESULTS: From April 23, 2018 to January 10, 2019 we screened 1589 encounters, 1559 (98.1\%) of which were identified via the report, $30(1.9 \%)$ via direct referrals. Of these, 855 had been discharged by time of screening. Of the remaining 734, $43(5.9 \%)$ patients were evaluated, $11(25.6 \%)$ of whom were started on buprenorphine in the hospital. Of the 32 who were not started, 16 $(50.0 \%)$ were referred to REACH, 4 of whom eventually started buprenorphine. Four (12.5\%) were referred to another OAT program. Of those started on buprenorphine in the hospital, 5 (45.5\%) attended an initial outpatient visit at REACH, $5(45.5 \%)$ were referred elsewhere for ongoing OAT, and 1 remains hospitalized, with $8(72.7 \%)$, $5(45.5 \%)$, and $4(36.4 \%)$ engaged in treatment at 30,60 , and 90 days, respectively.

CONCLUSIONS: Inpatient hospitalizations represent a 'reachable moment' to identify patients with OUD, initiate buprenorphine, and facilitate the transition to continued PC-based treatment. As hospitals see increasing numbers of opioid-related admissions, a structured partnership and facilitated transition between inpatient and outpatient providers may help expand access to OAT and increase engagement in care. These data provide preliminary evidence for the feasibility and efficacy of this collaborative model. 
"AN INCREDIBLE TOOL WITH MANY FLAWS." PRIMARY CARE PHYSICIANS' ATTITUDES TOWARD ELECTRONIC HEALTH RECORDS: AN INTERVIEW STUDY

Tania Moerenhout ${ }^{2}$ 2 ; Gary Fischer ${ }^{1}$; Marlies Saelaert ${ }^{2}$; An I. De Sutter $^{2}$; Veerle Provoost ${ }^{2}$; Ignaas Devisch ${ }^{2} .{ }^{1}$ University of Pittsburgh, Pittsburgh, PA; ${ }^{2}$ Ghent University, Ghent, Belgium. (Control ID \#3175488)

BACKGROUND: The electronic health record (EHR) has evolved into a complex multifunctional tool that facilitates the sharing of medical information with both patients and their health care providers, even across institutions. Although much has been written about positive and negative effects of the EHR on efficiency, safety, and physician burn-out, many questions remain unanswered regarding the ethical dimensions of the EHR and its effect on the patientdoctor relationship. The aim of this study is to understand how general internists (PCPs) integrate the EHR into their workflow and what ethical questions arise while sharing medical information.

METHODS: The first author held semi-structured interviews between February and May 2017 with 14 PCPs recruited through a purposive, convenience-based sampling process from an academic general internal medicine practice of 46 faculty and 46 residents in Pittsburgh, PA. The clinic uses Epic software with a patient portal. Data were analyzed against a theoretical background of postphenomenology (combining the empirical orientation of Science and Technology Studies with a more conceptual philosophical analysis) and moral mediation (examining how "technologies mediate moral decisions and actions" (Verbeek. Moralizing Technology. Understanding And Designing The Morality Of Things. )), using thematic analysis in an iterative process. Three interviews were coded by 2 coders and discussed until consensus was reached. The first author continued the coding process with the established code book, expanding with new codes as needed. The thematic analysis process adhered to the six phases described by Braun and Clarke of data familiarization, code generation, theme identification, theme revision, theme definition and production of the final report.

RESULTS: Four major themes and 1 minor theme were identified. Major themes: (1) PCPs describe the EHR as a medicine with side effects, for which they provide suggestions for improvements; (2) The transition to a digitally shared record raises ethical dilemmas related to autonomy and paternalism; (3) Although use of the EHR often disturbs rapport with the patient, it can support patient-doctor communication when it becomes an active part of the conversation; (4) The digitalization of doctor-doctor communication has a complex effect on the concept of trust. Minor theme: A shared record may cause health care workers (and their relatives) to avoid seeking help for sensitive issues.

CONCLUSIONS: By understanding physicians' coping strategies and suggestions for improvement, and by focusing on the active integration of the EHR into patient-doctor communication, EHR design could be adapted to improve quality of care. EHRs raise underexplored issues of autonomy vs paternalism regarding sharing information with patients. More research is needed to understand the unexpected effects of the technology on moral dimensions of the patient-doctor interaction, in particular on autonomy and trust.

\section{"I DIDN'T THINK IT WAS FAIR BECAUSE I'M NOT AN ABUSER": OPIOID TAPERING EXPERIENCES AMONG RA- CIAL AND ETHNIC MINORITIES IN A PRIMARY CARE SETTING \\ Hector R. Perez ${ }^{2}$; Ariana G. Pazmino ${ }^{2}$; Michele J. Buonora ${ }^{1}$; Joanna L. Starrels ${ }^{2} .{ }^{1}$ Albert Einstein College of Medicine and Montefiore Medical Center, Bronx, NY; ${ }^{2}$ Albert Einstein College of Medicine \& Montefiore Medical Center, Bronx, NY. (Control ID \#3184347)}

BACKGROUND: Substantial evidence exists that pain management practices differ by patient race and ethnicity, and that racial and ethnic minorities perceive their pain care as biased. However, prior studies have not examined the perspectives of racial and ethnic minorities on chronic opioid therapy (COT) about their experiences with opioid tapering.

METHODS: In this qualitative study, we conducted semi-structured 1:1 telephone interviews with patients who experienced opioid tapers in a primary care setting in a large urban health system in the Bronx, NY. Patients were eligible if they were at least 18 years old and were prescribed a stable dose of COT before undergoing a $30-100 \%$ dose reduction (opioid taper) between 2016 and 2017, as confirmed by manual chart review. Patients were ineligible if they had a history of cancer at the initiation of the taper or if they were not retained in care in the health system during the taper. Interview questions sought to understand patients' experiences and beliefs about opioid tapering, including perceived barriers and facilitators. Sociodemographics were collected by self-report and from the electronic medical record. Transcripts were coded and analyzed by two authors using a thematic analysis approach.

RESULTS: Of 43 patients contacted, 10 agreed to participate. Average age was 53.9, 5 were male, 6 were Black/African-American, and 4 were Hispanic. The overarching theme was participants felt unfairly targeted for a taper given their ongoing chronic pain; within this were three prominent sub-themes reflecting judgments derived from personal experiences. First, participants distinguished themselves from other patients who deceive providers to obtain opioids: "I know of a lot of people who only gets them to sell them, which makes it bad for people who really need it." Second, participants lacked trust in the healthcare system: "[The providers are] just telling me whatever to get a paycheck. They don't really care." Third, participants perceived themselves to have low risk for negative consequences of opioids: "If I'm in pain I'm going to take my medicine...I'm not that weak to sit there and...overdose." Key facilitators of successful tapering included support from friends, family, and providers: "[The taper] was a great experience...He's the best doctor I could ever ask for...He works for me...He's been with me the whole time."

CONCLUSIONS: In this sample of racial and ethnic minorities who experienced opioid tapers, we found that feeling unfairly targeted for a taper was a predominant theme. Participants described how their experiences with others who use opioids, with the healthcare system, and with their medications contributed to feelings of inequity. Future interventions for racial and ethnic minorities should address real and perceived inequities associated with tapering to promote patient engagement and facilitate successful tapering experiences.

\section{"IF YOU CAN'T TALK TO YOUR DOCTOR ABOUT IT, WHO CAN YOU TELL?" A QUALITATIVE STUDY OF PATIENT ACCEPTABILITY AND PREFERENCES FOR SOCIAL RISK SCREENING IN HEALTH SETTINGS.}

Elena Byhoff $^{1,2}$; Emilia De Marchis ${ }^{3}$; Nancy E. Adler ${ }^{4}$; Kelly M. Doran $^{5}$; Danielle Hessler ${ }^{3}$; Stephanie Ettinger de $\mathrm{Cuba}^{7}$; Eric Fleegler ${ }^{8}$; Nicholas Gavin ${ }^{9}$; Amy G. Huebschmann ${ }^{10}$; Stacy T. Lindau $^{11}$; Maria C. Raven ${ }^{12}$; Elizabeth L. Tung ${ }^{11}$; Alicia Cohen ${ }^{6}$; Susan Jepson ${ }^{17}$; Wendy Johnson ${ }^{13}$; Cara C. Lewis ${ }^{16}$; Eduardo Ochoa $^{14}$; Aric Prather ${ }^{3}$; Megan Sandel ${ }^{15}$; Richard Sheward ${ }^{15}$; Caroline Fichtenberg ${ }^{4}$; Laura M. Gottlieb ${ }^{4} .{ }^{1}$ Tufts Medical Center, Boston, MA; ${ }^{2}$ Tufts University School of Medicine, Boston, MA; ${ }^{3}$ University of California, San Francisco, San Francisco, CA; ${ }^{4}$ UCSF, San Francisco, CA; ${ }^{5} \mathrm{NYU}$ School of Medicine, New York, NY; ${ }^{6}$ Ann Arbor VA, Providence, RI; ${ }^{7}$ Boston University School of Medicine, Boston, MA; ${ }^{8}$ Boston Children's Hospital, Boston, MA; ${ }^{9}$ Columbia University Vagelos College of Physicians and Surgeons, Brooklyn, NY; ${ }^{10}$ University of Colorado School of Medicine, Aurora, CO; 
${ }^{11}$ University of Chicago, Chicago, IL; ${ }^{12} \mathrm{UCSF}$ School of Medicine, Berkeley, CA; ${ }^{13}$ La Familia Medical Center, Santa Fe, NM; ${ }^{14}$ University of Arkansas for Medical Sciences, Little Rock, AR; ${ }^{15}$ Boston Medical Center, Burlington, VT; ${ }^{16}$ Kaiser Permanente, Seattle, WA; ${ }^{17}$ Hennepin Healthcare, Minneapolis, MN. (Control ID \#3180063)

BACKGROUND: As research supporting potential benefits of social risk screening in health settings continues to emerge, there is little data on the patient perspective on social screening. The aim of this study is to describe the perspectives and preferences of patients and caregivers on being screened for social risks in diverse health care settings.

METHODS: As part of a larger mixed methods multi-site study, we conducted semi-structured interviews lasting approximately 30 minutes with patients or caregivers who had completed the Center for Medicare and Medicaid Innovation (CMMI) social risk screening tool. After completion of the screening questions, 5 randomly selected respondents from each of 10 study sites were invited to participate in an interview. Interviews were conducted in English or Spanish. The interview guide asked about reactions to social risk screening and screening acceptability, preferences on screening administration, prior experiences that informed perspectives, and expectations for social assistance. Interviews were recorded, transcribed and translated. Two coders used basic thematic analysis and constant comparative methods to identify codes, group codes into unified themes and map themes into domains of screening acceptability.

RESULTS: Fifty adult patients or caregivers participated in semistructured interviews across all study sites, which included 6 primary care clinics and 4 emergency departments. Respondents were $78 \%$ female, $36 \%$ Black, $32 \%$ Hispanic, $20 \%$ caregivers, and $71 \%$ reported having one or more social risk factor. There was broad consensus among interviewees across all clinical sites that social risk screening was acceptable. Several themes emerged: (1) respondents felt screening was the "right thing to do;" (2) respondents identified framing and compassionate approach as the most important aspects of administration; (3) respondents had insight into the connections between social risks and physical and mental health. Despite overall agreement that social risk screening is appropriate, respondents did not expect their health care team to address or resolve all of the identified issues. Interviewees felt referrals out to social services and resource sheets were adequate, and that there was benefit to the act of screening itself. Patients emphasized that screening should be done with empathy while protecting patient confidentiality.

CONCLUSIONS: Respondents agree that social risk screening is important, acceptable, and relevant to health. Respondents believed social risk screening would be most acceptable when implemented by a clinic staff member trained to ensure privacy and compassion. Despite published concerns about futility of social risk screening when social resources are inadequate, respondents expressed that they did not expect the health care system, and physicians in particular, to solve unmet social problems. Done appropriately, screening for social risk can build trust and strengthen relationships between patients and health care providers.

"LAUGH AND BACK OUT OF THE ROOM:" FEMALE PHYSICIAN STRATEGIES FOR DEALING WITH SEXUAL HARASSMENT BY PATIENTS

Amy H. Farkas $^{2,1}$; Cecilia Scholcoff ${ }^{2,1}$; Cynthia Kay ${ }^{1,2}$; Julie Lucas ${ }^{2,1}$; Sarah Nickoloff $^{2}, 1$; Kathlyn Fletcher ${ }^{2,1}$; Jeffrey L. Jackson ${ }^{1,2}$. ' Zablocki VAMC, Milwaukee, WI; ${ }^{2}$ Medical College of Wisconsin, Milwaukee, WI. (Control ID \#3183047)

BACKGROUND: Sexual harassment of providers by patients has not received as much attention as harassment from other sources. Our purpose was to assess the types of harassment encountered, level of discomfort and strategies used by providers to deal with patient sexual harassment.
METHODS: We used a mixed-method approach combining a survey and qualitative, semi-structured interviews at a Midwestern academic medical center and affiliated VA. The survey assessed provider characteristics, forms of sexual harassment experienced as well as a Likert-scaled assessment of the degree the behavior was bothersome. Semi-structured interviews assessed provider experience with sexual harassment, how they dealt with it, whether they reported it, and strategies they used to mitigate harassment. We used purposive sampling, grounded theory and duplicate coding to generate themes.

RESULTS: The average participant was 43 years old and graduated medical school 17 years ago (range 2-45). Some forms of verbal harassment were nearly universal (being mistaken for a nurse, remarks on appearance, questions about marital status, sexist jokes). Physical harassment was less common (90\% had experienced leering and standing too close, $44 \%$ had experienced suggestive exposure of patient's body). The commonly experienced forms of harassment were mostly rated as "bothered a little," while physical forms of harassment were nearly universally rated as "extremely bothersome." During interviews, respondents described predisposing factors that made harassment more likely, such as patient factors (cognitive impairment, older age), provider factors (experience), and cultural factors. Harassment took many forms and occurred across a spectrum, from compliments/comments about appearance to addressing questions to the males in the room rather than the female attending physicians, to sexual propositions or actual grabbing. These episodes impacted not only the targeted women, but also other patients who witnessed the events, other staff, and learners. The respondents identified several strategies for dealing with patient-initiated sexual harassment. These strategies included preventing it (modifying dress, having only male providers for certain patients), terminating it (using humor, redirecting the patient) and coping with it (debriefing with colleagues). Respondents often used nonconfrontational methods for diffusing situations, but later regretted not being more direct.

CONCLUSIONS: Some forms of verbal sexual harassment by patients are nearly universal, though are experienced as minimally bothersome. More serious forms of harassment are less common, and respondents rated these as extremely bothersome. Participants identified a number of factors that increase the likelihood of experiencing harassment as well as strategies for mitigating and managing it

\section{"MAY I PLEASE SEE YOUR INSURANCE CARD AND ID?": HOW PATIENTS WITH LIMITED-ENGLISH PROFI- CIENCY COMMUNICATE WITH ANCILLARY STAFF DURING THE PRIMARY CARE VISIT}

Lily Kornbluth $^{1}$; Celia P. Kaplan ${ }^{2}$; Leah S. Karliner ${ }^{1} .{ }^{1}$ UCSF, San Francisco, CA; ${ }^{2}$ University of California San Francisco, San Francisco, CA. (Control ID \#3165795)

BACKGROUND: Limited English Proficiency (LEP) is associated with health disparities which can be ameliorated with use of professional interpretation. Numerous studies have investigated communication between clinicians and patients with LEP. However, little is known about communication between ancillary staff and patients with LEP. This study describes communication patterns between front desk staff and medical assistants (MAs) and patients with LEP in an academic primary care setting.

METHODS: This descriptive study is part of the Language Access Systems Intervention (LASI) study on communication for patients with LEP conducted at a large, urban, academic primary care practice. In the LASI study, 735 patients with LEP (Spanish, Cantonese, and Mandarin-speaking) completed a telephone survey 
that included questions about language barriers with front desk staff and MAs. Following analysis of these survey data, we conducted semi-structured interviews with 6 front desk staff, 6 MAs, and 3 physicians in the practice to lend qualitative perspective to the patient survey results. Detailed notes were taken during the semi-structured interviews with subsequent thematic analysis.

RESULTS: Among the 735 participants with LEP surveyed, half $(49 \%)$ reported speaking in their preferred non-English language directly with bilingual front desk staff, and $38 \%$ directly with MAs. However, one-third (30\% with front desk, 32\% with MAs) used English to communicate. Among those using English, $76 \%$ rated their ability to speak English as 'not well' or 'not at all'. Professional interpreter use was minimal (3\%) with ancillary staff overall. Communication patterns varied by language, with Spanish-speakers using English more frequently than other Chinese-speakers $(51 \%$ vs $25 \%$ of Cantonese and Mandarin-speakers). In semi-structured interviews, front desk staff and MA's reported that often patients' limited English was sufficient for basic tasks, although they acknowledged that more complex tasks required professional interpretation (e.g. medication review). However, they rarely accessed professional interpretation due to perceived time constraints. Physicians commented that they took on extra tasks during visits for patients with LEP, such as medication reconciliation, because ancillary staff were not using interpreters and then not completing the tasks they normally would with English-speakers.

CONCLUSIONS: Our results highlight a previously undocumented communication challenge in ambulatory care: minimal professional interpreter use by front desk staff and MAs leading to many patients with LEP getting by with very limited ability to communicate in English. As a result, physicians feel the burden of extra tasks during already complex visits. Suboptimal communication with ancillary staff makes true team-based care challenging, and must be addressed in order to provide high quality, equitable care for LEP populations.

\section{"SO I DO HAVE A COCKTAIL. IT'S VERY POPULAR": NON-OPIOID MEDICATION AND SUPPLEMENT MISUSE AMONG OPIOID USERS EXPERIENCING HOMELESS- NESS}

Avik Chatterjee $^{4,}$ 1; Shankar Ramkellawan ${ }^{2}$; Diego B. Lopez ${ }^{3}$; Jessie M. Gaeta ${ }^{4}$; Kamala Smith ${ }^{4}$; Travis P. Baggett ${ }^{5,}{ }^{4}$. ${ }^{1}$ Harvard Medical School, Cambridge, MA; ${ }^{2}$ Harvard College, Boston, MA; ${ }^{3}$ Harvard Medical School, Boston, MA; ${ }^{4}$ Boston Health Care for the Homeless Program, Boston, MA; ${ }^{5}$ Massachusetts General Hospital, Boston, MA. (Control ID \#3175784)

BACKGROUND: While the impact of the opioid epidemic in the United States continues, certain aspects of the epidemic have changed, requiring updates in clinical practice. Recent evidence suggests that the practice of combining opioid use with nonopioid medications to alter the opioid high is common, though little is known about this phenomenon and how best to address it. METHODS: We recruited adults who reported current opioid use from a low-threshold engagement center targeting individuals experiencing homelessness in Boston, Massachusetts. We conducted semi-structured interviews to explore the practice of combining non-opioid medications and other substances with opioids. We used Borkin's immersion/crystallization approach to analyze transcripts to uncover themes.

RESULTS: Twenty-two individuals completed interviews. Mean age of participants was 36.3 years, and $36 \%(8 / 22)$ reported female gender. Fifty-nine percent (13/22) reported White race, 36\% (8/22) reported Black race, and 64\% (14/22) reported Latino ethnicity. Combining substances with opioids to alter the high was a common practice: "So I do have a cocktail. It's very popular." Participants reported use of clonidine, gabapentin, benzodiazepines, Phenergan, Adderall, Seroquel, barbiturates, Nyquil, Robitussin, and Tylenol PM as well as alcohol and candy. Participants most commonly reported purchasing these substances on the street, but less commonly reported stealing them or getting them from a doctor. Participants identified several purposes of combining substances: to augment the high ("I heard [gabapentin] gets you a higher high like really hits you so I wanted to get that"), to alter the high ("Never done mushrooms but [Robitussin is] similar to that"), or to offset the opioid high ("So, you know, the sugar [from candy] would lift up the high"). Importantly, participants also reported combining substances to treat psychiatric symptoms: "I use drugs to selfmedicate. If I [feel] depressed." Individuals commonly reported learning about combining substances "from people on the street" but also reported learning from the internet and television: "Ah, from TV. [Nyquil]'s supposed to make you sleepy so I thought it would give the same effect with the opioid." Safety was either not a concern or was outweighed by perceived benefits: "When I'm doing it I don't care about safety. I be wanting to feel nothing." Participants also felt that combining over-the-counter or prescription medications would be safe: "Not think about [safety] cuz it's not that strong like the pills. It's just for sleep."

CONCLUSIONS: Combining a wide variety of substances with opioids is common, and driven by a diverse range of motives. Clinicians who care for opioid-involved patients should consider screening for concurrent use of other substances and discussing the potential risks of this practice. Adequately diagnosing and treating psychiatric conditions may also decrease risky combinations of substances with opioids.

\section{\#METOO: A NATIONAL STUDY OF FORCED SEXUAL} INITIATION AMONG US WOMEN

Laura Hawks ${ }^{1}{ }^{4}$; Stephanie Woolhandler ${ }^{2}$; David Himmelstein ${ }^{3}$; David Bor ${ }^{1}$; Adam Gaffney ${ }^{1}$; Danny McCormick ${ }^{4}$. ${ }^{1}$ Cambridge Health Alliance, Cambridge, MA; ${ }^{2}$ Cambridge Hospital, New York, NY; ${ }^{3}$ City University of New York at Hunter College, New York, NY; ${ }^{4}$ Harvard Medical School / Cambridge Health Alliance, Cambridge, MA. (Control ID \#3185066)

BACKGROUND: The \#MeToo movement has highlighted the frequency of sexual assault. However, no previous studies have assessed the incidence of sexual assault during women's first sexual encounter, or its reproductive and general health sequelae.

METHODS: We analyzed data on female respondents to the 20112017 National Survey of Family Growth, a nationallyrepresentative, in-person survey conducted by the CDC. We excluded women with no history of vaginal intercourse. Using survey weights provided by the CDC, we assessed the number and characteristics of women who experienced forced sexual initiation (FSI), and the age of the male partner. We also examined the association between FSI and subsequent reproductive outcomes, gynecologic illnesses and other health measures. We used linear regression to assess continuous outcomes and logistic regression for binary outcomes. Models controlled for women's age, poverty status, race, current employment and place of birth; for reproductive outcomes we also adjusted for marital status and education.

RESULTS: Among women age 18-44 at the time of the survey $6.5 \%$ ( $n=993$, representing 3,351,733 nationally) reported FSI. The mean age at first intercourse for those experiencing FSI was 1.3 years younger than for other women, 16.6 years [95\% CI 15.817.4] vs. 17.9 [17.7-18.1]. The age discrepancy of first male partners was much larger, 27.0 [24.8-29.2] vs. 21.0 [20.6-21.3] years. $29.0 \%$ of women with FSI (vs. $12.2 \%$ without FSI) reported 
sexual initiation before age 15 and $6.0 \%$ of women with FSI (vs. $0.1 \%$ without FSI) reported sexual initiation before turning 11. Women who experienced FSI were somewhat less likely to be white, born in the US, college educated and have incomes above the poverty level, but all demographic groups reported substantial rates. Women who reported FSI had more lifetime pregnancies $(2.4$ vs. $1.9 \mathrm{p}<0.001)$ and lifetime sexual partners $(9.6$ vs. $7.4 \mathrm{p}<0.001)$, and were more likely to have an unwanted first pregnancy (OR 1.7 [1.3-2.2]) and an abortion (OR 1.4 [1.1-1.8]). FSI survivors had higher rates of gynecologic illnesses, e.g. endometriosis (OR 1.6 [1.1-2.3]), pelvic inflammatory disease (OR 2.2 [1.5-3.4]) and disorders of ovulation or menstruation (OR 1.8 [1.4-2.3]) than other women. Though uncommon in both groups, victims of FSI more frequently reported illicit drug use $(2.6 \%$ vs. $0.7 \%$, OR 3.6 [1.8-7.0]) in addition to fair or poor health (OR 2.0 [1.5-2.7]) and difficulty completing tasks due to a physical or mental health condition (OR 2.8 [2.0-3.9]).

CONCLUSIONS: Violent sexual initiation is common, and associated with adverse reproductive and general health outcomes, and may impact functioning later in life. These findings highlight the importance of trauma-informed care in clinical settings, and the urgent need to develop public health and other strategies to prevent sexual coercion and assault.

\section{BUPRENORPHINE AND THE TREATMENT DIVIDE BE- TWEEN WHITES AND BLACKS}

Pooja A. Lagisetty; Ryan Ross; Amy Bohnert; Michael Clay; Donovan Maust. University of Michigan, Ann Arbor, MI. (Control ID \#3184065)

BACKGROUND: Opioid overdoses are increasing amongst both Black and White populations, but receipt of medication treatment for opioid use disorders (OUD) may differ by race and income. Studies examining disparities in receipt of medications used to treat OUD have been limited to examining methadone and buprenorphine provided by opioid treatment programs (OTPs). However, most patients never seek specialty addiction treatment and are largely cared for in office-based settings. There have been no studies examining racial disparities in the receipt of buprenorphine in office-based settings, where the majority of patients seek care. Here we describe time trends in buprenorphine office-based visits by race and payment type in the US.

METHODS: We combined data from the National Ambulatory Medical Care Survey (NAMCS) and the National Hospital Ambulatory Medical Care Survey (NHAMCS) hospital outpatient departments (OPD) to examine visits where buprenorphine was prescribed between 2004-2015, aggregated into four-year periods. We estimated buprenorphine prescription rate estimates by race and payment. We used the most recent period (2012-2015) to test the association of race with receipt of buprenorphine with logistic regression adjusting for age, gender, payment method, and complex survey design elements.

RESULTS: Between 2004-2015, buprenorphine visits rose from $0.04 \%$ of all ambulatory visits to $0.36 \%$ and in total constituted an estimated 13.4 million visits between 2012-2015. By 2012-2015, buprenorphine was a prescribed medication during more visits of white patients than other races (12.7 million [CI 8.6 million - 16.8 million] versus 363,000 [CI 134,000 $594,000])$. Self-pay and private insurance were the most common payment methods across all years. The use of self-pay for buprenorphine visits dramatically increased from 585,568 [CI 0 - 1.3 million] visits in years 2004-2007 to 5.3 million [CI 2.5 million - 8.5 million] in 20122015. After accounting for payment, gender, and age, Black patients had significantly lower odds of receiving buprenorphine at their visits (adjusted odds ratio 0.23 [CI, 0.13-0.44].
CONCLUSIONS: Our findings demonstrate wide and growing disparities in the receipt of buprenorphine by race and insurance across the U.S. It is notable that, nationally, visits paid for by private insurance and self-pay have surpassed those paid for with public insurance despite recent improvements in addiction treatment coverage for Medicaid beneficiaries after the Affordable Care Act. This study provides alarming evidence of disparities in buprenorphine treatment for OUD. With rising rates of opioid overdoses, it is imperative that policy and research efforts specifically address racial and economic disparities in treatment access and engagement.

\section{INCREASING THE MEDICAL PIPELINE: ANALYSIS OF A SUMMER EDUCATION PROGRAM FOR PHILADELPHIA HIGH SCHOOL STUDENTS}

Krystal Hill; Catherine Raney; Heardley M. Murdock; Jaya Aysola. University of Pennsylvania, Philadelphia, PA. (Control ID \#3185558)

BACKGROUND: Mounting evidence reveals the benefits of diversifying our physician workforce, yet many minority and low socioeconomic populations remain underrepresented. In response, many academic medical centers have pipeline programs designed to provide educational opportunities to underrepresented populations. Yet, little is known about their effectiveness. Thus, we evaluated a wellestablished pipeline program to identify what student and program factors yield optimal benefit.

METHODS: We analyzed the impact of The Summer Mentorship Program-Medicine (SMPM), a high school medical education pipeline program at the University of Pennsylvania ( $n=12$ students) with the Kirkpatrick model, an established method to evaluate educational programs. We surveyed students to assess overall program satisfaction and the perceptions of learning across the following domains: academic enhancement, career discussion with medical students and physicians, health science lessons, patient care and procedures, mentorship and field experiences, and public health with communitybased problem solving. We examined the relationship between student demographics, academic characteristics, and student success defined by the following three outcomes: 1) program satisfaction 2) pre-post SAT scores and 3) 4-month follow-up self-reported SAT scores. We examined student demographic and academic factors such as race/ethnicity, sex, socioeconomic status, single parent home, college readiness index (rating of a high school's college preparatory curriculum), parental education, siblings in college, pipeline program rankings to gauge interest in medicine, grade point average (GPA), recommendation letters, and personal essays.

RESULTS: All students $(\mathrm{n}=12)$ were from low-income households and 10 of 12 would be the first in their families to attend college. A third were males and 25\% had GPAs $<3.00$. Students rating for overall program was 4.2 out of 5. Program domains with the highest rankings included health science lessons (4.48), patient care and procedures (4.48), and mentorship and field experiences $(4.55)(\mathrm{p}<0.05)$. Interquartile ranges for pre, post, and follow-up SAT scores were 613-788, 769-863, and 1000-1150, respectively $(\mathrm{p}<0.05)$. Significant predictors of higher follow-up SAT scores were the "college readiness index" and "ranked medicine first" $(\mathrm{p}=0.05)$. Notably, there was no statistically significant relationship between GPA and outcomes.

CONCLUSIONS: This study of a well-run, well-funded high school pipeline program reveals that hands-on activities such as patient care and procedures provide high program satisfaction and perceptions of learning. Our results suggest that students benefit from intensive standardized test preparation and that those with lower academic scores may derive equal benefit from and succeed in pipeline programs. Lastly, we found that holistic factors such as interest or motivation may better predict success in pipeline programs over traditional factors, such as GPA. 
INTEGRATING GERIATRICS INTO INTERNAL MEDICINE CORE CURRICULUM: AGING THEME

Alyson Michener; Jerry Johnson; Rachel K. Miller. University of Pennsylvania, Philadelphia, PA. (Control ID \#3185706)

BACKGROUND: In 2014, our internal medicine (IM) residency program moved to a " $6+2$ " block schedule and theme-based ambulatory curriculum. Geriatrics/Aging was designated a longitudinal theme to span all three years. We created an aging curriculum for IM residents that increased exposure to geriatric competencies and enhanced training in the care of older adults.

METHODS: We integrated an aging theme into the IM categorical and primary care outpatient curricula. In each year of training, we co-taught several didactics with other specialists to promote collaboration and sharpen the relevance of each topic. Those topics included PGY1 (categorical and primary care): Geriatric Assessment 1 (fall) \& 2 (spring) and capstone of spring Renal block (co-taught with renal), Hypertension guidelines for Older Adults (incorporating multi-morbidity). We reinforced that knowledge in the (mandatory) PGY2 ACE unit rotation and PGY3 geriatric community-based, service-learning experience. PGY2/3 (categorical) topics included: Geriatric Polypharmacy (co-taught with pharmacist), Hot Topics in Dementia, Shared Decision Making in Cancer Screening for Older Adults (co-taught with oncology) and Healthy Aging. Each talk was given 4 times to reach all learners. Additionally, the primary care residents had 5 geriatrics didactics per year, as above, and toured local senior sites. Before and after implementing the curriculum, we compared annual ACGME evaluation responses regarding the adequacy of geriatric training. We also evaluated resident performance on geriatric questions on the annual in-training exam.

RESULTS: The ACGME evaluations showed a significant increase in residents who assessed themselves as possessing adequate experience in geriatrics ( $71 \%$ prior to implementation in 2013-14 year versus $87.5 \%$ in the 2016-17 academic year). In 2017, the residents mean percent correct score was $77 \%$ (62 residents) of the geriatrics questions on the in-training exam - a 6\% increase from 2014. We also observed more residents choosing longitudinal rotations in geriatrics and geriatrics fellowships. On the in-training exam in 2014, residents mean percent correct scores in geriatric medicine was $70 \%$ compared to $77 \%$ correct in 2017.

CONCLUSIONS: An Aging curriculum is now standard in our IM curriculum. Given that other internal medicine programs have similar block schedules, this curriculum could be replicated and adapted. We will continue expanding the aging theme and evaluating the IM residents' care of older adults.

\section{LIFESTYLE INTERVENTION PROGRAMS FOR ADULTS AT HIGH-RISK FOR TYPE 2 DIABETES: A PLATFORM TO REACH HIGH-RISK CHILDREN?}

Maya S. Venkataramani ${ }^{1}$; Tina L. Cheng ${ }^{2}$; Nisa Maruthur ${ }^{1} .{ }^{1}$ Johns Hopkins University School of Medicine, Baltimore, MD; ${ }^{2}$ Johns Hopkins, Baltimore, MD. (Control ID \#3175404)

BACKGROUND: Type 2 diabetes risk factors cluster in families, and family-oriented approaches to identify high-risk individuals and prevent disease can have a multi-generational public health impact. The National Diabetes Prevention Program (DPP) lifestyle intervention targets high-risk adults who meet body mass index (BMI) and other eligibility criteria. Eligible adults may have family members, including children, who are also at high-risk for type 2 diabetes. As such, the National DPP could provide a unique platform through which to identify, and intervene upon, diabetes risk across generations. To understand the potential multi-generational reach of familyoriented approaches to identifying and intervening upon high-risk adults and children, we explored the correlation between parental eligibility for the National DPP program and child weight status using nationally representative data.

METHODS: We analyzed nationally representative data from the National Health Interview Survey from 2016 and 2017, focusing upon sample children ages 12 through 17 years old for whom BMI data was available and who could be linked to a parent (selected as a sample adult in the survey). Child BMI was categorized as underweight/normoweight, overweight or obese, using CDC BMIfor-age criteria. Parental DPP eligibility was determined based upon National DPP eligibility criteria (being overweight/obese; and a selfreported diagnosis of prediabetes or gestational diabetes history). Using Pearson's chi-squared analyses, we explored the association between 1) parent and child BMI and 2) parental DPP eligibility and child BMI.

RESULTS: Our analytic sample consisted of 13,633,334 weighted (3905 unweighted) children, each linked to one parent. $50.1 \%$ of children and $61.6 \%$ of parents were females. $25.9 \%$ of children and $68.1 \%$ of parents were overweight or obese. Child and parent BMI were correlated (Chi-squared $\mathrm{p}<0.001$ ): over one-fifth of obese parents had at least one obese child compared to only $5 \%$ of underweight/normoweight parents. $9.5 \%$ of parents were likely eligible to participate in the National DPP. Parental DPP eligibility was positively correlated with child overweight/obese status (Chi-squared p <0.001): nearly 1 in $5(19.8 \%)$ of likely eligible parents had a child who was obese, compared to $11.0 \%$ of ineligible parents. Overall, more than one-third of likely eligible parents in the sample had at least one child who was overweight or obese.

CONCLUSIONS: In this nationally representative sample, parent and child BMI were positively correlated, as was parental eligibility for diabetes prevention programming and child BMI. This highlights the potential of leveraging the national platform for adult diabetes prevention to identify and reach high-risk children through familyoriented, multi-generational programming. Limitations include the use of self-reported weight and height (potentially leading to miscategorization at extremes of BMI), and confirming these associations with measured BMI is an important next step.

\section{MAJOR CAUSES OF DEATH AMONG TRIBAL COMMUNI- TIES IN SOUTH DAKOTA}

Stephanie Sun; Leila H. Borowsky; Matthew Tobey. Massachusetts General Hospital, Boston, MA. (Control ID \#3186016)

BACKGROUND: In the Great Plains (ND, SD, NB, and IA), the American Indian/Alaska Native (AI/AN) life expectancy is 10 years less than the US total population. The health disparities facing AI/ AN populations have been attributed to systemic discrimination, poverty, and remoteness, among other factors. Over the past 60 years, health disparities between $\mathrm{AI} / \mathrm{AN}$ and white populations have decreased everywhere except the Great Plains, where they have increased. Efforts by tribal leaders, policymakers, and healthcare experts to address AI/AN health relies on data that reflects the needs of their tribal communities. Studies that have reported state and regional data may miss nuances between reservations. We collected mortality data of individual reservations in South Dakota (SD). By focusing on local data, we found both areas of similarity and uniqueness across SD reservation health profiles.

METHODS: We used CDC Wonder data to collect the top 15 leading causes of death from 1999-2017 by county for all 10 SD reservations. Crude death rates per 100,000 and number of deaths were obtained and compared to that of SD and the US. All ages, genders, and races were included. Crude death rates for all reservations were averaged and compared to the top 15 leading causes of death for SD, and for the USA. 
RESULTS: Generally, the mortality rate of SD reservations was higher than the total state, which was higher than that of USA. Notable exceptions include Alzheimer's disease, where the mortality rate of SD reservations equalled that of all of SD, which in turn was greater than that of USA. The top two causes of death on South Dakota reservations are diseases of the heart and malignant neoplasms, with crude death rates of 245 and 211, respectively. This was similar to the top two causes of death in SD and USA. Unintentional accidents, intentional self-harm (suicide), and chronic liver disease were ranked higher in SD reservations. Nephritis, nephrotic syndrome and nephrosis, septicemia and Parkinson's disease were ranked higher in USA and SD. Mortality data of reservations varied widely. Two reservations, Lower Brule and Ponca, consistently had the lowest crude death rates of all SD reservations. Three reservations, Pine Ridge, Rosebud, and Yankton, consistently had the highest crude death rates of all SD reservations. Pine Ridge and Rosebud, two neighbouring reservations, were the sole reservations with assault (homicide) as a leading cause of death.

CONCLUSIONS: These findings point to common ground where national and state-wide efforts can be focused. This data also demonstrates the unique needs of different communities. A deeper look at the reservation-level data demonstrated heterogeneity amongst health profiles of different reservations, highlighting the importance of targeted interventions tailored to each reservation's needs. Future studies will examine the factors influencing these differences, and will examine the trends in mortality data over time for each reservation.

\section{THE POTENTIAL IMPACT OF UNIVERSAL MEDICAID EXPANSION ON ECONOMIC AND RACIAL DISPARITIES IN COVERAGE}

Elisabeth Poorman ${ }^{1}$; Hugo Torres ${ }^{2}$; Danny McCormick ${ }^{3}$. ${ }^{1}$ Cambridge Health Alliance, Cambridge, MA; ${ }^{2}$ Cambridge Health Alliance, Somerville, MA; ${ }^{3}$ Harvard Medical School / Cambridge Health Alliance, Cambridge, MA. (Control ID \#3185857)

BACKGROUND: While the Affordable Care Act (ACA) narrowed racial and socioeconomic disparities in coverage, uninsurance remains substantial in these groups. Currently, 14 states have not expanded Medicaid. Little is known about the number of racial/ethnic minorities or low-income adults that would gain coverage under universal Medicaid expansion.

METHODS: We analyzed BRFSS, a nationally representative telephone survey, for adults 18-64 in the 2 years prior to (20122013) and the 2 years following (2015 and 2016) ACA implementation. We calculated the pre-to post ACA percentage point difference in coverage gains between Medicaid expansion (MES) and non- expansion (NES) states. Using logistic regression and predictive margins, we adjusted for age, sex, education, marital and employment status and 3 state-level factors: 1) the use of a state vs federal insurance exchange; 2) state per capita federal funding for insurance enrollment support; and 3) the pre-ACA percentage of the state that was Medicaid eligible. We multiplied the adjusted difference by the population of the NES and applied weights provided by the BRFSS to project number of newly insured total, black, Hispanic, low-income adults and chronic disease patients if all states expanded Medicaid.

RESULTS: Unadjusted coverage gain were 2.3 percentage points higher in MES following the ACA $(1.7,3.0)$. The adjusted difference was 1.8 percentage points $(1.3,2.4)$, representing a projected gain in coverage for $1,354,514$ Americans. For those with a chronic disease, there was an adjusted 2 percent greater increase in coverage in MES, representing a projected gain in coverage for 614,798 people. There was a 2 percent $(1.2,2.9)$ increase for Blacks in MES (241,573). Hispanics experienced a 5.2 percentage point $(4.3,6.2)$ gain in $\operatorname{MES}(698,510)$. For low income adults, the adjusted difference in coverage was $4.7 \%$ (3.7, 5.7) $(1,178,960)$.

CONCLUSIONS: More than a million Americans stand to gain insurance coverage if all states expanded Medicaid. Most gains would be among low-income adults, and racial minorities and thus would likely further reduce existing wide disparities in coverage for these groups.

\section{THE WELL-BEING OF SWISS GENERAL INTERNAL MEDICINE RESIDENTS}

Brigitta I. Zumbrunn ${ }^{1}$; Odile Stalder ${ }^{2}$; Andreas Limacher ${ }^{2}$; Maxime Schmutz ${ }^{1}$; Peter E. Ballmer ${ }^{3}$; Stefano Bassetti ${ }^{4}$; Edouard Battegay $^{5}$; Juerg H. Beer ${ }^{6}$; Michael Brändle ${ }^{7}$; Daniel Genné ${ }^{8}$; Daniel Hayoz ${ }^{9}$; Christoph Henzen ${ }^{10}$; Lars C. Huber ${ }^{11}$; PierreAuguste Petignat ${ }^{12}$; Jean-Luc Reny ${ }^{13}$; Peter Vollenweider ${ }^{14}$; Drahomir Aujesky ${ }^{1} .{ }^{1}$ Bern University Hospital, Bern, Switzerland; ${ }^{2}$ University of Bern, Bern, Switzerland; ${ }^{3}$ Kantonsspital Winterthur, Winterthur, Switzerland; ${ }^{4}$ University Hospital of Basel, Basel, Switzerland; ${ }^{5}$ University Hospital Zurich, Zurich, Switzerland; ${ }^{6}$ Cantonal Hospital of Baden, Baden, Switzerland; ${ }^{7}$ Cantonal Hospital of St. Gallen, St. Gallen, Switzerland; ${ }^{8}$ Cantonal Hospital of Biel, Biel, Switzerland; ${ }^{9}$ HFR Hopital Cantonal, Fribourg, Switzerland; ${ }^{10}$ Cantonal Hospital of Lucerne, Lucerne, Switzerland; ${ }^{11}$ City Hospital Triemli Zurich, Zurich, Switzerland; ${ }^{12}$ Hospital of Valais, Sion, Switzerland; ${ }^{13}$ Geneva University Hospitals, Geneva, Switzerland; ${ }^{14}$ Lausanne University Hospital, Lausanne, Switzerland. (Control ID \#3184797)

BACKGROUND: Physician well-being is not only associated with empathy and professionalism, it has also a direct impact on productivity, quality of care, and patient safety. Residency training is a challenging and particularly stressful period in the life of physicians. We therefore assessed the well-being of Swiss general internal medicine (GIM) residents as well as associated personal and work-related factors.

METHODS: We conducted an anonymous electronic survey among GIM residents from 13 Swiss teaching hospitals in June 2018. Participants were asked to indicate personal and work-related characteristics and to complete the Physician WellBeing Index (PWBI), which measures 6 domains (burnout, depression, stress, fatigue, and mental and physical quality of life). We explored the association between a reduced well-being (PWBI 5 points) and personal and work-related factors using multivariable mixed logistic regression. We used spearman's rank correlation coefficient to examine the relationship between the PWBI and job satisfaction, self-reported medical errors, suicidal ideation, and intention to leave clinical practice.

RESULTS: The response rate was 54\% (472/880). Although $95 \%$ of residents were at least partially satisfied with training quality and $91 \%$ with their job autonomy and the job overall, $19 \%$ of participants had a reduced well-being, $60 \%$ felt burned out, $57 \%$ reported emotional problems, and $21 \%$ had career choice regret. Age (OR 1.20, 95\% CI 1.06-1.35), having children (OR 0.10, $95 \%$ CI $0.02-0.41),<2.5$ personally rewarding work hours per day (OR 3.01, 95\% CI 1.62-5.61), low satisfaction with training quality (OR 2.40, 95\% CI 1.34-4.31), and low satisfaction with job autonomy (OR $2.42,95 \%$ CI 1.36-4.28) were significantly associated with reduced well-being, but not working hours, administrative workload, and satisfaction with income. We found significant, weak to moderate correlations between the PWBI and job satisfaction $\left(\mathrm{r}_{\mathrm{s}}=-0.54, \mathrm{p}<0.001\right)$, medical errors $\left(\mathrm{r}_{\mathrm{s}}=0.18, \mathrm{p}<0.001\right)$, suicidal ideation $\left(\mathrm{r}_{\mathrm{s}}=0.12, \mathrm{p}=0.009\right)$, and the intention to leave clinical practice $\left(\mathrm{r}_{\mathrm{s}}=0.38, \mathrm{p}<0.001\right)$. 
CONCLUSIONS: Our survey reveals a mixed picture. Although the vast majority of Swiss GIM residents are satisfied with many aspects of their job, approximately $20 \%$ appear to have a reduced well-being or career choice regret and around $60 \%$ feel burned out or report emotional problems. Having few rewarding work hours and a low satisfaction with training quality and job autonomy are the most significant predictors of reduced well-being. Given the human costs of physician distress to both patients and physicians, health care organizations have the shared ethical and professional responsibility to improve physician well-being, for instance by providing adequate support structures and staffing levels.

\section{1-ITEM BURNOUT MEASURE CORRELATES WELL WITH EMOTIONAL EXHAUSTION DOMAIN OF BURNOUT BUT NOT DEPERSONALIZATION}

Tabor E. Flickinger; Rachel H. Kon; Rebecca Jacobsen; Justine E. Owens; John Schorling; Margaret L. Plews-Ogan. University of Virginia, Charlottesville, VA. (Control ID \#3171873)

BACKGROUND: Burnout is a serious concern in medical education and practice with negative impact on trainees' professionalism and patient care. Accurate measurement of burnout is necessary for medical education scholarship and intervention evaluation. The Maslach Burnout Inventory (MBI) is a validated 22-item tool with three domains of emotional exhaustion (EE), depersonalization (DP), and personal accomplishment (PA). A 1-item burnout measure is faster and easier to administer than the full MBI and is available at no cost. However the correlation between a 1-item measure and the full MBI has not been established for physicians-in-training. We aimed to compare measurement of burnout by these two tools among medical students.

METHODS: All medical students at our academic medical center receive an online survey annually. Since 2014, this survey has included the full MBI to measure burnout. In the 2017-2018 academic year, we added the 1-item burnout measure, previously used in the Physician Work Life Study (PWLS). This item asks respondents to rate their level of burnout with responses from 1 (no burnout) to 5 (severe burnout). Previously established cut-offs for high scores were used for both measures. Scores on the 1-item PLWS burnout measure and the full MBI domains of EE and DP were compared using Pearson correlation and Chi square tests.

RESULTS: Of the 631 surveys sent, 287 students completed the 1item PWLS burnout question and the full MBI (46\% response rate). Mean scores on EE were 21.6 (SD 10.8) and on DP 6.4 (SD 5.4). Mean score on the 1-item PLWS measure was 2.17 (SD 0.726). Scores on EE and DP domains were moderately correlated with each other $(\mathrm{r}=0.472, \mathrm{p}<0.001)$. Scores on the 1 -item PWLS measure showed strong correlation with EE scores $(\mathrm{r}=0.665, \mathrm{p}<0.001)$ but weak correlation with DP scores $(r=0.160, p=0.007)$. High burnout was reported by $34 \%$ of students for EE (scores above 27 ) and $25 \%$ for DP (above 10). On the 1-item PWLS measure, 21.3\% of students reported high burnout ( 3 and above). Students with high scores on the 1-item PLWS measure were more likely to have high EE than low/ medium EE ( $86 \%$ vs $19 \%, \mathrm{p}<0.001)$ but no significant difference on high vs low/medium DP ( $31 \%$ vs $24 \%, \mathrm{p}=0.248)$.

CONCLUSIONS: The 1-item PWLS measure correlated better with EE than with DP domains of burnout. When asked by the 1-item measure to rate their own level of burnout, respondents may primarily express how drained or fatigued they feel, which corresponds well to EE. However, DP appears to measure a different concept which captures the ability to treat patients with empathy. DP is a particularly worrisome aspect of burnout because it may undermine the formation of therapeutic relationships with patients and how well future physicians deliver patient-centered care. DP may not be well evaluated by the 1 -item
PWLS burnout measure, as compared to the full MBI, and should be used and interpreted with caution by medical educators and researchers.

\section{MONTH OUTCOMES: UTILIZING REMOTE GLUCOSE MONITORING TO DELIVER CONTINUOUS, PATIENT- CENTERED CARE AND IMPROVED OUTCOMES}

Elizabeth B. Kirkland ${ }^{1}$; Jingwen Zhang ${ }^{1}$; Justin Marsden ${ }^{2}$; William P. Moran ${ }^{2} .{ }^{1}$ MUSC, Charleston, SC; ${ }^{2}$ Medical University of South Carolina, Charleston, SC. (Control ID \#3186342)

BACKGROUND: Remote patient monitoring (RPM) is an accessible tool for chronic disease management. This technology improves access to care and provides up-to-date data for provider action. While there is a growing body of evidence to suggest that diabetic and hypertension control improves at 6months with home monitoring, there is less evidence for effectiveness or sustained improvement at 12 months follow up time. Meta-analyses of remote interventions for other chronic diseases, such as obesity, reveal promising improvement up to 6 months, but insignificant change at 12 months. We seek to determine whether remote patient monitoring of glucose over $12 \mathrm{months}$, with provider engagement to allow for medication changes in the inter-visit period, will produce sustained improvements in hemoglobin A1c (hbA1c) at 12 months or longer.

METHODS: Over 600 patients across the state of South Carolina enrolled in a remote monitoring program utilizing an easy-to-use, 2-in-1 automated device with blood pressure and glucose monitoring capability. The device transmits data directly to a secure database through SIM technology for healthcare provider review, obviating the need for Bluetooth, cords, or other ancillary devices. Patients were eligible for participation if they had an hbAlc of 8 or higher within 21 days of enrollment. Providers were encouraged to view these incoming data and to make adjustments to diabetes and/ or hypertension treatment plans at their discretion. HbAlc measurements were obtained at baseline, 6-, and 12-months after enrollment and compared via paired t-tests (baseline to $6 \mathrm{mo}, 6 \mathrm{mo}$ to $12 \mathrm{mo}$, and baseline to $12 \mathrm{mo}$ ).

RESULTS: We report on the changes in hbAlc as a marker of diabetes control over time. Blood pressure outcomes are not reported here as there was no blood pressure entry criteria. RPM was associated with significant reduction in hbAlc at 12 months, compared to baseline ( 8.8 vs 9.7 , respectively; $\mathrm{n}=$ $24 ; \mathrm{p}=0.05$ ). There was also a significant reduction in hbA1c at 6 months compared to baseline ( 8.8 vs 10.4 , respectively; $\mathrm{n}=106 ; \mathrm{p}=<0.0001$ ), but not at 12 months compared to 6 months ( 8.8 vs 8.9 , respectively; $n=23$; $p=0.78$ ). CONCLUSIONS: Based on these initial data, RPM leads to improved diabetes control which is sustained at 12 months. Data collection is ongoing and we expect to report 12 month findings for a sample size of approximately 100 patients by the time of presentation in May 2019. RPM is an effective tool for improving diabetes management and delivering inter-visit care to patients of diverse geographic and socioeconomic backgrounds, affording improved chronic disease control in a patient-centered fashion. The user-friendly design and the healthcare providers' ability to provide inter-visit care with this technology, coupled with the sustained benefit on chronic disease control as measured by hbAlc, suggests that this form of care can reduce barriers to care and improve outcomes for otherwise vulnerable and underserved populations.

\section{A BARBER-PHARMACIST INTERVENTION COULD REDUCE CVD EVENTS AT 10 YEARS IN HYPERTENSIVE NON- HISPANIC BLACK MEN: THE LOS ANGELES BARBERSHOP (LA BARBER) TRIAL}

Kelsey B. Bryant ${ }^{1}$; Brandon K. Bellows ${ }^{1}$; Dhruv S. Kazi ${ }^{4}$; Valy Fontil ${ }^{2}$; Joanne Penko ${ }^{3}$; Ciantel A. Blyler ${ }^{5}$; Kathleen Lynch $^{5}$; Norma B. Moy ${ }^{5}$; Joseph Ebinger $^{5}$; Florian Rader ${ }^{5}$; Kirsten Bibbins-Domingo ${ }^{3}$; Andrew E. Moran ${ }^{1}$. ${ }^{1}$ Columbia University Medical Center, New York, NY; ${ }^{2}$ University of California San Francisco, San Francisco, CA; ${ }^{3}$ University of California, San Francisco, San Francisco, CA; ${ }^{4}$ Beth Israel Deaconess, Boston, MA; ${ }^{5}$ Cedars Sinai, Los Angeles, CA. (Control ID \#3183887) 
BACKGROUND: Pharmacist-driven blood pressure (BP) management in barber shops showed $>20 \mathrm{mmHg}$ reductions in systolic BP over one year in Non-Hispanic black men with uncontrolled hypertension compared to the hypertension education-only control arm in the LA BARBER trial. However, the long-term potential to prevent cardiovascular disease (CVD) events using pharmacist-barber collaborations in this high-risk, vulnerable population is unknown.

METHODS: We used the microsimulation version of the CVD Policy Model to project the expected 10-year reduction in CVD events (stroke, myocardial infarction, or angina) if the LA BARBER intervention BP reduction persisted past the 12-month trial observation period. We simulated a hypothetical LA BARBER-eligible cohort of 10,000 Non-Hispanic black men sampled from the U.S. National Health and Nutrition Examination Surveys. Based on published literature, we assumed $43 \%$ of patients discontinued treatment after the first year in the base-case analysis. In an alternate scenario, all patients were fully adherent to medications for the entire 10 years. We assumed age-related changes in BP occurred regardless of treatment and that no other BP management occurred outside of the interventions during the time horizon.

RESULTS: The baseline characteristics of the simulation cohort were similar to the LA BARBER trial; mean baseline age was 54.1 years (LA BARBER 54.5 years) and mean baseline BP was $155 / 91 \mathrm{~mm} \mathrm{Hg}$ (systolic/diastolic) (LA BARBER 154/91 mm Hg). At one year, the model simulated mean BP reductions of $-31.6 /-16.7 \mathrm{~mm} \mathrm{Hg}$ in the barber-pharmacist intervention (LA BARBER -28.6/-17.8 mm Hg) compared with control of -7.4/-4.0 (LA BARBER -7.2/-3.3) [resulting control rates 68\% intervention (equal to LA BARBER), 7\% control (LA BARBER 11\%)]. At 10 years, the base-case analysis showed $29 \%$ hypertension control in the barber-pharmacist intervention arm compared to $0 \%$ in the control arm. In the full adherence scenario, control was $49 \%$ in the barber-pharmacist intervention arm and $2 \%$ in the control arm. Compared with the control arm, we projected about 300 CVD events averted over ten years in the barber-pharmacist intervention in the basecase scenario and 500 events averted in the full adherence scenario.

CONCLUSIONS: Our model predicted a reduction of about 300-500 CVD events in 10,000 LA BARBER-eligible Non-Hispanic black men over 10 years with the pharmacist intervention compared with an education-only control. A barber-pharmacist collaborative intervention delivered to NonHispanic black men with hypertension could potentially reduce CVD outcome disparities between Non-Hispanic black men and other U.S. ethnic groups. Further analyses will assess the cost-effectiveness and scalability of the labor-intensive BARBER hypertension treatment intervention.

\section{A COMMUNITY HEALTH WORKER PROGRAM TO REDUCE CARDIOVASCULAR RISK IN UNDERSERVED RURAL COMMU- NITIES}

Samuel Cykert ${ }^{1}$; Carmen Samuel-Hodge ${ }^{2}$; Audrina J. Bunton ${ }^{2}$; Sallie D. Allgood ${ }^{3}$. ${ }^{1}$ University of North Carolina, Chapel Hill, NC; ${ }^{2}$ The UNC Gilling's School of Global Public Health, Chapel Hill, NC; ${ }^{3} \mathrm{UNC}$ School of Nursing, Chapel Hill, NC. (Control ID \#3182956)

BACKGROUND: Cardiovascular disease (CVD) remains the leading cause of death throughout the U.S. The burden of CVD is particularly onerous in North Carolina which consistently ranks near the bottom tertile of CVD mortality among states. CVD-related death is worse in rural NC counties. Although no funds were appropriated, the creation of a community health worker (CHW) workforce as a vehicle for community outreach to improve prevention, adherence, and lifestyle was emphasized in the Affordable Care Act (ACA). In this report, we describe results from the Carolina Heart Alliance Networking for Greater Equity Project. The study objective was to evaluate the effectiveness of a CHWdelivered lifestyle program on intermediate health outcomes and lifestyle changes that could reduce CVD risk among underserved rural populations. CHWs were based in two public health departments (PHD) and two federally-qualified health centers (FQHC).
METHODS: Recruitment of participants to this pre-/post-test study involved two main strategies. FQHC-based CHWs were given a list of patients noted to have a CVD risk factor consistently uncontrolled. The PHD-based CHWs presented information sessions at community events and engaged interested individuals to participate. All consented individuals had their blood pressure measured. In the preparatory phase of the study, we started with modules from an evidence-based intervention targeting physical activity, a Mediterranean-like dietary pattern, medication adherence, and smoking cessation. We adapted program materials with input from a local community advisory board then developed a protocol for CHWs to deliver these modules to participants during 4 monthly sessions in participants' homes. Participants also received three phone calls for reinforcement mid-way between each session.

RESULTS: To date, 203 participants have completed the intervention. Mean age was 59 years (SD +/- 11.8); $91 \%$ were Black and $76 \%$ were women. At baseline, 72 participants had elevated blood pressure (systolic blood pressure (SBP) $>140 \mathrm{mmHg}$ or diastolic blood pressure $(\mathrm{DBP})>90 \mathrm{mmHg}$ ) despite receiving regular clinical care. Postintervention only 37 still had elevated blood pressures. The average decrease in SBP for all participants was $5 \mathrm{mmHg}(\mathrm{SD}+/-18)$ and DBP $2.4 \mathrm{mmHg}(\mathrm{SD}=/-11)$. With Mediterranean-like dietary advice (without specific caloric restriction), $60 \%$ of participants lost weight; average weight loss among all participants was $2.7 \mathrm{lbs}$. (SD +/- 17) over 3 months. Self-reported dietary changes included an average increase of fruit-vegetable servings of 0.86 per day (SD +/- 1.9) and a 0.5 increase in weekly intake of nuts (SD +/- 0.95). Physical activity increased by an average of 23 minutes per week (SD +/- 187).

CONCLUSIONS: CHW outreach in underserved rural communities effectively delivered an evidence based intervention that improved intermediate health measures and life-style likely to lead to long term CVD risk reduction.

\section{A COMMUNITY SUPPORTED AGRICULTURE INTERVEN- TION FOR HEALTH CENTER PATIENTS: A RANDOMIZED CLINICAL TRIAL}

Seth A. Berkowitz ${ }^{1}$; Jessica O'Neill ${ }^{2}$; Edward Sayer ${ }^{3}$; Naysha N. Shahid $^{4}$; Maegan Petrie ${ }^{3}$; sophie schouboe ${ }^{2}$; Megan Saraceno ${ }^{2}$; Rochelle Bellin ${ }^{2} .{ }^{1} \mathrm{UNC}$ School of Medicine, Chapel Hill, NC; ${ }^{2} \mathrm{Just}$ Roots, Greenfield, MA; ${ }^{3}$ Community Health Center of Franklin County, Greenfield, MA; ${ }^{4}$ Massachusetts General Hospital, Boston, MA. (Control ID \#3182962)

BACKGROUND: Socioeconomically vulnerable individuals often face poor access to nutritious food and bear a disproportionate burden of diet-related chronic illness. We sought to determine whether participation in a community supported agriculture intervention that subsidized healthy food could improve diet quality.

METHODS: We conducted a randomized clinical trial (NCT03231592) from May 2017 to December 2018. Adults at a community health center in central Massachusetts were eligible if they had a BMI $>25 \mathrm{~kg} / \mathrm{m}^{2}$ in the past year. Individuals were randomized to receive either subsidized participation in a community supported agriculture intervention (which provided a weekly box of farm produce from June to November) for 2 years, or usual care plus healthy eating information (control group). The primary outcome was the Healthy Eating Index 2010 (HEI) total score, and secondary outcomes were the HEI sub-scores. Assessment of HEI was made using three 24-hour recalls per participant per season. Higher total HEI score (range: 0-100) and sub-scores represent 'better' dietary quality. A 3 point difference in HEI total score is clinically significant. Intention-to-treat analyses compared HEI scores between the intervention and control group, accounting for repeated measures with generalized estimating equations. 
RESULTS: There were 122 participants for analysis. The mean age of participants was 50.3 (SD: 13.6) years, $82 \%$ were women, $88 \%$ were non-Hispanic white, and there were no significant differences in baseline characteristics between the intervention and control groups. Baseline HEI total score was 53.9 (SD: 15.3) in the control group and 55.1 (SD: 15.2) in the intervention group $(\mathrm{p}=.68)$. The intervention increased the mean HEI total score relative to the control group (4.3 points higher, $95 \%$ CI 0.5 to $8.1 ; p=.03$ ). Analyses of the HEI subscores showed that this was driven by greater consumption of vegetables and fruits, and lower consumption of 'empty' calories like sugar-sweetened beverages (Table).

CONCLUSIONS: A community supported agriculture intervention resulted in clinically meaningful improvements in diet quality for health center patients. Subsidized community supported agriculture may be an important intervention for vulnerable individuals.

\section{A COMPARISON OF APIXABAN IN PATIENTS WITH AD- VANCED CHRONIC KIDNEY DISEASE AND END STAGE RENAL DISEASE}

Rajendrakumar C. Patel ${ }^{2}$; Kyle James ${ }^{1}$; Jason $\mathrm{Cobb}^{2}$; Traci Leong ${ }^{3}$. ${ }^{\mathrm{T}}$ Emory Univeristy Hospital Midtown, Atlanta, GA; ${ }^{2}$ Emory University School of Medicine, Atlanta, GA; ${ }^{3}$ Emory University, Atlanta, GA. (Control ID \#3184895)

BACKGROUND: Many patients with end stage renal disease (ESRD) or advanced chronic kidney disease (CKD) have conditions for which the appropriate treatment is anticoagulation. These conditions include atrial fibrillation/flutter and deep vein thrombosis/ pulmonary embolism (DVT/PE). Classically, the oral anticoagulant of choice for patients with advanced renal disease and these conditions has been warfarin. The use of warfarin can be challenging, but newer direct oral anticoagulants are available for use. Apixaban, a direct oral anticoagulant, is potentially appropriate for use in patients with advanced renal disease as it is predominantly metabolized by the liver. Prospective studies of apixaban to date have excluded patients with advanced renal disease.

METHODS: A retrospective analysis by manual chart review was performed using data from a four-hospital system. Patients with diagnoses of either CKD 4, CKD 5, or ESRD who were ordered apixaban while hospitalized were identified. Hospitalizations occurred over 5.5 years. Patients were manually excluded if they never received a dose of apixaban or had an estimated glomerular filtration rate (GFR) greater than 30. Three independent physicians reviewed physician/provider/nursing notes, lab results, and medication administration records. Additionally, imaging reports were reviewed for therapeutic failures.

RESULTS: Of 508 encounters identified, 49 were excluded. Of the remaining 459 encounters, 171 had CKD and 288 had ESRD. There were 14 bleeding complications in the CKD group and 16 in the ESRD group $(p=0.36)$. The average number of red blood cell units transfused was 2.38 in the CKD group and 1.63 in the ESRD group (p $=0.027$ ). Deaths occurred in 4 CKD encounters and 7 ESRD encounters $(p=1.0)$. One death was attributed to bleeding and this was in the ESRD group. Regarding therapeutic failure, there were no strokes, DVTs, or PEs in the CKD group, but there were 4 strokes observed ( $p$ $=0.30)$ and 3 DVTs or PEs observed $(p=0.29)$ in the ESRD group. CONCLUSIONS: Our study suggests that there was no significant difference in risk of bleeding or therapeutic failure between CKD and ESRD patients. One strength of this study is its inclusion of multiple hospitals serving different populations. Limitations of this study include its retrospective nature and its restriction to the inpatient setting. An additional limitation is the use of encounter-level data rather than patient-level data. While randomized controlled trials are ultimately needed to properly address the safety of apixaban in CKD and ESRD, our study may be helpful to providers in their decisionmaking in the meantime.

\section{A CROSS-SECTIONAL STUDY USING CASE-BASED SKILLS ASSESSMENT TO MEASURE QUALITY OF INTEGRATED COMMUNITY CASE MANAGEMENT CARE DELIVERED BY VILLAGE HEALTH WORKERS IN BUGOYE, UGANDA} James S. Miller ${ }^{1}$; Rapheal Mbusa ${ }^{2}$; Stephen Baguma ${ }^{2}$; Palka R. Patel ${ }^{3}$; Michael Matte ${ }^{2}$; Moses Ntaro ${ }^{4}$; Shem Bwambale ${ }^{5}$; Sara MianMcCarthy ${ }^{6}$; Jessica Kenney ${ }^{1}$; Daniel Guiles ${ }^{1}$; Edgar M. Mulogo ${ }^{4}$; Geren Stone ${ }^{1}$. ${ }^{1}$ Massachusetts General Hospital, Cambridge, MA; ${ }^{2}$ Global Health Collaborative, Mbarara, Uganda; ${ }^{3}$ Indiana University School of Medicine, Indianapolis, IN; ${ }^{4}$ Mbarara University of Science and Technology, Mbarara, Uganda; ${ }^{5}$ Bugoye Health Center, Bugoye, Uganda; ${ }^{6}$ UNICEF, New York, NY. (Control ID \#3180333)

BACKGROUND: Village health workers (VHW) in 8 villages in Bugoye subcounty, Uganda provide Integrated Community Case Management (iCCM) care to children under 5 years old. In iCCM, VHWs assess and treat malaria, pneumonia, and diarrhea using a clinical algorithm. Study objectives included: 1) quantify VHWs' clinical performance and adherence to the clinical algorithm;2) assess impact of formal education on skills assessment performance; 3) compare VHW' performance as measured by the skills assessment exercise to prior clinical record review data (Miller et al. Malaria Journal 2018 17:99).

METHODS: All 36 VHWs agreed to complete the skills assessment exercise. This exercise included video cases depicting a caregiver of a sick child being interviewed, brief case vignettes portraying children with or without "danger signs" of severe illness, and reading mock rapid diagnostic tests (RDT) for malaria. Video cases were scored on completion of all steps in the clinical algorithm and a subset of "critical steps" required to achieve correct clinical management. Results were analyzed in Stata, with t-tests used to assess the association between formal education and performance.

RESULTS: Of the VHWs, 53\% were women; $31 \%$ had completed primary school only and $69 \%$ some or all of secondary school. For cases of uncomplicated diarrhea and pneumonia, completion of all correct steps was low (19\% and 6\%, respectively), due to failure to address all recommended health education topics; completion of critical steps was high (92\% and $97 \%$, respectively). In a case of fever with a negative RDT, $100 \%$ of VHWs completed all correct steps. In a case of pneumonia with danger signs, $56 \%$ of VHWs completed all correct steps, while $89 \%$ completed all critical steps. Inappropriate use of antibiotics or antimalarials was low in all cases $(0-3 \%)$. VHWs correctly classified $82 \%$ of brief case vignettes for children with or without danger signs. VHWs read $97 \%$ of mock RDTs correctly. There was no evidence of a difference in performance (measured by total correct management steps in the video cases and by a weighted overall performance metric) between VHWs with primary school education and with some or all of secondary school ( $p=0.76$ and $p=0.89$, respectively). Rates of completion of critical steps in the cases were similar to prior assessment via clinical record review for uncomplicated diarrhea (92\% vs. $97 \%$ ) and uncomplicated pneumonia ( $97 \%$ vs. $95 \%$ ) but quite different for fever with negative RDT (100\% vs. $44 \%)$.

CONCLUSIONS: VHWs often omitted recommended health education topics, but otherwise demonstrated high quality of care in the video cases, recognition of danger signs, and reading RDTs. Level of formal education did not appear to influence performance. The difference in performance between data sources for fever with negative RDT may have resulted from training prompted by the prior results, direct observation during this present study, or incomplete clinical record-keeping. 


\section{A DECISION AID ON MAMMOGRAPHY SCREENING FOR WOMEN AGED 75 AND OLDER REDUCES SCREENING} Mara A. Schonberg ${ }^{1}$; Roger B. Davis ${ }^{1}$; Mary Beth Hamel ${ }^{1}$; Gianna Aliberti $^{1}$; Alicia R. Jacobson ${ }^{1}$; Maria Karamourtopoulos ${ }^{1}$; Michele Hayes $^{2}$; Christina C. Wee ${ }^{1}$; Edward R. Marcantonio ${ }^{1}$; Carmen L. Lewis ${ }^{3}$; Christine Kistler ${ }^{2} .{ }^{1}$ Beth Israel Deaconess Medical Center, Boston, MA; ${ }^{2}$ University of North Carolina at Chapel Hill, Chapel Hill, NC; ${ }^{3}$ Univesrity of Colorado, Denver, CO. (Control ID \#3168057)

BACKGROUND: The utility of breast cancer screening in later life varies according to women's life expectancy, breast cancer risk, and preferences. To improve informed, value-concordant breast cancer screening in later life, we conducted a clustered randomized trial to test the effectiveness of a mammography decision aid (DA) containing information on outcomes of screening, life expectancy, competing mortality risks, and a values clarification exercise.

METHODS: We recruited English speaking women ages 75-89 years with an upcoming appointment with their PCP without a history of breast cancer or dementia from 11 diverse primary care practices in Massachusetts (MA) and North Carolina (NC). Eligible women were screened in the past 24 months but not in the past 6 months. A research assistant (RA) administered a pre-visit questionnaire to participants on their intentions to be screened, health, breast cancer risk factors, and sociodemographic characteristics. Before the visit, the patient's PCP was randomized to either the pamphlet DA (intervention arm) or to a home safety pamphlet (HS, the control arm); subsequent participants from each PCP remained in the same arm. After the visit, the RA administered a post-visit questionnaire on screening intentions and knowledge. Participants were followed for 18 months for receipt of screening (i.e., at least 2 years since last mammogram). To examine the DA's effects, we fit marginal logistic regression models using generalized estimating equations to allow for clustering by PCP. We included PCP panel size, site, patient life expectancy, and 5-year Gail breast cancer risk in our models.

RESULTS: Of 1,031 eligible women contacted, 541 (53\%) from 136 different PCPs consented to participate; 281 patients received the DA and 260 received HS. Patients in each arm were well-matched; $80 \%$ were from MA; $54 \%$ from community (vs. academic) sites; $38 \%$ had $<10$ year life expectancy; mean 5-year breast cancer risk was $2.2 \%$ $(+/-1.1 \%) ; 79 \%$ were non-Hispanic white; and $62 \%$ were college graduates. Post-visit, $87 \%$ of women who received the DA found it helpful and $95 \%$ would recommend it to a friend. Women in the DA arm were more likely to lower their intentions to be screened from pre to post visit ( $25 \%$ of women in the DA arm lowered their intentions vs. $17 \%$ in the HS arm, $\mathrm{p}=0.03$ ), were more knowledgeable about screening (mean knowledge score 6.1 vs. 5.3 [out of 10 questions], $\mathrm{p}<0.001$ ), and were more likely to have a discussion on mammography's pros and cons documented by their PCP (30\% vs. $12 \%, \mathrm{p}=0.001)$. After 18 months, $4 \%$ fewer in the DA arm were screened $(57 \%$ vs. $61 \%, p=0.03)$. In MA where $74 \%$ of women in the control arm were screened, the DA was associated with a $12 \%$ reduction in screening $(\mathrm{p}=0.006)$; we found no difference in $\mathrm{NC}$ where screening in the control arm was low (23\%).

CONCLUSIONS: Our mammography DA was perceived as helpful, improved older women's knowledge and discussions about screening, and led to fewer women aged 75-89 years being screened with mammography.

A FLUID DISCHARGE: LIQUID OPIOIDS AND OTHER RISK FACTORS THAT CONTRIBUTE TO EXCESSIVE OPIOID PRESCRIPTIONS FROM A HOSPITAL MEDICINE SERVICE Nicholas Iverson; Nader Najafi; Yumiko Abe-Jones; Margaret Fang; Kirsten Kangelaris; Priya A. Prasad; Catherine Lau. UCSF, San Francisco, CA. (Control ID \#3173256)
BACKGROUND: Recently enacted policies in multiple states have placed restrictions on opioid prescription days given the risk of development of chronic opioid therapy with prolonged opioid prescriptions. However, there are still patients who receive an excessive number of opioid prescription days after discharge from the hospital. We studied this population retrospectively to understand which patient-level risk factors contribute most to being an outlier of excess opioid prescribing on discharge from a hospital medicine service.

METHODS: We used the electronic health record to identify all patients hospitalized on the acute care medical service at a 600-bed tertiary care hospital from years 2012-2018. Patients were included if they received an opioid prescription both while hospitalized and on discharge and data were obtained on patient demographics, medication administration, and diagnosis codes. All opiate prescriptions were standardized to morphine milligram equivalents (MMEs). Days of opioids prescribed on discharge was calculated by the discharge prescription signature and number of pills. Patients were excluded if ICD9 or 10 codes for cancer-related pain or sickle cell disease were documented, or who were on hospice or followed by the palliative care service. Patients were categorized as receiving $<=14$ days of opioids, 14 to 30 days of opioids, or $>30$ days of opioids. We then performed a multivariable logistic regression to assess for factors associated with longer opiate prescriptions.

RESULTS: We identified 9,275 medical inpatients prescribed opioids both during the hospitalization and on discharge who were eligible for the analysis. By the discharge prescription signature, 6,001 (64.7\%) patients were prescribed less than 14 days, 2,688 (29.0\%) received 14-30 days, and $586(6.3 \%)$ received $>30$ days. Factors associated with receiving a $>30$ day supply include: being discharged with liquid opioids (OR 4.3 [3.4-5.4]), being on benzodiazepines prior to admission (OR 1.9 [1.62.6]), being male (OR 1.4 [1.1-1.6]), and having non-metastatic cancer (OR 1.3 [1.1-1.7]) (Figure 1). The maximum number of days prescribed was 1,875 days.

CONCLUSIONS: Patients prescribed liquid formulations of opioids were substantially more likely to have a prolonged duration of opiates supplied at discharge.

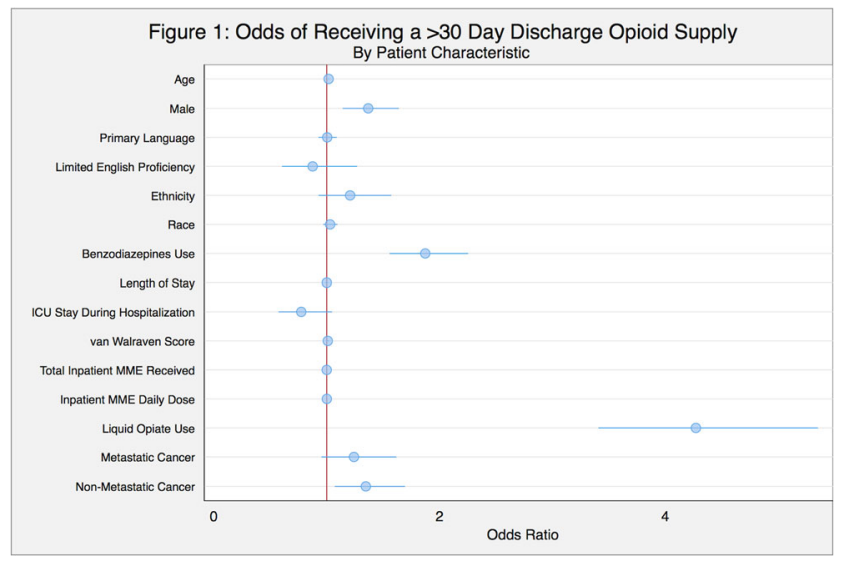

\section{A MIXED METHODS ANALYSIS TO EVALUATE EMERGENCY DEPARTMENT (ED) WAIT TIME DISPARITIES}

Jaya Aysola; Sean Foster; Mira Mamtani; Justin Clapp; Matthew Kearney; Eve Higginbotham. University of Pennsylvania, Philadelphia, PA. (Control ID \#3185263)

BACKGROUND: We identified a significant disparity in ED wait times among patients eventually admitted to the hospital. Evidence reveals ED wait times influence patient satisfaction, length of stay, care quality, and hospital costs. This study aimed to identify key contributors to this inequity. 
METHODS: We examined non-trauma patients admitted to the hospital that went through the ED from November 2017 to May 2018 $(\mathrm{n}=1531)$ and compared all time-stamped points along the patient's path from ED arrival to their departure to an inpatient floor, by race/ethnicity. We estimated with linear regression models the relationships between ED wait times, and the following patient- and EDlevel characteristics: race/ethnicity, age, gender, comorbid conditions, insurance status, primary language, having a primary care provider, a prior ED visit within the past 30 days, assigned triage score (ESI), same day outpatient visit, call-in referral, admitting service and unit, degree of ED overcrowding, and the presence of provider in triage. Guided from our findings, we then collected and analyzed data from: 1) semi-structured interviews with patients $(n=12) ; 2)$ general 2-hour observations of the ED ( $\mathrm{n}=8)$; and 3 ) provider observations and semistructured interviews $(n=9)$. Providers were assigned groups based on their wait-time disparities and we selected at least two providers from each group to participate in the study. Team members were blind to provider groupings until the analysis was complete. A minimum of $25 \%$ of the sampled from each data source was double- or triplecoded to ensure interrater reliability.

RESULTS: We isolated the disparity to between when the ED provider sees the patient and requests a bed. After adjusting for the patient- and ED-level characteristics noted above, racial/ethnic ED wait time disparity attenuated but remained significant. We found a consistent pattern of communication between providers (physician or nurse) and patients, depending on whether they were of similar race/ethnicity (concordant pair) or not (discordant pair). Among discordant pairs, providers were more likely to interrupt patients, ignore patient requests, and have less eye contact. Among concordant pairings, providers exhibited a greater level of advocacy, such as tracking down labs or a consultant. Providers without significant ED wait time disparities, articulated objective criteria, such as triage scores (ESI), for prioritizing which patients they see first. In contrast, only one provider from the groups with significant wait time disparities mentioned ESI, only to question its validity in prioritizing patients.

CONCLUSIONS: Our findings suggest that both provider-patient interactions and the provider's approach to prioritizing care play a significant role in ED wait time disparities. This study yields actionable provider-specific contributors to the wait time disparities that may apply to broader set of quality metrics.

\section{A MULTICENTER STUDY OF THE FORMAT AND CON- TENT OF INTERNAL MEDICINE MORNING REPORT}

Dan Heppe $^{1}$; Albertine Beard ${ }^{2}$; Brian Kwan ${ }^{3}$; Michael Ong ${ }^{4}$; Azadeh Lankarani-Fard ${ }^{4}$; Paul B. Cornia ${ }^{5}$; Matthew Tuck ${ }^{6}$; Craig G. Gunderson ${ }^{7} .{ }^{1}$ Eastern Colorado Veterans Health Administration, Denver, CO; ${ }^{2}$ Minneapolis VA, Minneapolis, MN; ${ }^{3}$ University of California, San Diego, San Diego, CA; ${ }^{4}$ UCLA, Los Angeles, CA; 5Seattle VA, Seattle, WA; ${ }^{6}$ Department of Veterans Affairs Medical Center, Washington, DC; ${ }^{7}$ Yale Medical School, West Haven, CT. (Control ID \#3185273)

BACKGROUND: Morning report has long been considered a core component of internal medicine residency training. There are multiple potential formats and topics for morning report and few prior studies have described the content and structure of contemporary morning reports.

METHODS: We conducted a prospective observational study of morning report at seven different Veterans Affairs academic medical centers. Each participating site observed a series of morning reports and collected data on the number and type of learners, the number and background of attendings, and the format of report. For case based reports the amount of time on different aspects of the case was recorded as well as the ultimate diagnosis when known. We also recorded other content areas such as medical literature, ethics, quality and safety, and high-value care.

RESULTS: We observed 136 morning reports from 7 different academic medical centers across the country. Reports included a mix of residents, interns and medical students, while $26.5 \%$ included non-physician learners. The most common attending was a hospitalist $(93.3 \%)$ followed by internal medicine sub-specialist $(43.3 \%)$ and general internist $(31.6 \%)$. Most were led by a chief resident $(83.8 \%)$. The most common format was case-based $(84.6 \%)$ with the remaining being a mix of EBM, journal club, and game based. Most case based reports were prepared $(86.1 \%)$ as opposed to "live" reports. On average, the most time was spent on differential diagnosis and testing $(12.0 \mathrm{~min})$, didactics $(9.5 \mathrm{~min})$ and history (7.3min). Most reports used PowerPoint (85.6\%) and $70.4 \%$ had more than 15 slides. Medical literature was only referenced in $35.3 \%$ of reports, most commonly as narrative reviews or professional guidelines. Original research was only discussed in $18.4 \%$ of reports and systematic reviews in $5.9 \%$. Psychosocial issues were discussed in $8.0 \%$ of reports, quality and safety in $7.3 \%$, and high value care in $7.3 \%$. Ethics was rarely discussed. No report went to the bedside. Of the 115 case based reports, 102 had known diagnosis and 13 were unknown. Of the known diagnoses, the most common categories were infectious disease (17), cardiology (15), nephrology (12) and rheumatology (8) which together accounted for more than half of the cases. Individual diagnoses were highly varied, with 92 diagnoses that occurred only once and 10 that occurred multiple times. $50 \%$ of diagnoses were rated as rare.

CONCLUSIONS: In our series, the primary format for report continues to be case based, which are primarily prepared prior to report by the chief resident and employ Power Point. One fourth of reports include non-physician learners. For case based reports, history, differential diagnosis, testing and didactics are the most emphasized areas. Few reports discussed ethics and none involved bedside teaching. The most common attending present was a hospitalist. Individual diagnoses were highly varied and favor rare diagnoses.

\section{A NATIONAL EVALUATION OF PATIENT PORTAL USE AS REPORTED BY PRIMARY CARE PROVIDERS TO THE CENTERS FOR MEDICARE AND MEDICAID SERVICE}

Jorge A. Rodriguez ${ }^{1}$; Stuart R. Lipsitz ${ }^{2}$; Courtney R. Lyles ${ }^{3}$; Lipika Samal ${ }^{2}$. ${ }^{1}$ Lawrence General Hospital, Somerville, MA; ${ }^{2}$ Brigham and Women's Hospital, Boston, MA; ${ }^{3}$ University of California San Francisco, San Francisco, CA. (Control ID \#3184455)

BACKGROUND: Patient portals expanded under the Meaningful Use (MU) Program, which provided over $\$ 35$ billion for them and led to provision of portals by $90 \%$ of providers. Yet this has not led to high uptake by patients, which has been attributed to sociodemographic factors in studies conducted at single institutions. The goal of our study was to correlate national data on broadband access with patient portal use, defined as number of patients who have used the portal to either view, download or transmit (VDT) information, after controlling for sociodemographic characteristics.

METHODS: We performed a retrospective study combining National Provider Identifier (NPI) data from 2016 MU public use files with NPI database to identify provider's address. By linking these addresses with Google Maps Application Programming Interface, the American Community Survey, US Department of Agriculture, and Federal Communications Commission Form 477 we attributed the level of broadband access to each provider's census 
tract. Broadband access was defined as the number of residential connections per 1,000 households by census tract. Our primary outcome was patient portal VDT for each primary care provider. We performed logistic regression with clustering by census tract. RESULTS: Our sample contained 67,686 primary care providers representing 40,653,351 patients across 14,974 census tracts. At the census tract level, the mean reported portal viewing was $28 \%$. Even after controlling for sociodemographic factors, the association between broadband access and portal use persisted (Table).

CONCLUSIONS: Despite nearly universal provision of patient portals, we found that only about a third of patients are using them. Previous studies at the healthcare system level have reported sociodemographic disparities in portal use. Our study provides the first national analysis of portal use and not only reaffirms this association but highlights the strong independent role of broadband access. Though significant efforts led to portal access, a focus on broadband, and patient training and input in development is needed to increase portal use and realize the benefits of patient facing technology.

\section{A NOVEL PATIENT-CENTERED APPROACH TO INTI- MATE PARTNER VIOLENCE IN PRIMARY CARE: RE- COVERING FROM IPV THROUGH STRENGTHS AND EM- POWERMENT (RISE)}

Megan R. Gerber ${ }^{1,}$ 2; Sara B. Danitz ${ }^{3}$; Katherine Iverson ${ }^{3,} 2$. Boston University, Jamaica Plain, MA; ${ }^{2}$ Boston University, Boston, MA; ${ }^{3}$ Women's Health Sciences Division, Jamaica Plain, MA. (Control ID \#3177218)

BACKGROUND: Intimate partner violence (IPV) is a public health problem common among women Veterans. IPV causes injury, chronic illness and health inequity, yet intervention in primary care remains challenging. Research shows that women prefer a self-directed approach to advocacy and recovery. To address this need, we developed "Recovering from IPV through Strengths and Empowerment" (RISE), a brief (30-60 min), innovative variable-length intervention for women with IPV. RISE is rooted in motivational-interviewing and empowerment principles and consists of 6 modules (Table) delivered in up to 6 sessions in primary care settings. Patients prioritize and select modules based on their unique needs. RISE was systematically developed and refined with iterative input from women Veterans and providers in the Veterans Health Administration (VA). We present preliminary results of the initial VA trial.

METHODS: Women with past-year IPV were recruited to participate in an open trial. Social workers and psychologists delivered RISE on-site, evaluation was conducted using a mixed methods approach. We collected pre- and post-treatment measures of psychosocial health (general self-efficacy [GSE], patient activation measure [PAM], valued living [VLQ], and depression [CES-D]). We also conducted semi-structured exit interviews with women after RISE receipt. Interviews were analyzed with a rapid qualitative approach. Enrollment is ongoing.

RESULTS: To date, 10 women Veterans have completed the trial (mean age $=29, \mathrm{SD}=12)$. The sample was $50 \%$ White, $40 \%$ Black, and $10 \%$ Latina. $80 \%$ opted to receive at least 5 sessions, and $60 \%$ of the sample chose to receive all 6 RISE sessions. From pre- to posttreatment, participants showed significant reductions in depressive symptoms and increases in self-efficacy, PAM, and valued living, with medium effect sizes across outcomes (Cohen's D range: .54 - .72). Women expressed high satisfaction with RISE, they 1) liked ability to choose the content delivered, 2) felt RISE provided validation of their IPV experiences, 3) were empowered to be more assertive, and 4) expressed that RISE improved understanding of the impact of IPV on their health, which promoted confidence in accessing additional services, congruent with increased PAM score.

CONCLUSIONS: Preliminary findings support the clinical utility and acceptability of RISE in primary care. Increases in selfefficacy, patient activation, and valued living scores suggest RISE may promote self-efficacy, quality of life, and even health equity as women are enabled to engage in care and access services. Recruitment continues, we anticipate a larger sample by Spring 2019. Future work includes an RCT which follows this open trial.

\section{A NOVEL PROGRAM TO COORDINATE CARE FOR SO- CIALLY VULNERABLE INDIVIDUALS WITH COMPLEX HEALTH NEEDS: EVALUATING STRUCTURE AND PRO- CESSES OF CONNECT 2 CARE}

Dailys Garcia-Jorda $^{2}$; Kerry McBrien ${ }^{1}$; Alicia J. Polachek ${ }^{2}$; Celise Ritter-Rattray ${ }^{2}$; Hasham Kamran ${ }^{2}$; Gabriel E. Fabreau ${ }^{1} .{ }^{1}$ University of Calgary, Calgary, AB, Canada; ${ }^{2}$ Cumming School of Medicine, University of Calgary, Calgary, AB, Canada. (Control ID \#3186129)

BACKGROUND: Background: Socially vulnerable individuals have higher mortality and acute care use than the general population and require specialized services to address complex health and social needs. Connect 2 Care (C2C), a mobile outreach program launched in November 2015, seeks to improve care coordination between acute and community care for socially vulnerable individuals with high acute care use, often also experiencing housing insecurity in Calgary, Canada. We are conducting a comprehensive mixed-method program evaluation to investigate the $\mathrm{C} 2 \mathrm{C}$ program's structure, processes and outcomes to inform its potential sustainability. We directed part of our qualitative evaluation to document program structure and processes, and understand frontline staff daily activities, perceptions and experiences of caring for their clients.

METHODS: Methods: We conducted observations of $\mathrm{C} 2 \mathrm{C}$ staff from February to June 2018. We sampled multiple settings and different times of the day and days of the week to examine the C2C team's: 1) structure, including infrastructure, personel and partnerships and; 2) processes, including daily activities, services, interactions, and challenges. Observations were triangulated with $\mathrm{C} 2 \mathrm{C}$ team members' qualitative semi-structured interviews, from October 2018. We conducted an inductive thematic analysis of observation field notes and interview transcripts and recorded themes that emerged using an iterative consensus process among all authors.

RESULTS: Results: We analyzed 36 cumulative hours of observation and nine interviews (three nurses, four health navigators, and two managers). Our analysis revealed how $\mathrm{C} 2 \mathrm{C}$ staff explicitly work to 'fill the gap' bestween acute and community care, and also between healthcare and social services (i.e., housing). System-level healthcare and social challenges became evident throughout the observations and interviews, as participants struggled daily to access necessary care and social services for their clients. The $\mathrm{C} 2 \mathrm{C}$ team's comprehensive healthcare and social system knowledge and commitment to their clients were core structural program elements, as were specified skills, training and intrinsic motivation of team members. These elements supported core program processes including client rapport building and navigation between many agencies and services. The program's novelty emerged as it addressed client's needs through a client-centred, harm-reduction approach, providing support, advocacy, and building relationships between acute and community care, and with other organizations. 
CONCLUSIONS: Conclusions: We identified core elements of $\mathrm{C} 2 \mathrm{C}$ program structure and processes, as well as strengths, challenges, and limitations that modulate it effectiveness. Our findings suggest core program elements are likely replicable but depend on both intrinsic qualities and motivations among program staff, as well as structural and interpersonal supports from the healthcare and social system broadly.

\section{A PEER OBSERVATION OF TEACHING PROGRAM TO OPTIMIZE FEEDBACK ACCEPTANCE BY FACULTY} Alice Tang ${ }^{1}$; Sydney $\mathrm{Katz}^{2}$; Stephanie J. Tang ${ }^{3}$; Kendrick Tang ${ }^{5}$; David Stern ${ }^{4}$. Weill Cornell Medical College, New York, NY; ${ }^{2}$ Weill Cornell, Brooklyn, NY; ${ }^{3}$ Weill Cornell Medicine NewYork-Presbyterian Hospital, Long Island City, NY; ${ }^{4}$ New York University, New York, NY; ${ }^{5}$ Memorial Sloane Kettering, New York, NY. (Control ID \#3184735)

BACKGROUND: Physicians are poor self-assessors in most domains, including teaching skills. However, self-assessment is essential for self-regulation and performance improvement through deliberate practice. The process by which one accesses, interprets, and accepts feedback is a complex process which involves a number of factors, conditions, and tensions. Optimizing these factors and conditions is essential for acceptance of feedback and improvement of skills. Peer observation of teaching (POT) has been shown to be a promising tool for improving teaching quality and confidence. The purpose of this phenomenological study is to explore the experiences of medicine faculty undergoing a POT program, consisting of in-person and video-based observation and feedback. The findings can be used to better understand how factors, conditions, and tensions can be optimized to enhance faculty self-assessment of teaching skills. Secondarily, the study aims to compare in-person with video-based peer observation and investigates how observing a peer's clinical teaching affects one's own teaching skills.

METHODS: A hermeneutic phenomenological approach was used to understand the "lived experiences" of faculty undergoing a novel POT program at an academic medical center in New York City. A comprehensive sample of ten participants was interviewed prior to and after completing the program. Participants were paired with a partner and each pair discussed teaching goals and observed one-another in both an in-person and video-based teaching session during ward rounds or precepting. Self-assessment, feedback, and goal setting followed each teaching observation. Interviews were transcribed and analyzed iteratively by two independent reviewers until thematic saturation was reached. Themes were presented to study participants during a focus group for member checking.

RESULTS: The median time as an attending physician for participants was 1.5 years, ranging between 0.5 to 5.5 years. Ninety percent of participants were observed during ward rounds. Features of the program that facilitated enhanced self-assessment and improved clinical teaching skills included the authenticity and structure of the program, partner credibility, matched educator competency, optimal balance between comfort and discomfort, and self-reflection. Many participants cited specific changes that they had made in their teaching behaviors as a result of this program.

CONCLUSIONS: The results of this study build upon the factors, conditions, and tensions identified in Sargent's informed selfassessment conceptual model and apply it to a faculty development program. Findings can aid other faculty development programs to optimize factors and conditions for feedback acceptance and improve clinical teaching skills through deliberate practice.
A PILOT STUDY OF A LOW-CARBOHYDRATE DIABETES PREVENTION PROGRAM AMONG ADULTS WITH PREDIABETES

Dina H. Griauzde ${ }^{1,2}$; Kaitlyn J. Patterson ${ }^{3}$; Tahoora Ansari ${ }^{1}$; Patti $\mathrm{Bihn}^{3}$; Samuel Shopinski ${ }^{3}$; Caroline Richardson ${ }^{1}$; Laura Saslow ${ }^{1}$. ${ }^{1}$ University of Michigan, Ann Arbor, MI; ${ }^{2}$ VA, Ann Arbor, MI; 3National Kidney Foundation of Michigan, Ann Arbor, MI. (Control ID \#3183918)

BACKGROUND: Individuals with prediabetes can prevent or delay progression to type 2 diabetes by losing $5 \%$ of their body weight. The Center for Disease Control and Prevention's National Diabetes Prevention Program (NDPP) is a lifestyle change program proven to help individuals lose weight through adherence to a low-fat, calorie-restricted diet. Yet, low-carbohydrate diets may be more effective for weight loss and glycemic control among individuals with prediabetes. This pilot study examined the feasibility, acceptability, and preliminary weight loss efficacy of a Low-Carbohydrate Diabetes Prevention Program (LC-DPP) among individuals with prediabetes.

METHODS: This single-arm mixed methods pilot study was conducted at a large, academic primary care clinic. Inclusion criteria were: BMI $25 \mathrm{~kg} / \mathrm{m}^{2}$ and prediabetes (defined as $\mathrm{HbAlc}$ $5.7-6.4 \%$ and absence of diabetes). Eligible participants were identified by chart review and invited to participate by postal letter. We adapted the dietary information in the NDPP's curriculum (16 weekly core sessions, 8 bimonthly/monthly post-core sessions) and trained a lifestyle coach to teach participants to follow a very low carbohydrate diet $(<35$ non-fiber grams of carbohydrates per day). Primary outcomes were feasibility (rate of recruitment) and acceptability (session attendance, qualitative interviews). Secondary outcomes were change in weight and hemoglobin A1c at 6- and 12-months.

RESULTS: Twelve percent of individuals (21/187) who were invited to participate enrolled in the study and had a mean age of 59 years, BMI of $34.2 \mathrm{~kg} / \mathrm{m}^{2}, \mathrm{HbAlc}$ of $5.9 \%$. Over half $(\mathrm{n}=12,57 \%)$ of participants were men. All individuals attended 3 or more core sessions and $67 \%(14 / 21)$ attended at least 9 core sessions. At 6 months, mean percent (SD) body weight loss was $4.5(5.0) ; 46 \%(10 / 21)$ of participants achieved $5 \%$ body weight loss and $27 \%(6 / 21)$ achieved $7 \%$ body weight loss. At 12 months, mean percent (SD) body weight loss was 5.9 (7.1); $43 \%$ of participants $(9 / 21)$ achieved $5 \%$ body weight loss and $36 \%(8 / 21)$ achieved $7 \%$ body weight loss. HbA1c decreased by an average of $0.1 \%$ at 6 months $(n=18)$ and by an average of $0.3 \%$ at 12 -months $(n=19)$ compared to baseline. Qualitative interviews $(n=13)$ revealed themes of subjective health benefits (e.g. improved sleep) beyond weight loss and relative ease of learning a low-carbohydrate lifestyle. Barriers included social pressures and concerns about high dietary fat intake. There were no significant changes in physical symptoms (e.g. thirst, constipation). One participant suffered an ischemic stroke.

CONCLUSIONS: A LC-DPP is feasible and acceptable among individuals with prediabetes. At 12-months, mean weight loss among our pilot program participants was greater than mean weight loss among participants in traditional NDPPs, as reported in metaanalyses $(5.9 \%$ vs. $4.0 \%)$. A large-scale comparative effectiveness trial is needed.

\section{A PILOT STUDY OF A PHOTO-BASED INTERVENTION TO PROMOTE DIETARY DISCUSSIONS AMONG OLDER ADULTS WITH MULTIPLE CHRONIC CONDITIONS IN PRI- MARY CARE}

Jane Jih; Ying Wang; Liliana Chacon; Jasmin Woo; Antony H. Nguyen; Tung T. Nguyen; Christine Ritchie. UCSF, San Francisco, CA. (Control ID \#3182021) 
BACKGROUND: We know little about how to best communicate with older adults about optimal dietary behaviors for the selfmanagement of multiple chronic conditions (MCC). In MCC selfmanagement, photos can promote efficient information exchange and activate patients to more effectively communicate their complex lived experiences. Based on this premise, we conducted a pilot study to assess the feasibility and acceptability of a photo-based communication intervention to promote dietary discussions among older adults with MCC in primary care.

METHODS: We recruited older adults age $60+$ with at least $2+$ concurrent chronic conditions and their primary care providers (PCPs) from an urban academic primary care practice. We recruited patient participants from clinic waiting rooms, PCP referrals, and from a telephone survey focused on food insecurity and MCC. Ten days before the study clinic visit, patients received a 25 -minute inperson training on photo-taking with a smartphone and then took photos in response to the prompt, "What aspects of your everyday life affect what you eat and how much you have to eat?" Patients received reminder calls or text messages to take 1-2 photos/day. One week before the visit, providers received a 5 minute in-person training about what to do when patients shared photos. Patients shared photos and their narrative to their PCP during the study clinic visit. Study clinic visits were audio-recorded. We also conducted separate audio-recorded patient and provider post-visit interviews to assess perspectives on the intervention. We used an iterative, grounded theory approach to analyze transcripts.

RESULTS: Nine patient-PCP dyads have completed the study to date. Patient participants were $44 \%$ female, $78 \%$ Latino or African American, and $67 \%$ completed high school education with mean age of 69 years old. PCPs on average had been in practice for 11 years since medical school (range 3-22 years) and saw on average 27 patients each week (range 7-60). 89\% of patient-PCP dyads completed the intervention as trained. Patients shared 6 photos (range 5-8) on average. Visits lasted 22-39 minutes with $14-47 \%$ (average $23 \%$ ) of the visit spent looking and discussing photos. $100 \%$ of patients definitely would like to share photos about health with their PCP again. $100 \%$ of PCP definitely [67\%] or probably [33\%] would like patients to share photos at future visits including photos related to medication use and home safety. Patient and PCP quotes illustrated the added value of the intervention (Table).

CONCLUSIONS: A pilot patient-centered photo-based intervention to promote diet-related discussions between older adults with MCC and their PCPs has shown high feasibility and acceptability. Future work will assess how a photo-based intervention can enhance patientPCP communication about other contextual factors important in chronic disease management.

\section{A PRAGMATIC TRIAL INTEGRATING ROUTINE SCREEN- ING FOR ATRIAL FIBRILLATION IN OLDER PATIENTS DURING PRIMARY CARE VISITS: INITIAL ENROLLMENT DATA FROM THE VITAL-AF TRIAL}

$\underline{\text { Steven J. Atlas }}{ }^{1}$; Jeffrey M. Ashburner ${ }^{1}$; Steven A. Lubitz ${ }^{1}$; Yuchiao Chang $^{1}$; Ana T. Trisini Lipsanopoulos ${ }^{1}$; Leila H. Borowsky ${ }^{1}$; Wyliena Guan ${ }^{1}$; Wei He ${ }^{1}$; Patrick T. Ellinor ${ }^{1}$; David D. McManus ${ }^{2}$; Daniel E. Singer ${ }^{1} .{ }^{1}$ Massachusetts General Hospital, Boston, MA; ${ }^{2}$ University of Massachusetts Medical School, Worcester, MA. (Control ID \#3185016)

BACKGROUND: Atrial fibrillation (AF) is often asymptomatic and sometimes diagnosed only at the time of a stroke. Efficient and scalable screening for AF may prompt appropriate initiation of oral anticoagulation to prevent strokes. A new pragmatic trial, VITAL-AF (ClinicalTrials.gov NCT03515057), will evaluate whether adding automated AF rhythm assessment as a vital sign among older adults during primary care visits is an effective new standard of care.

METHODS: The study is being conducted within an academic primary care network in the United States that uses a single electronic health record (EHR). Randomization is at the practice-level with 8 practices randomized to AF screening and 8 to usual care. Patients 65 years and older attending an outpatient visit in a participating site with a physician or nurse practitioner are eligible. Screening is performed by medical assistants, not research personnel. All eligible patients are approached while checking vital signs and those who agree are screened using a single-lead ECG device (AliveCor, US). Screening results are documented in the EHR and clinicians are notified about patients with possible AF. Clinicians can discuss screening results with patients and manage $\mathrm{AF}$ at their discretion. All single-lead ECGs are reviewed by a cardiologist. The primary study endpoint is incident AF during a 1-year enrollment period which began July 31, 2018. Secondary outcomes include new oral anticoagulation prescriptions, ischemic stroke and major hemorrhage. Outcomes are based on EHR documentation. With a sample size of 16,212 patients per group, a baseline AF incidence of $1.6 \%$ and an $85 \%$ screening rate, the study will have $80 \%$ power to detect a $0.42 \%$ difference in AF incidence.

RESULTS: As of November 30, 2018, 11089 eligible encounters have occurred in intervention practices, with 9621 (86.8\%) approached for screening by a medical assistant, and $8629(77.8 \%)$ completing screening. Encounter data across intervention sites show high rates with modest variation (see table). Encounters where screening was declined average $8.7 \%$ (range $3.6-13.0 \%$ by site). Among 8716 eligible patients with encounters, 7141 (81.9\%) have completed screening. Since patients are offered screening at all visits, $220(62.2 \%)$ not initially screened were screened at a subsequent visit.

CONCLUSIONS: VITAL-AF is the first randomized clinical trial to embed AF screening into routine primary care in the US. Initial enrollment data show that adding automated AF rhythm assessment as a new vital sign among older, at-risk adults at primary care visits is feasible and accepted by patients. Outcome assessment will determine whether it is an effective new standard of care.

\section{A PRE-IMPLEMENTATION STUDY OF HOSPITAL-BASED HEALTH PROFESSIONAL PERSPECTIVES ON PRESCRIB- ING OF MEDICATIONS FOR ALCOHOL USE DISORDER}

$\underline{\text { Paul Joudrey }}^{1}$; Benjamin Oldfield ${ }^{1}$; Kimberly A. Yonkers ${ }^{2}$; Patrick $\mathrm{O}^{\prime}$ Connor ${ }^{1}$; E. Jennifer Edelman ${ }^{1}$. ${ }^{1}$ Yale School of Medicine, New Haven, CT; ${ }^{2}$ Yale School of Public Health, New Haven, CT. (Control ID \#3186284)

BACKGROUND: Medications for Alcohol use disorder (MAUD) (acamprosate, naltrexone, and disulfiram along with off label gabapentin and topiramate) remain underutilized despite guideline recommendations and rising alcohol-related morbidity and mortality. Alcohol use disorder (AUD)-related hospitalizations are opportunities to initiate MAUD, but optimal implementation strategies are unclear. Using an implementation science framework, we assessed a multidisciplinary group of hospital-based health professional perspectives on prescribing MAUD among patients with AUD within the inpatient general medicine setting.

METHODS: We completed a pre-implementation mixed method study at Yale New Haven Hospital. We administered the first two scales (evidence and context) of the Organizational Readiness for Change Assessment (ORCA) survey. We evaluated median scores (five-point Likert scale, 1 = lowest readiness, 5 = highest readiness) across ten subscales to determine the highest and lowest subscale within the evidence and context scales. We conducted professionspecific focus groups among general medicine inpatient teams, 
guided by the Consolidated Framework for Implementation Research (CFIR) and purposively sampled for variation by discipline and experience. We analyzed focus group transcripts using directed content analysis in accordance with the CFIR framework. We used the constant comparative method to identify emerging themes across the entire sample.

RESULTS: We completed seven focus groups with all 57 participants completing an ORCA survey (eight nurses, six nurse practitioners, ten physicians, ten social workers, eight physician assistants, nine pharmacists, and eight psychiatrists). Among participants, the median age was $34,43(75 \%)$ were female, and had a median of four years of experience. ORCA evidence subscale results showed high research evidence (median 4.0) but low patient preference (median 3.5) for MAUD. Context subscale results showed staff culture (median 4.0) to be supportive of implementation but low availability of resources for implementation (median 3.0). We identified four emerging themes. Across disciplines, there was limited knowledge and experience regarding MAUD. There was concern about patient barriers to accessing care following discharge and perceived low patient engagement. Participants viewed long term abstinence as the goal of treatment rather than reducing harm by reducing use. Participants reported existing care support systems for other chronic diseases (i.e. tobacco use) could be adapted to support MAUD utilization.

CONCLUSIONS: Among hospital-based health professionals, a lack of knowledge, a lack of patient treatment access following discharge, perceived low patient engagement, and a focus on abstinence reduced inpatient utilization of MAUD. Implementation interventions should aim to adapt existing care support systems, increase provider understanding of these medications, and support harm reduction rather than abstinence as a treatment goal.

\section{A PRELIMINARY QUALITATIVE ANALYSIS OF FIBROMY- ALGIA CARE IN VA THROUGH NARRATIVES OF WOMEN VETERANS}

Kristina M. Bogdan ${ }^{1,}{ }^{2}$; Erica R. Scioli ${ }^{1,2}$; Megan R. Gerber ${ }^{3} .{ }^{1}$ VA Boston Healthcare System, Boston, MA; ${ }^{2}$ Boston University School of Medicine, Boston, MA; 3Boston University, Jamaica Plain, MA. (Control ID \#3186398)

BACKGROUND: Fibromyalgia (FM) is a chronic disorder more common in women. It is characterized by widespread bodily pain, fatigue, and poor sleep. Prior studies have concluded that participants often feel unheard, demoralized, and invalidated by healthcare providers. Women Veterans are a rapidly growing population in the Veterans Health Administration (VHA), and their rates of FM exceed that of the general population. A better understanding of women Veterans' perceptions of care will inform patient-centered approaches to FM. Thus, we conducted a mixed methods study to better understand women Veterans' experiences with and preferences for VA FM care. The qualitative portion of the study data are presented below.

METHODS: Six, in-person, focus groups of women Veterans, who used VA primary care and had medical record-confirmed FM, were conducted. A clinical psychologist conducted all 6 groups using a semi-structured interview guide. The focus groups were transcribed and analyzed using a hybrid thematic approach. Codes were identified and grouped into themes. A second investigator reviewed the identified codes for congruence and differences were resolved. Finally, a third researcher, who was present at all focus groups, reviewed the codes and themes to achieve final consensus.

RESULTS: 22 women Veterans with FM participated in 6 focus groups. The mean age was 51.3 (range $30-76, \mathrm{SD}=11.6$ ). Most of the patients were White $(54.5 \%)$ and unemployed (77.3\%). Most $(72.7 \%)$ had a diagnosis of posttraumatic stress disorder (PTSD). The following 4 dominant themes emerged: 1) Challenge of living with FM (women expressed profound fatigue and isolation as a result of unpredictable pain), 2) Invalidation (both healthcare providers and family/friends were dismissive of FM symptoms), 3) Health Behaviors (women expressed self-efficacy approaches to FM symptom management as well as avoidance of known triggers), 4) Trust in VA care (patients valued primary care providers [PCP] who listened and validated FM symptoms; patients expressed concern that their gender had resulted in inadequate FM care by other providers. When referring to participant VA treatment experiences, one said "I won't come in unless I'm desperate" while another illustrated a memory by saying "...they sent me to the pain management and the doctor told me that, you know, fibromyalgia wasn't a disease, it was mental."

CONCLUSIONS: Women Veterans encounter many challenges in functioning with FM and accessing care, they often felt disbelieved and invalidated. Many have developed knowledge of what triggers a flare and what helps them feel better. Women across focus groups expressed greater satisfaction and trust in VA care when their PCP showed interest in their condition and listened well. These findings support the need for a patientcentered approach to FM care.

\section{A PROACTIVE TEXT MESSAGING INTERVENTION WITH MEDICATION ADHERENCE SUPPORT TAILORED TO PRIMARY CARE POPULATIONS: A PILOT RANDOM- IZED TRIAL OF GETREADY2QUIT}

Gina R. Kruse $^{2,}{ }^{1}$; Elyse Park ${ }^{2}$, ; Yuchiao Chang ${ }^{2,}$ 1; Jessica Haberer $^{2,}{ }^{1}$; Lorien Abroms ${ }^{3}$; Naysha N. Shahid ${ }^{2}$; Sydney How$\operatorname{ard}^{2}$; Jennifer Haas ${ }^{2,1}$; Nancy A. Rigotti ${ }^{2}{ }^{1}$. ${ }^{1}$ Harvard Medical School, Boston, MA; ${ }^{2}$ Massachusetts General Hospital, Boston, MA; ${ }^{3}$ George Washington University, Washington, DC. (Control ID \#3179241)

BACKGROUND: Text messaging is an effective aid to help with smoking cessation, but it is unclear how to integrate it effectively into primary care settings. We developed and tested an automated, proactive, text messaging program with 2 novel uses: 1) to enhance motivation for smokers not ready to quit and 2) to promote adherence to nicotine replacement therapy (NRT). We present feasibility and efficacy results for a 12-week pilot randomized controlled trial (RCT).

METHODS: We used proactive telephone calls to recruit adult daily smokers from 10/2017-10/2018 for a RCT. Randomization was stratified by clinic and smoker's plan to quit within 30 days. Enrollees were assigned to 1 of 4 groups: brief advice about treatment options and local services (BA), brief advice + text messages (TM), brief advice +2 -weeks of NRT by mail (NRT), or brief advice $+\mathrm{TM}+\mathrm{NRT}(\mathrm{TM}+\mathrm{NRT})$. Patients were surveyed by phone or email at 1-, 2-, 6- and 12-weeks.

RESULTS: We approached 1,544 potentially eligible patients, we reached $988(64 \%)$. Of these, $527(53 \%)$ declined, 32 (3\%) dropped out before randomization, $276(28 \%)$ were ineligible and $153(15 \%)$ of those reached were randomized. Of these, $54 \%$ were women, mean age 53 years, $76 \%$ white, $12 \%$ African-American, $6 \%$ Hispanic, $5 \%$ other races, $54 \%$ on Medicaid, mean 15 cigarettes/day and $75 \%$ smoked $<30$ minutes from awakening. Overall, $31 \%$ screened positive for alcohol use disorder (single-item measure), $13 \%$ for substance use (single-item measure), 35\% for depression (PHQ-2) and $55 \%$ for anxiety (GAD-2). Acceptance of treatments offered (BA, TM, and/or NRT) was $100 \%$. Retention at the 12 -week follow-up was $92 \%(n=140)$. A large majority of patients selfreported 1 quit attempt(s) (1oclinical outcome), with no differences by group compared to BA $(71 \%)$, TM $(89 \%, \mathrm{p}=0.13)$, NRT $(82 \%$, $\mathrm{p}=0.40)$, TM+NRT $(81 \%, \mathrm{p}=0.41)$. Past 7 -day abstinence at 12 weeks was reported twice as often in the NRT, TM, and TM+NRT 
groups, though differences were not significant compared to BA $(11 \%)$, TM $(29 \%, \mathrm{p}=0.13)$, NRT $(30 \%, \mathrm{p}=0.07)$, and TM+NRT $(22 \%, \mathrm{p}=0.35)$. Groups offered NRT by mail were more likely to report NRT use compared to BA $(51 \%)$, TM (63\%, p=0.47), NRT $(82 \%, \mathrm{p}=0.011)$, and TM+NRT $(76 \%, \mathrm{p}=0.049)$. There was no difference in days of NRT use over the first 2 weeks between NRT only (4.6 days of NRT use) or NRT+TM (3.0 days, $\mathrm{p}=0.13$ ).

CONCLUSIONS: This pilot RCT demonstrated feasibility as we recruited, treated, and retained smokers from a primary care network. Our uptake of $15 \%$ is comparable to other proactive outreach interventions for smokers. A proactive offer to have NRT mailed to patients' homes increased NRT use, even though many patients already had access to low-cost NRT from Medicaid. Adherence messages in the texting program did not increase NRT use. Proactively-delivered smoking cessation interventions that are offered outside office visits are feasible and hold promise for reducing smoking rates and promoting health in primary care populations.

\section{A PROGRESSIVE-STAGE MODEL OF PATIENT ENGAGE- MENT IN INNOVATION TEAMS: FROM VISITOR TO PART- NER TO ACTIVE CHANGE AGENT}

Sophie Higgins ${ }^{1}$; Mariam K. Atkinson ${ }^{3}$; Sara Singer ${ }^{2} .{ }^{1}$ Harvard T.H. Chan School of Public Health, Boston, MA; ${ }^{2}$ Harvard School of Public Health, Boston, MA; ${ }^{3}$ Harvard University, Boston, MA. (Control ID \#3183975)

BACKGROUND: Patient engagement is recognized as vital for improving healthcare delivery. Increasingly, patients are collaborating with teams working to redesign healthcare processes. Yet, there is limited understanding of the ways patients' social identity (i.e., the way patients and other team members view patients in relation to a particular group) evolves over the course of involvement in healthcare innovation teams. This study explored the evolution of patient identity on innovation teams to help teams optimize patient engagement in improvement efforts.

METHODS: In 3 interdisciplinary teams that included patients across 3 health systems participating in an innovation collaborative, we observed weekly team meetings over 18 months and conducted 16 interviews with team members (4 health system staff, 4 physicians/physician leaders, 4 system engineers, 3 patients, and 1 administrative manager). In interviews, respondents addressed targeted questions about patient engagement, including perceptions of patient contributions to team progress and the evolution of patients' role over time. Field notes and interview transcripts were coded and analyzed using a grounded theory approach to identity major themes.

RESULTS: Qualitative data analysis led to the development of a framework that illustrates the process by which patient social identity evolves from visitor to partner to active change agent over the course of an innovation project. The process is driven by team members' (including patients') view of a patients' role, wherein both patient and staff's (i.e., non-patient team members) views of a patient's role on the team lead the patient to enact this role, as staff team members engage in behaviors and activities that reflect these views. This dynamic interaction between patients' role enactment and staff activities results in patient contributions that impact the work of the team. Patient contributions, in turn, shape team members' view of patients' role in an iterative, virtuous cycle, as patient contributions lead patient and staff team members to view patients as possessing increased, essential knowledge to the work of the team. This process propels an evolution in the patient's social identity, progression through which is marked by an increased sense of patient team member integration, growing respect for their contributions, more responsibilities bestowed upon and assumed by patient team members, with patient contributions that move from responsive to proactive.

CONCLUSIONS: Patient and staff team member views of a patient's role in innovation teams drive the ways team members interact and the ways patients contribute to team work. Patient evolution requires conscious aim and willingness among all team members to set expectations and support progressive advancement of patients in the overall work of the team

\section{A QUALITATIVE STUDY OF PATIENTS WHO OVERDOSED IN THE CURRENT ENVIRONMENT OF RE- STRICTIVE OPIOID PRESCRIBING POLICIES}

Shane Mueller ${ }^{1}$; Jason M. Glanz ${ }^{1}{ }^{3}$; Steve Koester ${ }^{2}$; Anh P. Nguyen ${ }^{1}$; Ingrid A. Binswanger ${ }^{1,2}$. ${ }^{1}$ Kaiser Permanente Colorado, Denver, $\mathrm{CO} ;{ }^{2}$ University of Colorado Denver, Denver, $\mathrm{CO} ;{ }^{3} \mathrm{Colo}-$ rado School of Public Health, Denver, CO. (Control ID \#3184382)

BACKGROUND: Faced with the growing opioid overdose epidemic, government agencies, health systems and medical providers have developed and implemented laws and policies to reduce opioid prescribing and expand access to naloxone and substance use disorder treatment. Despite these efforts to reduce overdose risk and improve pain management, overdoses continue to occur at an unprecedented rate. The reasons for this persistently elevated rate remain unclear. We conducted a qualitative study of patients who experienced an overdose and their family members to better understand the factors that led to an overdose in this current environment.

METHODS: We conducted semi-structured interviews with patients from a large integrated care organization in Colorado who experienced an opioid overdose in 2017 or 2018. Patients were identified using ICD codes for overdose and medical charts were reviewed to confirm the overdose. Patients were recruited and asked to invite family members who were present at the time of the overdose to participate in the interview. Interviews explored patients' history of pain, perceived risks and benefits of opioids, circumstances that led to the overdose and actions taken in response to the overdose. A constant comparative method of inductive and deductive analysis was used to code transcribed interviews and generate themes.

RESULTS: We enrolled 9 patients who experienced an opioid overdose, 8 of which involved a pharmaceutical opioid. Five patients were female and mean age was 46.1 years (range 22-65). Three family members of participants also enrolled. We identified 3 themes: The first theme was process failure - It appears as though some policies had limited reach. No patients or family members had picked up naloxone, nor were any patients in treatment for substance use disorder. The second theme was low risk perception Prior to experiencing the event, patients did not believe they were at increased risk and thought only patients who were using medications to "get high" were at risk. The third theme was increased pain leading to risky behaviors - Patients felt providers reduced opioid dose in response to prescribing policies. These dose reductions led to increased pain and feelings of powerlessness and desperation. To find relief, they took more opioids than prescribed or in combination with other medications and substances, leading to overdose.

CONCLUSIONS: In this qualitative study, we identified 3 major themes related to overdose risk. Our findings suggest that the reach of some preventive interventions, such as naloxone, are limited. Additionally, while these policies have significantly reduced opioid prescribing across the United States, they may also have unintended consequences. Further research should examine the adverse outcomes of these policies at a population level. 
A QUALITATIVE STUDY OF THE INFLUENCE OF LOSS AVERSION AND INCREASED SOCIAL PRESSURE IN PHYSICIAN PAY-FOR-PERFORMANCE

John W. Urwin ${ }^{4}$; Shireen E. Matloubieh ${ }^{1}$; Judy A. Shea ${ }^{3}$; Kristen L. Caldarella $^{1}$; Matt Walters ${ }^{1}$; Akriti Mishra ${ }^{3}$; Nichia McDowald ${ }^{5}$; Ezekiel J. Emanuel $^{1}$; Kevin G. Volpp ${ }^{1,2}$; Amol S. Navathe ${ }^{1,2}$. ${ }^{1}$ Medical Ethics and Health Policy, University of Pennsylvania, Philadelphia, PA; ${ }^{2} \mathrm{CMC}$ VA Medical Center, Philadelphia, PA; ${ }^{3}$ University of Pennsylvania, Philadelphia, PA; ${ }^{4}$ Perelman School of Medicine, University of Pennsylvania, Philadelphia, PA; ${ }^{5}$ Advocate Health Care, Chicago, IL. (Control ID \#3184326)

BACKGROUND: Payers are increasingly using behavioral economic principles to design reimbursement programs, despite little evidence regarding the efficacy of these principles on influencing physicians' clinical behaviors. We conducted a qualitative follow-up study to a randomized controlled trial testing how loss aversion and increased social pressure in physician pay-for-performance ( $\mathrm{P} 4 \mathrm{P})$ influenced the motivations and behaviors of participating physicians.

METHODS: We interviewed 22 of the 33 physicians who participated in a previously reported randomized controlled trial using a standardized script. The trial included all physicians in an integrated physician network who treated patients with one of five chronic conditions (diabetes, asthma, COPD, CAD, or CHF). Seven participants were in the "active control" arm whose incentive was the same as the year prior; nine were in the loss aversion arm and had the option to withdraw up to $50 \%$ of their expected bonus early (averaging $\$ 19,292$ ); and twelve were in the increased social pressure arm and had $50 \%$ of their bonus based on group performance, rather than $30 \%$. Transcripts from these interviews were coded by two trained observers and analyzed using nodal analysis with NVivo 11.

RESULTS: The most frequently discussed topics were the financial salience of the interventions (3.6 references per interview), changes in personal motivations ( 2.3 references per interview), and changes in group dynamics (2.0 references per interview). Participants in the increased social pressure arm demonstrated significantly increased group cohesiveness and individual empowerment that were associated with changes in clinical behavior, including offering additional testing and arranging for closer follow-up. Participants in the loss aversion arm demonstrated little change in their clinical behavior. The ineffectiveness of this arm was attributed to a lack of participation in the voluntary incentive - only two of the eleven physicians withdrew any money - rather than a lack of salience of the principle of loss aversion to physicians necessarily. Barriers to success included clerical burdens of P4P and using outcomes measures rather than process measures for performance bonuses.

CONCLUSIONS: Incorporation of increased social pressure into physician $\mathrm{P} 4 \mathrm{P}$ incentive programs can lead to significant changes in physicians' personal motivations, group dynamics, and clinical behaviors. Lack of participation in pre-funded bonuses may indicate limitations to feasibility of using voluntary loss aversion in physician P4P programs.

\section{A QUALITY IMPROVEMENT PROJECT TO INCREASE COMPLIANCE WITH DIABETES MEASURES IN AN ACA- DEMIC OUTPATIENT SETTING}

Jordan Bushman $^{1}$; Subhash Edupuganti ${ }^{2}$; Pradeep Kaminoulu ${ }^{1,}$; Alexandra Halalau ${ }^{1,2}$. ${ }^{1}$ Beaumont Health, Troy, MI; ${ }^{2}$ Oakland University William Beaumont School of Medicine, Rochester, MI. (Control ID \#3165173)

BACKGROUND: Diabetes is one of the most common chronic diseases in the United States, present in nearly $9.4 \%$ of the US population. 1 As part of internal medicine residency training, medical residents are often first-line primary care providers in residency clinics and thus have an important role in providing appropriate diabetes care for these patients. Consequently, American Diabetes Association (ADA) sets forth annual guidelines on preventative measures that can help prevent or delay the onset of more severe complications of diabetes mellitus. 2 However, previous studies describe suboptimal care amongst residents with regards to preventative care in diabetic patients, including at our own clinic prior to the onset of this study. 3 The aim of our project is to improve diabetic care preventative measures, with $\mathrm{HbA} 1 \mathrm{c}$ as our primary outcome and other core measures as secondary outcomes, to comply with the ADA recommendations in our resident clinic through the implementation of quality improvement interventions via PDSA (Plan-DoStudy-Act) cycles.

METHODS: Our resident clinic consists of 76 residents working in 8 different teams at Beaumont Hospital - Royal Oak. In November 2016, baseline data was obtained on 538 patients with diabetes, on core ADA measures (HbA1c, foot examinations, lipid panel, etc.). 5 teams developed a quality improvement intervention plan to improve their diabetes care in the clinic and the remaining 3 teams served as controls. Within the next year, PDSA sessions took place every 3 months where teams analyzed their core measure results up to that date and brainstormed strategies to better implement their plans. In November 2017, post-intervention data was collected on the same diabetes core measures.

RESULTS: With HbA1c as our primary outcome, there was paradoxically a slight increase in HbAlc levels in both intervention and control groups post-PDSA cycle (change in HbAlc was +0.09 in intervention, +0.32 in control groups, $\mathrm{p}=0.174)$. With secondary outcomes, the change in outcome measures were as follows: eye examinations $(+5 \%$ in intervention vs. $-7 \%$ in control, $\mathrm{p}<0.01)$, foot examinations $(+13 \%$ vs. $+5 \%, p=0.09)$, lipid panel $(+7 \%$ vs. $-5 \%$, $\mathrm{p}<0.01)$, microalbumin $(+4 \%$ vs. $+1 \%, \mathrm{p}=0.03)$, and $\mathrm{HbAlc}(+8 \%$ vs. $+5 \%, \mathrm{p}=0.24)$.

CONCLUSIONS: While there was not an improvement in HbA1c values, there were significant improvements in the rates of ADA recommended examinations and laboratory tests performed by residents in the intervention groups. One resident team implemented an intervention involving protected half-day blocks for its residents to identify overdue examinations and consequently had the largest improvements in its quality indicators, thus serving as a potential intervention to replicate in future studies. Given our study results, we believe that quality improvement interventions improve preventative care for patients with diabetes in residency clinics.

\section{A RANDOMIZED CONTROLLED TRIAL TO REDUCE JOB BURNOUT USING AN INTER-PROFESSIONAL FACILITAT- ED DISCUSSION GROUP INTERVENTION}

Jonathan Ripp $^{1}$; Ching See Lau ${ }^{1}$; Kyle W. Prochno ${ }^{2} .{ }^{1}$ Icahn School of Medicine at Mount Sinai, New York, NY; ${ }^{2}$ Sidney Kimmel Medical College, Philadelphia, PA. (Control ID \#3184939)

BACKGROUND: Job burnout is common in medical professionals and has been found to correlate with depression, medical errors, productivity, and unprofessional behavior. Based on previous studies which have demonstrated that facilitated discussion reflection sessions may decrease burnout, we chose to examine whether such an intervention might similarly improve measures of well-being using a novel inter-professional participant format.

METHODS: Resident physicians, early-career nurses and social workers from the Mount Sinai Health System in New York, NY were recruited and randomized to either a small-group reflection intervention or a control arm. Intervention participants were invited to participate 1-2 times monthly over 6 months in groups of 6-8 for discussions guided by a curriculum found to be effective in reducing 
burnout among practicing physicians. Sessions were facilitated by experts in promoting discussion around work-related challenges between colleagues from different medical professions. Participants in both arms were asked to complete surveys before and after program participation which included measures of burnout, resilience, depression, and job satisfaction. Statistical analysis was performed to identify correlations of burnout and other outcomes at baseline and in relation to participation in the intervention or control arm.

RESULTS: The response rate for the baseline survey was $23 / 24$ (96\%) for the control arm and 18/19 (95\%) for the intervention arm. 22/24 (92\%) control arm participants and 16/19 (84\%) intervention arm participants completed the follow-up survey. There were no statistically significant changes in levels of burnout in either the intervention $(14 / 17(82,4 \%)$ pre- v. $13 / 17(76.5 \%)$ post, $\mathrm{p}=0.31)$ or control (17/22 (77.3\%) pre- v. 18/22 (81.8\%) post-, $\mathrm{p}=0.56)$ arms. At baseline, we did identify correlations between burnout and resilience $(-0.49, \mathrm{p}=0.002)$ and burnout and meaning in work $(-0.30, \mathrm{p}=0.04)$. CONCLUSIONS: Participation in an inter-professional facilitated discussion group intervention showed no impact on job burnout. As has been previously demonstrated, burnout was found to negatively correlate with baseline resilience and measures of job satisfaction. Our study was limited by its small size and variable participation among colleagues from different professions. Further studies are needed with larger cohorts to better elucidate the benefits of interprofessional facilitated reflection group discussion.

\section{A RANDOMIZED TRIAL OF PEER COMPARISON FEED-} BACK TO IMPROVE GUIDELINE-BASED PRIMARY CARE Amol S. Navathe ${ }^{2,1}$; Kevin G. Volpp ${ }^{2,}{ }^{1}$; Amelia M. Bond ${ }^{3}$; Kristin Linn $^{4}$; Kristen L. Caldarella ${ }^{2}$; Andrea B. Troxel ${ }^{5}$; Jingsan Zhu ${ }^{2}$; Lin Yang $^{2}$; Shireen E. Matloubieh ${ }^{2}$; Elizabeth E. Drye ${ }^{6}$; Susannah Bernheim $^{6}$; Emily Oshima Lee ${ }^{7}$; Mark Mugiishi ${ }^{7}$; Kim Takata ${ }^{7}$; Justin Yoshimoto ${ }^{7}$; Isaac Yuen ${ }^{7}$; Sheryl Okamura ${ }^{7}$; Michael Stollar ${ }^{7}$; Jeffrey Tom ${ }^{7}$; Michael Gold ${ }^{7}$; Ezekiel J. Emanuel ${ }^{2} .{ }^{1}$ CMC VA Medical Center, Philadelphia, PA; ${ }^{2}$ Medical Ethics and Health Policy, University of Pennsylvania, Philadelphia, PA; ${ }^{3}$ Healthcare Policy and Research, Weill Cornell Medical College, New York, NY; ${ }^{4}$ Biostatistics, Epidemiology and Informatics, University of Pennsylvania, Philadelphia, PA; ${ }^{5}$ New York University School of Medicine, New York, NY; ${ }^{6}$ Yale University School of Medicine, New Haven, CT; ${ }^{7}$ Hawaii Medical Service Association, Honolulu, HI. (Control ID \#3182764)

BACKGROUND: Peer comparisons, providing feedback on clinician performance relative to peers, have been tested successfully in narrow settings such as increasing guideline-based antibiotic and opioid prescribing. However, there are no studies evaluating the effectiveness of peer comparisons on broader quality measures in the setting of alternative payment models.

METHODS: We conducted a cluster randomized trial with the Blue Cross Blue Shield of Hawaii to examine the impact of providing peer comparison feedback to its primary care practitioners (PCPs) on the quality of care. This study included patients of 86 PCPs randomized to receiving peer comparisons plus individual feedback (intervention group) versus individual feedback alone (control group). Feedback was provided on quality, cost, and utilization performance. All PCPs were also simultaneously moved to a new population-based primary care payment system. The primary outcome was the probability of achieving national benchmark thresholds on thirteen primary care focused quality metrics that included preventative and chronic disease measures. We analyzed the primary outcome using a generalized linear model, adjusting for patient characteristics, PCP characteristics, baseline proportion of measures achieved by the patient, and a quality measure fixed-effect, clustering standard errors at the PCP.
RESULTS: The RCT included 27,930 patients and 31 PCPs in the control group and 46,694 patients and 55 PCPs in the intervention group. Patients nor PCPs exhibited large differences across groups, with small differences in demographics and panel size, respectively. In primary analysis, the patients in the peer comparisons intervention group experienced a 2.4 percentage point (pp) increase in the probability of achieving an eligible quality measure ( $95 \%$ CI 0.3 pp to $4.6 \mathrm{pp}, \mathrm{p}=0.03$ ). Secondary analysis of individual measures indicated that Breast Cancer Screening (3.9 pp, 95\% CI 0.2 to 6.0 pp, p<0.001), Cervical Cancer Screening (2.4 pp, $95 \%$ CI 0.01 to $4.8 \mathrm{pp}, \mathrm{p}=0.05$ ), Colorectal Cancer Screening (2.8 pp, 95\% CI 0.3 to 5.2 pp, p=0.03), Diabetes Care - Eye Exam (5.6 pp, 95\% CI 1.6 to $9.5 \mathrm{pp}, \mathrm{p}=0.006$ ), Diabetes Care - Medical Attention for Nephropathy (2.5 pp, 95\% CI 0.4 to $4.6 \mathrm{pp}, \mathrm{p}=0.02$ ) and Review of Chronic Conditions ( $4.9 \mathrm{pp}, 95 \%$ CI 0.0 to $9.8 \mathrm{pp}, \mathrm{p}=0.05$ ) likely accounted for the increased overall quality score. Other measures demonstrated trends toward differential improvement, but associations were not significant. Cost and utilization did not demonstrate differences between arms.

CONCLUSIONS: A peer comparisons intervention that displayed quality information in a real-time dashboard in the setting of a broad primary care payment system change improved quality scores by over 2 percentage points. This highlights the ability of peer comparisons to influence clinician practice in broad endpoints and is reassuring in light of new payment programs that have begun sharing comparative feedback.

\section{A RETROSPECTIVE REVIEW OF EPIC MYCHART UTILIZA- TION AMONGST PAYER CLASSES WITHIN A FEDERALLY QUALIFIED HEALTH CENTER NETWORK IN BROOKLYN NEW YORK}

Isaac Dapkins ${ }^{2}$; Rhett Pilao ${ }^{1}$; Neil A. Pasco ${ }^{1} .{ }^{1}$ NYU Langone Health, $\overline{\text { New York, NY} ; ~}{ }^{2}$ NYU, Brooklyn, NY. (Control ID \#3185686)

BACKGROUND: The Hitech Act of 2009 led to Federal funding on EHR incentives such as Advancing Care Information within MIPs and Meaningful Use. EPIC currently has a MyChart application which allows a patient to interface with their medical records and provider. The Family Health Centers (FHC) at NYU Langone is a network of 8 Federally Qualified Health Centers (FQHC) located in Brooklyn New York. The primary service area has a large immigrant population with $47 \%$ of the population reported as being foreign born, and a diverse payor mix with $12 \%$ of patients being self-pay/uninsured.

METHODS: Retrospective analysis was performed regarding 78,168 unique patients seen within the Family Health Center Network from January 2018 to December 3rd, 2018. Patient were identified by payor class and by utilization of MyChart. Given the diversity of healthcare plans afforded within New York State, payor classes were grouped into 7 broad categories: Medicaid/ Managed Medicaid, commercial, Medicare/ Managed Medicare, self-pay, no insurance, Child Health Plus and Medicaid Expansion (Affordable Care Act). Patient MyChart data abstraction within the EPIC Clarity database included whether the patient was enrolled and when the last date of activity occurred. Enrollment with activity versus enrollment without activity within the last calendar year was used to gauge whether the patient would be considered an active subject in this retrospective review.

RESULTS: Regarding percentage of patient enrolled in MyChart, the patient population most likely to enroll was found to be those who have commercial health plans at $41 \%$, with Medicaid expansion plans at $37 \%$, followed by Medicaid tied with self-pay coverage (23\%) and Medicare at $18 \%$. Utilization tells a different story with the highest utilizers found in the Medicare enrollees at $79 \%$, followed by Medicaid expansion at $78 \%$, then commercial plans at $77 \%$. The next tier of active users was found to be no coverage (67\%), self-pay (66\%) and Medicaid (61\%). Retrospective review with enrollment data was somewhat expected; high enrollment in patients with commercial plans and lower enrollment amongst Medicare beneficiaries. What was surprising was the utilization/activity data 
revealed an entirely different picture. Activity usage reflected two tiers. Patients who have Medicare utilize the application as much as patients who have commercial plans and Medicaid expansion. Despite connotations on patients who are self-pay or who have no coverage at all, these patients still use the application, with greater than $50 \%$ of those enrolled, actively using MyChartwithin the last calendar year.

CONCLUSIONS: As medical care becomes more immersed in webbased technologies, attention and opportunities exist for patients who traditionally were viewed as not having access nor inclination to use such technologies. Continued efforts should be maintained regarding enrollment regardless of the payor class or age.

\section{A SCOPING LITERATURE REVIEW OF PHYSICIAN SUICIDE} Sima Pendharkar ${ }^{1}$; Tiffany I. Leung ${ }^{2,2}$; Chwen-Yuen A. Chen ${ }^{3,3}$. ${ }^{1}$ Mount Sinai Health System, Brooklyn, NY; ${ }^{2}$ Maastricht University, Maastricht, Netherlands; ${ }^{3}$ Stanford University, Palo Alto, CA. (Control ID \#3142595)

BACKGROUND: Physician suicide is a global issue with significant consequences for workforce sustainability and for public health and disproportionately affects women physicians [1]. A clearer understanding of physician suicide is needed. A literature review would enable community learning towards preventing physician suicidal behaviors. The aim of this scoping review was to map the landscape of published research and perspectives on physician suicide.

METHODS: This scoping review was conducted following the Arksey and O'Malley framework as expanded upon and outlined within the Joanna Briggs Institute Reviewers' Manual. A broad research question was crafted by our team. The protocol was registered with PROSPERO. For the purposes of this review, the research question was broadly designed for gathering and analyzing all citations in scholarly journals which mentioned physician suicide specifically. The initial searches were executed on August 21, 2017 in Ovid MEDLINE, and October 11, 2017 in Ovid PsycINFO. Team members contributed seed articles which were analyzed by the librarian coauthor. Search terms and databases were selected and tested before the search strategy underwent a peer review process with two additional medical librarians. A subsequent sweep of the citation index, Scopus, was conducted on April 28, 2018. Inclusion criteria were English-language journal publications, including opinion/perspective publications, that focus primarily on physician suicide. Exclusion criteria were non-English or media publications. Data extraction was performed and topic annotations were applied to citations.

RESULTS: Query results totaled 1,596 citations; 348 studies passed the full-text review round. The earliest article is an editorial from 1903; more than $60 \%$ of articles were published in 2000 or later. There were 142 opinion articles (40.8\%). Of the remaining 206 articles, a variety of study designs were used; 84 used a crosssectional study design, which was most common. Authors from 37 countries were represented, although authors from the U.S. $(n=51)$ are the largest subset. Only 13 publications described interventions. Unexpected subpopulations described included immigrant physicians, physician pilots, and physicians who experienced war either as victims (e.g. Holocaust survivors who were physicians) or as wartime medics. One investigator studied the interpersonal psychological theory of suicidal behavior in physicians, which posits three necessary and sufficient precursors to death by suicide: (1) thwarted belongingness, (2) perceived burdensomeness, and (3) acquired capability.

CONCLUSIONS: Most journal articles about physician suicide were published recently - a promising reflection of growing awareness. Limitations include excluding non-English language articles, interventions that mitigate only burnout, and other media. Further research and advocacy is needed in support of interventions specifically targeted for physician suicide prevention. [1] Leung et al. SGIM Forum 41 (9): 4-5, 14
A SINGLE CENTRE RETROSPECTIVE STUDY COMPARING POST-OPERATIVE OPIOID PRESCRIBING PRACTICES BETWEEN CAESAREAN SECTIONS AND OPEN GENERAL SURGERY PROCEDURES

Tanya Girard ${ }^{1}$; Emily G. McDonald ${ }^{1}$; Natalie Dayan ${ }^{1}$; Miriam Harris ${ }^{1}$; Amira El-Messidi ${ }^{2}$; Todd C. Lee ${ }^{1}$; Sophie Gosselin²; David Fleiszer ${ }^{2}$; Andre Bonnici ${ }^{2}$; Eric Villeneuve ${ }^{2} .{ }^{1}$ McGill University, Montréal, QC, Canada; ${ }^{2}$ McGill University, Montreal, QC, Canada. (Control ID \#3181947)

BACKGROUND: Physician prescribing practices are contributing to the North America opioid crisis. Identifying practice variability may be an important step towards addressing the problem. The objectives of this study were to compare postoperative opioid prescribing practices between selected open abdominal and pelvic surgeries and highlight variations in practice.

METHODS: A retrospective cohort study of 927 patients who underwent a caesarean section (CS) or one of four open general surgery (GS) procedures performed between January and November 2017 at a university health centre in Montreal, Canada. Open GS procedures included appendectomy, cholecystectomy, hernia repair, and laparotomy for lysis of intra-abdominal adhesions. Post-operative opioids in daily morphine equivalent doses (MED) in hospital and at discharge were compared between CS and open GS procedures with similar expected postoperative pain and length of admission. Factors associated with the odds of receiving more than $50 \mathrm{mg}$ MED daily at discharge were explored via logistic regression.

RESULTS: 927 patients were included; 689 women underwent a CS and 238 (180 men and 58 women) underwent an open GS procedure. Patients undergoing CS were more likely to receive higher doses of daily postoperative opioids in the hospital than GS patients and the difference was more pronounced for men than women (80mg MED (IQR 80-80) for CS vs $15 \mathrm{mg}$ MED (IQR 0-60) for GS men and 45mg MED (IQR 0-90) for GS women; $\mathrm{p}<0.001)$. At discharge, patients who underwent a CS received lower median doses of opioids than GS patients (20mg MDE (IQR 20-20) vs 45mg MDE (IQR 40-60); $<<0.001$ ). The odds ratio of exceeding the Centre for Disease Control's (CDC) recommended daily maximum of 50mg of MED was 19.8 (CI 6.2-63.1) for GS men and 19.3 (CI 5.4-68.1) for GS women, with CS as the reference group.

CONCLUSIONS: In a study of post-operative opioid prescribing practices, when compared to general surgeons, obstetricians prescribed lower doses of opioids at hospital discharge and were less likely to exceed the CDC's safe dosing recommendations. Prescribing variability and discordance with recommended safe doses could be minimized by using preprinted discharge prescriptions for given procedures.

\section{A STUDY OF STANDARD DEVIATION OF NEXT AVAILABLE APPOINTMENTS}

Sumeet Brar ${ }^{2}$; Michael Hopkins ${ }^{1}$; David Margolius ${ }^{1} .{ }^{1}$ The MetroHealth System, Cleveland, $\mathrm{OH} ;{ }^{2}$ Case Western Reserve University, Cleveland, OH. (Control ID \#3177863)

BACKGROUND: Days until third next available appointment is a metric that describes backlog for appointments by counting the number of days until the third next available appointment on a clinician's schedule. The metric is widely used by health care systems as a measure of access to care. Third next is considered a more reliable metric because last-minute cancellations might distort the days until the first or second next. We tested this assumption by comparing the variability over time of days until first, second, third, and fourth next available appointments.

METHODS: We collected four variables - the number of days until first, second, third, and fourth next available appointments - for clinicians over a 20 -week period, in weekly increments. Clinicians were included if we had complete appointment data for all values. For clinicians who worked 
in more than one location, each location schedule was counted separately as the values were unique. Clinicians were physicians and advanced practice providers in all specialties, dietitians, audiologists, psychologists, and optometrists. All clinicians were employed by a multi-site tertiary health care system. We calculated the standard deviation from the mean for each variable for each clinician across all weekly values. We then averaged standard deviations, so that the outcome was an average standard deviation of days until first next, an average standard deviation of days until second next, and so on. We used a one-way ANOVA to compare the effect of counting out first, second, third, or fourth next available appointments on the standard deviation of the variables. We conducted post hoc analyses with the Tukey test.

RESULTS: Our study sample included 421clinicians working from 611 schedules. The mean first, second, third, and fourth next available appointment across all dates and clinician schedules was 20,24, 26, and 27 respectively. The mean standard deviation for each variable beginning with first next available was 10.7, 9.4, 9.0, and 8.8 days. An analysis of variance showed that the effect of measuring days until sequential available appointments on standard deviation was significant, $F(3,2440)=11.66, p<.01$. Post hoc comparisons with the Tukey test showed that standard deviations of first next available appointments differed significantly from standard deviations of each of the other metrics $(p<.01)$, but mean standard deviations from second, third, and fourth next available did not significantly differ from each other.

CONCLUSIONS: Days until first next available appointment is a less consistent measure from week to week than days until second, third, or fourth next available appointment. This study is the first to add evidence that second next is a more reliable metric than first next, but we found no statistically significant difference between the reliability of second and third next. Health care systems may choose to use days until second next available appointment as a more practical access metric.

\section{A SURVEY OF ATTITUDES AROUND OPIOID USE DISORDER AND PERCEIVED BARRIERS TO PROVIDING BUPRENORPHINE MAINTENANCE TREATMENT AMONG OUTPATIENT PRIMARY CARE PROVIDERS IN AN URBAN ACADEMIC MEDICAL SETTING}

Monique A. Tello ${ }^{1}$; Sarah E. Wakeman ${ }^{1}$; Stuart R. Lipsitz ${ }^{2}$; Lipika Samal ${ }^{2}$. ${ }^{1}$ Massachusetts General Hospital, Charlestown, MA; ${ }^{2}$ Harvard Medical School, Boston, MA. (Control ID \#3184407)

BACKGROUND: The US is experiencing an epidemic of opioid use disorder (OUD), which can be effectively treated with buprenorphine in the outpatient primary care setting. Primary care providers (PCPs) must complete 8 hours of training and apply for a waiver to prescribe buprenorphine, but only a fraction have done so, and not all prescribe. Our goal was to identify barriers that could be addressed through educational or logistical interventions.

METHODS: A 41-item self-report survey was created after a review of the literature and administered anonymously through the online service SurveyMonkey (San Mateo, CA). PCPs were asked to self-identify as waivered or non-waivered. PCPs were then queried about the magnitude of the issue in their geographic area and their practice, personal impact of the opioid epidemic, perceived effectiveness of OUD treatment modalities, and potential logistical barriers to training and prescribing buprenorphine (depending on their waiver status). All PCPs (physicians, physician assistants, and nurse practitioners) at two academic medical centers were asked to complete the survey. Results were characterized using descriptive statistics and compared across non-waivered and waivered respondents using logistic regression.

RESULTS: Responses were received from 100 PCPs who were predominantly MDs (93\%) and female (72\%); 69\% had not completed buprenorphine waiver training; $31 \%$ had (and $67 \%$ of those were actively prescribing). $99 \%$ of PCPs said that OUD was an issue in their geographic area, $94 \%$ reported seeing patients with OUD, and $18 \%$ reported that they had been personally impacted by the opioid epidemic. $84 \%$ of PCPs rated buprenorphine maintenance as moderately or very effective, the highest ranked of six OUD treatment options (including 12-step programs, outpatient counseling, inpatient rehabilitation, methadone maintenance, and extended-release naltrexone). Non-waivered PCPs were significantly more likely than waivered PCPs to say that they did not see enough patients with OUD to change their practice (OR 0.267, p=0.005) and were also more likely to express concern about allowing patients with OUD into their practice (OR 0.348, p=0.020). There were no other significant differences between those two groups (safety, moral acceptability, or diversion risk of buprenorphine, time, financial support, peer mentoring, psychosocial support staff, potential legal issues).

CONCLUSIONS: Surprisingly, non-waivered PCPs cite too few OUD patients to justify training, but the vast majority of them report having OUD patients, feel the opioid epidemic is an issue in their geographic area, and rate buprenorphine/naloxone maintenance as the most effective treatment option for OUD. Non-waivered PCPs were also significantly concerned about allowing patients with OUD into their practice. There were no other differences, which suggests attitudinal barriers (i.e. stigma) as the most appropriate target for intervention.

A SYSTEMATIC REVIEW OF THE CAUSES, IMPACT AND RESPONSE TO MORAL DISTRESS AMONG MEDICAL STUDENTS Susan B. Glick; Jacob Schulman; Max Harris; Daniel Pohlman. Rush Medical College, Chicago, IL. (Control ID \#3186130)

BACKGROUND: Moral distress occurs when one is aware of the moral and ethical course of action, yet is unable to perform it. Lack of resources and futile care are some of the many reported causes of moral distress among healthcare professionals, and may result in frustration, anger, a sense of powerlessness, job dissatisfaction and intent to leave the profession. These symptoms are strikingly similar to those of burnout and may be confused with it. Medical students may disproportionately experience moral distress, as they are well trained in the ideals to which the profession aspires, yet wield little power in the health care system. To prevent, diagnose and treat moral distress among medical students requires an understanding of the causes and impact of moral distress, as well as the response to it at the personal, professional and institutional levels.

METHODS: With the assistance of a medical librarian, we conducted a systematic review of the English language literature to assess studies that described moral distress among medical students. We searched OVID, CINAHL, PsycINFO, and ERIC from their inception through October 2018. We searched the reference sections of relevant review articles as well as all included studies for additional manuscripts. Eligible studies had to represent an original study; present data about the causes, impact, or response to moral distress; measure and report outcomes in medical students; and report findings in English. Titles and abstracts were reviewed. Articles not recommended for exclusion were reviewed in full. Following full text review, those articles that did not meet all four inclusion criteria were excluded.

RESULTS: The search yielded 63 articles. Following title, abstract and full text review, 6 studies were included. Five addressed situations that result in moral distress; two addressed its impact. No studies addressed the response to moral distress. Due to data heterogeneity, narrative synthesis was performed. Studies differed in their conclusions about which situations resulted in the greatest moral distress, including those with immediate potential to cause harm; covert, status-related and verbal abuse; communication issues, intra-team conflicts, resource allocation, and access to care; and experiences with geriatric patients. Role modeling was inversely correlated with student distress, as was action-taking. One study found repeated exposure to moral distress can result in either emotional desensitization or in worsening distress. Studies differed in their conclusions about whether moral distress disproportionately affected women or men. 
CONCLUSIONS: Little is known about the situations that result in moral distress among medical students and its impact. The literature is silent on the response to medical students' moral distress. Deepening our understanding of the causes, impact and response to moral distress among medical students is imperative to promote student wellness.

\section{A TRANSFORMATIVE APPROACH: MOTIVATIONAL INTERVIEWING CURRICULUM IMPROVES PATIENTS' HEALTHY BEHAVIORS WHILE ENHANCING LEARNING} Chavon Onumah ${ }^{1}$; Mihir Patel ${ }^{2}$; Matthew Mintz ${ }^{1}$; Namarik Alenezy ${ }^{3}$. ${ }^{\mathrm{T}}$ George Washington University, Washington, DC; ${ }^{2}$ George Washington University, Washington, MD; ${ }^{3}$ George Washington University SMHS, Washington, DC. (Control ID \#3184308)

BACKGROUND: Modifiable risk behaviors account for a considerable amount of morbidity and mortality. Motivational interviewing is a patient-centered method of guiding patients to modify health behaviors that can help address health disparities. As few as one MI session may be effective in enhancing readiness to change and promoting action towards change. MI curricula, is being implemented in medical training, however, data regarding curricular outcomes is limited to knowledge and confidence gained by students through the use of standardized patients and role-play exercises. Data is lacking on the student experience with MI curricula involving live patients and patient outcomes with one student- led MI session.

METHODS: Using Kolb's Experiential Learning Cycle as a Model, a 4-part MI curriculum, "Promoting Healthy Behaviors," was implemented in our longitudinal primary care clerkship to enhance learning in motivational interviewing. The curriculum includes self-directed learning, small-group exercises, standardized patient practice, AND a real-life clinical intervention (medication adherence, lifestyle modification, or substance cessation) with patient follow-up and reflection. The curricular initiative was implemented as a core requirement for all clinical clerkship students $(\mathrm{N}=155)$. Students completed anonymous surveys regarding their experience with the project and their respective patient's goal progress at the time of follow-up. Data was analyzed via quantitative and qualitative methods. Three faculty members coded qualitative responses independently through open coding. Disagreement was resolved by discussion and data recoded by a final coding scheme. The authors discussed the coded data to arrive at a final set of themes. RESULTS: $78 \%$ of patients reported progress towards their selfdirected health behavioral change goal established in the studentled MI encounter at 4 to 6 weeks post-encounter. From student reflections, the following themes across the knowledge, skills, and attitudes learning domains emerged: Meeting patients "where they are at" on the Stages of Change scale is key in promoting change. Behavioral change is difficult and takes considerable support and follow-up. Incremental changes, tapping into internal motivation, and addressing barriers aid in success. Motivational interviewing is an impactful way to promote healthy behaviors and important skill to learn.

CONCLUSIONS: The Promoting Healthy Behaviors project is an effective method of enhancing multi-dimensional medical student learning in motivational interviewing and engaging patients in behavior change and health promotion. A clinical intervention with follow-up helped students appreciate their potential impact, recognize the difficulties patients experience in moving towards behavioral change, and provided "immediate applicability" for skills learned through the in-class and standardized patient exercises. The high rate of patients reporting successful goal progress is encouraging, however, more outcome data is warranted.
ACCEPTABILITY OF SOCIAL RISK SCREENING TO PATIENTS AND CAREGIVERS

Emilia De Marchis ${ }^{1}$; Danielle Hessler ${ }^{1}$; Nancy E. Adler ${ }^{1}$; Elena Byhoff $^{2}$; Alicia Cohen ${ }^{3}$; Kelly M. Doran ${ }^{4}$; Stephanie Ettinger de $\mathrm{Cuba}^{5}$; Eric Fleegler ${ }^{6}$; Nicholas Gavin ${ }^{10}$; Amy G. Huebschmann ${ }^{7}$; Stacy T. Lindau ${ }^{8}$; Maria C. Raven ${ }^{1}$; Elizabeth L. Tung ${ }^{8}$; Caroline Fichtenberg $^{1}$; Susan Jepson ${ }^{11}$; Wendy Johnson ${ }^{12}$; Cara C. Lewis ${ }^{13}$; Eduardo Ochoa ${ }^{14}$; Ardis L. Olson ${ }^{15}$; Aric Prather ${ }^{1}$; Megan Sandel $^{5}$; Richard Sheward ${ }^{16}$; Laura M. Gottlieb ${ }^{9}$. ${ }^{1}$ University of California, San Francisco, San Francisco, CA; ${ }^{2}$ Tufts Medical Center, Boston, MA; ${ }^{3}$ University of Michigan, Ann Arbor VA, Ann Arbor, MI; ${ }^{4}$ NYU School of Medicine, New York, NY; ${ }^{5}$ Boston University School of Medicine, Boston, MA; ${ }^{6}$ Boston Children's Hospital, Boston, MA; ${ }^{7}$ University of Colorado School of Medicine, Aurora, CO; ${ }^{8}$ University of Chicago, Chicago, IL; ${ }^{9}$ UCSF, San Francisco, CA; ${ }^{10}$ Columbia University Vagelos College of Physicians and Surgeons, New York, NY; ${ }^{11}$ Hennepin Healthcare, Minneapolis, MN; ${ }^{12}$ La Familia Medical Center, Santa Fe, NM; ${ }^{13}$ Kaiser Permanente Washington Health Research Institute, Seattle, WA; ${ }^{14}$ University of Arkansas, Little Rock, AR; ${ }^{15}$ Dartmouth Medical School, Hanover, NH; ${ }^{16}$ Boston Medical Center, Boston, MA. (Control ID \#3141279)

BACKGROUND: Despite the health care sector's growing interest in systematic collection of social risk data, little is known about patient acceptability of health care-based social risk screening. Acceptability to patients and caregivers has implications for adoption, utility and sustainability. We aimed to explore acceptability of the Center for Medicare and Medicaid Innovation's (CMMI) social risk screening tool, which covers 5 actionable social domains: food, housing, utilities, and transportation security, and personal safety.

METHODS: Cross-sectional analysis of patients or caregivers of pediatric patients recruited from 6 primary care clinics (PCCs) and 4 emergency departments (EDs). Participants completed a tablet-based survey that included the CMMI social risk tool and questions related to both acceptability of social screening and predictors of acceptability. The primary acceptability measure was perceived appropriateness of screening in health care settings; a secondary outcome was comfort with including social risk data in electronic health records (EHRs). Multilevel mixed-effects logistic regressions were used to evaluate acceptability measures, clustering by site.

RESULTS: 969 patients/caregivers completed the survey; 952 (98\%) answered survey questions about acceptability. $72 \%$ of participants screened positive for at least 1 of 5 social risks. $80 \%$ of respondents reported screening was very or somewhat appropriate; $7 \%$ reported screening was very or somewhat inappropriate. $65 \%$ of participants reported being completely or somewhat comfortable with social risk data being included in EHRs; $18 \%$ reported being completely or somewhat uncomfortable. In the primary multivariable model, participants reporting higher acceptability of social risk screening had higher odds of previous social risk screening in a health care setting (aOR 1.80 [95\% CI 1.312.47]); not endorsing prior discrimination in a health care setting (aOR 1.45 [95\% CI 1.07-1.97]); being from a safety-net setting (aOR 1.85 [95\% CI 1.11-3.10]); and being recruited in a PCC (aOR 2.10 [95\% CI 1.014.32]). Participants who were more comfortable with including social risk data in EHRs had higher odds of previous social risk screening in a health care setting (aOR 1.56 [95\% CI 1.17 to 2.09]). There were no associations between specific social risk domains or cumulative number of social risks with either measure of acceptability. No additional respondent-level characteristics were associated with acceptability.

CONCLUSIONS: Across PCC and ED settings, a majority of respondents found social risk screening acceptable. Acceptability levels did not differ across individual health care settings or level of social risks, though we identified some potentially modifiable factors associated with 
acceptability. High rates of acceptability support health care-based social screening implementation efforts, although future research should explore how to improve acceptability in specific patient populations and acceptability of EHR integration.

\section{ACCESS TO TREATMENT FOR DRUG USE DISORDER AT U.S. HEALTH CENTERS: A NATIONAL STUDY}

Benjamin I. Bearnot ${ }^{1}$; Danielle R. Fine ${ }^{2}$; Nancy A. Rigotti ${ }^{1}$; Travis P. Baggett ${ }^{1}{ }^{1}$ Massachusetts General Hospital, Boston, MA; ${ }^{2}$ Massachusetts General Hospital, Brookline, MA. (Control ID \#3185426)

BACKGROUND: Drug use disorder (DUD) is a growing public health concern in the US, particularly among medically-underserved and lowincome populations. Treatment rates for DUD remain low despite the existence of evidence-based behavioral and pharmacologic treatments. Little is known about the barriers to accessing DUD treatment at federally-funded US health centers, which provide care to nearly 30 million vulnerable individuals annually. The aim of this study was to examine the continuum of DUD care, location of treatment delivery, and barriers to receiving treatment at Health Resources and Services Administration (HRSA) funded health centers.

METHODS: We analyzed data from adult respondents to the 2014 Health Center Patient Survey, a cross-sectional, nationally representative, in-person survey of HRSA health center patients conducted between 9/2014 and 4/2015 with a 3-stage sampling design. The main measure was self-reported symptoms of DUD, assessed using the WHO ASSIST questionnaire. Individuals with 2 symptoms were defined as having a diagnosis of DUD. We examined responses to a series of questions regarding receipt of DUD care, the location of this care, and the primary reason individuals were unable to access treatment. We conducted analyses in SAS using strata, cluster, and weight variables to account for complex sampling design. Percentages are weighted to reflect health center patients nationally. Rao-Scott 2 tests and t-tests were used to compare proportions and means, respectively, with a 2 -sided $\mathrm{P}$ value $<0.05$ for significance.

RESULTS: Among 5,547 participants, 374 (4.5\%) had current evidence of DUD. 293 individuals (85.3\%) had a single DUD, while $81(14.7 \%)$ had a DUD of 2 or more substances. Among respondents with DUD, the most commonly used substances were cannabis $(74.8 \%)$, sedatives $(21.1 \%)$, cocaine $(19.1 \%)$, stimulants $(15.5 \%)$ and opioids $(10.6 \%)$. $11.6 \%$ had ever used injection drugs. $49.7 \%$ reported discussing drug use with a health professional, $35.7 \%$ reported wanting or needing treatment, and $28.3 \%$ reported receiving treatment for DUD. Among 102 individuals who reported receiving DUD treatment, $37 \%$ received treatment that was delivered at a HRSA health center, while $63 \%$ sought treatment elsewhere. Among 41 respondents who wanted but did not receive DUD treatment, the most commonly reported reasons for not obtaining treatment were stigma, skepticism about treatment, logistical and financial barriers.

CONCLUSIONS: Symptoms of DUD were more common in this nationally representative study of community health center attendees than in other national surveys, although there are notable differences in methodology. Only half of individuals with DUD discussed their drug use with a health professional, and even fewer reported wanting, needing, or receiving treatment. Marked losses across the continuum of DUD care highlight the considerable barriers to routinely identifying, referring, and linking individuals to effective addiction care at U.S. health centers.

\section{ACUTE HEART FAILURE EXACERBATION AND INFLUENZA INFECTION}

Nicholas Daering; Lindsey Aurora; Lucas Maahs; Zachary Bauer; Ahmed Dakka; Leika Raychouni. Henry Ford Hospital, Detroit, MI. (Control ID \#3176759)
BACKGROUND: Influenza infection causes 3-5 million cases of severe illness annually and has been linked to exacerbations of congestive heart failure (CHF) as well as myocarditis. Patients with diagnosed CHF appear to have an increased risk of hospitalization during influenza season, but a relationship between serologically diagnosed influenza infection and acute heart failure (AHF) exacerbations has not been studied.

METHODS: This was a retrospective chart review studying patients with a diagnosis of CHF between April and October 2016 with specific focus on those presenting with AHF exacerbations until 2018. Descriptive data was collected regarding patient demographics, total number and dates of encounters, length of stay, presenting laboratory values, influenza testing results, intensive care admission, and outcomes including mortality. This data was divided based on influenza testing status and univariate analysis was applied to capture any association of peak influenza infection with peak AHF encounters, correlation of influenza infection with length of stay, and correlation of influenza infection with patient demographics. Descriptive statistics were gathered on all variables of interest. Statistical significance was set at $\mathrm{p}$-value $<0.05$. All analyses were performed using SAS 9.4.

RESULTS: Out of 1880 patients with a diagnosis of AHF exacerbation, there were two defined groups: influenza positive $(n=28)$, and influenza negative/not tested $(\mathrm{n}=1852)$. There was no significance noted amongst the rate of heart failure exacerbations between these two groups (Table 1, p-value 0.134). Out of all variables analyzed, only two demonstrated statistical significance between influenza positive and influenza negative/not tested groups. Diagnosis of pulmonary hypertension ( $\mathrm{p}$ value 0.013$)$ and coronary artery disease $(p=0.031)$ was more likely in influenza positive patients. No statistically significant correlation was found for the primary endpoint of influenza positivity with acute heart failure exacerbation.

CONCLUSIONS: This data comprised a significant portion of patients with AHF exacerbations with negative influenza tests or those that were not tested. This allows us to deduce that perhaps a larger cohort of patients may be required to determine if there is true statistical significance of additional variables and endpoints. These results also indicate a need for higher clinical suspicion of influenza infection in patients with heart disease, particularly coronary artery disease and pulmonary hypertension. Future studies may also focus on the effect of influenza treatment on hospital outcomes for cardiac patients.

\section{ADDING SOCIAL AND FUNCTIONAL DETERMINANTS OF HEALTH IMPROVES READMISSION RISK PREDICTION IN URBAN UNDERSERVED ADULTS HOSPITALIZED FOR ACUTE MYOCARDIAL INFARCTION}

Oanh K. Nguyen ${ }^{1,2}$; Anil N. Makam ${ }^{1,2}$; Christopher Clark ${ }^{3}$; Sameh Saleh ${ }^{1}$; Sandeep R. Das ${ }^{4}$; Ethan Halm ${ }^{5}$. ${ }^{1}$ UT Southwestern Medical Center, Dallas, TX; ${ }^{2}$ University of California, San Francisco, San Francisco, CA; ${ }^{3}$ Parkland Health \& Hospital System, Dallas, TX; ${ }^{4}$ University of Texas Southwestern Med Ctr, Dallas, TX; ${ }^{5}$ Univ of TX Southwestern Med Ctr, Dallas, TX. (Control ID \#3181967)

BACKGROUND: Although social and functional determinants of health (SFDoH) are increasingly recognized to contribute to increased 30-day readmission risk for cardiovascular illnesses, most health systems lack individual-level data to enable individual risk stratification and targeted interventions to address these needs. We sought to determine whether electronic health record (EHR) data in SFDoH domains collected as a part of routine inpatient care from a safety-net hospital could be used to enhance prediction of 30-day readmissions beyond traditional clinical risk factors alone among underserved adults hospitalized with acute myocardial infarction (AMI).

METHODS: We used Epic EHR data on AMI hospitalizations from 2014-2017 in an urban safety-net hospital in Dallas to predict non-elective 30-day readmissions to any of 75 hospitals in north Texas, using a 
regional all-payer hospitalization database.. We started with the AMI READMITS score, our previously validated model consisting of 7 clinical risk factors. Next, we derived and validated an enhanced model incorporating SFDoH predictors. EHR data for candidate SFDoH predictors included structured, semi-structured, and short free-text fields from demographics, encounter types, note types, and case manager, social worker, and nursing inpatient flowsheets. We selected novel predictors using stepwise backward selection with a threshold of $\mathrm{p} 0.20$, forcing in the composite AMI READMITS score. We conducted sensitivity analyses using forward selection, lasso regression, and random forest which resulted in similar models. We internally validated our final model using 5 -fold cross-validation and compared model performance to the AMI READMITS score.

RESULTS: Of 1,071 hospitalizations, 154 (14.5\%) were readmitted within 30 days. In addition to the composite AMI READMITS score, our final model included 9 SFDoH risk factors, including neighborhood poverty (aOR $1.39,95 \%$ CI $0.91-2.14$ ), address change in the past year (aOR $1.53,95 \%$ CI $0.96-2.46$ ), any opioid use in the past year (aOR 2.35, 95\% CI 1.36-4.05), history of durable medical equipment use in the past year (aOR 1.70, 95\% CI 1.02-2.82), and ADL impairments in feeding, bathing, and toileting in the past year (aOR 1.81 for all three impairments, 95\% CI 0.99-3.29). The AMI READMITS+SFDoH model had better discrimination than AMI READMITS alone (optimism-corrected Cstatistic 0.69 vs $0.62, p<0.001$ ), was able to predict a broader range of probabilities for readmission risk (3.6-72.7\% vs. $8.2-37.2 \%$ ), and improved net reclassification (categorical NRI of highest 2 risk quintiles vs. lowest 3 of $0.09,95 \%$ CI $0.00-0.18$ ).

CONCLUSIONS: Incorporating existing EHR data on social and functional determinants of health improved readmission risk prediction after an AMI hospitalization in an urban underserved population.

\section{ADDRESSING LATENT TUBERCULOSIS INFECTION TEST- ING AND TREATMENT IN ORANGE COUNTY, CALIFORNIA: BASELINE ASSESSMENT AT A LOCAL HEALTH CENTER}

Fayette K. Nguyen Truax ${ }^{1}$; Setie Asfaha ${ }^{2}$; Tessa Mochizuki ${ }^{2}$; Setie Asfaha $^{2}$; Julie Low ${ }^{3}$; Michael Carson ${ }^{3}$; Duc Nguyen ${ }^{4} .{ }^{1}$ Loma Linda University, Wildomar, CA; ${ }^{2}$ California Department of Public Health, Richmond, CA; ${ }^{3}$ County of Orange Health Care Agency, Santa Ana, CA; ${ }^{4}$ Nhan Hoa Health Center, Garden Grove, CA. (Control ID \#3185388)

BACKGROUND: Of the 3.2 million individuals in Orange County, California, an estimated $240,646(7.5 \%)$ are Vietnamese. Of these, 153,329 are non-U.S.-born individuals. In 2017, of the TB cases in Orange County, $89 \%$ were among non-U.S.-born individuals, of whom $42 \%$ were born in Vietnam. In Orange County, the estimated percentage of non-U.S.-born individuals with latent TB infection (LTBI) is $17.3 \%$ as compared to $1.6 \%$ in U.S.-born individuals. A key strategy to eliminate TB is to increase LTBI testing and treatment in non-U.S.-born populations at community clinic settings. We conducted a baseline assessment of barriers, facilitators and current practices surrounding LTBI care to identify opportunities for improvement.

METHODS: A local community clinic serving primarily Vietnamese individuals was chosen. To identify barriers and facilitators to LTBI care, Likert-type scale surveys were given to providers, 1 (strongly agree) to 7 (strongly disagree). To understand current LTBI practices, we abstracted charts for patients who had a TB test in 2017 and a subset of charts for patients who had a physical exam during August 1-14, 2017. Data were entered into REDCap and analyzed using SAS.

RESULTS: Eight of $10(80 \%)$ providers completed the survey. All respondents agreed that testing and treating patients born in countries with an elevated TB rate for LTBI will improve their patients' long-term health. Only four $(50 \%)$ providers were comfortable with LTBI drug regimens other than 9 months of isoniazid therapy. Four (50\%) providers were confident in their ability to convince patients to accept treatment. Three (38\%) providers did not feel they customarily had enough time to assess patients for LTBI. A query of the electronic health record showed a total of 270 patients had a tuberculin skin test (TST) ordered in 2017. Of these, $220(82 \%)$ had results documented: $42(19 \%)$ were positive, of whom $40(95 \%)$ had chest $x$-rays ordered, all of which were negative. Of the $40,3(8 \%)$ started preventive treatment, but no treatment completion was documented. Of the 124 patients who had a physical exam during August 2017, the median age was 54 (range 5-72) and 110 (89\%) were Asian. Of the 110, 102 (93\%) spoke Vietnamese as their primary language, and of those, 97 (95\%) had no prior treatment documented. Of those with no prior treatment, $8(8 \%)$ were tested for LTBI; of the 8,3 (38\%) had positive results; 2 had chest x-rays completed; 2 started treatment and 1 had treatment completion documented.

CONCLUSIONS: Our community clinic serving a high-risk population could improve on screening and treating patients for LTBI. Following these results, LTBI training for providers and ancillary staff were provided. Further, a new process for documenting TB risk factors has also been implemented. Further barriers should continue to be examined and addressed through training and provision of educational materials from local public health agencies. An ongoing partnership among key partners is encouraged to foster best practices.

\section{ADDRESSING OVERTREATMENT IN OLDER ADULTS WITH DIABETES: LEVERAGING BEHAVIORAL ECONOMICS AND USER-CENTERED DESIGN TO DEVELOP CLINICAL DECI- SION SUPPORT.}

Devin M. Mann; Sara K. Chokshi; Hayley Belli; Saul Blecker; Caroline Blaum; Roshini Hegde; Andrea B. Troxel. NYU School of Medicine, New York, NY. (Control ID \#3184949)

BACKGROUND: Older adults with diabetes continue to be overtreated despite current guidelines recommending less aggressive target A1c levels based on life expectancy. The suboptimal management of this vulnerable population could be due to physicians having conflicting beliefs regarding this guideline or simply lacking awareness, and changing these behaviors is challenging. Clinical decision support (CDS) within the electronic health record (EHR) has the potential to address this issue, but effectiveness is undermined by alert fatigue and poor workflow integration. Incorporating behavioral economics into CDS tools is an innovative approach to improve adherence to these guidelines while reducing physician burden, and offers the promise of improving care in this population. METHODS: We applied a systematic, user-centered approach to incorporate behavioral economic "nudges" into a CDS module and performed user testing in six pilot primary care practices in a large academic medical center. To build the nudges, we conducted: (1) semi-structured interviews with key informants $(n=8)$; (2) a two-hour design thinking workshop to derive and refine initial module ideas; and (3) semi-structured group interviews at each site with clinic leaders and clinicians to elicit feedback on the module components. Clinicians were observed using the module in practice; detailed field notes were collected and summarized by module idea and usability theme for rapid iteration and refinement. Frequency of firing and user action taken were assessed in the first month of implementation via EHR reporting to confirm that module components and reporting were working as expected, and to assess utilization.

RESULTS: Insights from key stakeholder and clinician group interviews identified the refill protocol, inbasket lab result, and medication preference list as candidate EHR CDS targets for the module. A new EHR navigator section notification and peer comparison message, derived from the design workshop, were also prototyped and produced. User feedback from site visits confirmed compatibility with clinical workflows, and contributed to refinement of design and content. The initial prototypes were first piloted at two sites, refined, and then activated at an additional four additional sites. Preliminary results for the six clinics indicate that 
over approximately 31 weeks: 1) the navigator alert fired 1047 times for 53 unique clinicians, and 2) the refill protocol alert fired 421 times for 53 unique clinicians. Reports for the other "nudges" are in development. CONCLUSIONS: Integrating behavioral economic nudges into the EHR is a promising approach to enhancing guideline awareness and adherence for older adults with diabetes. This novel pilot will demonstrate the initial feasibility and preliminary efficacy of this strategy and determine if a fullscale effectiveness trial is warranted.

\section{ADDRESSING SOCIAL ISOLATION TO IMPROVE THE HEALTH OF OLDER ADULTS: A RAPID EVIDENCE REVIEW CONDUCTED FOR THE AHRQ EPC PROGRAM}

Martha Gerrity; Stephanie Veazie; Jennifer Gilbert; Kara Winchell; Allison Schmidt; Robin Paynter; Jeanne-Marie Guise. Portland VA Research Foundation, Portland, OR. (Control ID \#3185567)

BACKGROUND: Addressing social determinants that lead to poor health outcomes in the aging U.S. population is a growing public health concern. Social isolation and loneliness in older adults is associated with worsening physical and mental health. Consequences of aging such as health deterioration, loss of family and friends, and reduced mobility can lead to social isolation and loneliness. We conducted a rapid evidence review evaluating the effects of interventions targeting social isolation/ loneliness in older adults.

METHODS: This topic was nominated to the AHRQ EPC Program for a rapid review by a healthcare system and was felt to be an important issue facing health systems, providers, community organizations, and the public. Streamlined systematic review methods were used, including conducting targeted searches in Ovid/Medline $®$, PsycInfo ${ }^{\circledR}$, and CINAHL $®$ from 2013 to 2018 for systematic reviews and from 2016 to 2018 for primary studies. Studies were evaluated using pre-specified inclusion criteria and assessed for risk of bias. Study characteristics were extracted, including measurements of social isolation/loneliness, health, health care utilization, and harms, and data were synthesized into evidence tables.

RESULTS: Sixteen studies (seven randomized controlled trials, eight pre-post, and one cross-sectional with post-test survey) totaling 17,656 participants age 60 or older were included. Eight were good- or fairquality studies. Four interventions reported a positive effect on social isolation/loneliness or health outcomes. A resistance training program that included nutrition, and psychosocial support reported improved functionality, depression, diet, and social capital, a physical/leisure activity intervention reported improved quality of life but not social support, and a group tai chi intervention and a facilitated group discussion intervention both improved loneliness but not health outcomes. Three of these four interventions reporting positive outcomes involved a health care professional and met more than once per week. Five of the sixteen studies reported on harms; none were clinically significant, and three reported on health care utilization.

CONCLUSIONS: Physical activity interventions to reduce social isolation show the most promise to improve the health of older adults, but the effects were inconsistent and the studies measured short-term outcomes. Overall we found striking variation in outcomes measured across studies and inconsistency in definitions for social isolation and loneliness. Just over a quarter of studies reported on harms and our search revealed very little information on healthcare utilization, a pressing issue for healthcare delivery systems. These findings suggest that health systems should rigorously evaluate their own programs and make their findings available in order to increase the evidence base on this pressing national issue.

\footnotetext{
ADHERENCE TO WEIGHT TELEMONITORING PREDICTS REDUCED EMERGENCY DEPARTMENT AND HOSPITAL UTILIZATION AMONG PATIENTS WITH HEART FAILURE
}

Sarah C. Haynes ${ }^{1}$; Daniel Tancredi ${ }^{1}$; Patrick S. Romano ${ }^{2} .{ }^{1}$ University of California, Davis, Sacramento, CA; ${ }^{2}$ UC Davis, Sacramento, CA. (Control ID \#3185710)

BACKGROUND: Weight telemonitoring may be an effective way to improve patients' ability to manage heart failure and prevent unnecessary utilization of health services. However, effectiveness of such interventions is dependent upon patient adherence to daily weighing. Adherence is complex and may change in response to triggering events, such as a hospital readmission or emergency department (ED) visit. Conversely, lower or higher adherence in a given week may help to predict events (rehospitalization, ED visits, or death) in the following week. The objectives of this study were 1) to compare adherence in the two weeks preceding an event to adherence in the two weeks following the same event and 2) to examine the relationship between adherence to daily weighing and the occurrence of events in the following week.

METHODS: The Better Effectiveness After Transition- Heart Failure (BEAT-HF) trial examined the effectiveness of an intervention involving wireless remote telemonitoring and health coaching, compared to usual care, for patients discharged to home after hospitalization for decompensated heart failure. Participants were patients aged 50 and older who were actively treated for decompensated heart failure and discharged to their home after the enrollment hospitalization at one of six academic medical centers in California. Participants were followed for 180 days and were instructed to transmit weight readings daily. For this secondary analysis we used Poisson regression to determine the within-person effects of adherence on the rate of events as well as the effect of events on subsequent adherence.

RESULTS: On average, participants adhered to daily weighing on $52.3 \%$ of possible days. 292 participants experienced at least one hospitalization or ED visit during the study period. After controlling for the number of previous events, the rate ratio for adherence decreased by a factor of 0.87 in the two weeks after an event compared with the two weeks prior to the event (95\% CI: 0.83-0.92, $\mathrm{p}=0.000$ ). Adherence also predicted events in the following week; an increase in adherence by one day decreased the rate ratio for experiencing an event by a factor of 0.88 (95\% CI: $0.86-0.91, \mathrm{p}=0.000)$.

CONCLUSIONS: Lower adherence in a given week is associated with increased risk of experiencing a rehospitalization, ED visit, or death in the subsequent week. Experiencing a rehospitalization or ED visit did not have the expected effect of triggering an increase in adherence; rather, events had the effect of diminishing adherence to daily weighing. Because lower adherence may in itself be a marker of heart failure exacerbation, telemonitoring programs should consider incorporating changes in adherence into the system's feedback structure in addition to changes in weight values. Furthermore, rehospitalizations and ED visits could be more effectively used as teaching moments to improve subsequent adherence to telemonitoring.

\section{ADOPTION OF AN EVIDENCE-BASED FALL PREVENTION PROGRAM IN LARGE ACADEMIC HOSPITALS: A MULTI- SITE QUALITATIVE STUDY OF FACILITATORS AND BAR- RIERS}

Srijesa Khasnabish $^{1}$; Eileen Carter ${ }^{2,3}$; Jason Adelman ${ }^{2}$; Lois Alfieri ${ }^{4}$; Mike Bogaisky ${ }^{5,6}$; Ann C. Hurley ${ }^{1}$; Mary Ellen Lindros ${ }^{4}$; Virginia Ryan $^{7}$; Maureen Scanlan ${ }^{4}$; Alexa Shelley ${ }^{2}$; Shao Ping $\mathrm{Yu}^{2}$; patricia dykes ${ }^{1,8}$. ${ }^{1}$ Brigham and Women's Hospital, Brighton, MA; ${ }^{2} \mathrm{New}$ York-Presbyterian Hospital, New York, NY; ${ }^{3}$ Colmbia University School of Nursing, New York, NY; ${ }^{4}$ Montefiore, Bronx, NY; ${ }^{5}$ Albert Einstein College of Medicine, Bronx, NY; ${ }^{6}$ Einstein Hospital (Montefiore Medical Center), Bronx, NY; ${ }^{7}$ Brigham and Women's Faulkner Hospital, Jamaica Plain, MA; ${ }^{8}$ Harvard Medical School, Boston, MA. (Control ID \#3162189) 
BACKGROUND: Inpatient falls lead to adverse outcomes and prolonged hospital stays. Fall TIPS (Tailoring Interventions for Patient Safety) is a patient-centered fall prevention program that provides clinical decision support. We previously tested this tool in a randomized control trial and demonstrated a $25 \%$ reduction in falls. We used qualitative methods to explore the facilitators and barriers to the adoption of Fall TIPS across three large academic health systems (Partners HealthCare, Montefiore Medical Center, and New YorkPresbyterian).

METHODS: A semi-structured interview guide based on the REAIM framework (reach, effectiveness, adoption, implementation, and maintenance) was used. We interviewed 50 patients and families and 71 staff members in focus groups in 2 phases (Phase 1: JulySept 2017 and Phase 2: May-June 2018 to validate Phase 1 themes). Upon verbal consent from participants, interviews were recorded, transcribed, and reviewed for accuracy. Transcripts were analyzed in NVivo (QSR International, London) using basic content analysis. RESULTS: Of the 50 patients and families interviewed, $30(60.0 \%)$ were 65 years, $30(60.0 \%)$ were female, and $22(40.0 \%)$ were white. Of the 71 staff members interviewed, $66(93.0 \%)$ were nurses; the mean (SD) age was $38.62(1.57), 64(90.1 \%)$ were female, and 25 $(35.2 \%)$ were white. Fall TIPS changed the paradigm of fall prevention. Pre-implementation, fall prevention consisted of one-size fits all approaches that were staff-driven and lacked actionability (e.g., falls bracelets for "high" risk fall patients). Post-implementation, patients were engaged in the identification and implementation of interventions to address individualized fall risk factors (e.g., walking assistance for patients with medical equipment). Dominant facilitators to the adoption of Fall TIPS included 1) staff motivation and engagement, driven by leadership's prioritization of fall prevention and recognition that prior fall prevention programs were ineffective; 2 ) patients welcoming their role in fall prevention and Fall TIPS bedside posters that facilitated patient engagement; and 3) information technology (IT) that supported the integration of Fall TIPS into staff workflows (e.g., clinical decision support tools that linked evidence-based interventions to fall risk factors). Dominant barriers included: 1) workarounds that threatened program sustainability (e.g. educating not engaging patients in fall prevention); and 2) difficultly engaging willful patients or those with cognitive impairments in fall prevention.

CONCLUSIONS: Barriers and facilitators to Fall TIPS included staff-level, patient-level and system-level (IT) considerations. Hospitals interested in adopting Fall TIPS should use hospital leadership to motivate staff in fall prevention, harness patients intrinsic desire to be engaged in their care, and use IT to support the integration of Fall TIPS into staff workflows.

\section{ADVANCE CARE PLANNING IN ADULTS AGED 85 AND OLDER IN A CONTINUING CARE RETIREMENT COMMU- NITY.}

Rebecca Mullan; Christina Tieu. University of Virginia, Charlottesville, VA. (Control ID \#3186018)

BACKGROUND: Advance care planning (ACP) is a process in which patients, in partnership with family and their healthcare team, discuss end of life care and make decisions regarding treatment preferences. These preferences are often documented in an advance directive (AD), with the goal being that patients receive care consistent with their values if they are unable to make decisions for themselves. This is particularly important in an older geriatric patient population (age 85 and older), who are at high risk of hospitalization. However, there is little information in the current literature regarding ACP and patient values in this population. We investigated the completion rates and qualitative components of advanced directives in a geriatric patient population aged 85 and older.
METHODS: We performed a cross-sectional chart review of a geriatric primary care practice based in a continuing care retirement community. Eligible patients were 85 years and older, living within the ambulatory and assisted living community, and receiving care at the clinic. Data abstracted included age, gender, comorbidities, hospitalization or emergency department visit within 90 days, and $\mathrm{AD}$ completed. Patients were considered to have an $\mathrm{AD}$ if there was a document uploaded and indexed in the Electronic Medical Record (EMR).

RESULTS: There were 192 patients who met the inclusion criteria. The mean age was 91.1 (SD 4.2), and 132 (69\%) were female. In terms of comorbidities, 51 (26\%) had coronary artery disease, $36(19 \%)$ had congestive heart failure, and $22(11 \%)$ had diabetes. There were $24(12 \%)$ patients who had been hospitalized or presented to the emergency department within 90 days. Of the 192 patients, 24 (12\%) had an AD uploaded in their EMR. In 4 of 24 patients, the $\mathrm{AD}$ included only a designated power of attorney (POA). In 18 of 24 patients (75\%), the $\mathrm{AD}$ included language directing a focus on comfort-based treatment in the event of imminent death. Two patients $(8 \%)$ included information about values to guide treatment decisions.

CONCLUSIONS: Advance directives were documented in the EMR in only a small percentage of patients age 85 and older. The majority of these directives included instructions for comfort-based care in the event of imminent death / medical futility, but only two included information regarding the patient's values to help direct medical care.

\section{ADVANCING EVIDENCE SYNTHESIS FROM EFFECTIVENESS TO IMPLEMENTATION: RECOMMENDATIONS FOR THE IN- TEGRATION OF IMPLEMENTATION MEASURES INTO EVI- DENCE SYNTHESIS APPROACHES}

Aaron A. Tierney $^{1,}{ }^{2}$; Marie Haverfield ${ }^{3}$; Mark McGovern ${ }^{4}$; Donna M. Zulman ${ }^{5}$. Stanford University School of Medicine, Mountain View, CA; ${ }^{2}$ Palo Alto VA Health Care System, Menlo Park, CA; ${ }^{3}$ Stanford University, Menlo Park, CA; ${ }^{4}$ Stanford School of Medicine, Palo Alto, CA; ${ }^{5}$ Stanford University and VA Palo Alto, Stanford, CA. (Control ID \#3168121)

BACKGROUND: The field of implementation science aims to address the time lag and quality chasm between discoveries that could improve health, and the widespread dissemination and adoption of those findings. To close this gap, there is a need for greater attention to the factors that influence dissemination and implementation of evidence-based practices. Evidence synthesis projects (e.g., systematic reviews) could contribute to this effort by collecting and synthesizing data relevant to dissemination and implementation. The objective of this paper is to recommend implementation measures and outcomes that should be considered in evidence syntheses of intervention studies and illustrate the application of these recommendations through a systematic review of doctor-patient communication interventions.

METHODS: Drawing on the implementation science literature, we describe ten implementation measures that should be considered when conducting evidence synthesis: acceptability, adoption, appropriateness, feasibility, fidelity, implementation cost, intervention complexity, penetration, reach, and sustainability. We describe opportunities to assess these constructs, for example by examining clinician satisfaction with an intervention (acceptability), participation/attrition rates (adoption), intervention complexity and time/resource demands (feasibility), the degree to which an intervention is implemented as planned (fidelity), and resources and time required to implement an intervention (implementation cost). As a case example, we describe a systematic review of 29 studies of interventions aimed at improving clinician-patient communication in clinical settings. Using the 29 studies, we illustrate opportunities to abstract the implementation measures using data that are frequently reported.

RESULTS: Among the 29 studies, there was variation in the proportion that reported information relevant to acceptability (48\%), adoption (86\%), appropriateness (66\%), feasibility (62\%), implementation cost (79\%), intervention complexity $(100 \%)$, penetration $(76 \%)$, reach $(86 \%)$, and 
sustainability (24\%). Approximately two-thirds (19/28) of studies included a measure of fidelity. While three studies reported a direct measure of cost, in all cases the measure reflected the impact of the intervention on cost, rather than implementation costs. Only 2 (7\%) studies contained information related to all 10 implementation measures.

CONCLUSIONS: To fully understand the potential value of an intervention, it is important to consider both its effectiveness and the process, demands, and resource requirements involved in implementation. While there is variation in the degree to which intervention studies currently report implementation factors, there is growing demand for this information. By incorporating implementation considerations, evidence synthesis projects can provide valuable contributions to the dissemination of effective interventions.

\section{ADVANCING REAL-WORLD GAINS IN EFFICACY THROUGH TECHNOLOGY AND SKILL BUILDING (TARGETS). A PILOT EVALUATION OF THE TIME2FOCUS MOBILE APPLICATION FOR DIABETES EDUCATION}

Alex H. Cho ${ }^{1,2}$; Bryan Batch ${ }^{1}$; Dan V. Blalock ${ }^{1}$; Susan Spratt ${ }^{1}$; Rowena Dolor ${ }^{1,2}$. ${ }^{1}$ Duke University School of Medicine, Durham, NC; ${ }^{2}$ Duke Primary Care Research Consortium, Durham, NC. (Control ID \#3185784)

BACKGROUND: Type 2 diabetes affects 30 million Americans, and is a significant cause of morbidity and mortality. Self-management support (SMS) is an important component of chronic illness care, and a key pillar of the Chronic Care Model. Face-to-face teaching and static patient education materials can suffer from not being interactive or compatible with mobile lifestyles. Digital applications ('apps') offer an alternative for providing SMS that is convenient, scalable and can incorporate principles of health behavior change. This pilot study tested the real-world deployment of a self-guided mobile app for diabetes education (Time2Focus), which utilizes evidence-based content and gamification to deliver an interactive learning experience across 12 levels, covering healthy eating, physical activity, and diabetes monitoring.

METHODS: Primary care providers in the southeastern US were approached for permission to invite their patients to participate. Eligible patients were 18-89 years old; had a diagnosis of Type 2 diabetes; hemoglobin A1c $8 \%$ and $<12 \%$ in the past 3 months; an active online patient portal account tied to the electronic health record (EHR); and access to an iOS or Android smartphone. Interested patients were emailed a baseline survey, and once this was completed, sent instructions for downloading the Time2Focus app (MicroMass; Cary, NC). They were then invited to begin using the app, taking a week to complete each of 12 levels, but could take longer. After completing all 12 levels, participants were sent a follow-up survey. Our primary outcome was pre-/post-change in hemoglobin A1c (A1c), using values from the EHR. Secondary outcomes included users' engagement with and rating of the app. This study was approved by the Duke University Health System IRB.

RESULTS: Of 1355 potentially eligible patients screened, 780 were ineligible, 169 declined, 205 were unreachable, and 201 were consented. 117 (58.2\%) participants completed <1 level, 84 (41.8\%) 1 level, 26 (12.9\%) completed 1-4 levels, 10 (5.0\%) completed 5-11 levels, and 48 (23.9\%) completed 12 levels. Those completing one or more levels had a mean pre-/ post- A1c change of $-0.41 \%$, compared to $-0.32 \%$ among those who did not. Unadjusted two-tailed t-test was not statistically significant $(p=0.73)$. Net Promoter Score (NPS) was 62.5 , indicating those who completed all levels of the app rated it highly and would recommend it to others.

CONCLUSIONS: Interest in using a diabetes education app may signal readiness for improved diabetes self-management, but did not always translate into initial use. The decrease in A1c observed in app users relative to non-users during this limited pilot study was not statistically significant. However, uptake and application of lessons learned from SMS may be delayed; in addition, sample size may have been an issue. Future research is needed to address how to increase engagement in SMS and investigate if follow-up over a longer period of time demonstrates significant change in outcomes like hemoglobin Alc.

\section{ADVERSE EVENTS EXPERIENCED BY PATIENTS HOSPITAL- IZED WITHOUT DEFINITE MEDICAL ACUITY: A RETRO- SPECTIVE COHORT STUDY}

Amanda Sekijima ${ }^{1}$; Cassandra Sunga ${ }^{1}$; Maralyssa Bann ${ }^{2} .{ }^{1}$ University of Washington School of Medicine, Woodinville, WA; ${ }^{2}$ University of Washington-Harborview Medical Center, Seattle, WA. (Control ID \#3162868)

BACKGROUND: There is evidence that physicians consider a variety of "non-medical" factors (e.g. lack of social support, barriers to access) in hospital admission decision-making out of concern for patient safety, and that patients are hospitalized even in the absence of a level of medical acuity warranting admission. The rationale underlying this decision may overvalue the safety of hospitalization based on an assumption that patients admitted without significant medical acuity are not subjected to the same risks as acutely medically ill patients. To our knowledge, this question has not been directly investigated. Our study sought to quantify adverse events (AEs) experienced by patients hospitalized without definite medical acuity in comparison to those who had a definitely medically appropriate indication for admission.

METHODS: This was a retrospective cohort study of 300 admissions from $2 / 1 / 2018$ to $6 / 1 / 2018$ in an urban academic medical center. We defined the exposure as hospitalization without definite medical acuity, as assessed by an attending physician faculty member upon admission. Chart review using the IHI Global Trigger tool method was used to measure AEs. UW IRB approval was granted for this study.

RESULTS: 748 admissions from the Emergency Department to a general medical floor were included, of which $513(68.8 \%)$ were considered definitely appropriate and $169(22.6 \%)$ were without definite medical acuity. A sample of 150 admissions from each was used to generate cohort groups. No statistically significant differences were observed between groups in terms of age, gender, race/ethnicity, or primary language. There was no difference in the percentage of admissions with AEs between the two groups $(27.3 \%$ vs. $29.3 \%$; RR $1.05,95 \%$ CI $0.82-1.34 \mathrm{p}=0.70)$ nor in AEs per 1000-patient days (76.8 vs. 70.4 ; IRR $=0.92$, 95\%CI 0.65-1.30, $\mathrm{p}=0.61$ ). Distribution of severity of AEs was also similar (see Table 1).

CONCLUSIONS: Our findings demonstrate that patients who are admitted without definite medical acuity are exposed to the same risk of experiencing an $\mathrm{AE}$ as those who have a definitely appropriate indication for admission. Further study into the underlying reasons for these admissions and refinement of the risk/benefits surrounding the decision to admit is warranted.

\section{AIM TO IMPROVE FOLATE ORDERING IN NORMOCYTIC AND MACROCYTIC ANEMIA AT LENOX HILL HOSPITAL}

Marcus Calderon $^{1,2}$; Andrew A. Moses ${ }^{2}$; Rebecca Mazurkiewicz ${ }^{3}$; Maria DeVita ${ }^{3} .{ }^{1}$ Providence Hospital, Canton, MI; ${ }^{2}$ Lenox HIll Hospital, New York, NY; ${ }^{3}$ Lenox Hill Hospital, New York, NY. (Control ID \#3185189)

BACKGROUND: Anemia is one of the most commonly diagnosed issues in the hospital setting, and the workup often is costlier than the treatment. Per the Choosing Wisely initiative in 2017, folate testing should not be ordered as it is cheaper to simply supplement it in macrocytic anemia and testing is not indicated in normocytic or microcytic anemia. Despite this, previous studies have shown that folate testing continues unabated in hospital settings. We hypothesized that folate testing was not ordered appropriately by the internal medicine service at Lenox Hill Hospital, a community hospital in New York City.

METHODS: We conducted a retrospective chart review including all adult patients admitted to the medicine service at Lenox Hill Hospital from July 2017 to January 2018 with Folate, Serum. Iron studies ordered at the 
same time in order to better select for patients who had folate ordered as part of an anemia work up as opposed to other indications. In this way iron testing was used as a surrogate for anemia analysis. Patients whose test was cancelled before resulting or with duplicate orders were excluded. Additionally, we administered an anonymous 3-question survey to housestaff for self-assessment and to determine the obstacles for ordering serum folate levels properly. An educational email and brief reminders in group settings were used to instruct regarding folate testing. The incentive was a free group meal if folate testing was lowered by $50 \%$ in a one-month test period in May 2018. These emails included the current literature about folate testing, the rationale behind our study and intervention, and a reminder about the incentive. The post-intervention chart review was conducted in a similar fashion to the first. Data was acquired from Sunrise, the hospital's electronic medical record and analyzed using MS Excel.

RESULTS: We identified 833 patients with an order for folate in the initial chart review, all with Mean Cell Volumes (MCV) less than 100 (normocytic to microcytic range). None of these had a folate level consistent with diagnosis of folate deficiency. Concurrent iron testing was performed in $472(57 \%)$. Thirty-five out of 60 residents (58\%) responded to the survey. Of those, only $8(23 \%)$ answered that they would order folate in a normocytic or microcytic patient, and $9(25 \%)$ answered that they would order folate no matter the MCV. In the post-intervention period of May 2018, 90 orders of serum folate in MCVs $<100$ were ordered, which was about a ten percent decrease from the average of the initial review period.

CONCLUSIONS: Our study was one of the first to look at proper anemia testing to exclude a test, namely folate, as a cost and blood saving measure for hospitals and patients. The initial review period showed that folate testing was an unnecessary expenditure and should not be taken as part of the routine workup for anemia. The cost of folate testing to the system and to the patient may seem negligible, but the follow up testing and blood draws can cause undue harm.

\section{AIMING FOR EQUITY: EXPLORING PATIENT PREFERENCES FOR ASSISTANCE WITH SOCIAL DETERMINANT OF HEALTH (SDOH) BARRIERS IN PATIENTS WITH UNCONTROLLED TYPE 2 INSULIN-DEPENDENT DIABETES (IDDM) SEEKING CARE AT A SAFETY-NET HOSPITAL}

Natalie K. Levy ${ }^{1}$; Agnes Park ${ }^{1}$; Daniela Solis ${ }^{1}$; Binhuan Wang ${ }^{3}$; Aisha Langford $^{3}$; Lu Hu${ }^{2}$; Erin Rogers ${ }^{4} .{ }^{1}$ NYU School of Medicine, Bellevue Hospital, New York, NY; ${ }^{2}$ New York University Langone Medical Center, New York, NY; ${ }^{3}$ New York University, New York, NY; ${ }^{4}$ NYU School of Medicine, New York, NY. (Control ID \#3180675)

BACKGROUND: Health equity can broadly be defined as giving people the opportunities and resources needed to maximize health regardless of socially determined circumstances. SDoH are economic and social conditions that lead to differences in health status. We practice medicine at a mission driven safety-net hospital and provide care to patients with uncontrolled type 2 IDDM. Understanding not only these patients' SDoH barriers, but also their preferences for assistance, is the first step in providing equitable help.

METHODS: We used surveys and interviews to learn about 3 themes: SDoH barriers that impacted the ability to care for one's diabetes, desirable service features of any assistance program, and the types of services patients feel are needed.

RESULTS: We learned that $84 \%$ of patients had $>1$ barrier to health care access, $54 \%$ were unable to pay for $>1$ essential item when it was needed, $53 \%$ reported $>1$ barrier in their built environment, $47 \%$ reported $>1$ issue with health literacy, and $37 \%$ shared that they only saw or talked to someone that they cared about or felt close to $<2$ times per week. In the process of defining SDoH barriers, we also learned about unhealthy behavior patterns: $69 \%$ of patients have inadequate fruit and vegetable consumption, $57 \%$ get no leisure-time physical activity, $48 \%$ sometimes or often miss a day of checking their blood sugar, $35 \%$ sometimes or often miss doses of their medications and $30 \%$ are unable to follow up with their doctor in the time frame they are given.
Despite their obstacles, patients felt that they didn't need "help". While they did share preferences on desirable service features, they volunteered very little regarding desirable types of services. Their lack of suggestions on service features was a finding in and of itself, warranting further exploration. In the end, we gauged interest in currently available resources at our hospital and in $\mathrm{NYC}$, as well as our team's ideas for potential new programs to tackle some of the unhealthy behavior patterns we learned about (see types of services below). The service features that were a priority for any assistance program were: doctor knows about the program (86\%), cost (87\%), program is inperson $(83 \%)$, program sends reminders $(74 \%)$, program is a group class (74\%), program is close to home (70\%). In terms of types of services, $70 \%$ of patients want to learn about Farmer's markets as a source to eat more produce, $65 \%$ want to use the pedometer program we are developing and up to $45 \%$ want to use Bellevue's new Diabetes Patient Navigator program for assistance with barriers to health care access, taking diabetes medications, and checking blood sugar.

CONCLUSIONS: Patients with uncontrolled type 2 IDDM at our safety-net hospital have significant SDoH barriers, multiple service feature preferences, and are interested in programs currently available and under development. Understanding patient preferences for assistance is a key step in creating solutions that provide equity for underserved patients in need.

\section{ALL-CAUSE MORTALITY AND NEUROLOGICAL OUTCOMES OF TARGETED TEMPERATURE MANAGEMENT AFTER CAR- DIAC ARREST: META-ANALYSIS}

Mohammed Abdalla $^{1}$; Abdelnasir Mohamed ${ }^{1}$; Wiam Mohamed ${ }^{2}$; Khlwd $\mathrm{Khtab}^{2}$. 'Saint Francis Hospital, Evanston, IL; ' ${ }^{\mathrm{U}}$ University of Khartoum, Khartoum, Sudan. (Control ID \#3185541)

BACKGROUND: Cardiac arrest carries a high mortality and morbidity. Different studies showed conflicting data regarding outcomes of targeted temperature management (TTM) for cardiac arrest. The purpose of this meta-analysis is to systematically determine the effect of TTM on all-cause mortality and neurological outcomes post cardiac arrest.

METHODS: We conducted a systematic search for randomized controlled trials in Pubmed, Cochrane \& ScienceDirect. Primary outcomes were neurological outcome and all-cause mortality.

RESULTS: Nine randomized controlled trials utilizing data for in-hospital and out-of-hospital cardiac arrest were selected for the meta-analysis. Number of patients included was 1,592. Mortality was lower in targeted temperature management group (OR 0.637, 95\% CI 0.436-0.93, p-value 0.019, $\left.\mathrm{I}^{2}=44.78 \%, \mathrm{n}=1,592\right)$. These results were statistically significant. Therapeutic hypothermia group also demonstrated reduction with regard to poor neurological outcomes (OR 0.582, 95\% CI 0.363-931, p-value 0.024, $\left.\mathrm{I}^{2}=56.79 \%, \mathrm{n}=1567\right)$. We conducted subgroup analysis after we excluded in-hospital cardiac arrest patients and results showed statistically significant reduction in poor neurological outcomes (OR $0.562,95 \%$ CI 0.331 $0.955, \mathrm{p}$-value $0.033, \mathrm{I}^{2}=61.78 \%, \mathrm{n}=1480$ ) and also showed mortality reduction in out-of-hospital cardiac arrest (OR 0.674, 95\% CI 0.454-999, $\mathrm{p}$-value $\left.0.049, \mathrm{I}^{2}=43.8 \%, \mathrm{n}=1,505\right)$.

CONCLUSIONS: Targeted temperature management after cardiac arrest may be associated with improvement in all-cause mortality and reduction in poor neurological outcome.

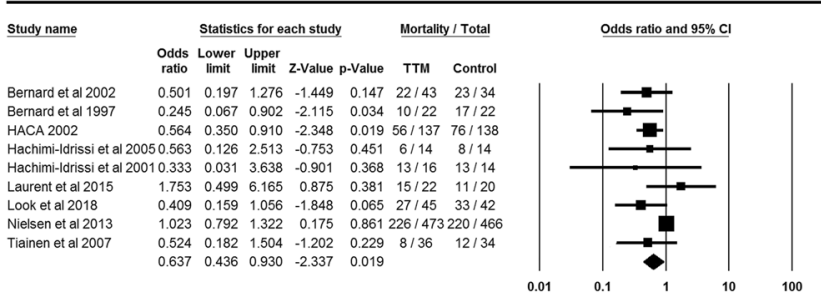


Forest plot for all-cause mortality of targeted temperature managmenet after cardiac arrest

\section{AMBIVALENCE TOWARD OPIOID TAPERING AMONG VET- ERANS ON LONG-TERM OPIOID THERAPY}

Anne Hale ${ }^{2}$; Evan Carey2; Lindsay B. Miller ${ }^{3}$; Charlotte Nolan ${ }^{4}$; Erin E. Krebs ${ }^{5}$; Joseph W. Frank ${ }^{1} .{ }^{1}$ University of Colorado School of Medicine, Aurora, $\mathrm{CO} ;{ }^{2}$ Veterans Affairs, Denver, CO; ${ }^{3}$ VA Eastern Colorado Healthcare System, Aurora, CO; ${ }^{4}$ Veterans Health Administration, Denver, CO; ${ }^{5}$ Minneapolis VA Health Care System, Minneapolis, MN. (Control ID \#3185906)

BACKGROUND: There is inadequate evidence of benefit from longterm opioid therapy (LTOT) and growing evidence of harms. Expert guidelines recommend weighing the risks and benefits of LTOT, and if risks outweigh benefits, recommend reducing or discontinuing LTOT. Many patients on LTOT report ambivalence about opioid medications but patient perspectives on opioid tapering are not well understood. To better understand patient perspectives on opioid tapering, we examined patient-reported importance, readiness, and confidence in their ability to taper opioid medications.

METHODS: We conducted a cross-sectional study within a national prospective cohort study, the Effects of Prescription Opioid Changes for Veterans (EPOCH) Study. The EPOCH Study identified Veterans on LTOT, defined as 150 days' supply in 180 days with no gaps $>40$ days, and 9,253 Veterans completed a baseline survey in 2015 ( $92 \%$ by mail, $8 \%$ by phone). We randomly selected 600 Veterans who met two criteria at EPOCH baseline: 1. completed mail survey; and 2. prescribed 50mg morphine equivalent daily dose (MED). We completed structured phone interviews at the 18-month EPOCH timepoint among 316 Veterans $(53 \%$ response rate). We assessed self-reported past year opioid tapering or discontinuation and 11-point scales of importance, readiness and confidence related to opioid tapering ("On a scale of 0 to 10 , where 0 is 'not at all important' and 10 is 'extremely important', how important do you think it is to cut down or stop using opioid medicines?"). Data on demographics, medications and diagnoses were obtained from the electronic health record. We used t-tests and chi-square tests to examine bivariate associations between past year opioid tapering and these 11-point scales responses.

RESULTS: The study sample was primarily age 60 years or older $(73 \%)$, male $(92 \%)$, and White $(87 \%)$, and the most common pain diagnoses were back pain $(76 \%)$ and other joint pain (46\%). Opioid medication doses at EPOCH baseline were 50-99mg MED (53\%), 100-199mg MED (37\%) and 200+mg MED (10\%). A majority (190/316, 60\%) reported tapering in the past year or discontinuing opioid medications. Responses ranged from 1-10 for all three scales. Individuals who reported past year opioid tapering or discontinuation reported significantly higher levels of importance, confidence and readiness related to opioid tapering (Table).

CONCLUSIONS: Veterans on long-term opioid therapy report ambivalence about opioid tapering or discontinuation. Measures of importance, readiness, and confidence are associated with prior opioid tapering experience. Future studies should examine the utility of these scales to support behavior change in the context of opioid tapering as well as the relationship between patient-reported ambivalence and subsequent opioid dose reduction.

\section{AMBULATORY HOLTER MONITORING VERSUS OUTPA- TIENT 12-LEAD EKG IN PATIENTS WITH CHRONIC ATRIAL FIBRILLATION \\ Anand Muthu Krishnan ${ }^{1}$; Usama Bin Nasir ${ }^{1}$; Gaurav Manek ${ }^{1}$; Supriya Tigadi $^{2}$; Heiko Schmitt ${ }^{2}$. ${ }^{1}$ University of Connecticut, Farmington, CT; ${ }^{2}$ UNIVERSITY OF CONNECTICUT, Farmington, CT. (Control ID \#3168575)}

BACKGROUND: Ambulatory Holter monitors are frequently used for evaluation of rate control in patients with persistent or permanent atrial fibrillation. The use of Holter monitors for this purpose is not mentioned in the current AHA/ACC atrial fibrillation guidelines. We sought to determine the correlation of average heart rate as reported by the 24 hour Holter monitor with that reported on clinic based 12-lead EKG

METHODS: We retrospectively searched the electronic Holter monitor database at the University of Connecticut John Dempsey hospital from the period of 2008 to 2018. The inclusion criteria were the presence of continuous atrial fibrillation throughout the Holter monitor, a resting outpatient 12-lead ECG showing atrial fibrillation that was performed within 30 days of the Holter monitor. Patients with paroxysmal atrial fibrillation, other atrial or ventricular rhythms on the Holter monitor or 12lead ECG were excluded from the study. Mean heart rate on the Holter monitor was compared to resting ventricular heart rate on 12- lead ECG. Statistical analysis was performed using JMP software.

RESULTS: In total, 227 patients were identified who underwent ambulatory Holter monitoring for the indication of atrial fibrillation. From the 227 Holters reviewed, 89 patients were identified to have persistent or permanent atrial fibrillation and have a 12-lead EKG performed within 30 days of Holter monitor. The Pearson's correlation coefficient for resting heart rate and mean Holter heart rate was 0.78 reaching statistical significance, $\mathrm{P}<0.0001$. (figure 1 )

CONCLUSIONS: In patients with permanent atrial fibrillation, mean heart rate from Holter monitors correlates with resting heart rates on 12lead EKG that was performed within 30 days of Holter monitor. These results indicate that Holter monitoring might not be necessary in evaluating heart rate in patients with persistent or permanent atrial fibrillation.

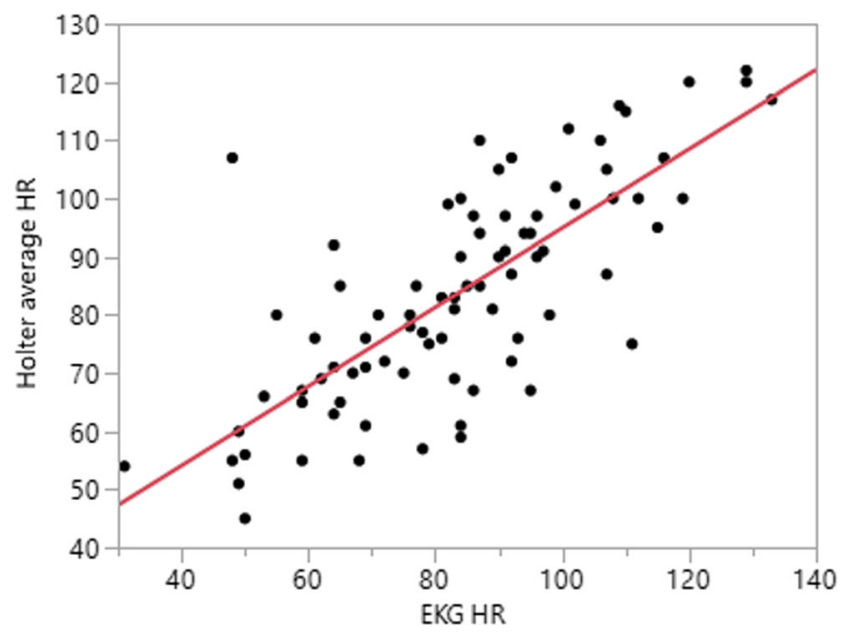

Figure 1

\section{AN ACCEPTABILITY STUDY OF HEALTHCARE NEED AND UTILIZATION IN STREET-BASED PERSONS WHO INJECT DRUGS IN CHICAGO}

Anna Solotskaya $^{1,2}$; Rachel Bernard ${ }^{2}$; Karim G. Khan ${ }^{2}$; Shaveta Khosla ${ }^{2}$; $\overline{\text { Antonio D. Jimenez }}{ }^{2}$; Stockton Mayer ${ }^{2} .{ }^{1} \mathrm{OHSU}$, Portland, OR; ${ }^{2}$ University of Illinois - Chicago, Chicago, IL. (Control ID \#3177350)

BACKGROUND: Injection drug use (IDU) is associated with significant morbidity and mortality. Persons who inject drugs (PWID) suffer from a number of psychiatric, chronic and acute illnesses. Despite their healthcare needs, this population has poor integration with primary care and preventative health services. As such, PWID are particularly difficult to study and have high burdens of disease.

METHODS: We confirmed the acceptability of conducting a research study through the use of a mobile outreach van with a population of 
PWID. This study assessed the perceived healthcare needs and healthcare utilization of a population of PWID and assessed the acceptability of providing preventative services and care through a mobile outreach van. In 2016 - 2017 we conducted a survey of PWID who utilize a mobile outreach van. We interviewed adult individuals with a history of recent IDU from various parts of the city of Chicago. A brief 5-10 min questionnaire was used to collect data.

RESULTS: 67 persons were enrolled in the study - 59 participants from the west side and 7 from the south side of Chicago. Mean age was 37 and $74.6 \%$ were male. $73 \%$ of respondents had recent contact with the medical system for conditions such as skin and soft tissue infections (SSTI), mood disorders, and drug and alcohol treatment. SSTIs were a major factor for seeking healthcare with many individuals requiring hospitalization and antibiotics. Mental health disorders were highly prevalent in this population. $91 \%$ of participants surveyed were attracted to receiving healthcare services in a mobile outreach setting.

CONCLUSIONS: Healthcare utilization is high in PWID for acute and chronic illness. Services aimed at reducing SSTIs and managing psychiatric illness may be especially effective at reducing healthcare costs and improving quality of life for PWID. This project demonstrates that it is acceptable and desired by a population of PWID to provide quick preventative services and healthcare through a mobile outreach vehicle.

\section{AN ANALYSIS OF HEALTHCARE UTILIZATION AMONGST UNDOCUMENTED PATIENTS 2014-2018}

Joseph Nwadiuko $^{1}$; Jashalynn German ${ }^{2}$; Kavita Chapla ${ }^{2}$; Frances Wang ${ }^{2}$; Sarah Polk ${ }^{2}$. ${ }^{1}$ University of Pennsylvania School of Medicine, Philadelphia, PA; ${ }^{2}$ Johns Hopkins School of Medicine, Baltimore, MD. (Control ID \#3180753)

BACKGROUND: The deportation of undocumented immigrants has increased since the 2016 presidential election. The increased rate of immigration enforcement might discourage undocumented patients from receiving healthcare.

METHODS: The following study analyzes primary care utilization amongst a pool of low-income, Medicaid ineligible patients and Medicaid controls across eight adult and pediatric clinics in a mid-Atlantic health system between 2014-2018. Medicaid ineligible patients were identified by membership in charity and sliding scale programs sponsored by the health system that provide primary care access to a predominantly $(92 \%)$ undocumented immigrant pool. Using a difference-in-difference approach, our goal was to determine whether there were any changes in the number of scheduled primary care visits between November 9, 2016 (the 2016 general election) and May 31, 2018, with January 1, 2014November 8, 2016 serving as a reference period. To be included in analysis, patients must have had a single visit between January 1, 2014October 31, 2015 covered by Medicaid or the charity/sliding program and not have died before the election. Visit records were drawn using the health system's electronic record system. Estimates were calculated using linear and Poisson mixed effect models, controlling for age and gender. RESULTS: There are 17,504 patients in the sample, 1,429 (8\%) in the cohort group and 16,075 (92\%) in the control group. The average pre-election number of visits per person per year was 4.54 in the control group and 2.55 in the cohort group. Compared to before the election, there was a decline to an average of $1.28(-49.6 \%)$ visits amongst cohort patients and an average of $3.06(-32.7 \%)$ visits amongst controls, for an adjusted difference-in-difference relative decline of $-13.3 \%$ (95\% CI $-21.9 \%,-3.77 \%, \mathrm{p}=0.007)$. This effect was exaggerated in adults (difference-in-difference -34\%, 95\% CI $-37.4 \%$, $-29.4 \%)$ and sliding-scale patients $(-20.4 \%, 95 \%$ CI $-34.9 \%,-0.27 \%)$. An adjusted time trend analysis showed however that visits in the cohort group fell from an average of 1.82 visits/quarter during the first quarter of 2014 to 0.34 visits per quarter in the final quarter of 2015 with little change between late 2016 and 2018. There was no significant difference-in-difference in noshow rates between groups $(+3.38 \%, 95 \%$ CI $-0.40 \%,+7.18 \%)$.
CONCLUSIONS: There is a larger decline in the number of visits in undocumented patients than Medicaid patients between 2014 and 2018, but it is not associated with changes around the 2016 election/2017 inauguration period. The true cause for the decline is unclear and needs further investigation. While there is no difference in clinic visits attributed to the election, the results do shed insight into substantial differences in the care trends of Medicaid and undocumented patients.

\section{AN AUDIT SURVEY OF ACCESS TO OFFICE-BASED BUPRENORPHINE TREATMENT IN STATES WITH HIGH RATES OF OPIOID-RELATED MORTALITY}

Tamara Beetham ${ }^{1}$; Brendan Saloner ${ }^{3}$; Sarah E. Wakeman ${ }^{2}$; Marema Gaye ${ }^{1}$; Michael L. Barnett ${ }^{1}{ }^{1}$ Harvard T.H. Chan School of Public Health, Boston, MA; ${ }^{2}$ Massachusetts General Hospital, Charlestown, MA; ${ }^{3}$ Johns Hopkins University, Baltimore, MD. (Control ID \#3181457)

BACKGROUND: While it is widely acknowledged that access to treatment for opioid use disorder (OUD) is a challenge for patients, little is understood about the diverse barriers that patients encounter in the process of seeking treatment due to appointment availability, wait times, and cost. Understanding the nature of these patient-facing barriers is crucial to design effective policy. To study these barriers, we contacted clinicians as "secret shoppers" requesting buprenorphine, a highly effective medication for OUD treatment.

METHODS: We performed an audit survey from August-October 2018 contacting randomly chosen publicly-listed buprenorphine prescribers twice, posing as uninsured or Medicaid-covered patients with active heroin use seeking buprenorphine treatment at the next available appointment. We used public directories of buprenorphine prescribers located in the six states/ jurisdictions (Massachusetts, Maryland, New Hampshire, West Virginia, Ohio, and the District of Columbia) with the highest opioid-related mortality in the United States in 2016 to define our sample. Rates of new appointments offered, whether buprenorphine prescription was possible at the first visit, wait times, and out-of-pocket cost for the first appointment were measured. We used linear regression models with clinician random effects to account for the clustering of outcomes within clinicians contacted twice.

RESULTS: Among 1,092 contacts with 546 clinicians, schedulers were reached for 849 calls ( $78 \%$ response rate), of whom $58 \%$ were offered a new appointment with a buprenorphine prescriber and $34 \%$ were offered an appointment with the possibility of buprenorphine prescription at the first visit. Callers with Medicaid coverage were less likely than uninsured/self-pay callers to be offered any appointment ( $54 \%$ vs. $62 \%$ of contacts, $\mathrm{p}=0.02)$ or possible buprenorphine at the first visit ( $27 \%$ vs. $40 \%$ of all contacts, $\mathrm{p}<0.001$ ). The median wait time to first appointment was 5 days (IQR 210) while the median additional wait to buprenorphine availability was 6 days (IQR 2-14). The median cost of appointment(s) up to induction for a patient paying cash was $\$ 250$ and $52 \%$ of contacts reported additional charges necessary for urine or other laboratory tests prior to induction.

CONCLUSIONS: Many buprenorphine prescribers did not offer new appointments or rapid buprenorphine access for callers with active heroin use, particularly those with Medicaid coverage. For self-pay patients, high out-ofpocket costs and recurring charges for laboratory testing were common. Unexpectedly, the primary barrier to buprenorphine access in this survey was not long wait times, but finding a clinician accepting new patients for rapid treatment. The co-existence of poor access with short wait times suggests that improving access to buprenorphine treatment may require efforts beyond increasing the number of waivered providers, such as better connecting patients to the existing supply of prescribers.

AN UNTAPPED POTENTIAL FOR AN INVISIBLE ECONOMY IN PRIMARY CARE? MAKING PATIENT PORTALS WORK FOR CAREGIVERS IN THE SAFETY-NET

Alejandra Casillas ${ }^{1}$; Anupama Cemballi ${ }^{2}$; Anshu Abhat ${ }^{3}$; Jennifer Portz ${ }^{6}$; Shobha Sadasivaiah ${ }^{4}$; Neda Ratanawongsa ${ }^{2}$; Wagahta Semere ${ }^{5}$; Courtney 
R. Lyles ${ }^{5} .{ }^{1}$ David Geffen School of Medicine @ UCLA, Los Angeles, CA; ${ }^{2}$ UCSF, San Francisco, CA; ${ }^{3}$ Harbor-UCLA Medical Center, Pasadena, CA; ${ }^{4}$ University of California San Francisco/Zuckerberg San Francisco General, San Francisco, CA; ${ }^{5}$ University of California San Francisco, San Francisco, CA; 6Colorado State University, Denver, CO. (Control ID \#3186037)

BACKGROUND: Vulnerable patients within safety-net settings are interested in accessing health information via patient portals, but are less likely to actually use one. Family and friends (in caregiver roles) are important resources to assist these types of patients (i.e. Medicaid recipients, older, and/or Limited English Proficient/LEP) in navigating health systems- and provider support may facilitate caregivers' portal engagement for these patients. We used qualitative methods to explore safety-net providers' perspectives on caregiver/proxy portal use.

METHODS: We conducted 40-60 minute semi-structured interviews with providers from three large California safety-net health systems. The interviews focused on providers' experiences with caregivers, caregiver roles, and how the portal could be leveraged as a tool to support caregivers in their responsibilities. Two coders analyzed the interview transcripts using both deductive and inductive approaches and established consensus regarding major themes. RESULTS: Of 17 participants interviewed, 4 specialized in geriatrics, and $>50 \%$ held a leadership/administrative role. The five themes highlight providers' recognition of potential benefits associated with caregiver portal use (Portal positioned to assist caregivers with healthcare tasks; Portal as a tool to directly support caregivers; Expanding portal functionality for caregiver use) and the specific challenges to caregiver portal engagement (Barriers to caregiver enrollment and use; Privacy and security in the caregiver-providerpatient triad). The table shows exemplar quotes.

CONCLUSIONS: Providers recognized the potential for portals to improve information delivery and communication - helping caregivers assist socially and medically complex patients in the safety-net. Further research is needed on assessing and supporting caregivers' interest and ability to access portals across health/digital literacy and English proficiency. Health systems must also address specific strategies to uphold patient preferences while maintaining privacy and security.

\section{ANALYSIS OF VISIT FACTORS ASSOCIATED WITH COLOREC- TAL CANCER SCREENING IN AN ACADEMIC OUTPATIENT CARE CENTER}

Kajali Mishra ${ }^{1}$; David E. Willens ${ }^{2}$; Danielle L. Heidemann ${ }^{2} .{ }^{1}$ Henry Ford Hospital, Detroit, MI; ${ }^{2}$ Henry Ford Health System, Detroit, MI. (Control ID \#3185949)

BACKGROUND: Colorectal cancer (CRC) is the third most commonly diagnosed malignancy and the second leading cause of cancer related death in the United States. CRC screening recommendations by all major organizations allow for either the use of stool-based tests such as fecal immunochemical test (FIT), fecal occult blood testing (FOBT) and multitargeted stool DNA (such as Cologuard) or visual tests such as Colonoscopy, CT Colonography, and flexible sigmoidoscopy. Prior literature has shown that screening services are inconsistently delivered across practice settings and continue to be underutilized. Most insurance cover preventative visits to accomplish screening goals and offer services in form of outreach, however, data is lacking on effectiveness of these visits. Likewise, interventions to increase CRC screening uptake have focused on modifying provider attitudes, although, studies have not addressed provider level and gender as a potential factor. Our primary objective was to assess the frequency and type of CRC screening offered by primary care doctors. Our secondary objective was to assess how type of visit and provider factors affect if screening was offered and the type of screening offered.

METHODS: A retrospective chart review of 2196 patients who were seen in an outpatient academic tertiary care center was performed. Patients between the ages 50-75 years old who had a primary care doctor and were seen in clinic between July 1, 2017 and July 31, 2018 were assessed. Screening offered was defined as either stool tests (FIT, FOBT and Cologuard) or visual tests (Colonoscopy and CT colonography).

RESULTS: A total of 2196 patients met our criteria. The mean age was 62.7 years; body mass index (BMI), $31.1 \mathrm{~kg} / \mathrm{m} 2$; females, $54.7 \%$. Cohort was divided into Group A (62\%), comprising of people who did not have CRC screening ordered and Group B (38\%), who had one or more CRC screening tests ordered. In Group B, some patients had more than one test ordered: $83.1 \%$ had Colonoscopy ordered, $10.7 \%$ had Cologuard ordered, $13.5 \%$ had FIT/FOBT ordered. The rate of colonoscopy completion was $14.7 \%$, and stool testing completion was 34-37.5\%. Out of 739 visits during which CRC screening was implemented, $90 \%$ were office visits. Surprisingly, having a preventative visit was not associated with an increased likelihood of having $\mathrm{CRC}$ screening test ordered $(\mathrm{p}=0.91)$. Among patients who had either test ordered, $65.6 \%$ of Colonoscopies or $48.4 \%$ of Stool tests were ordered by residents $(\mathrm{p}<0.001)$.

CONCLUSIONS: A significant amount of patients (62.3\%) that were seen by their primary care doctor had no CRC screening ordered. Having a wellness visit was not associated with having CRC screening tests ordered. Although colonoscopy was the most commonly ordered screening test, compliance to stool testing was twice as that of colonoscopy. Residents as provider were more likely to order colonoscopy compared to senior staff.

\section{ANEMIA PROFILE IN HEART FAILURE: HIGH PREVALENCE OF IRON DEFICIENCY AND SCOPE FOR CORRECTION. A PER- FORMANCE IMPROVEMENT PROJECT}

Radhika Gali $^{1}$; Doris Yang ${ }^{2}$; Aiman Zafar ${ }^{1}$; Beshoy Y. Adly ${ }^{3}$; Christina Elzahaby $^{3}$; Fatima Sarwar ${ }^{3}$; Hamza Ahsan ${ }^{3}$; Huma Ahmed ${ }^{3}$; Lakshmi Priyanka Mahali ${ }^{1}$; Mohamed Gabr $^{3}$; Nikita Fernandes ${ }^{1}$; Pooja Hebbal ${ }^{4}$; Romaisa Wadood ${ }^{3}$; Saliha Saleem ${ }^{1}$; Saketh Parsi ${ }^{3}$; Soumya Mamidi ${ }^{2}$; Sumaira Zareef ${ }^{3}$; Swathi Pabba ${ }^{1}$; Usman Ghani ${ }^{3}$; Dharmarajan S. Thiruvinvamalai ${ }^{5}$. ${ }^{1}$ Montefiore Medical Center, Bronx, NY; ${ }^{2}$ Montefiore medical center, Bronx, NY; ${ }^{3}$ Montefiore Medical Center/Albert Einstein College Of Medicine., Bronx, NY; ${ }^{4}$ MONTEFIORE MEDICAL CENTER, BRONX, NY; ${ }^{5}$ Montefioe Medical Center (Wakefield Campus), Bronx, NY. (Control ID \#3185676)

BACKGROUND: In the United States, Congestive Heart Failure (HF) is a common reason for hospitalization in those aged over 65 years. Modifiable or addressable risk factors for HF include anemia, chronic kidney disease (CKD), hypertension, obesity, diabetes, arrhythmias, hypothyroidism and others. Anemia is a prognostic indicator, associated with decreased functional capacity, reduced quality of life and worsened outcomes in those with HF.

METHODS: A resident team was trained to provide education to hospitalized HF patients regarding lifestyle, diet, fluids, medication adherence and more. The program participated in the CONNECT-HF multicenter trial, a PI project to track and prevent re-admissions within a month. A tool helped document data on demographics and comorbidity. 139 patients hospitalized with HF (September 2017 - December 2018) were identified; clinical profile and health outcomes were analyzed. Anemia was defined as $\mathrm{Hb}<12 \mathrm{~g} / \mathrm{dl}$ in females and $<13 \mathrm{~g} / \mathrm{dl}$ in males (WHO criteria). Our tool identified anemia evaluation (serum iron, ferritin, iron binding capacity, vitamins B12, folate and renal function), to enable appropriate management where possible.

RESULTS: Mean patient age: 70 years. Most hospitalizations occurred for age group 65 years (61\%), with 52\% males. 99 of 139 patients were anemic, mean $\mathrm{Hb} 11.4 \mathrm{~g} / \mathrm{dl}$. Of the 99 with anemia, $42 \%$ had iron deficiency anemia (IDA), $17 \%$ had anemia of chronic disease (ACD) and $30 \%$ had not undergone evaluation. Of those anemic, $41 \%$ had diastolic $\mathrm{HF}$ and $59 \%$ had systolic $\mathrm{HF} ; 32 \%$ had diabetes; $48 \%$ had CKD. Among those anemic, $4 \%$ had 3 or more co-morbidities (e.g. CKD, diabetes, cognitive impairment, etc). Of those with anemia and HF, $26 \%$ were re-hospitalized, whereas the readmission rate in those with HF without anemia was $22 \%$ ). Among those with irondeficiency, the readmission rate was $14 \%$. Those with anemia had more unplanned readmissions and longer inpatient stays (avr: 9.8 days, SD: 15), versus HF patients without anemia (avr. 6.4 days (SD: 4.33), ( P-value: 0.9). 
CONCLUSIONS: Our PI project brought awareness on the high prevlanence of anemia $(71 \%)$ in patients with heart failure. Iron deficiency was present in $42 \%$ of HF cases with anemia, but not statistically significant with longer hospital stay and re-admission rates. Anemia of iron deficiency in $\mathrm{HF}$ can be easily treated with oral or preferably intravenous iron, resulting in better outcomes (exercise performance, HF symptoms and quality of life). Going forward, our project highlights the importance of routinely evaluating patients with $\mathrm{HF}$ and anemia for iron deficiency anemia to consider corrective therapy. Additionally, evaluation for etiology of iron deficiency should be instituted

\section{ANTIBIOTIC AND ANTIVIRAL PRESCRIBING FOR HOSPITAL- IZED PATIENTS WITH RESPIRATORY VIRUSES}

Abhishek Deshpande; Pei-Chun Yu; Peter B. Imrey; Michael B. Rothberg. Cleveland Clinic, Cleveland, OH. (Control ID \#3185408)

BACKGROUND: Suspected respiratory infections are the leading cause of antibiotic prescription among hospitalized patients. A significant proportion of pneumonias are caused by viruses, yet it's unclear how often clinicians test for or treat viral pathogens in pneumonia patients. The objective of this study was to quantify the frequency of viral testing in hospitalized patients with pneumonia, measure the proportion with positive respiratory virus tests that are treated with antibiotics and antivirals and compare the outcomes of patients tested for suspected viral infection to those not tested.

METHODS: We included adult patients admitted with pneumonia from 2010 - 2015 to 175 US hospitals participating in the Premier Database and providing administrative and microbiological data. Pneumonia cases were identified via ICD9-CM codes as well as chest radiograph and antimicrobial treatment on the first hospital day. Patients were excluded if they had been transferred from another facility, had cystic fibrosis, or had a hospital length of stay (LOS) of 1 day. We also excluded patients with identical organisms in blood and urine. We compared characteristics, treatment and outcomes of patients who were tested for suspected viral infection to those not tested. Using logistic and linear regression models, as appropriate, we assessed the following outcomes; inpatient mortality, LOS and cost, adjusting for demographics, co-morbidities and treatments.

RESULTS: 178,895 patients met inclusion criteria. The overall viral testing rate increased from $10 \%$ in 2010 to $37 \%$ in 2015. In 2015, the viral testing rate varied by season from $65 \%$ in winter to $17.5 \%$ in summer. The rate also varied among hospitals, ranging from $10-72 \%$. Influenza virus was the most frequently tested (22.9\%) followed by respiratory syncytial virus (3.9\%), with a positivity rate of $10.6 \%$ and $2.1 \%$, respectively. Influenza virus accounted for $85 \%$ of the 5,318 patients with a positive viral test, followed by rhinovirus $(5.7 \%)$. Tested patients were younger (67 vs. 71 years), less likely to be admitted from SNF (6\% vs. $8 \%$ ) and had fewer comorbidities (2.9 vs. 3.4) (all comparisons $\mathrm{p}<0.001)$. On treatment day 3 , among patients with a positive viral test, $23 \%$ received antibiotics only, $15 \%$ antivirals only, and $56 \%$ received both. Compared to patients not tested, those tested were less likely to receive antibiotics $(79 \%$ vs. $89 \%, p<0.001)$. Viral testing was associated with reduced odds of in-hospital mortality; negative viral test (adjusted odds ratio $[\mathrm{aOR}] 0.80,95 \% \mathrm{CI} 0.76-84$ ) and positive viral test (aOR $0.71,95 \% \mathrm{CI}$ $0.62-84$ ). Testing was not significantly associated with LOS or cost.

CONCLUSIONS: In a large US inpatient sample hospitalized for pneumonia, only $37 \%$ of the patients were tested for viral pathogens in 2015 , but testing varied widely by hospital. Influenza testing was most common and most likely to be positive. Although a positive test was associated with antiviral treatment, it had a small impact on antibiotic prescribing.

ANTIHYPERTENSIVE TREATMENT TO LOWER CARDIOVASCULAR RISK AMONG POST-MENOPAUSAL WOMEN ON ESTROGEN REPLACEMENT THERAPY

Tran Nguyen; Talal Alzahrani; Jannet Lewis. George Washington University Hospital, Washington, DC. (Control ID \#3139875)
BACKGROUND: Approximately one in every three women between the ages of 40 to 59 , and two in every three women aged 60 and above in the United States have hypertension. For older women, estrogen is occasionally used to treat common menopausal symptoms, but increases the risk of cardiovascular events. Although the results of the original Antihypertensive and LipidLowering Treatment to Prevent Heart Attack (ALLHAT) trial showed that hydrochlorothiazide (HCTZ) diuretics are superior to calcium-channel blockers and angiotensin-converting enzyme inhibitors in preventing 1 or more major forms of cardiovascular disease (CVD), there are several reasons why physicians may prefer to avoid

HCTZ in postmenopausal women on estrogen. At least one study has shown that, among postmenopausal women, HCTZ was less well tolerated than moexipril and HCTZ is known to increase blood glucose while postmenopausal status by itself already increases risk of developing diabetes mellitus. The differences in CVD effects of the various first-step drugs specifically among post-menopausal women on estrogen therapy have not been studied and hence, will be investigated in this study.

METHODS: The data of the ALLHAT was utilized to examine the effects of amlodipine versus lisinopril compared to chlorthalidone in a subgroup of women on estrogen. These women were 55 years of age or older with hypertension and at least one other CVD risk factor from 623 North American centers $(n=2752)$. ANOVA test and student t-test were used to compare the baseline characteristics between subjects in each group of therapy. Hazard ratios and 95\% confidence intervals (CIs) were obtained from the Cox proportional hazards model to compare the clinical outcomes between the three antihypertensive groups. A composite endpoint of cardiovascular mortality and nonfatal myocardial infarction, stroke, coronary revascularization procedure, angina, heart failure, and peripheral vascular disease was used, as well as each CVD outcome individually and all-cause mortality. RESULTS: There were no significant differences in the baseline characteristics of the three treatment groups. Compared to chlorthalidone, the hazard ratios for the composite endpoint were 1.03 (95\% CI $0.94-1.13$, p-value $=0.55$ ) for amlodipine and 1.02 $(95 \%$ CI $0.93-1.12$, p-value $=0.64)$ for lisinopril. Likewise, hazard ratios for all-cause mortality as well as the individual CVD outcomes were not significantly different among either lisinopril or amlodipine compared to chlorthalidone.

CONCLUSIONS: Among women aged 55 years or above on estrogen, calcium channel blockers and angiotensin-converting enzyme inhibitors as initial antihypertensive therapy are associated with similar cardiovascular outcomes compared to thiazide diuretics. Therefore, for post-menopausal women on estrogen replacement therapy, physicians should not feel constrained to use HCTZ, but should instead choose initial anti-hypertensive medication taking into account individual patient's side effect profile and other comorbidities.

\section{APPOINTMENT TIMING AND OPIOID PRESCRIBING}

Michael L. Barnett ${ }^{1}$; Hannah Neprash ${ }^{2}$. ${ }^{1}$ Harvard T.H. Chan School of Public Health, Boston, MA; ${ }^{2}$ University of Minnesota School of Public Health, Minneapolis, MN. (Control ID \#3181483)

BACKGROUND: Rising opioid prescribing has been a major contributor to the national opioid crisis. Many observers have blamed rushed practice environments for high rates of opioid prescribing because opioids can be a "quick fix" for a visit where pain is a symptom. However, there is little evidence examining the magnitude of time pressure on prescribing decisions. We 
hypothesized that as the workday progresses and appointments run behind schedule, physicians may be more likely to prescribe opioids to simply "move on," which would be mitigated by scheduled breaks.

METHODS: Using data from athenahealth, a national electronic health record provider, we identified all previously scheduled primary care physician (PCP) appointments from 2013-14 for adult patients with no opioid prescriptions in prior 12 months. Our main outcome was opioid prescribing with secondary outcomes of physical therapy orders and nonsteroidal antiinflammatory drug (NSAID) prescriptions. We examined average within-physician changes in each outcome by the order of the appointment in the day (groups of 3 appointments from $1^{\text {st }}$ to $\left.21^{\text {st }}\right)$ and by appointment "lateness" (0-60 minutes late in 10minute increments). We also examined prescribing rates for visits immediately before and after scheduled breaks of 15 minutes or more. We used multivariable linear probability models adjusting for patient and visit characteristics and included physician fixed effects to control for time-invariant differences across physicians. RESULTS: Our sample included 408,579 primary care appointments with 2,653 PCPs. Several patient characteristics had statistically significant, but small differences by appointment order (e.g. patients seen in the first 6 appointments vs. the last 6 appointments of the day were less likely to have Medicaid insurance $4.8 \%$ vs. $5.3 \%, \mathrm{P}<0.001)$. The rate of opioid prescribing increased as the workday progressed (1st-3rd appointment: 4.2\% [95\% CI 4.1-4.3] vs. 19th-21st appointment: $5.1 \%$ [95\% CI 4.9-5.4], $\mathrm{P}<0.001$ ), a $22.5 \%$ relative increase. Opioid prescribing also increased as appointments ran behind, holding the time of the day constant $(4.3 \%$ vs. $5.6 \%$ for $0-9$ vs. $60+$ minutes late, $\mathrm{P}<0.001)$. Conversely, opioid prescribing fell after a scheduled break of more than 15 minutes, controlling for time of day $(5.0 \% \mathrm{vs}$. $4.7 \%$ before vs. after a break, $\mathrm{P}=0.006$ ). None of these relationships were present for physical therapy or NSAID prescribing.

CONCLUSIONS: In a large, national sample of primary care appointments, we found that within-provider opioid prescribing increased during the work day and with appointment lateness. Scheduled breaks mitigated this effect to a small degree. These results are consistent with the concept that time pressure, through multiple possible mechanisms, can influence physician decisionmaking. If similar patterns exist in other clinical scenarios, this phenomenon could have widespread relevance for public health and quality improvement efforts.

\section{APPROPRIATE DIAGNOSIS AND MANAGEMENT OF ACUTE CORONARY SYNDROME BY INTERNAL MEDI- CINE INTERNS: A NEEDS ASSESSMENT}

Akshai Subramanian; Patricia O'Sullivan; Lucas Zier. University of California, San Francisco, San Francisco, CA. (Control ID \#3181731)

BACKGROUND: Acute coronary syndrome (ACS) is common and associated with significant morbidity and mortality. We performed a needs assessment to determine the ability of internal medicine interns to accurately diagnose and manage ACS.

METHODS: We conducted a needs assessment using a test with open-ended questions that we administered to internal medicine interns at a large urban medical center four months into their training. We collected demographic data including completion of a cardiology rotation during residency. Using two cases (one demonstrating demand ischemia due to sepsis and one demonstrating ACS) we assessed interns' ability to diagnose ACS, differentiate ACS from other types of myocardial infarction (MI), manage these conditions with guideline-based therapies, and provide a pathophysiologic basis for each therapy. Ideal responses were determined by a consensus of two cardiologists resulting in a rubric with point values for correct responses. Three individuals scored a sample of responses to determine interrater reliability of the rubric. We descriptively analyzed the number of correct and incorrect responses. We used student's t-test and Fisher's exact test to determine differences between respondents who had completed cardiology rotations and those who had not. Incomplete assessments were excluded.

RESULTS: $81 \%(52 / 64)$ of learners completed the assessment. Interrater reliability for the scoring rubric was excellent (Cohen's for the 17 responses ranged from 0.74 to 1.0 ). The mean score was $49 \% \pm 19 \%$. In the demand ischemia case, $44 \%$ properly managed demand ischemia due to sepsis, $69 \%$ of respondents inappropriately treated ACS, and 10\% inappropriately managed both sepsis and ACS. In the ACS case, $15 \%$ of respondents provided complete guideline-directed medical therapy for ACS and $4 \%$ also provided accurate pathophysiologic basis for each therapy. Comparing respondents who had completed a prior cardiology rotation and those who had not, there were no significant differences between mean scores ( $53 \%$ vs. $46 \%, p>0.20)$, number of respondents properly managing demand ischemia $(\mathrm{p}>0.9)$, and number providing complete guideline-directed medical therapy for ACS ( $>00.9)$.

CONCLUSIONS: In our study, internal medicine interns performed poorly in appropriately diagnosing and managing ACS. Completion of a cardiology rotation was not associated with differences in these abilities. This suggests the need for improved educational methods to promote knowledge acquisition and retention when teaching ACS diagnosis and management.

\section{ARE RESIDENTS' TEST UTILIZATION PATTERNS ASSO- CIATED WITH THEIR COMMUNICATION SKILLS AND PATIENT CENTEREDNESS?}

Colleen Gillespie ${ }^{1}$; Eli Cahan ${ }^{3}$; Kathleen Hanley ${ }^{3}$; Andrew B. Wallach $^{2}$; Barbara Porter $^{3}$; Sondra Zabar ${ }^{3}$. ${ }^{1}$ NYU School of Medicine, Brooklyn, NY; ${ }^{2}$ Bellevue Hospital, New York, NY; ${ }^{3} \mathrm{NYU}$ School of Medicine, New York, NY. (Control ID \#3185214)

BACKGROUND: It is well documented that few ordered tests are "high value" a significant percentage of those ordered are "low-value." Residency offers an opportunity to teach high-value care and educational interventions to do so have been effective. However, the relationship between high-value care and residents' ability to communicate effectively with patients has not been explored. Ability to establish rapport, gather information effectively, and be patient-centered may impact residents' use of tests. We hypothesize that residents with poor skills in these areas may order tests less efficiently.

METHODS: Unannounced Standardized Patients (USPs) were introduced into residents' primary care clinics in a large urban, safety net hospital to portray 3 clinical scenarios: a well visit, a chief complaint of fatigue, and a diagnosis of asthma. Orders were extracted via chart review. Appropriateness of orders was determined by reference to United States Preventive Services Task Force (USPSTF) and clinical practice guidelines. Excessive tests were defined as not explicitly indicated for the scenario-indicated tests were the converse. Number of excessive and \% of indicated tests were calculated across the 3 visits for 48 residents. Communication skills in information gathering (5 items) and developing a relationship (6 items) and a patient-centeredness score (4 items: took a personal interest, answered all my questions) were computed as $\%$ of behaviorally anchored items rated as "well done" and included in regression models predicting test utilization. 
RESULTS: On average, residents ordered $15 \%$ of indicated tests (SD $9 \%, 0-38 \%$ ) across the 3 visits and a mean of 1.3 unnecessary tests (SD $1.7,0-6)$. In the regression model, the 3 skills explained $16 \%$ of variation in unnecessary tests $(\mathrm{p}=.047)$. Information gathering explained the greatest share $(8 \%, \mathrm{p}=.041)$. With all 3 variables in the model, patientcenteredness was positively associated with unnecessary tests (Std Be$\mathrm{ta}=.42, \mathrm{p}=.016$ ) and information gathering was negatively associated with unnecessary tests (Std Beta=-.34, p=.041). Mirroring these results, superutilizers ( 10 residents ordering $>=3$ excessive tests) had lower information gathering and relationship development scores than other residents (66\% vs $75 \%$ and $72 \%$ vs $76 \%$ ) but higher patient centeredness scores ( $80 \%$ vs $74 \%$ )-although differences were not significant.

CONCLUSIONS: Our findings suggest that information gathering skills may have a small influence on residents' ordering of excessive tests. Further research with larger samples (adequate power) will help clarify the effect sizes. If our results stand, interventions for high-value care should include information gathering skills and residency programs should continue to reinforce core communication skills training. In addition, our finding that patient centeredness was associated with ordering unnecessary tests suggests that residency programs could caution residents about conflating ordering of tests with patient-centeredness.

\section{ARE SAFETY NET AND SMALLER HOSPITALS BEING LEFT BEHIND UNDER NEW PAYMENT MODELS? IMPACT OF MEDICARE'S MANDATORY BUNDLED PAYMENT PROGRAM ACROSS DIFFERENT HOSPITAL GROUPS}

Michael L. Barnett ${ }^{1}$; Andrew Wilcock ${ }^{2}$; J. Michael McWilliams ${ }^{2}$; Arnold M. Epstein ${ }^{1}$; Ateev Mehrotra ${ }^{2} .{ }^{1}$ Harvard T.H. Chan School of Public Health, Boston, MA; ${ }^{2}$ Harvard Medical School, Boston, MA. (Control ID \#3184192)

BACKGROUND: In 2016, Medicare implemented the Comprehensive Care for Joint Replacement model (CJR), a national mandatory bundled payment model for lower extremity joint replacement (LEJR) in randomly selected metropolitan statistical areas (MSAs). Overall, intervention hospitals reduced spending by $3 \%$ vs. control hospitals with no impact on quality. However, there are concerns that hospitals with fewer resources, such as smaller hospitals and safety net hospitals serving a high proportion of vulnerable patients, cannot implement systematic changes needed to respond effectively to this new payment system.

METHODS: We conducted difference-in-differences analyses using Medicare claims from 2013-2017, comparing LEJR episodes in 75 MSAs randomized to mandatory participation in CJR (treatment MSAs) with episodes in 121 control MSAs, before vs. after CJR implementation. Our primary outcomes were institutional spending per LEJR episode (i.e. Medicare Part A), complication rates, the proportion of patients in the highest quartile of predicted spending (a measure of patient selection) and 90-day readmission rates. We categorized hospitals by what fraction of patients were on Medicaid or uninsured patients, as defined through Medicare's disproportionate share hospital (DSH) adjustment, number of beds and for-profit status. Within each subgroup of hospitals, we used linear regression models adjusted for beneficiary and procedure characteristics, and hospital fixed effects.

RESULTS: In 2013-2017 there were 280,161 LEJR procedures in treatment and 377,278 procedures in control MSAs. Hospitals with $<100$ beds had a smaller decline in institutional spending than those with $>250$ beds ( $-\$ 650$ or $2.7 \%$ vs. $-\$ 856$ or $3.1 \%$ relative decrease, $p<0.001$ ). Hospitals in the highest tertile of DSH percentage had a larger relative decline in institutional spending compared to hospitals in the lowest tertile $(-\$ 1,250$ or $4.1 \%$ vs. $-\$ 666$ or $2.8 \%$ relative decrease, $\mathrm{p}<0.001)$. For-profit hospitals had declines in savings twice as large as savings in non-profit hospitals $(-\$ 1,310$ or $5.2 \%$ vs. $-\$ 634$ or $2.4 \%$ relative decrease, $\mathrm{p}<0.001)$. There was no change or slight decreases in complications or 90- day readmissions across hospital subgroups. In addition, there was no statistically significant change in the proportion of patients with LEJR procedures in the highest quartile of predicted spending in any subgroup. CONCLUSIONS: We found little evidence that smaller or safety net hospitals were unable to respond to the bundled payment programs. The difference between the performance of smaller vs. larger hospitals was modest while safety net hospitals had larger spending reductions vs. other hospitals with no apparent negative impact on quality. Despite concerns that Medicare's payment reforms could negatively impact smaller or safety-net hospitals, we found little evidence that this occurred within the CJR program. These results may not generalize beyond LEJR procedures and should be replicated with other payment reforms.

\section{ASSESSING DOCUMENTATION OF CLINICAL REASONING IN ADMISSION NOTES OF PHYSICIANS WORKING IN HOS- PITAL MEDICINE}

Susrutha Kotwal $^{1}$; David Klimpl ${ }^{1}$; Sean Tackett ${ }^{2}$; Regina Landis ${ }^{3}$; Scott Wright ${ }^{1}$. Johns Hopkins University School of Medicine, Baltimore, MD; ${ }^{2}$ Johns Hopkins Bayview Medical Center, Baltimore, MD; ${ }^{3}$ Johns Hopkins, Baltimore, MD. (Control ID \#3140154)

BACKGROUND: High quality clinical documentation is essential for patient safety. Thoughtful clinical documentation transmits one's clinical reasoning and is considered to be a professional responsibility. There are no accepted standards for assessing documentation with respect to clinical reasoning. We therefore undertook this study to establish a metric to evaluate hospitalists' documentation of clinical reasoning in the assessment and plan (A\&Ps) section of admission notes.

METHODS: This was a retrospective study reviewing admission notes of hospitalists at 3 hospitals between January 2014 and October 2017. Admission notes were included for patients hospitalized with a diagnosis of either fever, syncope/dizziness, or abdominal pain. A total of 1130 admission notes were identified randomly; notes were excluded if they were not on the hospitalists' service, or if the diagnosis had been confirmed in the emergency department. So as to sample the notes of many providers, no more than 3 notes written by any single provider was analyzed. We developed the 'Clinical Reasoning in Admission Notes Assessment \& PLan' (CRANAPL) tool to assess the comprehensiveness of clinical reasoning documented in the A\&Ps of admission notes. The tool was iteratively revised during pilot testing; ultimately two authors scored all A\&Ps using the finalized version of the CRANAPL tool. These authors also assessed each A\&P using single item broad ratings: a 'global clinical reasoning' and a 'global readability/clarity' measure. All data were deidentified prior to scoring. Content, internal structure, and relation to other variables validity evidence were established.

RESULTS: The total number of hospitalists whose admission notes were evaluated was 120. 285 admission notes were reviewed across one community and two academic hospitals. The nine-item CRANAPL rubric includes elements for problem representation, uncertainty, differential diagnosis and plan, as well as items related to length of stay and disposition plans. The mean score for both raters for the total CRANAPL score was 6.4 (SD 2.2), and it varied significantly between hospital sites $(p<0.001)$. The ICC measuring inter-rater reliability for both raters for the total CRANAPL score was 0.83 (95\% CI 0.76-0.87). Associations between CRANAPL total scores, global clinical reasoning, and global score for note readability/clarity were statistically significant $(p<0.001)$. In multivariate regressions after adjusting for covariates, higher scores on the CRANAPL tool were seen among American Medical Graduates as compared to International Medical Graduates $(\mathrm{p}<0.05)$.

CONCLUSIONS: This study represents a first step to characterize clinical reasoning documentation in Hospital Medicine with real patient notes. With validity evidence established for the CRANAPL rubric, it can be used to assess the documentation of clinical reasoning skills of hospitalists and for providing feedback. This is an essential step to improve the diagnostic process and reduce medical errors. 


\section{ASSESSING ENGAGEMENT IN USER-CENTERED DESIGN OF A TEXT MESSAGING APP IN A DIVERSE, SAFETY-NET POP- ULATION}

Sarah Nouri ${ }^{1}$; Patricia Avila-Garcia ${ }^{2}$; Anupama Cemballi ${ }^{1}$; Urmimala Sarkar $^{1}$; Adrian Aguilera ${ }^{2}$; Courtney Lyles ${ }^{1} .{ }^{1}$ University of California, San Francisco, San Francisco, CA; ${ }^{2}$ University of California, Berkeley, Berkeley, CA. (Control ID \#3184645)

BACKGROUND: Healthcare systems are rapidly deploying digital tools with the goal of improving chronic disease management, yet few studies evaluate their usability by vulnerable populations. To understand barriers to lower rates of app use among low-income patients, we employed usercentered design (UCD) methods in the development of a new text messaging app. We describe here variations in engagement in design process, with specific attention on patients with limited health literacy (LHL), limited English proficiency (LEP), and limited digital literacy (LDL).

METHODS: We conducted 20 in-person, in-depth, semi-structured interviews in English or Spanish with primary care patients at a safety-net healthcare system. We used open-ended interviews and card sorting tasks to seek input about mobile phones and prioritize text messaging for health behaviors. We used open coding to (1) categorize mobile phone use and (2) evaluate engagement in the interview process. Engagement was defined by the frequency and quality of (1) feedback on existing text message content and (2) novel suggestions for text messages. Based on mobile phone use, we identified participants with LDL; we then quantitatively examined mobile phone use by LHL and LEP. We also evaluated engagement by LHL, LEP, and LDL with Wilcoxon rank sum tests.

RESULTS: Participants were $59( \pm 8)$ years old; $65 \%$ women, $90 \%$ nonwhite, $80 \%$ with limited LHL, $65 \%$ with LEP. All had depression and $70 \%$ had diabetes. Mobile phone use: Major themes related to texting networks and difficulty with basic (text messaging) and advanced (step tracking, location sharing) features of mobile phones. Nearly all had smartphones and regularly used text messaging, but 16/20 reported difficulty with text messaging due to inability to type, physical disability and low literacy. We identified 8 participants as having LDL; 7/8 also had LEP and/or LHL. Feedback: Quality of feedback ranged from yes/no answers to explanations of why a text message was liked or disliked. Frequency of comments was lower in all subgroups, with a significant difference between those with LDL vs adequate digital literacy $(\mathrm{p}=0.045)$. Novel suggestions: Quality of suggestions ranged from text messages that had already been presented to ideas for structural changes to the app. Frequency of suggestions made was lower in those with LEP or LDL ( $\mathrm{p}=0.02$ and 0.008 , respectively); there was no difference by HL.

CONCLUSIONS: Despite widespread mobile phone use, digital literacy barriers are common among vulnerable populations. Engagement was qualitatively and quantitatively lower among those with LHL, LEP, and LDL, suggesting traditional UCD methods (open-ended hypothetical interviews, card sorting tasks) may be less effective for those with literacy or language barriers. Different methods in UCD, such as direct observation, may be more productive in eliciting feedback and matching interventions with end users' needs and preferences.

\section{ASSESSING INTERPROFESSIONAL TEAM FUNCTIONING VIA DIRECT OBSERVATION IN A PRIMARY ARE SETTING} Alana Doonachar ${ }^{2}$; Lyndonna M. Marrast ${ }^{1}$; Aubrey Rogers ${ }^{3}$; Daniel J. Coletti ${ }^{4}{ }^{1}$ Zucker School of Medicine at Hofstra/Northwell, Kew Gardens, NY $;{ }^{2}$ Hofstra University, Hempstead, NY $;{ }^{3}$ Zucker School of Medicine at Hofstra/Northwell, Hempstead, NY; ${ }^{4}$ Northwell Health, Great Neck, NY. (Control ID \#3185574)

BACKGROUND: Improving Patient Access, Care, and Cost through Training (IMPACcT) is an interprofessional (IP) training program developed to enhance primary care training through an interprofessional team approach. Maintaining collaborative and efficient team interactions is essential to delivering quality care to our underserved patients. We sought to systematically review and evaluate the IP process, describe team dynamics, and assess adherence to clinical workflows including: 1) presession huddles; 2) trainee precepting processes, and 3) patient interactions.

METHODS: From March to July of 2018, four raters silently observed IMPACcT clinical sessions and rated behaviors using a tailored version of the Modified McMaster-Ottawa Scale: Team Assessment form. Raters completed quantitative assessments of team-based competency attainment in the following areas: patient communication, team collaboration, role clarification, collaborative patient-family centered approach, conflict management, and (overall) team functioning. Interactions were rated as to "below expected", "at expected" or "above expected" levels of quality. Raters also recorded field notes to add context to the ratings.

RESULTS: A total of 233 team assessment forms were completed. The majority of the observation were made of precepting events (67\%), compared to huddle ratings (14\%) and observations of patient interactions (19\%). The average huddle duration was 23 minutes and included an average of 6 IP team members. Examination of ratings indicated that $70 \%$ of the assessments were "at expected" competency level with $27 \%$ exceeding expectations. When assessing members' role and responsibilities, the results showed that $52 \%$ of the observations were at expected levels and $43 \%$ were above expected levels of competency. With regards to conflict management/resolution and team functioning capabilities, both competencies scored over $95 \%$ in meeting expectations and going above expected levels. In contrast, teams were less likely to promote a familypatient centered approach: only $43 \%$ of the observations demonstrated "at expected" levels and $30 \%$ of the assessments were rated as "below expected levels." Overall the global rating score of team assessment was $71 \%$ at expected and $28 \%$ above expected proficiencies.

CONCLUSIONS: We observed high levels of IP team functioning in a majority of observed domains during team huddles, precepting, and patient interactions. Differences in observations were expected given there is one daily huddle, but multiple precepting events and patient encounters. Results suggest the need to consider ways to incorporate more of a family-systems framework for considering patient strengths and needs. High competency ratings overall, however, are one indicator of success in our faculty development and learner training efforts in teambased care. Future plans include conducting "360 degree" evaluations to permit faculty and trainees to reflect on team processes interactively and engage in self-ratings of team effectiveness.

\section{ASSESSING KNOWLEDGE, PERCEIVED SUSCEPTIBILITY, SEVERITY, BENEFITS, BARRIERS, AND WILLINGNESS OF COLORECTAL CANCER SCREENING IN ARMENIA.}

Nika M. Harutyunyan ${ }^{1}$; Evelyn Abrami $^{2}$; Taron Torosian ${ }^{3}$; Shant Shekherdimian $^{4}$; Marine Hovhannisyan ${ }^{5}$; Armen Aboulian ${ }^{6}$; Hovhannes Hovhannisyan ${ }^{5}$; Garegin Dallaqyan ${ }^{5} .{ }^{1}$ UCLA, Sherman Oaks, CA; ${ }^{2}$ USC, Los angeles, CA; ${ }^{3}$ USC, Los Angeles, CA; ${ }^{4}$ UCLA, Los Angeles, CA; ${ }^{5}$ Yerevan State Medical University, Yerevan, Armenia; ${ }^{6}$ Kaiser, Los Angeles, CA. (Control ID \#3179997)

BACKGROUND: Colorectal cancer screening (CRCS) has been shown to be cost-effective and life-saving. To date, no organized screening programs for colorectal cancer (CRC) exist in Armenia. Furthermore, little is known about the population's knowledge and attitudes towards CRCS. The purpose of this study is to evaluate knowledge of CRC and CRCS, willingness to participate in various CRCS modalities and barriers to implementation of a successful CRCS program.

METHODS: Following IRB approval, previously validated survey questions using the Health Belief Model were translated and modified for local relevance. Convenience sampling was used at a representative number of outpatient clinics throughout the capital city of Yerevan. Surveys were administered to consenting adults between the ages of 40-65. 
RESULTS: Of 368 respondents (54\% female), $84 \%$ reported having knowledge of CRC, however only $22 \%$ percent had heard of tests that can detect CRC. Despite this, $91 \%$ of respondents felt that getting checked regularly increases the chances of finding CRC early, and that early detection leads to "excellent" or "good" outcomes. 19\% of respondents $(n=68)$ have worried about the possibility of having CRC, and of those, only half $(\mathrm{n}=35)$ sought the consultation of a physician regarding this concern. When asked whether they would be afraid of CRCS, 46\% answered "yes" $(n=87)$ or "unsure" $(n=81)$. The most common reasons for not undergoing screening were "I don't think it is necessary" $(n=221)$, "I didn't know I should get it" ( $n=166)$, "If I don't have symptoms I won't pursue it" $(\mathrm{n}=156)$, "I will do it later" $(\mathrm{n}=156)$ and "Screening and treatment costs would be too much" ( $\mathrm{n}=153)$. Only $7 \%$ of those surveyed stated that a provider has ever mentioned CRCS screening to them, but $76 \%$ were willing to undergo CRCS if their doctor recommended it. There was no strong preference towards any screening modality, however $78 \%$ would only undergo screening if free $(n=189)$ or less than $10,000 \mathrm{AMD} /$ $\sim$ 20USD $(n=96)$.

CONCLUSIONS: Despite the high self-reported familiarity with CRC, knowledge of CRCS in Armenia remains low. While most respondents were willing to undergo CRCS if they were advised to do so, significant barriers to voluntary screening exist. These findings suggest that targeted interventions to directly increase awareness of CRC and CRCS among the population, encouraging healthcare providers to discuss CRCS with patients, and keeping out-of-pocket costs for CRCS and CRC treatment at a minimum should be considered when designing organized CRCS in Armenia.

\section{ASSESSING THE ASSOCIATION OF CRIMINAL JUSTICE CON- TACT WITH WELLBEING}

Ram Sundaresh ${ }^{1}$; Brita Roy ${ }^{1}$; Youngmin $\mathrm{Yi}^{2}$; Carley Riley ${ }^{3}{ }^{4}$; Christopher Wildeman ${ }^{2}$; Emily A. Wang ${ }^{1}$. ${ }^{1}$ Yale School of Medicine, New Haven, CT; ${ }^{2}$ Cornell University, Ithaca, NY; ${ }^{3}$ University of Cincinnati College of Medicine, Cincinnati, OH; ${ }^{4}$ Cincinnati Children's Hospital Medical Center, Cincinnati, OH. (Control ID \#3185988)

BACKGROUND: Research has shown that mass incarceration has wide reaching effects on health and mortality. However, the impact of mass incarceration on broader emotional and social wellbeing has not been closely studied. The aim of this study is to assess how exposure to the criminal justice (CJ) system is associated with wellbeing.

METHODS: We used data from the 2018 FamHIS study, a nationally representative cross-sectional study of incarceration among family members ( $\mathrm{N}=2815)$. Of 4,401 who completed the screening about family incarceration, 1,806 individuals with- and 1,009 without- family incarceration experience were included in the study. CJ exposure was classified by each individual's highest level of personal CJ contact: none, stopped by police, stopped and frisked, arrested, incarcerated, or incarcerated multiple times. We measured wellbeing using the 100 Million Healthier Lives Adult Wellbeing Assessment, a set of validated items that measures thriving or suffering within five domains of wellbeing: physical health, mental health, social wellbeing, spiritual wellbeing, and overall life evaluation. We report frequencies of thriving and suffering at each level of CJ contact, and use logistic regression to compare wellbeing across CJ exposure levels, controlling for confounding by age, gender, race, income, housing type, employment status, education, and marital status.

RESULTS: Compared to those without personal CJ exposure ( $\mathrm{N}=1,575)$, any $\mathrm{CJ}$ exposure $(\mathrm{N}=1,229)$ was associated with lower rates of thriving $(74 \%$ vs $61 \%, \mathrm{p}<0.001)$ and higher rates of suffering ( $4 \%$ vs $8 \%, p=<0.001)$. Across all five domains of wellbeing, there was a progressive decrease in thriving and increase in suffering with increasing exposure to the system (table, $p<0.001$ for each trend). In adjusted models, a strongly decreased odds of overall thriving was only seen in those with any police stop (AOR 0.59, 95\% CI 0.41 - 0.87 ) or any incarceration (AOR $0.59,95 \%$ CI $0.4-0.85$ ), compared to those without CJ exposure.
CONCLUSIONS: Increasing exposure to the criminal justice system is associated with a dose dependent decrease in wellbeing in every domain. This highlights the need for clinical interventions in diversion and CJ reform to not only focus on incarceration, but also on reducing police contact to achieve population-level improvements in health.

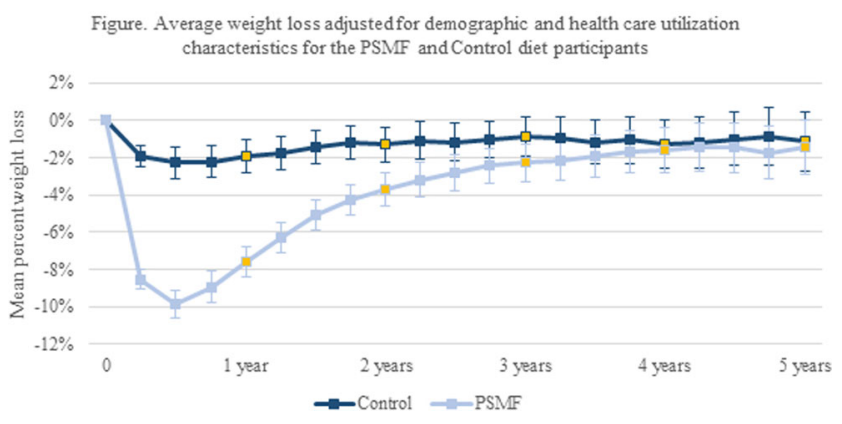

ASSESSING WHEN TO STOP OR SCALE BACK ROUTINE MEDICAL SERVICES: THE ASSURES STUDY

Eve A. Kerr ${ }^{1,2}$; Mandi L. Klamerus ${ }^{1}$; Adam Markovitz ${ }^{1,2}$; Tanner Caverly ${ }^{1}$, ${ }^{2}$; Jeremy Sussman ${ }^{1,2}$; Sameer D. Saini ${ }^{1,2}$; Lillian Min ${ }^{1,}{ }^{2}$; Steven J. Bernstein $^{2}$; Roger $\mathrm{Chou}^{3}$; Shannon E. Lohman ${ }^{1,4}$; Whit Froehlich ${ }^{1,2}$; Sarah E. Skurla ${ }^{1}$; David E. Goodrich1; TImothy Hofer ${ }^{1,2}$. ${ }^{1}$ VA Center for Clinical Management Research, Ann Arbor, MI; ${ }^{2}$ University of Michigan, Ann Arbor, MI; ${ }^{3}$ Oregon Health and Science University, Portland, OR; ${ }^{4}$ Wayne State University, Detroit, MI. (Control ID \#3184286)

BACKGROUND: Recommendations in current guidelines primarily focus on intensifying medical services. While Choosing Wisely (CW) has encouraged specialty groups to develop recommendations about overuse, to date no one has developed a systematic approach to identifying and validating recommendations for stopping or scaling back (de-intensifying) tests or treatments. We designed such an approach and applied it to a set of common ambulatory conditions.

METHODS: The identification of candidate recommendations for deintensification began with a systematic scan of guidelines/recommendations developed by selected specialty societies, the USPSTF, and CW. We limited our search to the following common clinical areas in routine ambulatory primary care: prevention and treatment of cardiovascular disease (CVD) and diabetes (DM), screening (non-CVD), and inappropriate medication use in adults. Each candidate recommendation was reviewed by physician investigators to: 1) verify it related to de-intensification and was relevant to routine primary care; 2) assess whether it would likely improve outcomes, impact patients, and be feasible to measure. An external advisory council prioritized the resultant recommendations. The prioritized recommendations, and supporting evidence, were considered by two multidisciplinary expert panels of physicians. Panel 1 (N=13 members) had expertise in CVD and DM; Panel $2(\mathrm{~N}=12$ members) had expertise in screening and medication use. We adapted the RAND/UCLA Appropriateness Method of two rounds of ratings with discussion between by employing virtual group participation via an internet collaboration tool plus teleconferences. The primary rating criterion was validity $(1-9 ; 9=$ recommendation definitely based on high-quality scientific evidence). The recommendations were classified according to the panel's median final rating and level of disagreement among panelists.

RESULTS: The systematic scan resulted in 947 potentially relevant recommendations. 538 were excluded by physician investigators as not meeting study criteria. 409 were grouped into 178 similar recommendations, with 37 prioritized by the advisory council. Following refinements to the recommendations, the two expert panels rated 44 de-intensification recommendations. $37(84 \%)$ were rated as valid (median validity rating between 7-9) without substantial disagreement. Recommendations classified as appropriate 
included: CVD (N=10; e.g., stop carotid artery stenosis screening in asymptomatic patients); $\mathrm{DM}(\mathrm{N}=4$; e.g., reduce $\mathrm{DM}$ medications in patients at highrisk for hypoglycemia); screening (non- CVD) $(\mathrm{N}=13$, e.g., don't screen for breast cancer in women with a limited life expectancy); and medication use ( $\mathrm{N}=10$; e.g., decrease benzodiazepines in older adults).

CONCLUSIONS: Recommendations for de-intensifying medical care can be systematically developed and validated using evidence curated by existing guideline efforts. These findings set the stage for planning implementation approaches to stop or scale back unnecessary services.

\section{ASSESSMENT OF INTERNAL MEDICINE RESIDENT PRE- PAREDNESS TO CARE FOR LESBIAN, GAY, BISEXUAL, TRANS- GENDER, AND QUEER/QUESTIONING PATIENTS}

Carl G. Streed ${ }^{1}$; Helene F. Hedian ${ }^{2}$; Amanda Bertram²; Stephen Sisson ${ }^{2}$. ${ }^{\mathrm{B}}$ Boston Medical Center, Boston, MA; ${ }^{2}$ Johns Hopkins University, Lutherville, MD. (Control ID \#3148706)

BACKGROUND: Recognizing the unique health needs of sexual and gender minorities (i.e. lesbian, gay, bisexual, transgender, queer/questioning individuals) is critical to providing competent and comprehensive health care. We aimed to assess resident knowledge of health care issues uniquely affecting sexual and gender minorities as well as the role of online casebased didactics to measure and improve knowledge in the diagnosis and treatment of these patients.

METHODS: We designed a multicenter online education intervention from December 2016 to April 2018. The study population consisted of 833 PGY1-3 residents at 120 internal medicine residency programs in the United States who completed 1018 tests. We developed a one-hour online module addressing sexual and gender minority (SGM) health. The test evaluated each resident in four categories: 1) terminology relevant to SGM patients; 2) health disparities and preventive care issues affecting SGM patients; 3) substance use and mental health issues unique to SGM patients; and 4) common sexually transmitted illnesses affecting SGM populations. Participants completed a pretest assessing sexual and gender minority (SGM) health knowledge. A didactic module reviewing diagnosis and management of these diseases was then completed, followed by a posttest.

RESULTS: Among 1018 resident responses, there was no difference between post-graduate year pre-test performance (PGY-1 52\%, PGY$250 \%$, PGY-3 51\%; p = 0.532) or post-test performance (PGY-1 $80 \%$, PGY-2 82\%, PGY-3 82\%; p =0.285). Pre-test and post-test performance of an online didactic module was the same across test categories and patient populations for PGY-1 vs. PGY-2 vs. PGY-3. Residents demonstrated an improvement between pre- and post-test knowledge.

CONCLUSIONS: Baseline knowledge of health issues of sexual and gender minorities, as assessed by pre-test performance, did not change during residency training. An online didactic module introduced trainees to critical issues regarding the care of these vulnerable populations until such curricula are required in training. Health disparities in LGBTQ communities may improve with improved physician training on clinical care of LGBTQ patients and families.

\section{ASSOCIATION BETWEEN ANTIBIOTIC USE AND ACUTE KIDNEY INJURY IN PATIENTS HOSPITALIZED WITH COMMUNITY-ACQUIRED PNEUMONIA}

${ }_{\text {Phuc H. Le }}^{1}$; Pei-Chun Yu ${ }^{1}$; Preethi Patel ${ }^{2}$; Andrei Brateanu ${ }^{2}$; Peter Imrey ${ }^{1}$; Michael B. Rothberg ${ }^{1}{ }^{1}$ Cleveland Clinic, Cleveland, $\mathrm{OH}$; ${ }^{2} \mathrm{CCF}$, Cleveland, OH. (Control ID \#3185606)

BACKGROUND: Acute kidney injury (AKI) is common during hospitalization for community-acquired pneumonia (CAP) and may be associated with specific antibiotics. The risk of AKI associated with vancomycin and piperacillin-tazobactam have been reported, but the association with other antibiotic classes is unknown. We aimed to estimate and compare the risk of AKI for various antibiotic combinations in a large sample of adults hospitalized for CAP.

METHODS: The study was a retrospective cohort analysis of the Premier Healthcare Database containing all admissions for 660 US hospitals from 2010-2015. We included adults aged 18 years hospitalized with CAP, defined as either a principal ICD-9 code of pneumonia or a principal diagnosis of sepsis or respiratory failure and a secondary code of pneumonia. We considered 8 different antibiotic combinations based on continuous use in the first 3 hospital days. Primary outcome was AKI, defined by ICD-9 codes 584.X. We excluded patients who were admitted to the ICU any time during hospitalization, had AKI codes documented on admission, or had dialysis in the first 2 days. We used mixed logistic regression with hospital as a random effect and adjusted for patient demographics, comorbidities, other treatments on day $0 / 1$, and hospital characteristics.

RESULTS: The total sample included 418,408 patients, $1.2 \%-3.6 \%$ of whom developed AKI. Compared to those without AKI, patients with AKI were older and more likely to be male, Black, and have Medicare, sepsis or aspiration pneumonia as principal diagnosis, and immune suppression. Patients with AKI also had a higher rate of in-hospital dialysis $(2.4 \%$ vs. $0 \%)$, discharge to hospice $(8.6 \%$ vs. $4.1 \%$ ), longer median length of stay (7 vs. 4 days) and higher mortality $(12 \%$ vs. $2.4 \%)$. Compared to a macrolide plus $3^{\text {rd }}$ generation cephalosporin, all other combinations except quinolones were positively associated with developing AKI. The combination of pipperacillin/tazobactam and vancomycin with or without quinolones was associated with the highest odds ratio (OR, 95\% CI: 2.55 (2.29- 2.83) and 2.46 (2.23- 2.72) respectively).

CONCLUSIONS: Broad spectrum antibiotics were associated with a higher odds of developing AKI compared to narrow spectrum regimens. The choice of empiric regimens for patients hospitalized with CAP should take into account this potential complication.

\section{ASSOCIATION BETWEEN BURNOUT AND INTELLI- GENCE MINDSET IN INTERNAL MEDICINE TRAINING PHYSICIANS}

Justin Chen ${ }^{1}$; Angela Fink ${ }^{2}$; Regina F. Frey ${ }^{2}$; Erin Solomon ${ }^{2}$; Stephen Fuest ${ }^{1}$; Rakhee Bhayani ${ }^{1}$; Patricia Kao ${ }^{1}$. 1Washington University in St. Louis School of Medicine, St. Louis, MO; ${ }^{2}$ Washington University in St. Louis, St. Louis, MO. (Control ID \#3180399)

BACKGROUND: Carol Dweck's theory of intelligence differentiates learners as individuals who characterize intelligence as a trait that can be increased through effective practice and perseverance (growth mindset) or one that is immutable and pre-determined (fixed mindset). Studies show that curriculums promoting a growth mindset have improved the academic performance of elementary to college learners, but its effect on graduate medical education is still not defined. This study aims to characterize the intelligence mindset of internal medicine (IM) residents, identify demographic risk factors associated with intelligence mindset, and ascertain if growth mindset education reduces burnout scores.

METHODS: This is a randomized control trial to study a fourphase educational intervention for IM residents at Washington University in St. Louis School of Medicine/Barnes-Jewish Hospital from 6/2018-6/2019. IM residents were randomized to receive an educational intervention on general wellness (control) or growth mindset (experimental). Participants will complete a survey including demographics, burnout risk factors, burnout measured by the Maslach Burnout Inventory (MBI) subscales, and intelligence 
mindset measured by the Theories of Intelligence Scale (TIS) and Mindset Assessment Profile (MAP) before and after the intervention.

RESULTS: $55 \%$ (86/155) of all IM residents and 100\% (52/52) of postgraduate year one residents participated in the first phase of the study. The proportion of fixed, indeterminate, and growth mindset were respectively $30.2 \%, 10.5 \%$, and $59.3 \%$ by the TIS and $7 \%, 62.8 \%$, and $30.2 \%$ by the MAP. There is a significant negative correlation between MAP and both the MBI emotional exhaustion $(p=0.006)$ and depersonalization $(p=0.05)$ scores and a positive correlation between MAP and MBI personal achievement scores $(\mathrm{p}=<0.001)$. The TIS was found to have a positive correlation with MBI personal achievement scores $(\mathrm{p}=0.04)$. Among the postgraduate year one residents, individuals from underrepresented minority groups were more likely to have a fixed mindset using the TIS ( $\mathrm{p}=0.05$ ).

CONCLUSIONS: There are approximately twice as many IM resident physicians with a growth mindset compared to a fixed mindset using the TIS and only $30 \%$ of IM residents carry a growth mindset using the MAP. MAP scores showed that residents with growth mindsets tended to report less burnout reflected by less emotional exhaustion and depersonalization and a greater sense of personal achievement. TIS scores were less sensitive to these associations but yielded consistent results. This study identifies a population of physicians where a growth mindset education may reduce burnout rates. If this curriculum is effective in reducing burnout, it may also reduce the consequences of burnout on physician performance, patient satisfaction, and healthcare utilization.

\section{ASSOCIATION BETWEEN HIGH DEDUCTIBLE HEALTH PLANS AND COST-RELATED NON-ADHERENCE TO MEDICA- TIONS AMONG PEOPLE WITH DIABETES: A NATIONAL STUDY \\ John Gaudet $^{2}$; Charlotte Rastas ${ }^{1}$; Ariel Majidi ${ }^{1}$; Drew Bunker ${ }^{2}$; Jaeyoung Yang $^{1}$; Shirin Karimi ${ }^{1}$; Vikas Gampa ${ }^{2}$; Laura Trueman ${ }^{1}$; Adam Gaffney ${ }^{1}$; Gaurab Basu ${ }^{1}$; Danny McCormick ${ }^{1}{ }^{1}$ Cambridge Health Alliance, Harvard Medical School, Boston, MA; ${ }^{2}$ Cambridge Health Alliance, Cambridge, MA. (Control ID \#3185828)}

BACKGROUND: Prices of diabetes medications, particularly insulin, have risen substantially in recent years. High-deductible health plans (HDHP), which require high out of pocket payments before coverage begins, have recently become the predominant type of commercial insurance. However, the impact of HDHPs on cost-related medication nonadherence (CRNA) among adults with diabetes nationally is not known.

METHODS: We analyzed data from the 2011 to 2017 National Health Interview Survey, a nationally representative survey of non-institutionalized adults in the United States. The study sample included privately insured adults age 18-64 with diabetes who were enrolled in either a HDHP or a traditional commercial health plan (THP). CRNA was assessed with 4 questions that asked if an individual 1) could not afford medication, or 2) skipped doses3) took less medicine or 4) delayed filling a prescription, in order to save money. We assessed the association between enrollment in an HDHP (vs. a THP) and each individual measure of CRNA as well as a composite measure (any individual measure) using multivariable linear probability models that controlled for age, sex, family size, race/ethnicity, marital and health status, functional limitation, education, income and employment status. We also assessed whether any CRNA was associated with 1 or more emergency room visits or hospitalization in the past year. Analyses were stratified by whether the individual reported insulin use.

RESULTS: Baseline demographic and health status indicators were similar between the 4,325 THP and 2,270 HDHP enrollees. Among those not taking insulin, HDHP enrollees were more likely than THP enrollees to: not fill a prescription $(13.0 \%$ vs $9.1 \%$, respectively; adjusted percentage point difference (AD), 3.6 [95\% CI, 1.0- 6.1]); skip medication doses ( $10.6 \%$ vs $8.1 \%$; $\mathrm{AD}, 2.5$ [95\% CI, $0.1-4.9$ ]; and to have any type (combined measure) of CRNA (19.2\% vs 15.0\%; AD, 3.5 [95\% CI, 0.5 6.6]. Higher percentages of enrollees in HDHPs also took less medication or delayed filling a prescription but these differences did not reach statistical significance. Among those taking insulin, $18 \%$ of THP and $25.3 \%$ of HDHP enrollees had any type of CRNA (AD, 6.1 [95\% CI, 1.0 -11.2]). Higher percentages of enrollees in HDHPs (vs THP) also reported CRNA for each individual measure but these did not reach statistical significance. Among those taking insulin, those reporting any CRNA were more likely to have at least 1 emergency department visit $(44.1 \%$ vs $24.2 \%$, respectively; $\mathrm{AD}, 13.8$ [95\% CI, $6.6-21.0])$ or hospitalization in the last year $(28.1 \% \mathrm{vs}$ $16.3 \%, \mathrm{AD}, 7.1$ [95\% CI, $0.9-13.4]$ ).

CONCLUSIONS: Nationally, HDHPs are associated with greater CRNA among people with diabetes, particularly those taking insulin. Lowering or eliminating deductibles, and lowering the price of insulin, could reduce CRNA among people with diabetes, and potentially reduce adverse outcomes of CRNA.

\section{ASSOCIATION BETWEEN MORTALITY AND FREQUENCY AND LIFETIME SPENT INCARCERATED AMONG US VET- ERANS WITH HIV}

Laura Hawks $^{2,1}$; Kathleen A. McGinnis ${ }^{3}$; Benjamin A. Howell ${ }^{4}$; Maria R. Khan $^{5}$; E. Jennifer Edelman ${ }^{4}$; Amy C. Justice ${ }^{6}$; Emily A. Wang ${ }^{4} .{ }^{1}$ Harvard Medical School, Boston, MA; ${ }^{2}$ Cambridge Health Alliance, Cambridge, MA; ${ }^{3}$ VA Pittsburgh Healthcare System, West Haven, CT; ${ }^{4}$ Yale School of Medicine, New Haven, CT; ${ }^{5}$ New York University School of Medicine, New York, NY; ${ }^{6}$ Yale University, New Haven, CT. (Control ID \#3180733)

BACKGROUND: Exposure to incarceration is associated with greater risk of mortality, especially following release from correctional facilities. Few studies have examined whether frequency of incarceration or amount of time spent incarcerated over one's lifetime is associated with increasing mortality. We used data from the Veterans Aging Cohort Study (VACS) to compare mortality by intensity of incarceration exposure among individuals with HIV. METHODS: HIV+ participants who provided a response to incarceration questions in the VACS survey from January 2011 to August 2017 were included $(\mathrm{N}=2,718)$, and mortality data were available through August 2018. We calculated and compared mortality rates per 100 person years (pys) by three incarceration measures: 1) ever/never; 2) number of times over lifetime; and 3) length of time over lifetime. We then used Cox Proportional Hazard models to determine whether the three incarceration measures are associated with increased mortality adjusting for demographics (race, sex, age), and then also adjusting for social and health-related factors (education, smoking, HCV, social support, hazardous drinking, and homelessness, past year drug use, and mental health disorders).

RESULTS: Participants' mean age was 56, ranging from 22 to 84 ; $66 \%$ were African-American, $19 \%$ white, and $9 \%$ Hispanic or other; $97 \%$ were male, $38 \%$ reported current cigarette smoking, $36 \%$ were $\mathrm{HCV}+, 55 \%$ reported exposure to incarceration. There were 465 mortality events over a mean follow-up time of 5.8 years. Unadjusted mortality rates increased with incarceration exposure for all three incarceration measures and were 2.7 per 100 person years (pys) for those never incarcerated vs 3.6 for those ever incarcerated. In models adjusted for demographics, ever incarceration was associated with an AHR of 1.36 [1.13-1.66]. Number of times incarcerated was associated with mortality in models adjusted for demographics with AHRs of: 1.1 [0.9-1.5], 1.45 [1.2-1.8], and 1.64 [1.2-2.2] for 1, 2-5; 6+ times, respectively. Length of time incarcerated was also associated with mortality in models adjusted for demographics with AHRs of: 1.1 [0.91.5], 1.4 [1.1-1.9]. 1.6 [1.2-2.2], and 1.6 [1.1-2.2] for <1 month, 1 to 11 months, $1-5$ years, and $>5$ years, respectively. After further adjustment for social and health-related factors, the associations were no longer statistically significant.

CONCLUSIONS: Among individuals living with HIV, increasing exposure to incarceration (both frequency and total length of time spent incarcerated) was associated with increased mortality in unadjusted analyses and models adjusting for demographics. However, this association was not seen after additional adjustment for social and health-related factors, suggesting some covariates may be mediators or confounders. 


\section{ASSOCIATION BETWEEN NEOPLASTIC MENINGITIS AND BIOLOGIC FALSE-POSITIVE CSF-VDRL}

Terin Sytsma; Elitza Theel; Zelalem Temesgan; Michel Toledano. Mayo Clinic, Rochester, MN. (Control ID \#3183048)

BACKGROUND: Neurosyphilis should be considered in patients with encephalopathy, chronic meningitis, and progressive cranial neuropathies. While there is no gold standard diagnostic test for neurosyphilis, the cerebrospinal fluid venereal disease research laboratory (CSF-VDRL) is considered highly specific for the disease. Neoplastic meningitis has been a rarely reported cause of false-positive CSF-VDRL, but the frequency is unknown. This study sought to quantify the rate and determine the cause(s) of CSF-VDRL false-positivity.

METHODS: This retrospective chart review of all CSF-VDRL testing in patients presenting to a tertiary care center between January 1, 1994 and February 28, 2018 assessed the rate of false-positive CSF-VDRL results and underlying condition(s) in patients with falsely reactive CSF-VDRL. Demographic and clinical information for all false-positive CSF-VDRL cases were summarized. Descriptive statistics were used to calculate rates of positive, true-positive and false-positive CSF-VDRL results.

RESULTS: Among 34,336 CSF-VDRL tests performed, 66 (0.19\%) were positive. Of 66 positive CSF-VDRL tests, 50 (75.8\%) were truepositives and $16(24.2 \%)$ were false-positives. Fourteen individual patients had a false-positive CSF-VDRL result: nine males, five females, ranging in age from 14 to 82 years old. None had positive serologic syphilis testing. Nine of 14 patients with false-positive CSF-VDRL results (64.3\%) had neoplastic meningitis: six (42.9\%) from metastatic carcinoma (five primary lung cancers and one esophageal cancer), two (14.3\%) from astrocytoma with meningeal involvement, and one (7.1\%) from lymphomatosis. Four of the 14 patients with false-positive testing had a repeat CSF-VDRL; two were repeatedly positive, and both of these had meningeal carcinomatosis due to metastatic lung carcinoma. The two patients with negative repeat CSF-VDRL did not have neoplastic meningitis.

CONCLUSIONS: A majority of patients with false-positive CSF-VDRL testing in this study were ultimately diagnosed with neoplastic meningitis. Neoplastic meningitis is a frequent cause of false-positive CSF-VDRL results, and should be considered in patients with a positive CSF-VDRL but negative syphilis serology. The VDRL assay detects antibodies, including cardiolipin, and anticardiolipin antibodies have been reported in lung and central nervous system malignancies. Thus it is possible that the CSF-VDRL assay reacts with these antibodies. The two patients in this study with positive repeat CSF-VDRL testing had meningeal carcinomatosis, further suggesting that the false-positive CSF-VDRL test in patients with neoplastic meningitis represents a biologic false-positive. All patients with false-positive CSF-VDRL results in this study had negative serologic testing for syphilis, but serologic testing was not always available prior to CSF-VDRL results. This highlights the importance of considering pretest probability when ordering and interpreting CSF-VDRL testing.

\section{ASSOCIATION BETWEEN OVERALL MATERNAL HEALTH STATUS AND FAMILY RESILIENCE: RESULTS FROM A NA- TIONAL SURVEY}

Sitara Soundararajan ${ }^{1}$; Shyla Jagannatha ${ }^{2}$; Jaya Aysola ${ }^{3} .{ }^{1}$ Drexel University College of Medicine, Philadelphia, PA; ${ }^{2}$ Janssen Research \& Development, Raritan, NJ; ${ }^{3}$ University of Pennsylvania, Philadelphia, PA. (Control ID \#3138305)

BACKGROUND: The relationship between maternal health and health outcome of offspring has been studied extensively. However, there is sparse data available regarding the association between overall maternal health and the health, functionality, and productivity of the family unit. More specifically, measures such as family resilience in the context of maternal health are not well understood. The objective of this study was to determine if overall maternal health status is associated with family resilience.

METHODS: We analyzed data from the 2016 National Survey of Children's Health (NSCH), the only national survey assessing the health of children ages 0-17 in the United States. The independent variable in this study was overall physical and mental maternal health status. The main outcome of interest was presence of family resilience. Other outcomes of interest for this study included parental aggravation and adverse childhood experiences. We estimated the association between maternal heath status and family resilience with a multivariate logistic regression model, adjusting for the following covariates: current health insurance status, family structure of child's household, child race, highest education of adult in household, income level, and primary household language.

RESULTS: Compared to mothers whose physical and mental health are not excellent or very good, mothers who had excellent or very good physical and mental health statuses have families that showed significantly better adjusted rates for family resilience measures. Results suggest that mothers with excellent or very good physical and mental health have higher odds of having families that exhibit resilience [adjusted odds ratio (AOR) 2.790, CI [2.409, 3.231]], compared to mothers whose physical and mental health are not excellent or very good. Controlling for protective factors (e.g. supportive neighborhoods, emotional help with parenting) did not change the significance of the results [adjusted odds ratio (AOR) 2.617, CI [2.245, 3.051]]. Parental aggravation and adverse childhood experiences were also identified as possible mediators of the relationship between overall maternal health status and family resilience.

CONCLUSIONS: The study's results indicate that improved maternal health status is associated with higher odds of family resilience, even after adjusting for potential confounders. These findings suggest that overall maternal health may have a unique role in shaping the way families respond to adversity. Interventions addressing the health of our nation's mothers may have potential in improving the manner in which US families withstand and rebound from adversity.

\section{ASSOCIATION BETWEEN PARTICIPATION IN BUNDLED PAYMENTS FOR MEDICAL CONDITIONS AND CHANGES IN SPENDING AND UTILIZATION: A 3-YEAR ANALYSIS OF THE BUNDLED PAYMENTS FOR CARE IMPROVEMENT INITIA- TIVE \\ Joshua Rolnick $^{2}$; Joshua M. Liao ${ }^{1,2}$; Xinshuo $\mathrm{Ma}^{2}$; Ezekiel J. Emanuel ${ }^{2}$, ${ }^{2}$; Eric Z. Shan ${ }^{2}$; Jingsan Zhu' ${ }^{2}$; Erkuan Wang ${ }^{2}$; Qian Huang ${ }^{2}$; Amol S. Navathe ${ }^{3,2}$. ${ }^{1}$ University of Washington, Seattle, WA; ${ }^{2}$ University of Pennsylvania, Philadelphia, PA; ${ }^{3} \mathrm{CMC}$ VA Medical Center, Philadelphia, PA. (Control ID \#3182757)}

BACKGROUND: Bundling payments for an episode of care have shown savings for lower joint replacement surgery, but are less wellstudied for medical conditions. An early investigation of Medicare's Bundled Payments for Care Improvement (BPCI) initiative examined five medical conditions, finding no changes in spending or utilization after a mean seven months of follow up. We extend this work with longer-term data and different model specification to investigate BPCI participation for four high-volume conditions: congestive heart failure (CHF), pneumonia, acute myocardial infarction (AMI), and chronic obstructive pulmonary disorder (COPD).

METHODS: We performed a difference-in-differences analysis of episodes in 2011-2016 Medicare claims. Episodes attributed to BPCI physician groups were removed to avoid bias. BPCI participant hospitals were propensity- matched with up to 5 non-participants by condition. Outcomes included standardized total episode spending (primary) and spending by category, readmission rates, and mortality. Models were adjusted for patient and market characteristics, as well as hospital and quarter fixed effects. Ordinary least squares models were used for utilization and 
generalized linear models with a log-link function and gamma distribution for spending.

RESULTS: From October 2013-January 2016, 185 hospitals joined the CHF bundle, 137 joined COPD, 145 joined pneumonia, and 97 joined AMI (238 total). 223 BPCI hospitals (293,899 baseline episodes, 92,801 treatment) were matched to 1,074 control hospitals $(433,082$ baseline episodes, 392,537 treatment). Mean follow-up per hospital-condition was 22 months. Baseline age and sex were similar across groups and the Elixhauser risk index was 20.3 BPCI vs. 19.3 controls $(\mathrm{p}<0.001)$. Total baseline spending was $\$ 20,667$ BPCI vs. $\$ 19,799$ controls $(\mathrm{p}<0.001)$. The parallel trends assumption was met. In adjusted analyses, BPCI participation was associated with a significant risk-adjusted total spending increase of $0.9 \%$ (95\% CI $0.4 \%-1.4 \%$, p-value $<0.001$ ) and an increase in physician fees of $1.2 \%$ (95\% CI $0.6 \%-1.8 \%$, p-value $<0.001$ ). There were non-significant increases of $1.6 \%$ (95\% CI $-0.6 \%-3.9 \%$, pvalue $=0.16)$ for $\mathrm{SNF}$ and $1.5 \%$ (95\% CI $-0.6 \%-3.7 \%$, p-value $=0.17)$ for readmission spending. Participation was associated with significant increases of $0.3 \%(95 \% \mathrm{CI} 0.01 \%-0.62 \%$, p-value $=0.04)$ in readmission rate and $0.4 \%(95 \%$ CI $0.08 \%-0.62 \%$, p-value $=0.01)$ in SNF discharges. Mortality was unchanged.

CONCLUSIONS: Contrary to program expectations, BPCI participation was associated with an increase in total spending, likely due to increases in physician fees. Readmissions increased. Bundled payment programs for medical conditions may not achieve the intended effects and may even cause harm. While further work is needed, these findings may caution policymakers given the new BPCI-Advanced program.

\section{ASSOCIATION BETWEEN THE USE OF CLINICAL DECISION SUPPORT AND PERFORMANCE ON QUALITY MEASURES BY PRIMARY CARE PROVIDERS: EVIDENCE FROM THE MEANINGFUL USE AND PHYSICIAN QUALITY REPORTING SYSTEM PROGRAMS}

Jorge A. Rodriguez ${ }^{1}$; Jonathan E. Fried ${ }^{2}$; Stuart R. Lipsitz ${ }^{3,}$; ; Adam B. Landman $^{3}$; Lipika Samal ${ }^{3,2}$. ${ }^{1}$ Lawrence General Hospital, Lawrence, MA; ${ }^{2}$ Harvard Medical School, Boston, MA; ${ }^{3}$ Brigham and Women's Hospital, Boston, MA. (Control ID \#3185517)

BACKGROUND: Federal quality reporting programs stopped measuring the use of clinical decision support (CDS) after 2016. We sought to determine whether successful implementation of at least five CDS interventions, as reported to the Meaningful Use (MU) program, was correlated with higher quality of care, as measured by the Physician Quality Reporting System (PQRS).

METHODS: We obtained provider-level performance rates on eight process measures reported to PQRS in 2016 by 24,686 primary care providers (PCPs) from the Physician Compare Individual Eligible Professional (EP) Public Reporting data file. We used the MU Stage 2 EP Public Use File to assess their CDS use (defined as using 5 or more CDS; providers opted in). We then used logistic regression clustered by EP to assess the relationship between CDS use and performance on quality measures.

RESULTS: Only $31.7 \%$ of PCPs reported using CDS to fulfill MU requirements and $0.85 \%$ explicitly reported not using it. $67.45 \%$ did not report on this measure and were excluded. There was a significant positive association between CDS use and influenza vaccination. There was no statistically significant association between CDS use and rates of advance care plan use, avoidance of high-risk medications in the elderly, pneumococcal vaccination, documentation of current medications, tobacco cessation counseling, osteoporosis screening, or colorectal cancer screening. CONCLUSIONS: Influenza vaccination rates were higher for providers that used CDS than for those who did not. We did not find associations for the other measures. However, EPs were not required to report on CDS and very few providers explicitly reported a lack of use, which limited our sample size. EPs were not asked to report which type of CDS they had implemented. Elimination of CDS measures from pay for performance (P4P) programs such as the Promoting Interoperability Program may reduce incentives to invest in CDS for quality and safety. Further, our ability to draw meaningful conclusions are limited by the Centers for Medicare \& Medicaid Services' (CMS) incomplete reporting of PQRS data. CMS should make complete, de-identified PQRS data available to identify ways to improve $\mathrm{P} 4 \mathrm{P}$ programs.

\section{ASSOCIATION OF ADHERENCE WITH CHRONIC OBSTRUC- TIVE PULMONARY DISEASE AND COMORBID HYPERTEN- SION AND DIABETES MEDICATIONS}

Grace McInerney $^{1}$; Rachel O'Conor ${ }^{3}$; Michael S. Wolf ${ }^{3}$; Juan Wisnivesky ${ }^{2}$; Alex Federman ${ }^{1} .{ }^{1}$ Icahn School of Medicine at Mount Sinai, New York, NY; ${ }^{2}$ Mount Sinai School of Medicine, New York, NY; ${ }^{3}$ Northwestern University, Chicago, IL. (Control ID \#3174967)

BACKGROUND: Non-adherence with chronic obstructive pulmonary disease (COPD) medications is common among older adults, but it is unknown whether COPD self-management behaviors are associated with adherence patterns to medications for comorbid conditions like hypertension or diabetes.

METHODS: To determine the association of COPD medication adherence with adherence to medications for comorbid illnesses, we performed a crosssectional analysis of data collected from a cohort of COPD patients with comorbid hypertension or diabetes. A sample of 282 adults ages 40 years and older with COPD were recruited from primary care practices in New York, NY, and Chicago, IL. Medication adherence was measured using the Medication Adherence Rating Scale (MARS), a validated 10-item self-reported measure designed to minimize social desirability bias. Each item is rated on a 5-point Likert scale, with higher scores indicating greater adherence. Participants with a MARS score of 4.5 or greater were classified as having good adherence. We calculated the Pearson correlations for MARS scores for COPD and hypertension, COPD and diabetes, and hypertension and diabetes.

RESULTS: The mean age was 68 (SD 9), 59\% were female, 38\% were black and $17 \%$ were Hispanic; $95 \%$ were English language proficient. Overall, $98 \%$ of participants had comorbid hypertension and $35 \%$ diabetes; $33 \%$ of participants had both comorbid diabetes and hypertension. Adequate COPD medication adherence was reported by $53 \%$ of patients, $70 \%$ for hypertension medications, and $61 \%$ for diabetes medications. Medication adherence for COPD and hypertension medications $(\mathrm{r}=0.43, \mathrm{P}<0.0001)$ and hypertension and diabetes medications $(\mathrm{r}=0.43, \mathrm{P}=0.0001)$ were strongly correlated. However, adherence to COPD and diabetes medications $(r=0.12$, $\mathrm{P}=0.29$ ) were not correlated.

CONCLUSIONS: Associations of medication adherence behaviors varied across different comorbidities. Understanding the underlying basis for different patterns of adherence to medications in different therapeutic classes may provide insight into new ways of facilitating self-management of medications among patients with multimorbidity.

\section{ASSOCIATION OF CHRONIC PAIN AND MYOCARDIAL IN- FARCTION}

Jigar J. Patel ${ }^{1}$; Talal S. Alzahrani ${ }^{2}$; Shaneke Weerakoon ${ }^{2}$; William Borden $^{2}$. ${ }^{1}$ George Washington University, Washington, DC; ${ }^{2}$ The George Washington University, Washington, DC. (Control ID \#3142581)

BACKGROUND: Chronic pain affects many patients and is the primary reason for visits to primary care clinics and emergency departments. In previous studies, chronic pain has been linked to mortality and cardiovascular disease. This study further explores this 
relationship by analyzing the association between chronic pain and myocardial infarction using a national database.

METHODS: The National Health Interview Surveys of 2017 ( $\mathrm{n}=$ $26,445)$ were used to examine the cross-sectional association between frequency of pain (never, some days, most days, every day) and myocardial infarction in a single logistic regression model that also included demographics [age, gender, Body Mass Index (BMI)] and health characteristics (smoking, hypertension, diabetes, hypercholesterolemia and exercise).

RESULTS: Participants who reported having pain every day were more likely to be female ( $60 \%$ vs. $52 \%)$ and older (61 vs. 49 years) than participants who reported never having pain. The rates of daily smoking ( $23 \%$ vs. $9.6 \%$ ), diabetes ( $24.9 \%$ vs. $8 \%$ ), hypertension (63.1\% vs. $29.2 \%)$, hypercholesterolemia $(50.4 \%$ vs. $27.4 \%)$, and myocardial infarction (10.9\% vs. $2.6 \%$ ) were significantly higher in participants who reported having pain every day compared to subjects who reported never having pain. The logistic regression model showed that reporting daily experience of pain was independently associated with increased odds of having had a myocardial infarction $(\mathrm{OR}=2.19,95 \%$ CI $1.69,2.83, \mathrm{p}<0.001)$ as was experience of pain most days $(\mathrm{OR}=2.08,95 \%$ CI 1.49-2.91, $\mathrm{p}<0.001)$, and experience of pain some days $(\mathrm{OR}=1.51,95 \% \mathrm{CI}=1.24-1.83, \mathrm{p}<0.001)$ compared to never having pain after adjusting for demographics and health characteristics.

CONCLUSIONS: The frequency of chronic pain is significantly associated with the rate of myocardial infarction after adjusted for cardiovascular risk factors including age, gender, BMI, hypertension, diabetes, hypercholesterolemia, and lack of exercise. Future studies are needed to evaluate the efficacy of managing pain in reducing myocardial infarction.

ASSOCIATION OF E-CIG USE AND NON-PRESCRIBED MEDICATION USE: RESULTS FROM THE PATH SURVEY Eric Mortensen; Nkiruka Atuegwu; Kathryn Bentivegna; Mario F. Perez; Cheryl Oncken. UConn Health, Farmington, CT. (Control ID \#3177619)

BACKGROUND: Use of electronic cigarettes ("e-cigs") is increasing in the US, but little is known about their association with other high-risk behaviors. The purpose of this study was to examine the association between e-cig use and a participant's report of the use of pain killers, sedatives, or tranquillizers that were not prescribed to them, within a nationally representative sample of adults.

METHODS: We used the waves 1-3 of the Population Assessment of Tobacco and Health (PATH) survey adult data $(\mathrm{N}=23,670)$. We defined current e-cig use as daily or some days use of e-cigs by participants in wave 1 . We then used logistic regression to examine the association between current e-cig use and the use of nonprescribed pain killers, sedatives, or tranquillizers in the last 30 days, or 12 months, after adjusting for the covariates including demographics (age, gender, marital status, income, prior education), use of combustible tobacco and other nicotine products, use of illicit drugs, and psychiatric symptoms. Replicate weights and balanced repeated replication methods were utilized to account for the complex survey design.

RESULTS: We compared the 2,639 current e-cig users to the 21,024 non-users. After adjusting for the potential confounders, the use of ecigs is associated with significantly higher use of non-prescribed pain killers, sedatives, or tranquillizers in the last 30 days (odds ratio [OR] 1.30, 95\% confidence interval [CI] 1.14-1.48) and last 12months (OR 1.31, 95\% CI 1.15-1.49).

CONCLUSIONS: Our findings demonstrate that e-cig users have significantly higher odds of non-prescription use of pain killers, sedatives, or tranquillizers in the future. Clinicians should be aware of this association and screen for the inappropriate use of prescription medications in their patients who regularly use e-cigs.

\section{ASSOCIATION OF HEALTHCARE TRUST AND DISCRIMI- NATION WITH SCREENING FOR VIOLENCE AND GUN OWNERSHIP IN CLINICAL SETTINGS}

Madison R. Wilson; Monica E. Peek; Nichole Smith; Tyrone A. Johnson; Elizabeth L. Tung. University of Chicago, Chicago, IL. (Control ID \#3184488)

BACKGROUND: Growing political discord over gun violence in the U.S. has resulted in national conversations about the role of healthcare in screening for violence and gun ownership. While some states have enacted laws limiting physicians' ability to screen patients for gun ownership, multiple medical associations encourage physicians to implement routine screening practices. However, few studies have documented the extent to which healthcare providers are implementing screening practices; and fewer have examined the relationship between these types of screening practices and patient-oriented outcomes, such as healthcare trust and perceived healthcare discrimination.

METHODS: We recruited a sample of 506 adult patients, ages 35 years, from 2 medical clinics on the South and West Sides of Chicago. Surveys were administered using Computer-Assisted Personal Interviewing software on tablets. Healthcare relationship trust (HCR Trust Scale) and discrimination in medical settings (DMS Scale) were measured using validated items and normalized to 100 -point scales. Healthcare screening for (1) community violence and (2) gun ownership/safety were measured using items adapted from national surveys ("Have you ever discussed [community violence / gun ownership or safety] with your physician or another health care professional?"). We used generalized linear regression models to independently assess HCR Trust and DMS scores as functions of healthcare screening items, adjusting for age, gender, race/ethnicity, education, insurance, and employment status.

RESULTS: The cooperation rate (COOP2) was $61 \%$. The mean age of participants was 60 years (SD 13.0); the majority were female (71\%) and black $(75 \%)$. Although many $(62 \%)$ had completed some college, almost two-thirds were unemployed (64\%). Few patients $(15 \%)$ had ever discussed community violence with a healthcare provider; even fewer (5\%) had ever discussed gun ownership or safety. Mean HCR Trust was $78.1 \pm 17.7$ and mean DMS was $5.2 \pm 1.2$ (100-point scale). While screening for community violence was associated with a $5.1 \%$ higher HCR trust score (95\% CI, 0.1 to 10.1), screening for gun ownership/safety was not associated with higher HCR trust. Conversely, while there was no association between screening for community violence and DMS scores, screening for gun ownership/safety was associated with a $6.8 \%$ higher DMS score (95\% CI, 1.4 to 12.2 ), compared to those who had not been screened.

CONCLUSIONS: While screening patients for exposure to community violence was associated with higher healthcare trust, screening for gun ownership/safety was not, and may even be associated with perceptions of healthcare discrimination. As healthcare systems begin to consider screening for gun ownership/safety, implementation in low-income, minority settings should be cognizant of historically unfair targeting and profiling of residents.

\section{ASSOCIATION OF MEDICARE SKILLED NURSING FACILITY COPAYMENTS WITH HIGHER DISCHARGE RATES OF ETH- NIC MINORITIES AND SOCIOECONOMICALLY VULNERA- BLE PATIENTS}

Paula Chatterjee $^{1}$; Mingyu Qi ${ }^{1}$; Norma B. Coe ${ }^{1}$; R Tamara Konetzka ${ }^{3}$; Rachel M. Werner ${ }^{2} .{ }^{1}$ University of Pennsylvania, Philadelphia, PA; ${ }^{2}$ University of Pennsylvania and Philadelphia VA, Philadelphia, PA; ${ }^{3}$ University of Chicago, Chicago, IL. (Control ID \#3185721) 
BACKGROUND: Medicare fully pays for post-acute care provided by skilled nursing facilities (SNFs) for the first twenty days within a benefit period. However, on the twenty-first day, patients become responsible for a daily copayment of over $\$ 150$. This may represent a significant financial burden for some patients, motivating discharge from SNFs based on finances rather than recovery. SNFs may also prematurely discharge some patients to avoid the risk of providing partially uncompensated post-acute care and bad debt. However, patterns of SNF discharge as they relate to these Medicare payment policies are unknown.

METHODS: We performed a retrospective cohort study of Medicare beneficiaries continually enrolled in fee-for-service Medicare with acute care hospital stays resulting in discharge to SNF from 2012-2016. For each SNF discharge, we computed the SNF benefit day on discharge based on the number of SNF benefit days used in that benefit period. We compared patient characteristics across benefit days, including age, sex, race/ethnicity, a count of 31 Elixhauser comorbidities, and ZIP-code-level socioeconomic status (from the American Community Survey), including median household income, poverty rate and unemployment rate. We compared differences using two-tailed t-tests

RESULTS: More patients are discharged from SNF on day 20 compared to days 19 or 21 (2.93\% on day $19,5.01 \%$ on day 20 , and $2.69 \%$ on day 21). Patients discharged from SNF on benefit day 20 were younger $(79.0$ years among patients discharged on day 20 compared to 80.0 years on day 19 and 81.3 on day $21, \mathrm{p}<0.01)$. They were also more likely to be Black/ Hispanic (12.6\% on day 20 compared to $8.1 \%$ on day 19 and $7.5 \%$ on day $21, \mathrm{p}<0.01)$, and live in areas of high poverty $(15.3 \%$ on day 20 compared to $13.8 \%$ on day 19 and $13.4 \%$ on day $21, \mathrm{p}<0.01$ ) with lower median household incomes $(\$ 56,586$ on day 20 compared to $\$ 60,443$ on day 19 and $\$ 61,619$ on day $21, \mathrm{p}<0.01)$.

CONCLUSIONS: Medicare beneficiaries are more commonly discharged from SNFs on benefit day 20 than days 19 or 21, and those discharged on day 20 are more likely to be racial/ethnic minorities and of lower socioeconomic status compared to beneficiaries discharged either before or after benefit day 20. SNFs may be preferentially discharging vulnerable patients to avoid the risk of assuming responsibility for defaulted payments. Whether these practices impact patient outcomes remain unknown, but nonetheless raises concerns about the implications of this practice.

\section{ASSOCIATION OF NUMBER OF UNIQUE HOSPITALISTS AND WORKLOAD WITH PATIENT OUTCOMES: AN ADMINISTRA- TIVE DATA ANALYSIS ACROSS 4 HOSPITALS}

Henry J. Michtalik ${ }^{1,2}$; Peter J. Pronovost ${ }^{2}$; Jill A. Marsteller ${ }^{1,3}$; Joanne E. Spetz ${ }^{4}$; Daniel E. Ford ${ }^{2,}{ }^{3}$; Eric Howell ${ }^{1,2}{ }^{2}$; Daniel Brotman ${ }^{2} .{ }^{1}$ Johns Hopkins Medicine, Baltimore, MD; ${ }^{2}$ School of Medicine, Johns Hopkins University, Baltimore, MD; ${ }^{3}$ Johns Hopkins School of Public Health, Baltimore, MD; ${ }^{4}$ University of California, Los Angeles, San Francisco, CA. (Control ID \#3180462)

BACKGROUND: Hospitalists play a central role in patient care but little research has considered whether their workload or handoffs affect care quality. This study examined the impact of hospitalist workload and number of unique hospitalists involved in the patient's care on quality of care and patient communication.

METHODS: Measured in 4 hospitalist programs within a regional healthcare network, the main outcome was hospitalist workload averaged over a patient's hospitalization as determined from billing data. For example, if a patient had a 2-day hospitalization and was seen by a hospitalist with 12 patients day 1 and a different hospitalist with 14 patients day 2, the average workload the patient was exposed to was 13 patients and 2 unique hospitalists. Adjusting by site, we used regression to analyze average workload and its association with all-cause 30-day readmissions, hospital-acquired conditions, in-hospital mortality, and the 3 HCAHPS physician communication questions. We adjusted for patient demographics (age, race, sex), severity of illness (APR-DRG SOI; range 1-4), observed length of stay (LOS), percentage of weekdays, and number of hospitalists. We also examined the effect of number of unique hospitalists involved in the patient's care on outcomes.

RESULTS: We analyzed 26,661 admissions at 4 hospitals from July 1, 2016 to June 30, 2017. The median patient LOS was 3 days [interquartile range (IQR): 2, 5]. The patient population was $60 \%$ Caucasian, $28 \%$ African American, 53\% female with median age of 67 years [IQR: 52, 80] and APR-DRG severity of illness 3 [IQR: 2, 3]. The median number of unique hospitalists was 2 [IQR: 2, 3] with workload of 11 patients per day [IQR: 8, 13]. All-cause 30-day readmissions, hospital-acquired conditions, and in-hospital mortality were $13.2 \%, 1.4 \%$ and $1.2 \%$ respectively. We found no significant association between hospitalist workload and readmissions, in-hospital mortality, or patient communication after adjustment. Counterintuitively, a higher workload was associated with a lower odds of hospital-acquired condition $(\mathrm{p}<0.001)$. In contrast, even a small increase in the number of unique attending hospitalists increased the odds of having a hospital-acquired condition (OR: 1.23; 95\% CI: 1.14 , $1.34 ; \mathrm{p}<0.001)$. The odds of patients reporting that the hospitalist carefully listened or clearly explained care also significantly decreased $(\mathrm{p}<0.01$, $\mathrm{p}<0.05$ respectively).

CONCLUSIONS: There was no significant association between hospitalist workload and the outcomes, except for hospital-acquired conditions. In contrast, increased number of unique hospitalists was associated with an increase in odds of a hospital-acquired condition and adversely affected listening and understanding. This suggests that transitions of care and communication, not necessarily workload, are associated with some adverse outcomes. Potential practice interventions to mitigate this may include standardizing handoffs and communication, and improving continuity with longer scheduling blocks.

\section{ASSOCIATION OF PRIMARY CARE CLINIC APPOINTMENT TIME WITH CLINICIAN ORDERING AND PATIENT COMPLE- TION OF BREAST AND COLORECTAL CANCER SCREENING Esther Y. Hsiang ${ }^{2,3}$; Shivan Mehta ${ }^{2,1}$; Dylan Small ${ }^{2}$; Charles Rareshide ${ }^{2}$; Christopher K. Snider ${ }^{2}$; Susan C. Day ${ }^{2}$; Mitesh Patel ${ }^{2,}{ }^{1}$. ${ }^{1}$ Crescenz VA Medical Center, Philadelphia, PA; ${ }^{2}$ University of Pennsylvania, Philadel- phia, PA; ${ }^{3}$ John Hopkins Medical School, Philadelphia, PA. (Control ID \#3139446)}

BACKGROUND: Early cancer detection can improve clinical outcomes, but cancer screening rates in the United States are often suboptimal. As clinic days progress, primary care providers (PCPs) may fall behind schedule resulting in shorter rushed interactions with patients scheduled later in the day, and face increased decision fatigue. Our objective was to evaluate the association of primary care clinic appointment time with clinician ordering and patient completion of breast and colorectal cancer screening. METHODS: We performed a retrospective analysis of 33 primary care practices in Pennsylvania and New Jersey. We evaluated adults eligible for breast or colorectal cancer screening and visiting with their PCP between September 1, 2014 and August 31, 2016. Primary outcome was clinician ordering of the screening test during the visit. Secondary outcome was patient completion of the tests within 1 year of the visit. To evaluate the outcomes by clinic appointment time, we grouped appointment times by the hour. In the adjusted analysis of patient-visit level data, we used PROC LOGISTIC in SAS to fit a conditional logistic regression, where the conditioning was on the PCP. The models were adjusted for patient demographics, insurance, Charlson comorbidity index, clinic visit type, fixed effects by practice site, year and calendar month, and a covariate for each appointment hour.

RESULTS: Among the 19,254 patients eligible for breast cancer screening, the mean age was 60.2 years, $60.7 \%$ were white, and $28.5 \%$ were black. Screening test order rates were highest at $8 \mathrm{am}$ at $63.7 \%$, declined throughout the morning to $48.7 \%$ at $11 \mathrm{am}$, increased to $56.2 \%$ at $12 \mathrm{pm}$, and then 
declined to $47.8 \%$ at $5 \mathrm{pm}$ (Adjusted Odds Ratio [aOR] for linear trend: 0.94, 95\% CI: $0.93-0.96, P<.0001)$. Trends in screening test completion rates were similar beginning at $33.2 \%$ at $8 \mathrm{am}$ and declining to $17.8 \%$ at $5 \mathrm{pm}$ (aOR: 0.95, 95\% CI: 0.94-0.97, $P<.0001$ ). Among the 33,468 patients eligible for colorectal cancer screening, the mean age was 59.6 years, $55.8 \%$ were female, $66.2 \%$ were white, and $21.8 \%$ were black. Screening test order rates were $36.5 \%$ at $8 \mathrm{am}$, declined to $31.3 \%$ by $11 \mathrm{am}$, increased at $12 \mathrm{pm}$ to $34.4 \%$, and then declined to $23.4 \%$ at $5 \mathrm{pm}$ (aOR: $0.94,95 \%$ CI: $0.93-0.95$, $P<.0001)$. Trends in screening test completion rates were similar beginning at $28.0 \%$ at $8 \mathrm{am}$ and declining to $17.8 \%$ at $5 \mathrm{pm}$ (aOR: $0.97,95 \%$ CI: $0.96-$ $0.98, P<.0001)$.

CONCLUSIONS: Clinician ordering of cancer screening tests significantly declined as the clinic day progressed. Patient completion of cancer screening tests within 1 year of the visit was also significantly lower as the primary care appointment time was later in the day. Future interventions targeting improvements in cancer screening should consider how time of day influences these behaviors.

\section{ASSOCIATION OF RESIDENT PERFORMANCE METRIC FEED- BACK FOR DIABETIC CARE IN PRIMARY CARE CLINICS AND ACHIEVEMENT OF PERFORMANCE METRICS}

Timothy J. Brown ${ }^{1}$; Salahuddin Kazi ${ }^{1}$; Tyler Miller ${ }^{2}$; Hyemi Chong ${ }^{2}$. ${ }^{1}$ UTSW, Dallas, TX; ${ }^{2}$ VA North Texas Healthcare System, Dallas, TX. (Control ID \#3185806)

BACKGROUND: Performance feedback during residency is a critical aspect of training. In the primary care clinics, feedback on chronic disease management is important but may not be readily available. Our residency program is divided into 5 "firms" sharing a clinic week in a "4+1" format in which the residents do 4 weeks of inpatient rotations followed by 1 week of clinic. Approximately half of the program has primary care clinic at the VA. The VA has developed guideline-based targets to improve the care of diabetic patients consisting of an annual hemoglobin A1c, achieving A1c $<9 \%$, targeting blood pressure to $<140 / 90 \mathrm{mmHg}$, targeting LDL $<100 \mathrm{mg} / \mathrm{dL}$ or prescription of a moderate-intensity statin, annual retinopathy referral, and annual nephropathy screening. However feedback related to progress towards these metrics is not readily available to the residents. We sought to improve rates of achievement of these metrics by providing residents with their performance data.

METHODS: Data were obtained prospectively from a continuouslyupdated EMR-based database. A natural experiment was designed to determine the effect of feedback on achievement of performance metrics. One of the five firms was blinded to this project (no feedback) and the other four firms received weekly email feedback with their performance metrics and an average composite score. All residents in the feedback group were provided data, however only senior residents PGY2 and higher were included in the analysis to account for patient "rollover" from the previous graduating class. After four completed clinic weeks spanning 20 weeks in the academic calendar, data were analyzed. Comparisons were made using two-tailed paired t-tests with alpha of 0.05 to determine if feedback of performance metrics improved achievement of the performance metrics.

RESULTS: Forty-one senior residents were included in the feedback group and ten senior residents were in the no feedback group. Feedback was associated with a mean decrease in composite score for diabetes care of $3.3 \%$ [95\% confidence interval $(\mathrm{CI}) 1.38-5.26 \%$ decrease, $\mathrm{p}=0.009]$ compared to no feedback. Similarly, feedback was associated with a $3.75 \%$ increase in the number of diabetic patients with hemoglobin A1c $>9$ [95\% CI $2.26-5.23 \%$ increase, $\mathrm{p}=0.002$ ] and with an $8.2 \%$ decrease in diabetic patients with $\mathrm{LDL}<100 \mathrm{mg} / \mathrm{dL}$ or on moderate intensity statin [95\% CI 4.7$11.8 \%$ decrease, $\mathrm{p}=0.03$ ) compared to no feedback. No difference was noted in rates of annual hemoglobin A1c screening, retinopathy screening, nephropathy screening, or achievement of blood pressure $<140 / 90 \mathrm{mmHg}$. CONCLUSIONS: In this analysis, providing residents with feedback on their diabetic patients did not improve whole-panel achievement of institutional performance metrics. These results could be affected by potential crossover of patients within the primary care clinic, relatively short follow-up and unknown uptake of feedback by the residents. Further attempts at improvement are ongoing.

\section{ASSOCIATION OF SOCIAL VULNERABILITY WITH TREAT- MENT BURDEN AND MEDICATION ADHERENCE AMONG PA- TIENTS WITH HYPERTENSION}

Hani A. Abi ${ }^{1}$; David Eton ${ }^{2}$; Mark Linzer ${ }^{3}$; Elizabeth A. Rogers ${ }^{1} .{ }^{1}$ University of Minnesota, Minneapolis, MN; ${ }^{2}$ Mayo Clinic, Rochester, MN; ${ }^{3} \mathrm{Hen}-$ nepin County Medical Center, Minneapolis, MN. (Control ID \#3186055)

BACKGROUND: Hypertension is a condition that more than 100 million Americans-nearly $1 / 3$ of adults-suffer from. New hypertension guidelines in the United States increase the number of patients who require treatment and the number of medications taken. This contributes to treatment burden, or the work of self-management and its impact on function and well-being, which may lead to decreased medication adherence. This study examined the associations between treatment burden, medication adherence, and social vulnerability measures among patients with hypertension.

METHODS: We conducted a cross sectional survey of patients within two Minnesota medical systems who had at least two chronic medical conditions. The mailed survey battery consisted of the Patient Experience with Treatment and Self-management measure (PETS), an 11-domain measure of patient-reported treatment burden; four validated measures of social vulnerability (disease management self-efficacy, healthcare provider relational quality, health literacy, and healthcare financial difficulties); and a single-item measure of medication adherence. For those respondents with hypertension, we calculated the correlation between PETS domains, social vulnerability measures, and medication adherence using Spearman's Rho. RESULTS: The study population $(n=254)$ was on average 67 years old, $54 \%$ female, $27 \%$ non-white, $67 \%$ having some college education, and $26 \%$ employed. Sixty-six percent reported taking 5 or more prescription medications and $13 \%$ reported low medication adherence. We found that higher treatment burden was correlated with lower patient perceived self-efficacy, healthcare provider relational quality, and health literacy, and with higher financial difficulty $(\mathrm{p}<0.05$ on all 11 PETS domains). Higher treatment burden was also correlated with lower medication adherence $(\mathrm{p}<0.05$ on 6 of 11 PETS domains). Lower medication adherence was correlated with lower healthcare provider relational quality, patient perceived self-efficacy, and health literacy; and with higher financial difficulty.

CONCLUSIONS: Patient-reported treatment burden is associated with lower medication adherence and greater social vulnerability among patients with hypertension. Lower medication adherence is associated with greater social vulnerability. Adding medications to follow the new hypertension guidelines may increase treatment burden, decrease medication adherence, and disproportionally affect socially vulnerable populations. Clinicians and future researchers should consider this in efforts to curb disparities in hypertension outcomes.

\section{ASSOCIATION OF STATE LAWS MANDATING PRESCRIPTION DRUG MONITORING PROGRAM USE BY PRESCRIBERS ON DISCONTINUATION OF CHRONIC OPIOID THERAPY IN VETERANS}

Jonathan Arnold ${ }^{4}$; Xinhua Zhao ${ }^{1}$; Florentina Sileanu ${ }^{2}$; Maria K. Mor ${ }^{2}$; John P. Cashy ${ }^{2}$; Patience Moyo ${ }^{3}$; Carolyn T. Thorpe ${ }^{2}$; Chester Good ${ }^{2}$; Thomas R. Radomski ${ }^{4}$; Michael J. Fine ${ }^{2}$; Walid F. Gellad ${ }^{2} .{ }^{1}$ VA Pittsburgh HealthCare System, Pittsburgh, PA; ${ }^{2}$ VA Pittsburgh Healthcare System, Pittsburgh, PA; ${ }^{3}$ Brown University School of Public Health, Providence, RI; ${ }^{4}$ University of Pittsburgh School of Medicine, Pittsburgh, PA. (Control ID \#3168221)

BACKGROUND: Several states have mandated that prescribers check prescription drug monitoring programs (PDMPs) prior to opioid 
prescribing and periodically during therapy. Despite concerns about inappropriately abrupt opioid discontinuation resulting from this policy, no studies have evaluated the extent to which this occurs. Our objective was to evaluate the impact of PDMP mandates on discontinuation of chronic opioid prescribing for Veterans managed in the Veterans Affairs Administration (VA) Healthcare System.

METHODS: We conducted an interrupted time-series (ITS) study of opioid discontinuation in Veterans without cancer diagnoses in 4 states that implemented a PDMP use mandate from 2010-2014: Ohio (OH; Nov 2011), West Virginia (WV; Jun 2013), Kentucky (KY; Jul 2012), and Tennessee (TN; Apr 2013). We used Veterans in neighboring states without a mandate as controls. We built monthly cohorts of Veterans with 305 days of a VA-prescribed opioid in the prior 365 days. We defined discontinuation as a 90day period without a VA prescribed opioid. For each state with a PMDP mandate and its pooled control we estimated monthly discontinuation rates adjusted for patient-level demographic and clinical covariates. We calculated the difference between the mandate states and their controls; and we used these differences in our ITS models with offset and slope-change terms at the time of the mandate. We included 12-months prior to and following the mandate in each state. All reported percentages represent absolute percentage points.

RESULTS: We built monthly cohorts for 4 PMDP mandate and 12 neighboring control states (3-7 controls for each mandated state). Monthly cohort sizes ranged from 4,733-8,909 Veterans in the mandate states and $11,985-41,163$ in the controls, a total of $2.5 \mathrm{M}$ veteran-months. The average adjusted discontinuation rates in the 12 months prior to (after) the mandates were $0.7(0.9) \%$ in $\mathrm{OH}, 0.7$ $(0.7) \%$ in WV, $0.8(1.3) \%$ in KY, and $0.8(0.9) \%$ in TN. There was no difference in the pre-mandate discontinuation rate between mandate states and controls. TN was the only state with an increasing baseline trend in discontinuation compared to its control of $0.02 \% /$ month $(\mathrm{p}=0.03)$. KY's mandate was associated with an immediate $1.03 \%(\mathrm{p}<0.001)$ increase in discontinuation rate. Mandates in KY and $\mathrm{TN}$ were associated with a decreasing trend in discontinuation of $-0.09 \%(p=0.001)$ and $-0.05 \%(p<0.001)$ per month respectively. There was no change associated with the mandates in $\mathrm{OH}$ or WV. CONCLUSIONS: For VA managed Veterans, state laws mandating prescriber PDMP use had little absolute effect on discontinuation of chronic opioid prescribing. We did observe a clinically significant but short lived increase in opioid discontinuation in KY immediately following its mandate, however the overall discontinuation rate remained low. These results suggest that PDMP use mandates did not cause abrupt discontinuation of long-term opioid therapy within VA.

\section{ASSOCIATION OF THE IMPLEMENTATION OF THE PATIENT-CENTERED MEDICAL HOME WITH QUALITY OF LIFE IN PATIENTS WITH MULTIMORBIDITY}

Linnaea C. Schuttner ${ }^{1,3}$; Ashok Reddy ${ }^{1,3}$; Ann-Marie Rosland ${ }^{2,}{ }^{4}$; Karin M. Nelson ${ }^{1,3}$; Edwin Wong ${ }^{1,3}$. ${ }^{1}$ VA Puget Sound Healthcare System, Seattle, WA; ${ }^{2}$ VA Pittsburgh, Pittsburgh, PA; ${ }^{3}$ University of Washington, Seattle, WA; ${ }^{4}$ University of Pittsburgh School of Medicine, Pittsburgh, PA. (Control ID \#3165054)

BACKGROUND: The patient-centered medical home (PCMH) has clinical benefits for patient with multimorbidity, yet the association with patient-reported outcomes such as health-related quality of life (HRQoL) is unknown. Therefore, we examined the association of the Veterans Affairs (VA) implementation of the PCMH model, the Patient Aligned Care Team (PACT), and HRQoL in multimorbid adults.

METHODS: We performed a retrospective cohort study of multimorbid patients $(2$ or more chronic diseases in 2 or more body systems, $\mathrm{n}=22,095$ ) who responded to a patient experiences survey in 2013-2014 and received VA primary care in 2012. PCMH implementation at a clinic was measured in 2012 with the validated PACT Implementation Progress Index $\left(\mathrm{PI}^{2}\right)$; Higher $\mathrm{PI}^{2}$ scores indicate better performance on 8 domains (access; care continuity; care coordination; comprehensiveness; selfmanagement support; communication; shared decision-making; and team-based care). HRQoL was measured from the patient survey with Short Form-12 physical and mental component scores (PCS, MCS). Surveys were weighted to the VA population. Generalized estimating equations, adjusted for patient and clinic characteristics, were used to model HRQoL against 1) quintiles of $\mathrm{PI}^{2}$ as the primary exposure, 2) highest and lowest versus mid-quartiles of $\mathrm{PI}^{2}$ in exploratory analyses of the $8 \mathrm{PI}^{2}$ domains, and 3 ) an interaction analysis of patients with and without hospitalizations in 2012.

RESULTS: The cohort was mostly male (96\%) and on average 68 years old with 4.4 chronic diagnoses. Compared to patients seen at the lowest-scoring $\mathrm{PI}^{2}$ clinics, receiving care in the highest $\mathrm{PI}^{2}$ clinics was associated with a 3.0 point higher adjusted marginal mean PCS [42.7 vs. 39.7, $\mathrm{P}<0.001)$ ], but a 1.2 point lower MCS [36.3 vs. $35.1, \mathrm{P}=0.04]$. Patients with prior hospitalizations seen in clinics with higher compared to lower $\mathrm{PI}^{2}$ scores had a 2.6 point greater MCS $(\mathrm{P}=0.02)$; Those without prior hospitalizations seen in higher compared to lower $\mathrm{PI}^{2}$ clinics had an MCS that was 1.6 points lower $(\mathrm{P}<0.01)$. Better performance on the communication and shared decision-making domains was significantly associated with a higher mean PCS (1.0 points higher for average versus lowest $\mathrm{PI}^{2}$ for communication, $\mathrm{P}=0.018 ; 0.9$ points higher for average versus lowest $\mathrm{PI}^{2}$ for shared decision-making, $\mathrm{P}<0.01$ ).

CONCLUSIONS: Multimorbid patients seen in clinics with greater PCMH implementation reported a clinically significant higher physical HRQoL, which may be due to better patient-provider communication and shared decision-making. Care from higher performing clinics was associated with a small but not clinically significant lower score for mental HRQoL, which may be related to disease severity as measured by hospitalizations. Our findings add HRQoL to the known benefits of the PCMH model.

\section{ASSOCIATION OF USE OF THE NEJM KNOWLEDGE+ PRODUCT AND PERFORMANCE ON THE ABIM IM-MOC EXAM}

Michael Healy ${ }^{1,2}$; Roy Phitayakorn ${ }^{1,}{ }^{2}$; Carl Gustaf Stefan Axelsson $^{1}$; Matthew O'Rourke ${ }^{2}$; Lauren M. Duhigg ${ }^{3}$; Bradley Gray ${ }^{3}$. ${ }^{1}$ Massachusetts General Hospital, Harvard Medical School, Boston, MA; ${ }^{2}$ NEJM Group, Waltham, MA; ${ }^{3}$ American Board of Internal Medicine, Philadelphia, PA. (Control ID \#3165130)

BACKGROUND: Internal medicine physicians who are completing their American Board of Internal Medicine Internal Medicine Maintenance of Certification (ABIM IM-MOC) requirements must eventually pass a knowledge assessment (i.e., MOC examination). Although physicians have many options to choose from as they prepare for this assessment, including the NEJM Knowledge+ Internal Medicine Board Review (NEJM Knowledge+) product, there exists no research related to the use of this or other similar study aids and MOC examination performance.

METHODS: To address this gap, we compared ABIM IM-MOC examination performance among similar physicians who did and did not use NEJM Knowledge+. Our physician sample included 177 physicians who attempted an ABIM IM-MOC examination from 2014-2017 and completed at least 75\% of the NEJM Knowledge+ product prior to the ABIM IM-MOC examination and 177 matched 
case-controls who did not use the NEJM Knowledge+ product. One limitation of our analysis is that physicians may have not used NEJM Knowledge+ exclusively (e.g., they could have also taken an exam review course). Physicians were eligible for the study if they were nongrandfather general internists. Case- control matched characteristic included sex, birth country (U.S. vs. international), medical school country (U.S. vs. international), year of MOC examination, MOC cycle number $\left(1^{\text {st }}\right.$ vs. $\left.{ }^{2 n d}\right)$, examination attempt number within the MOC cycle (1 vs. 2 ) and decile ranked score on the first attempt at the initial IM certification examination. Performance on the ABIM IM-MOC examination, which was the primary outcome of interest, was operationalized as indicators for 1) passing the ABIM IM-MOC examination, 2) having an examination score in the top quartile of the ABIM IM-MOC examination and 3) being in the bottom quartile on the ABIM IM-MOC examination, focusing on top and bottom quartile ranks. Quartile cut-offs were calculated across all physicians attempting the examination within a year.

RESULTS: When comparing NEJM Knowledge+ users to non-users on passing the MOC examination, the difference was $9.6 \%(\mathrm{p}=<0.001)$ with a regression adjusted difference of $10.6 \%(\mathrm{p}=<0.001)$. When comparing NEJM Knowledge+ users to non-users on having an examination score in the top quartile on the MOC examination, the difference was $9.6 \%(\mathrm{p}=0.016)$ with a regression adjusted difference of $10.7 \%$ ( $\mathrm{p}=0.009$ ). Further, when comparing NEJM Knowledge+ users to non-users on being in the bottom quartile on the MOC examination, the difference was $-9.6 \%(\mathrm{p}=0.003)$ with a regression adjusted difference of $-10.8 \%(\mathrm{p}=<0.001)$.

CONCLUSIONS: Physicians who were users of NEJM Knowledge+ had better performance on ABIM's IM-MOC examinations than similar physicians who were not users.

\section{ASSOCIATIONS BETWEEN AGE DISPARITIES IN TYPE 2 DIABETES AND RATES OF DIABETES-RELATED HOSPITAL USE AND DIABETIC COMPLICATIONS}

David C. Lee $^{1,1}$; Ta'Loria Young ${ }^{2}$; Christian A. Koziatek ${ }^{1}$; Christopher J. Shim ${ }^{3}$; Marcela Osorio ${ }^{1}$; Andrew J. Vinson ${ }^{1}$; Joseph Ravenell ${ }^{1}$; Stephen P. Wall ${ }^{1}{ }^{1}$ New York University School of Medicine, New York, NY; ${ }^{2}$ Touro College of Osteopathic Medicine, New York, NY; ${ }^{3}$ California Northstate University College of Medicine, Elk Grove, CA. (Control ID \#3138339)

BACKGROUND: Current guidelines for diabetes screening start at age 45 , but disparities in certain subgroups exist and poor diabetic outcomes are known to cluster in specific neighborhoods. The objective of this study was to quantify disparities in the age distribution of patients with type 2 diabetes by sex, race/ethnicity, and geographic location. We also studied how patient age relates to diabetes-related hospital use and development of diabetic complications.

METHODS: Using all-payer hospital claims data, we performed a cross-sectional analysis of patients with type 2 diabetes. Our study included patients in New York City as identified by geocoded home address. Patients aged 10 to 100 years old were identified as having type 2 diabetes based on diagnosis codes from emergency claims data from 2011-2015. Our main measures included the estimated prevalence of type 2 diabetes at each year of life, the age distribution of patients as stratified by decade, and the comparison of patient age in geographic hotspots of frequent diabetes-related hospital use and diabetic complications.

RESULTS: We identified 576,306 unique patients diagnosed with type 2 diabetes, which represented over half of all cases in New York City. Minority subgroups were on average 5.5 to 8.4 years younger than nonHispanic White patients. Males with type 2 diabetes were 2.6 years younger than females. At 45 years of age, the estimated prevalence of type 2 diabetes was $10.9 \%$ among Black patients compared to $5.2 \%$ among White patients. In our geospatial analyses, patients with type 2 diabetes were on average 5.9 years younger in hotspots of diabetes-related emergency department use and inpatient hospitalizations. The average age of patients with type 2 diabetes was 1.5 to 2.2 years younger in hotspots of microvascular diabetic complications.

CONCLUSIONS: We identified profound disparities in the age of patients with type 2 diabetes among minorities and in neighborhoods with poor health outcomes. The younger age of these patients may be due to earlier onset of diabetes and/or earlier death from diabetes-related complications. Our findings demonstrate the need for geographically targeted interventions that promote earlier diagnosis and better glycemic control to reduce disparities in diabetes burden.

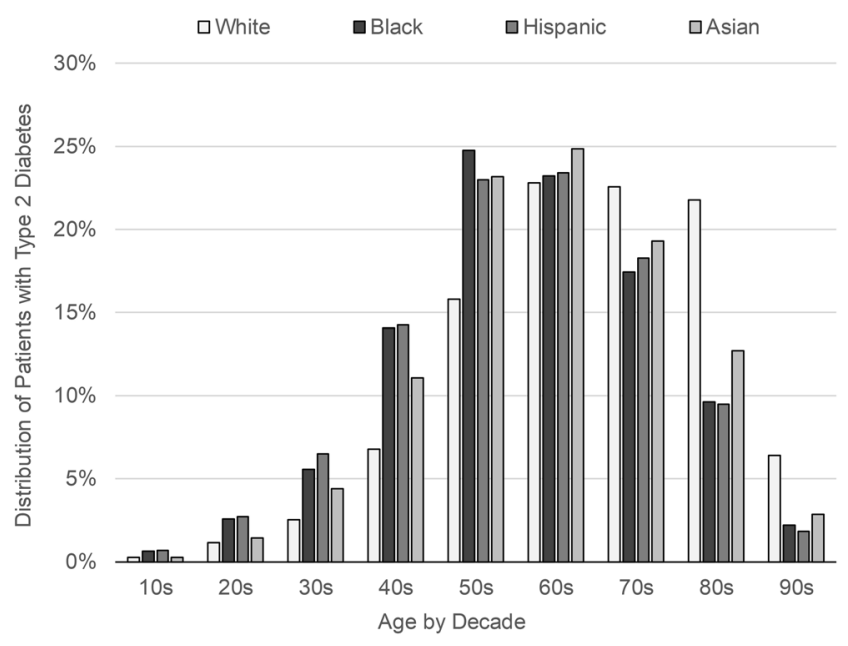

Age Distribution of Patients with Type 2 Diabetes by Race and Ethnicity

\section{ASSOCIATIONS BETWEEN NEIGHBORHOOD AND PO- LICE VIOLENCE, HYPERVIGILANCE, AND HYPER- TENSION AMONG RACIAL/ETHNIC MINORITIES IN CHICAGO}

Nichole Smith ${ }^{1}$; Dexter Voisin ${ }^{1}$; Joyce P. Yang ${ }^{2}$; Elizabeth L. Tung ${ }^{1} .{ }^{1}$ University of Chicago, Chicago, IL; ${ }^{2}$ Stanford University School of Medicine, Palo Alto, CA. (Control ID \#3183498)

BACKGROUND: Hypervigilance, a state of heightened awareness and watchfulness, is a consequence of violence that has been linked to negative psychosocial outcomes. While hypervigilance has been well-documented in Veteran populations, it has been poorly quantified in community populations that may experience chronic exposure to violence. Moreover, the long-term health effects of hypervigilance are not well understood. The objective of this study was to examine community and police violence, their association with hypervigilance, and the health consequences of hypervigilance in a sample of predominantly racial/ethnic minority adults.

METHODS: Primary care patients $(\mathrm{N}=506)$ were recruited from clinics in 2 epicenters of violent crime and surveyed using computer-assisted personal interviewing software. Lifetime violence exposure was measured using the Brief Trauma Questionnaire (BTQ), which included items for being a direct victim of community violence, or a witness, friend or relative of someone who died violently. BTQ items were also adapted to query participants about exposure to police violence. Hypervigilance was measured using the Brief Hypervigilance Scale. Linear regression models were used to assess 
hypervigilance scores as a function of violence exposure type, controlling for age, gender, race/ethnicity, educational attainment, insurance status, and employment status. Survey results were then paired with the electronic health record to examine associations between hypervigilance scores and blood pressure.

RESULTS: The sample included patients from an academic medical center $(n=419)$ and a federally-qualified health center $(n=87)$ in Chicago. Mean age was 60 years $(\mathrm{SD}=13)$. The majority identified as Black/African-American (75\%) or Hispanic/Latino (14\%). More than half $(56 \%)$ were insured by Medicaid/Medicare. Two-thirds $(62 \%)$ reported prior experience with community violence, and onethird $(30 \%)$ reported prior experience with police violence. Of the 263 participants who reported a previous police stop, 19\% met criteria for the stop being a traumatic event. The mean hypervigilance score in our population was $54.7 \%(\mathrm{SD}=28.1), 5.7 \%$ higher than the score correlated with PTSD in the validation sample. While experience with community violence was marginally associated with a $5.5 \%$ higher hypervigilance score (95\% CI, 0.2-10.8), experience with police violence was associated with a $9.8 \%$ higher hypervigilance score (95\% CI, 4.1-15.5). Among participants who reported a police stop, classification as a traumatic event was associated with an $18.6 \%$ higher hypervigilance score (95\% CI, 10.3-26.9). A hypervigilance score in the highest quartile was associated with higher systolic blood pressure (10.3 mmHg; 95\% CI, 4.3-16.2).

CONCLUSIONS: Neighborhood and police violence may have important linkages to both psychological and physical health. Understanding these linkages may help to inform both neighborhood policing practices and healthcare responses to violence in at-risk communities.

\section{ASSOCIATIONS BETWEEN REASONS FOR HOSPITALIZA- TION IN THE ELDERLY AND ONE-YEAR MORTALITY}

Chana A. Sacks; Katrina Armstrong; Thomas McCoy. Massachusetts General Hospital, Boston, MA. (Control ID \#3185288)

BACKGROUND: Optimal clinical decision making among the elderly is challenged by uncertain and potentially inaccurate understanding of life expectancy. Because clinicians can conflate reversibility of the condition prompting a hospitalization with longer-term likelihood of survival, determining 1-year mortality following common reasons for hospitalizations could improve clinicians' understanding of patient prognosis and clinical decision making, as well as the accuracy of information provided to patients and their families. METHODS: We conducted a retrospective cohort study, analyzing data from individuals discharged from Medicine services at 2 academic medical centers between January 1, 2005 and December 31, 2016. We included all patients 70 years of age or older who were admitted for 10 common diagnoses and survived to hospital discharge. We divided age into categories: 70-79 years, 80-89 years, and 90 and older. The primary outcome was death within 1 year after discharge as reported by public records (a composite from the Massachusetts Department of Public Health and Social Security Death Index).

RESULTS: The cohort included 36,291 hospitalizations of 23,071 unique individuals. $51 \%$ were male; $83 \%$ were white, and mean age was 81 years. The analysis included 116,404 person-years of follow up, with a median follow-up of 876 days. Overall, $26.3 \%$ of the cohort had died within 1 year of discharge. The table describes the variation in 1-year mortality based on age and reason for admission. CONCLUSIONS: There was substantial variation in 1-year mortality based on age and reason for admission. For example, people in their 70s admitted for a urinary tract infection had a higher 1-year mortality than those in their 90 s admitted with syncope. Accurate prognostication is important to inform clinicians, patients, and their families as they plan for the future and navigate decisions about goals of care.

\begin{tabular}{|l|c|c|c|}
\hline \multirow{2}{*}{} & \multicolumn{3}{|c|}{$\begin{array}{c}\text { Percent of patients } \\
\text { who died within 1 } \\
\text { year after discharge }\end{array}$} \\
\hline \multicolumn{1}{|c|}{$\begin{array}{l}\text { Reason for } \\
\text { Admission }\end{array}$} & $70-79$ & $80-89$ & $90+$ \\
\hline Syncope & $6 \%$ & $10 \%$ & $22 \%$ \\
\hline $\begin{array}{l}\text { Nonspecific chest } \\
\text { pain }\end{array}$ & $6 \%$ & $10 \%$ & $19 \%$ \\
\hline Acute MI & $11 \%$ & $20 \%$ & $33 \%$ \\
\hline COPD & $19 \%$ & $27 \%$ & $30 \%$ \\
\hline Gl hemorrhage & $20 \%$ & $24 \%$ & $39 \%$ \\
\hline Pneumonia & $25 \%$ & $27 \%$ & $34 \%$ \\
\hline UTI & $26 \%$ & $30 \%$ & $41 \%$ \\
\hline CHF & $26 \%$ & $33 \%$ & $46 \%$ \\
\hline $\begin{array}{l}\text { Acute renal } \\
\text { failure }\end{array}$ & $29 \%$ & $40 \%$ & $46 \%$ \\
\hline $\begin{array}{l}\text { Aspiration } \\
\text { pneumonitis }\end{array}$ & $40 \%$ & $44 \%$ & $57 \%$ \\
\hline
\end{tabular}

The percent of patients in each age group, for each reason for admission who died within 1 year after discharge.

ASSOCIATIONS BETWEEN SOCIAL RISK FACTORS AND
DIALYSIS FACILITY PERFORMANCE IN THE END-STAGE
RENAL DISEASE QUALITY INCENTIVE PROGRAM Andrew C. Qi; Anne M. Butler; Karen E. Joynt Maddox. Washington University School of Medicine, Saint Louis, MO. (Control ID \#3139408)

BACKGROUND: Medicare's End-Stage Renal Disease Quality Incentive Program (ESRD QIP) is a mandatory pay-for-performance program for U.S. dialysis facilities. While analyses of similar valuebased payment programs in other settings have shown performance to be related to social risk factors such as poverty and access to care, it is unknown whether the ESRD QIP displays similar patterns. We aim to determine whether dialysis facility performance and associated financial penalties under the ESRD QIP are associated with facility-level social risk factors.

METHODS: Retrospective, cross-sectional analysis of 6,314 U.S. dialysis facilities using data from the United States Renal Data System, American Community Survey, and Medicare for the 2018 ESRD QIP payment year. We used linear and logistic regression models to determine whether social risk factors, including poverty, race/ethnicity, and neighborhood income were related to performance and risk of receiving a penalty.

RESULTS: Facilities located in low-income ZIP codes and with high proportions of black patients had worse performance under the ESRD QIP and higher rates of penalization. In a multivariate model accounting for key facility-level characteristics, significant effects 
persisted for facilities in the bottom quintile of ZIP code income (odds ratio $[\mathrm{OR}]=1.53, \mathrm{p}=0.002$, vs. top quintile) and those in the top quintile of proportion of black patients served $(\mathrm{OR}=1.69$, $\mathrm{p}<0.001$, vs. other facilities). Among other covariates examined, independent (versus chain) status, large facility size, and urban location were the strongest structural predictors of poor performance and penalties.

CONCLUSIONS: Dialysis facility performance and financial penalization may be influenced by social risk factors potentially independent of the quality of care provided. Future research is needed to elucidate the interactions between sociodemographic factors and outcomes under the ESRD QIP and to determine appropriate adjustments to pay-for-performance program design to account for social risk.

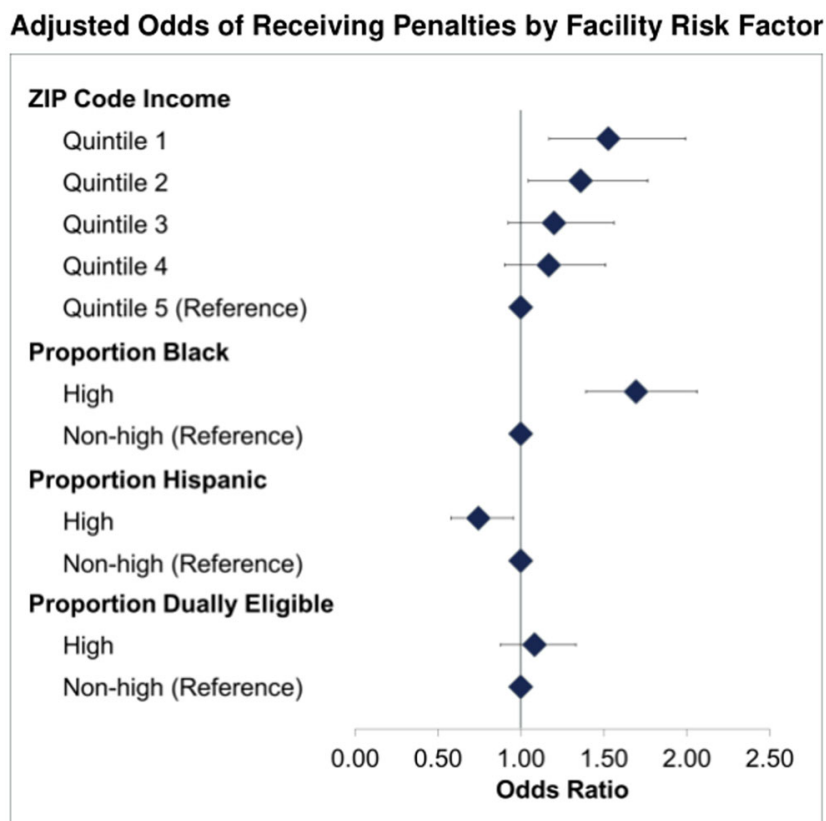

ASSOCIATIONS OF HEALTH LITERACY, PATIENT ACTIVATION, AND PATIENT-PROVIDER COMMUNICATION REGARDING HIGHER-RISK MEDICATION USE

Marina Arvanitis ${ }^{1}$; Laurie A. Hedlund ${ }^{1}$; Laura M. Curtis ${ }^{1}$; Stephanie Batio ${ }^{1}$; Stacy Bailey ${ }^{1}$; Danielle M. McCarthy ${ }^{1}$; Michael K. Paasche-Orlow ${ }^{2}$; William G. Adams ${ }^{2}$; Mary J. Kwasny ${ }^{1}$; Amy Moore $^{1}$; Michael S. Wolf ${ }^{1} .{ }^{1}$ Northwestern University, Chicago, IL; ${ }^{2}$ Boston University, Boston, MA. (Control ID \#3185951)

BACKGROUND: Health literacy and patient activation have been independently associated with a variety of health outcomes and behaviors. Despite these independent associations, both are thought to affect health, at least in part, by way of communication between patients and healthcare providers. We sought to better understand the similarities and differences in associations between health literacy, patient activation, and patientprovider communication in the context of prescribed medication.

METHODS: Baseline data was analyzed from an NIDDK-funded, 2arm, patient-randomized controlled trial of a medication risk communication and surveillance strategy. Participants were adults cared for at one of 7 federally qualified health centers, who received a new prescription or dose of a medication considered to be of higher risk per FDA labeling. All participants completed the Consumer Health Activation Index (CHAI), the Newest Vital Sign (NVS), and modified versions of the supplemental items of the Consumer Assessment of Health Providers Survey (CAHPS) to evaluate patient-perception of the extent and quality of verbal counseling on medications.
RESULTS: In all, 581 participants completed both the health literacy (NVS) and patient activation (CHAI) assessments at baseline. Participants' mean age was 50,71\% were African-American, and $40 \%$ reported an income of less than $\$ 10,000 /$ year. The majority had low to moderate patient activation and limited health literacy ( $84 \%$ and $68 \%$ respectively). There was no correlation between CHAI and NVS total scores (rho = $-0.05, \mathrm{p}=0.20)$ overall. However, the majority $(67 \%)$ of those with limited health literacy also reported CHAI scores consistent with low to moderate activation $(\mathrm{p}=0.05)$. Compared to those with high activation, participants with low to moderate activation were less likely to report having received instructions from their provider about how to take their newly prescribed medication ( 330 vs $72, p=0.04$ ), or had possible side effects explained (192 vs 46, $\mathrm{p}=0.05$ ). We did not observe similar differences among participants across health literacy levels. By contrast, those with limited health literacy were more likely than those with adequate health literacy to report their provider had suggested ways to help them remember to take their medication (146 vs $41, \mathrm{p}=0.0007$ ). Neither patient activation nor health literacy was associated with patientprovider communication regarding risks, benefits, and patient opinions regarding medication use.

CONCLUSIONS: Patient activation and health literacy were associated with different patient-reported patterns of provider communication regarding high-risk medication use, and neither were associated with higher-level discussions about risks, benefits, or patient preferences. Ongoing, longitudinal analyses will help determine the directionality of these associations, and help to better target communication interventions to providers, or patients depending on their level of activation and/or health literacy.

\section{ATRIAL FIBRILLATION IS ASSOCIATED WITH INCREASED IN-HOSPITAL MORTALITY IN PATIENTS WITH CLOSTRIDI- UM DIFFICILE INFECTION.}

Dipesh Ludhwani $^{1}$; Maryna Shayuk ${ }^{1}$; Karam Khaddour ${ }^{1}$; Belaal Sheikh ${ }^{2}$. ${ }^{1}$ Rosalind Franklin University of Medicine and Science at NorthwesternMcHenry hospital, McHenry, IL; ${ }^{2}$ Chicago Medical School, McHenry, IL. (Control ID \#3176126)

BACKGROUND: Clostridium Difficile Infection (CDI) and Atrial Fibrillation (AF) are common medical entities encountered in the hospital. Both conditions warrant hospital admission and have been well established as major health care burdens of the society. Previous studies have identified advanced age as a poor prognostic factor for CDI. Large-scale studies analyzing outcomes of CDI in presence of concomitant AF are lacking.

METHODS: All patients with age greater than 18 years and admitted under the primary diagnosis of CDI (ICD-10-CM code A04.72) $(\mathrm{n}=66,602)$ were identified in 2016 National Inpatient Sample (NIS). ICD-10 codes I48.0, I48.1, I48.2, I48.3, and I48.4 were used to further categorize patients into two groups based on the presence or absence of AF. Patients with CDI and without previous history of AF served as the reference population. Using multivariate logistic regression model, confounders were adjusted and in-hospital mortality was compared for both groups. Additionally, the odds ratios were calculated for other complications such as acute renal failure (N17), hypovolemic shock (R57.1) requiring vasopressor support (3E033XZ), and acute systolic or diastolic heart failure exacerbation (I50.21, I50.23, I50.31, and I50.33). Multivariate linear regression analysis was done to compare the total length and cost of hospital stay for both groups.

RESULTS: Amongst 66,602 admissions for CDI, 8668 patients were found to have a secondary diagnosis of AF. Patients with CDI and AF had a $29 \%$ increase odds of in-hospital mortality as compared to the reference population (OR-1.29, 95\% CI=1.19-1.41, $\mathrm{p}<0.0001)$. These patients were also noted to have a higher probability of acute renal failure, acute heart failure exacerbation and hypovolemic shock requiring vasopressor support (see table 1). Mean length and cost of the hospital stay were higher for patients with $\mathrm{CDI}$ and $\mathrm{AF}$. 
CONCLUSIONS: History of AF in patients admitted with CDI is associated with longer complicated hospital course and increased inhospital mortality. Resuscitation for hypovolemia and acute renal failure tends to occur at the expense of worsening heart failure. Treatment strategy for such patients should include a multidisciplinary team approach and intensive monitoring with a close eye on hemodynamic status.

\section{ATTITUDES TOWARDS GENETICS TRAINING AMONG IN- TERNAL MEDICINE HOUSE STAFF}

Raja Chandra Chakinala2; Steven Murphy1; Akshay Khatri2; Leanne Forman2; Jagjit Khosla2; Kartik Dhaduk2; Melissa Gennarelli2; Rebecca Newman2; Christopher Nabors2. 1New York Medical College, Valhalla, NY; 2New York Medical College at Westchester Medical Center, Valhalla, NY. (Control ID \#3163528)

BACKGROUND: The availability of genetic testing information is increasing. Perceptions regarding its utility vary among practicing providers. In this study, we sought to determine the attitudes and practices of residents in training towards genetic testing.

METHODS: Categorical trainees at a tertiary care center completed a baseline survey (12-2017 to 4-2018) followed by an educational intervention based on materials from the Genetics Education Canada Knowledge Organization (4 hours of lecture; 3-hours online training) and then a post-training survey (5-2018).

RESULTS: Baseline response rate was 80\% (36/45): PGY-1 (16, 44\%), PGY-2 (10, 28\%), PGY-3 (10, 28\%). 72\% (26) had taken a medical school genetics course; most reported prior training to be insufficient $(21,61 \%)$. $28 \%$ (10) ordered a genetic test once/month or once/year $22 \%$ (8). Common tests were for thrombophilia (52\%), BRCA (20\%) and hemochromatosis $(8 \%)$. Respondents believed patients would be interested in genetic testing $(61 \%, 22)$, that testing is useful $(61 \%, 22)$ and within 5 years would improve outcomes $(50 \%, 18)$, but could lead to insurance discrimination $(36 \%, 13)$. $61 \%$ (22) knew BRCA transmission mode, frequencies of BRCA mutations among breast cancer patients $(67 \%, 24)$ and the percentage of persons with Lynch Syndrome who would develop colorectal cancer $(72 \%, 26)$. A posttraining pilot survey was offered to those who attended all lectures $(\mathrm{N}-15)$. Of those, 11 responded. Post-intervention results differed in a number of respects. Perceived frequency of testing increased to $36 \%$ monthly and to $45 \%$ yearly. $72 \%$ and $18 \%$ reported they were somewhat or much more able to manage genetic testing after training. Pre-training/post-training mean self-confidence ratings (low 1 to high 5 ) in the ability to elicit information were $(3.46 / 3.64, \mathrm{p}=0.08)$, to interpret results $(2.77 / 3.45, \mathrm{p}=0.29)$, to evaluate usefulness $(2.69 / 3.27, \mathrm{p}=0.04)$, to order test/referral $(2.49 / 3.45, \mathrm{p}=0.07)$, to discuss risks/benefits $(2.37 / 3.45, \mathrm{p}=0.02)$ and to provide counselling $(2.46 /$ $3.36, \mathrm{p}=0.10$ ). Following training, $73 \%$ believed their training was either sufficient/more than sufficient compared to $60 \%$ prior to training. Medical knowledge related to hereditary cancers was comparable pre/postintervention, while all residents agreed (post-intervention) on the importance of taking a detailed family history to interpret tests related to late onset Alzheimer's disease.

CONCLUSIONS: In this study, internal medicine residents were receptive to learning more about genetic testing and believed results of genetic tests would become more important as a guide to treatment in the next five years. Following a core set of lectures and online training, most respondents felt more confident in managing genetic test information and reported ordering genetic tests more frequently. Medical knowledge related to hereditary cancers was similar following training. Due to the small post-intervention pilot sample size, results will require further study for confirmation.

\section{AVAILABILITY OF GROCERY DELIVERY IN AREAS WITH LIMITED FOOD ACCESS: AN EIGHT-STATE ANALYSIS Eric Brandt ${ }^{3}$; David Silvestri ${ }^{3}$; Jerold Mande ${ }^{1}$; Joseph S. Ross ${ }^{2}{ }^{1}$ Tufts University, Boston, MA; ${ }^{2}$ Yale University School of Medicine, Woodbridge,}

CT; ${ }^{3}$ Yale University School of Medicine, New Haven, CT. (Control ID \#3184225)

BACKGROUND: The United States Department of Agriculture (USDA) Supplemental Nutrition Assistance Program (SNAP) provides federallyfunded nutritional economic support to qualifying low-income individuals and families to help reduce financial barriers to food access. Nevertheless, about one fourth of the 15 million households receiving SNAP nationwide currently reside in USDA-defined "foods deserts" — census tracts with belowmedian incomes, low grocer availability, and limited vehicular access. To expand food access, the 2014 Farm Bill authorized an Online Purchase Pilot (OPP) enabling SNAP recipients to spend benefits on groceries online. However, benefits cannot be used to pay for grocery delivery fees, potentially limiting the impact of online purchasing for increasing access to grocers, particularly in areas with limited vehicular access. Accordingly, we sought to quantify the proportion of USDA-defined food deserts and SNAP households in OPP states serviceable by existing grocery delivery networks via online grocers.

METHODS: We used the USDA Economic Research Service Food Access Research Atlas database to identify food deserts in OPP states (AL, IA, MD, NE, NJ, NY, OR, WA). The US Housing and Urban Development United States Postal Service ZIP code crosswalk data allowed census tract to zip code conversion. Using the USDA Food and Nutrition Service SNAP retailer database, we identified all retailers with $>5$ stores in study states and determined if they delivered to a ZIP code using manual retailer website review. Food desert census tracts were classified as fully-, partially-, or notdeliverable, according to whether all, some, or no corresponding ZIP codes fell within delivery areas, respectively. Primary outcomes were the proportion of food deserts and SNAP households serviceable by grocery delivery, stratified by urban status.

RESULTS: Food deserts comprise $9.5 \%(\mathrm{n}=1,250)$ of census tracts and $18.4 \%(\mathrm{n}=506,863)$ of SNAP households in OPP states. Grocery delivery is available fully to $85.9 \%(\mathrm{n}=1,074)$, partially to $2.6 \%(\mathrm{n}=32)$, and not at all to $11.5 \%(n=144)$ of food desert census tracts, and to $87.3 \%(n=442,643), 2.7 \%$ $(n=13,696)$, and $10.0 \%(n=50,524)$ of SNAP households within food deserts, respectively. Among rural food desert tracts $(n=59)$ grocery delivery is available fully to 0 , partially to $27.1 \%(\mathrm{n}=16)$, and not at all to $72.9 \%$ $(n=43)$, while in urban food deserts $(n=1,191)$, grocery delivery is available fully to $90.2 \%(\mathrm{n}=1,074)$, partially to $1.3 \%(\mathrm{n}=16)$, and not at all to just $8.5 \%$ $(\mathrm{n}=101)(\mathrm{p}<0.001)$.

CONCLUSIONS: Expanding SNAP benefits for grocery delivery in OPP states could increase access to grocery stores for roughly 7 of 8 food desert census tracts and corresponding SNAP households, with greatest impact in urban areas. Planned OPP expansion nationwide could consider financing grocery delivery, which if coupled with incentives that direct consumers to highest-quality food choices could increase the utility of online grocery purchasing.

BARRIERS AND FACILITATORS OF SELF-MANAGEMENT BEHAVIORS AMONG PATIENTS WITH MULTIPLE CHRONIC CONDITIONS: A MIXED-METHODS INVESTIGATION

Rachel O'Conor ${ }^{1}$; Kimberly A. Muellers ${ }^{2}$; Andrea Russell ${ }^{1}$; Sarah Wright ${ }^{1}$; $\overline{\text { Sara Thompson }}^{2}$; James W. Griffith ${ }^{1}$; Michael S. Wolf ${ }^{1}$; Juan Wisnivesky ${ }^{3}$; Alex Federman $2 .{ }^{1}$ Northwestern University, Chicago, IL; ${ }^{2}$ Icahn School of Medicine at Mount Sinai, New York, NY; ${ }^{3}$ Mount Sinai School of Medicine, New York, NY. (Control ID \#3177569)

BACKGROUND: Multimorbidity challenges disease management among older adults, yet self-management programs are often condition-specific. We investigated how individuals managing multiple chronic conditions (MCC) navigate their conditions and identify any difficulties they face in their management.

METHODS: We conducted a mixed-methods study using semi-structured interviews among a subset of patients $(n=18)$ participating in a cohort study 
( $n=302)$ of adults with COPD, hypertension, and diabetes. Participants selfreported their nutrition using the Hypertension Self-Care Activity Level scale, and medication adherence for each of the three conditions using the Medication Adherence Report Scale, and were categorized as good (adequate adherence to all medications), mixed (adequate and poor adherence across medications), or poor (poor adherence to all medications). Qualitative interviews explored patients' experiences with MCC and their self-care strategies. Four investigators used content and constant comparative analysis to analyze transcripts and compared quantitative and qualitative data.

RESULTS: Among the subsample, the mean age was 65 years, $49 \%$ were male, 50\% black, and 8, 7, and 3 individuals demonstrated good, mixed, and poor adherence. Individuals reported an overall health management strategy (taking medications, engaging in physical activity, avoiding food high in fat, salt, or sugar, and eliminating tobacco), as opposed to managing each condition separately. Individuals with good or mixed adherence described a daily medication routine that facilitated regular medication taking behaviors. Walking was universally recognized as beneficial, but few engaged in it regularly, due to co-morbidities or inclement weather. Those who did had access to fitness centers with senior programming. Regarding their diet, patients reported avoiding unhealthy foods, rather than seeking nutritious options. While many were aware that they should eat healthy, quantitative data found only 2 patients had high diet quality and qualitative data illustrated many misperceptions regarding what constituted healthy foods (i.e. eliminating candy, yet drinking soda frequently). Individuals with mixed adherence sought to maintain their mental well-being through mental calmness. Many reported coexisting mental illness and managing their behavioral health was critical to their health. Lastly, intrinsic motivation to adopt healthy lifestyle behaviors and adhere to prescribed medications did not vary across adherence groups; however, socioeconomic stressors were common among most participants and challenged their ability to follow through with their entire self-care regimen.

CONCLUSIONS: We found patients with MCC were highly motivated and aware of the lifestyle modifications they needed to make, but behaviorally, many may only require small adjustments in order to support behavior change. A tailored approach to patients' personal barriers (social, behavioral, misperceptions) may improve self-management programs.

\section{BARRIERS AND FACILITATORS TO ENGAGING HIGH-NEED VETERAN PATIENTS: REFLECTIONS OF KEY STAKE- HOLDERS IN VA'S PRIMARY CARE INTENSIVE MANAGE- MENT (PIM) TEAMS}

Alicia Bergman ${ }^{1}$; Susan E. Stockdale ${ }^{2}$; Marian L. Katz ${ }^{3}$; Donna M. Zulman ${ }^{4}$; Evelyn T. Chang ${ }^{5}{ }^{1}$ VA Greater Los Angeles, North Hills, CA; ${ }^{2}$ Greater Los Angeles VA Healthcare System, Sepulveda, CA; ${ }^{3}$ Veterans Health Administration, North Hills, CA; ${ }^{4}$ Stanford University and VA Palo Alto, Stanford, CA; ${ }^{5}$ VA- Greater Los Angeles, Los Angeles, CA. (Control ID \#3184399)

BACKGROUND: Patient engagement among complex high-need patients in intensive primary care programs can be challenging, as many patients experience medical, social, and behavioral barriers to active participation in their care. The Veterans Health Administration (VA) has implemented and tested intensive primary care programs called PACT Intensive Management (PIM). Our goal was to describe barriers and facilitators to engaging high-risk patients by conducting a qualitative analysis of VA PIM staff members' interviews.

METHODS: Semi-structured interviews with VA PIM staff members and program leaders regarding their experiences with and reflections on PIM. Interviews were recorded, transcribed, and analyzed to identify patient engagement barriers and facilitators. Twenty-nine interdisciplinary staff members who care for complex high-need patients, including physicians, nurses, psychologists, and social workers.

RESULTS: Participants described two main types of barriers and facilitators: those related to patient characteristics and those related to provider/ system level characteristics. Patient-level characteristics that facilitated engagement in PIM and their healthcare included: having a pre-existing motivation to improve one's health, having certain chronic conditions and already adhering to a regimen, being a "frequent flier" (e.g., multiple visits per week), being lonely and welcoming human contact, having a desire for extra assistance and interaction, and having been a prior recipient of homebased primary care services and being habituated to healthcare staff entering homes. Provider/system or other-level characteristic-related engagement facilitators included providers making home visits and assessing a patient's home environment, providers having successfully established relationships with patient, patient's family members, connecting patients with other programs and services (e.g., VA or non-VA), having mental health services offered in the primary care clinic (e.g., assisting patients to avoid stigma). Patient-level characteristics that posed a barrier to engagement included: an active substance use disorder, mental health conditions (e.g., depression, anxiety), and non-adherence to recommended treatment regimens. Provider/system or other-level characteristic-related engagement barriers included the time intensive nature of the PIM work, providers changing (e.g., rotating residents), provider compassion/empathy fatigue and/or not understanding psycho-social issues, and obstruction of care by the patient's caregiver.

CONCLUSIONS: Staff in intensive primary care programs identified numerous patient- and provider/system-level factors that influence the engagement of complex, high-need patients and may serve as targets for focused interventions. Increased awareness of barriers and facilitators to patient engagement among high-risk patients may inform intervention design for intensive primary care programs and other services for highneed patients.

\section{BARRIERS TO WELLNESS: FACTORS CONTRIBUTING TO PROVIDER BURNOUT IN AN URBAN ACADEMIC PRACTICE}

Kira Y. Xie; Stephanie Duarte; Gabi Schiller; Lauren Peccoralo. Icahn School of Medicine at Mount Sinai, New York, NY. (Control ID \#3185936)

BACKGROUND: Medical provider burnout is now recognized as an increasingly prominent problem threatening the integrity of the medical profession. Compared to other professions, physicians are $36 \%$ more likely to experience symptoms of burnout, with high rates of burnout in primary care providers. Burnout can negatively impact many factors such as productivity, patient trust, and provider mental health. While providers have many possible sources of burnout, the unique milieu of stressors in each clinic environment must be assessed to ensure key contributors are attended to. In our study, we analyzed free text survey responses in order to best identify challenges to provider wellness at an urban academic continuity practice.

METHODS: Based on the American Medical Association's STEPS Forward program, our Division of General Internal Medicine formed a faculty wellness committee, which created an anonymous survey with quantitative and qualitative components. The aim of the qualitative survey was to assess providers' major barriers to wellbeing and suggestions for improvement. In this study, the qualitative responses were analyzed using a grounded theory approach. The first reviewer used inductive and deductive strategies to encode statements and then generate overall categories for barriers to well-being. A second reviewer independently assigned survey responses to the categories generated by the first reviewer to determine inter-reviewer reliability. Discrepancies were resolved via discussion between the reviewers and the PI.

RESULTS: The inter-rater reliability was $49 \%$. The most often cited factors contributing to provider burnout in order of frequency were lack of teamwork and communication, poor work flow, insufficient staffing, malfunctioning equipment, limited work space, and staff not functioning at the top of their licenses. Examples highlighting limited space, low staff functioning, and equipment malfunction include: "Space cramped, difficult to find space to informally gather as team", "I am often doing things outside 
the scope of my job...but most of the rest of the non-physician staff view their roles as having very clear boundaries", and "A provider should not be looking for staples, fixing printers and rooming patients." Other barriers to well-being were misaligned leadership values, lack of continuing education resources, missing supplies, heavy workload, and insufficient rewards or incentives.

CONCLUSIONS: Faculty in an urban academic center reported a number of barriers to well-being on a qualitative survey. The main factors were poor team cohesiveness and work flow, environmental dysfunction, and insufficient staffing. Limitations of the study include small sample size, qualitative analysis, and lack of generalizability to other clinics. However the utility of this analysis is that it can by hypothesis generating for future quantitative work, and it can provide ideas for potential interventions to improve wellbeing for faculty in this clinic.

BEHAVIORAL HEALTH INTEGRATION IN VA PATIENTCENTERED MEDICAL HOMES AND CLINICAL QUALITY OF MENTAL HEALTH CARE

Lucinda B. Leung ${ }^{1,}$; Edward P. Post ${ }^{2}$; Erin L. Jaske ${ }^{3}$; Kenneth B. Wells ${ }^{4}$; Lisa V. Rubenstein ${ }^{5} .{ }^{1}$ UCLA David Geffen School of Medicine \& West LA VA Medical Center, Los Angeles, CA; ${ }^{2}$ VA Ann Arbor Healthcare System and University of Michigan, Ann Arbor, MI; ${ }^{3}$ Department of Veteran Affairs, Seattle, WA; ${ }^{4}$ UCLA, Los Angeles, CA; ${ }^{5}$ RAND and UCLA, Medford, OR; ${ }^{6}$ VA Greater Los Angeles, Los Angeles, CA. (Control ID \#3179178)

BACKGROUND: Behavioral health integration in primary care is effective but challenging to disseminate and implement in healthcare systems. Over the past decade, the Primary Care-Mental Health Integration (PCMHI) (i.e., care management, specialist collaborative care) and Patient Aligned Care Teams (PACT) (i.e., patient-centered medical home) initiatives transformed VA primary care clinics nationally into team-based care models to improve care for patients with psychiatric conditions. With increasing use of PC-MHI services over time, mental health care has accordingly shifted from specialty to PACT/primary care settings. We examined the combined effects of PC-MHI and PACT initiatives on quality of mental health care among VA primary care patients.

METHODS: We examined 828,050 primary care patients in 396 VA clinics mandated to offer PC-MHI services nationally (10/1/2013-9/30/ 2016). We calculated each clinic's PC-MHI penetration (i.e., the proportion of assigned primary care patients seen by a PC-MHI provider) and PACT implementation Progress Index $\left(\mathrm{PI}^{2}\right)$ (i.e., measure of core PACT components [e.g., access, care coordination]) annually. Trained chart abstractors reviewed patient records for 11 process quality measures for psychiatric illness screening and management. In multilevel logistic regressions, we used clinic PC-MHI penetration to predict odds of meeting each quality measure, controlling for $\mathrm{PI}^{2}$, regional healthcare system, clinic characteristics (e.g., size, rurality), and patient characteristics (e.g., age, gender).

RESULTS: Clinic screening rates for depression, post-traumatic stress disorder (PTSD), and alcohol misuse were high (on average, 96\%, 99\%, and $97 \%$ ). Averages rates of clinician follow-up within 24 hours following abnormal screening were 64\% (depression), 79\% (PTSD), and 79\% (alcohol misuse). In adjusted models, there were no PC-MHI or $\mathrm{PI}^{2}$ effects observed among all mental health care quality indicators. There were no observed differences in care quality due to differences in gender, racialethnicity, homelessness, physical comorbidity, and serious mental illness. There were slight improvements to care quality related to patient age - timely follow-up of abnormal depression (Odds ratio[OR] $=1.01$; $\mathrm{p}=.04)$ and PTSD (OR=1.01; $=.01]$ screening).

CONCLUSIONS: Although mental health treatment uptake has historically been poor in primary care, VA primary care patients continue to receive high quality mental health care (i.e., screening and timely followup). Without changing care quality, $\mathrm{PC}-\mathrm{MHI}$ actively engaged more patients in care and reduced their reliance on specialty-based mental health resources. We observed no differences in VA clinical care quality for patients of racial-ethnic minorities, nor for vulnerable patients (i.e., homeless, serious mental illness). Results examining VA's national dissemination of PC-MHI and PACT models highlight remaining mental health quality gaps and encourage continued model optimization to improve mental health care processes in primary care.

\section{BEYOND THE BOOM: HEPATITIS C AND PEOPLE WHO IN- JECT DRUGS AT A SUBURBAN TERTIARY ACADEMIC MEDI- CAL CENTER 2016-2017}

Kerim Odekon; Kalie L. Smith; Silvia L. Bronson; Luis A. Marcos. Stony Brook Medicine, Stony Brook, NY. (Control ID \#3184694)

BACKGROUND: In 2004, adults aged 40-60, the 1945-1965 "Baby Boomer" birth cohort comprised 74\% of NYS Hepatitis C (HCV) cases. By 2014, a bi-modal distribution emerged with Baby Boomers' share of $\mathrm{HCV}$ cases falling to $45.1 \%$, and a new second peak of 20-40-year olds accounting for $38 \%$ of all HCV cases (2016 NYS DOH HIV Epidemiological Profile). In 2016 Stony Brook Hospital, a Tertiary Academic Hospital in Long Island NY, instituted a successful opt-out HCV screening and linkage to care initiative targeted to all persons born between 1945-1965. Long Island (comprising Nassau and Suffolk Counties) is an epicenter of the national opioid use and overdose epidemic. This study aims to look beyond Baby Boomers to quantify Hepatitis $\mathrm{C}$ screening rates for high risk and hard to reach suburban people who inject drugs (PWID) born after 1965.

METHODS: This study is a retrospective chart review 1/1/2016-11/30/ 2017 at Stony Brook University Hospital to identify Opioid Related Visits (ORV) and patients with a history of injection drug use. Charts were selected based on discharge ICD-10 codes related to opioid use. ER visits and hospital admissions were classified as ORV if associated with nonprescribed opioid use and 1) infection, 2) overdose, and/or 3) altered mental status. Individual charts were electronically queried using chart search function for the following keywords: Heroin, Cocaine, IVDA, IVDU or IV Drug to identify people with a hospital visit related to non-prescribed opioid use and/or history of injection drug use.

RESULTS: This study identified 1,030 Opioid Related Hospital Visits made by 684 unique people born after 1965 with a history of injection drug use. Only $24 \%$ of these individuals had any hepatitis $\mathrm{C}$ testing. $57 \%$ of these visits were for opioid overdose. For individuals who presented with an opioid overdose and documented history of injection drug use, only $19 \%$ had a hepatitis $\mathrm{C}$ test on record within the study period. Among this group, testing was more frequently associated with age and female gender, with only $15 \%$ of men aged 18-29 having any hepatitis $\mathrm{C}$ testing compared to $32 \%$ of women aged 30-44 and 45-51.

CONCLUSIONS: In July 2018, NYS Governor Cuomo announced a declaration to "end hepatitis C in New York State." The CDC recommends testing anyone who has injected drugs for $\mathrm{HCV}$. The relatively low $\mathrm{HCV}$ screening rates for young people with a recent Opioid Related Visit and history of injection drug use suggests the need to expand HCV screening programs to target PWID as a necessary first step towards eradicating HCV virus in Long Island. Stigma around injection drug use, along with suburban geography pose unique challenges for outreach, screening, harm reduction, and treatment. Healthcare facilities must move beyond Baby Boomers and target screening and harm reduction efforts towards people who inject drugs to eradicate Hepatitis $\mathrm{C}$.

BLENDED COLLABORATIVE CARE FOR TREATING SYSTOLIC HEART FAILURE AND CO-MORBID DEPRESSION: 12-MONTH PRIMARY OUTCOMES FROM THE HOPEFUL HEART TRIAL $\underline{\text { Bruce L. Rollman }}{ }^{1}$; Amy M. Anderson ${ }^{1}$; Kaleab Abebe ${ }^{1}$; Kwonho Jeong ${ }^{1}$; Bea Herbeck Belnap $^{1}$; Ravi Ramani2; Matthew Muldoon ${ }^{2}$; Jordan F. Karp ${ }^{1}$. ${ }^{1}$ University of Pittsburgh, Pittsburgh, PA; ${ }^{2}$ UPMC, Pittsburgh, PA. (Control ID \#3185822) 
BACKGROUND: Heart failure (HF) affects 6.6 million Americans with over 650,000 newly diagnosed cases and 330,000 deaths annually. Yet despite improvements in care, HF mortality is essentially unchanged in recent years. One potential reason is depression. It is co-morbid in nearly $40 \%$ of hospitalized HF patients and linked to reduced healthrelated quality of life (HRQoL) and increased mortality, but often goes untreated. The NHLBI-funded Hopeful Heart Trialis the first study to evaluate the impact of a collaborative care program for treating depression in HF patients.

METHODS: From 3/14 to 10/17, we screened inpatients with systolic HF (ejection fraction (EF) 45\%) and NYHA class II-IV symptoms for depression using the Patient Health Questionnaire (PHQ-2) at 8 Pittsburgh-area hospitals. Two weeks post-discharge, we telephoned screen-positive patients (PHQ-2 (+)) to administer the PHQ-9 and randomized those who scored 10 to either their PCPs' "usual care" (UC) or to one of two nurse-provided, 12-month telephone-delivered collaborative care programs for treating: (1) HF and depression ("blended"); or (2) HF alone (attention control (AC)). Study nurses met with clinicianinvestigators for weekly case review sessions and then gave treatment advice to patients and their PCPs. To facilitate comparisons, we also randomly selected a cohort of non-depressed HF patients (PHQ-2()/PHQ-9 <5). At baseline, we collected sociodemographic and clinical data, and then monitored outcomes through periodic blinded telephone assessments and vital status determination.

RESULTS: Of the $756 \mathrm{HF}$ patients recruited (629 depressed; 127 nondepressed; mean age: 64, 56\% male, 73\% White, mean EF 28\%), those depressed had worse mental HRQoL (SF-12 MCS, mean: 40.1 vs. 60.5), physical function (Kansas City Cardiomyopathy Questionnaire): 40.5 vs. 76.8), and mood (PROMIS Depression: 60.0 vs. 41.8) than those nondepressed (all $\mathrm{P}<0.001$ ), but were otherwise similar medically. Compared to UC, "blended" care patients reported a 0.34 ES improvement on the SF-12 MCS, our primary outcome measure (95\% CI: $0.13-0.57)$, and $0.47 \mathrm{ES}$ improvement on the PROMIS-D (0.72-0.22) at 12-months. Compared to AC, "blended" care patients also reported improved PROMIS-D (0.24 ES; 0.44-0.04), but not SF-12 MCS scores (0.09 ES; P=0.33). We confirmed 12-month vital status on all 756 patients and identified 95 deaths $(82$ depressed, 13 non-depressed); all-cause mortality for "blended", AC, UC, and non-depressed control patients was similar at $0.13,0.14,0.15$, and 0.10 per person-year of observation, respectively ("blended" vs. AC: $\mathrm{P}=0.86$; "blended" vs. UC: $\mathrm{P}=0.65$; baseline depressed vs. nondepressed: $\mathrm{P}=0.39$ ). CONCLUSIONS: "Blended" collaborative care for treating both HF and depression improves HRQoL and mood symptoms more than physicians' UC at 12-months following hospitalization. Analyses are ongoing and we will present additional data on the impact of our interventions on health services utilization, mortality, and other outcomes.

\section{BRCA TESTING IN MASSACHUSETTS AMONG WOMEN WITH PRIVATE INSURANCE OR MEDICAID, 2011-2015}

Lydia E. Pace ${ }^{1}$; Christopher F. Baum ${ }^{2}$; Krisztina Horvath ${ }^{2}$; Sahitya Raja ${ }^{3}$; Jessica Cohen ${ }^{4}$; Summer S. Hawkins ${ }^{2}$. ${ }^{1}$ Brigham and Women's Hospital, Boston, MA; ${ }^{2}$ Boston College, Chestnut Hill, MA; ${ }^{3}$ Brigham and Women's Hospital, Somerville, MA; ${ }^{4}$ Harvard T.H. Chan School of Public Health, Boston, MA. (Control ID \#3185293)

BACKGROUND: There are growing concerns about inequitable access to and uptake of genetic medicine in the United States. BRCA1/2 testing has increased among privately-insured women, likely due to declining test costs, increased public awareness, and the Affordable Care Act's requirement that most private insurers cover testing without cost-sharing for highrisk women. Select state Medicaid programs cover BRCA testing, including Massachusetts' Medicaid program, MassHealth, but BRCA testing rates among Medicaid-insured women have been very rarely examined. We compared rates and trends in BRCA testing from 2011-2015 in Massachusetts between privately-insured and MassHealth- insured women.
METHODS: We used the Massachusetts All-Payer Claims Database to identify insurance claims for BRCA tests between January 1, 2011 and June 30, 2015 among women aged 18-64 with private insurance or MassHealth who had at least 12 months of continuous insurance enrollment before any BRCA claim. We identified whether women had breast or ovarian cancer as identified in ICD9/10 codes within 12 months before testing. We calculated monthly testing rates and mean monthly testing rates per year. We calculated absolute and relative differences in mean monthly rates between privately-insured and MassHealth-insured women and used linear trend modeling to examine trends in relative rate differences across years. Our study was considered exempt by the Boston College Institutional Review Board.

RESULTS: Overall, testing rates were lower among MassHealthinsured women compared with privately-insured women in all years, but mean monthly rates increased from 3.7 per 100,000 in 2011 to 14.7 per 100,000 in 2015 among MassHealth-insured women and from 9.3 to 18.4 per 100,000 in privately-insured women. The absolute mean monthly rate difference between privately- versus MassHealth-insured women declined from 5.6/100,000 (2011) to $3.8 / 100,000$ (2015). The relative difference declined from 2.5 to 1.3 , converging by $31 \%$ per year $(\mathrm{p}<.001)$. Trends and differences between groups varied by age. Among women aged 18-34, by 2015 the BRCA testing rate among MassHealth-insured women (7.9/ $100,000)$ was higher than among privately-insured women (5.1/ $100,000)$. The proportion of women without breast or ovarian cancer diagnoses obtaining BRCA testing was stable at $6 \%$ in both groups across years.

CONCLUSIONS: From 2011-2015, BRCA1/2 testing rates were lower among MassHealth-insured women compared with privatelyinsured women in Massachusetts. However, the difference decreased over time, and the difference was smaller among younger women. Our study suggests that in Massachusetts BRCA testing is not utilized equally among publicly- and privately-insured populations, though this gap may be narrowing. Future research should investigate contributing factors and examine other states.

\section{BREAST CANCER SCREENING OUTCOMES USING DIGI- TAL BREAST TOMOSYNTHESIS: EVIDENCE FROM A CONTEMPORARY COHORT}

Ilana B. Richman ${ }^{1}$; Jessica R. Hoag ${ }^{2}$; Kelly A. Kyanko ${ }^{3}$; Xiao Xu ${ }^{4}$; Regina Hooley ${ }^{1}$; Susan Busch ${ }^{2}$; Cary Gross ${ }^{1} .{ }^{1}$ Yale School of Medicine, New Haven, CT; ${ }^{2}$ Yale University, New Haven, CT; ${ }^{3} \mathrm{NYU}$ School of Medicine, New York, NY; ${ }^{4}$ Yale University School of Medicine, New Haven, CT. (Control ID \#3185799)

BACKGROUND: Digital mammography has imperfect sensitivity and specificity for detecting breast cancer. Digital breast tomosynthesis (DBT) may improve upon 2D mammography by reducing the need for subsequent imaging (known as recall) and improving cancer detection. DBT has been rapidly adopted, but whether the benefits reported in early studies of DBT are generalizable to the broader populations in which the technology is now used is not known. The goal of this study was to compare the effectiveness of DBT versus 2D mammography for breast cancer screening in a large contemporary population.

METHODS: We identified DBT and 2D screening mammograms from administrative claims data using a validated algorithm. We used data from the Blue Cross Blue Shield Axis, a large commercial claims database accessed via secure portal. Screening outcomes included recall (defined as subsequent imaging), biopsy, and cancer detection following screening. Screen detected cancers were defined according to a validated algorithm as invasive cancers detected within 120 days of screening. We also evaluated the rate of potential interval cancers, defined as cancers diagnosed between 120 and 365 days after 
screening. We used multivariable models to compare screening outcomes among women screened with DBT versus 2D mammography, adjusting for age, hospital referral region, residence in a metropolitan area, year, and time since last mammogram. We also performed agestratified analyses.

RESULTS: Our sample included 2,460,359 women who underwent mammography between 1/1/2015 and 12/31/2016 across all 50 states. Overall, $22 \%$ of screening exams used DBT. In adjusted analyses, DBT was not associated with a difference in recall rate compared to 2D mammography (121 vs 119 per 1000 screening exams, $\mathrm{p}=0.27$ ). DBT was, however, associated with a higher biopsy rate (23 vs 18 per 1000 exams, $\mathrm{p}<0.001)$ and a higher cancer detection rate (4.1 vs 3.0 per 1000 exams, $\mathrm{p}=<0.001)$. Despite a higher cancer detection rate, DBT was not associated with a reduction in potential interval cancers compared to $2 \mathrm{D}$ mammography ( 1.3 vs 1.2 per 1000 exams, $\mathrm{p}=0.007$ ). In age stratified analyses, results were similar across age groups.

CONCLUSIONS: Consistent with prior studies, we found that DBT is associated with a higher cancer detection rate compared to $2 \mathrm{D}$ mammography. In contrast to previous studies, though, we found that DBT did not reduce recall and was associated with a higher biopsy rate. Our findings have implications for the value of DBT, which may more effectively detect breast cancer, but is more expensive than 2D mammography and may generate additional invasive testing.

\section{BUILDING A PARTNERSHIP TO IMPROVE HOSPITAL CARE AND CARE TRANSITIONS FOR PERSONS EXPERIENCING HOMELESSNESS}

Sarah A. Stella $^{1,2}$; Lisa Thompson ${ }^{3}$; Tammy Bellofatto ${ }^{3}$; Sarah E. Leslie $^{1}$; Ellen Sarcone ${ }^{1,}{ }^{2}$; Erin Bredenberg ${ }^{2}$; Lilia Cervantes ${ }^{1,2}$. ${ }^{1}$ Denver Health, Denver, CO; ${ }^{2}$ University of Colorado School of Medicine, Denver, CO; ${ }^{3}$ Colorado Coalition for the Homeless, Denver, CO. (Control ID \#3162606)

BACKGROUND: Research which includes the perspectives of persons experiencing homelessness is needed to inform the development of meaningful interventions to improve hospital care and care transitions for this group. Our objective was to establish a Community Advisory Panel (CAP) to better understand the perceptions of people experiencing homelessness and key community stakeholders with respect to hospitalization and care transitions, and to develop a shared vision regarding opportunities/priorities for improvement through future programs and research.

METHODS: To establish our CAP, we partnered with leaders at the Colorado Coalition for the Homeless, a non-profit organization that provides housing, integrated healthcare, and other supportive services to those experiencing homelessness. We developed mutual partnership goals and a framework for shared oversight. We jointly recruited CAP participants, seeking to include both individuals experiencing homelessness, and community stakeholders with relevant experience providing care to this group. Additional selection criteria were: age 18 years or older; English-speaking, and able to attend the majority of 6 panel meetings. Participants received a meal and small stipend for their participation at meetings. All meetings were jointly facilitated by lead academic and community partners. Plans were developed a priori with input from community partners, and refined through analysis of emerging themes and through shared decision-making with participants. With participants' consent, meetings were audio-recorded and transcribed verbatim to assist the synthetization of information, iterative refinement of concepts, and qualitative analysis. De-identified transcripts were coded in Atlas.ti utilizing codes derived a priori from theory and inductively through identifying emerging themes.
RESULTS: A total of 12 individuals were recruited for the CAP. They included: 6 individuals with lived experience of homelessness who had direct experience with hospitalization and care transitions; and 6 community stakeholders with relevant experience caring for homeless individuals while hospitalized or in the community. The CAP met 6 times from July 2018- January 2019. We identified 4 important themes: 1) trauma encountered through the everyday experience of homelessness and/or through healthcare interactions; 2) empathy and other aspects of the patient-physician relationship; 3) moral distress and burnout among healthcare providers and other personnel caring for persons experiencing homelessness; 4) and the availability, coordination, and integration of services after discharge to respite, shelters or other locations.

CONCLUSIONS: Engaging individuals with lived experience of homelessness and key community stakeholders in research results in a better understanding community needs and priorities with respect to hospital care and care transitions, and thus more meaningful translational research to address the significant, known health inequities faced by this community.

BUILDING AN AMBULATORY INFRASTRUCTURE TO REDUCE HOSPITALIZATIONS AMONG CHRONIC KIDNEY DISEASE PATIENTS: A SYSTEM-WIDE VALUE APPROACH TO HIGH COST POPULATIONS

Reshma Gupta $^{2}$, ; Samuel A. Skootsky ${ }^{1}$; Katherine L. Kahn ${ }^{2}$; Lucia Chen $^{2}$; Sitaram Vangala ${ }^{2}$; James Wilson ${ }^{2}$. 1UCLA, Los Angeles, CA; ${ }^{2}$ David Geffen School of Medicine at UCLA, Los Angeles, CA. (Control ID \#3161044)

BACKGROUND: Chronic kidney disease (CKD) is a leading cause of spending nationally. With an imperative to provide accountable care across ambulatory and inpatient settings, there is a shift toward risk-sharing contracts that reward reductions in total spending achieved through coordinated, quality care. The alignment of primary and specialty care teams provides strategies to facilitate patients receiving timely, needed services in the most appropriate, cost-effective setting. As part of an enterprise-wide transformation to improve healthcare value, UCLA identified condition-specific, high cost patients and adapted a new patientcentered approach to improve quality and care coordination. Recognizing that advanced (stage 4 or 5) CKD patients experienced frequent emergent hospitalizations to initiate dialysis and treat catheter dysfunction, UCLA Health implemented a pathway for advanced CKD patients attributed to the UCLA primary care network. We evaluated the impact of this initiative on health system utilization.

METHODS: Between October and December 2016, an interdisciplinary, multispecialty team co-created and implemented care pathways for the advanced CKD patient population that involved: 1) clinically meaningful longitudinal data management, 2) proactive care management to align clinical and social service care across primary and multi-specialty settings, and 3) strategies to enhance access to lower-cost interventional radiology care sites. We used internal EHR and claims data across eight payers to define utilization outcomes (i.e. hospitalizations, inpatient bed days, 30-day all-cause readmissions, and emergency department (ED) visits). Using an 18-month pre-intervention period to control for baseline bias, we conducted descriptive and interrupted time series regression analyses, to evaluate the impact of the intervention on outcomes.

RESULTS: Of 1442 advanced CKD patients, the estimated rate of hospitalizations per 1000 patient-years was 712 at the beginning of the pre-intervention period (95\% CI: 610-832), and declined at 
an estimated monthly rate of $2 \%$ during this period $(95 \% \mathrm{CI}$ $0.8 \%-3.2 \%$ ). By contrast, the estimated rate of hospitalizations per 1000 patient-years declined at an estimated monthly rate of $5 \%$ during the post-intervention period (95\% CI: $3.4 \%-7.4 \%$ ). This acceleration in the monthly rate of decline after the intervention was statistically significant $(p=0.004)$. CKD patients also had a nonsignificant $4 \%$ faster reduction in monthly inpatient bed days (4.8\% [95\% CI: $2.5 \%-7.0 \%$ ] versus $8.6 \%$ [95\% CI: $4.7 \%-12.3 \%$ ], $\mathrm{p}=0.10)$ and $2 \%$ faster reduction in ED visits $(0.9 \%$ [95\% CI: $-0.1 \%-2.0 \%$ ] versus $2.7 \%$ [95\% CI: $0.9 \%-4.3 \%$ ], $\mathrm{p}=0.09$ ) from pre- to post-intervention periods. There was no difference in 30day readmissions $(\mathrm{p}=0.26)$.

CONCLUSIONS: By creating care pathways that link primary and specialty care teams with increased ambulatory infrastructure, health systems have potential to enhance care coordination in association with reductions in health costs.

CAN A LARGE SCALE QUALITY IMPROVEMENT INTERVENTION DESIGNED TO REDUCE CARDIOVASCULAR RISK ALSO ATTENUATE BLACK -WHITE DISPARITIES IN HYPERTENSION CONTROL?

Samuel Cykert ${ }^{1}$; Crystal W. Cene ${ }^{1}$; Darren Dewalt ${ }^{1}$; Justin Trogdon ${ }^{2}$. ${ }^{1}$ University of North Carolina, Chapel Hill, NC; ${ }^{2}$ UNC Gilling's School of Global Public Health, Chapel Hill, NC. (Control ID \#3183099)

BACKGROUND: Hypertension affects more than 70 million people in the US and increases the risk of all-cause mortality, heart disease, stroke, and chronic kidney disease. There are significant racial disparities in hypertension control. Compared to Whites, Blacks have a higher prevalence of hypertension $(40.1 \%$ vs. $27.4 \%$ ). Additionally, they are less likely to achieve blood pressure control. The lack of control in the Black population accounts for half the excess cardiovascular disease (CVD) mortality observed in Blacks and 5\% of the overall racial difference in potential life-years lost. Heart Health Now (HHN) is an AHRQ sponsored initiative designed to reduce CVD risk for adults served by small, North Carolina primary care practices. This report examines the effect of this intervention on hypertension disparities. METHODS: We conducted a pre-post evaluation of change in blood pressure control comparing White with Black patients. Clinical data were derived from daily electronic health record uploads from 219 practices. To be eligible, practices had to have 10 or fewer clinicians. The intervention consisted of provision of onsite practice facilitation to implement QI strategies and use of a CVD population management dashboard. Adult patients with an ICD-9 or ICD-10 diagnosis of hypertension aged 18 to 85 years were included. We examined the overall hypertension control (SBP $<140$ and DBP $<$ 90) gap between Black and White patients at baseline, the percentage of practices that had gaps, and whether these differences changed after the 12 -month intervention. The analyses controlled for practice demographics, patient centered medical home status, practice disruptions, and measured change capacity.

RESULTS: 219 practice sites caring for 116,952 White and 46,406 Black hypertensive patients. Average \# providers per site was 7. Fiftynine $\%$ of practices were clinician-owned; $29 \%$ were federally qualified health centers; $52 \%$ were rural. The average payer mix was Medicare $28 \%$, Medicaid $16 \%$, dual $10 \%$, uninsured $12 \%$, commercial insurance $30 \%$, and "other" $4 \%$. Clinicians averaged 21 patient visits per day. At baseline, $57.8 \%$ of Black patients' hypertension was controlled compared to $64.2 \%$ of Whites $(\mathrm{p}<0.001)$. Post-intervention these proportions were $60.1 \%$ and $66.3 \%$, respectively $(\mathrm{p}<0.001)$. While hypertension control for Blacks improved (over 1000 individuals newly controlled), racial disparity remained. On the practice level, there was no racial difference in hypertension control in $22 \%$ of practices at baseline; the majority $(78 \%)$ of practices had a disparity favoring Whites $(\mathrm{p}<0.001)$ that was unchanged post-intervention.

CONCLUSIONS: Although widespread implementation of an intensive, practice-based, QI intervention improved hypertension control for Black and White patients, it did not reduce disparities. Within practice disparities were predominant. Research examining practices with no disparities and devising socio-culturally tailored strategies appear to be necessary next steps to close racial gaps.

\section{CAN AN INTENSIVE AMBULATORY CARE INTERVENTION IMPROVE THE EXPERIENCE OF HIGH-UTILIZING PA- TIENTS?: 6-MONTH OUTCOMES OF THE SUMMIT RAN- DOMIZED CONTROLLED TRIAL.}

Brian Chan ${ }^{1,}{ }^{2}$; Samuel T. Edwards ${ }^{4,}{ }^{1}$; Matthew Mitchell ${ }^{2}$; Christina Nicolaidis $^{3,5}$; Devan Kansagara ${ }^{4,5}$; Philip T. Korthuis ${ }^{5}$; Somnath Saha ${ }^{4}$. ${ }^{1}$ OHSU, Portland, OR; ${ }^{2}$ Central City Concern, Portland, OR; ${ }^{3}$ Portland State University, Portland, OR; ${ }^{4}$ Portland VA Medical Center, Portland, OR; ${ }^{5}$ Oregon Health \& Science University, Portland, OR. (Control ID \#3186182)

BACKGROUND: People experiencing homelessness, co-morbid chronic medical conditions, and substance use disorder (SUD) make up a disproportionate number of high-cost, high-need patients at risk of frequent acute care utilization. Intensive ambulatory care unit ("A-ICU") interventions aim to improve patient engagement, patient experience and quality of care, and reduce high-cost utilization.

METHODS: This is a preliminary analysis of a randomized trial of SUMMIT, an A-ICU for high-utilizers at a federally qualified health center that serves patients with high rates of homelessness and substance use. SUMMIT is a stand-alone A-ICU team consisting of care coordinators, an addictions-boarded physician, social workers, complex care nurse, pharmacist, and team manager, with a low staff-to-patient ratio and increased appointment flexibility. Patients were eligible to be referred to SUMMIT if they had 1+ hospitalizations in the prior 6 months, or had $2+$ chronic medical conditions and/or active substance use or a mental health condition. After consent and a baseline survey, patients were randomized to enroll in SUMMIT immediately or to join the SUMMIT wait list while remaining in usual care (patient-centered medical home model). We assessed patient experience and functional status using ambulatory consumer assessment of healthcare providers and systems (CAHPS) and the 12-item short form survey (SF-12) at baseline and at six months. We also examined average number of hospital admissions at 6 months, average length of stay (LOS), emergency department (ED) visits, primary care visits, and behavioral health visits.

RESULTS: Of the first 102 patients enrolled, 52\% $(\mathrm{n}=53)$ were randomized to SUMMIT immediately. Average age was 53.8 years $(+/-10.1)$, the majority $(64.7 \%)$ were male, $87.3 \%$ had experienced homelessness, $56.9 \%$ had a SUD history. Baseline utilization for enrolled patients was 3.0 admissions, $4.5 \mathrm{ED}$ visits, 8.1 primary care visits, and 2.9 behavioral health visits in the prior 6 months. At six months patients in SUMMIT reported improved CAHPS ratings in communication ( $81 \%$ "top-box" vs $63 \%, \mathrm{P}=.001)$ and care coordination $(60 \%$ vs $43 \%, \mathrm{P}=0.01)$, and improved mental health composite SF-12 scores (48.1 vs $41.8, \mathrm{P}=0.006$ ) versus control patients. The SUMMIT patients had more PCP visits (14.1 vs $5.8, \mathrm{P}<.001)$ and tended to experience fewer hospitalizations ( $1.8 \mathrm{vs}$ 2.1, $\mathrm{P}=0.25)$, shorter $\operatorname{LOS}(13.0$ vs $16.0, \mathrm{P}=0.2)$, more $\mathrm{ED}$ visits 3.7 vs $2.8, \mathrm{P}=0.36$ ), and more behavioral health visits (5.8 vs $5.1, \mathrm{P}=0.1$ ).

CONCLUSIONS: The SUMMIT program improved patient experience, improved mental health symptoms, and increased primary care engagement at six months. We did not observe significant changes in inpatient or ED utilization, possibly due to our small sample size and limited time frame. Future analyses will evaluate 12-month outcomes and will assess whether certain subgroups of high-utilizing patients benefit most from A-ICU care. 
CAN AN INTERPROFESSIONAL EDUCATION INITIATIVE AFFECT QUALITY OF CARE AND HEALTH CARE UTILIZATION? Samuel T. Edwards ${ }^{1}$; Elizabeth R. Hooker ${ }^{2}$; Rebecca Brienza ${ }^{3}$; Bridget $\overline{\text { O'Brien }}^{10}$; Hyunjee Kim ${ }^{4}$; Stuart Gilman ${ }^{5}$; Lillian Gelberg ${ }^{6}$; Sarah Shull ${ }^{7}$; nancy Harada ${ }^{5}$; Meike Niederhausen ${ }^{2}$; Samuel King ${ }^{5}$; Elizabeth Hulen ${ }^{11}$; Mamta K. Singh ${ }^{8}$; Anais Tuepker ${ }^{9} .{ }^{1}$ Boston VA Healthcare System, Boston, MA; ${ }^{2}$ Portland VA Medical Center, Portland, OR; ${ }^{3}$ VA Connecticut Healthcare System, West Haven, CT; ${ }^{4}$ Oregon Health \& Science University, Portland, OR; ${ }^{5}$ US Dept of Veterans Affairs, Washington, DC; ${ }^{6}$ UCLA, Los Angeles, CA; ${ }^{7}$ Portland VA, Portland, OR; ${ }^{8}$ Case Western Reserve University, Cleveland, OH; ${ }^{9}$ VA Portland Health Care System/Oregon Health and Science University, Portland, OR; ${ }^{10} \mathrm{UCSF}$, San Francisco, CA; ${ }^{11}$ VA Portland Health Care System, Portland, OR. (Control ID \#3185305)

BACKGROUND: Interprofessional education (IPE) has been shown to improve learner proficiencies, but the effect of IPE interventions on patient level outcomes is unknown. The Department of Veterans Affairs' (VA) Centers of Excellence in Primary Care Education (CoEPCE) is a multi-site IPE initiative that integrates the post graduate training of physicians, nurse practitioners, pharmacists and psychologists in VA academic primary care clinics. In this study, we estimated the impact of CoEPCE implementation on quality of care and health care utilization.

METHODS: We performed a retrospective cohort study using VA electronic health record data. We identified patients cared for by clinician trainees in five CoEPCE sites, and patients cared for by clinician trainees in regionally matched non-CoEPCE academic primary care clinics, from 7/1/2008 - 6/30/ 2015. Outcomes included seven measures of quality of care: annual hemoglobin Alc [HbAlc], poor Alc control [ $<9 \%$ or unmeasured], and annual renal test in diabetes mellitus; prescription of a high risk medication in patients $>65$ years; hypertension control $(<140 / 90)$; timely mental health referrals, and primary care mental health integrated visits; and 3 measures of health care utilization: emergency department (ED) visits, all cause hospitalizations and hospitalizations for ambulatory care sensitive conditions (ACSC). We compared changes in outcomes from the three years before $(7 / 1 / 2008-6 / 30 / 2011)$ to the four years after (7/1/2011-6/30/2015) initiative launch, between CoEPCE patients and non CoEPCE patients using a difference in differences approach. Models adjusted for demographics, comorbidities, year and years under VA care, and standard errors were clustered by site. Alternative comparison groups were used as sensitivity analyses.

RESULTS: We identified 49,279 CoECPE clinician trainee patient-years (mean age 59.3 [sd 15.2] years, mean Elixhauser comorbidity score 12.9 [sd 15.1]) and 58,407 non CoEPCE clinician trainee patient-years (mean age 61.8 [sd 15.3] years, mean Elixhauser comorbidity score 13.8 [sd 15.7]). Compared to non-CoEPCE patients, CoEPCE patients had improvements in the proportion with poor control of HbAlc (-4.6 percentage points [pp], $\mathrm{p}<0.001)$, annual renal testing $(+3.2 \mathrm{pp}, \mathrm{p}=0.016)$, patients over age 65 receiving a high risk medication $(-2.3 \mathrm{pp}, \mathrm{p}=0.005)$ and timely mental health referrals $(+1.6 \mathrm{pp}, \mathrm{p}=0.002)$. CoEPCE patients had fewer ED visits (proportion with $>1$ ED visit in year $-3.7 \mathrm{pp}, \mathrm{p}<0.001)$, hospitalizations $(-0.8 \mathrm{pp}$ had $>1$ hospitalization in year, $\mathrm{p}=0.007)$, and hospitalizations for an $\operatorname{ACSC}(-0.4$ $\mathrm{pp}, \mathrm{p}=0.014)$. Sensitivity analyses with alternative comparison groups yielded similar results.

CONCLUSIONS: The CoEPCE initiative was associated with improved quality of care and reduced health care utilization. Interprofessional education can impact patient level outcomes and may potentiate delivery system reform efforts.

\section{CAN PATIENT-PROVIDER INTERPERSONAL INTERVENTIONS ACHIEVE THE QUADRUPLE AIM OF HEALTH CARE?: A SYS- TEMATIC REVIEW}

Marie Haverfield $^{1}$; Aaron A. Tierney ${ }^{2}$; Rachel Schwartz ${ }^{3}$; Michelle Bass ${ }^{1}$; Cati Brown-Johnson ${ }^{4}$; Dani L. Zionts ${ }^{5}$; Nadia Safaeinili ${ }^{3}$; Jonathan G. Shaw ${ }^{5}$; Sonoo Thadaney ${ }^{1}$; Gabriella Piccininni ${ }^{6}$; Karl Lorenz ${ }^{7}$; Steven Asch ${ }^{7}$; Abraham Verghese ${ }^{1}$; Donna M. Zulman ${ }^{8} .{ }^{1}$ Stanford University, Menlo Park, CA;
${ }^{2}$ Stanford University School of Medicine, Mountain View, CA; ${ }^{3}$ Stanford University, Stanford, CA; ${ }^{4}$ Stanford School of Medicine, Stanford, CA; ${ }^{5}$ Stanford University School of Medicine, Stanford, CA; ${ }^{6}$ Stanford University, Toronto, ON, Canada; ${ }^{7}$ VA/Stanford, Menlo Park, CA; ${ }^{8}$ Stanford University and VA Palo Alto, Stanford, CA. (Control ID \#3166752)

BACKGROUND: Human connection is at the heart of medical care. The extent to which such connection can be taught or improved has been the subject of much research, but questions remain as to the effectiveness of these interventions. We synthesized the evidence for interpersonal interventions to improve patient-provider interactions and downstream health outcomes.

METHODS: We searched PubMed, EMBASE, and PsycInfo (January 1997 to August 2017) for randomized controlled trials and controlled observational studies that examined the association between patient-provider interpersonal interventions and at least one outcome measure of the Quadruple Aim. We also mined applicable systematic reviews to identify additional relevant citations. Using Covidence, abstractors worked in pairs to cull information about the content and methods of each article, assessed the quality of research, and graded levels of evidence. We characterized the evidence related to intervention target (provider-only vs. provider-patient dyad), the objective of the intervention, the type and duration of intervention training, and the Quadruple Aim outcomes.

RESULTS: 77 out of 21,835 studies met all design and outcome inclusion criteria. The methodological quality of research was moderate-to-high for the majority of studies. Most interventions focused on enhancing provider communication skills (29\%). Among the 44 studies that reported a positive patient experience outcome, $50 \%$ used a provider-focused intervention, suggesting patients do not have to be involved in the intervention to improve their perceptions of care. Population health was often improved following an interpersonal intervention, and studies measuring improvements in patient health often corresponded with improved patient experience. Similarly, provider well-being (e.g., burnout, communication confidence, stress) improved from enhanced patient-provider interpersonal interactions. One of the most common types of interventions that positively influenced provider experience was clinician training using a communication content focus, which was associated with improvements in communication, satisfaction, and perceived provider friendliness. Few studies evaluated overall cost outcomes, but several reported reduced utilization and prescription rates. Few interventions increased visit length, and interventions with lower demands on provider time and effort were generally as effective as those with higher demands on provider time.

CONCLUSIONS: We found good evidence that interpersonal interventions aimed at improving patient-provider interactions also improve patient and provider experience, and weaker evidence that they improved population health or cost. Quality improvement efforts might focus on simple low demand interventions, as they were equally effective.

\section{CAPTURING THE REST: INPATIENT INTERVENTION STUDIES FOR NON-ADHERENT HOSPITALIZED WOMEN TO IMPROVE THEIR BREAST CANCER SCREENING ADHERENCE}

Waseem Khaliq ${ }^{1}$; Regina Landis ${ }^{2}$; Eric Howell ${ }^{2}$; Scott Wright ${ }^{3} .{ }^{1}$ Johns Hopkins University School of Medicine, Ellicott city, MD; ${ }^{2}$ Johns Hopkins, Baltimore, MD; ${ }^{3}$ jhusom, Baltimore, MD. (Control ID \#3150766)

BACKGROUND: More than a third of hospitalized women are overdue for breast cancer screening and at high risk for developing breast cancer. We evaluated the feasibility of two separate interventions for nonadherent hospitalized women to improve their adherence to breast cancer screening recommendation. First intervention consisted of inpatient breast cancer screening education, scheduling an outpatient mammography prior to hospital discharge, a reminder phone call and a small monetary incentive for screening test. The second intervention consisted of inpatient breast cancer screening education and offering inpatient screening mammography. 
METHODS: For both intervention studies women aged 50-75 years who were hospitalized to a general medicine service and were nonadherent to breast cancer screening were approached for enrollment. Socio-demographic, reproductive history, family history for breast cancer, and medical comorbidities data was collected for all patients. Chi square and unpaired t-tests were utilized to compare characteristics among women who did and did not get screening.

RESULTS: First intervention was conducted among 30 hospitalized women who were non-adherent to breast cancer screening at Johns Hopkins Bayview Medical Center, the mean age for study population was 57.8 years, mean 5 -year Gail risk score was 1.68 , and $57 \%$ of women were African American. Only one third of the enrolled women $(n=10)$ went to their pre-arranged appointments for screening mammography. Not feeling well enough after the hospitalization and not having insurance were reported as main reasons for missing the appointments. Second intervention was conducted among 101 hospitalized women non-adherent to breast cancer screening at Howard County General Hospital, the mean age for this study population was 59.2 years, mean 5-year Gail risk score was 1.63 , and $29 \%$ of women were African American. More than two-third of the enrolled women $(n=79)$ under went in-patient screening mammography. All women who underwent screening mammography during inpatient stay were extremely satisfied. Neither the ordering hospitalists nor the nurses taking care of these women reported any concerns or misgiving. Convenience of having a screening mammography while in-patient stay was reported to be a facilitator of completing the screening test.

CONCLUSIONS: Although both interventions were successful in improving awareness and adherence to breast cancer screening, inpatient mammography intervention had much higher impact on breast cancer screening adherence. Because hospitalization creates the scenario wherein patients are in close proximity to healthcare resources, at the same time that they are reflecting upon their health status, strategies could be employed to counsel, educate, and motivate these patients towards health maintenance. Future studies need to evaluate the inpatient feasibility of other common cancer-screening tests to overcome the significant barriers to compliance with screening for hospitalized population.

\section{CARDIOVASCULAR HEALTH OF FOREIGN-BORN VET- ERANS IN THE VETERANS HEALTH ADMINISTRATION}

Karen Wang ${ }^{1}$; Christine Ramsey ${ }^{3}$; Carol Oladele ${ }^{1}$; Sally G. Haskell ${ }^{2}$; Cynthia Brandt ${ }^{1} .{ }^{1}$ Yale University School of Medicine, New Haven, CT; ${ }^{2}$ Yale University, VA CT, and VA Central Office, Madison, CT; ${ }^{3}$ Corporal Michael J. Crescenz Veterans Affairs Medical Center, Philadelphia, PA. (Control ID \#3180109)

BACKGROUND: Foreign-born individuals have served in all major military conflicts, including recent conflicts in Iraq and Afghanistan. There are a half-million veterans who are foreignborn, yet we have limited knowledge about their health. Our objective is to describe the socio-demographic and cardiovascular-health risk factors and outcomes among the foreign-born veterans of the recent conflicts

METHODS: We analyzed data from the Women Veterans Cohort Study, a study of current-era veterans who used Veterans Health Administration (VHA) services between September 11, 2001 and November 19, 2014. Our sample included veterans whose place of the birth was identified as outside of the United States and its territories. We examined prevalence of socio-demographic and cardiovascular-health risk factors and outcomes by United Nations (UN) geographic regions using chi-squared tests. Risk factors included age, sex, race/ethnicity, marital status, education, obesity, smoking history, and diagnoses of diabetes, hypertension, major depressive disorder (MDD), and post-traumatic stress disorder (PTSD). Outcomes were diagnosis of coronary artery disease (CAD), congestive heart failure (CHF), cardiovascular disease (CVD), and stroke

RESULTS: Our sample included 66,940 veterans who are foreign-born (14\% women; $55.7 \%$ aged 21-29; $28 \%$ white, $17.1 \%$ black, and $27.4 \%$ Hispanic). Over half were married and $98.6 \%$ had at least a high-school education. Thirty percent $(\mathrm{N}=20,440)$ had data on country of birth. Among these, $8.2 \%$ were born in North America, 33.2\% in Central America, 38.6\% in Asia, $18.1 \%$ in Europe, and $1.3 \%$ in Melanesia, Micronesia, and Polynesia. Almost $28 \%$ percent of all foreign-born veterans were obese, and $30 \%$ were current smokers. Four percent were diagnosed with diabetes; 9.6\% with MDD; $13.1 \%$ with hypertension; and $26.5 \%$ with PTSD. Age, race/ethnic, and gender of Veterans differed significantly by region, as did the prevalence of obesity, smoking history, diabetes, hypertension, and PTSD $(\mathrm{p}<0.001$ for all). Veterans from Melanesia, Micronesia, and Polynesia had the highest prevalence of obesity (54.9\%) and current smoking along with Europe (43.3\% for both). Prevalence of hypertension $(24.4 \%)$ and diabetes $(9.4 \%)$ were highest in veterans born in Asia; and prevalence of PTSD was highest in veterans from Central America (31.8\%). Overall prevalence of cardiovascular outcomes among foreign born veterans were: CAD $0.77 \%$, CHF $0.11 \%$, CVD $0.24 \%$, and stroke $0.4 \%$. Prevalence of CAD differed significantly by region $(\mathrm{p}<0.001)$.

CONCLUSIONS: Foreign-born veterans originate from different regions of the world and are at risk for preventable cardiovascular outcomes. One tenth to one-third of individuals in specific regions had modifiable risk factors, such as obesity, current smoking, and hypertension. This VHA data presents a unique opportunity to further examine health differences between foreign-born and USborn individuals

\section{CARE FOR AMERICA'S ELDERLY AND DISABLED RE- LIES ON IMMIGRANT LABOR}

Leah Zallman ${ }^{1,2}$; Karen Finnegan ${ }^{1}$; David Himmelstein ${ }^{3,}{ }^{2}$; Stephanie Woolhandler ${ }^{3,}{ }^{2}$. ${ }^{1}$ Institute for Community Health, Malden, MA; ${ }^{2}$ Cambridge Health Alliance/Harvard Medical School, Cambridge, MA; ${ }^{3}$ City University of New York at Hunter College, New York, NY. (Control ID \#3184574)

BACKGROUND: As our nation wrestles with immigration policy and how to care for an aging population, data on immigrants' role in the provision of long term care can inform both debates. Previous studies have examined immigrants' role as direct care workers, but have not examined those hired by private households (or by non-medical facilities such as senior housing) to assist elderly and disabled persons, or undocumented immigrants' role in the provision of these services.

METHODS: We use detailed occupation and industry data from the Current Population Survey (CPS) 2018, which provides nationally representative data reflecting 2017 events. We estimate the number of immigrant health care workers, long-term care workers and non-formal caregivers, and direct care workers. We use a previously developed methodology to impute immigration status.

RESULTS: We find that immigrants accounted for $18.2 \%$ of all health care workers in 2017, and $23.5 \%$ of workers in long-term care and the non-formal caregiving sector. More than one quarter $(27.5 \%)$ of direct care workers were immigrants and $30.3 \%$ of nursing home housekeeping and maintenance workers were immigrants, over one third of whom were undocumented. Although legal noncitizen immigrants accounted for $5.2 \%$ of the U.S. population, they comprised $9.0 \%$ of direct care workers, including $16.0 \%$ of non-formal sector workers. 
CONCLUSIONS: In light of the current and projected shortage of direct care workers, our finding that immigrants already fill a disproportionate share of such jobs suggests that policies curtailing immigration are likely to compromise the availability of care for elderly and disabled Americans.

CARE MANAGEMENT FOR THE EFFECTIVE USE OF OPIOIDS (CAMEO): A RANDOMIZED TRIAL

Matthew J. Bair ${ }^{1,2}$; Samantha D. Outcalt ${ }^{1}$; James E. Slaven ${ }^{2}$; Kurt Kroenke $^{3}$; Carol Kempf ${ }^{1}$; Alan Zillich ${ }^{4}$; Teresa Damush ${ }^{1}$; Chandan Saha $^{2}$; Dustin D. French ${ }^{5}$; Erin E. Krebs ${ }^{6} .{ }^{1}$ VA Center for Health Information and Communication, Indianapolis, IN; ${ }^{2}$ Indiana University School of Medicine, Indianapolis, IN; ${ }^{3}$ Indiana University, Indianapolis, IN; ${ }^{4}$ Roudebush VA Medical Center, Indianapolis, IN; ${ }^{5}$ Northwestern U, Chicago, IL; ${ }^{6}$ Minneapolis VA Health Care System, Minneapolis, MN. (Control ID \#3185091)

BACKGROUND: Chronic low back pain is common and accounts for an enormous burden in patient suffering, diminished quality of life, disability, and health care costs. Many options are available to treat chronic low back pain, yet management remains difficult. The objective of the CAMEO Trial was to compare the effectiveness of pharmacological treatment and optimization vs. cognitive behavioral therapy for Veterans with chronic lower back pain on chronic opioid therapy.

METHODS: CAMEO was designed as a 2-arm, randomized clinical trial. The study setting was 5 general medicine clinics at a Veteran Affairs Medical Center and 2 community based outpatient clinics. Participants included 261 Veterans with chronic low back pain of at least moderate intensity who were on chronic opioid therapy at baseline. Enrollment occurred from August 2011 to December 2014, with 12-month follow-up completed by December 2015. The pharmacological (PHARM) arm involved nurse care management focused on analgesic treatment and optimization according to an algorithm. The behavioral (BEHAV) arm involved cognitive behavioral therapy delivered by clinical psychologists. The primary outcomes were pain impact, pain intensity, and pain interference as assessed by the Brief Pain Inventory (BPI).

RESULTS: The mean age participants was 57.9 years, $92 \%$ men, $73 \%$ White and $21 \%$ Black. 131 participants in the PHARM arm and 130 participants in the BEHAV arm were included in the primary analysis. At 12 months, the adjusted mean decrease from baseline in the BPI total score (pain impact) was 0.64 points (Standard deviation $[S D]=0.22$ ) in the BEHAV group and 1.14 points $(\mathrm{SD}=0.23$ ) in the PHARM group (between group difference of 0.5 points, $p=0.0423$ ). Scores were adjusted for baseline BPI total score, depression (PHQ-9), and gender. At 12 months, BPI pain intensity decreased by 0.40 points in the BEHAV group $(\mathrm{SD}=0.19)$ and 1.02 points $(\mathrm{SD}=0.20)$ in the PHARM group (between group difference of $0.62, p=0.0044)$. The mean decrease from baseline in the BPI interference score was 0.71 points in the BEHAV group and 1.19 points in the PHARM group (between group difference of 0.48 points; $p=$ 0.0846). There were no differences in secondary outcomes (depression, anxiety, PTSD, and health-related quality of life) across study arms.

CONCLUSIONS: Both treatment groups improved, but improvement was modest. A nurse care management intervention focused on pharmacological management reduced pain impact and intensity more than a behavioral intervention involving cognitive behavioral therapy. While the between-group differences were statistically significant, these differences may not be clinically significant. To improve treatment effects combination therapy is likely needed, especially for patients with complex chronic low back pain.

CARE MANAGEMENT PLUS: TIME-LIMITED LONGITUDINAL RN CARE MANAGEMENT

Corinne Rhodes ${ }^{1}$; Sarah LaMar ${ }^{3}$; Andrew Maier ${ }^{4}$; Susan C. Day ${ }^{2} .{ }^{1}$ University of Pennsylvania Health System, Philadelphia, PA; ${ }^{2}$ University of
Pennsylvania, Philadelphia, PA; ${ }^{3}$ Penn Medicine, Philadelphia, PA; ${ }^{4}$ UPenn, Philadelphia, PA, PA. (Control ID \#3185836)

BACKGROUND: We developed and implemented a longitudinal, proactive care management $(\mathrm{CM})$ program targeting our highest risk patients as part of Comprehensive Primary Care Plus (CPC+) Medicare innovation grant in 3 urban academic clinics. Goals were to decrease utilization and achieve patient and primary care provider (PCP) goals. We hypothesized decreased utilization during enrollment would persist after graduation.

METHODS: Our central CM model was overseen by a RN manager and physician lead. Patients enrolled in a 6-month intervention with a two-step process including EPIC risk score including registries, utilization, social determinants of health (SDH) proxy measures and then underwent PCP review. Care managers $(\mathrm{CM})$ preformed a standardized intake including modifiable barriers of care and collected patient centered goals. CM proactively followed patients and communicated to PCP or designated providers via EPIC and brief weekly meetings. Patients graduated at 6 months, sooner if not engaged, or extended enrollment at discretion of PCP. This interim analysis looks at 192 patients enrolled with 6 months of post-graduation utilization data. Descriptive data shows patients approached, enrolled, engaged, and graduated and completed goals with means and standard deviations (SD). We examined combined ED and hospital utilization by unadjusted and apriori adjusted longitudinal person-clustered mixed regression models. We randomly matched control patients with recent utilization at a 1:1 ratio by clinic, age, sex, race, and Charlson comorbidity score.

RESULTS: 326 patients were approached, 84 (25.8\%) did not enroll; $30 \%$ of enrolled were not engaged and did not graduate, and $10 \%$ died, moved, or entered skilled care by end of intervention. Enrolled patients' mean age was 64.9 (SD 14.6), 60.9\% female, $89.1 \%$ Black, $25.5 \%$ Medicaid or Managed Medicaid, with average Charlson score of 7.6 (SD 3.3). 175 (91.1\%) set goals and 68.2\% achieved a goal. Combined relative utilization decreased by $33.1 \%$ from 1.6 events 6 months prior, 1.07 events during 6-month enrollment and 1.09 events 6 months after enrollment, $\mathrm{p}<0.0001$ for unadjusted and adjusted models. We matched patients with recent utilization by age, sex, clinic, race, and Charlson score. The model for the 190 matched patients showed no significant change in utilization during the same 6-month periods (unadjusted $p=0.85$; adjusted $p=0.85$ ). Combined models showed significant intervention status $(\mathrm{p}=0.0015)$ and intervention*time interaction $(\mathrm{p}=0.0058)$ in unadjusted and adjusted models.

CONCLUSIONS: Our central CM program reduced utilization by $33.1 \%$ during and after program graduation, with $68.2 \%$ of patients reaching a stated goal. Intervention patients had higher utilization at enrollment than matched control samples, which could indicate regression to mean, however control patients showed no regression to overall population mean from their initial utilization (mean utilization events for population $=0.19$ events SD 0.56 ).

\section{CAREER CONSEQUENCES FOR MEDICAL STUDENTS} EXPERIENCING SEXUAL HARASSMENT

Emmanuelle B. Yecies ${ }^{1,2}$; Melissa McNeil ${ }^{3}$. ${ }^{1}$ University of Pittsburgh School of Medicine, Pittsburgh, PA; ${ }^{2}$ VA Pittsburgh Healthcare System, Pittsburgh, PA; ${ }^{3}$ University of Pittsburgh Medical Center, Pittsburgh, PA. (Control ID \#3185051)

BACKGROUND: Recent studies have demonstrated the high prevalence of sexual harassment in medicine, with $30 \%$ of female physicians and over $15 \%$ of medical students endorsing sexual harassment. There is also increasing recognition that the spectrum of sex discrimination includes microaggressions. However, it remains unclear what consequences sexual harassment has on the attitudes and career trajectories of women in the field of medicine. We aimed to identify the effect of sexual harassment on 
student attitudes towards medicine and specialty choices.

METHODS: An in-person cross-sectional survey was administered to incoming interns at UPMC about their experience with sexual harassment in medical school. The survey was adapted from a previously-published validated instrument (Sexist MESS) and included questions about attitudes following the experience of sexual harassment. The Likert scale answers to these questions were analyzed using chi-squared statistics to determine differences between male and female respondents.

RESULTS: 185 graduates of U.S. medical schools returned the survey (100\% response rate), with 182 completing both demographics and attitudes sections. Interns represented the full spectrum of specialties and graduated from medical schools across the country. 92 respondents identified as female, and 90 as male. The statement "Negative experiences related to my gender caused me to feel bad about coming to work" yielded answers of agree or strongly agree in $8.7 \%$ of women and $2.2 \%$ of men. $8.7 \%$ of women and $0 \%$ of men agreed with "Negative experiences related to my gender caused me to doubt my choice of medicine as a profession." $10.9 \%$ of women and $2.2 \%$ of men agreed with "Negative experiences related to my gender contributed to changing the specialty I pursued" $(\mathrm{p}<0.001$ for all). Of those respondents who identified a specialty they switched from due to negative gender-related experiences, women most frequently identified surgical fields (5 of 9 responses). Men exclusively identified Obstetrics-Gynecology (3 of 3 responses).

CONCLUSIONS: Students of both genders reported career consequences to negative gender-related experiences. Women are four times more likely to report negative outcomes related to sexual harassment, and these experiences impact their specialty choices, particularly in surgical fields. Addressing sexual harassment in medical school and building strong support systems will be crucial to ensuring that students can thrive in all specialties.

CAREGIVER STRAIN IS ASSOCIATED WITH MEDICATION ADHERENCE AMONG CAREGIVERS WITH DIABETES: THE REASONS FOR GEOGRAPHIC AND RACIAL DIFFERENCES IN STROKE (REGARDS) STUDY

Alexandra King ${ }^{3}$; Joanna Bryan ${ }^{1}$; Monika M. Safford ${ }^{1}$; Catherine Riffin ${ }^{1}$; Ronald Adelman ${ }^{1}$; David Roth ${ }^{2}$; Madeline R. Sterling ${ }^{1}$. ${ }^{1}$ Weill Cornell Medical College, New York, NY; ${ }^{2}$ Johns Hopkins University, Baltimore, MD; ${ }^{3}$ NewYork Presbyterian Cornell, New York, NY. (Control ID \#3185462)

BACKGROUND: Caregiver strain is associated with adverse effects on caregivers' own health, particularly if the caregiver has cardiovascular disease. Less is known about the effects of caregiving strain among those with diabetes, a disease which has high self-care demands. Using a national sample, this cross-sectional study examined the effect of caregiver strain on caregivers' diabetes self-care behaviors.

METHODS: This study was conducted using data from the REGARDS study, a national, observational, longitudinal cohort of 30,239 black and white adults aged 45 years. A baseline telephone interview that included health behaviors was followed by an in-home visit collecting physiologic data, medication adherence, and dietary information. Participants who reported having diabetes and being a caregiver were included. Caregiver strain was assessed with, "How much of a mental or emotional strain is it to provide this care?" Responses were categorized as no strain, some strain, or high strain. Diabetes self-care was assessed across 4 domains (diet, exercise, smoking status, and medication adherence) and a composite self-care score which summed performance across these 4 domains (low [0-3], medium [4-5], and high [6-8]). The association between caregiving strain and diabetes self-care score was examined with multivariable logistic and ordinal regression, adjusting for demographic, clinical, physical and mental function, and caregiving covariates.

RESULTS: The sample included 795 caregivers with diabetes of mean age 63 years; $69 \%$ were women, $56 \%$ were black, and $18.4 \%$ reported high levels of caregiver strain. Being a woman, low income, low selfreported mental and physical health, high levels of stress, lack of social support, and spending 30 hours a week caregiving were associated with high caregiver strain. For diabetes self-care behaviors, compared to those with no or some strain, high caregiver strain was associated with less physical activity $(\mathrm{p}<0.05)$, low medication adherence $(\mathrm{p}=0.002)$, and overall worse self-care with lower composite self-care scores $(\mathrm{p}<0.02)$. In unadjusted models, high caregiver strain was associated with less physical activity (OR: 0.67 [95\% CI: 0.46, 0.97]), low medication adherence (OR: $0.53,[0.35,0.82])$, and less optimal self-care overall $(0.57$, $[0.36,0.91])$. After adjustment for sociodemographic data and cholesterol, the association between high strain and lower overall self-care persisted (aOR 0.59 [0.36, 0.97].) After adjustment through all models, only the association between high strain and low medication adherence persisted (aOR: 0.52, [0.30, 0.89]).

CONCLUSIONS: These findings suggest that caregiving may interfere with caregivers' ability to take their own medications. Longitudinal studies are needed to determine the impact of this finding on health outcomes among diabetic caregivers. Additionally, increased awareness of the ramifications of caregiver strain on self-care is needed among medical providers and caregivers.

\section{CAREMORE'S INNOVATIVE MODEL OF CARE FOR HIGH- RISK MEDICAID PATIENTS IN THE MID-SOUTH: PRELIMI- NARY OUTCOMES AND LESSONS LEARNED}

Mary E. McBride $^{1}$; Sandeep Palakodeti ${ }^{2}$; Laura R. Sprabery ${ }^{1}$; James E. Bailey ${ }^{1}$. ${ }^{1}$ University of Tennessee Health Science Center, Memphis, TN; ${ }^{2}$ CareMore Health, Memphis, TN. (Control ID \#3185955)

BACKGROUND: CareMore (CM), an integrated health plan and care delivery system, is seen as an innovator in improving outcomes and patient-centered care for the Medicaid population. Their model focuses on using extensivists in hospital to clinic transitions and a care team to provide support for patients. This study documents the early impact of the model and describes strengths and weaknesses from the perspective of personnel.

METHODS: This cross-sectional mixed-methods study evaluated early experience with the $\mathrm{CM}$ model which uses capitated payment from one Medicaid managed care organization (MCO) to serve some Medicaid patients in Shelby Co., TN. Assignments were non-random, with purposeful assignment of high-risk patients. Claims data for March '17-April ' 18 were used to compare outcomes between $\mathrm{CM}$ and non-CM patients, stratified by eligibility (e.g. Temporary Assistance for Needy Families [TANF], Aged/Blind/Disabled Non-Dual [ABD ND], \& Long-Term Supportive Services [LTSS]), age, and gender. Outcomes were: number of ED visits, hospitalizations, hospital days, and specialist visits per 1000 members per year. We employed qualitative key informant interviews to evaluate the strengths and weaknesses of the model. Interviews with staff facilitated discussion of ways CM serves patients well, achieving lower hospitalization rates, what differentiates them from other clinics, motivation for working at $\mathrm{CM}$, and suggestions for improvement.

RESULTS: $\mathrm{CM}$ served $22.4 \%$ ( $\mathrm{N}=7637$ ) of the MCO's patients during the period. CM ED visits were lower for TANF 21-44 F (\% difference [ ] -14.9), TANF 21-44 M ( -34.5), TANF 45+ ( -31.6), and ABD ND ( $-25.1)$; specialist visits were similarly lower. Hospitalizations were lower for TANF 21-44 F ( -2.1), TANF 45+ (-42.4), and ABD ND ( -13.6). $\mathrm{CM}$ had similar reductions in hospital days in these groups. Eighteen employees completed interviews. Positive themes identified include holistic approach, case management, transitions of care, relationships with patients, extensivists, and wraparound team. They identified areas in which CM performed better: holistic approach, case management, relationships with patients, and wraparound team. Employees identified areas needing improvement: wait time, transportation, walk-ins, and accessing specialists. 
CONCLUSIONS: This study provides early evidence that the CM model is improving outcomes and reducing costs for Medicaid patients in Shelby Co. Despite serving sicker patients, CM generally achieved better results for TANF and ABD ND patients: less ED visits, hospitalizations, hospital days, and specialist visits, resulting in lower health care use. Other providers performed better for members ages 14-20 and for LTSS members. Staff feel they are building relationships, yielding improved outcomes and cost savings. This study suggests that the model has potential to reduce costs and improve care for Medicaid patients. Future work is needed to further assess key components of the model and its added impact on clinical outcomes.

\section{CASCADES FOLLOWING LOW-VALUE CARE: PRE- OPERATIVE ELECTROCARDIOGRAM FOR CATARACT SURGERY}

Ishani Ganguli ${ }^{1}$; Claire Lupo ${ }^{1}$; Alexander J. Mainor ${ }^{2}$; Stephanie Raymond $^{2}$; Qianfei Wang ${ }^{2}$; E. John Orav ${ }^{1}$; Chiang-hua Chang ${ }^{2}$; Nancy E. Morden ${ }^{3}$; Meredith Rosenthal ${ }^{4}$; Carrie H. Colla ${ }^{2}$; Thomas D. Sequist ${ }^{1}$. ${ }^{1}$ Brigham and Women's Hospital, Chestnut Hill, MA; ${ }^{2}$ The Dartmouth Institute for Health Policy and Clinical Practice, Lebanon, NH; ${ }^{3}$ The Dartmouth Institute of Health Policy and Clnical Practice, Lebanon, NH; ${ }^{4} \mathrm{TH}$ Chan Harvard School of Public Health, Boston, MA. (Control ID \#3183156)

BACKGROUND: Little is known about downstream health care use triggered by low-value services. Measurement of such "cascades" is essential to understanding the full impact of low-value care. We described cascades (tests, treatments, visits, hospitalizations, and new diagnoses) following a common low-value service: pre-operative electrocardiogram (EKG) for patients undergoing cataract surgery.

METHODS: We used a random 20\% sample of Fee-for-Service Medicare beneficiaries age 66 years or older without known heart disease who underwent cataract surgery 2014-2015. We compared beneficiaries who received a pre-operative EKG to those who did not. Our primary outcome was cascade event rates and associated charges in the 90 days following pre-operative EKG (or in a matched timeframe for the comparison group). Among those who received a pre-operative EKG, we determined patient, physician, and area-level characteristics associated with experiencing a potential cascade.

RESULTS: Among 110,183 cataract surgery recipients, $11.3 \%$ $(n=12,408)$ received a pre-operative $E K G$; of those, $14.4 \%$ $(n=1,789)$ had at least one potential cascade event. Those who received a pre-operative EKG experienced 5.11 [95\%CI, 3.966.25] additional events per 100 beneficiaries relative to the comparison group. This included 2.18 [95\% CI, 1.34-3.02] tests, 0.33 [95\%CI, 0.19-0.46] treatments, 1.40 [95\%CI, 1.18-1.62] new patient cardiology visits, and 1.21 [95\%CI, 0.62-1.79] new cardiac diagnoses. Payment for the additional services was $\$ 559$ per Medicare beneficiary [95\%CI, \$342-\$775], or \$35 million annual$1 y$, in addition to the $\$ 3.3$ million paid for the pre-operative EKGs across all Medicare beneficiaries. Among pre-operative EKG recipients, those who were older (Adjusted Odds Ratio (AOR) 1.43 for 75-84 years old vs. 66-74 years old [95\%CI, 1.29-1.59]), had more chronic conditions (AOR 1.19 for each additional Elixhauser condition [95\% CI, 1.15-1.23]), lived in more cardiologist-dense areas (AOR 1.06 [95\% CI, 1.03-1.10]), or had their pre-operative EKG performed by a cardiac specialist (AOR 1.29 [95\%CI, 1.131.48]) were more likely to experience a potential cascade.

CONCLUSIONS: Care cascades following pre-operative EKG for cataract surgery are infrequent but costly. We may see substantial savings from policy and practice interventions to reduce both low- value services and the cascades that follow. Payers might incentivize beneficiaries to see clinicians with lower rates of low-value ordering while health systems might use clinical decision support or specialist e-consultations. This claims-based study is limited by clinical details that would confirm intentions behind billed services, so we used conservative estimates of cascade events relative to a comparison group. We did not capture other cascade elements such as complications from cascade procedures or psychological or financial harm to patients. Further work might explore these aspects of cascades and examine cascades following other low value events.

CASE MANAGEMENT AS A SOLUTION TO ADDRESSING HEALTH RELATED SOCIAL NEEDS: COMPETING RATIONALES AND UNINTENDED CONSEQUENCES

Elena Byhoff ${ }^{1}$; Lauren A. Taylor ${ }^{2} .{ }^{1}$ Tufts Medical Center, Boston, MA; ${ }^{2}$ Harvard Business School, Boston, MA. (Control ID \#3182959)

BACKGROUND: Health care systems are building capacity to address health related social needs. Community based organizations (CBOs) are strategically investing in Case Management (CM) activities to better position themselves as partners with health care systems to address health related social needs. Little is known, however, about how $\mathrm{CBO}$ define $\mathrm{CM}$ and what consequences might be of significant investment in $\mathrm{CM}$ by the social service sector.

METHODS: We used mixed methods to understand how Massachusetts CBO leaders envision partnership with health care systems to address unmet social needs. We recruited executives and senior staff working in social service sectors identified as priorities by policy-makers and health care administrators. Participants completed a 15-item survey on organizational structure and scope of services, including a variety of $\mathrm{CM}$ services. Participants also took part in semi-structured interviews lasting 1 hour. We used a snowball sampling strategy to recruit additional participants until achieving thematic saturation. Interviews were recorded, transcribed and inductively coded to identify themes highlighting CM strategies.

RESULTS: Leadership and executive staff $(n=46)$ from CBOs across Massachusetts represented 9 social services sectors: food, housing, community centers, legal services, transportation, workforce development, domestic violence, and early childhood education. Survey responses (response rate 87\%) indicated that $\mathrm{CM}$ services were described as core organizational functions across nearly all participating CBOs. CM services included screening for social needs and referring to appropriate organizations, assessing eligibility for services and application assistance for services or entitlements. Qualitative data analysis suggested that CBO leadership noted several ways in which $\mathrm{CM}$ was seen as a high-value strategy, explaining the popularity of $\mathrm{CM}$ services statewide. The perceived mechanisms through which $\mathrm{CM}$ produced value were: (1) $\mathrm{CM}$ improves patient care by delivering high touch interventions for complex patients; (2) $\mathrm{CM}$ allows CBOs to grow their organizational footprint by increasing billable services and referral capacity; and (3) CM facilitates inter-organizational partnership to reduce overall system complexity and navigate patients more effectively to needed resources. Nevertheless, interviewees expressed explicit concern that over-investment in CM services may medicalize social service delivery and increase demand and wait-times for already limited social services. Investment in CM services by both health care and many $\mathrm{CBOs}$ may also create $\mathrm{CM}$ redundancy, thereby unintentionally overburdening patients most in need of simplified care and assistance with multiple competing CMs. 
CONCLUSIONS: Without agreed upon rationales as to how CM should be provided and scaled, CM may actually add complexity and cost to the $\mathrm{CBO}$ ecosystem. A framework for investment in $\mathrm{CM}$ as a tool for brokering clinical-community linkages may be warranted.

\section{CHALLENGES AND OPPORTUNITIES IN ENGAGING PRIMA- RY CARE PROVIDERS IN BRCA TESTING: EARLY RESULTS FROM THE BFOR STUDY}

Lydia E. Pace ${ }^{1}$; Nadine Tung ${ }^{2}$; Jada G. Hamilton ${ }^{3}$; Camila Gabriel ${ }^{4}$; Yeonsoo S. Lee ${ }^{1}$; Sahitya Raja ${ }^{1}$; Colby Jenkins ${ }^{4}$; Anthony J. Braswell ${ }^{7}$; Kelly Morgan ${ }^{3}$; Jeffrey Levin ${ }^{3}$; Jeremy Block ${ }^{3}$; Susan Domchek ${ }^{6}$; Katherine Nathanson ${ }^{6}$; Heather Symecko ${ }^{6}$; Beth Karlan ${ }^{5}$; Daniella Kamara $^{7}$; Kenneth Offit ${ }^{3}$; Judy Garber ${ }^{4}$; Nancy L. Keating ${ }^{8,} 1$. ${ }^{1}$ Brigham and Women's Hospital, Boston, MA; ${ }^{2}$ Beth Israel Deaconess Medical Center, Boston, MA; ${ }^{3}$ Memorial Sloan Kettering Cancer Center, New York, NY; ${ }^{4}$ Dana-Farber Cancer Institute, Boston, MA; ${ }^{5}$ David Geffen School of Medicine at UCLA, Los Angeles, CA; ${ }^{6}$ Perelman School of Medicine, University of Pennsylvania, Philadelphia, PA; ${ }^{7}$ Cedars-Sinai Medical Center, Los Angeles, CA; ${ }^{8}$ Harvard Medical School, Boston, MA. (Control ID \#3182765)

BACKGROUND: Engaging primary care providers (PCPs) in genetic testing could increase access to these services. The BRCA Founder Outreach (BFOR) study offers online education and no-cost BRCA1/2 founder mutation testing to individuals of Ashkenazi Jewish descent in 4 U.S. cities, with results review and management by participants' PCPs or genetics specialists. We sought to understand participants' interest in engaging PCPs in results disclosure, PCPs' willingness to disclose results, and PCPs' attitudes about and experience with genetic testing.

METHODS: BFOR participants were asked if they wished to receive BRCA results from their PCP or BFOR-affiliated genetics specialists; negative results with no further evaluation needed were disclosed via mail. We are surveying the first 125 invited PCPs from each city about their experiences and preferences for involvement in genetic testing and the first 100 PCPs who disclose results about their disclosure experience. We used descriptive and Chi-square statistics.

RESULTS: By December 2018, of 2497 participants enrolled, 792 (33\%) requested their PCP disclose results; the others requested a BFOR genetics specialist. Of 763 invitations sent to 585 unique PCPs (some PCPs had $>1$ patient enrolled), PCPs agreed to disclose results $45 \%$ of the time (237 PCPs). Among 471 PCPs surveyed to date, 214 (45\%) have responded. Respondents were more likely than non-respondents to agree to disclose results $(59 \%$ vs. $30 \%, \mathrm{p}<0.001)$. Most respondents $(91 \%)$ had been asked by patients about BRCA testing in the past year. Overall, $76 \%$ reported feeling very or somewhat well-qualified to recommend BRCA testing, $60 \%$ felt very or somewhat well-qualified to share results, and $60 \%$ noted genetic testing was or could be easily incorporated into their practice. The most frequently identified major obstacles were genetic testing costs, lack of insurance coverage for testing, and lack of counseling experience. Most PCPs (78\%) were interested in further education in BRCA risk assessment and testing. Among the 68 respondents who reported declining to share results with their patients, the most common reasons were limited knowledge and belief this should be done by a specialist. In the post-disclosure survey, of 81 PCPs who disclosed positive results or negative results requiring further evaluation, $42(53 \%)$ have responded. Most $(74 \%)$ felt very comfortable sharing results; $26 \%$ felt somewhat comfortable. Among the subset who disclosed positive results $(n=5), 4$ felt somewhat and 1 felt very comfortable. CONCLUSIONS: Early BFOR results suggest challenges and opportunities for engaging PCPs in genetic testing. Only one-third of participants nominated their PCP to disclose their BRCA results, and over half of those PCPs declined. PCPs described barriers to integrating genetic testing in their practice, but most who disclosed results felt comfortable doing so. Ongoing work will examine factors associated with participants' and PCPs' preferences to guide interventions to educate and support PCPs.
CHALLENGES TO ABNORMAL MAMMOGRAM FOLLOW-UP: PERSPECTIVES FROM PRIMARY CARE PROVIDERS

Leah S. Karliner ${ }^{1}$; Karla Kerlikowske ${ }^{2}$; Nancy Burke ${ }^{3}$; Sarita Pathak ${ }^{1}$; Steven Gregorich $^{1}$; Jennifer Livaudais-Toman ${ }^{1}$; Celia P. Kaplan ${ }^{1}{ }^{1}$ University of California, San Francisco, Greenwich, CT; ${ }^{2}$ University of California San Francisco, San Francisco, CA; ${ }^{3}$ UC Merced, Merced, CA. (Control ID \#3186174)

BACKGROUND: Timely follow-up of an abnormal mammogram result can help avoid a delay in breast cancer diagnosis and, in turn, a more advanced cancer. Our prior research demonstrated that facilities with longer follow-up times have the expectation that the referring provider is primarily responsible for communication of abnormal results and ensuring women undergo appropriate follow-up. We set out to understand the primary care physician (PCP) perspective on communication responsibility and abnormal mammogram follow-up.

METHODS: Survey of all PCPs in San Francisco (SF) about their experiences with communication and care coordination of abnormal mammogram results and diagnostic follow-up. We used names and emails of PCPs practicing in academic, public health, and Veterans Affairs Medical Center settings combined with searches of publicly available health plan websites to identify PCPs actively practicing in SF. PCPs completed the survey online or by mail. RESULTS: Of 588 eligible PCPs, 300 (51\%) completed the survey: $64 \%$ were general internists; $33 \%$ were in private practice, $25 \%$ public safety net, $26 \%$ academic practice, and $16 \%$ staff model HMO (Kaiser or VA). Participants graduated from medical school on average $21.9 \pm 11.8$ years previously. PCP patient panels had a mean of $65 \%$ (range $0-100 \%$ ) minority and $33 \%$ (range $0-100 \%$ ) LEP patients. Only $24 \%$ of PCPs felt they were solely responsible for notifying a woman of her abnormal mammogram result and need for follow-up, $20 \%$ felt radiology was solely responsible, and $56 \%$ felt it was a shared responsibility of the PCP with radiology. This was similar with regard to perceived responsibility for notifying a woman of her radiologyguided biopsy results (33\% PCP only, $47 \%$ shared PCP \& radiology; $20 \%$ radiology only). Forty-four percent reported having no formal system for tracking the need for - and completion of - diagnostic testing after an abnormal mammogram. Important barriers were cited as impacting PCPs' ability to track women for follow-up: no formal tracking system (33\%), competing time demands (34\%), no dedicated staff to support tracking (26\%). Only $25 \%$ of PCPs felt well equipped to explain to a woman what to expect during a core biopsy; $60 \%$ reported women sometimes/very often asked questions about breast biopsy that they lacked the expertise to answer. A third (31\%) of PCPs found it somewhat/very difficult to discuss abnormal mammogram or biopsy results with women who have a low education level, $23 \%$ with women from a very different cultural group from their own, and $47 \%$ with non-English speaking women.

CONCLUSIONS: Our data suggest the need for 1) standardized protocols to optimally delineate responsibilities of PCPs and radiologists, and 2) health system investment in formal tracking and dedicated staff to ensure timely, equitable care for all women after an abnormal mammogram or breast biopsy. Additionally, many PCPs would benefit from education on breast biopsy and training on communication across differences.

\section{CHANGES IN ECONOMIC AND CLINICAL OUTCOMES UN- DER CMS MANDATORY BUNDLED PAYMENTS FOR JOINT REPLACEMENTS}

Derek A. Haas ${ }^{1,2}$; Xiaoran Zhang ${ }^{1,2}$; Robert S. Kaplan ${ }^{2,}{ }^{1}$; Zirui Song ${ }^{3}$, ${ }^{4}$. ${ }^{1}$ Avant-garde Health, Boston, MA; ${ }^{2}$ Harvard Business School, Boston, MA; ${ }^{3}$ Harvard Medical School, Boston, MA; ${ }^{4}$ Massachusetts General Hospital, Boston, MA. (Control ID \#3186135)

BACKGROUND: In 2016, CMS launched its first mandatory bundled payment model, the Comprehensive Care for Joint Replacement (CJR) model, through randomizing metropolitan statistical areas (MSAs) into the program. 
This study evaluates changes in key economic and clinical outcomes associated with the CJR after 2 full years.

METHODS: We used a difference-in-differences model to compare Medicare spending, quality of care, utilization, and patient characteristics in episodes of lower extremity joint replacements between propensity-score matched CJR and non-CJR hospitals. The propensity score was constructed with patient and hospital characteristics. Using 100\% Medicare Part A data and 5\% Medicare Part B data from January, 2014 through March, 2018, we used a linear model adjusted for age, sex, risk, and hip fracture status. We used an intention-to-treat framework, in which we studied episodes in all 75 MSAs initially randomized into treatment relative to control. We also estimated the local average treatment effect among 67 MSAs that received the full CJR.

RESULTS: We identified episodes for lower extremity joint replacement beginning with 815 hospitals in MSAs that were randomly assigned to the CJR and 955 hospitals in MSAs that were randomly assigned to control. Within a joint replacement episode, Medicare Part A claims spending accounted for nearly $90 \%$ of total spending in both treatment and control. After propensity score matching, there were 157,828 primary lower extremity joint replacement episodes in the CJR group and 180,594 episodes in the control group. The CJR led to a decrease of $\$ 582$ per episode $(\mathrm{p}<0.001)$ in Medicare Part A claims spending, a 2.5\% savings on claims driven by a $5.5 \%$ decline $(\mathrm{p}<0.001)$ in 90-day post-acute care spending, concentrated in skilled nursing facilities and inpatient rehabilitation facilities. No significant changes in hospital length of stay, readmissions, complications, 30-day or 90-day mortality, volume of episodes, or patient characteristics were found.

CONCLUSIONS: The CJR was associated with reduced Medicare Part A claims spending over 2 years, largely through lower post-acute spending, without detectable changes in the volume of inpatient admissions for primary hip and knee replacements, length of stay, complication rates of hip and knee replacements, 90-day readmissions, mortality out to 90 days, or characteristics of beneficiaries in whom the bundled payment was triggered. Lower episode spending was driven by a decrease in 90-day post-acute care spending, concentrated within skilled nursing facilities and inpatient rehabilitation facilities. Estimated savings on claims, while consistent with changes in provider behavior, may not have exceeded the reconciliation payments to hospitals reported by CMS to date. The CJR model is the first wide-scale mandatory bundled payment model implemented by CMS in a randomized fashion. Mandatory bundled payments may serve as a useful model for policy efforts to slow spending without harming quality.

\section{CHANGES IN ENROLLEES' HEALTH STATUS AFTER MEDIC- AID EXPANSION}

Susan D. Goold ${ }^{2,1}$; Renuka Tipirneni ${ }^{2,}{ }^{1}$; Edith C. Kieffer ${ }^{1,1}$; Tammy Chang ${ }^{1,}$ ${ }^{1}$; Matthias Kirch ${ }^{1}$; Corey Bryant ${ }^{2}$; Erica Solway ${ }^{1}$; Sunghee Lee ${ }^{1}$; Zachary Rowe $^{4,5}$; Erin Beathard ${ }^{1}$; Sarah J. Clark ${ }^{1,1}$; Jennifer Skillicorn ${ }^{1,}$; John Z. Ayanian $^{1,2}$; Jeffrey T. Kullgren ${ }^{3,1}$. ${ }^{1}$ University of Michigan, Ann Arbor, MI; ${ }^{2}$ University of Michigan, Keego Harbor, MI; ${ }^{3}$ Ann Arbor VA Healthcare System and University of Michigan, Ann Arbor, MI; ${ }^{4}$ Friends of Parkside, Detroit, MI; ${ }^{5}$ Urban Research Center, Detroit, MI. (Control ID \#3177579)

BACKGROUND: Evidence about the impact of Medicaid expansion on health has been mixed, and has largely come from comparing expansion to non-expansion states. We assessed the impact of Michigan's Medicaid expansion, the Healthy Michigan Plan (HMP), on the health of enrollees over time.

METHODS: From January-November 2016, the Healthy Michigan Voices telephone survey was conducted in English, Arabic and Spanish with 4,090 HMP beneficiaries aged 19-64 with 12 months of HMP coverage and 10 months in a Medicaid health plan (RR $53.7 \%$ ). Sampling was stratified by income and region. Respondents in 2016 who consented to be re-contacted (96.4\%) were eligible for the 2017 HMV Follow-Up Survey, conducted from March 2017 to January 2018 (RR 83.4\%). Surveys measured demographics, health status, access to and use of health care, health risks and behaviors.
Analysis included weights for sampling probability and nonresponse. Comparisons between 2016 and 2017 included only those who responded to both surveys.

RESULTS: Of 2017 HMV follow-up survey respondents $(n=3,104)$, $76.8 \%$ were still enrolled in HMP ("current enrollees") and $23.2 \%$ were no longer enrolled at the time of the 2017 survey. Most enrollees reported that, in the past year, their physical health had improved $(29.6 \%)$ or stayed the same $(56.1 \%)$, their mental health had improved $(28.4 \%)$ or stayed the same $(58.6 \%)$, and their oral health had improved $(21.0 \%)$ or stayed the same $(60.7 \%)$. Over one-third $(36.4 \%)$ reported in 2017 that their health was excellent or very good. The proportion of all enrollees who reported fair or poor health decreased from $30.7 \%$ in 2016 to $27.0 \%$ in 2017 (aOR=0.66, $\mathrm{p}<.001)$. The largest decreases in reports of fair/ poor health from 2016 to 2017 were observed in enrollees who were Hispanic (from $28.3 \%$ to $21.5 \%$ ), non-Hispanic Black (from $31.5 \%$ to $26 \%$ ), from the Detroit Metro area (from $30.7 \%$ to $24.9 \%$ ), and with an income 0$35 \%$ FPL (from $37.6 \%$ to $32.3 \%$ ). The mean number of days of poor physical health in the past month decreased from 2016 to 2017 from 6.9 to 5.7 (Coeff $=-1.32, \mathrm{p}<.001)$. Fewer days of poor physical health were found in both current enrollees (from 7.0 to 5.6) and former enrollees (from 6.8 to 5.8), those with a chronic condition (from 8.2 to 6.8), those with two or more chronic conditions (from 9.9 to 8.5), those with a mental health condition (from 9.5 to 8.0 ), and those with a substance use disorder (from 10.0 to 8.1 ). There was no statistically significant decrease from 2016 to 2017 in the number of days of poor mental health or the number of days of usual activities missed due to poor physical/mental health.

CONCLUSIONS: Medicaid expansion in Michigan was associated with improvements in self-reported health and days of poor physical health, including for enrollees with chronic physical and mental health conditions.

CHANGES IN HEALTH INSURANCE COVERAGE BY OCCUPATION AFTER THE AFFORDABLE CARE ACT

Sumit Agarwal ${ }^{1}$; Anna Goldman ${ }^{2}$; Benjamin D. Sommers ${ }^{3} .{ }^{1}$ Brigham and Women's Hospital, Somerville, MA; ${ }^{2}$ Cambridge Health Alliance, Cambridge, MA; ${ }^{3}$ Harvard School of Public Health, Brookline, MA. (Control ID \#3185500)

BACKGROUND: We sought to assess changes in health insurance coverage across occupations after implementation of the Affordable Cart Act (ACA).

METHODS: Using data from the American Community Survey from 2010 through 2017, we examined changes in presence and type of insurance coverage before and after the ACA. We focused on five standard occupation groups: managers and professionals (e.g. chief executives, physicians), service workers (e.g. food servers, barbers), sales and office workers (e.g. cashiers, secretaries), farming and construction workers (e.g. farmers, plumbers), and industrial and manufacturing workers (e.g. machinists, truck drivers). We used a linear regression model to estimate unadjusted and adjusted changes in the following categories of coverage: uninsured, Medicaid, employer-sponsored insurance (ESI), and directly purchased insurance (includes ACA Marketplaces).

RESULTS: Uninsured rates before the ACA and coverage gains since 2014 varied significantly by occupation (Figure). Managers and professionals were the most likely to receive coverage through their employer $(82.7 \%$ prior to the ACA). Those in farming and construction $(56.4 \%)$ and in service $(52.0 \%)$ were the least likely to receive coverage through their employer. There were modest gains in ESI among all groups, ranging from 1.9 to 3.1 percentage points. The bulk of the gains in coverage after 2014 were due to increases in Medicaid and directly purchased insurance. Service workers experienced a 4.3 percentage-point increase in directly purchased insurance and 5.8 percentage-point increase in

Medicaid. There were also large gains for those in sales and office 
(2.8 percentage points and 3.2 percentage points), farming and construction (2.8 percentage points and 3.0 percentage points), and industrial and manufacturing ( 2.8 percentage points and 3.3 percentage points). There were smaller gains for managers and professionals ( 0.8 percentage points and 1.3 percentage points). All p-values were $<0.001$.

CONCLUSIONS: The largest gains in coverage associated with the ACA occurred among non-professional workers, and these positive effects on coverage were not due to crowd-out of ESI. Policies that reduce public insurance enrollment or disrupt the ACA's health insurance Marketplaces will have adverse effects on those who have less access to employer-sponsored insurance.

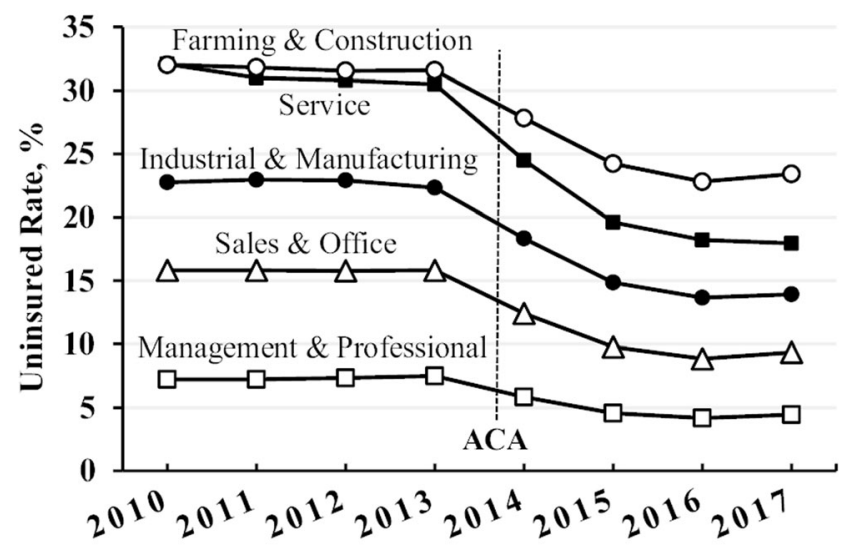

CHANGES IN HOSPITAL ACQUIRED CONDITIONS AND MORTALITY ASSOCIATED WITH THE HOSPITAL ACQUIRED CONDITION REDUCTION PROGRAM

Emily Arntson ${ }^{1,2}$; Justin B. Dimick ${ }^{2}{ }^{3}$; Usha Nuliyalu ${ }^{3}$; Joshua Errickson ${ }^{3}$; Tedi A. Engler ${ }^{1}$; Andrew Ryan ${ }^{1,3}$. ${ }^{1}$ University of Michigan School of Public Health, Ann Arbor, MI; ${ }^{2}$ University of Michigan Medical School, Ann Arbor, MI; ${ }^{3}$ University of Michigan, Ann Arbor, MI. (Control ID \#3184751)

BACKGROUND: Hospital acquired conditions (HACs) are common, costly, and deadly. Despite recent reductions, HACs still occur at a rate of 121 events per 1,000 discharges. Building on previous payment reforms to reduce HACs, CMS announced the Hospital Acquired Condition Reduction Program (HACRP) in August 2013. The program - largely focused on surgical admissions - reduces Medicare payments by $1 \%$ for hospitals in the worst performance quartile of targeted hospital-acquired conditions. Penalty criteria are based on absolute performance levels for a relatively narrow set of measures. However, rates of HACs are highly sensitive to hospital coding practices. As a result, the HACRP's effect on both targeted and downstream clinical outcomes is uncertain.

METHODS: Using an interrupted time series design, we estimated models with linear splines to test for changes in hospital-acquired conditions and 30-day mortality before the Affordable Care Act (ACA), after the ACA, and after the HACRP. To assess whether improvements may be related to coding practices, we examined changes in the percentage of hospital-acquired conditions that were coded as present-on-admission. We used data from the $100 \%$ MedPAR Files for Medicare patients undergoing surgery and discharged from eligible hospitals between January 1, 2009 and August 31, 2015 ( $\mathrm{n}=8,857,877$ ).

RESULTS: Patients experienced hospital-acquired conditions at a rate of 13.39 per 1,000 discharges (95\% confidence interval $\{\mathrm{CI}\}$, 13.1 to 13.68$)$ in the pre-ACA period. This rate declined after the
ACA was passed and declined further after the HACRP was announced (adjusted difference in annual slope, 1.34 [95\% CI, - 1.64 to -1.04]). Adjusted 30-day mortality was 3.69 (95\% CI, 3.64 to 3.74 ) in the pre-ACA period among patients receiving surgery. 30-day mortality declined after the ACA (adjusted annual slope - 0.04 [95\% CI, -0.05 to -0.02$]$ ) but was flat after the HACRP (adjusted annual slope -0.01 [ $95 \%$ CI, -0.04 to 0.02$]$ ). While the use of coding for present-on-admission slightly increased after the HACRP was announced, coding changes did not explain reductions in hospitalacquired conditions.

CONCLUSIONS: Overall, hospital-acquired conditions targeted under the HACRP declined at a greater rate after the program was announced. The HACRP was not associated with declines in 30-day mortality. This suggests that either: 1) hospitals are artificially reducing reported $\mathrm{HAC}$ rates and not improving downstream clinical outcomes, such as mortality, or 2) hospital-acquired conditions are not sufficiently common or mechanistically related to major harm such that improvement will drive a mortality reduction. Further investigation, especially into hospitals' HAC reduction mechanisms, is needed. Our findings are instructive to policymakers seeking to improve patient safety. CMS should continue to invest in robust systems to capture valid HAC measures and externally audit these data in order to ensure their integrity for value-based payment programs.

\section{CHANGES IN PERCEPTIONS OF DISCRIMINATION IN HEALTHCARE: AN ANALYSIS OF THE CALIFORNIA HEALTH INTERVIEW SURVEY 2003-2017}

Lucy Schulson $^{1}$; Ziming Xuan ${ }^{3}$; Michael K. Paasche-Orlow ${ }^{2}$; Alicia Fernandez ${ }^{4}$. ${ }^{1}$ Boston University/ Boston Medical Center, Boston, MA; ${ }^{2}$ Boston University, Boston, MA; ${ }^{3}$ Boston University School of Public Health, Boston, MA; ${ }^{4}$ University of California San Francisco, San Francisco, CA. (Control ID \#3185334)

BACKGROUND: Research in the early 2000s in California demonstrated that racial and ethnic minorities, immigrants, and those with limited English proficiency (LEP) experienced high rates of discrimination in healthcare. Less is known how patients' perceptions of discrimination in healthcare have changed for these groups.

METHODS: We used data from the 2003, 2005, 2015, 2016, and 2017 waves of the California Health Interview Survey (CHIS) to determine if rates of perceived discrimination in healthcare have changed. We identified those as having experienced discrimination if they responded "yes" to the question, "Was there ever a time when you would have gotten better medical care if you had belonged to a different race or ethnic group?" and reported this occurred within the last five years. Our primary exposure of interest was survey year dichotomized as combined 2003 and 2005 and combined 2015, 2016, and 2017. We used jack-knife replicate weights provided by CHIS and performed chi-square analyses comparing perceptions of discrimination in 2003-2005 vs. 20152017 and multivariate logistic regression comparing discrimination in late vs. early years controlling for race, poverty level, education, insurance status, usual source of care, self-reported health, and LEP. We also performed sub-analyses by racial/ethnic groups, immigrant status, and LEP status.

RESULTS: There were 84,088 participants in 2003-2005 with $5.97 \%$ of the population reporting recent discrimination in healthcare and 63,242 participants in 2015-2017 with $3.98 \%$ of the population reporting recent discrimination in healthcare $(\mathrm{p}<.0001)$. Latinos and Asians experienced less discrimination in healthcare in 2015-2017 vs. $2003-2005(6.58 \%$ vs. $12.73 \%$ p $<.0001 ; 4.74 \%$ vs. $7.37 \%$ $\mathrm{p}=.00770)$. Rates were stable among whites and African-Americans ( $2.26 \%$ vs. $2.61 \%$ p $=0.25 ; 13.84 \%$ vs. $13.14 \%$ p=0.75). Fewer immigrants and LEP individuals reported discrimination in healthcare 
in $2015-2017$ vs. $2003-2005$ (6.16\% vs. $12.10 \%$ p<.0001; $7.69 \%$ vs. $15.84 \% \mathrm{p}<.0001)$. In adjusted analyses, perceptions of discrimination in healthcare decreased in 2015-2017 compared to 2003-2005 (OR 0.608 , CI $0.519,0.712, \mathrm{p}<.0001)$. There was a significant race by year interaction for Latinos (OR 0.584, CI 0.422, 0.809, p=.0012), but not for Asians or African-Americans (OR 0.767, CI 0.456, 1.289, $\mathrm{p}=0.664$; OR 1.11, CI 0.69, 1.794, $\mathrm{p}=0.664)$. There was a significant immigrant by year interaction and LEP status by year interaction (OR 0.594, CI 0.440, 0.801, p=.0007; OR 0.671, CI 0.499, 0.902, $\mathrm{p}=.0083$ ).

CONCLUSIONS: Perceptions of discrimination in healthcare in California decreased between 2003- 2005 and 2015-2017 but only for Latinos, immigrants, and LEP individuals. African Americans reported consistently high rates of discrimination. Interventions specifically targeted at reducing discrimination are still necessary.

\section{CHANGES IN THE COLORECTAL CANCER INCIDENCE AND MORTALITY IN NEW YORK CITY IN THE CONTEXT OF INCREASED SCREENING, 2003-2015}

Jennifer J. Brown ${ }^{3}$; Charles Asumeng ${ }^{3}$; David Greenwald ${ }^{2}$; Matthew A. Weissman ${ }^{1,2}$; Ann Zauber ${ }^{4}$; Jared Striplin ${ }^{3}$; Justin M. List ${ }^{5}$; Shannon M. Farley ${ }^{3}$; Sidney Winawer ${ }^{4} .{ }^{1}$ Mount Sinai Beth Israel, New York, NY; ${ }^{2}$ Icahn School of Medicine at Mount Sinai, New York, NY; ${ }^{3}$ New York City Department of Health and Mental Hygiene, New York, NY; ${ }^{4}$ Memorial Sloan Kettering Cancer Center, New York, NY; ${ }^{5}$ New York City Health + Hospitals, New York, NY. (Control ID \#3179909)

BACKGROUND: Incidence rates for new colorectal cancer (CRC) cases have been falling $2.6 \%$ each year over the last 10 years, while mortality rates have been falling $2.4 \%$ each year over 2006-2015, nationally. CRC screening has increased nationally during this time. The New York Citywide Colon Cancer Control Coalition (C5) in partnership with the New York City (NYC) Department of Health and Mental Hygiene promoted CRC screening from 2003 to present. Here, we report rates of change and trends in CRC incidence and mortality in the context of changes in colonoscopy screening rates from 2003 to 2015 in NYC.

METHODS: Age-adjusted CRC incidence and mortality rates were calculated using the New York State Cancer Registry and NYC Vital Statistics Data. Screening rates for CRC by colonoscopy were estimated using the NYC Community Health Survey. We used the Joinpoint Regression Program to determine annual percent change (APC) for incidence and mortality and screening curves.

RESULTS: There was a $29 \%$ decrease in age-adjusted CRC incidence from 54.1 to $38.4 / 100,000$ and a $35 \%$ decrease in the CRC mortality rate from 21.0 to $13.7 / 100,000$, from 2003 to 2015, Figure 1. In 2015 Blacks had higher CRC mortality rates $(14.9 / 100,000)$, compared to Hispanics $(11.2 / 100,000)$ and Asians $(10.5 / 100,000)$. In 2015 CRC mortality rates were more than twice as high in Central Harlem and the South Bronx neighborhoods compared to Chelsea and Union Square. Asian/Pacific Islanders had significantly lower CRC screening, at $58.8 \%$ compared to Blacks (63.9\%), Hispanics $(64.3 \%)$ and Whites $(64.7 \%)$. Overall, screening rates increased from $41.7 \%$ to $69.9 \%$ from 2003 to 2015 . Using single point estimates, $\mathrm{CRC}$ screening increased at $\mathrm{APC}=15.65$, from 2005 to $2009 \mathrm{APC}=$ 3.69 , and from 2009 to $2015 \mathrm{APC}=0.80$.

CONCLUSIONS: A decreasing burden of CRC was evident from lower incidence and mortality rates from 2003 to 2015. During this time screening colonoscopy rates in NYC increased after a citywide public health effort. While these inverse trends are going in the right direction, the persistent disparities for certain NYC neighborhoods and racial/ethnic groups demonstrate a need for continued targeted public health efforts in CRC prevention.

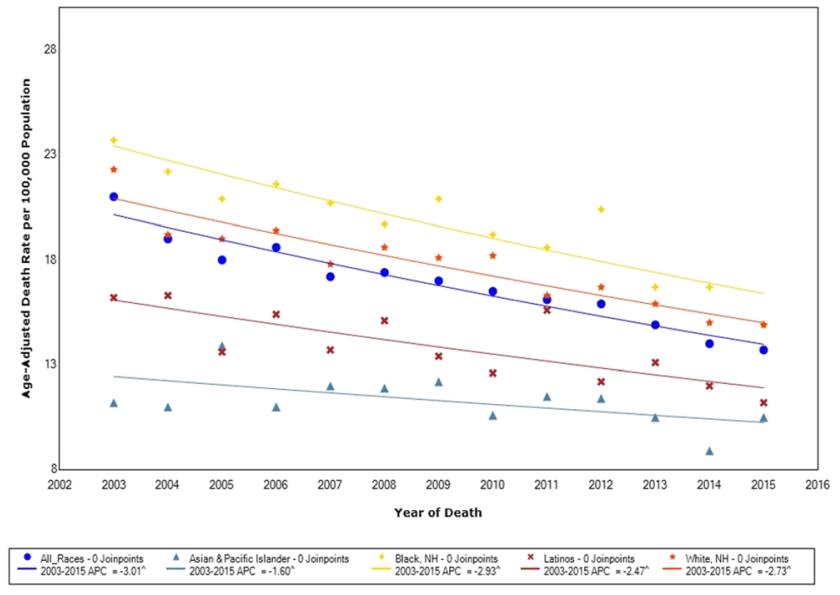

CRC Mortality Rate per 100,000 NYC Population, 2003 to 2015, Age-Adjusted: NYC Vital Statistics Data

\section{CHANGES IN VETERANS' COVERAGE AND ACCESS TO} CARE FOLLOWING THE AFFORDABLE CARE ACT

Alan T. Kelley ${ }^{1,2}$; Renuka Tipirneni ${ }^{1}$; Helen Levy ${ }^{1}$. ${ }^{1}$ University of Michigan, Ann Arbor, MI; ${ }^{2}$ VA Ann Arbor Health System, Ann Arbor, MI. (Control ID \#3172950)

BACKGROUND: Insurance coverage for nonelderly veterans improved following coverage expansions of the Affordable Care Act (ACA). However, whether improvements in coverage have translated into improved access to healthcare has not been previously reported.

METHODS: Using data from the Behavioral Risk Factor and Surveillance System, self-reported insurance status and access to care among nonelderly veterans ages 18-64 were compared in the three-year periods before and after ACA coverage expansions went into effect. Measures of self-reported insurance status, general access to care (cost-related delays in receiving care), and access to primary care (report of having a usual source of care and report of having a checkup in the last 12 months) were included. Difference-in-differences analysis was used to compare changes in Medicaid expansion states to non-expansion states between the time periods. Pre-specified subgroup analyses were performed for veterans with low socioeconomic status (SES) and fair to poor health (poor health).

RESULTS: Among all nonelderly veterans, uninsurance decreased 4.4 percentage points $(12.6 \%$ to $8.2 \%, P<0.05)$, and cost-related delays in care declined 1.9 percentage points $(11.9 \%$ to $10.0 \%$, $P<0.05)$. Report of no checkup within 12 months declined slightly ( $28.1 \%$ to $26.7 \%, P<0.05)$ but there was no significant change in report of a usual source of care. Compared to all nonelderly veterans, veterans with low SES or poor health had greater declines in uninsurance and cost-related delays in care; however, differences in report of no checkup within 12 months or usual source of care were non-significant. When comparing Medicaid expansion states to non-expansion states, differences in uninsurance rates were negligible $(<1 \%)$ and differences in all access measures were mixed and mostly non-significant.

CONCLUSIONS: Uninsurance and cost-related delays in care decreased, especially among nonelderly veterans with low SES and poor health. However, changes in primary care access were mixed. Additional study is needed to understand why gains in coverage among veterans did not translate into larger gains in access. 
CHARACTERISTICS AND INJURY MECHANISMS OF VETERAN PRIMARY CARE SUICIDE DECEDENTS WITH AND WITHOUT DIAGNOSED MENTAL ILLNESS; 20002014

Joseph A. Simonetti ${ }^{1}{ }^{2}$; Rebecca I. Piegari ${ }^{3}$; Charles Maynard ${ }^{4}$; Lisa Brenner ${ }^{1,}{ }^{6}$; Alaina M. Mori ${ }^{4}$; Edward P. Post ${ }^{7}{ }^{8}$; Karin M. Nelson ${ }^{4},{ }^{9}$; Ranak Trivedi ${ }^{5,}{ }^{10}$. ${ }^{1}$ Veterans Health Administration, Denver, CO; ${ }^{2}$ University of Colorado School of Medicine, Aurora, $\mathrm{CO} ;{ }^{3}$ Department of Veterans Affairs, Canandaigua, NY; ${ }^{4} \mathrm{VA}$ Puget Sound Healthcare System, Seattle, WA; ${ }^{5}$ Center for Innovation to Implementation, Palo Alto, CA; ${ }^{6}$ University of Colorado, Aurora, CO; ${ }^{7}$ VA HSRD, Ann Arbor, MI; ${ }^{8}$ University of Michigan Medical School, Ann Arbor, MI; ${ }^{9}$ University of Washington, Seattle, WA; ${ }^{10}$ Stanford University, Palo Alto, CA. (Control ID \#3184627)

BACKGROUND: Suicide is the 10th leading cause of death in the United States, and rates increased among most groups from 1999-2014. Suicide prevention programs largely focus on identifying and treating mental health diagnoses (e.g., depression) because they are well-known, prevalent, and potent predictors of suicide. However, no study has assessed what proportion of suicide decedents lacked such diagnoses within a primary care population.

METHODS: We conducted a retrospective cohort study of Veterans Health Administration (VHA) primary care patients who died by suicide from 2000-2014. We identified Veterans receiving primary care using data from the VHA Corporate Data Warehouse (CDW). These records were then linked to the US Department of Veterans Affairs and Department of Defense Joint Suicide Data Repository to identify those who died by suicide (ICD-10 codes: X60-X84, Y87.0) and their mechanism of suicide (e.g., firearm injury). Using ICD-9 codes available in the CDW, we categorized suicide decedents by whether they had a history (yes/no) of any of the following mental health diagnoses: depression, post- traumatic stress disorder, anxiety disorder, substance use disorder, schizophrenia or bipolar disorder. CDW data were also used to characterize decedents' sociodemographic and clinical characteristics, including total physical health diagnoses and healthcare utilization. We compared differences between those with and without any mental health diagnosis using chi-square or Student's T-tests. RESULTS: Of the 9,550,726 veterans identified, $28,812(1.1 \%)$ died by suicide. Of those, $12,740(44.2 \%)$ had no history of a mental health diagnosis. In comparison to those with mental health diagnoses, decedents with no mental health diagnoses were more likely to be older ( 68 vs. 57 years, $\mathrm{p}<0.01)$, male $(98.2 \%$ vs. $95.4 \%, \mathrm{p}<0.01)$, and married or partnered $(49.4 \%$ vs. $35.9 \%$, $\mathrm{p}<0.01$ ), were less likely to have a service-connected disability $(57.4 \%$ vs. $72.8 \%, \mathrm{p}<0.01)$, and had a higher number of physical health diagnoses $(2.63$ vs. $2.59, \mathrm{p}<0.01)$. In the year prior to death, decedents without mental health diagnoses had fewer primary care visits, mental health visits, emergency department visits, and hospitalizations. Those without mental health diagnoses were more likely to die by firearm injury $(78.8 \%$ vs. $59.9 \%, \mathrm{p}<0.01)$.

CONCLUSIONS: From 2000-2014, nearly half of VHA primary care patients who died by suicide did not have an antemortem mental health or substance-related diagnosis. Veterans without mental health diagnoses who died by suicide were more likely to be older, married, male, without service-related disability benefits, and had a high number of physical health diagnoses and were more likely to die by firearm injury. Suicide prevention efforts within primary care relying solely on the identification of mental health or substance-related diagnoses may be insufficient in preventing a large proportion of veteran suicides.
CHARACTERISTICS AND QUALITY OF ROTATIONSPECIFIC RESIDENT LEARNING GOALS: A PROSPECTIVE STUDY

Adam P. Sawatsky; Andrew J. Halvorsen; Paul Daniels; Sara Bonnes; Meltiady Issa; John T. Ratelle; Christopher Stephenson; Thomas J. Beckman. Mayo Clinic, Rochester, MN. (Control ID \#3175489)

BACKGROUND: Identifying learning goals is part of self-directed learning, and is triggered by the revelation of gaps in knowledge. While learning goals are part of self-directed learning, residents struggle with setting learning goals. Faculty can support selfdirected learning through supporting resident learning goal development. We sought to characterize the topics and quality of residents' learning goals.

METHODS: We conducted a prospective mixed-methods study of 153 internal medicine residents, assessing 455 learning goals for general medicine inpatient rotations during the 2016-2017 academic year. For each learning goal, we coded themes, competencies and learning domains, and categorized themes into topic categories. We assessed quality using the Learning Goal Scoring Rubric (LGSR), an instrument with evidence of validity for content, internal structure and relationship to other variables. It has 4 items (specific goal, important goal, realistic multisource plan, and measurable outcome), each scored from 0-3, with a total possible score of 12 . We compared learning goal topic categories, competencies, learning domains, and quality between first and second months of postgraduate (PGY) 1 residents and between PGY-1 and PGY-3 residents. For PGY-1 residents, we compared mean overall and item scores between month 1 and 2 using a paired t-test. To compare PGY-1 and PGY-3 mean overall and item scores, we used an independent 2-sample ttest. We assessed factors associated with learning goal completion and coded themes regarding barriers and facilitators to learning goal completion.

RESULTS: The overall response rate was $80 \%$. The top three learning goal categories were patient management, specific diseases related to general medicine, and teaching skills. There were no significant changes in learning goal characteristics between PGY-1 months ( $\mathrm{p}$ 0.04). There were differences between PGY-1 and PGY-3 residents' learning goals in patient management (28\% vs $6 \%$; $\mathrm{p}<.001)$, specific disease conditions ( $19 \%$ vs $3 \% ; \mathrm{p}<.001)$, and teaching skills $(2 \%$ vs $56 \% ; \mathrm{p}<.001)$. There was no difference in the LGSR score between PGY-1 months (1.63 vs. 1.67 ; $=0.82$ ). The PGY-3 learning goals were of higher quality than PGY-1 learning goals for the "specific goal" item (1.38 vs. 0.98, $\mathrm{p}=0.005$ ), but not for other items or overall (all p 0.02). Residents reported $85 \%(297 / 347)$ learning goal completion, with main drivers being experience $(88,28 \%)$, intentional effort $(128,41 \%)$, and external guidance $(98,31 \%)$.

CONCLUSIONS: Residents identified a broad array of topics for learning goals. While PGY-1 residents developed learning goals in medical knowledge and patient care, PGY-3 residents developed learning goals for more integrative abilities like team management and teaching skills. Residents' learning goal quality was low, reflecting the need for more guidance from faculty members and residency programs to support residents' selfdirected learning.

CHARACTERISTICS OF CANCER SURVIVORS IN OUTPATIENT SAFETY NET CLINICS

Megan Hoopes ${ }^{1}$; Teresa Schmidt ${ }^{1}$; Nathalie Huguet ${ }^{2}$; Kerri Winters-Stone $^{2}$; Heather Angier ${ }^{2}$; Miguel Marino ${ }^{2}$; Jackilen Shannon $^{2}$; Jennifer DeVoe ${ }^{2}$. ${ }^{1}$ OCHIN, Portland, OR; ${ }^{2}$ Oregon Health \& Science University, Portland, OR. (Control ID \#3179117) 
BACKGROUND: Little is known about the prevalence of cancer survivors seen in primary care settings. To provide comprehensive healthcare and care coordination, it is important for primary care providers to understand the utilization patterns, clinical complexity, and specific needs of this growing population.

METHODS: This retrospective, observational study used electronic health record (EHR) data from 586 safety net clinics, namely community health centers (CHCs), in 20 states, from 2014-2016. CHCs serve a patient population that is largely publicly insured or uninsured, and underrepresented in research. Cancer survivors were defined as adults with a visit to a $\mathrm{CHC}$ and a history of malignant cancer; we identified and matched these patients in a 1:3 ratio to patients without a cancer history. We characterized cancer history (primary site, age at diagnosis, time since diagnosis) and compared survivors to their matched counterparts without a cancer history on demographic characteristics, medical complexity, and healthcare utilization. Generalized estimating equation models were used to compute adjusted odds ratios and $95 \%$ confidence intervals for select utilization indicators.

RESULTS: We identified 45,779 cancer survivors from a population of 1.2 million adult $\mathrm{CHC}$ patients (prevalence=3.4\%). Most cancer survivors were female $(60.8 \%)$, with a median age of 61 . The leading cancer sites were breast (28.7\% of female survivors), prostate $(22.9 \%$ of males), cervical ( $8.8 \%$ of females), colorectal (7.1\% of both sexes), melanoma (5.8\%), non-Hodgkin lymphoma $(4.3 \%)$, and lung and bronchus (4.1\%); sex-stratified rankings agreed with national statistics. The median age at diagnosis was 58 (mean=56.9, $\mathrm{SD}=14.5$ ). Compared to matched patients without a cancer history, survivors had been established with their clinic longer (mean=3.6 years vs. 3.0), had more $\mathrm{CHC}$ visits per year (mean=10.8 vs. 7.8 ), were more likely to be assigned a primary care provider ( $86.6 \%$ vs. $83.2 \%$ ), and were less likely to be uninsured $(13.6 \%$ vs $21.6 \%, \mathrm{p}<.001$ for all). Cancer survivors had much higher medical complexity, including substantially higher rates of hypertension, depression, chronic obstructive pulmonary disease, diabetes, and drug/alcohol abuse disorder diagnoses $(\mathrm{p}<.001$ for all). In covariate-adjusted analyses, cancer survivors had $18 \%$ higher odds of having any opioid prescribed and $22 \%$ higher odds of chronic opioid therapy, relative to matched cancer-free counterparts $(\mathrm{p}<.001$ for both).

CONCLUSIONS: Cancer history is an important health factor in primary care, as it impacts screening and follow-up recommendations. We found clear differences between $\mathrm{CHC}$ patients with a history of cancer and those without. Recognizing and improving the identification of cancer survivors in outpatient EHRs may improve care coordination and appropriate follow-up care.

\section{CHARACTERISTICS OF PATEINTS WITH DUAL DIAG- NOSIS OF SARCOIDOSIS AND RHEUMATOID ARTHRI- TIS IN COMPARISION TO ISOLATED SARCOIDOSIS OR RHEUMATOID ARTHRITIS.}

Lisa Zickuhr ${ }^{2}$; Ruhani Desai ${ }^{1}$; Rula Hajj-Ali ${ }^{3}$; Ryan Incledon ${ }^{3}$; Jean Schils ${ }^{3}$. ${ }^{1}$ Cleveland clinic foundation, Cleveland, $\mathrm{OH}$; ${ }^{2}$ Washington University, St. Louis, MO; ${ }^{3}$ Cleveland Clinic Foundation, Cleveland, OH. (Control ID \#3159949)

BACKGROUND: Sarcoidosis and Rheumatoid Arthritis (RA) uncommonly coexist. We propose to understand the features unique to patients with dual diagnosis in comparison to those with isolated sarcoidosis or RA.

METHODS: We retrospectively searched the electronic medical record at Cleveland Clinic from 2004-2017 for ICD9 and ICD10 codes pertaining to sarcoidosis and RA. Patients were included in our analysis if they had a biopsy-proven diagnosis of sarcoidosis and/or met the 2010 American College of Rheumatology/European League Against Rheumatism criteria for RA. Patients prescribed tumor necrosis factor inhibitors (TNFi) were excluded, as TNFi can cause a granulomatous reaction and induce sarcoidosis. Agematched controls with sarcoidosis or RA were selected randomly via retrospective chart review in a 1:1:1 ratio. Demographic, clinical, and radiographic information were collected and compared among the three groups using ANOVA, Krukal-Wallis, Pearson's chi-square, and Fisher's exact tests.

RESULTS: 15 patients met the criteria for the dual diagnosis of sarcoidosis and RA. Patients with dual diagnosis and isolated RA were predominantly female, while patients with isolated sarcoidosis were predominantly male. Those with dual diagnosis presented with lower pulmonary stages compared to isolated sarcoidosis cases, and their sharp scores were lower than isolated RA patients. Despite this, patients with dual diagnosis had more frequent anticyclic citrullinated peptide (anti-CCP) serologies than isolated RA cases.

CONCLUSIONS: We identified 15 patients carrying both diagnoses, the largest cohort described to date. Those with dual diagnosis were demographically most similar to isolated RA cases, yet they had less joint damage as demonstrated with lower Sharp scores despite more frequent anti-CCP positivity. They also presented with lower pulmonary stages based on the Siltzbach Classification System compared to patients with isolated sarcoidosis. These findings suggest that anti-CCP antibodies may play a role in the pathogenesis of overlapping sarcoidosis and RA. They also suggest that dual diagnosis may protect against severe joint and lung manifestations of the diseases. However, this retrospective study does not account for prior use of disease modifying agents, which may account for the lower disease activity in patients with both diagnoses.

CHARACTERISTICS OF PATIENTS WHO RECEIVE OPIOIDS DURING AN ACUTE MEDICAL HOSPITALIZATION Kaitlin Keenan ${ }^{2}$; Michael B. Rothberg ${ }^{1}$; Simon W. Lam ${ }^{1}$; Shoshana J. Herzig ${ }^{3}$; Vicente J. Velez ${ }^{1}$; Kathryn A. Martinez ${ }^{1}$. ${ }^{1}$ Cleveland Clinic, Cleveland, OH; ${ }^{2}$ Cleveland Clinic Lerner College of Medicine, Cleveland, OH; ${ }^{3}$ Beth Israel Deaconess Medical Center, Brookline, MA. (Control ID \#3184213)

BACKGROUND: Patients often report pain during medical hospitalizations, yet few guidelines exist regarding recommended use of opioids. Nearly half of medical inpatients receive opioids, however little is known about who receives them. The objective of this study was to identify characteristics associated with opioid receipt during an acute medical hospitalization.

METHODS: This is a retrospective observational study of patients at Cleveland Clinic Hospitals in 2016. The sample included patients with an acute medical hospitalization between 2-30 days who reported pain and excluded ICU stays and pre- or postoperative hospitalizations. For patients with multiple hospitalizations in 2016, one admission was randomly selected. All data came from the electronic health record. Prior opioid use was defined as any opioid prescription in the 30 days before hospitalization. Opioid receipt was defined as any opioid prescription during the hospitalization and analyzed dichotomously. Patient characteristics included age, gender, race, and smoking status. Clinical characteristics included maximum pain score during hospitalization, history of chronic pain or psychiatric diagnosis, length of stay and prior opioid use. Differences in the odds of opioid receipt were assessed using mixed effects logistic regression, accounting for clustering by discharging physician. The model was then stratified by prior opioid receipt. 
RESULTS: The sample included 15,201 patients; $56 \%$ female, $70 \%$ white and mean age was 70 years (Standard Deviation (SD):16); $54 \%$ had a history of chronic pain, $38 \%$ had a history of a psychiatric diagnosis, and $16 \%$ had prior opioid use. The average maximum pain score was $6.3(\mathrm{SD}=3.7)$. Overall, $66 \%$ of patients received opioids during their hospitalization. In the overall mixed effects model, opioid receipt was associated with male gender (OR: 1.16, 95\% CI: [1.08 - 1.25]), maximum pain score (OR per point: 1.31, CI: [1.29 - 1.33]), history of chronic pain (OR: 1.45, CI: [1.25 - 1.68]), and prior opioid use (OR: 1.84, CI: [1.58 - 2.14]). Black patients were less likely to receive opioids than whites (OR: 0.67, CI: [0.59 - 0.77]) and patients 65 years and over were less likely to receive opioids than those under 50 years (OR: $0.76, \mathrm{CI}$ : $[0.66-0.86])$. Patients with a history of a psychiatric diagnosis were less likely to receive opioids than without (OR: 0.78, CI: [0.72 - 0.84]). In the model stratified by prior opioid use, there was no difference by race in the odds of opioid receipt for those with prior use, but among those without prior use, black patients were less likely to receive opioids than whites (OR:0.55, CI: [0.49 -0.63]).

CONCLUSIONS: Receipt of opioids during an acute medical hospitalization varies by clinical and patient characteristics, including race and gender. Guidelines to support evidence-based opioid prescribing during medical hospitalizations are needed to promote appropriate pain management for all patients.

\section{CHARACTERISTICS OF PHYSICIAN PRACTICES AND HOSPITALS IN MEDICARE'S NEW VOLUNTARY BUN- DLED PAYMENT PROGRAM}

Joshua M. Liao ${ }^{3,}$; Joseph R. Martinez ${ }^{1}{ }^{1}$; Eric Z. Shan ${ }^{1}$; Jack

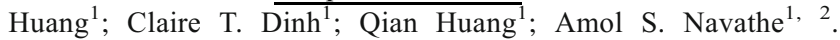
${ }^{1}$ University of Pennsylvania, Philadelphia, PA; ${ }^{2} \mathrm{CMC}$ VA Medical Center, Philadelphia, PA; ${ }^{3}$ University of Washington, Seattle, WA. (Control ID \#3179531)

BACKGROUND: Medicare reinforced its commitment to voluntary bundled payments via the new Bundled Payments for Care Improvement (BPCI)-Advanced program. Since launching in October 2018, BPCI-Advanced has enrolled 1,547 hospital and physician group practice (PGP) participants, making it the largest voluntary bundled payment program to date. Therefore, it is important for policymakers and health care organizations to understand characteristics of BPCI-Advanced participants, particularly those "newly" attracted to bundled payments (i.e. those that did not previously participate in BPCI). Given little existing information about physician practices in bundled payments, such insight is particularly important for the PGPs that comprise nearly half of all BPCI-Advanced participants.

METHODS: We used Medicare data to identify PGPs and hospitals that participated in BPCI Model 2 or BPCI-Advanced as of October 1, 2018. We categorized participants as those continuing participation from BPCI into BPCI-Advanced ("continuing") and those newly participating in bundled payments via BPCIAdvanced ("new"). We evaluated the distribution of 557 (408 new, 149 continuing) BPCI-Advanced PGPs and 832 (662 new, 170 continuing) BPCI-Advanced Hospitals across markets nationwide, defining "new markets" as those with new, but no continuing or BPCI only, participants. Data from Physician Compare, Hospital Compare, and the American Hospital Association were used to compare characteristics of PGPs and hospitals, respectively, by continuing versus new participation status. Categorical variables were compared using Chi squared tests and continuous variables were compared using $t$ tests, Kruskal-Wallis, and Wilcoxon rank sum tests. Statistical tests were 2tailed and significant at an $=0.05$.
RESULTS: There was geographic variation in participation, with BPCIAdvanced engaging 66 new markets. Most were located in central rather than coastal US regions. New and continuing PGP participants differed with respect to several characteristics. New groups were less likely to be multispecialty (96.9\% versus $99.9 \%)$, hospital-affiliated $(91.2 \%$ versus $97.3 \%$ ), and urban (96.4\% versus $98.1 \%$ ) ( $\mathrm{p}<0.001$ for all). New PGPs also employed different and more diversely represented specialty types than continuing groups. Among BPCI-Advanced hospitals, new participants were smaller (mean hospital beds 276 versus $341, \mathrm{p}=0.001$ ), lower volume (mean annual Medicare admissions 4,740 versus 6,861, $\mathrm{p}<0.001$ ), and less likely to be not-for-profit $(62.1 \%$ versus $71.6 \%, \mathrm{p}=0.04)$ and teaching $(58.7 \%$ versus $76.9 \%, \mathrm{p}<0.001)$ (Table). New and continuing hospitals did not vary with respect to safety-net status, financial measures, or readmission rates.

CONCLUSIONS: Differences between new and continuing PGP and hospital participants suggests that BPCI-Advanced at least in part prompted some new organization types to engage in Medicare bundled payments rather than only attracting "more of the same".

\section{CHARACTERISTICS OF USE OF SELF-MONITORING OF BLOOD GLUCOSE IN PATIENTS WITH NON-INSULIN DEPEN- DENT TYPE 2 DIABETES}

Kaitlin Keenan; Elizabeth Pfoh; Anita D. Misra-Hebert; Michael B. Rothberg. Cleveland Clinic, Cleveland, OH. (Control ID \#3185182)

BACKGROUND: For patients with Type 2 Diabetes (T2D) not on insulin, self-monitoring of blood glucose (SMBG) does not reduce $\mathrm{HbA1}$, and has been identified by SGIM as low value care. Knowing which patients are using SMBG and whether rate of use differs across physicians can inform interventions to reduce its use.

METHODS: This retrospective study included adults with T2D who had a primary care visit at an integrated health center between 2013 and 2015. Patients were excluded in years they received an insulin prescription. Each year, we defined SMBG use based on receiving 1 prescription for blood glucose strips. We calculated the number of strips prescribed per day by multiplying the quantity of strips by the number of refills and dividing by 365 . A hierarchal multivariate logistic regression clustered within patient and physician tested the relationship age, sex, race, smoking status, insurance, duration of diabetes, HbA1c value, Charlson comorbidity index and use of SMBG. To assess provider variation, we calculated the mean and $95 \%$ confidence interval (CI) for the rate of SMBG prescriptions for each provider with 30 patients. Physicians were classified as low prescribers if their 95\% CI was below the mean prescribing rate and high prescribers if their $95 \% \mathrm{CI}$ was above.

RESULTS: Our sample included 41,257 people who had a mean age of 63 , were $51 \%$ female and $75 \%$ white. Use of SMBG increased slightly from $26 \%$ in 2013 to $29 \%$ in 2015 ( $<<0.01)$. In $2015,48 \%$ of SMBG users were prescribed less than one strip per day. SMBG users had a higher HbA1c than non-users (7.2 vs $6.7, \mathrm{p}<0.01)$ but had been diagnosed with diabetes for a similar number of years (3.6 vs 3.5). In the regression model government insurance (vs private adjusted odds ratio (AOR) 1.52, 95\% CI:1.39-1.65), years with diabetes (AOR: 1.03, 95\%CI: 1.02-1.04 per 1 year), increased HbAlc (AOR: 1.50 , 95\%CI: 1.46-1.52 per 1 point) and higher Charlson comorbidity index (AOR: $1.08,95 \% \mathrm{CI}$ : $1.05-1.11$ per 1 point) were significantly associated with SMBG. Among patients with newly diagnosed with T2D in 2013, SMBG use was similar in 2013 and 2015 ( $26 \%$ vs $28 \%, p=0.12$ ). SMBG users had higher HbAlc values than nonusers (difference $=0.42$ in $2015, \mathrm{p}<0.01$ ). On average, physicians prescribed SMBG to $29 \%$ of their T2D patients not using insulin (range: $0.03 \%$ to $73 \%$ ); $30 \%$ of physicians were high 
prescribers and 25\% were low prescribers. Fifty-three percent of patients of high prescribers had T2D more than five years compared to $38 \%$ of patients of low prescribers $(\mathrm{p}<0.01)$.

CONCLUSIONS: There is substantial variation in rates of SMBG by physicians. The association of $\mathrm{HgA1C}$ with SMBG suggests physicians may believe that SMBG will lower $\mathrm{HgA1C}$. Private insurance co-pays may discourage SMBG.

\section{CHARACTERIZING FATIGABILITY IN HOSPITALIZED PATIENTS WITH ANEMIA}

Micah T. Prochaska; Cyrus Alavi; Sarah Bradbury; David Meltzer. University of Chicago, Chicago, IL. (Control ID \#3161008)

BACKGROUND: Fatigability describes how fatigued a patient is at any given level of activity, and is measured as the degree of fatigue a patient experiences after performing a specific amount of activity. The National Institute of Aging has suggested that fatigability is an important measure in patients with conditions where fatigue is a prominent symptom, such as hospitalized patients with anemia. This is because fatigue is the primary symptom of anemia, and both hospitalization and anemia-related fatigue are associated with declines in activity and functional capacity. As such, measuring fatigability in hospitalized patients with anemia could provide hospitalists information about the severity of patients' fatigue, and how fatigue may be interfering with their functional capacity. Moreover, fatigability could be used to predict future functional decline or disability post hospitalization. However, fatigability has not been previously described in hospitalized patients with anemia. The purpose of our study was to establish the clinical reliability of fatigability, by characterizing the association between fatigability and patient's clinical (i.e. $\mathrm{Hb}$, comorbidities) and demographic characteristics.

METHODS: From 6/2017-1/2018, hospitalized general medicine patients with a $\mathrm{Hb}<10 \mathrm{~g} / \mathrm{dL}$ were approached for an inpatient interview at hospital admission. Fatigability was measured using the Pittsburgh Fatigability Scale (PFS). The PFS contains 10 questions that measure fatigue in the context of specific activities. PFS scores range from 0-50, with higher scores indicating greater fatigability (higher fatigue at lower activity). Patients' hemoglobin $(\mathrm{Hb})$ values and clinical data were abstracted from hospital administrative data. Patients who received a transfusion prior to the PFS measurement were analyzed separately since transfusion affects $\mathrm{Hb}$ and could confound the association with fatigability. Linear regression was used to test the association between fatigability (PFS) as the dependent variable, and patient $\mathrm{Hb}$ and Charlson Comorbidity score (CCS) as predictor (independent) variables, controlling for age, race, and gender.

RESULTS: 467 patients completed the inpatient PFS. The median age of the sample was 55 (IQR 39-68) years old, 61\% $(n=293)$ were female, $77 \%(n=360)$ were African American, with a median CCS of 2 (IQR 1-5). The median PFS score of the sample was 33 (IQR 25-38), and the median $\mathrm{Hb}$ was $8.9 \mathrm{~g} / \mathrm{dL}$. In regression models, a $\mathrm{Hb} 7-8 \mathrm{~g} / \mathrm{dL}(\mathrm{b}=2.7, \mathrm{p}=0.04)$ compared to $\mathrm{Hb} 9-10$, and higher $\mathrm{CCS}(\mathrm{b}=0.8, \mathrm{P}<0.001)$ were associated with increased fatigability.

CONCLUSIONS: Severity of anemia and number of comorbidities are predictive of higher fatigability (higher fatigue at lower activity) during hospitalization. This suggests that fatigability is reliably associated with expected clinical characteristics during hospitalization, and could be measured and used by hospitalists to clinically evaluate the severity of a patient's fatigue, and how that fatigue may be affecting their functional capacity.
CHARACTERIZING GENDER-BASED DIFFERENCES IN BURNOUT AMONG PHYSICIANS IN AN ACADEMIC MEDICAL CENTER

Lisa Rotenstein ${ }^{1}$; Elizabeth Harry ${ }^{1}$; Paige Wickner ${ }^{1}$; Anu Gupte ${ }^{1}$; Bridget Neville $^{1}$; Stuart R. Lipsitz ${ }^{1}$; Elizabeth Cullen ${ }^{1}$; Ronen Rozenblum $^{1}$; Thomas D. Sequist ${ }^{2}$; Jessica Dudley ${ }^{1,}{ }^{1}$. ${ }^{1}$ Brigham and Women's Hospital, Boston, MA; ${ }^{2}$ Partners Healthcare System, Boston, MA. (Control ID \#3185855)

BACKGROUND: Burnout, a syndrome that develops in response to chronic job-related stressors, is a critical issue affecting modern physicians. Burnout has negative effects on healthcare practice and workforce availability. We sought to characterize gender-based differences in burnout among physicians at an academic medical center and to understand contributors to burnout in this population.

METHODS: In Spring 2017, an adaptation of the Stanford Physician Wellness Survey was administered to all clinical faculty (defined as actively practicing medicine) at Brigham and Women's Hospital, an academic medical center affiliated with Harvard Medical School. This survey was designed by the Physician Wellness Academic Consortium. It included validated measures of aggregate burnout (defined as a combination of emotional exhaustion and interpersonal disengagement) and professional fulfillment, as well as a single question on self-defined burnout. Demographics and data regarding previously characterized burnout drivers were also collected. Generalized estimating equations clustered by department (chi-squared or t-tests) were used to compare demographics, drivers, and outcomes between gender, while content analysis was used to analyze free-text comments.

RESULTS: Our population included 1,145 clinical faculty (610 $(53.2 \%)$ men and $535(46.8 \%)$ women; $65 \%$ response rate). A majority were under age 50 . The largest departments represented were medicine $(26.9 \%)$ and general surgery $(10.6 \%)$. Female physicians were younger than male counterparts $(\mathrm{p}=0.02)$ and trended towards lower academic ranks $(\mathrm{p}=0.06)$. They were significantly more likely to report burnout than male physicians (227 $(43.3 \%)$ vs. $199(33.5 \%) ; \mathrm{p}=0.02)$. This finding held for self-defined burnout $(45.8 \%$ of females vs. $32.3 \%$ of males; $p<0.01)$. Female physicians were less likely to report professional fulfillment (183 $(34.5 \%)$ vs. $298(49.7 \%) ; \mathrm{p}<0.01)$. They had lower ratings of perceived appreciation $(\mathrm{p}<0.01)$, schedule control $(\mathrm{p}<0.01)$, work environment diversity $(\mathrm{p}=0.01)$, diversity and quality of departmental recruiting $(\mathrm{p}=0.02)$, and salary/benefits $(\mathrm{p}=0.02)$. Although there was not a difference in female vs. male physicians' intention to leave the institution within two years, females were more likely to report a departure would be driven by considerations around mentoring/leadership, schedule flexibility, workload expectations, and dual careers (all $\mathrm{p}<0.05)$; qualitative analysis of free-text comments furthered these conclusions.

CONCLUSIONS: Female physicians practicing in an academic medical center experience higher levels of burnout and lower professional fulfillment than their male counterparts. They perceive lower levels of appreciation, schedule control, and diversity, and have decreased satisfaction with compensation practices. Further research is needed to understand which factors most robustly predict burnout, and which interventions can mitigate these phenomena in an academic medical population.

CHARACTERIZING HIGH RISK ATRIAL FIBRILLATION PATIENTS IN A SAFETY NET INSTITUTION TO IDENTIFY TRENDS THAT MAY HELP IMPROVE ANTICOAGULATION GUIDELINE ADHERENCE AND STROKE PREVENTION

Benjamin Leon ${ }^{5}$; Carlos Irwin Oronce ${ }^{2}$; Joel C. Marrs ${ }^{3}$; Sarah L. Anderson $^{4}$; Tara B. Vlasimsky ${ }^{1}$; Samuel D. Richesin ${ }^{1}$; Carolyn A. Valdez ${ }^{1}$; Rebecca Hanratty ${ }^{1} .{ }^{1}$ Denver Health, Denver, CO; 
${ }^{2}$ University of Rochester Medical Center, Rochester, NY; ${ }^{3}$ University of Colorado Skaggs School of Pharmacy and Pharmaceutical Sciences, Aurora, CO; ${ }^{4}$ University of Colorado Skaggs School of Pharmacy \& Pharmaceutical Sciences, Aurora, CO; ${ }^{5}$ University of Colorado, Denver, CO. (Control ID \#3179221)

BACKGROUND: Atrial fibrillation (a-fib) is associated with significant morbidity and mortality, particularly due to stroke risk. Oral anticoagulation $(\mathrm{OAC})$ has been shown to reduce the risk of stroke and mortality in patients with a-fib. However, the use of OAC remains underutilized, as nearly $40 \%$ of eligible patients are not on OAC. Little has been published characterizing OAC utilization in the highest risk groups, particularly in underserved populations. The objective of this evaluation is to assess utilization of OAC among patients with a-fib in a safety net health system and characterize the highest risk groups to identify trends that may help improve anticoagulation guideline adherence.

METHODS: ICD-9 codes were used to identify patients with a-fib and at least one visit with a primary care provider from July 2012 through June 2014. CHA2DS2VASc and HAS-BLED scores were calculated using ICD-9 codes, medication lists, recent vital signs and demographics. High risk patients were defined as CHA2DS2-VASc score $>2$. Among patients at the highest risk of stroke, clinical characteristics of those not on $\mathrm{OAC}$ were compared to those who were on OAC in a cross sectional design.

RESULTS: A total of 867 patients with a-fib were identified, a large proportion of whom were at high risk for stroke with a CHA2DS2VASc score $>2$ ( $85 \%$ of total a-fib population). Of this high-risk group, $35 \%$ of patients were not on OAC. When comparing the high-risk groups based on OAC status, there was no significant difference between the two groups based on many baseline characteristics including age, race, gender, renal function or liver function. In addition, average HAS-BLED scores were similar between the two groups. High-risk patients who were not on OAC were less likely to have heart failure and more likely to be prescribed an anti-platelet agent (both $\mathrm{p}<0.05$ ). There were a significantly greater proportion of patients with a CHA2DS2VASc score of 2 not on OAC. However, no significant difference was seen in OAC status between CHA2DS2VASc scores for those with higher scores (CHA2DS2VASc $>3$ ).

CONCLUSIONS: Despite high-risk status, over one-third of these patients were not on OAC. Patients not receiving OAC were more likely to be prescribed an anti-platelet agent and less likely to have heart failure, but bleeding risk as assessed by the HAS-BLED score was not associated with OAC status. More patients with CHA2DS2VASc scores of 2 were not anticoagulated, which may be due to patients recently entering that risk category, either by age or other comorbidities, and not having their risk reevaluated. There may be unmeasured factors that explain why these patients were not on $\mathrm{OAC}$, which could guide future interventions to help improve guideline adherence.

\section{CHARACTERIZING INPATIENT ADMISSIONS FOR NATIVE AMERICANS CARED FOR OUTSIDE THE INDIAN HEALTH SERVICE}

Joshua N. Wadlin ${ }^{1}$; Matthew Tobey ${ }^{2}$; Scott Wright ${ }^{3} .{ }^{1}$ Johns Hopkins School of Medicine, Baltimore, MD; ${ }^{2}$ Massachusetts General Hospital, Boston, MA; ${ }^{3}$ jhusom, Baltimore, MD. (Control ID \#3179541)

BACKGROUND: Native Americans (NAs) in the US experience substantial health disparities such as the highest prevalence of type 2 diabetes of any racial or ethnic group and a lower life expectancy than the general population. Out of estimated 5.2 million NAs in the US, 3.7 million qualify for Indian Health Service (IHS) benefits, but only 2.2 million receive care from IHS. The IHS periodically publishes data on health trends which helps to identify areas of need and targets for future interventions. These data do not include NAs who do not receive care at the IHS. The remaining millions of NAs represent a cohort whose healthcare data are poorly understood. We sought to describe reasons for admissions and related outcomes for NAs compared to whites in the US.

METHODS: We used data from the 2014 National Inpatient Sample (NIS), a representative sample of hospital discharges across the US which excludes episodes of care at federal hospitals including IHS. We conducted a descriptive analysis, exploring the most common diagnoses for inpatient admission for NAs and compared differences in outcomes for NAs with those of whites. We identified the 10 most common reasons for adult, non-obstetric admissions for NAs, stratified by age (adults 18 to 64 and 65 or older), and compared these to results for whites, exploring differences in reasons for admission. We then evaluated the outcomes of in-hospital mortality, length of stay (LOS), and hospital charges for admissions with one of the 10 most common diagnoses for NA. We compared outcomes between NAs and whites using univariate regression for LOS and charges and chi-squared tests for mortality, each stratified by age group.

RESULTS: The most common diagnoses for NAs $<65$ were septicemia, alcohol-related, complication of diabetes, mood disorder, and skin/soft tissue infection. The most common diagnoses for 65 and older were septicemia, pneumonia, CHF, osteoarthritis, and COPD. In an unadjusted model for those $<65$ with one of the top 10 diagnoses, compared to whites $(n=1,704,805)$, NAs $(n=20,124)$ had a higher mortality $(2.33 \%$ v $1.59 \%$, $\mathrm{p}<0.001)$, a longer mean LOS ( $5.25 \mathrm{v} 5.03$ days, $\mathrm{p}=0.01)$, and lower mean total charges $\$ 40,416 \mathrm{v} \$ 43,032, \mathrm{p}=0.007)$. For those 65 or older, mortality was not significantly different between NAs $(n=9,768)$ and whites $(\mathrm{n}=1,836,672)$, but NAs had a longer mean LOS (5.21 v 4.89 days, $\mathrm{p}$ $<0.001)$ and lower mean total charges $(\$ 45,357 \mathrm{v} \$ 48,792, \mathrm{p}=0.002)$.

CONCLUSIONS: This analysis identified the top reasons for adult, nonOB admission among NAs receiving care outside the IHS. It also identified differences in outcomes for NAs compared to whites with the same primary diagnosis. Because length of stay is a critical driver of hospital charges, it is noteworthy that NAs had longer LOS yet lower charges. The higher mortality for NA adults $<65$, suggests a particularly vulnerable population. Poor access to care and other social determinants of health may explain some of these variations, but more research is needed to understand these differences.

\section{CHARACTERIZING TRENDS IN PRIMARY CARE VISITS USING NAMCS 2007-2015}

Aarti Rao ${ }^{1}$; Zhuo Shi ${ }^{2}$; Kristin N. Ray ${ }^{3}$; Ateev Mehrotra ${ }^{2,}$; ; Ishani Ganguli $^{4}{ }^{1}$ Icahn School of Medicine, New York, NY; ${ }^{2}$ Harvard Medical School, Boston, MA; ${ }^{3}$ University of Pittsburgh, Pittsburgh, PA; ${ }^{4}$ Brigham and Women's Hospital, Chestnut Hill, MA; ${ }^{5}$ Beth Israel Deaconess Medical Center, Boston, MA. (Control ID \#3162916)

BACKGROUND: Supporting primary care has been a focus of policy reforms in the United States. However, recent evidence suggests a decline in primary care visits per capita. We examined factors underlying the decline and in particular, the role of changing physician practice.

METHODS: Using 2007-2015 National Ambulatory Medical Survey (NAMCS) data, we examined visits to a primary care physician (PCP) (i.e. internal medicine, family practice, or pediatrics) by patients 18 years old. We calculated visits per capita by dividing number of visits by annual US Census estimates for each population subgroup and used rolling averages to minimize yearly fluctuations (e.g. 2008 estimate included 2007-2009 data). We estimated visit rates stratified by patient demographic groups. We examined visit duration and the number of diagnoses and medications addressed per visit. We also examined visit disposition and the percentage of PCPs using email consults. For each outcome, we used ordinary leastsquares regression to estimate linear time trends and assessed statistical significance at $\mathrm{p}<0.05$. Analyses were performed using Stata version 14.1. RESULTS: Our weighted sample represented 2.2 billion PCP visits. Over 9 years, visit rates declined by 18.9 visits per 100 patient years $(-0.04$ per year; $95 \%$ CI $-0.05,-0.02)$ with similar declines across sex and age groups. We 
found increases in visit duration ( 0.41 min per year; $95 \% \mathrm{CI} 0.20,0.64)$ and in number of diagnoses $(0.04$ per year; $95 \%$ CI $0.01,0.06)$ and medications $(0.12$ per year; $95 \%$ CI $0.06,0.16)$ addressed. We found a decline in visits per capita in which PCPs recommended follow-up $(-0.03$ per year; 95\% CI $-0.04,-0.02)$. A larger proportion of PCPs reported using email consultations over time (1.7\% per year; $95 \%$ CI 1.2,2.2).

CONCLUSIONS: Over time PCP visits were longer, more issues were addressed per visit, and physicians were less likely to suggest follow-up. These findings suggest that the declining PCP visit rate may be partially explained by PCPs providing more comprehensive in-person care and using more non-face-to-face care such as email. Our findings are limited by physician self-report and potential changes in billing practice that may contribute to a rise in self-reported diagnoses.

CHRONIC CONDITIONS AND PHYSICAL FUNCTIONING: DEVELOPMENT OF A NEW ICD-CODED MULTIMORBIDITY INDEX FOR USE IN ADMINISTRATIVE CLAIMS DATA

Melissa Y. Wei ${ }^{1,2}$; Jamie Luster ${ }^{1}$; David Ratz ${ }^{3}$; Kenneth Langa ${ }^{1,2}$. ${ }^{1}$ University of Michigan, Ann Arbor, MI; ${ }^{2}$ Institute for Healthcare Policy and Innovation, University of Michigan, Ann Arbor, MI; ${ }^{3}$ Veterans Affairs Ann Arbor Healthcare System, Ann Arbor, MI. (Control ID \#3179958)

BACKGROUND: Most older adults have multimorbidity, the coexistence of multiple chronic conditions, that profoundly affect physical functioning. Physical functioning is a universal health value that strongly predicts poor health outcomes and is linked to multimorbidity in aging adults. However, it is not consistently available in administrative data. We previously developed and validated a multimorbidity index that weights self-reported physician-diagnosed diseases by physical functioning. Although the multimorbidity-weighted index (MWI) has been extensively validated, its use is currently limited to self-reported conditions. Herein, we aimed to develop an ICD-coded MWI (MWI-ICD) for use in administrative claims data and compare its performance with traditional multimorbidity metrics.

METHODS: We linked Health and Retirement Study (HRS) and Medicare claims to develop a multimorbidity index of ICD-coded conditions (MWI-ICD) for administrative data. Medicare-eligible HRS participants with at least two outpatient claims or one inpatient claim within two years contributed repeated measures of ICD-9-Clinical Modification chronic conditions and a modified Short Form-36 10-item physical functioning scale between 20002012. Mixed effects models adjusted for age and all other ICDcoded chronic conditions were used to obtain regression coefficients for conditions on physical functioning. Conditions that were inversely associated with physical functioning and stably estimated were retained in the final model. MWI-ICD was calculated by summing the physical functioning-weighted conditions for each participant. We compared the distribution and performance of MWI-ICD with a simple (i.e., unweighted) disease count.

RESULTS: We included 71,756 observations from 17,478 participants with mean age 70.3 (SD 7.2) years and 56.0 (SD 30.3) physical functioning units. We considered 409 ICD-coded chronic diseases and conditions in the initial analysis and narrowed this to 143 in the final model. Diseases varied several-fold in their impact on physical functioning (median 1.3, range 0.03-17.3). The top prevalent conditions were hypertensive disease $(73 \%)$, osteoarthosis $(43 \%)$, other disorders of kidney and ureter, urethra and urinary tract, and bladder $(41 \%)$, and coronary atherosclerosis $(36 \%)$. ALS, allergic arthritis, female genitourinary cancers, hemiplegia/ paraplegia, and Parkinson disease had the greatest impact on physical functioning. The mean MWI was 14.0 (median 10.3, range 0-100.1) and mean simple disease count was 8.3 (median 6 , range $0-40$ ).

CONCLUSIONS: ICD-coded chronic conditions from Medicare claims had wide-ranging associations with physical functioning. MWI had a wider distribution than simple disease count, suggesting that a simple disease count is unlikely to capture the full impact of multimorbidity on physical functioning. A newly-developed multimorbidity index based on ICD-coded chronic conditions is feasible and readily implemented in administrative claims data.

\section{CLINICAL ARCHITECTURE INTERVENTION INCREASES TOBACCO CESSATION IN PRIMARY CARE}

Richard Altman ${ }^{2}$; Melanie D. Whittington ${ }^{3}$; Lauren Drake ${ }^{2}$; Hillary Chrastil ${ }^{3}$; Carmen L. Lewis ${ }^{1}$. ${ }^{1}$ Univesrity of Colorado, Denver, CO; ${ }^{2}$ University of Colorado, Lone Tree, CO; ${ }^{3}$ University of Colorado, Aurora, CO. (Control ID \#3180280)

BACKGROUND: Tobacco cessation is a high value intervention but clinician counseling in primary care practices is suboptimal. Our goal was to determine whether a clinical architecture intervention that includes reliable medical assistant (MA) tobacco data sent to clinicians' notes at the point of care is associated with an increase in tobacco cessation in primary care.

METHODS: We implemented the clinical architecture intervention in one internal medicine clinic and compared outcomes to two other primary care practices in the same health care system. The clinical architecture intervention paired MA data collection on current tobacco status with a clinician "action" tool. The clinician action tool pushed MA information to the assessment and plan section of the clinician's note, acting as a prompt for the clinician during the visit. The action tool had a drop-down menu with documentation for 1) tobacco cessation addressed and plan description 2) tobacco use not addressed, and 3) "wildcard" so that clinicians could provide their own documentation. Using electronic health record data, we identified patients who had an index visit within the practices from $4 / 2017$ to $4 / 2018$ and who reported tobacco use. We compared self-reported tobacco use for these patients at follow up visits between $90-179$ days and 180 to 359 days from the index visit in intervention and control practices. Propensity score matching (on age, gender, zip code, and duration of follow-up) was used to match patients at the intervention practice to comparable patients in the two control practices. Logistic regression was used to estimate the association between the intervention and tobacco use at the first follow-up visit (90-179 days) and the second follow-up visit (180-360 days). A difference in differences specification was used to control for temporal trends. Standard errors were clustered at the provider level.

RESULTS: We identified 538 patients that used tobacco at the index visit in the intervention practice and 1,647 tobacco users in the control practices. One hundred sixty-six patients $(31 \%)$ in the intervention and 500 patients $(31 \%)$ in the control practices had a first follow up visit. For the second follow up visit, $142(26 \%)$ and $388(24 \%)$ patients had a follow up visit in the intervention and control practices, respectively. The intervention was associated with a $10 \%(\mathrm{p}=0.006)$ reduction in tobacco use at the first follow-up visit and a $6 \%$ reduction at the second follow-up visit $(\mathrm{p}=0.121)$.

CONCLUSIONS: Our clinical architecture intervention was associated with a statistically significant reduction in reported tobacco use at the first follow-up visit. A reduction, although not quite significant, was also observed at the second follow-up visit. Having actionable tobacco data for clinicians within the assessment and plan at the point of care may help to facilitate tobacco cessation for primary care patients.

CLINICAL IMPACT OF LAUNCHING A NEW PALLIATIVE CARE SERVICE AT A REGIONAL HOSPITAL: THE GRAND STRAND MEDICAL CENTER EXPERIENCE DR. TYLER KILGORE; DR. ELIJAH ENGWALL; DR. VINOD NAMBUDIRI 
Tyler Kilgore; Elijah Engwall; Vinod E. Nambudiri. Grand Strand Medical Center, Myrtle Beach, SC. (Control ID \#3182877)

BACKGROUND: Inpatient Palliative Care (PC) services have been shown to reduce cost and increase patient satisfaction in cases that vary in clinical nature and course. However, data suggests that PC teams are underutilized, whether by delays in appropriate consultation or lack of involvement entirely. We sought to characterize the clinical impact of a PC service team in the first months of introduction at a regional medical center by examining timing of consultations, changes in patient code status, and patient destination at discharge.

METHODS: We conducted a retrospective, descriptive chart review analysis of all cases on which PC was consulted from its introduction in 3/2016 through 12/2016. 213 cases were reviewed. Patient demographics, diagnoses, length of stay (LOS), LOS prior to PC consultation, code status and destination at discharge were reviewed. Information was compared to national data available from the National Palliative Care Registry.

RESULTS: $82 \%$ of patients consulted were Caucasian and $13 \%$ African American, similar to the overall demographics at our institution. $40 \%$ of all patients who received an inpatient PC consult expired during hospitalization; $37 \%$ were discharged to home or hospice, and $22 \%$ were discharged to another medical facility. The most common primary diagnoses for individuals who received PC consults were Hematologic/Oncologic disease (25\%), Neurologic disease (23\%), Cardiopulmonary disease (17\%), Infectious disease $(11 \%)$, and Trauma/Overdoses $(9 \%)$. Change in code status was observed frequently; nearly $25 \%$ of patients who received a PC consultation had their code status changed to DNR and over $50 \%$ of individuals had DNR statuses prior to consultation. In no case was a DNR patient changed to Full Code. Following PC consultation, the vast majority of patients (86\%) did not undergo any further life prolonging procedures such as intubation, blood transfusion, paracentesis, PEG tube, or cardiac catheterization.

CONCLUSIONS: Our data demonstrates the multifaceted impact of a new PC consult team in a regional hospital. Palliative care consultation was associated with marked reductions in life prolonging procedures and frequent change of code status to DNR. During the study period, the inpatient mortality rate of the $\mathrm{PC}$ service was twice that reported in national registries. This likely indicates a tendency for a new PC service to be consulted later in the course of care than the national average, and speaks to ongoing needs to address underutilization of PC services for end of life care. Finally, nearly $10 \%$ of consults at our center were for trauma/overdose, a category not featured in the national palliative care registry, and may speak to local demographics and the profound impact of the opioid crisis on shaping care delivery locally.

CLINICAL TRIAL EVIDENCE SUPPORTING FDA APPROVAL OF NEW DRUGS OVER THREE DECADES, 1995-2017

Audrey Zhang ${ }^{3}$; Jeremy Puthumana ${ }^{1}$; Nilay Shah ${ }^{2}$; Joseph S. Ross ${ }^{1}{ }^{1}$ Yale University School of Medicine, New Haven, CT; ${ }^{2}$ Mayo Clinic, Rochester, $\mathrm{MN} ;{ }^{3}$ New York University School of Medicine, New York, NY. (Control ID \#3183972)

BACKGROUND: The US Food and Drug Administration (FDA) possesses regulatory flexibility in determining the evidence necessary to support new drug approval. This has increasingly been exercised through special regulatory programs, such as Accelerated Approval and Breakthrough Designation. These permit the use of different clinical evidentiary standards to support approval, such as surrogate markers of disease (e.g. biomarkers) rather than clinical (e.g. overall survival) endpoints. This study examined whether clinical trial evidence supporting FDA approval of new drugs has changed over three decades.

METHODS: We performed a cross-sectional analysis of all new drugs (small molecule and biologic) approved by the FDA between 1995-1997, 2005-2007, and 2015-2017. Using the publicly-available Drugs@FDA database, we identified drug approvals and classified them by therapeutic area and use of special regulatory programs. Next, we identified the pivotal efficacy trials supporting each drug's approval, and characterized use of randomization, double-blinding, choice of comparators and endpoints, number of participants, and trial duration. Comparisons between time periods were performed using chi-square and Kruskal-Wallis tests. Analyses were repeated aggregated by indication and stratified by product therapeutic area and use of special regulatory programs.

RESULTS: We identified 273 new drugs approved by the FDA in these 3 periods, representing 339 indications supported by 802 pivotal efficacy trials. The proportion of approvals receiving priority review increased from 19951997 to $2015-2017$ (34\% vs. $61 \%$; $\mathrm{p}<0.001$ ), as did those receiving an orphan designation ( $13 \%$ vs. $38 \%$; $<<0.001)$. Rates of accelerated approval did not differ $(11 \%$ vs. $17 \% ; \mathrm{p}=0.23)$. The most common therapeutic area for approvals differed over time (infectious disease [34\%] in 1995-1997 vs. oncology [27\%] in 2015-2017; $\mathrm{p}=0.002$ ). The median number of pivotal trials supporting an indication decreased from 2 (IQR 2-3) to 1 (IQR 1-3; $\mathrm{p}<0.001)$. The proportion of indications based on at least one pivotal trial using an active comparator decreased ( $57.3 \%$ vs. $30.2 \%$; $<<0.001)$, whereas use of only single-arm trials increased $(4.0 \%$ vs. $17.0 \%$; $=0.005)$. The median number of enrolled patients per indication remained steady (774 [IQR, 464-1314] vs. 816 [IQR, 199-2112]; $\mathrm{p}=0.47$ ). The proportion with at least one pivotal trial of at least 6 months' duration increased $(25.8 \%$ vs. $46.2 \%$; $\mathrm{p}=0.006$ ). Differences in pivotal trial characteristics over time were consistent in analyses stratified by therapeutic area and use of special regulatory programs.

CONCLUSIONS: Clinical trial evidence supporting new drugs at the time of approval has changed over time, in part reflecting increasingly flexible evidentiary standards. This was consistent regardless of use of special regulatory programs. Diminished quantity and quality of premarket evidence suggests an increasing need for continued evaluation after market approval.

CLINICIAN-EDUCATOR CAREER PATHS: THE IMPACT OF ADVANCED EDUCATION TRAINING ON ACADEMIC SUCCESS AND CAREER SATISFACTION

Daniella A. Zipkin ${ }^{1}$; Subha Ramani²; Corrie A. Stankiewicz ${ }^{3}$; Margaret C. $\mathrm{Lo}^{4}$; Alia Chisty ${ }^{5}$; Irene Alexandraki ${ }^{6}$; Maria A. Wamsley ${ }^{7}$; Scott D. Rothenberger ${ }^{8}$; Kwonho Jeong ${ }^{8}$; Carla Spagnoletti ${ }^{8}{ }^{1}$ Duke University, Durham, NC; ${ }^{2}$ Brigham and Women's Hospital, Harvard Medical School, Boston, MA; ${ }^{3}$ University of Pennsylvania SOM, Narberth, PA; ${ }^{4}$ University of Florida College of Medicine, Gainesville, FL; ${ }^{5}$ Temple, Philadelphia, PA; ${ }^{6}$ Florida State University College of Medicine, Tallahassee, FL; ${ }^{7}$ University of California, San Francisco, San Francisco, CA; ${ }^{8}$ University of Pittsburgh, Pittsburgh, PA. (Control ID \#3184925)

BACKGROUND: Clinician-educator career paths are varied. Some faculty pursue additional training in medical education through programs offered by professional medical organizations, certificate programs, clinician-educator fellowships, or advanced degrees in education. It is unclear whether such preparation impacts academic advancement, job satisfaction, or leadership opportunities.

METHODS: Using a mixed-methods approach we explored the impact of medical education training on subsequent academic achievements, leadership, and job satisfaction among clinician-educators in internal medicine. We distributed an online survey to the Society of General Internal Medicine (SGIM) membership in the fall of 2017, and conducted two in-person focus groups of a subset of survey respondents at the 2018 SGIM Annual Meeting. We used multivariate analysis for quantitative data and thematic analysis of qualitative data. We created an "intensity of training" metric to categorize the type of additional training, with clinician-educator fellowships and Master's in medical education considered to be highest intensity, longitudinal training courses as intermediate intensity, and local faculty development as low intensity.

RESULTS: 198 clinician-educators completed the survey. 64\% were female with $59 \%$ in the $36-55$ year age range. $16 \%$ had a Masters degree in medical education. $42 \%$ had completed fellowship with $67 \%$ of those being in 
education, and $87 \%$ percent had participated in some faculty development in education, with the majority reporting local training opportunities. Women were more likely than men to have completed a fellowship and/or Master's degree in medical education. $(\mathrm{p}=0.029)$. Advanced training was associated with more publications ( $\mathrm{p}=0.002$ ), lectures or workshops at the regional, national or international level $(\mathrm{p}=0.001)$, and regional or national committee memberships $(\mathrm{p}=0.04)$. Having an education leadership role was not associated with additional training, with the exception of a Dean's office role $(\mathrm{p}=0.029)$. Over $70 \%$ of respondents indicated a positive impact of additional training on career achievement and job satisfaction, with greater positive perception in those with higher levels of additional training $(p<0.001)$. Qualitative data exploring factors viewed as influencing clinician-educators' career advancement were grouped under the major thematic categories of: (1) passion for teaching, (2) institutional culture and (3) advanced training and its impact. Themes within these categories suggest variation in institutional and generational expectations regarding carving out time for education, promotion, and obtaining leadership roles. Detailed description of themes will be provided in the full abstract.

CONCLUSIONS: Clinician-educators participate in a wide array of additional training opportunities. Advanced training is associated with scholarly productivity and perceived job satisfaction, but not acquisition of most leadership roles. Variations in institutional culture may explain this discrepancy.

\section{CLINICIAN-LEVEL VARIATION IN THREE MEASURES REPRESENTING OVERUSE BASED ON THE AMERICAN GERI- ATRICS SOCIETY CHOOSING WISELY STATEMENT}

Theresa A. Rowe ${ }^{1}$; Ji Young Lee ${ }^{1}$; Tiffany Brown ${ }^{2}$; Stephen Persell ${ }^{3} .{ }^{1}$ Northwestern University, Chicago, IL; ${ }^{2}$ Northwestern University Feinberg School of Medicine, Chicago, IL; ${ }^{3}$ Northwestern University, Evanston, IL. (Control ID \#3173753)

BACKGROUND: The extent of clinician-level variation in the overuse of testing or treatment in older adults is not well understood. The objective is to examine the clnician-level variation on three measures of overuse in older adults.

METHODS: The study was a retrospective analysis of two process and one outcome measures in adults aged 65 years and older evaluated in outpatient primary or immediate care within a health system affiliated with an academic medical center between July 1, 2016 and June 30, 2017. Subjects: Clinicians: Primary or immediate care clinicians caring for adults aged 65 years and older. Patients: Women and men aged 65 years and older being seen in a primary or immediate care clinic. Measures: The primary outcomes of interest were three electronic clinical quality measures taken from electronic health record data representing potentially inappropriate use of medical services in older adults: prostate specific antigen screening in men age 76 and older (PSA), urine culture or urinalysis for non-specific reasons in women age 65 and older (UC/UA), and hemoglobin A1c less than 7.0 in adults age 75 and older with diabetes treated with insulin or oral hypoglycemic medication (DM). Physicians, advanced practice nurses and physician assiastants with at least 10 eligible cases were included in the analysis.

RESULTS: There were 42 to 144 clinicians (mostly physicians) and 665 to 5933 patients included in the analysis per measure. Variation at the physician level was highest for PSA (intraclass correlation coefficient $[\mathrm{ICC}]=0.27)$, followed by UC/UA $(\mathrm{ICC}=0.18)$, and lowest for DM $(\mathrm{ICC}=0.024)$. The percentage of patients with overuse attributed to the lowest and highest overusing quartile of clinicians were: $8 \%$ and $54 \%$ for PSA, $3 \%$ and $35 \%$ for UC/UA and $13 \%$ and $49 \%$ for DM, and the odds ratio of overuse in highest quartile compared to the lowest were 99.3 (95\% CI 43 to 228), 15.7 (10 to 24 ), and 6.0 (3.3 to 11$)$, respectively.

CONCLUSIONS: Within the same health system, overuse of testing and treatment in older adults varied greatly across clinicians, particularly for the process measures examined.
COLLECTIVE VULNERABILITY IS ASSOCIATED WITH HIGHER PERCEIVED DISCRIMINATION, PAIN, AND DEPRESSION IN VETERANS WITH OSTEOARTHRITIS Utibe R. Essien ${ }^{1,2}$; Ada O. Youk ${ }^{3,2}$; Said Ibrahim ${ }^{4}$; Leslie R. Hausmann ${ }^{2}$. ${ }^{1}$ University of Pittsburgh School of Medicine, Pittsburgh, PA; ${ }^{2}$ VA Pittsburgh Healthcare System, Pittsburgh, PA; ${ }^{3}$ University of Pittsburgh, Graduate School of Public Health, Pittsburgh, PA; ${ }^{4}$ Weill Cornell Medicine, New York, NY. (Control ID \#3177582)

BACKGROUND: Perceived discrimination is associated with physical and mental health outcomes, including chronic pain and depression, and plays a role in racial health disparities. However, little is known about how the intersection of race and other factors for which one might experience discrimination (collective vulnerability), such as gender, education, income, weight, or disability, impact health. Our aim was to examine the additive effects of six vulnerable group memberships on perceived discrimination, pain, and depression and to test whether perceived discrimination mediates the impact of collective vulnerability on pain and depression.

METHODS: Our sample included patients at two large VA medical centers who enrolled in a randomized controlled trial testing a positive psychological intervention for chronic pain. Participants were white and African American Veterans aged 50 years reporting symptomatic knee osteoarthritis (OA). At baseline, we measured perceived discrimination using the Everyday Discrimination Scale; severity of pain using the pain subscale of the Western Ontario and McMaster Universities Osteoarthritis Index (WOMAC); depression severity using the Personal Health Questionnaire Depression Scale (PHQ-8); and demographic variables (self-reported gender, age, race, income, education, and employment). We abstracted body mass index (BMI) from medical records. We assigned binary indicators of vulnerability to participants with each of the following characteristics: African American race, female gender, high school education or less, $<\$ 20,000$ income, disabled employment status, and morbid obesity (BMI $>40$ ). We then summed individual indicators to capture each participant's number of vulnerabilities. We conducted separate linear regression models to assess the relationship between the number of vulnerabilities and measures of perceived discrimination, pain, and depression. Lastly, we used Sobel's test of mediation to examine perceived discrimination as a mediator of the relationships between collective vulnerability and pain and depression.

RESULTS: Among the 517 participants (47.8\% white, 52.2\% African American), the average number of vulnerabilities was $1.66(\mathrm{SD}=1.17)$. Number of vulnerabilities was significantly associated with perceived discrimination $($ Coef $=0.41,95 \%$ CI $0.18,0.65)$, pain $($ Coef $=4.14$, $95 \%$ CI $2.92,5.37)$ and depression severity $($ Coef $=1.13,95 \% \mathrm{CI}$ $0.74,1.51)$. Although perceived discrimination significantly explained $16.9 \%$ of the association between vulnerabilities and depression ( $\mathrm{p}$ value $<0.01$ ), it did not mediate the association with pain ( $p$-value $=0.10$ ).

CONCLUSIONS: Among Veterans with chronic pain from OA, collective vulnerability is associated with higher levels of perceived discrimination, pain, and depression. Perceived discrimination also partially mediates the relationship between vulnerabilities and depression. Further understanding of how vulnerabilities combine to worsen physical and mental health outcomes is warranted.

COLORECTAL CANCER SCREENING: IMPACT OF PROVIDER FACTORS AND STATE-LEVEL COLORECTAL CANCER SCREENING RATES ON MULTI-TARGET STOOL DNA TESTING COMPLIANCE AMONG MEDICARE BENEFICIARIES Rebecca Swartz ${ }^{1}$; Emily Weiser ${ }^{1}$; Philip Parks ${ }^{1}$; Jack Van Thomme ${ }^{1}$; Paul Limburg ${ }^{1,2}$; Barry M. Berger ${ }^{1}{ }^{1}$ Exact Sciences, Madison, WI; ${ }^{2}$ Mayo Clinic, Rochester, MN. (Control ID \#3180325) 
BACKGROUND: The multi-target stool DNA (mt-sDNA) test is a noninvasive colorectal cancer (CRC) screening test included in United States Preventive Services Task Force, American Cancer Society, and Multi-Society Task Force guidelines for average-risk adults ages 50 years and older. Mt-sDNA testing is performed by a single national reference laboratory (Exact Sciences Laboratories LLC [ESL], Madison, WI) and has an embedded patient navigation system (compliance program) supporting test completion through patient education, reminders, outreach, and multilingual support. Provider specialty and state-level CRC screening rate associations with mt-sDNA test compliance in the Medicare population is reported here. Medicare covers mt-sDNA screening with no out-of-pocket expenses, mitigating the potential impact of financial barriers on compliance.

METHODS: Medicare beneficiaries ages 65-85 years with a valid mtsDNA order from September 1, 2016-August 31, 2017 were identified from the ESL database. Compliance was defined as completion of mtsDNA testing (positive or negative result) within 365 days of order date. Time to compliance (TTC) was days from order date to completion of mt-sDNA testing. Associations between compliance and provider specialty were evaluated using descriptive statistics. Mt-sDNA compliance was compared for providers in states having the highest (ME [75.9\%], CT [75.8\%], and MA [75.3\%]) and lowest (MS [59.9\%], OK [58.8\%], and NM [58.5\%]) overall CRC screening rates. ${ }^{1}$ RESULTS: The mt-sDNA test compliance rate was $71.1 \%$ for the study population $(\mathrm{N}=368,494)$. Internal medicine (IM) and family medicine (FM) practitioners ordered $74.6 \%$ of mt-sDNA tests, with a TTC of 27 days for both specialties. IM practitioners had a $70.7 \%$ compliance rate, and FM practitioners had a $71.4 \%$ compliance rate. Compliance was highest $(78.4 \%)$ and median TTC was lowest $(25$ days) for mt-sDNA tests ordered by gastroenterologists. Compliance in states with high $(>75 \%)$ and low $(<60 \%)$ CRC screening rates were similar. Mt-sDNA test compliance rates for ME, CT, and MA, respectively, were $73.7 \%, 68.2 \%$, and $68.3 \%$ (median TTC of 28,28 , and 29 days). The mt-sDNA test compliance rates for MS, OK, and NM, respectively, were $70.1 \%, 72.3 \%$, and $72.6 \%$ (median TTC of 25,26 , and 28 days).

CONCLUSIONS: These data suggest that provider specialty is associated with mt-sDNA test compliance. Gastroenterologists had higher compliance rates than IM and FM practitioners, possibly due to differences in patient factors (eg, patients completing gastroenterology referrals may have more motivation to complete CRC screening or a greater sense of urgency) and/or provider factors (eg, patient appreciation of specialty knowledge). Mt-sDNA test compliance was similar in states with both high and low overall CRC screening rates. Broader adoption of mt-sDNA testing may help improve participation among Medicare beneficiaries, especially in states with lower than average screening rates.

\section{COMBINED PATIENT - PROVIDER ENGAGEMENT SYSTEM AND MHEALTH INTERVENTIONS IMPROVED MEDICA- TION ADHERENCE FOR MINORITY AND LOW-INCOME PATIENTS WITH DIABETES COMPARED TO MHEALTH ONLY: THE OFFICE-GUIDELINES APPLIED TO PRACTICE PROGRAM}

Khader Herzallah; Amari Ellsworth; Zane Alroshood; Ngoc Nguyen; Karen Kelly-Blake; William Hart-Davidson; Ling Wang; Sherif Elkinany; Ade B. Olomu. Michigan State University, East Lansing, MI. (Control ID \#3183109)

BACKGROUND: Approximately $21-42 \%$ of patients with diabetes (DM) do not take their medications as prescribed. Current implementation studies suggest that mHealth interventions alone may not be enough to improve patient health outcomes. Our objective was to determine the impact of a combined patient activation program
(Office-Guidelines Applied to Practice $\{$ Office-GAP $\}$ ) + mHealth in improving medication adherence compared to mHealth alone for minority and low-income populations.

METHODS: Office-GAP intervention included: 1) Patient activation group visit 2) Physician training for patient activation, and 3) Decision support checklist used in real time in the office. mHealth intervention was teaching patients to send and receive text messages; patients received daily messages appropriate to their diagnosis and medications for 14 weeks along with appointment reminders. Diabetic patients with A1c $>8$ or with uncontrolled hypertension (BP $>130$ / 80) attending Internal Medicine Residency Clinics were recruited into the study. Patients were consecutively assigned to combined OfficeGAP + mHealth group (Green) or mHealth only group (White) based on the resident clinic designation. After a group visit, patients followed up with two physician visits over a 4-month period. Medication adherence was compared at baseline and follow-up by two validated adherence scales: 1) Adherence to Refills and Medications Scale (ARMS), a 14-item scale with two subscales for taking medications as prescribed and refilling medications on schedule, and 2) Morisky Medication Adherence Scale (MMAS), a 4-item self-report measure of adherence to all prescribed medications.

RESULTS: Seventy-one patients enrolled in the pilot study; 37 were Office-GAP + mHealth (Green) arm and 34 mHealth alone (White) arm at baseline. At 4 months follow-up, 20 patients from Green and 18 patients from White completed ARMS. Nineteen from Green and 19 from White completed MMAS. Regression analysis for ARMS show that after adjusting for relevant clinical variables, from baseline to 4 months follow-up, ARMS in Green group decreased significantly with 1.74 points on average $(\mathrm{P}$-value $=0.008)$ signifying better adherence to refills and medications. In White group, ARMS increased by 0.256 points ( $\mathrm{P}$-value $=0.80$ ). Compared with White group, ARMS in Green group are 2.0 points lower from baseline to follow-up (Pvalue $=0.09$ ). Poisson regression results for MMAS show that from baseline to follow-up, the Incident Rate Ratio (IRR) of MMAS in Green group is $0.762(\mathrm{P}=0.05)$, while IRR in White group is 1.09 $(\mathrm{P}=0.44)$. Compared with White group, IRR of MMAS in Green group from baseline to follow-up is $0.72(\mathrm{P}=0.045)$ indicating improved medication adherence.

CONCLUSIONS: Combined Office-GAP + mhealth significantly improved adherence to refills and to all prescribed medications compared to mhealth alone. Our study suggests that the combined intervention shows promise to improve health outcomes for patients with diabetes.

\section{COMMUNICATING CARDIOVASCULAR RISK TO HIGH RISK CANCER SURVIVORS: A MIXED METHODS PILOT STUDY OF A STATIN RISK COMMUNICATION TOOL}

Nirupa J. Raghunathan ${ }^{2}$; Emily Zabor $^{3}$; Kevin Oeffinger ${ }^{1}$; Emily S. Tonorezos ${ }^{2}$; Deborah R. Korenstein ${ }^{2} .{ }^{1}$ Duke University, Durham, NC; ${ }^{2}$ Memorial Sloan-Kettering Cancer Center, New York City, NY; ${ }^{3} \mathrm{Me}-$ morial Sloan Kettering Cancer Center, New York, NY. (Control ID \#3182633)

BACKGROUND: Over a million survivors of childhood, adolescent, and young adult cancer live in the US; many were treated with radiation therapy (RT). RT with fields including the coronary arteries is a risk factor for cardiovascular disease (CVD). Survivors may be unaware of their high CVD risk or how to mitigate it; tools are needed to improve understanding. The Institute of Medicine recommends decision aids to optimize patient discussions of therapies. We developed and pilot tested a risk communication tool to improve shared decision making in high-risk survivors regarding CVD risk reduction with statin therapy. We included quantitative and qualitative arms to inform development and testing of the tool. 
METHODS: The statin risk communication tool was adapted from a previously validated tool. Participants were recruited from Memorial Sloan Kettering Cancer Center into two arms: control CVD risk discussion vs. tool-facilitated discussion. All patients were at increased risk for CVD due to history of chest RT. The post-visit survey used Likert-like scales to explore patient perceptions of statins and included knowledge questions and a decisional conflict scale. This feasibility and acceptability study used descriptive statistics and was not powered for significance. Semi-structured interviews with intervention arm participants explored the shared decision-making process.

RESULTS: Median participant $(n=46)$ age was 45 . Most intervention patients $(22 / 24,92 \%)$ and $50 \%(11 / 22)$ of controls found statin information acceptable while $31 \%(7 / 22)$ of the control arm selected "not applicable" regarding information acceptability. Most participants were unaware of their CVD risk or statin side effects. In semistructured interviews participants found the tool helpful to visualize risk and aid physician conversations; they expressed apprehension at starting a statin and comfort in decision-making.

CONCLUSIONS: This risk communication tool was acceptable. Many receiving usual care often not recall a conversation, suggesting an absent or forgettable discussion. Knowledge of CVD and statins was poor. Qualitative data suggested the tool improved decisional clarity and comfort.

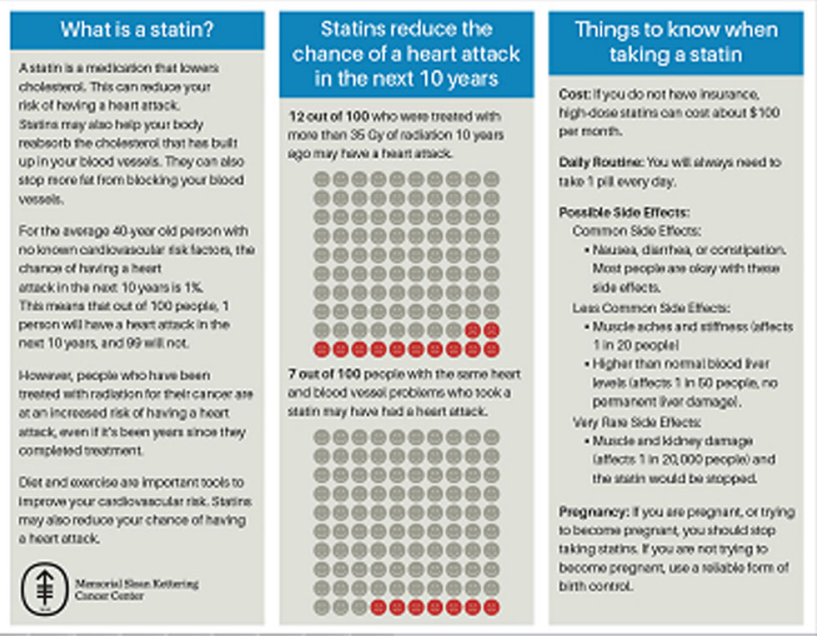

Statin Risk Communication Tool

\section{COMMUNITY PHARMACY MEDICATION REVIEW, DEATH AND READMISSIONS AFTER HOSPITAL DISCHARGE}

Lauren Lapointe-Shaw ${ }^{1,}{ }^{1}$; Chaim M. Bell ${ }^{1,}{ }^{2}$; Peter C. Austin ${ }^{3,}{ }^{1}$; Petros Pechlivanoglou ${ }^{1}$ 6; Lusine Abrahamyan ${ }^{1,}{ }^{4}$; Ping $\mathrm{Li}^{3}$; Noah M. Ivers $^{1,}{ }^{5}$; Donald Redelmeier ${ }^{1}{ }^{7}$; Lisa Dolovich ${ }^{1}$. ${ }^{1}$ University of Toronto, Toronto, ON, Canada; ${ }^{2}$ Sinai Health System, Toronto, ON, Canada; ${ }^{3}$ ICES, Toronto, ON, Canada; ${ }^{4}$ Toronto Health Economics and Technology Assessment (THETA) Collaborative, Toronto, ON, Canada; ${ }^{5}$ Women's College Hospital, Toronto, ON, Canada; ${ }^{6}$ SickKids Research Institute, Toronto, ON, Canada; ${ }^{7}$ Sunnybrook Health Sciences Centre, Toronto, ON, Canada. (Control ID \#3180233)

BACKGROUND: In-hospital medication review has been linked to improved outcomes after hospital discharge, yet there is little evidence to support the use of community pharmacy-based interventions as part of transitional care. MedsCheck, a program of medication reconciliation and review by community pharmacists, was introduced throughout the province of Ontario in 2007. Our objective was to determine if discharged patients receiving MedsCheck had lower rates of subsequent death or readmission than patients not receiving it.

METHODS: This was a population-based retrospective cohort study of eligible patients discharged home from an Ontario hospital from April $1^{\text {st }} 2007$ to September $16^{\text {th }} 2016$. Eligible patients were at least 66 years of age on the day of hospital discharge and filled a prescription at a community pharmacy within 7 days of discharge. Propensity score matching was used to ensure comparability of groups with respect to demographics, comorbidities, previous healthcare usage, hospitalization, hospital and pharmacy characteristics. The exposure of interest was receipt of MedsCheck, a publiclyfunded medication reconciliation and review encounter provided by community pharmacists. The primary outcome was time to death or readmission, with the latter defined as an emergency department visit or urgent re-hospitalization, within 30 days of MedsCheck receipt. Secondary outcomes were time to adverse drug event and the 30-day count of outpatient physician visits.

RESULTS: Of 879,497 unique patients discharged, 77,459 (8.8\%) received a MedsCheck. Of these, 67,163 patients $(86.7 \%)$ were propensity scorematched 1:1 to patients who did not receive a MedsCheck. Patients who received MedsCheck had a lower risk of 30-day death or readmission than controls ( $23.4 \%$ vs $23.9 \%$, HR $0.97,95 \%$ CI $0.95-1.00, \mathrm{p}=0.02)$, driven by a decreased risk of death $(1.7 \%$ vs $2.1 \%$, HR $0.79,95 \%$ CI $0.73-0.86)$ and rehospitalization ( $11.0 \%$ vs $11.4 \%$, HR $0.96,95 \%$ 0.93-0.99). There was no significant difference in 30-day return to the emergency department (22.5\% vs $22.8 \%$, HR $0.99,95 \%$ CI 0.96-1.01). There was no difference in adverse drug events associated with MedsCheck status (1.5\% vs $1.5 \%$, HR 1.03, 95\% CI 0.94-1.12). MedsCheck recipients had more outpatient visits than controls (mean 2.11 vs 2.09 , RR 1.01, 95\% CI 1.00-1.02, $\mathrm{p}=0.02$ ).

CONCLUSIONS: Among older adults filling a prescription after hospital discharge, receipt of a pharmacy-based medication reconciliation and review encounter was associated with a reduced risk of death or readmission in the following 30 days. Due to the possibility of unmeasured confounding in this observational study, experimental studies are needed to confirm this finding.

\section{COMPARING MEDICAID AND MARKETPLACE COVERAGE: EFFECTS ON HEALTH CARE UTILIZATION, OUT-OF-POCKET SPENDING, AND COSTS}

Heidi Allen ${ }^{3}$; Sarah Gordon ${ }^{4}$; Dennis Lee ${ }^{5}$; Benjamin D. Sommers ${ }^{1,2}$. ${ }^{1}$ Harvard School of Public Health, Brookline, MA; ${ }^{2}$ Brigham \& Women's Hospital, Boston, MA; ${ }^{3}$ Columbia University, New York, NY; ${ }^{4}$ Brown University, Providence, RI; ${ }^{5}$ Harvard University, Jamaica Plain, MA. (Control ID \#3185045)

BACKGROUND: After the 2018 election, several states appear poised to expand Medicaid under the Affordable Care Act. The Trump administration is encouraging states to prioritize expanding private insurance instead, and some states have proposed moving Medicaid beneficiaries to private coverage. In this context, understanding the tradeoffs between these coverage types is critical. We leverage a unique dataset from Colorado's Medicaid and Marketplace agencies to compare the effects of insurance type on coverage duration, utilization, and health care costs.

METHODS: Using income data provided at the time of application for Medicaid or Marketplace coverage, merged with Colorado's All Payer Claims Database from 2014, we examined utilization, out-ofpocket spending, and total costs using a regression discontinuity (RD) approach. Our sample contained non-pregnant individuals ages 1964, with incomes between $75 \%$ and $400 \%$ of the Federal Poverty Level (FPL), enrolled in Medicaid or Marketplace coverage in 2014 $(\mathrm{N}=206,810)$. Comparing individuals just below versus just above the eligibility threshold of $138 \%$ of FPL, we assessed the impact of coverage type on these health-care related outcomes. 
RESULTS: Our preliminary findings are threefold. First, income just below the Medicaid eligibility threshold led to 6.9 months of additional Medicaid coverage per year, while those just above the threshold experienced 6.3 months more of Marketplace coverage. Second, utilization patterns differed significantly. Those eligible for Medicaid were 3.4 percentage-points more likely to visit the Emergency Department (ED) each year (sample mean 24\%) than those eligible for Marketplace coverage, but were 6.3 percentage-points less likely to have an outpatient visit (sample mean 60\%). Medicaid also led to a 4 percentage-point decrease in the likelihood of a prescription fill (sample mean 58\%). We found no statistically significant differences in hospitalization rates. Third, costs were significantly higher in Marketplace coverage, despite the slight reduction in months covered. Annual spending per person was $\$ 707$ higher in Marketplace coverage than Medicaid, representing a 35\% increase compared to the sample mean (\$2034). After standardizing prices to Medicaid rates, costs were similar in the two groups, indicating that different prices (not volume) were responsible for the cost difference between the two coverage types. Out-of-pocket spending (not including premiums) was even more disparate. Marketplace beneficiaries spent \$105 more per person than Medicaid enrollees, more than a fourfold increase compared to the Medicaid average (\$25.50).

CONCLUSIONS: Medicaid results in higher coverage rates than Marketplace insurance, but less outpatient care and more ED care. However, Medicaid coverage is substantially less costly both to enrollees and to society. These results have important implications for policymakers considering alternative approaches to coverage expansion.

\section{COMPARING THE NATIONAL COST-EFFECTIVENESS OF ALTERNATIVE DIABETES SCREENING STRATEGIES}

Rahul Dadwani ${ }^{1}$; Mohammed Reza Skandari ${ }^{3}$; Lawrence Phillips ${ }^{2}$; Mary Rhee $^{2}$; Neda Laiteerapong ${ }^{1} .{ }^{1}$ University of Chicago, Chicago, IL; ${ }^{2}$ Emory University School of Medicine, Atlanta, GA; ${ }^{3}$ Imperial College of London, London, United Kingdom. (Control ID \#3168022)

BACKGROUND: With the high costs of diabetes in the United States, it is important to identify cost-effective strategies for Diabetes Mellitus screening. Currently, the American Diabetes Association (ADA) recommends the use of two of the following four tests to screen for diabetes: fasting plasma glucose (FPG), glycated hemoglobin (HbAlc), oral glucose tolerance test (OGTT) or random plasma glucose (RPG) if symptoms of hyperglycemia are present. However, recent studies have suggested that RPG values at lower screening thresholds than recommended by the ADA, and the glucose challenge (GCT) that is currently utilized for gestational diabetes screening, can be used for Type 2 diabetes screening. To assess the utility of these tests, this study models a one-time universal diabetes screening of the U.S population using alternative screening strategies.

METHODS: Using a lifetime Markov Monte Carlo simulation model (US Type 2 Diabetes Policy Model), this study examined the costeffectiveness of all 16 combinations of two of five possible tests [FPG (>125 mg/dl), HbAlc (>6.4\%), OGTT (>199 mg/dl), GCT (>160 mg/dl) and RPG (>120 mg/dl) values collected for clinical purposes besides diabetes screening (opportunistic screening)]. We estimated lifetime costs and quality adjusted life years (QALYs) for each combination of screening tests for a nationally-representative sample of U.S adults (National Health and Nutrition Examination Survey 2013-2014) at high risk of developing diabetes according to United States Preventative Services Task Force criteria.

RESULTS: There was no statistically significant difference in either lifetime costs or QALYs between any of the 16 screening strategies. Lifetime costs ranged from $\$ 78,333$ (FPG followed by HbA1c) to $\$ 82,059$ (RPG followed by GCT) per person screened. QALYs ranged from 19.6274 (OGTT followed by GCT) to 19.6287 (HbA1c followed by OGTT or FPG).
CONCLUSIONS: This study suggests that diabetes screening strategies that utilize opportunistic RPG values at a lower threshold (>120 mg/dl) than currently recommended by the ADA ( $>200 \mathrm{mg} / \mathrm{dl}$ and symptoms of hyperglycemia) or the GCT may be comparable in effectiveness to current screening modalities. This is important because RPG values may already be clinically available for certain patients and, thus, a more convenient initial diabetes screening tool compared to tests that require the provider to make a conscious decision to screen his or her patient. Future studies that model the effect of diabetes screening should examine the impact of early intensive glycemic control and the subsequent "legacy effect" of early diabetes diagnoses.

\section{COMPARING THE PERFORMANCE OF SELF-REPORT MEDI- CATION ADHERENCE MEASURES AMONG PATIENTS WITH LIMITED LITERACY AND ENGLISH PROFICIENCY}

Lauren Opsasnick; Scott Hur; Laura M. Curtis; Stephanie Batio; Guisselle Wismer; Michael S. Wolf. Northwestern University, Chicago, IL. (Control ID \#3185776)

BACKGROUND: Recent debates have ensued with regard to the use of certain self-report measures of medication adherence. Despite valid criticisms concerning social desirability of patient self-report, concerns around the validity of such assessments among lower literate, and more limited English proficient adults have also been raised by health services researchers. We sought to examine the concordance of different types of available self-report, medication adherence tools, and compare their construct validity within a sample of these more vulnerable, at-risk patient populations.

METHODS: 314 English and Spanish-speaking patients with type 2 diabetes, ages 32-80 at baseline, were recruited from 11 primary care clinics across Chicago. The study population was $92 \%$ low health literate (measured by the Newest Vital Sign), and 54\% of patients had limited English proficiency. At baseline, patients underwent a structured interview where they were administered a measure intentionally designed for lower literate audiences (Patient Medication Adherence Questionnaire, PMAQ), as well as the Morisky Scale, and a demonstrated proper use task based on the prescription sigs. At a 6 month follow-up interview, an objective assessment of adherence was performed via pill count for each of the patients' diabetes and hypertension medicines. Additionally, the proportion of days covered (PDC) was obtained for a subset of patients who used a partnering national retail pharmacy (Walgreens). For this analysis, agreement and Kappa statistics were calculated between the three self-report adherence measures. Logistic regressions were conducted to assess if the baseline self-report measures predicted PDC or pill count. Additionally, a generalized linear model was performed to measure the impact of self-report adherence on glycemic control (HbAlc).

RESULTS: Agreement between PMAQ and proper use was measured at 56\% (Kappa $=0.10$ ), whereas agreement between PMAQ and Morisky was $17 \%$ and agreement between proper use and Morisky was 23\% (Kappa= $-0.15 \&-0.03$, respectively). In multivariable analyses modeling pill count adherence and controlling for relevant covariates, PMAQ was associated with significantly higher odds of pill count adherence $(\mathrm{OR}=2.8 ; 95 \% \mathrm{CI}$ : $1.4,5.7 ; \mathrm{p}<0.01)$. Proper use and Morisky had no significant associations with pill count. Additionally, multivariable analyses examining HbA1c as the outcome showed that PMAQ was significantly associated with a decline in $\mathrm{HbA} 1 \mathrm{c}(=-0.68 ; 95 \%$ CI: $-1.09,-0.24 ; \mathrm{p}<0.01)$. Again, proper use and Morisky had no significant associations with HbAlc. Futher multivariate modeling of PDC adherence was conducted on the Walgreens sub-sample, but results were insignificant due to the small sample size.

CONCLUSIONS: In a primarily low literate patient population, the PMAQ performed well as a means to predict pill count adherence, as well as clinical outcomes such as HbA1c. Conversely, the Morisky scale and demonstrated proper use task were found to lack construct and predictive validity. 
COMPARISON OF ELECTRONIC CONSULTATION RECOMMENDATIONS FROM A COLLECTIVE OF CLINICIANS VERSUS BOARD-CERTIFIED SPECIALISTS

Elaine C. Khoong ${ }^{1}$; Sarah Nouri ${ }^{1}$; Delphine Tuot ${ }^{2}$; Valy Fontil ${ }^{1}$; Urmimala Sarkar ${ }^{1} .{ }^{1}$ University of California - San Francisco, San Francisco, CA; ${ }^{2}$ University of California, San Francisco, San Francisco, CA. (Control ID \#3185062)

BACKGROUND: Electronic consultations (e-consults) have improved timely access to specialists, but adoption remains incomplete. In areas with inadequate number of specialists, primary care providers may have limited access to e-consults/in-person specialty consultations. Preliminary studies suggest that leveraging the collective intelligence (CI) of multiple clinicians, or the shared intelligence that emerges from collaboration, may provide similar benefits to e-consults. We assessed the accuracy and harm of $\mathrm{CI}$ recommendations vs. specialist recommendations in e-consult cases.

METHODS: We collected actual endocrinology, neurology, gynecology econsult cases from a single, integrated healthcare system. Case information was placed on an electronic CI platform, where a board-certified specialist then responded. If cases had gold-standard recommendations (defined as recommendations agreed upon by both the e-consult and CI platform specialists), we then identified a CI by soliciting responses from nonspecialty clinicians through the CI platform. The platform aggregated responses into a CI output, which ranked all recommendations. We analyzed the CI output after at least five clinicians responded. The primary outcome was accuracy of $\mathrm{CI}$ output against gold-standard recommendations. Accuracy was defined at four levels: focused (all gold-standard recommendations ranked highest); expanded (all gold-standard recommendations ranked high); partial (some gold-standard recommendations ranked high); or none. For the top ten recommendations in the CI output, we assessed as a binary outcome the potential for meaningful harm among non-gold standard recommendations. Two research team clinicians independently assessed accuracy and harm and reached agreement.

RESULTS: At total of 227 clinicians answered 33 cases (11 endocrine, 10 neurology, 12 gynecology). A median of 11 clinicians (interquartile range [IQR]: 8-15) responded to each case. Table 1 shows accuracy of the CI output. The number of potentially harmful recommendations per case was: endocrinology (median 1, IQR 1-3); neurology (median 2, IQR 1-2); gynecology (median 2, IQR 1-3)

CONCLUSIONS: The CI of multiple clinicians is not equivalent to boardcertified specialist recommendations, but it can provide useful recommendations. Some CI recommendations have potential for harm, and clinicians should be wary of higher-risk diagnostic tests/therapies. Future research should address refining CI to safely meet gaps in specialist access.

\begin{tabular}{|l|c|c|c|}
\hline $\begin{array}{l}\text { Level of } \\
\text { Accuracy }\end{array}$ & Endocrine & Neurology & Gynecology \\
\hline Focused & $0 / 11(0 \%)$ & $2 / 10(20 \%)$ & $4 / 12(33 \%)$ \\
\hline Expanded & $2 / 11(18 \%)$ & $1 / 10(10 \%)$ & $4 / 12(33 \%)$ \\
\hline Partial & $3 / 11(27 \%)$ & $4 / 10(40 \%)$ & $2 / 12(17 \%)$ \\
\hline None & $6 / 11(55 \%)$ & $3 / 10(30 \%)$ & $2 / 12(17 \%)$ \\
\hline
\end{tabular}

Table 1: Accuracy of Collective Intelligence Recommendations vs Specialist Recommendations for Electronic Consultation Cases

CONCORDANCE OF BELIEFS ABOUT CHRONIC OBSTRUCTIVE PULMONARY DISEASE, HYPERTENSION, AND DIABETES AMONG PATIENTS WITH MULTIMORBIDITY

Grace McInerney $^{1}$; Rachel O'Conor ${ }^{2}$; Michael S. Wolf ${ }^{2}$; Juan P. Wisnivesky ${ }^{3}$; Alex Federman ${ }^{1} .{ }^{1}$ Icahn School of Medicine at Mount Sinai, New York, NY; ${ }^{2}$ Northwestern University, Chicago, IL; ${ }^{3}$ Mount Sinai School of Medicine, New York, NY. (Control ID \#3184189)
BACKGROUND: Beliefs about chronic obstructive pulmonary disease (COPD) and its treatments are important because they influence COPD medication adherence. Comorbidities are common among COPD patients, but it is unknown whether health and medication beliefs for COPD are associated with beliefs for comorbid chronic diseases. Identifying common belief patterns would potentially provide efficient targets for promoting better self-management behaviors for patients with multimorbidity.

METHODS: To test the association of COPD illness and medication beliefs with illness and medication beliefs specific to hypertension or diabetes in patients with multimorbidity, we performed a cross-sectional analysis of data collected from COPD patients with comorbid hypertension or diabetes. A sample of 282 adults ages 40 years and older with COPD were recruited from primary care practices in New York, NY, and Chicago, IL. Beliefs about COPD, hypertension, and diabetes were measured using the Brief Illness Perception Questionnaire (BIPQ), with wording adapted to each condition. Higher scores indicate a more threatening view of the illness. Beliefs about COPD, hypertension, or diabetes medications, were measured in two domains (necessity, concerns) using the 10-item Beliefs about Medicines Questionnaire (BMQ). Correlation coefficients were calculated for the associations of beliefs (COPD and hypertension, or COPD and diabetes).

RESULTS: The mean age was 68 (SD 9), 59\% were female, 38\% were black and $17 \%$ were Hispanic; $98 \%$ had hypertension and 35\% had diabetes, and $33 \%$ had both comorbidities. Beliefs about illness threat were highly correlated for COPD and hypertension $(r=0.52, \mathrm{P}<0.0001)$, and COPD and diabetes $(r=0.45, \mathrm{P}<0.0001)$. The correlation was similarly high for COPD and hypertension medication concerns $(r=0.64, \mathrm{P}<0.0001)$, COPD and diabetes medication concerns $(r=0.61, \mathrm{P}<0.0001)$, and COPD and hypertension medication necessity beliefs $(\mathrm{r}=0.56, \mathrm{P}<0.0001)$, but less so for COPD and diabetes medication necessity beliefs $(r=0.31, P=0.004)$. CONCLUSIONS: Patients with COPD and comorbid hypertension or diabetes have mostly consistent beliefs about their diseases and the medications used to treat them. This consistency may provide opportunities to utilize patients' illness perceptions and medication beliefs to find strategies that facilitate improved self-management of multiple chronic illnesses.

\section{CONCURRENT USE OF SULFONYLUREAS AND INTERACTING ANTIMICROBIALS IN THE UNITED STATES: NAMCS 2006-2015}

Scott J. Pilla; Nisa Maruthur. Johns Hopkins University, Baltimore, MD. (Control ID \#3162727)

BACKGROUND: Hypoglycemia is the most common serious adverse effect of diabetes treatment and is associated with significant morbidity and mortality. Sulfonylureas, the most common oral diabetes treatment after metformin, have interactions with a number of antimicrobials which substantially increase the risk of hypoglycemia when prescribed together. In this study, we provide the first nationally representative estimates of the concurrent use of sulfonylureas and interacting antimicrobials, and the indications for interacting antibiotic prescriptions.

METHODS: We used the 2006-2015 National Ambulatory Medical Care Survey (NAMCS), an annual sample of visits to office-based physicians, and National Hospital Ambulatory Medical Care Survey (NHAMCS), an annual sample of visits to hospital emergency departments, and outpatient departments through 2011. Together, these provide nationally representative estimates of U.S. physician visits. At each visit, physicians reported the patient's chief complaint and up to four additional reasons for the visit, as well as the medications ordered, supplied, administered, or continued. This study included visits for adults aged 18 years or older with concurrent use of a sulfonylurea and antimicrobial (excluding topical therapies). The primary outcome was use of antimicrobials interacting with sulfonylureas: clarithromycin, fluconazole, fluoroquinolones, metronidazole, or sulfamethoxazole-trimethoprim. We also examined whether the 
reason for visit included diagnoses for which antibiotics (i.e. excluding antifungals) are almost always indicated (Tier 1), sometimes indicated (Tier 2), or not clearly indicated (Tier 3) using the algorithm developed by Fleming-Dutra et al. (JAMA 315(17): 1864-1873, 2016). Results are national estimates of patient visits (presented as the yearly average) or proportions.

RESULTS: Between 2006-2015, there were 2.6 million yearly visits (95\% CI 2.2-3.0) for patients using sulfonylureas and antimicrobials, of which interacting antimicrobials were used in $38.9 \%$ (95\% CI, $32.9-45.2)$ or 1.0 million visits (95\% CI 0.8-1.2). Most visits with use of interacting antimicrobials occurred in ambulatory care offices $(0.9$ million, 95\% CI 0.7-1.2); a minority occurred in other care settings. The most commonly used interacting antimicrobials were fluoroquinolones $(60.3 \%$ of interacting antimicrobials, $95 \% \mathrm{CI}$ 51.3-68.7) and sulfamethoxazole-trimethoprim (20.9\%, 95\% CI 14.8-28.7). Among visits using interacting antibiotics, $69.0 \%(95 \%$ CI 55.1-80.1) had no appropriate indication for antibiotic use (Tier 3); 9.1\% (95\% CI 6.0-13.7) and 21.9\% (95\% CI 11.3-38.2) had Tier 1 and Tier 2 indications, respectively. The most common Tier 1 indication was urinary tract infection $(6.8 \%$ of interacting antibiotic visits, 95\% CI 4.3-10.4).

CONCLUSIONS: Concurrent use of sulfonylureas and interacting antimicrobials is common in U.S. ambulatory practice and likely contributes to hypoglycemic events. Reducing inappropriate antibiotic prescribing may help alleviate this problem.

\section{CONSOLIDATION IN THE DIALYSIS INDUSTRY AFTER BUNDLED PAYMENT REFORM}

Caroline Sloan ${ }^{1,2}$; Abby Hoffman ${ }^{1,3}$; Matthew Maciejewski ${ }^{2,1}$. Cynthia Coffman $^{1,2}{ }^{2}$; Linda L. Sanders ${ }^{1}$; Richard Hirth ${ }^{4}$; Shoou-Yih D. Lee ${ }^{4}$; Virginia Wang ${ }^{1,2}$. ${ }^{1}$ Duke University, Durham, NC; ${ }^{2}$ Durham VA Medical Center, Durham, NC; ${ }^{3}$ University of North Carolina Gillings School of Global Public Health, Chapel Hill, NC; ${ }^{4}$ University of Michigan, Ann Arbor, MI. (Control ID \#3183020)

BACKGROUND: Dialysis facilities currently treat almost 500,000 patients with end-stage renal disease (ESRD) and have experienced accelerating consolidation over the last two decades. By the late 2000 s, two for-profit chains controlled over $70 \%$ of the dialysis market, leading to concerns about further market concentration. The impact of Medicare's 2011 prospective payment system (PPS) for dialysis on consolidation in the dialysis industry is largely unknown. We evaluate whether rates of acquisition among dialysis facilities that were independently-owned or affiliated with small chains were higher in the post-PPS period compared to the pre-PPS period.

METHODS: We conducted a retrospective cohort study of nonfederal US outpatient dialysis facilities that were independentlyowned or affiliated with small chains $(<20$ facilities) for at least one year between 2008 and 2013. These facilities were deemed to be at highest risk of acquisition by large dialysis organizations. We used data from the Center for Medicare and Medicaid Services, the US Renal Data System and the Area Health Resource File to evaluate facility and market characteristics before (2008-2010) and after (2011-2013) the PPS. The outcome of interest was change in dialysis facility ownership (i.e. acquisition) before and after implementation of the PPS. We used a generalized estimating equation with a logit link to examine the association between the PPS and facility acquisition, adjusting for dialysis facility and regional demographic characteristics.

RESULTS: Overall, 1686 dialysis facilities (27\% of all facilities in the US) were at risk of acquisition for at least one year of the entire study period; $59 \%$ were independently-owned and $41 \%$ were affiliated with small chains. Almost a quarter of at-risk facilities $(23 \%)$ were new to the dialysis market during the study period. The rate of acquisitions increased from $4 \%$ (66/1495 facilities) before PPS to $8 \%(106 / 1412$ facilities) after PPS. After adjusting for all covariates, the odds of acquisition increased after the PPS was introduced (odds ratio 1.72, $95 \%$ confidence interval 1.24-2.39).

CONCLUSIONS: Medicare payment reform was associated with increased acquisition of independent and small chain-affiliated facilities by large chains, possibly because large dialysis organizations are better poised to bear the financial risk associated with the PPS. Consolidation in the dialysis industry may be acting as a buffer against reduced revenue and increased financial risk in the post-PPS era. As the number of nonchain-affiliated dialysis facilities has dwindled in recent years, two large dialysis organizations have gained an increasing amount of market power. Closer scrutiny and additional policy interventions are needed to ensure sufficient diversification and competition in the dialysis industry.

\section{CONSUMER EXPERIENCES WITH PRIVATE HEALTH INSUR- ANCE PROVIDER NETWORKS}

Kelly A. Kyanko ${ }^{1}$; Susan Busch ${ }^{2} .{ }^{1}$ NYU School of Medicine, New York, NY; ${ }^{2}$ Yale University, New Haven, CT. (Control ID \#3181557)

BACKGROUND: Provider networks are an important tool for private health insurers to control costs and ensure quality care for their enrollees. However, provider networks have been criticized for inaccurate directories, limited or narrow choice of providers that omit highly rated "star" hospitals and may lead to undesired or surprise out-of-network care, and disruption of continuity of care if a provider leaves the network or a patient changes plans. We conducted a nationally representative survey to examine consumer experiences and preferences with provider networks in private health insurance plans.

METHODS: Internet survey conducted in 2018 with participants in the GfK KnowledgePanel®, a probability-based online research panel designed to be representative of the U.S. population. The sample included 2,059 English-speaking US adults aged 18 to 64 years enrolled in private health insurance with a provider network and used an outpatient health care provider in the last year.

RESULTS: $74 \%$ agreed that their insurer had made enough in-network providers available. A significantly greater proportion of respondents rated protection from inpatient surprise bills as extremely important or very important from their plan as compared to inclusion of top rated "star" hospitals in the plan network (77\% versus 59\%, p<0.01). Among those with a choice of plan, $60 \%$ of respondents tried to determine if a specific provider was in-network before choosing a plan, and just over half $(57 \%)$ reported that the result of the search affected their choice of plan. Of the $46 \%$ who used the provider directory once enrolled in the plan, $36 \%$ reported a problem either with inaccurate provider contact information or with a listed provider not actually taking their insurance or not taking new patients. Among the $16 \%$ who had a provider leave their network, $62-66 \%$ had their relationship with the provider disrupted and switched to new provider. Another 10-12\% simply stopped treatment.

CONCLUSIONS: Consumers in private insurance plans report satisfaction with the breadth of their provider networks, however problems remain in the accuracy of provider directories and disruption in care from provider turnover. More respondents rated protection from surprise inpatient out-of-network bills as an important plan attribute compared to inclusion of top rated hospitals in the network. Insurers may consider in their product design strong consumer preferences for protections from surprise out-of-network bills and the inclusion of a specific provider in the network in their choice of a plan. In addition to monitoring network adequacy and accuracy of provider directories, additional policy efforts may be needed to ensure and continuity of care due to providers leaving a network. Addressing these provider network issues can preserve their function as a cost saving tool without compromising access and the consumer experience. 
CONTEXTUAL AMBIGUITIES IN THE INTERPRETATION OF CODE STATUS ORDERS: AN ACTIONABLE TARGET FOR IMPROVING HOSPITAL CODE STATUS POLICIES

Jason N. Batten ${ }^{1}$; Jacob A. Blythe ${ }^{1}$; Sarah Wieten ${ }^{1}$; Stephanie M. Harman $^{1}$; Miriam P. Cotler ${ }^{5}$; Joshua B. Kayser ${ }^{3}$; Karin PorterWilliamson ${ }^{4}$; Elizabeth Dzeng ${ }^{2}$; David Magnus ${ }^{1}$. ${ }^{1}$ Stanford University, Stanford, CA; ${ }^{2}$ University of California, San Francisco, San Francisco, $\mathrm{CA} ;{ }^{3}$ University of Pennsylvania, Philadelphia, PA; ${ }^{4}$ University of Kansas, Kansas City, KS; ${ }^{5}$ California State University, Northridge, Northridge, CA. (Control ID \#3186011)

BACKGROUND: Code status orders (e.g., Do-Not-Resuscitate) are designed to succinctly and accurately convey patient preferences for cardiopulmonary resuscitation and other life-sustaining treatments (LSTs). Hospitals have adopted differing code status policies that vary in the number of code status options provided, their definitions, and the institutional ordering process (e.g., design of forms and menus). We sought to explore the ramifications of these differences in policy design for communication among medical team members.

METHODS: We designed a multi-institutional mixed methods study, selecting seven institutions to achieve variation in geographic location (western, midwestern, and eastern United States), type of institution (community, government, academic), and code status policy (i.e., what code status options are available and how they are defined). At each institution, we reviewed code status policies and menus in the electronic health record and conducted 60-90 minute semistructured interviews with physicians. We purposively sampled physicians based on specialty (internal medicine, palliative care, and critical care) and training level (attending physicians and trainees), for a total of 30 interviews. Using techniques from grounded theory, we analyzed transcripts to explore challenges in interpreting the intended meaning of code status orders.

RESULTS: Whether or not a physician who interprets a code status order arrives at its intended meaning (i.e., the meaning of the physician who authored the order) depends on many contextual factors outside of the order itself. We inductively identified six such contextual factors, which often remain implicit and may not be known by the interpreting physician: (1) the author's judgment of the patient's overall condition, (2) the author's intention in not addressing a potentially relevant LST, (3) the location of the patient in the hospital, (4) the clinical indication(s) for which the author intended the order to apply, (5) the purpose of the institutional code status policy, and (6) the author's identity and training level. Institutional policies and ordering practices vary in whether these contextual factors are explicitly addressed, thus aiding or hindering physicians in correctly interpreting the intended meaning of a code status order.

CONCLUSIONS: Failure to arrive at the intended meaning of a code status order is a threat to patient autonomy and a likely source of harm to patients. The contextual ambiguities that underlie these failures may be amenable to intervention through the careful design of hospital code status policies and ordering practices.

\section{CONTRACEPTION ACCESS THROUGH DIRECT TO CONSUM- ER TELEMEDICINE}

Radhika Rastogi ${ }^{4}$; Michael B. Rothberg ${ }^{1}$; Laura D. Lipold ${ }^{3}$; Mark N. $\operatorname{Rood}^{2}$; Kathryn A. Martinez ${ }^{1}{ }^{1}$ Cleveland Clinic, Cleveland, OH; ${ }^{2} \mathrm{Cleve}-$ land Clinic Foundation, Chagrin Falls, $\mathrm{OH} ;{ }^{3} \mathrm{Cleveland}$ Clinic, Beachwood, $\mathrm{OH} ;{ }^{4} \mathrm{Cleveland}$ Clinic Lerner College of Medicine, Cleveland, OH. (Control ID \#3184250)

BACKGROUND: Direct to consumer (DTC) telemedicine connects patients with physicians via mobile applications, generally serving as a substitute for urgent care. Access to care is an often-reported barrier to obtaining hormonal contraception. Because visits are virtual and insurance is not required, use of DTC telemedicine could expand access to contraceptives. The objective of this study was to characterize requests for and provision of contraceptives in a large nationwide DTC telemedicine service.

METHODS: Data come from the AmericanWell DTC telemedicine platform and includes encounters conducted between July 2016 and July 2018. Patients accessing the telemedicine system provided gender, age and insurance information, if applicable (out-of-pocket visits cost \$59). Via free-text field, patients stated their reason for accessing care. Two independent reviewers categorized patient-reported call reason based on keywords (e.g. "birth control," "refill," etc.), and also recorded if the patient cited problems with access to care for contraception. Prescriptions were determined via National Drug Codes associated with encounters, defined as drugs designated as contraceptives by the Food and Drug Administration. Promotional coupons for free or reduced cost visits on the platform are sometimes available. Whether a patient used a coupon for the visit was assessed. We described patients requesting and/or receiving contraception on the telemedicine platform in terms of patient-reported reason for accessing care, insurance information, and coupon use.

RESULTS: Of 159,033 telemedicine encounters with female patients during the study period, 652 reported their call reason was related to contraception and 718 received contraception. The mean age was 29 years (Standard Deviation=7.2). Of those with a contraception-related call reason, $78 \%$ received contraception. Based on the patient-reported call reason, $56 \%$ stated they were seeking a new birth control prescription and 33\% were requesting refills. Six percent of patients reported problems with access to contraception, including insurance changes or difficulty seeing a provider. Patients requesting refills were more likely to receive contraceptives compared to those requesting new prescriptions ( $90 \%$ versus $79 \%, \mathrm{p}<0.001)$. Of the $5 \%$ requesting emergency contraception, $89 \%$ received it. Patients requesting or receiving contraception were less likely to report insurance information than other telemedicine users $(78 \%$ versus $85 \%, \mathrm{p}<0.001)$ and were more likely to use a coupon for free or reduced-cost care ( $15 \%$ versus $8 \%, \mathrm{p}<0.001)$

CONCLUSIONS: Requests for contraception are relatively rare in DTC telemedicine, yet, of those seeking contraception, most received it. Greater use of coupons and lower insurance reporting suggests DTC telemedicine may be addressing access issues for some patients. While appropriateness is unknown, DTC telemedicine is likely a reasonable alternative to outpatient care for most women seeking either new contraceptive prescriptions or refills.

\section{CONTRASTING OPINIONS BETWEEN PRIMARY CARE PRO- VIDERS AND HOSPITALISTS ON COMMUNICATIONS NEED- ED AT HOSPITAL DISCHARGE}

Patrick Ryan ${ }^{1,2}$; Chi Zheng ${ }^{3,2}$; Anna Furniss ${ }^{4}$; John Rice ${ }^{4}$; Amy G. $\overline{\text { Huebschmann }}^{2}, 4$; Elizabeth A. Bayliss ${ }^{5,}{ }^{4} .{ }^{1}$ Denver Health and Hospital Authority, Denver, CO; ${ }^{2}$ University of Colorado School of Medicine, Aurora, CO; ${ }^{3}$ Denver Health, Denver, CO; ${ }^{4}$ Adult and Child Consortium for Outcomes Research and Delivery Science, Aurora, CO; ${ }^{5}$ Kaiser Permanente and University of Colorado, Denver, CO. (Control ID \#3184748)

BACKGROUND: Clinical care has become less integrated since the advent of the hospitalist movement in the late 1990s, and is particularly prone to errors during transitions of care. With increased use of electronic health records (EHRs), there are now more options for primary care providers (PCPs) and hospitalists to communicate. The goal of this study was to assess PCP and hospitalist behaviors and attitudes regarding communication with one another at time of discharge.

METHODS: We performed a cross-sectional, web-based survey in June 2018 of PCPs and hospitalists, employed at two tertiary care health systems with shared EHRs between inpatient and outpatient settings. The survey contained 30 items addressing provider behaviors and attitudes towards communication methods and preferences, interprofessional care, transition of responsibility, and the value of collaboration between PCPs and hospitalists. 
RESULTS: Of 439 potential participants, 191 (45\%) responded; 34\% were hospitalists. Only $5 \%$ of hospitalists, compared to $25 \%$ of PCPs, still participate in the opposite area of clinical care (outpatient vs. inpatient, respectively; $\mathrm{p}<0.01$ ). At least $70 \%$ of each group preferred electronic communication methods. Over $96 \%$ in both groups agreed that mutually developing a transition-of-care plan avoids errors in care delivery, and nearly $80 \%$ of each group agreed that interprofessional collaboration through scholarly work and interdepartmental meetings improves the quality of patient care and fosters collaboration. The majority of both PCPs and hospitalists $(>80 \%)$ agreed that patients receiving interprofessional care from PCPs and hospitalists are more prepared for discharge than those who do not. Nearly all PCPs (99\%) agreed that communicating the need for the PCP to take responsibility for inpatient test results upon discharge was important, compared to $89 \%$ of hospitalists $(p=0.03$ ). However, $47 \%$ of hospitalists felt that developing a transitions of care plan is excessively time consuming, compared to $16 \%$ of PCPs ( $\mathrm{p}<0.01)$. Only $17 \%$ of PCPs and $3 \%$ of hospitalists felt that patient care is adequately discussed between PCPs and hospitalists $(\mathrm{p}=0.01)$.

CONCLUSIONS: PCPs and hospitalists value interprofessional relationships and collaboration to provide high-quality care to patients. However, current practice structures make it difficult to find time to build these relationships, particularly for hospitalists. Given the preferred use of electronic messages to coordinate care between hospitalists and PCPs, EHR communications that specify care-transition needs may be a low-burden option to foster more collaboration between PCPs and hospitalistshowever, the optimal content and delivery of care-transition communications require further study. Additionally, standardized agreements between PCP and hospitalist groups in their respective responsibilities in transitionsof-care may reduce errors during this critical time.

\section{CONTROLLER MEDICATION USE AND EXACERBATIONS AMONG ADULTS WITH ASTHMA IN HIGH-DEDUCTIBLE HEALTH PLANS}

Alison Galbraith $^{1,3}$; Dennis Ross-Degnan ${ }^{1}$; Fang Zhang ${ }^{1}$; Xin Xu ${ }^{1}$; Anna Sinaiko ${ }^{2}$; Jamie Wallace ${ }^{1}$; Ann C. Wu ${ }^{3}$; James F. Wharam ${ }^{1}$. ${ }^{1}$ Harvard Medical School and Harvard Pilgrim Health Care Institute, Boston, MA; ${ }^{2}$ Harvard TH Chan School of Public Health, Boston, MA; ${ }^{3}$ Boston Children's Hospital, Boston, MA. (Control ID \#3185381)

BACKGROUND: High-deductible health plans (HDHPs) are increasingly common and require enrollees to pay annual individual deductibles of more than $\$ 1,000$ before the health plan will being to pay for health care, often including medication costs. We sought to examine the impact of HDHP enrollment on asthma controller medication use and oral steroid bursts compared to enrollment in traditional health plans for adults with asthma. METHODS: We selected adults aged 18-64 years who were enrolled in commercial coverage from a large national insurance carrier between 2004 2014 and had at least 12 months of enrollment in a traditional plan (baseline period) and 12 months of follow-up after either switching to a HDHP (intervention group) or staying in a traditional plan with low or no deductible (control group). Enrollees with a choice of plans were excluded to minimize bias due to selection. Our sample included all enrollees who had HEDIS-defined persistent asthma prior to the baseline year. We implemented a difference-in-difference (D-in-D) comparison of asthma care utilization between enrollees switching to a HDHP and controls staying in a traditional plan. We employed coarsened exact matching using baseline characteristics to select control groups. We analyzed changes in rates of 30-day fills of controller medications (inhaled corticosteroids [ICS], leukotriene inhibitors [LTI], or ICS-long-acting beta agonists [ICS-LABA]) and rate of asthma exacerbations (defined as oral steroid bursts) between the HDHP and control groups.

RESULTS: We identified 18,908 adults with persistent asthma who were switched to HDHPs, and 214,261 matched adult controls. Sixty-four percent of adults had no controller fills in the baseline year. Compared to controls, enrollees in HDHPs had relative decreases in 30-day fill rates for any controller (D-in-D: $-2.9 \%$ [95\% CI $-4.8 \%--1.0 \%]$ ) and for ICSLABA $(-3.1 \%$ [95\% CI $-5.8 \%--0.5 \%])$. There were no significant differences observed in rates of ICS or LTI fills, and no significant differences in rates of oral steroid bursts.

CONCLUSIONS: Regular utilization of asthma controller medications is low for adults with persistent asthma. HDHPs were associated with reduced use of controller medications overall, especially ICS-LABA. There were no differences in rates of asthma exacerbations as measured by oral steroid bursts. Regular use of important preventive asthma medications may be compromised in HDHPs, where patients must pay a greater (and increasingly often the full) amount out-of-pocket for medications for an expensive class of drugs, particularly ICS-LABA. Differences in controller fill rates between HDHP and traditional plan enrollees were modest, suggesting that HDHP enrollees are spending significantly more out-of-pocket on asthma medications each year, putting them at risk of financial burden.

\section{COPING WITH COMMUNITY VIOLENCE: A MIXED METHODS STUDY OF ADULT RESPONSES TO CHRONIC TRAUMA}

Tyrone A. Johnson; Nichole Smith; Madison R. Wilson; Monica E. Peek; Elizabeth L. Tung. University of Chicago, Chicago, IL. (Control ID \#3184635)

BACKGROUND: Community violence continues to be a public health issue in many high-poverty, urban communities. Clinical strategies on providing care for violence-impacted patients are limited, as little research has examined how patients process and cope with violence. This mixed methods study aimed to characterize and quantify associations between violence exposure and common coping responses, including gun ownership. METHODS: In 2017, we conducted, recorded, and transcribed focus groups and interviews with adults 35 years old $(\mathrm{N}=51)$ from 2 epicenters of violent crime in Chicago. Codes and themes were generated using grounded theory and the constant comparison method. In 2018, we additionally surveyed adult patients $(\mathrm{N}=506)$ using computer-assisted personal interviewing software. Participants were queried on coping strategies, using the Brief COPE normalized to a 100-point scale, as well as validated items about gun ownership. Lifetime violence exposure was measured using the Brief Trauma Questionnaire. We used generalized linear models to assess coping strategies as a function of violence exposure, controlling for age, gender, race/ethnicity, education, insurance, and employment. We also used identical model specifications to assess health behaviors as a function of positive or negative coping strategies.

RESULTS: Mean age was $60.2 \pm 12.7$ years. Participants were predominantly female $(72 \%)$ and non-Hispanic black $(76 \%)$. Two-thirds reported a prior exposure to community violence (62\%), either as a direct victim $(41 \%)$ or as an indirect witness, friend, or family member (49\%). Some reported owning a gun (19\%) or actively carrying a gun (12\%), citing protection as the primary reason $(77 \%)$. As one participant shared, "A lot more guys do carry a gun... for defense." Prior exposure to community violence was associated with 3.1 times higher odds of carrying any weapon $(95 \% \mathrm{CI}$, 1.6-6.0) and 2.8 times higher odds of carrying a gun (95\% CI, 1.3-6.1). A high level of stress due to violence was associated with a 29.3-point increase in implementing any coping strategy, positive or negative (95\% CI, 22.935.8). However, participants reported implementing positive strategies (mean score 45.5) more often than negative strategies (mean 21.7, $p$ $<.001$ ). For instance, exposure to community violence was significantly associated with increased coping by distraction ( 8.8 points; $95 \%$ CI, 1.715.9); one respondent explained the need to "plan fun... (to) try to lift your spirits." Higher positive coping was associated with more days of physical activity (1.03 days; $95 \%$ CI, 0.03-2.04), but not more days of fresh fruit and vegetable consumption or medication adherence.

CONCLUSIONS: Exposure to community violence was associated with both positive and negative coping strategies, including gun ownership. 
Understanding how patients cope with community violence may inform healthcare strategies and intervention targets in high-risk populations.

\section{CORRELATION BETWEEN BILIRUBINEMIA AND ACUTE KID- NEY INJURY IN PATIENTS WITH CIRRHOSIS}

Adrienne Lenhart ${ }^{2}$; Patrick Brown ${ }^{1}$; omar khan ${ }^{1}$; Maryam Alimirah ${ }^{1}$; Hafsa Abdulla ${ }^{1}$; Reena Salgia ${ }^{1}$; Junior Uduman ${ }^{1} .{ }^{1}$ Henry Ford Health System, Detroit, MI; ${ }^{2}$ Henry Ford Hospital, Detroit, MI. (Control ID \#3182074)

BACKGROUND: Acute kidney injury (AKI) in the setting of cirrhosis is associated with significant morbidity. However, data is limited regarding the role that hyperbilirubinemia plays in renal injury. One proposed mechanism is direct renal tubular injury from bilirubin and bile salts, but this is not well elucidated. Using a tertiary care population, we aimed to evaluate whether serum bilirubin levels predict the likelihood of AKI in patients with cirrhosis, as well as predict the probability of renal recovery in this population.

METHODS: Hospitalized patients with cirrhosis, with and without AKI, were retrospectively identified from January 2012 - December 2015. AKI was defined per KDIGO criteria and classified into HRS type 1, HRS type 2, and/or acute tubular necrosis (ATN). Other etiologies of AKI were excluded. Data concerning patient demographics, MELD-Na score, peak total serum bilirubin during hospitalization, change in total serum bilirubin from baseline, peak serum creatinine ( $\mathrm{SCr}$ ) during hospitalization, change in $\mathrm{SCr}$ from baseline, and nadir estimated glomerular filtration rate (eGFR) were analyzed. In patients with AKI, renal recovery was evaluated after 6 months of follow up and was defined as a return of $\mathrm{SCr}$ towards baseline or a lack of dialysis dependence.

RESULTS: A total of 200 cirrhotic patients were included. The majority were Caucasian $(70 \%)$, male $(62 \%)$, and had alcoholic cirrhosis $(61 \%)$ as the etiology of their liver disease. Of these, $57 \%$ had AKI, while $43 \%$ did not. The average peak total bilirubin was significantly higher in patients with AKI compared to patients without AKI $(13.6 \mathrm{mg} / \mathrm{dL}$ vs. $5.0 \mathrm{mg} / \mathrm{dL}$ respectively; $\mathrm{p}<0.001)$. The change in bilirubin from baseline was significantly higher in patients with AKI compared to patients without AKI $(8.7 \mathrm{mg} / \mathrm{dL}$ vs. $2.5 \mathrm{mg} /$ $\mathrm{dL}$ respectively; $\mathrm{p}<0.001)$. Patients' peak bilirubin level also correlated with their peak $\mathrm{SCr}(\mathrm{p}<0.001)$, change in $\mathrm{SCr}(\mathrm{p}=0.004)$, and nadir eGFR ( $\mathrm{p}=0.004)$ during hospitalization. There was no significant difference in peak bilirbuin levels between HRS type 1, HRS type 2, and ATN. While peak bilirubin level was unable to predict the likelihood of renal recovery among patients with AKI, a lower MELD-Na score was predictive of renal recovery ( $\mathrm{p}=0.015$ ).

CONCLUSIONS: Higher bilirubin levels predicted both an increased likelihood of and severity of AKI, manifested as both higher $\mathrm{SCr}$ and lower nadir eGFR. Elevated bilirubin also correlated with the development of both HRS and ATN, suggesting that hyperbilirubinemia may play a role in the pathogenesis of AKI, irrespective of etiology. Measured bilirubin did not predict renal recovery in cirrhotic patients with AKI. Further data on the association between hyperbilirubinemia and AKI can guide clinicians to risk stratify and plan initiation of dialysis. Identification of the pathogenic mechanisms may also lead to directed therapy.

\section{CORRELATION BETWEEN SELF-ESTEEM AND INTER- NAL MEDICINE CLERKSHIP GRADES}

Rebecca B. Forman ${ }^{1}$; Monica Malviya ${ }^{1}$; Masrur A. Khan ${ }^{1}$; Keara English $^{1}$; William Southern ${ }^{2}$; Amanda Raff ${ }^{2}$; Tulay Aksoy ${ }^{3} .{ }^{1}$ Albert Einstein College of Medicine, Bronx, NY; ${ }^{2}$ Montefiore, Bronx, NY; ${ }^{3}$ Montefiore Medical Center, Bronx, NY. (Control ID \#3184122)

BACKGROUND: Medical student performance in clinical clerkships is assessed in a variety of ways using both clinical evaluations and standardized exams. Studies have shown that the clinical score can be influenced by factors including gender, age, and personality characteristics such as agreeableness, neuroticism, and extraversion. There have been very few studies, however, investigating the effect of self-esteem on clerkship grades. Our study assessed the relationship between medical students' selfesteem and internal medicine clerkship grades.

METHODS: Over the course of two academic years, an optional survey including the Rosenberg Self-Esteem Scale (RSES) was sent to all medical students in the internal medicine clerkship at our institution. Their responses were recorded and associated with their internal medicine clerkship grades. The de-identified data was provided to the research team. This study was deemed exempt by the Einstein Institutional Review Board. RSES is composed of ten statements answered on a 4-point agree/disagree scale. Responses are graded out of 40, with scores below 15 indicating low selfesteem. The scale has been shown to have both internal validity and test-retest reliability, both in general and in the field of higher education specifically. Chi-squared analysis was performed to assess for correlation between self-esteem scores and clerkship grades. Regression analysis was also performed to investigate correlation between individual survey answers and clerkship grades.

RESULTS: 356 students were eligible to participate. 97 responded ( $27 \%$ response rate), and 94 provided information to allow linking to clerkship grades; the remaining 3 were assumed to have received a non-honors grade for the purpose of analysis. $51(53 \%)$ of the respondents were female, $45(46 \%)$ were male, and $1(1 \%)$ identified as non-binary. The average age was $26.4 \pm 2.6$. 56 $(60 \%)$ received either a High Pass or a Pass, and $38(40 \%)$ received Honors in the clerkship. $11(11 \%)$ had a low self-esteem score, and $86(89 \%)$ had a self-esteem score within the normal range. Of the people with low self-esteem, $8(73 \%)$ received a non-honors grade, and $3(27 \%)$ received honors. Of people with normal self- esteem, 51 (59\%) received a non-honors grade, and $35(41 \%)$ received honors. The Pearson chi-squared score was 0.74 , with a p-value of 0.39 . Further analysis was conducted on individual questions, with no p-values reaching significance.

CONCLUSIONS: No significant correlation was found between self-esteem and an honors grade in the internal medicine clerkship. While this may indicate that grades are not affected by self-esteem, the results are hampered by a low response rate and a relatively small number of students with low self-esteem. A study with a larger population is necessary to further investigate this question.

\section{CORTICOSTEROID USE FOR ACUTE RESPIRATORY TRACT INFECTIONS IN DIRECT TO CONSUMER TELE- MEDICINE}

Evan L. Dvorin ${ }^{3}$; Michael B. Rothberg ${ }^{1}$; Mark N. Rood ${ }^{2}$; Kathryn A. Martinez ${ }^{1} .{ }^{1}$ Cleveland Clinic, Cleveland, $\mathrm{OH} ;{ }^{2}$ Cleveland Clinic Foundation, Chagrin Falls, OH; ${ }^{3}$ Ochsner Medical Center, Jefferson, LA. (Control ID \#3182928)

BACKGROUND: Systemic corticosteroids are not indicated for acute respiratory tract infections (ARTIs), but may provide some symptom improvement for sinusitis and pharyngitis. Research in the outpatient setting shows substantial variation in corticosteroid use for ARTIs by physician and region, with the highest use in the South. ARTIs are the most common diagnosis in direct to consumer (DTC) telemedicine, yet use of corticosteroids in this setting is unknown. The objective of this study was to characterize corticosteroid prescriptions for ARTIs in a large nationwide DTC telemedicine platform. 
METHODS: Data for this study come from the AmericanWell DTC telemedicine platform. Encounters with patients 18 years and older were conducted by board-certified physicians between July 2016 and July 2018. ARTI diagnoses were identified based on ICD10 codes. Encounters resulting in an inhaler prescription or coded with an asthma and/or COPD diagnosis were excluded. Oral corticosteroid and/or antibiotic receipt was determined by National Drug Codes associated with each encounter. Patient and physician geographic location were categorized by U.S. Census region, based on state of residence. To ensure stability of estimates at the physician level, physicians with $<10$ RTI encounters were excluded. Mixed effects logistic regression was used to assess differences in the odds of corticosteroid prescription, accounting for clustering by physician and geographic region. Models included specific ARTI diagnosis, patient age and geographic region, and physician specialty and geographic region. The model also accounted for antibiotic receipt.

RESULTS: The sample included 96,152 ARTI visits with 472 physicians, the majority of whom $(58 \%)$ were board certified in Family Medicine. Most encounters were for sinusitis (45\%), followed by pharyngitis (12\%). Overall, $11 \%$ of ARTI visits resulted in a corticosteroid prescription. Of those, $33 \%$ also received an antibiotic. The mean prescribing rate for individual physicians ranged from $0 \%$ (7\% never prescribed a corticosteroid) to $63 \%$. In the adjusted model, compared to ARTI encounters with Northeastern physicians, those with Southern physicians had higher odds of resulting in a corticosteroid prescription (OR:4.37;95\%CI:2.756.93). Compared to visits with Family Medicine physicians, those with Internal Medicine physicians were more likely to result in a corticosteroid prescription (OR:3.89; 95\%CI:3.17-4.78). Receipt of a corticosteroid was associated with lower odds of antibiotic receipt (OR: 0.29;95\%CI:0.28-0.31).

CONCLUSIONS: Prescription of corticosteroids for ARTIs in DTC telemedicine varied widely by physician. Regional variation suggests that prescribing is driven by physician preference rather than patient demand. Some physicians appear to substitute corticosteroids for antibiotics, but given adverse effects of corticosteroids, this practice should be discouraged.

\section{COST \& UTILIZATION OUTCOMES FOR A TELEMEDICINE- BASED URGENT CARE CLINIC OFFERED TO EMPLOYEES AT A LARGE ACADEMIC MEDICAL CENTER}

Krisda H. Chaiyachati $^{1,2}{ }^{2}$; Christopher K. Snider ${ }^{2}$; Nandita Mitra ${ }^{2}$; Ann Marie Huffenberger ${ }^{3}$; Bill Hanson ${ }^{2,}{ }^{3}$; Gregory Kruse ${ }^{3}$; Katy Mahraj $^{2,}{ }^{3}$; Shivan Mehta ${ }^{2}, 2$; David A. Asch ${ }^{1,2}$. ${ }^{1}$ The University of Pennsylvania, Philadelphia, PA; ${ }^{2}$ University of Pennsylvania, Philadelphia, PA; ${ }^{3}$ University of Pennsylvania Health System, Philadelphia, PA. (Control ID \#3185179)

BACKGROUND: Employers are increasingly turning to telemedicine services to address low acuity urgent care needs among their insured employees. These efforts aim to reduce costs, but their effectiveness is unknown. Since July 2017, Penn Medicine has offered a free telemedicine-based urgent care clinic (FirstCall) to all health system insured employees and adult dependents, an estimated 50,000 covered lives. FirstCall is staffed 24/7, 365 days per year by Penn Medicine nurse practitioners who conduct livetelemedicine appointments and coordinate follow-up care. We evaluate the impact of FirstCall on health care costs.

METHODS: This study is a retrospective claims-based analysis comparing per episode costs for employees using FirstCall with employees treated conventionally for the same diagnoses between July 2017 to September 2018. To account for selection bias, we conducted 1:3 nearest neighbor matching between FirstCall and
non-FirstCall encounters using propensity scoring based on the encounter diagnosis, season, and employee's gender, age, employee or beneficiary status, median income of employee's ZIP code, and Charlson Comorbidity Score. Once matched, an employee's future encounters were excluded from matching. We used a generalized linear regression model to estimate per episode cost differences incurred within 3 days adjusting for employees' prior costs and utilization. As sensitivity analyses, we repeated these measures within 7-day and 30-day time windows. To estimate whether FirstCall substituted for conventional care, rather than inducing unnecessary care, we compared encounter rates for respiratory tract infections (the top diagnosis at $39 \%$ of all visits) before and after FirstCall was available using an interrupted time series analysis.

RESULTS: Our cohort included 1,335 FirstCall and 3,965 matched non-FirstCall users. Each FirstCall encounter avoided an average of $\$ 215$ ( $\$ 161$ to $269 ; \mathrm{p}<0.001$ ) due to fewer visits to conventional care settings such as outpatient clinics, urgent care centers, and emergency departments. Results were unchanged with extended time windows. Rates for respiratory tract infection visits did not increase after FirstCall was offered.

CONCLUSIONS: FirstCall visits resulted in significant per episode cost savings. FirstCall's operational costs are fixed, therefore FirstCall's per episode costs are volume sensitive (i.e., more visits, lower per episode costs). Based on these estimates, FirstCall is budget neutral for Penn Medicine when 13 or more visits occur per day. These favorable per episode costs might be undermined if FirstCall's convenience induced utilization for symptoms that otherwise would have been ignored. However, these concerns are not supported given similar utilization rates for respiratory tract infections before and after FirstCall. Telemedicine services may be financially promising for academic medical centers with employer-sponsored health plans who offer in-house telemedicine services and care coordination as an employee benefit.

\section{COST COMPARISONS OF OPIOID USE DISORDER TREATMENT PATHWAYS}

Marc Larochelle ${ }^{1}$; Omid Ameli ${ }^{2}$; Sarah E. Wakeman ${ }^{3}$; Christine Chaisson $^{2}$; Jeffrey McPheeters' ${ }^{2}$; Francisca Azocar ${ }^{4}$; Darshak Sanghavi ${ }^{2}$; William Crown ${ }^{2} .{ }^{1}$ Boston University School of Medicine and Boston Medical Center, Boston, MA; ${ }^{2}$ OptumLabs, Cambridge, MA; ${ }^{3}$ Massachusetts General Hospital, Charlestown, MA; ${ }^{4}$ Optum Behavioral Health, San Francisco, CA. (Control ID \#3185417)

BACKGROUND: Nearly 3 million Americans have opioid use disorder (OUD). The relative cost of initial OUD treatment strategies is unknown. We aimed to compare total cost of care across common OUD treatment pathways in a large, nationally representative sample.

METHODS: We conducted a retrospective cohort analysis using the OptumLabs ${ }^{\circledR}$ Data Warehouse of de-identified claims for privately insured and Medicare Advantage enrollees in a large U.S. health plan. We included individuals 16 years with OUD and contiguous enrollment for three months before and after index OUD diagnosis date. We identified OUD as 1 inpatient or 2 outpatient claims for opioid dependence or 1 claims for opioid dependence/use/abuse plus experiencing an overdose, medication for opioid use disorder, detox, or injection-related infection. We examined treatments received in the 12 weeks after cohort entry and clustered subjects into one of six pathway designations: 1) no treatment, 2) inpatient detox/rehab with/without medication for OUD, 3) buprenorphine/methadone, 4) naltrexone, 5) only behavioral health inclusive of intensive outpatient ( $\mathrm{BH}$ - intensive), and 
6) only behavioral health including other types of services ( $\mathrm{BH}$ other). The primary outcome was total cost of care, based on paid claims, in the 90 days after cohort entry. We used generalized linear models adjusting for age, sex, race, insurance type, and baseline comorbidities.

RESULTS: We identified 42,232 individuals with OUD between $1 / 1 / 2015$ and $9 / 30 / 2017$. The most common treatment pathway was $\mathrm{BH}$ - other $(52.7 \%)$, followed by buprenorphine/methadone $(19.1 \%) ; 5.9 \%$ received no treatment (Table). Unadjusted mean 90 day costs were $\$ 16,885$ for those who received no OUD treatment. Compared with no treatment, adjusted cost ratios ranged from 0.53 (95\% CI:0.50-0.56) for buprenorphine/ methadone to 1.98 (95\% CI:1.87-2.10) for detox/rehab (Table).

CONCLUSIONS: In this large cohort of individuals with OUD and commercial or Medicare Advantage insurance, receipt of buprenorphine/methadone was associated with lower total 90 day costs compared with no treatment or other treatment options. Improving access to buprenorphine and methadone as the initial treatment for OUD may decrease costs compared with inpatient or outpatient behavioral health approaches.

\section{COST-EFFECTIVENESS OF AN ELECTRONIC HEALTH RECORD-BASED INTERVENTION TO PREVENT WEIGHT REGAIN}

Cindy L. Bryce ${ }^{2}$; Heather Tomko ${ }^{2}$; Kathleen Mary Mctigue ${ }^{2}$; Jonathan Arnold ${ }^{4}$; Gary Fischer ${ }^{2}$; Bethany Barone Gibbs ${ }^{2}$; Rachel Hess $^{3}$; Kimberly Huber ${ }^{2}$; Laurey Simkin-Silverman ${ }^{2}$; Dana Tudorascu ${ }^{2}$; Molly Conroy ${ }^{1}$. ${ }^{1}$ University of Utah, Salt Lake, UT; ${ }^{2}$ University of Pittsburgh, Pittsburgh, PA; ${ }^{3}$ University of Utah, Salt Lake City, UT; ${ }^{4}$ University of Pittsburgh Medical Center, Pittsburgh, PA. (Control ID \#3183039)

BACKGROUND: Maintaining intentional, healthy weight loss poses a major behavioral health challenge, yet it is key to preventing and managing weight-related diseases such as diabetes and heart disease. Few studies have compared alternative approaches for avoiding weight regain after intentional loss and evaluated their cost effectiveness.

METHODS: Maintaining Activity and Nutrition through Technology-Assisted Innovation in Primary Care (MAINTAIN-pc) was a randomized controlled trial of primary care patients $(n=196)$ who had intentionally lost at least $5 \%$ of their body weight. It compared the impact of an EHR-based self-monitoring system, either alone (tracking-only) or with personalized coaching, on weight regain. Participants recorded weight, plus diet/exercise information (calories, fat grams, steps, other physical activity) over time. Using 24-month data for costs (coaching time, participant monitoring frequency) and outcomes (weight change, quality of life using SF-36 data), we developed a Markov model to assess the cost- effectiveness of coaching relative to tracking-only for avoiding weight regain. Other parameters (hourly wages) relied on publicly available data.

RESULTS: Frequent self-monitoring was associated with better weight outcomes, and the coached participants monitored more consistently than tracking-only participants. Both groups reported good quality of life regardless of weight, resulting in only small gains in quality-adjusted life-years (QALYs) with coaching. When used in the analyses, we initially defined the effectiveness measure as percent weight regain and calculated a base case incremental cost-effectiveness ratio (ICER) for coaching relative to tracking-only that was less than $\$ 500$ per percent weight regain avoided (societal perspective). For the reference case using QALYs, the ICERs were $\$ 23,740$ and $\$ 4,940$ per QALY gained from societal and health care system perspectives, respectively.
Results were robust in sensitivity analyses - the main driver of costs is not personnel time, but rather participant time devoted to consistent monitoring. This was varied widely in probabilistic sensitivity analyses, and coaching was found to be cost-effective in $58 \%$ of model iterations (societal perspective) and $64 \%$ of model iterations (health care system perspective), based on an acceptability threshold of $\$ 100,000$ per QALY gained.

CONCLUSIONS: Maintenance of intentional, healthy weight loss is essential for realizing long-term health benefits. Although only minimal differences in quality of life between the two groups were reported, more weight regain was seen in the tracking-only group. Modeling the projected outcomes for longer time horizons would help inform overall health benefits and economic costs resulting from maintenance of intentional weight loss. Deployment of technologies that reduce the time needed to monitor health behaviors could also be useful for improving the costeffectiveness of the coaching intervention.

\section{COST-EFFECTIVENESS OF USING FINANCIAL INCEN- TIVES TO IMPROVE GLYCEMIC CONTROL: RESULTS OF A PILOT RCT}

Leonard E. Egede $^{1}$; Rebekah J. Walker ${ }^{1}$; Clara E. DismukeGreer $^{2}$; Heather Prigmore ${ }^{1}$; Joni S. Williams ${ }^{1}$; Aprill Z. Dawson ${ }^{1}$; Jennifer A. Campbell ${ }^{1}$. ${ }^{1}$ Medical College of Wisconsin, Milwaukee, WI; ${ }^{2}$ Medical University of South Carolina, Charleston, SC. (Control ID \#3185393)

BACKGROUND: Behavioral economics research theorizes the utility of providing financial incentives to overcome a natural tendency to discount the consequences of poor health behaviors. Given the daily self-management required for good glycemic control, individuals with diabetes may benefit from use of financial incentives in an effort to promote regular activities that improve glycemic control. However, the cost of using financial incentives for the benefit of improved glycemic control has not been tested. The aim of this project was to determine the costeffectiveness of three financial incentive structures in obtaining a $1 \%$ drop in $\mathrm{HbA} 1 \mathrm{c}$.

METHODS: 60 African Americans with type 2 diabetes were randomized to one of three financial incentive structures and followed for 3 months. Group 1received a single incentive at 3 months for A1c reduction, group 2 received a two-part incentive for home testing of glucose and A1c reduction at 3 months and group 3 received a multiple component incentive for home testing, attendance of weekly telephone education classes and A1c reduction at 3 months. The primary clinical outcome was A1c reduction within each arm at 3 months, and cost for each arm was calculated based on the cost of the intervention, cost of primary care, ER, and other health care visits during the 3-month time frame, and cost of work days missed from illness. Incremental cost effectiveness ratios (ICER) were calculated based on the intervention cost for each arm to achieve a $1 \%$ drop in $\mathrm{HbA} 1 \mathrm{c}$ and were bootstrapped with 1,000 replications.

RESULTS: Incentive structures led to significant reductions in A1c at 3 months with a mean reduction for group $1=1.25 \%$, mean reduction for group $2=1.73 \%$, and mean reduction for group $3=1.74$. Mean per person costs for each group were calculated at $\$ 3,765$ for group $1, \$ 4,649$ for group 2, and $\$ 3,315$ for group 3 . The incremental cost effectiveness ratio to decrease A1c by $1 \%$ was $\$ 1,100$ for all three arms, however, bootstrapped standard errors differed with group 1 having twice the variation around the ICER coefficient as groups 2 and 3. ICERs were statistically significant for groups 2 and $3(\mathrm{p}<0.001)$ indicating they are cost effective interventions for achieving a $1 \%$ drop in HbA1c. 
CONCLUSIONS: Given ICERs of prior diabetes interventions range from $\$ 1,000-\$ 4,000$, a cost of $\$ 1,100$ per $1 \%$ decrease in $\mathrm{HbA} 1 \mathrm{c}$ is a promising intervention, suggesting costs incurred through financial incentive systems have long term cost savings in health for diabetes populations. Multi-component financial incentive structures seem to have less variation in the cost effectiveness, and result in larger over drops in $\mathrm{HbA1c}$ suggesting more frequent and smaller incentives may be more effective in initiating behavior change than larger but less frequent incentives.

\section{COUNT YOUR PENNIES: COSTS OF MEDICAL RESIDENT DEVIATION FROM CLINICAL PRACTICE GUIDELINES IN USE OF TESTING ACROSS 3 UNANNOUNCED STANDARD- IZED PATIENT CASES}

Eli Cahan ${ }^{2}$; Kathleen Hanley ${ }^{2}$; Andrew B. Wallach ${ }^{1}$; Barbara Porter ${ }^{2}$; Lisa Altshuler ${ }^{2}$; Sondra Zabar ${ }^{2}$; Colleen C. Gillespie ${ }^{2}$. Bellevue Hospital, New York, NY; ${ }^{2}$ NYU School of Medicine, New York, NY. (Control ID \#3185399)

BACKGROUND: Diagnostic tests account directly for 5\% of healthcare costs, but influence decisions constituting $70 \%$ of health spending. Only $5 \%$ of ordered labs are actually "high value," depending on clinical circumstances. Low-value tests, defined as not appropriate for a given clinical scenario, are ordered in one in five clinic visits. Up to $\$ 750$ billion is spent on these low-value tests, contributing to the estimated one-quarter to one-third of healthcare spending is on wasteful services. We sought to quantify test-specific low-value ordering behaviors in urban outpatient clinics across three standardized patient cases.

METHODS: Unannounced standardized patients (USPs - highly trained actors portraying patients with standardized case presentations) were introduced into medicine residents' primary care clinics in a large urban, safety net hospital over the past five years. The USPs simulated three common outpatient clinical scenarios: a "Well" visit, a visit with a chief complaint of "Fatigue," and a visit with a diagnosis of "Asthma." Diagnostic orders were extracted via retrospective chart review for these standardized visits. For each scenario, appropriateness of diagnostic testing was determined by reference to United States Preventative Services Task Force (USPSTF) and relevant specialty society clinical practice guidelines (CPGs). "Wasteful" (over-ordered) tests were defined as those not explicitly indicated for the given scenario. Costs were derived from GoodRx.com according to local ZIP codes.

RESULTS: The most commonly wasteful tests for the Asthma case were $\mathrm{CBC}$ (8\% of 170 visits) and Chem-7 (6\%), though the relative risk of over-ordering TSH was $3.8 \mathrm{x}$ that of other scenarios. The most commonly over-ordered tests for the Fatigue case were LFTs (14\% of 148 visits) and HBV (5\%), with LFTs ordered up to 15-fold more frequently than in other scenarios. The most commonly over-ordered tests for the Well case were BMP (35\% of 124 visits), CBC (15\%), LFTs (15\%), and HBV (11\%) ordered at rates up to $6.3 \mathrm{x}, 2.0 \mathrm{x}, 14.2 \mathrm{x}$, and $7.4 \mathrm{x}$ higher than other scenarios. Finally, the average per patient excess costs were $\$ 8.27(+/$ \$1.76), \$6.79 (+/- \$4.5), and \$23.5 (+/- \$9.34) for Asthma, Fatigue, and Well cases respectively.

CONCLUSIONS: Inappropriateness in test ordering patterns were observed through USP simulated cases. Certain tests (CBC, BMP, LFTs, and HBV) were more likely used wastefully across cases. Between cases, specific tests were ordered in an inappropriate manner (such as TSH for Asthma, LFTs for Fatigue, and BMP for Well visits). The per patient direct cost of low value testing rose above \$20 per visit for the Well visit, though the Fatigue case exhibited the most variation. Notably, this excludes downstream (indirect) costs inestimatable from standardized encounters alone. Knowledge of wasteful utilization patterns associated with specific clinical scenarios can guide interventions targeting appropriate use of testing.
CULTURE AND COGNITION? THE ASSOCIATION BETWEEN ACCULTURATION AND SELF-REPORTED MEMORY PROBLEMS AMONG MIDDLE-AGED AND OLDER LATINOS IN THE NATIONAL HEALTH AND EXAMINATION SURVEY (NHANES), 1999 TO 2014

Alejandra Casillas ${ }^{1}$; Li-Jung Liang ${ }^{2}$; Stefanie D. Vassar ${ }^{2}$; Arleen F. Brown ${ }^{2}$. ${ }^{1}$ David Geffen School of Medicine @ UCLA, Los Angeles, CA; ${ }^{2}$ UCLA, Los Angeles, CA. (Control ID \#3186113)

BACKGROUND: The Immigrant Paradox suggests that immigrant Latinos are "protected" from negative health outcomes, like cognitive decline, versus US-born and/or acculturated Latinos. However, eight waves from the Hispanic Established Populations for the Epidemiologic Study of the Elderly recently showed that older age at migration (i.e. less acculturated) was associated with higher risk for cognitive decline. Other studies suggest that the effects of acculturation on health vary between Latino ethnic groups (i.e. Mexican vs. non-Mexican/other Latino). We examined the association between a well-established acculturation scale and self-reported memory problems (a "warning sign" for cognitive decline) over multiple time periods among Latinos in a nationally representative sample.

METHODS: These analyses included 5038 NHANES participants 45 years, in four 4-year time periods, between 1999-2014, who reported their race/ethnicity as Latino/Hispanic and were further categorized as "Mexican" or "other Latino." The primary outcome was self-reported memory problems (yes vs. no). An acculturation score (0/lowest - 3/highest) was calculated by giving 1 point for each of these characteristics: being born in the United States, speaking predominantly English, and living in the United States 20 years. We used generalized linear regression models with appropriate sample weights accounting for unequal probabilities of selection, oversampling, and non-response to examine the association between acculturation and memory problems, across ethnicity and age groups.

RESULTS: There was no difference in memory problems between the two ethnic groups for both middle aged (age 45-64 years) and older Latinos (age 65 years). Acculturation scores were higher for the Mexican groups for both age groups, across all time periods. Linear regressions showed a significant negative association between acculturation score and memory problems in older Latinos in three of the four latter time periods (range of estimates: -0.56 to -0.28 ; $p$-values $=0.05$ ). When examining older Latinos by Mexican vs. other Latinos, acculturation and self-reported memory problems remained negatively correlated in both ethnic groups.

CONCLUSIONS: We observed that acculturation scores were negatively associated with self-reported memory problems (higher score $\rightarrow$ lower \% memory problems) among older Latinos, suggesting that acculturation may be protective against one of the early "warning signs" for cognitive decline. This finding is counter to the traditional theory of the Immigrant Paradox, wherein acculturation is linked to worsened health outcomes. These results point to a need for more aggressive efforts to address cognitive decline among less acculturated, older Latinos. Finally, the effects of acculturation on self-reported memory problems may be related to factors such as education, socioeconomic status and other social determinants, and warrant further investigation.

\section{CURRENT LANDSCAPE OF CENTRALIZED IIS-BASED REMINDER/RECALL TO IMPROVE IMMUNIZATION RATES FOR ADULT IMMUNIZATIONS}

Dennis Gurfinkel $^{1}$; Laura P. Hurley ${ }^{2}, 1$; Alison Saville ${ }^{1}$; Brenda Beaty ${ }^{3,}$; Heather Roth ${ }^{4}$; Amanda Dayton ${ }^{5}$; Alison $\mathrm{Chi}^{5}$; Allison Kempe ${ }^{1,6}{ }^{1}{ }^{1}$ University of Colorado, Aurora, $\mathrm{CO} ;{ }^{2}$ Denver Health, Denver, $\mathrm{CO} ;{ }^{3}$ Unviersity of Colorado, Aurora, CO; ${ }^{4}$ Colorado Department of Public Health and Environment, Denver, CO $;{ }^{5}$ American Immunization Registry Association, Washington DC, DC; ${ }^{6}$ University of Colorado and Children's Hospital Colorado, Aurora, CO. (Control ID \#3185546) 
BACKGROUND: Adult immunization information is increasingly being uploaded into Immunization Information Systems (IIS) nationally; the National Vaccine Advisory Committee has called for adult immunization documentation in IIS as part of their Standards for Adult Immunization Practice. Centralized IIS-based Reminder/Recall (C-R/R) performed by an IIS to centrally send out reminders to individuals about needed immunizations has been successful at increasing childhood immunization rates and is a promising approach to increasing adult immunization rates. Little is known about IIS managers' experiences regarding $\mathrm{C}-\mathrm{R} / \mathrm{R}$ for adult vaccines and perceived barriers to its use and sustainability.

METHODS: The objective of this study is to describe among IIS managers: 1) IIS capabilities related to performing $C-R / R$, 2) previous $C-R / R$ experience and future interest in conducting $C-R / R$ for adult vaccines, and 3) perceived barriers to $C-R / R$ use and sustainability. A survey was developed in collaboration with the American Immunization Registry Association (AIRA). Surveys were sent electronically to all IIS managers in the U.S. and territories via AIRA's member network in July 2018. Up to 6 email and phone call follow-ups to non-responders occurred across a two-month period. A $\$ 25$ gift card was offered to respondents.

RESULTS: Fifty-seven of 62 IIS managers (92\%) responded to the survey. All but one IIS reported the capability of receiving adult immunization data from various immunization providers. For $24 \%$ (13/55) of IIS, immunization reporting from primary care practices and public health departments for adult immunizations is mandated. Eighty-three percent (45/54) of respondents reported they have the capability to identify eligible patients needing immunizations at a county level and $76 \%(41 / 54)$ at a zip-code level. Sixtyone percent $(34 / 56)$ of IIS have ever conducted C-R/R. Of the 25 who responded, 9 (36\%) had done C-R/R so for adult vaccines. Thirty-four percent $(19 / 56)$ reported they are "very likely" to conduct C-R/R in the next 6 months; 28\% (7/25) for adult vaccines. The most commonly reported barriers to performing $\mathrm{C}-\mathrm{R} / \mathrm{R}$ were lack of staff (78\%), competing demands (76\%), and cost (63\%). Lack of support from leadership was a minor barrier $(7 \%)$

CONCLUSIONS: Most IIS have the capabilities to perform C-R/R within a geographical area and contain adult vaccination data. The majority of IIS have at least tried $\mathrm{C}-\mathrm{R} / \mathrm{R}$, although a minority for adult vaccines. Sustaining and expanding $\mathrm{C}-\mathrm{R} / \mathrm{R}$ may require further training from successful programs regarding best practices for efficient $\mathrm{C}-\mathrm{R} / \mathrm{R}$ methods in order to limit staff involvement and costs. Successful programs for adult $C-R / R$ should be disseminated and replicated by other IIS.

DECLINING USE OF PRIMARY CARE AMONG COMMERCIALLY INSURED ADULTS IN THE US, 2008-2016

Ishani Ganguli ${ }^{1}$; Zhuo $\mathrm{Shi}^{2}$; Aarti Rao ${ }^{3}$; Kristin N. Ray ${ }^{4}$; Ateev Mehrotra ${ }^{2}$. ${ }^{1}$ Brigham and Women's Hospital, Chestnut Hill, MA; ${ }^{2}$ Harvard Medical School, Boston, MA; ${ }^{3}$ Icahn School of Medicine, New York, NY; ${ }^{4}$ University of Pittsburgh, Pittsburgh, PA. (Control ID \#3183630)

BACKGROUND: Primary care is a key component of the healthcare system and U.S. healthcare reform efforts, yet per capita visit rates to primary care physicians (PCPs) are declining. Using national commercial claims data, we explored potential mechanisms underlying this decline.

METHODS: We examined 2008-2016 claims data for adults (18-64 years old) enrolled with a large commercial insurer for at least one month in a given year. We determined annual visit rates to physicians and advanced practitioners (APs) providing primary or specialty care and to alternative settings (telemedicine, urgent care, retail clinic, emergency department). We stratified results by member demographic and clinical characteristics, baseline county-level visit rates, and visit characteristics including primary diagnosis type. We estimated linear trends for each subgroup; two-sided $\mathrm{p}$ values were significant at $<0.005$ (given multiple testing) unless otherwise specified.

RESULTS: Over the 8-year-period, PCP visit rates declined by $26.2 \%$ (167.5 visits per 100 member-years in 2008, 123.6 in 2016). More adults had no visits in 2016 (32.5\% of adults) than in 2008 (25.6\%). The PCP visit decline was driven by problem-based visits $(-40.8$ visits, $26.5 \%$ decline) while preventive visit rates increased (+6.4 visits, $42.7 \%$ increase). The decline was larger for visits addressing low-acuity conditions ( -15.2 visits, $45.4 \%$ decline) than for those addressing chronic conditions ( -11.4 visits, $29.2 \%$ decline; $\mathrm{p}=0.01)$ and larger in counties with higher PCP visit rates in 2008 (-43.7 visits, $11.5 \%$ decline in top quartile of counties vs. 3.1 visits, $1.3 \%$ increase in bottom quartile $\mathrm{p}=0.69$ ). When examining member characteristics, we found larger decline among those living in a zip code with median income $0-200 \%$ of federal poverty level (FPL) (-45.9 visits, $26.4 \%$ decline vs -22.0 visits, $14.2 \%$ decline for $>400 \%$ FPL). Among members with a medical claim in a given year, the decline was greater for those without a chronic condition (-23.3 visits, $4.9 \%$ decline vs. -14.8 visits, $1.7 \%$ decline for those with a chronic condition). Over this period, a greater share of PCP visits were subject to deductible $(+11.7$ visits, $140 \%$ increase), while mean, inflation-adjusted out-of-pocket costs increased by $\$ 9.89$ per visit (33.6\% increase). We observed a rise in visit rates to other clinicians (APs, +9.6 visits; specialists, +4.1 visits $\mathrm{p}=0.98$ ) and settings (+9.1 visits), though these increases did not fully offset the decrease in PCP visits.

CONCLUSIONS: The decline in PCP visit rates was only partly explained by greater use of alternative clinicians and settings. Declines were larger for healthy members, for low-acuity condition visits, and in regions with higher-baseline visit rates, suggesting some of the foregone visits may have been unneeded. The larger decline among low-income patients coupled with the rise in deductibles and out-of-pocket costs imply financial barriers deterring PCP visits. Further work is needed to explore mechanisms in other populations.

\section{DEMENTIA IS AN INDEPENDENT RISK FACTOR FOR CARE FRAGMENTATION AMONG OLDER ADULTS}

Sara Turbow ${ }^{1}$; Mohammed K. Ali ${ }^{2}$; Nikkil Sudharsanan ${ }^{3} .{ }^{1}$ Emory University School of Medicine, Atlanta, GA; ${ }^{2}$ Emory University Rollins School of Public Health, Atlanta, GA; ${ }^{3}$ Harvard T.H. Chan School of Public Health, Boston, MA. (Control ID \#3178904)

BACKGROUND: Of the over 35 million hospital admissions annually in the United States, 5 million result in readmissions, and over $25 \%$ of readmissions occur at hospitals different from the one the patient was previously discharged from. These fragmented readmissions are associated with poor outcomes including a nearly two-fold higher risk for mortalityand additional $\$ 2000$ in healthcare costs per admission. Patients with Alzheimer's Disease and related dementias (ADRD) are more likely to be readmitted, but it is unknown if these readmissions are more likely to be fragmented or if dementia is itself a risk factor for fragmented readmissions. METHODS: We analyzed data from the 2014 National Readmissions Database. Our analysis included adults 65 years old with an ICD-9 diagnosis code for ADRD. We defined fragmentation of care as a readmission to a hospital different from the one the patient was previouslydischarged from. We limited our analysis to the first two admissions in 2014. We estimated and compared prevalence of care fragmentation among older adults with and without ADRD. We compared demographic and clinical characteristics of ADRD and non-ADRD patients using chi-squared and t-tests. We used multivariable logistic regression to examine the relationship between ADRD and interhospital care fragmentation, adjusted for demographic and clinical variables. We used SAS 9.4 (Cary, NC) for all data analysis.

RESULTS: There were a total of 1,050,660 patients aged 65 years with readmissions in 2014. Of those, $8.0 \%$ had a diagnosis of ADRD. The prevalence of fragmented readmissions was higher for individuals with ADRD than those without $(26.3 \%$ v $23.0 \%, \mathrm{p}<0.001)$ (Table 1$)$. In unadjusted regression models, dementia was associated with $19 \%$ higher odds of fragmented readmissions (95\% CI 1.17-1.21) that persisted after adjustment for clinical and socio-demographic variables (Table 1). 
CONCLUSIONS: Older adults with ADRD, who are already at risk for poor outcomes post-hospitalization, are at higher risk for readmissions to nonindex hospitals compared to those without ADRD. Further research on the causes and effects of fragmented care for patients with ADRD is needed to reduce the health and economic burden for this important and growing patient population.

\section{DEPRESSION SCREENING OF ADULT ASIAN PATIENTS IN AN URBAN SAFETY-NET PRIMARY CARE CLINIC}

Jennifer Dong ${ }^{1,2}$; Mamadou Bah ${ }^{3}$; Sarika Modi ${ }^{1,2} .{ }^{1}$ NYU Langone Health, New York, NY; ${ }^{2}$ Bellevue Hospital, New York, NY; ${ }^{3}$ Monroe College, New York, NY. (Control ID \#3186002)

BACKGROUND: Depression and suicide rates among Asian Americans are high, making them the only racial group in New York City for whom suicide is one of the top ten causes of death. The underutilization of mental health services by Asians compared to other ethnic groups is a disparity likely attributed to the strong stigma around mental illness and poor selfrecognition of depression. Universal depression screening in primary care has led to increased detection and treatment of depression in all patients. We sought to understand the prevalence of depression through completion of the Patient Health Questionnaire (PHQ2) screener among Asian primary care patients at a large, urban, tertiary safety-net medical center.

METHODS: In our cross-sectional study we analyzed a registry of patients who identified their race as "Asian" and visited our primary care clinic (Bellevue Hospital Adult Primary Care Clinic) from July through December 2016. Chart review was performed to assess the completion and response rates of the Patient Health Questionnaire (PHQ2) and determine prevalence of depression. When possible, we identified countries of origin for each patient.

RESULTS: We identified a total of 1105 Asian patients. Regions of origin included East Asia (27.7\%), Indian Subcontinent (29.3\%), Southeast Asia (25.2\%). Countries most represented were China, Bangladesh, the Philippines, and Tibet. For $11 \%$ of patients, there was no documentation of country of origin. PHQ2 completion rates by country were comparable to our overall clinic screening rate of $64 \%$ : China $65.0 \%$, Bangladesh $67.5 \%$, Philippines $64.1 \%$, Tibet $61.3 \%$. The rates of positive PHQ2 scores were: China $6.4 \%$, Bangladesh 7.3\%, Philippines 4.9\%, Tibet 7.4\%, comparable to the national depression rate of $8 \%$. For English-speakers compared to non-English speakers, the rates of PHQ2 completion varies: China $65.8 \%$ vs. $64.9 \%$, Tibet $68.8 \%$ vs. $57.1 \%$, Bangladesh $64.0 \%$ vs. $70.8 \%$, Philippines $63.1 \%$ vs. $80.0 \%$. Of the positive PHQ2 screens, $72.7 \%$ of the Chinese patients had PHQ9 scores 10 or higher, indicating moderate depression or worse, as did $92.3 \%$ of Bengali, $16 \%$ of Tibetan, and $60 \%$ of Filipino patients. CONCLUSIONS: Disparities in mental health screening and treatment continue to affect the Asian immigrant population, in part driven by culture and language differences. In our large, safety-net primary care clinic, the completion rate for depression screens among Asians are similar to the clinic's overall monthly PHQ2 screening rate of $64 \%$. This analysis indicates that Asian patients in our clinic who screen positive on the PHQ9 may have moderate to severe depression, consistent with the phenomenon that depression in Asians is often under-detected, and therefore undertreated. Further studies are needed to assess the role of culture and language on depression screening for each Asian sub-group. We are conducting further analysis to review the rates of depression diagnosis and treatment patterns in this population.

\section{DERIVATION AND VALIDATION OF A RISK ASSESSMENT MODEL FOR DRUG-RESISTANT PATHOGENS IN COMMUNITY-ACQUIRED PNEUMONIA}

Michael B. Rothberg ${ }^{1}$; Pei-Chun $\mathrm{Yu}^{1}$; Peter K. Lindenauer ${ }^{2}$; Sarah Haessler ${ }^{2}$; Peter Imrey ${ }^{1} .{ }^{1}$ Cleveland Clinic, Cleveland, OH; ${ }^{2}$ Baystate Medical Center, Springfield, MA. (Control ID \#3186282)
BACKGROUND: Antibiotic resistance is a major concern for patients hospitalized with community-acquired pneumonia (CAP). Risk factors for organisms resistant to recommended CAP therapy are known, but there are few validated risk assessment models for identifying such patients. We derived a risk assessment model using a large sample of CAP patients with blood or respiratory cultures. We then validated our model and compared it to the Drug Resistance in Pneumonia (DRIP) score.

METHODS: This cross-sectional study assessed adult patients admitted with pneumonia from 2010-2015 to 168 US hospitals that provided administrative and microbiologic data to Premier, Inc. Patients were identified by a combination of ICD-9 codes, chest $\mathrm{x}$-ray, and antibiotic treatment. We also required patients to have blood or respiratory cultures performed by hospital day 1 . We used stepwise multiple logistic regression to screen potential predictors of infections resistant to CAP therapy (ceftriaxone/azithromycin or levofloxacin): sociodemographics (age, sex, race, marital status); admissions, antibiotics and drug resistance in the past year; comorbidities, and severity of illness. For parsimony, we culled predictors based on prevalence, odds ratio, and clinical and statistical judgment. We developed our models in an $80 \%$ derivation set, reserving a $20 \%$ simple random sample for model validation. We then compared our model's receiver operating curve characteristics in the validation set to those of the DRIP score.

RESULTS: The derivation set contained 110,087 patients, of whom 2985 (2.7\%) had cultures that grew organisms resistant to CAP therapy. A 21predictor stepwise model was reduced to a 14-predictor model. Factors associated with antibiotic resistance in the reduced model were previous admission to hospital or cultured organism resistant to CAP therapy in the past year; admission from a skilled nursing facility; pressure ulcer or wound care; low functional status; chronic pulmonary disease; paralysis; ICU admission; SOFA score; vasopressors; mechanical ventilation; and male sex. Alcohol abuse predicted lower risk of resistance. The model had a c-statistic of 0.70 in the derivation set. In the validation set the model's c-statistic was 0.69 vs. 0.61 for the DRIP score. Predicted probabilities across deciles of risk ranged from $1.5 \%$ to $8.5 \%$ and calibration was good.

CONCLUSIONS: A risk assessment model with 14 predictors had good calibration and moderate discrimination. It outperformed the DRIP score in the validation set. After further validation, the model could be used in clinical practice to identify patients who would benefit from empiric broad spectrum antibiotic coverage.

\section{DESCRIBING THE CLINICAL APPROACH TO DIAGNOSIS AND TREATMENT OF PATIENTS WITH HYPOACTIVE SEXUAL DE- SIRE DISORDER}

Sharon J. Parish ${ }^{1}$; Zainab Shahpurwala ${ }^{2}$; Rahul Ravindranath ${ }^{2}$; Amod Athavale $^{2}$; Nandini Hadker ${ }^{2}$; Michelle Lim-Watson ${ }^{3}{ }^{1}$ Weill Cornell Medical College, New York, NY; ${ }^{2}$ Trinity Partners, Waltham, MA; ${ }^{3}$ AMAG Pharmaceuticals, Inc., Waltham, MA. (Control ID \#3184893)

BACKGROUND: Hypoactive sexual desire disorder (HSDD) is defined as persistent diminished or lack of sexual desire accompanied by distress and affects approximately $10 \%$ of US women aged 18-44 years. Despite the high prevalence of HSDD, there is a limited understanding of the condition among physicians. The aim of this study was to understand the clinical approach to diagnosis, treatment, and unmet needs of patients with HSDD.

METHODS: Data from 312 medical charts from 103 US board-certified obstetricians/gynecologists ( $\mathrm{OB} / \mathrm{GYNs})$, psychiatrists, and primary care physicians, which included family and internal medicine physicians, were collected and analyzed using an IRB-approved case report form that mimicked a typical patient chart. Physicians also completed an IRB-approved web-based questionnaire. Physicians treated/managed 2 patients diagnosed with HSDD and 5 patients diagnosed with female sexual dysfunction (FSD) each month. Inclusion criteria for medical charts were females 18 years old who had been diagnosed with HSDD.

RESULTS: Among the 103 physicians, $41 \%$ were OB/GYNs, $18 \%$ were family medicine physicians, $14 \%$ were internal medicine physicians, and 
$28 \%$ were psychiatrists; $16 \%$ reported a subspecialty in sexual medicine. Most primarily practiced in an office-based setting (92\%) and spent most of their time engaged in patient care. Physicians reported a high level of comfort diagnosing and treating HSDD and initiated conversations about overall sexual health approximately half of the time. Medical charts for premenopausal $(57 \%)$ and postmenopausal patients (43\%) were analyzed; their mean age was 45 years. The time to diagnosis from the first physician visit for HSDD symptoms was approximately 4 months. The Decreased Sexual Desire Screener (DSDS) was used in only $14 \%$ of the medical charts to diagnose HSDD, and the Female Sexual Function Index and Female Sexual Distress Scale-Revised were used in only $2-4 \%$ of the medical charts to assess sexual function. While $72 \%$ of physicians agreed that HSDD is a medical condition, 30-40\% did not associate hallmark symptoms of HSDD with the condition. Additionally, challenges exist in their ability to distinguish the symptoms of HSDD from other comorbid conditions, including other sexual problems such as decreased libido (83\%), anorgasmia (56\%), and sexual aversion disorder (50\%), which are frequently associated with HSDD. Physicians reported a high level of unmet need regarding efficacious treatments for HSDD and reported only moderate levels of satisfaction with currently utilized treatments for HSDD.

CONCLUSIONS: While physicians agree that HSDD is a medical condition, a gap remains in the timely and appropriate diagnosis of HSDD using validated tools such as the DSDS and in differentiating HSDD from other FSDs. Additionally, there are a lack of efficacious treatment options for the treatment of HSDD. More education is needed to help physicians better identify HSDD symptoms and appropriately diagnose the condition.

\section{DESIGN OF A MULTIFACETED STRATEGY TO INCREASE UP- TAKE OF THE USPSTF HYPERTENSION SCREENING RECOM- MENDATIONS IN A PRIMARY CARE NETWORK: USING THE BEHAVIORAL CHANGE WHEEL FRAMEWORK TO MAP BAR- RIERS TO THEORY-INFORM INTERVENTION COMPONENTS. Nathalie Moise ${ }^{1}$; Erica Phillips ${ }^{2}$; Jacob Julian ${ }^{1}$; Anusorn Thanataveerat ${ }^{1}$; Carmela Alcantara $^{3}$; Eileen Carter $^{3}$; Joseph Schwartz ${ }^{1}$; Siqin Ye ${ }^{1}$; Andrea T. Duran $^{1}$; Daichi Shimbo ${ }^{1}$; Ian M. Kronish ${ }^{1} .{ }^{1}$ Columbia University Medical Center, New York, NY; ${ }^{2}$ Weill Cornell Medical College, New York, NY; ${ }^{3}$ Columbia University, New York, NY. (Control ID \#3185313)}

BACKGROUND: The U.S. Preventive Services Task Force (USPSTF) now recommends out-of-office blood pressure (BP) testing to exclude white-coat hypertension prior to hypertension diagnosis. Despite improved availability and coverage of home and 24-hour ambulatory BP monitoring (HBPM, ABPM), both are infrequently used to confirm diagnoses. Few if any studies of interventions to increase the use of out-of-office BP testing exist. We describe a theory-informed process for developing an implementation strategy to improve the uptake of out-of-office testing for hypertension diagnosis.

METHODS: We used the Behavior Change Wheel framework, a multistep process for mapping barriers to theory-informed behavior change techniques. First, a multi-disciplinary team (medicine, psychology, nursing) conducted geographically diverse provider focus groups $(n=63)$ to explore barriers to provider referral for out-of-office testing. Second, the team used content analysis to categorize barriers according to capability, opportunity, and/or motivation constructs (COM-B model). Third, the team independently mapped COM-B barriers to corresponding intervention functions (i.e., a list of 9 broad categories of ways an intervention can change behavior, e.g., education), and behavioral change techniques (i.e., a standardized language for describing the active ingredients in behavior change interventions). Lastly, the team conducted key informant interviews $(n=12)$ with practice administrators, medical directors, providers, medical assistants, financial advisors, and nursing staff to refine and finalize intervention components.

RESULTS: Top ranked provider barriers to completing out-of-office BP testing included: lack of knowledge about the guideline (capability); limited time to instruct patients about HBPM, costs of HBP devices, and challenges with accessing ABPM testing (opportunity); and concerns about ability to get patients to successfully and accurately complete tests and whether benefits of testing outweigh costs (motivation). We determined that the most feasible, effective behavioral change techniques would include delivering information about the consequences of out-of-office testing, demonstration/instruction on how to perform out-of-office HBPM and ABPM testing, feedback on completion rates of out-of-office testing, and adding objects to the environments/prompts and cues via a computerized clinical decision support (CDS) tool along with a culturally-tailored, locally accessible ABPM testing service.

CONCLUSIONS: In theory, a multicomponent implementation strategy of provider education, reminders, and decision support coupled with an ABPM testing service would be needed to implement out-of-office BP testing for the diagnosis of hypertension. We are currently testing the effect of our locally-adapted intervention on the change in the proportion of eligible patients who complete out-of-office BP testing in a 1:1 cluster randomized trial across 8 socioeconomically diverse clinics.

\section{DESIGN THINKING TO IMPROVE PATIENT EXPERIENCE}

Erin M. Rainosek ${ }^{1}$; Erika Bowen ${ }^{1}$; Thomas Pederson ${ }^{1}$; Musarrat Nahid ${ }^{1}$; Temple Ratcliffe ${ }^{3}$; Luci Leykum ${ }^{2} .{ }^{1}$ UT Health San Antonio, San Antonio, TX; ${ }^{2}$ STVHCS / UTHSCSA, San Antonio, TX; ${ }^{3}$ UTHSCSA, San Antonio, TX. (Control ID \#3173061)

BACKGROUND: Hospitalization can be overwhelming for patients. Diagnostic uncertainty is common, and patients interact with many professionals who present information that may seem confusing. We bring a design thinking perspective to creating more patient-responsive hospital care, allowing their stories to drive the creation of a human-centered experience. Design thinking is about understanding needs and generating multiple ideas to create innovative, far reaching solutions. It focuses on how the system is experienced by the user. By using a rapid-cycle approach of obtaining input followed by ideation, prototyping, and testing, we offer a unique approach to improving experience and outcomes.

METHODS: We sought to explore patients' experiences of hospitalization from a design thinking perspective. As part of an ongoing collaborative care implementation, we interviewed patients, asking about their needs, communication, family involvement, and understanding of care plan. Patients were identified by a case manager based on cognitive function, acuity of illness, and English fluency. Interviews were recorded and transcribed. We adopted the design thinking method from IDEO, an international design firm, and coded interviews for unmet expectations, workarounds, tensions, problems, and needs. We aggregated responses with similar content into themes that allow us to identify opportunities for innovation.

RESULTS: We utilized interviews of 45 patients conducted over 3 months. Transcripts were coded by two members of the research teams, who had $74 \%$ agreement. Disagreements were resolved by consensus. Unmet expectations included patients' perception that information was not explained, or their expectation that physicians have answers to all of their questions, not recognizing that there might be diagnostic or treatment uncertainty. A key problem related to understanding important information versus day to day "noise." Patients used workarounds to better understand the care plan. An example includes self-checking consistency across the team to ensure accuracy in their care. Specific areas identified for improvement included understanding the care plan during admission, medicine reconciliation, involving family and caregivers, health literacy, coordination of services across specialties, and continuity of care transitions.

CONCLUSIONS: Design thinking offers a novel approach that focuses on understanding patients' experience as the basis for improving care delivery. Using patients' perspectives enables us to identify opportunities for innovation and ways to approach those opportunities that we would not otherwise discover by learning not just what patients say and do, but how they think and feel. In turn, we can understand their motivation for health in 
terms of personal priorities rather than disease. These insights will allow us to better address their actual needs. Our work demonstrates the feasibility and utility of applying this framework to understanding hospital care from patients' perspectives.

\section{DETERMINANTS OF PRIMARY HEALTHCARE UTILIZATION AMONG LGBT INDIVIDUALS IN HUDSON VALLEY REGION}

Suman Pal; Nicole Zagelbaum; Anila Kumar; Alina Kifayat; Christopher Nabors; Rebecca Newman. Westchester Medical Center, Valhalla, NY. (Control ID \#3184495)

BACKGROUND: The Lesbian, Gay, Bisexual and Transgender (LGBT) community represents a vulnerable population that has historically faced disparities in healthcare. Young adult members of the LGBT community are more likely to have mental health issues such as anxiety, depression and substance abuse, attempt suicide, be homeless, and have high rates of transmission of sexually transmitted infections including human immunodeficiency virus (HIV). They also face barriers to primary healthcare and preventive services due to fear of bias, social isolation, and fear of victimization. The United States department of health and human services Healthy People 2020 initiative aims to reduce disparities and improve LGBT health. In keeping with that goal, we aim to understand the facilitators and barriers to primary healthcare utilization for LGBT community in the Hudson Valley region.

METHODS: We utilized a cross-sectional study design using an anonymous online survey modified from the Gelberg-Anderson model of healthcare utilization among vulnerable populations. The survey was administered to a convenience sample of volunteers from the mailing list of the "LOFT" LGBT Community Services Center, a local LGBT organization, via email link to an online survey (SurveyMonkey (B). Data were analyzed using descriptive statistics. The study was approved by the New York Medical College Institutional Review Board (IRB).

RESULTS: In the first two weeks the survey was open, 26 persons responded. Of these, self-reported gender identities were: cisgender male (55\%), cisgender female (33\%), transgender (5\%, FTM), transgender $(5 \%$, MTF) and 8 individuals who chose not to reveal their gender identity. With respect to sexual preferences, $20 \%$ identified as lesbian, $40 \%$ as gay, $12 \%$ as bisexual, $16 \%$ as asexual and $12 \%$ as heterosexual. While $87 \%$ of respondents believed that they should follow up with a primary care provider (PCP ) regularly, 30\% did not currently have a PCP. Of respondents who had a PCP, $35 \%$ were not comfortable discussing their sexual orientation and sexual well-being with that provider. In choosing an LGBT friendly PCP, the most important factors were prior positive experience with a provider and positive reference from friends or family. Access to mental health services was the second most important factor in choosing a LGBT friendly clinic. Other important factors were the use of appropriate gender and LGBT friendly language in verbal and written communication. Distance to LGBT friendly PCP and fear of discrimination were identified as key barriers to accessing healthcare by our respondents.

CONCLUSIONS: The results of this study indicate that factors related to LGBT community access to and comfort with primary care identified in prior published reports are also present in the Hudson Valley of New York. Data from this study will be utilized to make changes to the Patient Centered Medical Home (PCMH) at the Westchester Medical Center Adult Primary Care Center to better serve the local LGBT population.

DEVELOPING CHRONIC OPIOID USE AND OPIOID USE DISORDER FOLLOWING THE FIRST OPIOID PRESCRIPTION IN A POPULATION OF EMERGING ADULTS

J. Deanna Wilson ${ }^{1,1}$; Kaleab Abebe ${ }^{1}$; Kevin Kraemer ${ }^{2}$; Elizabeth Miller ${ }^{3}$; David Kelley ${ }^{4}$; Julie M. Donohue ${ }^{5} .{ }^{1}$ University of Pittsburgh, Pittsburgh, PA; ${ }^{2}$ University of Pittsburgh Medical Center, Pittsburgh, PA; ${ }^{3}$ Children's Hospital of PIttsburgh of UPMC, Pittsburgh, PA; ${ }^{4}$ Pennsylvania
Department of Human Service, Harrisburgh, PA; ${ }^{5}$ University of Pittsburgh Graduate School of Public Health, Pittsburgh, PA. (Control ID \#3179164)

BACKGROUND: As one out of every five deaths among young adults is now a result of opioid-related morbidity and mortality, it is critical to understand prescription opioid use in adolescent and young adult (AYA) populations as it is the first exposure to opioids for many young people. We aim to characterize a population of AYA following the first opioid prescription to identify risk factors for chronic opioid use and time to opioid use disorder (OUD).

METHODS: We conducted a retrospective cohort analysis using data from Pennsylvania Medicaid enrollees from 2007-2016. We used National Drugs Codes in pharmacy claims to identify those age 10-21 years at time of the first opioid prescription. We included those with 6 months of continuous enrollment in Medicaid before and 12 months following the initial prescription. We used International Classification of Diseases, Ninth/ Tenth Revision codes to define depression, anxiety, attention deficit disorder, and substance use disorders, including OUD, in the year following the initial prescription. Chronic opioid use was defined as receiving three or more opioid prescriptions. We used a mixed effects logistic regression model to estimate odds of chronic opioid use following the initial opioid prescription, and frailty time-to-event models to estimate time to first OUD diagnosis after accounting for county-level clustering using SAS. This study was deemed exempt by University of Pittsburgh IRB.

RESULTS: Among the 189,477 AYA who received an initial opioid prescription, the mean age at first prescription was 16.52 years (SD 2.80). Enrollees had high rates of comorbid mental illness with 24,637 (13\%) diagnosed with depression, attention deficit hyperactivity disorder $(\mathrm{N}=25,681 ; 14 \%)$, or anxiety $(10,719 ; 6 \%)$. By 12 months, approximately $14.3 \%(\mathrm{n}=18,864)$ received two or more prescriptions following the initial opioid prescription and $6.2 \%(n=8,087)$ were given a diagnosis of OUD. Racial and ethnic minorities were less likely to both receive chronic opioids (OR 0.627 for Hispanic and OR 0.691 for Non-Hispanic Black) and develop OUD (HR 0.396 for Hispanic and 0.403 for Non-Hispanic Black). Females were more likely to receive chronic opioids (OR1.316) but less likely to be given a diagnosis of OUD than males (HR 0.887). Increases in duration of first opioid script from mean of 4.51 days and dosage from mean of 57.7 morphine milligram equivalents per day were associated with increased odds of chronic opioid use and also elevated risk of OUD.

CONCLUSIONS: Our findings demonstrate even short opioid prescriptions in a population of previously opioid-naive young people are associated with high rates of chronic opioid prescribing. Over 1 in 20 AYA received an OUD diagnosis within 12 months of the first opioid prescription suggesting rapid escalation of opioid use and misuse in this population. Even short, low-dose opioid prescriptions seem to infer risk to vulnerable populations of emerging adults.

\section{DEVELOPMENT AND EVALUATION OF A PREDIABETES DE- CISION AID ON PATIENT-CENTERED OUTCOMES}

Matthew J. O'Brien ${ }^{1}$; Kenzie A. Cameron ${ }^{1}$; Namratha Kandula ${ }^{1}$; Geoffrey C. Williams ${ }^{2}$; Angie Fagerlin ${ }^{3}$; Ronald T. Ackermann ${ }^{1} .{ }^{1}$ Northwestern University, Chicago, IL; ${ }^{2}$ University of Rochester, Rochester, NY; ${ }^{3}$ University of Utah, Salt Lake City, UT. (Control ID \#3185982)

BACKGROUND: Eighty four million American adults have prediabetes, making diabetes prevention a public health priority. Despite the availability of two evidence-based treatments to prevent diabetes in this population - intensive lifestyle interventions (ILI) and metformin — neither is widely used. Patient-level barriers to initiating treatment include limited awareness or knowledge about prediabetes, inaccurate perceptions of their risk of developing diabetes, and low motivation to adopt treatment. Decision aids are the most widely studied method for communicating risk information and presenting treatment options, yet examples do not exist for prediabetes. 
METHODS: With national stakeholders in diabetes prevention, our group developed a prediabetes decision aid and evaluated its impact on patientcentered outcomes. Inclusion criteria were age 18 years, prediabetes according to standard glycemic definitions, and BMI $25 \mathrm{~kg} / \mathrm{m} 2$. Exclusion criteria were diabetes, dementia, and previous use of ILI or metformin. This pretest-posttest trial of 40 primary care patients tested whether exposure to the decision aid impacted the following 3 outcomes: 1) knowledge about diabetes prevention, 2) decisional conflict; and 3) intention to adopt ILI and/or metformin. Each of the outcomes was assessed with widely used, validated questionnaires. We used paired t-tests and McNemar's tests to examine pretest-posttest differences in the continuous and dichotomous study outcomes, respectively.

RESULTS: The study participants were 95\% female and 68\% Hispanic/ Latino, with a mean age of 46.5 years. Exposure to the decision aid increased knowledge about diabetes risk, but the pre-post change was not significant. Mean decisional conflict scores were significantly decreased from pretest $(31.0 \pm 15.7)$ to posttest $(20.9 \pm 17.1), \mathrm{p}<0.001$. There were also significant reductions in all of the subscale scores for decisional conflict. The proportion of participants who intended to adopt ILI increased from $70 \%$ at baseline to $88 \%$ after exposure to the decision aid $(\mathrm{p}=0.02)$. There was no significant change in the proportion of participants who expressed intention to initiate metformin. The performance of the decision aid did not differ in subgroups defined by sex, language of administration (English vs. Spanish), or educational attainment ( high school completion vs. lower).

CONCLUSIONS: This study demonstrates the feasibility and preliminary effectiveness of using a decision aid for diabetes prevention in primary care. Brief exposure to the decision aid resulted in modest improvements in knowledge about diabetes risk, significant reductions in decisional conflict, and significant increases in participants' intention to adopt ILI. Collectively, these findings suggest that our prediabetes decision aid is promising and may improve treatment decisions among patients with prediabetes in primary care.

\section{DEVELOPMENT OF A NOVEL MEASURE OF TIMELY ACCESS TO PRIMARY CARE SERVICES}

Matthew R. Augustine ${ }^{2,1}$; Adam J. Batten ${ }^{3}$; Karin M. Nelson ${ }^{3,}$; Peter J. Kaboli ${ }^{6,5}$. ${ }^{1}$ Icahn School of Medicine at Mount Sinai, New York, NY; ${ }^{2}$ Department of Veterans Affairs, New York City, NY; ${ }^{3}$ Department of Veterans Affairs, Seattle, WA; ${ }^{4}$ University of Washington, Seattle, WA; ${ }^{5}$ Carver College, Iowa City, IA; ${ }^{6}$ Deparment of Veterans Affairs, Iowa City, IA. (Control ID \#3169176)

BACKGROUND: Timeliness is a critical component of access and optimizing patient-centered care. Current objective measures of timely access to primary care (PC) may not account for patient-specific urgency or reflect modern scheduling practices. Our objective was to develop a novel measure for identifying clinic performance in fulfilling next-day and walk-in requests.

METHODS: We performed a retrospective, longitudinal analysis identifying clinic level success rates in fulfilling timely care requests within 48 hours from June 2014 to June 2017. We analyzed VA administrative data from 174 PC clinics located in 8 regional Veteran Integrated Service Networks. We defined a timely care request as any appointment type designated as walk-in or first next available. Successful fulfillment of timely care included a completed encounter within 48 hours and could occur in the same clinic with any PC provider, a different clinic within the same facility or regional network, a non-VA visit paid by VA, hospital admission, or via secure message or telehealth visit. Failure to provide timely care was determined if the patient was not seen within 48 hours, the appointment was cancelled by the clinic, or the appointment was fulfilled in the emergency department for conditions considered PC treatable as designated by a validated algorithm. We used Bayesian hierarchical regression to model the annual timely care success rate within each clinic, while controlling for age, sex, race, rurality, and patient risk. For risk, we used Nosos, a co-morbidity score that predicts costs. In addition, we also tested two- and three-way interactions between rurality, patient risk, and time.

RESULTS: From 2014 to 2017, the number of timely care requests increased $17 \%$ from 240,191 to 280,874 with $32-40 \%$ of all patients requesting timely care. Of all timely care requests, $85-90 \%$ were successfully fulfilled: $98 \%$ by VA with $61-64 \%$ in PC, $31-33 \%$ in other VA clinics, and $3-4 \%$ in VA mental health clinics. Non-VA care and secure messaging fulfilled $2 \%$ and $1 \%$, respectively. Of the 174 clinics, we identified a mean overall success rate of $87-88 \%$ with an upper bound of $95-97 \%$. The distribution of success widened overtime with lower bound of the Bayesian credible interval decreasing from $72 \%$ to $64 \%$. Fourteen clinics $(11 \%)$ exhibited significant variability in their baseline timely care success rate and another 6 clinics had significantly decreasing success rates over time. All two and three-way interactions were not significant.

CONCLUSIONS: We developed a novel metric of timely care, which included patient perceived urgency, modern scheduling practices, and multiple care delivery modalities. Robust models identified a high level of timely care success across VA clinics and identified clinics struggling to deliver timely care. While further validation is needed, this measure offers a novel procedure to assess access while providing real-time, actionable information to clinical leadership.

\section{DEVELOPMENT OF COMMUNICATION SKILLS ACROSS THE UME - GME CONTINUUM}

Amanda Mari; Ruth Crowe; Kathleen Hanley; Deirdre Apicello; Norkila Sherpa; Lisa Altshuler; Sondra Zabar; Adina Kalet; Colleen C. Gillespie. NYU School of Medicine, New York, NY. (Control ID \#3185402)

BACKGROUND: The core Entrustable Professional Activities medical school graduates should be able to perform on day 1 of residency provides a framework for readiness for residency. Communication skills are an essential foundation for these core EPAs and yet there have been few studies that describe communication competence across the UME-GME continuum. We report on our OSCE-based assessment of communication skills from the first few weeks of medical school to the first year of medicine residency.

METHODS: Assessment of communication is consistent in our OSCE program across UME and GME. Domains include Information Gathering (5 items), Relationship Development (6 items), and Patient Education (3 items) and these are assessed via a behaviorally anchored checklist (scores $=\%$ well done) that has strong reliability and validity evidence. In this study, we report on 3 multi-station OSCEs: the Introductory Clinical Experience (ICE) OSCE that occurs within the first weeks of medical school; the high-stakes, pass/fail Comprehensive Clinical Skills Examination (CCSE) OSCE that is fielded after clerkship year; and the Medicine Residency Program's PGY 1 OSCE. Across 3 classes of medical school (2014-2016) we have complete data for the 24 students who continued on in our Medicine Residency (and who provided consent to include their educational data in an IRB-approved registry). Analyses focus on differences in communication skills over time and between cohorts and the relationship between communication skills measured in medical school and those assessed in residency.

RESULTS: Communication scores show significant improvement through medical school (but not into residency) in Information gathering $(\mathrm{ICE}$ mean $=56 \%$; CCSE mean $=76 \%$; PGY1 mean=77\%) $(\mathrm{F}=11.54$, $\mathrm{p}<.001, \mathrm{ICE}<\mathrm{CCSE})$ and in relationship development (ICE $=59 \%$; $\mathrm{CCSE}=78 \%$; $\mathrm{PGY} 1=74 \%$ ( $\mathrm{F}=10.68, \mathrm{p}<.001, \mathrm{ICE}<\mathrm{CCSE})$. Mean patient education skills, however, increase significantly across all 3 time points ( $32 \%$ to $50 \%$ to $65 \% ; \mathrm{F}=31.00, \mathrm{p}<.001$ ). Patterns are similar across cohorts except that the Class of 2016 means increase from CCSE to PGY1. Regression analyses show that CCSE information gathering scores are more strongly associated with PGY performance than ICE scores (Std Beta=.32 vs .06), while for relationship development, it is the 
ICE scores that are more strongly associated (Std Beta=.40 vs .24). ICE and CCSE patient education skills have associations with PGY1 skill of similar size (Std Beta=.30 and .28).

CONCLUSIONS: Findings, despite the small sample, suggest a clear developmental trajectory for communication skills development and that information gathering and patient education skills may be more influenced by medical school than relationship development. That communication skills seem to level out in PGY1 highlights need for reconsolidation as clinical complexity increases. Results can inform theory development on how communication skills develop and point to transitions where skills practice/feedback may be particularly important.

\section{DIABETES PREVALENCE AND CONTROL IN THE UNIN- SURED OF THE TAMPA BAY AREA}

Hadi Joud $^{1}$; Sabbir Mirza ${ }^{1}$; Eslam Mohamed ${ }^{2}$; Hozaifa Tabbaa ${ }^{1}$; Mouaz Tabbaa $^{1}$; Laurie Woodard ${ }^{1}$; Rahul Mhaskar ${ }^{1}$; Chighaf Bakour ${ }^{1}$; Sayeef Mirza ${ }^{1} .{ }^{1}$ University of South Florida, Tampa, FL; ${ }^{2}$ Lake Erie College of Osteopathic Medicine, Bradenton, FL. (Control ID \#3181454)

BACKGROUND: Diabetes is a common disease in many populations but places an especially heavy burden on the uninsured or underinsured, who are likely to utilize free healthcare services. These services, including free clinics, may provide patients with suboptimal treatment for diabetes and its many important comorbidities, such as hypertension and hyperlipidemia. The prevalence, management, and control of diabetes in uninsured or underinsured populations are not well studied and have only recently been examined in the Tampa Bay area. This study aims to characterize the prevalence and management of diabetes as well as predictors of good diabetes control in the uninsured of the Tampa Bay area.

METHODS: The study data was collected through a retrospective chart review of adult diabetic patients seen in nine different free clinics in the Tampa Bay area in 2016 and 2017. Sociodemographic variables, including race, ethnicity, age, and gender were collected along with chronic disease diagnoses, treatments, and relevant lab results. The data was analyzed in SAS and a logistic regression analysis was run to identify predictors of good control.

RESULTS: Out of 6815 patients seen in nine free clinics, 1323 patients were reported to be diabetic (19.41\%). 787 of those $(59.5 \%)$ had uncontrolled diabetes, defined by a HbA1C value of greater than $7 \%$. The mean HbA1C for the study population was $7.98 \%$. Demographically, the population was found to be mostly White $(76.3 \%)$, Hispanic $(52.4 \%)$, and female $(54.1 \%)$. The mean BMI for diabetics was 32.3 , with $59 \%$ of the population being obese and only $11.9 \%$ being of a normal weight. Compared to poorly controlled diabetics, those with good control were more likely to be female (59\% vs. $56 \%$ ), employed ( $58 \%$ vs. $50 \%$ ), of normal weight $(13.1 \%$ vs. $11.5 \%)$, and to have never smoked (67\% vs. $62 \%$ ). Logistic regression analysis showed that unemployment is a significant predictor of poor control $(\mathrm{OR}=1.92,95 \%$ CI $1.03,3.60)$ after adjusting for age, gender, race, comorbidities, and BMI. When stratified by gender, unemployment is a significant predictor of poor control in males $(\mathrm{OR}=4.13,95 \%$ CI $1.36,12.5)$, but not in females $(\mathrm{OR}=1.40,95 \%$ CI $0.62,3.18)$.

CONCLUSIONS: Diabetes control is suboptimal in uninsured people visiting free clinics in the Tampa Bay Area, as evident by $\mathrm{HbA} 1 \mathrm{C}$ levels. Unemployment appears to be a significant predictor of poor control in male diabetics, but not in females. Further research is needed to understand the mechanisms underlying this difference. Nutrition education initiatives may be beneficial in improving control of diabetes and decreasing the high rates of obesity by addressing the patients' lifestyles. Additionally, improving diabetes management programs at free clinics and equipping them with more resources may improve the quality of care and patient compliance.

\section{DIAGNOSIS CODES VS. LABORATORY VALUES FOR IDEN- TIFICATION OF ACUTE KIDNEY INJURY}

Amrit Vasdev ${ }^{1,2}$; Rachel Giblon ${ }^{1}$; Joel A. Hickman ${ }^{1}$; Arnar J. Jónsson ${ }^{3}$; Runolfur Palsson ${ }^{3}$; Kianoush Kashani ${ }^{1}$; Bjorg Thorsteinsdottir ${ }^{1,1}$. ${ }^{1}$ Mayo Clinic, Rochester, MN; ${ }^{2}$ University of Minnesota, Minneapolis, MN; ${ }^{3}$ Landspitali University Hospital, Reykjavik, Iceland. (Control ID \#3185265)

BACKGROUND: Acute Kidney Injury (AKI) is a common syndrome especially in the context of multi-morbidity and can result in serious adverse outcome including mortality and chronic kidney disease (CKD). There have been concerns about the lack of sensitivity of billing codes in identifying this important complication. Therefore we sought to identify AKI episodes based on creatinine values in the electronic medical record and test its ability to detect AKI episodes as compared to ICD9 diagnosis code and chart review.

METHODS: Using a population based registry database from the Rochester Epidemiology Project, we identified patients $>65$ years of age who had a serum creatinine measured between 1996-2006. For each patient, each creatinine value was assessed for the presence of AKI using a previously validated macro, following, Kidney Diesase Improving Global Outcome (KDIGO) guidelines, defined as an increase in serum creatinine $0.3 \mathrm{mg} / \mathrm{dl}$ in less than 48 hours or an increase from baseline of $0.3 \mathrm{mg} / \mathrm{dl}$ using older creatinine values on file. We used AKI based on chart review as gold standard.

RESULTS: Of 29,494 patients identified there were 18,024 times ICD9 billing diagnosis code for Acute Kidney Injury was used in 6292 patients. ICD billing codes were only $55 \%$ sensitive compared to AKI diagnosis based on the laboratory based macro. Using chart review as the gold standard this algorithm had a positive predictive value of $64 \%$ and negative predictive value of $91 \%$.

CONCLUSIONS: Diagnosis codes alone miss almost half of all AKI cases. Using an automated search based on the KDIGO guidelines shows high agreement with the previously validated code and significantly increases the sensitivity while maintaining high specificity. Our findings suggest that billing codes may be insufficiently sensitive to assess patient complexity across population and that one should be careful to draw firm conclusions, change policy or make practice recommendations solely based on big data based on billing diagnosis.

\section{DIAGNOSTIC PITFALLS: OPERATIONALIZING A NEW PAR- ADIGM TO UNDERSTAND AND PREVENT DIAGNOSTIC ER- RORS}

Harry Reyes Nieva ${ }^{1,2}$; Elise L. Ruan ${ }^{1,3}$; Mayya Volodarskaya ${ }^{1,4}$; Sumit Agarwal $^{1}$; Adam Wright ${ }^{1,}{ }^{2}$; Hardeep Singh ${ }^{5}$ Gordon D. Schiff ${ }^{1,2}$. ${ }^{1}$ Brigham and Women's Hospital, Boston, MA; ${ }^{2}$ Harvard Medical School, Boston, MA; ${ }^{3}$ Tufts University School of Medicine, Boston, MA; ${ }^{4}$ University of Chicago Pritzker School of Medicine, Chicago, IL; ${ }^{5}$ Michael E. DeBakey Veterans Affairs Medical Center and Baylor College of Medicine, Houston, TX. (Control ID \#3184699)

BACKGROUND: Most diagnostic errors are rooted in cognitive and system failures with multifactorial origins. This complexity requires innovative approaches to better anticipate, detect and prevent them. We developed a new construct — diagnostic pitfalls — to account for both system and cognitive issues and create awareness of what can go wrong in diagnosis. We mined various data sources to uncover diagnostic errors and distilled disease-specific clinical pitfalls to analyze their types and patterns. METHODS: We collected data from multiple sources to identify diagnostic error cases and delineated potential pitfalls, which we defined as clinical situations, patterns, or vulnerabilities related to errors leading to missed, delayed, or wrong diagnosis. Sources included a literature review, AHRQ Web M\&M cases, institutional ambulatory M\&Ms and risk management reports, focus groups with specialists, and primary care 
malpractice closed claims for 5 years from 2 leading malpractice insurers in MA. To provide a framework for characterizing diagnostic errors, we used two previously published taxonomies Diagnosis Error Evaluation and Research (DEER) and Reliable Diagnosis Challenges (RDC). We performed an iterative thematic content analysis to derive both general and disease-specific diagnostic pitfalls. From qualitative review of these pitfalls, we created a taxonomy of generic types of pitfalls and then classified pitfalls from each case into one or more of these categories.

RESULTS: We identified a total of 549 diagnostic pitfalls. The most common diagnoses for which diagnostic errors and pitfalls were identified included cancer ( $\mathrm{n}=186)$ : colon (46) lung (43), prostate (34), breast (33), thyroid (7), melanoma (6), bladder (5), liver (5), Hodgkin's (4), renal (3); followed by other diagnoses: myocardial infarction (29), sepsis (16), stroke (16), pulmonary embolism (11), appendicitis (4), chronic heart failure (4), diabetes mellitus (4), disk herniation (3). Analysis of the top 10 diagnosis' pitfalls $(\mathrm{n}=241)$ included: failures in follow-up $(28 \%)$, limitations of test or exam not appreciated (26\%), disease A mistaken for disease B (24\%), risk factors not adequately appreciated (16\%), atypical presentation (15\%), counter-diagnosis cues overlooked (10\%), communication failures between primary care and specialists (7\%), urgency not fully appreciated (3\%), chronic disease presumed to account for new symptoms (2\%), evolving symptoms not monitored (2\%).

CONCLUSIONS: Diagnostic pitfalls is a new and useful construct to identify disease-specific pitfalls from diagnostic error cases. Analysis identified generic types of pitfalls and a taxonomy to categorize them, thus revealing the relative frequency of certain types of pitfalls. This work can serve as the basis for heightening situational awareness (e.g. interventions to detect and warn clinicians of pitfall situations) and building additional cognitive safety nets to prevent harm from falling into these traps.

\section{DIASTOLIC DYSFUNCTION IN PATIENTS WITH CHRONIC OBSTRUCTIVE PULMONARY DISEASE A META-ANALYSIS} Angelina Zhyvotovska ${ }^{2}$; Denis Yusupov ${ }^{1}$; Haroon Kamran ${ }^{2}$ 1, Samy McFarlane $^{2}$; Tarik Al-Bermani ${ }^{2}$; Samir T. Kumar ${ }^{2}$; Rishard Abdul ${ }^{2}$. ${ }^{1}$ SUNY Downstate Medical Center, Brooklyn, NY; ${ }^{2}$ SUNY Downstate, Brooklyn, NY. (Control ID \#3159469)

BACKGROUND: Chronic obstructive pulmonary disease (COPD) is an important cause of morbidity and mortality around the world. Estimates show that in 2030 COPD will become the third leading cause of death worldwide. Additionally heart failure is a disease of great morbidity and mortality whose presence is estimated to increase by $46 \%$ from 2012 to 2030. Whether left ventricular diastolic dysfunction (LVDD) is a comorbidity in patients with COPD varies widely in literature. The association between DD and COPD is an important one to know given that dyspnea is a complaint shared between both illnesses. The objective of this metaanalysis was to determine if the prevalence of the diastolic dysfunction is increased in chronic obstructive pulmonary disease (COPD) patients as evidenced by echocardiographic parameters.

METHODS: We used a time-and-language-restricted search strategy to identify 4912 studies. 4905 were excluded, and we did a systematic review and meta-analysis of the seven remaining case control studies conducted to compare echocardiographic parameters to diagnose diastolic dysfunction in patients with COPD and controls.

RESULTS: A total of 7 studies with 764 subjects were included. The total of 4905 articles were excluded based on not meeting our inclusion criteria, case control studies performed after the year 2000. Duplicates were excluded. Eighteen studies meeting the inclusion criteria were reviewed in full; 9 articles were removed due to not clearly stating the findings, and two studies were removed because the results for their control groups were unclear. Therefore, a total of 7 studies were included $(n=764)$. Our study found the prevalence of diastolic dysfunction was higher among COPD subjects versus control group patients (OR; 1.446,
C.I: $1.063-1.829$, Z: $7.399 ;$ p: 0.000$)$. The heterogeneity observed for the pooled analysis was Q-value; $17.855 \mathrm{df}(\mathrm{Q})$; 5 P-value: 0.003, I-squared; 71.997; Tau2; 0.699, suggesting appropriate study selection and moderate heterogeneity. There were no differences in left ventricular ejection fraction.

CONCLUSIONS: Patients with COPD are more likely to have echocardiographic parameters of diastolic dysfunction. The diastolic dysfunction in this population, based on our data, is likely due to lung hyperinflation, chronic hypoxia, and ventricular interdependence: ventricular remodeling and deviation of the interventricular septum towards the left ventricle. Our study confirms the association between DD and COPD and sheds light that new treatment strategies that focus on preventing further cardiac dysfunction may improve mortality in COPD. Thus, more studies are needed to ascertain the effect of the treatment of COPD for the prevention of DD, a growing health burden.

DID THAT PCP PRESCRIBE THAT DRUG? CONCEPTUAL AND METHODOLOGICAL CHALLENGES IN DEFINING PRESCRIPTIONS INITIATED BY PCPS

Diana E. Norwich ${ }^{1,}$; William L. Galanter ${ }^{3}, 3$; Jennie B. Jarrett ${ }^{3}$; Maria Mirica $^{2}$; Diane L. Seger ${ }^{2}{ }^{4}$; Julie Fiskio ${ }^{2}$; Tewodros Eguale ${ }^{5}$, 2; Alejandra Salazar ${ }^{2}$; John P. Cashy ${ }^{6}$; Jennifer A. Hale ${ }^{6}$; Jeffrey A. Linder ${ }^{7}$; Bruce Lambert ${ }^{8}$; Suzanne Falck ${ }^{9}$; Lynn A. Volk ${ }^{4}$; Gordon D. Schiff ${ }^{2},{ }^{10} .{ }^{1}$ Tufts University School of Medicine, Boston, MA; ${ }^{2}$ Brigham and Women's Hospital, Boston, MA; ${ }^{3}$ University of Illinois at Chicago, Chicago, IL; ${ }^{4}$ Partners HealthCare System, Somerville, MA; ${ }^{5} \mathrm{MCPHS}$ University, Boston, MA; ${ }^{6} \mathrm{VA}$ Pittsburgh Healthcare System, Pittsburgh, PA; ${ }^{7}$ Northwestern University Feinberg School of Medicine, Chicago, IL; ${ }^{8}$ Northwestern University, Evanston, IL; ${ }^{9}$ University of Illinois Hospital and Health Sciences System, Chicago, IL; ${ }^{10}$ Harvard Medical School, Boston, MA. (Control ID \#3185583)

BACKGROUND: To understand the prescribing behaviors of primary care physicians it is necessary to identify their newly initiated prescriptions. While several studies invoke the concept of an initial prescription, definitions vary, and no standard criteria have been defined. As part of a multi-institutional study to understand variation in conservative prescribing practices, we explored and developed approaches to defining and identifying newly initiated prescriptions, attempting to account for various potential sources of error.

METHODS: We studied EMR prescription data from all PCPs $(\mathrm{N}=60)$ at University of Illinois at Chicago (UIC) and a sample $(\mathrm{N}=23)$ from Brigham and Women's Hospital $(\mathrm{BWH})$ ordered in 2016-2017. After excluding refills, we identified the first patientprescription occurrence during the study period. Data for the preceding two-year period was then reviewed to identify any prior prescriptions. We then sought to identify additional issues that could result in erroneously labelling a prescription as "new."

RESULTS: Of 65,114 prescriptions written by PCPs at BWH, 29,647 (46\%) were identified as newly initiated, while 79,419/ 161,062 (49\%) were newly initiated at UIC. Reviewing the data we identified three additional issues needing to be refined in the algorithm to accurately classify a new prescription: (i) excluding patient-reported prior medications, (ii) accounting for first-time visits, where pre-existing drugs may be misinterpreted as new, and (iii) how to handle drugs (e.g. selected antibiotics) with primarily acute indications that should be considered new even though their record had evidence of prior prescriptions. For issue (iii), a review of prescription data for 19 medications (12,808 total prescriptions) with both acute and chronic indications, showed that 7 were prescribed acutely $>80 \%$ of the time at both institutions, suggesting that prescriptions for these drugs can generally be considered new, while 
the remaining 12 may require specific indications-based rules for categorization as new vs. pre-existing.

CONCLUSIONS: This analysis confirms the importance of developing and adhering to a consistent, measurable, defensible, and reproducible definition of an initial prescription because nearly half of all PCPs' prescriptions in our dataset were not actually newlyinitiated by the prescribing PCP. Using EMR rather than claims data both adds clinical richness and introduces methodological challenges (since longitudinal ordering and fill data is lacking) that must be addressed-most notably, accounting for patient-reported medications, accounting for first-time visits, and medications with both acute and chronic indications. Efforts to determine the percentage of prescriptions in these challenging categories is underway. Addressing these challenges is key not only for informing a definition of a newly-initiated prescription, but also for highlighting the data elements that must be accounted for to facilitate accurate analyses.

\section{DID THE HOSPITAL READMISSION REDUCTION PRO- GRAM RESULT IN DELAYED READMISSIONS JUST AF- TER 30 DAYS?}

Joseph Martinez ${ }^{1,2}$; Ashwin Nathan ${ }^{3,2}$; Jay Giri ${ }^{3,}$ 2; Amol S. Navathe ${ }^{1,2}$. ${ }^{1}$ University of Pennsylvania, Philadelphia, PA; ${ }^{2}$ Leonard Davis Institute, Phialdelphia, PA; ${ }^{3}$ Hospital of the University of Pennsylvania, Philadelphia, PA. (Control ID \#3183402)

BACKGROUND: The Hospital Readmission Reduction Program (HRRP) was enacted in March 2010 and imposed financial penalties beginning in October 2012 for hospitals with higher-than-expected 30-day readmissions for acute myocardial infarction (AMI), congestive heart failure (CHF) and pneumonia. The HRRP has been associated with reduced readmissions for these conditions, but recent evidence has also suggested an increase in mortality for heart failure and pneumonia as an unintended consequence. Because of strong hospital responses to the HRRP, we examined whether hospitals postponed readmissions around the 30-day threshold, resulting in a spike in "delayed" readmission just after 30 days.

METHODS: Hospital admissions for AMI, CHF, and pneumonia were identified between January 2007 and October 2015 using the Medicare Provider Analysis and Review (MedPAR) database, containing admissions for all fee-for-service Medicare enrollees. Stroke was studied as a falsification endpoint because it was not included in HRRP. As in prior literature, we divided the study period into three phases: the pre-HRRP phase (January 2007 to March 2009), the implementation phase (April 2009 to September 2012), and the penalty phase (October 2012 to October 2015). We used an interrupted time-series analysis to examine whether there was an increased probability of readmissions between days 31-35 compared to days 25-30. We estimated Poisson and Quasi-Poisson with slope and intercept changes and adjustments for seasonality by condition and compared the changes in slope for AMI, CHF, pneumonia and stroke in the three phases.

RESULTS: A total of $68,020,501$ admissions were included in this analysis. For AMI, the slopes for readmissions between days 31 to 35 as compared to days 25 to 30 over time were $-0.003,0.001$ and -0.0003 for the three phases, respectively. The change in slope between the pre-HRRP and penalty phase was $0.003(\mathrm{p}=0.31)$. For pneumonia, the slopes for readmissions between days 31 to 35 as compared to days 25 to 30 over time were $0.002,-0.0004$ and 0.001 for the three phases, respectively. The change in slope between the pre-HRRP and penalty phase was $-0.001(\mathrm{p}=0.74)$. For CHF, the slopes for readmissions between days 31 to 35 as compared to days 25 to 30 over time were $0.001,-0.0002$ and -0.001 for the three phases, respectively. The change in slope between the pre-HRRP and penalty phase was $-0.001(\mathrm{p}=0.29)$. For stroke, the changes in slope between days 31 to 35 as compared to days 25 to 30 were $0.002,0.0004$ and -0.002 for the three phases, respectively. The change in slope between the pre-HRRP and penalty phase was $-0.004(\mathrm{p}=0.11)$.

CONCLUSIONS: Using a national sample of Medicare enrollees, we found no association between the start of HRRP and higher likelihood of readmissions for AMI, CHF, or pneumonia just after 30 days. This suggests that hospitals did not differentially delay readmissions from just before to just after 30-days for AMI, CHF, pneumonia or stroke in response to HRRP.

\section{DIFFERENCES IN ED DESTINATION OF EMS TRANSPORT OF CO-LOCATED PATIENTS BY RACE/ETHNICITY}

Amresh D. Hanchate ${ }^{1}$; Michael K. Paasche-Orlow ${ }^{2}$; William E. Baker $^{2}$; Meng-Yun Lin ${ }^{3}$; Souvik Banerjee ${ }^{1}$; James Feldman ${ }^{1} .{ }^{1}$ Boston University School of Medicine, Boston, MA; ${ }^{2}$ Boston University, Boston, MA; ${ }^{3}$ Boston Medical Center, Boston, MA. (Control ID \#3176475)

BACKGROUND: National evidence indicates systematic differences in hospitals where racial/ethnic minorities receive care, with most care obtained in a small proportion of hospitals. As a large proportion of patients hospitalized for acute conditions arrive by emergency medical services (EMS), we examined for patterns in emergency department (ED) destination of EMS transported patients by race/ethnicity. To adjust for residential segregation by race/ethnicity, we used a national sample of Medicare enrollees purposively sampled to enable comparison of transport destination of patients from the same zip code.

METHODS: Using administrative data for a national sample of Medicare enrollees (2006-2012), we selected zip codes with a sizable count (>10) of Hispanic, (non-Hispanic) black and (non-Hispanic) white enrollees and obtained data on all ED visits using EMS. Identifying the most frequent (modal) ED destination for EMStransported white patients from each zip code as the "reference ED" destination, we defined concordance with reference ED as the main outcome. We estimated differences in concordance rates by race/ethnicity, overall and for subgroups by demographics, clinical condition, socioeconomic status and proximity to multiple EDs. We used zip code level fixed effects to limit comparison of patients from same zip code. A secondary outcome was EMS transport to a safetynet ED, defined as the top quartile of hospitals by Medicaid patient share in each hospital referral region. We performed similar analysis of ED visits without using EMS.

RESULTS: In our study cohort of 864,750 enrollees from 4,175 selected zip codes, there were 458,701 ED visits using EMS. Overall $61.3 \%$ of white patients were transported to the reference ED; this rate was lower among black $(-5.3 \%$; 95\% confidence interval [CI], $-6.0 \% \%$ to $-4.6 \%)$ and Hispanic $(-2.5 \%$; $95 \%$ CI, $-3.2 \%$ to $-1.7 \%)$ patients. A similar pattern was found among subgroup of high-risk patients with acute myocardial infarction, heart failure, pneumonia, stroke, sepsis and GI bleed. Concordance rates were lower in areas with multiple nearby EDs; in the largest 16 US cities, concordance rate was $46.1 \%$ among whites, and lower among blacks $(-9.3 \%$; $95 \%$ CI, $-10.9 \%$ to $-7.7 \%)$ and Hispanics $(-2.7 \% ; 95 \%$ CI, $-4.5 \%$ to $-0.8 \%)$. As reference ED is usually the most proximate ED, bypassing of nearest ED was common among all race/ethnic groups. In all areas, black and Hispanic EMS patients were more likely to be transported to a safety-net ED compared to co-resident white patients. Parallel analyses of concordance rates of ED visits without using EMS indicated significantly lower rates relative to those for EMS transported ED visits.

CONCLUSIONS: We found variation by race/ethnicity in destination ED for EMS transport patients, with black and Hispanic patients more likely to be transported to a safety-net hospital relative to 
whites. The extent to which these differences arise from factors other than proximity (e.g., patient preference) need to be examined.

\section{DIFFERENCES IN NARRATIVE LANGUAGE IN EVALUA- TIONS OF MEDICAL STUDENTS BY THE GENDER AND UNDER-REPRESENTED MINORITY STATUS OF MEDICAL EDUCATORS}

Alexandra E. Rojek ${ }^{1}$; Joanne W. Yim ${ }^{1}$; Sarah Lisker $^{1}$; Rebekah Gardner $^{2}$; Raman Khanna ${ }^{1}$; Karen E. Hauer ${ }^{1}$; Catherine R. Lucey ${ }^{1}$; Urmimala Sarkar ${ }^{1}$. ${ }^{1}$ University of California, San Francisco, San Francisco, CA; ${ }^{2}$ Brown University, Providence, RI. (Control ID \#3186008)

BACKGROUND: Prior studies have shown significant differences in narrative evaluations associated with the gender and underrepresented minority (URM) status of medical students, even among students receiving the same grade. Limited qualitative studies in other educational settings suggest that male and female evaluators may assess students differently based on the student's gender. It remains poorly described, however, whether in medicine the gender and URM status of the evaluator influences the narrative text of evaluations.

METHODS: We set out to identify and enumerate text descriptors in medical student evaluations, particularly the differences in language depending on evaluator gender and URM status, and whether these differences persisted after adjustment for student gender and URM status. We collected clerkship evaluations from core clinical rotations at two medical schools, parsed the deidentified text of evaluations with a neural network algorithm, and employed natural language processing to quantify differences in the narrative text.

RESULTS: There were 86,655 evaluations, 41,629 (48\%) by male evaluators and $45,026(52 \%)$ by female evaluators; 10,049 $(12 \%)$ were by URM evaluators, and $70,799(82 \%)$ were by nonURM evaluators. We found significant differences in how students were described depending on the gender and URM status of their evaluator, and a subset of these differences remained significant after adjustment for students' gender and URM status. Of 1,312 descriptors identified in all evaluations, the usage frequency of descriptors was significantly different by evaluator gender for $152(11.5 \%)$ words, and by evaluator URM status the usage frequency differed significantly for 76 (5.7\%) words. Of the 100 most commonly used descriptors, 64 were used significantly differently by evaluator gender, and 45 were used significantly differently by evaluator URM status. For example, female evaluators used "wonderful" more than men $(p<0.001)$, while men used the term "superb" more often $(p<0.001)$. URM evaluators used the term "mature" $(\mathrm{p}<0.001)$ more often, and nonURM evaluators the term "impressive" $(\mathrm{p}<0.001)$. When adjusting for student URM status, there were minimal differences among URM students, although non-URM students were described differently depending on URM status of their evaluator.

CONCLUSIONS: While many of the descriptors used are shared, we find that there were preferred vocabularies used by male and female evaluators tied to evaluators' genders regardless of students' gender, and subsets of preferred vocabularies when segmenting by students' gender. When interrogating evaluators' and students' URM statuses together, we find few differences in how evaluators describe URM students, but preferred sets of descriptors for how evaluators describe non-URM students. These findings raise concern for an additional set of biases in narrative evaluation of students that is not directly related to students' clinical performance and may have implications in their career paths.
DIFFERENCES IN PRIMARY CARE ACCESS, PROVIDER TRUST, AND DISCLOSURE OF SAME-SEX BEHAVIORS TO PRIMARY CARE PROVIDERS AMONG MEN WHO HAVE SEX WITH MEN IN THE UNITED STATES

Christopher B. Stults ${ }^{3}$; Christian Grov ${ }^{2}$; Kathryn Anastos ${ }^{1}$; Elizabeth A. Kelvin ${ }^{2}$; Viraj V. Patel ${ }^{1}$. ${ }^{1}$ Montefiore Medical Center/ Albert Einstein College of Medicine, New York, NY; ${ }^{2}$ City University of New York, New York, NY; ${ }^{3}$ Baruch College, New York, NY. (Control ID \#3186080)

BACKGROUND: Engaging in primary care is important to ensure appropriate healthcare. Men who have sex with men (MSM) may be less likely to access primary care and engage fully, but little is known about factors associated with primary care engagement in MSM. Therefore, we assessed characteristics associated with having a primary care provider (PCP), and among MSM with a PCP, trust in and disclosure of same-sex behaviors to a PCP.

METHODS: In 2016, 4,239 MSM (aged 18+, reporting 1 sex act with a man in past 5 years) from across the US, recruited via diverse social media sites and in-person, completed an online survey including demographic/ behavioral characteristics, health literacy and (1) having a $P C P$, and among those with a PCP, (2) trust in their PCP (using Wake Forest Physician Trust Scale, dichotomized at the mean), and (3) disclosure of same-sex behaviors to their PCP. We used multivariable logistic regression with GEE to identify characteristics associated with each of the 3 outcomes. All results adjusted for age, race/ethnicity, HIV status, state, urbanicity, and recruitment source and shown as (Adj Odds Ratio,95\% CI).

RESULTS: Participants were from all 50 US states and distribution was roughly equivalent across age groups. Over half identified as white $(59.8 \%), 19.1 \%$ as Latino, $10.6 \%$ as black, and $3.4 \%$ as Asian. Most identified as gay $(79.7 \%)$ or bisexual $(17.6 \%) .18 .2 \%$ reported being HIV-positive, $62.8 \%$ HIV-negative-not on pre-exposure prophylaxis (PrEP), 11.5\% HIV-negative-on PrEP and 7.6\% unsure of their HIV status. Most (86.3\%) had a PCP. The odds of having a PCP was lower $(\mathrm{p}<0.01)$ among those who were younger $(0.09,0.03-0.3$; ref: $65+)$, HIV-negative $(0.08,0.04-0.15$; ref: HIV+) or -unknown $(0.05,0.03-0.1)$, single $(0.7,0.5-0.8)$, or with low medical literacy $(0.7,0.5,0.9)$. Of those with a PCP $(n=3,658), 45.3 \%$ had low trust in their provider, which was associated with $(\mathrm{p}<0.01)$ being younger (0.6, 0.4-0.9), Asian (0.67, 0.45-1; ref: white), bisexual (0.7, 0.6-0.8; ref: gay), HIV-negative-not on PrEP (0.5, 0.4-0.6; ref: HIV+), HIVunsure $(0.3,0.2-0.4)$, or with low medical literacy $(0.4,0.3-0.5)$. Finally, among those with a PCP $(n=3,658), 24.5 \%$ had not disclosed same-sex behaviors to their provider. Non-disclosure was associated with $(\mathrm{p}<0.01)$ being Asian $(0.5,0.3-0.8)$, bisexual $(0.2,0.2-0.3)$ or straight $(0.05,0.02-0.1)$, HIV-negative or -unknown $(0.09,0.06$ $0.14)$, having no sex partners in past 3 months $(0.6,0.5-0.8$; ref: $>1$ partner), recruited from hookup sites, low medical literacy $(0.7$, $0.5,0.9)$, and having low trust in their PCP $(0.6,0.5-0.7)$.

CONCLUSIONS: We found a number of factors associated with having a PCP and, among those with a PCP, we identified a number of predictors of primary care engagement, indicated by trust in a PCP and disclosure of same-sex behaviors. Research is needed to evaluate if increasing patient-provider trust and creating an enabling environment for disclosing same-sex behaviors can improve primary care engagement among diverse MSM populations.

\section{DIFFERENCES IN RISK FACTORS BETWEEN GENDERS FOR PATIENTS READMITTED WITH ACUTE PANCREATI- TIS A RETROSPECTIVE COHORT STUDY}

Neal Mehta ${ }^{1}$; Gilbert-Roy B. Kamoga ${ }^{2}$; Khaled Khasawneh ${ }^{1}$; Ron T. Varghese ${ }^{2}$. ${ }^{1}$ White River Health System, Batesville, AR; ${ }^{2}$ White River Medical Center, Batesville, AR. (Control ID \#3184581) 
BACKGROUND: In the United States, Acute Pancreatitis (AP) is the most frequent discharge diagnosis within Gastroenterology [1]. Those that have been admitted within 30-days have increased mortality at the 1-year mark [2]. Previous literature suggests that males have higher propensity for readmission for AP [3]. We sought out to determine the 30-day all-cause readmission rate for adults (age $>=18$ ) readmitted with a principal diagnosis of AP and compare the risk factors for males and female.

METHODS: We utilized Agency of Healthcare Research and Quality's (AHRQ) 2014 Nationwide Readmission Database which includes 14.9 Million discharges across 22 states to identify admissions with a principal diagnosis of AP (ICD-9 577.0). Applicable admissions were all adults (age $>=18$ ) with an index hospitalization discharge from January 1 to November 30, 2014. Patients who died during index admission or those with missing covariates were excluded. All-cause including AP readmissions within 30-days of an AP admission were analyzed. Statistical analysis was completed with Stata 15 (StataCorp, College Station, TX) with p-values $<0.05$ considered statistically significant. A univariate and multivariate analysis of data collected was completed using both odds ratio and chi square tests for significance. Predictors for readmission were determined using a multivariate logistic regression model following sequential step-wise elimination of covariates including demographics, comorbidities, hospital characteristics, length of stay (LOS), and the modified Elixhauser Comorbidity Index.

RESULTS: A total of 112,374 patients met criteria for inclusion. Of which, there was 16,939 readmissions (overall rate $15.07 \%$ ) within 30-days of the index admission. Of the 16,939 readmissions, $7,180(42.38 \%)$ had a principal diagnosis of AP. Males were readmitted for AP at a rate of $44.84 \%$ while females were readmitted at $39.48 \%$. Multivariate analysis showed that increased odds for 30-day readmission for acute pancreatitis (both genders) were admissions on weekend, younger, disposition at discharge (other facility, short term hospital, and leaving AMA), longer LOS, and admitted to medicine compared to surgery. For males, increased odds were admissions on weekend, younger, disposition at discharge (other facility, short term hospital, and leaving AMA), LOS between 7 and 14 days, and admitted to medicine. For females, increased odds were admissions during the week, younger, disposition at discharge (short term hospital, home with home health care, and AMA), longer LOS ( $>7$ days), and admitted to medicine.

CONCLUSIONS: Male have a higher readmission rate for AP compared to females with slight differences in risk factors particularly admissions on weekend and LOS. Better understanding and development of interventions for individuals with risk factors and high-risk for readmission may help to reduce future morbidity and mortality. Sources: [1] Lankisch, Lancet, 2015. [2] Lee, Pancreas, 2016. [3] Vipperla, Clin Gastroenterol Hepatol, 2014.

DIFFERENCES IN THE EFFECT OF PRIMARY CARE MENTAL HEALTH INTEGRATION ON HEALTHCARE UTILIZATION AMONG MEN AND WOMEN VETERANS

Lucinda B. Leung ${ }^{1,}{ }^{5}$; Lisa V. Rubenstein ${ }^{2}$; Edward P. Post ${ }^{3}$; Ranak Trivedi ${ }^{4}$; Sabine Oishi ${ }^{5}$; Alison Hamilton ${ }^{5}$; Jean Yoon ${ }^{6}$; Erin L. Jaske ${ }^{7}$; Elizabeth M. Yano ${ }^{8}$. ${ }^{1}$ UCLA David Geffen School of Medicine \& West LA VA Medical Center, Los Angeles, CA; ${ }^{2}$ RAND and UCLA, Medford, OR; ${ }^{3}$ VA Ann Arbor Healthcare System and University of Michigan, Ann Arbor, MI; ${ }^{4}$ VA Palo Alto Health Care System/Stanford University, Menlo Park, CA; ${ }^{5}$ Veterans Administration, Los Angeles, CA; ${ }^{6}$ Palo Alto VA, Menlo Park, CA; ${ }^{7}$ Department of Veteran Affairs, Seattle, WA; ${ }^{8}$ VA Greater Los Angeles Healthcare System, Sepulveda, CA. (Control ID \#3179172)
BACKGROUND: Evidence-based collaborative care models, such as VA's Primary Care Mental Health Integration (PC-MHI), can improve patient access to treatment of depression and anxiety, which are more prevalent among women. Access to PC-MHI care varies among women Veterans, because VA primary care organization may differ by gender (e.g., separate clinics for men and women). We examined whether greater primary care clinic penetration in PC-MHI care over time was associated with changes in healthcare utilization, particularly mental health visits, differently for women and men.

METHODS: We examined 448,455 female and 4,928,638 male patients receiving primary care between October 1, 2013 and September 30, 2016 from 396 hospital- or community-based VA clinics required to offer on-site PC-MHI services. In this national retrospective longitudinal cohort study, we calculated annual clinic PC-MHI penetration as the percent of assigned primary care patients seen by a PC-MHI provider. We used clinic PC-MHI penetration in multivariate regression models to predict specialty mental health visits (including PC-MHI visits) and other healthcare visits (i.e., primary care, other non-mental health specialty care, telephone, hospital stays). Models controlled for year, clinic, level of PACT implementation, utilization-related patient characteristics (e.g., age, gender, chronic comorbidities), and an interactive effect between gender and PC-MHI penetration.

RESULTS: Women, compared to men, had twice the rate of diagnosed depression ( $27 \%$ vs $15 \%$; $=.04)$ and anxiety (17\% vs $8 \% ; \mathrm{p}=.5)$. Women, on average, had higher rates of outpatient specialty mental health $(1.7$ times; $\mathrm{p}<.001)$ and primary care $(1.3$ times; $\mathrm{p}<.001)$ visits than men. Adjusted regressions showed, among women compared to men, 1 percentage-point greater clinic PC-MHI penetration was associated with a decrease in average specialty mental health visits $(-38 \%$, vs $+39 \%)$, other specialty visits $(-48 \%$, vs $-13 \%)$, and hospital stays $(-74 \%$, vs $+2 \%)$, but an increase in primary care visits $(+22 \%$, vs $+40 \%)$, for patients per year (all P's<.0001). There were no observed gender differences in PC- MHI effects on telephone care.

CONCLUSIONS: Women Veterans have high rates of depression and anxiety, which can be effectively treated by PC-MHI models. Greater primary care clinic penetration in PC-MHI was associated with higher outpatient specialty mental health utilization among men, but lower utilization among women Veterans. While PC-MHI care models may increase engagement in outpatient services for men, greater clinic penetration in PC-MHI services may instead be shifting mental health care (and perhaps other non-mental health specialty care and acute care) to primary care providers for women Veterans. With increasing patient choice in where to receive care, the VA must tailor medical care to the needs of rising numbers of women patients. More research is needed to understand gender differences in mental healthcare access and utilization related to $\mathrm{PC}-\mathrm{MHI}$.

\section{DIRECT OBSERVATION OF PATIENT EDUCATION BY THE HEALTHCARE TEAM ON THE DAY OF DISCHARGE} Shreya P. Trivedi ${ }^{1}$; Sara Corderman ${ }^{2}$; Caroline Katzman ${ }^{1}$; Mark D. $\overline{\text { Schwartz }}{ }^{1}$ Leora I. Horwitz ${ }^{1}$. NYU School of Medicine, New York, NY; ${ }^{2}$ SUNY Downstate College of Medicine, Brooklyn, NY. (Control ID \#3179319)

BACKGROUND: The transition of care from hospital to home is a vulnerable time for patients, often leaving individuals with a suboptimal understanding of the care plan, which can lead to post-hospitalization morbidity and readmissions. Prominent national medical societies and health services interventions have identified important domains to be addressed on discharge. Yet, 
there remains variable implementation of these high value discharge practices and often little transparency surrounding roles of medical team members at discharge. Direct field observation from the perspective of the patient is critical to inform gaps in the delivery of high quality discharge care. This study is the first to capture the discharge process by the entire healthcare professional team from the patient perspective.

METHODS: Purposeful sampling was used to select patients designated for "discharge by noon" with complex care plans. On the day of discharge, a research assistant sat at the bedside of a single consented patient from 6am until time of discharge, and recorded all communication and time spent on discharge education. Field notes were deductively analyzed for how patients were educated on key domains: medications, appointments, self-management of illness, symptom expectations, red flags, teach back and patient activation.

RESULTS: To date, 15 patients with a total of 92 hours of observations have been conducted. On average, interns spent about 4.5 minutes with the patient on discharge and less than 1 minute on discharge education. Nurses had variable discharge practices, which ranged from asking the patient to read printed discharge information to reading the discharge instructions to the patient. While most patients were told about medication changes, $40 \%$ were not told the purpose of medication changes. Similarly, the majority of patients were not told the purpose of follow-up medical appointments or pending results. There was limited education surrounding the self-management of diseases, symptom expectations or red flags. When provided, discharge education was one-sided; no patients were engaged through teach-back or patient activation. One patient was asked about potential barriers to adherence with the care plan. The majority of discharge communication was on practical steps of leaving the hospital, not preparing the patient to care for their themselves at home.

CONCLUSIONS: There is significant opportunity for improved discharge techniques to enhance safety and quality of care for patients leaving the hospital - including teach-back and patient engagement. Interventions must be implemented to increase patient education, particularly interprofessional ones that clarify assumptions on each other's roles. Further studies on effective communication strategies as well as systems redesign that foster patient-centered discharge education are imperative.

\section{DISPARITIES IN COMMUNICATION AMONG THE INPA- TIENT HOMELESS POPULATION AT A SAFETY-NET HOSPITAL}

Cristina Vellozzi-Averhoff ${ }^{1}$; William W. Thompson ${ }^{2}$; Claudia Vellozzi $^{3}$; Ike Okosun ${ }^{4}$; Kathy Kinlaw ${ }^{1}$; Jada C. Bussey-Jones ${ }^{1}$. ${ }^{1}$ Emory University, Atlanta, GA; ${ }^{2}$ Reproducible Scientific Solutions, LLC, Atlanta, GA; ${ }^{3}$ Grady Memorial Hospital, Atlanta, GA; ${ }^{4}$ Georgia State University, Atlanta, GA. (Control ID \#3177752)

BACKGROUND: Homelessness in the United States has a long history of stigma with little to no objective data on communication or care quality for the homeless. This research looks at the implications of stigma to determine whether the homeless population experiences disparities in care and communication during inpatient hospitalizations in a safety-net hospital.

METHODS: We administered a modified Hospital Consumer Assessment of Healthcare Providers and Systems (HCAHPS) survey to 112 age- and sex-matched homeless and nonhomeless adults at a university-affiliated safety-net hospital from December 2017 through March 2018 and performed a retrospective review of medical records of the survey participants. Linear regression models were used to assess differences in responses to survey subscales, length of stay, and other process of care measures.

RESULTS: Homeless participants trended toward poorer ratings for all HCAHPS subscales, reaching significance for the Communication about Medications subscale, with a mean score of 1.2 (95\% CI 0.48 - 1.76) points lower compared to nonhomeless sample. Length of stay and general process of care measures were not significantly different between homeless and non-homeless participants.

CONCLUSIONS: In an urban safety-net hospital, disparities in communications regarding medications between hospital staff and patients were found based on housing status, which may reflect systemic implicit bias of providers.

\section{DISPARITIES IN DISPOSITION AND LENGTH OF STAY IN HOSPITALIZATIONS FOR SERIOUS INFECTIONS WITH AND WITHOUT OPIOID USE DISORDER}

June-Ho Kim ${ }^{1,}{ }^{2}$; Danielle R. Fine ${ }^{3}$; Lily $\mathrm{Li}^{1}$; Simeon D. Kimmel $^{4}$; Joji Suzuki ${ }^{1}$; Christin N. Price ${ }^{1}$; Matthew Ronan ${ }^{5}$; Shoshana J. Herzig ${ }^{6}$. ${ }^{1}$ Brigham and Women's Hospital, Boston, MA; ${ }^{2}$ Ariadne Labs, Brigham \& Women's Hospital and Harvard T.H. Chan School of Public Health, Boston, MA; ${ }^{3}$ Massachusetts General Hospital, Boston, MA; ${ }^{4}$ Boston Medical Center, Boston, MA; ${ }^{5}$ VA Boston, Hopkinton, MA; ${ }^{6}$ Beth Israel Deaconess Medical Center, Brookline, MA. (Control ID \#3185253)

BACKGROUND: Patients with opioid use disorder (OUD) and serious bacterial infections, which often require prolonged courses of intravenous antibiotics, may face disparities in hospital and post-acute care, potentially leading to more discharges against medical advice (AMA), longer hospital stays, and higher costs. We compared differences in disposition, length of stay (LOS), and hospital charges between hospitalizations of patients with serious infections with and without OUD.

METHODS: We analyzed a nationally representative cohort of U.S. hospitalizations using the 2016 National Inpatient Sample. We included all adult (age 18+) hospitalizations with a primary ICD-10 diagnosis code for endocarditis, osteomyelitis, septic arthritis, or epidural abscess. The exposure of interest was OUD, identified as a secondary code. Outcomes were LOS, discharge disposition, and total hospital charges. Baseline characteristics were compared using chi-square tests and t-tests for categorical and continuous variables, respectively. Multivariable models were adjusted for patient, hospitalization, and hospitallevel characteristics. We used multivariable logistic and linear regression to estimate odds ratios for dispositions and differences in LOS and hospital charges, respectively.

RESULTS: In 2016, there were 91,015 hospitalizations in the U.S. for serious infections, 7,115 of which were associated with OUD. Patients with OUD were younger (mean age 41 vs 59 ), female (44 vs $35 \%$ ), and white (73 vs $69 \%$ ). More had Medicaid (58 vs $18 \%$ ) or were uninsured ( 10 vs $5 \%$, all $\mathrm{p}<0.01)$. Fewer hospitalizations with OUD ended in discharge to home (48 vs $66 \%, \mathrm{p}<0.01$ ); similar proportions were discharged to a post-acute care facility (30 vs $30 \%, p=0.96)$; and more were discharged AMA (20 vs $3 \%$, $\mathrm{p}<0.01)$. Adjusted for covariates, hospitalizations with OUD had lower odds of discharge home (adjusted odds ratio, aOR $0.37,95 \%$ CI $0.31,0.43)$ and higher odds of discharge to a post- acute care facility (aOR 1.76, 95\% CI 1.48, 2.09) or AMA (aOR 3.08, 95\% CI $2.47,3.83)$. Hospitalizations with OUD had longer mean lengths of stay (14 vs 8 days, $\mathrm{p}<0.01$ ), higher average total hospital charges $(\$ 109,476$ vs $74,400, p<0.01)$, and lower average daily charges $(\$ 8,454$ vs $9,856, p<0.01)$. After accounting for potential confounders, 
hospitalizations with OUD still had an average 3.3 days longer length of stay $(\mathrm{p}<0.01)$.

CONCLUSIONS: U.S. hospitalizations in 2016 for serious infections in patients with OUD had significantly longer lengths of stay and greater associated costs, along with higher odds of discharge to a post-acute care facility or AMA as opposed to home, compared to those without OUD. Further studies elucidating the source of these disparities and interventions to address them (such as more access to post-acute care facilities, inpatient substance use treatment, transitional addiction clinics, and outpatient parenteral antibiotic therapy) may improve equity of care and lower costs.

\section{DISPARITIES IN PHYSICIAN-PATIENT NONVERBAL COMMUNICATION AND INFLUENCE ON PATIENT AD- HERENCE}

Kedous Mekbib; Carol Oladele; Marcella Nunez-Smith. Yale University, New Haven, CT. (Control ID \#3186084)

BACKGROUND: Physician-patient communication is a quality of care indictor that has demonstrated associations with patient experience, satisfaction, and healthcare outcomes. Findings show that physician-patient communication varies across patient sociodemographic groups and contributes to healthcare disparities. This body of research has primarily focused on verbal physicianpatient communication. Few studies have investigated the influence of nonverbal physician-patient communication on healthcare disparities. This study aimed to examine differences in physician nonverbal communication among diverse patient groups and to determine the relationship between nonverbal communication and patient adherence.

METHODS: Data from the Patient-reported Experiences of Discrimination in Care Tool (PreDict) Study was used for the current analysis. The PreDict study includes a self-administered survey of recently discharged adult patients from a sample of US hospitals. Patients were asked about their most recent inpatient experiences using de novo items and previously validated scales. Nonverbal communication was measured by asking patients if their doctors were distracted when talking, stayed physically distant, and made eye contact with them. Our outcome, patient adherence, was measured using items that assessed whether patients were following discharge instructions or taking medications as prescribed. Chi-square tests were used to compare differences in nonverbal communication by race, ethnicity, total household income, and level of education. Logistic regression models were used to determine associations between nonverbal communication and patient adherence, adjusting for potential confounders.

RESULTS: Ninety-one percent of respondents reported their doctor was not distracted, $71.8 \%$ reported their doctor was not physically distant, and $88.6 \%$ of respondents reported their doctor made eye contact. Ninety-seven percent of respondents said they followed discharge instructions and $89.2 \%$ of patients took their prescribed medications. Bivariate analyses showed that lower income and non-white patients reported physicians maintained less eye contact and were distracted when talking to them. Multivariate results showed that patients who reported that doctors maintained less eye contact had 2.54 times lower odds of adhering to discharge instructions. Findings for medication adherence were not statistically significant.

CONCLUSIONS: These findings demonstrate that nonverbal communication matters to patients and is an important factor to consider in efforts to improve patient adherence. Findings also suggest the potential role of implicit bias in disparate physician- patient communication experiences among low-income and minority patients. This study highlights the need for increased provider awareness of nonverbal behaviors that contribute to poor healthcare outcomes and disparities.

\section{DO CLINICIAN-EDUCATORS FEEL THEY ARE ADE- QUATELY MENTORED: A MIXED-METHODOLOGY STUDY}

Attila Nemeth $^{1}$; Alia Chisty ${ }^{2}$; Carla Spagnoletti ${ }^{3}$; Corrie A. Stankiewicz ${ }^{4}$; Subha Ramani ${ }^{5} .{ }^{1}$ Case Western Reserve University, Cleveland, OH; ${ }^{2}$ Temple, Philadelphia, PA; ${ }^{3}$ University of Pittsburgh, Pittsburgh, PA; ${ }^{4}$ University of Pennsylvania SOM, Narberth, PA; ${ }^{5}$ Brigham and Women's Hospital, Harvard Medical School, Boston, MA. (Control ID \#3184642)

BACKGROUND: Being mentored is key to professional advancement, job satisfaction and institutional faculty retention. While many institutions have formal mentoring programs, it is unclear if clinician-educators (CE) feel that such programs adequately address professional development.

METHODS: A mixed methods approach explored the mentoring experiences and perceptions of general internal medicine CEs, focusing on the adequacy and quality of being mentored. To gather quantitative and qualitative data, an online survey was distributed to self-identified CEs within the Society of General Internal Medicine (SGIM) in the fall of 2017, and two in-person focus groups of interested survey respondents were held at the SGIM Annual Meeting in 2018. Pearson Chi-squared analysis was used for quantitative data and thematic analysis was conducted on qualitative data.

RESULTS: 192 CEs completed the survey. 64\% of respondents were women, $79 \%$ were between $30-55$ years, $68 \%$ were at the Assistant or Associate professor rank. $81 \%$ of women vs $59 \%$ of men had at least 1 active mentor $(\mathrm{p}<0.001) .40 \%$ had $>3$ active mentors, and $28 \%$ had none. $66 \%$ of respondents agreed or strongly agreed that they received adequate mentoring, and $94 \%$ agreed or strongly agreed that mentoring was beneficial for career advancement. $80 \%$ agreed or strongly agreed that being mentored contributed to increased academic products. $79 \%$ either agreed or strongly agreed that mentoring challenged them to be better CEs. Gender comparisons of results indicated more women faculty sought out their own mentors ( $64 \%$ vs $44 \%, \mathrm{p}<0.01$ ), had mentors outside of their home institution ( $34 \%$ vs $18 \%, \mathrm{p}<0.05)$, and used peer mentoring ( $33 \%$ vs $16 \%, \mathrm{p}<0.01)$. Women used mentoring sessions to discuss career advancement $(71 \%$ vs $42 \%, \mathrm{p}<0.001)$, job satisfaction $(48 \%$ vs $28 \%, \mathrm{p}<0.01)$, developing educational products $(66 \%$ vs $41 \%, \mathrm{p}<0.01)$, personal/professional life balance ( $45 \%$ vs $27 \%, \mathrm{p}<0.01)$, negotiating tactics $(28 \%$ vs $4 \%, \mathrm{p}<0.01)$, and deciding which opportunities to pursue (66\% vs $46 \%$, $\mathrm{p}<0.01)$. Gender congruence between mentor and mentee was important. $70 \%$ of responding women had a primary mentor who was female, and $68 \%$ of men had a primary mentor who was male $(p<0.01)$. We discovered the following key themes from our qualitative data: (1) an effective mentor is vital for career advancement; (2) peer mentoring offers unique benefits for professional growth; (3) there are distinct challenges in mentoring CEs; and (4) it is unclear whether mentoring is a teachable skill.

CONCLUSIONS: Female CEs appear to be significantly more proactive in seeking mentors inside and outside their institutions and engaging in peer mentoring. Even if institutions have formal faculty mentoring programs, mentoring that addresses unique needs of clinician educators is necessary for their career advancement and job satisfaction. 
DO HOSPITALIZATIONS DISRUPT LOYALTY TO AMBULATORY CARE PROVIDERS?

Laura F. Gingras ${ }^{3}$; Jennifer Chester $^{3}$; Sanjai Sinha ${ }^{2}$; Lisa M. Kern'; Mangala Rajan ${ }^{3}$. ${ }^{1}$ Weill Cornell Medical College, New York, $\mathrm{NY} ;{ }^{2}$ weill cornell medical college, New York, NY; ${ }^{3}$ Weill Cornell Medical College, New York City, NY. (Control ID \#3185484)

BACKGROUND: Hospitalizations can be disruptive events, which result in new diagnoses, new treatments, and new providers for patients. The effect of hospitalizations on ambulatory care patterns, though, is not known. That is, it is not known whether patients return post-discharge to their prehospitalization ambulatory provider, or whether they follow up with the new providers they met in the hospital. The answer to this question has important implications for value-based purchasing contracts, which assign patients to providers based on ambulatory visit patterns yet hold providers accountable for all of their patients' care (including hospitalizations elsewhere).

METHODS: We conducted a retrospective cohort study (2014-2016) of commercially insured adult patients ( 18 years) who were attributed to a physician organization (PO) by a commercial payer for the purpose of a reimbursement contract. We included patients who were hospitalized in 2015 and, using chi-squared tests, analyzed their patterns of ambulatory care 6 months before vs. 6 months after the hospitalization. Using logistic regression, we determined if seeing a PO provider 6 months before the hospitalization was associated with seeing a PO provider 6 months after, adjusting for the location of the hospitalization, patient age, patient gender, and the type of admission (emergency or elective).

RESULTS: Of the 569 patients in our sample, all of whom were attributed to the PO, $311(55 \%)$ were hospitalized at the affiliated hospital, whereas the remainder $(\mathrm{N}=258,45 \%)$ were hospitalized elsewhere. There was no difference in the proportion of patients with at least 1 ambulatory visit to any provider before vs. after the hospitalization $(p>0.10)$. However, patients who were hospitalized elsewhere were significantly less likely to have at least 1 visit with a PO provider in the 6 months before the hospitalization, compared to those hospitalized at the affiliated hospital $(54 \%$ vs. $73 \%$, p < 0.0001$)$. Patients who were hospitalized elsewhere were also significantly less likely to have at least 1 visit with a PO provider in the 6 months after the hospitalization, compared to those hospitalized at the affiliated hospital $(61 \%$ vs. $80 \%, \mathrm{p}<0.0001)$. CONCLUSIONS: Hospitalizations did not change patterns of ambulatory care (that is, before vs. after the hospitalization). However, patients who were hospitalized elsewhere had less loyalty to the PO both before and after hospitalization than patients hospitalized at the affiliated hospital. This study raises important questions about whether attribution logic should be affected by hospitalizations and, separately, how well POs need to monitor their patients for hospitalizations elsewhere.

\section{DO PATIENT SATISFACTION SCORES VARY BY PHYSICIAN RACE/ETHNICITY? AN EXPLORATION IN DIRECT TO CON- SUMER TELEMEDICINE}

Kathryn A. Martinez ${ }^{1}$; Kaitlin Keenan ${ }^{2}$; Radhika Rastogi ${ }^{2}$; Joud Roufael $^{3}$; Mark N. Rood ${ }^{4}$; Michael B. Rothberg ${ }^{1}{ }^{1}$ Cleveland Clinic, Cleveland, $\mathrm{OH} ;{ }^{2}$ Cleveland Clinic Lerner College of Medicine, Cleveland, $\mathrm{OH} ;{ }^{3}$ Kent State University College of Public Health, Kent, OH; ${ }^{4}$ Cleveland Clinic Foundation, Chagrin Falls, OH. (Control ID \#3183105)

BACKGROUND: The U.S. physician workforce is increasingly diverse and foreign medical graduates are common. Patient satisfaction is often publicly reported and can influence physician payment. Racial/ethnic bias against non-white physicians has been reported but not widely studied. The objective of this study was to assess differences in patient ratings of physicians in a large direct to consumer (DTC) telemedicine platform, by physician race/ethnicity.

METHODS: cross-sectional study of encounters conducted between July 2016 and July 2018 on the AmericanWell telemedicine platform. Four independent and blinded reviewers of different racial/ethnic backgrounds categorized physicians into racial/ethnic groups based on name (e.g. "Juan Ramirez" would be coded as Hispanic, "Preethi Gupta" would be coded as Indian) and publicly available photos. Physicians for whom no consensus was reached were excluded. Patient satisfaction was assessed by the telemedicine system immediately following each encounter, measured on scales of 0 to 5 stars, analyzed continuously. To maximize stability of satisfaction estimates for each physician, only those with at least 20 encounters were included. Mixed effects linear regression was used to assess differences in satisfaction scores by physician race/ethnicity, accounting for clustering by physician. Patient covariates included age, gender and geographic region. The model also controlled for prescription receipt and coupon use for free or reduced-cost care, as both are highly associated with patient satisfaction with telemedicine.

RESULTS: The sample included 119,016 encounters with 390 physicians. Fifty-nine percent of physicians were categorized as White American, 14\% Indian, 7\% Black American, 7\% Hispanic, 6\% Arab and 6\% Asian. For both White and Black American physicians, the unadjusted mean satisfaction score was 4.8, for both Arab and Hispanic physicians it was 4.7, and for both Indian and Asian physicians it was 4.5. In the adjusted mixed effects linear model, compared to White American physicians, satisfaction scores for Asian physicians were 0.24 points lower (95\%CI:-0.26, -0.22), scores for Indian physicians were 0.24 points lower (95\%CI:-0.26, -0.22), scores for Arab physicians were 0.16 points lower (95\%CI:-0.18, -0.14), scores for Hispanic physicians were 0.05 points lower (95\%CI:-0.08, -0.02 ), and scores for Black American physicians were 0.03 points lower (95\%CI:-0.04, -0.01).

CONCLUSIONS: All groups of non-White American physicians were rated worse than White American physicians, with particularly lower scores for Arab, Indian, and Asian physicians. Even small differences in satisfaction scores can impact physician payment and reputation. Understanding whether lower scores for non-White American physicians were influenced by patient bias or differences in quality and/or practice style is critical.

\section{DO PATIENTS WITH SUBCLINICAL HYPOTHYROIDISM WHO SUFFER FROM GREATER SYMPTOM BURDEN BENEFIT FROM LEVOTHYROXINE THERAPY? FURTHER ANALYSIS OF THE RANDOMIZED PLACEBO-CONTROLLED TRUST TRIAL}

Maria de Montmollin $^{1,2}$; Martin Feller ${ }^{1,2}$; Shanthi Beglinger ${ }^{1,2}$; Alex $\overline{\text { McConnachie }^{3} \text {; Cinzia Del Giovane }}{ }^{2}$; Rosalinde K. Poortvliet ${ }^{4}$; Drahomir Aujesky $^{1}$; Ian Ford ${ }^{3}$; Simon P. Mooijaart ${ }^{4}{ }^{4}$; Torquil Watt ${ }^{5}$; Rudi G. Westendorp ${ }^{6}$; Patricia M. Kearney ${ }^{7}$; Jacobijn Gussekloo ${ }^{4,}{ }^{4}$; David J. Stott ${ }^{8}$; Nicolas Rodondi1, ${ }^{1}$; Douglas Bauer'. ${ }^{1}$ Bern University Hospital, Bern, Switzerland; ${ }^{2}$ Institute of Primary Health Care (BIHAM), University of Bern, Bern, Switzerland; ${ }^{3}$ Institute of Health and Wellbeing, University of Glasgow, Glasgow, United Kingdom; ${ }^{4}$ Leiden University Medical Center, Leiden, Netherlands; ${ }^{5}$ Copenhagen University Hospital Herlev, Copenhagen, Denmark; ${ }^{6}$ University of Copenhagen, Copenhagen, Denmark; ${ }^{7}$ University College Cork, Cork, Ireland; ${ }^{8}$ Institute of Cardiovascular and Medical Sciences, University of Glasgow, Glasgow, United Kingdom; ${ }^{9}$ University of California, San Francisco, CA. (Control ID \#3170456)

BACKGROUND: A recent large trial and meta-analysis of all RCTs found that levothyroxine does not improve hypothyroid symptoms or quality of life among adults with subclinical hypothyroidism (SCH). However, those who report higher symptom burden prior to treatment might still benefit. The aim of this analysis is to determine whether levothyroxine, compared to placebo, improves hypothyroid physical symptoms and tiredness among older adults with $\mathrm{SCH}$ and higher symptom burden.

METHODS: We analyzed data from adults 65 years with persistent $\mathrm{SCH}$ (TSH 4.6-19.9mIU/L for $>3$ months, normal fT4) included in the TRUST trial, a multicenter, double blinded, placebo-controlled, randomized trial. 
Participants were randomized to levothyroxine or matching placebo including mock titration. The main outcomes were the change in 1-year Hypothyroid Symptoms score and Tiredness score in the Thyroid-Related Quality-of-Life Questionnaire. Both scores were self-reported (range 0100 , higher scores indicate more symptoms) with a 9 point difference considered clinically significant. In this subgroup analysis, we focus on participants with a baseline Hypothyroid Symptoms score $>30$ or Tiredness score $>30$.

RESULTS: At baseline, 132 adults (20.7\%) had Hypothyroid Symptoms scores $>30$ (mean age of 75 years, $64 \%$ women, mean \pm SD TSH $6.2 \mathrm{mIU} / \mathrm{L}$ \pm 1.65 and mean Hypothyroid Symptoms score 45.2 \pm 14.6$)$ and 209 (32.8\%) had Tiredness scores $>30$ (mean age of 75 years, $63 \%$ women, mean TSH $6.2 \mathrm{mIU} / \mathrm{L} \pm 1.65$ and mean Tiredness score $48.9 \pm 14.9$ ). After 1 year, among those with a Hypothyroid Symptoms score $>30$ at baseline, the mean score was 34.9 in the placebo and 32.8 in the levothyroxine group; and in those with a baseline Tiredness score $>30,43.1$ and 41.3 respectively. 1-year outcomes were similar between the levothyroxine and placebo groups (adjusted between-group difference -0.5 , 95\% CI: -6.8 to $5.8, \mathrm{p}=0.9$, Figure 1 ; and $-0.7,95 \%$ CI: -6.1 to $4.8, \mathrm{p}=0.8$, respectively). Among all TRUST participants $(n=737)$, baseline scores of the two main outcomes (as continuous variables) did not modify the effects of levothyroxine vs. placebo on Hypothyroid Symptoms (interaction $\mathrm{p}$ value $=0.85)$ or Tiredness $(\mathrm{p}=0.65)$.

CONCLUSIONS: In this secondary analysis of older adults with $\mathrm{SCH}$ and higher symptom burden at baseline, levothyroxine therapy did not improve hypothyroid symptoms or tiredness.

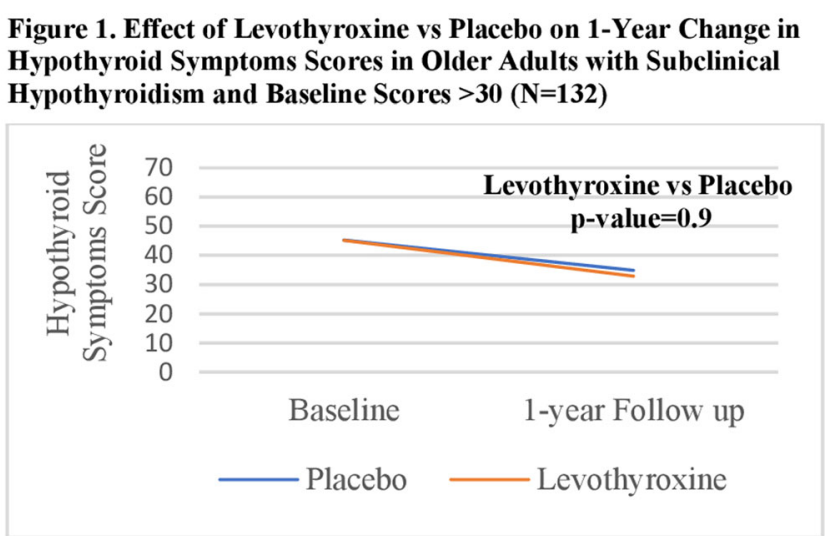

\section{DO QUALITY IMPROVEMENT PROJECTS AFFECT WORK- FORCE BURNOUT?}

Robert G. Badgett; Brent A. Duran. KU School of Medicine - Wichita, Wichita, KS. (Control ID \#3181672)

BACKGROUND: Burnout among healthcare professionals is a threat to both workforce and patient well-being. Based on Spreitzer's Integrative Model of Human Growth at Work, execution of quality improvement (QI) projects could be posited to reduce burnout if the workforce gains control over a dysfunctional worksite or to increase burnout if the workforce loses autonomy. This meta-analysis explores the link between workforce participation in the design of QI or practice redesign initiatives and impact on levels of burnout.

METHODS: We included studies published through January 2019 that executed QI projects, measured burnout, and had either concurrent or historical controls.

RESULTS: Six controlled studies with 6842 healthcare professionals were included. $66 \%$ of professionals were in the VA PACT study. One study, the Healthy Work Place (HWP) trial, was randomized. All interventions included components of workflow improvement. Two studies used a controlled before and after design to compare changes in burnout in intervention vs control clinics (Group Health Cooperative and Sutter Clinic). Meta- analysis showed no overall effect on burnout (odds ratio: 0.95 ; $95 \%$ CI 0.74 to 1.23); however, heterogeneity was substantial ( $\mathrm{I} 2=72 \%)$. Whether healthcare professionals had local control over project design significantly modulated the results: studies granting local control tended to reduce burnout (odds ratio 0.77 ; $95 \%$ CI 0.57 to $1.03 ; \mathrm{I} 2=44 \%$ ) whereas studies not clearly granting local control tended to increase burnout (odds ratio $1.29 ; 95 \%$ CI 0.91 to $1.81 ; \mathrm{I} 2=80 \%$ ). Additional findings include: 1 ) the Sutter clinic found that interventions did not affect burnout when the workforce had control over implementation, but the same interventions significantly increased burnout when the workforce did not have control, 2) the HWP found that workflow interventions significantly reduced burnout, 3) two studies found a significant association between the degree of practice redesign and freedom from burnout, and 4) Group Health reported a positive financial return on investment. A qualitative study by Parker advocated for a hybrid approach to QI that merges central and local control of implementation. Studies with patient outcomes reported either significant benefit (VA PACT, Group Health, Sutter Clinic) or mixed results (HWP). CONCLUSIONS: Burnout can be avoided, and likely reduced by both workforce involvement in implementation decisions and also addressing workflow. The recommendation for a hybrid strategy is consistent with the High-Performance Work Practices of the Agency for Healthcare Research and Quality and is consistent with complexity leadership theory (CLT). In CLT, the role of management includes data gathering, sensemaking of the data, and dissemination of information. Limitations to this analysis include relying on mostly non-registered, observational studies. The benefit to patient outcomes tends to be positive.

\section{DOES A FOCUSED TRAINING IMPROVE INTERNAL MEDI- CINE RESIDENTS' SYSTEM- AND PRACTICE-BASED CONFI- DENCE IN INTEGRATED BEHAVIORAL HEALTH CARE?}

Christine A. Pace ${ }^{1}$; Lindsay B. Demers ${ }^{2}$; Carly Taylor ${ }^{5}$; Evonne Yang ${ }^{3}$; Cara Fuchs $^{3}$; Robert Joseph ${ }^{3}$; Daniel P. Alford ${ }^{4} .{ }^{1}$ Boston University School of Medicine, Boston, MA; ${ }^{2}$ Boston University, Boston, MA; ${ }^{3}$ Boston Medical Center, Boston, MA; ${ }^{4}$ Boston University School of Medicine and Boston Medical Center, Boston, MA; ${ }^{5}$ Tufts University School of Medicine, Boston, MA. (Control ID \#3185720)

BACKGROUND: Successful implementation of integrated behavioral health (IBH) requires physicians to take on new system-based and clinical responsibilities that are not well covered in residency training. The Boston Medical Center General Internal Medicine practice, where almost 100 residents receive ambulatory training, began an IBH program in 2014. Due to the large number of educational priorities for the residency program, we had limited time with the residents and developed a focused training. We sought to evaluate its effectiveness in improving residents' IBH systemand practice-based confidence.

METHODS: The training was an hour-long lecture with case discussion, focused on screening and intervening for depression and unhealthy substance use, and best practices for co-management with behavioral health clinicians. For example, we discussed interpretation of screening results, brief interventions for risky alcohol use, and behavioral health tools in our electronic record, and we modeled warm hand-offs to IBH clinicians. We trained four groups of residents, with each session led by a primary care clinician, psychologist or psychiatrist, and an IBH social worker. The training evaluation included a pre- and immediate-post-survey of residents assessing confidence in IBH processes and clinical skills. Changes in confidence were assessed using a five-point scale ranging from "Not at all Confident" $=1$ to "Completely Confident" $=5$. A series of non-parametric sign tests appropriate for Likert-type data was used.

RESULTS: 31 residents completed the training with 27 (87\%) completing the surveys. 17 were in their first year, 7 in their second, and 3 in their third. In order to assess changes in confidence from pre- to post-, a series of nonparametric sign tests, appropriate for Likert-type data, was used. We found statistically significant gains in confidence in all areas assessed. 
CONCLUSIONS: A focused internal medicine resident training combining lecture with case discussion was effective in increasing residents confidence in incorporating IBH into their outpatient practice. In a training environment with multiple competing priorities, efficient approaches that address systems-based practice are particularly important.

\section{DOES A HIGHER LEVEL OF EDUCATION TRANSLATE INTO A LOWER PREVALENCE OF CARDIOVASCULAR DISEASE}

Taher M. Tayeb $^{1}$; Talal Alzahrani ${ }^{2}$; Marco Mercader ${ }^{2} .{ }^{1}$ The George Washington University, Arlington, VA; ${ }^{2}$ The George washington University hospital, Washington, DC. (Control ID \#3141282)

BACKGROUND: Education is one of the determinants of health as described by the WHO, and it is one of the primary determinants of Socio-Economic Class (SEC), a higher level of education is associated with a higher level of SEC. Few researchers have addressed the link between the level of education and cardiovascular disease. However, there remains a need for a national study to determine the relationship between the level of education and cardiovascular disease. In this study, we investigated the effect of the level of education on the rate myocardial infarction (MI) and cardiovascular risk factors including diabetes mellitus, hypertension, hypercholesterolemia, and smoking status.

METHODS: The National Health Interview Surveys of $2016(n=73,353)$ were utilized to measure the effect of level of education on MI and other risk factors. Subjects were assigned into four groups based on the level of education. The first group labeled as no education till the 12th grade, the second group identified as General Education Diploma (GED) or a high school diploma, the third group included subjects with some college, associate degree or bachelor, and the fourth group designated for subjects with master's degrees, professional degrees, or doctorates. Logistic regression models were used to examine the association between level of education and cardiovascular risk factors. The final model examined the association between level of education and MI after adjusting for demographics and cardiovascular risk factors.

RESULTS: A higher level of education was associated with lower odds of having cardiovascular risk factors. The final logistic regression model revealed that subjects with GED and high school education (OR 0.80 , 95\% CI 0.65-0.97; $\mathrm{p} 0.027)$, subjects with some college degree/ bachelor degree (OR 0.74, 95\% CI 0.61-0.90; $\mathrm{p}<0.003$ ), and subjects with master degrees or professional degrees (OR $0.58,95 \%$ CI $0.43-0.78$; $p<0.001$ ) have lower odds of having MI compared to the first group after adjusting for other risk factors including age, gender, diabetes mellitus, hypertension, hypercholesterolemia, and tobacco use.

CONCLUSIONS: This study unveiled that a higher level of education is associated with a lower rate of myocardial infarction and cardiovascular risk factors.

\section{DOES AN INDEX COMPOSED OF ROUTINE LABS DISCRIMI- NATE RISK OF MORTALITY BETTER THAN THE CHARLSON INDEX}

Christopher T. Rentsch ${ }^{1,}$; Janet P. Tate ${ }^{2}$; Laura Tarko ${ }^{4}$; Jacqueline Honerlaw $^{4}$; Kelly Cho ${ }^{4}$; ; Yuk-Lam $\mathrm{Ho}^{4}$; Amy C. Justice ${ }^{2,}$. $^{3}{ }^{1}$ London School of Hygiene \& Tropical Medicine, London, United Kingdom; ${ }^{2}$ Yale School of Medicine/VA Connecticut Healthcare System, New Haven, CT; ${ }^{3}$ Yale School of Public Health, New Haven, CT; ${ }^{4}$ VA Boston Healthcare System, Boston, MA; ${ }^{5}$ Brigham and Women's Hospital/ Harvard Medical School, Boston, MA. (Control ID \#3175306)

BACKGROUND: The diagnostic code adaptation of the Charlson Comorbidity Index has been used as a mainstay adjustment for overall disease burden by thousands of investigators for decades. Now that electronic health records (EHR) make large scale access to laboratory data possible, we ask whether the Veterans Aging
Cohort Study (VACS) Index, based on routinely available clinical laboratory data, provides superior discrimination of mortality to that of the Charlson Index.

METHODS: We conducted an analysis using data from the Department of Veterans Affairs Million Veteran Program, a volunteer cohort of $>500,000$ patients providing full access to their EHR data. Baseline was set at enrollment. Patients were followed up to five years. The VACS Index was calculated from hemoglobin, ALT, AST, platelets, creatinine, hepatitis C status, albumin, white blood count, body mass index, and age. The Charlson Index was calculated from age and ICD-9/10 diagnostic codes for acute myocardial infarction, congestive heart failure, peripheral vascular disease, cerebral vascular accident, dementia, pulmonary disease, connective tissue disorder, peptic ulcer, mild and severe liver disease, diabetes and diabetic complications, paraplegia, renal disease, cancer and metastatic cancer, and HIV/AIDS. Values for VACS Index and codes for Charlson Index were assessed in the year prior to baseline. We compared discrimination ( $C$-statistics) and model fit (AIC) between Cox models assessing 30-day, 6month, 1-year, and 5-year mortality using VACS Index and Charlson Index scores as the sole predictor. We then compared $C$-statistics of models assessing 1-year mortality stratified by sex, age, and race/ethnicity.

RESULTS: Among 313,691 participants with laboratory data, median age was 64 years (IQR 55-70), 91\% were male, 67\% were European-American, and $21 \%$ were African-American. At 30 days, 6 months, 1 year, and 5 years, there were 370 (1.4 per 100 personyears [PY]), 1,383 (2.4/100 PY), 8,106 (2.7/100 PY), and 28,889 deaths $(3.1 / 100 \mathrm{PY})$, respectively. $C$-statistics were significantly higher for VACS Index than Charlson Index at every time interval (C, 0.812 [95\% CI, 0.789-0.836] vs. $C, 0.759$ [95\% CI, 0.733-0.786] at 30 days; $C, 0.765$ [95\% CI, 0.762-0.768] vs. $C, 0.752$ [95\% CI, $0.749-0.755$ ] at 5 years) and among men, participants aged 45 years, and European-Americans. Model fit was better for VACS Index than Charlson Index at every time interval. Of note, a benefit for Charlson is that there are no missing data since conditions are assumed absent if not coded; $C$-statistics for Charlson among those without laboratory data were consistent with those having laboratory data (data not otherwise shown).

CONCLUSIONS: The VACS Index, based on routinely available laboratory data, provides superior discrimination of mortality compared to that of the Charlson Index based on ICD codes. Now that EHRs can provide laboratory data on a large scale, the Charlson Index should no longer be the preferred method of risk adjustment.

\section{DOES DIABETES EDUCATION INFLUENCE GLYCOSYLAT- ED HEMOGLOBIN?}

Pooja Bhargava $^{1}$; Linda Keller-Doyle ${ }^{1}$; Eric Green ${ }^{2} .{ }^{1}$ Mercy Catholic Medical Center, Philadelphia, PA; ${ }^{2}$ Mercy Catholic Medical Center, Darby, PA. (Control ID \#3180218)

BACKGROUND: Even though patient education is considered an essential component of diabetes management, little is known about the effectiveness of formal diabetes management classes.

METHODS: Mercy Catholic Medical Center (MCMC) offers a fourweek class in self-management of diabetes. The classes are free, taught by certified diabetes educators, and are offered at times believed to be convenient for members of our community. The curriculum addresses diabetes pathophysiology, self-management of glucose, healthy eating, medications, healthy coping, and the benefits of exercise. We evaluated the impact of diabetes education on a cohort of patients who attended diabetes classes between 2015 and 2018. Patients had glycosylated hemoglobin (Hgbalc) measured before and after classes as part of routine care. We limited our analysis to 
patients who had recorded Hgbalc before and 3-6 months after taking the classes. We used a paired t-test to determine statistical significance.

RESULTS: Of 296 patients completed diabetes education between 2015 and 2018, 139 patients (46\%) met inclusion criteria. The mean age was 58, and 58\% were female. The mean Hgba1c was $7.2 \pm 1.4$ prior to the class and $6.6 \pm 1.1$ at the next measured Hgba1c at least 3 months after taking the class $(\mathrm{p}<.001$ for change). No significant difference was seen in impact of intervention between men $(-0.57$ $\pm 1.4)$ or women $(-0.59 \pm 1.1)$.

CONCLUSIONS: In this patient population, diabetes education classes produced a statistically significant and clinically meaningful reduction in Hgbalc. Our findings are limited by our inclusion criteria for patients with pre- and post-education Hgbalc; this population had a relatively low baseline Hgbalc and the impact of the behavioral intervention may be exaggerated by unmeasured confounders that also led to adherence with lab monitoring. Future research should be done to assess the impact of diabetes education classes on other populations.

\section{DOES HOSPITAL TEACHING STATUS INFLUENCE RISK FACTORS AND INCIDENCE RATE OF 30-DAY READMIS- SION FOR ATRIAL FIBRILLATION}

Ankitha Antony ${ }^{1}$; Ron T. Varghese ${ }^{1}$; Gilbert-Roy B. Kamoga ${ }^{2}$; Khaled Khasawneh ${ }^{1}$; Neal Mehta ${ }^{1}{ }^{1}$ White River Health System, Batesville, AR; ${ }^{2}$ White River Medical Center, Batesville, AR. (Control ID \#3184827)

BACKGROUND: Atrial Fibrillation (AF) is a common arrhythmia accounting for nearly $1 / 3$ of all cardiac rhythm admissions with hospital admissions for AF increasing by $70 \%$ over the past several years [1]. Furthermore, previous literature suggests that teaching hospitals may have higher readmission rates compared to nonteaching hospitals [2]. We sought out to determine the 30-day allcause readmission rate for adults (age $>=18$ ) admitted with a principal diagnosis of AF and compare the risk factors for teaching and non-teaching metropolitan hospitals.

METHODS: We utilized Agency of Healthcare Research and Quality's (AHRQ) 2014 Nationwide Readmission Database which includes 14.9 Million discharges across 22 states to identify admissions with a principal diagnosis of Atrial Fibrillation using ICD-9 code 427.31. Applicable admissions were all adults (age $>=18$ ) with an index hospitalization discharge between January 1 to November 30, 2014. Patients who died during index admission and those with missing covariates were excluded. Index admissions to nonmetropolitan hospital were excluded. All-cause including Atrial Fibrillation readmissions within 30-days of an Atrial Fibrillation were analyzed. Statistical analysis was completed with Stata 15 (StataCorp, College Station, TX) with $\mathrm{p}$-values $<0.05$ considered statistically significant. A univariate and multivariate analysis of data collected was completed using both odds ratio and chi square test for significance. Predictors for readmission were determined using a multivariate logistic regression model following sequential stepwise elimination of covariates including demographics, comorbidities, hospital characteristics, length of stay (LOS) for index admission, and the modified Elixhauser Comorbidity Index.

RESULTS: A total of 127,156 patients met criteria for inclusion. There was 21,384 readmissions (overall rate $16.82 \%$ ) within 30 days of the index admission. Of the 21,384 readmissions, 5,589 $(26.17 \%)$ had a principal diagnosis of Atrial Fibrillation. Metropolitan teaching hospitals had an overall readmission rate of $14.43 \%$ compared to $14.32 \%$ of non-teaching metropolitan hospitals (not statistically significant). Multivariate analysis showed that for metropolitan hospitals in general, younger age, weekend admissions, association with renal failure, Medicaid insurance, different disposition at discharge (including leaving AMA, going home with home health or to a short-term hospital or other facility) all increased the odds for 30-day readmission from Atrial Fibrillation.

CONCLUSIONS: In general, nearly 1 in 6 patients discharged with a principal diagnosis of Atrial Fibrillation will be readmitted within 30 days. Metropolitan teaching and non-teaching hospitals have similar readmission rates and profile. Provider awareness of risk factors for readmissions in metropolitan hospitals will allow for the development of interventions to decrease future morbidity and mortality. References: [1] Anter, Circulation, 2009. [2] Mueller, Med Care, 2013.

\section{DOES HOSPITAL TEACHING STATUS INFLUENCE RISK FACTORS AND INCIDENCE RATE OF 30-DAY READMIS- SION FOR CONGESTIVE HEART FAILURE IN THE UNIT- ED STATES?}

Shoaib Khan ${ }^{1}$; Ron T. Varghese ${ }^{1}$; Gilbert-Roy B. Kamoga ${ }^{2}$; Khaled Khasawneh ${ }^{1}$; Neal Mehta ${ }^{1}{ }^{1}$ White River Health System, Batesville, AR; ${ }^{2}$ White River Medical Center, Batesville, AR. (Control ID \#3185493)

BACKGROUND: Congestive Heart Failure (CHF) admissions continue to rise with nearly 1 Million hospitalizations annually [1]. Furthermore, previous literature suggests that teaching hospitals may have higher readmission rates compared to non-teaching hospitals [2]. We sought out to determine the 30-day all-cause readmission rate for adults (age $>=18$ ) admitted with a principal diagnosis of $\mathrm{CHF}$ and compare the risk factors for readmission at teaching and non-teaching metropolitan hospitals.

METHODS: We utilized Agency of Healthcare Research and Quality's (AHRQ) 2014 Nationwide Readmission Database which includes 14.9 Million discharges across 22 states to identify admissions with a principal diagnosis of CHF using ICD-9 codes (402.01, 402.11, 402.91, 404.01, 404.11, 404.03, 404.13, 404.91, 404.93 and 428.XX). Applicable admissions were all adults (age $>=18$ ) with an index hospitalization discharge between January 1 to November 30, 2014. Patients who died during index admission and those with missing covariates were excluded. All index admissions to non-metropolitan hospital were excluded. All-cause including CHF readmissions within 30-days of a CHF readmission were analyzed. Statistical analysis was completed with Stata 15 (StataCorp, College Station, TX) with p-values $<0.05$ considered statistically significant. A univariate and multivariate analysis of data collected was completed using both odds ratio and chi square tests for significance. Predictors for readmission were determined using a multivariate logistic regression model following sequential step-wise elimination of covariates including demographics, comorbidities, hospital characteristics, length of stay (LOS) for index admission, and the modified Elixhauser Comorbidity Index.

RESULTS: A total of 354,681 patients met criteria for inclusion. Of which, there was 78,588 readmissions (overall rate $22.15 \%$ ) within 30-days of the index admission. Of the 78,588 readmissions, $31,030(39.48 \%)$ had a principal diagnosis of CHF. Metropolitan teaching hospitals had an overall readmission rate of $22.203 \%$ compared to $22.06 \%$ at non-teaching metropolitan hospitals with the difference not statistically significant. Multivariate analysis showed that for metropolitan hospitals in general increased odds for 30day readmission from CHF were weekend admissions, younger, any different disposition at discharge than routine (other facility, short term hospital, with home health, and leaving AMA), Medicaid as payer, central metropolitan areas with population $>1$ million, longer LOS, and lower household income. 
CONCLUSIONS: With almost 1 in 4 patients discharged with a principal diagnosis of CHF will be readmitted within 30 days. Metropolitan and non-teaching hospitals have similar readmission rates and profile. Provider awareness of risk factors for readmissions in metropolitan hospitals will help in planning interventions to decrease morbidity and mortality. References: [1] Roger. Circ Res. 2013. Aug 30;113(6):646-59. [2] Mueller. Med Care. 2013 Jul;51(7):567-74

\section{DOES HOSPITAL TEACHING STATUS INFLUENCE RISK FACTORS AND INCIDENCE RATE OF 30-DAY READMIS- SION FOR DIABETIC KETOACIDOSIS?}

Ron T. Varghese; Diana G. Jacob; Gilbert-Roy B. Kamoga; Khaled Khasawneh; Neal Mehta. White River Medical Center, Batesville, AR. (Control ID \#3184547)

BACKGROUND: Nearly 1 in 10 individuals in the United States have Diabetes Mellitus [1]. One potential preventable complication is Diabetic Ketoacidosis (DKA). Better understanding of the risk factors for readmissions of DKA will allow the development and implementation of specific patient-centered interventions to decrease future readmissions. Furthermore, previous literature suggests that teaching hospitals may have higher readmission rates compared to non-teaching hospitals [2]. We sought out to determine the 30-day all-cause readmission rate for adults (age $>=18$ ) admitted with a principal diagnosis of DKA and compare the risk factors for teaching and non-teaching metropolitan hospitals.

METHODS: We utilized Agency of Healthcare Research and Quality's (AHRQ) 2014 Nationwide Readmission Database which includes 14.9 Million discharges across 22 states to identify admissions with a principal diagnosis of DKA related ICD-9 diagnosis $(250.10$ - 250.13) associated with both Type 1 and Type 2 Diabetes Mellitus. Applicable admissions were all adults (age $>=18$ ) with an index hospitalization between January 1 to November 30, 2014. Patients who died during index admission and those with missing covariates were excluded. In addition, all index admissions to non-metropolitan hospital were excluded. All-cause readmission including DKA within 30-days of DKA were analyzed. Statistical analysis was completed with Stata 15 (StataCorp, College Station, TX) with $\mathrm{p}$-values $<0.05$ considered statistically significant. A univariate and multivariate analysis of data collected was completed using both odds ratio and chi square test for significance. Predictors for readmission were determined using a multivariate logistic regression model following sequential step-wise elimination of covariates including demographics, comorbidities, hospital characteristics, length of stay (LOS) for index admission, and the modified Elixhauser Comorbidity Index.

RESULTS: A total of 59,840 patients met criteria for inclusion. Of which, there was 11,635 readmissions (overall rate $19.44 \%$ ) within 30-days of the index admission. Of the 11,635 readmissions, $6,329(54.40 \%)$ had a principal diagnosis of DKA on the readmission. Metropolitan teaching hospitals had an overall readmission rate of $19.32 \%$ compared to $19.66 \%$ of non-teaching metropolitan hospitals which was not statistically significant. Multivariate analysis showed that for both teaching and non teaching metropolitan hospitals increased odds for 30day readmission from DKA were female, younger, leaving AMA, Medicare as payer, longer LOS, having renal failure and admitted to a hospital that was either non-profit or investor-owned compared to a government entity.

CONCLUSIONS: Almost $20 \%$ of all patients discharged with a principal diagnosis of DKA will be readmitted within 30 days. Metropolitan teaching and non-teaching hospitals have similar readmission rates and profile. Targeted interventions in patients at risk for readmission will help to decrease future morbidity and mortality.

DOES MEDICAID EXPANSION FOR VETERANS RESULT IN MORE DUAL USE OF VA AND NON-VA ACUTE HEALTH CARE SERVICES?

Patrick O'Mahen ${ }^{1,}{ }^{2}$; Laura A. Petersen ${ }^{1,2}$. ${ }^{1}$ Michael E. DeBakey VA Medical Center, Houston, TX; ${ }^{2}$ Baylor College of Medicine, Houston, TX. (Control ID \#3184082)

BACKGROUND: Medicaid expansion may offer multiple sources of care for Veterans eligible for the VA, which creates potential dual use and may risk poorer care-co-ordination and health outcomes. Our goal is to examine how pre-Affordable Care Act state-level Medicaid expansions affect how many Veterans had a hospital admission or used emergency department (ED) at both Veterans Health Administration (VA) and non-VA Medicaid funded care.

METHODS: To account for confounders, we used a differencein-difference approach to determine associations between Medicaid expansion and utilization among low-income Veterans ages 18-64 to eliminate age-related Medicare-eligible Veterans. We studied states that expanded Medicaid (New York, Arizona) to adults in 2001 or 2002 and paired them with demographically similar non-expansion states (Pennsylvania, New Mexico, Nevada) to isolate changes in ED visits and hospital admissions. We used ordinal logistic regression to separately estimate the proportion having hospital admissions or ED visits at both VA and non-VA facilities paid by Medicaid. We stratified by gender, race, and number of comorbid conditions. We controlled for age and distance to the nearest VA. Data sources were the VA Corporate Data Warehouse and the Medicaid Analytic Extract files. The study period encompassed $1,405,667$ person years and represents every Veteran age 18-64 living in one of these five states for at least one year from 1999 to 2006 and qualified for VA care due to low income.

RESULTS: For women, Medicaid expansion was associated with an increase of 0.3 percentage points in the proportion of Veterans having hospital admissions at both Medicaid and VA facilities in a given year (99\% CI -0.22 to 0.83 ) and 1.14 points in ED visits (.62 to 1.62$)$. Men saw increases of 0.46 points for admissions $(0.24$ to $0.68)$ and 0.87 for ED visits (0.73 to 1.01$)$. Among whites, expansion was associated with an increase of 0.64 points in proportions of Veterans having dual admissions ( 0.39 to 0.87 ) and 1.19 points in ED visits (0.62 to 1.66). Non-whites saw decreases of 0.01 points for admissions ( -0.45 to 0.43$)$ and an increase of 0.46 for ED visits (0.23 to 0.69$)$. Among Veterans with no comorbid conditions, expansion was associated with an increase of 0.7 points in persons having dual admissions ( -0.55 to 0.85 ) and 0.70 points in ED visits (0.62 to 1.66). Veterans with three or more comorbid conditions saw decreases of 0.09 points for admissions $(-0.55$ to 0.38$)$ and increases of 0.62 for ED visits ( 0.27 to 0.98$)$.

CONCLUSIONS: Medicaid expansion associates with small or no increased dual use of VA and Medicaid-funded services, suggesting problems like care coordination arising from dual use may be limited. Further work should explore dual use among vulnerable groups. This information may help project usage for both ACA and VA Choice programs, which expand access to non-VA care.

DOES REMOVING FINANCIAL INCENTIVES LEAD TO DECLINES IN MEASURED PERFORMANCE? INSIGHTS FROM MEDICARE ADVANTAGE STAR RATINGS PROGRAM 
Rachel O. Reid ${ }^{1,2}$; Susan M. Paddock ${ }^{1}$; Cheryl Damberg ${ }^{1} .{ }^{1}$ RAND Corporation, Boston, MA; ${ }^{2}$ Brigham and Women's Hospital, Boston, MA. (Control ID \#3167588)

BACKGROUND: As policymakers face pressure to reduce measurement burdens, understanding what happens to measure performance after de-incentivization is important. The Centers for Medicare \& Medicaid Services (CMS) publishes 5-star quality ratings for Medicare Advantage plans and pays Quality Bonus Payments to highly-rated 4- and 5-star plans. The Star Ratings incorporate measures from various sources [e.g., Healthcare Effectiveness Data and Information Set (HEDIS), Consumer Assessment of Healthcare Providers and Systems (CAHPS), Health Outcomes Survey (HOS)]. CMS has added and removed Star Rating measures over time; removed measures are still monitored and reported on cms.gov, but no longer contribute to bonus payments and are not publiclyreported on Medicare Plan Finder. No prior study has examined performance on measures removed from the Star Rating program.

METHODS: We examined 201 Medicare Advantage plans that received Star Ratings (i.e., Local Coordinated Care Plan (CCP), Medical Savings Account, Private Fee-for-Service, Regional CCP), were continuously present without mergers between 2009 and 2018, and had at least 1000 enrollees each year from 2009 to 2016. We excluded Puerto Rico plans. We assessed mean plan performance on Part C HEDIS, HOS, and CAHPS measures continuously reported to CMS for the 2009 to 2018 Star Ratings, excluding measures removed from the Star Ratings before 2012 or those temporarily removed for 1 or 2 years. We identified 13 measures that continuously contributed to Star Ratings and 6 measures removed from Star Ratings in 2012 or 2013.

RESULTS: Among the 6 measures removed from the Star Ratings, performance improved over the entire measurement period from 2009 to 2018 (from +2.2 percentage-points for "Doctors Who Communicate Well" to +8.7 percentage-points for "Testing to Confirm Chronic Obstructive Pulmonary Disease") and after removing the measure from Star Ratings (from +0.9 percentage-points for "Access to Primary Care Doctor Visits" to +6.7 percentage-points for "Testing to Confirm Chronic Obstructive Pulmonary Disease"). Among the 13 measures remaining in the Star Ratings between 2009 and 2018, performance trends varied from -0.3 percentagepoints for "Getting Needed Care" to +21.6 percentage- points for "Osteoporosis Management in Women who had a Fracture". We did not observe declines in performance after measures were removed from the Star Ratings, compared to before removal and to performance on measures that remained incentivized in the Star Ratings.

CONCLUSIONS: Removing measures from the Medicare Advantage Star Ratings public reporting and financial incentives program did not result in performance declines, nor did performance trends differ from measures remaining in the Star Ratings. Payers and policymakers considering removing measures from public reporting or financial incentive programs should not necessarily anticipate worsening performance.

\section{DOES TRAINING MATTER? ATTENDING PHYSICIANS' CORE CLINICAL SKILLS DO NOT APPEAR TO BE ANY BETTER THAN THOSE OF THEIR RESIDENTS.}

Khemraj Hardowar; Lisa Altshuler; Colleen C. Gillespie; Jeffrey Wilhite; Harriet Fisher; Sarah Chaudhary; Kathleen Hanley; Sondra Zabar. NYU School of Medicine, New York, NY. (Control ID \#3185414)

BACKGROUND: Considerable resources are put into training physicians to be effective providers after residency. Practicing physicians are generally assumed to be more effective and more efficient than resident physicians who are still undergoing training. We capitalize on a unique opportunity to test that hypothesis using the controlled methodology of Unannounced Standardized Patients (USPs), Standardized Patients sent into clinical environments to systematically assess provider skills in the context of a standardized clinical scenario. Due to last minute scheduling changes, a small sample of attending physicians ended up seeing USPs we had intended to send to residents. In this study, we report on comparisons between how these attending physicians performed in terms of their patient centeredness, patient activation, assessment, and communication skills in comparison to residents.

METHODS: 6 USP visits were delivered to primary care clinics in an urban safety net hospital from 2009 to 2015 . Of those $700+$ visits, visits were completed inadvertently with 16 attendings. We selected the 16 attendings with at least 4 years of post-graduate experience and then matched them with 2 resident visits based on hospital, time period, and USP visit type ( $n=32$ residents). In all visits, USPs completed a behaviorally anchored post-visit checklist that assessed patient centeredness (4 items), patient activation (2 items), visit-specific assessment (10 items), and communication skills including information gathering (4 items), relationship development (5 items) and patient education (3 items). Items were rated as not done or partially done vs. well done and summary scores were calculated as $\%$ well done. Mean scores for attendings and matched residents were compared using t-tests.

RESULTS: Resident and attending scores on patient centeredness (68\% vs $73 \%$ ), patient activation ( $44 \%$ vs $38 \%$ ), assessment ( $53 \%$ vs $51 \%$ ), patient education ( $49 \%$ vs $52 \%$ ), information gathering ( $71 \%$ vs $78 \%$ ) and relationship development ( $70 \%$ vs $73 \%)$ did not significantly differ $(p>.05)$. Nor did we see any substantial differences in variances or find any outliers. CONCLUSIONS: In our matched sample of residents and attendings, there were no significant differences by training level for any of the assessed clinical skills. While we viewed the inadvertent scheduling of USP visits with attendings as an opportunity to investigate the impact of training, our study is limited by the small sample size and whether we were able to create good matches. Findings may reflect ceiling effects (our checklists are too hard) or expertise-reversal effects (experts can skip some elements of the interaction and still arrive at the correct diagnosis and treatment plan). Further research, if our mistakenly-assessed attending sample increases, could explore the influence of PGY level and of patient load as attendings carry substantially heavier patient panels and see more (and probably more complex) patients per day then residents

\section{DOES URBAN OR RURAL HOSPITAL LOCATION INFLUENCE RISK FACTORS AND INCIDENCE RATE OF 30-DAY READMIS- SION FOR CONGESTIVE HEART FAILURE IN THE UNITED STATES?}

Michael Andryka ${ }^{1}$; Ron T. Varghese ${ }^{1}$; Gilbert-Roy B. Kamoga ${ }^{2}$; Khaled Khasawneh ${ }^{1}$; Neal Mehta ${ }^{1}$. White River Health System, Batesville, AR; ${ }^{2}$ White River Medical Center, Batesville, AR. (Control ID \#3185559)

BACKGROUND: Congestive Heart Failure (CHF) admissions continue to rise with nearly 1 Million hospitalizations annually [1]. Furthermore, previous literature suggests that hospital characteristics such as location affect CHF hospitalization outcomes [2]. We sought to determine the 30-day allcause readmission rate for adults (age $>=18$ ) admitted with a principal diagnosis of $\mathrm{CHF}$ and compare the risk factors for readmission at urban and rural hospitals.

METHODS: We utilized Agency of Healthcare Research and Quality's (AHRQ) 2014 Nationwide Readmission Database which includes 14.9 Million discharges across 22 states to identify admissions with a principal diagnosis of CHF using ICD-9 codes (402.01, 402.11, 402.91, 404.01, 404.11, 404.03, 404.13, 404.91, 404.93 and 428.XX). Applicable admissions were all adults (age $>=18$ ) with an index hospitalization discharge between January 1 to November 30,2014. Patients who died during index admission and those with missing covariates were excluded. Rural and Urban classification was determined using the 2013 NCHS Urbal-Rural Classification Scheme. All-cause readmissions within 30-days of an index admission were analyzed. Statistical analysis was completed with Stata 15 (StataCorp, College Station, TX) with p-values $<0.05$ considered statistically significant. A univariate and multivariate analysis of data collected was completed using 
both odds ratio and chi square tests for significance. Predictors for readmission were determined using a multivariate logistic regression model following sequential step-wise elimination of covariates including demographics, comorbidities, hospital characteristics, length of stay (LOS) for index admission, and the modified Elixhauser Comorbidity Index.

RESULTS: A total of 389,466 patients met criteria for inclusion. Of which, there was 85,831 readmissions (overall rate $22.03 \%$ ) within 30-days of the index admission. Index admission to an urban hospitalization had a readmission rate of $22.22 \%$ compared to $20.94 \%$ from rural hospitals $(\mathrm{p}<0.001)$. Multivariate analysis showed that admissions to rural and urban hospitals each had a higher likelihood of readmission if they were admitted on the weekend, any disposition other than "routine," adults $<65$, longer LOS, renal failure comorbidity present and admitted to a medical service. In addition, Medicaid as a payer increased the odds for readmission at urban hospitals.

CONCLUSIONS: Almost 1 in 4 patients discharged with a principal diagnosis of CHF will be readmitted within 30 days. Index admissions to urban hospitals have as higher readmission rate than rural hospitals, but the risk factors appear to be similar. Further investigation is necessary to elucidate the reasons for the disparity in readmission rates between urban and rural hospitals, which may prove useful in developing interventions for populations at risk to reduce future morbidity and mortality. References: [1] Roger, Circ Res, 2013. [2] Joshi, Congest Heart Fail, 2004.

\section{DON'T JUST DO SOMETHING, SIT THERE! ASSESSING THE IMPACT OF SITTING AT THE BEDSIDE ON PATIENTS' SATIS- FACTION WITH THEIR DOCTORS}

Anna K. Donovan ${ }^{1}$; Carla L. Spagnoletti ${ }^{1}$; Scott D. Rothenberger ${ }^{2}$; Raquel Forsythe ${ }^{1}$; Jennifer Corbelli ${ }^{1}{ }^{1}$ University of Pittsburgh School of Medicine, Pittsburgh, PA; ${ }^{2}$ University of Pittsburgh, Pittsburgh, PA. (Control ID \#3182767)

BACKGROUND: Improving patient satisfaction remains an important yet elusive endeavor for academic medical centers. One study demonstrated that hospitalist attending physicians in a non-teaching hospital had higher-rated communication skills when sitting compared to standing at the bedside during rounds ${ }^{1}$. It is unclear whether these data can be applied to resident-led multidisciplinary team rounds, during which $7-8$ providers round together. We hypothesized that if resident team leaders sat during morning rounds that patient satisfaction would improve without impacting rounding efficiency.

METHODS: We performed a cluster-randomized trial with crossover in which internal medicine (IM) and surgery residents served as their own controls. Each resident team leader (IM N=18; Surgery N=8) was assigned to sit or stand on a chair at the bedside during alternating weeks in May and June of 2018. Research assistants administered electronic surveys to 5 randomly selected patients per team per week for the study period of 8 weeks. In addition, all IM interns and residents $(\mathrm{N}=54)$ from participating teams were invited to complete a post-rotation survey about their perceptions of sitting vs. standing during rounds.

RESULTS: A total of $18 \mathrm{IM}$ and 8 surgery resident team leaders participated. Surveys were collected from 583 patients. There was no statistically significant difference in patient perception of physician skill in giving complete information, using plain language, showing interest in patients and their problems, or giving patients time to ask questions (all $\mathrm{p}>0.05$ ). Results from IM and surgery did not differ. IM residents (response rate $80 \%$ ) felt that rounding duration did not differ. Most indicated that sitting at the bedside was already their standard practice.

CONCLUSIONS: When randomizing medicine and surgery residents to sit or stand during team rounds, we found no difference in patient perceptions of physician communication. While sitting positively impacted attending hospitalists' patient-rated communication skills when rounding alone ${ }^{1}$, this was not replicated with resident-led team rounds. We suspect that one member sitting, while all others stand, is not sufficient to impact the patients' perception of the entire encounter. Further, patients rated resident communication highly in both groups, so a ceiling effect may have prevented us from detecting any difference. We speculate that sitting during one-on-one patient encounters (e.g. initial history- taking, breaking bad news), rather than team-based encounters, may have greater impact in teaching hospitals. Significance: Standing was non-inferior to sitting during rounds. Our results suggest that initiatives to optimize patient communication and satisfaction in teaching hospitals should be focused elsewhere. References: 1. Merel et al, JHM, 2016.

ECONOMIC OPPORTUNITY, AUTOMOTIVE PLANT CLOSURES, AND DRUG OVERDOSE MORTALITY: A QUASIEXPERIMENTAL ANALYSIS

Atheendar Venkataramani ${ }^{1}$; Elizabeth Bair ${ }^{2}$. ${ }^{1}$ University of Pennsylvania, Philadelphia, PA; ${ }^{2}$ University of Pennsylvania, Philadelpha, PA. (Control ID \#3185703)

BACKGROUND: There is considerable debate over the role of economic factors in driving rising drug overdose mortality in the U.S. Recent theories suggest that drug overdose mortality may arise as a result of fading economic opportunities. However, standard economic measures, such as unemployment rates, explain little of the variation in drug overdose mortality. We examined the effects of U.S. automotive plant closures in the 2000s and onward. Automotive plant closures are salient shocks to economic opportunity that can shift future expectations around economic opportunity.

METHODS: Our primary outcome were annual, county-level, age-adjusted drug overdose mortality rates for 20-64 year old adults for the period 19992015 (obtained from the CDC). We identified all automotive plants in operation in 1999, whether they closed, and the year of closure. We defined exposure to automobile plant closures at the commuting zone-level, which are groups of counties where people live and work. The sample of interest consisted of counties with high shares of manufacturing workers. We used a quasi-experimental, event-study approach, comparing mortality rates in each individual year before and after a plant closure in that commuting zone to drug overdose mortality rates in counties in commuting zones where plants remained open, adjusting for county and year fixed effects. As a falsification test, we estimated the same model for non-manufacturing counties.

RESULTS: Our sample included 120 manufacturing counties in 39 commuting zones. Drug overdose mortality rose in each year following a plant closure (Figure): by ten years drug overdose mortality rates were $42 \%(95 \%$ CI: $25 \%, 59 \%$ ) higher in closure vs. non-closure counties. Both sets of counties followed similar trends prior to plant closures and we found null estimates for non-manufacturing counties. The findings were driven by men, and not explained by differential migration rates.

CONCLUSIONS: Automotive plant closures had substantial effects on drug overdose mortality, explaining over $35 \%$ of the overall rise in the study counties between 1999-2015. We have currently obtained restricted mortality data to examine differences across age, gender, race, and education groups.

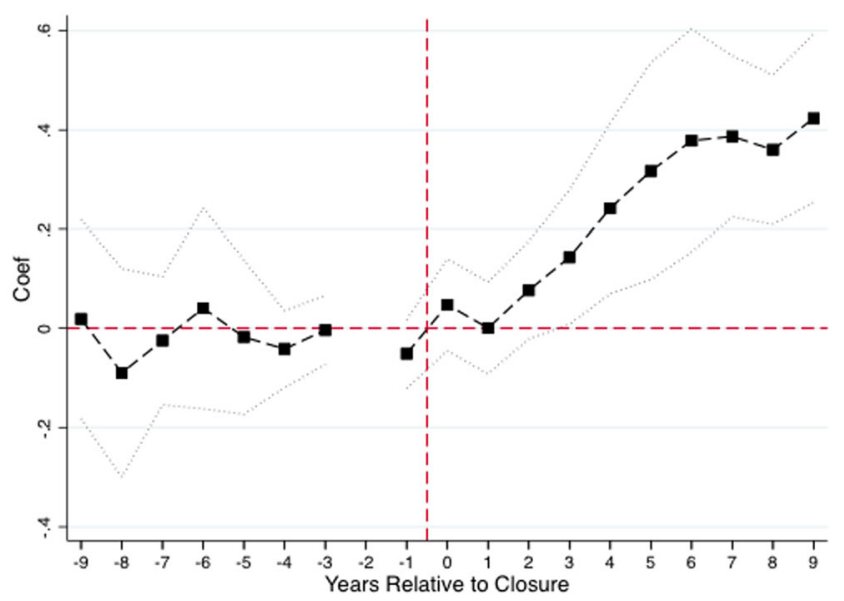

Event study model estimates 
EFFECT OF A SCORING RUBRIC ON THE REVIEW OF SCIENTIFIC MEETING ABSTRACTS

Nia S. Mitchell ${ }^{1}$; Kelly Stolzmann ${ }^{2}$; Jolie B. Wormwood ${ }^{3,}{ }^{4}$; Lauren V. $\overline{\text { Benning }}{ }^{1}$; Amy Linsky ${ }^{2}, 2$. ${ }^{1}$ Duke University, Durham, NC; ${ }^{2}$ VA Boston Healthcare System, Boston, MA; ${ }^{3}$ Bedford VA Medical Center, Bedford, MA; ${ }^{4}$ University of New Hampshire, Durham, NH. (Control ID \#3184121)

BACKGROUND: Rubrics provide guidance for objective measurement of performance to decrease or eliminate subjective judgement. In 2014-2016, the Society of General Internal Medicine (SGIM) Annual Meeting scored scientific abstracts based upon four criteria: 1) importance of research question; 2) strength and appropriateness of methods; 3 ) validity of conclusions; and 4) clarity and concision. Reviewers were instructed to score each criterion from 1 - $7(1=$ lowest and 7 = highest $)$, with little other guidance. The SGIM 2017 Annual Meeting program committee renamed the last criterion "quality of writing" and added detailed descriptions for each score within each criterion to provide more guidance for reviewers. The purpose of the current study is to estimate the effect of the standardized scoring rubric on the use of the entire range of scores and on reliability between reviewers.

METHODS: We evaluated scores for all abstracts submitted to SGIM annual meetings pre-rubric (2014-2016) and post-rubric (2017-2018). For each abstract, we calculated a mean and standard deviation across reviewers for each of the four individual criteria scores and the composite score (i.e., the average of the four criteria scores). We used general linear mixed modeling, nesting reviewers within abstracts, to determine if there was a difference in mean criteria and composite scores by year. Intraclass correlation coefficients (ICC) estimated inter-reviewer agreement.

RESULTS: There were 3,523 abstracts with 22,242 submitted reviews. The Table indicates the number of abstracts submitted, total number of reviews, mean (SD) number of reviewers per abstract, the mean (SD) of the composite scores, and ICCs in each of the five years. Composite scores in pre-rubric years were statistically significantly greater than composite scores in postrubric years (5.0 pre-rubric vs. 4.8 post-rubric; $\mathrm{p}<0.0001$ ). Repeat analyses for each of the four criteria similarly demonstrated similar statistically significant differences pre- and post-rubric $(\mathrm{p}<0.0001)$. There was no apparent difference in ICCs between pre-and post-rubric years.

CONCLUSIONS: A detailed scoring rubric successfully shifted scores closer to the mid-point of the scoring range, but it did not increase interreviewer reliability. Objective evaluation of abstract submissions is an important component to ensure the rigor and quality of the information selected for presentation. Efforts should continue to refine and use tools such as rubrics to improve the scoring of abstracts.

EFFECT OF A SPECIALTY-INITIATED E-CONSULT ON PROVIDER UNLEARNING AND PSYCHOLOGICAL REACTANCE IN DEIMPLEMENTATION OF A LOW-VALUE MEDICATION FOR COPD Christian D. Helfrich ${ }^{1}$; Toral J. Parikh ${ }^{3}$; Laura Feemster ${ }^{5}$; Seppo Rinne ${ }^{2}$; Renda S. Wiener ${ }^{2}$; Barbara Majerczyk ${ }^{1}$; Edmunds M. Udris ${ }^{4}$; David Au ${ }^{1}$. ${ }^{1}$ VA Puget Sound Healthcare System, Seattle, WA; ${ }^{2}$ Edith Nourse Rogers Memorial VA, Bedford, MA; ${ }^{3}$ Puget Sound VA Healthcare System, Seattle, WA; ${ }^{4}$ VA Puget Sound Health Care System, Seattle, WA; ${ }^{5}$ VA Puget Sound/ University of Washington, Seattle, WA. (Control ID \#3178515)

BACKGROUND: Learning health systems need to reduce low-value care as well as increase high-value care. One approach is to utilize specialists to engage at a population health level for patients in primary care. However, primary care providers (PCP) may feel their autonomy is threatened if specialists deliver unsolicited recommendations. We tested a proactive econsultation to target low value practices among patients with chronic obstructive pulmonary disease (COPD). We sought to understand the effect of the e-consult on PCP unlearning of ineffective care for patients with mild to moderate COPD and psychological reactance to receipt of the e-consult. METHODS: Attending PCPs at 13 VHA primary care clinics were surveyed prior $(n=138)$ and after $(n=137)$ the e-consult intervention as part of a provider-level clustered randomized trial. The goals of the e-consult were to de-implement guideline-discordant use of inhaled corticosteroids (ICS) and improve safety. Intervention PCPs received a proactive e-consult with an unsigned order reflecting pulmonologist recommendations. Control PCPs received no specialist input. We assessed whether e-consults promoted unlearning of overuse of ICS by increasing awareness of ICS harms and evidence for effectiveness of substitute medications (long-acting bronchodilators, LABD), and if e-consults had the unintended effect of causing irritation and skepticism (i.e., psychological reactance).

RESULTS: Surveys were completed by 26 intervention and 20 control PCPs at baseline and 24 intervention and 24 control PCPs at follow-up (34\% and $35 \%$ response rates respectively). The proportion of PCPs who correctly agreed that $\mathrm{LABD}$ were effective in reducing $\mathrm{COPD}$ exacerbations increased by $30 \%$ for intervention PCPs ( $42 \%$ at baseline to $72 \%$ at follow-up) vs. $3 \%$ for control PCPs (55\% to 58\%). The proportion who correctly agreed that ICS results in higher risk of pneumonia increased by $17 \%$ for intervention PCPs (50\% to $67 \%$ ) vs. declining by $2 \%$ for control PCPs (60\% to $58 \%)$. Finally, the proportion who incorrectly agreed that LABD use has higher risk of cardiovascular events relative to ICS decreased by $8 \%$ for intervention PCPs (58\% to $50 \%$ ) vs. increasing by $19 \%$ for control PCPs (36\% to 55\%). Followup surveys asked PCPs how they felt when receiving information and advice about reducing use of ICS for patients with COPD. 25\% of intervention PCPs and $4 \%$ of controls felt irritated or annoyed. No PCPs felt angry. $8 \%$ of intervention PCPs and $0 \%$ of controls reported criticizing the information. $21 \%$ of intervention PCPs and $13 \%$ of controls could think of points that go against efforts to reduce ICS use among patients with COPD. Finally, $17 \%$ of intervention PCPs and $0 \%$ of controls reported skepticism of efforts to reduce ICS use among patients with COPD.

CONCLUSIONS: We found evidence of unlearning: intervention PCPs had increased awareness of ICS risks and of LABD as effective alternatives. However, we found some evidence of reactance such as annoyance, counter-arguing and skepticism.

EFFECT OF A WIDESPREAD IMPLEMENTATION OF A STRATEGY USING CARDIOVASCULAR RISK STRATIFICATION AND PRACTICE COACHING ON HIGH RISK ADULT PATIENTS RECEIVING CARE IN SMALL NORTH CAROLINA PRACTICES

Samuel Cykert1; Michael Pignone ${ }^{2}$; Thomas C. Keyserling ${ }^{3}$; Darren Dewalt ${ }^{1}$; Crystal W. Cene ${ }^{1}$; Justin G. Trogdon ${ }^{4} .{ }^{1}$ University of North Carolina, Chapel Hill, NC; ${ }^{2}$ Dell Medical School, Austin, TX; ${ }^{3} \mathrm{UNC}-$ Chapel Hill, Chapel Hill, NC; ${ }^{4}$ University of North Carolina at Chapel Hill, Chapel Hill, NC. (Control ID \#3185185)

BACKGROUND: Cardiovascular disease (CVD) remains the leading cause of death in the U.S. and is particularly devastating in North Carolina (NC) counties associated with the "Stroke Belt". Despite this outlook, adoption of new evidence remains slow. An example of this is use of the November 2013 American College of Cardiology (ACC) guidelines on cholesterol management which include 10-year ASCVD risk calculation. Barriers such as poor capability of certified electronic health records (EHRs) to automate risk scores and to measure guideline adherence persist. Heart Health Now (HHN) is an AHRQ-sponsored initiative designed to evaluate the effect of risk stratification, practice facilitation, and quality improvement (QI) techniques on CVD risk for adults served by small, North Carolina primary care practices. In this report, we assess the impact of the intervention on ASCVD 10-year risk for patients found to be high risk at baseline.

METHODS: We conducted a pre-post assessment of ASCVD risk for high risk patients that were identified using a CVD population management dashboard. In addition to the dashboard, the intervention consisted of onsite practice facilitation over 12 months to help develop QI strategies and workflows that serially engage high risk patients. Risk reduction approaches included statin prescribing for patients defined by the 2013 ACC guidelines, aspirin prescribing for individuals $<60$ yrs. old with ASCVD 10-year risk > $10 \%$, and hypertension treatment. Tobacco cessation was also advised but did 
not factor into post-intervention calculations. Regression analyses using patient characteristics and rural location were performed to identify factors more strongly associated with risk reduction in the study population.

RESULTS: A total of 437,556 patients in 219 small NC primary care practices received ASCVD risk scores and 183,346 (41.9\%) had a calculated 10 -year risk $>10 \%$. 36,520 were associated with practices that did not complete data submission for the full intervention yielding 146,826 available for analysis. $66 \%$ of these patients were White, $24 \%$ Black, 3\% Hispanic, $54 \%$ male, and $52 \%$ of rural residence. At baseline, the mean ASCVD risk score for the high risk group was $23.6 \%$ (SD +/- 12.8\%). After the intervention the mean score for this group was $18.1 \%$ (SD +/- 11.7\%). 52.2\% of high risk patients experienced risk score reductions. In regression analysis, younger patients (OR 1.1, 95\% CI 1.08 - 1.15), Blacks (OR 1.2, 95\% CI 1.18 - 1.26), and rural residents (OR 1.3, 95\% CI 1.27 - 1.34) benefited a little more from the intervention.

CONCLUSIONS: A one-year intervention utilizing CVD risk-stratification coupled with practice coaching resulted in significant risk reductions for a large population of high risk patients cared for by small primary care practices. These results suggest that informatics support and practice facilitation can strongly drive the use of new evidence to, specifically, reduce CVD risk and, generally, to promote health and improved outcomes.

\section{EFFECT OF MANDATED BREAST DENSITY REPORTING LEG- ISLATION ON WOMEN'S AWARENESS AND KNOWLEDGE OF BREAST DENSITY}

Kelly A. Kyanko'; Jessica R. Hoag' ${ }^{2}$; Susan Busch ${ }^{2}$; Jenerius Aminawung ${ }^{3}$; Xiao Xu ${ }^{2}$; Ilana B. Richman ${ }^{2}$; Cary Gross ${ }^{4}{ }^{1} \mathrm{NYU}$ School of Medicine, New York, NY; ${ }^{2}$ Yale University, New Haven, CT; ${ }^{3}$ Yale University School of Medicine, New Haven, CT; ${ }^{4}$ Yale School of Medicine, New Haven, CT. (Control ID \#3182670)

BACKGROUND: To date, 35 states have enacted dense breast notification (DBN) laws mandating that mammogram reports include language informing women of risks related to dense breast tissue. We conducted a nationally representative survey to determine the impact of these laws on women's awareness of breast density, knowledge of its association with the risk of breast cancer and sensitivity of mammography, and anxiety around breast cancer screening.

METHODS: Internet survey conducted in 2018 with participants in the GfK KnowledgePanel囚, a probability-based online research panel designed to be representative of the U.S. population. The sample included 2,539 Englishspeaking US women aged 40 to 59 years without a personal history of breast cancer who had ever had a mammogram in their lifetime.

RESULTS: Enrollment was closed when a predetermined number of panelists screened in, resulting in a completion rate of $68.2 \%$. Overall, $41.3 \%$ of women reported that they were informed of having dense breasts. A significantly greater proportion of women residing in DBN states were informed of having dense breasts as compared to women residing in non-DBN states ( $43.6 \%$ versus $32.7 \%, \mathrm{p}<0.01$; adjusted analyses OR $1.74,95 \%$ CI: 1.37 2.22). Only $23.0 \%$ of women overall knew of the increased risk of breast cancer with dense breasts, with no significant difference between women in DBN states and non-DBN states. Similarly, $68.0 \%$ of women correctly answered that dense breasts make it more difficult for mammography to detect cancer, with no significant difference seen by residence in a DBN state. There was also no association between residing in a DBN state and reporting breast cancer-related anxiety.

CONCLUSIONS: In a large nationally representative survey, we found that women living in states with mandated breast density reporting laws were more likely to be aware of having dense breasts. However this was not accompanied by an increase in knowledge that dense breasts are associated with a higher risk of breast cancer and limit the ability of mammography to detect cancer. Improving readability and understandability of notification language around the clinical implications of dense breasts, or other forms of educational efforts such as mass media, may help meet this goal. Efforts may also best be directed toward informing conversations between women and their providers, such as additional research to clarify the benefits and harms of supplemental screening and tools to engage and educate women.

\section{EFFECT OF MARYLAND BEHAVIORAL HEALTH HOMES ON CANCER SCREENING IN WOMEN WITH SERIOUS MENTAL ILLNESS}

Karly A. Murphy ${ }^{1}$; Gail L. Daumit ${ }^{1,2}$; Elizabeth Stone ${ }^{1}$; Sachini Bandara ${ }^{2}$; Alene Kennedy-Hendricks ${ }^{2}$; Craig E. Pollack ${ }^{1,2}$; Beth McGinty ${ }^{2,}{ }^{2}$. ${ }^{1}$ Johns Hopkins University School of Medicine, Baltimore, MD; ${ }^{2}$ Johns Hopkins Bloomberg School of Public Health, Baltimore, MD. (Control ID \#3184170)

BACKGROUND: People with serious mental illness (SMI) die 10-20 years earlier than the general population, primarily due to physical health conditions; cancer is the second leading cause of death. Behavioral health homes (BHHs), which integrate primary care into specialty mental health settings, have proliferated recently with the goal to improve management and care coordination of physical health. This study sought to evaluate the effect of the Maryland Medicaid BHH program on preventative cervical and breast cancer screening in women with SMI.

METHODS: We used Maryland Medicaid administrative claims data from October 1, 2012-December 31, 2016. We included 6,059 women between ages 21-64 years with SMI, who participated in a psychiatric rehabilitation program (PRP) which indicates significant functional impairment as a result of mental illness, and were continuously enrolled in Medicaid during the study period. Of these, 1,408 women were BHH participants, and 4,651 were non-BHH participants and served as the comparison group. In one year intervals, we measured whether women ages 21-64 years received pap smears or women ages 50-64 years received screening mammography. For our analysis, we used marginal structural modeling with inverse probability treatment weighting applied to person-time periods. This approach allowed us to accommodate rolling enrollment of participants into a $\mathrm{BHH}$ and to adjust for baseline covariates (age, race, psychiatric diagnosis) and time-varying covariates (residence, disability, substance use, managed care organization, comorbidity, PRP characteristics). We used logistic regression to estimate the effect of BHH enrollment on cancer screening and the predicted probability of receiving screening.

RESULTS: Among 6,059 women in the study, the mean age was 41.1 years $(\mathrm{SD}=11.2)$ with $58.1 \%$ black race, $32.7 \%$ with schizophrenia and $55.1 \%$ with bipolar disorder, $24.8 \%$ with substance use disorder, and $57.3 \%$ with disability. The predicted annual cervical cancer screening rate was $32.6 \%$ for women enrolled in a $\mathrm{BHH}$ and $29.3 \%$ for women not enrolled in a $\mathrm{BHH}$ after adjustment for baseline and time-varying covariates. The predicted annual breast cancer screening rate was $28.5 \%$ for women enrolled in a $\mathrm{BHH}$ and $24.3 \%$ for women not enrolled in a BHH after adjustment for baseline and time-varying covariates. Women who were enrolled in a BHH were $27 \%$ (adjusted Odds Ratio [aOR] 1.27, 95\% CI 1.04-1.55) more likely to receive pap smears and $17 \%$ (aOR 1.17, 95\% CI 1.04-1.31) more likely to receive mammography.

CONCLUSIONS: Maryland's Medicaid BHH program increased cancer screenings in women with SMI. However, annual cancer screening rates among the SMI population remain lower than annual screening rates in the general population (cervical cancer $42 \%$, breast cancer $43 \%$ ). This suggests that integrated care models, such as BHHs, can improve preventative care for the SMI population, but future work is needed to improve health equity between SMI and non-SMI populations.

EFFECT OF PRACTICE FACILITATION ON PREVENTIVE CARDIOLOGY IN PRIMARY CARE: A PRACTICE-RANDOMIZED, COMPARATIVE EFFECTIVENESS STUDY

Yaw A. Peprah ${ }^{1}$; Tiffany Brown ${ }^{1}$; Jody Ciolino ${ }^{2}$; Randy Hountz ${ }^{3}$; Karen Iversen $^{4}$; Linda Murakami ${ }^{5}$; Jennifer Potempa ${ }^{6}$; Luke Rasmussen ${ }^{2}$; Theresa L. Walunas ${ }^{2}$; Jesi Wang ${ }^{7}$; Chen Yeh ${ }^{2}$; Abel Kho'; Stephen Persell ${ }^{1}$. 
${ }^{1}$ Northwestern University Feinberg School of Medicine, Chicago, IL; ${ }^{2}$ Northwestern University, Chicago, IL; ${ }^{3}$ Purdue Healthcare Advisors, West Lafayette, IN; ${ }^{4}$ AllianceChicago, Chicago, IL; ${ }^{5}$ American Medical Association, Chicago, IL; ${ }^{6}$ Telligen, Oak Brook, IL; ${ }^{7}$ MetaStar, Madison, WI. (Control ID \#3184154)

BACKGROUND: Healthy Hearts in the Heartland (H3) is a quality improvement (QI) program focused on preventive cardiology in primary care practices in the Midwest using practice facilitation. Clinical targets included the "ABCS" quality measures: (A) Aspirin/antiplatelet therapy for ischemic vascular disease, (B) Controlling High Blood Pressure, (C) Statin Therapy for the Prevention and Treatment of Cardiovascular Disease, and (S) Tobacco Use: Screening and Cessation Intervention.

METHODS: Practices from Illinois, Indiana, and Wisconsin were randomized to 12 months of facilitator support aimed at implementing either point-ofcare (POC) QI strategies alone or POC plus population management $(\mathrm{POC}+\mathrm{PM})$ strategies. Randomization was done in four waves, with a modified constrained process used to ensure within-wave balance. Practices in the POC arm received coaching and encouragement to select improvement strategies to address care at office visits. Practices in the POC+PM arm received coaching to use $\mathrm{POC}$ approaches and population health management strategies. Practice surveys included items about EHR functionality, perceived ability to address barriers to QI, Change Process Capability Questionnaire (CPCQ) summary score for preventive cardiology improvement, and each practice's patient population. Quality measures were collected in three ways: directly from EHRs using Physician Quality Reporting System (PQRS) measures, using popHealth (an open-source quality measure database and reporting engine using data exported from the practices' EHRs), or manual chart review (when PQRS and popHealth not possible). Primary analyses compared the performance on each ABCS measure at baseline and 12 months among all practices using a paired t-test. We then compared the mean values of the ABCS measures at 12 months across study arms controlling for baseline values.

RESULTS: 2281 practices were assessed; 583 were ineligible; 1472 declined, would not schedule a recruitment visit or were unreachable; 226 practices were enrolled and randomized. Study arms were balanced across measured baseline practice characteristic. 179 practices contributed follow-up 12-month data. Overall, performance on ABCS measures was greater at 12 months compared to baseline: (A) 4\% (95\% CI, 2 to 6), (B) 4\% (2 to 6), (C) $5 \%$ (3 to 7), (S) $5 \%$ (2 to 7), $\mathrm{P}<0.001$ for each. Over 12 months, the adjusted difference-in-differences for the POC+PM arm versus POC was: (A) $2 \%(-2$ to 5), (B) $-1 \%$ (-4 to 3), (C) 3\% (0 to 7), and (S) $2 \%$ (-2 to 6); $\mathrm{p}>0.05$ for all. CPCQ improved slightly overall, mean change 0.30 ( 0.09 to 0.51 ) but did not differ across study arms.

CONCLUSIONS: Among participating practices, practice facilitator-led QI was associated with modest improvements in preventive cardiology measures, but we did not find convincing evidence that adding the option to implement population management approaches was superior to point-of-care strategies alone.

\section{EFFECT OF SOCIODEMOGRAPHIC FACTORS ON UPTAKE OF A PATIENT-FACING INFORMATION TECHNOLOGY FAMILY HEALTH HISTORY RISK ASSESSMENT PLATFORM}

R. R. $\mathrm{Wu}^{2}{ }^{2}{ }^{1}$; Rachel A. Myers ${ }^{2}$; Adam Buchanan ${ }^{3}$; David Dimmock ${ }^{4}$; $\overline{\text { Kimberly G. Fulda }}{ }^{5}$; Susanne Haga ${ }^{2}$; Irina V. Haller ${ }^{6}$; Melissa L. Harry ${ }^{6}$; Catherine McCarty ${ }^{7}$; Joan Neuner ${ }^{8}$; Tejinder K. Rakhra-Burris ${ }^{2}$; Nina Sperber $^{9}$, ${ }^{\text {; Corrine Voils }}{ }^{10,}{ }^{11}$; Geoffrey S. Ginsburg ${ }^{12}$; Lori A. Orlando ${ }^{13}$, ${ }^{2}$. ${ }^{1}$ Veteran Health Affairs, Durham, NC; ${ }^{2}$ Duke University, Raleigh, NC; ${ }^{3}$ Geisinger Health, Danville, PA; ${ }^{4}$ Rady's Childrens Institute for Genomic Medicine, San Diego, CA; ${ }^{5}$ University of North Texas Health Science Center, Fort Worth, TX; ${ }^{6}$ Essentia Health, Duluth, MN; ${ }^{7}$ University of Minnesota Medical School, Duluth campus, Duluth, MN; ${ }^{8}$ Medical College of Wisconsin, Milwaukee, WI; ${ }^{9}$ Duke University, Durham, NC; ${ }^{10}$ University of Wisconsin School of Medicine and Public Health, Madison, WI; ${ }^{11}$ William S.
Middleton Memorial Veterans Hospital, Madison, WI; ${ }^{12}$ Duke Center for Applied Genomics \& Precision Medicine, Durham, NC; ${ }^{13}$ Duke, Durham, NC. (Control ID \#3163018)

BACKGROUND: Patient-facing family health history $(\mathrm{FHH})$ based risk assessment platforms have been shown to increase identification of at-risk individuals within the general population and alter practice patterns to address at-risk individuals' needs. We sought to ascertain differences in utilization of one such tool, MeTree, across a diverse patient population. Given patients' range of experiences and comfort with IT-based health applications, knowledge of and sharing of health information between family members, health information-seeking, cultural practices, and other patient demographics, we hypothesized that differences in uptake might exist. In this paper, we report our findings regarding 1) the degree to which uptake of the intervention varied by patient demographics and 2) the impact of socio-demographics on variations in utility of the platform as measured by the quality of the data collected. METHODS: This was a type III hybrid implementation-effectiveness trial in four diverse healthcare systems across the United States. The trial was administered almost exclusively online, including consent, baseline survey, and risk assessment completion. Subjects were any adult (age 18) scheduled for a routine primary care appointment at a participating clinic. Measures included participant demographics, personal and family health history, health literacy and information seeking. We used multi-variate logistic regression to model the effect of sociodemographic and other factors on study progression. Quality of FHH data entered as defined as relatives: 1) with age of onset reported on relevant conditions; 2) if deceased, with cause of death and 3) age of death reported and 4) percentage of relatives with medical history marked as unknown was analyzed using grouped logistic fixed effect regression.

RESULTS: 2,514 participants consented, mean age 57 and $10.4 \%$ minority. Multi-variate modeling showed progression through study stages was more likely for younger ( $p$-value $=0.005)$, more educated $(p$-value $=0.004)$, nonAsian ( $p$-value $=0.009)$, and female ( $p$-value $=0.005$ ) subjects. Those with lower health literacy or information-seeking confidence were less likely to complete the study (p-value $<0.001$ and 0.03 respectively). Most significant drop-out occurred during the risk assessment completion phase. Overall, quality of FHH data entered was high: relatives' condition's age of onset reported $87.9 \%$, relative's cause of death $85.6 \%$ and age of death $93.8 \%$, and relative's medical history marked as unknown $19.8 \%$ of the time.

CONCLUSIONS: A demographically diverse population was able to complete an IT-based risk assessment but there were differences in attrition by sociodemographic factors particularly related to age and education level. More attention should be given to ensure end-user functionality of health IT and leverage electronic medical records to lessen patient burden.

EFFECT OF THYROID HORMONE REPLACEMENT ON FATIGABILITY IN OLDER ADULTS WITH SUBCLINICAL HYPOTHYROIDISM: A RANDOMIZED PLACEBO CONTROLLED TRIAL Mirah J. Stuber ${ }^{1}$; Elisavet Moutzouri ${ }^{1,2}$; Martin Feller ${ }^{1,}{ }^{2}$; Cinzia Del Giovane ${ }^{1}$; Manuel R. Blum ${ }^{2,3}$; Nancy W. Glynn ${ }^{4}$; Douglas Bauer ${ }^{5}$; Jacobijn Gussekloo $^{6,6}$; Vera J. McCarthy ${ }^{7}$; Patricia M. Kearney ${ }^{7}$; Drahomir Aujesky ${ }^{2}$; Rudi G. Westendorp ${ }^{8}$; David J. Stott ${ }^{9}$; Nicolas Rodondi ${ }^{1,2}$. ${ }^{1}$ University of Bern, Bern, Switzerland; ${ }^{2}$ Inselspital, Bern University Hospital, Bern, Switzerland; ${ }^{3}$ Stanford University School of Medicine, Stanford, CA; ${ }^{4}$ University of Pittsburgh, Pittsburgh, PA; ${ }^{5}$ University of California, San Francisco, CA; ${ }^{6}$ Leiden University Center, Leiden, Netherlands; ${ }^{7}$ University College Cork, Cork, Ireland; ${ }^{8}$ University of Copenhagen, Copenhagen, Denmark; ${ }^{9}$ University of Glasgow, Glasgow, United Kingdom. (Control ID \#3185049)

BACKGROUND: Global fatigue is one of the most commonly reported symptoms in subclinical hypothyroidism (SHypo) and one of the main reasons for prescribing levothyroxine. The recent TRUST trial done by our group indicated no benefit on global fatigue from levothyroxine replacement in SHypo. Given that the treatment of SHypo remains controversial, we analyzed the effect of levothyroxine replacement in patients with SHypo 
using a new fatigability scale that measures both physical and mental domains. Perceived fatigability anchors tiredness to a set of activities and has been shown to be a more sensitive measure than global fatigue.

METHODS: The TRUST trial was a double-blind, randomized, placebocontrolled, parallel-group trial involving adults aged 65 and diagnosed with SHypo defined as the presence of persistently elevated TSH levels (4.6-19.9 $\mathrm{mU} / \mathrm{L}$ ) with free thyroxine within the reference range. The present substudy on fatigability included participants from Switzerland and Ireland. Our outcome was the between-group difference in perceived physical and mental fatigability after twelve months using the Pittsburgh Fatigability Scale (PFS), a 10-item self-administered questionnaire, that measures both physical and mental fatigability (scores range from 0 to 50 , higher score=higher fatigability). Multivariate linear regression models were adjusted for levothyroxine starting dose, sex, site and baseline scores in a modified intention to treat population.

RESULTS: This study included 230 participants (mean age: 73.4 years; $44.4 \%$ female). The mean \pm SD TSH was $6.2 \pm 1.9 \mathrm{mIU} / \mathrm{L}$ at baseline and after 1 year decreased to $3.1 \pm 1.3 \mathrm{mIU} / \mathrm{L}$ in the levothyroxine group vs. $5.3 \pm 2.3$ $\mathrm{mIU} / \mathrm{L}$ in the placebo group $(\mathrm{p}<0.001)$. At baseline, mean physical PFS score was $14.7 \pm 9.3$ in levothyroxine group and $11.1 \pm 9.1$ in placebo group; mean mental PFS score was $7.4 \pm 8.0$ and 5.1 \pm 6.9 , respectively. The mean physical PFS score at 12 months (adjusted for baseline score) was $13.7 \pm 0.7$ for levothyroxine group and $13.6 \pm 0.7$ for placebo group ( $\mathrm{p}=0.88$ ); mean mental PFS score was $5.5 \pm 0.6$ and $6.5 \pm 0.7$, respectively ( $\mathrm{p}=0.26$ ). After adjustment for covariates, we found no significant difference in the mean follow up scores at 1 year after levothyroxine replacement on perceived physical fatigability (between-group difference $=0.2 ; 95 \%$ confidence interval $[\mathrm{CI}]=-1.8$ to $2.1 ; \mathrm{p}=0.88$ ) nor on mental fatigability (between-group difference $-1.0 ; 95 \% \mathrm{CI}=-2.8$ to $0.8 ; \mathrm{p}=0.26$ ).

CONCLUSIONS: Our substudy extended the TRUST trial's findings by examining the novel constructs of physical and mental fatigability. Levothyroxine replacement in SHypo was not associated with change in physical fatigability nor with change in mental fatigability. Therefore, fatigue does not seem to be a good indication to prescribe levothyroxine in SHypo.

\section{EFFECTIVE DEPRESSION MANAGEMENT IN A COLLABORA- TIVE CARE MODEL: COMPARING RESIDENTS AND ATTEND- ING PHYSICIANS IN AN ACADEMIC GENERAL MEDICINE CLINIC.}

Jessica Ristau $^{1,2}$; Celia P. Kaplan²; Douglas Bauer²; Mitchell D. Feldman²; Maki Aoki ${ }^{2}$; Rosemary Lam ${ }^{2}$; Maria E. Garcia $2 .{ }^{1}$ UCSF, San Francisco, CA; ${ }^{2}$ University of California, San Francisco, San Francisco, CA. (Control ID \#3176032)

BACKGROUND: The Collaborative Care Model (CCM) is effective for depression care. Yet there is limited information regarding CCM implementation in academic centers. This study examines whether patients with resident primary care physicians (PCPs) achieve similar outcomes as those with attending PCPs.

METHODS: This prospective cohort study assessed potential differences in a combined resident and attending academic general medical practice between PCP type (resident versus attending) and depression identification and follow-up. CCM included multidisciplinary team-based engagement including a psychiatrist consultant, social worker and behavioral health assistant using systematic universal screening, validated clinical rating scales and population-based registries to facilitate active management with the PCP. The study included all patients seen from October 2017 to October 2018 with a PHQ9 10 and a second PHQ9 $>0$ at least 6 weeks later $(\mathrm{N}=149$ of $>6,000$ patients screened). We used the electronic medical record to assess primary outcomes (PHQ9<10, PHQ9 decrease by 50\%, and/or remission), and secondary outcomes (referrals to psychotherapy and/or antidepressant prescriptions) at the end of the study period.

RESULTS: The population was diverse (69\% non-white), primarily English-speaking (92\%), had mostly public insurance (71\%), and had a high medical complexity score (62\%, clinic-specific index that includes chronic illnesses, medications, online messages). Patients with resident PCPs $(n=23)$ were younger (age 45 vs. $56, \mathrm{p}<0.001$ ) with higher rates of Medicaid $(20.3 \%$ vs. $7.8 \%, \mathrm{p}=0.025)$, compared to those with attending PCPs $(\mathrm{n}=18)$. At baseline, patients averaged PHQ9 score 15.8, 14 visits in the health care system per year, and time between PHQ9 scores was 6.2 months, with no difference by PCP type. Patients with an attending PCP were more likely to improve by all metrics (see Table 1), despite no differences in the secondary outcomes (therapy referrals and antidepressant prescriptions). Notably, the patients with attending PCPs were more likely to have their initial positive screening visit with their PCP $(81.1 \%$ for attendings vs. $62.7 \%$ for residents, $\mathrm{p}=0.012$ ) rather than a covering provider.

CONCLUSIONS: In a diverse urban academic clinic, the depression CCM demonstrated lower effectiveness for patients of resident PCPs compared to those with attending PCPs despite similar process measures.

\section{EFFECTIVE TEACHERS OF INVASIVE BEDSIDE PROCE- DURES: A MULTI-INSTITUTIONAL QUALITATIVE STUDY}

Diana J. Kelm ${ }^{1}$; Jennifer L. Ridgeway ${ }^{1}$; John T. Ratelle ${ }^{1}$; Adam P. Sawatsky ${ }^{1}$; Alexander S. Niven ${ }^{1}$; Anna K. Brady ${ }^{2}$; Jakob McSparron ${ }^{4}$; Margaret Hayes ${ }^{3}$; Kannan Ramar ${ }^{1}$; Thomas J. Beckman ${ }^{1}$. Mayo Clinic, Rochester, MN; ${ }^{2}$ Oregon Health and Science Center, Portland, OR; ${ }^{3}$ Beth Israel Deaconess Medical Center, Boston, MA; ${ }^{4}$ University of Michigan, Ann Arbor, MI. (Control ID \#3178534)

BACKGROUND: Invasive bedside procedures (IBPs) in the intensive care unit (ICU) are often performed urgently, putting patients at substantial risk. Complications from IBPs are preventable and related to operator proficiency. Residents receive IBP education, yet there is limited research on what constitutes an effective IBP teacher. We conducted a multi-institutional qualitative study of Pulmonary and Critical Care Medicine (PCCM) faculty and fellows to better understand their perspectives on the characteristics of effective IBP teachers.

METHODS: Focus groups (FGs) were conducted at Mayo Clinic and Oregon Health and Sciences University. Two FGs were held with fellows and two with faculty. FGs were facilitated by a trained investigator, audiorecorded, and transcribed verbatim for analysis. Themes were identified inductively and then compared against constructs from social and situated learning theories. Data was analyzed between and across professional groups. Qualitative research software (NVivo, QSR International) was used to facilitate data organization and create an audit trail of the analysis process. A multidisciplinary research team was engaged to minimize interpretive bias. RESULTS: Seventeen fellows and eighteen faculty participated. Inductive analysis revealed 3 themes related to attributes of effective IBP teachers: observed behaviors, personal characteristics, and influence on the learning environment. Observed behaviors (representative quotes) included leading pre-procedure huddles to assess learner experience with IBPs and defining expectations ("Assess the learner... to figure whether they can proceed"), along with debriefing and providing feedback ("A good procedural teacher gives feedback after the procedure ... reinforcing what you liked ... and what could make it go more smoothly"). Personal characteristics included procedural competency, calm demeanor, and demonstrating trust ("I think it's just personality ... some people seem to be composed," "They stay calm ... especially in high-pressure situations."). Important environmental factors were creating safe learning climates, reducing distractions, and focusing on the procedure ("The best procedural teacher ... no matter what was going on outside ... remained focused," "In a crowded room and intense situation ... I literally whisper in the learner's ear, use the needle like this").

CONCLUSIONS: PCCM fellows and faculty cited a range of personal and behavioral characteristics of effective IBP teachers, such as checking in with residents and debriefing on procedures; 
remaining calm and providing encouragement; and creating safe environments and ensuring focus on IBPs. The potential risk of complications during IBPs underscores the need for procedural competency, yet this study found a notable emphasis on the interpersonal, as opposed to technical, aspects of successful IBP teachers. These findings should inform future curricula on teaching IBPs in the ICU.

\section{EFFECTIVENESS AND COST-EFFECTIVENESS OF MAILED FECAL IMMUNOCHEMICAL TESTS FOR COLO- RECTAL CANCER SCREENING IN A SAFETY NET HEALTH SYSTEM}

Brennan Lanier ${ }^{1}$; Nicole Kluz ${ }^{1}$; Charu Juneja ${ }^{1}$; Todd Olmstead ${ }^{2}$; Michael Pignone ${ }^{1,}{ }^{1}$. ${ }^{1}$ Dell Medical School, Austin, TX; ${ }^{2}$ LBJ School of Public Affairs, Austin, TX. (Control ID \#3184162)

BACKGROUND: Colorectal cancer screening rates are suboptimal, especially in uninsured and low-income patients served in Federally Qualified Health Centers (FQHC). Mailed Fecal Immunochemical Testing (FIT) has been demonstrated to increase screening rates in vulnerable patients, but the optimal means of ensuring uptake and cost-effectiveness is unclear. Human-Centered Design can help improve the effectiveness of patient-directed interventions like mailed FIT. We sought to evaluate the effectiveness and cost-effectiveness of a novel mailed FIT intervention based on Human-Centered Design principles.

METHODS: Methods: As part of a larger study to implement mailed FIT in an FQHC system, we sought to test the effectiveness and cost-effectiveness of different approaches to encouraging uptake of mailed FIT. Our basic intervention consisted of a generic mailing packet that included an introductory letter in plain language, the FIT itself, easy to read instructions, and a postagepaid lab mailer, supplemented with written and text messaging reminders from the FQHC system. Our enhanced intervention was developed in collaboration with the Dell Medical School Design Institute for Health. It included simplified instructions with pictorial guidance that emphasized ease of use, a graphic redesign, enhanced packaging (cardstock pouch that allowed the FIT to be placed in the bathroom as a visual cue), and the same written and text-based reminders. We conducted multiple rounds of small tests of change to evaluate the return rate (effectiveness) of mailed FIT within 60 days. We then used modeling to examine the cost effectiveness of the standard and enhanced mailers in terms of costs per additional patient screened. Cost parameters were based on supply costs and detailed observations of labor required, valued at the wage rate. We prospectively defined a cost-effectiveness threshold for adoption of $\$ 50$ per additional patient screened.

RESULTS: Uptake of the basic mailer and reminders was $22.5 \%$ at an average cost of $\$ 5,374$ per 500-patient mailing. Uptake of the enhanced mailer was $27.5 \%$ at a cost of $\$ 7,225$ per 500 -patient mailing (difference $5.0 \%$ points uptake and $\$ 1851$ costs). The cost per additional patient screened was $\$ 47.77$ for the basic intervention mailer compared with no intervention. The incremental cost-effectiveness of the enhanced mailer was $\$ 74.05$ per additional patient screened. If the unit cost of the FIT pouch could be reduced from $\$ 2.22$ to $\$ 1.01$, or if the uptake rate of the enhanced intervention was $31.8 \%$ or greater, then the enhanced mailer would become cost-effective.

CONCLUSIONS: Mailed FIT is an effective and cost-effective population health strategy for increasing CRC screening in safety net patients. As compared to the standard mailed FIT process, our enhanced mailer increases the response rate, but is not costeffective with current unit costs. Additional testing of lower cost variants based on human-centered design is recommended as a next step.
EFFECTIVENESS AND SAFETY OF DABIGATRAN ETEXILATE IN PATIENTS WITH NEWLY DIAGNOSED ATRIAL FIBRILLATION FROM THE UNITED STATES: ANALYSIS OF THE GLOBAL REGISTRY ON LONGTERM ORAL ANTITHROMBOTIC TREATMENT IN PATIENTS WITH ATRIAL FIBRILLATION STUDY

Alex Spyropoulos ${ }^{2}$; Brian Olshansky ${ }^{3}$; Shihai Lu ${ }^{1}$; Gregory Y. $\mathrm{Lip}^{4}{ }^{8}$; Menno Huisman ${ }^{5}$; Christine Teutsch $^{6}$; Jonathan L. Halperin ${ }^{7}{ }^{1}$ Boehringer Ingelheim Pharmaceuticals Inc., Ridgefield, CT; ${ }^{2}$ The Donald and Barbara Zucker School of Medicine at Hofstra/Northwell, New York, NY; ${ }^{3}$ University of Iowa, Carver College of Medicine, Iowa, IA; ${ }^{4}$ Institute of Cardiovascular Science, University of Birmingham, Birmingham, United Kingdom; ${ }^{5}$ Leiden University Medical Center, Leiden, Netherlands; ${ }^{6}$ Boehringer Ingelheim International GmbH, Ingelheim, Germany; ${ }^{7}$ Mount Sinai Medical Center, New York, NY; ${ }^{8}$ Liverpool Centre for Cardiovascular Science, University of Liverpool and Liverpool Heart and Chest Hospital, Liverpool, United Kingdom. (Control ID \#3184986)

BACKGROUND: In randomized clinical trials, dabigatran etexilate (DE) has demonstrated efficacy and safety in the prevention of stroke in non-valvular atrial fibrillation (NVAF). However, long-term, real-world prospective data are limited. The Global Registry on Long-Term Oral Antithrombotic Treatment in Patients with Atrial Fibrillation (GLORIA-AF) is an ongoing, 3-phase, prospective, global registry assessing real-world data on usage and outcomes of antithrombotic treatments for stroke prevention in patients with newly diagnosed NVAF who are at a risk of stroke.

METHODS: Clinical and composite outcomes from the GLORIA-AF Phase II trial comprising baseline evaluation and 2-year follow-up for United States (US) patients taking DE $150 \mathrm{mg}$ twice daily were assessed. Outcomes per 100 patient-years ( $95 \%$ confidence interval) were calculated. A small number of patients who were receiving DE $75 \mathrm{mg}$ twice daily were excluded.

RESULTS: Of the 591 eligible patients with newly diagnosed NVAF prescribed DE $150 \mathrm{mg}$ twice daily (mean age, 69.4 years; mean $\mathrm{CHA}_{2} \mathrm{DS}_{2}$-VASc score, 3.0; mean HAS-BLED score, 1.3), 590 had taken $1 \mathrm{DE}$ dose. The table shows incidence rates for clinical and composite outcomes.

CONCLUSIONS: These data are the first long-term, prospectively collected real-world data of a US cohort with newly diagnosed NVAF treated with DE. The results, consistent with clinical trials, indicate a favorable benefit-risk profile of DE $150 \mathrm{mg}$ twice daily in US patients with newly diagnosed NVAF.

EFFECTIVENESS OF A BRIEF INTERVENTION TO INCREASE RAPID PRIMARY CARE FOLLOW-UP AMONG AFRICAN AMERICAN PATIENTS WITH UNCONTROLLED DIABETES Emily M. Mylhousen ${ }^{1}$; Elizabeth A. Tolley ${ }^{2}$; Satya Surbhi ${ }^{1}$; Patti Smith $^{1}$; Carolyn Riordan ${ }^{1}$; James E. Bailey ${ }^{2}$. ${ }^{1}$ University of Tennessee Heath Science Center, Memphis, TN; ${ }^{2}$ University of Tennessee Health Science Center, Memphis, TN. (Control ID \#3185131)

BACKGROUND: Diabetes management requires primary care engagement. Without a usual source of care, patients with diabetes have higher risks of uncontrolled disease, hospitalizations, and early death. Patients in medically underserved areas (MUA) are at especially high risk for inadequate diabetes management during transitions of care. This study sought to assess: 1) feasibility of assisting hospitalized patients to obtain rapid primary care follow-up 2) effectiveness of a brief intervention to encourage rapid primary care follow-up and 3) challenges patients face in scheduling follow-up care after hospitalization. 
METHODS: Three-part study of adult patients with uncontrolled diabetes including: 1) cross-sectional study- patient experience getting care, 2) pilot pragmatic randomized controlled trial - intervention to improve primary care follow-up and 3) secret shopper assessment- ease of primary care appointment making. Patients were enrolled at hospitalization to one of 3 major hospitals of a non-profit health system serving MUA in the Mid-South. Inclusions: African-American, $\mathrm{HbA} 1 \mathrm{C}>8 \%$, and $>1$ chronic health condition. Exclusions: pregnancy, psychiatric, or cognitive impairment. The baseline crosssectional survey was conducted upon enrollment. All patients received pamphlet on importance of rapid PCP follow-up. The intervention group also was given an appointment within 14 days of anticipated discharge. Hold times and new patient appointment availability were recorded. Primary outcomes included self-reported PCP attendance within 7 and 14 days. Secondary outcomes included patient-reported difficulty getting needed care as well as hold times and new patient appointment availability for primary care follow-up.

RESULTS: Six African American patients with uncontrolled diabetes were enrolled in each trial arm $(n=12)$. Characteristics: mean age 54, $67 \%$ male, $75 \%$ high school education or less, $25 \%$ with regular PCP, $66.7 \%$ with usual source of preventive and mean of 2 hospitalizations in past 6 months. Patients reported delay of care due to inability to get through on the phone (42\%), inability to make appointment (50\%) and long wait times $(42 \%) .80 \%$ of primary care clinics reported new patient availability. Clinics with call centers $(n=3)$ had long hold times (mean 14 mins, 16 secs). PCP follow-up was similar across groups-0\% follow-up within 7-days, $20 \%$ within 14 days.

CONCLUSIONS: This pilot study demonstrates patients with uncontrolled diabetes in MUA have difficulty getting prompt outpatient follow-up care. Vulnerable patients commonly experience difficulty getting scheduling appointments. Hold times for scheduling PCP visits are long and serve as a barrier to rapid follow-up. We found that hospital assistance with appointment making is no panacea - assistance did not improve PCP follow-up. This study suggests that primary care capacity, accessibility, and integration with hospital networks are fundamental problems that must be addressed to adequately serve our most vulnerable patients.

\section{EFFECTS OF A REFINED EVIDENCE-BASED TOOLKIT ON MEDICATION RECONCILIATION QUALITY AND SAFETY AT MULTIPLE HOSPITALS: RESULTS OF THE MARQUIS2 STUDY \\ Jeffrey Schnipper $^{1,2}$; Harry Reyes Nieva ${ }^{1,2}$; Meghan Mallouk ${ }^{3}$; Amanda} S. Mixon ${ }^{4}$; Stephanie Rennke ${ }^{5}$; Eugene S. Chu ${ }^{6}$; Stephanie Mueller ${ }^{1,2}$; G. R. Smith ${ }^{7}$; Mark V. Williams ${ }^{8}$; Tosha B. Wetterneck ${ }^{9}$; Jason Stein ${ }^{10,}{ }^{11}$; Anuj K. Dalal ${ }^{1,2}$; Stephanie Labonville ${ }^{1}$; Anirudh Sridharan ${ }^{12}$; E. John Orav $^{1,13}{ }^{13}$; Brian Levin ${ }^{1,14}$; Catherine Yoon ${ }^{1}$; Marcus Gresham ${ }^{1}$; Jenna Goldstein ${ }^{3}$; Sunil Kripalani ${ }^{15}$. ${ }^{1}$ Brigham and Women's Hospital, Boston, MA; ${ }^{2}$ Harvard Medical School, Boston, MA; ${ }^{3}$ Society of Hospital Medicine, Philadelphia, PA; ${ }^{4}$ VA Tennessee Valley Healthcare System and Vanderbilt University, Nashville, TN; ${ }^{5}$ University of California San Francisco, San Francisco, CA; ${ }^{6}$ UTSW, Dallas, TX; ${ }^{7}$ Northwestern University, Chicago, IL; ${ }^{8}$ University of Kentucky HealthCare, Lexington, KY; ${ }^{9}$ University of Wisconsin School of Medicine and Public Health, Madison, WI; ${ }^{10}$ Centripital, Atlanta, GA; ${ }^{11}$ 1Unit, Atlanta, GA; ${ }^{12}$ Howard County General Hospital, Columbia, MD; ${ }^{13}$ Harvard T.H. Chan School of Public Health, Boston, MA; ${ }^{14}$ SUNY Downstate Medical School, Brooklyn, NY; ${ }^{15}$ Vanderbilt University Medical Center, Nashville, TN. (Control ID \#3183344)
BACKGROUND: The Multicenter Medication Reconciliation Quality Improvement Study (MARQUIS1) demonstrated that a medication reconciliation best practices toolkit decreased total unintentional medication discrepancies in 5 hospitals. Incorporating lessons learned, we implemented a refined toolkit in a more diverse sample of hospitals.

METHODS: After a formal application process, we selected 18 sites to implement the revised toolkit. This quality improvement (QI) project offered 17 intervention components. One of 8 hospitalists trained in physician-mentored QI coached each site via monthly calls and 1-2 site visits during an 18-month intervention. Each site's QI team assessed local practices, identified improvement opportunities, and implemented toolkit components. The primary outcome was unintentional medication discrepancies in admission or discharge orders, measured by comparing them to a gold standard medication history taken by trained pharmacists, supplemented with medical record review. We used interrupted time- series (ITS) analysis to determine effects of the intervention over time, adjusting for baseline temporal trends. We modeled the number of discrepancies per patient using multivariable Poisson regression adjusted for patient covariates with total number of medications as a model offset and clustered by site. We also conducted on-treatment analyses to determine the effects of a patient receiving at least 1 intervention.

RESULTS: All 18 sites implemented 1 to 15 toolkit components. One site did not collect enough data to be included in analyses. The other 17 sites (4947 patients) saw a steady decline in medication discrepancy rates from approximately 3 discrepancies/ patient to 1 discrepancy/patient (Figure 1). In ITS analysis, sites saw a $5 \%$ relative decrease in discrepancies/month over baseline temporal trends (adjusted incidence rate ratio 0.95 (95\% CI 0.93 $0.97 ; \mathrm{p}<0.001)$. Patients who received at least 1 patient-level intervention had $53 \%$ fewer discrepancies than those who did not receive any [aIRR $0.47(0.45-0.49) ; \mathrm{p}<0.001]$.

CONCLUSIONS: A multicenter medication reconciliation QI initiative using a refined best practices toolkit was associated with a large reduction in unintentional medication discrepancies.

Statistical process control chart of effects of MARQUIS2 intervention on unintentional medication discrepancies

Total medication discrepancies per patient

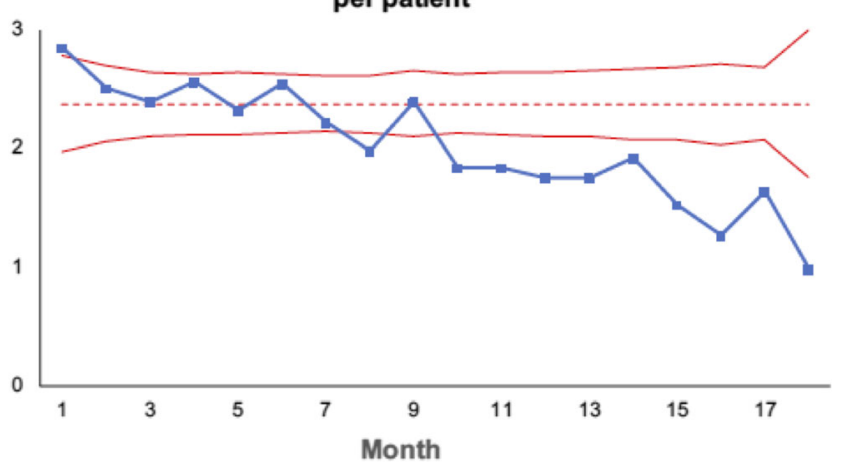

EFFECTS OF GLUCAGON-LIKE PEPTIDE-1 RECEPTOR A G ONISTS VS. PLACEBO AND OTHER ANTIHYPERGLYCEMIC AGENTS IN PATIENTS WITH TYPE 2 DIABETES: A META-ANALYSIS OF RANDOMIZED CONTROLLED TRIALS.

Jason T. Alexander ${ }^{1}$; Erin M. Staab ${ }^{1}$; Wen Wan ${ }^{1}$; Melissa Franco ${ }^{1}$; Alexandra Knitter ${ }^{1}$; Enkhe-Tuyaa Montgomery ${ }^{1}$; Celeste Thomas ${ }^{1}$; Valerie G. Press ${ }^{1}$; Meltem Zeytinoglu ${ }^{1}$; Elizabeth L. Tung ${ }^{1}$; 
Kathryn E. Gunter ${ }^{1}$; Sanjay Jumani ${ }^{1}$; Brittany Bindon ${ }^{1}$; Shari Bolen $^{2}$; Nisa Maruthur ${ }^{3}$; Elbert S. Huang ${ }^{1}$; Louis Philipson ${ }^{1}$; Neda Laiteerapong ${ }^{1}$. ${ }^{1}$ University of Chicago, Chicago, IL; ${ }^{2}$ MetroHealth/ Case Western Reserve University, Cleveland, OH; ${ }^{3}$ Johns Hopkins University School of Medicine, Baltimore, MD. (Control ID \#3180182)

BACKGROUND: Recent evidence from large randomized trials suggests that a new class of medication, the glucagon-like peptide-1 receptor agonists (GLP1RAs), may reduce mortality in addition to lowering hemoglobin A1c in patients with type 2 diabetes (T2D). Even with this evidence, treatment algorithms continue to list metformin monotherapy as first-line. ${ }^{1}$ Understanding the magnitude of benefits of GLP1RAs compared to placebo and other antihyperglycemic agents may lead to significant changes in clinical algorithms. We quantified the effects that GLP1RAs have on hemoglobin A1c and mortality by conducting a systematic review and meta-analysis of all randomized trials of this new drug class. ${ }^{1}$ Davies MJ, D'Alessio DA, Fradkin J, et al. Management of hyperglycemia in type 2 diabetes, 2018. A consensus report by the American Diabetes Association (ADA) and the European Association for the Study of Diabetes (EASD). Diabetologia. 2018 Dec;61(12):246198.

METHODS: We searched PubMed and Scopus (inception to March 2018) for randomized trials of at least 52 weeks duration enrolling adults with T2D that compared GLP1As with placebo or a different antihyperglymeic agent. Two reviewers independently extracted data to ensure accuracy, and differences were resolved by a third reviewer and discussion if necessary. Primary outcomes were change in hemoglobin A1c and all-cause mortality. Effect sizes and standard errors were calculated using the Hedges' g method and Mantel-Haenszel method for hemoglobin A1c and mortality, respectively. Pooled analyses with both fixed and random effect models were performed using the generic inverse variance method. Heterogeneity for each outcome was assessed with the Cochran's Q test and $\mathrm{I}^{2}$ statistic.

RESULTS: A total of 31 randomized controlled trials comprising 46,912 patients were included in the primary analysis. Using a random effect model, patients who received a GLP1RA had a lower hemoglobin A1c when compared to placebo or other antihyperglycemic agent (mean effect size $=-0.28,95 \% \mathrm{CI}-0.32$ to -0.23 ), however heterogeneity was high (Cochran's $\left.\mathrm{Q}=144, \mathrm{I}^{2}=0.79\right)$. Patients receiving GLP1RAs also had lower all-cause mortality ( $\mathrm{OR}=0.87,95 \% \mathrm{CI} 0.81$ to 0.95$)$, and results were not heterogeneous (Cochran's $\mathrm{Q}=14, \mathrm{I}^{2}=0$ ).

CONCLUSIONS: In this systematic review and meta-analysis, patients who received GLP1RAs when compared to placebo or other antihyperglycemic agent had significantly lower hemoglobin A1c levels and mortality rates. Subsequent analyses of changes in cardiovascular risk factors, adverse events, and costs will be needed to help inform future healthcare policy decisions.

\section{EFFECTS OF INTENSIVE PRIMARY CARE ON HIGH-NEED PATIENT EXPERIENCES: FINDINGS FROM A VETERANS AF- FAIRS RANDOMIZED QUALITY IMPROVEMENT TRIAL}

Donna M. Zulman ${ }^{1,3}$; Evelyn T. Chang ${ }^{2}$; Ava Wong ${ }^{3}$; Jean Yoon ${ }^{3}$; Susan E. Stockdale ${ }^{4}$; Michael Ong ${ }^{5,2}$; Lisa V. Rubenstein ${ }^{6}$; Steven Asch $^{1,3}$. ${ }^{1}$ Stanford University, Stanford, CA; ${ }^{2}$ VA- Greater Los Angeles, Los Angeles, CA; ${ }^{3}$ Palo Alto VA, Menlo Park, CA; ${ }^{4}$ Greater Los Angeles VA Healthcare System, Sepulveda, CA; ${ }^{5}$ UCLA, Los Angeles, CA; ${ }^{6}$ RAND and UCLA, Medford, OR. (Control ID \#3184123)

BACKGROUND: Intensive primary care programs have been implemented across diverse healthcare settings to address the needs of patients with medical, behavioral, and social complexity. This study sought to determine how these programs influence patient satisfaction, perceived access, care coordination, trusting relationships.
METHODS: In 2014, the VA launched a national demonstration program to augment the Patient Aligned Care Team (PACT) medical home with PACT-Intensive Management (PIM) programs for high-need patients. Veterans in five VAs were identified based on VA-based hospitalization risk scores $90^{\text {th }}$ percentile and a recent hospitalization or ED visit. A random sample were allocated to PIM between 9/1/2014 and 6/1/15; the remaining patients received usual PACT care. Patients in PIM were offered services such as health coaching, medication reconciliation, care coordination with specialists, and home visits from a dedicated team staffed by primary care providers, social workers, psychologists, nurses, and/or other support staff. We contacted 2,566 PIM and PACT patients to complete a mail survey between 6/3/16 and 12/16/16. The survey included measures of satisfaction with VA services, perceived access, care coordination, patient-centered care, having a trusted provider, and the Patient Assessment of Chronic Illness Care (PACIC). Responses were compared for PIM vs. PACT patients using multivariable logistic and mixed models with sitelevel fixed effects, adjusting for age, sex, race/ethnicity, education, income, functional status, marital status, living alone, social support, loneliness, and health literacy. Veterans who reported participating in PIM were also queried about their experiences with the program.

RESULTS: 759 PIM and 768 PACT patients responded to the survey (response rate 60\%). Patients randomized to PIM were more likely than those in PACT to report that they have a VA healthcare provider who helps coordinate their care $(\mathrm{AOR}=1.17 ; \mathrm{P}=0.031)$, that they were asked about their health goals $(\mathrm{AOR}=1.23 ; \mathrm{P}=0.011)$, and that they have a VA provider whom they trust $(\mathrm{AOR}=1.31 ; \mathrm{P}<0.001)$ and who respects them (AOR=1.17; $\mathrm{P}=0.032$ ). PIM patients were also more likely to report 10 out of 10 on satisfaction with primary care $(\mathrm{AOR}=1.20$; $\mathrm{P}=0.017)$, however other effects on satisfaction and access did not achieve statistical significance. Among the 281 Veterans who reported current or previous enrollment in PIM, most agreed or strongly agreed that the team responded quickly to health concerns $(80 \%)$, helped them get VA care that they needed (82\%), helped them meet their health goals $(70 \%)$, and improved their overall VA care $(72 \%)$.

CONCLUSIONS: The VA's intensive primary care program was wellreceived by participants and had a positive influence on patient experiences with care coordination, trust, and provider relationships, but did not have a significant impact on most patient-reported access and satisfaction measures.

\section{EFFECTS OF MEDICAID EXPANSION ON AVOIDABLE MOR- TALITY IN KENTUCKY IN COMPARISON TO NORTH CARO- LINA}

Maryam L. Famouri ${ }^{1}$; Julia Rushing ${ }^{2}$; Ramon Velez ${ }^{1}$. Wake Forest Baptist Medical Center, Winston-Salem, NC; ${ }^{2}$ Wake Forest University Medical Center, Winston-Salem, NC. (Control ID \#3185459)

BACKGROUND: In 2014, the Affordable Care Act (ACA) expanded Medicaid eligibility for most adults with incomes up to 138 percent of the poverty level, which resulted in large decreases in uninsured rates in both expansion and non-expansion states, especially in low income populations. Studies have indicated that Medicaid expansions prior to the ACA have led to decreases in all-cause mortality; however, the ACA Medicaid expansions' effect on health care amenable mortality is less clear. To explore this relationship, we compared health care amenable mortality rates in Kentucky, an expansion state, with North Carolina, a non-expansion state.

METHODS: All mortality data were obtained from the CDC WONDER online database, and all demographic data were extracted from the American Community Survey. We defined 2010-2013 as the pre-reform period and 2014-2016 as the post-reform period. Individuals who were not between the ages of 15-64 during the pre or post-reform period were excluded from the analysis. Demographic characteristics and health care amenable age-adjusted mortality rates were collected at the county level. The criteria for health care amenable diseases were based on a definition by 
McKee and Nolte. A generalized linear model was developed for ageadjusted health care amenable mortality in both states, adjusting for demographics including median income, gender, marital status, race, unemployment rate, and other socioeconomic factors. The average age-adjusted mortality rate for the pre-intervention period (2010-2013) was also used as a covariate in the model to ensure that the comparisons were balanced beginning with the intervention period.

RESULTS: All demographic characteristics significantly varied between the two states $(\mathrm{p}<0.05)$. Between 2010-2016, age-adjusted health care amenable mortality increased by $6.25 \%$ in Kentucky, while North Carolina saw a modest decrease of $0.7 \%$ during the same time period. Crude ageadjusted mortality rate in the post-reform period in Kentucky was 184.9 per 100,000 as compared to 176.2 in the pre-reform period. Crude ageadjusted mortality in North Carolina was 148.1 per 100,000 in the postreform period and 147.6 in the pre-reform period. In the generalized linear model, both states showed increasing age-adjusted mortality rates with time, but Kentucky had a sharper increase (188.92 to 204.13, p-value $<0.0001$ ) in comparison to North Carolina (178.61 to 181.84 , pvalue $<0.0001$ ) during the post-reform period.

CONCLUSIONS: This analysis suggests that the ACA Medicaid expansions either had no effect or resulted in increased mortality in the state of Kentucky. Given that age-adjusted health care amenable mortality has been steadily increasing in Kentucky since 2010, it is possible that increased insurance coverage has not provided the long-term medical management necessary to amend outcomes. We plan to conduct additional studies to better characterize the relationship between Medicaid expansion and mortality in the United States.

\section{EFFECTS OF PATIENT-CENTERED MEDICAL HOMES TEAM- WORK ON PRIMARY CARE STAFF SATISFACTION WITH CARING FOR HIGH-RISK PATIENTS}

Alexis K. Huynh ${ }^{1}$; Donna M. Zulman ${ }^{2}$; Susan E. Stockdale ${ }^{3}$; Alissa Simon $^{1}$; Lisa S. Meredith ${ }^{5}$; Gulrez Azhar ${ }^{5}$; Evelyn T. Chang ${ }^{4}$. ${ }^{1}$ Veterans Affairs, Los Angeles, CA; ${ }^{2}$ Stanford University and VA Palo Alto, Stanford, CA; ${ }^{3}$ Greater Los Angeles VA Healthcare System, Sepulveda, CA; ${ }^{4}$ VA- Greater Los Angeles, Los Angeles, CA; ${ }^{5}$ RAND Corporation, Santa Monica, CA. (Control ID \#3184137)

BACKGROUND: Caring for complex patients at high-risk for hospitalization may be stressful for patient-centered medical homes (PCMH) care teams. Augmenting or replacing PCMH with intensive primary care (IPC) teams may improve care for high-risk patients. IPC teams specialize in caring for vulnerable populations such as HIV, homeless, intensively managed patients, and women. Little is known about extent to which IPC teams support PCMH staff and improve satisfaction. We examine the impact of IPC teams on PCMH staff perception satisfaction with help received to care for high-risk patients and overall job satisfaction within the context of the PCMH.

METHODS: Data consisted of a cross-sectional survey of primary care providers and nurses $(\mathrm{N}=291)$ at five Veterans Health Administration (VHA) PCMHs in 2016. Outcome measures: satisfaction with help received to care for high-risk patients and overall job satisfaction. Predictors: cohesion among core PCMH teamlet members, availability and perceived care coordination with IPC team and value of PCMH team members, and ease of coordination with PCMH neighbors (e.g., medical and mental health specialists). Covariates: staff exposure to high-risk patients, and staff characteristics (age, gender, provider type and primary care setting), and PCMH team staffing status. We performed bivariate and multiple logistic regressions, weighted for nonresponse.

RESULTS: In fully adjusted models, team cohesion among core PCMH teamlet members $(\mathrm{OR}=1.79 ; \mathrm{p}=0.01)$, perceived care coordination with IPC team $(\mathrm{OR}=1.29 ; \mathrm{p}<0.01)$, ease of coordination with medical subspecialists $(\mathrm{OR}=4.05 ; \mathrm{p}=0.01)$, and spending $>20$ hours per week providing face-to-face care $(\mathrm{OR}=2.64 ; \mathrm{p}=0.01)$ were significantly associated with improved satisfaction with help received to care for high-risk patients. Staff with greater perceived workload for high-risk patients had significantly decreased satisfaction with help $(\mathrm{OR}=0.80 ; \mathrm{p}=0.03)$. PCMH cohesion $(\mathrm{OR}=4.64 ; \mathrm{p}<0.01)$ and perceived care coordination with IPC team $(\mathrm{OR}=1.15 ; \mathrm{p}=0.03)$ were also significantly associated with improved overall job satisfaction.

CONCLUSIONS: Improving PCMH team functioning may be more important than staffing levels in improving staff satisfaction. Care coordination with IPC team that is prompt, frequent, and contain helpful communication may improve PCMH staff satisfaction. Findings suggest that interventions should focus their efforts on improving PCMH teamwork and functioning.

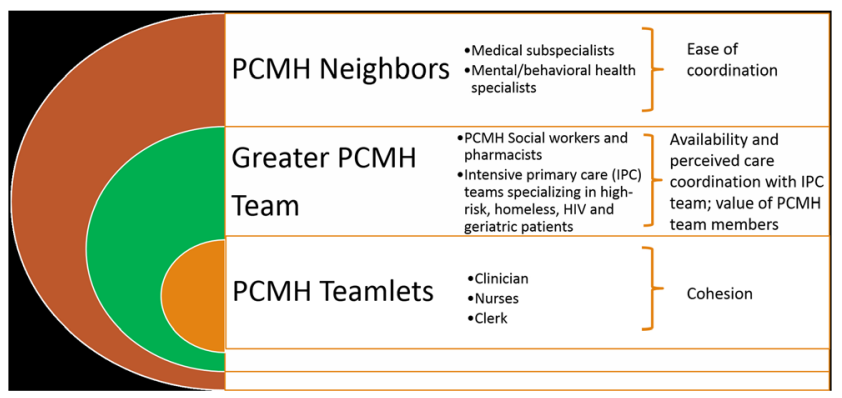

EFFECTS OF SODIUM-GLUCOSE COTRANSPORTER-2 INH I B I T O R S V S P L A C E B O A N D O T H E R ANTIHYPERGLYCEMIC AGENTS IN PATIENTS WITH TYPE 2 DIABETES: A META-ANALYSIS OF RANDOMIZED CONTROLLED TRIALS.

Jason T. Alexander ${ }^{1}$; Erin M. Staab ${ }^{1}$; Wen Wan $^{1}$; Melissa Franco ${ }^{1}$; Alexandra Knitter $^{1}$; Enkhe-Tuyaa Montgomery ${ }^{1}$; Celeste Thomas ${ }^{1}$; Valerie G. Press ${ }^{1}$; Meltem Zeytinoglu ${ }^{1}$; Elizabeth L. Tung ${ }^{1}$; Kathryn E. Gunter $^{1}$; Sanjay Jumani ${ }^{1}$; Brittany Bindon ${ }^{1}$; Shari Bolen ${ }^{2}$; Nisa Maruthur $^{3}$; Elbert S. Huang ${ }^{1}$; Louis Philipson ${ }^{1}$; Neda Laiteerapong ${ }^{1}$. ${ }^{1}$ University of Chicago, Chicago, IL; ${ }^{2}$ MetroHealth/Case Western Reserve University, Cleveland, OH; ${ }^{3}$ Johns Hopkins University School of Medicine, Baltimore, MD. (Control ID \#3185893)

BACKGROUND: Recent evidence from large randomized trials suggests that a new class of medication, the sodium-glucose cotransporter-2 inhibitors (SGLT2Is), may reduce mortality in addition to lowering hemoglobin A1c in patients with type 2 diabetes (T2D). Even with this evidence, treatment algorithms continue to list metformin monotherapy as first-line. ${ }^{1}$ Understanding the magnitude of benefits of SGLT2Is compared to placebo and other antihyperglycemic agents may lead to significant changes in clinical algorithms. We quantified the effects that SGLT2Is have on hemoglobin A1c and mortality by conducting a systematic review and meta-analysis of all randomized trials of this new drug class. ${ }^{1}$ Davies MJ, D'Alessio DA, Fradkin J, et al. Management of hyperglycemia in type 2 diabetes, 2018. A consensus report by the American Diabetes Association (ADA) and the European Association for the Study of Diabetes (EASD). Diabetologia. 2018 Dec;61(12):2461-98.

METHODS: We searched PubMed and Scopus (inception to March 2018) for randomized trials of at least 52 weeks duration enrolling adults with T2D that compared SGLT2Is with placebo or a different antihyperglycemic agent. Two reviewers independently extracted data to ensure accuracy, and differences were resolved by third reviewer and discussion if necessary. Primary outcomes were change in hemoglobin A1c and all-cause mortality. Effect sizes and standard errors were calculated using the Hedges' g method and Mantel-Haenszel method for hemoglobin A1c and mortality, respectively. Pooled analyses with both fixed and random effect models were performed using the generic inverse variance method. Heterogeneity for each outcome was assessed with the Cochran's Q test and $\mathrm{I}^{2}$ statistic. 
RESULTS: A total of 26 randomized controlled trials comprising 43,119 patients were included in the primary analysis. Using a random effect model, patients who received a SGLT2I had a lower hemoglobin A1c when compared to placebo or other antihyperglycemic agent (mean effect size $=-0.29,95 \%$ CI -0.38 to -0.20 ), however heterogeneity was high (Cochran's Q $=297, \mathrm{I}^{2}=$ 0.92). Patients receiving SGLT2Is also had lower all-cause mortality (OR 0.77, 95\% CI 0.67-0.90), and results were not heterogeneous (Cochran's $\mathrm{Q}=23, \mathrm{I}^{2}=0$ ).

CONCLUSIONS: In this systematic review and meta-analysis, patients who received SGLT2Is when compared to placebo or other antihyperglycemic agent had significantly lower hemoglobin A1c levels and mortality rates. Subsequent analyses of changes in cardiovascular risk factors, adverse events, and costs will be needed to help inform future healthcare policy decisions.

\section{EFFICACY OF A MULTI-LINGUAL MOBILE APP TO IN- CREASE HEPATITIS B AND C SCREENING AMONG ASIAN AMERICANS}

Tung T. Nguyen ${ }^{1}$; mandana khalili ${ }^{1}$; Janice Y. Tsoh ${ }^{1}$; Judith M. Walsh $^{2}$; L. E. Goldman ${ }^{1}$; Arcadi Kolchak ${ }^{3}$; Ginny gildengorin ${ }^{1}$; Ching Wong ${ }^{1}$; Ivy Lau ${ }^{1}$; Vi-Van Nguyen ${ }^{1} .{ }^{1}$ University of California San Francisco, San Francisco, CA; ${ }^{2}$ UCSF, San Francisco, CA; ${ }^{3}$ San Francisco Hep B Free, San Francisco, CA. (Control ID \#3185711)

BACKGROUND: There are few studies of technological interventions to address health promotion and patient-provider communication for Asian Americans, who have significant health disparities, including high rates of chronic hepatitis B but low screening rates. There are also few interventions to promote screening for hepatitis $\mathrm{C}$ among the appropriate risk groups in this population. METHODS: Using stakeholder-engaged methods, we developed an iPad app in English, Cantonese, Mandarin, and Vietnamese with: questions about personal risks and beliefs related to hepatitis $\mathrm{B}$ and $\mathrm{C}$ and their screening; tailoring audio-visual messages delivered by doctors to address patient's responses; and a printout summarizing the patient's situation and recommendations to give to primary care providers (PCPs). PCPs and their patients at two health systems were cluster randomized by provider to the intervention or comparison arm. Eligible patients were Asian Americans age 18+, spoke one of the languages, and no history of hepatitis B screening. In both arms, PCPs received a list of patients with no testing for hepatitis B every 6 months. Prior to a PCP visit, intervention participants used the app while comparison participants learned about nutrition and physical activity. Data sources included electronic health record (EHR) and surveys conducted prior to, immediately after, and 3 months after the visit. Analyses were conducted using an intent-to-treat approach and accounting for provider clusters. The dependent variables were. Generalized linear mixed-effects models (GLMM) were constructed to test the hypotheses that the intervention increased patientprovider discussion and receipt of hepatitis $\mathrm{B}$ or $\mathrm{C}$ screening in the appropriate risk groups.

RESULTS: The intervention $(\mathrm{N}=270)$ and comparison $(\mathrm{N}=182)$ participants were similar at baseline, with an overall mean age of 56.8 years $(64.0 \%$ female, $79.7 \%$ foreign-born, $44.8 \%$ limited English proficient). At post-visit, intervention patients were more likely to report discussing hepatitis B $(70.4 \%$ vs. $16.5 \%)$ or $\mathrm{C}$ $(62.6 \%$ vs. $12.6 \%)$ with their providers. At 3-month, EHR data showed higher test receipt $(\mathrm{p}<0.001)$ among intervention participants for hepatitis B $(37.8 \%$ vs. $7.7 \%)$ and hepatitis C (30.3\% vs. $6.0 \%)$. In multivariable analyses, the intervention was very effective in increasing test receipt of hepatitis $\mathrm{B}(\mathrm{OR}=7.5 ; 95 \%$ Confidence Interval [CI] 3.6, 15.5) and hepatitis $\mathrm{C}(\mathrm{OR}=10.5 ; 95 \%$ CI $3.0,36.8)$.

CONCLUSIONS: A multi-lingual mobile app led to more viral hepatitis discussion and screening test receipt. Health information technology can improve healthcare quality among Asian Americans if designed appropriately.

EFFICACY, CLINICALLY MEANINGFUL RESPONSE, AND EARLY EFFECT OF FREMANEZUMAB IN PATIENTS WITH CHRONIC MIGRAINE

Stephen D D. Silberstein ${ }^{1}$; David W. Dodick ${ }^{2}$; Joshua M. Cohen ${ }^{3}$; Ronghua Yang ${ }^{3}$; Melissa Grozinski-Wolff ${ }^{3}$; Xiaoping Ning ${ }^{3}$; Andrea Hinds $^{3}$; Verena Ramirez Campos ${ }^{3}$. ${ }^{1}$ Jefferson Headache Center, Philadelphia, PA; ${ }^{2}$ Mayo Clinic, Phoenix, AZ; ${ }^{3}$ Teva Pharmaceuticals, Frazer, PA. (Control ID \#3185665)

BACKGROUND: Migraine is a highly prevalent and disabling neurological disorder. Chronic migraine $(\mathrm{CM})$, defined as 15 or more headache days per month, at least 8 of which are migraine days, has a substantial negative impact on patients' lives. Preventive migraine treatment is recommended in patients with $\mathrm{CM}$, and preventive therapeutics with an early onset of efficacy may improve the outcomes of patients with CM. Fremanezumab is a fully humanized monoclonal antibody (IgG2 a) that selectively targets calcitonin gene-related peptide (CGRP) and is approved for the preventive treatment of migraine in adults. This analysis of the HALO CM trial assessed the onset of efficacy of fremanezumab for the preventive treatment of CM.

METHODS: HALO CM was a multicenter, randomized, doubleblind, placebo-controlled, parallel-group Phase 3 study evaluating the efficacy and safety of fremanezumab for the preventive treatment of CM. A diagnosis of CM was confirmed during a 28-day pretreatment period. Adults with $\mathrm{CM}$ were randomized to receive either subcutaneous fremanezumab quarterly $(675 \mathrm{mg}$ at baseline and placebo at Weeks 4 and 8), fremanezumab monthly (675 $\mathrm{mg}$ at baseline, and $225 \mathrm{mg}$ at Weeks 4 and 8), or matching placebo over a 12-week period. The primary endpoint the mean change in the average number of headache days of at least moderate severity: a day in which headache pain lasted 4 consecutive hours and had a peak severity of at least a moderate level, or an acute migraine-specific medication (triptans or ergots) was used to treat a headache of any severity or duration.

RESULTS: A total of 1130 patients were randomized to receive either fremanezumab quarterly $(n=376)$, fremanezumab monthly $(n=379)$, or placebo $(n=375)$. Fremanezumab significantly reduced the mean number of monthly headache days of at least moderate severity during the 12 -week treatment period, for both fremanezumab quarterly (least-squares mean change: -4.3 days; $P<0.0001)$ and fremanezumab monthly $(-4.6$ days; $P<0.0001)$ compared with placebo ( -2.5 days). Response to treatment was seen during the first week of treatment (all-fremanezumab group [n=750]: -1.1 days; $P<0.0001$ vs placebo: -0.5 days). During the 12 -week treatment period, there were reductions in the number of days with any acute headache medication use (quarterly: -3.7 days; monthly: -4.2 days; both $P<0.0001$ vs placebo: -1.9 days) and the number of migraine days (quarterly: -4.9 days; monthly: -5.0 days; both $P<0.0001$ vs placebo: -3.2 days), and a greater proportion of patients who received fremanezumab achieved at least a 50\% reduction in headache days of at least moderate severity (quarterly: $38 \%$; monthly: $41 \%$; both $P<0.0001$ ), as compared with those who received placebo (18\%).

CONCLUSIONS: Fremanezumab is effective for the preventive treatment of $\mathrm{CM}$, with responses seen within the first week of treatment. 
EFFICACY, CLINICALLY MEANINGFUL RESPONSE, AND EARLY EFFECT OF FREMANEZUMAB IN PATIENTS WITH EPISODIC MIGRAINE

David W. Dodick ${ }^{1}$; Stephen D D. Silberstein ${ }^{2}$; Joshua M. Cohen ${ }^{3}$; Ronghua Yang $^{3}$; Melissa Grozinski-Wolff ${ }^{3}$; Xiaoping Ning ${ }^{3}$; Sissy Easo-Joseph ${ }^{3}$; Carolina Burgos-Vega ${ }^{3}$. ${ }^{1}$ Mayo Clinic, Phoenix, AZ; ${ }^{2}$ Jefferson Headache Center, Thomas Jefferson University, Philadelphia, PA; ${ }^{3}$ Teva Pharmaceuticals, Frazer, PA. (Control ID \#3185671)

BACKGROUND: Migraine is a prevalent and disabling neurological disorder. The most common form of migraine is episodic migraine (EM), defined as fewer than 15 headache days per month. Patients with 3 or more headaches per month are at risk for progression to chronic migraine $(\mathrm{CM})$. Preventive migraine treatment is recommended in EM patients with 4 or more headache days per month with some impairment, and preventive therapeutics with early onset of efficacy may improve patient outcomes. Fremanezumab is a fully humanized monoclonal antibody (IgG2 a) that selectively targets calcitonin gene-related peptide (CGRP) and is approved for the preventive treatment of migraine in adults. This analysis of the HALO EM trial assessed the onset of efficacy of fremanezumab for the preventive treatment of EM.

METHODS: HALO EM was a multicenter, randomized, double-blind, placebo-controlled, parallel-group Phase 3 study evaluating the efficacy and safety of fremanezumab for the preventive treatment of EM. Adults with EM, confirmed during a 28-day pre-treatment period, were randomized to receive either subcutaneous fremanezumab quarterly (675 $\mathrm{mg}$ at baseline and placebo at Weeks 4 and 8), fremanezumab monthly (225 mg at baseline, Weeks 4 and 8), or matching placebo over a 12-week period. The primary endpoint was the mean change from baseline in the monthly average number of migraine days: a day with 2 consecutive hours of a headache meeting criteria for migraine, probable migraine, or when acute migraine-specific medication (triptans or ergots) was used to treat a headache.

RESULTS: A total of 875 patients were randomized to receive either fremanezumab quarterly $(n=291)$, fremanezumab monthly $(n=290)$, or placebo $(n=294)$. Fremanezumab significantly reduced the mean number of monthly migraine days during the 12-week treatment period, for both fremanezumab quarterly (least-squares mean change: -3.4 days; $P<0.0001)$ and fremanezumab monthly $(-3.7$ days; $P<0.0001)$ compared with placebo ( -2.2 days). Response to treatment was seen during the first week of treatment (quarterly: -0.8 days; monthly: -0.9 days; both $P<0.0001$ vs placebo: -0.3 days). During the 12 -week treatment period, there were reductions in the number of days with any acute headache medication use (quarterly: -2.9 days; monthly: -3.0 days; both $P<0.0001$ vs placebo: -1.6 days), and a greater proportion of patients who received fremanezumab achieved at least a 50\% reduction in migraine days (quarterly: $44 \%$; monthly: $48 \%$; both $P<0.0001$ ), as compared with those who received placebo $(28 \%)$.

CONCLUSIONS: Fremanezumab is effective for the preventive treatment of migraine, with responses seen during the first week of treatment.

\section{ELDERLY PATIENTS' AND THEIR CAREGIVERS' PERSPEC- TIVES ON MEDICATION VALUE: A QUALITATIVE STUDY} Aimee N. Pickering ${ }^{1}$; Megan E. Hamm ${ }^{2}$; Joseph T. Hanlon ${ }^{2,}{ }^{3}$; Carolyn T. Thorpe $^{3}$; Walid F. Gellad ${ }^{3,}$ 2; Thomas R. Radomski ${ }^{2,}{ }^{3}$. ${ }^{1}$ University of Pittsburgh Medical Center, Pittsburgh, PA; ${ }^{2}$ University of Pittsburgh School of Medicine, Pittsburgh, PA; ${ }^{3}$ VA Pittsburgh Healthcare System, Pittsburgh, PA. (Control ID \#3184453)

BACKGROUND: Health systems are increasingly implementing deprescribing interventions to reduce elderly patients' use of unnecessary or harmful medications. Shared decision making between prescribers and patients is an essential component of successful deprescribing, yet patients' and caregivers' perspectives on medication value and their willingness to stop a medication are poorly understood. Our objective was to identify the most significant factors that impact the perceived value of a medication from the perspective of patients and caregivers.

METHODS: We conducted 6 focus groups ( 3 of patients; 3 of caregivers), each consisting of 6-8 community-dwelling adults aged 65 years, or their respective caregivers, who had been prescribed 5 medications in 2018. We sampled participants with diverse sociodemographic backgrounds and varying forms of health insurance. We explored participants' views on factors that enhance or diminish their perception of a medication's value and motivate their decision to discontinue its use. Focus groups were audio-recorded and transcribed verbatim. We developed a codebook that 2 members of the research team independently applied to each transcript, with discrepancies adjudicated by the principal investigator. We then conducted a thematic analysis to identify salient themes.

RESULTS: We identified 3 key themes. First, participants cited effectiveness as the primary factor that caused them to consider a medication to be of high value and worth taking. Patients appraised effectiveness based upon their perceived improvement in symptoms, such as pain, or objective improvement in clinical values, such as blood pressure. Caregivers considered these factors but also based their assessment upon a prescriber's recommendation without directly observing clinical improvement. Second, patients and caregivers considered a medication to be of low value and would stop taking it if it adversely affected their quality-oflife. Participants most frequently cited severity of side effects, discomfort associated with administration, or inconvenience in taking the medication. One caregiver noted, "if it's causing... debilitating pain or nausea... then I don't know if it is worth it... it's sacrificing your quality of life in your older years..." Third, while factoring less prominently, participants also cited a medication's cost as a consideration in determining its value, especially if it caused them to make other material sacrifices.

CONCLUSIONS: We identified a complex interplay of factors, including perceived effectiveness, impact on quality of life, and cost as playing central roles in patients' and caregivers' views of a medication's value and their willingness to discontinue its use. Our findings will allow health systems to incorporate a patient-centered assessment of value into systems-based deprescribing interventions and enable prescribers to more meaningfully engage older patients in shared decision making when deprescribing unnecessary or harmful medications.

\section{ELECTIVE HIP AND KNEE REPLACEMENT: IMPACT OF SO- CIAL DETERMINANTS ON POST-ACUTE CARE USE AND OUTCOMES}

Shawna N. Smith ${ }^{1}$; Ashley Snyder ${ }^{1}$; David Ratz ${ }^{2}$; Laurence F. McMahon ${ }^{1}$; Jennifer Meddings ${ }^{1} .{ }^{1}$ University of Michigan, Ann Arbor, MI; ${ }^{2}$ Veterans Affairs, Ann Arbor, MI. (Control ID \#3182513)

BACKGROUND: The Hospital Readmissions Reduction Program (HRRP) includes elective total knee and hip arthroplasty (TKA/THA). We assessed how claims-based variables used for Medicare riskadjustment and pre-procedure measures of social determinants of health, mobility, activities of daily living (ADL), and pain are associated with the post-acute care (PAC) type first provided after discharge and postprocedure outcomes

METHODS: Retrospective cohort study of Medicare claims was performed for index TKA/THA admissions 2010-2014 as defined by HRRP, and all PAC 90 days post-discharge for adults age 65 years with elective primary TKA/THA, merged with the closest biennial Health and Retirement Study (HRS) survey waves to measure pre-procedure social determinants of health, and pre- and post-procedure self-reported mobility limitations, ADL limitations, and pain. We examined social and clinical determinants as well as pre-post procedure changes in mobility, ADLs, and pain associated with 3 categories of PAC: home health rehabilitation $(\mathrm{HH})$, outpatient rehabilitation (OutptRehab), and skilled nursing facility 
or inpatient rehabilitation (SNFInptRehab). Bivariate and mixed models examined associations between social and clinical factors and first PAC discharge, and differences in pre-post-procedure change by PAC.

RESULTS: 977 TKA/THA hospitalizations (977 patients, 708 TKAs, 269 THAs) were identified with pre- and post-HRS survey data. Overall, patients post-procedure reported 0.19 fewer mobility limitations $(\mathrm{p}=0.12)$, 0.02 more ADL limitations ( $\mathrm{p}=0.77)$, and fewer reported pain (55\% preversus $44 \%$ post-procedure, $\mathrm{p}<0.001) .851(87 \%)$ had Medicare PAC claims. Patients without PAC claims were younger, more likely married, had single procedure, but otherwise no different from those with PAC claims. The 276 discharged to $\mathrm{HH}$ were younger, more likely married, men, with children, fewer comorbidities, with single procedures compared to the 262 discharged to SNFInptRehab. Those discharged to $\mathrm{HH}$ were younger, more likely married, with fewer comorbidities compared to the 313 discharged to OutptRehab. PAC was not associated with preprocedure pain, ADL or mobility limitations, wealth or race. Rates of readmission within 30 days were significantly higher for SNFInptRehab (7.6\%), compared to $\mathrm{HH}(2.9 \%)$, but not significant compared to OutptRehab (5.4\%). Full mixed effects models found no significant differences between HH, OutptRehab, and SNFInptRehab cohorts by pre-post changes in mobility, ADLs, or pain, though pre-post procedure pain improved significantly within each cohort; there was small but statistically significant mobility improvement for SNFIntptRehab. CONCLUSIONS: PAC after TKA/THA differed by patient age, gender, comorbidities, and having spouse or children, but unexpectedly not by pre-procedure pain, mobility, and ADLs. Failure to address social determinants of health in policies aiming reduce PAC costs may reduce effectiveness or risk adverse outcomes.

\section{ELIMINATING LOW-VALUE PRE-OPERATIVE CARE IN VIR- GINIA: A STATE'S CALL TO ACTION}

John N. Mafi ${ }^{1,2}$; Beth A. Bortz ${ }^{3}$; Kyle Russell ${ }^{4}$; Sitaram Vangala ${ }^{5}$; ChiHong Tseng ${ }^{5}$; Joshua M. Liao ${ }^{6}$; Bruce E. Landon ${ }^{7}$; Catherine Sarkisian ${ }^{8}$; A. M. Fendrick ${ }^{9} .{ }^{1}$ David Geffen School of Medicine at UCLA, Los Angeles, CA; ${ }^{2}$ RAND Corporation, Santa Monica, CA; ${ }^{3}$ Virginia Center for Health Innovation, Henrico, VA; ${ }^{4}$ Virginia Health Information, Richmond, VA; ${ }^{5}$ University of California, Los Angeles, Los Angeles, CA; ${ }^{6}$ University of Washington, Seattle, WA; ${ }^{7}$ Harvard Medical School, Boston, MA; ${ }^{8}$ UCLA and Greater Los Angeles VA, Los Angeles, CA; ${ }^{9}$ University of Michigan Health Systems, Ann Arbor, MI. (Control ID \#3182816)

BACKGROUND: For decades, randomized-controlled trials have repeatedly proven that routine pre-operative care for patients undergoing low-risk surgery offers little value; yet, low-value pre-operative care still occurs far too frequently. Using Virginia's All Payer Claims Database (APCD), we examine the prevalence and costs of low-value pre-operative care in order to inform a state-sponsored, randomized intervention to reduce low-value care.

METHODS: Using the 2017 Virginia APCD, we evaluated the use and costs of (1) low-value pre-operative laboratory testing, (2) low-value EKGs, chest x-rays, and pulmonary function testing (PFT), and (3) lowvalue cardiac imaging and cardiac stress testing among asymptomatic, low-risk patients undergoing low-risk surgery (e.g., cataract surgery), The Virginia APCD includes administrative claims data on state residents insured under traditional Medicare, Medicare Advantage, Medicaid, and commercial insurers, and it also divides the state into 5 major regions: Northern, Northwest, Central, Eastern, and Southwest. We defined lowvalue pre-operative care using the Milliman Health Waste Calculator, which applies Choosing Wisely ${ }^{\circledR}$ campaign recommendations and evidenced-based clinical guidelines to administrative claims data. For instance, a screening EKG in an asymptomatic, low-risk patient undergoing cataract surgery would be deemed low-value; in contrast, if the patient has chest pain or a recent myocardial infarction, then an EKG would be considered appropriate. We estimated costs by calculating the average amount of money per service paid to a health care provider across all payers (including patients' out-of-pocket spending).

RESULTS: Among 5.4 million Virginia beneficiaries, 613,242 (11\%) received $1,017,104$ pre-operative services in 2017 . Of these services, $487,561(48 \%)$ were determined to be low-value, which cost the state $\$ 227$ million in unnecessary spending on pre-operative care (an estimated $0.8 \%$ of total health care spending in Virginia). Northern Virginians received the highest proportion of low-value pre-operative services: $53 \%$ of the pre-operative services were low-value (compared to $45-49 \%$ across the other four regions). Low-value pre-operative laboratory testing, low-value EKGs/chest x-rays/PFTs, and low-value cardiac imaging/ cardiac stress testing comprised approximately $94 \%, 0.2 \%$, and $6 \%$ of the volume and approximately $96 \%, 0.2 \%$, and $4 \%$ of the spending on low-value pre-operative care respectively.

CONCLUSIONS: Low-value pre-operative care-particularly preoperative laboratory testing - is highly common and costly in Virginia. As state governments face mounting pressure to lower health care spending, low-value pre-operative care represents an important target for local interventions to improve quality and safely lower the costs of care.

\section{EMERGENCY DEPARTMENT VISITS AND HOSPITAL ADMIS- SIONS FOR OVERDOSE IN A STATE WITHOUT MEDICAID EXPANSION 2016-2017}

Kathleen Fairfield $^{1,}{ }^{1}$; Kimberly Murray ${ }^{1}$; Timothy B. Plante ${ }^{2}$; Eric Maughan ${ }^{1}$; Susan Richardson ${ }^{3}$; Valerie S. Harder ${ }^{3}{ }^{1}$ Maine Medical Center, Portland, ME; ${ }^{2}$ University of Vermont Larner College of Medicine, Burlington, VT; ${ }^{3}$ University of Vermont, Burlington, VT. (Control ID \#3185302)

BACKGROUND: Opioid use disorder increased to epidemic proportions in the past decade, and Maine has one of the worst overdose rates in the US. State legislation was passed in 2016 to reduce provider opioid prescribing through mandated checking of a Prescription Drug Monitoring Program, increased provider education, and limits on amount of opioids that could be prescribed. Simultaneously, medication assisted therapy (MAT) and illicit drug use were rising in the state. This study aims to identify the effect of these policies and changes on patterns of overdose in 2016 and 2017.

METHODS: We used Maine's all-payer claims database to identify $1,299,537$ people aged $15+$ with insurance eligibility between 2016 and 2017. We examined the extent and timing of emergency department (ED) visits and inpatient hospital admissions for overdose from opioids and unspecified drugs in Maine, and variations across age groups (15-24, 25$44,45-64,64+)$. We used descriptive statistics to examine differences between groups.

RESULTS: We observed a total 5,498 overdose events resulting in an ED visit or hospital admission in 2016 and 2017. These episodes decreased slightly, from $2859(0.026 \%)$ in 2016 to $2639(0.024 \%)$ in 2017. We observed significant differences in the rates of overdose by age category. Of the 5,498 overdose events, more events occurred in those aged 45 to 64 (38\%), followed by those aged 25-44 (29\%), aged $65-84(21 \%)$, and the least in the group aged 15 to $24(12 \%)$. Only the youngest age group (15 to 24 year olds) experienced increases in overdose from 2016 to 2017 (Figure). A higher proportion of events occurred in patients with Medicaid or dual Medicare/Medicaid coverage (54\%) compared to those with Medicare only (27\%) or commercial insurance (19\%). CONCLUSIONS: ED visits and hospital admissions for drug overdose decreased during 2016 to 2017 in Maine, in all age groups except the youngest. Overdoses disproportionally affected the Medicaid population. As Maine now begins Medicaid expansion and MAT programs expand, overdose may further decrease in the Medicaid population. Continued efforts to describe the groups most at risk of overdose may inform outreach, prevention, and policy efforts. 
Rates of drug overdoses in Maine 2016-2017 Among Insured Patients Age 15 and older by Age Category (Data from Maine All Payer Claims)

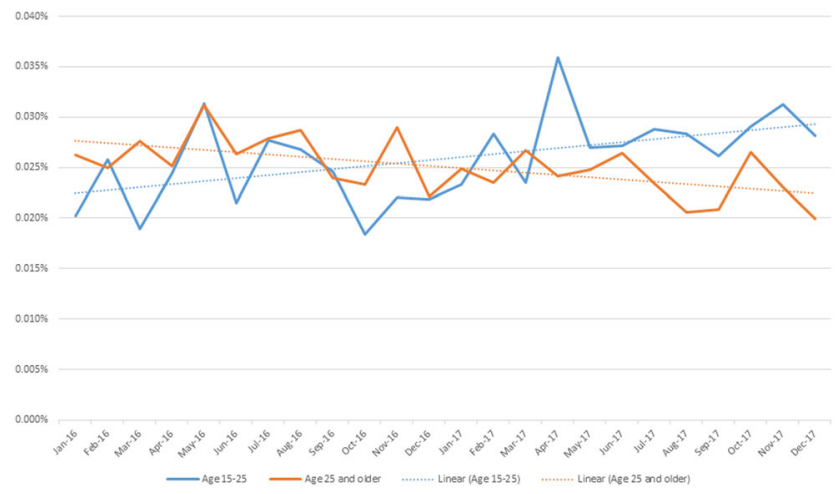

EMOTIONAL COMMUNICATION IN AMBULATORY ENCOUNTERS: HOW DOES PHYSICIAN RESPONSE TO PATIENT EMOTION IMPACT SUBSEQUENT COMMUNICATION AND VISIT LENGTH?

Mary Catherine Beach ${ }^{1}$; Jenny Park ${ }^{1}$; Dingfen Han ${ }^{1}$; Hsin-Chieh Yeh ${ }^{1}$; Somnath Saha ${ }^{2} .{ }^{1}$ Johns Hopkins University, Baltimore, MD; ${ }^{2}$ Portland VA Medical Center, Portland, OR. (Control ID \#3186096)

BACKGROUND: It is widely cited that responding directly to patient emotions is an efficient use of time and that patients are less likely to repeat their emotional concerns if they've felt adequately understood. Because this assertion is based on limited evidence, we sought to verify it.

METHODS: We audio-recorded 41 clinicians interacting with 342 unique patients in an HIV clinic, transcribed each encounter, and used the Verona Coding Definitions of Emotional Sequences (VR-CoDES) to categorize and time-stamp patient emotional expressions and clinician responses. VRCoDES, categorizes patient expressions as either cues (hints about emotion) or concerns (clear emotional expressions). Clinician responses are categorized as (1) providing vs. reducing space for the patient to talk further and (2) explicit vs. non-explicit in terms of addressing the emotion. Responses that reduce space are subdivided into those that provide information to the patient and those that block the patient from speaking further. We used random intercept multilevel logistic and linear regression models (accounting for clustering of emotional expressions within encounters and of patients within clinicians), evaluating whether clinician responses were associated with the time in the visit that the emotion was expressed, with subsequent patient repetition of the emotion, and with subsequent visit length.

RESULTS: The mean visit length was 30.4 minutes, with 1028 emotional expressions ( 750 cues and 228 concerns). Most clinician responses provided space $(81 \%)$ and most were not explicit $(56 \%)$. As each minute of the visit passed, clinician responses were less likely to provide space (OR 0.96 95\% CI 0.95-0.98) and more likely to be explicit (OR 1.02, 95\% CI 1-1.03). Emotions were more likely to be repeated (OR 2.33, 95\% CI 1.66-3.27) when clinicians provided space, and less likely to be repeated (OR 0.61, 95\% CI 0.47-0.80) when clinicians were explicit. When clinicians gave information in response to emotion, the patient was less likely to repeat the emotion immediately (OR $0.50,95 \%$ CI $0.33-0.75$ ) but more likely to repeat the emotion (OR 2.31, 95\% CI 1.17-4.55) later in the visit. In adjusted analyses, visit length was shorter $(=-0.28,95 \%$ CI-0.54 0.02 ) when clinicians provided space for patients, and longer when clinicians gave information in response to patients' emotions ( $=0.42,95 \% \mathrm{CI}$ $0.11-0.73)$. There was no association between the explicitness of clinician response and visit length.

CONCLUSIONS: These results support the widely-held belief that patients are less likely to repeat emotions when clinicians respond explicitly to them, and visit lengths are reduced when clinicians allow space for emotional discussion. Giving information to patients in response to their emotions may result in later patient repetitions, and an increase in visit length.

\section{EMPLOYER AND EMPLOYEE PERSPECTIVES ABOUT A VALUE-BASED BENEFIT DESIGN: DECISION-MAKING FOR OFFERING FREE MEDICINES FOR CHRONIC ILLNESS} Vanessa Simiola ${ }^{1}$; Dennis Ross-Degnan ${ }^{2}$; Connie M. Trinacty ${ }^{1} .{ }^{1}$ Kaiser Permanente, Honolulu, HI; ${ }^{2}$ Harvard Medical School and Harvard Pilgrim Health Care Institute, Boston, MA. (Control ID \#3186146)

BACKGROUND: Failure to take medications as prescribed has been linked to several negative health consequences for patients with diabetes. To improve health outcomes for patients with chronic illness, Kaiser Permanente, Hawaii offers a value-based insurance design (VBID) to select large employer accounts that waives all copays for essential medications and supplies for patients with chronic diseases. Since most Americans under age 65 have medical coverage through their workplace, employers play a prominent role in efforts to lower healthcare costs and increase high quality care through their coverage choices.

METHODS: Using qualitative interviews, this study seeks to understand employer perspectives and decision making for offering VBIDs within their medical insurance benefit plan. Patient surveys were conducted and linked to employer interviews to assess concordance of patient and employer perceptions. We conducted 8 interviews with representatives from five employer groups currently enrolled in a VBID program. Transcribed interviews were analyzed from a grounded theory perspective. Survey data were obtained from 287 adults with diabetes actively receiving free medicines via the VBID program.

RESULTS: Diabetes prevalence in these employers ranged from 2.2-9.8\% $(M=5.5 \%)$. All employer groups $(\mathrm{N}=5)$ reported use of consultants to assist in determining benefits offered to employees and to complete cost-benefit analyses. Several employer representatives reported belief that VBIDs would be viewed differently amongst various facets of their insured members with the most interest amongst retirees $(n=3)$ and the least interest among youngest employees (e.g., ages 18-35; $n=2$ ). Employers $(n=4)$ also regarded the program as improving not only health behaviors within their employees but seeing effects impact families as well. Indeed, several employers provided anecdotes about how their employees demonstrated overall more confidence in their ability to manage their health and were teaching their family members how to improve health outcomes through healthy eating, health screening and overall diabetes management. These results were congruent with findings from patient surveys, where on a scale of $0-10$, patients reported high confidence in their ability to maintain or improve glucose levels $(M=6.64)$ in the next 6 months, and nearly $80 \%$ reported taking medications as prescribed daily.

CONCLUSIONS: Both employers offering the VBID program and employees using the VBID program reported positive outcomes in the presence of program use. Employers noted observable changes in employee behavior and employees identified confidence in their ability to manage their diabetes in the future. Results of this study demonstrate VBIDs can result in an intergenerational transmission of knowledge and behavior change within families. This study provides important information from both employer buyers and employee consumers on VBID plans that can shape the future development of VBID programs and increase uptake in commercial plans.

EMPOWERING PATIENTS TO ACT: USING GRAPHIC MEDICINE TO IMPROVE OUTPATIENT ENGAGEMENT WITH THE EHR

Tyrone A. Johnson; Jacqueline Nichols; Meera Dhodapkar; Maria L. Alkureishi; Wei Wei Lee. University of Chicago, Chicago, IL. (Control ID \#3185964)

BACKGROUND: Electronic health record (EHR) use can have positive and negative effects on the patient-doctor relationship and communication. 
Unfortunately, providers receive little training on EHR-related communication behaviors, and no prior studies have assessed if advocacy interventions can increase patient-EHR engagement. Given that graphic art has been effective in health education, our study aimed to evaluate the impact of graphic medicine comics in promoting patient-centered EHR use and patient engagement during outpatient office visits.

METHODS: Educational comics for patients and providers were developed using best practices from a systematic review on the effects of EHR use on doctor-patient communication, and qualitative studies on patient perceptions of the EHR. Each comic highlighted three best practices for patient-centered EHR use; patient comics advised asking to see the screen, being involved with computer use, and calling for providers' attention during periods of EHR-related distraction. Provider comics were made visible in provider workspaces for the duration of the study and highlighted three best practices: screen sharing, valuing the EHR, and utilizing patient educational resources. Patients attending appointments at the University of Chicago Primary Care Group were recruited for the study. Comics were given to patients at check-in and they were asked to complete a post-visit survey. Participants were randomly selected to complete a follow-up phone survey 1-2 months after the visit.

RESULTS: Response rate of the initial post-visit survey was 84\% (198/ 236). $79 \%$ rated provider communication behaviors positively, $67 \%$ agreed provider communication improved compared to prior visits, and $70 \%$ agreed the comic effectively encouraged patient-EHR involvement. Decreased education level was positively correlated with increased engagement in selfadvocacy behaviors, including asking to see the screen ( $\mathrm{rs}=.273, \mathrm{p}<.001$ ), asking to be involved ( $r s=.244, p=.003$ ), and calling for providers' attention ( $r s=.162, \mathrm{p}=.049$ ). Female patients were more likely to ask to be involved with their doctor's computer use ( $\mathrm{z}=2.18, \mathrm{p}=.030$ ). On phone follow-up, $90 \%$ (45/50) remembered the comic; $46 \%$ recalled at least one best-practice communication behavior. When asked about subsequent doctors' visits, patients reported increases in asking to see the screen (median 3 vs. 4, $\mathrm{z}=2.746, \mathrm{p}=0.010$ ).

CONCLUSIONS: This is the first study to evaluate the impact of graphic medicine on adult patients' EHR-related impressions and behaviors. Comics were effective in encouraging patient involvement, and follow-up indicates lasting impacts over time. The comic may be especially effective with female patients and populations of low-to-average educational level.

\section{ENDOSCOPIC ULTRASOUND-GUIDED FINE NEEDLE ASPIRA- TION OF PANCREATIC METASTASES: A RETROSPECTIVE STUDY}

KEVIN LAMM; Andrew Dries; Jason Lewis; Tarun Narang. Carolinas Medical Center, Charlotte, NC. (Control ID \#3184882)

BACKGROUND: Metastatic lesions to the pancreas are an uncommon but increasingly prevalent problem, accounting for $2 \%$ of pancreatic malignancies. Surgical resection is often recommended for medically fit patients with a single lesion, but in an era with rapid emergence of new chemotherapies and biologic agents, it has not been well established which modality offers the best survival. Lung, breast, renal, skin, and gastrointestinal origins are the most common metastatic primaries. It is postulated that pancreatic metastases may directly invade the ductal epithelium, causing patients to present similarly to pancreatic adenocarcinoma. We report a cohort of cases at a single medical center that utilizes endoscopic ultrasound-guided fine needle aspiration (EUS-FNA) for diagnosis of metastatic lesions to the pancreas.

METHODS: Medical records were abstracted for patients who underwent EUS-FNA with a diagnosis of pancreatic mass. Data regarding demographics and clinical outcomes were recorded from patients with metastatic primaries.

RESULTS: There were a total number of 1,472 EUS-FNA procedures performed for evaluation of pancreatic mass from 2007- 2017. Twenty-two $(1.5 \%)$ of the masses were found to be from metastatic primaries. Nineteen were diagnosed with immunocytology after EUS-FNA. Small cell carcinoma of the lung was the most common histopathology (7 of 22; $32 \%)$. In the remaining patients, the primary histopathology was lung adenocarcinoma $(n=5)$, renal cell carcinoma $(n=4)$, adenocarcinoma of the breast $(n=1)$, liposarcoma $(n=1)$, neuroendocrine tumor of the rectum $(n=1)$, melanoma ( $n=1)$, osteosarcoma $(n=1)$, and squamous cell carcinoma of the lung $(n=1)$. EUS-FNA generated 7 new diagnoses of cancer including small cell carcinoma of the lung and adenocarcinoma of the lung. The most common location for metastatic lesions was the pancreatic head ( 8 of 22; $36 \%$ ). Four masses were found in the tail of the pancreas, and all were found to be small cell carcinoma of the lung. Five of the patients have documented one year survival and one with five year survival. Surgery was performed in three patients. Hospice was pursued by nine of the patients, all with metastases from the lung.

CONCLUSIONS: Pretherapeutic diagnosis of pancreatic masses due to metastatic primaries is necessary for proper management by the multimodal treatment team. As the treatment of cancer continues to rapidly evolve, EUSFNA with immunocytology can assist with the diagnosis of pancreatic metastases and have a major clinical role.

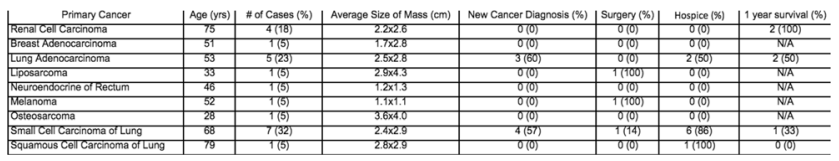

ENGAGING PATIENTS TO PROMOTE DEPRESCRIBING OF POTENTIALLY INAPPROPRIATE MEDICATIONS

\section{Amy Linsky ${ }^{1,2}$; Nancy R. Kressin ${ }^{1}$; Barbara Bokhour ${ }^{3}$; Amy Rosen ${ }^{2}$; Kelly} Stolzmann ${ }^{2}$; Steven R. Simon ${ }^{2} .{ }^{1}$ Dept of Veterans Affairs and Boston University, West Roxbury, MA; ${ }^{2}$ VA Boston Healthcare System, Boston, MA; ${ }^{3}$ ENRM Veterans Affairs Medical Center, Bedford, MA. (Control ID \#3185250)

BACKGROUND: While efforts exist to prevent initiation of medications with low likelihood of benefit, fewer initiatives target medication dose reduction or discontinuation, a process known as deprescribing. While some believe providers have primary responsibility for deprescribing, patients can also facilitate deprescribing. We sought to understand how medicationspecific brochures activate Veterans to discuss medications with their primary care providers and affect deprescribing of targeted medications.

METHODS: We identified Veterans at one Veterans Affairs Medical Center (VA) with primary care appointments over a 3-month period eligible for one of two cohorts: 1) chronically prescribed a proton pump inhibitor (PPI), or 2) diabetes $(\mathrm{DM})$ at risk of hypoglycemia and prescribed either insulin or a sulfonylurea. Each subject was mailed a VA-tailored EMPOWER ("Eliminating Medications through Patient Ownership of End Results") brochure two weeks prior to their appointment. Patients who did not attend their appointment were excluded from analyses. Chart review identified our two outcomes: 1) documentation of discussion about the target medication (possible vs. no), and 2) target medication prescription changes (dose increase or no change vs. dose decrease or discontinuation ["deprescribing"]). Control subjects were patients seen in primary care in the month prior to the intervention who would have been eligible. Additional variables included demographics and select comorbidities and medications. Descriptive statistics examined patient characteristics and prevalence of the two outcomes. Chi-square tests examined the association of receiving brochures with discussions and deprescribing. Within each cohort, we used chi-square tests to assess relationships between patients' characteristics with each outcome.

RESULTS: There were 254 Veterans who received a brochure and attended their primary care appointment (217 in PPI, 37 in DM) and 95 Veterans in the control group (80 in PPI,15 in DM). Subjects were predominantly male $(94 \%)$, age $>65$ years $(80 \%)$, and white $(82 \%)$. There was a possible discussion for $1 / 95(1.1 \%)$ control vs. 32/254 (12.6\%) intervention subjects $(\mathrm{p}=0.001)$. Target medications were deprescribed for $4(4.2 \%)$ control vs. 37 $(14.6 \%)$ intervention subjects $(\mathrm{p}=0.008)$. No medication or comorbidity was statistically associated with deprescribing except within the PPI cohort, 
where gastroesophageal reflux disease was statistically associated with deprescribing, with 24 (20\%) of those with reflux experiencing deprescribing compared to $11(6.25 \%)$ of those without reflux ( $\mathrm{p}=0.0004)$.

CONCLUSIONS: Targeted distribution of medication-specific brochures in advance of a primary care appointment increased both discussions about and deprescribing of potentially inappropriate medications. Engaging patients can lead to changes in clinicians' prescribing practice, specifically for deprescribing. Stopping or reducing inappropriate medication has the potential to improve quality, patient safety, and efficiency.

\section{ENGAGING USERS IN THE DESIGN OF AN MHEALTH, TEXT MESSAGE-BASED INTERVENTION TO INCREASE PHYSICAL ACTIVITY AT A SAFETY-NET HEALTHCARE SYSTEM}

Patricia Avila-Garcia ${ }^{4}$; Sarah Nouri ${ }^{2}$; Anupama Cemballi ${ }^{1,3}$; Urmimala Sarkar ${ }^{1,3}$; Courtney Lyles ${ }^{1,3}$; Adrian Aguilera ${ }^{4} .{ }^{1}$ UCSF, San Francisco, CA; ${ }^{2}$ University of California, San Francisco, San Francisco, CA; ${ }^{3}$ Zuckerberg San Francisco General Hospital, San Francisco, CA; ${ }^{4}$ UC Berkeley, Berkeley, CA. (Control ID \#3183046)

BACKGROUND: Mobile health (mHealth) interventions using textmessages are a promising approach to increasing physical activity in vulnerable populations. This participatory design study outlines the structure to address both content adaptation as well as implementation processes of a physical activity intervention delivered via text-messaging among low-income English and Spanish speakers.

METHODS: Qualitative data was collected in three stages, including one focus group and two rounds of 10 in-depth, semi-structured interviews with primary care patients at a safety-net healthcare system. Discussions focused on participant's overall experience with using text messages as part of depression treatment, influences and perceptions of physical activity, and mobile phone use. We open coded for themes within the transcripts and documented each associated change in the intervention protocol.

RESULTS: The sample was $69 \%$ female, mean age 56.2 years (range 3672); $62 \%$ interviewed in Spanish, $65 \%$ identified as Hispanic/Latino(a), and $46.2 \%$ reported less than a high school education. All had depression and $62 \%$ had diabetes. Specific barriers that emerged in our intervention design included low literacy and only basic use of mobile phones in everyday life, in combination with a high prevalence of co-morbid health conditions and limited mobility. These were each addressed with a specific content or implementation change in the overall intervention design (Table).

CONCLUSIONS: Conducting participatory research with end users of an mHealth intervention to increase physical activity allowed us to tailor and modify components of the text message content and format and ensure that the final intervention will engage participants most effectively.

\section{ESTIMATING RISK OF RECURRENT GASTROINTESTINAL BLEEDS OVER PATIENTS AND TIME}

Matthew A. Pappas. Cleveland Clinic Foundation, Cleveland, OH. (Control ID \#3179153)

BACKGROUND: Following an anticoagulation-associated gastrointestinal hemorrhage (GIB), whether and when to resume anticoagulation is controversial. Patient-level recurrence risk is difficult to predict with accuracy, but time-based recurrence risk may be more predictable. To better inform clinical impressions and future decision-analytic models, we set out to estimate the decrease in GIB recurrence risk over time among patients with atrial fibrillation.

METHODS: Using the Nationwide Readmissions Database (NRD), a large administrative dataset of hospital readmissions, we created a two- stage prediction model, with the first stage predicting patient-level risk of recurrence and the second stage predicting timing, conditional on recurrence. In the first stage, we used standard logistic regression and tested patient characteristics that have been previously associated with recurrent hemorrhage and that are captured in administrative datasets (e.g., cirrhosis, end-stage renal disease, number of chronic conditions). In the second stage, we compared standard survival functions (exponential, Weibull, Gompertz, lognormal, and loglogistic), selecting the survival function that best represented risk of recurrent GIB over time according to Akaike and Bayesian information criteria (AIC and BIC).

RESULTS: Our first-stage model demonstrated reasonable calibration across a 3.5 -fold gradient of risk but discriminated poorly (c-statistic: 0.55 ). A Weibull survival function accurately explained the declining risk of recurrent GIB over time. A survival curve and Weibull model are shown in the Figure. Of patients who sustain recurrent GIB, around $50 \%$ will have done so by day $55,80 \%$ will have done so by day 129 , and $95 \%$ will have done so by day 235 .

CONCLUSIONS: Using a large, nationally-representative administrative sample of patient readmission, we have estimated the risk of GIB recurrence, across patients and over time. Our patient-level discrimination was poor; datasets with physiologic parameters may be better able to predict patient-level risk. But regardless of patient characteristics, risk over time is highly predictable. Decisions to resume anticoagulation after GIB may be better tailored according to time than according to patient-level risk.

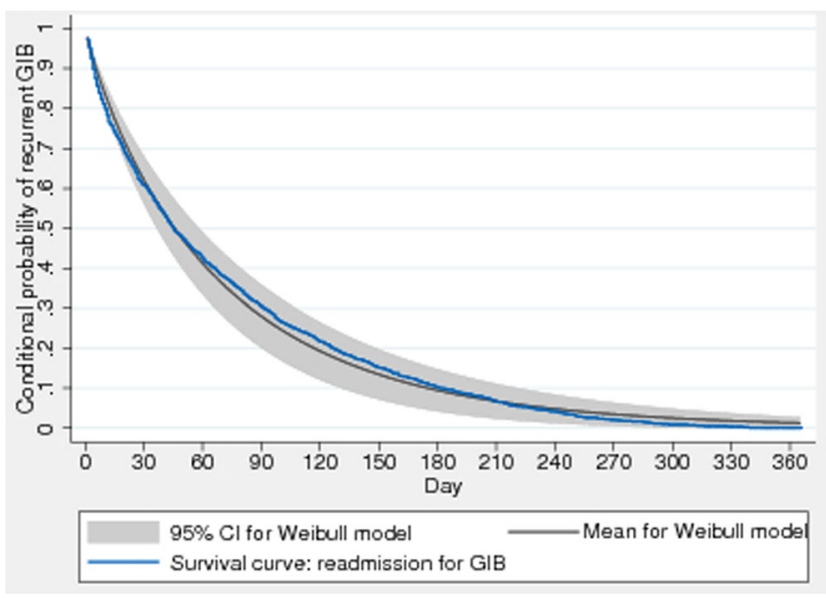

ESTIMATING THE IMPACT OF A MEDICALLY-TAILORED MEAL INTERVENTION FOR FOOD INSECURITY ON HEALTHCARE UTILIZATION: A NEAR/FAR MATCHING ANALYSIS

Seth A. Berkowitz ${ }^{1}$; Jean Terranova ${ }^{2}$; Liisa Randall ${ }^{3}$; Kevin C. Cranston $^{3}$; David B. Waters ${ }^{2}$; John Hsu ${ }^{4}$. ${ }^{1} \mathrm{UNC}$ School of Medicine, Chapel Hill, NC; ${ }^{2}$ Community Servings, Boston, MA; ${ }^{3}$ Massachusetts Department of Public Health, Boston, MA; ${ }^{4}$ Massachusetts General Hospital, Boston, MA. (Control ID \#3152343)

BACKGROUND: Poor access to nutritious food is associated with increased healthcare use and costs, but whether interventions to improve food access can reduce this is unknown. We sought to determine whether participation in a medically-tailored meal intervention is associated with fewer subsequent hospitalizations.

METHODS: We conducted a retrospective cohort study using near/far matching instrumental variable analysis. This analytic approach combines matching on measured characteristics with instrumental variable analysis to account for unmeasured characteristics. We used linked data from the 2011-2015 Massachusetts All-Payer Claims Database (APCD), and Community Servings, a not-for-profit organization delivering medically-tailored meals (MTM). Individuals were eligible for MTM on the basis of being 
chronically ill and at nutritional risk (inability to afford the foods needed to manage their condition). Among those with $360+$ days of APCD data, we matched MTM recipients with non-recipients based on demographic, clinical, and neighborhood characteristics. The MTM intervention comprised weekly delivery of ready-toconsume meals tailored to individual medical needs as determined by a registered dietician nutritionist. Distance from the MTM program was the instrumental variable. The primary outcome was inpatient admissions. Secondary outcomes were admission to a skilled nursing facility and healthcare costs (from medical and pharmaceutical claims).

RESULTS: There were 807 eligible MTM recipients. The instrumental variable passed standard tests of instruments (first stage F-statistic: 219; improved covariate balance with standardized mean difference $<0.2$ for all covariates). Prior to matching, and compared with non-recipients in the same area, healthcare utilization, healthcare cost, and comorbidity were all significantly higher in recipients. For example, pre-intervention mean inpatient admissions were 1.6 (SD: 6.5) in MTM recipients versus 0.2 (SD: 0.8 ) in non-recipients $(\mathrm{p}<.0001)$, and mean healthcare costs were $\$ 80,616.80$ (SD: $\$ 312,336.56$ ) versus $\$ 16,138.42$ (SD: $\$ 68,737.55$ ) ( $\mathrm{p}<.0001$ ). Recipients were also significantly more likely to have HIV (21.9\% vs. $0.7 \%, \mathrm{p}<.0001)$, cancer (37.9\% vs. $11.3 \%, \mathrm{p}<.0001)$, and diabetes mellitus ( $33.7 \%$ vs. $7.0 \%$, $\mathrm{p}<.0001)$, compared with non-recipients. In 'near/far' matching analyses, MTM receipt was associated with significantly fewer inpatient admissions, skilled nursing facility admissions, and healthcare costs (Table).

CONCLUSIONS: Participation in a medically-tailored meals program was associated with fewer hospital and skilled nursing admissions, and less overall medical spending.

\section{EVALUATING THE CASCADE OF CARE FOR LATENT TUBER-} CULOSIS INFECTION AT AN URBAN PRIMARY CARE CLINIC Amy M. Beeson $^{3,1}$; Jonathan S. Schultz ${ }^{3,1}$; Joshua M. Gannon ${ }^{2,1}$; Anne Frank $^{3,1}$; Kaylynn Aiona ${ }^{4}$; Michelle Haas ${ }^{4,5}$; Julie Venci ${ }^{3,1}$; Timothy P. Newton ${ }^{6} .{ }^{1}$ Federico Peña Southwest Family Health Center, Denver Health, Denver, CO; ${ }^{2}$ Denver Health, Denver, CO; ${ }^{3}$ University of Colorado, Denver, CO; ${ }^{4}$ Denver Metro Tuberculosis Program, Denver, CO; ${ }^{5}$ University of Colorado School of Medicine, Aurora, CO; ${ }^{6}$ University of Colorado School of Medicine, Englewood, CO. (Control ID \#3186203)

BACKGROUND: Screening and treatment of latent tuberculosis infection (LTBI) is a key strategy for elimination of TB in the United States. Screening and treatment based in primary care clinics may be beneficial for patients and allow for a broader reach than centralized TB clinics alone; however, the optimal strategy for scaling up testing and treatment in this setting is uncertain. From 2016-2018, providers at Denver Health's Federico Peña Clinic developed a new protocol for clinic-based LTBI treatment. We report on the current status of the LTBI treatment cascade at our clinic.

METHODS: A retrospective chart review was performed from April 11, 2016 to Dec 18, 2018 to identify patients at risk of LTBI based on birth in a high incidence country, defined as active TB incidence of $>20 / 100,000$. Among these, patients with an interferon-gamma release assay (IGRA) test result were identified. Charts of patients with positive IGRA results were then reviewed in depth to characterize the treatment cascade, including evaluation for active TB, clinical decision to treat or not, treatment initiation (defined as an initial prescription for one of three LTBI treatment regimens) and treatment completion (defined as documentation of the final monthly monitoring visit). When patients did not start treatment or discontinued treatment without documentation of clinical rationale (such as pregnancy or low risk of progression to active TB), they were considered lost to follow up.

RESULTS: There were 94,859 patient visits during the evaluation period, including 23,102 individual patients from 120 different countries. Of these, $6,566(28 \%)$ were identified as at-risk for TB. 1,523 (23\%) of these were screened for LTBI with an IGRA, of which 172 tests were positive
(11\%). Among patients with positive IGRA, 153 (88.9\%) were evaluated with a chest X-ray, of which $145(94.7 \%)$ did not show evidence of active TB, while 7 were indeterminate and one identified active TB. Of those with negative x-rays, 97 (66.9\%) initiated treatment, 53 (36.5\%) completed treatment and $25(17.2 \%)$ are currently on treatment. Among those with positive IGRA who did not initiate treatment $(\mathrm{N}=75,43.6 \%)$, a clinical decision not to treat was documented in 43 cases $(57.3 \%)$ while $25(33.3 \%)$ were lost to follow-up. Of 21 women with positive IGRA during pregnancy, only one had a plan to initiate treatment, while 6 remained pregnant, precluding treatment initiation, and a majority (14) were lost to follow up post-partum.

CONCLUSIONS: While urban primary care clinics may contribute meaningfully to large-scale LTBI testing and treatment efforts, these interventions are complex. We noted significant gaps in both testing and treatment at the Peña clinic, with the largest gap in testing individuals at risk. Forthcoming improvement efforts may include standardized screening, strengthening of the clinic-wide protocol to increase treatment initiation when indicated after a positive IGRA result, and targeted efforts to improve post-partum follow-up.

\section{EVALUATING THE EFFECT OF REPORTING PAIN ON CARE FOR ELEVATED BLOOD PRESSURE}

Elizabeth Pfoh; Alexander Chaitoff; Kathryn A. Martinez; Kaitlin Keenan; Michael B. Rothberg. Cleveland Clinic, Cleveland, OH. (Control ID \#3185204)

BACKGROUND: Physicians may consider elevated systolic blood pressure (SBP) in the presence of pain to be physiologic and put off medication intensification. Our objective was to identify whether reporting pain was associated with SBP and delaying medication intensification.

METHODS: We included adult primary care patients seen in an integrated health system in 2015. Pain was identified by asking ""Are you having pain associated with your visit today?". We defined acute pain as lasting 7 days and severe pain as a rating 7/10. We defined elevated SBP as $140 \mathrm{mmHg}$. Medication intensification was defined as a dosage increase or additional antihypertensive medication 1 day of the visit. We performed mixed-effect regression models adjusted for age, sex, race, marriage, insurance status and repeated visits. For patients with elevated $\mathrm{SBP}$, a linear regression was used to identify the association between pain and SBP. Logistic regression was used to identify the association between pain and medication intensification after also adjusting for SBP. We used linear regressions to identify the association between pain at the $1^{\text {st }}$ visit and change in SBP between the $1^{\text {st }}$ and $2^{\text {nd }}$ visit, after further adjustment for initial SBP and medication intensification.

RESULTS: In total, 17,357 people had 1 elevated SBP and a pain score on the same day. Patients had a mean age of 65 , were majority women $(60 \%)$, white $(77 \%)$ and married $(59 \%)$. Patients reported pain at $26 \%$ of visits, acute pain at $5 \%$ and severe pain at $12 \%$. There was no significant difference in SBP when comparing patients who did vs. did not report pain, nor was there a difference in SBP in patients reporting acute vs. chronic pain ( $p>0.05)$. Patients reporting severe pain had slightly higher SBP than those reporting moderate pain ( $=1.2 \mathrm{mmHg}$; 95\% CI: $0.7,1.7)$. Compared to patients without pain, the adjusted odds ratio (AOR) of medication intensification with pain was 0.61 (95\% CI: 0.57-0.66). The odds of medication intensification was lower for patients with acute pain vs. chronic pain (AOR: 0.53; 95\%CI: 0.45-0.64), but similar for severe vs. moderate pain (AOR: 0.90; 95\% CI: $0.79-1.03$ ). Adjusted change in SBP at the $2^{\text {nd }}$ visit was slightly greater for patients who initially reported pain vs patients who reported no pain ( =-1.0mmHg; 95\%CI: -1.80, -0.19). Change in SBP was not different for patients who reported acute vs chronic pain, severe vs. moderate pain, or pain that resolved vs. persisted ( $\mathrm{p}>0.05$ for all comparisons).

CONCLUSIONS: In primary care, severe pain is minimally associated with uncontrolled hypertension, but physicians were less likely to intensify antihypertensive therapy in the presence of any pain. Reduction in SBP at the 
following visit was only slightly greater for patients who had pain initially. Physicians should not consider pain when deciding about medication intensification for hypertension.

\section{EVALUATION OF USE OF LONG-ACTING INSULIN IN INPA- TIENTS PER AMERICAN DIABETES ASSOCIATION (ADA) GUIDELINES}

Richa Panara $^{1}$; Ethan Molitch-Hou ${ }^{1}$; Erika Brechtelsbauer ${ }^{2}$; Shanza Ashraf ${ }^{2}$; $\overline{\text { Susan Henry }}^{2}$; Karen Robinson ${ }^{2}$; Mary Beth $\mathrm{Clark}^{2}$; Haritha Katakam²; Ingrid M. Pinzon ${ }^{2}$; Dhaval Desai ${ }^{2}$; Maria Klimenko ${ }^{2}$; Hasan Shabbir ${ }^{2}$. ${ }^{1}$ Emory University School of Medicine, Atlanta, GA; ${ }^{2}$ Emory University, Atlanata, GA. (Control ID \#3185690)

BACKGROUND: The current American Diabetes Association (ADA) guidelines recommend against using correctional insulin alone for inpatient blood glucose control in patients with diabetes mellitus. The use of correctional insulin alone inadequately controls hyperglycemia, leads to larger swings in blood glucose when compared to scheduled insulin use, and increases hospital complications. Basal insulin use has been recommended for inpatient glycemic control for more than a decade. We analyzed the frequency of prescribing guideline-recommended basal insulin for hospitalized patients with a blood glucose greater than $180 \mathrm{mg} / \mathrm{dL}$ within our healthcare system.

METHODS: All hospital encounters at four hospitals within a quaternary healthcare system involving patients above the age of 18 admitted to the medical and surgical wards from September 1, 2017 to September 30, 2018 with an inpatient blood glucose greater than $180 \mathrm{mg} / \mathrm{dL}$ were reviewed. Information was obtained from a clinical data warehouse. Patients in critical care units, pregnant patients and patients with an admission diagnosis directly influencing insulin use such as hyperglycemia, hypoglycemia, ketoacidosis, and hyperosmolar states were excluded from the analysis.

RESULTS: A total of 11,752 patient encounters were analyzed. Of these encounters, $42 \%$ were started on basal insulin during the hospitalization ( $2 \%$ Basal alone, 3\% Basal/Bolus, 44\% Basal/Bolus/Correctional, 51\% Basal/ Correctional.) $39 \%$ were treated with lone correctional insulin, and $17 \%$ were not started on insulin of any kind. Basal insulin was used more commonly if admitted by a Medicine service compared to Surgical service ( $45 \%$ vs. $25 \%$ $\mathrm{p}<0.01$ ), the patient was on home basal insulin (90\% vs. $60 \% \mathrm{p}<0.01)$, the patient had an A1c $>9 \%$ ( $85 \%$ vs. $43 \% \mathrm{p}<0.01)$, or an order set was used ( $70 \%$ vs. $30 \%$ p < 0.01$)$. The use of basal insulin resulted in more patient days with hypoglycemia $(<70 \mathrm{mg} / \mathrm{dL}, 0.64 \mathrm{~d}$ vs. $0.29 \mathrm{~d}$, p $<0.01)$ and hyperglycemia ( $>299 \mathrm{mg} / \mathrm{dL}, 2.3 \mathrm{~d}$ vs. $0.5 \mathrm{~d}, \mathrm{p}<0.01)$.

CONCLUSIONS: This study showed that ADA guidelines for inpatient insulin dosing are being underutilized in multiple hospitals within a quaternary healthcare system. Only $42 \%$ of patients were prescribed basal insulin during hospitalization. While hyperglycemia and hypoglycemia were worse in the basal insulin group, this group was more likely to have worse glucose control prior to admission. Implementing a guideline-derived inpatient order set could increase prescribing of a basal-bolus insulin regimen.

EVIDENCE REVIEW: MODIFIABLE RISK FACTORS AND INTERVENTIONS TO PREVENT OR DELAY LONG-TERM NURSING HOME PLACEMENT FOR ADULTS WITH IMPAIRMENTS Wei Duan-Porter ${ }^{3}$, 2; Kristen Ullman ${ }^{3}$; Christina Rosebush ${ }^{3}$; Lauren McKenzie $^{3}$; Nancy Greer $^{3}$; Kristine E. Ensrud ${ }^{3}{ }^{4}$; Edward Ratner ${ }^{1,2}$; Tetyana Shippee ${ }^{4}$; Joseph Gaugler ${ }^{4}$; Timothy Wilt ${ }^{3,}{ }^{2}$. ${ }^{1}$ Minneapolis VA Health Care System, Minneapolis, MN; ${ }^{2}$ University of Minnesota Medical School, MInneapolis, MN; ${ }^{3}$ Minneapolis VA, Minneapolis, MN; ${ }^{4}$ University of Minnesota, Minneapolis, MN. (Control ID \#3185374)

BACKGROUND: To help adults with physical or cognitive impairments remain in community settings, national efforts have sought to improve access to home and community-based services (HCBS) and rebalance funding. In this review of reviews, we examined modifiable risk factors for long-term nursing home placement (NHP) and intervention effects on preventing or delaying NHP.

METHODS: Multiple databases were searched, and additional references sought from experts. Eligible systematic reviews (SR) examined frailty, other risk factors, and various interventions. Abstracted data included: study characteristics; target population; NHP ascertainment; and risk factor or intervention category. Using predetermined criteria, we prioritized a subset of SR for further abstraction of detailed results: meta-analysis or qualitative synthesis of effects, moderation effects, datasets used, NHP ascertainment, quality of included studies, and overlap in studies.

RESULTS: Of 7014 unique citations, we found eligible 67 SRs (20 on risk factors, 47 for interventions); all included older adults with impairments, or at risk for impairments due to age or medical burden. Eighteen SR were high quality, 10 were umbrella reviews, and 46 included randomized controlled trials. Few SR discussed how studies assessed NHP; those that examined this question found that most studies used participant (or family) reports or had unclear NHP ascertainment. Main results from the subset of eligible SR (6 for risk factors, 20 for interventions) are summarized in Table.

CONCLUSIONS: Comprehensive case management and caregiver support showed some benefit, although results were inconsistent. Future studies should compare effects of interventions that target similar factors in alternative settings (eg, home vs. clinic), and consider follow-up periods $>2$ years.

\section{EVOLUTION OF NURSING ROLES AND NURSE-PHYSICIAN COLLABORATION DURING IMPLEMENTATION OF VA'S PATIENT-CENTERED MEDICAL HOME: KEY STAKEHOLDER PERSPECTIVES}

Alicia Bergman ${ }^{1}$; Danielle Rose ${ }^{2}$; Linda Kim ${ }^{3}$; Alison B. Hamilton ${ }^{4}$; Susan E. Stockdale ${ }^{5}$ VA Greater Los Angeles, North Hills, CA; ${ }^{2}$ VA GLA Healthcare System, Sepulveda, CA; ${ }^{3}$ VA-Greater Los Angeles, Los Angeles, CA; ${ }^{4}$ VA Greater Los Angeles Healthcare System, Los Angeles, CA; ${ }^{5}$ Greater Los Angeles VA Healthcare System, Sepulveda, CA. (Control ID \#3184426)

BACKGROUND: Effective nurse-physician collaboration is essential for providing quality health care, but long-standing relationship challenges have been reported between these professions. Recent studies have explored the impacts of the patient-centered medical home (PCMH) model on primary care providers and teams, but a targeted investigation into nurses' and physicians' respective roles and interprofessional dynamics with one another is missing. Veterans Health Administration (VHA) implemented PCMH in 2010. The goal of this study was to explore primary care provider (PCP) and nurse $(\mathrm{RN})$ interprofessional collaboration against the backdrop of evolving $\mathrm{RN}$ roles during PCMH implementation.

METHODS: Qualitative interviews were conducted with 47 key stakeholders (e.g., primary care and nursing leaders) as part of a PCMH implementation evaluation in 3 VA healthcare systems. Interviews were recorded, transcribed, and analyzed to identify major themes regarding RN roles and RN-PCP interprofessional collaboration.

RESULTS: Key stakeholders perceived that: 1) PCPs and RNs understood PCMH RN roles differently; 2) PCPs expected RNs to work above the top of their license; 3) RNs were not working at the top of their license; and 4) PCPs were coping with deviations from a traditional physician/RN model. Differences in understanding of the $\mathrm{RN}$ roles were perceived to derive from separate reporting structures between PCPs and RNs, and nursing roles being translated and practiced differently across different PCMH teams. With regard to perceived PCP expectations, key stakeholders noted that some nurses took on extra work (beyond their scope) in order to appease PCPs. Other key stakeholders described how RNs were not working at the top of their license for reasons including PCP territoriality, inadvertent creation of clerical work hindering dedication of sufficient time to higher functioning tasks, 
and/or a lack of prior exposure to and/or experience with higherlevel tasks (e.g., chronic disease management). Key stakeholders also recounted observing physician resistance to the less hierarchical and more team-oriented model of the RN-PCP relationship promoted in $\mathrm{PCMH}$, and they recalled instances of power struggles as physicians resisted evolving $\mathrm{RN}$ autonomy and/or new reporting structures.

CONCLUSIONS: Key stakeholders had diverging perspectives on the nature of $\mathrm{RN}$ tasks being performed, but most agreed that there was confusion in PCMH over RN roles; many perceived that a breakdown of the traditional $\mathrm{PCP} / \mathrm{RN}$ hierarchical structure was being met with resistance by some physicians. A key aim of PCMH models is to use team-based approaches to improve patient care, but challenges to RN-PCP collaboration can make implementation of such models more difficult. These findings highlight areas for improvement and can guide the development of future trainings.

EXAMINATION OF INTERNAL MEDICINE, PEDIATRIC AND FAMILY MEDICINE RESIDENTS' PERCEPTIONS, SKILLS, AND PRACTICES IN DELIVERING CROSSCULTURAL CLINICAL CARE

Eun Ji Kim ${ }^{1}$; Lyndonna M. Marrast ${ }^{1}$; Omolara T. Uwemedimo ${ }^{3}$; Shari Jardine ${ }^{2}$; Johanna Martinez ${ }^{1}{ }^{1}$ Zucker School of Medicine at Hofstra/Northwell, Manhasset, NY; ${ }^{2}$ Northwell Health, New Hyde Park, NY; ${ }^{3}$ Zucker School of Medicine at Hofstra/Northwell, New Hyde Park, NY. (Control ID \#3185108)

BACKGROUND: Social determinants of health (SDH) encompass conditions arising from where people are born, live, learn, and work. $\mathrm{SDH}$ affect more than half of patient health outcomes and the presence of health harming needs have been associated with poor health outcomes. The purpose of this study is to assess previous education in SDH-related topics and examine the translation of resident education into preparedness, skillfulness, and clinical practice to address patients' SDH.

METHODS: In the beginning of the academic year, Internal Medicine, Pediatrics, and Family Medicine residents were asked to complete a survey containing questions about sociodemographic background, cross-cultural characteristics, and prior education or training in SDH. The survey also contained questions about self-perceived preparedness, skillfulness, and clinical experience addressing SDH. We conducted descriptive analysis of resident's responses to the survey and examined characteristics associated with skillfulness.

RESULTS: The study included 160 residents from Internal Medicine $(n=87)$, Pediatrics $(n=63)$, and Family Medicine $(n=10)$. The study sample respondents self-identified as $36.9 \%$ White, $6.9 \%$ Black, $8.1 \%$ Hispanic, $36.9 \%$ Asian, and $11.2 \%$ Other. About a quarter $(27.5 \%)$ of residents reported coming from a disadvantaged background (low income, limited English proficient, or attending a school with high dropout rate). A majority of the residents spoke another language other than English (58.8\%) and had a parent who was born outside of the US $(65.0 \%)$. Residents reported prior education or training in health disparities $(75.6 \%), \mathrm{SDH}(83.8 \%)$, and community health $(57.5 \%)$. Despite the majority of the residents reporting prior education in various cross-cultural topics (reported range $63.1-98.1 \%$ ), there were wide variations in perceived preparedness in addressing patients with cross-cultural differences $(24.4 \%$ prepared to care for patients with a distrust of healthcare system and $51.3 \%$ prepared to care for patients with limited English proficiency or from a different culture). Very small percentages of residents regularly asked patients about social needs, including homelessness $(4.1 \%)$, affordable housing $(2.7 \%)$, food insecurity $(0.0 \%)$, and unhealthy living conditions $(9.5 \%)$. Residents perceived level of skill in providing cross-culture care increased when they had received medical school education in social history, including identifying 1) how well a patient can read or write English, 2) religious beliefs that might affect clinical care, 3) cultural customs that might affect clinical care, and 4) how a patient makes decisions with other family members.

CONCLUSIONS: Medical school teaching in cross-cultural care is associated with improved self-perception of skill in addressing patients SDH among residents. Graduate medical education that is inclusive of cultural competency, social justice, and health equity may improve trainees' perceptions, readiness, and skill for clinical practice adept in addressing SDH.

\section{EXAMINATION OF THE ASSOCIATION BETWEEN LIFE- COURSE SOCIOECONOMIC STATUS, ACCULTURATION, AND HYPERTENSION AMONG UNITED STATES IMMI- GRANTS WITH AND WITHOUT DIABETES}

Aprill Z. Dawson; Rebekah J. Walker; Leonard E. Egede. Medical College of Wisconsin, Milwaukee, WI. (Control ID \#3185017)

BACKGROUND: More than 42 million immigrants live in the United States (US), and are more likely to work low paying jobs, live in poverty, and lack health insurance. It is known that ethnic minorities are disproportionately affected by hypertension and diabetes. Immigrants face additional challenges in disease management due to barriers to accessing care, limited English proficiency, and cultural differences. Therefore, the aim of this study was to understand the relationship between life-course socioeconomic status (SES), acculturation, and hypertension among US immigrants with and without diabetes.

METHODS: Data from the New Immigrant Survey, a multicohort study of documented US immigrants, was analyzed. Logistic regression with hypertension as the outcome was used to investigate the relationship between acculturation \& life-course SES in adult US immigrants with and without diabetes. The acculturation variable included measures of English proficiency (understand, speak, read, write) and length of residence in the US ( $>15$ years). Life-course SES included father's education, mother's education, number of siblings, individual's level of education, income, and employment status. Hypertension and diabetes were measured by self-report.

RESULTS: 8533 US immigrants, 317 of which had diabetes were included in the study. 659 immigrants without diabetes had hypertension, and 121 with diabetes had hypertension. Unadjusted models found a significant association between life-course SES and hypertension in those without diabetes $(\mathrm{OR}: 0.83 ; \mathrm{p}<0.001)$ and with diabetes (OR:0.58; $\mathrm{p}<0.001)$. After adjustment for age, sex, race/ethnicity, and marital status significance only remained among immigrants with diabetes (OR:0.68; $\mathrm{p}=0.033$ ). Acculturation was significantly associated with hypertension among immigrants without diabetes in the unadjusted model (OR:1.35; $\mathrm{p}=0.001$ ), however significance did not hold after adjusting for demographics.

CONCLUSIONS: US immigrants with diabetes and with higher life-course SES were found to have reduced risk of having hypertension whereas this relationship was not seen in immigrants without diabetes. Additionally, acculturation was not identified as a significant predictor of hypertension among US immigrants with or without diabetes. Similar to US-born adults, our findings support the idea of socio-economic status across the life-course being associated with the development of chronic disease in US adults. Additionally, these results suggest that low SES immigrant populations with diabetes may be at high risk for hypertension and should be the focus of culturally tailored interventions. 
EXAMINING FACTORS ASSOCIATED WITH NICOTINE REPLACEMENT THERAPY USE AMONG ASIAN AMERICAN MALE SMOKERS

Fiona Ng${ }^{1,}{ }^{4}$; Hallen $\mathrm{Pham}^{1,}{ }^{4}$; Janet $\mathrm{Chu}^{2,}{ }^{4}$; Ginny gildengorin ${ }^{1,4}$; Susan Stewart $^{5,}{ }^{4}$; Alice Guan ${ }^{1,}{ }^{4}$; Edgar $\mathrm{Yu}^{3}$, ${ }^{4}$; Angela Sun ${ }^{6,}$; Joyce Cheng $^{6,}{ }^{4}$; Tung T. Nguyen ${ }^{2,4}$; Janice Y. Tsoh ${ }^{3,4}{ }^{1}{ }^{1}$ University of California, San Francisco, San Francisco, CA; ${ }^{2}$ UCSF, Livermore, CA; ${ }^{3}$ University of California San Francisco, San Francisco, CA; ${ }^{4}$ Asian American Research Center on Health, UCSF, San Francisco, CA; ${ }^{5}$ University of California, Davis, Davis, CA; ${ }^{6}$ Chinese Community Health Resource Center (CCHRC), San Francisco, CA. (Control ID \#3161742)

BACKGROUND: Compared to the average prevalence of smoking in California (16\%), smoking prevalence remains disproportionately high among Vietnamese (30\%) and Chinese (27\%) men with limited English proficiency. Asian Americans are less likely to use and to receive nicotine replacement therapy (NRT) prescriptions, an evidence-based smoking cessation intervention. Additional barriers to NRT-use in this population include misconceptions of its side effects, skepticism of its effectiveness compared to willpower alone, and preference for non-pharmacological strategies. This study examined factors associated with NRT-use among Asian American male daily smokers.

METHODS: Participants consisted of dyads formed by a Chinese or Vietnamese daily smoker and a non-smoking family member. In this randomized controlled trial, dyads received a family-centered education intervention taught by lay health workers (LHW) on either smoking cessation $(n=166)$ or nutrition and physical activity $(n=159)$. Bivariate and multivariable regression analyses were used to identify factors associated with NRT-use reported at 3-months after enrollment. Treatment group, demographics, and the following baseline InformationMotivation-Behavioral Skills Model (IMB) constructs were examined: information (harms of tobacco use, effectiveness of NRT), motivation (family support, discussion with doctor, intention to quit), and behavioral skills (24-hour quit attempt, self-efficacy).

RESULTS: The sample consisted of 163 Vietnamese and 162 Chinese men who smoked daily at the time of enrollment (mean age $=54.7$ ). All but one participant were foreign born; 98\% spoke limited English; 74\% had high school or less education. On average, the participants have smoked for 28.3 years with a mean of 9.4 cigarettes smoked daily; $46 \%$ had no intent to quit in 6 months; and $49 \%$ smoked 30 minutes after waking (indicating high nicotine dependence). At 3 months post-enrollment, smoking cessation group yield higher NRT-use than the control group $(12 \%$ versus $6.9 \%)$ [(AOR=3.28, $\mathrm{p}=.01)]$. In multivariable analysis, independent of group assignment the following IMB constructs were associated with NRT-use: motivation (frequent encouragement from family member to use cessation resources $[\mathrm{AOR}=2.04, \mathrm{p}<.01]$, discussion with doctor about smoking $[\mathrm{AOR}=5.24, \mathrm{p}<.01]$, intention to quit 6 months [AOR=2.57, $\mathrm{p}=.049]$ ) and behavioral skills (at least one 24-hour quit attempt in the year prior to study [AOR=3.69, $\mathrm{p}=.01]$ ).

CONCLUSIONS: While LHW-led family-based smoking cessation intervention increased NRT-use among Chinese and Vietnamese male smokers, utilization remains low. Information on the harms of tobacco and efficacy of cessation resources alone were insufficient. Our findings underscore the importance of healthcare providers promoting NRT-use with an understanding of personal and cultural barriers. Efforts to improve NRT-use among this population should also involve empowering family members to discuss cessation resources with smokers.

EXAMINING THE ASSOCIATION OF SOCIAL DETERMINANTS OF HEALTH WITH 30-DAY READMISSION AND MORTALITY IN OLDER VETERANS WITH CONGESTIVE HEART FAILURE

Charlie M. Wray ${ }^{1,2}$; Marzieh Vali ${ }^{3}$; Louise Walter ${ }^{1}$; Wendy Chapman ${ }^{4}$; Brett South ${ }^{4}$; Amy L. Byers ${ }^{1,}{ }^{2}$; Salomeh Keyhani ${ }^{5}$. ${ }^{1}$ University of
California, San Francisco, San Francisco, CA; ${ }^{2}$ San Francisco VA Medical Center, San Francisco, CA; ${ }^{3}$ NCIRE, San Francisco, CA; ${ }^{4}$ University of Utah, SLC, UT; ${ }^{5}$ University of California at San Francisco, San Francisco, CA. (Control ID \#3185361)

BACKGROUND: The Veterans Health Affairs (VA) closely tracks 30-day readmission and mortality rates among patients hospitalized for congestive heart failure (CHF). While previous work has identified multiple factors that are predictive of readmission and death, social determinants of health $(\mathrm{SDOH})$ are often not examined due to the difficulty in extracting this information from clinical databases. We previously extracted four SDOH (see Table) that are commonly associated with poor clinical outcomes. We examined whether SDOH were associated with 30-day readmission and mortality in the VA's integrated health care system.

METHODS: We randomly selected 1500 elderly (65 years and older) Veterans hospitalized in the VA with a primary admission diagnosis of $\mathrm{CHF}$ in 2012. We identified 25 candidate variables associated with the outcomes of interest in CMS hospital profiling models. SDOH were independently extracted by two reviewers from patient charts. We examined the association of each clinical variable with the outcome of interest (30-day readmission or 30-day mortality) and retained variables significant in univariate analysis $(\mathrm{p}<0.1)$ in subsequent analyses. Using multivariate logistics regression, we examined the association between the four SDOH and 30-day hospital readmission and 30-day mortality after adjusting for age and clinical factors.

RESULTS: Of the 1500 patients, $312(21 \%)$ were readmitted while 85 $(6 \%)$ died within 30 days of discharge. In multivariate analysis, lack of social support (OR 5.37; 95\% CI 1.53-19.81), higher ALaRM Score (OR 1.01 ; $95 \%$ CI $1.00-1.02$ ), or renal failure (OR 1.39; 95\%CI 1.05-1.83) were associated with higher 30 -day readmissions. Of note, marginal housing (or receipt of housing services) appeared to decrease a patients' propensity to be readmitted to the hospital (OR 0.24 ; 95\% CI $0.03-0.91$ ). CONCLUSIONS: Of the four SDOH we examined, only 'lack of social support' was shown to be associated with higher 30-day readmission, and none were associated with 30-day mortality. Patients with marginal housing (or who were received housing services) were less likely to be readmitted. The VA's extensive outpatient and social safety net programs may be attenuating the deleterious effects SDOH commonly have on health outcomes in other settings. Future work should focus on how these $\mathrm{SDOH}$ effect other, younger patient populations within the VA, and what social services may be mitigating risk.

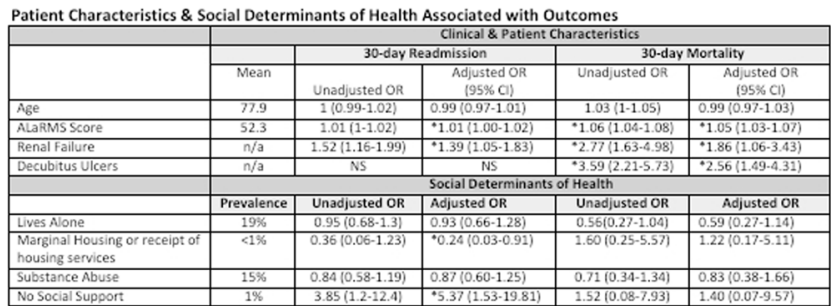

Note: Only clinical variables that were significantly associated with outcome were included in the table. Multivariate analyses also controlled for age and the following comorbid conditions: PCI, CABG, CAD, valvular heart disease, arrhythmias, stroke, renal failure, COPD, pneumonia, diabetes, dementia, functional disability, metastatic cancer, major osychiatric disorder, chronic liver disease, severe hematologic disorder iron deficien depression, ESRD, decubitus skin ulcers. OR: Odds Ratio: ALaRMS: Acute Laboratory Risk of Mortality Score. $P<0.05$, NS: Not significant in univariate analysis

EXAMINING THE AWARENESS OF UNHEALTHY WEIGHT AMONG CHINESE AMERICAN AND VIETNAMESE AMERICAN IMMIGRANTS

Hallen Pham ${ }^{1}$; Fiona $\mathrm{Ng}^{1}$; Janet $\mathrm{Chu}^{1}$; Ginny gildengorin ${ }^{1}$; Susan Stew${\overline{\operatorname{art}^{2}}}^{2}$ Edgar Yu${ }^{1}$; Alice Guan ${ }^{1}$; Angela Sun ${ }^{3}$; Joyce Cheng ${ }^{3}$; Tung T. Nguyen ${ }^{1}$; Janice Y. Tsoh ${ }^{1} .{ }^{1}$ University of California, San Francisco, San Francisco, CA; ${ }^{2}$ University of California, Davis, Sacramento, CA; ${ }^{3} \mathrm{Chi}-$ nese Community Health Resource Center (CCHRC), San Francisco, CA. (Control ID \#3147717) 
BACKGROUND: The World Health Organization (WHO) recommends lowering the cutoff for overweight body mass index (BMI) for Asians from 25 to 23 due to disproportionately higher risks of having diabetes among Asians with BMI 23. Self-awareness of being overweight or obese correlates positively with healthy weight management. This study aims to examine the factors associated with lack of awareness of unhealthy weight among Chinese American and Vietnamese American immigrants.

METHODS: This study analyzed pre-intervention survey data from 680 participants enrolled in a randomized trial to promote smoking cessation and healthy eating. Participants were male daily smokers accompanied by a non-smoking family member to form a smoker-family dyad. All participants were asked "Do you think your current weight is at a healthy range?" BMI was computed from self-reported height and weight. Participants whose BMI 23 but self-perceived their weight to be in a healthy range were considered as unaware of their unhealthy weight. For the subset of participants with BMI 23, bivariate and multivariable logistic regression models using generalized estimating equation to account for within-dyad clusters were conducted to identify correlates of being unaware of one's unhealthy weight.

RESULTS: Participants included 346 Chinese Americans and 334 Vietnamese Americans: $53.5 \%$ male, $99.3 \%$ foreign born, $95.8 \%$ limited English proficiency, and $41.9 \%$ had less than a high school education. About half of participants had BMI 23 (50.2\%, n=319). Among those with BMI 23, 46.7\% had BMI between 23 and 24.9, which would have been categorized as healthy weight by the standard BMI cutoff of 25 . Of those with BMI 23, 58.9\% were unaware of their unhealthy weight, and only $22.3 \%$ reported discussing weight with their doctor within the last year. Among those who discussed weight with their doctor, $36.6 \%$ remained unaware of being overweight or obese. In multivariable analysis, factors associated with lack of awareness of being overweight or obese included being male ( $\mathrm{OR}=2.96,95 \% \mathrm{CI}$ : 1.58-5.53), perceiving good/excellent general health $(\mathrm{OR}=2.95,95 \% \mathrm{CI}: 1.66-5.23)$, having BMI between 23 and 25 ( $\mathrm{OR}=4.37,95 \% \mathrm{CI}: 2.62-7.27)$, and not discussing weight with their doctor $(\mathrm{OR}=3.13,95 \% \mathrm{CI}: 1.69-5.82)$.

CONCLUSIONS: A majority of Chinese American and Vietnamese American immigrants with BMI 23 lacked awareness about being overweight. Those who did not discuss weight with their doctors were at increased odds of overweight unawareness, but some remained unaware even after discussion with their doctor. These findings underscore the need for targeted interventions to help patients understand healthy weight range and weight management to prevent and improve health outcomes for diseases associated with being overweight or obese. Interventions should also engage healthcare professionals to increase weight discussions with their Asian patients utilizing recommended BMI cutoff of 23.

\section{EXAMINING THE REPLETION REFLEX: THE ASSOCIATION BETWEEN SERUM POTASSIUM AND OUTCOMES IN HOSPI- TALIZED PATIENTS WITH HF}

Kevin F. O'Sullivan $^{3,2}$; Mohammad A. Kashef ${ }^{1,3}$; Alexander B. Knee ${ }^{3,3}$; Alexander S. Roseman ${ }^{3}$; Penelope S. Pekow ${ }^{3}$; Mihaela S. Stefan ${ }^{3,3}$; Meng-Shiou Shieh3; Quinn R. Pack ${ }^{3,}$; Peter K. Lindenauer ${ }^{3,}$; ; Tara $\mathrm{Lagu}^{3,3}$. ${ }^{1}$ Hamden and Franklin Cardiovascular Associates, Springfield, MA; ${ }^{2}$ University of Massachusetts Medical School, Worcester, MA; ${ }^{3}$ University of Massachusetts Medical School-Baystate Medical Center, Springfield, MA; ${ }^{4}$ Baystate Medical Center, Springfield, MA. (Control ID \#3184657)

BACKGROUND: Many physicians monitor their hospitalized patients' potassium levels daily and replete as needed to maintain serum potassium $4.0 \mathrm{mEq} / \mathrm{L}$. The evidence basis for this practice is relatively weak. We aimed to evaluate the association between serum potassium levels and outcomes in patients admitted with heart failure (HF).

METHODS: We identified patients with acute HF exacerbations admitted to hospitals that contributed to an electronic health record (EHR)- derived dataset in the years 2010-2012. In a subset of patients with normal serum potassium $(3.5-5.0 \mathrm{mEq} / \mathrm{L})$ at the time of admission, we averaged serum potassium values within a 72-hour exposure window during and just after admission. We categorized average serum potassium during this window as less than $4.0 \mathrm{mEq} / \mathrm{L}$ (low normal), $4.0-4.5 \mathrm{mEq} / \mathrm{L}$ (medium normal), or greater than $4.5 \mathrm{mEq} / \mathrm{L}$ (high normal). We created multivariable models examining the association between these categories and outcomes (inpatient mortality, length of stay, and admission to ICU after the "exposure window"), adjusting for patient demographics, comorbid conditions, and presenting severity (using the Laboratory-based Acute Physiology Score (LAPS-2), which includes admission blood urea nitrogen and creatinine levels as well as anion gap).

RESULTS: We included 4,995 patients, of which 2,080 (41.6\%), 2,326 (46.6\%), and $589(11.8 \%)$ were in the low, medium, and high cohorts, respectively. The $<4.0 \mathrm{mEq} / \mathrm{L}$ and $4.0-4.5 \mathrm{mEq} / \mathrm{L}$ groups were relatively similar in terms of age, sex, illness severity (LAPS-2), and comorbidity score. Compared to these groups, the group with mean potassium $>4.5$ $\mathrm{mEq} / \mathrm{L}$ group had higher admission creatinine, more chronic kidney disease, more deficiency anemias, and more chronic obstructive pulmonary disorder. Overall, 3.7\% $(\mathrm{n}=187)$ of patients died during the hospitalization, 2.4\% ( $\mathrm{n}=98)$ were admitted to ICU after the exposure window and median length of stay was 5.6 days. After adjustment, we observed a trend towards higher risk of mortality $(\mathrm{OR}=1.51 ; 95 \% \mathrm{CI}=0.97$ to 2.36$)$ and ICU transfer (OR $=1.78 ; 95 \% \mathrm{CI}=0.98$ to 3.26 ) and a statistically significant longer length of stay (median of 0.57 days; $95 \% \mathrm{CI}=0.21$ to 0.93 ) in patients with a potassium level of $>4.5 \mathrm{mEq} / \mathrm{L}$.

CONCLUSIONS: In this large observational study of patients hospitalized with an acute HF exacerbation, we found no association between low normal serum potassium and negative outcomes. Compared to those with mean potassium values $<4.0 \mathrm{mEq} / \mathrm{L}$, potassium values of $>4.5 \mathrm{mEq} / \mathrm{L}$ were associated with worse outcomes, but this was attenuated after adjustment. This suggested that factors beyond potassium values influenced the observed relationship. Because daily potassium repletion represents a huge cost in time, money, and effort, to physicians, nurses, hospitals, laboratories, and healthcare payers, future pragmatic trials could attempt to disentangle the associated risks and benefits of potassium repletion and identify the best treatment methods going forward.

\section{EXECUTIVE PHYSICALS IN THE VALUE ERA: ARE TOP MEDICAL CENTERS LEADING THE WAY?}

Deborah R. Korenstein; Maha Mamoor; Peter Bach. Memorial Sloan Kettering Cancer Center, New York, NY. (Control ID \#3182494)

BACKGROUND: Executive physical programs are one to two-day comprehensive evaluations offering "preventive" testing/screening. Program fees cover all included testing; subsequent services in response to findings are billed to insurers. Amidst widespread efforts to improve healthcare value, executive physicals have been criticized as wasteful and non-evidence-based, but no studies have described their offerings. As top-ranked academic hospitals may be viewed as role-models in appropriate care, we set out to describe the prevalence of executive physical programs in top US academic hospitals and to characterize included non-laboratory testing.

METHODS: We searched websites of the 20 hospitals included in the US News \& World Report 2018-2019 hospital "honor roll" for information about executive physical programs and recorded prices and services offered. When information was incomplete, we performed Google searches and telephoned representatives to inquire about programs. When hospitals provided different packaged services based on age or price, we recorded information for each package separately. We divided services into 7 domains: cardiovascular risk review, cardiac screening, ENT/ Ophthalmology, cancer screening, lung disease screening, bone health, and total body CT. We assessed the appropriateness of each service based on US Preventive Services Task Force (USPSTF) guidelines as: 
recommended, non-recommended (NR), risk- or age-based (RB), and insufficient evidence (IE). For these determinations we assumed all patients were under age 65 . The study was deemed exempt by the MSKCC IRB

RESULTS: Seventeen (85\%) hospitals offered executive physicals. We identified services included in 20 packages across 13 hospitals; information was unavailable for 4 hospitals. Package price ranged from $\$ 1700$ $\$ 9400$. The most commonly offered services were screening ECG (included in $90 \%$ of packages), hearing exam $(75 \%)$, and stress testing $(70 \%)$; all are NR. Across packages, cancer screening was the most common domain, with 48 cumulative services (12 [25\%] NR), followed by cardiac screening ( $n=32$, all NR), ENT/ophthalmology ( $n=25$, all IE), and lung disease screening $(n=19$, all NR). Overall, among unique services offered in any package $(n=16), 1(6.3 \%)$ was recommended by USPSTF, $6(37.5 \%)$ were NR, 5 (31.3\%) were RB, 3 (18.8\%) were IE, and 1 was not addressed by USPSTF. Notably, few hospitals included pap/pelvic exam (54\%), which is recommended by USPSTF, or abdominal ultrasound for AAA screening (23\%) (deemed RB per USPSTF), none offered lung cancer screening (RB), and 1 offered total body CT.

CONCLUSIONS: Most top academic hospitals offer executive physical programs; public information on these programs is incomplete. Price varies, non-recommended services are common, and some recommended services are omitted. With growing emphasis on value, leading hospitals should align executive physical offerings with best evidence.

\section{EXPANDING SUPPLY OF MEDICATION ASSISTED TREAT- MENT TO UNDERSERVED AREAS: BUPRENORPHINE WAIVERS FOR ADVANCED PRACTICE PROVIDERS}

Michael L. Barnett ${ }^{1}$; Dennis Lee ${ }^{1}$; Richard Frank ${ }^{2} .{ }^{1}$ Harvard T.H. Chan School of Public Health, Boston, MA; ${ }^{2}$ Harvard Medical School, Boston, MA. (Control ID \#3181480)

BACKGROUND: There are large segments of the country where access to evidence-based treatment for opioid use disorder (OUD) is limited by the lack of clinician supply. To help address the limits of OUD treatment capacity, the Comprehensive Addiction and Recovery Act (CARA) passed in July 2016 enabled nurse practitioners and physicians' assistants (NP/PA) to obtain federal waivers to prescribe buprenorphine, an effective medication for treating OUD, after 24 hours of training. We examined the take-up and use of these waivers by NPs and PAs and their association with scope of practice laws.

METHODS: We obtained the date and county of buprenorphine waiver approval for all clinicians including NP/PAs from 2005-2017 from the Substance Abuse and Mental Health Services Administration, merged with publicly available county demographic characteristics and 2016 opioid mortality data. We captured buprenorphine prescribing from the publicly available Drug Enforcement Agency databases. Using linear regression, we estimated the associations in characteristics of counties with the highest vs. lowest quartiles of waivered NP/PAs per capita by the end of 2017. Next, we estimated a county- or 3-digit ZIP- quarter level linear regression with geographic fixed effects to quantify changes postCARA in buprenorphine prescribing per capita. We compared growth in these outcomes between states with fully independent vs. restricted scope of practice laws for NP/PAs as of 2016.

RESULTS: By the end of 2017, 3,682 NPs and PAs in 812 counties representing $75 \%$ of the US population obtained buprenorphine waivers, including 39 counties with no buprenorphine-waivered providers before 2017. Among counties with any waivered NP/PAs, there were 6.2 vs. 0.6 waivered NP/PAs per 100,000 in counties in the top vs. bottom quartiles of NP/PA waiver adoption. Top quartile counties had significantly higher opioid-related mortality per 100,00 in 2016 than bottom quartile counties (18.4 vs. 13.2, adjusted $p<0.001$ ). By the end of 2017, counties in states with full vs. restricted scope of practice had over twice as many waivered NP/PAs per 100,000 (2.2 vs. 0.9, p<0.001). Annual buprenorphine prescribing per capita grew faster in 2017 after CARA passage than from 2005-2016 (15.2\% faster annual growth vs. national average, $\mathrm{p}<0.001$ ), but there was no difference in growth between states with full vs. restricted scope of practice regulations $(\mathrm{p}=0.27)$.

CONCLUSIONS: One year after CARA passage, thousands of NP/PAs obtained buprenorphine waivers despite the 24-hour training requirement, many of whom were in counties with no prior buprenorphine providers. There was greater adoption in states where NP/PAs could practice independently, though buprenorphine prescribing did not grow faster in states with full scope of practice laws in 2017. The growth of NP/PAs as buprenorphine providers suggests that they could serve a crucial role in expanding access to OUD treatment nationally.

\section{EXPECTATIONS AND EXPERIENCES WITH PRESCRIBING CLINICIANS AMONG SHORT-STAY PATIENTS IN SKILLED NURSING FACILITIES}

Kira Ryskina $^{1}$; Kierra A. Foley ${ }^{1}$; Jason H. Karlawish ${ }^{1}$; Joshua Uy ${ }^{1}$; Briana Lott ${ }^{2}$; Erica Goldberg ${ }^{1}$; Nancy Hodgson ${ }^{1} .{ }^{1}$ University of Pennsylvania, Philadelphia, PA; ${ }^{2}$ Veteran Affairs, Philadelphia, PA. (Control ID \#3169816)

BACKGROUND: One in four Medicare beneficiaries discharged from hospitals to skilled nursing facilities (SNFs) for post-acute care (shortstay) are re-hospitalized or die within 30 days. While prescribing clinicians (physicians, nurse practitioners, and physician assistants) are considered integral to improving care coordination and transitions in care, little is known about the role of SNF prescribing clinicians in the experience of patients who transition from hospitals to post-acute care in SNFs. Our objectives in this study were: 1) to describe patient and caregiver expectations and experiences with SNF prescribing clinician care among patients receiving post-acute care in these facilities, and 2) to elicit thematic differences in patient and caregiver experiences among patients with and without cognitive impairment as well as among white patients and black patients.

METHODS: We conducted a qualitative study using semi-structured patient interviews at five diverse (i.e., size, setting, and prescribing clinician staffing) SNFs between January and August 2018. Forty-eight patients transferred to the SNFs for short-term care 5-10 days prior to the interview were eligible to participate. All participants were asked to identify a primary caregiver who was contacted by telephone to complete the interview as well. Thematic analysis of the transcripts was performed to detect recurrent themes and divergent ideas with a focus on modifiable prescribing clinician-level factors that might improve patient experience with post-acute care in SNFs. Analysis was stratified by patient cognitive impairment (measured by the Montreal Cognitive Assessment (MOCA) test) and race. Member checks were conducted as a validation technique. RESULTS: The major theme that emerged was misleading expectations about the prescribing clinician (for example, patients expected to continue seeing the doctor who was treating them during the preceeding hospitalization). A majority of subjects $(85 \%)$ reported transient interactions with their SNF prescribing clinician. Most (95\%) had limited knowledge of the prescribing clinician(s) in SNFs and $60 \%$ reported feeling disempowered to discuss their care with the clinician(s). Patients with lower MOCA scores and minorities were more likely to report feeling disempowered.

CONCLUSIONS: Our findings identify specific areas for improvement in the delivery of care for patients discharged to SNF for postacute care after a hospitalization. In the context of growing reliance on SNFs as a post-acute care destination after hospital discharge, especially among patients with cognitive impairment, and increasing specialization of prescribing clinicians in SNF practice (i.e. the emergence 'SNFists'), better understanding of patient and caregiver expectations of prescribing clinician care in SNFs is needed to inform policy and local practices related to prescribing clinicians in SNFs. 
EXPERIENCES OF SEX TRAFFICKING VICTIMS IN HEALTHCARE SETTINGS

Joseph Shin $^{1}$; Vivian Pender ${ }^{3}$; Karen Mathewson ${ }^{4}$; Monika M. Safford ${ }^{2}$. ${ }^{1}$ Weill Cornell Medicine, Brooklyn, NY; ${ }^{2}$ Weill Cornell Medical College, New York, NY; ${ }^{3}$ Weill Cornell Medicine, New York, NY; ${ }^{4}$ New York University, New York, NY. (Control ID \#3183293)

BACKGROUND: It is estimated that up to $88 \%$ of sex trafficking victims in the United States have had contact with one or more health professionals during the period of time they were trafficked. As victim identification remains low, little is known about the characteristics of these healthcare encounters or the experiences of victims. The purpose of this study is to explore the experiences of domestic sex trafficking victims in order to identify potential barriers to and opportunities for improved victim identification, disclosure, and linkage to appropriate services.

METHODS: A qualitative study involving a series of focus groups with survivors of domestic sex trafficking was conducted. Study participants were English-speaking women, age 18 years or older, survivors of child and adolescent domestic sex trafficking. Participants were recruited from a single community-based organization in New York City. Transcripts of the focus groups were analyzed using a grounded theory approach, and key concepts and themes were identified.

RESULTS: Four focus groups were conducted including a convenience sample of 28 total participants with a mean age of 22 (range: 18-27), and an average age of initiation into trafficking of 14.8 years (range: 11-18). Participants were mostly Black $(n=13)$, Hispanic $(n=5)$, and Mixed-race $(n=8)$. They reported seeking healthcare from a wide variety of locations and practice settings, for a variety of medical problems and symptoms: 1) physical trauma/injuries, 2) mental health, 3) gynecologic and reproductive health, 4) substance-use related and 5) neurologic. Six key themes emerged that characterized their healthcare experiences. Generally, trafficking victims experience high levels of 1) healthcare fragmentation; 2) stigma and disrespect during healthcare visits; 3 ) high burdens of disclosing trafficking experience; 4) narrowed expectations for effective assistance; 5) conflict between self-perception of agency and victimization; and 6) a greater receptivity to peer support.

CONCLUSIONS: Although sex trafficking victims appear to have frequent contact with healthcare providers, these encounters are often characterized by negative experiences and interactions. These may represent major contributors to the current gaps in care and limited effectiveness of screening and service linkage strategies for trafficking victims. Interventions that enhance trauma-informed care, stigma-free engagement, and peer support while reducing the personal, legal and administrative burdens of disclosure and perceived threats to agency or security may lead to improved victim identification and assistance.

EXPERT RECOMMENDATIONS ON IMPROVING TRAUMASENSITIVE PRIMARY CARE DELIVERY FOR WOMEN VETERANS WITH HISTORIES OF MILITARY SEXUAL TRAUMA

Alicia Bergman $^{1}$; Elizabeth M. Yano ${ }^{2}$; Joya G. Chrystal ${ }^{3}$; Bevanne BeanMayberry ${ }^{4}$; Alison B. Hamilton ${ }^{3} .{ }^{1}$ VA Greater Los Angeles, North Hills, $\mathrm{CA} ;{ }^{2}$ VA Greater Los Angeles Healthcare System, Sepulveda, CA; ${ }^{3} \mathrm{VA}$ Greater Los Angeles Healthcare System, Los Angeles, CA; ${ }^{4}$ VA Greater Los Angeles HSR\&D Center, Sherman Oaks, CA. (Control ID \#3184446)

BACKGROUND: The rate of military sexual trauma (MST) among regular primary care (PC) women Veteran (WV) users in the VA is high. History of sexual trauma is associated with depression, anxiety, PTSD, adverse impacts on medical management of chronic conditions, and discomfort with routine preventive exams. Ensuring delivery of VA's medical home care that is sensitive to sexual trauma histories is therefore critical. However, there is a need for additional expert guidance for primary care providers on the delivery of trauma-sensitive primary care. Our goal was to explore VA subject matter expert (SME) perspectives on critical aspects and best practices of trauma-sensitive primary care.

METHODS: We conducted interviews with 30 VA SMEs (Women's Health Medical Directors [n=10], Military Sexual Trauma Coordinators $[\mathrm{n}=10]$, and Women's Mental Health Champions $[\mathrm{n}=10])$ in $10 \mathrm{VA}$ Medical Centers regarding their experiences with and expertise on trauma-sensitive PC. Interviews were recorded, transcribed, and analyzed to identify trauma-sensitive best practices for PCPs to promote feelings of physical and psychological safety during general PC exams.

RESULTS: Participants discussed various verbal and non-verbal strategies PC providers can use in general exams to promote feelings of physical safety and psychological safety among WV patients who have histories of sexual trauma. Strategies for physical safety included ensuring the patient is fully clothed during discussions, giving the patient a wide girth of space, asking permission before any physical contact, not coming up to the patient from behind, and proactively asking about the patient's comfort regarding specialty procedures before making a referral (e.g., if sedation is ok). Participants also shared numerous strategies that PC providers can use to facilitate feelings of psychological safety. For example, displaying good bedside manners was described as important to building a foundation of trust and positive emotions (e.g., making eye contact, greeting patient in a comforting manner), as was giving the patient options for how to proceed with care (e.g., "If you prefer that a female provider do $\mathrm{A}, \mathrm{B}$, and $\mathrm{C}$, that's ok with me"). Additional strategies for encouraging emotional comfort included calling patients by name, using shared decision-making, making sure the patient is ok with medical students observing exams or procedures, and employing careful and intentional (non-judgmental) phrasing for asking questions and recommending tests (e.g.,STD or HIV test).

CONCLUSIONS: Participants highlighted various concrete trauma-sensitive tips and strategies for how VA PC providers can promote feelings of physical and emotional safety among WV patients who have histories of sexual trauma. Increasing awareness of pragmatic trauma-sensitive care strategies can improve PC provider sensitivity and care delivery. The translation of recommended strategies into practice-wide changes will require multilevel action to operationalize and implement them.

EXPLORING HOW SCREENING AND REFERRAL FOR SOCIAL DETERMINANTS OF HEALTH AFFECTS USAGE OF HOSPITAL AND COMMUNITY RESOURCES THAT HELP WITH SOCIAL NEEDS: A PILOT STUDY

Pablo Buitron de la Vega ${ }^{1}$; Kelsi Carolan ${ }^{2}$; Marna Stack ${ }^{4}$; Nancy R. Kressin' ${ }^{5}$ Stephanie Losi ${ }^{3}$; Linda Sprague Martinez ${ }^{2}$; Alana Ewen $^{3}$; Haeyeon Hong ${ }^{7}$; Daniel Sylvester ${ }^{7}$; Heloisa DeCarvalho ${ }^{6}$; Arvin Garg ${ }^{3}$. ${ }^{1}$ Boston Medical Center / Boston University, Dorchester, MA; ${ }^{2}$ Boston University School of Social Work, Boston, AL; ${ }^{3}$ Boston Medical Center, Boston, MA; ${ }^{4}$ Boston University School of Medicine, Boston, MA; ${ }^{5}$ Dept of Veterans Affairs and Boston University, West Roxbury, MA; ${ }^{6}$ Boston University School of Medicine, Chelsea, MA; ${ }^{7}$ Boston university, Boston, MA. (Control ID \#3172757)

BACKGROUND: Social determinants of health (SDOH) affect risk and outcomes from chronic diseases. Yet, there have been few systematic and sustained clinical strategies and processes for addressing SDOH. An Electronic Health Record-based SDOH screening and referral model called THRIVE, adapted from the WECARE model for pediatrics, has been implemented at Boston Medical Center (BMC) adult primary care since October 2017. This study examined the potential impact of THRIVE on usage of 
Hospital and Community Resources that Help with Social Need among BMC adult primary care patients.

METHODS: Mixed methods telephone survey to explore usage of resources that help with social needs. 57 adult patients (English and Spanish speaking) that went through THRIVE from October 2017 to March 2018 were called 4-8 weeks after presenting to their primary care visit. A comparison group of 37 participants that presented to BMC primary care from July to October 2017 (prior to THRIVE implementation), were also called.

RESULTS: Of the 57 THRIVE participants, 15 (26\%) endorsed the need for assistance connecting with resources (including housing, food, medicines, transportation, utilities, employment and education). Of the 57 patients, $14(25 \%)$ attempted to connect with some type of resource and $17(30 \%)$ indicated that they received help with a social need since their last visit. Of the 37 surveyed patients in the comparison group, two (5\%) indicated that their doctor had referred them to resources, $5(14 \%)$ attempted to connect with some type of resource and $4(11 \%)$ indicated that they have received help with a social need since their last visit. THRIVE participants reported high levels of difficulty connecting with housing resources. Additionally, they described having an easier time connecting with BMC resources including the BMC pharmacy and food pantry. Qualitative analysis of open ended questions for both groups showed that reaching out by phone but not hearing back from resources was the main challenge when trying to receive help. Additional obstacles included having to reapply for the same resources multiple times, language barriers, health and transportation barriers and importantly, limited knowledge of available resources. The most consistent piece of feedback that both groups provided was that healthcare professionals should ask patients about SDOH resource needs and provide resource or referral information - as part of primary care visits.

CONCLUSIONS: The THRIVE participants attempted to connect with resources more often than those in the control group. Although both group participants attempting to connect successfully received resources, and several described Boston Medical Center itself as a source of assistance, patients experience multiple obstacles to obtaining resource assistance. The results indicate that patients are interested in physicians and/or healthcare professionals proactively asking about potential resource needs as part of office visits.

\section{EXPLORING MEDICATION ADHERENCE FOR OHIO MEDIC- AID ENROLLEES WITH HYPERTENSION IN AN ERA OF TIGHTER TARGETS: WHERE ARE THE OPPORTUNITIES FOR THE GREATEST IMPACT?}

Shari Bolen ${ }^{1,2}$; Suparna Navale ${ }^{2}$; Siran Koroukian ${ }^{2}$; Michelle Menegay ${ }^{3}$; Douglas Einstadter ${ }^{4}$; Jackson Wright ${ }^{2}$; Randall D. Cebul ${ }^{2}$; Adam T. Perzynski $^{5}$; Catherine Sullivan ${ }^{5}$; Wanda Ali-Matlock ${ }^{6}$; Jonathan Lever ${ }^{6}$; Michael Konstan ${ }^{2}$; Dushka A. Crane ${ }^{3}$; Allison Lorenz ${ }^{3}$; Michael Nau ${ }^{3}$; Melissa Nance ${ }^{7}$; Stephen Albanese ${ }^{7}$; Donald Wharton ${ }^{7}$; Mary Applegate $7 .{ }^{1}$ MetroHealth/Case Western Reserve University, Cleveland, $\mathrm{OH} ;{ }^{2}$ Case Western Reserve University, Cleveland, OH; ${ }^{3}$ Ohio Colleges of Medicine Government Resource Center, Columbus, $\mathrm{OH} ;{ }^{4}$ MetroHealth Medical Center, Cleveland, OH; ${ }^{5}$ Case Western Reserve University at MetroHealth, Cleveland, OH; ${ }^{6}$ Better Health Partnership, Cleveland, OH; ${ }^{7}$ The Ohio Department of Medicaid, Columbus, OH. (Control ID \#3185055)

BACKGROUND: Although prior studies have utilized claims data to evaluate medication adherence, utilization and cost for adults with hypertension, few studies have combined clinical and administrative data to explore factors associated with medication adherence and blood pressure (BP) control. In an era of tigher BP targets, this study combines recent electronic health record (EHR) data from Ohio safety net primary care clinics linked with Medicaid claims data to explore these relationships and provide potential opportunities for improving medication adherence and BP control.

METHODS: Ohio Medicaid enrollees aged 64 or younger with a diagnosis of hypertension and seen at one of six safety net primary care clinics participating in a statewide hypertension quality improvement project during 2017-18 were included in this retrospective observational study. We conducted 2 sequential logistic regression analyses: 1) a baseline analysis for medication adherence adjusted for age, race, sex, selected comorbidities, and days supply of medication; and 2) an analysis for BP control with the same adjustments and also adjusted for medication adherence. We defined medication adherence as a medication possession ratio (MPR) of $80 \%$.

RESULTS: Of 1,038 Ohio Medicaid enrollees seen at one of 6 clinics during 2017- 18, 56\% were under good blood pressure (BP) control (most recent $\mathrm{BP}<140 / 90 \mathrm{~mm} \mathrm{Hg}$ ). Patients had a mean age of 50 (SD 10) and were diverse (46\% African American, $48 \%$ white, and 58\% female). About $1 / 3$ of uncontrolled patients had no claims for BP medications during the study period. Of 729 patients who had filled 1 BP medications, $68 \%$ were considered adherent (MPR 0.8). Those with controlled BP had higher medication adherence compared to those with uncontrolled BP ( $75 \%$ vs $57 \%$ respectively). Due to coverage policies, most patients ( $88 \%$ ) received a 30 -day supply of their BP medications, with only $10 \%$ and $2 \%$ of patients receiving $>30$-day or 90-day supply respectively. In adjusted models, medication adherence was associated with better BP control (OR 2.1; 95\% CI 1.5-2.9). Age was positively associated with medication adherence (OR 1.8; 95\% CI 1.0-3.2 comparing 55-64 versus 18-34 years) but was not associated with better BP control after adjusting for adherence. Receiving more than a 30 day supply of BP medication had a suggestion of an association with better medication adherence (OR 1.3; $95 \%$ CI 0.8 to 2.3), and was associated with better BP control (OR 2.0; 95\% CI 1.2-3.3). African Americans (versus whites) had lower medication adherence and lower BP control (OR 0.5; 95\% CI 0.3-0.7 and OR $0.6,95 \%$ CI 0.4-0.8 respectively).

CONCLUSIONS: Non-adherence was common and strongly associated with worse BP control. Programs targeted at younger hypertensive adults and African Americans with uncontrolled BP could have the greatest impact. The effect of changing policy to permit a 90-day supply of medications should be further evaluated.

EXPLORING PATIENT PERSPECTIVES ON THE FEASIBILITY AND ACCEPTABILITY OF MEDICATION ADHERENCE SUPPORT SERVICES FOR HYPERTENSION AT A PRIVATE HOSPITAL IN KAMPALA, UGANDA

Rachel Wilkinson ${ }^{1}$; Evan Garden ${ }^{1}$; Rose C. Nanyonga ${ }^{4}$; Allison Squires ${ }^{2}$; Jeremy I. Schwartz ${ }^{3}$; David J. Heller ${ }^{1,}{ }^{1}$. ${ }^{1}$ Icahn School of Medicine at Mount Sinai, New York, NY; ${ }^{2}$ New York University, New York, NY; ${ }^{3}$ Yale School of Medicine, New Haven, CT; ${ }^{4}$ Clarke International University, Kampala, Uganda. (Control ID \#3185654)

BACKGROUND: Hypertension (HTN) is the leading risk factor for death not only in the United States but also in low- and middle-income countries. In Uganda, HTN affects $24 \%$ of adults, but the primary care system has struggled to control it. Only $7.7 \%$ of Ugandans with hypertension are aware of their diagnosis, and less than half of those persons receive treatment with medication. Moreover, less than half of persons on HTN treatment achieve blood pressure control, suggesting substantial barriers to medication adherence even among patients with adequate care access. As HTN treatment programs scale up across Uganda, identifying and solving barriers to adherence within these programs becomes increasingly crucial. This study explored patient perspectives on acceptability of medication adherence interventions for HTN at International Hospital Kampala (IHK) in Uganda. 
METHODS: Using a qualitative descriptive design, we conducted 42 key informant interviews with hypertensive patients probing beliefs about the causes of medication nonadherence, and identifying viable adherence support tools to mitigate them. We explored the acceptability of support strategies validated in similar contexts, such as: daily text reminders, educational materials on hypertension, monthly group meetings (i.e. "adherence clubs") led by patients or providers, one-on-one appointments with providers, and modified drug dispensing at the hospital pharmacy. An open coding approach using NVIVO software structured the analysis. Themes and categories emerged iteratively and were solidified through consensus building processes.

RESULTS: Two themes emerged regarding causes of and solutions to adherence barriers: medication access and care engagement. Engagement barriers included lack of HTN symptoms, lack of peer or family support, interest in alternative or traditional medicine, forgetfulness, and disease denial; access barriers included distance to clinics and medication cost and availability. Patients were most supportive of interventions designed to further engage them in care, such as educational materials and adherence clubs. Patients praised their convenience (i.e. educational materials are portable and customizable) and peer support (i.e. learning from successfully adherent peers). Text message reminders and one-onone counselling also received support because of convenience and high levels of provider trust respectively.

CONCLUSIONS: Patients are motivated to control their blood pressure and are supportive of interventions designed to actively engage them in their care, suggesting that adherence clubs and educational materials may be successful tools for improving adherence in this population. Pilot testing of adherence clubs is underway; further work will directly evaluate its impact on blood pressure control. If effective, these interventions have the potential to improve medication adherence in similar contexts globally.

\section{EXPLORING PHYSICIAN EXPERIENCES ENGAGING CAREGIVERS IN THE SAFETY-NET}

Wagahta Semere ${ }^{1}$; Anupama Cemballi ${ }^{1}$; Alejandra Casillas $^{2}$; Neda Ratanawongsa ${ }^{1}$; Shobha Sadasivaiah ${ }^{1}$; Courtney Lyles ${ }^{1} .{ }^{1}$ University of California San Francisco at Zuckerberg San Francisco General Hospital, San Francisco, CA; ${ }^{2}$ University of California Los Angeles, Los Angeles, CA. (Control ID \#3185330)

BACKGROUND: Physicians experience communication gaps with caregivers, which create care coordination challenges that may be compounded when caring for patients in the safety-net who have limited resources and language barriers. However, knowledge of physician interactions with caregivers in this setting is limited. The objective of this study is to explore physician experiences with caregivers in the safety-net.

METHODS: We conducted 40-60 minute semi-structured interviews with 16 primary care providers from three California safetynet hospital systems (San Francisco Health Network, Los Angeles Department of Health Services, and Alameda Health System). Interviews explored provider engagement with caregivers in the safety-net setting. We analyzed interview transcripts using both deductive (i.e., guided by the interview guide) and inductive approaches (i.e., open coding) then established consensus regarding major themes and exemplary quotes.

RESULTS: Of 16 primary care providers interviewed, 4 were geriatricians. We identified four themes describing provider experiences with caregivers: (1) the diversity of caregivers, (2) visit and inter-visit communication strategies, (3) engaging caregivers in patient care, and (4) language and literacy barriers (Table). Providers noted a distinction between formal, paid caregivers and informal (often family) caregivers that coordinate patient care. Most interactions with caregivers were described in the context of clinic visits, where providers frequently encountered language and literacy barriers. Providers expressed that caregivers in the safetynet displayed limited confidence engaging in care management.

CONCLUSIONS: Providers in the safety-net understood caregivers to be a diverse group, including both formal and informal caregivers, providing multiple supportive roles on behalf of patients. In order to better leverage caregivers in patient care, providers in the safety-net may benefit from targeted resources to overcome language and literacy barriers and to specifically empower caregivers.

\section{EXPLORING PROVIDER PERSPECTIVES ON THE FEASI- BILITY AND ACCEPTABILITY OF MEDICATION ADHER- ENCE SUPPORT SERVICES FOR HYPERTENSION AT A PRIVATE HOSPITAL IN KAMPALA, UGANDA}

Evan Garden $^{1}$; Rachel Wilkinson ${ }^{1}$; Rose C. Nanyonga ${ }^{2}$; Allison Squires ${ }^{3}$; Jeremy I. Schwartz ${ }^{4}$; David J. Heller ${ }^{1,}{ }^{1}$. ${ }^{1}$ Icahn School of Medicine at Mount Sinai, New York, NY; ${ }^{2}$ Clarke International University, Kampala, Uganda; ${ }^{3}$ New York University, New York, NY; ${ }^{4}$ Yale School of Medicine, New Haven, CT. (Control ID \#3185640)

BACKGROUND: Hypertension (HTN) is the most common contributor to mortality in Uganda, and cardiovascular disease (CVD) is the leading cause of death. Although access to HTN screening and treatment is a major contributor to this burden, medication adherence is also a barrier to disease control. Previous work demonstrates that primary care providers in Uganda struggle to aid patients with HTN medication adherence, as patients lack symptoms and disease information, and face financial obstacles. Several interventions to improve HTN medication adherence in sub-Saharan Africa (SSA) exist in similar settings, but the feasibility and acceptability of these options for care providers, especially within the private sector, is unclear.

METHODS: Using a qualitative descriptive approach, we explored provider perspectives on challenges to HTN medication adherence, as well as the feasibility and acceptability of various forms of medication adherence support. Data collection occurred through key informant interviews with 15 providers (8 medical officers, 7 nurses) at International Hospital Kampala (IHK), a private hospital in Uganda. We specifically asked about potential interventions, including daily SMS (text) reminders; educational materials; monthly group meetings ("adherence clubs") led by patients or providers; one-on-one appointments with providers; and modifying drug dispensing at the hospital pharmacy. Two coders analyzed the interviews using a general content analysis approach, where themes and categories emerged iteratively.

RESULTS: Providers' beliefs on adherence determinants centered on two themes: patient access (i.e. cost, distance from care centers) and patient engagement (i.e. lack of knowledge of HTN causes and treatment, lack of support systems), aligning with patients' views in related interviews. Providers especially supported adherence clubs, citing improved patient engagement through peer support and increased provider-patient interactions. They also endorsed patient educational materials due, but cited limited patient literacy as a barrier. Daily SMS reminders were deemed convenient, particularly for patients who lived far from IHK. Providers considered staffing concerns as the major barrier to implementing these interventions, but also cited program finances, patient participation, and scheduling concerns.

CONCLUSIONS: Providers viewed adherence support interventions as feasible and acceptable additions to HTN care at IHK, but cited financial cost and human resources as barriers. Providers' views regarding HTN 
adherence initiatives centered on both improving access to care and increasing patient engagement, whereas patients' beliefs centered primarily on engagement. HTN adherence clubs, developed based on patients' and providers joint perspectives, could substantially improve patient engagement with and access to HTN care; design and implementation of this initiative at IHK is ongoing.

\section{FACTORS AFFECTING HEALTHY EATING AND EXERCISE AMONG LESBIAN AND BISEXUAL LATINA WOMEN AND DISCUSSING HEALTHY LIFESTYLE WITH THEIR PRIMARY CARE PROVIDERS}

Julie Grutzmacher $^{1}$; Jessica I. Mora ${ }^{1}$; Katherine Ordonez ${ }^{1}$; Monica Vela ${ }^{2}$; Seeba Anam ${ }^{1}$; Arshiya A. Baig ${ }^{1} .{ }^{1}$ University of Chicago, Chicago, IL; ${ }^{2}$ University of Chicago Pritzker School of Medicine, Chicago, IL. (Control ID \#3177734)

BACKGROUND: Latina lesbian and bisexual women experience high rates of overweight and obesity compared to their white counterparts; however few studies have explored the factors that influence healthy eating and exercise in this population and their experiences discussing healthy lifestyle with their primary care providers.

METHODS: We conducted semi-structured one-on-one interviews in English and Spanish with 20 lesbian and bisexual cisgender Latina women in Chicago. Questions focused on identity and intersectionality; relationships with healthcare providers, the mechanism of disclosure and shared decision making; particularly involving decisions about healthy and unhealthy diet, and exercise. Examples of these questions include: "Tell me about how your identities (racial/ethnic and sexual orientation/gender identity) affect your thoughts about healthy and unhealthy eating," and "Have you ever talked with a healthcare provider about healthy and /or unhealthy eating, exercise and/or body image?" The interviews were later transcribed verbatim. A codebook was developed, transcripts were double coded and entered into NVIVO, and themes were analyzed using modified Grounded Theory. Fifteen of the interviews were conducted in English and five in Spanish.

RESULTS: Of the 20 participants, the average age was 33 years old. Fourteen or $70 \%$ of them identified as lesbian and six $(30 \%)$ identified as bisexual, pansexual, or queer. All of them identified as a cisgender woman and as Latina/Hispanic. Participants indicated that cultural traditions and norms (i.e. carnitas, tortillas, chorizo) can pose as barriers to healthy eating patterns. On the other hand, some participants stated that being part of the queer community has endorsed healthier eating patterns and exposed them to healthy foods not endemic to their popular cultural traditions (i.e. vegan diets). With regards to physical activity, participants' cultural heritage, comfort and safety, and stereotypes about certain sports (i.e. soccer or softball) influenced their decisions around physical activity more so than their sexual minority identities. Participants described the stress of having to educate their providers on queer lifestyle, which they described as awkward and wasting time where they could otherwise be asking questions of their own. The recommendations were for providers to take a patient-centered, individualized counseling approach and engage them in dialogue about healthy eating and exercise.

CONCLUSIONS: Identity and behavior is inextricably connected. Participants' decisions around eating and exercise were heavily impacted by their multiple intersecting identities (race/ethnicity, gender, sexual orientation, SES and employment status). Raising awareness among providers about intersectionality and factors that influence patients' decision making is an essential place to start for reducing disparities and further supporting the health equity of this population.
FACTORS AFFECTING YOUNG GAY MEN'S PREFERENCE FOR SEXUAL ORIENTATION- AND GENDER IDENTITY-CONCORDANT PROVIDERS

Stephanie E. McLaughlin ${ }^{2}$; Cary Blum ${ }^{2}$; Angelina Gomes ${ }^{2}$; Carolyn Drake ${ }^{2}$; Colleen Gillespie ${ }^{2}$; Richard Greene ${ }^{2}$; Perry Halkitis ${ }^{1}$; Farzana Kapadia ${ }^{1}$. ${ }^{1}$ New York University, New York, NY; ${ }^{2}$ New York University School of Medicine, New York, NY. (Control ID \#3184662)

BACKGROUND: A relative dearth of literature exists on preferences of young gay male patients have regarding the sexual orientation and gender identity (SOGI) of their healthcare providers. Further research in this area is warranted to better serve the young MSM population.

METHODS: Data collection: A sample of 800 young adult gay men completed a brief survey on healthcare preferences between 2015-2016. Participant inclusion criteria were: age 18-29, male gender, self-identified gay sexual orientation, living in US for 5+ years, and being a resident of the New York City metropolitan area. Only participants who reported having a current PCP provided information on preferred PCP characteristics (i.e. male and/ or LGBT). Data analysis: Multivariable logistic regression models were built to assess factors associated with participant preference for an LGBT or male PCP. Covariates for inclusion were considered based on prior literature as well as those identified as significant in bivariate logistic regression analyses. Backward model selection with variance inflation factor (VIF) analysis was used to eliminate collinearity and arrive at the most parsimonious models.

RESULTS: In this sample, $\mathrm{n}=614$ men $(77 \%)$ reported having a PCP. Of those 614 with a PCP, $42 \%$ indicated a preference for male PCP, $36 \%$ preferred a gay or LGBT PCP, and a total of $20 \%$ preferred a male-LGBT provider. A preference for consolidated care and distrust in the health system were associated with preference for a sexual orientation concordant PCP. Preference for sexual orientation concordance was strongly associated with preference for gender concordance, and vice versa. Minority race was also found to be associated with preference for a gender-identity concordant (male) PCP.

CONCLUSIONS: Gay men who wish to discuss their overall health and sexual health with their primary care provider (ie, receive consolidated care) tend to prefer a LGBT provider. This is also true of gay men who distrust the healthcare system, possibly because they anticipate these providers will provide more culturally sensitive care. A surprising association was found between minority racial background and preference for a gender concordant provider. Further research is warranted to explore the factors giving rise to this finding.

Table 2. Summary of OLS regression predicting preference for PCP SOGI concordance

\begin{tabular}{lcc}
\hline Variable & $\begin{array}{c}\text { Prefer gay or LGBT PCP } \\
\text { OR }(95 \% \mathrm{CI})\end{array}$ & $\begin{array}{c}\text { Prefer male-identified PCP } \\
\text { OR }(95 \% \mathrm{CI})\end{array}$ \\
\hline Age 24-29 & $1.02(0.703-1.47)$ & $0.981(0.684-1.40)$ \\
Minority race & $0.865(0.537-1.39)$ & $2.02(1.24-3.27) * * *$ \\
HIV positive & $0.981(0.684-1.40)$ & $1.20(0.734-1.97)$ \\
Distrust in health system & $1.59(0.922-2.73) *$ & $0.928(0.540-1.59)$ \\
Prefer consolidated care & $1.72(1.01-2.92) * *$ & $0.958(0.587-1.50)$ \\
Prefer male-identified PCP & $2.61(1.81-3.74) * * *$ & $\mathrm{~N} / \mathrm{A}$ \\
Prefer gay or $L G B T P C P$ & $\mathrm{~N} / \mathrm{A}$ & $2.60(1.81-3.37) * * *$ \\
\hline Observations & 552 & 552 \\
\hline Pseudo- $\mathrm{R}^{2}$ & 0.0482 & 0.0502 \\
\hline$* * * p \leq .005, * * p \leq .05,{ }^{*} p \leq 0.1$ & &
\end{tabular}


FACTORS ASSOCIATED WITH INCREASED RISK PERCEPTION FOR DEVELOPING TYPE 2 DIABETES IN WOMEN WITH RECENT GESTATIONAL DIABETES Jacinda M. Nicklas ${ }^{1}$; Geraldine Skurnik ${ }^{2}$; Ellen W. Seely ${ }^{2} .{ }^{1}$ University of Colorado School of Medicine, Aurora, CO; ${ }^{2}$ Brigham and Women's Hospital, Boston, MA. (Control ID \#3184760)

BACKGROUND: Women with gestational diabetes (GDM) have a $30-70 \%$ risk for developing type 2 diabetes. Although women with a history of GDM can decrease their risk for type 2 diabetes through weight loss and increased physical activity, prior studies indicate that many women with a history of GDM do not engage in risk reducing behaviors. Perception of personal risk for type 2 diabetes may influence participation in these risk reduction behaviors. We sought to identify factors associated with increased risk perception for developing type 2 diabetes in women with recent gestational diabetes.

METHODS: For the Balance After Baby Intervention 2 (BABI-2) trial, we recruited women with GDM during pregnancy or just after delivery. We asked participants to come in at 6 weeks postpartum for a baseline study visit. We measured weight and height, and women completed questionnaires detailing demographic, family history and medical history, as well as the Edinburgh Postnatal Depression Scale and the Risk Perception Survey for Developing Diabetes. All measurements and questionnaires were conducted before randomization into the intervention trial. For this analysis we included all women answering a risk perception question about their risk for getting diabetes within the next 10 years. We then categorized women into almost no or slight perceived chance vs. moderate or high perceived chance for developing diabetes. We conducted univariate analyses, and modeled the odds of increased risk perception for type 2 diabetes using multivariable logistic regression. We included all variables with a $\mathrm{p}$ value $<.2$ from the univariate analyses in the initial model, and then used backward selection to fit a final model.

RESULTS: Our study included 181 women (mean $33 \pm 5.3$ years; $48 \%$ White, $17 \%$ African-American, 22\% Asian, $13 \%$ other race, with $35 \%$ Hispanic). $48 \%$ of women spoke a primary language at home that was not English and $54 \%$ of women had graduated from college. $31 \%$ of women reported household incomes of $<\$ 35,000$ per year. $28 \%$ had had GDM in a previous pregnancy, and $43 \%$ had a first degree relative with type 2 diabetes. The mean BMI at the study visit was $31.3( \pm 5.9) \mathrm{kg} / \mathrm{m}^{2}$. Factors included in the final model associated with a moderate to high perception of risk for diabetes included having a first degree relative with type 2 diabetes (OR 2.9, 95\% CI 1.5-5.5) and identifying as Hispanic or Latino (OR 2.5, 95\% CI 1.3-4.9). Having a history of GDM in a previous pregnancy and education status were not retained in the final model.

CONCLUSIONS: Among women with recent gestational diabetes, identifying as Hispanic/Latino and having a first degree relative with type 2 diabetes were associated with increased risk perception for developing diabetes. Further research should address how risk perception in this population influences participation in interventions designed to reduce risk for type 2 diabetes and whether employing unique strategies addressing risk perception can improve outcomes.

\section{FACTORS ASSOCIATED WITH LONG-TERM RETENTION IN BUPRENORPHINE BASED ADDICTION TREATMENT PRO- GRAMS: A SYSTEMATIC REVIEW}

Amy J. Kennedy ${ }^{3}$; Jessica S. Merlin ${ }^{3}$; Charles B. Wessel ${ }^{2}$; Rebecca Levine $^{1}$; Iman Hassan ${ }^{3}$; Kendall Downer ${ }^{1}$; Megan Raymond ${ }^{4}$; Deborah Osakue $^{1}$; Jane M. Liebschutz ${ }^{3}{ }^{1}$ University of Pittsburgh Medical Center, Pittsburgh, PA; ${ }^{2}$ University of Pittsburgh, Pittbsurgh, PA; ${ }^{3}$ University of Pittsburgh, Pittsburgh, PA; ${ }^{4}$ University of Pittsburgh School of Medicine, Pittsburgh, PA. (Control ID \#3170853)
BACKGROUND: The average length of opioid agonist therapy with buprenorphine (BUP) for opioid use disorder is less than 6 months. We conducted a systematic review to determine what treatment level factors (dose, treatment setting and behavioral therapies) were associated with longer retention in BUP treatment.

METHODS: We searched Medline, Embase and the Cochrane Database of Systematic Reviews in February 2018. Articles were restricted to randomized-controlled trials on human subjects, written in English, and contained $>24$ weeks of objective data on retention in BUP treatment. We assessed whether dose of BUP, treatment setting, or co-administration of behavioral therapy were associated with retention rates.

RESULTS: Over 14,000 articles were identified. Twenty-two articles met final inclusion criteria, describing a total of 13 studies (Figure 1). There was significant heterogeneity in the measurement of retention. Measures included days in treatment $(\mathrm{n}=10)$, urine drug testing for $\mathrm{BUP}(\mathrm{n}=2)$, and a combination of days in treatment and plasma level testing for BUP $(n=1)$. Three studies compared doses of BUP between 1-8mg and showed significantly higher rates of retention with higher doses ( $p$-values $<0.01$ ). All other studies in our review utilized maintenance BUP doses between $8 \mathrm{mg}-24 \mathrm{mg}$ daily, without comparison. No study found a significant difference in retention between BUP alone and BUP plus behavioral therapy ( $\mathrm{p}$-values $>0.05$ ). Starting BUP prior to initiation in outpatient treatment programs (inpatient induction or within criminal justice settings) was significantly associated with retention in BUP treatment (pvalues 0.009 and 0.005 respectively).

CONCLUSIONS: Setting of treatment initiation and higher BUP dose are associated with improved long-term treatment retention. More data on BUP treatment programs is needed as well as a standardized approach to defining retention in BUP treatment programs.

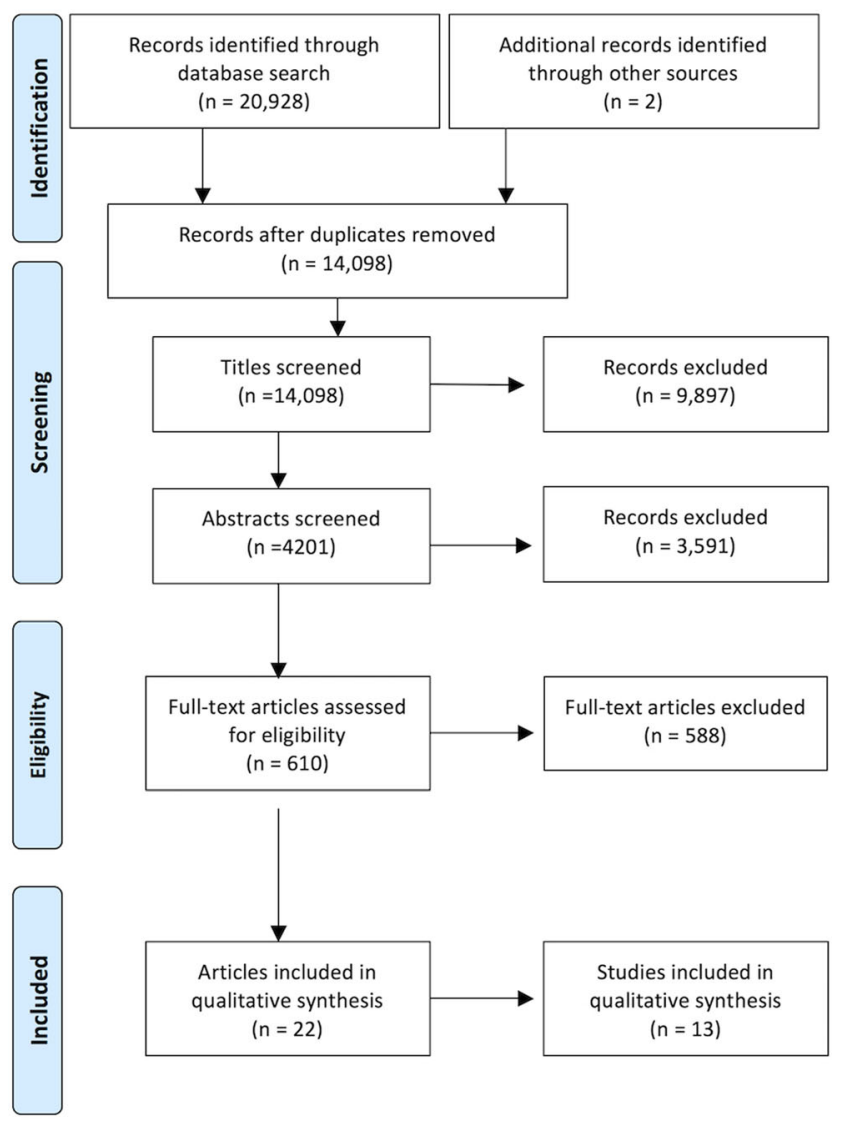

Figure 1. Flow diagram of the selection process for study inclusion. 
FACTORS ASSOCIATED WITH OPIOID DISCONTINUATION IN CHRONIC OPIOID THERAPY

Jason M. Glanz ${ }^{1}{ }^{2}$; Stanley $\mathrm{Xu}^{1}$; Komal J. Narwaney ${ }^{1}$; Susan M. Shetterly $^{1}$; Jo Ann Shoup ${ }^{1}$; Jennifer Lyden ${ }^{3}$, ${ }^{4}$; Ingrid A. Binswanger ${ }^{1,4}$. ${ }^{1}$ Kaiser Permanente Colorado, Denver, CO; ${ }^{2}$ Colorado School of Public Health, Aurora, CO; ${ }^{3}$ Denver Health, Denver, $\mathrm{CO} ;{ }^{4}$ University of Colorado School of Medicine, Aurora, CO. (Control ID \#3186067)

BACKGROUND: There is limited evidence on the safety and effectiveness of opioid tapering practices in pain management. In addition, there is a lack of data describing tapering practices and outcomes in routine clinical care. We sought to to examine characteristics associated with opioid discontinuation among patients receiving chronic opioid therapy (COT).

METHODS: We conducted a matched cohort study using electronic health record data from an integrated health system in Colorado. We identified health plan members 18 years between 2006 and 2017, who had 90 days continuous enrollment and received COT ( 3 opioid prescriptions in 90 days). Among these patients, we identified periods of opioid discontinuation, defined as 3 months without any opioid dispensings. Using calendar time, patients who had been discontinued were frequency matched to patients who did were not discontinued. We conducted two logistic regression analyses examining 11 demographic and clinical characteristics. The first compared patients with 1 period of discontinuation to patients without a discontinuation. The second analysis compared patients who had a period of discontinuation but resumed opioid therapy to patients who remained discontinued while enrolled in the health plan.

RESULTS: We identified a cohort of 14,419 patients who received COT during the observation period. Over a mean follow-up of 4.8 years, 8,518 (59\%) were never discontinued, 2,740 (19\%) stayed discontinued, 2,234 (15\%) were discontinued and resumed opioids, and 927 (6\%) were discontinued and disenrolled from the health plan. In the first analysis, the following variables were positively associated with being discontinued: young age, white, history of overdose, mental health diagnosis, alcohol and drug use disorders, long acting opioids, and low opioid dose (Table). In the second analysis, the following variables were positively associated with sustained discontinuation: young age, drug use disorder, and low opioid dose. Receipt of a benzodiazepine was negatively associated with sustained discontinuation.

CONCLUSIONS: Among patients on COT, periods of opioid discontinuation were common. Discontinued patients frequently resumed opioids, and baseline patient characteristics were significantly associated with opioid discontinuation patterns. The results suggest that it may be easier to discontinue patients on low opioid doses, and sustained discontinuation may be difficult with patients prescribed benzodiazepines.

\section{FACTORS ASSOCIATED WITH STATE LEGISLATOR SUP- PORT FOR MEDICAID WORK REQUIREMENTS}

Jane M. Zhu ${ }^{1}$; Renuka Tipirneni ${ }^{2}$; David Jones ${ }^{3}$; Michael Fichman ${ }^{4}$; David Grande ${ }^{4}$. ${ }^{1}$ Oregon Health and Science University, Portland, OR; ${ }^{2}$ University of Michigan, Ann Arbor, MI; ${ }^{3}$ Boston University School of Public Health, Boston, MA; ${ }^{4}$ University of Pennsylvania, Philadelphia, PA. (Control ID \#3185422)

BACKGROUND: As of early 2019, seven states (AR, IN, KY, ME, MI, $\mathrm{NH}, \mathrm{WI}$ ) have Medicaid waivers approved by the Centers for Medicare and Medicaid Services (CMS) to implement work or community engagement requirements in exchange for coverage, and more states are considering or seeking similar waivers. This study examined district-level demographic and socioeconomic characteristics associated with state legislators' support for Medicaid work requirements.

METHODS: Using a database from the National Conference of State Legislators (NCSL), we surveyed active state legislators $(n=7,340)$ on their views of Medicaid policy proposals, including work requirements, selected from approved and pending Medicaid section 1115 waiver applications. The survey instrument was pilot-tested with current state legislators and federal legislative staff to ensure policy relevance of questions and non-partisan language. Post-survey, responses were linked to demographic and socioeconomic data from the 2016 American Community Survey. We used descriptive analyses to assess views by political party, and multivariable logistic regressions to analyze legislator- and district- level factors that were associated with support for work requirements.

RESULTS: Overall, 894 (12.2\%) state legislators completed the survey, a response rate consistent with those in other studies of elite-level actors. Non-respondents were more likely to be non-health care committee members, Republican, male, and from the Midwest region. Republicans were more likely than Democrats to support work eligibility requirements for Medicaid ( $84.8 \%$ vs. $26.7 \%$ ). Supporters of work requirements tended to be Republican (OR 27.75, 95\% CI: 15.69-49.08, $\mathrm{p}<0.0001$ ), reside in rural areas (OR 2.43, 95\% CI: 1.48-3.99, $\mathrm{p}<0.0001)$, and have higher levels of Medicaid enrollment in their districts (OR 1.34 for each higher quartile of district-level Medicaid enrollment, 95\% CI: 1.04-1.71, $\mathrm{p}=0.021$ ). Legislators who supported work requirements also tended to represent districts with higher percentages of white constituents $(\mathrm{OR}=0.68$ for each higher quartile of white population, $95 \%$ CI: $0.52-0.89, \mathrm{p}=0.004$. State participation in Medicaid expansion was not associated with legislators' views on Medicaid work requirements, nor was legislator membership in a health-related legislative committee.

CONCLUSIONS: Among state legislators, support for Medicaid work requirements is more strongly associated with political party affiliation than with other legislator or district-level characteristics. As state legislators become increasingly influential health policy actors, future research should probe more deeply into how views and knowledge about specific policies are cemented, including the influence of constituencies and their needs.

FACTORS INFLUENCING PATIENTS IN MEDICALLY UNDERSERVED AREAS TO CHOOSE THE EMERGENCY ROOM INSTEAD OF PRIMARY CARE FOR NON-URGENT CONDITIONS Rosita N. Ekem; Satya Surbhi; Leonard J. Harris; James E. Bailey. University of Tennessee Health Science Center, Memphis, TN. (Control ID \#3183980)

BACKGROUND: Patients often go to the emergency department (ED) for non-urgent conditions, that can be managed in primary care (PC) settings. Past research suggests that ED care for nonurgent conditions may result in unnecessary testing, elevated costs, and missed opportunities for essential primary and preventive care. ED use for non-urgent conditions is especially prevalent in medically underserved areas (MUA). This study explored patient experiences, perceptions, barriers to $\mathrm{PC}$ access, and preferences regarding $\mathrm{ED}$ and primary care use. We also examined factors would encourage patients with non-urgent conditions to choose primary care over ED.

METHODS: A self-administered cross-sectional survey was given to a convenience sample of adults triaged as semi-urgent or nonurgent conditions in the ED of a large non-profit healthcare system during June-July of 2018. Consent was obtained before patients completed the survey in private exam rooms. Exclusion criteria included pregnancy, cognitive impairment, non-English speaking, under police supervision, or hospital admission. The survey was designed to compare experiences in the ED and PC, collect demographics, patient perception of illness severity, and factors that affect non urgent usage of the ED. Descriptive analyses were conducted to describe the study population, examine the most common barriers to PC access, and identify factors that would encourage patients to choose primary care over the ED. 
RESULTS: Of 125 eligible patients approached for this study, 89 agreed to participate (response rate $71.2 \%$ ). Participant mean age was 36.6 years. Most were female (50\%), African-American ( $88.4 \%$ ), and had $2 \mathrm{ED}$ visits in the past year $(56.6 \%)$. Also, $70 \%$ had less than a high school education, low health literacy $(53.6 \%)$, and a yearly income of $<\$ 20,000(75.3 \%)$. Most patients regarded their condition as urgent $(32.6 \%)$ or emergent $(33.7 \%)$. One-third reported not having a PCP $(35.3 \%)$ and using the ED as a usual source of care when sick $(39.7 \%)$. Patients cited long wait times $(31.4 \%)$, untimely appointments (29.4\%), inconvenient clinic hours (21.2\%), and transportation difficulties (18.8\%) as barriers to care. Patients cited improved accessibility, acceptability, provider technical quality, and getting appointments on short notice as factors that would encourage use of $\mathrm{PC}$.

CONCLUSIONS: Patients in MUA commonly perceive their conditions as urgent or emergent and desire patient-centered care when they need it, in convenient locations, and at low out-of-pocket cost. Difficulty contacting providers by phone, making timely appointments, long wait times, and high out-of-pocket costs are barriers that discourage utilization of PC. Patients cite that improved PC accessibility, accommodation, acceptability, and provider technical quality increased PC attractiveness. This study provides insight for health systems on how to increase value of care for medically underserved patient populations by improving access, affordability, and quality of PC.

\section{FACULTY DEVELOPMENT IN MEDICAL EDUCATION IM- PACTS CLINICIAN EDUCATORS' ROLE IDENTITY AND SENSE OF COMMUNITY}

Penelope Lusk ${ }^{1}$; Kevin Hauck ${ }^{1}$; Verity Schaye ${ }^{3}$; Neil Shapiro ${ }^{4}$; Khemraj A. Hardowar ${ }^{5}$; Sondra $Z_{\text {abar }}^{3}$; Anne Dembitzer ${ }^{2,}{ }^{1}$. ${ }^{1} \mathrm{NYU}$ Langone, Brooklyn, NY; ${ }^{2}$ VA Health and Harbor, New York, NY; ${ }^{3}$ NYU School of Medicine, New York, NY; ${ }^{4}$ Dept. Of Veterans Affairs, New York, NY; ${ }^{5} \mathrm{NYU}$, New York, NY. (Control ID \#3183943)

BACKGROUND: Faculty development programs (FDP) in medical education can increase clinician educators' (CE) confidence in teaching and improve their teaching skills. The impact of FDP on faculty's role as educators and sense of an educator community is less well understood. Identification with a community of educators (COE) can enhance teaching in the workplace along with personal and professional growth. We evaluated the impact of participation in the Education for Educators program (E4E) on these issues. E4E is a yearlong FDP designed to enhance teaching confidence and skill in a variety of venues; improve ability to assess learners; promote an environment of academic inquiry with trainees at different levels; and create a COE.

METHODS: An annual needs assessment of key stakeholders including medical school deans, program directors, and participants forms the basis for the E4E curriculum. The program begins with a Group Observed Structured Teaching Experience (GOSTE) followed by three 3-hour workshops which pair a clinical and teaching topic. After each workshop, participants complete "commitment to change" statements and take part in peer-to-peer (P2P) observations wherein participants observe each other teaching in their usual teaching environment. The program concludes with structured debriefs and an assessment of participants' perception of their role as educators and their sense of an educator community. Participants reported how participation in E4E impacted their teaching and what new skills they implemented. Structured phone conversations assessed the same information one-year after completing the program.

RESULTS: Fifty-one CEs completed the program in two cohorts (2016-17 and 2017-18), 60\% of whom were women. Participants included 20 hospitalists and 31 subspecialists, averaging 8 years in practice (range 1-28) and spending an average of $63 \%$ of their time in patient care (range 10-100\%). Thirty-eight participants (75\%) completed the immediate post-program debrief sessions. Participants reported a renewed identification with their role as an educator. They cited a change in perspective to become more reflective and focused on teaching and recognized that their teaching skills can in fact be improved. Many reported time constraints as a barrier to teaching. They noted an increased identification with their COE, stating that they now had peers and mentors with whom to discuss teaching challenges. To date, phone interviews have been completed with three participants at one-year of follow-up. The preliminary results show a sustained impact on educators' roles and belonging to a COE. They also reported ongoing use of specific skills including resilience strategies, and planning teaching sessions.

CONCLUSIONS: Longitudinal FDP in medical education for CE can lead to a greater appreciation for the role of an educator, and identification with a COE. Investment in longitudinal FDP may have lasting impact on the clinical learning environment and the identity of faculty as an educator.

FACULTY DEVELOPMENT NEEDS FOR THE AMBULATORY CLINICIAN EDUCATOR: RESULTS OF THE AMBULATORY FACULTY RECRUITMENT, RETENTION, AND REMUNERATION IN GRADUATE MEDICAL EDUCATION SURVEY BY THE AAIM EDUCATION COMMITTEE

Jonathan Tolentino $^{1}$; Isitri Modak ${ }^{2}$; Margaret C. Lo ${ }^{3}$. ${ }^{1}$ Stony Brook University, Cincinnati, OH; ${ }^{2}$ Methodist Health System, Grand Prairie, TX; ${ }^{3}$ University of Florida College of Medicine, Gainesville, FL. (Control ID \#3186358)

BACKGROUND: With expansion of the Affordable Care Act, GME directors must create robust ambulatory teaching environments. Successful redesign of ambulatory education requires highyield faculty development (FD) programs with clear understanding of the FD needs of ambulatory educators. While literature cites various FD programs, little exists on the FD priorities of ambulatory faculty. Tasked by AAIM, our descriptive survey reports key FD skills needed in ambulatory education.

METHODS: After several teleconferences and Delphi processes, AAIM Education committee members drafted a needs-assessment survey to address the barriers and strategies for effective ambulatory teaching and FD. Survey asked the value of 20 specific ambulatory FD skills and 5 specific venues to effectively teach these skills to residents in the ambulatory setting, based on 5point Likert scale. Request for survey participation was posted bimonthly from August-Nov 2017 on listservs of APDIM, APM, SGIM and AAIM Ambulatory Faculty focus group. Participation was anonymous and voluntary. De-identified results were analyzed via descriptive statistics from STATA software. University of Oklahoma HSC provided IRB approval.

RESULTS: 217 ambulatory faculty completed the survey. Most were core ambulatory resident educators $(76 \%)$ at academic medical centers (65\%). [M1] Highest-valued FD skills for ambulatory teaching were bedside teaching $(77.5 \%)$; clinical reasoning $(76 \%)$; and goal-setting, evaluation, and feedback (71.6\%). Least-valued FD skills were concept mapping $(14.3 \%)$, research methods $(19 \%)$, and large group teaching $(20.1 \%)$. No differences exist between respondents from academic medical centers (AMC) or community-based programs for all skills except for promotion of clinician educators $(p<0.05)$, which held less value to communitybased faculty. Respondents preferred small group workshops as the delivery mechanism for learning most FD skills over didactic sessions, webinars, online modules, or conferences. 
CONCLUSIONS: This cross-sectional survey adds to the literature by identifying high-yield ambulatory skills to help GME directors tailor their FD programs based on faculty types and engage ambulatory faculty to teach housestaff. Ambulatory FD programs should target teaching skills most useful in one-on-one precepting relationships (e.g. bedside teaching, feedback, and goalsetting/mentorship) and deliver such skills in small group interactive workshops. Survey data do not support FD skills esoteric to ambulatory educators such as concept mapping, research methods, or promotion for community-based faculty. Further work must focus on effective ambulatory FD models to teach these skills in low- and high-resource institutions.

\section{FAMILY CAREGIVER PERSPECTIVES ON LONG TERM CARE PLANNING}

Steffi Shilly ${ }^{1}$; Vanessa Chicas ${ }^{1}$; Jillian Shotwel1 ${ }^{1}$; Caren Steinway ${ }^{1}$; Sophia Jan ${ }^{2}$. ${ }^{1}$ Cohen Children's Medical Center at Northwell Health, New Hyde Park, NY; ${ }^{2}$ Northwell Health, New Hyde Park, NY. (Control ID \#3175394)

BACKGROUND: Although many family caregivers of individuals with intellectual/developmental disabilities (IDD) worry about their relative's future, few develop solid long term care (LTC) plans. This lack of LTC planning may lead to crises and emotional trauma for concerned loved ones, unexpected dilemmas for siblings or extended families, and unwanted or inappropriate emergency placement.

METHODS: We conducted five focus groups in two states with parents and siblings of individuals with IDD. Before each focus group, participants were asked to complete a survey that included basic demographic information, the Waisman Activities of Daily Living Scale (W-ADL), and Scales of Independent BehaviorRevised, Problem Behavior Scale (SIB-R). Caregivers were asked about perceived barriers and facilitators of LTC planning and preferences for tools to promote LTC planning among families like theirs. Focus group recordings were transcribed verbatim and coded using the constant comparative method to identify major themes.

RESULTS: Twenty-seven family caregivers, 19-74 years old (mean age, $39.59)$, participated in focus groups. Family caregivers were predominantly female $(56 \%)$ and White $(81 \%)$. Caregivers resided in Delaware (44\%), Pennsylvania (48\%), and New Jersey (7\%). Education level and yearly household income varied among family caregivers. The individuals with IDD, ages 14-40 years old (mean age, 23.24) lived in various states: Delaware $(\mathrm{n}=12,44 \%)$, Pennsylvania $(\mathrm{n}=12,44 \%)$, New Jersey $(\mathrm{n}=2,7 \%)$, and Missouri $(\mathrm{n}=1,4 \%)$. Of the individuals with IDD, $78 \%$ $(\mathrm{n}=21)$ identified as White, $15 \%(\mathrm{n}=4)$ as Black, and $4 \%(\mathrm{n}=1)$ as Asian. Almost half (48\%) of the individuals with IDD had a Medicaid Waiver and $63 \%$ had 1 problem behavior. The mean W-ADL score was 1.209 , meaning that most individuals need assistance with activities of daily living. Focus groups revealed that having an advocate (i.e., a support coordinator or government official), networking (such as with other caregivers), emotions (i.e., hope or fear), adverse events (i.e., eviction) and the experience or personal background of a caregiver (i.e., having a medical background) facilitated planning. Lack of information, lack of appropriate resources (i.e., medical providers inexperienced in caring for adults with IDD), emotions (i.e., uncertainty of the future), finances, and difficulty in systems navigation (i.e., difficulty obtaining Medicaid) were identified as barriers to planning. These barriers and facilitators are especially prominent when most services are lost during transition to adulthood. Family caregivers mentioned that a website would be a useful resource to help with LTC planning.

CONCLUSIONS: LTC planning is influenced by the coordination of many complex factors, including emotions, knowledge, and resources. Interventions for LTC planning may improve quality of life, independence, and personal choice of adults with IDD and their caregivers.

\section{FASTING STATUS OF PATIENTS UNDERGOING AMBULA- TORY LABORATORY TESTING}

Eva Tseng ${ }^{1}$; Jodi B. Segal ${ }^{2}$; Nisa Maruthur ${ }^{2} .{ }^{1}$ Johns Hopkins University SOM, Baltimore, MD; ${ }^{2}$ Johns Hopkins University School of Medicine, Baltimore, MD. (Control ID \#3185039)

BACKGROUND: Many population-level studies use glucose values from EMR data to identify patients with prediabetes and diabetes. Since diagnostic criteria are based on fasting glucose, investigators often assume patients are fasting, particularly if phlebotomy was in the morning. We sought to assess the validity of the assumption that outpatient, morning measurements are fasting measures.

METHODS: We surveyed adult patients (age 18 years) presenting for phlebotomy at the Johns Hopkins Outpatient Center during $7 \mathrm{am}-12 \mathrm{pm}$ over 2 weeks in November 2018. Patients selfadministered a 4-question written survey, provided by the registration staff, about their fasting status, whether the ordering provider instructed them to fast, whether they were getting a glucose and/or cholesterol test, and what time they presented to the lab. We defined fasting status as "nothing to eat or drink 8 hours before the test except for water." We estimated a sample size of $n=500$ would provide a relatively tight confidence interval around the estimates (i.e., $95 \%$ CI within $5 \%$ on either side of the estimate).

RESULTS: Of 506 survey respondents, 49\% reported they had fasted. Patients presenting earlier vs. later were more likely to be fasting (Figure) with $64 \%$ fasting between $7-8$ am compared to $31 \%$ fasting between $11 \mathrm{am}-12 \mathrm{pm}$ ( $\mathrm{p}$ trend $<0.001$ ). Among those who had fasted, $49 \%$ reported their provider advised them to fast, and the remainder had not. Of those recommended by their provider to fast, the majority (91\%) had fasted. Overall, more respondents who reported they were having a glucose and/or cholesterol measurement fasted $(75 \%)$ than those having other testing $(37 \%), p<0.001$. The prevalence of fasting among those reporting a glucose or cholesterol measurement before $10 \mathrm{am}$ was $80.2 \%$ (95\% CI, 71.9 to 86.9 ).

CONCLUSIONS: About half of patients presenting to a large, academically affiliated lab in the morning between $7 \mathrm{am}$ and $12 \mathrm{pm}$ had fasted. Glucose and cholesterol measures before 10am are more likely than not to be fasting. The reported range of fasting patients may help investigators explore the impact of misclassification of patient fasting status in population-level studies. Consideration of other variables may also enhance the accuracy of using ambulatory glucose data for population studies of diabetes and prediabetes.

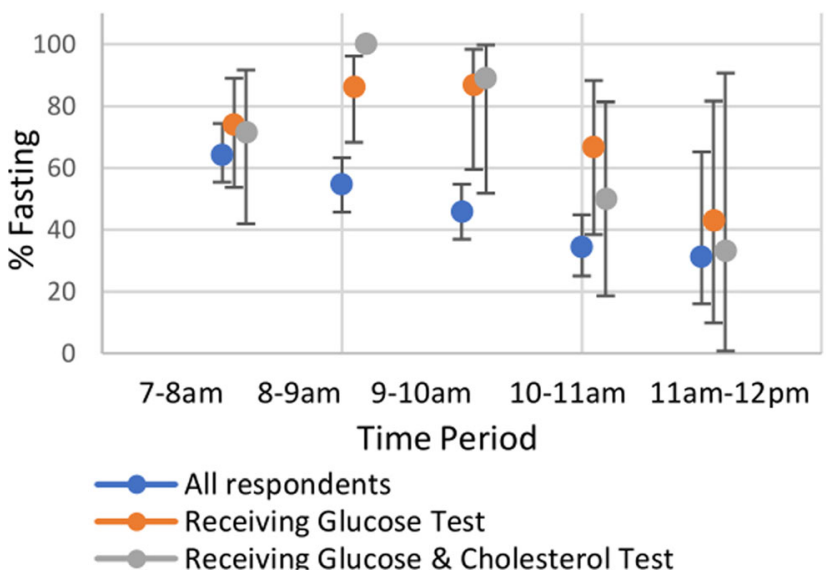


FEMALE SPEAKER REPRESENTATION AT INTERNAL MEDICINE RESIDENCY CONFERENCES

Alicia M. Corwin ${ }^{2}$; Paula Chatterjee ${ }^{1}$; Maria Yialamas ${ }^{3} .{ }^{1}$ University of Pennsylvania, Philadelphia, PA; ${ }^{2}$ Brigham and Women's Hospital, Brookline, MA; ${ }^{3}$ Brigham and Women's Hospital, Boston, MA. (Control ID \#3185701)

BACKGROUND: Morning reports and noon conferences are two forums that make up the backbone of internal medicine residency curricula nationwide. Invited discussants are considered master clinicians, educators and/or researchers, and often become role models for clinical trainees. While it has been documented that women are less likely to be represented at departmental grand rounds nationally, little research has evaluated exposure to female speakers at the level of clinical trainees.

METHODS: We analyzed morning report and noon conference data from three large internal medicine residency programs for the 2017-2018 academic year in Boston, MA: Brigham and Women's Hospital, Massachusetts General Hospital, and Boston Medical Center. Discussants were categorized by gender, and we performed Student's t-tests to compare the prevalence of male and female speakers at the two types of conferences.

RESULTS: The average number of female residents in the training programs was $51.5 \%$. Morning reports were largely clinicallybased case reports, while noon conferences were mainly didactic lectures. For morning reports, approximately $2 / 3$ of speakers were men $(63.7 \%)$ while $1 / 3$ were women $(36.3 \%)$. Similar results were seen for noon conferences $(62.1 \%$ male and $37.9 \%$ female). These comparisons were statistically significant $(\mathrm{p}<0.05)$. The percentage of female discussants at morning report and noon conference fell $32.2 \%(+/-7.4 \%)$ and $31.2 \%(+/-7.9 \%)$, respectively, below the percentage of female constituents in each individual residency program.

CONCLUSIONS: Representation of female attending physicians in internal medicine residency educational conferences falls below the female composition of the resident audience. These results indicate that residents are not being exposed to a cohort of speakers that reflects their programs' gender demographics. These educational conferences often showcase clinical reasoning and medical leadership, in addition to providing an opportunity for role modeling for female trainees. Being exposed to female role models may reduce the effects of implicit bias during clinical training. Programs should examine mechanisms behind these disparities, and future research could examine whether programmatic changes impact how trainees envision medical leaders and perceived implicit biases.

FIDELITY TO KEY FEATURES OF EVIDENCE-BASED QUALITY IMPROVEMENT TO ACCELERATE PATIENTCENTERED MEDICAL HOME TRANSFORMATION IN THE VETERANS HEALTH ADMINISTRATION (VHA)

Susan E. Stockdale ${ }^{1,2}$; Alicia Bergman ${ }^{1}$; Alison B. Hamilton ${ }^{3,2}$; Danielle Rose ${ }^{1}$; Elizabeth M. Yano ${ }^{4}$; Lisa V. Rubenstein ${ }^{5} .{ }^{1}$ VA HSR\&D, Los Angeles, CA; ${ }^{2}$ University of California, Los Angeles, CA; ${ }^{3}$ VA Greater Los Angeles Healthcare System, Los Angeles, CA; ${ }^{4}$ VA Greater Los Angeles Healthcare System, Sepulveda, CA; ${ }^{5}$ RAND and UCLA, Medford, OR. (Control ID \#3179187)

BACKGROUND: Transforming primary care toward patientcentered medical home (PCMH) models requires cultural, techni$\mathrm{cal}$, and clinical innovation. Evidence-Based Quality Improvement (EBQI) engages research evidence, organizational data, and boundary-spanning organizational stakeholders in innovation. Previous studies in the VHA comparing EBQI to non-EBQI sites in a modified stepped-wedge design showed more rapid adoption of
PCMH features and reduced primary care provider burnout in EBQI sites. Little information is available, however, on EBQI implementation and fidelity to its key features. In this study, we assessed fidelity to three key EBQI features over four study years (2010-2014).

METHODS: EBQI for PCMH was rolled out in one Southern California/Nevada VHA region from April 2010-September 2014, and included 9 primary care practices and 7 cross-site, topicfocused workgroups. Key features included: 1) Leadership and frontlines priority setting process for primary care QI; 2) QI learning collaborative for front-line primary care and cross-site QI teams; and, 3) support for the use of data/evidence to inform QI. We reviewed project files to assess fidelity to these key features based on explicit criteria. We used qualitative analysis of 73 interviews conducted in Sept 2013-Feb 2014 and July 2014-January 2015 to assess stakeholder perceptions of EBQI. Participants included multilevel, interprofessional leaders; primary care providers; staff; and researchers.

RESULTS: Data showed high or medium fidelity for participation in the priority setting process, with 72 QI projects submitted by frontline QI teams between 2011 and 2014 and 24 interprofessional regional leaders participating in at least one of four rounds of project proposal review. 26 QI projects were prioritized by leaders; QI teams completed 21 projects and 16 projects resulted in tools/toolkits for spread. Participation in QI collaborative activities varied by site, but all sites attended in-person across-site collaborative learning sessions and biweekly telephone calls. Most QI teams showed high or medium fidelity for using data or evidence in their QI projects. Qualitative interviews identified bi- directional communication between leaders and QI teams, project management and data support for QI, and partnership with health services researchers as critical for achieving fidelity to EBQI features.

CONCLUSIONS: These findings suggest that participation in local primary care QI is enhanced by 1) systematically linking multilevel, interprofessional leadership to frontline innovators; 2) acrosssite communication and learning; and 3) availability of project management and data support. Learning health systems search for effective methods for developing evidence-based innovation and improvement agendas that span organizational boundaries and engage the frontlines. Our data support EBQI as a method for achieving these goals, advancing our understanding of complex QI interventions.

FINANCIAL IMPLICATIONS TO THE VETERANS AFFAIRS HEALTHCARE SYSTEM OF ALLOWING FOR 12MONTH DISPENSING OF ORAL CONTRACEPTIVE PILLS Colleen P. Judge-Golden ${ }^{1}$; Kenneth J. Smith ${ }^{1}$; Maria K. Mor ${ }^{2}$; Sonya Borrero ${ }^{1,2}$. ${ }^{1}$ University of Pittsburgh, Pittsburgh, PA; ${ }^{2}$ VA Pittsburgh Healthcare System, Pittsburgh, PA. (Control ID \#3164458)

BACKGROUND: The Veterans Affairs (VA) Healthcare System stipulates a 3-month maximum dispensing limit for all medications, including oral contraceptive pills (OCPs). However, emerging evidence from other practice settings suggests that 12-month OCP dispensing improves continuation, decreases coverage gaps between medication refills, and reduces unintended pregnancy. We aimed to evaluate financial implications for VA of a 12-month OCP dispensing option, with the goal of informing potential policy change.

METHODS: We developed a decision model from the VA (payer) perspective to estimate incremental costs of allowing the option to receive 12 months of OCPs, compared to 3 months (Figure). Perwoman average and total costs were estimated over a 1-year time horizon. Because a subset of VA-users incur co-pays for OCPs, 
base case analyses assumed that $50 \%$ opt to receive 12 months. Probabilities of OCP continuation, coverage gaps, pregnancy and pregnancy outcomes were drawn from published data. Costs of OCP provision and pregnancy-related care, and the number of women using OCPs were drawn from VA administrative data. One-way and probabilistic sensitivity analyses were performed to assess model robustness.

RESULTS: The 12-month dispensing option resulted in VA cost savings of $\$ 96.49$ per woman per year compared to 3-month dispensing, or $\$ 2,345,575$ total saved annually. Cost savings resulted from an absolute reduction of 34 unintended pregnancies per 1,000 women per year with 12month dispensing, or 827 unintended pregnancies averted annually. Expected cost savings with 12-month dispensing were sensitive to changes in the probability of an OCP coverage gap given 3-month dispensing, probability of pregnancy during coverage gaps, and the proportion of pregnancies paid for by VA. In probabilistic sensitivity analysis simultaneously varying all parameters over plausible ranges, the 12-month strategy was cost saving in $97 \%$ of model iterations.

CONCLUSIONS: Adoption of a 12-month OCP dispensing option is expected to produce substantial cost savings for VA while reducing unintended pregnancies experienced by women Veterans served by VA. The proposed policy change would better support women Veterans in meeting their reproductive goals, and is economically feasible for VA.

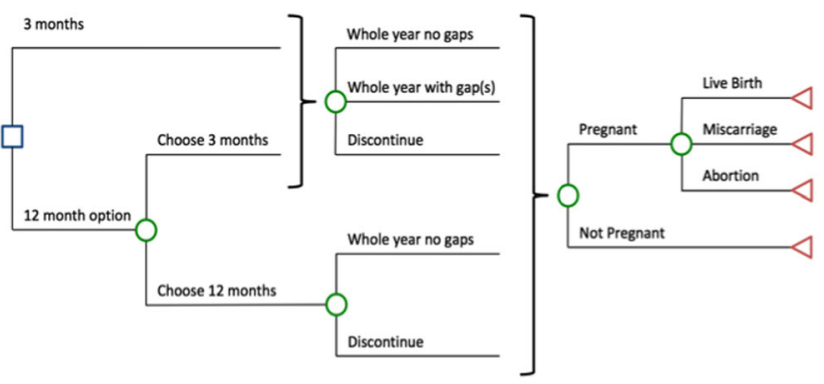

Figure. Decision model schematic

\section{FOOD INSECURITY AND HEPATIC FIBROSIS MEASURED BY TRANSIENT ELASTOGRAPHY AMONG WOMEN WITH AND AT RISK FOR HIV}

Ilya Golovaty ${ }^{1}$; Yifei Ma ${ }^{1}$; Sheri D. Weiser ${ }^{1}$; Jennifer C. C. Price ${ }^{1}$; Mark H. Kuniholm ${ }^{2}$; Howard Minkoff ${ }^{9}$; Audrey L. French ${ }^{3}$; Adaora Adimora ${ }^{4}$; Igho Ofotokun ${ }^{5}$; Margaret Fischl ${ }^{6}$; Deborah Konkle-Parker ${ }^{7}$; Eric Seaberg ${ }^{8}$; Phyllis C. Tien ${ }^{1} .{ }^{1}$ University of California San Francisco, San Francisco, CA; ${ }^{2}$ University at Albany, State University of New York, Rensselaer, NY; ${ }^{3} \mathrm{CORE}$ Center/Stroger Hospital of Cook County, Chicago, IL; ${ }^{4}$ University of North Carolina at Chapel Hill, Chapel Hill, NC; ${ }^{5}$ Emory School of Medicine, Atlanta, GA; ${ }^{6}$ Miami Center for AIDS Research, University of Miami Miller School of Medicine, Miami, FL; ${ }^{7}$ University of Mississippi Medical Center, Jackson, MS; ${ }^{8}$ Johns Hopkins Bloomberg School of Public Health, Baltimore, MD; ${ }^{9}$ Maimonides Medical Center, Brooklyn, NY. (Control ID \#3183408)

BACKGROUND: Non-alcoholic Fatty Liver Disease (NAFLD) will soon be the leading cause of chronic liver disease in people living with HIV. Household food insecurity (FI) may be an upstream determinant of metabolic risk. In a U.S. general population survey, very low food security was associated with increased NAFLD risk. Among women with HIV, FI is highly prevalent $(>30 \%)$ and has been linked to key risk factors for
NAFLD, including obesity and poor diabetes control. Studies of liver disease in the setting of HIV infection have been limited to the use of indirect serum markers of fibrosis that may be affected by HIV infection itself. We evaluated the relationship of FI with liver fibrosis estimated by transient elastography in a large cohort of women with and at risk for HIV. METHODS: We conducted a cross-sectional analysis of women with and without HIV from the multicenter Women's Interagency HIV study. Inclusion criteria were age $>18$ years, no active hepatitis $\mathrm{B}$ or $\mathrm{C}$ infection, not pregnant, and not reporting heavy alcohol use. FI was estimated using the US Department of Agriculture's Core Food Security Module (18-point scale). Since liver fibrosis may result from the cumulative stress of FI, we defined persistent $\mathrm{FI}$ as consistently reporting FI+ over an 18-month period ( $>2$ of previous 3 semi-annual visits); any FI ( 1 of 3 previous visits reporting FI+); and food secure (3 previous visits reporting no FI). Our primary outcome was liver stiffness (LS) measured using vibrationcontrolled transient elastography at a subsequent visit and analyzed continuously in kiloPascals $(\mathrm{kPa})$. We used multivariable linear regression, adjusting for socio-demographics (age, gender, ethnicity, education, household income), traditional metabolic risk factors (diabetes, body mass index, self-reported menopause status) and HIV status.

RESULTS: 1,042 women (744 HIV-seropositive; 82\% African-American) from 9 study sites completed a liver stiffness measurement and food security surveys. 19\% reported persistent FI and 15\% reported any FI in the past 18 months. Overall, $18 \%$ of the population had significant fibrosis (i.e. LS $>7.1$ $\mathrm{kPa}$; median $5.0 \mathrm{kPa}$; IQR: $3.9,6.6$ ) and $52 \%$ had BMI $>30 \mathrm{~kg} / \mathrm{m}^{2}$ (median $30.5 \mathrm{~kg} / \mathrm{m}^{2}$; IQR 26.0, 35.7). Compared to food-secure women, those reporting persistent FI had 5\% lower liver stiffness that did not reach statistical significance $(95 \% \mathrm{CI}:-11 \%, 1 \%)$, whereas there was little association of any FI with liver stiffness (1\%; 95\% CI: $-6 \%, 7 \%)$. After multivariable adjustment including metabolic factors and HIV status, the relationship between food security and liver stiffness remained unchanged. CONCLUSIONS: Contrary to expectations, we did not observe an independent association of FI with liver fibrosis among our population of women with or at risk for HIV. Using a highly specific measure for fibrosis, the overall high metabolic burden in this cohort may have masked an appreciable difference in liver stiffness by FI.

\section{FOSTERING PHYSICIAN PRESENCE IN PRIMARY CARE: FINDINGS FROM A HUMAN-CENTERED DESIGN PROJECT AND DELPHI PANEL}

Donna M. Zulman ${ }^{1,2}$; Marie Haverfield ${ }^{1,2}$; Rachel Schwartz ${ }^{1,2}$; Jonathan G. Shaw $^{1,2}$; Cati Brown-Johnson ${ }^{1,2}$; Dani L. Zionts ${ }^{1}$; Nadia Safaeinili1; Aaron A. Tierney ${ }^{1,2}$; Meredith Fischer ${ }^{1}$; Sonoo Thadaney ${ }^{1}$; Steven Asch ${ }^{1}$,

${ }^{2}$; Abraham Verghese ${ }^{1}$. ${ }^{1}$ Stanford University, Stanford, CA; ${ }^{2}$ VA Palo Alto Health Care System, Menlo Park, CA. (Control ID \#3180119)

BACKGROUND: Modern medicine has produced record levels of physician distress, as clinicians attempt to reconcile time constraints and administrative demands with empathy and caring. While structural changes are undeniably needed, there is evidence that certain physician actions and communication practices can lead to more meaningful and effective patient encounters. We sought to identify evidence-based practices through which physicians can adopt a state of presence - a purposeful practice of awareness, focus, and attention with the intent to understand and connect with patients. METHODS: We derived potential presence practices through a systematic literature review of effective interpersonal interventions ( $\mathrm{n}=77$ articles), followed by a human-centered design process that included clinical observations of 29 primary care encounters (at an academic medical center, a VA general medicine clinic, and a Federally Qualified Health Center), qualitative interviews with primary care physicians $(n=10)$ and patients $(n=29)$, and 
interviews with non-medical professionals whose jobs involve intense interpersonal interactions $(n=30)$. After synthesizing the evidence, we identified 13 preliminary presence practices. We then conducted a 3-round modified Delphi process with 14 national experts, including researchers, physicians, patients, caregivers, and health system leaders. The experts reviewed and rated the practices on three 9-point Likert scales [range -4 to +4 ] that reflected the potential impact on patient experience, potential impact on clinician experience, and implementation feasibility. We prioritized practices with median rantings 2 for all three criteria.

RESULTS: Eight practices received high ratings for implementation feasibility and the potential to positively impact patient and clinician experience: 1) Come prepared (review patient information before the encounter to focus and familiarize yourself with the patient you are about to see); 2) Take a moment to focus (incorporate a brief ritual into clinical practice to focus your attention on the impending patient encounter); 3) Position yourself (use positive open body language, e.g., sit down, lean in, orient your body towards the patient); 4) Listen without interrupting (avoid interjecting during a patient's opening description of health issues); 5) Elicit patient priorities and incorporate these priorities into the visit agenda; 6) "Walk in the patient's shoes" (consider a patient's sociocultural and life circumstances); 7) Focus on the positive (acknowledge specific patient efforts in a genuine and positive manner); and 8) Attend to patient emotions (assess and acknowledge patient feelings).

CONCLUSIONS: Through a novel process that combined a systematic literature review with human-centered design and Delphi methodology, we identified simple and concrete practices that facilitate clinician presence and connection with patients, and have the potential to improve patient and physician experience.

FREQUENCY OF INPATIENT DEATHS DUE TO MEDICAL ERROR: A SYSTEMATIC REVIEW AND META-ANALYSIS Benjamin Rodwin ${ }^{1,2}$; Victor P. Bilan ${ }^{1,2}$; Naseema B. Merchant ${ }^{1,2}$; Catherine G. Steffens ${ }^{2}$; Lori A. Bastian ${ }^{1,2}$; Craig G. Gunderson ${ }^{1,2}$. ${ }^{1}$ Yale School of Medicine, New Haven, CT; ${ }^{2}$ VA Connecticut Healthcare System, West Haven, CT. (Control ID \#3138948)

BACKGROUND: Since the Institute of Medicine report To Err Is Human in 1999, preventable inpatient deaths in the United States have been estimated as between 44,000 and 98,000 annually. A more recent review concluded that the number of preventable deaths was over 250,000 annually. Because inpatient deaths are believed to be commonly due to medical error, and therefore preventable, mortality rates are used for hospital rankings by the Centers for Medicare and Medicaid services (CMS) and the Veterans Administration. Given the uncertainty in reported rates of preventable mortality, we undertook a systematic review and metaanalysis of studies that directly assess rates of preventable mortality in hospitalized patients.

METHODS: A systematic search was conducted for studies that reported case series of hospitalized patients who died and used physician review to determine the number of deaths due to preventable medical errors. Our search included MEDLINE, EMBASE, Web of Science, and the Cochrane library. Study quality was assessed using the Newcastle-Ottawa Quality Assessment Scale. Mortality rates were pooled using random-effects meta-analysis.

RESULTS: 1,086 studies were screened for inclusion. Six studies including 11,455 deaths met study inclusion criteria. The overall pooled rate of mortality due to preventable error was 3.4\% (95\% CI 2.7\% - 4.2\%) (figure 1). Two studies reported rates of preventable mortality limited to patients expected to live longer than three months, which was $0.8 \%$ (95\% CI $0.3 \%$ - $1.9 \%$ ). In the United States, these estimates equate to approximately
21,450 preventable deaths annually and 5,720 deaths among patients with greater than three-month life expectancy.

CONCLUSIONS: The number of deaths due to medical error is much lower than previously reported. Furthermore, most of these preventable deaths appear to occur in patients with less than three months life expectancy. Because the vast majority of inpatient deaths are not due to medical error, overall hospital mortality rates are unlikely to be valid quality measures. Variation is more likely to reflect differences in patient characteristics and location of patient death. Our results also question the validity of using disease-specific mortality rates for quality measures and reimbursement, as currently done by CMS.

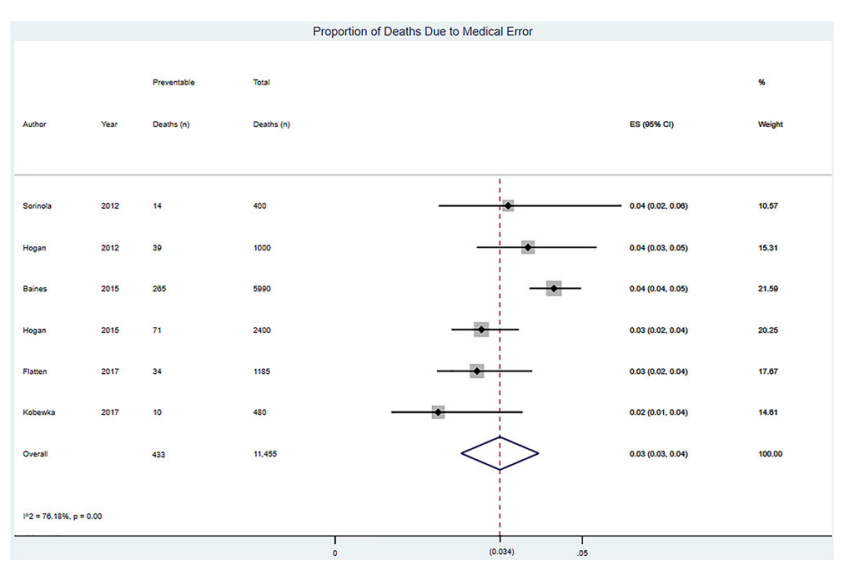

FREQUENTLY HOSPITALIZED PATIENTS' PERCEPTIONS OF FACTORS CONTRIBUTING TO HIGH HOSPITAL USE Kenzie A. Cameron ${ }^{1}$; Margaret Chapman ${ }^{2}$; Shandu Foster ${ }^{1}$; Lyndsey O'Hara ${ }^{1}$; Bruce L. Henschen ${ }^{1}$; Kevin O'leary ${ }^{1} .{ }^{1}$ Northwestern University Feinberg School of Medicine, Chicago, IL; ${ }^{2}$ Massachusetts General Hospital, Ipswich, MA. (Control ID \#3180056)

BACKGROUND: Frequently hospitalized patients pose unique challenges to existing medical and psychosocial support networks offered within the current model of health care delivery. Most studies focus on understanding this "high utilizer" population from the perspective of the clinician or health care system. We sought to obtain patients' perspectives of factors associated with onset and continuation of high hospital use.

METHODS: This qualitative study took place at a large, urban, 894-bed hospital. From December 2016-September 2017, we recruited adult patients admitted to the general medicine services and conducted one-on-one semi structured interviews. Eligible participants had two-unplanned 30-day inpatient hospital readmissions within the prior 12 months, and one or more of the following: 1 readmission in last 6 months, referral from a clinician, or 3 observation visits. Our interview guide sought to explore, from the patients' perspective, what factors contribute to patients becoming and continuing to be high-utilizers of hospital care. Digital audio recordings were transcribed verbatim, deidentified, and analyzed using an iterative inductive team based approach to coding. In our first cycle coding, coders used 
descriptive coding and subcoding to generate a preliminary codebook with code definitions. Our second coding cycle utilized pattern coding to identify consistency and associations both within and between transcripts. Participant recruitment, interviews, and analysis of transcripts continued until no new codes emerged and thematic saturation was achieved.

RESULTS: Overall, 26 participants completed interviews; $42.3 \%$ were female, $73.1 \%$ were non-white, and mean age was $47.0(\mathrm{sd}=15.3)$. The median number of admissions in past year was 8.5 (interquartile range 6.0 - 11.0). Four main themes emerged. First, major medical problems were universal but the onset of frequent hospital use varied, such that some participants had experienced periods of high hospital use throughout their lifetime, while others experienced high hospital use as an adult. Second, participants perceived fluctuations in their course of illness to be related to psychological, social, and economic factors. Social support was perceived as helpful and participants benefitted when providing social support to others. Third, episodes of illness could range from sudden onset to a gradual onset of symptoms, but generally seemed uncontrollable and often unpredictable to the participant. Fourth, participants strongly desired to avoid hospitalization and typically sought care only after selfmanagement failed.

CONCLUSIONS: Emergent themes pointed to factors that influence patients' onset of high hospital use, fluctuations in their illness over time, and triggers to seek care during an episode of illness. These findings enable patients' perspectives to be incorporated into the design of programs serving similar populations of frequently hospitalized patients.

FROM THE \#METOO FRONTLINES: INCOMING INTERNS REPORT A BREADTH OF EXPERIENCES RELATED TO SEXUAL HARASSMENT IN MEDICAL SCHOOL Emmanuelle B. Yecies ${ }^{1,}{ }^{2}$; Melissa McNeil ${ }^{3,}{ }^{2}$. ${ }^{1}$ University of Pittsburgh School of Medicine, Pittsburgh, PA; ${ }^{2}$ VA Pittsburgh Healthcare System, Pittsburgh, PA; ${ }^{3}$ University of Pittsburgh Medical Center, Pittsburgh, PA. (Control ID \#3184999)

BACKGROUND: The prevalence of sexual harassment in medicine has recently been in the spotlight, with up to $30 \%$ of female physicians reporting sexual harassment in the workplace. There is also increasing recognition that the spectrum of sex discrimination includes microaggressions, the prevalence of which have yet to be elucidated. Medical students are a particularly vulnerable population within medicine as they experience the widest power differentials and their ongoing grades and evaluations deepen the consequences of retaliation to reporting. With the influence of the \#MeToo movement, a few medical school deans have seen an increase in reporting, but most schools report stagnant (and low) reporting rates, indicating that sexual harassment may still be under-represented in the current reporting system. We aimed to study the ways in which medical students experienced sexual harassment in medicine.

METHODS: An in-person cross-sectional survey was administered to incoming interns at UPMC about their experience with sexual harassment in medical school. An optional question invited respondents to share experiences of sexual harassment in free-text form. The reports were examined for common themes.

RESULTS: 185 graduates of U.S. medical schools returned the survey, with 42 completing the optional free-text question ("If you are willing and interested in sharing any of your experiences, please use the space below"). Of these, 33 respondents identified as female, and 9 as male. Analysis of the responses revealed six recurring themes: (1) decision not to report, (2) unsuccessful reporting, (3) disproportionate "scut" work for female students, (4) inappropriate comments and touching, (5) repeat offenders with well-known reputations, and (6) male patients harassing female students. This last theme was particularly attributed to Veterans Administration (VA) patients.

CONCLUSIONS: More female students than male students shared negative experiences. They identified a breadth of experiences attributed to both colleagues/superiors and patients. Most importantly, many students identified the current reporting system as discouraging of reporting. Given the variety of experiences shared by incoming trainees, tackling sexual harassment must evolve from a single broad campaign to targeted approaches for each of these issues. The current reporting system appears to be insufficient and reforms are needed to develop an improved system that encourages and supports trainees to report inappropriate experiences.

FURTHER VALIDATION OF THE CONSUMER HEALTH ACTIVATION INDEX (CHAI) AMONG DIVERSE PRIMARY CARE SETTINGS

Guisselle Wismer ${ }^{1}$; Laura M. Curtis ${ }^{1}$; Lauren Opsasnick ${ }^{1}$; Scott Hur ${ }^{1}$; Stacy Bailey $^{1}$; Rachel O'Conor ${ }^{1}$; Marina Arvanitis ${ }^{1}$; Alex Federman ${ }^{2}$; Ruth Parker ${ }^{4}$; Amisha Wallia ${ }^{3}$; Michael S. Wolf ${ }^{1} .{ }^{1}$ Northwestern University, Chicago, IL; ${ }^{2}$ Icahn School of Medicine at Mount Sinai, New York, NY; ${ }^{3}$ Northwestern Medicine, Chicago, IL; ${ }^{4}$ Emory University, Atlanta, GA. (Control ID \#3185042)

BACKGROUND: Although there has been increasing interest in patient activation, few measures are publicly available and suitable for patients with limited health literacy. The Consumer Health Activation Index (CHAI) is a valid, reliable, and easily administered tool that can be used to assess health activation among a diverse adult population, including those with limited health literacy. This study was completed to further validate the CHAI in a more diverse different setting.

METHODS: We used data from two ongoing randomized controlled trials of community dwelling adults. 617 English and Spanish-speaking patients who had been prescribed 5+ medications were recruited in outpatient academic and federally qualified health center practices in New York City, NY and Chicago, IL. Outcomes included health literacy via the Newest Vital Sign assessment (NVS), regimen adherence (ASK-12), depression (PROMIS) and blood pressure. To confirm CHAI associations with health outcomes, we ran correlations between the CHAI and depression, regimen adherence, and blood pressure. We obtained the alpha, Eigen, and factor loadings range.

RESULTS: The mean age of participants was 60 years; $36.5 \%$ were men, $73.5 \%$ had a high school education or less, and $91.9 \%$ had an annual income of less than $\$ 30,000$. Nearly half $(41.5 \%)$ of participants had limited health literacy. The CHAI showed good internal consistency (alpha $=0.82$ ). The ten items included in the CHAI loaded onto a single factor (Eigenvalue: 3.24, 9 of 10 factor loadings ranged $0.45-0.66$; one item $<0.20$ ). A higher CHAI score was associated with fewer depressive symptoms $(\mathrm{r}=-0.34$, $\mathrm{p}<0.001)$ and greater medication adherence $(\mathrm{r}=-0.46, \mathrm{p}<0.001)$. No significant differences were noted by blood pressure $(r=0.04$, $\mathrm{p}=0.28$ ).

CONCLUSIONS: The CHAI tool is a reliable, valid measure of consumer health activation that demonstrated exceptional psychometric performance, although opportunities may exist for further 
reducing items. CHAI scores were also associated with patientreported outcomes, while not being associated with intermediary clinical outcomes. These results suggest the CHAI should still be considered a viable, publicly available alternative to existing activation measures.

GAPS IN INSURANCE COVERAGE AMONG LOWINCOME ADULTS AFTER THE AFFORDABLE CARE ACT Anna Goldman ${ }^{2}$, ; Benjamin D. Sommers ${ }^{3}$. ${ }^{1}$ Cambridge Health Alliance, Cambridge, MA; ${ }^{2}$ Havard T.H. Chan School of Public Health, Boston, MA; ${ }^{3}$ Harvard School of Public Health, Brookline, MA. (Control ID \#3185319)

BACKGROUND: Gaps or changes in health insurance coverage, known as "churning," is a common problem in Medicaid and has been shown to have adverse consequences. ${ }^{1,2}$ Little is known about the effect of the Affordable Care Act's (ACA) Medicaid expansion on churning rates. We used a difference-in-differences framework to compare coverage continuity for low-income adults in expansion vs. non-expansion states under the ACA.

METHODS: We analyzed data from the Medical Expenditure Panel Survey on monthly insurance status for the years 20112016 (the ACA's Medicaid expansion went into effect in 2014). Our study population $(n=14,370)$ included non-elderly adults ages 19-64 with family incomes under $138 \%$ of poverty who reported having Medicaid for at least one month. Our intervention group included adults residing in states that expanded Medicaid prior to December 31, 2015. Our control group included adults in states that did not expand Medicaid prior to the end of our study period. Individuals living in Montana and Louisiana, which expanded Medicaid during in 2016, were excluded. In our primary analysis, we examined changes in churning rates among Medicaid enrollees in expansion vs. non-expansion states before and after the ACA. The annual churning rate was defined as the proportion of Medicaid enrollees who moved from Medicaid coverage to no coverage or non-Medicaid at any point during the year. A second outcome was the proportion of Medicaid enrollees who experienced a coverage gap, defined moving from Medicaid coverage to no coverage. Lastly, to explore the impact of churning on access to care, we analyze the following outcomes in expansion vs. nonexpansion states: inability to get necessary care; delays in care; and delays in obtaining prescription drugs.

RESULTS: The annual churning rate among Medicaid enrollees in expansion states was $17.9 \%$ prior to the ACA, and it declined by 4.2 percentage points $(\mathrm{p}=0.02)$ in the post-ACA period compared to non-expansion states. Gaps in coverage were experienced by $14.0 \%$ of Medicaid enrollees in expansion states prior to the ACA, and this rate decreased by 4.1 percentage points $(\mathrm{p}=0.02)$ after Medicaid expansion. Access to care outcomes did not change significantly in association with expansion status in this population of Medicaid-enrolled adults.

CONCLUSIONS: Using a quasi-experimental approach, we found that churning rates and gaps in coverage for low-income adults decreased significantly in states that expanded Medicaid. Measures of access to care did not change significantly between the study groups. Previous research has documented that Medicaid expansion significantly reduced the uninsured rate among low-income adults. Our study makes a novel contribution in showing that some of this effect is due to reduced churning among existing beneficiaries, in addition to new enrollment. Mechanisms for the reduced churning rates due to Medicaid expansion include the ACA's higher income eligibility, uniform income cutoffs across eligibility groups, and better outreach.

GASPING FOR AIR: MEASURING PATIENT EDUCATION AND ACTIVATION SKILLSETS IN TWO CLINICAL ASSESSMENT CONTEXTS

Jeffrey Wilhite ${ }^{1}$; Kathleen Hanley ${ }^{1}$; Khemraj Hardowar ${ }^{1}$; Harriet Fisher $^{1}$; Lisa Altshuler ${ }^{1}$; Adina Kalet ${ }^{1}$; Colleen C. Gillespie ${ }^{1,2}$; Sondra Zabar ${ }^{1} .{ }^{1}$ NYU School of Medicine, New York, NY; ${ }^{2}$ NYU, New York, NY. (Control ID \#3185457)

BACKGROUND: Asthma education should focus on patient selfmanagement support. Objective structured clinical examinations (OSCEs), as measured by standardized patients (SPs), provide a controlled, simulated setting for timed competency assessments while Unannounced Standardized Patients (USPs) measure clinical skills transfer in real world clinical settings. Both enable us to assess skills critical for providing quality care to patients. Learners seeing USPs have added real world stressors such as clinical load. This study describes differences in education and activation skills in two assessment contexts.

METHODS: A cohort of primary care residents $(n=20)$ were assessed during two time points: an OSCE and a USP visit at an urban, safety-net clinic from 2009-2010. Residents consented to use of their de-identified routine educational data for research. The SP and USPs presented with the same case; a female asthmatic patient with limited understanding of illness management and concern over symptom exacerbation. Providers were rated using a behaviorally-anchored checklist upon visit completion. Competency domains assessed included patient education (4 items) and activation (4 items). Within the education domain, items included illness management, while the activation domain items assessed resident communication/ counseling style. Responses were scored as not done or well done. Summary scores (mean \% well done) were calculated by domain. OSCE vs USP means were compared using a paired samples t-test.

RESULTS: Residents were more likely to offer an oral steroid as treatment in the OSCE case (50\% vs. $35 \%$ for USPs), but performed better with USPs on most other items including domain scores. Residents seeing a USP scored significantly higher on five out of eight individual assessment items $(p<.05)$ including recommending a spacer, helping a patient understand their condition, making patients feel like they can take control of their own health, helping a patient understand illness management, and having a patient leave feeling confident in finding solutions independently. Inhaler technique was assessed rarely in either setting (OSCE: $15 \%$, USP: $5 \%$ ). Domain summary scores (\% well done) from the OSCE (activation: $12 \%$, education: $31 \%$ ) were lower than USP scores (activation: $84 \%$, education: $37 \%$ ), with differences in overall activation scores being significant $(\mathrm{t}(19)=-8.905, p<.001)$.

CONCLUSIONS: OSCEs are a widely accepted tool for measuring resident competency in a standardized environment but may be focused primarily on knowledge and technical skills. While SPs are trained to be as objective as possible, rater bias might impact scores. USPs may provide more nuanced assessments of communication skills in a setting with reduced time constraints. Next steps include examining attitudes toward OSCEs vs the clinical setting, looking at impact of provider gender, and examining settingspecific issues that promote or hinder high quality care. 
GENDER AND ACADEMIC HOSPITALISTS

Carrie A. Herzke ${ }^{1}$; Joanna M. Bonsall ${ }^{4}$; Amanda Bertram ${ }^{3}$; Hsin-Chieh $\mathrm{Yeh}^{2}$; Arielle Apfel ${ }^{3}$; Joseph Cofrancesco ${ }^{2} .{ }^{1}$ Johns Hopkins, Baltimore, MD; ${ }^{2}$ JHUSOM, Baltimore, MD; ${ }^{3}$ Johns Hopkins University, Baltimore, MD; ${ }^{4}$ Emory University School of Medicine, Atlanta, GA. (Control ID \#3180219)

BACKGROUND: Issues of gender inequity in recruitment, retention and advancement of women in academic medicine persist. Within GIM, there are fewer female division directors, first/last authors and presenters at national meetings. Academic Hospital ( $\mathrm{AH})$ medicine is a growing field and studies raise a concern about gender equity.

METHODS: A national survey via Qualtrics ${ }^{\circledR}$ of all leaders of $\mathrm{AH}$ programs associated with AAMC. Survey was developed via literature review, discussion/pilot with experts, in collaboration with the Society of Hospital Medicine. Domains included program/faculty information and perceptions about promotion. Gender of the leader was determined via website or telephone. Programs excluded if no identifiable primary teaching hospital or hospitalist program, or not staffed by university-affiliated physicians. Means, standard deviations, medians, and proportions were used to describe data distribution. Chi-square, Fisher's Exact test, and ttest were used in univariate analysis to compare respondents and nonrespondents, and to determine if leader and program characteristics were significantly associated with being in the lowest quartile of female faculty. Multivariable logistic regression was used to determine adjusted associations.

RESULTS: $58 \%$ response rate (78 of 135); no differences of non-responders in NIH funding, public v. private, geographic region or year established. Reported number of female and male faculty approximately equal. $73 \%$ of hospitalist leaders were male; $38 \%$ of male leaders were professors, no female leaders were professors. In univariate analysis, there were no gender associated differences regarding importance of scholarship for promotion but programs with the highest quartile of associate and professors had lowest quartile of female faculty. Other predictors of having lowest quartile of female faculty include less NIH funding, single promotion track, more time with learners, fewer total faculty, and fewer faculty ranked instructor. In the multivariable regression model, institutions with lowest quartile of $\mathrm{NIH}$ funding were significantly associated with the lowest quartile of female faculty (odds ratio $=3.54, \mathrm{p}=0.049$ ) after adjusting for covariates. Having lower female faculty was not associated with amount of protected time, GIM versus independent division/department, region, or number of faculty remaining in hospital medicine long-term.

CONCLUSIONS: This study demonstrated gender issues in academic hospital medicine, especially regarding leadership and rank. Though equal gender distribution of faculty with similar plans to remain in $\mathrm{AH}$, leaders are predominately male, no female leaders are professors and programs reporting higher numbers of female faculty were most likely to report lower rates of faculty at the associate and professor rank. As diversity benefits the tripartite mission, and can lead to more diverse role models for learners, faculty and patients, research on methods, initiatives and program that achieve gender equity are needed.

\section{GENDER DIFFERENCES IN AND PATTERNS OF HEALTH- RELATED COMMUNITY RESOURCE NEEDS AMONG ADULT PRIMARY CARE PATIENTS}

Jennifer A. Makelarski; Elizabeth L. Tung; Stacy T. Lindau. University of Chicago, Chicago, IL. (Control ID \#3185890)
BACKGROUND: As payment models shift from fee-for-service to valuebased, health systems are seeking evidence-based strategies for managing population health. The U.S. Centers for Medicare \& Medicaid Services is currently assessing an Accountable Health Communities (AHC) model which uses a standardized screener to assess and address, during clinical visits, health-related social needs (HRSNs) including food, housing, rent/utility support, interpersonal safety, and social support. The purpose of this study is to examine gender differences in acceptability of the AHC HRSNs screener and patterns of HRSNs among adult primary care patients.

METHODS: Participants were enrolled in the primary care waiting room of an urban, academic medical center 09/2017-07/2018. Study participants included adults ages 18 and older who spoke English or Spanish, were seeking primary care and were randomized to complete the AHC HRSNs screener and related acceptability questions via a tablet. Sociodemographic and health care characteristics, acceptability of the AHC screener, and selfreported HRSNs were described by gender and differences were examined using Student's t-test (continuous variables) or Chi-squared statistics (categorical variables).

RESULTS: Study participants were predominantly non-Hispanic black $(65.9 \%)$ and aged $35-64$ years old $(50.5 \%)$. There were no significant differences in sociodemographic characteristics between men and women. The majority of women and men reported excellent, very good or good health ( $82.4 \%$ and $64.3 \%$, respectively, $p$-value $=0.06)$. Acceptability of the AHC screener was high and similar among gender groups. Only a third of all participants had previously been asked about at least 1 HRSNs during a clinical visit in the last 12 months. Nearly two-thirds (63.4\%) of men and 44.6 of women reported at least $1 \mathrm{HRSN}$ (p-value, 0.10). Men were significantly more likely than women to report that they would like assistance with 1 social need (39.3 versus 16.4, p-value 0.02 ). The most common needs were food (28.8 and 40.7, women and men) and housing (28.4 and 42.9, women and men).

CONCLUSIONS: Acceptability of the AHC HRSNs screener and rates of HRSNs are high among men and women. As screening is adopted into clinical practice, low-intensity, scalable solutions to address HRSNs are needed. Men may be more receptive to assistance.

\section{GENERAL PRACTITIONER PERCEIVED WORKLOAD OF PA- TIENT CHARACTERISTICS}

Justin A. Charles ${ }^{1}$; Peder Ahnfeldt-Mollerup ${ }^{2}$; Jens Søndergaard ${ }^{2}$; Julia Logan'; Troels Kristensen 2, 2. 'Stony Brook University School of Medicine, Stony Brook, NY; ${ }^{2}$ University of Southern Denmark, Odense, Denmark. (Control ID \#3184696)

BACKGROUND: It is well known that patients with certain sociodemographic characteristics provide additional challenges to patient care and add to general practitioner (GP) workload. However, updated measurements of perceived workload in general practice are lacking. Understanding the contribution of different sociodemographic patient types to GP perceived workload may inform planning and resource allocation in general practice. This study aims to measure perceived workload from patients with different sociodemographic characteristics in general practice, and to reduce patient characteristics into underlying constructs and global, rounded measures of perceived workload.

METHODS: A web-based survey was distributed to a random, stratified sample of 1,196 Danish GPs. A part of it contained 
questions that measure perceived workload of 11 sociodemographic patient characteristics using a 0-9 Likert scale. Eight of the sociodemographic characteristics were adapted from the Care Need Index (CNI). Three new patient characteristics were added that are relevant to workload in the context of Danish General Practice: early pension, public transfer payment; outside the labor force in job center courses. Exploratory Factor Analysis (EFA) was conducted using principal-component factors method. Additionally, we created an overall perceived workload indicator, weighted by individual patient types.

RESULTS: GPs perceived foreign-born patients to contribute the most (6.82 out of 9) and children under 5 to contribute the least (3.59 out of 9) to their perceived workload. Male GPs perceived a higher workload than female GPs. Factor analysis revealed two stable underlying factors, entitled "Economically Disadvantaged" and "Difficult Communication". Weighted overall perceived workload was 5.49 (out of 9).

CONCLUSIONS: There is considerable variation in how different sociodemographic patient characteristics contribute to perceived GP workload. The suggested measures of overall perceived workload and underlying constructs of perceived workload may simplify how perceived workload is measured and be used to inform the organization of primary care delivery.

\section{GEOGRAPHIC PREVALENCE OF ALCOHOL AND BEN- ZODIAZEPINE CO-INVOLVEMENT IN OPIOID OVER- DOSE DEATHS, 2015-2017}

Marco Tori ${ }^{1}$; Timothy S. Naimi ${ }^{1}$; Marc Larochelle ${ }^{2}$. ${ }^{1}$ Boston Medical Center, Boston, MA; ${ }^{2}$ Boston University School of Medicine and Boston Medical Center, Boston, MA. (Control ID \#3181622)

BACKGROUND: 1. Compare rates of alcohol and benzodiazepine co-involvement in opioid overdose deaths (OOD) among US states and regions. 2. Describe differences in alcohol or benzodiazepine involvement in prescribed and illicit opioid overdose deaths. From 2015-2017 illicit opioids, mainly heroin and illicitly manufactured fentanyl, were responsible for a majority of OOD in the United States, while prescribed opioids were responsible for a minority. Sedating substances like alcohol and benzodiazepines can compound the respiratory depressant effect of all opioids. Polysubstance use is common among OOD and possibly contributes to excess mortality. The prevalence of alcohol and benzodiazepine co-involvement in OOD is not well described at the national or state level.

METHODS: Data from CDC WONDER Multiple-Cause-of-Death files for poisonings involving opioids as an underlying cause of death from 2015-2017 were used to calculate the prevalence of co-intoxication with benzodiazepines and alcohol, among opioid subtypes with respective ICD-10 codes.

RESULTS: For all OOD, state ranges of alcohol and benzodiazepine coinvolvement were $7.1 \%-28.6 \%$ and $5.2 \%-44.6 \%$, respectively. Ranges for alcohol co-involvement in illicit OOD were $8.3 \%-31.0 \%$ and $6.8 \%$ $27.2 \%$ in prescribed opioids. Ranges for benzodiazepine coinvolvement in illicit OOD were $3.8 \%-38.4 \%$ and $10.2 \%-54.7 \%$ in prescribed opioids. There is no correlation (Pearson co-efficient $r=0.075$ ) between benzodiazepine and alcohol co-involvement in all OOD by state. In 45 states with sufficient data, 35 had higher alcohol co-involvement in illicit OOD, and all states had higher rates of benzodiazepine coinvolvement in prescribed OOD.
CONCLUSIONS: Involvement of alcohol and benzodiazepines in OOD varies widely among states and opioid type but affects a large percentage of deaths. Alcohol co-involvement is more common in illicit OOD benzodiazepines more common in prescribed OOD. There is no relationship between state benzodiazepine and alcohol co-involvement in OOD.

\section{GET S-M-A-R-T！TEACHING STUDENTS GERIATRIC HEALTH-RELATED GOAL SETTING}

Vivianne Oyefusi ${ }^{1}$; Paula Leslie ${ }^{2}$; Rachel Jantea ${ }^{3}$; Pamela E. Toto ${ }^{4}$; Rollin M. Wright ${ }^{1}$. ${ }^{1}$ University of Pittsburgh School of Medicine, Pittsburgh, PA; ${ }^{2}$ University of Pittsburgh, Pittsburgh, PA; ${ }^{3}$ University of Pittsburgh Medical Center, Pittsburgh, PA; ${ }^{4}$ University of Pittsburgh School of Health and Rehabilitation Sciences, Pittsburgh, PA. (Control ID \#3185838)

BACKGROUND: Goal-setting, particularly in geriatrics and primary care, is essential to person-centered care. It is linked to improved outcomes, quality of life and health behavior change. While integral to training in some health professions, we suspected third year medical students (MS3) had not yet learned how to develop health-related goals. There is little published data on how skilled clinicians are at eliciting goals from patients despite the acknowledged importance. We piloted a tool to teach medical and other health sciences students how to develop healthrelated Specific-Measurable-Assignable-Realistic-Time-based (SMART) goals with community-dwelling older adults.

METHODS: Clinical students (including all MS3s) enrolled in an interprofessional geriatrics course sponsored by the University of Pittsburgh School of Medicine participated in a geriatric assessment skills fair. Each student attended 12 out of 18 possible skills stations, spending 12 minutes at each station and collecting skills cards to prepare them to do geriatric assessments at 9 communitybased health fairs in western Pennsylvania in October 2018. All students were required 1) to attend the SMART goals station where they practiced developing health-related SMART goals, and 2) to apply this new skill and elicit at least 1 SMART goal from each senior health fair participant, writing them on health assessment forms. We graded the goals according to the number of S-M-A-R-T elements represented. Without clinical information available, goals were presumed to be Realistic for all the senior participants. Goal statements were independently graded as meeting 0 to 4 elements by the skills station instructor (PL) and the course director (RW) for comparison.

RESULTS: 200 students from 9 health professions schools worked in teams of 2-3 to complete health assessments on 209 geriatric health fair participants, eliciting a total of 112 SMART goals. $49 \%$ of elicited goals had 3+ SMART elements. 83 goals were assignable, 71 were measurable, 55 were specific, and 22 were time-based. "Memory problems, blood sugar control, and fall risk" and "call ear doctor to make hearing aid appointment in the next week" were examples of poorly-written (0 elements) and well-written (all 4 elements) goals, respectively.

CONCLUSIONS: We designed a tool that successfully taught health sciences students to engage older people in person-centered, health-related goal-setting, a necessary skill for future careers in an environment that emphasizes high-value health care delivery. "Specific" and "time-based" appeared to be the most challenging of the SMART elements. We will use these findings to refine this education tool further. 
GOT CHILDCARE? THE RELATIONSHIP BETWEEN INSTITUTION-BASED CHILDCARE AND EMPLOYEE WELLBEING

Rachel Apple ${ }^{1}$; Lauren Samuels ${ }^{2}$; Christianne Roumie ${ }^{1} .{ }^{1}$ Vanderbilt, Nashville, TN; ${ }^{2}$ Vanderbilt University, Nashville, TN. (Control ID \#3183504)

BACKGROUND: For most parents, securing reliable and safe childcare ranks high on the list of parental priorities. Among health care professionals, there is increasing interest in wellness initiatives to combat growing rates of burnout. Despite the crucial role which finding and securing childcare plays in many parents' lives, few studies have examined the relationship between childcare and wellbeing at the level of a medical institution. The purpose of this study was to evaluate the relationship between utilization of institution-based childcare and employee wellbeing at a large, academic medical center.

METHODS: We conducted a cross-sectional analysis of data obtained from an online survey. Study participants were VUMC employees with children in a Vanderbilt-affiliated childcare center (institution-based childcare) or on the waitlist for Vanderbilt-affiliated childcare (non-institution-based childcare).

RESULTS: 558 respondents were predominantly female (78.0\%) and represented a fairly equal distribution between clinical and non-clinical job roles. The majority of respondents $(58.8 \%)$ reported having 1 child under the age of 6 years in childcare. There was a statistically significant difference in overall stress, with individuals having institution-based childcare reporting less overall stress, as well as less stress specifically related to finances, childcare, and personal health. There was no statistically significant difference between exposure groups related to job, relationship with significant other, or parental responsibilities.

CONCLUSIONS: This study demonstrated an association between childcare provision type and stress after adjusting for demographic factors and potential confounders such as job type, hours per week in childcare, and percent of income spent on childcare. These findings highlight the centrality of childcare to employees' wellbeing and ability to be productive in their jobs. Greater attention to the impact of parenthood on employee stress and wellbeing is warranted at an institutional level.

\section{GRADUATE MEDICAL TRAINEE (GME) INVOLVEMENT IN HOSPITAL MEDICATION SHORTAGE MANAGEMENT}

Andrew Hantel $^{1}$; Ashley Egan ${ }^{2}$; Trinh Nguyen ${ }^{2}$; Erin DeMartino ${ }^{2}$; Fay Hlublocky ${ }^{1}$; Mark Siegler ${ }^{1}$; Christopher Daugherty ${ }^{1}{ }^{1}$ The University of Chicago, Chicago, IL; ${ }^{2}$ Mayo Clinic, Rochester, MN. (Control ID \#3185894)

BACKGROUND: Hospital medication shortages in the United States (US) are associated with decreased patient quality and quantity of life. We recently conducted a national survey to investigate shortage management practices of US hospitals, which revealed widespread shortages of common medications and heterogeneous management and rationing practices. GMEs frequently manage inpatients with varying levels of supervision; internal medicine (IM) and anesthesia residents frequently utilize medications with known shortages to manage patients. Therefore, to understand the involvement of GMEs in shortage management, we conducted a survey of these residents at the University of Chicago Hospital (UCH).

METHODS: A 17-item survey was created to evaluate shortage management. Subjects included trainees at least one year into their program. Items were piloted for clarity and relevance. The survey was performed in person between 11/4/2018-1/12/2019. 2 tests were used to compare response differences by post-graduate year (PGY) and program. Subjects that responded to at least one question were included in the analysis. The UCH IRB approved the study.

RESULTS: A total of 123 GMEs were eligible; 85 completed the survey (response rate 69.1\%). Question completion was 100\%. 96.5\% reported experience in managing shortages. Of these GMEs, $90.6 \%, 88.4 \%$, and $75.2 \%$ reported managing shortages, using alternatives, and that shortages made clinically relevant differences, respectively, on an at least weekly basis. $47.1 \%$ reported that equivalent alternatives were available "half of the time" or less. When shortages occurred, $70.5 \%$ of GMEs reported discussing management with their attending "some of the time" or less; when asked to consider only clinically relevant shortages (CRS), this became $53.1 \%$. PGY2 residents and IM residents were more likely to discuss CRS frequently with their attending ( $2=21.3$ and $17.2, \mathrm{P}=0.05$ and 0.01 ). $49.1 \%$ reported discussing CRS with patients "some of the time" or less frequently. IM residents were more likely to discuss CRS frequently with their patients $(2=26.2, \mathrm{P}<0.01) .13 \%, 8.2 \%$, and $78.8 \%$ responded that shortages should be disclosed to patients always, never, or only when clinically relevant, respectively. Of those who preferred to disclose based on relevance, $45.9 \%$ would only disclose if shortages resulted in a moderate or major difference in efficacy or toxicity. $14.1 \%$ reported receiving shortage training and $91.8 \%$ reported that they would want training.

CONCLUSIONS: GMEs frequently manage clinically relevant shortages with little training and variable communication to their supervising physicians or to patients. Less experienced GMEs and IM GMEs are more likely to discuss management with their attendings and IM residents are more likely to discuss management with their patients, as compated to anesthesia GMEs. Further study and education of GMEs is warranted; therefore this survey is currently being performed with UCH emergency medicine GMEs and parallel programs at the Mayo Clinic.

\section{GREAT EXPECTATIONS: A SURVEY OF HOSPITAL PORTAL INFORMATION FOR PATIENTS}

Joy L. Lee ${ }^{1,2}$; Claire E. Williams ${ }^{2}$; Sean Baird ${ }^{2}$; Marianne S. Matthias ${ }^{3,2}$; Michael Weiner, ${ }^{4}$. ${ }^{1}$ Indiana University School of Medicine, Indianapolis, $\mathrm{IN} ;{ }^{2}$ Regenstrief Institute, Inc., Indianapolis, IN; ${ }^{3}$ Roudebush VAMC, Indianapolis, IN; ${ }^{4}$ Indiana University, Indianapolis, IN. (Control ID \#3149443)

BACKGROUND: Adequate access to health care, providers, and information, is a problem for many patients. Patient portals present an opportunity to expand patients' access to care, providers, and information online, most notably through access to electronic medical records and secure messaging. Yet clinicians have expressed concern about patients' "misuse" and "misunderstanding" of portals, especially secure messaging. It is not clear if and what expectations of portal use are communicated to patients. This study sought to identify what hospitals communicate to patients about portal use, to describe the information available to patients, and to assess ease in accessing this information.

METHODS: We randomly sampled 200 acute-care hospitals from the Centers for Medicare \& Medicaid Services Hospital Compare dataset, and searched the web to collect publicly available information about each hospital's patient portal. The web search was followed up with phone calls to the hospitals (up to 3 attempts each) to request any additional patientdirected materials (e.g., pamphlets) not included in the web search. Ease of access was defined as the number of mouse clicks from the hospital's main webpage needed to access the portals. A mixed-methods approach was used to evaluate and describe the data. 
RESULTS: Although 197 (99\%) of the 200 hospitals sampled reported meeting Meaningful Use standards, portal information could only be found for 178 (89\%) hospitals. Regarding ease of access, $74 \%$ of portals were accessible from the hospital homepage within 3 clicks; $48 \%$ offered portals in a language other than English. 108 (61\%) portals offered secure messaging, and all of these provided a disclaimer that messaging should not to be used for emergencies; 23 hospitals (20\%) posted the disclaimer in the terms and conditions. $33 \%$ of hospitals with secure messaging provided no additional guidance about messaging. Content analysis of hospital pamphlets revealed a focus on logistical information, portal features, and parameters of use. Logistical information (e.g., how to create an account) was the most thorough and most prevalent. Few hospitals provided guidance on expected behaviors or defined misuse beyond using secure messaging for urgent situations.

CONCLUSIONS: There is a discrepancy between providers' expectations of patients and the information available to patients regarding portal use. Although most hospitals have a patient portal, many fail to set expectations for its use, especially regarding secure messaging. Instead, educational materials largely focused on how to access the portals. To improve patient engagement and for the full benefits of patient portals to be realized while minimizing misuse, hospitals and clinicians need to provide more information and set clearer guidelines for patients.

\section{GROUP VISITS FOR ADVANCE CARE PLANNING FOR PA- TIENTS WITH HEART FAILURE}

Julia Bandini ${ }^{1}$; Alexis Coulourides Kogan ${ }^{4}$; Jessica Phillips ${ }^{1}$; Bonnie Olsen $^{4}$; Rebecca L. Sudore ${ }^{2}$; David Bekelman ${ }^{3}$; Sangeeta Ahluwalia ${ }^{1}$. ${ }^{1}$ RAND Corporation, Santa Monica, CA; ${ }^{2}$ University of California, San Francisco, San Francisco, CA; ${ }^{3}$ University of Colorado, Denver, CO; ${ }^{4} \mathrm{Keck}$ School of Medicine, University of Southern California, Los Angeles, CA. (Control ID \#3178411)

BACKGROUND: Background: Advance care planning (ACP) is an emotionally and cognitively challenging task, as patients are asked to consider their healthcare preferences in advance of their anticipated decline. We know little about how to encourage patients to engage in this intensive and time-consuming process in healthcare settings, in an efficient and effective manner. Group visits have potential to help patients identify their healthcare values and engage in ACP in a resource-efficient manner. They provide a forum for social learning and social support to empower patients to overcome common barriers to ACP and provide economies of scale that can address provider and system barriers to ACP. Disease-specific group visits may provide common ground for patients to begin thinking about their treatment options and prepare them for future 1on-1 discussions with their treating provider. We sought to understand whether a disease-focused group visit for ACP is a valuable, acceptable and feasible approach to ACP, including participant preferences for content, learning tools, group composition, and facilitation.

METHODS: Feasibility trial of five 90-minute group visits and 2 followup group visits, led by a geriatric psychologist, with 16 patients with heart failure (HF) and 11 caregivers $(n=27)$. Participants completed pre- and post-visit surveys including Likert-scale items on feasibility and acceptability and a 1-on-1 post-visit telephone interview to assess perceived value. Structured observations during the group visits further assessed process and feasibility.

RESULTS: Data from post-visit surveys indicated participants felt comfortable discussing ACP in a group, understood the information covered/ felt it was the right amount, and were able to identify and clarify their healthcare values. All participants noted they would recommend the group visit to family/friends. Of the 27 group visit participants, 20 (81\%) attended a second follow-up group visit covering additional ACP topics. Interview and observation data identified four key themes: participants leveraged others' experiences to identify and clarify preferences and potential choices; participants found value in mixed patient-caregiver groups for understanding alternative perspectives; the disease-focused nature of the visit and peer and social support aspect of the group provided participants with a safe space to share difficult experiences and explore their healthcare preferences and values; and the group visit motivated subsequent ACP steps such as completing an advance directive or sharing preferences with a provider or family member.

CONCLUSIONS: Disease-focused group visits could be an efficient health system intervention for engaging patients and caregivers in the otherwise time- and resource-intensive task of ACP. Policymakers should continue to explore ways to financially support the use of ACP group visits. A second phase of this study is underway to assess group visit effectiveness.

\section{GROWING INEQUALITY IN HEALTH EXPENDITURES FOR REPRODUCTIVE AGE WOMEN: THE INVERSE CARE LAW REVISITED}

Samuel L. Dickman ${ }^{1}$; Stephanie Woolhandler ${ }^{2}$; David Himmelstein ${ }^{3}$. ${ }^{1}$ University of California, San Francisco, Oakland, CA; ${ }^{2}$ Cambridge Hospital, New York, NY; ${ }^{3}$ City University of New York at Hunter College, New York, NY. (Control ID \#3181614)

BACKGROUND: Women of reproductive age (15-49 years) have lower incomes and greater healthcare needs than their male peers and may be especially vulnerable to policies that impose cost-sharing (e.g. copayments, deductibles). Death rates for such women have stalled recently, and maternal mortality has risen, raising concern about unmet healthcare needs.

METHODS: We analyzed population-wide healthcare utilization by compiling 801,622 person-years of data from 26 nationally representative surveys over 54 years: the 1963 and 1970 Surveys of Health Services Utilization and Expenditures; the 1977 and 1980 National Medical Care Utilization and Expenditure Surveys; the 1987 National Medical Expenditure Survey; and the 1996-2016 Medical Expenditure Panel Surveys. We tabulated all spending on health services, including out-of-pocket and insurance payments. We assessed income, self-reported health status, and inflation-adjusted spending decomposed into price and volume effects.

RESULTS: Health expenditures for reproductive-age women grew $335 \%$ between 1963 and 2016, from $\$ 1,182$ to $\$ 3,966$ in 2016 dollars. Expenditures for the wealthiest $20 \%$ of women accounted for most of the increase since 2000. The growing expenditure gap since 2001 was largely attributable to price increases, which rose $18 \%$ faster for high income than low income women. Outpatient visit rates also diverged; wealthy women had 1.2 more visits than poorer women in 1996, a gap that rose to 1.8 visits (95\% CI $1.3-2.3$ ) by 2016, despite persistent disparities in health status by income. Twenty-seven percent of poorer women in fair/poor health had total expenditures of less than $\$ 500$, an indicator of inadequate access to care, vs. $5.6 \%$ of affluent ones $(\mathrm{p}<0.05)$. ED visit rates were low overall, but higher for poorer than wealthier women: 0.30 vs. 0.13 per capita.

CONCLUSIONS: Medical expenditures and outpatient visits for reproductive age women have become increasingly concentrated among the affluent, a reallocation of health resources away from those with the 
highest medical need. Rising cost-sharing may discourage poorer women from seeking care, while diverging fees (i.e. prices) may discourage providers from serving them, to the detriment of population health.

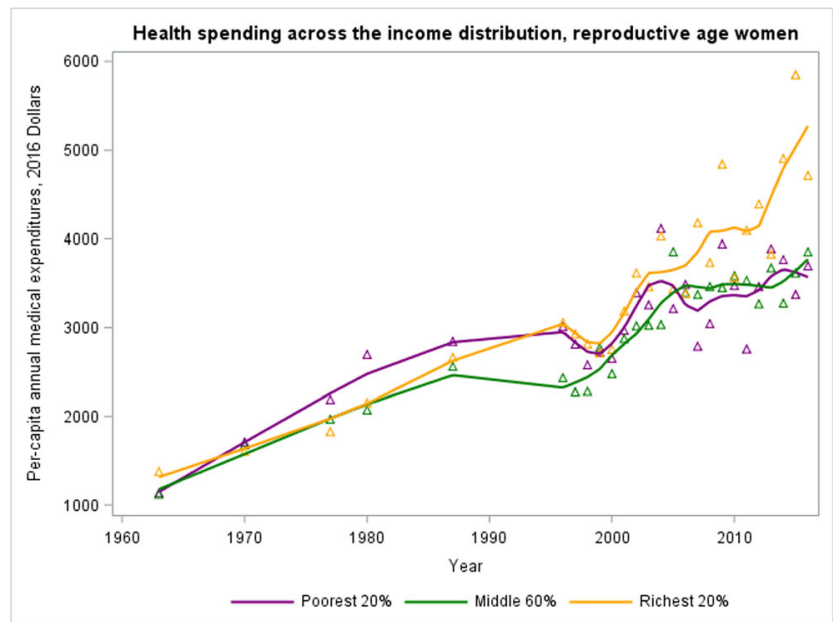

GUIDELINE-CONCORDANT CHRONIC OPIOID PRESCRIBING: DISCREPANCIES BETWEEN KNOWLEDGE, ATTITUDES AND CURRENT PRACTICE IN AN ACADEMIC PHYSICIAN PRACTICE

Mim Ari; Rebecca Chohlas-Wood; Neda Laiteerapong; Lisa M. Vinci; George Weyer. University of Chicago, Chicago, IL. (Control ID \#3186298)

BACKGROUND: Many Americans with chronic pain are prescribed chronic opioid therapy, which may improve patient quality of life and pain, but is associated with significant risk, namely opioid misuse, overdose and opioid-related death. In 2016, the CDC published its Guideline for Prescribing Opioids for Chronic Pain to help PCPs prescribe chronic opioids in a safe and effective way. In order to ensure that guidelines are distilled into clinical practice, it is essential to understand the current state of practice. The purpose of this study was to evaluate knowledge, attitudes and beliefs, and practice patterns of physicians in an academic primary care practice with regards to chronic opioid prescribing, in order to assess the need for quality improvement.

METHODS: Thirty-seven general IM physicians at a single academic medical center anonymously completed a survey in 2017 (95\% response rate). Physicians were surveyed about knowledge, attitudes and beliefs, and current practice patterns of key aspects of the CDC Guideline. A general sense of "regard" (biases, emotions, and expectations) for patients with chronic pain on long-term opioid therapy was also assessed using the Medical Condition Regard Scale. Data and descriptive analyses was compiled using REDCap software.

RESULTS: While $51 \%$ of physicians were aware of the key elements of the patient-provider agreement, and $75 \%$ believe them to be very useful, $70 \%$ never or rarely used them. Seventy percent of physicians knew how to access the PMP and $76 \%$ found the information helpful, however, only $8 \%$ checked PMP prior to initiating opioids. Sixty-five percent never performed urine drug screening; knowledge of how to interpret urine drug screening and beliefs of its usefulness were more varied. While most physicians (57\%) thought prescribing naloxone was very important, $73 \%$ of physicians had never prescribed it. Only $38 \%$ of physicians felt that working with patients with chronic pain on long-term opioid therapy was satisfying and $57 \%$ found these patients particularly difficult.

CONCLUSIONS: Our data indicate fairly high levels of knowledge and perceived usefulness of key CDC guideline recommendations. However, application of these guidelines was inconsistent. While the opioid epidemic has received significant publicity, formal education on chronic opioid therapy and support to implement the new guidelines has lagged behind. To tackle this complex issue and increase guideline adherence, a combination of policy changes, health system changes, and physicianlevel interventions is likely needed. In response to our findings, we have created a clinical practice guideline, a standardized patient-provider agreement, standardized documentation tools for the EHR, and didactic education. Future directions include resurveying physicians and assessing changes in practice using EHR data.

\section{HEALTH IT FOR HEALTH EQUITY: DEVELOPING A CHECK- LIST TO IMPROVE THE QUALITY OF EMR DATA COLLECT- ED TO INFORM HEALTH DISPARITIES INTERVENTIONS}

S. M. Ogunwole. Johns Hopkins University School of Medicine, San Antonio, TX; UT health San Antonio, San Antonio, TX. (Control ID \#3176435)

BACKGROUND: The electronic medical record (EMR) is a powerful patient data collection tool that can help organizations identify and monitor disparities in healthcare and health outcomes. This collection of data is often the first and most critical step needed to address health equity within clinical environments. Despite this, many organizations do not leverage their EMR to collect data that can inform and help prioritize interventions that reduce health disparities and improve health equity. We set out to develop a checklist that could identify items needed to ensure quality data capture from the EMR - a critical step for health disparities data collection.

METHODS: Following the World Health Organization (WHO) approach to developing surgical checklists; we assembled evidence on possible areas of improvement, drafted checklist items, solicited an expert review of the draft checklist, validated the checklist using patient and stakeholder feedback, and piloted the checklist at a clinical site. We found traditional quality improvement tools such as creation of a process map detailing the patient intake process, observation of the patient and intake staff interactions and creation of an Ishikawa diagram to identify implementation barriers to be most useful in assessing fulfillment of the checklist items and generating ideas for change based on identified deficits.

RESULTS: We established three domains of requirements for adequate EMR data capture. 1) standardization 2) inclusion of relevant 'metrics that matter'(i.e., social determinants of health) and 3) requirement to report and act on health disparities data. In addition, there were several tasks identified within each domain, leading to a final comprehensive "EMR equity checklist" composed of 21 items (see figure 1). On evaluation, we found that only 10/21 checklist items were met. The information from our quality improvement assessment in combination with clearly defined deficits from our checklist allowed generation of specific interventions (ex: intake staff education, EMR changes, and the creation of a Health Equity Taskforce) to address the identified checklist items that were not fulfilled.

CONCLUSIONS: An EMR equity checklist in conjunction with quality improvement tools, created a systematic framework to evaluate and improve the collection of data relevant to health disparities.

In order for Health Information Technology to function at a level that can inform our leadership about health disparities, the following elements have to be met:

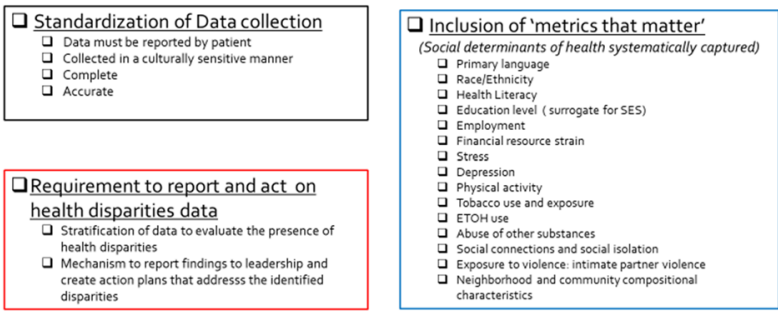


HEALTH LITERACY AND SOCIODEMOGRAPHICS ARE MORE IMPORTANT THAN PRIOR EXPERIENCE FOR ADVANCE CARE PLANNING KNOWLEDGE AMONG OLDER ADULTS

Sarah Nouri ${ }^{1}$; Deborah E. Barnes ${ }^{2}$; Aiesha Volow ${ }^{3}$; Ryan D. McMahan ${ }^{3}$; Margot Kushel $^{1}$; Chengshi Jin ${ }^{4}$; W. John Boscardin ${ }^{3}$; Rebecca L. Sudore ${ }^{3}$. ${ }^{1}$ University of California, San Francisco, San Francisco, CA; ${ }^{2}$ Univesity of California, San Francisco, San Francisco, CA; ${ }^{3}$ University of California, San Francisco; San Francisco Veterans Affairs Medical Center, San Francisco, CA; ${ }^{4}$ University of California San Francisco, San Francisco, CA. (Control ID \#3184653)

BACKGROUND: Engagement in advance care planning (ACP) is low among vulnerable populations, and limited knowledge about $\mathrm{ACP}$ has been suggested as a possible modifiable barrier. We aimed to determine the factors associated with ACP knowledge, including prior ACP engagement, health literacy, and sociodemographic factors. We hypothesized that prior ACP engagement would be associated with higher ACP knowledge, and limited health literacy would be associated with lower ACP knowledge.

METHODS: We used cross-sectional baseline data from two randomized controlled trials that enrolled 1400 English- and Spanish-speaking primary care patients aged 55 years with 2 chronic medical conditions from a Veterans Affairs and public hospital system in San Francisco. We assessed ACP knowledge, the outcome variable, with validated multiple-choice questions on a 0-7-point scale. We defined prior ACP engagement as documented legal forms and/or goals of care discussions in the medical record. Sociodemographic measures included age, gender, health literacy (validated s-TOFHLA scores dichotomized into limited vs adequate literacy), preferred language (English vs Spanish), education ( vs > high school), race/ethnicity, health status (5-pt Likert scale), finances (limited vs adequate), and social support (11-item validated survey, scores 11 to 55 ). We used Kruskall-Wallis tests and linear regression analysis to examine associations of prior engagement in ACP and sociodemographic factors with ACP knowledge.

RESULTS: Participants were $65( \pm 10)$ years old; $48 \%$ were women, $34 \%$ had limited health literacy, 32\% were Spanish-speaking, $47 \%$ had high school education, $70 \%$ were non-white, $45 \%$ had fair-to-poor health, and $21 \%$ had limited finances. In unadjusted analyses, ACP knowledge scores were higher for those with vs without prior ACP engagement (5.1 (SD 1.8) vs 4.8 (SD 2.0), p=0.02). In multivariate analyses adjusted for sociodemographic variables associated with ACP knowledge (gender, health literacy, preferred language, education, race, health status, finances, and social support), prior ACP engagement was no longer associated with knowledge ( $\mathrm{p}=0.8)$. Instead, limited health literacy was associated with 1.0point lower knowledge scores ( 4.7 vs 5.7, $\mathrm{p}<0.001$ ), Spanish language and education high school were associated with 0.6 -point lower scores $(5.1 \mathrm{vs}$ 5.7, $\mathrm{p}<0.001$ for each), and non-white race was associated with 0.4-point lower scores (5.3 vs 5.7, $\mathrm{p}<0.001)$. Higher social support was also independently associated with higher ACP knowledge scores $(0.12$ knowledge points per 1 social support point, $\mathrm{p}=0.004$ ).

CONCLUSIONS: Health literacy and sociodemographics are more important predictors of ACP knowledge than prior engagement in ACP. This study suggests that providing easy-to-understand, culturally appropriate ACP materials is paramount for vulnerable populations and should be reoffered even if patients have previously engaged in ACP.

\section{HEALTH LITERACY PHENOTYPES: COGNITIVE VS. NON- COGNITIVE LOW HEALTH LITERACY AND IMPACTS ON HEALTH STATUS}

Laura M. Curtis $^{1}$; Stacy Bailey ${ }^{1}$; Mary J. Kwasny ${ }^{1}$; Rachel O'Conor ${ }^{1}$; Julia Yoshino Benavente ${ }^{1}$; Michael Paasche-Orlow ${ }^{3}$; Alex Federman ${ }^{2}$; Michael S. Wolf ${ }^{1}$. ${ }^{1}$ Northwestern University, Chicago, IL; ${ }^{2}$ Icahn School of
Medicine at Mount Sinai, New York, NY; ${ }^{3}$ Boston Medical Center, Boston, MA. (Control ID \#3185415)

BACKGROUND: The influence of health literacy (HL) on personal health is well-established, and there have been consistent calls to action for health care systems to respond. The underlying HL construct reflects a variety of cognitive and psychosocial factors, but existing HL measures merely identify those with higher vs. lower skills. Understanding the underlying nature of the barrier(s) causing lower HL (e.g. cognitive impairment, limited healthcare knowledge/experience) could better inform healthcare system responses. We sought to investigate the prevalence of two such 'phenotypes' of low HL, cognitive vs. non-cognitive, and evaluate their associations with physical health.

METHODS: 900 adults between the ages of 55 and 74 receiving care at either an academic general internal medicine clinic or 1 of 5 federally qualified health centers in Chicago, IL were recruited for an ongoing study. Structured interviews were conducted that included three HL measures (REALM, TOFHLA, NVS) as well as a detailed psychosocial and neuropsychological battery that allowed for the determination of a cognitive impairment. In addition, participants completed the PROMIS Physical Functioning Short Form. The prevalence of low HL was calculated per test among all subjects, then stratified by those with and without a cognitive impairment to define the 'cognitive' and 'non-cognitive' phenotypes, respectively. Physical functioning t-scores corrected for the general population were then compared across HL levels, overall and stratified by these two health literacy phenotypes.

RESULTS: Among 877 participants with cognitive data, 201(23\%) were cognitively impaired and rates of low HL by REALM, TOFHLA, and NVS were $10 \%, 14 \%$, and $32 \%$ respectively. The prevalence of low HL varied by cognitive impairment status (cognitive phenotype: $66 \%$ vs. non-cognitive phenotype: $22 \% ; \mathrm{p}<0.001$ (by NVS)). Similar trends were noted by REALM and TOFHLA measures. Differences in mean (SD) physical function scores were noted across HL levels among all participants (NVS: low 44.1(9.1), marginal 47.1(8.8), adequate 50.1(8.1), p<0.001; TOFHLA: low 42.4(8.4), marginal 45.4(9.1), adequate 49.2(8.5), $\mathrm{p}<0.001$; REALM: low 42.6(9.1), marginal 45.5(9.2), adequate 48.7 (8.6), $\mathrm{p}<0.001)$. Significant, gradient trends with better physical health increasing with greater HL skills persisted in both phenotypes (NVS: (cognitive): low 42.4(9.3), marginal 46.8(10.0), and adequate 46.8(8.2), $\mathrm{p}=0.01$; (non-cognitive): low 45.6(8.7), marginal 47.1(8.5), and adequate 50.3(8.1), $\mathrm{p}<0.001)$.

CONCLUSIONS: This initial investigation into the possibility of multiple HL phenotypes suggests that even among cognitively 'normal' participants, limited HL can negatively impact physical health, albeit scores were still significantly higher than those with a cognitive impairment. This may directly inform the design of HL interventions, recognizing that different approaches will be needed to remediate different phenotypes of HL.

\section{HEALTH SYSTEM PARTICIPATION IN ALTERNATIVE PAY- MENT MODELS AND INSURANCE PRODUCTS}

Laura Kimmey $^{1}$; Rachel Machta ${ }^{1}$; David J. Jones ${ }^{1}$; Michael Furukawa ${ }^{2}$; Eugene C. Rich ${ }^{3}$. ${ }^{1}$ Mathematica Policy Research, Durham, NC; ${ }^{2}$ Agency for Healthcare Research and Quality, Rockville, MD; ${ }^{3}$ Mathematica Policy Research, Silver Spring, MD. (Control ID \#3186475)

BACKGROUND: In the past decade, consolidation of hospitals and physicians into local or regional health systems has increased in the U.S. Researchers, policymakers, health system leaders and others may be interested in studying health system characteristics and their relationship with performance. As part of AHRQ's Comparative Health System Performance (CHSP) Initiative, we developed new variables to describe whether health systems: (1) participated in alternative payment models (indirectly through participation by the systems' hospitals), including an Accountable Care 
Organization (ACO) or a Medicare bundled payment program and (2) offered an insurance product, including any insurance product or a Medicare Advantage (MA) product.

METHODS: We used data from Leavitt Partners' 2016 Torch Insight tool to construct measures of system participation in ACOs. We used publicly available data from the Centers for Medicare \& Medicaid Services (CMS) to construct a measure of system participation in a Medicare bundled payment model including the Comprehensive Care for Joint Replacement model and the Bundled Payments for Care Improvement initiative. We used the 2015 American Hospital Association Annual Survey to construct a measure of whether a system offered any insurance product and CMS's January 2016 MA Plan Directory to identify which systems offered an MA plan. The population was the 626 health systems in AHRQ's Compendium of U.S. Health Systems, 2016, and the system and non-system hospitals in the Hospital Linkage File. For the purposes of the Compendium, health systems include at least one hospital and at least one group of physicians that provide primary and specialty care and are connected with each other through common ownership or joint management.

RESULTS: In 2016, more than half of health systems (56\%) participated in an ACO contract with Commercial and Medicare contracts being the most common (42\% and $37 \%$, respectively) compared to Medicaid contracts $(10 \%)$. Nearly forty percent offered an insurance product with roughly twice as many systems offering an HMO or PPO product (31\% each) than indemnity fee-for-service product $(16 \%)$. Systems that participated in an alternative payment model or offered an insurance product tended to be larger, had a higher teaching intensity, and were investor-owned. System hospitals were more likely to participate in ACO contracts (44\% of system hospitals vs. $13 \%$ of non-system hospitals) or a Medicare bundled payment model ( $28 \%$ vs. $8 \%)$.

CONCLUSIONS: These results show that in 2016, health systems had relatively high rates of participation in alternative payment models and insurance products, but with potentially important variation by system characteristics. This work illustrates how the Compendium of U.S. Health Systems can be used to study variation in health systems' characteristics and determine which characteristics are associated with better performance.

\section{HEPATITIS C SCREENING MAY BE INADEQUATELY PER- FORMED IN ADULTS SEEKING PREVENTIVE HEALTH CARE: NON-AFRICAN AMERICANS ARE LESS FREQUENTLY TEST- ED THAN AFRICAN AMERICANS}

Ali Khan; Anna Stachura; Jenny Dave; Bedoor Alabbas; Praphopphat Adhatamsoontra; Abdullah Al-Asaad; Arun Muthiah; Daniel Szvarca; Karan Chawla; Nadeem Tabbara; Tina Boortalary; Andres Garza; Marie Borum. George Washington University, Washington, DC. (Control ID \#3162861)

BACKGROUND: Despite US Preventive Services Task Force recommendations to perform Hepatitis C Virus (HCV) screening in adults born between 1945-1965, testing is inconsistent. All health care encounters provide an opportunity for HCV testing. Notably, adults pursuing colon cancer screening are interested in preventive care. Physicians who refer for or perform colon cancer screening have an opportunity to test for HCV. This study evaluated the rate at which adults undergoing screening colonoscopy had HCV testing and the physicians who ordered the HCV test.

METHODS: A retrospective chart review of all individuals born between 1945-1965 referred for colonoscopies during a one-year period at an academic medical center was performed. Patient age, gender, HCV screening status, and ordering physician were obtained. Individuals were excluded if they were not in the appropriate birth cohort. A confidential database was created using Microsoft Excel with statistical analysis set at $<0.05$. The IRB approved the study.

RESULTS: In 2205 patients, 920 (42\%) were male and 1285 (58\%) were female (mean age 61.7 years) with 1181 (54\%) African Americans and 930 (42\%) non-African Americans. 964 (43.7\%) had HCV screening. There was no significant difference in HCV screening based upon gender (402 $(43.7 \%)$ males, 562 (43.7\%) females; $\mathrm{p}=1.000)$. Non-African Americans were screened significantly less than African Americans (385 (41.4\%) and $555(47.0 \%)$ respectively; $\mathrm{p}=0.0111)$. Primary care physicians $(51.1 \%)$ performed significantly more HCV screening than hospitalists $(15.7 \%$; $\mathrm{p}=0.0001)$, gastroenterologists $(10.0 \% ; \mathrm{p}=0.0001)$ and other specialty physicians $(18.7 \%$; $\mathrm{p}=0.0001)$.

CONCLUSIONS: In this study, HCV screening in individuals born between 1945-1965 was inadequately performed, with less than half seeking preventive health care being tested. There was also a disparity in HCV screening between non-African Americans and African Americans. While this study is limited based upon single institution design, it provides a basis for the development of interventions to improve HCV testing. It is important that all individuals at risk for $\mathrm{HCV}$ be tested with elimination of any potential bias in screening. Primary care physicians and gastroenterologists have the potential to improve $\mathrm{HCV}$ testing when referring for or conducting colon cancer screening. Innovative approaches are needed to optimize preventive care delivery.

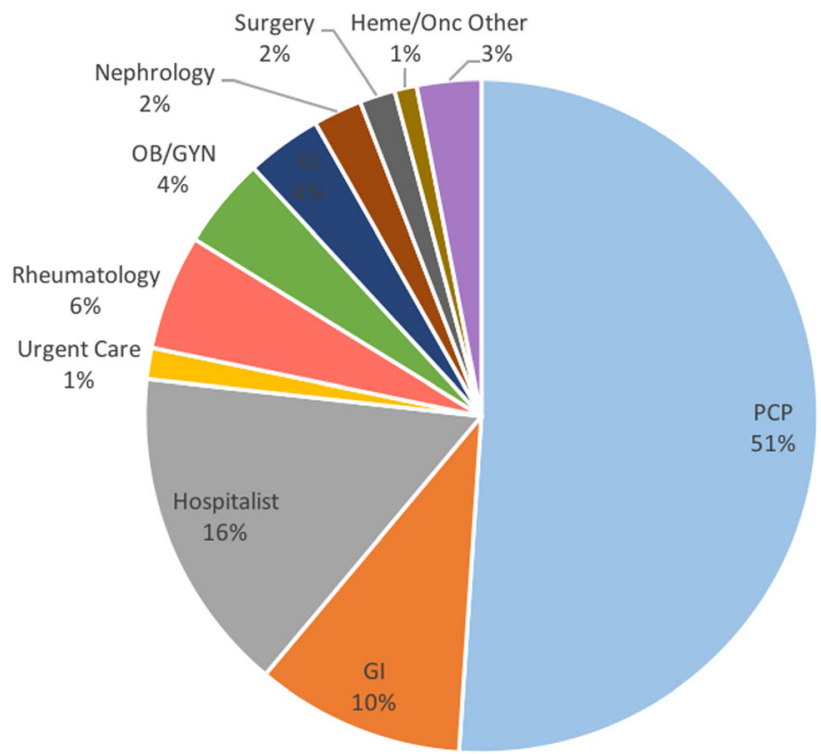

\section{HIGH VALUE CARE AND QUALITY IMPROVEMENT: A DEPRESCRIBING INITIATIVE IN RESIDENT PRIMARY CARE CLINICS}

Joel C. Boggan $^{2,}{ }^{1}$; Suchita S. Sata ${ }^{3}$; Alicia Clark ${ }^{4} .{ }^{1}$ Duke University Health System, Durham, NC; ${ }^{2}$ Durham VA Medical Center, Durham, NC; ${ }^{3}$ Duke University Hospital, Raleigh, NC; ${ }^{4}$ Beth Israel Deaconess Medical Center, Wellesley, MA. (Control ID \#3185986)

BACKGROUND: Patients taking multiple medications are often at risk of complications related to medication interactions. In addition, patients frequently desire to reduce the burdens of medication administration and costs related to complicated pharmaceutical regimens. In this quality improvement (QI) study, we sought to identify patients at high risk for polypharmacy in resident clinics and determine if education with auditand-feedback initiatives could increase rates of conversations about medication discontinuation and actual cessation.

METHODS: The three resident clinics at a large internal medicine program were included in this study. An online interactive experience to guide the project was developed utilizing Microsoft Sharepoint ${ }^{\mathrm{TM}}$. Junior and senior residents participated in this mandatory residency-wide project as part of regularly scheduled ambulatory blocks during each half of the academic year 2017-18. After Phase 1, residents reviewed up to 20 of their primary care encounters for rates of conversations related to medication 
discontinuation and actual discontinuation. Thereafter, residents were provided real-time statistics of their individual performance relative to peers at the clinic and residency level. Next, they were given the opportunity to set specific goals and methods for improvement. In Phase 2, chart review was re-performed with repeat real-time data presentation to assess whether auditand-feedback methods and goal setting increased rates of medication discontinuation conversations and actions.

RESULTS: Overall, 1,559 unique patient encounters were reviewed during the study period ( 853 pre-intervention and 706 post-intervention) by a total of 72 residents. On average, patients in both phases were receiving similar numbers of medications prior to their reviewed visit (11.1 meds in Phase 1 vs. 10.9 in Phase $2, p=0.58$ ). There were no statistically significant differences in the proportion of patients over the age of 65 years in each phase ( $42.7 \%$ vs. $47.0 \%, p=0.09$ ), nor the proportion deemed by resident primary care physicians to be at-risk for polypharmacy $(55.9 \%$ vs. $55.7 \%, \mathrm{p}=0.92)$. While rates of discussing cessation of a medication within a clinical encounter increased by $32.3 \%$ between phases $(28.1 \%$ in Phase 1 vs. $37.2 \%$ in Phase 2, $\mathrm{p}<0.01$ ), no similar increase in actual medication discontinuation was found $(19.1 \%$ vs. $20.8 \%$, $\mathrm{p}=0.39)$.

CONCLUSIONS: Audit-and-feedback QI methods led to an increase in conversations regarding medication discontinuation in resident primary care encounters; however, rates of actual medication discontinuation did not increase. These findings may suggest that, even in patients deemed at high risk of medication complications, many medications may be necessary to manage chronic medical conditions. Alternatively, patients may require multiple conversations or encounters to reduce or eliminate certain medications (such as proton pump inhibitors or opioids) given chronic symptoms.

\section{HIGHER PERCEIVED SOCIAL SUPPORT CORRELATED WITH LOWER BURNOUT IN MEDICAL STUDENTS}

Brittany Smith; Justine E. Owens; Tabor E. Flickinger; John Schorling; Margaret L. Plews-Ogan; Rachel H. Kon. University of Virginia, Charlottesville, VA. (Control ID \#3185641)

BACKGROUND: Each year, medical educators dedicate more effort towards preventing burnout in their medical trainees. The Maslach Burnout Inventory (MBI) assesses emotional exhaustion (EE), depersonalization (DP), and low sense of personal accomplishment (PA) as the three main determinants of burnout. Previous papers hypothesize personal life events, such as illness or death of family members may contribute to EE, and that workplace cynicism toward the chronically ill may be contributory to the development of DP. Other studies show social support from family, friends, fellow students, teachers, and social groups correlate negatively with mental health problems. We conducted a study to assess the relationship between perceived social support and burnout levels in medical students during each year in school to determine importance of structured social opportunities to promote trainees' wellness and reduce likelihood of burnout.

METHODS: All medical students at the UVA School of Medicine were sent an annual online survey from 2014-2018. The survey included the 22item MBI, demographic information, and five items using a 5-point Likert scale to assess students' perception of support provided by their family, friends, faculty members, administration, and deans. Pearson correlation was used to measure the relationship between perceived support and burnout domains (EE and DP) for each year of training.

RESULTS: Of 2509 students eligible, 1371 surveys were completed for all years with response rates of $48 \%, 42 \%, 45 \%$, and $44 \%$ for each year in school respectively. Fifty-two percent of participants who completed the survey were female. In year 1, of the five categories of support, perceived support from friends had the strongest inverse correlation with EE ( $\mathrm{r}=$ $-0.312, p<0.000$ ). In year 2 , perceived support from administration and faculty had a moderate inverse correlation with $\mathrm{EE}(\mathrm{r}=-0.353, \mathrm{p}<0.000$ and $r=-0.330, p<0.000$, respectively). In year 3 , perceived support from faculty and administration had moderate inverse correlations with $\mathrm{EE}(\mathrm{r}=-0.403, \mathrm{p}$ $<0.000$ and $\mathrm{r}=-0.398, \mathrm{p}<0.000$, respectively). In year 4 , perceived support from faculty and administration had moderate inverse correlations with $\mathrm{EE}$ $(\mathrm{r}=-0.401, \mathrm{p}<0.000$ and $\mathrm{r}=-0.317, \mathrm{p}<0.000$, respectively). Similarly categories of social support were significantly inversely correlated with $\mathrm{DP}$, but were more strongly correlated to $\mathrm{EE}$.

CONCLUSIONS: During the first year of training, perceived social support from friends seems to most strongly mitigate EE in medical students. However, as medical students enter their $3^{\text {rd }}$ and $4^{\text {th }}$ years in training, particularly when burnout is highest, perceived social support from faculty and administration seems to counter EE more. Further studies investigating students' specific definitions of social support from faculty, administration, and friends, may give educators insight into which social interactions are beneficial at lowering burnout levels at each stage of medical school.

\section{HOME HEALTHCARE DELIVERY IN HEART FAILURE:} WORKFLOW, CHALLENGES, AND POTENTIAL SOLUTIONS Madeline R. Sterling ${ }^{1}$; Nicola Dell ${ }^{2}$; Benedetta Piantella ${ }^{3}$; Jacklyn Cho ${ }^{4}$; Harveen Kaur'; Emily Tseng ${ }^{2}$; Fabian Okeke ${ }^{2}$; Mikaela Brown²; Peggy B. Leung ${ }^{1}$; Ariel F. Silva ${ }^{1}$; Amy L. Shaw ${ }^{1}$; Faith Wiggins ${ }^{5}$; Lisa M. Kern ${ }^{1}{ }^{1}$ Weill Cornell Medicine, New York, NY; ${ }^{2}$ Cornell Tech, New York, NY; ${ }^{3}$ New York University, New York, NY; ${ }^{4}$ Cornell University, Ithaca, NY; ${ }^{5} 1199$-Home Care Industry Education Fund, New York, NY. (Control ID \#3181586)

BACKGROUND: Reducing hospital readmissions for heart failure (HF) patients is a national priority. Data suggest that readmission rates are particularly high among HF patients who require home healthcare (HHC) after discharge. One possible reason is that the workflow of HHC may not be optimized. Since the exact workflow of HHC is not well described, pinpointing opportunities for interventions has been difficult. To better articulate the workflow of $\mathrm{HHC}$ for HF patients and identify challenges and opportunities for improvement, we elicited the perspectives of key stakeholders, including home health aides, who-despite being the minute-tominute observers of HF patients - have not been included in research.

METHODS: We conducted a qualitative study using a combination of interviews and focus groups with home health aides, nurses, HF patients, family caregivers, physicians, social workers, home care agency leaders, and policy experts in NYC. We used a combination of purposeful and convenience sampling. To elicit participants' experiences with $\mathrm{HHC}$ in HF, we used a semi-structured topic guide. We also asked selected participants to draw their understanding of HHC post-hospitalization workflow for HF patients. Interviews were recorded, transcribed and analyzed thematically. Drawings were synthesized into a final image.

RESULTS: 80 participants were recruited from 10 home care agencies, 1 academic medical center, and 1 nonprofit organization. Participants described $\mathrm{HHC}$ for HF patients occurring in 6 steps, with home health aides playing a main role: 1) Transitioning from hospital to home; 2) Recognizing clinical changes; 3) Making decisions; 4) Managing symptoms; 5) Asking for help; and 6) Calling 911. For each step, challenges and solutions were identified. In Step 1, participants reported that care was poorly coordinated among providers, with information lost in translation: "I send the discharge summary to the agency, but it never gets to the patient's aide." To address this, they proposed a handoff process between hospital and home providers. The leading issue for Steps 2, 3, and 4 was that despite providing the most patient care, home health aides do not receive HF training: "Picking up on symptoms and knowing what to do requires training." Participants recommended a HF training course for home health aides. In Step 5, participants were frustrated by communication: "I call the agency or the hospital, but I can't get through so I call 911!" Due to this, many felt tech-based innovations could improve connectivity for the escalation of care. Lastly, participants felt that tackling these issues could reduce 911 calls (Step 6) and potentially readmissions.

CONCLUSIONS: Our findings suggest that HHC for HF patients occurs in a series of discrete steps, each plagued with different challenges. Rather than a one-size-fits all approach, it is likely that a variety of different interventions will be required to optimize $\mathrm{HHC}$ delivery for $\mathrm{HF}$ patients in the postdischarge period. 
HOME MODIFICATIONS AND AGING IN PLACE: RESULTS FROM THE NATIONAL HEALTH AND AGING TRENDS STUDY Cynthia Schoettler ${ }^{1,}{ }^{2}$; Edward R. Marcantonio ${ }^{4}$; David M. Levine ${ }^{3}$. ${ }^{1}$ BIDMC, Boston, MA; ${ }^{2}$ Veteran's Health Administration - Boston, Boston, MA; ${ }^{3}$ Brigham and Women's Hospital, Boston, MA; ${ }^{4}$ Beth Israel Deaconess Medical Center, Boston, MA. (Control ID \#3183132)

BACKGROUND: Most older adults wish to remain in their own homes, termed aging in place. Numerous barriers to this exist, including functional limitations and housing not designed to meet the needs of persons with limited physical abilities. Addressing functional limitations with home modifications (HMs) may be a way for older adults to delay transition out of their home to a more institutionalized setting (e.g., nursing home or assisted living facility).

METHODS: The National Health and Aging Trends Study (NHATS) is a nationally-representative longitudinal prospective cohort of community dwelling Medicare beneficiaries aged 65 years and older. Data from 2011-2017 were analyzed. Cox proportional hazards survival analysis was used to compare time living in the community of those with HMs to those without HMs. HMs were defined as the presence of at least one of: bath grab bar, toilet grab bar, shower seat, raised toilet seat, stair lift, or entrance ramp. Models were: unadjusted, minimally adjusted (age, gender, race/ethnicity, income, and education), heavily adjusted (minimally adjusted variables plus partner status, census division, living alone, number in social network, Medicaid, Tricare, self-rated health, number of comorbidities, recent hospitalization, recent fall, worry about falls, short physical performance battery (SPPB) score, and number of activities of daily living (ADLs) requiring help), and function only (falls, worry about falls, SPPB score and ADL help).

RESULTS: Data from 10,949 participants were analyzed. $65 \%$ of the participants had at least one HM in place at time of study entry, this increased to $81 \%$ by the time of participant exit from the community. At study entry those with HMs were more likely to be older ( $42 \% 80 \mathrm{yrs}$ Vs $22 \%$ in the population without HMs; $\mathrm{p}<0.01$ ), female (59\% Vs 54\%; $<<0.01)$, Caucasian (69\% Vs $62 \%$; $p<0.01$ ), widowed ( $34 \%$ Vs $25 \%$; $p<0.01$ ), have more comorbidities (mean sum comorbidities 3.74 ; $95 \%$ CI 3.67 - 3.80 Vs 3.07 ; $95 \%$ CI 2.99 3.14), lower mean SPPB score $(6.57$; $95 \%$ CI $6.47-6.68$ Vs $7.94 ; 95 \%$ CI 7.80-8.08), and require help with ADLs (mean sum ADLs requiring help 1.36; 95\% CI 1.33-1.39 Vs 1.12; 95\% CI 1.10-1.14. Having a HM was associated with shorter time to institutionalization in unadjusted (HR 1.35; 95\% CI 1.211.51 ) and minimally adjusted (HR $1.88 ; 95 \%$ CI 1.44-2.46) models. The function only model showed longer time to institutionalization (HR 0.74; $95 \%$ CI $0.65-0.84$ ), but not in the heavily adjustment model (HR 1.30; $95 \%$ CI $0.92-1.84)$.

CONCLUSIONS: Multivariate survival analysis suggests that home modifications may delay transition to an institutionalized setting for those with reduced physical function. However, the effect of HMs may further be complicated by additional confounding by indication not adequately controlled for. More detailed study of the effect of home modifications on aging in place is needed.

\section{HOMELESS VETERANS RATE PRIMARY CARE MORE FAVOR- ABLY IN HOMELESS-TAILORED COMPARED TO MAIN- STREAM PRIMARY CARE CLINICS: A NATIONAL SURVEY Stefan Kertesz ${ }^{1,3}$; Audrey Jones ${ }^{5,6}$; Aerin DeRussy ${ }^{1}$; Lillian Gelberg ${ }^{2}$; Ann E. Montgomery $^{1,3}$; April E. Hoge ${ }^{1}$; Allyson Varley ${ }^{1,3}$; Sally Holmes ${ }^{1}$; Sonya Gabrielian $^{2}$; Kevin R. Riggs ${ }^{1,3}$; Erika Austin ${ }^{1,3}$; John R. Blosnich ${ }^{4}$; Adiseshu Gundlapalli ${ }^{5,}{ }^{6}$; Adam Gordon ${ }^{5,}{ }^{6}$; Young-il Kim ${ }^{1,}{ }^{3}$. ${ }^{1}$ Birmingham VA Medical Center, Birmingham, AL; ${ }^{2}$ VA Greater Los Angeles Health Care System, Los Angeles, CA; ${ }^{3}$ University of Alabama at Birmingham, Birming- ham, AL; ${ }^{4}$ Pittsburgh VA Medical Center, Pittsburgh, PA; ${ }^{5}$ VA Salt Lake City Health Care System, Salt Lake City, UT; ${ }^{6}$ University of Utah, Salt Lake City, UT. (Control ID \#3179350)}

BACKGROUND: People experiencing homelessness face challenges in accessing primary care and difficulties related to care coordination and stigma.
In 2012, the Veterans Health Administration (VHA) implemented a patientcentered medical home model for Homeless Experienced Veterans (HEVs): Homeless Patient-Aligned Care Teams (HPACTs). HPACTs tailor services with smaller panels, outreach, dedicated staff, and support for tangible needs, along with linkage to social and other services. Some HEVs use HPACTS and some use Mainstream Primary Care (PC). We compared patient experience in these two service models using a validated patient experience survey.

METHODS: We chose a stratified, random sample of 14,352 HEVs in HPACT and Mainstream PC at 26 VA facilities. Response rate was $40 \%$ based on 4 mailings and up to 5 phone calls. The validated 33-item Primary Care Quality-Homeless (PCQ-H) measured Patient-Clinician Relationship, Perceived Cooperation among Caregivers, Accessibility/Coordination, and Homeless-Specific Needs. We used mixed effect linear regressions to estimate mean differences in PCQ-H scores, controlling for age, gender, race/ethnicity, unsheltered status, chronic homelessness, \# of medical conditions, alcohol/ drug problems, psychiatric medication, monthly income, mental distress, social support and chronic pain, adjusting for facility as a random effect.

RESULTS: Compared to HEVs in Mainstream PC, HPACT users were younger, more often male, unsheltered and chronically homeless. They also had more drug/alcohol problems, lower social support, worse emotional distress, and lower income. PCQ-H scores were typically favorable (about 3 on a 1-4 scale). However, mean scores for HPACT were superior to Mainstream PC on all scales: Patient-Clinician Relationship (3.19 vs 3.08), Cooperation (2.79 vs 2.65), Access/Coordination (3.07 vs 2.95) and HomelessSpecific Needs (3.02 vs 2.84), all $\mathrm{p}<0.001$. These differences persisted when controlling for covariates (Figure). The largest magnitude of association between PCQ-H score and any predictor was the comparison of HPACT and Mainstream PC.

CONCLUSIONS: HEVs rated HPACT PC more favorably than Mainstream PC despite characteristics that could make care more challenging. This survey, the largest among homeless Veterans to date, suggests benefits from tailoring PC design to a vulnerable population.

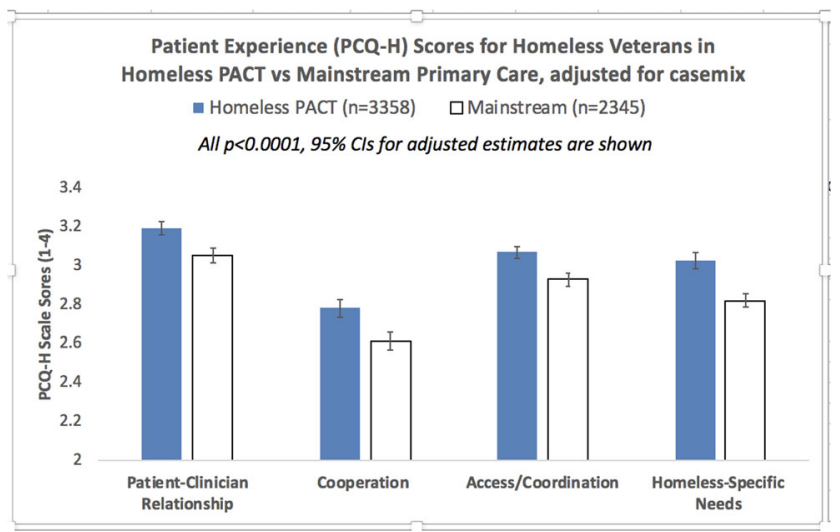

\section{HOSPITAL BASED ENGAGEMENT OF PATIENTS WITH OPIOID USE DISORDER}

Susan L. Calcaterra. University of Colorado, DENVER, CO. (Control ID \#3186908)

BACKGROUND: Individuals with opioid use disorder (OUD) have high hospitalization rates which presents an opportunity to initiate OUD treatment in the hospital. Hospitalists care for most hospitalized patients in the United States, yet little is known about their practices and beliefs regarding OUD treatment. We hypothesized that OUD treatment varies widely among hospitalists, however, with sufficient support and training, hospitalists would routinely initiate medications for OUD and link patients to addiction treatment. METHODS: From October through November 2018, we conducted an anonymous online survey of academic hospitalists across the US. We identified a convenience sample of Hospital Medicine Division Chiefs and requested they email the survey to their hospitalist peer networks using a snowball 
sampling technique. The survey queried three domains: current OUD treatment practices, perceptions and beliefs about treating patients with OUD, and hospital barriers and/or processes for OUD management. We rated responses on a 4-part Likert scale or true/false and report descriptive statistics. Response rates are unknown due to snowball sampling techniques with online surveys. RESULTS: Among 305 respondents, 295 completed the survey. Many respondents lived in the West $(n=121,56 \%)$ and practiced 5 years $(n=130$, $49 \%)$. Of the respondents, $82 \%(\mathrm{n}=238)$ reported they "always/often" cared for patients with OUD, however 50\% ( $\mathrm{n}=142)$ "rarely/never" screened for OUD. Hospitalists reported feeling "very/somewhat unsure" using DSM-5 criteria to diagnose OUD $(65 \%, \mathrm{n}=186)$, though $63 \%(\mathrm{n}=182)$ "strongly/ somewhat agree" that they have the clinical skills to screen, diagnose and refer patients to treatment. Furthermore, 65\% $(n=188)$ reported that they always/often" refer patients to treatment for their opioid use disorder. Hospitalists were less confident about the medical management of OUD. Only 25\% ( $\mathrm{n}=71)$ reported that they "strongly/somewhat agreed" that they were comfortable prescribing buprenorphine and 50\% ( $\mathrm{n}=140)$ "strongly/ somewhat agreed" that prescribing buprenorphine was outside of their scope of practice. Hospitalists reported "rarely/never" initiating buprenorphine in the hospital $(89 \% ; n=257)$ and "rarely/never" prescribed or recommended naloxone for opioid overdose reversal $(58 \% ; n=168)$. Hospitalists reported insufficient ancillary support to arrange for treatment referrals $(\mathrm{n}=174,6 \%)$ and lack of addiction specialty support $(\mathrm{n}=113,43 \%)$ as barriers to hospital-based OUD treatment. However, $86 \%(n=86)$ said they would routinely screen, diagnose and initiate treatment for hospitalized patients with OUD if they had access to an addiction specialist.

CONCLUSIONS: Hospitalists are well-positioned to provide lifesaving treatment to hospitalized patients with OUD. This could be accomplished by providing hospitalists with increased education regarding OUD, greater institutional support with dedicated ancillary staff to screen and refer patients to treatment, and access to an addiction specialist.

\section{HOSPITAL CONSERVATION STRATEGIES AND INPATIENT IN- TRAVENOUS OPIOID USE DURING THE CURRENT NATIONAL SHORTAGE}

Hemal N. Sampat; John Marshall; Christopher Fortier; Chana A. Sacks. Massachusetts General Hospital, Boston, MA. (Control ID \#3183314)

BACKGROUND: Due in part to manufacturing problems, the US is experiencing a shortage of intravenous (IV) opioids. In response, many hospitals developed conservation strategies. The impact of the ongoing shortage and resulting hospital-based conservation efforts on the use of IV opioids is unknown.

METHODS: We evaluated pharmacy dispensing records for morphine, hydromorphone, and fentanyl (the three most commonly used IV opioids) for all inpatient units at a single, large academic medical center. We divided the study period into two phases: January 29 through March 7, 2018 (the "preshortage" period before the hospital's conservation efforts began) and March 8 through June 3, 2018 (the "shortage" period for which data are currently available). We used descriptive statistics to determine the median number of dosage units of each opioid that was dispensed each week. Conservation efforts instituted on March 7 included a warning to prescribers in the electronic health record system about the shortage and recommendations about pharmacologic and non-pharmacologic alternatives to IV opioids. We tracked three hospital-level, patient-reported quality metrics during the study period, as measured by the Hospital Consumer Assessment of Healthcare Providers and Systems (HCAHPS) survey: overall patient satisfaction, communication about pain, and discussion of pain treatment.

RESULTS: During the study period, use of IV morphine decreased by $51 \%$, from a median of 1158 weekly dispenses (IQR 870-1523) in the pre-shortage period to a median of 572 (IQR 412-742) weekly dispenses during the shortage. Use of hydromorphone decreased by $36 \%$, from a median of 668 (IQR 568-1300) weekly dispenses to 426 (IQR 240-586) dispenses per week. The use of IV fentanyl increased by $26 \%$, with a median of 397 (IQR $357-$
432) dispenses per week to a median of 501 (IQR 456-523) dispenses per week. Quality metrics (assessed by top-box survey response) were similar during the study period: overall patient satisfaction ( $84 \%$ pre-shortage vs. $82 \%$ during the shortage), communication about pain (77\% vs. $73 \%)$, and discussion of pain treatment ( $80 \%$ vs. $79 \%$ ).

CONCLUSIONS: During the study period, there was a substantial reduction in overall use of IV opioids, with large reductions in IV morphine and hydromorphone use and a modest increase in IV fentanyl use. A larger sample over a longer duration of the shortage will be important to understand patients' perceptions of the associated changes. Conservation efforts developed during shortages may be useful to promote use of alternatives to IV opioids during non-shortage periods.

\section{HOSPITAL VARIATION IN DIAGNOSTIC TESTING OF PATIENTS ADMITTED WITH SYNCOPE}

Timothy Anderson; Grace A. Lin. University of California, San Francisco,

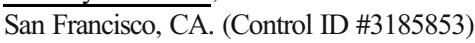

BACKGROUND: Syncope accounts for $1 \%$ of emergency department (ED) with an annual cost of over $\$ 2.5$ billion. Although advanced diagnostic testing for low risk patients with syncope is not recommended due to low yield, such patients are frequently hospitalized. Little is known about variation in diagnostic testing patterns between hospitals and regions.

METHODS: We used State Inpatient Databases to examine all adults hospitalized from the ED with an admitting diagnosis of syncope in 2013 from 10 states reporting detailed procedure utilization. We calculated rates of advanced cardiac testing (echocardiogram, stress test, or diagnostic catheterization) and neuroimaging (head CT or brain MRI) for each hospital. Predictors of receiving imaging were assessed using a hierarchical mixed-effect regression models adjusting for patient-, hospital-, and region-level factors. We estimated the proportion of variation in testing explained by differences among patients, hospitals, and regions using variation partition coefficients. The etiology of syncope was identified by primary discharge diagnoses.

RESULTS: We identified 45,541 patients (67\% age 65, 54\% female) admitted for syncope from 486 hospitals in 73 hospital referral regions (HRRs) from 10 states. Over two-thirds of patients received neuroimaging $(31,381,69 \%)$ and 20,051 (44\%) received advanced cardiac testing. Hospital-level rates of advanced cardiac testing varied widely (median hospital $48 \%$ of patients received testing, IQR $16 \%-82 \%$ ) as did neuroimaging rates (median $72 \%$, IQR $43 \%-84 \%$ ). There was no correlation between hospital-level rates of neuroimaging and cardiac testing (Spearmen correlation 0.09). After adjusting for case-mix, nearly half of the variation in hospital-level testing rates was explained by differences in hospital and regional practice patterns (Table). The majority of patients were discharged with unexplained syncope $(28,069,62 \%)$ and neither receipt of advanced cardiac testing nor neuroimaging was associated with an increased likelihood of being discharged with an identified cause of syncope ( $\mathrm{P}<0.001$ for both).

CONCLUSIONS: The performance of advanced cardiac testing and neuroimaging in patients admitted for syncope varies widely between hospitals, with nearly half of variation explained by differences in hospital and regional practice patterns. Wide variation in testing rates suggests that Choosing Wisely campaign goals of reducing low value testing are achievable but are likely to require targeted local interventions.

\section{HOSPITAL-LEVEL CARE AT HOME FOR ACUTELY ILL ADULTS: A QUALITATIVE EVALUATION OF A RANDOMIZED CONTROLLED TRIAL}

David M. Levine ${ }^{1}$; Julia Pian $^{2}$; Karthiyayini Mahendrakumar'; Agustina Saenz ; Jeffrey L. Schnipper ${ }^{1}$. 'Brigham and Women's Hospital, Boston, MA; ${ }^{2}$ Harvard Medical School, Boston, MA. (Control ID \#3184962)

BACKGROUND: Hospitals are the standard of care for acute illness management, but hospital care can be expensive, uncomfortable, and associated 
with adverse events. Substitutive hospital-level care in a patient's home has been shown to lower cost, utilization, and readmission. However, evidence to explain how and why home hospital accomplishes many of these results is lacking. To best elucidate the inner workings of the intervention, we report an in-depth qualitative evaluation.

METHODS: We performed a randomized controlled trial comparing usual hospital care versus acute care at home for patients admitted via the emergency department with any infection, exacerbation of heart failure, chronic obstructive pulmonary disease, asthma, or several other specified internal medicine diagnoses. Patients randomized home had nurse and physician home visits, IV medications, remote monitoring, video communication, and point of care diagnostic testing. Between 1 and 30 days after discharge from either arm, we administered semi-structured interviews to elicit detailed patient experience, using a convenience sample of patients until we reached thematic saturation. We analyzed responses using NVivo via the constant comparative method with 2 coders, with discrepancies resolved by a third.

RESULTS: Of the 60 eligible patients, we interviewed 15 home patients and 13 control patients ( $47 \%$ of all eligible patients); no sociodemographic differences existed between those who were and were not interviewed, although control patients were slightly younger (median age 72 vs 77 years). Patients' experience clustered around 3 main themes: clinician factors, factors promoting healing, and systems factors. Both groups noted positive experiences with their clinicians, although the home group had fewer negative comments regarding interactions with physicians, nurses, and aides. Compared to control patients, home patients described more thematic factors promoting healing: improved sleep, increased physical activity, improved social support from family, and better environmental comfort. Regarding systems factors, control patients described more difficulties during their admission from the emergency department to the hospital floor (compared to home patients' experience of hospital to home), more negative experiences with technology (e.g., alarms from monitors), and more logistical difficulties in receiving care during their admission (e.g., roommates). Both groups noted difficulties with the discharge process, citing difficulty carrying out the proposed plan after discharge.

CONCLUSIONS: Patients who received hospital-level care at home experienced fewer negative experiences with the clinical team, more factors promoting healing, and fewer systemic logistical hurdles. Both noted difficulty with discharge after hospitalization. To our knowledge, this is first qualitative analysis of home hospital care and will serve to provide insights and improvements for future home hospital efforts.

\section{HOSPITALIZATION, RE-HOSPITALIZATION, MORTALITY, AND UTILIZATION PATTERNS PREDICTED BY NOVEL HIGH-RISK PATIENT LATENT SUBGROUPS AND COMPLEXITY SCORES}

Xinhua Zhao ${ }^{1,4}$; Sandeep Vijan ${ }^{2,5}$; Matthew Maciejewski ${ }^{3,}$; Donna M. Zulman $^{7,8}$; Hongwei Zhang ${ }^{1}$; Joshua M. Thorpe ${ }^{1,9}$; Ann-Marie Rosland ${ }^{1,4}$. ${ }^{1}$ VA Pittsburgh Center for Health Equity and Promotion, Pittsburgh, PA; ${ }^{2}$ VA Center for Clinical Management Research, Ann Arbor, MI; ${ }^{3}$ Durham VA Medical Center, Durham, NC; ${ }^{4}$ University of Pittsburgh, Pittsburgh, PA; ${ }^{5}$ University of Michigan, Ann Arbor, MI; ${ }^{6}$ Duke University, Durham, NC; ${ }^{7}$ VA Palo Alto Health Care System, Menlo Park, CA; ${ }^{8}$ Stanford University, Stanford, CA; ${ }^{9}$ UNC, Chapel Hill, NC. (Control ID \#3165380)

BACKGROUND: Latent groups have been described among high-risk complex patients. Before such groupings are used to inform clinical care, the ability of such groups to predict distinct health outcomes and utilization patterns over time should be established.

METHODS: We previously used Mixture Item Response Theory (MixIRT), a hybrid classification-dimensional modeling approach, to define 6 clusters of chronic comorbidities and group-specific patient theta ('complexity') scores among 934,787 VA primary care patients whose predicted probability of hospitalization over 12 months using standard VA prediction algorithms was $\geqq 25 \%$ during any week in 2014 . Here we assess the associations of group membership and patient theta scores with rates of all-cause mortality, all-cause acute hospitalizations, and 30-day re-hospitalization in the year following group assignment, in regression models adjusted for patient demographic characteristics. We also examined detailed VA utilization patterns among groups.

RESULTS: $81 \%$ of patients were matched with a group at a probability $\geqq 80 \%$. High-risk patient groups were characterized by high levels of Substance Use Disorder (SUD, $11 \%$ of patients assigned), Cardiometabolic Conditions (CM, 21\%), Mental Health Conditions (MH,14\%), Pain \& Arthritis (PA, 16\%), Cancer (13\%), and Chronic Liver Disease (12\%). The overall rate of one-year mortality was $8 \%$ and VA hospitalization was $25 \% .17 \%$ of hospitalizations led to a readmission within 30 days. Mortality varied significantly by group (SUD=reference; AOR $(95 \% \mathrm{CI})$ : Liver $3.2(3.0,3.3)$, Cancer 2.3 (2.2, 2.4), CM 1.6 (1.5, 1.7), PA $1.06(1.02,1.11)$, and MH 0.7 (0.66, $0.73)$ ). One-year hospitalization rates ranged from 61 per 100 patients (Liver) to 31 per 100 patients $(\mathrm{MH})$ (adjusted incidence risk ratio (AIRR), SUD=reference: Liver 1.27, Cancer 0.95, CM 0.88, PA 0.75, MH 0.6, all $\mathrm{p}<0.001)$. Among all hospitalizations $(\mathrm{n}=253,933), 30$-day re-admission rate ranged from Liver 24\% to MH 15\%; (AIRR Liver 1.1, SUD reference, Cancer 0.99, CM 0.92, PA 0.82, MH 0.73, all $\mathrm{p}<0.001$ except Cancer $\mathrm{p}=0.69$ ). MixIRT derived patient theta scores independently predicted mortality (AOR 1.16, CI 1.15, 1.17), any hospitalization (AOR 1.17, CI 1.16, 1.17), and at least one 30-day re-hospitalization (AOR 1.29, CI 1.28, 1.30). Notable group utilization patterns included large variation in primary care use (range 5.4 PCP visits/year for MH to 4.2 for SUD, specialty care use (range 13 visits/ year for Cancer to 4.8 for SA), and ED visit rate (range 2.7 visits/year for SA to 1.9 for Cancer).

CONCLUSIONS: MixIRT models were able to assign high-risk patients, previously in a single group defined by a homogenously high risk score, to subgroups with distinct hospitalization, re-hospitalization, and utilization profiles. Intervention content and intensity could be tailored to group utilization profiles and patient theta scores. High rates of re-hospitalization were seen among groups not typically targeted in re-admission prevention programs (Liver, Substance Use).

\section{HOW DO ONLINE SITES OF ACADEMIC MEDICAL CENTERS PORTRAY THE HARMS AND BENEFITS OF LUNG CANCER SCREENING AND WHAT STEPS DO THEY RECOMMEND FOR PATIENTS}

Stephen Clark; Daniel S. Reuland; Daniel Jonas. University of North Carolina-Chapel Hill, Chapel Hill, NC. (Control ID \#3175218)

BACKGROUND: The USPSTF recommends lung cancer screening to adults aged 55 to 80 years who have a 30 pack-year smoking history and currently smoke or have quit within 15 years, but not before a shared decisionmaking discussion of benefits and harms. Many medical centers publish online information on screening, making the public aware of their ability to perform it. However, because websites could influence patient perceptions of screening, some experts have cautioned about overly optimistic portrayal of screening in patient-facing materials. We evaluated the online information for the reporting of harms and benefits as well as recommended next steps for patients.

METHODS: We performed a standardized web search for publicly available information on lung cancer screening at academic medical centers affiliated with any of the 152 US allopathic medical schools. A single author reviewed webpages for the reporting of benefits, harms, and recommended next steps. We used descriptive statistics to summarize the findings.

RESULTS: Eighty-one institutions (53.3\%) had publicly available information on lung cancer screening. Centers were more likely to report benefits than harms $(98.8 \%$ vs. $56.8 \%$, Table 1$)$. Only 19 (23.5\%) webpages specifically recommended patients weigh the benefits and harms of screening. Only 11 (13.6\%) mentioned overdiagnosis. Seventy-six (93.8\%) institutions listed follow up steps with only $16(19.8 \%)$ specifically indicating patients should discuss benefits and harms with a PCP. Three (3.7\%) stated harms and benefits would be discussed at a lung cancer screening specific appointment scheduled by the medical center. 
CONCLUSIONS: Among academic medical centers' online publicly available information for lung cancer screening, a balanced message of both benefits and harms was often not provided, and harms were commonly ignored. Many centers do not guide patients toward a discussion of harms and benefits with a patients' primary provider. Continued study and refinement of published information in this area is needed to optimize value concordant patient decision making for lung cancer screening.

\section{HOW DO RESIDENTS RESPOND TO UNANNOUNCED STAN- DARDIZED PATIENTS PRESENTING SOCIAL DETERMINANTS OF HEALTH?}

Fariha Ansari ${ }^{1}$; Harriet Fisher ${ }^{1}$; Jeffrey Wilhite ${ }^{2}$; Kathleen Hanley ${ }^{2}$; Colleen C. Gillespie ${ }^{1}$; Sondra Zabar ${ }^{1}$; Lisa Altshuler ${ }^{1} .{ }^{1}$ NYU School of Medicine, New York, NY; ${ }^{2}$ New York University School of Medicine, New York, NY. (Control ID \#3186372)

BACKGROUND: There is an increased awareness among healthcare professionals to discuss social determinant of health $(\mathrm{SDOH})$ information with patients. However, the awareness does not necessarily translate into effective response to the situation. In order to better understand the nuances in such conversations between patients and providers, we reviewed qualitative responses from Unannounced Standardized Patient (USP) portraying patients with $\mathrm{SDOH}$ concerns who were seen as part of a study to investigate healthcare teams' management of SDOH information.

METHODS: USPs, representing six different clinical cases, were seen by residents at an urban safety-net hospital. Each case had SDOH issues (financial and housing insecurity, social isolation), and USPs were trained to provide such information in a systematic fashion in response to provider questioning. After the encounter, USPs completed a behaviorally-anchored, standardized checklist, and also entered their impressions of the encounter in free text. The focus of this study was to evaluate these comments using a qualitative approach, focusing only on those that addressed SDOH. 258 visits occurred from 2017-present, and 209 relevant comments were analyzed.

RESULTS: Three general themes emerged: residents' openness to discussion of SDOH, their understanding of how these issues related to presenting concerns, and how they responded to those concerns. Some providers did not explore SDOH prompts, e.g. "I don't think she cut me off, but she quickly moved on to her next question without further delving deeper", while others were more responsive and supportive e.g., the provider "is very open to hearing my situation, I was able to fully explain my situation clearly." Such provider behavior impacted trust and connection, e.g., "Doctor X had good communication skills, but I felt like he didn't really hear my full story" There were variations in how well providers related $\mathrm{SDOH}$ to medical symptoms, e.g. "he completely ignored my concerns about mold at home" [asthma case] vs. "His questions centered around possible anxiety this (housing issue) might be causing me." After acknowledgement, fewer providers provided specific information or referrals to address the problem. This lack of follow-up seemed to leave USPs feeling uncomfortable. Both empathic comments and suggestions for actions influenced their sense of activation to manage their health post-visit. CONCLUSIONS: Data from the USP visits indicate that there is a range of attention to and follow up on patient presentation of $\mathrm{SDOH}$ needs by trainees in clinical settings. Issues of both general communication skills, awareness of connection between SDOH and health, and awareness of local resources impacted provider behavior, which then had an effect on relationship with patients. The complex issues involved in addressing SDOH highlights the diverse training needs for learners.

\section{HOW EMPATHY DIFFERS BY MEDICAL SCHOOL CLASS AND GENDER}

Mario A. Scarpinato ${ }^{1}$; Pinky $\mathrm{Jha}^{2}$; Kevin Schlidt ${ }^{1}$; Kate Stark $^{1}$; Daniel Roadman ${ }^{1}$; Devashish Joshi ${ }^{1}$; Sanjay Bhandari ${ }^{1} .{ }^{1}$ Medical College of Wisconsin, Milwaukee, WI; ${ }^{2}$ medical college of wisconsin, Milwaukee, WI. (Control ID \#3172868)
BACKGROUND: Studies have found that empathy in medicine (clinical empathy) can improve patient outcomes. These outcomes include reducing metabolic complications in diabetic patients, increasing their treatment compliance, and improving patient outcomes for the common cold. Despite empathy's beneficial value in medicine, other studies indicate that empathy significantly declines during the third year of medical school and attribute this decrease too many facets of students' lives: fear of making mistakes, training distress, demanding curriculum, conflicting time commitments, sleep loss, and a hostile work environment. Seeking to better understand student experiences with clinical empathy at the Medical College of Wisconsin (MCW), we surveyed third and fourth-year medical students (M3 and M4) using a self-report measure. Our goals were to determine the student's perception of empathy's role in medicine, the quality of teaching on empathy and the best way to teach empathy. We compared the results between M3 and M4 students and between genders.

METHODS: A survey was distributed to M3 and M4 students via a schoolwide email between August 2018 and September 2018. Responses were anonymous, and a response rate of $39 \%$ was achieved. A Chi-square analysis was performed to analyze differences between males and females as well as between M3 and M4 students.

RESULTS: $50 \%$ of M4 students $(n=38)$ believed that working with attending physicians increased their understanding of the importance of compassion and empathy in patient care which was significantly more than the $34.3 \%$ of M3 students $(n=36)(p$-value $=0.034) .100 \%$ of females $(n=78)$ and $89.2 \%$ of males $(n=91)$ believed that providing empathy and compassion in patient care improved patient outcomes $(\mathrm{p}=0.003)$. Gender preferences for empathy education methods also proved to be significantly different. Among females, $2.6 \%$ $(n=2)$ felt that auditorium lectures were an effective method to learn empathy in medical school while $10.8 \%$ of males $(n=11)$ felt it could be effective $(\mathrm{p}=0.035$ ). Additionally, $53.8 \%$ of females and $32.4 \%$ of males felt that standardized patient encounters such as the objective structured clinical exams (OSCE) would be an effective medium for empathy education ( $\mathrm{p}=0.004)$.

CONCLUSIONS: Our institution, and institutions across the country, can use this study to provide support for a change to education on empathy. Our data suggests that the current model of teaching empathy is not working for our students. The lack of awareness among medical students on the value of physician empathy is troubling. Our study provides direction for those changes and possible future intervention: increasing patient interaction and individualizing empathy education. As greater patient interaction appears to increase, not decrease, students' opinions on the value of empathy, and differences in female and male students' perceptions on the value of empathy and how they would like to be taught suggest the opportunity to personalize empathy education in the future.

\section{HOW LONG SHOULD IT TAKE TO PROVIDE HIGH QUALITY PRIMARY AND SECONDARY PREVENTIVE CARE IN THE ERA OF TEAM-BASED MEDICINE AND POPULATION HEALTH?} Justin Porter ${ }^{1}$; Cynthia M. Boyd ${ }^{2}$; Mohammed Reza Skandari ${ }^{1}$; Neda Laiteerapong . ${ }^{1}$ University of Chicago, Chicago, IL; ${ }^{2} \mathrm{JHU}$, Baltimore, MD. (Control ID \#3183683)

BACKGROUND: Primary care providers (PCPs) have always been expected to provide quality preventive and chronic care for each of their patients, but studies have shown that many preventive and chronic care gaps exist. To address these care gaps, new strategies have emerged including, populationlevel approaches for completing preventive care gaps and increasing numbers of non-physician providers in primary care. To understand their implications, we updated previous calculations of the total amount of time a PCP would need to provide preventive and chronic disease care for a panel of 2500 patients on their own, as well as with team-based and population level strategies.

METHODS: We calculated the amount of time per day a PCP would have to spend to complete preventive and chronic care, if the PCP 1) alone, 2) with team-based, 3) with population-level, and 4) with teambased and population-level strategies were used to complete care 
recommendations. We estimated times for a panel of 2500 patients with the age, gender, and racial distribution and chronic disease prevalence of the 2014 adult U.S. population. For preventive care, we used the list of United States Preventive Services Task Force Grade A/B recommendations for non-pregnant adults. We performed a literature review to ascertain the time necessary for a PCP and/or team member (e.g., nurse, medical assistant) to provide evidencebased care. We also collected evidence about population-level strategies for completing recommendations (e.g., mailers). For chronic care, we compiled a list of the 10 most prevalent diseases in adult primary care and performed a literature review to determine the number of PCP visits per year and time required per visit for each condition. We assumed that each chronic disease would require 10 minutes per visit if a specific time was not found.

RESULTS: For a panel of 2500 US patients, it would take a PCP 13.9 hours/day to complete preventive (10.6 hours/day) and chronic care (3.3 hours/day). If team-based and population-level strategies were employed, then PCPs would require 5.3 hours per day to complete preventive (2.0 hours/day) and chronic care (3.3 hours/ day). Preventive care counseling tasks formed the majority of physician time saved, with that time shifted to Dieticians (5.7 hours/day) and Counselors (1.7 hours/day). Population based interventions only saved 0.2 hours/day.

CONCLUSIONS: We found that providing high quality primary care in the U.S. continues to require direct patient contact for longer than the average workday. However, incorporating evidence-based team-based strategies would reduce the work necessary to more feasible levels and allow PCPs to have time to complete administrative tasks, conduct shared decision-making and care for patients with complex medical illness.

HOW MEDICAL EDUCATORS USE ONLINE CURRICULA: DATA ANALYTICS FROM A GRADUATE MEDICAL EDUCATION CURRICULUM DISSEMINATION WEBSITE

John Geyer ${ }^{2}, 1$; Anna Golob ${ }^{2,}{ }^{1}$; Cassandra Song ${ }^{3}$; Mayuree Rao ${ }^{1}$; Joyce E. Wipf $f^{2}, 1$. ${ }^{1}$ University of Washington, Seattle, WA; ${ }^{2}$ VA Puget Sound, Seattle, WA; ${ }^{3}$ VA Puget Sound Health Care System, Seattle, WA. (Control ID \#3183346)

BACKGROUND: Web-based asynchronous applications are increasingly used for dissemination of complex undergraduate and graduate medical curricula. Online analytic applications can provide curriculum developers with valuable data about usage, guiding future content revisions. A population health panel management medical education curriculum, developed at the Seattle Center of Excellence in Primary Care Education at VA Puget Sound (COE), includes electronic medical record best practice, inter-professional casebased discussions and chronic disease didactics. The curriculum, including competency domains and evaluation results of the curriculum, was ported to a website in December 2017 and has been disseminated to a limited group of national and international medical educators for the past 12 months with protected site access.

METHODS: Curriculum content was derived from a previously established population panel management series described elsewhere and in practice with VA-based residents in Internal Medicine and other health professions since 2011 in Seattle primary care and women's clinic. A web development software package was used to generate an HTML5/CSS website with video content hosted on YouTube and embedded in the site. Resources such as didactic PowerPoints, curricula handouts and computer screen shots were placed alongside 1-2 minute video clips. Google Tag Managerand Google Analytics were deployed to track user usage. Content was protected with SSH basic password protection.

RESULTS: 8 local and national sites at the VA and University of
Washington were given access to the website. 407 and 123 views of the home and password protected content pages occurred, respectively, from 1/1/18 - 12/28/18. Session duration averaged 1 minute and 49 seconds. Video content was viewed by 29 users with average view duration of 48 seconds per video. 21 users downloaded curricula resources. There have been positive reviews among a national cohort of medical educators. Comments include "easy to use interface" and "approachable content." One physician educator requested further clarification of one component of the curriculum regarding the integration of inter-professional providers.

CONCLUSIONS: About a fourth of users who visited the home page entered the password protected content domain. Current website restricted access likely limits the ability for users to access content due to password protection. Only a third of users viewed video content or downloaded resources. However, video content was viewed by many users at local and national COE teleconferences. To be determined will be how de novo users interact with site content. Comments, while positive, have directed efforts to clarify key points of curriculum. Future endeavors include broadening population for dissemination by removing password protection, revising content to increase user engagement and developing additional web-based curricula for other topics.

\section{HOW MUCH IS ENOUGH? PHYSICAL ACTIVITY FOR YOUNG ADULT CARDIOMETABOLIC HEALTH}

Heather R. Frank ${ }^{1}$; Hillary Mulder ${ }^{2}$; Karishma Sriram ${ }^{1}$; Taruni Santanam $^{1}$; Asheley C. Skinner ${ }^{3}$; Eliana M. Perrin ${ }^{3}$; Sarah C. Armstrong $^{3,}{ }^{3}$; Eric Peterson ${ }^{2}$; Charlene A. Wong ${ }^{3,2}$. ${ }^{1}$ Duke University, Durham, NC; ${ }^{2}$ Duke University Hospital, Durham, NC; ${ }^{3}$ Duke University School of Medicine, Durham, NC. (Control ID \#3175938)

BACKGROUND: The 2018 Physical Activity (PA) Guidelines recommend 150 minutes weekly for adults. It is unclear if this activity level matches cardiovascular benefits for young adults (YA) transitioning from adolescence. We aim to determine the doseresponse of PA measures on cardiometabolic health measures in YA. METHODS: We examined YA (20-29 years) in the 2003-2016 National Health and Nutrition Examination Survey. Primary exposures were objective (accelerometer) and self-report weekly mean minutes of moderate/vigorous physical activity (PA). Primary cardiometabolic outcomes were systolic blood pressure (BP), diastolic BP, total cholesterol, high-density lipoprotein (HDL), body mass index (BMI), and maximal oxygen uptake ( $\left.\mathrm{VO}_{2} \mathrm{Max}\right)$. The dose-response relationship between each PA exposure and cardiometabolic outcome was assessed with unadjusted spline analyses; each pairing was tested for non-linearity using natural cubic splines, with non-linear relationships converted to 2-part piece-wise linear splines. Sex stratified outcomes were modeled using multivariable linear regression adjusting for sociodemographic characteristics. Mean estimated change is presented for 150-minute PA dose increases.

RESULTS: YA (N=6587) were $50 \%$ female, $59 \%$ white, $13.4 \%$ black. Among females, a slope change of the unadjusted doseresponse curve for self-report PA occurred at 600 minutes for HDL, 725 minutes for BMI. With adjustment, a 150-minute increase in objective PA was associated with increased HDL of $5.4 \mathrm{mg} / \mathrm{dL}$ $(\mathrm{p}=0.04)$ and reduced BMI of $1.8 \mathrm{~kg} / \mathrm{m}^{2}(\mathrm{p}<0.001)$; a 150 -minute increase in self-reported PA was associated with increased HDL of $0.98 \mathrm{mg} / \mathrm{dL}(\mathrm{p}=0.01)$ and reduced BMI of $0.23 \mathrm{~kg} / \mathrm{m}^{2}(\mathrm{p}=0.02)$. Among males, the unadjusted dose-response curve slope changed for objective PA and BMI at 100 minutes. With adjustment, a 150minute PA increase was associated with a $\mathrm{VO}_{2}$ max increase of 5.4 $\mathrm{ml} / \mathrm{kg} / \mathrm{min}(\mathrm{p}<0.001)$. We found inconsistent associations between PA and systolic BP, diastolic BP, and total cholesterol for males and females. 
CONCLUSIONS: Achieving the advised 150 weekly minutes of PA was associated with improved $\mathrm{HDL}$ and $\mathrm{BMI}$ in females and $\mathrm{VO}_{2}$ max in males. Some relationships indicated lower marginal cardiometabolic benefit of PA after a certain point. Future guidelines can account for the unique gender dose-response relationships among young adults.

\section{Figure 1: Dose Response Relationship between Objective Physical Activity and BMI among Young Adults, Unadjusted Models}

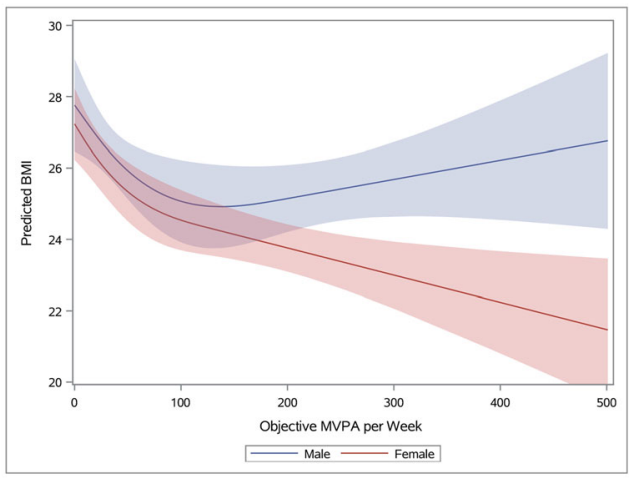

MVPA: Moderate-to-vigorous physical activity; BMI: Body Mass Index

\section{HOW PRECEPTORS IN AN INTERNAL MEDICINE CONTI-} NUITY CLINIC DEVELOP TRUST IN RESIDENTS

John Penner; Karen E. Hauer; Katherine Julian; Leslie Sheu. University of California, San Francisco, San Francisco, CA. (Control ID \#3186131)

BACKGROUND: Trust is a growing areas of interest because it informs supervision. Most research on trust focuses on the inpatient setting. However, trainee responsibilities, team structure, teaching, and trainee-supervisor relationships differ in continuity clinics. As medical education accreditation bodies increasingly emphasize the need for improved continuity clinic education, it is important to understand how trust develops in this setting to provide trainees developmentally-appropriate graded autonomy. We aim to understand how faculty preceptors in internal medicine continuity clinics develop trust in residents, and how trust affects supervision.

METHODS: This is a qualitative study using thematic analysis of semi-structured interviews with continuity clinic preceptors in an internal medicine residency program. Interviews address preceptors' views of trust, resident characteristics that build or erode trust, and supervisory behaviors that inform and vary with trust.

RESULTS: We have interviewed eight preceptors; data collection is ongoing. Preliminary results show preceptors determine trust through residents' knowledge of patients, clinical judgment, presentation organization, and reliability in follow-up. Longitudinal relationships and observation of residents both conducting patient encounters and handling virtual care (lab results, patient messages) inform trust. Resident openness about knowledge gaps and desire for feedback support trust development. With greater trust, preceptors promote resident autonomy by probing less about background information, patient history, and physical exam findings, providing less directive guidance on care plans, and shifting their focus towards teaching. Challenges to developing trust include preceptors' lack of familiarity and limited contact with residents' patients and limited preceptor continuity across resident clinic sessions. Despite noting their importance, preceptors cite difficulty in observing residents, especially their execution of virtual care, and engaging in feedback conversations as often as they would like to.

CONCLUSIONS: Factors unique to the continuity clinic setting, including longitudinal faculty interactions with residents and the growth of virtual care, influence trust formation. Areas of tension for preceptors lie in the fact that behaviors and interactions most conducive to trust development are also among the most difficult to observe and experience. Strategies to create continuity in oversight of in-person encounters and virtual patient care, foster longitudinal relationships, and normalize direct observation and feedback can align trust and supervision in continuity clinics of internal medicine residency programs.

\section{HOW SHOULD WE CARE FOR PATIENTS WITH HIV AND COMORBIDITIES? A MULTISITE QUALITATIVE STUDY OF HIV CARE IN US DEPARTMENT OF VETERANS AF- FAIRS}

Barbara G. Bokhour ${ }^{1,2}{ }^{2}$; Gemmae Fix ${ }^{1,}{ }^{2}$; Steven Asch ${ }^{8}$; Rendelle Bolton $^{1}$; Kelly Dvorin ${ }^{1}$; Allen L. Gifford ${ }^{9}{ }^{3}$; Justeen Hyde ${ }^{1}$; Keith McInnes ${ }^{1,2}$; Amanda Midboe ${ }^{7}$; Victoria A. Parker ${ }^{1,}{ }^{4}$; Avy A. Skolnik $^{1,5}$; Juliet $\mathrm{Wu}^{1}$; Michael Ohl ${ }^{6} .{ }^{1}$ Edith Nourse Rogers Memorial Veterans Hospital, Bedford, MA; ${ }^{2}$ Boston University School of Public Health, Bedford, MA; ${ }^{3}$ Boston University, Boston, MA; ${ }^{4} \mathrm{U}$. of New Hampshire, Durham, NH; ${ }^{5}$ University of Massachusetts Amherst, Amherst, MA; ${ }^{6}$ Iowa City VA Healthcare System, Iowa City, IA; ${ }^{7}$ VA Palo Alto Health Care System, Menlo Park, CA; ${ }^{8}$ VA/ Stanford, Menlo Park, CA; ${ }^{9}$ Boston VA Healthcare System, Boston, MA. (Control ID \#3185155)

BACKGROUND: HIV is a chronic manageable condition, however as patients age comorbidity management often becomes the highest priority. Whereas care for these patients previously was concentrated solely in infectious disease or HIV specialty clinics, there has been a shift towards co-management between HIV specialty care and primary care. It is unclear how patients in HIV specialty care only vs. comanagement fare with regards to HIV and comorbidity quality of care. Moreover, care provided in patient-centered medical homes $(\mathrm{PCMH})$ is associated with higher quality. Thus, we sought to understand how variation in HIV care delivery was aligned with PCMHprincipled care, and how comorbidity and HIV care quality relate to the structures and processes of care.

METHODS: We profiled 144 US Department of Veterans Affairs Medical Centers (VAMCs) on HIV quality (viral control), physical comorbidity care quality (e.g. HbA1C) and mental health care quality (e.g. guideline concordant depression care) using medical record data. We selected a purposive sample of 8 VAMCs high and low in these quality indicators. We conducted in-depth qualitative site visits, blinded to sites' quality designation, including: 1) patient interviews $(\mathrm{n}=60) ; 2)$ HIV care team interviews $(\mathrm{n}=60) ; 3)$ direct observation of clinic processes and team interactions $(\mathrm{n}=22)$; and 4$)$ direct observations of patient-provider clinical encounters $(n=45)$. We coded all using both a priori constructs from PCMH principles and emergent codes. We constructed individual site templates to reflect care according to PCMH principles, observed processes and structures of care. We then revealed quality profiles and examined relationships between templated descriptions and level of quality.

RESULTS: Across variation in sites' structures and process of care, three main findings emerged. First, the sites highest and lowest in both HIV and comorbidity quality demonstrated clear differences in provision of PCMH-principled care. The highest site provided greater team-based care, comprehensiveness of services, patient-centered care, data-driven care and engaged in continuous improvement. Second, sites with higher HIV care quality attended more to psychosocial needs, either via HIV clinic team-based care or through coordination with psychosocial services outside the clinic. Third, comorbidity quality was not directly related to PCMH principles. Rather, comorbidity care was better managed in sites that had explicitly created structures and consistent processes for such care, regardless of 
whether care was provided in HIV or primary care clinics.

CONCLUSIONS: Our multisite study using several sources of qualitative data shows that comorbidity and HIV care quality relate to different structures and processes of care. Providing care aligned with PCMH principles, integrating psychosocial needs into care, and establishing explicit consistent approaches to comorbidity management are critical for optimal HIV and comorbidity care quality and outcomes.

\section{HOW WELL DO NEWLY ARRIVED INTERNS INTERPRET ECG'S}

Leonardo L. Zelaya Castillo; Adam Horblitt; Gabriela Andries; zahava Farkas; Michael Karass; Jason Goutis; Rebecca Newman; Merita Shehu; Melissa Gennarelli; Leanne Forman; Srikanth Yandrapalli; Christopher Nabors. Westchester Medical Center, Valhalla, NY. (Control ID \#3161259)

BACKGROUND: Resident physicians are often the first clinicians to review an ECG and to make critical initial management decisions. Thus, it is essential that residents are able to rapidly interpret urgent findings on ECG.

METHODS: 17 ECG's which were believed to demonstrate either normality or a primary diagnosis were identified. This content was based upon the ECG types identified by Jablonover, and StagnaroGreen. 1 The ECG's were pilot tested by 3 chief residents and 2 faculty members for clarity and ease of diagnosis. All 34 interns were asked to take the test over a two day span during the second month of training without using any reference materials.

RESULTS: Details in table 1. Trainees rated their preparedness to interpret ECG's (1, not well to extremely well, 5) at mean 2.34 and how intensively their medical school had focused on ECG training (1, not intensively to very intensively, 5) at 2.58. In medical school 29 respondents $(88 \%)$ had a moderate or considerable amount of selflearning and $28(85 \%)$ a small or moderate amount of classroom teaching and lectures; 30 (94\%) had very little to only a moderate amount of hands on clinical training or small groups sessions 32 (97\%). Compared to those who had a moderate amount of classroom training in medical school, those who had very little scored lower (59\%, SD 25\%) versus Mean: $75 \%$, SD 17\%). Of those who rated their preparedness to interpret ECG's at the second highest or highest level on a five point scale, the mean score was $90 \%$, SD $10 \%$. Of those who believed their medical school focused on ECG training at the second highest or highest intensity, mean scores were $72 \%$ SD $19 \%$.

CONCLUSIONS: In this study, the ability to accurately identify important ECG patterns varied widely. Testing of early trainees may permit a targeted approach to teaching ECG's. Further study may permit an association to be identified between types of preresidency ECG training experiences and subsequent competence in ECG interpretation during residency training.

\section{HUNGER IS HEALTH: THE ASSOCIATION BETWEEN FOOD INSECURITY AND TYPE 2 DIABETES IN AN URBAN, SAFETY NET HOSPTIAL PATIENT POPULATION}

Meghana Anugu; Emily A. Kaltz; Jada C. Bussey-Jones. Emory University, Atlanta, GA. (Control ID \#3185801)

BACKGROUND: Food insecurity remains a pressing public health issue. Literature suggests food insecure individuals are at greater risk for developing type 2 diabetes mellitus (T2DM), a condition associated with increasing morbidity and mortality in the United States. We aim to estimate the prevalence of food insecurity and how it relates to T2DM status in an urban, safety net hospital setting.
METHODS: We conducted a cross-sectional survey of 323 patients in the Primary Care Center at Grady Memorial Hospital (GMH), in Atlanta, GA from January 2016 to March 2017. Survey included demographic information including age, sex, race, household income, number of people in the household, and zip code, items to assess food insecurity, SNAP utilization, and diabetes status. An affirmative response to either question in the USDA 2-item food security screener was classified as food insecure, which has been previously validated as $97 \%$ sensitive. Primary outcome was a combined variable of prediabetes and type 2 diabetes.

RESULTS: The overall prevalence of food insecurity among the study sample was $53.5 \%$. Considering diabetes status, food insecurity status was $37.5 \%$ and $62.5 \%$ among non-T2DM and T2DM patients, respectively. In multivariable logistic regression analyses, the odds of having diabetes was 2.6 times greater (95\% CI 1.7-4.1) among food insecure patients compared to food secure patients. The effect remained statistically significant after controlling for sex, race, and income [2.6 (95\% CI 1.5-4.4)]. Gender significantly modified the association between food insecurity and diabetes with an increased odds of diabetes among men with food insecurity [odds ratio 5.1 (95\% CI 2.5-9.8)] compared to women [odds ratio 1.5 (95\% CI 0.8 2.8)].

CONCLUSIONS: The prevalence of food insecurity in our sample was greater than four times the national average of $11.8 \%$. This likely reflects the disparities and needs facing the patients of an urban, safety net hospital setting. There is a clear association between food insecurity and T2DM in the patient population studied. Further, our finding that gender significantly modified food insecurity and T2DM may reflect the differences in coping mechanisms between men and women who are in poverty and food insecure. For example, it has been suggested in the literature that men who are food insecure are less likely to access SNAP services. Given the high prevalence of food insecurity and the association between food insecurity and T2DM, we assert that initiatives to address T2DM need to consider food insecurity as an important and potentially intervenable variable. Initiatives to address this may include screening for food insecurity in patients with diabetes and food as medicine as an adjunct to diabetic treatment plans.

\section{HYPERTENSION CARE DELIVERY IN RESOURCE- LIMITED SETTINGS: A LITERATURE REVIEW}

$\underline{\text { Daniel C. Mays }}{ }^{1}$; David J. Heller ${ }^{1}$; Rose C. Nanyonga ${ }^{2} .{ }^{1}$ Icahn School of Medicine at Mount Sinai, New York, NY; ${ }^{2}$ Clarke International University, Kampala, Uganda. (Control ID \#3185472)

BACKGROUND: Hypertension, along with other noncommunicable diseases, poses a significant burden of morbidity and mortality in low- and middle-income countries (LMICs) around the world. Health systems in LMICs are faced with the challenge of delivering care to prevent and treat such diseases with limited resources. There is a growing body of evidence on unique approaches to managing hypertension in resource-limited health systems, but these data have not been formally synthesized. This literature review aims to summarize high quality evidence through a systematic exploration of existing literature on hypertension care delivery in resourcelimited settings.

METHODS: We conducted a systematic literature search using primarily the PubMed and Scopus databases along with grey literature databases. Title and abstract keywords as well as Medical Subject Heading (MeSH) terms focused the search on interventions addressing non-obstetric systemic arterial hypertension in adults in LMICs throughout the Global South. Titles and abstracts were systematically reviewed and rated according to relevance to the study aim. The most relevant publications underwent a focused in-depth analysis. 
RESULTS: The search yielded 857 unique and relevant publications issued between 1970 and 2018. After title and abstract review, twenty highly relevant publications were selected for in-depth analysis, and among these were twelve original research studies and eight literature reviews. The original research studies evaluated interventions including task-shifting, community-based health insurance and clinical decision-making support tools to manage hypertension. The majority of these studies demonstrated significant blood pressure reduction in the intervention groups. Seven of the eight reviews were systematic and their topics included task-shifting, health-oriented mobile technology (mHealth), lifestyle change and community-based interventions to address hypertension. The strongest evidence supports, firstly, shifting care delivery tasks to non-physician health workers who address hypertension as part of organized teams with care escalation pathways according to clinical qualification. Secondly, there is strong evidence supporting decision-support tools and standardized clinical guidelines, commonly through novel utilization of technology.

CONCLUSIONS: Regarding hypertension care delivery in resource limited-settings, published evidence notably supports task-shifting within organized care teams and utilizing clinical decision-making support tools. Where health systems face limited capacity to manage the population burden of hypertension, these approaches were both feasible and effective. Given the range of contexts in which they were implemented, these approaches are likely applicable to a variety of health system settings in both resource-rich and resource-limited countries.

\section{HYPERTENSION MANAGEMENT IN AN INTERNAL MEDI- CINE RESIDENT CLINIC: A QUALITY IMPROVEMENT INITIATIVE}

Elaine Hutchison ${ }^{1}$; Charlotte Lin ${ }^{1}$; Christine Moorehead ${ }^{2}$; Emily D. Fondahn ${ }^{3}$. ${ }^{1}$ Washington University, St. Louis, MO; ${ }^{2}$ Barnes-Jewish Hospital, St. Louis, MO; ${ }^{3}$ Washington University, St Louis, MO. (Control ID \#3181892)

BACKGROUND: Hypertension is an important modifiable risk factor for cardiovascular disease. The Barnes-Jewish Hospital Primary Care Medicine Clinic is an academic internal medicine resident clinic affiliated with a tertiary referral center with an average 1960 patient visits per month. Clinic data shows about $62 \%$ of our patients have a diagnosis of hypertension. Our quality improvement project sought to improve the percentage of hypertensive patients with controlled blood pressure from a baseline of $60.7 \%$ to $80.0 \%$.

METHODS: We defined controlled blood pressure as less than systolic $140 \mathrm{mmHg}$ and diastolic $90 \mathrm{mmHg}$. Baseline and postintervention data were collected through our electronic medical record (EMR). Blood pressure data was not readily available prior to June 2018 due to a change in EMR platforms. Interventions using a Plan-Do-Study-Act (PDSA) model were developed and prioritized in several multi-disciplinary meetings. The first PDSA cycle involved changing the nurse hypertension clinic referrals from paper to electronic to increase use of this resource by residents. In this clinic, a nurse measures blood pressure, consults with a resident if blood pressure is uncontrolled, and educates patients regarding lifestyle changes and blood pressure goals. Patients can follow up weekly until they have reached their goal blood pressure. Clinic nurses, pharmacists, residents, attendings, and information technology were involved in making this change.

RESULTS: The baseline percentage of patients with controlled hypertension was $60.7 \%$ from June to mid-September 2018. In September, when the electronic referral system was implemented, the percentage of patients with controlled hypertension improved to $64.5 \%$ but dropped to $61.3 \%$ in October and $57.0 \%$ in November. The average number of visits scheduled in the nurse hypertension clinic monthly prior to EMR change was 150 . Post-EMR change, there were 55 scheduled visits in June 2018, increasing to 95 in September, 139 in October, and 127 in November. Improving blood pressure control through this program is complicated by a high no-show rate to these appointments: $43.6 \%$ in June 2018 increasing to $50.3 \%$ in October and $48.0 \%$ in November. Prior to June 2018, the average no-show rate was $37 \%$.

CONCLUSIONS: Our initial PDSA cycle increased the number of referrals to the nurse hypertension clinic to the pre-EMR transition baseline without sustained improvement in blood pressure control. We anticipate more substantial improvement as residents and staff become increasingly familiar with the referral process. The high noshow rate limits the effectiveness of this intervention and future PDSA cycles should consider this as an area for improvement. Our next PDSA cycles will focus on developing a hypertension documentation template within the EMR to facilitate communication between residents during transitions of care and distribution of ambulatory blood pressure monitors to patients.

\section{IDENTIFYING CAUSES OF MISSED COLONOSCOPY AP- POINTMENTS AT A SAFETY NET HOSPITAL}

Michael A. Yu; Gordon T. Robbins; Saraubh Chawla; Erica Heiman. Emory University School of Medicine, Atlanta, GA. (Control ID \#3183667)

BACKGROUND: In the US, colorectal cancer (CRC) remains the 4th most common cancer among men and women, and the 2nd leading cause of cancer deaths. CRC-related mortality has decreased due to better CRC screening rates. Nationally, $65 \%$ of the population eligible are up-to-date with recommended CRC screening. Grady Health System is a large, urban safety net hospital in Atlanta, GA. Overall CRC screening rates of Grady Primary Care Center patients is $55 \%$. In an effort to improve CRC screening rates, we have implemented multiple interventions including offering Fecal Immunochemical Tests, educating providers via resident-run teaching sessions, engaging patients in waiting room conversations about CRC screening options, and streamlining our EMR. Despite this, our screening rates are still below the national average. One issue identified was high no-show rates to colonoscopy appointments. No-show rates from 2 other safety net systems were $27-29 \%$. We hypothesize various contributors to this including scheduling issues, socioeconomic hardship, health illiteracy, challenges with completing colon prep, and inadequate resources for transportation. We explored this further by surveying patients with incomplete colonoscopy appointments.

METHODS: After we achieved IRB determination, we worked with the Grady Outpatient Endoscopy Center to identify patients who did not complete their colonoscopy appointments. We contacted these patients and, using a scripted questionnaire with open and closedended questions, surveyed them about why they were unable to complete their colonoscopies.

RESULTS: Our preliminary data reflects incomplete screening colonoscopies over a one-month period. There was a total of 30 incomplete colonoscopy visits and we were able to contact and survey 25 of these patients. Of these, $92 \%$ were no-shows, whereas the other $8 \%$ were incomplete prep or wrong arrival time. $92 \%$ of patients surveyed were aware of the appointments and similarly, $92 \%$ felt that they were provided with adequate notice. Transportation was cited as a problem for $56 \% .20 \%$ of patients missed due to personal health problems, while $28 \%$ missed due to family obligations or emergencies. $16 \%$ cited financial issues, including inability to afford copay or prep costs. $24 \%$ of patients had scheduling issues, such as doublebooked appointments. $20 \%$ of patients had problems with prep being incorrectly prescribed or not prescribed at all. 
CONCLUSIONS: At Grady, patients failed to attend colonoscopy appointments despite possessing knowledge of the schedule and adequate reminders. Patients cited transportation, personal health problems, finances, and family obligations as barriers. However, double booking or failure to prescribe prep suggest that improvements can be made in execution from the health system perspective. Furthermore, providing financial aid may benefit those unable to afford visit copay and colon prep costs. We hope to expand on this data and use it to implement changes to reduce no-shows and increase CRC screening rates.

\section{IDENTIFYING OUTLIER PRIMARY CARE PRACTICES TO REDUCE HIGH-COST UTILIZATION: A POSITIVE DEVI- ANCE APPROACH TO UNDERSTANDING THE PERSIS- TENCE OF HIGH-PERFORMANCE IN THE PATIENT- CENTERED MEDICAL HOME}

Ashok Reddy $^{2,}{ }^{1}$; Linnaea C. Schuttner ${ }^{2}$, ${ }^{1}$; Eric Gunnink ${ }^{2}$; Karin M. Nelson ${ }^{2,1}$. ${ }^{1}$ University of Washington, Seattle, WA; ${ }^{2}$ Department of Veterans Affairs, Seattle, WA. (Control ID \#3173936)

BACKGROUND: Veterans Health Administration (VHA) and Medicare measure primary care clinic performance based on rates of high-cost utilization including emergency department (ED) visits and hospitalizations. Yet, our understanding of high performing primary care clinics ("positive deviants") associated with low utilization rates is limited. Our primary objectives were to determine: (1) how positive deviant clinics who exhibited low rates of ED visits or hospitalizations in the baseline year persisted in subsequent years (2) the impact of adjusting for patient or clinic characteristics on the persistence of high performance among VHA primary care clinics.

METHODS: We used a cross-sectional design using annual data from 2012-2017 from the VHA Corporate Data Warehouse. We first calculated the observed over expected $(\mathrm{O} / \mathrm{E})$ ratio of hospitalizations (or ED visits) by clinic site for a baseline year. Expected utilization was calculated as patients per site multiplied by the national mean hospitalization or ED visits per year. We limited ED visit analysis to clinics with on-site EDs. We defined high-performance as the lowest $10 \%$ of clinics by $\mathrm{O} / \mathrm{E}$ ratio in a baseline year. Next, we determined the number of high-performing clinics that persisted in the top decile in the following 5 years. Finally, we used a mixed effects model to determine the impact of patient (age, sex, race/ethnicity, comorbidity) and clinic (size, location) characteristics.

RESULTS: We analyzed hospitalizations at 899 clinics and ED visits at 144 clinics. A greater proportion of patients at high-performing clinics were younger, white, urban residents, and were cared for at communitybased outpatient clinics. Of clinics initially identified as high-performing in 2012, we found 40 of 89 clinics for hospitalizations, and 9 of 25 clinics for ED visits were persistent high-performers. For this baseline year, highperforming clinics had on average 18 hospitalizations per 1000 patients and 229 ED visits per 1000. Between 2013-17, high-performing clinics had on average 14 hospitalizations and 221 ED visits per 1000. In 2012, clinics consistently below the top performers had 123 hospitalizations per 1000 patients and $661 \mathrm{ED}$ visits per 1000. Between 2013-17, these clinics had 116 hospitalizations and $668 \mathrm{ED}$ visits per 1000. After adjusting for patient and clinic characteristics, we did not find persistent primary care clinic performance outliers in either outcome of ED visit or hospitalizations.

CONCLUSIONS: Our findings suggest that many clinics persist as highperforming outliers with persistent low ED and hospital utilization. However, most of this persistence is related to non-modifiable patient and clinic-level characteristics. This implies that the usual next step in a positive deviance approach - qualitatively studying high performers to generate hypotheses about why these practices are successful - may not be useful in identifying strategies to reduce high-cost utilizations.

\section{IDENTIFYING RACIAL/ETHNIC DISPARITIES IN INTERHOS- PITAL TRANSFER}

Evan Shannon ${ }^{1}$; Jeffrey L. Schnipper ${ }^{1}$; Stephanie Mueller ${ }^{2} .{ }^{1}$ Brigham and Women's Hospital, Boston, MA; ${ }^{2}$ Brigham and Women, Boston, MA. (Control ID \#3185287)

BACKGROUND: Racial/ethnocultural inequities have been demonstrated throughout our health care system. Their presence among patients who undergo interhospital transfer (IHT) to receive more specialized care is poorly understood. We evaluated the association between patient race/ethnicity and IHT to identify potential disparities in this care transition.

METHODS: We performed a cross-sectional analysis of 2016 data from the National Inpatient Sample (NIS), the largest publicly available allpayer inpatient health care database in the United States which yields national estimates of hospital inpatient admissions. All adult patients were included. The primary outcome was IHT, defined as transfer to another acute care facility. The primary predictor was race, categorized as traditionally underserved racial/ethnic minority groups (Black, Hispanic, Native American) versus other (White, Asian, Other). We used logistic regression models to obtain odds ratio IHT by race, adjusting for patient and transferring hospital characteristics, including patients' age, sex, and insurance, median household income by zip code, mortality risk by diagnosis-related group (DRG), Elixhauser comorbidity index, hospital teaching status, region and bedsize. We performed secondary analyses using individual race/ethnicity categories.

RESULTS: There were a total of $6,037,727$ unique admissions in the cohort representing 25,238.650 admissions nationally. 120,396 (2.4\%) patients were transferred to an acute care facility. Among those transferred, $46.2 \%$ were female, $72.7 \%$ were white, $13.3 \%$ were Black and $8.4 \%$ were Hispanic. Minority patients had a lower odds of transfer compared to other groups (OR $0.75,95 \%$ CI $0.72-0.78$ ). Multivariable logistic regression adjusting for patient characteristics also revealed a lower odds of transfer (OR $0.83,95 \%$ CI $0.79-0.86$ ). However, when further adjusted for hospital characteristics, this association was no longer significant. In secondary analysis, Hispanic ethnicity was associated with lower adjusted odds of transfer compared to White patients (OR 0.94, 95\% CI 0.90-0.98).

CONCLUSIONS: In this nationally representative sample of patients, we found that minority race/ethnicity, and Hispanic ethnicity in particular, was associated with lower odds of IHT. These findings remained after adjusting for patient characteristics, but lost significance after also adjusting for hospital characteristics, suggesting that existing racial disparities may be mitigated in part by factors affecting the hospitals to which minority patients present. Additional research is needed to further explore the drivers of these inequities.

\section{IDENTIFYING RISK FACTORS FOR HYPOGLYCEMIA TO PREVENT ADVERSE OUTCOMES AMONG HOSPITALIZED PATIENTS}

Corinne F. Levitus ${ }^{3}$; Julie Anne L. Gemmill ${ }^{3}$; Leena Shah ${ }^{1}$; Marissa A. Wolfe $^{3}$; Alexander Gee ${ }^{3}$; David T. Harris ${ }^{2}$; Nancy A. Wichtendahl ${ }^{2}$; Barbara M. Mills ${ }^{2}$; Joshua D. Miller ${ }^{3} .{ }^{1}$ Montefiore Medical Center Albert Einstein, Paramus, NJ; ${ }^{2}$ SUNY Stony Brook University Hospital, Stony Brook, NY; ${ }^{3}$ Renaissance School of Medicine at Stony Brook University, Stony Brook, NY. (Control ID \#3162799)

BACKGROUND: Diabetes affects 30.3 million people in the US, and $25 \%$ of total hospital days are incurred by patients with diabetes. Among hospitalized adults, diabetes is considered a chronic secondary diagnosis. As the population of patients with diabetes grows, so does the potential for adverse events during hospitalization. Previous studies have shown that both hyper- and hypoglycemia are associated with poor outcomes in broad populations of hospitalized patients, including increased morbidity 
and mortality. Hypoglycemia occurs in $3.5-10.5 \%$ of patients on the general medical floors and in $12-18 \%$ among patients with diabetes, owing in part to focus on glycemic control in patients with diabetes. Moderate glycemic control prevents microvascular complications and may reduce long-term cardiovascular complications; however, results from the ACCORD trial have shown that very intensive glycemic control may increase risk of mortality. Risk of hypoglycemia is especially pronounced in elderly patients with advanced duration of diabetes. As the population of adults with diabetes continues to expand, emphasis should be placed on identifying patients at risk for hypoglycemia and individualizing treatment strategies and glycemic targets to avoid adverse outcomes. We sought to identify patients with hypoglycemia preceding acute clinical deterioration requiring urgent response by our institution's Rapid Response Team (RRT), a multidisciplinary modality that aims to rapidly assess, monitor, and reverse clinical deterioration.

METHODS: We performed a retrospective chart review of three years of data acquired by our institution's RRT. We identified 95 hospitalized patients who underwent a "rapid response" due to hypoglycemia (pointof-care capillary glucose or serum glucose $<70 \mathrm{mg} / \mathrm{dL}$ ). We performed a multivariate analysis to identify contributors to poor outcomes (defined as all-cause mortality) following a hypoglycemic event.

RESULTS: Patients found to have significantly higher all-cause mortality following RRT for hypoglycemia $(\mathrm{p}<0.05)$ were 65 years or older, had lower blood pressure, lower median hemoglobin A1c, higher AST and INR, longer hospital length of stay, were more likely to be NPO, and had a higher percentage of having an RRT in the morning. Patients who had a glucose level $<41 \mathrm{mg} / \mathrm{dL}$ at time of RRT had higher rates of insulin use during admission when compared to individuals with higher glycemia.

CONCLUSIONS: As the diabetes epidemic worsens and more patients are hospitalized with the disease, it is critical that clinicians identify and manage risk factors for hypoglycemia. Our study identified several risk factors that were associated with all-cause mortality. Our findings support previous research that tight glycemic control can be detrimental to elderly patients. Based on our results, further research is needed to identify systems-based strategies for risk-stratification and prevention of inpatient hypoglycemia and improvement of patient safety.

\section{IDENTIFYING SPECIFIC DRIVERS OF PHYSICIAN AND AD- VANCED PRACTICE PROVIDER BURNOUT ACROSS DIVI- SIONS IN A DEPARTMENT OF INTERNAL MEDICINE}

Elisha L. Brownfield; Benjamin Kalivas. MUSC, Charleston, SC. (Control ID \#3183979)

BACKGROUND: Burnout in physicians is well described and has consistently been shown to negatively impact the quality of care delivered, provider productivity and frequency of medical errors. The factors that contribute to burnout are numerous and their specific impact on providers can vary. Reducing burnout and in turn improving provider engagement are vital tasks of any division, department and health care system. Shanafelt et al. have laid out a structured approach identifying nine areas as drivers of burnout and deterrents of engagement. It is unclear how these drivers affect providers from different subspecialties across large departments.

METHODS: The Department of Medicine at a medium-sized academic health system administered the Maslach Burnout Inventory and Areas of Worklife survey and determined that a more detailed assessment of specific factors contributing to burnout was necessary. Using the framework laid out by Shanefelt et al., a focus group of departmental leaders created a survey and distributed this to 261 physician and advanced practice providers in the department. Likert scale assessment of 14 potential burnout drivers, as well as opportunity for free-text comments were included. Results were analyzed by division and compared across the department. Free text answers were independently reviewed by two team members for theme extraction and differences adjudicated.
RESULTS: The survey was completed in 2018 by $124 / 261(47.5 \%)$ eligible Department of Medicine providers. Among burnout drivers, productivity pressure and not being heard by leadership were consistently rated as "moderately-highly" contributing to burnout across all 9 divisions of the department. Following these top two concerns, there was greater heterogeneity in factors contributing to burnout between different divisions. The free-text comments were analyzed and used to create other potential action items.

CONCLUSIONS: The growing problem of physician burnout must be addressed at all levels of health care. In a recent systematic review, organization-directed interventions were associated with greater effectiveness at reducing burnout than those directed at individuals. There is limited literature supporting specific strategies at a departmental level. Internal medicine is a diverse field, with a variety of subspecialties, resulting in a wide variation in practice structures and personalities. Although some of the themes are consistent, our study supports the hypothesis that factors contributing to burnout are different between divisions. Undertaking this process has allowed our Department to generate a list of action items to pursue in the interest of mitigating burnout, and we will follow the impact on physician burnout over time.

\section{IMPACT OF A CANCER URGENT CARE CLINIC ON REGION- AL EMERGENCY DEPARTMENT VISITS}

Arthur Hong $^{1}$; Thomas Froehlich ${ }^{1}$; Stephanie Clayton Hobbs ${ }^{1}$; Simon Craddock Lee ${ }^{1}$; Ethan Halm ${ }^{2} .{ }^{1}$ University of Texas Southwestern Medical Center, Dallas, TX; ${ }^{2}$ Univ of TX Southwestern Med Ctr, Dallas, TX. (Control ID \#3159926)

BACKGROUND: Several cancer centers have created urgent care clinics (UCC) to address commonly anticipated issues that arise for adults with newly-diagnosed cancer. There is little rigorous evaluation of whether UCCs reduce emergency department (ED) visits, or serve as conduits to the ED. Interest in reducing early expenditures for cancer patients is spurred by the Oncology Care Model payment demonstration project from Centers for Medicare and Medicaid Innovation. Our institution's oncology practice created a UCC in May 2012, open from M-F, 8AM5PM.

METHODS: Interrupted time series analysis of regional ED visits generated within 180 days after a diagnosis of cancer. We linked cancer registry patients to a regional database of $98 \%$ of non-federal hospitals, to quantify near-comprehensive ED and hospital use. We estimated adjusted monthly ED visit rates, with a denominator of patient-months to account for patient deaths within the 180 -day period. We used a 6-month intervention phase-in (May-Oct 2012). We compared weekday ED visits to control weekend ED visits when the UCC was closed.

RESULTS: We identified 33,316 adults ages $18+$, contributing 17,835 ED visits within 180 days after cancer diagnosis, between January 1, 2009 and December 31, 2016. Median age was 60 years; $47.2 \%$ were female, $65.2 \%$ were non-Hispanic white, and $19.1 \%$ had advanced-stage cancer at the time of diagnosis. The monthly weekday ED visit rate was growing at 0.43 visits per 1,000 patient-months $(95 \% \mathrm{CI}: 0.29,0.57)$ prior to the UCC, then fell to 0.19 (95\% CI: $0.11,0.28)$ afterwards ( $\mathrm{p}=0.007)$. Monthly weekend ED visit rate growth was initially 0.08 (95\% CI: $-0.03,0.19)$ and was unchanged after the UCC, at 0.05 (95\% CI: -0.02, 0.13; $\mathrm{p}=0.533$ ). By December 2016, this amounted to a difference of 15.34 fewer weekday ED visits than predicted by pre-UCC trends $(95 \% \mathrm{CI}$ : $4.78,25.91)$. One out of every eight patients who visited the ED also visited the UCC.

CONCLUSIONS: Creation of an UCC was associated with a halving of the adjusted growth rate of ED visits, with no change in non-targeted ED visits. However fewer than 1 in 8 patients who visited the ED also used the UCC. This promising finding, despite use by only a limited proportion of patients, suggests the potential for further reductions in ED use. 
Monthly emergency department visit rates before and after creation of an urgent care clinic (UCC) for cancer patients

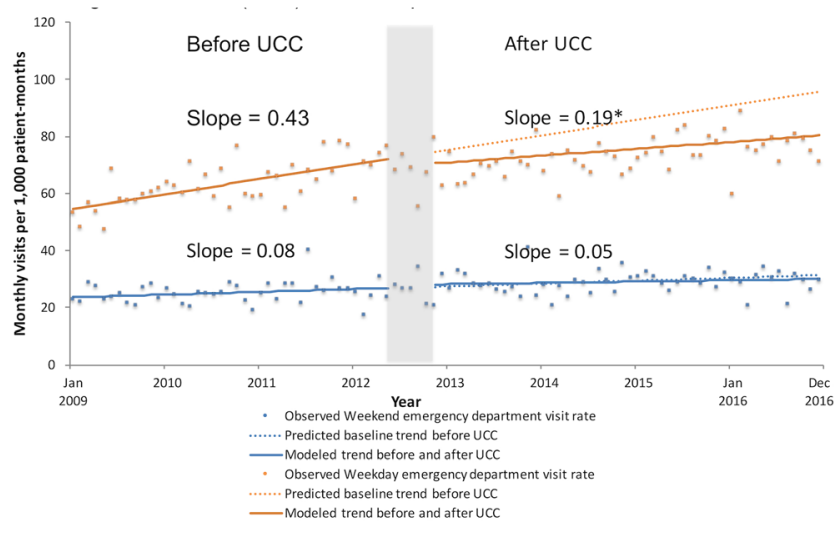

IMPACT OF A STATE OPIOID PRESCRIBING LIMIT AND ELECTRONIC MEDICAL RECORD ALERT ON ACUTE OPIOID PRESCRIPTIONS : A DIFFERENCE-INDIFFERENCES ANALYSIS

Margaret Lowenstein ${ }^{1,}{ }^{2}$; Erik Hossain ${ }^{3}$; Wei Yang ${ }^{1}$; David Grande $^{1,}{ }^{4}$; Jeanmarie Perrone ${ }^{1}$; Mark D. Neuman ${ }^{1}$; Michael Ashburn ${ }^{1}$; M. Kit Delgado ${ }^{1,}{ }^{1}$. ${ }^{1}$ Perelman School of Medicine at the University of Pennsylvania, Philadelphia, PA; ${ }^{2} \mathrm{C}$. Michael $\mathrm{J}$. Crescenz VA Medical Center, Philadelphia, PA; ${ }^{3}$ Penn Medicine, Philadelphia, PA; ${ }^{4}$ Leonard Davis Institute of Health Economics, Philadelphia, PA. (Control ID \#3155039)

BACKGROUND: One strategy thought to prevent chronic opioid use and misuse is judicious opioid prescribing for acute pain. Over half of US states have passed legislation limiting the duration of new opioid prescriptions for acute pain, but there is limited evidence about the impact of these policies. In May 2017, New Jersey enacted a required five-day limit on new opioid prescriptions. In response to the law, the University of Pennsylvania Health System (UPHS) also implemented a nonmandatory electronic medical record (EMR) alert in NJ to notify providers if prescriptions did not meet the five-day requirement. Our aim was to understand the impact of the policy and EMR alert on characteristics of new opioid prescriptions and potential unintended consequences.

METHODS: We evaluated changes in prescription characteristics for initial opioid prescriptions written for adults in UPHS ambulatory practices in both $\mathrm{NJ}$ and $\mathrm{PA}$ from one year prior to the implementation of the NJ prescribing limit (5/15/16-5/15/17) through 10 months after (5/16/17-3/15/18) using EMR data (Epic Systems). We excluded prescriptions for patients with cancer or enrolled in hospice or palliative care. The primary outcome was change in morphine milligram equivalent (MME) for new opioid prescriptions following the implementation of the law plus EMR alert. We also examined tablet quantity/initial prescription, refills within 30 days, utilization (clinic, ED, inpatient visits) and communication (telephone encounters) for these periods. We performed a difference-in-differences analysis comparing outcomes in $\mathrm{NJ}$ practices with control practices in PA, adjusted for patient and clinic characteristics and clustering by provider.

RESULTS: There were a total of 668 new prescriptions in NJ and 4368 in PA. Prior to the intervention, median MME/ prescription was $225 \mathrm{mg}$ in $\mathrm{NJ}$ and $150 \mathrm{mg}$ in $\mathrm{PA}$, and median quantity was 30 tablets in both states. After implementation, median MME in both states was $150 \mathrm{mg}$, and median quantity was 20 in NJ and 30 in PA. After adjustment for patient and practice characteristics, there was a greater decrease in both mean MME and tablet quantity in NJ relative to PA after implementation of the policy plus alert (-82.99 MME/prescription, 95\% CI -148.15 to -17.84 and -10.41 tabs/prescription, $95 \%$ CI -19.70 to -1.13). There were no significant differences for change in refills, telephone calls, ambulatory or emergency department visits, or admissions based on exposure to the policy and alert.

CONCLUSIONS: Implementation of a state prescribing limit accompanied by an EMR alert was associated with a $30 \%$ greater decrease in volume of opioids per new prescription in NJ compared to controls in PA without changes to rates of refills, calls, or visits. The combination of prescribing limit policies and EMR alerts may be an effective strategy to influence prescriber behavior. Further evaluation will be needed to evaluate the impact on downstream outcomes.

\section{IMPACT OF AN INTEGRATED CLINICAL PREDICTION RULE ON ANTIBIOTIC PRESCRIPTION RATES FOR ACUTE RESPIRATORY INFECTIONS IN DIVERSE PRI- MARY CARE SETTINGS.}

Devin M. Mann ${ }^{1}$; Rachel Hess ${ }^{5}$; Thomas McGinn ${ }^{2}$; Simon Jones ${ }^{1}$; Joseph Palmisano ${ }^{3}$; Safiya Richardson ${ }^{2}$; Sara K. Chokshi ${ }^{1}$; Catherine Dinh-Le ${ }^{1}$; Linda S. Park ${ }^{4}$; Rebecca G. Mishuris ${ }^{3}$; Paul Smith $^{4}$; Ainsley Huffman ${ }^{5}$; Sundas Khan ${ }^{2}$; David Feldstein ${ }^{4}$. ${ }^{1}$ NYU School of Medicine, New York, NY; ${ }^{2}$ Donald and Barbara Zucker School of Medicine at Hofstra/Northwell, Manhasset, NY; ${ }^{3}$ Boston University School of Medicine, Boston, MA; ${ }^{4}$ University of Wisconsin School of Medicine and Public Health, Madison, WI; ${ }^{5}$ University of Utah School of Medicine, Salt Lake City, UT. (Control ID \#3185043)

BACKGROUND: Clinical decision support (CDS) tools which incorporate clinical prediction rules (CPRs) have the potential to successfully deliver accurate information and guide decision-making at the point of care. Our previously validated integrated clinical prediction rule (iCPR) was designed to guide evidence-based treatment within an electronic health record for streptococcal pharyngitis and pneumonia based on chief complaints of sore throat, cough or upper respiratory infection. In initial testing at a single site, it resulted in high provider tool adoption (58\%) and decreased antibiotic prescribing rates $(35 \%)$ for acute respiratory infections. Our objective for this study was to assess the impact of this tool when adapted and implemented in diverse primary care settings.

METHODS: This was a randomized controlled trial including 33 primary care practices at two large academic health systems in Wisconsin and Utah. Between October 2015 and June 2018 providers in the intervention group were prompted to complete either Centor Score or Heckerling Rule for Pneumonia based on the chief complaint of the patient encounter. EHR data on provider and patient demographics, tool use rates, and antibiotic order rates from 541 providers and 100,573 monitored patient encounters were collected for analysis. Risk ratios, CIs, and P values are calculated from a generalized estimating equation log-binomial model adjusting for clustering of orders or visits by provider and using robust standard error estimators.

RESULTS: The tool was triggered 42,126 times among 214 intervention providers and was completed in $6.9 \%$ of eligible visits. The intervention and control groups prescribed antibiotics in $35 \%$ and $36 \%$ of visits respectively and were not significantly different. There were no differences in rates for rapid streptococcal test or chest $\mathrm{x}$-ray orders between groups (Strep: relative risk, 1.0; $\mathrm{P}=.11$; Pneumonia: relative risk, 1.8; $\mathrm{P}=.64)$.

CONCLUSIONS: In diverse primary care settings, the tool was not effective at reducing unnecessary antibiotic prescription and diagnostic testing. This outcome was possibly driven by low overall use of CDS tools highlighting the growing impact of "alert fatigue" and the need for 
new approaches to enhance provider engagement with CDS tools. New strategies for reducing the persistently high rates of inappropriate antibiotic prescribing for acute respiratory infections are needed. Novel approaches in future studies are necessary for reducing barriers to CDS tools in order to increase use and engagement.

\section{IMPACT OF COGNITION ON ILLNESS BELIEFS IN BREAST CANCER}

Kimberly A. Muellers ${ }^{1}$; Karisma Pantaleon ${ }^{1}$; Yael T. Harris ${ }^{2}$; Mita S. Goel $^{3}$; Jenny J. Lin ${ }^{1}{ }^{1}$ Icahn School of Medicine at Mount Sinai, New York, NY; ${ }^{2}$ Northwell Health, Great Neck, NY; ${ }^{3}$ Northwestern University, Chicago, IL. (Control ID \#3185328)

BACKGROUND: Older breast cancer (BC) survivors with diabetes (DM) are at higher risk for cognitive dysfunction as age, DM and cancer treatment are independently associated with worse cognitive function. Cognitive dysfunction may also affect survivors' beliefs about both cancer and comorbid illness, with consequences for disease management. We undertook this study to assess the relationship between cognitive function and beliefs about $\mathrm{DM}$ and $\mathrm{BC}$ among older $\mathrm{BC}$ survivors.

METHODS: We enrolled women $>55$ years with pre-existing DM who had completed first line chemotherapy or are taking hormone therapy for early-stage (I-IIIA) BC diagnosed in the past 5 years. Cognitive function was measured using the Weschler Memory Scale Logical Memory test to assess working memory and delayed recall and the Weschler Adult Intelligence Scale-IV Similarities test to assess executive function. Poor cognition was defined as scoring $<1$ standard deviation below the mean. Beliefs about DM and BC were assessed using the Illness Beliefs Questionnaire-Revised. DM medications beliefs were assessed using the Beliefs about Medications Questionnaire. Univariate analyses compared differences in cognition by age, race, ethnicity, education, income, and English ability. Mann-Whitney U tests were used to analyze associations between cognition and illness and medication beliefs.

RESULTS: To date, we have enrolled $51 \mathrm{BC}$ survivors. Mean age was 66.5 years; $29 \%$ were White, $31 \%$ were Black, $8 \%$ were Asian, and $18 \%$ were Hispanic. Survivors who identified as Asian or "other" race were more likely to have worse working memory $(\mathrm{p}=0.013)$ and delayed recall $(\mathrm{p}=0.042)$, and those who were Hispanic $(\mathrm{p}=0.009)$, had $<$ high school education ( $p=0.004)$ or household income $<\$ 2,000$ per month $(p=0.003$ ) had poorer executive function. Those with poor working memory perceived both $\mathrm{DM}$ and cancer as more cyclical in nature (DM: $\mathrm{p}=0.01$; $\mathrm{BC}$ : $\mathrm{p}=0.021$ ) and more emotionally impactful (DM: $\mathrm{p}=0.038 ; \mathrm{BC}: \mathrm{p}=0.026$ ). Additionally, poor working memory was associated with belief in less personal control over DM ( $\mathrm{p}=0.028)$, more consequences of DM $(\mathrm{p}=0.05)$, and more concern about DM medications $(\mathrm{p}=0.01)$. Poorer executive function was associated with belief in more cyclical nature of $\mathrm{DM}(\mathrm{p}=0.038)$ and $\mathrm{BC}(\mathrm{p}=0.04)$, as well as less perceived personal control $(\mathrm{p}=0.038)$ and treatment control $(\mathrm{p}=0.035)$ for $\mathrm{DM}$ but not $\mathrm{BC}$.

CONCLUSIONS: BC survivors with poor working memory and executive function tend to perceive both $\mathrm{BC}$ and $\mathrm{DM}$ as unpredictable and recurrent and as having more emotional impact. Poor cognition was also associated with other negative beliefs about DM but not BC. Memory and executive function are important for managing medication regimens and other aspects of both DM and BC care; however, DM self-management may pose a higher cognitive load than $\mathrm{BC}$. Understanding the impact of illness beliefs on self-management behaviors will be important for the design of interventions to improve care of survivors with comorbidities.

\section{IMPACT OF FREMANEZUMAB ON HEADACHE-RELATED DISABILITY AND QUALITY OF LIFE IN PATIENTS WITH MIGRAINE}

Richard B. Lipton ${ }^{1}$; Sanjay K. Gandhi ${ }^{2}$; Joshua M. Cohen²; Ronghua Yang $^{2}$; Melissa Grozinski-Wolff ${ }^{2}$ Xiaoping Ning ${ }^{2}$; Sissy Easo-Joseph ${ }^{2}$;
Carolina Burgos-Vega ${ }^{2} .{ }^{1}$ Albert Einstein College of Medicine, Bronx, NY; ${ }^{2}$ Teva Pharmaceuticals, Frazer, PA. (Control ID \#3185595)

BACKGROUND: Migraine is commonly seen in primary care settings and is the second-leading cause of years lived with disability worldwide. Migraine negatively affects patients' quality of life (QoL), has a broader impact on the family, and is a financial burden on society. Patients with chronic migraine (CM) have 15 headache days/month, 8 of which are migraine days. Patients with episodic migraine (EM) have $<15$ headache days/month. Preventive migraine treatment is recommended in patients with 4 headache days/month with some impairment. Fremanezumab is a fully humanized monoclonal antibody (IgG2 a) that selectively targets calcitonin gene-related peptide (CGRP) and has been shown in clinical trials to reduce the frequency, severity, and duration of headaches in patients with $\mathrm{CM}$; it is approved for the preventive treatment of migraine in adults. This analysis assessed the impact of fremanezumab on headache-related disability and QoL in patients with migraine.

METHODS: HALO CM and HALO EM were concurrent, multicenter, randomized, double-blind, placebo-controlled, parallel-group Phase 3 studies evaluating the efficacy and safety of fremanezumab for the preventive treatment of migraine. Adults with $\mathrm{CM}$ or $\mathrm{EM}$, confirmed during a 28-day pre-treatment period, were randomized to receive either subcutaneous fremanezumab quarterly $(675 \mathrm{mg}$ at baseline and placebo at Weeks 4 and 8), fremanezumab monthly (CM: $675 \mathrm{mg}$ at baseline, and $225 \mathrm{mg}$ at Weeks 4 and 8; EM: $225 \mathrm{mg}$ at baseline and Weeks 4 and 8), or placebo over a 12-week period. Headache-related disability was assessed via the six-item Headache Impact Test (HIT-6; score range 36-78) in CM and the Migraine Disability Assessment (MIDAS) questionnaire (score range 0270 ) in EM, with higher scores for either indicating greater disability. The Migraine-Specific Quality of Life (MSQoL) was used to assess how migraine affect daily functioning across three domains (range: 0-100; higher scores indicate better QoL): role function-restrictive (RFR), activity limits; role function-preventive (RFP), activity prevention; emotional function (EF), associated emotions.

RESULTS: In HALO CM, fremanezumab significantly reduced HIT-6 scores from baseline (quarterly [least-squares mean change]: -6.4; monthly: -6.8; both $P<0.001)$ compared with placebo $(-4.5)$ and significantly improved MSQoL RFR (quarterly: 20.7; monthly: 21.5 ; both $P<0.0001$ vs placebo: 14.7), RFP (quarterly: 16.8; monthly: 16.5 ; both $P<0.001$ vs placebo: 12.2), and EF (quarterly: 21.1 ; monthly: 20.9 ; both $P<0.05$ vs placebo: 17.0). In HALO EM, fremanezumab significantly reduced MIDAS scores from baseline (quarterly: -23.0 ; monthly: -24.6 ; both $P<0.01$ ) compared with placebo (-17.5). Significant improvements were seen in MSQoL RFR (quarterly: 20.6; monthly: 23.6; both $P<0.01$ vs placebo: 16.5), RFP (quarterly: 17.4; monthly: 18.9 ; both $P 0.001$ vs placebo: 13.8 ), and EF (quarterly: 21.8 ; monthly: $24.6, P<0.0001$ vs placebo: 19.2 ). CONCLUSIONS: Fremanezumab improved disability and QoL in patients with migraine.

IMPACT OF HIGH-DEDUCTIBLE HEALTH PLANS ON HIGHACUITY CARE AMONG PEOPLE WITH BIPOLAR DISORDER James F. Wharam ${ }^{2}$; Alisa Busch ${ }^{3}$; Jeanne Madden ${ }^{4}$; Fang Zhang ${ }^{2}$; Robert F. LeCates $^{2}$; Phyllis Foxworth ${ }^{1}$; Jamie Wallace ${ }^{2}$; Stephen B. Soumerai ${ }^{2}$; Dennis Ross-Degnan ${ }^{2}$; Christine $\mathrm{Lu}^{2} .{ }^{1}$ Depression and Bipolar Support Alliance, Chicago, IL; ${ }^{2}$ Harvard Medical School and Harvard Pilgrim Health Care Institute, Boston, MA; ${ }^{3}$ Harvard Medical School and McLean Hospital, Boston, MA; ${ }^{4}$ Northeastern University, Boston, MA. (Control ID \#3184516)

BACKGROUND: High-deductible health plans (HDHP) have recently become the predominant commercial health insurance product in the US. People with serious mental illness may be especially vulnerable to adverse outcomes under HDHPs but effects on high-acuity care among such populations are unknown. 
METHODS: We studied administrative claims data from a large national health insurer. We used a controlled interrupted-time-series design to examine employer-mandated transitions to HDHPs, minimizing selection bias. The intervention group comprised HDHP members with bipolar disorder age 12-64 included on a rolling basis between 2004-2014. HDHP members were enrolled for 1 year in a low-deductible ( $\$ 500)$ plan followed by 1 year in a HDHP ( \$1000). HDHP members were matched to contemporaneous individuals with bipolar disorder in low-deductible plans during both years based on multiple characteristics and using coarsened exact matching. Outcome measures included emergency department visits and hospitalizations for mental health and non-mental health diagnoses. We plotted monthly outcomes for the HDHP and control group to determine whether baseline trends were parallel, and we used difference-in-differences analysis with generalized estimating equations to estimate relative changes in the HDHP group, adjusting for multiple patient characteristics.

RESULTS: Our final sample included 3,264 HDHP members with bipolar disorder and 35,122 matched controls who stayed in low deductible plans throughout. Members who transitioned into HDHPs experienced a $-9.1 \%$ ( $95 \%$ confidence interval [CI]: - $17.7 \%,-0.4 \%$ ) pre-to-post reduction in nonmental health emergency department visits relative to controls, and a nonsignificant $3.0 \%$ change (95\% CI: $-22.8 \%, 28.9 \%$ ) in mental health-related emergency department visits. Estimated changes in non-mental health- and mental health-related hospitalizations among HDHP members were not statistically significant: $4.8 \%$ (95\% CI: $-14.1 \%, 23.7 \%)$ and $-13.8 \%(95 \%$ CI: $-32.1 \%, 4.4 \%)$, respectively.

CONCLUSIONS: People with bipolar disorder who were enrolled in HDHPs experienced reduced emergency department visits for non-mental health conditions but no statistically significant changes in mental healthrelated emergency department visits or hospitalizations of either type. Patients who suddenly face the high out-of-pocket costs of HDHPs may continue to prioritize acute care in the event of a mental health crisis. Changes in non-mental health emergency department visits raise the possibility that either members inappropriately reduce such care or that they identify more affordable alternatives to emergency department care. Policy makers, employers, and health insurers should remain cautious in encouraging HDHP uptake among people with serious mental illness.

\section{IMPACT OF HIGH-DEDUCTIBLE INSURANCE ON BREAST CANCER CARE AMONG RURAL WOMEN}

James F. Wharam ${ }^{1}$; Jamie Wallace ${ }^{1}$; Fang Zhang $^{1}$; Anita Wagner ${ }^{1}$; Christine Lu'; Larissa Nekhlyudov' ${ }^{1}$ Craig C. Earle ${ }^{3}$; Stephen B. Soumerai ${ }^{1}$; Dennis Ross-Degnan ${ }^{1} .{ }^{1}$ Harvard Medical School and Harvard Pilgrim Health Care Institute, Boston, MA; ${ }^{2}$ Dana Farber Cancer Institute, Boston, MA; ${ }^{3}$ Institute for Clinical Evaluative Sciences, Toronto, ON, Canada. (Control ID \#3184500)

BACKGROUND: High-deductible health plans are increasingly common but are associated with breast cancer care delays of up to 7 months among average commercially-insured women. High cost sharing might represent an especially large barrier to care among rural women who generally have challenging access to health care services and lower incomes. We hypothesized that rural women in high-deductible plans, when compared to rural women in more generous plans, would experience substantial delays in breast cancer services.

METHODS: We used 2004-2014 data from a large US health insurer and a before-after controlled time-to-event analytic approach. We applied the Rural-Urban Commuting Area Codes classification to identify women living in rural areas (codes 4 through 9). We included 32,105 rural women in high-deductible plans age 25-64 without evidence of breast cancer prior to the baseline period. These women were continuously enrolled for 1 baseline year in a low-deductible ( $\$ 500$ ) plan followed by up to 4 years in a high-deductible ( $\$ 1000)$ plan after an employer-mandated switch. The comparison group included 254,022 contemporaneous rural women whose employers offered only low-deductible plans and who were closely matched on baseline characteristics. Measures were times to first diagnostic breast imaging, first breast biopsy, incident early-stage breast cancer diagnosis, and first breast cancer chemotherapy. We estimated delays in care in the high-deductible and control groups at baseline and follow-up using a parametric regression survival time model with a Weibull distribution, adjusting for baseline characteristics.

RESULTS: We detected no differences in the timing of breast cancer care at baseline when both study groups had low-deductible plans. During follow-up, the delay among rural high-deductible members compared to rural low-deductible members was 1.6 months $(\mathrm{p}<0.01)$ for receiving first breast diagnostic imaging, 3.0 months $(\mathrm{p}<0.05)$ for undergoing first breast biopsy, 8.1 months $(\mathrm{p}<0.05)$ to incident breast cancer diagnosis, and 10.0 months $(\mathrm{p}<0.05)$ to breast cancer chemotherapy initiation.

CONCLUSIONS: Rural women who were switched to high-deductible insurance experienced substantial delays in diagnostic breast imaging, breast biopsy, early-stage breast cancer diagnosis, and chemotherapy initiation compared to rural women in low-deductible plans. Health insurers, primary care physicians, oncologists, and population health managers should carefully monitor breast cancer care among rural women enrolled in high-deductible health plans. Strategies such as value-based insurance and targeted outreach might be needed to prevent adverse outcomes.

\section{IMPACT OF INTERIM BUPRENORPHINE PRESCRIBING ON DISCHARGE FROM INPATIENT DETOX ON HOSPITAL READMISSIONS}

Cynthia Eleanya $^{1}$; Darius Rastegar ${ }^{2}$; Megan Buresh ${ }^{1}$. ${ }^{1}$ Johns Hopkins Bayview Medical Center, Baltimore, MD; ${ }^{2}$ Johns Hopkins University, Baltimore, MD. (Control ID \#3183854)

BACKGROUND: Maintenance treatment with buprenorphine has been shown to be an effective treatment for opioid use disorder (OUD), but the majority of Americans with OUD are not in treatment. Inpatient hospitalization provides an opportunity to identify patients with OUD and start them on treatment. The objective of this study was to determine if providing patients with a prescription for buprenorphine after discharge from inpatient treatment for withdrawal is associated with a decrease in 30 day hospital admissions and to determine factors associated with frequent hospital admissions.

METHODS: All patients admitted for inpatient withdrawal treatment at Johns Hopkins Bayview from October 2016 to December 2017, and discharged with a new script for buprenorphine were included. Wilcox Rank Sum was used to compare change in number of patients with pre vs. post intervention 30 day hospital admission. Student's T-test was used to compare pre vs. post intervention 30-day hospital admission rates.

RESULTS: A total of 57 patients received a new buprenorphine prescription during study period. 55 patients $(96.5 \%)$ had a hospital admission prior to their current admission and 14 (24\%) patients had a hospital admission in the 30 days after their current admission $(\mathrm{P}<0.001)$. 30-day hospital admission rate was $1.54 \%$ prior to the current admission and $0.51 \%$ after the current admission ( $\mathrm{p}<0.001)$.

CONCLUSIONS: Initiating buprenorphine prescription for patients with opioid use disorder after inpatient withdrawal treatment is associated with a significant decrease in 30-day hospital readmissions.

IMPACT OF MEDICAL HOME IMPLEMENTATION ON MISSED TEST RESULTS FOR DIAGNOSTIC EVALUATION OF CANCER Debra Choi ${ }^{1}$; Ashley N. Meyer ${ }^{1}$; Li Wei ${ }^{1}$; Daniel R. Murphy ${ }^{1}$; Hardeep $\underline{\text { Singh }^{2}} .{ }^{1}$ Baylor College of Medicine, Houston, TX; ${ }^{2}$ Michael E. DeBakey Veterans Affairs Medical Center and Baylor College of Medicine, Houston, TX. (Control ID \#3178465)

BACKGROUND: Lack of timely follow-up of test results can lead to delays in cancer diagnosis. We previously identified certain results to be high-risk for delays: positive fecal occult blood tests/fecal immunochemical tests 
(FOBT/FIT) and iron-deficiency anemia (IDA) for colorectal cancer (CRC); elevated serum alpha-fetoprotein (AFP) for hepatocellular carcinoma (HCC); and abnormal chest imaging for lung cancer. The VA implemented the patient-centered medical home $(\mathrm{PCMH})$ model in 2010-2011 to provide patient-driven, team-based care. We hypothesized that PCMH will improve timely follow up of abnormal test results nationally across the VA.

METHODS: To identify patients with potentially missed follow-up, we applied previously validated electronic trigger algorithms to the VA's national repository of electronic health record data. The triggers included patients with newly abnormal results, excluding patients for whom follow-up was not required or was completed within a recommended timeline. Positive predictive values were previously determined to be $56 \%, 55 \%, 82 \%$ and $61 \%$ for FOBT/FIT, IDA, AFP and chest imaging, respectively, and were higher than any known measures of diagnostic care gaps. Positive triggers acted as proxies for missed test results. We applied each trigger to all patients seen at 130 VA facilities across 18 national VA networks from 2006-2015 and derived yearly counts of trigger-positive patients at each network and site. Negative binomial regression models were applied to assess overall and yearly changes in number of trigger-positive patients while accounting for clustering by VA network, over-dispersion and correlation due to repeated measures. Final models were adjusted for VA networks and PCP visits.

RESULTS: After excluding patients not meeting inclusion criteria, we identified 5,887,006 patients from 2006-2015 with + FOBT/FIT, of which 713,035 (12\%) met trigger criteria (i.e. potentially missed). Of $37,762,419$ patients with IDA, 424,740 (1\%) met trigger criteria. Similarly, 888,033 patients received AFP tests, with 78,277 (9\%) patients meeting trigger criteria. An estimate of 10,947,742 patients received chest imaging exams, with 291,250 (3\%) meeting trigger criteria. The trigger positive mean proportion of potentially missed test results following PCMH implementation decreased for FOBT/FIT (37.7\% pre-PACT vs. 35.8\% post-PACT) and AFP tests (23.5\% pre- vs. $21.1 \%$ post), but increased for IDA (33.6\% pre vs. $35.1 \%$ post) and lung imaging ( $35.9 \%$ pre vs. $38.3 \%$ post) tests (all p values $<0.001$ ). The mean proportion of potentially missed test results overall was statistically unchanged (32.7\% pre-PACT vs. $32.6 \%$ post-PACT implementation).

CONCLUSIONS: Primary care medical home implementation does not appear to improve follow-up of abnormal test results that warrant cancer evaluation. Further contextual evaluation to explore lack of impact from this teamwork-based intervention is needed.

\section{IMPACT OF MEDICAL SCRIBES ON CLINICAL PRODUCTIVI- TY IN ACADEMIC PRIMARY CARE}

Anastasia Pozdnyakova; Neda Laiteerapong; Sandra Ham; Wei Wei Lee. University of Chicago, Chicago, IL. (Control ID \#3186237)

BACKGROUND: Primary care practices have been increasingly hiring medical scribes to assist with Electronic Health Record (EHR) documentation and to address physician burnout. However, little is known about the impact of scribes on productivity in primary care. The aim was to assess the impact of scribes on clinical productivity at an academic general internal medicine clinic.

METHODS: We piloted a 3-month scribe program at an academic general internal medicine clinic with six faculty and one full-time professional medical scribe. The scribe was present during $25-80 \%$ of total clinic sessions for each provider. The scribe drafted EHR notes for providers to review and sign. All clinic sessions for 2 months prior (baseline control) and during the 3-month pilot (without scribe (concurrent control) and without scribe (intervention) were included. The main outcome was the number of patients seen per clinic session. Secondary outcomes were rates of reconciliation of external information; review of problem list, medications, and immunizations; and patient time to checkout. Interrupted time series mixed models were used to test the scribe effect, controlling for weekday, AM/PM clinic session, and provider. We compared results for the baseline, concurrent control, and intervention groups.
RESULTS: The baseline period included 789 encounters; the pilot had 1,184 encounters, $579(48.9 \%)$ without scribe and $605(51.1 \%)$ with scribe. At baseline, providers saw an average of 6.85 patients per clinic session (95\% CI 6.27-7.48). With the scribe, this number increased nonsignificantly to 7.26 patients per clinic (CI 6.99-7.53, p=0.07). Rates of reconciliation of external information and review of the problem list were higher with the scribe than at baseline $(77.0 \%$ (CI $65.8-85.3 \%)$ vs. $68.8 \%$ (CI 56.8\%-78.7\%), $\mathrm{p}=0.002$ and $9.42 \%$ (CI $5.48 \%-15.7 \%$ ) vs. $6.10 \%$ (CI $3.11 \%-11.7 \%), \mathrm{p}<0.0001$, respectively). The percentage of encounters billed as a Level 4 or 5 Professional Fee Service increased from $91.0 \%$ ( $\mathrm{CI}=84.6 \%-94.9 \%$ ) at baseline to $95.3 \%$ (CI 91.4\%-97.5\%, p=0.005) without the scribe and $93.4 \%$ (CI 88.8\%-96.2\%, $\mathrm{p}=0.003$ ) with the scribe. The time to check-out declined non-significantly from 76.8 (CI=59.6-107.8) minutes at baseline to $70.1(\mathrm{CI}=58.7-86.8)$ minutes without the scribe and $66.4(\mathrm{CI}=60.2-74.1)$ minutes with the scribe. There were no differences for review of current medications or immunizations.

CONCLUSIONS: In this short pilot, we found evidence of more detailed review of patient charts, particularly for the problem list and reconciliation of external information. The number of patients seen per clinic session with a scribe and time to check-out suggested improvements but were not significant. These findings suggest that hiring scribes may improve productivity and documentation of care in the primary care setting; however, further research is needed.

\section{IMPACT OF RELIGION ON MEDICAL STUDENT PERCEP- TIONS AND INTENTION TOWARDS ABORTION}

Joseph S. Needleman $^{1}$; Shenila B. Lallani ${ }^{2}$; Cynthia H. Chuang ${ }^{1}$. ${ }^{1}$ Penn State College of Medicine, Hershey, PA; ${ }^{2}$ University of Alabama School of Medicine, Birmingham, AL. (Control ID \#3186077)

BACKGROUND: One in four U.S. women have an abortion during their lifetime, however $90 \%$ of U.S. counties currently do not have an abortion provider. The future of abortion care relies on a physician workforce who plans to incorporate abortion into their medical practice. This study was designed to describe medical student attitudes toward abortion and abortion provision. Religious affiliation influences attitudes toward abortion among the general public, but whether religious affiliation and religiosity is associated with abortion attitudes and practice intentions among future physicians is unknown.

METHODS: Students at the University of Alabama School of Medicine, Penn State College of Medicine, and University of Massachusetts Medical School were distributed a 26-item electronic survey designed to measure abortion attitudes, practice intentions, religious beliefs, and demographics. The main independent variable was whether religion was very important in the student's life. Logistic regressions were used to model three outcomes: 1) the belief that abortion should be legal in all/most cases, 2) career plan to incorporate abortion into practice, and 3) willingness to refer patients seeking abortion. Models controlled for religious affiliation, gender, intended specialty, intention to practice in a rural location, a rural childhood residence, in-state status, and year in school.

RESULTS: Of the 862 students who completed the survey (response rate $46 \%), 35 \%$ said religion was very important in their lives. The study sample was $31 \%$ Protestant, $16 \%$ Catholic, $3 \%$ Jewish, $12 \%$ other religion, and $37 \%$ had no religious affiliation. Sixty-four percent agreed that abortion should be legal in most or all cases, $25 \%$ intend to incorporate medical and/ or surgical abortion into their practice, and $86 \%$ would refer patients to other providers for abortion services. In the multivariable models, religion being very important in a student's life was a strong negative predictor of all 3 outcomes; those students were significantly less likely believe abortion should be legal in all/most cases (AOR $0.17,95 \%$ CI $0.11-0.25$ ), plan to incorporate abortion into their practice (AOR $0.24,95 \%$ CI $0.14-0.42$ ), or be willing to refer patients for abortion (AOR 0.10, 95\% CI 0.05-0.20). Catholic affiliation and male gender were also negative predictors of all 3 outcomes. 
CONCLUSIONS: Like the general public, the majority of medical students in our study said abortion should be legal in most or all cases. However, abortion attitudes were strongly associated with religiosity, religious affiliation, and gender, suggesting that personal beliefs, values, and experiences strongly influence abortion attitudes. Controversial topics in medicine are often avoided in medical student curricula, but we would encourage such topics be addressed openly and scientifically so student have the opportunity to reassess their attitudes in the context of medical evidence and patient experience.

\section{IMPACT OF WORKPLACE WELLNESS ON HEALTH AND ECONOMIC OUTCOMES: A RANDOMIZED CONTROLLED TRIAL}

Zirui Song ${ }^{1,2}$; Katherine Baicker ${ }^{3}{ }^{4}$. 'Harvard Medical School, Boston, MA; ${ }^{2}$ Massachusetts General Hospital, Boston, MA; ${ }^{3}$ University of Chicago, Chicago, IL; ${ }^{4}$ National Bureau of Economic Research, Cambridge, MA. (Control ID \#3186066)

BACKGROUND: Employers are increasingly investing in workplace wellness programs to improve employee health and decrease health care costs. However, little experimental evidence on the effects of such programs exists, particularly workplace-wide interventions that affect culture or norms. We examined the effect of a multi-component workplace wellness program, similar to wellness programs offered by employers in the U.S., using a randomized controlled trial.

METHODS: This randomized controlled trial spanned January 2015 through June 2016. We used random assignment at the worksite level to assess the effects both of working at an intervention worksite (intention-to-treat) and of participating in the programming (local average treatment effect). The wellness program was implemented at 20 randomly-selected treatment worksites (4,037 employees) and 140 randomly-selected control worksites $(28,936$ employees) at a large multi-site warehouse retail company. The program comprised 8 modules focused on areas of wellness including nutrition, physical activity, and stress reduction, with program implementation carried out by Registered Dietitians. We examined 4 pre-specified domains of outcomes: (1) self-reported health and behaviors; (2) clinical measures of health; (3) health care spending and utilization; and (4) employment outcomes. We assessed the heterogeneity of program effects by age and sex. We also conducted secondary analyses, including using a prespecified cohort of stably-employed workers, worksite-level analyses, and alterations in the statistical model. We assessed endogenous selection into program participation and into primary data collection.

RESULTS: There were 4,037 individuals at treatment worksites, 4,106 at primary control worksites (which received surveys and biometrics, but no wellness program), and 24,830 at secondary control worksites (business as usual). About $20 \%$ of the population was black and $18 \%$ Hispanic; $60 \%$ of workers were full-time. After 18 months, the wellness program led to a higher proportion of employees who reported engaging in regular exercise (8.3 percentage-points more, $\mathrm{p}=0.03$ ) and actively managing their weight (13.6 percentage-points more, $\mathrm{p}=0.02)$. These outcomes were 10.6 percentage-points $(\mathrm{p}=0.02)$ and 17.2 percentage-points $(\mathrm{p}=0.02)$ higher, respectively, for program participants. The program had no significant effects on clinical markers of health; spending or utilization; or absenteeism, tenure, or job performance. We found no evidence of differential selection into survey participation or biometrics between treatment and control on all baseline covariates.

CONCLUSIONS: This randomized controlled trial of a workplace wellness program found significant effects on self-reported health behaviors, but no significant effects on other outcomes after 18 months. Amidst a shortage of causal evidence on workplace wellness programs, these results from a randomized trial may temper the expectations of such programs to improve health or save money in the short term.
IMPACTS OF IMPROVED CONTINUITY OF CARE IN RESIDENT PRIMARY CARE CLINICS ON PATIENT OUTCOMES: A SYSTEMATIC REVIEW

Margaret Connolly ${ }^{1}$; Robert J. Fortuna ${ }^{1}$; Erin D. Snyder ${ }^{3}$; William G. Weppner ${ }^{2}$. ${ }^{1}$ University of Rochester, Rochester, NY; ${ }^{2}$ University of Washington, Boise, ID; ${ }^{3}$ University of Alabama at Birmingham, Birmingham, AL. (Control ID \#3186321)

BACKGROUND: Continuity of care has been established as an essential component of non-teaching primary care practices, resulting in improved patient and provider satisfaction, chronic condition management, and adherence with screening recommendations. The influence of continuity in resident practices on quality outcomes remains unclear. The goal of this systematic review is to summarize existing data addressing the effect of continuity of care in resident primary care clinic settings on measurable patient health outcomes.

METHODS: A systematic search was performed through PubMed to identify articles published prior to August 1, 2018 addressing continuity of care in resident primary care clinic settings including internal medicine, pediatrics, internal medicine and pediatrics, obstetrics and gynecology, and family medicine. Abstracts were then evaluated by two independent reviewers and excluded if not meeting strict criteria including English language, original research, focus on a primary care residency program as recognized by the American Council for Graduate Medical Education, and providing a definition and quantitative value for continuity. Through this process, 39 articles were identified as meeting all inclusion criteria and underwent full text review.

RESULTS: This review revealed significant variation in methods, definition of continuity, and endpoints measured in the existing literature. Study participants included an average of 273 residents (range 15000), 15 academic attending preceptors (range 4-66), and 13,021 patients (range 40-149,346). Additionally there was significant variability in the duration of time evaluated in each study, ranging from 1 week to 17 years (average 2.3 years). The Usual Provider of Care Measure, defined as an individual patient's percentage of visits with a single provider, was the most frequently used definition of continuity of care. However, this was used in only $49 \%$ of included studies. Furthermore, studies measured a variety of outcomes including diabetes and hypertension control, rates of screening colonoscopy and mammography, and emergency department utilization, and patient satisfaction. Results of multiple studies suggest improvement in diabetes control, hypertension control, and adherence to screening recommendations with improved continuity of care. However, these results were inconsistent and varied significantly between studies with multiple studies showing no effect.

CONCLUSIONS: Review of the existing literature revealed that increased continuity of care in resident primary care clinics may improve patient-specific health outcomes, most clearly in diabetes. However, significant differences in study design and outcomes make generalizing results difficult. Additional research is needed to delineate continuity's relationship to chronic medical disease management and utilization in resident clinics.

\section{IMPLEMENTATION OF AN EHR-BASED RISK-STRATIFIED PROSTATE CANCER SCREENING PROGRAM IN A PRIMA- RY CARE NETWORK.}

Anand Shah ${ }^{1}$; Robert C. Mills ${ }^{2}$; Devon Check ${ }^{1}$; Terry Hyslop ${ }^{2}$; Thomas Polascik ${ }^{2}$; Daniel George ${ }^{2}$; Steven Patierno ${ }^{2}$; Andrew J. Armstrong 2 ; Michael Ferrandino ${ }^{1}$; Glenn M. Preminger ${ }^{1}$; Rajan T. Gupta ${ }^{1}$; Robert Lee $^{1}$; Nadine J. Barrett ${ }^{4}$; John Ragsdale ${ }^{1}$; John Anderson $^{3}$; Kevin $\mathrm{Shah}^{3}$; Kevin Oeffinger ${ }^{2} .{ }^{1}$ Duke University, Durham, NC; ${ }^{2}$ Duke Cancer Institute, Durham, NC; ${ }^{3}$ Duke University Health System, Durham, NC; ${ }^{4}$ Duke University, School of Medicine, Durham, NC. (Control ID \#3159942) 
BACKGROUND: Prostate cancer screening recommendations and the proportion of men undergoing screening with a prostate specific antigen (PSA) test varies widely across the U.S., due largely to controversies regarding the harms and benefits of screening. As a consequence, screening rates are decreasing while the incidence of advanced stage prostate cancer is increasing, particularly among African American men. A multi-disciplinary group developed an age- and race-specific risk-stratified screening algorithm, combined with shared decision making, and using varying intervals of PSA follow-up testing. Our overarching goals were to identify clinically significant cancer while minimizing treatment of indolent cancer through a standardized practice model.

METHODS: We evaluated the impact of implementing this risk-stratified screening algorithm in February 2017 in the health maintenance section of our health system-wide EHR (electronic health record; Epic). We used a pre-post implementation study design, comparing the percent of men who met up-to-date screening criteria, based on the algorithm, from 2/1/16-2/1/ 17 (pre-implementation) to $2 / 2 / 17-2 / 21 / 18$ (post-implementation). Men were defined as 'met screening' if they had a PSA value recorded in our EHR within 27 months prior to their visit with their primary care provider (PCP). Descriptive statistics were performed followed by a one-sample prepost test for binomial proportion and a two- proportion $\mathrm{z}$ test.

RESULTS: During the pre- and post-implementation periods, 49,058 and 49,988 men, respectively, aged 40-74 were seen by 290 PCPs across 26 clinics (20.6\% African American, pre and post). Overall, the percent of men who met screening criteria based on the algorithm increased from $48.9 \%$ (pre) to $67.5 \%$ (post) $(\mathrm{p}<0.0001)$; for African Americans it increased from $54 \%$ (pre) to $71.5 \%$ (post). The percent of men who met screening criteria increased across all age groups: age $40-45,17 \%$ to $46 \%$; age $45-49,31 \%$ to $55 \%$; age $50-69,60 \%$ to $73.8 \%$; and age $70-74,51 \%$ to $67 \%$. Similarly, the percent of men who met screening criteria increased across all clinic sites and all PCPs. Importantly, while screening according to the algorithm increased, the volume of PSA test ordering did not change. In the preand post-implementation period, 27,100 and 27,500 PSAs were ordered, respectively. This stability in PSA volume was largely due to a decrease in annual testing among men in the pre-implementation period.

CONCLUSIONS: With the implementation of an EHR-based riskstratified algorithm, we observed a significant increase in meeting our screening criteria while avoiding a net increase in PSA test volume. This approach was successful in standardizing our network-wide approach to prostate cancer screening. Future efforts will evaluate costs and downstream outcomes of this strategy.

\section{IMPLEMENTATION OF FOOD INSECURITY SCREENING IN ADULT PRIMARY CARE PATIENTS}

Arjun Janardhan; Michael W. Latreille; Charles D. MacLean; Michelle Cangiano; Claudia Berger. The Robert Larner, MD College of Medicine at the University of Vermont, Burlington, VT. (Control ID \#3184866)

BACKGROUND: Social determinants of health (SDH) are increasingly recognized for their importance in driving health outcomes. Food insecurity (FI), a lack of regular access to adequate food, is prevalent in the United States, impacting an estimated $12 \%$ of households in 2017. The two-item Hunger Vital Sign (HVS), has demonstrated validity in identifying FI, though there is not agreement on how best to address FI in a healthcare setting. This study aimed to evaluate the implementation of a screening protocol for FI in adult primary care patients, and to investigate any downstream impacts on services delivered in patients who screened positive for FI.

METHODS: In December 2017, our institution added functionality for entering and tracking SDH via an Epic-based EMR. The tool, which incorporated the HVS, was developed based on the Health Related Social Needs Screening Tool by CMS. We instituted protocols for administering and entering the HVS component at two academic primary care sites in Vermont: a semi-urban adult internal medicine practice and a rural family medicine practice. Implementation plans were developed at both sites, each involving education for providers and staff, and pairing of the HVS with annual behavioral health screening. We then identified patients who screened positive for FI from January 1 through April 30, 2018 and retrospectively analyzed charts for services in place prior to positive screens, as well as for documentation and actions taken in response to positive screens.

RESULTS: A total of 1,188 patients were screened for FI during the study period, and 79 (7\%) screened positive. Of patients with FI, 41 (48\%) had some documentation of food or financial insecurity prior to screening. A total of $32(37 \%)$ were documented to have prior access to food insecurity services such as food stamps, food bank etc., and 19 (22\%) had been previously seen by a social worker embedded in the practice. At the time of positive screen, $26(31 \%)$ provider notes included documentation of FI. In response to a positive screen, 16 patients (19\%) were offered social work referral, of whom 12 (14\%) completed a visit.

CONCLUSIONS: In this brief pilot study, we found the implementation of a process for FI screening in primary care to be relatively straightforward, made so by pairing it with other annual health screening protocols and the presence of a function for entering SDH in Epic. The HVS screening tool was also successful in newly identifying FI, as evidenced by the absence of prior documentation in nearly half of food insecure patients. However, the paucity of subsequent documentation and the small fraction of patients who received targeted intervention, despite the education for providers and staff at both practices, indicate a need for further investigation around the best approach to addressing FI in a primary care setting, once it is identified.

\section{IMPLICATIONS OF USING AN ALTERNATIVE MEASURE TO ASSESS OPIATE DAYS SUPPLIED AT DISCHARGE}

Nicholas Iverson; Nader Najafi; Yumiko Abe-Jones; Kirsten Kangelaris; Margaret Fang; Priya A. Prasad; Catherine Lau. UCSF, San Francisco, CA. (Control ID \#3184807)

BACKGROUND: Development of opiate use disorder has been associated with prolonged opiate prescriptions. Conventionally, opiate-days supplied has been calculated by the discharge prescription signature. However, it is not clear whether the number of days supplied reflects the actual needs of the patient. We compared the days supplied using two metrics: one based on the discharge signature, and one adjusted for the amount of opiates required during the hospitalization.

METHODS: We used the electronic health record to identify all patients hospitalized on the acute care medical service at a 600-bed tertiary care hospital from 2012-2018 who received opiates during hospitalization and were discharged on an opiate. We excluded patients with ICD9 or 10 codes for cancer-related pain, sickle cell disease, or who were on hospice or followed by the palliative care service. We ascertained the total amount of opiates administered for each hospital day, standardized to morphine milligram equivalents (MMEs). The number of opiate-days supplied was first determined using the discharge prescription, based on the prescription signature and number of pills ordered. We then determined the number of opiate days supplied adjusted for the amount of opiates required by the patient during the last 24 hours of hospitalization. The difference in days supplied between the two measures was compared using t-tests.

RESULTS: We identified 6,902 patients with a baseline opiate prescription and 2,373 patients without opiates as an admission medication who were discharged on an opiate. The median MME per day of hospitalization was $57.7 \mathrm{mg}$ [IQR 21.1, 150.4], and $60 \mathrm{mg}$ [IQR 24, 165] for the last 24 hours. Using the metric adjusting for the opiate needs on the last 24 hours of hospitalization resulted in a mean days supplied of opiates that was 3.6 days longer than when using a standard days supplied metric (11.9 vs 8.3 days, $\mathrm{p}<0.001)$. Patients with a baseline opioid prescription were prescribed a median of 10 days on discharge by prescription signature, and 14 days based on hospital requirements $(\mathrm{p}<0.001)$. Patients without a baseline opioid prescription were prescribed a median of 5 days on discharge by 
prescription signature, and 7.1 days based on hospital requirements $(\mathrm{p}<0.001)$. Of patients prescribed $<7$ days of opioids by discharge prescription signature, 126 patients $(3.5 \%)$ received a $>30$ day prescription when adjusted for needs in the hospital. A total of 325 patients (8.0\%) without a baseline prescription were discharged on opioids despite requiring no opioids in the 24 hours prior to discharge.

CONCLUSIONS: Days on a prescription signature and a calculation of days based on hospital need are two significantly different mechanisms to calculate discharge opioid-days. The amount of opiates supplied to patients at discharge may exceed their actual clinical needs, since the frequency with which a patient takes opiates at home after discharge may be better reflected by the adjusted days model.

\section{IMPLICIT BIAS AMONG HOUSESTAFF IN THE SAFETY NET: INITIAL RESULTS OF A PROSPECTIVE COHORT STUDY} Giulia Leggett ${ }^{1}$; Nicholas Nelson ${ }^{1}$; Katherine Dang ${ }^{2}$; Kala Mehta ${ }^{2} .{ }^{1}$ Highland Hospital, Oakland, CA; ${ }^{2}$ Highland Hospital, Danville, CA. (Control ID \#3165223)

BACKGROUND: Implicit bias is an important contributor to health disparities. While a robust literature describes changes in empathy during medical training, little is known about implicit biases among resident physicians during training. The goal of our study is to assess the prevalence and evolution of implicit bias over the course of graduate medical training in a safety-net hospital serving one of America's most diverse communities.

METHODS: This is a prospective cohort study of resident physicians in a safety net hospital, who participated on a voluntary basis. Participants were assigned personalized codes to ensure anonymity over serial assessments, and given a survey which assessed explicit biases, as well as attitudes towards medical school admissions and racial, financial, and other biases in healthcare. They were then asked to take Implicit Association Tests (IAT) on race and skin tone, and to report their scores anonymously using their personalized code. Fischer exact test and Kruskal-Walis test were used to analyze whether any difference existed between PGY year with IAT, explicit bias and attitude results.

RESULTS: 17 residents participated. Implicit and explicit bias differed across PGY1 and PGY3 classes with results displayed in Table 1. There was no statistically significant relationship between IAT race categories and resident characteristics.

CONCLUSIONS: Acknowledging the limits of our study, our results suggest the intriguing possibility that implicit bias falls over the course of training within a safety net environment. We plan to evaluate this hypothesis through serial assessment of individual housestaff as they move through residency.

\section{IMPROVED CONTROL OF DIABETES ASSOCIATED WITH A MOBILE TECHNOLOGY INTERVENTION FOR DIABETES MANAGEMENT}

Aaron Baum $^{1}$; Erick R. Scott ${ }^{1}$; Pei Wang ${ }^{1}$; Christophe Hunt ${ }^{6}$; John W. $\overline{\text { Vona }^{6} \text {; Abby L. Schwartz }}{ }^{5}$ Ronald Tamler ${ }^{4}$; Laurie Edelman ${ }^{2}$; Victoria L. Mayer $^{3}$; Juan P. Wisnivesky ${ }^{4}$; Michael Berger ${ }^{6}$; Andrew Kasarskis ${ }^{1} .{ }^{1}$ Icahn School of Medicine at Mount Sinai, New York, NY; ${ }^{2}$ Mount Sinai Medical Center, New York, NY; ${ }^{3}$ Icahn School of Medicine at Mount Sinai, Brooklyn, NY; ${ }^{4}$ Mount Sinai School of Medicine, New York, NY; ${ }^{5}$ The Mount Sinai Medical Center, New York, NY, NY; ${ }^{6}$ Mount Sinai Health System, New York, NY. (Control ID \#3185573)

BACKGROUND: Mobile technology tools for diabetes management are of significant clinical and policy interest, as they offer a potentially low-cost and scalable platform for frequent monitoring and tailored engagement of patients with diabetes. We sought to determine whether a mobile technology intervention for diabetes management - comprising an electronic glucometer device that stores blood glucose data and reports data to patients and their primary care provider, with $24 / 7$ on call alert phone calls from nurses - achieved the objective of reducing glycated hemoglobin among a majority low-income and racial and ethnic minority population.

METHODS: The study population included adult Medicaid beneficiaries with a prior diagnosis of type 2 diabetes who were patients of a large, urban internal medicine primary care practice. We used electronic health records data from the Mount Sinai Data Warehouse from 2013-2017. All patients received usual care that included visits with a certified diabetes educator. Given non-random participation in the intervention, we used a differencein-difference analysis to compare within-patient changes over time in glycated hemoglobin among patients who enrolled in the intervention versus those who did not enroll, while using fixed effects to control for common time trends and time-invariant individual characteristics, such as age, sex, race/ ethnicity, and baseline health status. <style type="text/css"> RESULTS: 150 and 2,593 individuals met the criteria for the treated and control groups, respectively. The overall study population had a median age of 63.60 years old, was $64.59 \%$ female, and had an average baseline glycated hemoglobin of 7.96 percent. Relative to changes over time in the control group, among the treated group glycated hemoglobin was lower by -0.50 percent $(95 \% \mathrm{CI}-0.69,-0.31 ; \mathrm{p}<0.001)$ at 6 months, $-0.65(95 \% \mathrm{CI}$ $-0.92,-0.39 ; \mathrm{p}<0.001)$ at 12 months, and $-0.58(95 \%$ CI $-1.20,0.09 ; \mathrm{p}=$ $0.09)$ at 18 months. Males benefitted more than females. Estimated coefficients were not significantly different from zero in pre- treatment time periods, providing support for the key identifying assumption of parallel trends. Results were consistent in sensitivity analyses that constructed alternative control groups using coarsened exact matching or used patients who enrolled in the program later as controls for those who enrolled earlier. CONCLUSIONS: Lower glycated hemoglobin was associated with a mobile technology intervention for diabetes management. An important limitation of this study is that difference-in-differences analyses rely on the untestable assumption that in the absence of the treatment, the difference between treated and the control groups would have been the same after the intervention.

\section{IMPROVEMENT IN ABDOMINAL AORTIC ANEURYSM (AAA) SCREENING IN INTERNAL MEDICINE RESIDENT VETERAN'S ADMINISTRATION CONTINUITY OF CARE CLINIC.}

Roger D. Struble ${ }^{1,3}$; Christopher Koehn ${ }^{1,3}$; Iiro Honkanen ${ }^{1,3}$; Behnam Laderian $^{1,3}$; Noah Williford ${ }^{1,3}$; Amanda Heuszel ${ }^{1,3}$; Syndey Bowmaster ${ }^{1,}$ 3; Carly Kuehn ${ }^{2}$, ${ }^{3}$; Leslie Brettell ${ }^{3}$. ${ }^{1}$ University of Iowa Hospitals and Clinics, Coralville, IA; ${ }^{2}$ University of Iowa, Iowa City, IA; ${ }^{3}$ Iowa City VA Healthsystem, Iowa City, IA. (Control ID \#3187015)

BACKGROUND: Description of problem: The USPSTF and American Association for Vascular Surgery recommend one-time screening for AAA with ultrasonography in men ages 65 to 75 years who have ever smoked. In Iowa, $94.2 \%$ of a total 226,175 veterans are male and use the VA at a rate higher than the national average, which makes them a high priority target for AAA screening compliance. Objectives: Increase AAA screening compliance to at least $75 \%$ of eligible veterans over the course of the 2017-2018 academic year in Internal Medicine Resident Continuity of Care clinic.

METHODS: Interventions: Increase resident provider education on AAA screening recommendations through a presentation at conference and educational emails. Increase patient education regarding AAA and appropriate screening by distributing pamphlets in patient exam rooms at the primary care clinic. Measures: Current or former male smokers between the ages of 65-75 were identified from eight internal medicine patient panels at the Iowa City VA Health Care System. Screening compliance was measured as completed if abdominal duplex ultrasonography or CT scan of the abdomen identifying the aorta were performed for any reason. Data was obtained before and after the six-month quality improvement intervention. Statistical analysis was performed using $\mathrm{N}-1$ chi-squared test using MedCalc statistical software. 
RESULTS: Findings: A total of 86 patients were identified pre-intervention with a screening compliance of $66.2 \%$. Post intervention, a total of 70 veterans were identified with a significant increase in screening compliance to $82.9 \%(\mathrm{p}=0.0188)$.

CONCLUSIONS: Key Lessons: The group significantly increased AAA screening compliance after a comprehensive intervention targeting both providers and patients. By providing education to both providers and patients, we allowed for shared decision making, which increased screening rates. The veteran population is largely male with a high prevalence of smokers, making this a high impact intervention. Ultrasonography is the preferred screening test as it is noninvasive, low-cost, without radiation; with high sensitivity (94\%), specificity $(98 \%)$. CT was used in place of ultrasound if previously done as it has a high sensitivity (90\%) and specificity $(91 \%)$ for AAA, and additional ultrasound screening would be wasteful and unlikely beneficial. The USPSTF AAA screening recommendation was published in 2007. As more providers have become familiar with the guidelines, screening rates have increased. In 2012, a study at a large academic private medical center found an effective screening rate of $9.2 \%$. A 2015 study in a primary care VA clinic similar to our population found a screening rate of $30 \%$. Through intervention we were able to reach $82.9 \%$. It will be important to continue to educate both patients and providers to ensure that screening for AAA continues to be maintained at high rate.

\section{IMPROVING BLOOD PRESSURE CONTROL BY OPTIMIZA- TION OF GUIDELINE DIRECTED THERAPY FOR HYPERTEN- SION IN A PRIMARY CARE SETTING: QUALITY IMPROVE- MENT PROJECT}

Cirous Sadeghi; Hassan A. Khan; Alexander J. Tiemeier; Smita Y. Bakhai. University at Buffalo, Buffalo, NY. (Control ID \#3167676)

BACKGROUND: Our Internal Medicine Clinic (IMC) is an academic safety-net clinic, with its population comprised of $70 \%$ African Americans within an underserved region. It is estimated about $16 \%$ of the patients with hypertension (HTN) had BP at goal (less than $130 / 80 \mathrm{mmHg}$ ), according to the 2017 ACC/AHA HTN guidelines. It is estimated $70 \%$ of the patients have a diagnosis of HTN with a mean high body mass index (BMI) of 32 in IMC. The aim of this quality improvement (QI) project is to improve BP control to less than $130 / 80$ from a baseline of $16 \%$ to $50 \%$ over 18 months in patients between 40 to 80 years old.

METHODS: A multidisciplinary QI team performed root cause analysis using fishbone diagram and identified physician, patient and system related barriers to optimal BP control. The QI team included physician champions, nursing and ancillary staff, residents, a social worker, patients; and administrative and information technology staff. We performed 4 Plan-Do-StudyAct (PDSA) cycles. The major components of interventions included: 1) Leveraging health information technology, 2) nursing education on accurate BP measurement, 3) physician education regarding ACC/AHA guidelines and medication reconciliation; and 4) patient education. The outcome measure is the percentage of patients with $\mathrm{BP}<130 / 80$. The percentage of patients with Stage 1 and Stage 2 HTN; and HTN crisis were also monitored. Process measures included: 1) care guide use for DASH diet and lifestyle modification for patient education, and 2) completion of medication reconciliation. Balanced measures included increase patient wait time due to BP re-check and education; and time constraints for physicians during clinic visit. Data analysis was performed using a monthly run chart.

RESULTS: There was a steady increase and sustainable improvement of patients with BP nearing goal $(<130 / 80)$ during various PDSA cycles. Sustainable improvement in stage $1 \mathrm{HTN}$, achieved highest at $41.5 \%$ in November 2018. There was also sustainable increase in rates of medication reconciliation and care guide use during clinic visit. Table below represents data from May to November 2018.

CONCLUSIONS: Engagement and synergy of our multidisciplinary team were the key factors for the success of this QI. Future PDSA cycles will include: 1) assessment of patient's adherence to medications, appointments and identifying barriers for non-adherence, 2) continuous education and reminders to patients and physicians regarding optimal BP goal, and 3) home BP measurements with patient logs to confirm mask and white coat HTN.

\section{IMPROVING COMMUNICATION OF DISCHARGE PLANS AMONGST RESIDENTS, NURSES AND PATIENTS: A QUALITY IMPROVEMENT PROJECT}

Camille Hawkins; Arianne Clare C. Agdamag; Manya Gupta. Rush University Medical Center, Chicago, IL. (Control ID \#3186779)

BACKGROUND: Discharge of hospitalized patients is a multistep process, involving communication amongst numerous healthcare providers. Delays in discharge cause a backlog of patients in the ED and ICUs, leading to prolonged patient wait times and overcrowding in the ED. Furthermore, many discharges occur in the evening hours, when there is a covering provider. Our institution has no standardized protocol on relaying discharge information from residents to nurses and patients, other than a simple EMRgenerated discharge medication list. In this project, our goal was to determine the baseline perception of the discharge process and implement a mandatory, standardized discharge instruction checklist accessible to nurses, residents, and patients.

METHODS: Baseline surveys to determine problems with the discharge process were sent via email to nurses on general medicine floors and to all Internal Medicine residents. A discharge checklist was developed based on the issues identified. The checklist was launched during a resident safety conference, and we worked with our EMR representatives to make sure the list was included in patients' discharge paperwork, which is visible to all physicians, nurses, and patients. Residents were required to complete this checklist prior to printing the AVS.

RESULTS: The survey was completed by 86 nursing staff and 48 residents. For both residents and nurses, $>95 \%$ have encountered discharge related issues. For nurses, $84.9 \%$ found inconsistencies in discharge related documentation, $89.5 \%$ have been contacted by patients after discharge and the most common issues were: discharge medication, transportation, and follow-up. Among residents, $91.7 \%$ had been contacted by nurses during cross cover to clarify discharge plans, $95.8 \%$ were willing to complete a discharge checklist and the most common issues were: medication refills, opioid prescriptions and follow-up. Both nurses $(94.2 \%)$ and residents $(81.3 \%)$ agreed that a standardized discharge template would be beneficial. CONCLUSIONS: The majority of nurses and residents perceive a lack of standardized discharge communication. This ineffective communication can have a significant impact on a patient's health. The discharge related issues seen in the survey, specifically medication related questions, were similar to what have been reported in literature. Prior studies have also identified ineffective communication as the underlying reason for adverse events after discharge. The openness of nursing and residents in the implementation of a standardized checklist is key for improvement. Our checklist includes the reason for admission, medication changes, follow-up and contact person after discharge, all written in patient- friendly language. We hope that our checklist will provide clearer discharge plans for both patients and providers, and ultimately help prevent adverse events. To determine its efficacy, a postintervention survey is being developed for residents and nurses.

\section{IMPROVING SAFE OPIOID PRESCRIBING IN PRIMARY CARE: IMPACT OF A CLINIC-WIDE POLICY AND DATA DRIVEN PRE- SCRIBER FEEDBACK ON PRESCRIBING PRACTICE}

Trang Vu; Linda Wang; Eva Waite; Caroline Round; mary fishman. Icahn School of Medicine at Mount Sinai, New York, NY. (Control ID \#3184607)

BACKGROUND: Despite the development of safe opioid prescribing guidelines, prescription opioids continue to contribute to the opioid epidemic 
in the US. The Internal Medicine Associates Opioid Management Committee (IMA OMC), a multidisciplinary group of primary care providers and pain management specialists, was formed to promote safer opioid prescribing by primary care providers within an urban hospital-based primary care clinic. METHODS: In January 2018, the IMA OMC created and disseminated a guideline-based safe opioid prescribing policy, which included obtaining yearly patient-provider agreements (PPA) and routine urine toxicology. The committee also reviewed monthly electronic medical record (EMR) data on opioid and benzodiazepine prescriptions. In March 2018, providers received their individual prescription data electronically, highlighting patients receiving high dose opioid (morphine milligram equivalent, MME $>50$ ) or concomitant opioid-benzodiazepine prescriptions. Retrospective data analysis of prescription data from January and November 2018 were completed to observe any changes in opioid prescribing activity.

RESULTS: As compared to January 2018, in November 2018, there was an increase in total number of opioid prescriptions $(31.5 \%)$. However, after removing buprenorphine prescriptions, the number of non-buprenorphine opioid prescriptions only increased by $3.5 \%$. The average MME decreased from 52.6 to 47.7 . Furthermore, the number of patients with MME $>50$ decreased from 59 to 48 , and number of patients with MME $>90$ decreased from 38 to 34 . The number of patients with concomitant opioidbenzodiazepine prescriptions did increase slightly from 5 to 9 patients. The number of patients receiving opioid prescriptions with a PPA recorded in the EMR rose by $16.7 \%$, while the number of patients with at least one urine toxicology screen increased significantly by $197.6 \%$.

CONCLUSIONS: The dissemination of a clinic-wide safe opioid prescribing policy with direct data-driven prescriber feedback was associated with a reduction in high dose opioid prescriptions and increased utilization of PPAs and routine urine toxicology. While the total number of opioid prescriptions increased, the number of patients receiving opioid prescriptions with MME $>50$ and $>90$ decreased. This provides promising data on the efficacy of policy and data driven interventions to promote safer opioid prescribing.

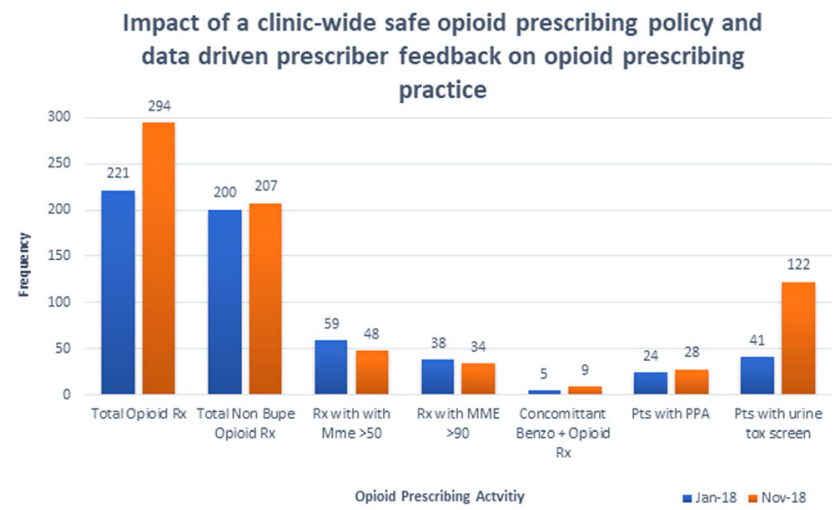

IMPROVING SHARED DECISION MAKING FOR ASIAN AMERICAN PACIFIC ISLANDER SEXUAL AND GENDER MINORITIES Stephanie Bi ${ }^{1}$; Kathryn E. Gunter ${ }^{1}$; Fanny Y. López Benítez ${ }^{2}$; Seeba Anam ${ }^{1}$; $\widehat{\text { Judy Y. Tan }^{3}}$; Danielle J. Polin ${ }^{1}$; Justin L. Jia ${ }^{4}$; Neda Laiteerapong ${ }^{1}$; Mai T. $\mathrm{Pho}^{1}$; Karen $\mathrm{Kim}^{1}$; Marshall Chin ${ }^{1} .{ }^{1}$ University of Chicago, Chicago, IL; ${ }^{2}$ Dominican University, River Forest, IL; ${ }^{3}$ University of California San Francisco, San Francisco, CA; ${ }^{4}$ Stanford University, Stanford, CA. (Control ID \#3180546)

BACKGROUND: Asian American Pacific Islander (AAPI) sexual and gender minorities (SGM) experience substantial mental health burden and face unique intersectional challenges in mental health burden and accessing high-quality mental healthcare. Shared decision making (SDM) values patient preferences and could be used to improve care for this population, but evidence is scant to inform SDM practices. We aimed to identify barriers and facilitators in SDM for AAPI SGM individuals, especially surrounding mental health, and to provide recommendations for providers.
METHODS: We conducted individual interviews $(n=40)$ and focus groups $(n=10)$ in Chicago and San Francisco to explore SDM experiences in the context of mental health, and multiple identities. An extensive codebook was iteratively amended by eight team members, and the transcripts were each randomly assigned to two coders. After secondary analysis, we developed a conceptual model through an inductive approach guided by a review of the literature.

RESULTS: Fifty-four percent of participants were gay, 33\% were queer, $10 \%$ were bisexual, $2 \%$ were asexual, and $2 \%$ did not use labels to describe their sexual orientation. Seventy-four percent of the participants were cis men, $6 \%$ were cis women, $8 \%$ were trans men, $2 \%$ were trans women, and $8 \%$ were gender nonconforming or two-spirited. Several racial/ethnic groups within AAPI were represented. Our conceptual model elucidates the patient, provider, and encounter-centered factors that may inform into SDM for AAPI SDM. Some participants shared the stigma of SGM identities and mental health in their AAPI families. Their AAPI and SGM identities were intertwined in impacting mental health. Some providers inappropriately controlled the visibility of the patient's identities, ignoring or overemphasizing them. Participants varied on whether they preferred a provider of the same race, and how prominently their AAPI and/or SGM identities affected SDM. CONCLUSIONS: Providers can improve the care they provide to AAPI SGM patients if they understand their identity-specific challenges in engaging in SDM. Providers should self-educate about AAPI and SGM history and intracommunity heterogeneity before the encounter, create a safe environment conducive to patient disclosure of SGM identity, and ask questions about patient priorities for the visit, pronouns, and mental health.

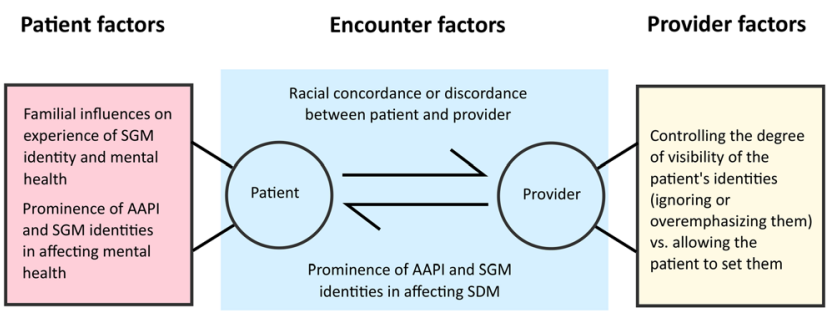

Conceptual model for shared decision making between providers and Asian American Pacific Islander (AAPI) sexual and gender minority (SGM) patients

\section{IN-HOME TECHNOLOGY TRAINING TO REDUCE SOCIAL ISO- LATION AND IMPROVE TABLET USE AMONG OLDER ADULTS: FINDINGS FROM THE TECH ALLIES PROGRAM}

Jessica Fields ${ }^{1}$; Anupama Cemballi ${ }^{1}$; Cathy Michalec ${ }^{2}$; Debbie Uchida ${ }^{2}$; Heather DeSmidt ; Jacqueline Cuellar ${ }^{4}$; Anna H. Chodos ${ }^{1}$; Courtney Lyles ${ }^{1}$. ${ }^{1}$ UCSF, San Francisco, CA; ${ }^{2}$ Little Brothers Friends of the Elderly, San Francisco, CA; ${ }^{3}$ Community Tech Network, San Francisco, CA; ${ }^{4}$ University of California, Berkeley, Berkeley, CA. (Control ID \#3180367)

BACKGROUND: Technology has the potential to increase feelings of social connectedness among older adults, but $1 / 3$ do not use the internet. We formed a community partnership called Tech Allies to provide iPads, internet access, and 1:1 technology training to isolated older adults. Our objective was to evaluate whether participation in the Tech Allies program affected loneliness and technology use among participants.

METHODS: Tech Allies was a partnership: Little Brothers Friends of the Elderly (LBFE, a volunteer organization providing home visits for isolated older adults), Community Tech Network (a digital literacy training organization), and the University of California, San Francisco. Participants were eligible for the study if they were (1) at least 65 years old, or if disabled, at least 50 ; (2) receive fewer than two social visits per month; (3) English speaking, and (4) able to provide informed consent. Participants were randomized into immediate training and a 2-month waitlist, to function as a 
control. LBFE volunteers visited participants weekly and provided 8 weekly lessons on iPad use. Surveys assessed loneliness, using the 3-item UCLA loneliness scale, and self-reported computer use and skills at baseline and follow-up. McNemar's tests were used to assess change over time in loneliness and technology use. A subgroup also completed in-home interviews discussing motivations for internet use and perceptions about technology and health.

RESULTS: 44 participants were randomized into immediate training and 39 into the waitlist. The sample was $51 \%$ female, mean age $75,73 \%$ income $<\$ 20 \mathrm{~K}$, and $46 \%$ nonwhite. Of the training group with complete data in Dec $2018(\mathrm{n}=24), 91 \%$ reported loneliness at baseline and $87 \%$ at follow-up $(\mathrm{p}=1.0)$, and $33 \%$ reported no technology use at baseline and $0 \%$ at follow-up $(\mathrm{p}=0.01)$. Of the waitlist group with complete data in Dec $2018(\mathrm{n}=28), 64 \%$ reported loneliness at baseline and $63 \%$ at follow up $(\mathrm{p}=1.0)$, and $57 \%$ reported no technology use at baseline and $64 \%$ at follow-up $(\mathrm{p}=0.63)$. The qualitative interviews showed isolation was a key driver of program participation, with high self-awareness about how technology may impact isolation. Participants expressed unmet needs regarding technology and health such as, "Sometimes I can't even make it [to the doctor]...I only have a caretaker. I can't drive myself. I don't even have a relative."

CONCLUSIONS: Though participation in Tech Allies did not result in change in loneliness, it did result in an increase in technology use. While other studies have evaluated small-scale digital training for older adults focused on health, this work is the first effort to our knowledge focused specifically on dissemination and implementation of digital training among this high-needs population. Our findings demonstrate that embedding training within existing community-based programs holds promise as a longitudinal and potentially sustainable mechanism to provide digital literacy training to older adults.

INADEQUATE REPORTING OF CO-INTERVENTIONS IN RECENT CARDIOVASCULAR CLINICAL TRIALS: A SYSTEMATIC REVIEW

Elisavet Moutzouri ${ }^{1,2}$; Luise Adam ${ }^{2}$; Martin Feller ${ }^{1,2}$; Lamprini $\overline{\text { Syrogiannouli }{ }^{1} \text {; Bruno R. da Costa }}{ }^{3,1}$; Cinzia Del Giovane ${ }^{1}$; Douglas Bauer ${ }^{4}$; Drahomir Aujesky ${ }^{2}$; Arnaud Chiolero ${ }^{1}$; Nicolas Rodondi ${ }^{1,2}$. ${ }^{1}$ University of Bern, Bern, Switzerland; ${ }^{2}$ Inselspital, Bern University Hospital, Bern, Switzerland; ${ }^{3}$ University of Toronto, Bern, Switzerland; ${ }^{4}$ University of California, San Francisco, CA. (Control ID \#3185147)

BACKGROUND: Co-interventions in a randomized controlled trial (RCT) is medical care given in addition to the tested intervention. If co-interventions are unbalanced across trial arms, the results may be biased. Co-interventions have to be adequately reported, particularly in RCTs at risk of bias or without full blinding. The aim of the present study was to describe the reporting of cointerventions, and to evaluate factors associated with the quality of their reporting in recent cardiovascular trials.

METHODS: We performed a systematic review of RCTs evaluating pharmacological interventions on cardiovascular outcomes, published in five high impact journals (New England Journal of Medicine, Lancet, Journal of the American Medical Association, British Medical Journal and Annals of Internal Medicine) from 1 January 2011 through 31 December 2016 (PROSPERO: CRD42018106771). Quality of reporting of co-interventions was evaluated independently by two reviewers, and was considered "inadequately reported" unless the authors reported 1) a list of co-interventions of interest across trial arms, or 2) explicitly stated the absence of potential cointerventions, in which cases it was considered "adequately reported". Furthermore, we assessed the association between quality of reporting and the blinding of participants and/or personnel, the risk of bias due to deviation of intended interventions, the adherence to study treatment, and the duration of follow-up.

RESULTS: Co-interventions were inadequately reported in 65 of 91 RCTs (71.4\%), with $38(41.8 \%)$ providing no information on co-interventions and $27(29.7 \%)$ only partial information. Adequate reporting was associated with adherence to study treatment (odds ratio (OR) 2.86; $95 \%$ confidence interval (CI) 1.01 to 8.04), a follow-up duration of less than 6 months (OR 4.25; $95 \% \mathrm{CI} 1.57$ to 11.47 ), but was not associated with blinding (OR $0.88,95 \% \mathrm{CI}$ 0.35 to 2.20) or risk of bias due to deviation of intended interventions (OR $2.34,95 \%$ CI 0.91 to 6.02 ), even though better reporting would have been expected in these RCTs.

CONCLUSIONS: Among recent major cardiovascular trials, over two thirds do not adequately report co-interventions, including among trials that are not fully blinded or judged to be at risk for bias. Co-interventions should be systematically reported, as they may be a source of bias, with a potential risk of marketing drugs that are less effective than what they appear in these trials.

\section{INAPPROPRIATE STATIN THERAPY ACCORDING TO ATH- EROSCLEROTIC CARDIOVASCULAR DISEASE (ASCVD) RISK; CAN WE DO BETTER?}

Raef A. Fadel; Guneet Ahluwalia; Courtnay Hughes; Shivani Sharma; Ahmad O. Aljamal; Jasmine Omar. Henry Ford Hospital, Rochester Hills, MI. (Control ID \#3178659)

BACKGROUND: Statin therapy targeted at reducing 10-year risk of stroke and heart attack has become a cornerstone for preventative health in the outpatient setting. Appropriate statin intensity prescription based on 10-year ASCVD risk calculation can lead to improved morbidity and mortality as outlined by current American College of Cardiology/American Heart Association (ACC/AHA) guidelines.

METHODS: We conducted an empiric observational study in August 2018 based on lab results of patients visiting the Henry Ford Hospital Academic Internal Medicine Clinic between January 2017 and December 2017. We calculated the 10-year ASCVD risk for the patients based on this data, and compared the ACC/AHA guideline recommended statin therapy with the one currently prescribed. The primary outcome was appropriateness of statin therapy based on ASVCD risk calculation. Our aim was to assess whether patients in the clinic setting are being adequately managed for ASCVD risk according to ACC/AHA guidelines.

RESULTS: Of the 2994 patients assessed, approximately 1548 patients were prescribed an inappropriate intensity of statin based on 10-year ASCVD risk calculation $(\mathrm{p}<0.001)$. For female patients, the odds of appropriate statin dose prescription increased by approximately $81.9 \%$ (odds ratio $1-1.819$ ) when compared to male patients (95\% CI 1.559-2.124). For black patients, the odds of appropriate statin dose prescription decreased by $32.2 \%$ (odds ratio 1-0.678) when compared to white patients (95\% CI 0.532-0.864). Asian patients were more likely to be on an appropriate statin dose as compared to non-Asians $(\mathrm{p}=0.022)$, and Hispanic patients were more likely to be on an appropriate statin dose as compared to non-Hispanics $(\mathrm{p}=0.005)$. Approximately 1245 patients currently taking high-intensity statin did not qualify for one based on 10-year ASCVD risk calculation as compared to 484 patients ( $\mathrm{p}$ $<0.001)$.

CONCLUSIONS: There is marked discrepancy in the guideline recommended statin therapy (based on 10-year ASCVD risk calculation) and currently prescribed statin, with gender and race serving as major variables. This data demonstrates a major opportunity for intervention on the part of primary care internists to improve patient outcomes in the outpatient setting.

INCORPORATING WALKING SPEED, THE FUNCTIONAL VITAL SIGN IN AN OUTPATIENT INTERNAL MEDICINE RESIDENT CLINIC. A PILOT STUDY

Sara E. Atwater ${ }^{1}$; Claudia L. Campos ${ }^{2}$; Elizabeth L. Tysinger ${ }^{1}$; Maria Cranston ${ }^{1}$. Wake Forest Baptist Health, Winston Salem, NC; ${ }^{2}$ Wake Forest University, WInston-Salem, NC. (Control ID \#3185565)

BACKGROUND: With rising healthcare costs, more pressure is being placed on primary care providers to provide efficient, yet accurate, thorough, 
and cost conscious care. Being able to quickly assess a patient's risk is critical to providers. One metric that risk stratifies and signals earlier health problems is walking speed. It has been described as the functional vital sign or sixth vital sign because of its correlation to mobility, morbidity, mortality and even cognitive function.

METHODS: The outpatient department internal medicine resident clinic at Wake Forest Baptist Health provides 10,000 visits per year. We conducted a pilot during July and August 2018 in patients presenting for a continuity clinic visit. The data collector, a high school volunteer, timed all patients from a starting mark near where all patients are weighed before being brought to the exam room. The stop mark was six meters beyond the starting mark and was denoted with an inconspicuous piece of tape on the railing along the wall. The six meter distance based on a literature review as well as trial and error. The nurses instructed the patients on walking at a comfortable pace. WS was measured with hand held stopwatches and patient's speed was recorded in meters per seconds $(\mathrm{m} / \mathrm{s})$.

RESULTS: During the two months of data collection, we measured and recorded walking speed in 36 patients with $61.1 \%$ female and $38.9 \%$ male participants. There was a wide patient age range from 24 to 75 years old with a mean age of 47.9 years old. The patient average WS was $0.9 \mathrm{~m} / \mathrm{s}$ with a range of 0.4 to $1.4 \mathrm{~m} / \mathrm{s}$. In addition, to the objective measures, information regarding the patient's experience of this measurement was also collected. The patients ranked the experience on a Likert scale of 1-5 with 1 being the most bothersome to 5 being the most pleasant. The average rating was 4.1. They were also asked to rank the helpfulness of this measure on a scale of 1-5 with 1 being the least helpful and 5 being the most helpful. The average score for this was 3.8. Five out of five nurses believed that it was an important metric, but were hesitant to incorporate in routine rooming flows without additional staff to help in the process.

CONCLUSIONS: Patients were very receptive to measuring their walking speed. Nurses were more reluctant given time constraints and rooming flows, but adjusted quickly to the measurement. Evidence continues to validate the prognostic and predictive values of walking speed, and accordingly, the measure's popularity as the "sixth vital sign" has only increased. As with any other vital sign, WS is a simple assessment that provides a wealth of information about underlying physiological processes. The value far out ways the cost, and resident internal medicine clinics should consider incorporating this vital sign into routine clinical practice.

\section{INDIVIDUAL BURDEN OF SOCIAL DETERMINANTS OF HEALTH (SDOH): A NEW RISK FACTOR FOR FATAL CORO- NARY HEART DISEASE}

Monika M. Safford ${ }^{1}$; Evgeniya Reshetnyak ${ }^{1}$; Madeline R. Sterling ${ }^{1}$; Joshua Richman $^{2}$; Paul Muntner ${ }^{2}$; Raegan Durant ${ }^{2}$; John Booth ${ }^{2}$; Laura C. Pinheiro ${ }^{3}$. ${ }^{1}$ Weill Cornell Medical College, New York, NY; ${ }^{2}$ University of Alabama at Birmingham, Birmingham, AL; ${ }^{3}$ Weill Cornell Medicine, New York, NY. (Control ID \#3184861)

BACKGROUND: SDOH are associated with poor health outcomes. One of these is death within 28 days of first presentation with coronary heart disease (CHD), also known as incident fatal CHD, which continues to be twice as common in blacks as whites. While much is known about risks associated with individual $\mathrm{SDOH}$, less is known about the burden of $\mathrm{SDOH}$ within individuals. We determined the association between the burden of withinperson SDOH and risk for incident fatal CHD.

METHODS: This prospective longitudinal study used data from the REasons for Geographic And Racial Differences in Stroke cohort. This population-based national sample of 30,239 community-dwelling black and white adults age $>45$ years was recruited from 2003-7. Baseline data came from telephone interviews and in-home visits. Telephone follow-ups were conducted every 6 months with retrieval of medical records and expert adjudication of suspected study endpoints including fatal CHD. The primary exposure was SDOH, guided by the Healthy People 2020 framework that includes five domains: social context (black race, social isolation [not seeing friends/family in the past month]), education ( $<$ High School), economic stability (annual household income $<\$ 35,000$ ), neighborhood and built environment ( $>25 \%$ residents in a zip code living below the Federal poverty line), and health/healthcare (lacking health insurance, living in a state with little public health infrastructure). Cox models estimated crude and adjusted associations between the number of within-individual SDOH and incident fatal CHD.

RESULTS: Of 22,152 participants free of CHD at baseline, 58.8\% were women and $42.0 \%$ were blacks. Over a median 9.2 years of follow-up, the incidence rate of fatal CHD events per 1000 person-years (and number of events) for the 4016 participants with $0 \mathrm{SDOH}$ was 1.18 (45); for the 5944 with $1 \mathrm{SDOH}$ was 1.25 (76); for the 4464 with $2 \mathrm{SDOH}$ was 1.97 (89); and for the 5024 with $>3 \mathrm{SDOH}$ was 2.85 (140). Compared to participants with 0 $\mathrm{SDOH}$, the crude hazard ratio (HR) for fatal CHD among those with $1 \mathrm{SDOH}$ was 1.18 (95\% CI $0.82,1.69)$, with $2 \mathrm{SDOH}$ was 2.02 (95\% CI $1.43,2.84)$, and with $>3 \mathrm{SDOH}$ was 3.00 (95\% CI 2.17, 4.15). Following adjustment for medical conditions and treatments, functional status, physiologic variables, and health behaviors, compared to participants with $0 \mathrm{SDOH}$, the adjusted HR among those with $1 \mathrm{SDOH}$ was 0.95 (95\% CI 0.66, 1.38), for those with $2 \mathrm{SDOH}$ was 1.31 (95\% CI 0.92, 1.87), and for those with $>3 \mathrm{SDOH}$ was 1.67 (95\% CI 1.18, 2.37). These findings were consistent for both men and women and older and younger participants.

CONCLUSIONS: An individual's burden of SDOH may be a new marker for risk of death at presentation with CHD. Many of the SDOH studied here are already part of the social history in a standard medical interview, allowing clinicians to quickly and easily identify patients who may benefit from the most aggressive CVD risk factor management until we gain better understanding of the mechanisms leading to these findings.

\section{INFLUENCE OF INDIVIDUAL-LEVEL NEIGHBORHOOD FAC- TORS ON HEALTH PROMOTING AND RISK BEHAVIORS IN THE HISPANIC COMMUNITY HEALTH STUDY/STUDY OF LA- TINOS (HCHS/SOL)}

Asmi Panigrahi $^{2,}{ }^{1}$; Jennifer Bayly ${ }^{2,}{ }^{3}$; Erik J. Rodriquez ${ }^{2}$; Eliseo J. PerezStable ${ }^{4}$. Rutgers New Jersey Medical School, Newark, NJ; ${ }^{2}$ National Heart, Lung, and Blood Institute, Bethesda, MD; ${ }^{3}$ Rutgers Robert Wood Johnson Medical School, Piscataway, NJ; ${ }^{4}$ National Institutes of Health, Bethesda, MD. (Control ID \#3185661)

BACKGROUND: Though people's place of residence is often tied to socioeconomic status, race, and ethnicity, the role of neighborhood environment as a determinant of health remains less clear. Neighborhood environment may be an especially important determinant that influences immigrant and minority health behaviors, particularly among US Hispanics/Latinos. We sought to understand the relationships between individual-level neighborhood factors and health promoting and risk behaviors in the most comprehensive study of Hispanics/Latinos in the US to date.

METHODS: We analyzed data from the Hispanic Community Health Study/Study of Latinos (HCHS/SOL) Sociocultural Ancillary Study which included 5313 men and women, 18-74 years old, recruited from San Diego, Chicago, Miami and New York City, and from Mexican, Puerto Rican, Cuban, Central American, Dominican and South American background. All participants underwent a baseline clinical exam and sociocultural survey including validated measures of neighborhood social cohesion and neighborhood problems. We used multivariable logistic regression to assess crosssectional and longitudinal relationships between participants' neighborhood factors and health behaviors, adjusting for age, gender, educational attainment and health insurance.

RESULTS: Participants had a mean age of 42.5 years (SD 0.38), 54.8\% were women, and $72.8 \%$ were foreign born. Mean perceived neighborhood problems were greater in US-born vs foreign-born participants [Mean $(\mathrm{SE})=12.9(0.16)$ vs $11.6(0.14), \mathrm{p}<0.001]$. Mean perceived neighborhood problems differed by site, and were highest in the Bronx, followed by Chicago, Miami and lowest in San Diego (13.5(0.21), 12.5(0.19), 
$11.5(0.21), 10.3(0.18), \mathrm{p}<0.001]$. However there was no significant difference between mean perceived neighborhood social cohesion in US-born vs foreign-born participants (15.8 vs 15.8), nor by study site (Bronx 15.6, Chicago 15.8, Miami 15.9, San Diego 15.9). Health protective behaviors were consistently less prevalent among foreign-born vs US-born participants, particularly colon cancer screening $(50.5 \%$ vs $71.5 \%)$, mammogram $(74.4 \%$ vs $84.4 \%$ ) and prostate cancer screening (51.9\% vs $63.3 \%)$. Health risk behaviors were also consistently less prevalent among foreign-born vs USborn participants, especially smoking ( $15.8 \%$ vs $31.4 \%)$, obesity ( $36.5 \%$ vs $49.9 \%$ ), and poor diet (16.8\% vs 36.6\%). Foreign-born participants with higher perceived neighborhood problems were less likely to meet physical activity recommendations $[\mathrm{OR}(95 \% \mathrm{CI})=0.74(0.58,0.95) \mathrm{p}<0.05)$. Foreignborn participants with less perceived neighborhood social cohesion were less likely to be adherent to pap smear guidelines $(\mathrm{OR}(95 \% \mathrm{CI})=0.48(0.23,0.98)$ $\mathrm{p}<0.05$ ).

CONCLUSIONS: Overall, neighborhood factors may significantly influence health behaviors of US Hispanics/Latinos, especially Hispanic/Latino immigrants. Neighborhood environment is important for health systems and providers to consider in prevention and treatment strategies that influence Hispanic/Latino health.

\section{INFLUENCE OF MIDLIFE COGNITIVE ACTIVITY ON LATE- LIFE COGNITIVE ACTIVITY, COGNITIVE FUNCTION AND FUNCTIONAL STATUS IN WI VETERANS}

Allison E. Cotter ${ }^{1}$; Jessica Kim ${ }^{1}$; Kia Semons-Booker ${ }^{1}$; Kate Sherman ${ }^{2}$; Rodney Sparapani ${ }^{3}$; Jeff Whittle ${ }^{4}$. ${ }^{1}$ Medical College of Wisconsin, Wauwatosa, WI; ${ }^{2}$ Milwaukee VA Medical Center, Milwaukee, WI; ${ }^{3}$ Medical College of Wisconsin, Milwaukee, WI; ${ }^{4}$ Zablocki VA Medical Center, Milwaukee, WI. (Control ID \#3185753)

BACKGROUND: Increased cognitive activity is associated with increased cognitive function in older populations. To test whether midlife cognitive activity might increase late life cognitive function, we studied older (age 68+) male Veterans participating in a longitudinal study of exercise and cognition.

METHODS: We measured current (late-life) cognitive activity using a validated survey (Wilson et. al) which includes such activities as reading books, newspapers or magazines and writing letters; we used a modified version of this survey to ask respondents to recall participation in similar activities at age 50 (midlife). We used standard tests (e.g., Brief Visuospatial Memory Test-Revised (BVMT-R), Wide Range Achievement Test (WRAT4)) to measure cognitive function. We measured functional status with the physical and mental component scores (PCS12, MCS12) from the Veterans Rand 12 item survey and PROMIS measures of physical and social function. We also asked, "What things influence how much time you spend on mental activity?". We first examined the association of midlife cognitive activity with current cognitive activity and functional status using simple correlations, then used linear regression to examine these same associations, controlling for demographics and other potential confounders.

RESULTS: The 78 participants were largely white (87\%), older average (mean age 74.8 years) and well educated (65\% had more than 12 years of education; $28 \%$ had a college degree). Most (67\%) were retired but only $24 \%$ had annual household income $<\$ 30 \mathrm{k}$. More education, higher income and white race were associated with increased current cognitive activity. The most common cognitive activity was reading; 76\% read "regularly" (more than 1 hour daily) at follow up and $68 \%$ reported doing so in midlife. While midlife cognitive activity was positively correlated with current cognitive activity $(\mathrm{p}<0.0001, \mathrm{r} 2=0.3123)$, there was no consistent relationship between mid-life cognitive activity and current cognitive function or functional status. Adjustment for potential confounders using multivariable analysis produced similar results. In our qualitative analysis, many respondents reported that much of their cognitive activities are embedded in everyday life (finances, healthcare). These activities are not captured in the cognitive activity measure that we used.
CONCLUSIONS: Higher levels of midlife cognitive activity were associated with more late-life cognitive activity but not improved function. The surprising lack of association between cognitive activity and cognitive function may reflect a healthy volunteer effect. Moreover, our qualitative work identified important cognitive activities that were not captured in our measure of cognitive activity. Our results do suggest that efforts to increase late-life cognitive activity may need to start earlier, when patterns of behavior are established.

\section{INFLUENCES OF PROVIDER GENDER ON UNDERLYING COM- MUNICATION SKILLS AND PATIENT CENTEREDNESS IN PAIN MANAGEMENT CLINICAL SCENARIOS}

Jeffrey Wilhite; Harriet Fisher; Khemraj Hardowar; Lisa Altshuler; Sarah Chaudhary; Sondra Zabar; Adina Kalet; Kathleen Hanley; Colleen C. Gillespie. NYU School of Medicine, New York, NY. (Control ID \#3185434)

BACKGROUND: For quality care, physicians must be skilled in diagnosing and treating chronic pain. Some studies have shown gender differences in how providers manage pain. And more broadly, female providers provide more patient-centered communication which in turn has been linked to patient activation and satisfaction with care. We explore, using Unannounced Standardized Patients (USPs), whether resident physician gender is associated with the core underlying skills needed to effectively diagnose and management chronic pain: communication, patient centeredness, and patient activation.

METHODS: We designed two USP cases and sent these undercover patients into primary care clinics at two urban, safety-net clinics. The USP cases were similar: a 30-35 y.o. male, presented as a new patient to the clinic with either shoulder pain induced by heavy lifting or knee pain due to a recreational sports injury. USPs completed a post-visit checklist that assessed patient satisfaction (4 items), patient activation (3 items), and communication skills (13 items) using a behaviorally-anchored scale (not done or partly done vs. well done). Summary scores were calculated for each of the three domains. Residents provided consent for their educational data to be used for research as part of an IRB-approved medical education registry.

RESULTS: A total of 135 USP visits ( 80 female providers, 55 male) occurred between 2012 and 2018. Female providers saw 41 shoulder pain and 39 knee pain cases while male providers saw 21 shoulder and 34 knee cases. ANOVA was used to assess differences in summary scores by provider gender (male vs female) and by case portrayed (knee vs shoulder). Skills did not differ significantly by whether knee or shoulder pain case. Gender effects were not seen for patient centeredness or for patient activation; however female providers performed significantly better at relationship development $(83 \%$ vs males $72 \%$ shoulder pain; $70 \%$ vs $66 \%$ knee pain case; $p<.001$ ) and information gathering ( $86 \%$ vs. males $72 \%$ shoulder pain; $79 \%$ vs $66 \%$ in knee case; $\mathrm{p}<.016)$. Male providers, however, performed slightly better in patient education and counseling (65\% vs $63 \%$ for shoulder and $38 \%$ vs $33 \%$ for knee cases; $\mathrm{p}<.001)$.

CONCLUSIONS: Developing a relationship and gathering information are critical to pain management and female residents performed better than male residents in these areas. Male providers performed slightly better than women in patient education and provider gender was not associated with any differences in patient centeredness or activation. In the future, we plan to link these underlying skills to pain management decisions, documentation and ultimately to patient outcomes. We suspect that patient activation may best be measured at follow-up, something not possible with our current USP methodology. Gender differences could be viewed as striking in the context of our relatively homogeneous sample (medicine residency program) and shared clinical environment/healthcare system.

INFLUENZA MANAGEMENT VIA DIRECT TO CONSUMER TELEMEDICINE

Kathryn A. Martinez; ${ }^{1}$ Mark N. Rood ${ }^{2}$; Michael B. Rothberg ${ }^{1} .{ }^{1}$ Cleveland Clinic, Cleveland, $\mathrm{OH} ;{ }^{2}$ Cleveland Clinic Foundation, Chagrin Falls, $\mathrm{OH}$. (Control ID \#3180272) 
BACKGROUND: Influenza is easy to clinically diagnose during an epidemic, and immediate treatment can shorten symptom duration. Direct to consumer (DTC) telemedicine connects patients with physicians 24 hours a day through mobile applications. Telemedicine may be an ideal setting in which to treat influenza, offering access to immediate care without exposing others to infection. Diagnosis outside of influenza epidemics requires rapid testing, which cannot be performed virtually, exposing patients to overtreatment. Patterns of care for influenza in DTC telemedicine are unknown. The objective of this study was to characterize care seeking, diagnosis and treatment for influenza in a large DTC telemedicine platform.

METHODS: This study uses data from the AmericanWell DTC telemedicine platform. Encounters were conducted with physicians between July 2016 and August 2018. Upon connecting with the telemedicine system, patients were asked to describe their call reason using a free text field. Whether they stated their call reason was influenza was assessed. Patients also provided age and insurance information, if applicable (visits cost \$59 out of pocket). Inlfuenza epidemics over the two-year study period were identified based on the Center for Disease Control's Weekly U.S. Influenza Map (December 24, 2016 to April 15, 2017 and December 2, 2017 to April 14, 2018) and encounters were categorized as taking place during an epidemic or not. Tamiflu prescriptions were identified via National Drug Codes associated with each encounter. We described patterns of care for influenza during epidemics versus not.

RESULTS: During the two year study period, 8,055 patients were diagnosed with influenza, of whom $16 \%$ were 18 years or younger, and $45 \%$ were between 19 and 39 years. Eighty eight percent of influenza diagnoses occurred during epidemics. Among those diagnosed with influenza, $80 \%$ received Tamiflu. The proportion of influenza patients receiving Tamiflu did not vary by epidemic status ( $80 \%$ during epidemics vs. $78 \%$ at other times). The majority of patients who were not diagnosed with influenza but nonetheless prescribed Tamiflu were diagnosed with viral illness, unspecified. Of the 8,112 patients who stated their call reason was influenza, $66 \%$ called during an epidemic. The proportion of these patients subsequently diagnosed with influenza varied by season: $52 \%$ were diagnosed with influenza during epidemics, versus $11 \%$ at other times.

CONCLUSIONS: Physicians treating patients with influenza symptoms were more likely to diagnose and treat patients during an epidemic. Prescription of Tamiflu at other times without rapid testing probably represents inappropriate care. DTC telemedicine represents a promising venue for treating influenza with antiviral medication, but provider education may be required.

\section{INITIATIVE TO IMPROVE COLORECTAL CANCER SCREEN- ING RATES AT A FEDERALLY QUALIFIED HEALTH CENTER}

Kyle T. Kunz; Swaroop Vitta; Kathryn Parker. Washington University in Saint Louis, Saint Louis, MO. (Control ID \#3185473)

BACKGROUND: Colorectal cancer (CRC) is the third-leading type of cancer and cause of cancer-related death for both men and women in the United States. Improved awareness and screening has led to earlier discovery and CRC-related deaths have been declining in recent decades. Based on guidelines, screening rates are still reported at only $63 \%$ nationally. Lower screening rates (40\%) and more frequent late stage discoveries occur in disadvantaged patient populations that are more apt to use federally qualified healthcare centers (FQHC). As a quality improvement initiative, we sought to increase $\mathrm{CRC}$ screening rates at a single $\mathrm{FQHC}$.

METHODS: This study was performed at a single multilocation FQHC. After gathering baseline data from July 2017 through October 2017, 4 PDSA cycles were conducted from October 2017 through June 2018 including (1) Provider education, Gemba walks, EHR (electronic health record) order set changes (2) Patient education with postcards and in-person nursing education (3) EHR reconfiguration and back-charting uncaptured results (4) Pre-shift huddle with medical assistants to identify patients with inadequate screening. All prior interventions were continued in addition to the subsequent interventions for each cycle. Data for screening results was gathered from Azara Healthcare, a community health center data reporting agency which draws from our EHR.
RESULTS: The baseline screening rate was $7 \%$ which ranked as the lowest for FQHCs in the state. Cycle 1 had the least impact with increased screening rates to $10 \%$ from Ocober 2017-January 2018. Cycle 2 from January 2018 through March 2018 increased screening rates from 10\% to 15\%. Cycle 3 had the most significant impact increasing screening rates from $15 \%$ to $26 \%$ from March 2018 through June 2018. Rates continued to increase from 26\% to 33\% during cycle 4 from June 2018 through December of 2018.

CONCLUSIONS: In a vulnerable patient population at single FQHC, CRC screening rates were improved and showed sustained rates with the above interventions. Consistent with prior quality improvement studies, EHR interventions provided the most significant increase likely due to noncaptured results while early education provided least improvement. Incorporation of the healthcare team with huddles also had improvement in screening results consistent with prior studies.

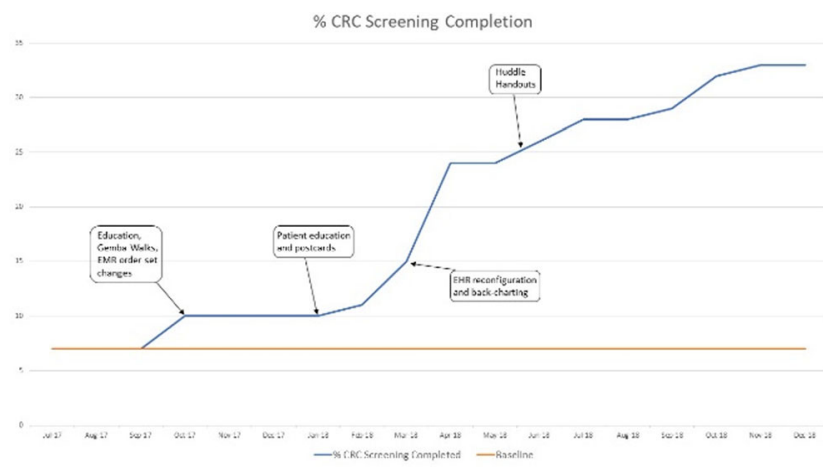

INTEGRATING AN ONLINE WEIGHT MANAGEMENT PROGRAM WITH POPULATION HEALTH MANAGEMENT SUPPORT IN PRIMARY CARE: PRELIMINARY RESULTS FROM THE PROPS STUDY (PARTNERSHIPS FOR REDUCING OVERWEIGHT AND OBESITY WITH PATIENT-CENTERED STRATEGIES)

Heather J. Baer ${ }^{1}$; Barbara A. De La Cruz ${ }^{1}$; Nyryan V. Nolido ${ }^{1}$; E. John Orav ${ }^{1}$; Kristina Secinaro ${ }^{3}$; Ronen Rozenblum ${ }^{1}$; Jason P. Block ${ }^{2}$; Katherine D. McManus $^{3}$; Florencia Halperin ${ }^{3}$; David W. Bates ${ }^{1} .{ }^{1}$ Brigham and Women, Boston, MA; ${ }^{2}$ Harvard Pilgrim Health Care Institute/Harvard Medical School, Boston, MA; ${ }^{3}$ Brigham and Women's Hospital, Boston, MA. (Control ID \#3185584)

BACKGROUND: Primary care providers often do not have sufficient time or training to counsel patients about weight. There is an urgent need for scalable, low-cost weight management strategies that can be easily implemented in primary care.

METHODS: We adapted an evidence-based online weight management program and integrated it with population health management support at primary care practices affiliated with Brigham and Women's Hospital (BWH), an academic medical center in Boston, Massachusetts. The online program consisted of educational sessions, structured meal plans, selfmonitoring tools, and automated feedback and reminders. The population health management strategy involved monitoring and support from nonclinical primary care staff. We conducted a pragmatic, cluster-randomized trial in 15 primary care practices ( 24 clinics) to compare the effectiveness of three strategies: 1) usual care, 2) online program alone, and 3) online program plus population health management support (combined intervention). Participants were ages 20 to 70, had body mass index (BMI) 27 and $<40 \mathrm{~kg} / \mathrm{m} 2$, and had hypertension or type 2 diabetes. They attended regular visits at their primary care practices over 18 months and completed surveys at enrollment and 6,12, and 18 months after enrollment. The primary outcome is weight change at 12 months after enrollment, calculated from weights measured at primary care visits. Secondary outcomes include changes in cardiovascular risk factors, weight-related quality of life, health status, physical activity, and diet. The 
12-month follow-up was completed on November 8, 2018, and the 18- month follow-up will be completed on May 8, 2019. Because follow-up is still ongoing, preliminary results are presented across the three groups, rather than separately by group.

RESULTS: A total of 840 participants were enrolled between July 2016 and August 2017 (326 usual care, 216 online program alone, and 298 combined intervention.) At enrollment, participants' mean age was 59.3 years, their mean weight was 203.1 pounds, and their mean BMI was 32.5 $\mathrm{kg} / \mathrm{m} 2 ; 60 \%$ of participants were female, $76 \%$ were white, $96.4 \%$ had hypertension, and $24.4 \%$ had type 2 diabetes. On average, participants lost weight, with a mean decrease of 4.7 pounds over 12 months. There were increases in weight-related quality of life (mean increase of 5.3 points on a 100-point scale), physical activity (mean increase of 5.4 minutes per week), and in excellent or very good health status (14\% increase) over 12 months, with small changes in cardiovascular risk factors and dietary factors.

CONCLUSIONS: It is feasible to integrate an online weight management program with population health management support in primary care. Preliminary results indicate that participants lost weight and had improvements in weight-related quality of life, health status, and physical activity over 12 months. Analyses comparing the effectiveness of the three strategies are forthcoming.

\section{INTEGRATING FINANCIAL COACHING AND SMOKING CES- SATION COACHING TO REDUCE HEALTH AND ECONOMIC DISPARITIES IN LOW-INCOME SMOKERS}

Erin S. Rogers ${ }^{1}$; Elizabeth Vargas ${ }^{1}$; Marc I. Rosen ${ }^{2}$; Miguel BarriosBarrios $^{1}$; Mittal Rana ${ }^{1}$; John Rezkalla ${ }^{1}$; Reynalry Rozon ${ }^{1}$; Christina Wysota ${ }^{1}$; Scott E. Sherman ${ }^{1,3}$. ${ }^{1}$ NYU Langone Health, New York, NY; ${ }^{2}$ Yale University School of Medicine, New Haven, CT; ${ }^{3}$ VA New York Harbor Healthcare System, New York, NY. (Control ID \#3185072)

BACKGROUND: Smoking rates are two times higher among people living in poverty. Low-income smokers face unique barriers to cessation, including high levels of financial distress. Reducing financial distress may improve cessation rates in this vulnerable population. Moreover, cessation of tobacco spending may further alleviate financial distress by freeing-up funds that could go toward essentials (e.g., food). We examined the efficacy of a program that integrates financial management coaching into smoking cessation coaching for low-income smokers.

METHODS: We recruited 359 smokers living below 200\% of the federal poverty level in New York City and randomized them 1:1 to receive up to 9 sessions of integrated financial management-smoking cessation coaching or usual care. The financial coaching aimed to help participants move from spending on cigarettes to spending on household essentials, and to help participants access financial resources. Participants completed surveys at baseline, 2 and 6 months to assess smoking and financial outcomes and treatment satisfaction.

RESULTS: Intervention patients were more likely to have made a quit attempt by 6 months than Control participants ( $81 \%$ vs. $66 \%, \mathrm{p}=.03$ ). Abstinence was significantly higher for the Intervention group at 2 months $(23 \%$ vs $9 \%, \mathrm{p}=.01)$ and 6 months (30\% vs. $10 \%, \mathrm{p}<.005)$. At 6 months, Intervention participants were less likely to report high levels of stress about their general finances ( $44 \%$ vs. $66 \%$ Control, $\mathrm{p}=.01$ ), high levels of worry about meeting monthly expenses (56\% vs $73 \%$ Control, $\mathrm{p}=.01)$, or high dissatisfaction with their present financial situation $(63 \%$ vs $75 \%$ Control, $\mathrm{p}<.05)$. Intervention participants were also less likely to report frequently living paycheck to paycheck (71\% vs $88 \%$ Control, $\mathrm{p}=.01$ ) or frequently being unable to afford leisure activities ( $51 \%$ vs $70 \%$ Control, $\mathrm{p}<.05)$. There was no group difference in the level of confidence in being able to pay for a $\$ 1,000$ financial emergency ( $71 \%$ low confidence for both). Among the $71 \%$ of Intervention participants who began counseling, $85 \%$ reported being very satisfied with the integrated counseling. Fifty-one percent reported that the number of counseling sessions they received was "just right," while 36\% reported that the number was "too few." Out of the participants who quit smoking, 100\% reported that quitting smoking helped them financially and $58 \%$ described achieving one or more of their post-quit financial goals.

CONCLUSIONS: Integrating financial coaching into our smoking cessation program was feasible and produced significantly higher abstinence rates and reductions in financial distress than usual care. Participants were highly satisfied with the integrated program and felt it helped them financially. Our integrated program can serve as model for addressing the unique needs of low-income smokers.

\section{INTENSIFICATION OF OLDER ADULTS ANTIHYPERTEN- SIVES AT HOSPITAL DISCHARGE LEADS TO SERIOUS AD- VERSE EVENTS WITHOUT LONG TERM BENEFIT}

Timothy Anderson ${ }^{1}$; Bocheng Jing $^{2}$; Charlie M. Wray ${ }^{3}$; Sarah $\mathrm{Ngo}^{2}$; Kathy Fung ${ }^{2}$; Molly Silvestrini ${ }^{2}$; Michael A. Steinman ${ }^{4}, 2$. ${ }^{1}$ University of California, San Francisco, San Francisco, CA; ${ }^{2}$ UCSF, San Francisco, $\mathrm{CA} ;{ }^{3}$ University of Chicago, Mill Valley, CA; ${ }^{4} \mathrm{SF}$ VA Medical Center, San Francisco, CA. (Control ID \#3165998)

BACKGROUND: Transient elevations of blood pressure (BP) are common in hospitalized older adults and often lead clinicians to discharge patients on intensified antihypertensive medication regimens, even when patients are hospitalized for non-cardiac conditions. This practice may expose patients to overtreatment, particularly those whose BP was previously at goal, however the clinical outcomes are unknown.

METHODS: We used national VA and Medicare data to examine veterans age $>=65$ years with hypertension who were hospitalized in a VA in 2011-2013 for common non-cardiac conditions. Using propensity score matching based on 120 demographic and clinical variables, patients discharged on intensified antihypertensives were compared to those who were not. Competing risk regressions were used to assess all-cause readmissions and serious adverse events (SAE) at 30 days and cardiovascular events at 1 year, accounting for the competing risk of death. The SAE outcome was a composite of emergency department (ED) visits and hospitalizations for injurious falls, syncope, hypotension, electrolyte abnormalities and acute kidney injury. The cardiovascular event outcome was a composite of ED visits and hospitalizations for acute coronary syndrome, stroke, heart failure, and hypertension. The secondary outcome of change in systolic BP was assessed within 1 year of discharge. Matching and analyses were repeated in the subgroup of patients with a pre-hospitalization SBP $<140 \mathrm{mmHg}$.

RESULTS: The matched cohort included 4056 patients, evenly divided among those who did vs did not receive antihypertensive intensifications, with excellent covariate balance (standardized mean differences for all covariates $<0.1$ ). Mean age was 77 years and $3 \%$ were female. At 30 days, patients receiving intensifications had a higher risk of SAE events [hazard ratio (HR) 1.41 (95\% CI, 1.06 to 1.88); number needed to harm (NNH) 63 (35-368)] and all-cause readmissions [HR 1.23 (95\% CI, 1.07 to 1.42); $\mathrm{NNH} 27$ (16-76)]. At 1 year, there was no difference in cardiovascular events [(HR 1.18 (95\% CI, 0.99 to 1.40)] and no difference in change in SBP among those who did vs did not receive intensifications [mean BP 134.7 vs 134.4 , difference-in-differences $0.6 \mathrm{mmHg}$ (-2.4 to 3.7)]. Among the 2244 matched patients with $\mathrm{SBP}<140$ prior to hospitalization, patients receiving intensifications had a higher risk of SAE events and readmissions at 30 days and cardiovascular events at 1 year $($ all $\mathrm{P}<0.05$ ). CONCLUSIONS: Among older veterans hospitalized for non-cardiac conditions, those discharged on intensified antihypertensives had no difference in BP control or cardiac events at 1 year but had a substantially increased risk of serious adverse events and readmission at 30 days, with a number needed to harm of up to 27 . Our findings indicate that the common practice of intensifying antihypertensive regimens during hospitalization poses greater risks than benefits and should be reserved for patients whose reason for hospitalization is related to BP control. 
INTER-HOSPITAL RETURN TRANSFERS: AN UNTAPPED OPPORTUNITY TO IMPROVE ACCESS TO TERTIARY CARE. Maleka Khambaty ${ }^{1}$; Stacia Birkland ${ }^{2}$; Keri Rateliff ${ }^{2}$; Michael Rhodes ${ }^{3}$; Karyn Baum ${ }^{3}$; Michael Usher ${ }^{1} .{ }^{1}$ University of Minnesota Medical School, Minneapolis, MN; ${ }^{2}$ Fairview - MHealth, Minneapolis, MN; ${ }^{3}$ University of Minnesota, Minneapolis, MN. (Control ID \#3185865)

BACKGROUND: More than a million people are transferred between hospitals on a yearly basis in the US. Patients are transferred to tertiary and quaternary care centers for specialty expertise, critical and surgical care, and multidisciplinary resources. Due to the increasing age, complexity, and need for subspecialty services, the demand for inter-hospital transfers is likely to rise, and efforts to efficiently manage this population is necessary. Internationally, regional hospital networks have arisen to streamline flow of patients across hospitals. Several studies have demonstrated safe return of adult patients with acute coronary syndrome, trauma, and cerebrovascular accidents to referring medical centers. In this study, we describe the current state of US inter-hospital return transfers in a large, multistate cohort of patients.

METHODS: We utilized the Healthcare Cost and Utilization Project's State Inpatient and Emergency Department Dataset, (FL, IA, VT, UT, and NY from 2011-2013), identifying transfers by matching patient ID (visitlinkID), age, gender, and transfer date. Our primary exposure variable was whether a patient was returned to the originating hospital following an inter-hospital transfer. Patient level factors such as age, gender, race, payer, and charslon comorbidity index, and admitting diagnosis were compared. Additionally, encounter and hospital differences were compared including length of stay, cost, inpatient mortality, hospital ownership and rural/urban location. Between group comparisons were made by chi-square and t-test where appropriate.

RESULTS: Of 358,354 patient transfers only $1.6 \%$ were returned to their originating hospital. These patients were older on average $(57.5 \mathrm{y}$ vs $61.9 \mathrm{y}$ $\mathrm{p}<0.001$ ), more likely to be non-white, with payment by Medicare, and a higher Charleston comorbidity index $(\mathrm{p}<0.001)$. Return transfers involved a markedly longer length of stay: total of 5 vs 23 days, higher total charges, $(\$ 48 \mathrm{~K}$ vs $\$ 274 \mathrm{~K} \mathrm{p}<0.001)$ and nearly twice the inpatient mortality $(6.7 \%$ vs $11.2 \%, \mathrm{p}<0.001)$. Hospitals involved in the coordination of return transfers, meaning they were responsible for accepting then referring, were more likely to be urban and for-profit (19.7\% vs $37.7 \%$ $\mathrm{p}<0.001)$. These differences remained consistent across several admitting diagnoses including acute myocardial infarction, cerebrovascular disease, and traumas.

CONCLUSIONS: Inter-hospital transfers are an understudied public health challenge. The unidirectional nature of transfers has the potential to over-burden tertiary care facilities as need increases. These data highlight the untapped potential to improve efficiency by which hospital-based subspecialty care is administered: return transfers: While other countries including Canada have shown return transfers after low risk procedures to be safe, this was not observed to occur in the US, where return transfers appeared to be a last resort for length of stay outliers.

\section{INTERHOSPITAL TRANSFER IS ASSOCIATED WITH IN- CREASED IN-HOSPITAL MORTALITY ON INTERNAL MEDI- CINE SERVICES}

Marc Heincelman ${ }^{1}$; Andrew Schreiner ${ }^{1}$; Elizabeth B. Kirkland ${ }^{2}$; Samuel O. Schumann ${ }^{1}$; Jingwen Zhang ${ }^{2}$; Patrick D. Mauldin ${ }^{1}$; Mulugeta Gebregziabher ${ }^{2}$; William P. Moran ${ }^{1} .{ }^{1}$ Medical University of South Carolina, Charleston, SC; ${ }^{2}$ MUSC, Charleston, SC. (Control ID \#3171238)

BACKGROUND: National administrative datasets have demonstrated worse outcomes among patients undergoing interhospital transfer (IHT); specifically increased risk-adjusted mortality, adverse events, cost, and length of stay, compared to patients admitted through the emergency department (ED). Although national administrative datasets are excellent for preliminary studies, limitations include the inability to adjust for disease-specific conditions and other patient level clinical characteristics. In this single center study, we investigate the independent association between IHT status and in-hospital mortality, after adjusting for diseasespecific conditions and other clinical variables not available in larger administrative datasets.

METHODS: We performed a retrospective cohort study of all adult patients admitted to Internal Medicine services from July 1, 2013 to June 30, 2014. The primary outcome was in-hospital mortality. Four Cox regression analyses were performed to examine the independent association between IHT status and in-hospital mortality, controlling for covariates that were potential confounders to the relationship between transfer and death. Model 1: IHT status, admit service. Model 2: IHT status, admit service, patient demographics. Model 3: IHT status, admit service, patient demographics, disease-specific conditions. Model 4: IHT status, admit service, patient demographics, disease-specific conditions, clinical variables.

RESULTS: Of the 7571 patients included in the cohort, $6 \%$ (438) experienced in-hospital mortality. IHT patients accounted for 38\% (165) of all inhospital deaths, despite accounting for only $18 \%$ (1393) of total admissions. In an attempt to determine whether IHT status was associated with inhospital mortality, we performed several Cox proportional hazard regression analyses. Model 1 demonstrated a hazard ratio (OR) of 1.876 for IHT status, after adjusting for admitting service. Model 2 demonstrated a hazard ratio of 1.842 for IHT status, after adjusting for admitting service and patient demographics. Model 3 demonstrated a hazard ratio of 1.810 for IHT status, after adjusting for admitting service, patient demographics, and disease-specific conditions. Model 4 demonstrated a hazard ratio of 1.564 for IHT status, after adjusting for admitting service, patient demographics, disease-specific conditions, and patient laboratory data and vital signs. The integrated time-dependent AUC for model 1 was 0.74, AUC for model 2 was 0.79, AUC for model 3 was 0.82 , and AUC for model 4 was 0.89 , consistent with a good prediction model.

CONCLUSIONS: After adjusting for patient-level clinical characteristics including vital signs, laboratory data, and individual comorbidities, IHT status remains independently associated with in-hospital mortality.

\section{INTERNAL MEDICINE RESIDENT MATRICULATION INTO CARDIOLOGY FELLOWSHIPS: ASSOCIATIONS WITH CA- REER INTENT, MEDICAL KNOWLEDGE, AND PUBLICA- TIONS}

Michael W. Cullen; Kyle W. Klarich; Andrew J. Halvorsen; Amy Oxentenko; Thomas J. Beckman. Mayo Clinic, Rochester, MN. (Control ID \#3182576)

BACKGROUND: Unique traits of internal medicine (IM) residents who matriculate into subspecialty fellowships, particularly cardiovascular (CV) diseases, are poorly understood. We sought to identify characteristics of IM residents who successfully match into CV fellowships.

METHODS: We conducted a retrospective cohort study of 8 classes of IM residents who matriculated into residency from 2007-2014 (graduating classes of 2010-2017). Residents not completing 3 years of residency were excluded. The primary outcome was a successful match to a CV fellowship within 1 year of completing IM residency. Independent variables included residents' licensing exam scores, research publications, medical school reputation, Alpha Omega Alpha (AOA) membership, declaration of intent to pursue $\mathrm{CV}$ in the residency application personal statement, performance during residency based on clinical evaluation, mini-clinical evaluation exercise (mini-CEX), and in-training exam (ITE) scores, and exposure to $\mathrm{CV}$ during residency based on time of first rotation and choice of a $\mathrm{CV}$ elective rotation. Data were analyzed by logistic regression.

RESULTS: Of the 339 included residents (59\% male; mean age 27) from 120 medical schools, 73 (22\%) matched to CV fellowship. At the time of residency application, $104(31 \%)$ had 1 publication, $38(11 \%)$ declared intention to pursue $\mathrm{CV}$ in their residency application personal statement, 
and $104(31 \%)$ were members of AOA. Prior to fellowship application, 111 (33\%) completed a CV elective rotation. At the completion of residency training, $108(32 \%)$ had 3 publications. Multivariable logistic regression revealed that declaration of intention to pursue CV (OR 6.4, 99\% CI 1.723.4; $\mathrm{p}<0.001$ ), completion of a CV elective (OR 7.3, 99\% CI 2.8-19.0; $\mathrm{p}<0.001$ ), percentile score on the CV portion of the PGY-2 ITE (OR 1.05, 99\% CI 1.02-1.08; $\mathrm{p}<0.001$ ), and publication of 3 manuscripts (OR 4.7, $99 \%$ CI 1.1-20.5; $\mathrm{p}=0.007$ ) were positively associated with matching to a CV fellowship. Overall PGY-2 ITE percentile score was negatively associated (OR 0.93, 99\% CI 0.90-0.97; $\mathrm{p}<0.001$ ). No associations existed with licensing exam scores, medical school reputation, AOA membership, timing of first $\mathrm{CV}$ rotation, mini-CEX scores, or clinical evaluations, both globally and during CV rotations.

CONCLUSIONS: Residents' matriculation into CV fellowships was associated with declaration of CV career intent, completing a CV elective rotation, CV medical knowledge performance, and research publications during residency. These findings may be useful to faculty members who are advising residents about pursuing careers in cardiology. The negative association between matching into CV fellowship and overall ITE knowledge performance may indicate excessive subspecialty focus during IM residency and requires further study.

\section{INTERNAL MEDICINE RESIDENT PROFESSIONALISM AS- SESSMENTS ARE ASSOCIATED WITH PATIENTS' OVERALL SATISFACTION WITH THEIR HOSPITAL STAY}

John T. Ratelle; Andrew J. Halvorsen; Jayawant N. Mandrekar; Adam P. Sawatsky; Darcy Reed; Thomas J. Beckman. Mayo Clinic, Rochester, MN. (Control ID \#3179008)

BACKGROUND: Successful training of internal medicine (IM) residents requires accurate assessments. Patients could provide useful assessments of IM residents in the hospital, yet there is little understanding of how contextual factors may impact on these assessments. Our objective was to explore the potential relationships between patient, resident, and hospital-related factors with standardized patient-of-resident professionalism assessment scores.

METHODS: This was a prospective cohort study of postgraduate year 1 (PGY-1) IM residents and the patients they cared for at Mayo Clinic from July 2015-June 2016. Hospitalized patients provided professionalism assessments of PGY-1 IM residents who were primarily responsible for their care using an instrument that was adapted from published measures. Assessments were obtained on hospital day \#4 to optimize the number of patient-resident encounters. Associations between patient-of-resident assessment scores and characteristics of residents (including validated measures of residents' performance and professional behaviors in other settings), patients, and hospital encounters were determined with multivariable modeling using generalized estimating equations to account for repeated assessments of residents. The threshold for statistical significance was $\mathrm{p}<0.01$.

RESULTS: A total of 409 unique patients assessed all 72 PGY-1 residents, with a mean (SD) of 5.7 (3.0) patient assessments per resident. Factor analysis of the 11-item instrument demonstrated two dimensions (Cronbach alpha): "Involvement and Respect" (0.95) and "Compassion and Rapport" (0.94). In the multivariable model, only patients' "top box" ratings of their hospital stay remained significantly associated with their assessments of resident professionalism $([\mathrm{SE}]=+0.7952[0.0788] ; \mathrm{p}<.0001)$. Notably, patient-ofresident assessment scores in the hospital were not significantly correlated with assessments of resident professionalism in other clinical and educational settings.

CONCLUSIONS: Patients' assessments of resident professionalism in the hospital were positively associated with patients' overall satisfaction with their hospital stays, but were not correlated with residents' professional behaviors in other settings. Residency programs should consider the potential limitations of patient evaluations before incorporating them into multi-source assessments of trainees.

\section{INTERNISTS' EXPERIENCE WITH TRAINING TO PRO-} VIDE MIFEPRISTONE: A QUALITATIVE ANALYSIS

Mindy Sobota ${ }^{2}$; Melissa Gosdin ${ }^{3}$; Jessica Beaman ${ }^{5}$; Suzan Goodman ${ }^{4}$; Maria Catrina D. Jaime ${ }^{3}$; E. Bimla Schwarz ${ }^{1}$. ${ }^{1}$ University of California, Davis, Sacramento, CA; ${ }^{2}$ Brown, Providence, RI; ${ }^{3} \mathrm{UC}$ Davis, Sacramento, CA; ${ }^{4} \mathrm{UCSF}$, San Francisco, CA; ${ }^{5}$ University of California, San Francisco, San Francisco, CA. (Control ID \#3186081)

BACKGROUND: Mifepristone (previously known as RU-486) has been a safe, and effective option for US women for almost 20 years; in 2016, the FDA issued an evidence-based update allowing use of abortion pills to terminate pregnancies less than 10 weeks gestation. Although $75 \%$ of the 1.2 million US women who seek abortion services each year do so before 10 weeks gestation, currently only $11 \%$ of US counties have a clinician who offers abortion services of any kind. As a result, many women must travel substantial distances to access abortion services, which is particularly difficult for low income women who are most likely to face undesired pregnancies.

METHODS: To understand barriers and facilitators to general internists' provision of medication abortion, a medical sociologist conducted semistructured phone interviews with 8 general internists who have sought training on how to offer mifepristone to patients seeking medication abortion. RESULTS: All participants reported feeling that reproductive health care is a fundamental component of primary care. Most felt comfortable offering counseling about contraception and miscarriage. However, participants reported having received limited training about abortion in medical school and even less training on abortion during Internal Medicine residency. Participating internists described medication abortion as a "simple-see one, do one, teach one" clinical service, but reported having needed to seek out independent training opportunities in order to learn how to offer medication abortion services to their patients. Participants noted that "compared to other new medications-like PrEP — mifepristone has just not been marketed to us or our patients." Participants further noted that accredited "continuing medical education (CME)" related to abortion or mifepristone was hard to find. For some participants, FDA regulations requiring that mifepristone be stocked in clinic (instead of in pharmacies) was a hurdle, while other participants noted they had been pleasantly surprised by how supportive clinic staff had been of adding mifepristone to the list of services their clinic offers. Participants noted that patients rarely asked for help managing undesired pregnancy until they routinely informed patients that "if this birth control pill fails...I have pills for that too." Internists reported that their patients greatly appreciated the convenience and privacy of obtaining mifepristone in familiar primary care settings. Participants felt that online training would be helpful to colleagues interested in offering mifepristone for medication abortion and miscarriage management.

CONCLUSIONS: Efforts are needed to increase training opportunities for general internists interested in providing mifepristone. Online training, especially if CME-accredited, may increase the number of internists able to provide mifepristone for medication abortion and miscarriage management.

INTERVENTIONS TO REDUCE AGGRESSIVE CARE IN THE LAST YEAR OF LIFE AMONG CANCER PATIENTS: A SYSTEMATIC REVIEW

Nauzley C. Abedini $^{1,}{ }^{1}$; Rachel K. Hechtman ${ }^{1}$; Achintya D. Singh ${ }^{2}$; Jason Mann $^{1}$; Rafina Khateeb ${ }^{1}$; Whitney A. Townsend ${ }^{1}$; Vineet Chopra ${ }^{1}$. ${ }^{1}$ University of Michigan, Ann Arbor, MI; ${ }^{2}$ All India Institute of Medical Science, New Delhi, India. (Control ID \#3152624) 
BACKGROUND: Aggressive end of life (EOL) treatments in advanced cancer patients are associated with low-value care and frequently lead to unnecessary hospitalizations. Since 2012, the National Quality Forum (NQF), American Society of Clinical Oncology (ASCO), and Centers for Medicare and Medicaid Services (CMS) have adopted EOL quality measures to reduce aggressive care. Using these measures, we conducted a systematic review of the literature to explore what interventions are associated with reductions in aggressive EOL care in cancer patients.

METHODS: We searched MEDLINE via Ovid, Cochrane, CINAHL, EMBASE, Scopus, and PsychINFO. We developed a comprehensive search strategy using the following concepts: EOL care, aggressive or intense care, and cancer. We included randomized control trials (RCTs), quasi-experiments, and observational studies. Studies that did not have a clear intervention or exposure variable and/or did not explicitly aim to reduce aggressive EOL care or improve quality of EOL care were excluded. We developed a taxonomy to organize our findings using the Systems Engineering Initiative for Patient Safety (SEIPS) model derived from human factors engineering. The study protocol was registered with the PROSPERO register of systematic reviews (CRD42018087528).

RESULTS: Of the 3,881 studies identified by our search, 271 were included based on title and abstract review, and 36 met the final inclusion criteria after full-text review. Five studies were RCTs. Thirty-two studies used validated or nationally endorsed measures of aggressive EOL care, while 26 used additional unendorsed or un-validated measures. Only 8 studies linked quality measures with patient- or caregiver-reported outcomes. Based on our taxonomy of SEIPS work system categories, we found that interventions targeting task-related factors were most common $(n=20)$. These included (1) referral to subspecialty palliative care or hospice; (2) interventions to improve communication and EOL discussions; and (3) primary care visits. Interventions targeting external factors, such as assessing the implications of a new policy for universal hospice care, were least common $(n=1)$. Meta-analysis was not performed due to heterogeneity of interventions and outcome measures. Interventions were highly variable in their ability to significantly improve measures of aggressive care at EOL in cancer patients.

CONCLUSIONS: Quality measures to reduce overly aggressive EOL cancer care, including hospitalizations, are used inconsistently by investigators. Interventions to improve aggressive EOL care have had mixed success. More studies are needed to assess the efficacy of interventions to reduce aggressive EOL care, particularly those that link quality measures with patient- and caregiver-reported outcomes.

\section{INTRODUCING VALUE-BASED CARE TO MEDICAL STU- DENTS: A CONTROLLED, PROSPECTIVE, COHORT STUDY OF AN EDUCATIONAL INTERVENTION.}

Sara-Megumi L. Naylor ${ }^{1}$; Rong Guo ${ }^{2}$; Allison L. Diamant ${ }^{2}$; Ming Lee ${ }^{2}$. ${ }^{1}$ UCSF, San Francisco, CA; ${ }^{2}$ UCLA, Los Angeles, CA. (Control ID \#3185759)

BACKGROUND: The ongoing crisis in US health care signals a critical need to educate medical students about quality, cost, and how they interact to create value. Some medical schools have incorporated value-based care (VBC) content in their curricula. Most available literature only provide descriptive information about curricular efforts and offer best practices; we currently do not know how these efforts specifically impact medical students when compared to a control group. To address this gap, our study evaluates an educational intervention that teaches medical students about VBC and compares intervention and control groups. We hypothesized that students who participated in the intervention would report higher performance related to VBC than those who did not, and that their higher performance would be sustained.

METHODS: We designed a prospective cohort study and received IRB exemption. We recruited third year medical students between 7/2017 and 4/2018. Out of 137 invited students, 122 (89\%) participated. The intervention sessions consisted of definitions, clinical cases, interactive activities, and resources that highlight VBC. The control sessions were shorter and focused on defining VBC. We collected retrospective pre- and post- survey data after all sessions, and again at the end of the academic year. The surveys used a 5-point Likert scale. Wilcoxon signed rank test and Wilcoxon rank sum test were utilized for data analysis.

RESULTS: We found significant improvement from pre- to post-test scores across all sessions and in all domains following a similar pattern $(p<0.001)$. The domain of knowledge improved the most and the domain of attitudes the least. When evaluating the change from pre-to post-test scores between intervention and control groups by domain, we found significant improvement in the intervention group compared to the control group in all domains, with more for the domains of knowledge, skills, and confidence $(p<0.001)$, and less for the domain of attitudes $(p<0.01)$. When comparing pre- and delayed post-test scores, both intervention and control groups showed significant within-group gains in all domains except knowledge, but we did not find significant difference between intervention and control groups.

CONCLUSIONS: Our study demonstrates that educating medical students in VBC with a concerted effort for students to develop personalized, concrete strategies in their current role as a medical student can increase their self-perceived knowledge, skills, attitudes, and confidence regarding VBC when compared to a control group that receives a general introduction on VBC. Our study design with a control group enriches our findings and gives us greater confidence in the efficacy of our intervention. Our goal is to continue to introduce VBC to medical students with educational interventions that we know have an impact on outcomes, and to help establish long term culture change among medical students to practice VBC in their careers.

\section{INVESTIGATING DIFFERENCES IN LENGTH OF STAY ON TEACHING AND NON-TEACHING HOSPITAL TEAMS}

Rebekah J. Walker; Jennifer Good; Kurt J. Pfeifer; Bradley H. Crotty; Mandy Kastner; Jennifer A. Campbell; Navdeep Gupta; Douglas Levine; Joan Neuner; Leonard E. Egede. Medical College of Wisconsin, Milwaukee, WI. (Control ID \#3185421)

BACKGROUND: The cost of healthcare in the U.S. is higher per capita than any other country with one of the largest drivers of cost being inpatient costs. With an average inpatient stay costing approximate $\$ 14,650$, many hospital systems are investigating ways to decrease lengths of stay while ensuring high quality care. To inform new interventinos, our Division of General Internal Medicine (GIM) aimed to investigate length of stay variability by team, as well as whether differences existed by hospitalist-only vs. hospitalist teaching teams.

METHODS: Inpatient encounters with GIM hospitalist oversight were extracted from the medical record over a 10-month period. Length of stay was calculated based on the time between hospital admission and hospital discharge with no removal of outliers. Encounters were assigned to teams based on the team assignment of the attending provider. In addition to comparisons by individual teams, teams were grouped based on whether they were teaching or non-teaching teams staffed by residents in addition to hospitalists. Observation teams and Advanced Heart Failure teams were not included in means of other non-teaching teams as length of stays were expected to differ for these groups. Since length of stay was not normally distributed, it was modeled first using generalized linear models with $\log$ link and secondly by quartile regression. Models were adjusted for age, gender, race/ethnicity, insurance coverage, Elixhauser comorbidity count, and whether the hospital stay included time spent in the ICU. RESULTS: When compared as individual teams, each teaching team had a significantly higher length of stay than a reference non-teaching team regardless of the non-teaching team selected (mean difference ranged from 1.1-1.5 days higher). There were no significant differences between individual non-teaching teams after adjusting for relevant covariates. 
When grouping teaching and non-teaching teams together into a mean length of stay, teaching teams again had a longer length of stay after adjustment (mean difference of 1.4 days higher). Based on quartile regression, there were no differences between teaching and nonteaching teams in the lowest quartile (mean stay of 1.34 days), however, teaching teams had longer lengths of stay in the middle and upper ends of the distribution (encounters with mean of 2.74 days and higher).

CONCLUSIONS: Based on these results, process improvement opportunities exist within teaching teams regarding length of stay. Hospitalists have begun discussions regarding differences in discharge process and ways to increase efficiency across teams.

\section{INVESTIGATING THE IMPACT OF VHA CLINICIAN BURN-} OUT ON PATIENT-REPORTED ACCESS TO PRIMARY CARE

Taona P. Haderlein ${ }^{1}$; Danielle Rose ${ }^{1}$; Lisa V. Rubenstein ${ }^{2}$; Susan E. Stockdale ${ }^{1}$. ${ }^{1}$ VA Greater Los Angeles Healthcare System, Sepulveda, CA; ${ }^{2}$ RAND and UCLA, Medford, OR. (Control ID \#3183108)

BACKGROUND: Burnout among healthcare professionals is associated with diminished job performance, poor mental and physical health, and increased employee attrition. While the Patient Centered Medical Home Model (PCMH) is associated with positive patient outcomes, the model requires the restructuring of primary clinic workflow and job roles. Past research has shown that organizational changes such as PCMH transformation heighten the risk for employee burnout. However, little is known is about subsequent impacts of clinician burnout on patients' experiences. The present study examines VHA clinician-reported burnout as a predictor of patient-reported access to primary care.

METHODS: We merged primary care clinician (PCC) data from the Veterans Assessment and Improvement Laboratory baseline clinician survey, collected from November 2011 through March 2012, with data from the Survey of Healthcare Experiences of Patients (SHEP) administered by RAPID. We included scores from three subscales of the Maslach Burnout Inventory - emotional exhaustion (EE), cynicism (CY), and professional efficacy (PE) - as our primary predictor variables. We used data from patients who were identified as members of the PCCs' patient panels. Our dependent variables were three SHEP items that assessed patient-reported access to 1) routine appointments, 2) urgent appointments, and 3) tests and treatments. Variable responses were dichotomized as $1=$ always and $0=$ sometimes, usually, or never. For each outcome variable, we conducted multilevel mixed-effect logistic regression analyses with patient data at Level 1 and PCC data at Level 2. EE, CY, and PE burnout subscales were included in the model as predictor variables. We also included provider and patient characteristics as fixed-effect covariates. We used a robust clustering method to address intercorrelation within healthcare systems.

RESULTS: We analyzed data from 161 primary care clinicians, and 4,171 Veteran patients. Patients of clinicians reporting high CY were less likely to report that they always obtained an urgent appointment (OR: .95, 95\% CI: .95-.96) or routine appointment (OR: .96, 95\% CI..92-.99) when needed. Patients of clinicians reporting high EE were more likely to report always getting urgent (OR: 1.02, 95\% CI: 1.01- 1.03) and routine appointments (OR: 1.01, 95\% CI:1.00-1.02) when needed. Low PE was associated with reduced patient-reported access to routine appointments (OR: .96, 95\% CI:.93.99). No association was found for access to tests and treatments.

CONCLUSIONS: The influence of burnout on patient-reported access to primary care appointments varies among burnout dimensions. $\mathrm{CY}$ and low PE had a negative impact on patient-reported access to primary care appointments, while EE was positively associated with patient-reported access. As past research has linked EE with increased workload, more work is needed to elucidate mechanisms of the relationship between clinician burnout and perceived access to care.
IS DIABETES OCCURRING AT YOUNGER AGES? SURPRISING RESULTS FROM NHANES 2001-16

Phuc H. Le ${ }^{1}$; Lu Zhang ${ }^{2}$; Anita D. Misra-Hebert ${ }^{1}$; Glen B. Taksler ${ }^{1}$; Michael B. Rothberg ${ }^{1} .{ }^{1}$ Cleveland Clinic, Cleveland, OH; ${ }^{2}$ University of Texas School of Public Health, Houston, TX. (Control ID \#3185652)

BACKGROUND: Diabetes affects $9.4 \%$ of US adults. The American Diabetes Association recommends screening asymptomatic patients at no later than 45 years old and overweight or obese adults with risk factors at any age. There is a common belief that diabetes is occurring more frequently in young adults, but whether the age at diagnosis has changed is unknown. We aimed to describe population-based trends in mean age at diabetes diagnosis from 2001-2016 among US adults.

METHODS: We conducted a retrospective analysis of National Health and Nutrition Examination Survey (NHANES) data from 2001-2002 through 2015-2016. We included participants aged 18 years and defined diabetes as having ever been told they had diabetes, HbA1C $>6.4 \%$, or fasting plasma glucose $>125 \mathrm{mg} / \mathrm{dL}$. Pregnant women and participants with missing data were excluded. Age at diabetes diagnosis was selfreported. For patients with previously undiagnosed diabetes, we assigned age at the interview as age at diagnosis. Diabetes duration was calculated as the difference between age at interview and age at diagnosis. Mean age at diagnosis and diabetes duration were estimated for each 2-year period and also stratified by patient characteristics and risk factors. Trends were assessed using linear regression. We used Stata 14.2, accounting for the complex survey design of NHANES.

RESULTS: The sample included 46,668 adults, 7,059 (15.1\%) of whom had diabetes. Mean age at diagnosis was 48.2 years and mean diabetes duration was 11.3 years, neither of which changed over time ( $p$ for trend $>0.05$ ). In all years, age at diagnosis was younger for those who were severely obese, poor, uninsured, Hispanic, or Black; had a family history of diabetes; or were current smokers or excessive alcohol users. Compared to 2001-2006, the number of patients diagnosed in 2013-2016 was $2 \mathrm{x}, 1.8 \mathrm{x}$ and $1.7 \mathrm{x}$ higher for ages 30,50 , and 70 years, respectively (figure).

CONCLUSIONS: Mean age at diagnosis did not vary throughout the study period. However, the absolute number of 30-year-olds diagnosed doubled, creating the appearance that diabetes was occurring at younger ages.

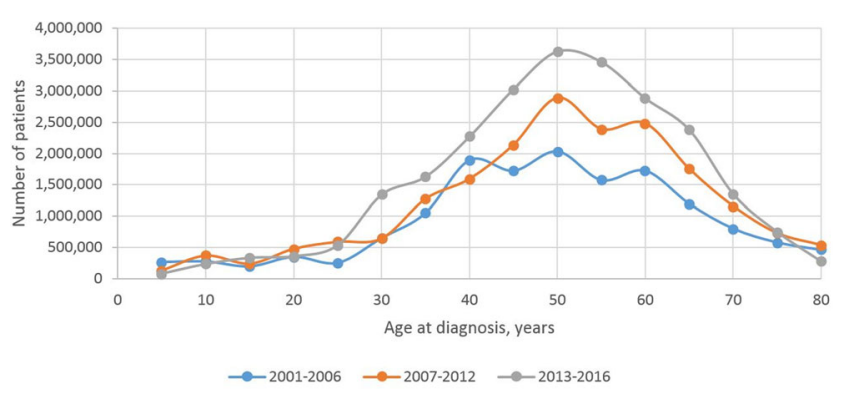

IS TAPERING OR DISCONTINUATION OF OPIOID THERAPY ACCELERATING OR TARGETING VULNERABLE POPULATIONS? EVIDENCE IN MEDICARE FROM 2011-2016

Michael L. Barnett ${ }^{1,3}$; Marema Gaye ${ }^{1}$; Hannah Neprash ${ }^{2} .{ }^{1}$ Harvard T.H. Chan School of Public Health, Boston, MA; ${ }^{2}$ University of Minnesota School of Public Health, Minneapolis, MN; ${ }^{3}$ Brigham and Women's Hospital, Boston, MA. (Control ID \#3181472)

BACKGROUND: Intense scrutiny of opioid prescribing from regulatory agencies and state governments has coincided with a $29 \%$ drop in opioid prescribing volume since 2011. Many observers fear that this trend reflects increasing rates of tapering or discontinuation of long-term opioid 
therapy for vulnerable patients. However, little evidence exists regarding trends in tapering or discontinuation of chronic opioid use or patient characteristics associated with de-escalation of therapy.

METHODS: We examined claims from a 20\% sample of Medicare beneficiaries with 24 months of continuous enrollment who initiated chronic opioid therapy from 2011-2016. We defined incident chronic opioid use for any beneficiary as $>60$ days of opioids supplied in each of 2 consecutive quarters. Exposures included "tapering": two consecutive quarters with $>25 \%$ decrease in average daily morphine equivalent dose from the prior quarter, and "discontinuation": two consecutive quarters with no opioid prescriptions filled. We examined the characteristics of beneficiaries associated with continuous chronic opioid use vs. those with tapering or discontinuation comparing them with ANOVA tests and Cox proportional hazard models to estimate changes in the risk of tapering or discontinuation by year of chronic opioid initiation controlling for beneficiary characteristics and state fixed effects.

RESULTS: Out of 4.8 million beneficiaries from 2011-2016, 268,940 (5.5\%) initiated an episode of chronic opioid use for a median of 1.5 (IQR 1.0-2.5) years. Among chronic opioid users, 72,877 (27.1\%) tapered and $11,365(4.2 \%)$ discontinued therapy. Compared to those with continued opioid use, beneficiaries with tapering or discontinuation were more likely to be eligible for Medicare through disability $(58.7 \%$ vs. $64.3 \%$ and $61.7 \%$, respectively, $\mathrm{p}<0.001$ ), and more likely to have a prior diagnosis of drug or alcohol use disorder $(1.6 \%$ vs. $2.4 \%$ and $2.2 \%, \mathrm{p}<0.001)$. However, compared to those with continued opioid use, those with tapering or discontinuation had similar or lower average chronic illness burden ( 0.93 vs. 0.94 and 0.87 mean number of Elixhauser comorbidities, $\mathrm{p}=0.009$ ). Tapering became slightly less common for chronic opioid episodes beginning later in the study period (adjusted hazard ratio for tapering 0.97 per year of initation, $95 \%$ CI 0.96-0.97) while the likelihood of discontinuation was stable (hazard ratio $0.99,95 \%$ CI 0.97-1.00).

CONCLUSIONS: From 2011-2016, a substantial proportion of Medicare beneficiaries used opioids for over $1 \frac{1}{2}$ years and close to $1 / 3^{\text {rd }}$ tapered or discontinued therapy. Discontinuation and tapering of chronic opioid therapy was stable or decreasing for beneficiaries initiating chronic opioid therpay over this period. There was no strongly consistent bias toward vulnerable populations: patients with discontinuation or tapering had more vulnerable features in some ways (e.g. disability, substance use) while in other ways they appeared similar or lower risk (e.g. chronic illness burden).

\section{IS THE PATIENT EXPERIENCE AT ODDS WITH TEACHING IN THE HOSPITAL? AN ANALYSIS OF PATIENT EXPERIENCE RATINGS OF TEACHING AND NON-TEACHING SERVICES}

$\underline{\text { Bradley H. Crotty }}^{1}$; Rebekah J. Walker ${ }^{1}$; Sneha Nagavally ${ }^{2}$; Jennifer A. Campbell $^{1}$; Jennifer Good ${ }^{1}$; Navdeep Gupta ${ }^{2}$; Mandy Kastner ${ }^{1}$; Douglas Levine $^{1}$; Joan Neuner ${ }^{1}$; Kurt J. Pfeifer ${ }^{1}$; Leonard E. Egede ${ }^{1} .{ }^{1}$ Medical College of Wisconsin, Milwaukee, WI; ${ }^{2}$ Medical College of Wisconsin, Milwaukee, Brookfield, WI. (Control ID \#3186359)

BACKGROUND: The experience of care is increasingly under scrutiny at academic medical centers (AMCs) with direct and indirect financial implications. A prior single-site study showed that hospitalist-only services had higher patient ratings of communication than teaching services. We sought to assess for differences in patient experience between teaching and non-teaching general medicine services using the standard patient experience survey (Hospital Consumer Assessment of Healthcare Providers and Systems or HCAHPS) given its use as a benchmark instrument

METHODS: We retrospectively reviewed data from HCAHPS for general medicine discharges between August 2016 and May 2018 at Froedtert Hospital, a 604-bed academic tertiary care center. The sample data is drawn from 845 HCAHP survey responses for discharges during the year 2017. We assessed differences in individual HCAHPS questions focused on communication, physicians, and discharge information between teaching and non-teaching teams by chi square. We dichotomized the overall rating as satisfied (score of 9) or unsatisfied $(<9)$. Prior to this, we compared illness severity using Charlson comorbidity counts and length of stay (LOS) between teaching and non-teaching services. Patients admitted to teaching and non-teaching services are admitted to the same medical floors.

RESULTS: During this period, there were 216 responses from individuals discharged from the teaching service and 561 responses from those discharged from the non-teaching service. The mean LOS was 4.61 and 4.2 respectively $(\mathrm{p}=0.3)$ and comorbidity counts were statistically similar (data not shown). For most questions, we did not observe significant differences according to teaching status. We saw no differences across responses for the overall hospital rating or personnel describing side effects, describing medication indications, explaining things in understandable terms, listening carefully, being treated with courtesy and respect, or getting information in writing, about symptoms or problems after discharge. Patients admitted to teaching teams were more likely to report discussions related to meeting needs upon hospital discharge (teaching services $90 \%$ vs non-teaching $82 \% \mathrm{p}=0.02$ ).

CONCLUSIONS: We identified that teaching services had similar patient experience scores on the standardized HCAHPS instrument as nonteaching services while having statistically equivalent comorbidity and lengths of stay. Teaching teams were rated higher in one communication domain. As hospitals seek to improve their patient experiences, ensuring that the teaching experience does not detract from the patient experience is critical.

\section{JOY IS NOT THE ABSENCE OF BURNOUT}

Kavya Sreevalsan $^{1}$; Maura J. McGuire ${ }^{1,2}$; Colleen Christmas ${ }^{1,}{ }^{2}$. ${ }^{1}$ Johns Hopkins Bayview Medical Campus, Baltimore, MD; ${ }^{2}$ Johns Hopkins School of Medicine, Baltimore, MD. (Control ID \#3140202)

BACKGROUND: Recently, the growing issue of professional burnout has received much attention. An understanding of the drivers of joy in medicine are only beginning to be elucidated. We aimed to understand the factors that promote joy in medicine in a group of teachers of medical students in primary care settings.

METHODS: We added an optional survey to an existing faculty survey for the primary care clerkship consisting of multiple choice and free responses related to the joy of teaching in the primary care setting. Burnout was assessed using the Mini Z Burnout survey, and results were dichotomized (burned out =rating of 3 or higher on this scale). Respondents rated on a scale from 1 (Always) to 5 (Never) how often they felt joyful during teaching. This item was dichotomized as 2 (joyful when teaching medical students) vs. 3 (not joyful when teaching medical students). We further asked respondents to describe examples of when they felt joyful during teaching encounters and elucidated themes using standard qualitative analyses. Finally, correlation between joy and burnout was analyzed using a chi-squared analysis. The study was approved through the Johns Hopkins Institutional Review Board.

RESULTS: Of the 118 faculty that were included, 43 (36\%) opted to respond our additional survey. Of these respondents, 20/43 providers experienced burnout (47\%). 32/43 respondents stated they felt joyful while teaching medical students in their practice. In describing a time the respondent felt joyful while teaching, 7 themes emerged: 1 . Bearing witness to student growth; 2. Professional Development; 3. Student Motivation; 4. Teacher-Student Relationship; 5. Joyful for Patient's Role in teaching; 6. Sharing Love for What You Do; and 7. Using Personal Expertise. There was no correlation between joy in teaching and burn out $(\mathrm{X} 2=0.0066, \mathrm{p}=0.935)$.

CONCLUSIONS: Levels of burnout (47\%) were consistent with other studies. The themes related to joy we identified in this study suggest that joy may stem from a fulfilling sense of purpose and identity. Relationships 
also appear to have a profound impact on one's perception of joy. We hope our study contributes to better well-being and ultimately can empower the medical system to make changes to promote joy in practice.

\section{LATERAL FEEDBACK IN ACCOUNTABLE CARE UNITS: RES- IDENT AND ATTENDING PERCEPTIONS OF INTERPROFES- SIONAL FEEDBACK}

Katarzyna Mastalerz ${ }^{1,2}$; Nikki Townsley ${ }^{2}$; Sarah Jordan ${ }^{2} .{ }^{1}$ Rocky Mountain Veterans Administration, Aurora, CO; ${ }^{2}$ University of Colorado, Aurora, CO. (Control ID \#3184115)

BACKGROUND: Feedback is an important driver of learner improvement. Physicians are expected to become effective members of interprofessional teams, but little is known about the quality of feedback exchanged between medical trainees and other healthcare professions. In order to understand inpatient team feedback interactions, we interviewed physicians on team based inpatient units called Accountable Care Units (ACU) about their perspectives on interprofessional feedback.

METHODS: We conducted in-person in-depth semi-structured interviews and focus groups with medicine residents and hospitalist attendings in a community teaching hospital between July 2017 and April 2018. The interview guide explored perceptions of interprofessional feedback. Residents that rotated on the ACU and supervising hospitalist attendings were eligible for inclusion. Interviews were audio-recorded, transcribed, and entered into qualitative software for organization (Atlas-ti.v8). During regular group meetings, we systematically analyzed interview transcripts and identified emerging themes using inductive thematic analysis. Saturation was reached.

RESULTS: We interviewed 41 medical residents and 10 hospitalist attendings $(\mathrm{n}=51)$. Residents were predominantly male $(65 \%)$ and Caucasian $(65 \%)$. Attendings were $50 \%$ female with an average of 9.5 years in practice. Three key themes emerged about feedback in interprofessional teams: 1) hierarchies and positionality inhibit resident feedback to interdisciplinary staff, 2) feedback from interprofessional staff is highly desired, 3) conditions that enable interprofessional feedback. Residents were reluctant to give feedback to members of other professions given the lack of familiarity with their roles or their workflow. One resident said: "I think there's this idea of separation of roles, like residents get feedback from their attendings and other residents, nurses get feedback from their charges." Hierarchies also constrained interprofessional staff from giving feedback to physicians, unless specifically asked. Feedback from hospital staff, especially from nurses, was highly desired by residents. An intern stated: "The feedback that's more important and will always be more important, is the direct feedback you get from nursing staff." Study participants recognized conditions on the ACU that enabled interprofessional feedback: a culture of knowing staff names due to common workflow, a clear expectation of interdisciplinary communication, and opportunities for "lateral" talk.

CONCLUSIONS: As we strive to integrate physicians into interprofessional teams, feedback from other healthcare professions is essential. Our study identified that interprofessional feedback is desired by physicians but constrained by professional siloes and hierarchies. Strategies that facilitate the exchange of interprofessional feedback, such as creating intentional integrated workflow and setting clear expectations around interprofessional communication, should be considered in future curricular design.

\section{LAW ENFORCEMENT AND IMMIGRANT HEALTH}

Amanda Bertram²; Derek W. Braverman ${ }^{2}$; Dana Goplerud ${ }^{2}$; Jaime M. La Charite ${ }^{2}$; Alexandra Norton ${ }^{2}$; Zackary Berger ${ }^{1}$. ${ }^{1}$ Johns Hopkins School of Medicine, Baltimore, MD; ${ }^{2}$ Johns Hopkins University, Baltimore, MD. (Control ID \#3183642)
BACKGROUND: US immigration policies and enforcement may make undocumented immigrants fearful of accessing healthcare. Although current immigration policies restrict enforcement in "sensitive locations" including health care facilities, there are reports of enforcement actions in such settings. It is unclear if healthcare professionals know how to respond to immigration enforcement at their facilities. We attempted to evaluate the experience of health professionals with workplace immigration enforcement, their knowledge and training regarding relevant policies, and their beliefs regarding which policies institutions should follow.

METHODS: We conducted a cross-sectional survey of providers via the member portal of the Society for General Internal Medicine. Providers were encouraged to forward this survey to other interested healthcare professionals.

RESULTS: Most survey respondents were attending physicians (69.1\%, $\mathrm{n}=29)$, with a further $21.4 \%$ listing additional administrative roles. A vast majority worked at academic medical centers $(90.5 \%$, $\mathrm{n}=38)$ and in outpatient settings $(83.3 \%, \mathrm{n}=38)$. Most lived in the Northeast $(40 \%, \mathrm{n}=17)$ and listed the majority language spoken by their patients as English $(85.7 \%, \mathrm{n}=36) .82 .5 \%$ of respondents were not aware of specific workplace policies regarding immigration-related law enforcement. Few respondents had received training regarding either immigration-related law enforcement $(4.76 \%, n=2)$ or law enforcement activity in general $(9.5 \%, n=4)$. Nearly 1 in 5 respondents reported that they were aware of immigration enforcement activities in or near their workplace $(19.1 \%, \mathrm{n}=18)$, and no staff members involved had received training beforehand. Only $24.3 \%$ of respondents considered their facility prepared to respond to immigration enforcement. Cited reasons include lack of training (35.5\%), lack of known policies (35.5\%), deference to immigration law enforcement $(9.5 \%)$, lack of awareness $(6.5 \%)$, or complete lack of preparedness (19.4\%). Responses to survey questions about policy awareness or training did not vary significantly by region, job description, practice setting or patient language. Awareness of enforcement activity differed significantly by practice setting, with $57 \%$ of inpatient providers and $11 \%$ of outpatient providers reporting enforcement activity at or near their place of work. CONCLUSIONS: Surveyed clinicians were largely unaware of workplace policies at their institution regarding responses to immigration enforcement. Few received relevant training in how to respond to immigration enforcement and most did not feel that their facility would be prepared to do so. Institutions should develop appropriate policies and procedures, educate and train staff, and engage with stakeholders to coordinate appropriate and ethical response to immigration enforcement in clinical settings.

\section{LET'S STEP UP THE WAR ON SUPERBUGS IN OUR HOSPI- TALS: EVALUATING METHODS TO REDUCE STETHO- SCOPE CONTAMINATION}

Marwa Moussa $^{1}$; Morris Jrada ${ }^{2}$; Amy otuonye ${ }^{1}$; Jesica Hayon ${ }^{2}$; Michael Phillips ${ }^{2} .{ }^{1} \mathrm{NYU}$ Langone, Staten Island, NY; ${ }^{2} \mathrm{NYU}$ Langone Health, Brooklyn, NY. (Control ID \#3167970)

BACKGROUND: Stethoscopes are recognized as a culprit of microbes that has been conclusively demonstrated to transmit microbes from one patient to another and from health care worker to patient. To curb infections, hospitals need to set more rigorous hygiene standards, identify methods to interrupt transmission and develop strategies on sterilizing the diaphragms of the stethoscopes. Furthermore, studies have shown that providers infrequently clean their stethoscopes. In one study, only $48 \%$ of providers cleaned their stethoscopes daily or weekly, $37 \%$ monthly and $7 \%$ reported that they had never cleaned their stethoscope. The objective of this study was to conduct a pilot study comparing efficacy of disposable diaphragm covers to no intervention, defined as their ability to reduce colony count of Methicillin Resistant 
Staphylococcus Aureus (MRSA) and reduce bacterial contamination on stethoscope diaphragm surfaces.

METHODS: This was a prospective pilot study using a randomized, controlled, single-blinded, crossover trial design, evaluating the effect of daily stethoscope disposable diaphragm covers vs. uncovered stethoscopes. Upon recruitment, residents on clinical rotations were randomized to receive one of two sealed opaque boxes. If a resident was randomized to the intervention arm, the package included instructions to begin with the covers. If a resident was randomized to the control arm, instructions were to begin with no covers. We instructed the participants to switch arms at 7 days. Laboratory methods: A sterile swab was rolled over the surface of the stethoscope's diaphragm from side to side in a streaking method. We used the chromagar MRSA plates (MRSASelect ${ }^{\mathrm{TM} I I}$ agar plates) to grow oxacillin resistant, nonenterococal gram positives and the non-selective blood agar plate. Cultures were obtained from each resident's stethoscope diaphragm at the end of every 7 day period. We performed a colony count in 24 hours and 48 hours of incubation.

RESULTS: We enrolled 37 residents, of whom 29 (70\%) completed both weeks of the trial. On the log-10 scale, the mean (range) colony count on plain agar was $1.5(0.0-3.7)$ during control and $1.6(0.0-3.0)$ using covers. For MRSA, the mean (range) log-10 colony count during control was $0.1(0.0-2.7)$ and $0.1(0.0-1.2)$ under covers. Overall, 7 $(11 \%)$ cultures were positive for MRSA during control and $6(9 \%)$ using covers. Using mixed models to account for within-subject and within-culture correlation, the covers increased colony count by 0.47 (95\% confidence interval, $-0.37-1.31$ ) in mean log-10 overall colony count, and increased risk of MRSA+ culture by 0.2 percent $(95 \%$ confidence interval, $-10.0-10.3)$.

CONCLUSIONS: This well designed study shows disposable diaphragm covers inadequate in reducing bacterial load. It is likely that this study was hindered by a small sample size, therefore a larger study to evaluate the ability of other methods to prevent cross transmission of MRSA and subsequent infections from the stethoscope diaphragm is needed.

\section{LET'S TALK CANNABIS: A QUALITATIVE STUDY OF VET- ERANS AFFAIRS PROVIDER AND CANNABIS DISPENSARY EMPLOYEE VIEWS ABOUT DISCUSSING CANNABIS WITH PATIENTS}

Vivian Christensen ${ }^{3}$; Shannon Nugent $^{3}$; Emilio Sulpizio ${ }^{2}$; Devan Kansagara ${ }^{1,2}$. ${ }^{1}$ Portland VA Medical Center, Portland, OR; ${ }^{2} \overline{\text { Oregon }}$ Health and Science University, Portland, OR; ${ }^{3}$ VA Portland Health Care System, Portland, OR. (Control ID \#3185489)

BACKGROUND: Medical and recreational use of cannabis has become increasingly common, and VA clinicians are asked to be prepared to engage in discussions about cannabis with their patients. This qualitative project sought to better understand provider attitudes, beliefs, and knowledge gaps regarding patients' use of cannabis with the ultimate goal of developing educational tools for clinicians to help them discuss cannabis use with their patients.

METHODS: We conducted in-depth, semi-structured interviews with 18 key informants (14 VA employees and 4 cannabis dispensary employees). Interviews were transcribed and analyzed using a predetermined/deductive framework. An inductive approach was then used to identify themes within each thematic framework.

RESULTS: The most common reasons patients seek advice about cannabis from their physicians is for chronic pain, nausea, depression, anxiety, PTSD, and sleep disorders. While clinicians feel comfortable having basic discussions about cannabis, their lack of specific knowledge about terminology, dose, modality, and strain results in hesitation to have in-depth discussions. Moreover, the continued federal distinction of cannabis as a Schedule 1 drug has led some providers to not engage in the topic altogether. While views vary, clinicians are generally open to the potential benefits of cannabis, though none view cannabis as risk free and most cite a need for more evidence from controlled clinical trials. Potential risks discussed include potential abuse, impact on comorbidities and/or mental health, and forgoing known effective treatments. Clinicians view medicinal cannabis through a traditional pharmacotherapeutic lens and cite lack of regulation regarding product quality and potency, and uncertainty about the potential for interaction with other prescription drugs as concerns. Dispensary employees are comfortable with the imprecise nature of cannabis as a therapeutic agent, believe that cannabis use is largely risk free, and believe that patient stories and employee experience are valuable sources of evidence that support the effectiveness of cannabis in treating symptoms of common diseases and conditions. Dispensary employees were eager to partner more with clinicians and wanted their customers to obtain guidance for cannabis use for medical purposes from their physicians. CONCLUSIONS: Cannabis has become widely available without the pharmacotherapeutic standardization and evidence to which clinicians are accustomed. The resulting uncertainty along with federal policy are barriers for clinicians to having discussions about cannabis with their patients. Many clinicians are open to the idea of medicinal cannabis, but have mixed feelings and concerns about safety. There are stark differences in how clinicians and dispensary employees view the use of medicinal cannabis, though dispensary employees appear open to collaboration with clinicians to improve patient safety.

\section{LET'S WORK TOGETHER: RESIDENTS' PERCEIVED ABILI- TY TO COMMUNICATE MANAGEMENT OF CHRONIC DIS- EASE WITH PATIENTS WITH LIMITED ENGLISH PROFI- CIENCY}

Mia Williams ${ }^{1}$; Brian Lewis ${ }^{2}$; Karen E. Hauer ${ }^{1}$; Alicia Fernandez ${ }^{1}$. ${ }^{1}$ University of California San Francisco, San Francisco, CA; ${ }^{2}$ University of Wisconsin, Middleton, WI. (Control ID \#3175486)

BACKGROUND: In the US there are $\sim 21$ million individuals with limited English proficiency (LEP) who are at risk for challenges in health care communication. These challenges can affect health outcomes. Although there is limited data on the ability of residents to communicate effectively with LEP patients, a previous study suggests that IM residents are dissatisfied with their ability to communicate with LEP patients. Determining local experience is important, as training hours and extent of exposure to LEP patients vary greatly across the country.

METHODS: We conducted a focused needs assessment to ascertain UCSF IM residents self-perceived patterns, strengths, and self-efficacy in communication with LEP patients via a vignette-based survey. The survey contained closed and open-ended questions. The latter were analyzed for themes by two researchers.

RESULTS: $41(77 \%)$ Internal Medicine residents responded to the questionnaire. Residents reported receiving little training in skills to communicate with LEP patients in spite of the fact that $45 \%$ of residents reported that $>25 \%$ of their outpatient encounters were with LEP patients. When residents compared their confidence in executing key tasks such as 'Identify patient's agenda' (100\% confident to very confident with English speakers vs. $74 \%$ with LEP patients) which increased in magnitude as tasks became more nuanced i.e. 'Understand patient's beliefs about diabetes' ( $87 \%$ vs. $18 \%$ ); this difference was statistically significant across all communication tasks. In the qualitative analysis, the most common barrier residents identified was time, followed by concern about cultural differences and uncertainty in working with the interpreter.

CONCLUSIONS: Our study found that UCSF IM residents have a significant lack of self-efficacy and satisfaction in the care of LEP patients; this gap worsens as the communication complexity of the task 
increases. Preliminary themes highlight systems-issues as areas that limit the ability to care for of patients who are LEP, but also note areas where education interventions could be helpful (ie: working with interpreters and understanding culture in the context of medical care). Future directions include focus groups to better characterize gaps in knowledge and assess where curricular programming could be implemented to improve resident self-efficacy and the quality of care for LEP patients.

\section{LEVERAGING COMMUNITY-BASED HEALTH PLANNING AND SERVICES (CHPS) PROVIDER INSIGHT AND EXPERI- ENCE FOR THE DESIGN AND IMPLEMENTATION OF AN INTERVENTION TO SCREEN AND TREAT CARDIOVASCU- LAR DISEASE IN NAVRONGO, GHANA: A QUALITATIVE STUDY}

Ethan P. Wood ${ }^{1}$; Katherine L. Garvey ${ }^{1}$; Edith Dambayi ${ }^{2}$; Denis A. Awuni $^{2}$; Raymond Aborigo $^{2}$; Elizabeth Jackson ${ }^{3}$; James Phillips ${ }^{3}$; Abraham Oduro ${ }^{2}$; David J. Heller ${ }^{1}{ }^{1}$. ${ }^{1}$ Icahn School of Medicine at Mount Sinai, New York, NY; ${ }^{2}$ Navrongo Health Research Center, Navrongo, Ghana; ${ }^{3}$ Columbia University in the City of New York, New York, NY. (Control ID \#3185082)

BACKGROUND: Cardiovascular disease (CVD) is the leading cause of morbidity and mortality worldwide as well as in the United States. CVD prevalence is rising in Ghana, and the shift in burden from communicable to non-communicable diseases poses a great challenge to fragile health systems in rural areas like Navrongo. The CommunityBased Health Planning and Services (CHPS) initiative has used nurseled primary care clinics and home visits to decrease child mortality, but does not currently screen or treat CVD or its risk factors. Our previous research in Navrongo identified lack of transportation, equipment, medicines and knowledge as barriers to CHPS CVD care. The goal of this study was to interview CHPS Community Health Officers (CHOs) and their supervisors, Sub-District Officers (SDOs), regarding locally feasible interventions to decrease CVD morbidity and mortality in Navrongo, Ghana based on these data.

METHODS: We conducted semi-structured interviews with $21 \mathrm{CHOs}$ and 10 SDOs from the Kassena-Nankana District, using a qualitative descriptive approach. Participants were asked for their reactions to our previous findings regarding barriers to CVD care. We then asked them to propose and discuss potential interventions, focusing on which interventions might be most feasible and why. The interviews were audio recorded, transcribed, coded and analyzed for themes. Two coders analyzed the interviews using a general content analysis approach, in which themes and categories emerged iteratively. Coding was completed using NVivo software.

RESULTS: Suggested interventions centered on provider and community education, staffing, and logistics. Respondents proposed CVD trainings for providers; community education at durbars (community gatherings); workforce augmentation; and improved resource and equipment accessibility, as well as nurse authorization to prescribe CVD medication, as important aspects of an intervention. Provision of necessary equipment for CVD screening and management was considered the most crucial intervention. CVD training for all clinical staff, including community health volunteers, was also frequently mentioned. CHOs and SDOs expressed enthusiasm for screening and treatment of CVD and its risk factors, citing a high burden of CVD within their communities, as well as optimism regarding its feasibility.

CONCLUSIONS: Clinical staff in this nurse-led primary care program propose several specific interventions to implement CVD care in Navrongo, and feel they are feasible and appropriate. These findings suggest a CVD intervention can be piloted and evaluated through CHPS in Navrongo. This intervention, once developed, implemented and evaluated, can potentially serve as a model for programs that rely on non-physicians to deliver care to rural communities.
LIFETIME RISK OF NON-FATAL FIREARM-RELATED INJURIES IN NEW YORK STATE: RACIAL AND SEX DISPARITIES $\mathrm{Yu}^{-T i e n ~} \mathrm{Hsu}^{1}$; David C. Chang ${ }^{1}$; Numa P. Perez ${ }^{1}$; Ya-Ching Hung ${ }^{1}$; Maggie Westfal $^{1}$; Cassandra M. Kelleher ${ }^{1}$; Peter Masiakos ${ }^{1}$; Chana A. Sacks ${ }^{2} .{ }^{1}$ Massachusetts General Hospital / Harvard Medical School, Boston, MA; ${ }^{2}$ Brigham and Women's Hospital, Boston, MA. (Control ID \#3179226)

BACKGROUND: Gun violence is an important public health problem in the US. While the rising national rate of firearm-related deaths is welldocumented, data regarding nonfatal firearm injuries and resulting hospitalizations are limited, and the cumulative lifetime risk of nonfatal firearm injuries is unknown.

METHODS: We performed a retrospective analysis using the New York Statewide Planning and Research Cooperative System (SPARCS), which includes all inpatient hospitalizations and emergency department presentations in NY state from 2005 to 2015 . We included all patients 15 years of age or older with firearm injuries, defined by ICD-9 and ICD-10 codes, and excluded in-hospital deaths. Projected lifetime risk of nonfatal firearm injuries per 100,000 persons was calculated as the sum of age-specific incidence rates across all age groups, and stratified by sex, race, and county.

RESULTS: A total of 12,699 firearm-related admissions for 12,381 unique patients were included in the study cohort. $2.9 \%$ of patients were shot more than once during the study period. The mean number of admissions was 1,155 per year (7.3 per 100,000 persons). Mean age at admission was $28.1(+/-11.7)$ years; most patients were male $(90.0 \%)$ and black $(65.1 \%)$. The overall lifetime risk for nonfatal firearm injuries was 87.3 per 100,000 persons. On average, males had a 9-fold increased lifetime risk of having nonfatal firearm injuries compared with females; blacks had a nearly 18 -fold increased risk compared with whites. Nonfatal firearm injuries were concentrated in urban counties, such as Albany and Onondaga, which includes Syracuse (Figure 1 shows lifetime risk of nonfatal firearm injuries by county in 2015). Albany had the highest lifetime risk in the state (157.0 per 100,000 people).

CONCLUSIONS: The lifetime risk of nonfatal firearm-related hospitalization in NY state was 87.3 per 100,000 persons. These data highlight substantial disparities, with men, blacks, and residents of urban counties having higher risks. Targeting prevention strategies to these high-risk populations is essential to reducing overall firearm-related injuries.

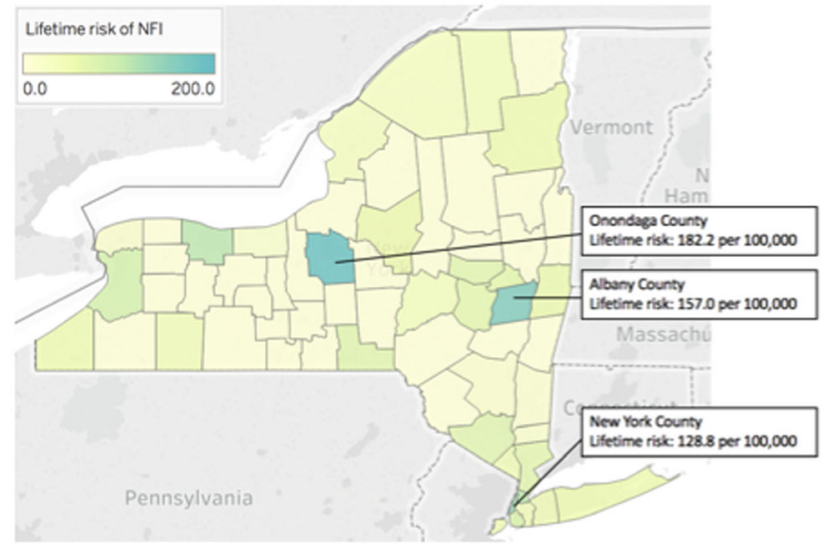

Figure 1: Lifetime risk of non-fatal firearm injuries per 100,000 persons in New York State by county in 2015

LIMITATIONS IN USING AREA UNDER THE RECEIVER OPERATING CHARACTERISTIC CURVE TO EVALUATE MODEL PERFORMANCE FOR LOW FREQUENCY OUTCOMES: APPLICATIONS TO NONCARDIAC SURGERY

Victor J. Lei ${ }^{1}$; Edward Kennedy ${ }^{2}$; ThaiBinh Luong ${ }^{3}$; Xinwei Chen ${ }^{1}$; Daniel Polsky $^{1}$; Kevin G. Volpp ${ }^{1,4}$; Mark D. Neuman ${ }^{1}$; John Holmes ${ }^{1}$; Lee Fleisher ${ }^{1}$; 
Amol S. Navathe ${ }^{1,4} \cdot{ }^{1}$ University of Pennsylvania, Philadelphia, PA; ${ }^{2}$ Carnegie Mellon University, Pittsburgh, PA; ${ }^{3}$ Penn Medicine, Philadelphia, PA; ${ }^{4}$ Veterans Health Administration, Philadelphia, PA. (Control ID \#3183310)

BACKGROUND: Predicting death in noncardiac surgery is important because although death is relatively rare, noncardiac surgeries are very common, with over 25 million procedures per year in the US. In this study, we examined how adding intraoperative data to baseline (pre-hospitalization) and preoperative data can improve prediction of postoperative mortality using the area under the receiver operating characteristic curve (AUROC) versus the area under the precision-recall curve (AUPRC).

METHODS: We conducted a retrospective cohort study of adult patients who underwent major noncardiac surgeries across four hospitals within an academic medical institution from January 2014 to June 2018. We used four modeling approaches to predict all-cause in-hospital mortality: logistic regression, naïve Bayes, random forest, and gradient boosted machine (GBM). Two feature reduction techniques were used to fit the logistic regression models (forwardselection and least absolute shrinkage and selection [LASSO]). 112 clinical variables were extracted and categorized as baseline, preoperative, or intraoperative. Variables were incrementally added to each model and performance was evaluated using AUROC and AUPRC. Though AUROC is an important indicator for model performance, it is not always aligned with how clinicians would use a prediction model. In noncardiac surgery, identifying patients at high risk of death is more critical than identifying patients who do not die. AUROC is immune to changes in class distribution because it is calculated only using sensitivity and specificity and therefore cannot distinguish between models that have equal sensitivity but different precision (positive predictive value). In our context, precision is more clinically meaningful than AUROC, and thus we also examine AUPRC.

RESULTS: Of the 74,147 patients, $512(0.78 \%)$ died in the hospital. The mean age of patients who died after surgery was significantly higher at 65 years vs 56 years for those who survived to hospital discharge. Additionally, patients who died were more likely to be male and covered by Medicare. AUROC increased as more features were added, but predictive performance was high with baseline and preoperative data (AUROC ranged from 0.831 [LASSO] to 0.917 [GBM]) and changes were small when adding intraoperative data (AUROC changes ranged from -0.049 [LASSO] to +0.059 [Logistic with forward selection], $\mathrm{p}<0.001$ for all). In contrast, AUPRC exhibited lower performance with baseline and preoperative data (AUPRC ranged from 0.045 [Naïve Bayes] to 0.235 [GBM]) and large improvements for some models (AUPRC changes ranged from -0.032 [LASSO] to +0.146 [Logistic with forward selection], $\mathrm{p}<0.001$ for all).

CONCLUSIONS: AUPRC is a more clinically meaningful measure than AUROC when predicting low probability events. Further, using AUPRC indicated that adding intraoperative data to baseline and preoperative data improved prediction of postoperative mortality, which would not have been apparent with AUROC.

\section{LIMITED ENGLISH PROFICIENCY AND EFFICACY OF SELF MANAGEMENT SUPPORT SYSTEMS}

Leah Machen; Delphine Tuot; Margaret Handley; Neil R. Powe. University of California, San Francisco, San Francisco, CA. (Control ID \#3184692)

BACKGROUND: Limited English Proficiency (LEP) is an important driver of health disparities, impaired medical comprehension, and high risk medication use. For many chronic conditions, patient-level interventions to prevent disease progression and its complications have focused on comorbidity management and self-management support (SMS) programs, which rely heavily on patient activation and communication to achieve improved health outcomes. As these interventions evolve, it is crucial to understand if these interventions are similarly applicable to LEP patients compared to English proficient patients.

METHODS: We conducted a retrospective analysis of the KARE (Kidney Awareness Registry and Education) randomized controlled pilot trial to assess the impact of a comprehensive SMS program on systolic blood pressure (SBP) among individuals with limited (vs. adequate) English proficiency. The 12-month language-concordant, culturally-sensitive SMS program consisted of automated telephone modules, low-literacy written patienteducational materials and telephone health coaching. Impact of the SMS program on change in SBP at 12 months by LEP status was determined using multivariable linear mixed models, controlling for socio-demographic variables and baseline SBP. To explore potential mechanisms underlying differential SMS program impact, engagement with the SMS program (i.e. patient use of educational materials, completion of a health coaching action plan, and duration of automated phone calls), by LEP status was also examined.

RESULTS: Of 137 KARE study participants 53 (38.7\%) reported limited English proficiency of which $45(85 \%)$ were Spanish speaking and $8(15 \%)$ Cantonese speaking. While many sociodemographic and clinical variables were similar between groups, individuals with LEP were more likely to be uninsured $(39.6 \%$ vs. $15.5 \%, \mathrm{p}=0.01)$ and have less formal education $(13.2 \%$ vs $47.6 \%$ attended some college, $\mathrm{p}<0.01)$. Overall, LEP patients randomized to SMS did not have a statistically significant change in SBP compared to English proficient patients randomized to SMS (estimated SBP change: $-4.5(-9.4,0.3) \mathrm{mmHg}$ for LEP, $\mathrm{p}=0.07$; and $-2.1(-8.6,4.3) \mathrm{mmHg}$ for English, $\mathrm{p}=0.51$. While LEP and English proficient patients similarly used educational materials provided ( $82 \%$ vs $87 \%, \mathrm{p}=0.69)$ and completed a health action plan ( $81 \%$ vs $74 \%, \mathrm{p}=0.49)$, participants with LEP had significantly longer average automated call duration $[3.3+/-1.4 \min$ vs. 2.2 $+/-1.1$ min, $\mathrm{P}<0.01$ ), indicating higher patient engagement.

CONCLUSIONS: Language concordant, culturally appropriate telehealth interventions can be a useful tool in reducing disparities within the LEP population with potential for greater patient engagement and at minimum similar impact on health outcomes compared to English proficient populations.

\section{LOCATION MATTERS: THE TYPE OF HOSPITAL UNIT CAN INFLUENCE SATISFACTION WITH PHYSICIAN COMMUNICA- TION.}

Douglas Levine ${ }^{1}$; Rebekah J. Walker ${ }^{1}$; Sneha Nagavally ${ }^{2}$; Leonard E. Egede $^{1}$; Jennifer Good ${ }^{3}$; Bradley H. Crotty ${ }^{3}$; Mandy Kastner ${ }^{1}$; Jennifer A. Campbell ${ }^{3}$; Navdeep Gupta ${ }^{4}$; Kurt J. Pfeifer ${ }^{1}$; Joan Neuner ${ }^{1} .{ }^{1}$ Medical College of Wisconsin, Fox Point, WI; ${ }^{2} \mathrm{MCW}$, Wauwatosa, WI; ${ }^{3}$ Medical College of Wisconsin, Milwaukee, WI; ${ }^{4}$ Medical College of Wisconsin, Milwaukee, Brookfield, WI. (Control ID \#3185787)

BACKGROUND: Patient satisfaction has been shown to predict compliance and is an integral part of providing high quality care. Payers such as Medicare now hold hospitals financially accountable for patient experience as measured by the Hospital Consumer Assessment of Healthcare Providers and Systems (HCAHPS) survey. Predictors of high HCAHPS scores are largely unknown. We sought to determine if the type of hospital unit (general medicine unit or specialty unit) influenced HCAHPS scores for physician communication.

METHODS: We utilized deidentified inpatient care satisfaction data collected from a large academic hospital through the HCAHPS survey from August 2016 through May 2018. Data included demographic information on the patient, discharge provider, length of stay, unit on which patient stayed and their responses to survey topics such as provider communication, overall satisfaction and physician care. Most patients cared for by general internal medicine (GIM) providers at this hospital are admitted to hospital units assigned to the Medicine service, but approximately $30 \%$ are admitted to units assigned to other hospital services. Patients of GIM physicians were therefore categorized based on their unit at discharge as medicine or nonmedicine. Only hospital stays with a discharging internist/hospitalist provider were included. The overall hospital rating $(0-10)$ was dichotomized to Not Satisfied (0-8) and Satisfied (9-10) categories. Chi square and t-tests were used to compare patients' hospital experience by whether they were located on a medicine or non-medicine unit. 
RESULTS: In this cohort of 1586 patients, $62 \%$ were 65 years or older, $56 \%$ were female, and $77 \%$ self-identified as white. The average overall hospital satisfaction score was 8.9 out of 10 and average length of stay was 4.6 days. Patients treated in non-medicine units had higher overall satisfaction than those in medicine units $(\mathrm{p}=0.02)$ and higher scores on the question about how often doctors listened to the patient carefully $(\mathrm{p}=0.002)$ Additional differences that showed a trend towards significance included how often doctors explained things in a way that was understood $(\mathrm{p}=0.06)$, and how often doctors treated the patient with courtesy and respect $(\mathrm{p}=0.06$ ). In all cases patients treated by GIM providers on non-medicine units responded more favorably than patients treated by GIM providers on medicine units.

CONCLUSIONS: This study demonstrates that the type of inpatient unit can influence both overall satisfaction and satisfaction with physician communication. Differences in the room environment, amenities, and nursing staffing and experience may explain why medicine patients were overall more satisfied when cared for on non-medicine units. These results can inform quality improvement work aimed at improving the patient experience.

\section{LONG-TERM TIME TRENDS OF OPIOID-RELATED HOSPITAL- IZATIONS AMONG VHA PRIMARY CARE ENROLLEES}

Eric Gunnink $^{1}$; Karin M. Nelson ${ }^{1,2}$; Erin L. Jaske ${ }^{1}$; Paul Hebert ${ }^{1,}$; ; ChuanFen Liu ${ }^{1,3}$. ${ }^{1}$ Department of Veterans Affairs, Seattle, WA; ${ }^{2}$ University of Washington, Seattle, WA; ${ }^{3}$ University of Washington School of Public Health, Seattle, WA. (Control ID \#3183347)

BACKGROUND: Drug overdose and opioid-related deaths continue to rise in the United States. While the volume of opioid-related hospitalizations was stable from pre-2000 to 2014, the primary diagnosis has shifted from opioid dependence or abuse to opioid or heroin poisoning. The Veterans Health Administration (VHA) cares for vulnerable veterans with high rates of substance use disorder. This study examined the long-term time trends in opioid-related hospitalizations among VHA primary care patients and by payer of the hospitalization, VHA vs Medicare.

METHODS: We conducted a repeated cross-sectional observational study between 2003-2015 using VHA administrative data and Medicare claims. Opioid-related hospitalizations were defined as hospitalizations with an opioid-related diagnosis as the primary diagnosis and were classified into three subgroups: opioid dependence or abuse, opioid poisoning, and heroin poisoning. The study sample included opioid-related hospitalizations in VHA and Medicare settings among VHA primary care patients from 2003 (3,980,807 patients with 3,736 hospitalizations) to 2015 (5,889,338 patients with 6,251 hospitalizations). Among 65+, there were 2,207,625 patients in 2003 with 134 opioid-related hospitalizations in VHA and 92 in Medicare and 3,013,686 patients in 2015 with 407 hospitalizations in VHA and 406 in Medicare. We examined long-term time trends in overall opioid-related hospitalizations, by subgroup of opioid-related hospitalization, and by race/ethnicity. We assessed the time trends by payer among 65 years or older. RESULTS: The rate of opioid-related hospitalizations rose from 2003 (0.85/ 1,000 patients) to 2015 (1.06/1,000 patients). We observed increased time trends for opioid poisoning ( 0.08 to $0.17 / 1,000$ patients) and opioid dependence or abuse ( 0.76 to $0.87 / 1,000$ patients), but a stable time trend for heroin poisoning ( 0.01 to $0.02 / 1,000$ patients). Whites contributed the most to the increase in opioid and heroin poisonings with the number of hospitalizations rising from 509 in 2003 to 1,726 in 2015. Among 65+, we observed similar increasing time trends in both VHA and Medicare hospitalizations. The rate of opioid-related hospitalizations rose from $0.06 / 1,000$ patients in 2003 to $0.14 / 1,000$ patients in 2015 in VHA and from $0.04 / 1,000$ patients in 2003 to 0.13/1,000 patients in 2015 in Medicare.

CONCLUSIONS: Like the general population, the opioid-related hospitalization rate increased over time among VHA patients, which was also observed in both VA and Medicare among 65+. However, opioid dependence or abuse and opioid poisoning accounted for most of the increase in VHA, rather than opioid and heroin poisoning observed in the general population. The integrated mental health care for substance use disorder is a key element of the VHA healthcare system. Future research should examine the impact of integrated mental health care on the long-term time trends of opioid-related hospitalizations.

\section{LONGITUDINAL CHANGES IN ENROLLEES' EMPLOYMENT AND STUDENT STATUS AFTER MICHIGAN'S MEDICAID EX- PANSION}

Renuka Tipirneni; John Z. Ayanian; Minal Patel; Edith C. Kieffer; Matthias Kirch; Corey Bryant; Jeffrey T. Kullgren; Sarah J. Clark; Sunghee Lee; Erica Solway; Tammy Chang; Adrianne N. Haggins; Jamie Luster; Erin Beathard; Susan D. Goold. University of Michigan, Ann Arbor, MI. (Control ID \#3179014)

BACKGROUND: Medicaid "community engagement" requirements (requiring work, school, job searching or community service) are being implemented by states for the first time, but the impact of coverage itself on enrollees' employment and school attendance is unclear. Michigan's Medicaid expansion, or Healthy Michigan Plan (HMP), expanded coverage to $\sim 680,000$ low-income individuals beginning in April 2014. Our objective was to assess longitudinal changes in enrollees' employment and student status after HMP enrollment.

METHODS: We conducted a telephone survey of 4,090 HMP enrollees in January-October $2016(\mathrm{RR}=53.7 \%)$ and a follow-up survey of respondents in March 2017-January $2018(\mathrm{~N}=3,104 ; \mathrm{RR}=83.4 \%)$. Sampling was stratified by income and geographic region. Survey items measured employment and student status; demographic characteristics were from Medicaid program files. Eligibility criteria for the 2016 survey were age 19-64, 12 months of HMP coverage and 10 months in a Medicaid health plan; those who agreed to be contacted for follow-up comprised the 2017 population. We used mixed models with time indicators to assess longitudinal changes in the proportion of enrollees who were employed or students, incorporating weights to adjust for sample design and nonresponse. Multivariable logistic regression models were used to identify predictors of being employed or a student.

RESULTS: Among all respondents, 54.3\% were employed and/or students in 2016 , increasing to $60.0 \%$ in 2017 ( +5.7 percentage points, OR 1.83, $p<0.001)$. In 2017, $76.8 \%$ were still enrolled in HMP and $23.2 \%$ no longer enrolled. Among those still enrolled in 2017, the proportion of employed/ students increased from $53.1 \%$ to $58.7 \%$ ( +5.6 percentage points, OR 1.78 , $p<0.001$ ); among those not enrolled in 2017, the proportion of employed/ students increased from $58.2 \%$ to $64.1 \%$ ( +5.9 percentage points, OR 2.03 , $p=0.002$ ). In multivariable analysis assessing predictors of employment/ student status, respondents were more likely to be employed if nonHispanic Black (aOR 1.74, $p=0.009$ ) or Hispanic (aOR 2.86, $p=0.012$ ) compared to non-Hispanic Whites, or if they had more education compared to high school (aOR 3.17 for some college, $p<0.001$; aOR 4.82 for Bachelor's degree or higher, $p<0.001)$. They were less likely to be employed if age 51-64 compared to age 19-34 (aOR 0.08, $p<0.001$ ). Improved health status (defined as fair/poor health in 2016 and good/very good/excellent health in 2017) was not associated with employment/student status in 2017 (aOR 0.93, $p=0.826$ ). CONCLUSIONS: Employment and school attendance among enrollees in HMP increased from 2016 to 2017. Black, Hispanic and younger adults were more likely to be employed or students in 2017. To our knowledge, this is the first study to assess longitudinal changes in employment and school attendance in Medicaid expansion enrollees. These findings suggest that, irrespective of requirements, Medicaid coverage itself may be associated with the desired outcomes of employment and school attendance.

LONGITUDINAL INVESTIGATION OF OLDER ADULTS' ABILITY TO SELF-MANAGE COMPLEX MEDICATION REGIMENS Stacy Bailey $^{1}$; Lauren Opsasnick ${ }^{1}$; Laura M. Curtis ${ }^{1}$; Alex Federman ${ }^{2}$; Julia Yoshino Benavente ${ }^{1}$; Rachel O'Conor ${ }^{1}$; Michael S. Wolf ${ }^{1}$. ${ }^{1}$ Northwestern University, Chicago, IL; ${ }^{2}$ Icahn School of Medicine at Mount Sinai, New York, NY. (Control ID \#3185667) 
BACKGROUND: Treatment burden, and specifically polypharmacy, increases with age. Older patients are increasingly being prescribed complex drug regimens that are challenging to self-administer safely and appropriately. Yet few studies have investigated the ability of older patients to correctly interpret and accurately dose multi-drug regimens over time. We sought to investigate whether medication self-management skills are maintained over a decade among a cohort of older primary care patients and what factors might predict declines in one's ability to self-manage complex drug regimens.

METHODS: An NIA-funded cohort study, known as 'LitCog,' was leveraged for this investigation. Beginning in 2008, LitCog enrollled 900 Englishspeaking adults, ages 55-74 at baseline, all receiving care at either an academic general medicine practice or one of 5 federally qualified health centers in Chicago, IL. At baseline (T1), subjects participated in a structured interview during which were given a standardized seven-drug medication regimen and asked to demonstrate how and when they would hypothetically take all of the medicine within a 24-hour period. To date, 273 LitCog participants have completed the same seven-drug regimen dosing assessment 10 years post-baseline (T4). We compared the mean number of dosing errors at $\mathrm{T} 1$ and $\mathrm{T} 4$ using a paired t-test and conducted a generalized linear model to assess the change in number of dosing errors over this decade-long period, adjusting for patient demographics, health literacy level, and key covariates. Patient health literacy skills were measured using the Newest Vital Sign.

RESULTS: Participants on average made 2.8 dosing errors (SD, 2.4; range, $0-21$ ) out of 21 potential errors at T1 and 5.0 dosing errors at T4 (SD 2.05; range $0-18)$ at $\mathrm{T} 2(\mathrm{p}<0.001)$. In a multivariate model controlling for relevant covariates, low and marginal health literacy (low: $=1.86 ; 95 \%$ confidence interval $[\mathrm{CI}]: 1.15-2.56, \mathrm{p}<0.001$; marginal: $=.55,95 \%$ CI: $0.15-1.09$, $\mathrm{p}=0.04)$, number of chronic conditions at baseline $(=0.16,95 \%$ CI: 0.15 , $0.30, \mathrm{p}=0.03)$, and baseline dosing errors $(=-0.79,95 \%$ CI: $-0.88,-0.70$, $\mathrm{p}<0.001)$ were all significant, independent predictors of changes in dosing errors over 10 years. Other sociodemographic variables (e.g. age, sex, race) were not significantly associated with dosing errors in bivariate or multivariate analyses.

CONCLUSIONS: Results indicate that regimen dosing errors were common among older patients and increased significantly over a decade of follow-up. Patients with limited literacy skills and those contending with multi-morbidity are at greater risk of making more errors, and consequently becoming less independent in their ability to self-manage multi-drug regimens over time. Findings emphasize the importance of routine counseling for older patients on appropriate medication use to ensure complex drug regimens are taken correctly and safely.

\section{LOW LIBIDO IN OLDER WOMEN: A QUALITATIVE STUDY}

Holly N. Thomas ${ }^{1}$; Megan Hamm ${ }^{1}$; Rachel Hess ${ }^{2}$; Sonya Borrero ${ }^{3}$; Rebecca C. Thurston ${ }^{1}$. University of Pittsburgh, Pittsburgh, PA; ${ }^{2}$ University of Utah, Salt Lake City, UT; ${ }^{3}$ University of Pittsburgh and VA Pittsburgh, Pittsburgh, PA. (Control ID \#3183183)

BACKGROUND: A majority of older women are sexually active and consider sex an important part of life. Low libido affects nearly half of women over 60 and is associated with poorer health, quality of life, and relationship satisfaction. The causative factors of low libido in older women are not well understood. In this study, we used a qualitative approach to explore factors that affect libido in older women with low sexual desire.

METHODS: We conducted 15 individual interviews and 3 focus groups (total $\mathrm{N}=36$ ). Included women were aged 60 years or older, were sexually active with a partner in the prior year, and screened positive for low libido using a validated instrument. Interviews and focus groups were led by trained facilitators using a semi-structured guide with open-ended questions regarding sex and desire. Interviews and focus groups were audio recorded and transcribed. A codebook was developed and refined by two analysts. Five interviews were double-coded to ensure accuracy. Codes were then assigned to all data and key themes were identified.
RESULTS: Mean age was 64.2 (range 60-71). All but two women were non-Hispanic White, $83 \%$ were married or living with a partner, and all but one identified as heterosexual. Women highlighted multiple factors that affected libido: (1) physical health, (2) life stressors, (3) psychological factors, and (4) partner factors. In terms of (1) physical health, women detailed how medical problems, low energy, bodily pain, prior gynecologic surgery, and menopause negatively impacted libido. Especially common were vaginal symptoms, including dryness, tightness, and pain. Women often used lubricants or vaginal hormone therapy to adapt to these symptoms, but voiced concerns about messiness, cost, and potential side effects. In terms of (2) life stressors, concerns regarding adult children and dying parents were common. In contrast, some women noted that retiring from work and children leaving the home gave them more time and freedom, resulting in a positive impact on libido. In terms of (3) psychological factors, women discussed concerns about attractiveness, body image, and mood that negatively impacted libido. In terms of (4) partner factors, higher intimacy and relationship satisfaction fostered increased libido, while partner erectile dysfunction (ED) had a negative impact. ED led to less satisfying sexual encounters for women, which in turn decreased their sexual interest. Fear of offending partners or partner defensiveness made communicating about ED challenging. The women noted that older women's sexuality is not discussed enough in our culture, and that speaking with peers about these issues is beneficial.

CONCLUSIONS: Healthcare providers should be aware that there are many factors that affect libido in older women, including physical health, life stressors, psychological factors, and partner issues. Assessment and treatment of low libido in this population requires a multifactorial approach.

\section{LOW NALOXONE PRESCRIPTIONS AMONG INDIVIDUALS AT HIGH RISK OF OPIOID-RELATED OVERDOSE DESPITE HEALTHCARE INTERACTIONS}

Sarah Follman ${ }^{1}$; Christopher Lyttle ${ }^{1}$; Vineet M. Arora ${ }^{2}$; P. Quincy Moore ${ }^{2}$; Mai T. Pho ${ }^{2}$. ${ }^{1}$ University of Chicago, Chicago, IL; ${ }^{2}$ University of Chicago Medical Center, Chicago, IL. (Control ID \#3168608)

BACKGROUND: As opioid-related mortality continues to rise, naloxone remains a critical intervention in preventing overdose death. Expanding access should be optimized to promote equity. Engaging providers to regularly prescribe naloxone is important to advocate for this vulnerable population. The primary goal of our analysis was to determine the extent to which patients at high risk of opioid overdose receive naloxone.

METHODS: Using Truven Health MarketScan ${ }^{\circledR}$, a national database of commercial insurance claims, we investigated naloxone prescriptions among high-risk patients accessing emergency, inpatient, and outpatient care. We identified patients between October 1, 2015 - December 31, 2016 and grouped them into three categories using ICD-10 codes: 1) patients with opioid misuse or dependence, 2) patients with opioid overdose, and 3) patients with both opioid misuse or dependence and overdose. We then linked patients in each group to their outpatient pharmacy claims. We compared naloxone pharmacy claims by opioid risk group using a ChiSquare test. Additional Chi-square tests characterized the groups across various demographic and healthcare utilization variables. We performed a multivariate logistic regression model to test the association between opioid risk group and naloxone claim, controlling for various demographic and healthcare utilization variables.

RESULTS: Of the 138,108 individuals identified as high-risk opioid users, 2,135 had naloxone claims, or $1.5 \%$ of the eligible patients identified. Differences in naloxone claims across opioid risk group were statistically significant $(\mathrm{p}<0.001)$. Only $1.5 \%$ of those with a diagnosis of opioid misuse/dependence and $0.8 \%$ of those with an opioid overdose diagnosis received naloxone. Of those with both an opioid misuse/dependence diagnosis and an opioid overdose diagnosis, only $4.6 \%$ received naloxone. Overall, $98.5 \%$ high-risk patients did not received naloxone, despite many 
interactions with the healthcare system including: 88,618 hospitalizations, 229,680 emergency department (ED) visits, 298,058 internal medicine visits, and 568,448 family practice visits. In the highest risk group, $96 \%$ of patients had at least $1 \mathrm{ED}$ visit.

CONCLUSIONS: Within our study population, patients at high risk for opioid overdose rarely received prescriptions for naloxone despite numerous interactions with the healthcare system. Healthcare visits remain a missed opportunity to provide naloxone. Efforts to support prescribing in emergency, inpatient, and in outpatient settings represent an important opportunity to improve naloxone access. This may include policies such as the implementation of naloxone standing orders, protection from civil liability for naloxone dispensing and use, and the addition of coprescribing recommendations to prescription opioid labeling. Overall, prescribing naloxone as a standard-of-care for high-risk patients addresses a critical patient-care need and helps advocate for this particularly vulnerable group.

\section{LOW PRIORITY OF OBESITY EDUCATION IN INTERNAL MEDICINE RESIDENCY PROGRAMS LEADS TO LACK OF PHYSICIANS' PREPAREDNESS TO EFFECTIVELY TREAT PA- TIENTS WITH OBESITY: RESULTS FROM THE U.S. INTER- NAL MEDICINE RESIDENCY PROGRAM OBESITY EDUCA- TION CURRICULUM BENCHMARK STUDY}

W. Scott Butsch ${ }^{1}$; Kathryn Robinson ${ }^{2}$; Ranita Sharma ${ }^{3}$; Julianne Knecht ${ }^{4}$; B. Gabriel Smolarz $4{ }^{4}$ Cleveland Clinic, Cleveland, OH; ${ }^{2}$ Penn Medicine Princeton Medical Center, Plainsboro Township, NJ; ${ }^{3}$ Rutgers Robert Wood Johnson Medical School, New Brunswick, NJ; ${ }^{4}$ Novo Nordisk, Inc., Princeton, NJ. (Control ID \#3177809)

BACKGROUND: Despite the obesity epidemic in the U.S., physicians are presently under-prepared to treat patients with this disease. This study sought to understand how obesity is currently taught in U.S. Internal Medicine (IM) residency programs. It serves as a benchmark for measuring the degree to which IM residency curricula incorporate topics pertaining to the recently developed Obesity Medicine Education Collaborative (OMEC) obesity competencies.

METHODS: In this cross-sectional study, IM residency program directors were invited to complete an online survey about their curriculum. During the summer of 2018, invitations were sent via postal mail to 501 accredited IM programs. Only one respondent was permitted from each program.

RESULTS: 81 IM residency programs, matching the geographic distribution of U.S. programs, participated in the survey. Although obesity was an intentional program objective for two-thirds of programs (67\%), only $2 \%$ of respondents believed their residents are "very prepared" to manage patients with obesity with nearly half of respondents reporting their residents are only "somewhat prepared". Formal rotation opportunities in obesity are limited, with approximately one-quarter of programs offering clinical non-surgical rotations in obesity. Most IM residency programs incorporate obesity throughout the curriculum in some form; however, at best, only one-third (35\%) of programs reported any one of the known core obesity competencies are covered to "a great extent". In addition, many programs reported psychosocial components of obesity $(40 \%)$, weight stigma and discrimination (44\%), etiological aspects of obesity $(65 \%)$ and pharmacological treatment of obesity $(43 \%)$ were covered only to a "very little extent" or "not at all". Competencies reportedly wellcovered to are more likely to be taught during teaching rounds, while those covered to a lesser extent are most likely to be taught informally. Lack of room in the curriculum and lack of faculty expertise are the greatest barriers to integrating obesity education. Less than half (40\%) of residency programs have discussed incorporating or expanding formal obesity education.

CONCLUSIONS: The obesity curriculum currently taught within U.S. IM residency programs does not adequately cover important aspects that address the growing epidemic. These results suggest obesity education is not enough of a priority for IM residency programs to evolve their curriculum to incorporate the number one leading health epidemic. Even though weight bias and stigma among medical students and practicing healthcare providers leads to suboptimal care and poor health outcomes, the topic is only covered to a limited extent within many residency programs. Recognizing the importance of providing residents with the education and skills they need to be prepared for managing and treating patients with obesity and formalizing them within IM residency programs will be helpful for graduating residents to be competent in addressing obesity.

\section{LOW-VALUE CARE AND CLINICIAN ENGAGEMENT IN THE MEDICARE SHARED SAVINGS PROGRAM: A SURVEY OF FRONTLINE CLINICIANS}

Adam A. Markovitz'; Michael Rozier ${ }^{3}$; Susan D. Goold ${ }^{2}$; John Z. Ayanian $^{2}$; Edward C. Norton ${ }^{2}$; Timothy Peterson ${ }^{1}$; John M. Hollingsworth ${ }^{2}$. ${ }^{1}$ University of Michigan School of Public Health, Ann Arbor, MI; ${ }^{2}$ University of Michigan, Ann Arbor, MI; ${ }^{3}$ St. Louis University College for Public Health and Social Justice, St. Louis, MO. (Control ID \#3185503)

BACKGROUND: Although the Medicare Shared Savings Program (MSSP) created new organizational incentives to improve health care value, Accountable Care Organizations (ACOs) have achieved only modest reductions in the use of low-value care. It is uncertain whether this reflects organizational failure to engage the frontline ACO clinicians charged with implementing ACO objectives. In this study, we asked: Have ACOs successfully engaged frontline clinicians? Is ACO engagement associated with clinicians' reported ability to implement recommendations against low-value care?

METHODS: We designed and administered a survey to clinicians in one of the ten largest MSSP ACOs in the country. Primary exposures included clinicians' participation in ACO decision-making, awareness of ACO incentives, perceived influence on practice, and perceived quality improvement. Our primary outcome was clinicians' reported difficulty implementing recommendations against low-value care (e.g., "Don't recommend cancer screening in adults with life expectancy of less than 10 years"). We used multivariable fixed-effect models to test the relationship between ACO engagement and reported difficulty implementing recommendations against low-value care. In these models, we compared clinicians of the same specialty (e.g., urology), type (e.g., physician), and provider organization (e.g., Michigan Medicine). We used multiple imputation and calibrated survey weights to reduce bias from missing data and survey non-response.

RESULTS: The analytic sample included 1,289 ACO clinicians: advanced practice providers $(29 \%)$, physician specialists $(27 \%)$, primary care physicians (18\%), internal medicine specialists (16\%), and surgeons $(10 \%)$. Response rate was $34 \%$. We found limited ACO engagement of frontline clinicians: Few clinicians participated in the decision to join the ACO (3\%). Few clinicians were aware of ACO incentives, including knowing the ACO was accountable for both spending and quality (23\%), successfully lowered spending $(9 \%)$, or faced upside risk only (3\%). Few agreed (moderately or strongly) the ACO changed compensation (20\%), practice $(19 \%)$, or feedback $(15 \%)$ or that it improved care coordination $(17 \%)$ or inappropriate care $(13 \%)$. Clinicians reported difficulty following recommendations against low-value care $18 \%$ of the time; clinicians reported patients had difficulty accepting recommendations $36 \%$ of the time. Increased ACO awareness (one standard deviation [SD]) was associated with decreased difficulty $(-2.3$ percentage point [p.p.], 95\% confidence interval $[\mathrm{CI}]:-3.8,-0.7)$ implementing recommendations, as was perceived quality improvement (1 SD increase, -2.1 p.p., 95\% CI, -3.4, -0.8). Participation in ACO decision-making and perceived influence on practice were not associated with recommendation implementation. 
CONCLUSIONS: ACO clinicians were broadly unaware of and unaffected by ACO objectives and activities. Limited engagement of ACO clinicians may hamper ACO efforts to reduce low-value care.

\section{LOW-VALUE CARE FOR 4 COMMON CONDITIONS WITHIN THE VETERANS HEALTH ADMINISTRATION} Thomas R. Radomski ${ }^{1,}{ }^{2}$; Yan Huang ${ }^{1}$; Seo Young Park ${ }^{1}$; Carolyn T. Thorpe $^{2}$; Joshua M. Thorpe ${ }^{2}$; Michael J. Fine ${ }^{2,}{ }^{1}$; Walid F. Gellad ${ }^{2,1}$. ${ }^{1}$ University of Pittsburgh School of Medicine, Pittsburgh, PA; ${ }^{2}$ VA Pittsburgh Healthcare System, Pittsburgh, PA. (Control ID \#3182678)

BACKGROUND: Low-value care is a major driver of wasteful health care spending and exerts physical, psychological, and financial harm upon patients. Whereas low-value care affects up to $43 \%$ of Medicare beneficiaries, less is known about its prevalence within VA. Our objective was to quantify the frequency and facility-level variation in the use of low-value diagnostic testing for Veterans managed within the Veterans Health Administration (VA).

METHODS: Among a 20\% random sample of all Veterans continuously enrolled in VA in Fiscal Year 2015 ( $n=1,022,987$ Veterans), we applied a claims-based metric used in Medicare to identify unnecessary diagnostic testing for 4 uncomplicated conditions: 1) low back pain, 2) acute sinusitis, 3) headache, and 4) syncope. Examples of such low-value care include low back imaging within 6 weeks of an initial back pain diagnosis without red-flag symptoms and maxillofacial CT imaging for acute uncomplicated sinusitis. For headache, we separately assessed low-value head imaging and electroencephalography (EEG). For syncope, we separately assessed low-value head imaging and carotid ultrasonography. For each condition, we determined the overall percentage of eligible Veterans who received low-value care and the range of low-value care use across 127 VA Medical Centers (VAMCs), adjusting for sociodemographic and VAMC characteristics. We used Pearson's correlation coefficient to determine the correlation between Veterans' use of different types of low-value care for the same condition within VAMCs, focusing on uncomplicated headache and syncope.

RESULTS: Of the 343,024 Veterans who had non-specific low-back pain in FY15, 19,736 (5.8\%) received low-value care, with a VAMC range of 3.6-7.7\%. Of the 52,889 Veterans who had acute sinusitis, $1,305(2.5 \%)$ received low-value care, with a VAMC range of $1.3-5.1 \%$. Of the 79,176 Veterans with uncomplicated headache, 6,904 (8.7\%) received low-value care (head imaging 6,786 (8.6\%) and EEG $167(0.2 \%)$ ), with an overall VAMC range of 6.2-14.6\%. Lastly, 23,776 Veterans experienced uncomplicated syncope, of whom 3,300 (13.9\%) received low-value care (head imaging 2,393 (10.1\%) and carotid ultrasound 1,390 (5.9\%)), with an overall VAMC range of 11.3-16.8\%. Undergoing different forms of lowvalue care for the same condition was weakly, but significantly, correlated for uncomplicated syncope (Rho $0.21, \mathrm{p}<0.019)$ but not headache (Rho $0.13, \mathrm{p}=0.157$ ).

CONCLUSIONS: In a national random sample of Veterans, we identified 2 to 5 -fold variation in the use of low-value diagnostic testing for 4 common conditions across VAMCs, after adjusting for differences in patient and VAMC characteristics. Veterans' receipt of low-value diagnostic testing for headache and syncope was weakly correlated within VAMCs. These findings highlight the importance of further characterizing the degree to which Veteran and facility-level factors drive these variations as a first step in developing facility-specific policies to mitigate the provision of low-value care.

\section{LOW-VALUE PROSTATE CANCER SCREENING WITHIN THE VETERANS HEALTH ADMINISTRATION}

Thomas R. Radomski ${ }^{1}$, 2 ; Yan Huang ${ }^{1}$; Seo Young Park ${ }^{1}$; Carolyn T. Thorpe $^{2}$; Joshua M. Thorpe ${ }^{2}$; Michael J. Fine ${ }^{2,}$ 1; Walid F. Gellad ${ }^{2,1}$. ${ }^{1}$ University of Pittsburgh School of Medicine, Pittsburgh, PA; ${ }^{2}$ VA Pittsburgh Healthcare System, Pittsburgh, PA. (Control ID \#3182875)
BACKGROUND: Prostate specific antigen (PSA) screening for prostate cancer is of low value in men with a limited lifespan. Because most patients managed in the Veterans Health Administration (VA) are older males, low-value PSA screening in VA is likely to be common and expose Veterans to excessive costs and unnecessary risks without measurable benefits. Our objective was to quantify the frequency and variation of low-value PSA screening across VA Medical Centers (VAMCs).

METHODS: We examined a national 20\% random sample of Veterans aged 75 using VA data from fiscal years (FY) 2014-2015. Using a claimsbased algorithm originally developed for use in Medicare, we defined lowvalue PSA screening as screening among men aged 75 without a history of prostate cancer. We calculated rates of low-value PSA screening in FY15 by VAMC, adjusting for sociodemographic and VAMC-level factors from FY14. We characterized variation across VAMCs using the adjusted median odds ratio (OR). We divided VAMCs into deciles of low-value PSA screening and calculated the adjusted OR of undergoing low-value screening for Veterans within each decile. To increase the specificity of our claims-based algorithm, we determined the prevalence of low-value PSA screening after excluding Veterans who underwent prostatectomy, had a prior PSA elevation, or had a clinical reason for PSA testing, such as hematuria. In subgroup analyses, we assessed the frequency of lowvalue PSA screening in Veterans at greatest risk of mortality based on a Gagne Comorbidity Index Score 3.

RESULTS: Among 214,480 Veterans aged 75 who received care in 127 VAMCs, 37,866 (17.7\%) received low-value PSA screening, with adjusted VAMC rates ranging from $3.3 \%-38.2 \%$. The adjusted median OR was 1.88 , meaning the median odds of receiving low-value PSA screening would increase by $88 \%$ were a Veteran to transfer his care to a VAMC with more low-value testing. Veterans at VAMCs in the top decile had an adjusted OR for receiving low-value PSA screening of 12.9 (CI 11.0-15.2) compared to those in the lowest decile. After excluding Veterans with a prior prostatectomy, PSA elevation, or acute symptoms, 31,556 (14.7\%) received a low-value PSA test, ranging from $2.0 \%-49.9 \%$ across VAMCs. Among Veterans with the greatest comorbidity burden $(n=23,377), 3,496$ $(15.0 \%)$ received a low-value PSA screening test $(1.7 \%-46.3 \%$ across VAMCs).

CONCLUSIONS: In a national cohort of older Veterans, more than 1 in 6 received low-value PSA screening, with greater than 10-fold variation across VAMCs. The frequency of low value PSA screening remained high even among Veterans without a clinical indication for testing and in those with the clearest limit to life expectancy. The overall prevalence and degree of VAMC-level variation we observed highlights the need to accurately identify and accounting for unique VAMC-level factors when developing interventions to mitigate the provision of low-value care.

\section{LOW-VALUE PROTON PUMP INHIBITOR PRESCRIPTIONS AMONG OLDER ADULTS AT A LARGE ACADEMIC HEALTH SYSTEM}

Michelle Chong ${ }^{1}$; Folasade May ${ }^{1}$; Katherine L. Kahn ${ }^{2}$; Edgar Coro$\mathrm{na}^{1}$; Liu Yang ${ }^{1}$; Margaret M. Mongare ${ }^{1}$; Vishnu Nair ${ }^{1}$; Eric Esrailian $^{1}$; Catherine Sarkisian ${ }^{3}$; John N. Mafi ${ }^{2},{ }^{4}$. ${ }^{1}$ David Geffen School of Medicine, University of California, Los Angeles, Los Angeles, CA; ${ }^{2}$ David Geffen School of Medicine at UCLA, Los Angeles, CA; ${ }^{3}$ UCLA and Greater Los Angeles VA, Los Angeles, CA; ${ }^{4}$ RAND Corporation, Santa Monica, CA. (Control ID \#3184539)

BACKGROUND: Proton pump inhibitor (PPI) drugs are associated with complications such as pneumonia or fractures. Older adults are particularly vulnerable to complications from long-term PPIs, which can also exacerbate polypharmacy and financial toxicity. In this context, we sought to characterize the prevalence of low-value PPI prescriptions among older adults, in order to inform a future randomized-controlled quality improvement (QI) intervention. 
METHODS: We created a cohort of patients aged 65 years receiving primary care at a large academic health system. Using electronic health record data, we identified patients prescribed any PPI. A multidisciplinary team (geriatrician, gastroenterologist, QI expert, two general internists) created evidenced-based criteria for appropriate vs low-value PPI prescriptions based on clinical guidelines and effectiveness literature. Supervised by a gastroenterologist and internist, an internal medicine resident conducted a manual chart review in a random sample of 100 patients prescribed a PPI to obtain demographic and clinical data. Specifically, we determined the reason for starting a PPI, duration of PPI use, whether the patient was taking the PPI, and the indication for long-term use ( $>8$ weeks) to assess whether the prescription was appropriate. Prescriptions were considered appropriate if we could identify any guideline-based indication for longterm use, and low-value otherwise. For example, if a patient had a diagnosis of Barrett's esophagus then a long-term PPI prescription would be considered appropriate; in contrast, if a patient continued a long-term PPI without a guideline-based indication and had no documented attempt to wean or discontinue, the prescription would be considered potentially low-value.

RESULTS: Among the 69,352 older adults in our cohort, $12.6 \%$ were prescribed a PPI. Of the sample of 100 patients prescribed a PPI, 59\% were female, $67 \%$ were White, and median age was 76 . The 3 most common reasons for starting a PPI were uncomplicated GERD (63\%), atypical GERD (7\%), and peptic ulcer disease (7\%). Four patients were not taking the PPI and were excluded from appropriateness assessment, and the rest $(n=96)$ were documented to be taking a long-term PPI. Of these, $30(31 \%)$ did not have a documented indication for long-term use, suggesting a lowvalue prescription. Most often $(n=19)$, a physician would initially prescribe a PPI for uncomplicated GERD (without a long-term indication) but would not attempt to wean or discontinue the PPI over time. The remaining $69 \%$ (66/96) of patients prescribed a long-term PPI had a guideline-based indication: peptic ulcer disease prophylaxis (56), erosive esophagitis (3), Barrett's esophagus (5), and esophageal stricture (2).

CONCLUSIONS: Physicians prescribed PPIs in one in eight older adults, and nearly one third of these prescriptions were potentially low-value. These findings represent an important opportunity for targeted interventions to improve quality, lower spending, and reduce patient harm.

\section{LUNG CANCER SCREENING: THE VIEWS OF PHYSICIANS} Celia P. Kaplan 1, 1; Margaret Lowenstein ${ }^{2,3}$; Maya Vijayaraghavan ${ }^{1,1}$; $\overline{\text { Judith M. Walsh }}^{1,}{ }^{1}$; Leah S. Karliner ${ }^{1,1}$. ${ }^{1}$ University of California, San Francisco, San Francisco, CA; ${ }^{2}$ Perelman School of Medicine at the University of Pennsylvania, Philadelphia, PA; ${ }^{3}$ Corporal Michael J. Crescenz VA Medical Center, Philadelphia, PA. (Control ID \#3185122)

BACKGROUND: Lung cancer is the leading cause of cancer death in the U.S. Screening high risk individuals with low dose CT (LDCT) reduces mortality and is recommended by the United States Preventive Services Task Force (USPSTF). The USPSTF and the Centers for Medicare and Medicaid Services recommend that eligible patients, together with their physicians, weigh benefits with limitations and risks to make an informed decision about whether or not to be screened. Little is known about the uptake of LDCT and shared decision-making with primary care physicians (PCPs). The goals of our study were to assess the practices, barriers, and facilitators of lung cancer screening among PCPs.

METHODS: California internal and family medicine PCPs were randomly selected though the American Medical Association Physician Masterfile. We conducted a self-administered (online or paper) survey between September 2017 and May 2018 and collected additional physician demographic information through the California State Licensing Board. The survey covered the following domains: physician's lung cancer screening practices, barriers and facilitators, perceived patients' barriers to lung cancer screening, and potentially useful tools to promote lung cancer screening.

RESULTS: Of the 886 selected participants, 368 (42\%) completed the survey. Over half of the respondents were female (54.4\%) and $42 \%$ were general internists. $11.8 \%$ of the physicians were Latino, $2 \%$ African American, and $37.7 \%$ Asian. Three-quarters of physicians reported offering patients lung cancer screening in the prior year (76.3\%). Only 35\% correctly reported the recommended ages for initiation of lung cancer screening, and $56.6 \%$ reported the correct age for discontinuation of screening. The two considerations most frequently cited when offering lung cancer screening were the potential mortality benefit and patient screening preference $(75.4 \%$ and $71.8 \%$, respectively). Most PCPs reported always discussing smoking cessation $(94.6 \%)$ and lung cancer risk factors $(89.8 \%)$ in relation to screening. With respect to lung cancer screening discussion barriers for physicians, the most frequently cited factor was the complexity of the visit (42.1\%). Concern about cost was the most frequently reported physician-perceived barrier to screening for patients $(34.9 \%)$. PCPs identified online shared decision-making tools integrated into the electronic health record as the most potentially useful counseling aid $(66.7 \%)$.

CONCLUSIONS: Our results reveal that while many PCPs offer lung cancer screening and discuss lung cancer risk and smoking cessation, there are significant knowledge gaps about screening recommendations. The most significant barriers to discussing lung cancer screening largely related to visit complexity and patient counseling, suggesting the need for EHRbased tools with easy integration into workflow in the primary care setting.

\section{MAJOR CARDIOVASCULAR OUTCOMES AMONG AT-RISK HIGH-DEDUCTIBLE HEALTH PLAN MEMBERS}

James F. Wharam $^{2}$; Fang Zhang ${ }^{2}$; Adrian F. Hernandez ${ }^{1}$; Christine $\mathrm{Lu}^{2}$; Xin $\mathrm{Xu}^{2}$; Jamie Wallace ${ }^{2}$; Dennis Ross-Degnan ${ }^{2}$; Joseph P. Newhouse ${ }^{3}$. ${ }^{1}$ Duke University, Durham, NC; ${ }^{2}$ Harvard Medical School and Harvard Pilgrim Health Care Institute, Boston, MA; ${ }^{3}$ Harvard Medical School, Boston, MA. (Control ID \#3184469)

BACKGROUND: The effect of high-deductible health insurance on major cardiovascular outcomes is unknown.

METHODS: We studied a 2004-2014 national sample of 63,363 highdeductible health plan members aged 40-64 years with diabetes, cardiovascular disease, hypertension, or hyperlipidemia, who were continuously enrolled for 1 year in a low-deductible ( $\$ 500)$ plan followed by 3 years in a high-deductible health plan ( \$1000) after an employer-mandated switch. We matched high-deductible health plan members to 539,445 contemporaneous controls who had the same cardiovascular risk factors and whose employers offered only low-deductible plans. Using a controlled interrupted-time-series design, we analyzed cumulative rates of myocardial infarction, stroke, and amputation. We modelled weighted cumulative event rates using aggregate-level segmented regression.

RESULTS: High-deductible health plan members experienced no detectable absolute change in myocardial infarction ( -0.9 events per 10,000 members, 95\% confidence interval: $-2.7,0.8)$ after 3 years of follow-up relative to the end of the baseline year compared to controls. Stroke and amputation declined by $3.4(-4.0,-2.9)$ and $6.7(-9.0,-4.4)$ events per 10,000 members, respectively, among high-deductible health plan members versus controls over the same period.

CONCLUSIONS: At-risk patients who were switched to high-deductible health plans did not experience increases in major cardiovascular outcomes over 3 follow-up years relative to similar members in low-deductible plans.

Figure: Cumulative rates of myocardial infarction, stroke, and amputation among at-risk HDHP and control group members.
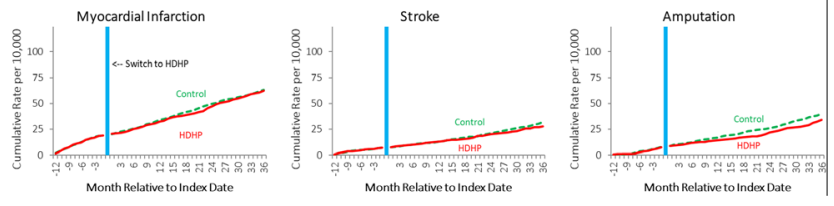
MAKING CONNECTIONS: NATIONWIDE IMPLEMENTATION OF VIDEO TELEHEALTH TABLETS TO ADDRESS ACCESS BARRIERS IN VETERANS

Donna M. Zulman ${ }^{1,2}$; Josephine Jacobs ${ }^{3}$; Cindie Slightam²; Amy J. Gregory $^{2}$; Tolessa Gurmessa ${ }^{2}$; Rachel Kimerling ${ }^{2}$, ${ }^{4}$; Daniel Blonigen ${ }^{2}$; Emily Wong ${ }^{2}$; John Peters ${ }^{5}$; Leonie Heyworth ${ }^{6,5}$. ${ }^{1}$ Stanford University, Stanford, CA; ${ }^{2}$ VA Palo Alto Health Care System, Menlo Park, CA; ${ }^{3}$ VA Health Economics Resource Center, Menlo Park, CA; ${ }^{4}$ VA National Center for Post-Traumatic Stress Disorder, Menlo Park, CA; ${ }^{5}$ VA Office of Connected Care/Telehealth Services, Washington, DC; ${ }^{6}$ VA San Diego Healthcare System, La Jolla, CA. (Control ID \#3180392)

BACKGROUND: In 2016, the VA launched an initiative to distribute video telehealth tablets to Veterans with health conditions, socioeconomic challenges, and geographic constraints that impede access to VA services. We sought to evaluate the implementation and effectiveness of this tablet distribution initiative.

METHODS: In a mixed methods evaluation guided by the RE-AIM framework, we examined the distribution and usage rates of 5,000 VAissued tablets shipped in 2016 and 2017. We characterized tablet recipients in terms of sociodemographics (i.e., age, sex, race/ethnicity, rural/urban status, distance to VA facility), chronic conditions, and health care utilization. We evaluated patient experience through a survey of tablet recipients upon tablet receipt $(n=2,120$ respondents, response rate $61 \%$ ) and after approximately 6 months of tablet use $(n=1,232$ respondents, response rate $61 \%$ ). Given high rates of mental illness among tablet recipients, we conducted a retrospective matched cohort analysis to assess how tablet receipt was associated with mental health care utilization, continuity of care, and clinical efficiency outcomes. We also examined implementation of tablet distribution through a survey of 68 facility telehealth coordinators and interviews with telehealth coordinators and regional leadership $(n=20)$. RESULTS: 89 VA facilities, spanning all 18 geographic regions, distributed tablets to 6,742 patients. Tablet recipients had an average age of 56 years, $53 \%$ lived in rural areas, $75 \%$ had a diagnosed mental illness, and almost half had six or more chronic conditions. Approximately 4 in 5 tablet recipients used the tablet during the evaluation period; $65 \%$ of video encounters were for mental health care; $10 \%$ for spinal cord injury; the remaining were for primary care, palliative care, rehabilitation, and other services. Among patient survey respondents, over 6 months there was an increase in the proportion who reported getting convenient care $(67 \%$ to $80 \%$ ), and many reported that the tablets saved them time (90\%), money (89\%), and helped them get care they couldn't otherwise (56\%). For Veterans with mental health conditions, relative to the matched control group, tablet recipients experienced an increase of 1.94 (SE, 0.27) psychotherapy encounters, 1.21 (SE, 0.07) medication management visits, and an $18.5 \%$ point (SE, 0.02) increase in their likelihood of meeting the VA quality metric for mental health care continuity in the six months posttablet receipt. Telehealth staff reported that the most common barriers to tablet distribution were insufficient training (59\%), staffing shortages (55\%), and provider disinterest (33\%).

CONCLUSIONS: VA's initiative to distribute video telehealth tablets to high-need patients reached many individuals with social and clinical access barriers, and had a positive impact on access to mental health care and patient experiences. Additional research is needed to understand the barriers and needs of the one in five tablet recipients who did not use their tablets.

\section{MAKING IT EASIER TO GET EVIDENCE INTO PRACTICE: AHRQ EVIDENCE-BASED PRACTICE CENTERS' (EPC) PILOT PROJECTS}

Celia V. Fiordalisi ${ }^{1}$; Allison Schmidt ${ }^{1}$; Martha Gerrity ${ }^{1}$; Stephanie M. Chang ${ }^{2}$; Amanda Borsky ${ }^{2}$; Lisa Hartling ${ }^{3}$; Thomas Trikalinos ${ }^{4}$; Gillian Sanders-Schmidler ${ }^{5}$; Mary Butler ${ }^{6}$; Emilia Flores ${ }^{7}$; C. Michael White ${ }^{8}$; Jennifer S. Lin ${ }^{9}$; Lisa V. Rubenstein ${ }^{10}$; Meera Viswanathan ${ }^{11}$; Annette M. Totten $^{12} ;$ Karen Robinson ${ }^{13} ;$ Jeanne-Marie Guise ${ }^{1} .{ }^{1}$ Portland VA Research
Foundation, Portland, OR; ${ }^{2}$ Agency for Healthcare Research and Quality, Rockville, MD; ${ }^{3}$ University of Alberta Evidence-based Practice Center, Edmonton, AB, Canada; ${ }^{4}$ Brown University Evidence-based Practice Center, Providence, RI; ${ }^{5}$ Duke University Evidence-based Practice Center, Durham, NC; ${ }^{6}$ University of Minnesota Evidence-based Practice Center, Minneapolis, MN; ${ }^{7}$ ECRI Institute-Penn Medicine Evidence-based Practice Center, Plymouth Meeting, PA; ${ }^{8}$ University of Connecticut Evidence-based Practice Center, Storrs, CT; ${ }^{9}$ Kaiser Permanente Research Affiliates Evidence-based Practice Center, Portland, OR; ${ }^{10}$ RAND Corporation Evidence-based Practice Center, Santa Monica, CA; ${ }^{11}$ RTI International-University of North Carolina (UNC) Evidence-based Practice Center at Chapel Hill, Chapel Hill, NC; ${ }^{12}$ Pacific Northwest Evidencebased Practice Center, Portland, OR; ${ }^{13}$ Johns Hopkins University Evidence-based Practice Center, Baltimore, MD. (Control ID \#3184508)

BACKGROUND: The Agency for Healthcare Research and Quality (AHRQ) Evidence-based Practice Centers (EPCs) produce rigorous and comprehensive systematic reviews to inform health care practice and policy. It is well-known that there is a gap in translating evidence into practice. EPCs conducted a series of pilot projects to develop novel translational products with the aim of increasing accessibility and usability of evidence in healthcare systems.

METHODS: EPCs partnered with a variety of healthcare systems stakeholders, to develop and pilot translational products intended to facilitate the uptake and implementation of evidence from EPC reports. EPCs evaluated their own translational products using various methods, including conducting interviews with health systems stakeholders, such as leadership, frontline staff, and patients. We analyzed and summarized findings across these pilot projects using three sources of information: qualitative narrative from each project, structured feedback provided via a standardized reporting form, and discussions during meetings.

RESULTS: The EPCs' nine translational products generally fell into three categories: dissemination products, interactive data visualizations, and implementation products. Dissemination products summarized results from systematic reviews and required on average 57 hours to develop (range: 2090 hours). Interactive data visualizations used technology or software to enable an interactive interface with findings of reports and required on average 152 hours to develop (range: 55-300 hours). Implementation products help health systems implement evidence into practice, and required on average 1,077 hours to develop (range:50-2850 hours). Products were reported to be useful when they presented important information in a concise format (graphical displays of information, tables, and 3-page summaries), allowed users to explore data relevant to them (interactive displays that could drill down to study level), provided contextual information for implementation, and/or could be directly implemented into electronic medical records or clinical care processes. Products were not found to be useful if they presented information on a topic that was not a priority or for an issue that was not pertinent/relevant to the health system at that time.

CONCLUSIONS: In the fast-paced complex environment of healthcare delivery, translational products that summarize the key elements of systematic reviews may improve health system and providers' access to evidence. Translational products should prioritize clear writing, meaningful tables and graphs, and tailor the evidence to the needs of the user. The EPC Program will continue these efforts in hopes to improve healthcare system's access to high-quality, unbiased evidence that can ultimately improve quality of care and promote evidence-based medicine.

MAKING THE HIDDEN MORE TRANSPARENT: A QUALITATIVE ANALYSIS OF MEDICAL STUDENTS' ENCOUNTER LOGS TO IDENTIFY THE SOCIAL DETERMINANTS OF HEALTH LIMITING OPTIMAL CARE

David Basile; Jed Gonzalo; Deanna Dubots. Penn State College of Medicine, Hershey, PA. (Control ID \#3185884) 
BACKGROUND: Health care delivery and health service research focuses on disease-related issues. However, increasing evidence suggests the social determinants of health (SDH) are directly associated with patient outcomes, but these factors are typically "hidden," and infrequently identified. This qualitative analysis explored the prevalence of a predetermined set of SDH experienced by a population of patients identified as "high risk" within one health system.

METHODS: All $1^{\text {st }}$-year medical students at Penn State College of Medicine serve as student patient navigators, working in interprofessional care teams and linked with patients who are identified as high risk. Following encounters, students complete detailed logs, addressing questions related to patient barriers and activities performed. A qualitative analysis of log data was performed using deductive and inductive techniques; a set of 22 SDH-related diagnostic codes was used (deductive) to investigate prevalence of each across all patients with reflexivity to identify potential codes (inductive) not within this pre-determined set. Two investigators performed the analysis (using NVIVO12 for data management), which involved independent coding and regular adjudication sessions.

RESULTS: A preliminary analysis of 300 logs related to 176 unique patients was performed, with the identification of 40 unique SDH codes. The five most-commonly identified SDH codes were: behavioral and mental health (7.2\%), health system coordination (7.2\%), transportation issues $(6.7 \%)$, elderly or disability $(6.3 \%)$, and lifestyle quality $(6.0 \%)$. The three least-commonly identified SDH codes were: poverty ( $0 \%)$, poor neighborhood composition $(0.1 \%)$, and cultural barriers $(0.1 \%)$.

CONCLUSIONS: These preliminary data help facilitate transparency regarding the often unforeseen SDH factors that may be placing patients at risk for poor health outcomes. Factors such care coordination, transportation, and lifestyle are less easily obtained through available methods, such as electronic health records or provider-based intake assessments. These results merit a re-evaluation in population health initiatives aimed at uncovering these barriers to improve care. Further screening by nurses and physicians for SDH needs to occur to allow for better points of care for patients. These codes can be utilized to help further achieve the goals of the Triple Aim in optimizing healthcare.

\section{MARIJUANA USE AND RISK OF CANCER: A SYSTEMATIC REVIEW AND META-ANALYSIS}

Mehrnaz Ghasemiesfe $^{4}$; Salomeh Keyhani ${ }^{3}$; Brooke Barrow ${ }^{1}$; Samuel Leonard $^{4}$; Deborah R. Korenstein ${ }^{1,2}$. ${ }^{1}$ Memorial Sloan Kettering Cancer Center, New York, NY; ${ }^{2}$ Weill Cornell Medical College, New York, NY; ${ }^{3}$ University of California at San Francisco, San Francisco, CA; ${ }^{4}$ San Francisco VA Medical Center, San Francisco, CA. (Control ID \#3177620)

BACKGROUND: Marijuana is the most widely used illicit substance in the United States; use is rising. Marijuana smoke shares carcinogens with tobacco smoke, and tetrahydrocannabinol (THC) from marijuana may have immunomodulatory effects that could lead to cancer. Rising marijuana use raises concerns about increases in the risk of developing cancer. We conducted a systematic review and meta-analysis to assess the effects of marijuana use on development of smoking-related (lung, head and neck, and urogenital) and other cancers.

METHODS: We searched 5 databases (PubMed, EMBASE, PsychINFO, MEDLINE and Cochrane Library) from January $1^{\text {st }}, 1973$ to June $11^{\text {th }}, 2018$. Two reviewers independently screened all titles and abstracts for inclusion and reviewed all observational and experimental full-text articles. We included studies in English, involving adults with 1 joint-year or more cumulative use (defined as "ever use") of marijuana, which reported development of cancer. Study characteristics and quality were assessed by 3 reviewers independently; strength of evidence for each outcome was graded by consensus. We conducted meta-analyses when there were 2 or more studies of the same design without high risk of bias (ROB), and when heterogeneity was low or moderate. In meta-analyses, we used random-effects models to calculate summary risk ratios with $95 \%$ CIs. Narrative synthesis was performed when meta-analysis was not possible.

RESULTS: We included 24 studies (18 case-control, 5 observational and 1 cross-sectional). Among case-control studies $(n=2)$, ever use of marijuana was not associated with head and neck squamous cell carcinoma (HNSCC) $\left(\mathrm{RR}=1.15,95 \% \mathrm{CI} 0.92-1.45, \mathrm{p}=0.23, \mathrm{I}^{2}=56 \%\right)$ or oral cancer $\left(\mathrm{RR}=1.14,95 \%\right.$ CI $\left.0.93-1.40, \mathrm{p}=0.20, \mathrm{I}^{2}=58 \%\right)$. However, $>8$ jointyears of use was associated with an increased risk of HNSCC $(\mathrm{RR}=2.20$, $95 \%$ CI $\left.1.07-4.50, p=0.03, I^{2}=81 \%\right)$. Pooled analysis of moderate ROB case-control studies $(n=3)$ demonstrated that ever use of marijuana was not associated with developing testicular germ cell tumor (TGCT) $(\mathrm{RR}=$ $1.07,95 \% \mathrm{CI} 0.87-1.30, \mathrm{p}=0.52, \mathrm{I}^{2}=60 \%$ ) compared to never- use, but > 10 joint-years use was associated with TGCT $(\mathrm{RR}=1.18,95 \%$ CI 1.00 $\left.1.40, \mathrm{p}=0.05, \mathrm{I}^{2}=0 \%\right)$, and non-seminoma TGCT $(\mathrm{RR}=1.51,95 \% \mathrm{CI} 1.17$ $-1.96, \mathrm{p}<0.01, \mathrm{I}^{2}=0 \%$ ). One prospective study found increased risk for lung cancer in marijuana smokers over a long follow-up period. Findings from 5 case-control studies of lung cancer were mixed; interpretation was limited by poorly described marijuana exposure, limited results on marijuana-only users, and low levels of exposure.

CONCLUSIONS: Low-strength evidence suggests smoking marijuana is associated with development of HNSCC and TGCT. Evidence on the association between marijuana use and other cancer types is insufficient. Long term studies in marijuana-only smokers are needed to better understand the effect of marijuana use on the development of lung, oral, and other cancers.

\section{MARKOV DECISION ANALYSIS OF THE COST-UTILITY FOR ANTICOAGULANT THERAPY IN PATIENTS WITH ATRIAL FIBRILLATION AT RISK FOR FALLS}

Eric K. Wong $^{1,2}{ }^{\text {; Christina Kosar }}{ }^{3,}{ }^{1}$; Harindra Wijeysundera ${ }^{1,}{ }^{4}$. ${ }^{1}$ University of Toronto, Toronto, ON, Canada; ${ }^{2} \mathrm{Li} \mathrm{Ka}$ Shing Knowledge Institute, St. Michael's Hospital, Toronto, ON, Canada; ${ }^{3}$ The Hospital for Sick Children, Toronto, ON, Canada; ${ }^{4}$ Sunnybrook Health Sciences Centre, Toronto, ON, Canada. (Control ID \#3185508)

BACKGROUND: Atrial fibrillation (AF) is a common cardiac condition in older adults that results in an increased risk of stroke. Anticoagulants decrease stroke risk associated with AF but increase bleeding risk. Older patients are often at high risk of falls, which further compounds bleeding risk. The purpose of this study was to determine the most cost-effective anticoagulant for older adults with $\mathrm{AF}$ at high risk of falling.

METHODS: A Markov cohort model was used to evaluate pharmaceutical options including aspirin, warfarin, and direct oral anticoagulants (DOACs; apixaban, edoxaban, dabigatran, rivaroxaban). The base case was a 70-year-old man with recently diagnosed AF and a fall in the last 12 months. The model used the Ontario (Canada) public payer perspective and a lifetime horizon. Outcomes included life years (LY), qualityadjusted life years (QALY), costs and incremental cost-effectiveness ratios (ICERs). One- and two-way sensitivity analyses were also conducted to evaluate model robustness.

RESULTS: The use of DOACs (11.81-12.01 LY) led to longer life expectancy than ASA (10.07 LY) and warfarin (10.94 LY). When utilities were included, DOACs (10.49-10.88 QALYs) remained superior to ASA and warfarin (8.61 and 9.72 QALYs respectively). The lowest lifetime cost was for apixaban at C\$207,249.42, which was lower than both ASA and warfarin (C\$217,071.45 and C\$209,031.26, respectively). Apixaban and dabigatran were the dominant strategies after evaluation of ICERs. Apixaban was the most cost-effective strategy as dabigatran had an ICER of C\$98,725.00/ QALY, which was above the cost-effectiveness threshold (C\$50,000/QALY). CONCLUSIONS: Apixaban is the most cost-effective anticoagulant in older individuals at high risk of falls from a public payer perspective. Apixaban had the lowest total lifetime cost compared to other anticoagulants. 
MD AWARE: QUALITATIVELY MEASURING THE IMPACT OF LONGITUDINAL RESILIENCY CURRICULUM AND WELLBEING SELF-ASSESSMENT TOOL AMONG MEDICAL STUDENTS

Kelly Crotty $^{1,2}$; Annie Robinson ${ }^{1}$; Colleen Gillespie ${ }^{3}$; Verity Schaye ${ }^{4}$; Katie Grogan ${ }^{4}$; Linda Tewksbury ${ }^{4} .{ }^{1} \mathrm{NYU}$, New York, NY; ${ }^{2}$ Deparntment of Veterans Affairs, New York, NY; ${ }^{3}$ NYU School of Medicine, Brooklyn, NY; ${ }^{4}$ NYU School of Medicine, New York, NY. (Control ID \#3163076)

BACKGROUND: To bolster medical student wellbeing and combat burnout, the NYU School of Medicine (NYUSOM) implemented a longitudinal resiliency curriculum coupled with a wellbeing self-assessment tool. We qualitatively studied the impact of this curriculum on knowledge, selfawareness, and behaviors related to wellbeing and resiliency.

METHODS: The MD AWARE curriculum was launched in August 2017 for the NYUSOM class of 2020. Six sessions were implemented at critical junctions of their training. Each session includes a short lecture, followed by a small group activity led by trained facilitators. At the start of each session, students complete an anonymous online self-assessment adapted from three validated assessment tools measuring different aspects of wellbeing and burnout. Students immediately receive scores with explanations and benchmarks of each and then debrief in their small group. After each MD AWARE session, students completed a retrospective pre/post evaluation survey. Focus Groups (FG) were held in December 2017 (after Sessions 1\&2) to gain richer insight into the impact of the curriculum and self-assessment tool. A purposeful sampling strategy with maximal variation was employed to recruit participants; 10 students participated in each FG. Qualitative data was gathered through the surveys and the FG. The FG were recorded and transcribed. Each FG had 2 project staff members and postsession debriefing. Member-checking was also used. Responses were subsequently coded and analyzed by two experienced faculty members (a third colleague assisted in theme triangulation). An iterative data analysis strategy was applied. Throughout the analysis, an audit trail, frequent memo writing and a reflexivity journal was maintained.

RESULTS: Themes: Community Building: Connecting with another student...it was helpful for my wellbeing Skill and Knowledge Acquisition and Application: The main sort of takeaway is you need to be aware of (Burnout) and if you need help there are resources Importance of Faculty Development: I think that a prep session between those who designed the curriculum and those who facilitate the small-groups could go a long way towards creating the environment I imagine was originally intended Value of Refection: The score didn't add much...It was more about the act of answering the questions than the number that came out of it NYU Administration Values Medical Student Wellbeing: Just the fact that NYU has this program and is making it part of orientation already speaks volumes about its priorities: that we matter

CONCLUSIONS: Thematic analysis of the impact of MD AWARE indicated that it provides concrete information on resources available to the students. Additionally, the students value both protected time with their peers and for self-refection. Lastly, although care must to be taken in selecting faculty to facilitate the small groups, the mere existence of the longitudinal curriculum signaled that the NYUSOM administration values medical student wellbeing.

\footnotetext{
MEANS SAFETY DISCUSSIONS FOR SUICIDE PREVENTION IN PRIMARY CARE: VETERAN AND VETERANS HEALTH ADMINISTRATION (VHA) PRIMARY CARE TEAM PERSPECTIVES Khaya D. Clark' ${ }^{2}$ Martha Gerrity ${ }^{1,7}$; Emily Kenyon ${ }^{2}$; Elizabeth Karras ${ }^{3,4}$; Joseph A. Simonetti ${ }^{5,}{ }^{6}$; Steven Dobscha ${ }^{2,}{ }^{7}$. ${ }^{1}$ VA Portland Health Care System, Portland, OR; ${ }^{2}$ Department of Veterans Affairs, Portland, OR; ${ }^{3}$ Center of Excellence for Suicide Prevention (VA), Canandaigua, NY; ${ }^{4}$ University of Rochester, Rochester, NY; ${ }^{5}$ Veterans Health Administration, Aurora, CO; ${ }^{6}$ University of Colorado School of Medicine, Aurora, CO; ${ }^{7}$ Oregon Health \& Science University, Portland, OR. (Control ID \#3180699)
}

BACKGROUND: Means safety (MS) counseling approaches have been developed in an effort to reduce deaths by firearms and other suicide methods. During MS (also known as lethal means) counseling, a clinician encourages the patient to limit access to potentially lethal means in case of a crisis or until the patient is no longer at elevated risk. While MS counseling can be delivered in any setting, it is time-intensive and rarely delivered in primary care. However, primary care is a critical place to intervene. Half of patients who die by suicide are seen in primary care in the month prior to death. We are developing a training program to facilitate brief MS discussions between primary care teams and Veterans with elevated suicide risk. The aim of this project was to identify main themes and language important in discussing MS with Veterans.

METHODS: This project was conducted at a large VHA Medical Center in the Pacific Northwest, and designated a quality improvement project by the institution. Four listening sessions were conducted with Veterans $(n=70)$ and four with primary care providers (PCPs), nurses, and other staff at clinics $(n=70)$ to understand stakeholder experiences with MS conversations and assess feasibility of staff discussing MS with Veterans in practice. Additional individual interviews were conducted with six Veterans and five PCPs. A grounded theory approach was used to analyze transcribed notes from the sessions.

RESULTS: Veteran and staff agreed that discussing means safety in primary care is appropriate and important, and may be beneficial for patients with elevated suicide risk. Staff felt that use of a team approach to MS discussionswould be optimal due to competing demands on PCPs. Veterans supported a team approach since nurses or other staff may have greater rapport and contact with them than PCPs. Veterans and staff felt there were opportunities to link MS discussions with VHA's emerging Whole Health prevention approach. Both groups felt that asking Veterans directly about firearm ownership ("do you own a firearm") is not an effective strategy for initiating MS conversations because it is a sensitive topic and may be perceived as a potential threat to $2^{\text {nd }}$ amendment rights. Indeed, Veterans acknowledged that they may not truthfully disclose firearms ownership, especially in the context of PCPs raising mental health concerns. Veterans reported they would feel more comfortable with MS conversations if 1) the rationale for discussing firearms in the context of a medical visit was provided; 2) they understood legal consequences of disclosure; and 3) the conversation was personalized, good eye contact was made, and staff refrained from documenting in the record during the conversation.

CONCLUSIONS: The findings suggest that training primary care teams to discuss MS with their patients is feasible. Primary care staff and Veterans have similar perspectives regarding MS which may enhance patient acceptance and usefulness of MS discussions.

MEASURING AND ANALYZING THE PERSONAL MEDICATION FORMULARY SIZE OF PRIMARY CARE PHYSICIANS

William L. Galanter ${ }^{1,}{ }^{1}$; Jennie B. Jarrett' ${ }^{1}$; Diana E. Norwich ${ }^{2,}{ }^{3}$; Maria Mirica $^{3}$; Diane L. Seger ${ }^{3},{ }^{10}$; Julie Fiskio ${ }^{3}$; Tewodros Eguale ${ }^{4,3}$; Alejandra Salazar $^{3}$; Walid F. Gellad ${ }^{5,6}$; John P. Cashy ${ }^{5}$; Jennifer A. Hale ${ }^{5}$; Jeffrey A. Linder $^{7}$; Bruce Lambert ${ }^{8}$; Suzanne Falck ${ }^{9}$; Lynn A. Volk ${ }^{10}$; Gordon D. Schiff ${ }^{3}{ }^{11} .{ }^{1}$ University of Illinois at Chicago, Chicago, IL; ${ }^{2}$ Tufts University School of Medicine, Boston, MA; ${ }^{3}$ Brigham and Women's Hospital, Boston, MA; ${ }^{4}$ MCPHS University, Boston, MA; ${ }^{5}$ A Pittsburgh Healthcare System, Pittsburgh, PA; ${ }^{6}$ University of Pittsburgh, Pittsburgh, PA; ${ }^{7}$ Northwestern University Feinberg School of Medicine, Chicago, IL; ${ }^{8}$ Northwestern University, Evanston, IL; ${ }^{9}$ University of Illinois Hospital and Health Sciences System, Chicago, IL; ${ }^{10}$ Partners HealthCare System, Somerville, MA; ${ }^{11} \mathrm{Har}-$ vard Medical School, Boston, MA. (Control ID \#3185286)

BACKGROUND: Prescribing decisions are fundamental to patient care and are responsible for substantial cost, harm, and benefit. Thus, variations in prescribing warrant scrutiny. As part of a large project analyzing prescribing practices at four institutions (Partners HealthCare System (PHS), Northwestern Medicine, University of Illinois at Chicago (UIC), and the Veterans 
Administration), we are profiling prescribing in the context of previously published principles of conservative prescribing. Here we present pilot data examining personal formularies of primary care physicians (PCPs) looking at the universe of drugs they initiate.

METHODS: We analyzed data from all PCPs at UIC $(\mathrm{N}=60)$ and a sample from PHS $(\mathrm{N}=23)$ totaling 226,176 prescriptions $(\mathrm{Rx})$ for patients seen by those PCPs in 2016-2017. An Rx was defined as new if there was no prior Rx by any prescriber since $1 / 1 / 14$. Formulary size was computed as the number of unique new drugs ordered by each physician in 2016-2017. Multivariate linear regression for UIC data were performed related to PCP panel characteristics: average age, $\%$ female, size, total visits, and PCP demographics: years since training, sex, general or family medicine.

RESULTS: PCPs started $46 \pm 18$ new drugs per 100 visits. The average formulary size was $228 \pm 96$ unique new drugs. Formulary size at PHS was $268 \pm 101$ versus $201 \pm 92$ at $\mathrm{UIC}(\mathrm{P}=0.02)$. Half $(49 \% \pm 12 \%)$ of these drugs were only prescribed once during the two-year period. At UIC, predictors of formulary size were general medicine compared to family medicine (30.8 \pm 11.9 meds, $\mathrm{P}=0.01)$; average panel age $(16.2 \pm 5.4$ fewer meds for every 10 years, $\mathrm{P}=0.004)$, and visits $(35.3 \pm 2.3$ meds when visits increased from 1500 to 2000 year, $\mathrm{P}<0.0001$ ). PCP sex, years since training and \% women in panel were not statistically significant. Comparing the average formulary at PHS and UIC, we found examples of variations in medications used. For example, oxycodone was used in $29 \%$ of new opiate Rx at PHS, but only $2 \%$ at UIC, while hydrocodone was used in $33 \%$ at UIC, but only $4 \%$ at PHS. CONCLUSIONS: There were differences in both the size and the medications that comprised the personal formularies of PCPs at the two institutions. Roughly half of the drugs initiated were ordered only once during the twoyear study period, suggesting that many PCPs are prescribing drugs for which they have limited experience in initiating. Variations in specific medication use within a class were noted and some were not evidencebased. We conclude it is feasible to compute personal formulary size at an institution level and that variability in this quantity is likely to be an important index of prescribing quality. Once known confounders such as number of visits are controlled for, comparing personal formularies between both individuals and institutions is likely to reveal important differences in prescribing patterns that may reflect variations in prescribing practices.

\section{MEASURING PHYSICIAN WORK}

Stephanie A. Eisenstat ${ }^{1,}{ }^{1}$; Michael Hu${ }^{2,}{ }^{1}$; Walter J. O'Donnell ${ }^{1,}{ }^{1}$; Retsef Levi ${ }^{2,1}$. ${ }^{1}$ Harvard Medical School, Boston, MA; ${ }^{2}$ Massachusetts Institute of Technology, Cambridge, MA. (Control ID \#3182417)

BACKGROUND: With the advent of electronic health records (EHRs), a substantial portion of Primary Care Provider (PCP) time is now spent on EHR tasks such as post-visit documentation, patient communication, staff messages, results management, etc. Although these tasks represent a significant portion of PCP time, time spent is generally not accounted for in physician compensation models such as fee-for-service or panel-based payment models. The goals of this study are to 1) establish a rigorous methodology for measuring physician time spent on EHR tasks and 2) investigate variability in how EHR work is distributed temporally and across physicians, patient type and medical conditions.

METHODS: All EHR click-level data were analyzed from a primary care practice within an AMC. The analyses were performed on the EHR data from October 2018 during which the physicians touched 5,062 patients in the EHR. Using click-level timestamps for all EHR activity, we developed an objective and easily replicable method to measure the amount of EHR time spent by a physician on any individual patient or task. We also used the practice's scheduling data to determine the amount of visit time physicians are spending with individual patients. Combining this information with panel attribution labels allows us to examine the distribution of PCP EHR time across different types of patients and across the time of day. In particular, we examine the time allocation of each PCP along three dimensions: 1) patient panel attribution, 2) tasks related to patients who did and did not have a PCP office visit and 3) whether the EHR work was performed after-hours (i.e., completed outside of the PCP's clinical scheduled bookable hours). The methods developed in this preliminary study are currently being expanded to additional primary care practices with click-level EHR data spanning 3 years.

RESULTS: Our analyses indicate that during one standard calendar month, the average PCP spent 290 hours completing EHR tasks. The average amount of time spent in office visits was 73 hours (both normalized to 1 full-time equivalent or FTE). On average, the division of total PCP EHR time spent between panel versus non-panel patients was $78 \%$ and $22 \%$, respectively. Similarly, the division of total PCP EHR time spent on patients with and without office visits was $71 \%$ and $29 \%$, respectively. Examining the temporal distribution of the EHR work, we observe that $>40 \%$ of PCP EHR time occurred after-hours.

CONCLUSIONS: PCP work is rapidly expanding beyond traditional office visits as the prevalence and complexity of EHR tasks continues to grow. Our findings indicate that the vast majority of PCP time has shifted away from patient facing activities and instead is now focused on EHR tasks. Developing the methodology to measure and categorize this EHR work is foundational to establishing a better understanding of modern PCP work. This is particularly important as health systems explore compensation models and strategies for improving physician work and engagement.

\section{MEASURING PRECONCEPTION WELLNESS WITHIN THE VETERANS HEALTH ADMINISTRATION (VHA)}

Deirdre A. Quinn ${ }^{1}$; Maria K. Mor ${ }^{1}$; Florentina Sileanu ${ }^{1}$; Xinhua Zhao ${ }^{1}$; Lisa S. Callegari ${ }^{2,5}$; Laurie C. Zephyrin ${ }^{4}$; Sonya Borrero ${ }^{1,3}$. ${ }^{1}$ VA Pittsburgh Healthcare System, Pittsburgh, PA; ${ }^{2}$ VA Puget Sound Healthcare System, Seattle, WA; ${ }^{3}$ University of Pittsburgh, Pittsburgh, PA; ${ }^{4}$ Department of Veterans Affairs, Washington, DC; ${ }^{5}$ University of Washington School of Medicine, Seattle, WA. (Control ID \#3184035)

BACKGROUND: More women in the United States (U.S.) are dying in or around childbirth now than 25 years ago. Despite increased attention to preconception care, lack of consensus about implementing such care or assessing the success of preconception care has stymied progress. In 2016, the Clinical Workgroup of the National Preconception Health and Health Care Initiative proposed nine wellness measures to assess the quality of preconception care in healthcare systems. As the largest integrated health care system in the U.S., the VHA is uniquely positioned to lead the way in implementing and evaluating efforts, including preconception care, to improve birth outcomes. This study is the first comprehensive assessment of VHA's performance on measures of preconception wellness.

METHODS: We examined national administrative data, including inpatient, outpatient, and fee-basis visits (visits to non-VA providers that are paid for by VA); lab and pharmacy data; and health factors screening data for women Veterans ages 18-45 with at least one pregnancy outcome (ectopic pregnancy, spontaneous abortion, stillbirth, and/or live birth) during fiscal years 2010-2015. After identifying outcomes, we estimated last menstrual period (LMP) from gestational age at the time of the outcome and used LMP as a reference point to assess eight of the nine preconception indicators at various timepoints (e.g., 3 or 12 months prior to LMP); one indicator, pregnancy intention, was not captured in VA administrative data.

RESULTS: We identified 26,556 pregnancy outcomes from 21,234 women Veterans. Just over half (56.6\%) were in non-Hispanic white women, $23.4 \%$ in non-Hispanic black women, and $10.9 \%$ in Hispanic women; the mean age at LMP was 29.8. The majority (77.1\%) of pregnancies ended in live birth; $22.4 \%$ resulted in spontaneous abortion or ectopic pregnancy, and $0.5 \%$ in stillbirth. Nearly one quarter $(23.7 \%)$ of pregnancies had no evidence of prenatal care; $44.4 \%$ of pregnancies had no documented tobacco use screening and $49 \%$ had no documented depression screening within 1 year prior to LMP; $29.5 \%$ of pregnancies occurred to obese women; and only $40.2 \%$ of pregnancies in women with pregestational diabetes had a documented optimal HbA1c measurement $(<6.5)$ in the year prior to LMP. Evidence of any 
STI screening in the year prior to 3 months post LMP was low, as was documentation of prenatal folic acid use; $95 \%$ of pregnancies appeared free from exposure in the year prior to LMP to medications under the Workgroup's six classes of teratogenic medications.

CONCLUSIONS: Despite the limitations of existing administrative data, monitoring standard measures of preconception wellness can provide benchmarks for improving women's health across systems and communities. Areas for intervention to improve women Veterans' preconception wellness were identified, including weight reduction, optimizing control of diabetes prior to pregnancy, and improved use and documentation of routine health screenings.

\section{MEDICAID EXPANSION AND PERFORMANCE ON ED THROUGHPUT MEASURES}

Anjali Bhatla; Jaya Aysola. University of Pennsylvania, Philadelphia, PA. (Control ID \#3183592)

BACKGROUND: Emergency department (ED) throughput is a key measure of hospital performance with public reporting of ED wait times metrics since 2012. Mounting evidence reveals the effects of ED wait times on inpatient length of stay, care quality, and hospital costs. However, we lack knowledge of the effect of Medicaid expansion on ED throughput measures. This study aims to determine if there is an association between state Medicaid expansion status and statewide ED throughput measures.

METHODS: First, we determined if the number of insured significantly increased over time in Medicaid expansion states versus nonexpansion states. Then, using CMS Hospital Compare data, we examined two ED throughput measures for patients admitted to the hospital: 1) the overall length of stay in the ED (ED1) as measured by the median time in minutes patients spent in the ED from their arrival to departure to inpatient floor; and 2) the boarding time (ED2), as measured by the median time in minutes patients spent in the ED from the time a provider requests a bed to departure to inpatient floor. For each metric, we used hospital-wide quarterly data, aggregated by state from 2012 to the first quarter of 2017. We conducted an interrupted time series analysis to evaluate the relationship between implementation of Medicaid expansion and ED performance metrics, as measured by quarterly average median ED wait times for Medicaid expansion states $(n=31)$ and non-expansion states $(n=19)$.

RESULTS: The percentage of insured individuals increased significantly $(\mathrm{p}=0.031)$ in states that expanded Medicaid. In 2012, the mean difference in ED1 between non-expansion states (241.6) and states that later adopted Medicaid expansion (281.2) was significantly different $(\mathrm{p}<0.001)$. In the first quarter of expansion, the average median total length of stay in the ED increased by 4.27 minutes in the nonexpansion states, as compared to a significantly greater increase of 10.14 minutes in average median wait times among the expansion states $(p=0.01)$. The average median boarding time (ED2) between non-expansion states (79.4) versus expansion states (103.7) differed significantly $(\mathrm{p}<0.001)$ in 2012 . In the first quarter of Medicaid expansion, ED2 increased by 1.97 minutes $(p=0.009)$ in nonexpansion states, and 3.2 minutes in expansion states $(p=0.22)$.

CONCLUSIONS: Since 2012, the total time spent in the ED (ED1) and boarding time (ED2) for patients admitted to the hospital have been significantly longer in Medicaid expansion states versus nonexpansion states. Our findings suggest that the influx of newly insured individuals through Medicaid expansion may have increased overall length of stay in ED for patients eventually admitted to the hospital in states that expanded Medicaid but had no significant impact on their average median boarding times. Future efforts should focus on delivering low-acuity care in settings outside of the ED to improve timeliness of care for patients requiring hospital admission from the ED.
MEDICAL ASSISTANT (MA)-FACILITATED CHRONIC DISEASE AND CANCER SCREENING IN A SAFETY NET CLINIC: TWOYEAR IMPACT OF A QUALITY IMPROVEMENT PROJECT

Kala Ghooray $^{1,2}{ }^{1}$; Jenny K. Cohen ${ }^{3}$. ${ }^{1}$ Highland Hospital, Berkeley, CA; ${ }^{2} \mathrm{UC}$ Irvine School of Medicine, Irvine, CA; ${ }^{3}$ Highland, Oakland, CA. (Control ID \#3176883)

BACKGROUND: Although screening can reduce morbidity and mortality from cancer and chronic disease, screening rates in minority and low-income populations continue to be low. Within primary care, medical assistants (MAs) play an integral role in patient education, advocacy, and routine health maintenance. We designed an intervention aimed to increase MAs knowledge and self-efficacy regarding screening through education and empowerment, with a goal of improving chronic disease and cancer screening rates. METHODS: As part of a 2016-2018 initiative to improve screening for chronic disease and cancer at an urban county outpatient Internal Medicine clinic, MAs were given more resources and time to conduct patient outreach. We created monthly education sessions to give MAs concrete tools for teaching and patient education. Topics included vaccines, hepatitis $\mathrm{C}$, diabetes, hypertension, colorectal, cervical, and breast cancer screening. Changes in MA knowledge were measured through pre- and post-surveys, and one cumulative end-of-year survey. We tracked screening order rates over the past 2 years to assess the interventions' impacts on patient care. MA knowledge and job satisfaction were assessed using surveys and a focus group. RESULTS: $83 \%$ (15/18) MAs participated in the pre- and post-surveys. Post-test scores improved following the sessions on cervical cancer screening (pre $=31 \%$, post $=55 \%, \mathrm{p}=0.06$ ), diabetes (pre $=49 \%$, post $=72 \%, \mathrm{p}=0.10$ ), and CRC screening (pre $=69 \%$, post $=85 \%, p=0.10$ ), reflecting increased knowledge about who should receive cervical cancer, diabetes, and CRC screening. Between $9 / 16$ and 11/18, there was an increase in order rates for cervical cancer screening $(65.81 \%$ to $70.48 \%)$, order rates for mammograms (52.04\% to $85.5 \%)$, completion rates for diabetic microalbumin/creatinine ratios $(58.05 \%$ to $83.26 \%$ ), and order and completion rates for FIT tests ( $74.98 \%$ and $60.95 \%$ to $75.54 \%$ and $62.31 \%$ ). The percentage of patients with diabetes and hypertension who had their blood pressure or HbA1c well controlled also improved during this time period $(59.45 \%$ and $64.41 \%$ to $77.51 \%$ and $68.35 \%$ ). We solicited free-response feedback from the MAs who enjoyed the sessions and appreciated feeling a sense of professional investment. While MAs felt they had the training necessary to take on an expanded role, the added time-pressure was a challenge.

CONCLUSIONS: Our primary care redesign model focusing on MA education and empowerment improved MAs knowledge about screening and was associated with modest improvements in order rates. In addition to education, the clinic's investment in their professional skills, team role, and time was critical. Although there have been improvements in order and completion rates for all screenings being tracked, those improvements have recently plateaued. This may be due to MA turnover and changes in staffing resulting in the loss of dedicated patient-outreach time, highlighting the need for continued education and the importance of protected time for patient-outreach.

\section{MEDICAL STUDENT EXPERIENCE WITH DIFFICULT CONVER- SATIONS DURING THE ACTING INTERNSHIP}

David Staudt ${ }^{1}$; Corrie A. Stankiewicz ${ }^{2}$; Laura Dingfield ${ }^{3} .{ }^{1}$ Hospital of the University of Pennsylvania, Philadelphia, PA; ${ }^{2}$ University of Pennsylvania SOM, Narberth, PA; ${ }^{3}$ University of Pennsylvania, Philadelphia, PA. (Control ID \#3181403)

BACKGROUND: Internal medicine residency program directors have identified communication skills as an important competency for incoming interns. ${ }^{1}$ Acting internships (AIs) are an opportunity for senior medical students to gain experience with higher-level communication skills prior to starting residency training. Little is known about students' experiences with difficult conversations during AIs. We surveyed students about their experience with several types of difficult conversations during their AI. 
METHODS: All students $(\mathrm{N}=105)$ who had completed a 4 week medicine $\mathrm{AI}$ at our institution in 2018 were sent a link to an electronic survey assessing their roles and attitudes with 5 potentially difficult conversations: discussions with upset patients/families, leaving against medical advice, breaking bad news, assessing code status and limiting/withdrawing life-sustaining treatments. Anonymous survey data was collected electronically via Qualtrics. Results were calculated using the total number of survey responses, including both complete and partially complete surveys.

RESULTS: Seventy-seven students completed the entire survey and 5 students submitted partial responses for a total of 82 survey responses (78\% response rate). Students most frequently reported participating in code status discussions (91\%) and discussions with upset patients/families (99\%). A majority of students reported holding these conversations without supervision at some point during their $\mathrm{AI}$ (52\%, code status and $74 \%$, upset patient/ family). While a majority of students reported having had training in talking with upset patients/families (83\%), less than half reported having had any training in assessing code status (45\%). By the end of the 4 week AI rotation, the majority of students reported confidence in their ability to independently talk with upset patients/families (74\%) and to independently assess code status (58\%).

CONCLUSIONS: Existing literature suggests that serial clinical exposure to code status discussions breeds false confidence without improvement in competency among residents. ${ }^{2}$ Our data suggest a similar phenomenon among medical students, with many students reporting confidence in leading difficult conversations despite only a short window of exposure, minimal to no formalized training and lack of oversight. Formal programs have been shown to improve trainee competency with durability in skill set over time., ${ }^{3,4}$ While our data is from a single institution, it highlights a significant confidence-skill gap with regard to communication skills during critical doctor-patient interactions. The number of partial responses $(\mathrm{n}=5)$ might represent another limitation, although this would tend to underestimate the magnitude of a confidenceskill gap. Further exploration of these trends and resulting evidence-based curricular development that spans trainee levels are essential.

\section{MEDICAL STUDENT PERSONALITY TRAITS AND CLINICAL GRADES IN THE INTERNAL MEDICINE CLERKSHIP}

Monica Malviya ${ }^{1}$; Rebecca B. Forman ${ }^{1}$; Masrur A. Khan ${ }^{1}$; Keara English ${ }^{1}$; William Southern ${ }^{2}$; Amanda Raff'; Tulay Aksoy ${ }^{3} .{ }^{1}$ Albert Einstein College of Medicine, Bronx, NY; ${ }^{2}$ Montefiore, Bronx, NY; ${ }^{3}$ Montefiore Medical Center, Bronx, NY. (Control ID \#3184959)

BACKGROUND: Evaluation of clinical performance is an important component of medical students' grades in $3^{\text {rd }}$ year clerkships. However, clinical evaluation is subjective and may be influenced by factors independent of clinical skills. We hypothesized that student personality traits might impact evaluator perception of clinical performance, thus impacting overall clerkship grade. Using the OCEAN personality trait scale (Openness, Conscientiousness, Extraversion, Agreeableness, Neuroticism), we examined the association of personality traits with grades in the $3^{\text {rd }}$ year internal medicine clerkship at a single institution.

METHODS: $3^{\text {rd }}$ year medical students enrolled in the medicine clerkship during the academic years of 2016-17 and 2017-18 responded to a questionnaire assessing their personalities in accordance with the OCEAN criteria. Student responses were linked to grade components, then de-identified data were provided to the research team for analysis. This study was deemed exempt by the Einstein Institutional Review Board. Grade components of the final clerkship grade include ward team clinical evaluations, preceptor evaluations, and NBME shelf exam score. Chi-squared and regression analyses were used to evaluate correlations between the OCEAN personality domains, individual personality traits, and clerkship grades.

RESULTS: 356 students were eligible to participate. 97 responded (27\% response rate) and 94 provided information to allow linking to clerkship grades and were included in the analysis. $51(53 \%)$ of the respondents were female, $45(46 \%)$ male, and $1(1 \%)$ identified non-binary. The average age was $26.4 \pm$
2.6. $56(60 \%)$ received either a High Pass or a Pass, and $38(40 \%)$ received Honors in the clerkship. An overall clerkship grade of honors was strongly correlated with a clinical performance above the median preceptor score $(\mathrm{p}=$ 0.001). No OCEAN domains correlated significantly with a final grade of honors. However, individual traits within the personality types were associated with an honors grade. "Deep thinking" correlated positively with honors $(\mathrm{p}=$ 0.006). "Shyness" correlated negatively with honors $(\mathrm{p}=0.054)$. "Moody" was a trait that correlated positively with honors, though was not significant. Traits that suggested a trend correlating negatively with honors but did not reach significance included "depressive thinking," "reserved," "worrying," and "enthusiastic." Increasing scores in the Openness domain were correlated positively with a grade of honors (OR 1.05, $\mathrm{p}=0.17$ ).

CONCLUSIONS: Significant correlations between clerkship honors and traits of "deep thinking" (positive) and "shyness" (negative) were observed, which are in the domains of conscientiousness and neuroticism, respectively. Response bias and power were limiting factors in the overall study, and could explain why other traits or personality types did not reach statistical significance in the final analysis. Further investigations are needed to assess whether clerkship grades are impacted by personality traits.

\section{MEDICARE'S BUNDLED PAYMENT FOR CARE IMPROVEMENT MODEL DID NOT CHANGE SKILLED NURSING DISCHARGE PATTERNS}

Jane M. Zhu ${ }^{1}$; Amol S. Navathe ${ }^{2}$; Yihao Yuan ${ }^{3}$; Sarah Dykstra ${ }^{2}$; Rachel M. Werner ${ }^{4}{ }^{1}$ Oregon Health and Science University, Portland, OR; ${ }^{2}$ University of Pennsylvania, Philadelphia, PA; ${ }^{3}$ University of Illinois at Chicago, Chicago, IL; ${ }^{4}$ University of Pennsylvania and Philadelphia VA, Philadelphia, PA. (Control ID \#3185492)

BACKGROUND: One potential response to bundled payment incentives is that hospitals may more carefully select where they refer patients after discharge, in order to concentrate discharges and improve care coordination with skilled nursing facilities (SNFs). We evaluated whether participation in Medicare's voluntary Bundled Payment for Care Improvement (BPCI) model was associated with changes in discharge referral patterns to SNFs, specifically number of SNF partners and discharge concentration.

METHODS: We used Medicare claims data from 2010-2015 to identify admissions for lower joint replacement surgery and the following medical conditions: congestive heart failure, renal failure, sepsis, pneumonia, UTI and kidney infections, chronic obstructive pulmonary disease, and stroke. We identified hospitals participating in Model 2 bundles, which comprises 95\% of BPCI participants, and matched hospitals based on hospital characteristics including urban/rural location, teaching hospital, hospital ownership, annual discharges to SNF, and certified bed count. Our analytic sample included 3,078 acute care hospitals and 14,866 Medicare-certified SNFs in the US, encompassing more than 47 million hospital discharges. We used differencein-differences analyses to assess changes in discharge patterns among BPCIparticipating hospitals, compared to matched control hospitals. Our two measures of hospital-SNF integration were: a hospital's number of SNF partners, or the number of unique SNFs to which a hospital discharged its patients for each half-year of the study period. The second was a hospital's discharge concentration in each half-year, based on the HerfindahlHirschman Index (HHI). Multivariable regression models with hospital fixed effects were estimated for each outcome and included a BPCI-hospital indicator, intervention period indicators, and the interaction between the two. RESULTS: Of the hospitals in our sample, 416 participated in BPCI, with the majority selecting into joint replacement episodes $(n=295)$. BPCI participation was not associated with any change in number of SNF partners (increase by $0.8 \mathrm{SNFs}$ among BPCI hospitals relative to non-BPCI hospitals, $95 \% \mathrm{CI}[-0.2,1.9], \mathrm{p}=0.11$ ), or in discharge concentration (increase in $\mathrm{HHI}$ of 0.2 among BPCI hospitals relative to non-BPCI hospitals, 95\% CI [-68.7, 69.1], $\mathrm{p}=0.36$ ). Results did not vary across clinical conditions, and were robust across duration of BPCI participation and with different comparison groups. 
CONCLUSIONS: BPCI participation was not associated with changes in the number of SNF partners or in discharge concentration relative to nonBPCI hospitals. Studies on bundled payment programs for lower joint replacement have demonstrated efficacy in cost containment with little adverse effects on quality, but how hospitals are achieving these results remains unclear. More research is needed to understand how hospitals are responding to bundled payment incentives and specific practices that contribute to improvements in cost and quality.

\section{MEDICATION CONCERNS PREDICT MEDICATION ADHER- ENCE IN LATINO PATIENTS WITH HYPERTENSION}

Kerstin Nolan; Mark Butler; Antoinette Schoenthaler. NYU School of Medicine, New York, NY. (Control ID \#3176485)

BACKGROUND: Latinos have the lowest blood pressure (BP) control rates in the US, which may be explained by lower rates of medication adherence versus other ethnic groups. While patient medication concerns have been shown to predict poorer adherence in hypertensive patients, empirical data in Latinos is non-existent.

METHODS: This study was a secondary analysis of a randomized controlled trial in 119 Latino patients with uncontrolled BP followed in a community-based practice. Patient medication concerns were assessed with the Concerns-specific subscale of the Beliefs about Medications Questionnaire; lower scores indicate greater concerns. Medication adherence was assessed with the 8-item Morisky questionnaire; higher scores indicate better adherence. Repeated measures analysis using linear mixed effects modeling was used to evaluate whether changes in medication concerns predicted changes in adherence overtime (Baseline, 3 and 6-months). Patient characteristics (e.g., age, gender, insurance) known to influence adherence and treatment group were treated as covariates in the model.

RESULTS: The mean age was 61 years (SD:11.2); $50 \%$ were women; $38 \%$ had annual household income $<\$ 20,000$, and $59 \%$ had less than a highschool degree. A majority of patients were of Puerto Rican or Dominican ancestry. At baseline, 51\% (SD:16.5) of the sample reported being adherent to their antihypertensive medications; the mean score on the Concerns scale was 3.07 (SD:0.5; range:1-5). We found a significant time by concerns interaction effect on adherence in multivariate analyses $(F(2,24)=1.61, p=0.04)$. Specifically, adherence improved at a greater rate overtime among patients that reported low medication concerns than those that reported high concern (Figure).

CONCLUSIONS: Concerns about antihypertensive medications is a potentially modifiable factor that affects adherence in Latino patients with uncontrolled BP. Interventions targeting patients' medication concerns may help to improve the low rates of adherence in this high-risk patient population.

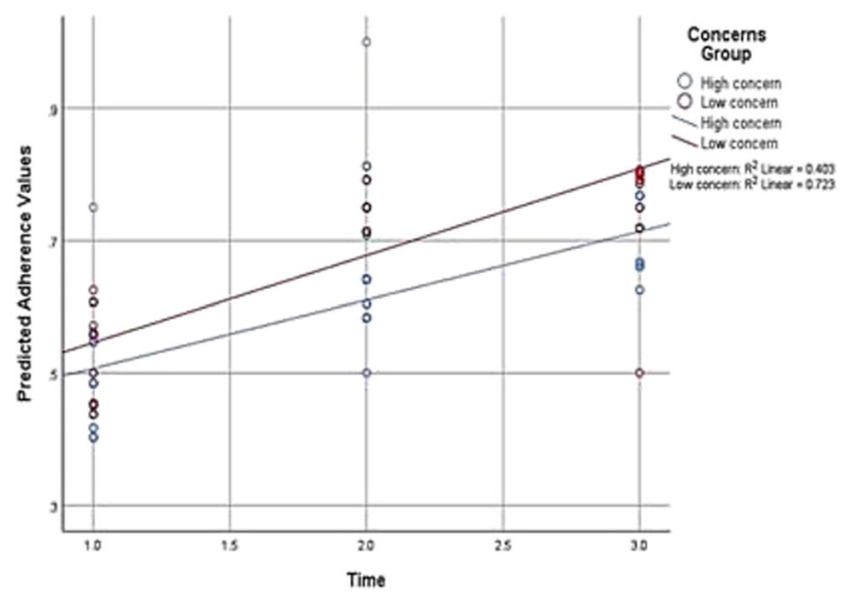

MENTAL HEALTH AND SELF-EFFICACY TO MANAGE CHRONIC HEALTH CONDITIONS AMONG NYC PUBLIC HOUSING RESIDENTS

Susan L. Creighton ${ }^{1}$; Jillian Diuguid-Gerber ${ }^{2}$; Katharine Lawrence ${ }^{1}$; Milna Rufin $^{2}$; Fred W. LaPolla ${ }^{3}$; Colleen Gillespie ${ }^{4}$; Noel Manyindo ${ }^{5}$; Rachel Dannefer $^{5}$; Lois Seidl ${ }^{5}$; Lorna Thorpe ${ }^{1} .{ }^{1}$ NYU School of Medicine, New York, NY; ${ }^{2}$ New York University, New York, NY; ${ }^{3}$ NYU School of Medicine, New York City, NY; ${ }^{4}$ NYU School of Medicine, Brooklyn, NY; ${ }^{5}$ NYC Deparment of Health, New York, NY. (Control ID \#3184616)

BACKGROUND: Self-efficacy to manage chronic conditions affects patients' health-related behaviors and interactions with the healthcare system and therefore influences health outcomes. Few studies have explored the complex relationships between mental health, self-efficacy, and management of chronic disease. A greater understanding of these interactions could inform successful community programming for marginalized populations such as public housing residents. Harlem Health Advocacy Partners is a community health worker (CHW) program designed to close health and social outcomes gaps in residents living in New York City Housing Authority (NYCHA). This study uses survey data collected for this initiative to explore the relationship between mental health and self-efficacy to manage chronic conditions among NYCHA residents with asthma, diabetes, and/or hypertension, and assess whether key variables such as connectedness to health care, social isolation and general health influence this relationship. METHODS: Five NYCHA housing developments were selected for the $\mathrm{CHW}$ intervention with five matched developments for comparison. Fourhundred adult residents with a chronic disease were recruited. Baseline intake interviews were conducted in person. Self-efficacy for managing chronic disease was measured with a 6-question scale. Depression was assessed using PHQ9 scores, a screen for the presence and severity of depression. Difficulty with mental health was assessed with questions on how difficult mental health problems made it to do work, take care of things at home, or get along with others. Bivariate analyses were conducted to assess the relationship between mental health and self- efficacy. A hierarchical linear regression model was run with mental health and other relevant variables (selected based on availability in the dataset and theoretical significance) as independent variables and self-efficacy as the outcome variable.

RESULTS: Self-rated general health predicted the greatest amount of variance in self-efficacy $(15.7 \%, \mathrm{p}<0.001)$. Mental health also contributed significantly; difficulty with mental health contributed $4.0 \%(p<0.001)$ and depressive symptoms contributed $1.1 \%(\mathrm{p}=0.03)$ to the variance in selfefficacy. Other variables, including demographics, type of insurance, connectedness to a primary care provider, and social isolation, were not associated with self-efficacy. Overall, the full model explained $22.5 \%$ of the variance in self-efficacy to manage chronic conditions.

CONCLUSIONS: NYCHA residents with mental health difficulty or depression represent a uniquely marginalized subpopulation of public housing residents, and were found to have lower self-efficacy than other residents, which may mean decreased ability to self-manage chronic medical conditions. Future research should explore relationships among mental health, self-management, and health care outcomes with the goal of augmenting targeted CHW interventions.

\section{MENTORSHIP OF UNDERREPRESENTED PHYSICIANS AND TRAINEES IN ACADEMIC MEDICINE: A SYSTEMATIC RE- VIEW}

Eliana Bonifacino ${ }^{1}$; Eloho Ufomata ${ }^{2}$; Amy H. Farkas ${ }^{3}$; Jennifer Corbelli ${ }^{2}$. ${ }^{1}$ UPMC, Pittsburgh, PA; ${ }^{2}$ University of Pittsburgh Medical Center, Pittsburgh, PA; ${ }^{3}$ Medical College of Wiscponsin, Milwaukee, WI. (Control ID \#3185553)

BACKGROUND: The US is becoming increasingly diverse from a racial and ethnic perspective; however, the physician workforce has continued to 
have disproportionately low numbers of medical school graduates from racial and ethnic groups that are described as underrepresented in medicine (URiM). Mentorship has been proposed as one way to increase the recruitment, retention and experience of URiM physicians and trainees. Our objective was to identify and describe mentoring programs for URiM physicians in academic medicine and to identify barriers and facilitators to success of such programs.

METHODS: We searched PubMed, PsycINFO, ERIC, and Cochrane databases following PRISMA guidelines in June 2017. We included original publications that described a mentoring program, exclusive of mentoring for a procedural skill, which included academic physicians or trainees from URiM backgrounds, and were conducted in the US. Two reviewers independently evaluated all records for eligibility, and abstracted data from included studies.

RESULTS: Our search returned 3308 results, of which 2632 references were excluded based on title and abstract. We performed a full text review on 669 manuscripts and 32 articles met our inclusion criteria. Of those, 13 programs were specifically created for URiM participants. Nine of the programs were developed for junior faculty, seven for medical students; two for residents and none for senior faculty. Frequently cited objectives of these programs were to improve research, diversify representation in specific specialty areas and recruit and retain URiM participants. The dyadic model of mentoring was the most common, however, several novel models were described which emphasized mentorship across different training levels. Program evaluations were primarily survey-based with high satisfaction scores, although some articles reported objective outcomes including publications, retention, and promotion.

CONCLUSIONS: This review describes a range of successful mentoring programs for URiM physicians. The traditional dyad model of mentorship remains common, though novel approaches aimed at improving the pipeline are emerging. Overall, the mentorship programs are met with high satisfaction regardless of mentor demographics, and can promote improvement in academic productivity, and recruitment and retention. Our review describes many mentoring programs for URiM physicians, most of which can be readily adapted by institutions depending on their local resources and goals to help promote and URiM physicians in academic medicine.

\section{META-ANALYSIS OF CT PULMONARY ANGIOGRAPHY YIELD IN US EMERGENCY DEPARTMENTS}

Safiya Richardson ${ }^{1}$; Anne Press ${ }^{2}$; Timothy Wigand ${ }^{3}$; Juliana A. Rosentsveyg, ${ }^{1}$; Patricia Driscoll ${ }^{3}$; Alexander O'Connell ${ }^{4}$; Saori Herman ${ }^{3}$; Janice Lester ${ }^{3}$; Sundas Khan ${ }^{3}$; Thomas McGinn ${ }^{3}$. ${ }^{1}$ Donald and Barbara Zucker School of Medicine at Hofstra/Northwell, Manhasset, NY; ${ }^{2} \mathrm{NYU}$ School of Medicine, New York, NY; ${ }^{3}$ Zucker School of Medicine at Northwell/Hofstra, Manhasset, NY; ${ }^{4}$ New York Presbyterian, New York City, NY. (Control ID \#3185364)

BACKGROUND: Every year about 2.4 million CT Pulmonary Angiography (CTPA) scans are performed to evaluate for pulmonary embolism in Emergency Departments (ED) in the United States. Each CTPA carries a $14 \%$ risk of contrast induced nephropathy and a lifetime malignancy risk that can be as high as $2.76 \%$. Incidental findings requiring diagnostic follow up are found in $24 \%$ of tests, increasing both costs and harms from repeat imaging. CTPA yield (percent of studies positive for PE) is a measure of appropriateness of use and has not been reviewed systematically. We conducted a systematic review and meta-analysis of CTPA yield in US EDs to establish a typical yield for our current practice environment and to determine if there have been changes to this yield over time.

METHODS: Cochrane, Embase, PubMed and Web of Science were searched as data sources. Observational cohort studies of adults were selected that measured CTPA yield in all ED patients referred for testing with clinical suspicion of PE. The titles and abstracts of studies identified by the search were independently reviewed by two reviewers. Studies were evaluated for exclusion criteria. Remaining studies were reviewed in full text by two independent reviewers. We extracted study characteristics and CTPA yield from the remaining studies. CTPA yields were estimated using random effects models. The I 2 test was used to assess heterogeneity in CTA yields among the studies.

RESULTS: Our search identified a total of 2,746 potentially relevant articles. Of those 296 remained after title and abstract review and 31 remained after full text review. The 31 studies included in our metaanalysis included a total of 42,670 patients. These studies were divided into three time periods for which CTPA yield was calculated. For time periods 1997-2002, 2003-2007 and 2008-2013 the yields were $11 \%$ (9\%, 14\%), 8\% $(6 \%, 9 \%)$ and $8 \%(6 \%, 10 \%)$ respectively. The studies were heterogeneous for all calculated pooled CTA yields ( $\mathrm{I} 2=82 \%, 94 \%$, and $86 \%$ respectively). CONCLUSIONS: This is the first study to systematically review CTPA yield in US EDs. Establishing a national benchmark for yield assists health systems seeking to monitor and increase CTPA yield as a part of quality improvement efforts. Future directions include further stratification by type of health system and analysis of non-US yields.

\section{METRICS OF RETENTION IN HIGH-RISK POPULATIONS: DE- CREASING "NO-SHOW" APPOINTMENTS THROUGH BEHAV- IORAL ECONOMICS}

Phillip Groden; Alexandra Capellini; Erica Levine; Bernard Ortega; Sandeep Kishore. Icahn School of Medicine at Mount Sinai, New York, NY. (Control ID \#3162708)

BACKGROUND: Studies have shown that a minority of the U.S. population - those often disconnected from care and beset with multiple chronic conditions - accounts for a substantial portion of annual health care spending. Interventions for these high-risk populations, such as Mount Sinai's Peak Health practice, aim to improve patient outcomes while reducing overall utilization and costs. "No-show" appointments directly oppose these goals by increasing the likelihood of ED utilization, inpatient admissions, and predictors of poor health. Highrisk interventions could therefore benefit from reducing no-show appointments by improving rates of patient retention. However, no standard for measuring retention exists within this setting, and reporting of retention rates across the literature for such interventions is sparse and inconsistent. Adoption of two previously reported metrics promises to capture a thorough picture of patient retention in high-risk populations: encounter adherence and encounter constancy. The application of these metrics is depicted through a study assessing the effects of a behavioral economics-based intervention on patient retention within Mount Sinai's Peak Health practice.

METHODS: The intervention consisted of specialized appointment reminder cards and clinic signage designed to improve the likelihood of successful patient encounters. At six months, appointment cards were transitioned to a computer format to promote provider adoption. Encounter adherence, a ratio of successful patient-provider interactions, is the number of completed encounters divided by all scheduled encounters, excluding those cancelled or rescheduled. Encounter constancy, a measure of consistent interactions across time, is defined as the number of 3-month intervals annually with at least one successful encounter (range $=0-4$ ), or the percentage of patients maintaining a specific constancy value.

RESULTS: At baseline, Peak Health's encounter adherence rate over six months was $74.7 \%$ (1282/1716); following introduction of the intervention, the encounter adherence rate improved over six months to $76.5 \%(1302 / 1701 ; p=0.2)$. At twelve months, encounter adherence will be compared to assess if use of the computer-based appointment reminders had an appreciable effect on retention, and encounter constancy will be compared to baseline to evaluate the effect of the intervention on the consistency of interactions across time.

CONCLUSIONS: Within the first six months, the behavioral economics-based intervention did not appear to significantly improve 
patient retention; this could reflect low adoption of the original appointment cards by Peak Health providers or the need for a more intensive intervention to improve retention above the rates described. Utilization of the encounter adherence and constancy metrics promises to fill salient gaps surrounding patient retention in this setting, while a renewed focus on retention may provide significant short and long-term benefits for high-risk interventions.

\section{MICROALBUMINURIA AS A MARKER TO ESTIMATE MA- JOR VASCULAR EVENTS IN STROKE PATIENTS: SYSTEM- ATIC REVIEW AND META-ANALYSIS}

Takayuki Yamada1; Satoshi Miyashita ${ }^{1}$; Hayato Mitaka ${ }^{1}$; Sera Satoi ${ }^{2}$; Svetlana Chernyavsky ${ }^{1}$. ${ }^{1}$ Mount Sinai Beth Israel, New York, NY; ${ }^{2}$ Nippon Medical School Hospital, Bunkyo-ku, Japan. (Control ID \#3181392)

BACKGROUND: Microalbuminuria is a marker of systemic endothelial dysfunction. It is known to be associated with coronary artery disease, stroke and cardiovascular mortality. However, it remains unclear whether or not microalbuminuria is related to major vascular events in patients with stroke.

METHODS: We conducted a systematic review and meta-analysis to evaluate the effect of microalbuminuria to estimate the risk of major vascular events in patients with stroke. PubMed, EMBASE and the Cochrane Library from inception to December 2018 were searched. Studies that compared stroke patients with microalbuminuria and nonalbuminuria were included. Primary outcome was major vascular events. All statistical analyses were performed using Comprehensive Meta-Analysis 3 (Biostat, Englewood, NJ, USA).

RESULTS: A total of five observational studies met the inclusion criteria. The pooled population consisted of 2,035 subjects. Microalbuminuria was associated with greater risk for major vascular events in patients with stroke (OR, 2.247; 95\% CI, 1.604 to $3.148 ; \mathrm{I}^{2}$, $0 \%$ ) (Upper figure). Subgroup analysis demonstrated that microalbuminuria was associated with greater risk for recurrent stroke in patients with stroke (OR, 1.924; 95\% CI, 1.228 to $\left.3.018 ; \mathrm{I}^{2}, 0 \%\right)$ (Figure 2). The rank correlation did not show significant publication bias $(p, 0.14)$ but Egger's test showed significant publication bias $(p, 0.033)$. CONCLUSIONS: Microalbuminuria provides good estimate of major vascular events including recurrent stroke in patients with stroke.

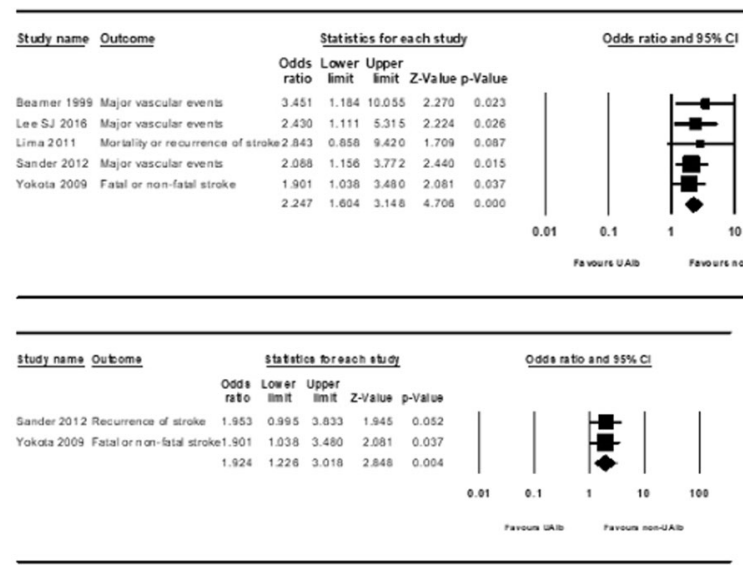

Upper figure: Forest plot of the association between microalbuminuria and cardiovascular events in patients with stroke. Odds ratio were presented as mean and standard deviation (SD). 95\% CI confidence interval. Lower figure: Forest plot for subgroup analysis of the association between microalbuminuria and stoke in patients with stroke. Odds ratio were presented as mean and SD.
MINIMAL CLINICALLY IMPORTANT IMPROVEMENT (MCII) IN KNEE OSTEOARTHRITIS: A POST HOC ANALYSIS OF A PROSPECTIVE, RANDOMIZED CLINICAL TRIAL OF DICLOFENAC SODIUM GEL (DSG) $1 \%$

Pamela Kushner $^{1,2}$; Bharat Patel ${ }^{3}$; Sandy Yacoub Garas ${ }^{4} .{ }^{1}$ University of

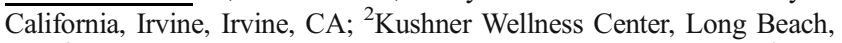
CA; ${ }^{3}$ GSK Consumer Healthcare, Weybridge, United Kingdom; ${ }^{4}$ GSK Consumer Healthcare, Warren, NJ. (Control ID \#3146561)

BACKGROUND: The minimal clinically important improvement (MCII) is defined as the smallest change in measurement that represents patients' perceptions of what they view as an important improvement of symptoms. Thus, the MCII represents an improvement of relevance in a clinical trial or the minimal meaningful change at an individual level.(1) The objective of this post hoc analysis was to determine the number and percentage of osteoarthritis (OA) patients achieving the MCII for pain, function, and stiffness in a previously reported clinical study of DSG $1 \%$.(2)

METHODS: The OA knee study was a 12-week, prospective, randomized, double-blind, multi-center, parallel group study that compared DSG $1 \%$ with vehicle in subjects with OA of the knee. The MCII for OA as determined and recommended by Tubach $\mathrm{F}$ et al (3) was applied to this study. The MCII responder, a relative improvement of $20 \%$ or more from baseline was analyzed using the Cochran-Mantel-Haenszel test stratified by center. Depending on MCII response in relation to vehicle, responder based on improvement of $30 \%$ or more was also analyzed. Time to first MCII response was analyzed using the log-rank test.

RESULTS: Consistent with other efficacy results previously reported for this OA knee study, statistically significant differences were evident at week 1 and were maintained through week 12 for this post hoc MCII analysis. Subjects achieving a MCII response with DSG vs vehicle at Week 1 were: Western Ontario McMaster Universities Osteoarthritis Index (WOMAC) pain, 66\% vs 55\%, $P=0.011$; WOMAC function $56 \%$ vs $42 \%, P=0.002$; WOMAC stiffness, $62 \%$ vs $48 \%, P=0.002$; POM, $66 \%$ vs $50 \%, P=0.0003$. All DSG responses remained statistically significantly different from placebo through Week 12 with the exception of WOMAC pain. WOMAC pain based on $30 \%$ improvement at Week 1 (55\% vs $42 \%$, $P=0.003)$ and Week 12 (66\% vs $56 \%, P=0.019)$, suggests that a higher MCII response criteria may be relevant to consider for WOMAC pain in the context of this specific study. Time to first MCII response was lower with DSG vs vehicle (10 vs 29 days) for all 4 measures: WOMAC pain, $P=0.0091$; WOMAC function, $P=0.0002$; WOMAC stiffness, $P=0.0076$; POM, $P=0.0002$.

CONCLUSIONS: The MCII is a new way of understanding clinically important improvement by taking the perspective of the patient into account. Using the MCII to express results in percentages is more clinically relevant and understandable for both the patient and the primary care physician.(1) This post hoc analysis represents the first time that the MCII has been applied to DSG clinical trial results. The majority of patients treated with DSG achieved MCII responses within 1 week which were sustained for 12 weeks, providing additional evidence for the efficacy of DSG in the treatment of OA of the knee. 1. Kvien TK et al. Ann Rheum Dis. 2007;66 Suppl 3:iii40-1. 2. Barthel HR et al. Semin Arthritis Rheum. 2009;39(3):203-12. 3. Tubach F et al. Arthritis Care Res (Hoboken). 2012;64(11):1699-707.

\section{MINORITY AND LOW-INCOME URBAN INTERNAL MEDI- CINE PATIENTS ARE LESS LIKELY TO HAVE A SCALE AT HOME FOR SELF-WEIGHING.}

Carolyn Bramante $^{1}$; Grace Lee ${ }^{2}$; Safira Amsili ${ }^{2}$; Jennifer A. Linde ${ }^{2}$; Sean $\overline{\text { Phelan }^{2} \text {; Wendy Bennett }}{ }^{2}$; Lawrence J. Appel ${ }^{3}$; Jeanne M. Clark ${ }^{3}$; Kimberly Gudzune ${ }^{3} .{ }^{1}$ University of Minnesota, Minneapolis, MN; ${ }^{2}$ Johns Hopkins, Baltimore, MD; ${ }^{3}$ Johns Hopkins University, Baltimore, MD. (Control ID \#3186035) 
BACKGROUND: Daily self-weighing has been shown to be an effective component of evidence-based weight management interventions. Daily self-weighing requires regular access to a scale, however no prior research has examined what proportion of primary care patients have a scale in their home. Our objectives were to understand if patients have a scale in their home, the characteristics associated with lacking a home scale, and patient willingness to self-weigh.

METHODS: We enrolled 216 patients from three general internal medicine clinics in Baltimore, MD from Dec 2017-Feb 2018 to complete a one-time cross-sectional survey. The three clinics served three different patient populations: mixed-income urban/suburban; mixed-income urban; and low-income urban. Our primary dependent variable of interested was access to a scale in the home ("Is there a scale available to weigh yourself in your home?" (Yes/No)). Our independent variables included demographics, insurance status, clinic setting, and self-weighing. We examined the association between home scale access and these factors using t-tests and $\mathrm{Chi}^{2}$ tests, as appropriate.

RESULTS: Our sample's mean age was 53 years, $71 \%$ were women, $71 \%$ identified as minorities (predominantly African American), and the mean body mass index (BMI) was $32 \mathrm{~kg} / \mathrm{m}^{2}$. Overall, $56 \%$ of patients had a scale in their home. There was no significant difference in home scale access by age, gender, insurance status, or BMI. Minority patients were less likely to have a home scale than white patients s (46\% vs $79 \%$, $\mathrm{p}<0.01)$. Patients from the urban clinic serving low-income communities were less likely to have a home scale than patients from the other two clinics $(35 \%$ vs $69 \%$ or $66 \%, \mathrm{p}<0.01)$. Patients without a home scale were less likely to self-weigh at least weekly than patients who had a scale at home $(13 \%$ vs $87 \%, \mathrm{p}<0.01)$. Patients with heart failure were not more likely to have a scale at home $(\mathrm{p}=0.27)$. Among patients who did not own a scale, the most common reason reported for not owning a scale at home was "Scales cost too much," (32\%); and $72 \%$ would weigh daily if they had a scale. Among all patients, $95 \%$ were willing to weigh weekly if a doctor recommended it, and $67 \%$ were willing to have their weights transmitted to clinic.

CONCLUSIONS: A substantial proportion (44\%) of all primary care patients do not have a scale in their home, but the lack of a scale was most common among minority patients and patients from a low-income urban clinic. Cost was the most common barrier to owning a scale. Most patients were willing to self-weigh, and even to have their weights transmitted to clinic, but lacking a home scale was associated with a lower likelihood of self-weighing. Because self-weighing is an important weightmanagement behavior, future research addressing factors that improve home scale access for patients could enhance weight management services in the primary care setting.

\section{MISSING PRIMARY CARE DESIGNATION IN SPECIALTY MENTAL HEALTH ELECTRONIC RECORDS CHALLENGES THE CARE COORDINATION OF PEOPLE WITH SERIOUS MENTAL ILLNESS}

Maria E. Garcia ${ }^{1}$; L. E. Goldman ${ }^{2}$; Marilyn Thomas ${ }^{1}$; Stephen Chan ${ }^{4}$; Fumi Mitsuishi $^{3}$; Dean Schillinger ${ }^{3}$; Christina Mangurian ${ }^{5}$. ${ }^{1}$ University of California, San Francisco, San Francisco, CA; ${ }^{2}$ University of California San Francisco, San Francisco, CA; ${ }^{3}$ UCSF, San Francisco, CA; ${ }^{4}$ University of California, Davis, Sacramento, CA; ${ }^{5}$ UCSF School of Medicine, San Rafael, CA. (Control ID \#3186123)

BACKGROUND: People with severe mental illness (SMI) such as psychosis, bipolar disorder, and major depressive disorder die on average 10-25 years earlier than the general population. Many people with SMI are prescribed medications that increase cardiovascular risk, yet patients with SMI are less likely to access primary care and to be screened for cardiovascular risk factors. National efforts to improve preventive care and the overall health of people with SMI have promoted care coordination care between specialty mental health and primary care. In this study, we assessed whether primary care clinics are accurately identified for patients in specialty mental health care settings in the public sector.

METHODS: Electronic record review was performed for patients within a large urban specialty mental health clinic who were enrolled in an intensive care management program. Analysis was limited to individuals within an integrated healthcare system that includes psychiatric, medical and laboratory services with separate electronic health records for mental health and medical clinics. 'Known primary care clinic' was defined as a matching clinic listed in the specialty mental health and medical electronic records. All others were designated as 'Unknown primary care clinic.' We assessed whether patient demographic factors predicted known primary care clinic using chi-square tests and whether having a known primary care clinic was associated with increased cardiovascular risk screening (for hypertension, obesity, diabetes, and/or hyperlipidemia through lab or biometric assessments).

RESULTS: Among 229 patients (66\% male; mean age 49; 36\% White, $30 \%$ Black, and 39\% Asian; $69 \%$ with a diagnosis of schizophrenia; $87 \%$ taking antipsychotics), $28 \%$ did not have a known primary care clinic. Sex, age, race, psychiatric diagnosis, and psychotropic medication use were not associated with primary care clinic identification. Among these patients in an intensive care management program, there was no difference in screening for cardiovascular risk factors for patients with known vs. unknown primary care clinic. Overall, patients had higher rates of biometric assessments (blood pressure $=71 \%$, obesity $=62 \%$ ) than laboratory assessments (diabetes $=47 \%$, hyperlipidemia $=40 \%$ ).

CONCLUSIONS: The high rate of unknown primary care clinic assignment in a sample enrolled in intensive care management directly challenges efforts to improve care coordination between primary care and specialty mental health for people with severe mental illness. In this sample of patients, known primary care clinic was not associated with increased cardiovascular risk screening, despite high use of medications with known cardiovascular risk factors. To improve care coordination for people with SMI, and the overall health of this vulnerable population, systems changes to ensure accurate primary care information and communication between providers will be needed.

\section{MISSING THE DEADLINE: HIGH RATES OF ABSENT AND UNTIMELY ACCESS TO SPECIALTY PALLIATIVE CARE IN PATIENTS WITH PERI-HOSPITAL MORTALITY AS A NOVEL QUALITY METRIC}

Christopher Schifeling; Stacy M. Fischer. University of Colorado, Denver, CO. (Control ID \#3181197)

BACKGROUND: Despite mounting evidence that specialty palliative care improves patients' symptoms, caregiver burnout and alignment of health care usage with patient preference, these services are likely underutilized. There is, however, a paucity of data on missed opportunities for specialty palliative care. This study sought to determine the rate of missed and delayed opportunities for specialty palliative care in patients who died in the hospital or within seven days of discharge.

METHODS: A retrospective analysis of patients from a large academic medical center with a Joint Commission awarded palliative care team was conducted. Included were adults who died in the hospital or within seven days of discharge and had prior encounters with the medical center in the last 12 months of life. Excluded were patients who had a sudden or obstetrics-related death. Data from the Colorado Department of Public Health linked with the electronic medical record determined time from first palliative care consultation to death. Chi squared analysis and multivariate logistic regression were used to identify patient variables (derived from the medical record) that were associated with presence of specialty palliative care.

RESULTS: The sample included 2150 patients with $81 \%$ of patients dying in the hospital. Only $34 \%$ of patients had palliative care consultation which was higher for patients with cancer (43\%) than those without 
cancer $(26 \%)$. Of patients with palliative care consultation the median time from first referral to death was eight days. Patients without palliative care referral had an average of 1.8 hospitalizations, 1.6 emergency room visits and 28 face-to-face clinic encounters in their last year of life. Specialty palliative care was positively associated with age less than 65 years old, white race, cancer, frailty, depression, chronic pain and having primary care visits in the last 12 months of life.

CONCLUSIONS: In spite of the general preference to avoid hospital deaths, patients who die in the hospital or shortly after discharge have a high degree of missed opportunities for specialty palliative care. This is a medical error that warrants greater attention. Using the rate of missed opportunities for specialty palliative care in this population as a quality metric will improve end-of-life care for patients', their caregivers and the entire healthcare system.

Histogram of patients per duration of palliative care

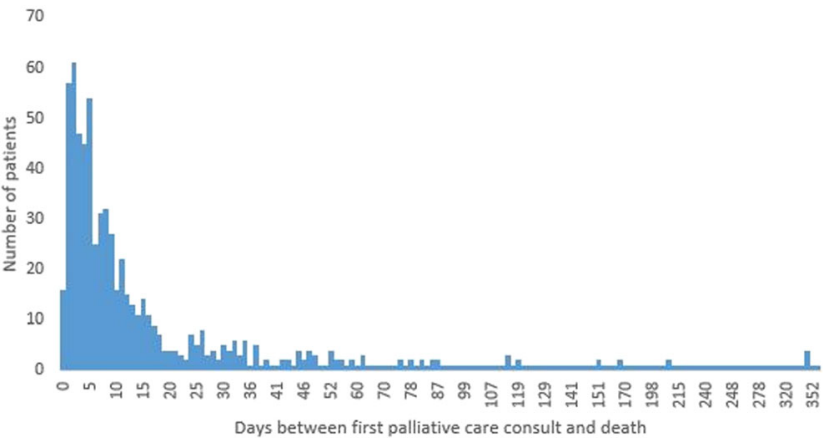

MITRAL LEAFLET CLIP PROCEDURE OUTCOMES: ANALYSIS FROM THE NATIONAL CARDIOVASCULAR DATA REGISTRY

Pedro Covas ${ }^{1}$; Talal S. Alzahrani ${ }^{2}$; James McCaffrey ${ }^{2}$; Joseph Krepp ${ }^{2}$; Ramesh Mazhari' ${ }^{2}$ Christian Nagy ${ }^{2}$; Jonathan Reiner ${ }^{2} .{ }^{1}$ George Washington University, Washington, DC; ${ }^{2}$ The George Washington University, Arlington, VA. (Control ID \#3159437)

BACKGROUND: Mitral regurgitation is one of the most common valvular diseases worldwide. Treatment for severe mitral regurgitation (MR) commonly involves surgical replacement of the mitral valve, but for those who are poor surgical candidates, another option has become mitral valve repair with a mitral leaflet clip procedure (MLCP). In this study, we conducted a descriptive analysis for all patients who underwent MLCP in the United States to investigate preoperative, intraoperative, and postoperative characteristics, techniques, complications, and clinical outcomes. METHODS: The National Cardiovascular Data Registry (NCDR) was used to summarize the baseline demographics and procedural quality measures of all patients who underwent MLCP between 2014 and 2017. The change in the demographics, mortality, complications, and clinical outcomes were summarized and visualized in graphs.

RESULTS: The number of subjects who underwent mitral valve repair increased from 1,023 subjects in 2014 to 5,075 in 2017. The overall mortality rates are relatively unchanged $(2.6 \%$ to $2.4 \%)$. However, there was an improvement in 30-day mortality $(4.5 \%$ to $4.0 \%)$. The rate of patients with low STS scores increased (34\% to 50\%). Overall, the rate of patients with functional MR who received mitral clips are low $(<10 \%)$. Of note, there has been a decrease in the number of patients who are 80 years old and older ( $62 \%$ to $56 \%)$. Intra-operatively, there were fewer complications $(14.6 \%$ to $12.2 \%)$ including fewer clips not deployed $(5.3 \%$ to $3.5 \%$ ) related to a decrease in leaflets not being able to be grasped. Interestingly, there was an increase in the percentage of patients with mitral valve pressure gradient $<=5 \mathrm{mmHg}(71.5 \%$ to $72.5 \%)$. Regarding 30-day follow-ups, our results have shown that residual mitral valve regurgitation (moderate or greater) has decreased from $39 \%$ to $5 \%$, with a decrease in the number of clips attempted per patient. NYHA class IV symptoms at 30 days decreased from $4.2 \%$ to $3.1 \%$. Finally, follow up echocardiograms changed in approach, with an increase in trans-thoracic (TTE) and a decrease in trans-esophageal (TEE) (86\% TEE in 2014 vs. $86 \%$ TTE in 2017).

CONCLUSIONS: Our interpretation shows a trend that MLCP continues to be a viable option for MR. The rate of procedures is expected to increase especially among younger patients with low STS scores and functional MR as results of the COAPT trial. We expect a continued decrease in complications and continued intra-operative success shown by improvement in mitral regurgitation.

\section{MORAL DISTRESS IN INTERNAL MEDICINE RESIDENTS}

Stephanie E. McLaughlin; Harriet Fisher; Colleen Farrell; Kathleen Hanley. New York University School of Medicine, New York, NY. (Control ID \#3184637)

BACKGROUND: Moral distress occurs when the ethically correct action cannot be taken because of internal or external constraints. High levels of moral distress are associated with burnout in medical providers. A better understanding of factors associated with high moral distress during post-graduate training is crucial to executing interventions to lower rates of burnout during residency.

METHODS: This is a mixed methods prospective observational cohort study that aims to enroll 90 internal medicine (IM) residents on a rolling basis from one IM residency program (12/2018-12/2019). Data is being collected by a series of 3 surveys over 1 year, using the previously validated Moral Distress Scale, to evaluate frequency and intensity of distress associated with specific situations experienced or witnessed by residents during training. This initial analysis, bivariate and multivariate regression of quantitative data from the first time point (survey \#1), investigated associations between moral distress scores and demographic, training-specific, and intrinsic personal factors of participants. Analysis of qualitative open-ended questions further explored causes of moral distress and as well as coping mechanisms employed by residents.

RESULTS: $32 \mathrm{IM}$ residents (44\% PGY-1, 28\% PGY-2 and 28\% PGY-3) have been enrolled thus far. 53\% of resident participants identify as male, $43 \%$ as female (and $3 \%$ as other). $66 \%$ were "Categorical" residents, 25\% "Primary Care (PC)," and 9.4\% "Preliminary Year" PGY-1 Interns. Mean and median moral distress scores were: 66.8 (SD 31.0) and 61 (range 16-132). In multivariate linear regression "PC" residents had scores 31 pt. higher compared to "Categorical" residents $(\mathrm{p}=0.009)$. Male residents had scores $25 \mathrm{pt}$ lower than female residents $(\mathrm{p}=0.008)$, and PGY year conferred an incremental score increase of 11 pt. per year $(\mathrm{p}=0.057)$. The model was adjusted for covariates: PGY-year, gender, age, and/or program type. Themes regarding causes of moral distress included: lack of resources, situations when patient care is dictated by cost-saving measures, and aggressive futile care. Coping mechanisms included: debriefing with team members or others outside of work, active individual reflection, exercise/yoga/meditation, participating in activities and social events outside of medicine, reflective writing/ journaling, and suppression and/or distraction.

CONCLUSIONS: In this preliminary analysis, residents in the PC track have higher average moral distress scores. It is unclear whether residents prone to more moral distress self select into this track or whether distress is related to differences in training between PC and categorical tracks. Additionally, more senior residents had average higher scores. This supports the theory of residual moral distress; an increasing amount of moral distress is experienced as a provider witnesses/experiences distressing events over time. Most coping strategies involve social connection and reflection. 
MORTALITY AMONG NORTHERN NEW ENGLAND ADULTS BY TOWNSHIP TYPE

Timothy B. Plante ${ }^{1}$; Insu Koh ${ }^{1}$; Dennis A. Plante ${ }^{1}$; David W. Ferguson ${ }^{3}$; Neil A. Zakai ${ }^{1}$; Kathleen Fairfield ${ }^{2}$. ${ }^{1}$ Larner College of Medicine at the University of Vermont, Burlington, VT; ${ }^{2}$ Maine Medical Center, Portland, ME; ${ }^{3}$ Waldo County General Hospital, Belfast, ME. (Control ID \#3186074)

BACKGROUND: Adults in rural areas die younger than adults in urban areas. Northern New England states are the most rural in America and comprise a greater proportion of adults in micropolitan areas, which are large towns within rural areas. Whether survival differs among these micropolitan areas is unknown. We sought to assess all-cause mortality by township type among Northern New England adults receiving their primary care at academic clinics.

METHODS: We downloaded structured electronic health record data from 86,733 adults receiving primary care at University of Vermont Medical Center-affiliated practices between October 1, 2010 and June 30, 2018 who were aged 18 at any point throughout the follow-up, censoring data prior to age 18. We applied Rural-Urban Commuting Areas (RUCA) codes to first documented ZIP codes to determine township type, defining RUCA codes 1-3 as urban, 4-6 as micropolitan rural, and 7-10 as small town rural. We calculated sex, age, and race-adjusted hazard ratios (HRs) for survival by township type. We visualized survival using KaplanMeier curves.

RESULTS: Among participants in the present analysis, mean (SD) age was 46 (19) years on $10 / 1 / 2010,56 \%$ were female, and $92 \%$ were white; $83 \%$ were urban, $9 \%$ were micropolitan rural, and $8 \%$ were small town rural. Over a median (IQR) follow-up of 4.6 (1.3-7.6) years, there were 2,651 deaths over $341,136$ person-years ( 0.8 deaths $/ 100$ person-years $)$. The mortality incidence rate was lowest among micropolitan rural $(0.4 / 100 \mathrm{P}-\mathrm{Y}$; $95 \%$ CI $0.3,0.4)$, then small town rural $(0.6 / 100 \mathrm{P}-\mathrm{Y} ; 0.5,0.7)$, and urban $(0.8 / 100 \mathrm{P}-\mathrm{Y} ; 0.8,0.9)$. When compared to micropolitan rural, the adjusted HR for mortality was 2.2 (95\% CI 1.9, 2.7; $\mathrm{P}<0.001)$ for urban and $1.8(1.4,2.3 ; \mathrm{P}<0.001)$ for small town rural. Survival curves are shown in Figure 1.

CONCLUSIONS: Adults living in urban and small town rural areas experience a disproportionate burden of mortality when compared to adults in micropolitan rural areas. Efforts to identify and modify the most important risk factors for mortality in these populations are important interventions to improve population health.

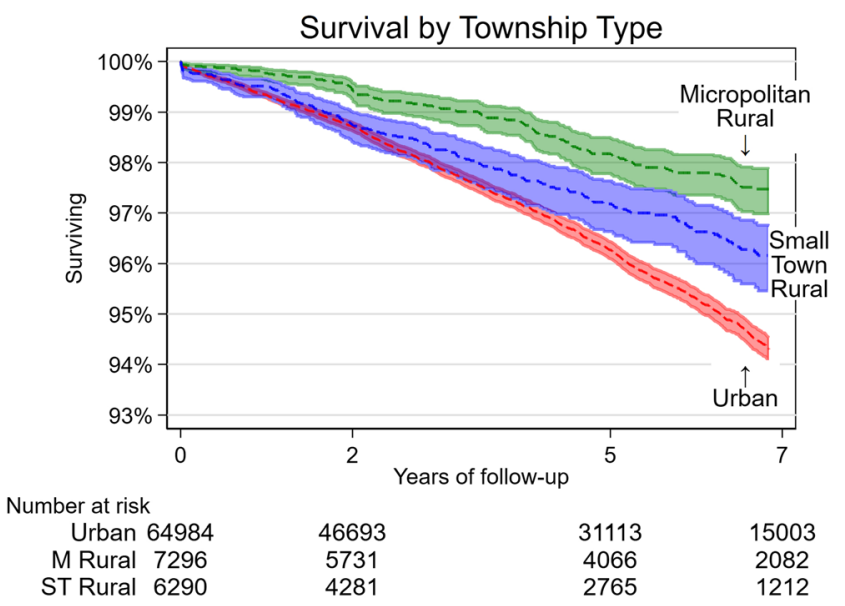

MORTALITY AT PRIVATE FOR-PROFIT AND PRIVATE NOT-FOR-PROFIT HEMODIALYSIS CENTERS: A SYSTEMATIC REVIEW AND META-ANALYSIS

Samuel L. Dickman ${ }^{1}$; Reza Mirza ${ }^{2}$; Maryam Kandi' ${ }^{2}$ Michael Incze $^{3}$; Rakhshan Kamran ${ }^{2}$; Raad Yameen ${ }^{2}$; Ying Zhang ${ }^{2}$; Arnav Agarwal $^{2}$; Lorin Dobiba ${ }^{2}$; Rachel Couban ${ }^{2}$; Steven Hanna ${ }^{2}$; Gordon Guyatt ${ }^{4}$. ${ }^{1}$ University of California, San Francisco, Oakland, $\mathrm{CA} ;{ }^{2}$ McMaster University, Hamilton, ON, Canada; ${ }^{3}$ Univeristy of California, San Francisco, San Francisco, CA; ${ }^{4}$ McMaster University, Dundas, ON, Canada. (Control ID \#3186034)

BACKGROUND: Most of the 630,000 Americans on hemodialysis for end-stage renal disease receive care at private for-profit (FP) or private not-for-profit (NFP) facilities. FP facilities comprise $79 \%$ of all dialysis providers and may lead to worse outcomes than NFP facilities.

METHODS: We searched ten databases (MEDLINE, HealthStar, EMBASE, PubMed, Cochrane Library, Web of Science, Proquest Dissertations and theses, ProQuest Business Premium Collection, Econlit, and CINAHL) for studies published between January 2001 to March 2018 comparing risk-adjusted mortality rates at FP vs. NFP hemodialysis facilities. We used similar criteria to a large 2002 meta-analysis on private dialysis outcomes: we included studies in any language, published and unpublished, with nonoverlapping data. Pairs of reviewers screened 8,876 titles and abstracts, of which 137 full-text articles were reviewed. Study characteristics, results, and risk of bias were assessed by two independent reviewers and discrepancies were resolved by consensus. We harmonized outcomes and conducted pooled fixed- and random-effects meta-analysis.

RESULTS: Seven observational studies, all from the US, were included in the final analysis. No randomized trials met inclusion criteria, and risk of bias was moderate to high. Point estimates in six studies showed worse risk-adjusted mortality rates at FP relative to NFP facilities; the single study with conflicting results compared free-standing FP dialysis centers with hospital-based NFP centers and thus may have been subject to residual confounding by severity of illness. Pooled fixed effects metaanalysis found an OR of mortality in FP relative to NFP facilities of 1.07 (95\% CI 1.05 - 1.09). Random-effects estimates were substantially similar. We found no evidence from more recent studies that mortality differences between FP and NFP dialysis centers have narrowed.

CONCLUSIONS: Patients at for-profit facilities face $6 \%$ greater odds of death annually than patients at not-for-profit facilities with similar risk profiles, a consistent pattern since the most recent comparable metaanalysis was published in 2002. As many as 5,000 deaths would be averted annually if mortality rates at FP facilities matched those at NFP facilities. Despite long-standing concerns about patient safety at FP facilities, public policies have failed to improve outcomes at FP dialysis centers or to limit their rising market share.

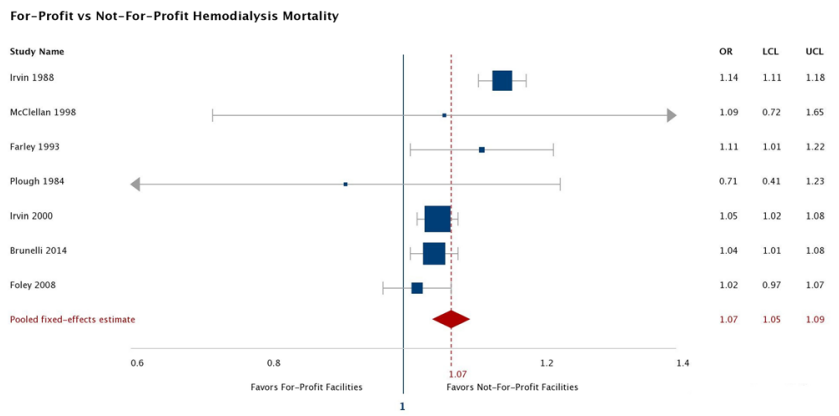


MOTIVATIONS FOR AND USE OF COMPLEMENTARY AND ALTERNATIVE MEDICAL MODALITIES FOR ACUTE PAINFUL CONDITIONS

Erica Morse ${ }^{1}$; Colleen Ross ${ }^{1}$; Stanley $\mathrm{Xu}^{1}$; Scott Clemensen ${ }^{2}$; Shaweta Babar $^{3}$; Heidi Clune ${ }^{2}$; Leonard Wisneski ${ }^{4}$; Melissa de Picciotto ${ }^{2}$; Ingrid A. Binswanger ${ }^{1} .{ }^{1}$ Kaiser Permanente, Aurora, $\mathrm{CO} ;{ }^{2}$ Kaiser Permanente, Boulder, $\mathrm{CO} ;{ }^{3}$ Kaiser Permanente, Denver, $\mathrm{CO} ;{ }^{4}$ University of Colorado, Aurora, CO. (Control ID \#3168415)

BACKGROUND: Opioids are commonly prescribed to manage acute pain. However, alternative approaches are needed. There is evidence suggesting that non-pharmacologic and complementary and alternative medical (CAM) modalities may be effective in helping patients manage painful conditions. In contrast to CAM use for chronic pain, there has been less research on the use of CAM for acute pain episodes, such as those presenting in urgent care settings. Our objective was to describe motivations for, beliefs about, and use of CAM among patients who accessed urgent care for a painful condition. We also sought to examine factors associated with CAM use after an urgent care visit.

METHODS: We developed an electronic survey measuring motivations for and beliefs about CAM use from the Attitudes and Beliefs About CAM instrument and questions about utilization of CAM one year before and 60 days after an urgent care visit. Adult members enrolled in the Kaiser Permanente Colorado Health Plan were recruited via email eight weeks after an urgent care visit for a painful condition between June and August 2018 and asked to complete the survey. We used descriptive statistics and unadjusted and multivariable logistic regression models to examine the association between prior CAM use, attitudes and beliefs about CAM, over-the-counter (OTC) medication use, self-reported prescription opioid use, and prescription non-opioid pain medication use, with CAM use after an urgent care visit.

RESULTS: There were 200 respondents to the survey (9\% of total recruited). They were mostly white $(86 \%)$ and female $(72 \%)$ presenting at urgent care with an acute $(82 \%)$ and painful $(88 \%)$ condition. Most participants believe CAM decreases emotional distress $(67 \%)$, helps reduce pain $(72 \%)$, and helps cope with the experience of having pain $(64 \%)$. Most were interested in using CAM modalities in urgent care if offered (73\%). Among those who used CAM after urgent care, most reported they used it to help reduce pain (81\%). A larger percentage of individuals with a history of CAM, used CAM following their urgent care visit compared to those without a history of CAM use ( $84 \%$ vs $17 \%$ ). The unadjusted models indicated individuals were more likely to use CAM services after an urgent care visit if they presented with an acute condition (OR 2.5, 95\% CI 1.1, 5.4). Pre-urgent care use of OTC medications (OR 1.1, 95\% CI 0.4 , 3.5 ), and prescription opioid (OR $0.7,95 \%$ CI $0.4,1.4$ ) and nonopioid pain relievers (OR $0.9,95 \%$ CI $0.5,1.9)$ were not significantly associated with post CAM use. Multivariable models demonstrate that prior CAM use was the only factor significantly associated with post urgent care CAM use (OR 25.5, 95\% CI 12.0, 53.9).

CONCLUSIONS: Our findings suggest that patients are interested in CAM services in urgent care settings to help manage acute pain. Patients are more likely to use CAM after an urgent care visit if they had a prior history of CAM use. Further research on the effectiveness of various CAM modalities for acute pain is needed.

NATIONAL SURVEY OF INTERNAL MEDICINE RESIDENTS: PERCEPTION OF SUPERVISION AT NIGHT

Jillian S. Catalanotti ${ }^{2}$; Alec O'Connor ${ }^{3}$; Kathlyn Fletcher ${ }^{1}{ }^{1}$ Medical College of Wisconsin/Milwaukee VAMC, Milwaukee, WI; ${ }^{2}$ The George Washington University, Washington, DC; ${ }^{3}$ William L. Morgan Professor of Medicine University of Rochester School of Medicine and Dentistry, Rochetser, NY. (Control ID \#3184235)
BACKGROUND: Balancing autonomy and supervision is one of the most difficult goals for residency training programs to achieve. Night time rotations historically have been opportunities for residents to experience greater autonomy, but increasing patient acuity and volume in many hospitals, coupled with greater awareness of the importance of resident supervision to ensure patient safety, have led to a rise in the number of teaching hospitals that employ in-house overnight hospitalists ("nocturnists"). We sought to understand residents' perspectives on the adequacy of overnight supervision and barriers to contacting their overnight supervising attendings.

METHODS: The Internal Medicine In-Training Examination (ITE) is a national voluntary examination administered annually in August and September. Each year, a select group of survey questions is administered at the end of the exam. In 2017, the ITE contained 7 questions about overnight supervision. We provide descriptive statistics of closed-ended questions. We performed content analysis of the open-ended questions and sorted the responses into categories based on the Systems Engineering Initiative for Patient Safety (SEIPS) model which identifies contributors to patient safety problems related to 5 factors: Tasks, tools/technology, people, organization, and environment.

RESULTS: There were 20,744 respondents, with 34\% PGY1s, 35\% PGY2s and $31 \%$ PGY3s. Fifty-seven percent were male, $93 \%$ were categorical internal medicine or primary care residents, and $62 \%$ were US medical graduates. Over half (53\%) reported having a supervising attending in the hospital overnight. Most residents (83\%) reported that their supervision at night was adequate to ensure patient safety "always" or "most of the time;" $11 \%$ reported "sometimes" and 6\% said "rarely" or "never." The 3 most common barriers to contacting attendings at night were: 1 ) wanting to make their own decisions, 2) not wanting to wake up an attending, and 3) the attending not being in the hospital. We asked what other barriers exist and categorized free text responses into each of the SEIPS categories. Programmatic culture discouraging residents from asking attendings for help (Organizational) was common. Other barriers included having access to other resources (e.g., fellows) (Organizational), being too busy to call (Task), believing they would not receive helpful input (Person), and previous attending behavior ("toxic, condescending, rude") discouraging calling (Person).

CONCLUSIONS: About half of medicine residents in 2017 reported access to in-hospital attending supervision overnight. Substantially more than half of residents feel adequately supervised at night. Many barriers to contacting attendings were identified. Program leaders should explore their program's culture to understand their residents' perceived barriers to seeking attending level supervision. Attending behavior and a culture that suggests it is "weak" to involve attendings when residents feel that they need them should be addressed.

\section{NATURAL LANGUAGE PROCESSING TO IDENTIFY PATIENTS WITH COGNITIVE IMPAIRMENT FROM THE ELECTRONIC HEALTH RECORDS}

Khalil Hussein $^{1}$; Tielman Van Vleck ${ }^{1}$; Lili Chan ${ }^{1}$; Girish Nadkarni ${ }^{1}$; Parul Agarwal $^{1}$; Monica Mindt ${ }^{2,}{ }^{1}$; Alex Federman ${ }^{1}$; Juan P. Wisnivesky ${ }^{1} .{ }^{1}$ Icahn School of Medicine at Mount Sinai, New York, NY; ${ }^{2}$ Fordham University, New York, NY. (Control ID \#3186148)

BACKGROUND: As the U.S. population ages, the prevalence of cognitive impairment among adults will rise, and early detection of persons with cognitive impairment will become a public health imperative to allow for early interventions to reduce the risk of progression to dementia and to facilitate the enrollment of persons with dementia into care programs. While ICD9/10 codes are highly specific for cognitive impairment, they have low sensitivity. Therefore, alternative tools to identify people at highest risk of developing cognitive deficits are needed. Natural language processing (NLP) is an automated process that can be applied to electronic health records (EHR) to search for patterns of words. Our aim was to determine the sensitivity and specificity of a NLP algorithm for identifying cognitive impairment in adults. 
METHODS: We utilized a NLP algorithm, CLiX NLP, to identify subjects with cognitive impairment using clinical notes from the Epic EHR and validated the algorithm using cognitive performance data from two cohort studies of older adults with asthma or COPD who were simultaneously enrolled into the BioMe Biobank at the Mount Sinai Hospital ( $\mathrm{n}=199)$. All subjects were prospectively administered the mini-mental status exam (MMSE); subjects with a score of $<24$ were considered cognitively impaired. Terms for cognitive impairment were selected from SNOMED Clinical Terms by two physician consensus. The NLP algorithm was used to parse all available notes from the EHR of subjects with the following restrictions: (1) For subjects with normal MMSE scores, only notes within 6 months before or after MMSE administration, and (2) For subjects with MMSE scores $<24$, all notes from 6 months prior to MMSE administration until December 2017. 12 additional subjects were excluded for having no encounters documented in the EHR during this time window. Additionally, performance of NLP was compared with manual chart review of 50 charts. Test statistics sensitivity, specificity, positive predictive value (PPV), and negative predictive value (NPV) were generated.

RESULTS: The mean age of subjects was 68 years old with standard deviation (SD) of 6.8. 25\% were male, 29\% Caucasian and 33\% AfricanAmerican. 54\% reported Hispanic ethnicity. Mean MMSE score was 24.8 (SD 3.8); $40 \%$ had MMSE<24. Mean MMSE scores were significantly lower in subjects identified as having cognitive impairment by NLP compared to those that were not, 21.9 (SD 3.9) vs. 24.7 (SD 3.7), respectively $(\mathrm{p}<.0001)$. Sensitivity of NLP for cognitive impairment using MMSE as a gold standard was $44.2 \%$, specificity $87.3 \%$; PPV $69.1 \%$, and NPV $70.8 \%$. Sensitivity of NLP for cognitive impairment using manual review as a gold standard was $84.6 \%$, specificity $62.2 \%$, PPV $44.0 \%$, NPV $92.0 \%$.

CONCLUSIONS: NLP can be used to identify older adults with cognitive impairment with moderate specificity. Refinement of these NLP methods may result in an effective approach to identify subjects with cognitive impairment from unstructured clinical electronic data sources.

\section{NEIGHBORHOOD VIOLENCE AS A DETERMINANT OF AC- CESS TO RESOURCES AND HEALTH STATUS ON THE SOUTH SIDE OF CHICAGO}

Maya Isaacsohn ${ }^{1}$; Monica E. Peek ${ }^{2}$; Nichole Smith ${ }^{2}$; Lydia A. Fu ${ }^{2}$; Elizabeth L. Tung ${ }^{2}$. ${ }^{1}$ Emory University, Atlanta, GA; ${ }^{2}$ University of Chicago, Chicago, IL. (Control ID \#3185482)

BACKGROUND: Neighborhood violence has been linked to negative health consequences, such as obesity and hypertension. Although numerous studies have investigated the relationship between neighborhood violence and access to outdoor physical activity resources, such as parks, fewer studies have examined the relationship to other health resources, such as fresh fruits and vegetables. The purpose of this study was to examine the relationship between neighborhood violence and use of community resources, including outdoor physical activity spaces, indoor gyms, grocery stores and pharmacies.

METHODS: Adult primary care patients $(\mathrm{N}=506)$ from two clinics in Chicago were recruited to complete a one-time survey. Surveys queried participants about use of community resources (i.e., "Do you usually use a [resource] within walking distance of your home?"). Survey responses were paired with objective, geocoded data from the Chicago Police Department. The number of fatal and non-fatal shootings were aggregated to the community area-level, as a proxy for violent crime exposure, and classified into 4 crime exposure risk quartiles. Participant addresses and body mass indexes (BMI) were retrieved from the electronic health record; obesity was defined as BMI $30 \mathrm{mg} / \mathrm{k} 2$. Logistic regression models were used to independently assess (1) use of community resources and (2) obesity status as a function of violent crime exposure. Final models adjusted for age, gender, race/ethnicity, education, and insurance status.

RESULTS: The cooperation rate was $61 \%$. Participants were predominantly female $(72 \%)$ and non-Hispanic black (76\%). Median number of shootings per community area was 58 (interquartile range, 21-128). Living in the highest quartile of violent crime was inversely associated with use of a grocery store $(\mathrm{AOR}=0.31 ; 95 \% \mathrm{CI}, 0.13-0.72)$ or pharmacy $(\mathrm{AOR}=0.20$; 95\% CI, 0.09 0.47 ) within walking distance from home. Associations with location of exercise were not significant. Use of a grocery store within walking distance from home was also inversely associated with obesity status $(\mathrm{AOR}=0.43 ; 95 \%$ CI, 0.26-0.70); associations with use of pharmacy and exercise resources were not statistically significant. Similarly, living in the highest quartile of violent crime was associated with obesity status ( $\mathrm{AOR}=2.76$; 95\% CI, 1.10-6.94).

CONCLUSIONS: Exposure to a higher number of neighborhood shootings was associated with poorer access to neighborhood resources, including grocery stores and pharmacies. Poorer access to grocery stores was also associated with obesity status. Contrary to expectations, access to exercise resources was not associated with violent crime or obesity status. Strategies to improve chronic disease management should consider neighborhood violence as a barrier to access - beyond access to exercise resources, but also access to food and medication resources.

\section{NET IMPACT OF SOCIAL DETERMINATS OF HEALTH ON CAR- DIOVASCULAR RISK FACTORS AND BEHAVIORS}

Ana M. Palacio ${ }^{1}$; Heidy Medina ${ }^{1}$; Maritza Suarez ${ }^{2}$; Leonardo Tamariz ${ }^{1}$. ${ }^{1}$ University of Miami, Miami, FL; ${ }^{2}$ university of miami, Miami, FL. (Control ID \#3185322)

BACKGROUND: Cardiovascular diseases disproportionately affect disadvantaged populations leading to calls to address social determinants of health $(\mathrm{SDH})$ as a preventive strategy. However, the limited information on the net impact that each SDH factor plays in CVD risk prevents the dissemination of SDH collection strategies across health systems. Thus the goal of our study is to create a weighed SDH score for cardiovascular risk using patient reported and geocoded SDH and to test the impact of each SDH factor on the Framingham risk score and on individual traditional cardiovascular risk factors.

METHODS: We conducted a retrospective cohort study of primary care patients at the University of Miami Health System who answered a SDH survey between September 16, 2016 and September 10, 2017. The survey included SDH domains recommended by the AHA position statement and by the National Academy of Sciences, Engeneering and Medicine. We selected the Framingham risk score (FRS) as well as all traditional cardiovascular risk factors as our outcome metrics. We calculated the SDH score for Cardiovascular risk by conducting linear regression of the FRS outcome using all the $\mathrm{SDH}$ variables. We used the beta coefficients of the regression to create the $\mathrm{SDH}$ score. We correlated the SDH Score in tertiles to the FRS risk categories and to the achievement of clinical targets for the traditional cardiovascular risk factors. We also evaluated the correlation of SDH to health behaviors such as smoking and physical activity.

RESULTS: We included 2,876 patients with a mean age of $53.8 \pm-15.8,61 \%$ were female, $9 \%$ were Black, $38 \%$ were Hispanic and $87 \%$ reported speaking English. The mean household income was 53,677 (42,813-67,760). The statistically significant beta coefficients in the FRS model corresponded to being born outside of the US but non-Hispanic, living alone, have a high social isolation score and a low geocoded-based median household income $(\mathrm{p}<0.01)$ The calculated SDH score ranged from 0 to 59. Increasing tertiles of SDH score were significantly associated with high FRS, with poor blood pressure and diabetes control and with sedentary lifestyle. In adjusted models, financial strain, health literacy, stress, education and median household income were significant correlates of weekly exercise while being Black, Hispanic, born outside the US and having low median household income were associated to obesity (table).

CONCLUSIONS: increasing values of a weighted SDH score are associated with increasing CVD risk suggesting that this approach could be a useful tool for risk stratification. The net impact of SDH varied by CVD risk factor evaluated, however, all SDH impacted at least one outcome and therefore should be included in SDH collection tools. 


\section{NEW MODELS FOR EARLY WARNING OF DECOMPENSATION AND DEATH IN AN ACUTE CARE GENERAL MEDICAL SET- TING.}

Russ Clay ${ }^{1,2}$; John Voss ${ }^{1}$; John E. Ainsworth ${ }^{2}$; Joel M. Schectman ${ }^{1}$; Andrew Barros ${ }^{1}$; Valentina Baljak ${ }^{2}$; Megan Pierce ${ }^{1}{ }^{1}$ University of Virginia, Charlottesville, VA; ${ }^{2}$ UVA Health System, Charlottesville, VA. (Control ID \#3180902)

BACKGROUND: Published vitals-based early warning tools may suffer from low positive predictive value (PPV) and low sensitivity (SENS) when applied clinically, and traditional methods of model evaluation do not fully represent clinical implementation trade-offs. We sought to 1) develop new early warning methods \& models for the acute care; 2) develop an improved method of evaluating the clinical model trade-offs.

METHODS: Data from 8,706 unique hospital General Medicine service stays from 7/2017 - 11/2018 were extracted from the EHR including vital signs, $\mathrm{CBC}$ and metabolic panels, and nursing assessments. Using these measures, we created excess risk (ER) and random forest (RF) models to predict mortality12-24 hours before death. For ER, we used Rothman's approach (2013) to estimate the non-linear excess risk of a death associated with input values and derive a total patient level excess risk of mortality. For the RF model, all input measures were used for training. For both models, the data was split into training $(70 \%)$ and test $(30 \%)$ partitions. We evaluated models by simulating their implementation on time-series structured historical data, incorporating a 6- hour lockout on model fires to mimic operational alerts, calculating the PPV, SENS and daily model fire rate. The bootstrap method was used to calculate confidence intervals. ER and RF model performance (including vitals, labs, and nursing data) was compared to the published NEWS model (vitals only) using traditional methods (AUC) as well as PPV/ SENS/Fire Rate (table).

RESULTS: Analysis demonstrated an AUC of 0.87 [0.865,0.874] for the ER, 0.847 [0.843. 0.851] for the RF and 0.806 [0.805, 0.808] for the NEWS model, showing superiority of both the ER and RF models to NEWS. For comparable fire rates of $\sim 4$ day (for a census of 80 patients), model ranking for SENS+PPV was ER $>$ RF $>$ NEWS.

CONCLUSIONS: This evaluation method of PPV/Sensitivity/Fire Rate indicates that the ER model outperformed the RF and NEWS models and that adding lab values and nursing assessments boosts the performance of the ER and RF models versus the vitals-based NEWS model. In evaluating model performance, sites will differ in terms of what are perceived to be optimal performance characteristics (PPV/Sensitivity/Fire Rate) given the trade-offs involved.

\section{NON-CONSENSUAL OPIOID TAPERING AMONG VETERANS RECEIVING LONG-TERM OPIOID THERAPY}

Joseph W. Frank $^{1,2}{ }^{2}$; Evan Carey ${ }^{2}$; Anne Hale ${ }^{3}$; Charlotte Nolan ${ }^{4}$; Erin E. Krebs ${ }^{5}{ }^{1}$ University of Colorado School of Medicine, Aurora, CO; ${ }^{2}$ Rocky Mountain Regional VA Medical Center, Aurora, CO; ${ }^{3}$ Veterans Affairs, Denver, CO; ${ }^{4}$ Veterans Health Administration, Denver, CO; ${ }^{5}$ Minneapolis VA Health Care System, Minneapolis, MN. (Control ID \#3185405)

BACKGROUND: There is inadequate evidence of benefit from long-term opioid therapy (LTOT) and growing evidence of opioid-related harms. Expert guidelines recommend assessment of benefits and risks of LTOT, and when risks exceed benefits, these guidelines recommend reduction or discontinuation of LTOT using individualized treatment plans. However, patients' goals may not align with recommendations to reduce or discontinue LTOT. We examined Veterans' experiences with non-consensual opioid dose reduction or discontinuation ("opioid tapering").

METHODS: We conducted a cross-sectional study within a national prospective cohort study, the Effects of Prescription Opioid Changes for Veterans (EPOCH) Study. The EPOCH Study identified Veterans on LTOT, defined as 150 days' supply in 180 days with no gaps $>40$ days, and 9,253 Veterans completed a baseline survey in 2015 ( $92 \%$ by mail, $8 \%$ by phone). We randomly selected 600 Veterans who met two criteria at EPOCH baseline:
1. completed mail survey; and 2. prescribed 50mg morphine equivalent daily dose (MED). We invited these individuals to participate in a structured phone interview at the 18-month EPOCH timepoint and completed 316 interviews (53\% response rate). Interviews examined Veterans' experiences with opioid tapering as well as domains including pain severity, function, mood, sleep, fatigue and social isolation. We obtained data on demographics, medications and diagnoses from the electronic health record. We used the chi-square test to examine bivariate associations with demographic characteristics, comorbid diagnoses and medication characteristics. All analyses were conducted using R (Vienna, Austria).

RESULTS: The study sample was $73 \%$ age 60 years or older, $92 \%$ male and $87 \%$ White. The most common pain diagnoses were back pain (76\%) and other joint pain (46\%). Opioid medication doses at EPOCH baseline were 5099mg MED (53\%), 100-199mg MED (37\%) and 200+mg MED (10\%). Overall, $68 \%$ reported fair or poor health status, and $51 \%$ reported pain severity that was slightly worse or much worse than 1 year ago. A majority (190/316, 60\%) reported tapering in the past year or discontinuing opioid medications. Past year opioid tapering was associated with White race $(\mathrm{P}=.02)$ and marital status $(\mathrm{P}=.01)$. Among participants who reported past year opioid tapering ( $\mathrm{N}=150), 50 \%$ reported that opioid medications were tapered "without your consent or against your wishes". In bivariate analyses, no patient characteristics were significantly associated with self-reported non-consensual opioid tapering.

CONCLUSIONS: Among Veterans prescribed high-dose long-term opioid therapy, a majority report recent dose reduction or discontinuation, and nonconsensual opioid tapering is common. Future studies should explore provider- and facility-level correlates of non-consensual opioid tapering and examine the effects of non-consensual tapering on the potential benefits and risks of opioid tapering.

\section{NON-OPIOID PRESCRIPTION ALTERNATIVE INITIATIVE (NOPAIN) - AN EDUCATIONAL INTERVENTION TO IMPROVE UTILIZATION OF NON-OPIATE ANALGESICS IN THE TREAT- MENT OF BACK PAIN.}

Scott Holmes ${ }^{1}$; Edward Ewen ${ }^{2} .{ }^{1}$ Christiana Care Health System, Philadelphia, PA; ${ }^{2}$ Christiana Care Health System, Newark, DE. (Control ID \#3177463)

BACKGROUND: The use of chronic opioid therapy (COT) has grown exponentially over the last 10 years, but there is currently no evidence for its use in the treatment of chronic back pain. The Non-Opioid Prescription Alternative Initiative (NOPAIN) focused on the effectiveness of an educational intervention to improve utilization of non-opiate pain medication. The study aimed to improve provider knowledge deficits of non-opioid adjuncts with an educational intervention based on the ACP's Clinical Practice Guideline for noninvasive treatment of low back pain. METHODS: NOPAIN is a prospective cohort study of patients seen in an urban Internal Medicine primary care clinic between April 2017 and December 2017. Individuals were identified by ICD-10 diagnosis of low back pain, with all demographics, diagnoses, and prescribing history obtained through the institution's electronic health record. A non-opioid prescription alternative protocol was created using the ACP's Clinical Practice Guideline for noninvasive treatment of low back pain. Education sessions took place during resident conferences and providers were then given ID badge cards summarizing the recommendations. The control group were the patients of attending physicians, whom did not receive the educational intervention. Data was then examined to determine whether prescribing patterns changed based on the NOPAIN intervention, evaluating the frequency of opioid versus non-opioid analgesic prescriptions. Comparisons between groups were evaluated using the related samples signed Wilcoxon rank test and Chi-square (SPSS 25.0).

RESULTS: Of the 8044 patients seen in 2017, 1053 (13.1\%) received opioid prescriptions, $133(1.7 \%)$ were identified as COT patients, and 96 $(1.2 \%)$ of these were diagnosed with back pain. Of this group, 40 patients were under the care of resident physicians, while 56 were treated 
by attending physicians. In the study period, the use of pain adjunct prescriptions (SNRI, Skeletal Muscle Relaxants, TCA, and Anticonvulsants) increased by $30 \%$ ( $\mathrm{p}<0.05$ ), along with a $22.5 \%(\mathrm{p}<0.05)$ increase in NSAID prescriptions. Additionally, the mean morphine equivalent dose (MED) per day decreased by $19.8 \%$ in the resident intervention group (64.9mg to $52.1 \mathrm{mg}, \mathrm{p}=0.85$ ). There were fewer changes in the prescription rates of pain adjunct medications among the 56 patients in the attending physician control group, with an increase by $12.5 \%$ ( $\mathrm{p}=$ 0.23 ). However, there was an increase in the mean MED per day by $8.2 \%$ (74.4mg to $81.1 \mathrm{mg}, \mathrm{p}=0.71)$.

CONCLUSIONS: This NOPAIN study suggests that when provided with medication guidelines and dosing recommendations, the prescribing of non-opioid alternatives increases in COT patients with back pain. Further study is needed to determine if the use of non-opioid analgesics results in lower MED in these patients. Provider education is a key component of clinical practice, and this data exemplifies the importance of continued provider education on non-opioid analgesic medications for low back pain.

\section{NON-PHARMACOLOGICAL THERAPY USE FOR CHRONIC PAIN AMONG US MILITARY VETERANS ON LONG-TERM OPIOID THERAPY}

Elizabeth S. Goldsmith $^{1,}{ }^{2}$; Richard F. MacLehose ${ }^{2}$; Agnes C. Jensen ${ }^{1}$; Barbara Clothier'; Siamak Noorbaloochi ${ }^{1,2}{ }^{1}$; Brian C. Martinson ${ }^{1,}{ }^{3}$; Melvin T. Donaldson ${ }^{2}$; Erin E. Krebs ${ }^{1,2}{ }^{1}{ }^{1}$ Minneapolis Veterans Affairs Health Care System, Minneapolis, MN; ${ }^{2}$ University of Minnesota, Minneapolis, MN; ${ }^{3}$ HealthPartners Institute, St. Paul, MN. (Control ID \#3180406)

BACKGROUND: Chronic pain is a major cause of disability in the US, and opioid prescriptions rose amid limited availability and awareness of other pain therapies. Though many nonpharmacological therapies (NPT) are effective for chronic pain, little is known about NPT use patterns among people prescribed opioid analgesics. We examined NPT use within a national sample of US military veterans on long-term opioid therapy for chronic pain.

METHODS: The Effects of Prescription Opioid Changes in Veterans $(\mathrm{EPOCH})$ study established a national cohort of 271,892 Veterans Affairs patients prescribed long-term opioid therapy for chronic pain. A representative sample received a survey including questions about past-year use of non-pharmacological therapies to treat or cope with chronic pain. We use descriptive statistics, generalized linear regression and latent class analysis to describe patients' self-reported NPT use patterns and to examine associations of NPT use with demographic and clinical characteristics and with patient-centered pain measures.

RESULTS: $8,891(65 \%)$ of 13,660 invitees completed the NPT questionnaire. Female sex and younger age were associated with use of every non-pharmacological therapy for chronic pain. Higher pain-related functional interference, higher prescribed opioid dose, and psychiatric diagnoses (depression, anxiety, PTSD) were associated with psychological skills-related NPT (meditation, relaxation, psychotherapy). Lower pain-related functional interference and lower physical comorbidity were associated with movement skills-related NPT (aerobic exercise, stretching/strengthening). Lower physical comorbidity was associated with manual therapy (acupuncture, chiropractic adjustment or manipulation, massage).

CONCLUSIONS: Findings can inform implementation and clinical research on non-pharmacological therapies for chronic pain, and raise questions about health system-related factors. Further analyses will examine how participants' NPT use relates to painrelated functional interference, opioid dosing, and quality of life outcomes over time.

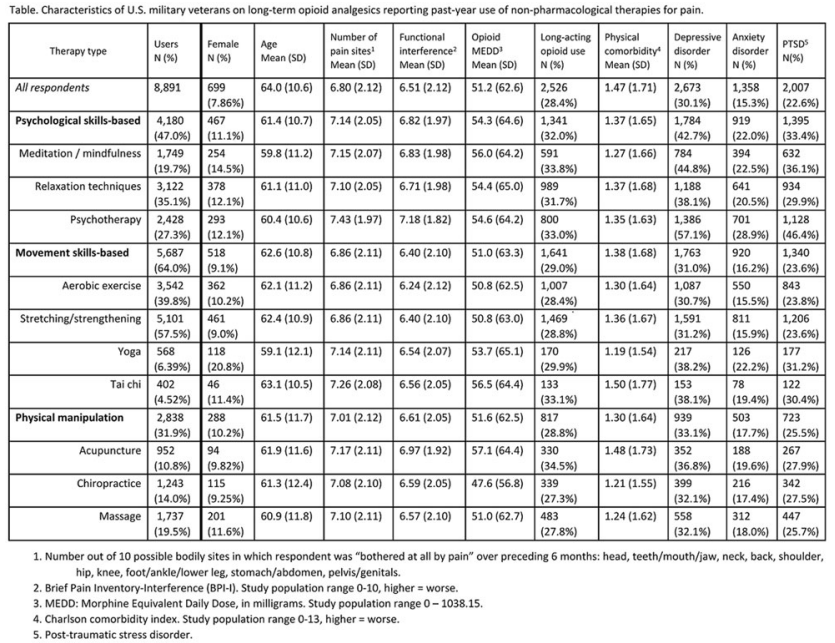

Characteristics of U.S. military veterans on long-term opioid analgesics reporting past-year use of non-pharmacological therapies for pain.

\section{NON-VISIT-BASED AND NON-INFECTION-RELATED ANTIBI- OTIC USE AMONG PRIVATELY INSURED PATIENTS DURING 2016-2018}

Michael A. Fischer ${ }^{1}$; Mufaddal Mahesri ${ }^{4}$; Joyce $\mathrm{Lii}^{2}$; Tiffany Brown ${ }^{3}$; Jeffrey A. Linder ${ }^{3}$ ' Brigham \& Women's Hospital, Boston, MA; ${ }^{2}$ Brigham and women's hospital, Boston, MA; ${ }^{3}$ Northwestern University Feinberg School of Medicine, Chicago, IL; ${ }^{4}$ Brigham and Women's Hospital, Boston, MA. (Control ID \#3178400)

BACKGROUND: Appropriate antibiotic prescribing is an important goal for outpatient safety and quality improvement. Antibiotic prescriptions written outside of visits lack in-person clinician assessment, which may increase the risk of overuse. Similarly, when antibiotics are prescribed at a visit but no diagnosis of infection is documented then assessment of quality of care may be limited. We measured the frequency with which antibiotics were prescribed without an associated clinician visit or without an infection-related diagnosis in a large cohort of privately insured patients.

METHODS: We identified all filled antibacterial, antibiotic prescriptions from April 1, 2016 to June 30, 2018 by patients who were enrolled in a large private health insurance plan (OPTUM/Clinformatics). We identified all medical claims in the 7 days prior to the antibiotic prescription and defined a non-visit-based prescription (NVBRx) as occurring when the patient had no encounter with a clinician in those 7 days. We assessed whether the proportion of NVBRx differed by patient or antibiotic characteristics. We also examined whether the diagnosis codes for the clinician visits were for infectious conditions. Given the massive sample size - all comparisons were statistically significant - we considered differences of $5 \%$ clinically significant.

RESULTS: We identified 22.3 million filled antibiotic prescriptions, dispensed to a total of 8.6 million patients. Half of the prescriptions (52\%) were dispensed to patients 17-64 years old and $61 \%$ were dispensed to female patients. For 6.9 million prescriptions $(31 \%)$ there were no claims for clinician visits in the prior 7 days. NVBRx prescribing rates were not clinically different by gender (women, 30.7\%; men, $31.3 \%)$, but were lower for children than for adults (0-17 years old, $15.9 \%$; 18-64 years old, $32.8 \%$; > 65 years old, 33.9\%). NVBRx rates were considerably lower in the South than in all other regions (South, $22.5 \%, 31-34 \%$ in other regions). Among the 5 most commonly- 
prescribed antibiotic classes, the NVBRx rate was highest for penicillins (35.9\%), followed by sulfa antibiotics $(30.0 \%)$, quinolones $(26.0 \%)$, cephalosporins $(25.0 \%)$, and macrolides $(24.7 \%)$. Of prescriptions associated with a clinician visit, $22 \%$ (4.9 million/22.3 million) were not associated with a diagnosis code for an infectious condition.

CONCLUSIONS: A large fraction of antibiotic prescriptions -millions in absolute terms- were filled without evidence of accompanying clinician visits. NVBRx occurred across the major classes of outpatient antibiotics, was more likely in adults, and occurred much less often in the South region. NVBRx may lack clinician assessment and, along with prescribing without an infectious diagnosis code, may increase the risk of adverse effects. Interventions should be developed to address these safety and quality concerns.

\section{NOT YET STANDARD: USE OF STANDARDS FOR ADULT IMMUNIZATION PRACTICE AMONG PRIMARY CARE PHY- SICIANS}

Laura P. Hurley $^{1,}{ }^{2}$; Sean T. O'Leary ${ }^{2},{ }^{5}$; David K. Kim ${ }^{3}$; Lori A. Crane $^{4,2}$; Michaela Brtnikova ${ }^{2}$, 5; Brenda Beaty ${ }^{2}$; Amy Parker Fiebelkorn ${ }^{3}$; Megan C. Lindley ${ }^{3}$; Allison Kempe ${ }^{2,5}$. ${ }^{1}$ Denver Health, Denver, CO; ${ }^{2}$ Adult and Child Consortium for Health Outcomes Research and Delivery Science (ACCORDS), Aurora, CO; ${ }^{3}$ Centers for Disease Control and Prevention, Atlanta, GA; ${ }^{4}$ University of Colorado Anschutz Medical Campus, Aurora, CO; ${ }^{5}$ Children's Hospital Colorado, Aurora, CO. (Control ID \#3165051)

BACKGROUND: In 2014 the National Vaccine Advisory Committee (NVAC) updated Standards for Adult Immunization Practice ("Standards") which consists of (1) assessing immunization needs at every visit, (2) strongly recommending needed vaccines, (3) administering vaccines or referring patients for vaccination, and (4) documenting receipt of vaccinations in an immunization information system (IIS). Our objectives were to 1) assess the extent of implementation of the Standards by primary care physicians and 2) describe the characteristics of primary care physicians who reported implementing the Standards.

METHODS: In December 2015-January 2016, we administered an Internet and mail survey to national networks of 480 general internist (GIM) and 455 family physician (FP) members of the American College of Physicians and American Academy of Family Physicians, respectively. Stocking was considered a proxy for administering. We performed multivariable logistic regression to examine associations between adhering to the Standards and demographic and practice characteristics.

RESULTS: Response rate was $68 \%$ for GIM (324/480) and $64 \%$ for FP (293/455). $32 \%$ of GIM and $25 \%$ of FP had read the NVAC Standards. The majority of GIM $(57 \%)$ and FP (54\%) reported assessing immunization needs at every visit. Table 1 shows physician recommendations for, stocking of, and stocking or referring for several recommended adult vaccines. $27 \%$ of GIM and $46 \%$ of FP reported documenting vaccinations in an IIS. $6 \%$ of GIM and $11 \%$ of FP reported adhering to all components of the Standards. Characteristics associated with adhering to all four Standards included FP specialty (OR 2.04, 95\% CI 1.09-3.84) and having an electronic clinical decision support system to determine if a patient needs a seasonal influenza vaccine (OR 2.70, 95\% CI 1.27-5.74).

CONCLUSIONS: While the majority reported stocking almost all recommended adult vaccines and several had read the Standards shortly after publication, very few primary care physicians are implementing all components of the Standards for routinely recommended adult vaccines. FP might be more likely to adhere to the Standards because they are more familiar with IIS as a result of using them for childhood vaccines. Development of electronic systems to support full implementation of the Standards may help improve adult immunization rates.
NOVEL RISK FACTORS FOR FALLS IN OLDER ADULTS EXPERIENCING HOMELESSNESS: RESULTS FROM THE HOPE HOME COHORT STUDY

Elizabeth Abbs ${ }^{1}$; Rebecca Brown ${ }^{2}$; David Guzman ${ }^{1}$; Claudia Ponath ${ }^{1}$; Lauren M. Kaplan ${ }^{1}$; Margot Kushel ${ }^{1,}{ }^{1}$. ${ }^{1}$ University of California, San Francisco, San Francisco, CA; ${ }^{2}$ University of Pennsylvania, Perelman School of Medicine, Philadelphia, PA. (Control ID \#3180782)

BACKGROUND: The median age of homeless adults is increasing, with half 50 years. Homeless older adults are at high risk for developing geriatric conditions, including falls, prematurely. Despite high morbidity and mortality, little is known about risk factors for falls in this population. Among older adults, risk factors include individual factors (e.g. cognitive and functional impairment, sensory disabilities, alcohol use, and social isolation) and environmental factors (e.g. uneven surfaces, lack of lighting). People experiencing homelessness have a high prevalence of established fall risk factors and face complex environmental conditions. In addition, they have a high prevalence of potential novel risk factors, including living unsheltered, drug use, and physical assault. In a longitudinal cohort of homeless older adults, we examined the prevalence of and risk factors for falling.

METHODS: We recruited 350 homeless adults aged 50 in Oakland, California. We interviewed participants every 6 months for 3 years about falls, housing and shelter status, health status, health-related behaviors and social support. We assessed prevalence, frequency, and severity of falls. Using generalized estimating equations (GEE), we assessed risk factors for falling one or more times in the prior 6 months over the course of 3 years. In addition to established risk factors (race/ethnicity, sex, age, chronic diseases, functional status, urinary incontinence, use of assistive devices), we assessed potential novel risk factors (spending any nights unsheltered, illicit substance use, and experiencing physical assault).

RESULTS: Of the 350 participants, the majority were men (77.1\%) and black $(79.7 \%)$. The median age was 58 (IQR 54.0, 61). At the baseline interview, one-third $(33.7 \%, 118)$ reported having fallen in the prior 6 months. Of those who fell, over one-quarter $(28.0 \%, 33 / 118)$ experienced 4 or more falls and one-third $(33.1 \%, 39 / 118)$ required medical evaluation. At study follow-up visits, between 23.1 to $31.2 \%$ of participants reported a fall. In GEE models, established risk factors (non-black race, women, older age, prior stroke, functional impairment, urinary incontinence, use of assistive devices) were associated with having fallen. In addition, novel risk factors, including spending any nights unsheltered, (AOR $=1.42,1.10-1.83)$, cannabis use $(\mathrm{AOR}=1.93,1.47-2.55)$, opioid use $(\mathrm{AOR}=1.64,1.02-2.65)$, and experiencing physical assault (AOR $=1.67,1.18-2.37$ ) were associated.

CONCLUSIONS: Older homeless adults experience a high prevalence of falls. While established risk factors increase the odds of falls, environmental stressors and novel risk factors also contribute risk. Fall prevention efforts in older homeless adults should target those at highest risk. Interventions focused on modifiable risk factors, such as housing, exposure to violence, and substance use could reduce morbidity and mortality due to falls.

\section{OBESITY, ANGIOTENSIN AXIS BLOCKADE AND ACUTE KID-} NEY INJURY IN ORTHOPEDIC SURGERY

Matthew Hennrikus ${ }^{3}$; William Hennrikus ${ }^{4}$; Erik B. Lehman ${ }^{2}$; Katelyn $\mathrm{McCann}^{5}$; Eileen Hennrikus ${ }^{1}$. ${ }^{1}$ Penn State College of Medicine, Hershey, PA; ${ }^{2}$ Pennsylvania State University, Hershey, PA; ${ }^{3}$ Tulane University Medical School, New Orleans, LA; ${ }^{4}$ Walter Reed National Military Medical Center, Bethesda, MD; ${ }^{5}$ University of Massachusetts Medical School, Worcester, MA. (Control ID \#3163103)

BACKGROUND: Acute kidney injury (AKI) occurs with a 7-11\% incidence following adult orthopedic surgery. It remains controversial whether perioperative angiotensin axis blockade ( $\mathrm{AAB}$ ) with angiotensin converting enzyme inhibitor (ACEI) or angiotensin receptor blocker (ARB) drugs contributes to AKI. We examined the relationships amongst $\mathrm{AKI}, \mathrm{AAB}$, and obesity in orthopedic surgery. 
METHODS: We reviewed the medical records of 1783 adult elective orthopedic surgery patients during the time period when patients were instructed to take their $\mathrm{AAB}$ drugs the morning of surgery. The primary outcome was postoperative AKI. A multivariable logistic regression was applied to statistically and clinically relevant variables in the entire group, an obese cohort (BMI>30) and a non-obese cohort (BMI <30).

RESULTS: Obesity increased the likelihood of developing AKI postorthopedic surgery (odds ratio $(\mathrm{OR})=1.86, \mathrm{p}=0.028$ ). The use of $\mathrm{AAB}$ increased the likelihood of developing post-operative AKI only in the nonobese cohort $(\mathrm{OR}=3.30, \mathrm{p}=0.030)$. When ACEI and ARB were evaluated individually, only the ACEI in the non-obese patients had a statistically significant odds ratio of developing post-operative $\mathrm{AKI}(\mathrm{OR}=3.49, \mathrm{p}=0.018)$. CONCLUSIONS: Obesity is an independent risk factor for AKI postorthopedic surgery. Adipose tissue contains a complex renin-angiotensin system (RAS), functioning independently of systemic RAS. Adiposeassociated RAS may contribute to the oxidative stress and inflammation induced by surgery, resulting in AKI. ARBs, by targeting the specific receptor involved in the oxidative and inflammatory pathway, may be protective of this stress. Non-obese patients, with low adipose RAS activity, who take preoperative ACEI appear to be more susceptible to AKI. Obesity and the different target receptors of ARBs vs. ACEIs may be contributing to the discordant findings in the literature regarding $\mathrm{AAB}$ in the perioperative patient

\section{OBJECTIVE \&AMP; SUBJECTIVE SOCIAL STATUS RELATE TO CANCER SCREENING BEHAVIOR}

Daliz Cruz ${ }^{1}$; Radhi Yagnik ${ }^{1}$; Kezhen Fei $^{1}$; Emily J. Gallagher ${ }^{2}$; Nina A. Bickell ${ }^{1} .{ }^{1}$ Icahn School of Medicine at Mount Sinai, New York, NY; $\overline{{ }^{2} \text { Icahn }}$

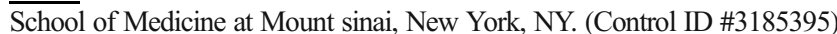

BACKGROUND: Objective and subjective measures of socioeconomic status (SES) are not interchangeable. Perceived SES (pSES) is associated with health status and behaviors independent of objective SES measures such as income and education. As many people refuse to answer survey questions about income, proxy measures may provide similar data. We assessed the relationship between breast cancer patients' perceived and objective SES with their cancer screening behaviors.

METHODS: Data were collected as part of a large prospective study evaluating the role of insulin resistance in women with newly diagnosed breast cancer. Patients were presented a ten rung ladder (The MacArthur Scale of Subjective Social Status tool that captures perceived SES) and were asked to place themselves where they stand in their communities. Low community pSES ranged from 1-8 and high pSES from 9-10. Objective SES measures included income and education; mammography and pap screening were assessed. Income $>\$ 100,000$ was classified as high income; education was dichotomized as college graduate. Comparisons used chisquare for categorical variables, t-tests for continuous variables. Logistic multivariate models were run.

RESULTS: Of 944 breast cancer patients with an average age of $57 \pm 12.0$ years, $81 \%$ were white and $19 \%$ black, $64 \%$ graduated college and $41 \%$ reported an income of $>\$ 100,000 /$ year. $18 \%$ of patients did not report an income. The median pSES= 8 (IQR: 7-8), 20\% had high pSES $(>8)$, and $80 \%$ had lower pSES ( 8 ). There were no racial differences observed in pSES (high pSES: $21 \%$ black vs $20 \%$ white; $\mathrm{p}=0.7$ ). More patients with high pSES graduated college ( $76 \%$ vs $61 \%, \mathrm{p}=0.0002)$ and had income $>\$ 100,000 /$ year $(61 \%$ vs $36 \%, \mathrm{p}<.0001)$ than patients with lower pSES. Of the objective SES, patients with higher income $(>\$ 100,000 /$ year) were more likely to undergo both breast and cervical cancer screening (75\% vs $58 \%, \mathrm{p}<.0001)$ as compared to patients with lower income $(>\$ 100,000 /$ year). There was no difference by college education on receipt of both breast and cervical cancer screening (66\% vs $64 \%, \mathrm{p}=0.5$ ). Of perceived SES, patients with high pSES had higher breast and cervical cancer screening rates $(78 \%$ vs $63 \%, \mathrm{p}=0.0002$ ) as compared to patients with low pSES.
CONCLUSIONS: Income and perceived SES are positively associated with cancer screening behaviors; education is not. Given some patients' reluctance to report income, perceived SES may offer an acceptable option to measure SES and its relationship to health behaviors.

\section{OBSTRUCTIVE SLEEP APNEA AND PAIN INTENSITY AMONG VETERANS OF RECENT WARS}

Wardah Athar ${ }^{2}$, ${ }^{\text {; }}$ Mary E. Card ${ }^{2}$; Antonios Charokopos ${ }^{2}$; Catherine G. Steffens $^{1}$; Kathleen M. Akgün ${ }^{2,1}$; Craig G. Gunderson ${ }^{2}$; Joseph L. Goulet ${ }^{1}$; Eric C. DeRycke ${ }^{1}$; Cynthia Brandt ${ }^{1}$; Lori A. Bastian ${ }^{2,}{ }^{1}$. ${ }^{1}$ VA Connecticut Healthcare System, West Haven, CT; ${ }^{2}$ Yale School of Medicine, New Haven, CT. (Control ID \#3185867)

BACKGROUND: Both obstructive sleep apnea (OSA) and pain are highly prevalent among Veterans. While the specific link between OSA and pain remains unknown, research suggests that OSA patients experience hyperalgesia due to sleep fragmentation and/or hypoxia that enhances sensitivity to pain, promotes inflammation, and advances spontaneous pain. Further, OSA is associated with the development and progression of many painful conditions such as headaches, temporomandibular disorders, and fibromyalgia. Most studies examining the association of OSA and pain focused on older populations, necessitating further research concerning the relationship between OSA and pain among younger patients. This study investigates if those patients diagnosed with OSA are more likely to report moderate/severe pain intensity compared to those patients without OSA in a young cohort of Veterans of recent wars (Operations Enduring Freedom/ Iraqi Freedom/New Dawn [OEF/OIF/OND]).

METHODS: We conducted a cross-sectional analysis of a cohort study of $\mathrm{OEF} / \mathrm{OIF} / \mathrm{OND}$ Veterans who had at least one visit to a Veterans Health Administration primary care clinic between 2001 and 2014. Diagnoses, including OSA, were identified using ICD-9 codes from electronic medical records. Current pain intensity, based on the $0-10$ numeric rating scale, was categorized as no/mild pain (0-3) and moderate/severe pain (4-10), and we included only those pain scores recorded after an OSA diagnosis was made. Covariates included age, sex, race, mental health diagnoses (substance use, PTSD, depression, and anxiety), headache diagnosis, body mass index (BMI), and current or former smoking status. Multiple imputation was performed to generate values for missing variables.

RESULTS: We identified 858,226 Veterans in the OEF/OIF/OND cohort. The mean age was 30 years, $10.6 \%$ carried a diagnosis of OSA, and $32 \%$ reported moderate/severe pain intensity. Compared to Veterans without an OSA diagnosis, Veterans with an OSA diagnosis were more likely to report moderate/severe pain intensity $(\mathrm{OR}=1.26$; $95 \%$ confidence intervals 1.24 1.28), after controlling for covariates including: age, gender, race, substance use, major depression, post-traumatic stress disorder, headache diagnosis, smoking status, and BMI.

CONCLUSIONS: We discovered a clinically significant association between OSA diagnosis and pain intensity, showing that Veterans with OSA have greater odds of comorbid moderate/severe pain. Due to the high prevalence of chronic pain in younger Veterans, this study highlights the need to understand the impact of OSA diagnosis and treatment on pain intensity and to then develop interventions to identify and treat for OSA among Veterans with pain.

\section{OLDER ADULTS' PERSPECTIVES ON HEALTH AND HEALTHCARE: A VIEW FROM PUBLIC HOUSING}

Brooke R. Wagen; Elizabeth Jacobs; Whitney Williams. University of Texas at Austin, Austin, TX. (Control ID \#3167188)

BACKGROUND: Geriatric health needs are projected to increase over the coming decades given demographic trends in the United States and the world. Many social service sectors have started strategizing how to address this trend, including public housing authorities. Recognizing the interplay 
of housing and health, public housing authorities have a 'health in all policies' perspective, yet many have not fully investigated the needs of their older residents. In partnership with a local housing authority, we conducted a qualitative study to better understand older residents' concept of health and healthcare, including what helps them maintain health and wellness.

METHODS: We conducted semi-structured interviews with English and Spanish-speaking adults 65 years residing in one of two public housing complexes. Questions were designed to elicit resident's thoughts and perspectives on health, healthcare, housing, problem-solving, and community. Participants were recruited in person at each site with personal invitations and at resident council meetings. Interviews were conducted in participant's homes or private meeting places, audio-recorded, and transcribed verbatim. Spanish interviews were translated into English. We used grounded theory to analyze the data.

RESULTS: We interviewed 23 residents. They ranged in age from 65-85 years; 12 identified as Hispanic, 5 African American, and 6 non-Hispanic white; $43 \%$ were men. We identified multiple themes. First, older adults residing in public housing equate health with their ability to move and 'feel good'; the presence of multiple chronic conditions does not, for them, define health. Instead, they frame health questions in terms of what they are able to do for themselves, including choices in eating, moving, interacting with others, or following doctor's recommendations. Additionally, the majority stated they have no challenges in getting what they need for their health, despite the fact that all have three or more chronic diseases. They attribute the lack of challenge to provision of public health insurance for older adults and supportive services now available to them, highlighting the difference from when they were younger. Those who mentioned challenges focused primarily on money and transportation, especially outof-pocket costs they can't afford.

CONCLUSIONS: We found that the older adult public housing residents in our study defined health as maintaining autonomy and mobility. Though socioeconomically limited, their approach to healthcare is independent; health challenges are framed in management terms with the self as the primary mover. Surprisingly, they identified few challenges to getting what they need for their health. Future efforts to improve older adult public housing residents' health should take into account their independence and resilience. Housing-health system partnerships that aim to impact this population will be best served by understanding their independent, management-oriented mentality.

\section{ONLINE DECISION AID USE LEADS TO DESIRE FOR LESS AGGRESSIVE MEDICAL TREATMENT AMONG PATIENTS WITH ADVANCED CANCER}

Michael J. Green ${ }^{1}$; Lauren J. Van Scoy ${ }^{2}$; Andrew J. Foy ${ }^{1}$; Anne Dimmock ${ }^{1}$; Erik B. Lehman ${ }^{3}$; Theresa Smith ${ }^{1}$; Benjamin Levi ${ }^{1} .{ }^{1}$ Penn State College of Medicine, Hershey, PA; ${ }^{2}$ Penn State University, Hershey, PA; ${ }^{3}$ Pennsylvania State University, Hershey, PA. (Control ID \#3185851)

BACKGROUND: To help ensure that patients receive medical treatment consistent with their desires, advocates of advance care planning (ACP) encourage individuals to articulate their wishes for medical treatment in advance of illness. While many patients prefer less rather than more treatment at the end of life, in the absence of clear documentation to the contrary, the medical profession's default position is to provide more aggressive treatment. Directive videos can alter decisions, but it is not known whether a nondirective, robust, online educational decision aid can affect treatment choices.

METHODS: This was a secondary analysis of a single center, single-blind RCT of an advance care planning intervention among patients with Stage IV cancer. Participants were randomized to an Intervention Group (Making Your Wishes Known, a values-neutral, non-directive, multimedia online decision aid) or Control Group (standard living will form plus an educational brochure). Subsequently, participants were provided 6 clinical vignettes and asked to indicate whether they would want or not want specific medical/surgical interventions in each situation. The primary outcome was participants' preferences for receiving 11 medical treatments (dialysis for $<1$ month; dialysis for $>1$ month; CPR; ventilator support for < 24 hours; vent for up to 1 month; vent for $>1$ month; feeding tube for up to 1 month; feeding tube for $>1$ month; medicine; surgery; and transfusion). Data were analyzed by summing responses to the 11 questions about the paradigmatic vignette (a moderate/severe stroke that would not improve after 1 year). A total treatment preference score was calculated and the median number of desired treatments was compared between groups using a Wilcoxon Rank Sum test. Logistic regression was used to compare between-group likelihood of wanting each specific treatment.

RESULTS: 200 individuals participated in the study. Overall, participants in the Intervention Group desired fewer life-sustaining interventions than those in the Control Group. With the opportunity to choose as few as 0 and as many as 11 interventions, the median number of interventions desired was 1 in the Intervention Group versus 5 in the Control Group $(p<0.001)$. For 7 of the 11 specific interventions, the Intervention Group was significantly less likely than Control to want aggressive treatment. Most notably, compared to Control, Intervention Group participants were less likely to want: $\mathrm{CPR}(\mathrm{OR}=3.2)$; short-term ventilator $(\mathrm{OR}=3.0)$; short-term dialysis $(\mathrm{OR}=2.6)$; surgery $(\mathrm{OR}=2.7)$; and transfusion $(\mathrm{OR}=2.6)$.

CONCLUSIONS: Individuals who use a robust, nondirective, educational ACP decision aid desire less aggressive medical treatment than those completing standard non-educational living will forms. These findings have implications not only for helping families and clinicians make end-of-life treatment decisions, but also potentially for reducing costs at the end of life.

ONLINE PORTAL USE BY ADULT GENERAL INTERNAL MEDICINE PATIENTS AT AN ACADEMIC MEDICAL CENTER

Alejandro Plana; Neda Laiteerapong. University of Chicago, Chicago, IL. (Control ID \#3185800)

BACKGROUND: Patient portals have the potential to improve patientprovider communication, increase patient satisfaction with care, and augment the efficiency and quality of healthcare. However, failing to sufficiently account for the work required to respond to portal messages will lead to underinvestment in staff and increase the risk of provider and staff burnout. This study sought to describe portal use across a healthcare system by adult patients seen at an academic general internal medicine clinic and assess associations between patient portal use and patient characteristics.

METHODS: All healthcare encounters for any adult patient with at least one encounter at an urban, academic general internal medicine faculty practice between June 2014 to February 2018 were collected. Patients were categorized as MyChart users if they had at least one MyChart encounter. We compared sociodemographics between MyChart users and non-users using analysis of variance, chi-square tests, and multivariate logistic regressions.

RESULTS: During the 3.75-year period, there were 968,484 encounters from 59,973 unique patients. 24\% $(\mathrm{N}=14,737)$ had at least one MyChart encounter and there was a mean 7283 MyChart encounters per year. 21\% $(\mathrm{N}=12,346)$ patients had used MyChart to request medical advice. $6 \%$ $(\mathrm{N}=3,647)$ requested an appointment and $4 \%(\mathrm{~N}=2,559)$ patients requested a medication refill. Over half (55\%) of MyChart users $(\mathrm{N}=7939)$ had 1 encounter. 3,668 patients (25\%) had 2, 1,719 patients had $3(12 \%)$, and 1,401 patients $(10 \%)$ had 4 MyChart encounters. MyChart encounters were distributed across a total of 1671 healthcare providers; $1380(83 \%)$ had completed 10 encounters, 174 (10\%) had completed 10 to 50 encounters, $35(2 \%)$ had completed 50-100 encounters, $43(3 \%)$ had completed $>100$ MyChart encounters. In multivariate regression, patients $>65$ years were less likely $(23.3 \%, 95 \%$ CI 22.0-24.6) and patients aged 35-50 years were more likely (33.0\%, CI 31.9-34.0) to use MyChart than patients less than 35 years old (28.4\%, CI 27.3-29.4). Men were less likely (14.7\%, CI 13.8 15.7) than women (23.3\%, CI 22.0-24.6) to use MyChart. Patients with 
private insurance were more likely $(23.3 \%$, CI 22.0-24.6) than those with Medicaid (8.9\%, CI 8.0-9.8) and Medicare (14.7\%, CI 14.1-15.3) to use MyChart. Whites were more likely (43.1\%, CI 41.5-44.9) than AfricanAmericans (23.3\%, CI 22.0-24.6), Asians (40.7\%, CI 38.3-43.2), and patients of other ethnicities (25.3\%, CI 23.3-27.3) to use MyChart.

CONCLUSIONS: Several vulnerable populations were less likely to use patient portals. Disparities in patient portal use may lead to significant differences in patient knowledge about their health and increased healthcare disparities in the future. In addition, patient portals contribute a large amount of work for physicians. Addressing the uncompensated work of patient portals is imperative such that primary care providers can provide high quality patient-centered care without increasing burnout.

\section{OPIOID GUIDELINE ADHERENCE AND DOSE REDUCTION IN A RESIDENT SPECIALTY CLINIC FOR PATIENTS WITH CHRONIC PAIN}

Laila Khalid $^{1}$; Serena Roth ${ }^{1}$; Gianni Carrozzi ${ }^{2}$; Chenshu Zhang ${ }^{1}$; Aaron Burkenroad $^{4}$; Joanna L. Starrels ${ }^{3}$. ${ }^{1}$ Montefiore Medical Center, Bronx, NY; ${ }^{2}$ montefiore medical center, Bronx, NY; ${ }^{3}$ Albert Einstein College of Medicine \& Montefiore Medical Center, Bronx, NY; ${ }^{4}$ ucla, Los Angeles, CA. (Control ID \#3185612)

BACKGROUND: In response to the challenges of providing guidelineadherent care to patients with chronic pain, the Power Over Pain (POP) Clinic was developed to address the dual aims of training internal medicine residents about judicious opioid prescribing and providing guidelineadherent care for patients on chronic opioid therapy (COT). Objective:To determine whether being seen in POP Clinic was associated with receipt of guideline adherent care and opioid dose reduction after 6 months.

METHODS: We conducted a retrospective study in an urban internal medicine teaching practice in Bronx, NY. Eligible patients were seen in POP Clinic at least once between 9/1/16 and 4/1/18 and were on COT ( 3 opioid prescriptions in past 6 months). Using manual chart review of electronic medical records and prescription drug monitoring program (PMP) data, outcomes assessed at the first POP visit (baseline) and 6 months included four measures of guideline adherence: 1) controlled substance agreement in the past year, 2) urine drug test in the past year, 3) PMP review at last opioid prescription, and 4) dispensing of naloxone; and opioid dose (total prescribed opioid dose in morphine-equivalent daily dose [MEDD]). We used McNemar tests and paired t-tests to compare guideline adherence and opioid dose at 6 months compared to baseline. RESULTS: Of 79 eligible patients, $67 \%$ were female, mean age was 59 years, $57 \%$ were Hispanic, $63 \%$ had back pain, mean PEG score was $7 / 10$ (moderately severe pain), and $39 \%$ had multiple visits to POP Clinic (range 1-6). At baseline, median opioid dose was 70 MEDD (range 2-495). From baseline to 6 months, guideline adherence increased significantly for having a controlled substance agreement, urine drug test, and naloxone; and mean opioid dose was reduced by from 70.3 to 55.1 MEDD (Table). There was no significant change in PMP review, which was high (79\%) at baseline. CONCLUSIONS: For patients with chronic pain on COT seen in a resident clinic specialty session, guideline adherence increased and opioid dose decreased within 6 months. Further study is needed to assess how guideline adherence and dose reduction are associated with patient-level outcomes such as pain, function, and quality of life.

OPIOID OVERDOSES BEFORE AND AFTER PRESCRIPTION POLICY REFORM IN NORTHERN NEW ENGLAND Timothy B. Plante ${ }^{1}$; Insu Koh ${ }^{1}$; Kathleen Fairfield ${ }^{2}$; Andrea Villanti ${ }^{1}$; Valerie S. Harder ${ }^{3}$. ${ }^{1}$ Larner College of Medicine at the University of Vermont, Burlington, VT; ${ }^{2}$ Maine Medical Center, Portland, ME; ${ }^{3}$ University of Vermont, Burlington, VT. (Control ID \#3186410)
BACKGROUND: The opioid epidemic has disproportionately affected adults living in rural states. Northern New England states legislated comprehensive policies aiming to limit opioid overprescribing habits among primary care providers. Whether these reforms reduced overdoses among a primary care population is unknown. We sought to compare the incidence rates of opioid overdose events among primary care patients before and after opioid reform policies in Northern New England.

METHODS: We included structured electronic medical record data from 70,267 adults receiving primary care at University of Vermont Medical Center (UVMMC)-affiliated practices between January 1, 2016 and June 30, 2018 who were aged 18 years during follow-up. Vermont's opioid reform policies were implemented on July 1, 2017. As anticipatory UVMMC policy changes preceded the enactment of the law, we considered the 6 months leading up to the law to represent the implementation of the policy and censored events in this time from the present analysis. We categorized time as pre-reform (January 1, 2016 - December 31, 2016; 12 months) and post-reform (July 1, 2017 - June 30, 2018 ; 12 months). We used ICD-9 and -10 codes to identify overdose events among inpatient and outpatient visits. We used Poisson regression to calculate the incidence rate ratios (IRR) to compare between time periods, adjusting for age, sex, race, and rurality status.

RESULTS: Among participants in the cohort (mean [SD] age 52 [19] years, $56 \%$ female, $92 \%$ white, $13 \%$ rural), there were 176 overdose events; 108 occurred in the pre-reform and 68 in the post-reform eras. In comparison with the pre-reform era, the adjusted IRR for post-reform was 0.62 $(0.46,0.85 ; \mathrm{P}=0.002)$.

CONCLUSIONS: Among primary care patients at an academic health network in Northern New England, implementation of comprehensive policy reforms aiming to curb the overprescription of opiates was associated with a reduced incidence of opioid overdose. Whether a similar pattern is observed among patients without primary care providers requires further analysis.

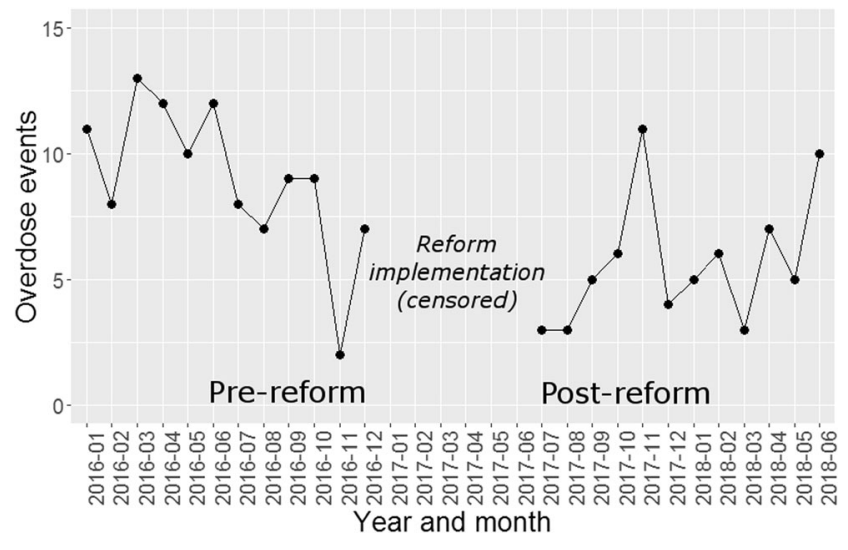

OPIOID PRESCRIBING - UMASS INTERNAL MEDICINE PRIMARY CARE: ASSESSMENT OF BASELINE PRESCRIBING PATTERNS AND PROVIDER NEEDS. JEEVARATHNA SUBRAMANIAN MD, JASON SHAFFER MS2, SHERI A KEITZ MD, PHD, GERTRUDE MANCHESTER MD, PHOEBE CUSHMAN MD, MS.

Jeevarathna Subramanian. UMass Memorial medical Center, Shrewsbury,

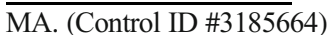

BACKGROUND: Central Massachusetts is one of the epicenters of the opioid crisis, Worcester County seeing an increase in opioid overdose deaths from 68 in 2000 to 226 in 2016. In 2017 Worcester was the only county in Massachusetts not to see a decrease in opioid overdose deaths. UMass Memorial Medical Center (UMMMC) is in the process of developing a standard approach for adherence to $\mathrm{CDC}$ guidelines on chronic 
opioid prescribing. Towards that goal, we aimed to create an opioid registry, to characterize patterns of opioid prescribing, conduct needs assessment in opioid prescribing at UMass Benedict Internal Medicine (IM) Primary Care clinic.

METHODS: 1) Patient registry: Working with our Office of Clinical Integration we created an Epic-based registry, tracking patients who received at least three opioid prescriptions in a six-month period (12/22/2017 - 06/22/2018). We used the Massachusetts Prescription Awareness Tool (MassPAT) and Epic data to assess baseline prescribing patterns, including morphine milligram equivalents (MME) per day, co-prescribing of opioids and benzodiazepines, early refills ( $<28$ days), presence of a pain agreement within the last year, urine toxicology testing within the last year, and presence of additional opioid prescribers. We maintained the registry as a secure password protected file 2) Needs assessment: We distributed a survey via SurveyMonkey to all 30 Benedict IM PCPs (26 MDs and 4 NPs), with responses on a scale of 1-4 and free text responses. We collapsed free text responses into themes.

RESULTS: We identified 450 long-term opioid users for whom we are primary prescribers. Eighteen percent received $>60 \mathrm{MME} /$ day, $25 \%$ received concurrent opioids and benzodiazepines, $22 \%$ received early refills. Of the 73 patients who received $>60 \mathrm{MME} /$ day, $53 \%$ did not have an updated pain agreement, $40 \%$ did not have a baseline urine toxicology. Majority of PCPs did not feel confident managing chronic non-malignant pain, identifying red flags for opioid misuse, interpreting urine toxicology results. Four main areas for which PCPs needed help were: choosing nonopioid options, following clinical guidelines, monitoring MassPAT, and managing challenging patients.

CONCLUSIONS: Baseline data demonstrate there is room for improvement with opioid prescribing patterns. With the creation of the electronic opioid registry, we have prescriber-level data and population-based approach towards improving opioid prescribing safety. We are working with IT analysts to allow the registry to be continually updated in real time. We will develop a multifaceted "peer support system" that centers around academic detailing, aiming to improve adherence to guideline. Our eventual goal is to disseminate our findings to other practices and contribute to a system-wide approach to opioid prescribing.

\section{OPIOID PRESCRIBING BEHAVIORS AMONGST SOUTH CAR- OLINA OPIOID PRESCRIBERS}

Samuel O. Schumann ${ }^{1}$; Jingwen Zhang ${ }^{2}$; Justin Marsden ${ }^{1}$; Patrick D. Mauldin'; William P. Moran ${ }^{1} .{ }^{1}$ Medical University of South Carolina, Charleston, SC; ${ }^{2}$ MUSC, Charleston, SC. (Control ID \#3186248)

BACKGROUND: Prescription opioids are the second leading cause of opioid-related death, and their non-medical use is a significant risk factor for transition to heroin use. Prescription Drug Monitoring Programs (PDMPs) allow prescribers, dispensers and regulators access to patientlevel details on controlled substances to reduce abuse and promote safe prescribing. This report outlines Class II - IV opioid prescribing behavior in South Carolina (SC) from 2010 - 2017 using de-identified prescription data collected the SC PDMP (SCRIPTS).

METHODS: This retrospective study uses seven 12-month de-identified SCRIPTS data sets from 2010 - 2017. Indicators for opioid prescribing were defined by the Centers for Disease Control and Prevention as a part of their Prescription Drug Overdose Prevention for States surveillance program. Rate of opioid prescribers was defined as the number of prescribers who prescribe Class II - IV opioids on any one prescription in a given quarter divided by the number of total number of SC prescribers who prescribed any one Class II-IV medication in that same given quarter. Rate of high-dose opioid prescribers was defined number of prescribers with 90morphine milligram equivalent (MME)/day prescribed on any one prescription in a given quarter divided by all prescribers who prescribed an opioid analgesic prescription identified in the numerator for the same given quarter. Tramadol and propoxyphene were excluded from the final analysis
RESULTS: Rate of opioid prescribers decreased from $75 \%$ in 2010 to $73 \%$ in 2017, while the absolute number of SC licensed prescribers who prescribed any Class II - IV medications remained. Rate of high-dose opioid prescribers decreased from $40 \%$ in 2010 to $27 \%$ in 2017. Percentage of high-dose opioid prescriptions also fell from $12.3 \%$ of all opioid prescriptions in 2010 to $9.6 \%$ in 2017.

CONCLUSIONS: Results show a decrease in the rate of opioid prescribers, high-dose opioid prescribers, and high-dose opioid prescriptions in SC from 2010 - 2017. This potentially signifies that either established SC prescribers stopped opioid prescribing or new SC prescribers were unwilling to prescribe opioids. While high-dose opioid prescribers fell by $13 \%$, the percent of all opioid prescriptions that were high-doses only fell by $2.7 \%$. This may indicate that patients are receiving high-dose opioids from a more concentrated pool of prescribers and reflects high-dose opioid tapering challenges. Rapid decreases in opioid prescribers should be monitored for unintended consequences, such as increases in transition to illicit opioids and accidental overdose. Future research should evaluate associated patient outcomes - including illicit drug use - and continue to monitor patterns of opioid prescribing. Special attention should be given to resource-poor areas of the state, which lack access to non-opioid pain management therapies, mental health services and medication assisted therapy providers.

\section{OPIOID PRESCRIBING PATTERNS FOR CHRONIC NON-} CANCER PAIN IN A PRIMARY CARE POPULATION

Alexander Chaitoff; Glen B. Taksler; Anita D. Misra-Hebert; Belinda

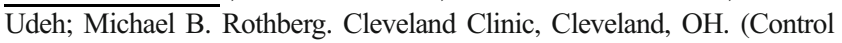
ID \#3185149)

BACKGROUND: In the midst of the opioid epidemic, guidelines and regulations are targeting outpatient opioid prescribing. While $50 \%$ of opioids are prescribed in primary care, prescription patterns within primary care populations have not been well explored. This study aimed to describe patterns in opioid prescribing to primary care patients with chronic noncancer pain.

METHODS: This was a retrospective study using data extracted from the electronic health record at one large integrated health system in the United States. Participants were primary care patients with chronic pain, defined as self-reported pain lasting $>3$ months on a routine pre-visit questionnaire, treated between January 2014 and December 2015. Patients with metastatic cancer were excluded. Opioid use was defined as receiving an outpatient opioid prescription. Opioids prescribed for at least two months in any three month period were classified as long-term. Opioid dose was measured in morphine milliequivalents per day (MME/day) averaged over each month a patient received a prescription. Predictor variables included demographic characteristics, pain severity measured using the numeric ranking scale-10, health insurance status, substance use behaviors, comorbidities, and receipt of non-opioid pain medications. Descriptive statistics were developed for pain medication use in the cohort. Multivariable regression models were used to explore the patient factors associated with receiving any opioid or a long-term opioid.

RESULTS: The sample included 24,127 patients with chronic pain. The sample had a mean age of 58.7 (standard deviation $(\mathrm{SD})=15.3$ ), and was majority female $(64.4 \%, n=15,414)$ and White $(77.4, n=18,668)$. Half of patients $(56.3 \%, \mathrm{n}=13,329)$ were prescribed an opioid. Patients received a median of 2 (interquartile range $(\mathrm{IQR})=2-3$ ) classes of non-opioid pain medications; $98.4 \%$ of patients who received an opioid also received another class of pain medication, and opioid users received more nonopioid pain medication classes than non-users ( 3 vs $2, p<0.001$ ). Only 204 $(0.8 \%)$ patients were prescribed an opioid continuously for 24 months. However, 6723 patients $(27.9 \%)$ received long-term opioids, with an average dose of $12.4 \mathrm{MME} / \mathrm{day}(\mathrm{SD}=44.43) ; 4.3 \%(\mathrm{n}=289)$ had an average dose $>50 \mathrm{MME} /$ day. Age and race were not correlated with receiving an opioid. Females were more likely to receive an opioid $(\mathrm{OR}=1.06$, 
95\% CI=1.01-1.12), but not long-term $(\mathrm{p}>0.2)$. Average pain severity was associated with receiving any opioid prescription (OR per point $=1.20$, $95 \% \mathrm{CI}=1.18-1.22$ ) and a long-term prescription (OR per point=1.23, $95 \% \mathrm{CI}=1.22-1.25$ ).

CONCLUSIONS: At one integrated health system, many patients with chronic pain received opioids, but few received continuous or high dose opioids, which may limit the impact of policies affecting prescribing in primary care. Patients receiving opioids also tried more non-opioid pain medications and reported higher pain severity, suggesting that opioids are reserved for difficult cases.

\section{OPTIMAL TRIGGERS FOR PULMONARY EMBOLISM CLINI- CAL DECISION SUPPORT}

Sundas Khan ${ }^{2}$; D'Arcy King ${ }^{2}$; Karina Calise ${ }^{1}$; Vishwanath Anantraman ${ }^{2}$; Jeffrey Solomon ${ }^{2}$; Thomas McGinn ${ }^{2} .{ }^{1}$ Hofstra University, Manhasset, NY; ${ }^{2}$ Zucker School of Medicine at Hofstra/Northwell, Hempstead, NY. (Control ID \#3185959)

BACKGROUND: Pulmonary embolism (PE) is a high-risk disease and providers often overestimate a patient's risk and order unnecessary diagnostic tests. Clinical decision support (CDS) can help identify specific variables associated with a patient's risk. As part of a larger ongoing trial, our research team identified order entry as a trigger point for CDS to evaluate PE in the Emergency Department (ED) setting. An optimal trigger is defined as the ideal time in a physician's workflow where the tool will alert them to a patient's risk. Based on experience in usability testing that led to high provider adoption rates of CDS (60\%), we hypothesized that ideal CDS would be flexible and trigger at multiple points. The aim of the study was to conduct usability testing on two different prototypes of a CDS tool based on the Wells' criteria which would present earlier in the clinical decision-making process.

METHODS: Two prototypes included a triage note and a pop-up agent; both consisted of a calculator, order set, and recommendations. Usability testing was conducted with a total of 7 participants, $3 \mathrm{ED}$ physicians and 4 triage nurses, at a tertiary care center within a large health system to evaluate the optimal design. The nurses and physicians were each asked to complete both versions of the tool. Following the completion of the tool, the study team conducted a debriefing session which included: the need for conversation between physician and nurse after using the tool, the amount of information provided by the tool, and if there were any interruptions in the clinical workflow. Following the session, the validated system usability scale (SUS) was administered for each version of the prototype to provide a basis for objective comparison.

RESULTS: Based on the SUS, providers and nurses cumulatively preferred the pop-up agent more $(76.7 \%)$ than the triage note (69.4\%). The positive comments for the pop-up agent prototype were that it helped providers get a quick recap on the patients' histories, it was easier to read, and centralized placement helped with easier navigation. The main negative comments for the triage note prototype was that it was not useful in a fast-paced environment such as the ED, content of the triage note was not unique, and to change the location of the note. Overall, providers stated that the tool would give them confidence in making decisions regarding the patient's treatment.

CONCLUSIONS: Usability testing was conducted on two CDS prototypes that triggered earlier in the clinical workflow than previous CDS. The pop-up agent was ranked higher and more effective as it had a different interface than the regular electronic health record, and it was a more user-friendly tool ultimately leading to increased utilization of the tool by providers. It is important for triggers to alert physicians at an optimal time with a user-friendly design. Our second phase of testing will be to compare the pop-up agent trigger to the order entry trigger.

\section{OPTIMIZING CANCER SCREENING IN WOMEN WHO WANT SOME, BUT NOT ALL, GUIDELINE-RECOMMENDED SCREEN- INGS}

Glen B. Taksler $^{1}{ }^{1}{ }^{1}$; Elisabeth F. Peterse ${ }^{2}$; Isarah T. Willems ${ }^{2}$; Kevin ten Haaf $^{2}$; Erik Jansen ${ }^{2}$; Inge de $\mathrm{Kok}^{2}$; Nicolien T. van Ravesteyn ${ }^{2}$; Harry J. de Koning ${ }^{2}$; Iris Lansdorp-Vogelaar ${ }^{2} .{ }^{1}$ Cleveland Clinic, Cleveland, OH; ${ }^{2}$ Erasmus Medical Center, Rotterdam, Netherlands. (Control ID \#3180304)

BACKGROUND: Middle-aged women often want breast and cervical cancer screenings but are hesitant to obtain colorectal and lung cancer screenings. We sought to evaluate how providers might best advise women on strategies to prevent overall cancer-specific mortality while still limiting screenings to $1-2$ per year.

METHODS: We modeled a cohort of females aged $50 \mathrm{y}$ with characteristics representative of the US population, utilizing 4 validated microsimulation models developed as part of the National Cancer Institute's Cancer Intervention and Surveillance Network (CISNET). We examined 45 strategies combining breast, cervical (PAP and HPV co-testing), colorectal (fecal immunochemical testing [FIT]) and/or lung cancer screenings where women obtained some, but not all, screenings recommended by the US Preventive Services Task Force (USPSTF) over their lifetimes. The primary outcome was lifeyears gained from all cancer screenings relative to full compliance with USPSTF recommendations ("optimal benefits"). Results were stratified by eligibility for lung cancer screening ("eligible/ineligible") and, proxying for overall interest in cancer screening, a woman's preference for cancer screening intensity (number and frequency of screenings).

RESULTS: Among women ineligible for lung cancer screening, the USPSTF recommended 3 cancer screenings: annual FIT, biennial mammography and cervical cancer screening every 5 years. Breast and cervical cancer screenings conferred only $48 \%$ of optimal benefits. However, women who were willing to introduce FIT could obtain just 1 screening/year and keep up to $88 \%$ of optimal benefits. The top-ranked strategy was alternating FIT and mammography, and omitting cervical cancer screening for ages $50 \mathrm{y}$. Among eligible women, lung cancer screening was essential; strategies omitting it provided $25 \%$ of optimal benefits. For these women, the USPSTF recommended annual lung and FIT, biennial mammogram and cervical cancer screening every 5 years until age $65 \mathrm{y}$. Breast and cervical cancer screenings provided only $12 \%$ of optimal benefits. Yet, just 2 screenings/year could provide up to $98 \%$ of optimal benefits. The top-ranked strategy was annual lung, alternate FIT and mammography, but skip every $3^{\text {rd }}$ mammogram (i.e., every $6^{\text {th }}$ year) for cervical cancer screening until age $65 \mathrm{y}$. For eligible women who only wanted 1 cancer screening/year, the top-ranked strategy was annual lung (omitting all other screenings), affording 73\% of optimal benefits. Sensitivity analyses were robust to receipt of colonoscopy instead of FIT, smoking history, cervical cancer screening history and background colorectal cancer risk.

CONCLUSIONS: Women who do not want all guidelinerecommended cancer screenings may be able to reduce screening intensity with minimal impact on overall benefits. However, colorectal cancer screening and (if eligible) lung cancer screening were essential. Providers might consider these results as part of a holistic cancer screening discussion.

\section{OPTIMIZING DISCHARGE SUMMARIES: A MULTI- SPECIALTY, MULTI-CENTER SURVEY OF OUTPATIENT PROVIDERS}

Jason Higdon $^{1}$; Aaron M. Silver ${ }^{2}$; Leigh Anne Goodman ${ }^{2}$; Romil Chadha $^{3}$; Michael Burton ${ }^{4}$; Venkataraman Palabindala ${ }^{5}$; Nageshwar Jonnalagadda $^{6}$; Abey Thomas ${ }^{4}$; Christopher M. O'Donnell ${ }^{7}$. ${ }^{1}$ Emory, Atlanta, GA; ${ }^{2}$ Banner University Medical Phoenix, Phoenix, AZ; ${ }^{3}$ University of Kentucky, Lexington, KY; ${ }^{4}$ UT Southwestern, Dallas, TX; ${ }^{5}$ University of Mississippi Medical Center, Jackson, MS; ${ }^{6}$ Baystate Health, Longmeadow, MA; ${ }^{7}$ Emory University, Atlanta, GA. (Control ID \#3182996) 
BACKGROUND: Hospital discharge is a complex and dangerous process. The emergence and rapid growth of the Hospitalist specialty with the simultaneous decline of traditional practice models complicates discharges. In light of the discontinuity, it is crucial to build reliable communication tools that facilitate transmission of critical information. The discharge summary is an essential piece of that transition and is often the only means of communication between the hospital and the outpatient provider. A well written, thorough, accurate, and timely discharge summary may help to prevent 30-day hospital re-admissions. Although the Joint Commission and the Center for Medicare and Medicaid Services both published guidelines for the structure of discharge summaries, it is unclear how outpatient providers use this information. In addition, formal training surrounding discharge summaries within residency programs has traditionally been lacking. Many studies demonstrate frequent inaccuracies or missing components from discharge summaries. They often lack information that outpatient providers may find most useful (e. g. reasoning behind medication changes) and are not completed prior to the posthospital visit.

METHODS: To further delineate the most useful elements of discharge summaries, we conducted a multi-center, multi-specialty survey of Internal Medicine, Family Practice, and Pediatric practitioners that assessed providers' preferences and how they utilized these resources.

RESULTS: In descending order, outpatient providers considered the hospital course, medications, discharge diagnoses, follow-up instructions, and HPI to be the most important components of discharge summaries. Labs and radiology were less important while weight bearing status, wound care, ancillary services, and consults were ranked lowest. Most outpatient providers read the summary the day of the appointment and $60 \%$ of respondents said it was the only source of communication with inpatient providers.

CONCLUSIONS: Using this information, it is feasible to create a standardized template that will increase efficiency of both hospitalists and outpatient providers and help to safely transition care. The most crucial elements that should be prioritized at the beginning of the summary are hospital course, complete medication list, discharge diagnoses, and history of present illness. Based on this large data set, we strongly believe that the creation of a standardized discharge summary template will lead to a more efficient and effective discharge process, with fewer readmissions and safer transition out of the hospital.

\section{OPTIMIZING MEDICAL STUDENT LEARNING EXPERI- ENCES ON HOSPITAL MEDICINE TEAMS-A QUALITATIVE STUDY}

Yogita Segon; Ankur Segon; Sun Young Jeong; Riley Westein; Michael Gehring. Medical College of Wisconsin, Brookfield, WI. (Control ID \#3185888)

BACKGROUND: As the landscape of healthcare changes, hospitalists are progressively at the fore front of medical training as educators for both students and residents. There is some literature suggesting that hospitalists are rated favorably by both house staff and medical students as better clinical educators on inpatient internal medicine rotation as compared to traditional non-hospitalist faculty. Some studies have raised concerns about possible negative impacts on autonomy and decision making, bedside teaching and subspeciality exposure of housestaff. However, impact of hospitalists on medical student education has received relatively less attention. Our study focuses on the educational experience of medical students and hospitalists on general inpatient hospitalist teams that are comprised of a hospitalist attending and advanced practice provider along with one to two medical students.

METHODS: This qualitative project involved one-on-one interviews with eleven third year medical students and seven hospitalist physicians who worked on a hospitalist service in last one year. A team of three third year medical students was deployed as interviewers to minimize pressure on student intervieews. Hospitalist physicians were interviewed by the author (hospitalist attending). Verbatim transcription was performed by three medical students and the author. All data was coded and analyzed by the author via thematic analysis.

RESULTS: Multiple themes emerged from these interviews. Student interviews generated themes around the ideal structure and content of rounds. In addition, student perceptions around the impact of attending and team-based variability on learning experiences were elucidated. The hospitalist attendings interviewed opined on barriers to providing a better learning experience for students. In addition, several learner and rotation based changes to optimize student experience were suggested by the hospitalists.

CONCLUSIONS: Data collected so far suggests that students appreciate variability in different attending's style of working and teaching and are willing to adapt as long as expectations are discussed beforehand. They really value dynamic feedback. The hospitalist attendings perceived high patient census as the biggest barrier to providing better learning experience for students. Changes recommended by them included more proactive involvement of students in the logistics of patient care and a desire for a set of recommended best practices to optimize learning experiences on hospitalist teams.

OUTCOMES OF INTENSIVE CARE MANAGEMENT FOR VETERANS AT HIGH RISK FOR HOSPITALIZATIONS IN YEAR 2 Evelyn T. Chang ${ }^{1,5}$; Jean Yoon ${ }^{2}$; Donna M. Zulman ${ }^{3}$; Lisa V. Rubenstein ${ }^{4}$; Michael Ong ${ }^{5,1}$; Susan E. Stockdale ${ }^{6}$; Angel Park ${ }^{2}$; Elvira E. Jimenez ${ }^{6}$; David Atkins ${ }^{7}$; Angela Denietolis ${ }^{9}$; Steven Asch ${ }^{8}$. ${ }^{1}$ VA- Greater Los Angeles, Los Angeles, CA; ${ }^{2}$ Palo Alto VA, Menlo Park, CA; ${ }^{3}$ Stanford University and VA Palo Alto, Stanford, CA; ${ }^{4}$ RAND and UCLA, Medford, OR; ${ }^{5}$ UCLA, Los Angeles, CA; ${ }^{6}$ Greater Los Angeles VA Healthcare System, Sepulveda, CA; ${ }^{7}$ Veterans Health Administration, Washington DC, DC; ${ }^{8}$ VA/Stanford, Menlo Park, CA; ${ }^{9}$ Primary Care, Veterans Health Administration, Washington, DC, DC. (Control ID \#3184217)

BACKGROUND: Like many healthcare systems, the Veterans Health Administration (VHA) is piloting programs to intensify primary care for patients at high risk for hospitalization. VHA operational leaders partnered with five demonstration sites and an evaluation team in a quality improvement initiative to determine whether augmenting primary care in a standard medical home model (called Patient Aligned Care Team, PACT) with Primary care Intensive Management (PIM) decreases healthcare costs. Thus far, PIM was cost-neutral in the first year; we further investigated health care costs in the second year after the intervention began to assess for potential cost-savings during Year 2.

METHODS: PIM programs included an interdisciplinary team, comprehensive patient assessment and evaluation, transitional care management, and care coordination services. We drew a random sample of 2210 Veterans who were at top $10^{\text {th }}$ percentile of risk for 90-day hospitalization based on a validated VHA risk algorithm and allocated half to PIM ( $n=1105)$. We excluded patients not either seen in a VHA emergency department visit or hospitalized during the past six months. We used an intent-to-treat design to compare the change in mean VHA inpatient and outpatient utilization and costs (including PIM expenses) per patient for the 12-month period before allocation and 12-month period at 13-24 months after allocation. We performed difference-in-difference regression analyses to detect changes in utilization and costs (using fixed effects adjusting for patient-level covariates).

RESULTS: PIM was associated with an increase in primary care $(p<0.001)$, care management $(p<0.001)$, telehealth $(p=0.002)$, mental health and substance use $(\mathrm{p}=0.01)$, and home care $\mathrm{p}=0.01)$ encounters, both in person and by telephone, compared to usual care in PACT. PIM was associated with a decrease in triage $(\mathrm{p}=0.02)$ and mental health assertive community treatment $(\mathrm{p}=0.03$ ) encounters compared to usual care in PACT. There was no significant difference in $E D$ visits $(\mathrm{p}=0.68)$. The mean costs of 
outpatient care in Year 2 were similar in the two groups $(\$ 19,609[\mathrm{CI}$ $\$ 18,621, \$ 20,596$ ] for PIM; $\$ 17,707$ [CI $\$ 16,586, \$ 18,827$ ] for usual care; $\mathrm{p}=0.12$ ). There was no significant difference in mean hospitalizations $(\mathrm{p}=0.17)$ and inpatient costs in Year $2(\$ 16,175$ [CI $\$ 10,896, \$ 21,455]$ for PIM; $\$ 15,027$ [CI $\$ 11463, \$ 18,592$ ] for usual care; $p=0.79$ ). Total mean health care costs were similar between the two groups during Year 2 $(\mathrm{p}=0.47)$.

CONCLUSIONS: Current intensive care management approaches that target patients solely based on high risk of hospitalization like PIM may increase primary care and mental health services. However, they are unlikely to reduce acute care use in VHA, a setting with a robust patient-centered medical home model. Additional work is needed to develop strategies to identify a subset of high-risk patients whose admissions are truly preventable and to design the strategies that will prevent them.

\section{OUTCOMES OF PERSONALIZED VERSUS GUIDELINE-BASED BREAST CANCER SCREENING: A SIMULATION MODEL}

Yiwey Shieh ${ }^{1}$; Laura J. Esserman ${ }^{1}$; Martin Eklund ${ }^{2} .{ }^{1}$ University of California, San Francisco, San Francisco, CA; ${ }^{2}$ Karolinska Institutet, Stockholm, Sweden. (Control ID \#3180851)

BACKGROUND: Breast cancer screening is costly and resourceintensive, yet current guidelines disagree on the optimal starting age and timing of screening. Personalized screening, or screening tailored to individual breast cancer risk, has been proposed as an improvement on guideline-based screening. WISDOM (Women Informed to Screen Depending on Measures of Risk) is an ongoing randomized trial comparing personalized to annual screening. To project the population-level outcomes of personalized screening, we constructed a simulation model comparing personalized to guideline-based screening strategies across advanced (Stage IIB+) cancers, false positives, negative biopsies, and cost.

METHODS: Our simulated cohort included 100,000 women aged 40-74 with demographic and risk factor distributions based on the U.S. screening population. We modeled the WISDOM approach to personalized screening where recommendations are based on the results of genetic mutation testing and 5-year risk estimates from a clinical risk model modified by a polygenic risk score containing 76 genetic variants. Each simulated woman was randomly assigned a clinical and genetic risk profile, which were used to generate a 5-year risk estimate. This was then used to assign a starting and stopping age, frequency, and modality (MRI vs. mammogram) of screening. We compared the aggregate outcomes over a 1-year time window between personalized screening and 3 strategies based on U.S. professional society guidelines (Table).

RESULTS: All screening strategies resulted in similar rates of advanced cancers (Table). However, the biennial, hybrid, and personalized strategies resulted in fewer false positives and biopsies compared to annual screening, and at lower cost. Though aggregate outcomes were similar between the hybrid and personalized strategies, the average 5-year risk of women assigned to biennial screening was lower under the personalized strategy, $1.4 \%$ vs. $2.0 \%$. Similarly, the average 5 -year risk of women recommended for adjunctive MRI under the personalized strategy was higher than that of the hybrid strategy, $10.4 \%$ vs $4.2 \%$.

CONCLUSIONS: Personalized screening results in a similar incidence of advanced cancers as annual screening while reducing false positives, negative biopsies, and cost. Compared to other guideline-based strategies, personalized screening better allocates screening resources by identifying higher-risk women for more intensive screening, and lower-risk women for less intensive screening.

OUTPATIENT TRANSTHORACIC ECHOCARDIOGRAM REFERRAL PRACTICES: INDICATIONS AND PREVALENCE OF FINDINGS

Patrick Miller; Michael Yang; Monvadi B. Srichai. MedStar Georgetown University Hospital, Washington, DC. (Control ID \#3185605)
BACKGROUND: Transthoracic echocardiography (TTE) is a widely used tool, and often the first imaging test performed for evaluation of patients with signs or symptoms suggestive of underlying cardiac disease. However, there is a paucity of epidemiologic data describing ordering practices and echocardiographic findings among these patients in the primary care setting. This study aims to characterize the most common indications for TTE referral in the outpatient setting, and the prevalence of significant findings in patients without a known history of heart disease.

METHODS: This was a retrospective, single center study. Consecutive outpatient TTE order requisitions were reviewed from 10/1/18 to 11/6/18. We included patients without cardiac history referred for specific clinical questions, including abnormal electrocardiogram (EKG), chest pain, dyspnea, edema, murmur, and hypertension. We excluded those referred for stress test, chemotherapy, pre-operative assessment, or follow-up of known cardiac disease including cardiomyopathy or valvular disease. Data were collected on ICD-10 code indication, past medical history, and relevant data from TTE final report.

RESULTS: Of 259 TTEs reviewed, 91 met inclusion criteria with $37.4 \%$ men, $62.6 \%$ female and a mean age of 54.4 years at the time of study. The most common indication was abnormal EKG in 20 patients (22.0\%). The next most common indications were dyspnea (13.2\%), palpitations $(7.7 \%)$, murmur $(7.7 \%)$, edema (7.7\%), syncope (6.6\%), hypertension $(5.5 \%)$, and chest pain $(5.5 \%)$. Eighty-three patients $(91.2 \%)$ had normal left ventricular (LV) function, including 72 (79.1\%) patients with completely normal TTEs. The most common abnormal finding was valvular disease (9.9\%), followed by decreased LV systolic function ( $8.8 \%)$. See Table.

CONCLUSIONS: At our institution, outpatients without cardiac history are most commonly referred for TTE due to abnormal EKG. Of those referred for TTE, the prevalence of abnormal findings was $20.9 \%$. The most common abnormal finding was valvular disease.

\section{Prevalence of Disease for Outpatient TTE $10 / 1 / 18$ to $11 / 6 / 18$}

\begin{tabular}{lcc} 
TTE Finding & $\#$ & Prevalence \\
\hline Normal LV Function & 83 & $91.2 \%$ \\
Valvular Disease & 9 & $9.9 \%$ \\
$\quad$ AV Thickening & 4 & $4.4 \%$ \\
MV Thickening & 2 & $2.2 \%$ \\
$\quad$ Mod-Sev AS & 1 & $1.1 \%$ \\
$\quad$ Mod-Sev AR & 1 & $1.1 \%$ \\
$\quad$ Mod-Sev MR & 1 & $1.1 \%$ \\
$\quad$ Mod-Sev MS & 0 & $0.0 \%$ \\
Abnormal LV Function & 8 & $8.8 \%$ \\
$\quad$ LVEF 40-50\% & 6 & $6.6 \%$ \\
$\quad$ LVEF 30-40\% & 2 & $2.2 \%$ \\
$\quad$ LVEF <30\% & 0 & $0.0 \%$ \\
LV Wall Motion Abnormalities & 4 & $4.4 \%$ \\
Abnormal RV Size & 4 & $4.4 \%$ \\
Abnormal RV Function & 3 & $3.3 \%$ \\
Abnormal LV Size & 1 & $1.1 \%$ \\
Pericardial Effusion & 1 & $1.1 \%$ \\
RV WMA & 0 & $0.0 \%$ \\
\# of Patients wl any Abnormality & 19 & $20.9 \%$ \\
Total \# of Patients & 91 & \\
Total \# of Findings & 113 &
\end{tabular}

Note: \# of findings is greater than total \# of patients bc some patients have more than one finding 
OUTREACH TO PROMOTE MANAGEMENT OF CARDIOVASCULAR RISK IN PRIMARY CARE AMONG RHEUMATOID ARTHRITIS PATIENTS SEEN IN RHEUMATOLOGY PRACTICE

Dawid Lipiszko $^{1}$; Ji Young Lee ${ }^{1}$; Darcy Majka ${ }^{2}$; Yaw A. Peprah ${ }^{1}$; Michael Schachter ${ }^{3}$; Eric Ruderman ${ }^{1,}{ }^{3}$; Stephen Persell ${ }^{1,}{ }^{3}$. ${ }^{1}$ Northwestern University, Evanston, IL; ${ }^{2}$ DuPage Medical Group, Westmont, IL; ${ }^{3}$ Northwestern Memorial Healthcare, Chicago, IL. (Control ID \#3182867)

BACKGROUND: Cardiovascular disease (CVD) is the leading cause of death in rheumatoid arthritis (RA) with CVD risk increased 1.5-2.0-fold above the general population. Despite recommendations for aggressive risk factor management in RA, physicians identify and manage cardiovascular risk factors less often in RA patients compared to patients without RA. Previously, we tested a multifaceted quality improvement intervention consisting of clinician education, clinical decision support at the point-ofcare, performance measurement and feedback to rheumatologists, and individualized mailed recommendations to patients to measure CVD risk factors and to address hypertension, cholesterol treatment, or smoking when applicable. This combined intervention led to considerable increases in the proportion of RA patients with all major CVD risk factors assessed but did not lead to significant improvements in CVD risk factor management or control. Therefore, we added a proactive outreach intervention aimed at promoting primary care treatment of CVD risk factors among RA patients to an existing set of interventions in order to attempt to improve primary care treatment of CVD risk factors in RA patients. We tested the impact of this strategy among RA patients identified through their rheumatology practice.

METHODS: Through electronic health record searches, we identified RA patients who were potential candidates for hypertension treatment initiation or intensification, statin therapy (based on age and risk) or a smoking cessation intervention. A non-clinician care manager contacted RA patients by phone and mail on behalf of the patients' rheumatologists, provided information about the identified CVD risk factor(s), recommend follow up with primary care physicians (PCPs), sent correspondence to PCPs, and followed up with patients approximately 4 weeks later to see what actions had been taken or barriers encountered. Information was shared with the patient's rheumatologist via EHR. We repeatedly measured preventive cardiology quality indicators from electronic health record data and compared pre-intervention to intervention time periods using interrupted time series methods.

RESULTS: During the 6-month intervention period, the proportion of RA patients age 40 to 75 prescribed a statin rose from $18.4 \%$ to $23.8 \%$, and the rate of increase was significantly greater than during the preceding time period (1.06 percent per month greater than the prior period, $\mathrm{P}<0.001)$. Rates of increase in hypertension diagnosis and control also improved more rapidly during the intervention time period (1.05 and 2.09 percent per month greater, $\mathrm{P}<0.001$ for each) and reversed preceding negative trends.

CONCLUSIONS: Implementing proactive non-clinician phone and mailed outreach to encourage primary care-based treatment of CVD risk factors was associated with measurable increases in statin prescribing, hypertension diagnosis, and hypertension control. Smoking was not affected.
OVERDOSE IS COMMON AMONG HOMELESSEXPERIENCED VETERANS: NEW NATIONAL SURVEY DATA

Stefan Kertesz $^{1}{ }^{4}$; Adam Gordon ${ }^{2}$, 3 ; Aerin DeRussy ${ }^{1}$; April E. Hoge ${ }^{1}$; Ann E. Montgomery ${ }^{1}$; Kevin R. Riggs ${ }^{1,}{ }^{4}$; Sally Holmes ${ }^{1}$; Erika L. Austin ${ }^{1}$; Lillian Gelberg ${ }^{5}$, ; Sonya Gabrielian ${ }^{5,}{ }^{6}$; Allyson Varley, ${ }^{1}$; Adiseshu Gundlapalli ${ }^{2}{ }^{3}$; Young-il Kim ${ }^{1,}{ }^{4}$; Audrey Jones ${ }^{3}$. ${ }^{1}$ Birmingham VA Medical Center, Birmingham, AL; ${ }^{2} \mathrm{VA}$ Salt Lake City Health Care System, Salt Lake City, UT; ${ }^{3}$ University of Utah, Salt Lake City, UT; ${ }^{4}$ University of Alabama at Birmingham, Birmingham, AL; ${ }^{5}$ VA Greater Los Angeles Health Care System, Los Angeles, CA; ${ }^{6}$ UCLA, Los Angeles, CA. (Control ID \#3184324)

BACKGROUND: Overdose (OD) is a frequent cause of death among homeless individuals. Prevention of overdose in primary care (PC) could depend on identifying people who have survived or witnessed OD. We measured the prevalence of both events among homeless-experienced veterans (HEVs) in primary care and assessed risk factors for overdose in the largest survey of HEVs conducted to date.

METHODS: A stratified, random sample of 5,666 homelessexperienced users of Veterans Administration primary care services was recruited for survey through sequential mailings (prenotification letter, survey, postcard reminder) followed by telephone outreach (recruitment pool 14,352, with $40 \%$ response rate). The survey queried OD "where you needed to go to the emergency room or get medical care right away" in the past 3 years, and separately, witnessing such an event. Additional items assessed current homelessness, substance use problems based on the Two-Item Conjoint Screener, and validated measures for social support, mental distress, chronic pain, receipt of psychiatric medication, count of medical conditions, and general self-reported health. Bivariate analyses (chi-squared and t-tests) and multivariable logistic regression were used to identify characteristics associated with self-reported overdose.

RESULTS: Among HEVs, $15.1 \%(\mathrm{n}=869)$ reported witnessing an OD and $6.6 \%(\mathrm{n}=378)$ reported experiencing OD. The latter (ODs experienced) more commonly involved alcohol (3.4\% of total, $51 \%$ of ODs) than drugs $(2.6 \%)$; however, $1.4 \%$ didn't endorse a substance, and $0.7 \%$ endorsed both alcohol and drugs. Among drugs, opioids were reported by $1.4 \%$ ( $22 \%$ of all ODs), and cocaine by $1.0 \%(15 \%)$. The following differences were significant at $\mathrm{p}<0.001$ : Persons reporting OD were more likely than those without OD to be white $(54.2 \%$ vs $40.9 \%)$, currently homeless $(20.4 \%$ vs $12.4 \%)$, to receive psychiatric medication $(57 \%$ vs $33 \%$ ), and to have witnessed another person OD (37\% vs $14 \%$ ). Additionally, patients with OD reported higher mental distress (11.0 vs 6.8 , scale $1-24)$, lower social support (3.8 vs 4.3 , scale $0-6)$, and a greater prevalence of alcohol (55\% vs $26 \%$ ) and drug problems $(31 \%$ vs $12 \%)$. In multivariable-adjusted models, characteristics associated with overdose included: white race (Odds Ratio, $\mathrm{OR}=2.3)$, current homelessness $(\mathrm{OR}=1.4)$, receipt of a psychiatric medication $(\mathrm{OR}=1.7)$, elevated mental distress $(\mathrm{OR}=1.1$ for +1-point on 24-point scale), and having witnessed overdose $(\mathrm{OR}=2.5)$; all $\mathrm{p}$ 's $<0.05$.

CONCLUSIONS: Compared to samples suggesting that 0.01 $0.1 \%$ of adults have experienced overdose, HEV's in PC report high rates of $\mathrm{OD}$, and 1 in 7 report having witnessed OD. Opioids are present in nearly $1 / 4$ of these events and alcohol in over $1 / 2$. 
These survey data suggest that PC providers for homelessexperienced persons may have a powerful opportunity to mitigate overdose risk in their patients by addressing polydrug, alcohol and prescription exposure, and to explore protection of others at risk in the patient's environment.

\section{OVERLAP BETWEEN MEDICARE'S ACCOUNTABLE CARE ORGANIZATIONS AND BUNDLED PAYMENTS} Joshua M. Liao $^{4,}{ }^{1}$; Sarah Dykstra ${ }^{3}$; Rachel M. Werner ${ }^{1,}$ 2; Amol S. Navathe ${ }^{1,2}$. ${ }^{1}$ University of Pennsylvania, Philadelphia, PA; ${ }^{2} \mathrm{CMC}$ VA Medical Center, Philadelphia, PA; ${ }^{3}$ The Wharton School, University of Pennsylvania, Philadelphia, PA; ${ }^{4}$ University of Washington, Seattle, WA. (Control ID \#3179510)

BACKGROUND: Accountable care organizations (ACOs) and bundled payments represent cornerstone payment models of Medicare's strategy to improve the value of care. As both models grow in prominence and scale, participating organizations in each may increasingly provide care to the same beneficiaries, posing important clinical and policy implications. However, no data about the magnitude and trend in overlap between ACOs and bundled payments exist.

METHODS: Using 100\% 2013-2016 institutional Medicare claims, Medicare Shared Savings Program (MSSP) ACO beneficiary attribution, and hospital participation data for the Bundled Payments for Care Improvement (BPCI) initiative, we identified care episodes for beneficiaries attributed to MSSP ACOs and receiving care at BPCI hospitals for its 48 (25 conditionfocused, 23 procedure-focused) episodes. For each episode, we defined 3 beneficiary groups: those attributed to MSSP who were hospitalized for an episode at BPCI hospitals participating in that episode (Overlap); those attributed to MSSP who were hospitalized for that episode at non-BPCI hospitals (MSSP-only); and those hospitalized at BPCI hospitals for a BPCI episode but who were not attributed to MSSP (BPCI-only). Medicare and US Census Bureau data were used to compare 246,392 Overlap, 2,824,898 MSSP-only, and 702,864 BPCI-only episodes with respect to sociodemographic, clinical and utilization characteristics. Categorical and continuous variables were compared using ${ }^{2}$ tests and $t$ tests, respectively. Statistical tests were 2-tailed and significant at $=0.05$.

RESULTS: The number of MSSP ACOs increased from 220 in 2013 to 432 in 2016. The number of BPCI hospitals increased from 9 to 389 over this period, peaking at 413 in 2015. Across episodes, Overlap patients were generally lower severity, though differences were small. The overlap as a share of ACO patients increased from $2.7 \%$ at the start of the study period to $10 \%$ at the end of 2016 across all clinical episodes, while overlap as a share of all bundled payment patients increased from $19 \%$ to $27 \%$. Overlap as a share of ACO patients varied by episode, ranging by the end of 2016 from 3\% for acute myocardial infarction and chronic obstructive pulmonary disease to $18 \%$ for automatic implantable cardiac defibrillator, and was greater for procedurefocused $(13 \%)$ than condition-focused $(9 \%)$ episodes. Similarly, overlap as a share of bundled payment patients was greater for procedure-focused (29\%) versus condition-focused (26\%) episodes, varying overall from $21 \%$ for spinal fusion episodes to $32 \%$ for lower extremity joint replacement and automatic implantable cardiac defibrillator episodes.
CONCLUSIONS: In the first description of overlap between ACOs and bundled payments, one in every ten MSSP patients received care - and in particular procedural care - under BPCI by the end of our study period, while over one in every four patients receiving care under BPCI was also attributed to MSSP.

\section{OVERVIEW OF THE CLINICAL DEVELOPMENT OF FREMANEZUMAB}

Stephen D D. Silberstein ${ }^{1}$; David W. Dodick ${ }^{2}$; Joshua M. Cohen ${ }^{3}$; Ronghua Yang ${ }^{3}$; Melissa Grozinski-Wolff ${ }^{3}$; Xiaoping Ning $^{3}$; Sissy Easo-Joseph ${ }^{3}$; Lindsey Noble ${ }^{3} .{ }^{1}$ Jefferson Headache Center, Thomas Jefferson University, Philadelphia, PA; ${ }^{2}$ Mayo Clinic, Phoenix, AZ; ${ }^{3}$ Teva Pharmaceuticals, Frazer, PA. (Control ID \#3185629)

BACKGROUND: Migraine is a highly prevalent neurological disorder and one of the leading causes of years lived with disability worldwide. Chronic migraine (CM) is defined by having 15 or more headache days per month, at least 8 of which are migraine days. Patients with episodic migraine (EM) have fewer than 15 headache days per month. Preventive migraine treatment is recommended in patients with 4 or more headache days per month with some impairment. Fremanezumab is a fully humanized monoclonal antibody (IgG2 a) that selectively targets calcitonin gene-related peptide (CGRP), a neuropeptide involved in the pathophysiology of migraine. Fremanezumab is approved for the preventive treatment of migraine in adults. The HALO trials assessed the efficacy and safety of fremanezumab for the preventive treatment of $\mathrm{CM}$ and EM.

METHODS: HALO CM and HALO EM were concurrent, multicenter, randomized, double-blind, placebo-controlled, parallelgroup Phase 3 studies evaluating the efficacy and safety of fremanezumab for the preventive treatment of $\mathrm{CM}$ and EM. Adults with CM or EM, confirmed during a 28-day pretreatment period, were randomized to receive subcutaneous fremanezumab quarterly $(675 \mathrm{mg}$ at baseline and placebo at Weeks 4 and 8), fremanezumab monthly (CM: $675 \mathrm{mg}$ at baseline, and $225 \mathrm{mg}$ at Weeks 4 and 8; EM: $225 \mathrm{mg}$ at baseline and Weeks 4 and 8), or matching placebo over a 12 -week period. The primary endpoint for HALO CM was the mean change in the average number of headache days of at least moderate severity: a day in which headache pain lasted 4 consecutive hours and had a peak severity of at least a moderate level, or an acute migrainespecific medication (triptans or ergots) was used to treat a headache of any severity or duration. The HALO EM primary endpoint was the mean change from baseline in the monthly average number of migraine days: a day with 2 consecutive hours of a headache meeting criteria for migraine, probable migraine, or when acute migraine-specific medication (triptans or ergots) was used to treat a headache.

RESULTS: In HALO CM, 1130 patients were randomized to fremanezumab quarterly $(n=376)$, fremanezumab monthly $(n=379)$, or placebo $(n=375)$, and 1034 patients $(92 \%)$ completed the study. In HALO EM, 875 patients were randomized to fremanezumab quarterly $(n=291)$, fremanezumab monthly $(n=290)$, or placebo $(n=294)$, and 791 patients $(90 \%)$ completed the study. On average, CM and EM patients were approximately 41 years of age, with 20 years since migraine diagnosis. Both $\mathrm{CM}$ and EM patients were highly disabled based on six-item Headache 
Impact Test (HIT-6) scores and Migraine Disability Assessment (MIDAS) scores.

CONCLUSIONS: The HALO trials were designed to provide flexible dosing options to establish the efficacy and safety of fremanezumab for the preventive treatment of migraine. The study population was representative of the real-world clinical patient population.

\section{OXYCODONE URINE DRUG TESTS IDENTIFY POTEN- TIAL MISUSE AND DIVERSION AMONG PATIENTS PRE- SCRIBED OPIOIDS FOR CHRONIC PAIN}

Marc Larochelle ${ }^{1}$; Ricardo $\mathrm{Cruz}^{2}$; Sarah Kosakowski ${ }^{2}$; Douglas Gourlay $^{3}$; Daniel P. Alford ${ }^{1}$; Ziming Xuan ${ }^{4}$; Erin E. Krebs ${ }^{5}$; Karen E. Lasser ${ }^{2}$; Jeffrey H. Samet ${ }^{6}$; Jane M. Liebschutz ${ }^{7}$. ${ }^{1}$ Boston University School of Medicine and Boston Medical Center, Boston, MA; ${ }^{2}$ Boston Medical Center, Boston, MA; ${ }^{3}$ Wasser Pain Management Centre, Toronto, ON, Canada; ${ }^{4}$ Boston University School of Public Health, Boston, MA; ${ }^{5}$ Minneapolis VA Health Care System, Minneapolis, MN; ${ }^{6}$ Boston University School of Medicine, Boston, MA; ${ }^{7}$ University of Pittsburgh School of Medicine, Pittsburgh, PA. (Control ID \#3185832)

BACKGROUND: Guidelines recommend urine drug testing (UDT) as one component of a risk monitoring strategy for patients prescribed opioids for chronic pain. Few studies have examined whether UDT results are associated with substance misuse or diversion.

METHODS: A retrospective cohort study using data from TOPCARE, a trial to promote guideline-concordant care for chronic pain patients prescribed opioids in four Boston, MA primary care clinics. The multimodal intervention included a nurse care manager who assisted physicians with UDT collection and interpretation. We included 2,111 oxycodone urine immunoassays collected over one year for 611 patients of primary care physicians randomized to the intervention. We reviewed all assays and identified $490(23.2 \%)$ with a result discordant from oxycodone prescription status (i.e., oxycodone negative during oxycodone prescription or oxycodone positive without active oxycodone prescription). For these 490, we abstracted detailed clinical data from the EMR, including UDT collection and interpretation notes from the intervention nurse care manager. Two addiction medicine experts adjudicated each oxycodone assay as follows: expected; equivocal (discordant assay without EMR evidence of misuse/diversion); or unexpected (discordant assay with EMR evidence of misuse/ diversion). We reconciled adjudication differences via discussion, and further categorized each patient as: expected (all expected), equivocal, or unexpected ( $>1$ unexpected).

RESULTS: Overall, we adjudicated $79.1 \%$ of the 2,111 oxycodone assays as expected, $7.3 \%$ equivocal, and $13.6 \%$ unexpected (Table). Of the 490 assays initially screened as having a result discordant from oxycodone prescription status, $58.8 \%$ were found to have EMR evidence of misuse or diversion and adjudicated as unexpected, $31.2 \%$ were adjudicated as equivocal, and $10.0 \%$ expected. For 406 (65.0\%) patients, all oxycodone assays were adjudicated as expected, and 127 patients $(20.3 \%)$ had at least one unexpected oxycodone assay.

CONCLUSIONS: An oxycodone urine immunoassay result discordant from prescription status offers clinical utility for identifying potential misuse or diversion. The urine test should be followed up with clinical assessment. Whether routine UDT improves patient outcomes remains unknown.
PAIN AGREEMENTS: TIME-TO-EVENT ANALYSIS OF SUBSTANCE MISUSE IN A PRIMARY CARE CHRONIC PAIN PROGRAM

Paul Chelminski ${ }^{1}$; Timothy Ives ${ }^{1}$; Ameer Ghodke ${ }^{2}$; Anna Austin ${ }^{4}$; Naishal Patel $^{3}$; Will Bennett ${ }^{1}$; Sharon Eshet ${ }^{1} .{ }^{1}$ University of North Carolina at Chapel HIll, Chapel HIll, NC; ${ }^{2}$ University of North Carolina, Cary, NC; ${ }^{3}$ UNC-Chapel Hill, Cary, NC; ${ }^{4} \mathrm{UNC}$ Chapel Hill School of Public Health, Chapel Hill, NC. (Control ID \#3171392)

BACKGROUND: Medication agreements (MA) are used to specify conditions for opioid use in chronic pain patients. MAs codify universal opioid prescribing precautions including urine toxicological testing (UTS), and describe treatment objectives, side effects, and responsibilities of the patient and provider. Violations of MAs include UTS positive for illicit or non-prescribed controlled substances, prescription alteration/ forgery, doctor-shopping, and diversion. To date, the time course of when a violation occurs after signing a MA is unknown. The aim of this study was to provide a time-to-event analysis of MA violations over a 15-year period within an academic internal medicine clinic.

METHODS: The University of North Carolina General Medicine Pain Service (GMPS) manages opioid-treated patients referred by their PCPs within an internal medicine clinic. All patients sign MAs. We performed a time-to-event analysis of MA violations from 2002 to 2017, treating discontinuation of care as a competing event, and examined demographic correlates.

RESULTS: We enrolled 937 patients in the GMPS from 2002 to 2017. The average age was $51 ; 58 \%$ were female; $69 \%$ were white. A total of $40.3 \%$ of patients had one or more violations during GMPS enrollment. Almost one-half of violations were due to inconsistent use of controlled substances. Thirty percent of patients had a violation within 365 days of signing the MA, and $20 \%$ had a violation within 90 days (Figure). Ten percent occurred after a year. Within 365 days of signing the MA, the incidence of violations was significantly higher among men compared to women ( $38 \%$ vs. $22 \%$ ), current compared to never of former smokers ( $41 \%$ vs. $21 \%$ ), and in patients with a DUI or drug-related offense prior to or after the violation $(52 \%$ vs. $28 \%)$.

CONCLUSIONS: MA violations are common in opioid-treated patients with chronic pain. A majority of violations are seen within three months of signing, but a substantial number of violations were detected at a year and farther out. Proxies for substance use disorders predicted an increased risk for violations. Given the individual and societal risks of opioid use disorders, MAs are valuable clinical tools whose application should not be relaxed over time.

Figure 1. Cumulative incidence function of time to first medication contract violation among pain service patients, 2002-2017 ( $(\mathrm{N}=1,210)$. Calculated using nonparametric estimators and assuming discontinuation of care at the pain and a competing risk and death as a censoring event.

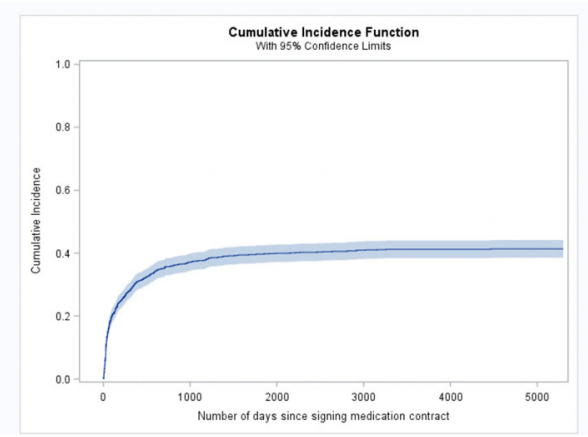


PAJAMA TIME: WORKING AFTER WORK IN THE ELECTRONIC HEALTH RECORD

$\underline{\text { Kanan Shah }}^{1}$; Harry S. Saag ${ }^{2,}{ }^{1}$; Leora I. Horwitz ${ }^{2,}{ }^{1}$; Paul Testa ${ }^{3} .{ }^{1}$ NYU Langone School of Medicine, Gibsonville, NC; ${ }^{2}$ NYU School of Medicine, New York, NY; ${ }^{3}$ NYU Langone Health, New York, NY. (Control ID \#3182665)

BACKGROUND: Electronic health record (EHR) documentation may contribute to burnout, especially for those with substantial clinical effort. We assessed whether clinical effort is associated with working in the EHR after work hours.

METHODS: We included all ambulatory physicians in a medicine specialty continuously practicing at any NYU Langone Health Faculty Group Practice site between May 1 and October 31, 2018. We quantified minutes logged into the EHR on days without scheduled appointments, and minutes logged into the EHR 30 minutes before and after appointments on days with scheduled appointments. We termed this time "work after work." We categorized physicians by their average number of days with appointments per week. Data were analyzed using SAS 9.4 (SAS Institute, Cary, NC). We calculated least squares means of fixed effects to account for heterogeneous variances, and compared means using Tukey's multiple comparison test. This study met institutional review board criteria for quality improvement work.

RESULTS: We included 300 physicians, of whom $28.6 \%$ were general internists. The average physician had 3 days/week with scheduled appointments, spent $114.9 \mathrm{~min}$ in the EHR on days without appointments, and spent $21.7 \mathrm{~min}$ in the EHR after work hours on days with appointments. Time spent in the EHR on days without appointments increased with the number of appointment days per week (14.7 min/unscheduled day for 1 day/week vs. $193.8 \mathrm{~min} /$ unscheduled day for $>4$ days $/$ week, $\mathrm{p}<0.001)$. Time spent in the EHR after hours on days with scheduled appointments did not significantly differ (Table 1).

CONCLUSIONS: All ambulatory physicians spend a substantial amount of time working in the EHR after hours and on unscheduled days (including weekends), but physicians with more clinical time were disproportionately burdened. The most clinically active spent an average of 2.8 hours in the EHR each unscheduled day. These findings add to concerns about EHR usability and documentation burden, particularly for busier clinicians. Our institution is now building dashboards to track work after work, offloading tasks to ancillary team members to reduce physician work burden, and exploring whether outliers would benefit from personalized technical assistance and training. Work after work analyses could be employed elsewhere to motivate similar improvements.

\section{Time spent on work after work, by clinical effort}

\begin{tabular}{|l|l|l|}
\hline $\begin{array}{c}\text { Days per week with } \\
\text { appointments }\end{array}$ & $\begin{array}{c}\text { Average work after } \\
\text { work (min/day) on } \\
\text { days with } \\
\text { appointments, } \\
\text { minutes }\end{array}$ & $\begin{array}{c}\text { Average work } \\
\text { (min/day) on days } \\
\text { without } \\
\text { appointments, } \\
\text { minutes }\end{array}$ \\
\hline$(0-1](n=7)$ & $28.9 \pm 11.7$ & $14.7 \pm 3.6$ \\
\hline$(1-2](n=17)$ & $12.8 \pm 5.5$ & $37.3 \pm 7.8$ \\
\hline$(2-3](n=59)$ & $20.6 \pm 3.3$ & $58.9 \pm 7.2$ \\
\hline$(3-4](n=113)$ & $20.3 \pm 2.2$ & $90.0 \pm 10.5$ \\
\hline$(4-5](n=104)$ & $24.5 \pm 2.7$ & $193.8 \pm 39.8$ \\
\hline & $p=0.34$ & $p<0.001$ \\
\hline
\end{tabular}

PARTNERING WITH ADVOCATES TO ASSESS BREAST DENSITY PATIENT EDUCATIONAL MATERIALS

Christine Gunn $^{1,}{ }^{2}$; Mark Kennedy ${ }^{3}$; Ariel Maschke ${ }^{1}$; Michael D. Fishman ${ }^{4}$; Margaret Hopkins ${ }^{5}$; Erica T. Warner ${ }^{5}$. ${ }^{1}$ Boston University, Boston, MA; ${ }^{2}$ Boston University School of Public Health, Boston, MA; ${ }^{3}$ Boston Public Health Commission, Boston, MA; ${ }^{4}$ Boston Medical Center, Boston, MA; ${ }^{5}$ Massachusetts General Hospital, Boston, MA. (Control ID \#3184896)

BACKGROUND: Mammographic breast density increases lifetime risk of breast cancer and reduces mammography sensitivity. Thus, 34 states enacted laws that require women be informed for their breast density. Evidence shows that relative to White women, Black women are more likely to have dense breast tissue, yet experience more anxiety and have less knowledge about their density. This is in part due to discordance between population health literacy levels and the grade levels at which breast density notifications are written. This study aims to 1) identify preferred educational content, format and opportunities for cultural tailoring and 2) characterize the readability and understandability of breast density information to improve the quality of breast density education for Black women.

METHODS: Members of the Pink \& Black Education and Support Network, a group of Black breast cancer patients/survivors in Boston, were invited to discuss breast density materials. We used a cognitive priming task in which women distributed 10 dots across six elements of communication, indicating the relative importance of each. Ratings guided the discussion of two sample materials from national breast cancer advocacy groups. We also compiled 21 publically-available educational materials designed for women with dense breasts. Readability was evaluated using the Flesch-Kincaid and Dale-Chall New Readability metrics. Understandability was measured using the Patient Education Material Assessment Tool (PEMAT), where higher scores suggest patients will be able to understand and act on the information provided. Scores were rated by two coders and discrepancies resolved in a 3-person consensus process.

RESULTS: Nine Pink \& Black members convened over 3 sessions. The cognitive priming task revealed that the most important elements of breast density education were "knowing what I can do about dense breasts", "being able to ask questions", and "getting information quickly". Consistent with readability analyses, women suggested current materials did not adequately convey a clear definition of breast density, but rather used complex medical jargon instead of simple language. Women advised briefing women in waiting rooms prior to their mammogram about breast density using informational videos. To decrease anxiety, women desired information via peers or health navigators, rather than a doctor. The mean Flesh-Kincaid grade for the 21 breast density materials was $9.7(5.5$ - 12.7). Thirteen were assessed at Grade 11-12 on the New Dale-Chall score, one Grade 10-11, and six Grade 9-10. The average PEMAT score was 66/100 (40-81).

CONCLUSIONS: We found that breast density education materials available to the public exceed the recommended reading level of 5-8th grade for health education materials, creating a barrier for patients' ability to use this information for breast cancer screening decision-making. Educational materials that respond to women's preferences are in development and will be presented to complement these analyses. 
PATIENT AND PROVIDER PERCEPTIONS OF DIABETES GROUP VISITS AT COMMUNITY HEALTH CENTERS

Samantha Mannion ${ }^{1}$; Erin M. Staab ${ }^{1}$; Sandra Ham ${ }^{1}$; Amanda Campbell ${ }^{3}$; $\overline{\text { Cynthia T. Schaefer }}^{2}$; Michael T. Quinn ${ }^{1}$; Arshiya A. Baig ${ }^{1} .{ }^{1}$ University of Chicago, Chicago, IL; ${ }^{2}$ University of Evansville, Evansville, IN; ${ }^{3}$ Midwest Clinicians' Network, East Lansing, MI. (Control ID \#3175222)

BACKGROUND: A pilot study in the Midwest with 6 community health centers (CHCs) defined diabetes group visits as shared medical appointments that include diabetes education in a group setting and individual visits with a medical provider. Providers and staff were given training and curriculum prior to implementing diabetes group visits at their health centers. This study analyzes patient and provider satisfaction with group visits, which is integral to their long-term viability and success.

METHODS: Adult patients at Midwestern health centers with an A1c of at least $8 \%$ were invited to participate in a 6-month group visit intervention. Surveys were administered to patients at baseline and at 6 months. Providers (primary care physicians, other clinicians, and non-clinical staff) were surveyed about the implementation of group visits 6 months after the first training session. Descriptive statistics were computed for survey responses using Excel. Eighteen semi-structured one-on-one interviews were conducted with providers 5-6 months and 9-10 months after the initial training session and coded for themes using HyperResearch.

RESULTS: Six CHCs had 27 total providers; 18 completed the 6 month survey. Of the 51 patients enrolled, 31 completed the 6-month survey. Ninety percent of patients reported "a lot" of overall satisfaction with group visits. Half (53\%) of patients felt "a lot" of support from other patients in the group and another 30\% felt "some" support. Three-quarters (77\%) of patients agreed that they liked having a longer visit "a lot." Providers and staff felt that group visits improved team dynamics by boosting staff morale (89\%) and fostering interdisciplinary collaboration (94\%). In interviews, providers felt that patients benefitted from interacting with the group and that they received more education and attention in a longer visit, especially a visit intended to focus solely on diabetes. Providers felt that implementation of group visits improved community relations for the health center. Only $39 \%$ of providers felt provider productivity had improved. Time was cited as the biggest challenge to implementation; this was commonly attributed to the initial time investment for a new program and to study-specific tasks such as chart abstraction.

CONCLUSIONS: Patients and providers participating in a diabetes group visit pilot program reported high satisfaction with the intervention. Patients benefited from interacting with other participants who shared their condition, and they enjoyed the increased education and attention they received in a longer group visit. Providers were excited to implement group visits as a way to better serve their patients and lead the way in healthcare innovation. Providers expressed a desire to continue group visits after the pilot program was finished and to expand them to other medical conditions. Other CHCs contemplating group visits should consider the up-front time commitment needed to organize group visits for the first time.

\section{PATIENT CENTERED MEDICAL HOME IMPLEMENTATION AND CHRONIC DISEASE CARE QUALITY IN PATIENTS WITH MULTIMORBIDITY}

Linnaea C. Schuttner ${ }^{1,2}$; Edwin Wong ${ }^{1,2}$; Ann-Marie Rosland ${ }^{3,4} ;$ Karin M. Nelson ${ }^{1,2}$; Ashok Reddy ${ }^{1,2}$. ${ }^{1}$ VA Puget Sound Healthcare System, Seattle, WA; ${ }^{2}$ University of Washington, Seattle, WA; ${ }^{3}$ VA Pittsburgh, Pittsburgh, PA; ${ }^{4}$ University of Pittsburgh School of Medicine, Pittsburgh, PA. (Control ID \#3165031)

BACKGROUND: Improving chronic disease management is central to the patient-centered medical home $(\mathrm{PCMH})$ and has been a demonstrated benefit of high-performing PCMH clinics. However, impacts on patients living with multimorbidity ( 3 or more diseases in 3 or more body systems) are less established. Thus, we examined the association between PCMH implementation and quality of care in multimorbid patients within an integrated health system.

METHODS: We conducted a retrospective cohort study among a sample of multimorbid adult patients $(\mathrm{N}=318,764)$ seen in a Veterans Affairs (VA) primary care clinic in 2012. We measured PCMH implementation in 2012 with the validated Patient Aligned Care Team Implementation Progress Index $\left(\mathrm{PI}^{2}\right)$. Higher $\mathrm{PI}^{2}$ scores indicate better performance on eight domains: access; continuity; care coordination; comprehensiveness; self-management support; patient-centered care; shared decision-making; and team-based care. Primary outcomes were binary patient-level measures of 16 quality metrics, including lipid, blood pressure, and glycemic control, medication adherence, and preventative care, 18 to 24 months after receiving care in a PCMH. We used generalized estimating equations to compare proportions of patients meeting the metric across clinics categorized by $\mathrm{PI}^{2}$ quintile, adjusting for patient and clinic characteristics, and ANOVA for tests of trend.

RESULTS: The cohort was mostly male (96\%) with an average age of 64.8 years and 5.1 chronic diagnoses. Receipt of care at clinics with higher PCMH implementation was associated with progressive odds of meeting the quality metric for 7 of 16 outcomes: diabetic patients with glycemic $\left(\mathrm{P}<0.0001\right.$ for test of trend across $\mathrm{PI}^{2}$ quintiles of clinics $)$ and lipid control $(\mathrm{P}=0.04)$; screening $(\mathrm{P}<0.005)$ and timely follow-up of positive screens $(\mathrm{P}=0.02)$ for $\mathrm{PTSD}$; ischemic heart disease patients with lipid control $(\mathrm{P}<0.01)$; and influenza $(\mathrm{P}=0.02)$ and pneumococcal $(\mathrm{P}<0.005)$ vaccination in older patients. No care differences were found for the remaining 9 metrics (diabetics with annual foot exam or blood pressure control, depression or alcohol screening or follow-up, heart failure patients on appropriate medications, hypertensive patients with blood pressure control, or influenza vaccines in non-elderly patients).

CONCLUSIONS: We found multimorbid patients receiving primary care at VA clinics with greater PCMH implementation received better clinical care across 7 of 16 quality markers, in particular adherence-related metrics (lipids, glycemic control), PTSD-related care, and vaccine rates in older patients. Care related to distinct tasks - such as a diabetic foot exam, may be less impacted by the level of PCMH implementation. Our findings are among the first to associate greater PCMH implementation with higher quality of chronic disease care for multimorbid adults within an integrated health system.

\section{PATIENT CONCENTRATION AMONG NURSING HOME PHY- SICIANS AND POST-ACUTE CARE OUTCOMES}

Kira Ryskina ${ }^{1}$; Rachel M. Werner ${ }^{2}$. ${ }^{1}$ University of Pennsylvania, Phila$\overline{\text { delphia, PA; }}{ }^{2}$ University of Pennsylvania and Philadelphia VA, Philadelphia, PA. (Control ID \#3185285)

BACKGROUND: Post-acute care in nursing homes (NHs), is common, costly and variable in quality. More and more generalist physicians are specializing in proving $\mathrm{NH}$ care, but whether these physicians care for a majority of patients in one or two facilities vs. a small propotion of patients in multiple facilities is unknown. Our objective was to measure the association between the degree to which $\mathrm{NHs}$ concentrate their patient care among a few versus many physicians who specialize in $\mathrm{NH}$ practice and patient outcomes.

METHODS: We analyzed 2,731,351 discharges by Medicare fee-forservice beneficiaries over 66 years of age from acute care hospitals to 15,013 NHs between 2012 and 2014. Physicians in generalist specialties with at least $90 \%$ of claims for $\mathrm{NH}$ care were considered $\mathrm{NH}$ specialists. Patients were assigned to the $\mathrm{NH}$ attending physician based on plurality of claims. The degree to which NHs concentrate care of their patients among a few or many physicians was measured by calculating a facility-level Herfindahl-Hirschman Index (HHI) as the weighted sum of each attending physician's share of the NH's patient population. We estimated the 
independent and joint effects of $\mathrm{NH}$ physician specialization and concentration on three outcomes: 30-day rehospitalization, 60-day episode Medicare payments (both derived from claims), and successful discharge to community (derived from the NH Minimum Data Set). To measure 60day episode of care payments we summed all facility and professional payments by Medicare Parts A and B (including hospital, NH, physician and other provider payments). Successful discharge to community was identified when a patient was discharged to the community within 100 days of admission to $\mathrm{NH}$ and remained alive and not readmitted to a hospital or $\mathrm{NH}$ for at least 30 days. All outcomes were adjusted for demographics (age, sex, race) and 72 clinical variables used by CMS for clinical measure riskadjustment. To account for heterogeneity across facilities, we included $\mathrm{NH}$ fixed effects. Outcomes were modeled using linear regression. Confidence intervals were adjusted for clustering within NHs.

RESULTS: The median HHI across U.S. nursing homes was 0.29 (IQR 0.19-0.44). Controlling for the degree of patient concentration among few vs. many physicians, patients under the care of physicians who specialized in $\mathrm{NH}$ care were less likely to be rehospitalized after 30 -days (14.54\% vs. $15.92 \%$; adjusted difference, $-1.38 \%, 95 \%$ CI -1.77 to -1.00$)$, more likely to be successfully discharged to the community ( $59.95 \%$ vs. $59.09 \%$; adjusted difference, $0.86 \%, 95 \%$ CI 0.40 to 1.33 ), but had higher 60 -day Medicare costs $(\$ 31,150$ vs. $\$ 30,875$; adjusted difference, $\$ 275 ; 95 \%$ CI $\$ 113$ to $\$ 437)$. These differences were consistent across different levels of patientphysician concentration.

CONCLUSIONS: NHs whose patients are treated by generalist physicians who specialize in $\mathrm{NH}$ practice may achieve marginally better patient outcomes at slightly higher costs.

\section{PATIENT DECISION-MAKING ABOUT USE OF HEALTH SAV- INGS ACCOUNTS}

Jeffrey T. Kullgren ${ }^{1,2}$; Elizabeth Q. Cliff ${ }^{2}$; Christopher Krenz ${ }^{2}$; Helen $\overline{\text { Levy }^{2}, 2 \text {; Brady T. }}$ West $^{2}$; A. M. Fendrick ${ }^{2,}{ }^{2}$; Angie Fagerlin ${ }^{3,}{ }^{4}$. ${ }^{1}$ VA Ann Arbor Healthcare System, Ann Arbor, MI; ${ }^{2}$ University of Michigan, Ann Arbor, MI; ${ }^{3}$ University of Utah, Salt Lake City, UT; ${ }^{4}$ Salt Lake City VA Medical Center, Salt Lake City, UT. (Control ID \#3184967)

BACKGROUND: Expansion of health savings accounts (HSAs) has been a centerpiece of recent federal health reform proposals. These accounts can be used by patients who are enrolled in a high-deductible health plan (HDHP) to save tax-free for health care expenses, with the policy goals of lessening patients' health care cost burdens and helping them be more discerning health care consumers. Despite the popularity of HSAs among many policymakers, little is known about which HDHP enrollees are more likely to have an HSA, what factors are associated with higher levels of saving in HSAs, and why patients with HSAs forego opportunities to contribute to their accounts.

METHODS: We surveyed 1,637 GfK KnowledgePanel participants who were 18 years of age and older and had been enrolled in an HDHP for at least a year (response rate 55\%). Respondents indicated whether they had an HSA and, if so, how much they had saved for health care in the past year. Individuals who had not saved for health care were asked why not. We used multivariable logistic regression to identify factors associated with having an HSA. Among individuals with an HSA, we identified the most frequent levels of saving for health care expenses and used multivariable ordered logistic regression to identify factors associated with higher levels of saving. Among individuals who had an HSA but had not contributed to their account, we identified the most frequent reasons why. All analyses used survey weights to generate nationally representative estimates.

RESULTS: Most individuals (58\%) reported having an HSA. Factors associated with having an HSA included having a graduate degree, no chronic conditions, and higher levels of financial literacy and health insurance literacy. Among individuals with an HSA, the most frequent levels of saving for health care expenses were $\$ 0$ (44\%), $\$ 2,000$ or more (22\%), and $\$ 500$ to $\$ 999$ (10\%). Factors associated with higher levels of saving in HSAs included older age, more education, and higher health insurance literacy. Among individuals who had an HSA but had not contributed to their account, the most common reasons were not needing health care services (45\%), already having enough savings to pay for health care (40\%), and not having considered saving for health care (36\%).

CONCLUSIONS: While most Americans enrolled in an HDHP have an HSA, nearly half of those with an HSA have not used their account to save for any health care expenses. Individuals with less education and lower health insurance literacy are less likely to have an HSA and, even when they do, to contribute to it. Federal efforts to expand HSAs may not achieve their policy goals of lessening patients' health care cost burdens and helping them be more discerning health care consumers without concurrent efforts to increase health insurance literacy and facilitate use of HSAs among individuals who are less likely to take up and contribute to these accounts.

\section{PATIENT EXPERIENCE IN A CLINIC-LEVEL BEHAVIORAL ECONOMIC INTERVENTION TO REDUCE OVERUSE OF LOW-VALUE TREATMENTS FOR CHRONIC CONDITIONS AMONG OLDER ADULTS}

Jeffrey T. Kullgren $^{1,2}$; Megan Slowey ${ }^{3}$; Hyungjin M. Kim ${ }^{2,}$, ; Joseph T. Colbert $^{2}$; Kerry A. Ryan ${ }^{2}$; Angie Fagerlin ${ }^{4}$, 5 ; Barbara Soyster ${ }^{2}$; Stuart Winston ${ }^{6}$; Jane Forman ${ }^{1}$; Eve A. Kerr ${ }^{1}{ }^{2}$. ${ }^{1}$ VA Ann Arbor Healthcare System, Ann Arbor, MI; ${ }^{2}$ University of Michigan, Ann Arbor, MI; ${ }^{3}$ Center for Health and Research Transformation, Ann Arbor, MI; ${ }^{4}$ University of Utah, Salt Lake City, UT; ${ }^{5}$ Salt Lake City VA Medical Center, Salt Lake City, UT; ${ }^{6}$ IHA, Ann Arbor, MI. (Control ID \#3184996)

BACKGROUND: The Choosing Wisely ${ }^{\circledR}$ campaign recommends that older patients avoid use of low-value care such as hypoglycemic diabetes medications to achieve a hemoglobin A1c $<7.5 \%$ and use of benzodiazepines and sedative-hypnotics for insomnia or anxiety. Yet, use of these lowvalue treatments is common, and it is unclear how to engage older patients and their providers to avoid such overuse while maintaining strong patientprovider relationships.

METHODS: We conducted an 8-month intervention in 8 primary care clinics from 2 health systems. Intervention clinic primary care providers (PCPs) were shown Choosing Wisely recommendations to avoid overtreatment of diabetes, insomnia, and anxiety among adults age 65 and older, and invited to commit their future selves (i.e., precommit) to following these recommendations by signing a commitment document. To provide a social incentive, precommitted PCPs had their photos displayed on clinic posters. Precommitted PCPs also received weekly emails with resources to help them and their patients avoid low-value treatments, and had access to point-of-care patient education handouts about overuse that were mailed to applicable patients prior to scheduled primary care visits. After primary care visits, we surveyed 354 older patients with diabetes, insomnia, or anxiety: 167 from the 8 intervention clinics and 187 from 7 control primary care clinics. Surveys asked patients whether at their visit they talked with their PCP about medications for their condition and, if so, changed their medications, and about their rating of and trust in their PCP. We used multilevel modeling to estimate differences in these outcomes between intervention and control clinic patients while accounting for PCP-level correlation.

RESULTS: Intervention clinic patients with diabetes were no more likely than control clinic patients with diabetes to have discussed their diabetes medications with their PCP, but conditional on discussing diabetes medications were more likely to report taking less diabetes medication after their PCP visit $(13.5 \%$ vs. $1.0 \% ; P=0.01)$. Intervention clinic patients with insomnia or anxiety were no more likely to report discussing or changing their medications for these conditions. There were no differences between intervention and control clinic patients in ratings of, or trust in, their PCPs.

CONCLUSIONS: A behavioral economic intervention that sought to engage PCPs and older patients to avoid use of low-value treatments for chronic conditions may have reduced use of medications for diabetes, but not for insomnia or anxiety, and did not adversely affect patients' ratings of, or trust in, their PCPs. These results suggest behavioral economic interventions can reduce overuse of low-value treatments for chronic conditions like diabetes 
without negatively affecting patient-provider relationships, but may need to be coupled with other approaches to reduce use of low-value treatments for more symptomatic conditions like insomnia and anxiety.

PATIENT NAVIGATION AMONG RECENTLY HOSPITALIZED SMOKERS TO PROMOTE SMOKING CESSATION: BASELINE RESULTS FROM A PILOT RANDOMIZED CONTROLLED TRIAI Lisa M. Quintiliani1 ${ }^{1,2}$; Ve Truong ${ }^{2}$; Jennifer Murillo ${ }^{2}$; Hasmeena Kathuria ${ }^{1,2}$; Belinda Borrelli ${ }^{3}$; Karen E. Lasser ${ }^{1}, 2$. ${ }^{1}$ Boston University, Boston, MA; ${ }^{2}$ Boston Medical Center, Boston, MA; ${ }^{3}$ Boston University Henry M. Goldman School of Dental Medicine, Boston, MA. (Control ID \#3176007)

BACKGROUND: Tobacco treatment interventions initiated during hospitalizations with supportive contacts after discharge promote continuity of treatment and enhance likelihood of cessation. However, to our knowledge, these interventions lack screening for and addressing health-related social needs. Such screening may improve intervention effectiveness: if social needs are addressed, patients may be better able to focus on cessation. Our objective is to present baseline and process data from a pilot study examining the feasibility of a tobacco treatment intervention that includes screening for health-related social needs among recently hospitalized smokers at a largesafety net hospital.

METHODS: In a two-group 3-month pilot randomized controlled trial, we assigned hospitalized smokers to either a control group (list of smoking cessation resources) or an intervention group (up to 10 hours of patient navigation over a 3-month period, in which a navigator screens for healthrelated social needs and discusses smoking with the patient and links patients to effective treatments and resources). Inclusion criteria were: 18 years old, hospitalized at the time of randomization, smoked 1 cigarette/day, had plans to ever quit smoking, spoke English, and owned a telephone. We assessed sociodemographics, smoking-related variables, and process data.

RESULTS: Of 38 individuals screened and found eligible, 36 (95\%) were enrolled. Participants $(n=36$, mean age $=53.9$ years, $61.1 \%$ Hispanic, $66.6 \%$ high school education or less, $80.5 \%$ Medicaid) smoked a mean of 12.2 cigarettes/day. Initially, our intervention protocol was to provide 2 hours of navigation over 1 month. However, only 3 of 7 participants received a minimum intervention dose (completion of the social needs screener and at least one conversation about smoking), as the navigator was unable to contact 1 participant and 3 participants were too sick (as reported by the patient or perceived by the navigator). Because so few participants received the minimum dose, we changed our protocol (allowing up to 10 hours of navigation over 3 months). Among those ( $\mathrm{n}=6$ ) who have completed the 3 -month intervention, 3 participants received the minimum intervention dose. The number of contacts per participant ranged from 9-16; contacts included texting, telephone, and home visits. Among the six participants who were screened for health-related social needs, all reported lack of employment, 2 reported trouble paying heating bills, and 1 reported not having a high school degree; 1 person requested follow-up services after completing the screener. CONCLUSIONS: While study enrollment was high among eligible patients, the feasibility of this intervention is limited by difficulty contacting recently hospitalized patients post-discharge due to illness. Future iterations of this intervention could introduce the navigator to the patient while the patient is still hospitalized. Future research should explore the acceptability of screening patients for health-related social needs.

PATIENT OPINIONS ABOUT DIGITAL MESSAGING FOR CLINICAL RESEARCH RECRUITMENT

Michael Cantor ${ }^{2,1}$; Michael Mishik ${ }^{3} .{ }^{1}$ NYU School of Medicine, New York, NY; ${ }^{2}$ Regeneron Pharmaceuticals, Tarrytown, NY; ${ }^{3}$ NYU Langone Health, New York, NY. (Control ID \#3142707)

BACKGROUND: Identifying and recruiting patients for clinical research remain major challenges for researchers. EHRs are playing larger roles in recruitment, from helping identify cohorts to alerting clinicians about potentially eligible patients. One major issue around using EHRs for recruitment is understanding the best way to manage the amount and type of messaging patients receive. Because research-related communications is a relatively new area, few institutions have set policies in this area. Before setting policy at NYU Langone Health, we chose to survey our patient population to better gauge their beliefs and to obtain data that would help us formulate a policy that aligns with patients' actual preferences rather than their hypothetical concerns.

METHODS: The NYU School of Medicine IRB determined that this project was exempt from review. We developed a 10-question survey in our institution's RedCAP system. The survey was developed with input from our institutional Research Governance Group, who gave input on both content and wording of the questions. We then obtained a random sample of 20,000 adult, active MyChart (our Epic ${ }^{\circledR}$ patient portal) users, and sent a survey invitation to 10,000 patients per week for two weeks. The survey was completely anonymous, and the invitation contained a direct link to the RedCap survey. We closed the survey after 3 weeks.

RESULTS: We received 2157 responses to the survey. $61.7 \%$ of respondents were female, $83 \%$ were white, and $11 \%$ identified as Hispanic/Latino. 2/3 of patients were within the $46-75$ age range. $72 \%$ of patients responded that they would be interested in participating in research studies, and $87 \%$ of all patients responded that they would be interested in receiving research-related messages. Responses about limits on the number of messages that patients receive were nearly evenly split, with $46 \%$ responding that there should not be a limit on the number of research-related messages received, and 39\% responding that there should be a limit. Over $90 \%$ wanted the option to opt-out of receiving further messages with each invitation. Opinion about the "right" number of research-related messages that a patient could receive in a particular time period was mixed, but were nearly evenly split between $1-2$ times a month and no limit on messages.

CONCLUSIONS: Developing and distributing the survey through the patient portal was a relatively straightforward process and was relatively quick to implement after institutional approval. Our survey revealed engaged patients who are interested in finding out more about research, and who wish to have input into and control over the number and types of research-related messages they receive. Performing a similar survey in a larger, more diverse population would help give a more accurate picture of broader patient preferences. Digital messaging can be an effective tool for research-related communications, and could be added to the current toolbox of general recruitment techniques.

\section{PATIENT STAKEHOLDER ENGAGEMENT IN RESEARCH: A SYSTEMATIC REVIEW TO DESCRIBE FOUNDATION PRINCI- PLES AND BEST PRACTICE ACTIVITIES}

James D. Harrison $^{1}$; Andrew Auerbach ${ }^{1}$; Krista Woodward ${ }^{4}$; Wendy Anderson $^{5}$; Maureen Fagan ${ }^{3}$; Martha Carnie ${ }^{2}$; Catherine Hanson ${ }^{3}$; Jm Banta ${ }^{6}$; Gina Symczak $^{6}$; Edmondo Robinson ${ }^{8}$; Jeffrey Schnipper ${ }^{7}$; Celene Wong ${ }^{2}$; Rachel Weiss ${ }^{1} .{ }^{1}$ University of California San Francisco, San Francisco, CA; ${ }^{2}$ Brigham and Women's Hospital, Milton, MA; ${ }^{3}$ University of Miami Health System, Miami, FL; ${ }^{4}$ Patient Centered Outcomes Research Institute, Washington DC, DC; ${ }^{5}$ Division of Palliative Medicine, University of California San Francisco, San Francisco, CA; ${ }^{6}$ Intensive Care Unit Patient \& Family Advisory Council, University of California San Francisco, San Francisco, CA; ${ }^{7}$ Department of Medicine, Brigham \& Women's Hospital, Boston, MA; ${ }^{8}$ Christina Care Health System, Delaware, DE. (Control ID \#3162491)

BACKGROUND: Health research is evolving to include patient stakeholders (patients, families and caregivers) as active members of research teams. The proliferation of frameworks describing the conceptual foundations underlying this engagement, and strategies detailing best practice activities to support this process has led to a diffusion of information. Therefore, the aims of this study are to identify, quantify and summarize 1) the foundational principles of patient stakeholder engagement in research and 2) best practice activities to support these efforts.

METHODS: We conducted a systematic review of the literature supplemented with review of the gray literature to create a narrative review. We accessed a publicly available repository of systematically identified literature of English- 
language articles from PubMED/MEDLINE related to patient stakeholder engagement in research. Two reviewers independently screened articles to determine their relevance. The contents of relevant articles were then reviewed, and data abstracted - specifically domains that were reported as foundational principles of patient stakeholder engagement in research and best activities that support the operationalization of engagement.

RESULTS: We identified 990 potentially relevant articles and reports of which 935 (94.4\%) were excluded and 55 (5.5\%) deemed relevant. Fifteen foundational principles and 25 best practice activities were identified. The most commonly reported foundational principles were "respect" $(\mathrm{n}=25,45 \%)$ and "equitable power between all team members" $(\mathrm{n}=21,38 \%)$. Creating "trust between patient stakeholders and researchers" was described in 17 (31\%) articles. Twenty-seven (49\%) articles emphasized the best practice of providing training and education for both patient stakeholder and researchers. Providing financial compensation for patient stakeholders' time and expertise was noted in $19(35 \%)$ articles. Nineteen articles (35\%) emphasized regular, bidirectional dialogue between patient partners and researchers as important for successful engagement. An overarching foundational framework summarizing key findings is shown in Figure 1.

CONCLUSIONS: Engaging patient stakeholders in research as partners presents an opportunity to design, implement and disseminate patient centered research. Our review creates an overarching foundational framework to facilitate authentic and sustainable partnerships between patient stakeholders and researchers.

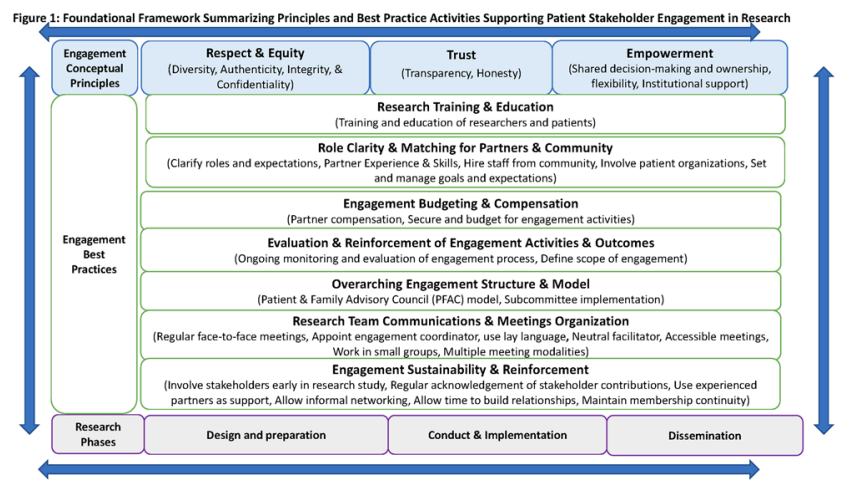

PATIENT-CENTEREDNESS OF THE MEDICATION RECONCILIATION PROCESS IN THE VA PRIMARY CARE SETTING Robin S. Mickelson²; Jea Young $\mathrm{Min}^{2}$; Susan Byerly²; Amanda S. Mixon ${ }^{1}{ }^{1}$ VA Tennessee Valley Healthcare System and Vanderbilt University, Nashville, TN; ${ }^{2}$ Department of Veterans Affairs, Nashville, Monteagle, TN. (Control ID \#3143013)

BACKGROUND: High quality medication reconciliation (MR) is critical to prevent medication errors. Inpatient MR toolkits have been developed, tested, and are efficacious; however, less is understood about the current outpatient MR process particularly the degree of patientcenteredness and patient engagement.

METHODS: We conducted an observational descriptive study from April to June 2018, in which we recruited 30 Veterans with non-urgent appointments and a medication list in their medical record from VA primary care clinics. We observed the primary care team conduct MR and assessed patient-centeredness, whether it (1) created a shared understanding; (2) explored the biopsychosocial context; (3) provided patientfriendly explanations; (4) encouraged active participation; (5) uncovered the patient's perspective; (6) produced decisions based on evidence, values, and feasibility; and, (7) created trust. We assessed patient-level subjective numeracy, health engagement, and interpersonal processes of care using validated measures. We conducted semi-structured interviews to evaluate patients' engagement in as well as barriers and facilitators to the MR process. Interviews were transcribed and imported into NVivo® software with the observation notes. Two researchers utilized the aspects of patient engagement and patient-centeredness to identify categories and sub-categories through directed content analysis.

RESULTS: The patients' median age was 70, 20\% African American, 7\% female. Patients had an average of 13.5 medications on their list. The mean subjective numeracy was high (5.3 on 1 to 6 scale), the mean perceived processes of care around medications was excellent (1.2 on 1 to 5 scale), and the mean engagement in health was high (6 on 0 to 7 scale). With regard to patient-centeredness and patient engagement, qualitative analyses revealed a MR process constrained by deficits in 3 areas: shared understanding, a standardized process, and tools to facilitate adequate information exchange. Patients misunderstand the purpose of the MR process, their role, and often had different goals regarding medications. Healthcare providers (HCP) did not always ask about adherence, explore biopsychosocial barriers and supports, or assess the patient's beliefs or understanding. During the medication review, generic names often confuse patients, although most providers used patient-friendly explanations to help patients understand the mechanism of action and side effects. Familiarity with the HCP, trust, and a friendly, unhurried attitude facilitated active patient participation in the conversation.

CONCLUSIONS: The outpatient MR process varied widely in its degree of patient-centeredness; therefore, opportunities to improve the patient's experience and efficiency of MR abound. To overcome barriers, tools that promote the systematic exchange of information, facilitate shared understanding, and promote patient-centeredness are needed to improve the outpatient MR process.

\section{PATIENT-REPORTED QUALITY OF CARE IN LOW- AND MIDDLE-INCOME COUNTRIES: A FOUR-COUNTRY SURVEY OF PATIENT EXPERIENCE}

June-Ho Kim ${ }^{2,}{ }^{1}$; Griffith A. Bell ${ }^{2}$; Hannah Ratcliffe ${ }^{2}$; Leah Moncada ${ }^{3}$; Stuart R. Lipsitz ${ }^{1,2}$; Dan Schwarz ${ }^{2}$; Lisa R. Hirschhorn ${ }^{4,2}$; Asaf Bitton ${ }^{2}$, ${ }^{1}$. ${ }^{1}$ Brigham and Women's Hospital, Boston, MA; ${ }^{2}$ Ariadne Labs, Brigham \& Women's Hospital and Harvard T.H. Chan School of Public Health, Boston, MA; ${ }^{3}$ RIWI Corp, Toronto, ON, Canada; ${ }^{4}$ Northwestern University Feinberg School of Medicine, Chicago, IL. (Control ID \#3185437)

BACKGROUND: Patient experience is a major tenet of high-quality health care but is rarely measured in low- and middle-income countries (LMICs). We assessed patient-reported experience, quality of care (QOC), and characteristics of people who sought care in four LMICs.

METHODS: We analyzed a 2016 web-based survey administered in India, Kenya, Mexico, and Nigeria. The main outcome was patientreported QOC, dichotomized into categories of "excellent" vs "not excellent." To quantify patient experience, we created a "responsiveness" index, derived from the WHO Health Systems Responsiveness framework, using questions assessing respect from providers, ability to understand the provider's advice, facility cleanliness, and rating of wait time. Covariates included age, sex, education, country, urban/rural status, person seeking care, reason for seeking care, type of provider, site of care, and facility type. We used multivariable Poisson regression to estimate adjusted prevalence ratios (aPR) for excellent QOC across levels of respondent characteristics and quintiles of responsiveness index.

RESULTS: From a survey population of $61,987,17,441$ respondents sought care in the past 6 months and 5,407 of those (31\%) completed the survey. Respondents were generally young $(86 \%$ age $<45)$, male $(67 \%)$, well-educated (57\% with post-secondary degree), and urban-dwelling (68\%). For their most recent care-seeking experience, respondents most often saw a specialist or primary care provider $(49 \%$ and $25 \%$, respectively). Wide inter-country variability was seen for care-seeking at private health facilities (65\% in India vs $45 \%$ in Mexico). Most patients in India and Mexico reported being part of a prepayment plan (i.e. receiving medical aid or insurance) in contrast to Nigeria and Kenya. Nigeria had the most respondents reporting excellent QOC (31\%) compared to only $19 \%$ in India and Mexico. In adjusted models, people living in rural areas were more likely to report excellent QOC compared to their urban 
counterparts (aPR 1.15, 95\% CI 1.02,1.31) while those who saw a specialist were more likely than those who saw a primary care provider (aPR 1.49, 95\% CI 1.27, 1.74). Respondents with prepayment plans (aPR $1.27,95 \%$ CI $1.13,1.43$ ) and those who sought care in a private facility (aPR 1.40, 95\% CI 1.23, 1.59) reported higher QOC. Mean responsiveness scores ranged from 0.64 to 0.66 on a scale of 0 to 1 in India, Kenya, and Mexico while Nigeria had a mean of 0.71. Respondents who experienced the highest responsiveness reported higher QOC than those who experienced lower responsiveness (aPR 8.38, 95\% CI 7.39, 9.50).

CONCLUSIONS: Higher patient-reported quality in four LMICs was associated with seeking specialty care compared to primary care and private sector care compared to public sector; having a prepayment plan; and experiencing higher responsiveness. Targeting interventions to improve health system responsiveness in settings of care with lower patientreported quality could improve patients' overall experience in LMICs.

\section{PATIENTS' REPORTS OF HARM FROM FAILURES OF CARE COORDINATION}

Lisa M. Kern ${ }^{1}$; Evgeniya Reshetnyak ${ }^{1}$; Lisandro Colantonio ${ }^{2}$; Paul Muntner $^{2}$; J. David Rhodes ${ }^{2}$; Lawrence P. Casalino ${ }^{1}$; Mangala Rajan ${ }^{1}$; Michael Pesko ${ }^{3}$; Jacqueline Marino ${ }^{1}$; Laura C. Pinheiro ${ }^{1}$; Monika M. Safford ${ }^{1}$. ${ }^{1}$ Weill Cornell Medicine, New York, NY; ${ }^{2}$ University of Alabama at Birmingham, Birmingham, AL; ${ }^{3}$ Georgia State University, Atlanta, GA. (Control ID \#3168018)

BACKGROUND: Previous studies show that some patients report dissatisfaction with the extent to which their providers coordinate their care. However, it has not been clear how significant any gaps in care coordination have been and whether they have led to undesirable outcomes. We sought to determine the frequency of patient-reported harm from perceived gaps in care coordination.

METHODS: We conducted a survey on care coordination within the REasons for Geographic and Racial Differences in Stroke (REGARDS) study, a population-based, ongoing, prospective cohort study of community-dwelling adults, who were recruited between 2003 and 2007. The 21-question care coordination survey module was administered to REGARDS participants 65 years old during a routine follow-up phone call between August 2017 and October 2018. The module asked about numbers of ambulatory visits and providers in the past year, perceptions of care coordination, and the occurrence of four outcomes (repeat tests, drug-drug interactions, emergency department (ED) visits, and hospitalizations). Participants experiencing outcomes were asked if they thought the outcomes were preventable with better care coordination. If a respondent reported poor care coordination in response to one or more (out of 7) questions on perceptions of care coordination, we considered the respondent to have reported a "gap" in care coordination. Among those who reported having 2 visits and 2 providers in the previous year (as a proxy for being at risk for problems with care coordination), we used logistic regression to determine associations between self-reported gaps in care coordination and selfreported outcomes that could have been prevented with better care coordination, adjusting for participants' demographic and clinical characteristics. RESULTS: Of 13,208 REGARDS participants approached, 10,418 (79\%) completed the care coordination survey module and were 65 years old. The average respondent was 77.2 years old (SD 6.9); $57 \%$ were women, and $36 \%$ were black. The average respondent reported having had 5.2 ambulatory visits (SD 6.4) across 2.8 ambulatory providers (SD 2.5) the previous year. Of those with 2 visits and 2 doctors the previous year $(\mathrm{N}=7,075), 38 \%$ reported one or more gaps in care coordination and $10 \%$ reported at least one outcome that could have been prevented with better care coordination (3\% reported repeat tests, $6 \%$ drug-drug interactions, $1 \%$ preventable ED visits, and $1 \%$ preventable hospitalizations). Participants with one or more gaps in care coordination were more likely to report preventable outcomes than those without gaps in care coordination (adjusted odds ratio $1.52 ; 95 \%$ confidence interval 1.24, 1.85).

CONCLUSIONS: Patients report that gaps in care coordination are common and that these gaps often lead to undesirable outcomes. Future interventions to improve care coordination should engage patients directly, as patients may be able to both identify the problem and contribute to a solution.

PEER-LED SOCIAL MEDIA BASED HEALTH CAMPAIGN: PERSONALIZED DIGITAL CONTENT AND STRATEGIES ASSOCIATED WITH HIGHER LEVELS OF ENGAGEMENT

Lawrence Gross ${ }^{1}$; Minia N. Rios Gutierrez ${ }^{2}$; Keith J. Horvath ${ }^{3}$; Viraj V. Patel $^{2}$. ${ }^{1}$ Albert Einstein College of Medicine, Bronx, NY; ${ }^{2}$ Montefiore Health System / Albert Einstein College of Medicine, Bronx, NY; ${ }^{3}$ University of Minnesota, Minneapolis, MN. (Control ID \#3179523)

BACKGROUND: The attributes of user-driven postings to a virtual community wall can influence engagement with online health interventions to support behavior change. Because techniques for optimal engagement of Black and/or Latinx men who have sex with men (BLMSM) are unknown, we analyzed the effects of digital post characteristics on engagement levels using data from a peer-delivered, social-media based HIV prevention intervention.

METHODS: 10 BLMSM peer-leaders participated in an online health intervention in New York City disseminating pre-determined tailored health messages ("posts") over six-weeks to 164 BLMSM participants from their Facebook networks in private Facebook groups. We categorized the standardized posts (developed by PLs) based on content types (video, image, infographic, text-only, or article) and engagement strategies used (posing a question, hashtag, tagging participants, emoji, celebrity references, and community [posts featuring people of color]). We calculated engagement scores by summing each post's likes and comments, and weighted by a ratio of total number of likes, comments, and potential viewers across all post. We then calculated mean engagement scores (MES) for each categorization and compared using ANOVA and multivariable regression (shown as $(95 \% \mathrm{CI})$ ).

RESULTS: There were a total of 459 posts, consisting of the following content types: 113 videos, 126 images, 67 infographics, 54 text-only and 99 articles. Distribution of engagement strategy was: 148 question, 120 hashtag, 35 tagging, 59 emoji, 10 celebrity, and 161 community. MES across all categories ranged from 8.8-59. When comparing posts by content type, text-only posts had the highest MES (23.1) and posts of articles had the lowest (8.8) $(\mathrm{p}=0.04)$. Among strategies, tagging participants had the greatest MES (59), while using an emoji had the lowest MES (16). MES increased with the number of strategies used (e.g., posts not using an identifiable strategy (MES=6.25) to posts using 4 strategies (MES=40.9)). In multivariable analyses of content-types, only text posts had significantly higher engagement $(0.14(0.05-0.24))$ than articles. In multivariable analysis of engagement-strategies, tagging $(0.49(0.39$ $0.58)$ ) and questions $(0.1(0.05-0.15))$ were more likely to have higher MES compared to not using an identifiable engagement-strategy $(p<0.01)$. Sensitivity analysis which considered the effect of PLs on MES did not change findings.

CONCLUSIONS: In an online health intervention for BLMSM, personalized content types and engagement strategies were most successful at eliciting greater engagement with materials. Text-only posts can be more easily tailored than other content types to address specific groups or individuals. Tagging and posing questions may increase engagement by making it socially desirable to respond to peers online. Online health interventions should consider personalizing digital contents to enhance engagement.

\section{PERCEIVED BARRIERS AND FACILITATORS TO CONTRA- CEPTIVE USE AMONG WOMEN VETERANS IN THE ECUUN STUDY}

Tierney Wolgemuth $^{1}$; Maris Cuddeback ${ }^{1}$; Keri L. Rodriguez ${ }^{3}$; Xinhua Zhao ; Sonya Borrero ${ }^{2} .{ }^{1}$ University of Pittsburgh School of Medicine, Pittsburgh, PA; ${ }^{2}$ University of Pittsburgh and VA Pittsburgh, Pittsburgh, PA; ${ }^{3}$ VA Pittsburgh Healthcare System, Pittsburgh, PA; ${ }^{4}$ VA Pittsburgh 
HealthCare System, Pittsburgh, PA. (Control ID \#3138503)

BACKGROUND: Despite coverage of the full range of effective contraceptive methods through the Veteran's Affairs Healthcare System (VA), women Veterans experience a high burden of unintended pregnancy, with proportions comparable to the general United States population. Although many studies evaluate factors affecting contraceptive use, little is known about influences specific to or prominent among women VA-users. This qualitative study seeks to characterize barriers and facilitators to contraceptive use experienced by women Veterans using VA services, as well as elicit their suggestions to improve VA contraceptive care.

METHODS: Semi-structured telephone interviews were conducted with a purposefully-selected national sample of 187 women Veterans aged 1844 at risk of unintended pregnancy who receive primary care in VA to explore their perspectives and experiences with contraceptive care. The sampling strategy sought to ensure that we heard the perspectives of women with potentially vulnerable demographic or clinical characteristics, including women in ethnic/ racial minority groups, and/ or with medical or mental health conditions or a history of military sexual trauma. Interviews were transcribed verbatim and transcript narratives analyzed using content analysis and the constant comparison method.

RESULTS: Five distinct themes emerged as barriers or facilitators to contraceptive use depending on participants' VA facility and provider, and participants offered suggestions to address issues pertaining to each theme. Most participants (56\%) noted low efficiency of care as a main factor, especially poor appointment access and difficulty navigating the VA system. Others (39\%) expressed that limited contraceptive counseling and patient education acted as barriers, and requested classes and support groups to enhance understanding. About a third of women (34\%) noted that low patient awareness of services impeded contraceptive care and suggested improved advertising to both servicewomen and Veterans. Some (32\%) stressed poor interaction with provider as a prominent barrier, specifically noting provider inattentiveness and lack of expertise. Finally, $31 \%$ noted feeling like an outsider at VA, and emphasized fostering a female-friendly environment by creating a separate space for women's clinics to remove discomfort associated with seeking contraceptive care.

CONCLUSIONS: These findings suggest that despite widespread access to low-cost contraception, many women Veterans experience barriers to accessing high-quality contraceptive care. While barriers and facilitators were unique to individual VA facilities and providers, women offered concrete suggestions that could make access to high-quality services more uniform, including enhancing patient education on contraceptive options; expanding advertising of contraceptive services to women Veterans; and providing a separate women's clinic to ensure women Veterans feel comfortable seeking contraceptive care.

\section{PERCEIVED SUPPORT DURING AND AFTER A WEIGHT LOSS MAINTENANCE INTERVENTION: SECONDARY ANALYSIS FROM THE MAINTAIN-PC TRIAL}

Skylar Larsen ${ }^{5}$; Maribel Cedillo ${ }^{1}$; Polina Kukhareva ${ }^{1}$; Cindy L. Bryce ${ }^{2}$; Bethany Barone Gibbs ${ }^{2}$; Jonathan Arnold ${ }^{3}$; Rachel Hess ${ }^{4}$; Laurey R. Simkin-Silverman ${ }^{2}$; Gary Fischer ${ }^{2}$; Molly Conroy ${ }^{1} .{ }^{1}$ University of Utah, Salt Lake, UT; ${ }^{2}$ University of Pittsburgh, Pittsburgh, PA; ${ }^{3}$ University of Pittsburgh Medical Center, Pittsburgh, PA; ${ }^{4}$ University of Utah, Salt Lake City, UT; ${ }^{5}$ Univesrity of Utah, Salt Lake City, UT. (Control ID \#3180303)

BACKGROUND: Many patients with obesity achieve significant weight loss only to regain. Weight loss has been well studied, but less is known about weight loss maintenance, including patients' perceptions of support received and desired.

METHODS: We conducted a secondary analysis of participant feedback data from the Maintaining Activity and Nutrition through TechnologyAssisted Innovation in Primary Care (MAINTAIN-pc), a randomized controlled trial of an electronic health record (EHR)-based intervention for weight loss maintenance. Participants were randomized into coaching or tracking groups. Both had access to EHR tools (flowsheets for tracking weight, diet, and physical activity, standardized surveys, and reminders) via the patient portal. The coaching group received personalized coaching via the EHR for two years. The primary care providers (PCP) of coaching group participants received real-time progress reports and notification of significant weight gain or loss. Participants provided feedback at 6, 12, 24, and 30 months. We evaluated responses about EHR ease of use and perceptions of sources of support after intervention (i.e., months 25-30) in both groups and preferences for communication and connectedness with coaches in the coaching group.

RESULTS: 136 (73.5\%) participants completed feedback at 24 months and $115(62.2 \%)$ at 30 months. Respondents were $74 \%$ female and $88 \%$ white. The majority of participants $(78.7 \%)$ indicated the EHR was "easy to use" ( $80 \%$ coaching; $77.3 \%$ tracking; $p=1.0$ ). Many coaching participants $(44.3 \%)$ preferred communicating through the EHR alone. However, additional 'in-person communication' was preferred by $41.4 \%$ of the coaching group (27.6\% individual, $31.0 \%$ group and $41.4 \%$ either). In addition, $71 \%$ of coaching group participants selected their coach as a source of support and $54.8 \%$ stated a moderate/strong connectedness with coach. PCPs were viewed as a source of support in $38.7 \%$ of coaching and $22.6 \%$ of tracking group $(\mathrm{p}=0.1)$ and health professionals ( $\mathrm{PCP}$ and other health care professionals (e.g., coaches, nurses)) were viewed as a source of support in $41.9 \%$ of the coaching group and $22.6 \%$ in tracking $(\mathrm{p}=0.046)$. The perception that 'no support was required after intervention' was indicated by $24.2 \%$ coaching and $24.5 \%$ tracking $(p=1.0)$. Finally, reported use of commercial online programs after intervention was low (4.8\% coaching; $7.5 \%$ tracking; $\mathrm{p}=0.83$ ).

CONCLUSIONS: An EHR-based format for a weight maintenance loss intervention is appropriate based on feedback about ease of use and perceptions of coach support and connectedness. Coaching participants were more likely to feel supported by a health professional than those in the tracking group. However, coaching participants also indicated a desire for in-person support in addition to online coaching. Future weight maintenance interventions may consider adding optional in-person support to remote communication.

\section{PERCEPTIONS AND PERFORMANCE OF LEARNERS IN AN INTERPROFESSIONAL COLLABORATIVE PRACTICE MOD-} EL

Alanna J. Diamond ${ }^{1}$; Stephanie Cornell ${ }^{1}$; Glennette Castillo ${ }^{1}$; Erika Bow$\mathrm{en}^{4}$; Lauren S. Penney ${ }^{5}$; Luci Leykum ${ }^{3}$; Temple Ratcliffe ${ }^{2}$. University of Texas Health - San Antonio, San Antonio, TX; ${ }^{2}$ UTHSCSA, San Antonio, TX; ${ }^{3}$ STVHCS / UTHSCSA, San Antonio, TX; ${ }^{4}$ UT Health San Antonio, San Antonio, TX; ${ }^{5}$ Veterans Health Administration, South Texas Veterans Health Care System (STVHCS), San Antonio, TX. (Control ID \#3184203)

BACKGROUND: With increased focus on Interprofessional education (IPE) and interprofessional collaborative practice (IPCP), more settings are implementing these care models. While patients appreciate IPCP, learner acceptance has been more variable. In 2015, we initiated an IPCP care model (Collaborative Care - CC), which incorporates nurses, physicians, students, pharmacists, social workers, physical therapists, patients and families. Early learner feedback noted improved communication with patients, families, and other professions, but also reflected educational concerns regarding medical knowledge and teaching on rounds. We enhanced orientation for residents and medical students, and after building our experience with CC, revisited learner perceptions in 2017. We expected learner perceptions of their educational experiences would improve. We also examined student NBME exam scores between traditional and $\mathrm{CC}$ teams to assess whether differences in medical knowledge as measured by NBME scores existed.

METHODS: We conducted semi-structured learner interviews and analyzed NBME scores. Interviews were conducted between August and October 2017. All interviews were recorded, transcribed, coded and 
inductively analyzed using MAXQDA software by three trained coders (AC, SC, GC). Themes were developed and compared to 2015. NBME Internal Medicine subject exam scores were compared between medical students on CC teams and non-CC teams from January 2015 through July 2018 using Student's t-test (Stata IC, version 15).

RESULTS: Interviewees included seven medical students (39\%), eight PGY-1 residents (44\%) and three upper level (PGY-2, PGY-3) residents $(17 \%)$. Themes previously found in the 2015 interviews were reiterated in 2017, including improved communication between patients and providers, feelings of uncertainty during new situations, strategies for adaptation, and loss of educational opportunities during rounds. Mean NBME scores were 75.82 for medical students on IPCP teams $(\mathrm{N}=103)$ and 76.32 for medical students on non-IPCP teams $(\mathrm{N}=661)$. No significant difference between means was found $(\mathrm{p}=0.57)$.

CONCLUSIONS: Learner concerns about educational loss in IPCP settings persisted despite equivalent NBME performance. While learners appreciated increased opportunities to improve communication skills with patients and other health professionals, they perceived these opportunities as trade-offs with profession-specific knowledge acquisition. While these concerns are not unique to IPCP (similar concerns have been reported in other profession-specific bedside rounding interventions), they are complex and difficult perceptions to improve. More work should be done to make evaluation of IPCP competencies similarly robust and valued compared with evaluation of profession-specific competencies including medical knowledge. Hidden curricular lessons regarding the relative decreased value of interprofessional competencies may also need to be addressed.

\section{PERCEPTIONS OF SOCIAL DETERMINANTS OF HEALTH AMONG INTERNATIONAL MEDICAL GRADUATES: A QUALI- TATIVE STUDY}

Raman Singhal; Aathira Sreenivasan; Sumaira Zareef; Grace Kajita. Montefiore Medical Center, Bronx, NY. (Control ID \#3184880)

BACKGROUND: In 2018, non-U.S. citizen International Medical Graduates (IMGs) filled over a quarter of internship slots in categorical internal medicine programs in the U.S. Little evidence exists to guide physician educators in teaching IMGs about the unique barriers to care faced by underserved communities in the U.S. It also remains unknown to what extent IMGs are aware of and interested in addressing the social determinants of health $(\mathrm{SDH})$ with their patients in the U.S.

METHODS: In this qualitative study, PGY2 IMG's from an Internal Medicine residency program in Bronx, NY were interviewed in one-hour long, semi-structured focus groups of 6 residents each. Interview questions sought to compare patient characteristics and health care delivery in home countries and the U.S., to understand how previous experiences affect IMGs' approach to patient care in the U.S. Perspectives on SDH in the U.S. were also elicited during focus groups, based on their one year of completed training here. Interviews were audio-recorded and professionally transcribed. Transcripts were coded and analyzed by 4 researchers, using a thematic analysis approach.

RESULTS: 24 PGY2 IMG residents (100\%) agreed to participate. Of these, $75 \%$ attended medical school in South Asia. Three overarching themes emerged. First, participants expressed judgments that U.S. patients do not value their health and mistrust doctors, unlike patients in their home countries: "(U.S. patients) have so many privileges and still, they don't take care of themselves. They are taking things for granted, and it's kind of frustrating sometimes."; "This was shocking to me when I came over here. People do not respect doctors at all. They think (we) are just trying to suck money out of their pockets and prolong their illness." Second, participants perceived social determinants of health to be less severe in the U.S. compared to their home countries: "The Bronx population may be poor, but people don't starve to death...back home, people do die of hunger." Third, participants were concerned that the responsibility for good health falls solely on the individual in the U.S. and acknowledge that patients may face various obstacles: "The reason they're missing appointments is because they're having two jobs or they're single parents taking care of their children, barely enough time to sleep." This is in stark contrast to the rich family support that is the norm for patients in home countries.

CONCLUSIONS: IMG resident patient care in the U.S. is strongly experienced in the context of patient care in home countries. IMGs are aware of $\mathrm{SDH}$ in both locations, and comparisons are commonplace. Although SDH are viewed as less prominent barriers to care in the U.S., residents are also able to empathize with the difficulties U.S. patients face in taking care of their health. This needs assessment will inform a curriculum in SDH tailored for IMG trainees, which will help them understand the scope of SDH specific to the U.S. and develop skills to assess and manage SDH.

\section{PERSPECTIVE-TAKING IN MEDICAL EDUCATION: A QUALI- TATIVE ANALYSIS OF INTERNAL MEDICINE RESIDENTS' AT- TITUDES TO A NOVEL CURRICULUM}

Brianna Rossiter ${ }^{1}$; Melissa McNeil ${ }^{1}$; Gaetan Sgro ${ }^{2} .{ }^{1}$ University of Pittsburgh Medical Center, Pittsburgh, PA; ${ }^{2}$ VA Pittsburgh Healthcare System, Pittsburgh, PA. (Control ID \#3186245)

BACKGROUND: Residency is marked both by intense knowledge acquisition, personal growth, and by increasing levels of burnout and cynicism. The latter erode empathy and undermine the altruistic motivations that led to a medical career path. Interactions with so-called "challenging" patients in the ambulatory setting are often cited as drivers of burnout and cynicism. Perspective-taking (PT) is a cognitive skill defined as "an understanding of other people's mental states" and has been studied as a way to cultivate empathy. The purpose of this study was to explore internal medicine residents' attitudes to the implementation of a PT exercise in an ambulatory curriculum. METHODS: The PT curriculum took place within the PGY3 Ambulatory rotation of the UPMC Internal Medicine Residency, which all PGY3 residents complete as part of their training. The curriculum included the PT writing exercise itself and a facilitator-guided, de-brief session. Residents were then invited to participate in an interview to explore their attitudes to the curriculum. Two researchers developed a code book based on the interviews, coded, and assessed inter-rater reliability. Thematic analysis was completed with consultation of a qualitative research group.

RESULTS: Thirteen residents completed interviews and were analyzed. Preliminary emerging themes include: appreciation for a novel approach to understanding challenging patients, ambivalence over the best way to engage in self-reflection in medical training, finding value in co-residents' contribution to the de-brief session, identification of PT as a way navigate challenging patient interactions, and defining empathy as an extremely important characteristic of a physician.

CONCLUSIONS: A clinical experience in PT, encouraging resident reflection, provides a novel approach for probing empathy, a challenging construct to teach in medical education. Residents appreciated the opportunity to reflect about challenging patient encounters and found value in de-briefing with their peers. These attitudes support the need for future work in using PT as a cognitive means for maintaining empathy in medical training.

\section{PERSPECTIVES ON DEPRESCRIBING CARDIOVASCULAR MEDICATIONS: A NATIONAL SURVEY OF INTERNISTS, CAR- DIOLOGISTS, AND GERIATRICIANS}

Timothy Anderson ${ }^{1}$; Parag Goyal $^{3}$; Michael A. Steinman ${ }^{2}$; Michael W. Rich ${ }^{4}$. ${ }^{1}$ University of California, San Francisco, San Francisco, CA; ${ }^{2}$ SF VA Medical Center, San Francisco, CA; ${ }^{3}$ Weill Cornell Medicine, New York, NY; ${ }^{4}$ Washington University School of Medicine in St. Louis, St. Louis, MO. (Control ID \#3186031)

BACKGROUND: Polypharmacy in older adults is prevalent and harmful. The practice of deprescribing to reduce harms from unnecessary medication use is an increasingly recognized approach to comprehensive, patient-centered care for older adults. Deprescribing may encompass stopping cardiovascular (CV) medications including statins, antiplatelets, and antihypertensives.

METHODS: To understand physician perspectives on deprescribing CV 
medications and how they may differ between specialties we conducted a cross-sectional survey of internists, cardiologists and geriatricians drawn from a random sample of members of the American College of Physicians (JuneAugust 2018). Physicians were surveyed on current deprescribing practices, reasons for deprescribing, barriers to deprescribing, and discussion of deprescribing with other providers. Respondents were asked to identify medications they would consider deprescribing in hypothetical clinical cases. RESULTS: The response rate varied between specialties: $26 \%$ for internists (182/771), $12 \%$ for cardiologists (87/717) and 26\% for geriatricians (184/ 711). Demographics were similar across groups except for fewer female respondants among cardiologists. In the month prior to being surveyed $83 \%$ of internists, $93 \%$ of geriatricians and $85 \%$ of cardiologists reported considering stopping a $\mathrm{CV}$ medication for a patient. However, all specialties were less like to report discussing discontinuing a $\mathrm{CV}$ medication with another provider $(39 \%, 55 \%$, and $41 \%$ respectively). Reported reasons for deprescribing were highly variable, with the most commmon reasons being medication side effects ( $>60 \%$ of respondents for all specialties) and among geriatricians limited life expectancy ( $>75 \%$ of respondants). Less frequently identified reasons for deprescribing included medication cost, polypharmacy, patient request, and lack of medication indication (all $<30 \%$ ). The most common barriers reported were similar across specialties and included concerns of patient reluctance and concerns of interfering with other physicians' treatment plans. In hypothetical cases, specialties were similarly likely to stop medications were patients reported specific side effects. Compared to internists, cardiologists were less likely and geriatricians were more likely to consider stopping CV medications when patients reported no concerns, a desire to decrease pill burden, a new diagnosis of metastatic malignancy, or a new diagnosis of dementia.

CONCLUSIONS: The majority of internists, cardiologists, and geriatricians reported consideration of deprescribing $\mathrm{CV}$ medications in their recent clinical practice, however reasons for deprescribing varied between groups which may reflect differing professional society guidelines. Common barriers included concerns of patient reluctance and concerns of interfering with other physicians' plans. These results indicate the need for additional efforts to develop communication strategies to improve the uptake of deprescribing.

\section{PHARM-MD; AN OPEN-LABEL, RANDOMIZED CONTROLLED PHASE II STUDY TO EVALUATE THE EFFICACY OF A PHAR- MACIST MANAGED DIABETES CLINIC IN HIGH-RISK DIABE- TES PATIENTS}

Alexandra Halalau ${ }^{1,2}{ }^{2}$ Jordan Bushman ${ }^{1}$; Jaspreet Hehar ${ }^{1}$; Janna Fett ${ }^{1}$; Scott Keeney ${ }^{3}$; Daniel Shelden ${ }^{4} .{ }^{1}$ Beaumont Health, Troy, MI; ${ }^{2}$ Oakland University William Beaumont School of Medicine, Rochester, MI; ${ }^{3}$ Baylor Scott \& White, Kileen, TX; ${ }^{4}$ University of Michigan, Ann Arbor, MI. (Control ID \#3165157)

BACKGROUND: Over 30 million Americans are currently living with diabetes and approximately 1.5 million cases are being diagnosed each year. The United States lags behind other developed countries on diabetes life expectancy and disease-related deaths. Previous studies demonstrated that for each $1 \%$ reduction in hemoglobin A1c (HbA1c), there was a corresponding 14\% reduction in myocardial infarction, $12 \%$ reduction in stroke, and a $37 \%$ reduction of microvascular complications. Our retrospective data showed that patients who received care in a pharmacy managed diabetes clinic (PMDC) had a decrease at six months in HbAlc of $3.2 \%$ versus $1.2 \%$ in the standard care cohort $(\mathrm{p}=0.044)$. Our hypothesis is that a PMDC will have a significant positive impact on diabetes measures and will result in higher quality care at a lower price.

METHODS: This phase II trial is currently enrolling patients and randomly assigning them to one of two groups: standard of care (SOC) or SOC+PMDC. The PMDC consists of six extra visits with the pharmacists that are focused on patient-identified diabetes management gaps, goals and medication adjustments. Inclusion criteria are: patients older than 18 years of age with a diagnosis of type 2 diabetes mellitus and a $\mathrm{HbA} 1 \mathrm{c}>9 \%$, who are established with a primary care resident and who have not been seen in the PMDC within the last 3 months. A sample size of 86 patients was calculated to detect a 1.0 mean difference in $\mathrm{HbAlc}$ between the groups with $80 \%$ power at a $\mathrm{p}<0.05$ significance. The primary outcome is the change in HbAlc, measured at 3, 6 and 12 months. Secondary outcomes include the impact on all diabetes core measures, patient quality of life, harms and cost impact related to the intervention.

RESULTS: At the time of preliminary analysis, 54 patients were enrolled, 28 in the intervention group and 26 in the control group. Baseline characteristics were not significantly different between the two groups. Mean $\mathrm{HbA} 1 \mathrm{c}$ at baseline was 11.5 in the intervention group and 11.0 in the control group. At 3 months, the mean $\mathrm{HbAlc}$ decreased to 8.0 in the intervention group versus 9.3 in the control group. Difference in means from baseline to 3 months is -3.5 and -1.7 in intervention and control group, respectively. Retinopathy screening was performed in 12 v. 18 patients in the intervention and control groups, respectively $(\mathrm{p}=0.045)$. No significant differences in remaining secondary outcomes were observed. CONCLUSIONS: If our study demonstrates improved patient outcomes with the utilization of pharmacists, a PMDC may become an integral part in the care of high-risk diabetic patients. This may help reduce the economic burden of the disease, improve life expectancy and disease related deaths. Complete data analysis will be presented at the conference.

PHARMACOTHERAPY FOR COCAINE USE DISORDER - A SYSTEMATIC REVIEW AND META-ANALYSIS

Brian Chan $^{1,2}$; Karli Kondo ${ }^{4,3}$; Michele Freeman ${ }^{4}$; Chelsea K. Ayers ${ }^{4}$; jessica Montgomery ${ }^{4}$; Robin Paynter ${ }^{4}$; Devan Kansagara ${ }^{3,}{ }^{4}$. ${ }^{1} \mathrm{OHSU}$, Portland, OR; ${ }^{2}$ Central City Concern, Portland, OR; ${ }^{3}$ Portland VA Medical Center, Portland, OR; ${ }^{4}$ Portland VA Healthcare System, Portland, OR. (Control ID \#3186054)

BACKGROUND: Currently there are no accepted FDA-approved pharmacotherapies for cocaine use disorder (CUD), though numerous medications have been tested in clinical trials. We conducted a systematic review and meta-analysis of the effectiveness of pharmacotherapy for cocaine use disorder.

METHODS: We searched multiple data sources (MEDLINE, PsycINFO, and Cochrane Library) through November 2017 for systematic reviews and randomized controlled trials (RCTs) of pharmacological interventions in adults with CUD. We combined the findings of trials with comparable interventions and outcome measures in random-effects meta-analyses. We assessed the risk of bias of individual trials and the strength of evidence for each outcome using standardized criteria. Outcomes of interest comprised abstinence, defined as 2 or more consecutive weeks with negative urine drug screens (UDS); overall use, analyzed as the proportion of UDS specimens that were negative; and retention, defined as the proportion of randomized patients who completed treatment.

RESULTS: We found 7 systematic reviews and 47 RCTs that met inclusion criteria studying 66 different drugs or drug combinations. Antidepressants were the most widely studied drug class (38 RCTs) but appear to have no effect on cocaine use or treatment retention. Increased abstinence was found with bupropion (2 RCTs: RR 1.63, 95\% CI 1.02 to 2.59), topiramate (2 RCTs: RR $2.56,95 \%$ CI 1.39 to 4.73 ), and psychostimulants (14 RCTs: RR 1.36, 95\% CI 1.05 to 1.77), though the strength of evidence for these findings was low. Antipsychotics improved treatment retention ( 8 RCTs: RR $1.33,95 \%$ CI 1.03 to 1.75 ) and the strength of evidence was moderate.

CONCLUSIONS: Most of the pharmacotherapies studied were not effective for treating CUD. Bupropion, psychostimulants, and topiramate may improve abstinence, and antipsychotics may improve retention. Future trials of medications with behavioral interventions may be warranted.

PHARMACOTHERAPY FOR METHAMPHETAMINE/ 
AMPHETAMINE USE DISORDER - A SYSTEMATIC REVIEW AND META-ANALYSIS

Brian Chan ${ }^{1,}{ }^{2}$; Karli Kondo ${ }^{4}$; Michele Freeman ${ }^{4}$; Chelsea K. $\overline{\text { Ayers }^{4} \text {; jessica Montgomery }}{ }^{4}$; Robin Paynter ${ }^{4}$; Devan Kansagara ${ }^{3}$, ${ }^{4}$. ${ }^{1}$ OHSU, Portland, OR; ${ }^{2}$ Central City Concern, Portland, OR; ${ }^{3}$ Portland VA Medical Center, Portland, OR; ${ }^{4}$ Portland VA Healthcare System, Portland, OR. (Control ID \#3186086)

BACKGROUND: Addiction to methamphetamine/amphetamine (MA/A) is an increasing public health problem. Currently, there are no accepted FDA-approved pharmacotherapies for MA/A use disorder, though numerous medications have been tested in clinical trials. We conducted a systematic review and meta-analysis of the effectiveness of pharmacotherapy for MA/A use disorder.

METHODS: We searched multiple data sources (MEDLINE, PsycINFO, and Cochrane Library) through November 2017 for systematic reviews (SRs) and randomized-controlled trials (RCTs) of pharmacological interventions in adults with MA/A use disorders. The outcomes of interest comprised abstinence, defined as 2 or more consecutive weeks with negative urine drug screens (UDS); overall use, analyzed as the proportion of UDS specimens that were MA/A-negative; and retention, defined as the proportion of randomized patients who completed treatment. We combined the findings of trials with comparable interventions and outcome measures in random-effects meta-analyses. We assessed the risk of bias of RCTs, and the overall strength of evidence for each outcome using standardized criteria.

RESULTS: 14 RCTs and 1 SR of pharmacotherapies for MA/A use disorder met our inclusion criteria. 14 different drugs were studied, including antidepressants, antipsychotics, psychostimulants, anticonvulsants, and opioid antagonists Although there was no evidence of benefit for psychostimulants overall, there was low strength evidence from 2 RCTs that methylphenidate may reduce MA/A use. Antidepressants had no effect on abstinence or retention based on moderate to high strength evidence. Studies of anticonvulsants, antipsychotics (aripiprazole), and opioid antagonists (naltrexone) provided either low strength evidence of no effect on the outcomes of interest, or insufficient evidence to draw conclusions. Many of the studies had high or unclear risk of bias.

CONCLUSIONS: Most pharmacotherapies were ineffective in treating MA/A use disorder. Novel pharmacotherapies specific to the neurobiology of MA/A use disorders need to be developed to supplement existing behavioral interventions for this emerging epidemic.

\section{PHYSICIAN CHARACTERISTICS CORRELATE WITH PA- TIENT READMISSION RATES}

Eileen Hennrikus ${ }^{1}$; Michael Skolka ${ }^{2}$; Mallory Schaffer ${ }^{3} .{ }^{1}$ Penn State College of Medicine, Hershey, PA; ${ }^{2}$ Penn State Health, Hershey, PA; ${ }^{3}$ University of Pittsburgh, Pittsburgh, PA. (Control ID \#3163053)

BACKGROUND: The 7- and 30-day patient readmission rates are tracked by hospitals and are used as a metric to measure physicians' quality of patient care. Several tools currently predict and prevent early readmission rates based on patient-specific characteristics. However, few studies have demonstrated if physician-specific characteristics play a role. The purpose of this study is to assess if physician characteristics correlate with patient readmission rates.

METHODS: In a five-year retrospective chart review, 31 internal medicine physicians and their hospital discharges were recorded for a total of 15,933 hospital discharges at one academic institution. Each physician's yearly 7-day, 8-30day (excluding the first 7 days), and 30-day readmission rates was compared to all the other physicians' rates. Each readmission rate was also correlated with years of post-graduate clinical experience, patient discharge volume, physician gender, and fiscal year. All analyses were performed using SAS 9.4.

RESULTS: The 7-day readmission rates did not correlate with the 8-
30 day rates $(\mathrm{p}=0.155)$. Individual physicians had significantly different rates for all readmission categories $(\mathrm{p}=0.001-0.010)$. However, the rates were not related to gender or years after post-graduate training. Physician patient volume was the only identified characteristic that significantly correlated with readmission rates. Physicians who discharged 100 patients in a year had a higher 7-day readmission rate, $8.24 \%(6.76,9.71)$, compared to physicians who discharged 101-200 (5.75\%, $\mathrm{p}=0.047)$, $201-300(5.83 \%, \mathrm{p}=0.033)$, or $301(5.49 \%, \mathrm{p}=0.024)$ patients in a year. The volume of patient discharges did not influence physician 8-30 day readmission rates.

CONCLUSIONS: Individual physician characteristics statistically correlate with patient readmission rates. A critical level of a physician's yearly hospital activity, as reflected by the number of patient discharges per year (>100) significantly results in lower 7-day readmission rates. Gender, post-graduate years of clinical experience, and fiscal year were not signigicant. Physician hospital activity did not affect 8-30 day readmissions, suggesting that the unidentified physician characteristics that influence the 8-30 day readmission rate are different than those physician characteristics that influence the more immediate 7-day readmission rates.

\section{PHYSICIAN HOME VISITS AFTER HOSPITAL DISCHARGE: A PILOT RANDOMIZED CONTROLLED TRIAL}

David M. Levine ${ }^{1}$; Kei Ouchi ${ }^{1}$; Mary R. Paz ${ }^{3}$; Kimberly P. Burke ${ }^{2}$; Jeffrey L. Schnipper ${ }^{1}$. 'Brigham and Women's Hospital, Boston, MA; ${ }^{2}$ Brigham and Women's Hospital, Brighton, MA; ${ }^{3}$ Brigham and Women's Hospital, BOSTON, MA. (Control ID \#3184860)

BACKGROUND: Transition from acute care to home is a time marked by new or worsening symptoms, readmission, and adverse events. Many interventions have attempted to improve the transition to home, including nurse, nurse practitioner, and pharmacist home visits, with inconsistent results. To our knowledge, no recently evaluated intervention has involved a physician home visit. Decades ago, physician home visits formed the backbone of care for the most vulnerable patients. We hypothesized that a physician home visit following discharge would reduce new or worsening symptoms and readmission.

METHODS: We performed a pilot randomized controlled trial of a single physician home visit within 4 days of discharge from an acute care episode vs usual care. We enrolled patients who were being discharged to home and whose inpatient care team felt for any reason a physician home visit would be of benefit. Patients had to live within 5 miles of the hospital. After enrollment, patients were randomized 1:1 to a physician home visit vs usual care. The content of the home visit was left entirely to the discretion of the physician, although s/he was provided materials for best practices in home visits and medication reconciliation based on prior research. The primary endpoint was having at least one new or worsening symptom at 30-days post-discharge, captured by a structured phone interview used in prior research. Our secondary endpoints included 30day readmission, 30-day emergency department (ED) utilization, 14-day PCP utilization, and readiness to transition via the Care Transition Measure (CTM)-3. We performed an intention-to-treat analysis and compared groups by Fisher exact test.

RESULTS: We enrolled 51 patients (26 home visits; 25 controls). Patients who received home visits had a median age of $65,65 \%$ were female, $81 \%$ underrepresented minorities, and $35 \%$ had less than a high school education. The control group was similar, except it had fewer underrepresented minorities $(68 \%)$. There was no difference in new or worsening symptoms at 30-days between the home visit (44\%) and control (42\%) group ( $\mathrm{p}=0.66$ ). The home visit group had nonsignificant trends toward fewer 30-day readmissions ( $23 \%$ vs $36 \%$, p=0.37), fewer 30-day ED presentations ( $8 \%$ vs $12 \%, \mathrm{p}=0.42$ ), and more often saw their PCP within 14 days ( $31 \%$ vs $25 \%$; $\mathrm{p}=0.39$ ). The CTM-3 rating was the same for both groups (median, 9 out of 12). Nine intervention patients $(35 \%)$ had medication changes as a result of their physician visit. 
CONCLUSIONS: In this pilot randomized controlled trial of a physician home visit following discharge from the hospital, patients who received a visit had a nonsignificant trend toward reduced readmission and ED presentation and better follow-up with their PCP, but no difference in self-reported symptoms or readiness to transition. To our knowledge, this is the first test of a physician home visit following discharge. Refinement of the intervention and replication in a larger cohort is needed.

\section{PHYSICIAN SATISFACTION WITH HEALTH PLANS: RE-} SULTS FROM A NATIONAL SURVEY

Natasha Parekh $^{1,2}$; Sheryl Savage ${ }^{3}$; Amy Helwig ${ }^{4}$; Patrick Alger ${ }^{3}$; Sandra McAnallen ${ }^{4}$; William H. Shrank ${ }^{4}$. ${ }^{1}$ University of Pittsburgh Medical Center, PIttsburgh, PA; ${ }^{2}$ UPMC Center for High-Value Health Care, Pittsburgh, PA; ${ }^{3} \mathrm{SPH}$ Analytics, Alpharetta, GA; ${ }^{4} \mathrm{UPMC}$ Insurance Services Division, Pittsburgh, PA. (Control ID \#3184380)

BACKGROUND: Physician satisfaction is associated with patient satisfaction, adherence to treatment recommendations, and quality. However, burnout is prevalent, and physician experience with health plans is likely a key contributor. Our existing knowledge of physician satisfaction with health plans, however, is limited, mixed and outdated. We therefore explored physician satisfaction with health plans, and assessed which physician and plan characteristics are associated with greater satisfaction. METHODS: We conducted a cross-sectional analysis of de-identified physician satisfaction surveys for US health plans in 2016. We included the following satisfaction domains that were rated by physicians on 5-point Likert scales as key outcomes of interest: overall health plan rating; financial issues; utilization and quality management; network/coordination of care; pharmacy issues; call center experience; provider relations; and recommendation of the sponsor plan to other physicians' practices. We assessed the association between the following characteristics and outcomes of interest using multivariable linear regression, weighted by the number of respondents: vertical (payer-provider) integration status, health plan size, practice size, provider type, and years of practice.

RESULTS: We analyzed surveys from 3,158 physicians on 74 health plans. We observed highest satisfaction in overall plan rating, finance, and call center domains (adjusted means 3.25), and lowest satisfaction in pharmacy (adjusted mean 3.02). The largest and smallest plans and vertically integrated plans had the highest satisfaction: $76 \%$ of physicians recommended vertically integrated plans while $66 \%$ of physicians recommended non-vertically integrated plans to others $(\mathrm{p}<0.001)$. Solo practitioners rated overall plan rating, finance, utilization/quality management and pharmacy domains more favorably than physicians in larger practices $(\mathrm{p}<0.001)$, while primary care physicians rated overall plan rating, finance, and utilization/quality management more favorably than specialists $(\mathrm{p}<0.001)$.

CONCLUSIONS: Our findings demonstrate that there is significant opportunity to improve physician satisfaction with health plans in all satisfaction domains, especially in pharmacy/formulary management. Since provider satisfaction is becoming increasingly recognized as a critical outcome, our findings suggest that health plans have a unique and important opportunity to improve physician satisfaction and highlight potential interventions such as prioritizing provider relationships, optimizing formulary management, reducing administrative burden, and providing resource support. Additionally, interventions could specifically target physicians in larger practices and nonvertically integrated and mid-size health plans. As physicians are required to adapt to a rapidly transforming healthcare landscape, it will be imperative for health plans to prioritize physician satisfaction moving forward.

PHYSICIAN-PHARMACIST COLLABORATIVE CARE MODELS TO PREVENT OPIOID MISUSE: A PILOT STUDY

Pooja A. Lagisetty ${ }^{1}$; Alex Smith $^{1}$; Suzanne Winters ${ }^{1}$; Michael A. Smith ${ }^{1}$; Hae Mi Choe'; Amy Bohnert ${ }^{1}$; Michele Heisler ${ }^{2} .{ }^{1}$ University of Michigan, Ann Arbor, MI; ${ }^{2}$ Ann Arbor VA/University of Michigan, Ann Arbor, MI. (Control ID \#3184050)

BACKGROUND: Clinical pharmacists embedded within primary care clinics have the potential to help manage medications used to treat chronic pain and opioid use disorders (OUD). However, their current scope of practice mainly consists of managing diseases such as diabetes and hypertension. Therefore, we sought to pilot and evaluate the feasibility and acceptability of a physician-pharmacist collaborative care model to assist with chronic pain and OUD management.

METHODS: The program consisted of an in-person pharmacist consultation as well as optional follow-up phone or in-person visits over a 4-month period in 2 large academic primary care practices. Eligible patients had chronic pain + a long-term prescription for opioids or for buprenorphine. The pharmacist reviewed participants' past pharmacologic and non-pharmacologic treatments and discussed the risks and benefits of the current medication regimen and potential alternatives. Recommendations were communicated back to the PCP via the electronic health record (EHR). Due to controlled substance regulations, the pharmacist did not have any prescriptive authority. We used mixed-methods to evaluate the model. These included baseline and follow up participant survey assessments on pain, co-morbid substance abuse disorders (SUDs, ED utilization, and naloxone and buprenorphine knowledge; EHR review of number of phone calls and clinic visits focused on opioids; and semi-structured interviews with 8 providers (7 MD and $1 \mathrm{PharmD}$ ) to assess feasibility and acceptability.

RESULTS: Between January and October 2018, 47 of the 182 patients who met eligibility criteria enrolled in the intervention, and 46 completed all follow-up assessments. Mean age was 55.8 yrs $(\mathrm{SD}=11.9)$, and $55 \%$ were women. 43 (91\%) of patients had received opioids over the past 6 months. The pharmacist recommended tapering opioids to $3(6 \%)$ of patients, adding or changing to a non-opioid pain medication to $30(64 \%)$ patients, and switching to buprenorphine for pain management in $20(43 \%)$ of patients. The number of patient calls and visits related to pain decreased from 10.4 during the 4 months prior to the initial pharmacist visit to 8.9 in the 4 months after that visit $(\mathrm{p}=0.059)$. Physicians found the intervention acceptable but wanted more guidance and support on using buprenorphine for pain. Patients felt that the in-depth conversations were helpful, but some reported lack of physician follow up to recommended medication changes.

CONCLUSIONS: This study demonstrated that using clinical pharmacists for patients with chronic pain and OUD management is feasible, acceptable, and shows promise in decreasing healthcare utilization related to opioid management. Policy changes to increase pharmacist prescriptive authority to co-manage controlled substances may increase physician willingness and confidence to carry out opioid tapers and prescribe buprenorphine.

\section{POINT-OF-CARE MOBILE APPLICATION TO GUIDE HEALTH PROFESSIONALS IN CONDUCTING SUBSTANCE USE SCREEN- ING AND INTERVENTION: A MIXED-METHODS USER EXPERI- ENCE STUDY}

Sandeep Kapoor $^{1,2}{ }^{\text {; }}$ Megan O'Grady ${ }^{3}$; Evan Gilmer ${ }^{3}$; Charles J. Neighbors ${ }^{3}$; Nancy Kwon ${ }^{4,2}$; Joseph Conigliaro ${ }^{1,2}$; Jonathan Morgenstern ${ }^{5,2}$. ${ }^{1}$ Northwell Health, New Hyde Park, NY; ${ }^{2}$ Zucker School of Medicine at Hofstra/ Northwell, Hempstead, NY; ${ }^{3}$ National Center on Addiction and Substance Abuse, New York, NY; ${ }^{4}$ Northwell Health - Long Island Jewish Hospital, New Hyde Park, NY; ${ }^{5}$ Northwell Health, Great Neck, NY. (Control ID \#3186087)

BACKGROUND: Well-documented barriers have limited the widespread, sustained adoption of screening and intervention for substance use problems in healthcare settings. Mobile health (mHealth) applications may address provider-related barriers; however, there is limited research on development and user experience of such applications. Despite being a major public health issue, substance use remains under addressed in healthcare settings. A 2017 report indicates only one in six binge drinkers in the US are asked about alcohol use and advised to cut down by a health professional. Screening, Brief Intervention, and Referral to Treatment (SBIRT) is a model that can be used in healthcare settings to identify and address risky substance use. Evidence for SBIRT efficacy has been demonstrated with the strongest evidence coming from studies conducted in primary care that target reductions in alcohol use. 
Better patient outcomes may be achieved by combining technology-based programs with in-person provider intervention. Several studies have shown that computer-guided, provider-delivered substance use interventions have equivalent or even better outcomes than patient self-administered computerized programs. However, most technology-based SBIRT programs described in the literature are patient self-administered and do not directly involve a healthcare provider. Therefore, because providers are an important piece of the puzzle from a clinical and technology adoption standpoint, their input on user experience is essential to the production of clinically relevant providerfocused mHealth SBIRT technology.

METHODS: The SBIRT app was designed for use by providers in conjunction with patients during a healthcare visit to assist the provider in assessing the patient's level of risk due to substances use and walk them through the steps of a brief intervention that is tailored to the patient's responses. This mixed methods study included think-aloud tasks, tasksuccess ratings, semi-structured interviews, and usability questionnaires (e.g., System Usability Scale [SUS]) to examine user experience among 12 health coaches who provide substance use services in emergency department and primary care settings.

RESULTS: The average rate of successful task completion was $94 \%$ and the mean SUS score was 76 out of 100 . Qualitative data suggested the app enhanced participants' capability to complete tasks efficiently and effectively. Participants reported satisfaction with the app's features, content, screen layout, and navigation and felt it was easy to learn, and could benefit patient interactions.

CONCLUSIONS: Overall results indicate acceptable user experience and usability for this provider-focused point-of-care mobile app for substance use screening and intervention as well as favorable potential for adoption by healthcare practitioners. Such mobile health technologies may help to address well-known challenges related to implementing substance use services in healthcare settings.

\section{POLYPHARMACY: PATIENT AND PHYSICIAN CONTRIBU- TION}

Niyati M. Gupta ${ }^{1}$; Bo Hu${ }^{1}$; Luke D. Kim² ${ }^{2}$ Simon W. Lam ${ }^{2}$; Giavanna Russo-Alvarez ${ }^{2}$; Michael B. Rothberg ${ }^{2}$. ${ }^{1}$ Cleveland Clinic Foundation, Cleveland, OH; ${ }^{2}$ Cleveland Clinic, Cleveland, OH. (Control ID \#3180369)

BACKGROUND: Polypharmacy is common among older patients. It is associated with more primary care visits, drug-drug interactions, fallrelated hospitalizations and mortality. Our objective was to study the burden of polypharmacy in an elderly population and identify patient factors associated with it, as well as assess the variation in individual provider's rates of polypharmacy.

METHODS: We conducted a retrospective cohort study of patients aged 65 years who had an ambulatory visit to an internal medicine or family medicine provider in 2015. For each patient, we extracted the following factors: demographics, comorbidities, medications (name, order date, sig and refills), and specialty visits; and details about their primary care provider (PCP). Pearson's chi-square tests were used to assess the association between polypharmacy and each patient factor. We then used linear regression to assess the association between patient factors and total medications. Using our regression model, we compared PCPs' adjusted rates of medication prescribing.

RESULTS: Our sample included 51,093 patients cared for by 681 physicians. Patients' mean age was 75.3 years, $59 \%$ were male, $84 \%$ were white, $33 \%$ had a BMI of $30 \mathrm{~kg} / \mathrm{m}^{2}$; and $47 \%$ were former smokers. Patients took an average of 7.9 medications (median 7, IQR 5 to 10): $24 \%$ patients took $<5,45 \%$ took $5-9,31 \%$ took 10 medications. Compared to patients taking $<10$ medications, those taking 10 medications were older (76 vs. 75 years), more often male (61\% vs. $58 \%$ ), Black (16\% vs. $11 \%$ ), not married ( $44 \%$ vs. $35 \%$ ), obese ( $47 \%$ vs. $34 \%)$, and had more comorbidities ( 8 vs. 4 ), specialists ( 9 vs. 5 ) and specialty visits ( 39 vs. 15 visits in the past 2 years). ( $\mathrm{p}<0.001$ for all comparisons) The following factors were independently associated with number of medications: age $(=0.10$ for age 86-90 years; 0.20 for $>90$ years), BMI ( $=1.2$ and 1.56 for BMI 35 39.9 and $40 \mathrm{~kg} / \mathrm{m}^{2}$ respectively), and Medicaid insurance ( $\left.=1.82\right)$. Organ transplant, cardiac and pulmonary conditions were the comorbidities most strongly associated with polypharmacy ( $=2.0,1.9$ and 1.6 respectively). Of the observed variance in number of medications, demographics accounted for $2 \%$, insurance status for $0.6 \%$ and co-morbidities for $33 \%$. Adjusted mean medications prescribed by physician ranged from 6.5 to 11.4 (median 8.3, IQR 7.7 to 8.9 ). Overall, $18 \%$ of providers had adjusted prescribing rates significantly higher and $17 \%$ had rates significantly lower than the mean.

CONCLUSIONS: Polypharmacy is common in primary care; $31 \%$ patients took 10 medications. Co-morbidities explain only $33 \%$ of the variance in number of medications, and there was extreme variation in physician prescribing rate that could not be explained by patient factors. More study is needed to understand physician attitudes that lead to polypharmacy in the elderly.

POST-DISCHARGE PATIENT-REPORTED DATA ON PAIN, APPETITE, HEART FAILURE SYMPTOMS AND MANAGEMENT AS PREDICTORS OF READMISSION OR ED VISITS

Jinying Chen ${ }^{1}$; Rajani Sadasivam ${ }^{1}$; Amanda C. Blok ${ }^{3}{ }^{1}$; Christine Ritchie $^{2}$; Catherine S. Nagawa ${ }^{1}$; Elizabeth Orvek ${ }^{1}$; Kanan Patel ${ }^{2}$; Thomas Houston ${ }^{1} .{ }^{1}$ University of Massachusetts Medical School, Worcester, MA; ${ }^{2}$ University of California San Francisco, San Francisco, CA; 3Bedford VA Medical Center, Bedford, MA. (Control ID \#3185384)

BACKGROUND: After a hospital discharge, patients with heart failure have higher rates of early repeat acute care use (i.e.: readmission or emergency department (ED) use within 30 days) than other patients. Current efforts on predicting risk of repeat acute care utilization rely on inpatient data from electronic health records. However, many changes occur after the transition to home. Post-discharge patient-generated data relevant to care transitions has had limited exploration. We examine the association between post-discharge patient-generated data and early repeat use of acute care.

METHODS: We used data from 156 patients with index hospitalization for chronic heart failure from a large tertiary hospital. For the first seven days after discharge, using a daily interactive voice response automated phone call to the home of the patient, we collected patient-reported health status, heart failure symptoms (dyspnea and edema), medication management, knowledge of healthcare follow-up plans, and other issues like pain and poor appetite. These patient-reported data from automated calls was reviewed by care transition nurses to determine whether a management call was required. Patients were also followed for 30 days after discharge to assess repeat acute care utilization. We examined the association between each patient-reported predictor variable and the 30-day outcome by using multivariable logistic regression, adjusting for race, marital status, disease burden, and also participation (number of automated phone calls completed) and the number of transition nurse's management calls.

RESULTS: Patients were on average 63 years old; $51 \%$ were AfricanAmerican, $52 \%$ were women, and $18 \%$ had less than high school education. Most patients (147 (94.2\%)) responded to all the automated phone surveys. At the end of 30-days post discharge, 30 (19\%) patients had again used acute care. After adjustment, patient-reported dyspnea, edema, medication management issues, and lack of understanding of follow-up were not associated with early acute care use. Overall health status (OR: $3.5 ; 95 \%$ CI: $1.1,11.6)$, pain (OR: $2.6 ; 95 \%$ CI: $1.1,6.2)$, or poor appetite (OR: 2.8 ; $95 \%$ CI: $1.1,7.4)$ were positively associated with 30 -day acute care utilization. Among 58 reports of pain in follow-up nursing notes, $2(3 \%)$ were cardiac pain, 39 (67\%) were non-cardiac pain (e.g.: leg pain, abdominal pain, knee pain, back/hip pain and headache), and 17 (29\%) with undetermined sources.

CONCLUSIONS: Patient-reported poor health status, pain (frequently non-cardiac), and poor appetite were positively associated with 30-day acute care utilization, and surprisingly, associations were stronger for these 
patient-reported data than for the common heart failure warning signs. These novel post-discharge markers require further study and consideration for incorporation into risk prediction to drive quality improvement efforts.

\section{POST-DISCHARGE RESOURCE USE AND 30-DAY UNPLANNED HOSPITAL READMISSIONS IN PATIENTS ADMITTED FOR HEART FAILURE}

Molly J. Horstman ${ }^{1,2}$; Javad Razjouyan ${ }^{1}$; Jacquelene Shahin ${ }^{2}$; Aanand D.

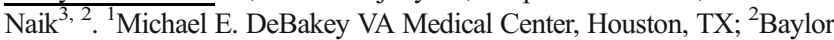
College of Medicine, Houston, TX; ${ }^{3}$ Michael E. DeBakey VAMC, Houston, TX. (Control ID \#3186013)

BACKGROUND: Patients have the opportunity to interact with the healthcare system following discharge using a range of communication methods. This study seeks to examine the role of different types of postdischarge encounters on unplanned hospital readmissions in patients with an index admission for congestive heart failure.

METHODS: This is a retrospective cohort study using a national Veterans Health Administration dataset of patients admitted with a principal diagnosis of congestive heart failure from 2012 to 2014. The outcome of interest was unplanned readmissions within 30 days of hospital discharge. The independent variables were post-discharge outpatient encounters up to 30 days following hospital discharge: primary care appointments, phone calls with primary care, phone calls with a 24-hour triage nurse, phone calls with a telemonitoring nurse, and cardiology appointments. Post-discharge outpatient encounters were recorded as being absent or present following discharge (dichotomous variable). For patients with a readmission, only encounters occurring between discharge from the index admission and the readmission were recorded. We used multivariable logistic regressions to determine the impact of post-discharge outpatient encounters on unplanned 30-day readmissions while controlling for age, race/ethnicity, Charlson/ Deyo comorbidity index, length of hospital admission, and number of admissions in the 12 months prior to the index admission.

RESULTS: There were 36,903 index admissions that met the inclusion criteria. The unadjusted, unplanned 30-day readmission rate was $19.2 \%$. Veterans with a readmission were older $(71.8$ (11.5) vs. 72.4 (11.2), $\mathrm{p}<0.027)$ and had more comorbidities (Charlson/Deyo Comorbidity Index, 2.2 (2.1) vs. 2.7 (2.2), p<0.001). Post-discharge encounters between patients and the primary care team were common (non-readmitted patients: $75.9 \%$ $(n=22,615)$ had at least one phone call with primary care, $62.4 \%(n=18,592)$ had at least one primary care appointment; readmitted patients: $71.5 \%$ $(n=5078)$ had at least one phone call with primary care, $42.0 \%(n=2,984)$ had at least one primary care appointment). The odds of an unplanned 30day readmission were lower for Veterans with a primary care appointment following discharge (Adjusted OR 0.44, CI 0.41-0.46), a cardiology appointment (Adjusted OR 0.50, CI 0.47-0.53), a phone call with a telemonitoring nurse (Adjusted OR 0.64, CI 0.59-0.69), or a phone call with primary care (Adjusted OR 0.77, CI 0.73-0.82). The odds of an unplanned readmission were higher for Veterans who had an ER visit (Adjust OR 30.60, CI 28.6-32.9) or phone call with a 24-hour triage nurse (Adjusted OR 1.17, CI 1.09-1.25).

CONCLUSIONS: Patients who are readmitted to the hospital use healthcare system resources differently than patients who are not readmitted. Learning Healthcare Systems may be able to use post-discharge outpatient encounters to identify patients at risk for hospital readmission.

\section{POTENTIAL FOR UNINTENDED CONSEQUENCES: THE ASSO- CIATION BETWEEN SMOKING AND BODY MASS INDEX AMONG PUBLIC HOUSING RESIDENTS IN BALTIMORE, MARYLAND}

Alejandra Ellison-Barnes ${ }^{1}$; Craig E. Pollack ${ }^{2}$; Jeanne M. Clark ${ }^{1}$; Kimberly Gudzune'. 'Johns Hopkins University, Baltimore, MD; ${ }^{2}$ Johns Hopkins University School of Medicine, Baltimore, MD. (Control ID \#3185870)

BACKGROUND: The newly implemented U.S. Department of Housing and Urban Development (HUD) rule requiring public housing to be smokefree may prompt some smokers to quit. While smoking cessation is generally desirable, it is associated with weight gain, and an excess burden of obesity already exists among public housing residents. Given little previous research on smoking and weight status in this population, our objective was to characterize the baseline association prior to the policy implementation.

METHODS: We conducted a cross-sectional analysis of survey data collected in 2014-2015 from randomly selected households of two public housing developments in Baltimore, MD (response rate 48\%). Our independent variable was self-reported smoking status (current vs. former/ never), and the dependent variable was measured body mass index (BMI). We used multivariate linear regression to examine the association between smoking status and BMI, adjusting for age, gender, physical activity level, added sugar intake, and housing development.

RESULTS: Respondents included 266 heads of household with mean age 44.5 (SD 12.4). Of these, $86 \%$ were women and $95 \%$ were Black. A majority $(63.2 \%)$ were current smokers. Seventy-five percent were overweight or obese, with unadjusted mean BMI of $32.6 \mathrm{~kg} / \mathrm{m} 2$ (SD 10.1). In the adjusted regression model, the mean BMI of current smokers was significantly lower than that of former/never smokers at $34.9 \mathrm{~kg} / \mathrm{m} 2$ versus $37.4 \mathrm{~kg} /$ $\mathrm{m} 2(-2.6 \mathrm{~kg} / \mathrm{m} 2,95 \%$ CI -5.0 to $-0.07, \mathrm{p}=0.044)$.

CONCLUSIONS: Among Baltimore public housing residents, the adjusted mean BMI of current smokers was significantly lower than that of former/never smokers after controlling for other risk factors, however it was still in the class I obesity range. Notably, nearly two-thirds of residents were current smokers and three-quarters were overweight or obese. Our results suggest that the new HUD smokefree rule has the potential to promote further weight gain that might lead to a higher risk class of obesity among smokers prompted to quit, highlighting the need to consider both of these prevalent risk factors in tandem in the setting of policy changes.

POTENTIAL IMPACT OF CHANGING PUBLIC CHARGE IMMIGRATION RULES ON CHILDREN WHO NEED MEDICAL CARE Leah Zallman ${ }^{1,3}$; Karen Finnegan ${ }^{1}$; David Himmelstein ${ }^{2,}{ }^{3}$; Stephanie Woolhandler $^{2}, 3$. Institute for Community Health, Malden, MA; ${ }^{2}$ City University of New York at Hunter College, New York, NY; ${ }^{3}$ Cambridge Health Alliance/Harvard Medical School, Cambridge, MA. (Control ID \#3184560)

BACKGROUND: In October, 2018, the Trump administration published a proposed rule change that would increase the chance of an immigrant being deemed a "public charge" and hence ineligible for legal permanent resident status or entry into the US. Under existing immigration policy, only immigrants who receive cash assistance or are institutionalized are considered to be "public charge". If these changes are finalized, immigrants' use of nonemergency Medicaid, Supplemental Nutrition Assistance Program, housing assistance and Medicare Part D low- income subsidy will be used to determine if they are "public charges". This change is expected to cause large numbers of immigrant parents to disenroll themselves and their children from safety-net programs, in large part due to fear and confusion over the rule even among immigrants to whom the rule does not apply.

METHODS: We analyzed a nationally representative sample from the 2015 Medical Expenditure Panel Survey. We estimated the number children "in need of medical care" who are at risk of disenrolling from Medicaid/ CHIP and compared them with Medicaid/CHIP-enrolled children who were not at risk. We include CHIP because many states use blended funding for Medicaid and CHIP, and Medicaid and CHIP beneficiaries often do not know which program is funding their health coverage. We defined "at risk of being disenrolled" as living in a household with any non-citizen adult, whether or not the child was a U.S. citizen. We defined children as being "in need of medical care" if they 1) were diagnosed with a medical condition such as asthma; 2) were disabled (functionally limited or disabled due to mental health condition); or 3) had used care in the 12 months (were prescribed medications, received care for illness or injury, or received any 
care, test or treatment). Finally, we modelled the number of children who would be disenrolled from Medicaid/CHIP under disenrollment scenarios similar to what occurred during welfare reform.

RESULTS: Medicaid/CHIP-enrolled children are at risk of being disenrolled due to public charge rule changes. 4.8 million of these children are "in need of medical care". Consistent with other data showing that immigrant families tend to be relatively healthy, children at risk of being disenrolled are somewhat healthier than other Medicaid/CHIP-enrolled children; they are less likely to have medical conditions (48.7 vs. $62.8 \%, \mathrm{p}<0.0001$ ), to be disabled (6.6 vs. $16.8 \%$, $\mathrm{p}<0.0001)$ or to have used care in the past year $(40.5$ vs. $50.8 \%, p<$ 0.0001 ). Our models predict that between 0.7 and 1.7 million children in "need of medical care" will likely be disenrolled from Medicaid/ CHIP if the new public charge rules are implemented.

CONCLUSIONS: The proposed public charge rules may cause many children "in need of medical care" to be disenrolled from Medicaid/ CHIP. The loss of insurance coverage could harm these children and contribute to future disability. TION OF INTEGRATION OF PERSONALIZED DATA AND SERVICES INTO PRIMARY CARE TO REACH HEALTH EQUITY

Nadia Safaeinili ${ }^{1}$; Cati Brown-Johnson ${ }^{2}$; Lisa Goldman Rosas ${ }^{3}$; Latha Palaniappan ${ }^{1}$; Marcy Winget ${ }^{4}$; Megan Mahoney ${ }^{3}$. ${ }^{1}$ Stanford University, Stanford, CA; ${ }^{2}$ Stanford School of Medicine, Stanford, CA; ${ }^{3}$ Stanford University, Palo Alto, CA; ${ }^{4}$ Stanford University Medical School, Stanford, CA. (Control ID \#3185585)

BACKGROUND: Precision medicine takes into account the complex interplay between genes, environment, and lifestyle by leveraging clinical, self-reported, genetic, and behavioral data to offer targeted approaches to prevention and treatment. Patient and provider attitudes toward implementation in primary care, however, are unknown.

METHODS: Primary care patients were enrolled in a precision health pilot and offered digital health (remote blood pressure, glucose, and weight monitoring with electronic health record integration), health coaching, pharmacogenetic, and genetic testing components. To evaluate the pilot, we summarized patient demographics, health status, and health outcomes using means and frequencies. Semi-structured interviews were conducted with patients and providers at baseline ( $\mathrm{n}=18$ and 7 , respectively) and 6 months ( $\mathrm{n}=9$ and 6 , respectively).

RESULTS: Of 67 patients invited to participate, 48 enrolled (72\%). Patients were diverse (4\% Black 27\% Asian, 19\% Latino); female (67\%); and with complex health needs (58\% cardiovascular (CV) risk, mean body mass index (BMI) $31 \mathrm{~kg} / \mathrm{m} 2)$. Patients reported mixed clinical benefit from precision health, though most felt participation supported their health goals and impacted their health and healthcare. Patient suggestions for improvement included fewer inperson clinical visits and the ability to select resources based on individual motivators. Providers reported that precision health resources were valuable, especially for complex patients, but sometimes resulted in misplaced emphasis on novelty rather than basics. The complexity of some resources also presented barriers, e.g., lengthy genetics test results and management of extensive digital health data. Interviewees identified three barriers to dissemination of precision health in primary care to address health disparities. First, the lack of racial/ethnic minority representation in genomics databases was perceived to reduce impact for those groups. Second, patients would need significant financial resources to participate outside a pilot, potentially exacerbating disparities for patients from low-income backgrounds. Third, providers struggled to incorporate precision medicine components into their work flow; these struggles may be magnified in safety net settings with fewer resources.
Overall, precision health was appealing to patients and providers. Workflow integration, however, slowed initial pilot adoption. Both providers and patients felt precision health would be difficult to scale due to cost and uncertain processes for data utilization.

CONCLUSIONS: Introducing precision health into a diverse primary care clinic was acceptable to all stakeholders and did not surface many concerns related to race/ethnicity. Furthermore, nearly half of the pilot's patients experienced a clinically-relevant finding that changed their treatment plan. Barriers and facilitators surfaced here should be addressed in future precision health interventions.

\section{PREDICTING FUTURE DISPARITIES FOR BLACK AND} WHITE US ADULTS LIVING WITH TYPE 2 DIABETES

Abena Appah-Sampong; Neda Laiteerapong. University of Chicago, Chicago, IL. (Control ID \#3179193)

BACKGROUND: Historically, Black patients living with Type 2 diabetes have had a higher risk for diabetes complications compared to White patients. However, control of individualized glycemic targets, blood pressure, and lipid levels have improved in the U.S. for patients living with Type 2 diabetes over the past decade. Therefore, we compared projected disparities in lifetime diabetes outcomes between two cohorts of Black and White US adults living with Type 2 diabetes.

METHODS: Survey Population: Non-Hispanic Black (NHB) and NonHispanic White (NHW) adults with self-reported diabetes diagnosed at $<18$ years old included in NHANES 2003-2004 (NHB: n=97; NHW: $n=183$ ) and in NHANES 2013- 2014 (NHB: $n=164$; NHW: $n=224$ ). Simulation model: The U.S. Type 2 Diabetes Policy Model (UST2D-PM) is a validated, Monte Carlo-based Markov model. The model utilizes individual patient-level information to forecast diabetes complications and risk of diabetes-related mortality. Outcomes: Lifetime risk of foot ulcer, blindness, renal failure, amputation, myocardial infarction, ischemic heart disease, and stroke; remaining life expectancy, by cohort year and race/ethnicity (NHB and NHW). Statistical Analysis: A multiple imputation procedure was utilized to address missing data. Model outcomes were weighted using NHANES survey weights. Results were compared by cohort year and by racial/ethnic subgroup using regression models.

RESULTS: There were not significant improvements in projected lifetime rates of diabetes outcomes overall or between racial subgroups between the 2003 and 2013 cohorts. The projected lifetime rate of amputation for the overall cohort was higher for the 2013 cohort compared to the 2003 cohort ( $6.2 \%$ vs. $5.5 \%, p=0.02)$, which reflected a higher projected lifetime rate of amputation for the 2013 NHW cohort vs the 2003 NHW cohort (6.2\% vs. $5.3 \%, \mathrm{p}=.01$ ). Also, the projected lifetime rate of renal failure was higher for the 2013 cohort than the 2003 cohort $(8.0 \%$ vs $5.7 \%, p=.04)$, which was driven by a $60.6 \%$ higher event rate for 2013 NHBs $(9.3 \%)$ compared to $2003 \mathrm{NHBs}(3.7 \%, \mathrm{p}=.01)$. This result was due to a longer average duration of diabetes in the $2013 \mathrm{NHB}$ cohort compared to the $2003 \mathrm{NHB}$ cohort $(9.4$ vs. 12.3 years, $\mathrm{p}=.03$ ).

CONCLUSIONS: Despite large scale efforts to reduce diabetes health disparities, our study suggests that racial disparities in the rates of complications from diabetes progression may not decline in the future. Greater efforts to ensure that patients achieve guideline-recommended risk factor control and targeted efforts to improve control in non-Hispanic Black patients are necessary to reduce future disparities in diabetes outcomes.

\section{PREDICTING LONG-TERM HEALTHCARE SPENDING THROUGH LONGITUDINAL MODELING IN MEDICARE PA- TIENTS}

Julie Lauffenburger; Mufaddal Mahersi; Niteesh K. Choudhry. Brigham and Women's Hospital, Harvard Medical School, Boston, MA. (Control ID \#3185020)

BACKGROUND: Current approaches to predicting healthcare costs 
generally rely on a single composite value of total spending and have modest predictive accuracy. Newer approaches that incorporate dynamic patterns of spending could improve accuracy and therefore costcontainment efforts by healthcare organizations. We recently showed that using group-based trajectory modeling enhanced the prediction of costly patients over a one-year time horizon; however, less is known about longerterm spending dynamics and potentially-modifiable risk factors for patient intervention.

METHODS: We used group-based trajectory modeling to empirically classify a random nationwide sample of Medicare beneficiaries 65 years enrolled by their spending patterns over a two-year period. We then assessed the ability to predict membership in each trajectory group using logistic regression and split-sample validation. Models were estimated using 1) baseline clinical and demographic factors and 2) only baseline factors that are potentially-modifiable through interventions.

RESULTS: In 329,476 patients, a 6-group trajectory model best described two-year longitudinal spending patterns (Figure), including high-cost (in red) and cost-bloomer (in orange) groups. Four spending groups could be highly predicted, including high-cost (validated C-statistic [C]: 0.887), cost-bloomers (i.e., lower spendings who later became more costly) (C: 0.722), rising (C: 0.810), and low-cost (C: 0.953). For cost-bloomers in particular, the most predictive potentially-modifiable factors were patients' number of medications, number of hospitalizations, and depression.

CONCLUSIONS: Examining a two-year time horizon using trajectory modeling improved the understanding of dynamic patterns, including the identification of a group of cost-bloomers and patients who are consistently high spenders. This approach could be adapted and implemented by healthcare organizations to improve costcontainment efforts.

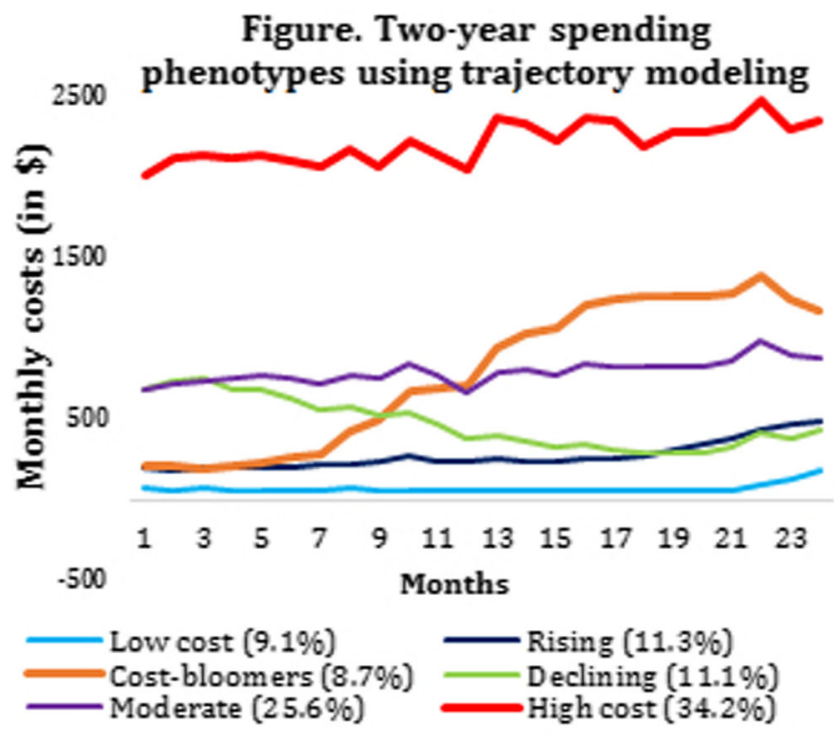

\section{PREDICTING UNMET VA PRIMARY CARE NEED USING ECO- NOMIC DISEQUILIBRIUM MODELS}

Edwin Wong ${ }^{1}$; Christopher Wilson ${ }^{1}$; Karin M. Nelson ${ }^{1,2}$. ${ }^{1}$ Department of Veterans Affairs, Seattle, WA; ${ }^{2}$ University of Washington, Seattle, WA. (Control ID \#3184178)

BACKGROUND: One of the key goals of the Patient-Centered Medical Home model is to improve access by providing care where and when patients need it. Recent reports have identified inadequate internal capacity as a key access barrier. There is currently limited evidence using rigorous methods to measure how much additional capacity is needed to meet veterans' health care needs. The objective of this study was to jointly estimate the supply and demand of VA primary care visits using econometric disequilibrium models to quantitatively estimate unmet primary care demand.

METHODS: This observational study examined a random sample of 51 VA medical centers across the U.S. Data were derived from VA administrative databases, linked with geographically aggregated data from the Area Health Resource File and the Centers for Disease Control and Prevention over the period January 2014 through September 2018. The outcome was primary care utilization defined as visits at VA clinic locations identified to provide primary care. We modeled the supply of VA primary care as a function facility-level primary care staffing, local area characteristics and seasonality. We modeled the demand of VA primary care as a function of patient enrollment, demographics and risk scores, a geographically varying measure of flu severity and seasonality. Many VA patients are exempt from copayments or other cost sharing, therefore we applied econometric disequilibrium models [Madalla and Nelson (1974)] to estimate the magnitude of excess primary care demand. These models jointly estimate parameters of the supply and demand equations, addressing the fact that observed number of primary care visits does not represent quantity demanded by veterans when excess demand exists. Joint supply and demand models were estimated separately for each facility using a maximum likelihood estimator and the unit of analysis was facility-month.

RESULTS: The average number of primary care visits per month provided by VA facilities decreased from $3,104(\mathrm{SD}=1,750)$ to 2,654 (SD=1,557) between January 2014 and January 2018. For 34 facilities, we did not reject the null hypothesis that the estimated number of visits demanded equaled visits supplied. For 8 facilities, the estimated number of visits demanded exceeded visits supplied by an average of $32 \%$. For the remaining 9 facilities, estimated visits demanded was less than visits supplied by an average of $22 \%$.

CONCLUSIONS: The estimated quantities of primary care supplied and demanded were balanced at most facilities. However, model estimates suggest the level of primary care needed by veterans exceeded resources at some facilities, while at other facilities, staffing likely exceeded veterans' primary care needs. Study findings inform the level of primary care staffing needed by facilities to fully meet expected primary care demand of VA patients. Models in this study also provide the opportunity to forecast staffing needs as conditions change over time.

PREDICTORS AND FOLLOW-UP USING THE CURRENT OPIOID MISUSE MEASURE IN PRIMARY CARE

Mathew Tsai ${ }^{1,2}$; MacLean D. Charles ${ }^{1,2}$. ${ }^{1}$ Larner College of Medicine, Burlington, VT; ${ }^{2}$ University of Vermont, Burlington, VT. (Control ID \#3178274)

BACKGROUND: Misuse of prescription opioids is a growing public health issue associated with adverse health outcomes. Clinical guidelines recommend formal risk assessment tools such as the Current Opioid Misuse Measure (COMM), a validated 17 question self-report survey assessing prior 30-day behavior associated with concurrent opioid misuse. However, little is known about the COMM's clinical utility among clinicians or the predictors of a high COMM score. To address these uncertainties, this study aimed to describe the consistency of use of the COMM and the distribution of COMM responses in a primary care population; to identify sociodemographic and clinical predictors of a positive COMM score; and to explore the association between a positive COMM score and increased clinical surveillance for opioid misuse.

METHODS: We performed a cross-sectional analysis of data from 
an academic medical center Epic-based clinical data warehouse. Institutional protocols, which follow $\mathrm{CDC}$ guidelines and State regulations require the annual use of consent and treatment agreements, risk stratification (e.g using the COMM), use of the prescription drug monitoring program (PDMP), and functional assessment. Study participants consisted of 859 adult patients on chronic opioid pain therapy who were not taking office-based opioid agonist treatment for substance abuse disorder and who had completed a COMM at an office visit during a three-year period from 1 January 2016 to 31 December 2018. The main outcome measure was the COMM score (positive if 9).

RESULTS: The mean COMM score was 4.2 (SD 4.5) and $13 \%$ of participants had a positive score. Additionally, $70 \%$ of the study population had a diagnosis of anxiety or depression and $50 \%$ were taking a concurrent gamma-aminobutyric acid (GABA) agonist. Regarding adherence to surveillance protocol, $21 \%$ of patients were overdue for a COMM. Patients were also overdue for an opioid patient consent form (32\%), opioid treatment agreement (40\%), PDMP lookup (17\%), formal functional assessment (28\%), a non-mandatory urine drug screen (32\%), and pill count (64\%). A multivariable model identified concurrent, weekly-orgreater use of GABA agonists $(\mathrm{OR}=1.595 \% \mathrm{CI} 1.0,2.3, \mathrm{p}=0.04)$ and severe mental illness $(\mathrm{OR}=1.795 \% \mathrm{CI} 1.0,2.8, \mathrm{p}=0.04)$ as significant predictors of a positive COMM score. No significant association was detected between COMM score and opioid surveillance measures.

CONCLUSIONS: The COMM is relatively easy to implement in primary care and is independently associated with severe mental illness and concomitant use of GABA agonist medication. However, the implications of a positive COMM score and the survey's clinical utility are still to be determined within these contexts.

\section{PREDICTORS OF ELIGIBLE PATIENTS' AGREEING TO LUNG CANCER SCREENING WITH LOW-DOSE COMPUTED TO- MOGRAPHY: A MULTILEVEL RETROSPECTIVE COHORT STUDY}

Joseph Leishman ${ }^{4}$; Angie Fagerlin ${ }^{5}$; Julie Lowery ${ }^{2}$; Renda S. Wiener ${ }^{3}$; Tanner Caverly ${ }^{1}{ }^{1}$ University of Michign School of Medicine, Ann Arbor, MI; ${ }^{2}$ VA Ann Arbor Healthcare System, Ann Arbor, MI; ${ }^{3}$ Carolinas Medical Center, UNC School of Medicine, Boston, MA; ${ }^{4}$ University of Michigan School of Public Health, Ann Arbor, MI; ${ }^{5}$ University of Utah School of Medicine, Salt Lake City, UT. (Control ID \#3186294)

BACKGROUND: The clinical reminders used in VA's Lung Cancer Screening (LCS) Demonstration project offered a unique opportunity to examine real-world LCS decision-making. Since baseline lung cancer risk and comorbidity/competing risk are key factors in determining absolute risk reduction with LCS, we conducted a retrospective cohort study to assess the extent to which these key factors played roles in decisions compared with factors unrelated to clinical benefit.

METHODS: Across the 8 demonstration project sites, we identified all patients without health-related exclusions who met LCS eligibility criteria $(>30$ pack-years, $<15$ years since quit and aged 55-80) and had an initial LCS reminder completed between 10/01/ $13-9 / 30 / 15$. We then fit a multilevel mixed-effects logistic regression model to assess predictors of a patient agreeing to annual screening (based on reminder data), adjusting for site and patient demographics.

RESULTS: Our cohort included 6,037 patients (mean age 67; 96.5\% male) who were considered appropriate candidates for LCS by their primary care providers and had documentation about whether they agreed to LCS after the initial provider discussion. The 2 variables related to net benefit (lung cancer risk and Charlson Comorbidity Index) were not associated with agreeing to screen, nor was gender. Two patient-level factors were significantly associated: age $(P<0.001)$ and distance from the patients' listed home address to the VA center offering LCS $(P=0.019)$. For example, men at the cohort mean for other model covariates (mean annual lung cancer risk of $0.7 \%$; Charlson score 1.12; and living 37.7 miles from the VA) were predicted to have a $78 \%$ probability of agreeing to screen at 57 years old but only a $60 \%$ probability at 77 . Likewise, a 67 year-old-man with the same lung cancer risk and comorbidity score was predicted to have a $70 \%$ probability of agreeing to screen if living 37.7 miles from the VA but only a $34 \%$ probability if living 137.7 miles away. The site where LCS was offered only explained $8 \%$ of the overall variance in agreeing to screen $(\mathrm{ICC}=0.08)$, but this still resulted in substantial site-level variation ranging from a rate of $38 \%$ of eligible patients agreeing to LCS to a rate of $79 \%$ across the 8 sites.

CONCLUSIONS: Baseline lung cancer risk and level of comorbidity were not associated with whether a patient chose to pursue LCS, even though these factors largely determine a patient's degree of benefit. While variation in agreeing to screen based on patient age and distance from the VA might appropriately reflect differences in patient preferences, the large variation across sites likely reflects more about provider preferences than patient preferences. We are currently studying the implementation of a risk-based LCS provider tool that aims to help primary care providers and screening coordinators tailor screening discussions to a patient's individualized lung cancer risk.

\section{PREDICTORS OF HEALTH INSURANCE LITERACY AMONG COMMUNITY HEALTH CENTER STAFF}

Cory Williams $^{1,}{ }^{3}$; Mellisa A. Pensa ${ }^{1,2}$; Douglas Olson ${ }^{1} .{ }^{1}$ Fair Haven Community Health Care, New Haven, CT; ${ }^{2}$ Yale University School of Medicine, New Haven, CT; ${ }^{3}$ AmeriCorps, New Haven, CT. (Control ID \#3178362)

BACKGROUND: Despite increased attempts to make health insurance more accessible and comprehensive, low literacy and numeracy prevents many from understanding aspects of health insurance. Health Insurance Literacy (HIL) is the degree to which an individual is able to find and assess health insurance information and navigate health plans. Disparities in HIL are correlated with delayed access to care and poorer health status. The primary goal of this research is to measure HIL among staff at a Federally Qualified Health Center (FQHC) and identify predictors of knowledge gaps.

METHODS: This is a cross-sectional study assessing HIL among staff at Fair Haven Community Health Care (FHCHC), an urban FQHC in New Haven, CT. In November 2018, all 212 staff members were emailed an anonymous survey and 91 (43\%) completed the survey, which was generated using Qualtrics ${ }^{\mathrm{TM}}$ software. Questions measured the participant's ability to identify and utilize various health insurance terms and topics. Descriptive statistics were calculated, and unadjusted analyses were used to examine potential associations between demographic, social, and health characteristics and HIL. We used Multivariate logistic regression to identify independent relationships to HIL. This study was approved by the FHCHC institutional review board.

RESULTS: Participants had a mean HIL score of 6.21 (out of 10 ), and characteristics that had a statistically significant difference in average score upon unadjusted analysis were race/ethnicity, education, role at $\mathrm{FHCHC}$, and self-reported health status (Figure). Upon multivariate regression, education and job role remained significant predictors of HIL.

CONCLUSIONS: The majority of staff had gaps in HIL, and HIL is significantly associated with education, professional status, race/ethnicity, and self-reported health status. Results of this study will be used to target staff and community training efforts.

Average health insurance literacy score (HIL) is represented in the 


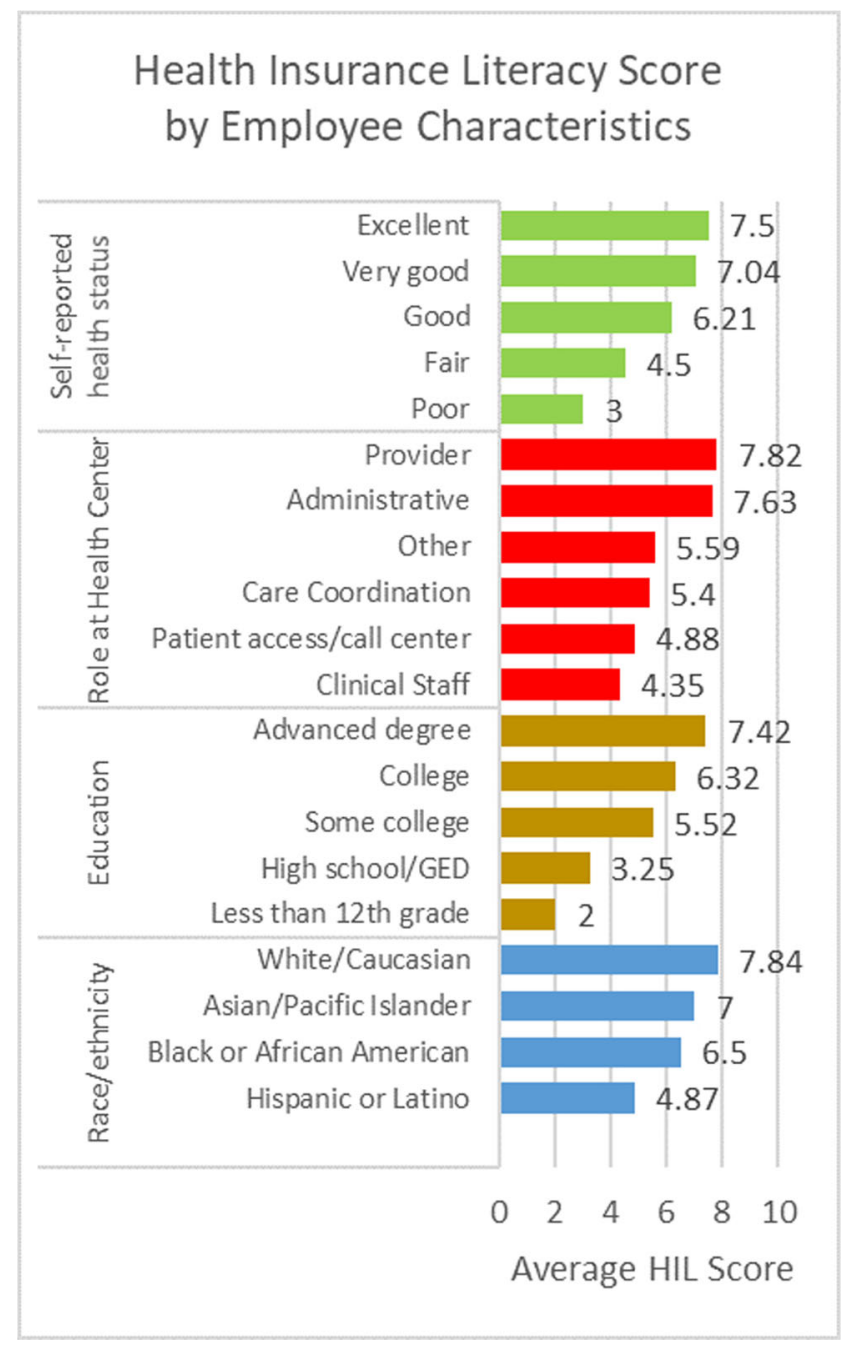

bars and organized by employee characteristic.

\section{PREDICTORS OF IATROGENIC HYPOGLYCEMIA IN TREATMENT OF HYPERKALEMIA}

Anh Do ${ }^{1}$; Sandra Naffouj ${ }^{1}$; Krishna Modi ${ }^{1}$; Rawan Hammoudeh ${ }^{1}$; Joseph Miller ${ }^{1}$; Mohammed F. Dabbagh ${ }^{2}$. ${ }^{1}$ Henry Ford Hospital, Detroit, MI; ${ }^{2}$ Henry Ford Hospital / Wayne State University, Detroit, MI. (Control ID \#3185737)

BACKGROUND: Hypoglycemia is a potential adverse outcome that could complicate the treatment of hyperkalemia with insulin administration. Treatment needs to be individualized for patients' specific characteristics to avoid iatrogenic hypoglycemia. Our study aimed to identify characteristics that could increase the risk of hypoglycemia.

METHODS: A retrospective study at a large tertiary institution was conducted from December 2016 to November 2017. A chart review was performed on adult patients who received subcutaneous insulin and intravenous glucose for treatment of hyperkalemia (potassium $>5$ $\mathrm{mmol} / \mathrm{L}$ ) while present in the emergency department or inpatient setting. Patients who received insulin for the treatment of hyperglycemia were excluded. Data including demographics, insulin dose, and laboratory values were collected. The cohort was divided into two groups; patients who developed hypoglycemic events (defined as serum glucose $<70 \mathrm{mg}$ /
$\mathrm{dL}$ ) and those who did not. Univariate and multivariate analysis was performed. The primary outcome was incidence of hypoglycemia. The secondary outcome was identifying predictors of hypoglycemia.

RESULTS: 1156 consecutive patients with hyperkalemia who were treated with insulin were analyzed. Mean age was 61 years, 59\% were males, and $61 \%$ were African American. 69\% had chronic kidney disease, and of those $32.7 \%$ were on hemodialysis. The mean dose of insulin administered was $0.105+0.05$ units $/ \mathrm{kg}$ for a median pre-treatment potassium level of $5.9 \mathrm{mmol} / \mathrm{L} .17 .8 \%$ of patients developed hypoglycemia $(\mathrm{N}=206)$. The median pre-treatment glucose level was $126 \mathrm{mg} / \mathrm{dL}$, and mean hypoglycemia level was $53 \mathrm{mg} / \mathrm{dL}$. On multivariate analysis age $(\mathrm{p}=0.0328)$, pre-treatment glucose level $(\mathrm{p}<0.0001)$, and insulin dosing $(\mathrm{p}=0.0032)$ were significant predictors of hypoglycemia. For every 10year increase in age, the risk of hypoglycemia increased by an odds ratio (OR) of 1.128 . For every $10 \mathrm{mg} / \mathrm{dL}$ increase in the pre-treatment glucose level, the odds of developing hypoglycemia were reduced by an OR of 0.929 . For every 0.025 units $/ \mathrm{kg}$ increase of insulin dosing above the 0.1 units/kg usually administered, the OR for hypoglycemia was increased by 1.006. There was a significant difference in the rates of hypoglycemic events between the emergency department (24.2\%) compared to the general practice units $(18.4 \%)$ and intensive care units $(12.8 \%)$ $(\mathrm{p}<0.001)$. There was no relationship between insulin dose and pretreatment glucose $\left(\mathrm{R}^{2}=0.0006\right)$.

CONCLUSIONS: Patients with older age, lower pre-treatment glucose, and higher insulin doses were at increased risk of developing hypoglycemia following treatment of hyperkalemia with insulin. Clinicians should more regularly evaluate baseline glucose levels when treating hyperkalemia with insulin to prevent iatrogenic hypoglycemia.

PREDICTORS OF LONG-TERM HIGH HEALTHCARE UTILIZATION AMONG NEW MEDICAID ENROLLEES

Somalee Banerjee ${ }^{1}$; Amy Alabaster ${ }^{3}$; Patricia Kipnis ${ }^{4}$; Alyce S. Adams ${ }^{2}$. ${ }^{1}$ Kaiser Permanente Oakland, Oakland, CA; ${ }^{2}$ Kaiser Permanente, Oakland, CA; ${ }^{3}$ Kaiser Permanente Northern California, Oakland, CA; ${ }^{4}$ Kaiser Health Plan, Oakland, CA. (Control ID \#3183708)

BACKGROUND: The 2014 Medicaid expansion with the Affordable Care Act (ACA) increased access to healthcare for underserved patients, but also increased cost. Interventions centering on care coordination for the highest healthcare utilizers or "super-utilizers" have not been effective in reducing cost because utilization typically normalizes over a year. There is limited knowledge about predictors of long-term utilization after a year. Identifying these predictors can help target populations most likely to benefit from case management and support. This study analyzes the association of diagnoses made at enrollment with the odds of super utilization and long-term super-utilization over the first two years in a cohort of newly enrolled Medicaid patients in a large integrated care system.

METHODS: This retrospective cohort study used electronic health record (EHR) data from Kaiser Permanente Northern California for 28,653 adults newly enrolled in Medicaid managed care between June and December 2014. The super-utilizer subgroup were patients in the top 5th percentile of total healthcare cost. Differences in baseline characteristics of super-utilizers and non-super-utilizers were determined using bivariate analysis. Multivariable logistic regression determined the independent association between superutilization in the first year (model 1) or super-utilization in the second year of enrollment (model 2) and comorbidities: mental health conditions (anxiety, depression, bipolar disorder or schizophrenia), substance use, cancer, BMI and other chronic diseases (Charlson comorbidity index greater than 2). Each model was adjusted for age, gender, race, English as first language, socioeconomic characteristics, dual enrollment, physician visits, and months enrolled. RESULTS: More super-utilizers $(1,428)$ had chronic mental and physical health conditions than the non-super-utilizer $(27,080)$ Medicaid population. Patients with the following comorbidities at enrollment had significantly increased odds of being super-utilizers over their first year of Medicaid enrollment after controlling for socio-demographics and enrollment: any 
mental health condition, chronic pain, active cancer, high Charlson comorbidity score, alcohol use disorder, and drug use disorder (Figure 1a). Among first-year super-utilizers, chronic pain, 2+ Charlson comorbidities, and schizophrenia were associated with second year super-utilization in adjusted models (Figure 1b).

CONCLUSIONS: Diagnoses of mental health conditions, substance use and other comorbid medical conditions at enrollment for Medicaid patients can predict super-utilization over the first year. Among super-utilizers, only patients with schizophrenia, chronic pain and comorbid medical illness showed significantly increased odds of long-term super-utilization over the second year. Focusing similar interventions on super-utilizer patients with risk factors for long-term utilization identified in this study could lead to improved quality and decrease of avoidable utilization.

\section{PREDICTORS OF MORTALITY IN PATIENTS TREATED WITH BUPRENORPHINE FOR OPIOID USE DISORDER}

Danielle R. Fine; Yuchiao Chang; Virginia Triant; Travis P. Baggett; Joshua Metlay. Massachusetts General Hospital, Boston, MA. (Control ID \#3179036)

BACKGROUND: Opioid replacement therapy (ORT) has been shown to reduce mortality in patients with opioid use disorder (OUD), yet mortality in those receiving ORT remains higher than in the general population. While several studies have evaluated risk factors associated with mortality in patients treated with methadone, there is limited research evaluating individuals treated with buprenorphine. The objective of this study was to identify risk factors for mortality in patients treated with buprenorphine for OUD.

METHODS: We conducted a retrospective cohort study utilizing a centralized clinical data registry within a multi-hospital health system in Boston, MA to identify adult ( 18 years) patients who were prescribed 2 consecutive buprenorphine prescriptions from January 1, 2007 to December 31, 2017. We abstracted baseline demographic, socioeconomic, and clinical characteristics from the clinical data registry. The primary outcome was all-cause mortality occuring by October 31, 2018. We identified deaths by linking the clinical data registry to both the National Death Index and the Massachusetts Registry of Vital Records and Statistics. We performed multivariable cox regression to identify baseline characteristics independently associated with all-cause mortality.

RESULTS: Of the 5,951 patients prescribed 2 consecutive buprenorphine prescriptions, the majority were white $(80.7 \%)$, male $(59.7 \%)$, with a mean age of 38.2 years at study entry. The all-cause mortality rate was 2.24 deaths per 100 person years. Baseline characteristics independently associated with an increased risk of all-cause mortality were: age (adjusted hazard ratio $[\mathrm{aHR}]=1.25$ per ten years of age; $95 \%$ confidence interval $[\mathrm{CI}]=1.17,1.33$ ), male gender $(\mathrm{aHR}=1.34 ; 95 \% \mathrm{CI}=1.12,1.60)$, congestive heart failure $(\mathrm{aHR}=1.92 ; 95 \% \mathrm{CI}=1.49,2.47)$, pulmonary disease $(\mathrm{aHR}=1.23 ; 95 \%$ $\mathrm{CI}=1.02,1.47)$, hepatitis $\mathrm{C}(\mathrm{aHR}=1.60 ; 95 \% \mathrm{CI}=1.28,2.01)$, chronic kidney disease $(\mathrm{aHR}=2.12 ; 95 \% \mathrm{CI}=1.17,3.83)$, malignancy (aHR=1.31; 95\% $\mathrm{CI}=1.03,1.68$ ), and a concurrent opioid prescription within 90 days of entering the study $(\mathrm{aHR}=1.28 ; 95 \% \mathrm{CI}=1.07,1.53)$. In addition, patients initially treated with buprenorphine as inpatients (vs. outpatients) had an independently increased risk of death $(\mathrm{aHR}=1.44 ; 95 \% \mathrm{CI}=1.20,1.74)$. Similarly, patients who entered the study from 2014-2015 (aHR=1.48; 95\% $\mathrm{CI}=1.09,2.01)$ and from $2016-2017(\mathrm{aHR}=1.70 ; 95 \% \mathrm{CI}=1.20,2.41)$ had an independently increased risk of death compared to those who entered from 2007-2009.

CONCLUSIONS: In a large cohort of patients with OUD treated with buprenorphine, older age, multiple medical comorbidities, a concurrent opioid prescription, and initial treatment as an inpatient were independently associated with mortality. Consistent with trends in national opioid-related deaths, mortality in our cohort was significantly higher from 2014 to 2017 . Clinicians should be aware of these risk factors when treating patients with buprenorphine and take steps to mitigate risk in this vulnerable population.

PRELIMINARY FINDINGS FROM THE HISPANIC SECONDARY STROKE PREVENTION INITIATIVE (HISSPI)
Olveen Carrasquillo $^{1}$; Jose G. Romano ${ }^{2}$; ChuanHui Dong ${ }^{2}$; Sonjia Kenya ${ }^{1}$; Stuti Dang. ${ }^{2}{ }^{1}$ University of Miami Miller School of Medicine, Miami, FL; ${ }^{2}$ University of Miami, Miami, FL. (Control ID \#3186396)

BACKGROUND: Latinos face a disproportionate stroke burden and are less likely to have stroke risk factors under adequate control. The objective of this randomized controlled trial is to evaluate the effectiveness of a Community Health Worker (CHW) intervention among Latino patients at risk of recurrent stroke.

METHODS: This is randomized clinical trial of 200 Latino stoke patients in South Florida whom had had a stroke within the last 5 years and were ambulatory (MRS $<=3$ ). Participants randomized into the CHW intervention receive health education, health care navigation and social services assistance through home visits and phone calls. Participants also receive daily text messages and are asked to send CHWs their daily home blood pressure readings. The primary outcome is change in systolic blood pressure at 12 months as assessed by trained RA using an automated cuff. Other secondary outcomes include changes LDL, A!C and adherence to ant-thrombotic/ antiplatelet medications.

RESULTS: Baseline characteristics were similar with respect to age $(61+/-$ $19.6 \mathrm{yrs})$, gender (68\% male), proportion having Medicaid (44\%), BMI $(28.1+/-15.0)$ and baseline BP $(127+/-118 \mathrm{mmHg})$. Intervention participants were more likely to have not completed High school (41\% versus $28 \%$ ). One average over one year, intervention participants received a mean of 7.7 home visits and 31.2 phone calls. Tabulated data on CHW activities showed the majority of CHWs interventions were focused on assistance with social service needs and patient navigation. $68 \%$ of patients in the intervention group agreed to receive text messages, and of those $42 \%$ responded to at least $50 \%$ of their daily text messages. In intention to treat analysis, at one year, of the first 131 enrolled participants, mean SBP was $7.7+/-13.5$ lower in the intervention group $(\mathrm{p}=0.02)$ and DBP was $5.0+/-1.9 \mathrm{mmHg}$ lower $(\mathrm{p}=.01)$. Adjusting for age, gender and baseline BP yielded similar results (SBP $6.4 \mathrm{mmHg}$ and DBP 3.1 lower, $\mathrm{P}=.04$ and .03 , respectively). At baseline over $50 \%$ of participants had an A1c under $6.5 \%$ and LDL under $100 \mathrm{mg} / \mathrm{dl}$. There were no changes in these two outcomes at one year.

CONCLUSIONS: HISPPI adds to the existing body of literature showing CHWs are an evidence-based approach to improving health outcomes among minority and underserved populations.

\section{PREP RRT: USING THE HOSPITAL AS A SITE TO IDENTIFY, EDUCATE AND REFER HOSPITALIZED MINORITY PATIENTS WITH ADVANCED CKD}

Milda R. Saunders ${ }^{2}$; Akilah King ${ }^{2}$; Eric Robinson ${ }^{1}$; Monica E. Peek ${ }^{3} .{ }^{1}$ University of Chicago Medical Center, Chicago, IL; ${ }^{2}$ University of Chicago, Chicago, IL; ${ }^{3}$ The University of Chicagoa, Chicago, IL. (Control ID \#3186059)

BACKGROUND: Education for patients with advanced chronic kidney disease (CKD) delays progression to ESRD and improve outcomes in renal replacement therapy (RRT) selection. Yet many minority patients face barriers in receiving pre-ESRD education. The hospital is an important site for patient education and linkage to care for patients with advanced CKD. Over $62 \%$ of patients are hospitalized in the year prior to dialysis initiation, and the hospitalization rate rises sharply in the three month prior to dialysis initiation. Thus, we developed and implemented an in-person education intervention for hospitalized African American patients with advanced CKD.

METHODS: Our pilot intervention, the Patient Referral and Education Program prior to Renal Replacement Therapy (PREP-RRT), was developed for hospitalized African American patients and was implemented at a minority-serving academic medical center where the inpatient general medicine population is more than $80 \%$ African American. We included hospitalized general medicine patients aged $18-75$ who self-identified as African American and who had an estimated glomerular filtration rate (eGFR) $<45 \mathrm{~mL} / \mathrm{min} / 1.73 \mathrm{~m} 2$. Participants were recruited through the 
Hospitalist Project, an ongoing clinical study that examines a variety of outcomes of hospitalized general internal medicine patients and includes administrative data, inpatient interviews and 30 day follow-up by phone. The PREP-RRT patient educator was an African American nephrology social worker trained in motivational interviewing techniques, CKD selfmanagement and RRT options. During a brief in-hospital session, she assessed patients' current knowledge, focused on their current positive self-care behaviors, assessed barriers and strategies for overcoming these barriers, and then provided knowledge about RRT. Participants were assessed both pre and post-intervention about their knowledge (using Kidney Disease Knowledge Survey (KiKs), attitudes and intent (using an investigator developed instrument) about CKD self- management and RRT options.

RESULTS: We recruited 46 patients over 9 months. Participants were $61 \%$ female, mean age 59.5 years. Overall half of patients $(56.5 \%)$ had CKD Stage 3B, 24\% had Stage 4 and 20\% had Stage 5 CKD. Despite having advanced CKD, only $33.4 \%$ believed they were extremely likely or likely to have kidney failure in the next 5 years. Patient knowledge using KiKs was significantly improved from $63.3 \%(15.2 / 24)$ to $80.2 \%$ $(19.3 / 24), p<0.05$. Participants also appeared to have increased knowledge of RRT modalities, but it reached statistical significance for peritoneal dialysis. There were no significant differences in patient reported intent to participate in CKD self-management or RRT selection.

CONCLUSIONS: PREP RRT was a feasible and effective way to identify, educate and refer hospitalized minority patients with advanced $\mathrm{CKD}$. Increasing patient intent for action around CKD self-care and RRT choice may require a more intensive intervention.

\section{PRESCRIBING ASSOCIATED WITH HIGH-RISK OPIOID EX- POSURES AMONG NON-CANCER CHRONIC USERS OF OPI- OID ANALGESICS IN A MEDICAID PLAN: A SOCIAL NET- WORK ANALYSIS}

Keiki Hinami; Kruti Doshi; Maria Torres; Steven Aks; John J. Shannon; William Trick. Cook County Health, Chicago, IL. (Control ID \#3184051)

BACKGROUND: The continued rise in fatalities from opioid analgesics despite a steady decline in the number of individual prescriptions directing 90 morphine milligram equivalents (MME)/day may be explained by patient exposures to redundant prescriptions from multiple prescribers. We evaluated prescribers' specialty and social network characteristics associated with high-risk opioid exposures resulting from singleprescriber high-daily dose prescriptions or multi-prescriber discoordination.

METHODS: We conducted a retrospective cohort study of prescribers with opioid analgesic prescription claims for non-cancer chronic opioid users in an Illinois Medicaid managed care program in 2015-16. We used social network analysis methods to construct a prescriber-beneficiary bimodal sociogram where an opioid analgesic prescription claim represented a tie. Owing to the network's cored structure, we assigned $k$-core levels to each prescriber; $k$-core is defined as the subset of the network involving prescriber nodes connected to at least $k$ beneficiary nodes. We used negative bionomial regression to assess sociometric position and prescriber specialty as risk factors for single-prescriber high-daily- dose prescriptions or multi-prescriber discoordinated prescriptions.

RESULTS: For 2280 beneficiaries, 36,798 opioid prescription claims were submitted by 3532 prescribers. Compared to $3 \%$ of prescriptions (involving $6 \%$ of prescribers and $7 \%$ of beneficiaries) that directed $90 \mathrm{MME} /$ day, discoordination accounted for a greater share of high-risk exposures $13 \%$ of prescriptions (involving $23 \%$ of prescribers and $24 \%$ of beneficiaries). The following specialties were at highest risk of discoordinated prescribing compared to internal medicine: dental [Incident Rate Ratio (95\%Confidence Interval): 5.9 (4.6, 7.5)], emergency medicine [4.7 (3.8, $5.8)]$, and surgical subspecialties $[4.2(3.0,5.8)]$. Social network analysis identified 2 small inter-connected prescriber communities of high-volume pain management specialists, and 3 sparsely connected groups of predominantly low-volume primary care or emergency medicine clinicians. In multivariate models, we found that the sparsely connected sociometric positions were a risk factor for high-risk exposures.

CONCLUSIONS: Low-volume prescribers in the social network's periphery were at greater risk of intended or discoordinated prescribing than interconnected high-volume prescribers. Interventions addressing discoordination among low-volume opioid prescribers in non-integrated practices should be a priority. Demands for enhanced functionality and integration of Prescription Drug Monitoring Programs or referrals to specialized multidisciplinary pain management centers are potential policy implications.

\section{PRESCRIPTION OPIOID USE INCREASES ODDS OF OBSTRUC- TIVE SLEEP APNEA}

Kevin Chen ${ }^{1,2}$; David A. Fiellin ${ }^{1}$; Henry Yaggi ${ }^{1}$; Lori A. Bastian ${ }^{2} .{ }^{1}$ Yale $\overline{\text { University S}}$ chool of Medicine, New Haven, CT; ${ }^{2}$ VA Connecticut Healthcare System, West Haven, CT. (Control ID \#3176031)

BACKGROUND: Prescription opioids can complicate obstructive sleep apnea (OSA) due to their ability to disrupt sleep architecture, induce central apneas, worsen upper airway obstruction, and deepen arousal thresholds. While continuous positive airway pressure (CPAP) is a standard treatment for OSA, it may not be optimal for opioid-related central apneas. Understanding patterns of prescription opioid use, OSA, and CPAP use may impact OSA screening and treatment in patients prescribed opioids. We sought to determine the association between prescription opioids, OSA, and CPAP use.

METHODS: Using the Women Veterans Cohort, we identified female and male veterans of recent wars who were prescribed opioids and the prevalence of OSA and CPAP, assessed by International Classification of Disease-9 and Current Procedural Terminology codes. Demographics and comorbidities were compared for patients across opioid prescription categories (none, acute, chronic - defined as being prescribed 90 annual opioid days). Bivariate and multiple logistic regression were used to test for associations between opioid prescription category, OSA, and CPAP. Known risk factors for OSA, including age, sex, obesity, smoking status, race, psychiatric disease, pain intensity (numeric rating scale: none $=0$, mild $=1-3$, moderate $=4-6$, severe $=7-10$ ), pulmonary disease, heart failure, stroke, traumatic brain injury, and substance use were controlled for.

RESULTS: A total of 1,149,874 veterans were assessed for inclusion. The mean age was $38.0 \pm 9.6(\mathrm{SD})$ years. 136,754 had any opioid prescription, and $29,034(2.5 \%)$ had chronic opioid prescriptions. $115,379(10.0 \%)$ veterans had a diagnosis of OSA, of which 41,033 (35.6\%) received CPAP. Compared to veterans without opioid prescriptions, those with opioid prescriptions were more likely to be older, female $(13.1 \%$ v $12.2 \%)$, obese, current or former smokers, have moderate or severe pain intensity, and have psychiatric and medical comorbidities ( $\mathrm{p}<0.01$ for all comparisons). Both acute (unadjusted OR 3.49 [95\% CI $3.43,3.54]$, aOR 1.82 [1.79, 1.86]) and chronic (OR 4.75 [4.62, 4.87], aOR $1.91[1.85,1.97])$ opioid prescriptions were associated with increased odds of having OSA compared to people who did not have opioid prescriptions. People who had opioid prescriptions also had higher odds of CPAP compared to those without (acute: OR 1.15 [1.11, 1.18], aOR 1.08 [1.05, 1.12]; chronic: OR 1.30 [1.24, 1.36], aOR 1.16 [1.10, 1.22]). CONCLUSIONS: As patients prescribed opioids are more likely to have OSA, clinicians should screen for sleep-disordered breathing in individuals being prescribed opioids chronically. And, since opioid use is associated with CPAP, the efficacy of CPAP in opioid-related sleep apneas should be further studied.

\section{PRESENCE: PHYSICIAN AND NON-PHYSICIAN INSIGHTS ABOUT THE ART OF HUMAN CONNECTION IN CLINICAL ENCOUNTERS}

Cati Brown-Johnson $^{2}$; Rachel Schwartz ${ }^{1}$; Amrapali Maitra ${ }^{2}$; Marie C. Haverfield $^{3}$; Aaron A. Tierney ${ }^{4}$; Jonathan G. Shaw ${ }^{2}$; Dani L. Zionts ${ }^{2}$; Nadia Safaeinili ${ }^{5}$; Sonoo Thadaney ${ }^{5}$; Abraham Verghese ${ }^{5}$; Donna M. 
Zulman ${ }^{6} .{ }^{1}$ Stanford University, Menlo Park, CA; ${ }^{2}$ Stanford University School of Medicine, Stanford, CA; ${ }^{3}$ Veterans Affairs, Menlo Park, CA; ${ }^{4}$ Stanford University School of Medicine, Mountain View, CA; ${ }^{5}$ Stanford University, Stanford, CA; ${ }^{6}$ Stanford University and VA Palo Alto, Stanford, CA. (Control ID \#3185651)

BACKGROUND: Physician disenchantment and burnout are driving demand for strategies that facilitate "clinician presence" and foster meaningful human connection during medical encounters. We sought to investigate the concept of presence through interviews with primary care physicians and diverse non-medical professionals from fields in which human connection is central.

METHODS: We conducted 40 semi-structured interviews with physicians $(\mathrm{n}=10)$ and non-medical professionals $(\mathrm{n}=30)$ to explore how they create connection, engage in interpersonal interaction, and build trust with individuals across different circumstances and contexts. Physicians originated from primary care clinics in three settings that include a community safetynet system, an academic medical system, and a Veterans Affairs facility. Non-medical professionals represented diverse fields, including protective services (e.g., police officer, firefighter), business (e.g., restauranteur, salesperson), management (CEO, school principal), education, art/design/entertainment (e.g., professional musician, documentary filmmaker), and social services (social worker, chaplain). Interviews were recorded and transcribed; we used a constant comparative method to code transcripts as a research team. Interview excerpts relevant to presence were independently analyzed by two qualitative researchers to generate core elements of presence. These elements were iteratively refined into a working definition of presence through team discussions and research meetings.

RESULTS: Data synthesis generated a definition for presence as, "a purposeful practice of awareness, focus, and attention with the intent to understand and connect with individuals/patients." For both medical and non-medical professionals, creating presence requires managing and considering time and environmental factors; for medical professionals in particular, this includes managing and integrating technology. Listening was described as central to creating the state of being present. Within a clinical setting, presence might manifest as a physician listening without interrupting, focusing intentionally on the patient, taking brief re-centering breaks throughout a clinic day; and informing patients when attention must be redirected to administrative or technology demands.

CONCLUSIONS: Clinician presence involves learning to step back, pause, and prepare to receive a patient's story. Building on strategies from physicians and non-medical professionals, clinician presence is best enacted through purposeful intention to connect, conscious navigation of time, and proactive management of technology and the environment in order to focus attention on the patient. Everyday practice or ritual supporting these strategies is paramount and could support physician self-care as well as physician-patient connection.

\section{PREVALENCE AND DETECTION OF COGNITIVE IMPAIRMENT IN PRIMARY CARE}

Laura M. Curtis ${ }^{1}$; Rebecca Mullen ${ }^{1}$; Rachel O'Conor ${ }^{1}$; Julia Yoshino $\overline{B e n a v e n t e}^{1}$; Mary J. Kwasny ${ }^{1}$; Marina Arvanitis ${ }^{1}$; Derin Cobia ${ }^{3}$; Alex Federman ${ }^{2}$; Michael S. Wolf ${ }^{1}{ }^{1}$ Northwestern University, Chicago, IL; ${ }^{2}$ Icahn School of Medicine at Mount Sinai, New York, NY; ${ }^{3}$ Brigham Young University, Provo, UT. (Control ID \#3185447)

BACKGROUND: Cognitive impairment can affect one's ability to effectively understand and independently self-manage health and healthcare. Despite incomplete guidelines regarding screening, primary care is an optimal place for detecting impairments, as it may inform clinical decision making, referrals, and opportunities to involve families and other caregivers in a patient's care. We sought to determine the prevalence of cognitive impairment among an ongoing cohort of community dwelling older adults and to determine whether any type of impairment was documented in patients' medical charts.

METHODS: 610 adults between the ages of 55 and 74 were recruited from a general internal medicine ambulatory care clinic in Chicago. Structured interviews were conducted at baseline between 2008 and 2010 and participants, to date, have been followed every 2.5-5 years. Impairment was determined using an extensive neuropsychological battery covering five domains and categorized as mild, moderate, or severe. ICD codes for dementia, mild cognitive impairment (MCI), and other cognitive-related deficits were extracted from participants' medical charts from enrollment date through December 2018. Detection was defined in those with cognitive impairment if an ICD code was documented prior to or within one year after impairment was assessed. Impairment and detection variables were compared by sociodemographic characteristics and health status.

RESULTS: Participants were 70\% female, 28\% African American, $64 \%$ White, had a mean (SD) age of 63.4 (5.5) at baseline and mean follow-up time of 7.1 (2.0) years. We detected impairment in $33 \%$ over the course of the existing study period post-baseline $(n=204) ; 52.5 \%$ of these had mild, $26.5 \%$ moderate, and $21.0 \%$ severe cognitive impairment. Impairment was associated with older age, lower education and income, and greater co-morbidity. In those with impairment, $30(15 \%)$ were detected within a year; an additional 35 $(17 \%)$ were detected an average of $5.2(\mathrm{SD}=2.5$; range 1.1-10.1) years later. Detection within a year was associated with older age (mean 68.5 (6.8) vs 65.0 (6.1), $\mathrm{p}=0.005$ ) and greater co-morbidity (mean 3.5 chronic conditions (1.8) detected vs. 2.8 (1.7) undetected, $\mathrm{p}=0.02)$ at the time of impairment. Detection was not associated with education or income.

CONCLUSIONS: Findings from this single-site investigation reveal a prevalence of cognitive impairment much higher than prior studies have reported. Rates of detection were exceptionally low, with more cases detected long after the patient may have exhibited worsening abilities. Further research is needed to better understand whether similarly high rates of impairment may be found in diverse primary care settings, and ways to improve case finding and referrals.

PREVALENCE AND IMPACT OF PRIOR TRAUMA EXPOSURE AMONG PATIENTS PRESENTING TO THE EMERGENCY DEPARTMENT WITH SUSPECTED ACUTE CORONARY SYNDROME

Justin Young $^{1}$; Adam Schweber ${ }^{1}$; Jennifer A. Sumner ${ }^{2}$; Laura Meli ${ }^{2}$; Ian M. Kronish ${ }^{2}{ }^{1}$ Columbia University Vagelos College of Physicians and Surgeons, New York, NY; ${ }^{2}$ Columbia University Medical Center, New York, NY. (Control ID \#3183625)

BACKGROUND: Trauma exposure is associated with higher rates of psychiatric morbidity, negative health outcomes, and greater vulnerability to retraumatization during acute medical care, particularly among patients who develop PTSD symptoms related to the trauma. Few studies have assessed the prevalence and impact of prior trauma exposure and associated PTSD symptoms in patients presenting in the emergency department (ED) setting for suspected acute coronary syndrome (ACS). We quantified (1) the prevalence of prior trauma exposure and PTSD symptoms among patients presenting to the ED for suspected ACS and (2) the risk of developing elevated PTSD symptoms after suspected ACS given the presence of elevated PTSD symptoms due to prior trauma.

METHODS: From 2014-2018, we enrolled a consecutive sample of patients presenting to the ED of an urban, academic medical center for suspected ACS. Prior trauma exposure was evaluated using the Life Events Checklist (LEC5), assessing lifetime exposure to 17 types of traumas. Patients also identified the most distressing prior event. Past-month PTSD symptoms associated with the most distressing event were assessed using the 17-item PTSD Checklist (PCL-S); PTSD symptoms due to the suspected ACS were again measured with the PCL-S at 1-month. A PCL-S score $>=34$ indicated elevated PTSD symptoms. Modified Poisson regression was used to estimate the relative risk of developing elevated PTSD symptoms associated with the suspected ACS event contingent on whether the patient had prior elevated PTSD symptoms. A variety of demographic and clinical variables were included as potential confounders.

RESULTS: Of 932 patients presenting to the ED for suspected ACS (mean 
age 60.8 years, $46.5 \%$ women, $56.7 \%$ Hispanic), $73 \%(n=712)$ had experienced at least one type of prior traumatic event. Among trauma-exposed participants, the median number of trauma types was 3.0 (range=1-16), with the most common types including transportation accident (32.2\%), natural disaster (29.7\%), and life-threatening illness/injury (20.4\%). $21 \%$ of participants $(n=196)$ had elevated PTSD symptoms due to their most distressing prior trauma. $42.2 \%$ of these patients with probable PTSD from prior trauma developed elevated PTSD symptoms due to the suspected ACS at 1-month follow-up. In contrast, only $9.6 \%$ of patients without elevated symptoms due to prior trauma developed elevated PTSD symptoms after suspected ACS. When adjusting for demographic and clinical variables, the adjusted relative risk was 4.1 (95\% CI: 3.0-5.6; p<.001).

CONCLUSIONS: Our findings indicate that among patients presenting with suspected ACS in an urban ED, there is a high prevalence of trauma exposure and associated PTSD symptoms. Patients with elevated PTSD symptoms due to prior trauma are at markedly increased risk of developing subsequent PTSD symptoms related to a suspected ACS event, suggesting that prior traumarelated psychopathology is associated with greater vulnerability to developing PTSD symptoms after a suspected ACS event.

\section{PREVALENCE AND PREDICTORS OF IMPOSTER PHENOME- NON AMONG HARVARD MEDICAL STUDENTS}

Alison M. Holliday $^{1}$; David E. Leaf ${ }^{3,1}{ }^{1}$; Galina Gheihman ${ }^{1}$; Cynthia M. Cooper $^{2}{ }^{1}$; Amy M. Sullivan ${ }^{1}$; Rebecca Karp Leaf ${ }^{2},{ }^{1}$. ${ }^{1}$ Harvard Medical School, Boston, MA; ${ }^{2}$ Massachusetts General Hospital, Boston, MA; ${ }^{3}$ Brigham and Women's Hospital, Boston, MA. (Control ID \#3179336)

BACKGROUND: The Imposter Phenomenon (IP) is a psychological construct characterized by feelings of inadequacy, drive for perfectionism, and fear of being discovered as a "fraud" by colleagues and supervisors. IP is known to limit performance and professional growth, and is associated with high rates of anxiety and burnout. Although IP is known to occur commonly among high-achieving individuals in various professional fields, limited data are available on IP among medical students.

METHODS: We conducted a cross-sectional web-based survey of all Harvard Medical and Dental School students to identify the prevalence and predictors of IP. We used the Clance Imposter Phenomenon Scale (CIPS), which is a validated instrument for assessing IP. CIPS consists of 20 questions based on a 5-point Likert scale, with a score of 62 or higher corresponding with experiencing IP and a score of 80 or higher corresponding with experiencing intense IP. The survey was distributed between June and August 2018 via an email distribution list. We collected demographic data including age, gender, race, ethnicity, medical school track (MD/PhD, Health Sciences and Technology/ physician-scientist, etc.), intended medical specialty, college major, whether the student took time off prior to medical school, and whether the student had a first-degree family member in medicine. We used logistic regression to identify predictors of IP.

RESULTS: Of the 753 students surveyed, 519 (68.92\%) responded, with $485(93.45 \%)$ completing all 20 CIPS questions. Among students who completed the CIPS questions, $285(58.76 \%)$ had a score of 62 or higher, corresponding with experiencing IP, and $72(14.85 \%)$ had a score of 80 or higher, corresponding with experiencing intense IP. Predictors of intense IP included female gender (odds ratio [OR] 1.95, 95\% confidence interval [CI] 1.15 to $3.31, \mathrm{p}=0.01$ ), time off prior to medical school (OR 1.77, $95 \%$ CI 1.07 to $2.93, \mathrm{p}=0.03$ ), and age (years, OR $1.11,95 \%$ CI 1.02 to 1.22 , $\mathrm{p}=0.02$ ). There was no association between intense IP and Caucasian race $(\mathrm{p}=0.40)$, having a first-degree family member who is a physician $(\mathrm{p}=0.76)$, or intention to pursue a surgical specialty $(\mathrm{p}=0.99)$.

CONCLUSIONS: IP is widespread among students at Harvard Medical and Dental School, particularly among women. Future research is needed to understand the relationship between IP and academic performance, as well as the progression of IP over time during residency, fellowship, and attending years.

PREVALENCE AND PREDICTORS OF PLEURAL EFFUSION
IN CONGESTIVE HEART FAILURE

Oreoluwa Oladiran; Rashmi Dhital. Reading Hospital, Wyomissing, PA. (Control ID \#3180664)

BACKGROUND: Pleural effusion is common in congestive heart failure (CHF) and usually portends a poor prognosis, however its prevalence and distribution is not well documented in literature. We sought to query a large national database to determine the prevalence, demographic and clinical factors associated with cardiogenic pleural effusions.

METHODS: We used National (Nationwide) Inpatient Sample database, the largest publicly available all-payer inpatient database in the US that contains $\sim 20 \%$ sample of all US hospitalizations ( $>7$ million hospital stays each year), to identify adult inpatients with the diagnosis of CHF and pleural effusion using ICD-9 codes. We excluded cases citing other possible causes of pleural effusion including liver cirrhosis, infectious and malignant effusions, and then compared various demographic and clinical factors among CHF in-patients with or without pleural effusion.

RESULTS: In the years 2012 and 2013, after excluding other common etiologies of pleural effusion, a total of 1,172,067 patients were admitted with CHF in the United States, out of which 40,319 (3.4\%) were coded for as pleural effusion. Heart failure patients with a pleural effusion were older than those without ( 74.9 versus 72.1 years, $\mathrm{p}<0.001$ ), more likely Caucasian $(76 \%$ versus $70 \%, \mathrm{p}<0.001)$. They were also found to have had a significantly increased length of stay $(9.0$ vs 5.4 days, $\mathrm{p}<0.001)$ and significantly higher inpatient mortality $(6.2 \%$ versus $3.2 \%, \mathrm{p}<0.001)$.

CONCLUSIONS: Among patients with heart failure, pleural effusion is uncommon, but is associated with higher lengths of stay and higher inpatient morbidity. Whether this is the reflection of poorer cardiac function or is an independent predictor of adverse outcomes is a matter for further study.

\begin{tabular}{|c|c|c|c|}
\hline & $\begin{array}{l}\text { CHF with pleural } \\
\text { effusion } \\
\mathrm{N}=201,595\end{array}$ & $\begin{array}{l}\text { CHF without } \\
\text { pleural effusion } \\
\mathrm{N}=5658739\end{array}$ & P-value \\
\hline $\begin{array}{l}\text { Mean age } \\
\text { (year } \pm \text { S.E) }\end{array}$ & $74.9 \pm 0.9$ & $72.1 \pm 0.6$ & \\
\hline $\begin{array}{c}\text { Age Categories }(\%) \\
18-34 \text { years } \\
35-64 \text { years } \\
>/-65 \text { years } \\
\end{array}$ & $\begin{array}{l}0.9 \\
19.6 \\
79.5 \\
\end{array}$ & $\begin{array}{l}1.1 \\
26.9 \\
72.0 \\
\end{array}$ & $\begin{array}{l}<0.001 \\
<0.001 \\
<0.001 \\
\end{array}$ \\
\hline $\begin{array}{l}\text { Gender }(\%) \\
\text { Male } \\
\text { Female } \\
\end{array}$ & $\begin{array}{l}48.9 \\
51.1\end{array}$ & $\begin{array}{l}48.2 \\
51.8\end{array}$ & $\begin{array}{l}<0.005 \\
<0.005\end{array}$ \\
\hline $\begin{array}{l}\text { Length of stay (days } \\
\text { SE) }\end{array}$ & 9.0 & 5.4 & \\
\hline Mortality (\%) & 6.2 & 3.2 & $<0.001$ \\
\hline $\begin{array}{c}\text { Race }(\%) \\
\text { Caucasians } \\
\text { African Americans } \\
\text { Others }\end{array}$ & $\begin{array}{l}76.8 \\
10.8 \\
12.4\end{array}$ & $\begin{array}{l}70.2 \\
18.3 \\
11.5\end{array}$ & $\begin{array}{l}<0.001 \\
<0.001 \\
<0.001 \\
\end{array}$ \\
\hline
\end{tabular}

PREVALENCE OF ASPIRIN USE FOR PRIMARY PREVENTION OF CARDIOVASCULAR DISEASE

Colin W. O'Brien; Stephen P. Juraschek; Christina C. Wee. Beth Israel Deaconess Medical Center, Boston, MA. (Control ID \#3175838)

BACKGROUND: Multiple practice guidelines have recommended use of aspirin (ASA) for primary prevention of cardiovascular disease (CVD) in adults at increased risk. However, three randomized control trials published in 2018 - ASCEND, ARRIVE and ASPREE - demonstrated minimal benefits and consistent bleeding risks, particularly for older adults. Our goal was to quantify aspirin use and characterize factors associated with use among US adults who take aspirin for primary prevention.

METHODS: We used the 2017 National Health Interview Survey (NHIS), a nationally representative in-person household survey, to examine rates of self-reported aspirin use to prevent heart disease among US adults aged 40 and older without CVD. We used multivariable logistic regression to 
characterize demographic and clinical factors associated with aspirin use. Analyses were weighted and adjusted using SAS 9.4 to account for the complex NHIS sampling design.

RESULTS: The mean age of our study sample was 57.5 years. Of this sample, $54 \%$ were women and $33 \%$ were non-white. There were $23.4 \%$ of adults 40 years without CVD taking a daily aspirin for the prevention of heart disease, representing approximately 29 million people. Among this population, $22.8 \%$ did so without a physician's recommendation. Aspirin use was higher with greater age: $7 \%$ of those aged $40-49$ without CVD reported aspirin use, compared to $46.2 \%$ aged 80 or older. After adjustment, older age and male sex were significantly associated with aspirin use (see table), as were Asian race (compared to white), diabetes, hypertension, hyperlipidemia, obesity, smoking, and having seen a doctor in the previous year. Peptic ulcer disease was not significantly associated with aspirin use, OR 0.90 (95\% CI 0.74 - 1.09).

CONCLUSIONS: Over 29 million US adults aged 40 and older without CVD use aspirin to prevent heart disease, including many octogenarians and adults with a history of peptic ulcer disease. This study highlights an important role for physicians to discuss the latest aspirin trials' findings with US adults to minimize risks and optimize benefits from aspirin use.

\begin{tabular}{lll}
\hline $\begin{array}{c}\text { Characteristic } \\
\text { of Interest }\end{array}$ & $\begin{array}{c}\text { Prevalence of } \\
\text { ASA Use (\%) }\end{array}$ & $\begin{array}{c}\text { Odds of ASA Use } \\
\text { (AOR*, 95\% CI) }\end{array}$ \\
\hline Sex & & \\
\hline Men & $25.5 \%$ & $1.32(1.18-1.48)$ \\
\hline Women & $21.8 \%$ & 1.00 \\
\hline Age & & \\
\hline $40-49$ & $7.0 \%$ & 1.00 \\
\hline $50-59$ & $18.4 \%$ & $2.52(2.11-3.01)$ \\
\hline $60-69$ & $34.7 \%$ & $5.55(4.59-6.71)$ \\
\hline $70-79$ & $44.6 \%$ & $7.71(6.35-9.35)$ \\
\hline $80+$ & $46.2 \%$ & $9.62(7.56-12.25)$ \\
\hline
\end{tabular}

*Also adjusted for race, BMI, HTN, HLD, DM, ulcer disease, smoking, and seeing a physician in the last year.

Aspirin Use Among Adults 40+ Without CVD

\section{PREVALENCE OF INTERNAL MEDICINE RESIDENTS WITH} AN ESTABLISHED PRIMARY CARE PROVIDER

Mark Connor; Robert L. Fogerty. Yale School of Medicine, New Haven, CT. (Control ID \#3185782)

BACKGROUND: The benefits of having an established primary care provider (PCP), especially in regards to decreased all-cause mortality and improved mental illness outcomes, is well established. CDC data indicates that the national averages for US men and women without a primary care physician are $28 \%$ and $17 \%$, respectively, and $19 \%$ and $10 \%$ in Connecticut. Recent healthcare trends have highlighted that millennials, those born between 1981 and 1996, are opting for more expedited and convenient access to medical care, thereby moving away from establishing and maintaining a primary care provider. The prevalence rate of resident physician trainees, the majority of whom are millennials, that have established care with a primary care doctor as well as their opinion towards having a PCP are currently unknown.

METHODS: An electronic twenty-question survey of privately insured, internal medicine residents with a response rate of $67.3 \%$ at a large academic center in Connecticut was utilized to determine various aspects of resident wellness including whether or not residents established care with a local primary care physician and their opinion regarding the utility of establishing care with a local primary care physician.
RESULTS: While $90 \%$ of the survey responders believe it is worthwhile to establish care with a local primary care physician, the survey found that $57 \%$ of resident physicians $(57.3 \%$ women, $57.1 \%$ men) had not established care with a local primary care provider. Per the CDC, the prevalence rates of Connecticut adults aged 25-34 years and 35-44 years not having a PCP are $30.6 \%$ (CI 26.7-34.4\%) and $17.9 \%$ (CI 15.0-20.9\%), respectively. These data suggest that internal medicine residents are establishing with primary care providers at a lower rate than age and geographically matched peers.

CONCLUSIONS: The survey suggests that although internal medicine residents believe it is valuable to establish care with a primary care provider, residents may be establishing with a primary care provider at a lower rate than age and geographically matched peers. These findings are of interest to residency programs directors due to concerns for wellbeing, including physician burnout of internal medicine residents. Additionally, these data suggest that there may be barriers to establishing care that warrant further investigation.

\section{PREVALENCE OF MULTIPLE CHRONIC CONDITIONS BY US STATE, 2017}

Daniel Newman; Erica Levine; Sandeep Kishore. Icahn School of Medicine at Mount Sinai, New York, NY. (Control ID \#3162709)

BACKGROUND: Understanding the prevalence of multiple (two or more) chronic conditions (MCC) is increasingly being recognized as a public health imperative. MCCs are associated with increased cost, increased burden of illness, and decreased quality of life. The objective of this research is to describe the prevalence and distribution of adults with MCC across the United States using 2017 data from the Behavioral Risk Factors Surveillance System (BRFSS).

METHODS: Prevalence of MCC (defined as having two or more of 12 diagnosed chronic conditions) was calculated for each U.S. state and territory overall as well as by sex and age.

RESULTS: Statewide prevalence of MCC ranged from $37.9 \%$ in the District of Columbia to $64.4 \%$ in West Virginia. Females had a higher prevalence than males in 47 of $53(89 \%)$ states and territories, and MCC prevalence increased with age in every state and territory.

CONCLUSIONS: The relative geographic and demographic distribution of MCC prevalence is largely consistent with prior research using data from the National Health Interview Survey (NHIS); however, overall prevalence estimates are noticeably higher due partly to the inclusion of high cholesterol, obesity, and depression as chronic conditions. This analysis is, to our knowledge, the first to analyze MCC prevalence across the United States using BRFSS data. Further, it extends and expands on prior research into the nationwide prevalence of MCC by incorporating more chronic conditions and using the most recent data available. These findings help to build an accurate and granular picture of the prevalence of multiple chronic conditions across the United States and will aid public health officials in creating programs targeted to their region.

\section{PREVALENCE OF POLYPHARMACY IN OLDER ADULTS - A CROSS-SECTIONAL ANALYSIS}

Michael I. Ellenbogen ${ }^{1}$; Peiqi Wang ${ }^{1}$; Heidi Overton ${ }^{1}$; Christine Fahim ${ }^{2}$; Angela Park ${ }^{1}$; William Bruhn ${ }^{1}$; Jennifer L. Carnahan ${ }^{3}$; Amy Linsky ${ }^{4}$; Seki Balogun'; Martin Makary ${ }^{1} .{ }^{1}$ Johns Hopkins School of Medicine, Baltimore, MD; ${ }^{2}$ Health Policy and Management, Baltimore, MD; ${ }^{3}$ Indiana University Center for Aging Research/Regenstrief Institute, Indianapolis, IN; ${ }^{4}$ VA Boston Healthcare, Boston, MA; ${ }^{5}$ University of Virginia, Charlottesville, VA. (Control ID \#3180213)

BACKGROUND: Polypharmacy in older patients increases the risk of medication-related adverse events and can be a marker of unnecessary care. We sought to describe the prevalence of polypharmacy among patients 65 years of age or older and identify factors associated with patient-level and physician-level polypharmacy prescribing. 
METHODS: We performed a cross-sectional analysis of $100 \%$ Medicare claims data from January 1 to December 31, 2016. All patients with continuous Medicare fee-for-service insurance (Parts A, B, and D) throughout 2016 who were 65 years of age or older and who were prescribed at least one medication for at least 30 days during this time were included. Each patient was attributed to the primary care physician (PCP) who prescribed them the most medications. PCPs treating fewer than ten patients were excluded. We defined polypharmacy as the highest number of concurrent medications at any point during the year. We characterized the distribution of PCPs prescribing by these metrics. We used hierarchical linear regression to study patient- and physician-level characteristics associated with high prescribing rates.

RESULTS: We identified 25,747,560 patients attributed to 147,879 PCPs. The patient-level mean concurrent medication rate was 5.6 (standard deviation 3.3). The physician-level mean number of concurrent medications was 5.6 (range 1.3-14.8; sd 1.08). The cutoff for high outlier physician was $7.7(=5.6+2 \times 1.08)$ concurrent medications per patient (Figure 1), which identified $4.1 \%$ of all physicians as outliers $(6,108$ physicians). A number of patient-level factors were associated with an adjusted increase in concurrent medications greater than 1.00 including: an Elixhauser score of 5 or more $(2.49$; $95 \%$ confidence interval 2.49$2.50)$, history of solid organ transplant (1.32; 1.27-1.37), diabetes (1.39; 1.39-1.40), and HIV/AIDS (1.89; 1.84-1.94).

CONCLUSIONS: Patterns of high prescribing to older patients are common and measurable at the physician-level. Addressing high outlier prescribers may represent an opportunity to reduce avoidable harm and excessive costs.

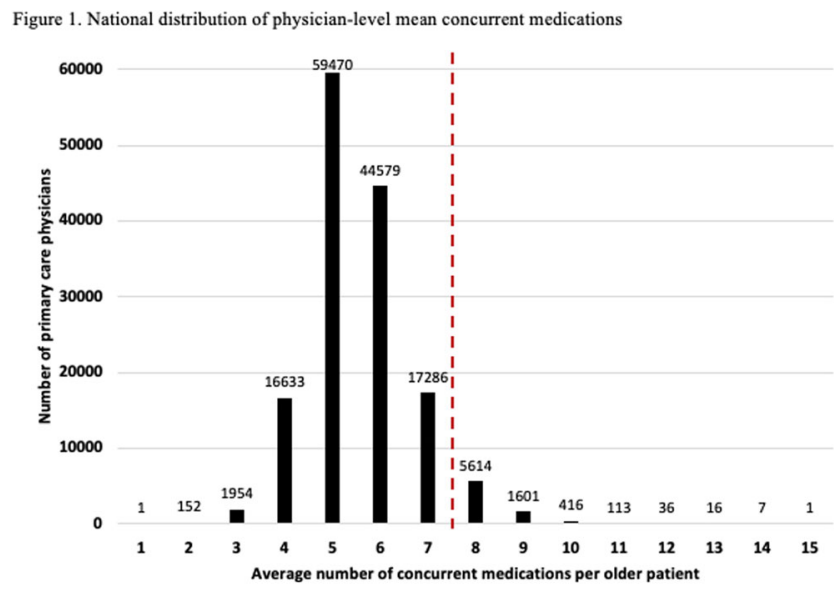

Note: Dashed red line indicated 2 standard deviations above mean number of concurrent medications $(7.7=5.6+2 \times 1.08)$

\section{PRIMARY CARE HOME VISITS AND HEALTHCARE UTILI- ZATION: A COHORT STUDY}

Xin Zhang; Jennifer Manggaard; Ondler Hinson Heather; Paul Takahashi. Mayo Clinic, Rochester, MN. (Control ID \#3181442)

BACKGROUND: While the outpatient office is the traditional care delivery model for primary care, primary care home visits are important to provide care for frail elderly patients. These patients have difficulty with functional limitations, socioeconomic reasons, and lack of transportation in coming into clinic. While some primary care providers and geriatric fellows perform home visits to provide care for those patients, but we do not know the healthcare utilization for this important group of patients. In this study, we aim to describe the patients who are served and describe emergency department (ED) visits and hospitalizations within 90 days of index home visit date.

METHODS: We evaluated all patients 65 years of age who had home visits and who were not enrolled in post-hospitalization transition, palliative or hospice programs from 2010-2017. Descriptive statistics were used to examine patient demographics and comorbidity health burden. ED visits and hospitalizations within 90 days post index home visit were collected. RESULTS: A total of 609 patients, 181 males and 427 females, were included. Average age at time of home visit was 85 years (65-105 years). Majority of the subjects were Caucasians $(561,92 \%)$ and a minority were married (196, 32\%). $43 \%$ of patients (260) were community dwelling, while the majority lived in assisted living. Most common medical conditions include hypertension (72\%), dementia (51\%), depression (34\%), cancer (28\%), osteoporosis $(28 \%)$, coronary artery disease $(25 \%)$, cardiac arrhythmia $(25 \%)$, diabetes $(21 \%)$, and congestive heart failure (18\%). 189 (31\%) patients had ED visits within 90 days; $68 \%$ (129) had 1 visit and 32\% (6) had 1 visit. Most common reasons for ED visit were fall (20\%), dyspnea (8\%), altered mental status (8\%), and musculoskeletal pain (8\%). 137 patients (22\%) had hospitalizations within 90 days; 90 (66\%) had 1 and 47 (34\%) had 1 hospitalizations. The main diagnosis for hospitalization includes infection (15\%), fall and/or fracture (12\%), behavioral disturbance (8\%), and heart failure (5\%).

CONCLUSIONS: In our patient population, $31 \%$ had ED visits and $22 \%$ had hospitalizations in 90 days after home visits. The 90 day hospitalization rate was $29.9 \%$ in a prior study on identified high risk patients who were involved in a post-hospitalization care transitions program at the Mayo Clinic 1. We found a similar 90 day hospitalization rate compared to that study, which suggests that our patients are similarly frail, medically complex, and prone to ED visits and hospitalizations. Further study is needed to improve care for this high risk population. 1. Takahashi PY, Naessens JM, Peterson $\mathrm{SM}$, et al. Short-term and long-term effectiveness of a post-hospital care transitions program in an older, medically complex population. Healthc (Amst). 2016;4(1):30-35.

\section{PRIMARY CARE PHYSICIANS' KNOWLEDGE OF THE CARDIO- VASCULAR EFFECTS OF DIABETES MEDICATIONS}

Jay H. Shubrook ${ }^{2}$; Jonathan Pak ${ }^{1}$; George E. Dailey ${ }^{3}$. ${ }^{1}$ Boehringer Ingelheim Pharmaceuticals, Inc., Ridgefield, CT; ${ }^{2}$ Touro University, Fairfield, CA; ${ }^{3}$ Scripps Health, La Jolla, CA. (Control ID \#3176456)

BACKGROUND: Cardiovascular (CV) disease is a leading cause of death in patients with type 2 diabetes (T2D). Several CV outcomes trials (CVOTs) have shown that specific antidiabetes agents improve CV outcomes in patients with $\mathrm{T} 2 \mathrm{D}$ and high $\mathrm{CV}$ risk or $\mathrm{CV}$ disease. This survey examined knowledge and beliefs about CVOTs among US internal medicine (IM) and family physicians (FP).

METHODS: A 23-item survey was administered by WebMD, LLC. from Sept 18-20, 2018. The survey was sent to 47,534 Medscape members who were randomly selected across US regions and specialties (quota, 250 per specialty). A z-test was used for statistical comparisons. The Touro University California IRB deemed that the study protocol met exemption criteria (TUC IRB \#M-3118).

RESULTS: Overall, 702 physicians responded, 199 did not meet eligibility criteria and 503 completed the survey. Familiarity with CVOTs was low, with $8-42 \%$ of respondents indicating that they were moderately or extremely familiar with each of 14 CVOTs listed. Respondents reported that they were more familiar with TECOS, LEADER, EMPA-REG OUTCOME and CANVAS than other CVOTs $(P<.05)$. Also, 38\%, 33\%, 28\% and $11 \%$ correctly responded that EMPA-REG OUTCOME, LEADER, CANVAS and SUSTAIN-6, respectively, showed superiority vs placebo for the primary composite major adverse CV event [MACE] endpoint; $26 \%$ did not know. Similarly, $31 \%$ of respondents did not know which CVOTs showed superiority for CV mortality; $36 \%$ and $26 \%$ correctly chose EMPA-REG OUTCOME and LEADER. Nearly half did not know which CVOT showed superiority for heart failure hospitalization. More than half of respondents incorrectly thought that acute pancreatitis occurred significantly more frequently with DPP-4 inhibitors or GLP-1 RAs vs placebo. Only $8 \%$ knew that retinopathy occurred more frequently with semaglutide than placebo in SUSTAIN-6. CANVAS was not well recognized for its risk of lower limb amputations (36\% selected CANVAS alone). However, $69 \%$ recognized the increased rate of genital infections with SGLT2 inhibitors in EMPA-REG OUTCOME, CANVAS, or both trials. 
CONCLUSIONS: Findings suggest knowledge gaps among IMs and FPs regarding CVOT results, which has implication for the treatment of patients with $\mathrm{T} 2 \mathrm{D}$ and $\mathrm{CV}$ disease.

\section{PRIMARY CARE PROVIDER ATTITUDES AND PERSPECTIVES ON HEPATITIS C VIRUS (HCV) MANAGEMENT IN PRIMARY CARE}

Yvette Ryan; Aaron Null; Sopheap Na. Rocky Mountain Regional VA Medical Center, Aurora, CO. (Control ID \#3184621)

BACKGROUND: The limited availability of specialists to treat the 2.4 million Americans living with Hepatitis $\mathrm{C}$ virus $(\mathrm{HCV})$ has led to the idea of shifting $\mathrm{HCV}$ management to primary care providers (PCPs). Although a recent study demonstrated HCV treatment by PCPs is as safe and effective as treatment by specialists, it is not known how PCPs feel about adding HCV treatment to the burden of chronic diseases they already manage. In this qualitative study, we explored PCP attitudes and opinions towards managing $\mathrm{HCV}$ and how they view the role of clinical pharmacists in taking on this role.

METHODS: We performed semi-structured interviews of 10 PCPs at a VA Medical Center, representing PCPs at both the main campus and community clinics. We conducted interviews both in-person and via telephone, using purposive sampling. The interview guide addressed chronic disease management $(\mathrm{CDM})$ burden in primary care, facilitators and barriers to PCPs treating HCV, and how PCPs view clinical pharmacists in CDM within the VA system. We transcribed all interviews at time of the interview and maintained field notes. We utilized open coding with all interviews coded independently by two members of the team and reconciled coding differences jointly.

RESULTS: Of participating PCPs, 20\% thought that PCPs could take on HCV treatment and management in their current work environment. We identified themes of limited PCP access and provider education as the most prominent barriers to PCPs managing HCV treatment. Providers identified PharmDs as valuable partners in CDM, and most thought they can facilitate treatment of HCV in primary care. PCPs found clinical pharmacists helpful in improving patient access, patient safety, and were strong allies in team based care. Despite having HCV trained clinical pharmacists available to co-manage HCV screening and treatment in primary care, none of the participating PCPs were fully aware of this existing support structure. Some PCPs expressed concern that PharmD co-management of HCV treatment would limit their own knowledge and skill in treating HCV independently.

CONCLUSIONS: Although evidence suggests HCV treatment in primary care has similar outcomes to treatment by specialists, PCPs appear reluctant in taking on this responsibility. While HCV treatment in primary care is a promising strategy, health systems need to be cognizant of PCP access and the CDM burden PCPs already face. PCPs do value clinical pharmacist co-management of chronic diseases and further integration of such workflows may improve access, allowing more patients with HCV to be treated. Despite HCV trained PharmDs being in place at the VA, there is limited PCP knowledge about them and more effort needs to be performed to ensure dissemination and utilization of these clinical support structures.

PRIMARY CARE PROVIDER GENDER SENSITIVITY TOWARD WOMEN PATIENTS AFFECTS PATIENT CONTINUING USE OF CARE

Claire $\operatorname{Than}^{3}$; Donna L. Washington ${ }^{1}$; Danielle Rose ${ }^{2}$; Ismelda Canelo ${ }^{4,}$; Elizabeth M. Yano ${ }^{3}$. ${ }^{1}$ VA Greater Los Angeles Healthcare, UCLA, Los Angeles, CA; ${ }^{2}$ VA GLA Healthcare System, Sepulveda, CA; ${ }^{3}$ VA Greater Los Angeles Healthcare System, Sepulveda, CA; ${ }^{4}$ VA Greater Los Angeles Healthcare System, North Hills, CA. (Control ID \#3183133)
BACKGROUND: Provider gender sensitivity means that providers are empathetic towards women patients and understand their health care needs. Gender sensitivity has assumed increasing importance in health care as it can affect women patients' experiences with care. Women veterans (WVs) are an extreme numerical minority of patients $(<10 \%)$ seen at the Veterans Health Administration (VA), and about $20-25 \%$ of them discontinue using VA within three years of first VA care use. A high rate of WVs leaving VA raises concerns on their experiences with VA care. Literature suggests that WVs are more likely to report poor satisfaction with VA care and to not use VA care when VA providers are not sensitive to their needs. We evaluated whether provider gender sensitivity is associated with patient discontinuating use of VA care.

METHODS: We used cross-sectional surveys to measure primary care provider (PCP) gender sensitivity based on agreement with 10 items, e.g. "VA should not be expected to provide special health services for women". We surveyed 281 PCPs at 12 VA Medical Centers between September 2014 and June 2015. A total of 94 PCPs completed the surveys (33\% response rate). We identified WV patients cared for by these PCPs in VA outpatient clinics between January 2013 and December 2014. We defined patient discontinuity from PC as having no in-person or telephone visits to any VA PC clinics within three years following their last visits to PCPs in the surveys, i.e. from their last visits between 2013 and 2014 to December 2017. Our analyses included 7,155 WVs who were seen by these PCPs between 2013 and 2014 and were alive through 2017. We used multivariate logistic regression to evaluate the relationship between PCP gender sensitivity and WV discontinuity from VA PC.

RESULTS: Overall, 32\% of WVs in our sample had no visit to PC over three years. Multivariate regression showed that higher PCP gender sensitivity was significantly associated with lower odds of patient discontinuity from VA PC (Adjusted OR=0.80, 95\% CI: 0.70, 0.91), but this association differed by age group. Among WVs in the 18-39 age group, higher PCP gender sensitivity predicted lower odds of WV discontinuing PC use (AOR $=0.64,95 \%$ CI:0.44, 0.95). Among WVs in the 40-49, 5059 and $65+$ age groups, gender sensitivity was not associated with care discontinuity. Among WVs in the 60-64 age group, higher gender sensitivity predicted lower odds of patient discontinuing PC use (AOR $=0.59$, 95\% CI: 0.37, 0.95).

CONCLUSIONS: PCP gender sensitivity was associated with patient's continuing use of VA PC, especially among younger WVs. Future efforts to improve and/or maintain high PCP gender sensitivity can help ensure continuity of care for women patients. An understanding of reasons for their care discontinuity, e.g. not needing care or receiving care elsewhere, may help inform efforts to increase or maintain WVs use of VA care.

\section{PRIMARY CARE PROVIDER PERSPECTIVES ON CALCULAT- ED VERSUS ADJUDICATED RISK STRATIFICATION IN PRI- MARY CARE: A QUALITATIVE STUDY}

Brian Ricci ${ }^{1}$; David Dorr ${ }^{2} .{ }^{1}$ Oregon Health and Science University, Portland, OR; ${ }^{2} \mathrm{OHSU}$, Portland, OR. (Control ID \#3185751)

BACKGROUND: Risk stratification (RS) is a process by which a population of empaneled patients are assessed for risk of poor health outcomes by an algorithm with or without a human review process (known as adjudication). RS computer algorithms seek to predict healthcare utilization, costs, morbidity and mortality, but are often limited to only specific factors calculable in the original data, such as from an electronic health record (2). However, confidence in RS processes are increased when computer-derived scores are adjudicated by providers with patient-specific knowledge (3). Using a natural health reform experiment, our objective was to identify factors that are considered by providers during the adjudication process.

METHODS: In the Comprehensive Primary Care+ $(\mathrm{CPC}+)$ initiative from the Center for Medicare and Medicaid Innovation, RS with 
adjudication was required to identify high needs patients, especially those at risk of exacerbations leading to hospitalization or ED utilization. For our study we used the Epic Risk Score tool which has been validated to specifically predict hospitalizations and ED utilization (AUC 0.63-0.78 at three different sites). Participating primary care providers in one $\mathrm{CPC}+$ practice received instruction on the RS tool prior to participation in this study. We used a think aloud protocol and structured interviews with providers as they adjudicated their own patient panels. We used a modified immersion/crystallization approach to analyze responses into factors and themes.

RESULTS: Ten PCPs participated, adjudicating a total of 2500 patients. Overall providers felt that RS was helpful in identifying patients at risk for ED visits or hospitalization. Providers cited self-management skills (health literacy, engagement with care plans, adherence to medications, etc.), availability of support systems and severity of disease as factors that were considered in the risk adjudication process. Providers felt that the computer algorithm did not accurately predict risk among patients with a history of psychological trauma, intimate partner violence, substance use disorders or mental health diagnoses. According to providers, the number of preventable and actionable emergency department (ED) visits and hospital admissions was not always accurately predicted by RS, leading to inappropriate elevation in calculated risk scores. Providers felt that human review/ adjudication is required to predict what patients would benefit from additional clinic resources (e.g., nurse care management)

CONCLUSIONS: Though providers described benefit of RS in terms of identifying those patients at risk for ED utilization or hospitalizations, they felt that RS did not accurately identify severity of disease or social support systems. Human review is required for consideration of the social determinants of health and to identify high need patients that would benefit from additional clinic resources.

\section{PRIMARY CARE PROVIDERS' PERSPECTIVE ON IMPLEMENTING RISK-BASED LUNG CANCER SCREENING FOLLOWING ACADEMIC DETAILING}

Sarah E. Skurla ${ }^{1}$; Julie Lowery ${ }^{1}$; Nathan J. Leishman ${ }^{1}$; Angie Fagerlin ${ }^{1,3}$; Tanner Caverly ${ }^{1,2}{ }^{1}$ Department of Veterans Affairs, Ann Arbor, MI; ${ }^{2}$ University of Michigan Medical School, Ann Arbor, MI; ${ }^{3}$ University of Utah, Salt Lake City, UT. (Control ID \#3184252)

BACKGROUND: Tailoring lung cancer screening (LCS) discussions based on individualized lung cancer risk (risk-based screening) is inherently patient-centered and prevents lung cancer mortality while avoiding excessive testing. However, this approach is new to most primary care providers (PCPs). To understand barriers to and facilitators of implementing a provider tool to aid risk-based LCS, we conducted academic detailing and follow-up qualitative interviews with PCPs.

METHODS: We detailed 105 PCPs at 7 VAs from Jun-Oct 2018. Detailing visits focused on the rationale for taking a risk-based approach to LCS and how to use the web-tool with eligible patients to inform riskbased screening discussions. The detailer took notes of PCP feedback during the visit and we conducted follow-up semi-structured phone interviews with 29 PCPs, 2-4 weeks following the visit. Audio recorded interviews lasted $\sim 20$ minutes and were transcribed verbatim. We used NVivo v12 to conduct a rapid content analysis and created comprehensive summaries to identify themes/patterns.

RESULTS: During the detailing visit, PCPs expressed major concerns about the lack of organizational direction/support regarding LCS, though many felt the tool seemed useful for selecting appropriate candidates. Of the PCPs completing follow-up interviews, many saw value in the tool's ability to 1) shape clinician feelings about LCS and 2) guide a useful approach to LCS discussions. They also felt the tool enhanced their ability to share information about individualized risk and the pros/cons of screening. However, at follow-up few PCPs (6 of 29) had used the tool with an actual patient. Limited time in clinic was perceived as a key barrier by almost all PCPs. Most PCPs reported needing only 1 to 2 minutes to discuss LCS but voiced frequently not having even 1-2 minutes during a visit, due to patient-specific needs that were higher priority (e.g., acute complaints), or organizational priorities (e.g., performance measures). Even small additions to workflow (e.g., inputting risk factors into the tool) were too time consuming. As well, some PCPs maintained a dichotomous view of the LCS decision (i.e. screen if eligible vs don't screen if not), so they did not see a need for a risk-based approach to LCS. PCPs supported several ideas to overcome barriers. They had firm opinions about cutting tool content substantially, better integrating the tool into the EMR, and automating the risk calculation. Engaging non- PCP providers or patients in data entry and initial tool use could help, but there are concerns it may not fit into team workflows and that only a minority of patients would participate.

CONCLUSIONS: Even when PCPs see value in a risk-based approach to LCS and a tool to support that approach, they have major challenges implementing it in clinic. Future risk-based tools need to better integrate into workflows and help PCPs understand how to prioritize LCS among other competing demands.

\section{PRIMARY CARE SATISFACTION WITH COLLABORATIVE CARE SERVICES: THE ROLES OF CARE MANAGEMENT AND TECHNOLOGY}

Lucinda B. Leung $^{1,2}{ }^{2}$; Alexander S. Young ${ }^{3}$; Leonie Heyworth ${ }^{4,}$; Danielle Rose ${ }^{2}$; Susan E. Stockdale ${ }^{2}$; A. Laurie Graaff ${ }^{6}$; Timothy Dresselhaus ${ }^{7,}{ }^{4}$; Lisa V. Rubenstein ${ }^{8} .{ }^{1}$ UCLA David Geffen School of Medicine \& West Los Angles VA Medical Center, Los Angeles, CA; ${ }^{2}$ VA Greater Los Angeles Healthcare System, Los Angeles, CA; ${ }^{3}$ VA \& UCLA, Los Angeles, CA; ${ }^{4}$ VA San Diego Healthcare System, La Jolla, CA; ${ }^{5}$ Office of Connected Care/Telehealth, Washington, DC, DC; ${ }^{6}$ VISN 22 (VA Desert Pacific Healthcare Network), Gilbert, AZ; ${ }^{7}$ VA San Diego Healthcare System, San Diego, CA; ${ }^{8}$ RAND and UCLA, Medford, OR. (Control ID \#3179140)

BACKGROUND: Aiming to improve access to mental health care, collaborative care models embed nurse care managers and mental health specialists to provide services in primary care. A decade after initiation of the Veterans Health Administration's (VA) Primary Care - Mental Health Integration (PC-MHI), representing the nation's largest implementation of such models, we know little about primary care perceptions of these services. Coordination between primary care and mental health specialties is crucial to collaborative care models, and PC-MHI care management and technologies may facilitate specialist communication and urgent care coordination. We examined primary care clinic leader satisfaction with care for various mental and behavioral health conditions and its relationships with key PC-MHI program features - traditional care management and newer information communication technology.

METHODS: This is a cross-sectional key informant organizational survey about PC-MHI among VA primary care clinic leaders (e,g., physicians, nurse practitioners) in Southern California, Arizona, and New Mexico (VA region with the greatest penetration of PC-MHI services) from February to May 2018 ( $n=65$, 94\% response rate). The survey assessed primary care clinic leaders' satisfaction with PC-MHI care, care management availability, and sufficiency of PCMHI information communication technology (telemedicine, electronic referral/consultation, instant messaging). Using $\mathrm{Chi}^{2}$ tests and regressions, we examined relationships between PC leaders' satisfaction and PC-MHI features of care management and of information communication technology $(3$-item scale, $=0.81)$, controlling for clinic size, location, and case-mix.

RESULTS: Primary care clinic leaders were often "very satisfied" with care for target PC-MHI conditions of anxiety (71\%) and depression (69\%), but not for other conditions (37\% alcohol misuse, $19 \%$ pain). Availability of PC-MHI care management was significantly associated with satisfactory depression $(\mathrm{p}=.02)$ and anxiety 
$(\mathrm{p}=.02)$ services. Although PC-MHI information communication technology was rated as only "always sufficient" in $<20 \%$ of sites, it was positively associated with satisfaction for suicide risk management $(\mathrm{p}=.002)$. These associations persisted after adjusting for clinic characteristics.

CONCLUSIONS: Primary care clinic leader satisfaction with PC-MHI care for depression and anxiety was generally high, but not for other common behavioral health conditions. Traditional care management was positively associated with satisfaction in treatment of targeted conditions (i.e., depression, anxiety). While not yet universally accessible among VA primary care clinics, newer PC-MHI information communication technologies were positively associated with satisfaction in time-sensitive suicide evaluation. Increasing availability of PC-MHI features (i.e., care management for common conditions, technology for time-sensitive suicide evaluation) may further practice transformation toward model integration.

\section{PRIMARY CARE SOCIAL NETWORKS AND CANCER PRE- VENTION BEHAVIORS}

Jaya Aysola; Chang Xu; Marilyn M. Schapira. University of Pennsylvania, Philadelphia, PA. (Control ID \#3185397)

BACKGROUND: Critical interest remains in how health care delivery models can leverage social networks, given the mounting evidence of the influence of social networks on the promotion of cancer prevention behaviors, such as diet and exercise. Yet, there lacks studies on both how to measure and seed social networks in the primary care setting. The objective of this study was twofold: 1) to evaluate the feasibility of collecting social network data from primary care patients and 2) to determine the social network characteristics of primary care patients enrolled in our study.

METHODS: We recruited 48 primary care patients as Egos in our network using both in-person waiting room and telephone recruitment. Patients that agreed to participate were designated egos in our network and scheduled for a telephone-administered survey on healthy behaviors and cancer prevention knowledge. That survey began with name generator questions, where we asked egos to name up to six family members and/or friends (alters) without qualifiers, followed by questions eliciting who attends his/her primary care practice, lives locally, and/or discusses health matters with $\mathrm{him} / \mathrm{her}$. Subsequent sampling and recruitment involved snowball sampling to collect data on social networks. We contacted up to two of the six named alters and at least one of the secondary alters (alters' nominated alters), giving preference to those who utilize primary care at the same practice as the ego or at one of the other study sites. A monetary incentive was provided upon completion of the study. We calculated enrollment rates of egos, comparing in-person versus telephone recruitment, as well as alter enrollment rates. We then assessed the density, reciprocity, and transitivity our social network as well as within ego-networks the degree of homophilly of cancer prevention knowledge, obesity, and smoking.

RESULTS: Ego in-person recruitment was more successful than telephone recruitment, with a contact rate of $38 \%$ as compared to $4 \%$. Enrollment rates were $57 \%$ and $65 \%$ were egos and alters, respectively. Study completion rates for egos was $97 \%$ and $100 \%$ for alters. The mean (SD) age of participants was 51 (17). About $31 \%$ of participants had a high-school diploma or less, $42 \%$ reported an annual household income of less than 25,000 , about $38 \%$ had governmental insurance, and about $62 \%$ selfidentified as black. The edge density of our social network was 0.01 , the degree of reciprocity and transitivity was 0.82 and 0.74 respectively. Egonetwork homophily varied by attribute.

CONCLUSIONS: We found snowball recruitment and name generator techniques twere successful in collecting social network data among primary care patients. This study yields key insights into how organizations can measure primary care patient networks and those network characteristics.

PROGNOSTIC ACCURACY AND DISCORDANCE AMONG PATIENTS, CAREGIVERS, AND PHYSICIANS IN ADVANCED CANCER kirti malhotra ${ }^{1}$; Joshua Fenton ${ }^{1}$; Guibo Xing ${ }^{1}$; Paul Duberstein ${ }^{2}$; Ronald Epstein ${ }^{3}$; Richard L. Kravitz ${ }^{1}$ ' UC Davis, Sacramento, $\mathrm{CA} ;{ }^{2} \mathrm{U}$ Rochester, Rochester, NY; ${ }^{3} \mathrm{U}$ of Rochester, Rochester, NY. (Control ID \#3186638)

BACKGROUND: In caring for patients with advanced cancer, shared clinical decision making relies on common understanding of prognosis among patients, caregivers, and physicians. However, prior studies have shown considerable discordance in prognostic estimates. The purpose of this analysis was to assess and compare the accuracy of two-year prognostic estimates provided by patients, caregivers and oncologists. We also evaluated various factors associated with prognostic accuracy.

METHODS: This is a secondary observational analysis of data obtained from the Values and Options in Cancer Care (VOICE) Study (a cluster Randomized Controlled Trial). The study included 38 oncologists, 263 patients with advanced non-hematologic cancer, and 193 of their caregivers from clinics in Sacramento and Western New York. We evaluated prognostic discrimination and calibration within each group (oncologists, patients, caregivers) using the c-statistic and chi-square statistic respectively. We also computed an index of prognostic accuracy by subtracting the two-year prognosis as predicted by patients, caregivers and oncologists (on a 0 , $0.10,0.25,0.50,0.75,0.90,1.0$ scale) from the vital status at two-years (whether the patient lived two years; yes $=1$ or no $=0$ scale). Finally, we constructed multinomial logistic regression models to assess demographic, clinical and attitudinal factors associated with prognostic accuracy.

RESULTS: Oncologists had better prognostic discrimination as compared to both patients $(\mathrm{p}=0.001)$ and caregivers $(\mathrm{p}=0.03)$ : $\mathrm{C}$-statistics for oncologists, patients, and caregivers were 0.81 [95\% CI: 0.76-0.86], 0.62 [95\% CI: $0.55-0.68$ ], and 0.72 [95\% CI: 0.65-0.78] respectively. Oncologists also had superior calibration; their predictions of two-year survival were similar to actual survival $(p$-value $=0.17)$. In contrast, patients' $(p$-value $=0.0001)$ and caregivers' ( $p$-value $=0.003$ ) predictions differed significantly from actual survival. Approximately half of patients' and caregivers' predictions $(50.0 \%$ and $46.0 \%$, respectively) were classified as unduly optimistic while most of oncologist predictions were classified as realistic $(62.0 \%)$. Non-white race and higher levels of social well-being were associated with over-optimism among patients $(\mathrm{p}<0.05)$.

CONCLUSIONS: Our findings suggest that oncologists have good discriminatory ability in their two-year survival predictions and their predictions are well-calibrated. In contrast, patients and caregivers have somewhat inferior discriminatory ability and their predictions are poorly calibrated. In addition, patients and caregivers predictions tend to be more optimistic as compared to the oncologists. Various patient factors such as race, ethnicity, and social and existential well-being may play a role in shaping prognostic accuracy. These findings may help general internists play a more effective role in mediating prognostic discussions and interpreting prognostic information for patients with advanced cancer and their families.

\section{PROMOTING SHARED DECISION-MAKING: LEVERAGING THE PHYSICIAN MORAL COMPASS OR IMPROVING THE WORKPLACE?}

Loren Saulsberry; Madison Wilson; Monica E. Peek. The University of Chicago, Chicago, IL. (Control ID \#3185200)

BACKGROUND: Shared decision-making (SDM), an essential tenet of patient-centered care, has increasingly been promoted by physician organizations and payers. SDM has potential to improve health outcomes, reduce healthcare costs and address health disparities. To date, studies have not compared the relative impact of internal motivation(i.e. physicians' moral compass) and external workplace factors(e.g. population health management(PHM) strategies such as longer physician visits for complex patients) in promoting shared decision-making between patients and their physicians. We evaluate how physicians' internal motivation and features of their clinical environments relate to provider intentions to practice SDM with their patients. 
METHODS: Multivariable logistic regressions of a cross-sectional survey of practicing U.S. physicians $(n=176)$ including medical and surgical specialties identified from the American Medical Association(AMA) Physician Masterfile. Primary care physicians were oversampled, and anesthesiologists and radiologists with limited direct patient care were excluded. Surveys from physicians were collected February-July 2018. The main outcome measure was shared decision-making, as defined based on a response option in a clinical vignette. Independent variables included: a) physician personal/ moral responsibility to address health disparities and b) the population health management (PHM) resources of the clinical practice (e.g. types of providers, information technology, and patient support tools). Regression analyses were adjusted for physician demographics (e.g. race/ethnicity, gender, age, political affiliation), physician specialty, and clinical practice characteristics which included practice size and practice type(e.g. group/ staff model HMO or hospital-based practice). All analyses determined statistical significance based on two-tailed $\mathrm{p}$-values of $<0.05$.

RESULTS: Physicians' reporting a sense of personal responsibility to address health disparities had a statistically significant association with expressing intentions to engage in SDM behaviors. Overall, physicians' reporting a sense of personal responsibility to address health disparities were more likely than physicians lacking that sense of responsibility to report SDM intentions $(\mathrm{OR}=3.10,95 \% \mathrm{CI}: 1.40-6.84, \mathrm{p}<0.01)$. PHM resources within a practice were not associated with SDM intentions.

CONCLUSIONS: A physician's individual sense of responsibility is a strong predictor of provider intent to engage patients in shared decisionmaking. Clinical environments with an infrastructure including PHM resources alone may not enhance physicians' equal use of SDM across all types of patients. Interventions focused on expanding PHM resources in clinical practice or implementing broader health system changes to address health disparities should identify clinicians with high ratings of internal motivation (i.e. moral compass) as champion physician leaders to help ensure equitable practices, such as SDM, across patient populations.

\section{PROVIDER "HOTSPOTTERS:" INDIVIDUAL RESIDENTS DEMONSTRATE DIFFERENT PATTERNS OF TEST UTILIZA- TION ACROSS 3 STANDARDIZED CASES \\ Eli Cahan ${ }^{2}$; Kathleen Hanley ${ }^{2}$; Barbara Porter $^{2}$; Andrew B. Wallach ${ }^{1}$; Lisa} $\overline{\text { Altshuler }^{2}}$; Colleen C. Gillespie ${ }^{2}$; Sondra Zabar ${ }^{2}$. ${ }^{1}$ Bellevue Hospital, New York, NY; ${ }^{2}$ NYU School of Medicine, New York, NY. (Control ID \#3185368)

BACKGROUND: Inter-provider variability is a major source of low-value care. The dissemination of clinical practice guidelines (CPGs) has targeted this variability, yet $44 \%$ of physicians are non-adherent to CPG. This may be due to factors including exceptionalism and incentive misalignment that present a conflict between comprehensiveness and prudence in work-up. A subset of super-utilizers are notable outliers: fewer than $0.5 \%$ of physicians account for $10 \%$ of healthcare costs. Super-utilizers order labs, request consults, order imaging, and prescribe medications at rates 30\%, 140\%, $14 \%$, and $25 \%$ higher than the general population. We sought to quantify provider-specific low-value test ordering behaviors across three cases.

METHODS: Unannounced standardized patients (USPs) were trained for standardized simulation of three clinical scenarios: a "Well" visit, a chief complaint of "Fatigue," and a diagnosis of "Asthma." USPs were introduced into medicine residents' clinics in a large urban, safety-net hospital. Diagnostic orders were extracted via retrospective chart review. Scenariospecific appropriateness of diagnostic testing was determined by referencing United States Preventative Services Task Force (USPSTF) and specialty society CPGs. "Excessive" tests were those not explicitly indicated for a given scenario in either USPSTF or society CPGs (versus "indicated" tests). "Discretionary" tests were those conditionally indicated, pending patientspecific factors (such as hemoglobin A1C, pending BMI).

RESULTS: One or more excessive tests were ordered in $44 \%, 22 \%$, and $17 \%$ of Well $(n=124)$, Fatigue $(n=148)$, and Asthma $(n=148)$ encounters respectively. Percent of orders that were excessive were $18 \%, 8 \%$, and $10 \%$, respectively. On average, $1.3(+/-1.7)$ excessive orders were made. Within each case, rates of excessive ordering were positively correlated with rates of indicated and discretionary ordering, and negatively correlated with rates of omitting indicated tests. For example, in Fatigue, the correlation between excessive and indicated orders was 0.38 , between excessive and discretionary orders rates was 0.59 , and between excessive and omitted-indicated tests was -0.25 (all $\mathrm{p}<0.05$ ). A similar, statistically-significant pattern was found for the other two cases. $10(21 \%)$ and $4(8 \%)$ of 48 residents completing all scenarios demonstrated excessive ordering at rates atleast 1 and 2 standard deviations above the mean, respectively.

CONCLUSIONS: Introducing USPs representing clinical scenarios revealed marked inter-provider variability. Positive associations between rates of excessive, discretionary and indicated ordering suggest tendencies for comprehensiveness over prudence. Over one-fifth of residents completing all 3 cases were high-utilizers, and nearly one in ten were super-utilizers. Awareness of provider-level ordering tendencies can guide education and interventions supporting appropriate diagnostic use.

PROVIDER AND PATIENT EXPERIENCES DEIMPLEMENTING INHALED CORTICOSTEROIDS

Toral J. Parikh ${ }^{6,1}$; Krysttel Stryczek ${ }^{2}$; Chris Gillespie ${ }^{3}$; George Sayre ${ }^{6,}{ }^{1}$; $\overline{\text { Laura Feemster }}^{6}$, ${ }^{1}$; Edmunds M. Udris ${ }^{6}$; Barbara Majerczyk ${ }^{6}$; Seppo Rinne $^{4,}{ }^{7}$; Renda S. Wiener ${ }^{5,}$; Christian D. Helfrich ${ }^{6,}{ }^{1}$; David Au ${ }^{6,}{ }^{1}$. ${ }^{1}$ University of Washington, Seattle, WA; ${ }^{2}$ VA Northeast Ohio Healthcare System, Cleveland, OH; ${ }^{3}$ Dept. of Veterans Affairs, Bedford, MA; ${ }^{4}$ Edith Nourse Rogers Memorial VA, Bedford, MA; ${ }^{5}$ ENRM VA Hospital, Bedford, MA; ${ }^{6}$ VA Puget Sound Healthcare System, Seattle, WA; ${ }^{7}$ Boston University, Boston, MA. (Control ID \#3180241)

BACKGROUND: De-implementing medications may present challenges for providers and patients. Little research has assessed specific instances when a medication is discontinued. We sought to understand the experiences of primary care providers (PCP) and patients when de-implementing inhaled corticosteroids (ICS) in patients with mild-to-moderate COPD, in whom ICS are not recommended.

METHODS: We conducted a mixed-methods analysis for a multi-site, provider-randomized quality improvement project testing a proactive previsit e-consultation from a pulmonologist recommending stopping the ICS if appropriate. Control and intervention PCPs were interviewed preintervention, and intervention PCPs were interviewed post-intervention. Patient interviews were held within 3 months after the clinic visit. We conducted inductive and deductive thematic analysis of the qualitative data. All participating PCPs ( $\mathrm{n}=137$ at 13 VHA primary care clinics) received a post-intervention survey. Forty-eight PCPs returned surveys (24 intervention and 24 control, response rate: $35 \%$ ). We completed 14 pre-intervention interviews; 6 post-intervention interviews; and 6 patient interviews. Postintervention interviews are ongoing for PCPs and patients.

RESULTS: In pre-intervention interviews, PCPs were reluctant to discontinue an ICS if the patient perceived a benefit: "Some of my patients have wondered if they truly need to stop something that seems to be working for them." PCPs also had misgivings about making a change when the ICS wasn't causing an acute problem, "deep down there's a little hesitation that if someone is doing well, why rock the boat?" In post-intervention interviews, PCPs reported patients were receptive to the medication change, especially in the context of potential harm: "If they don't need the medication, there's not much point in prescribing something that's only going to do them harm...most of my patients were very receptive to that." One PCP reported patient reluctance: "they feel like something is working very well so they don't want to change it, even though they may not have the exact indication for it." Patients often reported deferring to their PCPs: "I just do what the doctor says so they don't yell at me" or "whatever the doctors think, because they have more schooling than I've got." However, one patient described ICS as "a security blanket . . . just to have it here in case." In post intervention surveys, $54 \%$ of PCPs reported having proposed discontinuing or reducing an ICS prescription for a patient in the past 6 months. Of this 
group, $90 \%$ reported that their patients responded somewhat or very receptively, and $80 \%$ discontinued the ICS or switched to another medication.

CONCLUSIONS: While PCPs were initially concerned with how patients would respond to discontinuing an ICS, post-intervention interviews and surveys suggested patients were receptive. For future de-implementation strategies, it may be possible to use these findings to address provider concerns regarding anticipated patient reactance.

\section{PROVIDER DECISION-MAKING FOR PROSTATE CANCER} SCREENING: A QUALITATIVE STUDY

Julian Brunner ${ }^{1,2}$; Emmeline Chuang ${ }^{2}$; Elizabeth M. Yano ${ }^{1,2}$; Narissa J. Nonzee ${ }^{2,4}$; Jeremy Shelton ${ }^{3}{ }^{1}$ VA Greater Los Angeles Healthcare System, Sepulveda, CA; ${ }^{2}$ UCLA, Los Angeles, CA; ${ }^{3}$ VA Greater Los Angeles Healthcare System, Los Angeles, CA; ${ }^{4}$ UCLA Fielding School of Public Health and Jonsson Comprehensive Cancer Center, Los Angeles, CA. (Control ID \#3185310)

BACKGROUND: Prostate-specific antigen (PSA) screening remains controversial, and harms resulting from over-diagnosis are common. In the optimal age group (50-69 years) the benefits are contested, and among men over 70, benefits are even less clear. Yet screening persists among men who are unlikely to benefit, and the factors motivating primary care providers' (PCPs') screening decisions remain poorly understood. Our objective was to examine PCP decision-making about prostate cancer screening and explore reasons for potentially inappropriate screening.

METHODS: We conducted semi-structured interviews with 25 PCPs from hospital-based and community-based VA clinics around the greater Los Angeles area. We conducted thematic analysis of interviews with deductive and inductive techniques, using the interview guide as a starting point for coding.

RESULTS: Most PCPs identified age as the primary factor informing their screening decisions. Several consider life expectancy (at any age), and some use an age cutoff but make exceptions for men in exceptional health. Other prominent factors were family history and race. Less-common factors included previous PSA levels, previous PSA trajectories, agent orange exposure, and testosterone supplementation. Many PCPs reported that they discontinue PSA screening at age 70 , but this practice was not universal. The most common reason for potentially inappropriate screening was difficulty applying guidelines precisely, despite general familiarity with guideline recommendations - for example, over- relying on age as a proxy for life expectancy, or overestimating the magnitude of a given risk factor in comparison to potential harms. Several PCPs reported ordering screening for patients who demand it, but reported that such demands are rare ("maybe less than 5\% does that ever happen"). A small minority of PCPs were distrustful of relevant guidelines, for example, questioning the generalizability of extant research, ("the department of urology here has a fairly conservative approach, so the likelihood that they are going to get unnecessary morbidity is lower than previous studies"). Complete lack of awareness of relevant guidelines was extremely rare. PCPs voiced the most support for guidelines from the United States Preventive Services Task Force, citing its credibility as an unbiased source. A smaller group preferred specialty society guidelines, citing urologists' expertise in prostate cancer.

CONCLUSIONS: Our findings help explain the reasons that PSA screening persists among patients unlikely to benefit. This information is an important precondition for designing interventions to reduce overuse and improve screening decisions. Interventions that help providers weigh competing risk factors may be more effective than interventions that presume a lack of awareness among PCPs, or presume overwhelming patient demand.

PROVIDER FACTOR IMPACTING THE SCREENING FOR HEPATITIS C INFECTION: A QUALITY IMPROVEMENT INITIATIVE kavya Kelagere Mayigegowda ${ }^{1}$; Rinad Tabbalat ${ }^{1}$; Kathryn Jobbins ${ }^{2}$. ${ }^{1}$ Baystate Medical Center, Springfield, MA $;{ }^{2}$ Baystate Health, Springfield, MA. (Control ID \#3184406)

BACKGROUND: Chronic hepatitis $\mathrm{C}$ virus $(\mathrm{HCV})$ is a major cause of liver-related deaths in the United States. The CDC estimates that about $50 \%$ of Americans with HCV infection are unaware of their infection status. Therefore a need exists for increased screening to identify people with unrecognized infections. There is a recent consensus that patients of advanced practitioners and those of female providers receive better preventative care. The aim of our retrospective cohort study was to determine how these factors impact screening for HCV at a community level.

METHODS: Data were extracted from our institution's electronic charts between October 2016 and October 2018. Patients born between 19451965 and had at least two visits to our primary care clinic were included. 3200 eligible visits were identified and patients who were already screened for $\mathrm{HCV}$ prior to the study period were excluded. A total of 1265 visits met our criteria. Visits were then categorized into resident group, advanced practitioner group and attending group. There were 800 patients in the resident group, 91 in the advanced practitioner group, and 374 in the attending group. These groups were further categorized into male and female providers. Screening for HCV was measured by laboratory testing for $\mathrm{HCV}$ antibody.

RESULTS: HCV screening rate for all providers in our clinic is $54 \%$. The screening rate in the advanced practitioner group is higher than the resident group $(64 \% \mathrm{v} / \mathrm{s} 52 \% \mathrm{p}=0.03)$. The screening rate in the advanced practitioner group is similar to the attending group $(64 \% \mathrm{v} / \mathrm{s} 58 \% \mathrm{p}=\mathrm{NS})$. The screening rate in the overall female provider group is higher than the male provider group $(52 \% \mathrm{v} / \mathrm{s} 57 \% \mathrm{p}=0.04)$. See table 1 for screening rates in different groups further categorized into male and female providers.

CONCLUSIONS: Based on our data, we conclude that female providers and advanced practitioners are more likely to provide screening for $\mathrm{HCV}$ infection. These results could be skewed by the fact that most of our advanced practitioners are females. These findings have prompted us to further gather data from our advanced practitioners and female providers on strategies that could be adopted to improve HCV screening throughout our community practice. Furthermore, need exists for identifying physician and patient related barriers to testing for $\mathrm{HCV}$, as the overall $\mathrm{HCV}$ screening rate in our clinic is suboptimal.

PROVIDER KNOWLEDGE, ATTITUDES, AND PRACTICE REGARDING CANNABIS: RESULTS FROM A NATIONAL VETERANS HEALTH ADMINISTRATION SURVEY

Devan Kansagara $^{4,}$; ; Megan O. Iacocca ${ }^{4}$; William Becker ${ }^{2}$; Matthew J. Bair ${ }^{3}$; Benjamin J. Morasco ${ }^{4}$. ${ }^{1}$ Oregon Health and Science University, Portland, OR; ${ }^{2}$ VA Connecticut Healthcare System, West Haven, CT; ${ }^{3}$ Center for Health Information and Communication, Indianapolis, IN; ${ }^{4}$ VA Portland Health Care System, Portland, OR. (Control ID \#3170354)

BACKGROUND: Cannabis is increasingly available and used for medical and recreational purposes due to legal changes and broader cultural acceptance. The Veterans Health Administration (VHA) recommends that clinicians be prepared to discuss cannabis with their patients, though they cannot certify medical marijuana use due its classification as a schedule I substance. Clinicians are allowed to make individualized decisions regarding opioid therapy in those also using cannabis. Few studies have assessed provider knowledge, attitudes and practice regarding cannabis. METHODS: We developed a 47-item survey to assess VHA primary care providers (PCPs)' knowledge, attitudes, and practice regarding patients' use of cannabis. Survey items were derived from: 1) systematic review data, 2) multidisciplinary expert group discussion, and 3) interviews with clinicians and cannabis dispensary staff. We pilot tested draft surveys with clinicians and made modifications. We conducted age- and 
gender-adjusted logistic regression analyses to examine the association of cannabis legality in respondents' states with survey responses. The anonymous survey was sent electronically to primary care chiefs in the VHA nationally, who could then distribute locally to PCPs.

RESULTS: We received 263 completed surveys from PCPs in 39 states and the District of Columbia: $47 \%$ of respondents were female, $70 \%$ white, and the mean age was 50 years; $38 \%$ supported legalization of medical cannabis in all states. A substantial minority were very or somewhat uncomfortable with the following: addressing patient questions about cannabis (33\%), identifying cannabis use disorder (35\%), and talking to patients about recreational cannabis use $(25 \%)$. There were knowledge gaps among a substantial minority in specific areas including terminology, psychoactive effects of cannabis components, VHA policy, and evidence regarding benefits and harms of cannabis. A majority of respondents $(68 \%)$ were likely or very likely to taper opioids if urine drug testing was positive for THC. More respondents from states in which cannabis was illegal (OR 4.7, 95\% 2.2-10.8) or was recreationally illegal (OR 5.7, 95\% CI 2.3-10.8), reported being likely or very likely to taper opioids than participants residing in states where cannabis was legal for medical and recreational purposes.

CONCLUSIONS: This is the first survey to assess VHA PCPs' knowledge, attitudes, and practice regarding cannabis. We found many PCPs had knowledge gaps regarding cannabis, and felt uncomfortable discussing key aspects of cannabis use with their patients. PCPs from states in which cannabis was legal for recreational use were less likely than PCPs from non-legal states to taper opioids in patients with urine drug screens positive for THC. These results suggest a need for targeted educational efforts, as well as more robust guidance and evidence regarding management of patients using prescription opioids and cannabis concomitantly.

\section{PROVIDER PERCEPTIONS OF TELEMEDICINE VIDEO VISITS TO HOME IN A VETERAN POPULATION}

Joseph N. Martinez ${ }^{2}$; Laura Samples ${ }^{2}$; Yodit Beru² ${ }^{2}$ Meghan Rochester ${ }^{2}$; Anders Chen ${ }^{1,2}$; John Geyer ${ }^{1,}{ }^{2}$. ${ }^{1}$ VA Puget Sound, Seattle, WA; ${ }^{2}$ University of Washington, Seattle, WA. (Control ID \#3183959)

BACKGROUND: To serve the geographically diverse population enrolled in primary care at VA Puget Sound Health Care System providers use several telehealth modalities including remote patient monitoring and mobile health applications. Clinical video telemedicine to home (CVTH) is a new tool offered that allows clinic-based providers to complete synchronous video clinical encounters via a smartphone, computer or VA issued iPad to patients at home. To date, few primary care providers are using CVTH. As part of a telemedicine project in the VA Center of Excellence in Primary Care Education and the University of Washington IM Residency program QI immersion block, a survey about attitudes and barriers of CVTH adoption was designed by Internal Medicine R2s and disseminated to clinicians at Seattle Primary Care Clinic.

METHODS: A 12-question Likert-type multiple choice survey was generated with input from senior clinical leadership, CVTH users from other disciplines and IT personnel. Study participants included faculty MDs and Nurse Practitioners (NPs), NP and Internal Medicine MD residents and Pharmacists. Content domains include respondent's attitudes, perceived barriers and likely patient-use-scenarios with respect to CVTH. All responses were collected electronically and anonymously via REDCap software over a period of 7 days in November 2018.

RESULTS: 45 responses of 85 surveys sent were received (52\%) (18 MD/NP faculty, $18 \mathrm{MD}$ and $2 \mathrm{NP}$ residents, 7 Pharmacists). 2 respondents had previously used video telemedicine and 35 had previously used Skype or Facetime. When asked which clinical scenarios were most amenable to CVTH, more than half of respondents were very likely to somewhat likely to use it for depression (69\%), tobacco use (84\%), chronic pain (67\%), hypertension (89\%), and diabetes (93\%). 69\% percent of respondents were very unlikely to somewhat unlikely to use
CVTH on visits heavily dependent on physical exam techniques such as musculoskeletal (MSK) complaints. 84\% of respondents somewhat or strongly agreed that internet connection or a patient's technological competence would limit implementation of CVTH. Less than $20 \%$ of respondents strongly agreed or somewhat agreed that their own technological competency would be a barrier.

CONCLUSIONS: Limited data exists about provider perceptions regarding primary care telemedicine. Our survey data will help improve adoption of CVTH in primary care. Given respondents concern about using CVTH for MSK related encounters, a training on video physical exam techniques is being developed with input from other professions that have already robustly implemented CVTH. Given respondents concern that Veterans' technological competency would limit incorporation of CVTH, a pre-visit checklist is being developed to certify patient technological familiarity before an initial CVTH primary care visit occurs. Next steps include surveying Veterans to gauge interest of CVTH, identifying administrative barriers and re-assessing provider perception overtime.

\section{PROVIDER PERSPECTIVES ON BARRIERS AND FACILITA- TORS OF LONG-ACTING REVERSIBLE CONTRACEPTIVE USE IN A LARGE HEALTH SYSTEM}

Anna M. Leone $^{1}$; Kelly Williams ${ }^{3}$; Elizabeth C. Swart ${ }^{3}$; Sonya Borrero ${ }^{1}$; Natasha Parekh ${ }^{2} .{ }^{1}$ University of Pittsburgh, Pittsburgh, PA; ${ }^{2}$ University of Pittsburgh Medical Center, PIttsburgh, PA; ${ }^{3}$ UPMC Center for HighValue Health Care, Pittsburgh, PA. (Control ID \#3180705)

BACKGROUND: In August 2012, the Affordable Care Act's contraceptive mandate required insurance plans fully cover at least one contraceptive method type from each of 18 FDA-approved method categories. Though a primary goal of the mandate was to eliminate cost as a barrier to accessing the full range of contraceptive methods including long-acting reversible contraception (LARC) (i.e., intrauterine devices and implants), only $12 \%$ of women in the United States utilized LARC methods in 2014. Qualitative interviews were conducted with LARC providers in a large health system in Western Pennsylvania, with nationally comparable rates of LARC usage, to understand practice- and system-based barriers and facilitators to LARC access.

METHODS: We conducted semi-structured interviews with LARC providers who inserted at least three LARCs in 2017. Using claims data, we compiled a list of 107 eligible providers and conducted a total of 30 total interviews. Providers were defined as "high-uptake" if they administered more than 42 LARCs in 2017 (the median) and "low-uptake" if they administered less than 42 LARCs in 2017. Interviews were recorded and transcribed verbatim. Two coders developed a codebook based on an iterative review of transcripts and then applied themes to remaining transcripts, holding regular meetings to adjudicate coding disagreements. Directed content analysis was utilized throughout the analytic process.

RESULTS: Preliminary themes focused primarily on practice-level barriers and facilitators, as well as one system-level barrier. Providers described several practice-level barriers to LARC access, including, (1) policies requiring women to return for a second appointment to complete LARC insertion and (2) policies requiring women to return while menstruating to complete LARC insertion. Providers mentioned practice-level facilitators to LARC access, including, (1) availability of ancillary clinical staff and (2) patient education materials. Time in provider schedules and practice stocking of the LARC method were mentioned as both facilitators and challenges to LARC access, and both were practice- dependent rather than standardized across all offices represented in the sample. Requiring insurance pre-authorization prior to insertion was commonly described as a system-based barrier to LARC access.

CONCLUSIONS: Patient access to LARC methods can be complicated by clinic-level barriers that are heavily practice-dependent, in addition to system-level barriers. These preliminary findings demonstrate heterogeneity across participating providers and practices regarding their LARC provision policies. Results will provide guidance for practice- and system- 
level changes that can be implemented to standardize guidelines for LARC provision and reduce practice- and system-level barriers to LARC access. Further analysis will focus on differences in themes between "high-uptake" and "low-uptake" providers and years in practice.

\section{PROVISION OF PREVENTIVE HEALTH SERVICES TO US WOMEN}

Shelby McLaughlin-Patel ${ }^{1}$; E. Bimla Schwarz ${ }^{2} .{ }^{1}$ University of California $\overline{\text { Davis, Sacramento, CA; }}{ }^{2}$ University of California, Davis, Sacramento, CA. (Control ID \#3185980)

BACKGROUND: The US Preventive Services Task Force (USPSTF) provides age-specific recommendations regarding a wide range of screening and counseling services. In 2014, the American College of Obstetricians and Gynecologists' Well-Woman Task Force developed age-specific recommendations to guide the care of women during well-woman visits. We assessed alignment between US provision of preventive health services to women and these recommendations.

METHODS: The National Ambulatory Medical Care Survey (NAMCS) collects data from non-federally employed office-based physicians in the United States. In 2015 (the most recently available data), NAMCS assessed provision of 23 preventive health services. We examined provision of these 23 preventive health services to women by age group (18-25, 26-39, 40-50, 51-65, 66-80, 81+ years), whether the provider identified as the patient's primary care provider (PCP), visit type, and clinician training (internal medicine, family medicine, gynecology).

RESULTS: In 2015, NAMCS collected data on 28,322 US ambulatory visits, of which 13,887 were made by women. Of these, $17 \%$ were with clinicians who identified as the patient's PCP; $19 \%$ were "preventive health" visits. Visits to a clinician who identified as the woman's PCP were not more likely to include preventive health services than visits to other clinicians; visits identified as "preventive health visits" were not more likely to include any preventive services than other visit types. Preventive counseling of any kind (i.e, on diet \& nutrition, exercise, weight reduction, tobacco use, prevention, alcohol abuse, substance abuse, prevention of sexually transmitted infections) was documented for less than $10 \%$ of visits made by women of any age group. The preventive service most commonly performed was a pelvic exam. Of the 803 visits to Internists, 1303 to family physicians, and 2246 to gynecologists, pelvic exam was documented for $6.5 \%, 2.8 \%$ and $45 \%$, respectively. In contrast, counseling about diet and nutrition was documented for $24 \%$ of visits to Internists, $17 \%$ of family physicians and $12 \%$ of gynecologists. For women aged 18-50, there were 5,598 visits. Of these, 1,247 (78\%) were to an ObGyn.

CONCLUSIONS: US women of all ages appear to be receiving preventive health services relatively rarely. Although it is possible that women received preventive services at other visits not documented in the NAMCS survey, few US women make more than 4 visits to their PCP each year. Given the time limitations inherent in current models of primary care, there is a need to prioritize preventive services. More attention should be given to preventive health counseling, with less time spent on pelvic exams for asymptomatic women, as the USPSTF has determined there to be insufficient evidence to support screening pelvic exams of asymptomatic women. Although this is a snapshot of ambulatory visits, the findings from this descriptive study support prior studies and calls for a stronger coordination of care between a woman's PCP and ObGyn.

PROVISION OF UTILITY SHUTOFF-PROTECTION ILLNESS CERTIFICATE LETTERS AT AN URBAN SAFETYNET HOSPITAL, 2009-2018

Karen E. Lasser ${ }^{1,}{ }^{3}$; Heather E. Hsu ${ }^{1,}{ }^{3}$; Erin M. Ashe ${ }^{1}$; Pablo Buitron de la Vega ${ }^{1}$; Ziming Xuan ${ }^{2}$; Michael Silverstein ${ }^{1,3}$. ${ }^{1}$ Boston Medical Center, Boston, MA; ${ }^{2}$ Boston University School of Public Health, Boston, MA; ${ }^{3}$ Boston University School of Medicine, Boston, MA. (Control ID \#3165439)
BACKGROUND: In Massachusetts, utility companies are prohibited from terminating service to low-income households where occupants present a "Utility Shutoff-Protection Illness Certificate" letter confirming that a household member is seriously ill. It is unclear, however, how many patients receive these letters from their medical providers and whether current efforts to screen for healthrelated social needs are identifying these patients. Our objective was to characterize the provision of such letters at Boston Medical Center, the largest safety-net hospital in New England.

METHODS: Using data extracted from the electronic health record (Logician and Epic), we performed descriptive analyses examining patient demographics and medical comorbidities among all unique adult patients (age $>18$ years) at Boston Medical Center with a "Utility Shutoff-Protection Illness Certificate" letter signed by a medical provider from 2009-2018. Among patients who received letters within the past year (when screening for health-related social needs was implemented among adults seen in general internal medicine, family medicine, obsterics-gynecology, the breast center, and the HIV clinic), we examined the proportion who were screened for having difficulty paying utility bills.

RESULTS: 2981 adult patients received a letter over the study period. The mean age of patients was 52 (sd 15.5); 66\% were nonHispanic black, $15 \%$ non-Hispanic white, and 5\% Hispanic of any race. An additional $12 \%$ had missing race data. Over two-thirds of patients were women. Patients most frequently resided in three highrisk Boston neighborhoods: Dorchester (31\%), Roxbury (11\%), and Mattapan (7\%). Most patients spoke English (86\%), while 6\% spoke Spanish and an additional 5\% spoke Haitian Creole. Most patients had government insurance (42\% Medicaid, 37\% Medicare), while $18 \%$ were commercially insured. Patients frequently had significant comorbid medical illness, including congestive heart failure (19\%), COPD (48\%), and diabetes (41\%). Among the 754 patients who received a letter in the past year, only $25 \%$ (185) were screened for having difficulty paying utility bills. Among these 185 patients, only 47\% (87) screened positive.

CONCLUSIONS: Many patients who receive care at an urban safetynet hospital are at risk for having their utilities shut off, yet current efforts to screen for health-related social needs detect only a fraction of these patients. When a patient presents with a request for a "Utility ShutoffProtection Illness Certificate" letter, this should be considered a sentinel event. Such an event should trigger referral of a patient to clinic staff who can connect the patient to fuel assistance and other resources.

PSYCHOMETRIC PROPERTIES OF THE ALTARUM CONSUMER ENGAGEMENT (ACE) MEASURE IN PATIENTS WITH PREDIABETES

Yelba Castellon-Lopez ${ }^{1}$; Kia Skrine Jeffers ${ }^{1}$; O. Kenrik Duru ${ }^{2}$; Tannaz Moin $^{3}$; Carol Mangione ${ }^{4}$; Keith Norris ${ }^{2}$; Gerardo Moreno ${ }^{2}$; Ron D. Hays $^{2} .{ }^{1}$ University of California, Los Angeles, Los Angeles, CA; ${ }^{2}$ UCLA, Los Angeles, CA; ${ }^{3}$ UCLA/VA Greater Los Angeles, Los Angeles, CA; ${ }^{4}$ David Geffen School of Medicine at UCLA, Los Angeles, CA. (Control ID \#3184777)

BACKGROUND: The Prediabetes Informed Decision and Education (PRIDE) study utilized pharmacist-delivered shared decision making to help patients with prediabetes choose lifestyle change, metformin, or both as a diabetes prevention strategy. All PRIDE participants selfadministered 12 items from the Altarum Consumer Engagement (ACE12) measure to assess activation at baseline, 4 months, and 12 months. The aims of this project were to evaluate the psychometric properties of the open-source ACE-12.

METHODS: We used electronic medical record data to identify overweight/obese patients with prediabetes between 18 and 74 years of age. We conducted exploratory factor analysis using polychoric 
correlations, and determined the number of factors based on scree plots and the Tucker and Lewis reliability coefficient. We fit graded response models to obtain item characteristic curves (ICC). We assessed construct validity using Cohen's d for correlations (small effect size defined as $\mathrm{r}=0.100$; medium effect size $r=0.243$; and large effect size $r=0.371$ ). We fit a bifactor model to evaluate whether there was support for an overall 12-item ACE score. Analyses were done with SAS, version 9.3 (SAS Institute), and the R Statistical Computing Environment (Vienna, Austria).

RESULTS: Participants $(n=515)$ had a median age of $58 ; 56 \%$ were female; 17\% Hispanic, $83 \%$ non-Hispanic (46\% White, 15\% African American, $19 \%$ Asian/Pacific Islander, $1 \%$ Native American, $19 \%$ Other). The scree plot and Tucker and Lewis reliability coefficient $(0.95)$ suggested 3 underlying factors: "Commitment to Health"; "Doctor Choice"; and "Learning About Health". Patients with a high school education or lower were less activated on the commitment scale $(r=0.352, p=0.002)$, choice scale $(\mathrm{r}=0.333, \mathrm{p}=0.008)$, and overall ACE score $(\mathrm{r}=0.301, \mathrm{p}=0.011)$. Baseline A1c was not correlated with activation. Depressive symptom scores correlated with our revised commitment scale and the overall score. Specifically, those who scored 5 or higher on the PHQ-9 had lower commitment scale scores $(r=0.352, p=<0.001)$ and lower overall ACE score $(r=0.222$, $\mathrm{p}=0.012$ ). Patient-reported health had a large correlation with patient activation ( $\mathrm{r}=0.590, \mathrm{p}=<0.001$ ). Patients who reported that their health was less than "very good" scored lower on the commitment scale $(r=0.375$, $\mathrm{p}=<0.001)$ and learning about health $(\mathrm{r}=0.266, \mathrm{p}=<0.001)$. The bifactor model provided support for an overall ACE score, though more work needs to be completed in this area.

CONCLUSIONS: Our analyses of the ACE measure in prediabetes suggest it is generally psychometrically sound. This study contributes to the literature by expanding the validity of open-source tools that measure patient engagement, as an alternative to existing proprietary options.

\section{PSYCHOSOCIAL DISTRESS IN ONCOLOGY PATIENTS IN THE SAFETY-NET: IDENTIFYING POPULATIONS AT RISK}

Theora B. Cimino ${ }^{1}$; Margot Albert'; Kiana Said²; Leslie Safier ${ }^{2}$; Anne Kinderman $^{2}$; Heather Harris ${ }^{2}$. ${ }^{1}$ University of California at San Francisco, San Francisco, CA; ${ }^{2}$ San Francisco General Hospital, San Francisco, CA. (Control ID \#3138442)

BACKGROUND: Heightened distress levels can lead to adverse outcomes in cancer patients, including decreased satisfaction with care, poorer adherence to recommended therapy, and diminished quality of life. Studies on distress screening among cancer patients have largely focused on Caucasian, English speaking, and relatively affluent patient populations. The purpose of this study is to examine the prevalence, predictors, and experience of psychosocial distress among cancer patients within a diverse, urban, and multi-lingual safety-net setting and to assess distress level and ability to access supportive resources at follow up.

METHODS: Participants included a cross-sectional sample of cancer patients age 18 at a medical oncology clinic at an urban public hospital, between December 2014 and June 2016, who completed distress screening at their first oncology visit. Distress levels and sources of distress were obtained using a modified version of the National Comprehensive Cancer Network (NCCN) distress thermometer (a 0-10 scale, 10 = extreme distress) and problem list. Demographic data were abstracted by review of medical records. Patients who reported moderate to severe levels of distress were contacted for telephone follow up 1-12 months after initial screening to reassess distress levels and ability to access referred resources.

RESULTS: 200 of 317 eligible patients (63\%) completed sychosocial distress screening and were included in the study. Of screened patients, $61 \%$ reported clinically significant distress scores. Results from logistic regression analysis indicated that identifying as African-American, having a history of psychiatric illness, and reporting problems with money, worry, sleep, memory or homelessness were associated with higher self-reported psychosocial distress. Cantonese/Mandarin-speaking patients reported lower levels of psychosocial distress compared to other groups. Telephone follow up was completed for 39 (42\% of eligible patients) and showed a decrease in distress scores from a mean of 6.5 at initial screening to 4.5 at follow up. At follow up, patients utilized only $44 \%$ of referrals to supportive services made at the initial visit. $54 \%$ of paitents cited barriers to access such as lack of knowledge of the referral, not hearing back from the resource, or needing more information - as the reason they did not utilize a particular service.

CONCLUSIONS: Our cohort had a greater prevalence of significant psychosocial distress than most other studied populations, with persistently elevated distress levels and difficulty accessing supportive services at follow-up. Our study identifies groups that may be at higher risk for clinically significant distress and persistent distress, and suggests that closer follow-up and assistance for patients who report high distress levels may be needed.

\section{PURPOSE IN LIFE AND LIFE SATISFACTION MODERATE THE ASSOCIATION BETWEEN MULTIMORBIDITY AND SO- CIAL PARTICIPATION AMONG U.S. MIDDLE-AGED AND OLDER ADULTS}

Jamie Luster ${ }^{1}$; David Ratz ${ }^{2}$; Melissa Y. Wei ${ }^{1,3}$. ${ }^{1}$ University of Michigan, Ann Arbor, MI; ${ }^{2}$ Veterans Affairs Ann Arbor Healthcare System, Ann Arbor, MI; ${ }^{3}$ Institute for Healthcare Policy and Innovation, University of Michigan, Ann Arbor, MI. (Control ID \#3179961)

BACKGROUND: High social participation is associated with improved happiness, health-related quality of life (HRQOL), lower chronic disease incidence, and all-cause mortality. Multimorbidity is associated with low physical and mental HRQOL; one potential mechanism may be through low social participation. We aimed to quantify the association between multimorbidity and social participation and identify potential moderators: purpose in life, and life satisfaction.

METHODS: Participants were noninstitutionalized US adults 51 years of age from the Health and Retirement Study (N=20918). Multimorbidity was quantified through a validated Multimorbidity-Weighted Index (MWI) that weights self-reported chronic conditions by physical functioning. MWI was examined as a time-varying predictor biennially between 2010-2014 and divided into quartiles (lower score indicated lower multimorbidity). Social participation was a continuous composite score of the frequency of participation in 21 activities in 2014 (range 21-147, lower score indicated more frequent participation in more activities). We used multiple linear regression to examine the association between MWI and social participation, adjusting for age, sex, race/ethnicity, education, employment status, household net worth, and marital status. We examined purpose in life (range 1-6, low to high) and life satisfaction (range 1-7, low to high) as effect measure modifiers for the association between MWI and social participation.

RESULTS: The final sample included 7529 participants with complete exposure and outcome data. Participants had a mean MWI of 7.9 (SD 6.5), social participation score of 101.4 (SD 16.1), purpose in life score of 4.5 (SD 1), and life satisfaction score of 5.0 (SD 1.5). The most frequently reported activities were watching television, reading, and using a computer. In the adjusted model, highest MWI quartile participants reported the lowest social participation $(=2.8,95 \% \mathrm{CI}=1.8-3.8)$ compared to those in the lowest MWI quartile, equivalent to participating in 1 less activity weekly. There were significant interactions between MWI and purpose in life $(\mathrm{p}=0.004)$ and MWI and life satisfaction $(\mathrm{p}=0.04)$. For all quartiles of MWI, participants with high purpose in life or life satisfaction scores had significantly greater social participation compared to those with low scores. For those in the highest MWI quartile, having high purpose in life or life satisfaction amounted to an additional 1 activity weekly.

CONCLUSIONS: High multimorbidity using a validated MWI is associated with low social participation among middle-aged and older adults. This relationship was modified by both strong purpose in life and high life satisfaction, which each buffered against lower social participation among adults with any degree of multimorbidity. Social 
participation is an important measure of HRQOL that is reduced with increased multimorbidity but may be improved by high purpose in life and life satisfaction.

\section{PURSUING THE DIAGNOSTIC ODYSSEY: PATTERNS OF RESIDENT TEST UTILIZATION DIFFER FOR PREVENTIVE VERSUS DIAGNOSTIC WORK-UP}

Eli Cahan ${ }^{2}$; Kathleen Hanley ${ }^{2}$; Barbara Porter ${ }^{2}$; Andrew B. Wallach ${ }^{1}$; Lisa Altshuler ${ }^{2}$; Sondra Zabar ${ }^{2}$; Colleen C. Gillespie ${ }^{2}$. ${ }^{1}$ Bellevue Hospital, New York, NY; ${ }^{2}$ NYU School of Medicine, New York, NY. (Control ID \#3185316)

BACKGROUND: Low-value tests, defined as inappropriate for a given clinical scenario, are ordered in one in five clinic visits. Residents tend to over-order diagnostic tests to "minimize uncertainty" of presenting cases, even though these tests are not useful according to Bayesian statistics; a pursuit deemed the "Ulysses syndrome". Simultaneously, evidence suggests residents misuse preventive tests in half of relevant clinical scenarios. We sought to quantify ordering behaviors in urban primary care clinics across three unannounced standardized cases.

METHODS: Unannounced standardized patients (USPs) were trained for standardized simulation of three clinical scenarios: a "Well" visit, a chief complaint of "Fatigue," and a diagnosis of "Asthma." USPs were introduced into medicine residents' clinics in an urban, safety-net hospital. All electronic orders were extracted via chart review. Scenario-specific appropriateness of diagnostic testing was determined by referencing United States Preventive Services Task Force (USPSTF) and society clinical practice guidelines (CPGs). "Preventive" tests (such as lipid panels or hemoglobin A1C) were derived from USPSTF guidelines whereas "Diagnostic" tests (such as pulmonary function testing for Asthma or heterophile antibodies for Fatigue) were from CPGs. "Excessive" tests were those not explicitly indicated for a given scenario in either USPSTF or CPGs (versus "indicated" tests).

RESULTS: Indicated tests were ordered in 29\% of Well (124 encounters), $16 \%$ of Fatigue (148 encounters), and $12 \%$ of Asthma (170 encounters) cases. One or more excessive tests were ordered in $44 \%, 22 \%$, and $17 \%$ of Well, Fatigue, and Asthma encounters respectively. The distribution of indicated and excessive tests for preventive versus diagnostic purposes varied by case: In Well visits, the majority (71\%) of excessive testing was in pursuit of a diagnosis, while three-quarters of indicated testing was for preventive purposes. In Fatigue and Asthma visits, the reverse patterns were true: the majority of indicated tests ordered were diagnostic ( $81 \%$ and $68 \%$, respectively) while the majority of excessive tests were preventive (78\% and $63 \%$, respectively).

CONCLUSIONS: Introducing USPs to resident clinics revealed that, for patients presenting without a chief complaint (Well visit), residents successfully ordered less than one-third of indicated tests, and over $75 \%$ of inappropriately ordered tests pursued a diagnosis. For patients presenting with chief complaints (Fatigue and Asthma), rates of appropriate ordering were even lower (16\% and 12\%), and tended to overlook preventive care. In these cases, inappropriate tests tended to be ordered for preventive purposes. Awareness of resident mis-utilization of preventive and diagnostic testing in distinct clinical circumstances can guide educational efforts towards evidence-based care and resource stewardship.

QUALITATIVE ANALYSIS OF A SOCIAL DETERMINANTS OF HEALTH (SDH) FOCUSED HOME VISIT CURRICULUM FOR INTERNAL MEDICINE RESIDENTS

Iman Hassan ${ }^{1}$; Amy J. Kennedy ${ }^{1}$; Flor de Abril Cameron ${ }^{2}$; Megan E. $\mathrm{Hamm}^{2}$; Thuy Bui ${ }^{2}$; Carla L. Spagnoletti ${ }^{1}$; Maggie K. Benson ${ }^{1}$. University of Pittsburgh Medical Center, Pittsburgh, PA; ${ }^{2}$ University of Pittsburgh, Pittsburgh, PA. (Control ID \#3186303)

BACKGROUND: Few categorical internal medicine residencies offer curricula on SDH, despite its impact on patient health. We sought to enhance residents' awareness of SDH via a home visit curriculum.
Following a small group session on neighborhood health effects and electronic tools to address SDH, residents identified one of their clinic patients for which they conducted a virtual and physical neighborhood assessment and $\mathrm{SDH}$-focused home visit.

METHODS: To assess curriculum impact, we conducted five 60-min focus groups of 3 to 5 residents within 2 weeks of the home visit. A total 19 residents participated. An independent, trained facilitator guided the discussion with a question script. Sessions were audio-recorded and transcribed. Using the Editing approach, a codebook was developed along with a qualitative expert. Two coders independently applied the codes to all transcripts using ATLAS.ti 8.0. Discrepancies were adjudicated until full agreement reached. Themes were then identified.

RESULTS: (1) Residents reported the curriculum offered a unique, experiential opportunity to see how patients' neighborhood and SDH impact their health. A resident said, "doing a home visit, you get to see it and that makes it really come to the fore-front of your mind, you don't have to imagine, you see it and then you realize 'oh I should be thinking about xy and z'. This is more helpful than someone saying think about social determinants." (2) Residents cited the neighborhood-mapping component of the virtual assessment as applicable to future patients. They said, "The benefit...was to get us thinking about the patient in the context of his surroundings, rather than just in the context of his or her home" and in clinic "actually seeing how far away [the neighborhood] is from the clinic would probably inform my care a little bit better without taking any extra time." (3) Residents felt the curriculum enhanced their conceptualization of SDH and offered concrete tools applicable to other patients. A resident said, "I think this entire exercise was useful in that now I feel like I know some resources better... feeling like I have something to offer them I think will increase the likelihood that I will bring these things up, with anybody." Another said, "I feel that the home visits do give me such a different, a better sense of my patients in general, that now in my clinic after having done these, I do spend so much more time just being like, "Okay what neighborhood do you live in? Do you live in a house, an apartment?...I ask all sorts of weird questions that I didn't used to ask. Just because I do feel like it's helpful to have a better sense of who they are and where they are."

CONCLUSIONS: Our curriculum prompted greater reflection on and engagement with SDH among residents. They valued the experiential nature and identified components applicable to other patients. Neighborhood and home visits, with an emphasis on local resources, can be successfully used to help residents incorporate SDH into clinical care.

\section{QUALITATIVE ASSESSMENT OF TWO APPROACHES TO IMPLEMENTING CLINICAL DECISION SUPPORT}

Susan Stork ${ }^{1}$; Jonathan Austrian ${ }^{2}$; Saul Blecker ${ }^{1} .{ }^{1}$ NYU School of Medicine, New York, NY; ${ }^{2}$ NYU, New York, NY. (Control ID \#3186048)

BACKGROUND: Clinical decision support (CDS) systems can be valuable resources in chronic disease management, but provider utilization of these tools and their integration into workflow remains suboptimal. We used a user-centered design approach to build a CDS recommending evidence-based therapy for heart failure in an inpatient setting. We implemented two versions of the CDS: an interruptive (pop-up) alert and a noninterruptive alert displayed in a provider checklist activity. In a prior study, we found that the interruptive alert was more effective than the noninterruptive alert but suffered from a high dismissal rate. The purpose of this study was to understand provider's perceptions of factors impacting CDS utilization following its implementation.

METHODS: We performed a qualitative study following implementation of two versions of a CDS at an academic medical center. We recruited providers who had either version of the CDS triggered in the prior 24 hours and obtained feedback through semi-structured interviews. Interviews were recorded, transcribed, and double-coded. We performed a constant comparative analysis of the transcripts and developed a coding scheme informed by the Five Rights of CDS combined with Proctor's 
outcomes for implementation research framework. We recruited participants until thematic saturation was achieved.

RESULTS: Fourteen providers participated in interviews. In general, providers found the CDS triggered for appropriate patients, provided the right information to determine appropriateness of recommendations, and had good usability. At least four providers believed they were not the right person to receive the alert, reporting that they primarily participated in cross-coverage, worked in a setting where these medications were typically contraindicated, or were already fully compliant with evidencebased medications. Providers complained that the interruptive alert led to workflow disruption and that frequently they would "just need to click through all of this." Nonetheless, many providers still preferred the interruptive version of the alert because they were either: 1) unaware of the non-interruptive alert in the checklist; 2) "don't use the provider checklist" where the non-interruptive alert resided; or 3) were unaware of the provider checklist.

CONCLUSIONS: We found that CDS was generally found to be acceptable, although the interruptive version of the alert was limited by disruptions in workflow. The interruptive alert was ultimately more successful as providers were unaware of the existence of the non-interruptive alert. Furthermore, they infrequently used the provider checklist, a native EHR feature for non-interruptive alerts and messages. Our findings suggest that incorporating user-centered design features can lead to success of an interruptive alert. Furthermore, future efforts to implement noninterruptive alerts must incorporate approaches to increase awareness of the alert and encourage changes in workflow to monitor alert lists.

\section{QUALITATIVE STUDY OF PRIMARY CARE PHYSICIANS' REASONS FOR NOT FOLLOWING GERIATRIC CHOOSING WISELY RECOMMENDATIONS}

Theresa A. Rowe ${ }^{1}$; Tiffany Brown ${ }^{2}$; Ji Young Lee ${ }^{1}$; Stephen Persell ${ }^{3}$. ${ }^{1}$ Northwestern University, Chicago, IL; ${ }^{2}$ Northwestern University Feinberg School of Medicine, Chicago, IL; ${ }^{3}$ Northwestern University, Evanston, IL. (Control ID \#3184036)

BACKGROUND: The Choosing Wisely Campaign identifies several areas to reduce unnecessary testing and treatment in older adults seen in primary care. Objective: To examine clinicians' views on the decision to: (1) screen asymptomatic men aged 76 using prostate specific antigen (PSA) (2) order urine studies in women aged 65 years without genitourinary specific symptoms and (3) de-intensify insulin or oral hypoglycemic medications in type 2 diabetes with a hemoglobin A1C $<7.0 \%$.

METHODS: Design, Setting, Participants: In this semi-structured interview study, we interviewed primary care physicians caring for adults aged 65 years recruited from primary care clinics within a large academic health system. Main Outcome and Measure: We transcribed the audiorecorded discussions and analyzed the transcripts using standard techniques of qualitative content analysis to identify major themes.

RESULTS: Fourteen participants were interviewed, 57\% were women. We identified several themes. First, most clinicians reported knowledge of these recommendations and could state reasons for not ordering these tests or for de-escalating treatment. Second, physicians relied heavily on patient preference when considering a potentially unnecessary test or treatment. This was particularly true for prostate cancer screening. For example, one physician stated, "Some patients are very insistent or expectant that they would want to continue to get testing." Another said, "If I couldn't educate him enough to reason with him then I think I would order it to make sure patient is less anxious." Third, there appeared to be an underestimation of harm associated with potentially unnecessary testing or treatment and potentially over-valuing clinical benefits. Most participants reported the concern of not wanting to miss a diagnosis when deciding whether to order testing, but few clinicians verbalized the harms associated with these tests. For example, clinicians voiced the concern of missing urosepsis or early prostate cancer, but few acknowledged the downsides of identifying a prostate cancer early in an older man with limited life expectancy or harms associated with using antibiotics for asymptomatic bacteriuria. Fourth, there was an acknowledgment of the lack of time needed to adequately discuss not performing a test like a screening PSA. Finally, clinicians were resistant to change. For example, clinicians articulated not wanting to deescalate medication for diabetes if the patient was doing well, despite understanding the harms associated with over treatment.

CONCLUSIONS: Although the Choosing Wisely campaign provides guidance on reducing over testing and treatment in older adults, clinicians may not follow these recommendations because of underlying psychological drivers. Understanding these drivers and developing approaches to physician behavior change that address these issues may help to improve evidence-based care for older adults.

\section{QUALITY AND SPENDING IN THE FIRST YEAR OF THE POPULATION-BASED PAYMENTS FOR PRIMARY CARE (3PC) SYSTEM IN HAWAII}

Amol S. Navathe ${ }^{2,}{ }^{1}$; Ezekiel J. Emanuel ${ }^{2}$; Amelia M. Bond ${ }^{3}$; Kristin Linn $^{4}$; Kristen L. Caldarella ${ }^{2}$; Andrea B. Troxel ${ }^{5}$; Jingsan Zhu ${ }^{2}$; Lin Yang ${ }^{6}$; Shireen E. Matloubieh ${ }^{2}$; Elizabeth E. Drye ${ }^{7}$; Susannah Bernheim ${ }^{7}$; Emily Oshima Lee ${ }^{8}$; Mark Mugiishi ${ }^{8}$; Kim Takata ${ }^{8}$; Justin Yoshimoto ${ }^{8}$; Isaac Yuen $^{8}$; Sheryl Okamura ${ }^{8}$; Michael Stollar ${ }^{8}$; Jeffrey Tom ${ }^{8}$; Michael Gold ${ }^{8}$; Kevin G. Volpp ${ }^{2,1}$. ${ }^{1} \mathrm{CMC}$ VA Medical Center, Philadelphia, PA; ${ }^{2}$ Medical Ethics and Health Policy, University of Pennsylvania, Philadelphia, PA; ${ }^{3}$ Weill Cornell Medical College, Healthcare Policy and Research, New York, NY; ${ }^{4}$ Biostatistics, Epidemiology and Informatics, University of Pennsylvania, Philadelphia, PA; ${ }^{5}$ New York University

School of Medicine, New York, NY; ${ }^{6}$ University of Pennsylvania, Philadelphia, PA; ${ }^{7}$ Yale University School of Medicine, New Haven, CT; ${ }^{8} \mathrm{Ha}-$ waii Medical Service Association, Honolulu, HI. (Control ID \#3183125)

BACKGROUND: In response to modest impact from quality programs, we worked with the Blue Cross Blue Shield of Hawaii to design and implement the Population-based Payments for Primary Care (3PC) system. This system was designed using behavioral economic insights and implemented in a staggered rollout across all Medicare, Medicaid, and commercial members starting in April 2016.

METHODS: We analyzed 2012-2016 claims and quality registry data for 77,225 HMSA members attributed to 107 primary care physicians (PCPs) and 4 organizations (POs) participating in the 1st wave of the 3PC and for 222,233 members attributed to 312 PCPs in 14 POs that continued in fee-for service in 2016 but had staggered 3PC start dates thereafter. We used a propensity-weighted, difference-in-differences design to compare riskadjusted changes in quality, cost, and utilization among patients in the $3 \mathrm{PC}$ group to those in the non-3PC group - after confirming parallel preintervention trends. We used generalized linear models with an identity link for quality, $\log$ link and gamma distribution for cost, and log link and negative binomial distribution for utilization, with standard errors clustered by PCP. RESULTS: The groups exhibited small baseline differences in demographics, risk scores, and socioeconomic characteristics. Adjusted analysis indicated an association between the 3PC group and increases in quality in 2016 (differential change in risk-standardized quality scores of 2.3 percentage points (p.p.), $95 \%$ CI 2.1 to 2.6 p.p., p<0.001). In secondary analyses, performance on 5 quality measures improved, 2 measures worsened, with no changes for six measures (see Figure). There were significant differential reductions in primary care cost $(-3.9 \%, 95 \%$ CI $-4.8 \%$ to $-2.9 \%, \mathrm{p}<0.001)$, but not total cost of care $(1.0 \%, 95 \%$ CI $-1.3 \%$ to $3.4 \%, \mathrm{p}=0.39$ ). Prescription drug cost differentially increased by $20.1 \%$ (95\% CI $10.2 \%$ to $32.5 \%, \mathrm{p}<0.001$ ). Changes in utilization by category were similar in magnitude to changes in cost by category.

CONCLUSIONS: The 3PC was associated with improvements in quality in its first year. Reduction in primary care visits and costs indicate shifts in practice patterns away from visit-based care. This study offers early evidence on the feasibility and effectiveness of shifting to a population-based primary care payment system across commercial, Medicare, and Medicaid 
populations in a fragmented market, which may have generalizable implications for other markets with similar characteristics nationwide.

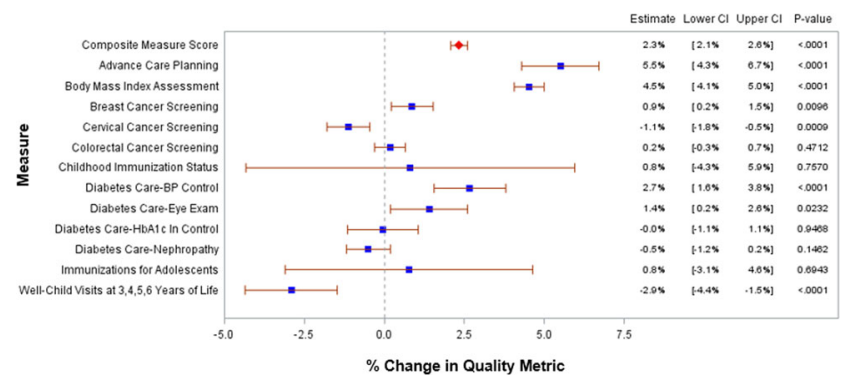

RACE/ETHNICITY AND SEX-RELATED DIFFERENCES IN ANTICOAGULATION IN PATIENTS NEWLY DIAGNOSED WITH ATRIAL FIBRILLATION

Utibe R. Essien $^{2,1}$; Jared W. Magnani ${ }^{3}$; Inmaculada Hernandez ${ }^{4}$; Michael $\overline{\text { J. Fine }}{ }^{2}$; Walid F. Gellad ${ }^{5} .{ }^{1}$ University of Pittsburgh School of Medicine, Pittsburgh, PA; ${ }^{2}$ VA Pittsburgh Healthcare System, Pittsburgh, PA; ${ }^{3}$ University of Pittsburgh Medical Center, Pittsburgh, PA; ${ }^{4}$ University of Pittsburgh, Pittsburgh, PA; ${ }^{5}$ A Pittsburgh/University of Pittsburgh, Pittsburgh, PA. (Control ID \#3183148)

BACKGROUND: Oral anticoagulation (OAC) is associated with stroke risk reduction in patients with atrial fibrillation (AF). Black individuals and women with $\mathrm{AF}$ have higher stroke rates, these disparities largely due to lower use and efficacy of traditional warfarin-based OAC in these groups. Less is known about race/ethnicity and sex-related differences in novel direct-acting oral anticoagulant (DOAC) use in patients with AF. Our aim was to compare OAC and DOAC initiation by sex, race, and ethnicity in a real-world, national cohort of patients with AF.

METHODS: We used claims data from a 5\% sample of Medicare beneficiaries to identify patients with newly-diagnosed AF between 2013-2014. We excluded those without continuous Medicare enrollment. The primary outcome was the initiation of any OAC (warfarin and DOAC- apixaban, dabigatran, rivaroxaban) after $\mathrm{AF}$ diagnosis based on prescription fill data. Among OAC initiators, we compared DOAC vs. warfarin use. We used logistic regression to assess the association between sex, race/ethnicity (white, black, Hispanic) and OAC initiation, adjusting for sociodemographic factors, comorbidities, and stroke risk $\left(\mathrm{CHA}_{2} \mathrm{DS}_{2}\right.$-VASc score).

RESULTS: The cohort of 47,952 patients with AF included 17,935 women, 3282 blacks, and 1958 Hispanics. Overall OAC initiation was low $(49.2 \%$ whites, $48.1 \%$ blacks, and $47.5 \%$ Hispanics). After adjusting, blacks (odds ratio (OR) 0.84; 95\% CI, 0.78-0.91) and Hispanics (OR 0.90; 95\% CI, 0.82$0.99)$ were less likely than whites to initiate any OAC. Women were less likely than men to initiate any OAC, OR 0.59 (95\% CI 0.55-0.64). Among OAC initiators, DOAC use was low ( $35.8 \%$ whites, $29.3 \%$ blacks, and $40.0 \%$ Hispanics). After adjusting, blacks were less likely to initiate DOACs than whites, OR 0.80 (95\% CI 0.71-0.90); the odds of DOAC initiation did not differ for Hispanic and white patients; OR 1.10 (95\% CI 0.95-1.27). There was no gender difference in DOAC use among OAC initiators, OR 0.98 (95\% CI 0.88-1.10).

CONCLUSIONS: In a national cohort of Medicare beneficiaries with newly diagnosed AF, OAC initiation was lower in blacks, Hispanics, and women. Among OAC initiators, blacks were less likely to initiate DOACs, with no differences identified by Hispanic ethnicity or gender. These findings, in a real-world cohort of medically insured patients, show that blacks with $\mathrm{AF}$ were less likely to receive novel DOACs - easier to use, potentially safer, and more effective than warfarin in stroke prevention. Identifying modifiable causes of treatment disparities is needed to improve the quality of care for all patients with $\mathrm{AF}$.

RACIAL AND ETHNIC DIFFERENCES IN THE MEDICAL TREATMENT OF OPIOID USE DISORDERS WITHIN THE VA HEALTHCARE SYSTEM FOLLOWING NON-FATAL OPIOID OVERDOSE
Utibe R. Essien $^{2,1}$; Florentina Sileanu ${ }^{3}$; Jane M. Liebschutz ${ }^{4}$; Chester Good ${ }^{2}$; Xinhua Zhao ${ }^{5}$; Maria K. Mor ${ }^{2}$; Michael J. Fine ${ }^{2}$; Walid F. Gellad ${ }^{6} .{ }^{1}$ University of Pittsburgh School of Medicine, Pittsburgh, PA; ${ }^{2}$ VA Pittsburgh Healthcare System, Pittsburgh, PA; ${ }^{3}$ VA Pittsburgh, Pittsburgh, PA; ${ }^{4}$ University of Pittsburgh Medical Center, Pittsburgh, PA; ${ }^{5}$ VA Pittsburgh HealthCare System, Pittsburgh, PA; ${ }^{6}$ VA Pittsburgh/University of Pittsburgh, Pittsburgh, PA. (Control ID \#3184992)

BACKGROUND: There has been slow adoption of medication-assisted treatment (MAT) in the US, despite substantial morbidity and mortality from opioid misuse and overdose. Even after non-fatal opioid overdose, research shows that few enter addiction treatment, and opioid prescribing patterns frequently are unchanged. Little is known about race/ethnicity differences in care after non-fatal overdose, despite known disparities in opioid prescribing. Our aim was to assess the association of race and ethnicity on receipt of MAT and prescription opioids before and after a non-fatal overdose in a national cohort of patients from the Veterans Health Administration (VA).

METHODS: Using data from the VA Corporate Data Warehouse and Pharmacy Benefits Management Services, we identified all Veterans enrolled in VA from 2011-2013 who had a hospitalization or emergency department visit for non-fatal opioid overdose based on established ICD-9 codes. We excluded patients in hospice care, those with missing or "other" race/ethnicity, and those who died within 7 days of the overdose date. We categorized race/ethnicity as white, black, or Hispanic. Our primary outcomes were: 1) MAT prescription (buprenorphine, naltrexone) or 1 methadone clinic visit within 30 days after overdose, and 2) change in opioid prescription fills within 30 days after overdose from the 30 days before. We used logistic regression, adjusting for age, to test whether MAT receipt after overdose differed by race/ethnicity and used difference-in-differences analyses to test whether change in opioid prescribing after overdose differed by race/ethnicity.

RESULTS: Among 5746 Veterans with a non-fatal overdose, $14.3 \%$ were black, $4.2 \%$ were Hispanic, and $7.5 \%$ women; mean age (SD) was 60.2 (13.7) years. After overdose, $3.3 \%$ of Veterans received any MAT, with no difference observed comparing black Veterans to white, adjusted odds ratio (aOR) 1.32, 95\% CI 0.90,1.92. Hispanic Veterans were more likely than whites to receive MAT after overdose, aOR 1.87 (95\% CI 1.04, 3.37). Before overdose, opioid prescriptions varied by race/ethnicity $(65.8 \%$ of white Veterans, $59.6 \%$ black and $62.9 \%$ Hispanic, $\mathrm{p}$ value $<0.01)$. After overdose, prescription opioid use decreased significantly to $49.3 \%$ in whites $(-16.5 \%$ absolute difference), $44.5 \%$ in blacks (-15.5\% absolute difference), and $46.4 \%$ in Hispanics (15.9\% absolute difference). These decreases in opioid prescription after overdose did not differ between groups (overall pvalue 0.88 ).

CONCLUSIONS: In a national cohort of Veterans with a non-fatal opioid overdose, overall MAT receipt was low with slightly higher rates among Hispanic Veterans compared to whites. After overdose, opioid prescribing decreased across all groups with no difference observed by race /ethnicity. Further examination of racial and ethnic disparities in post-overdose care in contemporary and other real-world patient cohorts is vital to improve quality of care for all patients.

\section{RACIAL DISPARITIES IN CLINICIAN RESPONSES TO PA- TIENTS' EMOTIONAL CONCERNS}

Jenny Park $^{1}$; Mary Catherine Beach ${ }^{1}$; Dingfen Han ${ }^{1}$; Somnath Saha ${ }^{2}$. ${ }^{1}$ Johns Hopkins University, Baltimore, MD; ${ }^{2}$ Portland VA Medical Center, Portland, OR. (Control ID \#3184737)

BACKGROUND: Prior studies have demonstrated racial differences in the quality of patient-provider communication, which may contribute to disparities in patient experience, treatment adherence, and clinical outcomes. Attending to patients' emotions (e.g., with empathic statements) is a particularly important aspect of communication, yet few studies have focused on potential disparities in how emotions are handled in clinical encounters. In a previous study of patients newly 
enrolled in HIV care, we observed that clinicians were less likely to address the emotional concerns of African American patients compared to whites. In the current study, we aimed to confirm these findings in a separate group of patients already established in HIV care. METHODS: We enrolled 41 HIV clinicians and 342 Englishspeaking adult patients receiving HIV care at an urban academic medical center. We audio-recorded one visit for each enrolled patient, transcribed the recordings, and coded them using the Verona Coding Definitions of Emotional Sequences (VR-CoDES). In VR-CoDES, clinician responses to patients' emotional expressions are categorized as (1) explicit vs. non-explicit in terms of addressing the emotion, and (2) providing vs. reducing space for the patient to talk further. Responses that provide space are subdivided into those that are passive (allow the patient to continue speaking with back-channeling such as "uh-huh"), actively explore the issue, or express empathy. Responses that reduce space are subdivided into those that provide information to the patient and those that block the patient from speaking further (e.g. switching topics). We used random intercept multilevel logistic regression, which accounted for clustering of emotional expressions within encounters and of patients within clinicians, to assess associations of race and patterns of emotional talk.

RESULTS: Patients were predominantly African American (76\%) or white $(21 \%)$. Clinicians were mostly white $(66 \%) ; 15 \%$ were African American. Among the 228 patients whose visits included emotional talk, African Americans were less likely to spontaneously express emotion during the visit (OR 0.50; 95\% CI 0.29-0.85). Clinicians were more likely to explicitly address emotional concerns expressed by African American patients (OR 1.56; 95\% CI 1.11-2.20), but they were less likely to provide space for the patient by offering passive responses (OR 0.56 ; 95\% CI $0.37-0.84$ ), and more likely to block African American patients from talking further about the emotional issue (OR 2.47; 95\% CI 1.08-5.67). Clinician race was not associated with response type.

CONCLUSIONS: Our findings confirm those of our prior study, demonstrating that clinicians tend to be less responsive to the emotional concerns of African American patients. Further research should explore reasons for this finding, its potential impact on patient experience and outcomes, and interventions to improve emotional communication across racial/ethnic groups.

\section{RACIAL DISPARITIES IN LOWER EXTREMITY AMPUTA- TION AMONG PATIENTS WITH PERIPHERAL ARTERIAL DISEASE OR DIABETES: A SYSTEMATIC REVIEW AND META-ANALYSIS OF PUBLISHED STUDIES}

Yvette Portilla ${ }^{1}$; Binhuan Wang ${ }^{2}$; Sundar Natarajan ${ }^{3} .{ }^{1}$ New York University Langone Medical Center, New York, NY; ${ }^{2}$ New York University, New York, NY; ${ }^{3}$ VA New York Harbor Healthcare System, New York City, NY. (Control ID \#3162692)

BACKGROUND: Amputation dramatically lowers quality of life and increases risk of mortality in peripheral arterial disease (PAD) or diabetes. Some studies report an increased lower extremity amputation (LEA) risk for black patients compared to white patients, though others report no disparity, particularly in persons with diabetes. We conducted a meta-analysis using PRISMA (Preferred Reporting Items for Systematic Reviews and Meta-Analysis) guidelines to examine the risk of LEA in black and white adults with PAD and/or diabetes.

METHODS: We systematically searched PubMed and EMBASE databases for cohort or case-cohort studies that included blacks and whites with PAD and/or diabetes and reported risks for LEA. A quality assessment was conducted using the Newcastle-Ottawa Scale (NOS). In addition to presenting the overall risks for amputation attributed to blacks, we conducted a meta-analysis of patients who had a previous amputation and examined the odds that the amputation was primary, defined as an amputation without any previous attempt at revascularization. A Jackknife sensitivity analysis evaluated the effect of individual articles on the estimate.

RESULTS: After the database search, we identified 570 articles. 13 articles met the inclusion criteria, 9 of which were pooled in the metaanalysis. Among diabetic patients, the unadjusted odds ratio (OR) for black patients ( $n=6$ studies) receiving a LEA was 1.35 (95\%CI, 1.051.75) compared to white patients [Figure below]. The Goldberg and Helmer articles had the greatest effect on the pooled estimate. The adjusted risks among black diabetic patients also suggested an increased risk but the large heterogeneity prevented pooling. Similarly, while the risk for LEA among PAD patients could not be quantified, the forest plot indicated an increased risk among blacks. The pooled unadjusted risk for primary amputation ( $\mathrm{n}=3$ studies) among black patients was high (OR, 1.86; 95\% CI, 1.35-2.56). Even when using the adjusted OR, the risk was attenuated but remained significant (OR 1.17 ; $95 \%$ CI, 1.09-1.26).

CONCLUSIONS: Black patients with PAD or diabetes are at an increased risk for a LEA as well as for primary amputation. Future studies should evaluate mediators of this disparity and examine ways to eliminate it.

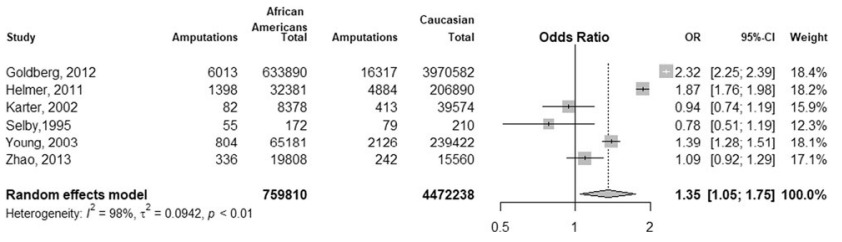

RANDOMIZED CONTROLLED TRIAL OF A RELATIONAL AGENT TO REDUCE RISKY ALCOHOL USE

Steven R. Simon ${ }^{1}$; Nicholas Livingston ${ }^{1}$; Molly Sawdy ${ }^{1}$; Catherine Yeksigian $^{1}$; Timothy Bickmore ${ }^{2}$; Shuo Zhou ${ }^{2}$; Richard Saitz ${ }^{3}$; Nancy R. Kressin ${ }^{1}$; Amy Rubin ${ }^{1} .{ }^{1}$ VA Boston Healthcare System, Boston, MA; ${ }^{2}$ Northeastern University, Boston, MA; ${ }^{3}$ Boston University and Boston Medical Center, Boston, MA. (Control ID \#3186360)

BACKGROUND: Risky drinking and alcohol use disorders are present among $20 \%$ to $40 \%$ of Veterans. Although the efficacy of brief intervention and referral to specialty care for risky drinking is well established, implementation of these approaches often falls short, owing to multiple competing demands on primary care providers (PCPs) and other barriers. A health-information technology (HIT)-based intervention that mimics a live human interaction may offload PCPs and may be more palatable to patients than direct interaction with their provider. We therefore conducted a randomized controlled trial (RCT) of a Relational Agent, a human character on a computer screen that talks with the patient, to reduce risky drinking and promote referral to specialty care.

METHODS: We conducted usability testing to tailor a previously pilottested Relational Agent to Veterans' preferences. We enrolled 179 Veterans who screened positive on the Alcohol Use Disorders Identification Test (AUDIT-C) and randomly allocated them to receive the Relational Agent Intervention (RAI) plus Treatment as Usual (TAU) vs. TAU. The RAI consisted of two 15-minute interactive sessions with the Relational Agent, the first occurring at the time of study enrollment, which coincided with a previously scheduled primary care office visit, and the second arranged for one month later. The intervention incorporated Motivational Interviewing, responsibility, empathy, and support of self- efficacy. Telephone follow-up three months after enrollment measured average weekly drinks (primary outcome measure), calculated from the Quick Drink Screen (QDS). We also ascertained referral to specialty care for treatment (secondary outcome measure), which we validated through chart review. RESULTS: Veterans in the RAI group ( $\mathrm{N}=90)$ and in the TAU group $(\mathrm{N}=89)$ were similarly distributed with respect to age, sex, race/ethnicity, employment, and presence of VA-recognized disability. At baseline, mean 
average weekly drinks (AWD) was lower in the RAI group compared with the TAU group (20.6 vs. 27.2, p<0.05). At three months follow-up, mean AWD was 12.4 in the RAI group and 16.0 in the TAU group. Using generalized multilevel mixed modeling to account for baseline differences, the reduction in AWD was similar in the two groups. For the secondary outcome, 26 subjects in RAI (29\%) were referred for specialty treatment, compared with 1 in the TAU $(1 \% ; \mathrm{p}<0.05)$.

CONCLUSIONS: In this primary-care based RCT, a tailored HIT-based intervention engaged Veterans and resulted in increased referrals for specialty treatment of alcohol-use disorder. Relational Agent interventions, now shown to be effective in various health care settings, may be a useful tool to incorporate in primary care; this approach may be tested through other channels, such as web-based platforms and mobile devices, for broader impact on risky drinking and other sensitive health conditions.

\section{RANDOMIZED TRIAL OF GROUP PROBLEM SOLVING FOR} CARDIOVASCULAR RISK REDUCTION

David Edelman $^{1}$; Laura Wray ${ }^{2}$; Jason Nieuwsma ${ }^{1}$; Cynthia Coffman ${ }^{1}$; George L. Jackson ${ }^{1}$; Jennifer M. Gierisch ${ }^{1}$; Margaret Dundon ${ }^{3}$; Amy S. Jeffreys $^{1}$; Rhonda M. Merwin ${ }^{4}$; Karen A. Juntilla ${ }^{1}$; Courtney WhiteClark $^{1}$; Christina L. Vair ${ }^{5}$; Michael Owings ${ }^{2}$; Amy C. Harris ${ }^{1}$; Corrine I. Voils ${ }^{6} .{ }^{1}$ Durham VA Medical Center, Durham, NC; ${ }^{2}$ Buffalo VA Medical Center, Buffalo, NY; ${ }^{3}$ VA National Center for Health Promotion and Disease Prevention, Durham, NC; ${ }^{4}$ Duke University, Durham, NC; ${ }^{5}$ Salisbury VAMC, Salisbury, NC; ${ }^{6}$ University of Wisconsin, Madison, WI. (Control ID \#3179399)

BACKGROUND: Shared medical group interventions have been shown to improve outcomes among patients with a common disease or disorder. We sought to determine if a group prevention intervention based on problem-solving theory could reduce cardiovascular risk among patients at risk for cardiovascular disease but without a common risk factor.

METHODS: Patients at 2 VA Medical Centers with at least 5\% 10-year risk of a major cardiovascular event as measured by Framingham Risk Score (FRS), and with at least $2 \%$ of that risk reversible, were randomized to Group Prevention Coaching (GPC) vs. usual care (UC). GPC comprised 6 group visits among 5-15 patients and 6 individual telephone calls over 18 weeks; the content was derived from Problem Solving Therapy. The group leader, a clinical psychologist, taught patients core skills to improve at least one risk behavior, usually by healthier eating, increasing physical activity, or smoking cessation. Group session content was reinforced with individual telephone calls between each patient and a healthcare coach trained in motivational interviewing. In group visits and individual calls, patients set SMART goals and discussed barriers to achieving these goals along with solutions to these barriers. Primary outcome was FRS; secondary outcomes included the Patient Activation Measure (PAM), range 0-100 points, and the Social Problem Solving Inventory (SPSI), rang 0-139 points. We analyzed differences between GPC and UC at 6 months using an intention-to-treat approach and linear mixed models (LMM), adjusting for group clustering in the GPC arm.

RESULTS: We randomized 202 patients to GPC and 199 to usual care. Mean age was 62.4 years, $10 \%$ were women, and $32 \%$ were black. Mean 10 -year risk of cardiovascular event was $29.2 \%$ by FRS; baseline mean PAM and SPSI scores were 60 and 105, respectively. Follow-up rate at 6months was $62 \%$ in GPC and $64 \%$ in usual care. GPC patients attended a mean of 3.4 (of 6 ) group sessions; $43.6 \%$ attended 5 or 6 groups, and $14.9 \%$ did not attend any. Patients completed a mean of 3.9 (of 6 calls); $57.4 \%$ completed 5 or 6 calls, and $14.4 \%$ completed no calls. Among GPC patients, $47.7 \%$ chose to work on improved eating or weight loss, $17.9 \%$ chose to increase physical activity, $27.8 \%$ chose to quit smoking, and $6.6 \%$ chose another strategy for improving risk (medication adherence or cessation of non-tobacco substance). In the LMM analyses, there was no difference at 6 months between GPC and UC patients in FRS (mean difference $0.7 \%, 95 \% \mathrm{CI},-1.4 \%, 2.8 \%$ ), PAM score (mean differences 1.3 points, $95 \%$ CI $-1.3,3.9$ ), or SPSI (mean difference 1.6 points,
95\% CI -0.8, 4.0).

CONCLUSIONS: Our problem-solving-based group prevention intervention failed to improve cardiovascular risk, as measured by Framingham Risk Score (FRS), possibly due to low patient engagement in the intervention.

\section{RAPID ENGAGEMENT OF PATIENTS WITH SUBSTANCE USE DISORDERS THROUGH EXPEDITED TRIAGE (RESET): A JAIL IMPLEMENTATION STUDY}

Lello Tesema $^{1,2}$; Alia Moore ${ }^{1,2}$; Christy Rico ${ }^{2}$; Michael Hochman ${ }^{2,2}$; Stephanie Navarro ${ }^{3}$; Albert J. Farias ${ }^{3,2}$. ${ }^{1}$ Los Angeles County Department of Health Services, Los Angeles, CA; ${ }^{2}$ University of Southern California, Los Angeles, CA; ${ }^{3}$ University of Southern California Keck School of Medicine, Los Angeles, CA. (Control ID \#3165072)

BACKGROUND: Approximately $2 / 3^{\text {rd }}$ of all inmates in the Los Angeles County (LAC) jail system suffer from substance use disorder, predominantly alcohol-related, and many will experience forced withdrawal following incarceration. Jail facilities often lack standardized methods for identifying patients who are in alcohol withdrawal or at risk of withdrawing, and treatment is commonly de-centralized, resulting in significant mortality and morbidity. In an effort to mitigate adverse outcomes associated with alcohol withdrawal in custody, the LAC jail system established a pilot detoxification (detox) unit and developed a novel triage-to-treatment pathway to identify incoming patients in acute or subacute alcohol withdrawal; treat using the evidence-based CIWA protocol; and refer for Medication Assisted Treatment (MAT). Our study aims to present preliminary clinical effectiveness data for this pathway.

METHODS: This hybrid effectiveness/implementation study uses a quasi-experimental design to compare data from an intervention site (LAC men's jail) and a control site that has not implemented the pathway or detox unit (LAC women's jail). Our study population includes males age 18 who present to jail with a reported alcohol use history and a CIWA score $>8$ or alcohol consumption within the prior 72 hours; and incarcerated females age 18 who were treated with the CIWA protocol. Variables include time from jail intake to first treatment dose; incidence of decompensated withdrawal; and number of referrals for MAT. Preliminary data were collected via chart abstraction of 5\% of all patients admitted to the detox unit (intervention site) or treated with CIWA (control site) between June 2018 to November 2018 .

RESULTS: Of 454 charts reviewed, $20 \%$ of patients were female and $80 \%$ were male. There was no statistical difference between intervention and control sites in the mean CIWA score on initial presentation (3.74, $95 \%$ CI $[3.42,4.05]$ versus $3.80,95 \%$ CI [3.20, 4.40]), or the proportion of patients admitted to the detox unit that had one or more CIWA scores $>8$ (19.6\% versus $20.7 \%), p=0.48$.). However, there was a significant difference between the intervention and control site in the proportion of patients who had 2 or more scores $>8$ during treatment $(9.4 \%$ vs $4.3 \%$, $\mathrm{p}<0.052)$. Ninety-seven percent of patients at the intervention site received an initial treatment dose within 24 hours of intake compared to $71 \%$ at the control site $(\mathrm{p}<0.001)$. Over the 5 month study period, 3 patients from the intervention site were transferred to the hospital for decompensated withdrawal, compared to 0 patients from the control site. Thirty-three percent of patients at the control site were referred for MAT, and none from the control site.

CONCLUSIONS: Preliminary results suggest that the pathway effectively expedites identification and treatment of suspected alcohol withdrawal and increases MAT referrals. Future analyses will explore acceptability and feasibility of this pathway.

RATES AND SOCIO-DEMOGRAPHIC CORRELATES OF FOOD INSECURITY AMONG NEW YORK CITY TOBACCO USERS Christina Wysota $^{1}$; Scott E. Sherman ${ }^{1,2}$; Elizabeth Vargas ${ }^{1}$; Erin S. Rogers ${ }^{2} .{ }^{1}$ NYU Langone Health, New York, NY; ${ }^{2}$ VA NY Harbor Healthcare System, New York, NY. (Control ID \#3180058) 
BACKGROUND: Tobacco remains the leading cause of preventable death and disease in the U.S. Tobacco users are at increased risk of food insecurity, and the co-occurrence of tobacco use and food insecurity may place individuals at especially high risks of poor health. The objective of this study is to identify rates and socio-demographic correlates of food-insecurity among low-income tobacco users in the New York City area.

METHODS: We used baseline survey data from a large randomized controlled trial testing a smoking cessation intervention for smokers living below $200 \%$ of the federal poverty level (FPL) to calculate the proportion of smokers experiencing food insecurity, as measured by the USDA 6-item food security model. We used bivariable and multivariable logistic regression to identify participant factors associated with food insecurity.

RESULTS: Overall, 55\% of participants were food insecure, with $27 \%$ reporting very high food insecurity. Food insecurity did not significantly differ by gender, race, education, or employment status ( $p>$.05). Marital status tended to be a protector against food insecurity. Participants who reported being separated, widowed, or divorced, were more likely to be food insecure than those who reported being married or cohabitating ( $\mathrm{AOR}=2.24,95 \% \mathrm{CI}$ : 1.07-4.72). Additionally, participants who reported being never married had almost three times greater odds of experiencing food insecurity than married or cohabitating participants $(\mathrm{AOR}=2.90,95 \%$ CI: 1.40-6.00). Participants who fell below $100 \%$ of the FPL were almost two times more likely to experience food insecurity than those with income above the FPL (AOR $=1.95,95 \%$ CI: 1.12-3.40).

CONCLUSIONS: Most low-income tobacco users were experiencing food insecurity. Having been separated, divorced, widowed or never married is a significant predictor of food insecurity. Social networks and removing social isolation may be a protector against food insecurity among this unique sample of low-income NYC smokers. Future interventions which seek to alleviate food insecurity may benefit from targeting the poorest and socially isolated smokers.

\section{RATES OF NO-SHOWS IN AN URBAN PRIMARY CARE CLINIC: DEFINING THE PROBLEM}

Elizabeth Pines ${ }^{1}$; Patty McGraw ${ }^{3}$; Vishal Patel ${ }^{1}$; Gretchen Rickards ${ }^{2}$, ${ }^{1} .{ }^{1}$ Christiana Care Health System, Newark, DE; ${ }^{2}$ Christiana Care Health System, Kennett Square, PA; ${ }^{3}$ Christiana Care Health Services, Newark, DE. (Control ID \#3156677)

BACKGROUND: No-show rates among primary care practices in the United States vary from $5 \%$ to $55 \% .^{1}$ Missed appointments have been associated with increased acute care utilization, ${ }^{2,3,4,5}$ reduced access for other patients, ${ }^{6}$ worse health outcomes, ${ }^{2,4}$ and decreased provider productivity. ${ }^{6,7}$ Continuity clinic is a required component of residency training and missed appointments translate into missed educational opportunities. Few studies have looked at the differences between no-shows among residents and attendings as well as internal medicine (IM) residents and family medicine (FM) residents in the context of primary care. The aim of this study was to examine the association between no-show rates at an urban IM and FM residency continuity clinic based upon provider characteristics (i.e., specialty type and postgraduate year) and appointment type as well as the financial implications no-show appointments have on our clinic.

METHODS: We performed a retrospective chart review of all scheduled appointments from July 1, 2016 to December 4, 2017 of adult patients at an urban IM and FMresidency continuity clinic. Data was obtained from a single large regional mid-atlantic health care system. A no-show appointment was defined as an appointment where the patient failed to appear and did not cancel prior to the appointment time. Lost clinic revenue was calculated based on the following billing codes: 99204, 99213, 99214,
99243, 99396. Chi-square test was employed to analyze differences between IM providers and FM providers.

RESULTS: During the study period, there were 58,890 discrete visits. The overall no-show rate was $18.9 \%(\mathrm{n}=11,137)$, which differed by provider type with IM residents having the highest no-show rate (29.0\%) when compared to FM residents (26.3\%), IM nurse practitioners $(19.1 \%)$, and all attendings (17.9\%). No-show rate by postgraduate year was significantly different only among categorical IM residents with the later postgraduate years having lower no-show rates $(\mathrm{p}<0.0001)$. For both the IM and FM practices, hospital discharge follow-up appointments had the highest no-show rate (34.7\% and $38.5 \%$, respectively) while preoperative appointments had the lowest $(9.1 \%$ and $17.6 \%$, respectively). The clinic had an estimated $\$ 2,092,260$ in lost revenue from no-show appointments during the 18-month study period.

CONCLUSIONS: This study identified significant differences in noshow rates between residents and attendings as well as IM residents and FMresidents. Hospital discharge follow-up appointments had the highest no-show rates among all residents. The next step of this project is to develop and implement a survey to assess patient barriers to attending appointments with emphasis on transportation, financial, and scheduling concerns. Subsequently, the chart review and survey data will be combined to develop targeted interventions to reduce no-show rates.

\section{RE-FRAMING OPERATIONAL DECISIONS IN A VALUE- BASED FUTURE: HOW THE LARGEST U.S. PUBLIC HEALTH SYSTEM IS THINKING ABOUT CAPITAL FOR QUALITY IM- PROVEMENT INITIATIVES}

Hannah B. Jackson; Jeremy O'Connor; Theodore Long. NYC Health + Hospitals, New York, NY. (Control ID \#3185494)

BACKGROUND: With ongoing payment reform, there is a continued movement away from fee-for-service to value-based payment (VBP) systems. Often, large hospital systems only account for direct service revenue when deciding whether or not to implement a program. However, with newer payment models such as ACOs as well as Medicaid value-based initiatives, a newer paradigm is emerging that determines program viability based on direct impact on metrics tied to payment. In this exercise, we examined whether it would be feasible to hire new clinical pharmacists to help with medication management for patients with uncontrolled diabetes in five of our outpatient clinics. To assess this, we modeled the capital costs and net income associated with program implementation with and without inclusion of VBPs.

METHODS: We did a cost analysis study to ascertain the feasibility of implementing a diabetes quality improvement (QI) initiative across five clinical sites within the NYC Health + Hospitals public health care system, which cares for a population that is largely uninsured or publicly insured. We defined our population as the number of patients with uncontrolled diabetes (defined as A1c $>8.0$, "quality metric") at each site. We identified nine metriclinked payment opportunities within our Medicaid managed care plans or linked to state incentive programs and determined the number of our defined population that was eligible for each measure. Next, we calculated the required percent change in a quality metric for the system to be eligible for the VBPs and compared this to the expected impact of our pharmacist program, using conservative estimates from other implementation studies of ambulatory clinical pharmacist integration. With these numbers, we assessed the net operating income/cost with versus without the addition of various potential VBPs.

RESULTS: When we accounted only for direct revenue (without VBPs), we predicted the pharmacy program to generate a net income of $\$ 764,429$ in one year. In contrast, with VBPs, we predicted the program to generate up to $\$ 40,195,906$ in one year. As an example, in a subset of patients eligible for one of the VBPs, improvements of 
$2 \%$ or $5 \%$ in the metric at the targeted sites would result in systemwide improvements of $0.8 \%$ or $2.1 \%$, respectively, which would surpass the eligibility threshold for a VBP of $\$ 20,000,000$. In another example, if we achieved the goal metric for just 222 more patients, the system would be eligible for a VBP of $\$ 817,950$.

CONCLUSIONS: Implementing a diabetes QI program in a valuebased era could be associated with a significant increase in revenue in a safety net system - an increase that can be used to support the hiring of additional providers for these programs. Thus, when considering whether or not to implement QI programs, health systems should account for VBP revenue, often tied to small changes in performance, to fully capture the financial impact.

\section{REACH AND EFFECTIVENESS OF VIRTUAL DIABETES PREVENTION PROGRAMS IMPLEMENTED AT NATIONAL SCALE}

Ilya Golovaty $^{1}$; Sandeep Wadhwa ${ }^{2}{ }^{3}$; Ronli Levi ${ }^{1}$; Hilary

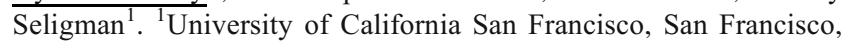
CA; ${ }^{2}$ University of Colorado School of Medicine, Aurora, CO;

${ }^{3}$ Solera Health, Phoenix, AZ. (Control ID \#3180379)

BACKGROUND: Nearly a third of US adults have prediabetes, $5-10 \%$ of whom will develop diabetes annually. Sustained weight loss via intensive lifestyle programs based on the Diabetes Prevention Program (DPP) may reduce this risk by half. Virtual DPP, as an alternative to in-person DPP, offers the promise of more rapid scaling but has not been rigorously evaluated. We examined the reach and impact of virtual DPP implemented at national scale.

METHODS: This longitudinal analysis includes data from 20,709 adults referred to 9 virtual DPP providers through a private platform that manages performance-based claims among multiple payers. We included adults age $>18$ at high risk for diabetes by CDC criteria who were referred to DPP from 2015-2017. The table defines our implementation outcomes (all binary) by categories of Reach, Engagement, and Effectiveness. Multivariable logistic models adjust outcomes for participant (age, gender, race/ethnicity) and setting (state, household income and rurality) characteristics.

RESULTS: Participants had a mean age of 49 and were $74 \%$ women and $60 \%$ white. The mean BMI was $34 \mathrm{~kg} / \mathrm{m}^{2}$. Reach: In multivariable models, there was an incremental decrease in odds of enrollment by increasing age group (18-44 yo referent, 45-64yo AOR 0.77, [95\% CI] 0.73-0.82, >65yo AOR 0.50, 0.45-0.55). Rural participants were slightly more likely than urban participants to enroll (AOR 1.10, 1.00-1.22). Engagement: $61 \%$ of participants interacted with the program at week 24 among those enrolled. There was an incremental increase in odds of 24-week engagement by increasing age group (18-44 yo referent, $45-64$ yo AOR $1.39,1.28-1.51,>65$ yo AOR 2.07, 1.76-2.44). Effectiveness: $28 \%$ of participants achieved $>5 \%$ weight loss between week 24 and 52 among those who enrolled. Odds of achieving 52-week $>5 \%$ weight loss increased as age group increased.

CONCLUSIONS: Maintained weight loss with virtual DPP was modest compared to national in-person DPP, but the high reach suggests broad population impact is possible, particularly among younger and more rural participants. As CMS expands reimbursement to performance-based DPP, rigorous examination of virtual platforms will be critical to inform national policy regarding acceptability of virtual DPP as an alternative to in-person DPP.

REASONS FOR NON-VISIT-BASED ANTIBIOTIC PRESCRIBING

Adriana Guzman ${ }^{3}$; Tiffany Brown ${ }^{3}$; Ji Young Lee ${ }^{3}$; Michael A. $\overline{\text { Fischer }}^{1}$; Mark W. Friedberg ${ }^{2}{ }^{4}$; Jeffrey A. Linder ${ }^{3}$. ${ }^{1}$ Brigham \&

\begin{tabular}{|c|c|}
\hline Implementation Measures & n (\%) \\
\hline \multicolumn{2}{|l|}{ Referred $(n=20,709)$} \\
\hline Reach (Enrolled/Referred) & $10,888(53 \%)$ \\
\hline \multicolumn{2}{|l|}{ Enrolled at Week $1(\mathrm{n}=10,888)$} \\
\hline $\begin{array}{l}\text { Engagement } \\
\text { (Interaction at Week 24/Enrolled) } \\
\text { Effectiveness } \\
\text { ( } \geq 5 \% \text { weight loss } \geq \text { Week 24/Enrolled) }\end{array}$ & $3,086(28 \%)$ \\
\hline \multicolumn{2}{|l|}{ Completed at Week $52(n=4,487)$} \\
\hline $\begin{array}{l}\text { Effectiveness } \\
\text { ( }>5 \% \text { weight loss at Week } 52 / \text { Completed) }\end{array}$ & $1,241(28 \%)$ \\
\hline
\end{tabular}

Women's Hospital, Boston, MA; ${ }^{2}$ RAND, Boston, MA; ${ }^{3}$ Northwestern University Feinberg School of Medicine, Chicago, IL; ${ }^{4}$ Brigham and Women's Hospital, Boston, MA. (Control ID \#3184238)

BACKGROUND: Non-visit-based antibiotic prescriptions (those that occur outside of an in-office encounter) account for over $20 \%$ of all ambulatory antibiotic prescriptions. Little is known about the reasons for non-visit-based antibiotic prescriptions, but the information in electronic health records (EHR) may offer insights. We conducted a retrospective cohort study in a large academic health system to understand reasons for non-visit-based antibiotic prescribing.

METHODS: We queried the EHR of a large, integrated health system to identify all oral, antibacterial antibiotics prescribed from November to December 2018. We excluded antibiotic prescriptions that occurred within 7 days after an in-person office encounter at the prescriber's clinic; to patients with a recently completed or scheduled procedure; to patients with a recent abnormal urine test result; and if there was an infection-related ICD-10 code associated with the antibiotic order. For the remaining non-visit-based antibiotic prescriptions, we randomly sampled a subset and manually reviewed the EHR to identify reasons for the prescription. If no EHR justification could be identified, we contacted clinicians, primarily via email, to complete a survey asking why they prescribed the antibiotic.

RESULTS: During the study, there were 13,686 antibiotic prescriptions, $12,100(88.4 \%)$ were associated with in-person visits at the prescriber's clinic. Of the 1,586 non-visit-based antibiotic prescriptions, 598 (38\%) were excluded because of a procedure or abnormal urine result and 159 $(10 \%)$ had a documented infection-related diagnosis associated with the antibiotic order. After exclusions, 829 (52\%) of non-visit-based prescriptions remained unexplained and 741 of these prescriptions were randomly sampled for review. Chart reviewers found an explanation in the EHR for $683(92 \%)$ prescriptions. The most common reasons - not mutually exclusive - were patient reported symptoms $(460 ; 67 \%)$, continuation of a previous clinical problem $(124 ; 18 \%)$, resulted labs or imaging studies $(117 ; 17 \%)$, or upcoming international travel $(103 ; 15 \%)$. The most common symptoms documented were cough $(170 ; 37 \%)$, sinus symptoms $(160 ; 35 \%)$, and urinary symptoms $(94 ; 20 \%)$. Continuation of a previous clinical problem was often related to an in-person encounter with a different clinician $(88 ; 71 \%)$ and often a response to persistent symptoms, such as cough and sinus symptoms. To date, we have received survey responses from 17 of 45 clinicians. Clinicians' stated reasons include poor documentation (e.g., antibiotic was for a symptom or an infection diagnosis not entered into the EHR), patient travel, or the antibiotic was for a prescriber's family member.

CONCLUSIONS: Non-visit based antibiotic prescribing is generally a response to patient-reported symptoms though it may also occur for a variety of other reasons. A high proportion of non-visit-based antibiotic 
prescribing may be inappropriate.

\section{REDEFINING THE ROLE OF PRIMARY CARE PROVIDERS IN CARE OF LIVER TRANSPLANT RECIPIENTS: A CASE STUDY OF THE PRIMARY-SUBSPECIALTY CARE INTERFACE}

Amy D. Lu ${ }^{1,2}$; Scott D. Rothenberger ${ }^{3}$; Gary Fischer ${ }^{3}$; Michael Dunn ${ }^{3}$; Peggy B. Hasley ${ }^{3} .{ }^{1}$ San Francisco VA Medical Center, San Francisco, $\mathrm{CA} ;{ }^{2}$ University of California San Francisco, San Francisco, CA; ${ }^{3}$ University of Pittsburgh, Pittsburgh, PA. (Control ID \#3185093)

BACKGROUND: A major challenge within the primary-subspecialty care interface is defining clear roles and expectations for the relationship between primary care provider (PCP) and subspecialist. As liver transplant recipients (LTRs) are surviving longer, transplant-related metabolic conditions such as, hypertension (HTN), diabetes (DM), dyslipidemia and chronic kidney disease (CKD) have become a major source of non-graft morbidity and mortality. For LTRs, the optimal involvement of PCPs in managing these conditions is unclear. We sought to characterize perceived responsibilities of PCPs and transplant hepatology providers (THP) in the co-management of post-transplant metabolic conditions and determine the effect of PCP perception of responsibility on clinical quality of care in a shared cohort of LTRs.

METHODS: We surveyed PCPs and THPs (surgeons, hepatologists, and physician extenders) regarding the care of post-transplant metabolic conditions in their shared cohort of 56 LTRs $>1$ year posttransplant (mean 11 years \pm 8 ). PCPs and THPs rated their agreement using a 5 -point Likert scale ( $2=$ strongly agree to $-2=$ strongly disagree) on statements describing ideal PCP involvement in managing the 4 metabolic conditions, analyzed using two-sample t-tests. PCPs also ranked their level of involvement for each LTR under their care, which was analyzed in relation to clinical outcomes abstracted from chart review including BMI, blood pressure, hemoglobin A1C, screening for retinopathy and CKD, and appropriate statin use via generalized estimating equations for binary data.

RESULTS: A total of 23 THPs (76\%) and 32 PCPs (94\%) completed the surveys. THPs felt PCPs should have primary responsibility for the management of HTN (1.55 \pm 0.80$)$, DM (1.45 \pm 0.86$)$, and dyslipidemia $(1.27 \pm 0.98, \mathrm{p}<0.001)$ in liver transplant recipients. Similarly, PCPs felt that subspecialists should not be primarily responsible for managing HTN $(-1.34 \pm 0.83)$, DM $(-1.31 \pm 0.9)$, dyslipidemia $(-1.47 \pm 0.88)$. Both groups held mixed opinions regarding the management of CKD (PCP $0.59 \pm 1.01$ vs THP $-0.27 \pm 1.16$ ) A majority of THPs (57\%) reported frequent concern about missed care while $26 \%$ PCPs shared the same sentiment. In terms of gaps in quality of care, $51 \%$ (20/39) of hypertensive patients in our cohort had uncontrolled HTN, 34\% (19/56) did not have regular screening for CKD, and 56\% (22/39) where not prescribed statins despite being in a statin benefit group. There were no significant differences in quality of care between PCPs who endorsed 50\% responsibility for care compared to those who endorsed $<50 \%$.

CONCLUSIONS: Both PCPs and THPs agree that PCPs should assume primary responsibility in the management of HTN, DM, and dyslipidemia in LTRs. Gaps remain in quality for these conditions but were not associated with PCP perception of responsibility. Future research should address barriers to achieving high-quality chronic disease management for transplant recipients and methods to improve communication between PCPs and subspecialists.

\section{REDUCING RESIDENT ADMINISTRATIVE BURDEN USING A REMOTE SCRIBE FOR DISCHARGE SUMMARIES}

Shilpa Sharma; Arielle Medford; Daniel Restrepo; Nilay Patel; Aksharananda Rambachan; Kathleen M. Finn. Massachusetts General Hospital, Cambridge, MA. (Control ID \#3141270)

BACKGROUND: Administrative burden is widely recognized as detrimental in medical education. The iCOMPARE study found residents spent $67 \%$ of their time on indirect patient care including interacting with the electronic chart. In order to reduce administrative burden, we implemented and evaluated the use of a remote scribe to write initial hospital courses in the Epic discharge module on the general medical service in a large internal medicine residency program.

METHODS: A time motion study was initially performed to assess the amount of time residents spent writing hospital courses. Next, we partnered with IKS Health, a scribe company based in India, whose physicians overnight reviewed resident progress notes and drafted a hospital course, which resident physicians subsequently edited and signed the next morning. To standardize the hospital course, IKS Health was provided with templates along with key findings to include for the 20 most common medical problems. A second time motion study was performed to assess the amount of time residents spent editing the IKS Health hospital courses. Once the program was established, in order to assess quality, we randomized for comparison $200 \mathrm{IKS}$-written/resident-edited hospital courses and 200 resident only-written hospital courses. Ten resident reviewers were hired, blinded to the writers, and asked to rate the hospital courses on five quality metrics: conciseness, clarity, detail, follow-up, and overall quality. Scores for each metric were averaged for comparison. Each resident evaluator served as their own control by assessing an equal number of intervention and control group hospital courses.

RESULTS: Editing the IKS hospital courses saved an average of 20 minutes per summary when compared to residents writing them in their entirety. The average time for a resident to write a hospital course was $30 \mathrm{~min}$ and editing took $10 \mathrm{~min}$. The IKS summaries scored higher on 4 out of 5 quality indicators: clarity, detail, follow-up, and overall. Average scores for IKS written/resident edited vs resident written hospital course were: conciseness 3.06 vs 3.36 ( $\mathrm{p}<0.01$ ), clarity 3.32 vs 3.29 ( $\mathrm{p}=0.88)$, detail 3.47 vs $3.24(\mathrm{p}=0.02)$, follow-up 3.37 vs $3.14(\mathrm{p}=0.01)$, and overall quality 3.34 vs $3.24(\mathrm{p}=0.30)$.

CONCLUSIONS: These results suggest implementation of a remote scribe for resident hospital courses is feasible and does not affect note quality. Blinded residents rated the IKS hospital courses higher than resident summaries in overall quality and in the subcategories of clarity, detail, and follow-up. With 20 minutes saved per summary this can reduce the administrative burden on residents especially with multiple discharges per day. The ultimate aim of this project was to reduce administrative burden on residents to create more time for residents to spend teaching, learning, and caring for patients. Investigating how residents use the time saved on discharge paperwork will be a compelling next step of the study.

\section{RELATIONSHIP BETWEEN BURNOUT, SPIRITUALITY, AND EMPATHY AMONG PHYSICIANS}

Anna-Carson Rimer Uhelski ${ }^{3}$; Mukta Panda ${ }^{1}$; Rehan Qayyum ${ }^{2} .{ }^{1}$ University

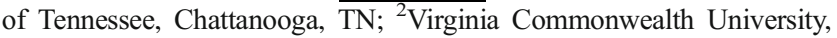
Richmond, Virginia, Richmond, VA; ${ }^{3}$ University of TN Health Science Center College of Medicine, Memphis, TN. (Control ID \#3167101)

BACKGROUND: Physician burnout has been suggested to be positively associated with degree of spirituality and negatively associated with empathy. Sparse data with mixed results are available. Our objective was to determine if physician burnout is related to spirituality and with empathy. METHODS: Email survey of faculty physicians, residents, and medical students using a questionnaire about demographic data and three validated tools; burnout tool (Oldenburg Burnout Inventory [OLBI]), spirituality tool (Daily Spiritual Experience Scale [DSES]), empathy tool (Interpersonal Reactivity Index [IRI]). Each item of the survey tools was scored and mean scores were calculated as recommended by survey authors. Linear regression was used to examine the relationship between the two burnout domains of OLBI (exhaustion and disengagement), spirituality, four domains of IRI (perspective taking, fantasy scale, empathetic concern, and personal distress), and total IRI without and with adjustment for age, gender, race, and faculty status.

RESULTS: Of the 101 participants, 47\% were females. Mean(SD) exhaustion score was $3.2(0.7)$, disengagement $3.0(0.6)$, spirituality $2.8(1.3)$, and total 
IRI score was 2.2 (0. 4). In unadjusted and adjusted analyses, we found no association between both domains of OLBI and DSES or total IRI scores. Of the IRI domains, we found a strong but negative association between both domains of OLBI and the personal distress domain but not with other domains. In adjusted models, for each one-point increase in the personal distress domain, OLBI exhaustion score decreased by $0.40(95 \% \mathrm{CI}=-0.68$ to $-0.12 ; \mathrm{P}=0$. $005)$ and physician disengagement decreased by $0.20(95 \% \mathrm{CI}=-0.47$ to -0 . 003; $\mathrm{P}=0.047$ ) (Figure).

CONCLUSIONS: We did not find an association between physician burnout and spirituality, total empathy, or empathy domains except for the personal distress domain. Personal distress is a tendency to experience distress and discomfort in response to extreme distress in others. Given that this is a cross sectional study, further research is necessary to establish causality.

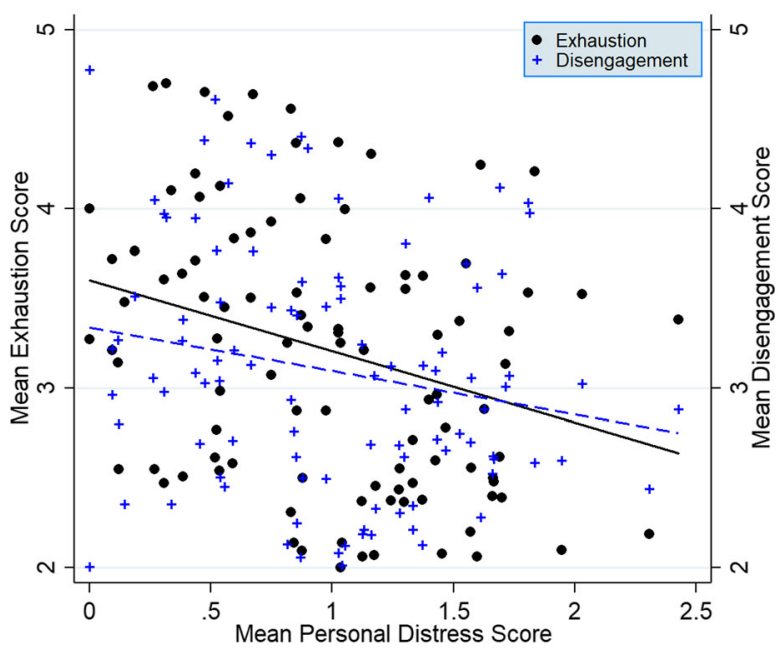

RELATIONSHIP BETWEEN FOOD AND HOUSING INSECURITY ON DIABETES SELF-CARE AND QUALITY OF LIFE IN ADULTS WITH DIABETES

Elise Mosley-Johnson ${ }^{1}$; Jennifer A. Campbell ${ }^{1}$; Rebekah J. Walker ${ }^{1}$; Sneha Nagavally ${ }^{2}$; Leonard E. Egede ${ }^{1}$. ${ }^{1}$ Medical College of Wisconsin, Milwaukee, WI; ${ }^{2} \mathrm{MCW}$, Wauwatosa, WI. (Control ID \#3186405)

BACKGROUND: Socioeconomic status (SES) has been shown to influence health, however, the aspects of care most strongly associated with social determinants of health related to SES, such as homelessness and food insecurity, are not yet well understood. The aim of this analysis was to examine the relationship between food and housing insecurities, diabetes self-care and quality of life.

METHODS: Data from 16,091 adults with diabetes in the nationally representative Behavioral Risk Factor Surveillance System (BRFSS), years 2014-2015 were used. The primary predictor variables were food insecurity, defined as being worried or stressed in the past 12 months about buying food; and housing insecurity, defined being worried or stressed about paying rent/mortgage in the past 12 months. The primary outcome variables included quality of life (health status, physically unhealthy days, mentally unhealthy days, days of poor health influencing activities) and diabetes self-care (HbAlc test in past 12 months, receiving diabetes education, daily foot checks, daily blood glucose monitoring, and physical activity in the past month). Unadjusted linear and logistic regression models were run for predictor variable and each outcome variable. Each model was then adjusted for demographics, socioeconomic status, and comorbidity count.

RESULTS: In this sample, $31.4 \%$ were food insecure and $38.1 \%$ had housing insecurity. Though all outcomes were significant in unadjusted models, after adjustment, housing insecurity was significantly associated with lower health status $(-0.22$, CI $-0.29,-0.15)$, more physically $(1.44$, CI $0.39,2.48)$ and mentally unhealthy days $(1.64$, CI
$0.43,2.85$ ). Food insecurity showed similar relationships (health status: 0.29 , CI $-0.36,-0.22$; physically unhealthy days: $1.16, \mathrm{CI}$ $0.12,2.20)$ and was associated with more mentally unhealthy days $(2.47$, CI 1.22,3.72). In regard to diabetes self-care, after adjustment both housing and food insecurity were significantly associated with lower odds of having an eye test (housing OR: 0.78, CI 0.64,0.94; food OR: 0.77 , CI $0.63,0.95$ ) and lower odds of physical activity (housing OR 0.86 , CI $0.74,0.99$; food OR 0.81 , CI $0.70,0.95$ ). Food insecurity was also associated with lower likelihood of having diabetes education (OR 0.79, CI 0.65,0.97). Conversely, housing instability was associated with higher odds of daily foot checks (OR 1.27, CI 1.03; 1.57) and food insecurity was associated with higher odds of daily blood glucose monitoring (OR 1.33, CI 1.05; 1.69).

CONCLUSIONS: In adults with diabetes, housing and food insecurity was associated with lower quality of life and poor self-care behaviors. Diabetes interventions should incorporate the influence of housing and food insecurity on quality of life and obtaining specialty care, such as eye exams. Interventions could also focus on improving opportunities for physical activity.

\section{RELATIONSHIP BETWEEN HOSPITAL READMISSION AND THE USE OF TRANSITIONAL CARE MANAGEMENT (TCM) SERVICES GUIDELINES AT PCP POST-DISCHARGE VISITS} Salina Bakshi ${ }^{1,2}$; Jeffrey Schnipper ${ }^{2,}{ }^{\text {; }}$ Lipika Samal ${ }^{2,2}$. ${ }^{1}$ Massachusetts General Hospital, Boston, MA; ${ }^{2}$ Harvard Medical School, Boston, MA. (Control ID \#3185895)

BACKGROUND: Transitions of care after hospital discharge are becoming increasingly important under new accountable care organization (ACO) payment models. While many patients are scheduled for follow-up appointments with their primary care physicians (PCPs) after hospital discharge, there are no standard of care guidelines for these often complex visits. Medicare's Transitional Care Management (TCM) billing codes reimburse providers for a set of recommended tasks for post-discharge visits. These tasks include components such as reviewing the discharge summary, reviewing pending diagnostic tests or treatments, providing patient and family education, and arranging for community resources. In this study, we aimed to assess if utilizing these guidelines is associated with reduced hospital readmissions.

METHODS: Patients admitted to medical or surgical units at two hospitals were eligible for the PCORI Transitions Study if they received primary care at one of 18 primary care practices within the Partners ACO. We selected patients in this study who were discharged from the hospital to home between October 2013 and September 2015 and had a post-discharge PCP visit within 14 days. We conducted a nested case control study within the larger trial, comparing readmitted patients with a sample of non-readmitted patients. We examined post-discharge notes for eight TCM elements based on Medicare's TCM Services Guidelines. We calculated a "TCM score," which was the unweighted sum of the number of TCM elements documented during the visit. We then used logistic regression to determine if there was any relationship between TCM score and readmission status. We adjusted the model to account for Elixhauser comorbidity index and number of visits to the PCP office from the prior year.

RESULTS: We reviewed 64 post-discharge visits, representing 34 different physicians and all 18 practices, including 27 readmitted patients and 37 non-readmitted patients. In unadjusted analyses, we found that readmitted patients had higher TCM scores on average than non-readmitted patients ( 4.9 v. 3.5 out of $8, p=0.004$ ). After adjusting for Elixhauser score and visits per year, an increase of 1 point in the TCM score was associated with a $52 \%$ increased likelihood of readmission (OR 1.52, 95\% CI 1.11,2.07, $\mathrm{p}=0.009$ ).

CONCLUSIONS: Counterintuitively, we found that patients that 
have higher TCM scores and are more likely to be readmitted, even after adjusting for intensity of primary care and medical comorbidities. We hypothesize that we were unable to control for confounding by indication, i.e., that PCPs provide (or at least document) more intensive transitional care for patients whom they believe are at higher risk for readmission. A randomized controlled trial is likely necessary to demonstrate whether TCM activities are effective in improving outcomes after hospital discharge. In addition, other methods of measuring the activities which take place during postdischarge visits may be needed.

\section{RELATIONSHIP BETWEEN MENTAL HEALTH AND OPI- OID MISUSE IN PEOPLE LIVING WITH HIV AND CHRON- IC PAIN}

Emma Richard $^{1}$; Ariana G. Pazmino ${ }^{1}$; Ana Alicia De La Cruz ${ }^{1}$; Chinazo Cunningham $^{1}$; Hector R. Perez ${ }^{1}$; Chenshu Zhang ${ }^{1}$; Yuting Deng ${ }^{2,}{ }^{1}$; Joanna L. Starrels ${ }^{1}{ }^{1}$ Albert Einstein College of Medicine \& Montefiore Medical Center, Bronx, NY; ${ }^{2}$ Montefiore Health System, Bronx, NY. (Control ID \#3185372)

BACKGROUND: People living with HIV (PLWH) have a high prevalence of chronic pain and prescription opioid use and misuse. Anxiety, depression, social isolation, and PTSD are common in PLWH and all patients with chronic pain. However, little is known about the relationship between these mental health conditions and prescription opioid misuse in PLWH who have chronic pain.

METHODS: This is a cross-sectional analysis of baseline data from 100 PLWH with chronic pain enrolled in a longitudinal observational study between May 2017 and December 2018. The primary outcome, opioid misuse, was assessed using the Current Opioid Misuse Measure (COMM, scored out of 68, 9 indicates misuse). The COMM assesses self-reported signs and symptoms of intoxication, emotional volatility, poor response to medications, addiction, healthcare use patterns, and problematic medication behavior. Four separate multivariate linear regression models were constructed to examine the relationship between each of 4 mental health variables with opioid misuse: anxiety (Brief Symptom Inventory anxiety subscale, scored out of 24), depression (Center for Epidemiologic Studies Depression Scale, scored out of 30), social isolation (PROMIS measure, scored out of 32), and PTSD (PTSD Checklist-Civilian Version, scored out of 25). Age, race, gender, and whether patients' had 3 opioid prescriptions 21 days apart in 6 months (chronic opioid therapy $[\mathrm{COT}])$ were covariates. A fifth model included all mental health variables.

RESULTS: Of 100 PLWH and chronic pain, 44 were currently prescribed opioids. Of the 44 , the mean age was $55,47.7 \%$ were male, $52.3 \%$ were African American and $34.1 \%$ were Hispanic, and $52.3 \%$ were on COT. The mean COMM score was 13 and $65.9 \%$ of participants met the threshold for misuse. In separate multivariate linear regression models, anxiety, depression, social isolation, and PTSD were each positively associated with opioid misuse (Table). When all mental health variables were included in the same model, only anxiety remained significantly associated with opioid misuse (adjusted beta=1.08, CI: 0.14-2.03, $\mathrm{R}^{2}=0.51, \mathrm{p}<0.05$ ).

CONCLUSIONS: Among PLWH with chronic pain who are prescribed opioids, anxiety, depression, social isolation, and PTSD were positively associated with opioid misuse using the self-reported COMM, and anxiety appears to be the most robust predictor of opioid misuse as measured by the COMM. However, future investigation is needed to validate which of these conditions are most strongly associated with misuse using objective measures of misuse. These findings suggest that each of these conditions should be evaluated when considering risks of opioids in PLWH with chronic pain.
SATISFACTION AFTER IMPLEMENTING TEAM-BASED PRIMARY CARE FOR COMPLEX PATIENTS: A LONGITUDINAL SURVEY ANALYSIS

Jonathan Weiner; Jodi McCloskey; Connie S. Uratsu; Richard W. Grant. Kaiser Permanente Northern California, Oakland, CA. (Control ID \#3178460)

BACKGROUND: Chronic stress from challenging work experiences may contribute to physician burnout. Caring for patients with complex medical and social issues may be one such stressor. We examined changes in self-reported physician stress and work satisfaction after implementing a team-based care management program for complex patients.

METHODS: Primary care physicians (PCPs) from a large integrated care system in Northern California completed surveys just prior to and one year after the implementation of a team-based (PCP, nurse, pharmacist, social worker, coordinator) program designed to support primary care for complex patients. The surveys prompted PCPs to consider their 10-15 most challenging patients and to report levels of stress and overall work satisfaction. The follow-up survey also asked PCPs to rate the helpfulness of eight different program services (e.g. medication management, care coordination, social services, support for elderly issues, and mental health). We compared changes in self-reported stress (decrease, no change, or increase) and changes in work satisfaction (increase vs. decrease or no change) from baseline to follow-up. We also compared changes in stress level and work satisfaction according to higher vs. lower PCP ratings of the helpfulness of care management services. We used logistic regression to control for gender, age, work experience, and baseline PCP panel complexity.

RESULTS: We examined 100 survey pairs for this analysis. Baseline and follow-up surveys were collected $11.9 \pm 1.6$ months apart (response rate $88 \%)$. Two-thirds $(65 \%)$ of PCPs were women; mean age was $47.8( \pm 8.2)$ years, and mean years' clinical experience was $20( \pm 8.6)$ years. At baseline, $84 \%$ of PCPs reported moderate or extreme stress, and $22 \%$ were moderately or extremely satisfied with their work experience. After one year, half (49\%) of PCPs reported unchanged stress levels, one third $(33 \%)$ had increased stress levels, and only $18 \%$ reported decreased stress. However, over this period, half of PCPs (51\%) reported improved work satisfaction. PCPs reporting decreased stress levels were more likely to highly rate help with medication management (aOR 2.44; 95\% CI 1.10$5.40)$ and identifying additional patient resources $(2.61 ; 1.15-5.90)$ as important benefits of the care management program. PCPs reporting increased satisfaction were more likely to highly rate help with all eight care management services ( aORs ranging from 3.14 to $5.75, \mathrm{p}<0.01$ ).

CONCLUSIONS: Implementation of a team-based coordinated care program to support primary care of complex patients was associated with increased PCP-reported work satisfaction. However, these changes were insufficient to significantly improve overall PCP work-related stress. These results underscore the challenge of addressing the multi-factorial problem of stress-related PCP burnout.

RELATIONSHIP OF HOME HEALTH CARE AFTER DISCHARGE FROM SKILLED NURSING FACILITIES WITH READMISSION AFTER HEART FAILURE HOSPITALIZATION Himali Weerahandi ${ }^{1}$; Haikun $\mathrm{Bao}^{7}$; Jeph Herrin ${ }^{2}$; Kumar Dharmarajan ${ }^{3}$; Joseph S. Ross ${ }^{4}$; Simon Jones ${ }^{5}$; Leora I. Horwitz ${ }^{6} .{ }^{1}$ NYU Langone, New York, NY; ${ }^{2}$ Yale University School of Medicine, Charlottesville, VA; ${ }^{3}$ Clover Health, Jersey City, NJ; ${ }^{4}$ Yale University School of Medicine, Woodbridge, CT; ${ }^{5}$ NYU, NY, NY; ${ }^{6}$ YU School of Medicine, New York, NY; ${ }^{7}$ Yale University, New Haven, CT. (Control ID \#3160011)

BACKGROUND: Discharge to skilled nursing facilities (SNF) is common in patients with heart failure (HF). The goal of a SNF stay is to improve functional status to allow patients to return home safely. However, the second transition from SNF to home may also be risky. Here, we examine the association between receipt of home health care (HHC) and readmission risk among patients discharged from SNF to home following HF hospitalization. 
METHODS: We examined all Medicare fee-for-service beneficiaries 65 and older admitted 2012-2015 with a HF diagnosis discharged to SNF then subsequently discharged home. The primary outcome was unplanned readmission within 30 days of SNF to home discharge, using CMS's HF readmission methodology. We plotted time to readmission with Kaplan-Meier curves and compared these groups with a log-rank test. Then, we compared time to readmission using an adjusted Cox model; this model included a frailty term to account for correlation of patient outcome by SNF.

RESULTS: There were 67,585 HF hospitalizations discharged to SNF and subsequently discharged home; 13,257 (19.6\%) were discharged with HHC, $54,328(80.4 \%)$ without. Patients discharged home from SNF with HHC had lower 30-day readmission rates than patients discharged without $\mathrm{HHC}(22.8 \%$ vs $24.5 \%, \mathrm{p}<0.0001)$. Kaplan-Meier curves demonstrated that patients discharged home from SNF with HHC have a longer unadjusted time to readmission. Of those readmitted within 30 days, median time to readmission for those discharged home from SNF with $\mathrm{HHC}$ was 11 days and 9 days for those discharged home without HHC $(\mathrm{p}<0.0001)$. After risk-adjustment, patients discharged home with HHC still had a lower hazard of 30-day readmission.

CONCLUSIONS: Patients who received HHC were less likely to be readmitted within 30 days compared to those discharged home without HHC. This is unexpected as patients discharged with HHC likely have more functional impairments and therefore at higher readmission risk. Since patients requiring a SNF stay after hospital discharge may have additional needs, they may be especially likely to benefit from restorative therapy through HHC; however only about $20 \%$ received such services.

\section{RESIDENT PERCEPTIONS OF BUPRENORPHINE-NALOXONE PRESCRIBING POST ADDICTION CLINIC IMPLEMENTATION} Deepa R. Nandiwada ${ }^{1}$; Judy Chertok ${ }^{2}$; Benjamin Larson ${ }^{3}$; David Lieberman'; Marc Shalaby ${ }^{4}$. ${ }^{1}$ University of Pennsylvania, Ardmore, PA; ${ }^{2}$ University of Pennyslvani, Philadelphia, PA; ${ }^{3}$ Lankenau Medical Center, Philadelphia, PA; ${ }^{4}$ Perelman School of Medicine at the University of Pennsylvania, Philadelphia, PA. (Control ID \#3186020)

BACKGROUND: There have been a growing number of residency buprenorphine-naloxone clinics with the goal that this should become a part of regular chronic care delivery in a primary care practice. The Penn primarycare-focused residency programs (Primary Care Internal Medicine and Family Medicine) have developed buprenorphine-naloxone residency clinics. The goal of this study is to compare the impact on patient perceptions, role and comfort of residents incorporating this into their scope of practice, and likelihood of including this into their clinical practice as attending physicians. METHODS: Residents participated in at least two half-day clinic sessions, seeing between 6 to 10 patients in a half day. All residents participated in waiver training and had an additional two didactic sessions on the topic. Surveys were provided to primary care residents and family medicine residents between April to June 2018. At this time primary care residents had all participated in 2 sessions of the clinic and were asked to rate changes in attitudes, knowledge, and perceptions post implementation. Family medicine residents had not begun their clinic yet and so baseline data was gathered.

RESULTS: 14 residents participated in the survey. Using a Wilcox-rank sum comparison we had significant changes $(p<0.01)$ with a change from Strongly Disagree to Agree in comfort prescribing. Other findings included being moree likely to recommend prescribing buprenorphine-naloxone and increased comfort being a PCP for a patient with opioid addiction. Residents strongly agreed that after participation they felt more empathy for this patient population. Finally they felt more empowered to care and enjoyed caring for this patients with addiction (Disagree to Strongly Agree). Key barriers pre implementation identified were lack of mental health care, logistics, and challenging patients.

CONCLUSIONS: While this represents a very limited data set, it shows that residents attitudes, knowledge, and comfort changed significantly post a direct patient care experience with patients actively prescribing buprenorphine naloxone. We are in the process of comparing this data to the family medicine pre and post data to see if carries across

\begin{tabular}{|l|l|l|l|}
\hline Question & $\begin{array}{l}\text { Median Pre- } \\
\text { Implementati } \\
\text { on }\end{array}$ & $\begin{array}{l}\text { Median } \\
\text { Post } \\
\text { Implement } \\
\text { ation }\end{array}$ & P value \\
\hline I am comfortable prescribing: & 1 & 3 & 0.01 \\
\hline I am very likely to recommend starting: & 2 & 4 & 0.01 \\
\hline I know which patients are good candidates & 2 & 4 & 0.01 \\
\hline $\begin{array}{l}\text { I am comfortable being the PCP for a patient } \\
\text { with opioid addiction }\end{array}$ & 2 & 3 & 0.01 \\
\hline $\begin{array}{l}\text { I enjoy caring for patients on } \\
\text { buprenorphine-naloxone }\end{array}$ & 2.5 & 4 & 0.01 \\
\hline $\begin{array}{l}\text { I feel like I made a different in my patients } \\
\text { life by prescribing buprenorphine-naloxone }\end{array}$ & & 3.58 & \\
\hline $\begin{array}{l}\text { I have more empathy for patients with } \\
\text { addiction }\end{array}$ & & 4 & 0.01 \\
\hline I feel this fits in the role of a PCP & 3 & 4 & 0.01 \\
\hline $\begin{array}{l}\text { I feel empowered to care for patients with } \\
\text { addiction }\end{array}$ & 2 & 4 & \\
\hline
\end{tabular}

\section{RESIDENTS IDENTIFY GAPS IN MEDICAL EDUCATION RE- GARDING THE OPTIMAL PROCESS FOR OUTPATIENT RE- FERRALS}

Masha J. Slavin ${ }^{1}$; Mangala Rajan ${ }^{2}$; Lisa M. Kern ${ }^{2} .{ }^{1}$ New YorkPresbyterian/Weill Cornell, New York, NY; ${ }^{2}$ Weill Cornell, New York, NY. (Control ID \#3180908)

BACKGROUND: Referrals from one physician to another in the ambulatory setting are routine, happening multiple times a day for any given physician. It is widely acknowledged that referrals should be accompanied by relevant clinical information about the patient being referred. However, previous work has shown that relevant clinical information is often missing at the point of care. Consequences of missing clinical information may include missed or delayed diagnosis, delayed treatment, unnecessary testing, and drug interactions. Whether residents are taught to provide adequate clinical information at the time of referral has not been well studied. Similarly, residents' attitudes toward providing clinical information at the time of referral and their experiences of the consequences of any subsequent missing information are unknown. Therefore, we sought to assess internal medicine residents' attitudes toward and experiences with outpatient referrals.

METHODS: We conducted a cross-sectional survey in OctoberDecember 2018 of all internal medicine residents affiliated with a large, urban academic internal medicine residency program. We used a novel survey instrument that included 13 questions about attitudes toward and experiences with outpatient referrals; each question had responses on a 5 point Likert scale. The survey also included 5 demographic questions. We used descriptive statistics to characterize the results.

RESULTS: We received completed surveys from 122 out of 132 residents (92.4\% response rate). Thirty-four percent of respondents were interns, $34 \%$ in post-graduate year 2 , and $31 \%$ in post-graduate year 3 . While $83 \%$ of residents reported that it is "always" important to provide the clinical reason for a referral, only $11 \%$ stated that they "always" provide a sufficient amount of clinical information for the consulting provider when making a referral. Forty-eight percent of residents "never" email, message, or call the consulting physician to explain a case beyond using the electronic health record's referral order template. Nine percent of residents "strongly agree" that residency provides sufficient training in knowing when to refer a patient, and $8 \%$ "strongly agree" that residency provides sufficient training in what information to provide the consulting physician.

CONCLUSIONS: These results highlight a discrepancy between the amount of information residents believe they should provide at the time of a referral and the amount they actually provide. Many residents felt that they had not received adequate training during residency on when to refer 
patients and what clinical information to convey. These results suggest that a formal curriculum is needed during residency on optimal referral processes, especially since trainees are unlikely to receive this education after they finish their training and because the referral habits developed during training will likely be carried forth into their medical careers.

\section{RESTRICTIONS ON PRIMARY CARE ACCESS FOR PATIENTS RECEIVING OPIOIDS}

Pooja A. Lagisetty; Nathaniel Healy; Claire Garpestad; Renuka Tipirneni; Amy Bohnert. University of Michigan, Ann Arbor, MI. (Control ID \#3184093)

BACKGROUND: New policies, including mandated prescription drug monitoring programs, have achieved an intended reduction of opioid prescribing. However, policies may create barriers that lead physicians to stop prescribing opioids entirely. Patients may thus encounter difficulties finding primary care providers (PCPs) willing to care for them if they take opioids. We aimed to assess provider willingness to accept and continue prescribing opioids to a new patient with pain and to determine if this differs across payer types.

METHODS: We randomly selected 667 Michigan primary care clinics between June-September 2018. Via calls, we surveyed clinics about the number and type of prescribers, insurances accepted, appointment availability, and whether their providers use medications to treat opioid-use disorders (OUD). Clinics accepting new patients with both Medicaid and private insurance were deemed eligible for inclusion and received a second call from a simulated patient, randomized to either having Medicaid or Blue Cross Blue Shield (BCBS) insurance. Research assistants (RAs) posed as the child of an adult woman needing a new primary care appointment. In this script, RAs revealed the patient's health insurance and asked, "Before we get too far, is it okay if my mother takes opioids for pain?" RAs documented each clinic's willingness to provide care. Clinic characteristics were summarized via descriptive statistics. Generalized logistic regression models estimated the odds of new opioid patient acceptance by insurance type, clinic size, and availability of OUD medications. RESULTS: Of the 667 clinics screened, 194 were eligible for study inclusion (29\%). Ninety-four clinics (48\%) were randomized to Medicaid and $101(52 \%)$ to BCBS. Of these clinics, $41 \%$ stated that their providers would not prescribe opioids to the simulated patient. $42 \%$ of clinics were willing to set up an initial appointment. 33 clinics (17\%) requested more information prior to making a decision. Compared to single-provider clinics, clinics with $>3$ providers were more likely (OR 2.99 [95\% CI 1.48-6.04]; $\mathrm{p}=0.002$ ) to accept new patients on opioids. There was no difference in access based upon insurance status (OR 0.92 [95\% CI 0.52$1.64] ; \mathrm{p}=0.78$ ) or whether the clinic offers OUD treatment (OR $1.10[95 \%$ CI 0.45-2.69]; $\mathrm{p}=0.83$ ).

CONCLUSIONS: Forty-one percent of clinics were not willing to schedule an appointment for a new patient requesting opioids for chronic pain. Insurance type was not related to willingness to accept a new patient on opioids. These findings may reflect clinicians' discomfort with managing opioid therapy for chronic pain or treating patients with OUD. Larger clinics may have more pain and behavioral health resources, making it easier for providers to care for this population. PCP's reluctance to prescribe opioids may have unintended adverse health outcomes, including increased transition to illicit opioid-use and reduced access to treatment for other comorbidities.

RETROSPECTIVE EVALUATION OF RENAL MEDICATION RELATED CLINICAL DECISION SUPPORT (CDS) ALERTS AND OVERRIDES IN THE INPATIENT SETTING POST IMPLEMENTATION OF A COMMERCIAL EHR.

Sonam N. Shah ${ }^{1,2}$; Diane L. Seger ${ }^{3,1}$; Mary Amato ${ }^{1,2}$; David W. Bates ${ }^{1,}$ ${ }^{4}$. ${ }^{1}$ Brigham and Women's Hospital, Jamaica Plains, MA; ${ }^{2}$ MCPHS
University, Boston, MA; ${ }^{3}$ Partners Healthcare System, Wellesley, MA; ${ }^{4}$ Harvard University, Boston, MA. (Control ID \#3180151)

BACKGROUND: Preventable adverse drug events (ADEs) are injuries from medications due to errors. One of the most common medication errors is inappropriate dosing for patients with renal insufficiency. Studies suggest about a third of inpatients with renal insufficiency receive incorrect doses of medications that should be dose-adjusted. Given their association with an increase in hospital length of stay, costs, and morbidity and mortality, efforts have been made to reduce ADEs resulting from these dosing errors. Medication-related CDS have been identified as an effective way to reduce ADEs. We analyzed the appropriateness of renal medication dose CDS alert triggers, number of overridden alerts, and the potential harm that is caused.

METHODS: We performed a retrospective evaluation of renal medication dose CDS alerts in the inpatient setting of a large academic medical center from 5/2017 to 4/2018. Included patients had a creatinine above 3.0 and an overridden renal CDS alert trigger $(n=1352)$. The primary outcome was appropriateness of the alert trigger and overrides assessed by two reviewers. Secondary outcomes included incidence of ADEs that resulted from the overridden alert based on documentation in the patients' medical record.

RESULTS: Of 1369 alert triggers identified that required renal dose adjustments, those involving medications given for diagnostic procedures or not administered to the patient were excluded, leaving 1352 alerts considered relevant for evaluation (98.8\%). Of these alert triggers, 83 (6.1\%) were considered appropriate and 1269 (93.8\%) were considered inappropriate. Override rates for the renal CDS alert triggers was $100 \%$, with $1291(95.6 \%)$ of the overrides of considered appropriate, and 61 $(4.44 \%)$ considered inappropriate. Appropriate overrides were largely due to the following medications: KCL $(51.3 \%)$, chlorothiazide $(28.6 \%)$, aspirin $(21.0 \%)$, cholecalciferol $(19.3 \%)$, magnesium oxide $(11.1 \%)$, and ergocalciferol (10.6\%). Inappropriate overrides were largely due to the following medications: duloxetine $(16.7 \%)$, phenazopyridine $(11.7 \%)$, fenofibrate $(6.67 \%)$, cetirizine $(6.67 \%)$, and alendronate $(5.0 \%)$. Identification of ADEs potentially associated with inappropriate CDS overrides was uncommon $(n=2)$. Further analysis is underway to identify if any ADEs occurred for appropriate CDS overrides.

CONCLUSIONS: Nearly all renal medication dose alert triggers were considered inappropriate; thus, the appropriateness of overrides was high. The risk for alert fatigue could be minimized by increasing the specificity and clinical significance of displayed alerts, while decreasing the number of insignificant alerts. Specific recommendations which could help improve the accuracy of these alerts included the differentiation of the dose prescribed, whether the patient is currently receiving hemodialysis, and patient specific factors such as ordered monitoring lab values.

RIGHT DIAGNOSIS, WRONG DRUG: ANTIBIOTIC PRESCRIBING PATTERNS FOR ACUTE SINUSITIS IN AN INTERNAL MEDICINE RESIDENT CLINIC

Ryan Kipp ${ }^{1}$; Eliana Hempel ${ }^{2}$; Gregory Caputo ${ }^{2}$; Thomas W. Abendroth ${ }^{4}$; John van Harskamp ${ }^{3}$; Nicholas Duca ${ }^{2} .{ }^{1}$ Penn State College of Medicine, Hershey, PA; ${ }^{2}$ Penn State Health Milton S. Hershey Medical Center, Hershey, PA; ${ }^{3}$ Penn State Hershey Medical Center, Hershey, PA; ${ }^{4}$ Penn State Milton S. Hershey Medical Center, Hershey, PA. (Control ID \#3185550)

BACKGROUND: Acute rhinosinusitis (ARS) accounts for over 23 million outpatient visits each year. The majority of these infections are viral in etiology, and do not require antibiotic treatment. It is estimated that antibiotics are inappropriately prescribed for patients with ARS in $41 \%$ of cases. Inappropriate antibiotic use exposes patients to drug-related adverse events, and promotes bacterial resistance. Though inpatient antibiotic stewardship programs have proved impactful, ambulatory antibiotic stewardship programs are not well documented. As a first step to planning 
interventions that will improve ambulatory antibiotic prescribing, this study aims to assess the antibiotic prescribing patterns for acute bacterial rhinosinusitis (ABRS) in an Internal Medicine resident clinic.

METHODS: Three investigators reviewed 102 charts for all encounters between October 1, 2015 and December 31, 2018 in which an ICD-10 code for ABRS was used and an antibiotic was prescribed. Utilizing the Infectious Disease Society of America (IDSA) guidelines, we developed a chart review tool to (1) determine appropriateness of antibiotic prescribing by confirming the diagnosis of ABRS, and (2) assess the appropriateness of the selected antibiotic, dose, and duration.

RESULTS: 96\% ( $n=98)$ met the IDSA criteria for diagnosis of ABRS, reflecting that an antibiotic was appropriately prescribed. Of those cases, only $41 \%$ followed IDSA recommendations regarding correct antibiotic selection, dose, and duration $(n=42)$. Of the patients with documented beta-lactam antibiotic allergy who were appropriately prescribed an antibiotic $(n=31)$, only $55 \%$ received a recommended second-line agent $(n=17)$. Amongst the cases in which the choice to prescribe an antibiotic was appropriate, azithromycin was the most frequently inappropriately selected antibiotic $(26 \% ; n=25)$. In cases in which antibiotic selection was appropriate, incorrect dosage accounted for $12 \%(n=7)$ of inappropriate prescriptions, and excessive treatment duration accounted for $32 \%(n=19)$.

CONCLUSIONS: Our study revealed that the decision to prescribe an antibiotic for ABRS was appropriate $96 \%$ of the time, demonstrating that residents are often effectively able to identify the signs and symptoms of ABRS. However, residents made errors in selection, dose and/or duration of the antibiotic in $58 \%$ of the cases $(n=59)$. This suggests that inappropriate antibiotic prescription lies not in the choice to prescribe, but in the details of the prescription. Moreover, only $57 \%$ of patients who received a second-line antibiotic had a documented allergy. Together, this data sheds light on the highest yield areas for improvement. Next steps for intervention include development of educational materials and electronic health record-based clinical decision support tools aimed at improving appropriate antibiotic selection.

\section{RISK FACTORS FOR 30-DAY READMISSION AFTER IN- FECTIVE ENDOCARDITIS USING NATIONWIDE READMISSIONS DATABASE}

Anthony T. Kunnumpurath ${ }^{1}$; Neal Mehta $^{2}$; Ron T. Varghese ${ }^{3}$. ${ }^{1}$ White River Health Systems, Batesville, AR; ${ }^{2}$ White River Health System, Kingsport, TN; ${ }^{3}$ White River Medical Center, Batesville, AR. (Control ID \#3181997)

BACKGROUND: The annual incidence of infective endocarditis, infection of the native/prosthetic cardiac heart value, endocardial surface and/or implanted cardiac device, has increased from a yearly incidence of 11 to 15 per 100,000 from 2000 to 2011 [1,2]. Infective Endocarditis is associated with considerable in-hospital mortality $(20 \%)$ and post-initial hospitalization mortality (30\%) [1]. Better understanding of the risk factors for readmissions of Infective Endocarditis may help to decrease morbidity and mortality. We sought out to determine the 30-day all-cause readmission rate for adults (age $>=18$ ) admitted with Infective Endocarditis and the associated predictors of readmissions.

METHODS: We utilized Agency of Healthcare Research and Quality's (AHRQ) Health Care Utilization Project's (HCUP) 2014 Nationwide Readmission Database which includes 14.9 Million discharges across 22 states accounting for $51.2 \%$ of the total U.S. population and $49.3 \%$ of all U.S. hospitalizations to identify admissions with a Infective Endocarditis related ICD-9 diagnosis (421.0, 421.1, and 421.9). Applicable admissions were all adults (age >= 18) with an index hospitalization discharge between January 1 to November 30, 2014. Patients who died during index admission and those with missing covariates were excluded. All-cause including Infective Endocarditis readmissions within 30-days of a infective endocarditis associated index admission were analyzed. Statistical analysis was completed with Stata 15 (StataCorp, College Station, $\mathrm{TX}$ ) with p-values $<0.05$ considered statistically significant. A univariate and multivariate analysis of data collected was completed using both odds ratio and chi square test for significance. Predictors for readmission were determined using a multivariate logistic regression model following sequential step-wise elimination of covariates including demographics, comorbidities, hospital characteristics, length of stay (LOS) for index admission, and the modified Elixhauser Comorbidity Index.

RESULTS: A total of 14,705 patients met criteria for inclusion. Of which, there was 3,842 (26.13\%) readmissions within 30-days of the index admission. Multivariate analysis showed that the predictors of 30-day readmission were younger age, female, disposition at discharge (other facility or against medical advice), Medicare as payer, length of stay, admission to a medical compared to surgery team, and presence of three comorbidities (complicated diabetes, perivascular disease, and renal failure).

CONCLUSIONS: Greater than 1 in 4 patients discharged after an infective endocarditis admission were readmitted within 30 days. Physician awareness and development of targeted interventions for individuals with risk factors and high-risk for readmissions may help decrease future morbidity and mortality.

ROOM FOR IMPROVEMENT: PRIMARY CARE PHYSICIANS' USE OF EVIDENCE-BASED STRATEGIES TO IMPROVE ADULT IMMUNIZATION

Laura P. Hurley $^{1,4}$; Sean T. O'Leary ${ }^{2,}$; Megan C. Lindley ${ }^{5}$; Lori A. Crane $^{2,}{ }^{4}$; Jessica R. Cataldi ${ }^{2,}{ }^{4}$; Michaela Brtnikova ${ }^{2,}{ }^{4}$; Brenda Beaty ${ }^{4}$; Allison Kempe ${ }^{3,4}$. ${ }^{1}$ Denver Health, Denver, CO; ${ }^{2}$ University of Colorado Anschutz Medical Campus, Aurora, CO; ${ }^{3}$ University of Colorado and Children's Hospital Colorado, Aurora, CO; ${ }^{4}$ ACCORDS University of Colorado Anschutz Medical campus and Children's Hospital Colorado, Denver, CO; ${ }^{5}$ Centers for Disease Control and Prevention, Atlanta, GA. (Control ID \#3178595)

BACKGROUND: Adult immunization rates are low nationally and little is known regarding the use of evidence-based strategies to increase rates by physicians serving adults. Our objectives were to assess among general internists (GIM) and family physicians (FP): 1) strategies used to improve adult immunization rates, and 2) reported use of strategies in 2013 vs. 2015/16.

METHODS: We administered Internet and mail surveys to two distinct nationally representative cross-sectional samples of GIM and FP derived from American College of Physicians and American Academy of Family Physicians memberships in June-October 2013 and December 2015-January 2016. Surveys were sent to 839 and 935 physicians in 2013 and 2015/16, respectively. Chi-square analyses were used to compare responses between the two surveys.

RESULTS: The response rate was $66 \%$ in both surveys $(553 / 839$ in 2013 and 617/935 in 2015/16); 19 physicians were removed in 2013 and 15 in 2015/16 because they did not administer vaccines. Table 1 shows reported use of strategies to improve vaccination for the two time periods for seasonal influenza and the other recommended adult vaccines. Several strategies, at least for seasonal influenza vaccine, were more commonly reported in the later survey except for written clinical decision support systems (written surveys to determine a patient's vaccine needs).

CONCLUSIONS: With the exception of standing orders for seasonal influenza specifically and electronic CDSS for all adult vaccines, the minority of physicians is using evidence-based strategies to improve adult vaccinations, but use of many strategies has improved over time. Bidirectional interfaces with IIS may facilitate assessing all adult vaccines in an IIS, and that, along with the expanded use of standing orders for all adult 
vaccines, may improve adult immunization rates.

\section{SAFETY OF ANTIMOTILITY AGENT USE DURING TREAT- MENT FOR CLOSTRIDIOIDES DIFFICILE INFECTION IN MALIGNANT HEMATOLOGY INPATIENTS}

David Sterken; Carla Kuon; Rae Wannier; Jeffrey Wolf; Priya A. Prasad. UCSF, San Francisco, CA. (Control ID \#3183429)

Table 1. Physician Reported Use of Strategies to Improve Adult Vaccination, 2013 and 2015/2016 Surveys

\begin{tabular}{|c|c|c|c|}
\hline & $\begin{array}{c}2013 \\
\mathrm{~N}=534 \\
\%\end{array}$ & $\begin{array}{c}2015 / 2016 \\
\mathrm{~N}=602 \\
\%\end{array}$ & $P$ value ${ }^{*}$ \\
\hline \multicolumn{4}{|l|}{ Standing orders } \\
\hline Seasonal influenza & 72 & 84 & $<.0001$ \\
\hline All other recommended adult vaccines & 10 & 16 & 0.002 \\
\hline \multicolumn{4}{|l|}{ Assessment and feedback } \\
\hline Seasonal influenza & 11 & 16 & 0.02 \\
\hline All other recommended adult vaccines & 11 & 12 & 0.63 \\
\hline \multicolumn{4}{|l|}{ Use IIS to assess vaccination status } \\
\hline Seasonal influenza & 19 & 26 & 0.006 \\
\hline All other recommended adult vaccines & 23 & 25 & 0.30 \\
\hline \multicolumn{4}{|l|}{ Use IIS to record vaccination } \\
\hline Seasonal influenza & 21 & 32 & $<.0001$ \\
\hline All other recommended adult vaccines & 23 & 32 & 0.0005 \\
\hline \multicolumn{4}{|l|}{ Written CDSS } \\
\hline Seasonal influenza & 21 & 17 & 0.19 \\
\hline All other recommended adult vaccines & 18 & 15 & 0.19 \\
\hline \multicolumn{4}{|l|}{ Electronic CDSS } \\
\hline Seasonal influenza & 52 & 61 & 0.002 \\
\hline All other recommended adult vaccines & 51 & 53 & 0.50 \\
\hline \multicolumn{4}{|l|}{ Reminders sent to all patients due for vaccine } \\
\hline Seasonal influenza & 14 & 24 & $<.0001$ \\
\hline All other recommended adult vaccines & 6 & 9 & 0.16 \\
\hline
\end{tabular}

* $p$-value represents a comparison between responses from the two survey time periods using chi-square analysis

ization Information System (a.ka immunization registry)

CDSS=Clinical Decision Support System

BACKGROUND: Guidelines on the treatment of Clostridioides (Clostridium) difficile infection (CDI) have historically recommended avoiding antimotility agents (AAs) in patients with active CDI based on theoretical concerns that administration of AAs may precipitate serious adverse events such as toxic megacolon in these patients. These recommendations, however, are based on limited and conflicting data. We previously reported preliminary data suggesting the potential safety of AAs in patients with CDI. The current study builds on our prior work using a larger, more robust dataset to describe the use of AAs and adverse gastrointestinal events in patients hospitalized on the malignant hematology service who developed CDI.

METHODS: We used the electronic health record of an 800-bed academic teaching hospital to identify all inpatients on the malignant hematology service from April 21, 2012 through September 21, 2017 with one or more positive C. difficile stool toxin assays during their admission. We assessed for administration of AAs (loperamide, atropine/diphenoxylate, or opium) within 14 days after CDI diagnosis as well as for any subsequent adverse gastrointestinal events (toxic megacolon, ileus, or bowel obstruction) during the admission by ICD-10 codes. We then manually reviewed all cases of $\mathrm{CDI}$ in which an adverse event occurred to determine whether the event occurred within 72 hours after AA administration. Multivariable logistic regression was used to assess the association of adverse events with AAs, adjusting for the propensity to receive AAs, patient characteristics, and markers of disease severity.

RESULTS: We identified 350 patients with CDI, all but 3 of whom received appropriate antibiotic treatment for CDI. $38 \%$ were female. $15 \%$ were Asian, $4 \%$ were black, and $57 \%$ were white. $19 \%$ were Hispanic. $13 \%$ went to the ICU during admission. Mean age was 52.9 years. Mean length of stay was 26.4 days. AAs were used in $27 \%$ of cases - or in 95 patients total - and within 48 hours of CDI diagnosis in 33\% of these cases. The risk of adverse events did not differ between patients who received AAs and those who did not $(2.1 \%$ vs. $2.0 \%$, adjusted odds ratio
0.45 [95\% CI: 0.08 - 2.70]). Patients who received AAs had more days of diarrhea (4.94 vs. $2.20, \mathrm{p}<0.001)$, though this was not significant in the adjusted analysis (relative risk 1.14 [0.94 - 1.37]). There were no significant differences in the number of fluid boluses given, the total volume of IV fluid given, or the number of wound care consults in adjusted or unadjusted analyses.

CONCLUSIONS: AAs were administered to $27 \%$ of patients in the first 14 days after CDI diagnosis and were not associated with a significantly increased risk of gastrointestinal adverse events. This evidence suggests that it is safe to use AAs in malignant hematology inpatients who are being treated for CDI, even soon after CDI diagnosis.

\section{SCOPE AND CORRELATES OF CHRONIC NON-CANCER PAIN IN PRIMARY CARE}

Alexander Chaitoff; Glen B. Taksler; Anita D. Misra-Hebert; Belinda Udeh; Michael B. Rothberg. Cleveland Clinic, Cleveland, OH. (Control ID \#3185076)

BACKGROUND: Up to 100 million adults in the United States (US) suffer from chronic pain. However, prevalence estimates from older national samples may not reflect the population of chronic pain patients in primary care who would be affected by policies aimed at curtailing opioid use. The objective of this study was to identify patient factors correlated with reporting chronic pain during primary care visits.

METHODS: This retrospective study used data extracted from the electronic health record at a large integrated health system in the US. Participants were adults treated between January 2014 and December 2015. Patients with metastatic cancer were excluded. The main outcome measures-self-report pain duration, location, and severity as measured by the numeric ranking scale-10--are routinely documented during primary care visits. Chronic pain was defined as lasting $>3$ months. Predictor variables included demographics, health insurance status, substance use, and comorbidities. Frequencies were used to describe the scope of chronic non-cancer pain in the cohort. Multivariable regression models were used to assess the patient factors associated with reporting chronic pain.

RESULTS: The dataset included 820,028 visits made by 141,635 patients, $24,127(17.0 \%)$ of whom reported at least one period of chronic pain. Patients with chronic pain had more primary care visits during the study period than those who did not report chronic pain (7.2 vs. 4.6, $\mathrm{p}<0.001)$. Pain was most commonly reported in the back $(n=7293)$, the lower extremities $(n=7194)$, and the upper extremities $(\mathrm{n}=5190)$. The median pain severity was $6 / 10$ (interquartile range $(I Q R)=4.0-8.0$ ), and $39 \%$ involved a severity 7 . Pain differed significantly by location; it highest when reported as generalized and lowest when reported in the breast $(\mathrm{p}<0.001)$. In a multivariable model, chronic pain was associated with being female $(\mathrm{OR}=1.16$, $95 \% \mathrm{CI}=1.13-1.20)$, Black vs. White $(\mathrm{OR}=1.66,95 \% \mathrm{CI}=1.59-1.73$,$) ,$ older age (per 10 years, $\mathrm{OR}=1.05,95 \% \mathrm{CI}=1.03-1.06$ ), lower income (per $\$ 10,000 ; \mathrm{OR}=0.98,95 \% \mathrm{CI}=0.97-0.99$ ), and being divorced $(\mathrm{OR}=1.10,95 \% \mathrm{CI}=1.05-1.16)$ or married $(\mathrm{OR}=1.10,95 \% \mathrm{CI}=1.06-$ $1.14)$ vs. single. Those with chronic pain were more likely to have Medicare $(\mathrm{OR}=1.87,95 \% \mathrm{CI}=1.76-1.99)$ or Medicaid $(\mathrm{OR}=1.11$, $95 \% \mathrm{CI}=1.07-1.16)$ vs. private insurance. Certain health behaviors, including being a current smoker vs. never smoker $(\mathrm{OR}=1.35$, $95 \% \mathrm{CI}=1.28-1.41)$ and illicit drug use $(\mathrm{OR}=1.24,95 \% \mathrm{CI}=1.14-$ $1.35)$, were associated with chronic pain, but any alcohol use was associated with lower odds of reporting chronic pain $(\mathrm{OR}=0.87$, $95 \% \mathrm{CI}=0.84-0.89)$. Finally, having a mental health comorbidity $(\mathrm{OR}=1.52,95 \% \mathrm{CI}=1.48-1.57)$ or higher Charlson comorbidity index (per point; OR=1.08, 1.07-1.09) was associated with chronic pain. CONCLUSIONS: In a large health system, nearly one in five primary care patients reported chronic pain. Patients historically subjected to disparities are more likely to have chronic pain and to be affected by policies related to its treatment. 
SCREENING FOR IMMIGRATION-RELATED HEALTH CONCERNS IN A FEDERALLY QUALIFIED HEALTH CENTER: A MIXED METHODS STUDY

Elena Byhoff ${ }^{1,5}$; Emilia De Marchis ${ }^{2}$; Sofia Halperin-Goldstein ${ }^{5}$; Laura M. Gottlieb ${ }^{3}$; Amy LeClair ${ }^{1}$; Keith Nokes ${ }^{4}$. ${ }^{1}$ Tufts Medical Center, Boston, MA; ${ }^{2}$ University of California, San Francisco, San Francisco, CA; ${ }^{3} \mathrm{UCSF}$, San Francisco, CA; ${ }^{4}$ Greater Lawrence Family Medical Center, Lawrence, MA; ${ }^{5}$ Tufts University School of Medicine, Boston, MA. (Control ID \#3180137)

BACKGROUND: Changes to the public charge rule may make undocumented immigrants less likely to present for health care or report unmet social needs for fear of losing benefits. Immigrationrelated concerns can impact health and are an important consideration while caring for an immigrant community. We aim to examine the acceptability of screening for immigration-related health concerns within the context of social risk screening in a Federally Qualified Health Center (FQHC) serving a predominantly immigrant (Central America and Caribbean) patient population.

METHODS: This study used a mixed-methods approach. Participants were randomly screened with one of two social risk screening tools. Both tools asked about housing stability, food security, material hardship and transportation. One tool included a question about "any health or stability concerns related to immigration status." The other tool, developed by the Center for Medicare and Medicaid Innovation, did not include an immigration health question. Patients or caregivers waiting for a non-urgent primary care visit were asked to participate. After completing the social risk assessment, respondents were invited to participate in a 30minute semi-structured interview. The interview focused on patients' understanding of the screening questions, acceptability and comprehensiveness of the screening tool, and perceptions about appropriateness of social risk screening in a health care setting. Interviews were transcribed, translated, and coded to identify themes. Patients were recruited until thematic saturation was reached. All study activities were conducted in either English or Spanish.

RESULTS: A total of 201 screens were completed (100 included immigration question), and 20 patients (10 in each arm) agreed to an interview. There were no significant differences between groups in age, gender, race/ethnicity, or caregiver status. Of those screened for immigration, $11 \%$ reported an immigration-related health or stability concern, with no skipped responses. Interview themes showed high acceptability for immigration-related screening, including specific mentions of heightened political concern, health relevance, and the doctor as a trusted ally. In both arms, interviewees felt that social risk screening generally was acceptable in a clinic setting. Those not in the immigration arm mentioned that screening for immigration-related concerns was important. Respondents saw clinics as a "safe space" to discuss immigration concerns in comparison to "government" spaces, like WIC, SNAP, and Medicaid offices.

CONCLUSIONS: Social risk screening is acceptable in an FQHC serving a predominantly immigrant community. Questions about immigration are timely, important, and relevant, and can be considered when implementing social assessments in communities where there are high levels of trust in providers. With proposed changes to public charge, FQHCs may serve as meaningful allies in serving immigrant patients with social risk.

SECURE MESSAGING THROUGH POSITIVELINKS: EXAMINATION OF ELECTRONIC COMMUNICATION IN A CLINIC-AFFILIATED SMARTPHONE APP FOR PATIENTS LIVING WITH HIV

Tabor E. Flickinger; Sabrina Swoger; Marika Grabowski; Karen S. Ingersoll; Rebecca Dillingham. University of Virginia,
Charlottesville, VA. (Control ID \#3182989)

BACKGROUND: Keeping patients engaged in care may require provider communication between visits. Electronic patient portals are available, but often underutilized. However, many patients now use smartphones daily and patient-provider messaging using these phones may be a promising option. PositiveLinks (PL) is a clinic-affiliated smartphone-based platform, designed to enhance retention in HIV care and tailored to our socio-economically disadvantaged non-urban population. PL includes a secure messaging feature to connect patients to their HIV care team. Our aim was to analyze messages exchanged between patients, staff, and HIV care providers through PL to determine the usage, content, and function of communication through secure messaging between clinic visits.

METHODS: We examined messages exchanged through PL from November 2017 through January 2018. The initial codebook was developed using an open coding strategy, then applied independently by two coders and refined until excellent reliability was achieved with a kappa statistic of 0.89 . Topic codes categorized messages as app-related or care-related, which could be medical or social. Function codes categorized messages by their purpose of information exchange or rapport-building. Qualitative analysis was performed using Dedoose software with descriptive statistics of code frequencies and code co-occurrence.

RESULTS: Of the 1474 PL messages analyzed, $44 \%$ were sent by PL staff, $38 \%$ by patients, and $18 \%$ by providers, while $61 \%$ were received by patients, $22 \%$ by providers, and $17 \%$ by PL staff. Message topics included app-related (57.6\%), medical care (34.3\%), and social concerns (12.4\%). App-related messages addressed technical difficulties, software updates, or coordinating phone payments. Medical messages included information, medications, appointments, outreach, and care coordination for physical and mental health. Social messages related to insurance, transportation, housing, food, utilities, disability, finances, and work. Function coding showed that $87.3 \%$ of messages contained information exchange and $33.8 \%$ contained rapport-building. Information exchange messages were utilitarian in nature while rapport-building messages included psychosocial components, such as addressing emotions, offering thanks or appreciation, or providing personal content beyond informationsharing. Messages sent by providers were most likely to contain rapportbuilding at $54.8 \%$.

CONCLUSIONS: PL messaging was used to address medical and social needs in a vulnerable population and to connect patients with care providers. Electronic communication offers an opportunity to exchange practical information and also to strengthen patient-provider relationships. Next steps include further analysis of socioemotional tone in the messaging and impact on engagement in care, provider attitudes, and patient outcomes. Secure messaging through a clinic-affiliated smartphone platform could enhance patient-centered care between clinical visits.

SEEING I TO EYE TOGETHER: AN EDUCATIONAL CAMPAIGN DESIGNED TO IMPROVE PHYSICIAN-PATIENT COMMUNICATION AMONG INTERNAL MEDICINE RESIDENTS

Robin Ortiz ${ }^{3}$; Meron Hirpa ${ }^{1}$; Aparna Sajja ${ }^{1}$; Jesse McDermeit ${ }^{1}$; Alison Agrawal $^{1}$; Megan Tschudy ${ }^{3}$; Stephen Berry ${ }^{2}$; Kimiyoshi Kobayashi ${ }^{1}$. ${ }^{1}$ Johns Hopkins Hospital, Baltimore, MD; ${ }^{2}$ Johns Hopkins School of Medicine, Baltimore, MD; ${ }^{3}$ Johns Hopkins University School of Medicine, Baltimore, MD. (Control ID \#3175978)

BACKGROUND: Sitting at the bedside improves patients' perception of their interactions with their physicians and improves patient satisfaction and outcomes. Despite these data, prior work shows that medicine interns sit during only $9 \%$ of observed physician-patient interactions. We aimed to design an educational curriculum to improve physician-patient communication and decrease resistance to sitting at the bedside.

METHODS: A needs assessment among Internal Medicine residents at an urban academic medical center was conducted anonymously using an 
electronic survey. The survey was designed to assess knowledge gaps in residents' perceptions and self-reported behaviors around sitting during inpatient encounters. Informed by survey results, an evidence-based educational campaign was designed to address the observed deficits in resident knowledge regarding time and communication in physicianpatient encounters.

RESULTS: The needs assessment yielded 96 responses of 151 residents surveyed (64\% response rate). Residents who reported spending enough time at the bedside were also likely to report that their patients could identify them as their doctor $\left(\mathrm{r}_{\mathrm{s}}=0.28, \mathrm{p}=0.006\right)$. The majority of residents $(63 \%)$, however, felt they rarely or never spent enough time at bedside. Although $69 \%$ of respondents felt that sitting during an admission history was important, only $30 \%$ reported doing this. Residents (26\%) also felt that sitting would make the patient encounter longer, even though there was no difference in reported time completing an admission history between those who reported sitting and those who reported standing ( $\mathrm{t}=-1.38, \mathrm{p}=0.17)$. Therefore, an educational campaign entitled "S.I.T: Seeing I to Eye Together" was designed with the following teaching points: 1) Sitting increases patient perception of time spent at the bedside, 2) Sitting does not increase actual bedside time, and 3) Sitting enhances patient adherence and satisfaction, as well as physician communication. Education was disseminated through flyers and peer-led didactic presentations. Additionally, since $69 \%$ of residents felt sitting during attending-led rounds is important, attending physicians were asked to emphasize the teaching points during teaching rounds on inpatient Internal Medicine teams.

CONCLUSIONS: Survey results confirmed expected knowledge deficits, specifically as they pertained to time as a barrier to patient-physician communication. The learning objectives of the "S.I.T." educational campaign may address a key deficit in Internal Medicine resident education related to optimizing physician-patient communication through sitting and, further, has larger implications for improving hospital performance on patient satisfaction

\section{SEEING I TO EYE TOGETHER: AN EDUCATIONAL CAM- PAIGN DESIGNED TO IMPROVE PHYSICIAN-PATIENT COM- MUNICATION AMONG INTERNAL MEDICINE RESIDENTS} Robin Ortiz ${ }^{1}$; Meron Hirpa ${ }^{1}$; Aparna Sajja ${ }^{1}$; Jesse McDermeit ${ }^{1}$; Alison Agrawal $^{1}$; Megan Tschudy ${ }^{1}$; Stephen Berry ${ }^{2}$; Kimiyoshi Kobayashi ${ }^{1}$. ${ }^{1}$ Johns Hopkins University School of Medicine, Baltimore, MD; ${ }^{2}$ Johns Hopkins School of Medicine, Baltimore, MD. (Control ID \#3175893)

BACKGROUND: Sitting at the bedside improves patients' perception of their interactions with their physicians and improves patient satisfaction and outcomes. Despite these data, prior work shows that medicine interns sit during only $9 \%$ of observed physician-patient interactions. We aimed to design an educational curriculum to improve physician-patient communication and decrease resistance to sitting at the bedside.

METHODS: A needs assessment among Internal Medicine residents at an urban academic medical center was conducted anonymously using an electronic survey. The survey was designed to assess knowledge gaps in residents' perceptions and self-reported behaviors around sitting during inpatient encounters. Informed by survey results, an evidence-based educational campaign was designed to address the observed deficits in resident knowledge regarding time and communication in physicianpatient encounters.

RESULTS: The needs assessment yielded 96 responses of 151 residents surveyed (64\% response rate). Residents who reported spending enough time at the bedside were also likely to report that their patients could identify them as their doctor $\left(\mathrm{r}_{\mathrm{s}}=0.28, \mathrm{p}=0.006\right)$. The majority of residents $(63 \%)$, however, felt they rarely or never spent enough time at bedside. Although $69 \%$ of respondents felt that sitting during an admission history was important, only $30 \%$ reported doing this. Residents (26\%) also felt that sitting would make the patient encounter longer, even though there was no difference in reported time completing an admission history between those who reported sitting and those who reported standing $(\mathrm{t}=-1.38, \mathrm{p}=0.17)$.
Therefore, an educational campaign entitled "S.I.T: Seeing I to Eye Together" was designed with the following teaching points: 1) Sitting increases patient perception of time spent at the bedside, 2) Sitting does not increase actual bedside time, and 3) Sitting enhances patient adherence and satisfaction, as well as physician communication. Education was disseminated through flyers and peer-led didactic presentations. Additionally, since $69 \%$ of residents felt sitting during attending-led rounds is important, attending physicians were asked to emphasize the teaching points during teaching rounds on inpatient Internal Medicine teams.

CONCLUSIONS: Survey results confirmed expected knowledge deficits, specifically as they pertained to time as a barrier to patient-physician communication. The learning objectives of the "S.I.T." educational campaign may address a key deficit in Internal Medicine resident education related to optimizing physician-patient communication through sitting and, further, has larger implications for improving hospital performance on patient satisfaction.

\section{SEEKING APPLES, FINDING ORANGES: MISMATCH BETWEEN PERCEIVED BARRIERS AND GENERATED SOLUTIONS DUR- ING THE SCOPING OF TWO CLINICAL INITIATIVES}

Eli Cahan ${ }^{1}$; Cati Brown-Johnson ${ }^{1}$; Nadia Safaeinili ${ }^{1}$; kimberly brunisholz ${ }^{2}$; Brenda Reiss-Brennan ${ }^{2}$; Marcy Winget ${ }^{1} .{ }^{1}$ Stanford University Medical School, Stanford, CA; ${ }^{2}$ Intermountain, Salt Lake City, UT. (Control ID \#3185499)

BACKGROUND: Across disciplines, nearly two-thirds of efforts devoted towards organizational change fail. In healthcare, uptake of research to the clinic takes over a quarter-century, at which point significant deviations from study protocols are often required. As a result, even following uptake, patients may receive only half of recommended services, and interventions received often demonstrate low clinical response rates. The Consolidated Framework for Implementation Research (CFIR), developed to facilitate uptake of new services, defines domains salient to successful integration including features of the clinical and institutional environments, as well as individual providers. It is not clear that organizations experience barriers or generate solutions with equal frequency across domains. We characterized expected barriers and solutions proposed when preparing for implementation of two distinct initiatives across two healthcare systems.

METHODS: Initiatives for early treatment of (a) substance use and (b) physical therapy were scoped conceptually at six primary care clinics across one academic and one non-academic health system. Before introducing these initiatives, qualitative assessments of barriers to implementation were captured via interviews with clinic staff and providers. Ideas to facilitate implementation were solicited. Feedback was coded into six thematic categories, addressing: connectivity, workflow, internal resources (such as site-specific care protocols), external resources (such as system-wide electronic resources), teamwork/culture, and individual mindset. Percentages of comments on perceived barriers and ideas proposed were calculated for each of these categories.

RESULTS: For substance use treatment, $26 \%$ of barriers reported were workflow related, $16 \%$ involved internal resources, $22 \%$ involved external resources, and $22 \%$ related to individual mindsets. $40 \%$ and $30 \%$ of solutions addressed workflow and internal resources respectively, however only a combined $15 \%$ of solutions related to external resources and mindsets. Similarly, for physical therapy, while $49 \%$ of barriers arose from external resources and mindsets, only $13 \%$ of proposed solutions addressed these categories. Meanwhile, $73 \%$ of solutions addressed the $36 \%$ of problems arising from barriers in workflow and internal resources. CONCLUSIONS: Organization of qualitative feedback following conceptual introduction of two clinical initiatives revealed a mismatch between perceived barriers and proposed solutions to implementation. While expected barriers were fairly evenly distributed amongst 4 key categories, solutions proposed by clinic providers and staff disproportionately addressed workflow and internal resources rather than mindsets and external resources. Observation of distribution and mismatch between perceived barriers and solutions can help inform institutional 


\section{SELF-WEIGHING FREQUENCY AND ASSOCIATED CHARAC- TERISTICS AMONG VETERANS OF THE WARS IN IRAQ AND AFGHANISTAN.}

Carolyn Bramante $^{1}$; Anne Joseph ${ }^{1}$; Jennifer A. Linde ${ }^{1}$; Charles J. Billington ${ }^{2}$; Steven $\mathrm{Fu}^{3}$; Rachel Widome ${ }^{1}$. ${ }^{1}$ University of Minnesota, Minneapolis, MN; ${ }^{2}$ Minneapolis VA Medical Center, Minneapolis, MN; ${ }^{3}$ Minneapolis VA Health Care System, Minneapolis, MN. (Control ID \#3185915)

BACKGROUND: Rates of obesity are high among Operation Enduring Freedom/Operation Iraqi Freedom (OEF/OIF) Veterans. Self-weighing is a promising tool for weight loss and maintenance. Daily self-weighing at home can promote weight loss, likely by raising one's awareness of current body weight and behaviors associated with excess caloric intake. Multiple professional organizations recommend that clinicians advise patients to weigh themselves regularly for management of obesity and overweight. Our objective was to determine the frequency that OEF/ OIF Veterans report engaging in self-weighing, and to explore demographic, behavioral, and mental health correlates of selfweighing.

METHODS: A random sample of 2,000 OEF/OIF veterans who had interacted with the Minneapolis VA Health Care System were mailed surveys about health-related behaviors in 2012. We conducted a retrospective analysis of this cross-sectional survey dataset. Our outcome of interest was the answer to "During the past 30 days, how many times did you weigh yourself?" We explored correlates using univariate analyses (t-test for continuous variables and $\mathrm{chi}^{2}$ tests for categorical variables), and examined associations between selfweighing and income, body mass index, health behaviors, mental health indicators, weight satisfaction, with adjustment for age, gender, race, education, and employment status.

RESULTS: Of the 2,000 veterans who were mailed the survey, 953 (48\%) responded, and 918 of these $(96 \%)$ answered the question about self-weighing. Of the $918,45 \%$ were female; $74 \%$ married; and $90 \%$ white, 5\% black, and 5\% "other." The mean age was 35 years, $44 \%$ had a college degree or more, and $62 \%$ were employed. Overall, 546 participants (59\%) self-weighed less often than weekly; $153(17 \%)$ selfweighed weekly; and 219 (24\%) self-weighed more than once per week. In univariate analysis, respondents who self-weighed more than weekly were more likely to have a college degree or more $(55 \%$, $\mathrm{p}<0.01)$, more likely to be female $(45 \%, \mathrm{p}<0.01)$, less likely to report high satisfaction with weight $(36 \%, \mathrm{p}=0.03)$, and more likely to prepare meals with vegetables $(77 \%, \mathrm{p}<0.01)$. There were no statistically significant correlations between self-weighing and mental health indicators. In multivariate analysis, participants who prepared meals with vegetables were more likely to self-weigh more than weekly (OR $1.9,95 \%$ CI 1.2-2.4), and there were no associations between selfweighing more than weekly and feeling downhearted, nervous, calm, or confident in one's ability to handle problems.

CONCLUSIONS: Most OEF/OIF veterans are not engaging in the important weight-management tool of self-weighing at least weekly. Stress and anxiety are often invoked as potential negative outcomes of self-weighing at home; however, we found no associations between self-weighing more than weekly and indicators of poor mental health in this cross-sectional survey. Self-weighing may be a behavior to encourage in this population for improved weight management.

SETTING HIGH STANDARDS FROM THE START: AN EXPERIENTIAL FACULTY ORIENTATION TO INTRODUCE INSTITUTIONAL EXPECTATIONS AROUND COMMUNICATION AND PATIENT SAFETY

Sondra Zabar ${ }^{1}$; Mara McCrickard ${ }^{1}$; Kinga Eliasz ${ }^{1}$; Deborah Cooke ${ }^{1}$; Katherine A. Hochman ${ }^{1}$; Andrew B. Wallach ${ }^{1,2}$. ${ }^{1}$ NYU School of Medicine,
BACKGROUND: Newly recruited clinicians have heterogeneous backgrounds and experiences and need a substantive introduction to their new institution's patient communication expectations and safety culture and standards for clinician performance. We describe a unique onboarding program designed to ensure that newly hired clinicians receive actionable, behaviorally specific feedback from the patients' perspective to support a satisfying transition to the new work environment, enhance patient experience and reduce the need to punitively react to complaints once they have started.

METHODS: During the 2-hour onboarding, participants complete 3, 10minute Objective Structured Clinical Exam cases designed to assess how they address a medical error, manage the patient's discharge goals of care, and respond to an impaired learner. During each encounter, participants interact with highly trained Standardized Patients (SPs) or Standardized Learners (SLs) who use behaviorally-anchored checklists to evaluate provider performance on communication and case-specific skills. Following each encounter, participants complete a self-assessment while the SPs/SLs complete a behavior-specific checklist, after which the two discuss the encounter and the SL/SP provides confidential and actionable feedback. At the end, participants are encouraged to set individual learning goals to implement in their daily work, complete a program evaluation, and engage in a debrief with experienced facilitators. Participants also receive their SP checklists in addition to an institutional guide containing relevant resources and contacts.

RESULTS: Over 2 years, 57 faculty members representing 6 clinical sites participated in the onboarding program. They are heterogeneous with respect to general and case specific performance on these SP/SL cases. For example, $86 \%$ adequately elicited the SP/SLs story during the discharge case compared to $66 \%$ in the other two cases, $77 \%$ addressed pain management (a key patient goal), while $44 \%$ did not discuss important medication side effects. Participants have universally found this onboarding to be useful and relevant; $98 \%$ agreed/strongly agreed that the program was an effective way to reinforce good habits in patient and learner communication, $96 \%$ felt it enhanced confidence about their ability to communicate effectively, and $96 \%$ felt it reinforced the institutional culture of safety. All 56 participants who completed the evaluation agreed/strongly agreed that the event was engaging and well-designed, and 93\% felt it was a good use of their time and would recommend the program.

CONCLUSIONS: Traditional orientations are not well recalled and do not address knowledge and skills in real-time. Although it requires additional resources, participants are enthusiastic about our low-stakes introduction to the institution's expectations. This program sets high standards and introduces a new model for skills-based onboarding which may lead to measurably improved patient outcomes.

\section{SHOULD I STAY OR SHOULD I GO? INTER-RELATION BE- TWEEN JOB TURNOVER INTENTION, BURNOUT, WELL- BEING, AND JOB SATISFACTION}

Shannon A. Scielzo; Rachel Bonnema; Ethan Halm. Univ of TX Southwestern Med Ctr, Dallas, TX. (Control ID \#3184650)

BACKGROUND: Much attention has been paid to the rising epidemic of provider burnout and its adverse effects on the wellness of individuals and cost to health systems. Replacing a physician who leaves is estimated to cost $\$ 500,000$, so understanding the determinants of job turnover (not just burnout) and addressing them is paramount. While many factors are hypothesized to influence burnout, well-being, job satisfaction and job turnover, the empiric inter-relationship among these constructs, how to best measure them, and factors most strongly predictive of them is less well studied.

METHODS: An anonymous, web-survey was sent to providers in GIM, Med-Peds, Palliative Care and Geriatrics clinicians working in a large, urban academic medical center. The survey included demographics, the MiniZ burnout tool (including the 1 item burnout question (MZB), 1 overall job satisfaction item (Sat), and 3 subcomponents, Meaning - M, Work-Life Balance - WLB, and Control/Influence -CI), Maslach Burnout Inventory 
(MBI, subscales of Emotional Exhaustion - EE, Personal Accomplishment PA, and Depersonalization - D), 1 item Well-Being Fuel Gauge (WBFG, a visual analogue scale from 'empty $=0$ to full tank $=100$ ') and job Turnover Intention (TI, plans to leave). Correlation matrices, factor analysis, and multiple regression was used to identify inter-relationships, factor structures, and independent predictors of TI, MZB, Sat, and WBFG.

RESULTS: Overall, 57\% completed the survey ( $\mathrm{N}=40 ; 75 \% \mathrm{MDs}, 25 \%$ NPs). Overall MZ burnout was 35\%, The mean WBFG was 64 out of 100 ; $62 \%$ of respondents were satisfied with their job, and $15 \%$ said they had plans to leave. Convergent validity was high with high correlations among MZB, MBI, WBFG, Sat, and TI measures ( $r=.51$ to .82 , $\mathrm{p}<.01$ ). Multiple regression entering all constructs $(\mathrm{MZB}, \mathrm{WBFG}$, Sat, TI, EE, PA, D, CI, WLB) found that different outcomes had different independent predictors. For turnover intention and burnout, the most robust multivariable predictor was the Well-Being Fuel Gauge. For job satisfaction, it was control/ influence. For the Well-Being Fuel Gauge, it was control/influence \& Work Life Balance ( $=0.4$ to 0.5 and $p<.05$ for all).

CONCLUSIONS: The single item Well-Being Fuel Gauge was the best tool tool for predicting Turnover Intention and Burnout, exhibited excellent psychometric properties, and is eaiser to monitor more frequently than annual, multi-item burnout surveys. The primary drivers of WellBeing Fuel Gauge scores were Control/Influence and Work-Life Balance. Control/Influence also strongly influenced job satisfaction. Strategies to improve provider well-being and reduce turnover should target ways to bolster control/influence and work-life balance.

\section{SIMPLE PREDICTORS OF MORTALITY IN ACUTE PANCRE- ATITIS}

Ashley M. Scott ${ }^{1}$; Mohammad Baidoun ${ }^{1}$; Naureen Farook ${ }^{1,}{ }^{1}$; Harold $\overline{\text { Gomez Acevedo }}^{1}$; Sandeep K. Bhatti ${ }^{1}$; Jeffrey Dueweke ${ }^{1}$; Vidhya Nair ${ }^{1}$; Hadi Mohammed ${ }^{1}$; Angie Adhami ${ }^{1}$; Guneet Ahluwalia ${ }^{1}$; Sudarshan Hebbar ${ }^{2}$; Joseph Miller ${ }^{1,}{ }^{1}$. ${ }^{1}$ Henry Ford Hospital, Detroit, MI; ${ }^{2}$ Calcimedica, La Jolla, CA. (Control ID \#3178613)

BACKGROUND: Various historical studies have found that hypoxia portends a poor prognosis in patients hospitalized with acute pancreatitis. The objective of this study is to test early respiratory compromise on hospital presentation as a mortality predictor in acute pancreatitis. Secondary aims of the study were assessing prognostication abilities of the quick sequential organ failure assessment (qSOFA) and hypoalbuminemia in acute pancreatitis.

METHODS: This is a retrospective observational study based on adult patients hospitalized for acute pancreatitis. The data were collected from eight standalone emergency departments and five hospitals within Henry Ford Health System of Southeast Michigan. Patients with lipase levels less than three times the upper limit of the normal laboratory range were excluded. The primary outcome was inpatient mortality. Univariate and multivariate logistic regression was performed to determine predictors of adverse outcomes. The main variable of interest was respiratory compromise, defined as an initial $\mathrm{SpO} 2$ of $92 \%$ on presentation. We combined other key variables on presentation to derive an area under the curve (AUC) for predicting mortality.

RESULTS: The study included 2,090 patients, mean age 55.5 years (SD 17.5 ) and $51.5 \%$ female. The median presenting lipase was $976 \mathrm{U} / \mathrm{L}$ (IQR 281-2500). Death was uncommon $(n=34,1.6 \%)$. Via univariate analysis, highly significant predictors of mortality were $\mathrm{SpO} 292 \%$, qSOFA score 2, and low albumin. Patients with a presenting $\mathrm{SpO} 292 \%$ had $9.2 \%$ mortality, in contrast to a $1.3 \%$ mortality rate among those with an $\mathrm{SpO} 2$ above $92 \%$ on presentation (OR 7.5, 95\% CI 3.1-17.7). Those with a qSOFA score 2 suffered 9.8\% mortality, compared to an average $1.3 \%$ mortality rate among those with a qSOFA of 0 or 1 (OR 8.6, 95\% CI 3.9-18.9). Adjusting for age, gender, race, leukocytosis, hematocrit, and major comorbidities, qSOFA 2 remained a significant predictor of mortality (OR 2.6, 95\% CI 1.7-4.1, $\mathrm{p}<0.001$ ). Low albumin (OR 2.7, 95\% CI 1.2-6.2, p 0.02) and $\mathrm{SpO} 292 \%$ (OR 2.6, 95\% CI $1.0-7.1, \mathrm{p} 0.05$ ) also remained significant predictors. By combining qSOFA with the presence of low albumin and $\mathrm{SpO} 292 \%$ into a novel early acute pancreatitis score (EAPS), the EAPS score had good accuracy for predicting mortality (AUC 0.80, 95\% CI 0.72-0.89).

CONCLUSIONS: Independent predictors of mortality on initial presentation include low albumin, $\mathrm{SpO} 292 \%$, and a qSOFA score 2. Perhaps these simple prognosticators can guide clinical practice by more precisely identifying those with acute pancreatitis who are more critically ill and could benefit from closer monitoring.

SMOKING CHARACTERISTICS AND QUIT BEHAVIORS AMONG HEALTH CENTER ATTENDEES WITH SUBSTANCE USE DISORDERS: A NATIONAL STUDY

Danielle R. Fine; Benjamin Bearnot; Travis P. Baggett. Massachusetts General Hospital, Boston, MA. (Control ID \#3169220)

BACKGROUND: Despite high smoking rates in patients with substance use disorders (SUDs), tobacco dependence in this vulnerable population has been undertreated. While current clinical practice guidelines recommend simultaneous treatment of substance use and tobacco dependence, there is limited research evaluating adherence to this guideline on a national level. The objectives of this study are to (1) describe smoking characteristics in patients with and without SUDs at US safety-net health centers, (2) assess differences in smoking-related quit behaviors between individuals with and without SUDs, and (3) evaluate the association between SUD treatment and receipt of advice to quit smoking.

METHODS: We analyzed data from 5,592 adult ( 18 years old) respondents to the 2014 Health Center Patient Survey, a nationally representative crosssectional survey of individuals who receive care at federally funded health centers within the US. The main exposures were having a current alcohol use disorder (AUD) or drug use disorder (DUD) based on responses to the Alcohol, Smoking, and Substance Involvement Screening Test (ASSIST). We first evaluated smoking characteristics (frequencies of being a current or former smoker) in those with and without AUD and DUD. We then performed multivariable logistic regression to assess the relationship between AUD/DUD and smoking-related quit behaviors (past-year desire to quit, pastyear quit attempt, plans to quit in the next 6 months, and being a former smoker among ever smokers [i.e. quit ratio]), and to evaluate the association between past-year AUD/DUD treatment and receipt of advice to quit smoking.

RESULTS: Sixty-four percent of health center patients with AUD and 55\% with DUD were current smokers compared to $25 \%$ and $27 \%$ without AUD and DUD, respectively. Among lifetime ever-smokers, those with AUD were significantly less likely to be former smokers than those without AUD (adjusted odds ratio $[\mathrm{aOR}]=0.30 ; 95 \%$ confidence interval $[\mathrm{CI}]=0.14,0.65$ ). Current smokers with AUD had higher odds of wanting to quit smoking in the past year $(\mathrm{aOR}=2.88 ; 95 \% \mathrm{CI}=1.19,7.05)$ but were not more likely to have made a past-year quit attempt ( $\mathrm{aOR}=1.02 ; 95 \% \mathrm{CI}=0.57,1.84)$. Additionally, there was no association between AUD or DUD and plans to quit smoking in the next 6 months $(\mathrm{aOR}=1.09 ; 95 \% \mathrm{CI}=0.49,2.42 ; \mathrm{aOR}=0.63 ; 95 \% \mathrm{CI}=0.25$, 1.58 , respectively). Smokers with SUD who engaged in AUD treatment $(\mathrm{aOR}=0.31 ; 95 \% \mathrm{CI}=0.05,2.01)$ or DUD treatment $(\mathrm{aOR}=0.97 ; 95 \%$ $\mathrm{CI}=0.21,4.44)$ in the past year were not more likely to have received advice to quit smoking.

CONCLUSIONS: Consistent with prior research, individuals with SUDs had high smoking rates, and smokers with AUD had a higher desire to quit smoking but a lower quit ratio than those without AUD. SUD treatment did not increase the likelihood of receiving advice to quit smoking. These findings suggest a missed opportunity to provide tobacco cessation counseling during SUD treatment as recommended by clinical practice guidelines.

SOCIAL DETERMINANTS OF HEALTH AND MISSED CLINIC VISITS: FACTORS UNDERLYING WASTED RESOURCES IN VA AMBULATORY CARE?

Charlie M. Wray ${ }^{1,}{ }^{2}$; Marzieh Vali ${ }^{3}$; Amy L. Byers ${ }^{1,}{ }^{2}$; Salomeh Keyhani ${ }^{4}$. ${ }^{1}$ University of California, San Francisco, San Francisco, $\mathrm{CA} ;{ }^{2}$ San Francisco VA Medical Center, San Francisco, CA; 
${ }^{3}$ NCIRE, San Francisco, CA; ${ }^{4}$ University of California at San Francisco, San Francisco, CA. (Control ID \#3185022)

BACKGROUND: Medical access failures, specifically missed clinic visits (MCV), are a significant resource drain on the Veterans Health Administration (VA). The VA reports that $15-18 \%$ of scheduled primary care appointments $(\sim 3$ times higher than in the private sector) are not completed. VA strategic goals include improving the timeliness and effectiveness of care - with missed clinic visits representing a highpriority area. While previous appointment history, and patient demographics are shown to be associated with patients' propensity to 'no-show', no previous work has examined the association of social determinants of health (SDOH) with MCV. Our goal was to assess the association of four SDOH (lives alone, lack of social support, substance abuse and marginal housing) with MCV among Veterans hospitalized for congestive heart failure $(\mathrm{CHF})$.

METHODS: We randomly identified 1500 elderly (65 and older) Veterans hospitalized with a primary diagnosis of CHF in 2012. SDOH were identified and extracted through chart abstraction. We determined the number of MCV (primary care, general internal medicine, geriatrics, and cardiology) in the year prior to admission. MCV were dichotomized into: 1 and $2 \mathrm{MCV}$. Multivariate logistic regression was used to examine the effect of the four SDOH on MCV rate; adjusting for age, psychiatric disorders, and the other $\mathrm{SDOH}$

RESULTS: Of the 1500 patients, $1282(85 \%)$ had 1, while $218(14 \%)$ had $2 \mathrm{MCV}$. Patients with $2 \mathrm{MCV}$ had higher prevalence of all four SDOH compared to those with $1 \mathrm{MCV}$, while psychiatric disorders were equally present in both groups. In multivariate analysis, living alone (OR 1.71, 95\% CI 1.21-2.41), marginal housing (OR 6.93, 95\%CI 2.88-17.38), a history of substance abuse (OR 1.48, 95\%CI 1.01-2.12) were significantly associated with having $2 \mathrm{MCV}$ than patients without these risk factors. Higher age was associated with a lower risk (OR 0.96, 95\%CI 0.94-0.98) of having $2 \mathrm{MCV}$.

CONCLUSIONS: SDOH are more common among Veterans who have high MCV rates in the VA. Primary care redesign strategies tailored to Veterans with a higher burden of SDOH may help improve delivery of care, reduce wasted resources, and improve access to care for all Veterans. SOCIAL ISOLATION, LONELINESS, AND VIOLENCE EXPOSURE IN OLDER ADULTS

Elizabeth L. Tung; Kathleen A. Cagney; Louise C. Hawkley; Monica E. Peek. University of Chicago, Chicago, IL. (Control ID \#3177732)

BACKGROUND: Social isolation, a key predictor of mortality in the

Characteristics and Missed Clinic Visit Outcomes in Veterans 65 and older Hospitalized for Congestive Heart Failure

\begin{tabular}{|l|c|c|c|}
\hline \multicolumn{4}{|c|}{ Descriptive Characteristics } \\
\hline & $\begin{array}{c}\text { Prevalence among } \\
\text { cohort }\end{array}$ & $\begin{array}{c}\leq 1 \mathrm{MCV}, \\
(\mathrm{N}=1282)\end{array}$ & $\begin{array}{c}\geq 2 \mathrm{MCV}, \\
(\mathrm{N}=218)\end{array}$ \\
\hline Age, years $( \pm \mathrm{SD})$ & -- & $78( \pm 8.7)$ & $75( \pm 7.9)$ \\
\hline Lives Alone & $19 \%$ & $225(18 \%)$ & $58(26 \%)$ \\
\hline Marginal Housing & $1.5 \%$ & $9(<1 \%)$ & $14(6 \%)$ \\
\hline Substance Abuse & $16 \%$ & $179(14 \%)$ & $53(24 \%)$ \\
\hline Lacks Social Support & $<1 \%$ & $7(<1 \%)$ & $5(2 \%)$ \\
\hline Psychiatric Disorders & $25 \%$ & $323(25 \%)$ & $54(25 \%)$ \\
\hline & \multicolumn{3}{|c|}{ Multivariate Analysis } \\
\hline & \multicolumn{3}{|c|}{ Odds of $\geq 2$ Missed Clinic Visit } \\
\cline { 2 - 4 } & Unadjusted Analysis & Adjusted Analysis \\
\hline Age & ${ }^{*} 0.96(0.94-0.97)$ & ${ }^{*} 0.96(0.94-0.98)$ \\
\hline Lives Alone & ${ }^{*} 1.70(1.21-2.36)$ & $* 1.71(1.21-2.41)$ \\
\hline Marginal Housing & ${ }^{*} 9.68(4.19-23.52)$ & $* 6.93(2.88-17.38)$ \\
\hline Substance Abuse & ${ }^{*} 1.97(1.39-2.78)$ & $* 1.48(1.01-2.12)$ \\
\hline Lacks Social Support & ${ }^{*} 4.27(1.25-13.49)$ & $2.21(0.54-8.04)$ \\
\hline Psychiatric Disorders & $0.97(0.69-1.37)$ & $0.91(0.64-1.27)$ \\
\hline
\end{tabular}

Notes: *indicates $\mathrm{P}<0.05$, multivariate analysis controlled for age, psychiatric disorders, and other $\mathrm{SDOH}$ covariates. Substance abuse includes: Alcohol or Drug Abuse
U.S., may be heightened in communities affected by violence. For instance, qualitative studies have documented that older adults who live in high-crime communities often report confinement to their homes due to safety concerns. However, few studies have quantified associations between social isolation and community violence exposure in at-risk populations.

METHODS: Adult patients $(\mathrm{N}=506)$ were recruited from clinics in 2 epicenters of violent crime in Chicago, and surveyed using computerassisted personal interviewing software. Social isolation was measured using validated items for social network characteristics, living arrangements, social participation, social support, and loneliness; all measures were normalized to 100-point scales. Lifetime violence exposure was measured using the Brief Trauma Questionnaire, which queried participants about being a direct victim of violence, a witness to violence, or a close friend/ family member of someone who died violently. We used generalized linear models to assess social isolation measures as a function of violence exposure, controlling for age, gender, race/ethnicity, education, insurance, and employment. We also used identical model specifications to assess health behaviors as a function of above- or below-average loneliness scores.

RESULTS: Mean age was $59.6 \pm 13.0$ years. Participants were predominantly female $(71 \%)$ and non-Hispanic black (75\%). Two-thirds reported a prior exposure to community violence $(62 \%)$, either as a direct victim $(41 \%)$ or as an indirect witness, friend, or family member (49\%). Prior exposure to community violence was associated with a 7.8-point reduction $(95 \% \mathrm{CI}$, -13.2 to -2.5 ) in perceived social support from friends; a reduction in perceived social support from family was not significant. Although mean social network size was similar between exposed ( $2.4 \pm 1.7$ persons) and unexposed groups $(2.2 \pm 1.7$ persons $)$, prior exposure to community violence was associated with a 8.0-point increase in perceived loneliness $(95 \%$ CI, 3.5 to 12.4). Living arrangement and social participation did not differ between groups. An above-average loneliness score was associated with fewer days per week of fresh fruit and vegetable consumption (-0.5 days; $95 \% \mathrm{CI},-1.0$ to -0.1$)$ and medication adherence $(-0.6$ days; $95 \% \mathrm{CI},-1.0$ to -0.2 ); and more days per week of binge alcohol consumption ( 0.4 days; $95 \% \mathrm{CI}, 0.1$ to 0.6$)$ and using tobacco products ( 0.5 days; $95 \% \mathrm{CI}, 0.1$ to $0.9)$. Physical activity did not differ between groups.

CONCLUSIONS: Exposure to community violence was associated with less social support and higher loneliness in this high-risk sample of urbandwelling adults; loneliness was associated with adverse health behaviors. At a time when public health leaders are calling for solutions to the "epidemic of loneliness," identifying populations at highest risk for loneliness may help to target interventions and ensure equitable access to social and medical support.

\section{SOCIAL NETWORK ANALYSIS OF FIREARM SHARING AMONG US STATES}

Sae Takada ${ }^{1}{ }^{1}{ }^{2}$; Kristen Choi $^{1}$; Shaw Natsui ${ }^{1}$; Altaf Saadi ${ }^{1}$; Liza $\overline{\text { Buchbinder }}^{1}, 2$; Molly C. Easterlin ${ }^{1,3}$; Frederick Zimmerman ${ }^{4} .{ }^{1}$ UCLA National Clinician Scholars Program, Los Angeles, CA; ${ }^{2}$ U.S. Department of Veterans Affairs, Los Angeles, CA; ${ }^{3}$ Cedars Sinai Medical Center, Los Angeles, CA; ${ }^{4}$ UCLA Fielding School of Public Health, Los Angeles, CA. (Control ID \#3185910)

BACKGROUND: There is growing concern that the movement of firearms across state lines may decrease the effectiveness of state-level firearm-restricting policies. Yet how firearms cross state lines and how state-level firearm policies affect cross-state movement have not yet been widely explored. The purpose of this study was to characterize the flow of firearms between states in a social network analysis and examine how state-level firearm policies affect level of firearm movement.

METHODS: We used the Bureau of Alcohol, Tobacco, Firearms, and Explosives firearm trace data from 2012 to 2016 to create a network of firearm flow between states, defined according to "origin states" (state where a given firearm is purchased) and "destination states" (state where a given firearm is recovered). We used social network analysis and considered these networks at three thresholds of firearm flow: at least 1 , at least 50 , and 
at least 100 firearms per year. The study examined all 50 states in the United States, excluding the District of Columbia and US territories. For each state, we calculated the number of destination states for which it served as the origin state, as well as the number of origin states for which it served as the destination state. We used fixed effects linear regression models to explore the relationship between the number of states for which a given state was the origin of firearms and state-level firearm policy, adjusting for poverty rate, population density, and hunting license rate as a proxy for gun ownership. We used a similar model to examine the relationship between the number of states for which a given state was the destination of firearms.

RESULTS: Over the 5-year period, in an average state, firearms recovered were traced back to 41 outside origin states. States served as the destination for firearms from as few as 16 origin states (Vermont) to as many as all 50 states (California and Florida). States served as the origin for firearms for as few as 18 states (Rhode Island) and as many as all 50 states (California). In a regression controlling for the other covariates, the number of states for which a given state served as the origin was negatively associated with the number of restrictive firearm policies in the state, while the number of states for which a it served as the destination was positively associated with the restrictive firearm policies in the state.

CONCLUSIONS: States with a greater number of restrictive firearm policies were more often destinations of firearms from outside states. The same states were less often the origin of firearms recovered in outside states. State-level firearm policies may not sufficiently address firearms that cross state lines. The effectiveness of firearm-restricting policies at the state level is complicated by a network of firearm sharing among states. Without national policy solutions, the impact of state-level restrictions on firearms may be muted.

\section{SOCIAL NETWORK CHARACTERISTICS AND OBESITY- RELATED BEHAVIOR AMONG HISPANIC IMMIGRANTS}

Jane W. Njeru; Mark L. Wieland; Janet Okamoto; Paul J. Novotny; Luz Molina; Graciella Porraz-Capetillo; Irene G. Sia. Mayo Clinic, Rochester, MN. (Control ID \#3182195)

BACKGROUND: Social networks influence behaviors through multiple mechanisms, including shared social activities and social support and are significant contributors to the obesity epidemic. Social network interventions targeting behavior change leverage these mechanisms. Social networks and obesity-related behaviors have not been described yet among Hispanic immigrant groups in the US. The overall goal of this study was to describe social networks and obesity-related characteristics among Hispanic immigrants in a mid-size Midwest US city, to inform a community based participatory researchderived nutrition and physical activity social network intervention, targeting behavior change to decrease obesity rates and cardiovascular risk.

METHODS: Setting and Participants: Hispanic immigrants in a mid-size city in SE MN Data collection: Conducted by bilingual study team members at community locations Measures: Demographics, general health measures, biometric (BMI), socio-behavioral and network measures Data analysis: Descriptive statistics were used to report the survey and biometric data. Logistic models were used to describe the basic associations of participants and network demographics with binary characteristics such as being overweight or being likely to lose weight. Network data was analyzed to identify nodes and ties and visualize the network. UciNet, Gephi, and R were used to calculate network metrics, conduct more complex network analysis and to visualize the social network.

RESULTS: Of the 610 Hispanic participants, $81 \%$ were overweight/ obese. The network had 1,998 named nodes with 2,354 ties between these nodes. Modularity of the network was high at 0.94 . $32 \%$ of named alters were perceived to be overweight/obese. Compared to normal weight respondents, the mean percentage of overweight/obese network members was higher for overweight/obese respondents $(33.7 \%$ vs. $28.7 \%$, $\mathrm{P}=<0.001)$. Participants who stated that they were likely to lose weight reported more positive social norms for obesity [mean (SD)] (2.4 (0.8) vs. 1.5 (0.8), $\mathrm{P}=<0.0001$ ), social support (3.2 (1.1) vs. $2.6(1.2), \mathrm{P}=<0.0001$ ), and more social cohesion (3.3 (1.4) vs. 3 (1.3), $\mathrm{P}=0.008)$ than participants who were not likely to lose weight. Findings were similar for participants whose social contacts were trying to lose weight compared to participants whose social contacts were not. If most or all of overweight/obese participant's social contacts were trying to lose weight the odds of stating they were likely or very likely to lose weight was four times higher compared to other participants (OR 4, CI 2.2-7.2).

CONCLUSIONS: In this community, obesity, obesogenic behaviors, weight loss intentions, social norms for weight loss and social support clustered by ascertainable networks. A social network intervention targeting behavior change for weight loss is feasible.

\section{SOCIAL SERVICE TOUCHPOINTS AS OPPORTUNITIES TO SCREEN WOMEN AT HIGH RISK FOR TYPE 2 DIABETES}

Maya S. Venkataramani; Nisa Maruthur. Johns Hopkins University School of Medicine, Baltimore, MD. (Control ID \#3184578)

BACKGROUND: Women with a history of gestational diabetes are at increased risk for type 2 diabetes, but many do not receive recommended testing for diabetes following pregnancy, in part due to limited interactions with the healthcare system. This is particularly true for low-income women. These women may have more regular contact with social service providers, including Head Start and WIC. Leveraging contact with these services could provide an opportunity to reach these women to enhance awareness of risk and encourage testing. We examined the proportion of mothers with gestational diabetes who accessed WIC or Head Start services who had not received recent diabetes screening.

METHODS: We analyzed data from the 2016 and 2017 National Health Interview Survey. Our analytic sample consisted of sample females ages 18 to 45 , linked to at least one child (age 17 or younger). We excluded women with a diagnosis of diabetes or prediabetes. We analyzed 2 groups: 1) those who received WIC services/had a child who received WIC (in the past calendar year); and 2) those with a child currently enrolled in Head Start. In each group, we characterized the prevalence of self-reported gestational diabetes, and the proportion of these women who had diabetes testing in the past 3 years. As women may have reported testing associated with pregnancy, we conducted sensitivity analyses using a definition of recent screening that could exclude testing during pregnancy (e.g, within 2 years if the youngest child was 2).

RESULTS: A total of $6661(29,628,017$ weighted) women between the ages of 18 and 45 could be linked to a child; $90.8 \%$ (26,890,789 weighted) did not report a history of diabetes or prediabetes. Among these women, $16.2 \%$ (937 unweighted; 4,343,424 weighted) reported WIC receipt for either themselves or their child. $7.5 \%$ (456 unweighted; 2,016,894) had a child currently enrolled in Head Start. (2.5\% reported both). Among women who had accessed WIC, $10.6 \%$ reported a history of gestational diabetes. $9.9 \%$ of these women did not report recent diabetes screening. $8.8 \%$ with a child in Head Start reported a history of gestational diabetes, and $8.2 \%$ of these women did not have testing within 3 years. In sensitivity analyses that could exclude recent pregnancy related testing, $35.0 \%$ (in WIC) and 21.2\% (in Head Start) did not report recent screening.

CONCLUSIONS: About 1 in 12 women with a history of gestational diabetes and recent contact with WIC or Head Start had not been screened for diabetes in the past 3 years, and this proportion maybe 3 to 4 -fold higher (if self-report included pregnancy-related testing). More precise estimates are difficult to ascertain due to limitations in pinpointing if testing occurred during pregnancy and the use of self-reported measure. However, the results suggest that leveraging contact with these programs could enhance appropriate follow-up among low-income, high-risk women who may have limited interactions with the healthcare system.

SOCIODEMOGRAPHIC CHARACTERISTICS OF PATIENTS WHO USE HUMAN VERSUS ANALOGUE INSULIN IN A NATIONALLY REPRESENTATIVE PATIENT SAMPLE

Alexandra M. Mapp; Jennifer N. Goldstein. Christiana Care Hospital, Newark, DE. (Control ID \#3186403) 
BACKGROUND: Human insulin (Regular, Neutral Protamine Hagedorn (NPH), 70/30 Regular/NPH mix), is available over-thecounter at a relatively low cost. Recent reports in the news media have indicated that some patients with limited to no insurance coverage rely on human insulins for treatment. Such patients are also at risk for poor diabetes outcomes. Using a nationally representative, publically available data set, we sought to examine whether there were sociodemographic differences in patients who use human insulin versus insulin analogues and whether the type of insulin used was associated with differences in glycemic control or hospitalization.

METHODS: This study was a cross-sectional analysis of the National Health and Nutrition Examination Survey (NHANES), a publicly available data set compiled and maintained by the Center Disease Control and Prevention's (CDC) National Center for Health Statistics (NCHS). Data was selected from four survey cycles (2007-2014) for participants who had interview and examination data, were 21 years and older, and reported current use of insulin. Participants were divided into three groups based on insulin formulation (human only, analogue only, and combination human/analogue insulins). Outcomes of interest were $\mathrm{HbA1C}$ and selfreported hospital utilization in the past 12 months. With insulin type as a predictor of interest, we controlled for potential confounders that were associated with our outcomes of interest.

RESULTS: Out of 802 respondents who report current insulin use, 698 reported the type of insulin used (human only $n=162$; analogue only $\mathrm{n}=500$; and human/analogue combination $\mathrm{n}=36$ ). In our bivariate analysis we found that respondents who used human insulin were more likely to identify as non-Hispanic African American than non-Hispanic White ( $38.9 \%$ vs $28.8 \%, p=0.002$ ) and were less likely to be college graduates (9.9\% vs $14.6 \% \mathrm{p}=0.024)$. Although not statistically significant, respondents who reported an insurance lapse in the past year and those with marginal food security were more likely to report use of human insulin compared to analogue insulin $(8.5 \%$ vs $3.8 \% \mathrm{p}=0.087 ; 14.9 \%$ vs $11.8 \%$ $\mathrm{p}=0.236$ ). There was no significant association between insulin type and glycemic control as measured by HbA1c. After multivariate adjustment, use of human insulin was not associated with differences in $\mathrm{HbA} 1 \mathrm{c}$ or rate of hospitalization.

CONCLUSIONS: In a nationally representative sample of patients with diabetes who use insulin, we found that use of human insulin was more common among patients who had characteristics associated with low socioeconomic status, such as minority race, lower education, unstable insurance and food insecurity. However, we did not observe differences in outcomes. Our findings suggest that human insulins may offer similar glycemic control, potentially at lower cost. However, further research should be conducted to understand outcomes for specific populations of patients of low socioeconomic status who appear to use human insulins at higher rates.

\section{SOLO SE VIVE UNA VEZ (YOU ONLY LIVE ONCE): A CAM- PAIGN TO IMPROVE HIV TESTING AMONG LATINX IN BALTI- MORE}

Harita Shah; Suzanne M. Grieb; Alejandra Flores-Miller; Kathleen Page. Johns Hopkins University, Baltimore, MD. (Control ID \#3162751)

BACKGROUND: Of the 263,000 Latinx with HIV in the US, one fifth are unaware of their infection. Consistent with national trends, Baltimore City Health Department (BCHD) data show that HIV-infected Latinx in Baltimore are more likely to be diagnosed late than any other racial/ethnic groups. In prior studies, we demonstrated prominent barriers to HIV screening among Latinx in Baltimore include HIV stigma, cultural/linguistic discordance, lack of insurance, and fear of deportation. Interventions that include complementary individual and community components have been shown to improve HIV testing rates; thus, we partnered with BCHD to create the Solo Se Vive Una Vez campaign to increase HIV screening rates among Latinx in Baltimore. The present study assessed reach of the campaign and its impact on people's decision to get tested.

METHODS: From July to December 2018, we implemented the Solo Se
Vive Una Vez campaign across Baltimore. On an individual level, the campaign centers on a website (solovive.org) addressing barriers to testing and offering rapid HIV testing with bilingual outreach workers. On a community level, the campaign was advertised through social media, dating apps, community events, buses, billboards, and radio. We conducted cross sectional surveys of Latinx adults obtaining HIV testing at the BCHD clinic and outreach before $(n=78)$ and after $(n=315)$ the campaign launch. Surveys assessed demographics, HIV testing history, exposure to the campaign, and influence of the campaign on the decision to get tested. Sexual risk behaviors and PrEP information were obtained from BCHD medical records by trained research assistants. Characteristics of the population exposed to the campaign versus not exposed were compared using t-test and chi squared analyses.

RESULTS: 34\% (106) of respondents surveyed after the campaign launch reported exposure to the Solo Se Vive Una Vez campaign, compared to $0 \%$ in the baseline surveys. Of those exposed to the campaign, $86 \%$ reported that it positively influenced their decision to get tested. Respondents who had campaign exposure had significantly higher numbers of sexual partners and rates of paying drugs/money for sex $(\mathrm{p}<0.05)$. The most common channels for exposure included Facebook, the website, and community events. 9,790 unique users have visited the website to date, and 127 unique users requested HIV testing through the website. $89 \%$ of Latinx surveyed had never heard of PrEP and were provided PrEP information.

CONCLUSIONS: Solo Se Vive Una Vez is Baltimore's first Spanishlanguage public health campaign promoting HIV screening among Latinx. It achieved comparable rates of exposure to other campaigns addressing HIV testing in Latinx. The campaign positively influenced the majority of testers exposed to it to get tested, including Latinx with high risk sexual behaviors. Based on the survey cohort, an estimated 29\% got tested due to the campaign; BCHD surveillance data is currently being tabulated to measure effect on testing rates for Latinx in Baltimore.

\section{SOURCE OF DIABETES KNOWLEDGE INFORMATION AND ITS IMPACT ON SELF-MANAGEMENT BEHAVIOR AMONG OLDER US ADULTS.}

Jennifer A. Campbell ${ }^{1}$; Emma Garacci ${ }^{2}$; Leonard E. Egede ${ }^{1} .{ }^{1}$ Medical College of Wisconsin, Milwaukee, WI; ${ }^{2}$ Meidcal College of Wisconsin, Milwaukee, WI. (Control ID \#3177601)

BACKGROUND: Diabetes Self-Management (DSM) is a key part of diabetes care and critical to maintaining optimal outcomes. Effective DSM requires knowledge, skills, and understanding of lifestyle changes to address modifiable risk factors that lead to diabetes complications. Diabetes knowledge information can be accessed from a number of sources outside of the diabetes care team including internet, magazines, television, and friends. Understanding how these sources of knowledge for diabetes may impact DSM behaviors has important implications for diabetes care and DSM Education practice.

METHODS: Data from 1,901 adults from the Health and Retirement Survey (HRS) 2003 diabetes cohort was used. The outcome, DSM behaviors included medication adherence, diet, and blood sugar testing. The primary independent variable was source of diabetes information. This included patients reporting how often they received diabetes information from the following: television, internet, newspaper or magazines, books, and friends. Survey logistic regression models tested the likelihood of performing recommended DSM behaviors, and whether source of information was associated with DSM behaviors. The model controlled for age, gender, race, education, and marital status.

RESULTS: The mean age of the population was 70 years. The majority of the population were non-Hispanic White (76\%), and $44 \%$ of participants had at least a high school diploma. Patients reporting the internet as a source of diabetes information was significantly related to lower odds of medication adherence for taking recommended medication (OR 0.37; CI 0.21-0.65), as well as taking recommended dose (OR 0.43; CI 0.24-0.76). Patients reporting television (OR .68; CI 0.51-0.91), newspapers/magazines (OR 
0.55; CI 0.42-0.73), and friends (OR 0.62; CI 0.47-0.82) as a source of diabetes information was related to lower odds of following a recommended healthful eating plan compared to those not using these sources for diabetes information. Conversely, reporting books as a source of diabetes knowledge was related to $74 \%$ higher odds of eating 5 or more servings of fruits/vegetables (OR 1.74; CI 1.222.49), 53\% higher odds of testing blood sugar as recommended (OR 1.53; CI 1.04-2.25), and 35\% higher odds of following a healthful eating plan (OR 1.35; CI 1.05-1.73).

CONCLUSIONS: Overall, sources of diabetes information had a differential relationship with DSM behaviors. Patients reporting the use of books as a source of diabetes information had significantly higher likelihood of following a healthful eating plan, following recommended diet for fruits/vegetables, and were more likely to test their blood sugar as recommended by their physician. Taken together, these results provide new information on the impact that sources of diabetes information may have on DSM behavior. Incorporating effective sources of information into the DSM Education and care plan may improve DSM, specifically for blood sugar monitoring.

\section{SPENDING AND COST-SHARING ON OUT-OF-NETWORK CARE IN THE UNITED STATES, 2008-2016}

Zirui Song ${ }^{1,2}$; Jacob Wallace ${ }^{3} .{ }^{1}$ Harvard Medical School, Boston, MA; ${ }^{2}$ Massachusetts General Hospital, Boston, MA; ${ }^{3}$ Yale School of Public Health, New Haven, CT. (Control ID \#3186093)

BACKGROUND: Out-of-network medical care has garnered increasing attention. While patients are becoming more aware of the costs associated with out-of-network care, little is known about how spending on out-of-network care has evolved over time in the United States. This study analyzes trends in in-network and out-of-network spending and cost-sharing in the largest segment of the United States - people with employer-sponsored health insurance.

METHODS: We analyzed in-network and out-of-network spending and cost-sharing for a large nationwide cohort of employers that continuously contributed data to the Truven database from 2008-2016. All individuals were enrolled for at least 1 year. Main outcomes were in-network and outof-network medical spending and cost-sharing, as well as the percent of spending and cost-sharing out-of-network. We estimated changes in the share of spending that was out-of-network using an ordinary least squares model adjusted for age, sex, risk, insurance plan, and region. We conducted subgroup analyses by provider specialty and category of service. RESULTS: Our population comprised 27,833,040 individuals nationwide, averaging 33.8 years of age, $52 \%$ female, and a DxCG risk score of 0.9 . The unadjusted share of medical spending that was out-of-network was $7.0 \%$ in $2008-2010$ and $6.1 \%$ in $2014-2016$, a decline of 0.10 percentage-points per year over the study period $(\mathrm{p}=0.02)$. Among outpatient services, the share of spending out-of-network was stable or declined for all categories except laboratory services, where it increased from $5.2 \%$ in $2008-2010$ to $11.5 \%$ in $2014-2016$ ( 0.80 percentage-point increase per year, $\mathrm{p}<0.001)$. Among professional services, the share of spending out-of-network was stable or declined among all specialties except hospitalists and pathologists, in which it rose by $0.54(\mathrm{p}=0.01)$ and 0.31 percentage-points per year $(p<0.001)$, respectively. Both prices and cost sharing were substantially higher when a service was provided out-of-network than in-network.

CONCLUSIONS: Contrary to some of the public discourse, the share of spending out-of-network has been relatively stable for the past decade in the employer-sponsored population (half of the United States population). However, some areas of medical care, particularly laboratory services, have seen a sharp rise in out-of-network spending and cost-sharing. These results are consistent with qualitative evidence that laboratory services may be increasingly contracted to large regional or national suppliers which are out-of-network for many insurers. To our knowledge, this is the first national evidence of these troubling upward trends in the share of laboratory spending out-ofnetwork. Extending consumer protections to these settings is one policy solution. Alternative proposals such as capping out-ofnetwork prices at a percentage of in-network prices, reference pricing, or protecting patients from balance-billing also warrant consideration as patients and employers struggle with out-of-network bills.

\section{SPENDING AND QUALITY AFTER 3 YEARS OF MEDICARE'S VOLUNTARY BUNDLED PAYMENTS FOR JOINT REPLACEMENT SURGERY}

Amol S. Navathe ${ }^{1,2}$; Ezekiel J. Emanuel ${ }^{1,}{ }^{1}$; Atheendar Venkataramani $^{1,1}$; Qian Huang ${ }^{1}$; Atul Gupta ${ }^{3}{ }^{1}$; Claire T. Dinh ${ }^{1}$; Eric Z. Shan ${ }^{1}$; Dylan Small ${ }^{1,}$; Norma B. Coe ${ }^{1,}{ }^{1}$; Erkuan Wang ${ }^{1}$; Xinshuo $\mathrm{Ma}^{1}$; Jingsan Zhu ${ }^{1}$; Deborah Cousins ${ }^{1}$; Joshua M. Liao ${ }^{4,1}$. ${ }^{1}$ University of Pennsylvania, Philadelphia, PA; ${ }^{2} \mathrm{CMC}$ VA Medical Center, Philadelphia, PA; ${ }^{3}$ The Wharton School, University of Pennsylvania, Philadelphia, PA; ${ }^{4}$ University of Washington, Seattle, WA. (Control ID \#3179521)

BACKGROUND: Based on early savings from the Bundled Payments for Care Improvement (BPCI) program, Medicare has continued to scale bundled payments for lower extremity joint replacement (LEJR) episodes nationwide through new voluntary programs such as BPCI-Advanced. However, despite growing participation in voluntary LEJR bundles, there is a paucity of evidence about their long-term impact on spending and quality.

METHODS: We used 2011-2016 Medicare claims to identify a sample of 205,655 Medicare beneficiaries undergoing LEJR at 244 BPCI hospitals operating in 123 markets and 53,711 fee-for-service Medicare beneficiaries undergoing LEJR at 244 propensity-matched Non-BPCI hospitals operating in 98 markets. We used a difference-in-differences method with propensity matching of hospitals not participating to those participating in BPCI, and hierarchical multivariable generalized linear models with quarter and hospital fixed-effects, to evaluate the association between hospital participation in LEJR bundles under BPCI and changes in spending and quality over a three-year period. This approach also used an instrumental variable (IV) that used historical hospital referral patterns to instrument for observed hospitalization at a hospital participating versus not participating in BPCI. This IV mitigated confounding from changes in unobservable patient characteristics after hospitals started in BPCI. We also evaluated associations by program period and participant wave. Parallel trends were confirmed. Analyses were performed using SAS, version 9.4. Statistical tests were 2-tailed and significant at $=0.05$. RESULTS: Over 3 years, BPCI participation was associated with a $1.5 \%$ decrease in average episode spending ( $95 \%$ CI $-2.1 \%$ to $-0.9 \%, \mathrm{p}<0.001)$, with reductions occurring in the early $(1.8 \%$ decrease in average episode spending, $95 \% \mathrm{CI}-2.5 \%$ to $-1.1 \%$, $\mathrm{p}<0.001$ ) but not late program period ( $1.0 \%$ decrease, $95 \% \mathrm{CI}-2.6 \%$ to $0.5 \%, \mathrm{p}=0.19)$. Savings were driven by performance of first wave (differential change of $-2.0 \%, 95 \%$ CI $-2.6 \%$ to $-1.3 \%, \mathrm{p}<0.001$ ) but not second wave participants (differential change of $-0.02 \%, 95 \%$ CI $-1.7 \%$ to $1.7 \%, p=0.98$ ). Savings were $30 \%$ lower than naïve regressions without the IV. BPCI participation was not associated with changes in 4 quality measures.

CONCLUSIONS: Participation in LEJR Bundles under BPCI was associated with sustained episode savings accounted for by early participants but not associated with changes in quality. These findings support Medicare's decision to continue scaling up bundled payments via voluntary participation through BPCI-Advanced, while also suggesting tempered expectations for participants in its second wave. Our findings also quantify the effect of unobserved patient selection in voluntary LEJR bundles - a major policy concern - but corroborate true associated savings in spite of such selection. 
AND ATTITUDES ABOUT HEALTH EQUITY

Arshiya A. Baig ${ }^{1}$; Erin M. Staab ${ }^{1}$; Jefferine $\mathrm{LI}^{1}$; Wen Wan ${ }^{1}$; Scott Cook $^{4}$; Joel Jackson ${ }^{1}$; Amanda Campbell ${ }^{3}$; Cynthia T. Schaefer ${ }^{2}$; Michael T. Quinn ${ }^{1}$. ${ }^{1}$ University of Chicago, Chicago, IL; ${ }^{2}$ University of Evansville, Evansville, IN; ${ }^{3}$ Midwest Clinicians' Network, East Lansing, MI; ${ }^{4}$ The University of Chicago, Chicago, IL. (Control ID \#3185297)

BACKGROUND: Little is known about the spillover effect of quality improvement training on staff knowledge and attitudes about health equity.

METHODS: As part of a 5-year cluster randomized trial to assess the effect of diabetes group visits on patient glycemic control, we trained 26 providers and staff from 7 health center sites across 6 Midwestern states. The 2-day in-person training was led by a team of academic and health center staff and focused mainly on how to implement group visits for adults with diabetes. The training included a video clip on National Standards for Culturally and Linguistically Appropriate Services in Health and Health Care (CLAS Standards), a presentation on trends in disparities in healthcare quality and an overview of healthcare care disparity interventions, and one presentation on understanding stereotypes and bias. Participants were surveyed before and after the training and answered questions on a 5-point Likert-type scale ranging from "strongly disagree" to "strongly agree." We used descriptive statistics were to summarize responses and paired t-tests to determine change from preto post-training. We used 2-sample t-tests to examine differences in responses between those with and without prior cultural competency training and the diversity of patient population served at their site.

RESULTS: From pre to post training, participants had improvements in perceived benefits of the group visit model of care $(\mathrm{p}=0.001)$, knowledge of potential barriers to implementing diabetes group visits $(\mathrm{p}=0.03)$, and confidence in implementing diabetes group visits at their site $(\mathrm{p}=0.04)$. Staff reported an increase in the potential of group visits to reduce racial/ ethnic disparities in diabetes outcomes ( $\mathrm{p}=0.03$ ) from pre to post training. Staff were also more familiar with the CLAS Standards ( $p<0.0001$ ), better able to describe racial/ethnic disparities in diabetes prevalence and outcomes $(\mathrm{p}=0.002)$ and reported knowing steps their center could take to address racial/ethnic health disparities $(\mathrm{p}=0.04)$. Changes in knowledge of CLAS or health disparities or perceptions of the impact of group visits on disparities did not differ significantly between those who participated in cultural competency regularly during the past 10 years vs. those who participated less or not at all. There was a greater increase in knowledge of steps to take to address racial/ethnic health disparities for staff at health centers with fewer racial/ethnic minority patients.

CONCLUSIONS: Health center staff demonstrated improvements in knowledge and confidence in implementing diabetes group visits. Staff also noted that group visits had the potential to improve racial/ethnic disparities in diabetes outcomes in their patient population and learned steps that their health centers could take to address disparities. Future studies need to assess the impact of staff QI training on racial disparities on patient health outcomes.

\section{SPILLOVER EFFECTS OF THE MEDICARE BUNDLED PAY- MENTS FOR CARE IMPROVEMENT PROGRAM AMONG NON-MEDICARE PATIENTS}

Joshua M. Liao $^{3,}{ }^{1}$; Ezekiel J. Emanuel ${ }^{1,}{ }^{1}$; Akriti Mishra ${ }^{1}$; Jingsan Zhu ${ }^{1}$; Claire T. Dinh ${ }^{1}$; Deborah Cousins ${ }^{1}$; Xinshuo $\mathrm{Ma}^{1}$; Joshua Rolnick ${ }^{1}$; Amol S. Navathe ${ }^{1,2}$. ${ }^{1}$ University of Pennsylvania, Philadelphia, PA; ${ }^{2} \mathrm{CMC}$ VA Medical Center, Philadelphia, PA; ${ }^{3}$ University of Washington, Seattle, WA. (Control ID \#3179513)

BACKGROUND: Early evidence suggests that voluntary bundled payments for joint replacement surgery under the Bundled Payment for Care Improvement (BPCI) program were associated with episode savings without decrements in quality. Practice redesign in response to BPCI may benefit all patients, not just Medicare fee-for-service beneficiaries. Understanding the presence and magnitude of such "spillover effects" is critical to understanding bundled payment as a mechanism for improving value. Therefore, we examined the impact of hospital BPCI participation on episode quality and costs for commercially insured patients, including those in Medicare Advantage, receiving joint replacement surgery.

METHODS: We used data from Medicare to identify hospitals participating in BPCI for joint replacement surgery (BPCI hospitals) from 2013 Quarter 3 to 2016 Quarter 4 and associated data from the Health Care Cost Institute (full claims activity of $25 \%$ of all insured US individuals younger than 65), to construct care episodes for commercially insured patients encompassing hospitalization and 90-days of post-discharge care. Our sample consisted of 21,470 commercially insured patients undergoing joint replacement surgery during hospital BPCI participation and 266,916 commercially insured patients undergoing joint replacement surgery outside of hospital BPCI participation. There were 287 BPCI hospitals and 2,044 non-BPCI hospitals. After confirming parallel trends, we used a difference-in-differences (D-in-D) method and hierarchical multivariable generalized linear models to evaluate changes in hospital length of stay (LOS), 90-day readmissions rate, and episode spending at BPCI hospitals before and after participation, versus changes at non-BPCI hospitals. Covariates adjusted for age, gender, Elixhauser risk score, dual eligibility status, and insurance product, and including quarter and hospital fixedeffects and a time-varying indicator of BPCI participation. Analyses were performed using SAS, version 9.4. Statistical tests were 2-tailed and significant at $=0.05$.

RESULTS: Several characteristics, including case-mix, age distribution, and dual-eligibility status, varied between patients cared for at BPCI versus non-BPCI hospitals. Over a 3-year period, hospital participation in BPCI was associated with a $2.8 \%$ decrease in average episode spending ( $95 \%$ CI $-3.5 \%$ to $-2.2 \%, \mathrm{p}<0.001$ ). Hospital BPCI participation was also associated with decrease in 90-day readmissions (D-in-D -1.3\%, 95\% CI $-1.9 \%$ to $-0.8 \%$ ) and hospital LOS (D-in-D $-8.8 \%, 95 \%$ CI $-9.9 \%$ to $-7.8 \%$, $)(\mathrm{p}<0.001$ for both).

CONCLUSIONS: Hospital participation in the Medicare BPCI program was associated with decreases in episode spending, hospital LOS and 90day readmissions for commercially insured patients receiving joint replacement surgery. Our findings suggest that hospitals may implement broad care redesign that benefits all patients regardless of insurance coverage and reimbursement through bundled payments.

\section{SPINA BIFIDA ACTION RESEARCH: ASSESSING THE NEEDS OF INDIVIDUALS WITH SPINA BIFIDA TRANSITIONING TO ADULT-ORIENTED CARE}

Ashley Jenkins $^{1,2}{ }^{2}$; Diane Burns ${ }^{3}$; Rhonda Morrison ${ }^{3}$; Brittany Spicer ${ }^{2}$; Lisa Vaughn, ${ }^{2,4}$; Jason Woodward ${ }^{2,1}$. 1 University of Cincinnati Medical Center, Cincinnati, OH; ${ }^{2}$ Cincinnati Children's Medical Center, Cincinnati, $\mathrm{OH} ;{ }^{3}$ Spina Bifida Coalition of Cincinnati, Cincinnati, $\mathrm{OH} ;{ }^{4}$ University of Cincinnati, Cincinnati, OH. (Control ID \#3179146)

BACKGROUND: Individuals with spina bifida (SB) experience gaps in care when transitioning from pediatric- to adult-oriented care. Our objective was to identify facilitators and barriers of successful transition to adult-oriented care for individuals with SB.

METHODS: We utilized concept mapping, a participatory research method that involves structured steps including: 1) community definition and prompt development, 2) idea generation, 3) sorting ideas, 4) visual representation of data, and 5) interpretation. We recruited from SB community stakeholder groups: individuals aged 12 years and older with SB, parents/caregivers, pediatric and adult healthcare providers, and community organizations. A purposeful sample of stakeholders generated ideasto three open-ended transition-related prompts (e.g. To be a healthy adult living with SB I think I need). We edited ideas to reduce redundancy and ensure clarity. A subset of stakeholders sorted responses into groups of similar ideas using a web-based platform. We used multidimensional scaling and hierarchical cluster analysis to generate a concept map based on the sorted data, reviewing to ascertain the appropriate number of 
clusters and their meaning

RESULTS: We recruited 97 stakeholders (individuals with SB 32\%, caregivers $21 \%$, healthcare providers $29 \%$, community organizations $18 \%$ ). Idea generation, followed by editing, revealed 90 unique ideas. After stakeholder sorting, subsequent analysis revealed a seven-cluster concept map (Figure 1) showing how the arrangement (i.e. location and distance between) of the 90 ideas, illustrated by points, have meaning. Each of the seven clusters represents ideas sorted together due to conceptual similarity. Clusters emphasized two key conceptual domains: healthcare access (Clusters 3, 5, 6, and 7) and development of skills that foster empowerment and independence when living with SB (Clusters 1, 2, 4).

CONCLUSIONS: SB community stakeholders identified complex needs relevant to successful transition for individuals with SB from pediatric- to adult-oriented care. Concept map generation allows all SB stakeholders to easily interpret the relationship between ideas, as well as the perspectives of other community members. Both will inform future development of contextually relevant interventions.

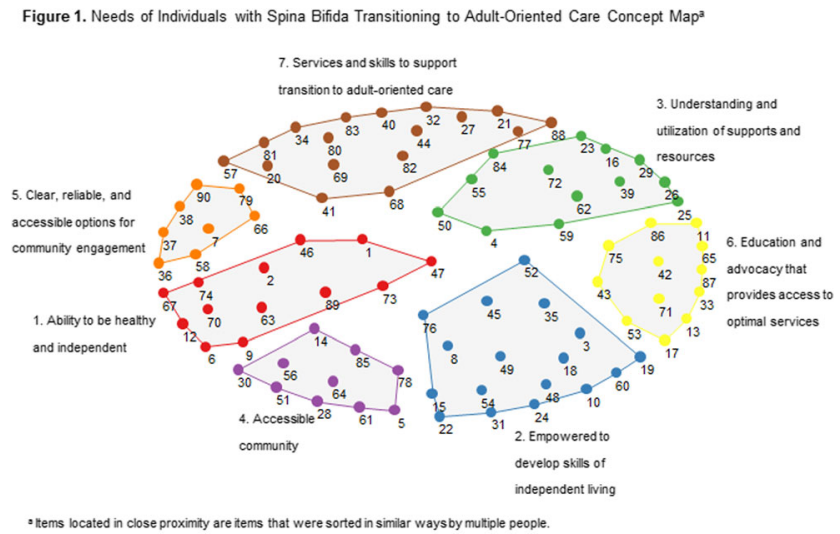

\section{SPIROMETRY UTILIZATION IN AN URBAN HEALTH CEN-} TER

Prithwijit Roychowdhury $^{1}$; Amulya Amirneni ${ }^{2}$; Jasdeep Badwal ${ }^{4,}$; Dilpreet Singh ${ }^{5}$; Alexander B. Knee ${ }^{2}$; Tara Lagu ${ }^{3} .{ }^{1}$ University of Massachusetts Medical School, Sturbridge, MA; ${ }^{2}$ Baystate Medical Center, Springfield, MA; ${ }^{3}$ Baystate Medical Center, Florence, MA; ${ }^{4}$ Allergy \& Immunology Associates of New England, Greenfield, MA; ${ }^{5}$ Arthritis Treatment Center, Springfield, MA. (Control ID \#3184668)

BACKGROUND: Asthma affects an estimated 39.5 million people in the United States with a total cost of more than $\$ 50$ billion. Although experts recommend that spirometry is performed for all patients in whom the diagnosis of asthma is being considered and as follow-up at least once every two years after an initial diagnosis is made, the few studies on the subject suggest that spirometry is widely underutilized in the diagnosis of asthma. There have been no studies examining longitudinal use of spirometry in asthma care. We, therefore, examined use of spirometry for both diagnosis and follow up and identified predictors of spirometry in a cohort of primary care patients within one large health system.

METHODS: We conducted a retrospective study of adult patients who had a diagnosis of asthma and an appointment in the year of 2014. We used diagnosis codes to randomly identify 200 patients from community health centers and another 200 from private practices. We collected billing records and extracted charts to identify patient characteristics, asthma severity, and the presence or absence of spirometry. We attempted to characterize diagnostic vs follow-up spirometry, but we were unable to do so due to poor documentation in the medical record. We further examined the process of spirometry, including use of the methacholine challenge.

RESULTS: Among our sample of 400 randomly selected subjects, we excluded 6 because they did not have a diagnosis of asthma, resulting in a final analytic dataset of 394 patients. Mean (SD) age was 52 years (13) with a large proportion of the sample being white $(61 \% ; n=239)$ and female
$(72 \% ; n=284)$. A total of $75 \%$ ( $n=297$ ) of patients had never had a prior visit with a pulmonologist or allergist. Of the 97 subjects who did see a specialist, $33(34 \%)$ saw a pulmonologist and $74(76 \%)$ saw an allergist. Of the 394 patients, $208(53 \%)$ received spirometry at any time during the follow-up period, and $186(47 \%)$ did not. For patients that received spirometry $(n=208), 29.3 \% \quad(n=61)$ received a methacholine challenge at any time and of these patients, $83.6 \%(n=51)$ showed a positive result, suggesting asthma/airway hyperreactivity. Seeing a specialist was the strongest predictor of whether a patient received spirometry or not: over $90 \%$ of those who had a specialist visit received spirometry, compared to $40 \%$ of those who did not have a specialist visit.

CONCLUSIONS: Despite the existence of guidelines set forth by experts, we found that spirometry is underused in both the diagnostic evaluation and subsequent monitoring of asthma. Among those who did receive spirometry, diagnostic tools such as the methacholine challenge were rarely used. The chance of receiving spirometry was strongly associated with seeing a specialist, which suggests a need to better incorporate spirometry into routine primary care for patients with asthma.

\section{SPOKESPERSON-PATIENT CONCORDANCE IS STRONGLY AS- SOCIATED WITH PATIENTS CHOOSING TO ACCEPT MEDI- CAL CARE NEAR THE END-OF-LIFE}

Michael J. Green ${ }^{1}$; Benjamin H. Levi ${ }^{1}$; Lauren J. Van Scoy ${ }^{1}$; Ashley Bucher' $^{1}$; Renee R. Stewart ${ }^{1}$; Anne Dimmock ${ }^{1}$; Erik B. Lehman ${ }^{2}$; Andrew J. Foy ${ }^{1,2}$. ${ }^{1}$ Penn State College of Medicine, Hershey, PA; ${ }^{2}$ Pennsylvania State University, Hershey, PA. (Control ID \#3185775)

BACKGROUND: A fundamental premise of surrogate decision-making in the context of serious illness is that spokespersons should accurately represent patients' wishes when patients cannot speak for themselves. Prior research using clinical vignettes showed that spokespersons' concordance with patient treatment preferences is imperfect $(\sim 50-70 \%)$ but factors associated with concordance are not well understood. We assessed whether spokesperson-patient concordance is associated with patient preference to accept vs decline medical interventions.

METHODS: This is a post-hoc analysis of a prospective trial of advance care planning (ACP) among adults with advanced, severe illness (class III/ IV heart failure; stage III/IV COPD; stage 4/5 kidney disease; or advanced cancer). The study took place at two tertiary care centers (Hershey, PA and Boston, MA) and the main outcome was spokesperson's concordance with patient preferences. After completing an ACP intervention, participants were asked whether they would 'accept' or 'decline' potentially life-saving interventions by responding to 28 questions related to 6 clinical vignettes. Spokespersons were separately asked to indicate which treatments they thought the patient would want. For all questions, patients' responses were classified as choosing to 'accept' or 'decline' treatment, and agreement with spokesperson's response was assessed as a dichotomous variable (yes/no). Multivariable logistic regression was performed for each question to assess whether 'accepting treatment' was associated with agreement after adjusting for study site, diagnosis, and prior advance directive.

RESULTS: 267 spokesperson-patient dyads were included and 7294 paired-responses were analyzed. Patients' mean age was 64 and $46 \%$ were female; spokespersons' mean age was 56 and $75 \%$ were female. Spokesperson-patient concordance was greater when patients 'accepted' versus 'declined' treatment, with significant differences in 24 of 28 questions. The overall median concordance rate was $87 \%$ when patients 'accepted,' and $44 \%$ when patients 'declined' treatment, and the median difference was $46 \%$. On multivariable testing, 'accepting' treatment was a significant, independent predictor of spokesperson-patient concordance for 24 of 28 questions. The overall median adjusted odds ratio was 10.9 when patients 'accepted' treatment. By contrast, no correlation was found with patients' or spokespersons' age, sex, race, ethnicity, religion, marital status, education, employment status, study site, prior experience with ACP, or patients' underlying medical condition.

CONCLUSIONS: Spokesperson-patient concordance is strongly 
associated with patient preference to accept medical treatment. This finding suggests that spokespersons may have a bias toward action or treatment to prolong life. Such a bias could lead to overuse of medical treatments when patients desire less care near the end-of-life. Future studies may further examine biases of spokespersons.

SPREADING PRIMARY CARE 2.0: A QUALITATIVE EVALUATION OF READINESS FOR A TEAM-BASED CARE MODEL IN AN ACADEMIC MEDICAL SYSTEM

Nadia Safaeinili ${ }^{1}$; Cati Brown-Johnson ${ }^{2}$; Jonathan G. Shaw ${ }^{3}$; Megan Mahoney ${ }^{4}$; Marcy Winget ${ }^{5}$. ${ }^{1}$ Stanford University, Stanford, CA; ${ }^{2}$ Stanford School of Medicine, Stanford, CA; ${ }^{3}$ Stanford University School of Medicine, Stanford, CA; ${ }^{4}$ Stanford University, Palo Alto, CA; ${ }^{5}$ Stanford University Medical School, Stanford, CA. (Control ID \#3185635)

BACKGROUND: In 2016, Stanford Healthcare developed a new model of primary care called Primary Care 2.0, which includes: 1) medical assistants (MAs) taking on advanced roles including scribing, population health management, and care-coordination; 2) team-based care, including an advanced practice provider and additional MA resources; and 3) an extended interdisciplinary care team (i.e. behavioral health, pharmacy, nutrition). The model has been implemented in one clinic with plans to spread to four additional primary care clinics. We conducted a qualitative assessment of perceived barriers and facilitators to implementation to inform the planned spread of the model.

METHODS: We collected data via semi-structured interviews with staff and providers $(n=26)$ and day-long observations of clinic teams and workflows at five primary care clinics. Detailed field notes were taken. Interview audio, transcripts, and notes were analyzed and synthesized in a rapid qualitative approach, the Lightning Report. We used the Consolidated Framework for Implementation Research (CFIR) to structure the coding of the data and predictions about factors for successful implementation.

RESULTS: Providers and staff reported factors related to the CFIR construct networks and communication to be most supportive of spread, including: strong clinic management, medical assistant wellness, and open communication within teams. Interviewees also felt that an explicit commitment to open and nonjudgmental communication may be protective against change fatigue during spread. Regularly scheduled times for teams to debrief and surface concerns were described as essential. All levels of the team reported benefits when the forum for debriefs took place as interprofessional "case conferences", allowing all to ask questions and receive support. Finally, providers perceived medical assistant wellness as critical for implementing and spreading a successful team-based care model. Providers and staff anticipated barriers that mapped primarily to the CFIR constructs available resources and access to knowledge and information. Reported barriers to spread included perceived inequality of workload distribution among team members of the same level, lack of understanding of workflows and roles within the new model, and instability of staffing for medical assistants and clinic management. Additional barriers focused on: 1) operations, including space needed for a co-located team environment; 2) time available for regularly-scheduled case conferences; and 3) difficulty recruiting appropriate staff in a compressed timeframe.

CONCLUSIONS: Based on our analysis, we predict that the following CFIR constructs will be key factors in spread success: networks and communication, specifically practices around open interprofessional communication and support of medical assistant wellness; access to knowledge and information about spread workflow and role changes, and available resources.

STATINS FOR PRIMARY PREVENTION: WE'RE NOT DOING IT ALL THE TIME

Connie Park; Jeffrey Le; Rebecca Mazurkiewicz. Hofstra Northwell Lenox Hill Hospital, New York, NY. (Control ID \#3185008)

BACKGROUND: Atherosclerotic Cardiovascular Disease
(ASCVD) is the leading cause of death worldwide and its complications remain expensive to treat. As per the 2013 American Heart Association and American College of Cardiology guidelines on the treatment of hypercholesterolemia to reduce ASCVD risk in adults, in patients without existing ASCVD, LDL cholesterol over 190, and diabetes, a moderate intensity statin is recommended if the 10-year ASCVD risk is $7.5 \%$ or greater using the ASCVD risk calculator. Given the complicated nature of this guideline, our hypothesis was that many of our patients are not appropriately on statins for primary prevention.

METHODS: This study consisted of chart review of patients seen by internal medicine residents from Lenox Hill Hospital from October 2017 to December 2017, aged 40-79 with a lipid panel resulted within the past year. We excluded patients with preexisting ASCVD, LDL over 190, diabetes, documented statin allergy, AST or ALT three times upper limit of normal, HIV, end stage renal disease, and duplicate encounters within our date range. Variables collected included age, gender, race, lipid panel results, blood pressure reading on the date of lipid panel, current anti-hypertensive medications, smoking history, current statin therapy, and reasons documented in the visit note for why patient was not on a statin. In addition, Lenox Hill medicine categorical residents were surveyed anonymously and electronically, asking what they believed to be the barriers to prescribing statins. The answer choices were as follows: time constraints, knowledge that a statin was necessary, other, or patient refusal.

RESULTS: Of the 113 patients who met inclusion criteria and did not meet exclusion criteria, $31(27.5 \%)$ were not on appropriate statin therapy for primary prevention of ASCVD. Of the 31 patients, $74 \%$ did not have a reason documented as to why they were not on appropriate statin therapy. For those who did have documentation, reasons included patient decision, conservative measures first for risk reduction, statin to be addressed at a follow up visit, and patient lost to follow-up. Twenty-three residents answered the survey. Residents most commonly cited time constraint $(n=13,56.5 \%)$ as the reason why they did not prescribe statins to patients. Others were patient refusal $(\mathrm{n}=5,21.7 \%)$, knowledge that a statin was necessary $(n=3,13.0 \%)$, or other $(n=2,15.4 \%)$.

CONCLUSIONS: Our chart review confirmed that the main barrier at our clinical site to having patients on statin therapy for primary prevention appears to be from the clinician's side. This was confirmed by surveying a group of residents. This is in contrast to what is in the literature, which is a more patient-centered issue of compliance. We will explore possible interventions to increase the rates of statin therapy in the future. One idea would be to include documentation of ASCVD risk score for every patient aged 40 and 79 at every visit, with the corresponding statin recommendation.

\section{STETHOSCOPE HYGIENE: USING CULTURES AND REAL- TIME FEEDBACK WITH RAPID BIOLUMINESCENCE SCORES TO CHANGE BEHAVIOR}

Jurgen Holleck $^{1,2}$; Sheldon Campbell ${ }^{1,2}$; Hedib Alrawili ${ }^{3}$; Cynthia Frank ${ }^{1,}$ ${ }^{2}$; Naseema Merchant ${ }^{1,2}$; Benjamin Rodwin ${ }^{4}$; Shaili Gupta ${ }^{1,2}$; Daniel G. Federman $^{1,2}$; John J. Chang ${ }^{1,2}$; Mario F. Perez ${ }^{5}$. ${ }^{1}$ Yale University School of Medicine, Southbury, CT; ${ }^{2}$ VACT Healthcare System, West Haven, CT; ${ }^{3}$ Quinnipiac University, Hamden, CT; ${ }^{4}$ Yale School of Medicine, New Haven, CT; ${ }^{5}$ Conn Health, Farmington, CT. (Control ID \#3149573)

BACKGROUND: Stethoscope contamination is comparable to that of our hands. Guidelines recommend disinfection between patients, yet this is rarely done. Previously, an educational intervention at our institution did not improve low rates of disinfection. Therefore, we aimed to 1) gain a better understanding of beliefs and barriers to stethoscope hygiene and 2) determine whether showing providers what is growing on their stethoscopes via culture or bioluminescence technology alters perceptions and improves stethoscope hygiene.

METHODS: Our study utilized a pre/post intervention design involving 
medical students, housestaff, and attendings between January and March 2018. Providers were given the opportunity to 1) culture their stethoscopes before and after disinfection with alcohol swabs, alcohol based hand rub (ABHR), or hydrogen peroxide wipes or 2) swab their stethoscopes for bioluminescence-based ATP testing before and after disinfection. Pre and post surveys were administered. Primary outcomes were observed stethoscope and hand hygiene rates. Secondary outcomes were survey responses exploring beliefs, barriers, and self-reported disinfection rates. Bacteria speciation, colony forming units (CFU), and bioluminescence scores pre and post disinfection were also tracked.

RESULTS: 1,245 observed hand hygiene opportunities found that compliance improved from $72.5 \%$ to $82.3 \%$ ( $\mathrm{p}<0.001$ ). 590 observed encounters revealed no significant change in stethoscope hygiene rates which were $10 \%$ at baseline and $5 \%$ post intervention $(\mathrm{p}=0.08)$. However, provider perceptions of the importance of stethoscope hygiene improved from 8.5 to 9.3/10 $(\mathrm{p}=0.04)$ and self-reported stethoscope hygiene rates trended up 56\% to $67 \%(\mathrm{p}=0.06)$. The impact of seeing bioluminescence scores on cleaning behavior rated equal to that of stethoscope cultures: 7.1vs 7.2/10 ( $p=0.89)$. $\mathrm{CFU}$ reduction with alcohol swabs, $\mathrm{ABHR}$, and hydrogen peroxide wipes was equivalent $(\mathrm{p}=0.21)$.

CONCLUSIONS: Showing providers what is growing on their stethoscopes via cultures and bioluminescence technology improved "buy in" regarding the importance of stethoscope hygiene. Although self-reported rates of stethoscope hygiene trended up, observed rates were lower and failed to show improvement, suggesting a gap between what providers think they are doing and what they actually are doing. Hand hygiene however, improved by $13.5 \%$ ( 9.8 percentage points).

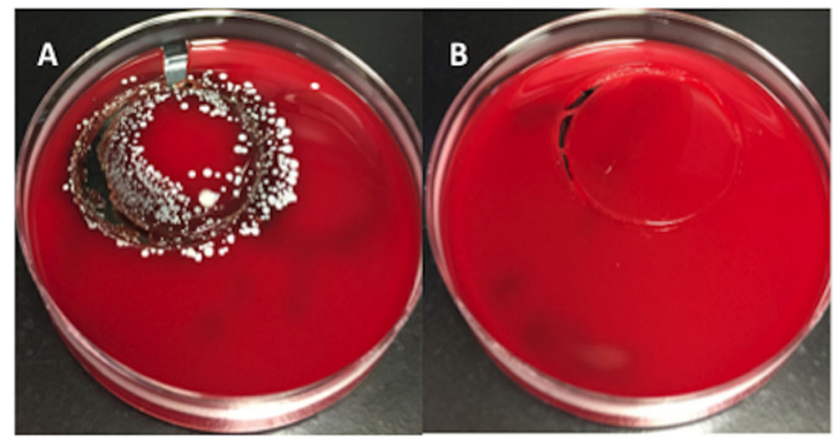

Stethoscope culture before (A) and after (B) disinfection.

\section{STRATEGIES FOR SHORT AND LONG-TERM PATIENT EN- GAGEMENT AMONG HIGH-NEED PATIENTS: REFLECTIONS OF KEY STAKEHOLDERS IN VA'S PRIMARY CARE INTEN- SIVE MANAGEMENT (PIM) TEAMS}

Alicia Bergman $^{1}$; Susan E. Stockdale ${ }^{2}$; Marian L. Katz ${ }^{3}$; Donna M. Zulman ${ }^{4}$; Evelyn T. Chang ${ }^{5}$. ${ }^{1}$ VA Greater Los Angeles, North Hills, $\mathrm{CA} ;{ }^{2}$ Greater Los Angeles VA Healthcare System, Sepulveda, CA; ${ }^{3}$ Veterans Health Administration, North Hills, CA; ${ }^{4}$ Stanford University and VA Palo Alto, Stanford, CA; ${ }^{5}$ VA- Greater Los Angeles, Los Angeles, CA. (Control ID \#3184414)

BACKGROUND: A recognized barrier to the success of intensive outpatient primary care programs for high-need patients is patient engagement, i.e., patients' active engagement in their own healthcare. However, research is lacking on how different strategies may be more conducive to different temporal phases of program involvement or lead to shorter or longer-term engagement. Our goal was to conduct a qualitative analysis of the Veterans Health Administration (VA) intensive primary care program (PIM) to learn about team members' successful patient engagement strategies, in order to describe the strategies by temporal phase of the patients' PIM program involvement, and by which strategies may facilitate shorterterm engagement versus longer-term self-sustaining patient engagement. METHODS: Semi-structured interviews were conducted with twenty- nine VA PIM team members (e.g., primary care providers, nurses, psychologists, social workers, dietitians) and primary care and program leaders. Interviews were recorded, transcribed, and analyzed to identify a novel organization of successful patient engagement strategies.

RESULTS: Some patient engagement strategies were particularly conducive to enabling immediate shorter-term engagement at the beginning phase of their program involvement, such as identifying with patients and general rapport-building, and setting guidelines/boundaries for what team member can and cannot do. During the ongoing phase of program involvement, other shorter-term "enabler" engagement strategies included scheduling appointments for patients, co-attending or connecting patients with primary care or specialty care appointments, assisting patients to get medication refills, providing or facilitating transportation to appointments, facilitating provider availability, and visiting patients when hospitalized. Other engagement strategies described by participants were potentially more conducive to longer-term and self-sustaining engagement; those employed at the beginning of patient program involvement included goal-setting, taking a whole-person health approach, conducting motivational interviewing, and educating patients on the wheel of health. Engagement strategies conducive to longer-term/self-sustaining patient engagement during the ongoing phase of program involvement included understanding psychiatric reasons that impede patient progress, medication management/education, encouraging patients to schedule/cancel appointments, coordinating/problem solving with the patient's caregiver/s or family, clinical dietitian advice, and collaborative decision-making.

CONCLUSIONS: Some engagement strategies were best employed at the beginning phase of patient program involvement while others fit well with ongoing involvement. Within both phases there were engagement strategies that seemed to facilitate either longer-term or shorter-term patient engagement. These findings can facilitate engaging primary care patients on a longer-term level, towards self-sustaining self-management.

STRATEGIES TO REDUCE ELECTRONIC HEALTH RECORD INBOX BURDEN: A MIXED METHODS STUDY

Daniel R. Murphy ${ }^{1,2}$; Tyler Satterly ${ }^{1,}{ }^{2}$; Traber Giardina ${ }^{2,}{ }^{1}$; Dean F. Sittig $^{3}$; Hardeep Singh ${ }^{2,1}$. ' Baylor College of Medicine, Houston, TX; ${ }^{2}$ Michael E. DeBakey VA Medical Center, Houston, TX; ${ }^{3}$ University of Texas, Houston, TX. (Control ID \#3168054)

BACKGROUND: Electronic health record (EHR) inbox notificationrelated workload leads to information overload and is cited as a contributing factor for job dissatisfaction and physician burnout. Better understanding of physicians' inbox requirements and workflows could optimize inbox designs, enhance efficiency, and reduce safety risks from notification overload.

METHODS: We conducted a mixed-methods study to identify strategies to enhance EHR inbox design and workflow. First, two reviewers performed a secondary analysis of national survey data collected in 2010 of all Department of Veterans Affairs (VA) primary care practitioners (PCP) to identify major themes in responses to a free-text question soliciting suggestions to improve EHR inbox design and workflows. We then conducted expert interviews of clinicians at five health care systems to understand existing optimal strategies to improve efficiency and situational awareness related to EHR inbox use. Themes from survey data were cross-validated with interview findings.

RESULTS: We analyzed responses from 2104 PCPs who completed the free text inbox question (of 5001 PCPs who responded to survey) and used an inductive approach to identify five themes: (1) Inbox notification content should be actionable for patient care and relevant to recipient clinician, (2) Inboxes should reduce risk of losing messages (3) Inbox functionality should to be optimized to improve efficiency of processing notifications, (4) Team support should be leveraged to help with EHR inbox notification burden, (5) Sufficient 'protected' time should be provided to all clinicians to process EHR inbox notifications. We subsequently interviewed 15 clinicians and identified 11 unique strategies 
corresponding to each of these 5 themes, including disabling certain automated for-your-information messages, making use of future reminder features to prevent information loss, using data entry automation features in the EHR, delegation of tasks to non-provider team members, and processing messages in a distraction-free environment.

CONCLUSIONS: Feedback from practicing end-user clinicians provides robust evidence to improve EHR inbox content and designs and organizational workflows and policies. Given the emerging efficiency and safety risks related to the EHR inbox, both EHR developers and health systems should work together on high-priority strategies identified.

\section{STRATIFICATION OF CHEST PAIN OBSERVATION PATIENTS USING THE HEART SCORE TO REDUCE UNNECESSARY CARDIAC TESTING}

Shelly L. McGurk ${ }^{1}$; Kevin Glenn ${ }^{1}$; Ethan Kuperman ${ }^{2} .{ }^{1}$ University of Iowa Hospitals and Clinics, Iowa City, IA; ${ }^{2}$ University of Iowa Carver College of Medicine, Iowa City, IA. (Control ID \#3185628)

BACKGROUND: Patients admitted to the observation unit with chest pain have a high utilization of cardiac diagnostic testing (e.g. stress testing). Current practice does not incorporate the consistent use of a risk-stratification tool. HEART score is a validated tool that accurately identified low-risk chest pain patients in the Emergency Room in past studies. The object of this study was to determine if the HEART score could identify patients on a medical observation unit who would not benefit from further cardiac testing.

METHODS: This was a retrospective, observational cohort study at a single academic medical center observation unit. Over a 12-month period, study data were collected and managed using REDCap. Data collection was done via manual chart review of patients with a chief complaint of chest pain. The HEART score was calculated using the history and physical, ED notes, EKG, and laboratory values. The endpoint was major adverse cardiac event (MACE), which was defined as acute myocardial infarction (AMI), percutaneous coronary intervention (PCI), surgical revascularization, or death within six weeks of the index visit. The type and results of cardiac diagnostic testing were also abstracted. Specificity, sensitivity, and frequency of cardiac testing were used for analysis.

RESULTS: There were 282 patients identified within the time period, $151(54 \%)$ female and $131(46 \%)$ male. The groups were dichotomized into low risk (HEART score 0-3) and elevated risk (HEART score $>3$ ). There were $115(41 \%)$ patients in the low-risk group and $167(59 \%)$ patients in the elevated-risk group. The MACE rate for the low-risk group was $2(1.7 \%)$ and $17(10.1 \%)$ for the elevated-risk group, $(\mathrm{p}=.005)$. The two MACE events in the low-risk group were PCI and 17 MACE events in the elevated-risk group included 6 AMI, 11 PCI, 1 CABG, and 1 death. The death in the elevated-risk group was non-cardiac due to sepsis related to peritonitis/appendicitis. The HEART score sensitivity was $89 \%$ (95\% CI: 66-98\%) and specificity $43 \%$ (95\%CI: $37-49 \%$ ). Stress testing was performed 70/115 (60.9\%) for the low-risk group and 102/167 (61\%) for the elevated-risk group $(\mathrm{p}=.89)$. The low-risk group had $58 / 68$ $(85.3 \%)$ normal stress test results and 10/68 (14.7\%) abnormal, 2 were not completed. The elevated-risk group had 67/102 (65.7\%) normal stress test results and 35/102 (34.3\%) abnormal results. There was a significant difference in the stress test results by group, $(\mathrm{p}=.005)$.

CONCLUSIONS: The HEART score identified a patient population at low risk for adverse cardiac events who received diagnostic testing at a similar rate as the elevated risk group. Incorporation of the HEART score into the admission protocol will provide decision support for clinicians and help improve decision-making with regards to cardiac testing for chest pain. This could reduce unnecessary diagnostic testing and decrease wasteful spending.

\section{STRENGTHENING THE FOUNDATION OF CLINICAL REASON-} ING: A RESIDENT-LED INITIATIVE

Stephen G. Philip; Bruno Alvarez Concejo; Melanie Holtrop; Sarah Collins; Stephanie Brinker; Reeni A. Abraham. University of Texas Southwestern,
Dallas, TX. (Control ID \#3175168)

BACKGROUND: Deficits in clinical reasoning skills have come into focus as a concern behind high rates of diagnostic medical errors in the United States. Since the foundation for a physician's diagnostic skill is laid in medical school, we sought to strengthen clinical reasoning by instituting a 4-week curriculum taught to medical students by internal medicine residents under faculty supervision. This study reports the initial effect of our curriculum on the students' subjective comfort with clinical reasoning.

METHODS: During the core internal medicine clinical rotation, students attended a 4-week course led voluntarily by a senior internal medicine resident. Each week, students analyzed a clinical case with guidance from the resident, and each session highlighted 1-2 concepts: illness scripts, problem representations, diagnostic schemas, likelihood ratios, semantic qualifiers, and awareness of bias. Cases became progressively more challenging to facilitate synthesis of earlier concepts. Simultaneously, the teaching skills of residents were observed and critiqued by internal medicine faculty using a rubric. Students completed a survey about their comfort level with and use of clinical reasoning both prior to and after the course.

RESULTS: 160 survey responses were received - 105 pre- and 55 postcourse. Students had a median 6 months of clinical rotations prior to the concurrent internal medicine clerkship, and their median age was 24 years. Before the course, $53 \%$ of students felt either comfortable or very comfortable altering their diagnosis based on new information, increasing to $80 \%$ after the course. Before the course, $60 \%$ reported having a clinical reasoning framework, increasing to $96 \%$ after the course, the majority using illness scripts ( $50 \%$ pre vs. $69 \%$ post ). Before the course, $5 \%$ of students felt comfortable or very comfortable using likelihood ratios, increasing to $58 \%$ after the course. CONCLUSIONS: Our outcomes evaluation reflects an increase in medical students' confidence using key components of clinical reasoning, and a large increase in students reporting the use of a framework - the majority adopting illness scripts. This is significant as failed reasoning and the lack of a standardized approach to clinical problems account for a large percentage of diagnostic error. Our goal is to reinforce a method of reasoning that will be used by students in the future; however, the question whether our curriculum has an objective impact on clinical reasoning has yet to be answered with script concordance testing data. Finally, this data provides evidence that residents can be effective teachers of clinical reasoning to medical students. Since residents provide a large proportion of a student's clinical training, formal instruction in the diagnostic process so residents can teach others might have exponential benefits in reducing diagnostic error for any healthcare system.

\section{STRIVES: ENGAGING MINORITY URBAN TEENS TO LEAD SOCIAL MEDIA CAMPAIGNS TO PROMOTE CLINICAL RE- SEARCH CAREERS IN THEIR COMMUNITIES}

Christianah Ogunleye $^{1}$; Jeanne M. Farnan ${ }^{2}$; Shannon K. Martin ${ }^{2}$; Audrey L. Tanksley ${ }^{3}$; Samantha Ngooi ${ }^{2}$; Laura Ruth Venable ${ }^{4}$; Samantha Anderson $^{2}$; Jhonatan Marte ${ }^{1}$; David Meltzer ${ }^{2}$; Vineet M. Arora ${ }^{5}{ }^{1}$ University of Chicago Pritzker School of Medicine, Chicago, IL; ${ }^{2}$ University of Chicago, Chicago, IL; ${ }^{3}$ Heartland Alliance Health, Chicago, IL; ${ }^{4}$ Univeristy of Chicago, Chicago, IL; ${ }^{5}$ University of Chicago Medical Center, Chicago, IL. (Control ID \#3170345)

BACKGROUND: One driver of poor health equity is underrepresentation of certain minority groups as leaders of clinical research studies. A major problem is the lack of exposure minority communities have to clinical research careers. This study aimed to assess whether high achieving minority youth enrolled in a pipeline program could create a social media campaign to promote interest in clinical research in their communities.

METHODS: Spreading Teen Research Inspired Videos to Engage Schoolmates (STRIVES) aimed to engage teens in a pipeline program to develop a targeted social media marketing campaign to boost interest in clinical research careers. The pipeline students conducted focus groups of their communities to inform themes to best promote clinical research. The 
students enrolled their friends to be study subjects. Friends were sent a survey to assess baseline interest in clinical research careers. The pipeline students scripted, storyboarded and filmed a short video to share on social media with a campaign hashtag. After the campaign launch, subjects were asked how they saw the video, their perceptions of the video and their postinterest in clinical research careers. Subjects who did not see the video through the online campaign were invited to watch as part of the study. Interest change scores were calculated using differences in Likert responses to "how interested are you in a career in clinical research?" An ordinal logistic regression model tested the association between watching a peershared video, perception of entertainment and interest change score controlling for underrepresented minorities in medicine (URM) status (Black, American Indian/Alaska Native, Native Hawaiian or Pacific Islander), gender and baseline interest in medical or clinical research careers.

RESULTS: From 2014-2017, 325 subjects were enrolled as part of 4 distinct campaigns: \#WhereScienceMeetsReality, \#RedefiningResearch, \#DoYourResearch and \#LifeWithoutResearch. Two-thirds [66\% (214)] were female, $31 \%$ (101) were Hispanic, and 23\% (75) were URMs. Over half(180) of subjects watched the video via the campaign, $77 \%(n=227)$ found the video entertaining, and $28 \%(\mathrm{n}=92)$ had baseline interest in clinical research. Interest change scores were approximately normally distributed with a median change score of 0 . The ordinal logistic regression model found that subjects who viewed the video from a peer (OR 1.56, 95\% CI 1.00-2.44, $\mathrm{p}=0.049$ ) or found the video entertaining (OR 1.36, 95\% CI 1.01-1.82, $\mathrm{p}=0.041$ ) had greater odds of increasing interest in a clinical research career. Subjects with higher baseline interest in medicine (OR 1.55, 95\% CI 1.28-1.87, p<0.001) also had greater odds of increasing interest in clinical research.

CONCLUSIONS: Authentic and relevant peer-created messages spread via video on social media can increase interest in clinical research careers among diverse youth. Peer-driven social media campaigns should be explored as a way to promote equity, engagement, and advocacy in minority communities.

\section{STUDY HABITS AMONG RESIDENTS AND ATTENDINGS AT A SINGLE ACADEMIC MEDICAL CENTER}

Casey N. McQuade ${ }^{1}$; Michael G. Simonson ${ }^{1}$; Kristen A. Ehrenberger ${ }^{2}$; Amar Kohli ${ }^{3}$. ${ }^{1}$ University of Pittsburgh Medical Center, Pittsburgh, PA; ${ }^{2}$ UPMC, Pittsburgh, PA; ${ }^{3}$ UPMC, Mars, PA. (Control ID \#3186176)

BACKGROUND: Modern physicians face increasing clinical demands, decreasing the time available to learn during and outside of work. We sought to characterize study patterns and the use of electronic study resources among residents and academic physician staff at an academic medical center. We hypothesized that residents would use electronic resources more frequently than attendings.

METHODS: Qualtrics surveys were emailed to 290 trainees and 165 faculty from the Departments of Medicine, Pediatrics, and MedicinePediatrics at UPMC. Survey participation was optional and not compensated. Questions covered demographic data, study time, resource usage, and time spent on social media. Means are reported \pm standard deviation for continuous variables. Relationships between variables were tested using non-parametric testing and regression analysis.

RESULTS: Responses were obtained from 118 trainees (41\% response rate), ranging from PGY-1 to PGY-6, and 110 attending physicians (67\%). Respondents were $60 \%$ female $(\mathrm{n}=131)$. The median age range was $25-34$ years (IQR, 25-34 years to 45-54 years). Social media use was reported by $162(86 \%)$. On average, participants studied for $15 \mathrm{~min}-1$ hour/day (IQR, 5$15 \mathrm{~min}$ to $15 \mathrm{~min}-1$ hour) on $4.0 \pm 1.8$ days/week. Increased participant seniority was correlated with more studying time/day $(\mathrm{p}=0.0274)$ and more positive views on how essential studying was to learning ( $\mathrm{p}=0.0002)$. The most popular study resources were websites $(n=196)$, journal articles $(n=180)$, and textbooks $(n=143)$. Increased seniority was also correlated with more frequent use of textbooks $(\mathrm{p}=0.046)$, quick references $(\mathrm{p}=0.022)$, and websites like Uptodate.com $(\mathrm{p}=0.0001)$ but was inversely correlated with journal article use $(\mathrm{p}=0.012)$. Social media use was associated with more frequent use of journal articles (OR 1.64, 95\% CI 1.06-2.55).

CONCLUSIONS: Time spent studying between residents and faculty was not significantly different, although attendings more frequently used print or online reference materials for extracurricular studying while residents more frequently read journal articles. The correlation between journal articles and social media use may suggest that social media increases physician exposure to and uptake of primary literature. This cross-sectional analysis reveals previously undescribed trends in resident and faculty study habits including determinates of exposure to the primary literature. These results could be used to tailor future educational interventions to increase supplemental learning and use of evidence-based practices in academic practice settings.

\section{SUBCLINICAL THYROID DYSFUNCTION AND DEPRESSIVE SYMPTOMS: AN INDIVIDUAL PARTICIPANT DATA ANALYSIS OF PROSPECTIVE COHORT STUDIES}

Lea Wildisen ${ }^{2}$; Martin Feller ${ }^{2,}{ }^{\text {; }}$ Elisavet Moutzouri ${ }^{2,}{ }^{1}$; Shanthi Beglinger ${ }^{2}$, ${ }^{1}$; Cinzia Del Giovane ${ }^{2}$; Anne R. Cappola ${ }^{7}$; Bjørn O. Åsvold ${ }^{3}$; Stephan Bakker $^{8}$; Graziano Ceresini ${ }^{12}$; Robin P. Dullaart ${ }^{8}$; Luigi Ferrucci ${ }^{9}$; Hans Grabe $^{10}$; Matthias Nauck ${ }^{10}$; Stella Trompet ${ }^{5}$; Henry Völzke ${ }^{10}$; Rudi G. Westendorp $^{4}$; Jacobijn Gussekloo ${ }^{5}$ 5; Robin P. Peeters ${ }^{11}$; Drahomir Aujesky ${ }^{1}$; Douglas Bauer ${ }^{6}$; Nicolas Rodondi ${ }^{2}{ }^{1}$. ${ }^{1}$ Inselspital, Bern University Hospital, Bern, Switzerland; ${ }^{2}$ University of Bern, Bern, Switzerland; ${ }^{3}$ Norwegian University of Science and Technology, Trondheim, Norway; ${ }^{4}$ University of Copenhagen, Copenhagen, Denmark; ${ }^{5}$ Leiden University Medical Center, Leiden, Netherlands; ${ }^{6}$ University of California, San Francisco, CA; ${ }^{7}$ University of Pennsylvania School of Medicine, Philadelphia, PA; ${ }^{8}$ University of Groningen, Groningen, Netherlands; ${ }^{9}$ National Institute on Aging, Baltimore, MD; ${ }^{10}$ University Medicine Greifswald, Greifswald, Germany; ${ }^{11}$ Erasmus Medical Center, Rotterdam, Netherlands; ${ }^{12}$ University Hospital of Parma, Parma, Italy. (Control ID \#3182648)

BACKGROUND: Subclinical thyroid dysfunction may be associated with several negative health outcomes; including depressive symptoms. However, evidence for this association is conflicting across a large number of studies, in part due to inconsistencies in definitions and cut-offs. Analysis of individual participant data (IPD) is needed to understand the role of subclinical thyroid dysfunction in the development of depressive symptoms and to inform practice and guidelines.

METHODS: We conducted a systematic review of cohort studies to assess the association between subclinical hypothyroidism or subclinical hyperthyroidism and depressive symptoms. We requested individual participant data from cohorts identified through a search of EMBASE and MEDLINE databases (up to November 2018) and through the Thyroid Studies Collaboration (www.thyroid-studies.org). The outcome was depressive symptoms at first follow-up, measured on any validated depression scale. For better clinical interpretation we transformed all scores into the Beck Depression Inventory (BDI) scale (range: 0-63, higher numbers indicating more depressive symptoms, minimal clinically important difference: 5 points) by calculating conversion factors. Subclinical hypo- and hyperthyroidism were defined as elevated thyroid stimulating hormone (TSH) ( $4.5 \mathrm{mlU} / \mathrm{L})$ and reduced TSH $(<0.45 \mathrm{mlU} / \mathrm{L})$ respectively, in combination with normal free thyroxine levels. We performed a two-stage IPD analysis. In each cohort, we estimated the mean difference (MD) in depressive symptoms scores between those with subclinical hypo- or hyperthyroidism and euthyroid controls adjusted for depressive symptoms at baseline. Further, we adjusted the multivariable linear regression analysis for age, sex and length of followup. We pooled the study effects estimates by using a random effects model. Heterogeneity was assessed by $\mathrm{I}^{2}$. The protocol is registered on PROSPERO (ID: CRD42018091627).

RESULTS: Among six cohorts, we analyzed data from 24,962 participants $(65 \%$ female, mean age $61 \pm 13.3$ years, subclinical hypothyroidism $\mathrm{N}=2,534$, subclinical hyperthyroidism $\mathrm{N}=684$, mean follow-up time $7.8 \pm 4.6$ years). At baseline, there was no difference in BDI scores between subclinical hypothyroidism (10.67), subclinical hyperthyroidism (10.78) and controls (10.25). After a mean 
follow-up time of $7.8 \pm 4.6$ years BDI scores did not differ between participants with subclinical hypothyroidism and controls (pooled MD $0.07,95 \%$ CI -0.25 to $0.40, \mathrm{I}^{2} 0.0 \%$ ) nor between participants with subclinical hyperthyroidism and controls (pooled MD -0.004 , $95 \%$ CI -0.57 to $0.56, \mathrm{I}^{2} 2.5 \%$ ). The results remained robust in sensitivity analyses, excluding participants taking thyroid medication $(\mathrm{N}=23,602)$.

CONCLUSIONS: In this large study of prospective cohorts, neither subclinical hypo- nor hyperthyroidism were associated with an increased development in depressive symptoms. Depressive symptoms seem not to be a good indication for thyroxine therapy if no overt thyroid dysfunction is present.

TAKING SOCIAL MEDICINE FROM FREE TIME TO PRIME TIME: SCREENING FOR SOCIAL DETERMINANTS OF HEALTH IN A LARGE URBAN PRIMARY CARE CLINIC

Daniel Gore ${ }^{1}$; Emily B. Phelps ${ }^{1}$; Zayd Hayani ${ }^{1}$; Coralie Pardo ${ }^{1}$; Leah Petrucelli ${ }^{1}$; Shunei Asao ${ }^{1}$; Adrienne Blackmon ${ }^{2}$; Eve Escalante $^{2}$; Nousheen Meherally ${ }^{2}$; Christopher Nolan ${ }^{2}$; Charles F. Sweitzer $^{2}$; Ritika Dhawan ${ }^{1}$; Elizabeth S. Davis ${ }^{2} .{ }^{1}$ Rush University, Chicago, IL; ${ }^{2}$ Rush University Medical Center, Chicago, IL. (Control ID \#3185815)

BACKGROUND: Patients at Rush University Internists (RUI) experience high rates of chronic diseases often influenced by social factors, but these social factors are infrequently discussed during appointments. This project aims to better identify, document, and address social determinants of health $(\mathrm{SDoH})$ during medical appointments.

METHODS: Medical students piloted a SDoH screening protocol in RUI, a primary care practice in Chicago's West Side. The project assessed feasibility and optimization of student-led SDoH screening in primary care. This project was embedded in a larger institutionwide effort to increase $\mathrm{SDoH}$ screening. The SDoH questionnaire contains ten items addressing food security, housing, transportation, health insurance, and PCP access. When patients screen positive, resources are provided through a community resource database, NowPow, linked to the electronic medical record (Epic). NowPow identifies community resources close to where each patient lives. The protocol includes escalation to a licensed clinical social worker when needed. Screenings were assessed for average time spent, as well as number of patients screening positive for the various $\mathrm{SDoH}$ metrics. Domains of positive screens were characterized and analyzed, and patient feedback was elicited.

RESULTS: Between March and May of 2018, 43 patients were screened by six students, with the average screening time of five minutes. Fifty-six percent of patients (26) screened positive for one or more SDoH: food insecurity (17), utilities interruptions (14), transportation issues (9), insurance status (5), housing insecurity (2). Twelve patients were referred to social work. Overall, patients endorsed these efforts, as one stated "I'm glad you're asking these questions." Based on these experiences, the students involved in the pilot developed a student-run service learning program in which each year, a new interprofessional group of students is trained to screen for SDoH. In the 2018-19 school year, 30 students were trained and began screening patients in primary care. These students also partner with Rush-wide screening initiatives to screen patients in community outreach settings including health fairs.

CONCLUSIONS: SDoH screening in primary care is feasible, and rates of needs related to social determinants of health may exceed providers' expectations. Student screening offsets the workload of other staff. Institutional support of SDoH screening helps with stakeholder buy-in and accessing resources for patients. However, student schedules may limit screening large volumes of patients.
Further exploration is needed to determine the most sustainable way to screen and connect patients with resources.

\section{TEACHING TRANSITIONS OF CARE: WHO OWNS IT?}

Patrick Ryan $^{1,2}$; Chi Zheng ${ }^{3,2}$; Alisha Skinner ${ }^{3,2}$. ${ }^{1}$ Denver Health and Hospital Authority, Denver, CO; ${ }^{2}$ University of Colorado School of Medicine, Aurora, CO; ${ }^{3}$ Denver Health, Denver, CO. (Control ID \#3184758)

BACKGROUND: Transitions of care are of increased focus and attention due to the financial impact of hospital readmissions on healthcare settings under the Hospital Readmission Reduction Program. However the amount of education dedicated towards transitions of care is not well quantified or described. In this study, we assessed what capacity and where residents receive transitions of care education.

METHODS: We developed a 21 question survey to assess where residents receive transitions of care teaching, the format of the instruction and their attitudes towards receiving further instruction in this arena. We distributed the survey to a singe internal medicine residency program with 180 residents having tailored curriculums including a hospitalist track, primary track and categorical tracks.

RESULTS: A total of 64 residents (35\%) respond to the survey with $92 \%$ reporting that they had rarely or never received transitions of care didactics during their inpatient rotations. Similarly, $89 \%$ of respondents reported rarely or never receiving such didactics during their outpatient rotations either. All respondents reported understanding transitions of care to be important to safe and high quality patient care and $98 \%$ reported resident education being important to very important in their residency education. Most respondents (57\%) felt moderately confident in their transitions of care planning while $39 \%$ felt slightly confident. None of the respondents felt very confident in their transitions of care planning. $90 \%$ of respondents had some interest in learning more about transitions of care; however, only $57 \%$ of respondents stated they would be interested in a rotation dedicated to learning more about transitions of care. Those who anticipated careers as hospitalists were more likely to be interested in a rotation dedicated to transitions of care compared to those pursuing careers as specialists or PCPs, $76 \%$ vs $45 \%$ vs $50 \%$, respectively $(\mathrm{p}<0.03)$. Respondents described their future career paths as predominantly specialty bound (39\%) compared to hospitalist $(30 \%)$ and primary care $(25 \%)$. The distribution of respondents was similar across years of training at 30\% for interns, $38 \%$ for second years, and $32 \%$ for third year residents.

CONCLUSIONS: Although transitions of care and readmission reduction have become of increasing importance to institutions to provide higher quality patient care and reduce healthcare costs, it appears that there is not much attention paid to resident education of this critical arena. Even as residents identify transitions of care to be of importance to their education and patient care, only slightly more than half of responding residents are interested in taking a curriculum dedicated to this field. Further study is necessary in the most effective ways of teaching transitions of care to resident physicians.

\section{TECHNOLOGY UTILIZATION IN PATIENTS WITH CIR- RHOSIS AND PREFERRED FEATURES OF DIGITAL HEALTH MANAGEMENT TOOLS}

Patricia Pringle; Thomas J. Wang; Bradley Green; Madeline Marx; Jasmine Ha; James M. Richter. Massachusetts General Hospital, Boston, MA. (Control ID \#3154013)

BACKGROUND: Evidence-based guidelines have made important advances in cirrhosis care; however, many of these guidelines are not sustained in practice. Technology represents a promising tool to improve guideline compliance and health care delivery. We aimed to 
assess cirrhotic patients' access to, utilization of, and comfort with technology, as well as the preferred features of a digital health tool. METHODS: We performed a mixed-methods study of cirrhotic patients with high healthcare utilization, those readmitted within 90 days for a cirrhosis-related complication. Patients were identified from the inpatient hepatology consult census. We obtained disease data from chart review and performed an in-depth semi-structured interview. Two investigators iteratively read and coded interview transcripts for themes using NVivo 11.0.

RESULTS: Of 134 cirrhotic inpatients identified from June to November 2018, 40 patients met inclusion criteria and agreed to participate. Of the 40 participants, $26(65 \%)$ were male, mean (SD) age was 58.4 years (11.7), with a mean (range) MELD of 22 (10-46). Thirty-one (78\%) patients own a Smartphone and $25(63 \%)$ patients have regular access to a computer. Smartphone apps are preferred by $23(58 \%)$ patients, while computers are preferred by $3(7.5 \%)$ patients. When asked if they would use a Smartphone app daily to manage their cirrhosis, $24(60 \%)$ reported yes, $10(25 \%)$ no, and $6(15 \%)$ maybe. Patients are interested in a Smartphone app that could: communicate with their physician $(32,80.0 \%)$, send medication notifications $(25,63 \%)$, transmit diagnostic results and appointment reminders $(30$, $75 \%)$, educate about cirrhosis $(31,78 \%)$, and offer low salt diet recipes $(25$, $63 \%)$. Of 25 patients with prior hepatic encephalopathy, 21 (72\%) would play a Smartphone game if it detected cognitive decline. Of 22 patients with volume overload, $19(86 \%)$ would use a Smartphone app to transmit weight data to their doctor. Common themes from qualitative data include a willingness to learn more about digital tools, a desire to learn about liver disease and communicate with providers via digital tools, and an eagerness to utilize digital tools if it could improve health (see Table).

CONCLUSIONS: Among cirrhotic patients with an early readmission at our center, most have Smartphones and would be willing to use one to manage their disease. Future technology interventions should be tailored to the specific use patterns and preferences of this population.

\section{TEMPORAL TRENDS IN DISCHARGE OPIOID PRESCRIBING FROM AN INPATIENT MEDICAL SERVICE}

Nicholas Iverson; Nader Najafi; Yumiko Abe-Jones; Priya A. Prasad; Margaret Fang; Kirsten Kangelaris; Catherine Lau. UCSF, San Francisco, CA. (Control ID \#3184800)

BACKGROUND: In March of 2016, the CDC released guidelines recommending shorter duration of opioid prescriptions. While these guidelines have the potential to influence hospitalist practice, it is not clear whether discharge prescribing patterns have changed. We examine changes in discharge opioid prescribing over time from an inpatient medical service at a single academic center.

METHODS: We used the electronic health record to identify all patients hospitalized on the acute care medical service at a 600-bed tertiary care hospital from 2012-2017, and stratified by whether they were on opioids at the time of admission according to provider medication reconciliation records. Patients were excluded if they had an ICD9 or 10 code for cancer-related pain or sickle cell disease, or who were on hospice or followed by the palliative care service. We calculated the number of opioid days supplied to the patient at discharge based on the discharge prescription signature and number of pills. All opioid prescriptions were standardized to morphine milligram equivalents (MMEs). We then calculated the median opioid days supplied at discharge by month, from 2012 to 2017. Multivariable Poisson regression analysis was used to assess temporal trends in likelihood of opiate prescription and median days supplied, with an interrupted time series approach to test for a significant change at the intervention point of March 2016.

RESULTS: We identified 19,759 medical inpatients who received opiates during their hospitalization, $23 \%$ of whom were not on opioids prior to admission. A total of 10,653 patients were discharged on opiates. The proportion of patients who were discharged with an opiate prescription declined over time (32.2\% in 2012 to $25.6 \%$ in 2017, p $<0.001$; Figure 1).
The median number of opiate days prescribed also declined over time ( 8.3 in 2012 to 7.5 in 2017, $\mathrm{p}=0.02$ ). The decline in median opioid days from 2016 to 2017 was statistically significant in patients taking opioids prior to admission (10 to 9.3 days, $\mathrm{p}=0.04$ ). Though the median days prescribed on discharge in patients without baseline opiate use remained at 5 days from 2012 to 2017 , there was a slight statistically significant decline in overall trend $(\mathrm{p}=0.02)$. After adjusting for patient level factors - age, sex, race, ethnicity, language, interpreter requirement, length of stay, ICU stay during admission, and van Walraven Comorbidity Index - we continued to find a significant decrease in opioid days supplied at discharge in all patients, with a significant decline post-March 2016 (rate ratio $=0.93, p<0.001$ ).

CONCLUSIONS: The percent of patients discharged on opioids from a hospital medicine service has declined over time, including a substantial drop after the release of the 2016 CDC guidelines. The median number of days among patients discharged on opioids has also declined over time. Patients on opioids at the time of admission are discharged with higher median days of opioids than those not previously on opioids - a trend that is ongoing.

\section{TERMINAL DIGIT BIAS AND BLOOD PRESSURE CONTROL IN AN ACCOUNTABLE CARE ORGANIZATION}

Kelsey B. Bryant ${ }^{1}$; Siling Li ${ }^{1}$; Yiyi Zhang ${ }^{1}$; Siqin $\mathrm{Ye}^{1}$; Brandon K. Bellows ${ }^{1}$; $\overline{\text { Teryl K. Nuckols }}^{2}$; Marie Krousel-Wood ${ }^{3}$; Jonas Green ${ }^{2}$; Andrew E. Moran ${ }^{1}$. ${ }^{1}$ Columbia University Medical Center, New York, NY; ${ }^{2}$ Cedars-Sinai Medical Center, Los Angeles, CA; ${ }^{3}$ Tulane University, New Orleans, LA. (Control ID \#3178947)

BACKGROUND: Accountable Care Organizations (ACOs) reimburse health systems based on performance, including blood pressure (BP) control (proportion controlled BP among hypertensive patients using last measurement of year). We hypothesized that terminal digit bias - intentional or unintentional rounding preference when measuring $\mathrm{BP}$ - misclassifies BP control.

METHODS: ACO patients were from 2 Columbia University outpatient Medicine practices in 2013-2016. BP is manually measured and recorded by providers using anaeroid sphygmomanometers in one practice. In the other, BP is automatically measured and manually recorded by medical assistants using oscillometric devices. Using last BP measurement/year in electronic health records, we compared systolic BP distributions and proportion controlled $(<140 / 90 \mathrm{mmHg})$ in hypertensives and normotensives combined. We identified terminal digit preference by examining BP distributions. We corrected for it by creating smoothed distributions reflecting the expected frequency of terminal digits $(10 \%$ in the absence of bias), and reassessed mean BP and proportion controlled BP.

RESULTS: 337,408 BP observations (65.01\% manual group) represented 24,643 unique Medicare Shared Savings Plan ACO patients ( $90.37 \%$ manual group). The manual BP practice had mean patient age 72 years old and $54 \%$ female; the automated BP practice had mean age 68 and $64 \%$ female. BPs in the manual BP practice exhibited terminal digit preference bias, which was eliminated by correction (Figure). Digit preference was less apparent in the automated measurement practice (data not shown). Percent ACO patients with controlled BP changed little in both populations after correction for terminal digit preference bias [all changes to $\%$ controlled $<2 \%$; chi squared $\mathrm{p}<$ 0.001 manual BP practice, $\mathrm{p}=0.64$ automated BP practice].

CONCLUSIONS: Terminal digit preference bias occurred in a practice where BP is measured manually, but not where it is measured automatically. Terminal digit preference bias had minimal effect on proportion with controlled systolic BP and likely did not impact the ACO performance benchmark. Nonetheless, biased BP measurement may lead to incorrect diagnoses and treatment decisions. Automated 
BP measurement appears to minimize this bias.

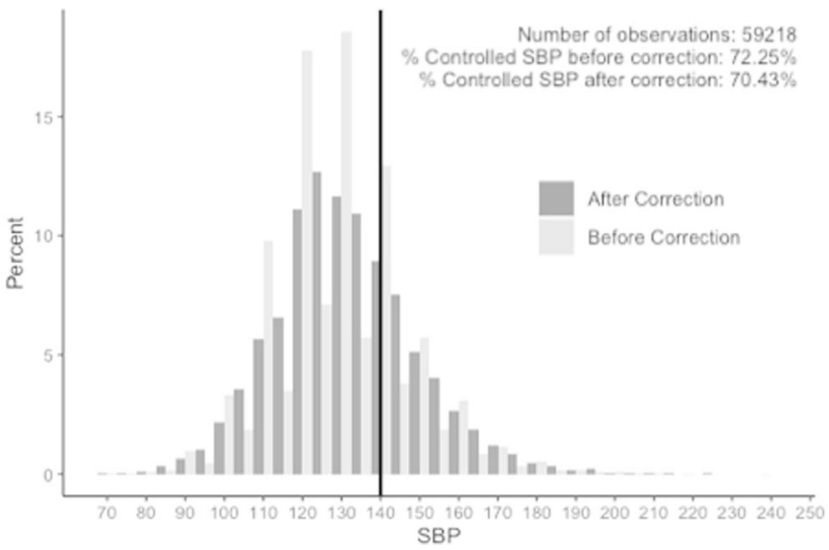

\section{TEST ORDERING AND RESULT ACKNOWLEDGEMENT PRACTICES AT TWO CANADIAN ACADEMIC HEALTH CEN- TRES}

Keith M. Lee ${ }^{1}$; Peter Cram ${ }^{2},{ }^{1}$. ${ }^{1}$ University of Toronto, Toronto, ON, Canada; ${ }^{2}$ University of Iowa, Toronto, ON, Canada. (Control ID \#3184584)

BACKGROUND: Physician test ordering practices have been shown to differ according to physician demographics characteristics and practice setting. Few studies have compared inpatient test ordering intensity between physicians from different specialties. Similarly, there is a paucity of data examining the utilization of electronic test result acknowledgement systems despite strong evidence that these systems can reduce "missed" test results. Moreover, the vast majority of studies come from the US. We examined physician test ordering and result acknowledgement practices at two large Canadian academic hospitals.

METHODS: Using electronic health record (EHR) data, we identified all patients hospitalized on 5 different clinical services (Critical Care, General Surgery, General Internal Medicine, Nephrology, and Orthopedics) at Toronto General Hospital and Toronto Western Hospital between February 1, 2017 and February 28, 2017. We obtained data including patient demographics, hospital length of stay, hematology and biochemistry test orders and associated results, and result urgency level (categorized as normal, abnormal, or critical). We reviewed EHR data regarding whether tests were acknowledged by the ordering physician in his/her electronic "in-box" at any time after the results were finalized. We compared testing intensity (tests ordered per-patient per-admission and per-day), result urgency, and acknowledgement status between clinical services using two-sided Chi-squared tests.

RESULTS: Our 5 clinical services had a total census of 1571 patients over our study window. Mean length of stay was 15.0 days. The mean number of hematology-plus-biochemistry tests per-patient per-admission was lowest for Orthopedics (106.9) and highest for Critical Care (537.1). In contrast, the mean number of hematology-plus-biochemistry tests perpatient per-day was lowest for Critical Care (6.7) and highest for General Internal Medicine (23.4). 7.4\% of all tests were reported as critical, ranging from $2.6 \%$ in Nephrology to $8.8 \%$ in General Internal Medicine. $27.2 \%$ of all test results were reviewed by a physician using the EHR "inbox" acknowledgement function. $38.5 \%$ of tests for patients admitted to General Internal Medicine were reviewed using the "in-box" function, as compared to $9.9 \%$ in General Surgery, $13.3 \%$ in Orthopedics, $15.5 \%$ in Nephrology, and $28.9 \%$ in Critical Care.

CONCLUSIONS: Test ordering intensity varies markedly across specialty. In our health system, a significant proportion of tests are flagged as critical, raising concern of alert fatigue particularly for General Internal Medicine where critical results are most common. Physician utilization of our result acknowledgement system was low, which is concerning given evidence of the substantial problem of overlooked (or "missed") test results.

\section{THE "KIND CARE BUNDLE" - A TRANSITION TOWARD COM-} PASSIONATE CARE

Cynthia M. Cooper ${ }^{1,2}$; Galina Gheihman ${ }^{2} .{ }^{1}$ Massachusetts General Hospital, Boston, MA; ${ }^{2}$ Harvard Medical School, Boston, MA. (Control ID \#3180251)

BACKGROUND: Negative behaviors in practicing clinicians are often learned during medical training through observation and mimicry - the socalled "hidden curriculum." Compassion and empathy are inconsistently reinforced in exchange for efficiency. This devaluing of kindness and connection may contribute to clinician burnout and result in suboptimal patient care. To counteract this trend, we developed a curriculum emphasizing self-reflection and teaching concrete verbal and non-verbal behaviors for showing attention, consideration, and compassion in patient interactions. The "Kind Care Bundle" was taught to medical students immediately prior to the start of their core clinical rotations. METHODS: The Kind Care session was taught at Massachusetts General Hospital in Aug-Sept 2018 over 5 weeks.

Groups consisted of 7-8 students. In each 3-hr. session, students first recalled a time when they felt cared for or witnessed compassionate care. These experiences were discussed to identify verbal and non-verbal demonstrations of care. The "Kind Care Bundle" was introduced with the Three Es: Entry, Encounter and Exit. The Three Es outline concrete behaviors for showing attention, consideration and compassion in patient interactions. Using question prompts, pairs of students interviewed inpatients to practice the Three Es and solicit patients' perspectives on compassionate care. Finally, students debriefed after the interviews and completed a postsession feedback survey. Mixed-methods analysis was used to evaluate Likert scale statements and free-text responses.

RESULTS: 37 (92.5\%) of 40 students completed the feedback form. The session organization was evaluated as 'Excellent' by $73 \%$ of students and 'Very Good' by $27 \%$. The session relevance was evaluated as 'Excellent' by $81 \%$ of students and 'Very Good' by $16 \%$. Students reported the bundle filled an educational gap. Major themes in the qualitative analysis included: (1) appreciation of the sessions' emphasis on Concrete Behaviors: 'It's hard to teach 'being kind'... but these concrete actions can be useful and important to put into action,' (2) the Importance of Empathy: 'Getting the patient's perspective on Kind Care is clutch' and (3) the Opportunity to Reflect on one's own experience of compassion: 'A light bulb went off! I was like 'duh' that's how we need to treat patients. 'Motivations for using the bundle in the future included Impact on Patient Experience, Clinical Success, Professional Ideals and Self-Care.

CONCLUSIONS: The Kind Care curriculum is an educational activity that prompts students to reflect on their own experiences of compassionate care and practice a bundle of concrete behaviors to improve student/patient interactions. The pilot session was highly rated by pre-clinical students at Massachusetts General Hospital. A next step is expansion to all pre-clinical students at Harvard Medical School. It may have use in other student and clinician populations and at other institutions.

\section{THE ART OF PRECEPTING: INNOVATIVE STRATEGIES TO PREPARE SENIOR RESIDENTS FOR CLINIC PRECEPTING}

Rebecca Davis ${ }^{3}$; Ehren K. Dancy ${ }^{2}$; Emily Sandberg ${ }^{4}$; Deepa R. Nandiwada $1 .{ }^{1}$ University of Pennsylvania, Ardmore, PA; ${ }^{2}$ Hospital of the University of Pennsylvania, Philadelphia, PA; ${ }^{3}$ Thomas Jefferson University, Philadelphia, PA; ${ }^{4}$ University of Pennsylvania, Philadelphia, PA. (Control ID \#3185994)

BACKGROUND: Our aim was to improve senior resident confidence regarding the transition to attending through a reverse direct observation preceptor curriculum. The curriculum is two pronged first with a direct observation component where residents observe attendings with guided facilitation sheets followed by attending observation of senior residents 
precepting medical interns (junior precepting). Although the concept of "junior precepting" is not new, the idea of a facilitated direct observation to better prepare residents for the junior precepting experience is novel.

METHODS: Residents and attendings in an internal medicine primary care track were involved in the development of a reverse direct observation precepting curriculum During resident observation of attendings, an assessment tool was created to evaluate the attending's preceptor "toolkit" highlighting microskills from the one-minute preceptor as well as a guided one on one discussion with the preceptor about their personal practices. Residents were then asked to commit to trying something they observed during their scheduled junior precepting sessions. The assessment tool included open ended questions regarding attending precepting style on topics such as attending chart review time/verification, presence vs absence of documentation during precepting, and use of electronic for teaching. RESULTS: Six attendings were observed by six different senior residents in the primary care program for a total of 30 observed encounters. In analysis of the attending preceptor toolkit, the median attending practice was to seek a commitment to plan as well as reinforce clinical decisions done well with correction of mistakes in half of the cases. Probing learner thought process for arrival of plan commitment was not done during the majority of encounters. Qualitatively attending style at this clinic was split between writing notable past medical history and listening to the resident while reviewing the chart. \The majority of attendings spent less than 5 minutes chart reviewing. The tech most often used centered around EMR optimization tips to streamline guidelines into management. Under the tips and behaviors section, resident observers most often cited specific phrasing that helped to elicit further information from the learner without creating a negative atmosphere for learning. In this single site assessement of this novel reverse direct observation precepting curriculum, initial data suggests that resident observers may benefit from observation sessions both prior to and following their own mock-precepting sessions to better prepare them for a junior precepting experience.

CONCLUSIONS: In this single site assessement of this novel reverse direct observation precepting curriculum, initial data suggests that resident observers may benefit from observation sessions both prior to and following their own mock-precepting sessions to better prepare them for a junior precepting experience.

\section{THE ASSOCIATION BETWEEN OPIOID DOSE FLUCTUATION AND INCIDENT HEROIN USE}

Ingrid A. Binswanger ${ }^{1,2}$; Komal J. Narwaney ${ }^{1}$; LeeAnn M. Quintana ${ }^{1}$; Stanley $\mathrm{Xu}^{1}$; Susan M. Shetterly ${ }^{1}$; Jennifer Lyden ${ }^{4,}{ }^{2}$; Jo Ann Shoup ${ }^{1}$; Jason M. Glanz ${ }^{1,3}$. ${ }^{1}$ Kaiser Permanente Colorado, Aurora, CO; ${ }^{2}$ University of Colorado School of Medicine, Aurora, CO; ${ }^{3}$ Colorado School of Public Health, Aurora, CO; ${ }^{4}$ Denver Health, Denver, CO. (Control ID \#3185991)

BACKGROUND: Opioid guidelines recommend tapering opioids for chronic pain if the risks of treatment outweigh the benefits. However, attempts to taper opioids can lead to dose fluctuation. Tapering attempts can cause increased pain and withdrawal symptoms, which may lead providers to increase the dose again or prompt individuals to seek illicit opioids to control their pain. Dose increases could enhance the euphoric effects of opioids, increasing the risk for addiction and heroin use. Thus, dose fluctuations may increase the likelihood that individuals seek heroin. We sought to examine the association between opioid dose fluctuation and incident heroin use.

METHODS: We conducted a matched nested case-control study in a Colorado-based integrated health system. We first defined a cohort of health plan members 18 between January 2006 and May 2018 on chronic opioid therapy (COT; 3 opioid dispensings in 90 days). The date of the $3 \mathrm{rd}$ opioid dispensing was the index dose date. Within the cohort, we identified heroin use that came to medical attention in the electronic health record using text string searches. This was followed by detailed medical record review by trained abstractors to verify heroin use and estimate the date of heroin onset, which represented the index date. Incident cases of heroin use after COT initiation in continuously enrolled patients were matched to up to 20 controls who did not have evidence of heroin use on milligrams morphine equivalents (MME), calendar time, and length of follow-up. The exposure of interest was dose fluctuation, defined as greater than the median of the standard deviation of the dose over each control patient's follow-up (8.6 $\mathrm{MME}$ ). Conditional logistic regression was used to estimate matched odds ratios (mOR) and 95\% confidence intervals (CI), adjusting for age, sex, race/ethnicity, insurance type, mental health diagnosis, and mean MME in the 3 months prior to the index date.

RESULTS: In the cohort of 23,144 patients receiving COT, the text string search identified $1,731(7.5 \%)$ patients with mention of heroin use. After medical record review, we identified 261 confirmed cases for whom we could estimate a heroin onset date. Among these, the first occurrence of heroin use occurred after COT started in 52 cases. Three cases could not be matched to a control. Cases $(n=49)$ were more likely than controls $(n=866)$ to be young, male, Latino, and insured by Medicaid. Dose fluctuation was strongly and independently associated with the risk of heroin use (mOR=5.87, 95\% CI, 2.05-16.27).

CONCLUSIONS: Among patients prescribed COT, we identified a strong association between opioid dose fluctuation and the risk of heroin use. Our measure of heroin use was based on documentation in addiction treatment and other medical settings. We did not evaluate whether fluctuation in dose exacerbated prior or current heroin use. When managing COT, physicians should consider heroin use as a potential sequela of dose fluctuation.

\section{THE ASSOCIATION BETWEEN SLEEP OPPORTUNITY AND INCIDENCE OF DELIRIUM IN GENERAL MEDICINE INPA- TIENTS}

David Sterken ${ }^{1}$; Rae Wannier ${ }^{2}$; Priya A. Prasad ${ }^{2}$; Yumiko Abe-Jones ${ }^{1}$; Nader Najafi ${ }^{1}$; Margaret Fang ${ }^{1}$; Kirsten Kangelaris ${ }^{1}$; Stephanie E. Rogers ${ }^{1}$; Sara C. LaHue ${ }^{1}$; Vanja Douglas ${ }^{1}$; Ari Hoffman ${ }^{1} .{ }^{1}$ UCSF, San Francisco, CA; ${ }^{2}$ UCSF, Berkeley, CA. (Control ID \#3184403)

BACKGROUND: Overnight interruptions in the hospital are thought to worsen sleep and increase patients' risk of delirium, but this relationship has not been well-quantified. Our group has developed a novel metric of overnight interruptions called "sleep opportunity." Using this metric, we conducted a single-center retrospective analysis to describe the association between overnight interruptions and the incidence of delirium in general medicine inpatients.

METHODS: Using our Epic-based electronic health record, we identified all admissions to the adult general medicine service at an 800-bed urban teaching hospital from April 9, 2017 to November 30, 2018. We excluded patients who were delirious on admission, had a Glasgow Coma Scale $<10$ within 24 hours of admission, or were in the intensive care unit during admission. The primary outcome was incident delirium, defined as the first positive score on the Nursing Delirium Screening Scale (NuDESC), which is a validated delirium screening tool that is assessed each shift as part of routine nursing care with score 2 defined as positive. The primary predictor was sleep opportunity per night, defined as the longest continuous block of time without interruptions by hospital staff between 10:00 PM and 6:00 $\mathrm{AM}$, calculated using timestamps for tasks such as vital signs, medications, and procedures. We developed a multivariable mixed-effects logistic regression model to assess the relationship of quartiles of sleep opportunity to the incidence of delirium during the following 24 hours and adjusted for confounders using a propensity score for quartiles of sleep opportunity. This propensity score included age, Elixhauser Comorbidity Index, diagnosis-related group weight, admission AWOL score (screening tool for risk of developing delirium), prior diagnosis of dementia by ICD codes in previous encounters, whether vital signs were ordered to be checked overnight, and whether a delirium-prevention order set had been ordered. We also included level of care as a fixed effect in our model.

RESULTS: We identified 8,913 eligible admissions during our study period with 20,278 eligible patient-nights. The mean sleep opportunity per patient-night was $4.98(\mathrm{SD}=1.75)$ hours. The incidence of delirium was 
$10.39 \%$ ( $\mathrm{SD}=0.32$ ) of admissions. Compared to the highest quartile of sleep opportunity (6.57-8.00 hours), the lowest quartile of sleep opportunity (0.93-3.62 hours) was associated with a significantly increased odds of incident delirium (adjusted odds ratio 1.80 [95\% CI: 1.11-2.93]). The middle quartiles (3.62-4.67 hours and 4.67-6.57 hours) were not significantly different from the highest quartile (adjusted odds ratios $1.36[0.84$ $2.22]$ and 1.56 [0.98-2.46], respectively).

CONCLUSIONS: Lower sleep opportunity overnight in the hospital was associated with an increased risk for incident delirium, highlighting the clinical importance of minimizing unnecessary interruptions. These results also show that our sleep opportunity metric has clinically meaningful correlates.

\section{THE ASSOCIATION BETWEEN SUBCLINICAL HYPOTHYROID- ISM AND CARDIOVASCULAR RISK FACTORS ACCORDING TO AGE AND GENDER - AN INDIVIDUAL PARTICIPANT ANALYSIS OF LARGE INTERNATIONAL COHORTS}

Manuel R. Blum ${ }^{1,3}$; Oliver Baretella ${ }^{1,2}{ }^{2}$; Lea Wildisen ${ }^{2}$; Elisavet Moutzouri $^{1,2}$; Cinzia Del Giovane ${ }^{2}$; Douglas Bauer ${ }^{4}$; Anne R. Cappola $^{5}$; Bjørn O. Åsvold ${ }^{6}$; Jacobijn Gussekloo ${ }^{7}$; Massimo Iacoviello $^{8}$; Giorgio Iervasi ${ }^{9}$; Misa Imaizumi ${ }^{10}$; Stefan Weiler ${ }^{11}{ }^{12}$; Kay-Tee Khaw ${ }^{13}$; Salman Razvi ${ }^{14}$; José A. Sgarbi ${ }^{15}$; Henry Völzke $^{16}$; John P. Walsh ${ }^{17}$; Rudi G. Westendorp ${ }^{18}$; Drahomir Aujesky ${ }^{1}$; Martin Feller ${ }^{1,2}$; Nicolas Rodondi ${ }^{1,2}{ }^{2}{ }^{1}$ Inselspital, Bern University Hospital, Bern, Switzerland; ${ }^{2}$ Institute of Primary Health Care (BIHAM), University of Bern, Bern, Switzerland; ${ }^{3}$ Stanford University School of Medicine, Stanford, CA; ${ }^{4}$ University of California, San Francisco, San Francisco, CA; ${ }^{5}$ University of Pennsylvania School of Medicine, Philadelphia, PA; ${ }^{6}$ Norwegian University of Science and Technology, Trondheim, Norway; ${ }^{7}$ Leiden University Medical Center, Leiden, Netherlands; ${ }^{8}$ University of Bari, Bari, Italy; ${ }^{9}$ National Research Council, Institute of Clinical Physiology, Tuscany Region G. Monasterio Foundation, Pisa, Italy; ${ }^{10}$ Radiation Effects Research Foundation, Nagasaki, Japan; ${ }^{11}$ University Hospital Zurich, Zurich, Switzerland; ${ }^{12}$ National Poisons Information Centre, Tox Info Suisse, Associated Institute of the University of Zurich, Zurich, Switzerland; ${ }^{13}$ University of Cambridge, Cambridge, United Kingdom; ${ }^{14}$ Newcastle University, Institute of Genetic Medicine, Newcastle upon Tyne, United Kingdom; ${ }^{15}$ Faculdade de Medicina de Marília, Marília, Brazil; ${ }^{16}$ University of Greifswald, Institute for Community Medicine, Clinical-Epidemiological Research, Greifswald, Germany; ${ }^{17}$ The University of Western Australia, Crawley, WA, Australia; ${ }^{18}$ University of Copenhagen, Copenhagen, Denmark. (Control ID \#3169994)

BACKGROUND: Subclinical hypothyroidism (SHypo) has been linked to an increased risk for coronary heart disease (CHD). Moreover, SHypo has been associated with several modifiable cardiovascular risk factors, but population-based data on these associations are inconsistent. Also, the impact of hormonal substitution in SHypo on CHD remains unclear with the lack of adequately powered trials. By pooling individual participant data from large prospective cohort studies, this international cross-sectional study assessed the association between SHypo and cardiovascular risk factors across wide age ranges to help clarify the possibly increased risk for cardiovascular events in patients with SHypo.

METHODS: Cardiovascular risk factors (arterial hypertension, dyslipidemia, inflammatory status as determined by hs-CRP) as primary outcomes were assessed in euthyroid (TSH 0.45-4.5 mU/ 1) and participants with SHypo (TSH $>4.5 \mathrm{mU} / 1$, normal free thyroxine) from large cohorts within the Thyroid Studies Collaboration (www.thyroid-studies.org). Participants on exposure-/outcome-modifying medications [antihypertensives, lipid-lowering drugs, antidiabetics, and (anti-)thyroid medication] were excluded and analyses stratified by gender due to significant interactions. Multilevel mixed-effects linear regression models adjusted for age and smoking status were used.

RESULTS: Among 50,045 participants (mean age 58, range 18100 years, $59 \%$ women, $27 \%$ current smokers) from 13 international cohorts, 2,832 (6\%) individuals had SHypo. All associations significantly differed by gender ( $p$ for interaction $<0.001$; HDLcholesterol $p$ for interaction=0.03), except for hs-CRP ( $p$ for interaction=0.60). Systolic blood pressure was higher with SHypo vs euthyroidism in women $(138 \pm 10$ vs $135 \pm 11 \mathrm{mmHg}, p<0.01)$, but not in men $(138 \pm 7$ vs $138 \pm 7 \mathrm{mmHg}, p=0.70)$ as was total cholesterol (women $6.10 \pm 0.56$ vs $6.03 \pm 0.52 \mathrm{mmol} / 1, p<0.001$; men $5.41 \pm 0.53$ vs $5.74 \pm 0.41 \mathrm{mmol} / 1, p=0.39$ ). With SHypo HDLcholesterol levels were lower (women $1.48 \pm 0.06$ vs $1.51 \pm 0.05$ $\mathrm{mmol} / 1, p<0.001$; men $1.16 \pm 0.04$ vs $1.23 \pm 0.04 \mathrm{mmol} / 1, p<0.001$ ) and triglyceride levels higher (women $1.50 \pm 0.24$ vs $1.38 \pm 0.22$ $\mathrm{mmol} / 1, p<0.001$; men $1.68 \pm 0.31$ vs $1.67 \pm 0.24 \mathrm{mmol} / 1, p<0.05)$ in both women and men, while LDL-cholesterol levels were slightly higher only in women $(4.16 \pm 0.56$ vs $4.12 \pm 0.55 \mathrm{mmol} / \mathrm{l}$, $p<0.001$; men $3.68 \pm 0.51$ vs $3.96 \pm 0.44 \mathrm{mmol} / 1, p=0.83)$. hs-CRP levels were similar in SHypo vs euthyroidism for both genders (women $2.01 \pm 0.70$ vs $1.91 \pm 0.67 \mathrm{mg} / 1, p=0.24$; men $1.81 \pm 0.67$ vs $1.64 \pm 0.68 \mathrm{mg} / \mathrm{l}, p=0.39$ ). Cardiovascular risk factors did not significantly differ across age groups.

CONCLUSIONS: SHypo is associated with elevations of traditional cardiovascular risk factors (but not hs-CRP levels) that differ according to gender, but not age. However, the differences may not be of clinical relevance as they are small. Therefore, cardiovascular risk factors do not appear to be a strong argument to substitute SHypo.

\section{THE ASSOCIATION OF LANGUAGE PREFERENCE AND OUTCOMES IN HISPANICS WITH ATRIAL FIBRILLA- TION}

Ephraim Leiderman $^{1}$; Faraj Kargoli ${ }^{2}$; Jeremy Miles ${ }^{1}$; John Fisher $^{2}$; Kevin Ferrick ${ }^{2}$; Andrew Krumerman ${ }^{2}$. ${ }^{1}$ Jacobi Medical Center, New Rochelle, NY; ${ }^{2}$ Montefiore Medical Center, Bronx, NY. (Control ID \#3179846)

BACKGROUND: According to the U.S. Census Bureau in 2011, $21 \%$ of Americans speak a language other than English at home. Of non-English speakers, $13 \%$ identify as Spanish-speaking primarily. This likely results in many Spanish-speaking individuals visiting physicians who cannot communicate with them in their primary language. The association between language preference and outcomes in atrial fibrillation is unknown.

METHODS: This study analyzed the association of language preference with length of hospital stay and all-cause mortality in Hispanics admitted with atrial fibrillation. This is a retrospective study of Hispanic patients aged 18 years or older admitted with a primary diagnosis of atrial fibrillation between the years 2000 and 2010. Deaths were ascertained from the social security index as well as records form the New York Department of Health. Multivariable logistic regression models were used to determine predictors of survival adjusted for age and comorbidities.

RESULTS: A total of 957 patients were identified with a mean follow up of 3.7 years. $476(50 \%)$ patients reported Spanish as their primary language. Overall mortality was higher in the Spanish-speakers relative to English-speakers. (34\% vs 26\%, p < $0.001)$. After adjusting for age and co-morbidities, Hispanic Spanish-speakers did not have increased mortality when compared to Hispanic English-speakers (OR: 1.063, CI: 0.777-1.454). However, Spanish language preference was associated with a significantly increased length of hospital stay (4.50 days (95\% CI 4.20$4.80)$ vs 5.45 days (95\% CI 5.01-5.89)).

CONCLUSIONS: This study illustrates the importance of recognizing the association of an increased length of hospital stay and Spanish language preference in hopes of improving the efficiency 
of atrial fibrillation management and decreasing the cost of hospitalization.

THE ASSOCIATION OF SHARED DECISION-MAKING FOR DIABETES PREVENTION WITH BLOOD PRESSURE CONTROL AND HYPERTENSION MEDICATION USE: 12MONTH RESULTS FROM THE PREDIABETES INFORMED DECISION AND EDUCATION (PRIDE) STUDY O. Kenrik Duru ${ }^{1}$; Norman Turk ${ }^{1}$; Tannaz Moin ${ }^{2}$; Kia Skrine Jeffers ${ }^{3}$; Yelba Castellon-Lopez ${ }^{3}$; Chi-Hong Tseng ${ }^{4}$; Amanda V. $\mathrm{Vu}^{5}$; Keith Norris ${ }^{1}$; Carol Mangione ${ }^{5} .{ }^{1}$ UCLA, Los Angeles, CA; ${ }^{2}$ UCLA/VA Greater Los Angeles, Los Angeles, CA; ${ }^{3}$ University of California, Los Angeles, Los Angeles, CA; ${ }^{4}$ ucla, Los Angeles, CA; ${ }^{5}$ David Geffen School of Medicine at UCLA, Los Angeles, CA. (Control ID \#3185754)

BACKGROUND: Intensive lifestyle change (e.g., the Diabetes Prevention Program/DPP) and metformin both reduce type 2 diabetes risk. Shared decision-making (SDM) may help patients select the option for diabetes prevention best aligned with their preferences. The PRIDE study of SDM for diabetes prevention resulted in greater uptake of both lifestyle change and metformin, as well as greater weight loss at 12 months. In the current analyses, we examined the effect of PRIDE on change in blood pressure (BP) control and hypertension (HTN) medication use from baseline to 12-month follow-up.

METHODS: PRIDE is a cluster-randomized trial of SDM for diabetes prevention within a large regional health system. PRIDE enrolled overweight/obese adults with prediabetes (HbAlc 5.7$6.4 \%$ ) from 10 randomly selected SDM intervention clinics, and used propensity score matching to identify control patients from 10 randomly selected usual care clinics. Intervention participants were invited to participate in a face-to-face SDM visit with a pharmacist who used a decision aid (DA) to engage participants in possible options for diabetes prevention; DPP, DPP +/- metformin, metformin only, or usual care. The endpoints for the current analyses were change from baseline to 12 months in 1) BP control defined by $<140 / 90$ and 2) number of HTN medication classes. To assess BP at 12 months, we used the closest available BP measured in clinic within 9-16 months of baseline. We classified HTN medications into 13 classes: thiazide diuretics, loop diuretics, potassium-sparing diuretics, aldosterone receptor blockers, beta-blockers, ACEs, ARBs, direct renin inhibitors, non-dihydropyridine CCBs, dihydropyridine CCBs, alpha blockers, centrally acting medications and direct vasodilators. Endpoints were extracted from the local electronic health record. Analyses were adjusted for clinic but no other covariates.

RESULTS: PRIDE included 515 intervention participants and 1,296 controls with measured BP at baseline, of whom 185 intervention and 552 control participants had an existing HTN diagnosis. We had complete data on 12 month BP values for 456 intervention participants (88.5\%) and 1,046 control participants $(80.7 \%)$. As compared to control participants (72.4\%), intervention participants $(77.4 \%)$ were more likely to have controlled blood pressure at baseline $(\mathrm{p}=0.04)$. However, there was no statistical difference in change of blood pressure control between groups over time $(\mathrm{p}=0.82)$. Among participants with diagnosed HTN, there was no difference in the mean number of anti-hypertensive medication classes taken by intervention (1.58) and control (1.56) patients at baseline $(\mathrm{p}=0.87)$ There was also no statistical difference in the change in number of antihypertension medication classes between groups over time ( $\mathrm{p}=0.27)$.

CONCLUSIONS: A prediabetes SDM intervention led by pharmacists was not associated with improvements in BP control or differences in the number of HTN medications at 12 months, in an intention-to-treat analysis. THE CONFIDENT COUNSELOR: THE IMPACT OF A CULINARY NUTRITION CURRICULUM ON MEDICAL STUDENTS' READINESS TO DISCUSS HEALTHY LIFESTYLE
INTERVENTIONS WITH THEIR PATIENTS

Nathan Wood ${ }^{1}$; Rebecca Gleit ${ }^{2}$; Diane L. Levine ${ }^{3} .{ }^{1}$ Wayne State University, $\overline{\text { New York, NY; }}{ }^{2}$ Stanford University, Stanford, CA; ${ }^{3}$ Wayne State University, Detroit, MI. (Control ID \#3181165)

BACKGROUND: The World Health Organization estimates that $80 \%$ of non-communicable diseases could be prevented if 4 key lifestyle practices were followed, the first of which is a healthy diet. For many, education on this tenet comes from their physician. However, as few as $20 \%$ of medical schools currently offer a dedicated nutrition course, and physicians perform lifestyle counseling in as few as $34 \%$ of their patient encounters. We hypothesized that an engaging culinary nutrition course intervention would better equip medical students to counsel their future patients on a healthy diet.

METHODS: With all first-year medical students at our school eligible, 10 participants enrolled in a voluntary, 4-part, 8-hour culinary nutrition course comprised of culinary-nutritional instruction and hands-on cooking in small groups. Assessment surveys were completed immediately prior to, immediately following, and 2 months after the intervention. Surveys included selfreported measures of attitudes regarding teaching healthy diet principles and an objective evaluation of culinary knowledge. The primary outcome was within-subject changes in attitude regarding counseling patients on the tenets of a healthy diet. Secondary outcomes included within-subject improvements and sustainability of culinary knowledge.

RESULTS: Overall survey completion was $99 \%$ across the 3 time-points. At baseline, the participants reported - on a 10-point scale $(10=$ highest $)$ high confidence in their ability to counsel patients on practicing a healthy diet based on their medical knowledge (mean 6.0 [95\% confidence interval 5.1, 6.9]) and nutritional knowledge $(5.9[5.3,6.5])$ and low confidence based on their culinary knowledge $(4.5[3.3,5.7])$. Immediately post-intervention, participants' confidence in their nutritional knowledge (standardized mean difference 2.12 [95\% confidence interval 1.02, 3.21]) and culinary knowledge $(1.99[0.92,3.06])$ significantly improved. There were no statistically significant differences between the immediately post-intervention and 2 months post-intervention timepoints $(P=0.189,0.494)$. Overall, the participants reported feeling more prepared to counsel patients on a healthy diet immediately post-intervention compared to baseline $(1.93[0.87,2.99])$, and this did not change significantly 2 months later $(P=0.322)$. Scores on the objective test of culinary knowledge were significantly improved at both immediately post-intervention $(3.20[1.84,4.56])$ and 2 months post-intervention (1.09 $[0.12,2.05])$ compared to baseline.

CONCLUSIONS: A culinary nutrition pilot course improved medical students' culinary-nutritional knowledge and confidence in counseling patients. Gains were maintained for 2 months post-intervention. Adding a culinary nutrition course to medical school curricula may result in improved abilities of students to provide dietary counseling. Further studies are needed to confirm results.

THE DOCTOR SAYS YOU CANNOT HAVE IT: A QUALITATIVE STUDY OF NON-PRESCRIPTION BUPRENORPHINE USE AMONG PARTICIPANTS AT HARM REDUCTION AGENCIES Benjamin Hayes $^{1}$; Andrea Jakubowski ${ }^{1}$; Aaron D. Fox ${ }^{2} .{ }^{1}$ Montefiore Medical Center, New York, NY; ${ }^{2}$ Montefiore Medical Center, Bronx, NY. (Control ID \#3185960)

BACKGROUND: Buprenorphine is an effective opioid use disorder (OUD) treatment. Barriers to care limit access to formal buprenorphine treatment, but people with OUD may use diverted buprenorphine (i.e., acquired without a prescription) to "self-treat" OUD. Few studies have explored diverted buprenorphine use in detail. This qualitative study investigated why people use diverted buprenorphine instead of seeking prescriptions. METHODS: In-depth, semi-structured interviews were conducted at harm reduction agencies in New York City. Participants were adults with selfreported OUD and prior buprenorphine use. Transcribed interviews were independently coded by three investigators, who generated preliminary themes from the coded data. Thematic analysis entailed iterative reading of 
transcripts with identification of common patterns and relationships between themes.

RESULTS: Of the 17 participants, most were latinx, mean age was 42, all primarily used heroin, and one had an active buprenorphine prescription. The overarching goal of all participants was to maintain control over their drug use without being reliant on healthcare providers. We identified 3 primary themes that reflect this goal: 1 . Diverted buprenorphine is useful as a short-term tool to moderate heroin use. Participants used diverted buprenorphine as either: a) bridging to prevent opioid withdrawal when heroin was unavailable, or b) temporarily unwanted, or self-detox, to assist in stopping heroin use. 2. Seeking a prescription for buprenorphine was less desirable than use of diverted buprenorphine for two main reasons: The barriers to care: "They told me they want to send me something in the mail, but I never received nothing. I bought [suboxone] on my own. If I'm going to go through a lot of things [a prescription is] not better"; or concerns that the prescription could be withdrawn: "Something will happen that they will shut you off and then you have to withdraw. That's why I went to the heroin because I could find it anytime I wanted. With suboxone, you cannot do that once the doctor says you cannot have it." 3 . The pharmacologic effects of buprenorphine were not ideal for some participants. This fell within two common scenarios: participants who were not ready to stop heroin use, and those concerned that buprenorphine would not prevent withdrawal. 4. Buprenorphine may be taken for euphoric effects, but this was during limited circumstances.

CONCLUSIONS: Participants expressed motivations to maintain control over their drug use and sometimes used diverted buprenorphine to meet these objectives. Some participants desired to continue heroin use, which limited engagement in formal buprenorphine treatment. Other participants expressed concerns that prescribed buprenorphine was difficult to access or could be withdrawn by the prescriber, which made diverted buprenorphine more desirable. Though buprenorphine treatment may not meet the needs of some people with OUD, those seeking buprenorphine should have easy and reliable access to care.

THE EDUCATION CENTERED MEDICAL HOME IMPROVES PRIMARY CARE TRAINING AND MAY MITIGATE BURNOUT: A RANDOMIZED MEDICAL EDUCATION TRIAL

Bruce L. Henschen ${ }^{1}$; David T. Liss ${ }^{1}$; Blair Golden ${ }^{2}$; Kenzie A. Cameron $^{1}$; Jennifer Bierman ${ }^{3}$; Elizabeth R. Ryan ${ }^{4}$; Diane Wayne ${ }^{3}$; Daniel Evans ${ }^{1}$. ${ }^{1}$ Northwestern University, Chicago, IL; ${ }^{2}$ Northwestern McGaw, Chicago, IL; ${ }^{3}$ Northwestern University Feinberg School of Medicine, Chicago, IL; ${ }^{4}$ Indiana University School of Medicine, Gary, IN. (Control ID \#3184582)

BACKGROUND: Traditional medical student clinical clerkships lack continuity of care, are associated with erosion of student empathy, and may increase burnout. Longitudinal clerkships may improve these outcomes, but rigorous studies are lacking. The Education-Centered Medical Home (ECMH) is a four-year, longitudinal, team-based primary care clerkship emphasizing continuity with patients, peers, and preceptors. We investigated effects of the ECMH on student attitudes and behaviors in a randomized medical education trial.

METHODS: We randomized students from two successive incoming classes to ECMH or to an enhanced Individual Preceptorship (IP). In $\mathrm{ECMH}$, teams of 16 students (4 per class) spend four years in the same primary care clinic, working in pairs with a single outpatient preceptor to care for a medically-complex patient panel. In IP, students spend two years one-on-one with a preceptor, followed by a traditional primary care clerkship in the third year. ECMH students continue their longitudinal clinic while completing other clerkships in the third year. Otherwise, students' curricular hours and learning objectives are equal. We surveyed students four times (baseline, M1,
M2, and M3 year) using the Maslach Burnout Inventory (MBI); the Patient-Physician Orientation Scale (PPOS); the C3 survey assessing the 'hidden curriculum;' and the Attitudes Toward Health Care Teams (ATHCT) scale. We analyzed results using an intent-to-treat approach.

RESULTS: 329 students were randomized; 316 (96\%) completed surveys. In regression analyses controlling for demographics and baseline interest in primary care or pediatrics, ECMH participation resulted in significantly greater continuity of care (mean \# patients actively followed per student, $2.2 \mathrm{ECMH}$ vs. $0.3 \mathrm{IP}, \mathrm{p}<0.001$ ), greater perceptions of positive role modeling from preceptors and peers (C3 mean, 4.6 ECMH vs $4.3 \mathrm{IP}, \mathrm{p}<0.001$ ), and more positive attitudes toward health care teams (ATHCT mean, 3.2 ECMH vs 3.0 IP, p=0.007) compared to IP across all three years. Regardless of initial assignment, $75 \%$ of students would recommend ECMH to a hypothetical incoming medical student. Regarding burnout, there was no effect on emotional exhaustion or cynicism, but ECMH students reported improved professional efficacy over three years (MBI-PE 4.1 ECMH vs 3.9 IP, p = 0.02). ECMH was associated with increasing professional efficacy across the study duration.

CONCLUSIONS: In a randomized medical education trial, students in ECMH reported positive effects on their learning environment, improved attitudes toward team-based care, and accelerated professional development. A four-year, outpatient clerkship model, layered onto a 'traditional' block clerkship curriculum, can produce improvements in primary care education and may promote student resiliency.

THE EFFECT OF A DIGITAL HEALTH INTERVENTION ON DECREASING BARRIERS AND INCREASING FACILITATORS IN COLORECTAL CANCER SCREENING IN VULNERABLE PATIENTS

Nancy M. Denizard-Thompson ${ }^{1}$; Kathryn Weaver $^{2}$; Doug $\mathrm{Case}^{2}$; John G. Spangler ${ }^{3}$; David P. Miller ${ }^{2}$. ${ }^{1}$ Wake Forest University School of Medicine, Winston-Salem, NC; ${ }^{2}$ Wake Forest School of Medicine, Winston-Salem, NC; ${ }^{3}$ Wake Forest School of Medicine, Winston Salem, NC. (Control ID \#3178634)

BACKGROUND: Colorectal cancer (CRC) is a major health problem for vulnerable populations. Understanding the factors that influence receipt of screening is paramount to improving CRC screening rates. In our randomized control trial conducted in a vulnerable population, we found an absolute $15 \%$ increase in CRC screening among patients randomized to the Mobile Patient Technology for Health-CRC (mPATH-CRC) program, an iPad application that informs patients of screening need, helps make a screening decision, allows "self-order", and sends electronic messages to help complete the test. We now investigate what factors led to the success of the intervention and mediated the common known barriers and facilitators of CRC screening.

METHODS: All data for the present analysis was collected as part of a randomized-controlled trial to determine the impact of mPATH-CRC on colorectal cancer screening within 24 weeks. Participants were enrolled from 6 community-based primary care practices between June 2014 and May 2016 and randomized to either usual care or mPATH-CRC. Six potential mediators were considered. These include the ability to form a screening decision $(\mathrm{Y} / \mathrm{N})$, the intent to have colorectal screening in the next month $(\mathrm{Y} / \mathrm{N})$, patient/doctor discussion about colorectal screening $(\mathrm{Y} / \mathrm{N})$, perceived benefits to screening (1-5), perceived barriers to screening (1-5), and self-efficacy (1-5). We used the method proposed by Lacobucci to assess the significance of the mediation, and logistic regression was used to determine which patient characteristics were associated with screening. 
RESULTS: 450 participants who were between the ages of 50 and 74 and due for colorectal screening were randomized. A total of 408 patients had complete data. In unadjusted analyses, participants in the mPATH arm were twice as likely to complete screening compared to the control arm (OR 2.70, 95\% CI 1.664.39). After adjusting for all potential mediators, the odds ratio for mPATH-CRC fell to 1.68 and was no longer statistically significant $(95 \%$ CI 0.98 - 2.88). Significant mediators of screening were perceived benefits, self-efficacy, ability to form a decision, and the occurrence of a patient/doctor screening discussion. The most powerful mediator was the occurrence of a patient/doctor discussion. In the full logistic regression model, only two factors were associated with completing screening: the occurrence of a patient/doctor discussion (OR 6.25, 95\% CI 2.85-13.7) and lower perceived barriers (OR 1.55, 95\% CI 1.00-2.40).

CONCLUSIONS: In summary, mPATH-CRC doubled the proportion of patients who completed CRC screening in a vulnerable patient population. The mPATH program influenced screening by affecting patients' attitudes, knowledge, and beliefs about screening and triggering healthcare provider discussions. The most powerful mediator was engaging in a patient/doctor discussion, highlighting that digital health interventions like mPATH are an important adjunct to the patient/doctor relationship but not a replacement.

\section{THE EFFECT OF A PATIENT DECISION AID ON DECI- SIONAL QUALITY AMONG PATIENTS WITH LUMBAR DISC HERNIATION}

O. Kenrik Duru ${ }^{1}$; Christopher Saigal ${ }^{1}$; Sylvia Lambrechts ${ }^{1}$; Richard Hwang ${ }^{2}$; Aaron Reyes ${ }^{2}$; Lorna Kwan ${ }^{1} .{ }^{1}$ UCLA, Los Angeles, CA; ${ }^{2}$ David Geffen School of Medicine, Los Angeles, CA. (Control ID \#3185687)

BACKGROUND: Among patients with low back pain and sciatica, the most common underlying condition is lumbar disc herniation $(\mathrm{LDH})$. As there are several potential potential first-line treatments, LDH is considered a preference-sensitive condition such that providers should elicit patients' goals and values to help them select the treatment option that best aligns with their preferences. In this study, we compared decisional conflict in patients with lumbar disc herniation who completed an internet-based Patient Decision Aid (PDA) prior to their treatment consultation with patients not using a PDA.

METHODS: Patients seeking treatment for LDH at L4-L5 and/or L5-S1 were offered an internet-based PDA prior to their specialist consultation with a spine surgeon or physiatrist, while patients in usual care were not offered the PDA. All patients completed a post-visit questionnaire, either in person or by phone, which included validated questions from the SURE test for clinical practice, Decisional Conflict Scale (DCS) and the Shared Decision Making Questionnaire. We performed bivariate analyses to examine the association between decisional conflict and measures of decisional quality.

RESULTS: Overall, $40 \%$ in the implementation group $(n=87)$ and $47 \%$ in the usual care group $(n=174)$ reported decisional conflict after their visit $(p=0.253)$, and the implementation group was more likely to know the treatment options than the usual care group $(91 \%$ vs. $83 \%, p=0.004)$. Among patients who reported decisional conflict, the implementation group was more likely to know benefits and risks of the treatment options compared to the usual care group $(72 \%$ vs. $41 \%, p=0.003)$. However, no differences were seen in other comparisons, including satisfaction with the decision, discussion of treatment options with the doctor, or inclusion in the treatment decision. Within the implementation group, patients without decisional conflict were more likely to know treatment options ( $96 \%$ vs $82 \%, \mathrm{p}=0.011)$, expect to stick with their decision $(96 \%$ vs $71 \%, \mathrm{p}=0.003)$, be satisfied with their decision ( $96 \%$ vs. $71 \%, \mathrm{p}=0.003)$, discuss treatment options with their doctor $(92 \%$ vs. $59 \%, \mathrm{p}<0.001)$, and feel included in the treatment decision $(98 \%$ vs. $67 \%, \mathrm{p}<0.001)$ compared to those with decisional conflict.

CONCLUSIONS: Use of a PDA prior to specialty consultation for LDH is associated with an increased likelihood of knowing the different treatment options for this condition, and among those with decisional conflict, greater knowledge of the benefits and risks of each treatment option compared to those in the usual care group. This analysis demonstrates that use of an internet-based PDA for LDH treatment options may not be sufficient in itself to enhance decisional quality among patients with decisional conflict. Further investigation is needed to identify subsets of patients who benefit most from PDA implementation and to enhance strategies that improve shared decision-making.

\section{THE EFFECT OF RED BLOOD CELL TRANSFUSION ON FATIGABILITY IN HOSPITALIZED PATIENTS WITH ANEMIA}

Micah T. Prochaska; Rilwan Babajide; Sarah Bradbury; David Meltzer. University of Chicago, Chicago, IL. (Control ID \#3160994)

BACKGROUND: Anemia is a common condition in hospitalized patients, and fatigue is the primary symptom of anemia. Anemiarelated fatigue is associated with decreased health-related quality of life and poor functional outcomes. Therefore, an important objective of treating anemia with red blood cell transfusion may be to reduce fatigue and increase patient activity. While data from previous studies suggests that transfusion improves fatigue in hospitalized patients with anemia, these studies did not examine whether transfusion and changes in fatigue may also effect patient's activity levels. This is important because if transfusion reduces fatigue, patients may become more active. Thus, the effect of transfusion on patients' fatigue in the context of their activity, a measure known as fatigability, may be a more accurate measure of the benefit of transfusion than fatigue alone. To date, there are no data on the effect of transfusion on fatigability. The objective of this study was to determine whether transfusion during hospitalization improves fatigability 30 days post-discharge.

METHODS: From June 2017-January 2018 hospitalized general medicine patients with a Hemoglobin $(\mathrm{Hb})<10 \mathrm{~g} / \mathrm{dL}$ were approached for an inpatient interview and a 30-day post-discharge phone interview. Fatigability was measured using the Pittsburgh Fatigability Scale (PFS) at both times points. PFS scores range from 0-50, with higher scores indicating greater fatigability (more fatigue at lower activity levels). Patients were categorized into 4 groups based on their inpatient level of fatigability (high or low) and $\mathrm{Hb}(<8 \mathrm{~g} / \mathrm{dL}$ or $8 \mathrm{~g} / \mathrm{dL})$. A "change in fatigability" score was calculated by subtracting PFS scores obtained during the post-discharge phone interview from the inpatient PFS score. Clinical and demographic data were obtained from hospital administrative data. Independent sample t-tests were used to compare changes in fatigability within each of the 4 groups between patients who received a transfusion and those who did not.

RESULTS: 140 participants completed both the inpatient and postdischarge interview. There were no significant baseline differences between those who were transfused and those who were not [(age 53 vs. 53 ; $p=0.94)$, (female $67 \%$ vs. $59 \%$; $\mathrm{p}=0.39$ ), (African American $74 \%$ vs. $69 \%$; $\mathrm{p}=0.76$ ), (Charlson Comorbidity Index 2 vs 2, p=0.59), (median in-hospital PFS 32 vs. $32 ; \mathrm{p}=0.97)$. For patients with high in-hospital fatigability $(\mathrm{PFS}<32)$ and a $\mathrm{Hb}<8 \mathrm{~g} / \mathrm{dL}$, transfusion was associated with improvement in fatigability 30 days after hospital discharge, compared to patients not transfused (14.8 vs $2.2, \mathrm{p}=0.02$ ). The effect of transfusion was not statistically significant in any other group. 
CONCLUSIONS: Transfusion is associated with improvements in fatigability after hospital discharge for patients with high inpatient fatigability and low $\mathrm{Hb}$. This suggests that for highly fatigued hospitalized patients with anemia, transfusion may both reduce fatigue and increase activity.

\section{THE EFFECT OF STARTING THE PROTEIN SPARING MODI- FIED FAST ON WEIGHT CHANGE OVER 5 YEARS: A COHORT ANALYSIS}

Elizabeth $\mathrm{Pfoh}^{1}$; Gilbert Lowenthal ${ }^{1}$; Laura Jeffers ${ }^{1}$; Bartolome Burguera ${ }^{1}$; $\overline{\text { Derrick Cetin }^{1}}$; Bo Hu ${ }^{1}$; Niyati M. Gupta ${ }^{2}$; Michael B. Rothberg ${ }^{1} .{ }^{1}$ Cleveland Clinic, Cleveland, $\mathrm{OH} ;{ }^{2}$ Cleveland Clinic Foundation, Cleveland, $\mathrm{OH}$. (Control ID \#3185236)

BACKGROUND: Ketogenic diets have been highlighted as a way to lose weight while experiencing reduced hunger. The protein-sparing modified fast (PSMF) diet induces ketosis and rapid weight loss but is difficult to maintain. Our objective was to track weight change for individuals initiating a PSMF or contrasting diet for up to five years.

METHODS: This was a retrospective cohort study of patients in a large integrated health system between 2007 and 2014. We included patients who discussed starting the PSMF diet or had a serum uric acid and a registered dietician visit within 30 days. The main predictor was starting the PSMF diet vs another diet. The main outcome was percent weight change from baseline, calculated quarterly. Patients were censored at their final weight or if they underwent bariatric surgery. After fitting a regression model adjusted for demographic, medication use and specialist visits, we compared the effect of diet on weight change at each time point.

RESULTS: Of 1,419 eligible patients, 879 (62\%) started the PSMF. The PSMF group was slightly younger ( 52 vs. $54, \mathrm{p}<0.01$ ), with a higher BMI (41.9 vs. 40.4, p<0.001). In the adjusted analysis, patients in the PSMF diet group averaged $2.7 \%$ more weight loss than patients in the other diet group (95\%CI: -3.5, -2.0, p<0.001) (Figure). PSMF patients lost more weight initially, but by year 4 , there was no difference between the diets. Compared to the control diet, patients starting the PSMF were more likely to experience $5 \%$ weight loss at 1 year ( $55 \%$ vs $20 \%, \mathrm{p}<0.001)$ and 3 years, $(33 \%$ vs. $23 \% \mathrm{p}<0.05)$, but not 5 years( $34 \%$ vs $30 \%, \mathrm{p}=0.16$ ).

CONCLUSIONS: In clinical practice, the PSMF reliably achieves rapid weight loss in the first six months, but only a small percentage of patients are able to maintain significant weight loss long-term.

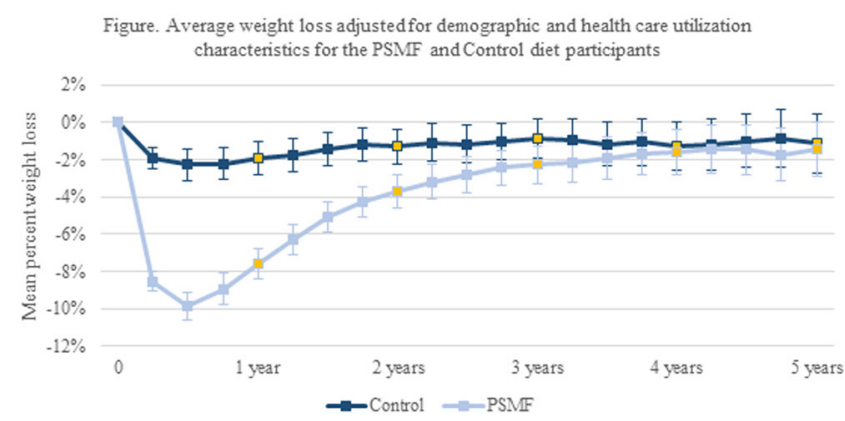

THE FINANCIAL COST OF INTERPROFESSIONAL AMBULATORY TRAINING: WHAT'S THE BOTTOM LINE?

Lauren Block $^{1}$; Adam Block ${ }^{2}$; Joseph Conigliaro ${ }^{3}$; Nancy A. LaVine ${ }^{3}$. ${ }^{1}$ Northwell Health, Lake Success, NY; ${ }^{2}$ New York Medical College, Valhalla, NY; ${ }^{3}$ Northwell Health, New Hyde Park, NY. (Control ID \#3181791)

BACKGROUND: The goal of interprofessional training programs is to improve clinical care and trainee interest in primary care careers by cultivating a cooperative, patient-centered team culture. Initiation and sustainability of interprofessional training programs is predicated upon familiarity with the benefits and costs associated with program operation. We sought to quantify the additional costs associated with running an interprofessional training program by comparing faculty and staff costs to a traditional residency training program at the same training site.

METHODS: The IMPACcT (Improving Patient Access, Care, and cost through Training) program at Northwell Health provides team-based clinical practice, interprofessional (IP) education, and career mentoring to internal medicine residents, medical students, and physician assistant, pharmacy and health psychology trainees. Trainees are supervised by physician, pharmacy, PA, and psychology faculty, with support from a dedicated medical assistant and a patient coordinator. A total of 10 residents are trained annually alongside 60 other IP trainees. Focusing on the additional staffing needs and faculty ratios, we compared annual costs associated with the IP clinic to those of an adjacent traditional clinic which trains 60 residents per year. Both programs meet ACGME staffing requirement for resident supervision, operate on a 4+1 schedule, have equivalent numbers of patient care sessions and have otherwise equivalent overhead costs. As an equal number of patients were seen per resident per week in the IP and traditional models, productivity was considered equivalent. Calculations were made using U.S. Department of Labor median salaries by profession for the state of New York.

RESULTS: Faculty and staff to resident ratios were higher in the IP model than the traditional residency training model. The interprofessional model cost $\$ 11,390$ more per resident per year than the traditional model, a total of $\$ 113,897$ annually for the ten residents in the program. Main costs were reflective of the higher faculty/staff to resident ratios, including additional salary of $\$ 60,292$ for pharmacy, $\$ 15,272$ in psychology, $\$ 22,172$ in physician (faculty:resident 1:2 IP vs 1:2.5), \$8485 in coordinator (1:2 IP vs 1:3), and $\$ 7676$ in medical assistant salaries (1:2 IP vs 1:3). Benefits of improved quality, training and patient experience were not studied in this analysis.

CONCLUSIONS: Operating an interprofessional training program costs an additional $\$ 11,390$ per resident per year, largely in higher faculty and staff to resident ratios and the services of psychology and pharmacy faculty in the IP training clinic. Given this was an interprofessional training program, these costs also funded the training of additional pharmacy, PA, and psychology students. In an academic setting, some of these costs may be funded by grants and other agreements.

\section{THE FIRST 2 YEARS OF THE ACA: QUALITY, COST, UTILIZA- TION, AND PATIENT EXPERIENCE}

David M. Levine ${ }^{1}$; Jeffrey A. Linder ${ }^{2}$; Bruce E. Landon ${ }^{3} .{ }^{1}$ Brigham and Women's Hospital, Boston, MA; ${ }^{2}$ Northwestern University Feinberg School of Medicine, Chicago, IL; ${ }^{3}$ Harvard Medical School, Boston, MA. (Control ID \#3185562)

BACKGROUND: The Affordable Care Act (ACA) expanded insurance coverage to Americans earning less than $400 \%$ of the federal poverty level (FPL) via subsidies for private insurance and, in states that elected it, Medicaid expansion. Prior work has shown resulting improvements in insurance rates and experience with care and decreased high-burden spending among the poor. No nationally-representative analysis has examined the association of the ACA with quality, cost, utilization, and patient experience. METHODS: We analyzed data from the 2011 to 2015 Medical Expenditure Panel Survey (annual response rate: 48\%-55\%), a nationally representative annual survey of non-institutionalized Americans and their respective clinicians, hospitals, pharmacies, and employers. We compared Americans, 18 to 65 years old, earning greater than $(n=29248)$ or less than $(n=71405)$ $400 \%$ of the FPL before (2011 to 2013) and after (2014 and 2015) the ACA using a difference-in-differences (DiD) approach with adjustment for 20 sociodemographic covariates. For quality and experience outcomes, we examined previously published composites based on 43 individual measures: 5 "high-value" care composites (e.g. cancer screening); 3 "low-value" care composites (e.g. inappropriate imaging); an overall patient experience rating; a physician communication composite; and an access-to-care composite. For cost outcomes, we examined total cost of care and out-of-pocket expenditures. For utilization, we examined outpatient, emergency, and inpatient encounters and prescribed medicines. 
RESULTS: Over 2 years of implementation, Americans earning < $400 \%$ FPL compared to $>=400 \%$ FPL received more high-value diagnostic and preventive testing $(\mathrm{DiD},+1.8 \%$; $\mathrm{p}<0.01$ ), but no difference in cancer screening, diabetes care, counseling, or medical treatments. Americans earning $<400 \%$ FPL had similar low-value antibiotic use and medical treatments, but worse low-value imaging ( $\mathrm{DiD},-3.1 \%$; $\mathrm{p}=0.04$ ), improved global health care ratings ( $\mathrm{DiD},+2.2 \% ; \mathrm{p}=0.04)$, and improved physician communication ratings ( $\mathrm{DiD},+2.8 \% ; \mathrm{p}<0.01$ ) with non-significantly better access to care $(\mathrm{DiD},+2.1 \% ; \mathrm{p}=0.14)$. There were no significant differences in utilization of preventive visits ( $\mathrm{DiD},+2.1 \%$; $\mathrm{p}=0.06$ ), inpatient, outpatient, emergency, or pharmacy services, nor in the cost of care (total cost $\mathrm{DiD},+\$ 233 ; \mathrm{p}=0.38$; total out of pocket cost, $-\$ 47 ; \mathrm{p}=0.15$ ).

CONCLUSIONS: In its first 2 years among Americans earning $<400 \%$ FPL, the ACA improved diagnostic and preventive testing and patient experience, worsened low-value imaging, and had minimal to no impact on other quality composites, cost, or utilization. Despite decreasing the uninsurance rate by over $25 \%$, the ACA to date has had a relatively limited impact on the quality of care experienced by lower income Americans. Future efforts are needed to assure access and quality for this population as significant disparities in quality persist.

\section{THE HIGH COST OF LOW VALUE CARE}

Thomas McGinn ${ }^{1}$; Stuart Cohen ${ }^{1}$; Sundas Khan ${ }^{1}$; Safiya Richardson ${ }^{2}$; Michael Oppenheim ${ }^{1}$; Jason J. Wang ${ }^{1}$ ' Zucker School of Medicine at Hofstra/Northwell, Manhasset, NY; ${ }^{2}$ Donald and Barbara Zucker School of Medicine at Hofstra/Northwell, Manhasset, NY. (Control ID \#3186288)

BACKGROUND: A fundamental problem with health care delivery in the United States is that a large percentage of the care delivered is not indicated and often harmful. In many clinical situations there is readily available evidence to inform proper decision making to reduce unnecessary testing and treatments, but that evidence is not utilized. Our research team developed a clinical decision support (CDS) tool based on the Wells' criteria for pulmonary embolism (PE) in which computed tomography pulmonary angiography (CTPA) is not recommended in low probability patients. As part of a cost analysis, we retrospectively characterized the rate of incidental findings on CTPAs performed despite the CDS recommendations.

METHODS: A retrospective analysis was conducted on patients evaluated for suspected PE in the emergency department at two tertiary academic hospitals between August 1, 2015 to July 31, 2017. A fellowship trained cardiothoracic radiologist performed a manual review of the reports of low and very low risk CDS patients with a CTPA result to account for incidental findings. Low risk patients were defined as a negative D-dimer during the visit $(<500 \mathrm{ng} / \mathrm{mL})$ and very low probability patients was determined based on a negative D-dimer and discharged home. For each follow up test recommended, the cost was estimated through their current procedural terminology (CPT) code using the 2018 Medicare physician fee schedule for area 2.

RESULTS: There were 12,759 patient visits representing 11,836 patients. For the 11,836 patients, 5,068 were patients in the low probability category and 3,645 were in the very low probability category. Of the very low probability group, 877 had a CTPA within 24 hours after a negative D-dimer. Review of the follow up recommendations for the 877 low probability patients resulted in $79.8 \%(700 / 877)$ with no follow up, $17.6 \%$ (154/877) had 1 item follow up recommended, $2.5 \%$ (22/877) had 2 items recommended, and $0.1 \%$ (1/877) had 3 items follow up recommended. The total costs of performing 877 CTPAs was $\$ 317,140.74$ and the follow up recommendations cost was $\$ 53,619.79$. If all tests were performed it represented an additional $17 \%$ cost increase compared to only performing the initial CTPA.

CONCLUSIONS: Avoiding unnecessary medical testing is important for several reasons including cost to the patient, health systems, and country. A more concerning issue regarding expense and harm is the potential for unnecessary follow up tests performed which could have been avoided if the initial test had never been performed. The cost estimates were limited by using Medicare standard fees which may underestimate the actual procedure cost and additional evaluation is needed to establish the true cost of unnecessary follow up testing. In addition, CDS tools built to estimate pretest probability of PE and discourage the CTPA use in low-risk patients have been shown to improve the CTPA yield. Seamlessly integrating evidence into care is imperative in the reduction of unnecessary tests.

\section{THE I-HOPE STUDY: IMPROVING HOSPITAL OUTCOMES THROUGH PATIENT ENGAGEMENT}

James D. Harrison $^{1}$; Esther Avitia $^{6}$; Michelle Archuleta ${ }^{2}$. JIm Banta ${ }^{3}$; Joy $\overline{B e n n}^{4}$; Marisha Burden ${ }^{2}$; Vineet Chopra ${ }^{5}$; Rebecca Coker ${ }^{6}$; Shaker Eid ${ }^{7}$; Margaret Fang ${ }^{1}$; Kathlyn Fletcher ${ }^{8}$; Julie K. Hagan ${ }^{7}$; Jawali Jaranilla ${ }^{9}$; Monalisa Mullick ${ }^{10}$; Christopher Nyenpan ${ }^{11}$; Lali Silva ${ }^{4}$; Melissa Wurst $^{10}$; Georgiann Ziegler ${ }^{5}$; Luci Leykum ${ }^{6,12}$. ${ }^{1}$ University of California San Francisco, San Francisco, CA; ${ }^{2}$ Denver Health, Denver, CO; ${ }^{3}$ Intensive Care Unit Patient \& Family Advisory Council, University of California San Francisco, San Francisco, CA; ${ }^{4}$ Minnesota Hospital Association, Saint Paul, MN; ${ }^{5}$ University of Michigan, Ann Arbor, MI; ${ }^{6}$ University of Texas Health at San Antonio, San Antonio, TX; ${ }^{7}$ Johns Hopkins Bayview Medical Center, Baltimore, MD; ${ }^{8}$ Medical College of Wisconsin/Milwaukee VAMC, Tbd, AL; ${ }^{9}$ HealthEast Care System, Saint Paul, MN; ${ }^{10}$ Washington University in St. Louis, Saint Louis, MO; ${ }^{11}$ Society of Hospital Medicine, Washington DC, DC; ${ }^{12}$ South Texas Veterans Health Care System, San Antonio, TX. (Control ID \#3162633)

BACKGROUND: Issues with the quality and safety of hospital care have been well-documented. However, what is less understood are the perspectives of patients, families, caregivers, and other stakeholders regarding what they feel are the most important targets of improvement research. We systematically engaged a broad sample of these groups to solicit their input on important questions and to develop a prioritized agenda to drive Hospital Medicine research and quality improvement.

METHODS: The study was led by 9 researchers, 8 patients, and 2 administrators recruited from 8 academic medical centers, who formed a Steering Committee that managed the project. We applied a collaborative and consultative approach to stakeholder engagement based on validated methods from the James Lind Alliance. This included multiple and iterative stages to our project such as: 1) a multi-pronged, inclusive patient and stakeholder engagement strategy; 2) an online survey of patients and stakeholder organization leadership and members; 3 ) rigorous qualitative thematic analysis; 4) a two-day in-person meeting with patients and stakeholders to prioritize the submitted survey questions and plan dissemination efforts.

RESULTS: In total, 499 respondents (including 267 healthcare providers, 244 patients and caregivers and 63 researchers) from 47 organizations submitted a total of 782 high-priority research questions and suggestions for improving hospital care. These questions were categorized into 73 distinct topics, 53 that were health-system related and 20 disease-specific. Thematic analysis of categories revealed 36 commonly submitted question domains. Following a two-step prioritization process involving the study team and representatives from 30 stakeholder organizations, 11 questions were identified as top priorities (Table 1).

CONCLUSIONS: Through a dynamic and collaborative stakeholder engagement process, key patient and family-centered research questions have been identified and developed. These results can help drive a national conversation in the Hospital Medicine community about how to address these priority areas and should inform stakeholder agencies about key areas of research focus. 


\section{THE IMPACT OF A NOVEL STEPWISE APPROACH TO PRUNING MEDICATIONS IN AN INTERDISCI- PLINARY POLYPHARMACY CLINIC}

Guibin Li; Kelli Barnes; Sara Woods; Stuart Beatty. The Ohio State University, Columbus, OH. (Control ID \#3184613)

BACKGROUND: Polypharmacy refers to the use of multiple medications by a single patient. Undesirable effects of polypharmacy include adverse drug events, medication interactions, poor compliance and increased costs. Several implicit (judgement based) and explicit (evidence based) criteria are utilized to evaluate polypharmacy (Beers, STOPP/START, and Medication Appropriateness Index). However, implicit criteria are time consuming and not feasible for routine clinical practice, while explicit criteria do not accurately evaluate medication effectiveness. A comprehensive yet personalized approach is needed. The aim of this study is to assess the impact of a step-wise approach in an Interdisciplinary Polypharmacy Clinic on reducing medication burden.

METHODS: A single institution prospective cohort study was conducted. The participants included were 18 years old and on 6 chronic medications. All terminally ill or severely demented participants were excluded. The interdisciplinary team included a geriatrician, a pharmacist and a social worker. All participants underwent a 3-step approach to evaluate polypharmacy (Figure). By the end of the visit, the participant was counseled on all medication changes and received a personalized medication record. Quality of life improvements were evaluated with the Short Form-12 Quality of Life health survey (SF-12) at baseline and 1month follow-up. The participant's pre- and post-intervention medication usage and quality of life was compared.

RESULTS: A total of 118 patients were included in the cohort. The mean age was 62.9 years. The mean number of medical comorbidities was 10.5 . Prior to intervention, participants had a mean of 15.65 medications compared to a mean of 13.44 after intervention $(\mathrm{p}<0.001)$, with an average reduction of 2.25 medications. Among the medications discontinued, $80.0 \%$ were NSAIDs, and $31.9 \%$ had anticholinergic effects. SF-12 data was collected for 89 patients. SF-12 scores trended toward slight improvement at 1 month in both the physical component $(\mathrm{p}=0.101)$ and mental component $(\mathrm{p}=0.626)$.

CONCLUSIONS: Our study demonstrates polypharmacy can be systematically reduced through a simple stepwise interdisciplinary approach. Importantly, there were no negative effects on quality of life after medication reduction.

\section{Steps to "Pruning" Polypharmacy}

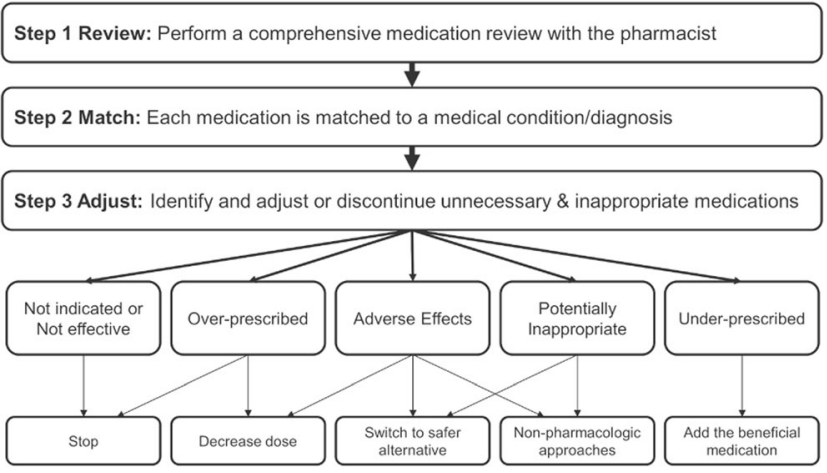

THE IMPACT OF GENDER ON INPATIENT MORTALITY OF HYPERTENSIVE LATINO PATIENTS ACROSS CKD STAGE 3 TO ESRD IN THE UNITED STATES.

Ricardo R. Correa ${ }^{1}$; Rodrigo Aguilar ${ }^{2}$; carmen Cervantes $^{3}$; Mark Nader ${ }^{2}$. ${ }^{\mathrm{T}}$ University of Arizona, Phoenix, AZ; ${ }^{2}$ Marshall University, Hungtinton, WV; ${ }^{3}$ AVentura Hospital, Hollywood, FL. (Control ID \#3167834)

BACKGROUND: Hypertension and chronic kidney disease are two of the most important risk factors for cardiovascular disease, a major cause of death in the US population. The impact of gender in this equation remains unclear, more so, on how it affects the Hispanic population. Studies comparing the outcomes and difference in inpatient mortality between male and female with hypertension and CKD are sparse. Our aim was to determine if gender in Latino population affect the inpatient survival rate among hypertensive patients across different CKD stages.

METHODS: Data was extracted from the 2005 to 2012 Nationwide Inpatient Sample (NIS). Using propensity score matching, female hypertensive with chronic kidney disease (stage 3, 4, 5 or ESRD) patients were matched with hypertensive males at a 1:1 ratio. We compared inpatient mortality, both crude mortality and mortality per CKD stage, length of stay and total hospital charges between male and females. Analyses were performed using SAS version 9.3 (SAS Institute, Cary, NC, USA).

RESULTS: Among 227,923 hospitalized hypertensive patients, 118,132 $(51.83 \%)$ were males and 109,791 (48.17\%) females. $18.76 \%, 10.31 \%$, $3.85 \%$ and $67.17 \%$ were Females with CKD 3 to ESRD respectively. Males across CKD stages 3 to ESRD were $19.47 \%, 9.54 \%, 3.63 \%$ and $67.43 \%$ respectively. In-hospital crude mortality was significantly higher for males compared to group of females before match, (3.89 vs $3.74 \mathrm{p}=$ 0.05 ), interestingly, mortality was higher after match for females (3.85 vs $3.79 \mathrm{p}=0.48)$. Mean length of stay for hypertensive females were significantly higher than males, irrespective of CKD stage (6.74 vs 6.70 days, $\mathrm{p}=0.001)$, however at the male group had an expense of higher hospital charges $(63,686$ vs 61,667 dollars, $\mathrm{p}=0.001)$.

CONCLUSIONS: Latino hypertensive men with any stage of CKD are more at risk of dying then their counterpart women and have higher hospital charges. Further studies are needed to explore these associated genders in difference outcomes in this population.

\section{THE IMPACT OF MEDICARE STAR RATING ADHERENCE MEASURES ON MEDICATION ADHERENCE FOR TARGETED AND NON-TARGETED MEDICATIONS}

Natasha Parekh ${ }^{1,2}$; Kiraat D. Munshi ${ }^{3}$; Inmaculada Hernandez ${ }^{4}$; Walid F. Gellad $^{1,5}$; Rochelle Henderson ${ }^{3}$; William H. Shrank ${ }^{2}$. ${ }^{1}$ University of Pittsburgh Medical Center, PIttsburgh, PA; ${ }^{2}$ UPMC Center for HighValue Health Care, Pittsburgh, PA; ${ }^{3}$ Express Scripts Holding Company, St. Louis, MO; ${ }^{4}$ University of Pittsburgh, Pittsburgh, PA; ${ }^{5}$ VA Pittsburgh/ University of Pittsburgh, Pittsburgh, PA. (Control ID \#3183269)

BACKGROUND: In 2012, Medicare incorporated medication adherence for oral antidiabetics, renin-angiotensin system (RAS) antagonists, and statins as highly weighted measures in star rating calculations. In the same year, health plans began receiving Quality Bonus Payments for achieving higher star ratings. It is unclear how these policy changes affected adherence to medications targeted by these policies and whether any impact spilled over to other chronic disease medications.

METHODS: We performed quasi-experimental interrupted time series analyses with multivariable segmented linear regression models to assess monthly changes in medication adherence (as measured by proportion of days covered) over a seven-year period from 2010 to 2016 using Medicare administrative claims data from a large pharmacy benefits manager. We conducted two separate sets of analyses: the first examined whether 
policy changes affected medication adherence for the 3 targeted therapy classes, and the second assessed the association of policy changes with improvements in adherence to five chronic disease therapy classes not considered in star ratings calculations (thiazides, beta blockers, calcium channel blockers, non-statin anti-hyperlipidemics, and levothyroxine). For the second analysis, we further compared adherence between beneficiaries who concomitantly used and did not use star rating medications.

RESULTS: We studied 240,811 Medicare beneficiaries on oral antidiabetics, 500,958 on RAS antagonists, 471,135 on statins, 464,910 on beta blockers, 392,762 on thiazides, 397,359 on calcium channel blockers, 165,151 on nonstatin anti-hyperlipidemics, and 323,803 on levothyroxine. There was a significant increase in monthly adherence for all star rating and non-star rating medications after $2012(\mathrm{p}<0.001)$. Adherence for oral antidiabetics, RAS antagonists, and statins was $11.2 \%, 8.1 \%$, and $3.7 \%$ higher than it would have been in the absence of star ratings policy changes, respectively $(p<0.001)$. The change in adherence for non-star rating anti-hypertensives and anti-hyperlipidemics was higher among those concomitantly on star ratings medications compared with those who were not $(p<0.001)$. Levothyroxine adherence trends did not significantly differ between those using and not using star rating drugs.

CONCLUSIONS: Incentivizing medication adherence in the star rating program was effective in increasing adherence to medications targeted by the policy change and also non-targeted medications that treat the same diseases. As policymakers seek to determine the optimal number and type of quality measures for improving health services delivery without increasing administrative burden, it is important to consider that incentives not only can improve performance of targeted measures, but can also spill over to promote improved performance in related outcomes. Our findings can ultimately inform future approaches to quality measure development and implementation.

\section{THE IMPACT OF OVERLAP BETWEEN MEDICARE'S VOLUN- TARY ACCOUNTABLE CARE ORGANIZATIONS AND BUN- DLED PAYMENTS ON THE COSTS AND QUALITY OF CARE} Amol S. Navathe $^{1,}{ }^{3}$; Sarah Dykstra ${ }^{2}$; Joshua M. Liao ${ }^{1,}{ }^{1}$; Rachel M. Werner $^{3,1}$. ${ }^{1}$ University of Pennsylvania, Philadelphia, PA; ${ }^{2}$ The Wharton School, University of Pennsylvania, Philadelphia, PA; ${ }^{3} \mathrm{CMC}$ VA Medical Center, Philadelphia, PA. (Control ID \#3179179)

BACKGROUND: While both voluntary accountable care organizations (ACOs) and bundled payments have demonstrated promising early results, it remains unclear to what extent outcomes are affected when the two models overlap (i.e., when patients receive care from organizations participating in both models).

METHODS: Our analysis focused on episodes for lower extremity joint replacement surgery (LEJR) and 5 medical conditions (acute myocardial infarction, congestive heart failure, chronic obstructive pulmonary disease, sepsis, pneumonia). We used 2011-2016 Medicare claims and program data to categorize beneficiaries attributed to Medicare Shared Savings Program (MSSP) ACOs into those who were hospitalized for 1 of the 6 episodes at hospitals in the Bundled Payments for Care Improvement (BPCI) initiative (Overlap) versus nonBPCI hospitals (MSSP-only). We used a difference-in-differences method and hierarchical generalized linear models among 68,548 Overlap and 988,933 MSSP-only patients receiving care at 529 ACOs, 402 BPCI hospitals, and 3,114 non-BPCI hospitals to evaluate differential changes with respect to 90-day episode outcomes: (1) postdischarge institutional care spending (combination of skilled nursing, inpatient rehabilitation, and acute hospital facilities) (2) discharge to an institutional post-acute care (PAC) provider, and (3) readmissions. Spending models used a log link and gamma distribution while others used an identity link and normal distribution. Covariates included patient characteristics (age, sex, Elixhauser comorbidities) as well as hospital, ACO, and quarter fixed effects. We clustered standard errors by hospital and used an $=0.05$.
RESULTS: Across 6 episodes, mean beneficiary age and sex did not vary between Overlap and MSSP-only groups, while differences in mean Elixhauser mortality score were significant, but small. For LEJR episodes, institutional post-discharge spending was $8.1 \%$ lower $(95 \% \mathrm{CI}-12.9 \%$ to $-3.4 \%, \mathrm{p}<0.001)$ and institutional PAC provider use was $2.6 \%$ lower $(95 \%$ CI $-3.3 \%$ to $-1.8 \%, \mathrm{p}<0.001)$ among Overlap patients compared to MSSPonly patients. Readmissions were unchanged $(0.3 \%, 95 \% \mathrm{CI}-0.2 \%$ to $0.9 \%, \mathrm{p}=0.20$ ). Across medical condition episodes, Overlap patients had $7.1 \%$ greater post-discharge institutional spending (95\% CI $2.8 \%$ to $11.3 \%, \mathrm{p}<0.001$ ) and $2.1 \%$ more readmissions (95\% CI $1.4 \%$ to $2.8 \%$, $\mathrm{p}<0.001$ ) compared to MSSP-only patients, but no differential changes in discharge to institutional PAC $(0.2 \%, 95 \% \mathrm{CI}-0.4 \%$ to $0.7 \%, \mathrm{p}=0.59)$.

CONCLUSIONS: Among ACO-attributed patients, admission to a BPCI hospital was associated with lower post-discharge institutional spending and PAC utilization for LEJR but not medical conditions. This suggests that ACOs may benefit when attributed beneficiaries are admitted to bundled payment hospitals for some episodes (i.e., LEJR), but not others.

\section{THE IMPACT OF PENNSYLVANIA'S MANDATED PRESCRIP- TION DRUG MONITORING PROGRAM PORTAL ON NEW- START OPIOID PRESCRIBING TO OPIOID-NAIVE PRIMARY CARE PATIENTS WITHOUT CANCER.}

Jonathan Arnold; Scott D. Rothenberger; Kevin Kraemer; Janel Hanmer. University of Pittsburgh, Pittsburgh, PA. (Control ID \#3184972)

BACKGROUND: Prescription drug monitoring programs (PDMPs) are in use by most states to identify patients at high-risk for adverse events from opioid prescribing. Pennsylvania (PA) deployed a PDMP portal in August 2016 and mandated its use by prescribers as of January 2017. Behavioral economics suggests that increasing the burden of prescribing will decrease the prescribing rate, independent of patient risk, a concern shared by patient and provider groups. Our objective was to evaluate the impact of PA's PDMP mandate on opioid prescribing in an opioid-naïve patient population by primary care providers (PCPs).

METHODS: We conducted an interrupted time-series study of ambulatory opioid prescribing at a large regional health system. We included 14 months prior to the PA PDMP portal availability (July 2015-August 2016) and 14 months following its mandated use (January 2017-February 2018). We included all patient visits to an internal or family medicine practice with a pain-related diagnosis by patients who had been previously established in primary care. We excluded patients with a cancer diagnosis or evidence of a prior opioid prescription within 12 months. We used generalized linear mixed models including random effects at the provider and practice levels and fixed-effects for baseline trend, post- PDMP offset and post-PDMP trend change. The CDC released its guideline for prescribing opioids in chronic pain in March 2016 and we included fixed effects in our analysis as post-CDC offset and post-CDC trend change. We evaluated several outcomes included percentage of visits with an opioid prescription, quantity of opioids prescribed per visit in milligram morphine equivalent (MME), and quantity of opioids prescribed per prescription. A review of state and institutional policies did not identify other significant policy changes occurring at the same time as the PDMP portal deployment and mandate.

RESULTS: We included 384,440 patient visits with 808 providers at 272 practices. Opioids were prescribed in $3.6 \%$ of all visits, an average of 18.3 MME per visit and 509 MME per prescription. The prescribing rate was decreasing at a relative $2.6 \%$ per month prior to the PDMP $(\mathrm{p}<0.001)$ without a change associated with the PDMP mandate. The PDMP mandate was not associated with any change in the quantities of opioid prescribed per visit or per prescription. The CDC guidelines were associated with a relative $8.5 \%$ decrease in the rate of opioid prescribing $(p=0.045)$, no change in the baseline trend in prescribing rate, and no change in the quantities of opioid prescribed per visit or per prescription. 
CONCLUSIONS: The deployment of a PDMP portal in PA and its mandated use by providers was not associated with a change in the rate or quantity of opioids prescribed by PCPs to opioid-naïve patients with non-cancer pain. There is no evidence that the increased burden by mandating use of the PDMP changed PCP prescribing behaviors for this opioid-naïve patient population.

\section{THE IMPACT OF PRICE CHANGES ON PROVIDER BEHAV- IOR: EVIDENCE FROM SHARP CHANGES IN MEDICARE PROFESSIONAL AND FACILITY FEES}

Zirui Song. Harvard Medical School, Boston, MA; Massachusetts General Hospital, Boston, MA. (Control ID \#3186116)

BACKGROUND: Understanding how providers respond to fee changes is central to health policy. The price of medical care differs by site of care - services delivered in a facility setting garner a "professional fee" for the physician and a "facility fee" for the hospital, the sum of which typically exceeds the single fee paid in an office setting. This study evaluates a natural experiment in Medicare prices using a sharp change in fees for coronary stents.

METHODS: In 2013, Medicare lowered the professional fee for a patient's initial stent, while raising the professional fee for additional stents. Simultaneously, Medicare raised the facility fee for all stents. This study examines its effect on multiple margins of physician response - spending, volume, site of care, stent choice, patient selection, offsets on inpatient stenting, and spillovers onto the commercially-insured population 4 years into the policy. It uses 2009-2016 Medicare claims and segmented regression adjusted for age, sex, risk, secular trends, seasonality, cost-sharing, and geography. Standard errors were clustered by MSA.

RESULTS: The population included 405,836 Medicare beneficiaries nationwide and 9,222,719 individuals with employer-sponsored insurance. On average over 4 years, the Medicare policy led to an increase in spending of $\$ 2.36$ per beneficiary per year on cardiac stents, a $15.6 \%$ increase over preintervention spending levels $(\mathrm{p}<0.001)$. This was driven by increased spending on drug-eluting stents in the facility setting $(\$ 2.02$ per beneficiary per year increase, $\mathrm{p}<0.001$ ). The volume of stents placed increased, on average, 0.63 stents per 1,000 beneficiaries per year ( $7.9 \%$ increase, $p<0.001)$, with drugeluting stents in the facility setting explaining this growth. The quantity of patient-days during which stenting took place increased by 52 days per 1,000 beneficiaries per year $(1.1 \%$ increase, $p=0.001)$, and stents placed per patientday increased by 0.28 per year $(p=0.008)$ over the 4 years. The share of patients stented without active chest pain increased, whereas that with active chest pain declined. Beneficiary cost-sharing remained stable. In terms of offsets, inpatient volume decreased by 0.04 hospitalizations per 1,000 beneficiaries per year $(p<0.001)$. Commercially-insured populations saw an increased spending of $\$ 0.47$ per enrollee per year (10.4\% increase vs. preintervention, $\mathrm{p}<0.001$ ) - smaller than the Medicare effect due to lower volume and price increases.

CONCLUSIONS: Medicare's cut in physician fees and increase in facility fees led to increased spending, driven by changes in site of care, treatment choice, treatment intensity, and share of patients with stable coronary artery disease stented. Moreover, Medicare faced the full costs of this policy; hospitalizations were partially offset, and smaller increases in spending and volume among commercially-insured populations took place. Altering Medicare prices exerts multiple downstream effects on provider behavior with potentially important unintended consequences.

\section{THE IMPACT OF SOCIAL MOVEMENTS ON TEAM-BASED PRIMARY CARE: A QUALITATIVE ASSESSMENT OF PER- CEPTIONS OF \#METOO AND \#BLACKLIVESMATTER}

Cati Brown-Johnson ${ }^{1}$; Nadia Safaeinili ${ }^{2}$; Jonathan G. Shaw ${ }^{1}$; Marcy Winget $^{3}$; Megan Mahoney ${ }^{4}{ }^{1}$ Stanford University School of Medicine, Stanford, CA; ${ }^{2}$ Stanford University, Stanford, CA; ${ }^{3}$ Stanford University Medical School, Stanford, CA; ${ }^{4}$ Stanford University, Palo Alto, CA. (Control ID \#3185638)
BACKGROUND: Social movements such as \#metoo and \#blacklivesmatter can impact the practice environment. Implementation science frameworks posit that the environment surrounding interventions can impact them. Studies of the impact of national policy and social movements on workplace culture have been conducted in medical contexts, but systematic reports are limited. These national conversations may be particularly relevant to team-based primary care interventions, which bring together healthcare staff with diverse training and backgrounds, and may be primarily supported by female medical assistants (MAs). To our knowledge, this project is the first research-based exploration of the impact of \#metoo and \#blacklivesmatter on team-based primary care.

METHODS: Qualitative interviews $(\mathrm{n}=26)$ were conducted as part of an evaluation of team-based care across 5 academic practices. Interviewees (providers, including nurse practitioners/physician assistants $n=12$; MAs $n=10$; management $\mathrm{n}=4$ ) were asked how, if at all, \#metoo or \#blacklivesmatter impacted interactions with staff or patients. Interviews were recorded; audio files and transcripts were coded for emergent themes by two researchers.

RESULTS: Interviewees were predominately women $(n=22)$, as these clinics were largely staffed by women. Some interviewees had not heard of \#metoo or \#blacklivesmatter ( $\mathrm{n}=6$ ). Perceived impacts of \#metoo included team meetings to discuss sexual harassment, implementing protocols to curb harassing behavior by patients, and utilizing case conferences to debrief the emotional toll of hearing "sad stories" from female patients who had experienced trauma. A small minority of interviewees reported perceiving that male colleagues were dismissive of female staff concerns, or "just didn't understand." Providers reported noticing increased clinical focus on supporting transgender and non-binary patients as a possible outgrowth related to the \#metoo movement. Perceived impacts of \#blacklivesmatter were reported less frequently. The majority of interviewees responded to questions about \#blacklivesmatter by noting that they treat everyone the same. A mix of MAs and providers reported tailoring their behavior for nonwhite patients, with one provider noting that she is more likely to attend to complaints from both non-white patients and women as a result of these national conversations. Another provider reported asking new patients "How has healthcare been for you? Have you had barriers in the past?" due to awareness that race/ethnicity can impact healthcare access.

CONCLUSIONS: Our novel exploratory study demonstrates that national movements can impact team-based primary care. Next steps include investigating how conversations around movements like \#metoo or \#blacklivesmatter might be leveraged to contribute to safer working environments for staff and more equitable care for patients.

\section{THE IMPACT OF THE AFFORDABLE CARE ACT MEDICAID EXPANSION ON RACIAL/ETHNIC AND SEX DISPARITIES IN HIV TESTING: NATIONAL FINDINGS FROM THE BEHAVIOR- AL RISK FACTOR SURVEILLANCE SYSTEM}

Anitha Menon; Payal Patel; Renuka Tipirneni. University of Michigan, Ann Arbor, MI. (Control ID \#3180729)

BACKGROUND: More than half of Americans have not been tested for HIV in their lifetime, and over a third of all HIV diagnoses are made less than a year before the progression to AIDS. While the cause of low testing rates is multifactorial, a major barrier to universal HIV testing is lack of universal access to healthcare. The Affordable Care Act (ACA) Medicaid expansion led to large coverage gains in states that expanded, which may translate to improvements in health outcomes. Prior work has shown that overall HIV testing rates increased after the ACA. This study assessed the differential impacts of ACA Medicaid expansion on racial/ethnic (e.g. Black race) and racial/ethnic-sex (e.g. white females) disparities in HIV testing.

METHODS: The group primarily targeted by the 2014 Medicaid expansion - low-income ( $138 \%$ of the federal poverty level) adults ages 19-64 who are non-pregnant and non-disabled - was studied using the Behavioral Risk Factor Surveillance System (BRFSS) data from all 50 States and the District of Columbia. A quasi-experimental study design was used with triple difference-in-differences (DDD) logistic regression analyses to 
assess the differential impacts of race/ethnicity groups and race/ethnicitysex groups. All 30 states and D.C. that expanded Medicaid by December 2015 were included in the treatment group and all 20 non-expansion states were included in the control group. Outcomes were 1) ever having received an HIV test and 2) having received an HIV test in the last year.

RESULTS: Overall, Medicaid expansion was associated with a statistically significant increase in the probability of both ever having received an HIV test and having received an HIV test in the last year, consistent with prior work. However, following the Medicaid Expansion, Black females were less likely to have increased rates of ever having HIV testing (65\% pre-expansion and $59 \%$ post-expansion) when compared to a white male reference group (33\% pre-expansion and 34\% post-expansion) and across other race/ethnicity-sex groups $(\mathrm{p}=0.016$ ). Findings were similar for HIV testing in the last year.

CONCLUSIONS: Medicaid expansion increased the overall probability of HIV testing among low-income, nonelderly adults, but it had differential impacts among different race/ethnicity-sex categories. Black females were the least likely to experience an increased probability of receiving an HIV test following the expansion. Though they had higher baseline HIV testing rates than white men, the negative trend in testing rates is concerning as Black females are disproportionately affected by HIV. Moreover, as the CDC recommends that all adults be tested for HIV at least once, these findings demonstrate that not all groups are approaching this testing goal. These data should guide further research on the drivers of HIV testing disparities, changes in HIV testing practices for clinicians, and policy changes that aims to expand insurance coverage and HIV testing in the United States.

\section{THE IMPACT OF THE BALANCING INCENTIVES PROGRAM ON INFORMAL CAREGIVER BURDEN IN THE UNITED STATES}

Rebecca E. Anastos-Wallen ${ }^{1}$; Paula Chatterjee ${ }^{1}$; Rachel M. Werner ${ }^{2}$. ${ }^{\mathrm{T}}$ University of Pennsylvania, Philadelphia, PA; ${ }^{2}$ University of Pennsylvania and Philadelphia VA, Philadelphia, PA. (Control ID \#3185793)

BACKGROUND: There are an estimated 34.2 million adults in the US who provide informal care to sick family members or friends. These caregivers are known to have higher rates of stress and poorer health than their non-caregiver peers. The Balancing Incentives Program (BIP), established under the Affordable Care Act, provided federal funding for states to shift long-term care out of institutional settings and into the home. However, the effect of BIP on informal caregivers is unknown. We compared changes in caregiving activities and measures of wellbeing among caregivers in states that participated in BIP compared to control states.

METHODS: As BIP states shifted more care into the home, we hypothesized that caregivers in those states might provide more frequent care. Given increased support services at home, we also hypothesized that caregivers might have improved wellbeing. To test these hypotheses we studied respondents to the American Time Use Survey who self-identified as caregivers and either lived in a state that participated in BIP or in a state that was eligible to participate but did not (non-BIP). We used quasiexperimental design with difference-in-difference regression to assess the likelihood of a respondent being a caretaker, frequency of caregiving, and wellbeing measures related to time slept and time spent on self- care.

RESULTS: From 2011-2017, we identified 4,311 informal caregivers in non-BIP states and 7,316 in BIP states. Caregivers in BIP states were more likely to report providing daily care after BIP implementation $(3.1 \%$, $\mathrm{p}=0.04)$. Consistent with the goals of the program, caregivers in BIP states had some improvement in markers of wellbeing (gains in sleep of 13.9 minutes, $p=0.01$; gains in time spent on self-care of 12.0 minutes, $\mathrm{p}=0.03$ ) compared to control caregivers. These effects were more pronounced among caregivers with more than a high school education (18.3 minutes of sleep, $p=0.004)$, annual family incomes greater than $\$ 50,000$ (14.4 minutes of sleep, $\mathrm{p}=0.03$ ), and who were employed full time (14.5 minutes of sleep, $\mathrm{p}=0.03$ ).

CONCLUSIONS: Caregivers in BIP states were more likely to provide daily care than caregivers in non-BIP states after BIP took effect. They also were able to spend more time on self-care and sleep compared to peers in non-BIP states. Further efforts to shift long term care from institutional to home-based settings may have a positive effect on informal caregivers. While continued support for the BIP program may serve to mitigate stressors faced by this at-risk population, caregivers with higher levels of education and income may experience these benefits disproportionately.

\section{THE INCIDENCE OF DIAGNOSTIC ERROR IN HOSPITAL- IZED ADULTS: A SYSTEMATIC REVIEW AND META- ANALYSIS}

$\underline{\text { Victor P. Bilan }}^{1,2}{ }^{\text {; }}$ Benjamin Rodwin ${ }^{1,2}$; Naseema Merchant ${ }^{1,2}$; Craig G. Gunderson ${ }^{1,2}$. ${ }^{1}$ Yale School of Medicine, New Haven, CT; ${ }^{2}$ VA Connecticut Healthcare, West Haven, CT. (Control ID \#3182980)

BACKGROUND: Achieving a correct and timely diagnosis is fundamental to medical practice, but the diagnostic process is fraught with potential for error. The National Academy of Medicine's 2015 report Improving Diagnosis in Health Care identified diagnostic error as the next "quality chasm" to be addressed by the patient safety movement. Nonetheless the actual incidence of diagnostic error remains uncertain due to difficulties in measurement and the heterogeneity of approaches used, including autopsy studies, reviews of malpractice claims, and surveys of physicians and patients. We performed a systematic review of studies that assess rates of diagnostic error using physician review of case series of hospitalized patients.

METHODS: A systematic literature search was conducted for studies that reported series of hospitalized patients and used physician review to determine the frequency of diagnostic error. Our search included MEDLINE, EMBASE, and the Cochrane library from database inception through October 3, 2018. The rates of diagnostic error from individual studies were pooled using randomeffects meta-analysis. Heterogeneity was estimated using the $\mathrm{I}^{2}$ statistic.

RESULTS: 6,137 studies were screened for inclusion. Twenty studies met inclusion criteria of which sixteen were of consecutive admissions representing 93,619 patients and were included in the primary meta-analysis. The pooled incidence of diagnostic error was $0.8 \%(95 \% \mathrm{CI}, 0.5 \%$ to $1.1 \%)$.

CONCLUSIONS: Whereas previous estimates have suggested that all adults will experience clinically significant diagnostic errors during their lifetimes, the results of this study suggest that diagnostic errors are a real but relatively infrequent cause of adverse events in hospitalized patients, as determined by retrospective chart review of consecutive patient series. Most studies did not further stratify diagnostic errors according to clinical context or actual missed diagnosis; this information will be necessary to inform future efforts at improving the diagnostic process.

\begin{tabular}{|c|c|c|c|c|c|c|}
\hline \multirow[b]{2}{*}{ Author } & \multirow[b]{2}{*}{ Year } & \multirow{2}{*}{$\begin{array}{l}\text { Total } \\
\text { Admissions }\end{array}$} & \multicolumn{2}{|l|}{ Diagnostic } & \multirow[b]{2}{*}{ ES $(95 \% \mathrm{Cl})$} & \multirow{2}{*}{ \% } \\
\hline & & & Errors & & & \\
\hline Laape & 1991 & 30195 & - & & $0.00(0.00,0.00)$ & 850 \\
\hline Thomas & 1999 & 17732 & + & & $0.01(0.00,0.01)$ & 8.36 \\
\hline Davis & 2003 & 6579 & 85 & $\rightarrow$ & $0.01(0.01,0.02)$ & 7.66 \\
\hline Baker & 2004 & 3745 & $\rightarrow$ & & $0.00(0.00,0.01)$ & 7.97 \\
\hline Forster & 2004 & 502 & 6 & $\rightarrow$ & $0.01(0.01,0.03)$ & 3.55 \\
\hline Sari & 2007 & 1006 & $\rightarrow$ & & $0.01(0.00,0.01)$ & 6.06 \\
\hline Aseveroengschai & 2009 & 576 & 14 & & $0.02(0.01 .0 .04)$ & 2.47 \\
\hline scop & 2009 & 1967 & 20 & + & $0.01(0.01 .0 .02)$ & 6.54 \\
\hline Letaier & 2010 & 620 & 8 & $\rightarrow$ & $0.01(0.01,0.03)$ & 3.83 \\
\hline Wilson & 2012 & 15548 & 244 & $\mp$ & $0.02(0.01,0.02)$ & 8.06 \\
\hline Wang & 2015 & 2950 & $\leftarrow$ & & $0.00(0.00,0.00)$ & 8.43 \\
\hline Wong & 2015 & 141 & 2 & & $0.01(0.00,0.05)$ & 123 \\
\hline Rater & 2016 & 1574 & 16 & $1+$ & $0.01(0.01,0.02)$ & 6.18 \\
\hline Aranaz-Andres & 2017 & 11379 & 73 & & $0.01(0.01,0.01)$ & 827 \\
\hline Aranaz-Andres & 2017 & 1088 & $\rightarrow$ & & $0.01(0.00 .0 .011)$ & 6.56 \\
\hline Hellion & 2017 & 1007 & $\rightarrow$ & & $0.01(0.00 .0 .01)$ & 6.32 \\
\hline \multicolumn{3}{|c|}{ Overall $\left(1{ }^{2}=9497 \%, p=0.00\right)$} & & & $0.01(0.01 .0 .01)$ & 100.00 \\
\hline
\end{tabular}


THE INFLUENCE OF GEOGRAPHIC DISPERSION ON OUTCOMES OF HOSPITALIZED MEDICINE SERVICE PATIENTS

Rachel Kohn; Michael O. Harhay; Brian A. Bayes; Hummy Song; Scott D. Halpern; Meeta P. Kerlin; S R. Greysen. University of Pennsylvania, Philadelphia, PA. (Control ID \#3139478)

BACKGROUND: Specialty wards (e.g., cardiology or oncology wards) group clinically similar hospitalized patients in efforts to improve outcomes and costs. When these wards exceed capacity, subsequent patients overflow to "geographically dispersed" alternate wards. Geographic dispersion has been associated with care inefficiency and worse outcomes on specialty wards, but has not been studied in a large, diverse sample of hospitalized medicine service patients.

METHODS: We performed a retrospective cohort study in 3 diverse University of Pennsylvania Health System (UPHS) hospitals. We included all medicine, family medicine, and geriatric service patients admitted from emergency departments (EDs), intensive care units (ICUs), or other wards in 2014 and 2015. Data were obtained from Penn Data Store, a UPHS clinical data warehouse that includes all payers. The primary exposure was placement on non-medicine wards, i.e., "geographical dispersion." We excluded patients who switched from medicine to nonmedicine wards and vice-versa $(\mathrm{n}=427[2 \%])$. The primary outcome was hospital length of stay (LOS). The secondary outcomes were hospital discharge to home and skilled nursing facilities (SNFs) and in-hospital mortality. We performed mixed effects linear and logistic regression models with a random effect to account for clustering by hospital. We first performed univariate analyses of geographical dispersion and each outcome separately. We then performed separate multivariable regression models, adjusting for age, gender, race, ethnicity, insurance type, body mass index, number of Elixhauser comorbidities, days spent in ICUs, number of ward transfers during hospitalization, number of previous inpatient, ICU, and ED visits during the prior 12 months, numbers of unique medications and procedures during hospitalization, the Centers for Medicare and Medicaid Services four-level severity risk adjustment (mild, moderate, major, severe), and hospital admission source.

RESULTS: The study population included 19,032 visits among 18,956 patients in 33 wards. Median age was 60 years, (IQR 45-74), 55\% were female, and $61 \%$ were black. Thirty-three percent of patients were geographically dispersed. Median LOS was 4 days (IQR 2-6), 77\% of survivors were discharged home, $14 \%$ were discharged to SNFs, and $10 \%$ died. In multivariable regression, geographically dispersed patients had longer LOS (1.1 days, 95\% CI 1.1-1.2, $\mathrm{p}<0.0005)$ and higher odds of discharge to SNF $(\mathrm{OR}=1.1,95 \%$ CI 1.0-1.2, $\mathrm{p}=0.04)$. Geographically dispersed patients had lower odds of discharge to home but this was not statistically significant (OR=0.9, 95\% CI 0.9-1.0, $\mathrm{p}=0.08$ ). In-hospital mortality did not differ between the groups $(\mathrm{OR}=1.0,95 \%$ CI 0.6-1.5, $\mathrm{p}=0.9)$.

CONCLUSIONS: Geographically dispersed hospitalized medicine patients had significantly increased LOS ( $>1$ day longer) and higher odds of discharge to SNF. Future studies are needed to confirm these findings and explore underlying mechanisms of these associations.

\section{THE INTERACTION OF NEIGHBORHOOD ENVIRONMENTS AND GENETIC RISK FOR TYPE 2 DIABETES: RESULTS FROM MESA AND CARDIA}

Paul Christine $^{1}$; Jennifer A. Smith ${ }^{1}$; Bhramar Mukherjee ${ }^{1}$; Sara Adar ${ }^{1}$; Kari Moore $^{2}$; Alain Bertoni ${ }^{3}$; Penny Gordon Larsen ${ }^{4}$; Wei Zhao ${ }^{1}$; Mercedes R. Carnethon ${ }^{5}$; James B. Meigs ${ }^{6}$; Laura Rasmussen-Torvik ${ }^{5}$; Leslie Lange ${ }^{7}$; Myriam Fornage ${ }^{8}$; Sharon Kardia ${ }^{1}$; Ana Diez-Roux ${ }^{2}$. ${ }^{1}$ University of Michigan, Ann Arbor, MI; ${ }^{2}$ Drexel University, Philadelphia, PA; ${ }^{3}$ Wake Forest, Winston-Salem, NC; ${ }^{4}$ University of North Carolina, Chapel Hill, NC; ${ }^{5}$ Northwestern University, Chicago, IL; ${ }^{6}$ Massachusetts General Hospital, Boston, MA; ${ }^{7}$ University of Colorado, Aurora, $\mathrm{CO} ;{ }^{8}$ University of Texas Health Science Center, Houston, TX. (Control ID \#3186418)
BACKGROUND: Genetic studies have documented the impact of genes on risk of type 2 diabetes (T2D). Similarly, a substantial body of research has demonstrated the impact of neighborhood environments on T2D risk by shaping health behaviors. However, no studies have examined whether genetic risk for T2D may be modified by neighborhood environments.

METHODS: We analyzed the interaction between genetic risk (summarized via genetic risk score [GRS]) and neighborhood environment in relation to T2D in 5704 individuals from the Multi-Ethnic Study of Atherosclerosis (MESA) from 2000-2012, and in 2609 individuals from the Coronary Artery Risk Development in Young Adults (CARDIA) cohort from 1985-2011. Neighborhood environment included the availability of supermarkets/fruit and vegetable markets and recreational establishments, and neighborhood socioeconomic status (SES). Intervalcensored survival analyses were used to estimate hazard ratios (HRs) for pooled prevalent and incident T2D associated with neighborhood environments, GRS, and their interaction. Additive and multiplicative joint effects were estimated to examine the independent associations of genetic risk, neighborhoods, and their interaction on T2D risk.

RESULTS: In models with continuous exposures, associations between genetic risk and T2D were modestly weaker in individuals residing in neighborhoods with more supermarkets/fruit and vegetable markets and recreational establishments (multiplicative interaction p-values, 0.03 to 0.19). Models with dichotomized exposures showed similar results. For instance, MESA participants jointly exposed to above-median genetic risk $(\mathrm{HR}=1.19 ; 95 \% \mathrm{CI}[0.99,1.39])$ and below-median availability of recreational establishments (HR=1.52; 95\% CI $[1.26,1.78])$ had an observed joint HR that was greater than expected under additive interaction (observed HR=2.00 [95\% CI 1.67, 2.32]; additive interaction p-value, 0.02), indicating synergism between the two exposures. Results for neighborhood SES were mixed.

CONCLUSIONS: Residing in neighborhoods with more healthpromoting resources may decrease the risk of T2D in genetically susceptible individuals. While much emphasis has been placed on using genetic risk to focus on individual prevention, this study suggests that T2D prevention efforts focused on modifying neighborhood environments may represent another fruitful, population-based approach.

\section{THE INTERSECTION BETWEEN PROFESSIONALISM AND INCLUSION: A MIXED METHODS ANALYSIS}

Dominique Alexis ${ }^{1}$; Matthew Kearney ${ }^{2}$; Jaya Aysola ${ }^{1}$; Eve Higginbotham ${ }^{1} .{ }^{1}$ University of Pennsylvania, Philadelphia, PA; ${ }^{2}$ Drexel University, Philadelphia, PA. (Control ID \#3183100)

BACKGROUND: Little is known about how to effectively operationalize professionalism in our evolving landscape throughout healthcare systems and academic environments. Moreover, to date, how effectively inclusion is integrated into the development of policies and practice related to professionalism is unknown. Building an inclusive environment for all requires a deeper understanding of how we redefine and apply professionalism standards to meet the needs of a diverse workforce. The objective of this study aimed to examine the intersection between inclusion and professionalism.

METHODS: We examined survey data $(\mathrm{n}=3500)$ as well as submitted narratives $(n=56)$ in a mixed-methods analysis designed to evaluate professionalism within healthcare learning and working environments. We first evaluated the relationship between respondent demographic characteristics and their responses to the following professionalism questions: 1) I have considered changing jobs due to inappropriate, disruptive, or unprofessional behavior by a co- worker or supervisor; 2) I value institutional initiatives, policies, and/or educational resources related to professional behavior in the workplace; 3) My institution supports a culture of professionalism. Thereafter, we coded and analyzed a subset of submitted narratives $(n=56)$ submitted by employees and/or students that referenced professionalism from our cultural audit of inclusion to identify emerging themes. 
RESULTS: Of the 3500 respondents, $11 \%, 64 \%$, and $10 \%$ self-identified as non-Hispanic Black, female, and LGTBQ, respectively. In comparison to white males, females and minorities placed greater value on institutional policies and educational resources related to professionalism. All respondents agreed that the institution supports a culture of professionalism, however, women, underrepresented minorities, and LGBTQ groups were significantly more likely to consider leaving after experiencing unprofessional behavior. Analysis of the narratives revealed a set of themes including causes and outcomes across a spectrum of professional-to-unprofessional experiences. Key contributors to unprofessional behaviors that emerged include a hostile environment, silent bystanders, the lack of boundaries and respect, and unequal application of standards. Narratives also revealed the effects of unprofessionalism on physical health, pressure to conform, and attrition.

CONCLUSIONS: A mixed methods analysis of survey data and submitted narratives providing an assessment of organizational inclusion revealed diverse individuals with varied cultural perspectives perceive and experience professionalism in the workplace differently compared to others. Based on the disparate responses from women, underrepresented minorities, minorities, and LGBT, there is unequal application of professional standards. Our findings highlight the need to revisit how organizations set professionalism standards, and ensure they are inclusive and applied equitably.

\section{THE LACK OF FORMAL EDUCATION ON OUTPATIENT BILLING}

Azra Bhimani; Sun Yoo. UCLA, Los Angeles, CA. (Control ID \#3159529)

BACKGROUND: A growing number of resident physicians are entering the primary care workforce every year. Upon graduating, they face the responsibility of independently and accurately billing for clinical services. Studies show there is inadequate training on billing for outpatient services during residency. Inaccurate billing can lead to financial repercussions and issues with compliance, and underutilization of annual wellness visits can lead to more out of pocket expenses for patients. We hypothesized that a majority of residents in the UCLA internal medicine training program lack the confidence to bill accurately in the outpatient setting and would be interested in receiving formal education on this topic. We also hypothesized underutilization of Medicare Wellness Exam (MWE) codes and Transitional Care Management (TCM) codes.

METHODS: We created two 10-question surveys for residents and faculty in the Internal Medicine program at UCLA over a 2-month period. The surveys included questions on confidence in billing encounters, differentiating 99213 and 99214 codes, utilizing MWE and TCM codes, and interest in a formal curriculum on billing. We also looked at billing data from 2,096 encounters at these clinics over a 4-month period to assess the difference in types of codes billed between PGY-levels.

RESULTS: 32 residents and 15 faculty physicians working at 3 UCLA Internal Medicine clinics participated in each survey. 2,096 encounters from 2 of these clinics over a 4-month period were analyzed. Less than $10 \%$ of residents felt very or extremely confident in their ability to bill encounters accurately. Over $80 \%$ of faculty felt they needed to change resident billing codes to more accurately reflect encounters. $93 \%$ of residents and $100 \%$ of faculty felt that residents would benefit from formal education on billing. Of the 2,096 encounters billed, only $3 \%$ were billed as TCM visits and $1 \%$ as MWE visits.

CONCLUSIONS: As more residents are pursuing primary care careers, a growing aspect of their practices will include coding. Multiple studies, including ours, have shown lack of confidence and errors in practice amongst residents when it comes to coding for outpatient services. In addition, TCM and MWE codes were being underutilized. Based on our study and numerous others, it is integral for training programs to incorporate formal education on outpatient billing.
THE LONG-TERM IMPACT OF SIMULTANEOUS PARTICIPATION IN MEDICARE ACCOUNTABLE CARE ORGANIZATIONS AND BUNDLE PAYMENTS ON OUTCOMES FOR LOWER EXTREMITY JOINT REPLACEMENT

$\underline{\text { Joshua M. Liao }}^{4,}{ }^{\text {; Ezekiel J. Emanuel }}{ }^{1,1}$; Atheendar Venkataramani ${ }^{1,1}$; Qian Huang $^{1}$; Atul Gupta ${ }^{2}$; Claire T. Dinh ${ }^{1}$; Eric Z. Shan ${ }^{1}$; Dylan Small ${ }^{1}$, ${ }^{1}$; Norma B. Coe ${ }^{1,1}$; Erkuan Wang ${ }^{1}$; Xinshuo $\mathrm{Ma}^{1}$; Jingsan Zhu ${ }^{1}$; Deborah Cousins ${ }^{1}$; Amol S. Navathe ${ }^{1,3} \cdot{ }^{1}$ University of Pennsylvania, Philadelphia, PA; ${ }^{2}$ The Wharton School, University of Pennsylvania, Philadelphia, PA; ${ }^{3} \mathrm{CMC}$ VA Medical Center, Philadelphia, PA; ${ }^{4}$ University of Washington, Seattle, WA. (Control ID \#3179243)

BACKGROUND: Based on savings from the Bundled Payments for Care Improvement (BPCI) program, Medicare continues to scale voluntary lower extremity joint replacement (LEJR) bundles among hospitals nationwide via new programs. Concurrently, a growing number of hospitals have participated in voluntary Accountable Care Organizations (ACOs) through initiatives such as the Medicare Shared Savings Program (MSSP). Because ACOs and bundled payments share the goal of containing costs while maintaining or improving quality, hospitals participating in LEJR bundles may achieve better performance and synergies from simultaneous "co-participation" in ACOs.

METHODS: Hospitals participating in MSSP ACOs and BPCI Model 2 for LEJR bundles were categorized as being in BPCI ("Bundles Only"), MSSP ("ACO Only"), both ("Co-Participant"), or neither ("Non-Participant"). Using 2011-2016 Medicare claims and two-stage least squares instrumental variable regression to mitigate confounding from changes in unobservable patient characteristics, we evaluated the association between co-participation and changes in episode spending and quality (mortality, unplanned readmissions, LEJR complications, ED visits) over 3 years among 671,288 fee-for-service Medicare beneficiaries receiving LEJR at acute care hospitals nationwide. The sample included 212 Bundles Only, 448 ACO Only, 105 Co-Participant, and 1,943 Non-Participant hospitals. Historical hospital referral patterns before BPCI began were used to generate the predicted probability of hospitalization for LEJR at an eventual BPCI hospital, which was used as an instrument for actual hospitalization for LEJR at a BPCI hospital. Standard errors were computed using bootstrapping and statistical tests were 2-tailed using an $=0.05$ for significance.

RESULTS: Across hospital groups, there were small differences in beneficiary (e.g., case-mix, prior hospital and institutional post-acute care utilization) characteristics. Hospital characteristics (e.g., size, ownership and teaching status, market share) also varied. Over 3 years, average episode spending decreased at both BPCI Only (2.9\% decrease in average episode spending, $95 \%$ CI $-3.4 \%$ to $-2.5 \%)$ and Co-Participant $(2.8 \%$ decrease in average episode spending, $95 \% \mathrm{CI}-3.4 \%$ to $-2.1 \%$ ) hospitals compared to Non-Participant hospitals ( $\mathrm{p}<0.001$ for both). BPCI Only hospitals also had lower unplanned readmission rates than Non-Participant hospitals $(1.1 \%$ decrease, $95 \%$ CI $-1.4 \%$ to $-0.7 \%$, p $<0.001)$. Compared to BPCI Only hospitals, Co-Participant hospitals did not have differential changes in episode spending but did have higher unplanned readmission rates ( $1.1 \%$ increase, $95 \%$ CI $0.58 \%$ to $1.7 \%, \mathrm{p}<0.001)$.

CONCLUSIONS: Compared to participation in BPCI Model 2 only, coparticipation in MSSP ACOs was not associated with LEJR episode savings but was associated with higher rates of unplanned readmissions. Our findings support current Medicare policy that does not encourage co-participation.

THE NET BENEFIT OF ORAL ANTICOAGULATION AMONG OLDER ADULTS WITH ATRIAL FIBRILLATION

Sachin J. Shah ${ }^{1}$; Margaret Fang ${ }^{1}$; Daniel E. Singer ${ }^{2}$; Kenneth Covinsky ${ }^{1}$; Alan S. Go ${ }^{3}$; Mark H. Eckman ${ }^{4}{ }^{1}$ University of California, San Francisco, San Francisco, CA; ${ }^{2}$ Massachusetts General Hospital, Boston, MA; ${ }^{3} \mathrm{Kai}-$ ser Permanente Northern California, Oakland, CA; ${ }^{4}$ University of Cincinnati, Cincinnati, OH. (Control ID \#3178343) 
BACKGROUND: Guidelines categorize all patients aged 75+ with atrial fibrillation (AF) as "high risk for ischemic stroke" thus warranting anticoagulation. While age increases the risk of ischemic stroke, it also increases the risk of hemorrhage and death from other causes; the latter two are not accounted for in guidelines recommendations. The age at which there is equipoise between benefit and harm when accounting for the risk of hemorrhage risk and death from other causes is not known.

METHODS: We examined all patients 75 years with incident AF in the Anticoagulation and Risk Factors in Atrial Fibrillation - Cardiovascular Research Network cohort. In a patient-level analysis, we accounted for patients' stroke risk (CHA2DS2-VASc score), hemorrhage risk (HASBLED score), and age-, sex-, comorbidity-adjusted life expectancy. Using an established 28-state Markov state transition model, we estimated the quality-adjusted life years (QALYs) with 3 strategies: warfarin, dabigatran, no anticoagulation. In each monthly cycle, patients face a chance of stroke, hemorrhage, and death from a competing cause. Stroke or hemorrhage leads to any of 3 health states: death, mild or severe neurologic sequelae, symptom resolution. The simulation runs for the life expectancy of the patient. We used an established threshold of 0.1 QALY to define minimal clinical benefit.

RESULTS: 14946 patients were included with a median age of 81 years and CHA2DS2-VASc score of 4. With warfarin, at age 87, the median patient gained 0.09 QALYs (IQR 0.04, 0.16) relative to no treatment. With dabigatran, at age 90 , the median patient gained 0.10 QALYs (IQR $0.06,0.16)$ relative to no treatment.

CONCLUSIONS: Accounting for the risk of stroke, hemorrhage, and death from competing causes, for the median patient anticoagulation with warfarin results in equipoise at age 87 and with dabigatran at age 90. Consensus guidelines should emphasize shared decision making for all patients considering anticoagulant therapy, particularly when treating the oldest patients.

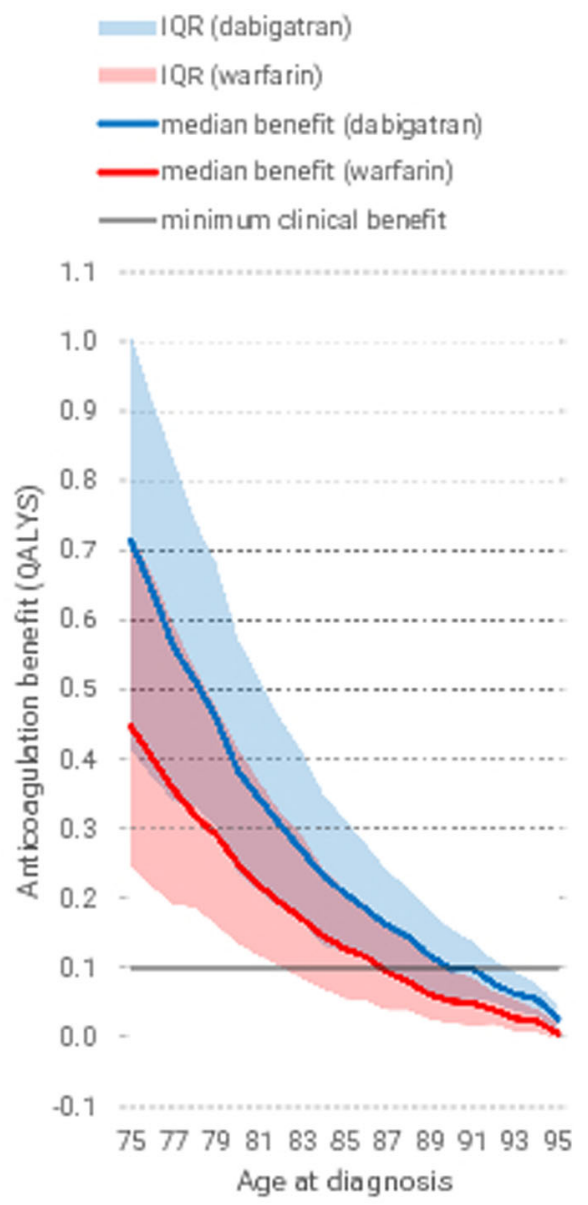

THE OBESITY PARADOX AND ACUTE KIDNEY INJURY IN ORTHOPEDIC SURGERY PATIENTS

Matthew Hennrikus ${ }^{2}$; William Hennrikus ${ }^{6}$; Erik B. Lehman ${ }^{3}$; Michael Skolka ${ }^{4}$; Katelyn McCann ${ }^{5}$; Eileen Hennrikus ${ }^{1}{ }^{1}$ Penn State College of Medicine, Hershey, PA; ${ }^{2}$ Tulane University Medical School, New Orleans, LA; ${ }^{3}$ Pennsylvania State University, Hershey, PA; ${ }^{4}$ Penn State Health, Hershey, PA; ${ }^{5}$ University of Massachussetts Medical School, Worcester, MA; ${ }^{6}$ Walter Reed National Military Medical Center, Bethesda, MD. (Control ID \#3163093)

BACKGROUND: The Obesity Paradox postulates that obesity, counterintuitively, is protective against mortality in certain chronic conditions such as chronic kidney disease and congestive heart failure. This study examined the effects of obesity and acute kidney injury (AKI) on length of hospital stay and on two year mortality.

METHODS: We retrospectively reviewed the electronic medical records of all adult elective orthopedic surgery patients over two years in a large academic hospital. The 1783 patients who met inclusion criteria were divided into obese (body mass index, BMI > 30, $\mathrm{n}=1123$ ) and non-obese groups (BMI < 30, n=660). Demographics, comorbidities, and perioperative variables were included in multivariate logistic regression analyses with AKI, LOS, and two year mortality as primary outcomes in all, obese and non-obese patients. Two-year mortality data was obtained from hospital electronic records and the social security death index.

RESULTS: AKI developed in 5\% ( $\mathrm{n}=90)$ of the post-surgical orthopedic patients. Obese patients had a 1.86 times higher odds (95\% CI 1.07-3.22, $\mathrm{p}=0.028$ ) of developing AKI than non-obese patients. AKI increased length of hospital stay in both obese $(1.28 \pm 0.38$ days, $\mathrm{p}=0.001)$ and non-obese patients $(1.61 \pm 0.78$ days, $\mathrm{p}=0.039)$. The adjusted odds of 2 year mortality were increased by AKI (OR 2.08, 95\% CI 1.03-4.22, $\mathrm{p}=0.041$ ) but decreased by obesity (OR 0.53 , CI $0.33-0.85, \mathrm{p}=0.009$ ). Of patients who developed AKI, only the non-obese patients had statistically significant higher likelihood of 2-year mortality (OR 3.869, CI 1.26-11.84, $\mathrm{p}=0.018$ ).

CONCLUSIONS: In adult elective orthopedic surgery patients, obese patients had an increased risk of AKI, and patients who developed an AKI had longer hospital stays. However, obesity was a protective factor for 2year mortality for all post-surgical patients including patients who developed an AKI.

\section{THE OPIOID EPIDEMIC AND THE HOMEBOUND OLDER ADULT: A SURVEY OF HOME-BASED PRIMARY CARE PROVIDERS}

Vassiliki Pravodelov. Boston University School of Medicine, Boston, MA. (Control ID \#3184513)

BACKGROUND: The Centers of Disease Control and State Public Health Departments have guidelines to regulate controlled substances. Though interventions to adhere to these guidelines are available in the ambulatory setting, none exist in the home-based primary care setting. This study aims to examine the attitudes, knowledge and trends of geriatrics providers in regards to prescribing and monitoring controlled substances in home care.

METHODS: The study took place at a large urban safety-net hospital. The study team administered an anonymous 57 -item survey. The subjects were 17 geriatrics providers, including registered nurses, nurse practitioners, and physicians, who practice in the home care setting.

RESULTS: The survey response rate was $100 \%$. Approximately $60 \%$ of responders noted that $>30 \%$ of their patients have chronic nonmalignant pain (CNMP). Almost all providers believed that diversion (82\%) and misuse $(100 \%)$ of prescription opioid pain medication in the community served by the practice is a moderate/serious problem. $82 \%$ reported that they were aware of the state regulations regarding controlled substance 
prescribing, but less than half of the providers $(43 \%)$ responded correctly to specific questions about the content of these regulations. 53\% noted that they use the state prescription monitoring program on at least a monthly basis. $82 \%$ felt confident in their ability to identify red flags of drug use disorder in patients prescribed controlled substances. Providers seldom signed Patient Provider Agreements (10\%) and rarely ordered urine/saliva drug testing (2\%). $41 \%$ of providers were confident to negotiate a Patient Provider Agreement while just $17 \%$ of providers were confident to interpret urine/saliva drug tests. Pill counts only occurred when the providers suspected a problem. Providers acknowledged many barriers in adhering to current state regulations including a knowledge deficit, lack of cooperation from patients/caregivers, lack of resources, and difficulty in obtaining urine toxicology samples due to comorbidities. CONCLUSIONS: This study highlights current attitudes and practices of geriatrics home-based primary care providers in regards to controlled substance prescribing and monitoring. It suggests that although CNMP is a common problem among patients, providers often feel that they cannot comply with all the recent federal and state regulations for several reasons. Furthermore, it suggests that though older adults have been on the margins of the opioid epidemic, primarily due to lack of data, opioid misuse and diversion is viewed as a significant problem in this population. The results of this study may serve as a stepping stone in developing efficient and effective plans for safely prescribing and monitoring controlled substances in the geriatrics home care setting.

\section{THE OSA PATIENT AND CAREGIVER EXPERIENCE OF PAP. A QUALITATIVE ANALYSIS OF ONE-ON-TWO SEMI- STRUCTURED MOTIVATIONAL INTERVIEWS}

NAZIA NAZ S. KHAN ${ }^{1}$; Ade B. Olomu ${ }^{2}$; Shireesha Bottu ${ }^{1}$; Margaret Roller ${ }^{1}$; Robert C. Smith ${ }^{1} .{ }^{1}$ MICHIGAN STATE UNIVERSITY, East Lansing, MI; ${ }^{2}$ Michigan State University, East Lansing, MI. (Control ID \#3185019)

BACKGROUND: The prevalence of Obstructive Sleep Apnea (OSA) is increasing. Positive Airway Pressure (PAP) is the mainstay therapy for OSA. Despite its several benefits that include improvement in sleep quality, daytime symptoms of fatigue, drowsiness, and quality of life, reduction in blood pressure and decrease the risk of traffic accidents, nearly $25 \%$ of patients discontinue PAP use within 2 weeks. There is some evidence that partner involvement in education regarding OSA and PAP therapy may help improve PAP adherence. The aim of this qualitative analysis was to identify OSA patients' preferences, partner experiences, barriers and facilitators to adherence to PAP, and to assess understanding of the educational content delivered during a group visit (GV).

METHODS: 28 interventional arm patients (from a larger study) with a new diagnosis of OSA and their caregiver participated in the $60-90$ minutes in-person GV conducted from 1-17 weeks of receiving the PAP machine. Thirteen GVs ( 1 to 3 patients with their caregiver/GV) were held during the course of the study. A qualitative thematic analysis was conducted on the one-on-two (Research assistant with patient and caregiver) semi-structured motivational interview, which was the fourth and last part of the intervention during the GV. The first three sessions of the GV included i) an Interactive Educational Session focused on education on OSA, its effect on health, PAP and its effects, and the importance of family/partner support; ii) peer coaching from an OSA patient; iii) handson experience with the PAP machine from a respiratory therapist. The 1015 minutes interview with the patient and caregiver focused on obtaining the personal and emotional history, providing support, assessed understanding of the OSA and negotiated plan and commitment.

RESULTS: Of the 28 participants, 16 were female and 12 were male. Mean age was 58 years. Of the 28 caregivers, 21 spouses, 2 sons/daughter, 1 friend, 1 sister and 3 were identified as girlfriend/significant other/ partner of the patient. The qualitative analysis considered patients' attitudes, behavior, and emotional effects of their OSA experience associated with the four themes: OSA symptom and diagnosis, using the PAP machine, perceptions about the GV, and factors that determine PAP adherence. Patients had positive, negative or mixed emotions towards the different aspects of the OSA diagnosis and PAP machine. The sense of relief and the desire to live a long life were the two positive underlying emotions; fear and frustration of the OSA diagnosis and PAP machine are the two negative emotions, experienced in varying levels of intensity.

CONCLUSIONS: We concluded that patients' and caregivers' positive experiences of PAP could be enhanced by a patient-centered interaction and that it was important to explicitly address their fears and concerns to further enhance the use of PAP.

\section{THE PATIENT-CENTERED MEDICAL HOME IN GOVERNMENT-BASED AND INTEGRATED DELIVERY AND FINANCE SYSTEMS: A SYSTEMATIC REVIEW}

Clark A. Veet ${ }^{1}$; Inmaculada Hernandez ${ }^{5}$; Thomas R. Radomski ${ }^{3}$; Christopher A. D'Avella ${ }^{2}$; Charles B. Wessel ${ }^{4}$; William H. Shrank ${ }^{1}$; Elizabeth C. Swart ${ }^{1}$; Natasha Parekh ${ }^{2} .{ }^{1}$ UPMC, Pittsburgh, PA; ${ }^{2}$ University of Pittsburgh Medical Center, PIttsburgh, PA; ${ }^{3}$ University of Pittsburgh School of Medicine, Pittsburgh, PA; ${ }^{4}$ University of Pittsburgh, Pittbsurgh, PA; ${ }^{5}$ University of Pittsburgh, Pittsburgh, PA. (Control ID \#3164140)

BACKGROUND: As healthcare reimbursement moves from volume to value-focused, new delivery models aim to coordinate care and improve quality. A popular model, the patient-centered medical home (PCMH), aims to promote focused, well-coordinated, and accessible healthcare to improve outcomes and decrease costs. Prior studies have shown mixed outcomes regarding the ability of PCMHs to achieve these objectives. Additionally, it is unclear whether PCMHs operating within governmentbased systems or integrated delivery and finance systems (IDFS) where healthcare payers and providers are vertically integrated impact utilization and cost outcomes.

METHODS: We performed a systematic review of clinical trials and observational studies that identified $\mathrm{PMCH}$ or equivalent interventions. Ten reviewers performed screening, data extraction, and risk of bias assessment. Primary outcomes included emergency department (ED) visits, hospital admissions, outpatient visits, and total cost of care. We categorized PCMHs as operating within government systems (including Veterans Affairs and Indian Health Service), IDFS, and non-IDFS.

RESULTS: A systematic search of PubMed, the Cochrane Library, and Embase from 2004 to April 2017 yielded 13,939 unique publications. 64 studies were included. $29 \%$ were government systems, $24 \%$ were IDFS, and $47 \%$ were non-IDFS. Utilization outcomes were reported in $74 \%$ of studies and included ED visits, hospitalizations, or outpatient visits. Of the 35 studies reporting ED visits, 17 (48\%) showed lower ED utilization. ED visits decreased in $41 \%$ of 11 government studies, $75 \%$ of 8 IDFS studies, and $38 \%$ of 16 non-IDFS studies. Of the 31 studies on admissions, 11 (35\%) showed decreased admissions. Admissions decreased in $50 \%$ of 12 government studies, $28 \%$ of 7 IDFS studies, and $25 \%$ of 12 non-IDFS studies. PCP visits decreased in $8(47 \%)$ of 17 studies. PCP visits increased in all 6 government studies while specialty visits decreased in $50 \%$ of 4 government studies. Among IDFS studies, PCP visits decreased in all 4 studies while specialty visits decreased in 1 (33\%) of 3 studies. Among 7 non-IDFS studies, PCP visits increased in $42 \%$ of studies while only $14 \%$ showed decreased specialty care. Economic outcomes were reported in 25 (39\%) studies, most commonly as total cost of care. Reduced costs were observed in $60 \%$ of 5 government studies, $50 \%$ of 8 IDFS studies, and $33 \%$ of 12 non-IDFS studies.

CONCLUSIONS: Our study uniquely assessed whether PCMH model effectiveness differed between government systems, IDFS, and nonIDFS. ED visits decreased most within IDFS, while hospital admissions decreased most within government systems. Notably, government systems observed an increase in PCP visits. It is unclear if this change reflects the effectiveness of the PCMH model or patient characteristics. Cost 
reductions were reported in less than half of studies. This study has important implications for understanding how system-based factors affects the success of PCMH interventions.

\section{THE PERCEIVED HEALTH IMPACT OF SIGNIFICANT PLACES AMONG AFRICAN AMERICAN ADULTS WITH CAR- DIOVASCULAR RISK FACTORS}

Michelle J. White ${ }^{1}$; Stephanie Hoover ${ }^{2}$; Katelyn Holliday ${ }^{1}$; Nicole Robinson-Ezekwe ${ }^{2}$; Leah Frerichs ${ }^{3}$; Kiana Bess ${ }^{2}$; Anissa Williams ${ }^{2}$; Giselle M. Corbie-Smith ${ }^{2} .{ }^{1}$ Duke University, Durham, NC; ${ }^{2}$ University of North Carolina, Chapel Hill, NC; ${ }^{3}$ University of North Carolina at Chapel HIll, Chapel Hill, NC. (Control ID \#3183908)

BACKGROUND: Environmental factors have been demonstrated to contribute to cardiovascular (CV) disparities among African American adults in the American South. Research has focused on observed built environment characteristics surrounding participants' place of residence, or pre-determined constructs (e.g., walkability). However, the built environment is perceived and experienced differently by individuals in ways that may moderate its influence on self management behaviors, yet limited research has explored such perceptions in-depth. We sought to examine African American adults' perceptions of the places that have significance in their lives and how these places impact their health. METHODS: We conducted a thematic analysis of African American adults $(\mathrm{N}=25)$ in two North Carolina counties considered to be at the highest level of disadvantage based on multiple economic factors. Participants were recruited from a community-based behavioral $\mathrm{CV}$ risk reduction intervention. All had at least one risk factor for $\mathrm{CV}$ disease. We asked participants to identify significant places including where they spent the most time, meaningful places in their day to day lives, and healthy and unhealthy places on local maps. Using these maps as a reference during semi-structured interviews, participants described the health impact of each location. Data were transcribed verbatim and coded using NVivo 12.

RESULTS: The average age of participants was $63(\mathrm{SD}=10)$ and $88 \%$ were female. Places participants defined as meaningful and places where they spent the most time included churches and relatives' homes. Healthy places included their homes and local gyms. Unhealthy places included fast food restaurants and relatives' homes where unhealthy food was served. Participants illustrated the impact of these places on diet and physical activity by describing both the physical characteristics (i.e. presence of a running track; menu of healthy food) and the social characteristics and influences within the place (i.e. attending church with people who are trying to live a healthy lifestyle; caring for grandchildren at home who prefer fast food). Participants also described how they "spread the word" about lessons acquired from the intervention within their significant places.

CONCLUSIONS: Participants identified several significant places that impacted their health and described how their health behaviors within significant places were influenced by both physical and social characteristics. As we seek to improve CV interventions for African Americans in the American South, it will be important to further assess the effect of significant places beyond place of residence and how the social and physical characteristics of these places interact to influence CV outcomes. Better understanding of social influence, particularly the manner in which healthy behaviors are reinforced, hindered, and disseminated within significant places, could be leveraged to improve the reach of $\mathrm{CV}$ interventions.

THE POCUS SUPERVISION SAFETY GAP: ATTENDING PHYSICIAN KNOWLEDGE IN POINT-OF-CARE ULTRASOUND LAGS BEHIND INTERNAL MEDICINE RESIDENTS

James E. Anstey ${ }^{2}$; Stephanie Conner ${ }^{2}$; Meghan T. O'Brien ${ }^{3}$; Farhan Lalani ${ }^{1}$; Trevor Jensen ${ }^{2}$. ${ }^{1}$ University of California San Francisco, San Francisco, CA; ${ }^{2} \mathrm{UC}-\mathrm{San}$ Francisco, San Francisco, CA; ${ }^{3} \mathrm{UCSF}$, San Francisco, CA. (Control ID \#3185369)
BACKGROUND: Point-of-Care-Ultrasound (POCUS) is increasingly recognized as a useful tool in hospital medicine. POCUS training opportunities for US Internal Medicine residents have been rapidly expanding, but education for hospitalist attendings may lag behind creating a potential safety gap in supervision. We developed a test assessing four domains of knowledge within routine applications of POCUS used in hospital medicine and compared attending and resident physician performance at a single academic teaching hospital.

METHODS: Hospital medicine faculty experienced in POCUS developed a 15-item test designed to assess the application of POCUS in hospital medicine practice. The test focused on four domains of use: basic knowledge, image interpretation, clinical integration, and understanding of limitations. The test questions were developed to address common usecases for POCUS and be performed in a short time ( $<10$ minutes). An invitation to complete the test was sent by email to all internal medicine residents $(n=180)$ and hospital medicine attendings $(n=97)$. Respondents were instructed not to guess, and each question included an option "Not sure". Test results (\% correct) were compared for attendings and residents using unpaired t-tests and analysis of variance.

RESULTS: A total of 58 residents (32\% response rate) and $41(41 \%)$ attendings completed the test. The mean resident score was $55.6 \%(95 \%$ CI 49.8- 61.3) and mean faculty score was $45.3 \%$ (95\% CI 27.9- 54.1) with a mean difference of $10.3 \%$ (95\% CI $0.32-20.3 ; p=0.022)$. Attendings performed significantly worse than residents in the domains of basic knowledge (mean difference $22.0 \%$ [95\% CI 9.1- 34.9], $\mathrm{p}=0.001$ ), image interpretation (mean difference $10.8 \%$ [95\% CI 0.2 21.5], $\mathrm{p}=0.046$ ), and understanding of limitations (mean difference $14.7 \%$ [95\% CI 1.7- 27.8], $\mathrm{p}=0.027$ ). Additionally, respondent's scores differed by level of clinical experience. Interns scored a mean of $45.5 \%$, and senior residents $61.7 \%$. Faculty of $0-3$ years' experience scored a mean of $59.3 \%$, $4-6$ years $51.6 \%, 7-10$ years $33.3 \%$, and faculty with $>10$ years' experience, a mean of $23.6 \%,(\mathrm{p}=0.0002$; ANOVA).

CONCLUSIONS: Our study revealed that, while resident physicians at our institution have limited knowledge and require ongoing supervision, our hospital medicine faculty had a significantly lower baseline of POCUS knowledge than the residents they supervise. Specifically, hospitalists were more deficient in domains of basic knowledge, image interpretation, and importantly, understanding of limitations. Hospitalists more recently out of training scored higher than those with more attending experience, which may reflect the recent wave of POCUS training in internal medicine resident programs. As resident physicians continue to gain valuable new skills in POCUS, this data argues for comprehensive faculty curricula with emphases on supervision, appropriate use, and knowledge of limitations of POCUS use in hospital medicine to address and prevent this supervision safety gap.

\section{THE PREVALENCE AND CAUSES OF DELAYED ENDOS- COPIES FOR HOSPITALIZED PATIENTS}

Molly A. Kantor; Sara Lewin; Kendall Beck; Ryan David; Helge Eilers; Bertina Herman; Cameron Locke; Scott Pasternak; Sajan Patel; Najwa ElNachef. University of California, San Francisco, San Francisco, CA. (Control ID \#3184503)

BACKGROUND: Hospitalized patients often require urgent gastrointestinal endoscopy, and timely procedures are associated with better patient outcomes. However, there is limited literature describing the timeliness of inpatient endoscopic procedures and potential causes of delay. To address this, we conducted a single-center observational study of all inpatient endoscopies to characterize the prevalence and causes of delay.

METHODS: We identified all inpatients planned to undergo endoscopy at our 600-bed urban academic medical center using the electronic health record and paper endoscopy unit records. All endoscopies were performed as part of routine clinical care. We obtained the procedure request date and procedure completion date; a delay was defined as any difference 
between these dates. For all identified cases of delay, a gastroenterologist or hospitalist reviewed the medical chart and indicated the reason(s) for delay using a structured data collection tool. For each requested endoscopy, the type of endoscopy and sedation, indication for endoscopy, and length of delay were also collected.

RESULTS: During the study period, 715 endoscopies were requested and $680(95.1 \%)$ were performed; $35(4.9 \%)$ were cancelled. Most (303, $44.6 \%$ ) endoscopies were esophagogastroduodenoscopies (EGDs), followed by combined EGD-colonoscopies $(173,25.4 \%)$, flexible sigmoidoscopies $(107,15.7 \%)$, and colonoscopies $(79,11.6 \%)$. Of completed endoscopies, 88 (12.9\%) were delayed. The most common causes of delay are shown in Table 1 . The majority of delays were avoidable (66, $75.0 \%)$, due to scheduling delays $(44,50.0 \%)$ and issues with bowel preparation $(18,20.5 \%)$. The most common unavoidable delays were due to clinical instability $(11,12.5 \%)$. Endoscopies were delayed for an average of 1.4 days (1-8 days, median 1) representing 120 days of delay. CONCLUSIONS: Delays in endoscopy at a single academic medical center were common, and most delays were due to avoidable causes. Interventions to reduce delay should target scheduling delays and issues with bowel preparation.

Table 1: Causes of Endoscopy Delay

\begin{tabular}{|c|c|}
\hline Reason for Endoscopy Delay & $\mathbf{n}(\%)$ \\
\hline Avoidable Delays & $66(75.0 \%)$ \\
\hline Schedule delay & $44(50.0 \%)$ \\
\hline Anesthesia schedule delay & $11(12.5 \%)$ \\
\hline Endoscopy schedule delay & $1(1.1 \%)$ \\
\hline Unspecified schedule delay & $32(36.4 \%)$ \\
\hline Issues with bowel preparation & $18(20.5 \%)$ \\
\hline Patient unable to tolerate preparation & $10(11.4 \%)$ \\
\hline Poor bowel preparation & $8(9.1 \%)$ \\
\hline Other competing procedures & $5(5.7 \%)$ \\
\hline Unavoidable delays & $15(17.0 \%)$ \\
\hline Clinical instability & $11(12.5 \%)$ \\
\hline Violated ni per os requirement & $2(2.3 \%)$ \\
\hline Refused procedure & $1(1.1 \%)$ \\
\hline Other clinical reason & $8(9.1 \%)$ \\
\hline Unknown & $7(8.0 \%)$ \\
\hline
\end{tabular}

\section{THE PREVALENCE OF DIAGNOSTIC ERROR IN SEVEN-DAY READMISSIONS OF GENERAL MEDICAL PATIENTS}

Molly A. Kantor $^{1,2}$; Katie Raffel ${ }^{1,2}$; Peter Barish ${ }^{1,2}$; Armond Esmaili ${ }^{1,3}$; Hana Lim ${ }^{1,4}$; Feifei Xue ${ }^{1,2}$; Sumant Ranji ${ }^{1,4}$. ${ }^{1}$ University of California, San Francisco, San Fransisco, CA; ${ }^{2}$ University of California, San Francisco, San Francisco, CA; ${ }^{3}$ San Francisco VA Medical Center, San Francisco, CA; ${ }^{4}$ Zuckerberg San Francisco General Hospital, San Francisco, CA. (Control ID \#3180388)

BACKGROUND: Diagnostic errors have been cited as a potential contributor to hospital readmissions, particularly early readmissions (e.g. within 7 days), but little is known about their frequency and characteristics. We performed structured chart review of all medical patients readmitted within 7 days of discharge from a single academic medical center to describe the prevalence of diagnostic error and characterize contributing factors.

METHODS: Our study included all patients discharged from the inpatient medical service and re-admitted to our 600-bed urban academic medical center within 7 days to any service from 1/1/18-9/30/18. Each case underwent independent structured review by two trained boardcertified internists, followed by adjudication to achieve consensus. Cases of discrepancy were reviewed by the larger diagnostic review team. Diagnostic errors were identified using the modified "Safer Dx Instrument," a validated tool to identify diagnostic error in primary care visits. When identified, errors within the diagnostic process were then characterized using the Diagnostic Error Evaluation and Research (DEER) taxonomy tool, an error classification system. Multiple contributing factors could be identified in the same admission. For all cases identified to contain diagnostic error, the discharging physician(s) from the index admission was contacted to provide further details of the clinical decision-making and circumstances that may have contributed to the error, which was then used to refine diagnostic error characterization.

RESULTS: During the study period, we identified 261 cases of 7-day readmission (4.6\% of 5663 admissions), of which 258 (98.9\%) were reviewed. A total of $18(7.0 \%)$ admissions contained diagnostic error. In 17 of the 18 cases $(94.4 \%)$, the error was judged as having "moderate" impact (such as short-term morbidity, increased length of stay, or invasive procedure), and 1 had no impact. In the DEER taxonomy, the most common culprit areas within the diagnostic process contributing to error were in Laboratory/Radiology Tests (25) and Assessment (17). The most common subcategories contributing to error were failure/delay in ordering needed test(s) [10,56\%], erroneous clinician interpretation of test(s) [6, $33 \%]$, and suboptimal weighing/prioritizing of finding or diagnosis [6, $33 \%$ ]. Diagnostic errors represented a breadth of diagnoses; no single diagnosis was repeated among the 18 identified errors.

CONCLUSIONS: The prevalence of diagnostic error among 7-day medical readmissions was $7 \%$, similar to prior estimates. Nearly all diagnostic errors had moderate clinical impact. Identified diagnostic errors represented a breadth of diagnoses; thus, disease-specific diagnostic improvement efforts may be of limited utility. The most common underlying drivers of diagnostic error were related to clinician diagnostic reasoning, highlighting the importance of efforts to augment diagnostic reasoning.

\section{THE RELATIONSHIP BETWEEN SEXUAL ASSAULT HISTORY AND GYNECOLOGIC EXAM DISTRESS AND DELAY AMONG WOMEN VETERANS IN THE VA}

Elisheva Danan $^{1}{ }^{2}$; Michele Spoont ${ }^{1}$; Julian Brunner ${ }^{3}$; Elizabeth M. Yano ${ }^{3}$. ${ }^{1}$ Minneapolis VA Health Care System, Minneapolis, MN; ${ }^{2}$ University of Minnesota Medical School, Minneapolis, MN; ${ }^{3}$ VA Greater Los Angeles Healthcare System, Sepulveda, CA. (Control ID \#3185977)

BACKGROUND: Sexual assault affects 1 in 3 U.S. women and has lifelong consequences for women's health that can include sexually transmitted infections, pregnancy, posttraumatic stress disorder (PTSD), and a more than two-fold higher cervical cancer risk (OR 2.6). Routine cervical cytology (pap) testing has reduced the U.S. incidence of cervical cancer by over $50 \%$ in the past thirty years. However, women who have experienced sexual assault face barriers to completing cervical cancer screening related to anxiety, fear, exam discomfort, and access to care. Invasive medical exams can also trigger an exacerbation of PTSD symptoms. Risk of sexual assault and barriers to pap testing may be intensified for women Veterans, as compared to non-Veterans. During military service, $25 \%$ of women experience military sexual trauma (MST), which is associated with multiple poor health outcomes and health risk behaviors. MST can also affect women Veterans' relationship with the VA healthcare system. We hypothesize that, compared to women Veterans with no sexual assault history, women Veterans with a history of lifetime sexual assault are more likely to experience high levels of distress related to pelvic exams, and are more likely to report ever delaying a pap test or gynecologic exam.

METHODS: We analyzed data from a 2015 survey of women Veterans who use primary care and/or women's health services at 12 VAs in 9 states. We compared characteristics of women Veterans with and without 
a history of lifetime sexual assault. Next, we conducted two hierarchical logistic regression analyses to examine associations of lifetime sexual assault with our outcomes: 1) distress associated with pelvic exams, and 2 ) ever delaying a pap test or gynecologic exam due to distress. Covariates were added to the model in conceptual blocks that included demographics, physical health, and health system factors.

RESULTS: Our sample included 1,152 women with a mean age of 49, of whom 675 (58\%) reported lifetime sexual assault. Compared to women with no history of sexual assault, women with a history of sexual assault were older, had poorer self-rated physical health, were more likely to be current or former smokers, and had higher rates of positive screens for anxiety/depression and PTSD. In the full regression model, lifetime sexual assault was significantly associated with reporting a high level of distress related to pelvic examinations (OR 2.3, 95\% CI 1.5-3.5), and with reporting ever delaying a gynecologic exam due to distress (OR 1.9, 95\% CI 1.3-3.0).

CONCLUSIONS: Among women Veterans, sexual assault is significantly associated with pelvic exam distress and with delay of gynecologic exams. Trauma-sensitive primary care practices are needed to reduce discomfort, distress and anxiety around pelvic examinations in order to improve patient experiences and completion of critical preventive screening.

\section{THE RELATIONSHIP OF LONELINESS TO SYMPTOM BUR- DEN, INTENSITY OF CARE, AND ADVANCE CARE PLAN- NING AT END OF LIFE IN OLDER AMERICANS}

Nauzley C. Abedini; HwaJung Choi; Melissa Y. Wei; Kenneth Langa; Vineet Chopra. University of Michigan, Ann Arbor, MI. (Control ID \#3156831)

BACKGROUND: Lonely adults are prone to a variety of poor health outcomes, including accelerated functional and cognitive decline, depression, and premature death. As a result, lonely adults may be prone to higher health-related suffering, triggering increased healthcare utilization and possibly undesired aggressive care, particularly as they approach end of life (EOL). However, little is known about the EOL experience of this population. We aimed to quantify the relationship of loneliness to symptom burden, intensity of care, and advance care planning in older adults at EOL.

METHODS: We conducted a pooled cohort analysis of lonely and not lonely decedents in the Health and Retirement Study (HRS) from 2004 to 2014, a nationally representative study of individuals over the age of 50 . Proxy informants were interviewed regarding decedents' EOL experience within 2 years of a participant's death. We defined loneliness using the 3 item Revised University of California Los Angeles (R-UCLA) Loneliness Scale derived from a decedent's most recent HRS interview prior to death. We assessed baseline characteristics of lonely versus not lonely individuals, including demographics, socioeconomic status, multimorbidity, depressive symptoms, and measures of social network (including partner status) and social connection (reliance on others for support). We used multivariable logistic, ordinary least squares, and negative binomial regression where appropriate to examine the independent association between loneliness and (1) total symptom burden in the last year of life; (2) measures of intense EOL care; and (3) advance care planning.

RESULTS: Of 3,065 decedents, 33\% were lonely. Loneliness was independently associated with total symptom burden at EOL $(\beta=0.16,95 \%$ CI 0.05-0.28; $\mathrm{p}=0.005$ ), after adjusting for demographics, socioeconomic status, multimorbidity, depressive symptoms, and measures of social network and social connection. Lonely decedents had higher odds of receiving life support in the last 2 years of life (AOR 1.52, 95\% CI 1.03-2.24; $\mathrm{p}=0.03$ ). However, loneliness did not have an independent association with other measures of EOL care intensity, including place of death, hospitalizations, ICU admissions, nursing home utilization, or hospice use. Lonely individuals had higher odds of engaging in EOL care discussions with their next of kin (AOR 1.70, 95\% CI 1.13-2.54; $\mathrm{p}=0.01$ ) and assigning a durable power of attorney (DPOA) (AOR 1.51, 95\% CI
1.01-2.24; $\mathrm{p}=0.04$ ), but had similar rates of documenting discussions and participating in EOL decisions.

CONCLUSIONS: Lonely older adults suffer from increased symptoms at EOL despite higher advance care planning and similar, if not higher, EOL healthcare utilization. These associations were independent of objective social network and social connection. Targeted interventions for lonely individuals are needed to improve EOL care and quality of life.

\section{THE RELATIVE CONTRIBUTIONS OF HOSPITAL VS. SKILLED NURSING FACILITY QUALITY ON PATIENT OUTCOMES}

$\underline{\text { Paula Chatterjee }}{ }^{1}$; Mingyu Qi ${ }^{1}$; Rachel M. Werner ${ }^{2}{ }^{1}$ University of Pennsylvania, Philadelphia, PA; ${ }^{2}$ University of Pennsylvania and Philadelphia VA, Philadelphia, PA. (Control ID \#3185716)

BACKGROUND: Medicare will begin financially penalizing skilled nursing facilities (SNFs) with higher-than-expected readmission rates. Despite enthusiasm for the goal of reducing unnecessary readmissions from SNFs, some have raised questions about whether SNF readmissions are truly under the control of SNFs or whether they are more likely attributable to the quality of care received at the hospital from which the patient was discharged. We compared the relative contributions of hospital and SNF quality on readmission rates while controlling for unobserved patient characteristics.

METHODS: We performed a retrospective cohort study of Medicare beneficiaries with two hospitalizations followed by SNF admissions from 2010-2014. We divided beneficiaries into two groups: (1) patients who went to different hospitals but were discharged to the same SNF after both hospitalizations; and (2) patients who went to the same hospital but were discharged to different SNFs. The primary exposure was hospital-level and SNF-level quality, using a lagged measure of 30-day riskstandardized readmission rates for each. We estimated the risk-adjusted probability of readmission in the two groups of patients using a linear probability model with patient-level fixed effects.

RESULTS: We identified 232,243 patients who changed hospitals but not SNFs, and 276,095 who changed SNFs but not hospitals. We found that patients who went to the lowest-performing quartile of hospitals (highest risk-standardized readmission rate) had a $1.5 \%$ higher likelihood of readmission $(\mathrm{p}<0.001)$ compared to patients who went to the highestperforming hospitals (lowest risk-standardized readmission rate). In contrast, SNF performance had a larger impact on the likelihood of patient readmission. Patients who went to the lowest-performing SNFs had a $3.1 \%$ higher risk of readmission $(\mathrm{p}<0.001)$ compared to patients to went to the highest-performing SNFs.

CONCLUSIONS: The association between SNF quality and patient outcomes was larger than the association between hospital quality and patient outcomes among post-acute care patients. Holding post-acute care providers accountable for their quality may be an effective strategy to improve SNF quality and prevent readmissions among a comorbid and costly patient population. While some policymakers have raised concerns about the magnitude of post-acute care expenses, there may be a benefit to patients by prioritizing high quality post-acute care.

\section{THE RETIREMENT MILESTONE: HOW CAN WE HELP OLDER ADULTS MAKE THE TRANSITION?}

Jennifer Shiroky; Chirstine Caufield-Noll; Alicia I. Arbaje. Johns Hopkins Bayview Medical Center, Baltimore, MD. (Control ID \#3185125)

BACKGROUND: Healthcare providers are in a unique position to help older adults transition into and optimize their health in retirement, yet there are few guidelines to assist providers. Over 40 million adults are approaching retirement; this major life milestone often leads to significant changes in psychological, social, financial, and physical dimensions of health. The objective of this study was to conduct a scoping review of 
research on strategies and best practices for healthcare providers to help older adults thrive during retirement.

METHODS: For this scoping review, electronic databases (PubMed; Google Scholar; PsycINFO (EBSCO); and Scopus) were searched using keyword and controlled vocabulary terms including retirement, physicians, physician-patient relationship, counseling, and guidance. No date limitations were used. English-language articles focusing on those 55 years of age and older planning to or recently leaving the labor force who were screened or counseled by their healthcare providers about how to maintain or improve their health during this transition were included. All study types were included.

RESULTS: Database searches retrieved 530 citations. One hundred ninety-six citations were for abstract review and six article met criteria for full-text review. We found that the majority of articles focused on retirement from the medical profession or on measuring a particular intervention in the retired population. Few commented on the actual act of retirement. Importantly, no articles addressed how healthcare providers could help older adults in preparing for and moving through retirement. The few articles that did discuss the importance of addressing this transition were opinion pieces from the 1950s-1980s.

CONCLUSIONS: Surprisingly, the majority of literature focused on physician retirement, rather than on how healthcare providers can assist older adults with their retirement. There was essentially no literature evaluating strategies and best practices for healthcare providers to address the complexities of retirement and its effect on older adults' physical and mental health. Retirement, as it currently exists in the healthcare literature, is a potential untapped treasure trove of opportunities for new healthcare interventions to optimize older adults' health. This is especially critical as a large portion of the US population prepares to retire under turbulent times for social security and public and private insurance coverage. Future research can assess if and how healthcare providers approach retirement with older adults, what factors help older adults thrive in retirement, and what tools are needed for healthcare providers to use to address retirement during healthcare visits.

\section{THE ROLE OF FAMILY ON DIABETES MANAGEMENT FOR LATINO ADULTS}

Olivia Jordan ${ }^{1}$; Amanda Benitez ${ }^{3}$; Deborah L. Burnet ${ }^{2}$; Michael T. $\overline{\text { Quinn }}^{2}$; Arshiya A. Baig ${ }^{2} .{ }^{1}$ University of Chicago Pritzker School of Medicine, Chicago, IL; ${ }^{2}$ University of Chicago, Chicago, IL; ${ }^{3}$ Enlace Chicago, Chicago, IL. (Control ID \#3184542)

BACKGROUND: Mexican Americans have one of the highest prevalence of diabetes within the U.S. Hispanic community with $18.3 \%$ affected. Diabetes self-care behaviors are essential to diabetes management and often performed in the context of family support. However, few studies explore this relationship in Mexican-Americans. Our study aims to better understand how family influences diabetes self-care management in the Mexican-American community and how family is impacted by a loved one's diagnosis of diabetes.

METHODS: We analyzed qualitative data from semi-structured interviews with Latino adults with diabetes and focus groups with adults with diabetes and their family members conducted in a predominantly Mexican-American immigrant Chicago community. Interviews and focus groups were conducted in Spanish by trained, bilingual research staff and transcribed and translated to English. Data was coded using a modified template technique using Hyperresearch.

RESULTS: Data was collected from a total of 71 participants. The majority were diagnosed with diabetes ( $82 \%$ ), from Mexico $(86 \%)$, and female ( $80 \%)$. Participants with diabetes described a lack of social support from family, family not knowing what it is like to live with diabetes, the negative effect of diabetes on the family relationship, and difficulty maintaining changes when with family. Many participants with diabetes had difficulty motivating family to engage in a healthy lifestyle with them ("[My family is] used to eating differently; some of them want to eat what I eat and others do not.") Even so, family was involved in day-to-day management of diabetes and provided emotional, informational, instrumental and companionship support. Suffering or death of family with diabetes and preventing diabetes in family were frequently mentioned as the main motivator to improve diabetes control. Many participants with diabetes shared information from diabetes education programs that led to positive behavioral changes in some family members. Family members noted that family responsibilities sometimes interfered with providing adequate support for their loved one with diabetes. Most family members expressed that diabetes affected their wellbeing and that of the whole family. Family members had a desire to learn more about the disease because they often did not know how to help ("You know, he's your dad and you're trying to at the same time please him but also in our culture, you respect your elders and you can't always tell them no.")

CONCLUSIONS: While lack of support and engagement from family members can undermine successful diabetes management, many participants had immediate family members available to support them. Most family members wished to learn more about how to help. Future patient education interventions with Mexican Americans with diabetes should use a culturally competent approach to educate family members on how to adopt supportive strategies to aid patients' diabetes self-management and implement behavioral changes at the family level.

\section{THE SUPPORTIVE CHURCH ENVIRONMENT SCORE: A NOV- EL SCORE TO QUANTIFY HOW SUPPORTIVE A CHURCH SETTING IS TOWARDS LOWERING CVD RISK FACTORS}

Shanna A. Tucker ${ }^{1}$; Priscilla Amanquah ${ }^{3}$; Rosio Ramos ${ }^{2}$; Naa-solo Tettey ${ }^{4}$; ${ }_{\text {Holly S. Andersen }}^{4}$; Erica Phillips ${ }^{2} .{ }^{1}$ New York Presbyterian- Weill Cornell, New York City, NY; ${ }^{2}$ Weill Cornell Medicine, New York, NY; ${ }^{3}$ Hunter College, New York City, NY; ${ }^{4}$ Ronald O. Perelman Heart Institute, New York-Presbyterian Hospital, New York City, NY. (Control ID \#3185360)

BACKGROUND: Age-adjusted obesity prevalence and cardiovascular disease (CVD) risk factors continue to disproportionately affect African Americans (AA). Multiple studies have shown that faith-based interventions can improve many of these factors, such as fruit and vegetable $(\mathrm{F} \& \mathrm{~V})$ intake. The development of a quantitative measure of the supportive health environment of churches, or the correlation of that support with church members' CVD risk behaviors have not been studied. The goal of this study was to develop and test a quantitative measure of how supportive a church's environment is towards promoting lifestyle habits that decrease CVD risk, termed the Supportive Church Environment Score (SCEC).

METHODS: Predominantly AA churches were enrolled in a 10-week faith-based cardiovascular risk reduction program led by trained lay health educators. Program participants completed a pre and post assessment inclusive of measures of CVD risk behaviors and 11 activities about the church environment. One point was assigned to each activity for a maximum score of 11. Higher scores suggest participants perceive that their church promotes CVD health activities. We evaluated the relationship between each participant's SCEC score and their daily F\&V intake, physical activity, and blood pressure control. We also compared the average SCES score between churches and average $F \& V$ intake between churches.

RESULTS: At the time of this analysis 9 churches (136 participants) completed the baseline assessment. The mean age was 62.5 (SD 1.2), $80 \%$ AA, $83 \%$ women, $34 \%$ married and $97 \%$ had a high school education or higher. The mean servings of F\&V were 1.7 for both. Table 1 depicts the number of participants (n) per church and average SCES. While there was a significant difference between mean SCES $(p<0.001)$, there was no significant difference between mean daily $\mathrm{F} \& \mathrm{~V}$ intake between churches. We found no correlation between baseline perceived individual SCEC with daily F\&V intake, physical activity level or blood pressure.

CONCLUSIONS: We were able to develop a quantitative measure of health promotion activities for faith-based organizations. This early analysis of baseline data is limited by a small sample size and a pervasively 
low consumption of $\mathrm{F} \& \mathrm{~V}$ overall regardless of the supportive church environment. A larger sample size, and other drivers of F\&V intake, such as income, education and knowledge of the benefits of F\&V intake, will need to be factored into the analysis.

\section{TIMELY PRIMARY CARE FOLLOW-UP AND 30-DAY READ- MISSION RATES: A RETROSPECTIVE ANALYSIS FROM A RESIDENT-BASED PRIMARY CARE CLINIC}

Niloofar Latifi; Robert N. Stuver; Tara M. Skorupa; Jorge Fradinho; Rebecca Glassman; Kelly L. Graham. Beth Israel Deaconess Medical Center, Boston, MA. (Control ID \#3184619)

BACKGROUND: The transition in care from hospital to ambulatory setting is a vulnerable period for patients. Previous analyses on whether timely post-discharge visits with a primary care provider (PCP) reduce readmissions have shown mixed results. The primary aim of our project was to investigate the hypothesis that a primary care visit within 14 days of discharge leads to a reduction in 30-day readmission rates.

METHODS: We performed a retrospective analysis of all Medicare and Medicare Advantage patients discharged from our hospital from 10/1/2017 - 9/30/2018 who have a PCP at our hospital-based primary care practice. Patients discharged to rehab or a long-term care facility were excluded. Patients were evaluated for having a timely post-discharge primary care appointment, defined as occurring within 14 days of discharge, in accordance with the Medicare Transitional Care Management period. They were further categorized based on our predictor variable of having an appointment scheduled at time of discharge, and then if this appointment was kept or cancelled/missed. The primary outcome was a readmission within 30 days of discharge. We used a chi square test to determine if rates of readmissions were significantly different between groups.

RESULTS: Our study population included a total of 1069 patients. At discharge, 585 patients $(55 \%)$ had a primary care visit scheduled within 14 days. The 30-day readmission rate for patients with a kept appointment was $17 \%$, compared to $21 \%$ for cancelled/missed appointments, and $36 \%$ in patients without a scheduled appointment at time of discharge $(\mathrm{P}<0.001)$. For patients who saw a specialist but not a PCP within 14 days of discharge, the 30 -day readmission rate was $28.8 \%$, versus $19.5 \%$ for those who saw both a specialist and a PCP $(\mathrm{P}=0.52)$ (Table 1).

CONCLUSIONS: A statistically significant reduction in 30-day readmission rates was observed in patients who are seen by a PCP within 14 days of discharge compared to those who do not keep their appointment or do not have an appointment scheduled at discharge. There is a decrease in 30-day readmission rates when patients are seen by a PCP in addition to a specialist that did not reach statistical significance. Our study demonstrates the importance of timely PCP follow up within the 14-day postdischarge period, as well as the benefit to scheduling appointments for patients at the time of discharge to reduce 30-day readmission rates.

TOWARDS VIRAL HEPATITIS ELIMINATION: AN INNOVATIVE STRATEGY TO SCREEN AT-RISK POPULATIONS IN THE EMERGENCY DEPARTMENT WITH LINKAGE TO PRIMARY CARE

Su H. Wang ${ }^{1}$; Ruth P. Brogden ${ }^{1}$; Eric Handler ${ }^{2}$; Briana Marcantonio ${ }^{3}$; Alyssa Gallipani ${ }^{4}, 5$. ${ }^{1}$ Saint Barnabas Medical Center, Florham Park, NJ; ${ }^{2}$ Saint Barnabas Medical Center, Livingston, NJ; ${ }^{3}$ Saint Barnabas Medical Center, West Orange, NJ; ${ }^{4}$ Fairleigh Dickenson University, Madison, NJ; ${ }^{5}$ RWJBarnabas Health, West Orange, NJ. (Control ID \#3184952)

BACKGROUND: Globally, deaths from viral hepatitis (VH) surpass $\mathrm{HIV}$, malaria and tuberculosis. In the US, VH is a large driver of rising hepatocellular carcinoma rates. However, with hepatitis B vaccines \& treatment and hepatitis $\mathrm{C}$ curative therapies, the World Health Organization has deemed that $\mathrm{VH}$ can be eliminated by 2030. Massive scale-up in screening is required to increase the current low diagnosis rates, and shifting of care from specialists to primary care is critical to achieving elimination. Both CDC and USPSTF recommend screening for $\mathrm{VH}$ in those at-risk: people born in 1945-1965 should be screened for hepatitis C (HCV) and foreign-born people from hepatitis B (HBV) endemic countries should be screened for HBV. We set up automated VH screening in the Emergency Department (ED) to reach the at-risk and vulnerable in New Jersey, where $21 \%$ of the residents are foreign-born and provided navigation and $\mathrm{VH}$ care in a primary-care

METHODS: Electronic Health Record (EHR) modifications include an algorithm for eligibility with date of birth and country of birth. If bloodwork is ordered by a provider, $\mathrm{VH}$ lab test(s) are automatically generated: $\mathrm{HCV}$ antibody ( $\mathrm{HCV} \mathrm{Ab}$ ) with reflex to $\mathrm{HCV}$ RNA and/or a hepatitis B surface antigen ( $\mathrm{HBsAg}$ ). Alerts notify nursing of patient eligibility and positive tests. The patient navigator $(\mathrm{PN})$ receives real time alerts of positive tests to contact patients, meeting them in person or calling to educate and arrange follow-up. The PN has direct access to schedule patients at a primary care practice of internists trained in $\mathrm{VH}$ care. Team meetings of physicians, pharmacist and navigators are held to discuss patient status, including those undergoing HCV cure.

RESULTS: Since March 2018, 7,550 patients have been screened for $\mathrm{HCV}$, with 243 (3.2\%) HCV Ab+ and 84 (1.1\%) with current infection. Fifty-six percent (47) were newly diagnosed and $54(64.3 \%)$ linked to care. HCV RNA+ patients were $61 \%$ male with a mean HCV RNA 2,987,000 IU/mL. Twenty five HCV patients were seen at our practice with 13 initiating HCV therapy for 8-12 weeks. So far, 9 (70.0\%) have undetectable HCV RNA at week 4, and $3(12 \%)$ with confirmed cure post-therapy. For HBV, 6,466 were tested with 76 (1.2\%) infected, 34 (44.7\%) newly diagnosed, and $42(55.3 \%)$ linked to care. Haiti, Jamaica and Ecuador were the top countries-of-origin for those screened, and Haiti, China and Ghana, the top countries of the HBV-infected patients.

CONCLUSIONS: Automated VH testing in the ED is a model for efficient, large-scale screening of at-risk patients, many difficult to reach due to barriers in language, cultural or healthcare access. Navigation is important to provide transition-of-care from the ED to appropriate followup. Primary care providers can manage VH patients, and a healthcare team approach can facilitate successful HCV cures

\section{TOXIDROME PATTERNS AND OUTCOMES IN A MEDICALLY- SUPERVISED OVERDOSE PREVENTION PROGRAM FOR HOMELESS PEOPLE IN BOSTON}

Gabriel R. Wishik-Miller ${ }^{1,2}$; Jessie M. Gaeta ${ }^{1,2}$; Melanie W. Racine ${ }^{1}$; James J. O'Connell ${ }^{1,3}$; Travis P. Baggett ${ }^{1,3}$. ${ }^{1}$ Boston Health Care for the Homeless Program, Boston, MA; ${ }^{2}$ Boston University School of Medicine, Boston, MA; ${ }^{3}$ Massachusetts General Hospital, Boston, MA. (Control ID \#3183938)

BACKGROUND: In response to rising opioid overdose deaths among homeless individuals, Boston Health Care for the Homeless Program (BHCHP) opened the Supportive Place for Observation and Treatment (SPOT) in 2016. SPOT provides medical monitoring and harm reduction services to people who are intoxicated and sedated, often because of concurrent use of opioids and other sedating substances. To inform the development and dissemination of this unique care model, our objective was to characterize toxidrome patterns and describe care outcomes of SPOT users.

METHODS: We analyzed data on adult (age 18-64 yrs) visits to SPOT between 1/1/17-12/31/17 in which the following measures were recorded: blood pressure, heart rate, respiratory rate, pulse oximetry, and Inova Sedation Scale level. Other measures included self-reported age, sex, and substances consumed, including opioids, non-opioid sedatives (e.g. benzodiazepines), stimulants (e.g. cocaine), cannabinoids, and alcohol. Care outcomes included duration of visit, administration of oxygen or naloxone, and manner of discharge. We used k-means cluster analysis to characterize toxidromes based on vital sign patterns. We used multinomial logistic regression to assess the demographic and substance consumption predictors 
of toxidrome cluster membership. We used linear and logistic regression to examine the association between cluster membership and care outcomes.

RESULTS: 156 visitors had 305 episodes of care. $72 \%$ of episodes reported opioid consumption and $63 \%$ reported consumption of a nonopioid sedative. Cluster analysis revealed 3 toxidrome patterns. Cluster A ( $26.6 \%$ of episodes) had mild sedation and normal vital signs. Cluster B (44.5\%) had higher levels of sedation and non-hypoxic vital sign abnormalities. Cluster C (28.9\%) was comparable to cluster B with the addition of hypoxia. In multivariable modeling, consumption of non-opioid sedatives significantly predicted membership in cluster B (aOR $2.75,95 \%$ CI 1.40-5.40) or cluster $\mathrm{C}$ (aOR $3.38,95 \%$ CI $1.48,7.70)$ as opposed to cluster A. Older age (aOR 1.54 per 10 years, 95\% CI 1.00-2.35) and female gender (aOR $2.51,95 \%$ CI $1.03,6.10$ ) were significantly associated with membership in cluster C vs. A. Cluster B (mean 4.4 hrs) and cluster $\mathrm{C}$ (mean $4.8 \mathrm{hrs}$ ) episodes were longer than cluster A episodes (mean $2.3 \mathrm{hrs}, \mathrm{p}<0.001$ for both). Cluster $\mathrm{C}$ episodes were more likely to require supplemental oxygen. Only 3 episodes required naloxone. Most episodes $(85.7 \%)$ ended in discharge to one's own care, without differences by cluster. No deaths were observed.

CONCLUSIONS: Among patients of a medically-supervised overdose prevention program, reported use of non-opioid sedatives strongly predicted more complex clinical courses. Despite this, few episodes required hospital transfer, very few required naloxone, and no deaths occurred. The SPOT experience suggests that many drug toxidromes can be managed effectively in the outpatient setting with early identification and appropriate monitoring and intervention resources.

\section{TRAINEES AND TRANSITIONS TO POST-ACUTE CARE: EVI- DENCE FOR IMPROVEMENT}

Julia E. Limes ${ }^{1}$; Catherine Callister ${ }^{1}$; Robert E. Burke ${ }^{2}$; Eric A. Young ${ }^{2}$; Christine D. Jones ${ }^{3} .{ }^{1}$ University of Colorado, Denver, CO; ${ }^{2}$ Denver VA Medical Center, Denver, CO; ${ }^{3}$ University of Colorado, Denver, Aurora, CO. (Control ID \#3186051)

BACKGROUND: A significant proportion of patients are discharged from the hospital with post acute care supports including skilled nursing facilities (SNFs) and home health care (HHC) services. These patients are often medically complex, with a higher risk of readmission and significant implications if the discharge transition does not go smoothly. Little is known about resident knowledge and perceptions of SNF and HHC transitions.

METHODS: We developed a cross-sectional survey for residents to assess their knowledge, attitudes, and experience with discharging patients to SNF or HHC. The questions were based on prior qualitative and survey studies of discharge to SNF and HHC. A 36-question survey was sent to 193 internal medicine (IM) and medicine-pediatrics residents at a large academic IM training program. Respondents selected answers on a 4-point Likert scale and entered free-text comments.

RESULTS: A total of 100 (51.8\%) residents responded. Residents felt confident in their ability to effectively transition patients to SNFs $(80.8 \%$ agree or strongly agree) and HHC (65.6\% agree or strongly agree). Twothirds of residents identified the discharge summary as their sole method of communicating care instructions to SNFs, but only $19.2 \%$ of respondents reported always completing a discharge summary prior to discharge. In addition, only $43.4 \%$ usually or always provide a contact number for the inpatient team. In terms of knowledge about services provided at a SNF, 36.4\% of residents knew how much physical therapy (PT), occupational therapy (OT), or speech therapy is provided at a SNF and $32.3 \%$ knew how frequently nursing services are provided. The perceived method of communicating care instructions to $\mathrm{HHC}$ agencies was more variable, with $45.7 \%$ communicating via discharge orders, $35.1 \%$ through a discharge summary, and $19.1 \%$ with a phone call by the case manager. Only $34.4 \%$ of residents usually or always provide a contact number for the inpatient team at discharge. Residents had similar gaps in knowledge about the frequency of RN care (39.1\% knew the frequency) and PT/OT (39.4\%), the length of a HHC episode (37.5\%), and what is required for wound care orders $(46.8 \%)$. In the free response section, residents expressed that the most effective educational methods would include: more exposure to each setting, specific teaching by attendings and case managers with patients discharging to SNFs and HHC, and feedback about those patients after discharge.

CONCLUSIONS: Residents feel confident in their ability to effectively transition patients to SNFs and HHC but identify gaps in their knowledge about the frequency and type of care provided in each setting. Residents do not report a standardized practice for how to communicate care plans with SNFs and HHC agencies. We identified specific targets for educational and process interventions and the most effective educational methods.

TRANSDISCIPLINARY STRATEGIES FOR PHYSICIAN WELLNESS: QUALITATIVE INSIGHTS FROM DIVERSE FIELDS

Rachel Schwartz $^{1,2}$; Marie Haverfield ${ }^{3,2}$; Cati Brown-Johnson ${ }^{4}$; Amrapali Maitra $^{5}$; Aaron A. Tierney ${ }^{6}$; Shreyas Bharadwaj ${ }^{3}$; Jonathan G. Shaw ${ }^{7}$; Farzad Azimpour ${ }^{8}, 3$; Sonoo Thadaney ${ }^{3}$; Abraham Verghese ${ }^{1}$; Donna M. Zulman ${ }^{9} .{ }^{1}$ Stanford University, Stanford, CA; ${ }^{2}$ VA Palo Alto Health Care System, Menlo Park, CA; ${ }^{3}$ Stanford University, Menlo Park, CA; ${ }^{4}$ Stanford School of Medicine, Stanford, CA; ${ }^{5}$ Brigham and Women's Hospital, Harvard Medical School, Boston, MA; ${ }^{6}$ Stanford University School of Medicine, Mountain View, CA; ${ }^{7}$ Stanford University School of Medicine, Stanford, CA; ${ }^{8}$ MYIA Labs, San Francisco, CA; ${ }^{9}$ Stanford University and VA Palo Alto, Stanford, CA. (Control ID \#3165677)

BACKGROUND: While barriers to physician wellness have been well detailed, concrete solutions are lacking. We looked to professionals across diverse fields whose work requires engagement and interpersonal connection with clients. The goal was to identify effective strategies from non-medical fields that could apply to preserving physician wellness.

METHODS: We conducted semi-structured interviews with 30 professionals outside the field of clinical medicine whose work involves fostering effective connections with individuals. Participants were from diverse professions, including the protective services (e.g., police officer, firefighter), business/finance (e.g., restaurateur, salesperson), management (e.g., CEO, school principal), education, art/design/entertainment (e.g., professional musician, documentary filmmaker), community/social services (e.g., social worker, chaplain), and personal care/services (e.g., massage therapist, yoga instructor). Interviews covered strategies that professionals use to initiate and maintain relationships, practices that cultivate professional fulfillment and preserve wellness, and techniques that facilitate emotional presence during interactions. Data were coded using an inductive thematic analysis approach.

RESULTS: Professionals identified self-care strategies at both institutional and individual levels that support wellness. Institutional-level strategies include scheduling that allows for self-care, protected time to connect with colleagues, and leadership support for debriefing after traumatic events. Individual strategies include emotionally-protective distancing techniques, and engagement in a bidirectional exchange that is central to interpersonal connection and professional fulfillment.

CONCLUSIONS: Across diverse fields, professionals employ common institutional and personal wellness strategies that facilitate meaningful engagement, support collegiality, and encourage processing after intense events. The transdisciplinary nature of these wellness strategies highlights universal underpinnings that support wellbeing in those engaging in people-oriented professions.

\section{TRANSLATING CHRONIC KIDNEY DISEASE RESEARCH IN- TO PRIMARY CARE PRACTICE}

Cara Litvin $^{1}$; Steven Ornstein ${ }^{2}$; Paul Nietert ${ }^{1}$; Ruth Jenkins ${ }^{1} .{ }^{1}$ Medical University of South Carolina, Charleston, SC; ${ }^{2}$ The PPRNet Foundation, Charleston, SC. (Control ID \#3185329) 
BACKGROUND: Chronic kidney disease (CKD) is common in the primary care setting. Early interventions may prevent progression of renal disease and reduce risk for cardiovascular disease. The purpose of this study was to assess whether implementation of a primary care improvement model results in improved identification and management of CKD compared to usual care.

METHODS: This 18-month group randomized trial was conducted from November 1, 2016 to April 30, 2018 in 21 practices in 13 U.S. states. Seventy-one physicians and 41 midlevel providers participated. All practices received performance reports as frequently as monthly on a set of 11 $\mathrm{CKD}$ clinical quality measures (CQMs) reflecting clinical practice guidelines related to screening for albuminuria in at-risk patients, monitoring (eGFR, albuminuria and hemoglobin), use of ACE-inhibitors or ARBs, blood pressure and glycemic control, statin therapy, and avoidance of NSAIDs. To promote implementation of practice improvement strategies derived from prior research and adapted to overcome known barriers to CKD care, 11 practices randomized to the intervention group hosted an on-site visit and 2 follow up webinars, and sent 2 representatives to a "best practice" meeting. The primary outcomes were changes in practice adherence to CKD CQMs over the 18-month trial. Linear mixed-effects regression models were used to compare changes over time for each CQM between intervention and control practices. Qualitative analyses of data obtained from site visits, webinars and the meeting were used to assess each practice's fidelity to implementation of the improvement strategies.

RESULTS: There were statistically significant greater improvements in intervention practices for annual screening for albuminuria in patients with diabetes or hypertension (absolute change $22 \%$ in intervention group vs. $-2.6 \%$ in control group, $\mathrm{p}<0.0001)$ and annual monitoring for albuminuria in patients with $\mathrm{CKD}$ (absolute change $21 \%$ in intervention group vs. $-2.0 \%$ in control group, $\mathrm{p}<0.0001)$. Avoidance of NSAIDs in patients with CKD declined in both intervention and control groups, with a significantly greater decline in the control practices (absolute change $-5.0 \%$ in intervention group vs. $-10 \%$ in control group, $\mathrm{p}<0.0001$ ). There were no other significant changes found for the other CQMS. There was variable implementation of CKD-specific improvement strategies across the intervention practices.

CONCLUSIONS: Implementation of a primary care improvement model designed to improve CKD identification and management resulted in significantly improved care on 3 out of $11 \mathrm{CKD}$ CQMs compared to usual care over 18 months. Incomplete adoption of improvement strategies may have limited further improvement. Achieving improved adherence to other CKD guidelines likely requires a longer and more intensive intervention.

\section{TRAUMA EXPOSURE, HEALTH STATUS, AND DISEASE BUR- DEN AMONG MINORS SEEKING ASYLUM IN THE US}

Joseph Shin ${ }^{1}$; Lynne M. Rosenberg ${ }^{1}$; Kevin Ackerman ${ }^{2}$; Maria Minor ${ }^{2}$; $\overline{\text { Pooja Shah }^{3}}{ }^{1}{ }^{1}$ Weill Cornell Medicine, Brooklyn, NY; ${ }^{2}$ Weill Cornell Medical College, New York, NY; ${ }^{3}$ Weill Cornell Medicine, New York, NY. (Control ID \#3185350)

BACKGROUND: Over the past decade, there has been an unprecedented influx of immigrant children seeking asylum or other forms of humanitarian relief into the US. For example, between 2009 and 2014, there was a $1,437 \%$ increase in unaccompanied alien children apprehended at the Southwest Border from El Salvador, Guatemala and Honduras. Driven primarily by epidemic levels of domestic, gang, and gender-based violence, many of these children have experience multiple types of trauma in their country of origin, as well as additional trauma during migration and post-migration. Previous studies of immigrant, asylee, and refugee children identify a relationship between trauma exposure and poor mental health outcomes. Comparatively little is known about the trauma exposure and health status of this current wave of immigrant children, or the impact of more recent changes and conditions in their countries of origin, during migration, or related to US immigration policies and practices.
METHODS: This study is a retrospective analysis of completed forensic health evaluations for child and adolescent immigrants evaluated as part of Physicians for Human Rights' Asylum Program between January 2014 and April 2018.

RESULTS: Evaluations for 183 individuals were included in this analysis (114 male and 69 female), with an average age of 15 years at the time of evaluation. Most were from the "Northern Triangle" countries (89\%) comprised of El Salvador (36\%), Guatemala (19\%), and Honduras $(34 \%)$. They experienced multiple forms of trauma including direct physical $(78 \%)$ and sexual violence $(18 \%)$, threats of violence/death (71\%) and forced gang conscription (24\%), and witnessed acts of violence $(59 \%)$. The perpetrators were most often family (47\%) and gang members $(60 \%)$. Major vulnerability factors present in this group included parental separation $(66 \%)$ or parent death $(11 \%)$. Health evaluations revealed that $76 \%$ had at least one major mental health diagnosis, most commonly posttraumatic stress disorder (64\%), major depressive disorder (40\%), and anxiety disorder (19\%).

CONCLUSIONS: Child and adolescent asylum seekers often have multiple types of repeated trauma exposures resulting in significant medical and mental health consequences. It is essential to enhance culturally sensitive, trauma-informed care for these vulnerable populations, while limiting practices and policies that could contribute to further trauma exposure and negative health outcomes during all phases of migration.

\section{TRAUMA INFORMED CARE NEEDS ASSESSMENT OF INTER- NAL MEDICINE RESIDENTS}

Jenny X. Wen; Colleen Christmas; Anika A. Alvanzo. Johns Hopkins

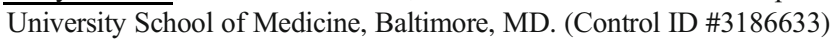

BACKGROUND: In the US, $60-90 \%$ of adults have experienced emotional trauma- defined as an event or series of events (such as abuse, loss, or chronic stressors) that negatively affect health. Trauma exposure is strongly associated with proportional increases in chronic diseases, behavioral health disorders, and risky behaviors. These negative sequelae disproportionately affect ethnic minorities and urban, underserved populations. Physicians and healthcare providers can play important roles in intervention or re-traumatization of victims. However, current standard medical training does not include trauma or Trauma Informed Care (TIC). We aimed to examine the knowledge, attitudes, perceived skills, and behaviors of Internal Medicine residents regarding managing patients with histories of trauma, as well as residents' desires for additional training in trauma informed care.

METHODS: We conducted an online survey of residents enrolled in 4 Internal Medicine programs in Baltimore, Maryland to assess the following behaviors: recognizing, screening, managing, and referring patients with trauma histories. The questionnaire was based on PREMIS (Physician Readiness to Manage Intimate Partner Violence Survey) and addressed residents' knowledge, attitudes, self-assessed preparedness, and close personal experiences with trauma. Nonparametric tests (KruskalWallis, Fisher's exact, and Wilcoxon rank sum) were used for analysis.

RESULTS: Of the 168 residents who responded to the survey (54\% response rate), $44 \%$ were female, 55\% White, $28 \%$ Asian, $4 \%$ Black and $13.2 \%$ unknown/other. Knowledge and preparedness were very limited. $83 \%$ percent of respondents underestimated trauma prevalence; $31.7 \%$ felt inadequately prepared to appropriately respond to positive disclosures. $59.5 \%$ reported they seldom asked about trauma in the past 6 months, and $8.8 \%$ never asked. Factors significantly associated with higher frequency of screening patients include greater perceived preparedness to identify, respond to, and refer patients ( $\mathrm{p}=.0001 .012)$; familiarity with referral resources $(\mathrm{p}=.005)$; comfort in discussing trauma with patients ( $\mathrm{p}=.003)$; and perceived faculty $(\mathrm{p}=.001)$ and workplace support $(\mathrm{p}$ $=.038$ ). $68.7 \%$ had previous training on some trauma-related topics in medical school, and $42.2 \%$ did in residency; $91.8 \%$ wished for additional training on trauma and trauma informed care. Differences among genders, races, years in training, and program sites were minimal. 
CONCLUSIONS: Internal Medicine residents in Baltimore had very limited knowledge about trauma prevalence and risks for comorbidities. Although most residents feel TIC is relevant to clinical practice, they feel unprepared to identify, respond to, manage, and refer patients with histories of trauma. Our results support the need for integration of trauma and Trauma Informed Care training into graduate medical education for Internal Medicine residents.

\section{TRENDS IN DIVERSITY OF MEDICAL SCHOOL MATRICU- LANTS RELATIVE TO THE US POPULATION}

Lanair A. Lett ${ }^{2}, 2$; Whitney Orji ${ }^{1}$; Jaya Aysola ${ }^{3,2}$; Ronnie Sebro ${ }^{2,} 2$. ${ }^{1}$ Perelman School of Medicine, University of Pennsylvania, Philadelphia, PA; ${ }^{2}$ University of Pennsylvania, Philadelphia, PA; ${ }^{3}$ Penn Medicine, Philadelphia, PA. (Control ID \#3184958)

BACKGROUND: Over the past several decades, there has been increasing effort to improve physician workforce diversity, including the institution of formal accreditation guidelines by the Liaison Committee of Medical Education (LCME) in 2009. Recently, several studies examining trends in diversity at the undergraduate, graduate and faculty level, have reported modest improvements in the proportions of racial/ethnic and sex groups within medicine. However, these studies fail to account for the shifting demographics of the US population. With this analysis, we provide a method of assessing the racial/ethnic diversity of medical school matriculants relative to the increasing diversity of the US population.

METHODS: The S-score, a measure of representation given a population distribution, was used to analyze the Association of American Medical Colleges data on self-reported race/ethnicity and sex of allopathic medical school matriculants, compared to population distribution of the medical school-aged population (20-34-year-olds), from 2002 to 2017. Linear trends over time were estimated using Pearson correlation.

RESULTS: The number of medical school matriculants increased from 16,488 to 21,326 between 2002 and 2017. During that time, proportions of 20-34-year-old Black, Hispanic, and Asian males and females in the US grew, while proportions of White males and females decreased. From 2002 to 2012, Black males ( $\mathrm{r}=0.83,95 \%$ CI: 0.46,0.96) and Black females ( $\mathrm{r}=0.9$, $95 \%$ CI: $0.65,0.97)$ were increasingly underrepresented amongst medical school matriculants. From 2013 to 2017, neither Black males ( $r=0.45,95 \%$ CI: $-0.71,0.95)$ nor females ( $r=-0.85,95 \%$ CI: $-0.99,0.14)$ experienced significant trends in representation. From 2002 to 2012, representation of Hispanic males varied without a significant trend $(r=0.45,95 \%$ CI: $-0.2,0.83$ ), while Hispanic females were increasingly underrepresented $(\mathrm{r}=0.86,95 \% \mathrm{CI}: 0.53,0.96)$. Both groups were underrepresented from 2013 to 2017 . White males were increasingly overrepresented from 2002 to 2012 ( $\mathrm{r}=-0.62,95 \% \mathrm{CI}:-0.89,-0.03$ ), while White females were increasingly underrepresented ( $\mathrm{r}=0.75,95 \%$ CI: $0.28,0.93)$. From 2013 to 2017, neither White males ( $\mathrm{r}=0.84,95 \%$ CI: $-0.16,0.99)$ nor White females ( $\mathrm{r}=-$ $0.86,95 \%$ CI: $-0.99,0.08)$ experienced significant trends in representation. Asian males and females were overrepresented from 2002 to 2017.

CONCLUSIONS: Despite increasing in number and proportion, Black and Hispanic students remain underrepresented amongst medical school matriculants when compared to the US population. To facilitate national progress in enrollment of underrepresented minority students, future studies should use the S-score to evaluate the efficacy of diversity initiatives at the local level to identify successful interventions that can be scaled. The S-score can be adapted to the institutional, regional, or state levels and used to track progress with better resolution than previously reported in national studies.

\section{TRENDS IN OBESITY AND MEDICAL EXPENDITURE AMONG WOMEN, 2008-2016: DIFFERENCES BY RACE/ETHNICITY}

Joni S. Williams; Sneha Nagavally; Leonard E. Egede. Medical College of Wisconsin, Milwaukee, WI. (Control ID \#3186374)
BACKGROUND: Two out of every three women in the U.S. have overweight or obesity, where annual medical expenditures exceed $\$ 520$ and $\$ 4870$, respectively. Evidence suggests women belonging to racial/ ethnic minority groups have a higher prevalence of obesity compared to Non-Hispanic White (NHW) women. Obesity results in significant morbidity, preventable mortality, and increased medical expenditures among women; however, evidence assessing trends in obesity and medical expenditure by race/ethnicity is limited. Therefore, the aim of this study was to examine racial/ethnic differences in obesity trends and medical expenditures among a nationally representative sample of women.

METHODS: Nine years of data (2008-2016) from the Medical Expenditure Panel Survey (MEPS) Full Year Consolidated File were used for the analyses. Cochran-Armitage tests were used to determine statistical significance of trends in obesity and mean expenditure for women. Two-part statistical modeling (Probit and Gamma General Linear Model (GLM)) with a combination of binomial and positive distributions was used to evaluate differences in medical expenditure. Covariates included age, education, marital status, insurance coverage, region, comorbidity count, and year. Data were clustered to 3 time points: 2008-2010, 2011-2013, 2014-2016. BMI levels were classified as underweight $(<18.5)$, normal ( $>=18.5$ - 24.9, overweight ( $>=25.0$ - 29.9), and obese ( $>=30.0$ - 134.7). Analyses were weighted for the U.S. population.

RESULTS: Cochran-Armitage tests showed trends in obesity from 2008 to 2016 differed significantly between Hispanic $(p<0.001)$, Non-Hispanic Black (NHB) $(\mathrm{p}<0.001)$, and Other $(\mathrm{p}<0.001)$ women compared to NHW women. Similarly, among obese women, trends in mean medical expenditures differed significantly between Hispanic $(\mathrm{p}<0.001)$, NHB $(\mathrm{p}<0.001)$, and Other $(\mathrm{p}<0.001)$ women compared to NHW women. From the two-part model, the Probit model indicated obese Hispanic $(-0.28(-0.35,-0.20))$, NHB $(-0.23(-0.31,-0.16))$, and Other $(-0.27(-0.42,-0.11))$ women were significantly less likely to spend on healthcare compared to NHW women, after adjusting for covariates. The Gamma GLM showed that among obese women with positive medical expenditure, Hispanic women spent $14 \%$ less ( $\$ 1145.87 ; \mathrm{p}<0.001)$, while NHB women spent $11 \%$ less $(\$ 940.03$; $\mathrm{p}<0.001$ ) compared to NHW women, adjusting for covariates. No significant differences in incurred medical expenditure were observed between obese Other and NHW women after adjusting for covariates.

CONCLUSIONS: These findings suggest the need for further understanding of the factors and underlying mechanisms associated with differences in trends for obesity among minority and NHW women and trends in medical expenditures between obese Hispanic, NHB, and NHW women to address these disparities and target the unique needs of women with overweight and obesity in the U.S.

\section{TRENDS IN SYSTOLIC BLOOD PRESSURE CONTROL AND MANAGEMENT IN THE UNITED STATES, 2006 TO 2015}

Cecilia Scholcoff $^{1,2}{ }^{2}$; Jeffrey L. Jackson ${ }^{1,2}{ }^{2}$ Cynthia Kay ${ }^{1,}{ }^{2}$; Sarah Nickoloff ${ }^{1,2}$. ${ }^{1}$ Zablocki VAMC, Milwaukee, WI; ${ }^{2}$ Medical College of Wisconsin, Milwauke, WI. (Control ID \#3179298)

BACKGROUND: Hypertension (HTN) is one of the most common conditions affecting the U.S. population and an important risk factor for cardiovascular disease, heart failure, stroke and kidney disease. In this study, we assess prescribing and counseling patterns as well as systolic blood pressure (SBP) control in United States primary care settings over the past 10 years.

METHODS: We analyzed data over one decade (2006-2015) from the National Ambulatory Medical Care Survey (NAMCS). We abstracted blood pressure readings among all visits and stratified SBP control as $<120 \mathrm{~mm} \mathrm{Hg}, 120-139 \mathrm{~mm} \mathrm{Hg}$ and $140 \mathrm{~mm} \mathrm{Hg}$. In addition, we abstracted whether the patient had a diagnosis of hypertension, the class and number of anti-hypertensive medications, whether anti-hypertensive medication was newly prescribed, and frequency of dietary and exercise 
counseling. Analyses were done using recommended study weights using the STATA SURVEY program (V15.1, College Station, Tx).

RESULTS: Among primary care visits between 2006 and 2015, the prevalence of hypertension was $25-35 \%$, gradually increasing over time ( $\mathrm{p}$ for trend $<0.001$ ). The highest rate of uncontrolled HTN (SBP $140 \mathrm{~mm}$ $\mathrm{Hg}$ ) was $25 \%$ in 2006. Subsequently, the rate of uncontrolled hypertension declined to $16-19 \%$ of visits for the remainder of the study period. In most years, nearly half of primary care patients diagnosed with hypertension were not receiving anti-hypertensive medications. About a third were treated with monotherapy, $20 \%$ with two medications and 5-10\% with three or more medications. Among patients on anti-hypertensive medications, the class of medication prescribed was consistent over the decade. Angtiotensin converting enzyme (ACE) inhibitors were used in 40-50\% of patients, calcium channel blockers, beta blockers and angiotensin receptor blockers (ARBs) were used in $25-35 \%$ of patients, and thiazide diuretics were only used in 14-17\% of patients. Patients with uncontrolled HTN received a new medication only $14-24 \%$ of the time. Overall, the rates of dietary and exercise counseling were low (7-15\% of visits), but slightly increased if patients had a diagnosis of hypertension, had SBP $140 \mathrm{~mm} \mathrm{Hg}$, or if hypertension was the primary reason for the visit.

CONCLUSIONS: Although we found that almost a fifth of patients had uncontrolled hypertension, our results are promising and show that blood pressure control is improving in the United States. We did identify areas that need continued attention, such as guideline-based prescribing, medication intensification and the use of counseling as treatment for hypertension.

\section{TRENDS OF HYPOGLYCEMIA-RELATED HOSPITALIZA-} TIONS IN VETERANS WITH DIABETES FROM 2005 TO 2016

Molly Zhao $^{1,2}$; Paul Conlin ${ }^{1}$; David Mohr ${ }^{3}$; Julia Prentice ${ }^{2}{ }^{1}$ Boston VA Healthcare System, Boston, MA; ${ }^{2}$ Boston University, Boston, MA; ${ }^{3}$ VA Boston Healthcare System, Boston, MA. (Control ID \#3186347)

BACKGROUND: One quarter of U.S. older adults with diabetes are potentially over-treated with glucose lowering medications that have a high risk of hypoglycemia. Individualizing hemoglobin A1c (A1c) target values has been recommended for managing diabetes (DM). This study examined trends of hypoglycemia-related hospitalizations across risk factors for hypoglycemia over time among veterans, a population with a T2D prevalence that is twice higher than the general population.

METHODS: This retrospective observational study used administrative data from the Veterans Health Administration to identify patients $(\mathrm{N}=484,285)$ diagnosed with $\mathrm{DM}$, aged 64 or older with 4 or more A1c tests during a three-year baseline period from 2005 to 2016 and at least one-year of post-baseline data. Hypoglycemiarelated hospitalizations were identified by any discharge diagnosis with ICD-9 codes from an algorithm validated by Ginde et al., 2009 and the corresponding ICD-10 codes. The frequency of such events was calculated across gender, race, age groups $(64-74 ; 75+)$, insulin and sulfonylurea use during the baseline period, baseline A1c levels $(<7 ; 7-7.9 ; 8-8.9 ;>=9) . \mathrm{X}^{2}$ tests were conducted for significance. The rate of hypoglycemia-related hospitalizations was calculated by dividing the number of hospitalizations for hypoglycemia by the number of overall hospitalizations in the same year among the sample.

RESULTS: A total of 9,020 unique patients had 10,639 hypoglycemiarelated hospitalizations from the start date of their baseline period to their date of death or the end of 2016. The rate of hypoglycemia-related hospitalizations declined from $2.3 \%$ to $1.8 \%$, the decline being most prominent from 2007 to 2015. The downward trend was similar across most of the sociodemographic and clinical parameters. Among the 9,020 patients, the frequency of patients hospitalized for hypoglycemia increased from $1.2 \%$ to $4.7 \%(\mathrm{P}<0.0001)$ from the lowest to highest baseline A1c range. Among the users of insulin and sulfonylureas, $3.8 \%$ and $2.2 \%$ were hospitalized for hypoglycemia compared to non-users at $1.3 \%$ and $1.6 \%$ ( $\mathrm{P}<0.0001)$, respectively. African American and Hispanic patients were hospitalized for hypoglycemia at a rate of $4.1 \%$, compared to $1.6 \%$ of the remaining sample $(\mathrm{P}<0.0001)$. The frequency of patients 75 years or older was $2.0 \%$ compared to the $1.8 \%$ of younger patients $(\mathrm{P}<0.0001)$.

CONCLUSIONS: The rate of hypoglycemia-related hospitalizations in veterans has declined since 2005, although African Americans, older individuals, insulin and sulfonylurea users and those with higher A1c levels remain at greater risk. These findings may inform efforts to individualize A1c target values to prevent potential overtreatment and complications of hypoglycemia.

\section{TRIAL DESIGN FOR INEFFECTIVENESS RESEARCH: A} MIXED-METHODS SURVEY

Kevin R. Riggs $^{1,2}{ }^{\text {; Joshua Richman }}{ }^{1,2}$; Stefan Kertesz ${ }^{3}$; Sara J. Knight ${ }^{4}$ 5. ${ }^{1}$ University of Alabama at Birmingham, Birmingham, AL; ${ }^{2}$ Birmingham VA Medical Center, Birmingham, AL; ${ }^{3}$ Birmingham VA Medical Center \& U. Alabama Birmingham, Homewood, AL; ${ }^{4}$ University of Utah, Salt Lake City, UT; ${ }^{5}$ VA Salt Lake City Health System, Salt Lake City, UT. (Control ID \#3184284)

BACKGROUND: Many widely used health services may be ineffective. Clarifying the effectiveness of these services could help reduce overuse, but the optimal trial design for evaluating ineffectiveness is uncertain. Some have advocated using equivalence trials (or closely related noninferiority trials), as demonstrating that placebo or an inactive control is equivalent to an intervention could support the claim that it is ineffective. The objective of this study was to determine whether experienced investigators favor superiority trials or equivalence trials for ineffectiveness research and their reasons for favoring that design.

METHODS: We conducted an online, mixed-methods survey. We crafted a vignette describing researchers planning to conduct a trial of a common intervention they hypothesized was ineffective. We identified experienced investigators by searching clinicaltrials.gov for trials registered MarchSeptember 2017 funded by federal sources that included an email address for the principal investigator. We emailed a link to the survey and two reminders to non-responders. Participants answered one multiple-choice question about which study design they favored, and one free-response question describing their reasoning. Participants were randomized to vignettes that varied in two domains: intervention (medication vs. placebo, or surgical procedure vs. sham procedure) and outcome (pain, or morbidity and mortality). We used content analysis to identify related reasons from free-response answers.

RESULTS: 139 participants completed the survey (completion rate $37.5 \%$ ). Overall, $56.8 \%$ favored superiority trials, $27.3 \%$ favored equivalence trials, and $15.8 \%$ were unsure. Responses did not differ by intervention ( $\mathrm{p}=0.56$ ) or outcome ( $\mathrm{p}=0.74) .95$ participants included a free text response to explain the reason for their selection. Themes identified for favoring superiority trials were: 1) evidence of superiority should be required to justify active treatment, 2) superiority trials are more familiar, 3) placebo should not be the comparator in equivalence trials, and 4) superiority trials require smaller sample sizes. Themes identified for favoring equivalence trials were: 1) negative superiority trials represent a lack of evidence of effectiveness rather than evidence of ineffectiveness, and 2) the research hypothesis should not be the same as the null hypothesis. Many responses were nonspecific or did not address differences between the trial designs, so did not contribute to themes.

CONCLUSIONS: Equivalence trials may have the potential to generate evidence supporting the ineffectiveness of some interventions, but do not appear to be favored by experienced investigators for this purpose at this time. General lack of familiarity with equivalence trials is a contributing factor. Other factors - such as uncertainty about the appropriateness of using placebo as a comparator - may represent misconceptions about equivalence trials. More debate is needed about the optimal design of ineffectiveness research. 
TWENTY-YEAR CHANGES IN UNMET HEALTH NEEDS OF NON-ELDERLY ADULT AMERICANS: A NATIONAL STUDY

Laura Hawks $^{1,2}$; David Himmelstein ${ }^{3}$; Stephanie Woolhandler ${ }^{4}$; David Bor $^{1}$; Adam Gaffney ${ }^{1}$; Danny McCormick ${ }^{5}{ }^{1}$ Cambridge Health Alliance, Cambridge, MA; ${ }^{2}$ Harvard Medical School, Cambridge, MA; ${ }^{3}$ City University of New York at Hunter College, New York, NY; ${ }^{4}$ Cambridge Hospital, New York, NY; ${ }^{5}$ Harvard Medical School / Cambridge Health Alliance, Cambridge, MA. (Control ID \#3185047)

BACKGROUND: Many recent studies have highlighted improvements in coverage and access to care attributable to the Affordable Care Act (ACA). However, a narrow focus on short term pre- to post-ACA changes may distract attention from longer-term trends in Americans' unmet health needs.

METHODS: We analyzed data on persons aged 18-64 from the 1998 and 2017 Behavioral Risk Factor Surveillance System (BRFSS), a telephonebased survey conducted in each US state and Washington D.C. that collects nationally-representative data for the non-institutionalized population. We used two measures of unmet health needs: 1 . being unable to see a physician when needed, due to cost; and 2. not having a check-up in the last two years. We assessed changes in insurance coverage and both indicators of unmet health needs from 1998 to 2017 for the overall population as well as for subgroups with specific health conditions. All analyses used weights that allow extrapolation to the US non- institutionalized population, and were also conducted stratified by insurance status. We estimated unadjusted differences (UD) in proportions between 1998 and 2017.

RESULTS: The sample included 105,886 respondents from 1998 and 301,752 from 2017. Between 1998 and 2017 the uninsurance rate among all non-elderly adults decreased from $16.9 \%$ to $14.8 \%$ (difference: -2.1 [95\% CI, $1.6-2.5]$; however, among young adults (aged 35-44), uninsurance increased by 1.2 percentage points [ $95 \%$ CI, 0.4 to 2.2]. Over the same 2-decade span, the proportion of respondents who reported being unable to see a physician due to cost increased by $4.3 \%$ [95\% CI, 3.8-4.7], from $11.4 \%$ to $15.7 \%$. Among all non-elderly adults this increased by $6.7 \%$ [95\% CI, 5.1-8.3] in the uninsured, and by 4.5\% [95\% CI, 4.1-4.9] among the insured. While this measure worsened for all racial/ ethnic groups, inequality persisted. In 2017, $18.8 \%$ [95\% CI, 17.9-19.7] of black and 21.9\% [95\% CI, 20.9-22.8] of Hispanic versus 13.3\% [95\% CI, 13.013.6] of white respondents reported this barrier to care. Additionally, 28.5\% [95\% CI, 27.2-29.7\%] of low income, and 39.6\% [95\% CI, 38.640.7] of uninsured respondents could not see a doctor when needed due to cost. This measure worsened to a similar degree among the chronically ill, including those who reported a diagnosis of hypertension, hyperlipidemia, diabetes, obesity, cardiovascular disease, binge drinking and smoking, and among those reporting poor health status. In contrast, the proportion of nonelderly adults who had received a check-up within the past 2 years did not change overall, regardless of coverage status.

CONCLUSIONS: Despite important short-term improvements in coverage and access to care under the ACA, coverage gains were less impressive over the longer term, and the proportion of non-elderly adults with unmet health needs either failed to improve or actually worsened in the last 2 decades. A reform more comprehensive than the ACA will likely be required to more fully address Americans' unmet health needs.

\section{U.S. ADULTS' PERSPECTIVES ON DECISION-MAKING ABOUT HEALTH INSURANCE, HEALTHCARE, AND EM- PLOYMENT NEAR RETIREMENT}

Renuka Tipirneni $^{1,}{ }^{1}$; Erica Solway ${ }^{1}$; Preeti Malani ${ }^{1,}{ }^{1}$; Jamie Luster ${ }^{1}$; Jeffrey T. Kullgren ${ }^{1,}{ }^{1}$; Matthias Kirch ${ }^{1}$; Dianne Singer ${ }^{1,}{ }^{1}$; Aaron M. Scherer ${ }^{2}$. ${ }^{1}$ University of Michigan, Ann Arbor, MI; ${ }^{2}$ University of Iowa Carver College of Medicine, Iowa City, IA. (Control ID \#3179040)

BACKGROUND: As U.S. adults approach Medicare eligibility at the age of 65, they face important decisions about health care and employment. Recent legislative, regulatory, and legal challenges to the
Affordable Care Act (ACA) may add new uncertainties to decisionmaking in this critical period. Our objective was to understand adults' perspectives on health insurance coverage, health care, and employment as they approach retirement age.

METHODS: In October 2018, a cross-sectional online survey was conducted using the Ipsos (formerly GfK) KnowledgePanel®, a large, nationally representative probability-based panel. This survey was conducted as part of the University of Michigan National Poll on Healthy Aging, a recurring online survey of participants age 50-80. The completion rate was $62 \%$ among panel members contacted to participate. This study included 1,028 community-dwelling U.S. adults, age 50-64. Analyses report descriptive and bivariate statistics incorporating survey weights. Measures included respondents' plans regarding current and future health insurance, medical care, and retirement decision-making. The margin of error was \pm 1 to 3 percentage points for questions asked of the full sample, and higher among subgroups.

RESULTS: Nearly half (45\%) of adults age 50-64 had little to no confidence in their ability to afford health insurance when they retire, and $27 \%$ had little or no confidence in ability to afford health insurance over the next year. Confidence in health insurance affordability during retirement was highest among ages 60-64, males, African-Americans, those with annual household incomes $>\$ 60,000$, higher educational attainment, and excellent or very good health. In the past year, $14 \%$ of respondents reported keeping a job and $11 \%$ delayed or considered delaying retirement specifically to have their employer-sponsored health insurance. $12 \%$ thought about or went without health insurance in the past year. $8 \%$ stated that they were waiting to get a medical procedure until they have Medicare coverage, and $13 \%$ had not gotten medical care in the past year because of cost concerns. More than two-thirds (68\%) of respondents were concerned about potential changes to health insurance due to changes in federal policies. Those age 60-64 and with lower incomes $(<\$ 60,000$ annually) were more likely to express these concerns.

CONCLUSIONS: Half of adults age 50-64 were worried about their ability to afford health insurance when they retire and most were concerned about how changes in federal policies could affect their insurance. Some even delayed retirement or kept a job to keep their employersponsored health insurance. While federal legislative efforts to change the ACA largely failed in 2018, these findings suggest that many adults age 50-64 still worry about potential future changes to their health insurance options. Health care policy solutions are needed to help patients near retirement better understand their coverage options and navigate health care in this critical transition period.

\section{UNDER-DISPENSING OF MEDICINES FOR NON- COMMUNICABLE DISEASES IN UGANDA AND ITS EFFECT ON PATIENTS: A CROSS-SECTIONAL MIXED METHODS STUDY}

Jeremy I. Schwartz ${ }^{1,3}$; Andrew Tusubira ${ }^{3,2}$; Rejoice Ngongoni ${ }^{4}$; Gidio $\overline{\text { Agaba }^{5} \text {; Christine Nalwadda }}{ }^{2,3} .{ }^{1}$ Yale School of Medicine, New Haven, CT; ${ }^{2}$ Makerere University College of Health Sciences School of Public Health, Kampala, Uganda; ${ }^{3}$ Uganda Initiative for Integrated Management of Non-Communicable Diseases, Kampala, Uganda; ${ }^{4}$ Stanford Medicine, Stanford, CA; ${ }^{5}$ Mulago National Referral Hospital, Kampala, Uganda. (Control ID \#3185863)

BACKGROUND: The prevalence of non-communicable diseases such as hypertension (HTN) and diabetes (DM) is rising rapidly in low-income countries. Access to medicines for these conditions is low, largely driven by low availability and high cost. Anecdotally, patients often do not receive the medicines prescribed for them at public sector health facilities. But the scale of such under-dispensing and its implications for patients have not been previously defined. In this study, we aimed to characterize the gap between prescribing and dispensing of these medicines in Uganda and to understand the effect of this gap on patients' ability to care for their chronic conditions. 
METHODS: We conducted a cross-sectional study at public-sector hospitals throughout Uganda, utilizing a parallel-convergent mixed methods design. We surveyed a convenience sample of patients at 20 hospitals that offer HTN/DM clinics and conducted in-depth interviews and focus group discussions with a purposive sample of patients at six of these clinics. All participants were adults presenting for HTN and/or DM care. Quantitative and qualitative findings were analyzed separately, their results compared and merged for interpretation. Survey data were analyzed using descriptive statistics. We defined the Prescribing-Dispensing Gap as prescribed doses minus dispensed doses divided by prescribed doses x100. Interviews were transcribed and coded and emergent themes were identified using the Framework method.

RESULTS: We surveyed 637 patients and interviewed 164 patients (36 in-depth interviews and 128 in 14 focus group discussions). Surveyed patients were prescribed a total of 82,591 doses, of which 47,300 (57.3\%) were not dispensed. $84 \%$ of patients $(n=535)$ did not receive all of their prescribed doses and 90 of $253(36 \%)$ patients contacted in follow-up had not obtained any remaining doses elsewhere. Interviewed patients reported medicines being out of stock and being dispensed less than what was prescribed. Patients noted that some medicines, primarily insulin, were often of low quality, leading to distrust and non-adherence. Patients were consistently advised to purchase non-dispensed medicines from private pharmacies, though financial barriers often prevented them from doing so. As a way of mitigating the frequent shortage of medicines, some patients reported organizing themselves to form informal "patient associations", localized to a few hospitals.

CONCLUSIONS: In this study, we found that $57.3 \%$ of prescribed doses of HTN and DM medicines are not dispensed at public-sector hospitals in Uganda due to stockouts and rationing and that many patients do not obtain remaining doses from private pharmacies due to financial barriers. This large Prescribing-Dispensing Gap has profound implications for patients' ability to consistently manage their chronic conditions. Future research should focus on improving access to medicines for chronic conditions, including through innovative patient-centered and patient-driven approaches.

\section{UNDERSTANDING GEOGRAPHIC VARIATION IN SYSTEMIC OVERUSE AMONG THE PRIVATELY INSURED}

Allison H. Oakes ${ }^{2}$; Aditi Sen ${ }^{2}$; Jodi B. Segal ${ }^{1,2}$. ${ }^{1}$ Johns Hopkins University School of Medicine, Baltimore, MD; ${ }^{2}$ Johns Hopkins University Bloomberg School of Public Health, Baltimore, MD. (Control ID \#3184881)

BACKGROUND: Overuse of services contributes importantly to the high spending on health care in the US. Annually, 14-25\% of Medicare beneficiaries and $4-8 \%$ of commercially-insured adults experience services that can be considered wasteful. We demonstrated that overuse within regions is stable longitudinally, which suggests that there are regional drivers of overuse. We aimed to characterize these drivers to inform interventions.

METHODS: We analyzed commercial insurance claims from 18-64 year olds. We calculated a Johns Hopkins Overuse Index (JHOI) score, semiannually, for each of the 375 Metropolitan Statistical Areas (MSA) in the U.S, from January 2011 to June 2015. The JHOI is a normalized measure of systemic health care services overuse that can describe overuse across the adult lifespan. To estimate associations between regional characteristics of the health care system and systemic overuse, we fit an OLS regression to model the JHOI as a function of regional characteristics with state and year fixed effects, clustered at the MSA. Regional characteristics were constructed from the Area Health Resource File and the American Hospital Association Survey. Each regional characteristic was conceptualized as describing: 1) resource availability, 2) healthcare services configuration, 3) market competition, or 4) prevailing local clinical practices. We controlled for demand-side covariates using data from the American Community Survey.
RESULTS: Across all years, the normalized JHOI identified 37 regions that were stable, markedly-overusing regions ( $>2$ S.D. above average) and 47 that were $>2$ S.D. below the average. Regional characteristics associated with higher JHOI scores, indicating more systemic overuse, included the total number of physicians per 1,000 residents $(\mathrm{p}=0.006)$ and a higher Medicare malpractice geographic price index, representing higher spending on malpractice insurance $(\mathrm{p}<0.001)$. Conversely, regions with a higher density of primary care physicians $(\mathrm{p}=0.006)$ and those with more hospital-based providers $(\mathrm{p}=0.029)$ had lower JHOI scores, indicating less systemic overuse. Relative to regions with lower competition in the both the hospital and insurance markets, results diverged when there was discordance in market power. Trending toward significance, areas with a competitive hospital market and a less competitive insurer market were associated with a 0.25 point increase in the JHOI $(\mathrm{p}=0.072)$ while areas with the inverse were associated with a 0.32 point decrease in the JHOI $(\mathrm{p}=0.053)$.

CONCLUSIONS: Regional variation in the clinician workforce and drivers of "practice culture" are associated with variation in systematic overuse of health care. Additional research is needed to understand the relationship between the JHOI and market competition. The role of primary care doctors in reducing health care overuse deserves further attention; new models of primary care delivery as stimulated by Centers for Medicare and Medicaid Services (CMS) may prove impactful on overuse of resources.

\section{UNDERSTANDING PRIMARY CARE PHYSICIAN'S INFORMA- TION NEEDS DURING INBOX MESSAGE PROCESSING}

Daniel R. Murphy ${ }^{1}$; Tyler Satterly ${ }^{1}$; Dean F. Sittig ${ }^{2}$; Hardeep Singh ${ }^{1}$. ${ }^{\mathrm{T}}$ Michael E. DeBakey Veterans Affairs Medical Center and Baylor College of Medicine, Houston, TX; ${ }^{2}$ University of Texas, Houston, TX. (Control ID \#3168062)

BACKGROUND: Primary care physicians spend a sizable portion of each day performing non-face-to-face care. Much of this activity involves processing notifications delivered via Electronic Health Record (EHR)based inboxes. Improving EHR inbox designs to better match information displays with physician's information needs and workflows can greatly improve the efficiency of notification processing. However, research on this topic is limited. To identify recommendations for future EHR inbox designs, we performed a study to understand the information physicians access and actions physicians take while processing inbox notifications.

METHODS: We conducted a task analysis by directly observing physicians at four large clinical practices as they processed their inbox notifications. During each session, we asked physicians to verbalize what actions they were taking in response to the messages and the rationale for these actions. We additionally recorded the information they viewed in the EHR. During analysis, notification types and actions taken were categorized (e.g., "test results," and "review medication list," respectively). The data was then analyzed to determine the actions most frequently performed while processing alerts.

RESULTS: We observed 25 physicians as they processed 147 notifications in their EHR inboxes. Notifications included 53 test results, 31 messages from staff members, 21 messages from other clinicians, 15 messages from patients, 13 refill requests, 5 system messages that a patient had not read a message sent to them, 4 co-signature requests, 3 inpatient records, and 2 self-reminders. While processing messages, physicians most frequently ( $10 \%$ of messages) performed the following actions: reviewed test results $(37 \%)$, send patient portal messages $(24 \%)$, documented in the patient's record (22\%), signed existing orders or notes $(20 \%)$, sent a message to another clinician or staff $(18 \%)$, called patients $(14 \%)$, reviewed their last progress note (13\%), review lab trends $(10 \%)$, and reviewed medications (10\%). Other activities (e.g., placing new orders), occurred in $<10 \%$ of messages. Physicians entered the full chart only $12 \%$ of the time.

CONCLUSIONS: We found that physicians performed a variety of actions while processing EHR-based notifications in their inboxes, 
primarily including review of various clinical data and communication with staff, patients, and other clinicians. When possible, physicians appeared to prefer accessing data within the inbox rather than enter the full record. To improve message processing efficiency for physicians, EHR vendors can ensure this relevant information is directly accessible from within the inbox and allow follow-up actions to be taken without entering the full record. Further research is needed to assess the impact of such changes on processing efficiency.

UNDERSTANDING RISK COMMUNICATION ABOUT FENTANYL USE IN HEALTH CARE SETTINGS: SUCCESSES AND OPPORTUNITIES FOR IMPROVEMENT

Christine Gunn ${ }^{1,2}$; Alexander Y. Walley ${ }^{3}$; Samantha F. Schoenberger ${ }^{1}$; Ariel Maschke ; Sarah Bagley ${ }^{1} .{ }^{1}$ Boston University, Boston, MA; ${ }^{2}$ Boston University School of Public Health, Boston, MA; ${ }^{3}$ Boston Univ, Boston, MA. (Control ID \#3173498)

BACKGROUND: Deaths due to fentanyl overdose continue to increase. Individuals who use opioids may not understand the risks associated with fentanyl use or know how to minimize their fentanyl overdose risk. Overdose risk communication, a process of information exchange to negotiate better risk-related behaviors and outcomes, has not been examined. This study explored overdose risk communication by assessing patient and care provider experiences in discussing fentanyl risks across 4 dimensions of risk communication.

METHODS: We purposively sampled equal numbers of men and women with past year fentanyl use in Boston for qualitative interviews. We also interviewed health care and community outreach practitioners ("providers"). We conducted a grounded content analysis of professionally transcribed interviews. Codes related to patient-provider risk communication about illicit fentanyl use assessed via 4 risk communication constructs: 1) providing information about the risk; 2) recommending the elimination of risky behavior (abstinence); 3) discussing safer use strategies (harm reduction); and 4) reassuring patients that care will continue regardless of adherence to recommendations. Codes were analyzed to build themes around these dimensions and compare strategies currently employed vs. those desired by each group.

RESULTS: Twenty people who use fentanyl (PWUF) and 7 providers were enrolled. Nineteen PWUF had experienced overdose; most attributed this to fentanyl. PWUF reported wanting information about fentanyl risks, but saw those with a history of addiction as the most credible source, not providers. Risk information was welcomed when it was delivered in a non-judgmental, "kind and courteous" manner, and the person did "not sugar coat it". When providers suggested abstinence, both groups stressed that "personal readiness" was essential for treatment. Yet, PWUF reported that referrals in post-overdose settings were uncommon: "They highly recommend to get into a detox, but they never try to make the effort to get me into a detox." All providers emphasized harm reduction as the main strategy to minimize overdose risk from fentanyl. Although providers expressed a willingness to continue care for patients with ongoing substance use, 14 PWUF had experienced stigma that undermined risk conversations, some being refused care: "One of my primary cares, he told me just to get out of his office. That was a family lifelong doctor. Now he will never see me again."

CONCLUSIONS: PWUF wanted to talk about overdose risks with those who had experienced addiction. While harm reduction messages were emphasized by providers, PWUF indicated that ongoing substance use in primary care is stigmatized. Building the capacity of medical practices to effectively counsel patients who use fentanyl in the midst of the opioid crisis is critical to engaging patients in health care. Partnering and employing peers with experience using illicit fentanyl is a promising strategy to improve fentanyl overdose risk communication.
UNDERSTANDING THE SOCIAL CONTEXT OF PUBLIC HOUSING: SOCIAL NETWORKS OFFER CHALLENGES AND ASSETS FOR CARDIOVASCULAR HEALTH

Benjamin Meza ${ }^{1}$; Craig E. Pollack ${ }^{2}$; Kimberly Gudzune ${ }^{3} .{ }^{1}$ Johns Hopkins, Baltimore, MD; ${ }^{2}$ Johns Hopkins University School of Medicine, Baltimore, MD; ${ }^{3}$ Johns Hopkins University, Baltimore, MD. (Control ID \#3185601)

BACKGROUND: The American Heart Association has identified the "mechanisms by which social networks affect health" as a significant knowledge gap and area for further inquiry because social networks have been shown to impact lifestyle factors such as diet and exercise. Yet little is published regarding the social networks of public housing residents and other low-income urban communities that suffer from a high prevalence of cardiovascular disease. The objective of this study was to describe the social network characteristics of a sample of public housing residents in Baltimore, MD.

METHODS: We used data from a cross-sectional survey of randomly selected households in two public housing complexes in Baltimore, MD from August 2014 to August 2015. Adult survey respondents answered an in-person survey of personal demographics, health, and health behaviors. After delineating up to 21 members of their social network, respondents answered detailed questions about 10 randomly selected members, including attributes of the respondent-member relationship and the frequency of interaction between members. We calculated network measures including network density (i.e., the extent to which all individuals in a respondent's social network are connected to each other) and the proportion of network members who are neighbors, live within Baltimore City, maintain daily contact, share a meal or snack, are family or friends, and provide material or emotional support.

RESULTS: The sample included 266 respondents (response rate 48\%). Mean age was 44.5 years, $86.1 \%$ were women, $95.5 \%$ Black, $66.2 \%$ were high school graduates, and $33.5 \%$ were unemployed. Respondents nominated a median of 15 network members (range 6-21). Network density was high when considering all nominated relationships $(0.57$, Standard Deviation [SD] 0.29) and even when selecting only frequent contacts $(0.25$, SD 0.24$)$. On average, the great majority of nominated members lived within Baltimore City (92\%, SD 16\%) yet only a small proportion lived within the same housing development (19\%, SD 20\%). Similarly, nearly all members were known to the respondent for greater than 12 months $(96 \%$, SD 12\%) and most maintained daily contact $(63 \%, \mathrm{SD}$ $31 \%$ ) but very few shared a meal or snack with the respondent on a daily basis $(8 \%$, SD $13 \%)$. The majority of members were family $(57 \%$, SD $28 \%$ ) as compared to friends (33\%, SD $26 \%$ ).

CONCLUSIONS: Our findings provide a social context for developing interventions that are sensitive to the local community. High network density has been shown to potentiate some interventions while hampering others; strong social ties but weak dietary ties could offer a space for a network-based intervention; and the predisposition toward local but minimal neighborhood contacts is critical for understanding how information may not readily diffuse through public housing communities.

\section{UNDERSTANDING VARIATION IN HOSPITALIZATION TRA- JECTORY AMONG HIGH COST, HIGH NEED PATIENTS EN- ROLLED IN THE COMPREHENSIVE CARE PHYSICIAN (CCP) PROGRAM}

Veena Sriram ${ }^{1}$; Lauren O. Wiklund ${ }^{2}$; Abbie Klein ${ }^{1}$; David Meltzer ${ }^{1}$; Joyce W. Tang ${ }^{1} .{ }^{1}$ University of Chicago, Chicago, IL; ${ }^{2}$ Michigan State University, East Lansing, MI. (Control ID \#3184971)

BACKGROUND: Decreasing hospitalization rates for high cost, high need patients is a major goal for practitioners and policymakers; however, existing interventions have had variable impact on utilization within their target groups, raising critical questions regarding what aspects of the intervention are working and for whom. The goal of this study is to explore patient and clinical staff perspectives on the factors underlying 
variation in hospitalization trajectories for patients enrolled in the Comprehensive Care Physician (CCP) program, a program which focuses on reducing hospitalization by offering patients care from an interdisciplinary team led by a physician who serves as both the patients' outpatient primary care physician and inpatient hospitalist.

METHODS: This is a qualitative study embedded within a randomized controlled trial (CCP versus usual care). In-depth interviews were conducted with a purposive sample of intervention arm patients experiencing decreasing hospitalization $(n=13)$ and persistent hospitalization $(n=9)$ and with CCP clinical staff $(n=8)$. Data were analyzed by a multi-disciplinary research team using inductive coding following the principles of General Thematic Analysis, and synthesized according to the Socio-ecological Model. All transcripts were coded by at least two team members; common themes were developed through discussion in face-to-face meetings.

RESULTS: Our analysis reveals three key themes. First, while patients tended to focus exclusively on individual-level factors (medical illness, selfcare behaviors) influencing their hospitalization trajectory, providers and staff highlighted a more complex, broader range of factors at the individual (mental health, substance use), interpersonal (social support) and societal levels (transportation, housing security). Second, both intervention patients and providers described the importance of program-related factors (coordination of care, continuity of care) in decreasing hospitalization frequency; however, neither patients nor providers described program-level factors as contributing to persistent hospitalization. Third, while providers noted the importance of strong patient-provider relationships in decreasing hospitalizations, patients, regardless of hospitalization frequency, highly valued a positive and trusting relationship with their provider.

CONCLUSIONS: Programs seeking to reduce hospitalization among high cost, high need patients would benefit from emphasizing individual-level interventions for patient-based self-care while simultaneously addressing interpersonal, organizational and societal-level issues. Given the value that patients place on trusting, positive relationships with providers, patients may appreciate a model that prioritizes longitudinal provider continuity over the addition of an increased number of short-term providers and staff as is characteristic of many current care management programs.

\section{UNDIAGNOSED CANCER RISK IN WOMEN UNDERGOING UTERINE ARTERY EMBOLIZATION FOR LEIOMYOMATA}

Jackie V. Rodriguez ${ }^{1}$; Rebecca Perkins ${ }^{1}$; Jake Roberts Morgan ${ }^{1}$; Susan $\mathrm{O}^{\prime}{ }^{1}{ }^{1}$; Eung-Mi Lee ${ }^{1}$; Benjamin Matthews ${ }^{1}$; Michael K. PaascheOrlow ${ }^{2} .{ }^{1}$ Boston University Medical Center, Boston, MA; ${ }^{2}$ Boston University, Boston, MA. (Control ID \#3180355)

BACKGROUND: Symptomatic fibroids were historically treated with hysterectomy. More recently, minimally invasive options for treating fibroids while preserving the uterus have been developed. Uterine artery embolization (UAE) is one of the most common techniques, and allows up to $80 \%$ of women to successfully treat their symptoms without hysterectomy. However, retaining the uterus raises the possibility of missing an occult cancer. We examined the likelihood of subsequent gynecologic cancer development in women undergoing UAE.

METHODS: Administrative data from 2007 to 2016 were obtained from MarketScan, a database of diagnostic and treatment data for over 60 million commercially insured individuals in the US. Our analysis was limited to women at least 18 years of age without prior diagnosis of cancer who underwent UAE for treatment of leiomyomata and had on average 4.2 years of follow-up. Records were electronically queried for any inpatient or outpatient diagnostic or procedure codes (ICD-9, ICD-10, and $\mathrm{CPT}$ ) related to uterine, cervical, tubal, or ovarian malignancies occurring after the procedure date through the end of follow-up. All codes for all charts identified through electronic query were then manually reviewed by a minimum of 3 coders, and discrepancies resolved by consensus. Diagnosis of cancer was defined as having at least two codes for gynecological malignancy submitted at least one week apart and/or those with malignancy codes linked to tissue pathology results. Descriptive statistics were generated for the final study sample.

RESULTS: Between 2007 and 2016 20,575 women underwent UAE. Fifty-one women met our criteria for exhibiting cancer after undergoing UAE (37/51, 73\% uterine cancer; $13 / 51,25 \%$ ovarian cancer; and, $1 / 51$, $2 \%$ cervical cancer). On average, women in our sample exhibited cancer within 2.3 years from the date of UAE with a standard deviation of \pm 2.14 years and a range of 17 days to 7.8 years. We estimated that 1 in 403 (95\% CI: 1 in 354 to 1 in 469) women undergoing UAE were at risk for developing a gynecological malignancy.

CONCLUSIONS: A small fraction of women undergoing UAE go on to develop gynecologic malignancies - the majority of which may have been avoided with hysterectomy. Women choosing uterine preservation with UAE for symptomatic fibroids should be aware that they might develop gynecologic malignancies in the future that could be prevented with hysterectomy.

\section{UNLOCKING IMPLICIT BIAS: IMPLEMENTATION OF AN IM- PLICIT BIAS WORKSHOP TO INCREASE RESIDENT PHYSI- CIAN AWARENESS OF PERSONAL IMPLICIT BIAS AND ITS EFFECT ON PATIENT CARE.}

Vy Pham ${ }^{1}$; Jabraan Pasha ${ }^{2}$; Jacob Murray ${ }^{1} .{ }^{1}$ OU-TU School of Community Medicine, Broken Arrow, OK; ${ }^{2}$ universit of oklahoma, Tulsa, OK. (Control ID \#3178438)

BACKGROUND: Unconscious attitudes, also known as implicit biases are ubiquitous and their effects are wide-ranging. From something as seemingly insignificant as the clutching of a purse in lieu of a passerby to something of potential great consequence such as the lack of a surgical referral, the fingerprints of implicit bias are on many of the decisions we make. However hard it is to conceive, physicians' patient care decisions are not immune to the influence of unconscious beliefs and attitudes, and the data showing the effect of implicit bias on healthcare disparities is growing. Mirroring the societal disparities seen in the criminal justice system and workforce, in healthcare, the divide is just as great. Given the strong data linking implicit bias to healthcare disparities, it is very important to promote awareness of implicit bias in not only healthcare, but also healthcare providers as individuals. Our study looked to increase resident awareness and confidence in managing unconscious attitudes and beliefs that may be affecting patient care.

METHODS: Participants were 53 internal medicine, pediatric and family medicine residents at OU-TU School of Community Medicine. Between August 2018 and October 2018, during Academic Grand Rounds, participants attended a 90-minute implicit bias workshop. The interactive workshop looked to define implicit bias, demonstrate its origins and show its societal impact via lecture, hands-on activities and small group discussion. Via a 7-point Likert scale, a pre and post-workshop questionnaire measured participants' awareness of implicit bias and confidence in discovering and managing implicit attitudes. Data was analyzed via paired T-test analysis.

RESULTS: There was a mean increase of resident physicians' awareness of general, personal implicit bias (mean increase of $0.938, \mathrm{P}<.001$ ) and awareness of implicit bias toward patients (mean increase $0.698, \mathrm{P}<.001$ ). The results also reflected an increase in confidence in discovering personal implicit bias (mean increase $0.358, \mathrm{P}<.001$ ) and in management of implicit attitudes discovered about patients (mean increase 0.321, $\mathrm{P}<.001$ ).

CONCLUSIONS: Our implicit bias workshop proved successful in increasing resident awareness of their vulnerability to unconscious attitudes and in increasing their confidence in recognizing and managing these same attitudes. Although increasing awareness, alone is not an adequate strategy for eliminating implicit bias and discriminatory practices that often follow, it has been shown to be effective in beginning to limit the unconscious bias. Paired with increased confidence in recognizing and managing these biases, this workshop appears to be a practical and effective first step towards combatting implicit bias. 
UNMET MATERIAL AND SOCIAL NEEDS AND PREEXPOSURE PROPHYLAXIS (PREP) FOR HIV PREVENTION ADOPTION: WHAT IS THE ASSOCIATION?

Tyler Andriano ${ }^{1}$; Minia N. Rios Gutierrez ${ }^{2}$; Julia H. Arnsten ${ }^{1}$; Viraj V. Patel ${ }^{1} .{ }^{1}$ Albert Einstein College of Medicine, Bronx, NY; ${ }^{2}$ Albert Einstein, Bronx, NY. (Control ID \#3185146)

BACKGROUND: Young Black/Latinx men who have sex with men (YBLMSM) have the highest rates of new HIV infections but low uptake of pre-exposure prophylaxis (PrEP)-a highly effective HIV prevention tool. While unmet material/social needs are associated with low uptake of biomedical prevention for diverse conditions, this association is unknown for PrEP. We investigated the effects of unmet needs on PrEP adoption in a sample of YBLMSM.

METHODS: We surveyed HIV-negative YBLMSM aged 18-29 in New York City at high-risk of HIV (eg, recent STI) recruited online $(n=143)$. Primary outcomes were 1) intention to use PrEP in the next month and 2) current PrEP use. We used the 17-item Family Resource Scale to measure unmet needs in three domains: basic (housing/food), health/social services, and economic, using a Likert scale ranged from $1=$ needs never met to $6=$ needs always met. For the full scale and each domain, we calculated the mean score and used bivariate logistic regression to test associations between outcomes and unmet needs (dichotomizing scores at having any unmet needs vs. needs always met).

RESULTS: Participants' mean age was 24.1( $\mathrm{SD} \pm 3)$ years. Most identified as male $(87 \%)$, gay $(76 \%)$, and Non-Hispanic Black $(63 \%)$ or Latinx/Hispanic $(37 \%)$. Some $(19 \%)$ had no health insurance and among the insured (79\%), $42 \%$ had Medicaid. Of those not on PrEP, 48\% (69/114) intended to start in the next month; 20\% (29/ 143) were currently on PrEP. In the full scale, 75 participants $(52 \%)$ had some unmet needs. Among the three domains, 36\% $(n=52)$ had unmet basic needs, $59 \%(\mathrm{n}=85)$ had unmet health/social needs, and $64 \%(n=92)$ had unmet economic needs. In bivariate analysis of the scale, participants were more likely to intend to use PrEP if they had more unmet needs in the full scale $(\mathrm{p}=0.03)$, basic $(\mathrm{p}=0.01)$, and economic (0.04) domains. Yet, for health/social needs, regardless of the level of unmet needs, participants had similar intentions to use PrEP. Likewise, regardless of unmet need levels overall or in each domain, current PrEP use was similar.

CONCLUSIONS: A high prevalence of unmet material/social needs existed among YBLMSM. Those with more unmet needs had a higher intention to use PrEP, but similar rates of PrEP use as those with met needs. Given the significant interest in PrEP use, reaching YBLMSM with greater unmet needs may help improve PrEP uptake.

\begin{tabular}{|c|c|c|c|}
\hline \multicolumn{4}{|c|}{$\begin{array}{c}\text { Table. Association of PrEP intention and current PrEP use with unmet } \\
\text { needs. }\end{array}$} \\
\hline & $\begin{array}{l}\text { Mean (SD) } \\
\text { Intending to take } \\
\text { PrEP in next } 30 \\
\text { days }(\mathrm{N}=69)\end{array}$ & $\begin{array}{c}\text { Mean (SD) } \\
\text { Not Intending to } \\
\text { take PrEP in next } \\
30 \text { days }(\mathrm{N}=45)\end{array}$ & OR $(95 \% \mathrm{Cl})$ \\
\hline Full Scale & $4.5(1.4)$ & $5.0(1.0)$ & $0.7(0.5,0.9)^{*}$ \\
\hline Basic Needs & $4.7(1.3)$ & $5.3(1.0)$ & $0.6(0.4,0.9)^{* *}$ \\
\hline Health Needs & $4.1(1.8)$ & $4.3(1.6)$ & $0.9(0.7,1.2)$ \\
\hline \multirow[t]{2}{*}{ Economic Needs } & $4.0(1.7)$ & $4.6(1.3)$ & $0.8(0.6,1.0)^{*}$ \\
\hline & $\begin{array}{l}\text { Currently Taking } \\
\operatorname{PrEP}(\mathrm{N}=29)\end{array}$ & $\begin{array}{l}\text { Not Taking PrEP } \\
(\mathrm{N}=114)\end{array}$ & \\
\hline Full Scale & $4.6(1.3)$ & $4.7(1.3)$ & $0.9(0.7,1.3)$ \\
\hline Basic Needs & $4.8(1.5)$ & $4.9(1.3)$ & $0.9(0.7,1.3)$ \\
\hline Health Needs & $4.0(1.7)$ & $4.2(1.7)$ & $0.9(0.7,1.2)$ \\
\hline Economic Needs & $4.2(1.6)$ & $4.2(1.6)$ & $1.0(0.8,1.3)$ \\
\hline
\end{tabular}

${ }^{*} \mathrm{p}<0.05,{ }^{*} \mathrm{p}<0.01$
UNMET MENTAL HEALTH NEED AMONG CHINESE AND LATINO PATIENTS IN PRIMARY CARE: THE INTERSECTION BETWEEN LANGUAGE, ETHNICITY, AND GENDER

Maria E. Garcia ${ }^{1}$; Ladson Hinton ${ }^{3}$; Steven Gregorich ${ }^{1}$; Jennifer Livaudais-Toman $^{1}$; Celia P. Kaplan ${ }^{2,}{ }^{1}$; Leah S. Karliner ${ }^{4,}{ }^{1} .{ }^{1}$ University of California, San Francisco, Greenwich, CT; ${ }^{2}$ University of California San Francisco, San Francisco, CA; ${ }^{3}$ University of California, Davis, Sacramento, CA; ${ }^{4}$ UCSF, San Francisco, CA. (Control ID \#3186068)

BACKGROUND: Although racial and ethnic minorities are more likely than whites to seek care for mental health symptoms in primary care, they are less likely to receive depression treatment. While prior studies have studied the independent contributions of race/ethnicity, gender and language on mental health disparities, the intersecting effects of these factors are unknown. This study examines the intersecting contributions of these factors to unmet mental health need among Chinese and Latino patients in primary care.

METHODS: As part of a study of communication and health outcomes in primary care, patients were interviewed via telephone in English, Chinese, or Spanish within 1 week of a visit. Two mental health outcomes were assessed: whether in the past year the patient 1) perceived a need for help with emotional or mental health symptoms ('mental health need'), and 2) had seen a primary care physician or a mental health professional for these symptoms. Among those who reported 'mental health need', we defined 'unmet mental health need' as those who had not used services for their symptoms. Main predictor variables included English proficiency (English proficient [EP] vs limited English proficient [LEP]), race/ethnicity, and gender. Descriptive statistics and chi-square tests estimated the prevalence of outcomes and examined differences by English proficiency and race/ethnicity. Regression models explored interaction effects among English proficiency, race/ethnicity, and gender on the 2 mental health outcomes, controlling for additional patient characteristics.

RESULTS: Among the 1149 participants (62\% women; 262 Chinese EP, 532 Chinese LEP, 172 Latino EP and 183 Latino LEP), 33\% reported mental health need. Women were more likely to report need than men (aOR 1.35, 1.03-1.79) regardless of race/ethnicity or English proficiency. There was a significant interaction between race/ethnicity and language proficiency. Among Chinese participants, those with LEP were more likely than those with EP to report mental health need (aOR 2.55, 95\% CI 1.73-3.76). While there was a trend in the opposite direction among Latinos, it was not statistically significant (aOR 0.64, 0.40-1.04). Among patients reporting mental health need, $41 \%$ had unmet mental health need. While there were no racial/ethnic differences in unmet need, there was a significant interaction between English proficiency and gender. Men with LEP, compared to those with EP, were more likely to have unmet need regardless of race/ethnicity (aOR 2.53, 1.06-6.04).

CONCLUSIONS: In a population of Chinese or Latino primary care patients, we found both high levels of mental health symptoms and unmet mental health need. Our results affirm the importance of universal depression screening that is inclusive of all populations and suggest that attention to intersecting patient characteristics can help identify those at highest risk of untreated symptoms (such as men with LEP) who would benefit from targeted interventions to ensure linkage to treatment.

\section{US MEDICAL SCHOOL APPLICANT EXPERIENCES OF BIAS ON THE INTERVIEW TRAIL}

Avik Chatterjee $^{1,2}$; Charlotte Greif ${ }^{3}$; Robert Witzburg ${ }^{4}$; Lori Henault ${ }^{5}$; $\overline{\text { Kristen Goodell }}^{4}$; Michael K. Paasche-Orlow ${ }^{6} .{ }^{1}$ Harvard Medical School, Cambridge, MA; ${ }^{2}$ Boston Health Care for the Homeless Program, Boston, MA; ${ }^{3}$ UT Southwestern, Dallas, TX; ${ }^{4}$ Boston University School of Medicine, Boston, MA; ${ }^{5}$ Boston Medical Center, Boston, MA; ${ }^{6}$ Boston University, Boston, MA. (Control ID \#3169424)

BACKGROUND: A diverse physician workforce will be necessary to meet the needs of the United States population, but US medical schools 
have been unsuccessful in attracting a diverse student body. Implicit bias is pervasive in medicine, including potentially in the medical school admissions process.

METHODS: We invited all 2018-2019 applicants who interviewed at one US medical school to complete the validated 8-item Everyday Discrimination Scale (EDS) asking about experiences of bias during their medical school interview experiences at any institution where they had interviewed. The survey asked participants the number of schools at which they had interviewed, and assessed covariates including their place of birth, race, ethnicity, native language, religious affiliation, sexual orientation, gender identity, disability status, veteran status and socioeconomic status.

RESULTS: Three hundred forty-seven (30\%) of 1175 interviewees completed the survey. Respondents reported having had a median of three interviews (range 1-16), and had a mean age of 23 (range 19-32). One hundred ninety $(54.8 \%)$ respondents reported White/Caucasian race, 83 $(23.9 \%)$ reported Asian race, $31(9.0 \%)$ reported mixed race, and 14 (4.0\%) reported Black or African-American race. Sixty-seven (19.3\%) reported Latino, Hispanic or Spanish ethnicity. One hundred ninety-eight $(57.1 \%)$ reported female gender and $144(41.5 \%)$ were male; $296(85.3 \%)$ reported their sexuality as straight. Most respondents $(334,96.3 \%)$ reported having no disability, and $60(17.3 \%)$ reported being from socially disadvantaged backgrounds. Participant demographics matching those of the broader interviewee pool. Seventy-two $(20.7 \%)$ respondents answered yes to one or more items on the EDS across their interview experiences. Gender, age, race, national origin, religion, and sexual orientation were all sources of discrimination. Among those responding affirmatively to a question on the EDS, 35\% reported experiencing bias at more than one institution. Those reporting bias had completed more interviews (5.2 vs. $3.9, \mathrm{P}<0.05)$ and were more likely to be Latino $(30.6 \%$ vs. $16.4 \%$, $\mathrm{P}<0.05)$. Two (14.3\%) Black/African-American respondents, $22(33 \%)$ Latino respondents, and 44 (22\%) women answered yes to at least one question on the EDS. Only three (4\%) reported the incident of bias to the medical school where it occurred - many participants reported telling friends or family members $(68 \%)$ or doing nothing (30\%).

CONCLUSIONS: One in five medical school applicants described experiencing bias during their interview process but very few reported such incidents. Latino ethnicity was associated with a higher likelihood of reporting bias, however, many of our subgroups were too small to allow meaningful subgroup analyses. Further work exploring applicant experiences of bias, how such experiences may affect medical school student body diversity, and how to decrease the frequency of incidents of bias is warranted.

\section{USABILITY OF M-HEALTH APPLICATIONS FOR SAFETY NET POPULATIONS}

Shreya Sharma $^{1}$; Rebecca G. Mishuris ${ }^{1}$; Katherine Gergen-Barnett ${ }^{1}$; Jack Maypole $^{2}$. Boston University School of Medicine, Boston, MA; ${ }^{2}$ Boston University School Of Medicine, Boston, MA. (Control ID \#3183095)

BACKGROUND: The number of smartphone users among diverse, lowincome communities is growing in the United States. As a result, mobile applications ("apps") are serving as an accessible means for health management. Unfortunately, little is known about how to evaluate health related apps or their usability features for diverse, low-income populations. We sought to define mobile health app features important for the needs of these populations and identify apps in the areas of chronic disease management and medication adherence that best included these features.

METHODS: In June 2018, online databases (PubMed, Embase) were searched to identify articles related to evaluating the usability of mobile applications in health and wellness. App domains evaluated in past studies were assessed to create a list of domains for rating apps relevant to diverse, low-income populations in this research study (Sarkar, Arnhold, Gordon, Stephan, Stowell, Vilardaga, Vu, Wen). The domains were categorized as: Usability, Population focus, Technology, and Clinical Impact. Each domain was given a weight related to importance in the usefulness of a mobile application by independent coders (RGM,KGB,JM) and a reviewer (SS) then adjudicated these weights. Ten apps in smoking cessation, diabetes management, and medication adherence were chosen to be rated by a coder (SS) in each domain with a score of 0 to 3 . Zero was assigned if the specific domain was unavailable, and 3 was assigned if the domain had high usability for the target population. The weight of each domain was incorporated into the domain's rating; the weighted scores across all domains were added to assign each app a final overall score. The apps with the highest overall scores were considered by a local patient advisory board for final recommendation of patient use.

RESULTS: The most important domains for diverse, low income communities include:languages available, literacy, graphics, multimedia, usability, patient centeredness, data entry mode, data exportability, cost, evidence based content, platform, connectivity requirement, social support provided, cultural sensitivity, privacy, updates, and a message or reminder function. Domains such as literacy and language had greater utility value than privacy and data entry. The top weight ranked apps for smoking cessation were Smoke Free- Quit Smoking Now and QuitNow! with an average score of 149.5/201 and 156/201; the apps for diabetes management were Glucosio and MyNetDiary with an average of 147/201 and 146/201; the apps for medication adherence were Medisafe and MyMeds with an average of 137.5/201 and 126/201.

CONCLUSIONS: Our research has shown that app domains relevant to low SES populations can be identified and used to rate and validate apps which specifically address these populations' needs. The next steps of this study are to implement the suite of apps in primary care clinics as "prescriptions" for patients and to determine uptake and impact of these specific mobile apps on health-related behaviors.

\section{USE OF A NATURAL LANGUAGE PROCESSING ALGORITHM TO PREDICT READMISSIONS AT A VETERANS AFFAIRS HOSPITAL}

Ryan D. Schulteis ${ }^{2}$; Joseph E. Lucas ${ }^{3}$; Joel C. Boggan ${ }^{1}$; David L. Simel ${ }^{2}$. ${ }^{\mathrm{T}}$ Duke University Health System, Durham, NC; ${ }^{2}$ Durham VAMC and Duke University, Durham, NC; ${ }^{3}$ Duke University, Durham, NC. (Control ID \#3186428)

BACKGROUND: Health systems have sought to predict hospital readmissions, with the hope of reducing readmissions through targeted interventions. The Department of Veterans Affairs (VA) developed an independently validated risk calculator with c-statistic of 0.65 used locally at Durham VA Medical Center (DVAMC) since 2010. We hypothesized including documentation from resident discharge summaries in a natural language processing (NLP) algorithm would improve the predictive accuracy for readmissions.

METHODS: All medicine discharge summaries over two academic years from 7/1/2016 through 6/30/2018 that included a calculated risk of readmission using the VA readmission risk calculator were collected from the VA electronic health record. We then extracted the calculated risk percentage of subsequent readmission within 30 days included within the summary text and excluded patients readmitted in less than 24 hours. Readmission events then were defined as subsequent admission to DVAMC within 30 days. An ROC curve was constructed to test the discriminatory power of predicted readmission risk from the calculator. For NLP, the set of discharge summaries was split into training and test sets. The text of each discharge summary was preprocessed and converted to a list of integer tokens and subsequently passed to a neural network. The network then outputs another predicted probability of readmission based upon the encoding of the discharge summary text - the "NLP Readmission Probability". We measured the predictive power of this NLP Readmission Probability with an additional ROC curve. Finally, logistic regression was used to add NLP prediction to the previous calculated risk to test for improved predictive ability. 
RESULTS: Overall, 4,306 discharge summaries were collected over the 2 -year period. The performance of the readmission calculator was similar to previous validation with a c-statistic of 0.62 . A vocabulary of all words occurring more than once across all summaries was used for NLP, for a total of 31,283 words. Subsequently, 2000 summaries were used to train the neural network iteratively, while 871 were used for validation. An additional 1435 summaries served as the test set. The accuracy of the NLP model for readmission was $82.3 \%$, which, given an overall readmission rate of $17.5 \%$, corresponded to a c-statistic of 0.56 . When combined with the calculated risk in a logistic regression model, the NLP algorithm did not add discriminatory power, with combined AUC of 0.62 .

CONCLUSIONS: Natural language processing using text from medicine discharge summaries did not provide additional discriminatory power when compared to a previously validated discharge risk prediction tool. The NLP algorithm offered little discriminatory power when used alone, with a AUC of 0.56. As many hyperparameters can be configured for NLP, we cannot rule out that a different combination would have created a model with higher predictive power.

\section{USE OF DIRECT-TO-CONSUMER TELEMEDICINE TO AC- CESS MENTAL HEALTH SERVICES}

Jessica Hohman $^{1}$; Kathryn A. Martinez ${ }^{1}$; Mark N. Rood ${ }^{2}$; Susannah L. $\overline{\text { Rose }^{1} \text {; Michael B. Rothberg }}{ }^{1} .{ }^{1}$ Cleveland Clinic, Cleveland, OH; ${ }^{2}$ Cleveland Clinic Foundation, Chagrin Falls, OH. (Control ID \#3184265)

BACKGROUND: Significant barriers to accessing mental health services exist, including limited insurance coverage, high costs, provider shortages, and long wait times. Direct-to-consumer (DTC) telemedicine connects patients and providers via mobile/web applications 24 hours a day, and insurance is accepted but not required. As such, DTC telemedicine has been proposed as a way to increase access to care for many common conditions, but little is known about its use for mental health care. The objective of this study was to characterize use of a large nationwide DTC telemedicine platform for mental health services.

METHODS: This is a retrospective cross-sectional study of encounters for mental health diagnoses conducted on the AmericanWell DTC telemedicine platform between July 2016 and July 2018. Mental health diagnoses were identified via ICD10-CM codes associated with encounters, and prescriptions were identified via National Drug Codes. Encounter measures included time of day, wait time, visit length, prescription outcome, and referral receipt. Patient measures included age, gender, whether they used a coupon for free or reducedcost care, and whether they provided insurance information. Patient satisfaction was ascertained at the conclusion of the encounter on scales of 0 to 5 stars. Provider characteristics included type (physician versus not) and specialty.

RESULTS: We examined 19,283 mental health encounters between 6,710 patients and 1,067 providers. Fifty-nine percent of patients were female, mean age was 35 years, $28.4 \%$ did not provide insurance information, and $11.8 \%$ used a coupon for free or reduced-cost care. Mean wait time was 5.6 minutes. Over $70 \%$ of encounters were for anxiety or depression, followed by bipolar disorder $(6 \%)$, ADHD (4\%), nicotine use disorders (2\%), alcohol use disorders $(2 \%)$, and sleep disorders (2\%). Schizophrenia and schizoaffective disorders accounted for $<1 \%$ of visits. Over half of encounters were with a therapist, psychologist, or licensed social worker; $20 \%$ were with primary care providers (PCPs) and 20\% with psychiatrists. Two percent resulted in referrals. Visit duration varied by provider type; median visit length with PCPs was 6 minutes vs. 16 minutes with psychiatrists and 47 minutes with therapists. Psychiatrists prescribed medication at a higher rate than PCPs (75\% vs. $70 \%$ of encounters). Patients reported high satisfaction (4.9/5) across all conditions. CONCLUSIONS: DTC telemedicine provides access to counseling and pharmacotherapy for a diverse range of common mental health conditions, particularly for younger users with mood disorders or ADHD, but the number of visits for other conditions was small. Most patients received prescriptions, and overall satisfaction was high. With growth, DTC telemedicine might facilitate care for patients in underserved areas.
USE OF INTERPRETERS FOR LIMITED ENGLISH PROFICIENCY PATIENTS WITH HIP FRACTURE

Jon Mong ${ }^{1}$; Shail Rawal ${ }^{1}$; Peter $\mathrm{Cram}^{2}$; Khalid Syed ${ }^{1} .{ }^{1}$ University of Toronto, Toronto, ON, Canada; ${ }^{2}$ University of Iowa, Toronto, ON, Canada. (Control ID \#3179311)

BACKGROUND: Informed consent (IC) is central to patient autonomy. Obtaining IC is difficult in the best of cases, and even more complex in patients with limited English proficiency (LEP). Professional interpreter use is standard of care when communicating with patients with LEP in Canada. Hip fractures are common and require urgent surgical repair for optimal outcomes, but also require that physicians obtain IC before surgery. Thus, obtaining IC in LEP patients with hip fracture could be challenging. We examined interpreter use in obtaining IC for LEP patients with hip fracture in an urban Canadian teaching hospital in a city where $48 \%$ of patients have a non-English mother tongue.

METHODS: We identified all patients admitted with hip fracture between January 1 2015-January 12018 to Toronto Western Hospital. We reviewed the electronic health record and selected all patients for whom English was not their preferred language. We excluded: 1) patients who had non-operative management of their hip fractures with no consent form; 2) patients for whom chart documentation was incomplete, precluding us from evaluating the measures described below. Using a standardized data abstraction tool we collected information on: whether our hospital consent form was signed; if the consent form was signed by the patient or a substitute decision maker (SDM); if there was documentation of interpreter use on the consent form, and if so, whether the interpreter was a professional employed by the hospital or ad-hoc (e.g., family member or hospital staff); and if an SDM signed the consent document, whether there was documentation that the patient was not capable (defined as documentation of: dementia; cognitive impairment; or delirium as measured by nurse administered Confusion Assessment Method). 10\% of charts were independently reviewed in duplicate.

RESULTS: Among 553 patients with hip fracture, we identified 134 (24\%) as LEP. 127 patients were included in the final analysis. Among those with LEP, 88 (69\%) were female (mean age 84 years). The most common languages were Portuguese (37 patients), Italian (33 patients), and Cantonese (9 patients). Among those who were LEP, 57 (45\%) signed their own consent forms, while $70(55 \%)$ had an SDM sign on their behalf. Of the 57 LEP patients who signed their own consent, 33 (58\%) lacked evidence of interpreter use, while $24(42 \%)$ had documented interpreter use. Of the 24 patients who had documented interpreter use, $4(17 \%)$ utilized a professional interpreter, while 20 (83\%) used ad-hoc interpreters. Of the 70 patients who had an SDM sign on their behalf, 11 (16\%) did not have documentation of lack of capacity.

CONCLUSIONS: In a Canadian teaching hospital serving a multicultural urban population we found significant gaps in utilization of interpreters for obtaining IC for patients with hip fracture.

\section{USE OF SOCIAL MEDIA TO IMPROVE CARDIOVASCULAR RISK FACTOR MODIFICATION IN THE CARDIOLOGY OUT- PATIENT SETTING}

Nina Liu; Zubiya Syed; Allan Chen. University of South Florida, Tampa, FL. (Control ID \#3138724)

BACKGROUND: Social media has become omnipresent and is a rapidly growing information source with widespread potential for patient education. There are no studies that examine the use of social media to improve cardiovascular risk factor modification. To address this gap, we studied the use of a social media educational program in an outpatient cardiology setting to improve patient knowledge and behavior related to cardiovascular health.

METHODS: Patients were prospectively enrolled at a single tertiary care center as part of routine outpatient visits. A baseline survey was given that 
assessed their knowledge and behaviors related to diet (including the Rapid Eating Assessment for Participants (REAPS) survey), exercise, risk factors and medication adherence. Patients elected to receive regular cardiovascular health related educational tips and resources either through a private Facebook group or email. The same survey was given after the follow-up period to assess changes in behavior and knowledge.

RESULTS: 51 patients were enrolled with mean follow-up period of 7.9 weeks. $31.4 \%$ were male with mean age of 55.8 years. There was a trend towards significant improvement in the REAPS score from baseline to follow-up ( $\mathrm{p}=0.05)$. Significant improvement was seen in knowledge related to weight/body mass index $(\mathrm{p}=0.04)$. In subgroup analyses, males rated their importance of personal exercise/fitness significantly higher after the follow-up period $(\mathrm{p}=0.02)$. Patients aged 60 years and older demonstrated a significant reduction from 5.7 to 3.6 hours/day of sitting/reclining from baseline to follow-up $(\mathrm{p}=0.01)$. No change was seen in medication adherence. At follow-up, $100 \%$ of participants reported learning from the program, 93\% reported perceived improvement in health, and $96 \%$ stated they would continue the program if available.

CONCLUSIONS: As the first of its kind, this study demonstrated that an educational program via a social media platform may improve patient behavior and knowledge related to risk factor modification in an outpatient cardiology setting. Further studies are needed to evaluate the potential of this adjunctive tool that requires little additional cost and resources.

\section{USER-CENTERED DESIGN AND USABILITY OF AN ELEC- TRONIC TOOL TO IMPROVE DEPRESSION TREATMENT IN PATIENTS WITH CORONARY HEART DISEASE.}

Jennifer Mizhquiri Barbecho ${ }^{1}$; Rachel Monane ${ }^{1}$; Martin McElhiney $^{3}$; Luis Blanco $^{1}$; Fereshte N. Kharazmi ${ }^{1}$; Emily M. Callanan ${ }^{1}$; Monica Polanco ${ }^{2}$; Drew Rabidoux ${ }^{2}$; Ian Kronish ${ }^{1}$; Siqin $\mathrm{Ye}^{1}$; Nathalie Moise ${ }^{1}{ }^{1}$ Columbia University Medical Center, Brooklyn, NY; ${ }^{2}$ New York Presbyterian, New York, NY; ${ }^{3}$ Columbia University Medical Center, New York, NY. (Control ID \#3182556)

BACKGROUND: Depressive symptoms in coronary heart disease (CHD) patients are common and associated with recurrent cardiovascular disease events and mortality. However, only $30 \%$ of depressed CHD patients are treated. Informed by behavior change theory and a user-centered design process, we explored treatment barriers and designed and conducted usability testing of a web-based tablet-delivered tool to enhance depression screening, referral and treatment initiation in CHD patients.

METHODS: An implementation team of physicians, patients, nurses, and administrators identified system requirements based on a national survey of $1500 \mathrm{CHD}$ patients and then designed and revised a patientfacing tablet interface of depression screening Patient Health Questionnaire-9 (PHQ-9), preference and barriers assessment, and video-assisted patient activation. We recruited 10 patients with documented CHD from hospital discharge and clinic codes to conduct usability testing. A research coordinator observed, recorded and guided participants through a usability protocol in which participants completed goaloriented tasks and a think-aloud protocol that assessed depression treatment attitudes and barriers and satisfaction with the tool using retrospective interview probing techniques. We employed descriptive statistics for quantitative responses and content analysis to code qualitative responses for acceptability and themes while iteratively refining the tool.

RESULTS: The average age of 10 participants was $62.7 \pm 7.5$ years; $60 \%$ were male, 50\% Hispanic/Latino, 30\% Black/African American; 60\% had at least mildly elevated depressive symptoms. All participants found the tool highly acceptable. Treatment barriers included stigma/privacy, gender/ cultural norms, fear of medications, lack of knowledge, and opportunities (transportation, insurance, time). Half remarked that worry, fear of death and changes in lifestyle, all exacerbated by fear-laden patient-physician communication, contributed to depressive symptoms. Tool facilitators included provision of information on the link between depression and heart disease and non-pharmacologic treatment options (e. g., cardiac rehab), normalization, depression treatment success stories, personalization, as well as coupling the tool with pamphlets and physician-initiated discussion about stress. The think-aloud protocol informed iterative updates to the tool, including addition of percent completion indicators, voiceover functionality, iconography, tailoring, and decision support.

CONCLUSIONS: We demonstrate how a multidisciplinary implementation team can use a theory-informed user-centered design process to yield a better understanding of participants' technology preferences. We contribute a novel interface to enhance CHD patient engagement in depression treatment. We will next test the effectiveness of this tool on depression screening and treatment in CHD patients in a stepped-wedge trial across 8 cardiology and primary care clinics.

\section{USING BILLING DATA TO COMPARE MEDICAL NEEDS AND UTILIZATION COSTS OF HOMELESS VS. NON-HOMELESS PATIENTS}

Benjamin Bovell-Ammon ${ }^{1}$; Allison Bovell-Ammon ${ }^{1}$; David Ko ${ }^{1}$; Christine A. Pace ${ }^{2,}{ }^{1}$; Thea James ${ }^{3}$; Megan Sandel ${ }^{1}$. ${ }^{1}$ Boston Medical Center, Boston, MA; ${ }^{2}$ Boston University School of Medicine, Boston, MA; ${ }^{3}$ Boston Medical Center, Cambridge, MA. (Control ID \#3185808)

BACKGROUND: Homelessness is associated with adverse health outcomes across the life course and complicates effective delivery of medical and behavioral health services. The growing share of value-based payment models increasingly incentivizes and creates opportunities for hospitals \& health systems to identify and intervene upon social determinants of health, including homelessness. The differences in healthcare utilization and costs between homeless patients vs. low-income non-homeless patients and the extent to which such differences might be impactable is understudied. This study aims to develop an easily replicable method for comparing prevalent health conditions and costs between homeless and non-homeless Medicaid-covered patients in a hospital's billing data.

METHODS: Hospital billing data from Boston Medial Center (BMC), New England's largest safety-net hospital, were acquired for patients in the Boston Accountable Care Organization (BACO, a Medicaid ACO) for any ambulatory, emergency department, and inpatient care at BMC between September 1, 2017-August 31, 2018. Patients were identified as homeless if they met at least one of the following criteria: had the ICD-10 code for homelessness (Z.59.0) in their billing record, receive primary care at Boston Healthcare for the Homeless, listed their primary address as a known homeless shelter or BMC. Socio-demographic factors, diagnoses, and costs were characterized and compared between homeless and non-homeless patients.

RESULTS: Among 20,640 patients enrolled in BACO who received health care services at BMC, $13.7 \%$ were homeless. Compared to nonhomeless patients, homeless patients in the study did not differ in age (avg 41.6y, s.d. $13.3 \mathrm{y})$, but they were significantly $(\mathrm{p}<0.01)$ more likely to be male $(61.9 \%$ vs. $37.3 \%)$, white $(38.1 \%$ vs $19.8 \%)$, and single/never married $(80.9 \%$ vs $72.3 \%)$ and had significantly $(p<=0.01)$ higher rates of vascular disease ( $30.9 \%$ vs $18 \%)$, CHF ( $4.7 \%$ vs $2 \%)$, diabetes $(16.3 \%$ vs $14.6 \%)$, COPD (10.3\% vs $3.6 \%)$, CKD (7.8\% vs $5.9 \%)$, schizophrenia (13\% vs $3.3 \%)$, bipolar disorder (19.5 vs $5.2 \%)$, and substance use disorder (65.8\% vs $18.7 \%$ ). Homeless patients had on average $\$ 28,657$ of health care costs per patient in the 12 month period while non-homeless patients had \$14,566 ( $<<0.01)$.

CONCLUSIONS: Among patients in a Medicaid ACO that received services at a major urban safety-net hospital, homeless patients had higher rates of major medical and behavioral health conditions and greater utilization of high cost services. This study demonstrates an easily replicable method by which a health care organization can define the scope of homelessness in their patient population and begin to estimate the excess health care costs that might be attributable to homelessness. Research that may help identify opportunities for developing interventions to stabilize this population, decrease cost and improve clinical outcomes. 
USING COMMUNITY ENGAGEMENT TO ADAPT AND IMPLEMENT AN EVIDENCE-BASED CVD INTERVENTION IN RURAL AFRICAN AMERICAN COMMUNITIES

Gaurav Dave ${ }^{1,1}$; Stephanie Hoover ${ }^{1}$; Leah Frerichs ${ }^{2,1}$; Madeline Hamilton $^{1}$; Kiana Bess ${ }^{\Gamma, 3}$; Hilary R. Jessup ${ }^{1}$; Brenda Boone ${ }^{4}$; Mysha Wynn ${ }^{5}$; Shirley McFarlin ${ }^{4}$; Project GRACE Steering Committee ${ }^{6}$; Giselle M. Corbie-Smith ${ }^{1,}{ }^{1}$. ${ }^{1}$ University of North Carolina School of Medicine, Chapel Hill, NC; ${ }^{2}$ Gillings School of Global Public Health, Chapel Hill, $\mathrm{NC} ;{ }^{3}$ University of Michican, Ann Arbor, MI; ${ }^{4}$ James McFarlin Community Development, Rocky Mount, NC; ${ }^{5}$ Project Momentum Inc., Rocky Mount, NC; ${ }^{6}$ Project GRACE, Rocky Mount, NC. (Control ID \#3184892)

BACKGROUND: Well known racial and geographic disparities in cardiovascular (CV) risk have led to terms such as a "stroke belt" and "mortality penalty" to describe the southeast US. While there has been improved overall CV mortality, these improvements have not been enjoyed by rural African Americans (AAs); in part because interventions have not addressed unique contextual and social factors that drive health disparities in rural minority communities. Using a Community-Based Participatory Research approach, we adapted PREMIER to Heart Matters, an evidence-based intervention to reduce $\mathrm{CV}$ risk, for rural AA communities in North Carolina. This abstract presents clinical and behavioral outcomes of a randomized controlled study at 6 months - the intensive intervention period.

METHODS: We recruited 143 AAs who resided in Edgecombe or Nash counties, had at least one risk factor for CVD, were 21 years or older, and consented to participate. Participants were recruited in clusters, each cluster representing a community- or faith-based organization, that were randomized into intervention $(n=71)$ and 6-month delayed intervention control groups $(n=72)$. Data on clinical measures (e.g. blood pressure (BP)), selfreported behaviors (e.g. physical activity) and demographics were collected at baseline, 6, and 12 months. Multivariate mixed model and regression analyses were used to estimate treatment effects. We controlled for clustering, age, and use of medications. The primary outcomes are change in systolic BP or diastolic BP, combined hypertension status, BMI, and HbAlc. The secondary outcomes are dietary and physical activity selfefficacy, social support, and vigorous physical activity.

RESULTS: Approximately three-fourths $(72.7 \%, \mathrm{n}=104)$ of the sample were female, with a mean age of 57.3 years. We found a statistically significant difference in BP status in the intervention group compared to the control group $(=0.38,95 \%$ CI $[0.05,0.71])$ at 6 months. While diastolic BP increased in both groups, the mean difference from baseline was statistically significant between the intervention $(1.93 \mathrm{mmHg})$ and control groups $(5.9 \mathrm{mmHg})$ at 6 months $(p=0.01)$. Self-efficacy to reduce salt increased significantly in the intervention group $(=0.31,95 \% \mathrm{CI}$ $[0.09,0.53])$. Similarly, the intervention group reported increased vigorous physical activity and increased social support for physical activity by friends and families $(p<0.05)$

CONCLUSIONS: The improvements in BP, reported physical activity, and sodium intake represent a meaningful reduction in major risk factors for CVD. More importantly, these findings build evidence regarding the importance of adapted interventions to address the complex aggregate of risk factors that contribute to CVD in rural minority communities. This work advances research on the implementation of evidence-based CVD interventions through community engagement.

\section{USING EXPERIENCE SAMPLING METHOD TO INVESTIGATE ENGAGEMENT OF TALENTED MINORITY URBAN YOUTH IN SCIENTIFIC RESEARCH PIPELINE PROGRAMS}

Jhonatan Marte ${ }^{4}$; John Cursio ${ }^{1}$; Samantha Ngooi ${ }^{2}$; Laura Ruth Venable ${ }^{3}$; Christianah Ogunleye ${ }^{4}$; Tyler Raclin ${ }^{3}$; Barbara Schneider ${ }^{5}$; David Meltzer ${ }^{2}$; Vineet M. Arora ${ }^{6} .{ }^{1}$ The University of Chicago, Chicago, IL; ${ }^{2}$ University of Chicago, Chicago, IL; ${ }^{3}$ Univeristy of Chicago, Chicago,
IL; ${ }^{4}$ University of Chicago Pritzker School of Medicine, Chicago, IL; ${ }^{5}$ Michigan State University, East Lansing, MI; ${ }^{6}$ University of Chicago Medical Center, Chicago, IL. (Control ID \#3184270)

BACKGROUND: Pipeline programs that promote equity by engaging minority youth to consider scientific careers are increasing, but novel evidence-based approaches for program evaluation are lacking. Experience Sampling Method (ESM) is a valid method to measure real-time engagement of teens in science classrooms that has not been applied to pipeline programs. We used ESM to compare engagement of minority youth participating in a hospital-based clinical research experience versus a traditional science program, and examine whether an ESM measure of engagement is associated with perceiving one's activities as related to future goals.

METHODS: Talented Chicago high school juniors were blocked randomized by sex and race to either the 6-week hospital-based program or a traditional science program. The hospital program included realistic research activities (patient interviews, chart reviews, team meetings). The traditional program offered lectures, labs, and field trips to science museums. Students were given watches that alerted them to complete a 40item ESM survey from the Sloan Study of Youth and Social Development. Students were alerted 5 times per day, Monday through Friday, during weeks 1 and 3 . The survey asked what students were doing and thinking (open-ended) and 3 Likert items that constitute an "optimal learning moment" (OLM), a valid ESM measure of engagement. We used a hierarchical linear model to test associations between program group and OLMs, controlling for year, week, gender, and race. We also examined the association between OLMs and perceiving one's activity as important to future goals.

RESULTS: From 2005-2015, we randomized 195 students to either the hospital (108) or traditional program (87) who completed $66.7 \%$ of alerts (7820/11680). Two-thirds were URM in medicine; over half (62\%) were female. Race, ethnicity, and gender did not differ significantly between groups. OLMs comprised $27.2 \%$ of alerts. In $54 \%$ of alerts, students reported being engaged in activities related to future goals. Assignment to the hospital-based program was associated with lower odds of OLMs during week $1(\mathrm{OR}=0.50,95 \% \mathrm{CI}=0.31-0.76, \mathrm{p}<0.001)$, but with greater odds in week $3(\mathrm{OR}=1.29,95 \% \mathrm{CI}=1.01-1.63, \mathrm{p}=0.036)$. Males had greater odds of OLMs $(\mathrm{OR}=1.52,95 \% \mathrm{Cl}=0.74-1.46, \mathrm{p}=0.015)$ and Hispanics had lower odds of OLMs $(\mathrm{OR}=0.52,95 \% \mathrm{CI}=0.68-1.98, \mathrm{p}=0.014)$. Regardless, OLMs were strongly associated with perceiving activity as important to future goals $(\mathrm{OR}=4.86,95 \% \mathrm{CI}=4.17-5.66, \mathrm{p}<0.001)$.

CONCLUSIONS: Although engagement was initially lower, students in the hospital-based research program were more engaged by week 3, highlighting the need for minority youth to acclimate to research environments. Efforts to improve engagement for females and Hispanics are warranted. Given that an ESM measure of engagement is associated with perceiving activities as important to future goals, ESM can be a relevant short-term outcome for evaluating pipeline programs.

\section{USING HUMAN-CENTERED DESIGN TO DEVELOP PRACTI- CAL, EVIDENCE-BASED INTERVENTIONS IN PRIMARY CARE}

Dani L. Zionts ${ }^{1}$; Cati Brown-Johnson ${ }^{1}$; Nadia Safaeinili ${ }^{1}$; Jonathan G. Shaw ${ }^{1}$; Lucie Richter ${ }^{2}$; Svava M. Atladóttir ${ }^{2}$; Donna M. Zulman ${ }^{1,3}$. ${ }^{1}$ Stanford University, Stanford, CA; ${ }^{2}$ Future Medical Systems, Sunnyvale, CA; ${ }^{3}$ VA Palo Alto Health Care System, Menlo Park, CA. (Control ID \#3185534)

BACKGROUND: Though many are working to address the wellness crisis resulting in record levels of physician burnout and patient dissatisfaction, it still takes $>15$ years for evidence-based innovations to integrate into practice. We sought to determine whether an evidence-based Human-Centered Design (HCD) methodology could be used to rapidly design a scalable solution to enhance provider presence and improve connection with patients. 
METHODS: HCD is a flexible, complex process utilizing evidence, intuition, and rapid, iterative prototyping to develop creative products and interventions. To approximate HCD in an academic setting while maintaining rigorous scientific standards, we leveraged a 3-stage HCD approach: Stage 1. Inspiration: a) Secondary research via a systematic review of interpersonal interventions in health care ( $\mathrm{n}=77$ studies); $b$ ) interviews and observations of diverse primary care encounters ( 29 observations across 3 clinics); c) interviews with professionals in non-medical fields to provide analogous inspiration $(n=30)$. Stage 2. Ideation: a) Delphi panel of 14 national experts to gather additional input on our "inspiration" output; $b$ ) journey mapping and framework development to scope design principles and opportunities (e.g., daily clinic flow from patient/provider lens); c) cocreation sessions with 10 physicians to develop initial prototypes. Stage 3 . Implementation: a) User feedback sessions with diverse providers inside and outside of clinic to test the feasibility and acceptability of preliminary intervention designs; b) live prototyping in varied clinics to assess implementation and maintenance metrics.

RESULTS: Over 16 months, we completed preliminary design work to develop an intervention focused on primary care. Stage 1 yielded evidencebased practices that influence patient and clinician experience. Provider interviews highlighted the importance of consistent meaningful connections with patients without extending clinic visit time. Interviews with nonmedical professionals illustrated universal approaches to protecting joy of practice in intense interpersonal interactions. Stage 2 highlighted key time points where intervening might positively impact connection in outpatient care, while also accommodating acutely busy periods such as just before a provider enters the room. It also surfaced the lack of opportunity for providers to exchange strategies to connect with patients. In Stage 3, HCD permitted ultra-rapid cycles of creation, piloting, evaluation, and iteration, surfacing ideas and determining feasibility of design prototypes with physician stakeholders in a matter of weeks.

CONCLUSIONS: We found that a design approach combining HCD methodology with a systematic literature review and Delphi process resulted in practical, acceptable, evidence-based intervention prototypes in less than 18 months. In the final stage of this project, the pilot intervention will be implemented and evaluated across multiple settings to determine the effect on patient and clinician experience.

\section{USING SIGNATURE INFORMED CONSENT TO IMPROVE PRESCRIBING LONG-TERM OPIOID THERAPY FOR CAN- CER PAIN: PERSPECTIVES OF PATIENTS AND INTERDISCI- PLINARY PROVIDERS}

Soraya Fereydooni ${ }^{2}$, ${ }^{1}$; Karleen Giannitrapani ${ }^{2}$, 9; Azin Azarfar $^{2}$; Peter Glassman $^{3,7}$; Maria J. Silveira ${ }^{4,8}$; Amanda Midboe ${ }^{5}$; Maria Zenoni ${ }^{6,10}$; Robert Pearlman ${ }^{11,}{ }^{12}$; William Becker ${ }^{6,}{ }^{10}$; Karl Lorenz ${ }^{2,9}$. ${ }^{1}$ Stanford University, Stanford, CA; ${ }^{2}$ VA Palo Alto Health Care System, San Jose, CA; ${ }^{3}$ VA Greater Los Angeles HCS, Los Angeles, CA; ${ }^{4}$ Ann Arbor VAMC HSRD, Ann Arbor, MI; ${ }^{5}$ VA Palo Alto Health Care System, Menlo Park, CA; ${ }^{6}$ VA Connecticut Healthcare System, West Haven, CT; ${ }^{7}$ University of California Los Angele, Los Angles, CA; ${ }^{8}$ University of Michigan, Ann Arbor, MI; ${ }^{9}$ Stanford University School of Medicine, Stanford, CA; ${ }^{10}$ Yale School of Medicine, New Haven, CT; ${ }^{11}$ Seattle VA Puget Sound Health Care System, Seattle, WA; ${ }^{12}$ University of Washington, Seattle, WA. (Control ID \#3185984)

BACKGROUND: Long-term opioids therapy (LTOT) for cancer pain is accompanied with numerous potential risks. As cancer survival becomes more common, the extension of written signature informed consent (SIC) for LTOT gains relevance. Our objective is to understand provider and patient perspectives on using SIC for LTOT in cancer patients.

METHODS: Design: Semi-structured interviews. Population: 20 interdisciplinary providers (primary care providers including general internists, oncologists, and advanced practice nurses) and 20 patients from two academically-affiliated Veteran Health Administration Medical
Centers. Analysis: Using a priori code from the bioethics literature, we employed deductive content analysis to understand provider perspectives and inductive constant comparison to understand patient perspectives.

RESULTS: Providers believed: 1) Autonomy: SIC is an educational tool "to get the patient involved in the decision." However, it may be perceived as a restriction on autonomy when "patients have to sign this form and agree or they're not going to get opioids." 2) Beneficence: SIC can promote patient-centered care by "setting the goals and the expectations right off the bat" to effectively and safely treat pain. 3) Non-maleficence: SIC can prevent potential harm by warning patients against potential adverse effects, divergence, and misuse. Conversely, it may divert providers' focus away from cancer-related issues and damage trust by "spending a lot of time on things that don't make sense to cancer patient," distress patients by "making them feel like criminals," and delay the process "because prescribers just don't feel like doing the work" of SIC. 4) Justice: Provider may reflect their biases by "Jump[ing] right into the consent with someone who's a past drug abuser" or has had "a history of suicide attempts or thinking." Global SIC requirement may minimize "patients thinking that you're singling them out" and ease the introduction of SIC in oncology clinics when "it's part of the clinical practice." 5) Dignity: Despite agreeing that SIC should "be demanded as an educational process," providers worried about SIC as a "one size fits all" approach that is "not designed for cancer patients." Emergent themes from patient data indicated that they want SIC: 1) to explain substance use disorder in simple language 2) to cover consequences of tapering and not tapering 3 ) to designate someone they can contact with questions 4) to provide simple parameters 5) to have written SIC information to be verbally explained 6) to warn against sensations of euphoria and potential triggers for overuse.

CONCLUSIONS: Having SIC as a standardized process for LTOT in patients with cancer may normalize SIC conversations; however, it is important to allow some room for clinician's autonomy to do what they perceive as best for patients. A systematic requirement of SIC for all patients is a complex proposition, and SIC needs to be tailored for patients with cancer pain on long-term opioid therapy.

\section{USING SIMULATION TO ASSESS COMPETENCY IN MEDI- CAL EDUCATION: OBSERVATIONS FROM AN INTERNA- TIONAL EXPERIENCE AT THE UNIVERSITAT INTERNACIONAL DE CATALUNYA}

Juan M. Salgado $^{1}$; Tiffany E. Schwasinger-Schmidt ${ }^{2}$; Montserrat Virumbrales Cancio ${ }^{3}$. University of Kansas School of Medicine, Wichita, KS; ${ }^{2}$ University of Kansas School of Medicine, Andover, KS; ${ }^{3}$ Universitat Internacional de Catalunya, Barcelona, Spain. (Control ID \#3181873)

BACKGROUND: In 2014 the AAMC published new guidelines for the expectations for medical graduates to include the core Entrustable Professional Activities (EPAs). The goal of EPAs was to establish a mechanism to address competency assessment in medical education and help decrease an emerging gap in student readiness to transition from medical school into residency training. Simulation offers a unique, integrated approach for educators to be able to assess medical student competence in clinical scenarios. At the Universitat Internacional de Catalunya (UIC) in Barcelona, Spain, the medical school has utilized the Comprehensive Centre for Advanced Simulation (CISA) to formulate a competency assessment for their Objective Structured Clinical Exam (OSCE) at the completion of medical school.

METHODS: The OSCE at UIC was analyzed as a case study from a student's perspective during a month long international medical experience in June 2017. Participants included CISA educators and technical staff, standardized patients, OSCE evaluators, and medical students. Core medical competencies included taking a medical history, physical examination skills, formulation of an appropriate assessment and plan, technical procedural skills, interprofessional interactions, communication skills, 
implementation of preventative medicine, and medical ethics. This exam comprised $50 \%$ of the medical students' grade in his or her final year of medical school. Assessment of student competency was evaluated by a review of student performance assessed by standardized checklists on the 20 different OSCE cases.

RESULTS: UIC has utilized the OSCE as a method of competencybased assessment since the 2013-2014 academic year. Overall class averages on the OSCE from 2014 to 2018 were $79 \%, 74 \%, 73 \%$, $71 \%$, and $74 \%$, respectively. Assessment and Plan scores were $81 \%$, $63 \%, 73 \%, 77 \%$, and $76 \%$, respectively. Medical Ethics scores were $58 \%, 81 \%, 82 \%, 85 \%$, and $67 \%$, respectively. Interprofessional Interaction scores were $72 \%, 84 \%, 65 \%, 62 \%$, and $70 \%$, respectively. A standardized overall OSCE score determined by the Medical Simulation director was used as a metric to determine competency.

CONCLUSIONS: This observational case study highlights the power of simulation to assess student competency with regards to skills necessary for residency training. As medical schools transition to EPA based assessments, the need for standardized measures to determine competency will become essential. The lessons learned from this case study of UIC's utilization of the OSCE at the completion of medical school can serve as a template for measuring medical students readiness for residency. Educators can track student performance and implement changes to curriculum to enhance student performance and ease the transition to clinical practice. From a student's perspective, simulation affords one the opportunity to translate classroom knowledge into clinical practice, and confidence can be instilled upon completion of competencies prior to entering residency training.

\section{UTILITY OF POINT-OF-CARE ULTRASOUND AS A TOOL FOR MEDICAL DECISION MAKING IN A RESOURCE-LIMITED SETTING}

Michelle Fleshner $^{1}$; Ayako W. Fujita ${ }^{2}$; DIVYA BHAMIDIPATI ${ }^{1}$; Steven Fox $^{1}$; Thuy Bui ${ }^{3} .{ }^{1}$ UPMC, Pittsburgh, PA; ${ }^{2}$ University of Pittsburgh Medical Center, PITTSBURGH, PA; ${ }^{3}$ University of Pittsburgh, Pittsburgh, PA. (Control ID \#3185226)

BACKGROUND: Point-of-care ultrasound (PoCUS) is a quick, inexpensive, and noninvasive tool for bedside diagnosis that is most effective when used to answer a specific clinical question. Few studies have looked at the effect of PoCUS on clinical diagnosis in resource-limited settings. This study investigated the ability of PoCUS to assist with medical decision making when used by Internal Medicine trainees in resourcelimited settings.

METHODS: This was a retrospective analysis of PoCUS studies conducted at a global health site through the University of Pittsburgh Medical Center (UPMC) Internal Medicine Residency program. A pilot study was performed in March of 2018 and a formal study is forthcoming in January - April 2019. Residents underwent a weeklong intensive ultrasound training course prior to traveling abroad and were then required to record and interpret ultrasound clips used in clinical practice. Equipment included Phillips Lumify cardiac and linear transducers. Prior to performing each scan, residents documented the "pre-PoCUS" diagnosis and clinical question. Afterwards, they recorded the "post-PoCUS" diagnosis and the ability of PoCUS to answer the clinical question and prioritize the differential diagnosis. Change in management was a secondary outcome. Expert and peer review of image acquisition and interpretation will be utilized for quality assurance. Data were analyzed using Microsoft Excel.

RESULTS: During the pilot study, a total of 58 studies were performed by 3 residents over 4 weeks. Twenty-five (43\%) were abdominal ultrasounds, $24(41 \%)$ were cardiac, and $9(16 \%)$ were pleural. Ultrasound scans answered the clinical question in $46(79 \%)$ studies, helped to prioritize the differential diagnosis in $51(88 \%)$ studies, and changed the diagnosis in $21(36 \%)$ studies. Finally, eighteen (31\%) ultrasound scans changed management, mostly by adding or removing medications. Efficacy of PoCUS scans was stratified by clinical question (Image 1). Data collection is ongoing through April 2019.

CONCLUSIONS: PoCUS is underutilized and has the potential to be extremely useful for medical decision making in areas with limited resources. PoCUS curricula for residents should focus on answering patient-specific clinical questions. Future directions include creating a peer-to-peer network for securely sharing and discussing PoCUS images and expanding this analysis to higher-resource settings in an internal medicine context.

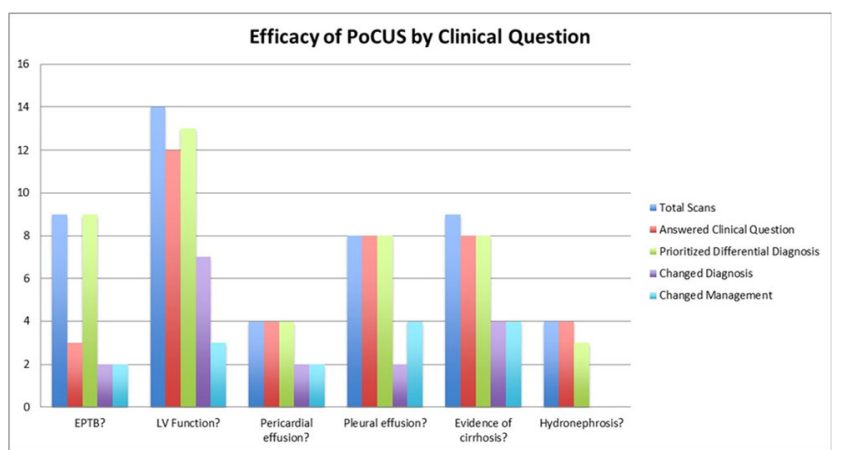

UTILIZATION OF ALLOGENEIC HEMATOPOIETIC CELL TRANSPLANTATION IN NEW YORK STATE, USA AND ONTARIO, CANADA

Samantha Hershenfeld ${ }^{1}$; Vicki Ling ${ }^{2}$; John J. Matelski ${ }^{1}$; Matthew Cheung ${ }^{1}$; Michael Paterson ${ }^{2}$; Peter Cram ${ }^{3}$. ${ }^{1}$ University of Toronto, Toronto, ON, Canada; ${ }^{2}$ Institute for Clinical Evaluative Sciences, Toronto, ON, Canada; ${ }^{3}$ University of Iowa, Toronto, ON, Canada. (Control ID \#3184706)

BACKGROUND: Hematopoietic Cell Transplantation (HCT) is a potentially curative treatment for multiple hematological and oncologic diseases. However, HCT is costly and resource-intensive. There is a general perception that the United States (US) offers improved access to many advanced therapies such as HCT, but also suffers from overtreatment. Alternatively, Canada is thought to suffer from protracted wait times and treatment delays, with advantages of greater equity and reduced disparities. Canada and the US share close geopolitical relationships and similar HCT indications. There have been no studies to our knowledge comparing utilization of HCT in the US and Canada.

METHODS: We used population level administrative health data from New York State (NY) and Ontario (ON) to identify all adults ( 18 years) who underwent inpatient allogeneic HCT between 2012-2015 using ICD9CM codes (NY) and CCI codes (ON). We compared ON and NY with respect to patient demographic characteristics, patient neighborhood income quintile (based on postal code of residence), and hospital characteristics using univariate techniques. We examined indications for HCT in NY and ON using ICD9 and ICD10 diagnosis codes. We compared HCT utilization rates in $\mathrm{NY}$ and $\mathrm{ON}$.

RESULTS: We identified $1361 \mathrm{HCT}$ procedures performed in NY and 593 procedures in ON. HCT recipients in NY were older than ON (mean age 51.5 vs 49.0 years; $p<0.001$ ). A significantly higher percentage of $\mathrm{HCT}$ in $\mathrm{NY}$ were repeat procedures relative to $\mathrm{ON}$. The most common indication for HCT in ON and NY was acute myeloid leukemia $(41.2 \%$ and $22.3 \%$ ). A higher percentage of HCT recipients in NY resided in affluent neighborhoods (defined as neighborhoods in the highest income quintile) compared to ON (32.0\% vs $26.9 \%$; $\mathrm{p}=0.026)$. Mean hospital HCT volumes were similar in NY and ON (34.8 vs 32.4 HCTs per-year; 
$\mathrm{p}=0.48)$. Utilization of HCT was 25.2 per-1 million population per- $y$ ear in $\mathrm{NY}$ and 16.4 per-1 million per-year in $\mathrm{ON}(\mathrm{p}<0.001)$. The magnitude of the NY-ON difference in utilization was larger for older patients.

CONCLUSIONS: We found significantly lower utilization of HCT in ON compared to NY. HCT recipients in NY also were significantly older and more likely to reside in high-income neighborhoods compared to ON HCT recipients. These differences should inform HCT policy in both jurisdictions.

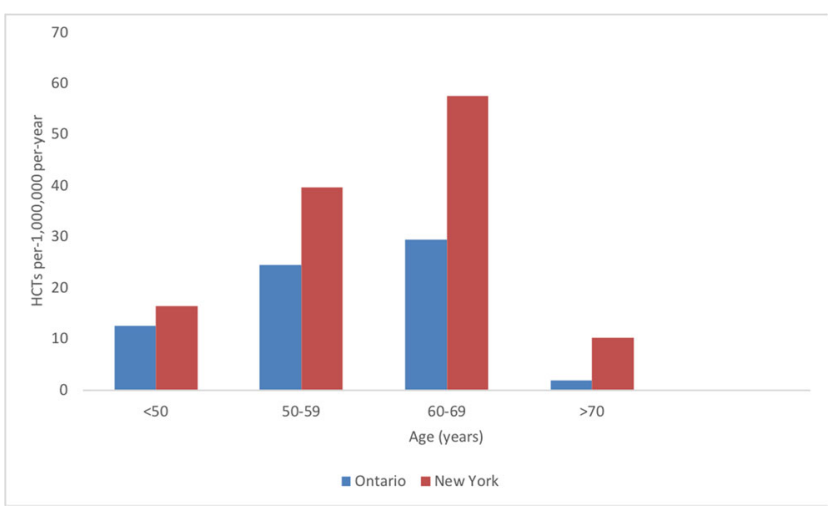

HCT utilization in ON and NY stratified by patient age

UTILIZATION PATTERNS, EXPENDITURES, AND ESTIMATED SAVINGS ASSOCIATED WITH ANALOG OR HUMAN INSULIN PRODUCTS IN THE MEDICARE PART D PROGRAM.

Jing Luo; Aaron Kesselheim. Brigham and Women, Boston, MA. (Control ID \#3185059)

BACKGROUND: High cost insulin analogs (e.g. glargine) are frequently used to treat patients with type 2 diabetes in the US. Recent evidence shows that switching older adults with type 2 diabetes from analog to lower cost human insulin (e.g. NPH or human premixed 70/30) is not associated with worsened glycemic control or increased risk of hypoglycemia and may result in cost savings for payors and patients. We sought to describe utilization patterns and expenditures for analog and human insulins separately in the Medicare drug benefit program and to estimate potential savings under 3 counterfactual scenarios between 2012 and 2016 .

METHODS: We used the Medicare Part D Spending Dashboard, which summarizes data available the Part D Prescription Drug Event (PDE) data to tabulate volume (where 1 dosage unit $=1 \mathrm{ml}=100 \mathrm{IU}$ insulin) and expenditures for human or analog insulin products from 2012 to 2016 . We included all standard strength insulin formulations except inhaled insulin. To estimate potential savings and informed by literature, we considered 3 counterfactual scenarios: 1) optimistic, where utilization in Part D was $70 \%$ human and $30 \%$ analog, 2) moderate, where utilization was 50\% human and 50\% analog and 3) pessimistic, where the overall proportion represented by analogs was $10 \%$ less than observed each calendar year. We report gross and net expenditures for the observed and counterfactual scenarios, after accounting for a class-specific $25 \%$ rebate. We inflated all dollars to 2016 using the CPI-U.

RESULTS: Our sample included 8 human and 19 analog insulin products. The number of beneficiaries increased from 4.1 million in 2012 to 5.2 million in 2016. Similarly, the volume of insulin dispensed increased from 389 million units to 486 million units. The mean units dispensed/ beneficiary was 94.8 (range 92.8 to 96.1 ). The proportion of insulin dispensed as analogs increased from $78 \%$ in 2012 to $83.6 \%$ in 2016 . Gross expenditures for all insulin products increased from $\$ 4.9$ billion in
2012 to $\$ 11.9$ billion in 2016 , a cumulative total of $\$ 43.7$ billion. The estimated 5-year savings under optimistic, moderate, and pessimistic counterfactual scenarios, inclusive of rebates, would have been $\$ 11.6$ billion, $\$ 7.2$ billion and $\$ 2.2$ billion, respectively.

CONCLUSIONS: Most insulins dispensed in the US Medicare drug benefit program were for analog products. Where it may be clinically appropriate, increased utilization of comparable human insulin products may result in substantial savings for Medicare.

\section{UTILIZING A NOVEL ADAPTIVE E-LEARNING PRODUCT WITHIN A LARGE INTERNAL MEDICINE RESIDENCY TRAINING PROGRAM}

Michael Healy ${ }^{1,2}$; Emil R. Petrusa ${ }^{1}$; Craig Noronha ${ }^{3}$; Carl Gustaf Stefan Axelsson $^{1}$; Ole-Petter R. Hamnvik ${ }^{4}, 2$; Melissa Elmore ${ }^{2}$; Leokadia Marchwinski ${ }^{2}$; Matthew O'Rourke ${ }^{2}$; Roy Phitayakorn ${ }^{1,2}$. ${ }^{1}$ Massachusetts General Hospital, Harvard Medical School, Boston, MA; ${ }^{2}$ NEJM Group, Waltham, MA; ${ }^{3}$ Boston Medical Center, Boston University School of Medicine, Boston, MA; ${ }^{4}$ Brigham and Women's Hospital, Harvard Medical School, Boston, MA. (Control ID \#3176864)

BACKGROUND: There is limited research about the impact of test preparation tools on subsequent resident performance on formal assessments. Question banks are a popular and widely used form of test preparation, and some studies suggest that question banks help learners reinforce knowledge and assess their individual learning needs. However, it is unclear how learners should use question banks to best maximize their knowledge retention. The objective of this pilot study was to examine how internal medicine residents utilized the NEJM Knowledge+ Internal Medicine Board Review (NEJM Knowledge+) product as an examination preparation tool and to measure their resulting performance on subsequent quizzes and exams.

METHODS: Participants were 69 residents (29 interns, 29 second-year residents, 10 third-year residents, and 1 chief resident) from a single internal medicine residency program. As compensation for participation, these residents were provided access to NEJM Knowledge+ starting in the beginning of the 2018 academic year to use independently for one year. Resident demographics, performance on a locally developed 60 question pre-test, and performance on the 300 question 2018 ACP Internal Medicine In-Training Examination were collected. Usage of NEJM Knowledge+ was monitored based upon the percent of progress made within the product. A correlation was computed between scores on the local test and the in-training exam. Finally, differences in the percent of progress made within NEJM Knowledge+ were calculated.

RESULTS: The correlation between performance on the local pre-test and the in-training exam was $0.53(\mathrm{p}=<0.0001)$, indicating a moderately positive relationship. Collectively, 48 residents $(69.6 \%)$ made progress within NEJM Knowledge+. Question sets in each of the 18 topics were completed, with the highest number of residents answering question sets correctly in cardiology $(n=43,62.3 \%)$, while the topic with the fewest was otolaryngology $(n=4,5.8 \%)$. There were no significant differences in progress within NEJM Knowledge+ between training years via the total number of question sets or the number of topic-specific question sets answered correctly.

CONCLUSIONS: The NEJM Knowledge+ product was used by a majority of the residents in this pilot study. Pre-test performance correlated with in-training examination scores, which opens the possibility of using the pre-test results to coach residents on effectively using the NEJM Knowledge+ product to address their existing knowledge gaps.

\section{UTILIZING GEOSPATIAL MODELS FOR IDENTIFICATION OF PATIENTS AT RISK FOR HEPATITIS C INFECTION}

Thomas Ludden $^{1}$; Lindsay Shade ${ }^{1}$; Jeremy Thomas ${ }^{1}$; Brisa Hernandez ${ }^{1}$; Sveta Mohanan ; Michael Leonard ${ }^{1}$; Mark Russo ${ }^{2}$; Jewell Carr ${ }^{1}$; Philippe Zamor ${ }^{2}$; Hazel Tapp ${ }^{1} .{ }^{1}$ Atrium Health, Charlotte, NC; ${ }^{2} \mathrm{CHS}$, Charlotte, NC. (Control ID \#3161058) 
BACKGROUND: Increased screening and the development of effective antiviral treatments have led to marked improvement in Hepatitis $\mathrm{C}$ (HCV) patient outcomes. However, many people in the US are believed to have undiagnosed HCV. Geospatial modelling using multiple high-risk variables including social determinants of health, age, and HCV prevalence provides an opportunity to identify at-risk populations in need of screening and linkage-to-care for HCV.

METHODS: We conducted a literature review to identify variables associated with patients at-risk for $\mathrm{HCV}$ infection. We separated the variables into two groups: HCV Prevalence and Social Determinants of Health. We collected data from publicly available sources and Atrium Health (formerly Carolinas HealthCare System), an integrated healthcare system based in Charlotte, $\mathrm{NC}$ with over 12 million patient encounters per year. We standardized the variables in the two groups and mapped out the overall combined index scores of each group at the census tract level in Mecklenburg County, NC.

RESULTS: 12 social determinants of health variables and 4 prevalence of $\mathrm{HCV}$ variables were identified. Between the two indexes, we identified 21 high risk census tracts that scored at least 2 standard deviations above the mean. Two census tracts overlapped between the two indexes; 11 were uniquely identified as high risk for prevalence of $\mathrm{HCV}$ and 8 were uniquely identified as priority tracts for social determinants of health.

CONCLUSIONS: Geospatial models identified a selection of census tracts that can be used to map high risk HCV populations. This will allow future targeted screening and linkage-to-care interventions for patients at high risk of $\mathrm{HCV}$.

\section{UTILIZING STAKEHOLDER PERSPECTIVES TO INFORM THE USER-CENTERED DESIGN OF THE RESOURCE ACCESS MAPPING PROJECT (RAMP), A MOBILE PHONE-BASED IN- TERVENTION TO IMPROVE ACCESS TO SOCIAL SERVICES}

Ashley Odai-Afotey ${ }^{1}$; Chaarushi Ahuja ${ }^{1}$; Trucmai Ton ${ }^{2}$; Danya Keene ${ }^{2}$; Mona Sharifi ${ }^{3}{ }^{1}$ Yale School of Medicine, New Haven, CT; ${ }^{2}$ Yale School of Public Health, New Haven, CT; ${ }^{3}$ Yale New Haven Hospital, New Haven, CT. (Control ID \#3168500)

BACKGROUND: Low-income and marginalized populations experience poorer health outcomes and lower access to health-promoting resources. The wide adoption of mobile phones in these populations could serve as a means to address systematic barriers to healthcare and resource access. In this study, we aim to explore resource needs and barriers to accessing services for social determinants of health among marginalized communities to inform the user-centered design of a mobile intervention. METHODS: Participants were purposefully recruited to participate in focus groups from low-income and marginalized populations through partnerships with three local community organization addressing mental health, food insecurity, and youth homelessness in New Haven, CT. We conducted focus groups located at these community-based organizations using a semi-structured moderator's guide. This guide was developed and iteratively refined by our qualitative research team to explore: (1) how participants navigate community resources, (2) barriers to resource access, and (3) perspectives on key requirements to optimize the usability and usefulness of a mobile application to improve resource access. We also reviewed prototypes of the mobile application to obtain more specific feedback. All focus groups were recorded, transcribed verbatim, and analyzed using a grounded theory approach.

RESULTS: To date, we have completed three focus groups with 20 participants (4-8/group) ages 19-64 years. Participants were 47\% male, $63 \%$ African-American, $42 \%$ had completed high school or less, $90 \%$ reported having access to a smartphone and out of those, $94 \%$ used "apps". Participants identified the primary sources of information on community resources as: disorganized and frequently inaccurate electronic resources, advertising at local community programs, and word of mouth. Barriers to resource access included lack of awareness, lack of centralized resources and outdated information, and distrust towards organizations and staff. Participants expressed that a mobile application to improve resource access should include comprehensive, up-to-date information, facilitate peer-to-peer communication and advocacy, and provide resources tailored to an individual's unique needs.

CONCLUSIONS: Engaging target end-users in the development of interventions is more likely to yield a design that will be effective and highly adopted. Focus group participants from marginalized populations described an existing resource system that is disorganized and information-limited and defined several modifiable barriers to accessing resources to address social determinants of health. A centralized mobile application could introduce standardization, improve the reliability and accuracy of information, capitalize on and expand existing social networks and forms of information sharing, and facilitate tailored resource recommendations.

UTILIZING STANDARDIZED DOCUMENTATION TO IMPROVE THE CLARITY AND EFFICIENCY OF PERIPROCEDURAL COMMUNICATION FOR INPATIENT VASCULAR INTERVENTIONAL RADIOLOGY PROCEDURES

Emma Simon; Erin McCaffrey; Masha Kuznetsova; Leora I. Horwitz; Eric Aaltonen. NYU School of Medicine, New York, NY. (Control ID \#3184932)

BACKGROUND: Hospitalized patients often undergo interventional radiology (IR) procedures, many of which require individualized pre-procedure preparation and post-procedure care. Internists caring for these patients may not be familiar with requirements for these patients, causing procedural delays or periprocedural adverse events. Clear communication between IR and internal medicine is therefore necessary, but is often lacking.

METHODS: We conducted qualitative interviews with hospitalists, house staff, nurses and IR staff to identify common breakdowns in periprocedural communication between IR and referring medicine units. Utilizing insights from these interviews, we identified essential elements for pre-procedure and post-procedure communication. These elements were added as fields in templated pre- and post-procedure IR notes (Table 1). Each standardized template contains 16 elements. Outcome measures included proportion of key elements included in IR notes, inpatient provider satisfaction, and frequency of phone calls into and out of IR before and after the intervention.

RESULTS: Before implementation of the standardized templates, preprocedure consult notes $(\mathrm{N}=25)$ contained an average of 3.5 key elements (typically a brief medical history, assessment and plan), while postimplementation $(\mathrm{N}=50)$, these notes contained an average of 15.3 elements $(\mathrm{p}<0.001)$. Similarly, post-procedure notes $(\mathrm{N}=25)$ contained an average of 4.7 elements (typically the names of the IR providers, a brief procedure description and patient condition) pre-intervention versus a mean of 15.0 elements post-intervention $(\mathrm{N}=50)(\mathrm{p}<0.001)$. Surveys of hospitalists pre- $(\mathrm{N}=17)$ and post-intervention $(\mathrm{N}=10)$ showed no significant difference in lack of confidence in preparing patients for IR procedures $(52.9 \%$ vs. $30.0 \%, \mathrm{p}=0.40)$, ineffective collaboration with IR ( $11.8 \%$ vs. $0 \%, \mathrm{p}=0.44)$, and not receiving clear recommendations ( $35.3 \%$ vs. $10 \%, \mathrm{p}=0.67$ ); however analyses were underpowered. Total calls into and out of VIR decreased $15.6 \%$ overall (mean decrease of 7.7 calls/weekday and 24.5 calls/weekend, $\mathrm{p}=.006$ ).

CONCLUSIONS: Standardizing pre- and post-procedure documentation can effectively increase the inclusion of key content, and this content may reduce internal medicine physician questions and concerns regarding periprocedural patient care.

\section{VALIDATING HOMELESS DATA FROM THE VETERANS HEALTH ADMINISTRATION ELECTRONIC HEALTH RECORD}

Kathleen A. McGinnis ${ }^{1}$; Melissa Skanderson ${ }^{1}$; Emily A. Wang ${ }^{2}$; Kathleen M. Akgün ${ }^{3}$; Mona Duggal ${ }^{1}$; Adam Gordon ${ }^{8}$; Vincent Marconi ${ }^{7}$; Keith 
McInnes $^{4}$; Christopher T. Rentsch ${ }^{5}$; Amy C. Justice ${ }^{6}$. ${ }^{1}$ VA Pittsburgh Healthcare System, West Haven, CT; ${ }^{2}$ Yale School of Medicine, New Haven, CT; ${ }^{3}$ Yale University, VACT West Haven, New Haven, CT; ${ }^{4}$ Dept Veterans Affairs, Bedford, MA; ${ }^{5}$ London School of Hygiene \& Tropical Medicine, London, United Kingdom; ${ }^{6}$ Yale University, West Haven, CT; ${ }^{7}$ Emory University, Atlanta, GA; ${ }^{8}$ The University of Utah, Salt Lake City, UT. (Control ID \#3184134)

BACKGROUND: Homelessness is especially prevalent among veterans and the Veterans Health Administration (VA) electronic health records (EHR) can be used as a research tool for understanding modifiable mediators of homelessness. We sought to validate homeless data in the VA EHR by comparing it with selfreported data from the Veterans Aging Cohort Study (VACS) survey, a confidential, self-administered survey of HIV+ and uninfected veterans.

METHODS: Using VACS survey items, we created two homeless variables - ever (prior to survey) and recent (w/in 4 weeks prior to survey) based on selfreport of having "been without a permanent address that you call home" or "stayed one or more nights in a shelter, on the street, in the park, or in an abandoned building". We also created two EHR homeless variables using the ICD-9 code v60.0 for "lack of housing": 1) homelessness any time prior to the survey, and 2) recent homelessness - within 30 days prior to or up to one week after the survey date. Agreement between survey and EHR variables were assessed using sensitivity and specificity, assuming the survey as the reference standard, and Cohen's kappa statistics. Positive predictive value (PPV) and negative predictive value (NPV) were also calculated.

RESULTS: There were 3734 HIV+ and 3791 uninfected VACS participants enrolled in VACS from 2000 to 2012, mean age was 50 years ( $\mathrm{sd}=9.4), 95 \%$ were male, and $65 \%$ were African-American. Ever and recent homelessness were reported by $47 \%$ and $17 \%$ on the survey, respectively. In the EHR, we identified $30 \%$ as ever homeless and $6 \%$ as recently homeless. Compared with self-report, using ICD-9 codes, sensitivity is low (53\%) but specificity is good (92\%) for EHR ever homeless (PPV=85\%, NPV=69\%, kappa = 0.46); similarly, sensitivity is low (26\%) and specificity is good (97\%) for EHR recent homelessness (PPV=68\%, NPV=87\%, kappa $=0.31$ ).

CONCLUSIONS: Agreement between EHR and survey homelessness is fair to moderate with good specificity. Although we can be confident that those identified as homeless are correctly identified, a large proportion of those who have experienced homelessness are not identified in the EHR. This work will be extended to incorporate evaluating other VA EHR sources of homeless information such as ICD-10 codes, clinic stop codes, and data collected using clinical reminders at clinic visits in order to develop and validate a robust measure for homelessness nationally across the VA. Identification of homelessness within the VA can be used in future studies to identify those at risk and to adjust for homelessness in research.

\section{VALIDATION OF OUR UNIQUELY DEVELOPED PREDICTION FORMULA OF FALL USING DIFFERENT POPULATION FROM ORIGINAL ONE USED IN ITS DEVELOPMENT: A SEC- OND REPORT}

Masaki Tago; Naoko E. Katsuki; Yoshimasa Oda; Hidetoshi Aihara; Shuichi Yamashita. Saga University Hospital, Saga, Japan. (Control ID \#3138735)

BACKGROUND: Previous studies have identified many risk factors for falls and several predictive formulae for falls have been developed. Hendrich II Fall Risk Model has been reported to be the most appropriate predictive formula with sensitivity of $70 \%$ and specificity of $61 \%$. They require examinations and techniques seldom used in routine clinical settings, as well as time-consuming assessment items, which poses a serious impediment to its use. Therefore, we have developed a simpler predictive formula using a regression coefficient acquired from the results of the multivariate analysis. We used two scales, the Japan Ministry of Health, Labour and Welfare (MHLW) bedriddenness rank and cognitive function score, which are both commonly used evaluation tools in Japan.
The formula was as follows: $y=(75$ years or older $) \times 6+($ male $) \times 4+$ $($ emergency admission $) \times 5+($ hypnotic $) \times 4+$ (history of falls $) \times 3+$ (bedriddenness rank of $\mathrm{J}) \times 12+$ (bedriddenness rank of $\mathrm{A}) \times 18+$ (bedriddenness rank of $\mathrm{B}) \times 20+($ bedriddenness rank of $\mathrm{C}) \times 17+($ cognitive function score of 2$) \times 4$. We validated the accuracy of our unique formula which we presented at the SGIM17, using other group setting than the one we originally used in its development.

METHODS: We retrospectively analyzed inpatients over 20 years old who were admitted to an acute care hospital in Japan, between April 2015 and March 2018. The data (including age, sex, the department to which the patient was admitted, emergency admission or not, whether the patient was transferred by ambulance, admission with a referral letter or not, taking hypnotics or not, bedriddenness rank and cognitive function score on admission, visual impairment and history of falls and strokes) were derived from the charts. We performed Receiver Operating Characteristic (ROC) analysis and the calculation of the likelihood ratio using our predictive formula and the data acquired in our study of falls of inpatients.

RESULTS: During the study period, 9344 patients were admitted, of whom 8967 were over 20 years old without unavailable data. The median age of 8967 inpatients was 77 years, $49.3 \%$ were men, and the median length of hospital stay was 9 days. The median calculated predictive score was 17. A total of 274 suffered falls, whose median age was 84 and median length of hospital stay was 25 days. The test of goodness of fit of the predictive formula using ROC curve analysis showed Area Under the Curve (AUC) of 0.766 (95\% CI: $0.744-0.789, P<0.001$ ), and sensitivity of $83.2 \%$ and specificity of $60.9 \%$ with the setting of cut-off point of 24 . The negative likelihood ratios with the cut-off points of 6 (the first quartile) and 10 were 0.04 and 0.10 , respectively.

CONCLUSIONS: We herein validated the accuracy of our previously reported predictive formula of falls of adult inpatients using bedriddenness rank and cognitive function scores, with different group setting from the one we originally used in its development showing higher accuracy of our formula than previously reported ones.

\section{VARIATION IN PRIMARY CARE SPENDING FOR MEDICARE PATIENTS IN AND OUT OF HEALTH SYSTEMS}

Rachel O. Reid ${ }^{1,2}$; Cheryl Damberg ${ }^{3}$; Mark W. Friedberg ${ }^{1,2}$. ${ }^{1}$ RAND Corporation, Boston, MA; ${ }^{2}$ Brigham and Women's Hospital, Boston, MA; ${ }^{3}$ RAND Corporation, Santa Monica, CA. (Control ID \#3185427)

BACKGROUND: Greater orientation toward primary care has been associated with better quality and lower costs of care. Health systems are growing in the United States and increasingly shape care delivery. In this study, we explore variation in primary care spending as a proportion of total spending among Medicare fee-for-service beneficiaries who seek care from health system-affiliated provider organizations (POs) versus unaffiliated POs, as well as across health systems.

METHODS: Using 100\% 2015 Medicare claims, enrollment, and provider data, we attributed $6,602,562$ beneficiaries to 10,863 health system POs and 9,902,449 beneficiaries to non-health system 88,600 POs, attributing beneficiaries to POs (identified by TIN) responsible for at least $30 \%$ of their evaluation and management service and identifying system-affiliated POs using the SK\&A provider database. We then assessed the percentage of their total medical and prescription drug spending on primary care. We defined primary care spending according to narrow and broad definitions of primary care providers (PCPs) and services. The narrow PCP definition included Family Practice, Internal Medicine, Pediatrics, and General Practice. The broad definition also included Nurse Practitioners, Physician Assistants, Geriatric Medicine, and Gynecology. Both excluded hospitalists. The narrow services definition included evaluation and management visits, preventive visits, care transition or coordination services, and in-office preventive services, screening, and counseling. The broad definition included all professional services billed by PCPs. 
RESULTS: Beneficiaries attributed to health system POs had less spending on primary care than those attributed to non-health system POs (e.g., $2.5 \%$ versus $3.3 \%$ for the narrow PCP and service definition). Among health systems, the primary care spending percentage varied according to the definition used (from $2.5 \%$ of total spending for the narrow PCP and service definition to $6.0 \%$ for the broad definitions), did not vary with the number of physicians in a system, and increased with the percentage of system physicians who were PCPs (ranging from $1.7 \%$ of spending for systems with less than $25 \%$ PCPs to $3.3 \%$ for systems with at least $75 \%$ PCPs, for the narrow PCP and service definition).

CONCLUSIONS: Compared to health system POs, non-health system POs have lower proportions of total spending on primary care. Health systems with greater primary care specialty composition have higher proportion of medical spending on primary care. As state policymakers working to establish minimum thresholds for the proportion of health care spending on primary care can be informed by information about the variation in primary care spending among health systems. Describing the proportion of spending devoted to primary care among POs can enable future research examining the relationship between primary care spending and the quality and efficiency of care.

\section{VARIATIONS IN THE USE OF SAFETY MEASURES AMONG HARVARD-AFFILIATED HOSPITALS}

Hojjat Salmasian ${ }^{1}$; Laura C. Myers ${ }^{2}$; Merranda Logan ${ }^{2}$; David M. Levine $^{1}$; Christopher Roy ${ }^{3}$; Michelle Frits ${ }^{1}$; Lynn A. Volk ${ }^{4}$; Ruth Akindele $^{1}$; Juliette Randazza ${ }^{1}$; Sevan Dulgarian ${ }^{1}$; David Shahian ${ }^{2}$; Elizabeth Mort ${ }^{2}$; David W. Bates ${ }^{1} .{ }^{1}$ Brigham and Women's Hospital, Boston, MA; ${ }^{2}$ Massachusetts General Hospital, Boston, MA; ${ }^{3}$ Mount Auburn Hospital, Cambridge, MA; ${ }^{4}$ Partners HealthCare, Wellesley, MA. (Control ID \#3178489)

BACKGROUND: The field of safety reporting has evolved since the Harvard Medical Practice Study was performed decades ago. As part of the new Harvard Safe Care Study, which is designed to emulate and expand upon that seminal study, one aim was to catalog the measures and triggers different Harvard-affiliated hospitals use to identify and monitor safety events, and to study variations in these processes by organization characteristics.

METHODS: Electronic surveys were sent to the designated quality/ safety leaders of 10 Harvard-affiliated hospitals that deliver both inpatient and outpatient care. The surveys asked if and how they collect and monitor an extensive list of 134 safety measures endorsed by regulatory bodies, expert groups, specialty boards, and/or the National Quality Forum. The measures were grouped by clinical domain into adverse drug events, critical care, deep vein thrombosis/pulmonary embolus, falls, general inpatient, general outpatient, hospital-acquired infections, maternal/perinatal, pressure injuries, surgical, and transitions in care. We analyzed within- and between-group agreement using Fleiss' kappa (using 0.3 and 0.6 as cutoffs for moderate and high agreement), stratifying sites based on hospital size ( $>400$ beds vs $<400$ beds) and academic status (non-teaching, graduate teaching, and major teaching). The consensus method was used for the between-group comparison. We also performed subgroup analysis of measures that are "required" to be reported to a regulatory agency such as the Center for Medicare and Medicaid Services (CMS) or a specialty board.

RESULTS: Overall, agreement among sites for measures they collected was low $(=0.148)$. Highest agreement was observed for measures related to pressure injury $(=0.554)$ and hospital-acquired infections $(=0.382)$. Lowest agreement was observed among maternal/peripartum $(=0.040)$ and surgical measures (0.048). Agreement was weak but larger within the group of large hospitals $(=0.272)$ and within the subgroup of major teaching hospitals $(=0.265)$. Of the 23 measures reported to governing agencies other than the CMS, sites reported that they actively collect an average of only $77 \%$ (range $=48-100 \%,=0.006$, p-value $=0.40)$.
CONCLUSIONS: We observed high variation among the studied Harvard-affiliated hospitals regarding which safety measures they collect, including those reported to governing agencies, which could not be simply explained based on hospital size and teaching status. This could be due to different monitoring needs and priorities at different sites, different institutional goals at the time of the survey, or disagreements about the usefulness of various measures. Notwithstanding the measures required by all payors and regulatory agencies, hospitals vary in the scope of metrics used to advance quality and safety in their institutions. Understanding the reasons behind the difference may inform the approach to improvement across sites.

\section{VETERAN PERSPECTIVES ON CANCER CARE COORDINATION}

Willi L. Tarver; Edward J. Miech; David Haggstrom. Roudebush VA Medical Center, Indianapolis, IN. (Control ID \#3186391)

BACKGROUND: Coordinating cancer care can prove especially challenging because of the complexity of both the disease and its treatment. Typically, the care of cancer patients involves multiple types of therapies and providers (surgery, radiation therapy, chemotherapy) and ongoing treatment of cancer and other health conditions. Cancer care coordination depends upon effective, regular communication among physicians, support staff, and the patient. The objective of this study is to explore Veteran patients' experiences of cancer care coordination in order to develop a deeper understanding of the issues Veterans consider important and actionable.

METHODS: Semi-structured interviews were conducted among patients diagnosed with cancer types and stages that commonly receive interdisciplinary care (e.g., colorectal [stage II and up], prostate [high risk], head and neck [all stages], and lung [all stages] cancer) were eligible to participate in the study. The constant comparison technique was used to identify emerging themes in the data.

RESULTS: Twenty-five Veteran cancer patients participated in this study. Our analysis identified four general categories representing different parts of the trajectory of the patient cancer care experience in need of coordination. Each phase had a distinct set of needs and challenges. Diagnosis: After receiving a diagnosis of cancer, Veterans reported initial feelings of shock and fear. PreTreatment: This phase was characterized as busy and chaotic, consisting of many appointments in different locations, and ultimately left many Veterans feeling a lack of control. Veterans repeatedly expressed an interest in having an early explicit understanding of their care, including the next steps and expectations. Onset of Treatment: Uncertainty about what to expect when receiving advanced treatments, like radiation and chemotherapy, was often exacerbated by poor provider-provider communication issues. Some participants identified novel solutions, such as proactively viewing the treatment area in advance. Ongoing Treatment: As treatment progressed, many Veterans experienced a growing familiarity with their routine, and simultaneously, began to regain a sense of control. Care Team: Nurse navigators were considered vital to cancer care coordination. This also marked a shift where many Veterans experienced a decrease in anxiety and worry. Veterans valued honesty and transparency from their providers which built a sense of trust.

CONCLUSIONS: Veterans expressed a need to have care coordination needs and steps more explicitly addressed early, as well as active mechanisms to meet those needs. The patient experience of coordination gradually improved over the course of the care trajectory, often facilitated by relationships fostered with members of the healthcare team. Exploring the perspectives of Veteran cancer patients can offer invaluable insight to aid cancer care providers and policy makers in developing programs to improve care coordination.

\section{VIDEO INTERVENTION TO IMPROVE INCIDENT REPORTING AMONG MEDICAL TRAINEES}

Jose R. Valery; Haythem Helmi; Aaron Spaulding; Xinxuang Che; Gabriel Prada; Natalia Chamorro Pareja; Pablo Moreno Franco; Fernando Stancampiano. Mayo Clinic Florida, Jacksonville, FL. (Control ID \#3184649) 
BACKGROUND: Efforts are being made across teaching hospitals to improve trainee involvement with incident reporting systems. Studies suggest that understanding barriers to incident reporting is essential in developing successful educational interventions. We previously reported on barriers to incident reporting within our institution which were lack of knowledge of how to report, concern about negative repercussions after reporting, lack of time to report and the belief that reporting is futile. This study was designed to address these specific barriers with a patient safety video distributed via email. Barriers were assessed using baseline and post-video surveys.

METHODS: The study was conducted after IRB approval. A 5-minute video was produced aiming to address the barriers noted above. Baseline and post-video surveys were developed after a literature review of similar surveys, review with a panel of patient safety experts, and pre-testing with a group of trainees. The surveys and video were distributed via email to 1144 trainees. Participation was anonymous and voluntary. T-tests assuming unequal variances were used to determine significance. A p-value of $<$ 0.05 was interpreted as significant.

RESULTS: The response rate to the baseline survey and video was $6.1 \%$. $41.4 \%$ of trainees who viewed the video responded to the follow up survey. Baseline and post-video survey results are shown in Table 1. Trainees indicating having knowledge to create an incident report increased from a baseline of $48.6 \%$ to $72.4 \%$ which was significant (pvalue of 0.013 ). Otherwise, there was no significant change in attitudes about incident reporting.

CONCLUSIONS: Using email to distribute a patient safety video targeting specific barriers to incident reporting with anonymous and voluntary participation yielded a low response rate. Although there was improvement in perceived knowledge among participants, there was no measured improvement in other attitudes measured about incident reporting. The same video delivered during an in-person, required, educational conference was previosuly studied where knowledge and attitudes about incident reporting significantly improved (manuscript in progress). Our data suggests that although email distribution is a practical way to deliver patient safety education, the impact is low if participation is voluntary and anonymous.

\section{VITAL-AF: A PRAGMATIC TRIAL INTEGRATING ROUTINE SCREENING FOR ATRIAL FIBRILLATION DURING PRIMA- RY CARE VISITS}

Steven J. Atlas ${ }^{1}$; Jeffrey M. Ashburner ${ }^{1}$; David D. McManus ${ }^{2}$; Yuchiao Chang ${ }^{1}$; Ana T. Trisini Lipsanopoulos ${ }^{1}$; Leila H. Borowsky ${ }^{1}$; Wyliena Guan $^{1}$; Wei He ${ }^{1}$; Patrick T. Ellinor ${ }^{1}$; Steven A. Lubitz ${ }^{1}$; Daniel E. Singer ${ }^{1}$. ${ }^{1}$ Massachusetts General Hospital, Boston, MA; ${ }^{2}$ University of Massachusetts Medical School, Worcester, MA. (Control ID \#3185071)

BACKGROUND: Atrial fibrillation (AF) is a common age-related arrhythmia associated with increased risk of ischemic stroke. Novel technology may permit efficient and effective AF screening among undiagnosed, at-risk individuals to reduce ischemic stroke through use of guideline-indicated oral anticoagulants (OAC). We designed VITAL-AF (ClinicalTrials.gov NCT03515057) as a pragmatic cluster-randomized trial in a large primary care network in the United States, with the primary objective of assessing the benefit of population-based screening for AF in older patients using a single-lead ECG as part of routine care.

METHODS: VITAL-AF was designed to be generalizable, implementable, and pragmatic. VITAL-AF includes all patients 65 years of age attending an outpatient visit in a participating site (including hospital-, community- and health center-based practices) with a physician or nurse practitioner, embeds screening as part of routine assessment of vital signs at each encounter, utilizes clinic medical assistants rather than research personnel to conduct screening, and assesses outcomes using electronic health record data. Practices were randomized to the screening intervention or control arm. Control practices continue with true usual care that may include pulse palpation and rhythm assessment based upon physician standard practice. Here we present historical data from the network about the size, visit patterns, and characteristics of the eligible population prior to trial enrollment starting July 31, 2018.

RESULTS: Overall, 142,291 patients were seen in network practices in the prior 3 years. There were 35,856 (25\%) eligible patients 65 years of age seen in 16 participating study sites (hospital, community and health center clinics) including 4,704 (13\%) patients with known AF. In 2017, 93\% of eligible patients 65 years completed at least one primary care outpatient visit, with $67 \%$ completing multiple visits. This population has many risk factors for AF including: $41 \% 75$ years of age, $43 \%$ male, $70 \%$ with hypertension, $19 \%$ with coronary artery disease, $18 \%$ with diabetes, and $8 \%$ with heart failure. Among those without prevalent AF on OAC, $93 \%$ had a CHA2DS2-VASc score 2 with $40 \%$ having a score 4 . The trial is in progress and subject accrual is expected to be complete as of late fall 2019.

CONCLUSIONS: VITAL-AF is a pragmatic, population-based trial of a screening program for undiagnosed $\mathrm{AF}$ in older at-risk patients within a primary care network. To emphasize generalizability, ease of implementation, and to facilitate screening uptake across a range of primary care clinics, VITAL-AF uses a single, age-based eligibility criterion. A small percentage of patients with low CHA2DS2-VASc score or with prevalent $\mathrm{AF}$ will be screened. Outcome assessment will evaluate rates of new AF detection and appropriate use of OACs among all patients 65 years of age seen in a participating clinic.

VOLUNTARY BUNDLED PAYMENT FOR CORONARY ARTERY BYPASS SURGERY: DO EFFECTS FOR JOINT REPLACEMENT EXTEND TO OTHER SURGICAL CONDITIONS? Joshua Rolnick $^{2}$; Joshua M. Liao ${ }^{1,2}$; Xinshuo Ma² Ezekiel J. Emanuel $^{2}$; Eric Z. Shan ${ }^{2}$; Jingsan Zhu ${ }^{2}$; Erkuan Wang ${ }^{2}$; Qian Huang'; Amol S. Navathe ${ }^{2,3}$. ${ }^{1}$ University of Washington, Seattle, WA; ${ }^{2}$ University of Pennsylvania, Philadelphia, PA; ${ }^{3} \mathrm{CMC}$ VA Medical Center, Philadelphia, PA. (Control ID \#3182898)

BACKGROUND: Episode-based payment is an increasingly common strategy to reduce spending. Medicare's voluntary Bundled Payments for Care Improvement (BPCI) program tested the impact for several surgical conditions. However, while evidence shows savings with BPCI for knee and hip replacement surgery, less is known about CABG, despite CMS's recent decision to reconsider a mandatory bundles program for CABG.

METHODS: We performed a difference-in-differences comparison using 2011-2016 Medicare claims. Episodes attributed to BPCI physician groups were excluded. CABG participants were propensity- matched with up to 15 non-participants. The primary outcome was standardized total episode spending. Secondary outcomes were changes in utilization and spending by category. Models were adjusted for patient characteristics, time-varying market characteristics, and hospital and quarter fixed effects. Ordinary least squares models were used for utilization and spending by category. A generalized linear model with a log-link function and gamma distribution was used for total spending.

RESULTS: From October 2013-October 2015, 49 hospitals enrolled in CABG. 43 participants (10,125 episodes baseline period, 4,688 treatment) were matched with 548 non-BPCI hospitals (30,133 episodes baseline, 29,273 treatment). Mean BPCI follow up was 24 months. There were no baseline differences in age or Elixhauser index. Baseline episode spending was $\$ 48,258$ BPCI group vs. $\$ 47,417$ controls $(p<0.001)$ and spending for post-acute institutional care was $\$ 5,366$ BPCI vs. $\$ 4,435$ controls $(\mathrm{p}<0.001)$. The parallel trends assumption was met for all outcomes. Difference-in-differences analysis showed BPCI participation was associated with a non-significant $1.9 \%$ increase (95\% CI $-4.7 \%-8.9 \%$, pvalue $=0.588$ ) in risk-adjusted total spending. Proportion of spending on physician fees decreased 5.1\% (95\% CI 6.3\%-3.9\%, p-value<0.001) and SNF spending showed a non-significant $1.3 \%$ decrease (95\% CI $-1.2 \%$ $3.7 \%$, p-value $=0.31$ ). Proportion of spending on post-acute institutional 
care significantly increased $5.1 \%(95 \%$ CI $1.9 \%-8.3 \%$, p-value $=0.002)$. There were no significant changes in readmission rate, ER visit rate, or mortality. However, discharge to post-acute institutional care increased $17.9 \%$ (95\% CI 6.0\%-29.8\%, p-value $=0.003$ )

CONCLUSIONS: BPCI participation was associated with shifts in spending by category but no significant total spending change. Utilization of post-acute institutional care after index hospitalization increased, but ER visits and readmissions did not change. Bundled payments in their current form may not produce savings for all conditions, raising questions about the likely impact of mandating bundled CABG payments.

\section{WASTE IN THE MEDICARE PROGRAM: 2015 NATIONAL ES- TIMATES OF LOW-VALUE SERVICE USE AND SPENDING} Rachel O. Reid ${ }^{1,2}$; John N. Mafi ${ }^{3,}{ }^{4}$; Cheryl Damberg ${ }^{4}$. ${ }^{1}$ RAND Corporation, Boston, MA; ${ }^{2}$ Brigham and Women's Hospital, Boston, MA; ${ }^{3}$ David Geffen School of Medicine at UCLA, Los Angeles, CA; ${ }^{4}$ RAND Corporation, Santa Monica, CA. (Control ID \#3185307)

BACKGROUND: Low-value health care services that offer no clinical benefit but expose patients to risk and expense are common in the US health care system. The Choosing Wisely campaign and other efforts have focused attention on waste since lowvalue service use and spending were last estimated in Medicare nationally. In this study, we provide updated national estimates of low-value service use and spending in the Medicare population using a broad set of low-value care measures.

METHODS: We included $100 \%$ of fee-for-service Medicare beneficiaries aged 65 and older who were continuously enrolled in Parts A, B, and D for at least 2 years in 2015: 14,024,927 beneficiaries. Using Medicare enrollment, medical claims, and prescription drug event data, we used the Milliman MedInsight Waste Calculator to assess 36 claims-based measures of low-value services applicable to the Medicare population, reflecting recommendations from the Choosing Wisely Campaign, the USPSTF, and other professional guidelines. We defined as low-value those services deemed "wasteful" or "likely wasteful" based on a sufficient claims history without a clinical rationale for the service, and calculated wasteful spending on those low-value services as the total allowed amount for the claim for that service.

RESULTS: Among included beneficiaries, 11,554,997 (82.4\%) had at least one service that could be considered potentially low-value (45.9 million services, 4.0 per capita). Of these, 6,307,792 (54.6\%) had at least one service ultimately deemed low-value, accounting for 13.5 million distinct services (2.1 per capita) and $\$ 4.7$ billion in wasteful spending ( $\$ 740$ per capita). The 3 low-value services most frequently received were: eye imaging testing without significant eye disease (3.4 million services, $25.5 \%$ of low-value services), baseline laboratory studies before elective low-risk surgery (2.8 million services, $21.1 \%$ of low-value services), and opioids for acute back pain (2.5 million services, $18.4 \%$ of low- value services) - comprising nearly two-thirds of wasteful services (64.9\%). The 3 services representing the greatest fraction of wasteful spending were: eye imaging testing without significant eye disease ( $\$ 1.5$ billion, $32.5 \%$ of wasteful spending), baseline laboratory studies before elective low-risk surgery ( $\$ 1.1$ billion, $24.0 \%$ of wasteful spending), and renal artery revascularization (\$528 million, $11.3 \%$ of wasteful spending) - comprising more than two-thirds of wasteful spending $(67.8 \%)$.

CONCLUSIONS: Delivery of low-value services remains common and costly in Medicare: most beneficiaries received at least one low-value service. However, utilization and spending are concentrated among a small subset of the measures assessed, suggesting targeted opportunities for waste-reduction. These findings suggest that targeted interventions to reduce this subset of low-value services could substantially reduce waste in Medicare and highlight the need for understanding of how best to design and implement such interventions.

\section{WELLNESS BEHAVIOR PROFILES OF INTERNAL MEDICINE AND PSYCHIATRY RESIDENTS}

Milad Memari; Margaret S. Chisolm; Sean Tackett; Anne Ruble; Scott Wright. Johns Hopkins Bayview Medical Center, Baltimore, MD. (Control ID \#3181174)

BACKGROUND: The ACGME requires that residency training programs (RTP) establish or bolster interventions that facilitate resident well-being, promote resilience, and guard against burnout. There is, however, limited data characterizing residents' participation or engagement in wellness promoting behaviors during their training to guide these initiatives.

METHODS: We surveyed a cohort of internal medicine and psychiatry residents regarding 16 self-care or wellness promoting behaviors over the preceding 30-days in December of 2017, dividing them into those done alone and those involving others.

RESULTS: 101 residents were invited to share their behavioral profiles, and most residents completed the entirety of the survey $(86 \%, n=87)$. Getting a good night's sleep (sleeping $>7$ hours consecutively) was the most frequent wellness supporting behavior that did not require others (averaged 14 times in prior 30 days). The most frequent wellness activities involving others included "cuddling" with another adult (16 times), calling family members (13 times), and sharing a meal with friends or family (11 times). Of the 16 behaviors studied, 13 were performed more frequently among residents compared to interns; two of these reaching statistical significance (sleeping $>7$ hours consecutively and attending a special event [e.g. sporting event, play, concert...], both $\mathrm{P}<0.05$ ).

CONCLUSIONS: This study offers a glimpse into the midyear self-care activities of interns and residents in 2 training programs. The wellness behaviors that were most commonly endorsed necessitated the involvement of friends and family. These basic wellness behavioral profiles of interns and residents may serve to inform the development of curricula and guidance from RTPs as mandated by the ACGME.

\section{WERE THE 2015 HYPERTENSION SCREENING RECOMMEN- DATIONS TAKEN WITH A GRAIN OF SALT? A RETROSPEC- TIVE STUDY ON CHANGES IN PHYSICIAN BEHAVIOR BE- FORE AND AFTER THE UPDATE TO THE 2015 USPSTF HY- PERTENSION GUIDELINES}

Deborah Fung ${ }^{1}$; Amani Allen ${ }^{2}$; Rosio Ramos ${ }^{3}$; Erica Phillips ${ }^{3}$; Nathalie Moise $^{4}$; Jacob Julian ${ }^{4}$; Ian Kronish ${ }^{4} .{ }^{1}$ New York Presbyterian Weill Cornell, New York, NY; ${ }^{2}$ Cornell University, Ithaca, NY; ${ }^{3}$ Weill Cornell Medical College, New York, NY; ${ }^{4}$ Columbia University Medical Center, New York, NY. (Control ID \#3179309)

BACKGROUND: Hypertension (HTN) is a major modifiable risk factor for cardiovascular disease (CVD) and is highly prevalent in the United States. Traditionally, two elevated office blood pressure (BP) measurements have been the mainstay of diagnosing HTN; however, many patients with elevated office BP do not have elevated out-of-office BPs. This phenomenon, known as white-coat HTN, confers lower risk of CVD, may lead to over-diagnosis of HTN, increased stress and anxiety for patients now labeled with a chronic illness, and unnecessary healthcare costs. In 2015, the U.S. Preventive Services Task Force (USPSTF) updated its HTN screening recommendations to emphasize the importance of ruling out white-coat HTN by obtaining ambulatory BP monitoring (ABPM) or home $\mathrm{BP}$ monitoring (HBPM) in adults with elevated office BP. The goal of this study is to evaluate the utilization of out-of-office BP monitoring via ABPM or HBPM by primary care providers before and after this update.

METHODS: We conducted a retrospective electronic health record review of scheduled visits between primary care providers from three diverse, urban academic-based clinical practices involving adult patients with elevated office BP but no prior diagnosis or treatment for HTN. Patient inclusion criteria were: age 18, office systolic BP $140 \mathrm{mmHg}$ and/or diastolic BP $90 \mathrm{mmHg}$, and no prior history of HTN or white-coat HTN. Exclusion criteria were: 
severely elevated BP 180/110 mmHg, history of stroke, coronary artery disease, peripheral artery disease, heart failure, left ventricular hypertrophy by echo, atrial fibrillation, and chronic kidney disease. Data pertaining to the patient demographics, and clinician BP management were extracted by medically-trained coders. Descriptive statistics were used to characterize the rate of out-of-office BP testing in 2014 versus 2016.

RESULTS: In 2014, of 150 visits with USPSTF HTN guideline-eligible patients (mean age 63 years, $51 \%$ female, $83 \%$ with SBP $140 \mathrm{mmHg}$ and $33 \%$ with DBP $90 \mathrm{mmHg}$ ), 1 patient was prescribed HBPM, none were referred for $\mathrm{ABPM}$, and 1 patient was diagnosed with $\mathrm{HTN}$ and started on $\mathrm{BP}$ medication. BP was not mentioned in $73 \%$ of visit notes, $\mathrm{BP}$ was to be rechecked next time in $24 \%$ of visit notes, and $\mathrm{BP}$ was to be measured through other methods (i.e. pharmacy) in 3\% of visit notes. In 2016, of 80 guideline-eligible patients (mean age 62 years, $58 \%$ female, $82 \%$ with SBP $140 \mathrm{mmHg}$ and $36 \%$ with DBP $90 \mathrm{mmHg}$ ), 1 patient was prescribed HBPM, none were referred for ABPM, and none were diagnosed with HTN. BP was not mentioned in $85 \%$ of visit notes, and BP was to be rechecked in $14 \%$ of visit notes.

CONCLUSIONS: The rates of ordering ABPM or HBPM for guidelineeligible patients remained low pre and post USPSTF guideline updates. This calls for the development of implementation strategies to increase the uptake of this important guideline. Additional interventions may be needed to reduce the predominant wait-and-see approach to elevated office BP.

\section{WHAT ASPECTS OF DIABETIC SELF-CARE ACTIVITIES IS MOST IMPACTED BY PATIENT ACTIVATION AND MHEALTH INTERVENTIONS? THE OFFICE-GUIDELINES APPLIED TO PRACTICE (OFFICE-GAP) PROGRAM.}

Sherif Elkinany; Zane Alroshood; Ngoc Nguyen; Karen Kelly-Blake; William Hart-Davidson; Ling Wang; Maya Al-Saghir; Khader Herzallah; Brandon Batarse; Ade B. Olomu. Michigan State University, East Lansing, MI. (Control ID \#3183056)

BACKGROUND: Office-Guidelines Applied to Practice (Office-GAP) is a patient activation intervention providing patient and physician training in shared decision-making (SDM) to improve communication and patient-physician relationships. Our objective was to determine aspects of diabetic self-care significantly impacted by the Office-GAP + mHealth intervention compared to mHealth alone.

METHODS: Office-GAP intervention included: 1) group visit 2) SDM physician training and 3) decision support checklist used in real time in the office. Mhealth intervention includes teaching patients to send and receive text messages; patients received daily messages appropriate to their diagnosis and medications for 14 weeks, along with appointment reminders and patients could respond to prompts and contact their providers via text. Seventy-four patients who completed the Summary of Diabetes Self Care Activities (SDSCA) at baseline were assigned to either Office-GAP + mHealth (39 patients) or mHealth only (35 patients). The SDSCA assesses six aspects of diabetic self-care: general diet, specific diet, exercise, blood-glucose testing, foot care, and smoking. Patients in both groups completed SDSCA at baseline, first (2months), and second (4months) follow-up visits. Generalized Estimating Equation for Poisson distribution is employed for statistical analysis.

RESULTS: There were significant improvements in general diet and foot care. For Office-GAP + mHealth, the average number of days/week following general diet was 2.69 at baseline, 3.77 at first follow-up and 3.77 at second follow-up. For mHealth only, the average was 3.23, 2.96 and 3.89 at baseline, first and second follow-ups respectively. Regression analysis showed that after controlling for age, gender, race, health insurance and BMI, from baseline to second follow-up, the incident rate ratios (IRR) for general diet in Office-GAP + mHealth group was 1.19 (P $=0.005$ ) while in mHealth only, from baseline to second follow-up, IRR for general diet was $1.10(\mathrm{P}=0.22)$. Compared with mHealth only group, IRR for general diet in Office-GAP + mHealth group was $1.08(\mathrm{P}=0.43)$.
For Office-GAP+ mHealth, the average number of days/week following foot care was 3.05, 4.80 and 4.95 at baseline, first and second follow-ups respectively. For mHealth only, the average following foot care was 3.23, 3.71 and 5.21 at baseline, first and second follow-up respectively. Controlling for age, gender, race, health insurance and BMI, the number of days/week following foot care in both groups increased significantly, with IRR of 1.30 in Office-GAP+ mHealth $(\mathrm{P}<0.01)$ and 1.19 in mHealth only $(\mathrm{P}<0.01)$. Compared with mHealth only, IRR for foot care in Office$\mathrm{GAP}+\mathrm{mHealth}$ was $1.09(\mathrm{P}=0.32)$.

CONCLUSIONS: Combined Office-GAP + mHealth showed significant improvement in patient general diet compared to mHealth alone. Both interventions showed significant improvement in number of days patients engaged in foot care with no significant differences between groups.

\section{WHAT DO PATIENT-CENTERED MEDICAL HOME (PCMH) TEAMS NEED TO BETTER MANAGE CARE FOR THEIR PA- TIENTS AT HIGH-RISK FOR HOSPITALIZATION OR MORTALITY?}

Susan E. Stockdale ${ }^{1,2}$; Marian L. Katz ${ }^{1}$; Alicia Bergman ${ }^{1}$; Donna M. Zulman ${ }^{3}$; Angela Denietolis ${ }^{4}$; Evelyn T. Chang ${ }^{5}$. ${ }^{1}$ VA HSR\&D, Los Angeles, CA; ${ }^{2}$ University of California, Los Angeles, CA; ${ }^{3}$ Stanford University and VA Palo Alto, Stanford, CA; ${ }^{4}$ Veterans Health Administration, Washington, DC; ${ }^{5}$ VA- Greater Los Angeles, Los Angeles, CA. (Control ID \#3179213)

BACKGROUND: Some research shows that intensive primary care (IPC) programs for high-risk patients may not generate cost savings. A recent 5-site IPC demonstration within the Veterans Health Administration (VHA) found that IPC programs resulted in greater use of outpatient services, but costs of the program and increased outpatient services were offset by decreased inpatient and emergency department utilization. Using existing PCMH teams to better manage the most complex patients in primary care through enhanced resources and training may be a less costly alternative to IPC programs. Our objective was to identify which IPC components could be incorporated into PCMH teams' workflow, and what additional resources, trainings, and staff PCMH teams would need to better manage their complex patients.

METHODS: As part of a qualitative evaluation of VHA's 5-site IPC demonstration, we conducted semi-structured interviews with PCMH teams (primary care providers, nurses, social workers; $n=24$ ), primary care $(n=14)$ and IPC program leaders $(n=6)$. We asked interviewees which components of IPC the PCMH teams could feasibly perform and what resources, trainings, or staffing PCMH teams would need. We conducted analysis of a priori themes using a rapid qualitative analysis approach.

RESULTS: Perspectives on which IPC components would be feasible for $\mathrm{PCMH}$ teams to perform varied by interviewee role. Most thought that PCMH teams could do clinic-based aspects of IPC, including more frequent calls/visits with patients, patient/caregiver education and psychosocial support, referrals to community services, and managing care transitions. Home visits and co-attending appointments requiring $\mathrm{PCMH}$ team members' physical absence from clinic were perceived as key IPC components but less feasible for PCMH teams. Use of existing home visit services buttressed with better collaboration between PCMH teams and these services may be more feasible than PCMH teams conducting home visits. Interviewees said that PCMH teams would need full staffing (including behavioral health, pharmacy, and other ancillary services) and more dedicated time for chart reviews and assessments, interdisciplinary team meetings, and patient follow-up. Some thought that additional staffing, especially RN care managers and social workers, or smaller panels would be needed. Most thought PCMH teams could use existing care management tools and resources, but would need more training in identifying and using them, and more training in social determinants of health and patient engagement/goal setting. 
CONCLUSIONS: PCMH teams may be able to adopt certain IPC practices for high-risk patients, such as more frequent contact, psychosocial support, and intensive management of care transitions, but are less suited for services such as home visits and co-attending appointments. PCMH teams need full staffing, enhanced collaboration with existing home care services, and training in use of existing tools and resources to optimally manage high-risk patients.

\section{WHAT DO PROVIDERS WANT TO KNOW ABOUT MEDICAL LEGAL PARTNERSHIP?}

Claire Popplewell $^{1}$; Johanna Martinez ${ }^{2}$; Anthony C. Serrano ${ }^{3}$; Omolara T. Uwemedimo ${ }^{6}$; Joseph Conigliaro ${ }^{4}$; Eun Ji Kim ${ }^{5} .{ }^{1}$ Northwell Health, Manhasset, NY; ${ }^{2}$ Northwell Health, Lake Success, NY; ${ }^{3}$ Maurice A. Deane School of Law at Hofstra University, Forest Hills, NY; ${ }^{4}$ North Shore LIJ Health System, New Hyde Park, NY; ${ }^{5}$ Northwell Hofstra School of Medicine, Lake Success, NY; ${ }^{6}$ Northwell Health, New Hyde Park, NY. (Control ID \#3170065)

BACKGROUND: Medical legal partnerships aim to address social determinants of health that require legal interventions to manage. Our healthcare system and an affiliated law school have recently partnered to create a Medical Legal Partnership (MLP) to assist our diverse patient population with various legal needs, including immigration, housing, and public benefit procurement. The MLP program was implemented in 2018 at ambulatory clinics where more than half of patients are racial/ ethnic minorities with Medicaid/Medicare or who are uninsured. We conducted a qualitative study using an open-ended survey to identify providers' experience with and need for MLP.

METHODS: We created a short survey to assess medical providers' attitudes about and previous exposure to MLPs. Participants were asked to complete this short survey during a departmental Grand Rounds session. We collected information about their role in the health system (attending physician, nurse practitioner, etc.) and years they had been in practice. The survey included items about previous exposure to medicallegal partnerships, what providers would like to learn about the Medical Legal Partnership, and how the Medical Legal Partnership could help their clinical practice. A qualitative analysis was performed to identify themes that arose from participants' responses.

RESULTS: 34 participants completed the survey. The majority of participants were attending physicians $(n=19,56 \%)$; other participants included residents/fellows $(n=10,29 \%)$, nurse practitioners $(n=2,6 \%)$, a student, and a pharmacist. Only one participant had previous experience with medical legal partnerships. Topics of interest about the MLP to participants included: (1) which patients were appropriate to refer, (2) what was the process to refer patients to the MLP program, and (3) what specific services were offered by the MLP. Three main themes emerged when participants were asked how the MLP would help their clinical practice: (1) assistance with immigration issues, (2) addressing social determinants of heath through increased access to services, and (3) improving patient wellness.

CONCLUSIONS: Most clinical providers in our health system had no exposure to medical legal partnerships in the past but they were interested about learning more about them and what the logistics were of our newlydeveloping MLP program. Providers were hopeful that the MLP would be able to address social determinants of health that impact many of our patients as well as provide support to patients dealing with immigration concerns or other legal issues.

WHAT DO WOMEN IN THE UNITED STATES THINK OF ANNUAL VERSUS BIENNIAL SCREENING MAMMOGRAPHY? RESULTS OF A NATIONAL SURVEY.

Mia Djulbegovic $^{2,3}$; Jessica R. Hoag ${ }^{1,7}$; Jenerius Aminawung ${ }^{1,}$; ; Susan $\overline{\text { Busch }}^{4,1}$; Xiao Xu ${ }^{1,7}$; Kelly A. Kyanko ${ }^{5}$; Cary Gross ${ }^{6} .{ }^{1}$ Yale University School of Medicine, New Haven, CT; ${ }^{2}$ National Clinician Scholars Program, Yale University School of Medicine, New Haven, CT; ${ }^{3}$ Veterans
Affairs Connecticut Healthcare System, West Haven, CT; ${ }^{4}$ Yale University School of Public Health, New Haven, CT; ${ }^{5}$ NYU School of Medicine, New York, NY; ${ }^{6}$ Yale School of Medicine, New Haven, CT; ${ }^{7}$ Yale Cancer Outcomes, Public Policy, and Effectiveness Research (COPPER) Center, New Haven, CT. (Control ID \#3183852)

BACKGROUND: In the United States, clinical practice guidelines (CPG) provide conflicting recommendations on whether screening mammography (SM) should be annual versus biennial. We assessed whether women who receive annual SM are aware that some CPG recommend biennial SM and whether they would be willing to change from annual to biennial SM.

METHODS: This is a cross-sectional study of women aged 40-59 years with no history of breast cancer and 1 SM. Women recruited by GfK KnowledgePanel®(a survey research firm that uses probability-based sampling) participated in an online survey in 2018. Survey items assessed whether women who reported annual SM use were aware that some CPG also recommend biennial SM and if they would be willing to change to biennial SM if their doctor recommended it. Women who were unwilling to switch to biennial screening were asked why. We used multivariable logistic regression to estimate sociodemographic factors associated with 1) awareness that some CPG recommend biennial screening, and 2) willingness to change from annual to biennial SM. The model adjusted for age, race, education, income, health insurance, history of dense breasts and geographic region. We conducted all analyses in Stata using poststratification weights to generate nationally representative estimates.

RESULTS: Of the women invited to participate, $68.2 \%$ completed the survey, of whom $60.7 \%$ reported receiving SM annually. Of these women, $50.9 \%$ were aware that some CPG also recommend biennial SM, and $42.8 \%$ were unwilling to switch to biennial SM. In adjusted analysis, women who were younger (age 40-49 versus 50-59 years, OR 0.66 [95\% CI 0.49, 0.88]) and those with lower levels of education versus those with graduate degrees (did not graduate high school, OR 0.36 [95\% CI 0.14, 0.93]; graduated high school, OR 0.31 [95\% CI 0.19, 0.50]; completed some college, OR 0.41 $[95 \% \mathrm{CI} 0.26,0.66])$ were significantly less likely to be aware that some CPG recommended biennial SM. However, we found no significant association between any sociodemographic factors with willingness to change from annual to biennial SM, including awareness that some CPG recommend biennial SM. The most common reasons cited by women who were unwilling to transition to biennial SM were that annual SM: is superior at detecting breast cancer $(46.1 \%)$, eases their mind $(22.9 \%)$, or is part of a regular health routine that they wouldn't want to change $(17.8 \%)$.

CONCLUSIONS: In this nationally representative survey, the majority of women received SM annually, approximately half of whom were unaware that some CPG recommended biennial SM, and half were unwilling to adopt biennial SM even if it was recommended by their physician. Future attempts to modify the frequency of SM use may depend on an improved understanding of the beliefs that hinder willingness to change from the existing annual approach to screening.

\section{WHAT FACTORS IMPACT PATIENT PERCEPTIONS OF PRI- MARY CARE ACCESS?: QUALITATIVE ANALYSIS OF VET- ERAN PATIENT INTERVIEWS}

$\underline{\text { Susan E. Stockdale }}^{1,3}$; Alicia Bergman ${ }^{1}$; Danielle Rose ${ }^{1}$; Michael McGowan $^{1}$; Lisa Edwards ${ }^{1}$; A. Laurie Graaff ${ }^{4}$; Timothy Dresselhaus ${ }^{2}$. ${ }^{1}$ VA GLA Healthcare System, Sepulveda, CA; ${ }^{2}$ VA San Diego Healthcare System, San Diego, CA; ${ }^{3}$ University of California, Los Angeles, CA; ${ }^{4}$ Veterans Health Administration, Gilbert, AZ. (Control ID \#3179169)

BACKGROUND: The 2014 "access crisis" within the Veterans Health Administration (VHA) revealed that some administrative access measures (e.g., appointment wait time in days) may be susceptible to "gaming", and emphasis has since shifted to performance measures based on patient perception surveys. Although some research shows patient survey-based 
measures are correlated with administrative measures, improvement efforts such as "open access" that target administrative measures may not result in improved patient perceptions. To inform improvement efforts, our objective was to understand what factors impact patients' perceptions of primary care access.

METHODS: We used a two-stage sampling approach, randomly sampling within 1 VHA region from among clinics with better than the regional average administrative access and worse than average patient survey ratings of access, stratified by clinic panel size ( 2 small, 1 medium, 2 large). We used quota sampling targeting 10 patients per clinic within 6 gender-by-age strata. We conducted 15-minute semi-structured telephone interviews (June-Sept. 2018) with 47 patients who had sought access to primary care for an urgent medical concern within the past 6-12 months. We analyzed qualitative data using a rapid qualitative analysis approach.

RESULTS: The response rate was $64 \%$. Overall, Veterans were satisfied with care received and most did not describe access as problematic. To obtain access, most reported first attempting to contact the primary care clinic by telephone. A few described strategies that involved multiple modes of contact (e.g., call first, then walk in if clinic doesn't call back). Of those who reported calling, a few reached the clinic directly, but most called an administrative call center. About half reported being seen in primary care or specialty within 1 week, but a few were unable to get needed care and sought care outside VHA. The most important aspect of access for most was getting a response back to messages or a timely appointment (urgent and routine). Several mentioned communication quality or ability to contact the clinic directly as important aspects of access, and several expressed concerns about provider/staff turnover.

CONCLUSIONS: Besides appointment availability, Veterans' perceptions may be shaped by many factors, including difficulty scheduling appointments (e.g., a multi-stage triage process involving the call center, care team, and/or nurse advice line), lack of timely response to requests/questions, and staffing turnover. While most Veterans had positive experiences using VHA, our results suggest that the cumulative "hassle factor" and the steps involved in getting care may affect Veterans' responses to survey questions about access. Improving Veterans' perceptions may require a multi-faceted, multi-level intervention, including improving response/resolution time, Veteran education about the multiple modes of accessing care, and provider and staff training in patient-centered communication.

\section{WHAT IF IT'S NOT SCREENING? HEPATITIS C ANTIBODY PERFORMANCE AND ABNORMAL LIVER TESTS}

Andrew Schreiner ${ }^{1}$; Jingwen Zhang $^{2}$; Patrick D. Mauldin ${ }^{1}$; William P. Moran ${ }^{1}{ }^{1}$ Medical University of South Carolina, Charleston, SC; ${ }^{2}$ MUSC, Charleston, SC. (Control ID \#3186057)

BACKGROUND: Hepatitis $\mathrm{C}$ virus (HCV) antibody (Ab) serologies are the tests of choice for $\mathrm{HCV}$ birth cohort screening given their high sensitivity and relatively low cost. However, how these tests perform in diagnosing $\mathrm{HCV}$ in patients with clinical signals of possible liver abnormality is less well known. We studied the performance characteristics of $\mathrm{HCV} \mathrm{Ab}$ testing in patients with abnormal liver chemistries.

METHODS: This retrospective study of administrative data investigates diagnostic testing for $\mathrm{HCV}$ in primary care patients with abnormal liver tests between 2007 and 2016 in an academic patient-centered medical home (PCMH). Patients seen in the PCMH at least once and possessing at least one abnormal liver test result in the electronic health record were included in the study. Abnormal liver tests included any elevation in bilirubin, aminotransferases, or alkaline phosphatase. Patients were evaluated for the first appearing HCV serologic testing results, including both $\mathrm{Ab}$ (ARCHITECT) and RNA tests, during the study period. The institution's laboratory incorporated reflex HCV RNA testing in response to positive $\mathrm{Ab}$ tests in 2015. The sensitivity and specificity of HCV Ab testing was analyzed in comparison to the gold standard of RNA testing results, when available. We conducted this analysis on patients with at least one abnormal liver test, and again on patients with at least 2 consecutive liver test abnormalities.

RESULTS: In total, 9,545 unique patients were studied, (Table 1). The 2,812 patients tested for HCV Ab yielded 335 (11.9\%) positive results. Unfortunately, $80(23.9 \%)$ of the $\mathrm{HCV} \mathrm{Ab}$ positive patients did not have HCV RNA testing documented. Of those with RNA follow-up, 205 of $255(80.4 \%)$ were positive. Only 54 patients with negative $\mathrm{HCV} \mathrm{Ab}$ tests had RNA testing, yielding $0(0.0 \%)$ positive RNA results. From the 309 patients with HCV Ab and RNA test results, the HCV Ab tests had a sensitivity of $100 \%$ and specificity of $51.9 \%$. We also looked at HCV Ab performance in the 5,364 patients with consecutive liver test abnormalities. Of these patients, 2,125 had HCV Ab testing and 301 (14.2\%) had positive results. From the 278 patients with consecutive liver test abnormalities and $\mathrm{HCV} \mathrm{Ab}$ and RNA results, the sensitivity of $\mathrm{HCV} \mathrm{Ab}$ testing was $100 \%$ and the specificity was $53.3 \%$.

CONCLUSIONS: HCV Ab testing is highly sensitive but poorly specific in primary care patients with abnormal liver tests. While such performance suits birth cohort screening, HCVAb testing (vs. RNA) in response to potential signals of liver abnormality may contribute to diagnostic error (i.e. false positives with patient worry and missing follow-up) and added costs. Future investigation with a cost-effectiveness analysis in similar patient populations is warranted.

\section{WHAT IS MISUSE? CLINICIAN CONSENSUS ON SECURE MESSAGING WITH PATIENTS}

Joy L. Lee ${ }^{1,3}$; Marianne S. Matthias ${ }^{2,}{ }^{3}$; Michael Weiner ${ }^{4,}{ }^{3}$. ${ }^{1}$ Indiana University School of Medicine, Indianapolis, IN; ${ }^{2}$ Roudebush VAMC, Indianapolis, IN; ${ }^{3}$ Regenstrief Institute, Indianapolis, IN; ${ }^{4}$ Indiana University, Indianapolis, IN. (Control ID \#3185246)

BACKGROUND: Secure messaging provides patients access to clinicians, care, and health information. Yet misuse of secure messaging by both patients and clinicians can lead to miscommunications and errors. We sought to uncover variations in provider approaches and responses to messaging, particularly their perceptions of appropriateness, and preferences for when to use secure messaging, to understand the potential to improve care via this mode of communication.

METHODS: This qualitative study targeted primary care clinicians from a Midwestern Veterans Affairs medical center and a tax-supported safety-net medical center. Participants were presented with five clinical vignettes and corresponding secure messages from hypothetical patients, asked to compose responses, and then interviewed about the messaging process, their preferences for how to respond, as well as their understanding of appropriateness. Recruitment is still ongoing and will continue until the findings reach saturation.

RESULTS: We uncovered key differences in clinicians' (13 physicians, 2 nurse practitioners, and 4 registered nurses) experiences with, and perceptions of, secure messaging. Clinicians disagreed about the circumstances for which responding to a secure message from patients by secure messaging was preferred over telephone calls with patients. Their rationales revealed differences in understandings of appropriate uses of secure messaging. There was most, but not complete, agreement that secure messaging should not be used to respond to a message from a patient with an urgent health issue (95\% preferred to call) or symptoms of depression (89\%). Clinicians disagreed regarding their use of secure messaging to discuss medication adjustments and laboratory results, and to respond to a dissatisfied patient. Although the 19 clinicians represented diversity in their approaches and secure messaging workload - half received 10 or fewer messages a week, and half received more - all indicated that the workload was manageable.

CONCLUSIONS: Among physicians, nurse practitioners, and nurses, this study revealed variations in approaches to secure messaging, particularly a lack of consensus about preferences and perceptions of appropriateness of secure messaging. Clinicians' perspectives about secure messaging can influence their patients' adoption and use of the technology. 
Further guidance on the appropriateness and effectiveness of the delivery of various facets of care through secure messaging may aid both clinicians and patients. Future work will explore patient understandings of appropriateness and misuse.

\section{WHAT MAKES A MEDICAL STUDENT LEADER? AN EX- PLORATORY STUDY OF STUDENTS' PERSPECTIVES ON LEADERSHIP}

Alison M. Holliday ${ }^{1}$; Galina Gheihman ${ }^{1}$; Erin E. Sullivan ${ }^{1}$; Amy M. Sullivan'; Rebecca Karp Leaf ${ }^{2}, 1$. ${ }^{1}$ Harvard Medical School, Boston, MA; ${ }^{2}$ Massachusetts General Hospital, Boston, MA. (Control ID \#3184898)

BACKGROUND: Physicians hold leadership roles within healthcare and within spheres of education, business, and government. Yet few medical students receive formal leadership training. While medical schools are beginning to recognize the need for a leadership curriculum, there is a gap in understanding of how students view themselves as leaders. We conducted an exploratory study to characterize medical students' perception of leadership and identify areas of improvement in leadership training at a top medical school.

METHODS: Two open-ended questions related to leadership were included as part of a larger cross-sectional survey between June and August 2018 at Harvard Medical School. We invited 753 students by email to complete a web-based survey with five follow-up reminders. We asked students to describe via free text responses: 1) characteristics and behaviors of successful medical students, and 2) what being a leader as a medical student means. We analyzed results in aggregate but separate from primary survey responses and used qualitative content analysis to identify major themes.

RESULTS: The primary survey response rate was 64\% ( $\mathrm{n}=485) ; 467(96 \%)$ of those participants completed the free-text responses. The scope and frequency of successful medical student characteristics and behaviors identified by respondents is shown in a word cloud. Qualitative content analysis to analyze what being a leader means to students revealed three major themes: 1) domains in which students could demonstrate leadership: classroom, clinical domain, and wider society; 2) leadership qualities: adaptable, emotionally intelligent, diligent; and 3) leadership practices: supporting others, taking initiative, staying true to one's values, demonstrating resilience and vulnerability, maintaining a healthy lifestyle, and showing humility, among others.

CONCLUSIONS: Medical students see an important role for student leaders in the classroom, the clinical setting, and within society at large. Curricula should be developed to build leadership skills and practices aligned with themes identified by students - fostering courage, resilience, vulnerability, self-care, integrity, and commitment to serving others. Practicing these qualities and behaviors may help individuals demonstrate student leadership, becoming effective team members, providing excellent clinical care, and maintaining their own sense of wellbeing.

Figure 1. Student responses to the open-ended query, "In your opinion, a successful medical student has the following characteristics and behaviors:"

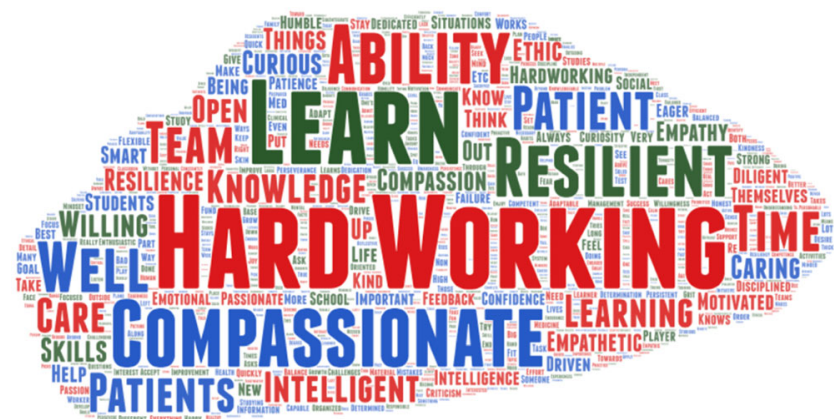

WHAT STUMPS PRIMARY CARE CLINICIANS? FIRST PHASE ANALYSIS OF DIAGNOSTIC UNCERTAINTY CASES

Lucia S. Sommers ${ }^{1}$; Viralkumar Vaghani ${ }^{2,3}$; Traber Giardina ${ }^{2,3}$; Hardeep Singh ${ }^{4}$. ${ }^{1}$ University of California, San Francisco, San Francisco, CA; ${ }^{2}$ Baylor College of Medicne, Houston, TX; ${ }^{3}$ Center for Innovations in Quality..., Houston, TX; ${ }^{4}$ Michael E. DeBakey Veterans Affairs Medical Center and Baylor College of Medicine, Houston, TX. (Control ID \#3148418)

BACKGROUND: Diagnostic difficulty is frequent in primary care, yet we know little about situations that present uncertainty to primary care practitioners (PCPs). We explored how PCPs discuss diagnostic conundrums with colleagues, frame dilemmas, and seek/receive support. These findings could advance understanding of knowledge/cognitive problems contributing to diagnostic error.

METHODS: We analyzed data from 459 uncertainty cases compiled by the first author while PCPs presented cases to peers in "Practice Inquiry Colleague Groups" conducted at 17 San Francisco-Bay Area PCP practices from 10/2002 to $9 / 2015$. Clinicians discussed patients where they encountered uncertainty of any type (e.g., diagnostic, ethical) for ideas and support. Meeting notes on each case included: 1)PCP presenter's uncertainty statement; 2) patient's medical history; and 3) Colleague Group's discussion summary. We assessed meeting notes for diagnostic uncertainty building on criteria developed in prior work on measuring uncertainty from EMR review. Specific data collected for analysis: presenters' uncertainty statement, abnormal physical exam, abnormal test/imaging findings; diagnoses/conditions under consideration by presenter; and patients' prior diagnoses/known conditions. Two authors analyzed data using a consensus approach to reconcile differences.

RESULTS: Of 459 cases discussed, 258(56\%) involved diagnostic uncertainty. Most cases involved women (61\%); median age decade 50-59 years. Most $(93 \%)$ had one/or more physical complaints and/or abnormal findings. (See Table.) Colleagues speculated about specific diagnoses/conditions contributing to uncertainty in 129 cases $(50 \%)$; of these, $27 \%$ were mental/behavioral/neurodevelopmental conditions. They discussed patients' prior diseases/ known conditions related to the uncertainty in $183(71 \%)$ cases; of these, $17 \%$ were mental/behavior/ neurodevelopmental conditions. Adverse events were reported in $14 \%$ of cases including 6 deaths.

CONCLUSIONS: Practice inquiry-based techniques illustrate unique diagnostic uncertainties faced by PCPs. Certain situations, such as patients with mental, behavioral or neurodevelopmental conditions, might pose particular diagnostic challenges. This small-group process also facilitated discussions of diagnostic error and patient harm. We now plan to analyze colleagues' responses to these cases

\section{First Phase Findings: What Stumps PCPs?}

\begin{tabular}{|c|c|}
\hline PCP Presenters' Data Category & $\# / \%$ Cases \\
\hline \multicolumn{2}{|l|}{ 1. Types of Diagnostic Uncertainties ( $N=258$ cases) } \\
\hline - $\geq 1$ non-pain, physical symptom & $182(71 \%)$ \\
\hline 21 abnormal lab/imaging finding & $87(34 \%)$ \\
\hline 21 pain symptom & $72(28 \%)$ \\
\hline $\begin{array}{l}\text { 2. Possible Diseases/Conditions (ICD-11) } \\
\text { ( } \mathrm{N}=171 \text { diseases/conditions) }\end{array}$ & \\
\hline - Mental, behavioral or neurodevelopmental disorders & $46(27 \%)$ \\
\hline - Diseases of the nervous system & $14(8 \%)$ \\
\hline - Diseases of the circulatory system & $14(8 \%)$ \\
\hline $\begin{array}{l}\text { 3. Past, Known Diseases/Conditions (ICD-11) } \\
\text { ( } N=402 \text { diseases/conditions) }\end{array}$ & \\
\hline - Mental, behavioral or neurodevelopmental disorders & $70(17 \%)$ \\
\hline - Endocrine, nutritional or metabolic diseases & $50(12 \%)$ \\
\hline - Diseases of the circulatory system & $42(10 \%)$ \\
\hline
\end{tabular}


WHEN LESS IS MORE: EVALUATION OF POSTPARACENTESIS ALBUMIN DOSAGE AND ADVERSE EVENTS IN HOSPITALIZED PATIENTS.

James E. Anstey $^{3}$; Claire Bainbridge ${ }^{2}$; Wendi Zhao ${ }^{1}$; Yumiko Abe-Jones ${ }^{3}$; Marissa $\mathrm{Hom}^{2}$; Anthony Wong ${ }^{2}$; Priya A. Prasad ${ }^{2}$; Margaret Fang ${ }^{4}$; Andrew Lai ${ }^{3}$. ${ }^{1}$ University of California San Francisco, San Francisco, CA; ${ }^{2}$ UCSF, San Francisco, CA; ${ }^{3}$ University of California at San Francisco, San Francisco, CA; ${ }^{4}$ University of California, San Francisco, San Francisco, CA. (Control ID \#3185261)

BACKGROUND: Large volume paracentesis is commonly performed in patients with refractory ascites but can induce hypotension and kidney injury. There is evidence that administering albumin at 6-8 grams per liter $(\mathrm{g} / \mathrm{L})$ of ascites removed during paracentesis can reduce these adverse events. Guidelines recommend this practice when $>2$ liters are removed in high-risk patients or $>5$ liters in any patient. Despite these guidelines, clinical practice varies widely potentially resulting in albumin overuse. We retrospectively analyzed the association of albumin dose and adverse events following large volume paracentesis in hospitalized patients at a single academic teaching hospital.

METHODS: Patient data were obtained from a registry of all procedures performed by a hospitalist procedure service at an academic teaching hospital from June 2013 to July 2018. We identified all patients who received large volume paracentesis $(>2 \mathrm{~L}$ ) and obtained electronic health record data on vital signs, laboratory tests, patient demographics, severity of illness, and albumin administration. We excluded patients with severe chronic kidney disease (serum creatinine $[\mathrm{Cr}]>3$ ), for whom albumin was not indicated, or who received $>25 \mathrm{~g} / \mathrm{L}$ of albumin (considered treatment for hepatorenal syndrome). Albumin administration was categorized as $6-8 \mathrm{~g} / \mathrm{L}$ vs $>8 \mathrm{~g} / \mathrm{L}$. We used multivariable logistic regression to test the association between albumin dose and acute kidney injury (AKI), defined as $>30 \%$ increase in serum creatinine over 72 hours postprocedure. We also examined mean change in systolic blood pressure (SBP) 24 hours post-procedure.

RESULTS: A total of 452 patients underwent $>2 \mathrm{~L}$ paracentesis (182 with $>5 \mathrm{~L}$ removed) and were eligible for analysis. One hundred fifty-four (34\%) were on the medicine service, $226(50 \%)$ on the liver transplant service, and $72(16 \%)$ on all other services. In all, $85(19 \%)$ patients received $6-8 \mathrm{~g} / \mathrm{L}$, and $367(81 \%)$ received $>8 \mathrm{~g} / \mathrm{L}$ of peri-procedural albumin. Patients who received $>8 \mathrm{~g} / \mathrm{L}$ of albumin were more likely to have a higher baseline $\mathrm{Cr}$ (1.18 vs 1.41 ; mean difference 0.23 [95\%CI: $0.08-0.37] \mathrm{p}=0.003)$, higher MELD score ( 18.5 vs 21.3 , mean difference 2.8 [95\% CI: 0.48-5.06] $\mathrm{p}=0.018)$ and be on the liver transplant service $(\mathrm{p}$ $<0.001$ ). Multivariate analysis showed albumin dosage was not associated with post-procedure AKI $(10.4 \%$ in $6-8 \mathrm{~g} / \mathrm{L}$ vs $16.9 \%$ in $>8 \mathrm{~g} / \mathrm{L}$, adjusted OR 1.24 [95\% CI: 0.52-2.99], $\mathrm{p}=0.625$ ). Post-paracentesis mean change in SBP also did not differ by albumin dose (mean SBP drop of 2.95 in 6-8 $\mathrm{g} / \mathrm{L}$ vs 4.36 in $>8 \mathrm{~g} / \mathrm{L}$, Coefficient $=0.71$ [95\% CI $-1.92-3.34$ ], $\mathrm{p}=0.594$ ).

CONCLUSIONS: Patients undergoing large volume paracentesis at our institution received widely varying doses of albumin, with the majority of patients receiving $>8 \mathrm{~g} / \mathrm{L}$. Albumin doses above current guidelines were not associated with a lower incidence of AKI or mean drop in SBP following paracentesis. Given the high cost of albumin and potential for volume overload, guideline-recommended dosing should be more consistently adopted.

WHEN THE TEAM WINS, YOU WIN: RESIDENT PERSPECTIVES ON IDENTITY FORMATION IN INTERPROFESSIONAL TEAMS AND LESSONS FOR GENERAL MEDICAL EDUCATION

Katarzyna Mastalerz $^{1,2}$; Sarah Jordan ${ }^{2}$; Nikki Townsley ${ }^{2} .{ }^{1}$ Rocky Mountain VAMC, Aurora, CO; ${ }^{2}$ University of Colorado, Aurora, CO. (Control ID \#3171551)
BACKGROUND: Forming team-oriented professional identities is essential to becoming effective team members in healthcare settings. However, little is known about how medical residents form professional identities in the context of interprofessional teams. In order to inform lessons for graduate medical education, we sought to understand residents' perspectives on and experiences with forming professional identities while working with interdisciplinary teams.

METHODS: We conducted a qualitative study using in-depth, semistructured interviews with medical residents working on Accountable Care Units (ACU, a geographic team-based unit) at one community teaching hospital between July 2017 and March 2018. Interviews explored perceptions of teamwork and professional roles and identities. All residents that rotated on the ACU were eligible for inclusion. Interviews were audio-recorded, transcribed, and entered into qualitative software for organization (Atlas-ti.v8). During regular group meetings, we systematically analyzed interview transcripts and identified emerging themes using inductive thematic analysis. Saturation was reached.

RESULTS: Twenty-five interns and 16 senior residents were interviewed $(\mathrm{n}=41)$. Resident participants were predominantly male $(65 \%)$ and Caucasian $(65 \%)$. Three key themes emerged: 1) negotiating new roles in team structures, 2) learning to self-advocate and integrate personal expertise into hierarchical team settings, and 3) bolstering professional identities by incorporating interprofessional expertise. Senior residents described new tensions in their identities as both learners and experts; interns described feeling 'responsible but powerless.' Interns and residents both practiced strategies to integrate their own growing expertise into interprofessional teams and to 'find their voice.' Senior residents recognized their dependence on interprofessional team members and sought out ways to defy existing hierarchies that constrained interdisciplinary teamwork. Interns struggled with balancing their lack of experience with the tenure of others, and with responding to the implicit expectation for physician leadership. Residents felt that their constant movement between rotations impaired authentic teaming. These perspectives inform several strategies for medical education that could support the creation of team-oriented professional identities for residents: longitudinal integrated programs, purposeful interprofessional shadowing experiences, and explicit leadership curricula.

CONCLUSIONS: Understanding resident professional identity formation provides insight into how residents integrate into interprofessional teams. Based on our study, residency is a time of a growing awareness of interprofessional work and its challenges. We suggest several education strategies that may improve resident interprofessional teaming skills and the formation of team-oriented professional identities.

\section{WHERE IS ALL THE MIXING HAPPENING? A VENUE-BASED ANALYSIS OF SEX NETWORKS IN BALTIMORE, MD}

Benjamin Meza ${ }^{2}$; Ian McCulloh ${ }^{2}$; Marc A. Johnson ${ }^{1}$; Ravikiran Muvva ${ }^{3}$; Carla Tilchin ${ }^{2}$; Jacky M. Jennings ${ }^{2}, 2$. ${ }^{1}$ Johns Hopkins University, Laurel, MD; ${ }^{2}$ Johns Hopkins University, Baltimore, MD; ${ }^{3}$ Baltimore City Health Department, Baltimore, MD. (Control ID \#3185626)

BACKGROUND: Baltimore has some of the highest numbers of new HIV and syphilis cases in the country. Nationally, STI prevention strategies often target specific priority populations (i.e., men who have sex with men [MSM], individuals who inject drugs [IDU], commercial sex workers [CSW], youth under 25 years old), yet studies show that transmission frequently occurs between different priority populations. Our objective was to use social network analysis to map the extent to which different physical and online spaces serve as sites of mixing and identify venues to focus limited prevention resources.

METHODS: We employed data from the Baltimore City Health Department on all new cases of HIV and syphilis reported in Baltimore City from 2009 to 2015. Data included the type of STI, demographic characteristics, priority population classification, self-reported high-risk behaviors, and sex partner meeting venues visited in the last 12 months. We created a 
social network in which venues were linked to one another via the individuals who reported those venues. We then applied a novel method to reduce the network's complexity, improving interpretability. Our method applies a Jaccard similarity algorithm followed by an irreducible backbone to derive a simplified version of the network that maintains important structural features. Using this network of sex partner meeting venues, we drew network diagrams to describe where mixing between priority populations was occurring.

RESULTS: The analytic set consisted of 932 new cases of HIV (31.9\%), syphilis (64.8\%), and HIV-syphilis coinfection (3.3\%). The median age at diagnosis was 27 years, $72.7 \%$ were Black, $72.5 \%$ were classified as MSM, $12.0 \%$ as CSW, and $4.5 \%$ as IDU. From a total of 1511 nominations, we identified 425 unique venues, including bars/clubs (13.2\%), internet/apps $(12.7 \%)$, and street addresses (53.6\%). The simplified network consisted of 259 venues and 356 links, with a single large interconnected group of 143 venues at its core. Unexpectedly, Facebook received the greatest number of nominations (17.6\% of all nominations) but was completely disconnected from this core group. Similarly, other large internet venues were highly nominated but were absent from central positions that would make them important mediators of transmission across the network. As expected, highly nominated venues were associated with greater mixing of priority populations, but exceptions to this pattern highlight unique qualities of certain venues, e.g., Craigslist was associated with a small, unconnected cluster of venues with multiple priority populations.

CONCLUSIONS: This study provides preliminary evidence that certain venues are important spaces for mixing between priority populations. We demonstrate a method for capturing difficult to measure local sex networks and reducing their complexity to identify discrete spaces where interventions may have the greatest impact on interrupting STI transmission.

\section{WHO HAS PRIMARY CARE IN THE UNITED STATES, 2002 TO 2015}

David M. Levine ${ }^{1}$; Jeffrey A. Linder ${ }^{2}$; Bruce E. Landon ${ }^{3} .{ }^{1}$ Brigham and Women's Hospital, Boston, MA; ${ }^{2}$ Northwestern University Feinberg School of Medicine, Chicago, IL; ${ }^{3}$ Harvard Medical School, Boston, MA. (Control ID \#3185704)

BACKGROUND: Primary care - first contact, comprehensive care that is coordinated and continuous - is considered an essential component of well-functioning health care systems. The clinical and demographic characteristics of Americans with primary care, and changes over time, are unknown.

METHODS: We analyzed 2002-2015 data from the Medical Expenditure Panel Survey, a nationally-representative annual survey of the noninstitutionalized US civilian population. We used a patient-centered definition of primary care to replicate the 4 "C's" of primary care, requiring that an individual had an identified physician for "new health problems" (first contact); "preventive health care, such as general checkups, examinations, and immunizations" (comprehensive); "ongoing health problems" (continuous); and "referrals to other health professionals when needed" (coordinated). To identify how the odds of having primary care changed between 2002 and 2015, we performed multivariable logistic regression controlling for over 20 sociodemographic characteristics, including clinical and health status. We also examined beliefs about health insurance over time.

RESULTS: In 2002, 77\% (95\% CI, 76 to 78) of Americans had primary care vs $75 \%$ ( $95 \%$ CI, 74 to 76 ) in 2015 ( $\mathrm{p}=0.01$ ), representing 4.2 million fewer Americans without primary care in 2015. Receipt of primary care increased with age, but younger Americans were less likely to have primary care in 2015 than 2002. For example, in 2015, 64\% of 30-yearolds had primary care compared to $71 \%$ in 2002 ( $\mathrm{p}<0.01)$. Americans less likely to have primary care included those who were male (aOR vs females, 0.61 [ $95 \%$ CI, 0.57 to 0.65]), Latino (aOR vs Whites, 0.72 [0.64-0.8]), resided in the South (aOR vs the West, 0.83 [0.74-0.94]), and poor (aOR vs high income, 0.60 [0.51-0.70]). Americans more likely to have primary care included those who resided in the Northeast (aOR vs the West, 1.49 [1.26-1.76]), were married (aOR vs not married, 1.23 [1.11-1.37]), privately insured (aOR vs uninsured, 3.08 [2.75-3.44]), and unemployed (aOR vs employed, 1.15 [1.05-1.26]). After adjustment, Americans overall were significantly less likely to have primary care in 2015 than in 2002 (aOR, 0.77 [0.69-0.84]). Other[BL1] predictors of having primary care were generally stable over time, except for race/ethnicity and census region. Between 2002 and 2015, Latinos increased their receipt of primary care by $15 \%(\mathrm{p}=0.04)$ and northeasterners decreased by $12 \%(\mathrm{p}=0.03)$. In $2015,46 \%$ (44-47) of those with primary care vs $27 \%$ (26-29) of those without primary care believed health insurance was worth the cost, and 68[BL2] \% (67-69) vs 40\% (38-42), respectively, believed they needed health insurance.

CONCLUSIONS: Significantly fewer Americans had primary care in 2015 than in 2002, particularly among younger Americans. To our knowledge, this is the first nationally-representative description of receipt of primary care and demonstrates an alarming trend toward a reduction in primary care health care relationships.

\section{WHO, WHAT, AND WHERE? MULTIPLE CHRONIC CONDI- TIONS IN A LOW-INCOME, VALUE-BASED URBAN COHORT: CLUSTERING FOR INTERVENTION}

Usnish Majumdar $^{1}$; Christophe Hunt ${ }^{2}$; Patrick Doupe ${ }^{1}$; Aaron Baum ${ }^{1}$; David J. Heller ${ }^{1}$; Rashi Kumar ${ }^{3}$; Robert Futterman ${ }^{3}$; Cother Hajat ${ }^{4}$; Erica Levine ${ }^{1}$; Sandeep Kishore ${ }^{1} .{ }^{1}$ Icahn School of Medicine at Mount Sinai, New York, NY; ${ }^{2}$ Mount Sinai Health Partners, New York, NY; ${ }^{3}$ Healthfirst, New York, NY; ${ }^{4}$ United Arab Emirates University, Al Ain, United Arab Emirates. (Control ID \#3185543)

BACKGROUND: In the US, patients with multiple chronic conditions ( 2 , MCC) account for more than $70 \%$ of all healthcare spending. Health systems have responded to this challenge with both clinical and financial innovations. Importantly, efforts to select sub-populations for intervention have been limited methodologically, and it remains difficult to compare and contrast the clinical and financial reforms enacted in different patient populations. Furthermore, literature on MCC patterns and trends among lower socioeconomic status and vulnerable populations is scarce, despite their carrying a disproportionately large share of chronic disease burden. In this study, we aim to (1) examine the burden of MCC in the low-income population served by an urban health system, and (2) propose a methodology to identify subpopulations of interest for both clinical and financial interventions.

METHODS: We used retrospective data from capitated Medicaid contracts in an urban health system. Combined data from 2012-2014 from 192,085 plan members were analyzed. Adults were categorized as having any number of chronic diseases from a list of 69 chronic conditions provided by the Agency for Healthcare Research \& Quality (AHRQ). After summarizing the demographics, geography, and prevalence of MCC within this population, we then explored groups of patients (clusters) using a novel methodology: We iteratively defined all $(26,495)$ potential clusters of patients as defined by a pair of chronic conditions, a sex, and an age group, and then ranked them by frequency, cost and ratios of observed to expected frequencies of co-occurring chronic conditions. We further explored the presence of certain clusters within bounded geographic "hot-spots" to suggest opportunities for co-localized specialty clinics.

RESULTS: $52.7 \%$ of the study population suffers from MCC, as compared to $42 \%$ nationally. The most frequent pair was hypertension and hyperlipidemia (18\% age-adjusted) and the most frequent trio was diabetes, hypertension and hyperlipidemia (9\% age-adjusted). Women aged 50-65 with hypertension and hyperlipidemia were the leading cost segment in the study population. Medicaid patients are concentrated geographically and MCC burden differs by locale. Of note, we find that anemia, pulmonary heart disease, congestive heart failure, and conduction disorders occur together more frequently than expected in geographically distinct locales. 
CONCLUSIONS: In this low-income, urban population, multiple chronic conditions are more prevalent than nationally. Furthermore, our identification of costly and co-occurring conditions in a capitated cohort of patients demonstrates potential for our novel methodology to inform decision-making in healthcare administration, especially under new value-based financing schemes. We argue this methodology has utility for health systems, financiers, and researchers working to address MCC, and provide a common approach to targeting populations for financial and clinical intervention.

\section{WITHIN AFRICAN-AMERICAN HETEROSEXUAL HIV- SERODISCORDANT COUPLES, AWARENESS OF PRE- EXPOSURE PROPHYLAXIS (PREP) AMONG HIV-POSITIVE PARTNERS PREDICTS PREP AWARENESS AMONG HIV- NEGATIVE PARTNERS}

Alicia Morehead-Gee ${ }^{1}$; Tamra B. Loeb ${ }^{2}$; Muyu Zhang ${ }^{2}$; Gail Wyatt ${ }^{2}$. ${ }^{\mathrm{T}}$ National Clinician Scholars Program at UCLA, Los Angeles, CA; ${ }^{2}$ UCLA, Los Angeles, CA. (Control ID \#3185748)

BACKGROUND: Although approximately 500,000 African-Americans qualify for pre-exposure prophylaxis (PrEP, a daily pill to prevent HIV infection) in the US, only $1 \%$ of African-Americans eligible for PrEP have received a prescription (CDC 2018), raising the issue of a possible lack of PrEP awareness among this population. This study examines whether sociodemographic factors, HIV-transmission-related behaviors, health systems experiences, and/or partner factors were associated with PrEP awareness among HIV-negative African-Americans in heterosexual HIV sero-discordant couples in South Los Angeles (LA).

METHODS: Cross-sectional baseline survey data was collected from 2013 to 2016 through "Eban II: Intervention for HIV Sero-Discordant Couples," an implementation study of a risk-reduction intervention for African-American heterosexual HIV sero-discordant couples. The primary outcome measure was PrEP awareness. Predictor variables included sociodemographic factors, HIV-transmission-related factors (including direct [condom use, multiple sex partners] and indirect [mental health disorders, history of trauma] factors), health systems experiences (including satisfaction with doctor and experiences of discrimination), and HIVpositive partner's PrEP awareness. Multiple logistic regression was used to create adjusted odds ratios (aOR) of PrEP awareness according to predictor variables.

RESULTS: Ninety-one (91) participants were enrolled in the study, including 46 (50.5\%) HIV-negative participants. Among HIV-negative participants, $32(69.6 \%)$ were male, average age was 51.9 years, 6 $(13.3 \%)$ were employed, and 43 (93.5\%) had health insurance. Eleven (24.4\%) HIV-negative participants were aware of PrEP. In examining sero-discordant couples as a unit, $6(14.0 \%)$ of 43 couples had shared PrEP awareness. In bivariate analyses, HIV-negative participants were more likely to be aware of PrEP if they felt that their doctor "understood their background and values" $(p=0.02)$ or if their HIV-positive partner was aware of PrEP ( $p=0.0008)$. Factors related to sociodemographics, HIV transmission risk, and other health systems experiences had no significant association with PrEP awareness. Controlling for age, gender and presence of a regular doctor, HIV-negative participants had higher odds of PrEP awareness if their HIV-positive partner was aware of PrEP (aOR 48.8, 95\% confidence interval [CI] 4.2 to 562.9).

CONCLUSIONS: Few HIV-negative African-Americans were aware of PrEP despite meeting criteria (HIV-serodiscordant relationship) to take PrEP. We found that HIV-positive partners' PrEP awareness significantly predicted HIV-negative participants' PrEP awareness, demonstrating the importance of PrEP education for people living with HIV in serodiscordant relationships. There was no association between PrEP awareness and factors related to sociodemographics or HIV transmission risk, which implicates a need to increase efforts to screen African-American patients for HIV risk and provide PrEP information to those at risk.
WORK REQUIREMENTS IN MEDICAID: RESULTS FROM THE FIRST YEAR OF ARKANSAS'S PROGRAM

Benjamin D. Sommers ${ }^{1,2}$; Anna Goldman ${ }^{1,4}$; Robert Blendon ${ }^{1}$; E. John Orav $^{2,1}$; Arnold M. Epstein ${ }^{3,2}$. 'Harvard School of Public Health, Brookline, MA; ${ }^{2}$ Brigham \& Women's Hospital, Boston, MA; ${ }^{3}$ Harvard School of Public Health, Boston, MA; ${ }^{4}$ Cambridge Health Alliance, Cambridge, MA. (Control ID \#3185359)

BACKGROUND: Six states have received approval to implement work requirements in Medicaid, and nine others have proposed similar requirements. Arkansas became the first state to implement this policy in June 2018, requiring beneficiaries ages 30-49 to report at least 80 hours a month of employment or other "community engagement" activities to maintain their coverage. The state began disenrolling people not meeting the reporting requirement in October 2018. We conducted a novel telephone survey in late 2018 to assess the policy's early effects.

METHODS: We conducted a random-digit dialing telephone survey of low-income adults in four states - Arkansas, Kentucky (which also plans to implement work requirements, but implementation has been blocked by a federal judge), Louisiana, and Texas - from November 8 to December 30,2018 . We assessed employment, other qualifying community engagement activities (e.g., job training, community service, and childcare), health insurance, and several measures of health care access in all four states. We also explored knowledge of and experiences with the work requirements among Arkansans; for this reason, we oversampled respondents in this state ( $\mathrm{n}=1501$ ), particularly $30-49$ year-olds subject to the requirements. We compared their responses to those from the other three states $(\mathrm{n}=1504)$, as well as historical data from a similar survey conducted in 2016 in these states $(n=2951)$, to estimate changes in outcomes before and after work requirements, using a difference-in-differences design.

RESULTS: $59 \%$ of 30-49 year-olds in Arkansas were aware of the new policy, with awareness lowest among those without a high school degree. Among those who were told by the state that they needed to report their employment status to maintain Medicaid, only $49 \%$ were doing so regularly. Medicaid coverage in Arkansas for $30-49$ year-olds decreased $(-15.1 \%, p=0.004)$ and uninsured rates increased $(+6.7 \%, \mathrm{p}=0.04)$ since 2016 , compared to those not subject to the new requirements. There were no significant changes in access to primary care or affordability of care associated with the policy thus far. Meanwhile, the policy to date has not lead to any significant change in employment, hours worked, or other community engagement activities. Notably, more than $95 \%$ of respondents were already spending at least 80 hours a month on required activities or appear to qualify for an exemption such as a disability.

CONCLUSIONS: The first six months of Arkansas's Medicaid work requirements led to significant coverage losses for low-income adults, without any detectible impact on employment or other community engagement behavior. Many adults subject to the requirements were not aware of them, and less than half were regularly reporting their activities to the state (putting them at risk for disenrollment), even though most respondents already met the policy's requirements. Future studies will be needed to explore this policy's longer-term impact.

\section{WORRY ABOUT MEDICAL EXPENSES AND ITS ASSOCIA- TIONS WITH PATIENTS' QUALITY OF LIFE IN END-OF- LIFE CANCER CARE}

Lauren Kelly ${ }^{1}$; Holly G. Prigerson ${ }^{2}$; Paul K. Maciejewski ${ }^{2} .{ }^{1}$ New York $\overline{\text { Presbyterian - Weill Cornell Medical Center, New York, NY; }{ }^{2} \text { Weill }}$ Cornell Medical College, New York City, NY. (Control ID \#3185602)

BACKGROUND: Burdensome medical costs disproportionately affect cancer patients compared to patients with other chronic illnesses. Cancer patients who are uninsured, a high proportion of whom are Black and Latino, face an even greater degree of financial hardship related to their 
medical care. A growing body of literature suggests that financial burden, or financial toxicity, has harmful effects on well-being and health-related quality of life (QoL). This study investigates patients' worry about paying medical expenses (WAPME) and its association with their QoL in end-oflife (EoL) cancer care.

METHODS: The sample includes patients $(\mathrm{N}=343)$ who participated in Coping with Cancer-II (CwC-II), a multi-site observational cohort study between 2010-2015 to examine racial and ethnic disparities in EoL cancer care. Measures included assessments of patients' sociodemographic characteristics (age, sex, race, ethnicity, education and health insurance status), degree of WAPME, and QoL (using the multi-domain McGill QoL Questionnaire). Unadjusted and adjusted odds ratios between patient WAPME and patient sociodemographic characteristics were estimated using ordinary and multiple ordinal logistic regression, respectively. Odds ratios between dichotomized patient QoL outcomes and patient worry about paying medical expenses, adjusted for patient sociodemographic characteristics (and, for some models, patient psychological well-being), were estimated using multiple logistic regression.

RESULTS: Patients who were less than 65 years of age $(\mathrm{OR}=2.67$, $\mathrm{p}<0.001)$, female $(\mathrm{OR}=1.60, \mathrm{p}=0.037)$, Black $(\mathrm{OR}=1.69, \mathrm{p}=0.039)$, Latino $(\mathrm{OR}=2.52, \mathrm{p}=0.003)$ and uninsured $(\mathrm{OR}=3.40, \mathrm{p}<0.001)$ were more likely to have WAPME. Adjusting for other sociodemographic characteristics, only patients' sex (female vs. male; $\mathrm{AOR}=1.65$, $\mathrm{p}=0.038$ ) and insurance status (uninsured vs. insured; $\mathrm{AOR}=2.46$, $\mathrm{p}=0.012$ ) remained significantly associated with patients' WAPME. Adjusting for sociodemographic characteristics, patients' WAPME was associated with worse psychological $(A O R=0.66, p<0.001)$ and existential ( $\mathrm{AOR}=0.75, \mathrm{p}<0.002$ ) well-being, as well as worse physical symptoms $(\mathrm{AOR}=0.77, \mathrm{p}<0.025)$ and worse overall $\mathrm{QoL}$ $(\mathrm{AOR}=0.70, \mathrm{p}<0.001)$. Patients' psychological well-being mediated the effects of WAPME on their existential well-being, physical symptoms QoL and overall QoL.

CONCLUSIONS: Health insurance disparities may explain racial and ethnic disparities in worry about paying medical expenses in EoL cancer care. Advanced cancer patients' WAPME has a direct, adverse association with their psychological well-being, and has indirect, adverse associations with their existential well-being, physical symptoms QoL and overall QoL. This investigation extends findings on financial toxicity in that it highlights the importance of patients' psychological well-being as a key (modifiable) mediator of the effects of financial distress on patients' physical and spiritual well-being.

\section{WORTH THE WEIGHT? RESIDENT ATTITUDES TOWARD DI- ET AND EXERCISE COUNSELING}

Adam Prince; Yashvi Patel; Rebecca Mazurkiewicz. Lenox Hill Hospital, New York, NY. (Control ID \#3185201)

BACKGROUND: Recent studies suggest nearly $80 \%$ of chronic diseases can be prevented through regular exercise, maintaining a healthy weight, healthy diet, and not smoking. We aimed to assess the attitudes, knowledge, and confidence of medicine residents toward lifestyle risk factor modification counseling in the outpatient setting.

METHODS: We sent an electronic, anonymous survey to categorical internal medicine residents of PGY1, PGY2, and PGY3 levels at Lenox Hill Hospital, a community hospital in New York, NY. This ten question survey was sent in February 2018, and assessed importance placed on lifestyle risk factor counseling, frequency of discussions in appropriate patients, comfort with counseling, familiarity with evidence-based behavioral change techniques, and confidence that patients would make changes based on counseling interventions.

RESULTS: Our survey received 41 out of 77 resident responses, including 10 PGY1, 16 PGY2, and 15 PGY3 residents; 23 respondents were male and 18 were female. Lifestyle counseling was rated as very or extremely important to $95 \%$ of respondents. Similarly, $78 \%$ stated that they usually or always discuss lifestyle risk factors in the outpatient clinic. With regard to comfort in providing counseling, $69 \%$ responded that they were very or extremely comfortable, but $32 \%$ rated their comfort as somewhat or slightly comfortable. For duration of counseling, $71 \%$ indicated that they spent $3-10$ minutes providing counseling per intervention. When asked about techniques for counseling, only $12 \%$ stated that they were familiar with evidence-based methods for behavior change. Lastly, only $7 \%$ of residents rated themselves as very or extremely confident that patients would make changes based on counseling, while $78 \%$ were slightly or somewhat confident, and $15 \%$ were not at all confident.

CONCLUSIONS: Residents at our institution place high importance on lifestyle risk factor counseling interactions and most feel comfortable having these conversations with applicable patients in the outpatient setting. However, residents do not appear to have a ready repertoire of evidence-based behavior change techniques, and do not feel that their counseling will be effective in leading to actual behavior change. This highlights the importance of teaching internal medicine residents evidence-based behavior change techniques. We are in the process of developing an educational intervention to provide our residents with tools for lifestyle risk factor modification counseling.

\section{YOU ARE WHAT YOUR SOCIAL NETWORK EATS: PUBLIC HOUSING SOCIAL NETWORKS AND FAST FOOD CONSUMPTION}

Benjamin Meza ${ }^{1}$; Craig E. Pollack ${ }^{2}$; Kimberly Gudzune ${ }^{3}$. ${ }^{1}$ Johns Hopkins, Baltimore, MD; ${ }^{2}$ Johns Hopkins University School of Medicine, Baltimore, MD; ${ }^{3}$ Johns Hopkins University, Baltimore, MD. (Control ID \#3162713)

BACKGROUND: High sodium diets, such as those that include fast food, are associated with cardiovascular disease (CVD), illnesses which occur at twice the rate among public housing residents as the general population. Perceived dietary patterns of one's social network members are theorized to set dietary norms and model practices that influence one's own dietary behaviors. As a basis for using social network principles as part of dietary interventions among public housing residents, our objective was to determine the association of high fast food intake among one's social network members and an individuals' own fast food consumption.

METHODS: We conducted a cross-sectional survey of randomly selected households in public housing complexes in Baltimore, MD from August 2014 to August 2015. Adult survey respondents answered an inperson survey of questions regarding their own diet, perceived dietary habits among their social network members, and the frequency of interaction with and among their social network members. Our dependent variable was self-reported frequency of fast food intake, dichotomized to eating fast food at least once daily or less than once daily. Our independent variable was the proportion of the respondent's social network who were perceived to consume fast food at least once daily. We used multivariable logistic regression to examine the association between the respondent's fast food consumption and consumption among their social network members, adjusted for individual demographic and health covariates as well as social network factors.

RESULTS: The sample included 266 respondents (response rate 48\%). Mean age was 44.5 years, $86.1 \%$ were women, $95.5 \%$ were Black, and $58.3 \%$ had a history of CVD (hypertension, myocardial infarction, cerebrovascular accident, or congestive heart failure). On average, $7.1 \%$ of respondents ate fast food daily themselves and the perceived proportion of network members who ate fast food daily was $10.5 \%$. After adjustment, there was a significant positive relationship between respondents eating fast food daily and a greater proportion of their social network members eating fast food daily (OR 1.18, 95\% CI [1.00, 1.38], $\mathrm{p}=0.034)$. 
CONCLUSIONS: We found a strong positive association between one's own consumption of fast food and fast food consumption in their social network. Our results highlight the importance of considering one's social network when designing dietary interventions, particularly among low income urban populations such as public housing residents at high risk of CVD.

\section{YOU CAN'T SCALE UNICORNS: EXPLORING COMMUNITY BASED ORGANIZATIONS' PERSPECTIVES ON HEALTH CARE'S SOCIAL DETERMINANTS OF HEALTH PROGRAMMING}

Elena Byhoff ${ }^{1}$; Lauren A. Taylor ${ }^{2}$. ${ }^{1}$ Tufts Medical Center, Boston, MA; ${ }^{2}$ Harvard Business School, Boston, MA. (Control ID \#3183013)

BACKGROUND: Policymakers are interested in the appropriate role for health care organizations in addressing social determinants of health (SDOH). Recent changes in Massachusetts Medicaid policy encouraged health care organizations to screen for and address SDOH deficits through Medicaid ACO models. Little is known about how community-based organizations (CBOs) view this new policy, nor how it may impact the social service delivery landscape.

METHODS: We conducted semi-structured interviews lasting 1 hour with representatives of health care systems participating in the Massachusetts Medicaid Accountable Care Organization (ACO) program and representatives from Massachusetts CBOs. We recruited representatives from the health care sector based on publicly available information on participation in the Medicaid ACO program. CBO recruitment focused on $\mathrm{SDOH}$ domains identified by health care interviewees as priorities. We used a snowball sampling strategy for recruitment until achieving thematic saturation. Interviews were transcribed and inductively coded to identify themes. We empaneled an advisory board of CBO representatives, academics, and health care representatives to provide feedback and ensure findings accorded with participant experiences.

RESULTS: Participants included health care executives $(n=8)$ at Massachusetts Medicaid ACOs and leadership $(n=46)$ from CBOs across Massachusetts. Health care interviewees were consistent in prioritization of SDOH domains (housing, food, transportation), concerns about scale and accountability among potential $\mathrm{CBO}$ partners, and ambivalence towards "owning" responsibility for $\mathrm{SDOH}$. On the contrary, CBOs were highly varied in their perspectives of health care's entry into SDOH programming. This variation presented both within and between SDOH domains. The CBOs expressed variation along key dimensions: (1) awareness of policy incentives to promote health care entering the SDOH space, (2) duration of experience in partnering with health care to provide social services in the community (3) enthusiasm for partnership with health care and (4) estimation of CBO's value to health care. CBOs with more experience partnering with health care were more skeptical of the potential for successful partnership, expressing specific concerns about conflicts of interest and misalignment of targeting priorities (covered lives vs whole community).

CONCLUSIONS: CBOs varied in identifying benefits of partnership with the health care sector to provide social services. Several cited concerns of medicalizing social services. CBO's limited partnership experience with the health care sector suggests that financial incentives may be necessary but insufficient to bridge the gap between the sectors. Expectations of what could be achieved through partnership diverged considerably. Our findings urge policymakers to carefully consider both stakeholder positions if and when they (a) devise policies for and (b) provide incentives to health care organizations to move into SDOH programming.

\section{CLINICAL VIGNETTE}

\section{"WHEN CLAP GETS YOUR HAND"}

Sarabjeet S. Suri; Justine Phifer; Andrea Porrovecchio. Montefiore Medical Center, Bronx, NY. (Control ID \#3184768)
LEARNING OBJECTIVE \#1: Recognize disseminated gonococcal infection (DGI) as a cause of polyarticular septic arthritis and more rarely, osteomyelitis.

LEARNING OBJECTIVE \#2: Assess all sexual risk factors, including oral sex as a source of gonococcal infection.

CASE: A 37-year-old man with well controlled HIV (undetectable viral load) presented with pain in his right wrist and left shoulder for 4 weeks. He never had fever, chills, rash, dysuria, or penile discharge. He endorsed having sex with men, but denied any sexual activity in the past year. On presentation, pertinent exam findings included swelling and tenderness of right wrist with limited active and passive range of motion. Left shoulder had tenderness with decreased range of motion. $\mathrm{X}$ ray of right wrist revealed erosion of the palmar aspect of the distal radius. Labs were significant for sedimentation rate of $110, \mathrm{c}$ reactive protein of 5.9, and a negative immunologic work up. Right wrist aspiration revealed a white count of $43 \mathrm{~K}$ with intra-op cultures revealing growth of Neisseria gonorrhoeae. Antibiotics were initiated and left shoulder fluid aspiration revealed a white count of $850 \mathrm{~K}$. On repeat questioning, patient revealed he performed oral sex approximately 7 weeks prior to onset of his wrist pain. MRI right wrist revealed osteomyelitis of the distal radius and ulna. An echocardiogram revealed no vegetation, blood cultures had no growth, and urine NAAT was negative for gonorrhea. He was treated with IV ceftriaxone \& clinically improved.

IMPACT/DISCUSSION: The most common etiologies of infectious arthritis in HIV patients include S. aureus and Streptococcal species. DGI is a far less common etiology of septic arthritis. It is usually seen in male patients under age of 40 with an estimated incidence of $0.5-3 \%$ in people infected with $\mathrm{N}$. gonorrhoeae. The incubation period is around 1-2 weeks with hematogenous spread occurring within 2-3 weeks post primary insult. Internists should be aware of risk factors for DGI, including complement deficiency, HIV, postpartum state, lupus, or any immunocompromised state and should take a thorough sexual history. DGI presents as arthritis dermatitis syndrome (tenosynovitis, dermatitis and polyarthralgia) or as purulent arthritis and results from intermittent bacteremic spread of gonorrhea (blood cultures may be negative). Synovial fluid analysis and anogenital/oral swabs should be obtained. Imaging of involved joints should also be performed to determine the extent of disease, as osteomyelitis is an uncommon but important sequela of DGI. Treatment in DGI involves joint drainage and 1-2 weeks of parenteral therapy with a third generation cephalosporins and a dose of azithromycin, with some requiring longer duration of parenteral therapy. CONCLUSION: DGI is on the differential diagnosis for polyarticular septic arthritis even in a patient with well controlled HIV. It is important to obtain complete sexual history and recognize that DGI may rarely lead to osteomyelitis.

\section{"WHEN A TICK GETS THE TICKER"}

Sarabjeet S. Suri ${ }^{1}$; Kristin A. Swedish ${ }^{2} .{ }^{1}$ Montefiore Medical center, Bronx, NY; ${ }^{2}$ Montefiore Medical Center, Bronx, NY. (Control ID \#3183953)

LEARNING OBJECTIVE \#1: Recognize that Lyme carditis can manifest from weeks to months after exposure

LEARNING OBJECTIVE \#2: Discuss how conduction abnormalities due to Lyme are variable and can include AV block, QT prolongation, bundle branch blocks and Ventricular tachycardia

CASE: A 34-year old man presented with dyspnea and palpitations for one week. He had no significant past medical history. He reported trouble walking up one flight of stairs, which was atypical, as he was otherwise an avid gym goer and outdoorsman. He had no significant family history, did not smoke or use drugs. He was bradycardic but otherwise hemodynamically stable. Physical exam was significant for irregular rhythm, bradycardia, and fading circular rash on left thigh. EKG revealed complete heart 
block. Basic labs were unremarkable, Lyme serology was positive. He reported hiking about four weeks prior to presentation. Echocardiogram (TTE) revealed decreased Ejection fraction (EF) at $40 \%$. He was treated with ceftriaxone. Within three days, his complete heart block converted to $1^{\text {st }}$ degree AV block. On follow up six weeks later, EKG revealed sinus bradycardia, TTE revealed normal EF.

IMPACT/DISCUSSION: There are only a few reversible causes of complete heart block: medication overdose with beta blockers, Lyme carditis and viral myocarditis. Lyme disease is a multisystem disease caused by infection with Borrelia burgdorferi. Cardiac involvement usually occurs within a few weeks or months of exposure. Lyme carditis is estimated to occur in approximately $1 \%$ of patients infected with Lyme. The pathophysiology of the disease is incompletely understood in humans, but animal studies have shown transmural inflammation in the connective tissue at the base of the heart, the basal interventricular septum, and perivascular regions including the aortic adventitia. The symptoms usually start within 4-6 weeks and may include palpitations, lightheadedness, syncope and chest pain. AV block is the most common manifestation of Lyme carditis ranging from $1^{\text {st }}$ degree $\mathrm{AV}$ block to complete heart block. Other manifestations include myopericarditis, LV dysfunction, and heart failure. Diagnosis is made via serologic testing with ELISA/western blot. The management involves hospitalization with telemetry monitoring in symptomatic patients (high grade block or first degree AV block with $\mathrm{PR}>300 \mathrm{msec}$ ). Lyme carditis is treated with intravenous antibiotics. Third generation cephalosporins are preferable; if patient is penicillin/ cephalosporin's allergic, desensitization should be performed. Patients should remain hospitalized until resolution of symptoms/blocks. The usual resolution occurs within one week of antibiotic therapy. Patient who are asymptomatic should be treated with oral antibiotics.

CONCLUSION: Detailed travel history and skin examination are important and can help pinpoint the diagnosis. Recognizing the reversible etiology of Lyme carditis helped prevent unnecessary intervention.

\section{A CASE OF THE B'S: B1, BLURRY VISION, AND BARIATRIC BERIBERI}

Tina P. Kapadia; Samir Bhalla. UCLA, Santa Monica, CA. (Control ID \#3186743)

LEARNING OBJECTIVE \#1: Recognize and diagnosis significant vitamin deficiencies in bariatric patients.

CASE: A 28 year old morbidly obese female status post gastric sleeve surgery two months prior presented to the hospital with falls and double vision for two days. Her surgery had resulted in a fifty pound weight loss. Her post operative period was complicated by intermittent bouts of nausea and vomiting and she was found to have pyloric stenosis requiring balloon dilation and botox injections. She developed a recurrent episode of nausea, vomiting and insufficient oral intake requiring around the clock Zofran. Approximately 1 week later she developed unsteadiness while walking with a sensation of falling associated with double vision and inability to focus eyesite. Physical examination demonstrated nystagmus with horizontal eye movements bilaterally, dysmetria with finger-to-nose testing, as well as an ataxic gait. Her strength, sensory and reflex examinations were otherwise normal, and labs noted a microcytic anemia. She was evaluated both neurology and ophthalmology and underwent CT and MRI imaging of the head and neck which were negative for acute findings. The patient was given IV fluids, multivitamin and high dose IV thiamine. Her symptoms rapidly improved with resolution of her diplopia and ataxia by hospital day 2. Labs later resulted in low levels of vitamin A, B1, iron, and serum ferritin. She was continued on bariatric vitamin supplementation and was discharged to home.

IMPACT/DISCUSSION: Micronutrient derangements are common following bariatric surgery. Thiamine (B1) deficiency is more commonly associated with malabsorptive procedures that decrease the surface area for nutrition utilization, metabolism, and absorption. B1 is typically absorbed in the duodenum, thus the mechanism for B1 deficiency in gastric sleeve surgeries has not been fully elucidated, although it is postulated that the stomach plays some key role in absorption. Some data shows that B1 can be depleted after only 20 days of inadequate supply. There have been numerous reports of acute thiamine deficiency in early post operative period in bariatric surgery patients leading to the term "bariatric beriberi." The term beriberi means "weakness" and refers to a spectrum of deficiencies in B1 including its effect on peripheral and central nervous systems. Only $16 \%$ of patients demonstrate the classic Wernicke's triad of encephalopathy, ophthalmoplegia and ataxia. Encephalopathy typically occurs more commonly than the other two features of the triad, interestingly this patient presented primarily with ataxia and ophthalmoplegia. Diagnosis mainly relies on clinical suspicion as there may be a delay in laboratory results, and brain imaging may not reveal any abnormal findings. Rapid recognition and treatment is important to prevent long term morbidity, or even mortality.

CONCLUSION: Thiamine deficiency can occur after gastric sleeve surgery and requires rapid treatment to prevent morbidity as in this case.

\section{FEVER IN A TRAVELER: DIAGNOSIS WITH THE PERIPHER-} AL SMEAR

PRIYANKA BHUGRA $^{1}$; Abhishek Maiti ${ }^{2}$; Richard Wu ${ }^{1}$; Alia N. Nazarullah ${ }^{1}$. ${ }^{1}$ University of Texas Health Science Center at San Antonio, San Antonio, TX; ${ }^{2}$ University of Texas MD Anderson Cancer Center, Houston, TX. (Control ID \#3181679)

LEARNING OBJECTIVE \#1: Parasitic infections should be high on the list of differential diagnoses of fever in a returning traveler, with relapses seen weeks to years after P. vivax and P. ovale infection.

LEARNING OBJECTIVE \#2: Peripheral blood smears are key to diagnosis and following the response to treatment after anti-malarial therapy.

CASE: A 50-year-old previously healthy woman presented with a 3 day history of fevers, chills and severe frontal headache in the evenings. She traveled from Rwanda to the United States 8 months ago. Physical examination was unremarkable. Laboratory studies were significant for thrombocytopenia (platelet count of $87 \times 10^{9} / \mathrm{L}$ ). A peripheral blood smear showed schizonts containing merozoites and intracellular ring forms, consistent with Plasmodium ovale infection. The patient was treated with artemether/ lumefantrine for 3 days. Her fevers, headache and thrombocytopenia resolved by third day of treatment. She was subsequently treated with primaquine for 14 days. At 3-month follow-up, the patient remained asymptomatic.

IMPACT/DISCUSSION: Malaria poses an important diagnostic challenge to the physicians in United States due to unfamiliarity with the disease and lack of specific clinical features in the early phase. Majority of the cases of malaria in US are associated with history of travel to the endemic areas including Africa, South-East Asia and South America. Relapse after initial infection can occur with P. vivax and P. ovale species of Plasmodium. This is due to the presence of the hepatic dormant hypnozoite stage that can last for weeks to several months or even years. Thus, a single infection can be responsible for series of waves of parasitemia or relapses by reactivation of hypnozoite stage. Early recognition of the disease is important to reduce mortality and prevent its spread in the community. Delay in diagnosis can lead to progression to severe disease with multi-organ failure and death. Examination of peripheral blood smear helps in prompt diagnosis of malaria, identification of the Plasmodium species and quantification of parasitemia. The treatment of non-falciparum malaria includes use of chloroquine or artemisinin-based combination therapy. The clearance of parasitemia is confirmed by serial blood smear examination after initiation of treatment. Patients with P. vivax and P. ovale need further treatment with 2-week course of primaquine to eradicate the liver hypnozoites and prevent future relapses. Tafenoquine is a newly approved anti-relapse drug which can be used as a single dose regimen in nonpregnant individuals. Testing for glucose-6-phosphate-dehydrogenase deficiency is mandatory prior to starting the anti-relapse therapy. 
CONCLUSION: Malaria in an important differential diagnosis in patients travelling from endemic areas. Late relapses of malaria can occur with P. vivax and P. ovale species. Early identification with peripheral smear examination is important for timely diagnosis and treatment.

\section{INCIDENTAL DIAGNOSIS OF POLYMYOSITIS IN PRIMARY CARE CLINIC}

Emily Fessler. Hospital of the University of Pennsylvania, Philadelphia, PA. (Control ID \#3177385)

LEARNING OBJECTIVE \#1: Develop an approach to asymptomatic abnormal liver function tests (LFTs) in the primary care setting

LEARNING OBJECTIVE \#2: Recognize the potential for depression to delay care-seeking and diagnosis

CASE: A 68 year old woman with hypertension and depression presents for a routine primary care visit. Her only complaint is discomfort with swallowing which she attributes to "chronic tonsillitis". Her exam is unremarkable and plan made for non-urgent ENT referral. In addition, her 10year ASCVD risk is $10.6 \%$, thus she is prescribed a high-intensity statin and ordered for baseline LFTs. Unexpectedly, her transaminases return markedly elevated with AST 322 and ALT 131 from normal 1 year prior. On further questioning, she denies alcohol use, strenuous activity, or herbal supplements. She returns for additional testing including hepatitis serologies, TSH, creatine kinase (CK), and right upper quadrant ultrasound. CK is elevated to 6196. With direct questioning she endorses several months of generalized weakness with difficulty climbing the stairs and raising her arms. Autoimmune labs reveal ANA $>1: 20480$. She undergoes thigh MRI with signal enhancement in the musculature, followed by muscle biopsy consistent with polymyositis. Anti-Ku antibodies are positive. She is seen by rheumatology and started on $60 \mathrm{mg}$ prednisone daily. CK remains elevated despite 4 weeks of prednisone, thus started on methotrexate and monthly IVIG. Barium swallow shows evidence of esophageal dysmotility. Screening for occult malignancy is negative. CK normalizes after 5 months of treatment and steroids tapered over 1 year.

IMPACT/DISCUSSION: Asymptomatic LFT elevation is often encountered in primary care. The first step in diagnosis is careful history including review of medications/supplements, alcohol use, and viral hepatitis risk factors. In patients with hepatocellular injury pattern it is important to consider non-hepatic sources including hyperthyroid, strenuous exercise and muscle injury; this is associated with a disproportionate elevation of AST>ALT. Further testing should be done in a stepwise manner (Schreiner 2018). Much of this process can be completed by a general internist prior to specialist referral. In addition, while it is acknowledged that depression can manifest as somatic complaints (Tylee 2005), the opposite is also true: depression can lead to underreporting of symptoms. For example, depression is associated with delayed care-seeking in both acute myocardial infarction and decompensated heart failure (Johansson 2011, Bundle 2006). Despite months of symptomatic weakness, had this patient not warranted LFTs for preventative care her polymyositis would likely have gone unrecognized until later in the disease process.

CONCLUSION: 1. Workup of abnormal LFTs should be done in a stepwise fashion based on history and injury pattern; differential should include both hepatic and non-hepatic sources. 2. Patients with depression require close attention to history and follow-up as they may delay careseeking despite new symptoms.

\section{KHAT GOT YOUR LIVER? A CASE OF KHAT-INDUCED LIVER INJURY}

Amanda Pechman; Jennifer P. Weintraub. Icahn School of Medicine at Mount Sinai, New York, NY. (Control ID \#3163629)

LEARNING OBJECTIVE \#1: Identify khat use as a risk factor for liver disease
LEARNING OBJECTIVE \#2: Apply khat-use screening questions to patients from countries where khat use is prevalent

CASE: A 31-year-old male with a history of cirrhosis presented to the emergency room with the chief complaint of altered mental status. He arrived with a note from his PCP stating a history of cirrhosis and concern for hepatic encephalopathy. On arrival to the emergency room the patient was reportedly confused and drowsy, without asterixis. His initial labs were normal except for a transaminitis, elevated ammonia, and negative urine toxicology. Initial imaging showed a normal head CT. This patient's only past medical history was cirrhosis of unknown etiology; as per his PCP he had a CT and MRI showing cirrhosis and an endoscopy showing small esophageal varices, and he had an upcoming appointment with hepatology for further workup. His social history was significant for tobacco use and frequent chewing of khat leaves, a stimulant native to East Africa and the Arabian Peninsula that is a common social practice in his home country of Yemen. He was given one dose of lactulose with improvement in his mental status and by the time he was admitted to the medicine service he was at his baseline. He was found to have negative hepatitis serologies, a negative alcohol level, as well as normal ceruloplasmin and iron studies. He was discharged to home with outpatient liver follow up. The patient saw a hepatologist as an outpatient the following month and underwent a transjugular liver biopsy that demonstrated chronic hepatitis, cirrhosis, and mild steatosis. The cause of his liver disease was deemed likely secondary to his khat use, and he was recommended to stop chewing khat to prevent further liver injury.

IMPACT/DISCUSSION: Khat-induced liver disease is a rare but important side effect of this substance. Though the mechanism of hepatotoxicity is unknown, there have been case reports of khat-induced liver damage. Khat use is a common social practice with an estimated 5-10 million users worldwide, particularly in the so-called khat belt countries of Ethiopia, Somalia, and Yemen. Given that there are over 300,000 immigrants from these nations in the U.S., it is likely that providers will encounter patients who use this substance and should thus screen for its use and recognize associated health risks. Risks of chewing khat include not only liver disease but also hypertension, tachycardia, stroke, decreased sperm count, and psychiatric disease. This case underscores the importance of taking a detailed social history that is both comprehensive and culturally sensitive to identify practices that can be harmful, and shows the importance of providing culturally competent care.

CONCLUSION: Chewing khat, a common social practice in Africa and the Middle East, can cause liver damage It is important to screen for khat use in patients from countries where this practice is common

\section{TB OR NOT TB: A CASE OF PLEURAL TB}

Nikita Fernandes; Lakshmi Priyanka Mahali; Kristin A. Swedish. Montefiore Medical Center, Bronx, NY. (Control ID \#3185875)

LEARNING OBJECTIVE \#1: Diagnose pleural tuberculosis (TB). LEARNING OBJECTIVE \#2: Recognize the importance of considering TB in the differential, even when pleural fluid may not reveal a typical presentation.

CASE: A 31 year-old woman from Honduras presented with moderate right sided pleuritic chest pain, aggravated by deep breathing for two weeks. She did not have cough, sputum production, or dyspnea. Patient reported recent travel to Honduras. Six months prior, she had a positive PPD. Chest X-Ray (CXR) at that time was normal; she did not receive any treatment. On examination, she had diminished breath sounds and dullness to percussion of the right lung field. CXR revealed moderate right pleural effusion and some sub-centimeter pulmonary nodules. A thoracocentesis revealed exudative effusion, lymphocyte predominant, and elevated Adenosine Deaminase (ADA) enzyme level; fluid cultures and cytology were negative. Patient underwent robotic video-assisted thoracic surgery (RVATS) with evacuation of the effusion and pleural biopsy. The pleural biopsy revealed multiple non-necrotizing granulomas. The acid fast bacilli stain was negative. The tissue cultures grew Mycobacterium TB. She was treated with 
Isoniazid, Rifampin, Pyrazinamide, and Ethambutol. Patient improved clinically and follows with pulmonology on outpatient basis.

IMPACT/DISCUSSION: Pleural TB is the most common extra pulmonary form of TB in adults, particularly in countries where the prevalence of the disease is high or moderate. However, pleural TB is under-reported, as negative pleural fluid mycobacterial cultures contribute to an underestimated incidence of disease. Although pulmonary TB can be a manifestation of primary infection, it is most commonly associated with reactivation of pre-existing foci. Tuberculous pleuritis develops from direct infection of pleura and lymph nodes as a result of a ruptured subpleural caseous focus in the lung. It is generally accepted that pleural TB results from a late hypersensitivity reaction to Mycobacterium Tuberculosis antigens. In patients with disseminated TB, the yield of pleural biopsy (77\%) is higher than the yield of AFB staining (43\%), PCR $(70 \%)$ or culture $(72 \%)$. In an instance of negative culture, such as in our case, biopsy can help increase the yield and decrease the time of diagnosis. Pleural biopsy can aid in diagnosis and treatment of TB.

CONCLUSION: This case illustrates the diagnostic difficulty of disseminated TB with atypical organ involvement. Culture is the gold standard for diagnosing TB, but is a long process and as $23 \%$ of TB in the United States is culture negative, the diagnosis sometimes relies on ruling-out other etiologies in the differential diagnosis and histologic examination.

\section{THE FORGOTTEN CONSEQUENCES OF THE OPIOID EPI- DEMIC: ADRENAL INSUFFICIENCY PRESENTING WITH HYPERCALCEMIA}

Christina Ryu. CPMC, San Francisco, CA. (Control ID \#3178626)

LEARNING OBJECTIVE \#1: Chronic opioid use is often overlooked as a cause of secondary adrenal insufficiency, but physicians should be aware of this increasingly common cause, especially with the current opioid epidemic.

CASE: A 54-year-old woman with history of opioid abuse presented with generalized weakness, anorexia and depressed mood. Labs showed a corrected calcium of $13.7 \mathrm{mg} / \mathrm{dL}$. Work-up revealed non-parathyroid hormone (PTH) mediated hypercalcemia with PTH $17 \mathrm{pg} / \mathrm{ml}$ in setting of non-elevated PTH related peptide (PTHrp), low 25-hydroxyvitamin D, low 1,25 dihydroxyvitamin $\mathrm{D}$, normal free light chain ratio, low vitamin $\mathrm{A}$ and normal thyroid function. Calcium normalized and symptoms improved with IV fluids, calcitonin, furosemide and zoledronic acid. Etiology of hypercalcemia remained unclear at discharge. She represented to the ED 1 day later with hypoglycemia (glucose $13 \mathrm{mg} / \mathrm{dL}$ ) and hypotension. Work-up revealed undetectable morning cortisol and ACTH levels, suggestive of secondary adrenal insufficiency. Unfortunately, cosyntropin stimulation test was not performed to confirm diagnosis prior to starting glucocorticoids. Pituitary MRI revealed a microadenoma measuring $5 \times 3 \times 3 \mathrm{~mm}$, but all other pituitary hormones were intact. Based on history and work-up, it was determined chronic opioid abuse was the most likely cause of her secondary adrenal insufficiency. The patient was started on steroids with immediate improvement.

IMPACT/DISCUSSION: Studies have shown that chronic opioid use affects cortisol secretion likely due to interactions in the hypothalamus-pituitary-adrenal (HPA) axis. Opioids affect the HPA axis at multiple levels, but the primary site of action is thought to be the hypothalamus as seen in a study of morphine's effect on rat hypothalamic fragments leading to the suppression of CRF-41 release. In studies of healthy volunteers, naloxone, an opioid antagonist, led to an increase in cortisol and CRH levels, suggesting that opioids exert tonic inhibition on the HPA axis. One study showed that acute administration of oral morphine led to suppression of serum basal cortisol and plasma ACTH. Further, though rare, adrenal insufficiency can cause hypercalcemia. The pathophysiology is unclear but appears to be related to increased bone resorption, volume contraction from adrenal insufficiency and increased proximal tubular calcium reabsorption in the setting of decreased kidney function.
CONCLUSION: This case highlights two points: adrenal insufficiency should be considered as a cause of unexplained non-PTH mediated hypercalcemia and the significant impact of chronic opioid use on the HPA axis. Given the current opioid epidemic, physicians need to recognize this commonly overlooked clinical entity.

\section{VITAMIN B12 DEFICIENCY PRESENTING AS HEMOLYTIC ANEMIA}

Suheil Albert Atallah-Yunes; Fatima Ali; Philip Brandt. UMASSBaystate, Springfield, MA. (Control ID \#3185780)

LEARNING OBJECTIVE \#1: Recognize hemolysis as a rare complication of Vitamin B12 deficiency

CASE: A 34-year-old male with no significant past medical history presented to the hospital with one month of progressive weakness and fatigue. He reported $11 \mathrm{~kg}$ weight loss in the previous 4 weeks. Physical exam did not find any palpable lymphadenopathy, jaundice or organomegaly. Blood work demonstrated a white cell count of $5.5 \mathrm{k} / \mathrm{ul}$, hemoglobin of $8.8 \mathrm{gm} / \mathrm{dl}$, platelet count of $190 \mathrm{k} / \mathrm{ul}$ with an MCV of $93 \mathrm{fl}$. CBC differential was significant for $2 \%$ myeloblasts. Hemolysis panel revealed an LDH of 4896 $\mathrm{U} / \mathrm{L}$, haptoglobin level less than 10 , total bilirubin of $2.5 \mathrm{mg} / \mathrm{dl}$ and indirect bilirubin of $2.1 \mathrm{mg} / \mathrm{dl}$. A Coomb's test was negative. Peripheral blood smear raised the concern for a bone marrow problem after dysplastic red blood cells with macroovalocytes, teardrop shaped cells and red blood cell precursors were observed. CT of the abdomen and pelvis did not demonstrate any splenomegaly or any evidence of a neoplastic process. Bone marrow biopsy findings were significant for megaloblastic changes and erythroid dysplasia. Cytogenetic analysis failed to highlight any abnormalities and hence the suspicion for a malignant process decreased. The negative Coomb's test and the low suspicion for microangiopathic hemolytic anemia in addition to the severely dysplastic myeloid cells raised the concern for nutritional deficiencies causing intramedullary hemolysis and bone marrow dysplasia. Evaluation then found a significantly low Vitamin B12 and high methylmalonic acid levels; $<150 \mathrm{pg} / \mathrm{ml}$ and $26 \mathrm{nmol} / \mathrm{ml}$ respectively. AntiParietal antibodies were elevated at 40. Atrophic gastritis was encountered on an EGD and gastric biopsy results demonstrated evidence of chronic gastritis all consistent with pernicious anemia, which eventually lead to the patient's intramedullary hemolysis.

IMPACT/DISCUSSION: Hemolysis is a very rare complication of Vitamin B12 Deficiency. Most of the cases of hemolysis due Vitamin B12 deficiency occur in patients with pernicious anemia. The pathophysiology is still unclear. The most commonly proposed mechanism for intramedullary and intravascular hemolysis caused by Vitamin B12 deficiency is ineffective erythropoiesis. Other proposed theories include elevated homocysteine levels in patients having vitamin B12 deficiency which could cause endotoxic injury and eventually microangiopathic hemolysis. Low Vitamin B12 levels indicate vitamin B12 deficiency however methylmalonic acid and homocysteine levels should be checked if suspicion is still high in the setting of normal Vitamin B12 level. Vitamin B12 replacement is the treatment for hemolysis related to Vitamin B12 deficiency.

CONCLUSION: Hemolysis is a very rare complication of Vitamin B12 Deficiency and should be suspected in patients presenting with a Coomb's negative hemolytic anemia and signs of Vitamin B12 deficiency under peripheral blood and bone marrow review. Given its rarity, physicians should have a high clinical suspicion for this rare complication.

\section{MM LUNG NODULE DEVELOPED INTO METASTATIC LUNG ADENOCARCINOMA IN 2 YEARS}

Mukesh Kumar ${ }^{1}$; Aiman Zafar ${ }^{2}$; Charles Fishman ${ }^{3} .{ }^{1}$ Montefiore Medical Center, Bronx, Pakistan; ${ }^{2}$ Montefiore Medical center, Yonkers, NY; ${ }^{3}$ Montefiore Medical Center, Bronx, NY. (Control ID \#3184227)

LEARNING OBJECTIVE \#1: To recognize the malignant potential of lung nodules even in young patients 
LEARNING OBJECTIVE \#2: To review criteria for early intervention for lung nodules in lower risk populations

CASE: A 30 year old man who presented appendicitis underwent abdominal CT scan and was incidentally found to have $12 \mathrm{~mm}$ irregularly bordered lung nodule. No prior chest imaging was available. Patient was discharged after appendectomy and was advised to have follow up imaging, but was thereafter lost to follow up. Two years later patient presented with productive cough and scant hemoptysis. A CT scan of the chest then revealed a $6.7 \mathrm{~cm}$ lung mass. Carcinoid tumor was suspected but octreotide scan was negative. A CT guided lung biopsy revealed adenocarcinoma positive for EGFR mutation. Three weeks later patient was admitted with headache and right arm weakness. Magnetic resonance imaging (MRI) of brain revealed left sided metastases in parietal and occipital lobe, confirming stage IV disease

IMPACT/DISCUSSION: Risk of a lung nodule being malignant depends on size, consistency and patient risk factors. The risk of malignancy in a nodule of $5 \mathrm{~mm}$ is $<1 \%, 5-9 \mathrm{~mm}$ is $2-6 \%, 8-20 \mathrm{~mm}$ is $18 \%$ and $>20 \mathrm{~mm}$ is $50 \%$. Solid nodules are at greater risk than semi solid and ground glass nodules. Significant risk factors include cigarette smoking, age $>35$ years, female gender, exposure to radiation or environmental toxins, pulmonary fibrosis, alcohol consumption and positive family history. According to Fleischner society guidelines a lung nodule $>8 \mathrm{~mm}$ should be either resected for biopsy in patients with risk factors for lung cancer, or followed up with CT chest in 3 months in low risk patients. In patients under 35 years old with no risk factors, the chance of a lung nodule proving to be malignant is $<3 \%$. It has also been noted that patients with nodules between $8-30 \mathrm{~mm}$ and concern of poor follow up (due to socioeconomic status, psychological issues, young age) should undergo either non-surgical biopsy or video assisted thoracoscopic surgery with wedge resection. Lung adenocarcinoma commonly presents as a pulmonary nodule. 5 year survival rate of stage IV lung adenocarcinoma is $<10 \%$ while for stage $\mathrm{I}$ it is $60-80 \%$. The patient in this case presented 2 years after the finding of an incidental nodule with cancer that had progressed to stage IV. We suggest that timely intervention with biopsy of the nodule on first presentation could have altered this course.

CONCLUSION: Guidelines should be used in the proper clinical context as a tool to help in patient management as exceptions always exist. In any patient, including young patients with no risk factors for lung cancer presenting with nodules $>8 \mathrm{~mm}$ in size, there should be significant clinical suspicion of malignancy. Follow up and tissue diagnosis are essential.

\section{A 'LOUSY' ANEMIA: SEVERE ANEMIA SECONDARY TO LICE INFESTATION}

Adina Wise; Hailey Gupta; Saad Khan; Seema Quraishi; Erica Grabscheid. Mount Sinai Beth Israel, Icahn School of Medicine, New York, NY. (Control ID \#3160816)

LEARNING OBJECTIVE \#1: Recognize chronic pediculosis corporis infestation as a potential cause of severe anemia.

CASE: A 26 year-old undomiciled man with an unknown past medical or psychiatric history presented with fatigue. Review of systems revealed no chest pain, shortness of breath, hemoptysis, palpitations, loss of consciousness, abdominal pain, melena, hematochezia or hematuria. Vital signs were all within normal limits (T: 98.7 F, HR: 73, RR: 19, BP: 108/72). Physical exam revealed overall poor hygiene, including an extensive infestation of pediculosis corporis (body lice). Patient's lice infestation was severe for at least several months according to documentation from prior ED visits. The patient displayed a disorganized thought process consistent with chronic mental illness. All other physical exam findings were within normal limits, including guaiac negative stool testing. Initial labs revealed hemoglobin of $2.9 \mathrm{~g} / \mathrm{dL}$, hematocrit $10.1 \%$, mean corpuscular volume $58 \mathrm{fL}$, RDW $19 \%$, platelet count $474 \mathrm{~K} / \mathrm{uL}$. Further workup for severe anemia revealed a ferritin of $6 \mathrm{ng} / \mathrm{mL}$, iron $6 \mathrm{ug} / \mathrm{dL}$, TIBC $280 \mathrm{ug} / \mathrm{dL}$, transferrin saturation $2.14 \%$, reticulocyte count 2\%, LDH $223 \mathrm{U} / \mathrm{L}$, haptoglobin $55 \mathrm{mg} / \mathrm{dL}, \mathrm{B} 12$ $423 \mathrm{pg} / \mathrm{mL}$ and folate $10.99 \mathrm{ng} / \mathrm{ml}$. Peripheral blood smear, Coombs test, bilirubin, parvo b19, EBV, CMV, lead level, hemoglobin electrophoresis and urinalysis were all normal. Anti-transglutaminase antibody was 11 $\mathrm{U} / \mathrm{mL}$ (nl 0-5). Upper and lower gastrointestinal endoscopy were offered, but the patient declined. Three permethrin treatments and a total body shaving were required to adequately treat the lice infestation. Patient received 5 days of intravenous iron sucrose followed by daily oral ferrous sulfate, with significant and rapid improvement in his Hgb to $10.4 \mathrm{~g} / \mathrm{dL}$.

IMPACT/DISCUSSION: Body lice infestations are particularly common among homeless and chronically mentally ill populations, and may go untreated for years while feeding off their host's blood. Thus, heavily infected individuals may suffer from clinically significant blood loss. A limited number of previous case reports have documented a possible causal relationship between severe anemia and chronic lice infestation in animals, as well as in human patients without access to adequate hygiene. This undomiciled patient presented with severe iron deficiency anemia (IDA) and chronic pediculosis corporis. A gastrointestinal bleed was unlikely given the negative fecal occult blood test and clinical presentation. Lab studies ruled out hemolytic, infectious, nutritional and hereditary etiologies (although anti-transglutaminase antibody was weakly positive, patient denied celiac disease symptoms while consuming a gluten-containing diet). This patient's IDA may, therefore, be attributable to the chronic pediculosis corporis infestation.

CONCLUSION: As body lice feed on human blood, long-term and heavy infestations could lead to severe anemia. The differential diagnosis for severe IDA should include blood loss secondary to chronic lice infestation in select patients.

\section{A 16 -YEAR DELAYED PRESENTATION OF ANTHRACYCLINE-INDUCED CARDIOMYOPATHY}

Ansh Johri ${ }^{1}$; Gloria Hwang ${ }^{1}$; Robert Wu ${ }^{1}$; Sally $\mathrm{Ng}^{1}$; Nicholas Duca ${ }^{2}$. ${ }^{\mathrm{T}}$ Pennsylvania State University, Hershey, PA; ${ }^{2}$ Penn State Health Milton S. Hershey Medical Center, Hershey, PA. (Control ID \#3146788)

LEARNING OBJECTIVE \#1: Describe the typical time courses and risk factors for anthracycline-mediated cardiotoxicity.

LEARNING OBJECTIVE \#2: Recognize the importance of long-term follow up of patients previously treated with anthracycline.

CASE: A 57-year-old female with breast cancer in remission, status post lumpectomy and 4 rounds of chemotherapy with doxorubicin, cyclophosphamide, paclitaxel, and a 5580 cGy dose adjuvant radiation therapy completed 16 years ago, presented with 1 week of severe exertional dyspnea. Vital signs were unremarkable, and physical exam revealed diffuse bilateral coarse crackles and jugular venous distension. Serum BNP was $7600 \mathrm{pg} / \mathrm{mL}$. Chest X-ray showed bilateral pulmonary interstitial edema, and transthoracic echocardiogram confirmed congestive heart failure $(\mathrm{CHF})$ with ejection fraction of 20-25\%, a dilated left atrium and left ventricle, and diffuse hypokinesis. Further ischemic workup was deferred in the absence of CHF risk factors, such as hyperlipidemia, hypertension, smoking history, and family history of cardiovascular disease. After 4 days of diuresis with furosemide $40 \mathrm{mg}$ b.i.d., the patient ambulated without dyspnea and was discharged on furosemide, lisinopril, and metoprolol for chronic CHF management.

IMPACT/DISCUSSION: Doxorubicin, an anthracycline, is commonly used to treat breast cancer, but it can also cause dilated cardiomyopathy. Cardiotoxicity typically occurs within 3 days of anthracycline therapy, but studies have shown cardiotoxicity up to 10 years later. A case report presented a 57-year-old female who developed diffuse cardiovascular dilation and CHF 17 years after doxorubicin therapy. The patient several features with our patient, such as age, gender, ejection fraction, cardiac morphology, and lack of cardiovascular risk factors. A major risk factor for anthracycline cardiotoxicity is a higher cumulative dose: one prospective study of 630 subjects found a greater incidence of CHF in patients when the cumulative dose exceeded $400 \mathrm{mg} / \mathrm{m}^{2}$. Concurrent cyclophosphamide chemotherapy, adjuvant radiation therapy, and female gender 
also increase this risk. This patient's gender, concurrent cyclophosphamide and radiation therapy, and multiple doxorubicin doses put her at greater risk for cardiotoxicity, resulting in $\mathrm{CHF}$ without traditional $\mathrm{CHF}$ risk factors. In patients treated with anthracycline, delayed-onset cardiotoxicity must be considered when the etiology of their CHF is not apparent. Patients with the previously mentioned risk factors for cardiotoxicity should therefore undergo routine echocardiographic assessment. There is controversy regarding testing frequency but since cardiotoxicity incidence is typically within 5 years post-treatment, it is reasonable to follow up yearly for 5 years and then every 2 years for the next 10 years. This way, cardiotoxicity can be identified early for these patients, reducing their risk of developing CHF.

CONCLUSION: -Anthracycline-mediated cardiotoxicity can have a delayed presentation. -Long-term follow up for high-risk patients can prevent CHF.

\section{A 65-YEAR-OLD WOMAN WITH NEW PANCYTOPENIA}

Tara M. Skorupa; Molly Brett. Beth Israel Deaconess Medical Center, Boston, MA. (Control ID \#3183154)

LEARNING OBJECTIVE \#1: Distinguish severe B12 deficiency from potential mimickers causing similar hematologic abnormalities, including acute leukemia and hemolytic anemia.

CASE: A 65-year-old otherwise healthy woman presented to her primary care physician with three 3 months of worsening dyspnea on exertion, fatigue, and gait instability, with 1 week of tingling in her fingertips. She endorsed 20-lb weight loss which she attributed to fatigue and anorexia. She denied recent travel or dietary changes and was not vegetarian. She denied smoking or illicit drug use and drank wine rarely. She was not taking any medications or supplements. Exam was unremarkable. Labs showed new pancytopenia with WBC 3.0 (ANC 1700), hemoglobin 7.2 with MCV 118, and platelets 134. All were normal when last checked 5 years ago. She was referred to the emergency room and admitted to the medicine service. Further workup revealed B12 $<150$ and elevated ferritin. Reticulocyte count showed hypoproliferation. Haptoglobin $<10$ and elevated LDH and indirect bilirubin suggested hemolytic anemia. Fibrinogen and INR were normal, and Coomb's test was negative. CT abdomen showed mild splenomegaly. Anti-intrinsic factor antibody was positive, consistent with pernicious anemia. She was started on cyanocobalamin injections, and her CBC normalized within one month. Her initial presenting symptoms of dyspnea, fatigue, and gait instability resolved as well.

IMPACT/DISCUSSION: This vignette describes a patient with exertional dyspnea, fatigue, and gait instability who was found to have new pancytopenia. The differential for acquired pancytopenia is broad and can be divided into disorders of production (related to marrow infiltration or failure), consumption, and sequestration ${ }^{1}$. Given the lifethreatening nature of several items on this differential, a finding of pancytopenia can lead to an invasive workup. In pancytopenic patients found to be B12 deficient, unnecessary diagnostic testing can often be avoided by treating the B12 deficiency. In our patient, macrocytosis with MCV 118 provided a major clue to her diagnosis. Vitamin B12 is a key cofactor in DNA synthesis, so B12 deficiency can lead to ineffective erythropoiesis and large, immature red blood cells, typically $>115 \mathrm{fL}^{1,2}$. In severe cases of B12 deficiency, all blood counts in the periphery can be low; paradoxically, the bone marrow is hypercellular ${ }^{2}$. Ineffective erythropoiesis can result in intramedullary hemolysis and early destruction of immature red cells and leads to a low reticulocyte count ${ }^{1}$. After initiation of treatment, patients with hematologic abnormalities typically show improvement in blood counts within 1-2 weeks, and complete resolution within 8 weeks ${ }^{1}$.

CONCLUSION: Severe B12 deficiency can cause pancytopenia and hemolytic anemia and can mimic acute leukemia and microangiopathic hemolytic anemia. For patients with clear B12 deficiency, macrocytic anemia, and mild leukopenia and thrombocytopenia, a trial of B12 supplementation is warranted before pursuing more invasive diagnostic testing.

\section{A CASE OF A PATIENT WITH EXTREMELY ELEVATED FER- RITIN AND SEPTIC SHOCK}

Brianna Knoll ${ }^{1}$; Pamela Boodram ${ }^{1}$; Taiye Odedosu ${ }^{2} .{ }^{1}$ New York University School of Medicine, New York, NY; ${ }^{2}$ NYU Medical Center, New York, NY. (Control ID \#3179286)

LEARNING OBJECTIVE \#1: Recgonizing hemophagocytic lymphohistiocytosis (HLH) in septic patients with ferritin levels greater than 20,000

CASE: A 35-year-old woman with a history of recurrent urinary tract infections (UTIs) complicated by nephrolithiasis presented with four days of fever and a sore throat. She was febrile to 103.1, blood pressure 99/62, and heart rate 100. WBC was 12, Hgb 8.6, creatinine 1.8, AST 304, ALT 92, and HIV, streptococcus, mononucleosis and EBV IgM testing were all negative. Urinalysis was consistent with a UTI, she was admitted, and started on antibiotics. A CT scan of the abdomen revealed a right, staghorn calculi with surrounding, ill-defined lesions in the parenchyma. Urology placed a right nephrostomy tube which drained frank pus. Other work-up revealed an $\mathrm{LDH}$ of 2,502, triglycerides of 474, ferritin of 31,000. An Interleukin 2 Receptor (CD25) soluble test was sent but bone marrow biopsy was deferred given improvement in clinical symptoms and laboratory data. Twelve days into her admission, the patient clinically deteriorated. Lactate was elevated to 5.7, LFTs and LDH began to rise, and ferritin was 24,000 from a nadir of 8,000. Repeat triglycerides were 1,210. She was transferred to the intensive care unit for septic shock, placed on vasopressors and was subsequently intubated. A bone marrow biopsy revealed histiocytosis in a scattered and cluster pattern and hemophagocytosis and hemophagocytic lymphohistiocytosis (HLH) was diagnosed. Treatment with dexamethasone and etoposide was initiated on day fourteen based on the modified HLH 94 protocol. Her CD25 soluble test later came back elevated. She was weaned of vasopressors, extubated and completed chemotherapy inpatient. She was discharged three months after presentation.

IMPACT/DISCUSSION: HLH is a rare, life-threating condition characterized by excessive immune activation that is most common in the pediatric population but can occur at any age. There are pre-disposing genetic defects and/or an immunological trigger such as infection, malignancy or rheumatologic disorder that have been linked to the development of HLH. Most commonly infectious HLH is reported in viral infections commonly EBV - but it is possible the diagnosis is being overlooked in bacterial infections that lead to sepsis. It is easy in sepsis to attribute cytopenia and rising LFTs to end organ damage from the sepsis itself, making it difficult to distinguish from HLH. Infection itself is also commonly associated with high ferritin levels making it even more difficult to distinguish from HLH. Without treatment, patients often only survive a few months and overall mortality is as high as $75 \%$. Appropriate, early treatment have shown remission rates up to $71 \%$.

CONCLUSION: Thus, this case report exemplifies the importance of investigating $\mathrm{HLH}$ as a possible contributor to end-organ damage in septic shock when extremely high ferritins are noted. Mortality is high in patients with HLH which makes timely diagnosis of the utmost importance.

\section{A CASE OF A RARE INFECTION WHICH MIMICS MALIGNANCY}

Belaal Sheikh. Chicago Medical School, McHenry, IL. (Control ID \#3185859)

LEARNING OBJECTIVE \#1: How to recognize clues which distinguish malignancy from a rare infection prior to lobectomy.

CASE: A 69 year old female with known emphysema and COPD on home oxygen presented with a 2 week complaint of progressive dyspnea, dry cough, and fever. She was an active smoker with a history of pulmonary nodules which presented intermittently on imaging. The patient also had a right middle and partial right lower lobe lobectomy 5 years 
ago after biopsy performed on 1 of 2 nodules present at the time raised a suspicion for possible non-small cell lung carcinoma. Multiple attempts at pathology and histology were nonspecific or negative on the remaining pulmonary nodules to confirm malignancy. Since the lobectomy the patient's condition worsened as she has recurrent pneumonia and worsening chronic respiratory failure. During this admission she was admitted with a diagnosis of acute on chronic hypoxic respiratory failure secondary to low lung volume from lobectomy and COPD exacerbation. Sputum cultures during this admission returned positive for Achromobacter xylosoxidans. Diagnosis was confirmed via bronchoscopy and the patient was started on ertapenem. Her condition improved and she was discharged on ertapenem for a total of 6 weeks of intravenous treatment. Follow up outpatient showed improvement in respiratory status and she has not since had pulmonary nodules appear on imaging.

IMPACT/DISCUSSION: Achromobacter xylosoxidans is a pulmonary infection which appears on imaging as speculated lung nodules mimicking malignancy. There have been a few reported cases of an unnecessary lobectomy due to misdiagnosis of Achromobacter xylosoxidans as non-small cell lung cancer. Though cases such as this are rare, it is important to differentiate Achromobacter xylosoxidans from malignancy as both may present with spiculated nodules on imaging. A clue favoring Achromobacter xylosoxidans infection is the intermittent nature of the nodules that come and go on imaging without explanation. This case highlights the importance of ruling out an acute or chronic infection presenting as neoplasia prior to any invasive procedures which may affect quality of life.

CONCLUSION: Achromobacter xylosoxidans is a rare gram negative catalase positive ubiquitous organism typically found in aqueous marine environments. The pathogen has a slow growing prevalence of $2.3 \%$ to $8 \%$ within healthcare facilities causing a prolonged, incapacitating pneumonia with a mortality rate of up to $50 \%$. Classically this organism gravitates to patients with cystic fibrosis due to the salty aqueous environment within their pulmonary parenchyma. It causes pneumonia in immunosuppressed individuals and presents as fleeting, spiculated, pulmonary nodules on imaging commonly mimicking pulmonary carcinoma. Successful identification and treatment of Achromobacter xylosoxidans can decrease morbidity, mortality, and unnecessary interventions for patients.

\section{A CASE OF ACUTE INTERSTITIAL NEPHRITIS RELATED TO SPIRONOLACTONE AND MENTAL HEALTH SUPPORTS IN A TRANSGENDER WOMAN}

Andrew Silapaswan ${ }^{1,2}$; Joseph J. Baker ${ }^{1,2}$. ${ }^{1}$ Beth Israel Deaconess Medical Center, Boston, MA; ${ }^{2}$ Fenway Health, Boston, MA. (Control ID \#3180797)

LEARNING OBJECTIVE \#1: Recognize acute interstitial nephritis (AIN) related to spironolactone

LEARNING OBJECTIVE \#2: Assess the impact of cross-sex hormone therapy (CSHT) interruptions on mental health in transgender patients

CASE: A 21-year-old transgender woman with history of depression presented with several weeks of nausea, vomiting, and poor oral intake. History was notable for CSHT maintained on estradiol and spironolactone since 2016. Review of systems was otherwise negative and physical exam was unremarkable. Initial labs showed acute kidney injury (AKI) with creatinine of 2.66 from baseline of $0.8-1.0$, and peaked at $4.6 \mathrm{mg} / \mathrm{dL} 10$ days later. She was admitted and received several liters of IV fluids, however did not have improvement in renal function. Urine sediment showed significant white cells, mild proteinuria, and white cell casts, although urine culture returned negative. Renal ultrasound was normal. Given the concern for AIN, spironolactone and estradiol were held. She ultimately underwent renal biopsy showing severe AIN, likely related to spironolactone. She completed a 2month steroid taper with recovery in renal function to baseline. She was then re-started on estradiol and her renal function remained stable. While off spironolactone and estradiol, she experienced worsening gender dysphoria requiring increased mental health supports.
IMPACT/DISCUSSION: AKI is a common inpatient presentation and the differential diagnosis in a patient with nausea and vomiting should include pre-renal AKI. However, intrarenal etiologies should be considered when patients do not respond to IV fluids. Diagnostic tools include urinalysis with microscopy, urine albumin to creatinine ratio, renal ultrasound to rule out obstruction, and renal biopsy if indicated. Multi-system adverse effects can occur with spironolactone including gynecomastia, nausea, and vomiting. Spironolactone can also cause dehydration leading to renal insufficiency and AIN. Treatment of AIN includes discontinuing the offending medication and in this case, the risks of resuming spironolactone were thought to outweigh the benefits. Comprehensive care of transgender patients should also focus on mental health and the provision of adequate behavioral health supports. Our patient had worsening gender dysphoria and suicidal ideation during the interruption in CSHT. She was ultimately re-started on estradiol, however providers must be equipped to provide additional mental health supports and appropriate pharmacotherapy if indicated. Our patient benefited from weekly behavioral health visits and ultimately referral for surgical therapy with orchiectomy as part of her gender affirmation.

CONCLUSION: This case illustrates potential adverse effects of spironolactone including renal insufficiency and AIN. Providers should be aware that interruptions in CSHT place transgender patients at higher risk for worsening mental health and should anticipate the need for increased mental health supports.

\section{A CASE OF ACUTE PANCREATITIS SECONDARY TO MILK- ALKALI SYNDROME IN A PATIENT WITH DIGEORGE SYNDROME}

Fareeha A. Khan ${ }^{1}$; Hunter Mwansa ${ }^{1}$; Basel Altaqi ${ }^{2} .{ }^{1}$ St. Vincent Charity Medical Center, Lakewood, OH; ${ }^{2}$ St. Vincent Charity Medical Center, Cleveland, OH. (Control ID \#3167255)

LEARNING OBJECTIVE \#1: Recognize hypercalcemia as a rare, but possible cause of acute pancreatitis.

LEARNING OBJECTIVE \#2: Recognize milk alkali syndrome causing hypercalcemia in patients with DiGeorge Syndrome, supplemented with extrinsic calcium

CASE: A 48-year-old female, with a past medical history of DiGeorge Syndrome and mental retardation was admitted to the Intensive Care Unit with a 2 days history of altered mental status. She had non-bloody, nonbilious vomiting, and non-bloody watery diarrhea in addition to poor appetite and anorexia. She had a remote cholecystectomy and no history of alcohol abuse. Home medications included hydrochlorothiazide, losartan, metformin, and supplementation with calcium acetate daily, calcitriol daily, calcium carbonate/vitamin D3 daily, and cholecalciferol daily. Physical exam was significant for Glasgow Coma Scale of 11, tachycardia, tachypnea and hypotension. Abdomen was distended and tender over the right upper quadrant and epigastric areas. Lab work revealed elevated white blood cell count and acute renal failure. Amylase and lipase were found to be elevated, as was serum calcium to $12.1 \mathrm{mg} / \mathrm{dL}$. Serum IgG4 antibodies were found to be within normal limits. Abdominal CT scan revealed an enlarged pancreas at the level of the head and body with ill-defined fluid and fatty stranding surrounding the pancreas. The patient was initially managed as a case of sepsis, however, later the diagnosis was confirmed and revised to acute severe pancreatitis. The etiology was established to be hypercalcemia due to Milk-Alkali Syndrome and HCTZ. The patient subsequently received hemodialysis due to ischemic acute tubular necrosis.

IMPACT/DISCUSSION: MAS has become the third leading cause of hypercalcemia in hospitalized patients, behind hyperparathyroidism and malignancy. [7] Our patient fulfilled four of the five major criteria for diagnosis of MAS except, metabolic alkalosis. [11] Pathophysiology of MAS includes a cyclic pathway where hypercalcemia is the main driving 
force of the entire cascade. [8] In this situation, differentiation from other causes of hypercalcemia is imperative. Our patient brings an interesting twist to this discussion due to her comorbid condition of DiGeorge Syndrome, deletion of chromosome 22q11.2, which inherently results in PTH deficiency, thus making patients prone to hypocalcemia. Our patient's intake of calcium is at par with the threshold described by Beall et al. of $>4 \mathrm{~g} /$ day. [5] This is the first case report of its kind, presenting pancreatitis due to milk-alkali syndrome in a patient with DiGeorge Syndrome, the picture here however is complicated with metabolic acidosis due to renal failure and lactic acidosis.

CONCLUSION: • Clinicians should consider Milk-Alkali Syndrome as a potential cause of acute pancreatitis and measure should be taken to address it. • Patients with DiGeorge Syndrome are prone to developing hypercalcemia due to continued extrinsic calcium supplementation.

\section{A CASE OF ACUTE PE-RICARDITIS}

Christine Chan ${ }^{1}$; Natalie Kappus ${ }^{2}$; Stefanie Reiff ${ }^{3} .{ }^{1}$ NYU Langone Health, Brooklyn, NY; ${ }^{2}$ NYU Langone Brooklyn, Brooklyn, NY; ${ }^{3}$ NYU Langone Hospital - Brooklyn, Brooklyn, NY. (Control ID \#3147048)

LEARNING OBJECTIVE \#1: Explain how the hemodynamics of pericarditis can produce the clinical manifestations (signs and symptoms) seen in pericarditis.

LEARNING OBJECTIVE \#2: Categorize the progressive electrocardiogram stages consistent with pericarditis.

CASE: A 70 year-old female with hypertension and diabetes presented to the hospital with a one-day history of non-positional, left-sided chest pain associated with shortness of breath and subjective fevers. Physical exam and vitals were unremarkable. Work up included three negative troponins, EKG in normal sinus rhythm (Figure 1), and D-Dimer of $1100 \mathrm{ng} / \mathrm{mL}$. CTA chest revealed a right subsegmental middle lobe filling defect consistent with pulmonary embolism for which patient was initiated on anticoagulation with resolution of chest pain. Approximately 24 hours after initial presentation the patient reported a new chest pain described as sharp, pleuritic, worse with lying flat and improved with sitting forward. Vitals revealed fever to $100.5 \mathrm{~F}$. Repeat EKG demonstrated diffuse ST elevations (Figure 2). Labs showed a negative troponin and ESR and CRP at $63 \mathrm{~mm} / \mathrm{hr}$ and $>190 \mathrm{mg} / \mathrm{L}$, respectively. Echo showed a trace pericardial effusion, normal ejection fraction, and no evidence of right heart strain. The patient was diagnosed with acute pericarditis and initiated on colchicine and NSAIDs with resolution of symptoms. Common causes for pericarditis including viral URI, TB, connective tissue disorders, and malignancy were ruled out with a negative review of symptoms, subsequent laboratory analysis and additional history from the patient's primary care provider. Given the unlikeliness of other etiologies, her acute pericarditis was felt to be secondary to her pulmonary embolism.

IMPACT/DISCUSSION: Between $80-90 \%$ of pericarditis cases are idiopathic or presumed to be of viral etiology. Given the relatively benign course of the majority of causes of pericarditis a definitive work up is often not performed. However, this case highlights an additional "can't miss" cause of pericarditis - pulmonary embolism. Although rare, postpulmonary embolism pericarditis is a well-documented phenomenon associated with elevated ESR, CRP, and low-grade fevers as in this patient's presentation. Proposed mechanisms include increased friction of an enlarged pulmonary artery and right ventricle against the pericardium and an immunologic response, similar to Dressler syndrome.

CONCLUSION: Despite being a known cause of pericarditis, pulmonary embolism is frequently overlooked or completely excluded from clinicians' differentials. Although the exact cause of pericarditis is often unknown, in patients with idiopathic pericarditis with no infectious signs, pulmonary embolism should always be considered based on history and physical exam findings to avoid missing a "can't miss" diagnosis.
A CASE OF AMIODARONE-INDUCED SWEET SYNDROME.

Aditya Singh; Junior Uduman. Henry Ford Hospital, Detroit, MI. (Control ID \#3183653)

LEARNING OBJECTIVE \#1: Recognize and differentiate Sweet syndrome (acute febrile neutrophilic dermatosis) from an infectious process. LEARNING OBJECTIVE \#2: Recognize amiodarone as a potential cause of drug-induced Sweet syndrome.

CASE: Our patient was a 41-year-old Caucasian male, with a past medical history of atrial fibrillation (Afib), non-ischemic cardiomyopathy (Ejection Fraction $9 \%$ ), and diabetes mellitus presented to the hospital with signs and symptoms of decompensated heart failure and skin lesions. $\mathrm{He}$ was febrile and had innumerable tender, pruritic, violaceous, annular plaques with central ulceration which had a contiguous centripetal spread from extremities extending to abdomen and chest over a period of 2 weeks. Careful medication review suggested recent re-initiation of amiodarone for Afib. Additional history revealed similar rashes in the past which were temporally related to amiodarone use. Interestingly each subsequent exposure resulted in increased severity and rapidity of skin lesions. Thought to be infectious in origin the patient had been treated with antibiotics each time, however, the patient identified improvement after stopping the offending drug. Differential diagnosis included amiodarone-induced adverse drug reaction, leukocytoclastic vasculitis, Sweet syndrome, IgA dermatosis, atypical infection or septic thrombi. Autoimmune serology was unyielding and empiric antibiotics failed to improve skin lesions. Confirmatory punch biopsy of the lesions revealed perivascular neutrophilic dermal infiltrate consistent with Sweet syndrome. Due to the severity of the disease and systemic involvement intravenous methylprednisolone was initiated, resulting in rapid clinical remission of symptoms, cutaneous lesions, and leukocytosis.

IMPACT/DISCUSSION: Sweet syndrome is characterized by a constellation of fever, neutrophilia, tender erythematous skin lesions with diffuse perivascular neutrophilic dermal infiltrate. It has been correlated with inflammatory bowel disease, antecedent respiratory/gastrointestinal infection, active malignancy, autoimmune disease, vaccines, and medications. Pathophysiologically hypersensitivity reaction is thought to play a role, given recurrence of the dermatosis with re-exposure of the offending agent and prompt response to corticosteroid therapy, as demonstrated in our patient. Amiodarone has been associated with various dermatological reactions, including photosensitivity, blue/grey hyperpigmentation and rarely vasculitis. The temporal association with amiodarone in our patient highlights amiodarone as a potential cause of drug-induced Sweet syndrome, which to the best of our knowledge has not been previously described in the literature.

CONCLUSION: Sweet syndrome can resemble an infectious process, usually, patients receive antibiotic treatments before glucocorticoid therapy, delaying definitive treatment. Hence clinicians should maintain a high suspicion of drug-induced Sweet syndrome in such patients, in order to improve patient-related outcomes.

\section{A CASE OF CLANDESTINE CANCER: INCREASING AWARE- NESS OF NON-HEMATOLOGIC CANCERS IN PATIENTS WITH ATAXIA TELANGIECTASIA}

Britney N. Bell ${ }^{1}$; Naomi Habib ${ }^{1}$; Mital Patel ${ }^{2} .{ }^{1}$ Creighton University Residency, Phoenix, AZ; ${ }^{2}$ University of Arizona/Dignity Health Cancer Center, Phoenix, AZ. (Control ID \#3186533)

LEARNING OBJECTIVE \#1: To discuss ataxia- telangiectasia and oncologic sequelae to better equip providers in the diagnosis of carcinomas in these patients

LEARNING OBJECTIVE \#2: To discuss the occurence the severity of gastric adenocarcinom in patients with ataxia-telangiectasia

CASE: A 25-year-old female with ataxia-telangiectasia (A-T), diagnosed at age 7, who presented with a 2-month history of progressive nausea and mid- 
abdominal pain, regurgitation of solids and liquids, and associated weight loss of 40lbs. Evaluation at an outside facility one month prior with an esophagogastroduodenoscopy (EGD) showed grade B esophagitis with hypertrophied gastric folds. Biopsies were negative for cancer. On current evaluation, her vital signs and basic laboratory data were normal, but she had some mild epigastric tenderness. Esophagram showed marked gastric slowing. CT scan of the abdomen revealed a diffuse gastric wall thickening. A repeat EGD showed worsening edematous, friable gastric folds without an obvious mass, concerning for gastric outlet obstruction. Biopsy confirmed this to be a poorly differentiated gastric adenocarcinoma. Staging laparoscopy with biopsies confirmed peritoneal metastatic disease. Her baseline performance was poor due to ataxia. Palliative chemotherapy was cautiously started on single agent 5-fluorouracil without severe side-effects and was escalated by adding a platinum agent, oxaliplatin as part of the modified FOLFOX regimen. She has been on this chemotherapy for the last 9 months (19 cycles) tolerating it well and has maintained stable disease on imaging.

IMPACT/DISCUSSION: A-T is an autosomal recessive chromosomal instability disorder characterized cerebellar ataxia, conjunctival telangiectasias, oculomotor apraxia, choreoathetosis, immunodeficiency, and increased risk of malignancy. The defect in DNA damage repair due to mutations in the ATM (ataxia-telangiectasia mutated) gene leads to early onset of nonHodgkin lymphoma and acute lymphoid leukemia. As patients with AT are living longer, there is an increasing, though under-recognized incidence of solid tumors including gastric cancer. In one literature review only 7 known cases of gastric cancer in patients with AT have been reported. Our patient had a diffuse gastric cancer which had metastasized to the peritoneum. Her diagnosis was delayed a few months due to her diffuse gastric involvement and she was metastatic at presentation. A heightened awareness of nonhematologic malignancies in patients with chromosomal instabilities can lead to diagnosis at an earlier, curable stage of cancer. Due to their inherent susceptibility to DNA breaks, these patients remain very sensitive to radiation and chemotherapy with toxic side-effects. Our patient has been cautiously escalated to a dual chemotherapy regimen and is tolerating treatment almost 10 months out from diagnosis.

CONCLUSION: A heightened awareness of non-hematologic malignancies in patients with chromosomal instabilities can lead to diagnosis at an earlier, curable stage of cancer

\section{A CASE OF CUSHING'S DISEASE SUSPECTED FROM THE EXACERBATION OF GLUCOSE INTOLERANCE AND DIAG- NOSED BY SURGERY.}

Toyohiro Miyata ${ }^{1}$; AOI YOGO ${ }^{1}$; Masaki Maeda ${ }^{2}$; Keisuke Kadooka ${ }^{2}$; Masahiro Masuzawa ${ }^{2}$. ${ }^{1}$ Kameda Medical Center, Kamogawa, Japan; ${ }^{2}$ Kameda Medical Center, Chiba, Japan. (Control ID \#3186227)

LEARNING OBJECTIVE \#1: Suspect Cushing's disease (CD) in patients with diabetes mellitus (DM) if glucose intolerance rapidly exacerbates.

LEARNING OBJECTIVE \#2: Recognize cases of CD on the basis of normal magnetic resonance imaging (MRI) findings.

CASE: A 50-year-old female with hypertension, hyperlipidemia, and type 2 DM was referred from a neighborhood clinic because of worsening $\mathrm{HbAlc}$ and blood pressure control. Four months before the current admission, she presented with bilateral leg edema, polydipsia, polyuria, and psychiatric symptoms, such as irritability and anxiety. Her HbAlc level increased from $6.5 \%$ to $10.4 \%$ and average blood pressure from 130 / 80 to $200 / 100 \mathrm{mmHg}$. She had no history of alcohol abuse or depression. Other signs and symptoms were suggestive of hypercortisolism, including moon face, buffalo hump, central obesity, wide purplish striae, bruising, and virializing features. Laboratory examinations revealed elevated $24-\mathrm{h}$ urine cortisol, and imcomplete suppression of serum cortisol levels with low-dose (1mg) dexamethasone suppression test. Additionally, the level of plasma adrenocorticotropic hormone was markedly elevated, suggesting CD. Further endocrine tests such as the high-dose dexamethasone suppression test, corticotropin-releasing hormone stimulation test, desmopressin test indicated pituitary lesion. On the other hand, 3-Tesla MRI with gadolinium enhancement indicated no evidence of a pituitary tumor. Finally, we performed bilateral inferior petrosal sinus sampling (BIPSS), which indicated pituitary lesion. Based on the result of BIPSS, endoscopic transsphenoidal surgery was performed despite no evidence of a pituitary tumor following 6 months of metyrapone and glucocorticoid replacement therapy. Microadenoma was found across both sides of the lower portion of the pituitary. Finally, symptoms such as glucose intolerance and bilateral leg edema were improved.

IMPACT/DISCUSSION: General practitioners may miss CD among patients with DM if they do not recognize the signs of hypercortisolism. Moreover, there is no single best approach for diagnosing CD, and MRI with gadolinium contrast enhancement identifies pituitary tumors in less than half of all patients with CD. For patients with suspected CD but no detectable pituitary lesions on MRI, BIPSS is recommended for confirmation. Surgical excision is required for definitive diagnosis as well as first-line treatment.

CONCLUSION: CD should be suspected in patients with sudden exacerbation of glucose intolerance, even if MRI findings are unremarkable.

\section{A CASE OF ERYTHEMA NODOSUM - HOW THE RESIST THE MILLION-DOLLAR WORKUP}

Max Ruge; Jordan Scharping; Michelle Sweet. Rush University Medical Center, Chicago, IL. (Control ID \#3184922)

LEARNING OBJECTIVE \#1: Discuss the various etiologies of erythema nodosum.

LEARNING OBJECTIVE \#2: Describe a high value workup for erythema nodosum.

CASE: A 53-year-old female with a history of mild pancytopenia presented to the ED with fatigue, chills, decreased appetite, myalgias, nonproductive cough, and a frontal headache that began 3 days prior. Shortly after symptom onset, she noticed multiple $2-5 \mathrm{~cm}$, erythematous, tender and raised nodules on her lower extremities and one on her left upper arm. In the ED, she was febrile (101.9), and her labs were significant for a CRP/ESR of 130/75, white blood count of 10.7, hemoglobin of 9.4, and platelets of 87. Lumbar puncture demonstrated $13 \mathrm{WBCs}$ (predominantly lymphocytes) with 82 RBCs. On the general medical floor, biopsy confirmed the nodules to be erythema nodosum. Chest CT, ANCA levels, autoimmune panel, complement levels, TSH, respiratory pathogen panel, serum protein electrophoresis, and urine protein electrophoresis had no significant abnormalities. Outpatient workup for pancytopenia had been unrevealing, so bone marrow biopsy was done that showed hypercellular marrow without evidence of malignancy. While her systemic symptoms improved, the nodules remained present and tender. She was discharged 5 days after admission with the diagnosis of idiopathic erythema nodosum. IMPACT/DISCUSSION: Erythema nodosum (EN) typically appears as raised tender nodules in the pretibial area. Most commonly, these lesions are idiopathic (55\%). However, EN can also be secondary to other diseases such as streptococcal pharyngitis (28-48\%), sarcoidosis (11$25 \%$ ), and drugs such as antibiotics or oral contraceptives (3-10\%). Other rarer causes include pregnancy, enteropathies, malignancy, and other infections. Obtaining a complete history is critical and should include recent illnesses, associated symptoms (particularly systemic symptoms, sore throat, or cough), and medication use. A thorough physical exam to evaluate for tonsillopharyngitis, abnormal breath sounds, or ocular abnormalities can help identify the underlying condition and direct testing. Excisional biopsy can confirm the diagnosis. Initial labs and imaging should include a CBC with differential, ESR, CRP, ASO antibodies, throat culture, and chest $\mathrm{x}$-ray. Further labs should be obtained only if the patient's history significantly increases suspicion for a rarer cause. For example, while malignancy is a rare cause of EN, this patient's history of cytopenia warranted a bone marrow biopsy. 
CONCLUSION: The massive differential associated with EN can tempt providers into the over-ordering of tests. However, to ensure high value care, a thorough history and physical exam combined with diagnostic discipline is critical as these nodules are idiopathic in $~ 55 \%$ of cases. We recommend obtaining a $\mathrm{CBC}$ with differential, ESR, CRP, ASO antibodies, throat culture, and chest $\mathrm{x}$-ray in patients with idiopathic EN, and further tests should be obtained only if the patient's history and physical exam significantly increase suspicion for an underlying condition.

\section{A CASE OF HEPATITIS LEADING TO INITIAL DIAGNOSIS OF SYSTEMIC LUPUS ERYTHEMATOUS}

$\underline{\text { Ryan Burkholder }}^{1}$; Patrick B. Harriss ${ }^{3}$; Daniella Lopes ${ }^{4}$; Hrishikesh Samant ${ }^{2}$. ${ }^{1}$ SU Health Sciences Center, Shreveport, LA; ${ }^{2}$ Lousiana State University, Shreveport, LA; ${ }^{3}$ LSU Health Sciences Center - Shreveport, LA, Shreveport, LA; ${ }^{4}$ Willis-Knighton Hospital, Shreveport, LA. (Control ID \#3184816)

LEARNING OBJECTIVE \#1: Distinguish features of lupus hepatitis after appropriate workup has excluded other common causes of transaminitis.

CASE: We present a $56 \mathrm{yr}$ old female with past medical history of Raynauds, MVP, and GERD who presents with a two week history of fatigue, malaise, anorexia, fever and jaundice. Physical exam was unremarkable except for generalized jaundice. Laboratory analysis revealed AST 1286, ALT 1191, total bilirubin 12.7, INR 1.2 , albumin 3.7 and WBC 5.8k. Platelets decreased to $125 \mathrm{k}$ and $\mathrm{WBC}$ decreased to $3.6 \mathrm{k}$ during hospital course. Further ultrasound and $\mathrm{CT}$ of the abdomen showed no obstructive process. Due to suspicious for a mononucleosis type picture, CMV serologies and an EBV viral panel were obtained which showed CMV both positive IgM and IgG, however CMV DNA PCR was negative. EBV serologies were positive for IgG and negative for IgM. Acute hepatitis panel, HIV, and anti liver-kidney microsomal screen, and anti Smith/RNP, anti-smooth muscle antibody were negative; However both ANA and dsDNA were positive with positive cytoplasmic reticular pattern observed which may be associated with antimitochondrial antibodies. Complements were mildly decreased. Since both AST and ALT remained elevated with no clear etiology, a liver biopsy was performed which showed predominantly lymphocytic inflammatory infiltrate with parenchymal areas demonstrating confluent hepatocellular necrosis consistent with lupus hepatitis. An immunohistochemical stain for cytomegalovirus was negative. Patient was treated as lupus and subsequently discharged home on an oral steroid regiment with rheumatology follow-up. Liver enzymes several weeks later were as follows: AST 26, ALT 16 and total bilirubin 0.8 .

IMPACT/DISCUSSION: Systemic lupus erythematosus (SLE) is an autoimmune disorder infecting multiple organs. Severe hepatic involvement is rare in patients with SLE, although $25-50 \%$ of patients will experience some form of transaminitis. In many cases, elevated transaminases are due to inciting factors such as hepatotoxic drugs, viral hepatitis or fatty liver disease. Viral infections can be triggering events in autoimmune disorders and CMV may have been an inciting factor that prompted acute liver injury in our patient.

CONCLUSION: Due to frequent multifaceted manifestations of liver diseases in SLE, an assessment of immunological, serological and virological markers should be performed. Autoimmune hepatitis (AIH) should always be considered in a patient with multiple positive autoantibody markers since it has a much more severe disease progression. Testing for AMA, ASMA, anti-liver kidney antibodies, and soluble liver antigen may be helpful in differentiating AIH. Finally, histopathology is the key method of diagnosis, and histopathological findings led to diagnosis of SLE in our patient.
A CASE OF HPV RELATED OROPHARYNGEAL SCC IN THE ABSENCE OF TRADITIONAL RISK FACTORS: A RISING CLINICAL ENTITY

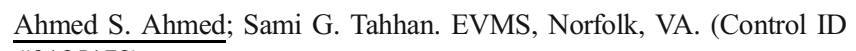
\#3185178)

LEARNING OBJECTIVE \#1: HPV-related oropharyngeal squamous cell carcinoma (OPSCC) represents a growing etiologically distinct subset of head and neck cancers with unique epidemiological, clinical, and prognostic characteristics. We report an interesting case of HPV related OPSCC in the absence of other identifiable risk factors.

CASE: A 49-year-old Caucasian male with no prior history of smoking or alcohol abuse presented in 2011 with complaints of asymptomatic left-sided neck swelling. Neck CT revealed a malignant appearing complex mass in the left neck, posterior to the submandibular gland. FNA biopsies revealed proteinaceous debris and were negative for malignancy. The patient was scheduled for surgical excision but was lost to follow up. In 2017, the patient presented again, now with complaints of a persistent left-sided neck mass. Examination revealed a firm lesion measuring approximately $3 \mathrm{x}$ $4 \mathrm{~cm}$ at the anterior border of his left SCM. Reimaging demonstrated a left palatine tonsillar mass with multiple multifocal ipsilateral cervical malignant appearing adenopathy concerning for metastatic malignancy from a palatine tonsil mucosal primary tumor. FNA biopsy was positive on immunohistochemistry for p16 HPV mediated OPSCC. The patient was treated with chemoradiation with a good clinical response.

IMPACT/DISCUSSION: Despite the decrease in tobacco use, the incidence of oropharyngeal cancer initially remained constant and then began to rise. Public health sources describe it as an upcoming epidemic. HPV related OPSCC seems to account for this incidence trend with one study suggesting that the annual number of HPV-associated oropharyngeal cancers in the United States will overtake the incidence of invasive cervical cancer cases by 2020. As in cervical cancer, oral HPV infection appears to be a sexually-acquired disease likely through oral sex thus reflecting societal changes in sexual behavior that have occurred over time in the developed world.

CONCLUSION: HPV infection, and in particular type 16 (HPV-16), is now recognized as a significant player in the onset of OPSCC. Diagnosis is made through Immunohistochemistry for p16 which is overexpressed and highly sensitive for HPV associated OPSCC. The p16 protein functions as a tumor suppressor preventing phosphorylation of the Retinoblastoma protein $(\mathrm{Rb})$ and affecting cell growth. Compared to non-HPV OPSCC, HPV associated tumors predominantly arise in the tonsillar region or the base of the tongue, it's more likely to present with an early-stage and usually have a better prognosis. The age of presentation tends to be younger peaking around 30 and 55 years with less exposure to the traditional risk factors of tobacco and alcohol. HPV positivity is less frequent in African Americans than in Caucasians with a threefold higher incidence in males than females. Treatment of HPV OPSCC includes a multimodality approach with chemotherapy, radiation therapy, and/or surgery with significantly better prognosis and five-year overall survival compared to HPV free OPSCC.

\section{A CASE OF KRATOM INDUCED CHOLESTASIS}

Randeep Kaur; Charles Siedlecki; Syed-Mohammed Jafri. Henry Ford Hospital, Detroit, MI. (Control ID \#3185390)

LEARNING OBJECTIVE \#1: Recognize that there are many uncommon and likely unrecognized drugs including prescription, over the counter and herbal remedies that can cause cholestasis

CASE: The patient is a 42 year old female with a history of polio, with no residual deficits, who presented with generalized symptoms of subjective fever, fatigue, nausea and poor appetite. She was being worked up in an outpatient setting however when her urine turned dark orange, she decided to present to the hospital. When she initially presented, she had elevated 
transaminases; alanine aminotransferase (ALT) 371, aspartate aminotransferase (AST) 171. Her ALT increased to 606 on recheck. Other labs included total bilirubin 3.3, alkaline phosphatase 298 and INR 0.97. Ultrasound of the abdomen showed a thickened gallbladder wall without murphy's sign and a normal evaluation of the liver. On initial questioning, she denied a previous history of liver disease, alcohol use, or tylenol use. Autoimmune liver panel, hepatitis and HIV screen was negative. Epstein-Barr virus and cytomegalovirus was negative. Alpha-1-antitrypsin, iron, ceruloplasmin were within normal limits. Upon further questioning of home medications, she reported kratom use for treatment of her chronic pain secondary to her past history of polio. Her duration of use of kratom was 4 months with her last use 4 weeks prior to presentation. She was diagnosed with herbal induced cholestasis by kratom. Her jaundice improved, liver enzymes trended down and she was discharged. Her one month outpatient follow up revealed normal liver enzymes with no further reported kratom use.

IMPACT/DISCUSSION: The herbal remedy kratom has been long used in traditional medicine most commonly for depression, anxiety and pain since the nineteenth century, however its impact on the liver is not well known. The mechanism by which kratom induced liver injury in this patient is unknown. The pattern of liver injury was mixed between hepatocellular and cholestatic suggesting that there might be multiple mechanisms of injury. Injury can present with generalized symptoms which can often times be mistaken for other pathologies which is why a thorough history is required to further understand and discontinue offending agents such as kratom. Although kratom was helping to alleviate pain in this patient, discontinuation was necessary to allow complete resolution of liver injury.

CONCLUSION: This case represented short term use of kratom which induced liver injury with subsequent resolution upon discontinuation. There are many oral agents that are still not fully understood or well known which cause liver injury. With this lack of knowledge, it remains important for internists to obtain thorough historys of everything that is ingested prior to presentation. With this information, better understanding and insight can be obtained to which agents should be avoided in the general population.

\section{A CASE OF LEVOFLOXACIN INDUCED PSYCHOSIS IN A YOUNG HEALTHY PATIENT}

Michael Muradian; Kaitlin Annunzio; Zachary Kaczmarek; Shaheena Khan; Kavita Naik; Pinky Jha. Medical College of Wisconsin, Milwaukee, WI. (Control ID \#3185210)

LEARNING OBJECTIVE \#1: To recognize levofloxacin as a cause of acute psychosis.

LEARNING OBJECTIVE \#2: Discuss the course and treatment of antibiotic induced psychosis.

CASE: A 23 year old African American female with a history recurrent submandibular abscesses treated with antibiotics presented to the hospital emergency department with anxiety, paranoia, visual hallucinations, and psychosis. She had been recently discharged from the hospital 4 days prior following incision and drainage with prescriptions for levofloxacin and metronidazole. Two days following discharge she developed paranoia and insomnia which progressively became more severe and the following day she developed visual hallucinations. On admission drug and toxicology screens were negative and a head CT showed no signs of intracranial pathologies. Labs showed no evidence of metabolic causes for altered mental status such as electrolyte abnormalities, hypoglycemia, or encephalopathies. The patient had no psychiatric history and her symptoms were not consistent with a primary psychiatric disorder. Given the lack of evidence for other causes of psychosis this raised concern it may be due to her antibiotics. The levofloxacin and metronidazole were promptly discontinued and she was then prescribed ceftriaxone. Over the following days additional testing including EEG were negative and no other causes of psychosis could be identified. She gradually improved and 3 days after discontinuing the antibiotics she had returned to baseline mental status.
IMPACT/DISCUSSION: Levofloxacin is in the fluoroquinolone class antibiotics and has activity against a wide range of organisms making it a commonly prescribed antibiotic. This medication is generally well tolerated; gastrointestinal distress is the most common adverse effect reported but the medication has an otherwise favorable side effect profile. Neuropsychiatric side effects are very rare and poorly described in the literature with psychosis only being reported in a few cases. It is believed that this effect may be related to possible inhibition of GABA receptors and some have reported that renal disease may increase the risk for these effects. The time of symptom onset in relation to the initiation of antibiotic treatment is highly variable which makes identifying the cause more challenging. These effects can be severe, but they are reversible within a few days of discontinuation of the drug. We present a case that highlights how otherwise healthy individuals with no significant risk factors or psychiatric history are susceptible. Medical providers should consider these poorly understood effects in any patient receiving levofloxacin who develops altered mental status or psychiatric symptoms.

CONCLUSION: Antibiotics should be considered as a cause of psychosis in patients with new symptoms. Patients with no significant risk factors can develop drug induced psychosis.

\section{A CASE OF LOSARTAN-ASSOCIATED GENERALIZED ERYTHRODERMA WITH FEVER}

Ruth Jobarteh ${ }^{2}$; Emily Peng ${ }^{2}$; Allan C. Gelber ${ }^{1}$; Paul O'Rourke ${ }^{1} .{ }^{1}$ Johns Hopkins University, Baltimore, MD; ${ }^{2}$ Johns Hopkins Bayview Medical Center, Baltimore, MD. (Control ID \#3185819)

LEARNING OBJECTIVE \#1: Recognize clinical features of a delayedtype drug reaction, including erythroderma, fever, transaminitis and eosinophilia (including DRESS syndrome)

LEARNING OBJECTIVE \#2: Manage a new diagnosis of DRESS syndrome

CASE: A 74-year-old disabled woman with multiple sclerosis and prior pulmonary embolism presented with a prominent truncal erythematous rash of 5 days duration. The rash spread to her arms, palms, buttocks and legs, became pruritic and desquamating, sparing only her face. On the day of admission to the Medicine Service, she developed a fever of $102.5^{\circ} \mathrm{F} /$ $39.2^{\circ} \mathrm{C}$ and was hospitalized. Home medications were warfarin, simvastatin, fluoxetine, methenamine, baclofen, tizanidine, furosemide and losartan, the latter begun the prior month. Examination was notable for BP 129/67 mmHg, HR 88 beats/min, Temp $36.7^{\circ} \mathrm{C}$, RR 22 breaths/min and $\mathrm{S} 02$ of $96 \%$ on $2 \mathrm{~L}$ of $\mathrm{O} 2$. There was marked diffuse blanchable erythroderma over the back, chest, scalp, breasts, abdomen, arms and legs. There was no mucositis, conjunctivitis, axillary or supraclavicular lymphadenopathy. Laboratory findings were significant for WBC 22.64 (peak eosinophil level 5\%), alkaline phosphatase 289, AST 299, ALT 230, bilirubin 0.4 and ESR 79. Urine and blood cultures were negative. Viral hepatitis serologies were negative. Upon admission, losartan was discontinued. Skin biopsy revealed focal neutrophilic infiltrate, dyskeratotic keratinocytes, diffuse spongiosis and subtle vacuolar alteration, without eosinophils. Oral prednisone $30 \mathrm{mg}$ thrice daily was commenced. Liver function tests trended down. By the third day of steroid therapy, the rash had substantially improved. She was discharged on a steroid taper with instruction to never resume losartan.

IMPACT/DISCUSSION: Delayed-type drug hypersensitivity reactions range in severity from skin eruptions alone, to the DRESS syndrome, to toxic epidermal necrolysis and can be life-threatening. Symptoms typically manifest 1-3 weeks after exposure to an inciting drug. Rash, fever, leukocytosis, eosinophilia and abnormal liver enzymes are hallmark findings. Skin manifestations in DRESS syndrome include diffuse erythroderma but can have various morphologies. It is critical to consider prescribed medication in the differential diagnosis of fever, skin rash and transaminitis. The next step is to terminate use of the putative culprit drug, consider steroid administration and furnish supportive skin care. Losartan has been linked to 
anaphylaxis, angioedema and to skin rashes. Yet, we identified no reports of losartan inducing overt DRESS. In our patient, once losartan was stopped and systemic corticosteroids were begun, clinical improvement ensued.

CONCLUSION: • Drug reaction, including DRESS syndrome, may result in diffuse erythroderma, fever, eosinophilia and transaminitis. • Cessation of the inciting medication and initiation of corticosteroids are the mainstays of therapy in DRESS.

\section{A CASE OF MEDICATION-INDUCED HYPOGLYCEMIA IN AN ELDERLY PATIENT WITH POORLY CONTROLLED TYPE 2 DIABETES}

Katharine Lawrence; Saif Laljee; Diana Randlett. NYU School of Medicine, New York, NY. (Control ID \#3184686)

LEARNING OBJECTIVE \#1: Recognize risks of hypoglycemia associated with sulfonylureas and thiazolidinediones

LEARNING OBJECTIVE \#2: Apply evidence-based guidelines for appropriate management of diabetes in elderly patients

CASE: A 72 year-old Spanish-speaking man with poorly controlled type 2 diabetes, hypertension, and newly diagnosed prostate cancer was bought by ambulance with confusion and weakness. He was found to have a fingerstick glucose of $30 \mathrm{mg} / \mathrm{dl}$. Additional workup was unremarkable and symptoms resolved with 50\% dextrose. He was admitted for symptomatic hypoglycemia. The patient reported taking multiple oral hypoglycemic agents, including glipizide twice daily, pioglitazone, metformin, and sitagliptan. Four months prior, pioglitazone was tripled due to A1c $10.4 \%$. $\mathrm{He}$ also revealed unintentional weight loss (possibly due to his cancer), and multiple home fingersticks in the $60 \mathrm{~s}$. Repeat A1c was $6.7 \%$. The sulfonylurea and pioglitazone were discontinued. The patient was educated on his medications, glucose targets, and symptoms of hypoglycemia. He was discharged with advice to follow with his primary physician.

IMPACT/DISCUSSION: Nearly 25\% of patients over age 65 have diabetes. Elderly diabetics are uniquely vulnerable, with higher risks of symptomatic hypoglycemia, increased hospitalizations, and higher overall associated morbidity and mortality. Polypharmacy, low health literacy, and language barriers contribute to their complex medical management. Recent studies have shown that tighter A1c control $(<7.0 \%)$ is associated with additional harms in geriatric patients, including a higher mortality rate. As a result, medical organizations have revised their glycemic control guidelines towards more personalized, patient-centered management. These guidelines include differential A1c goals (from 7.0-9.0\%) based on overall health, comorbidities, and life expectancy. Guidelines for geriatric diabetes care were updated in 2018 to include recommendations that overtreatment of diabetes should be avoided, medication classes at low risk of hypoglycemia are preferred, and complex medication regimens should be simplified to reduce hypoglycemia. Our patient illustrates the importance of each of these guidelines. With his age and comorbidities, including newly diagnosed cancer, he had indications for looser A1c goals. He was also at high-risk for medication-related adverse events, given his polypharmacy and limited health literacy. Metformin and sitagliptin would have been favored agents. Education on symptoms of hypoglycemia, as well as interpreting fingersticks, was crucial to prevent rehospitalization and improve health literacy.

CONCLUSION: Geriatric patients with diabetes represent a unique and vulnerable population. Guidelines for diabetes management in these patients are evolving, with increased emphasis on personalized, patient-centered management, including looser A1c goals for those with comorbidities or shorter life expectancy. Providers are encouraged to simplify medications and avoid polypharmacy to improve outcomes and save lives.

A CASE OF METASTATIC ACRAL LENTIGINOUS MELANOMA MASQUERADING AS HEMORRHAGIC CVA
Farukh Ikram; Leslie Cler; Haseeb Ikram; Syeda A. Raza. Methodist Dallas Medical Center, Plano, TX. (Control ID \#3185439)

LEARNING OBJECTIVE \#1: Recognize malignancy as a potential cause of intra-parenchymal hemorrhage

CASE: A 75 yo man with a history of hypertension presented with generalized weakness along with a severe, unremitting headache. The patient also reported a growth on his foot of unknown chronicity as well as back pain and urinary retention. The physical exam was significant for left-sided neglect and a large fungating mass on the plantar aspect of his left foot measuring approximately $5 \times 5 \mathrm{~cm}$. The mass had patchy areas of necrosis and was visibly bleeding. A stat CT head demonstrated a $4.6 \mathrm{~cm} \mathrm{x}$ $4.4 \mathrm{~cm} \times 3 \mathrm{~cm}$ frontoparietal hematoma. He was taken to the operating room by neurosurgery for evacuation of the hematoma and was found to have a mixed density intracerebral hemorrhage. Pathology of the solid component of the hemorrhage, along with biopsies of the foot mass, was sent for laboratory analysis. Histopathology was consistent with acral lentiginous melanoma. Tumor markers were positive for BRAF mutation. An intrathecal melanoma at T11-T12 of his spinal cord was identified via MRI for which he underwent laminectomy. Unfortunately, the patient's health continued to decline and he eventually elected for hospice.

IMPACT/DISCUSSION: This is a case of acral lentiginous melanoma (ALM) presenting as hemorrhagic CVA. ALM very rarely metastasizes to the brain making this a unique form of presentation for the disease. Furthermore, the sheer size of the mass - nearly golf-ball sized - is remarkable. Most lesions are much smaller, especially in respect to thickness. If the melanoma were not as prominent and the hematoma had been medically managed, the diagnosis could have been missed as there was no radiographic evidence for malignancy. This underscores the importance of including malignancy on the differential when a patient presents with intra-parenchymal hemorrhage. Patients should always be asked whether they have received age-appropriate cancer screening or have noticed any concerning skin growths or changes.

CONCLUSION: There are four subtypes of invasive cutaneous melanoma: 1) superficial spreading 2) lentigo maligna 3) nodular and 4) acral lentiginous. Superficial spreading melanoma accounts for approximately $70 \%$ of melanomas. Acral lentiginous melanoma (ALM) is the rarest subtype and accounts for $5 \%$ of melanomas. Although rare, it is the most common form of melanoma in darker skinned individuals and is typically found on the palm, soles, and feet. Definitive diagnosis is through histopathology. Testing should always be performed for V600 BRAF mutation. If results are positive, then the patient is eligible for immunotherapy and/or targeted therapy. Unfortunately, patients have an overall poor prognosis when presenting with advanced melanoma and CNS melanoma worsens prognosis even further. Stage IV melanoma has a median survival of 6 to 9 months and 5-year survival is estimated to be $10-20 \%$.

\section{A CASE OF MYCOSIS FUNGOIDES BULLOSA MIMICKING STEVENS-JOHNSON SYNDROME}

Sophia Zhang ${ }^{1}$; Hilary C. Strollo ${ }^{1}$; Sarah B. Merriam ${ }^{2}$. ${ }^{1}$ University of Pittsburgh, Pittsburgh, PA; ${ }^{2}$ University of Pittsburgh Medical Center, Pittsburgh, PA. (Control ID \#3185699)

LEARNING OBJECTIVE \#1: Recognize Symptoms of Mycosis Fungoides Bullosa

LEARNING OBJECTIVE \#2: Differentiating MFB from other acute vesiculobullous eruptions

CASE: A 74-year old veteran with a past medical history of gout was admitted for culture-negative septic arthritis and treated with IV vancomycin. On hospital day 3, he developed acute, diffuse blistering and desquamation of the trunk in the setting of recent allopurinol use. Review of symptoms was significant for a one-month history of bilateral palmar desquamation with pruritis and fatigue. He denied other new medications (i.e. NSAIDs, anticonvulsants, antibiotics aside from vancomycin), drug allergies, and a history 
of immunobullous disease or recent insect bites. The patient was afebrile with stable vital signs. Small flaccid, non-tender bullae with positive Nikolsky sign were present on the trunk, thighs, and intergluteal folds, covering less than $10 \%$ of the skin, sparing mucosa and eyes. CBC showed persistent lymphocytosis to $36 \times 10^{9} / \mathrm{L}$. Histopathology revealed intraepidermal deposits of atypical CD4+ lymphocytes forming Pautrier microabscesses. Direct immunofluorescence (IF) studies were negative. Peripheral blood flow cytometry revealed clonal T-cell receptor gamma gene rearrangement with corresponding bone marrow involvement on biopsy. CT abdomen/pelvis demonstrated widespread lymphadenopathy. The patient was diagnosed with Stage IVA2 cutaneous T-cell lymphoma (CTCL) and started on low dose oral methotrexate and topical hydrocortisone cream for symptomatic relief.

IMPACT/DISCUSSION: Mycosis fungoides bullosa (MFB) is a rare but aggressive variant of CTCL with a poor prognosis. It presents as a rapidly progressive desquamating vesiculobullous eruption, which can be mistaken for Stevens-Johnson Syndrome (SJS) especially in the setting of common pharmacologic culprits such as allopurinol. This case adds to the sparse literature on MFB and the differential diagnosis of acute vesiculobullous eruptions. Four criteria are recommended for diagnosis of MFB: 1) vesiculobullous lesions with or without typical MF lesions, 2) typical histologic features of MF (atypical lymphoid cells, epidermotropism, Pautrier's microabscesses) with intraepidermal blisters, 3 ) negative IF to rule out concomitant autoimmune bullous diseases, and 4) negative workup for other etiologies of vesiculobullous lesions (e.g., medications, infections). In our case, all diagnostic criteria were met with corresponding clinical and histologic findings. In cases of acute veisculobullous eruption, SJS is an important, rapidly fatal diagnosis to rule out. Our patient did not have ocular or mucosal involvement (present in $>90 \%$ of SJS patients), nor systemic symptoms associated with SJS. Histopathologic findings specific to MFB such as CD4+-predominant lymphocytes forming Pautrier's microabscesses can facilitate diagnosis.

CONCLUSION: MFB is a rare variant of CTCL with a poor prognosis and should be on the differential workup for vesiculobullous eruptions to ensure prompt diagnosis and treatment.

A CASE OF NONBACTERIAL THROMBOTIC ENDOCARDITIS IN A YOUNG PATIENT WITH ACUTE MYELOGENOUS LEUKEMIA

Rami Atallah $^{2}$; Nicholas Ojile ${ }^{2}$; Kamal Chamoun ${ }^{3}$; Mohinder Vindhyal ${ }^{1}$. ${ }^{\mathrm{T}} \mathrm{KU}$ - Wichita, Wichita, KS; ${ }^{2}$ University of Kansas Wichita, Wichita, KS; ${ }^{3}$ Case Western Reserve University, Cleveland, OH. (Control ID \#3185270)

LEARNING OBJECTIVE \#1: Recognize the causes and clinical features of nonbacterial thrombotic endocarditis.

LEARNING OBJECTIVE \#2: Recognize that nonbacterial thrombotic endocarditis is potentially underdiagnosed.

CASE: A 29 year-old female with a history of recurrent pancreatitis presented to her primary care physician complaining of severe dyspnea on exertion and fatigue. Outpatient laboratory work revealed severe anemia and the patient was sent to the emergency department for further evaluation. She denied any symptoms of bleeding at the time. Family history was remarkable for maternal grandfather with history of leukemia. Her physical examination was significant for skin pallor and tachycardia. Complete Blood Count (CBC) on presentation showed hemoglobin of 4 $\mathrm{g} / \mathrm{dl}$, platelets of $56 \times 10^{9} / \mathrm{L}$, and white blood cells (WBC) of $14 \times 10^{9} / \mathrm{L}$ with $40 \%$ peripheral blasts. A bone marrow biopsy confirmed the diagnosis of AML with complex karyotype, and negative NPM1 and CEBPA mutations. An initial echocardiogram revealed an ejection fraction of $55 \%$ with no valvular abnormalities. Blood cultures were obtained and she was started on broad spectrum antibiotics. The DIC panel was negative. Induction chemotherapy was then started using cytarabine and daunorubicin. After initiating chemotherapy, she developed bilateral infiltrates on chest X-ray and her respiratory status rapidly deteriorated requiring mechanical ventilation. Bronchoscopy was consistent with diffuse alveolar hemorrhage (DAH). Multiple sets of blood cultures in the setting of persistent neutropenic fever were repeatedly negative. Patient was on vancomycin, meropenem, acyclovir, and voriconazole. Suddenly, a week later, she developed neurological deterioration including absent brainstem reflexes, renal failure and left leg ischemia caused by an arterial clot in the deep femoral artery. Family discussion led to comfort care. Autopsy revealed fibrin vegetation on the aortic valve and tricuspid valve with massive ischemic infarct involving the left frontal, parietal and occipital lobes of the brain in addition to bilateral kidney and spleen infarcts. Bilateral pulmonary hemorrhage was also noted.

IMPACT/DISCUSSION: Nonbacterial thrombotic endocarditis (NBTE), formerly known as marantic endocarditis, is a rare condition characterized by deposition of sterile thrombi on heart valves with the absence of bacterial growth on blood cultures. NBTE increases the risk of systemic embolic events including stroke. Microscopically, NBTE results from agglutinated platelets mixed with strands of fibrin. NBTE is most commonly associated with advanced malignancy and systemic lupus erythematosus. In this case, NBTE has led to significant multi-organ failure including kidney failure, massive stroke and leg ischemia.

CONCLUSION: NBTE is uncommon but associated with poor outcomes. It is uncommonly reported in patients with AML and is often a postmortem diagnosis. NBTE is potentially underdiagnosed and should be considered in any cancer patient with an episode of arterial thromboembolism.

\section{A CASE OF OXALOSIS FOLLOWING ROUX-EN-Y BYPASS SURGERY}

John Paul Mikhaiel; Ryan M. Commins; Margot Wheeler. Georgetown University, Washington, DC. (Control ID \#3168633)

LEARNING OBJECTIVE \#1: Recognize the clinical presentation and risk factors for hyperoxalemia

CASE: A 61-year-old male with history of acute renal failure on hemodialysis and obesity, four years status post Roux-en-Y bypass, presented with episodic skin changes and paresthesia in his fingers and toes as well as hemoptysis. Six months prior to presentation, he was admitted for acute renal failure with renal biopsy revealing ethylene glycol crystals. In the preceding months, he also complained of sporadic day-long episodes of burning and paresthesia in his fingers and toes and three episodes of small volume hemoptysis. His skin exam revealed distal fingertips with necrotic ulcerations and slight purpura, and distal toes with necrotic ulcerations extending into the fat pads. Lung exam was clear to auscultation with diminished sounds at bases. While initial labs revealed an elevated CRP, follow-up infectious and immunologic studies were unremarkable. CT scan of the chest demonstrated diffuse ground-glass airspace opacities and confluent consolidation in the right upper lobe. Punch biopsy, taken during a stage of healing, of the skin ulcers was suggestive of thrombogenic vasculopathy. Finally, his plasma oxalate level was found to be $28.5 \mathrm{umol} / \mathrm{L}$, greater than $15 \mathrm{x}$ the normal limit, indicating hyperoxalemia. He was treated with dietary modification and initiation of cholestyramine.

IMPACT/DISCUSSION: An estimated 100 million American adults are obese, and over 200,000 bariatric surgery procedures were performed in 2017 alone. While Roux-en-Y bypass has been supplanted by the gastric sleeve as the most common bariatric surgery performed, Roux-en-Y was still performed over 40,000 times in 2017. This case highlights the postoperative complication of hyperoxaluria in malabsorptive gastric bypass procedures. Hyperoxaluria following Roux-en-Y gastric bypass is estimated at $20 \%$. While not completely understood, it has been suggested that the enteric hyperoxalemia that follows bariatric surgery is secondary to free fatty acid malabsorption. The excess free fatty acids and bile salts in the intestinal lumen bind to and saponify calcium, inhibiting the formation of calcium oxalate, and increasing absorption of soluble free oxalate. Hyperoxalemia may lead to nephrocalcinosis, often manifesting as nephrolithiasis or progressive renal insufficiency. Hyperoxalemia can also 
mimic systemic vasculitis, presenting with Raynaud's, acrocyanosis, livedo reticularis, diminished peripheral pulses, gangrene, and rarely, diffuse alveolar hemorrhage. In this patient, hyperoxalemia due to malabsorptive gastric bypass surgery is a unifying diagnosis for this patient's renal failure, peripheral gangrene, and diffuse alveolar hemorrhage.

CONCLUSION: While hyperoxaluria leading to calcium oxalate nephrolithiasis following malabsorptive gastric bypass is not uncommon, it is important to be aware of other systemic manifestations of hyperoxalemia, which can provide a unifying diagnosis in a challenging clinical presentation.

\section{A CASE OF POTENTIALLY FATAL EPISTAXIS}

PRIYANKA BHUGRA ${ }^{1}$; Abhishek Maiti ${ }^{2} .{ }^{1}$ University of Texas Health Science Center at San Antonio, San Antonio, TX; ${ }^{2}$ University of Texas Health Science Center at Houston, Houston, TX. (Control ID \#3187054)

LEARNING OBJECTIVE \#1: Pseudoaneurysms of the internal carotid artery are uncommon but potentially life-threatening cause of epistaxis.

LEARNING OBJECTIVE \#2: History of prior trauma, surgery or radiation are important clues to diagnosis. Early recognition and endovascular intervention can help improve morbidity and mortality.

CASE: A 42-year-old previously healthy man presented to the emergency room after having epistaxis and syncope. Patient reported having recurrent episodes of right epistaxis over past 2 weeks. Earlier in the day, he had 4-6 cupfuls of bleeding from his nose leading to lightheadedness and eventually passing out. He had a remote history of trauma with facial fractures after a motor vehicle accident 8 years ago for which he underwent surgery. On arrival, patient was hypotensive with blood pressure of $80 / 50 \mathrm{~mm} \mathrm{Hg}$ and tachycardic with a heart rate of 110 beats/ minute. Examination showed crusted blood in anterior nasal cavity. Laboratory studies showed hemoglobin of $6.4 \mathrm{~g} / \mathrm{dL}$. There was minimal improvement in blood pressure and hemoglobin with intravenous fluid resuscitation and blood transfusion. Furthermore, flexible nasopharyngoscopy revealed bleeding originating from the right sphenoid traveling down the posterior nasal cavity to choana and posterior oropharynx. Shortly after the scope was withdrawn, patient sneezed and started bleeding profusely from the right nare. At this point, anterior and posterior nasal tamponade were applied and patient was intubated for airway protection. An emergent angiogram identified a right cavernous internal carotid artery (ICA) pseudoaneurysm as the source of bleeding. Endovascular coil embolization of pseudoaneurysm was performed followed by placement of pipeline embolization device. Subsequently, patient became hemodynamically stable and was successfully extubated. The patient remained asymptomatic at 3-month follow up.

IMPACT/DISCUSSION: Majority cases of epistaxis are self-resolving. However, red flag signs like recurrent or intractable bleeding, heavy blood loss, hemodynamic instability, vision loss or accompanying neurologic deficits should raise concern for more sinister etiology. Pseudoaneurysms of ICA are uncommon but potentially life-threatening cause of epistaxis. These can occur as a result of blunt trauma, surgery or after radiation therapy to the head and neck region. Epistaxis due to post-traumatic pseudoaneurysms usually occurs within weeks of injury, but it may be delayed by years. This poses a significant diagnostic challenge to clinicians. Prompt control of bleeding and airway protection are crucial as rupture of ICA pseudoaneurysms can lead to catastrophic bleeding. Early recognition of this life-threatening condition is important as majority of cases are amenable to endovascular intervention.

CONCLUSION: Pseudoaneurysms of ICA can cause serious epistaxis with significant morbidity and mortality. Timely recognition and endovascular intervention can be life-saving.

A CASE OF PROSTHETIC VALVE DYSFUNCTION PRESENTING WITH DYSPHAGIA AND ACUTE TUBULAR NECROSIS
Yael Tarshish; Vidya Gopinath. Brown Medicine, Pawtucket, RI. (Control ID \#3185670)

LEARNING OBJECTIVE \#1: Recognize dysphagia as a side effect of mitral valve dysfunction and identify possible mechanisms

LEARNING OBJECTIVE \#2: Diagnose acute tubular necrosis (ATN) in the setting of hemolysis with minimal serum creatinine $(\mathrm{Cr})$ elevation CASE: A 76 year old female with history of five mitral valve replacements (last surgery: 12 years ago for hemolysis) presented with dark urine and intermittent difficulty swallowing for one month. She had been hospitalized two weeks prior for the same symptoms, but had negative esophagogastroduodenoscopy (EGD) and normal swallow evaluation and no valve abnormality on transthoracic echocardiogram. She was discharged home, yet continued to endorse worsening swallowing function and "coca cola" colored urine. Vitals: unremarkable. Pertinent exam findings: scleral icterus; crisp mitral sound. Significant labs: UA positive for 3 RBCs, Hgb 8.5, Cr 0.88, TBili 2, LDH 1961. Cardiology, hematology, nephrology and gastroenterology were consulted. Transesophageal echocardiogram demonstrated a small paravalvular leak with severe mitral regurgitation and left atrium (LA) dilatation. Barium swallow demonstrated severe esophageal dysmotility without barium passage to the lower esophageal sphincter. Hematology found no underlying erythrocyte abnormality. Spun urine revealed muddy brown casts and evidence of ATN despite normal Cr. ATN was attributed to hemoglobinuria from hemolysis and was treated with fluids. Hgb dropped to 6.6, requiring transfusions. She underwent a perivalvular leak repair with valvular plugs. Hemolysis resolved and dysphagia gradually improved.

IMPACT/DISCUSSION: Mitral valve dysfunction causes dysphagia through various potential mechanisms. LA enlargement can cause hoarseness through compression of the recurrent laryngeal nerve, and can impact esophageal functioning through compression of the vagus nerve. A study demonstrated association between LA size and esophageal transit with barium swallows demonstrating cardiac indentation of esophagus. Research in paroxysmal nocturnal hemoglobinuria $(\mathrm{PNH})$ proposes a mechanism by which hemolysis causes dysphagia: increased free hgb binds free nitrous oxide (NO), a modulator of smooth muscle function. Decrease in NO effects esophageal tone. Two case studies describe dysphagia associated with hemolysis caused by left ventricular assist device, providing possible explanations for the mechanism behind our patient's dysphagia. Hemolysis can cause an increase in free hgb resulting in urine dipstick positive for blood and sediment negative for RBCs. Free hgb degradation in the urinary space with release of heme pigment damages the kidneys, leading to ATN. ATN can cause minimal increase in serum $\mathrm{Cr}$ with evidence of kidney injury on urine microscopy. Management involves aggressive hydration.

CONCLUSION: Mitral valve dysfunction can manifest pathologically in disparate organ systems. Knowledge of uncommon sequelae can prompt more rapid diagnosis.

\section{A CASE OF PSEUDOHYPONATREMIA FROM SEVERE SEC-} ONDARY HYPERCHOLESTEROLEMIA

Leah Bettner ${ }^{1}$; Carleigh L. Benton ${ }^{2,1}$; Amy Yu ${ }^{1} .{ }^{1}$ University of Colorado Denver, Denver, CO; ${ }^{2}$ University of Colorado, Denver, CO. (Control ID \#3176728)

LEARNING OBJECTIVE \#1: Identify severe hypercholesterolemia from cholestasis as a cause of pseudohyponatremia.

LEARNING OBJECTIVE \#2: Diagnose pseudohyponatremia by measuring osmolar gap and whole serum sodium levels.

CASE: A 59 year-old man with diabetes and chronic pancreatitis was admitted with weight loss, poor oral intake, abdominal pain, and jaundice. Imaging detected a pancreatic mass and biliary ductal dilation concerning for malignancy. Labs were notable for a total bilirubin 
of $22 \mathrm{mg} / \mathrm{dL}$, direct bilirubin $14.1 \mathrm{mg} / \mathrm{dL}$, alkaline phosphatase 1,928 $\mathrm{U} / \mathrm{L}$, and serum sodium $119 \mathrm{mmol} / \mathrm{L}$. Initial hyponatremia was attributed to hypovolemia however sodium did not improve with intravenous fluids. Urine studies were consistent with the syndrome of inappropriate antidiuretic hormone secretion but sodium did not improve with fluid restriction. The calculated serum osmolar gap was noted to be high $(23 \mathrm{mOsm} / \mathrm{kg})$ and a subsequent lipid panel showed a total cholesterol of 1,289 mg/dL, LDL 1,059 mg/dL, HDL $184 \mathrm{mg} / \mathrm{dL}$, and triglycerides $228 \mathrm{mg} / \mathrm{dL}$. Lipid studies three months prior were normal. The diagnosis of pseudohyponatremia was confirmed with a normal whole blood sodium level of $135 \mathrm{mmol} / \mathrm{L}$. Ultimately, the patient underwent biliary stent placement and biopsy confirmed pancreatic adenocarcinoma. With biliary obstruction relief, the cholestatic labs improved but the hyperlipidemia (HLD) persisted. A statin was prescribed at the time of discharge and was later discontinued.

IMPACT/DISCUSSION: Hyponatremia is common in hospitalized patients and this case highlights the importance of broadening the differential diagnosis to include an otherwise relatively rare laboratory artifact of pseudohyponatremia in a patient presenting with obstructive cholestasis and secondary HLD. Furthermore, understanding that secondary HLD from cholestasis does not confer an atherosclerotic risk prevents unnecessary statin therapy. Our case highlights the importance of considering pseudohyponatremia from severe HLD in patients with hyponatremia and cholestasis. Patients with cholestatic disease may have poor oral intake leading to the assumption of hypovolemic hyponatremia. Calculating an osmolar gap can be a helpful first step, and if elevated, checking a lipid panel and whole blood sodium can help confirm the diagnosis of pseudohyponatremia. Cholestasis can be an important cause of secondary HLD. Case studies have identified an abnormal lipoprotein X (Lp-X) as the primary cholesterol carrier in patients with hepatobiliary and pancreatic cholestasis as opposed to LDL particles found in many patients with primary HLD. Lp-X is thought to have a negligible atherosclerotic effect, and thus statin therapy is not needed.

CONCLUSION: In patients with cholestasis and hyponatremia, early calculation of the osmolar gap and/or obtaining a whole blood sodium level can lead to an earlier diagnosis of pseudohyponatremia. Statin therapy is not necessary to prevent atherosclerosis in patients with secondary HLD from cholestasis.

\section{A CASE OF RAPID NECROTIC SKIN ULCERATIONS IN AN ESRD PATIENT WITH REPORTED ANTIPHOSPHOLIPID SYN- DROME ON WARFARIN}

Christopher Chen ${ }^{1}$; Henry C. Zheng ${ }^{1}$; Mikel Etchegaray ${ }^{2} .{ }^{1}$ Baylor College of Medicine, Houston, TX; ${ }^{2}$ MD Anderson Cancer Center, Houston, TX. (Control ID \#3186404)

LEARNING OBJECTIVE \#1: Review differential diagnosis of necrotic skin lesions

LEARNING OBJECTIVE \#2: Review pathophysiology, treatments, and recent developments in calciphylaxis

CASE: A 42 year-old woman with HTN, morbid obesity, ESRD on peritoneal dialysis due to recurrent $\mathrm{AV}$ fistula thromboses, tertiary hyperparathyroidism, reported anti-phospholipid syndrome on warfarin, three prior strokes presents with 2 months of worsening dyspnea, fatigue, bilateral leg swelling, 1-month painful non-healing ulcer of left ankle. Vitals and exam remarkable for fever, tachycardia, mild hypoxemia, bilateral rales and lower extremity edema with left anteromedial ulcer above the malleolus with clean base and serosanguinous drainage exquisitely tender to palpation. Initial labs showed leukocytosis, azotemia, and calcium-phosphorus product of 81 . Left tibial x-ray negative for osteomyelitis. Despite dialysis initiation, her ulcer rapidly worsened into widespread necrotic eschar tracking up bilateral thighs and lateral abdomen. Her ANA was $>1: 640$ speckled pattern but extensive autoimmune and infectious work-up all negative. Punch biopsy showed extensive dermal purpura, small intravascular fibrin thrombi. Unfortunately, patient refused deep tissue biopsy for further analysis. Given clinical picture, a diagnosis of calciphylaxis was reached. She was started on pulse dose steroids, empiric antibiotics, heparin drip, continued dialysis, aggressive wound care, pain control, and sodium thiosulfate infusions. She underwent 3.5 parathyroidectomy, steroids were tapered, and was transitioned to low dose apixaban. She was discharged to long term care facility for continued wound care, IV antibiotics, and continued dialysis without significant improvement to skin ulcerations.

IMPACT/DISCUSSION: The differential for necrotic skin lesions is broad and often overlapping many body systems. Calciphylaxis, characterized by microvascular calcification, inflammation, and thrombosis in the adipose tissue layer, is even amongst dialysis patients a rare event (4\%). With a 1 -year mortality of $45-80 \%$, patients are often bedbound with debilitating, uncontrollable pain. Diagnosis is mostly clinical based on risk factors, characteristic skin ulcers and eschar, and calcium phosphorus product $>70$. Biopsy is controversial given the need for deeper tissue sampling, pathergy risk, and high mortality rate with infection. No guidelines yet delineate standard work-up or treatments. Treatment is multifocal including aggressive wound care, pain control, electrolyte homeostasis, and sodium thiosulfate infusions. Underlying thrombophilia have been found in up to $80 \%$ of patients and highlights challenges in anticoagulation especially with co-morbid ESRD and risk of worsening with warfarin. Small studies suggest warfarin may have similar survival profiles to other anticoagulants.

CONCLUSION: Calciphylaxis is a rare but debilitating disease that is a challenge to effectively treat and in need of further trials based on recent evidence of common underlying associated thrombophilias.

\section{A CASE OF RECURRENT SINUSITIS}

Rebecca Angoff ${ }^{1}$; Vimal Jhaveri ${ }^{2}$; Marisa Jupiter ${ }^{2} .{ }^{1}$ Beth Israel Deaconess Medical Center, Brookline, MA; ${ }^{2}$ Beth Israel Deaconess Medical Center, Boston, MA. (Control ID \#3183857)

LEARNING OBJECTIVE \#1: Recognize the features and epidemiology of extranodal NK/T-cell lymphoma.

LEARNING OBJECTIVE \#2: Reassess the differential diagnosis when a patient's presentation no longer fits the illness script.

CASE: Mr. T is a 43-year-old male from Vietnam with a history of treated latent tuberculosis who presented with fevers, weight loss, and left facial swelling. In the weeks prior to admission, he had two Emergency Department (ED) visits due to fevers, headache, rhinorrhea, congestion, and left maxillary tenderness and was prescribed amoxicillin then subsequently amoxicillin-clavulanate without improvement of his symptoms. On his third ED visit, he also endorsed night sweats and a 6-pound weight loss. He had a CT that showed sinusitis of the left maxillary and anterior ethmoid sinuses and chest imaging that showed a $3 \mathrm{~cm}$ cavitary lung lesion in the right upper lobe. He was admitted and underwent a tuberculosis rule out. He had three negative sputums for acid fast bacilli and a negative nucleic acid amplification test. Otolaryngology performed an endoscopy which showed nasal polyposis. A nasal sinus aspirate grew pan sensitive Klebsiella pneumoniae on culture, and the patient was initiated on ceftazidime. He continued to have fevers so was transitioned to meropenem. His fevers persisted, and he underwent a biopsy of his nasal polyp, which seven days later, resulted in extranodal NK/T-cell lymphoma, nasal type. Therefore, he was transferred to the oncology service for treatment.

IMPACT/DISCUSSION: In retrospect, his night sweats, refractory symptoms to antibiotics and lack of history of prior sinusitis made this an atypical presentation of simple bacterial sinusitis and there were clues that could have possibly put his rare diagnosis on the differential. Extranodal NK/T-cell lymphoma is an aggressive lymphoma with tropism for the nasopharyngeal and aerodigestive tracts. Rarely, it can metastasize to the lung and cavitate as it did in this case. The disease is associated with EBV and is more common in patients from Asia. The SMILE regimen, 
which consists of dexamethasone, methotrexate, ifosfamide, Lasparaginase, and etoposide, while aggressive, has shown favorable outcomes. Mr. T is from Vietnam and presented with night sweats, sinusitis, and weight loss. When he was first admitted, there was appropriately high concern for mycobacterium tuberculosis. He then failed to improve on broad spectrum antibiotics, despite a sensitive isolate, and the team had to revisit the initial framing of the case by obtaining a tissue sample. This case highlights the importance of avoiding diagnostic anchoring by reassessing the differential diagnosis when a patient no longer fits the illness script for a disease.

CONCLUSION: In patients from Asia with persistent sinusitis and polyposis, extranodal NK/T-cell lymphoma should be on the differential. NK/T-cell lymphoma is associated with EBV, and it is treated with the SMILE regimen. When patients are not improving as expected, revisit the initial diagnosis.

\section{A CASE OF SIMULTANEOUS OCCURRENCE OF GRAVES' DISEASE(GD) AND SEROSITIS CAUSED BY SYSTEMIC LU- PUS ERYTHEMATOSUS (SLE) WITH ANTI-DSDNA ANTI- BODY NEGATIVE}

KENJI TSUKAMOTO. Okazaki city hospital, Okazaki, Japan. (Control ID \#3183771)

LEARNING OBJECTIVE \#1: It needs to pay attention to clinical condition of SLE when examining a patient with GD.

CASE: The case was for female at the age of 48 with no past medical history and no family medical history for thyroid disease and collagenosis. She visited a clinic in May 2018 due to edema and weight gain. She was given furosemide and levocetirizine. However, the symptom tended to be worsened with respiratory discomfort and she was hospitalized in October 2018. She had blood pressure $108 / 60 \mathrm{mmHg}$, pulse 86 beats/minute, respiration rate 15 times/minute, and body temperature 36.9 degrees Celsius. We recognized systemic pitting edema and lower bilateral respiratory sound without any other specific abnormal findings. There was no particular examination findings for SLE, but the patient had loose stool. The laboratory tests indicated C3 $69 \mathrm{mg} / \mathrm{dL} / \mathrm{C} 49 \mathrm{mg} / \mathrm{dL}$ for hypocomplementemia, 80x for antinuclear antibody, 1.60 for Lupus Anticoagulant (diluted Russell's viper venom time), less than $0.01 \mu \mathrm{IU} / \mathrm{mL}$ for thyroid-stimulating hormone(TSH), free T3 $5.70 \mathrm{pg} / \mathrm{mL}$, free T4 2.52 $\mathrm{ng} / \mathrm{dL}, 2.1 \mathrm{IU} / \mathrm{L}$ for TSH receptor antibody, and negative for anti-dsDNA antibody/anti-histone antibody, but no finding for other collagen diseases and infective diseases. There was no particular finding in the urinary test and also no test result to cause pleural effusion. Bilateral pleural effusion and a small amount of pericardial fluid were found in chest X-ray and echocardiogram respectively, but other sites indicated normal. Because we diagnosed simultaneous occurrence of GD and serositis caused by SLE, celecoxib and azosemide were initiated. For GD, potassium iodide $50 \mathrm{mg} /$ day was used in consideration of SLE-like symptom caused by antithyroid drugs. Although we could manage hyperthyroidism after 2 months, anti-dsDNA antibody turned out positive and serositis control was worsened. When prednisolone $30 \mathrm{mg} /$ day was initiated, serositis was improved after 1 month with weight loss.

IMPACT/DISCUSSION: In this case, serositis could simultaneously develop for a patient with SLE's anti-dsDNA antibody Negative and GD. It is reported that there can be a relation between SLE disease activity and dsDNA for a SLE patient, and serositis is also often found as a clinical finding in SLE's dsDNA antibody Negative. In the recent report, SLE could have development of complication with autoimmune thyroid disease(AITD) including GD and serositis might become a risk in case of SLE associated with AITD. On the basis of the findings in the present case, serositis could be considered as a clinical feature in the development of complication with SLE and GD. However, it still remains unknown regarding the clinical feature of AITD and SLE's dsDNA antibody Negative.
CONCLUSION: We experienced a case of simultaneous occurrence of GD and serositis caused by SLE with anti-dsDNA antibody Negative. Clinicians need to conduct medical examination with the consideration of possible SLE complication when examining a patient with GD.

\section{A CASE OF SKIN RASH AND SEPTIC ARTHRITIS}

Hasan Ahmad Hasan Albitar. Mayo Clinic, Rochester, MN, Rochester, MN. (Control ID \#3184470)

LEARNING OBJECTIVE \#1: Identify the clinical features of rat bite fever syndrome

LEARNING OBJECTIVE \#2: Recognize septic arthritis as a rare complication of rat bite fever

CASE: A 27-year-old lady presented to the emergency department with fever, chills and fatigue of 3 days duration. Physical examination revealed temperature of 39.6. Workup revealed neutrophil-predominant leukocytosis with a white blood cell count of $14 \times 10(9) / \mathrm{L}(3.4-9.6 \times 10(9) / \mathrm{L})$ in addition to elevated CRP of $277.8 \mathrm{mg} / \mathrm{L}(<8 \mathrm{mg} / \mathrm{L})$. On hospital day 3, the patient developed asymmetric polyarthritis involving the left $3{ }^{\text {rd }} \mathrm{MCP}$, left wrist, right shoulder and right ankle joints. In addition to this, the patient was noted to have a pustular skin rash involving the palms and soles. Screening tests for Neisseria gonorrhoeae, Chlamydia trachomatis, and HIV were negative. In addition to this, syphilis IgG antibody and Parvovirus B19 serology testing were negative. On further questioning, the patient reported that she has 3 pet rats and that she had a rat bite 2 days prior to onset of symptoms. Rat bite fever was suspected and the patient was started empirically on $2 \mathrm{~g}$ of IV ceftriaxone daily. Arthrocentesis of the left $3^{\text {rd }}$ MCP joint yielded purulent synovial fluid. Synovial fluid culture was not performed because of the small volume of synovial fluid obtained following during the procedure. Synovial fluid gram stain was negative but numerous white blood cells were noted. Blood cultures were obtained and they showed growth of Streptobacillus moniliformis in $4 / 6$ bottles confirming our initial suspicion of rat bite fever. Repeat blood cultures were negative. The patient had near complete resolution of her symptoms and was discharged on $2 \mathrm{~g}$ of IV ceftriaxone for a total of 2 weeks.

IMPACT/DISCUSSION: Rat bite fever (RBF) is a rare disorder most commonly caused by Streptobacillus moniliformis in the USA. The syndrome typically presents with fever and fatigue followed by migratory polyarthralgias and skin rash that involves the palms and soles. Septic arthritis is a rare complication of RBF with only 14 cases in adults repoarted in the literature. The reported mortality rate approaches $10 \%$. Penicillin is the drug of choice for treatment of RBF and ceftriaxone is a reasonable alternative. For patients with penicillin allergy, tetracyclines can be used

CONCLUSION: RBF is a disease caused Streptobacillus moniliformis. Septic arthritis is a rare complication of RBF. Given the potential for severe and fatal disease in patients with RBF, a therapeutic trial is indicated once the disease is suspected.

\section{A CASE OF STAPHYLOCOCCAL BACTEREMIA WITH IGA DOMINANT CUTANEOUS LEUKOCYTOCLASTIC VASCULI- TIS AND GLOMERULONEPHRITIS}

Joshua Allgaier $^{1,}$ 2; Osama Kandalaft ${ }^{1,2}$; Sehar Zulfiqar ${ }^{1,}{ }^{2}$. ${ }^{1}$ Baystate

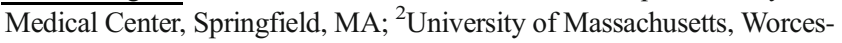
ter, MA. (Control ID \#3184018)

LEARNING OBJECTIVE \#1: To raise awareness of the rare presentation and clinical course of $\operatorname{IgA}$ mediated cutaneous vasculitis and acute glomerulonephritis associated with staphylococcal bacteremia

CASE: A 59 year old female with peripheral vascular disease and chronic right heel eschar was admitted for right ankle cellulitis. Blood cultures grew methicillin sensitive staphylococcus aureus (MSSA) and MRI identified extensive right ankle osteomyelitis. Cefazolin was started in place of ampicillin-sulbactam and vancomycin. On day 8 of her hospitalization, 
she developed a palpable, pruritic rash at her right knee that rapidly spread across her bilateral lower extremities, buttocks and abdomen. Renal function also declined abruptly, coinciding with the onset of the rash. Leukocytoclastic vasculitis (LCV) was suspected and the patient was started on high dose steroids. Cefazolin was the suspected trigger and was changed to oxacillin. Due to worsening renal function with anuria, hemodialysis (HD) was started. Serum IgA was elevated at $1255 \mathrm{mg} / \mathrm{dl}$ with normal $\mathrm{C} 3$ and $\mathrm{C} 4$. Renal biopsy revealed acute proliferative glomerulonephritis (GN) with IgA immune complex deposition. Other labs were negative for ANA, c-ANCA, and p-ANCA. Ultimately, her infected foot was amputated, while oxacillin and steroids were continued. The rash disappeared gradually over one week and HD was discontinued after 2 months with some recovery of her renal function.

IMPACT/DISCUSSION: Staphylococcus-associated GN is an uncommon cause of renal failure that can manifest as LCV, most commonly with methicillin resistant staphylococcus aureus. ${ }^{1}$ While most of these patients have $\mathrm{C} 3$, IgG, or IgM glomerular immune complex deposition, there is a less common $\operatorname{IgA}$ dominant or co-dominant form that has been increasingly recognized in the literature. ${ }^{3}$ Optimal evidence based treatment is unclear, but some data suggests that up to $70 \%$ of patients have a favorable response. ${ }^{2}$ Satoskar et al reported IgA dominant postinfectious GN should not be treated with steroids, but by removing offending infectious agents, ${ }^{4}$ whereas other limited evidence supports short steroid courses if renal failure persists. ${ }^{5}$

CONCLUSION: Our patient suffered with an atypical presentation of acute proliferative IgA-mediated GN and LCV related to MSSA infection ultimately leading to HD. This clinical constellation was identified near the end of her treatment course, progressing despite appropriate antibiotic coverage, source control with amputation and a course of high-dose steroids. We raise awareness to the limited recognition of this clinical entity, as earlier identification of this ambiguous syndrome may have led to improved recovery of her renal function by initiating earlier source control and steroids. Further awareness and recognition of this clinical entity may lead to stronger evidence-based guidelines on its management.

\section{A CASE OF SWELLING IN A FORGOTTEN JOINT}

Shane Murray; Ana B Arevalo; Gustavo Contreras Anez; Geeta Varghese. Mount Sinai St Luke's - West, New York, NY. (Control ID \#3183582)

LEARNING OBJECTIVE \#1: Recognize the clinical features of native joint Cutibacterium septic arthritis

CASE: 67-year-old Hispanic Male with a history of treated Hepatitis C presented to clinic with 6 months of chronic dull left shoulder pain. Pain was located over the medial shoulder, radiated to the neck and was associated with enlarging tender lump over the left clavicle. Social history revealed he was born and raised in Puerto Rico, and a history of heroin, cocaine and tobacco use. No prior surgery or history of trauma to the left shoulder. He denied symptoms consistent with an infectious or inflammatory process. Examination revealed a $2 \mathrm{~cm}$ tender, non-erythematous hard mass over medial third of the left clavicle. Ultrasound confirmed a nonspecific hypoechoic mass in the clavicle. MRI showed marked enlargement of the left sternoclavicular joint (SCJ) with effusion, synovitis, subchondral erosions and marrow edema consistent with an inflammatory arthropathy. Laboratory tests revealed an elevated ESR and CRP with otherwise normal CBC, QuantiFERON gold, CCP antibody, ANA, HIV 1/2 4th, Lyme antibody titers and Uric acid. The patient was subsequently referred to Rheumatology and Infectious Disease for further evaluation. Bone biopsy of the clavicular head was performed. AFB smear, culture and fungal culture tests were negative. Tissue cultures grew Cutibacterium Acnes. The patient was treated with Ceftriaxone two grams daily for a total of six weeks.

IMPACT/DISCUSSION: Cutibacterium species are a documented cause of joint infection following surgery but its role in native joint infections has only recently become established. Recent literature suggests that the species is underdiagnosed as a cause of septic arthritis due to its indolent course, frequent dismissal as a contaminant and specific culture requirements. One study looking at a group of patients with synovitis, acne, pustulosis, hyperostosis, osteitis syndrome (SAPHO), which frequently involved the SCCJ, found Cutibacterium acnes on bone cultures in two thirds of patients. While further research is needed to determine a role for the species in diseases specific to the SCJ, this case adds to the increasing body of literature that suggests that Cutibacterium infections are more prevalent than once thought. Cases usually lack signs of acute infection and have a prolonged course often leading to misdiagnosis. Infection should be suspected in patients presenting with atypical osteoarthritis symptoms or features of monoarticular chronic inflammatory arthritis. Arthrocentesis/biopsy and culture may prevent unnecessary invasive procedures in these patients.

CONCLUSION: Cutibacterium acnes septic arthropathy is an underdiagnosed entity that typically lacks symptoms of acute infection. One should consider the diagnosis in a patient with atypical osteoarthritis or chronic monoarticular inflammatory symptoms.

\section{A CASE OF THE FEEBLE LEG.}

Yulia Khodneva ${ }^{1}$; Meghan K. Black ${ }^{2} .{ }^{1}$ University of Alabama Birmingham, Birmingham, AL; ${ }^{2}$ University of North Carolina, Durham, NC. (Control ID \#3185085)

LEARNING OBJECTIVE \#1: Identify vitamin B12 deficiency presenting with unilateral pain and weakness.

CASE: A 61 year-old previously healthy African American man presented with a 5-week history of sharp, shooting pain in his right thigh, exacerbated with weight bearing, and not relieved with symptomatic treatment. Additionally, 2 weeks prior to presentation, he began experiencing difficulty holding onto things with his right hand. He also described multiple falls upon standing and inability to ambulate. $\mathrm{He}$ reported current cocaine and alcohol use. Vital signs were within appropriate limits. Cardiopulmonary and abdominal exam were unremarkable. He had limited range of motion in his right hip with tenderness to palpation. Sensation to light touch was diminished up to T2/T3 level bilaterally, with hyperreflexia and negative Babinski reflex. Strength was decreased in his right upper extremity in biceps, triceps, and wrist to $2 / 5$ and in the right lower extremity to $2 / 5$ throughout. Left extremities had intact strength. Muscle atrophy in both hands was noted. Hemoglobin and hematocrit were $13.1 \mathrm{~g} / \mathrm{dL}$ and $39.2 \%$ respectively with mean corpuscular volume (MCV) of $87.9 \mathrm{fL}$. White blood count and electrolytes were normal. Multiple imaging modalities were negative for stroke, but showed areas of nerve root compression with radiculopathy, without cord compression.. There were degenerative changes in hip and spine. Further testing revealed vitamin $\mathrm{B}_{12}$ level was $268 \mathrm{mmol} / \mathrm{mL}$ and methylmalonic acid (MMA) was $396 \mathrm{mmol} / \mathrm{mL}$. Folic acid was normal and antibody to intrinsic factor was negative. The patient was started on intramuscular cyanocobalamin and after 7 days had marked improvement in his pain and weakness and was able to walk with assistance.

IMPACT/DISCUSSION: Vitamin $\mathrm{B}_{12}$ deficiency is commonly encountered by the internist, but seldom does it become severe enough to present with extensive neurologic manifestations. It is especially difficult to recognize when it presents with asymmetrical extremity pain and weakness without megaloblastic anemia. While the differential for extremity pain and weakness is broad, the key components of this case leading to a diagnosis of $\mathrm{B}_{12}$ deficiency were the subacute onset, progressive weakness, sensory ataxia and bilateral hand muscle atrophy. Due to demyelination of the dorsal and lateral column of the spinal cord, vitamin B12 deficiency typically manifests with symmetrical combined motor and sensory deficits. However, like our case there have been reports of unilateral findings. The diagnosis of vitamin $\mathrm{B}_{12}$ deficiency is most often made by low serum levels, megaloblastic anemia and an elevated MMA. However, 5-10\% patients have normal or low normal B12 levels and $15 \%$ have a normal MCV. 
CONCLUSION: Patients with polysubstance abuse and poor nutrition are at risk of unrecognized vitamin B12 deficiency. Unilateral presentation of neuropathy and a normal vitamin B12 and MCV level make the diagnosis challenging.

\section{A CASE OF UNEXPLAINED COAGULOPATHY}

Amitabh Singh; Neil Evans; Jennifer Schmidt. Saint Louis University, Saint Louis, MO. (Control ID \#3185755)

LEARNING OBJECTIVE \#1: Recognize the public health emergency of Brodifacoum laced marijuanna

LEARNING OBJECTIVE \#2: Understand the mechanism of toxicity of brodifacoum and be able to manage toxicity

CASE: A 25-year-old male presented with bilateral lower quadrant abdominal pain radiating to his back starting one day prior to presentation. $\mathrm{He}$ also reported hematuria, decreased appetite. He denied new foods, medications, supplements or illicit substances and travel. On presentation, vital signs were normal. Abdomen was distended and tender. Computer tomography showed bilateral acute perinephric hemorrhage extending into the retroperitoneum and pelvis. Lab work showed $\mathrm{Hgb}: 10.1$, platelets 178, PTT $>212$, PT $>106$, INR $>10$. Urine drug screen was negative. The patient was admitted to the ICU and given fresh frozen plasma (FFP) and IV vitamin K. Workup showed factors II, IX, and X below 3 (normal range 65-175) and normal levels of factors V and VIII. Warfarin toxicity panel was positive for brodifacoum. PTT and INR trended down and normalized. Patient was discharged with oral vitamin K. On further discussion, the patient admitted to smoking synthetic marijuana.

IMPACT/DISCUSSION: As synthetic marijuana is not detected on standard urine drug screens, its use is increasing. Often, substances are added to increase or prolong its euphoric state. One such additive is brodifacoum, a coumadin-derived rodenticide. It inhibits the enzyme vitamin-K-epoxide reductase (VKOR), as mediator in the production of factor II, VII, IX, and X, resulting in low factor levels. Toxicity typically occurs by ingestion of laced-marijuana and symptoms directly correlate with amount ingested. Treatment is focused on repletion of Vitamin $\mathrm{K}$ with the addition of FFP for severe toxicity. During 2018, there have been publicized outbreaks of laced marijuana resulting in multiple deaths.

CONCLUSION: It is critical that internists are able to identify when patients present after ingestion of laced-illicit drugs - not only does this allow for appropriate workup and treatment, it is also essential in identifying public health emergencies.

\section{A CASE OF VITAMIN D MEDIATED HYPERCALCEMIA IN LYMPHOMA: CALCITONIN RESISTANCE?}

Priyanka Majety; Seda Babroudi; Feyza Erenler; Richard Siegel. Tufts Medical Center, Boston, MA. (Control ID \#3184551)

LEARNING OBJECTIVE \#1: There are multiple mechanisms of hypercalcemia in malignancies and treatment should be tailored accordingly to be effective

CASE: An 82-year-old male with history of hypertension presented with one month of generalized malaise, two weeks of shortness of breath and constipation. Physical exam and labs on admission were notable for moderate ascites, $\mathrm{Cr} 2.29 \mathrm{mg} / \mathrm{dL}, \mathrm{LDH} 253 \mathrm{IU} / \mathrm{L}$, Calcium (Ca) $12.8 \mathrm{mg} / \mathrm{dL}$, Hemoglobin $12.8 \mathrm{~g} / \mathrm{dL}$. Calcium level 6 months prior to admission was normal. Parathyroid hormone (PTH) was $7 \mathrm{pg} / \mathrm{mL}$ (low), Vitamin D 25OH D3 was 29ng/mL (normal). Workup was not suggestive of Multiple Myeloma. In the light of worsening renal function, he was started on Calcitonin that only decreased the Ca from $12.8 \mathrm{mg} / \mathrm{dL}$ to $12.6 \mathrm{mg} / \mathrm{dL}$. PTHrP was $14 \mathrm{pg} / \mathrm{mL}$ (normal) \& $1,25 \mathrm{OH}$ Vitamin D was $58 \mathrm{pg} / \mathrm{mL}$ which was inappropriately normal in the setting of suppressed PTH. There was no evidence of hyperthyroidism or Vitamin A toxicity. Paracentesis showed cloudy fluid and triglyceride level of $237 \mathrm{mg} / \mathrm{dL}$, consistent with chylous ascites raising the possibility of malignancy. CT abdomen revealed lymphadenopathy and inguinal lymph node biopsy confirmed angioimmunoblastic T-cell lymphoma. He received steroids as a part of the treatment, that decreased the $\mathrm{Ca}$ level. He was started on appropriate chemotherapy.

IMPACT/DISCUSSION: We discuss the management of hypercalcemia in a patient with new diagnosis of T-Cell lymphoma. Hypercalcemia occurs in about $20-30 \%$ of patients with cancer. It occurs with both solid tumors and hematologic malignancies. The most common cancers associated with hypercalcemia in the US are breast, renal, lung cancer \& multiple myeloma. The main mechanisms involved are parathyroid hormone related protein (PTHrP) secretion, osteolytic metastases with cytokine release, vitamin D-mediated and ectopic PTH (very rare). Our patient had non-PTH related hypercalcemia and a trial of calcitonin (which decreases serum Calcium level by inhibiting osteoclasts \& promoting bone resorption) failed, prompting that the mechanism here was not osteoclast mediated. His PTHrP level was normal ruling out humoral hypercalcemia. Although his $1,25 \mathrm{OH}$ Vitamin D level was normal, it was inappropriately high normal given the low PTH (responsible for 1hydroxylase enzyme upregulation) suggesting it is Vitamin D mediated. The hypercalcemia in lymphoma is due to PTH-independent extrarenal production of 1,25-dihydroxyvitamin $\mathrm{D}$ in malignant lymphocytes that causes increased intestinal absorption of Calcium. Steroids decrease the intestinal absorption of Calcium \& decrease calcitriol production in mononuclear cells. His Calcium level decreased to 10.1 after a course of steroids establishing the Vitamin D mediated mechanism. Anti-resorptive treatments are probably limited in treating hypercalcemia in lymphomas due to the underlying mechanism.

CONCLUSION: Understanding the pathophysiology and mechanisms of hypercalcemia in malignancy is important. This helps us choose the most effective treatment.

\section{A CASE REPORT DEMONSTRATING THE DIFFICULTIES IN DIAGNOSING ADULT TUBERCULOUS MENINGITIS}

Yi-Chia Wu $^{1}$; Laila Dadvand ${ }^{2} .{ }^{1}$ Mount Sinai Beth Israel, New York, NY; ${ }^{2}$ Icahn School of Medicine at Mount Sinai, Hoboken, NY. (Control ID \#3185333)

LEARNING OBJECTIVE \#1: Recognize the difficulties in diagnosing tuberculous meningitis

CASE: A 72 year-old female with history of hypertension and coronary artery disease presented with one month of worsening headache, described as a generalized sharp pain associated with nausea and vomiting. She had no neurological deficits, fevers, neck stiffness, confusion, nor recent travel. Vital signs were stable. Physical exam and labs were unremarkable; ESR and CRP were normal. Brain magnetic resonance imaging (MRI) with angiogram and venogram were also unremarkable. There was a concern for intractable migraine, so the patient was given several treatments, including benadryl, reglan, and toradol, without relief. Initial cerebral spinal fluid (CSF) showed lymphocytic pleocytosis, elevated protein, and normal glucose. CSF smear and culture were negative. A repeat CSF was performed a week later and revealed similar results except with elevated opening pressure and low glucose. The results were concerning for tuberculous meningitis (TBM). CSF adenosine deaminase (ADA) was obtained and was 2.1 $\mathrm{U} / \mathrm{L}$. On further questioning, the patient revealed her son had tuberculosis many years prior although she self-reported having had negative PPD testing. Given her history of tuberculosis exposure, non-resolving headache, and the CSF results, the patient was treated empirically for TBM with rifaximin, isoniazid, pyrazinamide, and ethambutol (RIPE) prescribed for six months. After a few doses of RIPE, the patient felt better and was discharged with follow-up at the tuberculosis clinic.

IMPACT/DISCUSSION: Diagnosing TBM is difficult given its nonspecific presentation in addition to slow and low sensitivity laboratory 
identification methods. Adult TBM often presents with worsening headache with vomiting and confusion for greater than 6 days. Studies show that TBM results in increasingly abnormal CSF composition and pressure with time, typically with high opening pressure, elevated leukocytes and protein, and low glucose. Current laboratory methods include CSF smear and culture, which are most commonly used. Although the acid-fast smear is quick and inexpensive, it requires about 10,000 organisms for a positive result, resulting in poor sensitivity. Studies have shown that a majority of TBM cases have a negative CSF smear. CSF culture takes 10-14 days to result and is highly dependent on laboratory expertise. Other methods include biochemical analysis, such as measuring CSF ADA, which helps to improve the diagnosis of TBM especially when bacterial meningitis is ruled out. ADA values $>10 \mathrm{U} / \mathrm{L}$ has high specificity $>90 \%$ for TBM; however, for $\mathrm{ADA}<10 \mathrm{U} / \mathrm{L}$, studies have demonstrated a wide range of sensitivities, which limit the diagnostic use of this test.

CONCLUSION: Therefore, given that there is a lack of reliable and rapid diagnostic tests for TBM and late treatment of TBM leads to poorer outcomes, one should consider treating TBM empirically if there is a suspicion for the disease.

\section{A COLD CASE OF A COLD ABSCESS}

Christine Park; Ian A. Downs; Rachel Simmons. Boston Medical Center, Boston, MA. (Control ID \#3185774)

LEARNING OBJECTIVE \#1: Recognize the broad differential of cold abscesses and utilize host risk factors in assessing a patient with an unusual infection

LEARNING OBJECTIVE \#2: Explore Klebsiella pneumoniae as a causative agent for a cold abscess

CASE: A 47-year old Vietnamese woman with a history of latent tuberculosis treated 21 years prior was admitted for a slow growing, tender, nonerythematous right anterolateral abdominal wall mass after a brief febrile respiratory illness 6 weeks prior. She was afebrile, hemodynamically stable and exam was unremarkable aside from the mass. Hematology and chemistry were unremarkable. CT scans of the chest, abdomen and pelvis showed pulmonary nodules, right middle lobe bronchiectasis, and large pleural, abdominal wall, and right upper quadrant subperitoneal rim-enhancing collections, concerning for a tracking abscess. Two induced sputum samples were negative for acid-fast bacteria. Cultures, cytology and pathology of aspirated abdominal wall fluid were negative for anaerobes, aerobes, fungi, mycobacteria or malignancy. Percutaneous drains were placed in the abdominal wall, and IV penicillin G was initiated for empiric Actinomyces species coverage. The abdominal wall collections improved but the pleural collection remained stable. Thoracentesis 25 days after initial presentation resulted in a culture positive for Klebsiella pneumoniae. Levofloxacin was started, and the abdominal wall mass improved, but with limited follow up.

IMPACT/DISCUSSION: Our patient presented with bronchiectasis and pleural effusion, which appeared to communicate with the "cold" intraabdominal and abdominal wall abscesses. Given her history, both tuberculosis and melioidosis were considered, but testing was negative. Acintomyces spp. infection is a cause of indolent pulmonary infections that can readily cross tissue planes, and this organism can be difficult to isolate from culture. After testing was unrevealing, Actinomyces treatment was started empirically but was ineffective. K. pneumoniae was the only agent isolated from numerous cultures. It is known to cause pulmonary, blood, intra-abdominal and urinary tract infections. In Taiwan, $21 \%$ of cases of lung abscesses were found to be due to $K$. pneumoniae and patients presented with $>30$ days of symptoms in 9 of 28 cases; however, the ability to cross tissue planes is not a typical feature, absent a necrotizing infection. Our ability to assess if K. pnuemoniae was the causative agent is limited due to lack of patient follow up. We would expect a response to fluoroquinolone treatment. Of note, although active pulmonary tuberculosis was ruled out and fluid and biopsy cultures were negative for mycobacteria, it is difficult to definitively rule out extrapulmonary tuberculosis.
CONCLUSION: $\bullet$ The differential for cold abscesses is broad, and may require extensive workup to confirm diagnosis $\bullet$ It is critical to rule out tuberculosis given the patient's immigration history, though extrapulmonary tuberculosis is difficult to exclude

\section{A COLD CASE OF DIGITAL ISCHEMIA}

Ryan Khodadadi; Muneeb Rehman; Absar Tahir; Delamo Bekele; Eric L. Matteson. Mayo Clinic, Rochester, MN. (Control ID \#3171697)

LEARNING OBJECTIVE \#1: Recognize type 1 cryoglobulinemia as a cause of digital ischemia in addition to other more common causes of vascular insufficiency.

LEARNING OBJECTIVE \#2: Distinguish between the differing types of cryoglobulins, their respective associations, and unique approaches to treatment. CASE: A 59 year-old Alaskan male with a history of nicotine dependence, coronary artery disease, hypertension, hyperlipidemia and multiple amputations presented for further evaluation of recurrent digital ischemia. A year prior, he experienced progressive bluish discoloration of the lower extremities with associated pain, swelling, and eventual digital necrosis resulting in the amputation of 3 digits despite normal vascular studies. Pathology revealed pauci-inflammatory small vessel occlusion by PASpositive homogeneous pink-purple material. Ten months after smoking cessation, he had recurrence of his symptoms with new left great toe necrosis, bilateral lower extremity ulceration, and intractable pain. Physical exam revealed amputated left $4 / 5$ th and right 5 th toes, black/blue discoloration of the left great toe, and periungual infarcts most prominent in the fingers without Raynaud's phenomenon. CT imaging, echocardiogram, and repeat vascular studies were unremarkable. Autoimmune serologies were negative but further workup revealed an elevated ESR and CRP, a SPEP with an increased M-spike in the gamma globulin portion, elevated total protein with elevated kappa free light chains (2.8) and a $10 \%$ type 1 cryoglobulin component.

IMPACT/DISCUSSION: Cryoglobulinemia is characterized by the precipitation of proteins including immunoglobulins and complement proteins in the blood below $37^{\circ} \mathrm{C}$. Type 1 cryoglobulinemic $(10-15 \%$ of cases) precipitate tends to be monoclonal in origin, related to an underlying lymphoproliferative disorder whereas a polyclonal cryoprecipitate is implicated in mixed cryoglobulinemia and classically associated with an underlying rheumatologic disorder or viral infections. Clinically, type 1 manifests with signs and symptoms of vascular insufficiency including digital ischemia, skin necrosis, and livedo reticularis. A diagnosis is established by laboratory isolation of cryoprecipitate and cryocrit measurement with treatment directed toward the underlying disorder to prevent hyperviscosity and further vascular injury.

CONCLUSION: It's important for the internist to recognize common causes of vascular insufficiency as well as type 1 cryoglobulinemia which is often driven by an underlying lymphoproliferative disorder, warranting further investigation and appropriately-targeted therapy. Prognosis and complications in patients with type 1 cryglobulinemia correlate with the underlying hematologic disorder and its treatment course. This patient's bone marrow aspirate and biopsy revealed a CD-20 negative kappaexpressing monoclonal plasma cell population. He ultimately underwent apheresis with resolution of cryoglobulinemia and treatment with prednisone followed by bortezomib for an underlying lymphoproliferative disorder (MGUS).

\section{A COMMONLY USED ANTIBIOTIC WITH A RARE, SEVERE, CUTANEOUS ADVERSE REACTION}

Shahrzad Shidfar. umassmemorial, Worcester, MA. (Control ID \#3186317)

LEARNING OBJECTIVE \#1: Recognize and distinguish clinical presentation of AGEP 
CASE: 44 year-old paraplegic man was admitted for left second toe osteomyelitis. He had open wound on left second toe, normal vital signs and white blood cell counts. He was started on vancomycin intravenously and underwent amputation of left second toe on hospital day3. His antibiotic was changed to doxycycline $100 \mathrm{mg}$ by mouth every 12 hours with the first dose given evening of operative day. The next morning patient complained of mild itchiness around the neck which he attributed to "not used to hospital sheets". There were no visible rash. He had normal vital signs and minimal pain at the surgical site. CBC showed leukocytosis of 11000 per microliter which was attributed to stress. Patient was discharged home on doxycycline. Later in that evening patient noted increase pruritus around the neck and upper body. The next morning (post-operative day 2) he noted erythmatous rash around the neck spreading to extremities and trunk. He also felt swollen painful "glands" on his neck. Patient was readmitted to the hospital. He had normal vital signs. There was a diffuse pink morbilliform rash with confluent erythema and scattered pustules over the back, chest and the medial aspects of bilateral thighs. There were 2 tender enlarged right-sided cervical nodes. There were neither genital or oral mucosal involvement nor facial edema. He had a Leukocytosis of 15000 per microliter without bandemia or eosinophilia. Liver function tests were normal. Skin punch biopsy showed acute generalized exanthematous pustulosis (AGEP). Doxycycline was discontinued and patient was started on triamcinolone $0.1 \%$ topical ointment twice daily.

IMPACT/DISCUSSION: Acute generalized exanthematous pustulosis (AGEP) is a rare, severe cutaneous adverse reaction characterized by the rapid development nonfollicular, sterile pustules on an erythematous base. It is attributed to drugs in the majority of cases with Antibiotics being considered as the most common cause. Fever and peripheral blood leukocytosis are usually present. The time period from drug exposure to reaction onset is typically within 48 hours, with antibiotics having a median of 24 hours. Generally, with prompt discontinuation of the offending agent and general supportive care, prognosis for patients with AGEP is excellent. Our patient was exposed to vancomycin and doxycycyline both but treatment with doxycycline started later than the treatment with vancomycin and signs and symptoms of AGEP appeared within 1 day after the start of doxycycline therapy which make doxycycline as more likely the cause of the AGEP.

CONCLUSION: Although AGEP is rare, its manifestations can be prone to misinterpretation. In contrast to most drug reactions, AGEP is manifested as pustules, thus, it is easily confused with other pustular eruptions and infectious processes. A thorough knowledge of the disease process of AGEP is important to avoid the dangerous continuation of the offending antibiotic.

\section{A COMPLEX CASE OF MYOCARDIAL BRIDGING}

Omar Hadzipasic ${ }^{1}$; Ahmed Nagy ${ }^{1}$; Adedotun Ogunsua ${ }^{2}$; Owusu Asamoah $^{2} .{ }^{1}$ University of Massachusetts Medical School, Shrewsbury, MA; ${ }^{2}$ University of Massachusetts Medical School, Worcester, MA. (Control ID \#3161489)

LEARNING OBJECTIVE \#1: Distinguish myocardial bridging from coronary artery disease

LEARNING OBJECTIVE \#2: Manage recurrent angina in myocardial bridging

CASE: A 58-year-old man with a history of hypertension and hyperlipidemia presented to the Emergency Department with anterior substernal chest pressure which started while he was cleaning his grill. EKG was notable for ST segment elevation in the anterolateral leads with elevated troponin to 25.5 . He underwent emergent coronary angiography which showed an anterolateral STEMI caused by a mid left anterior descending (LAD) $100 \%$ thrombotic occlusion and had successful thrombectomy and balloon angioplasty of the mid-LAD. He was started on Aspirin and Ticagrelor and treated with a Tirofiban IV infusion for 48 hours. Two days later, he underwent Ocular coherence tomography/Intravascular ultrasound (OCT/IVUS) of the LAD which demonstrated severe myocardial bridging in the mid-LAD. The patient presented to the hospital again two weeks later with the same chest pain. It was not relieved with nitroglycerin and he had not missed any doses of his DAPT therapy. EKG was notable for anterior lead Q waves with $\mathrm{T}$ wave inversions, troponin elevated to 6.02 . Repeat coronary angiography again demonstrated myocardial bridging of the mid-LAD without occlusion. Recommendation was made for maximizing his Metoprolol dose and continued treatment with Aspirin, Ticagrelor, and Atorvastatin.

IMPACT/DISCUSSION: Myocardial bridging is an uncommon medical disorder that must be considered in the differential diagnosis for recurrent anginal chest pain. In this condition, the coronary artery traverses through an intramyocardial course and is compressed during systole. Although most instances are of limited clinical significance, our patient had experienced repeat infarctions over time and developed an ischemic cardiomyopathy with reduced ejection fraction of $40 \%$. Medical therapy is aimed at reducing heart rate and myocardial contractility through the use of beta blockers and calcium channel blockers, as well as procedural intervention via coronary artery stenting. For persistent symptoms, surgical intervention with myocardial bridge resection can be performed. Importantly, nitrates are contraindicated given the risk for reflex increase in contractility. Although similar in presentation to acute coronary syndrome from classic coronary artery disease, myocardial bridging differs in medical and interventional therapy. This case highlights how crucial it is to recognize and distinguish myocardial bridging from coronary artery disease to avoid recurrence and sequelae of continued myocardial infarction.

CONCLUSION: Myocardial bridging is an uncommon condition which presents as acute coronary syndrome and can be diagnostically indistinguishable from myocardial infarction until coronary angiography is performed. Initial medical therapy is aimed at the use of beta blockers and calcium channel blockers. Nitrates are contraindicated given the risk for reflex increase in contractility.

\section{A COMPLICATED CASE OF PSEUDOMONAS ENDOCARDITIS PRESENTING AS A SUBARACHNOID HEMORRHAGE}

Pedro Covas ${ }^{1}$; Alexander E. Aldrich ${ }^{2}$; Adrienne N. Poon ${ }^{3} .{ }^{1}$ George Washington University, Washington, DC; ${ }^{2}$ The George Washington University Hospital, Washington, DC; ${ }^{3}$ George Washington School of Medicine and Health Sciences, Clifton, NJ. (Control ID \#3149722)

LEARNING OBJECTIVE \#1: Neurological sequela of infective endocarditis

LEARNING OBJECTIVE \#2: Diagnosis and treatment of Pseudomonas endocarditis

CASE: A 60 year-old woman with intravenous drug use (IVDU), presented with a week of cough, shortness of breath, and lower extremity edema. The patient was afebrile, tachycardic, and tachypneic. Lower extremity edema and bibasilar crackles were present, but had a normal cardiac examination. Labs were significant for leukocytosis to 18,000 , platelets of 69 , hemoglobin of 7.6, creatinine of 2.9, and BNP of 11,000. $\mathrm{CT}$ chest revealed bilateral septic emboli to the lungs and transthoracic echocardiogram (TTE) revealed vegetations on the tricuspid valve. Transesophageal echocardiogram (TEE) was unable to be performed. CT head revealed a subarachnoid hemorrhage (SAH). MRA was negative for aneurysm. Broad spectrum antibiotics were initiated for infective endocarditis (IE) and after blood cultures returned positive for P. aeruginosa, antibiotics were adjusted to meropenem and ceftazidine. Blood cultures remained positive for 10 days until renal function improved and gentimicin was added. Surgical management was not pursued given $\mathrm{SAH}$ and she completed 6 weeks of intravenous antibiotics. She was later readmitted for reoccurrence of $\mathrm{P}$. aeruginosa IE when TEE revealed an aortic vegetation and tricuspid vegetation. Her condition rapidly deteriorated and died from sepsis and multiorgan failure.

IMPACT/DISCUSSION: Pseudomonas Aeruginosa is a rare cause of IE seen most often with concomitant IVDU. Recognizing that IE may have multiple neurological sequelae is essential in management as treatment and prognosis varies significantly depending upon involvement. Ischemic 
strokes commonly result from embolization, but SAH is a rarer complication, especially spontaneous, as most result from mycotic aneurysm rupture. Pseudomonas endocarditis is a rare form of IE and treatment is not clearly established, but generally includes an aminoglycoside with a secondary pseudomonal agent and consideration of surgical resection of the vegetation. The role of echocardiography in IE is extremely helpful for diagnosis and TEE should be pursued if TTE not indicative. Our patient had progression of her IE with new left-sided involvement. Mortality is higher with left sided endocarditis and surgery should urgently be pursued if possible. We were able to confirm that the vegetation decreased in size with a repeat echocardiogram, but serial echocardiograms could be useful in surveillance with antibiotic therapy to confirm resolution of the vegetations as P. aeruginosa endocarditis has no established antibiotic duration. One can consider echocardiograms to confirm resolution to prevent reoccurrence, as it occurred with our patient. Further research is needed to establish optimal antibiotics therapy.

CONCLUSION: We present a case of Psuedomonas endocarditis that highlights recognizing SAH as an uncommon neurological sequelae of IE, treatment with the importance of an aminoglycoside, and the role of echocardiography in IE.

\section{A COMPLICATED CASE OF PV}

Hanyuan Shi. Tulane University School of Medicine, New Orleans, LA. (Control ID \#3185271)

LEARNING OBJECTIVE \#1: Demonstrate a complication of polycythemia vera

LEARNING OBJECTIVE \#2: Illustrate diagnostic testing for ischemic colitis

CASE: A 63-year-old female with a history of polycythemia vera (JAK2 mutation and hypertension presented with acute onset of diffuse colicky abdominal pain and hematochezia. She had no prior weight loss, weakness, or anorexia. The patient described liquid bowel movements with overt blood. She had been managed with aspirin $325 \mathrm{mg}$ daily, dipyridamole $50 \mathrm{mg}$ TID, hydroxyurea $200 \mathrm{mg}$ daily at home. She underwent monthly phlebotomies that had been uncomplicated per her outpatient hematology provider. Her vitals were stable, albeit for mild hypertension at 152/76. Initial labs were significant for WBC 6.9, hemoglobin 13.8, and platelets of 447. INR was normal at 1.1. CT abdomen/pelvis showed thickening of the gastroesophageal junction and adenomyomatosis. Infectious stool studies were negative. She had a screening colonoscopy two months ago that was negative for colonic abnormalities, only non-bleeding internal hemorrhoids. During her admission, she underwent a flexible sigmoidoscopy. Findings included congested, erythematous, inflamed and ulcerated mucosa in the descending colon, sigmoid, and rectum likely secondary to colon ischemia. The hematology service was consulted for phlebotomy and the patient had $500 \mathrm{ml}$ of blood removed with resolution of symptoms. Confirmation biopsies came back a week later showing descending colon and rectum with atrophic colonic epithelium consistent with ischemic changes. The biopsy was negative for evidence of chronic colitis, as well as negative for dysplasia or malignancy.

IMPACT/DISCUSSION: Polycythemia vera (PV) is a myeloproliferative disorder characterized by increase in red blood cell, white blood cell, and platelets. Thrombotic complications are unfortunately not rare, and can include cerebral infarction, thrombophlebitis, myocardial infarction, and ischemic colitis. Here we demonstrate an acute case of ischemic colitis (IC) (biopsy-proven) from PV, only the $4^{\text {th }}$ documented case in the literature, that occurred 1-2 months after a normal colonoscopy. Ischemic colitis from PV is reversible but requires quick and precise diagnostic effort. Although enhanced abdominal CT and CT enterography are the gold standards for IC, a colonoscopy or a flexible sigmoidoscopy can also be used for definitive histopathological diagnosis. In patients with PV, biopsies of colonic lesions can more accurately differentiate IC from chronic inflammatory bowel diseases. Treatment of IC relies on controlling the primary cause. In this case, that involved expedited phlebotomy for PV; other therapies have included anticoagulation with LMWH and initiation of mesalazine.

CONCLUSION: Internists are often asked to coordinate care from different subspecialty services. This case highlights a complication of polycythemia vera and the use of consultant services to aid in diagnosis and management of this patient.

\section{A COOL CASE REPORT: SPONTANEOUS PERIODIC HYPOTHERMIA}

Hayley N. Price; Jennifer Jacobs; Alexander Hajduczok; Ami DeWaters. Penn State Hershey Medical Center, Hershey, PA. (Control ID \#3185792)

LEARNING OBJECTIVE \#1: Describe the etiologies and evaluation of hypothermia.

LEARNING OBJECTIVE \#2: Discuss the pathophysiology of spontaneous periodic hypothermia and how that may guide treatment options.

CASE: A 66-year-old man with dementia was transferred to the hospital from a local skilled nursing facility with recurrent hypothermia. On presentation, he was obtunded and unable to provide a history. He had been hospitalized twice that same year with a similar presentation. An extensive workup had been done during those admissions and was unrevealing. On admission, his temperature was 32 degrees Celsius, with a pulse of 44 , and blood pressure of $92 / 56$. On physical exam, he was obtunded. Pupils were equal, round, and reactive to light; more complete neurologic exam was deferred due to inability for him to participate. Laboratory testing demonstrated chronic anemia with a hemoglobin of 7.2 and chronic kidney disease with a creatinine of 2.3, with a normal TSH and morning cortisol. A urine toxicology screen was negative. He was treated with a Bair Hugger blanket as well as supportive care. His temperature spontaneously returned to normal after two days and the Bair Hugger was discontinued. His mental status improved with resolution of hypothermia, and he was discharged back to his facility with the presumptive diagnosis of spontaneous periodic hypothermia.

IMPACT/DISCUSSION: Hypothermia has multiple etiologies presenting a diagnostic challenge. Causes of hypothermia are commonly divided into the following categories: exposure, metabolic, infectious, and central. Exposure can be both intentional, for instance in an operating room, or unintentional, such as accidental cold water immersion. These etiologies can be determined by history. Metabolic etiologies include hypothyroidism, adrenal insufficiency and hypopituitarism. Sepsis can result in hypothermia. Central causes include trauma or stroke. Therefore, the evaluation of hypothermia should include TSH, morning cortisol, full infectious work-up with cultures, and head imaging, including MRI. If no etiology is discovered, the differential may need to be expanded further, as in this case. Spontaneous Periodic Hypothermia is a rare cause of hypothermia characterized by recurrent episodes of hypothermia thought to be mediated by central nervous system pathology without a clearly identifiable brain lesion. Though the exact pathophysiology is unclear, the cyclic nature of episodes has led to the thought that the pathophysiology may be similar to epilepsy. Treatment with antiepileptic medications has been trialed with success in a number of case reports. Further research is necessary to determine if these medications are truly effective.

CONCLUSION: Evaluation of a patient with hypothermia mandates consideration of a broad differential, particularly in cases in which a clear etiology is not evident. Spontaneous Periodic Hypothermia is a rare cause of hypothermia that may respond to treatment with antiepileptic medications.

\section{A COUGH MAY JUST BE THE TIP OF THE ICEBERG}

Ian Cormier; Michael P. Smith. University of Nebraska Medical Center, Omaha, NE. (Control ID \#3186036)

LEARNING OBJECTIVE \#1: Develop an approach to liver abscesses LEARNING OBJECTIVE \#2: Recognize the association of pyogenic liver abscesses and occult malignancy 
CASE: A 61-year-old man presented with two weeks of cough. His cough was productive of yellow sputum with associated shortness of breath. He was initially diagnosed with a viral syndrome and treated conservatively. His symptoms worsened, and he developed new onset frontal headaches, fevers up to $103 \mathrm{~F}$, myalgias and chills. He had a past medical history of malignant melanoma excised in 2006 and pyogenic liver abscess (PLA) treated in 2008. The patient was afebrile with normal vitals. He had normal cognition, poor dentition, and diminished breath sounds with crackles noted in the right lung base. His white blood cell count was elevated with bandemia and a procalcitonin of 12 . Chest $\mathrm{x}$-ray revealed bilateral lower lung and right midlung infiltrates. The patient was given intravenous vancomycin and piperacillin-tazobactam and was admitted for community acquired pneumonia (CAP). He was transitioned to intravenous levofloxacin for CAP. Overnight, the patient became febrile to 102.8, tachycardic and tachypneic. Vancomycin and piperacillintazobactam were restarted. Blood cultures were drawn and a computed tomography (CT) of the chest, abdomen and pelvis revealed multiple bilateral pulmonary nodules with early cavitation concerning for septic emboli and a large multilocular hepatic hypodensity in the right lobe of the liver concerning for liver abscess. Gram stain of the abscess was positive for gram-negative rods. Anaerobic cultures grew Fusobacterium nucleatum. Blood cultures were negative. A peripherally inserted central catheter line (PICC) was placed and the patient was discharged on ampicillin-sulbactam along with an outpatient colonoscopy to evaluate for occult malignancy.

IMPACT/DISCUSSION: Pyogenic Liver abscesses, which are typically polymicrobial. Other causes of liver abscess include malignant, iatrogenic, and other infectious sources with overlap between the three categories being common. Many pyogenic liver abscesses result from portal vein pyemia, usually related to bowel leakage and peritonitis. Biliary, Pelvic, and other intraperitoneal sources are the most common source of infection in hepatic abscesses along with hematogenous spread. Fusobacterium spp. are anaerobic gram negative rods that occur as normal oral flora and have been associated with periodontal infections. However, they are uncommon causes of pyogenic liver abscesses. Pyogenic liver abscesses have a known association with GI malignancies and Fusobacterium nucleatum has recently been shown to promote colorectal carcinogenesis by affecting genes associated with the adenoma-carcinoma sequence. The oral cavity could be a potential source of infection for this patient, however, occult GI malignancy must be ruled out.

CONCLUSION: The general internist must diagnose and treat a pyogenic liver abscess and understand its implications for evaluation for malignancy

\section{A CURIOUS CASE OF CARDIAC TAMPONADE WITHOUT TACHYCARDIA}

LU CHEN ${ }^{1}$; Uta Guo ${ }^{3}$; PARAG MEHTA ${ }^{2}$; Tristan Tanner ${ }^{4} .{ }^{1} \mathrm{NEW}$ YORK PRESBYTERIAN - BROOKLYN METHODIST HOSPITAL, Brooklyn, NY; ${ }^{2}$ NewYork-Presbyterian BMH Hospital, Brooklyn, NY; ${ }^{3}$ SUNRISE GME CONSORTIUM, LAS VEGAS, NV; ${ }^{4}$ New York Presbyterian - Brooklyn Methodist, Brooklyn, NY. (Control ID \#3186251)

LEARNING OBJECTIVE \#1: Diagnosing and managing subacute cardiac tamponade

CASE: A 67-year-old female with a past medical history of hyperthyroidism and total thyroidectomy, presented with progressive confusion and respiratory distress over the past three months. Patient became more lethargic and confused over the past month, with episodes of hallucination. She became bed bound a week prior to admission. Patient stopped taking her levothyroxine over a year ago. On admission, she was afebrile at $98.0 \mathrm{~F}$, with blood pressure of 147/84 with heart rate of 70 . Physical exam was significant for lethargy, facial swelling, and macroglossia. Decreased breath sounds in the left lung base were noted along with bibasilar crackles. No murmurs, clicks, gallops or rubs were appreciated on the cardiac exam. Laboratory finding was significant for a serum sodium level of $133 \mathrm{mmol} / \mathrm{L}$, Thyroid Stimulating Hormone (TSH) at $58.5 \mathrm{ng} / \mathrm{dL}$, and Free Thyroxine (T4) of $0.19 \mathrm{ng} / \mathrm{dL}$. Electrocardiogram (EKG) showed low voltage QRS complexes with electrical alternans. Chest Radiography (CXR) was significant for consolidation, atelectasis or effusion in the area of left middle and lower lung region. Patient was started on levothyroxine and hydrocortisone. Echocardiogram (Echo) showed normal systole function of the left and right ventricles. No valvulopathy was identified. A large pericardial effusion with swinging of the heart in the pericardial sac was noted. Significant chamber collapse of both atria, as well as the right ventricle, was also noted. Inferior Vena Cava was dilated and showed no decrease in size with inspiration. Patient received pericardiocentesis with $1300 \mathrm{ml}$ serous straw-colored fluid drained. Analysis of pericardial fluid was negative for malignant cells. Acid-fast bacilli stain and culture were negative. Patient was discharged on levothyroxine. Serial TSH and T4 measurements over two months showed normalization of both hormone levels. Repeat echo showed diminishing pericardial effusion.

IMPACT/DISCUSSION: Subacute Cardiac Tamponade (SCT) develops over weeks to months and can have a subtle presentation. The most common sign of SCT is sinus tachycardia. Due to the slow development of the pericardial effusion, patients may not be hemodynamically compromised despite the presence of a large effusion. In our patient, tachycardia was possibly masked by baseline bradycardia from myxedema coma secondary to hypothyroidism. Additional physical findings associated with SCT include hypotension, pulsus paradoxus, and elevated jugular venous pressure. EKG, CXR, and Echo should be obtained to aid diagnosis and facilitate treatment.

CONCLUSION: Thorough physical exam and a high level of clinical suspicion are needed to prevent a delay in diagnosis of subacute cardiac tamponade, especially in the setting of myxedema coma.

\section{A CURIOUS CASE OF EOSINOPHILIA}

Elizabeth Thottacherry ${ }^{1}$; Ali Hassoun ${ }^{2} .{ }^{1}$ University of Alabama School of Medicine, Huntsville, AL; ${ }^{2}$ Alabama Infectious Diseases Center, Huntsville, AL. (Control ID \#3186072)

LEARNING OBJECTIVE \#1: Recognise rare presentations of eosinophilia

LEARNING OBJECTIVE \#2: Differentiate infectious, immunological and hematological causes of eosinophilia

CASE: A 62 year old female from India on dialysis for 2 years presented with chest pain occurring in dialysis. Chest pain was midsternal, occurring with dialysis and had started the week prior. Examination revealed an alert female, comfortable on room air and afebrile. Her cardiac exam was normal and no rashes or lymphadenopathy were noted. Labs reflected a white count of $43.7 \times 103 / \mathrm{uL}$ (reference: $4.1-12.2 \times 103 / \mathrm{uL}$ ) and absolute eosinophil count of $37.2 \times 103$ (reference: 0.0-0.5 x 103/uL). Her cardiac enzymes were unremarkable and her kidney function was at baseline. Blood and stool cultures, ova and parasite screens and $\operatorname{IgE}$ levels were sent as well as a strongyloides and filarial panel given her Indian background. While cultures and parasite screen were negative, her IgE levels resulted grossly elevated. Peripheral smear and flow cytometry were inconclusive. Cardiac investigation revealed nonobstructive coronary disease. Bone marrow biopsy resulted showing eosinophilia and neutrophilia lacking malignancy. The patient attempted to continue dialysis, however again experienced chest pain. At this point a variety of differentials were considered with a discussion about recent lifestyle changes. The patient noted that she recently changed dialyzer membranes from polyarylethersulfone to polysulfone. Steroid therapy was started and her dialyzer membrane was changed back with immediate cessation of symptoms. Her eosinophilia improved and a polysulfone allergy was recorded.

IMPACT/DISCUSSION: Eosinophilia can be categorised into primary/ clonal, secondary/reactive or idiopathic. Diagnosis can be challenging due to the differentials present. After a detailed history is obtained, initial work 
up includes a complete blood count, liver and kidney function, peripheral smear, c-reactive protein, erythrocyte sedimentation rate and bone marrow biopsy. While investigating the above case, causes of secondary eosinophilia were examined while ruling out primary eosinophilia. With the patient's subtropical background, infectious and parasitic causes were initially ruled out. Her elevate IgE prompted investigation into allergic causes and drugs. Primary clonal eosinophilia and malignancies were ruled out with a bone marrow biopsy. The temporal association of symptoms with dialysis led us to question changes in dialysis causing her symptoms. Polysulfone diasylate membranes are associated with increased incidence of hemodialysis associated eosinophilia with resolving eosinophilia noted after membrane change. The rapid response with oral steroids as well as changing the diasylate membrane further confirmed that the patient may be having an immune mediated reaction to the Polysulfone membrane.

CONCLUSION: Eosinophilia is seen in a variety of different clinical scenarios. A meticulous history and thorough investigation is key to forming a diagnosis and carrying out appropriate treatment.

\section{A CURIOUS CAUSE OF ABDOMINAL PAIN IN A PATIENT WITH ANAPLASMOSIS}

Julia Cooperman. Beth Israel Deaconess Medical Center, Boston, MA. (Control ID \#3173639)

LEARNING OBJECTIVE \#1: Diagnose and manage atraumatic splenic rupture non-operatively.

LEARNING OBJECTIVE \#2: Recognize atraumatic splenic rupture as a complication of tickborne illness.

CASE: A 35 year old gentleman with a history notable for a spontaneous splenic hemorrhage and splenectomy in 2012 presented to the emergency department with fevers, myalgias and arthralgias 5 days after a deer tick bite. He was febrile. Initial labs were notable for a normal WBC count with neutrophil predominance. No parasites were seen on a parasite smear. He started Ceftriaxone and was admitted to the medical service. Overnight he complained of 10/10 peri-umbillical pain, diarrhea, and emesis. Labs showed bandemia of $14 \%$ and hemoglobin drop from 13.2 to 9.2. CT scan with IV contrast showed a large amount of hemorrhagic free fluid in the left upper quadrant around the splenic remnant, with a curvilinear area of hyperdense blood highly suspicious for active hemorrhage. He received 2 units of packed red blood cells and underwent emergent splenic arteriogram which did not reveal a source of active hemorrhage. He remained hemodynamically stable and required no further blood transfusions. He was found to have a positive Anaplasma PCR. Patient defervesced and felt improved. The remainder of his tickborne panel was negative. He was discharged with oral doxycycline.

IMPACT/DISCUSSION: Atraumatic splenic rupture is uncommon. Its exact incidence is unknown and there are no systematic studies defining its clinical presentation, but it is an important entity to recognize as mortality can be up to $12 \%$. $^{1}$ Most common causes identified are neoplastic, infectious, and inflammatory. Clinical presentation can include left sided abdominal pain and/or left shoulder-tip pain from diaphragmatic irritation and signs of hemorrhage. Rupture is confirmed by either CT scan, ultrasound, or laparotomy in hemodynamically unstable patients. With increasing availability of angiography, current practice is trending toward non-operative management. ${ }^{2}$ Therefore, diagnosis and management of splenic rupture is an increasingly important skill for general medical providers. Patients may not present with abdominal pain or any history of trauma, which may delay diagnosis if clinicians are not on alert for this potentially fatal complication. One case of Anaplasmosis associated with splenic rupture has been described. ${ }^{3}$ There are more reports of Babesiosis associated with splenic rupture. ${ }^{4}$ Any practitioner may encounter a patient with tickborne illness and should be familiar with its potential complications. 1. Renzulli et al. (2009) 2. Miller et al. (2014) 3. Khan and Ali (2018) 4. Li et al (2018)
CONCLUSION: Atraumatic splenic rupture is a potentially fatal complication in conditions commonly encountered by general medical practitioners. Its management is increasingly non-operative, and as such, important for providers to diagnose and manage. It is also important to recognize atraumatic splenic rupture as a potential complication of tickborne illness, even in post-splenectomy patients.

\section{A DANGEROUS WEB}

Nikita Agrawal $^{1}$; Daniel Labovitz ${ }^{2} .{ }^{1}$ Montefiore Medical Center (Moses \& Weiler), New York, NY; ${ }^{2}$ Montefiore Medical Center, Bronx, NY. (Control ID \#3184787)

LEARNING OBJECTIVE \#1: Recognize carotid web as a cause of stroke.

LEARNING OBJECTIVE \#2: Recognize imaging findings consistent with carotid stroke and other diagnosis with similar findings.

CASE: A 42-year-old woman with no known stroke risk factors woke up with slurred speech and left arm paresis. MRI brain and MRA head/neck demonstrated MCA infarction with insular cortex involvement and mild stenosis of the proximal right cervical internal carotid artery. CTA of the carotid demonstrated a low density filing defect along the posterior wall of the proximal right internal carotid artery. Carotid web $(\mathrm{CaW})$ was suspected based on imaging features. She was placed on IV heparin followed by carotid enterectomy confirming the presence of a CaW, which was removed. Patient was discharged on dual antiplatelet therapy for a three month course and lifelong aspirin and statin.

IMPACT/DISCUSSION: Carotid web $(\mathrm{CaW})$ is an under recognized cause of stroke in young patients without classical risk factors. This case illustrates the importance of considering $\mathrm{CaW}$ as a cause of anterior circulation ischemic stroke. $\mathrm{CaW}$ is a variant of fibromuscular dysplasia that appears in the posterior aspect of the carotid bulb at the origin of the internal carotid artery. CaW can be diagnosed using imaging including US CTA, MRI or digital subtraction angiography (DSA). On imaging CaW appears as a shelf like structure. Whereas atherosclerotic lesions are irregular with a thickening of the carotid wall, $\mathrm{CaW}$ is distinguished by smooth borders of the lesion itself and of the vessel wall. Moreover, $\mathrm{CaW}$ can be mistaken for arterial dissections as the shelf on the web can appear as a dissection flap. In comparison arterial dissections classically affect the middle or distal cervical segments of the ICA and present with irregular borders. Moreover, dissections may include enlargement of the arterial diameter whereas $\mathrm{CaW}$ is associated with normal bulb shape. Currently epidemiologic data on $\mathrm{CaW}$ is limited as it remains an underrecognized pathology. At large, $\mathrm{CaW}$ is more common in young women. Currently there have not been studies examining the genetic propensity for the pathology. For ischemic strokes in CaWs, there is currently no optimal secondary stroke prevention strategy. Initial data has demonstrated that antiplatelet monotherapy may be insufficient in preventing recurrent ischemic events with reoccurrence of events occurring in nearly one third of patients. Revascularization with surgical resection has increasingly been used in prevention of carotid webs with no case reports of reoccurrence following resection. Carotid artery stenting has also been described as an alternate to surgical resection with a study demonstrating no reoccurrence in a 12 month follow up.

CONCLUSION: It is important to consider CaW as an etiology of stroke in young patients with limited risk factors. Given the life altering nature of strokes, prevention of repeat events with appropriate therapy is imperative. Strokes associated with CaW may be better managed with surgical intervention.

A DEADLY MYX: HYPOTHYROIDISM AND AMPHETAMINES Syeda A. Raza. Methodist Health Systems Dallas, Dallas, TX. (Control ID \#3168551)

LEARNING OBJECTIVE \#1: Recognize that myxedema coma can be precipitated by amphetamine use 
CASE: A 56- year old male presented with encephalopathy after being found unresponsive at home. On examination, patient's vitals were notable for blood pressure of $90 / 74 \mathrm{mmHg}$, pulse of 43 , and temperature of 97.6 degrees Fahrenheit. Physical exam revealed an obtunded, disheveled male with bilateral periorbital edema. Labs were notable for pancytopenia and elevated liver function tests. Further lab tests demonstrated TSH of $110 \mathrm{mU} / \mathrm{L}$ with Free T4 of $0.25 \mathrm{mU} / \mathrm{L}$. A subsequent urine drug screen was positive for amphetamines. Patient was admitted to the ICU and was treated for myxedema coma presumed to be triggered by recent amphetamine use. Patient was treated with warming blankets, IV hydrocortisone, and IV levothyroxine. He had improvement of his mentation and subsequent labs over the next few days showed resolution of pancytopenia and transaminitis. TSH also noted to downtrend appropriately. As patient became more alert, he was questioned and admitted to history of hypothyroidism with non-compliance to medication. He further reported recent use of amphetamine prior to hospitalization.

IMPACT/DISCUSSION: Myxedema coma is an extreme but rare manifestation of hypothyroidism, resulting in life-threatening secondary systemic decompensation. It is usually precipitated by an inciting event, such as infection, stroke, trauma, substance use, or cold exposure. In our case, the inciting event was noted to be secondary to recent amphetamine use. It is important for clinicians to develop broad differential diagnosis to prevent premature closure. Our patient's case was initially concerning for drug overdose but was found to be due to myxedema coma.

CONCLUSION: Myxedema coma is a medical emergency that necessitates immediate care. Management is necessary prior to confirming diagnosis as delay in treatment is associated with high mortality rates. It is important for clinicians to be aware of amphetamine use as a possible trigger for myxedema coma.

\section{A DIAGNOSIS OF MGUS, A COMMON BUT COVERT DISEASE, IN A PATIENT WITH RECENT MYOCARDIAL INFARCTION}

Daniel Clement ${ }^{1}$; Eun Ji Kim ${ }^{2} .{ }^{1}$ Northwell Health, Manhasset, NY; ${ }^{2}$ Northwell Hofstra School of Medicine, Lake Success, NY. (Control ID \#3172918)

LEARNING OBJECTIVE \#1: Diagnose MGUS and differentiate it from other plasma cell disorders

LEARNING OBJECTIVE \#2: Recognize the association between thrombotic disease, including myocardial infarction, and MGUS

CASE: 76 year-old African American female with diabetes mellitus, hypertension, hyperlipidemia, and coronary artery disease presented for establishment of care after a recent hospitalization for NSTEMI. She was a lifelong non-smoker and denied family history of malignancy. Physical examination was within normal limits. Laboratory studies were relevant for a hemoglobin of $10.5 \mathrm{~g} / \mathrm{dL}$ (normal 11.5-15.5) with normal platelets and leukocytes. Total protein was elevated at $8.6 \mathrm{~g} / \mathrm{dL}$ (normal $6.0-8.3$ ). The patient's liver function, creatinine, albumin, and calcium were within normal limits. Serum and urine protein electrophoresis were obtained because of her anemia and elevated protein level. These were relevant for an elevated level of Gamma-migrating paraprotein with $\mathrm{M}$-spike in the serum with no evidence of a paraprotein in the urine. The patient was referred to hematology for further evaluation. Repeat serum protein electrophoresis confirmed the presence of a paraprotein. A skeletal survey showed no evidence of lytic lesions. Given these findings, the patient was diagnosed with Monoclonal Gammopathy of Unknown Significance (MGUS). She is currently scheduled for a bone marrow biopsy.

IMPACT/DISCUSSION: Diagnosis of MGUS is defined as the presence of an M-spike on serum protein electrophoresis less than $3 \mathrm{~g} / \mathrm{dL}$, fewer than $10 \%$ plasma cells on bone marrow biopsy, and the absence of hypercalcemia, renal insufficiency, severe anemia, and bony lesions. MGUS is a common disease and affects higher percentages of elders (3.2\% 50 years or older, $5.3 \% 70$ years or older, and $7.5 \% 85$ years or older). The prevalence of this disease is also higher in the African
American population (in one study, $8.6 \%$ of the participants in this ethnic group had an M-spike). MGUS is important to diagnose because there is a $1 \%$ chance of progression to multiple myeloma, lymphoma, Waldenstrom's macroglobulinemia, or AL amyloidosis every year after diagnosis. Current guidelines do not recommend screening the general population for MGUS. However, patients who have elevated total protein or immunoglobulins, unexplained anemia, elevated ESR, hypercalcemia, or renal failure should be screened. Once a diagnosis is established, the patients should be monitored with regular laboratory studies. This should initially occur six months after diagnosis followed by every two to three years thereafter. In this case, having an NSTEMI increased our suspicion for hematologic disease as having MGUS is associated with a 2-fold increase in the risk of developing both arterial and venous thrombosis.

CONCLUSION: In elderly patients with anemia and elevated protein level, serum and urine protein electrophoresis should be performed to evaluate for plasma cell disorders such as MGUS. Subsequently, patients with MGUS should be monitored closely for progression to other hematologic diseases.

\section{A DYSFUNCTIONAL DIAGNOSIS FOR ABDOMINAL PAIN}

Kevin Zheng; Jay J. Chudow; Darlene LeFrancois. Montefiore Medical

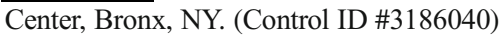

LEARNING OBJECTIVE \#1: Recognize sphincter of Oddi dysfunction as a common cause of post-cholecystectomy pain syndrome

LEARNING OBJECTIVE \#2: Diagnose functional biliary sphincter disorder using the Rome IV criteria

CASE: A 31-year-old obese woman presented five months ago with recurrent epigastric pain. She underwent cholecystectomy with pathology showing chronic cholecystitis. Since then, she presented and was admitted multiple times for similar, near daily epigastric pain. The pain was worse after meals, lasted for hours at a time, and did not improve with antacids or ranitidine. She denied smoking and rarely drank alcohol. Exam revealed tachycardia as well as tenderness in the epigastrium and RUQ. Liver tests were elevated with AST 552 and ALT 612. Bilirubin, alkaline phosphatase, and pancreatic enzyme levels were within normal limits. EGD revealed mildly erythematous gastric mucosa and CT abdomen and pelvis was unremarkable. Abdominal ultrasound and MRCP did not reveal ductal dilatations or stones. She underwent ERCP and had biliary sphincterotomy without preceding manometry. Her symptoms improved and she was discharged. She has not returned since for a similar issue.

IMPACT/DISCUSSION: Sphincter of Oddi dysfunction (SOD) should be suspected in patients with recurrent biliary-type pain after cholecystectomy. The prevalence of SOD among these patients is 9$14 \%$. After cholecystectomy, there is loss of a cholecystosphincteric reflex that is responsible for sphincter relaxation when the gallbladder is distended. This results in increased resistance to bile outflow and a subsequent rise in biliary pressure that is perceived as pain. Other theories include an increased sensitization of nociceptors to biliary pressure that remains after cholecystitis. The term functional biliary sphincter disorder (FBSD) is now used for patients with postcholecystectomy biliary pain and some objective findings of sphincter dysfunction. According to the Rome IV criteria, a diagnosis of FBSD is established when either epigastric or RUQ pain satisfies all of the following: 1) lasts for 30 minutes or longer, 2) recurs at different intervals (not daily), 3) is severe enough to interrupt daily activities or lead to an emergency department visit, 4) is not significantly related to bowel movements, and 5) is not significantly relieved by postural change or acid suppression. In addition, patients must have elevated liver enzymes or a dilated bile duct, but not both, and have absence of bile duct stones or other structural abnormalities. Sphincter manometry is recommended to predict response to treatment with sphincterotomy but is often forgone in clinical practice due to the risk of pancreatitis associated with ERCP. 
CONCLUSION: SOD is important to consider as a cause of postcholecystectomy pain syndrome. By the Rome IV criteria, FBSD is diagnosed by having biliary-type pain with either elevated liver enzymes or a dilated bile duct.

\section{A FAILING HEART: A NEW DIAGNOSIS OF HEART FAILURE DUE TO HYDROXYCHLOROQUINE-INDUCED CARDIOMYOPATHY}

Alexander Ghincea ${ }^{1}$; Justin Allsop ${ }^{2}$; Daniel W. Groves ${ }^{6}$; Mona Rizeq ${ }^{4}$; Monica P. Revelo ${ }^{5}$; Sridharan Raghavan ${ }^{3}$. ${ }^{1}$ University of Colorado School of Medicine, Denver, CO; ${ }^{2}$ University of Colorado, Aurora, CO; ${ }^{3}$ Denver VA Medical Center, Denver, CO; ${ }^{4}$ VA medical Center (ECHCS), Denver, CO; ${ }^{5}$ University of Utah, Salt Lake City, UT; ${ }^{6}$ University of Colorado School of Medicine, Aurora, CO. (Control ID \#3180736)

LEARNING OBJECTIVE \#1: Diagnose hydroxychloroquine-induced cardiomyopathy in patients with complex multi-organ-system medical history.

LEARNING OBJECTIVE \#2: Recognize the importance of early diagnosis and medication cessation in hydroxychloroquine-induced cardiomyopathy.

CASE: A 43-year-old woman presented with acute shortness of breath, dry cough, and chest pain. She had a history of seronegative inflammatory arthritis treated with hydroxychloroquine (HCQ) $200 \mathrm{mg}$ twice daily for 8 months and cystic lung disease. Pulse was $128 \mathrm{bpm}$ and oxygen saturation $89 \%$ on room air. She had distant heart sounds and diffuse inspiratory crackles. White cell count was $12.7 \times 10^{9} / \mathrm{L}$, hemoglobin $15.2 \mathrm{~g} / \mathrm{dL}$, and platelets $297 \times 10^{9} / \mathrm{L}$. Comprehensive metabolic panel was normal. ProBrain Natriuretic Peptide (pBNP) was $3539 \mathrm{pg} / \mathrm{mL}$, and troponin peaked at $0.05 \mathrm{ng} / \mathrm{mL}$. Infectious and autoimmune workup was negative. Ferritin was $91 \mathrm{ng} / \mathrm{mL}$ and thyroid stimulating hormone was 0.35 . She had sinus tachycardia with inferior T-wave flattening and lateral $\mathrm{T}$-wave inversions on electrocardiogram (ECG). Computed tomography revealed ground glass opacities and bilateral pleural effusions. Echocardiography revealed global systolic dysfunction without ventricular hypertrophy and an ejection fraction of $20-25 \%$. Cardiac magnetic resonance imaging (CMR) revealed dilated cardiomyopathy, and no focal inflammation, edema, infarction, or fibrosis. HCQ was discontinued, and her oxygen requirement resolved with diuresis. Coronary angiography was normal and endomyocardial biopsies (EMB) taken. She discharged in stable condition after optimization of guideline directed medications for heart failure. Histopathology and electron microscopy revealed non-specific myocyte hypertrophy, no evidence of iron or amyloid deposition, and no lymphocytic or granulomatous infiltrate, but did show myelinoid bodies consistent with HCQ toxicity.

IMPACT/DISCUSSION: HCQ is commonly used in patients with connective tissue diseases. Cardiotoxicity is rare, and is thought to be underrecognized. We present a patient without prior cardiac history who developed severe heart failure after only 8 months of HCQ therapy. High doses, long-term exposure, female sex, and preexisting heart disease are risk factors. Cardiac complications include conduction disease and restrictive or dilated cardiomyopathy. EMB with histopathology and electron microscopy is essential to the diagnosis, and should be undertaken in any patient receiving HCQ presenting with new or unexplained cardiac abnormalities. CMR and echocardiography are important adjuncts to the diagnosis. Withdrawal of HCQ is the cornerstone of management owing to potential reversibility of cardiac complications.

CONCLUSION: Identification of adverse drug reactions and timely intervention are paramount for internists. Screening with ECG and echocardiography should be performed in all patients receiving HCQ. HCQ should be discontinued in all patients with confirmed or suspected cardiotoxicity to reduce the morbidity and mortality of these devastating complications.

\section{A FATAL BUT UNCOMMON CAUSE OF HEADACHE}

Matthew Langston ${ }^{2}$; Michael T. Vest ${ }^{1} .{ }^{1}$ Christiana Care Health System, $\overline{\text { Wilmington, DE; }}{ }^{2}$ Christiana Care Health System, Newark, DE. (Control ID \#3185766)

LEARNING OBJECTIVE \#1: Consider etiologies of headache with potentially serious consequences.

LEARNING OBJECTIVE \#2: Treat cerebral venous thrombosis.

CASE: A 57-year-old female was brought to the emergency department found unresponsive at home. She missed work due to a headache the day of admission. She had a history of breast cancer diagnosed in 2007 and treated with chemotherapy and mastectomy. A bone scan three months prior revealed a sclerotic left parietal lesion suspicious for metastatic disease. She was having headaches for several months. Her outpatient evaluation included magnetic resonance (MR) imaging of head four months prior, revealing sinus opacification. She was felt to have sinus headaches and underwent a right maxillary antrostomy and ethmoidectomy one week prior to admission. She was intubated in the emergency department for airway protection, where a urine drug screen was positive for cocaine, cannabinoids, and benzodiazepines. Head computed tomography with angiography (CTA) showed lack of opacification of the intracranial dural venous sinuses with enhancement of cortical veins. Further evaluation with MR venography revealed venous hypertension and ischemia of the deep grey nuclei secondary to extensive venous thrombosis involving the torcula, deep venous system, and bilateral transverse sinuses. Anticoagulation with intravenous heparin was started. An emergent thrombectomy was performed and the evacuated thromboses were felt to be chronic due to their density. Despite this intervention, she developed progressive neurologic deterioration due to increased intracranial pressure and ultimately died. IMPACT/DISCUSSION: Headache is the most frequent symptom of cerebral venous thrombosis (CVT). High clinical suspicion for CVT is needed as general neuroimaging not protocolled appropriately may not detect this diagnosis and early diagnosis improves outcomes. Retrospective studies have indicated an incidence of cerebral venous thrombosis higher than previously expected. We suspect that her months of headaches were the result of CVT rather than sinus disease. Metastasis of recurrent malignancy could have played a role in her presentation. Treatment focuses on anticoagulation, however more recent endovascular therapies are being employed, as in our patient. This case underscores the importance of not prematurely attributing altered mental status entirely to substance abuse without searching for other causes in patients with positive urine drug screens.

CONCLUSION: Cerebral vein thrombosis is a challenging diagnosis as an uncommon etiology of headache. The inclusion of CVT remains an important consideration in the differential of an obtunded patient with headache history.

\section{A FATAL CASE OF SEPTIC SHOCK CAUSED BY ACINETOBACTER BACTEREMIA ACQUIRED FROM A PLATELET TRANSFUSION}

Christopher Nevala-Plagemann $^{1}$; Patrick Powers ${ }^{1}$; Mustafa MirKasimov $^{2}$; Richard Rose ${ }^{2} .{ }^{1}$ University of Utah, Salt lake City, UT; ${ }^{2}$ Veterans Affairs, Salt Lake City, UT. (Control ID \#3182679)

LEARNING OBJECTIVE \#1: Prompt initiation of broad spectrum antibiotic therapy is essential in suspected septic transfusion reactions

LEARNING OBJECTIVE \#2: Septic transfusion reactions are most commonly associated with platelet transfusion

CASE: A 67 year old male with a history of type II diabetes and alcoholic cirrhosis was admitted for medical optimization prior to a transarterial chemoembolization procedure for recently diagnosed hepatocellular 
carcinoma. The patient was feeling well at the time of admission. Admission vitals were unremarkable and his labs were notable only for chronic thrombocytopenia. In anticipation of his upcoming procedure, two units of platelets were ordered. Shortly after initiation of the first platelet transfusion, the patient complained of chills and was noted to have a temperature of $100.8^{\circ} \mathrm{F}$. The transfusion was stopped and the patient was administered acetaminophen and diphenhydramine. Approximately 1 hour later, the patient developed tachycardia, tachypnea, and hypotension. The patient was transferred to the intensive care unit where he was started on vasopressors and broad spectrum antibiotics. Within a few hours of admission to the ICU the patient developed severe disseminated intravascular coagulation (DIC). Blood cultures revealed Acinetobacter bacteremia. Cultures obtained from the unit of transfused platelets grew the same species of Acinetobacter. Antibiotic coverage was broadened to tobramycin and meropenem. Unfortunately, over the subsequent 24 hours, the patient's vasopressor requirements continued to rise. Given his clinical deterioration further aggressive treatment was felt to be unlikely to improve his condition. Aggressive care was withdrawn and the patient expired shortly after.

IMPACT/DISCUSSION: Septic transfusion reactions caused by bacterial contamination of blood products have long been known to be a problem in transfusion medicine. The risk of a sepsis is highest with the transfusion of platelets due to storage at room temperature. Bacterial contamination rate of platelets is around 1 in 5000 transfusions, with a risk of transfusion associated sepsis around 1 in 100,000 transfusions. Death related to transfusion of contaminated platelets remains rare with only 10 fatalities reported by the Food and Drug Administration from 2012 to 2015. Clinicians must be able to recognize the signs of a septic transfusion reaction which include fever, chills, hypotension, and DIC. It may be difficult to differentiate sepsis from other types of transfusion reactions such as acute hemolytic reactions. In order to assist with diagnosis, the blood bank should be contacted immediately so that proper laboratory testing can be performed, including $\mathrm{ABO}$ compatibility and culture of the transfused product. Administration of antibiotics should be considered in any decompensating post transfusion patient.

CONCLUSION: Septic tranfusion reactions are a relatively rare but important complication of blood product administration that clinicians must be able to recognize.

\section{A FEBRILE CASE OF PRIMARY HEPATIC NEUROENDO- CRINE TUMOR}

Manojna Konda $^{1}$; swathi subramany ${ }^{1}$; GAYATHRI KRISHNAN ${ }^{2,}{ }^{1}$; Richa Parikh ${ }^{1}$; Kulsum Bano ${ }^{1}$. ${ }^{1}$ University of Arkansas for Medical Sciences, Little Rock, AR; ${ }^{2}$ UAMS, Little Rock, AR. (Control ID \#3185685)

LEARNING OBJECTIVE \#1: Recognize that fever could be an atypical presentation of primary hepatic neuroendocrine tumor.

LEARNING OBJECTIVE \#2: Recognize that diagnosis can be challenging due to their common radiologic characteristics with other liver lesions.

CASE: A 54 year-old previously healthy male presented with one-week duration of fevers, abdominal pain, and diaphoresis. The patient was febrile to $102 \mathrm{~F}$ with otherwise normal vital signs. The patient was jaundiced on examination with tenderness in RUQ. Lab work showed white count of $17.4 \mathrm{k} / \mu \mathrm{l}$. LFTs were also elevated with total bili of $3.2 \mathrm{mg} /$ dL, Direct bili $1.6 \mathrm{mg} / \mathrm{dL}$, ALP $176 \mathrm{U} / \mathrm{L}$, GGT: $231 \mathrm{U} / \mathrm{L}$ AST: $52 \mathrm{U} / \mathrm{L}$ ALT: $40 \mathrm{U} / \mathrm{L}$. Abdominal CT showed multiple hypodense lesions in the liver concerning for abscesses. Empiric antibiotics were started as he was febrile with leukocytosis and imaging findings. Blood cultures, hepatitis, entamoeba and echinococcus tests were negative. Targeted FNA biopsy of one of the liver lesions was done. Final pathology revealed welldifferentiated neuroendocrine tumor and was negative for any organisms. Antibiotics were discontinued. Octreotide scan showed increased activity only in the liver with no abnormal activity outside the liver. This was suggestive of the patient having primary hepatic neuroendocrine tumor with multiple liver metastases. Serum chromogranin A was high at $50 \mathrm{ng} / \mathrm{mL}$, serum serotonin was elevated at $3333 \mathrm{ng} / \mathrm{ml}$ and urine 5HIAA was high at $156.9 \mathrm{mg} /$ day. The patient was started on somatostatin analogue lanreotide and mTOR kinase inhibitor everolimus with dramatic resolution of his fevers. His most recent imaging showed improvement in the size of his liver lesions.

IMPACT/DISCUSSION: This case adds to the literature about the rare presentation of primary hepatic neuroendocrine tumor which has previously not been described. It also highlights the importance of distinguishing various liver masses. While more than $80 \%$ of neuroendocrine tumors found in the liver are metastatic, primary hepatic neuroendocrine tumors are extremely rare with less than 100 cases reported worldwide. While most of the tumors are solitary and functionally silent, less than $10 \%$ cases have been described as being functional with flushing, diarrhea and wheezing. Although hypothetical, fevers may have been a result of high levels of seratonin. Diagnosis is often challenging based on radiological appearance as it can mimic other liver masses. Biopsy can confim the diagnosis. Octreotide scan is a useful tool in differentiating primary hepatic neuroendocrine tumors from other primary sites with liver metastases. While surgical resection is the preferred treatment, in cases of unresectable disease use of somatostatin analogues has shown to have good results with overall prognosis being better than other types of liver cancer.

CONCLUSION: Clinicians need to be aware of fever as an atypical presentation of primary hepatic neuroendocrine tumors. While challenging to diagnose by conventional imaging, exclusion of other primary lesions and liver biopsy is strongly warranted.

\section{A GREAT IMITATOR OF POSITIONAL VERTIGO}

Priya Chopra $^{1}$; Ericson Stoen ${ }^{1}$; Chi Zheng ${ }^{2} \cdot{ }^{1}$ University of Colorado School of Medicine, Aurora, CO; ${ }^{2}$ Denver Health, Denver, CO. (Control ID \#3158950)

LEARNING OBJECTIVE \#1: Recognize neurosyphilis as a cause of recurrent falls

LEARNING OBJECTIVE \#2: Understand the difference between otosyphilis and neurosyphilis

CASE: A 73-year-old woman presented after a fall at home resulting in a forehead hematoma in the setting of a four-month history of progressive dizziness and recurrent falls. She described the dizziness as "room spinning" exacerbated by changes in position; she also had a two-year history of right-sided tinnitus and progressive hearing loss. Her past medical history was notable for atrial fibrillation, type 2 diabetes mellitus with peripheral neuropathy, and hypertension. Medications included warfarin, NPH insulin, lisinopril, metoprolol, baclofen, tizanidine, and hydrocodone. Vital signs including orthostatics were normal. Cardiopulmonary exam was normal. Neurologic examination showed terminal dysmetria and deficits in bilateral lower extremity proprioception. Hemoglobin, glucose, troponin, TSH, B12, and HIV were all normal, and her INR was therapeutic. ECG showed sinus rhythm, and interrogation of the patient's loop recorder placed for her falls was unremarkable. CT and MRI of the brain were unremarkable. A prior audiometry report showed isolated right-sided neurosensory hearing loss. Treponemal pallidum antibody was positive, with subsequently positive RPR at 1:2 and a positive confirmatory FTA-ABS. Cerebrospinal fluid (CSF) protein was elevated at 58, and CSF VDRL was non-reactive. As she had a negative RPR 11 years prior and scored $18 / 25$ on a partially completed Mini- Mental State Examination, the patient was treated for neurosyphilis with continuous penicillin $\mathrm{G}$ infusion for 14 days with improvement in her vertigo and tinnitus but not hearing.

IMPACT/DISCUSSION: Over 800,000 patients are hospitalized each year due to falls, and the possible etiologies of recurrent falls are numerous. Our patient had several potential causes for her falls, and many of which were systemically excluded from the differential, but her neurologic and vestibular symptoms gave concern for neuro and oto-syphilis. 
Syphilis can present with both hearing loss and vertigo. This occurs when T. pallidum invades the cochleovestibular system resulting in otosyphilis, or the cerebrospinal fluid (CSF) resulting in neurosyphilis. In neurosyphilis, extra-otic symptoms such as impaired proprioception, stroke, and dementia may also be present. To differentiate neurosyphilis from otosyphilis, a lumbar puncture should be performed. Making this distinction is important, as the treatment may differ. The literature currently suggests intravenous penicillin $\mathrm{G}$ treatment for neurosyphilis; however, there is controversy regarding treatment for otosyphilis. Some studies suggest that three doses of intramuscular penicillin is sufficient, while other experts recommend the same treatment as neurosyphilis due to concerns of incomplete eradication.

CONCLUSION: This case exemplifies the importance of a thorough diagnostic evaluation after recurrent falls and peripheral vertigo, including ruling out "the great imitator."

\section{A HARBINGER OF MALIGNANCY: PARANEOPLASTIC CER- EBELLAR ATAXIA}

Michael H. Nguyen. California Pacific Medical Center, San Francisco, CA. (Control ID \#3185948)

LEARNING OBJECTIVE \#1: Recognize the clinical features that suggest a paraneoplastic process in patients with ataxia

LEARNING OBJECTIVE \#2: Manage patients with paraneoplastic ataxia

CASE: A 73 year old female with a remote 25 pack per year smoking history presented to an emergency room 12/26 with 3 weeks of headache, blurry vision, and slurred speech. She was discharged home with negative workup including a CT scan. She received an outpatient MRI on 12/29 that was negative for acute pathology and subsequent work up with an ophthalmologist was also unrevealing. One week later, she presented to CPMC on $1 / 2$ with progressive bilateral upper extremity weakness and ataxia. Lumbar puncture (LP) was only remarkable for elevated protein. She received a PET CT 1/3 that showed evidence of a $1.4 \mathrm{~cm}$ left upper lobe (LUL) nodule and hypermetabolism in her mediastinal lymph nodes. She underwent biopsy on $1 / 5$ that revealed nonsquamous cell lung cancer that is subsequently positive for PD-L1 expression (20\%). Her CSF also later comes back positive for Purkinje cell cytoplasmic antibody (PCA-1). At this point she was diagnosed with paraneoplastic cerebellar ataxia (PCA)PCA Over the ensuing months, she underwent plasma exchange $1 / 5-1 / 7$, external radiation $1 / 25-3 / 7$, and chemotherapy $1 / 31-4 / 4$ with no remission in her symptoms and was now wheelchair bound. A PET CT 4/10 reveals resolved hypermetabolism in her mediastinal lymph nodes but persistence of her $1.4 \mathrm{~cm}$ LUL nodule. A MRI 5/31 reveals cerebellar atrophy consistent with cerebellar degeneration. The patient underwent another round of plasmapharesis 6/12-6/20 with no improvement of her symptoms and she was discharged to hospice.

IMPACT/DISCUSSION: Ataxia is a common complaint in the ER. When imaging and initial laboratory tests are negative, differentials must be widened to include autoimmune and malignant processes. The cause of ataxia can even be a combination: in PCA, there is severe loss of cerebellar Purkinje cells as a result of onconeural antigens. This case demonstrates the diagnostic challenge of PCA and the devastating consequences of late treatment. The patient's negative MRI, negative LP, and acute presentation delayed her diagnosis of PCA. While it is a rare complication of malignancy, PCA often precedes the diagnosis of cancer and is a clue to diagnose malignancy at an early treatable stage. If given early enough, there is evidence for immunotherapy. However, in most cases the diagnosis is delayed rendering treatment ineffective. A patient with worsening ataxia over weeks with unremarkable brain imaging and laboratory tests must be considered for an autoimmune process. If there are any risk factors for malignancy, a paraneoplastic process should be • PCA commonly precedes the diganosis of cancer, marking a critical time to diagnose malignancy $\bullet$ It is thought the cebrebllar damage is irreversible in PCA because interventions are too late $\bullet$ If a patient presents with worsening ataxia with negative imaging and lab tests, an autoimmune process should be considered

\section{A HEALTHY HEART?}

Magued W. Haroun; Vladyslav Dieiev; Pakhi Goel; Karishma Kitchloo. Montefiore Medical Center, Bronx, NY. (Control ID \#3185968)

LEARNING OBJECTIVE \#1: Recognize prevalence of early repolarization pattern (ERP) on electrocardiogram (ECG) among young, healthy athletes

LEARNING OBJECTIVE \#2: Distinguish between ERP and ST segment elevation myocardial infarction (STEMI)

CASE: A 40 year-old Hispanic man presented with two episodes of palpitations. He had no significant past medical history; he was an athlete who ran three marathons during the preceding two-weeks period. Palpitations were non-exertional, lasted for ten minutes and resolved by the time of presentation. His initial ECG revealed STE in leads V3 and V4 that extended to lead V5 twelve hours later, and T-wave inversions (TWI) in leads III and V3 that extended to leads V4 and aVF six hours later and completely resolved on EKG at twelve hours. There were no ST depressions in reciprocal leads. Serial Troponin $\mathrm{T}$ was $<0.01$. Patient remained asymptomatic throughout the admission. Echocardiography revealed no abnormalities. Coronary angiography and left heart catheterization revealed patent coronary arteries.

IMPACT/DISCUSSION: Dynamic changes in ST segments is a worrisome clinical finding which raises suspicion for an acute coronary process but it is important to consider these findings in clinical context and to use specific ECG criteria. In our case, the ECG pattern and morphology, lack of chest pain, electrolyte disturbances or troponin elevation were consistent with ERP in anterior leads. Invasive evaluation confirmed absence of an underlying disease process which may have caused the changes described. ERP has always been considered a normal ECG variant. It is characterized by J-point ST elevation and manifests either as terminal QRS slurring (the transition from the QRS segment to the ST segment) or notching (a positive deflection inscribed on terminal QRS complex) associated with concave upward STsegment elevation and prominent $\mathrm{T}$ waves in at least two contiguous leads. ERP is more prevalent in competitive athletes and its prevalence increases after intense physical training. ERP is not associated with structural left ventricular measurement, including chamber volume or wall thickness, both of which are well-established components of the "athlete heart" suggesting that exercise-related ERP may be an isolated electric phenomenon that develops parallel to, but not as a result of, structural myocardial remodeling.

CONCLUSION: While STE may reflect occlusion of the coronary artery and ongoing necrosis of myocardium, in the appropriate clinical scenario other etiologies should be considered. Internists need to be able to discern ERP from STEMI and to expect a higher prevalence of ERP in athletes.

\section{A HEARTY INFECTION}

MATTHEW WEBER ${ }^{1}$; Pinky Jha ${ }^{2} .{ }^{1}$ Medical College of Wisconsin, Wauwatosa, WI; ${ }^{2}$ medical college of wisconsin, Milwaukee, WI. (Control ID \#3185978)

LEARNING OBJECTIVE \#1: Report a case of disseminated mucormycois involving lungs and heart in an immunocompromised patient. LEARNING OBJECTIVE \#2: Show prophylaxis choice can be a clue for earlier diagnosis of mucormycotic infections.

CASE: 57 YO F diagnosed with myelodysplastic syndrome. Admitted for pre-transplant chemotherapy and matched unrelated allogenic PBSCT. 
Prophylaxis included acyclovir, fluconazole, ciprofloxacin and Augmentin. Successful PBSCT on day 7. She began having sharp, pleuritic, left-sided chest pain. CXR showed lingular infiltrate in left upper lobe. CT showed bilateral ground glass and alveolar opacities over coarse interstitial disease. Broad infectious work was negative. She was switched from fluconazole to micafungin, and antibiotics broadened. On day 13 bronchoscopy showed normal lungs. BAL negative for bacterial, viral, and fungal infection. Day 14, began requiring 1-2 L of O2. Work of breathing increased as she developed atrial fibrillation with RVR and hypotension requiring norepinephrine. Upon arrival to ICU she was sedated, intubated, and started on norepinephrine drip. Amphotericin B was started. Lingula biopsy obtained showing rhizopus. On day 25 , CT surgery performed left upper lobectomy, resected pericardial fat, and pericardial window which revealed dark serous fluid. Pathology on the pericardial fat showed Rhizopus and Aspergillus, lung lobe showed Rhizopus. That night she became hypotensive, refractory to fluid, norepinephrine and vasopressin drips. Patient's family requested comfort cares only - the patient died a few hours later.

IMPACT/DISCUSSION: PBSCT patients are at high risk for infection. It is impossible to provide coverage for every possible infectious entity but being able to make quick a diagnosis can lead to early treatment and intervention. Mucormycosis is not typically the infection prioritized in prophylaxis selection. One study showed 875 patients with fungal infections: $43 \%$ with aspergillosis, $28 \%$ candidiasis, and $8 \%$ mucormycosis. The ASBMT Guidelines for Preventing Infectious Complications among PBSCT Recipients recommend prophylaxis targeted with fluconazole which has no mucor coverage. In patients at higher risks for mold infections (ie high risk for GVHD) they recommend voriconazole. Patients with voriconazole prophylaxis are actually more prone to breakthrough mucormycosis. Additionally, use of fluconazole or itraconazole therapy is associated with a fourfold increase in incidence of mucormycosis compared posaconazole. Pulmonary mucormycosis is a rapidly expanding disease that causes necrosis and can, on rare instances, invade the mediastinum and heart. Quick diagnosis can expand the window of treatment. Diagnosis requires histopathology evidence and positive cultures which often requires biopsy. Treatment involves surgical debridement and amphotericin B.

CONCLUSION: Using prophylaxis as a clue, providers can raise their index of suspicion for mucormycosis in the setting of progressive pneumonia in PBSCT patients.

\section{A HEMATOLOGIC EMERGENCY MASQUERADING AS AN NSTEMI}

Andrew B. Dicks; Bess Flashner; Jonathan D. Goldman. Beth Israel Deaconess Medical Center, Boston, MA. (Control ID \#3177560)

LEARNING OBJECTIVE \#1: Recognize an abnormal presentation of thrombotic thrombocytopenic purpura (TTP)

CASE: A 48 year old female with history of scleroderma and vitiligo presented with chest pain. The pain was sudden onset, sharp, and radiated to her back. There was no positional or pleuritic component, and no inciting event. Presenting vital signs and physical exam were unremarkable. ECG showed T-wave inversions in leads II and V1. Troponin-T and CK-MB trended upward, peaking at $0.28 \mathrm{ng} / \mathrm{mL}$ and $16 \mathrm{ng} / \mathrm{mL}$, concerning for NSTEMI. CTA chest demonstrated no evidence of pulmonary embolism or aortic dissection. Coronary angiography demonstrated no CAD. She was subsequently admitted to the medicine service for evaluation of her chest pain. Presenting labs were also notable for $\mathrm{Hgb} 9.5 \mathrm{~g} / \mathrm{dL}$ and platelet count $87 \mathrm{~K} / \mathrm{uL}$, down from $12.0 \mathrm{~g} / \mathrm{dL}$ and $264 \mathrm{~K} / \mathrm{uL}$ nine months prior. Additional work up demonstrated absolute reticulocyte count $0.18 \mathrm{~m} / \mathrm{uL}$, haptoglobin $<10 \mathrm{mg} / \mathrm{dL}, \mathrm{LDH} 516 \mathrm{IU} / \mathrm{L}$, and a peripheral blood smear with $2+$ schistocytes, consistent with microangiopathic hemolytic anemia. Given concern for TTP, ADAMTS13 activity level was sent. While awaiting the result, she was risk stratified for TTP using the PLASMIC score (defined by 7 variables including platelet count $<30 \mathrm{~K} / \mathrm{uL}, \mathrm{MCV}<90 \mathrm{fL}$, INR $<1.5$, creatinine $<2.0 \mathrm{mg} / \mathrm{dL}$ evidence of hemolysis, lack of active cancer, and lack of organ or stem cell transplant). She scored 6 out of 7, indicating high likelihood of TTP and was urgently started on plasma exchange and prednisone. with eventual addition of rituximab. Her laboratory parameters and chest pain improved with treatment, and she was discharged home with hematology follow up. ADAMTS13 activity returned at $<5 \%$, confirming the diagnosis of TTP. Her presentation of NSTEMI was felt to be the first clinical manifestation of her TTP.

IMPACT/DISCUSSION: TTP causes extensive microscopic thromboses of small blood vessels which can lead to dysfunction of nearly any organ system, including the cardiovascular system. Microvascular occlusions of the heart can result in MI, arrhythmias, heart failure, or cardiogenic shock. Cardiac dysfunction is a rare initial presentation of TTP as other sequelae are usually present, making this case an atypical and unique presentation of TTP. TTP has a high mortality rate and treatment should not be delayed if suspicion of TTP is high. ADAMTS13 activity may take several days to result, and the PLASMIC score can therefore be used to predict the likelihood of ADAMTS13 activity and guide initiation of therapy while awaiting definitive diagnosis (1). 1. Bendapudi PK, et al. Derivation and external validation of the PLASMIC score for rapid assessment of adults with thrombotic microangiopathies: a cohort study. The Lancet Haematology. 2017;4(4):e157-e164.

CONCLUSION: Patients with TTP can present with end organ damage of nearly any organ system, including the cardiovascular system. Treatment of TTP should not be delayed while awaiting definitive testing if probability of TTP is high based on PLASMIC score

\section{A HOLE IN OUR LOGIC: USING BAYESIAN PROBABILITIES TO OVERCOME ANCHORING BIAS}

Rachel P. Shah ${ }^{1}$; Meredith H. Lora ${ }^{2} .{ }^{1}$ Emory University School of Medicine, Atlanta, GA; ${ }^{2}$ Emory, Atlanta, GA. (Control ID \#3185695)

LEARNING OBJECTIVE \#1: Review anchoring bias and its effect on diagnostic error

LEARNING OBJECTIVE \#2: Apply Bayesian analysis to overcome anchoring bias

CASE: A 50-year-old homeless man with a recent incarceration presented to a hospital with hemoptysis. He reported two months of productive cough, pleuritic chest pain, and B symptoms. Physical exam showed temperature 39.2 degrees $C$, pulse 115 , oxygen saturation $96 \%$ on room air, and normal lung exam. Labs showed white blood cell count 19,000 ( $86 \%$ neutrophils) and negative HIV test. Chest CT revealed an $8 \mathrm{~cm}$ RUL cavitary lesion with surrounding consolidation and no associated lymphadenopathy. The differential diagnosis included tuberculosis (TB), necrotizing bacterial pneumonia, invasive fungal infection, and malignancy. Empiric community acquired pneumonia treatment was started. Respiratory culture grew two strains of Pseudomonas aeruginosa, interpreted by the team as superimposed pseudomonas infection on a cavitary lesion. Ciprofloxacin was added. Tuberculosis workup included three negative sputum acid fast bacilli (AFB) smears, negative sputum TB PCR, and weakly positive QuantiFERON-TB Gold. Due to suspicion for smearnegative TB, the patient was started on empiric RIPE therapy. He developed drug-induced liver injury and symptoms worsened. Bronchoscopy yielded negative AFB smear and TB PCR. BAL culture grew levofloxacin-resistant Pseudomonas. Repeat CT chest showed enlargement of the cavitation to $13 \mathrm{~cm}$. Patient was treated with cefepime for six weeks and underwent RUL lobectomy. Pathology showed organizing pneumonia without evidence of TB.

IMPACT/DISCUSSION: This case highlights the risk of anchoring bias in patients with classic presentations of disease. While there is a broad differential for cavitary lung lesions, this patient had a presentation typical 
of TB. Empiric treatment for TB was initiated and continued despite mounting evidence that decreased the probability of cavitary TB. This patient suffered from iatrogenic liver injury and a high-risk surgery that potentially could have been avoided through earlier diagnosis. About $75 \%$ of diagnostic errors have a cognitive component. Anchoring bias is the tendency for clinicians to stick with a diagnosis even as new contradictory information becomes available. To avoid anchoring, clinicians can employ Bayesian reasoning. While calculation of post-test probabilities using likelihood ratios can be daunting, a practical nomogram can aid in rapid utilization. If the pretest probability of $\mathrm{TB}$ in this patient was $70 \%$, the posttest probability of TB after three negative sputum AFB smears and PCR dwindled to $20 \%$, necessitating additional workup. Increased cognitive awareness through utilization of these probabilities can overcome anchoring bias and decrease errors.

CONCLUSION: 1. Cavitary lung lesions may result from microorganisms including but not limited to TB. 2. Anchoring bias can delay correct clinical diagnosis and increase risk of morbidity. 3. Bayesian analysis can be used to minimize the effects of anchoring bias.

\section{A HORSE IN A ZEBRA COSTUME}

Elaine Buckholtz; Michael P. Smith. University of Nebraska Medical Center, Omaha, NE. (Control ID \#3186127)

LEARNING OBJECTIVE \#1: Develop a systematic approach to the work-up for hypoglycemia.

LEARNING OBJECTIVE \#2: Understand the importance of early detection of factitious hypoglycemia

CASE: A 20 year-old woman presented with extreme lethargy and was found to have a blood sugar of 49. She was admitted for recurrent and profound hypoglycemia of unknown etiology that had been ongoing for several months. She also had admissions for seizures related to hypoglycemia in the past. Due to increased sensitivity to even small doses of insulin, the patient reports that she stopped taking basal insulin altogether three weeks ago, which was interesting given her history of type 1 diabetes and hereditary pancreatitis which required a pancreatectomy. She was found to have undetectable levels of pro-insulin and c-peptide, but an elevated level of insulin. Insulin antibody levels were also elevated. IGF-1 and IGF-2 were normal. ACTH stimulation test was normal. A CT abdomen showed a mass suspicious for a neuroendocrine tumor. A DOTATE scan was also mildly positive which increased suspicion for a neuroendocrine tumor. An exploratory laparotomy yielded a tissue sample of the mass that was negative for neuroendocrine tissue. The patient improved without further intervention and tolerated a modest regimen of insulin without recurrence of hypoglycemia. The patient suffered from severe depression with prior suicide threats in addition to difficult social circumstances. It became evident that she had several risk factors and exhibited signs concerning for factitious hypoglycemia.

IMPACT/DISCUSSION: Recurrent hypoglycemia, outside of an acutely ill patient, requires a systematic approach from the general internist. It is important to implement a methodical approach to hypoglycemia as the differential is vast and work-up can become complicated as well as costly. Certain drugs and alcohol should be ruled-out first as a cause. Adrenal insufficiency will usually present with symptoms outside of what would be typical for hypoglycemia. Distinguishing between endogenous versus exogenous insulin will also be a helpful clue, which can be assessed by measuring insulin and c-peptide levels in the blood. C-peptide levels will be high with endogenous secretion of insulin. A common cause of exogenous insulin would be accidental or surreptitious administration of insulin. If there is suspicion for factitious behavior, it is important to ruleout an insulinoma, as labs will appear similar when compared to excessive sulfonyurea administration. Identifying a person with factitious disorder can be elusive and the implications of missing this diagnosis are serious. Complications of hypoglycemia include seizures, coma, and even death. Additionally, the cost of searching for an alternative cause can be high and it may lead to invasive, unnecessary procedures.
CONCLUSION: The general internist needs to have an approach to hypoglycemia that minimizes unnecessary testing and interventions in order to rapidly diagnose and treat the appropriate etiology

\section{A LEGION OF THEIR OWN: A CASE OF LEGIONNAIRE'S DISEASE}

William Chastant ${ }^{1}$; Phillip S. Bell ${ }^{1}$; Robert Miller $^{2} .{ }^{1}$ Tulane University School of Medicine, New Orleans, LA; ${ }^{2}$ Tulane University Health Sciences Center, New Orleans, LA. (Control ID \#3184473)

LEARNING OBJECTIVE \#1: Discuss the rationale behind Legionella urine antigen testing.

LEARNING OBJECTIVE \#2: Describe the severity of Legionnaire's disease.

CASE: A 67-year-old native Hawaiian with a history of COPD and dietcontrolled diabetes presented with one week of progressively worsening dyspnea and weakness. He reported associated subjective fevers, a nonproductive cough, and episodes of drenching sweats. Additionally, he experienced multiple episodes of watery, non-bloody diarrhea for several days, which resolved the day prior to presentation. His dyspnea and weakness resulted in an inability to get out of bed. He denied drug or alcohol use. He worked as a groundskeeper at a naval base. On physical exam his temperature was 103, HR 102, BP 159/90, RR 20, O2 \% 95 on $2 \mathrm{~L} \mathrm{NC}$. He exhibited increased work of breathing, diffuse rhonchi bilaterally and was extremely diaphoretic, with pools of sweat forming behind his clavicles. His hemoglobin was normal. WBC count was 11.7 (4.810.8 ) with $19 \%$ bands. His sodium was 131 , and his AST and ALT were 148 and 105. CRP was 24.01 (nl 0-0.6). Rapid flu was negative. The rest of the chemistry panel was normal. Patchy, multi-focal, bilateral, lobar opacities were noted on initial chest x-ray. Piperacillin/tazobactam was initiated and Medicine was consulted for admission. Legionella and S. pneumoniae urine antigen, sputum cultures and blood cultures were ordered. Chest CT demonstrated multi-focal pneumonia with emphysematous changes and bronchiectasis. Given his structural lung disease, risk factors \& toxic appearance, levofloxacin was added for additional antipseudomonal coverage. He improved initially but later became increasingly dyspneic and required intubation. Legionella urine antigen resulted positive. His coverage was narrowed to levofloxacin. Blood and sputum cultures had no growth. The patient was subsequently extubated and discharged home with a diagnosis of Legionnaire's pneumonia.

IMPACT/DISCUSSION: The severity of Legionnaire's disease warrants consideration of Legionella spp. as the etiology of any pneumonia that presents to the hospital. Radiographically there is no distinction with typical pneumonia. The commonly taught distinguishing features of diarrhea, hyponatremia and elevated transaminases, while helpful, are often absent. Sputum culture remains the gold standard for diagnosis. However, up to $50 \%$ of patients with legionella pneumonia do not produce sputum, making a culture difficult to obtain. Legionella urinary antigen has a sensitivity of $70-80 \%$ with a specificity approaching $100 \%$. Therefore, multiple organizations recommending Legionella urine antigen testing in all patients with severe pneumonia.

CONCLUSION: In 2015, pneumonia was the $5^{\text {th }}$ most common principal diagnosis leading to hospitalization in the United States. $1-10 \%$ of these cases were caused by Legionella spp. with mortality rates reaching up to $10 \%$ even with treatment.

\section{A MALIGNANT CURE: SECONDARY NEOPLASM PRESENT- ING AS A CANCER OF UNKNOWN PRIMARY IN A HODGKIN'S LYMPHOMA SURVIVOR}

Carlos A. Lopez ${ }^{1}$; Nirupa J. Raghunathan ${ }^{2} .{ }^{1}$ Memorial Sloan Kettering Cancer Center, New York City, NY; ${ }^{2}$ Memorial Sloan Kettering Cancer Center, New York, NY. (Control ID \#3186118) 
LEARNING OBJECTIVE \#1: Recognize the increased risk of solid subsequent malignant neoplasms (sSMN) in patients that have received chest irradiation.

CASE: A 50 year old female presented for a routine exam and was noted to have new, painless abdominal distention. She had a previous history of Hodgkin's Lymphoma (HL) treated with mantle radiation therapy (RT) and chemotherapy (treated at age 24) and triple negative breast cancer treated with bilateral mastectomy and chemotherapy (treated at age 44). She had no exposure to tobacco, and the rest of her history was non-contributory. Her exam was notable only for a protruding soft abdomen without mass and a palpable liver edge. An abdominal and pelvic ultrasound identified a hypoechoic $2.6 \times 2.4 \times$ $2.1 \mathrm{~cm}$ mass separate from the liver within the right upper quadrant adjacent to the region of the pancreatic head. A CT of the abdomen and pelvis revealed a gastrohepatic node increased in size compared to prior imaging. Biopsy of the gastrohepatic mass suggested lung adenocarcinoma. A staging PET CT scan showed disease localized to the lymph node without evidence of a lung primary tumor, other lymph node involvement, or distant metastases, so she underwent local therapy via surgical resection of the lymph node only and was monitored with imaging every 3 months. CT scans of the chest, abdomen, and pelvis at 3,6, and 9 months were negative for evidence of progression; at 12 months, however, surveillance PET CT scan showed a new perihilar foci in the right lung with post-obstructive atelectasis suspicious for malignancy and FDG-avid enlarged lymph nodes in the infrahilar paraesophageal and portocaval regions. Biopsy of the infrahilar lymph node confirmed the diagnosis of metastatic lung adenocarcinoma. She was started on carboplatin, pemetrexed, and pembrolizumab.

IMPACT/DISCUSSION: This case highlights that survivors of childhood cancers who undergo RT are at high risk of developing solid subsequent malignant neoplasms, and that new symptoms or signs on physical exam should be investigated carefully. The cumulative incidence of developing sSMN at 40 years after diagnosis of HL is about $26.4 \%$. Women who receive chest RT are at high risk for breast cancer, with a cumulative incidence of breast cancer of about $45.3 \%$ by age 50 . Chest RT also increases the risk of lung cancer, with an estimated cumulative incidence of $2.2 \%$ by 40 years from diagnosis of HL. Though the risk of lung cancer conferred by RT appears to be higher than that of smoking in the general population, there is insufficient evidence at this time to recommend low-dose CT-imaging based screening in these patients. Notably, due to absence of a primary lung mass at the time of diagnosis, screening our patient with lung imaging would have proved unhelpful.

CONCLUSION: Cancer survivors exposed to RT should communicate and engage with their providers if they have new findings that may warrant investigation of disease reoccurrence or new sSMN, even in the absence of other traditional risk factors.

\section{A MYSTERY DIAGNOSIS IN A PATIENT WITH ALTERED MENTAL STATUS}

Cristina Nguyen. Alameda Health System - Highland Hospital, Oakland,

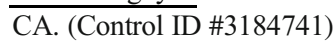

LEARNING OBJECTIVE \#1: Assess when to treat bacterial meningitis with adjunctive Dexamethasone.

LEARNING OBJECTIVE \#2: Recognize when to give antimicrobial chemoprophylaxis for bacterial meningitis.

CASE: A 51 year-old homeless man with a past medical history of methamphetamine use presented for altered mental status after being found lying in a bathtub. While en route, he complained of a headache. He had a temperature of $103.2 \mathrm{~F}$, heart rate of 112 beats per minute, and oxygen saturation of $98 \%$ on room air. Exam was notable for nuchal rigidity and decreased level of consciousness. His WBC count was 22,000. CT head revealed chronic bifrontal encephalomalacia and multifocal paranasal sinus fluid and mucosal thickening. Lumbar puncture revealed an opening pressure of $55 \mathrm{~cm} \mathrm{H2O}$. Cerebrospinal fluid analysis was remarkable for $1750 \mathrm{WBC}$ 's, $92 \%$ neutrophils, protein 578 , and glucose $<5$. Urine toxicology was positive for methamphetamine. The patient was stated empirically on intravenous Ceftriaxone and Vancomycin for presumed bacterial meningitis. Dexamethasone and Rifampin were also added as adjuncts for Streptococcal pneumonia treatment. All staff members treating the patient received a single dose of Ciprofloxacin for prophylaxis. On hospital day 5, the patient's altered mental status improved. He was also found to have a reactive, reactive trepenemal antibody, a serum RPR titer 1:64, and reactive CSF VDRL. However, the patient left against medical advice without treatment.

IMPACT/DISCUSSION: Understanding cognitive biases for the vulnerable population affects physicians' decisions. Implicit bias also plays a major role in health care settings in regards to socioeconomic disparities which affect clinical judgment and decision-making. Thus, considering the patients' perspectives, advocating for the vulnerable, and maintaining a broad differential for patients presenting with altered mental status will lead to improved health outcomes. Our patient had many possible causes of altered mental status, including intoxication, neurosyphilis, and bacterial meningitis. Based on studies, adjunctive Dexamethasone should be given at the same time or prior to antibiotics if there is a likelihood of pneumococcal meningitis to decrease the rate of hearing loss and mortality. It has no benefit if given after antibiotics. Unlike Vancomycin, Rifampin is not limited in crossing into the CSF by the decrease in inflammation with Dexamethasone and is often added when Dexamethasone is started. Both Vancomycin and Rifampin can be discontinued if the organism is susceptible to the cephalosporin. Postexposure chemoprophylaxis can prevent the spread of $N$. meningitidis, but not pneumococcal disease. It is indicated for those in close contacts with patients and is most effective within 24 hours of the exposure. Although healthcare workers are at increased risk of exposure, the absolute increase in risk is very small. Thus, prophylaxis is not recommended for those who have not had direct exposure to respiratory secretions

CONCLUSION: See above.

\section{A NEARLY-MISSED OPPORTUNITY: DIAGNOSING EPIDU- RAL ABSCESS DURING RESOLVING ENCEPHALOPATHY}

Steven Omansky $^{1}$; Patricia Dharapak ${ }^{2} .{ }^{1}$ Mount Sinai Beth Israel Hospital, $\overline{\text { New York, NY; }}{ }^{2}$ Mount Sinai Beth Israel, New York, NY. (Control ID \#3161458)

LEARNING OBJECTIVE \#1: To understand the incidence, risk factors, warning signs, and diagnostic process of spinal epidural abscess in the hospital setting.

CASE: A 59 year-old woman with uncontrolled diabetes mellitus and end-stage kidney disease on hemodialysis presented with altered mental status and a suprapatellar abscess. Following irrigation and debridement, her course was complicated by septic shock. She was found with highgrade methicillin-sensitive Staphylococcus aureus bacteremia, and her blood cultures ultimately sterilized with IV cefazolin therapy. Despite hemodynamic stabilization and resolution of leukocytosis, the patient remained stuporous. Further investigation revealed septic emboli on brain MRI as likely culprits. In the following days, her mental status gradually improved such that she could answer questions. While she had been evaluated by five different providers on weekend coverage, a new complaint of left lower extremity paralysis was only identified by the primary team. An emergent MRI of the spine revealed osteomyelitis and an epidural abscess at C5-6. Resultant cord compression and edema were also noted. The patient was urgently transferred to an affiliate hospital with the capability of neurosurgical intervention.

IMPACT/DISCUSSION: Finding S. aureus bacteremia in the hospital setting should provoke vigilance for its sequelae, especially in patients 
with risk factors such as diabetes mellitus and kidney disease on hemodialysis. S. aureus infection is the most common cause of spinal epidural abscesses through hematogenous spread; however, it is still rare and easily missed with an incidence of only $0.2-2.8$ per 10,000 hospital admissions. An MRI with gadolinium contrast is the most sensitive modality for epidural abscess diagnosis but unfortunately, many other clinical and laboratory findings are nonspecific if MRI is not possible. The typical symptoms of fever, back pain, and focal neurologic deficit are not always observed at presentation and are uncommonly found together. An altered mental status makes the diagnosis challenging when sensorimotor function or a review of systems cannot be reliably assessed. If the patient cannot follow commands, observation of bladder and bowel dysfunction or depressed reflexes can support a diagnosis of spinal cord injury, although non-specific for an epidural abscess. For these reasons, around half of patients with an admission for epidural abscess are initially misdiagnosed, and $4-22 \%$ of patients will suffer irreversible paralysis in part due to delays in appropriate treatment.

CONCLUSION: Patients with S. aureus bacteremia are at particularly high risk for morbid or fatal complications and require vigilant monitoring, even when blood cultures have sterilized. The potential for epidural abscess should not be forgotten, although diagnosis can prove elusive. In this setting, continued surveillance is imperative to assess for clinical developments, especially those undetected prior to recovery from altered mental status.

\section{A NEW ERA OF OPIOIDS - THE RISE OF FENTANYL IN US DRUG MARKETS}

Ashley Engel ${ }^{1}$; Amy H. Farkas ${ }^{1,2}{ }^{2} .{ }^{1}$ Medical College of Wisconsin, Milwaukee, WI; ${ }^{2}$ Milwaukee VA Medical Center, Milwaukee, WI. (Control ID \#3182666)

LEARNING OBJECTIVE \#1: Understand the initial approach to altered mental status

LEARNING OBJECTIVE \#2: Recognize the rise of fentanyl use in the United States

CASE: A 57-year-old male with a past medical history of substance abuse presented with altered mentation following a motor vechical accident. Family reported that he had been admitted to an outside hospital onemonth prior for unknown reasons. On exam the patient was oriented to person only with a blunted affect and minimal engagement. No focal neurologic deficits were noted. Initial work up including infectious, toxic, and metabolic causes of his altered mentation were unremarkable. MRI demonstrated extensive, confluent, symmetric, bilateral hyperintense T2 signal abnormalities. EEG showed a mild degree of slowing and encephalopathic changes. One week into his admission, records from the previous admission were obtained. Per those records, patient was admitted to the ICU after being found nonresponsive secondary to a fentanyl overdose with a resultant anoxic brain injury. Patient records did not reveal any fentanyl prescriptions in the past 7 years. Given his prior admission and anoxic brain injury it was felt that his mental status represented a new baseline as a consequence of his fentanyl overdose. Patient was ultimately transferred to a long-term care facility.

IMPACT/DISCUSSION: Altered mental status is a common presenting complaint in internal medicine. A thorough review of the patient's medical record can help direct work-up. Best practices suggest that all patients with altered mental status should begin with an assessment of hemodynamic stability, history of trauma, past medical and substance abuse history, and history of present illness. Work-up should progress in a stepwise fashion to include basic infectious testing, toxicology screening, head imaging, EEG, LP, and evaluation of psychiatric disorders. It is important for clinicians to recognize the clinical impact of the opiate epidemic. With nearly 70,000 overdose deaths in 2016 and 2.5 million Americans battling addiction, the rise of illicit manufactured fentanyl is an increasing public health concern. Fentanyl is the leading cause of opioid overdose related deaths in the United States. Heroin contaminated with fentanyl is 20-40x more potent and this increased potency increases the risk of overdose. Patients who survive overdose can be left with devasting lifelong health consequences.

CONCLUSION: 1. All patients with altered mentation should be assessed for metabolic, infectious, toxic, malignant, and neurologic causes 2 . Fentanyl is the leading cause of opiate overdose 3. Survivors of opiate overdose can be left with significant health consequences References: Hedegaard H, Bastian B. Drugs Most Frequently Involved in Drug Overdose Deaths: United States, 2011 - 2016. National Vital Statistics Reports. 2018; 67(9): 1-13. Xiao H, Wang Y, Xu T, Zhu H, Guo S, Wang Z, Yu X. Evaluation and Treatment of Altered Mental Status in Patients in the Emergency Department: Life in the Fast Lane. WJEM. 2012; 3(4): 270-277.

\section{A NON-CLASSICAL PRESENTATION OF CELIAC DISEASE}

Ammu T. Susheela ${ }^{1}$; Clyde L. Smith ${ }^{1,2}$. ${ }^{1}$ Beth Israel Deaconess Medical Center, Boston, MA; ${ }^{2}$ Brigham and Women's hospital, Boston, MA. (Control ID \#3186421)

LEARNING OBJECTIVE \#1: Weight loss can be an initial only symptom of celiac disease. Celiac disease can be challenging to diagnose in an atypical presentation of non-European descent patients.

CASE: A 35-year-old Ethiopian female is presented with $50 \mathrm{lb}$ weight loss over 7 months after her second pregnancy. Her appetite is decreased and significantly limited by bloating. She has history abdominal pain and black stools. Past history is significant for gastroesophageal reflux disease (GERD), thyroiditis, anemia, migraine headache, vitamin D deficiency, malaria, and human papillomavirus infection. Her family history is significant for mother diagnosed with diabetes mellitus and asthma. Physical examination was normal with the soft, non-tender and non-distended abdomen. Her free T4 was 0.9 , but her TSH was normal at 1.3. Liver enzymes were slightly elevated with ALT at 63, the rest of her liver function tests were normal. Peripheral smear showed anemia. Her hepatitis core antibody was positive and surface antibody was also positive indicating a previously cleared hepatitis B infection. Her ultrasound showed a hepatic cyst. She was however also tested for celiac disease and her Tissue Transglutaminase Ab, IgA was greater than 100 . Her esophagogastroduodenoscopy showed chronic focally active gastritis with marked architectural abnormalities-villous shortening/crypt elongation consistent with celiac disease. Surprisingly H-pylori was negative. A diagnosis of celiac disease was made. Patient education about celiac disease was done extensively. She was given counseling about food intake and was put on a gluten-free diet with a nutrition consultation. She was advised mind-body stress reduction. She has also advised a lactose-free diet. Her condition remarkably improved on putting on following a strict gluten-free- lactose-free diet. She started gaining weight and has no gastrointestinal distress. Her GERD symptoms completely subsided and she feels she is doing very well.

IMPACT/DISCUSSION: Celiac disease may present with a wide range of clinical symptoms and signs. Non-gastrointestinal symptoms have also been reported in patients. A new term called the silent celiac disease has been used to refer for patients who do not present with diarrhea and it has been reported that the silent variant of celiac disease is more common than the patients who present with diarrhea. Patients with one autoimmune disease is more susceptible to other autoimmune diseases such as celiac disease. Currently, the only treatment for celiac disease is the gluten-free diet. In this case, it took several months to diagnose this patient with celiac disease due to the non-classical silent presentation.

CONCLUSION: The presentation of celiac disease varies within a spectrum of classical symptoms to atypical and silent variety. This makes it a challenge to diagnose the disease in atypical presentations which can significantly delay the treatment and lead to benign and malignant complications. 


\section{A PARADOXICAL TURN OF EVENTS}

Jimmy Mao; Neha Nidhi; Terin Sytsma; Brody Slostad; Iftikhar Kullo. Mayo Clinic, Rochester, MN. (Control ID \#3179235)

LEARNING OBJECTIVE \#1: Recognize clinical and diagnostic features of paradoxical emboli (PDE) causing myocardial infarction (MI)

LEARNING OBJECTIVE \#2: Manage venous thromboembolism (VTE) in the setting of malignancy

CASE: A 71-year-old female with no known coronary artery disease (CAD) and recently diagnosed unprovoked pulmonary embolism in the setting of a probable right malignant ovarian mass found on subsequent imaging, on apixaban $5 \mathrm{mg}$ twice daily, presented to the emergency department with new onset chest pressure and shortness of breath. She was found to have a normal electrocardiogram (ECG) but elevated highsensitivity troponins ( $340 \mathrm{ng} / \mathrm{L}, 2$-hour at $580 \mathrm{ng} / \mathrm{L}, 6$-hour at $1051 \mathrm{ng} / \mathrm{L}$ ), loaded with aspirin $324 \mathrm{mg}$ plus clopidogrel $600 \mathrm{mg}$, and started on a high-intensity heparin nomogram for a presumed recurrence of VTE vs. new MI. A repeat ECG hours later showed new ST elevations in the inferior leads, and she was taken urgently to the cardiac catheterization lab. Coronary angiogram showed no evidence of significant atherosclerotic disease but revealed a distal occlusion in the left anterior descending (LAD) artery, suggestive of embolism. A transesophageal echocardiogram (TEE) revealed a patent foramen ovale (PFO) with a small bidirectional shunt at rest. A lower extremity ultrasound showed a new acute thrombus in the right gastrocnemius vein. These findings suggested that the etiology of the patient's symptoms was an MI secondary to a PDE to the LAD artery in the setting of likely apixaban failure. She subsequently was transitioned from apixaban to enoxaparin and underwent an exploratory laparotomy and right salpingo-oophorectomy days later. Histopathology revealed high-grade carcinoma of the right ovary with mixed serous, clear cell, and mucinous differentiation and peritoneal involvement. A follow-up appointment with medical oncology was made for further staging and management.

IMPACT/DISCUSSION: PDE to the coronary arteries should be considered in patients presenting with acute coronary syndrome (ACS) in the setting of recent VTE and minimal CAD risk factors. Once suspected, a TEE should be performed to assess for a PFO. Treatment should focus on manual aspiration of the thrombus if possible and prevention of recurrence with anticoagulation. Low molecular weight heparin (LMWH) has been shown to be superior to vitamin $\mathrm{K}$ antagonists in preventing VTE in cancer patients. Although emerging data suggest that certain direct oral anticoagulants (DOACs) are more convenient, affordable, and as effective as LMWH, VTE recurrence in the setting of medication compliance may warrant switching to a LMWH.

CONCLUSION: Patients with known VTE and minimal CAD risk factors presenting with ACS should be assessed for PDE to the coronary arteries. Medical management involves long-term anticoagulation. LMWH is preferred over vitamin $\mathrm{K}$ antagonists for VTE prevention in the setting of malignancy, while comparisons between LMWH and DOACs are currently undergoing investigation.

\section{A PATIENT BETWEEN THE CRACKS: NAVIGATING HEALTHCARE BETWEEN HOMELESSNESS AND INCARCERATION}

Maria Srour; Francis G. Wade; Jennifer Schmidt. Saint Louis University, Saint Louis, MO. (Control ID \#3184624)

LEARNING OBJECTIVE \#1: Recognize the challenges of providing quality health care to patients within the Medicaid coverage gap.

LEARNING OBJECTIVE \#2: Highlight healthcare access disparities between low income citizens in non-Medicaid expansion states and correctional facility inmates.
CASE: A 27-year-old male with Crohn's disease presented from a correctional facility with worsening enterocutaneous fistulae and abdominal wall abscesses. Prior to incarceration, the patient was homeless and uninsured with very limited access to healthcare. He was unable to afford the recommended immunomodulator therapy. Once incarcerated, he qualified for Department of Corrections healthcare coverage and sought care. Definitive treatment for his disease required surgery with temporary colostomy. Concerned about returning to prison with an ostomy bag, he opted for a biologic agent, the inferior treatment option. Treatment required complete bowel rest and total parenteral nutrition therapy, extending his admission to 107 days. Due to ongoing treatment needs, the correctional facility enacted a compassionate release, terminating his healthcare coverage and jeopardizing access to further care. Eventually, the patient was enrolled in Missouri Medicaid due to disability but not before suffering significant morbidity due to preventable complications of his Crohn's disease.

IMPACT/DISCUSSION: Medicaid expansion is a key provision of the 2010 Affordable Care Act (ACA). Medicaid was guaranteed previously only to the elderly, blind or disabled, children, pregnant women, and lowincome parents or caretakers if their income falls within certain limits of the federal poverty level (FPL). Medicaid expansion ensures eligibility for all adults aged 18-65 years old with incomes up to $138 \%$ of the FPL. 19 states, including Missouri, have not implemented Medicaid expansion, leaving a coverage gap for low-income adults who do not qualify for Medicaid nor the ACA's Marketplace subsidies. It remains a challenge for non-disabled low-income adults in non-expansion states to access reliable healthcare. Meanwhile, healthcare coverage for correctional facility inmates is guaranteed by the Missouri Department of Corrections. Our patient in the Medicaid coverage gap only became eligible for healthcare coverage once deemed a criminal. Paradoxically, his incarceration led him to choose an inferior treatment for his disease. His story highlights challenges in providing quality care to vulnerable populations in states without Medicaid expansion. It also illuminates an ethical dilemma about how our healthcare system addresses both poverty and criminality.

CONCLUSION: -Access to healthcare remains a challenge for many patients within the Medicaid coverage gap of non-expansion states. -Nondisabled, low-income adults without children in non-expansion states do not share the same rights to healthcare access guaranteed to correctional facility inmates. -Policy change in non-expansion states may lead to improved outcomes in low-income patient populations by improving healthcare access.

\section{A PATIENT WITH TWO UNCOMMON CONDITIONS: CAN THIS BE CAUSE AND EFFECT?}

Justin Oveyssi; Chester Choi; Alice Park. St. Mary Medical Center, Long Beach, CA. (Control ID \#3172389)

LEARNING OBJECTIVE \#1: Recognize the immunological manifestations of sarcoidosis.

LEARNING OBJECTIVE \#2: Understand the pathophysiology of cryoglobulinemia and glomerulonephritis.

CASE: A 53 year old male with a history of uveitis caused by sarcoidosis presented with acute kidney injury (AKI) with hypertension, proteinuria, hematuria, edema, and vasculitic skin lesions. Diagnostic testing revealed a normocytic anemia and positive rheumatoid factor (RF) and qualitative cryocrit. His complement levels were mildly decreased and his ANA, ANCA, RPR, HbsAg, HepC ab, and HIV tests were all negative. He underwent kidney biopsy showing a membranoproliferative (MPGN) pattern consistent with mixed cryoglobulinemia. Immunofluorescence microscopy showed positive staining in mesangial areas and capillary walls for IgG, IgA, IgM, C1q, C3, and both kappa and lambda light chains. Corticosteroid therapy was instituted.

IMPACT/DISCUSSION: Glomerulonephritis (GN) is an uncommon manifestation of sarcoidosis, which more often causes interstitial 
granulomatous nephritis (IGN). Cryoglobulinemia has not been previously reported with sarcoidosis. However, with the exclusion of other common causes of cryoglobulinemia and GN, the pathophysiology of sarcoidosis, which leads to a broad-based hypergammaglobulinemia, and the MPGN which can be caused by mixed cryoglobulinemia raise the likelihood that these conditions are linked. The immunoglobulins that may be produced include RF (an IgM directed against IgG) and this and others may act as cryoglobulins and may be the cause of this patient's GN. In a 2013 review of glomerular diseases associated with sarcoidosis, the GN often occurred years after the diagnosis of sarcoidosis; it could, however, precede the diagnosis, and it could occur in conjunction with IGN. Establishing a unifying diagnosis may be of benefit in terms of consolidating treatment and subsequent follow up. This may be the first case of documented mixed cryoglobulinemia, glomerulonephritis, and sarcoidosis.

CONCLUSION: Sarcoidosis is an uncommon disease and Idiopathic Membranoproliferative Glomerulonephritis with Cryoglobulinemia is even more rare; the probability that they would occur in the same patient is remote if they are independent variables. If they are dependent variables, the probability would be greater and such a conjoint occurrence could be consistent in our patient given the pathophysiology of these conditions. The difference may have relevance in terms of steroid treatment which may be indicated for extrapulmonary manifestations of sarcoidosis, whereas not all patients with idiopathic cryoglobulinemic MPGN require intervention with steroids.

\section{A PERNICIOUS MIMICKER: B12 DEFICIENCY PRESENTING AS SPLENOMEGALY, PANCYTOPENIA AND HEMOLYTIC ANEMIA}

Megan K. Cochran ${ }^{2}$; Angela Thinda ${ }^{1}$; Elieth Martinez ${ }^{1} .{ }^{1}$ UCSF Fresno, Fresno, CA; ${ }^{2}$ UCSD, La Jolla, CA. (Control ID \#3184924)

LEARNING OBJECTIVE \#1: Recognize the common and rare clinical manifestations of B12 deficiency

LEARNING OBJECTIVE \#2: Recognize clinical features of pseudothrombotic angiopathy secondary to B12 deficiency

CASE: A 76 year-old man with a mechanical mitral valve replacement and atrial fibrillation (on coumadin) presented with 1 month of dyspnea on exertion and 20 pound weight loss. Exam revealed irregular rhythm, scleral icterus, and a painless $23 \mathrm{~cm}$ spleen. Labs were notable for WBC 1.9, Hgb 4.5 (MCV 108), and PLT 62. INR was 5.9. Blood smear showed macrocytosis, schistocytes, tear drops, and hypersegmented neutrophils. The patient was transfused pRBCs/FFP and was given oral vitamin $\mathrm{K}$. INR decreased to 1.5. Hemolysis labs were positive (LDH 4500, haptoglobin $<30$, indirect bilirubin 5.2) and Coomb's test was negative. TTE showed a normal ejection fraction with normal mitral valve function. Bone marrow biopsy demonstrated a hypercellular marrow. Serum folate was normal while vitamin B12 was low at 62. Methylmalonic acid was elevated and intrinsic factor antibody was positive. After active bleeding was ruled out, the patient was started on coumadin with heparin bridge as he was also thrombogenic due to the mechanical valve and atrial fibrillation. He was started on B12 injections (1000 mcg daily) and was discharged in stable condition. The remainder of the workup was as follows: negative MDS FISH analysis, negative ADAMSTS13/PNH assays, and no clonal abnormality on cytogenetic analysis.

IMPACT/DISCUSSION: This case highlights several important diagnostic and management challenges. First, B12 deficiency manifests as a variable spectrum of disease. Although the classic features include megaloblastic anemia and neuropsychiatric symptoms, our patient presented with rare manifestations of splenomegaly, pancytopenia, and hemolysis. Second, the patient's elevated INR, hemolysis, and schistocytes seen on presentation prompted us to consider microangiopathic hemolytic anemia that led to an extensive workup such as obtaining ADAMSTS13 assay. This phenomenon is known as pseudo-thrombotic microangiopathy, and often leads to delay in diagnosis and delivery of blood products such as
FFP as seen in our patient. Our patient's subtherapeutic INR offered the secondary challenge of balancing the risk of anticoagulating a prothrombotic patient while ruling out a bleeding diathesis. Furthermore, while a hematological process was suspected such as myelodysplastic syndrome or myelofibrosis given his age, pancytopenia, and enlarged spleen, reversible and easily treatable etiologies should not be overlooked. Our patient's low serum B12 and elevated MMA levels ultimately lead to a diagnosis of pernicious anemia that was managed with B12 injections. CONCLUSION: B12 deficiency due to pernicious anemia can rarely present with pancytopenia, hemolysis, and splenomegaly Diagnostic evaluation of pancytopenia should emphasize testing for reversible and easily treatable conditions including B12 deficiency

\section{A PREEMPTIVE STRIKE AT SUSPECTED PRIMARY ALDO- STERONISM IN A MEDICALLY UNDERSERVED PATIENT}

Zachary S. Pinchover; Sam Bedri; Steven Taylor. Dell Medical School at the University of Texas Austin, Austin, TX. (Control ID \#3155089)

LEARNING OBJECTIVE \#1: Recognize the clinical presentation of primary aldosteronism (PA) in an acute care setting.

LEARNING OBJECTIVE \#2: Detail the evaluation and management of suspected PA in a medically-underserved patient.

CASE: A 29-year-old uninsured male with untreated hypertension, inadequate access to outpatient care, and frequent emergency department (ED) visits presented to the ED with a two-day history of headaches, blurry vision, chest pain, and shortness of breath. He denied muscle cramps, prescription medication use, illicit drug use, or family history of hypertension. His physical exam showed a blood pressure (BP) of 280/140 $\mathrm{mmHg}$, otherwise normal vital signs, and normal cardiopulmonary and neurologic exams. Initial lab studies revealed a low serum potassium (2.2 $\mathrm{mEq} / \mathrm{L})$ and elevated serum creatinine $(1.4 \mathrm{mg} / \mathrm{dL})$. ECG and serial troponin measurements ruled out acute MI. He was diagnosed with hypertensive emergency with acute kidney injury (AKI) and treated in an ICU setting with potassium repletion and continuous esmolol and nicardipine infusions. With suspicion for PA, morning serum samples for aldosterone and plasma renin activity measurement were collected. The patient was transitioned onto maximum doses of three antihypertensive agents and required continued potassium repletion. Though his AKI resolved his BP remained elevated and, on hospital day \#4, he voiced a desire to be discharged at a time his lab evaluation for PA was still pending. With high concern for PA, he was prescribed spironolactone with subsequent improvement in BP and serum potassium. He was discharged with follow up in a local clinic for the uninsured, and his lab evaluation later returned confirming high suspicion for PA. Confirmatory biochemical testing for PA was deferred, CT imaging showed a $2.3 \mathrm{~cm}$ right adrenal adenoma, and arrangements were made for right adrenalectomy.

IMPACT/DISCUSSION: PA is an often underrecognized cause of secondary hypertension that carries significant morbidity. Treatment-resistant hypertension and spontaneous hypokalemia are cardinal features in PA that should prompt a diagnostic evaluation for it. This can be costly, timeconsuming, and challenging, however. Medications, hypokalemia, and AKI can result in falsely elevated or suppressed results of biochemical tests used for PA case detection. Further, consensus guidelines indicate confirmatory testing — often to include adrenal venous sampling — should be pursued for many patients for whom PA is suspected based on biochemical case detection results. A patient-centered approach to the evaluation and treatment of PA is essential for medically-underserved patients for whom high clinical suspicion for this diagnosis exists.

CONCLUSION: Empiric treatment for PA may be considered in a medically-underserved patient with high clinical suspicion for PA while awaiting the results of biochemical tests for case detection. Confirmatory biochemical tests may be deferred in the setting of high clinical suspicion for PA. 


\section{A QUANDARY OF UNRESOLVING ORBITAL CELLULITIS}

PRIYANKA BHUGRA ${ }^{1}$; Abhishek Maiti ${ }^{2}{ }^{1}$ University of Texas Health Science Center at San Antonio, San Antonio, TX; ${ }^{2}$ University of Texas Health Science Center at Houston, Houston, TX. (Control ID \#3187157)

LEARNING OBJECTIVE \#1: IgG4-related disease is an immunemediated condition which can mimic infectious, malignant and inflammatory disorders.

LEARNING OBJECTIVE \#2: Histopathology is the key to diagnosis and timely diagnosis can prevent severe end-organ damage, disabling tissue fibrosis, and even death.

CASE: A 50-year-old woman with history of asthma presented to emergency room with progressive swelling of her left upper and lower eyelids for 4 weeks. Examination showed severe edema and redness of left eyelids. Pupils were normal. Visual acuity was 20/20 in both eyes. Fundus examination was normal. Laboratory studies were unremarkable. Computed tomogram of the orbits and sinus showed left preseptal and orbital cellulitis, chronic pansinusitis and left ethmoid osteomyelitis. Swab and tissue culture from nasal and sinus endoscopy grew methicillin-sensitive Staphylococcus aureus. No improvement was noted after 2 weeks of IV antibiotics. Repeat imaging showed further progression of the inflammatory changes in left orbit and ethmoid. At this point, a left caruncular orbitotomy with biopsy showed dense lympho-plasmacytic infiltrate and fibrosis. Immunohistochemistry showed diffuse IgG positivity with $>100$ $\mathrm{IgG} 4$ cells/HPF and polyclonal kappa and lambda light chains. Diagnosis of IgG4-related disease (IgG4-RD) was established and patient was started on IV methylprednisolone. Dramatic improvement was noted over next few days and she was discharged on an oral steroid taper. At 6-month follow-up, she remained asymptomatic.

IMPACT/DISCUSSION: IgG4-RD is a recently-recognized, immunemediated condition that is characterized by fibro-inflammatory lesions rich in IgG4-positive plasma cells. It can affect one or more organs with tumefactive lesions, synchronously or metachronously. It can masquerade as a neoplastic or inflammatory condition. The pathogenesis is poorly understood but involves multiple immune- mediated mechanisms with autoimmune as well as allergic components. Tissue diagnosis is the gold standard for diagnosis. The chief pathologic features include lymphoplasmacytic infiltration and storiform pattern of fibrosis. High number of IgG4 positive plasma cells on immunostaining is the hallmark for diagnosis. This may or may not be accompanied by elevation of serum IgG4 levels. All patients with symptomatic and active IgG4-RD require treatment. Majority cases are responsive to glucocorticoids which are the first line of treatment. Disease recurrence is common. Glucocorticoid sparing agents may be used in cases of contraindication to steroids, failure of steroids, or for remission maintenance but their efficacy has not been studied.

CONCLUSION: IgG-RD has a wide spectrum of presentation with single or multi-organ involvement. Early diagnosis can help prevent serious organ damage and death as most of the cases are responsive to steroids.

\section{A QUINKE COMPLICATION OF TRANSJUGULAR LIVER BIOPSY}

Grant Pickett; Nathan C. Nowalk. Baylor College of Medicine, Houston, TX. (Control ID \#3186241)

LEARNING OBJECTIVE \#1: Identify hemobilia as a potential complication of trans-jugular liver biopsy

LEARNING OBJECTIVE \#2: Formulate the appropriate management of hemobilia

CASE: A 56 year-old female with adult polycystic kidney disease and recurrent ascites underwent transjugular liver biopsy to evaluate for cirrhosis. Pre-procedural imaging was significant for multiple hepatic cysts, largest at $8 \mathrm{~cm}$ in diameter. Four core biopsies were collected with no immediate complications recognized. Following the procedure, the patient had several episodes of large volume hematemesis. Emergent esophagogastroduodenoscopy demonstrated a large amount of blood in the stomach and duodenum without a recognized source of bleeding. Despite blood transfusions, her hemoglobin continued to fall prompting further investigation. Endoscopic retrograde cholangiopancreatography showed active bleeding from the ampulla. Interventional Radiology performed angiography with embolization of a $1 \mathrm{~cm}$ pseudoaneurysm arising from the pancreaticoduodenal vessels. Following embolization, she demonstrated no further evidence of hemorrhage.

IMPACT/DISCUSSION: In patients undergoing liver biopsy, percutaneous liver biopsy is considered the gold standard. Contraindications to percutaneous biopsy include morbid obesity and ascites, such as seen in our patient. In these cases, a transjugular approach is considered, which some literature has reported to have comparable outcomes both for specimens and patient safety relative to a percutaneous approach. Hemobilia is a rare complication of transjugular liver biopsy with an incidence of $0.04 \%$. Quinke's triad of hemobilia is acute gastrointestinal bleed, jaundice, and right upper quadrant pain, although the full triad is uncommon. Definitive diagnosis of hemobilia is made by visualization of bleeding from the ampulla. As seen in our patient, acute hemobilia is most commonly iatrogenic from direct injury to intrahepatic blood vessels or bile ducts during instrumentation. Angiographic intervention is the preferred method of managing active hemobilia. In one study, success rates with angiography were greater than $95 \%$. However, this same study acknowledged that successful embolization will be dependent on the skill of the interventional radiologist.

CONCLUSION: Transjugular liver biopsy is the preferred method for patients with morbid obesity or ascites. It is important for physicians to be able to recognize post-procedural complications such as hemobilia and initiate early and appropriate management. In patients with hemobilia, first-line treatment is transcatheter arterial embolization, which has shown high rates of success.

\section{A RARE AUTO-IMMUNE-LIKE SYNDROME ASSOCIATED WITH NIVOLUMAB THERAPY}

Eugene N. Quaye. University of Oklahoma School of Community Medicine, Tulsa, OK. (Control ID \#3183619)

LEARNING OBJECTIVE \#1: Recognize autoimmune related adverse effects of check-point inhibitors

LEARNING OBJECTIVE \#2: Treat autoimmune related adverse effects of check-point inhibitors

CASE: A 63 yo male with medical history of atrial fibrillation, hypertension, and stage III metastatic melanoma on immunotherapy with nivolumab presented for evaluation of a five day history of dyspnea with onset shortly after his second chemotherapy treatment. He also endorsed orthopnea, paroxysmal nocturnal dyspnea, diplopia which resolved upon closing the right eye, and right eye lid droop at the time of presentation. Examination was significant for tachypnea, and tachycardia with an irregular rhythm. There were no neurological deficits. Laboratory work revealed AST of 818 and ALT of 358, troponin of 13.1 and CPK of 16,493. EKG showed atrial fibrillation with rapid ventricular rate. Treatment initiated with heparin drip, aspirin and ticagrelor. Hepatic thrombus was ruled out with testing. ECHO ruled out cardiac wall abnormalities. Acute intervention for possible ACS was decided against due to possible myocarditis associated with his immunotherapy. He had positive acetylcholine binding antibody, but anti-MUSK antibody returned negative. His troponin, CPK and transaminase levels improved after initiation of immune suppressive therapy with steroids and plasmapheresis. However, he had a decline in respiratory function necessitating intubation. He later developed GI bleeding due to biopsy proven duodenitis. His case was further complicated by varying degrees of heart block, bradycardia and ultimately hemodynamic instability. He failed to improve despite aggressive measures, ventilator support and continued steroid therapy. His 
family ultimately made the decision to pursue comfort care. The rapid improvement of his troponin, CPK and transaminitis with steroids and plasmapheresis, and positive acetylcholine binding antibody supported our suspicion for autoimmune involvement as a side effect of this immunotherapy.

IMPACT/DISCUSSION: Check point inhibitors such as nivolumab act by binding PD 1 receptor with subsequent blockade of PD 1 and PD 2 ultimately causing improved antitumor response that has been beneficial in the treatment of metastatic melanoma and renal cell carcinoma. However, nivolumab has been reported to cause an autoimmune like syndrome of myocarditis, hepatitis, myasthenia gravis, endocrinopathies and pneumonitis. Our case highlights an autoimmune-like syndrome of de novo myasthenia gravis, hepatitis, duodenitis and myocarditis with each having a less than $1 \%$ occurrence. Initial therapy involves the use of glucocorticoids and immunomodulators to curb further injury to the patient. Ultimately, supportive care remains the corner stone of medical therapy.

CONCLUSION: There is an increasing number of case reports describing autoimmune syndrome associated with check point inhibitors, thus early recognition and routine follow-up is critical in improving quality of life of patients initiated on therapy

\section{A RARE CASE OF C. DIFFICILE ENTERITIS; A COMMON BUG IN AN UNCOMMON PLACE}

Amreet K. Aujla $^{1}$; Shivaraj Patil ${ }^{1}$; Nha Duong ${ }^{1}$; Alexander Potashinsky ${ }^{2}$. ${ }^{\mathrm{T}}$ UConn Health, Hartford, CT; ${ }^{2}$ UConn Health, Farmington, CT. (Control ID \#3180776)

LEARNING OBJECTIVE \#1: To recognize signs and symptoms of atypical Clostridium Difficile infection (CDI) in order to improve disease related mortality and morbidity

CASE: Patient is a 55-year-old male with history of Crohn's disease status post total colectomy with end ileostomy on mesalamine therapy, chronic osteomyelitis on suppressive therapy with doxycycline, ESRD on hemodialysis who presented to the emergency department with symptoms of increased ostomy output and abdominal pain for two days. Physical exam was significant for tachycardia, hypotension, and a diffusely tender abdomen. The ileostomy was viable and a small amount of fluid noted in the ostomy bag. Laboratory examination revealed a white blood cell count of 10,900 cells $/ \mathrm{mm}^{3}$ and a serum lactate of $2.2 \mathrm{mg} / \mathrm{dL}$, with negative blood cultures. Stool studies from his stoma grew C. Dificile and he was treated with oral metronidazole with improvement in ostomy output. He was discharged with metronidazole to complete a 10-day course. Three months later, the patient presented with a four-day history of increasing ostomy output with watery stools and epigastric abdominal pain. Vitally, patient was hemodynamically stable and on physical examination, patient had epigastric abdominal tenderness. Laboratory examination were significant for a white blood cell count of 14,200 cells $/ \mathrm{mm}^{3}$. CT abdomen and pelvis with oral contrast showed bowel thickening with minimal stranding suggestive of enteritis along with enlarged peritoneal lymph nodes. Stool studies were positive for C. Difficile and lactoferrin. Patient was started on oral vancomycin and improved. He was discharged to complete a 12-week course.

IMPACT/DISCUSSION: Isolated small-bowel involvement is exceedingly rare but is more commonly seen in patients with colectomy. The diagnosis of $C$. difficile enteritis requires a high index of suspicion. As many patients may not initially raise suspicion of $C$. difficile infection, CT scan may provide supportive evidence. Ascites and fluid-filled small bowel in the presence of mild mesenteric stranding is often consistent with $C$. difficile enteritis. Analogous to colonic infection, antibiotics such as metronidazole and vancomycin are the corner stone of treatment, along with supportive therapy.

CONCLUSION: CDI is the major identifiable cause of antibioticassociated diarrhea and is responsible for $15-25 \%$ of all cases. CDI has increased in incidence, particularly among patients with inflammatory bowel disease. Normally manifesting as infectious colitis, small bowel involvement known as CDI enteritis is a very rare condition and is associated with a mortality rate of approximately $30 \%$. In addition, it is also coupled with increased morbidity, length of stay, health care cost and poor quality of life. CDI involvement of the small bowel is rare, however, it appears that the incidence is increasing. High index of suspicion and early identification of $C$. difficile in the small bowel is imperative for early intervention in order to improve outcomes.

\section{A RARE CASE OF GI BLEED ASSOCIATED WITH NEUROFI- BROMATOSIS TYPE 1}

Ricci Kalayanamitra ${ }^{1}$; Jose Chu Luo ${ }^{1}$; Zainab Shahid ${ }^{2}$; Neal Shah ${ }^{3}$; Ravi

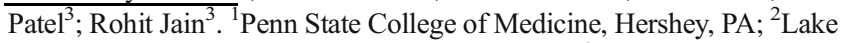
Erie College of Osteopathic Medicine, Erie, PA; ${ }^{3}$ Penn State Milton S. Hershey Medical Center, Hershey, PA. (Control ID \#3165746)

LEARNING OBJECTIVE \#1: Recognize the association between neurofibromatosis type 1 and gastrointestinal stromal tumor.

CASE: A 21-year-old male with PMHx of NF1 presented with SOB, lethargy, and 1 episode of melena. He was admitted to an OSH 2 weeks prior with similar symptoms and found to be anemic due to a GI bleed. Colonoscopy and EGD were negative. He was then scheduled for an outpatient capsule endoscopy but this was not done due to his abrupt rehospitalization at said $\mathrm{OSH}$, where he was found to be tachycardic and anemic with $\mathrm{Hgb}$ of $6 \mathrm{~g} / \mathrm{dl}$. He underwent an abdominal CT, was transfused with 3 units of pRBCs, and was transferred to our institution. Patient reported continued lethargy and denied abnormal bowel movements between his 2 hospitalizations. His abdominal and rectal exams were unremarkable and skin exam revealed cafe-au-lait spots on the right arm. $\mathrm{Hbg}$ and t-bili were $8.5 \mathrm{mg} / \mathrm{dl}$ and $2.3 \mathrm{mg} / \mathrm{dl}$, respectively. Vital signs were stable. The CT-scan from the OSH was initially reaffirmed as normal, but it was later re-examined with the patient's history of NF1 in mind. This revealed a $2.1 \times 2.1 \times 3.2 \mathrm{~cm}$ well-circumscribed ovoid mass adjacent to a loop of small bowel in the left upper quadrant, which was subsequently resected, biopsied, and confirmed as a low-grade GIST.

IMPACT/DISCUSSION: The differential diagnoses of patients who present with anemia are broad. In the setting of anemia and melena, CT-scans, EGDs, and colonoscopies are often ordered. If these exams are non-revealing, patients may be subjected to repeat studies or more specialized studies, such as capsule endoscopies and push or double balloon enteroscopies. This adds a physical and financial burden to the patient and may still fail to identify the underlying etiology of their symptoms. After admitting this patient to our institution, our plan was to repeat testing with a push enteroscopy and a capsule study. We were able to avoid this course by taking a thorough history of his NF1, researching its link to GIST, and correlating this with our suspicious findings on imaging. According to the American Society of Clinical Oncology, the annual incidence of GIST is 6.8 cases per million people in the US. Of these, 5 percent of patients have a familial AD syndrome, such as NF1, familial GIST syndrome and CarneyStratakis. In fact, patients with NF1 are 34 times more likely to develop GIST than unaffected individuals. Imatinib is an effective treatment for GIST; it has shown improvement in $54 \%$ of GIST patients within 6 months and an increase in survival of GIST patients from 18 to 57 months in a phase 2 clinical trial. Despite its rarity, providers should consider GIST in patients with a history of NF1 who present with GI symptoms, especially because there is an effective treatment for it.

CONCLUSION: -Have clinical suspicion for GIST in patients with NF1 presenting with GI symptoms. -The primary team should conduct thorough history taking and provide adequate contexts when ordering imaging. -GIST is treatable and therefore a prompt diagnosis is essential. 


\section{A RARE CASE OF GONOCOCCAL CELLULITIS IN A PATIENT WITH SEVERE SOFT TISSUE INFECTION}

Marissa Fox ${ }^{1}$; Veronica Scott ${ }^{1}$; Patrick Pham $^{2}$; Molara Alexis ${ }^{3}$; Thida $\mathrm{Win}^{3} .{ }^{1}$ University of California Riverside, Riverside, CA; ${ }^{2}$ University of California Riverside School of Medicine, Riverside, CA; ${ }^{3}$ St. Bernardine's Medical Center, San Bernadino, CA. (Control ID \#3184188)

LEARNING OBJECTIVE \#1: Inquire about sexual history and screen at-risk individuals for sexually transmitted infections.

LEARNING OBJECTIVE \#2: Assess for potential sexually transmitted infections in patients with evidence soft tissue infection with joint involvement

CASE: A 47 year old female with diabetes presented to the Emergency Department with progressive redness, pain, and swelling of the right foot and left knee over the course of five days. She had extensive erythema and swelling on the right foot as well as left proximal calf, left knee, and left distal thigh. She also experienced pain with flexion of the left knee. On admission she was afebrile and vital signs were stable. CT scan revealed gas along the subcutaneous tissue and deep in the fascial planes of the proximal calf and distal thigh along with joint effusion. Her laboratory studies were significant for an elevated White Blood Count of 28,700 . The patient was started on broad spectrum antibiotics and was evaluated by a general surgeon. She was taken to the Operating Room for incision and debridement to rule out necrotizing fasciitis. The surgeon noted extensive induration of the posterior calf and thigh with serous fluid without evidence of necrosis or purulence along the fascial planes. The debrided subcutaneous tissue from the Operating Room was sent to the laboratory for culture and returned with gram negative diplococci, that was later confirmed to be Neisseria Gonorrhea. Upon further assessment, the patient stated she was sexually active with multiple male partners and had inconsistent condom use in the past year. She was unsure of her last screening for sexually transmitted infections. She denied any symptoms of urethritis or vaginal discharge. Her urine gonococcal and chlamydia screen was negative, however, the patient had already received five days of IV Ceftriaxone which likely eliminated the infection prior to urine screening.

IMPACT/DISCUSSION: This case highlights a unique clinical finding that has scarcely been described in the literature. Given the patient's sexual history and lack of screening, she likely had an untreated gonococcal infection for an unknown period of time. We diagnosed her with severe cellulitis related to disseminated Neisseria gonorrhea infection. This highlights the importance of adequate sexual history taking in all sexually active patients as well as screening those at-risk individuals to prevent severe and life-threatening complications of untreated infections.

CONCLUSION: Gonococcal cellulitis is an extremely rare clinical finding that has been scarcely reported in the literature. Screening at-risk individuals for sexually transmitted infections can prevent detrimental complications of untreated infection.

\section{A RARE CASE OF INVASIVE NON-TYPHOIDAL SALMONEL- LA CAUSING EMPHYSEMATOUS OSTEOMYELITIS}

Rohan B. Parikh; Muhammad U. Khalid; Krupa K. Solanki; Lance Blau; Munir Shah. Western Reserve Health Education, Youngstown, OH. (Control ID \#3185931)

LEARNING OBJECTIVE \#1: We present a rare case of emphysematous vertebral osteomyelitis due to Non-typhoidal salmonella (NTS) in an immunocompetent adult. NTS commonly causes foodborne diarrheal illness but rarely causes fulminant invasive infections. There is a six fold higher risk of invasive infection with NTS when compared with other bacterial causes of gastroenteritis. There should always be a high degree of suspicion in presence of particular risk factors which would aid in reducing morbidity and mortality.
CASE: Our patient is a 51-year-old male who presented to the ED in acute distress with worsening back pain for four weeks. Associated symptoms included shortness of breath, sharp chest pain, high grade fever, and chills. His significant medical history included type II diabetes mellitus, hyperlipidemia, and peripheral vascular disease. His medications were aspirin, cilostazol, insulin glargine, losartan, and fenofibrate. He had a 30-pack year smoking history but denied any illicit drug use. He owned a mobile petting zoo and was in close contact with many animals on a daily basis. Examination revealed oxygen saturation of $84 \%$ on room air, bilaterally diminished breath sounds, and point tenderness around the first lumbar vertebra. His laboratory studies were significant for platelet count of 103 , ESR of 88, sodium of 129, creatinine of 1.94, and total bilirubin of 1.7. Blood cultures were drawn. Chest x-ray showed bilateral extensive multifocal airspace opacities, and an MRI of spine showed emphysematous osteomyelitis of the L1 vertebral body with small amounts of gas in the retroperitoneum. The patient was started on supplemental oxygen, vancomycin, cefepime, and levofloxacin. Over the next two days, his oxygen requirement increased, and he eventually required non-invasive ventilation in the ICU. On day four, blood cultures grew gram negative rods which finalized to pansensitive group D salmonella. He was treated with intravenous ceftriaxone for six weeks. Neurosurgical intervention was deemed unnecessary, as there were no signs of necrosis or abscess formation on serial imaging. At three-month follow up, the patient reported complete resolution of his symptoms.

IMPACT/DISCUSSION: Extraintestinal infections of NTS species are well reported in immunocompromised patients; however, they are rare in immunocompetent patients such as ours. Invasive NTS is also associated with diabetes and atherosclerosis. Along with our patient's comorbidities, repeated exposure to animals in his petting zoo likely culminated in his extraintestinal, invasive infection. Due to disease rarity, management of emphysematous osteomyelitis can prove difficult as optimal antibiotics and the need for surgery have not yet been established. Although there has been development in the field of invasive NTS vaccines, none have shown much promise in clinical trials.

CONCLUSION: There should be an increased awareness amongst physicians to reduce morbidity associated with NTS emphysematous osteomyelitis.

\section{A RARE CASE OF LIFE-THREATENING HYPONATREMIA FROM A SWOLLEN HEART}

Shaunak Mulani; Margaret C. Lo. University of Florida College of Medicine, Gainesville, FL. (Control ID \#3185939)

LEARNING OBJECTIVE \#1: Recognize the clinical presentation of acute hyponatremia secondary to pericardial tamponade

LEARNING OBJECTIVE \#2: Identify the risk factors and disease markers for acute hyponatremia secondary to tamponade

CASE: A 76-year-old female presented with three weeks of lightheadedness, substernal, non-radiating chest pain and self-reported syncopal episodes. Medical history includes rheumatoid arthritis and GERD. Exam revealed tachypnea, hypotension, irregularly irregular rhythm and rate (140s), jugular venous distension and 1+ pitting edema in bilateral legs. EKG showed new onset atrial fibrillation with rapid ventricular rate (RVR). CBC, BMP and TSH were normal on admission. ProNT BNP was elevated at 408. CTA Chest noted a moderately sized pericardial effusion but no PE. TTE showed a newly depressed EF of $35 \%$ and a pericardial effusion but no tamponade physiology. Patient was stabilized by diuresis for $\mathrm{CHF}$ and metoprolol for rate control. On hospital days 4-6, she developed worsening dyspnea, increasing hypoxia, recurrent hypotension refractory to IVF and atrial fibrillation with RVR refractory to IV metoprolol and digoxin. Labs noted acute kidney injury (AKI) and acute hypotonic hyponatremia worsening in 48 hours (creatinine 1.1$>2.1 \mathrm{mg} / \mathrm{dl}$, Na 136->122mEq). The hyponatremia did not respond to fluid restriction or salt tablets. Repeat TTE showed worsening pericardial effusion with tamponade physiology. Urgent pericardiocentesis removed 
$1200 \mathrm{~mL}$ bloody, exudative fluid likely due to recent anticoagulation for atrial fibrillation. Within 24 hours of pericardial drainage, her clinical picture improved with rapid resolution of hyponatremia and AKI.

IMPACT/DISCUSSION: Hyponatremia is a common electrolyte abnormality often caused by medications, hormonal imbalance, liver or cardiac failure. This case adds to the limited literature of acute hyponatremia from tamponade, often overlooked as another potential cause. It teaches the dangers of anchoring and premature closure as diagnostic heuristics in the work-up of hyponatremia. Diagnostic clues include abrupt clinical deterioration and rapid progression of hyponatremia refractory to standards of therapy. Literature suggests monitoring NT-pro BNP as a marker of disease severity in tamponade. Cases are mostly in malignancy and rarely hemorrhagic from anticoagulation or nonmalignant like our case. Pathophysiology is not well-understood but likely due to inappropriate release of ADH from the low-flow state of tamponade. Prompt pericardial drainage improves systolic blood pressure, decreasing $\mathrm{ADH}$ production, increasing ANP production and resulting in rapid recovery of hyponatremia, as cited in most cases.

CONCLUSION: Consider tamponade in cases of unexplained hyponatremia, particularly in the setting of malignancy, existing pericardial effusion and/or hyponatremia refractory to empiric treatment Reversal of hyponatremia after pericardial drainage is rapid and spontaneous so prompt diagnosis of tamponade is imperative and life-saving

\section{A RARE CASE OF RECURRENT ENDOCARDITIS DUE TO CANDIDA PARAPSILOSIS}

Daniel Tsang; Misa Hyakutake; Kevin Andujar. Mount Sinai Beth Israel, Bronx, NY. (Control ID \#3183552)

LEARNING OBJECTIVE \#1: Recognize the importance of long-term suppressive therapy in patients with a history of fungal endocarditis.

CASE: A 51 year old man with a history of active inhaled cocaine use and former intravenous heroin use presented with dyspnea and fevers for 2 weeks. Patient denied taking any medications other than methadone. He last used cocaine 3 days ago and quit intravenous heroin use 8 years ago. Physical exam was notable for mild jugular venous distention, a 3/6 systolic murmur, $1 / 6$ diastolic murmur, and $1+$ pitting edema in his lower extremities. No skin or nail abnormalities were observed. Laboratory test showed WBC 7.6 K/UL, BType Natriuretic 506.2 PG/ML. Urine toxicology was positive for cocaine and methadone. Chest X-ray showed pulmonary vascular congestion and interstitial edema. Transthoracic echocardiogram and subsequent transesophageal echocardiogram showed a mobile $1 \mathrm{x}$ $0.5 \mathrm{~cm}$ echodensity on the aortic valve and severe aortic regurgitation. All 3 sets of blood cultures that were drawn grew Candida parapsilosis. He underwent successful aortic valve replacement and completed a 3 week course of intravenous caspofungin. He was discharged with a 90 day supply of oral fluconazole and planned outpatient follow up. Approximately 4 months later, he presented again to the ED complaining of left sided abdominal pain and left chest pain. CT of the abdomen showed large areas of splenic infarctions. Transesophageal echocardiogram revealed multiple new vegetations on the bioprosthetic aortic valve with the largest measuring $3.4 \mathrm{~cm}$. He underwent successful redo aortic valve replacement. Blood cultures and cultures from the aortic root vegetations again grew Candida parapsilosis and he was continued with caspofungin. Upon further questioning, patient admitted that he had not been compliant with his suppressive fluconazole, only taking it for 2 weeks. He was eventually discharged with 6 weeks of IV caspofungin, followed by planned lifelong fluconazole therapy.

IMPACT/DISCUSSION: Recurrence rates of fungal endocarditis after surgical and antifungal treatment have been estimated to be around 30$40 \%$ and occur more frequently in those who stop suppressive therapy. There have been reported cases of recurrence several years after the initial valve replacement. Fungi have been shown to be present in replaced valves decades after placement. Given the rarity of fungal endocarditis (about $2 \%$ of all infectious endocarditis cases), there are no definitive guidelines on anti-fungal treatment duration after valve replacement. This report demonstrates a case of a rare infection and the possible effects of an abbreviated treatment course. Taking into account the high recurrence rates of fungal endocarditis, it is reasonable to consider lifelong suppressive therapy.

CONCLUSION: - There are no definitive guidelines as to treatment duration for fungal endocarditis because of its rarity. - Given the high mortality rate and high rate of recurrence, lifelong suppressive therapy should be considered for fungal endocarditis.

\section{A RARE CASE OF RECURRENT PNEUMONIA}

Belaal Sheikh; Dipesh Ludhwani. Chicago Medical School, McHenry, IL. (Control ID \#3185779)

LEARNING OBJECTIVE \#1: Important considerations when assessing causes of recurrent pneumonia.

CASE: A 63 year old Caucasian female presented to our hospital with a complaint of dyspnea for a few days. Her past medical history was significant for multiple sclerosis, quadriplegia and recurrent pneumonia. A month prior to presentation, the patient was discharged from the hospital on antibiotics for her third episode of aspiration pneumonia in the last 8 months. Patient had a normal bronchoscopy 6 months ago. Physical exam on admission revealed a low grade temperature with hypoxia and coarse breath sounds. Initial lab work showed an elevated leukocyte count of 15.4 and normal serum chemistry. CT chest revealed progression of bilateral chronic lower lobe infiltrates with right upper lobe infiltrates now lower in density compared to previous imaging. Patient was started on broad spectrum antibiotics and underwent bronchoscopy with Broncho-alveolar lavage and Transbronchial biopsy. Cytopathology from right upper lobe revealed reactive bronchial cells and foamy macrophages consistent with a diagnosis of Lipoid pneumonia. Careful investigation of her medication list revealed mineral oil use for chronic constipation. Mineral oil was stopped and patient was continued on supportive antibiotic therapy. Her symptoms improved and patient was discharged to a skilled nursing facility. Follow up CT scan performeded outpatient revealed a complete resolution of upper lobe infiltrates and improvement in lower lobe opacities.

IMPACT/DISCUSSION: Lipoid pneumonia should be considered in all cases of recurrent pneumonia especially in patients at high risk of aspiration. Detailed history and thorough medication review are imperative in making the diagnosis. CT scan in lipoid pneumonia characteristically shows fat attenuation as low as $-30 \mathrm{HU}$ within consolidative opacities. Presence of superimposed inflammation can confound diagnosis by increasing attenuation and obscuring fat component. Imaging with histologic confirmation is gold standard in diagnosis of lipoid pneumonia. Distinction between endogenous and exogenous pneumonia plays a big role in management of such cases. Endogenous pneumonia is commonly associated with lung malignancy. Treatment for lipoid pneumonia is supportive. Historically corticosteroids, immunoglobulins and whole lung lavage have all shown successful results.

CONCLUSION: Pneumonia remains a leading cause of mortality and morbidity worldwide. Lipoid pneumonia is a rare presentation of pneumonia seen in 1-2.5\% cases. Lipoid pneumonia results from accumulation of lipid in alveoli and can either be exogenous or endogenous in nature based on the source of lipid. Mineral oil with long chain saturated hydrocarbons can induce inflammatory reaction in alveoli. These oils are phagocytosed by macrophages and coalesce to form a mass also known as paraffinoma. Successful identification and treatment of lipoid pneumonia can decrease morbidity, mortality, and unnecessary interventions for patients. 


\section{A RARE CASE OF SYSTEMIC AMYLOIDOSIS: AN UNDERDIAGNOSED DISEASE}

Syeda A. Raza ${ }^{1}$; Somtochi I. Okafor ${ }^{2}$. ${ }^{1}$ Methodist Health Systems Dallas, Dallas, TX; ${ }^{2}$ UT Southwestern Medical School, Dallas, TX. (Control ID \#3185729)

LEARNING OBJECTIVE \#1: Recognize primary systemic amyloidosis in elderly patients with no evidence of preceding or coexisting disease in elderly patients

LEARNING OBJECTIVE \#2: Diagnose primary systemic amyloidosis based on clinical features and laboratory studies

CASE: An 81-year old man with no known past medical history presented to the hospital via emergency medical services for altered mental status. On physical exam, patient was awake but only oriented to person. He had multiple purpuric lesions around the eyelids and upper chest wall. He also had a distended abdomen with bilateral lower extremity pitting edema. Initial labs were notable for sodium of $117 \mathrm{mmol} / \mathrm{L}$ and a creatinine of $1.91 \mathrm{mg} / \mathrm{dL}$. He was admitted to the ICU for treatment of his hyponatremia that was corrected with $3 \%$ hypertonic saline. However, patient remained volume overloaded despite aggressive diuresis. A transthoracic echo showed normal systolic function but impaired diastolic relaxation. As the patient remained volume overloaded in setting of renal dysfunction, decision was made to initiate patient on hemodialysis. A renal biopsy was obtained showing Congo red positive amyloid deposition. During the hospitalization, the patient continued to deteriorate and developed an ileus that did not improve with supportive care. A subsequent endoscopy and colonoscopy were performed to help with decompression. Gastric, colonic, and rectal biopsies that were taken showed Congo red positive amyloid deposition consistent systemic amyloidosis. At this point the patient's clinical status had not improved. Multiple family meetings were held to discuss treatment and prognosis. However, the family revealed that the patient had advanced directives in place and would not want aggressive treatment. He was transitioned to palliative care and ultimately passed away.

IMPACT/DISCUSSION: Amyloidosis constitutes a plasma cell dyscrasia and consists of a subset of various diseases to which primary systemic amyloidosis belongs. Primary systemic amyloidosis involves deposition of monoclonal immunoglobin light chain proteins throughout the body tissues and typically occurs without existence of prior amyloid disease or concurrent chronic inflammatory conditions. Clinical manifestations often include macroglossia, mucocutaneous lesions, restrictive cardiomyopathy, kidney failure, and edema. Laboratory workup involves CBC, SPEP/UPEP, and biopsy of the affected organ to identify amyloid deposition. Treatment involves targeted therapy of affected organs as well as systemic chemotherapy. In rapidly progressive cases, in depth conversation with family regarding treatment, prognosis, and patient's wishes remain imperative to ensure effective communication and compassionate care.

CONCLUSION: Primary systemic amyloidosis may manifest as rapidly progressive systemic organ failure prompting immediate treatment. Treatment and management of primary systemic amyloidosis include a combination of targeted organ therapy and chemotherapy or palliative care depending on patient's wishes

\section{A RARE CASE OF THROMBOTIC MICROANGIOPATHY IN THE SETTING OF SEVERE HIV/AIDS PRESENTING AS ATYP- ICAL HEMOLYTIC UREMIC SYNDROME}

Garima Gupta. Jewish Hospital of Cincinnati, Blue ash, OH. (Control ID \#3186367)

LEARNING OBJECTIVE \#1: Thrombotic microangiopathy (TMA) is a clinical syndrome that can present as thrombotic thrombocytopenic purpura (TTP) or hemolytic uremic syndrome (HUS). Among these conditions, atypical HUS is now recognized to be a disease of alternative complement pathway dysregulation. TMA occurs when insults to the vascular wall of small vessels trigger formation of microvascular thrombosis. Clinically, patients with HUS present with thrombocytopenia, microangiopathic hemolytic anemia and fever. TMA is a known but rare complication of human immunodeficiency virus (HIV) and is suspected to play a role in the pathogenesis of HIV by direct endothelial cell damage. TMA is a medical emergency and can cause significant morbidity and mortality if not promptly diagnosed.

CASE: A 65-year-old male presented with a two-week history of generalized weakness and 20-pound weight loss. He was found to have acute kidney injury as well as pancytopenia. Few schistocytes were noted on the peripheral blood smear with elevated lactate dehydrogenase and low haptoglobin. The patient underwent plasmapheresis due to concerns of atypical HUS while his ADAMTS13 level was pending, which was later noted to be within normal limits. A renal biopsy was performed which showed thrombotic microangiopathy. The patient was immunized appropriately in anticipation to start eculizumab outpatient. The patient was brought to the hospital five days later with altered mental status. He was febrile and his laboratory studies demonstrated worsening pancytopenia and acute kidney injury. He was started on eculizumab inpatient. He required urgent hemodialysis due to worsening renal function. His HIV screen was positive with a CD4 count of less than 1 and viral load elevated at 1,400,000 copy/ml. Urine histoplasma antigen and serum antibodies were noted to be positive and yeast was seen on the peripheral blood smear. Patient was treated with amphotericin B for disseminated histoplasmosis. He was started on HAART after 21 days of therapy for histoplasmosis and discharged to a long term care facility.

IMPACT/DISCUSSION: In the setting of HIV associated TMA, AIDS defining illnesses can play a significant role in accelerating the terminal outcome of this disease. Our patient initially presented with non-specific symptoms and was diagnosed with atypical HUS which was confirmed with renal biopsy. It was not known until his readmission that he was discovered to be infected with HIV complicated by disseminated histoplasmosis. He was treated promptly and aggressively once his diagnosis was confirmed, which yielded positive outcomes.

CONCLUSION: Early treatment of HIV with HAART has led to a decrease in the development of TMA. However, it remains a life threatening condition that can present as a rare complication of HIV. Eculizumab is now considered an important treatment of complementmediated TMA, and patients with prompt diagnosis and treatment now have improved morbidity and mortality outcomes.

\section{A RARE CAUSE OF HEADACHE}

Chandralekha Ashangari ${ }^{1}$; Waqas Hafeez ${ }^{1}$; Anuja Kamat ${ }^{1}$; Praveen Tumula ${ }^{2} .{ }^{1}$ TTUHSC, Amarillo, TX; ${ }^{2}$ Texas Oncology, Amarillo, TX. (Control ID \#3181526)

LEARNING OBJECTIVE \#1: Leukemia, and leukostasis, in particular, is a very uncommon etiology of headaches in the emergency department. This case highlights the importance of keeping the differential diagnosis broad, taking an adequate and detailed history, and pursuing a more thorough workup when no etiology for subacute or chronic headaches is identified.

LEARNING OBJECTIVE \#2: This case also identifies hematologic malignancy as a potential cause of subacute headaches, one that may require emergent intervention.

CASE: A 56-year-old Caucasian female with a past medical history of chronic headaches presented to the emergency department with a thunderclap headache for 2 days, sudden in onset and continuous. The pain was aggravated with cough and bright light, briefly relieved with tylenol and was associated with vision disturbances. Vital signs and the physical examination were unremarkable at the time of presentation; a fundoscopic exam was normal. Laboratory studies showed a WBC count of 87100 with a neutrophilic predominance $(82.7 \%)$ with significant metamyelocytes. Serum LDH was 797. Lumbar puncture was done which ruled out meningitis with an opening pressure of $30 \mathrm{~cm} \mathrm{H} 2 \mathrm{O}$. Concern shifted to leukemia as the likely etiology of headache, with potentially a blast crisis causing leukostasis. Hematology/oncology were consulted. On repeat 
history after laboratory studies, the patient endorsed unintentional weight loss. Peripheral smear showed multiple mature leukocytes with occasional blasts and had findings consistent with chronic CML. Bone marrow biopsy and aspirate showed hypercellular marrow with features consistent of myeloproliferative neoplasm with only $1-2 \%$ blasts. BCR-ABL test was positive. The final diagnosis of chronic phase of CML was made and the patient was recommended starting on Imatinib.

IMPACT/DISCUSSION: Gross leukocytosis and subsequent leukostasis resulted in this patient's presentation of a chronic headache with vision changes. This is an uncommon headache etiology. The clinical manifestations of leukostasis most commonly manifest within the central nervous and respiratory systems. Neurologic symptoms range from headaches, tinnitus, or dizziness, to blurry vision, confusion, and decreased mentation. Respiratory symptoms are nonspecific as well, including dyspnea, hypoxemia, tachypnea, and respiratory failure. It is also possible to have manifestations of ischemia from leukostasis, including myocardial infarction, limb ischemia, and bowel ischemia. Marked leukocytosis in CML is most commonly attributed to a blast crisis but can result from advanced chronic disease.

CONCLUSION: Headaches are common, though leukostasis secondary to malignancy is an extremely rare etiology. Overall, a thorough history of the present illness with a physical examination can help diagnose even the rarest of disorders.

\section{A RARE COMPLICATION OF DIVERTICULITIS}

Brenden S. Ingraham; Maryam Mahmood. Mayo Clinic - Rochester, Rochester, MN. (Control ID \#3159402)

LEARNING OBJECTIVE \#1: Pylephlebitis should be considered when there is pain inconsistent with the primary site of infection or when the pain or fever persists despite treatment.

LEARNING OBJECTIVE \#2: Recognition is critical as pylephlebitis requires a prolonged duration of antibiotic therapy and sometimes systemic anticoagulation to prevent significant morbidity and mortality.

CASE: 59-year-old female with minimal medical history presented to the ED with severe left lower quadrant abdominal/pelvic pain and nausea. CT of the abdomen/pelvis showed findings of acute diverticulitis. She was admitted and managed medically with bowel rest and IV ciprofloxacin and metronidazole. She was discharged on oral antibiotics. Unfortunately, two days after discharge she returned to the ED with right upper quadrant pain and fever. Labs were notable for leukocytosis and transaminitis. CT showed portal venous gas, thrombus in the portal vein and inferior mesenteric vein (IMV), and a small abscess in the pelvis. She was initiated on IV ciprofloxacin, metronidazole, and heparin. Blood cultures grew candida albicans and C. dubliniensis, and anidulafungin was initiated. Despite antimicrobials, the pain worsened over the subsequent days with persistent fevers. She was broadened to meropenem and daptomycin. Repeat imaging showed septic thrombophlebitis, phlegmon around the IMV, and bowel ischemia without perforation. She was urgently taken to the OR for washout, colectomy, colostomy, and drain placement. Fluid collections drained intraoperatively grew Streptococcus anginosis, Lactobacillus rhamnosus, L. paracasei, and C. albicans. She was discharged on piperacillin-tazobactam IV for 3 weeks, anidulafungin for 6 weeks, and apixaban for 6 months.

IMPACT/DISCUSSION: Mesenteric pylephlebitis is suppurative thrombophlebitis of the portal vein. It is a rare diagnosis that complicates only $0.6 \%$ of intraabdominal infections. It is usually nonobstructive but can result in extension of the thrombus to the mesenteric and splenic veins, bowel ischemia, hepatic abscess, septic pulmonary emboli, and, rarely, acute portal hypertension with variceal hemorrhage. Bacteremia occurs in $44-88 \%$ of cases, and it is usually polymicrobial. The most common organisms are E. coli, Bacteroides fragilis, and streptococcus. CT with IV contrast is the best study for diagnosis and monitoring. Treatment includes 4-6 weeks of antibiotics. Anticoagulation is controversial but usually unnecessary. It may be considered if there is progression of the thrombus or persistent fevers/ bacteremia. Mortality ranges between $11 \%$ and $32 \%$.
CONCLUSION: Pylephlebitis is an exceedingly rare complication that can occur following infection of any portion of the GI tract drained by the portal system. It typically presents with right upper quadrant abdominal pain and fever in a patient with another intraabdominal infection. Recognition is critical as the infected thrombus requires a prolonged duration of antibiotic therapy and sometimes systemic anticoagulation to prevent significant morbidity and mortality.

\section{A RARE COMPLICATION OF EPIDIDYMOORCHITIS AND MANAGEMENT}

Jaepil E. Yoon ${ }^{2}$; Meghana Anugu ${ }^{1}$; Dave R. Gupta ${ }^{1}$; Rafael L. Jurado ${ }^{1}$. ${ }^{1}$ Emory, Atlanta, GA; ${ }^{2}$ Emory University School of Medicine, Atlanta, GA. (Control ID \#3186331)

LEARNING OBJECTIVE \#1: Recognize the clinical presentation, workup, and treatment of acute epididymoorchitis.

LEARNING OBJECTIVE \#2: Identify symptoms of testicular infarction and its management options.

CASE: A 64 year old male presents with acute onset, severe, left scrotal pain. Medical history was notable for benign prostatic hypertrophy requiring intermittent catheterization and prior urinary tract infections (UTIs). He denied nausea, vomiting, fever, urinary frequency, dysuria, or hematuria. Exam revealed left scrotal tenderness and edema. Laboratories were notable for Cr 1.2, WBC 14,000, and urinalysis with WBC 36 and RBC 111. Scrotal ultrasound (US) revealed a heterogeneous left epididymis with increased vascularity. Ceftazidime was initiated for presumed UTI. Urine culture grew Klebsiella pneumonia. He continued to report intermittent left scrotal pain and was diagnosed with acute epididymoorchitis. On hospital day four, he developed suprapubic tenderness. Repeat US revealed decreased echogenicity of the left testis with diminished, nearly absent vascular flow, consistent with testicular infarction. Surgical intervention was not recommended as continued fertility was not a concern and his right testis could provide adequate testosterone levels. He was treated with a two week regimen of oral Levofloxacin. Following resolution of his epididymoorchitis, urology recommended suprapubic catheter for prostatic rest and an open prostatectomy.

IMPACT/DISCUSSION: Acute epididymoorchitis (EO) is the most common cause of scrotal pain in men, accounting for 600,000 cases per year in the U.S. It presents as acute, unilateral scrotal tenderness and edema. EO is a clinical diagnosis, but Doppler US may reveal increased blood flow to an enlarged, thickened epididymis. In sexually active men aged 14 to 35 years old, Neisseria gonorrhea and Chlamydia trachomatis are the most common pathogens. In older men, pathogens are often enteric bacteria that have migrated into the vas deferens by retrograde flow of urine due to obstruction. Most episodes of EO resolve with appropriate antibiotic treatment. However, progression to testicular infarction may occur due to inflammation in the epididymis or spermatic cord, causing compression of the arterial supply to the testicle. While there are no standard guidelines for treatment of infarction due to EO, most episodes resolve with outpatient treatment. Therefore, clinicians must be aware of rare complications like testicular infarction in the treatment of EO, given its consequences on fertility. Serial or periodic examination of testicular blood flow by US can enable early detection of infarction. Once diagnosed, treatment recommendations are not clear, but urology should be consulted for consideration of surgical decompression.

CONCLUSION: EO can progress to testicular infarction in patients with persistent symptoms despite antibiotic therapy. Serial US assessment may allow early detection of infarction. Urology should be consulted.

\section{A RARE COMPLICATION POST-TRANSPLANT ATYPICAL HEMOLYTIC UREMIC SYNDROME (HUS)}

Seda Babroudi ${ }^{1}$; Cindy Varga ${ }^{2} .{ }^{1}$ Tufts Medical Center, Lincoln, MA; ${ }^{2}$ Tufts Medical Center, Boston, MA. (Control ID \#3179428) 
LEARNING OBJECTIVE \#1: Recognize the clinical features of atypical HUS

LEARNING OBJECTIVE \#2: Treat atypical HUS in a timely fashion

CASE: A 63 year-old Asian woman with a past medical history of end stage renal disease secondary to adult-onset polycystic kidney disease underwent deceased donor kidney transplant. She was initiated on tacrolimus, mycophenolate, and prednisone for immunosuppression. Post-transplant course was complicated by delayed graft function requiring dialysis, anemia, and thrombocytopenia. Laboratory studies were notable for elevated LDH, undetectable haptoglobin, and negative DAT. Peripheral smear revealed the presence of schistocytes, but ADAMTS13 was within normal limits and infectious work up was negative. Tacrolimus was discontinued without clinical improvement, so the patient was empirically started on eculizumab. The patient's renal function, anemia, and thrombocytopenia improved within 48 hours of initiation. Kidney biopsy later showed evidence of acute thrombotic microangiopathy (TMA) without rejection, and atypical HUS genetic panel was equivocal. The patient's graft function eventually recovered and she no longer needed dialysis.

IMPACT/DISCUSSION: This is a case of atypical Hemolytic Uremic Syndrome (HUS) in a post-renal transplant patient. Atypical HUS may be distinguished from classic HUS by the presence of renal failure, hemolytic anemia, and thrombocytopenia in the absence of an infectious source. TMA post-renal transplant is a complication that only occurs in approximately $0.5 \%$ patients annually, however it is associated with a $50 \%$ graft failure rate at two years and a $50 \%$ mortality rate at three years after diagnosis. Although calcineurin inhibitors have classically been implicated in the pathogenesis of atypical HUS post-transplant, calcineurin inhibitors are used commonly without incident. It has been postulated that calcineurin inhibitors, or even organ transplantation itself, act as triggers for acquired defects of the alternative complement pathway, which constitute the basis of genetic testing in suspected cases of atypical HUS. Genetic testing, however, remains neither sensitive nor specific in the diagnosis of atypical HUS. Given the risk of graft failure, it is imperative to recognize TMA syndromes early in the post-transplant course and withdraw all offending agents. If no clinical improvement is observed, and clinical suspicion of thrombotic thrombocytopenic purpura is low, empiric treatment for atypical HUS should be initiated. Eculizumab, an anti-C5 monoclonal antibody, is the treatment of choice for atypical HUS, and should be continued lifelong given the risk of graft failure and TMA syndrome recurrence.

CONCLUSION: Atypical HUS is a rare cause of post-transplant associated-TMA Early recognition of atypical HUS is crucial to preserve donor graft function First-line therapy for atypical HUS is the use of the monoclonal antibody eculizumab

\section{A RARE PRESENTATION OF CUSHING'S SYNDROME (CS): ECTOPIC ACTH SECRETION SECONDARY TO AN OLFACTO- RY NEUROBLASTOMA}

Sumedha Chablani; Andrea Carter. University of Pittsburgh Medical Center, Pittsburgh, PA. (Control ID \#3185431)

LEARNING OBJECTIVE \#1: Recognize typical physical exam and laboratory findings of hypercortisolism secondary to CS

LEARNING OBJECTIVE \#2: Diagnose and treat CS secondary to an ectopic ACTH secreting tumor

CASE: A 43 year-old man with a newly diagnosed olfactory neuroblastoma was admitted from home after he was noted to have hypokalemia (serum potassium level $2.1 \mathrm{mEq} / \mathrm{L}$ ) on pre-operative laboratory evaluation. Admission physical examination revealed hypertension (blood pressure $162 / 94 \mathrm{mmHg}$ ), facial plethora, fat deposition in the dorsocervical area, and anasarca. Initial laboratory testing revealed significant metabolic alkalosis (blood gas pH 7.55, PaCO2 $53 \mathrm{mmHg}, \mathrm{HCO} 346 \mathrm{mEq} / \mathrm{L}$ ) and hyperglycemia (serum glucose level $229 \mathrm{mg} / \mathrm{dL}$ ). Given these findings,
CS was suspected, and further testing showed an elevated random cortisol level of $112 \mathrm{ug} / \mathrm{dL}$ and serum ACTH level of $565 \mathrm{pg} / \mathrm{mL}$. A confirmation of CS was pursued with dexamethasone suppression testing, which failed to suppress the serum cortisol levels. Given our high suspicion of ectopic ACTH secretion, a pituitary MRI and CT of the chest, abdomen, and pelvis were performed to identify possible sources but these imaging tests failed to reveal additional masses beyond his olfactory neuroblastoma. As such, a presumptive diagnosis of CS secondary to an ACTH secreting olfactory neuroblastoma was made. Prior to tumor resection, patient's hypercortisolism was initially managed with ketoconazole and spironolactone, but he ultimately required an etomidate drip to decrease his serum cortisol levels. Patient underwent tumor resection with normalization of plasma cortisol and ACTH levels. Immunohistochemical testing is currently being performed to confirm whether the patient's olfactory neuroblastoma is positive for ACTH, and therefore the etiology of his CS. IMPACT/DISCUSSION: CS is a rare condition of hypercortisolism, which affects approximately two to three persons per million per year. $\mathrm{CS}$ can result from ACTH dependent or independent causes. The most common etiology of ACTH dependent CS is pituitary hypersecretion of ACTH, followed by ectopic secretion of ACTH by non-pituitary tumors. CS secondary to an olfactory neuroblastoma is very uncommon, with less than ten cases discussed in the literature. The diagnosis of CS secondary to an olfactory neuroblastoma includes immunohistochemical staining of the tumor, as well as clinical and laboratory improvement subsequent to tumor resection. While the mainstay of treatment is tumor resection, hypercortisolism in the preoperative period may be treated with ketoconazole, spironolactone, and if not adequately controlled, an etomidate drip, as was required for our patient.

CONCLUSION: This case highlights distinguishing physical exam and laboratory features of hypercortisolism secondary to CS. This case also demonstrates the presentation, diagnosis, and treatment of a rare form of $\mathrm{CS}$ secondary to ACTH secretion from an olfactory neuroblastoma, while underscoring the importance of pursuing a thorough work-up of CS to evaluate for alternative etiologies.

\section{A RARE RASH WITH A COMMON CAUSE: A CASE OF DRUG- INDUCED LINEAR IGA BULLOUS DERMATOSIS}

Malik Darwish; Joseph M. Rocco; Ayako W. Fujita. University of Pitts-

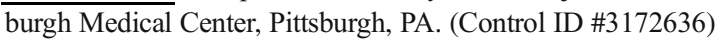

LEARNING OBJECTIVE \#1: Identify Linear IgA bullous dermatosis (LABD) as a rare blistering disease that requires direct immunofluorescence to confirm the diagnosis

LEARNING OBJECTIVE \#2: Recognize the most common offending agents of drug-induced LABD

CASE: A 73-year old woman with recent history of an intraabdominal infection on antibiotics presented with a blistering rash. Two months prior, she was admitted for acute pancreatitis complicated by polymicrobial peripancreatic abscesses, requiring drain placement and meropenem and vancomycin. After approximately one week of antibiotics, she developed an erythematous rash on her abdomen and legs with rapidly enlarging blisters. She was afebrile. Exam was notable for multiple tense bullae of various sizes filled with serous fluid on a base of tender, erythematous papules, with oral involvement. Nikolsky sign was negative. Total body surface involved was less than $20 \%$. Skin biopsy revealed neutrophilic subepidermal blisters with linear deposits of IgA along the basement membrane zone consistent with linear IgA bullous dermatosis. Vancomycin was suspected as the most likely precipitating factor, so it was stopped. She was also started on a highpotency topical steroid. She initially continued to form new bullae and erythema, but three days after withdrawing the vancomycin, she had rapid improvement in her rash with shrinking borders, reduced erythema, and cessation of new bullae formation.

IMPACT/DISCUSSION: Linear IgA bullous dermatosis (LABD) is a rare disease, with an incidence of less than 0.5 to 2.3 cases per million 
individuals per year. A classic pattern of presentation includes an acute eruption of an erythematous rash with tense bullae and mucosal involvement but negative Nikolsky sign. In the case of drug-induced LABD, onset usually begins within one month of drug initiation, and vancomycin has been identified as the offending agent in $46 \%$ of cases. Other drugs associated with LABD include lithium, captopril, and diclofenac. LABD is difficult to distinguish on exam due to its similar appearance to other blistering rashes that can present with bullae or vesicles, such as bullous pemphigoid or dermatitis herpetiformis. Therefore, skin biopsy is the gold standard. This includes both a biopsy of an entire vesicle or edge of a bullae for histology, as well as an unaffected, peri-lesional skin sample for immunofluorescence staining. Evidence of continuous linear IgA deposits along the basement membrane confirms the diagnosis. For idiopathic LABD, dapsone with high potency topical corticosteroids is first-line treatment. For drug-induced LABD, withdrawal of the offending agent leads to spontaneous resolution of the rash over several weeks, however new bullae may continue to form for a few days before improvement occurs.

CONCLUSION: Biopsy with immunofluorescence staining is necessary to diagnose and differentiate LABD from other blistering rashes. Vancomycin is the most common cause of drug-induced LABD.

\section{A RETURN TO THE FOLD}

Max Harris; Jacob Schulman; Susan B. Glick; Daniel Pohlman. Rush Medical College, Chicago, IL. (Control ID \#3186157)

LEARNING OBJECTIVE \#1: Recognize the importance of timely diagnosis of amyloidosis

LEARNING OBJECTIVE \#2: Diagnose light chain (AL) amyloidosis based on its clinical presentation and laboratory features

CASE: A 60 yo man with AS presented with edema of the legs, hands and face $\mathrm{x} 6 \mathrm{wk}$. The edema improved with elevation. There was no shortness of breath, PND or chest pain. He consulted his cardiologist. ECHO revealed moderate AS and EF 60\%. Furosemide was prescribed without improvement. PMH: Hypercholesterolemia. Medications: Metoprolol, atorvastatin, and aspirin. He worked in the entertainment industry and smoked tobacco. He appeared well. BP 99/69, HR 76, T 98.6. + Systolic ejection murmur without radiation to the carotids. No JVD. 1-2+ pitting edema 1/3 up the bilateral lower extremities. Exam was otherwise normal. Creatinine 0.92, albumin 1.5, cholesterol 465 . CBC normal. UA: protein $>300 \mathrm{mg} / \mathrm{dL}$, otherwise normal. Serum immunofix: monoclonal IgG lambda. Urine immunofix: monoclonal free lambda light chains. 24-hour urine protein $18 \mathrm{~g}$. BNP 18. Troponin 0.01. Renal ultrasound, skeletal survey: normal. Bone marrow biopsy: 5\% plasma cells, AL amyloid. Renal biopsy: amyloid nephropathy, $\mathrm{AL}$, involving glomeruli and vessels.

IMPACT/DISCUSSION: Amyloidosis is caused by clonal plasma cells that produce amyloid, a misfolded protein that deposits in and damages tissue. Deposition may be systemic or localized; $>75 \%$ is due to $\mathrm{AL}$ amyloid. To prevent irreversible organ damage, prompt diagnosis and treatment are needed. The combination of macroglossia and periorbital purpura is pathognomonic for AL amyloidosis, though uncommonly seen. Cardiac deposition causes restrictive cardiomyopathy with JVD and edema. Renal involvement most often presents with heavy proteinuria due to glomerular deposition, though may present as worsening renal function without proteinuria due to vascular deposits or as tubular dysfunction due to tubular deposits. Neurologic involvement causes both peripheral and autonomic neuropathy. Soft tissue infiltration causes salivary gland enlargement and muscular pseudohypertrophy. Diagnosis requires confirmation of a monoclonal plasma cell disorder via serum and urine immunofix electrophoresis and measurement of serum free light chains. Congo red staining establishes the presence of amyloid. Demonstration of organ involvement and fibril type are also required. Treatment focuses on eradication of the plasma cell clone. Newer therapies under study target the amyloid deposits.
CONCLUSION: Prompt diagnosis and treatment of amyloidosis can prevent irreversible organ damage. Light chain amyloidosis causes restrictive cardiomyopathy (JVD, edema), nephropathy (edema, proteinuria, renal insufficiency, RTA), neuropathy (peripheral/autonomic) and soft tissue infiltration (macroglossia, muscular pseudohypertrophy). Diagnosis requires serum and urine immunofix electrophoresis and measurement of serum free light chains to confirm a monoclonal plasma cell disorder; Congo red staining to confirm amyloid; evidence of organ involvement; and fibril type.

\section{A ROTTEN CASE OF HUS: THE EMERGING PATHOGENESIS OF SALMONELLA}

Sarah Kurz; Kristin Christensen; Selen Uman. University of Pennsylvania, Philadelphia, PA. (Control ID \#3185773)

LEARNING OBJECTIVE \#1: Recognize the value and importance of a complete clinical history

LEARNING OBJECTIVE \#2: Distinguish between forms of MAHA based on clinical and laboratory data

CASE: A previously healthy 27-year-old male presented with 1 week of nausea, vomiting, and abdominal pain, and was found to have a white blood cell count of $22 \mathrm{THO} / \mathrm{uL}$, hemoglobin of 18.8 $\mathrm{g} / \mathrm{dL}$, a platelet count of $214 \mathrm{THO} / \mathrm{uL}$, and a creatinine of 1.31 $\mathrm{mg} / \mathrm{dL}$. He underwent CT imaging which was notable for severe colitis from the cecum to the distal descending colon. He was started on broad-spectrum antibiotics for presumed colitis. The patient's history was remarkable for his girlfriend having similar symptoms following the consumption of eggs the couple had purchased and both eaten but her symptoms had resolved without intervention. Over the course of the next 4 days, patient developed rapidly progressive symptoms in addition to notable changes in his laboratory data, in particular thrombocytopenia with platelets of $34 \mathrm{THO} / \mathrm{uL}$, renal failure with creatinine of $4.01 \mathrm{mg} / \mathrm{dL}$, anemia with hemoglobin of $10.8 \mathrm{~g} / \mathrm{dL}$, and a persistent leukocytosis with white blood cell count of $19 \mathrm{THO} / \mathrm{uL}$. Based on his clinical decline, the syndrome of microangiopathic hemolytic anemia (MAHA) was entertained. The three most common forms of MAHA are Thrombotic thrombocytopenic purpura (TTP), Hemolytic uremic syndrome (HUS), and Disseminated Intravascular Coagulation (DIC). While all have different causes, each is life threatening and characterized by the triad of small-vessel hemolytic anemia, thrombocytopenia, and renal impairment. Our patient's evaluation for the etiology of MAHA included evaluation for TTP with ADAMTS13 (activity of 55\%, negative), DIC with slightly elevated fibrinogen of $463 \mathrm{mg} / \mathrm{dL}$ (low fibrinogen levels are seen in DIC), and a stool culture that was negative for EHEC O157:H7 via enzyme immune assay (EIA), but positive for salmonella via PCR testing. The patient remained in the ICU for 48 hours where he received 2 days of steroids. He received a total of 5 days of broad-spectrum antibiotics including Vancomycin, Zosyn, and Flagyl. Within the next few days, with continued fluids, his renal function improved, his WBC count downtrended and his platelets normalized. He was discharged home in good condition on Day 8.

IMPACT/DISCUSSION: This case illustrates the expanding body of clinical evidence that non-shiga toxin producing pathogens are capable of causing HUS. This growing link between non shiga-toxin producing pathogens and HUS opens opportunities for exploration of the underlying mechanisms and the potential role for novel therapies.

CONCLUSION: -A good clinical history is of high value especially when there is concern for an underlying infectious cause -Case reports indicate a sporadic association between salmonella gastroenteritis and HUS without clear etiology of pathophysiologic basis given lack of shiga-toxin production in salmonella infections 


\section{A SECOND OPINION FROM DR. GOOGLE: A FAMILY RE- QUESTS NON-BENEFICIAL TREATMENTS AT THE END OF LIFE \\ Kyle Cornell. Baystate Medical Center, Springfield, MA. (Control ID \#3186416)}

LEARNING OBJECTIVE \#1: Recognize a family's request for nonbeneficial treatment as well-intentioned advocacy for a dying loved one.

LEARNING OBJECTIVE \#2: Utilize multidisciplinary family meetings to address medical questions and provide supportive outlets for continued advocacy.

CASE: An active 56-year-old female with diet-controlled hyperlipidemia presented to our hospital after an out-of-hospital cardiac arrest secondary to anterior myocardial infarction. The time from her arrest to high-quality compressions was over 10 minutes. Subsequent interventions included coronary revascularization and targeted temperature management. There was evidence of cerebral edema on imaging and she remained comatose with progressive loss of neurologic function. She was declared brain dead by day 5 of hospitalization. The patient's family including husband, 3 teenaged children and many extended relatives were present and understandably desperate for hope. After reading through various online articles, they insisted that treatment with hyperbaric oxygen be tried for their wife/mother. In response, a multidisciplinary team was convened to help explain the futility of that treatment whilst recognizing the family's efforts in providing her the best care possible.

IMPACT/DISCUSSION: Ubiquitous internet access provides the general public with an abundance of research articles which can be misconstrued without appropriate medical expertise and context. Desperate family members are particularly vulnerable to this phenomenon. Though the use of hyperbaric oxygenation in some types of brain injury is under review, there was no evidence for benefit in this case. The persistence of such requests despite a medical explanation can be challenging. The care team's approach to such cases is critical to validate advocacy, answer medical questions, and maintain family rapport. Multidisciplinary team meetings have been shown to improve quality of patient centered care. A consensus amongst involved providers is the most helpful approach to address such family concerns and ensure consistency and quality of care.

CONCLUSION: The emotional trauma of a sudden and unexpected critical illness can manifest in various forms of patient advocacy from grieving family members. Frequent requests for non-beneficial treatment are often inspired by readily available internet searches and require careful attention to acknowledge intentions while explaining futility. Multidisciplinary team meetings with the family are instrumental in responding to these concerns and creating an appropriate care plan. Direct reference to the family's specific internet resources and a unified consensus amongst the patient's providers has been found to be most helpful. Repetitive requests may indicate denial as part of the complex grieving process. Such delicate situations require a provider's best patience and understanding.

\section{A SEVENTEEN-YEAR-OLD, IN HER FIRST TRIMESTER OF PREGNANCY, PRESENTING WITH A MYOCARDIAL INFARCTION}

Amitabh Singh ${ }^{1}$; David Evans ${ }^{2}$; Neil Evans ${ }^{1}$; Jennifer Schmidt ${ }^{1}{ }^{1}$ Saint Louis University, Saint Louis, MO; ${ }^{2}$ Unity Health, Searcy, AR. (Control ID \#3185862)

LEARNING OBJECTIVE \#1: Recognizing myocardial infarction in low risk patients

LEARNING OBJECTIVE \#2: Understanding alternate mechanisms of thrombus formation in the coronary vessels

CASE: A 17-year-old female G1P0, at eight weeks gestation, presented with severe, sharp, substernal chest pain occurring at rest. The pain began twenty minutes prior to arrival and is associated with dyspnea, diaphoresis, nausea and right arm pain and tingling. 1 Sublingual nitroglycerin relieved her pain. Vital signs were within normal limits. Initial EKG demonstrated normal sinus rhythm at $78 \mathrm{bpm}$, with a normal axis. and ST-segment elevation in leads II, III, AvF with reciprocal changes in leads V1-3. Labs showed CPK of $12 \mathrm{U} / \mathrm{L}$, troponin I $0.01 \mathrm{ng} / \mathrm{mL}$. Her urine drug screen was negative. Cardiac echocardiogram demonstrated hypokinesis of posterobasal and inferolateral segment of left ventricle. Subsequent coronary catheterization showed right-dominant circulation with complete occlusion of the distal circumflex artery (underwent balloon recanalization) and normal right coronary artery, left main coronary artery and the left anterior descending arteries Thirteen hours later troponin peaked at $29.83 \mathrm{ng} / \mathrm{mL}$. She was discharged and experienced no further pregnancy complications.

IMPACT/DISCUSSION: Myocardial infarctions are the leading cause of death amongst adults. The pathophysiology is thought to be due to atherosclerotic plaque rupture. Once a plaque ruptures there is exposure to underlying endothelial tissue. The exposure to sub endothelial collagen promotes platelet aggregation. An occlusive thrombus is formed leading to a transmural infarction which may be seen on EKG as ST- segment elevations. Plaque rupture accounts for a majority of MIs however other pathophysiological mechanisms of MI exist. The differential diagnosis for non-atherosclerotic myocardial infarctions include coronary vasospasm, vasculitis, thrombi, and coronary dissection. In the pregnant population, coronary dissection is a common cause of myocardial infarction. Roth et al. suggest the rate to be at $27 \%$ of all MIs in pregnant patients. This patient did not have coronary vasospasm, vasculitis, and coronary dissection. She had developed a thrombus in the absence of any triggering mechanisms.

CONCLUSION: We present a case of a 17-year female G1P0 at eight weeks pregnancy with no clinical risk factors, who presents for evaluation of chest pain. There is concern for misdiagnosis or failure to diagnose by Internists given her lack of risk factor

\section{A SHORT DIFFERENTIAL COMES UP SHORT IN A PATIENT WITH SHORTNESS OF BREATH}

Duy Ha; Steven G. McKee. University of Arkansas for Medical Sciences, Little Rock, AR. (Control ID \#3184724)

LEARNING OBJECTIVE \#1: Identify premature closure as a powerful cognitive bias when addressing common respiratory symptoms

LEARNING OBJECTIVE \#2: Utilize a broad differential diagnosis as a cognitive debiasing strategy

CASE: A 41-year-old male with chronic sinusitis and lung disease presented with worsening dyspnea and productive cough and internal medicine admission was requested for an exacerbation of chronic obstructive pulmonary disease (COPD). Chart review revealed two recent admissions treated as COPD flares. His chronic lung disease and episodic dyspnea began at age 29. Workup in the past included a normal alpha-1-antitrypsin level, pulmonary function tests with mid-flow-loop obstruction plus reduced diffusion capacity, and a non-diagnostic bronchial alveolar lavage (BAL). He reported night sweats, hemoptysis, and 10-pounds of weightloss. He admitted five pack-years of smoking but quit 10 years previously. Prior to current incarceration he worked at a fertilizer factory. On examination, the patient was afebrile and thin. His respirations had a prolonged expiratory phase and polyphonic wheeze. Standard laboratory testing was unremarkable. Computed-tomography displayed central bronchiectasis. Repeat BAL cytology resulted with eosinophilia, but all other BAL tests were negative. Serum Aspergillus fumigatus $\mathrm{IgE}$ was positive $(4.23 \mathrm{kU} / \mathrm{L})$ and serum IgE was elevated (3868 kU/L). Allergic bronchopulmonary aspergillosis (ABPA) was diagnosed and his symptoms improved with itraconazole and prednisone.

IMPACT/DISCUSSION: Though dyspnea and wheeze are common complaints to internists, rare diseases lurk among the etiologies. If our initial differential diagnoses are not robust, physicians can fall prey to 
premature closure and/or framing bias as likely occurred when this patient was repeatedly treated for COPD despite minimal smoking and a history of occupational hazards. ABPA is a form of hypersensitivity with airway damage triggered by A.fumigatus exposure. It is characterized by recurrent bouts of dyspnea, cough, and thick sputum. Wheeze, fever, and hemoptysis may occur. The diagnostic process of modern medicine still hinges on the practitioner's data gathering, hypothesis testing, and clinical reasoning. Yet, cognitive biases and flawed logic by physicians contribute to nearly one in five adverse events in healthcare. A thorough differential diagnosis is a key strategy to prevent errors, without which vital history and exam components will be overlooked and the provider will fail to consider alternative illnesses. Studies have shown that a broad differential diagnosis helps clinicians make timely, accurate diagnoses despite our limitations. As a course of habit, we find this a cost-effective method to avoid readmissions and enhance patients' quality of life.

CONCLUSION: Premature closure increases the risk of delayed or incorrect diagnoses. Establishing forced consideration of a variety of etiologies is an efficient strategy that can reduce diagnostic errors.

\section{A SOUR CASE OF SWEET SYNDROME}

Hao Chi; Lindsay Hammons. Mount Sinai Beth Israel, New York, NY. (Control ID \#3182560)

LEARNING OBJECTIVE \#1: Recognize clinical features that is required to establish the diagnosis of Sweet syndrome.

LEARNING OBJECTIVE \#2: Understand the three subtypes of Sweet Syndrome

CASE: A 30 year-old Japanese male with no significant past medical history presented with two days of diffuse painful erythematous papules/ plaques. The patient stated that the painful and pruritic papules appeared four days ago on his right knee and then spread to his upper and lower extremities, sparing his trunk, face, palms and soles. Last week, the patient recovered from a flu-like illness where he experienced achy joints, two episodes of watery, non-bloody diarrhea and a subjective fever. Twelve years ago, the patient had a similar episode of acute onset rash for which he was admitted to an outside hospital and treated for one day with an unknown medication. On exam, he was afebrile and his vital signs were stable. Physical exam was significant for diffuse, tender, raised erythematous papules/plaques 1-2 inches in diameter on the extensor surfaces of the lower extremity and extensor/flexor surfaces on bilateral upper extremities. Initial labs showed the following: ESR 84, CRP 432 and WBC 37. 8k/ $\mathrm{LL}$ with $83 \%$ neutrophils. A skin biopsy was completed; histology showed atypical subcutaneous neutrophilic dermatosis. These findings, in addition to an elevated CRP/ESR, WBC count $>8 \mathrm{k} / \mu \mathrm{L}$ and neutrophil percentage $>70 \%$, met the diagnostic criteria for Sweet syndrome. The patient was started on IV steroids with rapid improvement of his rash.

IMPACT/DISCUSSION: Sweet syndrome (SS), also known as acute febrile neutrophilic dermatosis, is a rare inflammatory disorder characterized by fever, neutrophilia and painful, edematous, and erythematous papules, plaques, or nodules on the skin. Often seen in 30 to 60 yearolds, Sweet syndrome is most commonly associated with the following three etiologies: (1) infection, especially upper respiratory and gastrointestinal illnesses, (2) malignancies, acute myelogenous leukemia being the most prevalent, and (3) drugs, most notably after treatment with granulocyte-colony stimulating factor. About $80 \%$ of infectionassociated SS cases are in women, whereas other types have no clinical gender bias. Speculated to be inflammatory in etiology, the exact pathogenesis of SS is still largely unknown. Factors theorized to contribute to the development of this disorder include hypersensitivity reactions, cytokine dysregulation, and genetic susceptibility. Genetically, it has been found that abnormalities in chromosome $3 \mathrm{q}$, seen in people of Japanese descent, is linked to an increased predisposition for SS, as suggested by this clinical case.
CONCLUSION: This case highlights the importance of considering Japanese descent as a genetic risk factor for Sweet syndrome. It is also vital for the treating physician to determine the underlying cause of the disease, as early detection of malignancy is paramount.

\section{A SPICE-Y CASE OF ANTIBIOTICS RESISTANCE}

Chelsea F. Dahl; Joseph M. Rocco. University of Pittsburgh, Pittsburgh, PA. (Control ID \#3185599)

LEARNING OBJECTIVE \#1: Understand the clinical significance of the AmpC plasmid and know the most common bacteria that carry this gene for inducible -lactamase resistance.

LEARNING OBJECTIVE \#2: Know the appropriate empiric antibiotic regimen to select when treating these organisms.

CASE: A 49-year-old man with history of alcoholic cirrhosis and chronic pancreatitis presented with obstructive pancreatitis requiring common bile duct stent placement. He was found to have a small hepatic liver abscess which was drained, and cultures grew pan-sensitive Citrobacter freundii complex and Streptococcus anginosus. His course was complicated by $S$. anginosus bacteremia, and therefore he received 2-week course of ceftriaxone. Three weeks later, he returned with fevers, night sweats, and worsening lower back pain. Blood cultures grew Citrobacter freundii and sensitivities now demonstrated resistance to ceftriaxone as well as ampicillin-sulbactam, cefazolin, ceftazidime, and cefuroxime. CT imaging revealed a new lumbar discitis and epidural abscess. CRP was 5.3. He was initially started on cefepime, but after Infectious disease was consulted, he was broadened to ertapenem due to concern for inducible ampC resistance. Blood cultures cleared after two days and he received a 6-week course of ertapenem. Upon completion, his CRP downtrended to 1.2 and interval MRI imaging revealed no epidural abscess.

IMPACT/DISCUSSION: A common mnemonic for bacteria known to carry the AmpC plasmid is the "SPICE" organisms which include Serratia, Pseudomonas, indole-positive Proteus, Citrobacter, and Enterobacter. However, this list is not complete and others including Acinetobacter, Providencia, and Morganella can carry AmpC as well. AmpC -lactamases encode a cephalosporinase commonly found on the bacterial chromosome of Enterobacteriaceae. Production of this enzyme can be inducible only after exposure to penicillin-based antibiotics, and routine microbiologic testing is not sensitive enough to detect them. The initial drug sensitivities may report that the organism is susceptible to penicillins and cephalosporins. Additionally, AmpC -lactamases are not inhibited by -lactamase inhibitors such as clavulanic acid. However, after starting these antibiotics, repeat sensitivities will demonstrate resistance as seen in our case. Therefore, initial antibiotic selection is essential and often requires a carbapenem, especially in severe infections. Third generation cephalosporins must be avoided. Cefepime and piperacillin-tazobactam have a lower chance of inducing resistance but should be avoided when source control is lacking as demonstrated in our case.

CONCLUSION: The "SPICE" bacteria are known to carry AmpC inducible -lactamase resistance which can lead to treatment failure despite the organisms initially appearing susceptible to cephalosporins. Broad empiric therapy is required which should include a carbapenem in severe infections.

\section{A SPONTANEOUS CASE OF ISOLATED CELIAC ARTERY DISSECTION}

SAMEER PATEL; Amr Salama; Michael R. DiSalle. Unity Hospital, Rochester, NY. (Control ID \#3184231)

LEARNING OBJECTIVE \#1: Manage the acute and follow-up care for a patient with spontaneous isolated celiac artery dissection (SICAD)

CASE: A 59 year old non-smoking gentleman presented with a 3 day history of evolving chest pain, dysphagia and upper abdominal pain. The left lateral posterior chest pain was first described as pleuritic, non- 
radiating, and stabbing in nature. The following day, the symptoms evolved into a radiating pain across the posterior chest between both shoulder blades. On presentation, he underwent a chest x-ray that was negative for any thoracic pathology, and had a negative cardiac workup (negative serial EKG's, negative troponin). Two days later, the patient presented back to the ED with unresolving abdominal pain, underwent a CT abdomen/pelvis, and was diagnosed with a focal dissection of the celiac artery with $50 \%$ of luminal narrowing. Vascular surgery recommended a non-surgical approach given the patient's hemodynamic stability. They recommended dual-antiplatelet therapy, blood pressure control, pain management, and serial abdominal examinations. The patient's symptoms improved and he was discharged with instructions to followup with the vascular team in the outpatient setting. He will be on dual antiplatelet therapy for 6 months, aspirin/ statin for life, and will need yearly imaging follow-up.

IMPACT/DISCUSSION: SICAD is a rare diagnosis that affects primarily males $(5: 1)$ with the average age of 55 years. The etiology is usually iatrogenic or secondary to atherosclerosis, trauma, pregnancy, fibromuscular dysplasia, inflammatory or infectious diseases, or a congenital disorder of the vascular wall. SICAD usually occurs between the intima and the external elastic layer, whereas in aortic dissection, the cleavage plane is between the first and the second layer of the intima. Most patients with SICAD are asymptomatic while some patients may present with abdominal pain due to involvement of the splenic, renal or superior mesenteric arteries causing ischemia. Patients with ruptured aneurysms can present acutely with bleeding while chronic dissections can present with symptoms of intestinal angina. An extensive literature on this subject shows that for the majority of cases of SICAD, conservative management and observation is appropriate. Conservative management typically consists of blood pressure control, imaging surveillance, and lifelong antiplatelet therapy $+/-$ a short course of anticoagulation therapy. If abdominal pain is persistent, endovascular stenting may stabilize or improve the pain, and surgical reconstruction can be done for aneurysmal degeneration. Close follow-up by surveillance imaging should be done in most cases and can be done by US to reduce radiation burden.

CONCLUSION: - SICAD is rare diagnosis with male predominance that can present with non-specific symptoms $\bullet$ The majority of stable patients with SICAD can be conservatively managed while surgical intervention can benefit patients with hemodynamic instability or persistent abdominal pain.

\section{A STIFF WOMAN}

FNU Srinithya ${ }^{1}$; Saikrishna Gadde ${ }^{2} .{ }^{1}$ Brookwood Baptist, Birmingham, AL; ${ }^{2}$ Ochsner Medical Center, Birmingham, AL. (Control ID \#3186163)

LEARNING OBJECTIVE \#1: Recognize clinical features and laboratory finding of Stiff Person Syndrome (SPS)

LEARNING OBJECTIVE \#2: Identify disorders associated with Stiff Person syndrome

CASE: A 37year old female with no significant past medical history presented with refractory stiffness and weakness. Initial symptom started 3 months ago with her right leg giving out while walking. She experienced a fall due to her symptoms, which resulted in fracture left foot. Denied similar episodes in the past. Social history and family history were unremarkable. Patient had unintentional weight loss of 15 pounds in last 4 months. Pap smear within the past year was negative. Review of systems was otherwise negative Vital signs were within normal limits on arrival. Cardiopulmonary and abdominal exam was without abnormalities. Neurological examination revealed mild stiffness; lower extremity stiffness greater than upper extremity; no rigidity. Deep tendon reflexes were 2+; cranial nerves, sensation, cognition were all intact. Breast exam was normal. Initial laboratory findings revealed a normal complete blood count, thyroid studies, comprehensive metabolic, serum vitamin B12, urinalysis, urine drug screen, serum thiamine and autoimmune panel.
CT brain and EEG were normal. Her symptoms were attributed to psychogenic non-epileptic seizures and sertraline was initiated. She developed serotonin syndrome due to the addition of Tramadol, trazadone and flexeril to setraline. MRI brain and pan spine revealed T2 hyperintensity within dorsal column in cervical spine and scattered foci on T2 flair within supratentorial white matter. Serum protein electrophoresis, CT chest abdomen pelvis with contrast and lumbar puncture were negative. Paraneoplastic workup revealed elevated titres for amphiphysin antibody indicative of SPS. The patient has since received IV immune globulin and methylprednisolone. Nuclear Medicine PET revealed abnormal nodes in the left axilla and a right upper lung nodule. Left axillary node biopsy was positive for Estrogen receptor carcinoma; suspicious for breast versus gynecologic malignancy.

IMPACT/DISCUSSION: This patient had profound stiffness and muscle weakness, which lead to the pursuit of testing for rheumatologic, neurologic, and paraneoplastic etiology Stiff person syndrome (SPS) is a rare, progressive neurolgical syndrome. It is caused by increased muscle activity due to decreased inhibition of the central nervous system. Symptoms include extreme muscle stiffness, rigidity and painful spasms. The syndrome affects twice as many women as men. Treatment aims to control symptoms and improve mobility, involves benzodiazepines, muscle relaxants, and/or intravenous immunoglobulins.

CONCLUSION: SPS is associated most commonly with breast cancer but has been linked to Hodgkin's lymphoma and small cell cancer and autoimmune diseases like diabetes mellitis, vitiligo and pernicious anemia. Treatment aims to control symptoms and improve mobility, and may involve benzodiazepines, muscle relaxants, and/or intravenous immunoglobulins

\section{A STROKE OF LUCK: SUPERIOR SINUS VENOSUS ATRIAL SEPTAL DEFECT DISCOVERED VIA A CRYPTOGENIC STROKE SHRUTI RAO MD, THOMAS WONG MD, ANDREW VU MD, SARA L. SWENSON MD}

Shruti Rao; Thomas J. Wong; Sara L. Swenson. California Pacific Medical Center, San Francisco, CA. (Control ID \#3186322)

LEARNING OBJECTIVE \#1: Findings such as an abnormal $\mathrm{P}$ wave axis, right axis deviation, and incomplete RBBB are indicative of a superior sinus venosus ASD on EKG. Internists should be aware of these specific findings, which can prompt a TEE and accelerate the often lengthy workup of cryptogenic stroke to ultimately repair the ASD.

CASE: A 33 year-old male developed acute onset of vertigo after abruptly turning his head to the right. He then experienced pain in the right posterior neck, binocular diplopia, aniscoria, and ataxia. CTA of head and neck was negative for dissection or obvious infarct or hemorrhage, however given persistent symptoms, patient received tPA with improvement. MRI showed a paramedian thalamic and midbrain stroke. His ECG showed an abnormal $\mathrm{P}$ axis with right axis deviation, and incomplete RBBB indicating cardiac pathology. The decision was made to use transesophageal echocardiography instead of transthoracic echocardiography with agitated saline contrast injection to search for intra-atrial pathology. The study revealed a large sinus venosus ASD of $1.7 \mathrm{~cm}$, left-to-right shunting, and a Qp:Qs ratio of 2.5, raising suspicion of a paradoxical embolism. The rest of his workup for alternative stroke etiology was negative. He eventually underwent Baffle repair of PAPVR to the left atrium and ASD closure. He recovered completely with no residual deficits and remains on antiplatelet therapy with aspirin.

IMPACT/DISCUSSION: The diagnostic workup of cryptogenic strokes is often lengthy. Interestingly, a test as simple as an ECG was able to reveal the most likely cause of this patient's stroke. Findings such as an abnormal $\mathrm{P}$ wave axis, right axis deviation, and potential RBBB are indicative of a superior sinus venosus ASD due to intra-atrial remodeling from left to right shunt across the defect. Using the patient's ECG prompted correct selection of TEE over TTE as superior sinus venosus atrial septal defect can be missed with TTE because of its posterior 
location. Presenting complications of large ASDs include right ventricular dilatation, tricuspid regurgitation, right heart failure, and pulmonary hypertension that can lead to right-to-left shunting. Indications for ASD closure include an embolic-appearing cryptogenic ischemic stroke and/or a large shunt (Qp:Qs ratio > 2) without pulmonary hypertension. Although his stroke was unfortunate, timely surgical correction of his ASD will prevent him from developing the more advanced complications.

CONCLUSION: Superior sinus venosus is a rare form of ASD that can have unique presentations, including cryptogenic stroke. Providers should be aware of the findings that are highly suggestive of superior sinus venosus ASD on electrocardiography as it can aid in the timely diagnosis and management of this rare cardiac condition.

\section{A SUBTLE PRESENTATION OF ADVANCED SMALL CELL LUNG CANCER}

Sara Vidovic; Pakhadi Buddhadev. Medical College of Wisconsin, Wauwatosa, WI. (Control ID \#3181473)

LEARNING OBJECTIVE \#1: Recognize when to suspect ectopic Cushing's syndrome (ECS)

LEARNING OBJECTIVE \#2: Distinguish ECS from other etiologies of Cushing's syndrome (CS)

CASE: A 66-year-old woman was admitted for hypokalemia $(1.6 \mathrm{mmol} / \mathrm{L})$ with associated metabolic alkalosis and hyperglycemia. She noted a few weeks of fatigue, generalized muscle weakness, and weight loss. She was a smoker and had a past medical history of chronic obstructive pulmonary disease and hypertension. Vital signs were significant for blood pressure of 197/87 mmHg. Physical examination showed skin atrophy and large bruises on the right hip. Remainder of exam was normal. Throughout admission patient was hypertensive despite increasing doses of three blood pressure medications. She was persistently hypokalemic requiring $120 \mathrm{mEq}$ potassium and 150-200 mg spironolactone daily. Renin activity and aldosterone level were normal. Adrenocorticotropic hormone (ACTH) $(136.1 \mathrm{pg} / \mathrm{ml})$, morning cortisol $(99.1 \mathrm{ug} / \mathrm{dl})$, and 24-hour urine free cortisol $(11,081.1 \mathrm{ug})$ were markedly elevated as were adrenal hormones dehydroepiandrosteronesulfate, testosterone, and androstenedione. MRI brain showed no evidence of pituitary lesion. Low dose dexamethasone suppression test was positive. CT chest, abdomen, and pelvis showed bulky necrotic mediastinal and right hilar adenopathy and possible lymphatic obstruction without discrete pulmonary mass. In the abdomen and pelvis were innumerable low-density hepatic lesions and a soft tissue nodule in the subcutaneous fat overlying the right gluteus musculature concerning for metastatic disease of unknown origin. High dose dexamethasone suppression test was positive suggesting ectopic ACTH secretion. Ultrasound-guided liver biopsy was consistent with small cell lung cancer (SCLC); salt and pepper chromatin with immunostaining positive for synaptophysin, chromogranin, and cytokeratin AE1/AE3.

IMPACT/DISCUSSION: CS occurs when the body is chronically exposed to high levels of glucocorticoids. CS can be divided into primary and secondary hypercortisolism depending on whether excess cortisol is dependent on ACTH. The differential diagnoses for secondary hypercortisolism include pituitary hypersecretion of ACTH and ectopic ACTH secretion from a neuroendocrine tumor. Ectopic ACTH secretion is responsible for $10 \%$ of all CS cases. Of these, $50 \%$ are attributed to neuroendocrine lung tumors including SCLC. Although it is well known that SCLC can secrete ACTH, only up to 5\% of SCLC cases are associated with ECS. As in this case, ECS may present with electrolyte disturbances and resistant hypertension rather than classic cushingoid traits like central obesity, acne, striae, facial plethora, and enlarged supraclavicular fat pads. This makes the diagnosis of ECS easy to miss, requiring high clinical suspicion. This suspicion is warranted as ECS may be the first manifestation of malignancy and is a poor prognostic factor in SCLC.

CONCLUSION: Diagnosis of ECS requires high clinical suspicion ECS may be the first sign of malignancy SCLC is a common culprit of ECS

\section{A TREATABLE MIMICKER OF METASTATIC PANCREATIC ADENOCARCINOMA}

John Xie; Mazen Zaarour. Tulane University School of Medicine, New Orleans, LA. (Control ID \#3184086)

LEARNING OBJECTIVE \#1: Recognize the clinical features of EBVpositive DLBCL with pancreatic involvement

LEARNING OBJECTIVE \#2: Differentiate prognosis of EBV-positive DLBCL with pancreatic involvement from pancreatic adenocarcinoma

CASE: A 62-year-old previously healthy man presented with a fivemonth history of worsening appetite, epigastric pain, and 10-lb weight loss. He had a 40-pack year smoking history. Labs revealed normal blood counts and liver enzymes, mildly elevated CA 19-9 at 49.7 (REF: $<35.1$ $\mathrm{U} / \mathrm{mL}$ ) and lactate dehydrogenase (LDH) level of $278 \mathrm{U} / \mathrm{L}$ (REF: $<201$ $\mathrm{U} / \mathrm{L}$ ), with normal CEA. Chest and abdominal CT revealed a $12 \mathrm{~cm}$ pancreatic mass, a $1.4 \mathrm{~cm}$ left upper lobe lung mass, numerous splenic lesions, two hypodense liver lesions, peritoneal carcinomatosis, and a large retroperitoneal mass. Given his clinical presentation, radiographic findings with peritoneal involvement, and elevated CA 19-9, the preliminary diagnosis was metastatic pancreatic adenocarcinoma. Surprisingly, biopsy of one of the liver lesions revealed Epstein-Barr virus (EBV)positive diffuse large B cell lymphoma (DLBCL). Rituximab, cyclophosphamide, doxorubicin, vincristine, and prednisone (R-CHOP) was immediately initiated, leading to an impressive clinical response with resolution of most of the patient's symptoms after just one cycle. A PET/CT after 2 cycles of treatment showed a significant decreased in size of the pancreatic and lung masses, along with resolution of the liver and spleen lesions. IMPACT/DISCUSSION: EBV-positive DLBCL is a rare subtype of Bcell neoplasm found in immunocompetent patients usually over 50 years of age. It accounts for $8 \%-15 \%$ of DLBCL in the Asian population and less than $5 \%$ in the Western population. EBV-positive DLBCL is an aggressive lymphoma subtype, as roughly $80 \%$ of patients present with extra-nodal involvement, which can include the stomach, eyelid, liver, lung, female genital tract, kidney, and testis. Although EBV-positive DLBCL of the pancreas is a known entity, only one case reported with isolated pancreatic involvement was identified. Based on earlier studies, it was believed that patients with EBV-positive DLBCL had a worse clinical course and survival compared to EBV-negative cases, especially in the Asian populations. However, recent data suggests that EBV positivity does not affect survival in North American patients with DLBCL. Median overall survival was 129 vs. 143 months for the EBV-positive and negative groups, respectively. Although there is no uniformly accepted treatment for EBV-positive DLBCL, the standard treatment for DLBCL is R-CHOP and recent studies suggest a better prognosis than originally believed, especially for North American patients with EBV-positive DLBCL.

CONCLUSION: Given the limited treatment options associated with metastatic pancreatic adenocarcinoma, tissue diagnosis is critical as alternative underlying pathology such as EBV associated DLBCL have a better prognosis with an appropriate chemotherapy regimen.

\section{A TWISTED CASE OF JAUNDICE}

Hung Hoang; Tara Norris. Methodist Dallas Medical Center, Dallas, TX. (Control ID \#3180005)

LEARNING OBJECTIVE \#1: Diaphragmatic hernia (DH) causing obstructive jaundice in the elderly is extremely rare, but it can occur.

LEARNING OBJECTIVE \#2: This condition can be managged surgically or non-surgically.

CASE: A 71-year-old woman presented to the hospital after her granddaughter noticed two days of worsening jaundice. The patient reported a one-week history of fatigue, early satiety, poor appetite, nausea, and vomiting. She also noted having tea-colored urine and a 30-pound unintentional weight loss during the previous eight months. She denied history 
of trauma, recent surgery, abdominal pain, fever, or chills. Upon admission, the patient was noted to be diffusely jaundiced over her entire body. Her liver function tests were elevated, and abdominal ultrasound demonstrated a mildly dilated common bile duct at $8 \mathrm{~mm}$, as well as intrahepatic ductal dilation. Magnetic resonance cholangiopancreatography (MRCP) demonstrated stomach, pancreas, small bowel and most of the colon to be above the diaphragm with abnormal orientation of the extrahepatic bile ducts extending superiorly to the duodenum located in the thorax. Computed tomography (CT) of thorax, abdomen, and pelvis revealed a large diaphragmatic hernia, and it confirmed presence of pancreas, small bowel, and colon in the thorax. Surgical repair of the defect was considered, however, due to the patient's poor functional status, the risks of surgery were determined to outweigh the benefits. Biliary decompression with stent placement was performed instead. This was accomplished percutaneously, as her anatomy was not amenable to an endoscopic approach. She tolerated the procedure well, and her jaundice and transaminitis subsequently resolved. Plan was made for stent replacement as needed for recurrence of jaundice.

IMPACT/DISCUSSION: DH can be congenital or traumatic. The vast majority of congenital diaphragmatic hernias (CHD) present in infancy or childhood and occur at a rate of less than 5 per 10,000 live births. The incidence of $\mathrm{CDH}$ found incidentally on $\mathrm{CT}$ in adults is $0.17 \%$, but the incidence of CHD leading to clinical presentation has not been reported. While several case reports of congenital DH causing obstructive jaundice can be found in the pediatric literature, only two published cases could be located describing this phenomenon in adults. One case was reported in a 19-year-old female and the other was reported in a 64-year-old female. Our patient reported no history of trauma therefore, her diaphragmatic hernia was presumed to be congenital. The treatment of choice is surgical correction of the hernia to decompress biliary obstruction while preventing bowel obstruction or ischemia and lung injuries.

CONCLUSION: Painless jaundice with early satiety and weight loss can be caused by compression of the common bile duct from a complication of a diaphragmatic hernia. Newly discovered anatomic anomalies are rare in elderly patients, but nonetheless should be considered by clinicians in the differential diagnoses when evaluating patients with jaundice.

\section{A UNIQUE CASE OF MIXED AUTOIMMUNE WARM AND COLD HEMOLYTIC ANEMIA WITH EXTRAVASCULAR HE- M O L Y S I S L E A D I N G T O C O N J U G A T E D HYPERBILIRUBINEMIA}

Shaheen Fatima; Adetoun Adeyemi; Eric Chang; Pooja Padigala; Muhammad Bilal; Balsam Elhammali; Nicolas Bakinde. Morehouse School Of Medicine, Atlanta, GA. (Control ID \#3186576)

LEARNING OBJECTIVE \#1: Recognize the unique features of mixed autoimmune hemolytic anemia

LEARNING OBJECTIVE \#2: Assess the need of involvement of multidisciplinary team for management of mixed autoimmune hemolytic anemia CASE: 25 year-old-female presented with fatigue, diffuse body pain, nausea/vomiting, fever for the past two days. History was significant for iron-deficiency-anemia. Labs revealed hemoglobin/hematocrit of 4.4/ 13.6, mean-corpuscular-volume: 107 , elevated reticulocytes-count, totalbilirubin: 26.7 , direct-bilirubin: 21.9 , leukocyte-count: 6.3 with neutrophilic predominance, lactate-dehydrogenase: 193, haptoglobin in normal range, elevated d-dimer/fibrinogen/NR (1.6)/procalcitonin, peripheral smear showed macrocytes and toxic granulation. Coombs-auto-antibody test for both warm and cold was positive, direct-anti-globulin testing (DAT) was positive. HIV screening test, rheumatoid-factor and parvovirus- panel were negative. $\mathrm{Hb}$ phenotype was not consistent with sickle cell/thalassemia. Hematology was consulted and 6 units of $\mathrm{pRBC}$ were transfused with a poor response, she then received IVIG (3 doses), epoetin-alfa, folic acid, bortezomib and high dose steroids with improvement in blood counts and hemolysis. Gastroenterology was consulted due to conjugated-hyperbilirubinemia and recommended abdominal imaging. CT-abdomen showed no evidence of biliary obstruction/cholecystitis, showed splenomegaly. US-abdomen was negative. CT-chest revealed mildly enlarged right axillary lymph node. Lymph node biopsy was negative for malignancy, flow cytometry had no evidence of clonal process. EBV qualitative PCR and EBV IgG were positive but IgM negative indicating likely past infection, hepatitis panel was negative, Mycoplasma IgM was negative. Positive ANA IFA of 1:80 (with negative ANA screen) which was assessed to be secondary to IV immunoglobulin. She improved throughout the hospital course, hemolysis resolved, hematocrit and hemoglobin (10.3/31.6) and was discharged with prednisone taper and hematology follow up.

IMPACT/DISCUSSION: This unique presentation of mixed-hemolyticanemia with conjugated-hyperbilirubenemia points towards similarity of mixed hemolytic anemia to warm-autoimmune-hemolytic-anemia (wAIHA) than cold-hemolytic-anemia (cAIHA). The importance of recognizing this is directly proportional to patient's clinical outcome as treatment for wAIHA is different from cAIHA. Although intravascular hemolysis is 10-fold greater than extravascular hemolysis, the slowed response to blood transfusion, no signs of hemolysis on the peripheral smear and conjugated hyperbilirubinemia should shift the focus to extravascular hemolysis. Early involvement of hematology and gastroenterology is crucial in such cases.

CONCLUSION: Only 7-8\% of all AIHAs show a DAT positive for IgG and $\mathrm{C} 3 \mathrm{~d}$, with the coexistence of warm autoantibodies and high-titer cold agglutinins (mixed AIHA). Further investigation is needed to delineate mixed type AIHA from wAIHA in terms of pathophysiology to better understand and treat this condition effectively.

\section{A UNIQUE CASE OF SPOROTRICHOID-LIKE SPREAD OF AN OPPORTUNISTIC INFECTIOUS ORGANISM}

Jodi-Anne Wallace; Hila Miskin; Anthony Cannella; Omar Taha. University of Florida, Gainesville, FL. (Control ID \#3148667)

LEARNING OBJECTIVE \#1: Recognize and manage severe cutaneous Mycobacterium chelonae infection

CASE: A 57 year-old woman with lupus nephritis and subsequent renal transplant failure on immune suppression was admitted for right forearm cellulitis. She noticed yellow exudate near her right thumbnail that spread to erythema of her hand and antebrachium, one month prior to presentation. She was evaluated by several clinicians and finally a dermatologist who prescribed clindamycin, cephalexin and trimethoprimsulfamethoxazole respectively. Dermatology also performed a biopsy with bacteria culture. Painful nodules began to spread up her forearm, and she presented to this hospital. She was immediately started on vancomycin. Infectious disease was consulted, who discovered she was an avid gardener and requested repeat biopsy adding AFB and fungal cultures. The first biopsy demonstrated filamentous Gram-positive organisms thought to be Nocardia. As a result, she was started on amikacin and trimethoprim-sulfamethoxazole. However five days later, the AFB culture of the repeat biopsy grew M. chelonae. Trimethoprim-sulfamethoxazole was exchanged for moxifloxacin and clarithromycin. Treatment was complicated by major side effects including QT prolongation. The regimen was adjusted with combinations of tobramycin, tigecycline, and imipenem/cilistatin. Unfortunately, control of the infection was not achieved and she expired.

IMPACT/DISCUSSION: This case adds to the few reports of severe cutaneous $M$. chelonae in an immunocompromised patient. It reinforces the importance of having opportunistic infectious organisms high on the differential when immunocompromised individuals present with chronic cellulitis.

CONCLUSION: $M$. chelonae is a rapidly growing species of nontuberculosis mycobacteria found in water and soil. Disseminated cutaneous $M$. chelonae manifests as abscesses or violaceous subcutaneous nodules. A sporotrichoid pattern in $M$. chelonae infections is not 
commonly seen. Diagnosis is often difficult as it involves differentiating Nocardia and nontuberculous mycobacteria. However, a specific diagnosis is needed as rapidly growing mycobacterium are usually notoriously difficult to treat. Treatment of $M$. chelonae is based on susceptibility patterns and involves use of antibacterial drugs including amikacin, tobramycin, clarithromycin, or moxifloxacin. In the setting of soft tissue infections, oral antibiotic therapy with two agents is recommended for at least 4 months. Treatment for severe cutaneous infections includes parental therapy with two different antibiotics until there is evidence of clinical improvement (usually 2-6 weeks) followed by 2 oral agents for at least 612 months. In this case of $M$. chelonae infection, it took time to isolate the correct bacteria, which delayed treatment. Also, prior medication exposures encouraged resistance, limiting treatment options. Ultimately, her gardening hobby mandated an expansion of the differential diagnosis to include Nocardia and nontuberculous mycobacteria.

\section{A WALK TO NOT REMEMBER}

Marcelle Tuttle ${ }^{1}$; Laura K. Snydman ${ }^{2} .{ }^{1}$ Tufts University School of Medicine, Somerville, MA; ${ }^{2}$ Tufts Medical Center, Boston, MA. (Control ID \#3184167)

LEARNING OBJECTIVE \#1: Recognize the presentation of HenochSchonlein Purpura and the heterogeneity of its presentation/severity.

LEARNING OBJECTIVE \#2: Recognize the pitfalls of anchoring bias.

CASE: A 61-year-old man with PMH significant for pre-diabetes presented to the emergency department with one week of arthralgias, night sweats, abdominal pain and constipation. He stated that these symptoms started several days after he went for a walk in the woods of New England and he noticed "tick bites" on his ankles bilaterally. Doxycycline was initiated for presumed tick-borne infection and he was discharged home. One day later, he represented to the ED as the rash had spread up to his thighs. A biopsy was taken at that time and he was again sent home. One week later, he returned to the ED with chest pressure and continued abdominal pain/constipation. CT demonstrated marked mural thickening of the duodenum and proximal jejunum consistent with enteritis and he was transferred to a tertiary care hospital. Labs on transfer were notable for CRP 104 and a serum IgA of 381 . He was started on solumedrol and Toradol for pain. Subsequently, immunofluorescence was performed on the biopsy sample and demonstrated Henoch Schonlein Purpura (HSP). He remained in the hospital for 12 days as his enteritis continued to cause him extreme pain requiring patient-controlled anesthesia. During this time he developed significant proteinuria with a protein to creatinine ratio of $8134 \mathrm{mg} / \mathrm{g}$ at its peak. At followup 4 months later he still had significant proteinuria which was treated with prednisone.

IMPACT/DISCUSSION: This case demonstrates the danger of anchoring bias. This cognitive error occurs when a clinician mistakenly latches onto a piece of information not relevant to a diagnosis. In this case, the walk in the woods and the patient's misinterpretation of his purpura as tick bites caused the clinician to assume that this was a tick-borne infection. However, his clinical picture did not fit with this diagnosis. Lyme can cause rash and arthralgias but does not usually cause abdominal pain or constipation. Rocky Mountain Spotted Fever could also be a consideration but is not generally found in New England. HSP typically presents in children less than 10 years old with palpable purpura, abdominal pain and arthritis. Renal involvement, including hematuria and proteinuria can also occur and is more commonly found in older children and adults. While most cases are self-limited, our patient required significant pain control as well as a course of steroids. The rate of recurrence of HSP is high, up to $40 \%$ in one year.

CONCLUSION: HSP is an uncommon disease in adults that presents with palpable purpura, abdominal pain and arthritis. Anchoring bias can occur when a clinician mistakenly believes a piece of information is of great importance.

\section{A YOUNG WOMAN PRESENTING WITH PANCYTOPENIA AND ISCHEMIC STROKE}

Michelle Lam. University of Washington, Seattle, WA. (Control ID \#3147866)

LEARNING OBJECTIVE \#1: Diagnose acute leukemia in a patient presenting with pancytopenia

LEARNING OBJECTIVE \#2: Manage acute promyelocytic leukemia in setting of coagulopathy

CASE: A 33 year old woman with history of hypertension initially presented with 5 days of nausea and abdominal pain and 1 day of newonset confusion. She takes no medications, lives with her parents, and has no history of any tobacco, alcohol or drug use. Brain MRI showed multiple right-sided cerebellar infarcts. She was found to be hypertensive to $200 \mathrm{~s} / 100 \mathrm{~s}$, lethargic and afebrile, with pancytopenia with WBC of 1.9 , hemoglobin of 10.5, platelets of 44 and elevated LDH at 437. She had normal creatinine and LFTs. ESR, complement, and ANCA testing were negative. Plasma exchange was initiated due to concern for atypical HUS/ TTP, however this was discontinued after no improvement in her symptoms. Initial transthoracic echocardiogram (TTE) imaging was negative for any vegetations and blood cultures were negative. She had hypofibrinogenemia $(<60)$ despite receiving multiple units of cryoprecipitate. She was transferred to a tertiary medical center and peripheral blood smear and bone marrow biopsy showed hypercellularity consistent with acute promyelocytic leukemia (APL). She was initiated on all-trans retinoic acid (ATRA) and arsenic treatment and was given repeated cryoprecipitate and platelet transfusions. Although initially alert with word-finding difficulties, she became more somnolent with withdrawal to pain only in her right upper extremity and later became obtunded, requiring intubation for airway protection. Repeat MRI imaging showed ongoing ischemic infarcts, including basilar artery occlusion. A repeat TTE showed a large aortic valve vegetation, with negative blood cultures. Despite concern for disseminated intravascular coagulation (DIC) and bleeding, given her ongoing cerebral infarcts, a heparin drip was initiated. Nevertheless, her infarcts progressed and due to poor longterm prognosis including blindness, inability to speak and hemiplegia, she was transitioned to comfort care after discussion with her parents and later died from complications related to APL.

IMPACT/DISCUSSION: Acute promyelocytic leukemia frequently presents with pancytopenia and coagulopathy including DIC. While bleeding from a coagulopathy is the most common presentation of APL, prothrombotic states are also seen in APL, with case reports showing acute myocardial infarction and ischemic stroke. ATRA is the main treatment for APL and can be curative, though it has been implicated in potentially worsening the prothrombotic state. In this case, the risks and benefits of treating DIC and avoiding bleeding complications had to be weighed with treating her ongoing thrombotic strokes.

CONCLUSION: A peripheral blood smear should always be reviewed in patients presenting with pancytopenia. Bleeding coagulopathies and thrombotic events are common presentations of APL. ATRA is the main treatment of acute promyelocytic leukemia and can be curative, though can contribute to prothrombotic states.

\section{A ZEBRA OF ADRENAL INSUFFICIENCY, WHAT WAS ONCE COMMON-NOW RARE}

$\underline{\text { Sean J. Godfrey }}^{1}$; Victoria Hudspeth ${ }^{2}$; Kelli Dunn ${ }^{2} .{ }^{1}$ Atrium Health, Morganton, NC; ${ }^{2}$ Atrium Health, Charlotte, NC. (Control ID \#3186373)

LEARNING OBJECTIVE \#1: Recognize atypical causes of persistent hypotension.

CASE: A 62 year old woman with a history of CKD, hyperlipidemia, and possible heart failure presented to the hospital with a 3 week history of worsening right knee pain. She was initially hypotensive at $68 / 48$. Her 
hospital course was complicated by renal failure, persistent hypotension, and hyponatremia. She received approximately 5 liters of fluid with no significant hemodynamic response. Initial labs showed sodium of 125 with normal range 135-145 and a creatinine of 3.63 with sodium levels hovering in the low 130 's throughout the next 8 days while systolic pressure remained less than 110. Endocrinology was consulted to evaluate the ongoing hypotension and hyponatremia 5 days into admission. Infectious disease was also consulted after it was later revealed that the patient had a history of TB with a positive ppd in 2014, treated with Isoniazid but she was unable to confidently state whether she completed treatment. Other lab work was remarkable for a positive ppd test, negative HIV, and random cortisol levels of 1.3 and 1.1. Cosyntropin stimulation test was very abnormal with cortisol results of 1.3 and 1.1 at 30 and 60 minute intervals (normal $>18$ ). ACTH on day 6 of admission was greater than 2000 , consistent with primary adrenal insufficiency. Abdominal CT without contrast on day 11 showed adrenal fullness with nodal calcifications consistent with post-infectious TB. Blood pressure and sodium improved on hydrocortisone $50 \mathrm{mg}$ three times daily. She was discharged on hydrocortisone $20 \mathrm{mg}$ in the $\mathrm{AM}$ and $10 \mathrm{mg}$ in the PM, fludrocortisone $0.1 \mathrm{mg}$ daily and the 4 drug combo for TB; Rifampin, Isoniazid, Pyrazinamide, and Ethambutol.

IMPACT/DISCUSSION: While TB was once a common cause of primary adrenal insufficiency, it is now most often autoimmune in the developed world, but there are rarely observed infectious causes, including TB, Fungal, and CMV. The extra-adrenal manifestations typically stem from pulmonary TB. When TB invades the adrenals, imaging of the adrenal glands initially shows hypertrophy, which progresses to fibrosis and calcification in almost half the cases. This adrenal hypertrophy is often a key marker for differentiating tuberculous adrenal insufficiency from autoimmune adrenalitis, in which case adrenals are usually atrophied. This case of primary adrenal insufficiency highlights an atypical cause that is now rare in the developed world. TB causes adrenal destruction in a gradual manner, targeting the medulla and cortex secondarily as illustrated here by this HIV-negative latent TB patient.

CONCLUSION: Tuberculous adrenal insufficiency typically presents in an insidious manner. This case demonstrates the importance of a thorough history and evaluation especially in a patient with persistent hypotension. Another key point is the importance of realizing that not all primary adrenal insufficiency is autoimmune.

\section{ABRUPT WORSENING OF DIABETES CONTROL ASSOCIAT- ED WITH NEW DIAGNOSIS OF ENDOMETRIAL ADENOCAR- CINOMA, WITH RETURN TO BASELINE FOLLOWING CURA- TIVE SURGERY}

${ }_{\text {Michael Goulet }}^{1}$; Zehra Hussain ${ }^{2} .{ }^{1}$ Christiana Care, Newark, DE; ${ }^{2}$ Christiana Care, Wilmington, DE. (Control ID \#3184521)

LEARNING OBJECTIVE \#1: Recognize malignancy in the differential diagnosis of a patient with abrupt worsening of diabetes or new-onset diabetes CASE: A 72 year old female with diabetes mellitus presented to the primary care office in January 2017 for diabetes follow up and with complaint of vaginal bleeding. Her diabetes had been well controlled for the past 12 years on metformin and basal insulin, but at this visit her A1c had increased to $8.7 \%$. The patient endorsed occasional dietary nonadherence but denied any lifestyle nonadherence, infectious symptoms, steroid use, or new medications. She was advised to follow a strict diabetic diet and monitor her blood sugars closely. For her postmenopausal bleeding, gynecologic workup over the next 2 months revealed a diagnosis of grade 2 endometrial carcinoma. She was scheduled for hysterectomy with bilateral salpingo-oopherectomy and staging. Over this time period, she reported persistent elevated blood sugars to the 300s-400s despite several increases in her basal insulin and the addition of mealtime insulin. Her pre-operative A1c increased to $>15 \%$. The surgical team initially hoped for improved glycemic control, but decided to proceed with surgery given the risks of delay. The patient noted improved blood sugars within days after her hysterectomy. A1c returned to $6.3 \%$ within 3 months and remained stable as her insulin was titrated down. As of January 2019 her diabetes is well controlled on metformin alone. Pathology had indicated stage 1 disease, and she remains cancer-free.

IMPACT/DISCUSSION: Our patient had a long standing history of well controlled diabetes with a clear worsening associated with a new diagnosis of endometrial cancer, and without any other known variables to explain the deterioration. Her blood sugars returned to baseline immediately following curative surgery, indicating that hyperglycemia was likely caused by her malignancy. Hyperglycemia is known to be a risk factor for the development of endometrial cancer, but to our knowledge hyperglycemia as a consequence of endometrial cancer has not been previously reported. Pancreatic malignancy is the only cancer that is known to induce insulin resistance and diabetes, and screening strategies have been suggested for older patients with new-onset diabetes. The mechanism is thought to be through hormonal mediators produced by pancreatic tumor cells. Adrenomedullin is one hormone that has been implicated in this paraneoplastic process. This hormone has also been shown to play a role in endometrial cancer, allowing for the possibility of an overlapping mechanism in endometrial cancer.

CONCLUSION: This case highlights the importance of taking a careful malignancy-focused history and physical in patients presenting with new or abruptly uncontrolled diabetes. Hyperglycemia has previously been described as an early sign of pancreatic cancer, and our case suggests it may be a sign of endometrial cancer as well.

\section{ABSENT TESTIS IN AN IMMIGRANT PATIENT}

Vishnu Manoranjan $^{1}$; David Feldstein ${ }^{2}$; Linda Baier ${ }^{3}$; Brian Lewis ${ }^{4}$. ${ }^{1}$ University of Wisconsin Hospital and Clinics, Madison, WI; ${ }^{2}$ University of Wisconsin School of Medicine and Public Health, Madison, WI; ${ }^{3} \mathrm{f}$ Medicine and Public Health, Madison, WI; ${ }^{4}$ University of Wisconsin, Middleton, WI. (Control ID \#3186199)

LEARNING OBJECTIVE \#1: Describe reasons for and possible contraindications to starting testosterone therapy in a hypogonadal male.

LEARNING OBJECTIVE \#2: Recognize the effects of social uncertainty on immigrant patients.

CASE: A 32 year-old male presents to establish care. His chief complaint is insomnia. He has difficulty falling asleep and is kept awake by racing thoughts. He and his wife are from Iran and he worries about their future in the US. He also mentions that he cannot feel his right testis. He thinks it was removed in his youth, but is unaware of the details. He also reports decreased energy and mood. Physical exam is unremarkable except for a lack of palpable testis on the right; left is present; no surgical scar. He is started on sertraline for anxiety. MRI shows absent right testis without evidence of undescended testis or previous procedure and an atrophic left testis. Testosterone levels are low and semen analysis shows azoospermia. At 6-week follow-up, sleep has greatly improved. Urology performs further evaluation and recommends karyotype testing and testosterone supplementation. Patient declines further testing and therapy, citing costs and uncertainty about future residence.

IMPACT/DISCUSSION: Endocrine Society guidelines recommend testosterone therapy in primary hypogonadism to induce secondary sex characteristics and to correct symptoms such as sleep disturbance, depression, and decreased energy, which were seen in this case. Other, more suggestive symptoms include erectile dysfunction, low libido, and lowtrauma fracture. Guidelines caution against testosterone use in patients who desire fertility in 6-12 months due to suppression of spermatogenesis. Caution is also advised in those with elevated hematocrit, thrombophilia, severe sleep apnea, severe lower urinary tract symptoms, uncontrolled heart failure, prostate cancer (or increased risk), or recent myocardial infarction. This case made me aware of the adverse effects of testosterone therapy, especially the negative effect on fertility, which I will now consider prior to prescribing. This case also highlights the de-stabilizing 
effects of uncertainty on immigrants. Grace et al. (2018) call this the "violence of uncertainty." Immigration is an inherently high-stress event; thus, anxiety, depression, and post-traumatic stress disorder may occur. Shifting immigration policies affect whether and how immigrants seek care. Those worried about finances or resident status may be less likely to follow-up or pursue long-term treatment. This case made me aware of the psychological and socio-political factors that may impact care.

CONCLUSION: Testosterone can correct hypogonadism symptoms and induce and maintain secondary sexual characteristics, but is contraindicated in those who desire fertility in the near term. Immigrant patients are often subject to social uncertainty, which can manifest as mental health disorders and difficulty with seeking and maintaining care.

\section{ACANTHAMOEBA ENCEPHALITIS IN HIV-INFECTED MALE PRESENTING WITH HEADACHE AND TONIC-CLONIC SEI- ZURES: A CASE REPORT}

Abigail C. Watts; Nonglin Mel. Emory University School of Medicine, Atlanta, GA. (Control ID \#3165946)

LEARNING OBJECTIVE \#1: Recognize the radiographic and pathologic features of Acanthamoeba encephalitis

LEARNING OBJECTIVE \#2: Formulate a differential diagnosis for space-occupying brain lesions in immunocompromised patients

CASE: A 27-year-old man with Acquired Immune Deficiency Syndrome (with a CD4+ cell count of 5 cells $/ \mathrm{mm} 3$ ) presented with tonic-clonic seizures and headache. Physical exam on admission did not reveal focal neurological deficits. Laboratory studies showed a WBC of $4.6 \mathrm{~K} / \mathrm{mcL}$ (12.7\% eosinophils). Magnetic resonance imaging (MRI) demonstrated a right frontoparietal peripherally enhancing lesion with mild surrounding edema and associated mass effect. Cerebrospinal fluid studies demonstrated neutrophil-predominant pleocytosis (WBC $147 / \mathrm{mcL}$ with 50\% neutrophils) with normal protein and glucose. Broad-spectrum therapy for potential bacterial, viral, and fungal infections was initiated; nonetheless, the patient developed increasing somnolence and new focal neurological deficits. Repeat MRI six days after admission demonstrated new, enlarging edematous and peripherally enhancing lesions favored to represent multifocal cerebritis. Craniotomy and open brain biopsy with resection of dura was performed ten days after admission. Pathology revealed lymphohistiocytic inflammation with amoebic necrotizing abscesses. Immunohistochemical and PCR testing confirmed Acanthamoeba infection. Despite multidrug treatment (with miltifosine, flucytosine, pentaminidine, sulfdiazine, fluconazole, and azithromycin), the patient expired on hospital day 16.

IMPACT/DISCUSSION: Acanthamoeba is a pervasive protozoal species found in soil, water, air, and dust; the organism has been associated with three infectious syndromes in humans: amoebic encephalitis, disseminated granulomatous amebic disease, and keratitis (especially in contact lense users). Unlike the fulminant infection caused by Naegleria fowleri, Acanthamoeba causes a subacute encephalitis that progresses over days to months. Acanthamoeba likely causes central nervous system infection in immunocompromised hosts through hematogenous spread after initial pulmonary or skin inoculation and subsequent penetration of the blood-brain barrier. Investigational therapies for Acanthamoeba encephalitis include surgical resection of lesions and combination drug therapy.

CONCLUSION: Amoebic encephalitis is an exceedingly rare cause of central nervous system infection with a mortality exceeding $90 \%$. Various protozoa have been implicated in central nervous system disease, including Naegleria fowleri, Balamuthia mandrillaris, and multiple species of Acanthamoeba. Little data exists regarding the clinical characteristics, radiographic findings, and treatment of Acanthamoeba encephalitis, as fewer than 150 cases have been reported globally since the condition was first described in the 1960s. Acanthamoeba encephalitis should be considered in immunocompromised patients presenting with central nervous system infection, as delayed recognition and treatment contribute to the condition's grave prognosis.
ACQUIRED HEMOPHILIA A DUE TO ALEMTUZUMAB TREATMENT FOR MULTIPLE SCLEROSIS

Jesse D. Faulk $^{1}$; Dennis Sehgal ${ }^{1}$; Jorge Diaz ${ }^{2}$; Pavan Panchavati ${ }^{3} .{ }^{1}$ University of Alabama at Birmingham, Huntsville, AL; ${ }^{2}$ Clearview Cancer Institute, Huntsville, AL; ${ }^{3}$ Huntsville Hospital, Huntsville, AL. (Control ID \#3185958)

LEARNING OBJECTIVE \#1: Diagnose acquired hemophilia A. LEARNING OBJECTIVE \#2: Treat acquired hemophilia A.

CASE: A 66-year-old Caucasian female presented to the ED due to bruising. Her bruising began 4 days prior, beginning on her hands and feet then involving her torso. She also admitted to hematuria for 4 days but denied melanotic stools, hematemesis, hematochezia, epistaxis, or joint swelling. She denied any recent trauma or history of easy bruising or bleeding. She has a medical history significant for multiple sclerosis and was treated nine months prior with alemtuzumab. She had a prior appendectomy and cholecystectomy without any significant bleeding. She has no family history of bleeding or clotting disorders. On examination, she was afebrile and normotensive. She had diffuse bruising. Her hemoglobin was $8.1 \mathrm{~g} / \mathrm{dL}$, platelet count was $381 \times 10^{3} / \mu \mathrm{L}$, and $\mathrm{WBC}$ was $11.7 \times 10^{3} / \mu \mathrm{L}$. Prothrombin time (PT) was $13.1 \mathrm{~s}$ and partial thromboplastin time (PTT) was 90.2 s. Factor 8 activity was $3 \%$ (normal: $50-150 \%$ ). A PTT mixing study was performed with PTT failing to correct. Lupus anticoagulant was not detected. Factor VIII inhibitor was detected. Computed tomography of chest, abdomen, and pelvis showed no malignancy. Antinuclear antibody was negative. She was diagnosed with acquired hemophilia A secondary to alemtuzumab therapy and started on prednisone and rituximab, which was changed to cyclophosphamide due to allergic reaction. Her PTT began to decrease, and she was discharged on prednisone and cyclophosphamide.

IMPACT/DISCUSSION: Acquired hemophilia A is an uncommon autoimmune disorder that is caused by an inhibitory autoantibody to factor VIII. Acquired hemophilia may be associated with certain medications, autoimmune disorders, or malignancy. Almost half of patients may not have an identified etiology. Alemtuzumab, a humanized monoclonal antibody targeting the surface molecule of CD52 of B and T lymphocytes, has been reported to cause autoimmune conditions, and limited case reports exist of this monoclonal antibody causing acquired hemophilia A. The usual presenting symptom is bleeding that may occur after a surgical procedure or spontaneously. Patients may present with mucocutaneous bleeding, such as epistaxis, gastrointestinal bleeding, hematuria, ecchymosis, or large hematomas. Acquired hemophilia is characterized by prolonged PTT with normal PT. A mixing test that results in uncorrected PTT differentiates acquired hemophilia from inherited factor deficiency, and a Bethesda assay diagnoses the presence of a factor VIII inhibitor. Desmopressin, factor VIII concentrates, or activated prothrombin complex concentrate can be used to control bleeding. In order to eliminate the inhibitor, immunosuppressive modalities, such as glucorticoids, cyclophosphamide, and rituximab, may be used.

CONCLUSION: Acquired hemophilia A is a rare but important cause of mucocutaneous bleeding and must be differentiated from inherited factor deficiencies. Alemtuzumab has been reported as a cause and must be identified to avoid re-exposure.

\section{ACUTE BACTERIAL MYOPERICARDITIS IN AN INTRAVE- NOUS (IV) DRUG USER.}

Juliann Koleszar ${ }^{1}$; Timothy Byrnes ${ }^{2} .{ }^{1}$ Summa Health System/Northeast Ohio Medical University Program, Akron, OH; ${ }^{2}$ Summa Health System, Akron, OH. (Control ID \#3186998)

LEARNING OBJECTIVE \#1: Recognize the clinical features of acute bacterial myopericarditis.

LEARNING OBJECTIVE \#2: Distinguish between pericarditis and myopericarditis. 
CASE: A 42-year-old Caucasian male with a history of IV drug use, alcohol abuse, hepatitis B and C, esophageal varices, and cirrhosis presented to the ER with two days of diffuse chest pain, 10/10 in intensity, sharp, nonradiating, and constant, associated with dyspnea and fever. It worsened with inspiration and lying supine and improved with sitting upright. Physical examination revealed a temperature of $39.1{ }^{\circ} \mathrm{C}$, pulse of 121 beats $/ \mathrm{min}$, respiration rate of $20 \mathrm{breaths} / \mathrm{min}$, and a blood pressure of $121 / 80 \mathrm{mmHg}$. Pulse oximetry on room air was $96 \%$ saturation. He appeared cachectic and chronically ill. Chest and cardiac auscultation revealed clear lungs, tachycardia with a regular rhythm, and no jugular venous distention, murmur, rub, or gallop. He had 2+bilateral pitting edema to the knee and abdominal ascites. Complete blood count showed $19.2 \times 10^{3} \mathrm{WBC} / \mathrm{uL}$ and $109 \times 10^{3}$ platelets/uL. Troponin was elevated at $1.2 \mathrm{ng} / \mathrm{mL}$. Electrocardiogram (EKG) showed diffuse ST elevations suggestive of pericarditis. CTA of the chest showed bilateral infiltrates concerning for pneumonia. Blood cultures were obtained and IV Vancomycin and IV Piperacillin/Tazobactam initiated. Transthoracic echocardiogram (TTE) revealed an ejection fraction of $65 \%$ and a moderate freeflowing pericardial effusion without evidence of tamponade. The patient underwent pericardiocentesis, $600 \mathrm{cc}$ of cloudy fluid was removed, and a pericardial drain placed. Blood and pericardial fluid cultures returned positive for MRSA. IV Vancomycin was continued for MRSA bacteremia and purulent myopericarditis. Pericardial cytology returned negative. The patient left against medical advice(AMA) but returned a few days later for worsening ascites and chest pain. Repeat TTE showed a small pericardial effusion. Repeat blood cultures returned negative. A six-week course of IV vancomycin was planned but the patient again left AMA.

IMPACT/DISCUSSION: A PubMed search using the terms MRSA and myopericarditis resulted in only two case reports of MRSA bacterial myopericarditis:a 41-year-old male with community-acquired pneumonia and a 48-year-old male with an initial elbow abscess. If the clinician encounters a septic patient who meets diagnostic criteria for acute pericarditis, broad spectrum antibiotic therapy should be initiated, and a TTE obtained to determine need for pericardiocentesis. MRSA bacterial myopericarditis is a rare entity, however with the rise of IV drug use within the US, its incidence is likely to increase.

CONCLUSION: Bacterial pericarditis is an infrequent finding in areas with low prevalence of tuberculosis, and purulent pericarditis is rarely diagnosed, comprising $<1 \%$ of all pericarditis cases. Patients meeting diagnostic criteria for pericarditis with positive cardiac biomarkers in the absence of ventricular dysfunction are further classified as having myopericarditis.

\section{ACUTE INFLUENZA B-RELATED ENCEPHALOPATHY}

Merza Nooraldin'; Muath Alsharif ${ }^{2}$; Mais $\mathrm{Abdou}^{3}$; Mazin Saadaldin ${ }^{4}$; Tareq Naguib ${ }^{3}$. ttuhsc, Amarillo, TX; ${ }^{2}$ Texas Tech University Health Science Center, Amarillo, TX; ${ }^{3}$ TTUHSC, Amarillo, Amarillo, TX; ${ }^{4}$ NWTHS, TTUHSC, Amarillo, TX. (Control ID \#3184711)

LEARNING OBJECTIVE \#1: 1-Discuss dagnostic criteria and clinical presentation of influenza-related encephalopathy. 2-To lower the threshold for diagnosing Influenza B-related encephalopathy in adults in the face of higher prevalence.

CASE: A 62-year-old male with prostate cancer treated with radiation therapy was admitted for hypertensive crisis with seizures and altered mental state. Although blood pressure improved, he deteriorated and developed oral apraxia. There was no cough, nasal discharge, sore throat, headache, arthralgia, or muscles aches. The temperature was $38.9 \mathrm{c}$, blood pressure $150 / 80 \mathrm{mmHg}$, pulse 106 beats/min, respiratory rate 21 breaths/ min, and oxygen saturation $96 \%$ on room air. The Glasgow Coma Scale (GCS) score was 13 with eye opening 4 and verbal response 4 . The rest of exam was unremarkable including normal pupillary reflexes and muscle tone of the neck. A nasopharyngeal swab was positive for influenza B rapid test, and Tamiflu was started, while spinal tap findings were all normal, including viral panels, ruling out meningoencephalitis. Subtle punctate acute cortical infarct involving both left parietal and right occipital lobes, appeared on MRI along with old left lacunar thalamic infarct and EEG suggested encephalopathy, all consistent with influenza Brelated acute encephalopathy. Due to brain damage and altered mentation, our patient was transferred to a skilled nursing facility upon discharge for rehabilitation and consideration for permanent placement.

IMPACT/DISCUSSION: Our patient was positive for Influenza B virus and met the diagnostic criteria of the Japanese research committee of influenza encephalopathy, which include severe impairment of consciousness with a sustained GCS score of 13 for more than 24 hours. The majority of cases are associated with influenza $\mathrm{A}$ in younger age but cases in adults are on the rise. Our case of influenza B-related encephalopathy in an older adult serves to raise awareness of this unusual condition.

CONCLUSION: Acute influenza B-related encephalopathy presents with various neurological complications but sharing common findings of seizure, fever and encephalopathy/encephalitis. The presence of influenza virus RNA in brain biopsy tissue, CSF, or even respiratory samples is diagnostic. The presence of brain lesions on MRI and influenza virus in CSF appear to be of prognostic value.

\section{ACUTE KIDNEY INJURY: LOOKING BEYOND THE URINARY TRACT}

Carine Davila; Nicholas Iverson; Irina Kryzhanovskaya; Sarah Schaeffer. University of California, San Francisco, San Francisco, CA. (Control ID \#3184723)

LEARNING OBJECTIVE \#1: Recognize gynecologic malignancy as a cause of obstructive uropathy in women

LEARNING OBJECTIVE \#2: Identify appropriate imaging modalities to evaluate for malignancy in women with obstructive uropathy

CASE: A 62-year-old woman with no known past medical history arrived at the emergency department obtunded after being found down at home. A friend revealed she had not seen a doctor in 20 years. She lives alone and does not use substances. On exam, she is afebrile, tachycardic to 120 , and hypertensive to $229 / 116$. She was arousable but disoriented, without clonus or asterixis. Initial labs showed potassium $6.5 \mathrm{mEq} / \mathrm{L}, \mathrm{BUN} 154$ $\mathrm{mg} / \mathrm{dL}$, and $\mathrm{Cr} 31.2 \mathrm{mg} / \mathrm{dL}$. A foley catheter was placed and drained $200 \mathrm{~mL}$ of light-yellow urine; urinalysis showed moderate blood and protein and urine toxicology was negative. Renal replacement therapy was initiated. A renal ultrasound after foley placement showed moderate bilateral hydronephrosis without apparent cause. A non-contrast CT abdomen/pelvis was performed to assess the cause of hydronephrosis, but noted only non-enlarged retroperitoneal lymphadenopathy, moderate ascites, and a uterine myoma. Ureteral stents were placed but failed to relieve the obstruction and the patient required bilateral nephrostomy tubes several days later. On hospital day five, a pelvic ultrasound was performed and showed endometrial thickening and an $8 \mathrm{~cm}$ calcified solid mass, concerning for malignancy. A cervical biopsy revealed squamous cell carcinoma; PET/CT demonstrated a hypermetabolic endometrial mass, abdominal carcinomatosis, and osseous metastases. The final diagnosis was cervical malignancy with retroperitoneal adenopathy causing bilateral complete obstructive uropathy, acute anuric renal failure, and uremic encephalopathy.

IMPACT/DISCUSSION: Though obstructive uropathy represents $10 \%$ of all cases of acute kidney injury (AKI), the incidence in women is one third to one half compared to men (Pichler 2010). Historically, a renal ultrasound is the first imaging study performed, as it can identify obstruction and at times an etiology of the obstruction. Licurse (2010) argues that this is not cost-effective given the low incidence of obstruction in all cases of AKI. When obstructive uropathy occurs in women, workup should include a prompt evaluation for malignancy, as it accounts for $72 \%$ of cases (Norman 1982). Renal ultrasound and CT abdomen/pelvis, as obtained in this case, do not always effectively evaluate for gynecologic 
malignancy. Pelvic ultrasound is a critical tool for evaluating female patients. Transvaginal ultrasound has been shown to be superior even to pelvic MRI for delineating cervical tumor (Alcazar 2014). Therefore, although $\mathrm{CT}$ is an easily-obtainable imaging modality, a transvaginal ultrasound is necessary to adequately evaluate a woman with urinary obstruction for gynecologic malignancy.

CONCLUSION: Malignancy accounts for nearly $75 \%$ of cases of obstructive uropathy in women. To better evaluate for gynecologic malignancy, a transvaginal ultrasound is more useful than CT or MRI.

\section{ACUTE ONSET TYPE 1 DIABETES MELLITUS IN A 95-YEAR- OLD PATIENT WITH METASTATIC MELANOMA}

Damond B. $\mathrm{Ng}^{1}$; Sara-Megumi Naylor ${ }^{2}{ }^{1}$. ${ }^{1}$ David Geffen School of Medicine at UCLA, Los Angeles, CA; ${ }^{2}$ Department of Veterans Affairs, Los Angeles, CA. (Control ID \#3162607)

LEARNING OBJECTIVE \#1: Demonstrate that T1DM is a rare, but critical side effect of anti-programmed cell death-1 (PD-1) antibodies. Endocrine disturbances can contribute to the morbidity of patients with malignancy.

LEARNING OBJECTIVE \#2: Appreciate that primary care providers can play a role in managing the side effects and weighing the benefits and risks of immune checkpoint inhibitors.

CASE: A 95 year-old man with a history of metastatic melanoma presenting for surveillance imaging was incidentally found to have a serum glucose of $428 \mathrm{mg} / \mathrm{dL}$. He had no past medical history of diabetes or known evidence of pancreatic metastases. His family history was notable for a daughter with type 1 diabetes mellitus (T1DM) diagnosed at a young age. For metastatic melanoma, he received 7 cycles of pembrolizumab ( $200 \mathrm{mg}$ every 3 weeks) for palliation. He reported polyuria, polydipsia, and weight loss for the last 3 months. He denied abdominal pain, nausea, and vomiting. His physical exam was pertinent for hypotension $(80 / 40 \mathrm{mmHg})$, normal respiratory effort, normal mentation, a normal abdominal exam without tenderness, and poor skin turgor. Laboratory evaluation revealed a normal anion gap and serum osmolality. There were no ketones in his urine and serum. Further studies demonstrated a hemoglobin A1c of $9.5 \%$, an inappropriately low serum C-peptide at $0.56 \mathrm{ng} / \mathrm{mL}(0.8$ 3.85 ), and an undetectable anti-GAD titer. Pembrolizumab was held and the patient was started on basal-bolus subcutaneous insulin. $\mathrm{He}$ successfully obtained glucose control with a follow-up hemoglobin A1c of $7.2 \%$ and normalization of his C-peptide.

IMPACT/DISCUSSION: Although pembrolizumab, a monoclonal antibody against PD-1, has improved survival in patients with metastatic melanoma, descriptions of endocrine disturbances are accumulating. Fortunately, most side effects are reversible, but early recognition and proper management are crucial.

CONCLUSION: Checkpoint inhibitor therapy has been associated with the acute onset of T1DM in approximately $0.2 \%$ of cases with a range of 1-52 weeks after initiation. This patient had the following glucose trend: $186 \mathrm{mg} / \mathrm{dL}$ at week $15,329 \mathrm{mg} / \mathrm{dL}$ at week 18 , and finally $428 \mathrm{mg} / \mathrm{dL}$ at week 21. There was no investigation or intervention until week 21. Timely evaluation might have led to pembrolizumab cessation and evaluation for T1DM rather than a presentation of a hyperglycemia crisis. Per recent guidelines, serum glucose should be measured at baseline and with each treatment cycle during induction for 12 weeks, then every 3 to 6 weeks thereafter. In a series of five cases of pembrolizumab associated T1DM, patients initially presented with hyperglycemia or diabetic ketoacidosis. All required insulin at diagnosis and remained insulindependent for diabetic control. The patient's advanced age, Do-NotResuscitate status, visual impairment and poor social support made this new diagnosis challenging. He was admitted overnight for insulin therapy and endocrine consultation. The risks and benefits of pembrolizumab were discussed and the drug was discontinued.
ACUTE RENAL AND SPLENIC INFARCTIONS AS THE INITIAL MANIFESTATION OF PAROXYSMAL ATRIAL FIBRILLATION (PAF).

Liangbo (linus) L. Shen ${ }^{1}$; Wei $\mathrm{Qi}^{1}$; Jose C. Duarte ${ }^{1}$; Anil Nagar ${ }^{1}{ }^{2}$; Naseema B. Merchant ${ }^{1,2}$. ${ }^{1}$ Yale University School of Medicine, New Haven, CT; ${ }^{2}$ VA Connecticut Health Care, West Haven, CT. (Control ID \#3184327)

LEARNING OBJECTIVE \#1: Diagnose uncommon embolic presentations of paroxysmal atrial fibrillation (PAF).

LEARNING OBJECTIVE \#2: Recognize the importance of cardiac monitoring and appropriate imaging studies in patients presenting with visceral emboli.

CASE: A 73-year-old man with a history of hypertension and diverticulosis presented to the emergency department with one day history of severe left lower quadrant (LLQ) abdominal pain and nausea. He denied dizziness, chest pain, palpitations, shortness of breath, hematuria, fever or diarrhea. Vitals were notable for hypertension (187/104 mmHg). Physical exam including cardiac was normal except for tenderness in LLQ. results of WBC, creatinine, LFT's and urinalysis (1+ protein, 1 white blood cell, and 4 red blood cells) were normal. Initial EKG showed normal sinus rhythm. A non-contrast abdominal CT showed no calculi in the urinary tract. Due to concern for acute diverticulitis and intestinal ischemia, he underwent a contrast enhanced abdominal CT scan showing acute infarctions in the left kidney and spleen. Anticoagulation was initiated due to a high suspicion of cardioembolic events as an underlying etiology. Subsequently, continuous telemetry showed occurrence of PAF, confirmed by a subsequent EKG. Patient's symptoms quickly improved, and he was discharged 3 days later in normal sinus rhythm on anticoagulation.

IMPACT/DISCUSSION: $\mathrm{n}$ patients with PAF, brain is a common site for cardiac emboli. Embolization to other organs is low (extremities: $1.22 \%$, mesenteric: $0.58 \%$, pelvic artery: $0.18 \%$, aorta: $0.14 \%$, renal: $0.04 \%$ Frost 2001). Splenic infarcts are rare and true incidence is unknown. AF is a common underlying etiology in patients presenting with renal infarction. Symptoms include flank or abdominal pain, nausea, vomiting, and fever. Lab abnormalities include elevated levels of LDH and WBC, hematuria and proteinuria. The common causes of splenic infarction include hematologic diseases (e.g. malignancy and sickle cell) and cardioembolic causes (Antopolsky 2009). Symptoms of splenic infarcts include leftupper-quadrant pain, fever, chills, nausea, vomiting, pleuritic chest pain, and left shoulder pain. Our patient presented with a normal cardiac exam, EKG, lab data and non-contrast abdominal CT but high clinical suspicion for a serious underlying abdominal condition prompted a contrast CT scan which confirmed the diagnosis and subsequent telemetry monitoring helped establish the diagnosis of PAF as a cause of his visceral infarctions. CONCLUSION: Acute renal and splenic infarctions are rare, but can manifest as the first and only presentation of PAF even in the absence of arrhythmia on initial cardiac exam or EKG. Hence for patients presenting with acute renal and splenic infarction, continuous telemetry monitoring is very important to diagnose PAF. Lastly, this case also demonstrates the value of using intravenous and oral contrast in patients suspected of having visceral embolic or ischemic events in the abdomen.

\section{ACUTE RENAL INFARCT A DELAYED OR MISSED DIAGNOSIS.}

Anthi Katsouli. Loyola University Medical Center, South Barrington, IL. (Control ID \#3168568)

LEARNING OBJECTIVE \#1: Recognizing acute renal infarct in patients with lower abdominal or flank pain and with risk factors for thromboembolism.

CASE: A 68-year-old man with past medical history of coronary artery disease with multiple stents placement, ischemic cardiomyopathy with 
ejection fraction $35 \%$ presented to the emergency department with sudden left-sided abdominal/flank pain and fever. Physical examination revealed normal vital signs, left lower quadrant and CVA tenderness, and no additional abnormalities. Laboratory investigations revealed mild leukocytosis (white blood count [WBC] $11.1 \times 10^{9} / \mathrm{L}$ ) and a normal serum creatinine. His liver function tests were mildly elevated (AST 80, ALT 58) with normal alkaline phosphatase, total bilirubin and lipase. Urine analysis performed and was negative for infection but showed trace blood with 0-2 RBC's. Computer tomography of the abdomen and pelvis showed a wedge-shaped area of decreased enhancement in the inferior left kidney concerning for infarct. Tele monitor revealed atrial fibrillation and electrocardiogram confirmed it. Patient was started on anticoagulation with heparin drip and underwent synchronized electrical cardioversion after transesophageal echocardiogram showed no left atrial appendage thrombus. Patient was discharged with oral apixaban and amiodarone.

IMPACT/DISCUSSION: Acute renal infarction involves occlusion of the arterial supply to the kidney and most commonly occurs as the result of thromboembolism. Incidence in patients presenting to hospital is estimated between 0.004 and $0.007 \%$. The average age at presentation is in the 6th to 7 th decades, with a similar incidence among the sexes. Bilateral infarcts occur in $2 \%$ to $20 \%$ of cases. Renal infarction nearly always occurs in patients with risk factors for thromboembolism, and patients may experience additional embolic sequelae. The most common risk factor is atrial fibrillation, present in $25 \%$ to $65 \%$ of cases. Other risk factors include valvular and ischemic heart disease, cardiomyopathy, known atherosclerotic disease, and prior thromboembolism. Cases have also been described in patients with heritable and acquired hypercoagulability, connective tissue disorders, endocarditis, and trauma.

CONCLUSION: Acute renal infarction is a rare condition. Recent advances in diagnostic imaging have increased the detection of renal infarction. The non-specific presentation of acute renal infarction has led to delayed diagnosis, but with the broad availability of high-quality computed tomography imaging, it is possible to identify cases early and initiate prompt treatment. Given the small numbers of cases described to date, quality evidence regarding treatment is lacking.

\section{ACUTE RENAL INFARCTION SECONDARY TO RENAL AR- TERY DISSECTION PRESENTING AS ACUTE ABDOMINAL PAIN}

Shiva Shankar Vangimalla; Zehra Dhanani; Hannie Batal; Jason Wexler. Medstar Washington Hospital Center, Washington, DC. (Control ID \#3186060)

LEARNING OBJECTIVE \#1: Recognize the clinical presentation of acute renal infarction (ARI) given its rarity and non-specific clinical presentation.

LEARNING OBJECTIVE \#2: Distinguish between various etiologies of ARI to initiate prompt treatment.

CASE: A 62 year female with past medical history of atrial fibrillation/ flutter status post ablation, complete heart block status post permanent pacemaker insertion, Stanford type A aortic dissection status post endovascular repair, hypertension presented with severe right flank pain, nausea and emesis since 1 day. Vital at admission were - blood pressure (BP) 230/106, heart rate 73 beats per minute, temperature $36.3 \mathrm{C}$. Initial lab work was notable for elevated white blood cell count (WBC) 11.5 $\mathrm{k} / \mathrm{uL}$, serum creatinine $0.64 \mathrm{mg} / \mathrm{dl}$, lipase 93 units/L and lactic acid 1.3 $\mathrm{mmol} / \mathrm{L}$. Urine analysis revealed specific gravity 1.013 , red blood cells 3 , WBC $<1$, negative leukocyte esterase, and no bacteuria. Computed tomography angiography (CTA) of abdomen/pelvis with IV contrast was concerning for occluded right renal artery with a large infarct and contrast enhancement of only a small portion of upper pole of right kidney. She was started on nicardipine and heparin drip for blood pressure control and anticoagulation immediately. Pain was controlled with fentanyl PCA. Vascular surgery did not recommend any acute surgical intervention as the kidney was unlikely to be salvageable due to the time of onset symptoms. Nephrology recommended continuing observation. Review of the CTA images showed occlusion of the right renal artery $2 \mathrm{~cm}$ from its origin and its supply from the false lumen suggestive of renal artery dissection. As her pain and renal function continued to improve over the next few days. She was transitioned to oral anti-hypertensive medications to keep her $\mathrm{BP}<130 / 70$. Hospital course was complicated by acute urinary retention and acute urinary tract infection secondary to her PCA use that resolved with insertion of foley catheter and antibiotic coverage. Her serum creatinine peaked at $1.80 \mathrm{mg} / \mathrm{dl}$ and improved to $0.63 \mathrm{md} / \mathrm{dl}$ at the time of discharge. She was resumed on oral eliquis for her other anticoagulation needs. At the time of discharge, her presenting symptoms resolved and had a good renal function.

IMPACT/DISCUSSION: ARI can be missed easily as there is a lack of awareness among the physicians including the radiologists given its rarity. Prompt identification of etiology may help guide treatment. Conservative management may be reasonable in patients with two functioning kidney and unilateral disease. Optimal treatment is uncertain given limited case reports and the absence of comparative studies.

CONCLUSION: High index of clinical suspicion is needed to diagnose ARI. An initial evaluation must include $\mathrm{CBC}$, serum creatinine and lactate dehydrogenase, urine analysis with culture and EKG. CT scan with contrast can help asses renal infarction. Reported treatment approaches include anticoagulation, endovascular therapy, open surgery, and observation depending on the etiology. Prognosis remains unknown.

\section{ACUTE RETROVIRAL SYNDROME PRESENTING WITH ACUTE LIVER INJURY}

Rinad Tabbalat; kavya Kelagere Mayigegowda; Shazia Samanani. Baystate Medical Center, Springfield, MA. (Control ID \#3186878)

LEARNING OBJECTIVE \#1: Recognize that acute antiretroviral syndrome can present with acute liver injury

CASE: A 23-year-old previously healthy male presented with 5 days of nausea, vomiting and diarrhea preceded by one day of flu-like symptoms. He denied any recent travel, exposure to sick contacts or consumption of possibly contaminated food or water. He reports being sexually active with one male partner and recent negative HIV test. On presentation he was febrile to 102.9F. Labs showed white blood cells of $3.1 \mathrm{k} / \mathrm{mm} 3$ with $38.4 \%$ neutrophils, platelet count $127 \mathrm{k} / \mathrm{mm} 3$, AST 1,075 units/L, ALT 1,012 units/L, alkaline phosphatase 71 units/L, and total bilirubin $0.3 \mathrm{mg} /$ dL. Initially there was concern for a possible tick born illness or acute viral hepatitis. Workup included Northeastern tick panel, viral panel for hepatitis $\mathrm{A}, \mathrm{B}$ and $\mathrm{C}$, infectious mononucleosis antibody, influenza $\mathrm{A}$ and $\mathrm{B}$, and RPR for syphilis which were all negative. Additional work up for acute liver injury included acetaminophen level, EBV and CMV serologies which were also negative. Given his high risk profile he was also tested for HIV 4th generation $\mathrm{Ab}-\mathrm{Ag}$ which came back positive. Absolute CD4 count was 246 and HIV viral load was greater than 10 million, confirming a diagnosis of acute retroviral syndrome. As the patient's viral load began to decrease, so did his transaminitis and shortly after he was started on antiretroviral therapy.

IMPACT/DISCUSSION: Signs and symptoms of acute HIV infection are nonspecific, making diagnosis difficult at times. Liver function test abnormalities are not uncommon in HIV patients but these are usually secondary to hepatotoxicity in those taking antiretroviral therapy (ART) or due to co-infection with viral hepatitis. Most clinical studies with severe elevation in liver enzymes ( $>5 \mathrm{X}$ the upper limit of normal) where seen in those taking ART. A review of the literature from the last 13 years found 31 cases of autoimmune hepatitis presenting in patients with different stages of HIV. Most of these were also in the setting of viral hepatitis and only one of these patients improved without use of steroids or immunotherapy making autoimmune hepatitis much less likely in our patient. 
CONCLUSION: Although this is less common, abnormalities in liver function tests and acute liver failure may be the first presentation for acute HIV infection. Given that HIV is not routinely tested in patients presenting with acute liver injury, it is important to have a high index of suspicion to prevent under detection and delays in diagnosis.

\section{ACUTE TOXIN MEDIATED RHABDOMYOLYSIS IN SETTING OF RECENT INITIATION OF SULFAMETHOXAZOLE- TRIMETHOPRIM THERAPY FOR URINARY TRACT INFECTION}

Dorjan Pantic; Michael Sperandeo; Linda Kurian. Hofstra Northwell School of Medicine, Manhasset, NY. (Control ID \#3141559)

LEARNING OBJECTIVE \#1: Identifying bactrim as a possible cause of rhabdomyolysis

CASE: A 41-year-old African American male with a past medical history of overactive bladder and benign prostatic hyperplasia presented to the ED with increasing proximal bilateral lower extremity muscle pain for 5 days and increasing difficulty with ambulation. The patient also complained of persistent hematuria, urgency and hesitancy for the 9 days. Prior to presentation, the patient saw his outpatient urologist and was diagnosed with a UTI for which he was prescribed TMP-SMX DS. On exam, patient was found to have lower extremity proximal muscle strength $4 / 5$ without point tenderness. Abdominal exam showed mild left lower quadrant and suprapubic tenderness to palpation. Physical exam was otherwise unremarkable. Initial laboratory analysis in the ED showed an AST 274 U/L, ALT $102 \mathrm{U} / \mathrm{L}$, and a CK 60665 U/L. Prior labs from his urologist showed normal AST/ALT and urine consistent with a UTI without laboratory evidence of rhabdomyolysis prior to initiation of TMP-SMX. The patient was admitted for non-traumatic acute rhabdomyolysis and UTI and had TMP-SMX discontinued. During the course of admission, the CK peaked on day 4 at $240000 \mathrm{U} / \mathrm{L}, \mathrm{AST}$ at 1200U/L and AST $1600 \mathrm{U} / \mathrm{L}$ prior to normalizing by day 8 . The symptomatic improvement preceded laboratory improvement by $\sim 3$ days. During admission the patient was evaluated for influenza, HIV and cocaine toxicity as possible other etiologies for rhabdomyolysis during his admission. These studies were negative. Epstein Barr Viral studies were consistent with a prior infection with immunity, without evidence of an acute viral process. Thyroid function tests and Glucose-6-Phosphate dehydrogenase studies were also normal.

IMPACT/DISCUSSION: Our patient is to our knowledge the fourth case of TMP-SMX induced rhabdomyolysis in a patient without HIV infection. Each of the three prior patients, as well as our most recent case were prescribed TMP-SMX for a presumed UTI. Our cases adds to the growing body of literature suggesting TMP-SMX as a cause of possible drug induced rhabdomyolysis and adds to the importance of a thorough medication review. CONCLUSION: The etiology of the rhabdomyolysis is varied and may be the result of toxin-mediated mechanisms, metabolic derangements or may develop secondary to other conditions like seizures, trauma and prolonged immobilization. In this case, we present a patient with suspected acute toxin-mediated rhabdomyolysis in the setting of new Trimethoprim-Sulfamethoxazole (TMP-SMX) therapy for urinary tract infection (UTI). Based on the time course of TMP-SMX initiation and the clinical improvement with discontinuation along the elimination of other possible hypoxic, biologic or physical/traumatic etiologies of acute rhabdomyolysis in our patient, we concluded that TMP-SMX was likely the insulting agent. To our knowledge, this marks the fourth case report of an otherwise healthy patient diagnosed with rhabdomyolysis secondary to TMP-SMX, further studies may be needed.

\section{ADRENALITIS AS A COMPLICATION OF HUMAN IMMUNO- DEFICIENCY VIRUS INFECTION.}

Omair Alam ${ }^{1}$; Robert Patton ${ }^{1}$; Manuel De La Rosa ${ }^{1}$; Rahul U. Nayak ${ }^{2}$. ${ }^{1}$ Inova Fairfax Hospital, Fairfax, VA; ${ }^{2}$ Virginia Commonwealth University, Richmond, VA. (Control ID \#3176777)
LEARNING OBJECTIVE \#1: Recognize adrenalitis as a complication of Human Immunodeficiency virus (HIV) infection.

CASE: A 25 year old male with high risk sexual behavior presented with shortness of breath, productive cough and fevers. He was found to be in hypoxic respiratory failure with multifocal opacities on chest imaging and empirically treated for community acquired pneumonia. Upon further testing, he was found to be HIV positive with elevated viral load and significantly decreased lymphocyte T-cells. Thus, he was started on empiric treatment with bactrim and prednisone for pneumocystis jiroveci pneumonia (PJP). Five days after discharge he presented to our hospital with new onset of severe epigastric abdominal pain, nausea and vomiting. He was found to have hyponatremia and hyperkalemia on laboratory studies. Chest and abdominal imaging demonstrated diffuse ground glass opacities in both lungs consistent with PJP infection and inflammatory changes in both adrenal glands. His signs and symptoms were concerning for adrenal insufficiency secondary to adrenalitis from HIV. His abdominal symptoms improved with fluid resuscitation, prednisone (glucocorticoid) and fludrocortisone (mineralocorticoid) therapy. However, his respiratory failure progressed to acute respiratory distress syndrome and septic shock secondary to severe PJP infection requiring ventilatory support, vasopressors and extracorporeal membrane oxygenation. Despite aggressive treatment he eventually succumbed to his illness and passed away 46 days later.

IMPACT/DISCUSSION: Clinically significant adrenal insufficiency is evident when 80 to $90 \%$ of the gland is destroyed; therefore, this is not only rare but also a potentially devastating consequence of HIV infection. In general, the approach to a suspected endocrinopathy in a patient with HIV should be similar to that of an immunocompetent individual. However, due to the body's adaptive changes to HIV infection the interpretation of the diagnostic studies can be difficult. The following should be kept in mind during the work up: - Cortisol levels may be present without elevated adrenocorticotropic hormone (ACTH) in the setting of infection related cytokine release. This may manifest as a blunted response to the ACTH stimulation test. - In a patient with elevated cortisol with Addisonian type clinical picture, consider peripheral glucocorticoid resistance due to HIV. - In a patient with HIV presenting malaise and non-specific abdominal symptoms, a broad differential should be kept in mind including adrenal insufficiency secondary to adrenalitis.

CONCLUSION: HIV endocrinopathies are well documented; however, with the ubiquity of antiretroviral therapy it is increasingly rare to see these clinical manifestations in practice. This case serves as a reminder to keep a broad differential diagnosis during an evaluation of a patient with HIV who presents with nonspecific abdominal symptoms found to have adrenal insufficiency due to adrenalitis.

\section{ADVOCATING FOR CONTINUATION OF HEPATITIS C (HCV) TREATMENT IN THE MASSACHUSETTS (MA) CORREC- TIONAL SYSTEM}

Sarah S. Roderick ${ }^{1}$; Juhee McDougal ${ }^{2,}{ }^{1}$; Alexandra Heinz ${ }^{1} .{ }^{1}$ Boston Medical Center, Boston, MA; ${ }^{2}$ Boston University Medical School, Boston, MA. (Control ID \#3176871)

LEARNING OBJECTIVE \#1: Demonstrate the need for advocacy for continuation of $\mathrm{HCV}$ treatment in incarcerated patients

LEARNING OBJECTIVE \#2: Recognize the heterogeneity of access to treatment and continuation of $\mathrm{HCV}$ treatment in the correctional system

CASE: Mr. S is a 34 year-old male with a history of chronic HCV and opioid use disorder (OUD) on buprenorphine-naloxone who presented to primary care for HCV treatment. Initial work up demonstrated mild portal fibrosis on fibroscan. After medication conseling, he was started on a twelve-week course of sofosbuvir-velpatasvir. Over the next three weeks, $\mathrm{Mr}$. S continued to be adherent to his regimen, and his OUD remained in remission. However, at his four- week follow up, our team was notified that he had been incarcerated. While incarcerated, Mr. S requested assistance 
from our team in order to continue his HCV treatment. Our social worker spent a considerable amount of time speaking with multiple health care providers within the correctional system. At first, his providers refused to continue treatment due to the high cost and because he tested positive for non-prescribed benzodiazepines and alcohol. Our team communicated that his medication had already been paid for, could be delivered to the prison free of charge, and it was imperative to continue his regimen in order to avoid developing treatment resistance. Ultimately, our request was denied, as the prison had a firm policy on not continuing treatment in patients with active substance use. Mr. S was released from prison two months later. Surprisingly, he was found to have achieved sustained virologic response after completing only four weeks of treatment.

IMPACT/DISCUSSION: Both in MA and throughout the United States (US), there appears to be a conservative approach towards initiation/ continuation of HCV treatment in the correctional system. This highlights a need for both advocacy and further education on the importance of continuing $\mathrm{HCV}$ treatment despite active substance use while incarcerated.

CONCLUSION: MA is one of the few states that has removed many of the barriers for initiation of HCV treatment; however, variations still remain based on the insurance payor and barriers still exist within the MA correctional system. In the US, few prisons initiate HCV treatment; some that do require patients to have at least advanced liver disease in order to start treatment. Outcomes are mixed when it comes to continuation of HCV treatment in prisons. Some providers continue treatment, but not without difficulty in terms of care coordination and the need to heavily advocate on behalf of the patient. Others may adopt an overall non-continuation approach to patients who continue to use substances, or they may altogether stop treatment due to high cost as prisons are not eligible for federal reimbursement. This case illustrates the need for further advocacy, education, and a coordinated approach towards continuation of HCV treatment for incarcerated individuals.

\section{AEROCOCCUS URINAE: A RARE CAUSE OF MITRAL VALVE ENDOCARDITIS}

Mallory Myers $^{1}$; Thanhnga Doan ${ }^{1}$; Daniel Gergen ${ }^{1}$; Rehaan Shaffie ${ }^{2}$. ${ }^{1}$ University of Colorado, Denver, CO; ${ }^{2}$ Denver Health Medical Center, Denver, CO. (Control ID \#3175216)

LEARNING OBJECTIVE \#1: Recognize Aerococcus urinae as a rare cause of infective endocarditis.

CASE: A 69-year-old man with no past medical history presented to the emergency department with two weeks of falls, difficulty urinating, and gross hematuria. On exam, he was afebrile and hemodynamically stable with a 3/6 systolic murmur, intention tremor, and shuffling gait. Labs revealed a white blood cell count of 19.4 , a beta-natriuretic peptide of 2,254 , and $3+$ blood in his urine. Urine and blood cultures grew 10,000100,000 colonies per $\mathrm{mL}$ of Aerococcus urinae. Head CT and MRI showed evidence of subarachnoid hemorrhages. Treatment began with two days of intravenous vancomycin but was switched to penicillin based on susceptibility studies in the literature. Blood cultures cleared on day three of treatment. An echocardiogram revealed a large mobile mass on the atrial side of the posterior leaflet of the mitral valve with mild to moderate regurgitation. During a right heart catheterization, the patient went into atrial fibrillation and several hours later had a stroke. MRI showed new multifocal infarcts that were likely embolic. Given the risk of additional embolic events, he was transferred to a cardiothoracic surgery center where he underwent a successful mitral valve replacement.

IMPACT/DISCUSSION: Aerococcus urinae is a gram-positive, catalasenegative coccus that produces alpha-hemolysis on blood agar. Though thought to have low pathogenicity, it is implicated in cases of urinary tract infections (UTIs), infective endocarditis (IE), and sepsis. These manifestations are rare, with an estimated UTI incidence of 54 per 1 million per year and a bacteremia incidence of 3 per 1 million per year. Risk factors for A. urinae infection include old age, male gender, and predisposing conditions such as diabetes mellitus, ischemic heart disease, urologic obstruction, and catheterization. This case illustrates A. urinae as a cause of IE with dangerous consequences. A. urinae possesses two important virulence factors for developing IE: biofilm formation and platelet aggregation. The aortic valve is most commonly affected, though the mitral and tricuspid valves can also be affected. Invasive A. urinae infections are associated with high morbidity and mortality. In a review of 29 A. urinae cases, 14 patients died within one week of hospital admission, 12 had arterial embolization events, 6 had septicemia, and 10 underwent mitral or aortic valve replacement. Therefore, prompt evaluation and treatment of patients found to have A. urinae bacteremia is crucial. The recommended treatment for A. urinae infection is penicillin or ceftriaxone. Adding an aminoglycoside has proven synergistic in numerous studies but should be weighed against the increased risk of adverse events.

CONCLUSION: A. urinae is a rare cause of infective endocarditis, especially in elderly men with predisposing health factors. In patients with A. urinae bacteremia, we recommend prompt evaluation for signs of infective endocarditis and arterial embolization.

\section{ALTERED MENTAL STATUS: SOMETIMES IT'S MORE THAN JUST THE DRUGS}

syed A. abbas; rizwan zafar; Karishma Kitchloo. Montefiore Medical center, Bronx, NY. (Control ID \#3186041)

LEARNING OBJECTIVE \#1: Recognize catatonia as a differential for patient presenting with altered mental status (AMS); early recognition and treatment may significantly reduce inpatient length of stay.

LEARNING OBJECTIVE \#2: Neuroleptics used to manage hyperactive delirium may precipitate catatonia.

CASE: A 71-year-old man from nursing home (NH) with history of Alzheimer's dementia presented with AMS. He was recently admitted for aggressive behavior and was started on valproic acid and quetiapine. Upon his return to $\mathrm{NH}$, he was found to be progressively more lethargic, bed bound, and mute. At baseline, he independently communicated and ambulated. On examination, his vital signs were stable, afebrile, lying flat in bed with arms crossed over the chest, unresponsive to verbal stimuli, minimally responsive to painful stimuli, with increased tone in all limbs. All baseline laboratory tests were negative including ammonia and valproic acid levels. Neuroimaging including CT head without contrast and MRI brain without contrast were done, both of which were negative for acute intracranial disease. His condition did not improve upon cessation of all neuroleptic and sedating agents. Electroencephalography was normal. Patient was given a lorazepam challenge, and thirty minutes later he was awake, sitting in bed, talking, and drinking water. He was started on oral lorazepam and discharged to $\mathrm{NH}$ with significant improvement.

IMPACT/DISCUSSION: In an acute medical setting, catatonia is seldom considered as a differential diagnosis for AMS, although prevalence ranges from $1.6-6.3 \%$. Due to its remarkably similar presentation to delirium, it is often misdiagnosed and inappropriately treated. Neuroleptics used to treat delirium may precipitate catatonia, prolonging the episode of AMS, and thus lengthening hospital stay. Catatonia is a clinical syndrome characterized by psychomotor symptoms including stupor, mutism, waxing flexibility, and posturing. Historically associated with psychiatric illness, catatonia is now recognized as a systemic medical syndrome, associated with medical, neurological, and psychiatric disorders. The exact pathophysiology remains unclear; studies suggest abnormal activity at the gamma-aminobutyric acid, glutamate and dopamine receptors. Medical catatonia warrants an expedient medical workup, and if unrecognized it may progress to potentially fatal syndrome of malignant catatonia. Lorazepam is the drug of choice and, in resistant cases electroconvulsive therapy is the treatment of choice. Neuroleptics should be used with caution. Remarkable phenotypic overlap between delirium and catatonia warrants further research to establish clear diagnostic and 
treatment guidelines to help clinicians treating medically and psychiatrically complex patients with catatonia.

CONCLUSION: Catatonia and delirium can often similarly present; the importance of a broad differential for altered mental status refractory to conventional treatment remains vital in appropriately managing patient care.

\section{ALTERED MENTAL STATUS: THE PARTY IS OVER}

Thomas John; Marjorie E. Bateman. Tulane University School of Medicine, Mandeville, LA. (Control ID \#3184074)

LEARNING OBJECTIVE \#1: Recognize clinical presentation of sedative-hypnotic withdrawal

LEARNING OBJECTIVE \#2: Identify barriers in uncovering substance abuse

CASE: A 32-year-old man presented after a 30 second tonic-clonic seizure. He was somnolent with normal vitals and no obvious neurologic deficits. The patient's family and friends provided his initial history. $\mathrm{He}$ had no recent infectious symptoms and was behaving normally until the seizure. Past medical history included narcolepsy and HIV for which he was on antiretroviral therapy. A recent CD4 count was 513. He had been drinking more alcohol than usual over the past several days but did not use illicit drugs. CSF showed elevated protein and WBC count with negative gram stain and culture. Urine drug screen was negative. CT and MRI brain were unremarkable. As the patient's mental status improved, he developed tachycardia and hypertension. On private interview, he reported taking gamma-hydroxybutyrate (GHB) daily for his narcolepsy and had been trying to wean himself off of it. He was treated with benzodiazepines for GHB withdrawal. After several days, he decided to leave against medical advice. He was counseled to follow up with a neurologist and discharged with diazepam and levetiracetam.

IMPACT/DISCUSSION: This patient's risk factors for substance abuse included HIV positive status, male gender, and young age. ${ }^{1}$ As GHB is the active ingredient in sodium oxybate, those who develop dependence also often have sleeping conditions such as narcolepsy. This patient's presentation of seizures, tachycardia, and hypertension is classic for sedative-hypnotic withdrawal. ${ }^{1}$ There are few cases of GHB withdrawal reported in the United States, where GHB is a Schedule I drug. Standard toxicology screens do not detect GHB, making abuse difficult to diagnose. ${ }^{1}$ This patient's GHB dependence was diagnosed after the patient revealed he has abused the drug to treat his narcolepsy. Fewer than half of patients with HIV have discussed their substance use with their providers. $^{2}$ A recent study revealed that current illicit drug use reduced patient comfort in discussing substance use. Patients with high selfefficacy had higher comfort in discussing substance use. ${ }^{2}$ Providers should ask patients about substance use and help them to develop the knowledge and skills for self-efficacy. Such skills may have enabled our patient to discuss his GHB dependence with a provider prior to presentation, potentially preventing his seizure.

CONCLUSION: Young age, male gender, and diagnosis of HIV have been associated with increased risk of substance abuse. GHB abuse results in high morbidity and mortality and cannot be diagnosed by urine drug screen. It is important to empower patients to make them comfortable with discussions about illicit substance use References: 1. Busardo FP, Jones AW. GHB Pharmacology and Toxicology. Curr Neuropharmacol. 2015; 13(1): 47-70. 2. Ray MK et al. Patient and Provider Comfort Discussing Substance Use. Fam Med. 2013; 45(2): 109-117.

\section{AMIODARONE: AN UNUSUAL CAUSE OF HEMOLYTIC ANEMIA}

Joel Thomas; Maniraj Neupane; Anita B. Leon-Jhong. UPMC, Pittsburgh, PA. (Control ID \#3163408)

LEARNING OBJECTIVE \#1: Recognize that amiodarone is a rare cause of drug-induced hemolytic anemia.
LEARNING OBJECTIVE \#2: Describe findings of hemolytic anemia on peripheral smear.

CASE: Ms. $Z$ is a 75 year old woman with mechanical mitral valve replacement on warfarin, atrial fibrillation on amiodarone, and achalasia who was admitted to the medicine service for esophageal dilation and peri-procedural heparin bridging. After the procedure her hemoglobin dropped from 11.8 to $6.9 \mathrm{~d} / \mathrm{L}$ over 11 days. Blood loss was initially attributed to a PICC-related upper extremity hematoma, but hemoglobin continued to fall even after the hematoma had stabilized. CT abdomen/ pelvis was negative for retroperitoneal bleed and stool hemoccult was negative. Hemolytic work-up was pursued and demonstrated elevated $\mathrm{LDH}$, undetectable haptoglobin, and peripheral blood smear withspherocytosis and reticulocytes. Her Coombs test was negative. Her medication list was reviewed and amiodarone was identified as the only agent with reported cases of hemolysis. Amiodarone was discontinued and she received 2 units of packed red blood cells for $\mathrm{Hb}$. $6.9 \mathrm{~d} / \mathrm{L}$. She was discharged to rehabilitation in stable condition and her hemoglobin stabilized around $10 \mathrm{~d} / \mathrm{L}$ over the next month.

IMPACT/DISCUSSION: Laboratory results suggestive of hemolysis include increased serum LDH and decreased haptoglobin levels. A peripheral blood smear should be obtained to confirm the diagnosis and identify possible etiologies. Reticulocytes are present in response to either hemolysis or acute blood loss. Heinz bodies and bite cells are suggestive of G6PD deficiency, and spherocytes are seen in hereditary spherocytosis or autoimmune hemolytic anemia. Other causes of hemolysis include sickle cell anemia, acute lead poisoning (basophilic stippling), diffuse intravascular coagulopathy (schistocytes). Drugs which are commonly associated with autoimmune hemolytic anemia include cephalosporins, penicillins, NSAIDs, levodopa, nitrofurantoin, dapsone, and quinine/ quinidine. In this patient, the negative Coombs test in conjunction with the spherocytes and LDH + haptoglobin suggestive of hemolysis was a peculiar finding, as the vast majority of drug-induced hemolysis is Coombs-positive. Postulated mechanisms for Coombs-negative druginduced hemolysis include antibody-free hemolysis by natural killer cells, low-affinity antibodies, and IgA-mediated hemolysis. Fortunately, the treatment of drug-induced anemia is straightforward: discontinuation of the drug and packed red blood cell transfusion as necessary.

CONCLUSION: Impact: This case broadens the differential for an unexplained drop in hemoglobin. The patient had red herrings of hematoma and a recent invasive procedure on anticoagulation. However, the undetectable haptoglobin and spherocytes suggested drug-induced hemolysis, and amiodarone was identified as the likely culprit.

\section{AN ATYPICAL CASE OF MILLER-FISHER SYNDROME PRE- SENTING WITH SEVERE DYSPHAGIA AND WEIGHT LOSS}

Kishan Patel ${ }^{1}$; Arjun Dhawan ${ }^{1}$; Eliezer Z. Nussbaum ${ }^{1}$; Jason Sico ${ }^{2,}$; Naseema B. Merchant ${ }^{1,3}$. ${ }^{1}$ Yale School of Medicine, Middlefield, CT; ${ }^{2}$ Yale School of Medicine, New Haven, CT; ${ }^{3}$ VA Connecticut Health Care, West Haven, CT. (Control ID \#3183461)

LEARNING OBJECTIVE \#1: Diagnose atypical presentation of Miller-Fisher Syndrome (MFS)

LEARNING OBJECTIVE \#2: Recognize dysphagia as an initial presentation of MFS

CASE: A 71-year-old male with smoking history was sent to the emergency department (ED) from the speech clinic after a failed outpatient swallow evaluation performed due to a 3-week history of dysphagia, soft voice and a $\sim 30 \mathrm{lb}$ weight loss. Initial physical exam was notable for hypophonia but follow up exam over the next 24-48 hours showed, dysarthria, diplopia, bilateral horizontal ophthalmoparesis, unilateral ptosis, bilateral weakness with hip flexion, ataxia and hyporeflexia, with normal upper extremity strength. CT head was normal and MRI brain showed periventricular and subcortical white matter T2 hyperintensity. Nerve conduction study of the facial nerve and lower extremities showed 
axonal sensorimotor polyneuropathy. Endoscopic speech evaluation also confirmed the severe dysphagia. CSF was notable for albuminocytologic dissociation (protein $49.9 \mathrm{mg} / \mathrm{dL}, 2$ nucleated cells), and serum anti-GQ1b antibodies were elevated (titer 1:200). The triad of ophthalmoplegia, areflexia, ataxia and CSF findings led to the diagnosis of the MillerFisher syndrome. The patient was treated with intravenous immunoglobulin $(0.4 \mathrm{~g} / \mathrm{kg})$ for 5 days, 3 weeks of enteral tube feeds and at his clinic visit 6 weeks post discharge, he was eating regular diet and all his symptoms had resolved.

IMPACT/DISCUSSION: Miller-Fisher Syndrome (MFS) is rare, estimated to occur in $1-7 \%$ of patients with GBS in the western hemisphere. Dysphagia to both solids and liquids is an uncommon presentation for MFS, and is more typically associated with botulism, myasthenia gravis, multiple sclerosis, and brainstem stroke. Estimates from prior studies suggest that only $2 \%$ of patients with MFS present with dysphagia as their primary complaint. Although other reports have identified dysphagia as a symptom of MFS, to our knowledge, this is the first report in the adult literature that demonstrates severe dysphagia with significant weight loss as the presenting complaint. Although the etiology of dysphagia in MFS reported in the literature is not clear, studies have shown positive immunostaining of human glossopharyngeal and vagal nerves with anti-GQ1b $\mathrm{IgG}$, suggesting that autoimmune neuropathy of these cranial nerves may play a role. Given the results of the FEES and nerve conduction studies, our patient likely had axonal damage to the vagus nerve, resulting in dysphagia. This case illustrates MFS can initially present with a predominant dysphagia. Secondly, other typical neurologic findings of MFS can be subtle and evolve over time.

CONCLUSION: Dysphagia can be the initial manifestation of MFS in some patients without any obvious deficits in the neurologic exam or neuro-imaging. Such patients should receive a thorough initial and follow up neurological exams, with particular attention to extraocular movements, reflexes, and gait, to rule out the possibility of MFS.

\section{AN ATYPICAL PRESENTATION OF ATYPICAL PNEUMONIA: LEGIONELLA CAUSING GUILLAIN-BARRE SYNDROME}

Abigail Entz; Sarah Gorgis; Junior Uduman. Henry Ford Hospital, Detroit, MI. (Control ID \#3186239)

LEARNING OBJECTIVE \#1: Recognize an atypical presentation of Legionella pneumonia

LEARNING OBJECTIVE \#2: Identify the association between Guillain-Barre variants and infectious syndromes

CASE: A 29 year-old-female with history of Systemic Lupus Erythematosus on plaquenil presented with two weeks of nausea, emesis, and cough. During that time she developed progressive numbness in bilateral lower extremities and began to experience falls at home. On presentation she was afebrile but hypoxic requiring intubation. She had no leukocytosis but did present with a moderate hyponatremia to 127 . Chest x-ray showed patchy infiltrates and urine legionella antigen was positive. She was started on treatment with moxifloxacin. Her weakness progressed to involve all extremities and respiratory muscles with decreased reflexes. A lumbar puncture was unremarkable with WBC 0, RBC 3 and protein 45 $\mathrm{mg} / \mathrm{dL}$. Electromyography (EMG) on day nine of hospitalization showed severe sensorimotor axonal polyneuropathy. She was treated with five days of intravenous immunoglobulin (IVIg) and seven sessions of plasmapheresis with limited improvement in weakness. The patient eventually required tracheostomy due to persistent weakness. We present an atypical case of legionella pneumonia causing Guillain-Barre Syndrome.

IMPACT/DISCUSSION: Legionella is an important cause of community acquired pneumonia, often with severe symptoms and a mortality rate up to $10 \%$. Patients with legionella pneumonia can present with extrapulmonary manifestations including emesis, diarrhea, chest pain, scant hemoptysis, and high fever. Mild neurologic symptoms including headache and encephalopathy are sometimes seen. There are few case reports of Guillain Barre variants associated with legionella pneumonia, though over two-thirds of patients with GBS have a proceeding respiratory or gastrointestinal illness. The weakness associated with GBS is typically progressive, symmetrical, and ascending- with maximal weakness occurring within two-four weeks from symptom onset. Though up to one-third of patients may require mechanical ventilation, nearly 80 percent make a near-complete recovery. Both IVIg and plasmapheresis have demonstrated in randomized controlled trials to shorten time to neurologic recovery in GBS, though the recovery phase can take several months and can require intensive rehabilitation.

CONCLUSION: Though weakness in critically ill patients is often attributed to critical illness myopathy, careful attention should be paid to timing, intensity, and progression of neurologic symptoms. Guillain-Barre syndrome has a known association with infections therefore clinical suspicion should remain high in patients with the combination of infection and rapidly progressive weakness.

\section{AN ATYPICAL PRESENTATION OF METASTATIC TESTICU- LAR CHORIOCARCINOMA}

Mrigank S. Gupta; Marium Khan. Medical College of Wisconsin, Wauwatosa, WI. (Control ID \#3186015)

LEARNING OBJECTIVE \#1: Recognizing a unique presentation of choriocarcinoma in young males.

CASE: A 29 year-old male with a past history of gastroesophageal reflux and epididymitis presented to the emergency department with a two day history of a band like abdominal pain. Pain was associated with retching emesis that was yellow gastric mucous in nature progressing to blood tinged. The patient denied any hematochezia, melena, or any recent weight loss but endorsed constipation. Home medications included ibuprofen and pantoprazole. Vital signs included temperature $98.5 \mathrm{~F}$, heart rate 105 beats per minute, blood pressure $131 / 72 \mathrm{~mm} \mathrm{Hg}$, respiratory rate 20 breaths per minute and oxygen saturation $95 \%$ on room air. Physical examination was significant for a healthy appearing young male with café au lait lesions on his back. Abdomen was tender to palpation in lower quadrants, firm, normal bowel sounds with no rebound tenderness. The remaining exam was nonfocal. Labs included white blood cell count 14.8e103 uL, Hgb $13.0 \mathrm{~g} / \mathrm{dL}$, platelet $273 \mathrm{e} 103 \mathrm{uL}$, lipase $20 \mathrm{units} / \mathrm{L}$, INR of 1.1 and basic chemistry within normal limits. Computed tomography chest and abdomen revealed a large central abdominal mass surrounding the inferior mesenteric artery with unclear origin, multiple lesions consistent with metastatic disease. The mass compressed the distal inferior vena cava, abdominal aorta, common iliac artery, and the right ureter causing right hydronephrosis. Recommendation from radiation oncology included obtaining a testicular ultrasound which revealed a punctate echogenic foci of the right testicle. Interestingly, the patient had a testicular ultrasound six months prior which showed a similar echogenic region on his right testicle which was lost to follow up. Tumor markers significant for Beta-hCG (human chorionic gonadotropin) 364,215 mIU/L and AFP (alpha fetoprotein) $15 \mathrm{ng} / \mathrm{ml}$. Liver biopsy illustrated syncytiotrophoblast and cytotrophoblasts, confirming stage 3 nonseminomatous germ cell tumor. A right ureteral stent was placed to relieve the obstruction and the patient was emergently started on VIP chemotherapy (etoposide, ifosfamide, and cisplatin).

IMPACT/DISCUSSION: Choriocarcinoma is a nonseminomatous germ cell tumor (NSGCT). It is seen in caucasian males in a bimodal distribution, ages 15 to 25 and great than 60 years of age. Choriocarcinoma produces large amounts of hCG and greater than $50,000 \mathrm{mIU} / \mathrm{L}$ is associated with a poor diagnosis. In adults, choriocarcinoma most frequently spread hematogenously to the lung and lymph nodes. In advanced cases, it can spread to liver, brain, and skin. Choriocarcinoma responds well to chemotherapy and should be started as soon as the diagnosis is made.

CONCLUSION: Early identification of testicular choriocarcinoma and initiation of chemotherapy is important in survival. Imaging modalities should be utilized if suspicion for malignancy is high or the patient's presentation is atypical. 
AN ATYPICAL PRESENTATION OF MRSA PNEUMONIA

Akanksha Vaidya. Emory University, Atlanta, GA. (Control ID \#3185817)

LEARNING OBJECTIVE \#1: Review the typical presentation of MRSA pneumonia

LEARNING OBJECTIVE \#2: Diagnose MRSA pneumonia in patients with atypical presentations

CASE: A 61- year- old woman with a history significant for asthma, possible sarcoidosis with chronic pulmonary nodules and hilar lymphadenopathy, and recent MRSA folliculitis, presented to the hospital with dyspnea. Patient reported fevers, chills, and rhinorrhea two weeks prior to presentation. The fevers and chills resolved, but she continued to have a cough productive of clear sputum and started having dyspnea on exertion that started four days prior to presentation. On hospital admission, she was afebrile, had a normal blood pressure, and had an oxygen saturation of $92 \%$ on room air. Her physical exam was notable for diffuse wheezing throughout bilateral lung fields. A chest $X$ ray showed scattered micronodular opacities that were unchanged from a prior $\mathrm{X}$ ray two years ago. She was started on treatment for an asthma exacerbation with steroids, azithromycin, and bronchodilators. Her symptoms failed to improve after 7 days of treatment, so a CT scan of the chest was ordered. The CT scan showed small cavitary changes in the right lower lobe, suggestive of a necrotizing bronchopneumonia. She underwent CT guided biopsy of the cavitation and was positive for MRSA that was resistant to clindamycin. She was treated for MRSA pneumonia with a 14 day course of doxycycline with improvement in her symptoms.

IMPACT/DISCUSSION: Community acquired MRSA pneumonia typically presents as a severe pneumonia and has a high mortality rate. Compared to hospital acquired MRSA, community acquired MRSA is more likely to produce the Panton-Valentine leukocidin toxin that forms cavitary lung lesions. Patients typically present with severe hypoxia, severe dyspnea, fever, and hemoptysis. Identifying patients with MRSA pneumonia is crucial in order to start timely antibiotic therapy. The patient in this case did not have severe symptoms that are usually seen in MRSA pneumonia cases. However, she did have some risk factors for MRSA pneumonia. Patients with MRSA pneumonia will typically have a preceding flu-like illness. Patients with a history of MRSA infection or colonization are also at risk for MRSA pneumonia. The patient presented in this case had both of these common risk factors. Recognizing these risk factors for MRSA pneumonia can help identify patients with atypical presentations of the disease and ensure that the appropriate antibiotic therapy is started for the patients.

CONCLUSION: This case illustrates an atypical presentation of community acquired MRSA pneumonia. While MRSA pneumonia usually has severe symptoms, the patient in this case had mild symptoms, which made it difficult to identify the disease. It is important to recognize risk factors for MRSA pneumonia to identify patients with atypical presentations of the disease.

\section{AN EMBOLIC ETIOLOGY OF NSTE-ACS}

Kristie Yang; Eli Wilber. Emory University, Atlanta, GA. (Control ID \#3184559)

LEARNING OBJECTIVE \#1: Recognize features of NSTE-ACS secondary to infective endocarditis.

CASE: A 58 year-old man presented with one day of exertional chest pain that began while moving a refrigerator. He described the mid-sternal pain as "twinges" radiating to the left arm and leg not alleviated by rest. Associated symptoms included shortness of breath, diaphoresis, and numbness in his left fingers. One week prior he had similar pain that resolved with rest. $\mathrm{He}$ had not seen a healthcare provider in 30 years, had no known medical problems, and reported no family history of heart disease. He was a 30 pack- year smoker and had poor dentition. Vitals were T 36.4, HR 79, BP 136/78, RR 18, SpO2 100\% on room air. He had a grade IV/VI blowing systolic apical murmur with radiation to the axilla; there were no S3 or S4 heart sounds, no jugular venous distension. His lungs were clear to auscultation; there was no lower extremity swelling. Admission labs showed troponin of 12.5 and BNP of 1080. EKG had no ST-segment or T wave abnormalities. Left heart catheterization revealed an occluded small left posterior descending coronary artery, thought to be embolic in nature, and otherwise normal coronary arteries. Transthoracic echocardiography showed mitral regurgitation and a mobile mass on the mitral valve, which was confirmed by transesophageal echocardiography. Two sets of blood cultures grew gram positive cocci in chains and the patient was started on vancomycin and ceftriaxone. The cultures speciated to Streptococcus salivarius and antibiotic regimen was narrowed to ceftriaxone. His remaining teeth were removed and he was discharged to complete two weeks of antibiotics prior to evaluation for mitral valve replacement.

IMPACT/DISCUSSION: Mechanisms of non-ST segment elevation acute coronary syndrome (NSTE-ACS) in patients without coronary artery disease include vasospasm, myocarditis, vasculitis, and embolic disease. This patient had mitral regurgitation and poor dentition, but lacked cutaneous manifestations of infective endocarditis (IE). He developed a fever at the same time that echocardiography suggested a valvular vegetation, which in conjunction with positive blood cultures for a typical organism made this a definite case of IE by the modified Duke criteria. Embolic events occur in $45 \%$ of patients with IE and most commonly affect the brain and spleen, not coronary arteries. New murmur or worsened preexisting murmur is reported in $48 \%$ and $20 \%$ of IE cases, respectively. In this case, an abnormal exam finding in a NSTE-ACS patient with unexpected angiographic results prompted additional workup leading to the correct diagnosis. While an unusual presentation for both NSTE-ACS and IE, this case reinforces a foundational principle of medicine that a stepwise approach to history and exam findings can lead to successful diagnosis.

CONCLUSION: Infective endocarditis commonly presents as either an embolic stroke or heart failure, however, rarely emboli to coronary arteries can lead to NSTE-ACS.

\section{AN IDIOSYNCRATIC CASE OF AMIODARONE-RELATED DRUG-INDUCED LIVER INJURY}

Michael Seidman; Christopher Bruti. Rush University Medical Center, Chicago, IL. (Control ID \#3186409)

LEARNING OBJECTIVE \#1: Evaluate an unusual presentation of amiodarone-related Drug Induced Liver Injury (DILI)

LEARNING OBJECTIVE \#2: Recognize clinical and historical factors contributing to the diagnosis of idiosyncratic DILI in medically complex patients

CASE: A 61-year-old man with known atrial fibrillation, diastolic heart failure, and stage III CKD presented with excessive bleeding following minor trauma to his toe. His medications included warfarin, diltiazem, metoprolol, and amiodarone, which was started 8 weeks prior. In addition to his minor wound, exam was notable for marked jaundice. He denied history of heavy alcohol use, hepatitis, IV drug use, recent travel, sick contacts, and was not sexually active. He felt otherwise well, noting improvement in his heart failure symptoms in recent months. In addition to supratherapeutic INR attributed to amiodarone-warfarin interaction, labs were notable for cholestatic liver enzyme derangement and elevated bilirubin. Warfarin and amiodarone were discontinued and he was admitted for work-up. A thorough infectious and immunologic workup was negative, and Imaging and MRCP were unrevealing. Given unclear etiology a liver biopsy was obtained, demonstrating cholestatic injury without steatosis, mallory bodies, or lamellar inclusions. Inflammatory changes were consistent with a variety of etiologies including cardiac, sepsis and DILI. Hepatic labs continued to worsen and in the 2 nd week of 
admission, the patient's renal function deteriorated with acute tubular necrosis, progressing to anuric ESRD requiring hemodialysis. The patient was evaluated for liver transplantation but declined given concurrent multiorgan failure. Over subsequent weeks the patient's liver function gradually improved and he was ultimately discharged.

IMPACT/DISCUSSION: While congestive hepatopathy was initially suspected, this patient's concurrent improvement in CV symptoms made cardiac etiology less likely as a primary mechanism. Amiodarone is a common antiarrhythmic, prescribed over 2.9 million times in the US in 2016. Amiodarone has a known propensity for hepatotoxicity, with $1 \%$ of patients developing clinically-evident liver disease annually. Amiodarone-related hepatotoxicity typically manifests as hepatocellularpattern injury, with biopsy demonstrating steatosis with Mallory bodies and lamellar inclusions. In this case, we characterize a potential idiosyncratic presentation of amiodarone-related DILI not yet represented in the literature. Diagnosis in this case was reached only through careful historytaking and negative work-up of alternatives.

CONCLUSION: Idiosyncratic drug toxicities may be subtle and difficult to differentiate in medically complex patients. Clinicians must be vigilant in their diagnostic work-up and maintain a high degree of suspicion for idiosyncratic DILI. Clinical understanding of adverse drug reactions is constantly evolving; only through proper diagnosis, characterization, and reporting of such injuries can we increase medical knowledge of unusual reactions to common medications.

\section{AN IMMUNOHEMOLYTIC PRESENTATION OF PERNICIOUS ANEMIA.}

Steven Omansky ${ }^{1}$; Patricia Dharapak ${ }^{2} .{ }^{1}$ Mount Sinai Beth Israel Hospital, New York, NY; ${ }^{2}$ Mount Sinai Beth Israel, New York, NY. (Control ID \#3162810)

LEARNING OBJECTIVE \#1: To understand the potentially overlapping etiologies for hemolysis in autoimmune pernicious anemia.

CASE: A 33 year-old US born woman with iron deficiency anemia presented after one episode of pre-syncope. She reported 3 weeks of progressive fatigue, shortness of breath, and pain in the chest, back, and hips. She denied abdominal pain, menstrual abnormalities, sick contacts, recent trauma, and blood disorders or malignancy in her family. She did not take any medications or herbal supplements and reported tolerating a normal, unrestricted diet. Her exam was remarkable for tachycardia and mucosal pallor at the conjunctiva and soft palate. Labwork revealed a hemoglobin of $4.5 \mathrm{~g} / \mathrm{dL}$, macrocytosis to $114 \mathrm{fL}$ with an RDW of $30.6 \%$, and platelet count of 108,000 per $\mu \mathrm{L}$. Her RBC count was $1.11 \mathrm{M} / \mu \mathrm{L}$ with a reticulocyte production index of $0.9 \%$. Packed red blood cells were transfused with an appropriate response. Additional work-up was significant for a markedly elevated LDH $(7035 \mathrm{U} / \mathrm{L})$ and reduced haptoglobin $(<8 \mathrm{mg} / \mathrm{dL})$. A peripheral smear showed schistocytes and anisocytosis, and a direct Coombs test was positive. Pulse dosed steroids were started for suspected autoimmune hemolytic anemia (AIHA). A serum folate level was normal, but a cobalamin level was undetectable. Subsequent methylmalonic acid and homocysteine levels were elevated, so daily intramuscular methylcobalamin was initiated. At the time of discharge, her hemoglobin had improved to $9.1 \mathrm{~g} / \mathrm{dL}$ and her presenting symptoms had resolved. Serum anti-intrinsic factor and anti-parietal cell antibody assays later returned positive and confirmed a diagnosis of autoimmune pernicious anemia (PA).

IMPACT/DISCUSSION: We present a case of combined AIHA and PA resulting in severe, symptomatic anemia. The prevalence of cobalamin deficiency is $<3 \%$ in adults $20-39$ years old with about $40 \%$ attributed to PA, but the incidence of PA with concurrent AIHA is unknown. A previous study of 865 patients with a positive direct Coombs test found PA in 3 lone subjects, and only 23 other case reports of dual-diagnosis have been published since 1951 . Some of these reports found transiently positive Coombs tests that resolved after cobalamin supplementation.
Conversely, PA can lead to hemolysis in the absence of AIHA less than $2 \%$ of the time due to rapid and ineffective erythropoiesis. This can be detected by the reticulocyte production index; if found low, as in our case, there is evidence of lysis within the bone marrow. Although rare, these examples highlight the importance of investigating multiple etiologies of hemolytic anemia, else risking incomplete treatment of the patient.

CONCLUSION: Cobalamin deficiency caused by autoimmunity often leads to PA and can expeditiously improve with steroids and cobalamin supplementation. PA is often associated with other autoimmune conditions, although concurrent presentation with AIHA is rare. Investigating all potential etiologies for hemolytic anemia minimizes the risk for incomplete treatment and relapse.

\section{AN INSIDIOUS ENCEPHALOPATHY: A CASE OF DISSEMINAT- ED COCCIDIOMYCOSIS}

Xin Zhang; Kyle McKenzie. Mayo Clinic, Rochester, MN. (Control ID \#3181400)

LEARNING OBJECTIVE \#1: Recognize signs and symptoms of coccidioidal meningitis

LEARNING OBJECTIVE \#2: Manage treatment of coccidioidal meningitis

CASE: A 74 year old non-immunosuppressed, male farmer from Minnesota was hospitalized with confusion. 6 months ago, he presented to the emergency department for 1 month of non-productive cough and intermittent fevers. Coccidioides antibody testing was obtained due to frequent travel to Arizona, but he did not receive news that it was positive. Over the next few months, he developed progressive dizziness, lethargy and confusion. MRI brain showed extensive basilar meningitis. Opening pressure was normal on lumbar puncture and CSF analysis was consistent with chronic meningitis. He was diagnosed with Coccidioidal meningitis after CSF Coccidioides PCR and anti-Coccidioides antibody returned positive. Evaluation for immunosuppressive conditions was negative. He was discharged on fluconazole. Two months later, he was hospitalized due to seizures. Repeat MRI demonstrated vasculitis associated with meningoencephalitis and an acute lacunar infarct. CSF specimen was obtained and showed no evidence of persistent meningitis. He was started on levetiracetam and dexamethasone for CNS vasculitis secondary to disseminated coccidioides. Despite the above treatment, he continued to have seizures, persistent encephalopathy, and progressive functional decline. He ultimately decided to pursue comfort cares and passed away 4 months after original diagnosis.

IMPACT/DISCUSSION: Coccidioidal meningitis is a rare presentation of disseminated coccidioidomycosis. It is associated with a high mortality rate of $95 \%$ within 2 years for untreated patients. The most common sign is persistent headache, but nonspecific symptoms and subacute presentation makes diagnosis difficult. Diagnosis can be made definitively by identifying Coccidioides in the CSF or presumptively by finding antiCoccidioides antibodies in the CSF. CNS findings include hydrocephalus, basilar meningitis and vasculitic cerebral infarction. The cerebral vasculitic infarctions occur in setting of inflammation of small and medium sized cerebral arteries and clinically affect $10 \%$ of patients. The IDSA recommends oral fluconazole $400-1200 \mathrm{mg}$ as initial treatment for coccidioidal meningitis. If treatment failure occurs, clinician can increase fluconazole dose, change azole, or use intrathecal amphotericin B. Of note, lifelong azole therapy is recommended to prevent recurrence. Corticosteroid has been shown to reduce recurrence of vasculitic cerebral infarctions in a retrospective study, but its use is controversial.

CONCLUSION: 1. Coccidioidal meningitis can present with hydrocephalus, basilar meningitis and vasculitic cerebral infarction. 2. Patients should remain on lifelong azole therapy. 3. In patients with cerebral infarctions, corticosteroid can considered to prevent recurrent vasculitic infarctions. 
AN INTERESTING PRESENTATION OF "HEART FAILURE"

Priyanka Ballal; Albin Oh. Boston Medical Center, Boston, MA. (Control ID \#3183312)

LEARNING OBJECTIVE \#1: Recognize a formidable diagnosis masquerading as acute decompensated heart failure.

CASE: A 61-year-old-female with a history of diastolic heart failure and atrial fibrillation presented to the emergency room with progressively worsening lower extremity edema, shortness of breath and orthopnea as well as a $15 \mathrm{~kg}$ weight gain over the last eight weeks. She noted the above symptoms despite faithfully taking her home diuretic as prescribed and adhering to a salt restricted diet. Her heart failure had been previously well controlled, with no hospital admissions for decompensation. Physical exam was consistent with volume overload as evidenced by jugular venous distention to $14 \mathrm{~cm}$, crackles over bilateral lung bases, marked abdominal distention, and pitting peripheral edema extending to midthigh bilaterally. Importantly, she was noted to have a large abdominal mass with ill-defined borders, palpable in all quadrants. A CT abdomen and pelvis was then obtained which revealed a large complex cystic structure measuring $15 \times 28 \mathrm{~cm}$ encompassing most of the abdomen and pelvis, compressing the inferior vena cava and abutting the inferior margin of the liver. Origin of the mass was unclear. This was followed by a dedicated MRI pelvis with contrast which did not visualize the left ovary, making the mass concerning for an ovarian primary. The patient was admitted to the medicine service, with general surgery and gynecology-oncology services consulted for operative management. On hospital day 2, the patient developed liver dysfunction with direct hyperbilirubinemia, elevated transaminases and alkaline phosphatase, concerning for congestive hepatopathy. After pre-operative cardiology clearance, she underwent exploratory laparotomy with left salphingooopherectomy, excision of the pelvic mass with omental dissection and lysis of adhesions. Patient tolerated the surgery with no major complications. Post operatively, however, she developed volume overload, thought to be due to peri-operative fluid resuscitation. She was diuresed with furosemide boluses over 4-5 days till she attained euvolumia. Her liver dysfunction completely resolved after surgery. The pathology report revealed benign mucinous cystadenoma with no evidence of malignancy in the omentum. Patient was discharged with physical rehabilitation services and is currently doing well.

IMPACT/DISCUSSION: This case illustrates the varied clinical presentation of an adnexal mass and the importance of a detailed physical examination, even when the diagnosis may seem apparent with just a thorough history. It is important to note that ovarian tumors do not present with menstrual symptoms, with the exception of sec cord tumors, and are often asymptomatic until their sheer size causes pressure related symptoms, like described in our case. Prognosis of benign ovarian tumors after operative management is excellent.

CONCLUSION: Timely diagnosis and operative management is key to avoid adverse outcomes in benign ovarian tumors.

\section{AN OFFAL ABSCESS: NON-ENDOCARDIT IS ERYSIPELOTHRIX BACTEREMIA AND ABSCESS FORMATION}

Kathryn Snyder ${ }^{1}$; Jennifer Keen ${ }^{2}$; LeeAnn M. Cox ${ }^{2} .{ }^{1}$ Indiana University School of Medicine, Carmel, IN; ${ }^{2}$ Indiana University School of Medicine, Indianapolis, IN. (Control ID \#3182931)

LEARNING OBJECTIVE \#1: Recognize patient characteristics that increase the risks for systemic infection with the zoonotic organism Erysipelothrix

LEARNING OBJECTIVE \#2: Recognize that while initial nonoperative management of epidural abscesses may be reasonable, close monitoring with early re-imaging for changing numbness, weakness or pain is necessary.
CASE: A 60 year old male with history of multiple myeloma (MM), alcohol abuse, and treated Hepatitis $\mathrm{C}$ was admitted to the hospital for unintentional acetaminophen overdose secondary to attempts at relieving three days of severe right-sided lower back pain. The pain became progressively worse over the prior three days with radiation down his right leg and an associated perception of "weakness" in his lower extremities. He denied fevers or any trauma/injury. He presented to the ED due to increased pain despite attempts to self-treat with large doses of acetaminophen, ibuprofen, and significant amounts of alcohol. Incidentally, he noted that about one week prior to his admission he had a generalized "purplish" rash that spontaneously resolved. On admission he was initially afebrile and without leukocytosis. His physical exam did not demonstrate any sensory deficits or overt asymmetrical weakness, though the exam was limited by his pain and intoxication. While his MM had been monitored and stable for a long time, his back pain was concerning and an MRI was obtained. Concurrently, he developed fevers, and blood cultures were drawn with initiation of broad spectrum antibiotics. The MRI was notable for a paravertebral and epidural abscess with osteomyelitis. Cultures from blood and the abscesses were initially reported as "diphtheroids" but later identified as E.rhusiopathiae. The infection progressed despite initial drainage thus urgent L3-5 laminectomy with debridement was completed. A transesophageal echo demonstrated no endocarditis, and the patient was treated with continuous IV Penicillin for eight weeks. Patient later shared he was a chef with frequent work-related hand injuries. He recalled a "cellulitis" of his left hand after making "head cheese" from a raw pig head a month prior to admission. This had been treated outpatient with a course of clindamycin and apparent clinical resolution. His bacteremia and abscesses were likely related to butchering the pig head.

IMPACT/DISCUSSION: This case demonstrates a rare presentation of Erysipelothrix infection manifesting as bacteremia with paravertebral and epidural abscesses, but lacking endocarditis. It additionally emphasizes the importance of appropriate surgical management and consideration of occupational exposures in the patient history.

CONCLUSION: Erysipelothrixis a rare, invasive pathogen in humans. It remains an important consideration in patients with relevant exposure history. A detailed, chronologic history is important to identify potential exposures. Non-operative management for epidural abscess is reasonable, but close monitoring and early re-evaluation is necessary.

\section{AN UNCOMMON CASE OF COMPLETE HEART BLOCK}

Anisha Contractor ${ }^{1}$; Deirdre Lewis ${ }^{2}$; Jason Phillippi ${ }^{2} .{ }^{1}$ UMMS-Baystate Medical Center, Easthampton, MA; ${ }^{2}$ Baystate Medical Center, Northampton, MA. (Control ID \#3185684)

LEARNING OBJECTIVE \#1: Recognize non-specific presentations of sarcoidosis

LEARNING OBJECTIVE \#2: Distinguish isolated cardiac sarcoidosis from systemic disease.

CASE: A 59-year-old African American male with past medical history of recurrent palpitations and hypertension on nadolol presented to the hospital with syncope, bradycardia, and new onset complete heart block. Laboratory data significant for pro-BNP elevation to $1254 \mathrm{pg} / \mathrm{mL}$, and rising ACE and ionized calcium levels to $75 \mathrm{U} / \mathrm{L}$ and $1.39 \mathrm{mM}$ respectively. Lyme C6 antibody negative. The patient failed emergent stabilization and required a permanent pacemaker. Electrophysiological and radiological evidence present prior to patient's presentation guided medical decision making to obtain cardiac PET. This study confirmed active sarcoid involvement of the distal aspect of the anterior wall of the heart, intraventricular septum, and inferior aspect of the right ventricle.

IMPACT/DISCUSSION: Sarcoidosis is an autoimmune disease characterized by non-caseating granuloma formation within multiple tissues, commonly affecting the lymphoreticular system, lungs, eyes and skin. Peak incidence of systemic disease among adults occurs between 20 and 39 , with female predominance. Though uncommon, sarcoidosis can 
present in patients over 60 with a similar gender predilection. Ethnic prevalence is higher among African American, Scandinavian and Japanese populations. Sarcoidosis can encompass diverse clinical presentations and as such lend itself to delayed diagnosis. Furthermore, there is no established diagnostic criterion; diagnosis relies on clinical and radiological evidence, pathology consistent with noncaseating granulomas, and exclusion of disorders with similar presentation and histopathology. Among patients diagnosed with sarcoidosis $5 \%$ will clinically manifest cardiac sarcoidosis (CS). The extent of left ventricular dysfunction is the most important predictor of prognosis among patients with clinically manifest CS, guiding treatment with steroids or assisted devices. CS may be further defined as systemic or confined to the heart, known as: isolated cardiac sarcoidosis (ICS). The rate of ICS is about $25 \%$ among cases of CS. Diagnosis of ICS involves excluding extra-cardiac involvement combined with cardiac MRI and PET. It is important to identify because patients with ICS have worse left ventricular systolic function at presentation compared to patients with systemic sarcoidosis and CS. Prognosis for patients with ICS is poor as patients are prone to ventricular arrhythmias and less event-free survival.

CONCLUSION: Sarcoidosis can be difficult to diagnose due to variable presentation. Early recognition of isolated cardiac sarcoidosis and extracardiac disease is crucial for prevention of critical complications.

\section{AN UNCOMMON CAUSE OF TERTIARY ADRENAL INSUFFICIENCY}

Zachary Reese; Shoshana J. Herzig; Gila Kriegel. Beth Israel Deaconess Medical Center, Brookline, MA. (Control ID \#3177781)

LEARNING OBJECTIVE \#1: Understand the physiology and causes of tertiary adrenal insufficiency

LEARNING OBJECTIVE \#2: Recognize common symptoms of carcinoid syndrome of neuroendocrine tumors

CASE: A 60-year-old female with history of hypertension presented with asymptomatic hypotension of 70/50. She noted a 10-pound weight loss in the preceding 6 months, and worsening diarrhea. Medications included lisinopril daily. She had a 60-pack year smoking history, and quit 6 months prior to admission. Family history was notable for metastatic melanoma in her mother. While inpatient, she was found to have orthostatic hypotension. A morning cortisol level was low at $1.7 \mathrm{ug} / \mathrm{dL}$ (nL 2-20 ug/dL), and responded appropriately to cosyntropin stimulation test $(20.2 \mathrm{ug} / \mathrm{dL}, 60$ minutes after cosyntropin administration). Serum ACTH was low-normal at $6 \mathrm{pg} / \mathrm{mL}(\mathrm{nL} 6-50 \mathrm{pg} / \mathrm{mL})$. A CT scan of the torso revealed hilar lymphadenopathy, bilateral lung masses, and retroperitoneal lymphadenopathy, all concerning for malignancy. MRI brain showed masses in the hypothalamus and parieto-occipital lobe. Biopsy of the hilar lymphadenopathy revealed well-differentiated neuroendocrine tumor. She was discharged on hydrocortisone. For treatment of her neuroendocrine tumor, she underwent radiation to the brain metastases, and was started on octreotide and everolimus. In follow-up, her orthostatic hypotension had resolved.

IMPACT/DISCUSSION: This case highlights an uncommon cause of tertiary adrenal insufficiency. Tertiary adrenal insufficiency arises when there is interference with secretion of corticotropin-releasing hormone (CRH) from the hypothalamus, leading to lower ACTH from the pituitary gland, and a decrease in cortisol release from the adrenal gland. Tertiary adrenal insufficiency is most commonly caused by abrupt cessation of chronic steroid administration, but can also be caused by invasion of the hypothalamus, as seen in this case. Treatment is aimed at treating the underlying cause, and administering glucocorticoids. Neurendocrine tumors (NETs) are neoplasms arising from epithelial cells of the gastrointestinal and/or bronchopulmonary tract. NETs release serotonin, histamine, and prostaglandins into the circulation. These can cause a constellation of symptoms, called carcinoid syndrome, which most commonly manifest as cutaneous flushing (85\%), and diarrhea (80\%). Carcinoid syndrome is commonly seen in metastatic neuroendocrine disease. While well-differentiated NETs typically metastasize to the liver, lymph nodes, and bones, they metastasize to the brain only $10 \%$ of the time. Treatment of metastatic NETs includes somatostatin analogues (e.g. octreotide) and mTOR inhibitors (e.g. everolimus), in addition to stereotactic radiation to brain metastases.

CONCLUSION: Metastatic disease to the hypothalamus can cause tertiary adrenal insufficiency, which is treated with steroids and treatment of the underlying malignancy. Cutaneous flushing and diarrhea are the most common symptoms of carcinoid syndrome, arising most commonly with metastatic neuroendocrine tumor.

\section{AN UNCOMMON CULPRIT OF NECK PAIN}

Islande Joseph ${ }^{1}$; Nida Waheed ${ }^{2}$; Keely Fischbach ${ }^{1} .{ }^{1}$ Univeristy of Florida, Gainesville, FL; ${ }^{2}$ University of Florida, Gainesville, FL. (Control ID \#3183332)

LEARNING OBJECTIVE \#1: Identify the clinical features and risk factors for Salmonella Osteomyelitis (OM)

LEARNING OBJECTIVE \#2: Diagnose and manage Salmonella OM CASE: A 59 year-old female with a medical history of scoliosis, depression, and ADHD presented to her PCP for bilateral neck pain after cleaning her home ceiling fan. Due to persistent neck pain and new numbness, tingling, and dull pain in her right index finger, an MRI was ordered and showed abnormalities in $\mathrm{C} 3, \mathrm{C} 4, \mathrm{C} 6$, and $\mathrm{C} 7$ concerning for $\mathrm{OM}$. She was transferred to our tertiary care center for a neurosurgical evaluation of C-spine OM. She denied any prior spinal trauma, IV drug use, diarrhea/foodborne illnesses, other weakness or numbness as well as changes in gait, bowel, or bladder function. Repeat cervical spine imaging showed $\mathrm{C} 5-\mathrm{C} 7$ with phlegmon involving the epidural space, narrowing the spinal canal and extending to her paravertebral soft tissue. Physical exam did not reveal point tenderness along her cervical spine, but she was placed in a c-collar per neurosurgery recommendations and routine neurological checks demonstrated no neurological deficit. Patient's labs revealed WBC and CRP values within normal limits, but her ESR was elevated at 48. Infectious disease was consulted and she was started on vancomycin and cefepime after interventional radiology performed a CT guided cervical disc aspiration. Aspiration biopsy culture was positive for pan-sensitive salmonella. TTE and abdominal ultrasound did not demonstrate concern for endocarditis or aortic aneurysm, respectively. Her antibiotics were de-escalated to ceftriaxone $2 \mathrm{gm}$ daily. She was discharged with continued administration of ceftriaxone for 6-8 weeks with weekly CBC with diff, creatinine, AST, ALT, ESR and hs-CRP as well as outpatient follow up with neurosurgery and infectious disease.

IMPACT/DISCUSSION: Salmonella OM is rare especially in an otherwise healthy individual. Salmonella is the causative organism in $0.45 \%$ of OM cases and salmonella OM itself accounts for as few as $0.8 \%$ of all salmonella infections. Salmonella typically effects the diaphysis of long bones, predominantly the femur and humerus. Histopathology of $\mathrm{OM}$ reveals necrotic bone with extensive resorption adjacent to an inflammatory exudate. It is predominantly seen in patients with hemoglobinopathies, including sickle cell disease or thalassemia. Due to the rarity of Salmonella OM, there are standardized antibacterial therapy regimes or surgical procedures. But chloramphenicol, third generation cephalosporins and fluoroquinolone are commonly used antimicrobials. Salmonella $\mathrm{OM}$ can be chronic, relapsing and difficult to treat.

CONCLUSION: Salmonella OM is rare. Further studies are needed to solidify treatment modalities. Early diagnosis and multidisciplinary management are necessary.

\section{AN UNCOMMON PRESENTATION OF SPLENIC MARGINAL ZONE LYMPHOMA AS SPLENIC INFARCTIO}

David Daniel ${ }^{2}$; Ribka Ayana ${ }^{1}$. ${ }^{1}$ Washington DC VA Medical Center, Washington DC, DC; ${ }^{2}$ George Washington University Hospital, Washington, DC. (Control ID \#3186075) 
LEARNING OBJECTIVE \#1: Articulate the differential diagnoses for splenic infarction

LEARNING OBJECTIVE \#2: Recognize the typical clinical presentation and indication for treatment of splenic marginal zone lymphoma

CASE: A 66-year-old man presented with an eleven-day history of severe, left upper quadrant abdominal pain. The pain was constant, non-radiating, exacerbated by inspiration and alleviated by NSAID use. His examination was notable for a non-tender abdomen and splenomegaly. Laboratory studies revealed mild leukocytosis (12.8), thrombocytopenia (72) and marginally elevated LDH. CT of abdomen and pelvis with intravenous contrast revealed enlarge spleen with an $8 \times 5 \mathrm{~cm}$ well defined hypodensity in the lower pole of the spleen consistent with splenic infarction. Vascular team recommended initiating heparin drip and obtaining abdominal CTA, which revealed patent splenic artery and vein. A transthoracic echocardiogram was also performed and was normal. Additional work up; including infectious (HIV Ab, t-spot, hepatitis panel), antiphospholipid panel, CT of chest, tumor markers (PSA, CA-19-9, CEA) was unrevealing. On day three of hospitalization, patient underwent a bone marrow biopsy, which revealed findings consistent with mature B-cell lymphoma, clinically consistent with splenic marginal zone lymphoma. Because the malignancy was symptomatic, patient was started on rituximab.

IMPACT/DISCUSSION: Splenic marginal zone lymphoma (SMZL) typically presents asymptomatically with splenomegaly, lymphocytosis, and cytopenia (anemia and thrombocytopenia). Rarely does SMZL present as a splenic infarction. This case represents one of the few reported cases in the literature. The differential diagnosis for splenic infarction includes hypercoagulable state, embolic disease, myeloproliferative disease, sickle cell disease, infectious mononucleosis, splenic trauma, or any condition that causes marked splenomegaly. SMZL is an uncommon indolent subtype of non-Hodgkin's lymphoma, making up less than $1 \%$ of all NHLs, which is characterized by infiltration of pre-existing lymphoid follicles and marginal zone differentiation in the spleen. Diagnosis is made based upon pathological findings by lymphocyte morphology, immunophenotype, cytogenetic analysis, and bone marrow histology. Not all patients with SMZL require immediate treatment because of its indolent nature. Treatment is only recommended in patients with symptoms related to splenomegaly such as abdominal discomfort or early satiety and patients with cytopenia because of bone marrow involvement. First line treatment for SMZL primarily involves rituximab monotherapy. Splenectomy is typically only considered in patients with severe localized abdominal symptoms due to splenomegaly as well as cytopenia due to splenic sequestration.

CONCLUSION: Splenic infarction is an atypical presentation of SMZL. It is important to consider SMZL in patients with splenic infarction, splenomegaly and lymphocytosis. Rituximab monotherapy is the primary treatment in patients with symptomatic SMZL.

\section{AN UNEXPECTED CASE OF WERNICKE'S ENCEPHALOPA- THY IN THE ABSENCE OF ALCOHOL USE}

Nancy Desai; Vassiliki Pravodelov; Won Lee. Boston Medical Center, Boston, MA. (Control ID \#3152943)

LEARNING OBJECTIVE \#1: Appreciate the possible causes of Wernicke's Encephalopathy (WE) in a patient without alcohol use

LEARNING OBJECTIVE \#2: Understand the diagnostic criteria associated with WE

CASE: A 68-year-old woman with hypertension, major depressive disorder and recent homelessness presented to the hospital with nausea, vomiting and weight loss for 2 months. Her symptoms were thought to be psychosomatic given her underlying psychiatric disease and an unremarkable prior work up. During her hospitalization, the patient became confused, unable to recall why she was in the hospital and confabulating. She denied prior substance use including alcohol. Exam was significant for a right beating nystagmus with slowed eye movements and a slow gait.
No ataxia was appreciated. Notably, she had an undetectable thiamine level $(<7 \mathrm{nmol} / \mathrm{L})$. Imaging was significant for symmetric FLAIR hyperintense signal abnormality in the bilateral thalami and central midbrain, suggestive of a toxic-metabolic process such as WE. She was started on intravenous thiamine supplementation and then transitioned to oral supplementation. A feeding tube was placed for nutritional support given her altered mental status, and the patient was discharged. The patient returned home after spending two weeks at a rehabilitation facility where her gastrointestinal symptoms as well as her cognitive, functional and nutritional status improved.

IMPACT/DISCUSSION: WE is a neurologic emergency secondary to thiamine deficiency requiring prompt treatment with thiamine repletion. It is commonly associated with alcohol use disorder; however, a number of other etiologies can result in acute thiamine deficiency including gastrointestinal disorders or surgeries, hemodialysis, malignancy, infection or prolonged nutritional deficiencies. WE is a clinical diagnosis classically associated with a triad of mental status changes, ataxia, and oculomotor dysfunction. As with our patient, this triad is not always present, and one can alternatively use Caine's criteria which has been shown to be more sensitive in identifying at risk patients. Caine's criteria require two of the following: dietary deficiencies, oculomotor abnormalities, cerebellar dysfunction and either an altered mental state or mild memory impairment. While plasma thiamine levels were low in this particular patient, they do not generally correlate with a diagnosis of WE. In terms of imaging, MRI is a more sensitive modality than CT and MRI findings typically include increased symmetrical T2 and FLAIR signals in the bilateral medial thalami, the mammillary bodies, tectal plate, periaqueductal area or around the third ventricle. There are no established guidelines in the United States regarding thiamine repletion, but it is recommended that immediate IV thiamine be given when WE is suspected.

CONCLUSION: Consider WE in a patient without history of alcohol use before attributing mental status changes to a chronic or irreversible cause. Clinical history and exam are key in identifying patients at risk for this disease.

\section{AN UNEXPECTED CAUSE OF CHEST PAIN}

Austin Rezigh; Nicholas Breitnauer. University of Colorado at Denver Anschutz Medical Campus, Aurora, CO. (Control ID \#3185874)

LEARNING OBJECTIVE \#1: Recognize the features of chronic cavitary pulmonary aspergillosis

CASE: A 43-year-old woman presented to a tertiary care center with dyspnea and acute worsening of chronic, left-sided, pleuritic chest pain. There was no preceding trauma, recent travel, or sick contacts. She did report fatigue and a productive cough but denied fever, chills, or weight loss. She had no past medical or surgical history and was on no medications. She was uncertain of her family history. She was a lifetime nonsmoker and denied alcohol and drug use. Socially, she was born in Somalia and had moved to the US $\sim 1$ year prior to presentation after spending 11 years in an Ethiopian refugee camp. Evaluation for tuberculosis, just prior to immigration, was negative. On arrival, vitals signs were unremarkable. Physical exam was notable only for decreased left-sided breath sounds. Initial laboratory work-up with CBC, BMP, D-dimer, and troponin were all within normal limits. EKG revealed normal sinus rhythm without ST segment changes. A chest radiograph showed absent lung markings on the left with a small layering effusion. Contrastenhanced computed tomography revealed right upper lobe bronchiectasis and near complete destruction of the left lung parenchyma with replacement by a large cavity. The Pulmonary and Infectious Disease services were consulted, following which the patient underwent bronchoalveolar lavage (BAL). While AFB cultures from the BAL returned negative, fungal culture returned positive for Aspergillus fumigatus. A corresponding serum Aspergillus fumigatus IgG level returned markedly elevated at $>100 \mathrm{U} / \mathrm{mL}$. Together, these findings were consistent with a diagnosis of chronic cavitary pulmonary aspergillosis (CCPA). The patient had a brief 
and uncomplicated hospital course. She was discharged on voriconazole $200 \mathrm{mg}$ BID with plans for a prolonged course. At last follow-up 3 months post-discharge, she was doing well.

IMPACT/DISCUSSION: CCPA, the most common form of chronic pulmonary aspergillosis, is characterized by: 1 . Serologic and/or culture evidence of Aspergillus, 2. One or more pulmonary cavities, and 3. At least three months of pulmonary and/or systemic symptoms - most commonly weight loss, cough (usually productive), shortness of breath, and hemoptysis. While generally occurring in immunocompetent patients, most have underlying lung disease - most commonly COPD or prior $+/$ - concurrent history TB/NTM infection. Important considerations in the differential diagnosis for CCPA include other cavitating infections, malignancy, granulomatosis with polyangitis, and pulmonary embolic phenomena.

CONCLUSION: Chronic cavitary pulmonary aspergillosis, the most common form of chronic pulmonary aspergillosis, should be considered in patients with respiratory $+/$ - constitutional symptoms and cavitary changes on lung imaging. The principal test to support this diagnosis is a positive Aspergillus IgG from the serum.

\section{AN UNEXPECTED PREGNANCY RESULT THAT LED TO THE DIAGNOSIS OF GASTRIC CANCER}

Jordonna Brown ${ }^{1}$; Odeth Barrett-Campbel1 ${ }^{1}$; Carol A. Luhrs ${ }^{2} .{ }^{1}$ SUNY Downstate Medical Center, Brooklyn, NY; ${ }^{2}$ Downstate Medical Center, Brooklyn, NY. (Control ID \#3185892)

LEARNING OBJECTIVE \#1: To diagnose gastric cancer in a nonpregnant woman with elevated hCG

LEARNING OBJECTIVE \#2: To recognize elevated hCG as an important prognostic factor in gastric cancer

CASE: A 47-year-old woman with a history of uterine fibroids presented with 2 weeks of vague intermittent abdominal pain, nausea and vomiting. She denied weight loss, early satiety or alteration in bowel habits and her last menstrual period was one week prior to presentation. Her physical examination revealed normal vital signs and generalized abdominal tenderness without rebound or guarding. Labs were significant for hemoglobin 7.7 (12-15g/dL), MCV 80.4 (80-96 fL/red cell), Iron 14 (40-155ug/ dl), TIBC 265 (240- $450 \mathrm{ug} / \mathrm{dl})$, ferritin 9.9 (12-150ng/dl), alkaline phosphatase 476 (40-100 U/L), positive urine hCG with serum hCG of $75.0(0-5 \mathrm{mIU} / \mathrm{ml})$. Transabdominal ultrasound showed an intramural uterine density and no evidence of an intrauterine pregnancy. CT abdomen-pelvis revealed ascites, multiple retroperitoneal, intraabdominal and pelvic lymphadenopathy, suspicious for metastatic disease or lymphoma and an ill-defined heterogenous density along the uterine fundus. A left para-aortic lymph node biopsy showed poorly differentiated metastatic adenocarcinoma with signet cell features suggestive of a gastric primary. Subsequent esophagogastroduodenoscopy and biopsy, confirmed poorly differentiated gastric adenocarcinoma. She received Cisplatin and 5-Fluorouracil with transient improvement in her HCG but unfortunately, she developed pleural effusions, profound thrombocytopenia, and pulmonary hemorrhage then succumbed to her illness within 3 months of diagnosis.

IMPACT/DISCUSSION: Gastric cancer is one of the leading causes of cancer mortality in the world and most patients present with advanced or widely metastatic disease. There are currently no specific tumor markers that have adequate sensitivity or specificity for diagnosis or follow-up of gastric cancer. However, serum levels of CEA, CA 125, CA 19-9, CA 724 and even less commonly hCG may be elevated in patients with gastric cancer and are associated with a poorer prognosis, though the use of these markers has not been standardized for clinical practice. The hormone hCG is used to monitor pregnancy and is a sensitive and specific marker for trophoblastic tumors of placental and germ cell origin. Outside of these contexts elevated levels of hCG are usually associated with malignancies such as breast cancer, leiomyosarcoma, pancreatic cancer, lymphoma, leukemia and gastric cancer. The role of hCG in the progression of gastric cancer remains unclear but its presence is usually consistent with a more poorly differentiated tumor and associated worse prognosis.

CONCLUSION: In the absence of pregnancy, elevated hCG is an ominous sign for malignancies that are not limited to trophoblastic tumors and include gastric cancer. The presence of hCG in gastric cancer signifies aggressive malignancy with significant prognostic implications.

\section{AN UNEXPLAINED CASE OF BILATERAL OPTIC NEURITIS}

Katie O'Brien ${ }^{1}$; Joshua Price ${ }^{1}$; Deirdre Lewis ${ }^{2} .{ }^{1}$ UMMS Baystate, Springfield, MA; ${ }^{2}$ Baystate Medical Center, Northampton, MA. (Control ID \#3184943)

LEARNING OBJECTIVE \#1: Generate a differential diagnosis for bilateral optic neuritis

LEARNING OBJECTIVE \#2: Recognize clinical features that suggest a diagnosis of CRION

CASE: A 19 year old previously healthy female presented to her optometrist's office with 2 weeks of progressive blurry vision. Her exam revealed elevated intraocular pressure and she was treated for glaucoma. Follow up 4 days later revealed improved pressures but deteriorating vision with complete loss of light perception and vision in her right eye. She was referred to the ED where CT and CTA of her brain were normal and she was admitted for further workup. The night of admission her left eye vision deteriorated resulting in complete bilateral vision loss with afferent pupillary defect on exam. Emergent LP revealed no evidence of multiple sclerosis (MS) or acute infection. MRI of her brain and spinal cord were significant only for asymmetric thickening and enhancement of the right optic nerve consistent with optic neuritis. There was no evidence of demyelinating disease. An autoimmune panel for neuromyelitis optica (NMO) antibodies was unrevealing. She had an otherwise normal neuro exam and was diagnosed with bilateral optic neuritis of unclear etiology. She improved slowly with 5 days of IV solumedrol. Two weeks after discharge she was seen by ophthalmology for recurrent left eye vision loss, diagnosed with left-sided optic neuritis, and treated with a second course of IV solumedrol. Six months later her vision had improved and her MRI was normal. Given her recurrent course and bilateral disease a diagnosis of Chronic Relapsing Inflammatory Optic Neuropathy (CRION) has been tentatively made.

IMPACT/DISCUSSION: The differential for bilateral optic neuritis is broad and includes inflammatory diseases such as MS and NMO. Less frequently it is seen in granulomatous diseases like sarcoidosis, infectious or post-infectious diseases, vasculitis, or can be idiopathic. In the setting of bilateral, recurrent optic neuritis without evidence of MS, NMO, or other autoimmune disease, a diagnosis of CRION should be considered. First described in 2003, CRION shares similar findings with NMO and MS. The current diagnostic criteria include objective evidence of loss of visual function, absence of autoantibodies associated with $\mathrm{NMO}$, contrast enhancement of the optic nerve on MRI, and a disease course typically characterized as responsive to steroid treatment but with tendency for steroid-dependent relapse with at least one relapse required for diagnosis. Compared to cases linked to MS, optic neuritis in CRION patients has a worse prognosis and can lead to permanent changes in retinal nerve layers that cause blindness. These long-term sequelae typically require treatment with long term immunosuppression different from those with MS which is why the diagnosis is so important.

CONCLUSION: CRION is a rare cause of bilateral optic neuritis that is distinct from MS and NMO and is associated with a poor prognosis without treatment.

\section{AN UNFORTUNATE CASE OF NONUREMIC CALCIPHYLAXIS}

Rawan Hammoudeh; Aeman Hana; Sarah A. Gorgis; Joshua D. Collins. Henry Ford Hospital, Detroit, MI. (Control ID \#3185741) 
LEARNING OBJECTIVE \#1: Recognize and distinguish calciphylaxis from other forms of ulcerating vasculitides in nonuremic patients.

CASE: A 74-year-old female smoker with a history of diabetes and peripheral artery disease (PAD) presents with painful persistent ulcers on her left arm and bilateral legs. She had a similar presentation one year ago and was diagnosed with thromboangiitis obliterans (Buerger's disease) after a biopsy of the leg ulcer showed inflammatory intraluminal thrombus. At that time, the patient was treated with antibiotics and counseled on smoking cessation. She successfully quit smoking, however skin ulcers continued to progress, which is unusual in Buerger's disease. She returned one year later with painful necrotic skin lesions involving the proximal legs, feet, and arms. Extensive rheumatological, hematological, endocrinological, vascular and genetic testing were all negative. A repeat punch biopsy was performed and revealed calcification and thrombosis of arterioles in the dermis and subcutaneous adipose tissue. Given extensive necrosis, she required bilateral transmetacarpal amputations.

IMPACT/DISCUSSION: We present a rare case of extensive nonuremic calciphylaxis in a patient with normal renal function. Calciphylaxis is an ischemic skin disorder recognized in patients with end-stage renal disease (ESRD). Our patient's history of heavy smoking, diabetes, and PAD gave suspicion to Buerger's disease, diabetic foot ulcerations, or ischemic ulcerations. It is essential to make the proper diagnosis, as treatment varies. The ulcers in Buerger's disease normally involve the distal legs and feet, whereas those in calciphylaxis involve the proximal extremities. Histologically, Buerger's disease has an intraluminal thrombus with relative sparing of the vessel wall and internal elastic lamina. This contrasts with calciphylaxis, which has thrombotic occlusion with dermal arteriolar calcification and subintimal fibrosis. Although rare, a systematic review revealed 36 reported cases of nonuremic calciphylaxis. The exact incidence of the disease is unknown. The only available treatment for calciphylaxis is sodium thiosulfate, but this is ineffective in patients with normal kidney function because it gets cleared by the kidneys too quickly to have any effect.

CONCLUSION: Calciphylaxis is a potentially lethal disorder that carries high morbidity and mortality if not recognized early. Although it is most commonly seen in patients with ESRD, clinical suspicion in non-uremic patients with the appropriate lesion distribution and histological findings is essential to early treatment. Further clinical research is needed to better understand non-renal etiologies and treatment.

\section{AN UNLIKELY CULPRIT: RENAL CELL CARCINOMA MASKED AS ACUTE ABDOMINAL PAIN}

Ara Vartanyan; Rachna Rawal. Saint Louis University, St. Louis, MO. (Control ID \#3185730)

LEARNING OBJECTIVE \#1: Recognize the importance of considering renal cell carcinoma (RCC) as a differential diagnosis in younger patients presenting with acute abdominal pain.

LEARNING OBJECTIVE \#2: Identify risk factors associated with RCC.

CASE: A 37-year old male with a history of hypertension presented for acute left sided flank pain while watching television. Additional symptoms included nausea, vomiting, and diaphoresis. He noticed dark-colored urine about 3 weeks ago followed by passing of blood clots in the urine for 2 weeks. He had no dysuria. He reported an intentional $40 \mathrm{lbs}$ weight loss over 5 months through lifestyle changes. On exam, the patient was hypertensive at 201/121, tachycardic at 103 BPM, obese (BMI 33.23), uncomfortable appearing, and exhibited moderate left lower quadrant and left flank tenderness. Labs were notable for an anemia of 9.8 from a baseline of 12.2 two months prior, with MCV 69.5, albumin 2.9, Hemoglobin A1c $11.2 \%$. There was no leukocytosis or increase in creatinine/ BUN. Urinalysis was significant for trace blood, 1+ protein, and 3+ glucose. Due to worsening abdominal discomfort, a CT abdomen \& pelvis with contrast was performed, which revealed a $3.7 \mathrm{~cm}$ mass-like lesion in the upper pole of the left kidney. MRI abdomen revealed a $9 \mathrm{~cm}$, nonhomogenously enhancing mass of the left kidney with direct extension into the left renal vein and inferior vena cava, left perinephric hemorrhage, and enlarged left para-aortic lymph nodes. These findings were concerning for RCC. A left open radical nephrectomy with IVC thrombectomy was performed. Pathology of the tissue revealed clear cell RCC, grade 3, without lymph node involvement. Surveillance imaging showed recurrent vena cava tumor thrombus and the patient was started on nivolumab/ipilimumab immunotherapy, which has resulted in decrease of thrombus size.

IMPACT/DISCUSSION: This case demonstrates the importance of considering RCC in younger patients and serves as a reminder to be cognizant of premature closure as physicians. This patient presented with acute flank pain, which is usually associated with acute processes rather than an insidious one such as RCC. His weight loss was a strong indicator of malignancy, but was confounded by his poorly controlled diabetes and reported history of intentional weight loss. Other risk factors for RCC included obesity and hypertension. Additionally, his work in a body shop may have exposed him to trichloroethylene, a chemical in grease removers that has been associated with RCC Approximately 5\% of patients with asymptomatic microscopic hematuria are found to have a urinary tract malignancy. By investigating further we treated this patient's cancer that could have ultimately caused more harm.

CONCLUSION: There are approximately 65,000 new cases of and 15,000 deaths from RCC, the most common form of kidney cancer, in the United States each year. Though RCC occurs predominantly in the sixth to eighth decades of life, signs such as weight loss, flank pain, and hematuria should raise red flags concerning for RCC.

\section{AN UNSUAL CASE OF SYNCOPE: INEXPLICABLE COR PULMONALE IN A 30-YEAR-OLD-MALE}

Ira Khanna $^{1}$; Eric Bondarsky ${ }^{2}$; Sharmila Ghosh ${ }^{1}$; Christopher Taurani ${ }^{1}$; Daniel Steinberg ${ }^{1} .{ }^{1}$ Mount Sinai Beth Israel, New York, NY; ${ }^{2}$ NYU, New York, NY. (Control ID \#3176468)

LEARNING OBJECTIVE \#1: Recognize clinical and diagnostic features of Pulmonary Tumor Thrombotic Microangiopathy

CASE: A 30-year-old male presented with 3 weeks of cough with hemoptysis, recent episode of syncope and decreased exercise tolerance for 6 months. Vitals in the ED: BP 134/84, HR 108, RR 18, Temp 98.1, $\mathrm{SpO} 295 \%$. Physical exam was normal. EKG showed TWI in v1-v4, bedside echo showed a dilated RV, labs showed troponin 0.704, BNP 363.7, D-dimer >20, LDH 328, ESR 108, CRP 83.9. CXR was unremarkable and pulmonary CTA-Dopplers were negative for PE/DVT but showed alveolitis and centrilobular nodules. Infectious and autoimmune workup was negative. TTE showed PASP 83 and RV dilation; V/Q scan showed low probability for PE. He had 3 more episodes of syncope with worsening dyspnea and a rising troponin. He was then transferred to the ICU, started on heparin drip for possible PE versus an NSTEMI and started on HFNC for hypoxic respiratory failure. RHC was suggestive of isolated RV failure. He was subsequently started on Dobutamine, Epoprostenol and inhaled NO. However, he went into PEA arrest and passed away despite resuscitative efforts. His autopsy found acute circulatory and respiratory failure secondary to Pulmonary tumor thrombotic microangiopathy (PTTM), RV hypertrophy and failure, and poorly differentiated gastric adenocarcinoma with metastases to mediastinal, perigastric and para-aortic lymph nodes.

IMPACT/DISCUSSION: Pulmonary tumor thrombotic microangiopathy is a rare but devastating complication in advanced metastatic cancers wherein tumor cells embolize into pulmonary arteries and induce activation of the coagulation cascade with intimal proliferation within small pulmonary arteries. Herbay et al first described a 3.3\% prevalence of PTTM in 630 cancer autopsies with gastric adenocarcinoma being most commonly associated. It usually presents as acute or subacute dyspnea with hypoxia and 
rapidly progressive Pulmonary Artery Hypertension (PAH) leading to cor pulmonale. Bloodwork may show non specific elevations of D-Dimer and LDH. Imaging is mostly negative, although CT may show dilated pulmonary arteries, V/Q scan may reveal peripheral perfusion defects and FDG PET may show uptake in lungs, however, smaller tumor deposits are likely to be missed. Histological diagnosis may be obtained via lung biopsy or pulmonary artery catheter blood aspiration. While PTTM remains primarily a post-mortem diagnosis, there have been a few cases where early diagnosis and treatment with pulmonary vasodilators, chemotherapy and anticoagulation has led to survival in months to years. In most of these cases patients had known cancer, however, cor pulmonale as the first clinical presentation is extremely rare. Had we considered PTTM, a pulmonary artery catheter blood aspiration could have been done during his RHC which could have prompted chemotherapy.

CONCLUSION: In patients with acute cor pulmonale without a clear cause, Pulmonary Tumor Thrombotic Microangiopathy from an occult malignancy should be considered.

\section{AN UNUSUAL CASE OF ALTERED MENTAL STATUS LEAD- ING TO A DIAGNOSIS OF PANCREATIC ADENOCARCINOMA}

Nina Kogekar; Julie Sung; Priya Sehgal; Mona Krouss. Icahn School of Medicine at Mount Sinai, New York, NY. (Control ID \#3178519)

LEARNING OBJECTIVE \#1: Recognize non-bacterial thrombotic endocarditis in patients with pancreatic adenocarcinoma or other malignancy

CASE: An 84 year old male with atrial fibrillation on apixaban and active smoker (25 pack-year history) presented with weakness and confusion for two weeks. On presentation, the patient had normal vital signs. Physical exam revealed irregularly irregular rhythm with mental status oriented only to name and place, not date. Detailed neurologic exam was notable for decreased coordination with irregular rapid alternating movements and inability to perform finger-to-nose, upward Babinski reflexes, and shuffling, wide-based gait. Laboratory studies were normal. A CT head showed no acute process. The patient continued to be increasingly altered, so MRI brain was obtained, which revealed several acute to subacute embolic infarcts in the bilateral cerebral and cerebellar hemispheres. This raised suspicion for endocarditis, and a transesophageal echocardiogram (TEE) found small mobile masses on the cusps of the aortic valve. A diagnosis of non-bacterial thrombotic endocarditis (NBTE) was made, given negative infectious work-up, and the patient was started on enoxaparin. CT angiography of the chest was performed to rule out pulmonary embolus during work-up, which incidentally showed a mass in the pancreatic head. MRCP showed a lesion at the posterior pancreatic head with upstream dilation of the main pancreatic duct. The pancreatic mass was biopsied which showed pancreatic adenocarcinoma (PAAD).

IMPACT/DISCUSSION: Our case is unique as the diagnosis of NBTE was made despite a seemingly benign initial presentation. Regardless of the incidental finding of the tubular structure in the pancreatic head, this patient with NBTE should have undergone workup of possible PAAD as this association has been increasingly reported in the literature. Awareness of the association is critical, as diagnosing NBTE in the context of malignancy alters the management plan, from antibiotics to anticoagulation.

CONCLUSION: NBTE, also known as marantic endocarditis, is caused by deposition of fibrin and platelet thrombi on heart valves. It occurs in the setting of a hypercoagulable state, often due to underlying adenocarcinoma. The incidence of NBTE in pancreatic cancer is not known. There have been few cases of ante-mortem diagnosis of NBTE with PAAD. Our case represents a rare instance of an ante-mortem diagnosis of NBTE as the initial presentation of underlying PAAD. The treatment of NBTE consists of anticoagulation with unfractionated or low-molecular weight heparin (LMWH). LMWH overall remains the treatment of choice over DOACs for malignancy-related thrombosis, and thus was chosen for treatment in this patient who was already on apixaban at the time of presentation. This case demonstrates that in patients with multiple cerebral infarctions, the diagnosis of NBTE and, subsequently, underlying malignancy should be included in the initial differential.

\section{AN UNUSUAL CASE OF BLEEDING GASTRIC VARICES SEC- ONDARY TO METASTATIC OVARIAN ADENOCARCINOMA}

Randeep Kaur; Reena Salgia; Crystal Seudeal; Eric Scher. Henry Ford Hospital, Detroit, MI. (Control ID \#3185409)

LEARNING OBJECTIVE \#1: Recognize the presence and different potential causes of gastric varices in a patient that does not have liver disease CASE: The patient is a 58-year-old Caucasian female with a history of metastatic ovarian cancer and no previous history of liver disease, who initially presented to the hospital with hematemesis, melanotic stools, lethargy and generalized weakness. She was found to have a hemoglobin of $5.7 \mathrm{~g} / \mathrm{dL}$, which dropped to $4.5 \mathrm{~g} / \mathrm{dL}$. Liver function tests at this time were within normal limits and testing for chronic liver disease was unrevealing. Abdominal ultrasound revealed patent hepatic vasculature and splenomegaly. An emergent esophagogastroduodenoscopy (EGD) was performed which showed isolated gastric varices located in the fundus (Sarin Classification, IGV1). MRI of the abdomen confirmed a hypovascular mass in the splenic hilum concerning for possible metastasis. Pathology confirmed metastatic ovarian cancer in the splenic hilum. After presentation at tumor board, the decision was made to perform splenic artery embolization (SAE) to decrease the inflow to the spleen with subsequent splenectomy. Proximal SAE and splenectomy was done without complication. The patient has continued her care with oncology for further treatment with Paclitaxol +/- Bevacizumab. Following the patient's splenectomy, she has reported no further episodes of hematemesis or melanotic stools.

IMPACT/DISCUSSION: Left sided portal hypertension (LSPH) causing gastric variceal bleeding is fortunately an infrequent cause of upper gastrointestinal hemorrhage. To the best of our knowledge, there has been one published case of metastatic ovarian cancer resulting in gastric varices which occurred 21 years following ovarian carcinoma surgery and adjuvant chemotherapy. This case demonstrates progression of ovarian metastatic disease despite chemotherapy status post debunking in the peritoneum, causing significant hemorrhage with complete resolution of bleeding gastric varices by splenic artery embolization, followed by splenectomy. It highlights the need for ongoing awareness regarding the evaluation and management of IGV in patients who do not have underlying liver disease. Ultimately, the finding of IGV on endoscopy in a noncirrhotic patient should prompt the investigation into other causative processes that could obstruct the splenic venous system.

CONCLUSION: Portal hypertension generally develops in the setting of liver cirrhosis, and can result in subsequent esophageal and/or gastroesophageal varices. LSPH, on the other hand, can arise in non-cirrhotic patients secondary to obstruction of the splenic vein, leading to isolated gastric varices. Given that most cases of LSPH remain asymptomatic, the incidence is likely $<5 \%$ of all portal hypertension cases. This is an uncommon case of metastatic ovarian adenocarcinoma to the splenic vein hilum causing outflow obstruction and resultant gastric variceal hemorrhage. This patient was ultimately treated with splenic artery embolization followed by splenectomy.

\section{AN UNUSUAL CASE OF ILEAL NEUROENDOCRINE TUMOR PRESENTING AS HEMATOCHEZIA}

Ahmed A. Elkafrawy ${ }^{2}$, Mahak Chauhan ${ }^{1}$; Fredy Nehme ${ }^{1}$. ${ }^{1}$ University of Missouri - Kansas City, Kansas City, MO; ${ }^{2} \mathrm{UMKC}$ School of Medicine, Mission, KS. (Control ID \#3186021)

LEARNING OBJECTIVE \#1: Recognize hematochezia as a rare presentation of Neuroendocrine Tumor of the small bowel. Assess small bowel bleeding with capsule endoscopy and double balloon enteroscopy. 
LEARNING OBJECTIVE \#2: Neuroendocrine tumors (NETs) are epithelial tumors with predominant neuroendocrine differentiation. The terminal ileum is the most common location for NETs. The symptoms of small bowel NETs are represented mainly by intermittent intestinal obstruction and carcinoid syndrome; however, hematochezia is a very rare presentation.

CASE: We present a 57-year-old Caucasian female with a past medical history of coronary artery disease with stents and alcohol abuse who presented with hematochezia. She was admitted 4 years ago with melena and low hemoglobin, she was diagnosed with erosive gastritis on EGD. She presented this time with recurrent episodes of hematochezia without hematemesis, melena or abdominal pain. She was on aspirin. Physical exam was unremarkable. Hemoglobin was $12.5 \mathrm{gm} / \mathrm{dl}$. EGD was normal and colonoscopy showed fresh blood through the colon but no active source of bleeding. Capsule endoscopy showed a mid-small bowel ulcer with fresh blood from mid to distal small bowel. Bleeding then appeared to have ceased spontaneously and hemoglobin stabilized. Anterograde double-balloon enteroscopy (DBE) was pursued for better visualization of the small bowel ulcer and was normal. A retrograde DBE revealed a $2.5 \mathrm{~cm}$ ulcerated mass in the terminal ileum. Biopsies showed focal nests of neuroendocrine tumor (NET). Surgical resection of $14 \mathrm{~cm}$ from the small bowel including the mass, wedge of mesentery and palpable lymph nodes was done. Pathology showed grade 1 of 3 well-differentiated NET invading the visceral peritoneum with lymph node metastasis. The patient recovered well and was discharged home to follow up with oncology.

IMPACT/DISCUSSION: This case highlights the importance of direct visualization of small bowel ulcers with enteroscopy despite cessation of bleeding. In our case, the suspected small bowel ulcer noted on capsule endoscopy was, in fact, an ulcerated NET. NETs of the small intestine represent the most common small bowel tumor. Metastasis to lymph nodes and liver are common at diagnosis and can lead to carcinoid syndrome. The diagnosis is often difficult due to their rarity and the nonspecific presentation. In fact, more than $50 \%$ of patients require at least 2 years for a diagnosis of NET after symptom onset while visiting on average 4 healthcare professionals. Therefore, a high index of suspicion must be maintained and one must be aware of all variable presentations.

CONCLUSION: NETs represent a rare but important pathological condition of the gut. While capsule endoscopy was an important tool in the diagnostic work up, balloon enteroscopy was the key to making the correct diagnosis. Desmoplastic reactions involving mesenteric vessels may lead to nonspecific abdominal pain, gastrointestinal bleeding, intermittent ischemia, or bowel obstruction.

\section{AN UNUSUAL CASE OF MENINGITIS CAUSED BY EPSTEIN- BARR VIRUS}

Parma Zahid ${ }^{1,4}$; Timothy C. Edmonds ${ }^{2,}{ }^{4}$; Sharon Rikin ${ }^{3,1}$. ${ }^{1}$ Montefiore Medical Center, Bronx, NY; ${ }^{2}$ Montefiore Medical Center, New York, NY; ${ }^{3}$ Montefiore - Albert Einstein College of Medicine, Bronx, NY; ${ }^{4}$ Albert Einstein College of Medicine, Bronx, NY. (Control ID \#3186338)

LEARNING OBJECTIVE \#1: Recognize the clinical features of Ebstein-Barr virus (EBV) meningitis

LEARNING OBJECTIVE \#2: The role of antiviral therapy in treatment of EBV meningitis

CASE: A 47 year old female with HIV (CD4 225) not currently on antiretroviral therapy presented with headaches, nausea, vomiting, and neck stiffness for four weeks. Associated symptoms included seventh nerve palsy, tinnitus and decreased hearing of the left ear. On exam, patient had a temperature of 100.8 , heart rate of 120 , papilledema, left ear hearing deficit, and neck stiffness. On admission, MRI brain was normal. A lumbar puncture showed opening pressure of $37 \mathrm{~mm} \mathrm{H} 2 \mathrm{O}$, WBC of 15 , and an elevated protein to 186 . Differential diagnosis for presumed aseptic meningitis included: varicella zoster virus (VZV) reactivation at the geniculate ganglion manifesting as Ramsay Hunt Syndrome or Lyme given cranial nerve findings. The patient was empirically started on acyclovir pending cerebrospinal fluid (CSF) results. CSF VZV PCR and Lyme antibody were negative. Results however showed CSF EBV PCR 1,100; serum EBV viral capsid antigen and antibody were also positive. As the patient's fever, headache, and hearing improved after initiation of acyclovir, treatment was continued with acyclovir IV for ten days, then with valacyclovir to complete a total of three weeks of treatment.

IMPACT/DISCUSSION: EBV meningitis is a rare illness and may present with cranial nerve deficits such as decreased hearing and facial nerve palsy along with other meningeal irritation signs. This case also reveals an opportunity to review therapeutic options for EBV-related illness. EBV should be considered in both immunocompetent and immunocompromised patients with viral meningitis/encephalitis. Supportive care is generally recommended; there is the potential for debilitating sequalae including increased intracranial pressure and death. We are aware of only one other reported case of EBV meningitis with hearing loss in which the patient was treated with hydrocortisone sodium phosphate and noted improvement in hearing acuity. While antivirals such as acyclovir are thought not to work well in vivo for EBV and thus are not recommended, there are case reports of using antiviral therapy for EBV meningitis/encephalitis. In these cases, patients treated with antiviral therapy were shown to have decreasing viral DNA in CSF and a corresponding resolution of their symptoms. Given that the literature only shows case studies of EBV meningitis treatment with antivirals, there is an opportunity for further research in this area.

CONCLUSION: EBV meningitis should be considered in the differential in patients who present with cranial nerve deficits. Antiviral treatment may be considered in EBV meningitis given observational studies which have been associated with improvement of disease manifestations.

\section{AN UNUSUAL CASE OF NEPHROGENIC DIABETES INSIPIDUS}

Michael Liu; Sandra Oza. Albert Einstein College of Medicine, Bronx, NY. (Control ID \#3177877)

LEARNING OBJECTIVE \#1: Recognize chronic obstructive uropathy as an etiology of nephrogenic diabetes insipidus.

CASE: The patient is a 53-year-old man with a past medical history of neurofibromatosis type 1 status post cervical and lumbar spinal surgeries, which were complicated by neurogenic bladder managed with intermittent catheterization, with which the patient was inadequately adherent. $\mathrm{He}$ presented with nausea, vomiting, diarrhea, and weakness prior to elective colonoscopy; the procedure was canceled and the patient was admitted. Laboratory studies were notable for: sodium $153 \mathrm{mEq} / \mathrm{L}$, creatinine 1.7 $\mathrm{mg} / \mathrm{dl}$, white blood cell count $15,500 / \mathrm{mm}^{3}$, and urinalysis revealing large leukocytes and $>100$ WBCs. The patient was started on $0.45 \%$ normal saline and $1 \mathrm{~g}$ ceftriaxone. The patient's hypernatremia did not improve with intravenous hypotonic fluids. Renal ultrasound revealed bilateral hydroureteronephrosis. A Foley catheter was placed, and on the subsequent nine days, the patient's 24-hour urine output ranged from $4.395 \mathrm{~L}$ to $10.65 \mathrm{~L}$. Urine osmolality was $107 \mathrm{mOsm} / \mathrm{kg}$, serum osmolality was 308 $\mathrm{mOsm} / \mathrm{kg}$, and serum antidiuretic hormone $(\mathrm{ADH})$ was $16.4 \mathrm{pg} / \mathrm{ml}$. Administration of $4 \mathrm{mcg}$ desmopressin subcutaneously resulted in an increase in urine osmolality of less than $40 \%$, and the diagnosis of nephrogenic diabetes insipidus (NDI) was made. The patient was started on hydrochlorothiazide $25 \mathrm{mg}$ once daily by mouth with a decrease in daily urine output to $3.7 \mathrm{~L}$.

IMPACT/DISCUSSION: NDI may be caused by lithium, hypercalcemia, hypokalemia, and genetic defects, but NDI secondary to chronic urinary tract obstruction is rare. Case reports have described NDI in patients with mechanical bladder neck obstruction secondary to prostate cancer and hypertrophy, ileal leiomyosarcoma, and rectal cancer. There has been only one reported case of NDI secondary to neurogenic bladder. In that case, a 48-year-old woman presented with polyuria of 4-6 L/day, and bladder catheterization led to improvement in symptoms. The 
theorized pathophysiologic mechanisms underlying NDI in patients with bladder outlet obstruction are increased cortical collecting pressures damaging tubular epithelium, thus altering the kidney's ability to respond to vasopressin. After restoring normal urine flow, polyuria and hyposthenuria continue since the tubular defect remains. Three possible mechanisms have been proposed: given the reduction in functional nephrons, each nephron has a higher glomerular filtration rate; there is decreased reabsorption of sodium and water at the proximal tubule; there is decreased water absorption in the collecting duct and descending limb of the loop of Henle. After relief of urinary obstruction, thiazide diuretics can be used to increase sodium and water reabsorption in the proximal tubule, thereby decreasing delivery to $\mathrm{ADH}$-sensitive sites in the collecting duct.

CONCLUSION: Chronic urinary obstruction, from both mechanical and anatomical causes including neurogenic bladders, should be considered in patients presenting with NDI.

\section{AN UNUSUAL CAUSE OF ABDOMINAL PAIN AND CONFU- SION IN A PATIENT WITH CIRRHOSIS}

Michelle Knees ${ }^{1}$; Kellen Hirsch ${ }^{1}$; Jason John ${ }^{1}$; Katarzyna Mastalerz ${ }^{2}$. ${ }^{1}$ University of Colorado School of Medicine, Denver, CO; ${ }^{2}$ VA Eastern Colorado Health Care System, Aurora, CO. (Control ID \#3138139)

LEARNING OBJECTIVE \#1: Recognize Chilaiditi sign and consider corresponding syndrome complications in any patient with cirrhosis, abdominal pain, and appropriate radiographic sign.

CASE: A 72-year-old man presented with three days of worsening confusion in the setting of known cirrhosis. Notably, he was recently treated at an outside hospital for dehydration and was discharged without his lactulose or rifaximin. Lactulose and rifaximin were restarted upon admission, with gradual improvement in his confusion. However, six days into his hospitalization, he developed acute abdominal pain, vomiting, and worsening confusion. Vital signs were within normal limits; his abdomen was distended and diffusely tender with voluntary guarding but without rebound tenderness. He was lethargic and alert only to person. An abdominal x-ray showed a distended large intestine under the right hemidiaphragm without clear signs of pneumoperitoneum. A subsequent $\mathrm{CT}$ abdomen/pelvis further delineated moderate stool burden and interposition of the large bowel between the diaphragm and liver. The radiologist's differential included Chilaiditi sign; given the patient's symptoms, a presumptive diagnosis of Chilaiditi syndrome leading to constipation and worsening hepatic encephalopathy was made, and the patient was treated conservatively with bowel rest, IV fluids, ondansetron, and additional lactulose. He soon had one large bowel movement with improvement of his abdominal pain and confusion.

IMPACT/DISCUSSION: Normally, the colon and liver are kept in place with suspensory ligaments; however, cirrhosis is a known risk factor for Chilaiditi syndrome due to liver atrophy disrupting the normal suspensory ligament anatomy. This can lead to intermittent bowel and liver interposition with resultant abdominal symptoms. The differential for acute abdominal pain is large. The danger of Chilaiditi syndrome resides less with the syndrome itself and more with mistaken diagnoses, which can include complete bowel obstruction, subdiaphragmatic abscesses, or pneumoperitoneum, all of which can lead to surgical intervention. When diagnosed correctly, uncomplicated Chilaiditi syndrome frequently resolves with only supportive treatment. Interestingly, our patient had a years-long history of intermittent abdominal pain and difficult-to-control hepatic encephalopathy. After diagnosing Chilaiditi syndrome, our team felt that his hepatic encephalopathy was so labile due to his inability to tolerate a consistent lactulose/rifaximin dosing regimen from symptoms related to this syndrome. To avoid unwarranted invasive interventions, recognition of this rare but important diagnosis should be kept in mind in any patient with cirrhosis, abdominal pain, and the appropriate radiographic signs.
CONCLUSION: Chilaiditi radiographic sign can indicate Chilaiditi syndrome and can be easily mistaken for pneumoperitoneum. Intermitent constipation resulting from Chilaiditi syndrome can complicate the disease course of hepatic encephalopathy.

\section{AN UNUSUAL CAUSE OF OBSTRUCTIVE JAUNDICE}

Chirag Buch ${ }^{2}$; Heather L. Briggs ${ }^{1} .{ }^{1}$ University of Texas Health Science Center at San Antonio, San Antonio, TX; ${ }^{2}$ UT Health San Antonio, San Antonio, TX. (Control ID \#3161334)

LEARNING OBJECTIVE \#1: Review clinical presentation of obstructive jaundice

LEARNING OBJECTIVE \#2: Recognize a rare complication of clip cholelithiasis after a laparoscopic cholecystectomy

CASE: 61 y/o Hispanic male with a PMH of HTN, HLD, DMII, gallstone pancreatitis $\mathrm{s} / \mathrm{p}$ cholecystectomy 3 years ago complicated by gallbladder fossa abscess s/p IR drainage presented with 3 weeks of acute onset nausea, vomiting, and abdominal pain. The RUQ pain is sharp, intermittent, worsens with food, and is relieved with vomiting. He endorses dark urine, acholic stools, chills, $10 \mathrm{lb}$ weight loss, and jaundice. He denies fever, neurological deficits, pruritus, hematochezia, and any symptoms of peripheral neuropathy. His admission laboratory tests were significant for elevated liver enzymes (AST 203, ALT 354, \& ALP 251) with hyperbilirubinemia (T Bili 10.0 and D Bili 5.9). CT scan showed possible migrated surgical clip. Gastroenterology was consulted and endoscopic retrograde cholangiopancreatography (ERCP) was performed, showing a prominent common bile duct with oblong distal filling defect. The patient underwent extraction of two stones (one which appeared to represent a concretion surrounding the migrated cholecystectomy clip) and subsequent ductal clearance/ decompression. The following day, he was tolerating a full diet and had resolution of his post-prandial symptoms, decrease in RUQ tenderness, and a decline of his liver enzymes and bilirubin.

IMPACT/DISCUSSION: The first laparoscopic cholecystectomy was performed in 1985 in Germany. Since then, this procedure replaced the conventional open cholecystectomy and became one of the most common operations performed. There are about 300,000 of these procedures performed each year for cholecystitis, symptomatic cholelithiasis, biliary dyskinesia, acalculous cholecystitis, gallstone pancreatitis, and gallbladder masses/polyps. Complications following this procedure occur less than $5 \%$ of the time. These include bleeding, infection, and injury to surrounding structures. Most patients can be managed with close follow up, but some require extensive hospitalizations that raise mortality, morbidity, and health care costs. Less than 100 cases of clip migration following laparoscopic cholecystectomy have been reported. Though the mechanism of surgical clip migration, gallstone formation around the clip, and its obstruction is unclear, thiscase highlights a rare complication in a patient who presented 3 years post cholecystectomy with classic symptoms of obstruction of the biliary system.

CONCLUSION: Surgical clip migration, a rare complication of laparoscopic cholecystectomy, should be considered part of the differential diagnosis in patients that present with clinical manifestation of choledocholithiasis post procedure. Urgent imaging and management should be considered.

\section{AN UNUSUAL CONSEQUENCE OF ALCOHOLISM AND RE- CURRENT PANCREATITIS}

MRUDULA THIRIVEEDI; Parekha yedla. UAB, Huntsville, AL. (Control ID \#3185898)

LEARNING OBJECTIVE \#1: Recognize an uncommon cause of ascites in patients with alcoholic liver disease and the treatment options available.

CASE: A 41 year old woman with history of alcoholism and recurrent pancreatitis presented with a three day history of abdominal pain and 
distension. She was seen in the emergency room a week ago for similar complaints, was diagnosed with cirrhosis and ascites, underwent paracentesis which was notable for a serum-ascites albumin gradient (SAAG) of 1.6 consistent with portal hypertension. During this presentation, physical exam was remarkable for ascites and diffuse tenderness to palpation. Laboratory studies were normal except for a low serum albumin of $2.1 \mathrm{~g} / \mathrm{dL}$, CT abdomen showed hepatic steatosis and cysts in the tail of the pancreas. Ascitic fluid analysis revealed total nucleated count of $414 /$ microliter, total protein of $3.1 \mathrm{~g} / \mathrm{dL}$ and a SAAG of 0.8 after which fluid amylase levels were checked and found to be elevated at $3671 \mathrm{U} / \mathrm{L}$. Endoscopic ultrasound was done for further evaluation of pancreatic cysts and the aspirate was noted to be benign. The patient was diagnosed with pancreatic ascites, underwent an endoscopic retrograde cholangiopancreatography (ERCP) with biliary sphincterotomy for choledocholithiasis. She came back 2 days later with worsening ascites. Fluid analysis again showed elevated amylase of 2475 U/L. Repeat ERCP revealed pancreatic duct leakage and a transpapillary stent was placed. The patient improved clinically and symptomatically over the next few days. At a follow up visit 2 weeks later, there was no recurrence of ascites. IMPACT/DISCUSSION: Most common causes of ascites are cirrhosis, malignancy and heart failure. Pancreatic ascites is rare and is usually from leakage of the pancreatic duct or pseudocysts. It is characterized by SAAG $<1.1$, ascitic amylase level $>1000 \mathrm{U} / \mathrm{L}$ and total protein $>3 \mathrm{~g} / \mathrm{dl}$. Goldstandard for diagnosis is ERCP showing pancreatic duct disruption. The most common cause of pancreatic ascites is chronic pancreatitis, other causes include acute pancreatitis and trauma. Estimated prevalence is $4 \%$ in patients with chronic pancreatitis and 6 to $14 \%$ in patients with pseudocysts. There are only a few case reports and case series available to guide the management of pancreatic ascites. Conservative therapy includes serial paracentesis, parenteral nutrition and octreotide which decrease pancreatic secretions facilitating fistula closure. Interventions include either endoscopic therapy or surgery as the last resort. ERCP with transpapillary stent placement is a viable option in most cases. Surgical procedures include partial pancreatectomy and pancreaticojejunostomy.

CONCLUSION: Pancreatic ascites should be considered in the differential diagnosis of recurrent ascites in a patient with history of alcoholism and pancreatitis. Growing evidence supports interventional therapy as an initial treatment strategy especially endoscopic transpapillary stenting of the pancreatic duct.

\section{AN UNUSUAL ETIOLOGY OF PNEUMOPERITONEUM}

Shayan Siddiqui; Assad Mohammedzein; Nooraldin Merza; Ahmed Taha; Juan Garrido. Texas Tech University Health Science Center, Amarillo, TX. (Control ID \#3147530)

LEARNING OBJECTIVE \#1: Recognize emphysematous pyelonephritis as a rare cause of pneumoperitoneum.

CASE: A 63 years old male presented with one week history of diffuse abdominal pain. He denied fever or dysuria. His medical history is significant for diabetes mellitus, Hypertension, coronary artery disease, congestive heart failure, chronic hepatitis $\mathrm{B}$ and $\mathrm{C}$ with liver cirrhosis. He injects heroin and snort cocaine. He was alert, had a temperature of 34.7 $\mathrm{C}$, heart rate of $66 \mathrm{bpm}$, and blood pressure of 147/69 mmhg. Abdomen was soft, diffusely tender, maximum at the right costovertebral angle, no rebound tenderness or rigidity, and bowel sounds were normal. No urethral discharge or penile ulcers. Laboratory tests showed WBC $3.0 \times 10^{3} /$ cumm, Hb $8.1 \mathrm{~g} / \mathrm{dl}$, platelet $32 \times 10^{3} / \mathrm{cumm}$, pyuria, and bacteriuria. Lactic acid was $3.9 \mathrm{mmol} / \mathrm{l}$. Toxicology screen positive for cocaine and opiate. Computed tomography (CT) abdomen showed free intraperitoneal air, foci of gas within the right renal parenchyma adjacent to the proximal right ureter and within the posterior portion of the bladder. The diagnosis of Emphysematous pyelonephritis (EP) was made. Blood cultures grew Extended Spectrum Beta Lactamase Escherichia Coli. He was treated with meropenem. Shortly after, he developed septic shock requiring intravenous fluids and vasopressor support. In view of his critical condition and comorbidities, he was not operated on, instead percutaneous right nephrostomy tube was placed. However, his condition continued to deteriorate and his family opted for hospice care.

IMPACT/DISCUSSION: EP is a severe necrotizing infection characterized by gas formation within or outside the renal parenchyma. Based on the pattern of gas extension on imaging, four types have been described in the literature. As the gas extended to para-renal space, our patient's type was III B, which has poor prognosis without surgical intervention. It is extremely rare that EP presents with pneumoperitoneum; only 3 cases have been described in the literature. All 3 patients required exploratory laparotomy, 2 required radical nephrectomy, the third underwent surgical drainage with nephron-conservative therapy. Other etiologies of pneumoperitoneum must be excluded, especially bowel perforation which often prompts exploratory laparotomy. Our patient was a high risk surgical candidate and he opted for percutaneous nephrostomy.

CONCLUSION: Emphysematous pyelonephritis is a life-threatening condition and it could very rarely present with pneumoperitoneum, to our knowledge this is the 4 th reported case in the literature. Such a finding should prompt an emergent exploratory laparotomy should the patient's condition permits.

\section{AN UNUSUAL PRESENTATION OF CEREBRAL MASS IN A PATIENT WITH HIV INFECTION}

Shadab Saboori; Leigh A. Medaris; Michael Leonard. Carolinas Medical Center, Charlotte, NC. (Control ID \#3185467)

LEARNING OBJECTIVE \#1: 1) Recognize unusual features of neurosyphilis in HIV infection

LEARNING OBJECTIVE \#2: 2) Diagnose intracranial mass lesions in HIV infection

CASE: A 23-year-old-male with a past medical history significant for untreated HIV infection presented to an outside hospital with a chief complaint of left-sided headache, left-sided hearing loss and bilateral blurred vision of two weeks duration. CT head without contrast showed a less than two centimeter left temporal parietal mass with mild mass-effect, and patient was transferred to our facility for neurosurgical and infectious diseases evaluation. He denied night sweats, chills, fever or weight loss, and he had no recent head trauma. Past medical history was significant for being diagnosed with HIV while seeking treatment for gonorrhea four years earlier. He never followed up and was naïve to antiretrovirals. He has a history of smoking but denies any drug use. His housing was unstable and he identified himself as bisexual. Neurological examination was significant for decreased left sided facial sensation, impaired left sided hearing, 4 out of 5 motor strength in left upper and lower extremity, and diminished light and pinprick sensation of left upper and lower extremity. The remainder of his exam was unremarkable. The RPR was reactive at 1:64 dilutions and treponema pallidum particle antigen was positive. Toxoplasma antibodies were negative. CD4 count was 121, and HIV 1 RNA viral load of 1,356. MRI brain revealed a solid nodular enhancing lesion in the left temporal lobe. Decision made to proceed with biopsy for further assessment and pathology revealed a fibrotic, lymphoplasmacytic infiltrate with a positive stain for spirochetes. Treatment was initiated with 14 days of IV penicillin G followed by two doses intramuscular benzathine penicillin one week apart. He was started on anti-retroviral therapy consisting of Descovy and Tivicay. At his one month follow-up visit, his symptoms were all improved. A repeat head CT 4 months after presentation showed complete resolution of the lesion.

IMPACT/DISCUSSION: Intracerebral gummas are a rare presentation of neurosyphilis that are seen mainly in HIV infection. HIV co-infection has been shown to alter the clinical course of syphilis, but despite an increased 
predisposition for neurosyphilis, cerebral gummas are rarely reported. Their presentation and imaging can be confused with tumors, abscesses, and other infectious lesions ie tuberculomas thus usually requiring pathology to make the diagnosis as seen in our case. Our patient showed a full recovery with radiologic evidence of complete resolution following treatment with IV penicillin $G$ and two doses benzathine penicillin. There are no specific treatment recommendations for syphilitic gummas, but we extrapolated based on recommendations for CNS syphilis.

CONCLUSION: 1) Syphilitic gummas should be considered in patients with mass lesions and evidence of latent syphilis 2) Recognize that syphilis can have unusual presentations in HIV infection

\section{AN UNUSUAL PRESENTATION OF GOUT: CHRONIC BACK PAIN.}

JACOB L. THOMAS ${ }^{1}$; Scott Friedberg ${ }^{2}$; Emmanuelle B. Yecies ${ }^{2} .{ }^{1}$ University of Pittsburgh, Pittsburgh, PA; ${ }^{2}$ University of Pittsburgh Medical Center, Pittsburgh, PA. (Control ID \#3186384)

LEARNING OBJECTIVE \#1: To recognize the symptoms and presentation of advanced gout

LEARNING OBJECTIVE \#2: To assess how bias limited the differential diagnosis of this patient

CASE: $\mathrm{Mr}$. C is a 68 year old white veteran with a history significant for diabetes mellitus, chronic kidney disease, gout, and chronic back pain who presented with acute worsening of his back pain. Patient reported $9 / 10$ pain in his upper back that radiated with a burning sensation down his right arm and over his right rib cage. The pain interfered with his sleep and he was unable to tolerate laying flat. He denied any other neurologic symptoms. Physical exam was notable for an obese male with mild diffuse thoracic paraspinal tenderness with otherwise normal, non-focal neurological exam. In terms of relevant medications, he was taking allopurinol and furosemide. Non-contrasted CT scan of the cervical and thoracic spine were obtained. Formal Radiology interpretation described a lesion adjacent to T8/9 with erosion into the bone, suspicious for a malignancy, but the evaluation was limited due to lack of contrast. Follow-up MRI with contrast revealed the erosion was smooth suggesting possible neurofibroma. Biopsy demonstrated tophaceous lesions consistent with gout.

IMPACT/DISCUSSION: Gout is a relatively common disease that affects between $3-6 \%$ of men and $1-3 \%$ of women in the developed world. It is caused by the deposition of monosodium urate crystals into joints and soft tissues which can cause an inflammatory response and pain. Risk factors for gout include obesity, chronic kidney disease, diuretic use, heavy alcohol use, and hyperuricemia, nearly all of which were present in our patient. As gout progresses, patients can develop tophi, granulomatous nodules that form in response to the crystalline deposits. Classically, tophi form in the joints of the hands, elbows, and feet, but they can form in any bone or soft tissue. Although the spine is an uncommon location, there are many case reports of spinal gout that can cause symptoms as severe as cord compression and radiculopathy. One review by Ribeiro da Cunha (2018) found over 100 reported cases of spinal gout. Despite this patient's known history of gout, it was not considered in the differential until the pathology report. Part of this can be attributed to authority bias and premature closure. In our case, authority bias manifested after the initial imaging when both Radiology and Neurosurgery expressed concern for malignancy. With malignancy being common and specialists suggesting this was a consistent presentation, premature closure was reached and no other diagnoses were considered. Representative restraint bias contributed as well. With his use of allopurinol and a lack of classic joint symptoms, we did not consider this unusual presentation of a common problem, and our illness script for gout was not triggered.
CONCLUSION: Gout is a common problem that can present with many atypical symptoms and should be considered for patients at risk.

\section{AN UNWELCOME SOUVENIR: RARE MYCOBACTERIUM IN- FECTION IN PATIENT WHO RECEIVED ABDOMINOPLASTY ABROAD}

Ann Wang; Alexa Yuen; Priya Nori; Matthew Shaines. Montefiore Medical Center, Bronx, NY. (Control ID \#3186304)

LEARNING OBJECTIVE \#1: Recognize the growing incidence of rare infections in patients participating in medical tourism

LEARNING OBJECTIVE \#2: Manage patients presenting with mycobacterium infections after undergoing surgical procedures abroad

CASE: A 49F presented to the hospital with abdominal pain and drainage. She had received an abdominoplasty and liposuction in the Dominican Republic 7 months prior. She reported normal healing until about 6 months post-operatively, when she began noticing a bump in her lower abdomen, and yellow drainage from her bellybutton. She then had worsening lower abdominal pain and presented to the hospital. Computed tomography scan of the abdomen and pelvis showed an intra-abdominal abscess measuring $7.2 \mathrm{~cm}$ at the largest dimension. She underwent ultrasound-guided drainage of the abscess and was empirically started on a 2 week course of amoxicillin-clavulanate at discharge. Cultures from the abscess were then found to be growing Mycobacterium senegalense. The Department of Health was notified, and the cultures were sent for sensitivity testing. Approximately 1 month later, she returned to the hospital with worsening abdominal pain and drainage. She was found to have a re-accumulation of the abscess. She again underwent drainage. By this time, sensitivities of the M. senegalense returned. The organism was found to be pan-sensitive, and she was started on a course of oral doxycycline and levofloxacin. She was discharged with a planned 6 week course, and follow-up in Infectious Disease clinic.

IMPACT/DISCUSSION: Up to 750,000 US residents per year travel abroad for medical care. This number will likely increase due to increasing ease of travel and perceived cost savings. The American Medical Association recommends that patients seek out internationally accredited medical facilities. However, standards for accreditation vary, and there is no evidence to suggest that outcomes are improved in accredited facilities. An outbreak of mycobacterial infections has been associated with medical tourism, particularly to the Dominican Republic. A report published in 2016 identified 21 cases in 6 US states in medical tourists to the Dominican Republic. Mycobacterium associated with medical tourism often have significant drug resistance, requiring long courses of multiple anti-microbials for effective treatment. In addition, surgical debridement is often necessary. Mycobacterium senegalense has primarily been identified as a bovine pathogen affecting cattle in East Africa. There have been rare case reports of human infection by M. senegalense, but primarily in immunosuppressed patients.

CONCLUSION: Patients who are considering obtaining surgical procedures abroad should be counseled on the risks of infection even in accredited facilities. Clinicians should maintain a high level of suspicion for mycobacterium infection for patients who present with wound infections after receiving surgeries abroad. As the medical tourism industry expands, rate of rare infectious complications will likely also increase.

\section{ANCHORING AND CONFIRMATION BIAS IN TERMINAL DIAGNOSES}

Matthew Lipinski ${ }^{1}$; Ronald Miller ${ }^{2}$. ${ }^{1}$ Penn State University at Hershey Medical Center, Hummelstown, PA; ${ }^{2}$ Penn State University at Hershey Medical Center, Hershey, PA. (Control ID \#3185714)

LEARNING OBJECTIVE \#1: Review anchoring and confirmation bias. LEARNING OBJECTIVE \#2: Discuss the importance of ruling out reversible causes before diagnosing a terminal illness. 
CASE: A 64 year-old man presented with new onset abdominal swelling. $\mathrm{He}$ has alcoholic cirrhosis and heart failure with preserved ejection fraction, and takes furosemide and spironolactone. Four liters of ascites was drained by paracentesis, and there was no spontaneous bacterial peritonitis. Creatinine was elevated to more than double his baseline, urine sodium was low. Hepatic duplex ruled out any venous thromboses. Diuretics were held due to concern for hepatorenal syndrome, and he was given large volumes of $25 \%$ albumin infusions. His creatinine continued to worsen, and he was started on midodrine and octreotide. Echocardiogram showed he was not a candidate for transjugular intrahepatic portosystemic shunt (TIPS) due to elevated right heart pressures. Given his thoracic aortic aneurysm, he was not a candidate for liver transplant. He was not interested in hemodialysis. In light of him being a candidate for neither TIPS nor liver transplant, he was given a terminal diagnosis of hepatorenal syndrome. Palliative Care was consulted, and the plan was for the patient to go home on hospice. We were consulted as the Nephrology service to help prognosticate regarding hepatorenal syndrome. We noticed he never received diuretics during his hospital stay. Given lack of diuretic trial and elevated right heart pressures, we did not feel that cardiorenal syndrome was fully ruled out. We recommended a trial of aggressive diuresis. Over the next three days his net output was several liters more than his net intake, and his creatinine markedly improved. After reaching euvolemia, echocardiogram was repeated and right heart pressures were remarkably lower. His creatinine returned to his baseline, and cardiorenal syndrome was determined to be the actual diagnosis. He was discharged home on higher doses of diuretics, and continues to do well with no further kidney issues.

IMPACT/DISCUSSION: Anchoring and confirmation bias occur when a clinician relies too heavily on an initial diagnosis and does not reevaluate the thought process as more clinical information becomes available. They are among the more common of the cognitive biases frequently encountered in medicine. These closely related biases can be incredibly detrimental to patient care, often leading to misdiagnosis. Since incorrectly diagnosing a terminal condition can lead to premature withdrawal of care and subsequent loss of potential time and health, it behooves us to constantly analyze our clinical data.

CONCLUSION: Cognitive biases can be difficult to avoid, and can cause patient harm if not corrected. Constantly reassessing the available clinical information from different angles helps prevent it. Any patient being given a terminal diagnosis requires a thorough re-evaluation of the clinical data, given the life-altering and potentially life-limiting impact of this news.

\section{ANCHORS AWAY: DIAGNOSING ACUTE HEPATITIS C IN A PATIENT WITH RECENT NONREACTIVE HEP C ANTIBODY AND HISTORY OF ACETAMINOPHEN INGESTION}

Raj Dalsania; Jill Allenbaugh. Temple University, Philadelphia, PA. (Control ID \#3160165)

LEARNING OBJECTIVE \#1: Cite the importance of the time course of an acute Hepatitis $C$ infection

LEARNING OBJECTIVE \#2: Name consequences of anchoring bias in clinical reasoning

CASE: A 29 year old Spanish-speaking male with a past medical history of intravenous drug use (last use 3 months ago), depression, and chronic back pain presented to the ED complaining 4 days of dark urine, abdominal pain, nausea, and vomiting. Pertinent labs included an elevated total bilirubin of 5.2, ALT 927 and AST 564 with normal alkaline phosphatase and PT, INR, PTT. The patient reported taking about 8-10 acetaminophen tablets a day along with 2-3 oxycodone/acetaminophen pills over the past 3 weeks in order to better control his back pain. A chart review showed a negative Hepatitis $\mathrm{C}$ antibody from 16 days prior. An acetaminophen level was $<10 \mathrm{ug} / \mathrm{mL}$, but there was high suspicion for acetaminophen overdose so the patint was admitted and started on NAC. Despite NAC treatment, the patient's LFTs rose, peaking at ALT 1143 and AST 679.
Further work up included an infectious work up which was negative for CMV, HSV, EBV, Hep A, and Hep B, and an autoimmune work up which revealed a positive smooth muscle antibody. A repeat Hep $\mathrm{C}$ antibody was positive with RNA of 752,428 . Based on these new findings, NAC was stopped, he was managed conservatively with symptom improvement, and he was discharged to follow up with Hepatology.

IMPACT/DISCUSSION: This case represents the importance of avoiding anchoring bias in medicine. The patient's presentation seemed like a "slam dunk" diagnosis with elevated liver enzymes, abdominal pain, excess acetaminophen use, and a recent negative Hep C antibody screen. However, the negative acetaminophen level, and lack of improvement on NAC forced a reevaluation of the patient that ultimately led to a conclusive diagnosis. Although the majority of patients acutely infected with Hepatitis $\mathrm{C}$ are asymptomatic, some experience jaundice, nausea, vomiting, and abdominal pain. These symptoms manifest anywhere from 2 and 26 weeks after Hepatitis C exposure with RNA being detectable by PCR within a few days, but anti-HCV antibodies needing weeks to become detectable. This patient had a negative HCV antibody just 16 days prior. At that time, the patient's immune system had not mounted a sufficient enough antibody response for detection and thus the diagnosis was almost missed. Had this diagnosis been missed again the patient would have had above $50 \%$ chance of developing chronic Hepatitis C and the sequela that comes with that diagnosis.

CONCLUSION: Anchoring bias is one of the most common clinical reasoning biases. This reinforces the importance of investigating all causes of a disease manifestation especially when clinical data does not support the suspected diagnosis. In this case, the negative acetaminophen level and continued worsening LFTs despite treatment led us to reevaluate the diagnosis and we were able to successfully diagnose this patient with acute Hepatitis $\mathrm{C}$ which reduced chances of transmission to others and allowed for treatment.

\section{ANCHORS AWAY: DRIFTING ON A SEA OF ASSUMPTIONS}

Tiffany L. Brazile ${ }^{2}$; Briana P. Patton ${ }^{1}$; Tanya Nikiforova ${ }^{2}{ }^{1}$ University of Pittsburgh School of Medicine, Pittsburgh, PA; ${ }^{2}$ University Of Pittsburgh Medical Center, Pittsburgh, PA. (Control ID \#3180789)

LEARNING OBJECTIVE \#1: Identify three essential tests to evaluate a patient with new-onset dyspnea

LEARNING OBJECTIVE \#2: Define framing, anchoring, and availability biases and identify how they can contribute to diagnostic error CASE: MG is a 60 y.o. female nurse who presented with one year of dyspnea. Over the last 3 months, her symptoms evolved to dyspnea, chest pressure, and fatigue with activity at 4 METS, as well as abdominal cramping and non-bloody emesis. Her history included subtotal colectomy, GI bleed, HTN, and left ventricular hypertrophy. MG initially presented to her cardiologist. On exam she was afebrile, had normal S1, S2 without murmurs or gallops, no JVD, and lungs were clear to auscultation. Her EKG showed a chronic left bundle branch block (LBBB). A stress cardiac MRI showed normal EF and absence of ischemic or infiltrative disease. MG was then referred to a pulmonologist. PFTs and CXR were negative. An ABG showed normal oxygenation and incidentally revealed a hemoglobin of 5 . She was subsequently hospitalized. Her labs were consistent with profound iron deficiency anemia (IDA). Upon further questioning, MG reported taking ibuprofen daily for the past 8 months and recent dark stools. EGD and flexible sigmoidoscopy were concerning for an occult GI bleed from her ileosigmoid anastomosis.

IMPACT/DISCUSSION: Blood loss is the most common cause of IDA worldwide and can be difficult to recognize if the source is not obvious. MG's presentation of exertional dyspnea, exercise intolerance, and angina are common symptoms of anemia, however, the biases of the patient and her physicians delayed diagnosis and treatment by 3 months. The workup for dyspnea depends on the history and physical exam, and almost always begins with a $\mathrm{CBC}, \mathrm{SpO}$, and EKG. Despite an unremarkable physical 
exam, the clinicians pursued a costly workup of cardiac and pulmonary etiologies and did not include anemia in their initial differential. Diagnostic error may result in up to 80,000 hospital deaths per year and is most commonly caused by cognitive biases. Three biases that affected this case are: Framing: MG assumed her symptoms were cardiac in nature and framed them in this context, which impacted how the clinicians viewed her case. Anchoring: The cardiologist may have relied too heavily on the patient's chest pain and LBBB, leading to a focused cardiac workup instead of exploring other etiologies. Availability: The specialists may have overestimated the likelihood of a cardiac or pulmonary cause of her dyspnea based on their familiarity with those diseases. Strategies to overcome these biases include recognition of these cognitive phenomena, enhanced communication among clinicians, and use of a cognitive forcing strategy to promote sound clinical reasoning.

CONCLUSION: Diagnostic error, most commonly caused by cognitive biases, remains an underestimated threat to patient safety and high-value health care. Acknowledging and assessing one's biases is an important first step in developing mindful practices to improve diagnostic accuracy and patient care.

\section{ANGIOEDEMA UNMASKING DIAGNOSIS OF COLORECTAL CANCER}

Niaz Memon; Kathleen Mccabe; Emily Silverman; Imran Naqvi. Mercy Health Jewish Hospital of Cincinnati, West Chester, OH. (Control ID \#3186379)

LEARNING OBJECTIVE \#1: Recognize various presentations of CRC.

LEARNING OBJECTIVE \#2: Understand the relationship between severe iron deficiency anemia and the possibility of developing chronic urticaria and acute angioedema

CASE: A 75 yo African American male with PMHx HTN, CKD, and DM-2 presents to the ED with tongue swelling, dysphagia, and shortness of breath. He tried epinephrine shot without relief. History includes previous episodes of angioedema over a one-year period with no triggering event. He was seen by allergist and was negative for complement disorders or allergies. He was not on ACE-I. Patient had unintentional weight loss of 50lbs and complained of nausea/vomiting. Denied significant family history. Physical exam revealed cachectic appearing male with tenderness to palpitation over abdomen. Labs showed hemoglobin of 8.3 with iron studies consistent with iron deficiency anemia. CT scan of abdomen revealed large mass within proximal ascending colon. Colonoscopy showed circumferential mass with biopsies consistent with adenocarcinoma. Patient underwent laparoscopic and did well post-operatively.

IMPACT/DISCUSSION: Angioedema is a common disorder that is seen in both children and adults. Typically, it is mast cell- and bradykininmediated. We introduce an elderly patient who has been intubated multiple times from anaphylaxis over a 1-year period without a clear underlying cause. There are reports that link iron deficiency anemia with the development of chronic urticaria leading to acute angioedema. Murine models suggest that the pathophysiology involves uninhibited histamine release by iron binding proteins. Though our patient was anemic, his workup was delayed due to larger concern for recurrent anaphylaxis. However, after treating the anemia, he showed resolution of the angioedema. This case highlights the various presentations of colorectal cancer, and we believe clinicians should include iron deficiency anemia as a differential for angioedema when other causes are absent.

CONCLUSION: Colorectal carcinoma with iron deficiency anemia may lead to the development of chronic urticaria presenting as acute angioedema

\section{ANKYLOSING SPONDYLITIS FLARE MASQUERADING AS RECURRENT DIGIT SWELLING}

Yen Cao; Ahl Jeffrey Caseja; John Cadavona. UNLV, Las Vegas, NV. (Control ID \#3186121)
LEARNING OBJECTIVE \#1: Recognize ankylosing spondylitis as a possible cause of recurrent digit pain and swelling

LEARNING OBJECTIVE \#2: Recognize the importance of careful history taking in the diagnosis ankylosing spondylitis flare in order to avoid delay in treatment

CASE: An 85 year old male with a medical history of hypertension, chronic back pain, hyperlipidemia, and uveitis, presented with diarrhea, knee swelling, and recurrent right third digit swelling. Patient reports a history of recurrent third digit swelling and was seen in the emergency department four times and treated for cellulitis with clindamycin. On evaluation, patient had right 3rd proximal interphalangeal pain with left knee swelling and bilateral ankle pain swellings. Further history taking reveals patient had history of recurrent left knee and ankle swelling, back and knee stiffness. Xrays show calcific enthesopathy of Achilles tendon of right ankle and left knee. Chart review revealed an old lumbar spine x-ray from two years ago that missed the documentation of classic bamboo spine with flowing ossification of annulus fibrosus and succinct syndesmophytes. Patient was treated with methylprednisolone IV followed by a prednisone taper on discharge with reported resolution of acute symptoms.

IMPACT/DISCUSSION: Ankylosing spondylitis is a chronic inflammatory disease that involves the axial skeleton, entheses, and peripheral joints. Ankylosing spondylitis has a male predominance of 2:1 with a peak age of diagnosis between age 20-30 years and onset can occur in adolescence. The most common presenting symptom is back pain but the disease can manifest as hip pain and peripheral arthritis. Active inflammation, or flares, can present with joint tenderness, particularly sacroiliac joints tenderness. Enthesitis has been reported to occur in $29 \%$ of patient and most often manifests as Achilles tendinitis while dactylitis occurs in about $6 \%$ of patient. Diagnosis of ankylosing spondylitis is non- specific. Clinical diagnosis of ankylosing spondylitis should be considered in patients even with later age onset who presents with continuous chronic back pain enthesitis and dactylitis. X-ray imaging can assists in diagnosis by revealing syndesmophyte formation, squaring of the vertebral bodies, vertebral endplate destruction, and Achilles tendinitis.

CONCLUSION: Ankylosing spondylitis can present with digit swellings or dactylitis. When the diagnosis of ankylosing spondylitis is suspected, a careful history taking is key making the correct diagnosis and guide further evaluations and treatment.

ANTI-MDA5-ASSOCIATED DERMATOMYOSITIS: AN UNCOMMON VARIANT OF INFLAMMATORY MYOPATHY

Shilpa Vasishta ${ }^{1}$; Joshua Khalili ${ }^{2}$; Nasheed Jamal ${ }^{3}$; Jay Persselin ${ }^{1}$. ${ }^{1}$ UCLA, Los Angeles, CA; ${ }^{2}$ UCLA School of Medicine/UCLA Health, Los Angeles, CA; ${ }^{3}$ UCLA, Losa Angeles, CA. (Control ID \#3186630)

LEARNING OBJECTIVE \#1: Recognize clinical features of antiMDA5-associated dermatomyositis

CASE: A 60-year-old female with no significant medical history presented with forty pounds of unintentional weight loss. Outpatient imaging, upper endoscopy, and age-appropriate cancer screening had been unrevealing. The patient reported dyspnea on exertion, proximal myalgias, generalized fatigue, and painful bilateral hand rash. Review of systems was otherwise negative. Vital signs, head, neck, cardiopulmonary, and abdominal exams were normal. Strength was $4 / 5$ in bilateral deltoids and iloapsoas. Skin demonstrated bilateral palmar papules and periungual ulcerations; joint exam was normal. Labs revealed pancytopenia (WBC 2.2, Hb 9.7, plt 135) with elevated inflammatory markers (ESR 65 , ferritin 1968) and positive ANA (1:640, speckled). Further serologies including dsDNA, Sm, RNP, SSA/B, Sc170, centromere, Jo1, Mi2, PL-7/ 12, Mi2, Ku, EJ, OJ, and SRP antibodies were normal. Workup was pursued for suspected dermatomyositis (DM). CK and aldolase were normal, however EMG of the right deltoid revealed irritable myopathy. CT chest showed bibasilar reticulation, and PFTs showed reduced DLCO of $53 \%$ predicted. Skin biopsy of the periungual ulcerations demonstrated 
vasculopathy with fibrin thrombi. Further myositis-specific serologic testing revealed elevated anti-melanoma differentiation-associated gene 5 (MDA5) antibody titers. The patient was thus diagnosed with antiMDA5-associated DM.

IMPACT/DISCUSSION: Anti-MDA5-associated DM is a unique entity characterized by absent or mild proximal myopathy and rapidly progressive interstitial lung disease (ILD). Though pathogenesis is unknown, MDA5 serves immune functions as a RIG I-like receptor in sensing viral antigens, suggesting a possible viral mediator. Anti-MDA5-associated DM was first noted in Japan and accounts for approximately $22 \%$ of DM cases in East Asian populations, versus 7-13\% of those in the US and Europe. Anti-MDA5-associated DM presents with tender palmar papules and digital ulcerations, with histologic features of vasculopathy. The condition is classically "amyopathic" or "hypomyopathic", given prominent skin findings in the presence of normal or only mildly reduced strength and normal CK. Significant weight loss may reflect esophageal dysfunction. Ferritin levels may be markedly elevated and correlate with disease activity. MDA5-associated ILD tends to progress rapidly and confers poor prognosis. Our patient presented with several hallmark features of anti-MDA5-associated DM. She is currently undergoing evaluation for immunosuppressive therapy. While optimal treatment remains unknown, case series note favorable responses to steroids, calcineurin inhibitors, cyclophosphamide, rituximab, and in refractory cases, IVIG or plasma exchange.

CONCLUSION: Anti-MDA5-associated DM is a unique entity to be considered in suspected DM cases with prominent cutaneous and pulmonary features with or without muscle involvement.

\section{ANTIBIOTIC-INDUCED RADIATION RECALL DERMATITIS}

Ian Garrahy ${ }^{1}$; Daniel Forman ${ }^{2}$; Kyle Macaulay ${ }^{1} .{ }^{1}$ Tower Health System, Wyomissing, PA; ${ }^{2}$ Tower Health, Reading, PA. (Control ID \#3186296)

LEARNING OBJECTIVE \#1: Radiation recall dermatitis is a rare inflammatory skin condition that occurs over a previously irradiated field most commonly triggered by chemotherapy.

LEARNING OBJECTIVE \#2: There are rare instances of antibioticinduced RRD.

CASE: Radiation recall dermatitis (RRD) is an inflammatory skin reaction that develops in a previously quiescent radiation field triggered most commonly by chemotherapy, particularly anthracyclines and taxanes. Radiation-recall dermatitis secondary to antibiotic therapy is quite rare. The patient is 61 year old female with a past history of squamous cell carcinoma of the left breast treated with neoadjuvant carboplatin, paclitaxel, and anthracycline chemotherapy followed surgery, then subsequent whole breast radiation to $6040 \mathrm{cGy}$. Eight years after completion of her radiation she developed diffuse redness of the left breast after two doses of nitrofurantoin taken for a urinary tract infection. On examination, vital signs were stable and she had an erythematous and excoriated rash on her left breast that clinically appeared to be cellulitis. Given her clinical history, coupled with the temporal relationship of starting an antibiotic, strong consideration was given for antibiotic-induced RRD. Nitrofurantoin was discontinued and the rash progressively resolved within several days. This case demonstrates an example of RRD which is the development of an acute inflammatory skin reaction of a previously irradiated area most commonly triggered by chemotherapeutic agents. This case highlights antimicrobial therapy as a rare cause of RRD and underscores the importance of considering RRD in a patient presenting with an acute rash over a previously irradiated area while on antimicrobial therapy.

IMPACT/DISCUSSION: RRD is an inflammatory skin condition that occurs over a previously quiescent irradiated field. It is most commonly caused by chemotherapeutic agents particularly the taxanes. However, this case higlights one of the rare instances in which antibiotics can cause RRD. CONCLUSION: RRD should be considered in all patients taking antibiotics who present with a rash over a previously quiesent irradiated field.
APPLICATION OF A NEW NONINASIVE DIAGNOSTIC APPROACH TO TRANSTHYRETIN CARDIAC AMYLOIDOSIS

Katryna Lim. Emory University, Atlanta, GA. (Control ID \#3185844)

LEARNING OBJECTIVE \#1: Recognize the unique clinical features of transthyretin amyloidosis.

LEARNING OBJECTIVE \#2: Consider a new noninvasive diagnostic approach to cardiac amyloidosis.

CASE: A 75 year-old African American male presented with two months of progressive dyspnea on exertion, orthopnea, and bilateral leg swelling. He had a history of carpal tunnel syndrome, hypertension, atrial fibrillation, chronic kidney disease, chronic heart failure with an ejection fraction of $25 \%$, and had an implantable cardiac defibrillator placed two years prior to this admission. Past workup included a left heart catheterization without coronary artery disease. He had been taking bumetanide, carvedilol, losartan, hydralazine HCL/isosorbide dinitrate, and apixaban. His blood pressure was $119 / 85$, heart rate 101 , respiratory rate 18 and oxygen saturation $98 \%$ on $2 \mathrm{~L}$ nasal cannula. He appeared comfortable sitting upright with jugular venous distention to the level of his mid-neck at 90 degrees. He had an irregularly irregular rhythm, an S3 gallop and a grade 3/6 systolic murmur at the left lower sternal border. Lung exam revealed bibasilar crackles. There was pitting edema to the knees bilaterally and his extremities were cool. Initial laboratory evaluated revealed sodium 138, potassium 3.7, BUN 48 and creatinine 2.29. BNP was 2,403 and troponin 0.13. EKG revealed atrial fibrillation with low voltage. Chest x-ray revealed bilateral pleural effusions. Echocardiogram revealed left ventricular ejection fraction of $10 \%$, grade 2 diastolic dysfunction, severely reduced right ventricular systolic function and a bright, thick myocardium. Further workup included a normal serum and urine immunofixation and serum free light chain assay. Nuclear bone scintigraphy findings were positive for transthyretin cardiac amyloid. Clinically, he worsened despite aggressive diuresis and inotropic support. He was discharged to home hospice.

IMPACT/DISCUSSION: Hospitalists commonly care for patients with chronic diastolic heart failure and evidence of hypertrophy; however, the etiology is not always clear. The prevalence of cardiac amyloidosis is surprisingly high and should be considered. Unique clinical features of transthyretin amyloidosis (the most common subtype) include bilateral carpal tunnel syndrome, spinal stenosis, peripheral neuropathy, and spontaneous biceps tendon rupture. Once cardiac amyloidosis is suspected and $\mathrm{AL}$ amyloidosis is ruled out, recent data support the use of nuclear bone scintigraphy to detect and confirm transthyretin cardiac amyloid. This is a noninvasive alternative to biopsy in making this diagnosis. We successfully confirmed that our patient had transthyretin cardiac amyloidosis using this approach.

CONCLUSION: Cardiac amyloidosis, specifically transthyretin substype, is common and often underdiagnosed. Thus, it is important for clinicans to recognize its unique clinical features, including carpal tunnel syndrome, spinal stenosis and biceps tendon rupture, and to consider using nuclear bone scintigraphy when making this diagnosis.

\section{APPRECIATING AMBIGUITY: ATYPICAL TYPHOID FEVER COUPLED WITH UNCERTAINTY IN SUSCEPTIBILITY TESTING}

Sarah W. Takimoto; Therese Chan Tack. University of California, San Francisco, San Francisco, CA. (Control ID \#3162818)

LEARNING OBJECTIVE \#1: Diagnose typhoid fever in a returning traveler.

LEARNING OBJECTIVE \#2: Recognize treatment failure due to antibiotic resistance despite reassuring susceptibility testing.

CASE: A 32-year-old man presented to the emergency room with one week of intermittent fevers that began after returning from Pakistan. He reported a previous sore throat, but had no localizing symptoms and was 
afebrile in the ED. He was diagnosed with a viral upper respiratory infection. Two weeks later, he presented again for ongoing fevers with a new $12 \mathrm{lb}$ weight loss. He denied cough, diarrhea, abdominal pain, or rash. He was afebrile with a normal CBC and BMP. He was discharged with plans for further outpatient work up. The next day he was called back for positive blood cultures. At this presentation, he was tachycardic and febrile to 39.4C. He was admitted and given one dose of piperacillintazobactam 4.5g IV. On Hospital Day 2, cultures grew Salmonella enterica serotype Typhi ( $S$. Typhi). The California Department of Public Health was notified and he was narrowed to ceftriaxone 2g IV QD x 4 days. He was discharged on Hospital Day 4 on ciprofloxacin 500mg PO BID x 10 days. One month later, the patient presented to his primary care physician with three days of fevers and fatigue. A blood culture was positive for $S$. Typhi. He was restarted on ciprofloxacin, and an e-consult was sent to infectious disease. Per infectious disease, susceptibility testing showed a ciprofloxacin minimum inhibitory concentration (MIC) of 0.5 $\mu \mathrm{g} / \mathrm{mL}$. This was reported as susceptible; however, a review of antimicrobial break points set by the US Food and Drug Administration revealed a recent change. Previously, $S$. Typhi was under Enterobacteriaceae $(<1$ $\mu \mathrm{g} / \mathrm{mL}=$ Susceptible), but increasing ciprofloxacin resistance resulted in new break points for extra-intestinal $S$. Typhi infections $(<0.06 \mu \mathrm{g} / \mathrm{mL}=$ Susceptible, $0.12-0.5 \mu \mathrm{g} / \mathrm{mL}=$ Intermediate, $>1 \mu \mathrm{g} / \mathrm{mL}=$ Resistant $)$. The report for ciprofloxacin was amended to intermediate. A comment on intermediate results stated that ciprofloxacin-treated patients may have delayed response or clinical failure. After reviewing this, the patient was started on ceftriaxone $2 \mathrm{~g}$ IV QD x 14 days. He had a resolution of symptoms and negative cultures.

IMPACT/DISCUSSION: $S$. Typhi is a gram-negative enteric rod that causes extra-intestinal infection, often referred to as typhoid fever. In the United States, 200 to 300 cases are reported each year with 80 percent in returning travelers. The infrequency with which providers encounter this disease creates a diagnostic challenge when patients present atypically. This is compounded by increasing ciprofloxacin resistance. Both early diagnosis and appropriate break point interpretation are critical to effective treatment of this communicable disease.

CONCLUSION: - Typhoid fever should be on the differential for an acute/subacute fever in a returning traveler. - Clinicians and laboratory workers need to be aware of increasing antibiotic resistance and resulting changes in susceptibility guidelines.

\section{AT A STUDENT-RUN FREE CLINIC, MULTIDISCIPLINARY ADVOCACY LEADS TO IMPROVED CANCER CARE}

Lisle Winston ${ }^{2}$; Ilana S. Lavina ${ }^{2}$; Sonia Iosim ${ }^{2}$; Pamela Charney ${ }^{1}$. ${ }^{1}$ Weill Cornell Medical College, New York City, NY; ${ }^{2}$ Weill Cornell Medicine, New York, NY. (Control ID \#3185925)

LEARNING OBJECTIVE \#1: Student's advocacy efforts are often multidisciplinary and time intensive, but can assure high quality patient care.

CASE: A 57-year-old G4P1031 woman with no past medical history presented to a student-run free clinic for her annual well-woman exam. She had menarche at age 14, menopause at age 49, and never used HRT. Family history included a sister diagnosed with stage III breast cancer three years prior. Of note, the patient had a BIRADS-2 mammogram 2 years prior, but had been unwilling to schedule interval screening breast imaging. The patient's physical exam, including breast and axillary lymph node exam, was unremarkable. Routine screening mammogram and ultrasound were scheduled. Mammography demonstrated a spiculated mass in the upper outer quadrant of the left breast, corresponding to an irregular vascular mass seen on ultrasound. Ultrasound-guided needle biopsy revealed invasive ductal carcinoma and DCIS, and the patient was scheduled for the next available appointment at the free clinic. During the interval 4 days, team members representing multiple disciplines collaborated to coordinate care for this patient. Medical student Clinical Directors arranged breast surgery appointments at two public hospitals and obtained discs of the breast imaging studies and pathology slides for the accepting breast surgeon. Simultaneously, team members worked with the affiliate hospital's social work and finance departments to re-enrolling the patient in health insurance because the patient desired to seek care from the same breast surgeon who had operated on her sister, who was in private practice. With effort, a internist who spoke her language was identified who would accept her limited insurance as well. When the patient arrived to her follow-up appointment, medical students used the SPIKES protocol to communicate the diagnosis. The patient's primary language was not English, but she had been paired with a volunteer firstyear medical student who spoke her primary language through our program aimed to maximize culturally competent care. This student played a key role in communication about the myriad of logistical arrangements. The patient was ultimately re-enrolled in health insurance. The same surgeon who operated on the patient's sister operated on the patient. She completed radiation therapy and is now taking hormone therapy. She now follows with a primary care phsycian near her home that speaks her language. Her prognosis is excellent.

IMPACT/DISCUSSION: Team members from several disciplines and levels of training, including medical students, attending physicians, social workers, and finance administrators, worked together to care for a vulnerable patient. After this case, our clinic implemented a "triage" system, which can be used when routine screening in a primary care setting detects life-threatening time-sensitive disease.

CONCLUSION: Effective advocacy for underserved patients often requires multi-disciplinary collaboration.

\section{ATELZOLIZUMAB INDUCED MYOCARDITIS ON A BACK- GROUND OF CARDIAC AMYLOIDOSIS}

Rahul Gupta ${ }^{1}$; Syed Zaid ${ }^{1}$; Amer Sayed ${ }^{1}$; Purva Ranchal ${ }^{2}$; Leonardo L. Zelaya Castillo ${ }^{1}$; Elliot Levine ${ }^{1}$; Julio Panza ${ }^{1} .{ }^{1}$ Westchester Medical Center, Valhalla, NY; ${ }^{2}$ westchester medical center, Valhalla, NY. (Control ID \#3174840)

LEARNING OBJECTIVE \#1: Diagnose cardiovascular complications related to immunotherapy

LEARNING OBJECTIVE \#2: Assess patients with myocarditis on background of amyloidosis

CASE: 81 year old man with history of stage 2B lung adenocarcinoma, treated with lobectomy, adjuvant chemotherapy with cisplatin followed by immunotherapy with Atelzolizumab presented to the hospital two weeks after his last infusion of Atelzolizumab with nausea, lightheadedness, diaphoresis and syncope. Vitals and physical exam were unremarkable. EKG demonstrated normal sinus rhythm with first degree AV block, right bundle branch block and left anterior fascicular block. Lab work was notable for troponin level of 19. Transthoracic echocardiogram revealed normal left ventricular systolic function, increased left ventricular wall thickness with an abnormal speckled myocardial appearance. Cardiac catheterization revealed one small vessel coronary artery disease which was not amenable for stenting. The patient subsequently developed slow sustained hemodynamically stable monomorphic ventricular tachycardia refractory to amiodarone and lidocaine. Cardiac MRI showed diffusely elevated T1 signal with apical wall motion abnormalities. Endomyocardial biopsy of the right ventricular septum revealed changes consistent with acute myocarditis, on a background of transthyretin-type cardiac amyloidosis. Patient was started on high dose intravenous methylprednisolone along with beta blocker therapy with resolution of the ventricular tachycardia. However, the patient subsequently developed complete heart block requiring temporary pacemaker. Unfortunately, six days later, the patient developed sudden pulseless electrical activity with cardiac arrest, and despite aggressive resuscitative efforts, the patient expired.

IMPACT/DISCUSSION: Atezolizumab is a monoclonal antibody against programmed death ligand-1 that has shown efficacy against multiple malignancies. Suppression of immune system with immune check points 
inhibitors (ICI) has also been associated with adverse effects on multiple organ systems, including the development of myocarditis, though it is very rare with reported incidence of $0.27-1.14 \%$. There is no evidence to suggest a patient with underlying cardiac amyloidosis is more or less susceptible to the development of an inflammatory process such as myocarditis, even when subjected to an unrestrained immune response. In fact, there is only one case report in the literature of combined myocarditis and amyloidosis. Here we have presented a unique case of a patient who developed acute myocarditis from treatment with ICI's and was found on endomyocardial biopsy to also have a background of cardiac amyloidosis.

CONCLUSION: This case illustrates a possible severe cardiovascular complication of immunotherapy, the utility of myocardial biopsy and possibly the predisposition of this complication by undiagnosed cardiac amyloidosis. Rapid diagnosis of myocarditis in patients with immunotherapy and treatment with steroid therapy might be critical to reduce the immune attack.

\section{ATORVASTATIN-TRIGGERED AUTOIMMUNE HEPATITIS}

M.Usman Ghani ${ }^{1}$; Harish Guddati ${ }^{2}$; Hiliary Hertan ${ }^{2} .{ }^{1}$ Montefiore Medical Center, Wakefield Campus, Bronx, NY; ${ }^{2}$ Montefiore Medical Center, Wakefield Campus, Bronx, NY. (Control ID \#3184053)

LEARNING OBJECTIVE \#1: -Recognize statins as a rare cause of autoimmune hepatitis

LEARNING OBJECTIVE \#2: -To differentiate autoimmune hepatitis from drug induced liver injury

CASE: A 65 year-old woman with primary hypercholesterolemia was admitted to the hospital for generalized weakness with new onset jaundice. She was prescribed atorvastatin $80 \mathrm{mg}$ daily 3 months prior to the onset of her symptoms. Labs revealed Alanine transaminase (ALT): 1786 U/L, Aspartate transaminase (AST): $2763 \mathrm{U} / \mathrm{L}$, Alkaline phosphatase (ALP): $1008 \mathrm{U} / \mathrm{L}$, and total bilirubin $10.4 \mathrm{mg} / \mathrm{dl}$ (direct $7.2 \mathrm{mg} / \mathrm{dl}$ ). Hepatitis serology was negative. Abdominal ultrasound and magnetic resonance cholangiopancreatography was negative for choledocholithiasis. Autoimmune workup was pursed at that time. Serum anti-nuclear antibody was negative; however smooth muscle antibody titers were positive at 1:80 dilutions. Immunoglobulin panel revealed increased serum IgG (3.5g). Liver biopsy was done due to persistently elevated liver enzymes, which revealed plasma cells mixed with eosinophils, neutrophils, lymphocytes, and neutrophils in the portal tracts, with focal centrilobular perivenular inflammation. There was no histologic evidence of fibrosis. Diagnosis of drug-induced AIH was made in view of absence of significant fibrosis, which was most likely triggered by atorvastatin. Atorvastatin was stopped and she was started on prednisone and azathioprine, with gradual normalization of liver tests.

IMPACT/DISCUSSION: Autoimmune hepatitis (AIH) is a chronic inflammatory disease of the liver with unknown etiology. Drugs such as minocycline, nitrofurantoin, and hydralazine are associated with AIH. Only a few cases of statin-induced AIH have been reported in literature. We describe a case of $\mathrm{AIH}$ that was potentially triggered by high-dose atorvastatin. Statins are commonly prescribed cholesterol lowering therapy, and are known to cause mild asymptomatic elevations in liver enzymes. In rare cases, especially with high doses, they may trigger AIH and liver failure. In this patient, presence of circulating auto-antibodies and hypergammaglobulinemia indicated an underlying autoimmune process. Liver biopsy was considered because of persistently high transaminases with bilirubin elevation despite discontinuation of atorvastatin. One of the helpful points in distinguishing AIH from drug-induced liver injury is absence of fibrosis and eosinophilic infiltration, like in our case. Treatment includes stopping the offending drug and immunosuppression with prednisone with or without azathioprine in severe cases.

CONCLUSION: Statins are rarely implicated in autoimmune hepatitis. Patients who are on statins and have persistently abnormal liver should be worked up for autoimmune hepatitis. The diagnosis is confirmed by serologic evidence of autoantibodies and gamma globulins, followed by liver biopsy.
ATYPICAL PRESENTATION OF CRYPTOCOCCAL MENINGOENCEPHALITIS IN A SARCOIDOSIS PATIENT.

Shreya Gor; Taimoor Khan; Deborah Alpert. Jersey shore university medical center, Neptune, NJ. (Control ID \#3179763)

LEARNING OBJECTIVE \#1: Appreciate the differential diagnosis of new neurologic symptoms in a sarcoidosis patient.

LEARNING OBJECTIVE \#2: Recognize the importance of lumbar puncture in establishing a diagnosis for neurologic symptoms in an immunocompromised patient.

CASE: 41 year-old male with a history of sarcoidosis presented with right upper extremity parasthesias, facial droop and slurred speech. He was previously treated with intermittent steroids and methotrexate, but had not received immunosuppressive therapy for two years. He had a normal complete blood count, metabolic panel and inflammatory markers. CT brain revealed a curvilinear diminished attenuation within the left temporal lobe. MRI brain demonstrated an abnormal area of enhancement in the left Sylvian fissure, concerning for venous thrombosis, meningoencephalitis or focal vasculitis; there was no leptomeningeal enhancement. MR venogram was suspicious for left transverse and sigmoid sinus thromboses and cerebral angiogram showed irregularity in left middle cerebral artery. Antiphospholipid antibodies were not detected. Neurological symptoms promptly resolved after empiric treatment with intravenous dexamethasone for presumed neurosarcoidosis. He refused lumbar puncture until hospital day 5. At that time, cerebrospinal fluid (CSF) demonstrated a white blood cell (WBC) count of $308 / \mathrm{mm}^{3}$ with $95 \%$ neutrophils (normal 0-5 WBC), glucose $18 \mathrm{mg} / \mathrm{dl}(40-60 \mathrm{mg} / \mathrm{dl})$, protein $115 \mathrm{mg} / \mathrm{dl}$ $(15-60 \mathrm{mg} / \mathrm{dl})$ and a positive cryptococcal antigen. Opening pressure was not obtained. Serum latex agglutination and CSF cultures were positive for Cryptococcus neoformans. HIV testing was negative and flow cytometry revealed lymphopenia with an absolute CD4 count of 293. Intravenous steroids were discontinued and the patient was treated with intravenous amphotericin and flucytosine. He was discharged on oral fluconazole for eight weeks

IMPACT/DISCUSSION: Cryptococcal meningitis typically presents as chronic meningitis with symptoms including headache, fever, lethargy, sensory and memory deficits, cranial nerve paresis, vision deficits and meningismus. Our sarcoidosis patient presented with symptoms mimicking a transient ischemic attack, and his presentation and imaging findings were initially attributed to neurosarcoidosis. Although neurologic symptoms responded to empiric high-dose steroids, CSF findings were concerning for infection and cryptococcal meningitis was diagnosed. Sarcoidosis patients are predisposed to opportunistic infections due to impairment of cell-mediated immunity and immunosuppressive therapy. Cryptococcal meningitis may be confused with neurosarcoidosis, as both may present with cranial mononeuropathy and meningeal involvement. Leonhard et al.(2016) reviewed 40 cases of cryptococcal meningitis in sarcoidosis patients and noted CD4 lymphopenia in all, similar to our patient.

CONCLUSION: It is crucial to have a high index of suspicion for infectious etiology in a sarcoidosis patient presenting with new neurological symptoms. Delay in diagnosis may lead to unfavorable outcomes.

\section{ATYPICAL PRESENTATION OF SEVERE HYPOTHYROIDISM AS CARDIAC TAMPONADE}

Saliha Saleem; Ejaz Shah; Colette Knight. Montefiore Medical Center, Bronx, NY. (Control ID \#3185724)

LEARNING OBJECTIVE \#1: Recognize cardiac tamponade as one of the presentations of severe hypothyroidism

LEARNING OBJECTIVE \#2: Distinguish between signs and symptoms of cardiac tamponade in a hypothyroid patient versus a classic presentation 
CASE: 46 year-old woman with hypertension and hypothyroidism came with shortness of breath (SOB) for 1 week. She reported being nonadherent to all medications. On presentation blood pressure (BP) was 202/112 mmHg, heart rate (HR) $62 \mathrm{bpm}$ and Oxygen saturation was 98\%. Physical examination was significant for bibasilar crackles. No pedal edema or JVD was found. Initial labs revealed normal troponin but elevated Pro Brain - Natriuretic Peptide. Thyroid function tests (TFT) showed TSH $>100 \mathrm{uU} / \mathrm{ml}$ and Free T4 $0.5 \mathrm{ng} / \mathrm{dl}$. Electrocardiogram (EKG) revealed sinus rhythm and nonspecific ST- T wave changes. Chest $\mathrm{X}$-ray was consistent with pulmonary vascular congestion. She was initially treated for hypertensive emergency with nitroglycerin drip and furosemide. Blood pressure improved but she continued to complain of SOB. Transthoracic Echocardiography (TTE) was done that showed left ventricular hypertrophy (LVH) and large pericardial effusion with evidence of early diastolic right heart collapse. Echo guided pericardiocentesis was done. Her symptoms improved drastically. She was restarted on levothyroxine and her antihypertensives.

IMPACT/DISCUSSION: Commonly reported cardiac manifestations of hypothyroidism include decrease in cardiac output, systolic and diastolic cardiac dysfunction, bradycardia and hypertension. Mild pericardial effusion is common, especially in cases of moderate to severe hypothyroidism. However, presentation of severe hypothyroidism as cardiac tamponade is rare. Our case reports an atypical presentation of hypothyroidism as cardiac tamponade and hypertensive emergency. Cardiac tamponade classically presents as Becks' triad of low blood pressure, distended neck veins and distant muffled heart sounds. Interestingly all these signs can be absent in patient with hypothyroidism which makes the diagnosis on clinical presentation even more challenging. Moreover, the classic EKG findings of cardiac tamponade: tachycardia, electrical alternans and low voltage EKG may be absent. Long-standing untreated hypothyroidism can result in increased arterial stiffness leading to hypertrophy giving LVH in EKG. Potential combination of LVH and large pericardial effusion can lead to a normal voltage EKG. Management of early tamponade is currently unclear and can involve medical management with close observation versus pericardiocentesis. Our case required pericardiocentesis for symptom control.

CONCLUSION: Severe Hypothyroidism if left untreated can present rarely with cardiac tamponade. Diagnosing cardiac tamponade in a hypothyroid person can be tricky and can delay diagnosis and treatment of this life-threatening presentation. Physicians should keep this rare presentation in mind while managing hypothyroid patients.

\section{ATYPICAL PRESENTATION OF SQUAMOUS CELL CARCINO- MA OF THE LUNG IN A NON-SMOKER}

Manasa Kalluri. Kern Medical Center, Bakersfield, CA. (Control ID \#3186030)

LEARNING OBJECTIVE \#1: Recognize risk factors for squamous cell carcinoma of the lung in a never smoker in order to decrease exposure

CASE: A 63-year-old Caucasian male with hypertension was admitted for worsening dyspnea and productive cough. The patient was a never smoker. He had a 40-year occupational history in mechanical special effects for the movie industry, which involved handling chemicals that included propane, asbestos, silica, and diesel fumes. His occupation also involved extensive foreign travel to Hong Kong, Mexico, Brazil, Philippines, Spain, and Africa. The patient had a 6-year history of chronic cough productive that was diagnosed as "post nasal drip". However, over time, sputum became a dry, thick, coarse dark brown material. In the past year his cough became associated with progessively worsening dypnea. One month prior to admission, he experienced chills, night sweats, and worsening cough for which he was given a course of levofloxacin with minimal relief. At time of admission, the patient's peripheral oxygen saturation level was $92 \%$ on $3 \mathrm{~L} / \mathrm{min}$ of supplemental oxygen. Physical examination revealed positive retractions and diffuse inspiratory and expiratory wheezes. Complete blood count and comprehensive metabolic panel were unremarkable. Chest X-ray showed innumerable small nodular opacities in the lungs bilaterally and patchy confluent alveolar densities in the right lower lobe. Chest CT angiography demonstrated mediastinal and confluent bilateral hilar adenopathy, with calcifications of the tracheal and bronchial cartilage rings. Bronchoscopy was performed and pathology results from biopsy of bronchotracheal tissue and bronchioalveolar lavage washings were significant for squamous cell carcinoma.

IMPACT/DISCUSSION: While smoking is the most important risk factor in lung cancers, approximately $10-15 \%$ of cases occur in never smokers. An even smaller percentage contribute to prevelence of squamous cell carcinoma of the lung. Lung cancers in never smokers differ from those in smokers in molecular profile and response to targeted therapy. Thus, the identification of environmental risk factors is important in diagnosing lung cancers. Environmental toxins including asbestos, radon, heavy metals and especially diesel fuel are all considerable risk factors for squamous cell carcinoma of the lung. This particular patient had a 40 year exposure to multiple enviromental toxins which placed him at increased risk. Low suspicion of lung cancer, because of he is a nonsmoker with an atypical presentation, may have have delayed diagnosis and initiation of treatment.

CONCLUSION: Importance of understanding occupational hazards patient may be exposed to and providing adequate counseling Awareness of atypical presentation of squamous cell carcinoma of the lung in non smoker

\section{B MINDFUL OF PERSISTENT HYPOGAMMAGLOBULINEMIA AFTER RITUXIMAB TREATMENT}

Laurie Edelman; Rachel Solomon. Icahn School of Medicine at Mount Sinai, New York, NY. (Control ID \#3184444)

LEARNING OBJECTIVE \#1: Recognize that hypogammaglobulinemia from rituximab can persist long after therapy.

LEARNING OBJECTIVE \#2: Identify patients at hight risk for recurrent infections who may benefit from ongoing immunoglobulin level monitoring.

CASE: A 59-year-old woman with history of stage 4 follicular lymphoma, treated with 6 cycles of rituximab-bendamustine followed by rituximab maintenance was observed by her PCP to have recurrent and refractory bacterial infections (including sinusitis, conjunctivitis, and a pneumonia) starting more than a year after completing treatment for her cancer. In between infections, the patient felt well. She denied weight loss, night sweats, fevers, and chills. There was no lymphadenopathy on exam. Objective weight remained stable. Labs including HIV, CBC, CMP and hemoglobin A1C were unremarkable; CT / PET was reassuring against recurrence of disease. However at 18 months post-treatment with rituxi$\mathrm{mab}$, an immunofixation panel revealed undetectable $\operatorname{IgA}$ and $\operatorname{IgM}$ and IgG 356 (700-1600). She was referred to an immunologist. Assessment for immunoglobulin functionality revealed low IgG subclass 1 levels. Her titers for MMR, Diphtheria, and pneumococcal IgG were undetectable despite prior vaccinations. A single dose of intravenous immunoglobulin (IVIG) quieted her infections for six months. She then developed severe, recurrent sinus infections. Administration of another dose of IVIG again showed benefit. She continues with active monitoring to determine appropriate treatment intervals.

IMPACT/DISCUSSION: Rituximab is a B-cell depleting monoclonal antiCD20 antibody used to treat Non-Hodgkin Lymphoma, Rheumatoid Arthritis and other autoimmune diseases. While nearly half of the patients who receive rituximab have a decrease in their immunoglobulin levels, very few manifest clinically with severe infections from hypogammaglobulinemia. A small subset will have persistent hypogammaglobulinemia beyond a year. Risk factors include older age, pre-treatment low IgG levels, concomitant chemotherapy, and multiple courses of rituximab. There is currently no guideline for monitoring immunoglobulin levels in patients treated with rituximab nor is there a defined treatment course. 
CONCLUSION: Primary care providers should have a low threshold to assess for hypogammaglobulinemia in patients treated with rituximab who present with recurrent or persistent infections. Immunology consultation and IgG therapy should be considered in patients with severe infections.

\section{BACTEREMIA AND RENAL/PERINEPHRIC ABSCESSES WITH LACTOBACILLUS JENSENII AND PREVOTELLA BIVIA IN A YOUNG WOMAN FOLLOWING URETERAL STENTING}

Abhinav Mohan ${ }^{1}$; Jacob Rubin ${ }^{2}$; Priyank Chauhan ${ }^{1}$; German E. Giese ${ }^{1}$; Juan C. Lemos Ramirez . 'Memorial Healthcare System, Pembroke Pines, FL; ${ }^{2}$ Florida Atlantic University, Fort Lauderdale, FL. (Control ID \#3182159)

LEARNING OBJECTIVE \#1: To describe predisposing factors leading to renal and perinephric abscesses caused by L. jensenii and P. bivia in a young immunocompetent individual.

LEARNING OBJECTIVE \#2: To present a successful antibiotic treatment regimen against $L$. jensenii and $P$. bivia.

CASE: We report a 26-year-old woman with history of renal cyst, recent nephrolithiasis with lithotripsy and ureteral stenting, hematuria with stent, and recent antibiotic use who presented to ED with right flank pain, fever, tachycardia, and leukocytosis. CT abdomen/pelvis showed right-sided renal and perinephric abscesses. Empiric meropenem was started. Renal abscess was percutaneously drained, and cultures grew Lactobacillus, Streptococcus anginosus and mixed anaerobes. Blood cultures grew Lactobacillus jensenii. Patient was switched to ceftriaxone and metronidazole and repeat blood cultures taken three days later were negative. One week later, repeat CT scan showed growing right perinephric abscesses. The largest was percutaneously drained and culture grew Prevotella bivia. CT urogram revealed no abnormal fistulous tracts. Patient clinically improved and was discharged on ceftriaxone and metronidazole with percutaneous drains in place. After 2 weeks, repeat CT scan showed near-complete resolution of all abscesses. Drains were removed and antibiotics discontinued. Patient was clinically asymptomatic until two months later, when she developed Gardnerella-positive bacterial vaginosis with undetectable Lactobacillus levels in the vaginal flora.

IMPACT/DISCUSSION: Lactobacillus jensenii and Prevotella bivia are normally protective, commensal organisms in the female genital tract. Clinical disease caused by these organisms is rare in young and/or immunocompetent persons; in fact, Lactobacillus infection is associated with high mortality due to frequent co-presentation with severe comorbid illness. In our patient, nephrolithiasis-induced urinary stasis, elimination of competing microflora with prior antibiotics, recent ureteral stenting, and history of renal cyst all created a favorable environment for $L$. jensenii abscess development. Subsequent clearance of $L$. jensenii, which normally inhibits $P$. bivia, then allowed for $P$. bivia abscess growth. Clinical resolution with ceftriaxone and metronidazole (along with interventional drainage) confirmed data from in vitro antibiotic susceptibility testing studies. Overtreatment of Lactobacillus may increase risk of bacterial vaginosis.

CONCLUSION: To our knowledge, there are no existing reports of a single patient with multiple, simultaneous abscesses in the renal system caused by $L$. jensenii and P. bivia, two normally commensal microorganisms. However, in our case, a series of factors led to a favorable environment for these organisms to produce clinical kidney infection in an immunocompetent patient. Resolution required targeted interventional drainage and appropriate antibiotic therapy.

\section{BACTERIAL MENINGITIS MASQUERADING AS ACUTE ST ELEVATION MI}

Eric W. Leland ${ }^{1}$; Rahul Dasgupta ${ }^{2} .{ }^{1}$ Summa Akron City Hospital, University Heights, $\mathrm{OH}, \mathrm{OH} ;{ }^{2}$ Summa Health, New Abany, OH. (Control ID \#3186440)
LEARNING OBJECTIVE \#1: Diagnose and treat CNS infections despite disparate symptomatology

LEARNING OBJECTIVE \#2: Quickly and effectively amend your treatment approach when presented with new information

CASE: 50 year old male with hypertension and moderate alcohol and tobacco use presents to the ER complaining of "pain all over" including headache which woke him from sleep, right sided chest, back, and leg pain. Upon arrival to the ER, he was hypertensive with a blood pressure of $178 / 128$ and bradycardic with a heart rate in the 40s. EKG showed ST elevation in leads II, III and aVF, and troponin was 13 . He was sent to the cath lab where a 100 percent right coronary artery occlusion was noted. Multiple attempts were made to cross the RCA lesion without success and therefore this was thought to be a chronic total occlusion. He was then sent to the ICU for post-procedure monitoring at which time his mental status precipitously deteriorated. He was intubated for airway protection and a team stroke was initiated. CT head showed no acute hemorrhage or cortical infarction and no other significant pathology. During this time, he became febrile and his level of consciousness continued to decline. Later on day hospital day two, his blood cultures showed positive streptococcus pneumoniae, and he was started on broad spectrum antibiotics with ampicillin, ceftriaxone and vancomycin. MRI showed cerebral edema concerning for meningitis which was confirmed with positive LP on hospital day 5 . His antibiotic regimen was deescalated to ceftriaxone after sensitivities resulted, and was prescribed six weeks of antibiotic therapy. His hospital course was further complicated by a GI bleed caused by duodenal ulcers which resulted in bi-hemispheric cortical ischemic stroke on MRI which resulted from acute anemia. A tracheostomy and PEG tube was placed 13 days into his hospitalization. He also developed $\mathrm{C}$ diff colitis and a re-bleed of his duodenal ulcers on hospital day 20. He was discharged 25 days after initial presentation to a rehab facility for further antibiotics and therapy.

IMPACT/DISCUSSION: This case highlights the importance of having a broad differential diagnosis for every patient, no matter how vague the complaint. This patient presented with non-focal complaints other than a headache that woke him up from sleep. The care team then was forced to pursue a cardiac pathology as the cause of his complaints due to EKG changes and elevated troponins. However, the true culprit of his illness was quickly identified less than 24 hours after presentation due to his declining mental status and worsening encephalopathy. The care team did well to swiftly and empirically treat his bacterial meningitis.

CONCLUSION: Although his hospital course was complicated by downstream effects of a long ICU, his prognosis very well could have been much graver had the care team not quickly identified a central nervous system infection. This case presentation reiterates the importance of having the ability to quickly amend one's diagnosis and resultant management.

\section{BACTRIM INDUCED HYPERKALEMIA: A WIDELY USED AN- TIBIOTIC WITH A SERIOUS ADVERS EFFECT}

Shahrzad Shidfar. umassmemorial, Worcester, MA. (Control ID \#3184337)

LEARNING OBJECTIVE \#1: Recognize hyperkalemia as a less common but clinically important adverse effect of hyperkalemia

LEARNING OBJECTIVE \#2: Assess need for antibiotic treatment even if it is a generally well tolearted antibiotic

CASE: A 53-year-old man with seizure disorder, hypertension controlled with lisinopril and enucleated right eye due to neurofibromatosis type 2 and chronic osteomyelitis of frontal bone presented with two-day history of worsening left-sided muscle twitching and pain. Pain was severe and aching from the left side of his face down to his leg. He described the aching as a "twitching" but without visible rhythmic motions. This was in conjunction with left lip numbness and associated dysarthria and worsening left-sided weakness. He had these symptoms before though they happened infrequently and lasted no more than five minutes. Now, the 
pain was reoccurring hourly and through the night. He had 3/5 flexion and extension strength in left upper and lower extremities and 4/5 flexion and extension in right upper and lower extremities. Reflexes were $1+$ bilaterally. He had a left-sided facial droop and was unable to open his left eye. There was whitish pus-appearing material in the right orbit but without orbital or periorbital erythema. Potassium was $7.7 \mathrm{mmol} / \mathrm{L}$, creatinine $1.19 \mathrm{mg} / \mathrm{dL}$ (baseline of $0.7-0.8 \mathrm{mg} / \mathrm{dL}$ ) and normal creatine kinase. Hyperkalemia resolved after receiving insulin, normal saline bolus, and furosemide. His presenting symptoms also improved in severity and he no longer felt the twitching nor the weakness. A more detailed review of his medications revealed that he had recently been started on Bactrim for presumed eye infection in the enucleated right eye a week prior to admission. He was evaluated by ophthalmology and otolaryngology and they concluded that presumed pus was in fact old necrotic tissue at the base of right orbit likely secondary to chronic osteomyelitis of his frontal bone which didn't require antibiotic treatment. His Bactrim was discontinued. Potassium level remained within normal limit.

IMPACT/DISCUSSION: Bactrim is commonly prescribed for a wide range of infections in the outpatient setting and is generally well tolerated. Given its ubiquity, it is important to recognize its many and varying adverse drug reactions. Hyperkalemia is a well-known side-effect of administering Bactrim though it happens less commonly than gastrointestinal upset or rash. Previous studies have demonstrated that hyperkalemia is much more likely to occur in patients receiving ACE inhibitors.

CONCLUSION: Hyperkalemia is a seroius adverse event associated with Bactrim and clinicains should consider laboratory monitoring of serum creatinine and potassium concentrations at baseline and during therapy in out patient setting with concomitant use of ACE inhibitors. This case exemplifies the importance of detailed history taking, medication administration, a thorough physical exam, as well as judicious antibiotic treatment to avoid patient harm.

\section{BAD TO THE BONE: A CHALLENGING DIAGNOSIS}

Letisha Ferris; Michael P. Smith. University of Nebraska Medical Center, Omaha, NE. (Control ID \#3185811)

\section{LEARNING OBJECTIVE \#1: Develop an approach to sterile osteomyelitis}

LEARNING OBJECTIVE \#2: Understand the diagnostic criteria and therapeutic options for SAPHO in order to prevent unnecessary and painful testing and treatments

CASE: A 76-year-old woman with past medical history of sero-negative polyarthritis presented with bilateral wrist pain and swelling. She had failed multiple immune-modulators. Synovial wrist biopsies were consistent with synovitis and osteomyelitis. She developed increased swelling, warmth, and a purulent drainage from her right wrist shortly after the biopsies prompting antibiotic treatment that resolved the purulence but joint pain and swelling persisted. She ultimately received multiple courses of antibiotics. There was never any significant culture growth. Wrist $\mathrm{x}$ rays revealed polyostotic destructive changes. MRI confirmed extensive osteolytic and cystic changes in multiple bones of both upper extremities. Ultrasound of her sternoclavicular joint revealed erosive disease without synovitis, effusion, or hyperemia, highly suggestive of SAPHO. There was hesitation to diagnose due to her lack of skin manifestations but given her lack of culture results and her clavicle imaging findings, Anakinra (an IL-1 inhibitor) was initiated. Disease progressed to involve bilateral elbows and left shoulder but when last seen, approximately one year since initiation of Anakinra, symptoms had completely resolved.

IMPACT/DISCUSSION: CNO or CRMO (thought to be the severe form of $\mathrm{CNO}$ ) is an auto-inflammatory disorder characterized by painful sterile bone inflammation that most commonly presents early in life and predominantly in females. SAPHO is a variant of this disease that exhibits skin manifestations prior to bone involvement. SAPHO is classically thought to be the adult version of $\mathrm{CNO} / \mathrm{CRMO}$ but has been described in adolescents.
Reliable serum markers for this disease have yet to be identified and routinely checked inflammatory markers may be elevated. Diagnosis is made by imaging, most reliably MRI. Whole body imaging is recommended when $\mathrm{CNO} / \mathrm{CRMO}$ or SAPHO are suspected as it can identify nonsymptomatic lesions. To the same effect, whole body imaging is used for treatment surveillance. Biopsy is warranted when the diagnosis is unclear. First-line therapy is non-steroidal anti-inflammatory drugs. Second-line therapies include steroids, bisphosphonates, and immunosuppressive agents. All these treatments have had mixed results, related to the fact that the pathogenesis of this disease remains unclear but likely involves multiple inflammatory pathways.

CONCLUSION: SAPHO, CNO, and CRMO are essential for the general internist to be aware of as these conditions are often misdiagnosed as bone cancer or infection leading to inappropriate interventions that carry morbidity and mortality risk. Conversely, bone sclerosis and subsequent deformity with disability can result when appropriate treatment is not provided.

\section{BARKING UP THE WRONG TREE: A CASE OF STREPTOCOC- CUS CANIS AND ANCHORING BIAS}

Josue R. Flores Balderas ${ }^{1}$; Evelyn C. Chan ${ }^{2} .{ }^{1}$ Medical College of Wisconsin and Affiliated Hospitals, Wauwatosa, WI; ${ }^{2}$ Medical College of Wisconsin, Milwaukee, WI. (Control ID \#3186044)

LEARNING OBJECTIVE \#1: Appreciate that $S$. canisis a rising zoonotic pathogen in humans.

LEARNING OBJECTIVE \#2: Recognize that anchoring bias can delay a correct diagnosis and lead to overlooking the significance of a physical exam finding.

CASE: A 67-year-old man presented with three days of progressive dyspnea at rest. On day four, over breakfast, he panted with no response to albuterol. He had a history of obstructive sleep apnea, asthma, and chronic venous insufficiency. His medications included fluticasone/ salmeterol, montelukast, and furosemide. In the ED he had a fever to 102.7 F, tachypnea to 34, and hypoxemia to 80 . Distressed, he spoke in short sentences. He had a neutrophilic leukocytosis to 19. Chest X-Ray and CT pulmonary angiography were unrevealing. His admission diagnosis was asthma exacerbation and sepsis due to community-acquired pneumonia. He was treated with BiPAP, broad-spectrum antibiotics, steroids and nebulizers. Two days later he reported pain in the medial right leg proximal to the ankle so sharp that he required support for transfers. On exam he was noted to have erythema in the lower right leg that was somewhat obscured by the hyperpigmentation seen in venous stasis dermatitis. Once blood cultures grew $S$. canis/dysgalactiae, the team asked if he had pets. He reported that he lived with four dogs who licked his legs. At that point that the team realized that the respiratory symptoms had arisen from sepsis due to $S$. canis cellulitis and sepsis with the portal of entry being the venous stasis dermatitis. Infectious diseased discouraged dog removal since the bacteria had likely colonized him. He went home on ceftriaxone to complete 14 days of antibiotics. Compression stockings and emollients were recommended. At follow-up his skin flaked as the cellulitis resolved.

IMPACT/DISCUSSION: As this case shows, anchoring bias can lead to errors in clinical judgment. Biased toward finding a pulmonary source for the respiratory distress, the team overlooked the significance of erythema on the leg and pet exposures in the social history. Anchoring bias is one of many cognitive errors such as premature closure and confirmation bias, an area of clinical reasoning that merits more attention as it can impact patient safety. This case also raises awareness of $S$. canis as a zoonotic pathogen. It is a commensal pathogen in the canine oral and genital flora that can cause opportunistic infections in humans such as cellulitis, pneumonia, urinary tract infections, osteomyelitis and infective endocarditis. The first case of $S$. canis was reported in 1997. As such, it is a relatively new pathogen that ought to be kept in mind as a potential source of infection in dog owners. 
CONCLUSION: - Anchoring bias can lead to cognitive error in diagnostic evaluations. - S. canis is an emerging zoonotic opportunistic infection that can cause sepsis.

\section{BE MINDFUL OF DISCREPANCIES BETWEEN GLUCOSE AND HEMOGLOBIN A1C MEASUREMENTS - THERE MAY BE IMPORTANT CONSEQUENCES FOR YOUR PATIENT}

Jeffrey R. Borgeson ${ }^{1}$; Alice Cusick ${ }^{2} .{ }^{1}$ Michigan Medicine, Fenton, MI; ${ }^{2}$ Michigan Medicine, Ann Arbor, MI. (Control ID \#3160743)

LEARNING OBJECTIVE \#1: Identify discrepancies between glucose measurements and hemoglobin a1c measurements

LEARNING OBJECTIVE \#2: Understand the possible causes for discrepant glucose and hemoglobin alc measurements

CASE: The patient is a 73-year-old woman with a history of asthma who presented for a routine health maintenance examination. At the time of the office visit she had no specific concerns. Specifically, she had no fatigue, jaundice, dyspnea, polyuria, polydipsia, or unexplained weight loss. Physical examination was normal. Screening laboratories revealed a fasted glucose of $140 \mathrm{mg} / \mathrm{dl}$, a hemoglobin alc level of $4.5 \%$, and a hemoglobin of $11.9 \mathrm{~g} / \mathrm{dl}$. Otherwise, the complete blood count, comprehensive metabolic panel, and lipid panel were normal. A repeat fasted glucose level was ordered and found to be $129 \mathrm{mg} / \mathrm{dl}$, confirming the diagnosis of diabetes. A repeat hemoglobin alc was $4.4 \%$. Fructosamine level was $265 \mathrm{micromol} / \mathrm{L}$ (high end of normal). Due to what appeared to be an inappropriately low hemoglobin a1c level, a lab investigation was initiated to explore causes of accelerated red blood destruction. This workup produced the following results: reticulocyte count $8.55 \%$ (high), LDH $266 \mathrm{IU} / \mathrm{L}$ (high), haptoglobin $<10 \mathrm{mg} / \mathrm{dl}$, total bilirubin $1.9 \mathrm{mg} / \mathrm{dl}$ (high), and a negative direct antiglobulin test (DAT). The peripheral smear showed polychromasia and numerous spherocytes. A CT scan of the chest, abdomen, and pelvis showed mild splenomegaly but was otherwise unremarkable. An osmotic fragility test showed increased erythrocyte osmotic fragility. The evidence of hemolytic anemia, presence of spherocytes on the manual differential, and positive osmotic fragility test confirmed the diagnosis of hereditary spherocytosis.

IMPACT/DISCUSSION: This case shows how the identification of a discrepancy between glucose and hemoglobin a1c measurements may lead to the discovery of a significant hematological condition. A fasted glucose level measurement of $140 \mathrm{mg} / \mathrm{dl}$ correlates to a hbalc of approximately $7 \%$. The measured hbalc of $4.5 \%$ in this case was therefore much lower than expected. This observation in turn led to the diagnosis of hereditary spherocytosis. Conditions that cause increased red cell turnover lead to a shorter lifespan for hemoglobin and subsequently less time for hemoglobin glycosylation, which in turn leads to misleadingly low hemoglobin a1c levels. Correspondingly, conditions associated with decreased red cell destruction may cause misleadingly high hemoglobin alc levels. In situations with such a discrepancy, a reasonable workup may include a fructosamine level to confirm the discrepancy, lab testing directed at hemolysis and other disorders of red blood cell metabolism such as iron and B12 deficiency, and imaging to look for splenomegaly.

CONCLUSION: When the hemoglobin alc is much lower than what would be expected based on an individual's glucose measurements, disorders associated with increased red cell turnover should be investigated.

BECOMING ADEPT IN AGEP: RECOGNIZING ACUTE GENERALIZED EXANTHEMATOUS PUSTULOSIS (AGEP) AS A DISTINCT SEVERE CUTANEOUS ADVERSE REACTION

Alaina C. Chodoff; Kristen M. Livesey; Margaret Shang. University of Pittsburgh Medical Center, Pittsburgh, PA. (Control ID \#3167664)

LEARNING OBJECTIVE \#1: Distinguish the features of a severe cutaneous adverse drug reaction
LEARNING OBJECTIVE \#2: Demonstrate the importance of a thorough physical examination for prompt diagnosis of acute generalized exanthematous pustulosis

CASE: A 84-year-old gentleman with a history of dermatomyositis, treated with prednisone and methotrexate, was admitted with a rapidly progressive pruritic skin eruption that began abruptly after taking clindamycin for a dental extraction. He was afebrile, normotensive and tachycardic. Skin exam revealed widespread erythematous papules coalescing into plaques across his neck, chest, back, abdomen and extremities. Discrete, non-follicular, pin-head sized pustules were scattered across his neck and upper chest. There was no mucosal membrane involvement or lymphadenopathy. Laboratory testing revealed a white cell count of 20,000 cells per microliter of blood of which $80 \%$ were neutrophils and $15 \%$ eosinophils. He had an acute kidney injury and liver function tests were normal. Cultures of the blood and urine were negative. Histology from a punch biopsy revealed focal parakeratosis and spongiosis with lymphocyte and neutrophil exocytosis, consistent with a pustular drug eruption. A diagnosis of AGEP was confirmed. The rash underwent desquamation and the patient gradually improved with supportive care.

IMPACT/DISCUSSION: AGEP, Stevens Johnson syndrome (SJS), toxic epidermal necrolysis (TEN) and drug rash with eosinophilia and systemic symptoms (DRESS) are categorized as severe cutaneous drug adverse reactions. A thorough physical examination enables detection of the early signs and symptoms of severe cutaneous adverse drug reactions including mucosal membrane involvement, lymphadenopathy, facial edema, blisters and high-grade fevers. Identification of the classic pinpoint, nonfollicular, pustules of AGEP is essential to distinguish this rash from other self-limited exanthematous eruptions. Skin biopsy is confirmatory for diagnosis. As pustular desquamation occurs, classically after 10 days, topical corticosteroids can be applied for symptomatic relief.

CONCLUSION: AGEP is a rare, idiosyncratic, T-cell-mediated, hypersensitivity drug reaction. It is distinguished by its rapid onset after administration of the offending agent, generally within 1-2 days. Over $90 \%$ of cases are drug-related, most commonly attributed to antibiotics, antiepileptic agents and antihypertensive agents. Although considered less severe than SJS-TEN and DRESS, there can be overlapping features including mucosal involvement, extensive skin blistering and severe systemic symptoms with end organ damage due to profound neutrophilia and subsequent tissue damage. Up to one-third of patients can also present with eosinophilia, further complicating the differentiation of AGEP. Treatment remains supportive with removal of the offending agent. Once AGEP is suspected, there is no evidence supporting the use of systemic corticosteroids.

\section{BEER-DRINKERS' CARDIOMYOPATHY REVISITED: COBALT TOXICITY AFTER A NON-METAL-ON-METAL HIP ARTHROPLASTY}

Daniel Davies; Panithaya Chareonthaitawee. Mayo Clinic, Rochester, MN. (Control ID \#3174131)

LEARNING OBJECTIVE \#1: Diagnose the cobalt toxidrome. LEARNING OBJECTIVE \#2: Recognize risk factors for cobalt toxicity.

CASE: A 59-year-old woman with new-onset ankle edema and fatigue presented for pre-operative evaluation prior to revision left hip arthroplasty. A cobalt prosthesis was placed 18 months ago after catastrophic failure of a ceramic-on-ceramic left hip arthroplasty, but subsequently developed "runaway wear". She denied other cardiovascular symptoms, but did note severe progressive hearing loss over the last eight months. A month later, she was diagnosed with hypothyroidism and was started on replacement. Her vision also markedly worsened despite normal ophthalmologic evaluations. She described minimal alcohol use and had no significant cardiovascular or neurologic family history. Physical examination was significant for regular tachycardia of $110 \mathrm{bpm}$, normal S1 and S2 without murmur, bilateral ankle edema, severe hearing and vision loss, no thyromegaly or nodules, and an 
otherwise normal exam. Basic laboratory evaluations, including $\mathrm{CBC}$, creatinine, and thyroid function studies, were normal. An ECG showed only sinus tachycardia. Review of recent brain MRI revealed no significant findings. Due to her signs of new-onset heart failure, an echocardiogram was performed, showing mild concentric left ventricular (LV) hypertrophy, LV enlargement, ejection fraction of $45 \%$, and resting regional wall abnormalities. A follow up stress myocardial perfusion imaging study was performed, which revealed no significant perfusion defects, together suggesting a new underlying cardiomyopathy. Due to her known "runaway wear" of the joint prothesis and her new constellation of heart failure, hearing loss, vision loss, and hypothyroidism, serum heavy metal levels were obtained with normal titanium, chromium, and markedly elevated cobalt at 953 (normal 0.0-0.9) $\mathrm{ng} / \mathrm{ml}$.

IMPACT/DISCUSSION: Our case describes joint prosthesis-related systemic cobalt poisoning. The cobalt toxidrome classically involves cardiomyopathy, peripheral neuropathy, hearing and vision loss, and hypothyroidism. Cobalt toxicity was first described in the 1965 in nutritionally deficient alcoholics after breweries began using it as an additive agent. A rare, but emerging, modern cause of toxicity is cobalt joint prothesis wear in the setting of retained ceramic particles from previous joint failure. Treatment includes joint replacement and occasionally chelation therapy.

CONCLUSION: Cobalt toxidrome includes cardiomyopathy, peripheral neuropathy, hearing and vision loss, and hypothyroidism Prosthetic joint failure is a new, emerging cause of cobalt toxicity

\section{BEHIND THE VEIL OF RENAL FAILURE}

Brian J. Kinsman; Justin B. Roy; Stephanie L. Harris. PennState Health

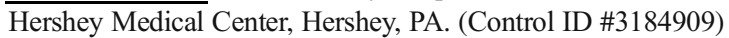

LEARNING OBJECTIVE \#1: Identify common clinical manifestations of multiple myeloma.

LEARNING OBJECTIVE \#2: Recognize overlap between endocrine disturbances in end stage renal disease and multiple myeloma.

CASE: A 66-year-old Caucasian male with a history of hip osteoarthritis, primary sclerosing cholangitis with cirrhosis, and end stage renal disease (ESRD) due to focal segmental glomerulosclerosis on hemodialysis (HD) presented with three days of acute on chronic right hip pain without an inciting trauma. Medications included lisinopril, bumetanide, sevelamer, calcitriol, and cholecalciferol. On examination, he was afebrile, blood pressure $205 / 95 \mathrm{mmHg}$, heart rate $69 \mathrm{bpm}$, respiratory rate 16 breaths $/ \mathrm{min}$. His right hip was swollen, euthermic, nonerythematous and diffusely tender. Attempts at right hip flexion, extension, or abduction elicited severe pain. Lower extremities were neurovascularly intact. The remainder of his exam was benign. X-ray of the right femur revealed an intertrochanteric fracture with associated lytic lesion. CT chest, abdomen, and pelvis also revealed lytic lesions in the left $9^{\text {th }}$ rib and iliac wing. Initial laboratories showed K $5.6 \mathrm{mEq} / \mathrm{L}, \mathrm{Ca} 9.7 \mathrm{mEq} / \mathrm{L}, \mathrm{PTH} 34 \mathrm{pg} / \mathrm{mL}$, 1,25-OH-Vit-D3 $16.5 \mathrm{pg} / \mathrm{mL}$, BUN $70 \mathrm{mg} / \mathrm{dL}$, serum creatinine $5.14 \mathrm{mg} /$ dL, Hgb 8.5 g/dL, MCV 91.3 fL, Albumin 3.7 g/dL, 2-microglobulin $15.47 \mathrm{mg} / \mathrm{L}$. Serum protein electrophoresis was negative for monoclonal immunoglobulins (Ig); however, urine fixation revealed elevated freemonoclonal Ig, and serum free- chain was elevated $35.3 \mathrm{mg} / \mathrm{dL}$ with a low : (0.13). This evidence led us to suspect a diagnosis of multiple myeloma (MM), rather than an alternative malignancy. HD was continued while inpatient and his femur surgically repaired. Subsequent results from biopsy of the lytic femur lesion revealed abundant -restricted, CD138+ plasma cells, confirming the diagnosis of MM.

IMPACT/DISCUSSION: The most common clinical manifestations of MM are anemia, bone pain, elevated creatinine, fatigue, hypercalcemia, and weight loss, which result from clonal plasma cell hyperproliferation and secretion of monoclonal Ig's and light chains. Although these manifestations of MM may also be attributed to ESRD-mediated secondary hyperparathyroidism, renal osteodystrophy, and impaired erythropoietin production. This man presented with worsening bone pain, anemia, elevated creatinine, and fatigue. He also had borderline hypercalcemia for a year prior to this presentation, which could be appropriate while taking calcitriol, cholecalciferol and sevelamer on HD. However, at presentation PTH was normal and 1,25-OH-Vit-D3 depressed, which hints hypercalcemia of malignancy masked by HD. Were it not for his pathologic fracture and lytic lesions, his MM may have progressed undiagnosed.

CONCLUSION: - Shared clinical manifestations to MM and ESRD include anemia, bone pain, elevated serum creatinine, fatigue, hypercalcemia, and weight loss. - Avoid anchoring on existing diagnoses in patients with multiple morbidities and new complaints.

\section{BENEATH THE SURFACE OF A PRURITIC, ERYTHEMATOUS RASH}

Megan K. Veglia; Meghan Thomas; Andrew Schreiner. Medical University of South Carolina, Charleston, SC. (Control ID \#3185477)

LEARNING OBJECTIVE \#1: Recognize the physical signs of dermatomyositis (DM) and how to distinguish them from systemic lupus erythematosus (SLE).

LEARNING OBJECTIVE \#2: Assess the therapeutic options for dermatomyositis and determine when intravenous immunoglobulin is a viable treatment.

CASE: A 60-year-old male smoker developed an erythematous, pruritic rash on his abdomen, face, and hands. Skin biopsy revealed hyperkeratosis, apoptotic cells, and a lymphocytic infiltrate with a crowded, band-like pattern. He was diagnosed with cutaneous lupus and treated with a topical corticosteroid. The rash spread and pruritis intensified despite corticosteroid use. He reported increased difficulty swallowing and myalgias so severe that he lost his job. Physical exam revealed erythema of the eyelids and cheeks, including the nasolabial folds. He had erythematous papules symmetrically over the elbows, knees, ankles, and the dorsal aspect of the metacarpophalangeal and interphalangeal joints. Strength was $4 / 5$ in his proximal musculature and $5 / 5$ elsewhere. His skin biopsy was reviewed with immunohistochemical staining and revealed many CD4+ and CXCR3+ cells. He was diagnosed with dermatomyositis and admitted to the hospital. Labs revealed a creatinine kinase of 781 and a sedimentation rate of 6. A swallow study showed silent aspiration. A PEG tube was placed for nutrition. He was treated with high dose intravenous steroids and IVIG for 2 days and discharged to inpatient rehabilitation on prednisone and azathioprine with monthly IVIG infusions.

IMPACT/DISCUSSION: Skin findings of DM are often mistaken for those of SLE. These two diseases are often initially evaluated and managed by general internists, and early diagnosis is crucial to a good prognosis. Classic DM is an idiopathic inflammatory myopathy characterized by proximal muscle weakness and a variety of skin manifestations, including Gottron's papules and heliotrope rash. Skin findings in DM can be distinguished from SLE by (1) the facial rash in DM involving the nasolabial folds; (2) the photo-eruption, which is pruritic in dermatomyositis but not in SLE. The hallmark treatment of DM involves glucocorticoids, and IVIG is used in resistant cases when there is severe dysphagia, or with severe, life threatening illness.

CONCLUSION: DM is an idiopathic inflammatory myopathy characterized by proximal muscle weakness and a variety of skin manifestations, most classically including Gottron's papules and a heliotrope rash. Skin findings in DM can be distinguished from SLE by (1) the facial rash in DM which, when present, involves the nasolabial folds, and (2) the photoeruption, which is pruritic in dermatomyositis unlike the photo-eruption seen in SLE. The hallmark of treatment of DM involves glucocorticoids, and IVIG is used in resistant/recurrent cases, when there is severe dysphagia, or with severe, life threatening illness.

BETA-BLOCKERS LEADING TO CARDIAC ARREST IN THYROID STORM, EVEN IN NORMAL HEARTS

Nooraldin Merza ${ }^{1}$; Phyo P. Kyaw ${ }^{2}$; Assad Mohammedzein ${ }^{1}$; Sameer Prakash $^{1}$; Ahmed Taha ${ }^{1}$; Manish Patel ${ }^{2} .{ }^{1}$ Texas Tech University Health 
Science Center, Amarillo, TX; ${ }^{2}$ Texas Tech Univ HSC Amarillo, Amarillo, TX. (Control ID \#3147482)

LEARNING OBJECTIVE \#1: Recognize thyroid storm as a potential cause of cardiac arrest.

LEARNING OBJECTIVE \#2: Recognize the potential risk of administering Intravenous beta blockers in thyroid storm.

CASE: A 24-year-old female with no past medical history was brought from jail due to altered mental status and abdominal pain for several days. She was febrile, tachycardic, tachypneic, drowsy and restless. She had dry mucous membranes and proptosis. $\mathrm{CBC}$ showed WBC $33.3 \times 10^{3}, 88 \%$ neutrophils, $\mathrm{Hb} 11 \mathrm{~g} / \mathrm{dl}$. pH 7.08, $\mathrm{PCO}_{2} 5$, anion gap 24, betahydroxybutyrate $78(0.2-2.8)$, lactate 1.2 , free $\mathrm{T}_{3} 23 \mathrm{pg} / \mathrm{ml}(2.2-6.2)$, free $\mathrm{T}_{4} 4.7 \mathrm{ng} / \mathrm{dl}(0.2-2.0), \mathrm{TSH}<0.01$ units $/ \mathrm{ml}$. EKG showed sinus tachycardia with early repolarization. A diagnosis of thyroid storm was made. She was intubated for airway protection and resuscitated with intravenous fluids, she also recieved hydrocortisone and propylthiouracil to block conversion of $\mathrm{T}_{4}$ to $\mathrm{T}_{3}$ and reduce synthesis of thyroid hormones respectively. Intravenous propranolol $1 \mathrm{mg}$ IV push was given, immediately after receiving propranolol, she went into asystole. ROSC was achieved after 4 minutes of cardiopulmonary resuscitation. She required more intravenous fluids and vasopressor support. Bicarbonate infusion was started for severe metabolic acidosis. Echocardiogram showed ejection fraction $70 \%$ with no hypokinesia; and Troponin I $1.3 \mathrm{ng} / \mathrm{ml}$ and trended down afterwards. Thyroid stimulating immunoglobulin and thyrotropin receptor antibodies were significantly elevated, confirming Graves' disease. Thereafter, she was stabilized and successfully extubated and was discharged on propranolol, methimazole and prednisone.

IMPACT/DISCUSSION: Although there is no definite diagnostic criteria for thyroid storm, Burch-Wartofsky score 45 is highly suggestive of thyroid storm. Our patient's score was 45 . No acute coronary syndrome was evident, despite the elevated troponin I, no ST-T changes were seen in EKG. The likely mechanism of Asystole is myocardial depression due to profound acidosis, worsened by propranolol-induced hypotension and bradycardia. Unlike most of the reported cases of cardiac arrest in thyroid storm after propranolol administration, when impaired left ventricular function was evident; our patient had a good left ventricle function with no hypokinesia.

CONCLUSION: Thyroid storm is a rare but a life-threatening condition with significant mortality rate. Beta blockers should be used cautiously in this setting due to potential circulatory collapse. It should be considered as a cause of cardiac arrest in the absence of other identifiable etiologies.

\section{BETWEEN THE BULLETS: A CASE OF MISSED OPPORTUNI- TY IN PRIMARY CARE}

Brittany Badesch ${ }^{1}$; Ivy Sledge ${ }^{2}$; Katherine Shaw ${ }^{2} .{ }^{1}$ Johns Hopkins Hospital, Baltimore, MD; ${ }^{2}$ Johns Hopkins Community Physicians East Baltimore Medical Center, Baltimore, MD. (Control ID \#3184104)

LEARNING OBJECTIVE \#1: Identify young adults who are at acute risk of death by gun violence.

LEARNING OBJECTIVE \#2: Describe the need to develop evidencebased strategies to minimize risk of death by gun violence for young adults in urban settings.

CASE: A 32 year-old African-American male with history of chronic left lower extremity pain after a gun shot wound and prior substance use disorder presents to establish primary care. He complains of a burning pain in his left lower extremity, radiating from his knee to his foot, associated with muscle spasms, hyperalgesia and allodynia. He takes Ibuprofen. Recently released from a year of incarceration, he is living with his girlfriend. He has a four year-old daughter. He is not working due to pain. He has $4 / 5$ strength of left foot plantar and dorsiflexion and a painful tingling sensation with touch of the medial left ankle. He endorses anxiety, but denies homicidal or suicidal ideation. He continued to follow in the clinic monthly and was referred to pain management. Adjustments to his medications improved his pain. He returned to work and increased involvement in his daughter's life. Six months after initial presentation, he was shot and killed.

IMPACT/DISCUSSION: Unfortunately, this is not a unique clinical presentation or outcome. In 2017, the year this patient was killed, there were 39,773 gun deaths in the U.S. Homicide is leading cause of death in 15-34 year old black males, accounting for 49\% of deaths for 15-24 yearolds compared to $7-8 \%$ in same-aged white males. The ACP's recent update of their Position Statement on reducing firearm deaths largely focused on prevention of mass casualty events and regulation of assault style weapons. Less guidance exists for prevention of everyday gun violence in urban settings, where poverty, unemployment, transiency, addiction, institutional racism and criminalization contribute to increased risk of death by firearm. The patient not only experienced many of these risk factors, but had elevated risk simply based on his previous gunshot wound. His enhanced risk and multiple visits to clinic before the fatal reinjury indicate potential missed opportunity to intervene.

CONCLUSION: Primary care teams are uniquely positioned to serve as navigators for community resources, yet care is limited by a lack of evidence-based practices aiming to address the complex social conditions that generate violence. Since this patient's death, the authors of this vignette, a medical assistant and resident doctor team, continue to encounter young black men who are at acute risk of death, but feel helpless to treat them without coordinated approaches to individualized intervention, community care, and policy change. The authors hope this case will highlight how primary care clinics could be potential staging grounds for interdisciplinary teams, including members of the community who have been affected by gun violence, to fight against this epidemic of young violent death.

\section{BEWARE THE DANGERS OF HOME REMEDIES}

Syeda A. Raza. Methodist Health Systems Dallas, Dallas, TX. (Control ID \#3185718)

LEARNING OBJECTIVE \#1: Recognize the dangers of common home remedies in the immunocompromised patients

LEARNING OBJECTIVE \#2: Recognize Burkholderia cepacia as an opportunistic pathogen in immunocompromised patients

CASE: A 68-year-old African-American woman with known history of refractory IgG kappa multiple myeloma presented with fever, congestion, and purulent drainage from both ears that had been progressively worsening over the past week. She initially attributed her symptoms to sinus infection and self-treated with a Neti Pot using non-sterile water. The Neti Pot was several months old and had not been disinfected since purchase. Physical examination revealed an acutely ill-appearing woman with obstructed visualization of both ears due to purulent drainage. The remainder of her physical examination was unremarkable and she did not have indwelling intravascular access. Computed tomography (CT) and magnetic resonance imaging (MRI) of the head and sinuses demonstrated fluid in the tympanic cavities and mastoid air cells bilaterally with paranasal sinus mucosal density and air fluid levels in the maxillary sinuses. Blood cultures grew Burkholderia cepacia susceptible to piperacillin-tazobactam and meropenem. She was treated with four weeks of intravenous (IV) piperacillin-tazobactam along with microdebridement of both ears. She responded well to therapy, repeat blood cultures were sterile, and she made a complete recovery.

IMPACT/DISCUSSION: Burkholderia cepacia is an aerobic gramnegative bacillus commonly seen in soil and moist environments and has propensity to survive in aquatic environments. Most cases of B. cepacia bacteremia reported in the literature are in patients with cystic fibrosis. However, B. cepacia has recently been identified as an important opportunistic pathogen in hospitalized and immunocompromised patients. There have been several studies reporting hospital outbreaks of B. cepacia emerging from a single contaminated source such as nebulizer 
solutions, medical devices, and intravenous solutions. There have even been reports documenting the growth of B. cepacia in water systems and colonizing bottled water. B. cepacia pseudobacteremia is also noted in numerous reports, but true bacteremia caused by B. cepacia is rare. In our case, the patient was not hospitalized in the ICU, did not have cystic fibrosis, did not have an indwelling line, and had not had invasive procedures. Instead, this was a unique case of B. cepacia isolation caused by probable environmental contamination after improper use of a Neti Pot with non-sterile water. It is important to note $\mathrm{B}$. cepacia is often resistant to many common antibiotics and therefore treatment plans can be complicated. Management primarily focuses on prevention of the infection.

CONCLUSION: Burkholderia cepacian has recently been identified as an important opportunistic pathogen in hospitalized and immunocompromised patients. It is important to educate immunocompromised patients about the dangers of common home remedies, which may increase their risk of infections.

\section{BILATERAL ADRENAL HEMORRHAGE PRESENTING WITH ACUTE ADRENAL INSUFFICIENCY IN A PATIENT WITH SUBDURAL HEMATOMA ON RIVAROXABAN}

Zachary S. Bugos; Fnu Abhishek. UPMC Mercy, Etna, PA. (Control ID \#3185728)

LEARNING OBJECTIVE \#1: Recognize ADRs associated with rivaroxaban

LEARNING OBJECTIVE \#2: Identify adrenal insufficiency in difficult clinical scenarios

CASE: 70yo morbidly obese F with PMHx of HTN, DM2, hypothyroidism and h/o spontaneous DVT now on rivaroxaban was admitted from OSH for SDH. Pt had been experiencing nocturnal HA and nausea $\mathrm{x} 2 \mathrm{~d}$, then while taking a shower the night before admission, suddenly felt weak, sat on bathtub, denies LOC or trauma. Later presented to ED, CT head revealed left tentorial SDH. Pt was transferred to our hospital for further management. Upon arrival to our ICU, factor complex was weight-based dosed for rivaroxaban reversal. Of note, pt has been on rivaroxaban for a year in setting of chronic LLE DVT, but was held on admission and no further $\mathrm{AC}$ was given for the duration of hospital course. Pt remained A\&O w/o focal deficits, transferred to the floors on HD3 for further monitoring of comorbidities. However, the AM of HD10, during routine nursing check, a rapid response was called for unresponsiveness. BP 51/38, agonal breathing and pulse ox $62 \%$. Initial concern for worsening SDH, pt was intubated and underwent CT head, which was unremarkable. Pt was transferred to ICU and started on norepinephrine plus vasopressin due to persistent hypotension refractory to IVF resuscitation. Initial workup focused on questionable infectious etiology causing septic shock, as pt had leukocytosis to $17 \mathrm{~K}$ but was afebrile. Due to unknown etiology, a CT abdomen/pelvis was performed which revealed b/l adrenal mass vs hemorrhage. Endocrine consulted, initiated stressdose hydrocortisone $50 \mathrm{mg}$ q $8 \mathrm{~h}$ plus fludrocortisone $0.05 \mathrm{mg}$ daily. Repeat $\mathrm{CT}$ abdomen/pelvis on HD12 confirmed the diagnosis of adrenal insufficiency caused by $\mathrm{b} / \mathrm{l}$ adrenal hemorrhage.

IMPACT/DISCUSSION: Rivaroxaban is part of a class of agents known as direct oral anticoagulants (DOACs). Rivaroxaban inhibits both free and bound Factor Xa, ultimately leading to interruption of both intrinsic and extrinsic pathways of the coagulation cascade. Common ADRs include prolonged bleeding from wounds or surgical sites, hematuria, epistaxis and melanotic stool. Notably, cerebrovascular or gastrointestinal sites are considered major bleed events, are acute and easily recognized by providers. However, rare delayed-onset complications, such as b/l adrenal hemorrhage leading to adrenal insufficiency, must be considered in patients presenting with worsening mentation, non-specific systemic symptoms and worsening lab findings. To the best of our knowledge, this is the first case report in which rivaroxaban was the implicated factor for both a cerebrovascular hemorrhage and a delayed $\mathrm{b} / \mathrm{l}$ adrenal hemorrhage in the same patient.
CONCLUSION: Adrenal hemorrhage should be considered in anticoagulated patients with refractory hypotension including new direct anticoagulants Diagnostic measures should never delay the treatment in the case of strong clinical suspicion of acute adrenal insufficiency Acute adrenal hemorrhage should be managed as an acute adrenal crisis with IV hydrocortisone

BILE IS VILE: CARDIAC COMPLICATIONS OF CHOLESTASIS Archana Sridhar; Sophie Barbant. Highland Hospital, Alameda Health System, Oakland, CA. (Control ID \#3184295)

LEARNING OBJECTIVE \#1: Recognize cardiac complications of elevated bile acid levels

LEARNING OBJECTIVE \#2: Treat arrhythmias caused by cholestatic liver disease

CASE: A 32-year-old woman at 38 weeks gestation was admitted to the obstetrics service for intrahepatic cholestasis of pregnancy (ICP). Prior to admission, the patient was seen in clinic reporting pruritis. Serum bile acid levels were ordered and returned elevated at $67 \mathrm{umol} / \mathrm{L}$, prompting admission for induction of labor (IOL). The patient's past medical history included an uncomplicated vaginal delivery 3 years prior. She took prenatal vitamins and had no allergies. On hospital day 2, after receiving misoprostol and oxytocin, internal medicine consultation was requested to evaluate her for sustained bradycardia. On examination, the patient appeared well with a heart rate of 35 beats per minute (bpm) and blood pressure of 95/60. Prior to IOL her heart rate was $56 \mathrm{bpm}$, and her outpatient baseline was 76-88 bpm. Cardiac examination revealed marked bradycardia; the remainder of her exam was unremarkable. Fetal monitoring was reassuring. An electrocardiogram showed sinus bradycardia and ultrasonography revealed normal cardiac structure and function. The differential diagnosis included a paradoxical vagal reaction from pain or positioning, medication side-effect, or thyroid dysfunction. These were considered unlikely given that her heart rate did not change significantly with positioning or contractions, she had received no anesthesia or medications known to cause bradycardia, and thyroid testing was normal. Elevated bile acid levels were ultimately felt to be driving her arrhythmia, and ursodeoxycholic acid (UDCA) therapy was started. Her heart rate improved to $58 \mathrm{bpm}$ one day after UDCA initiation and fetal delivery. In out-patient follow-up, her heart rate was $75 \mathrm{bpm}$.

IMPACT/DISCUSSION: ICP is known to cause stillbirth through placental transfer of bile acids leading to fetal bradycardia and cardiac arrest. The role of circulating bile acids on maternal cardiovascular health is less well-described, with few reported cases in the literature. Recent animal studies have shown an association between elevated bile acid levels and arrhythmias. Interruption of the cardiac cell membrane by hydrophobic bile acids may alter transcellular calcium exchange, one of the many proposed mechanisms of arrhythmogenesis. Treatment with UDCA, a water-soluble bile acid that decreases levels of hydrophobic bile acids, mitigates cardiotoxicity. Despite newer research examining the role of bile acids in inducing bradyarrhythmias, this relationship remains underrecognized by clinicians. Prompt identification is vital, as treatment with UDCA can prevent morbidity. Further study should explore whether UDCA may ameliorate cardiovascular dysfunction seen in liver cirrhosis, where bile acid secretion is impaired.

CONCLUSION: Elevated bile acid levels in cholestatic liver disease may cause bradycardia. UDCA treats arrhythmias associated with cholestasis.

\section{BILE, THOU SHALT NOT PASS! A CASE OF SYMPTOMATIC HEPATIC SARCOIDOSIS.}

Kamesh Gupta; Ali Shami; Vellayat Ali. Baystate Medical Center, Springfield, MA. (Control ID \#3179967)

LEARNING OBJECTIVE \#1: Describe the presentation of symptomatic hepatic sarcoidosis 
LEARNING OBJECTIVE \#2: Recognize treatment and outcomes for hepatic sarcoidosis

CASE: A 59 year-old African-American male presented to clinic with a several week history of pruritis, jaundice, nausea and weight loss. He had a medical history of hypertension and no history of significant alcohol, drug, or herbal supplement use. Physical examination revealed jaundice without signs of chronic liver disease and a benign abdomen without hepatosplenomegaly. Labs showed elevated liver chemistries including alkaline phosphatase $(475 \mathrm{U} / \mathrm{L})$, alanine aminotransferase $(88 \mathrm{U} / \mathrm{L})$, aspartate aminotransferase $(82 \mathrm{U} / \mathrm{L})$, total bilirubin $(3.6 \mathrm{mg} / \mathrm{dL})$ and direct bilirubin $(2.2 \mathrm{mg} / \mathrm{dL})$. Extensive hepatic workup was negative including viral hepatitis panel, alpha-1 anti-trypsin, carbohydrate antigen 19-9, as well as negative workup for IgG-4 related disease and other infectious causes for hepatitis. Ultrasound showed only hepatic steatosis, though further abdominal computed tomography (CT) showed an enlarged celiac axis lymph node $(>1 \mathrm{~cm})$ and portal lymphadenopathy. He underwent endoscopic ultrasound which was negative for bile duct dilatation, and fine needle aspiration cytology of the enlarged lymph node was non-diagnostic. An ultrasoundguided liver biopsy was therefore performed. This showed non-caseating granulomas and bile duct injury, highly suggestive of sarcoidosis. Sarcoidosis was further confirmed with CT of the chest showing mediastinal and hilar lymphadenopathy. There was also high activity of angiotensin converting enzyme in the blood. The patient was started on budesonide and 6-mercaptopurine therapy and responded well to treatment.

IMPACT/DISCUSSION: Sarcoidosis is a multi-system disease characterized by non-caseating granulomas in various organs. Hepatic involvement is common and seen in around $50-80 \%$ of patients, but the vast majority of those affected are asymptomatic. Approximately $35-40 \%$ of all cases may have abnormal liver chemistries. In some cases, however, hepatic sarcoidosis may present suddenly with symptoms and signs including jaundice, abdominal pain, nausea and vomiting. Hepatic sarcoidosis can lead to cholestasis, portal hypertension and cirrhosis. Extrinsic compression of the biliary tree due to sarcoid granulomas has been reported, as well as an association with increased risk of hepatobiliary cancers. Liver biopsy is the gold standard for diagnosis. Steroids are the mainstay of treatment though their efficacy is variable with only about one-third of patients achieving a complete clinical response. Definitive treatment is liver transplantation; however, recurrence of granulomatous disease is still possible in the transplanted organ.

CONCLUSION: Hepatic sarcoidosis is a common entity that may occasionally present as obstructive jaundice and other signs of liver injury.

\section{BONES AND STONES: RECOGNIZING HYPERPARATHY-} ROIDISM AS A SECONDARY CAUSE OF OSTEOPOROSIS

Callie Plafkin ${ }^{1}$; Elaine Pelley ${ }^{2}$. ${ }^{1}$ University of Wisconsin Hospital and Clinics, Madison, WI; ${ }^{2}$ University of Wisconsin School of Medicine and Public Health, Madison, WI. (Control ID \#3150221)

LEARNING OBJECTIVE \#1: Recognize primary hyperparathyroidism as a secondary cause of osteoporosis

LEARNING OBJECTIVE \#2: Suspect hyperparathyroidism in cases with disproportionate loss of bone at the distal radius, which can represent PTH-mediated loss of cortical bone

CASE: A 74-year-old woman with a history of osteoporosis, erosive seronegative arthritis, osteoarthritis status post bilateral hip arthroplasty, and a single bout of nephrolithiasis in 2010 presented to Osteoporosis Clinic in 2017 for further evaluation and management of bone health. Her initial dual-emission X-ray absorptiometry (DXA) scans in 2003 and 2009 had demonstrated only mild osteopenia, with spine T-scores of -0.7 and -1.1 , respectively. Due to intervening bilateral hip replacements, her next scan in 2013 measured bone density at the lumbar spine and, for the first time, the distal radius. The spinal bone density was statistically unchanged, but the distal radius had a T-score of -3.4. She was diagnosed with osteoporosis and started on therapy with weekly alendronate and supplementation with calcium and vitamin D. Labs were notable for intermittent mild hypercalcemia dating back to 2010. The reported worsening in T-score from 2009 to 2013 represented a function of the new measurement site, rather than a true decline in overall bone mineral density. The discrepancy in bone mineral density between the distal radius (primarily cortical bone) and the lumbar spine (primarily trabecular bone), in conjunction with her hypercalcemia and nephrolithiasis, was highly concerning for primary hyperparathyroidism. Further testing revealed an elevated parathyroid hormone (PTH) of $124 \mathrm{pg} / \mathrm{mL}$, normal renal function, normal 25-hydroxy-vitamin D level, and normal 24-hour urine calcium, consistent with a diagnosis of primary hyperparathyroidism (PHPT). She underwent left inferior parathyroidectomy, with subsequent normalization of PTH and serum calcium levels.

IMPACT/DISCUSSION: While the majority of cases of PHPT in the United States are diagnosed prior to the development of obvious skeletal manifestations via incidentally-noted lab abnormalities, PHPT remains an important secondary cause of bone mineral density loss. Excess PTH causes preferential demineralization of cortical bone, frequently manifesting as low bone density at the distal radius site. Such discrepancies should raise suspicion for hyperparathyroidism even in the absence of persistent hypercalcemia.

CONCLUSION: Disproportionately low bone density at the distal radius site compared to axial (spine, hip) sites should prompt evaluation for hyperparathyroidism. Early in its course, primary hyperparathyroidism can manifest as high-normal or intermittent hypercalcemia. Complications of bone loss and nephrolithiasis can occur prior to the development of persistent hypercalcemia.

\section{BRADYCARDIA: THE ONLY PARENCHYMAL MANIFESTA- TION OF RARE HSV-2 ENCEPHALITIS}

Daymon Peterson; Tanaz Salimnia. Wayne State University, Shelby Township, MI. (Control ID \#3183051)

LEARNING OBJECTIVE \#1: To recognize atypical presentations for improved outcomes of common central nervous system infections.

CASE: A 39-year-old male presented with debilitating headache and fever. Exam demonstrated overt meningismus, however extensive neurologic exam was unremarkable. He was febrile and bradycardic with significant leukocytosis. Lumbar puncture revealed lymphocyte predominate pleocytosis, and indices consistent with viral pathogen. He was hospitalized for empirical antimicrobial therapy, including Acyclovir. Over the first 48 hours, his clinical condition worsened. He developed profound bradycardia consistently in the 20 's and serial neurological exams remained unchanged. The following day he began exhibiting severe neuropsychiatric disturbances, lethargy, and loss of gustatory function. CSF testing confirmed HSV-2 by PCR. Patient ultimately responded to Acyclovir therapy in the following days, with parallel resolution of neuropsychiatric symptoms and bradycardia, however had permanent gustatory changes.

IMPACT/DISCUSSION: Although encephalitis often presents with easily identifiable phenotypes, diagnostic challenge arises when encephalitis is encountered in the setting of atypical parenchymal manifestations. Here we present bradycardia as a sign of parenchymal infection associated with pathognomonic involvement of the temporal lobes in HSV encephalitis. Bradycardia is not conceptualized as having relation to encephalitis or temporal lobes, however, bradycardia has previously been linked to temporal lobe dysfunction in other disease processes. Ictal bradycardia syndrome, is a rare event seen in epilepsy, where seizures are accompanied by cardiac arrhythmias, specifically bradycardia. This phenomenon almost exclusively is seen in epileptic disease localized to the temporal lobes and there is no evidence to suggest that this effect is limited to epileptiform activity. Additionally, HSV encephalitis can lead to seizures, suggesting the inflammation of encephalitic disease shares provoking features on temporal lobe neurons, and can trigger a similar cardiac response. Comparable temporal lobe insults with parallel results support a mechanism for which the parenchymal disruption of encephalitis manifests as brady-arrhythmias. 
CONCLUSION: Encephalitis is a devastating central nervous system infection that requires early antiviral therapy to optimize management for improved outcomes, however is dependent upon clinician detection of focal parenchymal dysfunction in the setting of CNS infection. Traditional encephalopathic features are well recognized, however concern should extend to atypical signs and symptoms that could represent brain tissue disfunction. The above case describes bradycardia as the presenting manifestation of encephalitis. HSV encephalitis shares the target insult location with the established Temporal-Lobe Epilepsy induced Ictal Bradycardia Syndrome, and suggest bradyarrhythmia as both a temporal lobe neuronal insult, and previously unrecognized encephalitis defining syndrome.

\section{BREATHING NOT PROPERLY? IT IS MORE THAN JUST HEART FAILURE}

Kendall Downer. UPMC, Pittsburgh, PA. (Control ID \#3185997)

LEARNING OBJECTIVE \#1: Illustrate the value of the negative likelihood ratio of BNP in patients with undifferentiated dyspnea

LEARNING OBJECTIVE \#2: Define anchoring bias and recognize the importance of cognitive forcing strategies

CASE: A 71 y/o man with a history of heart failure and COPD on chronic prednisone presented with two months of progressive dyspnea on exertion in addition to weight gain, lower extremity edema and nonproductive cough. He was recently hospitalized for a CHF exacerbation and did not restrict salt or fluid after discharge. He denied paroxysmal nocturnal dyspnea or orthopnea. Physical exam was notable for oxygen saturation $83 \%$ on room air. Crackles were present in all lung fields. No gallops or jugular venous distension was appreciated. Marked pitting edema was present in his lower extremities. Labs were notable for BNP 22. Chest xray showed patchy, bilateral lung opacities. He was treated for a $\mathrm{CHF}$ exacerbation with two rounds of diuresis without improvement in symptoms. At that time, the diagnosis was reconsidered, and a chest $\mathrm{CT}$ showed pulmonary fibrosis in a usual interstitial pneumonia pattern. His clinical trajectory was most consistent with an IPF flare as he progressed to respiratory failure and passed away a few days after presentation.

IMPACT/DISCUSSION: Dyspnea is a common complaint faced by internists in all clinical settings and has a large differential. That differential can be narrowed with clinical tools such as The Rational Clinical Exam that allow quick access to and comparison of likelihood ratios (LRs). The negative likelihood ratio of BNP for heart failure in patients with dyspnea in the ED is 0.11 . With a pre-test probability of $50 \%$, this patient's post-test probability of heart failure is $9.9 \%$. Other signs and symptoms with high predictive value include an S3 gallop, pulmonary venous congestion, and absence of cardiomegaly with LRs of 11,12 , and 0.33 , respectively. In contrast, PND, orthopnea and lack of JVD are of less predictive value, with LRs of only 2.6, 2.2 and 0.66 respectively. Failing to modify the initial clinical impression when discordant information arises - such as adjusting the post-test probability based on the BNP value - is termed anchoring bias. When contradictory data emerges, an appropriate next step is deliberate, systematic reconsideration of the diagnosis, called a cognitive forcing strategy. When these steps do not occur, there is an increased risk of diagnostic error and potential patient harm.

CONCLUSION: Physicians should be aware of the signs, symptoms and tests with the greatest predictive values in common conditions, including the powerful negative predictive value of BNP for heart failure in patients with dyspnea. When a highly predictive test is discordant with the initial clinical impression, physicians should deliberately consider potential biases and alternative diagnoses.

BROADENING YOUR DIFFERENTIAL FOR ABDOMINAL PAIN TO EXTRA-ABDOMINAL CAUSES

Nida Waheed; Kelsey Pan; James Smith; Zareen Zaidi. University of Florida, Gainesville, FL. (Control ID \#3185435)
LEARNING OBJECTIVE \#1: To recognize not all generalized abdominal pain stems from an intra-abdominal source

CASE: A 47-year-old triathlon runner presented to his primary care clinic due to abdominal pain that was initially epigastric and then generalized and associated with nausea, poor oral intake, and a 15-20 pound unintended weight loss over two months. Physical examination revealed generalized abdominal pain with no organomegaly, point tenderness, guarding, or rebound on palpation. The pain was not associated with referred pain, exercise, or gait abnormalities. Initial work up revealed a negative upper endoscopy and normal lipase. Due to persistent symptoms, a CT abdomen and pelvis was ordered and revealed a soft tissue mass in the right anterior quadriceps femoris muscle measuring $7.3 \times 8.3 \mathrm{~cm}$ with mass effect upon the vastus lateralis, vastus medialis, and rectus femoris muscles. A biopsy revealed high-grade synovial sarcoma, spindle cell type. He is currently being treated with radiation therapy and neoadjuvant ifosfamide and mesna.

IMPACT/DISCUSSION: The differential diagnosis for generalized abdominal pain is extensive, including IBD, IBS, viral gastroenteritis, constipation, malignancy, chronic mesenteric ischemia and more. Given the clinical presentation of unintentional weight loss, GI malignancy was high on the differential. Although the CT abdomen was unrevealing for intra-abdominal etiologies, it serendipitously led to the discovery of the synovial sarcoma of the right thigh. This case highlights the importance of understanding abdominal and quadriceps muscle anatomy, specifically their origin, insertion, and action. It is also important to pursue further imaging in the setting of "red-flag" symptoms, such as unintentional weight loss. There are no prior cases found in the literature of thigh masses presenting with abdominal pain. Both mass effect on the abdominal and quadriceps muscles and nerve irritation leading to referred pain along the abdominal sheath were considered to be the culprit for this patient's abdominal pain. Synovial sarcoma is a high-grade soft tissue sarcoma with a high mortality rate, making up 5 to 10 percent of all softtissue sarcomas. It commonly occurs in children and young adults. Synovial sarcomas often present as a chronic pain at the local tumor site prior to the appearance of a noticeable tumor bulge. Diagnosis is made by biopsy and mainstay treatment for non-metastatic sarcoma consists of surgical resection, followed by radiation and possible adjuvant chemotherapy. Large tumor size $(>5 \mathrm{~cm})$, along with high histologic grade, increased age, and invasion of bone or vessels are often associated with poor prognosis. Delay in diagnosis is common, and early recognition has significant prognostic impact.

CONCLUSION: Abdominal pain does not always stem from an intraabdominal source, and it is crucial to pursue further workup for suspected sources of malignancy, despite negative workup of the most likely source.

\section{BRODIFACOUM COAGULOPATHY IN A 23 YEAR-OLD WOM- AN AFTER SYNTHETIC CANNABINOID USE}

Evans Brown. Johns Hopkins Bayview, Baltimore, MD. (Control ID \#3185542)

LEARNING OBJECTIVE \#1: Recognize signs, symptoms, and historical details of Brodifacoum toxicity

LEARNING OBJECTIVE \#2: Understand basic treatment of Brodifacoum toxicity

CASE: A 23 year-old woman presented with a several day history of unexplained extremity bruising, bleeding from inside of her mouth/lips, epistaxis, and progressive menorrhagia. Review of systems was notable for headache and abdominal discomfort. She had no personal or family history of bleeding or clotting disorders. She reported smoking synthetic marijuana with friends two weeks prior to presentation. Her physical exam revealed scattered ecchymosis on all extremities, varying in size and color, and petechiae on her legs. There was a large amount of blood in the vaginal vault. Laboratory results were most notable for INR >9.6, and PTT 94.1. Cat scan of the head, abdomen, and pelvis revealed no acute internal 
bleeding. She was empirically treated for Brodifacoum exposure with high dose vitamin $\mathrm{K}$ for one month, and was transfused 1 unit of fresh frozen plasma. Laboratory analysis returned positive for Brodifacoum.

IMPACT/DISCUSSION: Brodifacoum is a long-acting and potent Vitamin K antagonist commonly used as a rodenticide. Since it was discovered in 1978, there have been reports of human exposure - predominantly via unintentional ingestion among children and intentional ingestion among adults causing significant coagulopathy and death. Recently there has been a dramatic increase in Brodifacoum coagulopathy related to contaminated synthetic cannabinoids. Per the CDC, as of $11 / 23 / 2018$, at least 320 people nationwide have presented to healthcare facilities with coagulopathy attributed to Vitamin-K antagonist coagulopathy from synthetic cannabinoid use. Brodifacoum coagulopathy presents a diagnostic challenge for clinicians in part because of the delay between exposure and symptom onset; the half-life of Brodifacoum is 16-24 days relative to 1737 hours for Warfarin. The most common presenting symptoms are spontaneous ecchymoses, mucocutaneous bleeding, and intramuscular hemmorage1. The most common cause of death associated with Brodicoum coagulatphy is intracranial hemorrhage. The mainstay of treatment for Brodifacoum coagulopathy is high dose vitamin $\mathrm{K}$, often with treatment courses that last months. Red-cell transfusion, 4-factor prothrombin complex concentrate (Kcentra), and fresh-frozen plasma can be used to reverse coagulopathy and treat bleeding depending on the severity of presentation. Brodifacoum is teratogenic and poses an additional risk to women of childbearing age. It crosses the placenta and increases the risk of bleeding for both mother and fetus.

CONCLUSION: Brodificaoum coagulopathy should be on the differential for any patient that presents with unexplained bleeding and coagulation abnormalities. Synthetic cannabinoid use should be part of history taking and risk factor analysis for all patients. The mainstay of treatment for brodifacaoum coagulopathy is high dose vitamin K, with typical treatment length of several month.

\section{BRUGADA UNMASKING IN THE SETTING OF CITALOPRAM OVERDOSE: A NOVEL ASSOCIATION}

Nicholas J. Beccarino ${ }^{1}$; Rishi K. Pandya ${ }^{2}$. NYU Langone Medical Center, Greenlawn, NY; ${ }^{2}$ NYU, New York, NY. (Control ID \#3164405)

LEARNING OBJECTIVE \#1: Review the Brugada phenotype and its classic pharmacologic triggers.

LEARNING OBJECTIVE \#2: Discuss the potential impact of citalopram in unmasking the Brugada phenotype.

CASE: A 25 year old man with depression was brought to our institution within thirty minutes of intentionally ingesting $125 \mathrm{mg}$ of citalopram. The patient was initially asymptomatic without significant abnormalities on EKG. An EKG the following morning showed ST elevations in V1-2 consistent with a type 1 Brugada pattern. The patient denied a personal or family history of syncope, palpitations, or sudden cardiac death. ICD placement was deferred considering the lack of high risk features. The patient's EKG normalized over the next few days and he was ultimately discharged on aripiprazole.

IMPACT/DISCUSSION: Brugada syndrome is an autosomal dominant condition associated with malignant ventricular arrhythmia and sudden cardiac death, most often caused by mutations in the alpha subunit of the cardiac sodium channel. Diagnosis requires characteristic EKG findings in the setting of unexplained syncope, ventricular tachycardia or fibrillation, or a family history of sudden cardiac death. While sodium channel blocking anti-arrhythmics such as procainamide are classically associated with the Brugada phenotype, tricyclic antidepressants, analgesics, and illicit drugs have also been implicated. Data regarding selective serotonin reuptake inhibitors (SSRIs) in Brugada syndrome are limited. Paroxetine, fluoxetine, and fluvoxamine have been shown to have sodium channel blocking properties with rare reports of worsening the Brugada phenotype although it is not clear how this correlates to cardiac risk. While it is recommended to avoid these agents in Brugada syndrome, no similar recommendations exist for Citalopram. Furthermore, there are no previous reports of an association between Citalopram and Brugada syndrome to our knowledge. In fact, very little data exists regarding the cardiac effects of Citalopram. One study of the impact of Citalopram on rodent myocardium noted a shortening of action potential duration, depression of plateau, and reduction of depolarization velocity consistent with sodium channel inhibition, although these findings have not been replicated in humans. Nevertheless, these data suggest citalopram may share the sodium channel blocking effects and impact on the Brugada pattern seen with other SSRIs.

CONCLUSION: Our case represents a young man who developed a Brugada phenotype after a relatively mild citalopram overdose highlighting the possible implication of citalopram in the exacerbation of the syndrome, presumably through an interaction with cardiac sodium channels. Further research on Citalopram's effect on the cardiac action potential and its association with Brugada syndrome is necessary to determine its safety profile within this high risk patient population.

\section{BULLOUS ERYSIPELAS WITH FAT HERNIATION}

Layla Al bizri; Yusuf Alalwan; Kutayba Alsafadi; Eric Scher. Henry Ford Hospital, Detroit, MI. (Control ID \#3181788)

LEARNING OBJECTIVE \#1: Recognize that fat herniation is a possible complication of erysipelas

LEARNING OBJECTIVE \#2: Distinguish bullous erysipelas from cellulitis or skin abscesses

CASE: A 68 year old female presented with acute onset left leg skin erythema and tenderness of two days duration. She attributed her symptoms following a sting to her left leg which woke her up from her sleep. She also reported mild systemic symptoms including fever and chills. Upon presentation she was found to be febrile and tachycardic. Inspection of the left leg showed a raised, well demarcated area of erythema and mild swelling. A presumptive diagnosis of erysipelas was made and the patient was started on a first-generation cephalosporin. Over the next two days her pain and erythema worsened. She began to form very large bullae which were filled with yellow, gelatinous material. She was initially treated with Cefazolin but was escalated to Vancomycin because of her worsening course. A soft tissue ultrasound ruled out any underlying collection of fluid. Dermatology was consulted on day two of admission and made a diagnosis of erysipelas with fat herniation secondary to elevated subcutaneous pressures from the inflammation. She was started on Prednisone to help reduce the inflammation and her antibiotics were switched back from Vancomycin to cefazolin. The Infectious disease service recommended treating for 14 days. During outpatient follow up in dermatology clinic, patient was noted to have resolution of leg pain and improvement of skin lesions after completion of antibiotic treatment.

IMPACT/DISCUSSION: Initially, the acute onset of symptoms: fever, pain, erythema and swelling with the appearance of a raised well demarcated skin lesion, made erysipelas the most likely diagnosis. However, the sudden appearance of bullae containing gelatinous-like material while on an appropriate antibiotic regimen forced the primary service to reconsider this diagnosis. Necrosis, abscess formation, deep thrombophlebitisis and hemorrhagic purpura are known complications of erysipelas that have been reported previously in the literature. Therefore, our reported case of fat herniation resembles a unique complication of erysipelas. According to the literature, fat herniation has been described in piezogenic papules which are benign mostly painless skin-colored papules made of fat associated with exercise, repetitive pressure forces, and connective tissue diseases. To our knowledge, cases of soft tissue infections with described fat herniation have not been reported before. The appearance of bullae on the epidermal surface with subcutaneous fat may have dissipated the pressure within the skin and prevented the formation of compartment syndrome within the patient's lower extremity. 
CONCLUSION: Erysipelas can present as a severe soft tissue infection creating a challenge for physicians in both diagnosis and management. Fat herniation represents a unique local complication of Bullous erysipelas.

\section{BULLOUS LUPUS RESEMBLING STEVENS-JOHNSON SYN- DROME (SJS) AS THE INITIAL MANIFESTATION OF SYS- TEMIC LUPUS ERYTHEMATOSUS (SLE)}

Juhi Bhargava. Medstar Washington Hospital Center, Washington, DC. (Control ID \#3176567)

LEARNING OBJECTIVE \#1: Recognize bullous lesions as the first manifestation of SLE to come to a timely diagnosis.

CASE: We describe a previously healthy 24-year-old woman who presented with vesiculobullous lesions raising concern for Stevens-Johnson syndrome (SJS) who was subsequently diagnosed with bullous lupus as an initial manifestation of SLE. 24 year old female with PMH of anxiety and depression presented with painful pruritic bullous rash for 2-3 weeks. Blisters appeared first on one of her extremities at the site of a recent tattoo and subsequently spread to her face, upper arms, abdomen and legs. The patient also endorsed fever and malaise. Of note, she presented to different ERs twice, where she was initially diagnosed with possible scabies and eventually with possible SJS and was subsequently transferred to our tertiary care facility for further management. Physical examination showed numerous tense bullae of varying sizes and circular ulcerated lesions with overlying hemorrhagic crust. Numerous oral ulcers and tongue papillitis were detected although no conjunctival or genital mucosal involvement. Symmetric inflammatory polyarthritis was present on joint exam. Infectious etiology including HSV and VZV were ruled out by negative viral serologies. Drug reaction and SJS-TEN was also considered given prior use of risperidone and involvement of skin and oral mucosa but this was thought less likely because the agent had been discontinued a month prior to onset of symptoms. Immunologic workup revealed positive ANA, anti-ds DNA, anti-Smith, anti U1RNP and low complement C3. A skin 'punch' biopsy revealed acute cutaneous bullous lesions. Direct immunofluorescence showed strong IgG, C3, IgA and IgM deposition on basement membrane consistent with bullous lupus vs epidermolysis bullosa aquisita (EBA). The clinical presentation (skin/oral lesions, arthritis and constitutional symptoms), positive SLE serologies and biopsy findings clinched the diagnosis of bullous lupus in the setting of SLE based on fulfillment of SLICC criteria. Treatment with prednisone and dapsone led to dramatic improvement.

IMPACT/DISCUSSION: Bullous lesions are seen in known cases of SLE but only rarely do they occur as the initial manifestation of SLE. This case highlights the importance of awareness of bullous lesions as the first manifestation of SLE. Furthermore, distinguishing BSLE from SJS-TEN is essential as the treatment and prognosis differs substantially. Our patient was repeatedly misdiagnosed which led to significant treatment delay and progression of her symptoms. Dapsone leads to rapid resolution of BSLE lesions with rituximab (B-cell depletion therapy) used as a second-line agent in refractory cases.

CONCLUSION: Bullous lesions may be the first manifestation of SLE and thus it is important to be cognizant of it to come to a timely diagnosis.

\section{BULLOUS PEMPHIGOID AS A CONSEQUENCE OF DPP-4 INHIBITORS}

Ryann Quinn; Frank Cacace. Northwell Health, Manhasset, NY. (Control ID \#3175569)

LEARNING OBJECTIVE \#1: Recognize bullous pemphigoid as a potential consequence of DPP-4 Inhibitors in patients with Diabetes Mellitus

CASE: A 91 year-old female with a history of type 2 diabetes mellitus presented to the emergency department with a pruritic rash worsened over the past six weeks. The rash started on her neck, progressed down her arms, chest, abdomen, back, and thighs. She also began to have tense blisters on her palms. She did not recall any triggering event prior to the rash, insect bites, new medications, or new skin products. She had previously gone to a dermatologist and was prescribed triamcinolone cream twice daily. Her medications included sitagliptin, which she had been on for 2 years, and glimepiride. Her vital signs were unremarkable. Exam revealed erythematous plaques with exfoliative scaling on her arms, back, abdomen, and legs, sparing skin folds, soles, and mucus membranes. She also had tense bullae on both palms. Labs significant for WBC $22 \times 10^{9} / \mathrm{L}$ with $24 \%$ eosinophils, hemoglobin A1c $7.8 \%$. Skin biopsy of lesion on right abdomen showed superficial perivascular infiltrate with lymphocytes and eosinophils, with direct immunofluorescence showing positive IgG and $\mathrm{C} 3$ staining in linear pattern at the dermoepidermal junction, consistent with bullous pemphigoid. Patient was started on an oral prednisone taper, changed to mycophenolate mofetil at discharge. Her diabetes regimen was changed to insulin and repaglinide, glimepiride and sitagliptin were discontinued. At 16 week follow up, patient had clear skin and eosinophilia resolved, patient continues on mycophenolate mofetil for a 3 month course.

IMPACT/DISCUSSION: Bullous pemphigoid is an autoimmune blistering disease that is most often seen in adults over 60 years old. There is a prodromal phase that can last weeks to months where pruritic eczematous or papular lesions may precede the development of tense bullae, therefore causing a delay in diagnosis. The gold standard for diagnosis is skin biopsy with direct immunofluorescence with linear IgG and/or linear C3 staining along the basement membrane. Bullous pemphigoid is associated with neurologic disorders, malignancy, and certain drugs (spironolactone, furosemide, several antibiotics). DPP-4 inhibitors are now commonly used agents in treating diabetes. The association between bullous pemphigoid and DPP-4 inhibitors has been demonstrated in case series, casecontrol studies, and registry studies, although the mechanism remains unclear. In reported cases, the development of bullous pemphigoid began 10 days - 5 years after exposure, seen across different drugs in this class. CONCLUSION: DPP-4 inhibitors are commonly used in primary care for management of diabetes. Primary care clinicians should be aware of the association between DPP-4 inhibitors and bullous pemphigoid. Patients who are taking these agents who present with a new rash suspicious of bullous pemphigoid should be referred for skin biopsy and the DPP-4 inhibitor should be discontinued.

\section{BUPRENORPHINE/NALOXONE INDUCTION IN A PATIENT WITH SEVERE OPIOID USE DISORDER COMPLICATED BY ACUTE HEPATITIS A}

Devin Oller. University of Kentucky, Lexington, KY. (Control ID \#3182891)

LEARNING OBJECTIVE \#1: Recognize the risks and benefits of buprenorphine/naloxone (BUP/NX) in patients with acute hepatitis A (HAV) and Opioid Use Disorder (OUD)

CASE: A 32 year-old woman with OUD presented to the hospital with abdominal pain, nausea and jaundice. She reported daily IV heroin and methamphetamine use in the month prior to presentation. On exam, vitals were within normal range and no confusion, asterixis, or ascites were noted. She scored 10 on the Clinical Opioid Withdrawal Scale. She had marked AST and ALT elevations (2897 and 2203 U/L, respectively), a total bilirubin (TBili) of $7.2 \mathrm{mg} / \mathrm{dL}$, and an INR of 1.5. HAV IgM was positive, HBV Surface Ag negative, HCV viral load negative, and acetaminophen level below assay. Urine drug screen showed amphetamine and fentanyl. Ultrasound of the abdomen revealed normal liver echogenicity and no gallstones. She was diagnosed with acute HAV. On hospital day 2, she was seen by the addiction consult team, and BUP/NX was recommended. She underwent a modified induction protocol, receiving a total of $4 \mathrm{mg}$ of BUP/NX on the first day of induction. Over 3 days her dose was titrated to $8 \mathrm{mg}$ daily. She tolerated the induction without 
issues; LFTs were stable at discharge. She was linked to an outpatient BUP/NX clinic. Two months following her discharge, she was seen in clinic and AST, ALT and TBili had returned to normal. Her OUD was in early remission on BUP/NX $16 \mathrm{mg} /$ day.

IMPACT/DISCUSSION: There are 3 FDA-approved medications for OUD (BUP/NX, Naltrexone, Methadone), but in patients with moderate or severe hepatic impairment BUP/NX initiation is not recommended. Our patient faced transportation and cost barriers for methadone maintenance, prompting a shared decision-making conversation about the risks and merits of BUP/NX in the setting of acute HAV. The team explained the risks of BUP/NX in patients with impaired liver function: worsened liver function, increased sedation with impaired BUP/NX clearance, and risk of withdrawal with increased exposure to naloxone component. ${ }^{1}$ The patient reported reduced overdose risk, prior success with BUP/ NX, and low cost as benefits. She tolerated BUP/NX without complications. 3 other cases of BUP/NX induction in the setting of acute HAV from our addiction consult team show similar tolerability of BUP/NX and stable LFTs at time of discharge - findings not previously described in the literature. Some OUD patients hospitalized with acute HAV can and should be offered BUP/NX. 1.Nasser et al. Pharmacokinetics of sublingual buprenorphine and naloxone in subjects with mild to severe hepatic impairment. Clin Pharm. 2015 Aug;54(8): 837-49

CONCLUSION: The risks of BUP/NX initiation in patients with acute hepatitis A may be significant-including worsened liver injury. The benefits of BUP/NX initiation may outweigh those risks for patients with stable LFTs and no altered mental status, especially when they lack other viable options for medication treatment.

\section{CALCIUM CONFUSION: HYPERCALCEMIA AND MENTAL STATUS CHANGES AS A HARBINGER OF METASTATIC MALIGNANCY}

Robert N. Stuver ${ }^{1}$; Dillon Schafer ${ }^{1}$; Alicia Clark ${ }^{2} .{ }^{1}$ Beth Israel Deaconess Medical Center, Boston, MA; ${ }^{2}$ Beth Israel Deaconess Medical Center, Wellesley, MA. (Control ID \#3176643)

LEARNING OBJECTIVE \#1: Initiate emergent management and workup for hypercalcemia.

LEARNING OBJECTIVE \#2: Recognize severe hypercalcemia of unknown etiology as a possible presentation of undiagnosed malignancy.

CASE: A 54-year-old male was found wandering the street. On initial examination, the patient was cachectic and disoriented. Laboratory testing showed total calcium of $14.8 \mathrm{mg}$ per deciliter $(\mathrm{mg} / \mathrm{dL})$. He was volume fluid resuscitated with subsequent normalization of his calcium within 48 hours. Additional laboratory studies showed low 25-hydroxyvitamin D, normal parathyroid hormone (PTH), and elevated PTH-related peptide (PTHrP). With normalization of calcium, the patient became fully oriented. He had no medical history but reported heavy alcohol use and one month of unintentional weight loss and nausea. Computed tomography of the chest showed an enhancing 2.8 centimeter gastroesophageal mass. Subsequent imaging showed additional lesions in the bones, liver, and brain. Biopsy revealed squamous cell carcinoma of the esophagus.

IMPACT/DISCUSSION: In this case, we present a man with confusion and hypercalcemia ultimately found to have metastatic cancer. The initial management and workup of hypercalcemia were critical in normalizing his neurological status and obtaining a diagnosis, and this case allows for a review of these fundamental principles. Severe hypercalcemia is a medical emergency. Initial treatment is aimed at correction via volume expansion with isotonic saline at rates of at least $200 \mathrm{~mL} /$ hour, titrated to maintain urine output of at least $100 \mathrm{~mL} /$ hour. Calcium should be monitored at least every 6 hours during this initial correction period. In symptomatic patients with initial calcium greater than $14 \mathrm{mg} / \mathrm{dL}$, calcitonin can be given. Hypercalcemia is never a normal physiological occurrence. The most common causes are parathyroid-related disease or malignancy, though rarer causes include vitamin $\mathrm{D}$ intoxication and granulomatous disease. Diagnosis is initiated by measuring PTH. If elevated, hyperparathyroidism is confirmed. If normal or low, levels of PTHrP, 1,25- and 1-hydroxyvitamin D should be obtained. Elevated vitamin $\mathrm{D}$ levels point towards granulomatous diseases or hypervitaminosis, whereas elevated PTHrP suggests humoral hypercalcemia of malignancy, such as in our case. Humoral hypercalcemia of malignancy is secondary to elevations in PTHrP, which acts via physiologic PTH pathways to increase bone resorption and calcium reabsorption in the distal tubule. In these cases, a thorough review of systems, along with ageappropriate cancer screening, should be completed in order to clue in on an underlying cancer. In summary, we present a case of "calcium confusion"-hypercalcemia and altered mental status - a clinical presentation that should always force the clinician to think of malignancy.

CONCLUSION: 1. Hypercalcemia is a medical emergency and should be treated with immediate, aggressive fluid resuscitation. 2. Severe hypercalcemia of unknown etiology should prompt an evaluation for underlying malignancy.

\section{CANAGLIFLOZIN-INDUCED EUGLYCEMIC DIABETIC KETOACIDOSIS IN A TYPE 2 DIABETIC}

Nour Chams. Emory, Atlanta, GA. (Control ID \#3185713)

LEARNING OBJECTIVE \#1: Definding and understanding euglycemic DKA and potential risk factors.

LEARNING OBJECTIVE \#2: Timely diagnosis of euglycemic DKA.

CASE: A 47-year-old man with a past medical history significant for diabetes mellitus type 2 presented to the emergency department for diffuse abdominal pain, nausea and vomiting of 2-day duration. He was recently started on canagliflozin $100 \mathrm{mg}$ daily for tighter glycemic control. On exam, the patient was hemodynamically stable with a benign physical examination except for lack of skin turgor and mild diffuse abdominal tenderness on deep palpation. Laboratory workup showed normal blood glucose, high anion gap metabolic acidosis, low bicarbonate, and high beta-hydroxybuterate. Further blood tests revealed hemoconcentration and normal kidney function, with no evidence of pancreatitis or liver disease. He was diagnosed with euglycemic DKA and treated following the DKA protocol and he was advised to discontinue canagliflozin.

IMPACT/DISCUSSION: Our case presents a patient with non-insulin dependent T2D who recently started canagliflozin and later developed euglycemic DKA, with canagliflozin as the most likely culprit after excluding all other causes of eDKA. SGLT2 inhibitors are a class of oral hypoglycemic drugs that block the reabsorption of glucose in the proximal renal tubules of the kidney leading to excretion of glucose in urine and thus decreasing blood glucose levels. The mechanism behind SGLT2 inhibitor-induced eDKA is not yet well studied. One of the proposed theories is that SGLT2 inhibitors reduce insulin secretion from pancreatic beta cells through its hypoglycemic effect thus stimulating lipolysis and the production of free fatty acids, which are then converted to ketones. Decreased insulin production also promotes the production of ketone bodies through the activation of carnitine palmitoyltransferase-I (CPT-I). Another mechanism through which SGLT2 inhibitors could cause eDKA is through stimulating the secretion of glucagon, either as a direct effect of SGLT2 inhibitors on pancreatic alpha cells or the secondary effect of decreased insulin secretion; glucagon contributes to the overproduction of ketones through increased CPT-I activity. Euglycemic DKA in the setting of SGLT2 inhibitor use develops mostly in individuals with type 1 diabetes and rarely occurs in type 2 diabetes. It is especially rare in those who still have insulin reserves. Our patient was a type 2 diabetic with negative GAD65 antibodies and normal C-peptide levels. These findings indicate that he might still have functioning pancreatic beta cells. Nevertheless, he still developed eDKA while using canagliflozin without having any known precipitating factors.

CONCLUSION: Given that SGLT2 inhibitors, which are widely used for tighter glycemic control, are associated with the development of eDKA in 
approximately 20 reported cases, this should trigger further investigations to better understand the mechanism and possible contributing factors

\section{CAPNOCYTOPHAGA CANIMORSUS: A NOT-SO-RARE CAUSE OF ZOONOTIC BACTERIAL MENINGITIS}

Patrick M. Azcarate ${ }^{1}$; Julie Caplow ${ }^{2} .{ }^{1}$ UCSF, San Francisco, CA $;{ }^{2}$ Northern Navajo Medical Center, Shiprock, NM. (Control ID \#3184599)

LEARNING OBJECTIVE \#1: Appreciate the challenge in diagnosing early meningitis

LEARNING OBJECTIVE \#2: Learn about an emerging cause of meningitis from dog bite exposure

CASE: A 58 year-old previously healthy woman presented to the emergency room with 1 day of fever, headache, and neck stiffness, and two weeks of a non-productive cough. She had no history of alcohol use, intravenous drug use, recent travel or sick contacts, but reported a dog bite to the left hand one week prior. Review of systems was otherwise negative. Exam was notable for a fever of $102.2 \mathrm{~F}$ and a healing puncture wound on her left $4^{\text {th }}$ finger. The WBC was $10,200 / \mathrm{mm}^{3}$ ( $84 \%$ neutrophils), and lumbar puncture (LP) was normal. After IV fluids her symptoms improved and she was discharged home with a diagnosis of an upper respiratory infection. She returned the following day with worsening symptoms and new bilateral hearing loss. She was febrile with a blood pressure of $86 / 70 \mathrm{mmHg}$ and a pulse of 102 . She appeared ill and lethargic with nuchal rigidity but an otherwise normal neurologic exam. WBC had increased to $14,500 / \mathrm{mm}^{3}$. Blood cultures were sent. She was admitted to the ICU and given acyclovir, vancomycin, ceftriaxone, and ampicillin. Repeat LP 48 hours after the first revealed WBC 158/ $\mathrm{mm}^{3}$ (73\% PMNs), RBC $530 / \mathrm{mm}^{3}$, glucose $46 \mathrm{mg} / \mathrm{dL}$, protein $112 \mathrm{mg} / \mathrm{dL}$ and negative gram stain consistent with meningitis. Three days later blood cultures grew gram negative rods, and antibiotics were changed to ceftriaxone and ampicillin. Negative workup included repeat blood cultures, HIV, CSF bacterial culture, and CSF testing forS. pneumoniae, H. influenzae, and Listeria. She completed ten days of antibiotics and the fever, headache and neck stiffness resolved. After thirty-five days, an outside lab identified the bacterium as Capnocytophaga canimorsus.

IMPACT/DISCUSSION: Once considered rare, Capnocytophaga canimorsus is now recognized as the second-most common infection transmitted by dog bites. Risk factors include asplenia, heavy alcohol use, and immunodeficiency. In half of cases there is a history of bite or saliva exposure from a dog and occasionally a cat. Both species have high prevalence of the bacteria in the oral cavity $(20 \%)$. Patients typically present with sepsis or meningitis, which may be complicated by hearing loss. The average incubation period is 6 days, but may take up to 30, and requires enriched agar making laboratory isolation difficult. Bacterium are most commonly isolated in blood cultures and rarely in CSF cultures. Patients with severe infections may be treated for 21 days with a third-generation cephalosporin and narrowed to clindamycin or amoxicillin-clavulanate. Immunocompromised patients who experience a dog bite should be prescribed amoxicillin-clavulanate for five days for prophylaxis.

CONCLUSION: -Initial LP may be normal in early bacterial meningitis. Consider repeat LP if no improvement after 48 hours. -In patients with a recent dog or cat bite and sepsis, Capnocytophaga canimorsus should be considered and blood cultures incubated for at least 2 weeks.

\section{CAPS: A CATASTROPHIC EVENT}

Sneha Patel. Montefiore Medical Center, Bronx, NY. (Control ID \#3185866)

LEARNING OBJECTIVE \#1: Recognize Catastrophic Antiphospholipid Syndrome (CAPS) as a rare cause of multiorgan failure LEARNING OBJECTIVE \#2: Review the diagnostic criteria and management of CAPS
CASE: A 41 year-old male was admitted after he was found unresponsive at home. On admission, the patient was found to have encephalopathy, acute liver failure, acute renal failure, and severe abdominal pain. Urine toxicology, ethanol levels and acetaminophen levels were negative. Infectious workup was unremarkable. Abdominal CT scan revealed renal and splenic infarcts, bowel ischemia and pneumoperitoneum. The patient underwent exploratory laparotomy, right hemicolectomy, and splenectomy. Liver biopsy showed large areas of thrombi formation. Workup for hypercoagulable state showed positive antinuclear antibody and lupus anticoagulant. ADAMSTS13, fibrinogen, PTT, and JAK2 levels were normal. Direct antiglobulin testing was negative. The patient was diagnosed with CAPS. He was immediately started on a course of intravenous immunoglobulin (IVIG), plasma exchange, methylprednisolone, and heparin. Rituximab was started soon after. The patient made a drastic recovery with improvement in liver and kidney function, and was discharged after 45 days of hospitalization.

IMPACT/DISCUSSION: Antiphospholipid syndrome (APS) is an autoimmune condition that is encountered by internists, often in the setting of recurrent thrombosis and/or pregnancy loss. An estimated $0.8 \%$ of patients with APS develop CAPS, a rare condition characterized by rapid development of multiorgan arterial and venous thromboses. With mortality close to $50 \%$, early diagnosis and aggressive therapy is crucial for management. CAPS may be diagnosed in individuals with a prior history of APS or can be the first manifestation of APS. Although rare, preliminary criteria for "definite diagnosis" of CAPS have been developed. The criteria require 1) involvement of three or more organ systems; 2) development of symptoms in less than one week; 3) confirmation of small vessel thrombosis by histopathology in at least one organ; and 4) presence of antiphospholipid antibodies. The diagnosis is further supported by ruling out other causes of multiorgan thrombosis, such as disseminated intravascular coagulation, heparin-induced thrombocytopenia, thrombotic microangiopathy, and myeloproliferative neoplasms. Management of this life-threatening syndrome requires urgent initiation of anticoagulation, high dose systemic glucocorticoids and use of plasma exchange, with or without IVIG. If resistant to standard therapy, rituximab or eculizumab can be added. Mortality rate is reduced to $30 \%$ with early and aggressive treatment. A retrospective study of 600 patients with CAPS showed that $66 \%$ do not have recurrent clotting or emboli.

CONCLUSION: CAPS is a rapid-onset rheumatologic emergency that causes multiorgan failure due to arterial and venous thromboses. Prognosis is extremely poor without treatment, but being aware of this syndrome may allow for prompt recognition and aggressive therapy.

\section{CARDIAC AMYLOIDOSIS AN OVERLOOKED CAUSE OF NEW ONSET HEART FAILURE IN THE ELDERLY}

Seda Babroudi ${ }^{1}$; James Udelson ${ }^{2} .{ }^{1}$ Tufts Medical Center, Lincoln, MA; ${ }^{2}$ Tufts Medical Center, Boston, MA. (Control ID \#3185923)

LEARNING OBJECTIVE \#1: Recognize key clinical features of that distinguish cardiac amyloidosis from other causes of heart failure

CASE: A 74 year-old Caucasian woman with a past medical history of MGUS, mitral valve regurgitation (MVR), and prior negative cardiac stress test with normal ejection fraction, presented with progressive fatigue and exercise intolerance. Blood pressure was 90/57, but other vitals were within normal limits. Exam was notable for jugular venous distension, symmetric crackles, and bilateral lower extremity pitting edema. Labs were notable for troponin 0.5 and pro-BNP 27,000, but TSH and CK were within normal limits. ECG showed normal voltage with no ischemic changes. Echocardiogram revealed an ejection fraction of $20-25 \%$, severe global hypokinesis of the left ventricle, and moderate to severe MVR. The patient was empirically treated with diuresis and ACE-inhibition, and subsequently became profoundly hypotensive. Endometrial biopsy obtained was positive for Congo red staining, consistent with cardiac amyloidosis. Later subtyping revealed AL amyloid, and the patient was noted to have nephrotic-range proteinuria. The patient passed away before chemotherapy could be initiated. 
IMPACT/DISCUSSION: It is estimated that there are approximately 1000 cases of cardiac involvement of AL amyloidosis per year in the United States, with a median age at presentation between 55 and 60 years. Prognosis is poor, with a median survival of 6 months from the onset of heart failure in the untreated population. Swift diagnosis and treatment is therefore crucial in potentially modifying the natural history of this disease. Clinical features suggestive of amyloidosis include the disproportionate degree of BNP elevation as compared to a patient's clinical presentation, intolerance to ACE-inhibition, and nephrotic-range proteinuria. It is postulated that the degree of BNP production in cardiac amyloidosis is secondary to abnormal hormonal activation of myocytes. Similarly, intolerance to ACE-inhibition has been attributed to autonomic involvement of systemic amyloid. Proteinuria is indicative of systemic involvement of disease. An additional feature of this case that may have assisted in the diagnosis included the patient's history of MGUS, a rare precursor of $\mathrm{AL}$ amyloidosis. The mainstay of treatment for $\mathrm{AL}$ cardiac amyloidosis is diuretics, chemotherapy, and stem cell transplant in select patients. Usual guideline-directed medical therapies for heart failure with reduced ejection fraction are often poorly tolerated.

CONCLUSION: In elderly patients with new-onset heart failure without significant valvular disease, who are low risk for ischemic heart disease, should be evaluated for cardiac amyloidosis Discrepant BNP elevation and intolerance to ACE-inhibition should be considered potential markers of cardiac amyloidosis

\section{CARDIOMYOPATHY UNMASKED BY PHEOCHROMOCYTOMA}

Younghwa Kwon; Niaz Memon; Yi Xiong. Mercy Health Jewish Hospital of Cincinnati, West Chester, OH. (Control ID \#3184610)

LEARNING OBJECTIVE \#1: Recognizing the rare presentations of pheochromocytoma

LEARNING OBJECTIVE \#2: Understanding the pathophysiology behind pheochromocytoma

CASE: A 53-year old female presented with palpitations, headache, and chest pain. Patient reported emesis and bilateral paresthesia. She recently had flu-like symptoms that resolved 6 weeks ago. Her BP was 182/87 $\mathrm{mmHg}$, and appeared diaphoretic. Labs revealed blood glucose of $497 \mathrm{mg} /$ $\mathrm{dL}$, troponin of $0.26 \mathrm{ng} / \mathrm{mL}$, Lactic acid of $6.9 \mathrm{mmol} / \mathrm{L}$, Anion Gap of 21, and $\mathrm{WBC}$ of $21.6 \mathrm{~K} / \mathrm{uL}$. Blood gas revealed metabolic acidosis. There were no ketones and A1c was 5.9\%. EKG showed anterior and lateral STsegment elevations, concerning for a ST-elevated myocardial infarction (STEMI). She was started on an insulin drip, heparin drip, and clopidogrel. Patient was transferred to the catheterization lab, which revealed normal coronary arteries, non-dilated cardiomyopathy with reduced ejection fraction of $40 \%$. She continued to complain of pounding palpitations followed by a hot flash sensation. These episodes had been ongoing for seven years. CT abdomen was obtained and showed right adrenal nodule. Plasma metanephrines were tested, which showed elevated metanephrines and normetanephrine. These were elevated on repeated labs, as well as in 24hour urine collection. She had robotic-assisted right adrenalectomy. Repeat plasma metanephrines 6 months later showed normalization.

IMPACT/DISCUSSION: Pheochromocytoma is able to present in a variety of ways. Typically, it presents as the classic triad: headache, sweating, and tachycardia. Rarely it can present as hypertension and insulin resistance. We present a case of a female who developed STEMI and underwent cardiac angiogram, which revealed clean arteries and resembled stress-induced cardiomyopathy. Further investigations showed elevated metanephrines and concern for pheochromocytoma. Though our patient had the classic triad, these symptoms were overlooked due to concerns for acute coronary syndrome and DKA (without history of diabetes mellitus). She also had leukocytosis and lactic acidemia with anion gap acidosis, suggesting infection. It is speculated that catecholamine can increase myocardial demand and cause coronary artery vasoconstriction, which may mimic ACS. Additionally, the hyperglycemia can be explained by alpha- 1 and beta- 2 adrenergic receptor-induced gluconeogenesis and glycogenolysis. This case highlights the various presentations of pheochromocytoma, and clinicians ought to include pheochromocytoma as a differential for acute coronary syndrome when other causes are unexplainable.

CONCLUSION: Pheochromocytoma can have various presentations due to various catecholamine induced pathways. When classic triad of sweating, headache and palpitation is present, especially in combination with hypertension, pheochromocytoma should always be on the differential diagnosis. Pheochromocytoma in the setting of "pheo crisis" can induce stroke, heart attack, organ failure, coma, and death. In this case, STEMI and DKA was demonstrated.

\section{CARDIOVASCULAR COLLAPSE AND SEIZURES: AN UNIN- TENDED QUETIAPINE OVERDOSE}

Elena Jimenez Gutierrez ${ }^{1}$; Spencer Winters ${ }^{2}$; Siobhan Proksell ${ }^{3} .{ }^{1}$ The University of Texas Health Science Center at San Antonio, San Antonio, TX; ${ }^{2}$ Bronson Medical Group, Kalamazoo, MI; ${ }^{3}$ University of Pittsburgh Medical Center Presbyterian Hospital, Pittsburgh, PA. (Control ID \#3145056)

LEARNING OBJECTIVE \#1: Identify rare presentation of quetiapine overdose as part of differential diagnosis for shock

LEARNING OBJECTIVE \#2: Recognize risks posed by concomitant use of antipsychotic agent and CYP3A4 inhibitor

CASE: A 58-year-old man presented with two days of confusion, tremors and seizures. Past medical history included schizophrenia, epilepsy, HCV infection and right hemiparesis due to ischemic strokes. He initiated dasabuvir, ombitasvir, paritaprevir and ritonavir (Viekira Pak) three days earlier. His epilepsy had been controlled on lamotrigine for years. Review of systems was unremarkable. Vital signs were notable for tachycardia to 120 and hypotension to 73/51. Physical exam revealed disorientation, miosis, dry oral mucosa, tremors in all extremities, normal reflexes, and no new focal neurologic deficits, meningeal signs, thrush, jugular venous distention or stigmata of cirrhosis. BMP was notable for hypokalemia, acute kidney injury, metabolic acidosis with high anion gap, and normal osmolal gap. VBG revealed mild respiratory acidosis. CBC showed mild anemia. Drug testing was positive for tetrahydrocannabinol. Hepatic function panel, PT/PTT, ammonia, lamotrigine, CK, troponin, cortisol, and D-dimer levels were unremarkable. Urine and blood cultures, HIV serology and CXR were negative. Head CT showed no acute process. EEG revealed only diffuse slowing. EKG showed sinus tachycardia, prolonged QTc, and no ST or T-wave changes. TTE showed hyperdynamic LV function without wall motion abnormalities (WMA) and no RV dysfunction. The quetiapine level was $1434 \mathrm{ng} / \mathrm{mL}$ (therapeutic range $100-500 \mathrm{ng} / \mathrm{mL}$ ).

IMPACT/DISCUSSION: Patient presented in distributive shock due to quetiapine overdose from interaction with ritonavir. Negative infectious work-up and no fever or leukocytosis made septic shock improbable. Cortisol levels ruled out addisonian crisis. Serotonin and neuroleptic malignant syndromes were unlikely given lack of muscular rigidity, abnormal reflexes and elevated CK levels. Normal osmolal gap and unremarkable toxicology screen did not favor toxic ingestions. No arrhythmias or ischemic changes on EKG, negative troponins, and preserved EF without WMA or severe valve insufficiency on TTE ruled out cardiogenic shock. Lack of significant hemorrhage and fluid depletion made hypovolemic shock unlikely. Given no evidence of massive pulmonary embolism, pneumothorax or pericardial tamponade on imaging, obstructive shock was improbable.

CONCLUSION: The case enforced the need to exclude other causes of shock and highlighted a rare presentation of overdose due to drug interactions. Patient experienced delirium, seizures, tachycardia, hypotension, hypokalemia and QTc prolongation as manifestations of quetiapine intoxication. Since quetiapine is metabolized by cytochrome P450 (CYP) 
3A4 and ritonavir is an inhibitor of CYP3A4, concomitant use caused toxicity in the patient. Given heightened use of antivirals for HCV therapy, it is important to be aware of potential interactions between these drugs and patients' current medications.

\section{CARPAL TUNNEL, A PRECURSOR TO CARDIOMYOPATHY}

Amanda Satterthwaite. Tulane University School of Medicine, New Orleans, LA. (Control ID \#3184032)

LEARNING OBJECTIVE \#1: 1. Recognize the clinical presentation of TTR cardiac amyloidosis

LEARNING OBJECTIVE \#2: 2. Differentiate light chain amyloidosis from TTR amyloidosis

CASE: A 73-year-old man with a 5-year history of non-ischemic cardiomyopathy presented with worsening lower extremity edema and arthralgias that resulted in immobilization. He has a previous history of bilateral carpal tunnel syndrome, atrial fibrillation, and chronic kidney disease. Exam revealed abdominal distention with ascites and bilateral pitting edema of the lower extremities extending to the knee. Laboratory studies are significant for BNP of $4000 \mathrm{pg} / \mathrm{mL}, \mathrm{BUN}$ and creatinine of $76 \mathrm{mg} / \mathrm{dL}$ and $2 \mathrm{mg} / \mathrm{dL}$, and troponin of $0.240 \mathrm{ng} / \mathrm{dL}$. Chest radiograph revealed an enlarged, boot-shaped cardiac silhouette. Echocardiogram revealed and ejection fraction of $25 \%$, severe concentric hypertrophy of the left ventricle, right ventricular dilation, severe bi-atrial dilation, and severe mitral regurgitation. Findings were concerning for infiltrative cardiomyopathy. A technetium pyrophosphate scan revealed diffuse uptake of radiotracer consistent with a diagnosis of TTR cardiac amyloidosis.

IMPACT/DISCUSSION: Transthyretin (TTR) is a tetrameric protein rich in beta strands that is present in human serum. It is synthesized primarily in the liver, as well as the choroid plexus, and retinal pigment epithelium. It is involved in the transportation of thyroxine and retinol-binding protein. Systemic deposition of TTR can lead to amyloidosis, particularly either wild type TTR amyloidosis, or mutant TTR amyloidosis. Mutant TTR is characterized by single point mutations that increase the likelihood of misfolding of TTR. Wild type TTR amyloidosis is sporadic, not associated with any specific biomarkers or mutations, and occurs almost exclusively in men over the age of 60 . This patient exhibits many clinical features that are key in recognizing TTR amyloidosis including the presence of atrial arrhythmia, ventricular septal and free wall thickening on echocardiogram without history of uncontrolled hypertension, right-sided heart failure, and presence of peripheral neuropathy (carpal tunnel). Nearly one half of patients with wild type TTR have associated carpal tunnel, that typically precedes cardiac sequelae by up to 8-10 years. Spontaneous symmetric peripheral neuropathy or carpal tunnel in an elderly man should raise suspicion for an amyloid process. Relative reported frequencies of light chain and TTR amyloid are 8 per million/year and 0.4 per million/year for mutant TTR. The prevalence of wild type TTR is largely unknown. The Tc-pyrophosphate scan is simple and effective imaging modality to differentiate the two types of amyloid, with a sensitivity and specificity of $97 \%$ and $100 \%$ for TTR amyloid.

CONCLUSION: It is important to distinguish light chain amyloidosis from TTR amyloidosis. Compared to light chain cardiac amyloidosis, TTR amyloid has a prolonged survival rate, decreased severity of illness, and different management.

\section{CASE REPORT: DISSEMINATED TOXOPLASMOSIS WITH} NONSPECIFIC GASTROINTESTINAL SYMPTOMS

Michael G. Gindi ${ }^{1,2}$; Angela Montoya ${ }^{1,2}$; Robert Mas ${ }^{1,2}$; Lyndsey Turbow ${ }^{1}$; Evan Sternberg ${ }^{1}$; Parisa Geraminegad ${ }^{1} .{ }^{1}$ Mount Sinai Hospital, Chicago, IL; ${ }^{2}$ Ross University School of Medicine, Miramar, FL. (Control ID \#3184897)

LEARNING OBJECTIVE \#1: Recognize clinical features of gastrointestinal and disseminated toxoplasmosis
CASE: A 36-year-old male with a PMH of HIV (on HAART) presented with one month of diarrhea, melena, anorexia, weight loss, fatigue and a week of non-bloody non-bilious emesis. On admission his vital signs, physical exam, CBC and CMP were unremarkable. His CD4 count was 206 cells $/ \mu$ land HIV viral load was 826 copies $/ \mathrm{mL}$. In the ED, the patient had an episode of emesis, urinary incontinence, left foot myoclonus and became unresponsive at which point he was intubated and transferred to the ICU. CT and MRI of the brain showed two ring-enhancing lesions (RELs) with surrounding edema and a $5.2 \mathrm{~mm}$ midline shift. CT abdomen and pelvis showed mild wall thickening of the proximal colon and a fluid filled distal colon. Cultures, hepatitis panel and serology for Cryptosporidium, Blastomyces, and Histoplasma were negative. IgG titers for Toxoplasma were positive, treatment was initiated and repeat MRI showed reduction in the size of the RELs and midline shift as well as full resolution of his gastrointestinal (GI) symptoms.

IMPACT/DISCUSSION: $T$. gondii is a protozoan parasite acquired via ingestion of oocysts in contaminated food or water. Most infections are asymptomatic as the immunocompetent can overcome the parasite burden. Latent infection exists in $15 \%$ of both HIV positive and negative populations and reactivation occurs in up to $30 \%$ of patients with a CD4 count $<100$ cells $/ \mu \mathrm{L}$. While $89 \%$ of patients with active toxoplasmosis have intracerebral manifestations, extra-cerebral toxoplasmosis (ECT) only has a prevalence of $1.5-2 \%$. The most significant risk factor for ECT is a CD4 count $<100$ cells $/ \mu \mathrm{L}$ and $41 \%$ of cases have concurrent CNS infection. While brain biopsy is the only way to definitively diagnose toxoplasmic encephalitis (TE), patients with positive $T$. gondii serology, brain imaging with multiple RELs, neurological symptoms and immunosuppression have a $90 \%$ probability of TE and treatment is usually initiated without further work up. Due to vague symptomology, ECT is difficult to diagnose and often leads to a delay in treatment. Symptoms of GIT include anorexia, nausea, vomiting and diarrhea and, while definitive diagnosis is by endoscopic biopsy, this test is invasive and time consuming. The prevalence of ECT is thought to be underestimated as post-mortem analysis of seropositive patients reveals higher rates of dissemination and extra-cranial manifestations.

CONCLUSION: Though the greatest risk of reactivation of $T$. gondii is a CD4 counts $<100$ cells $/ \mu \mathrm{L}$, this case demonstrates that toxoplasmosis must be considered in any immunocompromised patient with or without neurological symptoms. Due to the difficulty in diagnosis and the rapidly progressive, often fatal course of toxoplasmosis we suggest a presumptive diagnosis in immunocompromised hosts with positive T. gondii titers, GI symptoms, negative stool cultures and alternative serology. Early diagnosis is crucial for successful treatment and prevention of mortality.

\section{CENTRAL FEVER: THE PARKINSONISM-HYPERPYREXIA SYNDROME.}

Sarabjeet S. Suri ${ }^{1}$; Dharmarajan S. Thiruvinvamalai ${ }^{2} .{ }^{1}$ Montefiore Medical center, Bronx, NY; ${ }^{2}$ Montefiore Medical Center (Wakefield Campus), Bronx, NY. (Control ID \#3183034)

LEARNING OBJECTIVE \#1: Recognize that fever can result from causes other than infection, such as central fever.

LEARNING OBJECTIVE \#2: Understand the importance of home medication reconciliation, and fever resulting from not taking medications.

CASE: 74 year old woman sent from nursing home with hypotension and lethargy. She could not provide a significant history due to lethargy. Initial vitals: temperature of 99.3F, BP-67/31 mm Hg. She received intravenous fluid resuscitation without appropriate response and now required pressor support. The patient had a large decubitus ulcer with necrosis and was placed on broad spectrum antibiotics. Initial labs: WBC 25,000, creatinine of $1.9 \mathrm{mg} / \mathrm{dl}$ (baseline 0.6 ), lactate 5.5. X-Ray chest normal. The patient had a history of Parkinson's disease, atrial fibrillation and hypertension. She was on aspirin $81 \mathrm{mg}$, apixaban, selegiline, carbidopa-levodopa, amiodarone 
and metoprolol. On day 2, she required intubation for airway protection. Repeat x'ray chest revealed new left lower lobe opacity compatible with aspiration pneumonia. On day 3, she had sustained episodes of high grade fever $(107.9 \mathrm{~F})$ while on appropriate antibiotics for her wound and aspiration pneumonia. Neuroleptic malignant syndrome was less likely as she had normal muscle tone and normal creatinine kinase. She was given intravenous levodopa as she had missed 2 days of medication, and was given dantrolene following which she became afebrile within 2 hours.

IMPACT/DISCUSSION: Parkinsonism-hyperpyrexia Syndrome (PHS) was first described in 1981, initially termed dopamine withdrawal syndrome. It occurs following the sudden withdrawal of levodopa/carbidopa or reduction in dose. It resembles neuroleptic malignant syndrome with rigidity, pyrexia and altered consciousness. Rare causes include deep brain stimulation. Symptoms usually present 24 hours to 7 days after missed doses. Clinical features include hyperpyrexia, altered sensorium and leukocytosis. Rhabdomyolysis may occur. Complications include acute renal failure, aspiration pneumonia, pulmonary embolism and DIC. Pathophysiology may involve sudden depletion of dopamine. Mortality may be $4 \%$ mortality if recognized early, $16 \%$ if untreated. Treatment involves intravenous hydration, supplementation of dopamine orally or intravenously and close monitoring. Physical measures such as cooling therapy are helpful.

CONCLUSION: Hyperpyrexia(>106.7F) should prompt evaluation for central causes of fever. The case illustrates the importance of home medication reconciliation upon hospitalization; not taking medications contributed to fever in this case

\section{CEPHALOSPORIN-INDUCED HYPOPROTHOMBINEMIA VIA A WARFARIN-LIKE EFFECT}

Vikas Gampa ${ }^{1}$; Ariel Majidi ${ }^{1}$; Jason Moran ${ }^{2} .{ }^{1}$ Cambridge Health Alliance, Somerville, MA; ${ }^{2}$ Beth Israel Deaconess Medical Center, Boston, MA. (Control ID \#3183005)

LEARNING OBJECTIVE \#1: Cephalosporins can lead to coagulopathy from inhibition of the enzyme inhibited by warfarin

LEARNING OBJECTIVE \#2: Management of cephalosporin-induced coagulopathy is identical to that of warfarin toxicity

CASE: Ms. $J$ is an 82 year-old woman who was diagnosed with a T2N0M0 squamous cell carcinoma of the tongue and was admitted to the hospital for planned surgical intervention including tracheostomy, right hemi-glossectomy, neck dissection, and skin graft. History was notable for decreased po intake. She received standard pre-operative antibiotic prophylaxis with cefazolin. Pre-operative labs were unremarkable. There were no intra-operative complications. She received cefazolin as per protocol after her surgery. On post-op day 3 , she had excessive oozing at the incision sites. Labs were notable for decreased Hgb $11.1 \rightarrow 8.1$, platelets 125 , an elevated PT (40.8) with INR 3.8, baseline normal PT 11.7 and INR 1.1. PTT was normal. Thrombin time was normal at 15.3 and fibrinogen was elevated to 432. Amidst bleeding and coagulopathy, $10 \mathrm{mg}$ IV vitamin $\mathrm{K}$ was urgently administered. On a subsequent lab draw, levels of Factors II, VII, IX, and X were $45,26,111$, and 47 , respectively, and a mixing study was inconclusive for presence of an inhibitor - these modestly reduced Factor levels and inconclusive mixing study were likely the result of administration of vitamin $\mathrm{K}$ with partial correction of her coagulopathy by the time they were drawn. She was treated with additional IV vitamin $\mathrm{K}$, discontinuation of cefazolin, and avoidance of other cephalosporin antibiotics, with normalization of PT/INR and resolution of bleeding.

IMPACT/DISCUSSION: Antibiotics are often implicated as a cause of coagulopathy with prolongation of PT/INR and the mechanism is attributed to elimination of gut flora. Cephalosporins may pose a unique mechanism of inducing a coagulopathy via a warfarinoid effect. Certain cephalosporins contain side-chains that, released upon drug degradation, can directly inhibit Vitamin $\mathrm{K}$ epoxide reductase, impairing gamma-carboxylation and the ability to activate the vitamin-K dependent factors II, VII, IX, and X. This particular case illustrates that patients with a prolonged duration of decreased PO intake with reduction of vitamin $\mathrm{K}$ stores are prone to the hypoprothrominemic effect of certain cephalosporins. This case should raise caution in the use of cephalosporins in patients with poor nutritional status, or close monitoring of coagulation parameters.

CONCLUSION: Our patient underwent an uncomplicated surgical resection for SCC of the tongue, though she developed significant bleeding, likely in part due to a coagulopathy from cefazolin administration. Cephalosporins can cause hypoprothrombinemia and bleeding via a warfarin-like mechanism and may be more likely in patients who have decreased dietary intake of vitamin $\mathrm{K}$. Treatment is vitamin $\mathrm{K}$ repletion and cessation of the offending antibiotic; if bleeding is severe, more urgent normalization of PT can be achieved with administration of vitamin- $\mathrm{K}$ dependent factors.

\section{CERVICAL MYELOPATHY SECONDARY TO B12 DEFICIEN- CY FOLLOWING SLEEVE GASTRECTOMY SURGERY}

Murali K. Duggirala. Mayo Clinic, Rochester, MN. (Control ID \#3181431)

LEARNING OBJECTIVE \#1: B 12 deficiency is common following a bariatric surgery; although rare, this deficiency can result in serious complications such as cervical myelopathy.

LEARNING OBJECTIVE \#2: Patient education is crucial in preventing serious complications of bariatric surgery.

CASE: Patient is a 33-year-old woman presented for a 2 nd opinion regarding an outside diagnosis of multiple sclerosis. She underwent a sleeve gastrectomy surgery approximately 3 years ago. She weighed $115 \mathrm{~kg}$ prior to surgery and was able to lose $50 \mathrm{~kg}$. She was recommended B 12 injections monthly but she took only one after surgery. Later, she switched to oral B 12 supplementation. Two years after the surgery she had developed subacute onset of numbness affecting her fingertips bilaterally. Over the next several weeks her numbness had extended to both wrists and also experienced difficulty with walking. MRI imaging showed T2 hyperintensity restricted to the $\mathrm{C} 3-4$ region and CSF analysis was negative. Her B 12 levels were borderline at 153 . She was started on glucocorticoids for suspected multiple sclerosis or clinically isolated syndrome. Her symptoms did not improve and a followup MRI scan demonstrated a longitudinally extensive T2 hyperintense lesion now extending from C1-C7. Her copper levels, NMO antibodies, paraneoplastic panel were negative. She was diagnosed with cervical myelopathy from B 12 deficiency and was started on vitamin B 12 injections. She received $1000 \mathrm{mcg}$ of vitamin-B 12 intramuscularly once a day for 7 days then, once weekly for 4 weeks and then once monthly. At three month followup, her symptoms have completely resolved.

IMPACT/DISCUSSION: B 12 deficiency is very common following bariatric surgery and can occur in up to $64 \%$ of the patients. So, following the surgery replacement of B 12 is very important. Oral B 12 is not absorbed well in patients with bariatric surgery. Parenteral B 12 supplementation is commonly recommended. If sublingual B 12 is supplementation is chosen then periodic monitoring of blood B 12 level is recommended.

CONCLUSION: Cervical myelopathy can be a complication following a bariatric surgery. It is important to counsel patients about postoperative B 12 supplementation. With adequate supplementation of B 12 this serious complication can be prevented.

\section{CHALLENGING THERAPEUTIC APPROACH FOR CENTRAL DIABETES INSIPIDUS}

Maria del Mar Morales Hernandez ${ }^{1}$; Marie A. Landicho ${ }^{1}$; Ivan A. Serra$\mathrm{no}^{2,}{ }^{1}$; Kent Wehmeier ${ }^{1}$. University of Florida COM, Jacksonville, FL; ${ }^{2}$ University of Florida, Jacksonville, FL. (Control ID \#3186355) 
LEARNING OBJECTIVE \#1: Diagnose central diabetes insipidus LEARNING OBJECTIVE \#2: Recognize limitations in the symptomatology treatment of central diabetes insipidus with desmopressin CASE: A 34-year old female with history of hypertension, gastric bypass surgery, polycystic ovarian syndrome, and non-functional pituitary macroadenoma presented with chronic headaches for 3 years. Work-up showed $1.6 \mathrm{~cm}$ lesion within the rightward sella consistent with macroadenoma. Endocrine studies were consistent with non-functional adenoma. She underwent elective macroadenoma resection via transsphenoidal approach. Post operatory course complicated with increased urinary output of $8 \mathrm{~L}$ within 24 hours. Morning cortisol and thyroid function studies were found within normal limits at that time. The patient was started on oral desmopressin(dDAVP) and adjusted to $400 \mathrm{mcg}$ three times daily without improvement of polyuria and polydipsia. Oral desmopressin was changed to $1 \mathrm{mcg}$ of subcutaneous form of dDAVP twice daily with improvement of polyuria. The urinary output improved from of 6.6 liters to 3 liters in 24 hours. She was eventually discharged home in stable condition on subcutaneous desmopressin with close follow up with Endocrinology with plans to try intranasal form as outpatient.

IMPACT/DISCUSSION: Central Diabetes Insipidus symptomatology can be efficiently managed with hormone replacement with Desmopressin. Data had showed increased morbidity and mortality involved with inadequate treatment of Central Diabetes Insipidus. Individualization of treatment should be provided based on patient severity of polyuria, absorption and baseline conditions. Patients who are unable to tolerate intranasal or oral desmopressin should be provided with subcutaneous form. The deficiency of the antidiuretic hormone, vasopressin (AVP) causes Central Diabetes Insipidus (CDI). AVP deficiency results in uncontrolled diuresis. Complete deficiency can lead to polyuria exceeding 10 liters in 24 hours. CDI is treated with desmopressin, an analogue of arginine vasopressin (AVP) without vasopressor activity. Desmopressin can be administer intranasally, in an oral tablet form or in a parenteral formulation. Oral route is preferred by patients for their ease of administration but only $5 \%$ is absorbed on the gut and its absorption can decrease up to $50 \%$ if taken with meals. Intranasal form is contraindicated in cases of rhinitis or nasal mucosa edema. When patients have inadequate response to intranasal or oral forms of dDAVP, it should be given subcutaneously and the dose should be adjusted accordingly. Treatment should be continued for as long patient has CDI. Patient should be educated and closely monitor about signs of symptoms of hyponatremia while on DDAVP.

CONCLUSION: Prompt recognition and adequate administration of desmopressin can efficiently control symptoms of CDI. Route of dDAVP administration should be decided based on patient profile and clinical response.

\section{CHECK YOUR BIAS AT THE DOOR: A CASE OF ANCHORING BIAS IN REFRACTORY IMMUNE CHECKPOINT INHIBITOR- ASSOCIATED COLITIS.}

Nathan C. Nowalk; Grant Pickett. Baylor College of Medicine, Houston, TX. (Control ID \#3186250)

LEARNING OBJECTIVE \#1: Appraise a clinically challenging case for cognitive errors, specifically, anchoring bias

LEARNING OBJECTIVE \#2: Compose a diagnostic schema for inflammatory diarrhea in patients on immune checkpoint inhibitors

CASE: A 50 year-old female with high-grade urothelial carcinoma presented with two-week history of abdominal pain and diarrhea of smallvolume hematochezia. One month prior to symptom-onset, the patient received her first doses of nivolumab and ipilimumab. A sigmoidoscopy with biopsies was performed showing neutrophilic crypt microabscessesconsistent with diagnosis of immune checkpointinhibitor (ICI) associated-colitis. The patient received intravenous methylprednisolone and one dose of infliximab with improvement in symptoms. She was discharged on oral prednisone. Over the following four weeks her prednisone was tapered down. Six weeks after initial presentation, she again presented with identical symptoms of abdominal pain and diarrhea of small-volume hematochezia. She was again started on intravenous methylprednisolone, but symptoms did not improve as they had before. Repeat sigmoidoscopy with biopsy showed mucosal ulcers with "owl's eye" inclusion bodies consistent with diagnosis of cytomegalovirus (CMV) colitis. She was started on intravenous ganciclovir, followed by oral valacyclovir with resolution in symptoms at two-week follow-up.

IMPACT/DISCUSSION: Due to prior success and growing indications, the use of ICIs in treating cancer continues to increase at a rapid rate. As expected, the frequency of immune-related adverse events from these agents has also increased. Prior studies have shown that combining ICIs can increase the risk of adverse events up to 55\%. ICI associated-colitis remains the most commonly encountered severe adverse event and usually occurs within six weeks of initial dose. Diagnosis is established by endoscopy with biopsy. Management includes discontinuation of ICIs followed by immunosuppression. As is the case with other inflammatory bowel diseases, such as Crohn's disease, the exclusion of infectious diarrhea is paramount before initiating immunosuppression. CMV colitis is seen in these other inflammatory diarrheas but is only recently being recognized in ICI-associated diarrhea.

CONCLUSION: Anchoring bias is the cognitive error of retaining a single diagnosis based on a few case features and failing to integrate new information when appropriate. An extension of anchoring bias is diagnostic momentum, which describes the resistance to broaden a prior diagnosis made by an earlier provider. Both errors are unfortunately wellrepresented in our case. Our patient had an established diagnosis of ICI associated-colitis when she presented with her second inflammatory diarrhea in two months. Her recent decrease in steroids as an outpatient fortified the suspicion for recurrence of ICI associated-colitis. Unfortunate anchoring bias resulted in a therapeutic trial of steroids with no improvement in symptom severity.

\section{CHECKING THE CHECKPOINTS: A CASE OF TYPE 1 DIABE- TES FOLLOWING PD-1 INHIBITION IN A PATIENT WITH HIV ON HAART}

Michael S. Hughes ${ }^{1}$; Sara Bedrose ${ }^{2}$; Madhuri M. Vasudevan ${ }^{2}$; Marco Marcelli ${ }^{2}$. ${ }^{1}$ Baylor College of Medicine, Houston, TX; ${ }^{2}$ Michael E. DeBakey VA Medical Center and Baylor College of Medicine, Houston, TX. (Control ID \#3179491)

LEARNING OBJECTIVE \#1: Recognize type 1 diabetes mellitus as a rare but important complication of immune checkpoint inhibition

LEARNING OBJECTIVE \#2: Employ higher clinical suspicion for potential immune related adverse events in patients with HIV on checkpoint inhibitors

CASE: A 48-year-old man with HIV on HAART was diagnosed with Hodgkin's lymphoma and initiated the Programmed Death 1 (PD-1) immune checkpoint inhibitor, nivolumab. After 6 months, he reported polydipsia and polyuria. Labs revealed glucose $764 \mathrm{mg} / \mathrm{dl}$ and A1c $7.1 \%$. Low C-peptide and elevated GAD-65 antibody levels confirmed type 1 diabetes mellitus (T1DM), and he started insulin therapy. Nivolumab was continued for 12 more months. Despite discontinuation of nivolumab, he remains on insulin therapy.

IMPACT/DISCUSSION: While A1c may reflect average glycemia, it alone cannot distinguish T2DM from T1DM progression. Tests for cpeptide and autoantibodies for T1DM must be included when evaluating patients on PD-1 inhibitors. T1DM is a known immune related adverse event (irAE) following treatment with PD-1 inhibitors with reported $0.9 \%$ incidence. However, little is known about such irAEs in individuals with HIV treated with PD-1 inhibitors. Patients with HIV have higher autoantibody titers in active disease states or following HAART initiation, as seen in immune reconstitution. Moreover, in vitro PD-1 inhibition elevates HIV cell proliferation and immune hyperactivity. The challenge in 
patients with HIV on HAART and PD-1 inhibitors is unraveling whether autoantibody generation resulting in T1DM is potentiated by pre-existing HIV on HAART and/or provoked by immune response to PD-1 inhibition. CONCLUSION: Clinicians treating patients with immune checkpoint inhibitors should maintain a high index of suspicion for development of autoimmune disorders. Literature on the use of PD-1 inhibitors in HIV patients is limited and warrants further investigation. To our knowledge, this is the first reported case of T1DM following PD-1 inhibition in a patient with HIV on HAART therapy. Such patients are predisposed to immune reconstitution, and response to PD-1 inhibition may potentiate risk for autoimmune complications. Therefore, higher clinical suspicion for irAEs is merited in patients with HIV on PD-1 inhibitors.

\section{CHEST AND BACK PAIN WITHOUT ABDOMINAL PAIN AS AN INITIAL SYMPTOM OF ACUTE CHOLECYSTITIS}

Risa Hirata; Masaki Tago; Hidetoshi Aihara; Motoshi Fujiwara; Shun Yamashita; Naoko E. Katsuki; Shu-ichi Yamashita. Saga University Hospital, Saga city, Japan. (Control ID \#3147145)

LEARNING OBJECTIVE \#1: Acute cholecystitis can develop only with chest pain at the site of sternal bone and back pain without abdominal pain

CASE: A 64-year-old man was transferred by ambulance to our hospital because of sudden-onset clamping chest pain in the lower half of his sternal bone, upper back pain, vomiting, and cold sweating at rest. On admission, he was alert and had a blood pressure of $127 / 81 \mathrm{mmHg}$, regular pulse rate of 70 beats/min, respiratory rate of 20 breaths/min, and $\mathrm{SpO}_{2}$ of $94 \%$. The only abnormality on physical examination was spontaneous and percussion pain in the lower half of the sternal bone; no other signs of acute cholecystitis were present, such as abdominal tenderness, muscular guarding, or Murphy's sign on his abdomen. Laboratory findings on admission including complete blood count and hepatobiliary enzyme were normal except for slightly elevated CRP of $1.08 \mathrm{mg} / \mathrm{dL}$. Echocardiography and cardiac ultrasonography findings were normal. Abdominal ultrasonography only showed debris and slight thickening of the gallbladder wall. Abdominal computed tomography (CT) failed to reveal any abnormalities at that time. However, thoracic and abdominal CT was repeated 8 hours after admission because of persistent pain showing findings of acute cholecystitis such as thickness, a low-density area, and enhancement of the gallbladder wall. No abnormality of the common bile duct or intrahepatic biliary tract was found on magnetic resonance cholangiopancreatography. Although the patient's chest pain disappeared on the second hospital day, a fever and percussion tenderness on the liver appeared without Murphy's sign. The CRP level increased to $11.0 \mathrm{mg} / \mathrm{dL}$. He was successfully treated by ampicillin/sulbactam and discharged from our hospital on the 10th hospital day.

IMPACT/DISCUSSION: What does this case add to the literature? This case suggests that acute cholecystitis can develop only with chest pain without typical abdominal signs or abnormalities on imaging studies in the early phase of the disease.

CONCLUSION: Acute cholecystitis presenting only as chest pain without epigastric or right hypochondrial pain is rare. Verdon et al. reported that only 1 of 672 patients who visited outpatient clinics for evaluation of chest pain had acute cholecystitis. The chest pain in our patient was likely to be referred pain. Pain from abdominal viscera is transmitted through the greater splanchnic nerve arising from $\mathrm{T} 5$ to $\mathrm{T} 10$, which could cause pain at the same dermatome level. Our patient complained of pain in the lower part of the sternal bone innervated by $\mathrm{T} 5$ to $\mathrm{T} 7$, which was compatible with referred pain from acute cholecystitis. In conclusion, acute cholecystitis can begin only with chest pain, which is likely to be referred pain, without typical abdominal signs or abnormalities on imaging studies in the early phase of the disease.
CHIKUNGUNYA "FEVER" IN AN AFEBRILLE TROPICAL TRAVELER WITH INTRACTABLE BACK PAIN

Kyle Macaulay; Ian Garrahy; Jesse Sengillo. Tower Health System, Wyomissing, PA. (Control ID \#3186386)

LEARNING OBJECTIVE \#1: Chikungunya fever can present in many ways which often leads to a missed or delayed diagnosis.

LEARNING OBJECTIVE \#2: With the history of recent travel to a tropical island, Chikungunya fever should be considered as a diagnosis even with an atypical presentation.

CASE: Chikungunya fever is an arthropod-borne virus with prevalence up to $70 \%$ in some hyperendemic countries, and has recently spread to North America since its identification in Caribbean countries in late 2013. Diagnosis can be delayed or missed as the presentation varies from asymptomatic to typically severe arthralgias, high-grade fever, and maculopapular rash to chronic arthritis and death. A 57-year-old female presented to the emergency department with five days of flu-like symptoms, severe mid-thoracic back pain, and a transient urticarial-like rash after returning from Jamaica three weeks prior. She reported no sick contacts. The patient was afebrile with stable vital signs. She exhibited tenderness to palpation of the lumbar spine, and had no evidence of conjunctivitis or lymphadenopathy. Labs were significant for sodium of $123 \mathrm{mEq} / \mathrm{L}$, lymphocytic-predominant leukocytosis of $16,200 / \mathrm{uL}$ with $17 \%$ atypical lymphocytes, and a mild transaminitis (AST 78 units/L, ALT 100 units/L). MRI of the lumbar and thoracic spine was unrevealing. Serology for chikungunya IgM antibody was unequivocally high with 1.54 IV (normal: $<1.1$ IV) and undetectable IgG antibodies. Titers were negative for CMV, HIV and dengue. Patients with non-classic presentations and recent travel to endemic areas should have Chikungunya fever considered as part of their differential. While most common presentations will include fever and debilitating joint pain, Chikungunya fever should be considered even in the absence of in-hospital fever when either peripheral or axial joints are involved.

IMPACT/DISCUSSION: Chikungunya fever most commonly presents with severe arthralgias, high-grade fever, and maculopapular rash. It should be considered even in those who who are afebrile but who have joint pain and a tropical travel history.

CONCLUSION: Chikungunya fever is an arthropod-borne virus which has recently spread to North America since its identificaiton in Caribbean countries in late 2013. Its increasing prevalence and varied presentations make it a diagnosis that should be considered when a traveler presents with joint pain.

\section{CHRONIC ALCOHOL ABUSE: AN UNUSUAL PRESENTATION OF ANEMIA}

Benjamin Yao ${ }^{1}$; Kunal Patel ${ }^{2,}{ }^{1}$; Calen Frolkis ${ }^{2,1} .{ }^{1}$ Case Western Reserve University, Cleveland, OH; ${ }^{2}$ MetroHealth Medical Center, Cleveland, OH. (Control ID \#3185757)

LEARNING OBJECTIVE \#1: Diagnose and manage complications of acute/chronic alcohol abuse

LEARNING OBJECTIVE \#2: Recognize the classic triad of Zieve's syndrome

CASE: A 39-year-old female with a past medical history of chronic alcohol abuse, hyperlipidemia, and cirrhosis presents for readmission with swelling of the abdomen/lower extremities and worsening jaundice. She was admitted and discharged 2 week ago for jaundice and new acute anemia (Hgb 4.6g/dL, Hematocrit 13.3\%) after consuming 4 drinks a night for 2 weeks; suspected to be decompensated cirrhosis secondary to alcohol abuse with alcoholic hepatitis and iron deficiency anemia. The patient received 4 units of packed RBCs and started on 4-week Prednisolone $40 \mathrm{mg}$ daily regimen, vitamin repletion, and instruction for alcohol cessation. On readmission, the patient endorsed alcohol relapse and found 
to have worsening anemia with no evidence of acute bleed $(\mathrm{Hgb} 6.0 \mathrm{~g} / \mathrm{dL}$, Hematocrit $18.3 \%$, MCV $110 \mathrm{fL}$ ), thrombocytopenia (platelet $45 \mathrm{~K} / \mathrm{uL}$ ), worsening liver disease (Total bilirubin $15.2 \mathrm{mg} / \mathrm{dL}$, Direct $5.8 \mathrm{mg} / \mathrm{dL}$ ), transaminitis (AST 127IU/L, ALT 48IU/L, alkaline phosphatase 87IU/L, albumin $2.8 \mathrm{~g} / \mathrm{dL}$ ). She received 3 units of packed RBCs without expected incrementation. CT abdomen showed splenomegaly with significant perisplenic and splenorenal varices. Complete blood count showed elevated reticulocytes, elevated $\mathrm{LDH}$, and low haptoglobin; coagulation panel showed prolonged PT 19.2 seconds, INR 1.68, and prolonged PTT 37 seconds. A peripheral blood smear showed schistocytes, Burr cells, and no toxic granulations. She was diagnosed with hemolytic anemia consistent with Zieve's syndrome.

IMPACT/DISCUSSION: This case illustrates the unique presentation of Zieve's syndrome which is a triad of hemolytic anemia, hyperlipidemia, and jaundice. The initial concern with this hospitalization was disseminated intravascular coagulation (DIC), given the presentation of schistocytes and recurrent, persistent anemia. The pathophysiology for DIC is based on the presence of increased thrombin formation in patients suffering from severe liver cirrhosis resulting in decreased clotting factors, hypofibrinogenemia and thrombocytopenia. The key finding was the presence of splenomegaly on CT abdomen. Zieve's syndrome is a special type of alcoholic hepatitis in which alcohol consumption causes hemolytic anemia and schistocytes from splenomegaly. Dr. Leslie Zieve originally proposed elevated circulating lipid levels resulted in alterations in erythrocyte membrane composition, leading to increased susceptibility to hemolysis. Patient treatment is focused on cessation of alcohol. Most patients recover within 4 to 6 weeks after alcohol withdrawal. For patients who continue to drink alcohol, it may aggravate the illness and potentially be fatal as this patient was readmitted again with worsening anemia unresponsive to transfusion and transferred to hospice care.

CONCLUSION: Establish unbiased differential diagnosis of alcoholic, liver, and blood related pathologies that may include Zieve's syndrome based on presentation

\section{CLINICAL DILEMMA: MANAGING COMORBID CHRONIC} PAIN, SUBSTANCE USE DISORDER, AND MENTAL ILLNESS

Joel Sawady $^{1,2}$; Gina Stenhouse ${ }^{1} .{ }^{1}$ Cambridge Health Alliance, Cambridge, MA; ${ }^{2}$ Harvard Medical School, Boston, MA. (Control ID \#3184727)

LEARNING OBJECTIVE \#1: Formulate a long-term, patient-centered chronic pain management plan (including opioid tapering) for very high risk patients

LEARNING OBJECTIVE \#2: Manage the clinical and professional dilemma of inheriting patients on high-risk opioid regimens

CASE: 52 yo male with history of OUD, MDD, and PTSD with recent suicide attempt, who transitioned to a new PCP. His chronic pain originated after an episode of vertebral osteomyelitis resulting in extensive surgery. At the time of meeting his new PCP, the patient was managed on $260 \mathrm{MME} /$ day along with psychotropic medications including a benzodiazepine. The new primary care team, in collaboration with the patient and his mental health team, decided to pursue a gradual opioid taper. During the taper, the patient developed worsening anxiety and had difficulty managing psychosocial stressors. However, tapering also provided him GI relief and allowed him to become activated, goal-directed, and engaged in adaptive behaviors. The patient pursued neurosurgery reevaluation which led to revision of his spinal surgery. Unfortunately, despite pre-surgery coordination, the patient was discharged on an inappropriate opioid regimen. This was addressed and taper was resumed. Again, the patient experienced worsening psychological symptoms including poor sleep. Despite this, the patient continued to display improved affect compared to initial presentation and exercised more self-efficacy. At this point, two years into the taper, the patient does not have increased thoughts of illicit opioid use, but this is a continuing concern. We are discussing with the patient transitioning to buprenorphine for OUD and chronic pain.
IMPACT/DISCUSSION: Current guidelines recommend against starting high-dose opioids, particularly in high-risk patients. However, they offer little guidance on how to care for patients already on these regimens. Tapering may result in destabilization and poor outcomes. This case will complement the guidelines and provide clinicians with tools to manage these complex patients. Discussion points include: 1 . The literature on concurrent OUD and chronic pain, including the role of buprenorphine 2. The evidence base for tapering opioids 3. Psychiatric symptoms that may emerge during opioid tapering, particularly in the presence of mental illness 4. Practices used to build a collaborative, patient-centered care plan 5. Ethical dilemmas in a situation in which all options (continue prescribing, fast taper, and slow taper) present risks

CONCLUSION: Prior to tapering opioids in any patient, including those with history of OUD, the risks of an expedited taper should be reviewed and consideration should be given to a gradual, patient-centered tapering plan. Empathy and relationship-building are important at every step. A multidisciplinary approach is critical for patients with concurrent mental illness, substance use disorder, and chronic pain. This is particularly important when tapering opioids, as psychiatric symptoms may be unmasked.

\section{CLOSTRIDIUM DIFFICILE LIVER ABSCESS: AN UNUSUAL PRESENTATION}

Pramod Acharya ${ }^{1}$; Pinky $\mathrm{Jha}^{3}$; Aravind Seetharaman ${ }^{2}$; Mirza Baig ${ }^{1}$; $\overline{\text { Sanjay Bhandari }}{ }^{2} .{ }^{1}$ Medical College of Wisconsin, Pewaukee, WI; ${ }^{2}$ Medical College of Wisconsin, Milwaukee, WI; ${ }^{3}$ medical college of wisconsin, Milwaukee, WI. (Control ID \#3178480)

LEARNING OBJECTIVE \#1: To report/recognize a case of Clostridium liver abscess in a patient without history of C. difficile colitis.

LEARNING OBJECTIVE \#2: To review the diagnosis and management of C. difficile liver abscess.

CASE: An 83-year-old woman with history of appendicitis, renal stones, and cholecystitis (with previous cholecystectomy) presented to an outside hospital for right upper quadrant pain 4 months ago and was found to have a $6.7 \mathrm{~cm}$ liver abscess on imaging. Biopsy and culture confirmed liver abscess with only $\mathrm{C}$. difficile grown on the anaerobic culture. She denied previous history of $\mathrm{C}$. difficile colitis. She was treated with 7 weeks of oral metronidazole, with much difficulty taking the medication. She reported 17 pounds weight loss. About 2-3 months ago she began to develop pleuritic chest pain and cough. Imaging showed pleural effusion and possible empyema. Pleural biopsy showed organizing pneumonia with negative special stains. She was admitted to our hospital with 3 weeks of right chest pain and cough with yellow sputum. Upon presentation vitals were stable. Physical Exam showed decreased breath sounds at posterior right base. Admission lab results were unremarkable except for Right lower lobe consolidation and possible empyema. ID was consulted, and patient was treated with IV vancomycin. Cardiothoracic surgery was consulted for empyema. Pleuropulmonary complication was secondary to needle manipulation during transpleural approach of liver biopsy.

IMPACT/DISCUSSION: In contrast to typical C. difficile infection (CDI), extra-intestinal manifestation of CDI has been rarely reported. The frequency of extra-intestinal CDI comprises approximately $0.17 \%$ to $0.6 \%$ of all CDI. Long-term metronidazole therapy (6-8 weeks) is considered to be effective because of excellent penetration to the liver and susceptibility of isolated $C$ difficile strains. CDIs are becoming more frequent worldwide, however, the precise trend of extra-intestinal CDI is unclear. Our case is unique as the patient does not report history of C. difficile infection. This case further warns the treating physician of the possible risk of seeding of infection elsewhere on needle aspiration.

CONCLUSION: A greater accumulation of extra-intestinal CDI cases is necessary to determine its epidemiology for the proper treatment of extraintestinal CDI, including recurrent $\mathrm{C}$. difficile bacteremia and liver abscess. Needle track seeding may complicate liver abscess especially when the location of the abscess requires transpleural route of aspiration and increase morbidity. 


\section{CLOZAPINE: AN UNDER-RECOGNIZED CULPRIT}

Deeva Berera ${ }^{1}$; Peter Ting ${ }^{1}$; Benjamin Bier ${ }^{2} .{ }^{1}$ Icahn School of Medicine at Mount Sinai Hospital, New York, FL; ${ }^{2}$ Mount Sinai Hospital, New York, NY. (Control ID \#3186660)

LEARNING OBJECTIVE \#1: Recognize the clinical features of clozapine cardiotoxicity.

LEARNING OBJECTIVE \#2: Manage suspected clozapine cardiotoxicity.

CASE: 68 YOF with PMH of schizophrenia who was involuntarily admitted to inpatient psychiatry for worsening psychosis, unresponsive to titration of her outpatient medication regimen. She was started on clozapine for treatment of refractory schizophrenia with significant improvement. PMH: Obesity, osteoarthritis, schizophrenia PSH: None; Meds: Haldol 10mg BID, benztropine 1mg BID, clonazepam 1mg QHS $\mathrm{SH}$ : Lives with husband and son, no EtOH, tobacco, or illicit drug use. Employed as a part time supermarket clerk. On day 21 of admission, she was febrile to $103.1 \mathrm{~F}$, other VS stable. She denied cough, rhinorrhea, N/V/D. CBC WNL. Infectious work-up including blood and urine cultures, and viral respiratory panel were negative. On day 22 , she developed chest pain and shortness of breath. EKG was normal, however troponin 1.34. CXR showed mediastinal widening, follow-up CTA showed bilateral ground glass opacities concerning for pulmonary edema. She became acutely hypoxemic requiring HFNC and was transferred to medicine for management of suspected NSTEMI. Medicine course was complicated by worsening mental status and intubation for hypoxemic respiratory failure. She became acutely hypotensive during intubation requiring vasopressor support. A bedside echo showed poor LV function and severe mitral regurgitation. Her ScvO2 was $30 \%$ consistent with cardiogenic shock. Follow-up TEE post dobutamine initiation was negative for endocarditis and showed normal LV function with moderate MR. Psychiatry and cardiology collectively determined her acute decompensation was most likely secondary to clozapine induced cardiotoxicity.

IMPACT/DISCUSSION: Clozapine induced cardiotoxicity is a well known phenomenon in the psychiatric community, often limiting its use. However, data is limited to case reports and there are few systematic reviews or guidelines for clinicians on how to recognize and manage clozapine induced toxicities. In retrospect, this case was classic for clozapine toxicity, which usually occurs at a median of 3 to 8 weeks from drug initiation. Cardiotoxicities (ranging from myocarditis to cardiogenic shock) are thought to be secondary to up regulation of IL-2 and TNF mediated pathways causing a systemic inflammatory response. Clinicians should have a high suspicion for clozapine cardiotoxicity in patients with acute cardiac decompensation without any other risk factors and a low treshhold for performing urgent echocardiograms. Clozapine should be discontinued and cases should be reported to expand the existing body of literature.

CONCLUSION: -Clinical features of clozapine toxicity include fevers, tropinemia, and acute cardiac decompensation within 3 - 8 weeks of drug initiation. -The mainstay of treatment of clozapine-induced cardiotoxicity is cessation of clozapine, early echocardiography, and appropriate medical management of the resultant toxicity.

\section{COCAINE AND ALCOHOL MISUSE INDUCED RHABDOMYOLYSIS}

Claire Huang Lucas ${ }^{2}$; Geeta Varghese ${ }^{1}$; Paul El Tomb ${ }^{2}$; Joshua Berookhim ${ }^{2}$. ${ }^{1}$ Mount Sinai Beth Israel, New York, NY; ${ }^{2}$ Mount Sinai Saint Lukes-Roosevelt, New York, NY. (Control ID \#3175204)

LEARNING OBJECTIVE \#1: Diagnose and distinguish cocaine and alcohol use as common differentials of rhabdomyolysis

CASE: 60 yo male with history of chronic alcohol misuse (1 pint of vodka 3-4 times per week), daily cocaine inhalation, DM2, hyperlipidemia, presented with fatigue and proximal muscle weakness of all extremities for 4 weeks. His medication list was noteworthy for daily Atorvastatin $40 \mathrm{mg}$, ASA $81 \mathrm{mg}$, Lisinopril $40 \mathrm{mg}$ and Metformin 1,000 bid. He denied trauma, infection, strenuous exertion, prolonged immobilization, LOC and seizure. Physical exam revealed $4 / 5$ strength in all extremities and no skin rash. Labs showed CPK > 35,000, transaminitis (AST 4,555, ALT 447), and normal BMP, TSH, ESR, CRP. UA positive for protein $1+$, blood 2+, and no RBC. The patient was then directed to the ER and received IV and PO hydration and underwent muscle biopsy. Statins were ceased and a progressive steroid taper was started. At hospital discharge, CPK trended down to 6,000 with clinical improvement, however after resuming cocaine and alcohol use, CPK rose to 11,000 while on steroids. Muscle biopsy showed moderate myophagocytosis with no inflammatory infiltrate. Serology was negative for ANA, anti RNP, anti Smith, anti Jo, anti SSA, anti SSB, anti MSA. Final diagnosis was drug induced rhabdomyolysis.

IMPACT/DISCUSSION: Cocaine and alcohol abuse is a public health issue encountered in our daily practice. These patients are at greater risk of developing severe conditions, such as cardiovascular, CNS and liver diseases. Cocaine and alcohol use are rarely perceived as common differentials of rhabdomyolysis unless they are associated with a history of syncope, seizure, fall or focal muscle pain, none of which was reported by our patient. These rhabdomyolysis differentials are often overshadowed by statin use and extreme exertion. Cocaine and alcohol nefarious health effects are well documented. Specifically, cocaine has been reported to induce rhabdomyolysis in multiple case reports and one study on eel found a correlation between cocaine exposure and muscle swellingbreakdown. Alcohol misuse, acute or chronic, leads to pathological changes in the muscle, called alcohol-induced myopathy, found in over half of the alcohol abusers. Clinically, rhabdomyolysis is difficult to diagnose, half of biologically-proven rhabdomyolysis had no muscle related complains and cocaine-induced rhabdomyolysis has been shown to poorly correlate with symptomatic muscle related complains.

CONCLUSION: Given the significant potential complications induced by rhabdomyolysis, such as renal insufficiency, and electrolytes disturbances with risk of fatal cardiac arrhythmia and death, it is vital to learn to diagnose and distinguish cocaine and alcohol use as common differentials of rhabdomyolysis. Such cocaine and alcohol use should trigger a specific inquiry about any complaints of fatigue and muscle discomfort, and warrant a lower threshold to order CPK as part of the lab workup.

\section{COLITIS ON THE CAPE - RECOGNIZING RESISTANCE PAT- TERNS IN SHIGELLA ENTEROCOLIIS}

Timothy M. Bober ${ }^{1}$; Furkan Ertem ${ }^{2}$; Amar Kohli ${ }^{3} .{ }^{1}$ UPMC PresbyterianShadyside, Pittsburgh, PA; ${ }^{2}$ University of Pittsburgh Medical Center, Pittsburgh, PA; ${ }^{3}$ UPMC, Mars, PA. (Control ID \#3186230)

LEARNING OBJECTIVE \#1: Emphasize antibiotic treatment in Shigella enterocolitis

LEARNING OBJECTIVE \#2: Recognize resistance patterns among Shigella infections among men who have sex with men

CASE: Case: This was a 54 year-old male with history of HIV with undetectable viral load on HAART, diverticulosis, Hepatitis C, and chronic inflammatory sensory polyneuropathy who presented with 5 days of large loose bowel movements with small clots and neurological symptoms. He had traveled to Cape Cod, where he explored marshes and ate "bad tasting" lobster. He had unprotected receptive anal intercourse with two male partners. Patient denied fever/chills, excessive NSAID use, recent antibiotic treatment, or family history of inflammatory bowel disease. Review of systems was notable for 4 pound weight loss and neurologic complaints including headache, diplopia, and patchy numbness. The patient was afebrile $\left(36.8^{\circ} \mathrm{C}\right)$ and normotensive at $112 / 72$ with a heart rate of 72 beats per minute. Abdominal exam showed a soft, nontender, non-distended abdomen with right lower quadrant tenderness, normal liver span, no splenomegaly, and hyperactive bowel sounds. The differential included bacterial, viral, fungal, and parasitic etiologies. 
Preliminary stool studies were negative but stool culture revealed Shigella species resistant to ampicillin-sulbactam and Bactrim. CT Abdomen/ Pelvis with contrast demonstrated severe pan-colitis with trace pelvic fluid. Patient was treated with one day of ciprofloxacin and metronidazole and supportive treatment. He was then discharged to home.

IMPACT/DISCUSSION: This case highlights growing resistance patterns among Shigella species in the United States - especially among men who have sex with men. Shigella species cause 500,000 diarrheal illnesses annually in the $\mathrm{US}^{1}$ with growing resistance to ciprofloxacin, azithromycin, and bactrim. ${ }^{2}$ Empiric treatment for diarrhea is not generally advised, but Shigella infections merit treatment with 7-10 days Bactrim (7-10 days) or azithromycin (5 days). ${ }^{3,4}$

CONCLUSION: References: 1. Scallan, E., et. al. (2011). Foodborne illness acquired in the United States - major pathogens. Emerging infectious diseases, 17(1), 7. 2. Heiman, K. E., et. al. (2014). Notes from the field: Shigella with decreased susceptibility to azithromycin among men who have sex with men-United States, 2002-2013. Morbidity and Mortality Weekly Report, 63 (6), 132-133). 3. Shane, A., et. al. (2017). 2017 Infectious Disease Society of America clinical practice guidelines for the diagnosis and management of infectious diarrhea. Clinical Infectious Diseases, 65 (12), e45-e80. 4. AIDSinfo Guidelines for the prevention and treatment of opportunistic infections among HIV-exposed and HIVinfected children, 2013. Available at: http:/aidsinfo.nih.gov/guidelines/ Accessed 16 January 2019

\section{COMPASSIONATE USE OF A NEW INVESTIGATIONAL PAT- ENT FORAMEN OVALE CLOSURE DEVICE FOR PLATYPNEA-ORTHODEOXIA SYNDROME}

Bilal Saqi. Cleveland Clinic Foundation, Cleveland, OH. (Control ID \#3186329)

LEARNING OBJECTIVE \#1: To recognize role of Patent foramen ovale (PFO) closure devices not just for cryptogenic strokes, but for the treatment of hypoxic conditions.

LEARNING OBJECTIVE \#2: Managing a patient with an investigational PFO closure device on basis of compassionate use

CASE: 69 year old male with history of squamous cell carcinoma of the right lung status post pneumonectomy, mid-tracheal recurrence, pulmonary aspergillosis complicated by recurrent pleural effusions, rectal adenocarcinoma, COPD, transient ischemic attack (TIA) and left lower lobe subsegmental pulmonary embolus presented for a surveillance bronchoscopy. Upon arrival, he was hypoxic and found to have platypneaorthodeoxia syndrome. Echo with agitated saline revealed a PFO. Ejection fraction was $55 \%$ with mild right ventricular (RV) dilation with low normal systolic RV function. In the setting of large intracardiac shunt with hypoxia as well as history of prior TIA, we elected to close the PFO. His transesophageal echo images suggested a large defect with PFO diameter of $21 \mathrm{~mm}$ and a very mobile/aneurysmal septum and borderline aortic rims anteriorly, therefore we favored the Gore Cardioform $30 \mathrm{~mm}$ device over an Amplatzer septal occluder (ASO) device to minimize long term risk of erosion. During the procedure, the device was seen to dislodge from the septum and migrate into the left atrial cavity. Unfortunately, the defect was too large and this procedure was unsuccessful, and the devices were retrieved. He was not deemed to be a surgical candidate due to multiple comorbidities, but we were able to get Gore's investigational Cardioform device on compassionate use (currently only available in clinical trials, for which patient would not be a candidate). Patient was approved and successful PFO closure with a $44 \mathrm{~mm}$ Cardioform device was performed with marked improvement in oxygen saturation.

IMPACT/DISCUSSION: Patent foramen ovale (PFO) closure devices for cryptogenic strokes have been well debated leading to FDA approval, but they also have a role in treating hypoxic conditions. This was a unique example of using a new investigational device that the patient wouldnot otherwise qualify for, but asking for its use under compassionate basis. In the end, patients impovement in oxygen saturation was remarkable. This case shows the impact new PFO closure devices can play in PlatypneaOrthodeoxia Syndrome patients as well.

CONCLUSION: Use of the new Cardioform closure device for patients with PFO and hypoxia due to right to left shunt and anatomy leading to failure of older closure devices leads to functional improvement.

\section{CONCURRENT PRESENTATION OF ALL 3-STAGES OF LYME DISEASE}

Sona Franklin; Manisha Naik. St Mary Medical Center, Langhorne, PA. (Control ID \#3186215)

LEARNING OBJECTIVE \#1: Recognize 3-stages of Lyme disease may present concurrently

CASE: A 57-year-old Caucasian male, with no reported past medical history, presented to the Emergency Department complaining of intermittent subjective fevers for three-weeks and drenching night sweats, associated with an erythematous "splotchy" rash over his abdomen and legs, joint pain, swelling, and muscle aches. He also had diplopia two days prior to arrival with no improvement. He did not recall tick, mosquito or animal bites and denied previous episodes of similar symptoms. He had recently traveled to Florida and had spiking fevers prior to that trip. He admitted to outdoor garden work and denied sick contacts. The rash initially presented on his left ankle with swelling and minimal pain, but no pruritus. Family history was significant for father with rheumatoid arthritis and daughter with Celiac disease. Initial vital signs were blood pressure:144/94, pulse:77, respiratory rate:16, temperature:36.7 centigrade, oxygen saturation of $98 \%$ on room air. Physical exam was significant for left eye lateral gaze palsy, rightward gaze nystagmus, circular rash with central clearing on the abdomen, and erythematous rashes without central clearing on left lower back and bilateral thighs. Neurological exam revealed slight left eye droop but was otherwise unremarkable. Chest radiograph, magnetic resonance imaging and magnetic resonance angiogram of the brain, were all unremarkable. Swab for influenza A and B was negative, and urinalysis was negative for infection. Complete blood count revealed a leukocyte count of $12.1 \mathrm{~K} / \mathrm{UL}$ with $81 \%$ neutrophils. Creactive protein was $45.30 \mathrm{mg} / \mathrm{L}$. He was started on intravenous ceftriaxone awaiting Lyme serology analysis. Cerebrospinal fluid array was negative. Lyme panel revealed a positive Lyme IgG and IgM, Western Blot was positive for six $\operatorname{IgG}$ antibodies, and reactive for three $\operatorname{IgM}$ antibodies. He was subsequently discharged home to complete a $28-$ day course of intravenous ceftriaxone and methylprednisolone.

IMPACT/DISCUSSION: Lyme disease is prevalent in Bucks County, Pennsylvania, and is caused by Borreliella species, with B. burgdorferi as the primary agent in the United States. Stages of Lyme disease occur in three phases: early localized, early disseminated and late disease. Most often the phases occur in succession. Overlap may occur, with later stages of Lyme as the initial presentation. There are case reports of simultaneous presentation of two stages, ${ }^{1,2}$ but no published cases of all three stages presenting concurrently. We present a unique case where all three stages of Lyme disease present simultaneously.

CONCLUSION: Our case is an unusual presentation of all three stages of Lyme disease presenting concurrently in a patient located in a region with a high prevalence of Lyme. It is imperative to bring light to this unique phenomenon of Lyme disease, as unawareness of this presentation may lead to misdiagnosis. Greater attention will help ensure prompt and appropriate treatment of these patients.

\section{CONGENITAL HERNIA MAY NOT BE CONGENITAL ANYMORE}

Ravi K. Thimmisetty ${ }^{1}$; Kiran Chintam ${ }^{2}$; Venu Madhav Chirunomula ${ }^{2}$; Matthew Shoemaker ${ }^{2}$. ${ }^{1}$ Nephrology Associates, Cape Girardeau, MO; ${ }^{2}$ Southeast hospital, Cape Girardeau, MO. (Control ID \#3186369) 
LEARNING OBJECTIVE \#1: Diagnosis and treatment of rare presentation of congenital hernia

LEARNING OBJECTIVE \#2: Assessing the complications of Morgagni hernia

CASE: 53 year old healthy Caucasian male transferred from outside facility with hypoxic respiratory failure. He was complaining of progressive shortness of breath over 8 months before admission. Admission vitals were temperature of $98.2 \mathrm{f}$, blood pressure of $122 / 79 \mathrm{~mm}$ of $\mathrm{Hg}$, heart rate of 79 beats per minute, respiratory rate of 27 per minute, $96 \%$ oxygen saturation on BiPAP 14/8. Physical examination revealed mild to moderate acute distress, with decreased breath sounds on right side. Rest of the exam is with in normal. Laboratory results revealed $\mathrm{pH}$ of $7.173, \mathrm{pCO} 2$ is 108 , pO2 is 98.7 and bicarbonate of 39 , serum sodium of 139 , potassium of 5.0, bicarbonate 34 . rest of laboratory results were acceptable range. CT scan of chest demonstrated diaphragmatic hernia suggestive of foramen of Morgagni hernia and right hemithorax is filled with abdominal contents including small, large bowel and omentum, pushing the heart into left side of chest, pneumonia vs pneumonitis. Empiric antibiotics was started (Azithromycin and piperacillin/tazobactum) after drawing blood cultures. Patient got intubated for severe respiratory acidosis. Patient underwent emergent explorative laparotomy and repaired diaphragm with prolene mesh patch. Fiberoptic bronchoscopy showed thick white mucus in right bronchus intermedius which was aspirated. Chest tube was placed in right 6th intercoastal space. Patient underwent thoracotomy and decortication of right lower and middle lobes. Blood and sputum cultures were inconclusive. Subsequently patient was hemodynamically improved after finishing the antibiotics (both piperacillin/tazobactum and azithromycin). He got extubated and chest tube was removed once drainage was minimal.

IMPACT/DISCUSSION: Our case illustrates the rare presentation of congenital hernia. Patient was asymptomatic since birth and presented to the hospital with respiratory failure. Morgagni hernia is an anatomical defect in the anterior diaphragm that allow herniation of abdominal organs into the thorax. These hernia's were the rarest of the congenital diaphragmatic defects with a reported frequency of $1 \%$ to $5.1 \%$. Chest radiograph can show homogenous masses in the thorax with air fluid levels suggestive of hernia. CT scan is the investigation of choice for diagnosis of Morgagni's hernia. This case illustrates the need to act quickly when a potential diagnosis of a Morgagni's hernia is made. A missed diagnosis can lead to complications such as hemorrhage, obstruction, or strangulation. Assessing the hernia in a timely manner and acting appropriately will minimize the complications of Morgagni hernia and by doing so, we were able to save the patient

CONCLUSION: All health care professionals not limited to internists should be aware of this rare presentation and can prevent devastating complications by suspecting diagnosis by clinical, physical examination and imaging studies

\section{CONGESTIVE HEART FAILURE OR COULD IT BE SOME- THING ELSE?}

Saketh Parsi ${ }^{1}$; Lakshmi Priyanka Mahali ${ }^{2}{ }^{1}$ Montefiore Medical centre, $\overline{\text { Bronx, NY}} ;{ }^{2}$ Montefiore Medical Center, Bronx, NY. (Control ID \#3185224)

LEARNING OBJECTIVE \#1: Diagnosis of Right Atrial myxoma should be suspected in patients with symptoms mimicking congestive heart failure associated with weight loss.

LEARNING OBJECTIVE \#2: Right atrial myxoma can create prothrombogenic environment and lead to clot formation causing pulmonary embolism.

CASE: A 71 year-old woman with hypertension presented with progressive ascending bilateral leg swelling for one week. She also reported chronic dry cough, wheeze and dyspnea on exertion since over one month and unintentional weight loss of 40 pounds since over a year. She was hemodynamically stable. Physical exam revealed a diastolic murmur on the left sternal border. Diagnosis of heart failure was suspected and Transthoracic Echocardiography (TTE) was done, which surprisingly revealed severe right atrial dilatation and a pedunculated, mobile mass with multiple stalks (3.1 x $1.9 \mathrm{~cm}$ in longest dimensions) attached to interatrial septum, consistent with an atrial myxoma. Computed tomography $(\mathrm{CT})$ of chest revealed bilateral extensive pulmonary embolisms $(\mathrm{PE})$, with right heart strain, pulmonary arterial hypertension, and irregular filling defect within the right atrium (RA) corresponding with the myxoma. Anti-coagulation (AC) was started with lovenox and patient was discharged on apixaban with cardiothoracic clinic follow up.

IMPACT/DISCUSSION: Approximately 80 percent of myxomas are found in the left atrium; the remainders are in the RA. RA myxomas grow into the lumen and obstruct flow, producing hemodynamic changes that are similar to tricuspid stenosis. RA myxomas typically present with signs and symptoms of right heart failure (peripheral edema, hepatomegaly, and ascites) and cause diastolic murmurs. Constitutional symptoms like malaise, anorexia, fever, arthralgia, weight loss are more common in cases of cardiac myxomas due to the release of cytokine interleukin-6 (IL-6). In addition, tumor fragments may be released into the pulmonary circulation causing symptoms consistent with pulmonary embolism, as seen in our patient. Presence of an atrial septal defect results in shunting of venous blood into the systemic circulation causing hypoxemia or systemic emboli. Provoked $\mathrm{PE}$ is temporarily managed with $\mathrm{AC}$ and permanently managed by treating the cause. RA myxomas require prompt resection to prevent further embolization or cardiovascular complications, including sudden death.

CONCLUSION: In patient with heart failure symptoms, PE and associated significant weight loss other diagnoses like right heart myxoma, malignant pericardial effusion should be considered. Patients with right atrial myxomas should be managed with $\mathrm{AC}$, if no medical contraindications. However ultimate management is tumor resection.

\section{CORONARY ARTERY DISSECTION IN MULTIPAROUS POST- PARTUM PATIENT}

Kuroush Nezafati. Methodist Dallas Hospital, Arlington, TX. (Control ID \#3185805)

LEARNING OBJECTIVE \#1: Recognize spontaneous coronary artery dissection (SCAD) as an important and rare etiology of chest pain, especially in younger women with a history of recent emotional or physical stressor such as in peripartum/postpartum women.

LEARNING OBJECTIVE \#2: Recognize the range of management for SCAD depending on the severity of dissection and associated mortality with SCAD.

CASE: A 29-year-old G5P5 female with past medical history of preeclampsia during her 3rd pregnancy presented to the Emergency Department (ED) with substernal chest pain 18 days postpartum. Pain was pressure like, non exertional and radiated into bilateral upper extremities; she initially attributed her symptoms to anxiety, but with a 3rd episode of pain and her measured systolic blood pressure of $180 \mathrm{mmHg}$ at home, she sought medical attention. Evaluation in the ED showed normal chest radiograph, electrocardiogram (ECG) with ST segment depression in precordial leads concerning for subendocardial ischemia. Initial troponin was $0.739 \mathrm{ng} / \mathrm{mL}$. Troponin was $4.27 \mathrm{ng} / \mathrm{mL}$ six hours later for which patient had an angiogram which showed multiple dissections in left anterior descending (LAD) \& left circumflex with anteroapical hypokinesis but preserved ejection fraction. The most significant of these dissections was within the left anterior descending coronary which had extensive anterograde and retrograde involvement. Two long stents were placed in the LAD, one proximally extending up to left main coronary and one distally up to the distal third of the left anterior descending coronary. The left circumflex dissection showed no intimal flap and good wall integrity. Vessel branches off of the marginal branch also showed area of dissection with no wall compromise. The patient was placed on dual antiplatelet therapy along with a statin and beta blocker and recovered well. 
IMPACT/DISCUSSION: Spontaneous coronary artery dissection (SCAD) is an important and rare cause of acute coronary syndrome (ACS). This case demonstrates the importance of thorough workup of chest pain in patients with few to no comorbidities with abnormal ECG, elevated troponin levels, and recent stressor. Literature demonstrates SCAD carries with it significantly increased in-hospital mortality above other non-SCAD acute myocardial infarction causes. Patients with SCAD should at the least have long term medical management including hypertension management and use of a beta blocker, to avoid recurrence of dissection. This case also highlights the range of management depending on severity of dissection.

CONCLUSION: SCAD is an important and rare cause of ACS more commonly found in younger women who generally carry fewer comorbidities than other ACS patients. Yet, SCAD is associated with significantly increased mortality above other ACS causes. Depending on findings during angiogram, SCAD management can vary from conservative management (especially hypertension control and beta blocker use) to interventions including coronary stenting.

\section{CREATIVE SELF-EXPRESSION: ONE PRONG IN A MULTI- MODAL TREATMENT PLAN FOR ADDICTION}

Colleen Farrel1 ${ }^{1}$; Katie Grogan ${ }^{2} .{ }^{1}$ NYU Langone, New York, NY; ${ }^{2} \mathrm{NYU}$ School of Medicine, New York, NY. (Control ID \#3181441)

LEARNING OBJECTIVE \#1: Recognize the therapeutic benefit of creative self-expression in the treatment of addiction

CASE: Ms. D is a 37-year-old woman with history of opioid use disorder (using intravenous heroin), who presented to our hospital with severe neck pain. She was diagnosed with C-spine osteomyelitis and initiated on IV antibiotic therapy. Her course was complicated by cardiac arrest from heroin overdose (provided by a personal visitor). During her hospitalization, she was initiated on buprenorphine and met regularly with an art therapist. She created drawings and small sculptures that depicted her years-long struggle with heroin use, as well as emotional traumas that contributed to her use. Prior to discharge, she shared her artwork with her medical team, eloquently articulating how creating art helped her process her trauma and make a commitment to recovery.

IMPACT/DISCUSSION: Addictions are an increasingly pressing national health concern. Many elements of Ms. D's case - her early childhood trauma, her life-threatening medical complications - will likely be familiar to internists who care for patients with substance use disorders. This presentation will focus on a promising and understudied aspect of addiction treatment: creative self-expression. Creative self-expression can be joyful, relaxing, and distracting from other difficulties. It also empowers patients to create their own narratives of self, recognizes patients' subjective experiences, and provides means of communicating difficult experiences with the clinical team and others. There is a strong theoretical foundation for the use of self-expression as a means for improved wellbeing in the medical setting and in trauma recovery $(1,2)$. Current empirical data are encouraging but limited by small sample size, lack of objective metrics, and heterogeneity of art therapy modalities $(3,4)$. This presentation will introduce conference participants to the robust theoretical basis of creative self-expression in healing and recovery. This theoretical basis will then be applied to a discussion of Ms. D's case. The presentation will include photographs of Ms. D's artwork, for which she has provided her consent. 1. Frank AW. Just Listening: Narrative and Deep Illness. Fam Syst Heal. 1998;16(3):197-212. 2. Larkin M and Griffiths M. Experiences of Addiction and Recovery: the Case for Subjective Accounts. Addiction Research \& Theory. 2009;10: 281-311. 3. Uttley $L$ et al. The clinical and cost effectiveness of group art therapy for people with non-psychotic mental health disorders: a systematic review and cost-effectiveness analysis. BMC Psychiatry. 2015;15:151. 4. Schouten KA et al. The Effectiveness of Art Therapy in the Treatment of Traumatized Adults: A Systematic Review on Art Therapy and Trauma. Trauma Violence Abuse. 2015;16(2):220-228.

CONCLUSION: This case demonstrates the therapeutic potential of creative self-expression as part of a multi-modal treatment strategy for addiction.

\section{CUTANEOUS CRYPTOCOCCUS NEOFORMANS: A FATAL WARNING SIGN.}

Tania M. Kohal; Ahmad D. Dudar; Gillian Grafton. Henry Ford Hospital, Detroit, MI. (Control ID \#3162884)

LEARNING OBJECTIVE \#1: Understand the incidence of cutaneous manifestations of cryptococcal infections and its relation to systemic cryptococcal infection.

LEARNING OBJECTIVE \#2: Identify cryptococcal skin lesions.

CASE: An 81 year old woman presented to the medical intensive care unit as an outside transfer for escalation of care. Two months prior to this hospitalization the patient was fully independent and lived alone; however, she had a recent admission for renal failure which revealed an ANCApositive vasculitis with myeloperoxidase antibody elevation and crescentic pauci-immune glomerulonephritis on renal biopsy. She was discharged on a maintenance dose of prednisone, but began to clinically deteriorate shortly thereafter at home with decline in mental status and renal function. The patient was transferred from an outside hospital to our facility with acute encephalopathy and cellulitis. On arrival, physical exam revealed skin lesions on the bilateral upper extremities and right lower extremity that were violaceous and purpuritic as well as areas of induration and necrosis. Blood cultures revealed a gram positive bacteremia. Dermatology was consulted with the request of a skin biopsy given the patient's prior history. On the day of the skin biopsy the patient had further clinical deterioration with progressive hypoxia requiring intubation as well as renal failure and severe lactic acidosis. She was treated with broad spectrum antibiotics as well as anti-fungals; however her clinical status continued to deteriorate and eventually she expired within 48 hours of admission. Her skin biopsy revealed disseminated encapsulated yeast consistent with Cryptococcus neoformans.

IMPACT/DISCUSSION: Cryptococcus neoformans is a dimorphic fungus that is ubiquitous in the environment, found in soil, dust, wild and domesticated birds as well as a skin contaminant. Clinically, its primary manifestation is pulmonary infection; however, $10-15 \%$ of clinical manifestations are cutaneous. Cutaneous manifestations can be diverse including plaques, crusts, nodules, or microhemorrhages. When presented with an individual with cutaneous manifestations, the patient should be assumed to have systemic infection unless proven otherwise, and this case demonstrates that delay in treatment can become fatal. The diagnostic challenge in this case was identifying a patient with known immunocompromised status presenting with no other objective data of cryptococcal meningitis or pulmonary infection. The patient was started on antifungals; however, not with enough alacrity to become effective.

CONCLUSION: Cryptococcosis can be encountered around the world and the majority of its clinical manifestations will be elucidated via the common infectious routes; however, there are opportunities to diagnose cryptococcosis and treat patient faster in an attempt to reduce morbidity and mortality from this infection. One of these opportunities is assessment of new dermatological manifestations of unknown origin in at risk patients.

CUTANEOUS NECROSIS: A CASE OF CONCOMITANT ECTHYMA GANGRENOSUM AND CALCIPHYLAXIS

Neha Agarwal ${ }^{1}$; Sarah Schoenbrun ${ }^{1}$; Estebes Hernandez ${ }^{2} .{ }^{1}$ UCLA Ronald Reagan, Los Angeles, CA; ${ }^{2}$ David Geffen UCLA School of Medicine, Los Angeles, CA. (Control ID \#3179543)

LEARNING OBJECTIVE \#1: Recognize the clinical features of ecthyma gangrenosum and calciphylaxis 
LEARNING OBJECTIVE \#2: Manage ulcers due to calciphylaxis CASE: An 89 year-old female with a history of multiple myeloma (MM) not on therapy, chronic kidney disease stage IV, and diabetes presented to the emergency room with confusion and left lower extremity allodynia. Home medications included cholecalciferol, patiromer, and nephrovite. Physical exam was notable for temperature of $38^{\circ} \mathrm{C}$, suprapubic tenderness, and left lower extremity edema, allodynia, and an inability to bear weight. Labs demonstrated creatinine $3.08 \mathrm{mg} / \mathrm{dL}$ (near baseline), white blood cell count $50.0 \mathrm{k} / \mathrm{uL}$, and normal calcium, phosphorus and parathyroid hormone (PTH). Urinalysis was significant for $>100$ white blood cells cells/ $\mu \mathrm{l}$ and leg X-rays were unremarkable. Urine cultures grew resistant Klebsiella pneumoniae which was treated with 5 days of ertapenem. On hospital day 7 , the patient developed a $12 \times 6 \mathrm{~cm}$ stellateshaped purpuric plaque with an overlying tense bulla over the medial ankle. Pathology from two punch biopsies was consistent with ecthyma gangrenosum and Klebsiella pneumonia grew on wound cultures. Ertapenem was continued, but the ulcer developed central necrosis with undefined borders and persistent allodynia. A CT of the leg showed extensive subcutaneous edema with vascular calcifications. Given lack of clinical improvement and progressive necrosis, a repeat biopsy was performed on hospital day 12 , which noted vessel thrombosis and calcification consistent with calciphylaxis. The patient was started on biweekly sodium thiosulfate and hyperbaric oxygen therapy.

IMPACT/DISCUSSION: Cutaneous necrosis is associated with high rates of morbidity and mortality, necessitating early recognition and diagnosis. Among the various etiologies are ecthyma gangrenosum and calciphylaxis. Ecthyma gangrenosum, typically associated with Pseudomonas infections in immunocompromised bacteremic patients, has been increasingly reported in association with E. coli and Klebsiella in the absence of bacteremia. As in this case, it can predispose to calcipylaxis in at-risk patients. Calciphylaxis typically develops in patients with advanced renal disease, hyperparathyroidism and elevated calciumphosphate product. It has also been reported in patients with MM due to an acquired protein $\mathrm{S}$ deficiency. Treatment is typically multimodal and includes sodium thiosulfate, which is associated with clinical improvement but lacks conclusive evidence demonstrating efficacy, treatment of hyperphosphatemia, and wound care. Hyperbaric oxygen has been used as an adjunctive therapy to facilitate wound healing. This case highlights the diagnostic challenge with a necrotic ulcer, and cautions against premature diagnostic closure.

CONCLUSION: The differential for acute cutaneous necrosis includes ecthyma gangrenosum and calciphylaxis. Calciphylaxis has been described in patients with MM due to an acquired hypercoagulability state. Hyperbaric oxygen therapy improves healing and morbidity in calciphylaxis.

\section{CYBERCHONDRIASIS-RELATED HYPERVITAMINOSIS D}

Sally Namboodiri; Clifford D. Packer. Cleveland VAMC, Cleveland, OH. (Control ID \#3163956)

LEARNING OBJECTIVE \#1: Recognize the internet's influence on patients with illness anxiety disorders

LEARNING OBJECTIVE \#2: Review the symptoms and management of hypervitaminosis D

CASE: A 50-year-old white male presented with two weeks of constipation and anorexia. His past medical history included chronic kidney disease (baseline creatinine $1.6 \mathrm{mg} / \mathrm{dl}$ ), family history of Huntington's disease and an untreated anxiety disorder. He took numerous vitamin supplements. His physical exam was unremarkable. Labs showed creatinine $3.7 \mathrm{mg} / \mathrm{dl}$ (0.5-1.2), calcium $12.5 \mathrm{mg} / \mathrm{dl}$ (8.5-10.1), Vitamin D 258 $\mathrm{ng} / \mathrm{ml}$ (30-45), and PTH $<7 \mathrm{pg} / \mathrm{ml}$ (15-72). Abdominal computerized tomography showed multiple nonobstructive renal calculi. When asked about his vitamin usage, he said that he took 100,000 international units (IUs) of Ergocalciferol daily for many weeks. He was convinced that he had Huntington's disease due to symptoms of hand tremors and brain fog.
Based on information from the internet, he compulsively took supplements and varied his diet to improve his symptoms. When asked for specific sources, he cited a physician on Youtube with secondary progressive multiple sclerosis who failed multiple medications. After searching the internet for answers, she cured her disease with high doses of vitamin $\mathrm{D}$ and other supplements along with a special diet. The patient was admitted with acute kidney injury and hypercalcemia from hypervitaminosis D. With intravenous (IV) hydration and withholding of Ergocalciferol, his creatinine improved to $1.8 \mathrm{mg} / \mathrm{dl}$ and calcium to 9.7 $\mathrm{mg} / \mathrm{dl}$. Despite his subsequently negative genetic testing for Huntington's disease, he remained convinced that he had a serious illness which could only be controlled with high doses of supplements and special diets.

IMPACT/DISCUSSION: Hypervitaminosis D is a rare phenomenon that is most often due to prescription or medication formulation errors. Intoxication has been reported when doses exceed 50,000 IUs per day. Hypercalcemia occurs due to increased gut absorption of calcium, with resultant anorexia and constipation. Vitamin D's direct effect on renal vasoconstriction can lead to acute kidney injury. Chronic hypervitaminosis D causes kidney stones due to increased urinary calcium excretion. Calcitriol $(1,25$ $\mathrm{OH}$ Vitamin D)-induced hypercalcemia is easily treated with IV fluids and withholding Vitamin D due calcitriol's short half-life. In contrast to most cases of hypervitaminosis D, this patient's illness anxiety disorder led him to intentionally consume high doses of Vitamin D, a supplement whose health benefits are touted on the internet based on little scientific evidence. Treatment options for illness anxiety disorder, or "cyberchondriasis" in this patient's case, include cognitive-behavioral therapy and antidepressants.

CONCLUSION: Our patient's deliberate ingestion of high doses of Vitamin D led to hypercalcemia, acute kidney injury and nephrolithiasis. Inquiring about vitamin usage can unmask illness anxiety disorders which, if untreated, can lead to self-harm under the influence of the internet.

\section{D-MYSTIFYING HYPERCALCEMIA}

Theresa Wong; Katherine Julian. UCSF, San Francisco, CA. (Control ID \#3181407)

LEARNING OBJECTIVE \#1: Summarize the evaluation of new hypercalcemia (hyperCa)

LEARNING OBJECTIVE \#2: Describe the risk factors for hypervitaminosis D

CASE: ES is an 80 year old woman with hypertension and chronic tobacco use who presented for an annual checkup. She had no complaints except for a buttock rash. Review of systems was normal except for a $15 \mathrm{lb}$ weight loss over the last 1.5 years due to poor appetite. Her medications included HCTZ, duloxetine, and multiple vitamin supplements. Her vital signs were normal. On physical exam, she was thin and frail with a shallow sacral pressure ulcer. The rest of the exam was normal. A screening low-dose CT scan showed several pulmonary nodules 2$15 \mathrm{~mm}$ in size that were unchanged from prior CT. A routine BMP revealed a serum calcium of $13.5, \mathrm{Cr} 1.36$ and albumin 4.4. Her previous labs showed a calcium of 10.9 and a baseline $\mathrm{Cr}$ of 0.9 . She was sent to the ED where further workup showed 25-hydroxy vitamin D (25(OH)D) elevated to 115 and low PTH. TSH, free T4, PTHrP, and 1-25 dihydroxy vitamin D (1-25(OH)2D) were normal. She revealed she was taking calcium and vitamin D (vit D) supplements not noted in her medication list. She was discharged home after her $\mathrm{Cr}$ and $\mathrm{Ca}$ downtrended with fluids, and she discontinued her HCTZ, Ca, and vit D.

IMPACT/DISCUSSION: Hyperparathyroidism (hyperPTH) and malignancy are the most common causes of hyperCa, making up more than $90 \%$ of the cases. The first step of diagnosis is whether the hyperCa is PTH or non-PTH mediated. An elevated PTH is diagnostic of primary hyperPTH. A low PTH prompts further testing with PTHrP, vit D metabolites, SPEP, serum free light chains, TSH, free T4. PTHrP can be elevated in malignancy. 1-25(OH)2D can be elevated in lymphoma and granulomatous diseases. Drug-mediated effects are most commonly due 
to thiazides and calcium and vit D supplements. Miscellaneous causes of hyperCa include immobilization and dehydration. The tolerable upper intake level of vit D as defined by the Institute of Medicine is $4000 \mathrm{IU}$ daily. Published cases of hypervitaminosis D have involved intake of $>40,000$ IU daily. The serum concentration of $25(\mathrm{OH}) \mathrm{D}$ that results in hyperCa is undefined, though experts have estimated vit $\mathrm{D}$ intoxication as $>150 \mathrm{ng} / \mathrm{ml}$. Cases of toxicity have been reported in those taking megadoses of vit $\mathrm{D}$ as part of a fad diet or as replacement therapy in conditions such as malabsorption, renal osteodystrophy, osteoporosis, or psoriasis. In our case, the patient was taking $1000 \mathrm{IU}$ of D3 daily. Though her dose and serum levels were not expected to cause toxicity and hyperCa, multiple other factors likely increased her serum $\mathrm{Ca}$, including $\mathrm{Ca}$ supplementation, thiazide use, dehydration, and immobility.

CONCLUSION: The etiology of hyperCa can be quickly determined with history, medicine reconciliation, and a limited set of laboratory tests. Vit D toxicity has been reported with intake of $>40,000$ IU per day and $25(\mathrm{OH}) \mathrm{D}$ levels $>150$, but hyperCa can occur at lower levels with multiple seemingly benign contributing factors.

\section{DEVELOPMENT OF SEVERE RED RASH DUE TO VITAMIN DEFICIENCY YEARS FOLLOWING GASTRIC BYPASS SURGERY.}

Robert Case ${ }^{1}$; Jared Freitas ${ }^{2}$; Sean P. Bliven ${ }^{2} .{ }^{1}$ University of Florida, Gainesville, FL; ${ }^{2}$ University of Florida School of Medicine, Gainesville, FL. (Control ID \#3183336)

LEARNING OBJECTIVE \#1: Recognize vitamin deficiencies of zinc and B6 as causes of red rash after gastric bypass surgery.

CASE: A 39-year-old female presented with red, painful rash, confusion and severe dehydration. Her past medical history included alcohol abuse, $\mathrm{CHF}$, and untreated hep B and hep C infections. Her surgical history included gastric bypass surgery (Roux en-Y) 6 years prior to admission and both mitral and aortic valve replacement due to endocarditis. Her rash was bright red, blanchable and exquisitely tender with areas of blisters and active wheeping of fluid. The rash involved the lower half of the trunk, acrum and upper thighs. She also had angular chelitis, glossitis and pain within her mouth. She denied recent initiation of new prescription medications or supplements. Her labs revealed multi organ system dysfunction as exhibited by acute on chronic liver injury, acute kidney injury and early signs of DIC. She became increasingly confused with worsening hypotension leading to transfer to the ICU. Her rash was biopsied and returned suggestive of a vitamin deficiency. Her labs further supported this result with critical deficiencies in vitamin B6 and zinc. After repletion of these vitamins, the rash progressively improved over the course of several weeks.

IMPACT/DISCUSSION: This case represents an unusual presentation of vitamin deficiency after gastric bypass surgery. Both dermatology and infectious disease consultants felt her presentation was concerning for toxic shock syndrome on initial evaluation. Staphylococcal toxic shock syndrome was supported as a possibility based on the presence of the erythematous rash, hypotension and multiple organ damage. However, biopsy definitively ruled this condition out. Both zinc and vitamin B6 deficiencies are common after bariatric surgery. One paper found $75 \%$ of women to have zinc deficiency after this surgery. Notably, alcohol abuse is known to exacerbate zinc deficiency by increasing bodily losses. The distribution of her rash resembled that of a diaper rash of an infant spreading to the trunk is notably consistent with a distribution due to these mineral deficiencies. The presence of angular chelitis and glossitis is also consistent with low zinc and B6 levels. While her rash may be wellexplained by the vitamin deficiency, her acute presentation and sudden onset of the rash were surprising and left this diagnosis in doubt initially. This case demonstrates the variability with which these vitamin deficiencies may present clinically. Clinicians who encounter similar cases should have a low threshold to test for vitamin deficiencies and initiate treatment promptly if levels are low.
CONCLUSION: -Patients who previously underwent bariatric surgery presenting with a diffuse, red rash should be tested for vitamin deficiencies, especially vitamin B6 and zinc. -The rash associated with zinc and B6 deficiency may resemble toxic shock syndrome -Onset of a systemic rash may occur years after bariatric surgery and progress rapidly without clear provoking cause.

\section{DEVIC'S DISEASE AND TRANSVERSE MYELITIS: AN IMPOR- TANT PRESENTATION OF AN UNCOMMON DISEASE}

Samuel Shapiro; Benjamin Hayes. gwinnett medical center, Lawrenceville, GA. (Control ID \#3162774)

LEARNING OBJECTIVE \#1: Evaluate a patient with transverse myelitis

LEARNING OBJECTIVE \#2: Diagnose neuromyelitis optica (NMO) in a patient with transverse myelitis

CASE: A 55-year-old African American female with a past medical history of polymyositis presented to the emergency room with progressive ascending paresthesia of the lower extremities to the mid abdomen for 3 days. Review of systems was positive for bowel incontinence and negative for bladder incontinence, motor weakness, dysphagia, dyspnea, fever or chills. Her only medication was prednisone $5 \mathrm{mg}$ daily. Otherwise she did not have a significant family, surgical or social history. On physical exam, her vitals and major organ systems were normal except for the neurologic exam. She had loss of sensation to light touch and temperature distal to T5 and decreased vibratory sense of the feet. Otherwise motor, cognitive and cerebellar functions were intact. MRI of the brain and spine were significant for signal abnormality of the central gray matter from T4 to T8. The patient was diagnosed with transverse myelitis and started on high dose steroids. Serologic work up was positive for aquaporin-4 (AQP4) antibody specific for NMO or Devic's disease. Her symptoms improved and follow up MRI 3 months later showed significant improvement in the lesion.

IMPACT/DISCUSSION: Acute TM is a rare inflammatory disorder that can affect any portion of the spinal cord and cause major morbidity. It is considered a neurologic emergency and can present with motor, sensory and/ or autonomic dysfunction. The differential is broad and includes spinal artery infarcts, metabolic abnormalities (vitamin B12, copper), infection, neoplasm, neurodegenerative (multiple sclerosis (MS), neuromyelitis optica), and autoimmune (SLE, sarcoidosis, Sjogren's) disorders. The location of lesion(s) is an important early indicator of the possible etiologies. For instance, our patient had a central thoracic lesion more commonly seen with NMO or autoimmune disease as opposed to an anterior lesion from an anterior spinal artery infarct. MRI of the entire spinal cord is essential to evaluate extent of disease as multiple lesions can be seen with either MS or NMO. High dose steroids are the mainstay of treatment for neurodegenerative and autoimmune etiologies and should be started promptly. Our patient improved but for those with refractory disases plasma exchange is another option. Previously thought to be a form of MS, NMO differentiates itself by its necrosis and cavitations of both white and gray matter. Optic nerve involvement is also common but was absent in our patient. Anti-AQP4 antibody is pivotal in the diagnosis of NMO attributed to its high sensitivity and specificity.

CONCLUSION: TM has a broad differential that internists should be able to recognize early to initiate the appropriate treatment as it is paramount in reversing the neurologic deficits.

\section{DIABETIC AMYOTROPHY: A DISTINCT AND DISABILING NEUROPATHY}

Cindy Fang; David Rhee. NYU, Flushing, NY. (Control ID \#3139144)

LEARNING OBJECTIVE \#1: Understand diabetic neuropathy does not only include distal polyneuropathy

LEARNING OBJECTIVE \#2: Recognize the clinical features of diabetic amyotrophy 
CASE: An 80-year-old man with a history of Type 2 diabetes and chronic lumbar radiculopathy presented to the emergency room after four days of left thigh pain radiating from the groin and progressive left leg weakness, now needing a wheelchair. On exam, he had decreased strength in hip flexion and knee extension, decreased sensation to touch along his medial calf, and a muted patellar reflex. He also had decreased vibrational sense in the toes bilaterally. Labs revealed a hemoglobin A1C of $9.8 \%$. An MRI showed diffuse, extensive degenerative disease in the lumbar-sacral spine but no focal structural lesions such as epidural abscess or osteomyelitis. Electromyography showed evidence for diabetic amyotrophy as well as moderate polyneuropathy. After two months of strict glycemic control and physical therapy, the patient recovered some function and was ambulatory with a cane. IMPACT/DISCUSSION: Diabetic amyotrophy is also known as diabetic femoral neuropathy, diabetic proximal neuropathy, diabetic lumbosacral radiculoplexopathy, and Bruns-Gardland syndrome. Patients with this entity typically present with acute or subacute, severe unilateral pain and weakness in the proximal lower extremity, and may often have muscle wasting. While distal polyneuropathy is the most common type of diabetes-induced neuropathy that can affect more than $20 \%$ of all diabetic patients, diabetic amyotrophy is much rarer and affect less than $1 \%$ of patients. Because of its low prevalence, it is often misdiagnosed as lumbosacral radiculopathy. In this case, our patient's neurologic symptoms were initially assumed to be due to known lumbar radiculopathy, but a thorough exam localized the lesion to the left femoral nerve, which did not correlate with the imaging finding. The diagnosis of diabetic amyotrophy depended on a high level of suspicion, recognition of poorly controlled diabetes, and confirmatory testing with electromyography. Treatment mainly consists of pain management and glycemic control, but studies on steroids and immunoglobulin are on-going. The majority of patients spontaneously improve over months.

CONCLUSION: Diabetic amyotrophy is a rare yet debilitating complication of diabetes. Because diagnosis reply heavily on clinical recognition, it is important for internist to keep a high level of suspicion when diabetic patients present with asymmetric proximal neuropathy.

\section{DIABETIC MUSCLE INFARCTION: A CASE OF MISTAKEN IDENTITY}

Kirsten Salline; Lily Belfi; David Pisapia; Sydney Katz. Weill Cornell Medicine, New York, NY. (Control ID \#3181712)

LEARNING OBJECTIVE \#1: Review common etiologies of muscle pain and edema in diabetic patients.

LEARNING OBJECTIVE \#2: Discuss the presentation, diagnosis, treatment, and prognosis of diabetic muscle infarctions.

CASE: A 47-year-old woman with poorly controlled T1DM(HbA1c 12$14 \%$ ) presented to the ER for the third time in two weeks with persistent right thigh pain and swelling despite a seven-day course of cephalexin for presumed cellulitis. She denied lacerations, trauma, fevers, or recent immobilization. On exam, she was afebrile and the right thigh was edematous and indurated without erythema. Labs were notable for WBC 9.9/uL, CRP $9.3 \mathrm{mg} / \mathrm{dL}, \mathrm{ESR}>130 \mathrm{~mm} / \mathrm{hr}$, and CK $771 \mathrm{U} / \mathrm{L}$. A thigh ultrasound showed soft tissue edema without drainable fluid collection or DVT. She was treated with IV vancomycin and piperacillin/ tazobactam for a working diagnosis of pyomyosits. A right knee effusion was noted on hospital day three which raised concern for septic arthritis in the setting of adjacent pyomyositis. Synovial fluid revealed no organisms, a normal nucleated cell count, and negative cultures. MRI of the right knee and thigh showed increased T2 signal of the quadriceps musculature. Blood cultures drawn on admission were sterile. Despite imaging suggestive of pyomyositis, the patient did notimproveclinically with broad-spectrum antibiotics. A muscle biopsy was performed. Biopsy revealed diffuse myonecrosis consistent with diabetic muscle infarction (DMI). Muscle tissue cultures were sterile. Antibiotics were stopped and aspirin was started.
IMPACT/DISCUSSION: DMI is a rare and morbid complication of poorly controlled diabetes. Diagnosis can be delayed due to its rarity and similar presentation to soft tissue infections and VTE. DMI is an infrequent complication of diabetes that presents with acute pain and swelling of large muscle groups without constitutional symptoms, prior trauma, or simultaneous infection. Patients often have complications of poorly controlled diabetes at diagnosis. Diabetic microangiopathy or ischemia-reperfusion injury is thought to cause the muscle infarction. Associated knee effusions have been described a handful of times in cases of DMI. Diagnosis is made via imaging in association with clinical context. Routine labs are nonspecific, though ESR and CRP are often elevated. MRI is the imaging modality of choice, and on T2-weighted images infarcted muscle appears heterogenous and hyperintense. Definitive diagnosis is made with muscle biopsy; however, this has been associated with increased recovery time. Antiplatelet agents have been associated with a shorter recovery time and lower recurrence rate compared to surgery. Recurrent infarctions and increased mortality make for an overall poor prognosis.

CONCLUSION: DMI is an under-recognized and often misdiagnosed cause of muscle pain and edema in poorly controlled diabetic patients. Prognosis of DMI is poor and treatment is largely supportive, though antiplatelet agents are associated with better outcomes.

\section{DIAGNOSING A RARE CASE OF METFORMIN INDUCED LACTIC ACIDOSIS}

Evan R. Gordon-Wollin ${ }^{1}$; Minho $\mathrm{Yu}^{1,}{ }^{1}$; Ilho Kang ${ }^{1,}{ }^{2}$. ${ }^{1}$ Loma Linda Medical Center, Rancho Cucamonga, CA; ${ }^{2}$ Riverside University Health System, Moreno Valley, CA. (Control ID \#3168756)

LEARNING OBJECTIVE \#1: Recognize the features of Metformin Induced Lactic Acidosis (MILA).

LEARNING OBJECTIVE \#2: Treat MILA promptly and aggressively to improve patient outcomes.

CASE: This is a 65-year-old female with a history of hypertension and well-controlled diabetes mellitus type 2 on Metformin, who presented with non-bloody diarrhea 2-3 times per day, poor oral intake, and no urine output for 3-4 days. She denied any abdominal pain or fever. Despite not producing any urine for days, she continued to take her Metformin daily. Social, family, and surgical history were negative. Her other medications included Furosemide, Simvastatin, and Lisinopril. On physical exam she was afebrile with stable vital signs. The patient was in no acute distress, her abdominal exam was benign, and the rest of the physical exam was normal. Laboratory studies revealed a leukocytosis of 13.5 , lactate 13.3 $\mathrm{mmol} / \mathrm{L}$, procalcitonin $0.6 \mathrm{ng} / \mathrm{mL}$, creatinine $10.6 \mathrm{mg} / \mathrm{dL}$, bicarbonate 5 $\mathrm{mmol} / \mathrm{L}$, anion gap 35 , and a $\mathrm{pH}$ of 6.85 . Glucose was $56 \mathrm{mg} / \mathrm{dL}$, lipase $1,043 \mathrm{U} / \mathrm{L}$, and beta-hydroxybutyrate $5.2 \mathrm{mmol} / \mathrm{L}$. Point of care ultrasound suggested that the patient was not in distributive shock. The most likely explanation for the severe lactic acidosis was a Type B lactic acidosis due to toxic Metformin accumulation in the setting of acute renal failure precipitated by a pre-renal state. No severe infection was identified, and other causes of a Type B lactic acidosis were ruled out. The patient was admitted to the ICU not on antibiotics, not on vasopressors, and immediately underwent emergent hemodialysis. There was a rapid reduction of lactate to 1.6, and a return of renal function by the end of hospital day two. The patient was discharged home four days later.

IMPACT/DISCUSSION: While a Type A lactic acidosis due to systemic hypoperfusion is often at the top of the differential, one must consider a Type B lactic acidosis when the clinical picture and objective data do not fit. Although controversial, rare, and without standard diagnostic criteria, a severe lactic acidosis in the setting of Metformin use and renal failure should prompt consideration of MILA. In a large cohort study of 258,539 patients on Metformin, Eppenga et. al. (Diabetes Care, 2014) found a sixfold increase in lactic acidosis with eGFR $<60 \mathrm{~mL} / \mathrm{min} / 1.73 \mathrm{~m} 2$, and Lazarus et. al. (JAMA, 2018) found among 75, 413 patients a two-fold 
increase with eGFR less than $30 \mathrm{~mL} / \mathrm{min} / 1.73 \mathrm{~m} 2$. As seen with our patient, given the dialyzable nature of Metformin, rapid identification and treatment of MILA can have a good outcome. Although the mortality rate can still be high, Friesecke et al. (Critical Care, 2010) demonstrated that when compared to a similarly severe lactic acidosis of other origin, MILA has a significantly better prognosis.

CONCLUSION: It is important to evaluate all potential etiologies of lactic acidosis before deciding on management. Consider MILA when a patient presents with renal failure and severe lactic acidosis, and treat aggressively with emergent dialysis as indicated.

\section{DIAGNOSING EHLERS-DANLOS SYNDROME IN THE AGE OF IN-HOME GENETIC TESTING}

Cameron Bell; Lucy Guerra; Asa Oxner. University of South Florida, Tampa, FL. (Control ID \#3184484)

LEARNING OBJECTIVE \#1: Assess patients for Ehlers-Danlos Syndrome using the Beighton Scoring System.

LEARNING OBJECTIVE \#2: Recognize the existence of in-home genetic testing, it's role in patient care, and its limitations in diagnosing disease. CASE: A 47 year old female with no past medical history presented for evaluation of joint hypermobility. During yoga she noted a dislocation of her right shoulder and was concerned about a possible diagnosis of Ehlers-Danlos syndrome. She recalled joint hypermobility since birth as well as diagnosis of a cardiac murmur in her 20s. She also reported frequent bruising of her legs which led to her being removed from her home at age 9 over concerns of abuse. She reported similar symptoms in 3 out of 4 of her children. On physical exam, patient scored a 7/9 on the Beighton hypermobility score but was otherwise unremarkable. In home genetic testing showed collagen mutations. She was referred to a geneticist and rheumatology for further workup.

IMPACT/DISCUSSION: This case highlights the importance of considering Ehlers-Danlos syndrome and the use of the Beighton hypermobility scale in the differential diagnosis of patients with joint hypermobility and dislocations not expected by the level of activity. Patients are also increasingly turning to in-home genetic testing which has unproven benefit for clinical practice, causing providers to navigate discussion of results. Patients presenting with joint hypermobility, skin hyperextensibility and atypical joint dislocations should be evaluated for a diagnosis of Ehlers-Danlos Syndrome. Bowen et. al discuss the Beighton hypermobility score which examines hand, elbow, knee and spine mobility as a tool to evaluate patients for joint hypermobility with a score of 5/9 or greater suggestive of the diagnosis. Patients suspected of having EhlersDanlos based on the hypermobility score and clinical symptoms should be referred for confirmatory genetic testing, as well as a multidisciplinary team consisting of a Rheumatologist and Sport's Medicine physician. In today's healthcare environment, in home genetic testing is increasingly prevalent which provides unique challenges in the physician patient relationship. In an editorial by Burke et. al in 2016, it was suggested that among patients who discussed their results at appointments, many patients were unsatisfied with the interaction, 1/5 saying they were not satisfied at all. These tests should be used to assist with diagnosis but should not replace formal evaluation by a geneticist or clinical judgement.

CONCLUSION: The Beighton hypermobility score is a valuable clinical tool for evaluating joint hypermobility in the diagnosis of Ehlers-Danlos Syndrome. Internists should also be aware of patients' increased use of inhome genetic testing and work with patients to navigate their results.

\section{DIAGNOSING MYASTHENIA GRAVIS FROM ISOLATED RE- SPIRATORY FAILURE}

Anthony Higinbotham; Hongyang Pi; Kaveh Moghbeli. University of Pittsburgh Medical Center, Pittsburgh, PA. (Control ID \#3185767)
LEARNING OBJECTIVE \#1: Recognize respiratory failure as a potential manifestation of neuromuscular disease.

CASE: 78 year old female with a history of hypertension on atelonol who presented initially to an outside hospital for ptosis and respiratory insufficiency requiring intubation prior to transfer. Stroke workup was unrevealing. Initial labs on arrival to our hospital were notable for a normal comprehensive metabolic panel, a troponin-I elevation of 1.25 (peak 3.65) and lactate $6.5 \mathrm{mmol} / \mathrm{L}$. EKG and chest radiograph did not show any abnormalities. Patient was quickly weaned off sedation and was noted to follow commands. Physical examination was normal except for $4 / 5$ strength of her upper extremities and bilateral hip flexors. Family added that patient was recently diagnosed with polymyalgia rheumatica for progressive muscle weakness and had received a short course of steroids. She passed a spontaneous breathing trial, met all criteria for extubation, and was subsequently extubated. Within 30 minutes of her initial extubation, she was noted to be hypertensive and tachycardia, an episode of apnea was noted, and she became hypoxemic, ultimately requiring emergent re-intubation. Forty eight hours later, she again passed a spontaneous breathing trial via T-piece and met all criteria for extubation. She was extubated. A few hours later, she was noted to have minimal respiratory effort, neck weakness with inability to lift her head against gravity, and a depressed mental status. Blood work confirmed hypopneic respiratory failure requiring re-intubation. Point of care ultrasound revealed an akinetic left hemidiaphragm. A neuromuscular serologies and EMG confirmed a diagnosis of myasthenia gravis. Patient underwent treatment with IVIG and was able to be extubated successfully. IMPACT/DISCUSSION: Myasthenia gravis (MG) is the most common neuromuscular disorder characterized primarily by weakness and fatigability of ocular, bulbar, limb and respiratory muscles. These symptoms are due to autoimmune inhibition or destruction of the muscle fiber nicotinic acetylcholine receptor at the neuromuscular junction by autoantibodies. Certain medications may precipitate or exacerbate an episode of MG crisis: in this patient's case - atenolol, magnesium, and, as a possible paradoxical effect, corticosteroids. Involvement of the respiratory muscles is the most serious form of a MG crisis and can be life-threatening. In many patients, manifestations in the early stages of respiratory insufficiency could be transient and remit spontaneously. However the symptoms typically get worse and more persistent without treatment. In intubated patients, evaluation of diaphragmatic function can provide helpful information with regards to the appropriateness of extubation.

CONCLUSION: Respiratory failure could be a potential manifestation of neuromuscular disease. Avoid the medications that could exacerbate myasthenia gravis crisis.

\section{DIAGNOSTIC APPROACH TO ACUTE RHEUMATIC FEVER PRESENTING AS POLYARTHRITIS}

Julien Feghaly; Ariana Mooradian. St. Louis University Hospital, St. Louis, MO. (Control ID \#3162790)

LEARNING OBJECTIVE \#1: Diagnose acute rheumatic fever using the Jones criteria.

LEARNING OBJECTIVE \#2: Review the utility of throat culture, rapid streptococcal antigen test and streptococcal antibody titers in the diagnosis of ARF.

CASE: A 52-year-old male with a previous medical history significant for gout, hypertension, hyperlipidemia and alcohol abuse, presented with 9day history of gradually worsening polyarthritis. He was treated with indomethacin \& colchicine for a possible gout flare, with no improvement. Additionally, receiving vancomycin for concern of septic arthritis, having had an elevated temperature of $101.9^{\circ} \mathrm{F}$ and an elevated ESR \& CRP. The patient went on to report history of recent illness following contact with relative having Group-A Streptococcus pharyngitis. Further investigation revealed an elevated ASO titer \& normal anti-DNase B titer. A diagnosis of acute rheumatic fever was made, as the patient fulfilled the 
Jones criteria with 1 major feature (Polyarthritis) and 2 minor features (documented fever and an elevated ESR \& CRP). He was started on a 10day course of oral penicillin V. Transthoracic echocardiography was performed showing no abnormalities nor any signs of carditis.

IMPACT/DISCUSSION: The diagnosis of acute rheumatic fever is guided by the modified Jones criteria. The criteria require the presence of a preceding GAS infection, in addition to the presence of two major criteria or one major with two minor criteria. Further, supported by evidence of a GAS infection by throat culture, rapid streptococcal antigen test, or elevated or rising streptococcal antibody titers. Throat culture \& rapid antigen testing tend be inconclusive as they do not differentiate between chronic pharyngeal colonization and ARF, thus serologic evidence of elevated or rising anti-streptolysin O (ASO) and anti-DNase B titers is needed. ASO has been the most widely used antibody test for GAS, detected in more than $80 \%$ of patient with GAS pharyngitis, having a sensitivity of $72.7 \%$ \& specificity of $93.2 \%$ in post-streptococcal disease phase. Comparatively, anti-DNase B titer measurements had a lower sensitivity of $70.5 \%$ \& a similar specificity to ASO of $93.2 \%$. Yet, the greatest advantage of antibody testing has been the combined measurement of ASO and anti-DNase B titers which exhibits a higher sensitivity of $95.5 \%$ in identifying post-streptococcal disease; at the cost of a lower specificity $88.6 \%$. Once the diagnosis has been confirmed, further diagnostic testing is required to rule out carditis. This promptly performed by clinical examination, auscultating for new murmurs, and echocardiography, assessing for mitral or aortic regurgitation.

CONCLUSION: ARF may have varying presentation and needs to be considered when assessing polyarthritis. The combined measurement of ASO \& anti-DNase B titers demonstrates the highest sensitivity in the diagnosis of GAS. Cardiac examination followed by echocardiography is highly recommended in ARF.

\section{DIAGNOSTIC BIAS IN ACUTE KIDNEY INJURY: A CASE OF MPO-ANCA VASCULITIS ASSOCIATED WITH MULTIPLE MYELOMA}

Jammie Law; Eugeniya Golub; Bibi Ayesha. Montefiore Medical Center, Bronx, NY. (Control ID \#3185768)

LEARNING OBJECTIVE \#1: Recognize diagnostic bias when working up worsening kidney injury in patients with multiple myeloma.

LEARNING OBJECTIVE \#2: Illustrate an uncommon renal manifestation of multiple myeloma associated with MPO-ANCA vasculitis.

CASE: A 70 year old female presented with acute kidney injury (AKI). She had a past medical history significant for hypertension and multiple myeloma. Two months prior, urine protein/creatinine was significant for 1.2 grams of protein with a serum creatinine of $1.3 \mathrm{mg} / \mathrm{dL}$. Labs showed a normocytic anemia, thrombocytosis, and elevated ESR. SPEP notable for monoclonal protein detected in gamma region-IgM Kappa M. Urine microscopy was notable for 2 RBC casts. On follow-up, patient had a serum creatinine of $2.1 \mathrm{mg} / \mathrm{dL}$. Further laboratory workup was significant for a MPO-ANCA ( $>8 \mathrm{AI}$ ) and low C4. Given unclear diagnosis of AKI secondary to multiple myeloma versus MPO-ANCA vasculitis, renal biopsy was obtained. Renal biopsy showed pauci-immune focal segmental glomerulosclerosis consistent with ANCA-associated glomerulonephritis without evidence of plasma cell disease or granulomas. HIV, hepatitis, cryoglobulin, and abdominal fat pad biopsy for amyloid workup was negative. Given underlying hematologic malignancy, patient was determined to have an ANCA-vasculitis associated with multiple myeloma.

IMPACT/DISCUSSION: It is important for general internists to recognize diagnostic bias when working up worsening AKI in patients with multiple myeloma.

CONCLUSION: Multiple myeloma is a malignant clonal plasma cell proliferation commonly diagnosed in elderly adults. It classically presents with bone pain and lytic lesions, hypercalcemia, anemia, elevated serum protein usually $\operatorname{IgG}$ or $\operatorname{IgA}$, and renal involvement due to free light chain excretion (Bence Jones Protein). While renal complications of multiple myeloma are well known, the significance of red blood cell casts in this patient's earlier presentation should have prompted a full workup for suspected glomerulonephritis. An accurate diagnosis with renal biopsy and serologic testing is especially important to prevent worsening kidney injury, which can progress to ESRD within months. Serologic workup includes complement levels, ANCA, anti-GBM, ANA, anti-dsDNA, hepatitis, and SPEP. Interestingly, our patient had a positive MPOANCA and subsequent renal biopsy was consistent with a MPO-ANCA vasculitis. There is a possible immunogenic relationship that has been observed in serum between monoclonal gammopathies and ANCA. The presence of monoclonal immunoglobulins, through possible neutrophil activity, may be causal in the pathogenesis of vasculitis. However, there are few reports of ANCA-vasculitis coexisting with multiple myeloma and is unlikely to be encountered by the general internist. Thus, we present a case that demonstrates the importance of recognizing diagnostic bias and looking for disconfirmatory evidence in patients with multiple myeloma and worsening kidney injury.

\section{DIAGNOSTIC MIS-CU(E): A MISSED DIAGNOSIS OF COPPER DEFICIENCY}

Kayla Correia-Staudt ${ }^{1}$; Deepa R. Nandiwada ${ }^{2} .{ }^{1}$ Hospital of the University of Pennsylvania, Philadelphia, PA; ${ }^{2}$ University of Pennsylvania, Ardmore, PA. (Control ID \#3185681)

LEARNING OBJECTIVE \#1: Identify clinical manifestations of copper deficiency and highlight its place in a differential for neurologic and hematologic abnormalities.

LEARNING OBJECTIVE \#2: Recognize the importance of a thorough review of non-prescribed supplements and hygiene products a patient is using. CASE: A 55 year-old woman with recently diagnosed myelodysplastic syndrome (MDS) on azacitidine presented to our hospital with pancytopenia, paresthesias, and gait dysfunction. Prior to this presentation, she was admitted to another hospital for similar symptoms where MRI revealed a thoracic dorsal cord lesion, felt to be congenital. She was given intravenous immunoglobulin (IVIG) and discharged. Following, her gait dysfunction progressed, prompting presentation to our hospital. Her exam was notable for impaired proprioception and sensation of the lower extremities, which was felt to represent a posterior cord syndrome. Laboratory studies revealed stable pancytopenia; normal vitamin B12, folate, and vitamin E levels; negative HIV testing and undetectable rapid plasma reagin titers. Further testing revealed serum copper $<10 \mathrm{ug} / \mathrm{dL}$ and elevated zinc levels (181 ug/ $\mathrm{dL}$, normal 60-120). On further history, the patient endorsed years use of a high-zinc "immune-boosting" supplement and several months use of a zinccontaining dental paste for a loose crown. She was diagnosed with copper deficiency due to excessive zinc intake. Zinc products were stopped and she was started on copper supplementation with improvement in pancytopenia and neurologic symptoms.

IMPACT/DISCUSSION: Clinical manifestations of copper deficiency are somewhat similar to those of B12 deficiency. It most commonly presents as anemia and leukopenia with neurologic manifestations, including dorsal column myelopathy (sensory ataxia) and sensory peripheral neuropathy (with associated paresthesia). However, as in this patient, copper deficiency can mimic MDS, with pancytopenia, clonal abnormalities, and ringed sideroblasts on bone marrow biopsy. It is caused by malabsorption (most commonly post-gastric bypass) or excessive zinc intake (which inhibits intestinal absorption), and though rare, it is increasingly recognized following gastric bypass in those without adequate supplementation. In this case, the diagnosis was initially missed likely in part due to clinician unfamiliarity with this rare syndrome and potentially due to unidentified use of zinc-containing products which were only discovered with direct questioning. This case highlights the importance of a thorough supplement and dental product review, and also illustrates the need to question whether new diagnoses have alternative explanations. 
CONCLUSION: -Copper deficiency should be considered in the differential diagnosis for cytopenias and neurologic symptoms, particularly in those at risk for malabsorption or with high zinc intake. -Supplements and dental products should be considered in a differential for unexplained symptoms, and a medication reconciliation should include these substances.

\section{DIALYSIS DEPENDENT RENAL FAILURE AFTER HEALTH MOTIVATED DIETARY CHANGE IN A PATIENT WITH RE- MOTE BARIATRIC SURGERY}

Karl Gubler $^{2}$; Michael Odom ${ }^{2}$; Elena Lebduska ${ }^{3}$; Sandipani M. Sandilya ${ }^{1}$. ${ }^{1}$ United States Air Force, North Las Vegas, NV; ${ }^{2}$ United States Air Force, Nellis AFB, NV; ${ }^{3}$ University of Pittsburgh Medical Center, Denver, CO. (Control ID \#3181385)

LEARNING OBJECTIVE \#1: Assess the bariatric patient for nutritional needs and facilitate adherence to postoperative recommendations.

LEARNING OBJECTIVE \#2: Recognize potential complications of dietary nonadherence following bariatric surgery

CASE: An 81 year old female with hypertensive chronic kidney disease stage $3 \mathrm{~b}$ (baseline creatinine $1.4 \mathrm{mg} / \mathrm{dL}$ ) developed acute kidney injury (AKI) with creatinine 3.2 one week after starting losartan. She had undergone uncomplicated Roux en Y gastric bypass (RYGB) sixteen years prior. She reported several days of darker urine and loose green stool. Examination was unremarkable. Her BMI was 23. She had moderate proteinuria and a bland urinary sediment. Laboratory studies were otherwise unremarkable. Renal ultrasound showed structurally normal kidneys. Renal function declined despite hydration and she required three sessions of dialysis. Biopsy showed acute tubular necrosis secondary to oxalate nephropathy. Further history revealed recently increased consumption of oxalate rich vegetables like spinach and okra in an attempt to improve her diet. The patient was started on oral calcium carbonate and potassium citrate to promote dissolution of oxalate crystals. She also received dietary education on avoidance of oxalate rich foods. Her renal function recovered to baseline within three months.

IMPACT/DISCUSSION: Consumption of oxalate rich foods, high doses of vitamin $\mathrm{C}$, and RYGB increase the risk of oxalate nephropathy. RYGB causes enteric hyperoxaluria via increased absorption, increased endogenous oxalate synthesis, concentrated urine, and altered gut flora. Treatment includes dietary modification to decrease exogenous oxalate, oral calcium to reduce enteric absorption, hydration to dilute and citrate supplements to alkalinize the urine. RYGB reversal has been reported to reverse hyperoxaluria and kidney failure refractory to medical management. There has been research on oxalate degrading bacteria in rat models as a potential future treatment. Our case demonstrates that significant complications can arise even years after bariatric surgery, ironically from changes most patients would expect to promote health. It also shows that complete recovery may occur with diet and medication.

CONCLUSION: The primary care provider (PCP) should be aware of the risks of both deficiencies and toxicities after bariatric surgery, as these can occur many years later when patients are no longer seen in bariatric clinics. The PCP should reinforce the need for dietary adherence, and follow guidelines on monitoring vitamin and mineral levels to provide thorough care for this ever growing population.

\section{DIFFUSE CORONARY ARTERY ECTASIA: AN UNUSUAL} CAUSE OF ST-ELEVATION MYOCARDIAL INFARCTION

Ann Wang; Vikram Brahmanandam. Montefiore Medical Center, Bronx, NY. (Control ID \#3186244)

LEARNING OBJECTIVE \#1: Recognize a relatively uncommon cardiac anatomy which increases the risk of myocardial infarction

LEARNING OBJECTIVE \#2: Recognize the challenges in management of coronary artery ectasia
CASE: A 58 year old male with no significant medical history presented to the emergency department (ED) with acute onset substernal chest pain. An electrocardiogram (ECG) showed inferior ST-segment depressions, and cardiac troponins peaked at $1.05 \mathrm{ng} / \mathrm{mL}$ (normal $0.00-0.10 \mathrm{ng} / \mathrm{mL}$ ). Cardiac catheterization revealed diffuse coronary artery ectasia (CAE), with marked dilation of the right coronary artery (RCA), left anterior descending (LAD), and left circumflex arteries. The ectatic vessels, especially the distal RCA, demonstrated slow flow. He otherwise had mild coronary artery disease. A transthoracic echocardiogram (TTE) showed normal function without wall motion abnormalities. The patient was started on warfarin and aspirin, as well as a beta blocker and high dose statin. Three months after discharge, he again felt severe substernal chest pain while walking and presented to the ED. ECG showed an inferior ST-segment myocardial infarction. Of note, his international normalized ratio (INR) was subtherapeutic. Cardiac catheterization again showed slow flow in the ectatic RCA without an intervenable lesion. He was then placed on triple therapy with clopidogrel, aspirin, and warfarin. He has remained on triple therapy and without further cardiac symptoms, now ten months after his initial presentation.

IMPACT/DISCUSSION: CAE is characterized by dilation of diffuse segment(s) of the coronary arteries to at least 1.5 times the normal diameter. CAE is typically asymptomatic and incidentally discovered during cardiac catheterization or other imaging, and the incidence has been reported to be between $0.3 \%-5 \%$. The RCA is most commonly affected, followed by the LAD. The etiology is likely multifactorial. CAE is strongly associated with atherosclerotic disease, hypertension, and dyslipidemia. Connective tissue diseases, vasculitis, and iatrogenic vessel injury have also been implicated. Patients with CAE have a higher incidence of major adverse cardiac events (MACE), likely due to increased turbulent flow, stasis, and endothelial dysfunction in the ectatic vessels. In one study, patients who presented with myocardial infarction and were found to have CAE had a 3.25 fold greater risk of recurrent MACE after 49 months, compared to similar patients without CAE. Evidence to guide management of $\mathrm{CAE}$ is still scarce. One retrospective study has found that warfarin monotherapy significantly decreased adverse cardiac events in CAE patients compared to no anticoagulation. Another study showed that dual antiplatelet therapy halved the incidence of cardiac events in patients with CAE, compared to no anticoagulation. CONCLUSION: Coronary artery ectasia is relatively uncommon. $\mathrm{Pa}-$ tients with CAE are at greater risk for MACE and will likely benefit from anti-coagulation; however, further studies are needed to determine the optimal anticoagulation strategy.

\section{DISSECTING A CASE OF CHEST PAIN}

Deep Sangani; Aaron W. Sweeney. Tulane University School of Medicine, New Orleans, LA. (Control ID \#3184095)

LEARNING OBJECTIVE \#1: Recognize the clinical presentation of spontaneous coronary artery dissection

LEARNING OBJECTIVE \#2: Review management of spontaneous coronary artery dissection

CASE: A 57-year-old woman presented with sudden onset, sharp chest pain across her entire chest with radiated down both arms. There was associated nausea, vomiting, and palpitations. She did not have any prior cardiac history. Her 36-year-old son had a myocardial infarction the previous year. Two-millimeter ST elevations were identified in multiple leads on the EKG and the patient was taken for cardiac catheterization. Coronary angiography did not reveal any evidence of obstructive coronary disease, though a dissection of the middle left anterior descending coronary artery was noted. Echocardiogram returned with an ejection fraction of $30-35 \%$ along with moderate septal hypokinesis. Approximately four hours into her hospital course, chest pain recurred with dynamic EKG changes and she underwent coronary artery bypass grafting. 
IMPACT/DISCUSSION: Spontaneous coronary artery dissection (SCAD) is epicardial coronary artery dissection that is not associated with atherosclerosis, trauma, or iatrogenic causes. The primary mechanism that leads to myocardial injury is coronary artery obstruction secondary to a spontaneous formation of an intramural hematoma following disruption of the vessel wall or spontaneous hemorrhage arising from the vasa vasorum within the vessel wall. Middle aged women account for up to $95 \%$ of SCAD cases. For young women presenting with acute coronary syndrome, including those with pregnancy-related myocardial infarction, the prevalence of SCAD is $24 \%-35 \%$. Additional risk factors for SCAD include fibromuscular dysplasia (FMD), connective tissue disorders, systemic inflammatory disorders, pregnancy, hormonal therapy, intense valsalva, and intracranial aneurysms. Presentation of SCAD mimics ACS with chest pain that radiates to the arms or neck, nausea, vomiting, and troponin elevation. Patients with SCAD are at risk of being incorrectly diagnosed due to their young age or lack of atherosclerotic risk factors. If SCAD is suspected, then coronary angiography should be performed to evaluate atherosclerotic lesions that could require intervention. There is a high rate of technical failure with PCI and lack of protective effect against future dissection events. Beta-blockers should be considered for patients who have LV dysfunction, arrhythmias, or hypertension. ACE inhibitors and ARBs are recommended. However, statins are not recommended unless the patient has guideline-based indications for primary prevention of atherosclerosis. Experts are divided about the use of anti-platelet and anti-coagulants.

CONCLUSION: SCAD should be considered in the differential diagnosis of acute chest pain, especially in a young female patient who lacks traditional atherosclerosis risk factors. It is important to distinguish SCAD from ACS as clinical management differs.

\section{DISSECTING THE CAUSE OF ATRIAL FIBRILLATION: AN ATYPICAL PRESENTATION OF SPONTANEOUS CORONARY ARTERY DISSECTION}

Pedro Covas ${ }^{1}$; Talal S. Alzahrani ${ }^{2} .{ }^{1}$ George Washington University, Washington, DC; ${ }^{2}$ The George Washington University, Arlington, VA. (Control ID \#3159762)

LEARNING OBJECTIVE \#1: Recognizing atrial fibrillation as an uncommon presenting symptom of a spontaneous coronary artery dissection.

CASE: A 46-year-old woman with a history of hypertension presented with acute substernal chest pain and an episode of emesis. EKG done by paramedics demonstrated atrial fibrillation with a rapid ventricular response and aberrancy (EKG difficult to interpret as limb lead reversal is possible), which spontaneously resolved. Troponin upon arrival was 0.10 $\mathrm{ng} / \mathrm{ml}$, which was presumed to be secondary to her atrial fibrillation. However, her repeated troponin was peaked at $10.5 \mathrm{ng} / \mathrm{ml}$. Serial EKGs showed normal sinus rhythm with prolonged QTc and few fusion beats without ST-segment changes. Echocardiogram showed anterolateral and apical wall motion abnormalities, and cardiac catheterization revealed spontaneous coronary artery dissection (SCAD). Renal ultrasound showed normal kidneys without any evidence of renal artery stenosis, a sign of fibromuscular dysplasia. The patient was discharged with optimal medical therapy.

IMPACT/DISCUSSION: SCAD is a form of non-atherosclerotic myocardial infarction, that commonly affects young women. Chest pain, sudden cardiac death (SCD), STEMI, NSTEMI with or without EKG changes are common presentations of SCAD, but arrhythmias are relatively uncommon and sparsely noted in the literature. Although ACS can cause arrhythmias leading to SCD, atrial fibrillation has never been reported in young women with SCAD. Treatment involves optimized medical therapy, but PCI has been performed for persistent symptoms. SCAD is highly associated with fibromuscular dysplasia and connective tissue disorders, and proper screening should be performed.
CONCLUSION: This case highlights the importance of early recognition of atypical presentations of SCAD, especially atrial fibrillation, as this can lead to immediate medical therapy and interventions

\section{DISSEMINATED BLASTOMYCOSIS IN AN IMMUNOCOMPE- TENT PATIENT}

Anjeanette Mendez ${ }^{1}$; Kristen Corrao ${ }^{1}$; Joyce Okendo ${ }^{1}$; Mirza Baig ${ }^{1}$; Pinky Jha ${ }^{2}$; Sanjay Bhandari ${ }^{1} .{ }^{1}$ Medical College of Wisconsin, Milwaukee, WI; ${ }^{2}$ medical college of wisconsin, Milwaukee, WI. (Control ID \#3186117)

LEARNING OBJECTIVE \#1: Recognize an interesting and unusual case of disseminated blastomycosis in an immunocpenent male patient.

LEARNING OBJECTIVE \#2: Review the diagnosis and management of disseminated blastomycosis.

CASE: A 27 year-old incarcerated male presented with a 2-month duration of hemoptysis and cough. He denied any symptoms of weight loss, night sweats or fatigue. He denied any any sick contact and any travel prior to incarceration. He also complained of bilateral headache and neck pain along with light sensitivity. Physical examination was significant for cutaneous lesions on his forehead, left ear, bilateral cheeks and right forearm. He was initially started on intravenous vancomycin and ceftriaxone for possible pneumonia. Due to concern for pulmonary tuberculosis (TB), patient was placed in isolation until further work-up could be done. Infectious disease and Dermatology were consulted. Computed tomography $(\mathrm{CT})$ of the chest was obtained along with lumbar puncture, sputum cultures and skin biopsy. CT chest revealed a right lower lobe consolidative opacity with areas of cavitation as well as bilateral diffuse centrilobular nodules. TB was ruled out with negative sputum cultures $\mathrm{x} 3$ and QuantiFERON-TB Gold test. HIV test was negative. Urine was positive for presence of Blastomyces antigen and sputum cultures were positive for Blastomyces. CSF studies showed high protein $(170 \mathrm{mg} / \mathrm{dl})$, low glucose $(21 \mathrm{mg} / \mathrm{dl})$, and elevated leucocyte count $(371 / \mathrm{microL})$ consistent with central nervous system (CNS) involvement. Skin sample under direct microscopy revealed broad based budding yeast. Tissue punch biopsy from left ear lesion was positive for round yeast forms consistent with Blastomyces dermatitidis. Antibiotics were discontinued, and he was started on Amphotericin B intravenouslyto complete a 4-week course followed by a 12-month course of voriconazole.

IMPACT/DISCUSSION: Blastomcyosis is treated with an oral azole in mild to moderate disease or Amphotericin B in more severe presentations. In our patient who not only had pulmonary and cutaneous but also possible CNS involvement, the treatment of choice is Amphotericin B. He was initially on antibiotics which were discontinued once the appropriate diagnosis was made and was started on Amphotericin B. This case describes a rare presentation of disseminated blastomycosis in an immunocompetent patient and can help increase its awareness among clinicians.

CONCLUSION: Disseminated blastomycosis is an uncommon fungal infection especially in immunocompetent hosts. Our case highlights the need for a high index of suspicion for the diagnosis of blastomycosis regardless of the immunological status of a patient, and the importance of prompt diagnosis and treatment with the appropriate antifungal.

\section{DISSEMINATED CRYPTOCOCCUS IN MYASTHENIA GRAVIS}

Neil Evans; Amitabh Singh; Soumya Chatterjee. Saint Louis University, Saint Louis, MO. (Control ID \#3186140)

\section{LEARNING OBJECTIVE \#1: Delineate risk factors for Cryptococcus} neoformans

LEARNING OBJECTIVE \#2: Diagnosis Cryptococcus neoformans

CASE: 70 year old male farmer from Illinois with a past medical history of myasthenia gravis treated with 15 milligrams of prednisone daily and Cellcept 500 milligrams twice daily. He was transferred to the hospital 
after recent biopsy of necrotic skin lesion revealed Cryptococcus neoformans. Notably, he also presented with fatigue and new productive cough and dyspnea in addition to the skin lesion. Serum Cryptococcal antigen titer was 1:10. Subsequent lumbar puncture was negative for cryptococcal antigen and CT head was also negative. Due to persistent dyspnea and cough, a CT chest was obtained. CT revealed a new $2 \times 2 \mathrm{~cm}$ nodule. Concerned for disseminated Cryptococcal infection in the setting of chronic immunosuppression, he was treated with flucytosine and amphotericin B for 3 weeks. After treatment, the skin lesion resolved and repeat $\mathrm{CT}$ chest showed decreased size of the pulmonary lesion to 1.2 $\mathrm{x} 1.5 \mathrm{c} \mathrm{m}$. He was transitioned to oral fluconazole $400 \mathrm{mg}$ for 8 weeks followed by fluconazole $200 \mathrm{mg}$ for at least a year.

IMPACT/DISCUSSION: Cryptococcus neoformans has worldwide distribution. Cryptococcus neoformans is an invasive fungal infection that is prevalent in immunocompromised patients and represents an important cause of morbidity and mortality in this population. It's most common presentation is cryptococcal meningitis (15\% of all AIDS-related deaths globally). It can also present as pulmonary disease, skin, lymph node, and bone disease. Patients at risk include: AIDS with CD4 counts $<200$ cells/ $\mu \mathrm{L}$, patients on 20 milligrams of prednisone for at least 2 weeks, history of organ transplantation, malignancy, and liver disease. Screening for cryptococcal antigen should be driven by symptoms in the context of chronic immunosuppression. For patients with respiratory symptoms, cryptococcal antigen is a good first test. Symptoms concerning for meningitis like neurological deficits, severe headaches, or neck stiffness should also prompt a lumbar puncture. Also, if a high serum cryptococcal antigen titer ( $>1: 512)$ is found, the high infection burden increases the risk for extrapulmonary spread and seeding of the central nervous system and also requires a lumbar puncture. Look for high opening pressure, positive culture, or elevated cryptococcal antigen titer.

CONCLUSION: Cryptococcus neoformans infection can present in several different ways, and is an important pathogen to consider in at risk populations (chronic immunosuppressed state). Notably, patients can present with more than one manifestation of cryptococcal disease. Cryptococcal disease, if progresses to meningitis, is uniformly fatal, but shows good response to treatment with the appropriate induction regimen of amphotericin and flucytosine, and maintenance therapy with fluconazole.

\section{DISSEMINATED HISTOPLASMOSIS AS AN IMPORTANT DIF- FERENTIAL TO CONSIDER IN PATIENTS PRESENTING WITH HYPERCALCEMIA}

Jacob Burch; Lawrenshey Charles; Emir Kurtovic; Jason Liu; Sonya Gupta. Sparrow Hospital, Lansing, MI. (Control ID \#3185825)

LEARNING OBJECTIVE \#1: Recognize uncommon presentations of disseminated histoplasmosis

LEARNING OBJECTIVE \#2: Distinguish various etiologies of hypercalcemia

CASE: JM is a 62-year-old female with history of rheumatoid arthritis, on etanercept and methotrexate, who presented to the hospital for persistent RLL pneumonia, confusion, weight loss, and weakness. Examination showed jaundice, dry mucosal membranes, and generalized abdominal pain. She was found to have hypercalcemia with corrected value of 17.8, vitamin D 1,25 elevated at 157, PTH 13.4, PTHrp 0.5, pancytopenia, elevated transaminases, and AKI with $\mathrm{Cr}$ of 2.58. CT revealed mild mediastinal lymphadenopathy and persistent infiltrates in RLL. Patient was admitted and worked up for possible infectious versus malignant etiology. She was started on broad-spectrum antibiotics, later discontinued with negative cultures. ACE level elevated at 170. SPEP, direct coombs, IFE and bence jones protein were negative. Hypercalcemia improved following IV fluids, furosemide, calcitonin, and pamidronate. Bone marrow biopsy was performed due to concern for possible lymphoma and demonstrated disseminated histoplasmosis. Fungal studies resulted with fungal $\mathrm{Ab}$ negative, urine histoplasma $\mathrm{Ag}$ positive, and fungal precipitins positive for histoplasma. Patient was started on oral itraconazole. Following improvement in clinical status, she was discharged to a long-term acute care facility with plans for close follow-up.

IMPACT/DISCUSSION: Histoplasmosis is the most common endemic mycosis to cause hospitalization in the US. It is prevalent along the Ohio and Mississippi River Valleys. It can have dramatic clinical manifestations, including flu-like illness to severe and progressive disseminated infections. Individuals at high risk of infection include the immunocompromised, those at extremes of age, or patients with unusually heavy exposure. Infections can involve multiple organ systems resulting in adrenal insufficiency, hepatosplenomegaly, pancytopenia, GI tract ulcers, skin and CNS lesions, and lung pathology. Radiographic studies typically show patchy or nodular focal infiltrates and mediastinal or hilar lymphadenopathy. Disseminated histoplasmosis presenting with hypercalcemia has been described in the literature, but is considered a rare manifestation. If left untreated mortality is as high as $80 \%$, reduced to $25 \%$ with treatment. As evident in our case, Histoplasmosis is often initially mistaken as other infection, inflammatory disease, or malignancy. Atypical presentations can delay diagnosis and lead to poor outcome. Our case demonstrates the importance of keeping a broad differential in patients presenting with hypercalcemia.

CONCLUSION: Disseminated histoplasmosis can present with many features similar to malignancy Physicians should have a high index of suspicion for granulomatous disease, such as histoplasmosis, when encountering patients with hypercalcemia Unusual pathogens should be suspected in immunocompromised patients

\section{DISSEMINATED INTRAVASCULAR COAGULATION AS INI- TIAL PRESENTATION OF METASTATIC PROSTATE CANCER} Kavita Agrawal; Nirav Agrawal. Overlook Medical Center, Summit, NJ. (Control ID \#3162850)

LEARNING OBJECTIVE \#1: Diagnose disseminated intravascular coagulation (DIC) based on laboratory findings.

LEARNING OBJECTIVE \#2: Understand pathophysiology of DIC in prostate cancer. Treatment of prostate cancer induced DIC

CASE: A 70-year-old male presented with hematuria and bruising of arms and legs for 3 days. He complained of 40 pounds weight loss over the last 4 months. Initial blood tests showed prothrombin time of 25.1 seconds, INR 2.5, partial thromboplastin time 43.9 seconds, fibrinogen $60 \mathrm{mg} / \mathrm{dL}$, fibrin degradation products $>20 \mathrm{ug} / \mathrm{ml}$ and platelets $88000 / \mathrm{nL}$. The results were consistent with DIC. Patient was seen on the outpatient basis for complains of urinary frequency and hesitancy 6 months prior to this admission. During this time, prostate specific antigen (PSA) was found to be elevated at 241 ug/dL. However, patient was lost to follow up. On this admission, he had worsening PSA of $942 \mathrm{ug} / \mathrm{L}$. Computed tomography of abdomen pelvis showed enlarged prostate with mass effect on the bladder base, left side hydronephrosis and numerous enlarged pelvic lymph nodes. Bone scan of the whole body showed increased sclerosis of L3 vertebral body suggestive of osseous lesions. There was a concern for metastatic prostate cancer inducing DIC. He received fresh frozen plasma and cryoglobulin. He was started on Bicalutamide and leuprolide as inpatient pending pathologic diagnosis. Patient responded well to the therapy and labs were improving. The patient refused prostate biopsy. So, bone marrow biopsy was performed which revealed metastatic prostate adenocarcinoma. Our case highlights DIC as an initial manifestation of prostate cancer which is unusual.

IMPACT/DISCUSSION: DIC is a pathological systemic condition which occurs due to widespread activation of clotting factors causing bleeding and thrombosis. DIC does not occur in isolation. It is crucial to search for underlying secondary causes propagating DIC. This includes sepsis, trauma, pre-eclampsia, malignancies, acute hemolytic transfusion reaction and others. It is important to search for malignancies when there is no apparent underlying cause. Among malignancies, DIC is more commonly associated with acute promyelocytic leukemia, brain tumor 
and mucinous tumors such as ovarian, pancreatic and gastric. Our patient had prostate cancer induced DIC. The pathophysiology involves release of polyphophate containing protasomes from prostate gland which activates Factor XII leading to DIC. Management should focus on supportive measures to correct the coagulopathy and treatment of underlying cause. Our patient received anti-androgen therapy bicalutamide and leuprolide with improvement in PSA and DIC.

CONCLUSION: DIC can be an initial manifestation of underlying prostate cancer. It is important to monitor the patients with metastatic prostate cancer for signs and symptoms of development of DIC such as bleeding or low platelet counts. The management of DIC must be to control coagulopathy with supportive measures (such as platelet transfusion, cryoglobulin) and treatment of underlying cause.

\section{DISTINGUISHING BETWEEN ERYTHEMA MULTIFORME MAJOR AND SEVENS JOHNSON SYNDROME: A DIAGNOS- TIC CHALLENGE}

Amali Gunawardana ${ }^{1,2}$; Ribka Ayana ${ }^{1}$; Mary Maiberger ${ }^{1} .{ }^{1}$ Washington DC VA Medical Center, Washington DC, DC; ${ }^{2}$ George Washington University Hosptial, Washington, DC. (Control ID \#3184120)

LEARNING OBJECTIVE \#1: Recognize the clinical distinction between Erythema Multiforme Major (EMM) and Stevens-Johnson Syndrome (SJS)

LEARNING OBJECTIVE \#2: Recognize the difference in morbidity and mortality associated with EMM vs. SJS

CASE: A 62-year-old hospitalized male complained of a burning, tender blister on his upper lip. Three days prior, he was started on vancomycin and ertapenem for urinary tract infection and osteomyelitis though after, ertapenem was changed to meropenem for persistent fevers. Acyclovir was started for suspected herpes infection, but his lesions progressed. Dermatology evaluated the patient and noted multiple superficial ulcerations and erosions involving the upper and lower lips, buccal mucosa as well as thin fissuring of the lower lip. Oral examination revealed grayishwhite mucosal desquamation of the hard palate. In addition, he had focal erythematous, dusty plaques scattered over several digits on both hands. There was no ocular or genital involvement. Patient declined a skin biopsy. A clinical diagnosis of EMM was made, and vancomycin and meropenem were discontinued. Lesions slowly resolved and patient competed treatment course with clindamycin and aztreonam.

IMPACT/DISCUSSION: First, drug-induced EMM is an uncommon variant. Second, to our knowledge, there exist no cases of vancomycin or carbapenem induced EMM reported in the literature. Lastly, in early stages, severe EMM and SJS can be confused, though early clinical distinction is critical as management strategies vary, and SJS is associated with a higher morbidity and mortality.

CONCLUSION: EMM is a mucocutaneous eruption characterized by targetoid lesions in an acral and mucosal distribution, categorized into two subtypes (major and minor) based on the severity and number of mucosal sites involved. Most commonly caused by infections, such as herpes simplex virus infection and mycoplasma pneumoniae, it is less commonly triggered by medications such as sulfonamindes, NSAIDs, and anticonvulsants. EMM is a distinct clinical syndrome from SJS/toxic epidermal necrolysis (TEN) which are more severe desquamating mucocutaneous drug reactions. SJS and TEN are often drug induced reactions. The degree of mucosal involvement is typically limited to $1-2 \%$ of body surface area in EMM, up to $10 \%$ in SJS and greater than $30 \%$ in TEN. SJS is more likely associated with lesions starting on trunk and spreading distally in contrast to EMM, and lesions appear more like atypical targetoid macules or purpuric papules. SJS/ TEN patients are more likely to have fever, skin detachment, and ocular manifestations and require treatment with immunosuppressive. EMM is a clinical diagnosis, although biopsy can support clinical findings and help exclude other causes. EMM is usually selflimited and resolves in 3-5 weeks, though can reoccur.

\section{DOCTOR! MY EYES?}

Nooraldin Merza ${ }^{1}$; Assad Mohammedzein ${ }^{1}$; Nibras Talib-mamury ${ }^{1}$; James ". Walker ${ }^{2}$; Karen Cutts ${ }^{1} .{ }^{1}$ Texas Tech University Health Science Center, Amarillo, TX; ${ }^{2}$ Texas Tech, Amarillo, TX. (Control ID \#3147541)

LEARNING OBJECTIVE \#1: Recognize Miller Fisher Syndrome (MFS) as a variant of Guillain-Barre (GBS)

LEARNING OBJECTIVE \#2: Maintain low threshold to perform lumbar puncture in a young patient with unusual neurological presentation

CASE: A 49-year-old male with no significant medical history presented with sudden onset drooping of the left eyelid and diplopia looking into the right. He was recently treated for sinusitis with antibiotic. He was seen a week prior with upper and lower extremity numbness and unsteady gait, CT head was unremarkable, his symptoms improved but he was still using a cane for support. He denied any focal weakness or incontinence. He is married, works in foundation repair, and denies recent travel or tick bite. Vital signs were normal. He had left partial ptosis. Left eye was directed downward and outward and unable to adduct. Left eye pupillary reflex was intact. Other cranial nerves were normal. Had normal power and sensation, no cerebellar signs, absent deep tendon reflexes and slightly unsteady gait with inability to perform tiptoe walk. Romberg sign was negative. Laboratory workup was unremarkable. MRI showed no demyelinating lesion. Lumbar puncture showed normal cell count with total protein of $157 \mathrm{mg}$ / dl (Ref. Range 15-45). MFS was suspected, the patient was started on intravenous immunoglobulin for 4 consecutive days, with remarkable improvement in eye movement noted by the time of discharge.

IMPACT/DISCUSSION: MFS is a variant of GBS, characterized by the triad of ophthalmoplegia, ataxia and areflexia. Incomplete forms with 2 out of the three features do exist. CSF is often diagnostic with the characteristic cytoalbuminologic dissociation. Antibodies against GQ1b (a ganglioside component of nerve) are present in 85 to $90 \%$ of patients. Most of the patients do recover, with a mean recovery onset at 10 weeks. Immunomodulatory treatment has been used to hasten recovery. Intravenous immunoglobulin, as used in our patient, have proved equally effective to plasma exchange. The initial presentation of our patient was a bit challenging, especially with normal motor function and reflexes. Gait instability and recent upper respiratory symptoms may have misled to vestibular neuritis as a probable cause. MFS as the likely diagnosis was not apparent until after he was readmitted with partial third nerve palsy and areflexia.

CONCLUSION: This case illustrates how important the clinical judgment in assessing someone who presents with complex neurological complaints. In young patients like ours, it helps to maintain a low threshold to perform lumbar puncture and CSF analysis whenever there is uncertainty about the diagnosis.

\section{DON'T FORGET THE STEROIDS! MANAGEMENT OF A RARE ETIOLOGY OF PERICARDIAL EFFUSION}

Christopher Lee $^{1}$; Henry Bergquist ${ }^{1}$; Stefan Nguyen ${ }^{1}$; Sumit Patel ${ }^{1}$; Harveen Bergquist ${ }^{2}$; Enrique Ostrzega ${ }^{1}{ }^{1}$ University of Southern California, La Crescenta, CA; ${ }^{2}$ University of Southern California, Los Angeles, CA. (Control ID \#3183748)

LEARNING OBJECTIVE \#1: Recognize a rare etiology of pericardial effusions and possible treatment modalities

CASE: A 65-year-old male with a history of hypertension presented with 2 weeks of shortness of breath and dyspnea on exertion along with orthopnea and a dry cough. He denied any chest pain, weight loss, fevers, or night sweats. On examination, vital signs were stable and he was saturating well on room air. He had jugular venous distension, distant heart sounds, and lower extremity edema. Laboratory values were within normal with the exception of alkaline phosphatase $558 \mathrm{U} / \mathrm{L}$, AST 137 U/L, ALT $88 \mathrm{U} / \mathrm{L}$, T Bili $1.4 \mathrm{mg} / \mathrm{dL}$, D Bili $0.7 \mathrm{mg} / \mathrm{dL}$, and a mildly elevated pro-BNP to $804 \mathrm{pg} / \mathrm{ml}$. HIV and hepatitis panel was negative. 
Electrocardiogram revealed normal sinus rhythm with low voltages on the limb leads and chest x-ray showed cardiomegaly without evidence of pulmonary edema. Transthoracic echocardiogram showed a large circumferential pericardial effusion measuring $3.3 \mathrm{~cm}$ with early tamponade physiology. Right heart catheterization revealed elevated right and leftsided pressures (right atrial mean 17, right ventricular pressure 43/13, right ventricular end-diastolic pressure 17 , pulmonary artery pressure 42 / 23 , pulmonary capillary wedge pressure 22 ) with a pericardial pressure of $13 \mathrm{mmHg}$. Pericardiocentesis was performed with removal of $700 \mathrm{cc}$ of serous fluid, resulting in improvement of filling pressures. Fluid analysis showed an exudative effusion with 2,035 nucleated cells with a $58 \%$ lymphocytic predominance and adenosine deaminase elevated to 118.1 $\mathrm{U} / \mathrm{L}$ (normal less than 9.2 U/L). He was empirically treated with a fourdrug anti-tuberculous regimen and subsequent pericardial fluid and induced-sputum cultures grew Mycobacterium tuberculosis. He was continued on anti-tuberculous antibiotics but unfortunately, eventually succumbed to his disease 9 months later.

IMPACT/DISCUSSION: Although a more common diagnosis in developing countries, tuberculous pericardial effusions are rare in developed countries and are usually limited to immunocompromised individuals. Treatment involves anti-tuberculous antibiotic therapy with possible adjunctive glucocorticoid use. Pericardiocentesis may also be performed. Studies regarding adjunctive glucocorticoid use have yielded mixed results but data from meta-analyses have shown that corticosteroids may reduce death from all causes in HIV-negative patients, which usually result from complications of pericardial constriction. However regardless of treatment, mortality rates remain high.

CONCLUSION: This case demonstrates the importance of having a broad differential when working up pericardial effusions. Although tuberculosis is a rare etiology of pericardial effusions, rapid recognition and intervention with antibiotics and corticosteroids are important in management.

\section{DON'T MIND THE GAP}

Yuliana Noah ${ }^{1}$; Justine Phifer ${ }^{2}$; Sarah W. Baron ${ }^{2} .{ }^{1}$ Albert Einstein College of Medcine, Bronx, NY; ${ }^{2}$ Montefiore Medical Center, Bronx, NY. (Control ID \#3159904)

LEARNING OBJECTIVE \#1: Recognize various etiologies of an elevated globulin gap.

LEARNING OBJECTIVE \#2: Assess appropriateness of SPEP (serum protein electrophoresis) and avoid unnecessary testing.

CASE: A 56-year-old woman with type 1 diabetes presented with altered mental status in diabetic ketoacidosis. On admission, her total protein was $12.1 \mathrm{~g} / \mathrm{dL}$ with an elevated globulin gap of $8 \mathrm{~g} / \mathrm{dL}$ (albumin level $4.1 \mathrm{~g} / \mathrm{dL}$ ), hemoglobin $13.5 \mathrm{~g} / \mathrm{dL}$ and calcium $9.8 \mathrm{mg} / \mathrm{dL}$. HIV and HCV tests were negative. Serum protein electrophoresis (SPEP) revealed an elevated M-Spike of $2.7 \mathrm{~g} / \mathrm{dL}, \mathrm{IgG}$ of $5860 \mathrm{mg} / \mathrm{dL}$, and free light chain ratio of 232. Skeletal survey was negative for bony lesions. Subsequent labs demonstrated protein gap closure after DKA resolution. She was followed for a presumptive diagnosis of monoclonal gammopathy of undetermined significance.

IMPACT/DISCUSSION: The globulin gap is the calculated difference between total protein and albumin level. It accounts for serum proteins besides albumin, notably immunoglobulins. Etiologies of an elevated globulin gap include polyclonal gammopathies from liver disease, connective tissue disease, and chronic infection, and monoclonal gammopathies from plasma cell dyscrasias, like monoclonal gammopathy of undetermined significance (MGUS) and multiple myeloma. There are many etiologies of a globulin gap, not just malignancy, as evidenced by the closure of our patient's gap after resolution of her DKA. Ordering SPEP following any elevation of globulin gap is inefficient and expensive. At one institution, only $3 \%$ and $1 \%$ of 7,090 SPEP ordered resulted in diagnoses of MGUS and malignancies, respectively. A clinical decision rule was developed to increase the abnormal SPEP yield: 5 points for globulin gap $>4.1 \mathrm{~g} / \mathrm{dL}, 3$ points for age 60,2 points each for hemoglobin
$<12 \mathrm{~g} / \mathrm{dL}$ and male sex, and 1 point for eGFR $<60 \mathrm{~mL} / \mathrm{min} / 1.73 \mathrm{~m}^{2}$. A score of $11+$ corresponds to a specificity of $>90 \%$ for abnormal SPEP, while a score of $0-5$ had a negative predictive value of $\sim 97 \%$. Using this tool, our patient scored 6, making her borderline for whether SPEP was warranted. Monoclonal Gammopathy of Undetermined Significance (MGUS) is a premalignant condition with $\sim 1 \%$ potential for progression. The clinical significance of MGUS diagnosis has come into question as disease progression is rarely recognized prior to serious complications despite close monitoring. One study found that a majority of malignant transformation identified were a result of symptomatic presentations outside of regularly scheduled follow-up. Thus, overtesting is not only expensive but also may lead to ineffective management.

CONCLUSION: • Clinical decision rules should be utilized to determine appropriateness of SPEP testing. - Overtesting leads to unnecessary diagnosis and follow-up of MGUS.

\section{DOUBLE TROUBLE: SIMULTANEOUS CUTANEOUS LYM- PHOMAS IN AN AIDS PATIENT}

Albert Jang; Lucy Huo; Mahmoud Gaballa; Martha Mims. Baylor College of Medicine, Houston, TX. (Control ID \#3139823)

LEARNING OBJECTIVE \#1: Understand that patients with HIV may develop more than one type of lymphoma.

LEARNING OBJECTIVE \#2: Recognize the need to biopsy a new or worsening cutaneous lesion in the setting of a diagnosed cutaneous malignancy.

CASE: A 41-year-old woman with AIDS (on HAART, CD4 count 154/ $\mathrm{mm} 3$ with an undetectable viral load) and plasmablastic lymphoma (on EPOCH chemotherapy) presented following syncope. Two weeks ago, she noticed a new annular lesion with an ulcerating, necrotic center and an erythematous-violaceous border on her right thigh, similar to other lymphoma lesions on her legs. On admission, she reported four days of fever and increasing right thigh pain. Physical exam was notable for an overall cachectic and tearful appearance, with no hepatosplenomegaly and no palpable lymphadenopathy, and skin exam was significant for several scattered large patches of cutaneous lesions with granulation tissue and thick yellow exudate but no purulent drainage along the legs bilaterally. A CT scan showed an abscess underlying her new right thigh lesion. The patient underwent bilateral thigh wound incision and drainage, irrigation, and debridement, with biopsies taken. While waiting on pathology, the patient's chemotherapy regimen was changed to bortezomib followed by ICE regimen, presuming progression of the original plasmablastic lymphoma. Biopsy results revealed the new lesion was an extranodal NK/T-cell lymphoma, nasal type. Comparison to the original biopsy demonstrated a completely different immunohistochemical profile.

IMPACT/DISCUSSION: Patients with HIV have more than a 60-fold risk of developing non-Hodgkin's lymphoma. The increased risk is related to transforming properties of the retrovirus, immunosuppression, cytokine dysregulation, and opportunistic infections from lymphotrophic herpesviruses. Plasmablastic lymphoma, a rare and aggressive subtype of diffuse large B-cell lymphoma, comprises $2 \%$ of all HIV-related lymphomas. NK/T-cell lymphoma is a destructive and necrotic lymphoma, most commonly presenting in the nasal cavity but can manifest on the skin. Epstein-Barr virus plays a role in the pathogenesis of both lymphomas. No standard chemotherapy regimens exist for either lymphoma, and outcomes are often poor for both. In this case, bortezomib with ICE to treat the progressing plasmablastic lymphoma coincidentally became an option to treat the NK/T cell lymphoma, which is sensitive to platinum agents. When an HIV patient with lymphoma develops new lesions during treatment, it is important to biopsy the new lesion. Not only does an immunocompromised state place a patient with HIV at increased risk of infection and malignancy, chemotherapy may induce a new malignancy or transformation of the original malignancy. 
CONCLUSION: Treating two separate but concurrent aggressive malignancies, each with no standardized regimen, is extremely difficult. Physicians should maintain a high index of suspicion when a new lesion appears different from the others, and obtaining a new biopsy if possible should be done quickly.

\section{DRY GANGRENE AND ULCERS IN MULTIPLE DIGITS IN A PATIENT WITH TOBACCO USE}

Anu Kurl $^{1}$; Robin Klein ${ }^{2} .{ }^{1}$ Emory University School of Medicine, Hampton, GA; ${ }^{2}$ Emory, Atlanta, GA. (Control ID \#3185362)

LEARNING OBJECTIVE \#1: Recognize the clinical presentation of thromboangiitis obliterans.

LEARNING OBJECTIVE \#2: Discuss diagnosis of thromboangiitis obliterans and how to differentiate from other causes of digital ulcers

CASE: A 61 year-old female presents with three months of pain and discoloration of her left thumb. Three months ago, she injured the tip of her thumb after which it became progressively swollen, painful, discolored and ulcerated. She also noted ulceration of multiple other digits, which she attributed to prior injuries. Past medical history was notable for arthritis, tobacco use and cocaine use. Exam revealed dry gangrene of the left thumb, and digital ulcers on the left index finger, right fourth digit and fifth digit. Laboratory studies revealed a positive ANA and P-ANCA. Drug screen was positive for cocaine. MRI of her hand showed acroosteolysis with soft tissue swelling, necrosis, and diffuse swelling of the first digit with flexion contracture. LUE arteriogram was performed which showed digital corkscrewing consistent with thromboangiitis obliterans. She underwent amputation of her left first digit, was started on amlodipine, and underwent counseling to avoid cocaine and nicotine containing products. At one month follow up she described no new symptoms

IMPACT/DISCUSSION: We present a case which illustrates the classic presentation of thromboangiitis obliterans including digit ulceration, smoking history and characteristic imaging findings. It is important to consider this complication of smoking in patients presenting with such findings. While initial presentation for distal necrosis can be broad and can include different types of vasculitis or infection, using different modes of diagnosis can be helpful. Our patient had a positive ANA which can be present in multiple rheumatologic conditions, but given her smoking history and other diagnostic modalities such as the arteriogram the diagnosis of thromboangiitis obliterans was more likely. Thromboangiitis obliterans (also known as Buerger's disease) is a segmental inflammatory disease that is usually seen in small to medium sized arteries and veins. In this condition there is inflammatory intraluminal thrombus formation with sparing of the vessel wall. It is classically seen in patients who use tobacco products. Patients may present with dry gangrene and digit ulceration as seen in our case. Other presentations may include claudication or Raynaud's phenomena. Diagnosis is difficult and often requires evaluation for and exclusion of other etiologies such as connective tissue disease or embolus. Certain angiogram patterns are diagnostic of thromboangiitis obliterans including a 'corkscrew' appearance of arteries and 'tree root' appearance from collateral vessels

CONCLUSION: Thromboangiitis obliterans is an inflammatory vessel disease that affects patients with a history of tobacco abuse. In this case, the tobacco history combined with digit ulceration and classic imaging findings aided the diagnosis of thromboangiitis obliterans.

\section{DYSKERATOSIS CONGENITA MANIFESTING AS PULMO- NARY FIBROSIS: AN UNUSUAL PRESENTATION}

Sehar Zulfiqar; Joshua Allgaier; Osama Kandalaft. Baystate Medical Center, Springfield, MA. (Control ID \#3184535)

LEARNING OBJECTIVE \#1: Describe the presentation and clinical course of isolated pulmonary fibrosis as a manifestation of Dyskeratosis Congenita.
CASE: We present a case of a 45-year-old female from Puerto Rico who presented to the pulmonology clinic for a 3-year history of chronic cough. She had a prior diagnosis of Dyskeratosis Congenita (DC) and her sister had recently passed away from pulmonary complications related to DC. A CT scan revealed subpleural septal thickening and honeycombing with mild traction bronchiectasis. A Video-assisted thoracoscopic surgery (VATS) biopsy revealed patchy interstitial fibrosis with mild to moderate chronic inflammation suggestive of usual interstitial pneumonia. Over the next few months, she had an extensive workup, yielding a positive ANA with speckled pattern, anti-Ro antibodies, and MI-2 on a myositis panel. Her pulmonary disorder was attributed to antisynthetase-related interstitial lung disease and she was started on daily prednisone. While her symptoms improved, her PFTs showed a rapid decline with FVC decreasing from $2.30 \mathrm{~L}$ ( $64 \%$ predicted) to $1.85 \mathrm{~L}$ ( $47 \%$ predicted) over seven months. Further workup was conducted with a bone marrow biopsy negative for major abnormalities and telomere length testing significant for short telomere length in lymphocytes and granulocytes indicating a primary telomere disorder. The conclusion was made that she had progressive pulmonary fibrosis due to $\mathrm{DC}$, though without bone marrow involvement. She was evaluated for lung transplant, but unfortunately passed away prior to this occurring.

IMPACT/DISCUSSION: DC is a genetic multisystem disorder characterized by short telomeres for age with a highly variable phenotype defined by a triad of nail dysplasia, mucosal leukoplakia and patchy skin hyperpigmentation. A recent review of data from the DC registry demonstrated that idiopathic pulmonary fibrosis develops in about $20 \%$ of the patients with $D C^{1}$ and is characterized by cough, dyspnea, impaired gas exchange, and reduced lung volume as demonstrated by a reduced DLCO and/or restrictive pattern. The onset and progression of the manifestations of DC vary, with bone marrow failure thought to precede the development of pulmonary fibrosis. Pulmonary complications are the second most common cause of mortality in patients with DC after aplastic anemia but idiopathic pulmonary fibrosis in the absence of bone marrow failure is rare which makes this a unique but lethal presentation.

CONCLUSION: It is important to recognize the protean manifestations of Dyskeratosis Congenita and that Pulmonary fibrosis although an uncommon entity, can still occur and is life threatening. Early recognition is important as these individuals will require a comprehensive healthcare program including smoking abstinence/cessation, monitoring of disease progression with regular PFTs and early evaluation for lung transplantation as no other therapy has shown to be of benefit.

\section{DYSPEPSIA ON DOAC? THINK DILI!}

Rachel Solomon; Laurie Edelman; Aparna Sarin. Mount Sinai Medical Center, New York, NY. (Control ID \#3184611)

LEARNING OBJECTIVE \#1: Recognize that direct oral anticoagulants (DOAC) confer risk of liver injury.

LEARNING OBJECTIVE \#2: Identify patients who may benefit from hepatic monitoring on DOAC therapy.

CASE: A 68 year-old female with hyperlipidemia on statin was hospitalized and started on rivaroxaban for bilateral, submassive pulmonary emboli. Two weeks post-discharge she e-mailed her PCP complaining of two days of intermittent abdominal pain, nausea, and vomiting after eating chicken salad. Symptoms were improving and were attributed to gastroenteritis. Several days later, she reported resolution of nausea and vomiting, but persistent burning pain, waxing and waning, with occasional radiation to back. The symptoms felt similar to her prior dyspepsia; seltzer helped. An $\mathrm{H} 2$ blocker was started. A week later, she called with recurrent vomiting for one day, abdominal discomfort unchanged on ranitidine, and nausea worsened after taking rivaroxaban each day. In clinic, exam was notable for epigastric tenderness. Labs revealed significant transaminitis: ALT 609, AST 332, Alkaline Phosphatase 575. Liver sonogram and hepatitis serologies were unremarkable. Rivaroxaban was 
stopped immediately. The patient was transitioned to LMWH and warfarin. Within a month, labs normalized and symptoms resolved.

IMPACT/DISCUSSION: As DOACs gain traction, providers must be aware of adverse effects, including liver injury, increasingly described in the postmarketing literature. DOACs, used for treatment and prevention of venous thromboembolism and stroke prevention in non-valvular atrial fibrillation, carry similar overall bleeding risk to vitamin $\mathrm{k}$ antagonists, but offer advantages like fixed dosing, and freedom from laboratory monitoring and dietary restrictions. While early studies focused mainly on bleeding outcomes, rarer side effects - like drug-induced liver injury (DILI) have since been supported by pharmacovigilance and case reports and statistical analyses. Risk factors may include underlying liver disease and concomitant use of other potential hepatotoxins (e.g. alcohol, statins). Rivaroxaban seems to carry the highest risk, though no clear mechanism has been described. Manifestations of DILI related to DOACs range from asymptomatic transaminitis to liver failure. Symptoms like those seen in our patient - nausea, anorexia, abdominal pain - should prompt immediate evaluation. Early identification and withdrawal of the offending drug may prevent progression to cirrhosis or liver failure and is generally followed by recovery and normalization of transaminases.

CONCLUSION: Evolving evidence of DOAC-related DILIs should prompt caution in prescribing and management. Patients with liver disease or who consume other potential hepatotoxins may be at higher risk. Given risk of asymptomatic presentation, monitoring liver function in all patients initiated on DOAC therapy may be warranted.

\section{DYSPHAGIA LUSORIA: A RARE PATHOLOGY FOR A COM- MON AND CONCERNING PRESENTATION}

Megan Kosirog ${ }^{2}$; Farhad Ghamsari ${ }^{1}$; Victoria Behrend ${ }^{2}$; Kelley Wachsberg ${ }^{2} .{ }^{1}$ Feinberg School of Medicine, Chicago, IL; ${ }^{2}$ Northwestern University, Chicago, IL. (Control ID \#3186109)

LEARNING OBJECTIVE \#1: Recognize this congenital anomaly as a potential cause of dysphagia in an otherwise nonspecific presentation.

CASE: A 69 year-old man with controlled HIV, COPD and significant smoking history presented to the ED complaining of sore throat and solidfood dysphagia without discrete odynophagia, along with a 30-pound weight loss over 3 months. Physical exam, routine labs, and CXR were unrevealing. Last CD4 count 1 month prior was 964. Given the patient's risk factors and presentation, diagnostics focused around esophageal pathologies. An initial barium swallow demonstrated esophageal dysmotility approximately one-third of the way down the esophagus. No discrete ulcers or strictures were visualized. A subsequent esophagogastroduodenoscopy (EGD) corroborated the existence of a pulsatile vessel along the posterior esophageal wall resulting in mild esophageal compression without significant luminal narrowing. EGD was otherwise unremarkable. A CT chest was obtained to evaluate for extrinsic mass effect which revealed an aberrant right subclavian vessel coursing posterior to the esophagus. A Videofluoroscopic Swallow Study was conducted to further evaluate the degree of esophageal narrowing and functional effect on swallowing, both of which were reported as minimal. A conservative management approach was advised with the patient instructed to chew well, eat slower and utilize smaller bites. Nutritional supplements were also begun. At 1 month follow up the patient reported resolution of symptoms and a 5 pound weight gain was noted.

IMPACT/DISCUSSION: An aberrant right subclavian artery, also known as arteria lusoria, is a congenital abnormality of the aortic arch wherein the right subclavian artery originates distal to the left subclavian artery. Arising from the distal aortic arch, the aberrant subclavian typically passes behind the esophagus and back across the midline to perfuse the right arm. General population prevalence is between $0.5-1.8 \%$ with 70 $80 \%$ of patients being asymptomatic. When symptomatic, $91 \%$ experience dysphagia; termed dysphagia lusoria. Cough, chest pain, and Horner's syndrome have also been reported. While the exact mechanism of associated dysphagia is unknown, direct esophageal compression and/ or motility changes are suspected. Dysphagia lusoria is most often diagnosed via barium swallow study showing a diagonal defect between the third and fourth vertebrae, followed by CT scan and upper endoscopy to help both confirm diagnosis and exclude alternate pathology.

CONCLUSION: This case illustrates a rare congenital anomaly that is typically only discovered incidentally, but when symptomatic, most often presents with dysphagia. Recognition of this pathology and screening for extrinsic mass effect is important given the non-specific presentation. Further, endoscopy is generally insensitive and can result in diagnostic delay. If symptoms fail to respond to conservative management, treatment of an aberrant right subclavian artery typically involves correction with vascular surgery.

\section{DYSPNEA IN DISGUISE: A PANCREATIC DILEMMA}

Pooja Magavi; Emily Kim; Duc T. Do. Scripps Clinic/Scripps Green Hospital, La Jolla, CA. (Control ID \#3186103)

LEARNING OBJECTIVE \#1: Identify risk factors for pancreatic fistulas and the basic underlying pathophysiology.

LEARNING OBJECTIVE \#2: Recognize the general clinical features of pancreatic fistulas.

CASE: A 72-year-old female with a history of alcoholic pancreatitis and recent perforated diverticulitis requiring emergent sigmoid colectomy with colostomy presented to urgent care with progressive shortness of breath and associated lower extremity swelling. She was initially afebrile with a blood pressure of $91 / 51$, heart rate of 108 , respiratory rate of 20 and oxygen saturation of $91 \%$ on room air. She was ill appearing with increased work of breathing, crackles throughout her lung fields and pitting edema up to her shins bilaterally. Chest X-ray showed a moderate right-sided pleural effusion. Lower extremity Doppler ultrasound showed acute proximal deep vein thromboses bilaterally. She was admitted to the ICU and started on a heparin drip for presumed pulmonary embolism. Thoracentesis revealed an exudative effusion with a significantly elevated amylase level. A chest tube was placed for continuous drainage and octreotide was started. ERCP identified a pancreatic-pleural fistula. The patient's pancreatic duct was stented. Her chest tube output gradually decreased and further imaging showed stabilization of the pleural fluid, allowing discontinuation of octreotide and removal of the chest tube. She was ultimately discharged on day 27 of her hospitalization.

IMPACT/DISCUSSION: Pancreatic fistulas can develop when the pancreatic duct is disrupted because of pancreatic disease, surgery or trauma, with subsequent leakage of secretions into the adjacent space. The pancreatic duct may communicate with the pleural or peritoneal cavity or other viscera, forming an internal fistula, or with the skin, forming an external fistula. Internal pancreatic fistulas are most commonly associated with chronic alcohol-induced pancreatitis, as in our patient. Pancreaticpleural fistulas are rare, with an estimated incidence of $0.4 \%$. This case illustrates the potential complexity of an uncommon condition that can present in a routine fashion. The diagnosis can be made based on evidence of pancreatic duct disruption on MRCP/ERCP, with high levels of amylase in the extravasated pancreatic fluid. Management involves supportive care and endoscopic therapy, if needed. Pancreatic fistulas can lead to sepsis, malnutrition and other complications, such as skin excoriation. Our case serves as a reminder to constantly reassess care plans to address changes in an individual's clinical picture and to include pancreatic fistula in the differential diagnosis for patients with prior pancreatic injury.

CONCLUSION: A pancreatic-pleural fistula may initially manifest as a pleural effusion on chest X-ray. The differential diagnosis for any patient with a history of pancreatic injury who presents with new or unusual symptoms and fluid collections or drainage should include pancreatic fistula.

\section{EBV IN AIDS: WHEN THE LAMB BECOMES THE LION}

Alexandra J. Chastant; Phillip S. Bell; Shanti Rao. Tulane University School of Medicine, New Orleans, LA. (Control ID \#3184422) 
LEARNING OBJECTIVE \#1: Recognize the risk of lymphoma in HIV infected patients.

LEARNING OBJECTIVE \#2: Utilize EBV DNA quantification in the workup of localized lymphadenopathy in the AIDS population.

CASE: A 44-year-old Caucasian man with HIV presented with new severe upper back pain. Six weeks prior to presentation, he visited an urgent care for a right submandibular mass. He was given one week of prednisone and Augmentin without resolution of the mass. Three weeks prior to presentation, he noticed progressively enlarging masses on his neck, scalp, axilla, back and groin. He endorsed a nonproductive cough, headache, night sweats and $6 \mathrm{lb}$ weight loss. He denied any fevers, sick contacts, or substance abuse. He was diagnosed with HIV 24 years ago but had not been on ART for two years. He was afebrile on presentation. Physical exam was remarkable for diffuse, palpable, non-tender lymphadenopathy. Labs on presentation were remarkable for a CD4 count of 41 . Imaging revealed numerous soft tissue nodules throughout the chest, abdomen and pelvis measuring up to $7.7 \times 4.5 \mathrm{~cm}$, as well as multiple pleural lesions. ID was consulted and ART was initiated. Excisional lymph node biopsy was performed. Further workup revealed an EBV DNA: 78,000 copies/mL. Pathology resulted as diffuse large B-cell lymphoma, activated B-cell type. Oncology was consulted and chemotherapy was started with DA-EPOCH and intrathecal methotrexate.

IMPACT/DISCUSSION: Internists frequently encounter HIV-infected patients. Given that $25-40 \%$ of HIV-positive patients will develop a malignancy in their lifetime, a heightened suspicion for malignancy is critical. Compared to the general population, HIV infection significantly increases the risk for non-Hodgkin's Lymphoma (NHL). This increased risk becomes striking in the AIDS population where highly aggressive NHL accounts for the vast majority of lymphomas. The relative risk is 400 -fold higher compared to the general population. Unexplained localized lymphadenopathy can be reasonably managed with one month follow up in the immunocompetent patient. This same presentation in the AIDS patient, however, requires strong consideration of lymphoma given the propensity for highly aggressive malignancy. No guideline exists for how to manage this clinical scenario. Evaluation may be limited as further imaging or excisional biopsy can be costly and difficult to arrange in a timely fashion. EBV DNA quantification is routinely performed in certain post-transplant patients to monitor for PTLD; additional studies have shown a significant association with EBV and HIVassociated lymphomas. Further, studies show that EBV DNA from plasma correlates with active EBV disease.

CONCLUSION: Given the risk for NHL in HIV-infected patients, EBV DNA PCR may be a critical diagnostic tool in the evaluation of unexplained localized lymphadenopathy in AIDS and HIV.

\section{ECULIZUMAB: A LIFE SAVER IN POSTPARTUM ATYPICAL} HEMOLYTIC UREMIC SYNDROME

Mais Abdou $^{1}$; Muath Alsharif ${ }^{4}$; Sameer Prakash $^{2}$; Tarek Naguib ${ }^{3}$; Manish Patel ${ }^{1}$. ${ }^{1}$ TTUHSC, Amarillo, Amarillo, TX; ${ }^{2}$ Texas Tech University Health Sciences Center Amarillo, Amarillo, TX; ${ }^{3}$ Texas Tech University Health Sciences Center - Amarillo, Amarillo, TX; ${ }^{4}$ Texas Tech University Health Science Center, Amarillo, TX. (Control ID \#3184566)

LEARNING OBJECTIVE \#1: Recognize postpartum atypical hemolytic uremic syndrome (P-aHUS) as a rare but potentially fatal disease. Consider Eculizumab for P-aHUS treatment.

CASE: A 20 year-old 3rd week postpartum female with uncomplicated pregnancy presented with nausea, vomiting and few episodes of watery diarrhea since delivery. Vitals were normal. Physical exam was notable for trace pitting edema in the lower extremities. Laboratory studies showed $\mathrm{Hb}$ 8.5 , platelets 137, creatinine $21.8 \mathrm{mg} / \mathrm{dl}$ (normal baseline), BUN $128 \mathrm{mg} / \mathrm{dl}$, and albumin of $3 \mathrm{mg} / \mathrm{dl}$. Urinalysis showed $2+$ protein and $3+$ blood. LDH was $916 \mathrm{U} / \mathrm{L}$. Haptoglobin and fibrinogen were low with elevated D-dimer. Next day, the patient developed severe pulmonary edema. She was transferred to ICU and intubated for 9 days. Acute glomerulopathy was suspected and she was started on hemodialysis. Peripheral blood smear and kidney biopsy showed evidence of thrombotic microangiopathy. Complement C3 level was low and ADAMTS13 activity was 65\%. Atypical hemolytic uremic syndrome (aHUS) was diagnosed. Complement gene panel showed complement factor $\mathrm{H}(\mathrm{CFH})$ gene and plasminogen (BLG) gene mutations. Daily plasmapheresis was initiated with hematological improvement and normalization of platelet counts. However, this was not matched by concomitant renal improvement. After discussion with the patient about the limited but promising data of Eculizumab, she agreed to proceed. She was started on $900 \mathrm{mg}$ IV weekly for 4 weeks followed by $1200 \mathrm{mg}$ every second week thereafter. She significantly recovered and became dialysis independent within few weeks.

IMPACT/DISCUSSION: P-aHUS remains an under-recognized disease with life-threatening complications. It is caused by dysregulation of the alternative complement pathway due to gene mutations. It offers particular challenges in postpartum period given the broad differential. Many other conditions manifest as thrombotic microangiopathy causing delay in initiating appropriate treatment. Plasma exchange $(\mathrm{PE})$ was the treatment of choice. However, with increased understanding of the complement pathway activation role, the monoclonal antibody $\mathrm{C} 5$-inhibitor Eculizumab is being used recently. It showed considerable success and improved outcomes compared to PE. In our case, Eculizumab was highly effective and well tolerated. It resulted in remission and full recovery of kidney function.

CONCLUSION: Postpartum atypical hemolytic uremic syndrome (PaHUS) is a rare but potentially fatal disease that physicians should be aware of in the setting of acute kidney injury in postpartum period. Recently, Eculizumab is relatively new but promising treatment in PaHUS. Early diagnosis and treatment can be lifesaving.

\section{ECZEMA HERPETICUM: A RARE MEDICAL EMERGENCY}

Leena Pathikonda; Preetivi Ellis. Methodist Dallas Medical Center, Dallas, TX. (Control ID \#3159470)

LEARNING OBJECTIVE \#1: Recognizing eczema herpticum so treatment with Acyclovir will be started quickly to prevent morbidity and mortality

CASE: A 23-year-old woman with a history of eczema and asthma presented to the emergency department with fever and painful rash on her right chest that began three days prior to hospitalization. The day prior to the rash she spilled jalapeno juice on her chest and reported having diarrhea she attributed to food poisoning. A physical exam revealed a vesiculopustular rash on an erythematous base with draining serous fluid on the chest and extending down the right arm and abdomen. The rash did not follow a dermatomal pattern. A clinical diagnosis of irritant contact dermatitis with a superimposed bacterial infection was made. She was empirically treated with intravenous vancomycin and cefepime. Despite antibiotics, her condition worsened and the rash spread to her lips and eyelids. Due to rapid skin sloughing and mucosal involvement, there was concern for Stephen Johnson syndrome, toxic epidermal necrolysis, or necrotizing fasciitis. Dermatology was consulted on day two of hospitalization. The diagnosis of eczema herpeticum was made clinically based on widespread vesicles and her history of eczema. A Tzanck smear, varicella zoster PCR, herpes simplex PCR, and biopsies were obtained. While results were pending, intravenous acyclovir was initiated. The patient clinically improved after only twelve hours on appropriate therapy. After two day on intravenous acyclovir, she had rapid improvement of her skin lesions. HSV-1 PCR came back positive, and the pathology showed extensive ulceration and underlying acute and chronic inflammation with focal smudgy nuclear inclusions in ulcer base with associated immunoreactivity for HSV. This was consistent with a diagnosis of eczema herpeticum. She was discharged on oral acyclovir. 
IMPACT/DISCUSSION: Eczema herpeticum, also known as Kaposi varicelliform eruption, is a generalized vesiculopustular rash caused by herpes simplex virus and is usually diagnosed in children. The clinical presentation can mimic other diseases making eczema herpeticum a difficult diagnosis, but an important one, because of the high morbidity and mortality without treatment.

CONCLUSION: Eczema herpeticum presents with symptoms similar to other pathologies, and usually presents in children, it is rarely diagnosed in adults. It is even more difficult to distinguish in patients with poorly controlled eczema as in our patient. The delay in treatment can be life threatening, since the virus spreads quickly. This case illustrates the importance of quickly recognizing the presentation of eczema herpiticum to prevent delays in treatment with acyclovir.

\section{ELEVATED VITAMIN B12 IN A CASE OF PERNICIOUS ANEMIA}

Marcella Tschautscher; Ryan Giddings-Connolly. Mayo Clinic, Rochester, MN. (Control ID \#3164820)

LEARNING OBJECTIVE \#1: Diagnose pernicious anemia as a cause of macrocytic anemia in an adult with elevated vitamin B12 levels

LEARNING OBJECTIVE \#2: Recognize that anti-intrinsic factor antibodies can lead to false elevations in serum vitamin B12 levels

CASE: A 59 year old woman with a history of chronic venous insufficiency, hypothyroidism, attention deficit hyperactivity disorder, nicotine use disorder, and traumatic brain injury presented to her outpatient visit for evaluation of a new, right>left lower extremity lace-like, non-pruritic rash and paresthesias. She denied new or worsening lower extremity pain, fevers, fatigue, urinary or abdominal symptoms. Medications included dextromethorphan and levothyroxine. Physical exam was notable for livedo reticularis involving the lower extremities that was blanchable and improved with elevation. Lab work consisting of complete blood counts, blood smear, coagulation studies, ferritin, liver function tests, creatinine, creatine kinase, thyroid function studies, vitamin B12, ANA, and ANCA was notable for hemoglobin $6.9 \mathrm{~g} / \mathrm{dL}, \mathrm{MCV}$ 124.7 FL, macrocytic red blood cells platelet count $114 \times 10^{9} / \mathrm{L}$, and vitamin B12>1,400 ng/dL. A methylmalonic acid (MMA) level was obtained and returned elevated at $50 \mathrm{~nm} / \mathrm{mL}(\mathrm{ref}<0.4 \mathrm{~nm} / \mathrm{mL})$ consistent with vitamin B12 deficiency. She was started on intramuscular vitamin B12 $1000 \mathrm{mcg} / \mathrm{mL}$ monthly. Three months into treatment additional blood work was performed and notable of a positive for anti-intrinsic factor antibody and anti-parietal cell antibody $(59.6 \mathrm{U}$ (ref:<20 U)). Her hemoglobin had improved to $12.1 \mathrm{~g} / \mathrm{dL}$ with resolution of livedo reticularis.

IMPACT/DISCUSSION: Although this patient demonstrated vitamin B12 deficiency as demonstrated by elevation of her MMA level, it is intriguing that her vitamin B12 level was not only elevated, but above the upper limit of normal. Standard practice sways us to rely on the competitive-binding luminescence assay (CBLA) for quantitative cobalamin measurements. This specific assay has been shown to report false normal levels of vitamin B12 in a state of deficiency, and more specifically-in pernicious anemia. This inaccuracy is due to the assay's inability to inactivate the anti-intrinsic factor antibody which can pose as cobalamin. This finding was studied from stored serum in patients with B12 deficiency with all false normal cobalamin levels only occurring among those who tested positive for anti-intrinsic factor antibody. This case highlights the importance of measuring an MMA level when evaluating for vitamin B12 deficiency and the limitations in using the CBLA in quantifying cobalamin levels especially in the setting of pernicious anemia.

CONCLUSION: The MMA level remains essential in evaluating for vitamin B12 deficiency and should be used in routine clinical practice. The CBLA has its limitations in quantifying cobalamin levels and should be recognized for its capability of reporting false normal values in the setting of pernicious anemia.
ELSBERG SYNDROME: AN UNDER-RECOGNIZED CAUSE OF CAUDA EQUINA SYNDROME

Joel Burnett ${ }^{1}$; Christopher P. Terndrup ${ }^{2}$. ${ }^{1}$ Oregon Health \& Science University, Portland, OR; ${ }^{2}$ Oregon Health and Sciences University, Portland, OR. (Control ID \#3186102)

LEARNING OBJECTIVE \#1: Identify compressive and noncompressive causes of cauda equina syndrome.

LEARNING OBJECTIVE \#2: Recognize Elsberg syndrome - an atypical presentation of herpes genitalis.

CASE: A 62-year-old woman with chronic left lower extremity radiculopathy, chronic kidney disease, hypertension, and remote invasive ductal carcinoma in remission presented to urgent care with one week of chills, vaginal pain, dysuria, urinary retention, and constipation. She had a new sexual partner of 4 months. They did not use condoms. On exam, vitals were within normal limits. Genitourinary exam revealed vaginal erythema and multiple, bilateral ulcerative lesions of the labia. Urinalysis was unremarkable. Tests for Neisseria gonorrhea and Chlamydia trachomatis were negative. HSV culture of a genital lesion was positive. She was prescribed acyclovir and advised to follow up in one week. A week later she represented with improvement in the genital lesions and new complaints of perineal numbness and fecal and urinary incontinence. A detailed neurologic exam was not documented. CT abdomen and pelvis with contrast revealed anterolisthesis of L3-L4 causing narrowing of the thecal sac. She was referred to neurosurgery but failed to schedule an appointment. After 6 weeks, she presented to her primary care physician (PCP), reporting persistent but improved fecal incontinence and perineal paresthesia. Exam was notable for normal gait, $5 / 5$ lower extremity strength, decreased perineal sensation, and flaccid rectal tone. She was sent to the emergency department for urgent MRI and surgical evaluation. MRI spine without contrast redemonstrated L3-L4 anterolisthesis with moderate canal stenosis. She was evaluated by orthopedic surgeons who concluded that "her degree of stenosis [did] not explain her incontinence symptoms. Other causes should be investigated" thus she was discharged. After 3 weeks, she saw her PCP and reported continued improvement in fecal incontinence and paresthesia. After careful review of primary data and consideration of etiologies of CES, she was diagnosed with Elsberg syndrome - a self-limited cause of CES due to genital HSV infection - and was continued on acyclovir.

IMPACT/DISCUSSION: In this case, a woman with chronic spinal stenosis developed subacute CES after acute HSV infection, underwent spinal imaging and surgical evaluation, and was judged to have insufficient spinal stenosis to account for CES. Over time she experienced spontaneous symptom improvement. This presentation is best explained by Elsberg syndrome, a manifestation of a primary infection or reactivation of HSV-2 consisting of acute/subacute sacral radiculitis. The syndrome presents with CES of acute/subacute onset and recent or coexisting HSV infection in the absence of alternative CES causes. The syndrome is likely under-recognized. Epidemiological data is scarce.

CONCLUSION: While managing patients with acute or chronic genital HSV, physicians should be aware that cauda equina syndrome can present as severe manifestation of HSV infection.

\section{EMOTIONAL STRESS INDUCED SPONTANEOUS CORONARY ARTERY DISSECTION}

Victoria Gonzalez ${ }^{1}$; Mulham Shikh Hamdon ${ }^{2}$; Vesna Tegeltija ${ }^{1} .{ }^{1}$ Wayne State University, Rochester Hills, MI; ${ }^{2}$ Wayne State University, Shelby TWP, MI. (Control ID \#3185229)

LEARNING OBJECTIVE \#1: Spontaneous coronary artery dissection (SCAD) is an important yet often forgotten cause of ACS, especially in women presenting with acute myocardial infarction. Frequently associated with varying precipitating risk factors, we are presenting a case in which intense emotional stress alone led to dissection in the absence of other identifiable underlying predisposing risk factors. 
CASE: A 47-year-old woman with history of asthma and eczema and no cardiovascular risk factors is admitted for acute STEMI. She reported no home medications including hormonal therapy and denies smoking or illicit drug use. The night prior to her arrival, she had an emotionally intense argument with her boyfriend which she described as very stressful. The following morning, she was awoken by a severe substernal chest pain which radiated to the left chest and had 2 episodes of non-bloody emesis. EKG showed ST elevation in V1, V2, and V3 with no reciprocal changes and echocardiogram showed EF 50\% with apical hypokinesis. Immediate angiography then revealed SCAD of the proximal left anterior descending artery with TIMI 3 flow of the coronary tree. There was complete resolution of ST-segment elevation without intervention. The patient was treated with dual antiplatelet therapy and discharged with follow-up CT-angiography in 3 months.

IMPACT/DISCUSSION: This case is a unique example of nonatherosclerotic SCAD presenting in a patient without predisposing risk factors, solely precipitated by emotional stress. Once believed to be a very rare condition, $\mathrm{SCAD}$, has become an important identifiable cause of MI in women ages 43-52. The underlying etiology of SCAD is multifactorial, yet, up to $20 \%$ of cases are considered idiopathic. Typically, patients have an identifiable predisposing arteriopathy; fibromuscular dysplasia (FMD) being the most common. Others include postpartum status, multiparity, connective tissue disorders, systemic inflammatory conditions, and hormonal therapy. Most patients do not have conventional risk factors for CAD but often do have specific precipitating stressors which compound the underlying arteriopathy. Physical activity, heavy lifting, severe coughing or vomiting can cause cardio-circulatory stress through increased intrathoracic pressures and potential SCAD. Intense emotional stress can manifest differently through catecholamines acting on myocardial contractility or vasospasm and increased arterial shear stress leading to dissection. Interestingly, the patient presented in this case had no such arteriopathies. She was a null-parous non-smoker with no cardiac risk factors or significant family history. She used no medications, including hormonal therapy and denied illicit drug use. This patient lacked any history or evidence of other contributing causes.

CONCLUSION: A very emotional argument was the only indefinable precipitating stressor leading to SCAD in an otherwise healthy woman with no distinguishable arteriopathy.

\section{EOSINOPHILIA IN PULMONARY ADENOCARCINOMA AS AN EXTREMELY RARE PARANEOPLASTIC MANIFESTATION}

Muath Alsharif; Mohammad Islam; Mais Abdou; Thien Vo. Texas Tech University Health Science Center, Amarillo, TX. (Control ID \#3184731)

LEARNING OBJECTIVE \#1: Recognize eosinophilia as a rare finding with solid tumors. Diagnose lung cancer when eosinophilia is the main paraneiplastic feature.

CASE: A 67-year-old female with history of severe chronic obstructive pulmonary disease (COPD) presented with generalized weakness, malaise and dizziness for 1 month. She denied any history of hemoptysis, anorexia or weight loss. On physical examination, vitals were normal and respiratory system was notable for diminished breath sounds bilaterally with no overt crackles or rhonchi. No clubbing, cyanosis, or organomegaly appreciated. Laboratory investigations revealed white cell count of 17,200/ $\mu \mathrm{L}$ with $34 \%$ eosinophils, hemoglobin $11.5 \mathrm{~g} / \mathrm{dl}$, blood urea nitrogen 33 $\mathrm{mg} / \mathrm{dl}$, creatinine $2 \mathrm{mg} / \mathrm{dl}$. Computed tomography of thorax revealed $2 \mathrm{~cm}$ nodular like density along with the medial aspect of the right upper lobe abutting the mediastinum with multiple enlarged mediastinal and axillary lymph nodes. Metastatic lung carcinoma and lymphoma with paraneoplastic eosinophilia were the initial differentials. Fine needle aspiration of axillary lymph node was inconclusive for malignancy. Later, Mediastinoscopy with excisional biopsy was done and showed poorly differentiated pulmonary adenocarcinoma with metastatic carcinoma to lymph nodes. Acid fast, fungal smear and culture were all negative. Her eosinophilia was thought to be secondary to the lung malignancy as no other overt cause was found including allergic, parasitic infection, vasculitis, drug reaction and hematological malignancy.

IMPACT/DISCUSSION: Paraneoplastic eosinophilia is an unual manifestation that usually remains asymptomatic but it can be life threatening because of the multi organ involvement. It is found in less than $1 \%$ of all cancer population. The pathogenesis of tumor-associated eosinophilia is not well understood. Interleukin-5 (IL-5) is a cytokine that has been implicated in the development of eosinophilia in humans. However, there is little data associating IL-5 production with eosinophilia in the presence of tumor. In some case series, it was reported that removal of primary cancer, if possible, or combination of steroid and hydroxyurea are currently available options to address paraneoplastic eosinophilia. CONCLUSION: Our case reminds physician about the rare association of eosinophilia and pulmonary adenocarcinoma; a relationship which is not frequently reported in medical literature. Early recognition may play a crucial role in management plan and treatment outcome.

\section{EOSINOPHILS AT WORK: CAVITARY LUNG LESIONS IN A YOUNG NURSE}

Kajali Mishra; Shivani Sharma; Sarah Gorgis; Marcus Zervos. Henry Ford

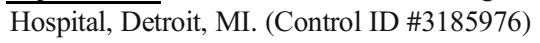

LEARNING OBJECTIVE \#1: Develop a differential diagnosis for cavitary lung lesions

LEARNING OBJECTIVE \#2: Diagnose and treat chronic eosinophilic pneumonia

CASE: A 35 year-old woman with recurrent sinusitis and development of asthma-like symptoms presented with two months of progressively worsening shortness of breath, wheezing and productive cough. She is an avid runner and noticed a significant decrease in her exercise capacity. She works as a CRNA in the bronchoscopy suite and her last PPD six months ago was negative. Vital signs were stable. Physical examination revealed bilateral wheezes. She had a leukocytosis of $13.3 \mathrm{~K} / \mathrm{uL}$ with eosinophilia (absolute eosinophils $2.30 \mathrm{~K} / \mathrm{uL}$ ). Computed tomography $(\mathrm{CT})$ thorax revealed pulmonary nodular densities with cavitations. A workup for infectious (tuberculosis, aspergillus, histoplasma, blastomycosis, coccidioides), vasculitis (pANCA, c-ANCA), and autoimmune (ANA, dsDNA, Anti-Smith, AntiRNP) disease was negative. Pulmonary function tests (PFTs) showed FEV1 of $103 \%$ with FEV1/FVC ratio of $73 \%$. The patient made symptomatic improvement on scheduled bronchodilators. Bronchoscopy revealed normal airways. Biopsy showed alveolar lung tissue with minimal inflammatory infiltrate, clusters of pigment-laden macrophages and minimally thickened alveolar septa. Bronchoalveolar lavage (BAL) was positive for $49 \%$ eosinophils and negative for infection. She was diagnosed with chronic eosinophilic pneumonia (CEP). The patient received inhaled corticosteroids and short acting beta agonists with symptomatic improvement. Repeat PFTs two weeks later showed improvement. CT thorax after 3 months showed improved ground glass opacities with stable pulmonary nodules. The patient endorsed complete resolution of symptoms at follow up and returned to her normal exercise capacity.

IMPACT/DISCUSSION: Chronic eosinophilic pneumonia is a rare disease which accounts for $2.5 \%$ of interstitial lung disease. It is an idiopathic disorder described by abnormal accumulation of eosinophils in the interstitium and alveolar spaces of the lung. Patients typically have a history of atopy, prolonged asthma-like symptoms, and exercise intolerance. IgE levels, peripheral eosinophilia, and elevation in inflammatory markers are nonspecific, but common. Bronchoalveolar lavage is essential to the diagnosis, as it can rule out infection and reveal alveolar eosinophilia. The BAL eosinophil count is almost always $>25 \%$, with more than $80 \%$ of patients having a BAL eosinophil count $>40 \%$. Glucocorticoids are the mainstay of management and patients often require long term systemic steroids. However, our patient made significant symptomatic improvement on inhaled steroids. 
CONCLUSION: CEP often presents with nonspecific respiratory symptoms which leads to delays in diagnosis. Early clinical suspicion upon recognition of marked peripheral eosinophilia can lead to timely imaging and procedures (BAL) that are required to make the diagnosis.

\section{EPIPERICARDIAL FAT NECROSIS- AN UNCOMMON CAUSE OF PLEURITIC CHEST PAIN}

Dimple Shah; Bijan Zisman. Donald and Barbara Zucker School of Medicine at Hofstra/Northwell, Manhasset, NY. (Control ID \#3184492)

LEARNING OBJECTIVE \#1: 1. Recognize epipericardial fat necrosis as a cause of acute onset chest pain.

CASE: A 59-year-old male with a medical history of Type 1 muscular dystrophy presented to the emergency room with acute onset of chest which began in the morning. He reports 10/10 left sided chest pain, sharp and pleuritic in nature. On admission, the patient's vital signs were within normal limits. His physical exam was significant for rhonchi in the left lower lobe, and upper and lower extremity weakness, with distal weakness greater than proximal. His EKG showed sinus rhythm with an incomplete right bundle branch block, unchanged from an EKG eight years prior. Cardiac enzymes, troponin T, CK and CKMB, were <.01, 30 and 1.0 respectively. His chest $\mathrm{x}$-ray revealed a left lower lobe opacity. Given an unrevealing EKG and negative cardiac enzymes, acute coronary syndrome was lower on the differential diagnosis, and patient underwent a CT angiogram of the chest. The CT showed fat stranding in the pericardial space concerning for epipericardial fat necrosis, and a small left sided pleural effusion; no pulmonary emboli were identified. The cardiothoracic surgery team was consulted for further evaluation of the fat necrosis, who advised to treat conservatively with NSAIDs. The patient's pain markedly improved on hospital day two and resolved by day three. His hospital course was complicated by aspiration pneumonia with development of a loculated pleural effusion requiring a video assisted thoracoscopy. A repeat CT scan of the chest four weeks later showed resolution of the epipericardial fat necrosis.

IMPACT/DISCUSSION: Epipericardial fat necrosis (EPFN) is a rare inflammatory disorder that causes acute onset pleuritic chest pain, lasting from hours to days. It can often be mistaken as a life-threatening disorder such as pulmonary embolism, pericarditis or myocardial infarction. Chest $\mathrm{X}$-rays can be clear or may demonstrate an opacity at the cardio-phrenic angle. A chest CT is the imaging modality of choice, which usually reveals a mass with fat attenuation, often encapsulated, adjacent to the pericardium with surrounding inflammatory changes. The pathophysiology is poorly understood, although it has been hypothesized it may be secondary to vascular pedicle torsion or pre-existing structural abnormalities. In the past, the diagnosis of EPFN required tissue diagnosis, but recent literature suggests a $\mathrm{CT}$ is enough to make the diagnosis. Although EPFN is an uncommon disease, it is especially relevant in the hospital setting where patients often present with acute chest pain. The treatment of EPFN is conservative and targeted towards pain control with NSAIDs. Resolution can be determined with a follow up CT scan.

CONCLUSION: EPFN in an uncommon cause of chest pain. It often presents as acute onset pleuritic chest pain, and should be included on the differential diagnosis of acute chest pain in patients who have been ruled out for life-threatening etiologies such as myocardial infarction or pulmonary embolism.

\section{ESOPHAGEAL NECROSIS: A RARE COMPLICATION OF DIA- BETIC KETOACIDOSIS}

Ricky A. Buckshaw $^{1}$; Ethan Stern ${ }^{1}$; Robert Andrews ${ }^{1}$; Frederic A. Stelzer ${ }^{1,2}$. ${ }^{1}$ Lehigh Valley Health Network, Allentown, PA; ${ }^{2}$ Eastern Penn Gastroenterology, Allentown, PA. (Control ID \#3155128)

LEARNING OBJECTIVE \#1: To be able to identify a high mortality complication of a fairly common medical problem.
CASE: A 50 year old male with past medical history of DM type I, gastroparesis, chronic pancreatitis, and Barrett's esophagus presented with non-radiating epigastric abdominal pain, coffee ground emesis, and nausea. He was vomiting frequently with severe pain for approximately two days. He denied any new medications, sick contacts, alcohol intake or intentional ingestions at the time. Arriving vitals were systolic BP 60 's 70 's, HR 98, GCS 12. Dried blood was present around the patient's mouth and tenderness in the epigastric area was present. He was given IV normal saline for volume resuscitation as well as emergent blood transfusions. He was started on empiric treatment for a GI bleed along with Zofran for persistent nausea. Initial lab work revealed hemoglobin 9.9, blood $\mathrm{pH}$ 7.34 , lactate 3.7 , glucose $>1000$, positive serum ketones, bicarbonate 14 , and anion gap 39. CT scan of his abdomen and pelvis revealed esophageal wall thickening, gastric distention with dilated fluid-filled esophagus, fatty liver, and chronic pancreatitis. He was later admitted to the MICU due to his critical clinical status. A diagnosis of diabetic ketoacidosis was made and DKA treatment protocol with insulin infusion, electrolyte replacement and frequent labs was started. Gastroenterology consult with EGD revealed the distal 2/3 of the esophagus having black, necrotic appearing mucosa with abrupt transition at the GE junction. No biopsies were taken due to the nature of the patient's presentation and risk of perforation. The patient was subsequently started on IV PPI and Carafate with gradual improvement of his symptoms.

IMPACT/DISCUSSION: Diabetic ketoacidosis (DKA) is a common complication of uncontrolled diabetes mellitus. Rarely, DKA can be complicated by acute esophageal necrosis (AEN) with the hallmark finding of circumferential black distal esophageal mucosa seen with endoscopy. Common presentation of AEN includes upper GI bleeding, nausea, vomiting, dysphagia, and syncope. Most commonly AEN is associated with hemodynamic compromise and low flow states, like what is encountered with the profound dehydration in patients with DKA. Those considered at greatest risk for developing AEN are men over age 50 with comorbid conditions such as DM, malignancy, hypertension, alcohol abuse, and CAD.

CONCLUSION: AEN is a rare complication of DKA and was discovered in this patient. Typical management involves fluid resuscitation, treatment of the underlying DKA, PPI, Carafate, and keeping the patient NPO. Although uncommon, AEN is an important condition to recognize as it is associated with mortality rates ranging from $15 \%-36 \%$. Also, $15 \%$ of survivors develop long term complications such as esophageal strictures or stenosis. Many of the symptoms of AEN are common presenting symptoms of DKA so clinical suspicion and evaluation in the correct context could prevent morbidity and mortality.

\section{EVEN THE ELDERLY CAN CONTRACT MENINGOCOCCAL} MENINGITIS - A CASE REPORT.

Yuriko Terada $^{1}$; Takafumi Kubota ${ }^{3}$; Suguru Matsuzaka ${ }^{4}$; Ivor Cammack ${ }^{2}$. ${ }^{1}$ Teine Keijinkai Hospital, Sapporo-shi, Japan; ${ }^{2}$ Keijinkai, Yoichi, Japan; ${ }^{3}$ Teine Keijinkai Hosipital, Sapporo, Japan; ${ }^{4}$ Teine Keijinkai Hospital, Sapporo, Japan. (Control ID \#3184849)

LEARNING OBJECTIVE \#1: Recognize that social activity in elderly individuals can put them at risk for contracting meningococcal meningitis. CASE: A 72-year-old female presented with a 2-day history of fever and sore throat. The fever got steadily worse, and she developed rigors. In the morning, she vomited so attended a nearby clinic, where her mental status deteriorated over 2 hours. She was transferred immediately to our hospital. Her past medical history was significant for hypertension, ischemic heart disease and osteoporosis. She was socially very active, participating in various activities. On examination, her temperature was 37.5 degrees Celsius, blood pressure 139/81, heart rate 96/min, respiratory rate 14-20/ min and oxygen saturations $96 \%$ on room air. Her Glasgow Coma Scale was E4V2M3. She had nuchal rigidity and a positive Kernig's sign. There was no rash. Blood tests showed white blood count $9860 / \mu \mathrm{L}, \mathrm{LDH}$ 
293U/L and C-Reactive protein $158.6 \mathrm{mg} / \mathrm{L}$. Cerebrospinal fluid (CF) gram stain showed gram-positive diplococci. CF microscopy showed white blood cells $483 / \mu \mathrm{L}$ (neutrophils $64 \%$ ), protein $463 \mathrm{mg} / \mathrm{dL}$, glucose $10 \mathrm{mg} / \mathrm{dL}$ and lactate $71 \mathrm{mg} / \mathrm{dL}$. Within an hour upon arrival, she was started on ceftriaxone $2 \mathrm{~g}$ intravenously (IV) every 12 hours, vancomycin $900 \mathrm{mg}$ IV every 10 hours, ampicillin $2 \mathrm{~g}$ IV every 4 hours and dexamethasone $8.25 \mathrm{mg}$ IV every 6 hours. On the second day, we changed to ceftriaxone treatment alone as her blood culture was positive for Neisseria meningitidis. 2 days after admission, her mental

status recovered. Although she had ongoing visual hallucinations at night, which led to insomnia. She also suffered cognitive dysfunction. She underwent intensive cognitive rehabilitation and her symptoms gradually resolved over 1-2 weeks.

IMPACT/DISCUSSION: In this case of a healthy 72-year-old female, it is uncommon to see sporadic meningococcal meningitis. Her only potential risk factor was her active social activities. She volunteered at nursing homes once a week, participated in an exercise program twice weekly in an elementary school and at our hospital and attended aqua jogging sessions. Crowding and close contact is common in these facilities, and it is therefore relevant to ask about social activities when seeing individuals suspected of meningococcal meningitis. Invasive meningococcal disease has a high mortality and adverse outcomes are common if treatment is delayed. Supporting the evidence that in community-acquired meningitis, there is a reduction in mortality and a benefit in terms of neurologic outcome with early administration of antimicrobial therapy, our case showed resolution of neurological symptoms after initiating antibiotic therapy within an hour of admission.

CONCLUSION: Social activities are risk factors for sporadic cases of meningococcal disease in elderly patients and immediate antibiotic therapy is vital to avoid neurological sequalae.

\section{EVEN WITH AN INTERPRETER, THINGS CAN GET LOST IN TRANSLATION}

Sean Burke; Indira Bhavsar. University of Virginia, Charlottesville, VA. (Control ID \#3183727)

LEARNING OBJECTIVE \#1: Understand the importance of cultural competency and health disparities in Latino populations

LEARNING OBJECTIVE \#2: Recognize the limitations of using a translator to conduct patient interviews

CASE: A 19-year-old male from Mexico with no past medical history presented to the hospital with abdominal pain. The interview was conducted by a telephone interpreter. He had two weeks of abdominal pain that was worse after eating and reported seeing a "sobador" who told him he had "empacho." His physical exam was notable for tenderness in the epigastrium and laboratory analysis was unremarkable. Imaging was pursued and revealed a jejunal filling defect concerning for a food bolus or other undifferentiated mass. He was admitted to the hospital. Small bowel follow through the next day was normal with passage of the mass. IMPACT/DISCUSSION: This case illustrates the challenges that arise when caring for non-English speaking patients despite the use of an interpreter. Notably, the patient recounted his recent interaction with a "sobador," a word that strictly translates to "masseuse" and his new diagnosis of "empacho" which had no English translation. Sobadores are folk healers who practice in Mexico and other Central American countries. They use manipulative therapies to treat common illnesses including musculoskeletal pain and gastrointestinal distress. The patient recounted his visit to a "sobador" and resulting diagnosis. Given the strict Spanish to English translation, the team understood that the patient visited a masseuse for his abdominal pain. Empacho is a traditional Latino illness well described in the literature. Characteristics include constipation, cramping, and vomiting. It is thought to be caused by dietary indiscretion. The most commonly agreed upon etiology is "blocked digestion" and many believe it is caused by a bolus of food that sticks to the wall of the intestine. Traditional treatment includes abdominal massage and herbal purgative teas. This case exemplifies multiple factors that can lead to disparities in health care. In retrospect, it is obvious that our patient had different cultural beliefs which he attempted to communicate during his initial interview. Given the translator's inability to provide correct translation and unawareness of folk illnesses, the team was unable to validate the patient's concerns. Factors such as these are routinely present during our interactions with minority populations further promoting disparities in the social determinants of health. Ongoing endeavors to foster cultural competence should be prioritized.

CONCLUSION: Latinos and other minority populations face multiple barriers leading to disparities in quality health care. Mistranslation can occur despite the use of certified interpreters. Empacho is a traditional Latino folk illness, often of organic etiology, that leads to abdominal pain, cramping, and emesis.

\section{EXPANDING YOUR DYSPNEA DIFFERENTIAL: A CASE OF AMIODARONE TOXICITY}

Tammy Tavdy; Dawn Zhao; Darlene LeFrancois. Montefiore Medical Center/Albert Einstein College of Medicine, Bronx, NY. (Control ID \#3185021)

LEARNING OBJECTIVE \#1: Recognize the pathophysiology of amiodarone toxicity, and the risk factors for its development

LEARNING OBJECTIVE \#2: Diagnose and manage amiodarone toxicity CASE: An 80-year-old woman with ischemic cardiomyopathy, mild COPD, and atrial fibrillation (on amiodarone $200 \mathrm{mg}$ for 14 months) presented with a two week history of progressive dyspnea and dry cough. One-month prior, the patient had been ambulating 5-6 blocks without difficulty. She is now unable to even ambulate within her one bedroom home. She did not have any sputum production, fevers, leg edema, or calf tenderness. She was afebrile, but hypoxic to $87 \%$ at rest. She had bibasilar rales with dullness to percussion. Lab results revealed no leukocytosis and a proBNP of 4580 (baseline). All microbiologic workup was negative. CXR revealed new bibasilar patchy opacities. In light of no clinical improvements with IV diuresis or antibiotics, a CT chest was performed, revealing hyperdense, ground-glass opacities of the lung and liver, findings highly specific for amiodarone toxicity. Amiodarone was stopped, and the patient was started on $60 \mathrm{mg}$ oral prednisone daily, low flow oxygen, and trimethoprim/sulfamethoxazole and alendronate prophylaxis. On follow-up within one month of discharge she improved - walking comfortably around her house without requiring supplemental oxygen.

IMPACT/DISCUSSION: Amiodarone is one of the most widely used antiarrhythmic medications in North America. The drug is an iodinecontaining compound that accumulates in several organs, most notably in the lungs and liver, leading to a direct toxic injury and an indirect immunologic reaction. Our case highlights that the presentation of amiodarone toxicity may be clinically indistinguishable from common pathologies of the lung or heart. The presentation is characterized by an insidious onset of non-productive cough with or without dyspnea, present in $50-75 \%$ of patients. Fever is also common, present in $20-30 \%$ of patients. CT imaging reveals bilateral ground glass opacities, which may also be seen in an acute CHF exacerbation or interstitial pneumonia. Age of patient, dose, and duration of amiodarone use are highly associated with the development of its toxicity. Incidence doubles to $\sim 5 \%$ when doses of $400 \mathrm{mg}$ or more are used daily. Patients over 60 years old, and those on amiodarone for 6-12 months have the highest risk of toxicity. In addition, an association between pre-existing lung disease and the development of amiodarone pulmonary toxicity has been reported. When amiodarone toxicity is diagnosed, the drug should be discontinued and steroids should be initiated. Treatment and recovery normally take months. In addition to clinical symptomatology, PFTs can be followed as a guide for recovery. In the setting of chronic steroid therapy, both antibiotic and bisphosphonate prophylaxis are appropriate therapies to begin. 
CONCLUSION: A high index of suspicion should be maintained when patients on amiodarone therapy present with new onset dyspnea, even when heart and lung disease may pre-exist.

\section{EXPECT THE UNEXPECTED: A RARE ETIOLOGY OF TRANS- VERSE MYELITIS}

Iman Afzal; Ivan Wong; Keerthana Keshava; Janaki Patel. New YorkPresbyterian Brooklyn Methodist Hospital, Brooklyn, NY. (Control ID \#3184761)

LEARNING OBJECTIVE \#1: Recognize the various underlying causes of Transverse Myelitis (TM).

LEARNING OBJECTIVE \#2: Diagnose and treat sarcoidosis with spinal cord involvement.

CASE: Our patient is a 53-year-old African American man who presented with worsening leg weakness, gait instability, and significant numbness radiating from his hips down to the toes and in his hands for 6 weeks. His physical examination was significant for decreased sensation in the hands, abdomen and lower extremities along with difficulty in tandem gait. Autoimmune work-up was negative, except for a mildly elevated ACE level. Magnetic resonance imaging of the brain and spine revealed severe spinal canal stenosis at C5-6 with loss of signal around the cord along with $\mathrm{T} 2$ hyperintensity in the spinal cord from C5-T1. A lumbar puncture was performed showing high protein, normal white blood cell count, normal cytology, and no oligoclonal bands. Given high suspicion for transverse myelitis he was given pulse dose steroids for five days. He had substantial improvement in his symptoms and was discharged home. At home, his symptoms of numbness and gait instability slowly worsened and he returned to the hospital. A computed tomography of the chest was done to further evaluate the elevated ACE level from prior admission and showed extensive mediastinal and bilateral hilar adenopathy. The patient was offered lymph node sampling via endobronchial ultrasound (EBUS) at a later date as he was simultaneously being evaluated by neurosurgery for his spinal cord stenosis. He subsequently underwent C5-6 anterior cervical discectomy and discharged home the next day. EBUS was performed with lymph node sampling taken from 4L, 7 and 11L. Histology was consistent with non-caseating granulomata supporting the diagnosis of sarcoidosis as the etiology behind the transverse myelitis. Given his persistent neurological symptoms despite surgery, he was started on Prednisone and showed excellent response to therapy.

IMPACT/DISCUSSION: Spinal cord involvement among patients with sarcoidosis is extremely rare at less than $1 \%$. Establishing the diagnosis of sarcoidosis causing TM can be difficult given the diverse manifestations of this disease process. Patients who present with signs of TM such as weakness, decreased sensory function, bowel or bladder dysfunction with no obvious underlying etiology should be evaluated for sarcoidosis. Diagnosis of spinal involvement of sarcoidosis includes MRI of the spine and sampling of the lymph nodes to confirm diagnosis. Initial treatment usually involves corticosteroids. Other treatments include other immunosuppressive agents such as azathioprine and infliximab.

CONCLUSION: Sarcoid is a rare but potential cause of TM. Establishing a diagnosis of spinal cord sarcoidosis is challenging in patients with no preceding diagnosis of Sarcoid. Evaluation of patients with no underlying explanation of TM and/or failure of initial treatment warrants further investigation for Sarcoid.

\section{EXPLAINING THAT DROP}

Pakhi Goel ${ }^{2}$; Vladyslav Dieiev²; Magued W. Haroun ${ }^{2}$; Karishma Kitchloo'. ${ }^{1}$ Montefiore Medical Center, Wakefield Campus, New York, NY; ${ }^{2}$ Montefiore Medical Center, Bronx, NY. (Control ID \#3186076)

LEARNING OBJECTIVE \#1: Diagnose beta blocker toxicity in patients using ophthalmic formulations.
LEARNING OBJECTIVE \#2: Recognize risk factors for beta blocker toxicity.

CASE: An 85 year-old man presented with a one-day history of profound weakness. He had a history of hypertension, chronic kidney disease (CKD), and cataracts. He did not report any other complaints, but reported being treated with repeated instillation of topical timolol into his right eye at the ophthalmologist's office earlier that day. His home medications included amlodipine and tamsulosin. His blood pressure was $70 / 50 \mathrm{mmHg}$ (baseline around 130/80). Electrocardiogram revealed sinus bradycardia with first-degree AV block. Labs were significant for potassium of 7.1 $\mathrm{mEq} / \mathrm{L}$. Later, his blood glucose dropped to $39 \mathrm{mg} / \mathrm{dL}$; he was treated with intravenous glucagon, with improvement in bradycardia, hypoglycemia, and hyperkalemia. Further cardiac workup did not reveal any structural heart abnormalities. Bradycardia resolved within 24 hours.

IMPACT/DISCUSSION: Beta blockers (BB) are commonly prescribed medications in the United States. Main indications include cardiovascular disease (oral forms) and intraocular hypertension (ophthalmic suspensions). Side effects of oral forms are common and well recognized, but clinically significant systemic side effects of ophthalmic suspensions may remain unrecognized. Some studies demonstrate increased risk of hospitalization for bradycardia one month after initiation of timolol eye drops. In our case, clinical presentation of acute weakness with simultaneous bradycardia and prompt response to glucagon is likely to be explained by systemic absorption of ophthalmic timolol. Underlying CKD and history of receiving timolol in high doses may have contributed to the toxicity. Approximately 80 percent of topically administered timolol is reported to drain through the nasolacrimal duct and is systemically absorbed. Although symptomatic bradycardia and hypoglycemia are well known side effects of topical timolol, hyperkalemia, which occurred in our patient, is uncommon. High dose intravenous glucagon and insulin therapy are recommended in cases of severe overdose, similar to the treatment for oral BB toxicity.

CONCLUSION: Systemic absorption of BB may lead to serious cardiovascular adverse events - symptomatic bradycardia, orthostatic hypotension, conduction disorders in the heart, syncope, and falls. Certain comorbidities (heart disease, COPD, cerebrovascular disease, CKD) may increase the risk of adverse reactions to ophthalmic BB.

\section{FACIAL TWITCHING UNMASKS UNDERLYING MYXEDEMA COMA}

Zachary S. Bugos; Fnu Abhishek. UPMC Mercy, Etna, PA. (Control ID \#3185837)

LEARNING OBJECTIVE \#1: Recognize subtle findings associated with myxedema coma

CASE: 29 year old woman with medical history of cerebral palsy, traumatic brain injury compounded by hypoxic brain injury secondary to polyethylene glycol poisoning, seizure disorder and hypothyroidism presented from long-term nursing facility for seizure activity presenting as "facial twitching." Emergency responders gave intravenous diazepam for witnessed repetitive facial twitching lasting 30 seconds per episode. Per report, her seizure disorder was well controlled on topiramate with last known seizure 4 years ago. On arrival to ED, she was hypothermic $\left(33.4^{\circ} \mathrm{C}\right.$ rectal), bradycardic (39-66 bpm) and hypotensive (SBP 70-90 $\mathrm{mmHg}$ ). Examination revealed an overweight, non-verbal patient with puffy facial features, non-palpable thyroid, cool skin, bilateral non-pitting lower extremity edema and only responsive to painful stimuli. Noncontrast head CT was unremarkable. Initially received IV antibiotics, fluid boluses with single pressor support and warming blanket for presumed septic shock. However, bloodwork significant for sodium $145 \mathrm{mmol} / \mathrm{L}$, creatinine $0.47 \mathrm{mg} / \mathrm{dL}$, ALT $202 \mathrm{IU} / \mathrm{L}$, AST $145 \mathrm{IU} / \mathrm{L}$, Alk phos $156 \mathrm{IU} /$ $\mathrm{L}$, albumin $3 \mathrm{~g} / \mathrm{dl}$, WBC count $4.3 \times 10^{9} / \mathrm{L}$, hemoglobin $11.6 \mathrm{~g} / \mathrm{dL}, \mathrm{MCV}$ $103.1 \mathrm{fL}$. TSH and AM cortisol were ordered due to suspicion for hypothyroidism vs adrenal insufficiency in setting of persistent shock requiring transfer to ICU. TSH was elevated to 11.1 and AM cortisol 
was $6.3 \mathrm{ug} / \mathrm{dL}$. Patient was administered $8 \mathrm{mg}$ IV dexamethasone and $200 \mathrm{mcg}$ IV levothyroxine with noted improvement in both vitals and mental status with cessation of seizures.

IMPACT/DISCUSSION: Myxedema coma is a rare, life-threatening, emergent presentation of hypothyroidism. It commonly presents in elderly females as depressed mental status along with hypothermia, hypotension, bradycardia and/or hypercapnia. Rarely, uncontrolled hypothyroidism can lower seizure threshold and contribute to onset of seizures as seen in this case. Laboratory indices that support the diagnosis, apart from elevated TSH with low free thyroid hormones, include hyponatremia, macrocytic anemia and elevated transaminases. Of note, the decreased AM cortisol level in our case was likely directly related to hypoalbuminemia, rather than adrenal insufficiency. Alteration in consciousness may be difficult to observe in patients with baseline impairment in cognition. However, this case elucidates the importance of recognizing and treating myxedema coma based on the characteristic presentation described above to avoid catastrophic and potentially fatal outcomes.

CONCLUSION: Myxedema coma is a rare, life-threatening, emergent presentation of hypothyroidism, making it important to recognize and begin treatment promptly to avoid sequela and death. Subtle clinical findings include hypothermia, hypotension, bradycardia and rarely, induction of seizure activity. Subtle lab findings suggestive of diagnosis include hyponatremia, elevated transaminases and macrocytosis

\section{FAILING TO DELIVER: IMPROVING TRANSITIONS OF CARE FOR PERIPART UM WOMEN WITH MEDICAL COMORBIDITIES}

Chelsea Wiltjer ${ }^{1}$; Meredith H. Lora ${ }^{2,}{ }^{1}$. ${ }^{1}$ Emory University, Atlanta, GA; ${ }^{2}$ Emory, Atlanta, GA. (Control ID \#3176067)

LEARNING OBJECTIVE \#1: Recognize the disparity which exists among African American women in the United States regarding postpartum mortality rates.

LEARNING OBJECTIVE \#2: Recommend a systems-based approach for addressing transitions of care between obstetrics and primary care to improve health care outcomes.

CASE: A 38-year-old African American female G1P0102 with past medical history of poorly-controlled hypertension, anxiety and chronic kidney disease underwent an emergent caesarian section to deliver identical twins due to pre-eclampsia. In the weeks following, while attending to her breastfed infants in the Neonatal Intensive care units, she continued to take her pre-partum antihypertensives. Six weeks after delivery, she was discharged from her obstetrician's office without adjustment of antihypertensives. Several days later, she was seen in urgent care for uncontrolled hypertension and her medications were adjusted. One week later, despite medication adherence and careful monitoring, she presented to the emergency department (ED) due to anxiety and uncontrolled hypertension. Her blood pressure reading upon arrival was 218/128 and she had evidence of a new acute kidney injury in addition to worsened proteinuria. She was admitted for intravenous anti-hypertensives. Over the next two months, she was hospitalized two additional times for uncontrolled hypertension.

IMPACT/DISCUSSION: This case highlights a woman who was actively engaged in her medical care, yet experienced a life-threatening complication of chronic hypertension due to a lack of primary care in the post-partum period. In recent years, high maternal morbidity and mortality rates within the United States have made national headlines. According to the Centers for Disease Control, black women within the U.S. have a three to four times higher maternal mortality rate, in comparison to their white counterparts. Cardiovascular and hypertensive disorders of pregnancy cause over one-fifth of these deaths. Management of chronic disease in the peripartum period can be challenging for new mothers and warrants close monitoring by a primary care provider. New mothers often struggle to navigate the system during this stressful period of their lives, and less than a third of women successfully establish with primary care in the year following childbirth. Small studies have demonstrated a significant improvement in establishment of primary care in postpartum women through a systems-based approach involving patient education and assistance. Improving this transition of care remains an important area of study to further determine how to best care for postpartum women with chronic medical disease.

CONCLUSION: - Among already high maternal mortality statistics affecting all women in the United States, a large disparity exists in the United States for African American Women. • Facilitating transitions of care between obstetrics and primary care through a systems-based approach remains an important step for providing quality care to those with complex medical disease.

\section{FALLING THROUGH THE CRACKS: A CASE OF SUBCLAVIAN STEAL SYNDROME}

Matthew J. Klebanoff ${ }^{2}$; Ashutosh Pathak ${ }^{2}$; John J. Chang ${ }^{4}$; Shaili Gupta ${ }^{1}$,

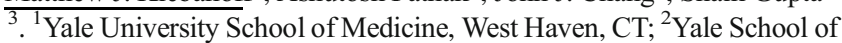
Medicine, New Haven, CT; ${ }^{3}$ Veterans Healthcare System of CT, West Haven, CT; ${ }^{4}$ Yale, West Haven, CT. (Control ID \#3160428)

LEARNING OBJECTIVE \#1: Recognize subclavian steal syndrome (SSS) as an uncommon but important cause of syncope.

LEARNING OBJECTIVE \#2: Identify signs of SSS that necessitate early surgical intervention.

CASE: A 63-year-old man with history of recurrent syncope and atrial fibrillation (AF) on warfarin presented to our hospital with headache. Within the last year, he had suffered several falls from syncope, which resulted in serious injuries, including jaw fracture and subdural hematomas (SDH). $\mathrm{He}$ used alcohol and marijuana daily. Prior workup for seizure activity, orthostatic hypotension, and vestibular dysfunction was negative; Holter monitor showed $0.4 \% \mathrm{AF}$ burden and rare supraventricular tachycardia. On our exam, the patient had regular heart rhythm, with no orthostatic changes or focal neurologic deficits. Laboratory findings were notable only for an INR of 4.9. CT head did not show any pathology. His medical record revealed a prior carotid ultrasound showing retrograde flow in the left vertebral artery, raising suspicion for SSS. The patient disclosed that minimal exertion (e.g., unloading a few items from the dishwasher) caused left arm pain and paresthesias, accompanied by headache and lightheadedness. He had interarm systolic blood pressure (BP) difference of $>40 \mathrm{mmHg}$. CTA neck confirmed total occlusion of the left proximal subclavian artery and patent bilateral internal carotid arteries. We sought revascularization, which was deferred in favor of further evaluation of arrhythmia and substance use as causes of syncope. One month later, the patient sustained more falls, leading to multiple vertebral fractures and SDH. An event monitor revealed many syncope events without arrhythmia. He later died in neurology ICU.

IMPACT/DISCUSSION: Our patient's history suggested multiple possible causes of syncope. Several prior workups failed to recognize SSS as a likely etiology. Our case underscores the importance of assessing each patient with fresh eyes and avoiding anchoring bias. It also highlights SSS as an uncommon but important cause of syncope. SSS results from steno-occlusion of proximal subclavian artery with subsequent retrograde flow in the ipsilateral vertebral artery. Radiological SSS is usually asymptomatic, but can present with dizziness, syncope, ataxia, and arm claudication. Symptomatic patients often have additional disease in anterior vasculature (e.g., carotid stenosis), causing inadequate collateral circulation. Severe symptoms can occur despite patent carotid arteries and should dictate the urgency of surgical intervention, which has a high success rate with low morbidity (AbuRahma et al. J Vasc Surg. 2000 Sep). Arm BP discordance $>40 \mathrm{mmHg}$ also correlates highly with the need for intervention (Labropoulos et al. Ann Surg. 2010 Jul).

CONCLUSION: In a patient with recurrent syncope, SSS should be included in the differential. Symptomatic SSS can occur despite patent carotid arteries. Clinicians should determine the urgency of revascularization in SSS based on clinical symptoms and signs, particularly arm BP discordance. 
FATAL IMMUNOSUPPRESSION: A CASE OF DISSEMINATED COCCIDIOIDOMYCOSIS PRESENTING AS HYDROCEPHALUS

Tyler J. Trahan; Diana Slawski; Ryan Eid; Patricia Greenstein; Margaret Hayes. Beth Israel Deaconess Medical Center, Boston, MA. (Control ID \#3176039)

LEARNING OBJECTIVE \#1: Distinguish between infectious and noninfectious etiologies of granulomatous lung disease.

LEARNING OBJECTIVE \#2: Describe the clinical features and diagnostic approach of disseminated fungal infection.

CASE: A 65-year-old man presented with pneumonia followed by three months of headaches after a brief stay in Arizona. Initial lumbar puncture revealed CSF with lymphocytic pleocytosis, increased protein, and low glucose. Cytology, infectious, and rheumatologic workup were unrevealing. Serum fungal cultures were negative. Serum Coccidioides IgG was negative. CSF Coccidioides tests were not sent. CT chest showed two pulmonary nodules. PET-CT revealed increased uptake in chest lymph nodes. Axillary lymph node biopsy showed noncaseating granuloma. Steroids were started for presumed sarcoidosis. After two weeks, patient developed diplopia and ataxia. CT head showed hydrocephalus. Repeat CT chest showed new reticulonodular opacities with hilar lymphadenopathy concerning for worsening sarcoid versus infection. He was transferred to a tertiary care facility for VP shunt placement and started on empiric antimicrobials. His course was complicated by worsening confusion and respiratory failure requiring intubation. Pathology from a transbronchial biopsy revealed fungal elements and he was immediately started on amphotericin and fluconazole; however he continued to deteriorate. After much discussion with the family, he was transitioned to comfort focused care, extubated, and died peacefully in the hospital. CSF and serum Coccidioides antibodies ultimately returned positive. Reevaluation of initial lymph node biopsy suggested necrotic granuloma.

IMPACT/DISCUSSION: The differential of granulomatous disease includes infectious and non-infectious etiologies. While sarcoidosis can cause granulomatous lung disease, it is a diagnosis of exclusion that relies on biopsy evidence of noncaseating granulomas and exclusion of infection. The most common organisms in pulmonary granulomas are mycobacteria and fungi. Coccioides is commonly found in the southwestern United States. Even brief exposures to endemic areas, such as changing airplanes in Phoenix or driving through the San Joaquin Valley, have been sufficient to cause infection. Thus, it is critical to obtain a detailed travel history. Serologic testing for Coccidioides should not be relied upon as it may be negative early in the course, and even persistently negative in immunocompromised patients. If meningitis is suspected in a patient with granulomatous lung disease, a lumbar puncture should be performed and CSF sent for fungal culture and antibody testing, as routine CSF and imaging abnormalities are nonspecific. The diagnosis of coccidioidal meningitis requires positive CSF antibodies, growth of the organism, or the presence of coccidioidal antigen.

CONCLUSION: Reevaluate infectious workup if a patient with granulomatous disease decompensates on immunosuppression. In suspected meningitis, test CSF for Coccidioides antigen/antibody, as serology alone may be unreliable.

\section{FEVER AND PULMONARY NODULES IN A RETURNING TRAVELER}

John K. Bui; Alice Tang. New York Presbyterian/Weill Cornell Medical Center, New York, NY. (Control ID \#3179254)

LEARNING OBJECTIVE \#1: Develop an approach to fever and respiratory symptoms in a returning traveler.

LEARNING OBJECTIVE \#2: Describe the diagnostic test characteristics for acute pulmonary histoplasmosis.
CASE: A previously healthy 20-year-old male recently returned from a 1week vacation to Cancun, Mexico. Two weeks after returning, he developed fevers, chills, headache, anorexia, myalgias, and productive cough. He felt well throughout his trip and denied raw food consumption, contact with animals, or freshwater exposures. He did not have any tuberculosis risk factors. The physical exam and laboratory results did reveal any abnormalities. A chest X-ray showed bibasilar opacities. CT of the head without contrast and LP were unremarkable. He was empirically treated for community acquired pneumonia but continued to develop fevers up to $40^{\circ} \mathrm{C}$ despite 2 days of antibiotics. A CT chest showed bilateral lung nodules and hilar adenopathy. Laboratory tests were unrevealing, including serum beta-D-glucan, Histoplasma urinary antigen, respiratory cultures, and serologies for Blastomyces, Coccidioides, and Histoplasma. A diagnostic bronchoscopy with transbronchial lymph node biopsy was performed showing necrotizing granulomas with organisms consistent with Histoplasma. The fevers, headache, and cough spontaneously resolved and the patient was discharged home.

IMPACT/DISCUSSION: When encountering fever and respiratory symptoms in a returning traveler, it is reasonable to first rule out common etiologies including common respiratory viruses and bacteria. However, the epidemiology and exposure history are integral in guiding the differential. Histoplasma is endemic in Cancun (CDC, WHO). He did not have any animal or fresh water exposures, making zoonotic infections less likely. Absence of eosinophilia made parasitic infections such as filariasis or strongyloidiasis less likely. Lack of immunosuppression in this previously healthy, HIV-negative patient eliminated opportunistic pathogens from the differential. CT findings were suspicious for fungal pneumonia, although lymphoma and sarcoidosis were also considered. Serologic tests for Histoplasma can have limited utility in acute pulmonary Histoplasmosis, since they may take over a month to become positive [1-3]. Histoplasma serum and urinary antigens have a sensitivity of $\sim 80 \%$ [3]. Fungal markers such as serum beta-D-Glucan has a sensitivity of $87 \%$ in disseminated Histoplasmosis, but likely lower in isolated pulmonary disease [4]. Respiratory cultures have a sensitivity of $\sim 41 \%$ [3]. Although a lung biopsy helped establish the diagnosis in this case, histopathology also has its limitations, including the poor sensitivity $(\sim 35 \%)$ and nonspecific morphology of Histoplasma [2,3,5]. CONCLUSION: Respiratory symptoms in a returning traveler should prompt work-up for common causes, while also considering epidemiologic exposures. The exposure history, presence or absence eosinophilia, immune status, and radiologic features can quickly narrow the differential.

\section{FEVER, LEUKOCYTOSIS, AND PAINFUL SKIN NODULES: A SWEET DIAGNOSTIC CHALLENGE}

Patrick Twohig; Jaclyn Rivington; Jennifer Hanrahan; David Soma. Case Western Reserve University - MetroHealth Medical Center, Cleveland, OH. (Control ID \#3185625)

LEARNING OBJECTIVE \#1: Recognize the relationship between Sweet Syndrome and other diseases

CASE: 60-year-old female presented with 4-days of right-sided anginal chest pain, palpitations, vaginal pain, and dysuria. Past medical history included CAD, type 2 diabetes, hypertension, essential thrombocytosis (JAK 2+), tobacco use and hepatitis C. She was febrile (T 103.8F), tachycardic, and hypoxic (SpO2 $80 \%$ on room air). She had tender right labial swelling without induration, fluctuance, or crepitus. The remainder of her exam was benign. Laboratory testing revealed: Leukocytosis (WBC 17,700 U/L with 22 bands), lactic acid 2.0, and D-dimer 707. CT abdomen/pelvis showed a complex 4 x $3 \mathrm{~cm}$ fluid collection involving the right labial fold. She was started on broad spectrum antibiotics and underwent incision and drainage. Intraoperative cultures were negative. Fevers persisted, and she developed acute respiratory distress syndrome (ARDS). Her labial lesion became increasingly necrotic with extension to the levator ani and urethra. Surgical pathology showed necrotic tissue but 
was otherwise non-specific. Despite multiple courses of anti-microbial therapy, her clinical condition worsened, ultimately requiring mechanical ventilation. On her $16^{\text {th }}$ hospital day, she developed new ulcerative skin lesions on her neck and R-wrist. She became increasingly hypotensive and transitioned to comfort care, with time of death called one day after skin biopsies revealed neutrophilic dermatosis consistent with Histiocytoid Sweet Syndrome (H-SS).

IMPACT/DISCUSSION: Only 100 cases of H-SS have been previously reported $^{5}$. We present the first case of H-SS that presented with a labial abscess. We performed a retrospective analysis using a de-identified clinical database of $>50$ million unique patients in the US. Odds ratios (OR) were calculated to determine the relationship between Sweet Syndrome (SS) and other diseases. Although she had no history of cancer, she did have essential thrombocytosis (ET) which increases the risk for SS (OR 19.1). SS can often manifest after infection. We suspect that the labial abscess was the sentinel event causing her SS and her ET increased her predisposition for H-SS. Our case has similarities to those in the literature and in our analysis. First, she was female (OR 3.5). Second, she had an underlying myeloproliferative disorder (OR 19.1). Third, her skin lesions were typical of H-SS - asymmetric tender red-violaceous nodules on the neck and arms ${ }^{1,3,5}$. Conversely, she was the first to present with labial ulcerations and chest pain. Most patients survive long enough to be treated, which often results in resolution of symptoms ${ }^{1,6}$. However, our patient expired prior to initiation of therapy. This severity of H-SS has not been previously documented.

CONCLUSION: Consider SS in patient's with history of hematologic disorders with constitutional symptoms, fever, and leukocytosis not responsive to antibiotics. Early identification and rapid treatment of SS can be life-saving

\section{FINDING A NEEDLE IN A FAT PAD: A CASE OF PRIMARY AMYLOIDOSIS COMPLICATED BY CARDIOMYOPATHYAND NEPHROTIC SYNDROME}

Ali Ali; Ali Saadi; Elieth Martinez; Sagar Wagle; Natasha Singh. UCSF Fresno, Fresno, CA. (Control ID \#3185700)

LEARNING OBJECTIVE \#1: Recognize the clinical features and diagnosis of AL amyloidosis

LEARNING OBJECTIVE \#2: Assessing for infiltrative cardiomyopathy in the absence of a biopsy

CASE: A 66 year old male presented with 6 months of progressive weight gain, edema and paroxysmal nocturnal dyspnea. Patient was otherwise healthy and not on medications. He was born in Mexico, but currently lived in Washington state. He did not smoke, drink or use drugs. Exam notable for +3 pitting edema in all extremities. Labs notable for serum albumin $1.5 \mathrm{~g} / \mathrm{dl}$, protein $4 \mathrm{~g} / \mathrm{dl}$ and corrected calcium of $8.7 \mathrm{mg} / \mathrm{dl}$. Complete blood count, liver function and renal function labs were otherwise normal. A 24 hour urine protein of $13 \mathrm{~g}$ was consistent with nephrotic syndrome. HbA1c was 6 . Serum immunofixation revealed trace free lambda monoclonal proteins with kappa to lamda ratio of 0.03 . Fat pad biopsy was negative for amyloid. Bone marrow biopsy showed 15$20 \%$ cellularity with lambda restriction without amyloid deposits consistent with plasma cell dyscrasia. Kidney biopsy showed AL ( lambda light chain ) amyloidosis predominantly glomerular with focal interstitial and vascular involvement. Bone survey was negative Patient also presented with persistent troponemia of $0.1 \mathrm{ng} / \mathrm{ml}$ without chest pain. BNP 375 . EKG showed sinus rhythm with low voltage in all leads. A transthoracic echocardiogram showed ejection fraction of $60-65 \%$ with moderate left ventricular concentric hypertrophy and impaired relaxation. Cardiac MRI with and without contrast showed abnormal myocardial gadolinium tissue kinetics and delayed enhancement consistent with amyloid deposition. Patient was diagnosed with AL amyloidosis complicated by nephrotic syndrome and cardiomyopathy. He was started on chemotherapy (CyBOR-D) and referred for stem cell transplant.
IMPACT/DISCUSSION: Light-chain (AL) amyloidosis is the most common form of primary systemic amyloidosis and normally associated with multiple myeloma, but can present as isolated plasma cell dyscrasia. It is a rare disorder with an incidence of nine per one million. The clinical syndromes can manifests as nephrotic-range proteinuria with or without renal dysfunction, hepatomegaly, congestive heart failure, and autonomic or sensory neuropathy. The diagnosis of $\mathrm{AL}$ amyloidosis requires tissue biopsy. In cases with limited organ involvement, biopsy of uninvolved sites, like fat pad, can have low sensitivity. Careful search for other organ involvement should be done as it impacts prognosis. In this case, the unexplained troponemia and low voltage lead to further workup and diagnosis of amyloid cardiomyopathy.

CONCLUSION: - Consider amyloidosis in cases of unexplained nephrotic syndrome - Unexplained low voltage with mild troponinemia is consistent with infiltrative cardiomyopathy, cardiac MRI can often be diagnostic in the absence of cardiac biopsy.

\section{FINDING PNEUMO: AN UNUSUAL PRESENTATION OF RE- CURRENT SPONTANEOUS PNEUMOTHORAX}

Samer N. Muallem; Ami DeWaters. Penn State Hershey Medical Center, Hershey, PA. (Control ID \#3184526)

LEARNING OBJECTIVE \#1: Identify catamenial pneumothorax as a cause of pneumothorax.

LEARNING OBJECTIVE \#2: Discuss treatment options to prevent recurrence of catamenial pneumothorax.

CASE: A 37-year-old woman with mild asthma presented to the internal medicine clinic one week after an emergency room visit for a spontaneous right apical pneumothorax which resolved on its own. She was asymptomatic in clinic, and the decision was made to monitor her condition. One month later, she presented to the emergency room with pleuritic chest pain and was found to have another small right-sided pneumothorax on x-ray. Computed tomography of the chest was obtained, and revealed no bleb disease to explain her recurrent pneumothorax. A thoracic surgeon evaluated her and performed an elective video-assisted thoracoscopic exploration. During this procedure, her right diaphragm was noted to have multiple fenestrations at the central tendon, as well as a small nodule which appeared consistent with endometriosis. She was thus diagnosed with catamenial pneumothorax. A patch was placed over the fenestrated area of the diaphragm, and mechanical pleurodesis was performed. She was started on oral norethindrone for progestin suppression, and had no further pneumothoraces.

IMPACT/DISCUSSION: Catamenial pneumothorax is an extremely rare condition caused by thoracic endometriosis, typically involving the parietal pleura, although the visceral pleura, diaphragm, or lung itself can also be involved. During menses, the aberrant endometrial tissue creates perforations in the pleura, resulting in the accumulation of air in the potential space. Symptoms of pleuritic pain or dyspnea classically present within three days of the onset of menstruation, but do not necessarily occur with every menstrual cycle. This condition is most common in women aged 30 to 40 , although it can occur in any post-menarchal woman. Given its rarity, catamenial pneumothorax is not classically taught in internal medicine training and is more often identified by subspecialists. Internists, especially primary care providers, are the patient's first point of entry into the healthcare system, so it is important for them to be aware of this entity and obtain a comprehensive gynecologic history when they suspect it. Once diagnosed with catamenial pneumothorax, patients should also be referred to thoracic surgery for evaluation. Typically, endometrial lesions will require resection, pleurodesis, and diaphragmatic repair to reduce the risk of further pneumothoraces. Following surgical intervention, these patients should be started on hormonal suppression with oral contraceptives as tolerated, and should continue medical therapy for at least 6-12 months, with close follow up. 
CONCLUSION: Internists should consider the diagnosis of catamenial pneumothorax in a female patient of childbearing age with recurrent episodes of pneumothoraces. Secondary prevention of catamenial pneumothorax often requires pleurodesis, resection of the endometriosis, and diaphragmatic repair, in addition to hormonal suppression.

\section{FINE NEEDLE ASPIRATION FOR DIAGNOSIS OF LYMPHADENOPATHIC KAPOSI SARCOMA IN THE HIV NEG- ATIVE PATIENT}

Kaitlyn E. Takach; Arie Szeinfeld; Eliran E. Zadikov; Lauren E. Menzies; Mohamed Aziz, MD FCAP. American University of the Caribbean School of Medicine, Cupecoy, Sint Maarten (Dutch part). (Control ID \#3185796)

LEARNING OBJECTIVE \#1: Fine needle aspiration (slides and cell block) is adequate for full diagnosis of Kaposi Sarcoma.

LEARNING OBJECTIVE \#2: Kaposi Sarcoma should be suspected even in a non-HIV positive patient when we have a suggestion of a vascular neoplasm in fine needle aspiration.

CASE: A 65 year-old Mediterranean man with history of colon cancer presented with right inguinal lymphadenopathy for several weeks. On physical exam, he was awake, alert and cooperative. Cardiopulmonary examination was normal. A $2.5 \mathrm{~cm}$ nontender, right inguinal lymph node was noted. Concern for metastatic colon cancer prompted sampling of the lymph node. Fine needle aspiration (FNA) was performed and cytologic analysis demonstrated fragments of atypical spindle cells in a background of polymorphous lymphocytes and extravasated blood. Cell block prepared from the cytology specimen demonstrated a spindle cell neoplasm with hyperchromatic elongated nuclei infiltrating lymphoid tissue and no morphologic evidence of epithelial cells. Immunohistochemical studies showed tumor positivity for HHV-8, CD31, CD34 and vimentin and were negative for cytokeratins, S100, p63, and desmin all of which was consistent with a diagnosis of Kaposi Sarcoma (KS) and not metastatic colon carcinoma. Testing for human immunodeficiency virus (HIV) was negative. Lymph node was excised and there was no evidence of recurrence or disseminated disease with a follow up for three years.

IMPACT/DISCUSSION: Kaposi sarcoma is the most common HIV associated malignancy, however, KS can be initiated through mechanisms other than viral, such as iatrogenic immunosuppression and elevated expression of cytokines and angiogenic growth factors. Although KS commonly presents as skin lesions, it can be associated with visceral involvement and may run an aggressive disseminated course. Cytologically, the nodal tumor is identical to skin KS, regardless of patient's age or clinical presentation. KS should be considered as a potential diagnosis for patients with lymphadenopathy of unknown etiology, even in those with a known HIV-negative status.

CONCLUSION: Fine needle aspiration can be used to adequately obtain lymph node samples as well as for the diagnosis of KS.

\section{FISTULIZATION OF HEMORRHAGIC PANCREATIC PSEUDOCYST}

Kelsey R. Grossman; Stacey Wolfson; Ravi Shah; Michael Janjigian. NYU School of Medicine, New York, NY. (Control ID \#3165653)

LEARNING OBJECTIVE \#1: Recognize pancreatic-enteric fistulization as a rare complication of pancreatitis

LEARNING OBJECTIVE \#2: Define the mechanisms for fistula formation in hemorrhagic pseudocysts

CASE: The patient is a 31-year-old female with a past medical history of systemic lupus erythematosus complicated by end stage renal disease on dialysis, and pancreatitis complicated by pseudocyst formation, presenting with acute onset abdominal pain, nausea, and vomiting. On presentation, she was afebrile and hemodynamically stable with exam notable for diffuse abdominal tenderness and voluntary guarding without rebound. $\mathrm{CT}$ abdomen and pelvis showed hemorrhage into the known pancreatic tail pseudocyst with interval enlargement from 8 to $12 \mathrm{~cm}$. CT Angiogram obtained four days after the onset of abdominal pain showed an open fistula between the pseudocyst and transverse colon, without evidence of active arterial bleeding. The patient then had two episodes of bright red blood per rectum. She became tachycardic and hypotensive with labs notable for a one-point hemoglobin drop from 9.7 to 8.5. She was triaged to the surgical ICU and underwent urgent exploratory laparotomy with transverse colectomy, partial pancreatectomy, and splenectomy, followed by staged colostomy creation.

IMPACT/DISCUSSION: Fistulization of pancreatic pseudocysts is an extremely rare complication, occurring in less than $3 \%$ of cases, and can involve a variety of abdominal organs. Connections with sources other than the colon often cause spontaneous drainage and resolution of symptoms, and thus do not require surgery. However, communication with the colon can lead to other complications such as hemorrhage and sepsis, as seen in this case, which require definitive management. A proximal diverting colostomy is standard practice in order to prevent further backup of fecal matter into the pseudocyst and prevent rupture into the abdominal cavity. Multiple mechanisms have been proposed to explain the cause of hemorrhage and rupture of pancreatic pseudocysts. These mechanisms include the presence of severe inflammation due to activated lytic enzymes of the pancreas, as well as persistent compression of blood vessels due to mass effect. Colonopancreatic fistula formation in the case of our patient with lupus is likely secondary to the pro-inflammatory environment compounded by high wall pressures and suppressed healing mechanisms due to chronic steroid use.

CONCLUSION: Given the gravity of a missed diagnosis, hospitalists must keep complications of pancreatic pseudocysts on their differential for patients with known pathology and acute abdominal pain. Management strategies differ based on the type of complication, however, urgent operative management is key in certain cases of fistula formation, especially when it involves the colon as in our patient.

\section{FLACCID PARALYSIS AS A COMPLICATION OF NEUROINVASIVE WEST NILE VIRUS}

Morris Jrada; Brandan Kovacs; Faryal Osman. NYU Langone Brooklyn, Brooklyn, NY. (Control ID \#3185474)

LEARNING OBJECTIVE \#1: Diagnose West Nile Virus (WNV) encephalitis

LEARNING OBJECTIVE \#2: Recognize neurologic symptoms seen in Neuro-invasive WNV

CASE: A 79 year old male with a past medical history of CAD s/p CABG, hypertension and hyperlipidemia presented with a 4 day history of generalized weakness, fevers, decreased oral intake, and confusion. He was accompanied by his wife, who denied any respiratory, skin or urologic changes, and reported the patient had been at baseline, tending his garden a couple of days prior. He had no recent travel, but had a sick contact in a grandchild who was diagnosed with an unknown virus. Upon admission, he was febrile to $39.5 \mathrm{C}$, hypotensive to $95 / 43$ with otherwise normal vital signs. Exam was notable for lethargy and proximal muscle weakness with diffuse hyporeflexia. CT scan of the head, chest X-Ray and urinalysis did not show acute abnormalities. He was started on antibiotics to cover for meningitis with improvement in his vitals, but not his mental status. The following day, a lumbar puncture was performed and cell count was notable for a white blood cell count of 344 with $76 \%$ neutrophils, 97 protein and 53 glucose, and negative gram stain. His course was complicated by an aspiration event that resulted in hypoxia and he was subsequently intubated. Additional CSF studies were then sent to the state lab, returning positive for WNV IgM the following week. The patient's hospital course was again complicated by a second aspiration event post extubation and required reintubation and eventually a tracheostomy and PEG placement. He 
continued to exhibit proximal and distal muscle weakness and an EMG was performed, which showed axonal and demyelination sensorimotor polyneuropathy. MRI of the spine showed enhancement of cauda equina consistent with Guillain Barre syndrome (GBS). Patient was started on plasmapheresis with improvement in his weakness.

IMPACT/DISCUSSION: The key aspect of this case was the atypical appearance of acute flaccid paralysis, a Guillain Barre-like picture, that developed in a case of confirmed West Nile Virus infection. Prior to 1996, WNV was associated with typical B symptoms. Following the NYC outbreak, neurological complications, including muscle weakness, became common. Another key point is the presentation difference between normal GBS and the flaccid paralysis seen in WNV infection. In standard GBS, weakness typically begins weeks after infection, while weakness in WNV occurs in the acute to subacute phase. As was present in this case, leukocytosis and elevated protein in CSF are both characteristic of WNV infection while typical GBS CSF shows elevated protein without leukocytosis, the classic albuminocytologic dissociation. While WNVassociated paralysis is a well-described phenomenon, it has not become commonplace knowledge in general medicine.

CONCLUSION: WNV often presents as a non-specific encephalitis that may be misdiagnosed as a bacterial pneumonia. It is imperative that WNV be part of the differential in an acutely ill patient presenting with muscle weakness.

\section{FOLLOW THE GREEN BRICK ROAD: AN UNEXPECTED} CAUSE OF ACUTE KIDNEY INJURY

Dennis C. Shi. Oregon Health \& Science University, Portland, OR. (Control ID \#3186722)

LEARNING OBJECTIVE \#1: Bile cast nephropathy should be considered as a possible cause when patients with liver disease present with AKI.

CASE: 64-year-old man with history of polysubstance use disorder and recently discovered pancreatic head mass with liver lesions presented with progressive jaundice and acute kidney injury in the setting of several months of progressive abdominal pain, distention, and weight loss. Physical exam was notable for jaundice, lower extremity edema and a distended abdomen. On admission, his creatinine was 2.01 increased from 0.8 the day prior. AST and ALT were elevated in the $100 \mathrm{~s}$, total bilirubin was 11.2 , and alkaline phosphatase was 822 . He was started on IV fluids, however creatinine continued to increase and patient was oliguric. $\mathrm{FeNa}$ was consistent with prerenal etiology. Renal US showed no evidence of hydronephrosis. TTE was unremarkable for hypo- or hypervolemia. Last contrast was two weeks ago. On day three of his hospitalization, Nephrology was consulted due to concern for bile cast nephropathy in the setting of acute obstructive jaundice. Urinalysis revealed large bile-stained granular casts consistent with bile cast nephropathy. Patient was switched to albumin with improvement of renal function.

IMPACT/DISCUSSION: Acute kidney injury in a patient with severe liver disease is often thought to be due to decreased kidney perfusion from intravascular volume depletion, acute tubular injury, or hepatorenal syndrome. Liver disease is also frequently complicated by hyperbilirubinemia, which can cause renal tubular injury as bile acids can act as endogenous nephrotoxins. Bile cast nephropathy is an important pathological factor that may account for renal dysfunction in some patients with liver disease. Though less common, literature has described numerous cases of bile cast nephropathy and reports that it is likely underdiagnosed. Typically, bile-case nephropathy presents in the setting of total bilirubin greater than 20. Interestingly, our patient's total bilirubin was only 13.6. However, bile casts were noted in the urine.

CONCLUSION: In summary, this case illustrates the importance of entertaining bile-cast nephropathy in the differential of AKI in patients with liver disease. AKI in this regard is caused by epithelial cell damage and tubular obstruction secondary to bile acid accumulation. Early identification is important for treatment, as correction of hyperbilirubinemia can address AKI, preventing further progression of renal disease.

\section{FOOD COMA: A CASE OF THYROTOXIC PERIODIC PARALYSIS}

Maryam Zamanian; Amanda Deis; Gena M. Walker. University of Pittsburgh Medical Center, Pittsburgh, PA. (Control ID \#3185432)

LEARNING OBJECTIVE \#1: Recognizing clinical characteristics of thyrotoxic periodic paralysis (TPP)

LEARNING OBJECTIVE \#2: Managing initial lab abnormalities in TPP CASE: A 20 year old male with no past medical history was admitted to the hospital after presenting to the ED with complaints of generalized weakness. He was in his usual state of health until the morning of admission when he woke up and was unable to stand up. He had some improvement over time and was able to walk to the ED with assistance. On presentation, his exam was notable for tachycardia and generalized weakness. Initial labs revealed a serum potassium of 2.2, with EKG demonstrating $U$ waves consistent with hypokalemia. He denied any diuretic or drug use, but did note recent high carbohydrate intake. Further laboratory evaluation revealed an undetectable TSH and elevated free T4, raising concern for thyrotoxic periodic paralysis (TPP) triggered by his recent dietary changes. No thyromegaly was noted on exam. Family history was notable for Graves' disease in his aunts with no known history of Asian heritage. He received potassium replacement therapy with complete resolution of symptoms. Endocrinology was consulted and further evaluation revealed an elevated TSI, with concern for possible Graves' disease. He was started on propranolol as prevention for further attacks and methimazole for his hyperthyroidism with plans to pursue further imaging and definitive treatment outpatient.

IMPACT/DISCUSSION: TPP is more common in Asian countries, with an estimated incidence of 1.8-1.9\% in thyrotoxic patients. In the US, there is significantly less exposure to this unique manifestation of hyperthyroidism. However, the incidence of TPP appears to be increasing in the US, making early recognition of this disease in all populations increasingly important. In particular, differentiation of TPP from the more common familial hypokalemic paralysis is essential to ensure appropriate treatment. Triggers of TPP attacks can include high carbohydrate meals, stress or exercise. Immediate management should involve potassium repletion to prevent cardiac complications with monitoring due to risk of rebound hyperkalemia during recovery. A non-selective b-blocker can be used in acute treatment and for recurrence prevention until a euthyroid state can be achieved. Ultimate treatment should focus on treating the underlying etiology of hyperthyroidism. References: Kung, Annie WC. "Thyrotoxic periodic paralysis: a diagnostic challenge." The Journal of Clinical Endocrinology \& Metabolism 91.7 (2006) Pothiwala, Pooja, and Steven N. Levine. "Analytic review: thyrotoxic periodic paralysis: a review." Journal of intensive care medicine 25.2 (2010)

CONCLUSION: Thyrotoxic periodic paralysis should be considered as a potential diagnosis in patients presenting with sudden weakness and hypokalemia. Hypokalemia in TPP should be repleted immediately but with close monitoring to avoid rebound hyperkalemia. Non-selective Bblockers can be used in prevention of recurrent attacks until euthyroid state has been achieved.

\section{FOR GOOD MEASURE: EXPLORING IMMUNODEFICIENCY IN PATIENTS WITH CMV RETINITIS}

Ryan Knodle. Boston Medical Center, Boston, MA. (Control ID \#3184884)

LEARNING OBJECTIVE \#1: Assess for alternative causes of immunodeficiency in non-HIV infected patients presenting with CMV retinitis LEARNING OBJECTIVE \#2: Recognize Good's syndrome as a potential diagnosis in patients with thymoma and "immunodeficiency-defining" illness CASE: An 82-year-old HIV-negative Chilean man with history of thymoma and bilateral retinal ischemia presented with acute loss of visual 
acuity in his left eye. He had been evaluated for an infectious cause of retinal ischemia two years prior and serologic work-up was negative for toxoplasmosis, tuberculosis, and syphilis, as was PCR testing of vitreous humor for varicella-zoster virus. He was lost to follow-up until the current presentation. His review of systems was negative for fever, weight loss, night sweats, headache, or diarrhea. On exam, he had mild conjunctivitis, equally reactive pupils, and grossly intact extraocular movements. His heart sounds were distant parasternally but normal at the apex. He had no lymphadenopathy. Ophthalmologic examination was concerning for occlusive vasculopathy and vitritis so paracentesis of the anterior chamber was performed and PCR confirmed the diagnosis of CMV retinitis. He was initiated on systemic ganciclovir and intravitreal foscarnet. Further investigation for immune deficiency was performed and he was found to be pan-hypogammaglobulinemic (IgA 54, IgG 183, IgM 13) with a relative CD4 lymphopenia (CD4\% 18, CD4:CD8 0.31). He did not have clinical evidence of systemic disease and was transitioned to oral valganciclovir at the time of discharge. A diagnosis of Good's syndrome was made on the basis of thymoma with pan- hypogammaglobulinemia, relative $\mathrm{CD} 4$ lymphopenia, and an immunodeficiency-defining diagnosis. IMPACT/DISCUSSION: Though CMV retinitis in the immunocompetent individual has been described, it is rare and should prompt clinicians to evaluate for causes of immunodeficiency. In the non-transplant, nonHIV-infected patient, this leaves the spectrum of primary immunodeficiency syndromes. In adults with thymoma, the differential must include Good's syndrome, an adult-onset combined B- and T-lymphocyte deficiency characterized by recurrent sinopulmonary infections from encapsulated organisms, as well as organ-specific viral infections. Laboratory findings include decreased B cells, pan-hypogammaglobulinemia, CD4 lymphopenia, and an abnormal CD4:CD8 ratio. Prognostically, the literature suggests poorer long term outcomes compared to patients with $\mathrm{X}$ linked agammaglobulinemia or combined variable immune deficiency. Treatment of CMV infection is identical to that in HIV-infected persons; management of Good's syndrome should include resection of thymoma and IVIG. Though the average age of patients at diagnosis is $40-50$, our patient (82) represents one of the oldest cases described in the literature.

CONCLUSION: CMV retinitis in immunocompetent patients is rare and should prompt investigation into causes of immunodeficiency. Good's syndrome should be considered in adults age $>40$ with thymoma and immunodeficiency.

\section{FOR STROKE'S SAKE: VARICELLA ZOSTER VIRUS (VZV) VASCULOPATHY}

Chengcheng Ye; Albert Anderson. Emory University, Atlanta, GA. (Control ID \#3186006)

LEARNING OBJECTIVE \#1: Emphasize stroke as a rare neurological complication of VZV

LEARNING OBJECTIVE \#2: Recognize empirical treatment with acyclovir and prednisone for VZV vasculitis

CASE: A 49-year-old African American man with a history of HIV/AIDS (CD4 66/6\%), hypertension, and VZV encephalopathy presented with progressive right sided weakness. He reported falls and dysarthria. $\mathrm{He}$ denied any fevers, chills, rashes, headaches, or visual changes. Examination revealed decreased strength and slowed coordination of the right arm and leg along with a right hemiparetic gait. MRI brain showed new subacute frontal lobe infarction and small left cerebellum infarctions. Transthoracic echocardiogram was normal. A cerebral angiogram demonstrated right posterior inferior cerebellar artery fusiform aneurysm and scattered segmental narrowing involving the mid to distal bilateral anterior and middle cerebral arteries concerning for vasculitis. Given his immunocompromised status, a lumbar puncture was performed with normal opening pressure. Studies revealed no pleocytosis, cerebrospinal fluid (CSF) protein of $45 \mathrm{mg}$ / $\mathrm{dl}$, normal CSF glucose, negative VDRL, HSV, JCV, CMV, toxoplasmosis, cryptococcus, and culture. Polymerase chain reaction (PCR) for VZV was negative but was positive for VZV IgG and IgM, confirming intrathecal production of anti-VZV antibodies. Acyclovir and prednisone were started, and patient's right sided weakness and motor function improved. Patient finished a 14-day course of acyclovir and prednisone and was discharged on valacyclovir for suppressive therapy.

IMPACT/DISCUSSION: The classic presentation of VZV vasculopathy is acute contralateral hemiplegia after ophthalmic zoster. Although our patient had no ophthalmic or dermatomal zoster, these absences should not deter a physician from pursuing a diagnostic evaluation for VZV especially in an HIV patient. Up to one-third of patients with VZV vasculopathy have no preceding rash. Importantly, the diagnosis of this treatable cause of stroke is often missed because one-third of patients have no zoster rash, one-third have normal CSF, and there is often a four-month delay from zoster to neurological symptoms. Characteristic data include MRI findings consistent with an ischemic or hemorrhagic infarction, and angiography reveals large and small vessel disease. CSF pleocytosis of less than 100 cells $/ \mathrm{mL}$ is common. Although a positive VZV PCR in the CSF is helpful, a negative PCR does not exclude the diagnosis. Only if both VZV PCR and VZV IgG antibody are negative can VZV vasculopathy be reliably excluded. Treatment for both immunocompetent and immunocompromised patients include acyclovir and prednisone for inflammation.

CONCLUSION: Clinical suspicion should be high for VZV vasculopathy as a cause of stroke especially in an immunocompromised patient. A negative CSF VZV PCR does not exclude the diagnosis, and early therapy should be initiated for this reversible cause of stroke.

\section{FORGOTTEN, BUT NOT GONE: A CASE OF LEMIERRE'S SYNDROME: THE FORGOTTEN DISEASE}

Margaret V. Spinosa; Twomey Kathleen; Fidelis Ojevwe. Penn State Hershey Medical Center, Palmyra, PA. (Control ID \#3186019)

LEARNING OBJECTIVE \#1: Recognize Lemierre's Syndrome as a potentially life-threatening illness if left untreated.

LEARNING OBJECTIVE \#2: Assess for multiorgan complications easily missed as features of Lemierre's Syndrome.

CASE: A 25 year old woman with neurofibromatosis presented with one day of nausea, vomiting, headache, diplopia and one month of sore throat and right ear pain. On physical examination, she was found to be hypotensive and tachypneic. She was unable to laterally abduct her right eye and her tongue deviated to the right with protrusion. She had an erythematous right tympanic membrane with effusion, bibasilar crackles and suprapubic tenderness. Initial laboratory studies were normal. Brain MRI and CT chest, abdomen and pelvis were ordered. Imaging revealed thrombosis of the right internal jugular vein extending into the right sigmoid, distal transverse and cavernous sinuses and inflammation of the internal carotid artery sheath. Right petrous apicitis, mastoid and middle ear fluid also was seen. Abdominal imaging showed a larger heterogenous pelvic mass. Chest imaging showed bilateral cavitating pulmonary lesions. An echocardiogram was notable for a small PFO and admission blood culture resulted positive for Fusobacterium necrophorum. The patient was examined with nasal laryngoscopy and found to have purulence in the Eustachian tub orifice and fullness of the right adenoid pad. She was treated with IV ampicillin-sulbactam. Interventional Radiology biopsied the pelvic mass which was notable for soft tissue inflammation with microabscesses and purulent exudate with no evidence of malignancy or neurofibroma. She completed a prolonged course of antibiotics and was started on anticoagulation due to extent of thromboses in her sinuses.

IMPACT/DISCUSSION: This case illustrates the potential for multiorgan consequences of Lemierre's Syndrome caused by Fusobacterium necrophorum, an anaerobe commonly found in the oropharynx and spread by septic thrombophlebitis of the internal jugular vein. Often a result of pharyngitis, in this case the inciting infection was likely acute otitis media. While septic emboli to the lung are a more 
common feature of this Syndrome, the patient's pelvic mass may have been a result of septic emboli due to presence of small patent foramen ovale. The development of neurologic symptoms, due to the extensive involvement of the venous sinuses, could be misdiagnosed as a primary neurologic process. Lemierre's Syndrome is now regarded as a rare occurrence due to the timely treatment of oropharyngeal infections. However, with increasing antibiotic resistance and lack of awareness of the diagnosis, a resurgence of Lemierre's Syndrome may be seen in the future. CONCLUSION: Lemierre's Syndrome is an easily missed diagnosis due to providers unfamiliarity with Fusobacterium infections. Atypical presentations of this Syndrome may delay appropiate therapy. Systemic involvement without early treatment is potentially life-threatening.

\section{FRIEND OR FOE? A CASE OF IMMUNOTHERAPY-INDUCED HYPOPITUITARISM AND EMPTY SELLA SYNDROME}

Jeremy Chang ${ }^{1}$; Jeffrey Tran ${ }^{1}$; Dina Kamel ${ }^{2}$; Arnab Basu ${ }^{2} .{ }^{1}$ LAC+USC, Wilmington, CA; ${ }^{2}$ LAC + USC, Los Angeles, CA. (Control ID \#3180700)

LEARNING OBJECTIVE \#1: Recognize that the use of novel treatments such as immune checkpoints inhibitors (ICIs) can lead to a wide spectrum of adverse effects, including endocrinologic complications.

CASE: A 63-year-old Caucasian woman with history of stage IVA lung adenocarcinoma presented for worsening fatigue. Her symptom started 2 weeks prior to admission with associated non-productive cough, myalgias, and multiple episodes of non-bloody, non-bilious emesis. She was previously started on the immune therapy nivolumab 1 year ago for treatment of her cancer. Infectious work-up on admission including cultures and imaging were unremarkable. Subsequently, several hormonal tests were ordered to evaluate for an endocrinologic etiology of her symptoms. The results were significant for a low TSH of $<0.01 \mathrm{mIU} / \mathrm{L}$, low free T3 of $1.1 \mathrm{ng} / \mathrm{dL}$, low free $\mathrm{T} 4$ of $2.4 \mathrm{ng} / \mathrm{dL}$, low morning cortisol level of $5.6 \mu \mathrm{g} / \mathrm{dL}$, and inappropriately normal adrenocorticotropic hormone (ACTH) of $8 \mathrm{pg} / \mathrm{mL}$. An MRI of the sella was performed which showed a partially empty sella without evidence of any pituitary lesion. These findings were consistent with hypopituitarism leading to adrenal insufficiency and primary hypothyroidism. The patient was started on oral hydrocortisone $20 \mathrm{mg}$ in the morning and $10 \mathrm{mg}$ at night with rapid improvement in her symptoms. On discharge, she was prescribed oral prednisone $80 \mathrm{mg}$ per day with a taper until follow-up with endocrinology.

IMPACT/DISCUSSION: The use of ICIs in the treatment of malignancies represents a monumental advancement in cancer therapy. However, the use of immunotherapy has also led to a wide range of adverse effects of varying severities. Endocrine toxicities occur infrequently during ICI use, but can include adrenalitis and thyroid dysfunction. Hypophysitis is an overall rare condition that refers to inflammation of the pituitary gland. While many subtypes were previously identified including lymphocytic, granulomatous, and plasmacytic, hypophysitis secondary to immunotherapy has emerged as a more recent etiology. Nivolumab-induced hypophysitis is an exceedingly rare phenomenon, occurring in $<1 \%$ of patients in several prior studies. The results of our patient's hormonal work-up indicated that her hypophysitis resulted in hypopituitarism with associated adrenal insufficiency and hypothyroidism. In addition, the presence of a partially empty sella on MRI was consistent with a secondary empty sella syndrome following the pituitary inflammation.

CONCLUSION: Given the potential complications of immunotherapy including hormonal dysfunction, it is crucial for practitioners to be aware of possible adverse effects when managing patients on novel therapies.

\section{FROM CLINIC TO THE CCU: A COMMON VIRAL SYNDROME WITH UNCOMMON MANIFESTATIONS}

Anne G. Montgomery. UCSF, San Francisco, CA. (Control ID \#3186010) LEARNING OBJECTIVE \#1: Recognize the clinical features of myocarditis and myopericarditis.
LEARNING OBJECTIVE \#2: Understand the necessary outpatient follow up for a patient with myocarditis.

CASE: A 32 year-old woman with no medical history presented to clinic with one week of flu-like symptoms and progressive myalgias. Her exam was notable for pronounced tenderness of the bilateral calves without rash, swelling, or weakness. Initial blood tests revealed a leukocytosis to 15.8 , creatine kinase of 735, CRP of 284 , and creatinine of 0.93 (her baseline). Given concern for a viral myositis, the patient was instructed to stay hydrated and return for repeat labs in 48 hours. Prior to follow-up, the patient called the clinic to report fever and the onset of mild pleuritic chest pain. She was instructed to present to the Emergency Department where an EKG revealed ST-segment elevation in leads I, II, and V1-V3 with depressions in II, aVR, and V1. A troponin was elevated at 4.65, and a transthoracic echocardiogram showed a small pericardial effusion and possible wall motion abnormalities. She underwent urgent coronary angiography, which showed no evidence of disease, but given concern for rapid progression of myocarditis, she was admitted to the CCU for further monitoring. Fortunately, she remained hemodynamically stable, and over the next several days her myalgias and inflammatory markers improved. She was discharged on a low-dose ACE inhibitor and beta-blocker with instructions to follow up with her primary care provider.

IMPACT/DISCUSSION: This case highlights the fact that the initial presentation of myocarditis and myopericarditis can be overshadowed by a patient's viral symptoms. Because myocarditis can rapidly progress to depressed cardiac function and even cardiogenic shock, it is important to recognize its early manifestations. In $80-90 \%$ of cases of pericarditis and myocarditis, the etiology is idiopathic and presumed to be viral in nature. These cases are often preceded by days to weeks of vague viral symptoms. Patients may have chest pain, and those with myocardial involvement will have elevated cardiac enzymes and/or evidence of depressed left ventricular function. Those with evidence of depressed left ventricular systolic function should be started on guideline-directed heart failure therapy once stable and followed closely in the outpatient setting. While this patient was overall well-appearing during her first outpatient visit even with markedly elevated inflammatory markers, the development of mild chest pain prompted the clinician to triage her more rapidly to the Emergency Department. She ultimately required ICU-level care for what started as a viral syndrome.

CONCLUSION: Myocarditis can start slowly as a subtle viral syndrome and progress rapidly to hemodynamic instability. All patients with evidence of myocarditis require inpatient admission and close outpatient follow up.

\section{FROM JAIL TO HOSPICE CARE}

Libia M. Vasquez ${ }^{1}$; Lucy M. Esteve ${ }^{2}$; Carolyn April ${ }^{3} .{ }^{1}$ University of Texas Health Science Center San Antonio, San Antonio, TX; ${ }^{2}$ UT Health San Antonio, San Antonio, TX; ${ }^{3}$ University of Texas Health Science Center at San Antonio, San Antonio, TX. (Control ID \#3139820)

LEARNING OBJECTIVE \#1: Recognize the barriers incarcerated patients face in a health care system

LEARNING OBJECTIVE \#2: Recognize "rule of rescue" (perceived duty to save endangered lives) practices do not result in better patient outcomes

CASE: A 61-year-old woman presented from county jail with nausea and vomiting for a week. In addition to dysuria, PO intolerance, chills, and abdominal pain. Urine and blood cultures grew E. Coli. She had bipolar disorder, type 2 diabetes, and hypertension. She lost 40-pounds in four months from early satiety and regurgitation after meals. EGD showed a friable mass at the GE junction, biopsy with poorly differentiated adenocarcinoma with signet cells. A week after diagnosis of GE cancer, she was compassionately released from the jail unit and moved to a regular floor. She was hospitalized for two months completing CT scans, PET scan, and diagnostic laparoscopy. She was diagnosed with stage IIa GE adenocarcinoma and was discharged to a homeless shelter with a 3-month supply 
of a nutritional drink unable to eat solid food. A couple months later, after reinstating Medicaid, she returned to the emergency department with similar symptoms. She was restaged, including laparoscopy, and esophageal stent was placed. Two weeks later additional imaging was requested in clinic. A month later, homeless and uninsured, she returned to the hospital. After her clinic appointment, she expected her chemotherapy to begin during hospitalization. CT scan of the chest showed numerous sclerotic foci throughout her vertebra. She was diagnosed with metastatic disease and was discharged to a nursing home with hospice care.

IMPACT/DISCUSSION: Her physicians, abiding by the "rule of rescue", ensured her cancer was diagnosed, staged and a treatment plan was formulated. Completing this work up as an inpatient was costlier than outpatient. A more constructive way of advocating is by working on resolving the barriers that result in an inability to access. Physicians cannot eliminate all barriers, mental health or homelessness, but adjusting the health care system for timely access would be less costly and effectively "rescue" patients.

CONCLUSION: This case is an example of inequity and a complicated wasteful health care system. A health care system in the Bronx created transition clinics for patients post-incarceration to prevent lack of health care after release. Prompt follow up would have prevented multiple hospitalizations and set up an easier path for her to follow. It is shown that much like in this case, treatment received during incarceration loses effect after release since patients are unable to receive continuous health care. Invasive diagnostic procedures directed by advocating physicians led to a treatment plan that never begun. It is our duty as physicians to not only advocate when patients are under our care but assist in creating policies for improved health care access. This patient had curable cancer with a clear treatment plan but ended up with a terminal illness because of a failing system.

\section{FROM ORATOR TO NON-VERBAL: A RARE CASE OF COGNI- TIVE DECLINE}

Saima M. Shikari; Chelsea Gordner; Aboorva Sudhakar. Baystate Medical Center, Springfield, MA. (Control ID \#3186407)

LEARNING OBJECTIVE \#1: Recognize Hashimoto's Encephalopathy (HE) as a differential in patients cognitive function decline

LEARNING OBJECTIVE \#2: Recognize need for urgent steroid treatment to prevent permanent cognitive deficits

CASE: A 61 year old female with past medical history of hypothyroidism and sickle cell disease who presented with 4 months of progressive memory loss, weakness intermittent nausea, vomiting and extreme fatigue after eating. GI workup including EGD, colonoscopy and labs for autoimmune inflammatory hepatic conditions were negative. Infectious disease and neurology were consulted and had ruled out tick borne illness, West Nile virus and paraneoplastic syndrome when she had rapid decline in cognitive function. At baseline patient was a college professor, within 3 days of admission she was unable to follow commands, non-verbal, incontinent, hallucinating and persistently drooling. EEG was abnormal due to excessive slowing and repetitive slow sharp transients and given the differential of Creutzfeldt-Jakob Disease LP was performed but CSF analysis showed no acute pathology. Due to persistent tachycardia and history of hypothyroidism, thyroid studies showed TSH 0.05 , Free T4 2.82 and Free T3 7.2. Endocrinology was consulted and ordered Thyrotropin receptor $\mathrm{Ab}<1.00$ and Anti-TPO Ab 869.7 leading to suspicion for HE. High dose steroids were started with rapid improvement in mental status within 24 hours and return to baseline in 3 days.

IMPACT/DISCUSSION: Hashimoto's Encephalopathy is a steroid responsive encephalopathy associated with autoimmune thyroiditis. It's estimated prevalence is $2: 100,000$ patients, with the majority being females in their $50 \mathrm{~s}-60 \mathrm{~s}$. The underlying pathogenesis is unknown but presentation is divided into two subsets. Subacute is described as multiple remitting and relapsing episodes of mental decompensation often accompanied by seizures. Chronic is described as a slower, gradual onset of cognitive decline, confusion and hallucinations with a subset of patients having rapid decline into coma. HE remains a diagnosis of exclusion with exact diagnostic criteria not established. However, four main features are accepted; encephalopathy associated with cognitive dysfunction and psychiatric features, presence of high titer anti-TPO Ab, exclusion of neurological disease and rapid response to steroid treatment. Of note, so far there does not appear to be a link between the severity of hypothyroidism or antibody levels and severity of HE. Literature review describes a percentage of patients left with permanent cognitive deficits therefore early treatment should be encouraged. Cases of reversible dementia are likely being missed due to lack of awareness of HE. This should be on the differential for patients presenting with cognitive decline.

CONCLUSION: - HE is a diagnosis of exclusion - Early initiation of therapy can prevent permanent cognitive deficits. - Severity of hypothyroidism does not correlate with severity or speed of onset of HE.

\section{FROM OVERDOSE TO BUPRENORPHINE INTAKE IN UNDER ONE HOUR!}

Alessandra Calvo-Friedman ${ }^{3}$; Meredith Lynn ${ }^{1}$; Angela Arbach ${ }^{1}$; Kathleen Hanley ${ }^{2}$; Sondra Zabar ${ }^{3}$. New York University, New York, NY; ${ }^{2}$ NYU, New York, NY; ${ }^{3}$ NYU School of Medicine, New York, NY. (Control ID \#3179270)

LEARNING OBJECTIVE \#1: Recognize and manage opioid overdose in a community health center setting

LEARNING OBJECTIVE \#2: Improve linkage to effective treatment for opioid use disorder after overdose

CASE: A 54 yo man was found unresponsive at the door of our community health center. Rapid Response was called and the patient was found to be unresponsive to sternal rub, with 6 breaths per minute, and pinpoint pupils. One dose of $4 \mathrm{mg}$ of intranasal naloxone was administered, and soon the patient was alert and oriented. He declined transfer to the ED but was amenable to observation, stating that he had just purchased his usual 3 bags and used them outside of his primary care clinic. His PMH was notable for 36 years of IV/intranasal heroin use, prior stroke, GERD, glaucoma, hyperlipidemia, lumbar radiculopathy, and tobacco use. He had one overdose in the 1990s, attempted detox several times and tried self-treating himself with methadone and buprenorphine. He lived with his girlfriend and was unemployed. His medications included cyclobenzaprine and ranitidine. The medical assistant from our addiction medicine clinic engaged the patient, who reported that the overdose scared him, and offered medication treatment which he accepted. The addiction clinic nurse and physician saw the patient that day and gave an initial buprenorphine prescription, instructions and follow-up appointment. He is now stable on buprenorphine $8 \mathrm{mg}$ daily.

IMPACT/DISCUSSION: The overdose described in this case represents one of three overdoses in the past month at our NYC health center. Urban health centers often serve as community hubs and may be seen as a safer place to use opioids. Overdoses at community health centers represent an important point of patient engagement in treatment for OUD. Treatment with opioid agonist therapy after overdose has been shown to reduce all-cause and opioid-related mortality. However, only a small percentage of patients receive medication therapy after overdose. (Larochelle et al. Annals of Internal Medicine. 2018) Initiation of medication treatment for OUD at the time of ED presentation has also been shown to improve engagement in treatment. (DOnofrio et al. JAMA 2015.) Institutional commitment to training all providers and staff to recognize the signs of opioid overdose and administer intranasal naloxone has direct impact on patient outcomes. Our experience with this case has demonstrated the importance of immediate engagement in care at the time of overdose. Having a team available at the time of overdose that cares for patients with addiction enabled us to quickly engage this patient in care and start medication therapy when he felt most receptive to treatment. 
CONCLUSION: Our case demonstrates two crucial steps for improving outcomes in opioid overdose: widespread availability of and training for intranasal naloxone use, along with community health sites equipped to treat patients with opioid use disorder at the time of overdose.

\section{FROM PSEUDOHYPOGLYCEMIA TO DIABETIC KETOACIDOSIS}

Saliha Saleem; Ejaz Shah; Syed Maisam Abbas; Colette Knight. Montefiore Medical Center, Bronx, NY. (Control ID \#3186254)

LEARNING OBJECTIVE \#1: Recognizing peripheral arterial disease (PAD) as possible etiology of pseudohypoglycemia

LEARNING OBJECTIVE \#2: Consider alternate sites/methods for glucose testing in patients with suspected pseudohypoglycemia

CASE: 76 year-old man with Diabetes mellitus, Peripheral Arterial Disease (PAD), Dementia was sent from Nursing Home (NH) to Emergency department (ED) for hypoglycemia ( $28 \mathrm{mg} / \mathrm{dl})$ and altered mental status. He was given 50\% Dextrose (D50) and glucagon injection in $\mathrm{NH}$ but continued to have recurrent hypoglycemia. In ED he was given D50 again, but his finger sticks remained less than $40 \mathrm{mg} / \mathrm{dl}$. He was otherwise hemodynamically stable. On examination he was less alert from his baseline and had decreased pulses in both upper extremities. Initial labs drawn in ED from a peripheral vein revealed Bicarbonate $12 \mathrm{meq} / \mathrm{l}$, Anion Gap 24, Blood Glucose level 604 mg/dl, White count 17k and Ph 7.17. Chest X- ray was consistent with pneumonia. He was started on insulin drip, intravenous fluids and antibiotics. Point of care (POC) site was changed from fingers to right shoulder; subsequent readings were found to be parallel to venous blood glucose readings. Within 24 hours he was transitioned to subcutaneous insulin regimen. His prior records showed evidence of PAD in both upper extremity vessels. Patient clinical status improved back to baseline.

IMPACT/DISCUSSION: Pseudohypoglycemia can be caused by Hypovolemic shock, Hypothermia, Raynaud's, Leukemia, and Polycythemia. PAD affects millions of people in United States, yet only one published case report identifies it as a cause of pseudohypoglycemia. Although PAD most commonly affects lower extremity arteries, it is not uncommon for upper extremities to also be involved. The pathophysiology of pseudohypoglycemia in PAD is almost same as for Raynaud and hypovolemic shock: decreased blood flow. [KS1] Atherosclerotic plaque formation decreases the blood flow to the respective extremity and digital microcirculation, blood stays in the capillary bed for a longer period, increasing glucose consumption, leading to finger stick glucose reading to be abnormally low. Capillary blood glucose measurement from finger tips is the main tool for the checking blood glucose levels. Other sites that can be used for POC glucose monitoring include palm, forearm, upper arm and thigh. These sites are especially useful when finger tips may be considered unreliable. Venous/arterial blood glucose measurement is the most reliable method for an accurate blood glucose reading. In cases of severe hypo/hyperglycemia, this reading should always be validated by alternate measures before aggressive management for hypo/hyperglycemia is initiated.

CONCLUSION: Pseudohypoglycemia should be considered whenever a low POC glucose is encountered. Use of alternate sites for POC glucose testing and checking venous/arterial blood samples can help differentiate between a true and pseudohypoglycemia.

FUNCTIONAL CARCINOID TUMOR ASSOCIATED WITH POLYCYTHEMIA VERA: A RARE DIAGNOSIS AND CHALLENGING MANAGEMENT DILEMMA

Shawnt Tosonian ${ }^{1}$; Zenia Sattar ${ }^{2} .{ }^{1}$ Eisenhower Medical Center, Glendale, CA; ${ }^{2}$ Eisenhower Health, Rancho Mirage, CA. (Control ID \#3181675)

LEARNING OBJECTIVE \#1: Recognize association of NET and polycythemia
LEARNING OBJECTIVE \#2: Identify risks of thrombosis management in this condition

CASE: A 69-year-old woman with duodenal neuroendocrine tumor (NET) and alcoholic cirrhosis presented with diarrhea. NET had been diagnosed 3 months prior, after 2 years of unexplained isolated diarrhea. Endoscopic ultrasound (EUS) had shown a submucosal lesion, with immunohistochemistry of well-differentiated NET (carcinoid tumor). She had no metastases nor functional hormonal syndrome. One week prior to admission, she developed new nocturnal diarrhea, abdominal pain, vomiting, cutaneous flushing, diaphoresis, and pruritis. On admission, vitals were normal; labs showed WBC $16.1 \mathrm{~K} / \mathrm{uL}$ and $\mathrm{Hgb} 19 \mathrm{~g} / \mathrm{dL}$. Abdominal CT (Fig 1) revealed duodenal enteritis and cirrhotic liver. Her presentation and labs [Table 1] suggested carcinoid crisis. Octreotide scan (Fig 2) revealed regional node metastasis. Symptoms improved with octreotide therapy and antacids, but facial plethora, pruritis, polycythemia, and leukocytosis persisted. Low serum erythropoietin indicated primary polycythemia vera (PV), confirmed by JAK2 mutation [Table 1]. She started therapeutic phlebotomy and cytoreductive hydroxyurea. Lower extremity DVTs were found, so enoxaparin was started, leading to large volume melena requiring emergent endoscopy. EGD showed bleeding from multiple duodenal ulcers and the ulcerated NET (Fig 3). Embolization of the gastroduodenal branch artery of the ulcer controlled the bleed; IVC filter was placed. With cirrhosis and low functional performance, she was deemed a poor surgical candidate. She was discharged and recovered well on outpatient follow up.

IMPACT/DISCUSSION: Carcinoid tumors have an age-adjusted incidence of 4.7/100,000. Increasing incidence in recent decades is likely due to improved detection. Most tumors are in the small intestine, with less than $3 \%$ of cases duodenal; only 5-7\% exhibit hormonal production. Only two cases of carcinoid tumor have been associated with $\mathrm{PV}$, one of which was also duodenal. Polycythemia is felt to be secondary; an association between NETs and primary polycythemias has not been established. Active functional carcinoid tumors with polycythemia have not been previously reported. Overlapping clinical manifestations of carcinoid syndrome and PV include facial plethora, pruritis, epigastric distress, and diarrhea; distinguishing these entities is difficult. Lab features can be misinterpreted; in our case, polycythemia and leukocytosis were attributed to hemoconcentration from diarrhea. Management is complicated by increased risk for thrombotic events, given malignancy and hyperviscosity. Risk for hemorrhage is also high, given carcinoid gastrin production.

CONCLUSION: Our case suggests an association between NET and polycythemia. Balancing thrombosis and hemorrhage risks is individualized; care should include multidisciplinary teams with use of serial gastrin levels, IVC filter, and early surgical evaluation.

\section{GASTROINTESTINAL AL-AMYLOIDOSIS PRESENTING AS PROTEIN LOSING ENTEROPATHY}

kavya Kelagere Mayigegowda; Rinad Tabbalat; Aditya Kalakonda. Baystate Medical Center, Springfield, MA. (Control ID \#3187027)

LEARNING OBJECTIVE \#1: Physicians should consider gastrointestinal amyloidosis in their differential when patients present with diarrhea and unexplained hypoalbuminemia.

LEARNING OBJECTIVE \#2: Understanding the pathophysiology of different clinical syndromes seen in gastrointestinal amyloidosis.

CASE: 82-year-old male with a history of CKD stage III and prostate cancer in remission presented with a four month history of worsening lower extremity edema, abdominal pain and watery diarrhea. Initial laboratory work-up was significant for a serum albumin of 2.0 and a normocytic anemia with hemoglobin of $7.5 \mathrm{gm} / \mathrm{DL}$. A stool infectious work-up to include Clostridium Difficile was negative. A CT scan of the abdomen done on admission showed pan-colonic wall thickening suggestive of colitis and mild abdominal ascites. Colonoscopy showed diffuse continuous abnormal vascularity, congestion, erythema, friability, 
inflammation and ulceration throughout the colon compatible with severe colitis. Biopsy showed diffuse mucosal and submucosal amyloidosis with a positive Congo red stain. Serum electrophoresis showed IgG kappa and lambda monoclonal gammopathy with elevated free lambda chain. Subsequent bone marrow biopsy showed plasma cell neoplasm with involvement of $9 \%$ of plasma cells and evidence of focal amyloid deposits. This was consistent with systemic AL light chain amyloidosis with likely lambda light chain deposition. Further work-up was done to evaluate for other organ involvement. Cardiac MRI showed minimal cardiac involvement and there was no evidence of proteinuria on urine analysis. Patient was initiated on chemotherapy with bortezumib and dexamethasone.

IMPACT/DISCUSSION: Clinical syndromes in gastrointestinal AL and AA amyloidosis are different due to the unique patterns of tissue involvement. AA amyloidosis causes Mucosal infiltration of amyloid protein, leading to diarrhea and malabsorption syndromes, while the muscularis mucosal deposition of AL amyloid often presents with constipation or pseudo-obstruction. Contrary to routine findings, our case of gastrointestinal AL amyloidosis presented with diarrhea and protein losing enteropathy. Mucosal erosions or ulcerations and submucosal hematomas are the most common endoscopic findings. The gold standard for diagnosis is with a biopsy with Congo red staining. The treatment of AL amyloidosis includes high-dose chemotherapy and hematopoietic stem cell transplantation. Patients with AL amyloidosis with gastrointestinal involvement have poor prognosis. Chemotherapy causes suppression of plasma cell clones and reduces the concentration of toxic light chains by suppression of plasma cell clones. This is necessary to improve organ function and prolong survival.

CONCLUSION: Physicians should consider gastrointestinal amyloidosis in their differential when patients present with diarrhea and unexplained hypoalbuminemia as prompt recognition and treatment is crucial.

\section{GASTROINTESTINAL BERIBERI CAUSES REFRACTORY LACTIC ACIDOSIS}

Zachary S. Bugos; Fnu Abhishek. UPMC Mercy, Etna, PA. (Control ID \#3185860)

LEARNING OBJECTIVE \#1: Recognize various causes of lactic acidosis

LEARNING OBJECTIVE \#2: Identify major risks for thiamine deficiency

CASE: 44yo F w/PMHx of EtOH abuse presented to ED w/ emesis. Pt reported 4d drinking binge $\mathrm{w} /$ unknown amount of vodka. By d5, she had episodes of non-bloody bilious emesis. Denied fever, chills, abd pain, diarrhea, sick contacts. While in ED, vitals $36.6^{*} \mathrm{~F}$, BP 137/59, HR 128, RR 19 satting $98 \%$ on RA. Labs revealed AG 32.4, bicarb 17, Cr 1.61 (baseline Cr 0.4); mild LFT abnormalities, unremarkable lipase and PT/ INR of 21.8/1.9; leukocytosis \& plt 570. UA, UCx, UDS \& ethanol were all unremarkable. Pt had persistent bilious emesis \& tachycardia. Received 2L NS + GI cocktail. With concern for sepsis, a lactate returned at 6.6. Pt started on D5NS $+20 \mathrm{mEq} \mathrm{KCl}$ at $75 \mathrm{cc} / \mathrm{hr}$. Admitted to floors for AKI \& IVF resuscitation. Following AM, pt reassessed s/p 3L IVF, appearing better $\mathrm{w} /$ minimal nausea. K 2.9, AG 17.9, AKI improved w/ down-trending creatinine to 1.31 . Repeat lactate still elevated to 6.6. Management revolved around EtOH keto-acidosis, however did not explain persistent hyperlactatemia. Over next $2 \mathrm{~d}$, lactate stayed elevated to $5.3,5.0$ and 4.0 despite receiving $7 \mathrm{~L}$ IVF over 3.5d. By HD4, pt was asx w/ near normals lytes and resolved AKI except for refractory hypokalemia and hypomagnesemia. With suspicion for rare causes of lactic acidosis, thiamine level found to be non-existent (lab value $<6$; range 8 $30 \mathrm{nmol} / \mathrm{L}$ ). Thiamine $500 \mathrm{mg}$ IV was given and lactate $\sim 12 \mathrm{hrs}$ later normalized to 1.5 . Dx of thiamine deficiency causing persistent lactic acidosis was made, and $\mathrm{pt} \mathrm{d} / \mathrm{c}$ home the following AM with thiamine.

IMPACT/DISCUSSION: Hyperlactatemia, a common occurrence in critically ill pts, results from accumulation of lactate $\mathrm{d} / \mathrm{t}$ impaired mitochondrial oxidation. Lactate is a marker of illness severity, higher levels correlate w/ worse outcomes. Elevated lactate is generally attributed to tissue hypoperfusion, however other causes of cell dysfxn can result in high levels, such as infection, liver dysfunction, severe trauma, uncommon ADRs \& in this rare case, $\mathrm{d} / \mathrm{t}$ thiamine deficiency Vitamin $\mathrm{B} 1$, aka thiamine, is a cofactor for enzymes involved in metabolism \& biosynthesis of neurotransmitters. Thiamine deficiency is a clinical $\mathrm{dx}$, as direct serum measurements are not indicative of thiamine status, as only $0.8 \%$ of total body stores are present in blood. Elevated blood pyruvate \& lactate measurements can be useful, however false positives in setting of sepsis \& cardiogenic shock are associated $\mathrm{w} /$ lactic acidosis, imitating thiamine deficiency. Refractory lactic acidosis s/p glucose load supports $\mathrm{dx}$ of thiamine deficiency, making it more practical to give thiamine $\&$ monitor for response.

CONCLUSION: Lactic acidosis is used to describe accumulation of lactate, a surrogate marker for hypo-perfusion \& cell dysfxn Major causes of elevation are attributed to hypovolemic states, sepsis or poor clearance (i.e. renal or liver failure) Thiamine, aka Vitamin B1, when deficient, causes lactic acidosis refractory to fluid resuscitation

\section{GENERAL PARESIS: A FORGOTTEN PSYCHIATRIC SYN- DROME OF NEUROSYPHILIS}

Joseph Boyle $^{1}$; Kelly White ${ }^{2} .{ }^{1}$ University of Colorado SOM, Denver, CO; ${ }^{2}$ University of Colorado SOM, Aurora, CO. (Control ID \#3150083)

LEARNING OBJECTIVE \#1: Recognize and diagnose neurosyphilis LEARNING OBJECTIVE \#2: Recognize emergent psychiatric symptoms may be due to neurosyphilis

CASE: A 61-year-old woman with a history of CIN I, seasonal allergies, low back pain, and homelessness presented to primary care clinic with 3 years of worsening mood lability, paranoid delusions, and increasingly confrontational behavior toward staff with frequent missed appointments. Attempted referrals to psychiatric care were unsuccessful and an accurate psychiatric diagnosis remained elusive. At a subsequent appointment, she complained of tingling pain in her hands and feet, but declined lab testing. Months later she presented with a new chronic headache and a c-spine CT report from an outside ED showing diffuse cervical lymphadenopathy. At that time, she agreed to testing and was found to have positive RPR, with normal $\mathrm{CBC}$, TSH, B12, and negative HIV Ab. Confirmatory testing showed a positive serum Treponema Ab, and LP showed 46 lymphocytes and positive VDRL. There were no notable neurological findings or palpable lymphadenopathy. The patient denied sexual contact for more than 20 years, and abstinence had been documented in the chart for at least 6 years. She was admitted to the hospital for treatment with IV penicillin. At the time of admission her MOCA was 23. She will be followed with serial cognitive exams, psychiatric evaluations, and CSF studies to document remission.

IMPACT/DISCUSSION: It is known that syphilis can go undiagnosed in the early stages before entering a long latent period. Screening recommendations exist for at risk populations including MSM and HIV+ individuals.1 US prevalence is increasing to $31 / 100,000$ since nadir in the year 2000.2 Only a small minority of cases are in women. Late symptoms were thought to have been largely eliminated since the antibiotic era but the psychiatric symptoms are frequently misdiagnosed.3 Late manifestations of syphilis include skin changes, neurological findings: including headache, psychiatric symptoms, and finally, dementia. The psychiatric symptoms are classically named general paresis, which can include emotional lability, memory deficits, and psychosis4, as seen in this patient. Diagnosis of neurosyphilis is made by CSF with increased lymphocytes and either Treponema Ab or VDRL. VDRL is more sensitive than RPR on CSF.5 The goal of treatment for general paresis is to halt progression to dementia, but reversal of symptoms is uncommon.6 1 . CDC, 2016. 2. CDC, 2017. 3. Yanhua W, BMC Psychiatry. 2016;16(1):16. 4. Mattei PL, Am Fam Physician. 2012;86(5):433-440. 5. Marra CM, Sex Transm Dis. 2012;39(6):453. 6. Hook EW. Syphilis. Lancet. 2017;389(10078):1550-1557. Ref abbrev due to space limits. 
CONCLUSION: Neurosyphilis may cause significant morbidity, and can be extremely challenging to diagnose. Consideration must be given to this important and potentially treatable cause of memory loss, psychiatric symptoms, and personality changes in all patients, not just those known to have an increased risk or known history of syphilis.

\section{GETTING TO THE HEART OF THE MATTER: A CASE OF SERONEGATIVE ANTIPHOSPHOLIPID SYNDROME}

Sonia D. Silinsky Krupnikova; Linda Yue; Jeffrey Tefft; Rodolfo Curiel. The George Washington University, Washington, DC. (Control ID \#3179062)

LEARNING OBJECTIVE \#1: Recognize seronegative antiphospholipid syndrome as a distinct clinical entity.

CASE: A 52-year-old gentleman with past medical history of lupus nephritis, non-ST-elevation myocardial infarction, venous thromboembolism (on anticoagulation), and peripheral vascular disease requiring arterial bypass grafting presented to the hospital with two days of left toe pain, fever and chills. Physical examination demonstrated gangrenous changes of the left second toe as well as nontender, nonblanching erythematous macules and patches along the plantar surface of the foot. Laboratory evaluation revealed prolonged partial thromboplastin time and elevated inflammatory markers without leukocytosis. Infectious serologies and final blood cultures were negative. Hypercoagulability studies, including antiphospholipid antibody titers and lupus anticoagulant, were normal. On imaging, he was found to have a patent bypass graft but was noted to have a $2.5-\mathrm{cm}$ vegetation on the tricuspid valve. He had no atrial septal defect. Intravenous antibiotics were initiated for four weeks without resolution, and the patient underwent surgical tricuspid valve replacement and toe amputation. Intraoperative pathology was notable for sterile vegetation and toes with arterial thrombus but no vasculitis nor organisms on Gram stain or final culture.

IMPACT/DISCUSSION: Antiphospholipid syndrome (APS) is a hypercoagulable state which often occurs secondary to systemic lupus erythematosus (SLE). The diagnostic criteria for APS require the presence of at least one clinical and one laboratory criterion. Clinical criteria include vascular thrombosis or pregnancy morbidity and laboratory criteria include the presence of lupus anticoagulant, anti-cardiolipin, or anti- 2-glycoprotein. APS also predisposes to the development of sterile thrombotic LibmanSacks endocarditis, which most commonly affects the mitral valve. Seronegative APS (SN-APS) is diagnosed when clinical criteria are met but serologic testing is persistently negative. Testing must be performed outside the contexts of acute thrombosis and treatment with anticoagulation, as these conditions can cause a transient loss or consumption of antiphospholipid antibodies. This patient's case was unusual for several reasons. His Libman-Sacks endocarditis was located on the tricuspid valve, a rare presentation. Moreover, his toe ischemia was the result of an arterial thrombus rather than embolism or ischemia from graft stenosis. Given his extensive history of hypercoagulability in the setting of known SLE, thrombosis secondary to SN-APS was the unifying diagnosis.

CONCLUSION: APS is a hypercoagulable state associated with SLE. It confers increased risk of developing Libman-Sacks endocarditis as well as peripheral thrombotic events which may mimic other clinical entities such as limb ischemia or embolism. SN-APS is a clinically important diagnosis to consider in patients with clinical manifestations of the syndrome but persistently negative serology.

\section{GLYCOGENIC HEPATOPATHY: AN UNDERRECOGNIZED CAUSE OF HEPATOMEGALY}

Jessica Sang. Carolinas Medical Center, Charlotte, NC. (Control ID \#3186268)

LEARNING OBJECTIVE \#1: Recognize that elevated plasma lactate levels can be part of the clinical presentation of glycogenic hepatopathy
LEARNING OBJECTIVE \#2: Review methods to distinguish GH from non-alcoholic fatty liver disease (NAFLD)

CASE: An 18-year-old female presented to the hospital after three-days of nausea, vomiting, and abdominal pain. She had a history of uncontrolled type 1 diabetes mellitus (HbA1c: 11.7\%) diagnosed at age 12 with recurrent hospital admissions for diabetic ketoacidosis (DKA). Her medications included insulin detemir 10 units daily and a 1:10 insulin to carbohydrate ratio of insulin aspart with meals. She reported nonbloody emesis and continuous right sided abdominal discomfort. On physical examination she was tachycardic. She had a distended abdomen that was moderately tender in the right upper and lower quadrants with palpable liver edge. Conjunctival icterus and jaundice were not present. Initial labs revealed: ALT $42 \mathrm{IU} / \mathrm{L}$, AST $54 \mathrm{IU} / \mathrm{L}$, INR 1.3, total bilirubin $0.6 \mathrm{mg} / \mathrm{dL}$, alkaline phosphatase $90 \mathrm{IU} / \mathrm{L}$, anion gap $19 \mathrm{mmol} / \mathrm{L}$, glucose $1,060 \mathrm{mmol} / \mathrm{L}$, lactic acid $8.9 \mathrm{mmol} / \mathrm{L}$, and urine ketones. CT abdomen and pelvis showed hepatomegaly. The liver measured $23.4 \mathrm{~cm}$ in length and extended to her left upper quadrant. No liver masses were visualized. Given the patient's history, lab results, and imaging the diagnosis highest on the differential for her hepatomegaly was glycogenic hepatopathy (GH) from poorly controlled diabetes. Both DKA and GH were treated with glycemic control. Her elevated lactic acid slowly trended down and she was discharged with close hospital follow-up.

IMPACT/DISCUSSION: $\mathrm{GH}$ is a complication of poorly controlled diabetes mellitus characterized by transient liver dysfunction with elevated liver enzymes. ${ }^{1}$ Patients with GH can be asymptomatic or can present with abdominal pain, hepatomegaly, recurrent DKA, and elevated lactate levels. ${ }^{1}$ Lactic acidosis can be present in patients even if they are not in DKA. ${ }^{2}$ The pathophysiology is not well understood. One hypothesis is that the reduction in gluconeogenesis and lack of conversion of pyruvate to glucose may raise lactate levels in the body. ${ }^{3}$ In regards to the hepatomegaly in $\mathrm{GH}$, it is caused by a reversible accumulation of excess glycogen in hepatocytes and can be misidentified as NAFLD on imaging. ${ }^{1}$ It is important to discriminate between them because NAFLD can progress to advanced liver disease. The gold standard for diagnosis is biopsy. ${ }^{4}$ Although ultrasound and CT cannot differentiate between the diseases processes, gradient-dual echo liver MRI can help distinguish hepatic glycogen in GH from the fat seen in NAFLD and is a less invasive alternative to biopsy. ${ }^{5}$ Treatment of GH is glycemic control and improvement of symptoms can be seen in days to weeks.

CONCLUSION: GH is an underrecognized complication of poorly controlled diabetes mellitus that can present with elevated lactic acid and hepatomegaly and can be misidentified as NAFLD. Gradient-dual echo liver MRI may help distinguish between these two entities.

\section{GOUT OF THE ORDINARY: CLAVICULAR GOUT IN HIV}

Jay Thetford ${ }^{1}$; Emil Heinze ${ }^{2}$; Hrishikesh Belani ${ }^{3} .{ }^{1}$ UCLA-Olive View, Los Angeles, CA; ${ }^{2}$ David Geffen School of Medicine at UCLA, Sylmar, CA; ${ }^{3}$ Olive View - UCLA Medical Center, Los Angeles, CA. (Control ID \#3184713)

LEARNING OBJECTIVE \#1: Tophi may involve axial joints and develop prior to active gout flares

LEARNING OBJECTIVE \#2: HIV infection is associated with hyperuricemia and gout

CASE: A 69 year-old male with HIV well-controlled on comprehensive anti-retroviral therapy (cART), HCV, HTN, and CKD stage 3 presented with 6 weeks of worsening pain in his right sternoclavicular (SC) joint. The patient noted clavicular bony enlargement over 1 year and denied any fevers, chills, night sweats, or unintentional weight loss. Physical exam revealed bony prominence of the right SC joint with tenderness to palpation, but without overlying erythema or warmth. MRI showed joint effusion, bone marrow edema, and bone erosions about the SC joint concerning for inflammatory arthritis or osteomyelitis. Arthrocentesis demonstrated extracellular birefrigent crystals consistent with urate crystals. Synovial fluid 
bacterial, fungal, and AFB cultures were all negative. Interestingly, the erythrocyte sedimentation rate (ESR) was only mildly elevated at $52 \mathrm{~mm} /$ $\mathrm{hr}$ and both serum uric acid, and white blood cell count (WBC) were within normal limits at that time. Soon after, he received an intralesional corticosteroid injection, which provided marked pain relief and significantly reduced the size, swelling, and tenderness of the SC joint.

IMPACT/DISCUSSION: While most episodes of gout occur in the lower extremities, gout may occur in nearly any joint in the body [1]. In this case, tophi formation occurred in the axial skeleton prior to onset of pain and the typical hallmarks of acute gout attacks were absent. Without these tell-tale clinical signs, the diagnosis of gouty arthritis was dependent on synovial fluid analysis, which is the gold standard [1]. While elevated serum uric acid, ESR, and WBC may help increase suspicion for gout, they are not necessary for diagnosis [1]. This case also highlights the association of HIV infection with hyperuricemia and gout [2]. This is thought to be due to a number of factors including increased T-cell turnover, HIV nephropathy, the creation of an inflammatory milieu, and, in some cases the effects from antiretroviral medication itself $[2,3]$. Didanosine, stavudine, zalcitabine, and ritonavir, for example, are all antiretrovirals associated with increased urate levels [2,4]. Interestingly, poorly controlled HIV and AIDS has been associated with hypouricemia due to malnutrition and increased renotubular losses; in patients started on cART may develop gout as part of an immune reconstitution inflammatory syndrome (IRIS) $[2,3,5,6]$.

CONCLUSION: Tophaceous gout may involve axial joints and develop in the absence of acute gout flares. Our patient was one such case, presenting with slowly developing tophaceous gout in his SC joint prior to any acute episodes. HIV infection and the antiretroviral therapy used to treat it can produce elevated serum uric acid levels. Our patient was on a number of ART medications, including ritonavir, which has been associated with elevated uric acid levels and possibly contributed to his unique presentation.

\section{GRANULOMATOSIS WITH POLYANGIITIS MANIFESTING AS SYMPTOMATIC SELLAR MASS IN A YOUNG WOMAN}

sachin gadani ${ }^{1}$; Fahid Alghanim ${ }^{1}$; rohini nadgir ${ }^{3}$; Annika Windon ${ }^{2}$; Lisa Rooper $^{2}$; Karan Desai ${ }^{1} .{ }^{1}$ Johns Hopkins Bayview Medical Center, Baltimore, MD; ${ }^{2}$ Johns Hoskins Hospital, Baltimore, MD; ${ }^{3}$ Johns Hopkins Hospital, Baltimore, MD. (Control ID \#3180086)

LEARNING OBJECTIVE \#1: Recognize that pituitary mass is a complication of GPA

LEARNING OBJECTIVE \#2: Diagnose hypopituitarism based on review of systems, physical, and labs

CASE: A 25 y.o. Puerto Rican woman with granulomatosis with polyangiitis (GPA) and known sellar mass presented to the ED with headache and vomiting. The day prior she developed left sided headache and fever, followed by nausea and profuse vomiting, symptoms she had been experiencing weekly for the past several months. Detailed review of systems revealed 8 months of amenorrhea, fatigue, cold intolerance, $201 \mathrm{~b}$ weight gain, and constipation. Physical exam showed no meningismus, papilledema, or visual field deficit. Lab studies showed TSH $0.06 \mathrm{u} / \mathrm{mL}$, free T4 $0.4 \mathrm{ng} / \mathrm{dL}, \mathrm{LH}$ $0.4 \mathrm{u} / \mathrm{mL}$, and FSH $2.5 \mathrm{u} / \mathrm{mL}$, consistent with hypopituitarism. These findings led to investigation of her known sellar mass. 10 months prior a cystic, nonenhancing sellar lesion was seen incidentally on MRI, thought to represent Rathke's cleft cyst. Repeat MRI showed notable enlargement, spread, and post-contrast enhancement, not consistent with Rathke's cyst. Diagnostic biopsy of the sellar mass demonstrated acute and chronic inflammation, reactive histiocytes, and necrobiosis, consistent with autoimmune inflammation related to GPA. She was treated with prednisone and cyclophosphamide. 2 months later the patient requested to be transitioned from cyclophosphamide to rituximab because of concerns about impaired fertility associated with cyclophosphamide.
IMPACT/DISCUSSION: Here we report the case of a woman who was found to have hypopituitarism due to a GPA-related sellar mass. A challenge in this case was the initial diagnosis of Rathke's cleft cyst based on the first MRI, which may have delayed accurate diagnosis of her pituitary lesion. This case sheds light on temporal imaging changes that such a lesion can undergo as it progresses, and underscores the nonspecific nature of imaging findings. Previously reported cases of pituitary GPA have showed varied MRI findings, with T1 hypo- or hyperintensity, T2 hyperintensity, and heterogeneous enhancement typical. When approaching a pituitary lesion in a patient with GPA suspicion for autoinflammation should remain high, and, in leiu of biopsy, careful consideration of patient history and disease progression is needed. Previous reports suggest that pituitary GPA lesions are often amenable to treatment. In one case series of 8 GPA patients with pituitary lesion all achieved remission after therapy with corticosteroids (CT) combined with either cyclophosphamide (CYC) or rituximab. In another series of 9 patients, all but one achieved remission after $\mathrm{CT}$ combined with secondary immunosuppression. Notably, however, prior studies have reported chronic hypopituitarism in many patients,

CONCLUSION: - GPA can cause symptomatic pituitary lesions, which may be associated with significant morbidity - The diagnosis of pituitary GPA is difficult to make on imaging alone - Routinely employing a thorough review of systems is critical to ensure the accurate diagnosis be made.

\section{GREEN URINE: BENIGN OR PATHOLOGIC?}

Ermias Sori. UT Health San Antonio, San Antonio, TX. (Control ID $\# 3186120)$

LEARNING OBJECTIVE \#1: Logical inexpensive evaluation of acute green urine color change.

CASE: A 55-year-old veteran presented to the hospital for worsening shortness of breath and severe hyponatremia found to have acute respiratory failure due to fluid overload treated with hemodialysis. His past medical history included alcoholic cirrhosis and hyperlipidemia. On exam, he was tachycardic(120 beats per minute), with reminder of exam unremarkable. On laboratory evaluation, he was noted to have a sodium level of $109 \mathrm{mEq} / \mathrm{L}$ that was suspected to be beer potomania. While on mechanical ventilation, he was sedated with a continuous propofol infusion. On day two, he developed green urine discoloration. Infectious work-up, especially for pseudomonas urinary tract infection, was negative. It was believed that the urine color change was suspected of propofol. The urine color changed back to normal after $12 \mathrm{hr}$ propofol discontinuation.

IMPACT/DISCUSSION: Urine discoloration is uncommon encounter in intensive care units. Many factors can cause green urine discoloration. The most common infectious etiology is Pseudomonas urinary tract infection. Other causes include Dyes such as Methylene blue, Biliverdin, Indigo blue and food colorings, and drugs including Propofol, amitriptyline, indomethacin, Cimetidine, metoclopramide, methocarbamol and promethazine. Clinicians must rule out infectious etiology on initial observation of green urine specially in intensive care setting. Propofolinduced urine discoloration is a rare occurrence. Blakey et al. reported incidence of less than $1 \%$. Propofol is metabolized in liver. It is conjugated to glucuronides and sulphate and subsequently excreted by kidney. These metabolites are biologically inactive and do not affect kidney function. It is reported that overflow of the extrahepatic elimination plays a critical role in green discoloration of urine. It has been reported that propofolrelated urine discoloration in a patient with fatal atypical intracerebral hemorrhage treated with hypothermia and osmotic therapy. It is known that glucuronosyltransferase and sulfotransferases enzymes play major role in phase II-drug metabolism in liver. In a cirrhosis and hypothermia state, the function of those enzymes is extensively compromised. As a result, overflow of extrahepatic elimination may be increased which will result in green urine. Our patient is a perfect example of this scenario. 
CONCLUSION: This case illustrates a case of acute discoloration of urine in cirrhotic patient after propofol infusion. Awareness of this benign side effect would help clinician to approach the cases in a cost-effective way.

\section{HAART-BROKEN: A CASE OF TENOFOVIR INDUCED FANCONI SYNDROME AND ASSOCIATED OSTEOMALACIA}

Kristin Berger; Carla L. Spagnoletti. University of Pittsburgh Medical Center, Lewisburg, PA. (Control ID \#3185752)

LEARNING OBJECTIVE \#1: Recognize and diagnose Fanconi syndrome in patients treated with tenofovir.

CASE: The patient is a 54-year-old male with HIV infection and chronic kidney disease who presented to the hospital due to 3 days of nausea and vomiting. He also reported months of diffuse bone pain and lower extremity myalgias. He reported adherence to his antiretroviral medication regimen including emtricitabine-tenofovir and lopinavir-ritonavir, which was unchanged for many years. His HIV viral load was undetectable and CD4+ count was 890 two weeks prior to presentation. Examination revealed an afebrile, normotensive male with diffuse $4 / 5$ strength in his extremities. Labs were remarkable for serum sodium 139, potassium 2.1, chloride 111, bicarbonate 16, creatinine 4.1, glucose 92, and phosphorus 2.1. Urinalysis was positive for $2+$ glucose and $1+$ protein. The patient's $\mathrm{CT}$ abdomen and pelvis demonstrated subacute bilateral rib and inferior pubic rami fractures. Fanconi syndrome was suspected given the presence of nonanion gap metabolic acidosis with associated hypokalemia, hypophosphatemia, glucosuria, and proteinuria. Given the suspected diagnosis, the patient's tenofovir was discontinued and he was rehydrated with intravenous fluids. His creatinine returned to his prior baseline of 2.1 by hospital discharge. He was seen in renal clinic at outpatient follow up for ongoing repletion of electrolytes and bicarbonate. Months following hospital discharge, his muscle and bone pains had resolved and his urinalysis had normalized.

IMPACT/DISCUSSION: Fanconi syndrome is characterized by a defect in the renal proximal tubules resulting in phosphaturia, glucosuria, tubular proteinuria, hypokalemia, and proximal renal tubular acidosis due to impaired bicarbonate reabsorption. Acquired Fanconi syndrome is most commonly due to adverse drug reactions, multiple myeloma, and heavy metal toxicity. Prolonged hypophosphatemia in patients with Fanconi syndrome may result in osteomalacia causing bone pain and myalgias as well as increased risk of fracture. ${ }^{1}$ Prognosis of tenofovir-associated Fanconi syndrome is generally good, with reversible renal toxicity following drug discontinuation. ${ }^{2}$ It is more likely to occur in patients who have underlying kidney disease. Patients benefit from electrolyte and bicarbonate repletion until the proximal tubule defect improves.

CONCLUSION: Tenofovir has a known association with proximal tubule dysfunction, including in HIV-uninfected patients taking the medication as HIV pre-exposure prophylaxis (PrEP). ${ }^{3}$ Increasing recognition of this potential adverse drug effect is critical for early discovery and drug discontinuation, particularly as increasing numbers of primary care doctors are prescribing tenofovir for PrEP. Delay in diagnosis may result in prolonged hypophosphatemia and subsequent osteomalacia in some cases. Regular renal function panel and urinalysis is recommended for patients treated with tenofovir for early detection of tubulopathy.

\section{HASHIMOTO ENCEPHALOPATHY: REVERSING ALTERED MENTAL STATUS WITH STEROIDS}

Jiyeon Son ${ }^{1}$; Ayako W. Fujita ${ }^{1}$; Joseph M. Rocco ${ }^{2} .{ }^{1}$ University of Pittsburgh Medical Center, Pittsburgh, PA; ${ }^{2}$ University of Pittsburgh, Pittsburgh, PA. (Control ID \#3177048)

LEARNING OBJECTIVE \#1: Recognize the diagnostic criteria and clinical features of Hashimoto encephalopathy.

LEARNING OBJECTIVE \#2: Identify differential diagnoses of rapidly progressive altered mental status without evidence of stroke or seizure.
CASE: A 75-year old man with history of heart failure, COPD, and prior stroke presented with two months of progressive confusion, aphasia and hypersomnolence. Patient was previously independent and could manage his finances without assistance. Symptoms were initially attributed to infection, and he received multiple courses of antibiotics without improvement. On exam, vital signs were normal, but he was minimally responsive. He was not oriented and not following commands. No rigidity or hyperreflexia were noted. Mild myoclonus was present. CT was unchanged from prior, and EEG was negative for seizure. MRI was not obtained due to his pacemaker. Symptoms continued to progress, and he developed new onset of seizures. Extensive workup was negative including B12, RPR, HIV, ANA and TSH. CSF showed elevated protein at $71 \mathrm{mg} / \mathrm{dL}$ but no cells. CSF viral PCRs and cytology were negative. However, his serum anti-thyroglobulin (TG) and anti-thyroid peroxidase (TPO) antibodies were elevated at $23 \mathrm{IU} / \mathrm{mL}$ and $484 \mathrm{IU} / \mathrm{mL}$, respectively. Given his clinical symptoms and elevated anti-thyroid antibodies, he was diagnosed with Hashimoto encephalopathy. Patient was treated with pulse steroids and IVIG with improvement in his mental status. However, steroids were discontinued due to complications including ischemic colitis and bacteremia. Family decided to focus on comfort care and he passed away peacefully shortly thereafter.

IMPACT/DISCUSSION: With rapidly progressive cognitive decline it is important to evaluate for reversible processes including occult metabolic, infectious, and autoimmune/paraneoplastic etiologies. Hashimoto encephalopathy is often responsive to steroids and can be reversible.

CONCLUSION: Hashimoto encephalopathy (HE), also known as steroid responsive encephalopathy, is believed to be immune mediated, rather than a direct effect of altered thyroid state on the CNS. Clinical manifestations include acute to subacute onset of confusion with alteration of consciousness. Other presentations include seizures, hallucinations, somnolence, myoclonus, and diffuse hyperreflexia with other pyramidal tract signs. HE can be diagnosed with elevated serum level of anti-TPO or anti-TG autoantibodies in the appropriate clinical setting. CSF typically has elevated protein concentration, EEG shows nonspecific slowing of background activity, and MRI is usually normal. It is imperative that other causes of rapidly progressive dementia are ruled out, including Creutzfeldt-Jakob disease, metabolic diseases, infectious meningoencephalitis, psychiatric disease, and autoimmune encephalitis. HE is treated with corticosteroids, and symptoms can be reversible over months. Other immunosuppressive treatments such as IVIG or plasmapheresis can be pursued but have not been well-studied.

\section{HASHING OUT THE UNKNOWNS OF THE CBD CRAZE}

Jessica Bass. Naples Community Hospital, Naples, FL. (Control ID \#3184977)

LEARNING OBJECTIVE \#1: Consider CBD toxicity in the differential when urine toxicology is negative.

LEARNING OBJECTIVE \#2: Recognize the potential for harm in CBD products sold OTC.

CASE: A 57 year old male with no history of substance abuse presented by ambulance to the hospital after being found by his coworkers acting strange, vomiting, and slurring his speech. He had legally purchased CBD gummies marketed for pain and anxiety relief at a gas station several hours prior. Vitals upon arrival were: T 36.8, HR 79, RR 12, BP 113/60 and $\mathrm{O} 2$ sat of $84 \%$ on RA that improved with arousal. Physical exam showed a morbidly obese man in no acute distress with a depressed level of consciousness but whom awoke to stimuli. Neuro exam was significant for dysarthric, hypophonic speech. ABG at that time showed $\mathrm{pH} 7.3$, pCO2 50.4 pO2 83.3 Lac 2.4. Labs were notable for WBC 17.8, Na 140, $\mathrm{K} 5.8, \mathrm{Cl} 109, \mathrm{CO} 2$ 25, BUN 21, Cr 1.2, CK 341, CKMB 4.4 and a comprehensive tox screen including cannabis was negative. Vitals 3 hours after presentation deteriorated, showing: HR 47, RR 8-12, BP 88/52, O2 $78 \%$. EKG revealed sinus bradycardia. The patient progressively became 
more obtunded and required constant stimuli in order to maintain his airway. CPAP was not administered due to continued emesis. The next morning, the patient was $\mathrm{A} \& \mathrm{O} \times 3$ and vitals had improved. Further history from the patient revealed he had consumed 2 packages of CBD gummies, totaling $370 \mathrm{mg}$ total of CBD (serving size on the package was $30 \mathrm{mg}$ ). He felt the products were healthy and safe based on packaging and did not believe they would have any adverse effects.

IMPACT/DISCUSSION: CBD is one of many cannabinoids found in marijuana and marijuana-derived products. It is generally considered safe unlike its more psychoactive counterpart, THC, which has been linked to seizures, respiratory depression, and cardiovascular complications. CBD has surged in popularity recently, being marketed in oils, capsules, and candies as a health supplement, claiming to treat everything from glaucoma to pain to preventing cancer. Most studies currently available do not look at isolated CBD nor their synthetic equivalents, and purity is not guaranteed, thus leading to unforeseen side effects and toxicities. Moreover, these compounds do not show on traditional toxicology screens, posing a diagnostic dilemma for physicians. This case of respiratory depression and cardiovascular compromise in a relatively healthy man is just one example of the importance of considering synthetic CBD toxicity in the differential, as little data is currently available for diagnosis or treatment. Furthermore, misleading marketing of such products poses a risk to public safety; thus educating patients when prompted is vital.

CONCLUSION: CBD toxicity is likely to be seen more frequently as access to unregulated products surges and given their enticing claims of safety and health benefits. Clinicians should be aware of the potential for serious harm for both diagnostic and patient-counseling purposes as well as to fuel further research in the area.

\section{HAVE YOU SEEN LIVER ENZYMES THIS HIGH IN CONGES- TIVE HEPATOPATHY: A CASE REPORT}

Laith Numan; Ahmed A. Elkafrawy; Andrew Tomaw; Khalil Abuamr. University of Missouri-Kansas City, Kansas City, MO. (Control ID \#3163001)

LEARNING OBJECTIVE \#1: Recognize that congestive hepatopathy can present with liver enzymes higher than three times the normal levels.

LEARNING OBJECTIVE \#2: Treat as soon as the etiology is identified to avoid irreversible damage to the liver, and trend the liver enzymes to assess the response.

CASE: A 33-year-old male previously healthy presented to the emergency department with epigastric pain of two weeks duration. At the same time, he noticed yellowish discoloration of his eyes. He denied acetaminophen use. On review of systems, he was complaining of increased shortness of breath and new lower limbs swelling. He denied any illicit drug use, excessive alcohol intake, recent travel, or mushrooms consumption. On physical examination, he had scleral icterus, bilateral crackles on auscultation, and lower limns pitting edema. On labs, his liver function test was trending up: AST(U/L) $883>2251$, ALT (U/L) $1334>2527$, and total bilirubin (mg/dL) $3.2>3.6$. Acetaminophen level was negative at presentation and 4 hours after presentation. International randomized ratio (INR) was trending up as well: $3.2>$ 3.6. Chest x-ray showed cardiomegaly. Computed tomography (CT) of the abdomen with contrast showed volume overload with small right pleural effusion, ascites and anasarca. There were no signs of liver ischemia or infarct on CT scan. Ultrasound Doppler of the liver showed patent vasculature and congestion of the inferior vena cava. Throughout his hospitalization, he remained normotensive. Hepatology team recommended to trend his liver enzymes, INR and to do neuro checks every 2 hours. Given his fluid overload, an echocardiogram was done, and it showed severely dilated left ventricle, ejection fraction of less than $20 \%$, severe global hypokinesis of the left and right ventricles, severe aortic regurgitation and bicuspid aortic valve. Cardiology team recommended to start the patient on intravenous furosemide twice daily, and then he underwent left heart catheterization which showed normal coronary arteries. After the first dose of furosemide, his liver enzymes and
INR started trending down till they reached normal levels. He was diagnosed with congestive hepatopathy that was due to congestive heart failure secondary to severe aortic insufficiency. The patient improved and was discharged on Spironolactone, Carvedilol, and Torsemide. He was scheduled for an outpatient aortic valve replacement.

IMPACT/DISCUSSION: Congestive hepatopathy is common with congestive heart failure, especially with right-sided heart failure. Typically, the liver enzymes will be elevated but not higher than three times the normal value. It is rare for congestive hepatopathy to presents with liver enzymes as high as this case. However, you always have to rule out other causes of acute transaminitis.

CONCLUSION: Identifying the cause of acute transaminitis is time sensitive, as the outcome could be catastrophic. Do not exclude congestive hepatopathy if the liver enzymes are very high, think about it if other physical signs of congestive heart failure are present.

\section{HE WHO STILL KNOWS SYPHILIS KNOWS MEDICINE}

Samantha R. Pop. Beth Israel Deaconess Medical Center, Boston, MA. (Control ID \#3186045)

LEARNING OBJECTIVE \#1: Recognize the clinical manifestations of secondary syphilis.

LEARNING OBJECTIVE \#2: Consider empiric treatment of patients with high clinical suspicion of secondary syphilis at risk for poor followup prior to laboratory confirmation of diagnosis.

CASE: A healthy 22-year-old woman presented to a primary care clinic requesting screening for sexually transmitted infections. She reported unprotected sexual intercourse with a male partner three months prior to presentation. On review of systems, she reported a new rash involving her palms and soles, patchy hair loss, and a painless genital lesion. She reported a recent history of fever, myalgias, and sore throat that had since resolved. Her exam was notable for multiple 0.5 to $1.5 \mathrm{~cm}$ annular red-brown erythematous papules and plaques with collarette of scale located on her palms and soles. She had bilateral mobile, non-tender anterior cervical and inguinal lymphadenopathy. Gynecologic exam showed a $0.5 \mathrm{~cm}$ gray-white papule on the left labia minora. Her physical exam findings and sexual history raised a high clinical suspicion for secondary syphilis and she was treated empirically with 2.4 million units of penicillin G benzathine intramuscularly during the initial encounter. Laboratory studies were significant for a rapid plasma reagin that finalized after 72 hours as reactive with a 1:64 titer. A reflexive treponemal antibody test was reactive confirming the diagnosis. HIV antibody testing was negative. Cervical specimens sent for nucleic acid amplification testing were positive for Chlamydia trachomatis and negative for Neisseria gonorrhea. She was treated with 1 gram of azithromycin PO for chlamydia co-infection. The diagnosis was reported to local and state departments of public health. The patient was scheduled for follow up at one month to monitor for resolution of symptoms and unfortunately was lost to follow up.

IMPACT/DISCUSSION: William Osler suggested "he who knows syphilis knows medicine" reflecting how common the disease caused by the spirochete Treoponema pallidum was during his tenure in the early $20^{\text {th }}$ century. The manifestations of secondary syphilis are varied and include constitutional symptoms such as fever, myalgias, pharyngitis, a generalized papulosquamous eruption involving the palms and soles, lymphadenopathy, condyloma lata (gray, moist anogenital lesions) and patchy alopecia or telogen effluvium. Now it is often thought of as a rarity, however, the reported rates of syphilis in the United States are rising. While, mostly attributed to an increased number of cases among men who have sex with men, within the last five years, the rates of primary and secondary syphilis among women have more than doubled. This increase is of particular concern given its association with congenital syphilis.

CONCLUSION: Syphilis is making a return and clinicians must recognize the manifestations of secondary syphilis and administer early treatment, especially in women of child-bearing age. 


\section{HELP, I'M FALLING! A CASE OF CEFEPIME-ASSOCIATED THROMBOCYTOPENIA}

Andraya Gower ${ }^{2}$; Meghana Gadgil ${ }^{1}$. ${ }^{1}$ Dell Medical School, Austin, TX; ${ }^{2}$ Dell Medical School, Oklahoma City, OK. (Control ID \#3186595)

LEARNING OBJECTIVE \#1: Recognize cefepime as a rare cause of thrombocytopenia

LEARNING OBJECTIVE \#2: Recognize the applications of the Naranjo scale for assessing adverse drug reactions

CASE: A 35-year-old female with no significant PMH presented with one day of right-lower quadrant abdominal pain radiating to the back associated with fever, hypotension, nausea and vomiting. Vital signs in the ER were: BP 89/55, temp 98.4, HR 70, RR 15. Physical exam was significant tenderness to palpation in RLQ without rebound tenderness, right CVA tenderness and suprapubic tenderness to palpation. Labs were significant for lactate of 4.15, moderate blood in urine, potassium of 2.7, anion gap of 15. Platelets were 171. Renal ultrasound revealed $\mathrm{R}$ sided mild hydronephrosis. MRI Abdomen/Pelvis showed R side hydronephrosis and hydroureter with $\mathrm{R}$ perinephric fat stranding. The patient was admitted to the ICU with severe sepsis from obstructive pyelonephritis likely secondary to obstructive renal calculus. The patient was started on ceftriaxone, but developed worsening hypotension and was broadened to cefepime overnight on day 1 of admission. The patient was on heparin for DVT prophylaxis. On day 2, platelets were found to be 79 ( $>50 \%$ drop) and continued to downtrend on day 3 down to 67[GMA1]. Her 4T score showed a low ( $<5 \%$ ") likelihood of HIT. The patient was switched to cefazolin on day 3 after $\mathrm{E}$ coli sensitivities resulted. Thrombocytopenia[GMA2] resolved with a platelet count of 139 on day 6. Ultimately, the patient received a percutaneous nephrostomy tube and was discharged on oral antibiotics.

IMPACT/DISCUSSION: Cefepime is a rare cause of thrombocytopenia in critically ill patients. Clinical trial data show cefepime-associated thrombocytopenia incidence between 0.1 - $1 \%$. Here, our patient developed thrombocytopenia with a low 4T score and an interesting temporal pattern related to the initiation of cefepime that prompted further investigation. The Naranjo scale assessing adverse drug reactions showed a possible relationship between cefepime and thrombocytopenia, which was further bolstered by the increase in platelets following discontinuation of cefepime. This case highlights the importance recognizing druginduced thrombocytopenia in the critically ill.

CONCLUSION: Cefepime is a rare cause but important of thrombocytopenia in the critically ill Application of a probability scale to test for possible adverse drug reactions can help elucidate etiologies of important clinical findings in the critically ill

\section{HEMATOGENOUSLY SPREAD SEPTIC THROMBOPHLEBITIS AS A CAUSE OF SPINAL EPIDURAL ABSCESS}

Mrunal Kulkarni; Kakageldi Hommadov; Ajinkya Kulkarni; Gul Madison. Mercy Catholic Medical Center, Yeadon, PA. (Control ID \#3186014)

LEARNING OBJECTIVE \#1: Spinal epidural abscess is a rare clinical diagnosis with incidence of 0.2 to 2 cases per 10,0000 hospital admissions and is considered a medical emergency.

LEARNING OBJECTIVE \#2: Delay in diagnosis is common due to non specific presentation and can result in permanent neurological deficits.

CASE: A 27 year-old African American female presented with fever, chills, urinary frequency, worsening pain in right hip and flank region for 2 days. Temperature $100.5 \mathrm{C}$, normal white cell count: 9.9 Thou/uL (4.511 Thou/uL). Urine analysis: bacteriuria, pyuria. CT abdomen (with contrast) unremarkable. With recent history of fall, Xray lumbar spine was done that showed no fracture. IV Ceftriaxone was started for clinically presumed urinary tract infection (UTI). Next day she continued to have lower back pain and also complained of pain, swelling of left arm where an IV access was obtained during her previous hospital admission for asthma exacerbation last week. Exam: tenderness over the lumbar spine region, mild proximal leg weakness (strength 4/5). Right sided straight leg raising test positive. Left arm: erythema, induration, tenderness, purulent drainage. Blood cultures grew Methicillin sensitive staphylococcus aureus (MSSA), 4/4 bottles. Antibiotic was switched to IV Cefazolin. Elevated Erythrocyte sedimentation rate: $70 \mathrm{~mm} / \mathrm{HR}(0-20$ $\mathrm{mm} / \mathrm{HR})$, C-reactive protein: $177 \mathrm{mg} / \mathrm{L}(<10 \mathrm{mg} / \mathrm{L})$. MRI lumbar spine: L3-L4 epidural absces, mass effect on exiting right L3 nerve root, L3-L4 right facet septic arthritis. CT Left arm: septic thrombophlebitis of cephalic vein, medial cubital vein, basilic vein. Patient underwent right L3-4 laminotomy for epidural abscess aspiration. Thrombophlebitic vein was excised for source control. Surgical cultures from both left arm and lower back, grew MSSA. Patient was treated with IV Cefazolin for 6 weeks with uneventful post-operative course and full functional recovery.

IMPACT/DISCUSSION: We present a rare case of spinal epidural abscess (SEA) in a young female with no history of diabetes, IV drug abuse or spinal instrumentation. Source of infection was her recent IV access. She had developed localized septic thrombophlebitis at the iv site, that had resulted in bacteremia and metastatic spread of the infection to the lumbar spine causing SEA. SEA was not considered as a differential initially as symptoms were masked by the clinical suspicion of UTI and a recent fall. Emergency surgical decompression and antibiotics are treatment of choice. This case led us to review our hospital policy on IV lines and appropriate changes were made for careful, timely monitoring in the future.

CONCLUSION: In appropriate clinical setting, it is important to consider spinal epidural abscess as a differential diagnosis even in the absence of classic risk factors and symptoms. Timing is a critical factor in determining ultimate prognosis, as a delay in diagnosis and treatment can result in permanent neurological deficits.

\section{HEMOLYSIS AND THROMBOCYTOPENIA IN THE SETTING OF COBALAMIN DEFICIENCY}

Jake A. Gibbons. University of Texas Medical Branch, Galveston, TX. (Control ID \#3139824)

LEARNING OBJECTIVE \#1: Recognize the clinical features of cobalamin deficiency.

LEARNING OBJECTIVE \#2: Differentiate pseudothrombotic microangiopathy from true microangiopathic hemolytic anemia.

CASE: A 65-year-old male presented with dyspnea beginning 3 months ago. Additionally, he endorsed 15-pound weight loss, fatigue, and decreased appetite. His family noticed yellowing in his eyes. He had no known past medical history. He described his diet as balanced, consisting of meat, eggs, milk, and vegetables. Review of systems was positive for intermittent heart palpitations. The patient had scleral icterus on physical examination. Complete blood count was significant for a decreased hemoglobin of $7.1 \mathrm{~g} / \mathrm{dL}$, decreased white blood cell count of $3.110^{\wedge} 3 / \mu \mathrm{L}$, and decreased platelet count of $9810^{\wedge} 3 / \mu \mathrm{L}$. Reticulocyte index was low at 0.36 . Mean corpuscular volume was high at $122 \mathrm{fL}$. Total bilirubin was elevated at $2.3 \mathrm{mg} / \mathrm{dl}$ with a predominance indirect bilirubin. Peripheral smear revealed schistocytes and hypersegmented neutrophils. Additional blood work showed an elevated lactate dehydrogenase and undetectable haptoglobin. Cobalamin level was found to be significantly decreased at $48 \mathrm{pg} / \mathrm{mL}$. Methylmalonic acid and homocysteine were both elevated. Intrinsic factor antibodies came back positive. Low cobalamin coupled with positive intrinsic factor antibodies suggested a diagnosis of pernicious anemia, which was confirmed by endoscopy. It was determined that the hemolysis and thrombocytopenia were both due solely to the cobalamin deficiency, as additional testing was negative. The patient was started on cobalamin replacement therapy. His laboratory abnormalities improved, he experienced improvement of his symptoms, and was discharged.

IMPACT/DISCUSSION: Cobalamin deficiency is relatively common with a prevalence of approximately $15 \%$ in patients 60 years and older. 
With that said, cobalamin deficiency presenting as pseudothrombotic microangiopathy with hemolytic anemia, schistocytes on peripheral smear, and thrombocytopenia is uncommon. A study of 201 patients with cobalamin deficiency showed that only $2.5 \%$ of those patients presented with pseudothrombotic microangiopathy. This particular presentation can pose a diagnostic challenge for clinicians, as it can mimic a true microangiopathic hemolytic anemia such as thrombotic thrombocytopenic purpura (TTP). An incorrect diagnosis of TTP, for example, could lead to admission to the intensive care unit and the unnecessary initiation of plasmapheresis. In patients with hemolysis, thrombocytopenia, and macrocytic anemia, cobalamin deficiency should be considered on the differential diagnosis.

CONCLUSION: Cobalamin deficiency can uncommonly present as pseudothrombotic microangiopathy with hemolytic anemia, schistocytes on peripheral smear, and thrombocytopenia. This presentation can resemble a true microangiopathic process such as thrombotic thrombocytopenic purpura. Correct diagnosis of pseudothrombotic microangiopathy is essential in avoiding mismanagement of the patient and unnecessary treatments.

\section{HEMOLYTIC ANEMIA AS AN UNUSUAL PRESENTATION OF B12 DEFICIENCY}

Margaret M. Mongare ${ }^{1}$; Arash Nafisi ${ }^{2},{ }^{1}$. ${ }^{1}$ University of Los Angeles California, Los Angeles, CA; ${ }^{2}$ Olive View - UCLA Medical Center, Encino, CA. (Control ID \#3181867)

LEARNING OBJECTIVE \#1: -Recognize sequelae of severe B12 deficiency, including hemolysis

LEARNING OBJECTIVE \#2: -Determine the etiology of B12 deficiency

CASE: A 44 year-old woman presents to clinic with generalized fatigue, dyspnea and easy bruising for one month. She notes 40 pound unintentional weight loss and chronic diarrhea over the past one year. She is otherwise healthy, has no known autoimmune disease, endorses a wellbalanced diet and denies use of medications, supplements, abdominal surgeries and recent travel. Patient was referred for hospital admission after she was found to be severely anemic. On presentation to the emergency department, her blood pressure was $126 / 74$, pulse 70 beats per minute with an exam significant for conjunctival rim pallor, multiples areas of ecchymosis on her lower extremities, abdomen without hepatosplenomegaly and no jaundice or lymphadenopathy. Laboratory studies were significant for pancytopenia and severe macrocytic anemia - Hemoglobin 6.8, MCV 137, Platelets 77K, WBC 3.5. Liver tests showed unconjugated hyperbilirubinemia (TB 1.4, DB 0.2) and urinalysis demonstrated urobilinogen. Hemolysis labs were significant for LDH of 1398, Haptoglobin < 15, D-Dimer 0.59, coombs negative and peripheral smear with hypersegmented neutrophils, few schistocytes. Additional workup revealed B12 < 146 with a negative intrinsic factor (IF) antibody. Folate, iron studies, stool cultures, stool ova \& parasites and celiac testing were normal. Hematology was consulted. Patient's severe anemia and pancytopenia with intramedullary hemolysis were attributed to severe B12 deficiency. She was treated with parenteral B12 and referred to gastroenterology for endoscopic evaluation. At three months follow up, patient was asymptomatic, felt well and all laboratory studies had returned to normal.

IMPACT/DISCUSSION: Severe anemia from intramedullary hemolysis is a rare manifestation of B12 deficiency that results from ineffective erythropoiesis, peripheral breakdown of fragile red blood cells and a pseudo-microangiopathy from homocysteine-induced endothelial damage. This patient's clinical symptoms along with her laboratory findings are supportive of the above processes. Pernicious anemia is the most likely cause of this patient's B12 deficiency despite a negative IF antibody, given IF sensitivity is only $50 \%$ and due to her responsiveness to B12 treatment, her age and lack of other identifiable causes or risk factors for B12 deficiency. Endoscopic evaluation can offer more definitive diagnosis.
CONCLUSION: -Severe B12 deficiency can cause hemolytic anemia and pancytopenia -Clinicians should maintain a high index of suspicious for pernicious anemia as the cause of B12 deficiency, even when IF antibodies are negative, particularly in the absence of other clinical risk factors for B12 deficiency.

\section{HEMOPHAGOCYTIC LYMPHOHISTIOCYSTOSIS: A FATAL DISEASE LEADING TO POOR PATIENT OUTCOMES SUMAIRA ZAREEF MD, MPH. DAVID FERNANDES MD MONTEFIORE MEDICAL CENTRE, DEPARTMENT OF IN- TERNAL MEDICINE.}

Sumaira Zareef. Montefiore Medical Centre, Bronx, NY. (Control ID \#3185643)

LEARNING OBJECTIVE \#1: Hemophagocytic lymphohistiocystosis (HLH) is an aggressive and life-threatening syndrome of excessive immune activation.

LEARNING OBJECTIVE \#2: Early intervention is the only tool that can help achieve better clinical outcomes

CASE: A 52-year old Ghanaian man without significant past medical history was brought to the ER for abdominal pain, cough, and multiple fever spikes above102. He was found to be in septic shock, liver failure and respiratory failure. He was intubated, and treated with broad spectrum antibiotics and multiple pressors. CT of chest, abdomen and pelvis revealed bilateral consolidations, hepatosplenomegaly and diffuse moderate abdominopelvic adenopathy. He had pancytopenia; Total leukocyte count was 2.4 $\mathrm{k} / \mu \mathrm{L}$ (4.8-10.8), Red blood cell count $3.5 \mathrm{ml} / \mu \mathrm{l}$ (4.5- 5.9), platelet count was $31 \mathrm{k} / \mu \mathrm{L}(150-400)$. His kidney function declined, and he was started on dialysis. His ferritin and triglycerides were elevated at $5104 \mathrm{ng} / \mathrm{ml}$ and $1071 \mathrm{mg} / \mathrm{dl}$ respectively. Microbiological testing for tuberculosis, Schistosoma, giardia, strongyloidiasis, malaria, cryptosporidium, HIV, and viral hepatitis panel were negative. He was started on dexamethasone in view of suspicion for Hemophagocytic lymphohistiocystosis (HLH) as he fulfilled five out of eight HLH 2004 criteria. He was medically too unstable to be started on platin based chemotherapy which is the definitive treatment for HLH. Bone marrow biopsy revealed trilineage hematopoiesis, without hemophagocytes. His medical condition worsened, and he passed away after cardiac arrest. His soluble CD25 level was elevated at 19475 unit/ml (45 -1105), and his natural killer cell (NK cells) function was reduced further solidifying HLH diagnosis.

IMPACT/DISCUSSION: HLH diagnosis requires molecular diagnosis/ genetic testing or fulfillment of at least five out of eight variables on diagnostic criteria. HLH-2004 diagnostic criteria includes fever more than $38.5 \mathrm{C}$; splenomegaly; peripheral blood cytopenias including two of the following (hemoglobin less than 9 $\mathrm{g} / \mathrm{dl}$, platelets less than $100,000 / \mu \mathrm{l}$ or ANC $1000 / \mu 1$ ); hypertriglyceridemia ( fasting triglycerides greater than $265 \mathrm{mg} / \mathrm{dl}$ ) and or hyperfibrinogenemia ( less than $150 \mathrm{mg} / \mathrm{dl}$ ) ; Ferritin more than $500 \mathrm{ng} / \mathrm{ml}$; hemophagocytes in bone marrow, spleen, lymph nodes or liver; low or absent NK cell activity, and elevated soluble CD25 ( soluble IL2 receptor alpha). The reported incidence of hemophagocytes on bone marrow examination ranges from 25 to $100 \%$. The sensitivity of NK cell activity and soluble CD-25 levels approaches $100 \%$ based on HLA 2004 diagnostic criteria. Therapy based on the HLH-94 protocol consists of eight weeks of induction therapy with etoposide (VP-16) and dexamethasone. If left untreated, patients with HLH survive only a few months due to progressive organ failure. Therapy should not be delayed while awaiting results of immunologic testing.

CONCLUSION: HLH is a potentially life threatening disorder of extensive inflammation which requires prompt recognition and early introduction of definitive therapy. 


\section{HEMOPHAGOCYTIC LYMPHOHISTIOCYTOSIS IN THE SET- TING OF SYSTEMIC LUPUS ERYTHEMATOSUS AND HISTOPLASMA CAPSULATUM}

Kavita Agrawal; Vijaya Kankanala; Nirav Agrawal. Overlook Medical Center, Summit, NJ. (Control ID \#3163090)

LEARNING OBJECTIVE \#1: Recognise hemophagocytic lymphohistiocytosis (HLH) based on clinical presentation and laboratory abnormalities

LEARNING OBJECTIVE \#2: Identify secondary causes of HLH Treatment modalities for HLH

CASE: A 28-year-old female with a history of systemic lupus erythematosus (SLE) presented with high grade fevers to $104 \mathrm{~F}$, right upper quadrant abdominal pain and nausea for 2 weeks. Based on the initial presentation, there was a concern for infectious etiology of the symptoms. There was no apparent source based on history and physical examination. She underwent work up including computed tomography (CT) chest, abdomen and pelvis, venous doppler, echocardiogram, tagged white blood cell scan and magnetic resonance cholangiopancreatogram (MRCP) which was negative. The symptoms were initially attributed to lupus flare. However, the hospital course was complicated by persistent fevers, pancytopenia and worsening transaminitis AST $211 \mathrm{IU} / \mathrm{L}$ and ALT $111 \mathrm{IU} / \mathrm{L}$. Blood work showed hemoglobin $(\mathrm{Hb})$ of $7.5 \mathrm{~g} / \mathrm{dl}$, platelet 79000/nl, elevated ferritin of 22,682 $\mathrm{ng} / \mathrm{ml}$, triglycerides $308 \mathrm{mg} / \mathrm{dL}$ and soluble IL-2R 12,820 pg/mL. Bone marrow biopsy showed normocellular bone marrow with hemophagocytosis. Based on the above findings, diagnosis of HLH was made. She was started on cyclophosphamide and pulsed dose steroids. Following the treatment, she had improvement of her symptoms and blood work and was discharged. However, four weeks after the discharge, she was readmitted with unremitting fevers for two weeks. She also had erythematous nodules on her back, thigh and legs. CT chest showed tree in bud nodularity pattern in left lung. Blood fungal cultures came positive for histoplasma capsulatum. She was treated with amphotericin for disseminated histoplasmosis. Blood work showed worsening serum ferritin $>40,000$ ng/ml, TG $291 \mathrm{mg} / \mathrm{dl}$, pancytopenia with $\mathrm{Hb} 7.5 \mathrm{~g} / \mathrm{dl}$, platelets 13,000/ml and IL-2R 18,910 pg/ml. This was consistent with the flare of HLH in the setting of SLE and disseminated histoplasmosis in immunocompromised patient. She was started on etoposide and dexamethasone. The patient showed consistent recovery of her symptoms and blood work.

IMPACT/DISCUSSION: HLH is an aggressive and life-threatening syndrome of excessive immune activation. HLH can occur as a sporadic or familial disorder or secondary to different conditions that disrupt the immune homeostasis. The most common conditions include malignancies, immunodeficiencies, infections and rheumatologic disease such as idiopathic juvenile rheumatoid arthritis, SLE, systemic sclerosis and mixed connective disease. Common findings include fever, rash, neurologic symptoms, elevated liver enzymes and ferritin. Treatment involves etoposide and dexamethasone given at tapering doses over eight weeks and therapies for underlying cause

CONCLUSION: HLH presents as febrile illness with multiorgan involvement HLH is a fatal disease that requires early recognition and assessment of secondary causes Urgent treatment is required and therapeutic modalities may differ accordingly to the underlying cause

\section{HEMORRHAGIC COMPLICATIONS OF PARACENTESIS}

$\underline{\text { Kayvon Sotoudeh }}^{1}$; Christopher Lee ${ }^{1}$; Andrew Young ${ }^{2} .{ }^{1}$ University of Southern California, La Crescenta, CA; ${ }^{2}$ Keck School of Medicine, Los Angeles, CA. (Control ID \#3184778)

LEARNING OBJECTIVE \#1: Recognize and treat a serious complication of paracentesis

CASE: A 32-year-old male with history of Child $\mathrm{C}$ alcoholic hepatic cirrhosis presented with worsening abdominal distension. His history was notable for alcohol and methamphetamine abuse. Vital signs were normal. Physical exam was remarkable for scleral icterus and jaundice. His abdomen was distended, without tenderness or rebound, with distended superficial abdominal veins and a positive fluid wave. Bilateral lower extremities were edematous. His initial laboratory values were notable for a sodium level of $109 \mathrm{mmol} / \mathrm{L}$ and a hemoglobin of $10.3 \mathrm{~g} / \mathrm{dL}$. Hypertonic saline, salt tablets, and diuresis were administered with resolution of hyponatremia. His ascites was managed with serial abdominal paracentesis. On hospital day 9, the patient underwent a third abdominal paracentesis with ultrasound guidance via the left lower quadrant with removal of 5 liters of sero-sanguinous fluid, consistent with his prior paracenteses. Post-procedural vital signs revealed a blood pressure of 112/63 $\mathrm{mmHg}$, heart rate 98 , respiratory rate 19 , and saturating well on ambient air. His initial post procedure hemoglobin was $7.5 \mathrm{~g} / \mathrm{dL}$, unchanged from before the procedure. His international normalized ratio (INR) was 2.13. Sixteen hours following the procedure, he became hypotensive to $83 / 40 \mathrm{mmHg}$ with a hemoglobin of $5.7 \mathrm{~g} / \mathrm{dL}$. The patient was transfused with 2 units of packed red blood cells and transferred to the intensive care unit where he was started on norepinephrine. He continued to require multiple transfusions with only minimal improvements in his hemoglobin. A CT scan of the abdomen and pelvis without contrast showed a dense collection of fluid within the greater omentum, likely representing a hematoma. On an angiogram performed by interventional radiology, active hemorrhage from a branch of the left inferior epigastric artery was revealed. The vessel was embolized. The patient's condition gradually improved. The patient was discharged on hospital day 16 in stable condition

IMPACT/DISCUSSION: Severe hemorrhage as a complication of abdominal paracentesis has been shown to occur in less than $1 \%$ of cases with an average time to symptom onset between 6 to 48 hours after paracentesis, with some cases occurring up to 1 week post-procedure. Types of hemorrhage include abdominal wall hematomas, hemoperitoneum, and pseudoaneurysm formation and are usually associated with damage to the inferior epigastric artery. Complication rates have not been associated with operator experience, elevated INR or thrombocytopenia. In cases of severe hemorrhage, transcatheter intervention has been shown to have significantly lower rates of mortality than surgical intervention (25\% versus $75 \%)$.

CONCLUSION: For hospitalists performing abdominal paracentesis, this case demonstrates the importance of rapid recognition of this complication. As in this case, Interventional Radiology is critical in managing the source of bleed in hemorrhage following abdominal paracentesis.

\section{HEPATITIS IN AN IMMUNOSUPPRESSED PATIENT}

Joseph S. Needleman ${ }^{1}$; Austin Portolese ${ }^{1}$; Abdul Basit ${ }^{2} .{ }^{1}$ Penn State College of Medicine, Hershey, PA; ${ }^{2}$ Penn State Hershey Medical Center, Hummelstown, PA. (Control ID \#3184394)

LEARNING OBJECTIVE \#1: Consider syphilis as an etiology of acute hepatitis in immunosuppressed patients

CASE: An African-American male in his 20s with human immunodeficiency virus (HIV) infection on highly active antiretroviral therapy (HAART), end-stage renal disease on dialysis, and type 1 diabetes presented with 10 days of fevers, nausea, vomiting, abdominal pain, and pruritus. He reported high-risk sex with other men, but denied drug and alcohol use. His exam was notable for scleral icterus, periorbital edema, and right upper quadrant pain. Alanine aminotransferase (ALT) was $127 \mathrm{u} / \mathrm{L}$ and aspartate aminotransferase (AST) was $106 \mathrm{u} / \mathrm{L}$, both $3 \mathrm{x}$ the upper limit of normal (ULN). Alkaline phosphatase (ALP) was 740u/L (56x ULN), total bilirubin 2.9 (2-3x ULN), and gamma-glutamyltransferase $1715 \mathrm{u} / \mathrm{L}$ (28x ULN). CD4+ count was 360 cells/MM3 and HIV viral load was undetectable. Viral hepatitis serologies were negative. Abdominal CT revealed biliary sludge and periportal edema without ductal dilation. Out of concern for drug-induced hepatitis and cholangitis his HAART medications, darunavir, ritonavir, and rilpivirine, started seven months prior, were held and he received piperacillin/tazobactam. Two days after 
admission the patient disclosed a painless penile chancre, and a reactive treponemal antibody screen with reflex rapid plasma reagin (RPR) was positive. He was diagnosed with syphilitic hepatitis and received the first of three intramuscular penicillin benzathine shots.

IMPACT/DISCUSSION: Syphilis infection rates in the United States have increased in recent years. The Centers for Disease Control and Prevention reports over 30,000 new cases of primary and secondary syphilis in 2017, with 7,000 cases in HIV-positive individuals, principally men who have sex with men. Syphilis-induced hepatitis is a rare manifestation occurring in an estimated $10 \%$ of immunocompetent patients, and is characterized by a disproportionately large elevation of ALP compared to ALT and AST. Standard stage-dependent antibiotics are the current recommended treatment. Of note, evidence has emerged that syphilitic hepatitis may develop in up to $38 \%$ of HIV-positive patients. Current recommendations for acute hepatitis include screening for Hepatitis $\mathrm{B}$ and $\mathrm{C}$ viruses. However, given this disproportionate rate of occurrence in HIV-positive patients, we recommend additional screening for syphilis when evaluating acute hepatitis in immunocompromised patients or high-risk patients with hepatitis secondary to an unclear etiology. As our patient shows, a thorough history and exam, as well as continued development of rapport, is vital to obtaining an early and accurate diagnosis

CONCLUSION: - Syphilitic hepatitis is an increasingly common etiology of acute hepatitis - It is characterized by ALP elevations significantly greater than AST and ALT - HIV-positive patients are particularly at risk for syphilitic hepatitis

\section{HEPATORENAL SYNDROME SECONDARY TO ABDOMINAL COMPARTMENT SYNDROME}

Merza Nooraldin ${ }^{1}$; Taylor Lindgren ${ }^{1}$; Deborah $\mathrm{Lin}^{2}$; Dylan Murray ${ }^{2}$; Tareq Naguib ${ }^{1}$. $t$ tuhsc, Amarillo, TX; ${ }^{2}$ Texas Tech University Health Sciences Center, Amarillo, TX. (Control ID \#3184720)

LEARNING OBJECTIVE \#1: 1-Recognize Increased intra-abdominal pressure (IAP) as a cause of acute kidney injury (AKI). 2-Identify massive ascites, increased IAP, and hepatorenal syndrome as complications of liver cirrhosis. 3-Demonstrate paracentesis as therapeutic management and treatment of increased IAP.

CASE: A 28-year-old Hispanic male with medical history significant for morbid obesity, recreational drug use, and alcohol-induced liver cirrhosis resulting in ascites presented to our facility complaining of increasing abdominal girth, nausea, generalized abdominal pain, and swelling. He reported decreased urine output over the past two days. CT of the abdomen showed a cirrhoticappearing liver without masses, massive ascites, and mild splenomegaly. Urine drug screen was positive for cocaine and opiates. Initial labs were significant for a total bilirubin of 10, ALP of 113, AST/ALT of 59/32, lipase of 205, and creatinine of 1.4. Baseline creatinine was around 0.9. An initial diagnosis of AKI due to hepatorenal syndrome (HRS) was made, and the patient was started on octreotide, midodrine, albumin, and furosemide. Serum creatinine improved initially to baseline before increasing to 2.2. IAP also increased, and a diagnosis of abdominal compartment syndrome (ACS) was made. An IR-guided paracentesis was performed, during which $6 \mathrm{~L}$ of fluid were removed. After the paracentesis, the patient's renal function returned to baseline.

IMPACT/DISCUSSION: HRS is defined as an association between liver disease and oliguric renal failure characterized by reduction in glomerular filtration rate in patients with liver cirrhosis and hepatic failure. There are several theories on its pathogenesis, but arterial vasodilation in the splanchnic circulation is a significant contributing factor. Decline in renal perfusion also plays a role, and increased IAP can contribute to this. Patients may recover from HRS following liver transplant. However, if the disease progresses, end-stage liver failure may result in ascites, which then further increases IAP and may advance to ACS, therefore, while the precise mechanism of HRS is unknown, increased IAP is one significant contributing factor.
CONCLUSION: Surgical treatment for increased IAP (particularly ACS) includes decompressive laparotomy, but non-surgical management for increased IAP is effective as well. This includes sedation and analgesia, avoidance of the head of bed $>30$ degrees, paracentesis, percutaneous drainage, nasogastric decompression, avoiding excessive fluid resuscitation, diuretics, hemodialysis, vasopressors, optimizing ventilation/ alveolar recruitment, and antibiotic therapy in septic patients. Physicians should be aware of the association between increased IAP, ACS, and HRS. Prevention of increased IAP and subsequent ACS can help avoid the development of HRS, which can then be treated via paracentesis.

\section{HEREDITARY ANGIOEDEMA FLARE MIMICKING CELLULITIS}

Praneet Kalkat ${ }^{1}$; Sara Wikstrom ${ }^{2} .{ }^{1}$ George Washington University, Washington, DC; ${ }^{2}$ George Washington University, Potomac, MD. (Control ID \#3184487)

LEARNING OBJECTIVE \#1: Consider hereditary angioedema in nonresolving erythema and edema of extremities

CASE: $60 \mathrm{y} / \mathrm{o}$ African-American woman with history of hereditary angioedema, hypertension, fibromyalgia, chronic lower back pain, and chronic lower extremity edema presenting with left lower extremity erythema, pain, and swelling of 2 days. She denied any recent trauma to the area. She also denied any abdominal pain, lip swelling or trouble breathing. She was afebrile and on physical exam had 2+ pitting edema of her left lower extremity with erythema, warmth and mild tenderness to palpation. Labs showed a normal white count. An ultrasound showed no evidence of thrombosis. She was discharged on a 7-day course of clindamycin for presumed uncomplicated cellulitis. She presented to the emergency room 6 days later now with bilateral lower extremity pain, erythema, and swelling despite clindamycin treatment. Labs again showed no leukocytosis. Given her bilateral presentation, no response to antibiotics and no leukocytosis concern for hereditary angioedema flare was raised. C1 esterase inhibitor was found to be low at $17.1 \mathrm{mg} / \mathrm{dl}$. She received 1 dose of Berinert IV 10-20 u/kg which she tolerated well with improvement of symptoms.

IMPACT/DISCUSSION: Hereditary angioedema is a rare autosomal dominant disease characterized by either low levels of $\mathrm{C} 1$ esterase inhibitor (type 1), as seen in our patient, or dysfunction of $\mathrm{C} 1$ esterase inhibitor itself (type 2). This genetic defect unleashes a break in the kallikrein-kinin pathway allowing for an increase in vasodilation and vascular permeability. During a flare, patients classically suffer from angioedema involving the layers of the gastrointestinal and upper respiratory tracts. We present an atypical presentation of hereditary angioedema, solely involving the skin of the lower extremities. Cutaneous attacks usually involve non-pitting edema with ill-defined margins that are non-pruritic and can be erythematous, but are usually not warm or tender to palpation as seen in our patient, likely leading to a delay in diagnosis. First-line treatment includes intravenous $\mathrm{C} 1$ esterase inhibitor, which our patient received with improvement in symptoms. As seen in our patient, it is important to consider non-infectious causes of erythematous and edematous lesions especially in the setting of no fever and leukocytosis. Earlier recognition can lead to unwarranted antibiotic use and rehospitalization. Finally, while patients with hereditary angioedema are usually screened for troubling upper respiratory or gastrointestinal symptoms it is important to screen for cutaneous symptoms in patients as well, which can be distressing for patients.

CONCLUSION: Consider hereditary angioedema in one's differential for a patient with non-resolving erythema and edema of extremities with no other signs of infection It is important to screen patients with hereditary angioedema for cutaneous symptoms

\section{HIDDEN ANSWER IN THE BONE MARROW}

Aivi Doan. University of Connecticut, Middletown, CT. (Control ID $\overline{\# 3186178)}$ 
LEARNING OBJECTIVE \#1: Recognize clinical manifestations and diagnosis of thrombocytopenia

LEARNING OBJECTIVE \#2: Understand the approach to adult with unexplained thrombocytopenia

CASE: 33 year-old female with past medical history of bipolar disorder on lithium and buproprion presented with one month of petechia rash evolving into ecchymoses without known trauma and several days of bleeding gums. On admission, CBC showed WBC 8.1, hemoglobin/ hematocrit 11.6/33.5, and platelet 11. Patient was started on dexamethasone and later switched to high dose $1 \mathrm{mg} / \mathrm{kg}$ prednisone based on working diagnosis of immune mediated thrombocytopenia. Bupropion was discontinued because of its implication in platelet antibody production and subsequent thrombocytopenia. Platelet did not improve with steroid; therefore a course of 5 days of IVIG-Privigen was started. Patient had intermittent epitaxis requiring transfusions. She also experienced menorrhagia and was started on medroxyprogesterone acetate. Her platelet remained low, and she received one dose of romiplostim. Her hospital course was complicated with acute intracranial hemorrhage along tentorium and posterior interhemispheric fissure likely subdural in location. Given the subdural hemotoma, patient was given one dose of rituximab. Bone marrow biopsy was performed. In the interim, she became agitated and not directable concerning for side effect of steroid and cessation of buproprion. She was also found to have emphysematous cystitis and Eschericiha coli bacteremia that might have contributed to the thrombocytopenia. Pathology from bone marrow was consistent with metastasis from breast cancer negative for progesterone, estrogen, and HER2/neu. Patient was started on chemotherapy of gemcitabine and carboplatin and steroid was stopped.

IMPACT/DISCUSSION: The case highlights the importance of recognizing uncommon cause of thrombocytopenia. On presentation, patient was severe thrombocytopenic with platelet about 11,000. Even though, thrombocytopenia is most often due immune mediated in relatively young and healthy individuals, alternative explanations should be investigated when the platelet does not improve with steroid or IVIG, which are considered first-line treatments. Patient's hospital course was complicated by acute intracranial hemorrhage, which is a rare, but can have devastating consequences. Therefore, any new headache in patients with severe thrombocytopenia should warrant a thorough neurological exam and likely an immediate CT head to rule out bleeding.

CONCLUSION: -Evaluate for wide range of explanations in patients with thrombocytopenia -Perform bone marrow biopsy in patients with persistent anemia and thrombocytopenia

\section{HIDE AND GO SEEK: A CASE OF VANCOMYCIN-RESISTANT ENTEROCOCCUS IN THE BONE MARROW}

Joannie M. Ivory ${ }^{1}$; Khadeeja Ashai ${ }^{1}$; Jennifer Schmidt ${ }^{2} .{ }^{1}$ Saint Louis University School of Medicine, St. Louis, MO; ${ }^{2}$ Saint Louis University, Saint Louis, MO. (Control ID \#3179388)

LEARNING OBJECTIVE \#1: Describe a case of VRE isolated in the bone marrow of a fungemic patient

LEARNING OBJECTIVE \#2: Recognize VRE in the bone marrow as a potential cause of fever of unknown origin

CASE: We present a 67-year-old woman with history of recurrent urinary tract infections (UTI) initially admitted to the intensive care unit (ICU) with sepsis secondary to Escherichia coli (E. coli). She completed a 14-day course of ceftriaxone for E. coli bacteremia. Despite adequate treatment, her cognitive function declined. The patient was somnolent, less talkative, stopped following commands, and often had a blank stare. Repeat blood cultures from hospital day six turned positive for Candida glabrata after five days. The patient was started on micafungin; she remained febrile without improvement in mental status. Subsequent blood cultures were negative. Due to her prolonged fever the team began a workup for fever of unknown origin (FUO) including evaluation for encephalitis, pulmonary, abdominal and pelvic infection sources, and malignancy. A left ureteral lesion (unable to biopsy due to active infection), was concerning for possible malignancy vs fungal ball. In addition, she was neutropenic with a white cell count of 3,200 per microliter, so we obtained a bone marrow biopsy. Bone marrow culture grew Vancomycin-resistant Enterococcus (VRE) at 11 hours. Once we obtained the bone marrow results, we immediately started daptomycin and patient fever resolved within 24 hours of first dose, along with improvement in mental status.

IMPACT/DISCUSSION: VRE emerged as an organism of importance in the 1980s, largely due to its prevalence as a hospital-acquired infection. It is most commonly found in the gastrointestinal tract, as well as the genitourinary tract, skin, and oral cavity. It can also cause bloodstream infection (i.e. bacteremia). The factors most associated with a high risk of VRE bacteremia include bone marrow and solid organ transplants (due to immunosuppression), cancer, prior antibiotic exposure and ICU admission. Healthy individuals are at low risk of VRE infection; however, healthy individuals can spread disease to those who are critically ill through contact or contaminated objects. In the hospital, VRE accounts for $\sim 35.5 \%$ of enterococcal infections, making it the second most common nosocomial infection in the United States. VRE bacteremia is typically treated with daptomycin or linezolid, with the former being preferred due to its bactericidal nature and low drug-to-drug interactions.

CONCLUSION: On review of the literature, there are no cases reported of VRE being isolated from the bone marrow of a non-transplant patient. However, clinicians should be aware of this organism's affinity for hematopoietic tissue. The work up for FUO can include a bone marrow biopsy, but does not always lead to a diagnosis. However, it is helpful when clinicians suspect the source of fever is malignancy, as it can detect limited production of cell lines, or even yield a source of infection.

\section{HIDING AMONGST THE RASHES-A CASE OF ECZEMA HERPETICUM IN A PATIENT WITH ACTIVE BENIGN FAMIL- IAL PEMPHIGUS}

Rachael A. Gordon ${ }^{1}$; Rebecca E. Mancoll ${ }^{2}$. ${ }^{1}$ University of Pittsburgh, Pittsburgh, PA; ${ }^{2}$ UPMC, Pittsburgh, PA. (Control ID \#3170197)

LEARNING OBJECTIVE \#1: Recognize eczema herpeticum in adults with underlying dermatological disorders.

CASE: A 75-year-old man with a history of benign familial pemphigus presented with a diffuse rash and fever. Eight days prior to admission, the patient developed pruritic, erythematous lesions on his genitals and inguinal folds, which he attributed to a benign familial pemphigus exacerbation. $\mathrm{He}$ applied topical clotrimazole/betamethasone dipropionate without improvement. Three days later, he developed a blistering rash over his right eye and mouth, along with worsening crusting plaques on his neck. The patient sought care at a local ED. He was given one dose of IV steroids and discharged on $40 \mathrm{mg}$ of prednisone for a presumed flare of his chronic disease. At follow-up with his PCP, he had a fever of $102.5^{\circ} \mathrm{F}$ and was referred back to the ED. There, his WBC was 8.5 , with $80.5 \%$ neutrophil predominance, but laboratory studies were otherwise unremarkable. Blood and urine cultures were obtained. He received ampicillin/sulbactam and vancomycin for presumed bacterial superinfection prior to admission. On arrival, he reported bilateral blurry vision, headache, nausea, and diarrhea. Physical exam was notable for multiple small ulcers under the tongue, buccal mucosa, and soft palate with tender, erythematous, crusted plaques over his head, neck, and groin. Vesicular lesions with red bases were superimposed on the aforementioned lesions. Ophthalmological exam was unremarkable. He was started on IV acyclovir due to concerns for disseminated varicella $(\mathrm{VZV})$ and continued on both antibiotics for possible bacterial superinfection. Blood and urine cultures remained negative. PCR of unroofed blisters at two different sites tested positive for herpes simplex virus (HSV)-1, suggesting a diagnosis of eczema herpeticum (EH). Antibiotics were discontinued on hospital day three. He was discharged on hospital day eight on valacyclovir to complete a total of 14 days of therapy. 
IMPACT/DISCUSSION: Though more common in children, EH can affect adults. Patients with bullous disorders are prone to superimposed HSV and VZV. Clinical and histological identification of these infections remains challenging in the context of the underlying skin disease. Failure to recognize EH delays the initiation of definitive antiviral therapy, increasing the risk of dissemination, multi-organ failure, and fatal outcomes. It remains controversial whether the use of topical and systemic steroids to treat bullous disorders, as in this case, potentiates extension of localized infection and increases the risk for systemic dissemination of HSV. Intriguingly, occult HSV, but not VZV, colonization has been reported in patients with bullous dermatitides. EH may be more common than previously appreciated in patients with bullous disorders.

CONCLUSION: • EH is a serious complication of benign familial pemphigus that can mimic the underlying disease. $\bullet$ For patients presenting with a presumed exacerbation of their bullous dermatitis, testing for HSV/VZV should be pursued.

\section{HIDING IN PLAIN SIGHT: PRIMARY CENTRAL NERVOUS SYSTEM LYMPHOMA WITH NO CONTRAST ENHANCE- MENT ON IMAGING}

Saboor E. Randhawa ${ }^{1}$; ahmed munir ${ }^{1}$; Daniel A. Ermann ${ }^{1}$; Ahmed Aly ${ }^{1}$; Carrie Valenta ${ }^{2} .{ }^{1}$ Creighton University, Omaha, NE; ${ }^{2}$ Creighton University School of Medicine, Papillion, NE. (Control ID \#3186150)

LEARNING OBJECTIVE \#1: Diagnose central nervous system lymphoma when there is no contrast enhancement on imaging studies.

LEARNING OBJECTIVE \#2: Assess for central nervous system lymphoma clinically based on symptoms and signs.

CASE: A 37-year-old female with history of migraines had frequent visits to the Emergency Department (ED) with severe progressive headaches. These were different in character from her migraines and associated with nausea, vomiting, photophobia and syncope. She was HIV negative and had no history of seizures or substance abuse. On her first ED visit, she had a syncopal episode with unremarkable neurological exam. and laboratory findings. CT without contrast showed non-specific hypodense areas with no evidence of acute abnormalities. MRI with contrast revealed extensive but non-specific changes suggestive of demyelination and inflammation. There were minimal patchy contrast enhancement areas that extended along the course of the perforator vessels in the basal ganglia on both sides, left greater than right. There was otherwise no contrast enhancement. She was readmitted within the same month for similar symptoms. Neurology was consulted. Cerebrospinal fluid (CSF) cell count, glucose and protein were within normal limits. Further investigations including CSF PCR for various pathogens for meningo-encephalitis were negative. The patient was started on a five-day course of steroids. A few immunoblasts were found on CSF fluid analysis. Peripheral blood smear did not reveal abnormal cells, and another LP was performed for CSF flow-cytometry to rule out malignancy. Due to the above inconclusive results, Neurosurgery suggested stereotactic brain biopsy which revealed Primary Diffuse Large B-Cell Lymphoma of the CNS. Repeat CT and MRI scans also did not reveal contrast enhancement. The patient was then started on appropriate chemotherapy with improvement in her symptoms.

IMPACT/DISCUSSION: Early diagnosis of PCNSL often requires a high degree of suspicion, as symptoms can evade diagnosis for many months. Presenting signs and symptoms vary, depending on the site of involvement. Evaluation of PCNSL should include imaging of the CNS, ideally with contrast-enhanced MRI. CT with contrast can be used if MRI is contraindicated. Tissue diagnosis should not be delayed and definitive diagnosis requires histopathological evaluation. In population-based studies, five-year survival for immunocompetent adults is approximately 30 percent. In another study, treatment delay (greater than 30 days after diagnosis) was the most important clinical variable associated with decreased survival, independent of baseline performance status. Non-enhancing lesions can delay the diagnosis, hence delaying the initiation of treatment.
CONCLUSION: - PCNSL can present without contrast enhancement, and delay in the treatment directly affects the survival of these patients. PCNSL must be in the list of differential diagnoses even when nonenhancing, diffuse lesions are seen on CT/MRI.

\section{HIT YOU WHILE YOU'RE DOWN: EARLY STENT THROMBO- SIS DUE TO HEPARIN-INDUCED THROMBOCYTOPENIA}

Elizabeth Oczypok; Joshua M. Haswell; Amar Kohli. UPMC, Pittsburgh, PA. (Control ID \#3186125)

LEARNING OBJECTIVE \#1: List risk factors for and clinical signs of early stent thrombosis

LEARNING OBJECTIVE \#2: Diagnose HIT based on established clinical and laboratory data

CASE: A 45-year-old man with hypertension and hyperlipidemia presented with chest pain. EKG revealed acute inferior wall ST-elevation myocardial infarction. Left heart catheterization showed a large occlusive thrombus in the right coronary artery, which was treated with three drugeluting stents. The patient was started on a heparin drip, aspirin, and clopidogrel for high-risk STEMI with large thrombotic burden. On hospital day 7, the patient developed chest discomfort and diaphoresis with several self-terminating runs of polymorphic ventricular tachycardia. Repeat catheterization revealed a large occlusive thrombus in the RCA at the site of previous stent placement. No further stenting was done. The patient's platelets were observed to acutely drop from 216 on hospital day seven to a nadir of 30 on hospital day nine. 4T score was 6 and heparin platelet factor-4 antibody OD was 1.658 (high probability of HIT). Serotonin release assay was negative. Patient was transitioned from heparin to argatroban on hospital day 9 , and his platelet counts recovered to baseline. He was discharged on hospital day 18 on aspirin, ticagrelor, and warfarin.

IMPACT/DISCUSSION: Heparin-induced thrombocytopenia is an uncommon, life-threatening, "can't miss" diagnosis. Cardiac patients are commonly exposed to heparin in the setting of acute myocardial infarction. Stent thrombosis can occur in up to $3.1 \%$ of STEMIs and can present with recurrent ischemic changes on EKG or arrhythmias such as polymorphic VT. Dual antiplatelet therapy and newer generation drug-eluting stents decrease rates of stent thrombosis, however, procedural characteristics such as emergent stent placement and residual thrombus near the stent, as well as patient factors such as low ejection fraction, underlying hypercoaguable state, and diabetes can increase stent thrombosis risk. If there is high clinical concern for HIT based on the 4T score, confirmatory testing should be sent, all heparin products should be stopped, and the patient should be anticoagulated with a non-heparin agent. In this case, the patient's $4 \mathrm{~T}$ score was elevated on hospital day 8 , but was not initially recognized, leading to a delay in treatment. Also, his serotonin release assay, considered the "gold standard" of HIT diagnosis, was negative, illustrating that false negatives are still possible in sensitive tests. Consideration of clinical context remains important.

CONCLUSION: 1) HIT should be considered as a possible cause of early stent thrombosis. 2) Stent thrombosis has a 30-day mortality rate of 10-25\% and has a high recurrence rate, necessitating close monitoring and careful choice of antiplatelet therapy. 3) Test characteristics of the 4T score, confirmatory immunoassay, and functional assay should be carefully considered when making clinical decisions about suspected HIT cases.

\section{HOG HUNTING GONE WILD: A RARE ZOONOSIS CAUGHT CLOSE TO HOME}

Syeda A. Raza. Methodist Health Systems Dallas, Dallas, TX. (Control ID \#3185804)

LEARNING OBJECTIVE \#1: Recognize infection with zoonotic organisms as a cause of sepsis in humans 
LEARNING OBJECTIVE \#2: Recognize importance of investigation of zoonotic staphylococcal infections to ensure correct species identification

CASE: A 57-year-old man with past medical history of hypertension and left partial nephrectomy for renal cell carcinoma presented to the emergency department with several days of fevers, diarrhea, and fatigue. On examination, the patient was lethargic and febrile to 103 degrees Fahrenheit with a blood pressure of $80 / 50 \mathrm{mmHg}$. His labs were notable for an elevated serum lactic acid of 2.06 with the remainder of labs otherwise unremarkable. Given complaints of diarrhea, a CT of his abdomen was performed showing enterocolitis. The patient was admitted for concern of sepsis and a set of blood cultures were collected on admission that ultimately grew Staphylococcus hyicus. He was started on fluids and IV Vancomycin resulting in improvement in his clinical condition. A subsequent trans-thoracic echocardiogram (TTE) and trans-esophageal echocardiogram (TEE) were negative for endocarditis. After the cultures grew this unusual organism, the patient was questioned more pointedly. He admitted recently visiting a farm where sausage had just been made from feral hogs. He had eaten the sausage just days prior to onset of illness and he further reported that he had undergone extensive dental work just prior to that meal. This additional history helped explain how our patient may have been infected from bacterial translocation/seeding to the blood.

IMPACT/DISCUSSION: Staphylococcus hyicus is a part of the natural flora of several animals. It is mainly involved in exudative epidermitis in pigs but has also been isolated from skin infections in horses, mastitis in cows, and even from a human wound infection from a donkey bite. On our review of literature, there was only one other case of S. hyicus bacteremia in a patient who came in close contact with his piglets. In our case, the patient's presumed source of infection came from consumption of wild hog meat after recent dental work.

CONCLUSION: This case highlights several important points for clinical practice when making the diagnosis. First, it is important to note that S. hyicus can present as sepsis in humans, especially those that are immunocompromised. Second, a thorough history is imperative to help with the clinical diagnosis. Third, $S$. hyicus is often misidentified as S. aureus. Therefore, further investigation is indicated in zoonotic staphylococcal infections to ensure correct species identification.

\section{HOLD YOUR HORSES: THIS AIN'T NO ORDINARY STREP}

$\underline{\text { Rui Jiang }}^{2,}{ }^{1}$; Michael Herscher ${ }^{3}$; Harish Jasti ${ }^{2}{ }^{1}$ The Mount Sinai Hospital, New york, NY; ${ }^{2}$ Icahn School of Medicine at Mount Sinai, New York, NY; ${ }^{3}$ The Mount Sinai Hospital, New York, NY. (Control ID \#3185046)

LEARNING OBJECTIVE \#1: Identify the clinical features of Streptococcus equi infections.

LEARNING OBJECTIVE \#2: Recognize the similarities to common features of other Streptococcus infections.

CASE: A 63yo woman presented with purulent drainage from a right breast mass. She first noticed the breast mass 8 years prior, but had declined work up at the time. A month prior to presentation, she had noted red discoloration overlying the mass and subsequently developed a draining wound. She also reported right upper extremity swelling, chills, shortness of breath, and dry cough. She owns and operates a horse-rescue farm, but denied any contact with sick horses over the past 1-2 months. On exam, she was afebrile with a HR of 116 . She had a $10 \times 20 \mathrm{~cm}$ irregularly-shaped wound with eschar and purulent drainage extending from the right breast into the axilla. Underlying muscles were visible. She had 3+ pitting edema extending from the right shoulder to hand. Labs were significant for WBC of 31.7 with $96.9 \%$ PMNs, and creatinine of 3.82, with unknown baseline. Blood cultures grew Strep. Equi in 4 out of 4 bottles. A CT chest showed a left pleural effusion, and possible osteomyelitis in the anterior ribs below the right breast mass. Thoracentesis revealed empyema with no malignant cells, and TTE did not show any valvular vegetation. Breast biopsy revealed poorly-differentiated duct carcinoma, ER/PR+, HER2-. She subsequently underwent chest tube placement for empyema and completed a 6-week course of antibiotics. She eventually accepted only hormone-modulating treatment for breast cancer with goals to be DNR/DNI.

IMPACT/DISCUSSION: Streptococcus equi (SE) infection is a zoonotic disease with a variety of clinical presentations. The subspecies zooepidemicus is a part of the normal flora of horse respiratory tract, and is the most commonly reported to cause human infections. Transmissions have mainly been reported by direct contact with horses, dogs, cats, cattle, sheep and goats. Epidemics have been reported through ingestion of unpasteurized milk and cheese from cows with mastitis. SE is a Lancefield C, beta-hemolytic Streptococcus that shares > 80\% DNA sequence as well as virulence factors with Strep. pyogenes (SP). It can cause rheumatic fever, glomerulonephritis, purulent arthritis, and skin infections similar to SP. In addition, pneumonia, bacteremia of unknown focus, meningitis, and more complex sequelae such as aortitis and endocarditis have been reported. Though all strains are penicillin sensitive, case-fatality rate can be as high as $63 \%$ in small outbreaks. Hand washing after handling animals has been proposed as the key intervention to prevent this infection.

CONCLUSION: Given that $30.5 \%$ of US households have a horse enthusiast and many more come in contact with barn animals, Strep. equi has the potential to become an emerging zoonosis with threat to human life. Prompt recognition of this infection with its similarities to that of Strep. pyogenes is important because appropriate management can lead to mitigation of its high mortality rates.

\section{HORSES DRESSED AS ZEBRAS - THE DIAGNOSTIC CHAL- LENGES OF AN OUTPATIENT WORKUP}

Joshua W. Harbaugh ${ }^{1}$; Claudia L. Campos ${ }^{2}$. ${ }^{1}$ Wake Forest University School of Medicine, Winston Salem, NC; ${ }^{2}$ Wake Forest University, WInston-Salem, NC. (Control ID \#3186423)

LEARNING OBJECTIVE \#1: Appropriate utilization of outpatient diagnostic workups in clinically complex patients

CASE: A 61-year-old Caucasian male presented to clinic for his first adult medical evaluation with complaints of progressive weakness. Along with this symptom, he experienced daily dry cough, dyspnea on exertion, fatigue and unintentional weight loss of thirty pounds over six months. He denied fever, chills, night sweats, chest pain, orthopnea, PND or edema. No changes in bowel or bladder function. He denied past medical history and took no medications or supplements. He is an 80 pack-year smoker with remote history of alcoholism and poly-substance abuse. He recounts severe life stress from losing his electricians job due to inability to climb ladders. Family history was unclear, but knew parents lived into their late 70 's. On physical examination, BP 143/90, pulse 68, RR 16, height 5' 8", 158lbs, Sp02 95\% on RA and 85\% with ambulation. Frail appearance with thinning hair. Lung examination revealed a barrel chest and diffuse expiratory wheezing. Cardiac examination: normal S1, S2, no murmurs and no JVD. Extremities: no edema, normal pulses, hands with digital clubbing. Neurological Examination: Hip flexion strength:3 of 5 bilaterally, 4+ elsewhere. Reflexes: 2+ symmetric.

IMPACT/DISCUSSION: The patient was diagnosed clinically with a chronic proximal myopathy, COPD with hypoxemia and unexplained weight loss concerning for underlying malignancy with possible paraneoplastic syndrome (presumed lung cancer). Supplemental oxygen and inhalers were prescribed for severe COPD. Results of his work-up spanned over four weeks and were unconventionally obtained by the patient. First, his CT Chest showed severe destructive centrilobular emphysema, no nodules or masses, severe coronary calcification and a small pericardial effusion. Then, he underwent EMG/NCS, his results pending at the time of his two-week follow-up when his complaints were unchanged. Laboratory analyses were notable for $\mathrm{Hb} 11.5, \mathrm{Na} 134, \mathrm{Cr} 1.79$, BUN 25, AST 41, Total Cholesterol 392, LDL 314, TG 291, HDL 30, 
normal B12, folate, Iron panel. TSH of 90.422 with free thyroxine $<0.03$. EMG was consistent with hypothyroid myopathy. Diagnosis was hypothyroidism and initiated on levothyroxine. High intensity statin for hypercholesterolemia. Work-up for chronic kidney disease, anemia and HTN was started but patient did not return to clinic and was not completed.

CONCLUSION: This case, largely suspenseful secondary to the labyrinthine outpatient workup, highlights challenges faced by internists embarking on ambulatory evaluations of weakness in medically complex patients. After CT excluded lung malignancy, and his EMG results and lab analysis were available, hypothyroidism was finally diagnosed. While thyroid myopathy is not rare, the multisystem physical and laboratory manifestations of hypothyroidism are perfectly showcased in this presentation. His weight loss remains puzzling but could be attributed to severe COPD (lung cachexia) or other malignancy.

\section{HOST BY AN ACCIDENT}

Rani Bhatia; Brett Styskel; Nautam Ganatra. Baylor College of Medicine, Houston, TX. (Control ID \#3185998)

LEARNING OBJECTIVE \#1: Assess exposure history and recognize potential endemic disease in a patient presenting with atypical infectious symptoms.

CASE: A 41-year-old male with non-alcoholic steatohepatitis presented with a week of high fevers to $105 \mathrm{~F}$, myalgias, abdominal pain, diarrhea, and 2 days severe headache associated with visual hallucinations. Vitals on presentation were $\mathrm{T} 102 \mathrm{~F}, \mathrm{BP} 93 / 55 \mathrm{mmHg}$, HR $120 \mathrm{bpm}$, and $\mathrm{SaO} 2$ 96\%. Physical exam was remarkable for nuchal rigidity, jaundice, diffuse abdominal tenderness without peritoneal signs, and hepatosplenomegaly. Lab results showed leukocytosis $10,300 / \mathrm{mm} 3$ with $91 \%$ PMNs, new thrombocytopenia $75,000 / \mathrm{mm} 3$, creatinine 1.6 (baseline 0.8 ) $\mathrm{mg} / \mathrm{dL}$, AST $139 \mathrm{IU} / \mathrm{L}$, ALT $178 \mathrm{IU} / \mathrm{L}$, ALP $236 \mathrm{IU} / \mathrm{L}$, total bilirubin $7.9 \mathrm{mg} /$ $\mathrm{dL}$, direct bilirubin $5.3 \mathrm{mg} / \mathrm{dL}$, lipase $88 \mathrm{U} / \mathrm{L}$, and lactate $1.8 \mathrm{U} / \mathrm{L}$. Influenza, RSV, C. difficile, HIV, hepatitis panel, acetaminophen level, hemolysis and disseminated intravascular coagulation labs were negative. CT scan of the head was unremarkable. Abdominal ultrasound showed no acute hepatobiliary pathology. Lumbar puncture showed white blood cell count 6 (neutrophil 80\%), protein $22 \mathrm{mg} / \mathrm{dL}$, and glucose $46 \mathrm{mg} / \mathrm{dL}$. Detailed history revealed that he spent an hour picking fleas off his pet dog eight days prior to the onset of fever. He also lived in a wooded area with opossums in his neighborhood. He was started on empiric doxycycline for presumed murine typhus with rapid improvement in his symptoms. Labs showed Rickettsia typhi $\operatorname{IgG}$ and IgM immunofluorescence assays $>1: 256$. All cultures as well as Leptospirosis, EBV, CMV antibodies and syphilis titer were negative.

IMPACT/DISCUSSION: In the US, the classic rat-flea-rat cycle as a reservoir for Rickettsia typhi was targeted by aggressive rat control in the 1940s. These efforts led to a decrease in annual cases of murine typhus from 5,000 to less than 100 by the $1980 \mathrm{~s}$. The infection is now seen mainly in suburban areas of Texas and California, and instead perpetuated by cat fleas (Ctenocephalides felis) that reside on opossums, dogs, and cats, with humans as the "accidental" host. Incidence of disease in Texas has been increasing over the last 15 years, with 519 cases in 2017 alone ( $40 \%$ increase from the year prior). Although murine typhus is generally a self-limited illness, the risk of complications and mortality may be up to $4 \%$ if treatment with doxycycline is delayed. Typhus presents a diagnostic challenge for clinicians, as symptoms are often nonspecific. In fact, the classic triad of fever, rash, and headache may only be seen in $33 \%$ of adults. Thus, awareness of murine typhus is important to ensure a timely diagnosis and reduce the risk of serious complications e.g. respiratory failure, aseptic meningitis, seizures, acute renal failure and septic shock.

CONCLUSION: -Murine typhus has increased in incidence in the US recently, with cat fleas as the new vector for $R$. typhi. -Awareness of murine typhus is important to ensure a timely diagnosis and reduce the risk of serious complications including mortality.

\section{HOW LOW CAN YOU GO? AN INTERESTING CASE OF HYPOGLYCEMIA}

Christopher Vercammen-Grandjean. St. Jude Heritage Medical Group, Orange, CA. (Control ID \#3162765)

LEARNING OBJECTIVE \#1: Recognize the problem of rebound hypoglycemia in diabetic patients with CKD or ESRD

LEARNING OBJECTIVE \#2: Learn how best to manage this problem with dietary changes

CASE: A 75 year old man with a history type 2 diabetes managed with insulin and sulfonylurea, end-stage renal disease recently started on hemodialysis, and obesity presented to a community hospital after a witnessed syncopal event at home. Prior to his loss of consciousness he had started to feel light headed and dizzy, but he quickly regained full consciousness after. His family checked his serum glucose and it was 37 , they had him drink orange juice and his symptoms resolved. He was then brought to the hospital. Later that evening a re-check of his glucose revealed a low of 67 and he was given orange juice, resulting in a rise in his blood sugar and resolution of symptoms of hypoglycemia. He was placed on a diabetic diet. In spite of this he continued to have frequent low blood sugars in the hospital ultimately requiring an infusion of dextrose containing normal saline. On further history, his family discussed how this had been an ongoing problem at home and that he was constantly eating candy bars throughout the day to avoid hypoglycemia, even after his insulin and sulfonylurea medications were discontinued. An endocrine consultation was then placed and he was started on a strict low carbohydrate diet without concentrated sugar, with higher protein and fat content. Low blood sugars were treated with combinations of complex carbohydrates and protein containing foods. Ultimately he was weaned off the dextrose infusion and his blood sugar stabilized. He continued the diet after discharge, at three month follow-up he had lost 40 pounds and had had no low blood sugars at home. His diet was liberalized without subsequent hypoglycemia.

IMPACT/DISCUSSION: Hypoglycemia in diabetic patients with chronic kidney disease is a well-recognized problem. (Rhee, 2018) As renal function worsens, insulin half-life lengthens, in combination with reduced renal gluconeogenesis and impaired metabolism of hypoglycemic medications. Hospitalizations for hypoglycemia can increase in the years leading up to initiation of renal replacement therapy. (Rhee, 2018) Thus it is important for the internist to recognized rebound hypoglycemia as described above, and intervene early to avoid unnecessary hospitalizations or prolonged hospital stays. Early consultation with endocrinology, a diabetes educator, and dietitian can help though further research is needed. In our case, a diet that limited concentrated sugars to almost none, and ensuring co-ingestion of protein and/or fat with carbohydrate helped avoid hypoglycemia.

CONCLUSION: Early recognition of normalizing blood glucose and the increasing risk of hypoglycemia in patients with CKD and diabetes is important to prevent adverse events for patients.

\section{HYDROXYCHLOROQUINE TOXICITY LEADING TO CARDI- AC ARREST IN A 23-YEAR-OLD}

Michael Grzeskowiak ${ }^{2}$; Daniel $\mathrm{Pu}^{1} .{ }^{1}$ Montefiore Medical Center, San Antonio, TX; ${ }^{2}$ University of Texas Health Science Center at San Antonio, San Antonio, TX. (Control ID \#3185812)

LEARNING OBJECTIVE \#1: Recognize that hydroxychloroquine (HCQ) can cause atrioventricular block, bundle branch block, and QT prolongation which can occur over weeks to years of treatment.

LEARNING OBJECTIVE \#2: Treat HCQ toxicity by discontinuing the medication and follow for the resolution of the toxicity, which does not always occur.

CASE: A 23-year-old woman with systemic lupus erythematosus (SLE) and end-stage renal disease (ESRD) presented to the hospital with 
hyperkalemia after missing dialysis and her HCQ for several months. Electrocardiograph (ECG) showed a normal QT interval and a transthoracic echocardiogram (TTE) revealed an ejection fraction of 55\%. She was subsequently restarted on dialysis and her home hydroxychloroquine. Three weeks after her admission she suffered a cardiac arrest from ventricular fibrillation during dialysis. Both pre-arrest and post-arrest labs were within normal limits. ECG showed no ischemic changes but revealed a prolonged QT interval of $600 \mathrm{msec}$. Repeat TTE revealed new severe biventricular failure with an ejection fraction of $15 \%$. HCQ was then discontinued and a cardiac biopsy revealed changes consistent with HCQ toxicity. Repeat TTE showed minimal improvement in ejection fraction and a subcutaneous implantable cardioverter defibrillator (ICD) was placed. Follow up TTE one month later showed continued severe left ventricular dilation with an ejection fraction of $25 \%$. However, ECG showed resolution of QT prolongation. The patient's mental status improved and she was discharged to acute rehabilitation.

IMPACT/DISCUSSION: Hydroxychloroquine (HCQ) has been associated with conduction disease including atrioventricular block, bundle branch block, and QT prolongation. Though these changes often take years to develop, they may rapidly worsen in patients on dialysis. If screening is not initiated and these changes are left unnoticed, the cardiotoxicities can lead to death.

CONCLUSION: HCQ is known to cause cardiotoxicities such as cardiomyopathies and conduction disturbances. These cardiotoxicities usually develop after long-term use with literature reviews demonstrating a mean of 10 years. Furthermore, the prognosis of HCQ cardiotoxicity ranges from complete improvement in cardiac function, to requiring cardiac transplant, to death. In our case, the QT interval demonstrated normalization following discontinuation of HCQ, as has been reported in prior cases. However, her cardiomyopathy did not improve and she required an ICD placement. With HCQ undergoing renal and hepatic excretion, toxicities can often be rapidly exacerbated in patients with renal insufficiency and can develop more rapidly than the 10-year average. Caution should be taken when starting or continuing patients on HCQ in patients on or nearing dialysis. Moreover, clinical suspicion should be high for cardiotoxicity in patients who have been on HCQ for many years. If caught early, these patients could reverse their cardiotoxicities and avoid premature death.

\section{HYPERAMMONEMIC ENCEPHALOPATHY IN ROUX-EN-Y GASTRIC BYPASS WITH AFFERENT LOOP SYNDROME}

Hanyuan $\mathrm{Shi}^{1}$; Kevin Lee ${ }^{2}$; Erik Johnsen ${ }^{1}{ }^{1}$ Tulane University, New Orleans, LA; ${ }^{2}$ Columbia University, New York City, NY. (Control ID \#3162974)

LEARNING OBJECTIVE \#1: Consider not only nutritional deficiencies in RNYGB patients but also encephalopathy and defects in the ammonia cycle

CASE: A 33-year-old female with elective gastric bypass surgery 10 years prior (Roux-en-Y, complicated by afferent loop syndrome requiring subsequent small bowel resection), untreated Hepatitis C (but persistently negative RNA titers), bipolar disorder initially presented to the hospital with rash on thighs and forearms thought to be due to drug allergy from furosemide. The patient was found to be encephalopathic and malnourished. Labs on admission were significant for INR 1.2 (0.9-1.1), total bilirubin 3.1 (0.1 - 1.2 $\mathrm{mg} / \mathrm{dl})$, alkaline phosphatase 238 (44-147 IU/L), ammonia 168 (15-45 u/dl), lactate $2.4(0.5-1.0 \mathrm{mmol} / \mathrm{L})$. Abdominal CT showed diffuse fatty infiltration of the liver but no signs of bowel ischemia. Ammonia peaked at $180 \mathrm{but}$ stayed elevated to 110-130 despite administration of optimal doses of rifaximin and lactulose. Found to have E. Coli UTI, treated with ceftriaxone. Stepwise decline in mental status along with worsening respiratory requirements led to intubation in the ICU. Imaging showed pulmonary edema and patient underwent diuresis with ethacrynic acid. She was extubated a few days later without complication and steadily improved in her mental status. Liver biopsy showed focal pericellular and perisinusoidal fibrosis on trichrome stain (stage 2/4), negative iron deposition, negative intracytoplamic inclusion, and negative reticulin stain. She had $>66 \%$ steatosis, some lobular inflammation, few ballooned hepatocytes for a non-alcoholic fatty liver disease activity score (NAS) of 5/8. Further work-up was significant for micronutrient deficiencies (Vitamin A, Vitamin D), low copper 38 (80-155 ug/dl), low zinc 24 (60-120 ug/dl).

IMPACT/DISCUSSION: A recent review noted a newly recognized, almost lethal syndrome related to hyperammonemia after Roux-en-Y gastric bypass (RYGB) surgery. 95\% of these patients are women. Previous cases have involved urea cycle deficiencies (ornithine transcarbamylase deficiency, carbamoyl phosphate synthetase deficiency) leading to impaired ureagenesis and elevated serum glutamine levels. Physiologic stressors can then predispose these non-cirrhotic patients to encephalopathy. In this patient's case, the trigger may have been an infection (UTI), nutritional changes (given hypoalbuminemia). Treatment includes lactulose, neomycin, arginine infusions, phenylbutyrate, citrulline, zinc supplements; the latter therapies work to remove nitrogen via alternative pathways in the ammonia cycle.

CONCLUSION: In RYGB, significant weight loss after surgery creates a catabolic setting where increased breakdown and subsequently deposition of nitrogenous waste in the liver can lead to encephalopathy. Our particular anatomically challenging case demonstrates life-threatening hyperammonemia that was exacerbated by a combination of liver disease and abnormal nitrogen processing from nutrition deficits.

\section{HYPERCALCEMIA AS THE ONLY MANIFESTATION OF COCCIDIOIDOMYCOSIS}

Mais Abdou' ${ }^{1}$ Nooraldin Merza ${ }^{1}$; Asrar Abdou $^{3}$; Kaylee J. Shepherd ${ }^{2}$. ${ }^{1}$ TTUHSC, Amarillo, Amarillo, TX; ${ }^{2}$ Texas Tech University Health Sciences Center, Amarillo, TX; ${ }^{3}$ St Joseph Mercy Oakland, Troy, MI. (Control ID \#3181584)

LEARNING OBJECTIVE \#1: Learn to include fungal infections such as coccidioidmycosis in your differential diagnosis of hypercalcemia. Recognize the diagnostic algorithm of hypercalcemia.

CASE: An 82 year-old male with medical history of hypertensive chronic kidney disease stage III presented to our hospital for near syncope during a routine outpatient laboratory draw. He reported generalized weakness, malaise, unintentional 30lbs weight loss, constipation, nausea and decreased appetite for several months. Vital signs stable and exam revealed palpable lymphadenopathy of the cervical chain. Laboratory workup showed normal white blood cell count and differentiation, hemoglobin $9.9 \mathrm{~g} / \mathrm{dl}$, calcium $12.1 \mathrm{mg} / \mathrm{dl}$ with ionized level $1.62 \mathrm{mg} / \mathrm{dl}$, creatinine 2.03 $\mathrm{mg} / \mathrm{dl}$ from a baseline of $1.3 \mathrm{mg} / \mathrm{dl}$, intact parathyroid hormone (PTH) $45 \mathrm{pg} / \mathrm{ml}$, PTH related peptide $8 \mathrm{pg} / \mathrm{ml}, 25$ hydroxyvitamin D

$45 \mathrm{pg} / \mathrm{ml}$, but an elevated 1,25 dihydroxyvitamin D at $60 \mathrm{pg} / \mathrm{ml}$. CT neck and chest showed cervical lymphadenopathy and thyroid nodule. Bone scan showed no lytic or destructive osseous lesion. Parathyroid sestamibi scan showed a focal abnormality, which correlated with the thyroid nodule detected on the CT scan. Fine needle aspiration pathology was consistent with benign follicular adenoma. Peripheral blood smear revealed moderate anemia with normal WBCs and no dysplastic features. Serum and urine protein electrophoresis were normal. Bilateral bone marrow biopsy showed normocellular marrow with no evidence of myeloma or malignancy. Cervical lymph node biopsy was consistent with granulomatous lymphadenitis with fungal spherules of Coccidioides immitis. There was no evidence of malignancy. On further history, he reported visiting rural Arizona frequently. The patient was placed on fluconazole $400 \mathrm{mg}$ daily with 3 month follow up showing significant symptomatic relief with calcium level down to $9.7 \mathrm{mg} / \mathrm{dl}$.

IMPACT/DISCUSSION: Hypercalcemia has been described in patients with granulomatous disorders, especially sarcoidosis and tuberculosis, but rarely with fungal granulomas like coccidioidomycosis. The mechanism of hypercalcemia in patients with coccidioidomycosis is unclear and there is 
no evidence of increased production of 1,25-dihydroxyvitamin D. Most cases reported hypercalcemia either due to disseminated disease or during the treatment phase. In our patient, his hypercalcemia was the main clinical finding that led to the diagnosis and interestingly his 1,25 dihydroxyvitamin D was elevated and hypercalcemia improved with antifungal treatment. This implies that more studies are needed to determine the underlying etiology for hypercalcemia in Coccidioidomycosis infections.

CONCLUSION: Coccidioidomycosis should be considered in the differential diagnosis of hypercalcemia. The mechanism of the hypercalcemia is unclear and more studies are needed. However, it appears to be independent of parathyroid hormone. Hypercalcemia in those patients is usually a reversible complication with treating the underlying infection.

\section{HYPERCALCEMIA FROM PNEUMOCYSTIS JIROVECII PNEUMONIA IN A RENAL TRANSPLANT RECIPIENT}

Alisha Hossain ${ }^{1}$; Vincent Lipari ${ }^{2}$; Snigdha Reddy ${ }^{2} .{ }^{1}$ Michigan State University College of Osteopathic Medicine, Exton, PA; ${ }^{2}$ Henry Ford Hospital, Detroit, MI. (Control ID \#3173935)

LEARNING OBJECTIVE \#1: Recognize clinical and laboratory features of Pneumocystis jirovecii pneumonia (PJP) in immunocompromised patients LEARNING OBJECTIVE \#2: Treat hypercalcemia in the setting of acute kidney injury

CASE: A 63-year-old male with a past medical history of a living unrelated donor renal transplant secondary to autosomal dominant polycystic kidney disease presented with 3 weeks of fatigue, persistent nonproductive cough, subjective chills, and worsening exertional dyspnea. Chest computed tomography showed ground-glass opacities and increased interstitial markings involving the left lung and right perihilar region without pleural effusion, pneumothorax, or cardiomegaly. He was empirically started on daily intravenous (IV) azithromycin and ceftriaxone due to suspected multifocal pneumonia. Acute kidney injury (AKI) with a creatinine of $3.4 \mathrm{mg} / \mathrm{dL}$ and BUN of $67 \mathrm{mg} / \mathrm{dL}$ was noted. He was admitted to the intensive care unit with hypoxic respiratory failure and escalation to broader spectrum antibiotics: vancomycin and piperacillin/ tazobactam. He was noted to have coarse breath sounds with wheezing on lung exam. Initial labs revealed a serum calcium $\left(\mathrm{Ca}^{+2}\right)$ of $12.0 \mathrm{mg} / \mathrm{dL}$. Vancomycin was subsequently changed to IV Linezolid. IV Clindamycin and Primaquine daily were added due to suspected PJP. Bronchoscopy with bronchoalveolar lavage revealed a white blood cell count of $103 / \mu \mathrm{L}$ with no organisms. PJP on polymerase chain reaction and elevated -Dglucan were noted. Antibiotics were then tailored to 3 weeks of Clindamycin and Primaquine to treat PJP. His $\mathrm{Ca}^{+2}$ increased to 13.5 $\mathrm{mg} / \mathrm{dL}$ likely due to PJP and AKI. Additional laboratory data revealed suppressed parathyroid hormone (PTH) levels at $15 \mathrm{pg} / \mathrm{mL}$ and elevated 1,25-dihydroxy-vitamin $\mathrm{D}\left(1,25-(\mathrm{OH})_{2} \mathrm{D}_{3}\right)$ at $156 \mathrm{pg} / \mathrm{mL}$. His hypercalcemia proved resistant to IV fluids followed by IV diuresis, ketoconazole, and calcitonin. However, his $\mathrm{Ca}^{+2}$ corrected to $10.0 \mathrm{mg} / \mathrm{dL}$ after administration of a denosumab injection.

IMPACT/DISCUSSION: This case demonstrates the intricate care and teamwork needed to care for a patient with a complicated clinical course. Approaching the treatment of hypercalcemia in a stepwise manner with attention to AKI resulted in improvement. Previously, hypercalcemia has been reported with PJP in many case reports. Ling et al. presented cases of PJP-induced hypercalcemia. It has been suggested that in PJP, interferon -induced alveolar macrophage activation of 1- hydroxylase converts 25$(\mathrm{OH})$ vitamin $\mathrm{D}$ to active $1,25-(\mathrm{OH})_{2} \mathrm{D}_{3}$. In turn, activated vitamin $\mathrm{D}_{3}$ increases $\mathrm{Ca}^{+2}$ absorption from the intestine and bone. PTH is suppressed as the normal inhibitory feedback mechanism is not affected.

CONCLUSION: We present a particularly resistant case of PJP-induced hypercalcemia, which resolved with a single denosumab injection. Reference: Ling, Jonathan, et al. "Hypercalcaemia preceding diagnosis of Pneumocystis jirovecii pneumonia in renal transplant recipients." Clinical kidney journal 10.6 (2017): 845-851.

\section{HYPERCALCEMIA IN SETTING OF MILK ALKALI SYNDROME}

Nihar Shah $^{1}$; Khary Edwards ${ }^{1}$; Toishi Sharma ${ }^{1}$; Jennifer Baldwin ${ }^{2} .{ }^{1}$ University of Connecticut, Hartford, CT; ${ }^{2}$ Univerisity of Connecticut, Farmington, CT. (Control ID \#3186273)

LEARNING OBJECTIVE \#1: Milk-alkali syndrome refers to a triad of hypercalcemia, metabolic alkalosis and acute kidney injury caused by the excessive ingestion of calcium and base - typically in the form of milk and calcium-containing antacids. It was common prior to the introduction of histamine-2 antagonists and proton pump inhibitors for the treatment of peptic ulcer disease. A resurgence has been seen now with the widespread availability of calcium supplements.

CASE: We present a case of a 49-year-old female who was sent by her primary care physician (PCP) after she was found to have a calcium level of $15.7 \mathrm{mg} / \mathrm{dL}$. She endorsed a one-week history of decreased appetite, generalized weakness and increased lethargy. Past medical history was significant for GERD for which she took $2 \mathrm{~g}$ of over-the-counter Calcium Carbonate daily over the past 30 years. She had also been started on a thiazide diuretic one month prior to her presentation. She denied any history of renal disease or malignancy. Physical exam was only significant for a psoriatic rash on her bilateral lower extremities. On admission, her laboratory results were significant for an elevated creatinine of $1.6 \mathrm{mg} / \mathrm{dL}$ (her baseline was $0.9 \mathrm{mg} / \mathrm{dL}$ ), Calcium of $14.8 \mathrm{mg} / \mathrm{dL}$, bicarbonate of $33 \mathrm{meq} / \mathrm{L}$. EKG showed a normal sinus rhythm with a QTc of $414 \mathrm{~ms}$. She was admitted for management with aggressive IV fluid hydration and she was also given one-time doses of calcitonin and zoledronic acid. Her hospital course was complicated by fever to 102.6 and diarrhea thought to be secondary to zoledronic acid. These symptoms resolved and at discharge, her serum calcium was $8.3 \mathrm{mg} / \mathrm{dL}$. She was started on a proton pump inhibitor and counseled to stop taking calcium carbonate tablets. Three days following discharge, she was sent by her PCP once more for assessment of a calcium level of $7.6 \mathrm{mg} / \mathrm{dL}$ and phosphate of $1.5 \mathrm{mg} / \mathrm{dL}$. She was treated with $1 \mathrm{~g}$ IV calcium gluconate and discharged. Laboratory results two days later showed a calcium of $8.1 \mathrm{mg} / \mathrm{dL}$.

IMPACT/DISCUSSION: Milk-alkali syndrome represents the third most common cause of hypercalcemia. Excessive intake of calcium leads to vasoconstriction of the afferent renal arterioles, decreased GFR and decreased ability to filter calcium. This is only exacerbated by introduction of a thiazide diuretic, further reducing calcium excretion. Zoledronic acid can cause delayed hypocalcemia as in this case, making close monitoring important. This case highlights the importance of a thorough medication history and the need for patient education on over the counter medication use.

CONCLUSION: This case highlights how fluid resuscitation forms the cornerstone of initial management of hypercalcemia and hightlights that use of bisphosphonates in the acute setting should only be initiated in refractory or severely symtomatic cases. It also re-emphasizes how dangerous over-the-counter medicines can be as well as the importance of thorough medication history and medication reconciliation to avoid potential dangerous drug-drug interactions.

\section{HYPERCALCEMIA OF MALIGNAN-D: A CASE OF VITAMIN-D MEDIATED HYPERCALCEMIA IN LYMPHOMA}

Maham Awan ${ }^{1}$; Shannon Devlin ${ }^{1}$; Simerjot K. Jassal ${ }^{1,2}$. ${ }^{1}$ University of California San Diego, San Diego, CA; ${ }^{2}$ VASD, San Diego, CA. (Control ID \#3186430)

LEARNING OBJECTIVE \#1: Recognize mechanisms of hypercalcemia in lymphoma

LEARNING OBJECTIVE \#2: Review treatments of hypercalcemia in malignancies

CASE: An 86-year-old man with splenic marginal zone lymphoma was admitted with hypercalcemia and acute kidney injury. Presenting symptoms 
included weakness and abdominal pain with exam notable for left upper quadrant tenderness. Admission labs revealed a corrected calcium level of $13.7 \mathrm{mg} / \mathrm{dL}$ and a creatinine of $2.62 \mathrm{mg} / \mathrm{dL}$ (baseline $1 \mathrm{mg} / \mathrm{dL}$ ). On review of history, hypercalcemia led to his diagnosis of lymphoma. He required prior admissions for treatment with fluid, furosemide, calcitonin, and bisphosphonates. Before his diagnosis, the patient was healthy and running two miles per day. Work-up to determine the etiology of his hypercalcemia showed low parathyroid hormone intact molecule and parathyroid hormone related protein, at $9 \mathrm{pg} / \mathrm{mL}$ and $12 \mathrm{pg} / \mathrm{mL}$ respectively. His urinary calcium was $9.9 \mathrm{mg} / \mathrm{dL}$. His vitamin D2 1,25 (OH)2 was $<8 \mathrm{pg} / \mathrm{dL}$ but his vitamin D3 $1,25(\mathrm{OH}) 2$ was found to be elevated at $91 \mathrm{pg} / \mathrm{mL}$. He was treated with fluids, furosemide, calcitonin and zoledronic acid. Despite these therapies, his calcium increased to abnormal levels and creatinine worsened each time fluids were stopped, requiring inpatient chemotherapy. Definitive treatment of his hypercalcemia required treatment of his lymphoma. He received rituximab, bendamustine, and high-dose steroids at the recommendation of oncology.

IMPACT/DISCUSSION: While hypercalcemia in lymphoma is rare, hypercalcemia of malignancy is common. Parathyroid hormone and Vitamin-D can be inappropriately upregulated and overproduced. The production of PTH-rP is the most common paraneoplastic syndrome leading to hypercalcemia in malignancy. Increased osteoclast activity is another cause, specifically relating to multiple myeloma. In this case, the etiology was vitamin $\mathrm{D}$ overproduction by macrophages associated with tumor cells in lymphoma, similar to that in sarcoidosis. The mechanism is increased production of 1-alpha-hydroxylase, the enzyme responsible for converting vitamin $\mathrm{D}$ to its active form, vitamin D3 1,25(OH)2. This case highlights the importance of understanding how cancers not typically associated with hypercalcemia are still subject to this phenomenon.

CONCLUSION: Multiple phenomena can lead to hypercalcemia in malignancy, including increased production of 1-alpha-hydorxylase. U1timately, definitive management of hypercalcemia of malignancy is treatment of the malignancy.

\section{HYPEREOSINOPHILIA - A DIAGNOSTIC DILEMMA}

Prarthna V. Bhardwaj ${ }^{1}$; Nakul Ravikumar ${ }^{2} .{ }^{1}$ Baystate Medical Center, Springfield, MA; ${ }^{2}$ UMMS - Baystate, Springfield, MA. (Control ID \#3178615)

LEARNING OBJECTIVE \#1: To recognize causes of hypereosinophilia. LEARNING OBJECTIVE \#2: To diagnose eosinophilic granulomatosis with polyangiitis when the presentation is confusing.

CASE: 69yo Caucasian man with severe asthma, chronic sinusitis, nasal polyps presented with parasthesias of $\mathrm{b} / \mathrm{l}$ lower extremities and hands; and diarrhea found to have eosinophilic leukocytosis (Total WBC - 42,000; Total eosinophil count - 28,700). He had a recent hospitalization flu like symptoms with transient bilateral consolidations on chest CT. He had traveled to Nicaragua 4 months prior. PE revealed subjective numbness in fingers and toes bilaterally; and an erythematous rash on his shoulder with surrounding hyperpigmentation that was grossly itchy. Strongyloides and trypanosoma $\mathrm{Ab}$; blood, sputum and stool cultures were negative. Bone marrow biopsy showed extensive infiltration with mature eosinophils. ABL-BCR, JAK 2, PDGFR, FGFR, CHIC -2, flow cytometry, cANCA, pANCA and ANA were negative with normal tryptase levels. EMG was normal. Skin biopsy of the rash was diagnosed as tinea. RAST was positive for aspergillus fumigatus with positive precipitin panel. Hospital course was complicated by new CHF with significant tropinemia (peak 7.03). Cardiac cath was negative for CAD. ECHO revealed newly reduced LVEF of $30 \%$. Cardiac MRI suggested an infiltrative process. He was put on steroids with improvement in counts and azathioprine later for steroid sparing for suspected eosinophilic granulomatosis with polyangiitis (previously Churg Strauss Syndrome) and is being followed by multiple specialties. He completed oral itraconazole for ABPA. On follow up, his symptoms have improved.

IMPACT/DISCUSSION: Hypereosinophilia is an elevation in eosinophil count above $1,500 / \mu \mathrm{L}$. It is divided into primary, secondary and idiopathic.
Secondary eosinophilia is non-clonal increase caused by infections, allergic conditions, autoimmune diseases and paraneoplastic syndromes. Main differentials were EGPA, allergic bronchopulmonary aspergillosis, clonal malignancy and idiopathic hypereosinophilic syndrome. While APBA can cause eosinophilia, involvement of organs other than the upper respiratory tract is uncommon. EGPA is classified as ANCA-positive and ANCAnegative. About $50-60 \%$ of EGPA show ANCA positivity. Based on American College of Rheumatology (ACR), patient

met 4 of the 6 criteria for EGPA despite negative ANCA. Cardiac involvement including CHF, myocarditis, pericarditis, myocardial ischemia and arrhythmias are the major cause of death and poor prognosis occurring in $27-47 \%$ cases. It is more common in ANCA-negative patients and directly correlates with eosinophilia.

CONCLUSION: ABPA can result in hypereosinophilia but is limited to organs in the upper respiratory tract. Despite ANCA negativity, if patient meets ACR criteria in the right clinical setting, EGPA can be confirmed. It is important to recognize the subtle differences between these entities causing hypereosinophilia in order to clinch the diagnosis and involve appropriate specialties to institute early treatment.

\section{HYPERGLYCEMIA, OBESITY, AND STIGMA: PHYSICIAN WEIGHT-BASED BIAS PREVENTING EARLY RECOGNITION OF RARE MEDICATION SIDE EFFECT.}

Pranav Shah ${ }^{1}$; Ahmed Yeddi ${ }^{1}$; Omnia Awad ${ }^{1}$; Diane L. Levine ${ }^{3}$; ijeoma Nnodim ${ }^{2} .{ }^{1}$ Detroit Medical Center, Plymouth, MI; ${ }^{2}$ wayne state university school of medicine, Westland, MI; ${ }^{3}$ Wayne State University, Detroit, MI. (Control ID \#3184572)

LEARNING OBJECTIVE \#1: Recognize how weight-based bias regarding obese patients can negatively impact health outcomes and contribute to disparities.

LEARNING OBJECTIVE \#2: Recognize iatrogenic causes of poorly controlled "lifestyle" diseases associated with obesity such as medication induced hyperosmolar hyperglycemic non-ketotic syndrome (HHNS).

CASE: 22-Year-old female with medical history of well controlled type 2 diabetes mellitus (HbA1c 6.9\%), morbid obesity (BMI 54) and pseudotumor cerebri status post ventriculoatrial shunt presents with worsening headaches for 3 days. A lumbar puncture showed mildly elevated opening pressure with normal cerebrospinal fluid analysis. She was started on acetazolamide $250 \mathrm{mg}$ twice daily with improvement of headaches. However, her blood glucose measurements became very high: 300$500 \mathrm{mg} / \mathrm{dl}$, requiring increasing insulin dosages. By day 4, she was requiring 490 units total dose of insulin, 7 times her home daily dose. We believed the patient was not adhering to a strict hospital ADA diet and was consuming additional carbohydrate-rich meals. However, upon further questioning, she denied this assertion. As her hyperglycemia remained resistant to insulin, multiple providers repeatedly questioned her adherence to the hospital diet. Eventually, a medication re-assessment resulted in the discovery of hyperglycemia as a rare side effect of acetazolamide, which was subsequently discontinued. Within 48 hours of cessation of acetazolamide, blood glucose levels normalized with a fasting glucose $70-120 \mathrm{mg} / \mathrm{dl}$, and post-prandial glucose $80-180 \mathrm{mg} / \mathrm{dl}$ and remained stable on her home insulin regimen.

IMPACT/DISCUSSION: Initial consideration for the patient's poorly controlled hyperglycemia was non-adherence to the hospital diabetic diet. Her denial of non-adherence was met with incredulity by the care team. Consequently, she endured a prolonged hospital length of stay, shame, and emotional distress. We learned a lesson about self-awareness of physician bias regarding obesity and how it can negatively impact outcomes and contribute to health disparities. Obesity is escalating in epidemic proportions with rising weight discrimination ( $>66 \%$ over the past decade). Many healthcare providers hold strong negative attitudes and stereotypes about people with obesity. Such attitudes influence interpersonal behavior and decision-making which may negatively impact care. Physicians should be 
mindful of their own biases and intentionally consider alternative causes including iatrogenic factors such as medication side-effects. In our case, hyperglycemia related to acetazolamide is a rare side-effect only seen in mouse models and the mechanism has not fully been clarified.

CONCLUSION: Obese patients suffer discrimination in the healthcare setting contributing to health disparities. Physician self- awareness of implicit bias can lead to a behavior change, "outside-the-box" thinking, and improved health outcomes. In rare cases, acetazolimide may induce severe hyperglycemia in patient's with diabetes mellitus

\section{HYPOCOPMPLEMENTEMIC URTICARIAL VASCULITIS SYN- DROME - A RARE CASE OF ANGIOEDEMA}

Kateryna Fedorov ${ }^{1}$; Chrisanna Dobrowolski ${ }^{2}$; Clement Tagoe ${ }^{2}$. ${ }^{1}$ Montefiore Medical Center, Bayside, NY; ${ }^{2}$ Montefiore Medical Center, Bronx, NY. (Control ID \#3184919)

LEARNING OBJECTIVE \#1: Identify patients with hypocomplementemic urticarial vasculitis syndrome

LEARNING OBJECTIVE \#2: Differentiate hypocomplementemic urticarial vasculitis syndrome from allergic reaction

CASE: 37-year-old female with systemic lupus erythematosus (SLE) presented with swelling of the lips and throat for 3 days. The swelling was accompanied by a husky voice, painful erythematous eruptions on her arms, legs, chest, back, as well as dull right knee and ankle pain. Within 48 hours the cutaneous eruptions changed in color to deep blue, and then resolved, leaving hyperpigmented macules. She has been having similar less severe skin eruptions and lip swelling episodes over the past year. Physical examination was significant for swelling over the right side of the upper lip, hyperpigmented macules on the eyebrows, nose, left cheek and ear, as well as bluish, circular macules on the left arm, right shoulder, and left posterior thorax. Leukocyte count was slightly elevated at $12.8 \mathrm{k} / \mathrm{uL}$, anti-dsDNA was elevated at $145 \mathrm{IU}$, and both $\mathrm{C} 3$ and $\mathrm{C} 4$ were low at $40 \mathrm{mg} / \mathrm{dL}$ and $4.4 \mathrm{mg} / \mathrm{dL}$ respectively. The patient was treated with for an allergic reaction but responded only briefly and soon developed shortness of breath and pulmonary edema. Further laboratory tests revealed $\mathrm{C} 1$ inhibitor $>100 \%, \mathrm{C} 1$ esterase inhibitor $28 \mathrm{mg} / \mathrm{dL}$, Tryptase $2 \mathrm{ng} / \mathrm{mL}$, all within normal range. Biopsy of the lesions showed leukocytoclastic vasculitis. Given prior diagnosis of SLE, new angioedema, urticaria, vasculitis, and arthralgias the patient was diagnosed with hypocomplementemic urticarial vasculitis syndrome (HUVS). She was treated with high-dose Solumedrol with complete resolution of all symptoms.

IMPACT/DISCUSSION: HUVS is an autoimmune condition in which IgG antibodies bind to the $\mathrm{Fc}$ region of $\mathrm{C} 1 \mathrm{q}$ triggering a type III hypersensitivity reaction, ultimately causing widespread endovascular damage. The most common signs and symptoms are reflected in the diagnostic criteria. For diagnosis patient needs to meet both major criteria: urticatia for 6 months and hypocomplementemia, and two of six minor criteria: venulitis of dermis, arthralgias, glomerulonephritis, uveitis or episcleritis, abdominal pain, and presence of C1q precipitin. Angioedema is an initial presentation in $50 \%$ of cases and is frequently mistaken for anaphylactic reaction. Urticarial rash is characterized by deep blue discoloration, pain, with resolution in 48 hours and residual dark skin pigmentation. Biopsy of the lesions classically reveal of leukocytoclastic changes. Many affected patients have other rheumatologic conditions, such as SLE. As awareness of HUVS in the medical community is low it is important to recognize patients who fail traditional treatment for allergic reactions and to broaden the differential diagnosis to include rare diagnoses such as HUVS.

CONCLUSION: HUVS is a rare condition that mimics atopic reactions. It is important to remain open minded when assessing SLE patients and broaden the differential diagnosis when a patient is not responding to standard treatments.

\section{HYPONATREMIA IN MALIGNANCY - IT'S NOT ALWAYS SIADH}

Vasilii Bushunow; Amar Kohli; Amar Bansal. UPMC, Pittsburgh, PA. (Control ID \#3168584)

LEARNING OBJECTIVE \#1: Explain physiology of hyponatremia in states with low effective arterial blood volume (EABV).

LEARNING OBJECTIVE \#2: Distinguish low EABV states from SIADH.

CASE: A 60-year-old man presented with several weeks of nausea, anorexia, and leg swelling. He also had several months of headaches with a biopsy negative for temporal arteritis. His PCP noted high liver enzymes. The patient had a forty-year smoking history without alcohol use or occupational exposure. Review of systems was positive for weight loss; negative for jaundice, cough, dyspnea or orthopnea, or seizures. Exam included a pulse of 90, BP of 107/71, no neurological deficits, cachexia, a right occipital nodule, dry mucous membranes, hepatomegaly, and lower extremity edema. Stigmata of chronic liver disease were absent. Labs included a $\mathrm{Na}$ of $118, \mathrm{~K}$ of 4.2 , bicarbonate of $20, \mathrm{Cl}$ of $85, \mathrm{Cr}$ of 0.5 , osms of 245 , and AST of 650. Urinalysis showed Na less than 10 and osms of 448. Thyroid hormones, cortisol, and aldosterone were normal. Imaging showed lesions concerning for metastatic disease, including the skull, bilateral lungs, and liver. The patient received $0.9 \%$ saline with worsening edema but no increase in sodium. Repeat urinalysis showed low sodium and high osmolarity. The patient left the hospital against medical advice but was readmitted after collapsing at home and soon died of lung and liver failure.

IMPACT/DISCUSSION: Hyponatremia requires integration of physical and laboratory findings to determine etiology and guide therapy. This patient's cachexia impeded the assessment of volume status causing diagnostic uncertainty. For me, this case reinforced the value of thorough daily exam to monitor volume status rather than relying solely on lab values. Hyponatremia involves up to $25 \%$ of ICU cases and is a sign of poor prognosis regardless of etiology. As the leading cause of hyponatremia in cancer care, SIADH was considered in this patient, but his hyponatremia was better explained by hepatic tumor burden leading to splanchnic vasodilation and low EABV. This term implies preserved or increased intravascular volume but a decreased ratio of blood in the arterial versus the venous circulation. Whether from low cardiac output or arterial vasodilation, low EAVB is perceived as hypovolemia, causing sympathetic and reno-humoral activation and non-osmotic ADH release. Increased aldosterone leads to further volume overload, while free water retention causes hyponatremia. In contrast, SIADH requires euvolemia, with normal urine sodium reflecting suppressed aldosterone. Some studies propose an interval increase of FENa by $>0.5 \%$ after a $2 \mathrm{~L}$ saline bolus as a diagnostic test for SIADH, but this was not assessed in this patient. Both SIADH and low EABV share inappropriately concentrated urine indicative of $\mathrm{ADH}$, but low urine sodium should prompt search for pathology decreasing EABV.

CONCLUSION: Differentiating low EABV from SIADH requires assessment of intravascular volume. Low urine sodium is a clue of perceived hypovolemia and excludes SIADH.

\section{IDIOPATHIC CHYLOTHORAX; IS IT A BENIGN OR MALIG- NANT DIAGNOSIS?}

Sandra Naffouj; Jasmine Omar. Henry Ford Hospital, Detroit, MI. (Control ID \#3180494)

LEARNING OBJECTIVE \#1: Diagnose idiopathic chylothorax LEARNING OBJECTIVE \#2: Manage chylothorax

CASE: A 29-year-old healthy male presented with acute onset of dyspnea and right-sided pleuritic chest pain. CT scan of the chest showed large right-sided pleural effusion. Thoracentesis was performed, draining 2 liters of turbid white pleural fluid and a chest tube was placed. Fluid 
analysis revealed exudative effusion with lymphocytic predominance and triglyceride (TG) of $1,100 \mathrm{mg} / \mathrm{dl}$ consistent with chylothorax. Pleural fluid cytology and cultures were negative for malignancy and infection. Patient was made nil per os, total parenteral nutrition (TPN) and IV octreotide were started. High-volume chyle leakage was persistent. Patient underwent lymphangiogram, which showed chyle leak from the thoracic duct (TD) at the level of T7-T8. He underwent embolization, however, output did not improve. Patient underwent video-assisted thoracoscopy (VATs) and TD ligation by thoracic surgery. Pleural biopsy was negative for malignancy. Serum LDH, alpha-fetoprotein, beta-human chorionic gonadotropin, HIV, acid-fast bacilli, fungal, and anaerobic cultures were all negative. Peripheral blood smear was negative for dysplasia and blasts. Full body CT did not show any evidence of malignancy. Output trended down, and chest tube was removed when chest $\mathrm{x}$-ray showed resolution of pleural effusion. Diet was advanced to low-fat, medium chain triglyceride (MCT) diet. There was no leak recurrence at 1- month follow-up.

IMPACT/DISCUSSION: Chylothorax is the presence of chyle in the pleural space with a TG level $>110 \mathrm{mg} / \mathrm{dl}$ or evidence of chylomicrons in the pleural fluid. Non-traumatic chylothorax is a rare condition and can be due to many disease processes. A thorough evaluation of the most common causes of non-traumatic chylothorax includes malignancy (lymphomas, lung and mediastinal cancers), infections (tuberculosis, fungal infections), and congenital disorders of the lymphatic system, which were negative in our case. Conservative management includes either nothing by mouth or a low-fat diet with MCTs, which are absorbed directly into the portal system, bypassing the TD to reduce chyle flow and promote healing. TPN is used to replace proteins, electrolytes, and deliver lipids directly into the bloodstream, thereby bypassing the lymphatic system and decreasing chyle flow. Octreotide is an adjunct to help reduce chyle absorption from the intestines. When conservative measures fail, there are many interventional modalities to consider, including TD embolization, TD ligation, or pleurodesis. For our patient, a combination of conservative and operative interventions was performed given high-output of chyle and absence of a clear etiology, resulting in leakage resolution.

CONCLUSION: It is crucial to perform a comprehensive assessment in non-traumatic chylothorax to exclude occult underlying etiology. Initial conservative management includes dietary modifications. If there is no improvement, interventions are available to repair TD and reduce chyle output.

\section{IF PSORIASIS ISN'T ENOUGH - A CASE OF ERYTHRODERMIC PSORIASIS}

Max Ruge; Jordan Scharping; Michelle Sweet. Rush University Medical Center, Chicago, IL. (Control ID \#3184916)

LEARNING OBJECTIVE \#1: Describe erythroderma and some of its common etiologies.

LEARNING OBJECTIVE \#2: Discuss the potential treatment options for erythrodermic psoriasis.

CASE: A 65-year-old female with a 1-year history of psoriasis presented with a worsening rash and associated chills, arthralgias, weight loss, and diarrhea. Initially the erythematous and scaly rash was localized to her arms and under her breasts, but two months ago, it began spreading and became significantly more erythematous, pruritic, and painful. Due to disease progression, she was enrolled in an experimental randomized double-blinded clinical trial where she was given either secukinumab (Cosentyx) or placebo one month prior to hospitalization. Subsequently, her symptoms continued to worsen to the point where she could not get out of bed. On admission exam, her temperature was $101.3^{\circ} \mathrm{F}$, heart rate was 103 , and she had diffuse confluent erythema with overlying scale covering her entire body. She was diagnosed with erythroderma due to psoriasis, likely due to medication withdrawal from starting placebo or changing to the study agent. She was prescribed triamcinolone wet wraps
BID and benadryl with significant relief of her symptoms. Infectious workup and assessment for electrolyte derangements were negative. She symptomatically improved and was discharged 5 days later.

IMPACT/DISCUSSION: Erythroderma is typically defined as erythema of over $75 \%$ of the body surface area and has multiple causes including psoriasis, atopic dermatitis, drug reactions, and numerous malignancies such as Sezary syndrome and other lymphomas. Erythrodermic psoriasis is the rarest subtype of psoriasis, yet it is critical to recognize given its significant mortality (9 to 64\%). Key triggers include infection or an abrupt change in steroids or anti-psoriatic agents. The severity of erythroderma is highly variable but potentially lethal as the degree of scaling and inflammation can lead to superinfection, electrolyte abnormalities, shock, or even ARDS. In cases with persistent severe symptoms or with hemodynamic or thermoregulatory instability, first-line treatment consists of either cyclosporin or infliximab along with topical steroids. In general, systemic corticosteroids should be avoided because steroid withdrawal has been associated with erythrodermic flares, although this is controversial. Supportive therapy is also critical given the risk for electrolyte and fluid abnormalities. In addition to basic labs, skin culture from open or crusted sites and blood cultures are also warranted in unstable cases. Our patient significantly improved after a 12-hour trial of topical therapy. However, if she had not improved or if her symptoms progressed, cyclosporin would have been started.

CONCLUSION: This case highlights an atypical and potentially lethal complication of psoriasis. While our patient improved with triamcinolone wraps and supportive care, severe cases that are refractory to topical therapies should be treated with cyclosporin or infliximab.

\section{IGA VASCULITIS ASSOCIATED WITH ACTIVE MESOTHELIOMA}

Bradley F. Woodman ${ }^{1}$; Ami DeWaters ${ }^{2} .{ }^{1}$ Penn State Health/Milton S Hershey Medical Center, Hershey, PA; ${ }^{2}$ Penn State Hershey Medical Center, Hershey, PA. (Control ID \#3185856)

LEARNING OBJECTIVE \#1: Describe the diagnostic signs and symptoms of adult-onset IgA vasculitis.

LEARNING OBJECTIVE \#2: Discuss the evaluation and management of adult-onset IgA vasculitis.

CASE: A 74 year old man with a recent diagnosis of desmoplastic-type malignant mesothelioma with recurrent pleural effusions presented prior to starting mesothelioma treatment with a one week history of painless purple lesions on his bilateral lower extremities and hand swelling leading to an inability to make a fist. The patient endorsed loss of appetite, but he denied abdominal pain or arthralgias. On physical exam, the patient had fine inspiratory crackles on auscultation, a non-tender abdomen, bilateral pitting edema of his upper extremities, and palpable purpura extending from his feet to his thighs bilaterally. Initial laboratory results were significant for a creatinine of $1.72 \mathrm{mg} / \mathrm{dL}$ from a previous baseline of $0.86 \mathrm{mg} / \mathrm{dL}$ one week prior. His erythrocyte sedimentation rate was 9.3 $\mathrm{mg} / \mathrm{dL}$, and his c-reactive protein was $75 \mathrm{~mm} / \mathrm{hr}$. He was admitted and treated with steroids, and a skin biopsy was diagnostic for IgA vasculitis. His renal function improved and he was discharged home on a steroid taper with topical steroids.

IMPACT/DISCUSSION: IgA vasculitis is rare in adults with only ten percent of cases occurring in adult patients. The tetrad of clinical features characteristic of IgA vasculitis is acute onset abdominal pain, arthritis or arthralgias, purpura, and renal involvement in the form of hematuria or proteinuria. The causes of $\operatorname{IgA}$ vasculitis are poorly understood, but it has been hypothesized that it could present as a paraneoplastic syndrome. In this case, it is possible that it presented as a paraneoplastic syndrome associated with mesothelioma. To evaluate and diagnose $\operatorname{IgA}$ vasculitis, a skin biopsy is necessary. A positive biopsy for IgA vasculitis has either a leukoclastic vasculitis or proliferative glomerulonephritis with predominantly IgA deposition. Treatment is mainly supportive, as it is a self- 
limiting disease in the majority of cases. Glucocorticoids are not routinely recommended for treatment. However, in patients with severe abdominal pain or significant renal dysfunction, glucocorticoids are used to reduce inflammation.

CONCLUSION: IgA vasculitis is a rarely seen disease in adults, and is characterized by purpura, abdominal pain, arthralgias, and kidney injury. Clinicians should consider IgA vasculitis with these symptoms and obtain a skin biopsy of the purpura to confirm the diagnosis. Treatment is mainly supportive.

\section{ILEAL NEOBLADDER, AN OFTEN OVERLOOKED CAUSE OF NON-ANION GAP METABOLIC ACIDOSIS.}

Henry C. Zheng; Christopher Chen; Katherine Timmins. Baylor College of Medicine, Houston, TX. (Control ID \#3186071)

LEARNING OBJECTIVE \#1: Recognize metabolic disturbances of ileal neobladders by understanding its physiology.

LEARNING OBJECTIVE \#2: Manage severe hyperchloremic non-gap metabolic acidosis in patients with ileal neobladders

CASE: A 75-year-old man with a history of bladder cancer status post radical cystoprostatectomy and ileal neobladder reconstruction 2 years prior presented with 2 weeks of malaise, and decreased urine output. He also notes a bug bite of the left hip which has now developed into a small, superficial abscess. He denies fevers, chest pain, shortness of breath, abdominal pain, nausea or vomiting. $\mathrm{He}$ was afebrile, normotensive, breathing 23 breaths/min, sating $98 \%$ on room air. He appeared lethargic but was without acute distress. A small $1 \times 2 \mathrm{~cm}$ superficial abscess of the left hip was seen without significant surrounding erythema, fluctuance or purulent discharge. Suprapubic discomfort on palpation was also noted. Workup was notable for $\mathrm{Na} 137 \mathrm{mEq} / \mathrm{L}, \mathrm{K} 4.0 \mathrm{mEq} / \mathrm{L}, \mathrm{Cl} 117 \mathrm{mEq} / \mathrm{mL}$, bicarbonate $7 \mathrm{mEq} / \mathrm{L}$, creatinine $4.19 \mathrm{mg} / \mathrm{dL}$, glucose $98 \mathrm{mg} / \mathrm{dL}$, ABG pH 7.19, PCO2 $11 \mathrm{mmHg}, \mathrm{PO} 298 \mathrm{mmHg}$ and lactate $1.3 \mathrm{mmol} / \mathrm{L}$. His anion gap is 13. A rapid response was called for acidemia with concerns of sepsis, however, given non-anion gap acidosis, a benign appearance of abscess, lack of fevers and normal lactate, suspicion for infection as primary etiology was low. A Foley catheter was placed for concerns of urinary obstruction and 1 liter of urine was collected immediately. An isotonic sodium bicarbonate infusion was started to treat the acidemia. Over the next 3 days, his non-gap acidosis resolved and creatinine downtrended. Blood cultures remained negative. He was discharged with plans to follow up in primary care clinic to determine the necessity of chronic bicarbonate replacement therapy in the future.

IMPACT/DISCUSSION: Mild metabolic derangements can occur in up to $15 \%$ of patients with ileal neobladder and severe acidosis occurs in $<1 \%$ of these patients overall. As the urine sits in the neobladder, the gastrointestinal mucosal sodium-hydrogen antiporter secretes sodium in exchange for hydrogen ions, and bicarbonate is excreted in exchange for hydrogen ion and chloride ion resulting in a hyperchloremic non-anion gap metabolic acidosis. Urinary retention results in severe acidosis due to prolonged absorption time of the urinary constituents. Treatment is focused on minimizing urine contact time with the reservoir mucosa. A Foley catheter should be placed to enhance urinary drainage. Though intravenous bicarbonate administration is generally not indicated for most types of anion-gap metabolic acidosis, it is reasonable to consider isotonic sodium bicarbonate infusion for these patients as they have chronic bicarbonate deficiency. Lastly, hypokalemia in the setting of severe acidosis requires close monitoring and repletion.

CONCLUSION: As neobladder surgeries become more common, it's increasingly important to understand the physiology and recognize the metabolic complications in order to initiate therapy in a timely manner.
IMMUNE CHECKPOINT INHIBITOR-INDUCED MYOCARDITIS PRESENTING AS COMPLETE HEART BLOCK

Tyler Mehegan ${ }^{1}$; Aarti Asnani ${ }^{2} .{ }^{1}$ Beth Israel Deaconess Medical Center, Brookline, MA $;{ }^{2}$ Beth Israel Deaconess Medical Center, Boston, MA. (Control ID \#3171120)

LEARNING OBJECTIVE \#1: Recognize fulminant myocarditis as an adverse effect of immune checkpoint inhibitors (ICIs).

LEARNING OBJECTIVE \#2: Manage complete heart block caused by ICIs.

CASE: The patient is a 79-year-old man with a history of high-grade pleomorphic bladder cancer status post cystoprostatectomy, recently started on adjuvant atezolizumab, who presented to the Emergency Department with one hour of lightheadedness, nausea, and pallor. His review of systems was otherwise negative. In the $\mathrm{ED}$, he was noted to have a heart rate of 24 and a blood pressure of 80/40. An electrocardiogram (ECG) showed complete heart block with a ventricular escape rhythm. Laboratory testing was notable for a troponin T level of $1.09 \mathrm{ng} / \mathrm{ml}$. A temporary transvenous pacemaker was placed, followed by placement of a permanent dual-chamber pacemaker. Coronary angiography, transthoracic echocardiography, and an infectious workup including Lyme IgG and IgM were all unremarkable. He was discharged with a diagnosis of senile conduction disease and a type II non-ST elevation myocardial infarction. Forty-eight hours later, he was readmitted with chest pain and an increased troponin $\mathrm{T}$ of $2.06 \mathrm{ng} / \mathrm{ml}$. In discussion with his oncologist, treatment was initiated for presumed atezolizumab-induced myocarditis with methylprednisone 1g IV Q24 hours and ultimately mycophenolate, resulting in improvement in his symptoms and cardiac biomarkers. Notably, repeat ECGs following immunosuppression demonstrated stable native conduction without pacemaker dependence.

IMPACT/DISCUSSION: Over the last decade, ICIs have drastically improved survival for patients with a variety of malignancies. Although generally well-tolerated compared to cytotoxic chemotherapy, immunerelated adverse events are common, including colitis, pneumonitis and hepatitis. ICI-induced myocarditis occurs less frequently, in less than $1 \%$ of patients treated with ICIs, but is often fatal. Despite aggressive immunosuppression, progression to fulminant myocarditis, multiorgan system failure, and death is common. As clinical presentations are variable and can be non-specific, a high index of suspicion for myocarditis in patients on ICI therapy is paramount. While complete heart block has been reported in the setting of ICI-induced myocarditis, there are no published data or guidelines regarding the need for permanent pacemaker implantation in this patient population. As demonstrated by this case, it is possible for native conduction to recover with immunosuppressive therapy in patients suspected to have ICI-induced myocarditis. A trial of temporary pacing may therefore be a reasonable approach while implementing an immunosuppressive strategy.

CONCLUSION: In patients on ICI therapy presenting with any new cardiac abnormality, a diagnosis of ICI-induced myocarditis should be considered. In ICI-induced myocarditis presenting as an advanced cardiac conduction disorder, a trial of aggressive immunosuppression may result in recovery of native conduction and obviate the need for a permanent pacemaker.

IMMUNE THROMBOCYTOPENIC PURPURA: AN UNCOMMON INITIAL PRESENTATION OF HUMAN IMMUNODEFICIENCY VIRUS

Ariana Mooradian; Gretchen Grosch. Saint Louis University, St Louis, MO. (Control ID \#3183925)

LEARNING OBJECTIVE \#1: Explore an unusual presentation of a common disease (HIV)

LEARNING OBJECTIVE \#2: Identify risk factors that increase likelihood of poor response to therapy in HIV patients with ITP 
CASE: A 49-year-old transgender female presented to the emergency department after a syncopal episode and reported weakness, shortness of breath, gingival bleeding, epistaxis, small red lesions and bruises on shins, and melena. She developed myalgias and foot pain three weeks prior and was taking naproxen $1-2 \mathrm{~g}$ daily. Patient denied change in weight, upper respiratory tract symptoms, headache, vision change, diarrhea, dysuria, and hematuria. Laboratory results revealed hemolobin of $4.9 \mathrm{~g} / \mathrm{dl}$, schistocytes $2+$, and platelets of $4 \times 10^{3} / \mathrm{uL}$. The patient was admitted to the intensive care unit where she was given 1 unit of packed red blood cells, 4 units of fresh frozen plasma and underwent plasmapheresis. She completed a 5 day course of dexamethasone and counts increased. Bone marrow biopsy performed revealed hyper cellular marrow with trilineage hematopoiesis without evidence of lymphoma and she was diagnosed with immune thrombocytopenic purpura (ITP). Human Immunodeficiency Virus (HIV) screening returned positive and CD4 count was found to be $493 \mathrm{cells} / \mathrm{mm}^{3}$ (21\%) with a viral load of $4,390,000$ copies. The patient was maintained on oral steroids until follow up with infectious disease clinic at which point highly active anti-retroviral therapy (HAART) was initiated.

IMPACT/DISCUSSION: The incidence of thrombocytopenia in HIV patients has been estimated at 6-15\% though early estimates were twice as high. Estimates of thrombocytopenia at diagnosis are much lower. The clinical entity of HIV-related thrombocytopenia usually presents during chronic infection or advanced immunosupression (CD4 <200) thus treatment of choice for this entity is antiretroviral therapy. Indeed, reports of thrombocytopenia after successful and sustained viral response is relatively uncommon. A retrospective analysis by Ambler et al. found that treatment of HIV alone was insufficient treatment of thrombocytopenia and nearly all patients treated for ITP required additional secondary treatment. Advanced age, anemia, intravenous drug use, and hepatitis infection have been indicated as risk factors for poor treatment response. Studies have found that there is no difference in risk between genders or ethnicities. Thrombocytopenia presenting early in HIV most resembles classic ITP, whereas presentation later in disease course may present more complicated. At this time, there has not been a consensus on HAART that is most effective for HIV-associated ITP.

CONCLUSION: HIV screening is part of standard preventative medicine. With advances in medication, early detection and initiation of antiviral therapy is paramount to best possible prognosis. Clinicians should consider HIV infection in any patient that presents with thrombocytopenia. Given current uncertainties as to optimal therapy, more research is warranted to establish a standard of care for HIV-associated ITP.

\section{IMPORTANCE OF RAPID DIAGNOSIS IN RAPIDLY PROGRES- SIVE DEMENTIAS: A CASE OF VITAMIN B12 DEFICIENCY}

Aradhana Verma ${ }^{2}$; Benjamin $\mathrm{Li}^{3}$; Michael Conte ${ }^{3}$; Katie Raffel ${ }^{1} .{ }^{1}$ University of California San Francisco, San Francisco, CA; ${ }^{2}$ California Northstate University, Elk Grove, CA; ${ }^{3}$ St. Mary's Medical Center, San Francisco, CA. (Control ID \#3185041)

LEARNING OBJECTIVE \#1: Review initial assessment for reversible causes of rapidly progressive dementia

LEARNING OBJECTIVE \#2: Recognize the neurologic sequelae and treatment trajectory of vitamin B12 deficiency

CASE: An independent 80 year-old man with well-controlled diabetes was brought in by family with a two-month history of progressively declining cognitive function, worsening vision, and visual and auditory hallucinations. The patient was no longer able to navigate city busses nor participate in basic self-care. The patient was afebrile, BP 126/48 and HR 54. On neurologic exam, he was oriented only to self, inattentive, and with deficits in all cognitive domains: executive function, abstraction, recall. He had impaired visual acuity. There were no strength or light touch sensory deficits. Reflexes were intact, and no myoclonus, rigidity, tremor, or cerebellar findings were noted. Complete blood count, chemistry panel, liver function tests, thyroid and coagulation profile were within normal limits. HIV, RPR, urine toxicology and heavy metal toxicology were negative. Infectious workup including urinalysis, blood cultures and CXR were unrevealing. MRI brain with contrast demonstrated small chronic microvascular ischemic changes. Cerebrospinal fluid analysis demonstrated protein of $49.2 \mathrm{mg} / \mathrm{dL}, 1 \mathrm{WBC} / \mathrm{mm}^{3}$, glucose $83 \mathrm{mg} / \mathrm{dL}$, absent oligoclonal bands and normal IgG index. HSV and CMV PCR and bacterial culture were negative. Vitamin B12 was $126 \mathrm{pg} / \mathrm{mL}$.

IMPACT/DISCUSSION: This clinical presentation constitutes a subacute rapidly progressive dementia that may have been caused by vitamin B12 deficiency. Cobalamin is required for the development and maintenance of myelination of the central nervous system. Deficiency leads to demyelination of the spinal cord, cranial and peripheral nerves, and white matter. While the most common neurologic complaints of vitamin B12 deficiency are paresthesia, numbness, and gait problems, our patient exhibited less common but known advanced symptoms including dementia, visual disturbances and psychosis. Notably, neurologic symptoms can occur in the absence of anemia. In adults with neurologic sequelae, parenteral administration of vitamin B12 is indicated. In contrast to relatively quick hematologic response to $\mathrm{B} 12$ repletion, neuropsychiatric improvement can take months to a year or even worsen transiently after treatment. Rarely, patients are reported to have residual long-term moderate to severe neurologic disability.

CONCLUSION: After sedation for lumbar puncture and MRI, the patient developed an acute on subacute encephalopathy, and the family chose to pursue comfort care. Prompt diagnosis of vitamin B12 deficiency as cause of subacute rapidly progressive dementia is critical as it is reversible and neurologic recovery is strongly associated with the severity and duration of symptoms prior to treatment. If index of suspicion for B 12 deficiency is high, methylmalonic acid and homocysteine should also be sent to detect early disease.

\section{IMPROVING MEDICATION RECONCILIATION}

April Ehrlich; Esther Oh. Johns Hopkins University, Baltimore, MD. (Control ID \#3185680)

LEARNING OBJECTIVE \#1: Recognize potential for error in medication reconciliation

LEARNING OBJECTIVE \#2: Identify ways to improve accuracy of medication reconciliation

CASE: Patient is a 79 year old woman with history of hypertension, stroke, asthma, chronic obstructive pulmonary disease, rheumatoid arthritis, myelomalacia of the cervical cord, lumbar stenosis, osteoporosis, and vitamin D deficiency who presented to a General Internal Medicine clinic for GI complaint. Her visit note mentions that she brought in her medications and a reconciliation was completed with a total of 14 medications. However, given concern for memory impairment a home visit was made. During the home visit, she presented a different list from another PCP with 12 medications and had difficulty presenting her medication bottles as there was no organization. She later came in for a dedicated medication reconciliation appointment, with her medications, and was found to have only 9 medications, was taking pills from 3 separate bottles of a single medication, and had incorrect pill counts. Further discussion revealed she frequently did not fill medications due to finances. Thus many of her medications were discontinued with the final medication list consisting of 4 medications in order to avoid polypharmacy and improve compliance.

IMPACT/DISCUSSION: According to the Institute for Healthcare Improvement adverse drug events remain a top patient safety priority. However, accurate medication reconciliation, especially in the geriatric population can be challenging. This challenge is highlighted by Project RED, a discharge intervention program at Boston Medical Center, that showed improvement in readmissions with comprehensive discharge planning including a medication reconciliation. Yet even in this structured environment, follow up phone calls by a pharmacist revealed that over $50 \%$ of those with completed reconciliations needed corrective action by a pharmacist. ${ }^{1}$ This 
indicates the need to determine ways to improve the accuracy of medication reconciliation in order to improve patient safety. This patient highlights the difficulty in obtaining an accurate medication list, especially in the geriatric population with concern for cognitive impairment, and shows how home visits and dedicated appointments can assist in this endeavor.

CONCLUSION: This case shows how the typical processes of doing a medication reconciliation in a general internal medicine clinic can lead to significant error, especially in the geriatric population. It also points to potential useful tools for addressing this challenge including home visits and dedicated clinic appointments for the purpose of medication reconciliation. Sources 1. Jack BW, Chetty VK, Anthony D, et al. A reengineered hospital discharge program to decrease rehospitalization: a randomized trial. Ann Intern Med. 2009;150(3):178-187.

\section{IN "LUE" OF OTHER SYMPTOMS: A CASE OF LUES MALIGNA IN A PATIENT WITH HUMAN IMMUNODEFICIEN- CY VIRUS}

Sherry Zhang ${ }^{1}$; Jeremy Chang ${ }^{2}$; Sindalisa Hean ${ }^{1}$; Eric Daar ${ }^{1} .{ }^{1}$ HarborUCLA Medical Center, Wilmington, CA; ${ }^{2}$ LAC+USC, Wilmington, CA. (Control ID \#3180886)

LEARNING OBJECTIVE \#1: Recognize that secondary syphilis may present as lues maligna with numerous manifestations, especially in patients with human immunodeficiency virus (HIV)

CASE: A 48-year-old male presented with 2 months of painful skin lesions, weight loss and fevers. The skin lesions initially appeared as small erythematous papules on his feet and lower shins, but became larger and spread to other sites on his body, including his arms, hands, trunk and face. In addition, the patient reported a 40-pound weight loss over the past year and fevers for several months. Vitals were notable for a temperature of 39.3 degrees. Physical exam was notable for multiple papular lesions with associated crusting and eschar throughout his bilateral upper and lower extremities, including the palms and soles, chest, back and face. An HIV antibody/antigen screen and confirmatory antibody test both returned positive, with CD4 count of 264. Rapid plasma reagin (RPR) titer was 1:64 with a positive fluorescent treponemal antibody absorption (FTA-ABS) test. Biopsy of one of the cutaneous lesions showed nonspecific ulceration and fibrosis as well as perivascular lymphocyte and neutrophil infiltration with negative stains for spirochetes, fungi and mycobacteria. The patient's clinical picture including physical exam, serologic testing, and biopsy results were consistent with lues maligna and secondary syphilis. The patient was given a single intramuscular injection of 2.4 million units of penicillin $G$ benzathine. Within a few days following this treatment, he experienced resolution of his fevers and shrinking of his skin lesions. The patient was subsequently started on anti-retroviral therapy with emtricitabine-tenofovir and dolutegravir for management of his HIV infection.

IMPACT/DISCUSSION: Lues maligna - also known as malignant syphilis - is a rare manifestation of secondary syphilis that predominantly presents in patients with HIV as well as in other immunocompromised individuals. It is characterized by ulcerative papular lesions and can also be associated with nonspecific symptoms such as fever, weight loss, joint pain, and malaise as seen in our patient. The cutaneous lesions may mimic viral exanthems or disseminated fungal infections, making visual diagnosis challenging. Although no spirochetes were seen on biopsy of our patient's skin lesion, this finding has low sensitivity in the diagnosis of lues maligna as pathology most often shows lymphocyte and neutrophil infiltration like in this case. The diagnosis of lues maligna was further supported by the prompt resolution of his fevers and skin lesions after a single dose of penicillin $\mathrm{G}$ benzathine.

CONCLUSION: As syphilis can have a myriad of clinical manifestations and mimic other conditions - therefore possibly clouding the diagnosis clinicians should consider lues maligna as a rare presentation of a common condition when in evaluating patients with ulceronodular skin lesions, in order to provide prompt diagnosis and treatment.

\section{IN-N-OUT: AN UNCOMMON CAUSE OF CHRONIC ABDOMI- NAL PAIN.}

Bradley N. Tun; Ricardo Cruz. Boston Medical Center, Boston, MA. (Control ID \#3180852)

LEARNING OBJECTIVE \#1: Review differential diagnosis and evaluation of chronic abdominal pain.

LEARNING OBJECTIVE \#2: Recognize internal hernia as a potential etiology of chronic abdominal pain and its potential complications.

CASE: 51 year old male with history of gastric adenocarcinoma who underwent subtotal gastrectomy with retrocolic Roux-en-Y reconstruction presented to the ED with acute on chronic abdominal pain. Prior to this visit he had been experiencing intermittent abdominal pain episodes since his surgery. Initially the pain was attributed to GERD. However, these episodes persisted and he underwent extensive evaluation including a referral to gastroenterology. He had an EGD that was positive for $\mathrm{H}$. pylori which was eradicated with quadruple therapy. He also had a colonoscopy and several CT scans during multiple ER visits that were all unremarkable. Leading diagnosis at the time was a functional abdominal pain syndrome. On this presentation, he described the abdominal pain as sharp, constant and associated with nausea, emesis and anorexia. His physical exam was concerning for peritonitis with lab studies notable for WBC of 15.4 and lactate of 2.7. CT abdomen pelvis showed swirling of vessels and mesentery in the abdomen with significant mesenteric edema concerning for mesenteric ischemia. He underwent an urgent laparotomy which showed an internal hernia with entire small bowel up to intracecal valve herniated through jejunal mesenteric defect. The internal hernia had caused a $15 \mathrm{~cm}$ segment of small bowel necrosis that required resection. He tolerated the surgery and was discharged home a week later.

IMPACT/DISCUSSION: Chronic abdominal pain is a common complaint in the outpatient setting; differential is broad and includes common causes such as peptic ulcer disease, GERD, gastritis and irritable bowel syndrome. Based on the history, physical and laboratory studies, imaging can be indicated such as an abdominal ultrasound, CT scan, MRI, endoscopy and ERCP. One uncommon cause of abdominal pain is an internal hernia. Causes of internal hernias include congenital anomalies due to improper intestinal rotation, atrophy of the omentum, inflammatory and ischemic processes of the peritoneal cavity and prior abdominal surgeries, Roux-en-Y being most common. Radiologic diagnosis of internal hernias is challenging since characteristic findings on CT scan are often missed as hernias can spontaneously reduce on their own. Complications of internal hernias include small bowel obstruction, intestinal ischemia from incarceration and strangulation of intestinal contents, and bowel perforation. Management of internal hernia is surgery to reduce hernia and to close the defect. In our patient, his internal hernia, as a result of his prior Roux-en-Y reconstruction surgery, had been causing his chronic abdominal pain.

CONCLUSION: Suspect internal hernia as a cause of chronic abdominal pain in a patient with a history of abdominal surgery, specifically Rouxen-Y gastric bypass.

\section{INCIDENTAL FINDING OF LEFT VENTRICULAR TRABECULATION IN THE SETTING OF CEREBROVASCU- LAR ACCIDENT}

Dolly Nam; Lillian Niakan; Brent Herndon; Jaison John. University of Texas at Austin Dell Medical School, Austin, TX. (Control ID \#3186357)

LEARNING OBJECTIVE \#1: To recognize how congenital persistence of deep trabeculae can lead to increased risk of thromboembolic events. CASE: A 37-year-old man with past medical history significant for hypertension, hyperlipidemia, and bipolar disorder presented to the emergency department with a two week history of right hand weakness and numbness and difficulty speaking. An MRI brain showed a left middle cerebral artery infarction. A CT angiography of his head and neck showed 
a subacute left middle cerebral artery distribution infarct and occluded intracranial left middle cerebral artery at the M1 segment. Subsequently, a TTE showed trabeculations in the left ventricle in the apex that were concerning for possible left ventricular non-compaction (LVNC). For definitive diagnosis, a cardiac MRI was performed and showed a noncompacted: compacted myocardium ratio of $<2.2$, and trabeculated mass $<20 \%$ of the total myocardial mass. A cerebral angiogram showed left M1 segment middle cerebral artery occlusion with good collateral circulation from anterior and posterior cerebral arteries. The cardiologist recommended a repeat cardiac MRI in two to three years to track progression of trabeculations. The patient was started on lifelong anticoagulation to prevent further embolic events.

IMPACT/DISCUSSION: Though guidelines vary based upon imaging modality, for cardiac MRI a ratio of non-compacted:compacted myocardium must be 2.3 and LV trabeculated mass must be $20 \%$ of global LV myocardial mass to meet the definition of LVNC (2). Although the patient does not meet the imaging criteria for LVNC, he is still at significantly higher risk for thromboembolic events than the general population. Involvement of the cardiologist, lifelong anticoagulation, and close outpatient follow up are three key points in this case that can potentially prevent and minimize the serious complications of this condition.

CONCLUSION: - LVNC is a congenital disorder that increases risk of thromboembolic events such as CVA - Deep trabeculations can likewise increase risk of thromboembolic events even in absence of diagnostic criteria for $\mathrm{LVNC}$

\section{INCREASING DIVERSITY UNCOVERS TUBERCULOSIS RARITY}

Marshall Meier ${ }^{1}$; Kanapa Kornsawad ${ }^{2} .{ }^{1}$ University of Texas Health San Antonio, San Antonio, TX; ${ }^{2}$ University of Texas Health Science Center, San Antonio, TX. (Control ID \#3184438)

LEARNING OBJECTIVE \#1: Recognize clinical features of pancreatic tuberculosis

LEARNING OBJECTIVE \#2: Assess need of invasive procedures for diagnosis

CASE: A 25-year-old man with no past medical history presented with five days of rapidly enlarging neck mass. He recently moved from India to San Antonio and noticed an unintentional weight loss of 20 pounds over the past month with a non-productive cough, nausea and vomiting. Denies neck pain, dysphagia and fever. Physical exam revealed a diffusely enlarged, non-tender anterior $5 \mathrm{~cm}$ neck mass without axillary or inguinal lymphadenopathy. The exam was negative for exophthalmos or abdominal tenderness. Laboratory studies showed TSH of 0.009 with free T4 of 1.4 and negative thyroglobulin, TSH receptor, and thyroperoxidase antibodies. Also found to have an elevated alkaline phosphatase to 135 . Computed tomography (CT) of the thyroid characterized multilobulated, complex cystic lesions with extension into the right thoracic inlet. CT abdomen revealed a $2.5 \mathrm{~cm}$ multilobulated complex cystic lesion in the head of the pancreas. Fine needle aspiration of the thyroid and paratracheal lymph nodes revealed +AFB with granulomatous inflammation and necrosis consistent with extrapulmonary tuberculosis (TB). The patient was treated with RIPE therapy and an MRI of the abdomen revealed complete resolution of both the paratracheal swelling and pancreatic mass at three months.

IMPACT/DISCUSSION: Pancreatic TB was first described in 1944, but little data exists regarding incidence within the United States. Abdominal $\mathrm{TB}$, which encompasses multiple organs and peritonitis, accounts for only about five to twelve percent of new cases per year. In India, a region with much higher overall prevalence, the incidence of pancreatic involvement is $8 \%$ of all abdominal cases. TB thyroiditis accounts for only $0.6 \%$ of all thyroid masses making this a unique presentation of extrapulmonary TB with both pancreas and thyroid involved. There is no universally accepted diagnostic or treatment strategy for pancreatic TB. For management of this patient, we followed an algorithm first suggested by Sharma et al. It utilizes imaging characteristics of the abdominal mass as well as biopsy proven TB in other non-pancreatic sites in order to avoid further unnecessary invasive tests. The use of fine needle aspiration for direct diagnosis of pancreatic TB has yielded variable results of $43-80 \%$. Based on this information and taking into account the patient's young age as well as no other risk factors, he was spared an invasive and potentially harmful procedure. This diagnosis requires a high index of suspicion but ultimately leads to appropriate treatment and sub-specialty follow up.

CONCLUSION: Tuberculosis can manifest within a multitude of different organs leading to puzzling symptoms and clinical presentations. By thoroughly investigating each abnormality, clinical context can guide a thorough workup while avoiding unnecessary and invasive procedures but still achieving favorable outcome and appropriate sub-specialty follow up.

\section{INDECISION BEFORE THE INCISION: DIFFICULT CHOICES BEFORE A WHIPPLE PROCEDURE}

Richard Pham. UPMC, Pittsburgh, PA. (Control ID \#3185842)

LEARNING OBJECTIVE \#1: Examine the variables affecting stable decision-making in advanced illness

LEARNING OBJECTIVE \#2: Identify social disparities among a minority group during end-of-life care

CASE: A 64-year-old African American woman is admitted with altered mental status and bacteremia while on total parenteral nutrition. On chart review, it was noted she was diagnosed with a benign pancreatic cystadenoma years ago and declined a curative Whipple due to her hesitancy regarding the operative risks. Over several years she had suffered functional decline, with multiple admissions for pancreatitis and difficulties with maintaining nutrition. Documented discussions revealed frequent uncertainty about her desires for artificial nutrition and invasive procedures going forward. The team notes this is her third admission for bacteremia this year, but this time the patient is unable to discuss her care preferences due to her mental status. A goals of care discussion is held with family, and her sister expresses concerns about the patient's worsening functional decline and reiterates her desires for no additional invasive procedures. Hospice planning is initiated. A few days later, the surgical team revisits the possibility of Whipple and a sudden change in treatment course is made. The patient and family decide to ultimately pursue Whipple and TPN is resumed. The patient is hospitalized for a total of two months and discharged without definite plans for surgery.

IMPACT/DISCUSSION: The patient expressed conflicting preferences through her disease course and as providers, we must be cognizant that preferences may be shaped by how we present treatment options. The way the surgery and medicine team communicated the illness course clearly resonated with the patient and family in opposite ways. Poor communication between providers resulted in poor communication with the patient. Poor communication is also a factor often cited as a possible source of health disparity in end-oflife planning. African Americans are statistically less likely to engage in hospice planning than white counterparts. In $2015,50.5 \%$ of Medicare white Americans engaged in hospice at the end of life while only $38 \%$ of African Americans did. African Americans are cited to undergo intubation and CPR more frequently than whites prior to death. Even among African Americans in hospice who have end of life preferences, $90 \%$ received care consistent with their wishes, while $95 \%$ of white Americans did.

CONCLUSION: -Making advanced decisions about an unknown future is a time of great stress and anxiety for patients. -Providers must communicate among each other to better ensure consistent communication to prevent inconsistent patient preferences -Health disparities among African Americans exist also in end-of-life care, and the onus is on physicians to recognize poor communication as a contributing factor. 


\section{INFERIOR MESENTERIC VEIN THROMBOSIS SECONDARY TO ACUTE SIGMOID DIVERTICULITIS A RARE COMPLICATION.}

Muqsita Nashat; Saba Mushtaq. Mercy Nazareth Hospital, Philadelphia, PA. (Control ID \#3178637)

LEARNING OBJECTIVE \#1: Diagnose and treat septic thrombophlebitis of inferior mesenteric vein secondary to acute diverticulitis.

CASE: A 50-year-old male presented to the emergency room with worsening left lower quadrant abdominal pain of two days duration associated with subjective fever and diarrhea. Three weeks prior to his presentation, he was diagnosed with acute diverticulitis confirmed by abdominal CT scan and completed a seven-day course of Ciprofloxacin and Metronidazole. Physical exam revealed a temperature of $36.4 \mathrm{C}$, pulse $136 / \mathrm{min}$, BP $140 / 85$, tenderness in the left lower quadrant without any peritoneal signs. There was no leukocytosis and a repeat CT of the abdomen showed worsening sigmoid diverticulitis with new septic thrombophlebitis of the inferior mesenteric vein extending to the Porto splenic confluence. Patient was admitted, placed on bowel rest, treated with intravenous Piperacillin/Tazobactam, fluids, Heparin drip and oral Vancomycin as his stool for C. diff toxin gene by PCR was positive. His blood cultures were negative and flow cytometry showed no evidence for a lympho or myeloproliferative disorder. Due to persistent abdominal pain, he underwent hemicolectomy and a coloproctostomy. Pathology of colon showed acute diverticulitis with pericolonic abscess. The patient was discharged home and advised to complete the remaining 14 days of oral Vancomycin and six-month course of Coumadin

IMPACT/DISCUSSION: Clinical cases of Inferior Mesenteric Vein thrombosis (IMV) complicating acute diverticulitis is rare and comprises of $4-11 \%$ of cases but carries a $15-23 \%$ risk of mortality. Acute diverticulitis can cause inflammation of IMV by peri colonic abscess and thrombosis occurs by inflammatory mediators causing endothelial injury and activation of coagulation cascade. Sigmoid diverticulitis is the most common causative etiology of thrombophlebitis of IMV accounting for $30 \%$ of cases, the remaining other causes are appendicitis (19\%), IBD $(6 \%)$, pancreatitis $(5 \%)$, infectious enteritis $(4 \%)$, bowel perforation and malignancies $(6 \%)$. There is high mortality in cases complicated by hepatic abscess or bowel ischemia. The clinical presentation of septic thrombophlebitis of IMV is insidious with vague symptoms delaying early diagnosis and therefore requires a high index of suspicion. CT scan of the abdomen with contrast is generally the first test of choice with direct visualization of endoluminal thrombus. Bacteremia is typically polymicrobial with E. coli, Bacteroides, Streptococcus Viridians, Proteus Mirabilis and Klebsiella Pneumonia being the most common pathogens. Sterile blood cultures in our patient can be explained by the prior use of antibiotics. Treatment with broad spectrum antibiotics and systemic anticoagulation often has a favorable outcome when compared to treatment with antibiotics alone

CONCLUSION: This case illustrates the fact that IMV thrombosis though a rare complication of acute diverticulitis requires a high index of suspicion for a prompt diagnosis and to prevent complications.

\section{INFLIXIMAB-INDUCED PULMONARY EMBOLISM.}

Areeg Bala ${ }^{1,2}$; Mahmoud Barbarawi ${ }^{1,2}$; Hoda Daher ${ }^{1,2}$; Ashok K. $\overline{\text { Kanugula }}^{1},{ }^{2}$; Ghassan Bachuwa ${ }^{3,}{ }^{2}$. ${ }^{1}$ Hurley Medical Center, Grand blanc, MI; ${ }^{2}$ Michigan State University, Flint, MI; ${ }^{3}$ hurley medical center, Flint, MI. (Control ID \#3186505)

LEARNING OBJECTIVE \#1: Recognize infliximab (Remicade) as a cause of pulmonary embolism.

CASE: A 20 years old African American male with a past medical history of diabetes type 1 and recently diagnosed Crohn disease 6 months prior presentation actively on mesalamine and infliximab infusions. His last infusion was shortly before the presentation. He presented to the emergency department with chest pain and back pain. On examination, he was tachycardic with a heart rate of 139. Laboratory investigations showed elevated D-dimer, EKG showed sinus tachycardia, and CT of the chest with contrast showed pulmonary embolism involving the left lower lobe segmental and subsegmental vessels. He denied any history of smoking, recent immobilization, surgery, travel, or any personal or family history of clotting disorders. He was diagnosed with provoked pulmonary embolism and started on IV heparin infusion. He showed improvement and was sent home with a subcutaneous anticoagulant for a period of 3 months.

IMPACT/DISCUSSION: Impact and Discussion: This case demonstrates the potential pulmonary embolism side effect of a commonly prescribed anti-TNF infliximab. Remicade, trade name for Infliximab, a chimerical monoclonal antibody that works against tumor necrosis factor TNF. It's used for a different array of autoimmune disease. It can't be given by mouth because the digestive system will destroy the drug, and hence, it's given in intravenous infusions. Although most patients treated with infliximab experience side effects related to suppression of the immune system, thromboembolic side effects are very low. The most common side effects are serious infections (fungal, bacterial, or viral), reactivation of tuberculosis, reactivation of hepatitis, drug-induced lupus, and increase the risk for certain types of cancer. A case report has identified the occurrence of pulmonary embolism during infliximab treatment. The mechanism by which infliximab causes pulmonary embolism is still not very clear and hence further research is warranted.

CONCLUSION: Although infliximab is associated with multiple common side effects, we should be aware of its thromboembolic aptitude. Close attention should be given to IBD patients started on infliximab infusion for any symptoms of pulmonary embolism or deep vein thrombosis DVT.

\section{INFLUENZA-A, A RARE CAUSE OF DIFFUSE ALVEOLAR HEMORRHAGE.}

Deep Patel. Brookwood Baptist Heatlth, Birmingham, AL. (Control ID \#3186005)

LEARNING OBJECTIVE \#1: Recognize that Influenza A is a rare but important cause of diffuse alveolar hemorrhage.

LEARNING OBJECTIVE \#2: Recognize the important aspects of diagnosis and treatment of Influenza A.

CASE: A 59-year-old African American male with a past medical history of hypertension, hemolytic anemia, thrombocytosis, and DVT presented with 1 day of fever up to $102 \mathrm{~F}$, shortness of breath, congestion, and palpitations. No prior surgical history. Family history significant for myocardial infarction in brother in his 60 's. Social history significant for prior tobacco use. Physical exam revealed increased work of breathing, bilateral wheezing, crackles, and tachycardia. Pertinent Lab findings included WBC of $12.1, \mathrm{H} \& \mathrm{H}$ 7.6/25.3, platelet count 93, Creatinine 1.58, lactic acid 4.0, troponin 0.141 , Procalcitonin 23.53.. Chest X-ray was concerning for bilateral pneumonia vs. pulmonary edema. Rapid Flu test was negative. Cultures were obtained and IV fluids, Azithromycin, and Piperacillin/tazobactam were started. Repeat X-ray was concerning for ARDS, so pulmonary service was consulted. Overnight his respiratory distress worsened and he was intubated. The next morning, he had bloody respiratory secretions and bronchoscopy revealed diffuse alveolar hemorrhage. Vasculitis labs were sent. Respiratory virus panel for Influenza A was positive and Tamiflu was initiated. Vasculitis work-up including ANA, ANCA, and Anti GBM was negative. Influenza was considered the cause of the diffuse alveolar hemorrhage.

IMPACT/DISCUSSION: Influenza-A - particularly H1N1 - is a viral illness associated with potential serious complications. One complication is diffuse alveolar hemorrhage. The first case of H1N1 was reported in March 2009 and by June 2009 it was a pandemic. It affected all ages, however death mostly occurred in those between 30-50 years of age. Although a rapid flu test and respiratory virus panel may be positive, real time reverse transcriptase polymerase chain reaction is the diagnostic 
method of choice. Even with a high index of suspension, clinical diagnosis is difficult because confirmatory testing may take up to a week. Oseltamivir is the treatment of choice. If started within 48 hours of symptom onset it reduces mortality and duration of hospitalization. If initial testing of influenza is negative in patients with alveolar hemorrhage then Influenza can be easily overlooked. In patients with alveolar hemorrhage H1N1 should be considered a differential as prompt empiric treatment is required.

CONCLUSION: This case highlights the importance of suspecting Influenza-A infection in a patient with diffuse alveolar hemorrhage, particularly when vasculitis work-up is negative. Although it is rare for influenza to cause diffuse alveolar hemorrhage, it certainly is possible. The physician should have a high index of suspicion as rapid flu tests may be falsely negative. Treatment with oseltamivir should be initiated promptly.

\section{INTESTINAL TUBERCULOSIS MIMICKING CROHN'S DISEASE}

Anira Iqbal $^{1}$; David T. Broome ${ }^{2}$; Rishi Rikhi ${ }^{2}$; Roop Kaw ${ }^{2} .{ }^{1}$ Chirayu Medical College and Hospital, Bhopal, India; ${ }^{2}$ Cleveland Clinic Foundation, Cleveland, OH. (Control ID \#3184017)

LEARNING OBJECTIVE \#1: Recognize and diagnose Intestinal tuberculosis(TB) mimicking Crohn's disease(CD), particularly in immigrants from TB-endemic countries.

CASE: A 73-year-old female Pakistani immigrant(1988) presented with 2 week history of bloody bowel movements, abdominal pain, fatigue, low grade fevers while on Tacrolimus and Prednisone, which she had been taking for 5 years post renal transplant. Colonoscopy showed terminal ileal inflammation with erosions, granularity, and ulcerations characterized as Crohn's disease and oral steroids were started. However, tissue biopsy demonstrated caseous granulomas consistent with TB. Steroids were discontinued after 5 days of initiation and she was switched to RIPE(Rifampin, Isoniazid, Pyrazinamide, Ethambutol), while continuing immunosuppressants, and discharged. A month later, she presented with recurrent symptoms. Pertinent findings were a fever of $39.3^{\circ} \mathrm{C}$ and right lower quadrant tenderness to deep palpation without signs of peritoneal irritation. Imaging displayed acute ileal inflammation of $8-10 \mathrm{~cm}$ with progressive ulceration. Fecal testing for acid fast bacilli(AFB) was positive. Serum RIPE levels were checked to ensure proper absorption. She was instructed to continue RIPE for 9 months and to follow up closely.

IMPACT/DISCUSSION: Intestinal TB and CD are chronic granulomatous diseases presenting similarly. With the reemergence of TB in the West due to the AIDS epidemic, differentiating them is of great importance. At-risk population for TB includes immigrants from endemic regions(Asian and African countries), alcoholics, prisoners and immunocompromised. Both conditions present with abdominal pain, fever, lethargy, bowel habit alteration and loss of appetite. CT scan in CD shows prominent ileal vasculature("comb sign"). In TB, the ileocaecal wall is asymmetrically thickened, with large nodes forming a mass. On colonoscopy, hypertrophic lesions with transverse ulcers suggest TB. CD has a cobblestone appearance with longitudinal deep ulcers and skip lesions. Fistulae are suggestive of $\mathrm{CD}$. Histopathologically, caseation necrosis with large granulomas $(>400 \mu)$ and AFB support $\mathrm{TB}$, as compared to small granulomas $(<200 \mu)$ in CD. Additional testing by stool AFB, Interferon gamma release assay for TB or Anti-Saccharomyces cerevisiae antibody for $\mathrm{CD}$ might corroborate diagnosis.

CONCLUSION: Physicians should be cognizant of Intestinal TB mimicking $\mathrm{CD}$ in at-risk population including immigrants from endemic regions, alcoholics, immunocompromised and prisoners. A combination of lab testing, imaging, colonoscopy and histopathology should be used to differentiate the two. Steroid therapy for a presumed diagnosis of $\mathrm{CD}$ will do more harm than good in individuals with Intestinal TB. References Sharma R, Madhusudhan KS, Ahuja V. Intestinal tuberculosis versus crohn's disease: Clinical and radiological recommendations. Indian J Radiol Imaging. 2016;26(2):161-72. Tandon HD, Prakash A. Pathology of intestinal tuberculosis and its distinction from Crohn's disease. Gut. 1972;13:260-9.
INTRAVASCULAR LARGE B-CELL LYMPHOMA PRESENTING AS HEMOPHAGOCYTIC LYMPHOHISTOCYTOSIS

Suheil Albert Atallah-Yunes ${ }^{1}$; Arthi Sridhar ${ }^{1}$; Riffat Sabir ${ }^{2} .{ }^{1}$ UMASSBaystate, Springfield, MA; ${ }^{2}$ Baystate Medical Center, Longmeadow, MA. (Control ID \#3186537)

LEARNING OBJECTIVE \#1: Recognize that intravascular lymphoma is very rare and mainly presents with non specific signs and symptoms LEARNING OBJECTIVE \#2: Recognize HLH as a presentation of lymphoma which could make treatment with chemotherapy more challenging CASE: A 66-year-old male with a past medical history of hypertension who was admitted to the hospital after being referred by his hematologist due to worsening of fever, chills and rigors. Prior to admission patient had an extensive work up for anemia, thrombocytopenia, fatigue and 15 pounds weight loss in 5 months duration. Outpatient work up ruled out an infectious etiology. CT scan showed hepatosplenomegaly and splenic lesions. Bone marrow biopsy was performed as an outpatient and preliminary findings were highly suggestive of large B-cell lymphoma within the sinusoids of the blood vessels pointing towards intravascular large Bcell lymphoma (IVBL). Upon admission, his vital signs revealed a high grade fever of $103 \mathrm{~F}$. Physical exam was positive for hepatosplenomegaly with no palpable lymph nodes. Blood work revealed a white cell count of $10.9 \mathrm{k} / \mathrm{ul}$, hemoglobin of $8.8 \mathrm{gm} / \mathrm{dl}$ and a platelet count of $37 \mathrm{~s} \mathrm{k} / \mathrm{ul}$. Given the high suspicion for intravascular large B-cell lymphoma, transjugular hepatic biopsy was performed and biopsy results confirmed large B-cells in the hepatic sinusoids. He was started on R-CHOP therapy. His ferritin level was elevated at $2000 \mathrm{ng} / \mathrm{ml}$ while IL-2 receptor levels were elevated at $18 \mathrm{~K}$ raising the suspicion for HLH in the setting of his worsening anemia, thrombocytopenia and hepatosplenomegaly. Patient was started on Dexamethasone for treatment of HLH and continued receiving chemotherapy for treatment of his IVBL.

IMPACT/DISCUSSION: IVL is a rare subtype of extranodal lymphoma and results from lymphocytic cell proliferation in the luminal vessels with minimal to no involvement of the surrounding parenchyma. Intravascular diffuse large B-cell lymphoma (IVBL) is the most common subtype. IVBL is classified into two: Classical IVBL and Asian Variant IVBL. The most common is the classical IVBL which usually occurs in western countries and mainly involves the CNS and the cutaneous tissue. Thus, patients present with CNS and cutaneous manifestation. Asian Variant (AV) IVBL is rare and tend to spare the CNS and the skin. These patients present with fever, malaise, anemia, thrombocytopenia, hepatosplenomegaly, hemophagocytosis, elevated liver function tests, elevated ferritin and hence HLH as it was seen in our patient. Theories support the release of macrophage stimulating factor and inflammatory markers mainly IL- 6 causing overactivation of the macrophages and so HLH. IVL is treated with the RCHOP therapy, if HLH is suspected then prednisone could be replaced with dexamethasone for treatment of HLH. CONCLUSION: Patients with IVL tend to present with nonspecific signs and symptoms in the usual absence of palpable lymph nodes. HLH is a rare presentation of IVL and could make treatment with chemotherapy more challenging due to the commonly seen cytopenias.

\section{IRON CLAD FEVERS OF UNKNOWN ORIGIN (FUO) IN A PA- TIENT WITH SICKLE CELL DISEASE}

Tessa Henry ${ }^{1}$; Steven G. McKee ${ }^{2} .{ }^{1}$ University of Arkansas for Medical Sciences, Little Rock, AR; ${ }^{2}$ University of Arkansas, Little Rock, AR. (Control ID \#3184536)

LEARNING OBJECTIVE \#1: Diagnose a rare cause of FUO in patients with sickle cell disease and iron excess.

CASE: A 32 year-old with sickle cell disease presented with fever and shortness of breath after multiple recent hospitalizations for pneumonia. Instead of several bouts of acute episodes, she divulged several months of 
intermittent fevers and productive cough. On examination she appeared ill and had tender, massive hepatomegaly. She had normal heart sounds, decreased breath sounds, and bilateral inspiratory crackles. White blood cell count was $15 \mathrm{~K} / \mathrm{uL}$, hemoglobin was $7.2 \mathrm{~g} / \mathrm{dL}$, liver function tests were elevated in a cholestatic pattern with alkaline phosphatase $721 \mathrm{IU} / \mathrm{L}$ and ferritin level was greater than $15,000 \mathrm{ng} / \mathrm{mL}$. She received a short course of broad-spectrum antibiotics without abatement of fever. Infectious workup for atypical and typical bacteria, fungi, Mycobacterium tuberculosis (MTB) and viral pathogens was negative. Computed-tomography (CT) displayed bilateral ground-glass opacities. The patient declined bronchoscopy. Hemophagocytic lymphohistiocytosis was entertained but she did not meet criteria. By hospital day 14, she remained febrile but had improvement of her respiratory status so she was discharged with plans for outpatient liver biopsy. The working diagnosis was adult onset Still's disease. Two weeks later she returned with shortness of breath and persistent fevers with exam unchanged apart from pitting edema to her knees. Evaluations for venous thrombus and embolism were negative and echocardiogram was non-diagnostic. Chest CT showed new pleural nodules with cavitation. Bone marrow, liver, and lung biopsies revealed noncaseating granulomas. Rare organisms were seen on acid-fast stain of the lung tissue. Standard testing for MTB plus PCR was again negative. Fever persisted despite antibacterial therapy so she was transitioned to azithromycin, ethambutol, and rifabutin. Blood transfusion was minimized and she received deferasirox for iron chelation. Lung biopsy cultures grew Mycobacterium intracellulare confirming disseminated infection and mandating one year of treatment.

IMPACT/DISCUSSION: Mycobacterium intracellulare is one species within the M. avium complex (MAC). MAC infections are a known complication of sickle cell disease in African populations with iron excess yet on review of the literature we found only four similar cases in North America.

CONCLUSION: This case highlights MAC as an important cause of FUO in a North American sickle cell patient with iron overload. The prevalence of nontuberculous mycobacterial infections is increasing in North America and all five cases of MAC infection in patients with sickle cell disease and iron overload have been reported since 2010. Thus MAC infection may be a growing risk for transfusion-dependent sickle cell patients on this continent. Recognizing the possibility of MAC infection in sickle cell patients with iron excess, despite locale, may lead to earlier diagnosis and treatment.

\section{IRON REPLACEMENT: A CAUTIONARY TALE}

Joy Tang; Gareth Hattersley. Medical College of Wisconsin, Milwaukee, WI. (Control ID \#3185220)

LEARNING OBJECTIVE \#1: Learn the symptoms, diagnosis and treatment for polycythemia vera.

CASE: A 68-year-old healthy male was found to have progressively decreasing mean corpuscular volume (MCV) on routine labs. Patient was asymptomatic. Vitals were unremarkable. Labs revealed a low MCV of $68.9 \mathrm{fL}$ (from $90.6 \mathrm{fL}$ three years ago), hemoglobin (hgb) of $15.8 \mathrm{~g} / \mathrm{dL}$, platelets (plt) of $445 \mathrm{~K} / \mu \mathrm{L}$, white blood cell (WBC) count of $12.8 \mathrm{k} / \mu \mathrm{L}$. Electrolytes and creatinine were unremarkable. Iron studies were consistent with pure iron deficiency. He was referred to gastroenterology given his progressive microcytosis and iron deficiency, concerning for occult bleed. EGD and colonoscopy were unremarkable, but did reveal a Billroth II anatomy, which was initially thought to be the etiology of his low iron. He was transfused with twelve doses of IV ferric gluconate $125 \mathrm{mg}$. Post-transfusions, he was noted to have elevated hgb of 18.8 $\mathrm{g} / \mathrm{dL}$. WBC and plts remained unchanged. Repeat iron level decreased to $37 \mu \mathrm{g} / \mathrm{dL}$. Patient was then referred to hematology. Serum erythropoietin was low at $1.0 \mathrm{mU} / \mathrm{mL}$. Ultrasound revealed splenomegaly and he was found to be positive for the JAK2 mutation. He was diagnosed with polycythemia vera (PV) and started on aspirin for prevention. Treatment was initiated with phlebotomies and hydrea.
IMPACT/DISCUSSION: PV is a rare, chronic myeloproliferative disease characterized by clonal proliferation of myeloid cells causing increased red cell mass. Symptoms include pruritus, headache, paresthesia, dizziness, visual disturbance and bleeding. Serum erythropoietin (epo) can be obtained to differentiate between primary and secondary polycythemia, with low epo pointing towards PV. Diagnosis can be made by fulfilling all major criteria or two major and the minor criteria. Major criteria include hemoglobin $>16.5 \mathrm{~g} / \mathrm{dL}$ in men and $16.0 \mathrm{~g} / \mathrm{dL}$ in women, bone marrow biopsy demonstrating hypercellularity with trilineage growth and presence of JAK2 V617F mutation or other functionally similar mutations. The minor criteria is subnormal serum erythropoietin. Currently there is no cure for PV. Management is based on risk stratification and can include starting aspirin for primary prevention, phlebotomy to maintain hematocrit $<45 \%$ and use of cytoreductive agents. PV can be difficult to diagnose due to confounding factors. Our patient had a classic presentation of PV that was initially mistreated due to concern for occult bleed based on his age, microcytosis and iron deficiency. Platelets are also frequently elevated in iron deficiency, making his diagnosis less apparent. Physicians should consider PV as a differential for patients who present with iron deficiency and inappropriately normal or elevated hemoglobin. Patients with PV should be carefully monitored as life-threatening complications can develop from vaso-occlusive causes or progression to myelofibrosis or leukemia.

CONCLUSION: Consider PV as a differential for patients who present with iron deficiency and inappropriately normal or elevated hemoglobin

\section{IS IGNORANCE BLISS? THE BIOPSY DILEMMA IN CIRRHOT- IC PATIENTS WITH GLOMERULONEPHRITIS}

Max Ruge; Jeffrey Bulger; Michelle Sweet. Rush University Medical Center, Chicago, IL. (Control ID \#3184888)

LEARNING OBJECTIVE \#1: Discuss the most common glomerular disorders seen in cirrhosis with a particular focus on IgA nephropathy.

LEARNING OBJECTIVE \#2: Describe two common methods of renal biopsy and the preferred method in a patient with decompensated cirrhosis. CASE: A 54-year-old male with history of alcoholic cirrhosis (MELD 18 on admission) presented from an outside hospital with decompensated cirrhosis and worsening acute kidney injury (AKI) likely from either hepatorenal syndrome or overdiuresis $($ FeUrea $=27.2 \%$ ). His diuretics were initially held at our hospital, and he received a $5 \%$ albumin infusion, which lowered his creatinine from 1.6 to his baseline of 0.85 . During his AKI workup, he was incidentally found to have nephrotic-range proteinuria with an estimated 24-hour protein excretion of $4.2 \mathrm{~g} /$ day and $>200$ $\mathrm{RBCs}$ on urinalysis. Some degree of proteinuria and hematuria had also been noted on all urine studies from prior admits dating back 2 months. Transjugular kidney biopsy was performed due to concern for underlying glomerular disease, and additional liver samples were obtained to reevaluate the stage of cirrhosis. The renal biopsy revealed findings consistent with $\operatorname{Ig}$ A nephropathy, and he was started on losartan. Shortly after the procedure, he was found to have the complications of a ruptured gallbladder and a slow bleed from the cystic artery that required Gelfoam embolization. He spent about 1 week in the MICU for treatment of his decompensated cirrhosis and complications of the biopsy and was ultimately discharged 20 days later.

IMPACT/DISCUSSION: Only a very small percentage of renal pathology in cirrhosis patients is the result of glomerular disease such as nephritic $(3.4 \%)$ or nephrotic syndrome $(2.2 \%)$, which most frequently includes IgA nephropathy, type I membranoproliferative glomerulonephritis, and mesangial proliferative glomerulonephritis. There is a welldocumented association between IgA nephropathy and liver cirrhosis likely because the damaged liver is not able to clear $\operatorname{IgA}$ complexes in circulation resulting in deposition in the kidneys. Furthermore, portal hypertension also appears to play a critical role in the development of IgA nephropathy in patients with cirrhosis. Both ACE inhibitors and 
ARBs are renal-protective in IgA nephropathy and have been shown to decrease proteinuria from this mechanism. Renal biopsy is difficult in this population due to the numerous clotting dysfunctions associated with cirrhosis leading to the elevated risk of complications such as perirenal hematoma. Transjugular biopsies are typically preferred over the traditional percutaneous route to mitigate some of these risks, even though adequate tissue is obtained only $78.6 \%$ of the time versus $90-95 \%$ for percutaneous route.

CONCLUSION: Given the risk of complications and the wellestablished relationship with IgA nephropathy, renal biopsy should be only obtained in cases of nephrotic-range proteinuria and hematuria if it may influence treatment such as evaluation for liver transplant or for rapidly progressive renal disease.

\section{IS THE HIGH WORTH THE TORSADES? A CASE OF LOPERAMIDE-INDUCED TORSADES DE POINTES}

Jaime de la Fuente; Jared Bird; Korosh Sharain; Vasken Keleshian; Nandan Anavekar. Mayo Clinic, Rochester, MN. (Control ID \#3161490)

LEARNING OBJECTIVE \#1: Identify loperamide as an important over-the-counter medication with abuse potential that can cause prolonged QT interval

LEARNING OBJECTIVE \#2: Recognize life threatening ventricular arrhythmias and their treatment

CASE: A 29-year-old female with a history of bile acid diarrhea and chronic abdominal pain presented for an outpatient esophagogastroduodenoscopy. Prior to the procedure, she developed severe nausea, vomiting, and dizziness, prompting direct admission to the general medicine service. Initial negative work up included: complete blood count, extended electrolytes, pregnancy test, urinalysis, urine drug screen, and serial troponins. ECG demonstrated a prolonged QTc interval of 549 msec. Upon further history, she revealed she had been consuming more than $200 \mathrm{mg}$ (100 pills) of loperamide daily, for over a year, to relieve her abdominal pain and diarrhea. Her last dose of loperamide was 48 hours prior to presentation. During hospital day one she developed palpitations and had a pre-syncopal episode. Review of her cardiac monitor revealed polymorphic ventricular tachycardia (PVT). She was promptly started on IV magnesium and isoproterenol to decrease the QTc interval and reduce the probability of further PVT. This initially abated further PVTs. However, while weaning down the isoproterenol she had recurrence of runs of PVT. Thus, IV lidocaine was added to her regimen. Significant QT interval prolongation continued despite loperamide discontinuation, raising the teams suspicion for an underlying congenital long QT2 syndrome. Thus, she was transitioned to nadolol without further occurrence of PVT. Nine days later patient was discharged from the hospital with a QTc of $460 \mathrm{msec}$ on nadolol. Further outpatient work up two months later, including electrocardiogram, stress test, and genetic testing, was negative for congenital long QT syndrome. QTc at that time was $430 \mathrm{msec}$. Patient's nadolol was discontinued. IMPACT/DISCUSSION: This case illustrates the importance of being familiar with over the counter QT interval prolonging agents, the possibility of loperamide being used as a drug of abuse, and the treatment of acquired PVT. It is believed that the mechanism of the prolonged QT by loperamide involves the inhibition of cardiac sodium and potassium channels. As demonstrated by this case, it is also critical to understand that the cardiac effects of loperamide, especially when abused and consumed for an extended period of time, will last much longer than the reported half-life of 11 hours. Additionally, congenital QT syndrome can potentially be unmasked when taking these agents, and as such should be maintained on the differential, and treated with beta blockade therapy in the outpatient setting.

CONCLUSION: Polymorphic ventricular tachycardia (PVT) is a life threatening arrhythmia resulting from a variety of mechanisms. For the general internist, it is important to recognize its initial managment and over the counter medications with abuse potential, like loperamide, which can lead to this serious arrhythmia.

\section{IS THERE A LAWYER IN THE HOUSE? THE PHYSICIAN ROLE IN LEGAL ADVOCACY}

Aliza Norwood; Alejandro Moreno. UT Dell Medical School, Austin, TX. (Control ID \#3184396)

LEARNING OBJECTIVE \#1: Recognize symptoms of panic disorder in an immigrant patient

LEARNING OBJECTIVE \#2: Apply the concept of the extended care team to legal services

CASE: A 27 year-old woman with a history of preeclampsia and depression presented with worsening paroxysmal left-sided chest pain for 3 years. Multiple prior visits that included CBC, CMP, TSH, and EKGs revealed no cause for her symptoms. She described the pain as sharp and non-radiating, usually lasting 15 minutes, and associated with lightheadedness and shortness of breath. She also reported occasional headaches, tremors, nausea, and cold sweats. Her vitals, including orthostatics, were stable. The exam was notable only for a heart rate of 50 beats/ min, and EKG confirmed sinus bradycardia. Labs were normal as in prior visits. A Holter monitor showed sinus bradycardia with rare supraventricular ectopic beats. Her PHQ9 score was 9. Further questioning revealed a long history of persecution that included child and sexual abuse. She fled from her country to the US while pregnant to escape growing threats against her life and that of her unborn child. She began to have flashbacks and nightmares and became socially withdrawn. She was diagnosed with panic disorder and referred to behavioral health; over the next 2 months she noted improvement in symptoms with counseling and mindful breathing exercises. Multiple attempts were made to contact her immigration lawyer by phone and email, with no response. The patient returned to clinic with worsening symptoms 3 months later after receiving a final deportation order. With the patient's permission she was referred to a nonprofit immigration advocacy firm and a pro-bono lawyer to explore possible avenues of relief. The immigration lawyer proposed a course of action which was limited to an extraordinary legal recourse given the time elapsed and the existing deportation order. The patient declined because immigration authorities would have detained her for several months until the trial proceedings were over.

IMPACT/DISCUSSION: Anti-immigration policies have been linked directly to negative mental health effects on immigrants including depression, anxiety, and PTSD. Evidence exists that threat of deportation increases the risk of mental illness. Physicians are increasingly relying on social workers and others in the extended care team to address the social determinants of health. There are already precedents to screen patients for abuse or domestic violence which can lead to social and legal referrals. While not a part of routine practice, we propose that doctors are under a similar obligation to ensure patients are referred to competent legal aid for immigration services if needed to protect physical and/or emotional health. If referred early, the negative consequences of inadequate legal counsel may be prevented.

CONCLUSION: Physicians should have a low threshold to screen for physical and mental effects of trauma in immigrant patients. Medicolegal partnerships can be a tool to aid patients who seek asylum.

\section{IS THIS BECAUSE I'M PREGNANT OR DID I JUST CUT MY TOE?}

Camille R. Bourgeois; M. Matias Iberico. Tulane University School of Medicine, New Orleans, LA. (Control ID \#3184152)

LEARNING OBJECTIVE \#1: Recognize the rising rate of invasive Group B streptococci (GBS) infections in non-pregnant adults

LEARNING OBJECTIVE \#2: Identify appropriate antimicrobial coverage for GBS infection

CASE: A 74-year-old woman presented with exertional dyspnea, orthopnea, chills, and rigors worsening over the preceding 24 hours. No 
cough or sputum production was reported. She was visiting from out of town and was inconsistently taking home medications for hypertension, diabetes, and atrial fibrillation. She had been walking more than usual and did not report chest pain but did develop a small toe laceration. Her blood pressure was found to be $210 / 110 \mathrm{mmHg}$ on arrival and temperature of 101.1F. Elevated JVP, bibasilar crackles, and bilateral lower extremity edema were present. The right great toe had a small superficial laceration without evidence of drainage or surrounding infection. She was treated for decompensated heart failure and suspected sepsis. Broad spectrum antibiotics were initiated. Blood culture reports returned 6 hours after the draw with group B streptococcus. Antibiotics were transitioned to IV ceftriaxone and she was discharged on cefadroxil. No other source of infection was identified, and bacteremia was attributed to the small toe laceration.

IMPACT/DISCUSSION: The CDC Active Bacterial Core surveillance estimates that 28,000 cases of invasive GBS occurred in 2014 and $93 \%$ occurred in non-pregnant adults. It is emerging as a frequent cause of invasive infection in non-pregnant adults with underlying chronic medical conditions. The main clinical presentations were bacteremias without apparent focus and soft tissue infections. One study reported that osteoarticular, skin and soft tissue infections account for $31 \%$ of manifestations and $45 \%$ of all patients studied experienced a complicating bacteremia. In about a quarter of the episodes of bacteremia studied, no source of infection identified. The most frequent risk factors associated were chronic kidney disease, cardiac failure, neoplasia history, ischemic heart disease, and diabetes mellitus. The high proportion of episodes of bacteremia indicates that blood cultures should be obtained in any elderly patient presenting with a skin and soft tissue infection or osteoarticular infection. GBS is susceptible to penicillin and amoxicillin, but these medications are only used for treatment about $29 \%$ of the time. Microbiology labs do not routinely test for sensitivities to GBS considering its consistent susceptibility to these antibiotics. It is important for internists to recognize the value of antibiotic stewardship and deescalate antimicrobial coverage accordingly when GBS is identified.

CONCLUSION: The incidence of invasive GBS infection is rising in the non-pregnant adult population. Penicillin is first line treatment for GBS infection and given the near uniform susceptibility, microbiology labs do not routinely run sensitivity testing on GBS species.

\section{IS THIS MUNDANE WORK? REFLECTIVE WRITING TO EMTIONAL WELL BEING.}

Manisha Bhide. University of Colorado Denver, Aurora, CO. (Control ID \#3167939)

LEARNING OBJECTIVE \#1: Reflect on daily work.

LEARNING OBJECTIVE \#2: Realize the importance of networking at regional and national level. Reflective writing and mentoring relationships play an important role in work satisfaction and emotional well being.

CASE: Boom ! Boom" The shots rang out, about to claim a life, but Ricky survived. "Rosie, why did you save me?" He croaked. "I want to die. I have nothing left to live for. Whatever mattered is all gone". "Ricky, He has given you a chance to lead a human life and you have to make the most of it. Until now you lived for yourself. Now, may be you can live for the society. There is so much to do. It is ultimately your relationship to Him that really matters." "In my busy clinic, I was running late again! I glanced at the paper in the door. "Paul P- BP followup." "This should be quick. It is just BP follow up. That should allow me to catch up" I thought erroneously. Paul unfolded his handkerchief from pocket and dabbed his eyes and unfolded his life story with it. He belonged to the era that still carried his handkerchief and ironed his jeans. Paul's wife of 40 years, Clara had a stroke. He spent every waking moment in the nursing home with Clara. He took it upon himself to clean her, dress her and take care of her personal hygiene every day for 18 years. She passed away in the middle of the night. I secretly felt relieved for Paul, thinking that his suffering was over. He could lead a new, near normal life. But, that aphasic expression, that contracted numb hand, that incontinent, sacral decubed relationship with Clara meant more to Paul than anything else in this world

IMPACT/DISCUSSION: I met "Dr. Young", my SGIM mentor 10 years ago when I was an eager junior faculty. He encouraged me to participate in National clinical content subcommittee and gave me a letter of recommendation for promotion. He was there with me in the reflective writing workshop. He has played a key role as a mentor and a guide in my professional development. I like the opening greeting that he uses inevery mentor-mentee meeting "Are you enjoying what you are doing?" Our residents do a relfective writing as a part of the continutiy clinic didiactics. These have served as a baseline for peer reviewed publication in JGIM, Annals, JAMA etc. Medical students are doing reflective writing as a part of hidden curriculum and learning professionalism. This helps them share their emotions like about the first death they encountered. Research has shown that this helps mental well being of trainees and professionals and gives them resilience.

CONCLUSION: Reflecting on each of these instances, every one of us needs to pour our heart and soul and passionately cherish our daily work like a prayer. Even in this world of electronic medical records, smart phones and virtual lives in social media, it is the human relationships that matter the most. It is through our routine, daily work that we are realizing our relationship with Him every moment of our lives and rising from mundane work to higher spiritual level.

\section{IT GETS WORSE BEFORE IT GETS BETTER: DELAYED POST HYPOXIC LEUKOENCEPHALOPATHY}

Stephen M. Stuart ${ }^{1}$; Maddie Bender ${ }^{1}$; Starr Steinhilber ${ }^{2} .{ }^{1} \mathrm{UAB}$, Birmingham, AL; ${ }^{2}$ University of Alabama at Birmingham, Birmingham, AL. (Control ID \#3185883)

LEARNING OBJECTIVE \#1: Define the clinical pattern of Diffuse Post-Hypoxic Leukoencephalopathy (DPHL)

CASE: A 58-year-old woman presents from a rehab facility for acute change in mental status. During a recent admission she was found down with profound hypoxia due to medication overdose, intubated, and experienced cardiac arrest during the hospitalization. She improved after one week in the intensive care unit and was verbal, mobile, and interactive at the time of discharge to the rehab facility. She then functionally declined within days and on admission to our facility was aphasic and encephalopathic. Exam notable normal vitals, minimal interaction, clonus with upgoing Babinski, diffuse rigidity with flexion of elbows, wrists, and hands, as well as mutism. Work up included a CT Head notable for subacute infarct that had been seen previously. Labs were unremarkable and infectious causes of encephalopathy were unrevealing other than a UA suggestive of UTI for which she was treated without improvement. EEG had generalized slowing with no seizure activity. Brain MRI revealed multiple white matter lesions consistent with demyelination. CSF fluid was normal. Given her imaging suggestive of demyelination, her clinical course of a hypoxic event with initial mental improvement followed by decompensation, exam findings, and otherwise negative work-up, the patient was diagnosed with DPHL. She slowly improved throughout her admission and became more alert, less confused and three months later she was independent on her ADLs with minimal neurologic deficits.

IMPACT/DISCUSSION: DPHL is a rare diagnosis that requires both clinical suspicion, specific imaging findings, and appropriate exclusion of other plausible diagnoses (1). The illness script includes a hypoxic insult to the brain followed by a lucid period with subsequent worsening of mentation. Lucid period reported at 2-40 days following recovery from initial hypoxic event (1). Subsequent clinical features include mutism, parkinsonism, bizarre behavior, frontal release signs, pathologic laughing, and hyperelexia. Radiographical findings include demyelination of the cerebral white matter. MRI is the best modality to visualize demyelination of white matter in the brain thought to be specific for DPHL (1). After a thorough work up, our team was without a diagnosis that could explain 
the patient's deficits. As we searched we learned about DPHL as a posthypoxic syndrome. We realized that the timing of her symptoms as well as clinical symptoms, EEG findings, and imaging findings matched the diagnosis. We watched as she recovered over time with supportive care. We hope this case helps recognize this rare entity as a potential diagnosis following hypoxic brain injury. Retrospectively detecting an initial recovery followed by a sudden deterioration in neurologic status will help move DPHL on clinician's differential.

CONCLUSION: Consider DPHL in patients with hypoxic brain injury followed by initial recovery and then delayed neurologic deficits.

\section{IT IS NOT COMMON COLD, IT IS AN UNCOMMON COLD IN AN ARIZONAN}

Alvaro J. Altamirano; Roshanak Habibi. Banner university medical center, Tucson, AZ. (Control ID \#3179495)

LEARNING OBJECTIVE \#1: To recognize an uncommon cause of pericardial effusion in a patient who failed outpatient treatment of upper respiratory tract infection.

CASE: 28 year-old male with no past medical history presented to emergency department in Tucson, Arizona with productive cough and progressive shortness of breath of one month. He was treated in urgent cares for upper respiratory tract infection with courses of levofloxacin, doxycycline, azithromycin, prednisone, fluticasone and ventolin; which did not improve his symptoms. Associated symptoms were nausea, fever, chills and night sweats. He was a nonsmoker, with no recent travel history, no sick contact and no tuberculosis (TB) exposure. He was from Korean descent, raised in Tucson. Social and family history were unremarkable. Abnormal laboratory data were white blood cell of 16 , erythrocyte sedimentation rate of 52, C-reactive protein of 78 and a positive serology for coccidioidomycosis (positive enzyme immunoassay for immunoglobulin $\mathrm{M}$ and $\mathrm{G}$ antibody with reflex confirmation). Coccidioides complement fixation titer was 1:32. Sputum culture, QuantiFERON TB gold, respiratory viral panel and human immunodeficiency virus testing were all negative. His chest $\mathrm{x}$ ray and commuted tomographic scan of chest and abdomen showed bilateral pulmonary infiltrates, hepatosplenomegaly, generalized lymphadenopathy and a moderate pericardial effusion. Echocardiogram also confirmed pericardial effusion with no signs of tamponade. EKG was normal. He was rehydrated and started on Fluconazole 400 milligram daily and 5 day course of Colchicine. He did not require pericardiocentesis or tissue biopsy, since the diagnosis seemed straight forward. His symptoms gradually resolved in course of few weeks and in Valley fever clinic follow up in Tucson his antifungal treatment continued for total of 6months.

IMPACT/DISCUSSION: Coccidioidomycosis, an infection with fungi Coccidioides immitis and C. posadasii, endemic of southwestern US is a rare disease, usually presented as community acquired pneumonia. Extrapulmonary manifestations are reported less than $1 \%$ in different studies. Pericardial involvement is unique presentation with less than thirty cases reported in literature. Presenting symptoms are usually nonspecific and resemble upper respiratory illness and pneumonia. Male sex and ethnicities such as Asians are more likely to develop disseminated disease. Any organ can be involved, with skin, lymph nodes, bones, and central nervous system being the most common extrapulmonary sites. Complement fixation titer ranges from 1:2 to $>1: 32$ in disseminated disease. Amphotericin B and fluconazole are treatment options. Misdiagnosis may lead to continued morbidity and in our case disseminated disease with potentially fatal cardiac involvement.

CONCLUSION: Pericardial effusion in our case could be life threatening if eventual Coccidioides screening was not sent. Pattern recognition of this infection in endemic areas should prompt an internist to send diagnostic studies early.
IT IS WHAT IT IS (UNTIL IT ISN'T): POLYMYALGIA RHEUMATICA AS A SUBSET OF GIANT CELL ARTERITIS

Mahmuda Khan ${ }^{1}$; Sara Abrahams ${ }^{4}$; Diane Horowitz ${ }^{3}$; Eun Ji Kim ${ }^{2}$; Nancy A. LaVine ${ }^{1} .{ }^{1}$ Northwell Health, New Hyde Park, NY; ${ }^{2}$ Northwell Hofstra School of Medicine, Lake Success, NY; ${ }^{3}$ Northwell Health, Manhasset, NY; ${ }^{4}$ Zucker School of Medicine at Hofstra/Northwell, Hempstead, NY. (Control ID \#3170068)

LEARNING OBJECTIVE \#1: Review diagnosic criteria for polymyalgia rheumatica (PMR) and giant cell arteritis (GCA).

LEARNING OBJECTIVE \#2: Assess for GCA in patients with PMR refractory to initial treatment.

CASE: 82 y/o man with coronary artery disease presented with abrupt right shoulder pain for 3 days. Abduction precipitated the pain, but it was improving. He returned 10 days later c/o of bilateral leg/shoulder pain, leg weakness, and fatigue. His ambulation was limited; he could not climb stairs. He denied fever, small joint pain, headache, jaw pain or visual changes. Exam noted pain with shoulder abduction $>90$ degrees and hip flexion, and upper/lower proximal muscle weakness. Being suspicious of polymyalgia rheumatica (PMR), ESR was obtained and was elevated at 49 (normal $<20$ ), ANA and anti CCP were negative. A diagnosis of PMR was made and prednisone $10 \mathrm{mg}$ /day was started. His pain improved slightly, but symptoms continued even with $20 \mathrm{mg}$. Despite absence of headaches/visual symptoms, the lack of improvement raised the concern for giant cell arteritis (GCA). Transcranial Doppler revealed concentric wall thickening of the right temporal artery, confirming a diagnosis of GCA. Prednisone was increased to $30 \mathrm{mg}$ daily with complete resolution of symptoms.

IMPACT/DISCUSSION: PMR is an inflammatory condition commonly diagnosed in older patients with proximal body aches, morning stiffness, and elevated ESR, which rapidly responds to low dose glucocorticoids (10$20 \mathrm{mg}$ prednisone/daily). GCA, another inflammatory condition of older adults, is associated with headaches, vision changes, jaw pain and elevated ESR, requiring higher dose prednisone $(40-60 \mathrm{mg} /$ day) with a goal of preventing vision loss. 40-60\% of GCA patients can have PMR like symptoms, and GCA should be suspected in patients with PMR who have an inadequate response to initial therapy. The gold standard for GCA diagnosis is temporal artery biopsy, thought with a significant false negative rate. Doppler US of the artery is an increasingly recognized noninvasive diagnostic tool; the "halo sign" (a hypoechogenic area of edema) is highly specific (80-97\%) for GCA. Although our patient lacked GCA symptoms (headaches, blurry vision, scalp tenderness, jaw pain), the lack of clinical improvement with appropriate steroids prompted further evaluation and diagnosis of GCA with treatment and clinical response.

CONCLUSION: This case is an important example of an atypical presentation of GCA, a "do not miss" diagnosis. The typical clinical picture of GCA includes headache, visual changes, or jaw pain, in addition to elevated inflammatory markers. Our patient presented with achiness, fatigue, and elevated ESR. Take Home Points: PMR can be the first presentation of GCA, which should be suspected in patients who do not respond to low-dose glucocorticoids. Doppler US showing concentric wall thickening of the temporal arteries ("halo sign") should be considered as an alternative diagnostic test to the gold standard of temporal artery biopsy for suspected subclinical GCA in patients with PMR.

\section{IT'S HAMMER TIME}

Ashley Kang; Chaya Abelow. Montefiore Medical Center, Bronx, NY. (Control ID \#3181268)

LEARNING OBJECTIVE \#1: Recognize signs and symptoms of hypothenar hammer syndrome

CASE: A 55-year old male smoker presented with left hand pain and dusky finger discoloration for one month. On exam he had distal dry gangrene of the left fourth and fifth digits with an absent ulnar pulse. Labs 
included ESR 42, CRP 6.6, normal complement levels, negative cryoglobulins, negative anti-phospholipid screen, normal urinalysis, and negative blood cultures. Echocardiogram showed no vegetations or thrombi. Upper extremity duplex revealed no flow in the distal ulnar artery. Angiogram showed distal ulnar occlusion, poor palmar arch filling, but with flow to all five digits. Upon further review of his occupational history, the patient stated that he is a construction worker and often uses drilling machinery. He was diagnosed with hypothenar hammer syndrome. He was initially treated with nifedipine and clopidogrel, but ultimately underwent amputation and botox injection.

IMPACT/DISCUSSION: Hypothenar hammer syndrome is a rare clinical entity that results from repeated trauma to the ulnar artery. It is typically described in middle-aged men whose occupation involves repetitive use of the hand. Metal workers, carpenters, auto mechanics, and constructions workers are some examples of those at increased risk. The ulnar artery branches off to the deep and superficial palmar arch at Guyon's canal. The superficial branch is the main blood supply for most fingers and crosses over the hypothenar muscles before penetrating the palmar aponeurosis. At this segment it is very susceptible to injury. Repetitive trauma can cause intimal damage and induce vasospasms, encouraging platelet aggregation and thrombus formation. Aneurysm may also form with damage to the arterial wall, limiting blood flow to the hand. Clinical presentation varies depending on the severity of damage. Patients may complain of a palpable mass, vasospasm, paresthesias, hypothenar pain, or coolness and discoloration of the fingertips. Generally, symptoms are unilateral and the thumb is spared. Severe disease presents as gangrene or eschar formation at the fingertips. Similar symptoms may be seen in Raynaud's disease, thromboangiitis obliterans, and connective tissue disorders, however, these patients typically have bilateral upper extremity involvement. Initial workup includes a thorough physical exam and Doppler ultrasound, but the gold standard test for diagnosis is angiography. Treatment may include lifestyle modifications, calcium channel blockers, antiplatelet agents, and Botox injection. Severe cases warrant vascular surgery.

CONCLUSION: Hand pain is a very common complaint in the outpatient population. Usually the underlying origin is musculoskeletal, but vascular injury should remain on the differential. A detailed review of occupational history and thorough physical examination are crucial. Physicians should be prudent to consider hypothenar hammer syndrome especially in patients that report occupational or recreational trauma to the hypothenar region.

\section{IT'S NOT A TUMOR! A CASE OF NECROTIZING SARCOID GRANULOMATOSIS}

Zachary G. Jacobs; James Clements. Oregon Health \& Science University, Portland, OR. (Control ID \#3171776)

LEARNING OBJECTIVE \#1: Recognize the clinical features of necrotizing sarcoid granulomatosis

LEARNING OBJECTIVE \#2: Describe the differential diagnosis for pulmonary granulomatous diseases

CASE: A 71-year-old female retired veterinarian with a history of seasonal allergies presented to the hospital with 5 weeks of worsening dyspnea on exertion, which now confined her to a recliner. She also reported a dry cough, orthopnea, anorexia, and a 40-lb weight loss over 3 months. She lived on a farm with numerous animals and vacationed in Mexico every year. She required $3 \mathrm{~L}$ oxygen at rest, and her $\mathrm{O} 2$ saturation fell to $74 \%$ with minimal activity. On lung exam she had diminished breath sounds and inspiratory crackles at the bilateral bases. Initial labs were notable only for mild anemia, hyponatremia, and low albumin. Chest CT revealed innumerable ill-defined pulmonary nodules diffusely as well as irregular intralobular septal thickening, bilateral pleural effusions, and mediastinal lymphadenopathy, which was thought to be consistent with lymphatic spread of tumor. Thoracentesis revealed an exudative effusion with negative culture and cytology. A bronchoscopy with endo- and trans-bronchial biopsies showed non-caseating granulomas with negative cultures (including acid-fast bacilli) and cytology. Additional laboratory testing included interferon-gamma release assay, anti-nuclear antibody, anti-neutrophil cytoplasmic antibody, and serologic studies for multiple fungal organisms, all of which were negative. A video-assisted thoracoscopic surgery (VATS) with wedge biopsy was performed, which revealed sarcoid-like granulomas with focal central necrosis and accompanying granulomatous inflammation involving pulmonary vasculature and lymphatics. The patient was diagnosed with necrotizing sarcoid granulomatosis (NSG). She was treated with highdose corticosteroids with marked symptomatic and radiographic improvement in less than one month.

IMPACT/DISCUSSION: NSG is a rare and poorly understood form of pulmonary granulomatous disease that was first described in 1973. Approximately 130 cases have been reported. It may present with fever, cough, dyspnea, chest pain, and weight loss, though patients are asymptomatic in as many as $25 \%$ of cases. Based on its tumor-like appearance, NSG is often mistaken for malignancy. Diagnoses such as sarcoidosis, granulomatosis with polyangiitis (GPA), tuberculosis (TB), and fungal infections are also on the differential, among others. NSG is characterized on histopathology by sarcoid-like granulomas, vasculitis, and a variable degree of necrosis. While no consensus guidelines for treatment exist, corticosteroids, lung resection, and watchful waiting have been employed. Prognosis is typically good.

CONCLUSION: 1 . NSG is a rare disease characterized by sarcoid-like granulomas, vasculitis, and necrosis. 2. It presents with diffuse lung nodularity and is often mistaken for malignancy. 3. The differential diagnosis includes sarcoidosis, GPA, TB, and fungal infections.

\section{IT'S NOT ALWAYS CANCER: ISCHEMIC COLITIS MASQUERADING AS COLONIC MASS}

SeQuoya Killebrew ${ }^{1}$; Michele D. Lewis ${ }^{2}$; Jonathan Lam ${ }^{1}$; Neema Patel ${ }^{1}$; Nancy Dawson ${ }^{1} .{ }^{1}$ Mayo Clinic, Jacksonville, FL; ${ }^{2}$ Mayo Clinic Florida, Jacksonville, FL. (Control ID \#3167686)

LEARNING OBJECTIVE \#1: Recognize risk factors associated with ischemic colitis.

CASE: A 72-year-old gentleman with past medical history significant for end-stage renal disease on intermittent hemodialysis, heart failure with reduced ejection fraction of 30-35\%, atrial fibrillation, and hypotension presented with one day of new lower abdominal pain. The day prior to onset of symptoms he had an episode of hypotension during dialysis. He reported a history of recurrent hypotension resulting in chronic use of midodrine. The patient stated the abdominal pain awoke him from his sleep, describing it as a severe pressure-like sensation. He had a history of chronic constipation, otherwise denied any episodes of nausea, vomiting, diarrhea, melena, or hematochezia. The patient had no history of nonsteroidal anti-inflammatory drug (NSAID) use. His last colonoscopy and EGD approximately 3 years ago reported normal. On admission, labs significant for hemoglobin 12.9, white blood cell count 13.9, platelets 126, and lactate 1.9. CT abdomen and pelvis without contrast revealed uncomplicated cecal colitis without evidence of obstruction and normal mesenteric vasculature. The patient was empirically started on antibiotics and placed on bowel rest. However, despite conservative management the patient's symptoms persisted and repeat $\mathrm{CT}$ abdomen and pelvis with contrast 3 days later revealed an eccentric, irregular cecal wall thickening concerning for malignancy with surrounding inflammatory stranding and edema. Subsequently the patient underwent colonoscopy the next day revealing an ulcerated partially obstructing large cecal mass that involved the entire cecum and the wall across from the ileocecal valve. Biopsies were collected during colonoscopy and colorectal surgery was consulted thereafter for presumed malignancy. However, there was no evidence of malignancy on histopathology. Repeat colonoscopy was completed for 
confirmation of the diagnosis, but final pathology revealed no evidence of malignancy. Ultimately, the patient continued to have severe right lower quadrant abdominal pain and underwent robotic ileocolic resection. Surgical pathology confirmed ischemic colitis.

IMPACT/DISCUSSION: This case represents a unique presentation of ischemic colitis presenting as a large colonic mass. Colonic ischemia may have a variable presentation ranging from mild to severe lower abdominal pain with or without hematochezia. Risk factors include elderly age, recent cardiovascular procedure, myocardial infarction, hemodialysis, hypercoagulable state, and medications. High suspicion and prompt diagnosis with appropriate imaging is crucial. Evaluation with colonoscopy can be a vital tool in confirming the diagnosis and determining ultimate therapy.

CONCLUSION: Ischemic colitis is the result of decrease blood flow to the colon that can be acute or chronic in onset and can have a nonspecific presentation. Providers should have high suspicion for the diagnosis when patients present with increased risk factors.

\section{IT'S NOT JUST A TEST QUESTION: VIBRIO VULNIFICUS NECROTIZING MYOFASCIITES}

Debapria Das; Andrea Ramirez Gomez; Rachna Rawal. Saint Louis University, St. Louis, MO. (Control ID \#3185996)

LEARNING OBJECTIVE \#1: Recognize the clinical features of primary bacteremia due to Vibrio vulnificus.

CASE: A 51- year old male with a past medical history of cirrhosis due to sarcoidosis, was admitted with worsening left lower extremity pain and swelling for two days. He remembered a pop in his left leg two days ago followed by severe left lower extremity pain the morning after. Additionally, he was unable to bear weight. On arrival, he was hemodynamically unstable requiring pressor support and broad spectrum antibiotics. His physical exam was notable for an obese male with swelling, tenderness to palpation, and crepitus in the left lower extremity. His abdomen was distended and he was not altered. His labs were remarkable for a leukocytosis of 35.7, lactic acidosis 8.48, and Cr 1.98 (baseline 1). A CT of his left lower extremity revealed nonspecific soft tissue edema without subcutaneous emphysema or fluid collection. Blood cultures were positive for Vibrio vulnificus. The patient had consumed oysters for dinner 10 days ago. He was started on piperacillin and doxycycline but continued to clinically decline. He required multiple debridements and ultimately due to severe myonecrosis needed an above-knee-amputation. He clinically improved after the amputation and his antibiotics were narrowed to minocycline and doxycycline. His hospital course was complicated by kidney failure due to acute tubular necrosis. He did not have complete renal recovery by time of discharge and required dialysis.

IMPACT/DISCUSSION: This case is an example of the presentation of primary V. vulnificus bacteremia complicated by necrotizing myofasciites associated with the consumption of oysters in cirrhotic patients. Over $90 \%$ of patients with primary septicemia report a recent history of consuming raw oysters, which have increased concentrations of V. vulnificus, especially in warm temperatures or with drops in salinity. When compared to other foodborne illnesses in the United States, V. vulnificus has the highest fatality at $51.6 \%$ of cases. $86 \%$ of diagnosed cases are male. Interestingly, $75 \%$ of infected patients present with bulbous lesions, which this patient did not have. However, V. vulnificus acutely leads to shock in one-third of patients with primary septicemia with mortality up to $90 \%$, requiring urgent diagnosis. Patient with MELD scores greater than 20 are associated with higher mortality compared to other cirrhotic patients. Although yearly reported cases are around 100, the CDC reports a 78\% increase in incidence since 1992, which may be due to an increasing vulnerable population and climate change.

CONCLUSION: Cirrhotic patients are well-known to suffer from unique medical complications. Additionally, one that is readily well-known but may not be as recognized due to a low prevalence is a severe infection from Vibrio vulnificus. We need to be cognizant of exposure in this population as prompt diagnosis could potentially be life-saving. Early diagnosis is crucial due to high risk of amputations and multiorgan failure.

\section{ITS ALL ON YOUR HEAD}

Alan J. Spanel; Michael P. Smith. University of Nebraska Medical Center, Omaha, NE. (Control ID \#3186139)

LEARNING OBJECTIVE \#1: Recognize the importance of source control in MRSA blood stream infections

LEARNING OBJECTIVE \#2: Review the treatment options when MRSA bacteremia fails to clear

CASE: A 38-year-old woman presented with a 3-day history of fevers and severe retro-orbital pain with diplopia. She was febrile to 104 , had left eye proptosis, severe periorbital edema, and inability to abduct the left eye. Multiple dry, non-purulent frontal scalp lesions without associated fluctuance were noted. The patient's medical history was significant for Sjogren's syndrome and autoimmune sensory ganglionopathy on chronic immunosuppression. She had recently received a scalp steroid injection for alopecia. Orbital MRI revealed bilateral cavernous sinus thrombosis. Her initial blood cultures were positive for MRSA and she was initiated on IV vancomycin. She became hemodynamically unstable despite sensitivity of her infection to vancomycin. Her antibiotic therapy was transitioned to IV daptomycin, again without clinical improvement and persistent bacteremia. She then became tachypneic with increased oxygen requirement and was found to have bilateral septic pulmonary emboli and pleural effusions. Linezolid was added. She then experienced worsening of her cranial nerve palsy and right arm weakness. A repeat orbital MRI demonstrated multiple acute infarcts of the cerebral hemispheres and internal capsule consistent with septic embolic stroke. Her treatment was broadened to three anti-staphylococcal drugs: IV daptomycin and ceftaroline with high dose oral Bactrim DS. Her bacteremia and daily fevers persisted. A PET CT scan was performed, demonstrating multiple scalp abscesses that were subsequently drained, leading to her bacteremia finally clearing. She was continued on a prolonged course of IV daptomycin and ceftaroline.

IMPACT/DISCUSSION: MRSA bloodstream infections often may take days or even weeks to resolve. In the setting of persistently positive blood cultures, antimicrobials are often adjusted based on susceptibility data to better target therapy. In this case, even multiple anti-staphylococcal agents were utilized. There are limited randomized controlled trial data regarding treatment of persistent MRSA bacteremia. Multiple case series and reports support the use of various regimens. A preferred regimen has not yet been identified. Regardless of the agents selected, the importance of source control cannot be overstated. This patient's blood cultures finally cleared only when her benign-appearing scalp lesions were drained surgically. A meticulous search for a persistent focus of infection can sometimes be the only solution.

CONCLUSION: MRSA infection is commonly seen by the general internist and its complications are numerous. Severe MRSA infections unresponsive to initial antimicrobial therapy carry a significant morbidity and mortality and treatment plans can be difficult to formulate.

\section{JAK2 V617F MUTATION: UNUSUAL CAUSE OF ACUTE POR- TAL VEIN THROMBOSIS.}

Hasan Mahbub; Nikita Fernandes; Yelena Averbukh. Montefiore Medical Center, Bronx, NY. (Control ID \#3186253)

LEARNING OBJECTIVE \#1: Learn manifestations and management of Portal Vein Thrombosis (PVT)

LEARNING OBJECTIVE \#2: Identify risk factors associated with PVT. CASE: A 29-year-old man admitted for abdominal pain for 7 days, severe generalized pain radiating to the back associated with vomiting and constipation alternating with diarrhea. Past medical history significant 
for Portal vein thrombosis (PVT) and superior mesenteric vein thrombosis, JAK2 V617F mutation and Protein S deficiency. Physical examination remarkable for tenderness over right and left lumbar regions. Lab tests revealed Hemoglobin $15.7 \mathrm{~g} / \mathrm{dL}, \mathrm{WBC} 11.9 \mathrm{k} / \mu \mathrm{L}$, Platelet $402 \mathrm{k} / \mu \mathrm{L}$, Lactic acid $3.4 \mathrm{mmol} / \mathrm{L}$, Coagulation studies within normal limits. Computed tomography $(\mathrm{CT})$ of abdomen revealed portal venous thrombosis, diffusely thickened colon and mild splenomegaly. Anticoagulation therapy was initiated with enoxaparin. Patient showed clinical improvement. Outpatient follow up with Hematology and Hepatology was arranged.

IMPACT/DISCUSSION: Prevalence of PVT is estimated to be $1 \%$ with $0.6 \%-16 \%$ occurring in asymptomatic liver disease and $35 \%$ in the setting of cirrhosis with hepatocellular carcinoma. PVT can present acutely with symptoms of acute abdominal pain, fever, ascites, or splenomegaly, while chronic PVT can be asymptomatic. Laboratory studies for evaluating PVT include liver function testing, $\mathrm{CBC}$ for erythrocytosis and testing for prothrombotic risk factors. Ultrasound is recommended as first-line imaging with an accuracy of $88 \%-98 \%$ and a specificity of $60 \%-100 \%$. CT and MRI additionally provide advantages of better visualization of anatomy and extension of occlusion, visualizing bowel ischemia, presence of malignancy, or cirrhosis. Treatment of PVT is directed towards reducing the advancement of thrombosis and preventing development of portal hypertension or intestinal infarction. Restoring portal venous flow in both partial and complete occlusion is achieved with anticoagulation (AC) in patients with prothrombotic states without contraindications to therapy. Because recanalization occurs between 4 to 6 months, it is recommended that anticoagulation be continued for at least 6 months, regardless of initial response to $\mathrm{AC}$ therapy. Choice of $\mathrm{AC}$ is low molecular weight heparin followed by long-term use of vitamin $\mathrm{K}$ antagonists with targeted INR of 2-3. Treatment beyond 6 months is dependent on patients with high recurrence risk. Endovascular interventions to restore portal flow have been utilized in patients that fail conservative management or demonstrate signs of bowel ischemia. Follow-up imaging is recommended every 6 months with ultrasound or contrast enhanced CT or magnetic resonance imaging (MRI).

CONCLUSION: • PVT should be considered as one of the differential diagnosis of abdominal pain in patients with risk factors like JAK2 mutations for developing a prothrombotic state. • If AC is not initiated, PVT can potentially lead to fatal complications like bowel ischemia.

\section{JUXTAPAPILLARY DUODENAL DIVERTICULITIS MASQUERADING AS A COMMON BILE DUCT MASS}

Leonard Walsh ${ }^{1}$; Andrew Groff ${ }^{2}$; Abraham Mathew ${ }^{3}$; Manpreet Singh ${ }^{1}$; Rohit Jain ${ }^{1}{ }^{1}$ Hershey Medical Center, Hershey, PA; ${ }^{2}$ Penn State Hershey College of Medicine, Hershey, PA; ${ }^{3}$ Penn State Hershey Medical Center, Hershey, PA. (Control ID \#3184595)

LEARNING OBJECTIVE \#1: Recognize duodenal diverticulitis as a differential diagnosis in patients presenting with abdominal pain, nausea, and vomiting who meet SIRS criteria and have indeterminate crosssectional imaging since delayed treatment can lead to severe complications.

LEARNING OBJECTIVE \#2: Recognize that duodenal diverticulitis in the absence of a leak should be treated with broad-spectrum antibiotics and endoscopic therapy in appropriately selected patients.

CASE: An 82-year-old Caucasian female with a past medical history of hypertension and coronary artery disease presented to the ED with a seven-day history of nausea, intermittent vomiting, and abdominal pain. She denied fevers, chills, or change in bowel habits. Vitals were significant for mild tachycardia and tachypnea. Physical exam revealed mild diffuse abdominal pain with normoactive bowel sounds and no rebound tenderness or guarding. Labs were notable for a mild leukocytosis of 12.0 WBC/uL. Computed tomography (CT) scan of the abdomen showed a thickened common bile duct (CBD) and $2.2 \mathrm{~cm}$ foci of gas in the distal $\mathrm{CBD}$ region that was concerning for a mass or abscess. The patient was started on IV levofloxacin and metronidazole. Gastroenterology performed an endoscopic ultrasound (EUS) and upper endoscopy, which showed a non-bleeding juxtapapillary duodenal diverticulum with purulent discharge and normal EUS findings. Disimpaction with an extraction balloon was performed followed by irrigation with normal saline. After endoscopy, the patient was transitioned to oral ciprofloxacin and metronidazole to complete a fourteen-day course of antibiotics. The patient was seen for follow-up 4 weeks after hospital discharge and was doing well with complete resolution of symptoms. Follow up abdominal CT showed an interval decrease in the size of the duodenal diverticulum with no inflammatory changes.

IMPACT/DISCUSSION: Duodenal diverticulae are relatively common incidental findings in adult patients between the ages of 50-70 however rarely become symptomatic. The associated complications such as infection and free perforation have the potential to be severe and thus early management is important. Diagnosis of diverticulitis based on presentation and imaging alone is often unclear since symptoms can be nonspecific and cross-sectional imaging can mimic other disease processes such as biliary or pancreatic pathologies. In such cases that are equivocal or fail conservative management, an upper endoscopic approach in the absence of free perforation is indicated for diagnosis and allows for therapies including debridement, irrigation with normal saline, and stent placement in select cases.

CONCLUSION: Duodenal diverticulitis is a rare complication of juxtapapillary diverticulae however it is important to promptly recognize and treat due to the risk of developing complications including a free perforation. Oftentimes diagnosis cannot be made by clinical presentation and cross-sectional imaging alone, and in such cases, upper endoscopy is indicated for diagnosis and treatment.

\section{KEEP AN EYE ON YOUR BONES}

Mahmuda Khan ${ }^{1}$; Eileen Kim ${ }^{1}$; Robin Koshy ${ }^{2} .{ }^{1}$ Northwell Health, New Hyde Park, NY; ${ }^{2}$ Northwell Health, Manhasset, NY. (Control ID \#3180211)

LEARNING OBJECTIVE \#1: Recognize ocular adverse effects of bisphosphonate use

LEARNING OBJECTIVE \#2: Manage bisphosphonate induced ocular disease with steroids

CASE: This is a 64 year-old woman with a past medical history of asthma, chronic urticaria, osteoporosis on risedronic acid who presented with acute right eye pain worsened with extraocular movements. Associated symptoms included fevers. On physical exam, she had right periorbital edema with erythema, episcleral injection with chemosis but no proptosis or resistance to retropulsion. She had leukocytosis (14.9), thrombocytosis (572k), and elevated CRP (1.9). Infectious and rheumatologic workup was negative. CT Maxillofacial revealed minimal preseptal soft tissue swelling with thickening of the conjunctiva, consistent with acute right conjunctivitis. MR of Head and Orbits showed no evidence for venous sinus or cortical vein thrombosis. She was started on ampicillin/sulbactam and linezolid without much improvement, which prompted concerns for bisphosphonate induced orbital inflammation. Only after the initiation of prednisone, she reported a significant improvement in the symptoms as well as objective examination findings of improving periorbital swelling, episcleral injection, and chemosis. Patient was discharged on a prednisone taper.

IMPACT/DISCUSSION: It is important to note that the differential diagnosis of patient's presenting with fevers and orbital erythema and swelling is not limited to infectious sources. Other etiologies include vascular, trauma, autoimmune, metabolic, neoplastic, and most importantly, medication induced inflammatory response. This case has taught me to have a higher level of suspicion for medication, specifically, bisphosphonate induced ocular manifestations. Common reactions to bisphosphonates include conjunctivitis, uveitis, episcleritis, sclertis, and keratitis. The mechanism of bisphosphonate-induced inflammatory events 
is unknown; however, alendronate and risedronate are nitrogen based aminobisphosphonates, which play a role in tumor necrosis factor and cytokine release. Increase in inflammatory mediators may increase risk of inflammatory disorders such as acute conjunctivitis. A retrospective cohort study showed patients who use oral bisphosphonates for the first time may be at higher risk of scleritis and uveitis compared to patients with no bisphosphonate use. Treatment includes discontinuation of bisphosphonates, depending on severity of ocular symptoms. A case report presented a patient whose diffuse conjunctival erythema did not respond to common first line topical agents- erythromycin, tobramycin, and dexamethasone. Severe cases with uveitis, scleritis, or global inflammation require systemic steroids and steroid eye drops, such as in our patient.

CONCLUSION: This case highlights the importance of accurate medication reconciliation both inpatient and outpatient settings. Internal physicians need to be aware of the potential ocular side effects of bisphosphates. Treatment in severe cases include topical and systemic steroids.

\section{KEEP YOUR EYE ON THROMBI IN RENAL CELL CARCINO- MA PATIENTS}

Kanishka G. Patel; David Yin; Sheira Schlair. Albert Einstein College of Medicine/Montefiore Medical Center, Bronx, NY. (Control ID \#3184717)

LEARNING OBJECTIVE \#1: Outline the differential diagnosis of an intracardiac mass in renal cell carcinoma

LEARNING OBJECTIVE \#2: Discuss differentiation and management of bland versus tumor thrombi

CASE: A 66 year old man with stage IV renal cell carcinoma (RCC), a non-occlusive IVC tumor thrombus and end-stage renal disease on hemodialysis via a right internal jugular permacath was admitted with a 5day history of fever and weakness. He had a temperature of 99.3, a WBC count of 17.2 and was found to have Klebsiella bacteremia. Physical exam revealed a new systolic murmur, which prompted echocardiogram, showing a large, mobile, multi-lobed structure in the right atrium likely attached to the permacath, suggestive of thrombus (could not rule out vegetation). Antibiotics and heparin were started. CT showed a chronic IVC tumor thrombus that did not extend into the SVC or right atrium, suggesting the right atrial mass was not tumor thrombus. Permacath was removed and to definitively rule out embolization of the right atrial thrombus, CTPE was performed, revealing a right lower lobe pulmonary embolism and warfarin was initiated.

IMPACT/DISCUSSION: RCC is a highly vascular tumor that can invade through the renal vein and into the IVC, with $10-15 \%$ of cases resulting in tumor thrombus formation. Less commonly (1\%), the tumor may extend into the right cardiac chamber. Accurate differentiation of bland from tumor thrombus is crucial for proper management. While both bland and tumor thrombi present as vessel filling defects, a malignant thrombus has continuity with the primary tumor, enhancement and vessel expansion on CT or MRI. Because a bland thrombus results from IVC compression by a renal mass, it presents as vessel narrowing on imaging. While nephrectomy and thrombectomy treat tumor thrombi, anticoagulation is used for bland thrombi. Though tumor thrombus dislodgement is a known complication of thrombectomy, the development of tumor emboli outside the lung in the absence of such manipulation is unclear. The differential diagnosis of an intracardiac mass in a septic RCC patient can span different etiologies including infectious (vegetation), malignant (primary tumor, metastases, tumor thrombus) and hematologic (bland thrombus). As imaging was strongly suggestive of a thrombus, several key details pointed to bland over tumor thrombus. Risk factors for thrombosis, including a hypercoagulable state due to malignancy as well as foreign body effect from the IVC tumor thrombus and permacath, and attachment of the mass to the permcath raised our suspicion for a bland thrombus. Moreover, lack of IVC thrombus extension into the SVC and right atrium suggested the mass was more likely bland thrombus. Thus, anticoagulation and antibiotics were given empirically.
CONCLUSION: This case demonstrates the importance of reviewing all clinical data when narrowing the differential diagnosis for an intracardiac mass. Moreover, identification of thrombi in RCC patients warrants subsequent differentiation of bland from tumor thrombus for effective management.

\section{KIKUCHI FUJIMOTO DISEASE: A RARE BUT IMPORTANT CAUSE OF LYMPHADENOPATHY}

Melanie Parikh ${ }^{1}$; Christina Thoonkuzhy ${ }^{2} .{ }^{1}$ Case Western Reserve University, Cleveland, OH; ${ }^{2}$ University Hospitals Cleveland Medical Center, Cleveland, OH. (Control ID \#3184869)

LEARNING OBJECTIVE \#1: Recognize the clinical features of Kikuchi Fujimoto Disease (KFD)

LEARNING OBJECTIVE \#2: Distinguish KFD from other causes of lymphadenopathy

CASE: A 30-year-old African American female with past medical history of asthma and Epstein-Barr virus, presented to the ED with an acutely worsened headache with associated fevers and chills, which were no longer responding to OTC ibuprofen. The patient reported a two-month history of chills, nausea, diffuse muscle pain, neck pain, throbbing headache, and morning joint stiffness, which would last for more than an hour. She had visited multiple emergency departments, but no definitive diagnosis had been made. Physical exam on admission was significant for tender posterior chain, preauricular, and anterior cervical chain lymphadenopathy, especially on the right side of the neck, severe headache and fevers reaching a Tmax of 104. Previous autoimmune workup showed positive ANA with a titer of 1:320, but all other specific autoimmune markers, including complement, ACE and ANCA were negative. Lumbar puncture did not show evidence of an infectious process, CT head and MRI brain were normal. A MRV was done to rule out cerebral thromboembolism, but evidence of benign intracranial HTN was seen. Patient was started on topiramate, with clinical improvement. Excisional biopsy was conducted which showed an infiltrate composed of immunoblasts, crescentic histiocytes and abundant apoptotic or karryohectic debris, thus making the diagnosis of Kikuchi-Fujimoto Disease.

IMPACT/DISCUSSION: Kikuchi-Fujimoto disease (KFD), or histiocytic necrotizing lymphadenitis, is a benign and self-limited disease that mainly affects young women, especially those of East Asian heritage. Patients typically present with localized lymphadenopathy, fever, and leukopenia in about $50 \%$ of cases. Although KFD is not very common, it should be included in the differential diagnosis of lymphadenopathy because its natural history and treatment differ dramatically from those of lymphoma, tuberculosis, and systemic lupus erythematous (SLE). The lymphadenopathy in KFD usually resolves spontaneously in 1-4 months without any treatment. The distinguishing factor is karryohectic debris on lymph node biopsy or aspiration. Without appropriate histologic diagnosis, a patient with KFD may undergo unnecessary testing and treatment for malignant lymphoma or tuberculosis. Once the diagnosis is made, it is important to maintain close follow-up with these patients to monitor for the development of SLE. KFD is known to occur in conjunction with SLE and some experts say that KFD can be an unusual presentation of SLE.

CONCLUSION: KFD should be considered in young patients presenting with unilateral cervical lymphadenopathy. Excisional biopsy is the key to diagnosis in these cases. Patients should be managed conservatively with close follow-up.

\section{KRATOM: A SO-CALLED LEGAL OPIOID}

David R. Henkin; Brendan Sullivan; Joshua S. Smith. Henry Ford Hospital, Detroit, MI. (Control ID \#3162271)

LEARNING OBJECTIVE \#1: Familiarize the reader of the substance called kratom 
LEARNING OBJECTIVE \#2: Assessment and management of intoxication, withdrawal and detoxification of kratom Recognize the legality and regulation of kratom in the U.S.

CASE: We present a case of a 25 year old male who presented to an inpatient rehabilitation and detoxification center requesting help for an addiction to kratom. He disclosed a history of IV heroin use 2 years earlier, which he stated he was able to wean himself from using kratom. He endorsed ingesting kratom powder up to 40 grams twice daily for the last 2 years. The patient recognized his tolerance and ultimately his addiction, but attempting to quit "cold turkey" caused him to develop symptoms of myalgias, chills, diarrhea, rhinorrhea, arthralgias and fatigue. Given his apparent tolerance and withdrawal symptoms similar to that of a true opioid withdrawal, he was managed with a buprenorphine taper, which adequately allowed him to successfully complete detoxification.

IMPACT/DISCUSSION: The substance called kratom has recently gained more recognition on the streets and in admissions to detoxification centers. Kratom (Mitragyna speciosa) is a plant indigenous to Southeast Asia and Thailand. At different concentrations of its leaves, ingestion will produce both stimulant and opioid-like effects, with studies showing its mode of action by binding mu opioid receptors. One online "Kratom User Guide" suggests that 1-5 grams elicit a stimulant effect and higher doses of 5-15 grams cause opioid-like effects. Intoxication at higher doses in long-term users or in acute toxicity has been reported to cause seizures and hepatotoxicity. The CDC has even traced kratom to a multi-state outbreak of salmonella. There is a noticeable increase in the number of articles referencing kratom, as well as an increasing amount of vendors and general information websites for kratom in the past few years which may suggest a greater overall demand.

CONCLUSION: Kratom is an addictive substance whose use has actually been outlawed in many Southeast Asian countries. In the US, kratom is legally regulated as an herbal product under US law, FDA and DEA policies, and is therefore considered a legal substance in the United States. That being said, the Drugs and Chemicals of Concern list states, "There is no legitimate medical use for kratom in the U.S." In 2016, the DEA planned to classify kratom as a schedule I drug; however the DEA received significant challenges from the public and ended up releasing a withdrawal of notice of intent. In the time of writing this, kratom is still not a scheduled drug. As it remains easily accessible in local tobacco shops and online, we must be aware of its existence and use pattern as well as its effects, abuse potential and addictive properties.

\section{LACTIC ACIDOSIS: WHO IS TO BLAME?}

Mohamed Gabr; Isaac Leader. Montefiore Medical Center (Wakefield Campus), Bronx, NY. (Control ID \#3183342)

LEARNING OBJECTIVE \#1: Recognize lactic acidosis as a common finding in inpatient settings

LEARNING OBJECTIVE \#2: Identify causes of lactic acidosis and rule out serious etiologies

CASE: A 68 year-old man presenting with chest pain was found incidentally to have lactic acidosis. His past history included diabetes mellitus, ischemic heart disease, chronic hepatitis $\mathrm{C}$, alcohol abuse, and cocaine abuse. His last reported cocaine use was two days prior to presentation and last alcohol use was the morning of presentation. Physical examination was unremarkable except for mild abdominal tenderness. Myocardial infarction was ruled out with negative troponins. Lactic acid level was $13.2 \mathrm{mmol} / \mathrm{L}$ (normal $<2$ ), with a $\mathrm{pH}$ of 7.2 and a sodium bicarbonate level of $15 \mathrm{mmol} / \mathrm{L}$ (normal 22-28). Blood glucose level was normal and the anion gap was 36. Liver function tests revealed an AST of $76 \mathrm{U} / \mathrm{L}$ (normal <50) and ALT of $50 \mathrm{U} / \mathrm{L}$ (normal <40). Urine drug screen was positive for cocaine and blood ethanol level was normal. Computed tomography scan revealed no evidence of bowel ischemia. Review of systems and bloodwork were not suggestive of any infectious processes, and vital signs were normal except for elevated blood pressure. Home medications were aspirin, atorvastatin, amlodipine, famotidine, and metformin; metformin was held at admission. His symptoms gradually improved and lactic acid levels returned to normal. He was counseled regarding alcohol and cocaine cessation and metformin was discontinued.

IMPACT/DISCUSSION: Lactic acidosis is defined as serum $\mathrm{pH}$ of less than 7.35 with a lactate level greater than $4 \mathrm{mmol} / \mathrm{L}$. It is commonly classified into type A acidosis, occurring as a result of tissue hypoxia with overproduction of lactic acid, or type B acidosis due to causes unrelated to hypoxia. Causes of type A acidosis include sepsis, shock, cardiac failure, hypovolemia, and other causes of hypoperfusion. Physical examination, vital signs, and other signs of tissue perfusion such as urine output and mental status may provide hint. Type B acidosis may be present in ethanol intoxication, hepatic dysfunction due to chronic alcoholism, malignancy, mitochondrial dysfunction, or due to medications (e.g. beta agonists, antiretrovirals, linezolid, and propofol). It may also occur in diabetes mellitus, whether in the setting of diabetic ketoacidosis or due to medications such as biguanides. D-lactic acidosis is another rare cause that occurs as a result of fermentation of carbohydrates by gut bacteria in cases of malabsorption or short bowel syndrome. In our patient's case, it appeared that several factors might have contributed to his type B acidosis, including alcohol use, drug use, liver disease, and metformin.

CONCLUSION: Physicians should promptly aim to identify the etiology of lactic acidosis. Type A lactic acidosis may indicate the presence of a more serious process that warrants urgent treatment. After confidently ruling out Type A, Type B acidosis can be considered through a careful review of patients' medical histories, habits, and medications.

\section{LAMBL'S EXCRESCENCES MASQUERADING AS ENDOCARDITIS}

Navid Berenji ${ }^{1}$; Abdulrahman S. Museedi ${ }^{1}$; Ermias Sori ${ }^{1}$; Omar Sheikh ${ }^{1}$; Rushit Kanakia ${ }^{2}$. ${ }^{1}$ UT Health San Antonio, San Antonio, TX; ${ }^{2}$ UTHSCSA, San Antonio, TX. (Control ID \#3186025)

LEARNING OBJECTIVE \#1: Recognize importance of anticoagulation in Lambl's excrescences

LEARNING OBJECTIVE \#2: Assess role of Positron Emission Tomography scans in complex cases of infective endocarditis

CASE: A 58-year-old woman with hypertension and multiple cerebrovascular accidents presented with fever and headache. Patient had recently presented with left upper extremity weakness, headache and blurry vision for which evaluation led to revealing several foci involving bilateral hemispheres on brain MRI. As part of the work up, trans-esophageal echocardiography (TEE) revealed small echodensities on aortic and mitral valves concerning for vegetations in setting of septic emboli. Thorough work up for infectious endocarditis, including blood cultures and serology, as well as autoimmune work up was negative. Diagnosis of culturenegative endocarditis was given and patient was started on broad- spectrum antibiotics with termination of anticoagulation therapy, as she was previously on Apixaban. She continued to have episodic fevers and a new rash, which biopsy revealed to be due to medication reaction secondary to antibiotics. Repeat TEE one month later revealed stabilized subcentimeter echodensities in aortic and mitral valves, suspecting Lambl's excrescences (LE) over septic or thrombotic emboli. Positron emission tomography (PET) scan was negative on the vegetation and potential metastatic sites to include brain lesions. Patient was started on warfarin.

IMPACT/DISCUSSION: Lambl's excrescences are small filamentous strands, which extend from the margins of the valves, especially mitral and aortic valves. LE, previously thought to be benign due to degenerative process, have been associated with few cases of embolic strokes. Given risks associated with surgery and obtaining a biopsy of these lesions, echocardiography remains a valuable tool in distinguishing LE. LE is a diagnosis of exclusion thus thorough work up is granted for every patient. In our patient, nonbacterial thrombotic endocarditis such as marantic 
vegetations and Libman-Sacks endocarditis, antiphospholipid syndrome, and other connective tissue disorders were ruled out. Additionally, PET scan was used in addition to Dukes criteria to exclude distant septic emboli and infective endocarditis. This can be an additional tool in the diagnosis of infective endocarditis in complex cases.

CONCLUSION: Currently, there are no definitive guidelines regarding management of LE. There are different reports of management for LE with anticoagulation therapy, dual antiplatelet therapy, and neither of antiplatelet nor anticoagulation therapy altogether. Given our patient with cerebrovascular events secondary to LE and other reported cases with similar presentations, we recommend anticoagulation with Warfarin due to lack of sufficient data on non-vitamin $\mathrm{K}$ antagonist oral anticoagulants. Surgical extraction is typically reserved for patients with recurrent cerebrovascular accidents while on anticoagulation therapy.

\section{LATE COMPLICATIONS AFTER AORTIC ROOT REPLACE- MENT IN A PATIENT WITH MARFAN SYNDROME - AN EMERGENT DIAGNOSIS.}

VenkataSivaKumar Pajjuru. Creighton University, Omaha, NE. (Control ID \#3186451)

LEARNING OBJECTIVE \#1: Recognize the complications of aortic root replacement in patients with Marfan syndrome.

LEARNING OBJECTIVE \#2: Assess the risk by Careful follow up studies after the surgical procedure to diagnose such complications.

CASE: A 62-year-old female with Marfan syndrome who previously had an aortic root replacement with Bentall valve procedure and the mechanical valve conduit at the age of 34 presented with chest pain. One week before she underwent elective ureteroscopy and bilateral ureteral stent placement for bilateral hydronephrosis. Three days after the procedure she developed chest pain with nausea and diaphoresis. Patient denies fevers, chills, cough; pain is not radiating to back/shoulders. She is not a smoker or drinks alcohol. Her past medical history includes CVA at the age of 38, hyperlipidemia, recurrent nephrolithiasis, UTI's. Family history includes hypertension, type ii $\mathrm{dm}$ in mother and father, Marfan syndrome in her son. She is on warfarin for life long anticoagulation for the mechanical aortic valve. Pulses is symmetrical over both extremities, hemoglobin is stable at $8 \mathrm{mg} / \mathrm{dl}$, Initial EKG was negative for ischemic changes, but Troponin was elevated. Echocardiogram showed normal EF and mechanical aortic valve. CTA chest showed leaking pseudoaneurysm with perigraft hemorrhage involving the ascending aorta, could originate from the right coronary ostium. Anticoagulation was held, increased beta-blocker dose to control heart rate (as the patient developed Atrial Fibrillation with RVR during inpatient) and to decrease shearing stress on the aorta. Cardiothoracic surgery recommended urgent surgical repair of aortic pseudoaneurysm.

IMPACT/DISCUSSION: The Bentall procedure is considered the gold standard in the treatment of patients requiring aortic root replacement. The vast majority of Bentall procedures involve the replacement of the aortic root with a mechanical valve conduit. Pseudoaneurysm formation at the coronary ostia or at the distal aortic suture line is a frequently reported sequela of the Bentall procedure. Pseudoaneurysms of the ascending aorta occur in from $7 \%$ to $25 \%$ of patients in whom the original inclusion/wrap technique of the Bentall procedure has been applied. The main causes of pseudoaneurysm formation are tension on suture lines, particularly those involving the coronary ostia. These pseudoaneurysms tend to remain small and asymptomatic, although they on occasions enlarge, cause compression of vital neighboring structures, can rupture, as in our patient, mandating urgent surgical intervention.

CONCLUSION: Elective aortic root replacement or repair in patients with Marfan syndrome is of paramount importance in order to prevent aortic dissections. Lifelong imaging of the entire aorta, using MRI or computed tomography, is needed to prevent devastating complications. Timely diagnosis and management of these complications
(Pseudoaneurysm rupture, Aortic dissections, Aorto-atrial fistula) are warranted as they are life-threatening.

\section{LEGAL DRUGS WITH ILL EFFECTS: A CASE OF KRATOM AND PHENIBUT USE}

Christine Soran ${ }^{1}$; Shin-Yu Lee ${ }^{2}$; Soraya Azari ${ }^{3}{ }^{1}$ University of California San Francisco, San Francisco, CA; ${ }^{2}$ San Francisco General Hospital \& Trauma Center, San Francisco, CA; ${ }^{3}$ University of California, San Francisco, San Francisco, CA. (Control ID \#3187163)

LEARNING OBJECTIVE \#1: Recognize patient perceptions and reasons for use of Kratom and Phenibut

LEARNING OBJECTIVE \#2: Utilize familiar medications for treatment of Kratom and Phenibut Use

CASE: The patient is a 30 year old male with a history of anxiety who presented to primary care asking for help stopping Kratom. The patient used 12 grams of powder Kratom 3 times daily for the 5 years for anxiety symptoms. Two years ago, the patient stopped utilizing an internet protocol hydrocodone taper but resumed use 2 weeks later. With cessation he experiences insomnia, restlessness, and nausea. He also reports taking daily Phenibut purchased on the internet for anxiety and low energy. His only prescribed medication was Citalopram. Physical exam was unremarkable and urine drug screen was negative for all illicit substances. The patient started on buprenorphine/naloxone (BNX) for Kratom use with resolution of his withdrawal symptoms. For Phenibut, he declined baclofen treatment. High resolution mass spectrometry on week 2 of treatment showed no active metabolites of Kratom but did show Phenibut. He has completely stopped using Kratom and tapered Phenibut use to 1-2 times weekly.

IMPACT/DISCUSSION: Kratom is a plant native to Southeast Asia with dose dependent actions on the monoaminergic system and at the mu-opioid receptors (Hassan, Neuro \& Bio Reviews, 2013). Recreational use of Kratom in the United States has increased; its purchase is legal in most states. Kratom is promoted on the internet for chronic pain, energy, and detox off opioids. Case reports describe the efficacy of BNX for kratom dependency (Khazeli, J Addict Med. 2018). Patients describe symptoms of anxiety and depression following cessation (Singh, J Psyhchoactive Drugs. 2018). This patient had prominent anxiety symptoms, likely due to his chronic condition made worse by kratom withdrawal, driving his co-consumption of phenibut. Phenibut is a gammaaminobutyric acid type B agonist that is legally available from online retailers (Owen, Drug Al Rev. 2016). It has anxiolytic and euphoric effects and has been implicated overdose cases (O'Connell, Amer J of Med. 2014). Case reports describe syndromes of dependence and withdrawal characterized by anxiety, agitation, insomnia, and tremors (Ahuja et al. Case Rep Psychiatry, 2018) and can be treated with Baclofen. There are no case reports that we are aware of describing patients with misuse of both substances simultaneously. These medicines are not detected by traditional testing and providers are unaware of patients' use of herbal medicines (Lee, J of Clin Onc, 2014). This case illustrates the importance of taking a thorough history for use of licit and illicit substances. This case illustrates low perceived risk in the public domain of legal substances. Providers and patients will need education about these products to help influence policy.

CONCLUSION: Patients using Kratom or Phenibut perceive low risks but experience dependence and withdrawal Successful treatment of Kratom use with BNX and Phenibut with Baclofen is possible

LESS IS MORE: REVERSAL OF VALVULAR DISEASE USING PATIENT-CENTERED CARE WITHOUT DEVICE THERAPY

$\mathrm{LU} \mathrm{CHEN}^{1}$; Uta Guo ${ }^{3}$; PARAG MEHTA ${ }^{2} .{ }^{1}$ NEW YORK PRESBYTERIAN - BR $\overline{O O K L Y N ~ M E T H O D I S T ~ H O S P I T A L, ~ B r o o k l y n, ~ N Y ; ~}$ ${ }^{2}$ NewYork-Presbyterian BMH Hospital, Brooklyn, NY; ${ }^{3}$ SUNRISE GME CONSORTIUM, LAS VEGAS, NV. (Control ID \#3186255) 
LEARNING OBJECTIVE \#1: Recognize and treat functional mitral regurgitation (MR) complicated by heart failure (HF) with without valve replacement CASE: A 71-year-old African American female with a past medical history of diabetes mellitus and hypertension presented to the Emergency Department with progressively worsening dyspnea at rest for two weeks. Admission blood pressure was $185 / 107 \mathrm{mmHg}$, heart rate was 120 , and respiratory rate was 37 . Physical examination was significant for volume overload with elevated jugular venous pressure, bibasilar crackles, and lower extremity edema bilaterally. Laboratory studies showed a Brain Natriuretic Peptide level of $1023 \mathrm{pg} / \mathrm{mL}$. Diuresis was initiated with intravenous furosemide. During her hospitalization, a transthoracic echocardiogram (TTE) revealed an LV ejection fraction (LVEF) of $25 \%$, enlargement of LV internal diameter (LVID) and severe eccentric MR. Findings were confirmed with transesophageal echocardiogram and coronary angiography. Angiography also revealed normal coronary vessels. Metoprolol and Lisinopril were initiated following diagnostic studies. Over the next few months, the patient was followed closely at our HF clinic and medications were optimized according to guideline directed therapy. Communication and encouragement were provided using secured exchanges via patient portal in our Electronic Health Record. This allowed home blood pressure monitoring as well as functional assessment between clinic visits. At four months follow-up, the patient reports exertional dyspnea after walking three blocks. TTE showed similar LVEF, but the reduction in both MR severity and LVID. Cardiac Resynchronization Therapy was deferred at this time. In nine months, the patient can ambulate symptom-free for six long blocks. TTE showed LVEF of $69 \%$, trace MR, and normalization of LVID. Patient continues to follow up at our clinic with no exacerbation or readmission.

IMPACT/DISCUSSION: As Internal Medicine residents, we frequently encounter hospitalized patients with poorly controlled comorbidities. Often, we attempt to optimize patient care by offering the latest innovation. The art of patient-centered care is lost in the whirlwind of technological advancement. Time and effort from both physician and patient should be allocated to optimize guideline-directed medical therapy fully. This approach resulted in a success story of patient engagement and encouragement to achieve quality, cost-effective and patient-centered care.

CONCLUSION: Patient-centered care is a cost-effective therapy that resulted in near complete reversal of MR and LV remodeling using medication optimization without device therapy.

\section{LET SLEEPING NODES LIE: A RARE CAUSE OF MASSIVE HEMOPTYSIS}

Kyle M. Koster; Zachary Sherman; Shira A. Sachs. Weill Cornell Medicine, New York, NY. (Control ID \#3181479)

LEARNING OBJECTIVE \#1: Recognize the varied clinical presentations of broncholithiasis

LEARNING OBJECTIVE \#2: Review treatment modalities for broncholithiasis

CASE: A previously healthy 27 year-old man from Tennessee presented with massive hemoptysis. He experienced sudden onset dyspnea and nonproductive cough, followed by large volume hemoptysis requiring intubation in the field. Computed tomography angiography of the chest revealed scattered ground glass opacities, bibasilar consolidations, and a 3.0 by 2.1 centimeter calcified, subcarinal lymph node. The lymph node was attributed to prior histoplasma exposure found on laboratory workup. Multiple bronchoscopies were notable for dark blood in the trachea extending into the right main stem bronchus and old clots in lower airways, without a clear bleeding source. The patient was ultimately extubated without subsequent episodes of hemoptysis. A final bronchoscopy was performed, revealing a round, white lesion protruding from the wall of the right main stem bronchus, consistent with broncholithiasis. However, a pulmonary Dieulafoy's lesion could not be excluded. The patient was discharged with outpatient follow-up and underwent bronchial artery embolization.
IMPACT/DISCUSSION: Broncholithiasis occurs when calcified lesions are present within the bronchus, usually due to erosion of a calcified lymph node into the airway. Calcified lymph nodes are typically the result of a prior inflammatory or infectious process; tuberculosis is the most common cause worldwide, but histoplasmosis is the most common cause in endemic areas. Potential complications of broncholithiasis include hemoptysis and airway obstruction. Computed tomography can be used to identify calcified lesions in the chest, though diagnostic bronchoscopy should be performed to directly visualize suspected broncholithiasis. However, bronchoscopy may be limited in the setting of active inflammation or bleeding. Definitive treatment should be pursued to prevent repeat decompensation. Options include bronchial artery embolization, endobronchial resection, or lobectomy. In our patient, a Dieulafoy's lesion, a vascular anomaly characterized by a tortuous, dysplastic arteriole in the submucosa, remained a consideration, and therefore bronchial artery embolization was performed.

CONCLUSION: Broncholithiasis is a rare cause of hemoptysis, usually due to calcified lymph nodes eroding into the airway. Patients may present acutely with hemoptysis or airway obstruction. Prior histoplasmosis infection is the most common cause in North America. Internists should consider this diagnosis in patients with unexplained hemoptysis or airway obstruction and an appropriate exposure history.

\section{LIFE IN A SHELTER: HEART FAILURE READMISSIONS IN THE HOMELESS}

Christina Elzahaby $^{1}$; Dharmarajan S. Thiruvinvamalai ${ }^{2} .{ }^{1}$ Montefiore Medical Center, Bronx, NY; ${ }^{2}$ Montefioe Medical Center (Wakefield Campus), Bronx, NY. (Control ID \#3183676)

LEARNING OBJECTIVE \#1: Identify barriers for outpatient health care access in homeless patients.

LEARNING OBJECTIVE \#2: Propose a plan to reduce hospital readmissions and improve healthcare access in such patients

CASE: A 57-year- old homeless man living in a shelter with history of uncontrolled hypertension, heart failure (HF), cocaine and alcohol addiction and schizophrenia presented with dyspnea, exercise intolerance and bilateral edema. He had difficulty maintaining access for outpatient care and adherence to diet, fluid restrictions and medications, depending on emergency visits for health-care needs. Examination: Blood pressure was 190/70 mm Hg, basal rales; $2+$ bilateral pitting edema; proBNP elevated; chest $\mathrm{x}$-ray revealed heart failure. Echocardiogram confirmed dilated cardiomyopathy, left ventricular hypokinesis and ejection fraction of $28 \%$. Cardiac cath was advised, but was refused. Intravenous diuretics, metoprolol and lisinopril helped treat acute heart failure. He was educated utilizing the $\mathrm{HF}$ re-admission prevention program. On discharge, he was lost to follow up, failing to keep outpatient appointments. Attempts to reach the patient or his shelter supervisor were futile. Four weeks later, he was readmitted for another episode of acute HF.

IMPACT/DISCUSSION: The US Department of Housing and Urban Development (HUD) estimates 63,559 homeless people live in NYC (2018). A majority of homeless live in a shelter that provides a temporary residence and sometimes a gateway for permanent housing after long waiting list; these shelters often lack facilities and services to help patients receive appropriate outpatient care. Cardiovascular disease is a major cause of death in homeless adults, who may be smokers, at risk for uncontrolled hypertension and diabetes, psycho social stress and substance use, and medication non-adherence. Homeless patients have higher rates of using acute care resources like ED visits and hospitalizations, and often sign out against medical advice; they have higher 30 day readmissions to hospital impacting costs and quality of life. These patients need close follow up, education, communication with shelter services, and social support, to fulfill transportation and other needs. Medical respite programs can offer medical, nursing and accommodation for homeless persons discharged from acute hospital stays, who are sick to take care of themselves in a shelter, to help reduce hospital re-admissions. 
CONCLUSION: - Homeless patients in a shelter have poor access to health care and fulfill their needs through acute care facilities, increasing costs of care. - Once hospitalized, efforts should target outpatient community integration to reduce re-admissions rate, including support, telephone calls, appointment reminders and transportation, in collaboration with social worker or case managers. - Simplify medication and dietary regimens to improve adherence.

\section{LIMITATIONS OF BISAP SCORE IN PREDICTING SEVERE ACUTE PANCREATITIS (SAP)}

Eshandeep S. Boparai; Nishant J. Trivedi. Kaiser Permanente Medical Center - San Francisco, San Francisco, CA. (Control ID \#3183756)

LEARNING OBJECTIVE \#1: Predict Severe Acute Pancreatitis when BISAP Score (Bedside Index for Severity in Acute Pancreatitis) is reassuring, less than or equal to 2

LEARNING OBJECTIVE \#2: Recognize utility of imaging in diagnosis of necrotizing pancreatitis

CASE: A 43-year-old male with a history of chronic alcohol use presented to the clinic for acute, severe nausea and epigastric pain. Labs showed transaminitis: with aspartate aminotransferase (AST) $226 \mathrm{U} / \mathrm{L}$, alanine aminotransferase (ALT) $514 \mathrm{U} / \mathrm{L}$ and total bilirubin at $1.7 \mathrm{mg} / \mathrm{dl}$. Lipase was within normal limits at $58 \mathrm{U} / \mathrm{L}$ and a gallbladder ultrasound was unremarkable. Two days later, the patient presented to the Emergency Department after an episode of non-bloody, non-bilious emesis at work and severe abdominal pain. The patient reported waves of crippling epigastric pain radiating posteriorly, that were worsened by oral intake. Physical exam was notable for severe distress, sinus tachycardia to 110 , and an abdominal exam significant for guarding and diffuse tenderness. Lab values were notable for White Blood Cell count elevation to 33,200 and a Hemoglobin of $16.5 \mathrm{~g} / \mathrm{dl}$, suggestive of hemoconcentration. Elevation in AST to $318 \mathrm{U} / \mathrm{L}$, ALT to $644 \mathrm{U} / \mathrm{L}$, alkaline phosphatase to 278 $\mathrm{U} / \mathrm{L}$, and total bilirubin to $2.4 \mathrm{mg} / \mathrm{dl}$ was noted, with lipase now elevated to $20,297 \mathrm{U} / \mathrm{L}$. The abdominal ultrasound remained unremarkable. However, a CT scan of the abdomen and pelvis with IV and PO contrast revealed large amounts of peripancreatic fat stranding, enlarged edematous pancreas with poor enhancement of majority of parenchyma consistent with necrotizing pancreatitis. Despite significantly abnormal lab and CT scan findings, the BISAP score was 2 , indicating a lower rate of complications and mortality. However, the patient went on to develop septic shock requiring antibiotics and vasopressors for blood pressure support, with a prolonged ICU hospitalization complicated by pancreatic fluid collections and abscesses needing multiple surgical drainage procedures and necrosectomies.

IMPACT/DISCUSSION: In the past decade, the BISAP score has offered internists prognostic insight for predicting severe acute pancreatitis, particularly in the first 24 hours. A score of 2 or lower is associated with a lower rate of mortality (2\% and lower) and complications whereas a BISAP score of 3 and greater has been associated with a steep rise in mortality (5-20\%), organ failure, and necrosis. Despite BISAP's clinical utility, a patient who presents with acute, necrotizing pancreatitis in its earliest stages may have a reassuring BISAP score and be subsequently mismanaged.

CONCLUSION: Though the BISAP score has very good predictive performance for SAP, this case reiterates the potential danger of overreliance on a single predictive scoring system. A thorough clinical assessment - history, physical exam, lab-work and imaging modalities, especially a CT scan - should be utilized for appropriate triage and management of a patient with acute pancreatitis

\section{LINEZOLID INDUCED INTERSTITIAL LUNG DISEASE}

Tanaz Salimnia $^{1}$; Daymon Peterson ${ }^{1}$; Neeharika Ralh ${ }^{1}$; Leonard John$\overline{\text { son }^{2} .}{ }^{1}$ Wayne State University, Rochester, MI; ${ }^{2}$ Ascension St. John, Detroit, MI. (Control ID \#3185839)
LEARNING OBJECTIVE \#1: Linezolid is a newer class of medication with this being the first reported case of it causing interstitial lung disease as a side effect.

LEARNING OBJECTIVE \#2: The mechanism of linezolid induced lung injury is believed to be due to the reduction in antioxidation ability and the subsequent formation of free radicals.

CASE: A 34-year-old male with a prior history of injection drug use, hepatitis $\mathrm{C}$, chronic tobacco use and seizures presented to the emergency department for a syncopal episode with associated urinary incontinence thought to be due to seizure. The patient reported that he had developed a nonproductive cough and dyspnea two weeks prior with no associated fever, chills or sick contacts. He had been taking linezolid for three weeks for a right lower extremity infection. At presentation, he was tachypneic, tachycardic and hypoxic. Upon examination, he appeared dyspneic, his lungs were clear and his right lower extremity demonstrated excoriations without active soft tissue infection. His WBC was 13.9 with predominant neutrophils and chest $\mathrm{X}$-ray demonstrated diffuse interstitial changes bilaterally. His linezolid was held and two days later, the patient's cough, hypoxia and dyspnea had resolved, WBC had normalized and repeat CXR showed significant improvement. The patient was readmitted 2 months later for an acute exacerbation of chronic lung disease and his CXR continued to be clear.

IMPACT/DISCUSSION: Linezolid has a wide side effect profile but has not been reported as a cause of DILD. The proposed etiology of DILD is based on free radical damage which is the same mechanism as bleomycin induced pneumonitis. Linezolid induces a reduction in antioxidation ability and the subsequent formation of free radicals. Levels of superoxide dismutase (SOD) and catalase (CAT) in the plasma were decreased after linezolid treatment, which provided the first clinical evidence that linezolid can regulate antioxidant enzyme release. This would in turn cause alveolar cell destruction resulting in pulmonary inflammation. The diagnosis of DILD is made clinically and is mainly based on excluding other causes. Eosinophilia may or may not be present. Treatment involves discontinuation of the offending agent and in acute cases, symptoms tend to resolve within 24 to 48 hours. Patients should also be cautioned to avoid repeat exposure to the offending agent.

CONCLUSION: Linezolid is a newer class of antibiotic in the oxazolidinone class with advantageous pharmacokinetics making it quite potent for numerous infections. However, as a newer drug class, unrecognized side effects are being discovered. This is the first reported case of linezolid causing DILD and clinicians should be aware of this potential side effect.

\section{LITHIUM INDUCED ENCEPHLOPATHY}

ejaz shah ${ }^{1}$; Saliha Saleem ${ }^{2} .{ }^{1}$ montefiore medical center, Bronx, NY; ${ }^{2}$ Montefiore Medical Center, Bronx, NY. (Control ID \#3186431)

LEARNING OBJECTIVE \#1: Assess lithium induced encephalopathy in elderly

LEARNING OBJECTIVE \#2: Recognizing lithium as a controlled substance.

CASE: 65 year old man with Bipolar Disorder (on lithium and valproate) presented with altered mental status (AMS). As reported by family he was progressively lethargic and somnolent since 2 months. At baseline he was well oriented. His last office visit was 2 months back with his Primary care provider (PCP) with blood work showing normal kidney function and was started on lithium. On presentation to hospital he was oriented to self only and rest of neurological examination was non-focal. Pertinent labs were: Creatinine of $1.50 \mathrm{mg} / \mathrm{dl}$ (Normal level -0.5 to $1.1 \mathrm{mg} / \mathrm{dl}$ ) and Lithium level $2.8 \mathrm{mmol} /$ lit (Normal level 0.6 to $1.2 \mathrm{mmol} / \mathrm{lit}$ ). On admission CT head was unremarkable. Electroencephalography (EEG) was consistent with diffuse cerebral dysfunction, worse in the left fronto-temporal region. presence of triphasic waves raising the possibility of a metabolic encephalopathy and is a reported finding in lithium toxicity. Lithium was held. 
Nephrology recommended aggressive intravenous hydration. Lithium levels trended down, $2.8 \rightarrow 1.8 \rightarrow 1.3 \rightarrow 0.8 \mathrm{mmol} / \mathrm{lit}$, with subsequent improvement of renal function and mental status to baseline. Lithium was taken off from his psychiatry medication list and was discharged home.

IMPACT/DISCUSSION: Lithium has many medical and psychiatric uses. Common uses include but are not limited to migraine headaches, alcoholism and bipolar disorder. Toxicity from lithium can occur by overdose especially from alteration in its clearance by the kidney. Known presentation with lithium overdose include ataxia, lethargy and tremors. Risk factors associated with toxicity include age $>60$ years, derranged kidney function and drug- drug interaction. Our patient was otherwise healthy elderly male with new onset derranged kidney functions that led him to have encephlopathy. Lithium prescriptions usually comes from psychiatrist, and once started lithium levels should be monitored monthly along with creatinine levels. Unfortunately, our patient had a controlled substance prescribed by a PCP and was not monitored. We as physicians should be cautious in prescribing such medications, and once prescribed regular neurological exams, drug levels and creatinine should be monitored.

CONCLUSION: Lithium is associated with serious but reversible side effects. As a physician extra care should be implemented after prescribing it. Patient physical examination and blood tests should be monitored strictly.

\section{LOOKING BACKWARDS: A 1940S HERBAL REMEDY FOR OPIOID WITHDRAWAL IN 2018}

Maximillian Bourdillon ${ }^{1}$; Nathan Pham ${ }^{1}$; Andrea Alonso ${ }^{2}$; Nicolette Oleng ${ }^{1} .{ }^{1}$ Boston Medical Center, Boston, MA; ${ }^{2}$ Boston University School of Medicine, Boston, MA. (Control ID \#3183661)

LEARNING OBJECTIVE \#1: Highlight the abuse potential of unregulated homeopathic substances

LEARNING OBJECTIVE \#2: Educate providers on the identification and management of kratom withdrawal

CASE: A 37-year-old woman with a history of polysubstance use disorder (alcohol, opiates without intravenous drug use in the last year) presented to the emergency department for right hand cellulitis and alcohol withdrawal. She reported drinking 5-10 beers daily; last drink on the morning of admission. She reported tremors, diaphoresis, and nausea. She received multiple doses of benzodiazepines using the CIWA (Clinical Institute Withdrawal Assessment) tool for alcohol withdrawal with initial improvement of her symptoms. However on the floor, symptoms of diaphoresis and tremulousness persisted while her exam was notable for dilated pupils and frequent yawning. Given this presentation and prior history of opiate dependence and treatment with suboxone, the patient was placed on COWS (Clinical Opiate Withdrawal Scale) for which she scored for moderate withdrawal. A urine drug screen showed trace amounts of buprenorphine. On admission, patient denied opiate use in the six months prior to admission. However, she disclosed a 6-month daily use of an herbal tea with kratom as a home remedy for depression and anxiety. Kratom has pharmacological interaction with the opioid system and opioid withdrawal was precipitated with abrupt cessation during this admission. With the help of addiction medicine consult team, she was treated for opioid withdrawal with suboxone, with symptomatic improvement.

IMPACT/DISCUSSION: Kratom (Mitragyna speciosa) is an herb indigenous to Southeast Asia with dose-dependent stimulant and opiate-like properties. It has been used as an over the counter treatment of opioid withdrawal dating to the 1940 s. It is traditionally chewed but can be powdered and mixed in liquid. There is scant literature demonstrating its efficacy in treating opiate dependence. While there are increasing reports of lethal overdose and other adverse effects, our understanding of kratom is limited to a few case reports or series. The DEA has even considered scheduling kratom. While kratom's popularity has been rising via internet vendors and smoke shops in the US, it has remained relatively unknown in healthcare thus rendering providers ill equipped to screen and manage the overdose or withdrawal from this substance. The active ingredient in kratom is mitragynine. At low doses of 1-5 grams of raw leaves, mitragynine has stimulant effects and can cause increased alertness, physical energy, and euphoria. At high doses of 5-15 grams it is an agonist on the $\mathrm{Mu}$ and Kappa receptors resulting in analgesia and relief from opioid withdrawal, which explains how cessation can precipitate opioid withdrawal, as described in this case.

CONCLUSION: -Kratom has abuse potential as an anxiolytic and analgesic agent -The potential of kratom in the treatment of opioid withdrawal or as maintenance therapy requires further inquiry, particularly in the current opiate use crisis

\section{LOOKING BENEATH THE SURFACE - DIFFERENTIAL DIAG- NOSES FOR SKIN FINDINGS IN A PATIENT WITH HIV}

Angela O. Suen; Eliana Bonifacino. UPMC, Pittsburgh, PA. (Control ID \#3163108)

LEARNING OBJECTIVE \#1: Understand how framing bias can limit the differential for patients with HIV.

LEARNING OBJECTIVE \#2: Recognize a common noninfectious dermatologic complication of HIV.

CASE: A 55-year-old man with substance use disorder and untreated HIV presents with a chronic diffuse hyperkeratotic crusting rash. Patient reports a recent history of not taking his antiretrovirals due to increased cocaine use and instability of his living situation. His rash started several months ago on his bilateral lower extremities and spread to his hands, feet, trunk, extremities, and face. It is a highly pruritic and painful rash. On examination, there were diffuse hyperkeratotic plaques distributed on the palms, soles, and extremities with areas of hyperpigmented nodules. His CD4 count was measured at 4 . Given a clinical picture concerning for Norwegian scabies, patient was started on ivermectin. The patient underwent skin scraping which was negative for scabies mites. He subsequently underwent biopsy which showed psoriasiform dermatitis. HHV-8 was negative. Patient was treated with daily moisturization and wrapping, and experienced improvement in symptoms. Patient was eventually started on darunavir/cobicistat and tenofovir alafenamide/ emtricitabine prior to discharge.

IMPACT/DISCUSSION: The skin is tightly connected to the immune system and a compromised immune system (especially in Tcell function) leads to clinically apparent skin disease. This patient has psoriasis in the setting of untreated HIV, which is an extreme presentation of a relatively common illness. His CD4 count, and homelessness, may have caused framing bias and limited the differential to infectious etiologies commonly seen in HIV: viral pathogens such as HSV, molluscum contagiosum, HPV, VZV; bacterial pathogens such as bacillary angiomatosis, syphilis; fungal pathogens such as Cryptococcus, mycobacterial infection of the skin, or infection by microorganism (such as bedbugs, parasites, scabies, lice). Psoriasis is a common complication of HIV which is not frequently discussed. One proposed mechanism is that the relative decrease in CD4 T-cells causes a relative increase in CD8 memory T-cells which are associated with onset and exacerbation of psoriasis. Patients with HIV tend to have severe psoriasis seen with low CD4 counts. There are no curative therapies for psoriasis and treatment is largely supportive. It is thought that optimizing HIV/AIDS with anti-retroviral therapy can lead to a significant reduction in symptoms.

CONCLUSION: Many illnesses commonly treated by internists have dermatologic manifestations. In the setting of HIV/AIDS, though appropriately, infectious etiologies of rashes should be considered, it is important for generalists to know that noninfectious rashes like psoriasis are also common. This case also highlights how framing bias can influence the approach to patients with HIV/AIDS by limiting the diagnosis to infectious etiologies. 


\section{LOOKING BEYOND THE VIRUS: SOCIAL DETERMINANTS OF HEALTH AND HEPATITIS B}

Ilana Prior. Weill Cornell Medical College/New York Presbyterian, New York, NY. (Control ID \#3185377)

LEARNING OBJECTIVE \#1: Using one patient's story, identify social factors that contribute to poor outcomes in HBV

CASE: 54M with chronic hepatitis B (HBV) infection (HBeAg neg) presented with abdominal distension. He was found to have AST/ALT 1000s, INR 3.9, and hep B viral load >170,000,000 IU/mL. PMH: He was diagnosed with HBV in 2009 and started on entecavir. He subsequently lost his Medicaid, including prescription coverage, due to his income exceeding the state maximum. He had stopped his antivirals 2 years prior due to cost. Social history: Born in Taiwan, he immigrated to the US in 1995. He was a naturalized citizen. He spoke no English; his wife was conversational. He was paid under the table for his $60+$ hours/week working at a restaurant. He did not have insurance. Hospital course: Despite treatment with NAC and tenofovir, he developed progressive liver failure with altered mental status and renal failure requiring the ICU, intubation, and CVVH. He underwent orthotopic liver transplant on hospital day 8. His family applied for emergency Medicaid; he received post-transplant immunosuppressives via pharmaceutical assistance programs. He was eventually discharged to rehab. His Medicaid application was approved post-discharge.

IMPACT/DISCUSSION: As outcomes in patients with liver disease improve, focus has shifted towards identifying the social determinants that contribute to their health. Our patient had three notable factors which played a role in his need for transplant. First, his ethnicity: HBV disproportionately affect minorities, specifically Asians, in the US. Despite efforts to target Asians for HBV screening and treatment, mortality from HBV remains 8 times higher in Asians than whites in America. Second, language barriers: although his wife could not understand complex medical discussions in English, she often found herself acting as his interpreter for these conversations. Patients with limited English proficiency are more likely than English speaking patients to experience adverse events that are the result of communication errors. Many of these patients feel that their doctors do not explain things clearly. Finally, losing prescription benefits and access to his antivirals resulted in viral reactivation and acute liver failure. Public insurance coverage (vs private) has been associated with increased mortality post-liver transplant. This patient required a transplant organ for a disease that is both preventable and manageable once contracted, and is at higher risk for post-transplant mortality given his current insurance status. His highly morbid outcome was largely the result of his ethnicity, language, and insurance status. These are all areas that should both be acknowledged when caring for patients with similar socioeconomic factors, and targeted for improvement as we attempt to achieve universal health equity.

CONCLUSION: Ethnicity, language barriers, and insurance status can be significant contributors to morbidity in hepatitis B patients

\section{LOST AT C: THE MYSTERIOUS CASE OF RECURRENT BLEEDING}

Laya Reddy. Scripps Clinic, San Diego, CA. (Control ID \#3185145)

LEARNING OBJECTIVE \#1: Recognize signs and symptoms of vita$\min \mathrm{C}$ deficiency

LEARNING OBJECTIVE \#2: Identify those at risk of vitamin C deficiency

CASE: This is a 25-year-old man with autism spectrum disorder who presented with 1-day of generalized weakness and left knee swelling. This patient had two recent hospitalizations in the month prior to presentation. Initial hospitalization was due to traumatic left knee hemarthrosis. The second admission was due to recurrent hemarthosis with a hemoglobin drop from 8.1 to 6.8. Orthopedic surgery did not feel the patient's anemia could be attributed solely to blood loss in left knee joint. Further workup was consistent with mixed picture of iron deficiency and anemia of chronic disease. He presented for a third admission with similar presentation. Past medical history was significant for autism. He also had protein-calorie malnutrition with a body mass index of 13 and had been eating 21 eggs per day for the past four months with no additional form of nutrition. He had no significant family history of bleeding disorders. Physical exam was significant for an ill-appearing cachectic male with a sallow appearance. Patient's left knee was with mild swelling and ecchymosis to lateral thigh as well as decreased range of motion due to pain. The anterior aspects of his bilateral upper and lower extremities with scattered perifollicular papules. Initial labs significant for anemia with hemoglobin of 6.7. Given that bleeding diathesis workup was unrevealing, further investigation was done in conjunction with patient's skin findings and diet devoid of vitamin $\mathrm{C}$, which is non-existent in eggs. This patient's vitamin $\mathrm{C}$ level was found to be undetectable and patient was diagnosed with scurvy. He was initiated on vitamin C supplementation with 500 milligrams daily for 3 months. With supplementation, patient's symptoms and anemia improved to baseline within 2 months.

IMPACT/DISCUSSION: Scurvy can mimic an acquired coagulapathy. Though scurvy is more common in underdeveloped countries, prevalence in the United States has been as high as $7.1 \%$ in the past few years. At risk populations include children with autism spectrum disorder who habitually eat a highly selective diet devoid of fruits and vegetables along with drug abusers, impoverished, and the elderly. Symptoms of scurvy generally occur within months of a vitamin $\mathrm{C}$ deficient diet. The disease is characterized by pronounced cutaneous signs such as petechiae, perifollicular hemorrhage, and bruising. Hemarthrosis and anemia can occur as seen in this patient. The mechanism of anemia is thought to be due to inappropriate folate and iron absorption and utilization and in some cases hemolysis and gastrointestinal bleeding.

CONCLUSION: In the high-risk populations of some developed nations, an estimated $25 \%$ of men and $16 \%$ of women are deficient in vitamin C. It is vital to keep scurvy on the differential in high-risk, nutritionally deficient patients presenting with hemarthrosis. Distinct cutaneous findings can give clues to diagnosis.

\section{LUSH LASHES AND LYMPHEDEMA: A RARE HERITABLE DISORDER UNDERLYING A COMMON COMPLAINT}

Matthew T. Brown; Laura Ragmanauskaite; Chengcheng Ye; Robin Klein. Emory University School of Medicine, Atlanta, GA. (Control ID \#3186110)

LEARNING OBJECTIVE \#1: Emphasize lymphedema distichiasis as a rare cause of bilateral lower extremity edema

LEARNING OBJECTIVE \#2: Recognize hereditary syndromes as etiologies for presenting illnesses if the classic work-up is unrevealing

CASE: A 59 year old female presents with four months of progressive swelling associated with dyspnea and generalized weakness. She first noticed bilateral leg swelling following a pedicure and was treated for a lower extremity cellulitis. However, her symptoms did not resolve, and her edema progressed proximally to involve her thighs and lower abdomen. Past medical history was notable for obesity and gastric sleeve surgery in 2014. Exam revealed smooth, pitting edema in her bilateral lower extremities extending to her lower abdominal wall. In addition to a circular left ankle plaque, a dimpling pattern similar to peau d'orange induration was noted at both thighs with brawny discoloration. There was no JVD, S3, or displaced PMI. Her lungs were clear to auscultation. No organomegaly or fluid wave on abdominal exam. Laboratory revealed normal creatinine and albumin. Urine protein was negative at $15 \mathrm{mg} / \mathrm{dL}$. Echo revealed an EF of $65 \%$, normal cardiac anatomy and no diastolic dysfunction. Ultrasound was negative for lower extremity thrombosis. CT abdomen showed only reactive inguinal lymphadenopathy without 
masses, liver abnormalities, or ascites. Trial of diuresis did not change her edema. Given her lower extremity plaque and skin changes, dermatology was consulted. Detailed exam revealed a double row of eyelashes or distichiasis. With further inquiry, she endorsed frequent conjunctivitis. She was presumptively diagnosed with Lymphedema distichiasis and referred for further testing.

IMPACT/DISCUSSION: Lymphedema distichiasis is a rare autosomal dominant genetic disorder due to a mutation in the FOXC2 gene. It is characterized by early-onset lymphedema and distichiasis or aberrant eyelashes, present in $94 \%$ of patients. Distichiasis ranges from a single aberrant eyelash to a full row of extra lashes along the inner lining of the lid causing corneal irritation or recurrent conjunctivitis in up to $75 \%$ of patients. Other associations include ptosis, varicose veins, and even cardiac abnormalities like tetralogy of fallot. Our case illustrates a classic presentation of a rare genetic disease presenting in adulthood. Lower extremity swelling is a relatively common symptom with a broad differential. However, while our initial systems-based evaluation was thorough, it failed to provide a clear clue to a diagnosis. This case illustrates to need to be mindful that hereditary diseases may present in previously well adult patients.

CONCLUSION: Lymphedema distichiasis is a rare genetic disorder for bilateral lower extremity lymphedema. Importantly, as internists, we often do not think of undiagnosed hereditary diseases in evaluating adult patients. Therefore, if the classic work-up is unrevealing, this genetic disease needs to considered in the differential.

\section{LYMPHOEPITHELIAL CYST: A RARE CASE OF RAPIDLY PROGRESSIVE ABDOMINAL PAIN ASSOCIATED WITH LARGE MULTILOCULATED INTRA-ABDOMINAL CYST}

Faith Villanueva; Joydeep Chakraborty; Maryam Sattari. University of Florida, Gainesville, FL. (Control ID \#3163324)

LEARNING OBJECTIVE \#1: Evaluate and manage large, symptomatic abdominal cysts with uncommon radiographic/pathologic features.

CASE: A 24-year-old man with no significant past medical history presented with left upper quadrant abdominal pain for 3 days. The pain was severe, progressive, non-radiating, increased with movement and eating, associated with subjective fever, and without antecedent abdominal trauma. He denied personal or family history of pancreatitis, pancreatic disease, or neoplasm. His social history was notable for heavy alcohol use and binge drinking. Physical exam was remarkable for severe left lower quadrant abdominal tenderness with palpable mass without peritoneal signs. Serum studies, including lipase and carbohydrate antigen 19-9 (CA 19-9), were unremarkable. Imaging (CT and MRI) revealed normal pancreas and pancreatic duct but showed a $6 \times 12 \times 18 \mathrm{~cm}$ multiloculated cystic lesion in the left upper quadrant adjacent to the pancreatic tail. Cytology and histopathology obtained from endoscopic ultrasoundguided fine-needle aspiration (EUS-FNA) showed nonspecific fragments of fibrous tissue without evidence of malignancy. Fluid analysis for amylase and CEA was inconsistent with pseudocyst or mucinous neoplasm. He underwent surgical fenestration of the cyst without complication, and final pathology was consistent with lymphoepithelial cyst. His symptoms significantly improved post-operatively with marked radiographic decrease in mass size.

IMPACT/DISCUSSION: With the recent advent of improved diagnostic and interventional modalities, consensus on evaluation and management of symptomatic abdominal cystic lesions is a continuously evolving field that remains challenging for internists. Lymphoepithelial cysts are benign, rare lesions often found incidentally, and usually require no intervention. However, this patient's cyst was causing severe symptoms and his history of alcohol use as well as size of the mass were concerning for malignancy. Ultimately, complete diagnostic workup including serum tumor markers, cross-sectional imaging, EUS-FNA with fluid analysis, cytology, and pathology was necessary as therapeutic intervention was etiologydependent. Timely diagnosis and therapeutic intervention required a multidisciplinary approach and coordination with interventional gastroenterology, pancreatobiliary surgery, radiology, and pathology.

CONCLUSION: Lymphoepithelial cysts are benign lesions that can be managed conservatively if asymptomatic. However, due to their rarity and often incidental identification, these lesions can pose a unique diagnostic and therapeutic challenge for internists and be misdiagnosed as neoplasms. Thorough multi-disciplinary evaluation with imaging as well as histologic analysis and correlation are essential to rule out other pathologic etiologies such as malignancy and avoid unnecessary extensive surgical intervention.

\section{MALABSORPTIVE CIRRHOSIS: A RARE COMPLICATION OF DUODENAL SWITCH}

Raphael Rabinowitz ${ }^{1}$; Tracey Martin ${ }^{2}$; David M. Feldman ${ }^{1,1}$; Benjamin Verplanke ${ }^{1} .{ }^{1} \mathrm{New}$ York University Langone Health, New York, NY; ${ }^{2}$ Weill Cornell Medical Center, New York, NY. (Control ID \#3161245)

LEARNING OBJECTIVE \#1: Recognize protein malnutrition and cirrhosis as potential complications of biliopancreatic diversion with duodenal switch (BPD-DS).

CASE: A 37-year-old man with a history of severe obesity status post laparoscopic BPD-DS presented with diffuse swelling. The patient was admitted six months previously for severe protein-calorie malnutrition requiring initiation of total parental nutrition (TPN). During that admission, he was found to have elevated liver enzymes and ascites. Workup for autoimmune, infectious, and hereditary etiologies of cirrhosis was unremarkable; a liver biopsy showed steatosis without evidence of alcoholic hepatitis or cirrhosis. He now complained of abdominal distension and lower extremity edema that had progressed over several weeks, requiring multiple large-volume paracenteses. He endorsed past heavy alcohol use, but denied recent exposure. On physical examination, he was grossly anasarcic with a distended abdomen and appreciable fluid wave, 3+ pitting edema to the hips, and scrotal edema. His admission Model for End-Stage Liver Disease (MELD) score was 14. Repeat biopsy demonstrated prominent portal fibrosis and focal nodularity indicative of advanced-stage cirrhosis, with an interval decrease in steatosis from his previous biopsy. The rapidity of fibrosis and reversal of fatty change suggested the etiology was his bariatric surgery. He was treated with intravenous diuretics with improvement in his anasarca. At one-month follow-up, he had a stable MELD and diuretic-responsive ascites. He has been approved for liver transplant evaluation, with plans to reverse his bypass prior to transplant.

IMPACT/DISCUSSION: BPD-DS is classically associated with improvement in hepatic function due to reversal of nonalcoholic steatohepatitis (NASH). However, case reports of hepatic failure following BPD-DS do exist. Clinicians should be alert to this complication and monitor postsurgical patients closely for signs of hepatic decompensation.

CONCLUSION: BPD-DS is the most effective bariatric surgery technique for sustained weight loss in the super-obese (BMI $>50 \mathrm{~kg} / \mathrm{m}^{2}$ ). Nevertheless, its widespread adoption has been limited by technical complexity and concerns over vitamin deficiencies and malnutrition. Loss of hepatotrophic factors due to protein malnutrition has been advanced as a mechanism to explain its contribution to the development of cirrhosis. With the increasing prevalence of obesity and the documented effectiveness of BPD-DS for sustained weight loss, this surgery will likely become more commonplace. Awareness of this potential complication and vigilance to ensure adequate protein intake, with aggressive intervention - including the initiation of TPN - to preserve nutritional status is paramount to effective management of BPD-DS patients post-operatively.

MANAGEMENT OF GAMMA-HYDROXYBUTYRATE (GHB) WITHDRAWAL IN A HOSPITALIZED ORTHOPEDIC PATIENT Hannan Braun; Miriam Harris; Zoe M. Weinstein. Boston Medical Center, Boston, MA. (Control ID \#3181804) 
LEARNING OBJECTIVE \#1: Recognize GHB withdrawal signs and symptoms

LEARNING OBJECTIVE \#2: Manage GHB addiction and withdrawal CASE: A 40-year-old man slipped at home and presented to the ED after feeling his right leg snap. Imaging with X-ray and CT-angiography showed spiral shaft fractures of the distal tibia and fibula complicated by entrapment of the anterior tibial artery. His medical and social history were significant for HIV (CD4 484), bipolar disorder, chronic pain, polysubstance use (reported cocaine, cannabis, MDMA, and crystal methamphetamine use), severe alcohol use disorder in remission for two years, and osteoporosis (of unknown origin). Home medications included alprazolam $0.5 \mathrm{mg}$ TID prn, asenapine $10 \mathrm{mg}$, baclofen $20 \mathrm{mg}$ BID and hydroxyzine $25-50 \mathrm{mg}$ prn. He is sexually active with men. Surgical repair was delayed when he became increasingly hypertensive, tachycardic, and agitated. Despite receiving alprazolam $1.5 \mathrm{mg}$, hydroxyzine $25 \mathrm{mg}$, baclofen $20 \mathrm{mg}$, and lorazepam $1 \mathrm{mg}$, his symptoms persisted, and he developed visual hallucinations. A workup for organic causes of delirium including serum toxicology was negative. Urine toxicology was positive only for medications given and substances reported in the patient's history. During an additional addiction-focused history, he denied alcohol use but reported daily GHB. His agitation required ICU care. He was initiated on a phenobarbital protocol for GHB withdrawal, with additional haldol and dexmedetomidine for his protracted delirium. On hospital day 4 , he underwent a successful intramedullary nailing of the femoral and tibial fractures. He received his last dose of phenobarbital on day 7 and was transitioned to home baclofen, a recommendation by the addiction consult service given its potential utility for GHB treatment.

IMPACT/DISCUSSION: GHB is a central nervous system depressant used recreationally (mainly in a liquid oral form) as a party drug for its effects of euphoria, sedation, amnesia, and sex-stimulation. While there is low use $(<1 \%)$ in the general population, high rates (up to $20 \%$ ) have been reported among club-going gay and bisexual men. GHB withdrawal includes autonomic nervous system hyperarousal and hallucinations. It remains difficult to treat: GHB is a GABA-B receptor agonist, while alcohol and its withdrawal treatments (benzodiazepines and phenobarbital) are GABA-A agonists. Long-acting benzodiazepines are the mainstay of treatment for GHB withdrawal, with barbiturates considered in withdrawal resistant to benzodiazepines or complicated by psychosis. While originally prescribed to our patient for pain, baclofen - a GABA-B agonist - is currently being studied for its potential to treat GHB use disorder.

CONCLUSION: -GHB withdrawal is similar in presentation to alcohol, presenting with tremor, diaphoresis, and agitation - though with a more rapid onset of neuropsychiatric symptoms. -GHB withdrawal is lifethreatening. A thorough addiction history is required for patients with suspected substance use and acute delirium, as GHB is not identified in standard toxicology screening.

\section{MASQUERADE OR MISS? A CASE OF UNDIAGNOSED NEUROSYPHILIS}

Justin Nichols $^{1}$; Nikita Donti ${ }^{2}$; Jennifer N. Goldstein ${ }^{3}$; Ribu Mathew ${ }^{4}$. ${ }^{1}$ Christiana Care Health System, Philadelphia, PA; ${ }^{2}$ Christiana Care Health Systems, Wilmington, DE; ${ }^{3}$ Christiana Care Health System, Newark, DE; ${ }^{4}$ Sidney Kimmel Medical College, Jefferson University, Philadelphia, PA. (Control ID \#3180821)

LEARNING OBJECTIVE \#1: Rule out reversible causes of psychosis. LEARNING OBJECTIVE \#2: Diagnose neurosyphilis in individuals with significant risk factors.

CASE: A 35-year-old African American previously healthy male presented with progressive waxing and waning encephalopathy, confusion, delusions, hallucinations, and amnesia. One year prior to presentation, he had been diagnosed with schizophrenia, hospitalized and started on antipsychotics without improvement. The patient developed difficulty with activities of daily living, night sweats, weight loss, ataxia, tremors and visual changes. Social history was significant for unprotected male sexual encounters, prompting STD testing by his primary care provider. Testing returned positive for HIV (CD4 count of 169), high RPR titers (1:2048 dilutions) and a positive Treponema pallidum particle agglutination assay (TPPA). He was admitted to the hospital for workup and treatment of suspected neurosyphilis. On exam, the patient was alert and oriented only to person with fine hand tremors and a narrow-based shuffling gait. A lumbar puncture revealed elevated protein, leukocytosis, positive Treponema pallidum antibody and elevated VDRL titer (1:16), confirming neurosyphilis. Cryptococcal antigen, JC virus and meningoencephalitis PCR panel were negative. Brain MRI showed cortical atrophy. The patient was treated with a fourteen-day course of IV penicillin G, followed by one dose of IM benzathine penicillin $G$ with improvement in mental status and affect. He was started on antiretroviral therapy and discharged with follow up at the HIV clinic.

IMPACT/DISCUSSION: Neurosyphilis is a great imitator, and early neurological involvement may be seen more frequently in individuals with HIV. Before making a diagnosis of schizophrenia or other psychiatric conditions, reversible causes must be ruled out. A thorough social history is required to assess for risk factors such as HIV status, male gender, and men who have sex with men. Our patient was ultimately diagnosed with AIDS and late-stage neurosyphilis, likely general paresis, after appropriate testing. Since neurosyphilis can occur during any stage of syphilis, a lumbar puncture should be performed when neurological and ophthalmic signs are present or the index of suspicion is high. Elevated protein ( $>45$ $\mathrm{mg} / \mathrm{dL}$ ), white blood cells ( $>5$ cells/microL) and a positive CSF-VDRL confirms the diagnosis of neurosyphilis. Patients should be promptly treated with IV penicillin with test of cure lumbar puncture every six months after treatment until resolution of CSF pleocytosis. Patients with HIV are at increased risk for treatment failure.

CONCLUSION: Strongly consider neurosyphilis on the differential in patients who present with neuropsychiatric symptoms and appropriate risk factors. Confirm the diagnosis of neurosyphilis with a lumbar puncture. Treat neurosyphilis with IV penicillin G for 10-14 days, with subsequent confirmatory testing.

\section{MASQUERADING MYOSITIS}

Nirav Antao; Andrea Ramirez Gomez. St.Louis University Hospital, St Louis, MO. (Control ID \#3186835)

LEARNING OBJECTIVE \#1: Identify the different types of idiopathic inflammatory myositis (IIMs) and diagnostic criteria for the new classification

LEARNING OBJECTIVE \#2: Review the clinical presentation of antisynthetase syndrome

CASE: A 53 year old woman presented with one month of diffuse myalgias, generalized weakness and shortness of breath. She had previously presented to an outside hospital ER on three separate occasions where she was initially treated with multiple antibiotic courses for suspected bronchitis and then briefly admitted for "double pneumonia" due to CT findings of multifocal bilateral reticulonodular pulmonary infiltrates. However, her symptoms never really improved, which prompted evaluation at our institution. Here, physical exam revealed coarse bilateral breath sounds with $3 / 5$ bilateral grip strength and 3/5 strength in proximal and distal bilateral lower extremities. Labs revealed elevated CK 1276, LDH 584, aldolase 34, ESR 91, CRP 11.7 and ANA 1:320 speckled pattern. As her labs, imaging and constellation of symptoms were suggestive of an underlying autoimmune/ rheumatologic process, a myositis panel was ordered which revealed +anti Jo-1 and + anti SSA. Due to concomitant persistent shortness of breath and hypoxemia in the setting of above CT, she underwent a bronchoscopy which showed diffuse alveolar hemorrhage. She was thus diagnosed with overlap myositis and more specifically "antisynthetase syndrome" which is the most representative form of this group. She was started on steroids after which azathioprine was added as an outpatient with significant improvement in her symptoms. 
IMPACT/DISCUSSION: Anti-synthethase syndrome is characterized by autoantibodies targeting aminoacyl tRNA synthetases, such as antiJo1, and is predominantly associated with interstitial lung disease. Diagnosis is achieved by a combination of clinical manifestations, presence of autoantibodies and biopsy findings. Other studies like EMG and muscle MRI are also useful in diagnosis. Treatment for inflammatory myopathies remains a challenge due to their heterogeneity, thus a multidisciplinary approach is warranted.

CONCLUSION: Inflammatory myopathies are a group of autoimmune disorders characterized by muscle inflammation, but often accompanied by extra-muscular involvement including skin, joints and lung, as seen in our patient. Recent discovery of autoantibodies and advances in pathophysiology have led to the development of a new classification criteria subgrouping IIMs in 5 types. These share some clinical presentations, but also have specific patterns of muscle involvement, distal organ affection or paraneoplastic syndrome association, which makes accurate diagnosis of upmost importance for adequate treatment and prognosis.

\section{MASS EFFECT: A CASE OF BILIARY OBSTRUCTION}

Alexander Katz ${ }^{1}$; Michael Crawford ${ }^{1}$; Deepa Bhatnagar ${ }^{2} .{ }^{1}$ Tulane University School of Medicine, New Orleans, LA; ${ }^{2}$ Tulane University, New Orleans, LA. (Control ID \#3184480)

LEARNING OBJECTIVE \#1: Demonstrate a rare cause of biliary obstruction

LEARNING OBJECTIVE \#2: Discuss the clinical spectrum of IgG related disorders

CASE: A 71-year-old man presented with 3 days of intermittent abdominal pain and nausea. His past medical history was pertinent for a recent episode of biliary pancreatitis. He did not report alcohol abuse or a history of gallstones, and his only medications included hydrochlorothiazide, rosuvastatin and losartan. Vitals were normal and epigastrium was tender. Liver enzymes revealed AST 220, ALT 320, alkaline phosphatase of 508 with total bilirubin of 2.8. Lipase was normal. Ultrasound revealed gallstones and common bile duct dilation of $6-7 \mathrm{~mm}$ but without clear obstruction. CT abdomen/pelvis revealed a $1 \mathrm{~cm}$ pancreatic mass and terminal ileum thickening with ileocolic lymph nodes. CA-19 was 264 and IgG was elevated to 1907. MRI and EUS were done to further evaluate pancreatic lesion and showed no evidence of pancreatic mass. Liver biopsy showed no fibrosis or malignancy. Lower endoscopy showed no intraluminal mass in the ileum. Ultimately, the mesenteric nodule biopsy was performed along with cholecystectomy which revealed IgG related sclerosing disease of both the gallbladder and mesenteric nodule. ERCP with stent placement was performed for biliary drainage. The patient was treated with steroids with improvement in symptoms.

IMPACT/DISCUSSION: Internists frequently encounter hepatobiliary pathology when caring for patients. In this case, the patient's clinical picture was more consistent with biliary obstruction. After more common causes were ruled out, including autoimmune hepatitis, an obstructive pancreatic lesion and gallstones, more advanced diagnostics were performed. IgG related disease was suspected based on elevated $\mathrm{IgG}$, recent history of pancreatitis and CT imaging findings. However, primary sclerosing cholangitis and cholangiocarcinoma can also present with biliary obstruction. Therefore, tissue samples were obtained from both the gallbladder and mesenteric nodules. This biopsy revealed a plasma cell infiltrate and storiform fibrosis, characteristic histology of IgG disease. IgG related diseases represents a spectrum of disorders that most commonly include autoimmune pancreatitis and IgG related sclerosing cholangitis, but it can affect most other organs. The clinical presentation is variable depending on the organ involved but when it involves the biliary system, it often mimics more common causes of cholecystitis, cholangitis and pancreatitis. While our patient presented without pancreatitis, which occurs in up to $30 \%$ of cases of IgG related sclerosing cholangitis, it is likely that his most recent episode represented autoimmune pancreatitis as well.
CONCLUSION: IgG related sclerosing cholangitis is the most common extra-pancreatic manifestation of autoimmune pancreatitis and is an important syndrome for internists to recognize, as it frequently mimics more common manifestations of biliary pathology.

\section{MASSIVE ABDOMINAL WALL HEMATOMA}

Eric H. Lee; Andrew Lai. UCSF, San Francisco, CA. (Control ID $\# 3144128)$

LEARNING OBJECTIVE \#1: To identify abdominal wall hematoma as a hemorrhagic complication of paracenteses.

CASE: A 45-year-old man with decompensated alcoholic cirrhosis presented to the emergency department with abdominal pain following a routine outpatient paracentesis. Physical examination revealed a distended abdomen with diffuse left lower quadrant tenderness. Initial laboratory tests revealed a hemoglobin level of 9.3 grams per deciliter (reference range, 14-18). Computed tomography of the abdomen and pelvis revealed a new abdominal wall hematoma $(22 \times 9 \times 6.5 \mathrm{~cm})$. He was initially admitted to an inpatient medicine wards service for monitoring. However, within 24 hours he was transferred to the intensive care unit for hemodynamic monitoring and large volume blood product transfusions. Due to evolving disseminated intravascular coagulation and new renal replacement therapy requirement, he was transferred to another hospital for expedited liver transplantation evaluation. On arrival, the patient's Model for End-Stage Liver Disease (MELD-Na) score was 39. Repeat imaging (Panel A, black arrow) showed a rapidly expanding hematoma (35 x 28 x $18.5 \mathrm{~cm}$ ) with abdominal mass effect. He underwent successful embolization of the left inferior epigastric artery, but later developed a spontaneous perihepatic hemorrhage (Panel B, white arrow) that led to hemodynamic collapse. He was subsequently transitioned to comfort care and died five days after transfer.

IMPACT/DISCUSSION: Paracentesis is a core internal medicine bedside procedure. While paracentesis complications are rare (1\%), hemorrhagic complications can be life-threatening, especially in those with endstage liver disease. IR-guided embolization of the inferior epigastric artery may halt hemorrhagic progression.

CONCLUSION: Abdominal wall hematomas are the most common hemorrhagic complication of paracenteses.

\section{MECHANICAL TRICUSPID VALVE THROMBOSIS PRECIPI- TATED BY INCARCERATION}

Daniel Brady; Aaron W. Sweeney. Tulane University School of Medicine, New Orleans, LA. (Control ID \#3180130)

LEARNING OBJECTIVE \#1: Diagnose mechanical valve thrombosis with appropriate imaging modalities

LEARNING OBJECTIVE \#2: Recognize that incarceration can precipitate medical illness

CASE: An incarcerated 36 year old woman presented with acute onset, substernal chest pain. It was constant, sharp and radiated to the left axilla. Taking a deep breath and laying flat made it worse, while sitting forward provided relief. She denied cough, lightheadedness, leg swelling and dyspnea. Medical history included tricuspid valve endocarditis as a result of intravenous heroin use for which she received mechanical valve replacement four years prior. She denied drug use for three years. Her medications included warfarin; however, she was incarcerated four days prior and had not received any medications since. Her physical exam was unremarkable with no murmur or click. Laboratory data was relevant for a subtherapeutic INR and negative serial troponins. Her electrocardiograms had no changes from prior. She was started on a heparin drip. A transthoracic echocardiogram revealed moderate regurgitation and severe stenosis of the tricuspid valve. Leaflet motion was not well visualized; however, a transesophageal echocardiogram the following day revealed immobility 
of all leaflets, concerning for prosthetic valve thrombosis. Fluoroscopy was performed and revealed minimal motion of the mechanical leaflets, confirming the diagnosis. She received thrombolytic therapy with tissue plasminogen activator and had subsequent resolution of symptoms. Repeat echocardiogram showed resolution of regurgitation and stenosis. She was discharged on therapeutic Warfarin.

IMPACT/DISCUSSION: This case illustrates the diagnosis and successful management of mechanical tricuspid valve thrombosis. Although there is extensive literature on left-sided prosthetic valvular thrombosis, there is little published and no current guidelines on this same phenomenon for right-sided prosthetic valve thrombosis. The case highlights how different imaging modalities are crucial in confirming the diagnosis. It also demonstrates successful management of this under-reported clinical situation. Limited data suggest that thrombolytic therapy is effective for these patients, as demonstrated by this case. With the rise of the opiate epidemic with concomitant increase in prevalence of infectious endocarditis necessitating heart valve replacement, the investigation into this condition is certainly warranted. The case also highlights flaws in our correctional system. The majority of cases of prosthetic valve thrombosis are due to subtherapeutic anticoagulation, and in this case was directly attributable to the withholding of medication during incarceration. It certainly calls for improvements in the medical management of incarcerated patients.

CONCLUSION: Mechanical tricuspid valve endocarditis is a rare clinical presentation that warrants further investigation. Incarcerated patients are at risk for inappropriate medical management that may lead to serious complications.

\section{MEDIASTINAL GERM CELL TUMOR (MGCT) AS INITIAL PRESENTATION OF KLINEFELTER SYNDROME (KS)}

Sarah S. Roderick; Aaron R. Kaufman; Cara Guenther. Boston Medical Center, Boston, MA. (Control ID \#3165765)

LEARNING OBJECTIVE \#1: Recognize cough, dyspnea, fevers, and weight loss as presenting symptoms of a mGCT in a young and healthy patient. LEARNING OBJECTIVE \#2: Recognize the relationship between KS and the elevated risk of $\mathrm{mGCT}$.

CASE: A 23-year-old man with no prior medical history presented with three months of cough, fevers, chills, night sweats, weight loss and pleuritic chest pain. The patient had immigrated from Colombia ten years ago and was recently exposed to tuberculosis (TB). His exam were notable for mild tachycardia and absent breath sounds in the left lung fields. Chest CT identified a large anterior mediastinal mass with central necrosis, compressive left lung atelectasis, and lateral displacement of the heart and mediastinum. PET CT showed hypermetabolic activity of the mass, the L2 and L4 vertebrae, and multiple lymph node chains. Labs demonstrated a negative interferon-gamma release assay (IGRA) and HIV, normal beta-HCG, and an elevated AFP. The mass was biopsied and pathology was consistent with yolk sac tumor. On testicular exam, the patient was noted to have small testes. Given recent diagnosis of a mGCT, suspicion was increased for underlying KS. Subsequent scrotal ultrasound visualized small volume testes. Labs showed an elevated FSH and LH, low testosterone, and normal estradiol and prolactin. Further testing demonstrated a 47,XXY karyotype, confirming diagnosis of KS. The patient was initiated on ifosfamide/ etoposide/cisplatin (VIP). He was subsequently discharged with plan for three additional chemotherapy cycles along with surgical evaluation for possible resection of the remaining mass.

IMPACT/DISCUSSION: With a new diagnosis of mGCT in a young and healthy patient with small testes, it is important to consider the association between $\mathrm{KS}$ and increased risk of mGCT.

CONCLUSION: Given symptoms of cough, dyspnea, fevers, night sweats, pleuritic chest pain, and weight loss in an otherwise young and healthy individual from Colombia, TB is high on the differential. However, given the patient's negative IGRA and large mediastinal mass seen on imaging, it's also important not to miss malignancy. In young adult men, the most common type of mediastinal malignances is GCT, including nonseminomatous GCT, with yolk sac tumors being the most common. An elevated AFP is present in a majority of patients, while a small minority present with an elevated B-hCG. GCT tends to be metastatic on presentation; therefore, treatment is with chemotherapy (VIP) followed by resection of any residual mass. Prior literature has illustrated that patients with KS are at an elevated risk of developing GCT. The classic phenotype includes tall stature, gynecomastia, small testes, and psychosocial impairments. Interestingly in our patient with karyotype-confirmed diagnosis, the only phenotypic finding was small testes. Identification of $\mathrm{KS}$ has importance for fertility counseling, especially in the setting of prechemotherapy decision-making (e.g. sperm banking).

\section{MELANOMAS MASQUERADING AS LIPOMAS, AND THE MORBID CONSEQUENCES}

Matthew Lipinski ${ }^{1}$; Diane Hershock ${ }^{2} .{ }^{1}$ Penn State University at Hershey Medical Center, Hummelstown, PA; ${ }^{2}$ Penn State University at Hershey Medical Center, Hershey, PA. (Control ID \#3185560)

LEARNING OBJECTIVE \#1: Describe the variety of clinical presentations of melanoma.

LEARNING OBJECTIVE \#2: Discuss the prognosis and treatment for metastatic melanoma.

CASE: A 22 year-old woman presented with several months of multiple progressively enlarging subcutaneous nodules. The week prior to admission, she had also developed diplopia and nausea. She does not go outside often, and uses sunscreen when she does Two siblings have had biopsyproven lipomas in the past. There is no history of skin cancer in her family. On physical exam, she had multiple small flesh-colored, freely mobile nodules with irregular borders that were present on her right axilla, left arm, back, and chest wall. There was a large five centimeter flesh-colored nodule on her right scalp. There were two one centimeter blue-pigmented nodules in her left axilla. Skin biopsy of a nodule revealed metastatic melanoma. MRI of her brain revealed a four centimeter lesion in the right occipital lobe with radiographic signs of metastatic melanoma.

IMPACT/DISCUSSION: Skin lesions are encountered frequently by internists, and are a common reason for patients to make an appointment. Superficial spreading melanoma presents as intensely dark macules with irregular borders on the skin surface. This is by far the most common type of melanoma, therefore many physicians associate melanoma only with this presentation. However, melanomas can have multiple colors, or be non-pigmented and resemble subcutaneous nodules. Nodular melanoma, especially when skin-colored, usually has the worst prognosis; this is partly due to delay in recognition. The ABCDEs of melanoma (asymmetry, border irregularity, color abnormalities, large diameter, enlarging rapidly) can be applied to any concerning skin lesion. Any rapidly growing skin lesion warrants a discussion with the patient about potential biopsy. Metastatic melanoma portends a very poor prognosis for patients. Treatments have evolved from the prior poorly efficacious chemotherapy, and now center around immunotherapy with inhibitors of CTLA- 4 and the PD- 1 receptor. Despite these advances, the overall average prognosis is still approximately two years.

CONCLUSION: Due to its many possible clinical presentations and high morbidity and mortality, internists need to have a high clinical suspicion for melanoma when presented with abnormal skin findings. Treatment options have improved but overall survival is still very poor.

\section{MESENTRIC PANNICULITIS: A RARE CAUSE OF EPIGAS- TRIC PAIN.}

Nikita Fernandes ${ }^{1}$; Lakshmi Priyanka Mahali ${ }^{1}$; Hiliary Hertan ${ }^{2}$. ${ }^{\mathrm{T}}$ Montefiore Medical Center, Bronx, NY; ${ }^{2}$ Montefiore Medical Center, BRONX, NY. (Control ID \#3185993) 
LEARNING OBJECTIVE \#1: Expand the differential diagnosis for epigastric pain.

LEARNING OBJECTIVE \#2: Diagnose mesenteric panniculitis.

CASE: A 52 year old woman with history notable for gastroesophageal reflux disease, hypertension and cholecystectomy was admitted for severe epigastric pain and bloating that had been worsening over one week. Patient was seen at the Gastroenterology clinic in the past for persistent epigastric pain since two years and patient was treated with proton pump inhibitors (PPI) and sucralfate. Patient had persistent epigastric pain which was refractory to PPI treatment. Patient had not taken NSAIDs in the past. Ultrasonography of the abdomen did not reveal any abnormality. EGD done one year ago revealed gastritis. Computed tomography (CT) of the abdomen and pelvis during this hospital visit revealed mesenteric panniculitis and patient was started on NSAIDs. After receiving treatment with NSAIDs for two weeks, patient improved symptomatically.

IMPACT/DISCUSSION: Mesenteric panniculitis is a rare inflammatory condition characterized by acute and chronic non-specific inflammation of the adipose tissue of the intestinal mesentery. The disease affects Caucasian men more commonly, with a male-to-female ratio of 3:1. Our patient is unique in that she is a woman and she is of Hispanic origin. As of 2016, less than 300 cases have been reported. It may be associated with a variety of conditions such as prior abdominal surgery, malignancy, autoimmune disease, infections and gall stones. Our patient was found to have a history of cholecystectomy for cholelithiasis. The pathogenic mechanism is unknown, however non-specific immune response to a variety of stimuli has been suggested. Clinical presentation is nonspecific, but abdominal pain is reported to be the most common symptom. Symptom duration may be twenty-four hours to two years. It presents mainly in the sixth to seventh decade. Clinical examination and laboratory test are usually not helpful in the diagnosis but help to exclude more common conditions. CT scan is the investigation of choice. CT scan may reveal soft tissue masses, prominent lymph nodes, inflammation and/or fibrosis of the mesentery. Treatment options are based on case reports as the condition is rare. NSAIDs, steroids, azathioprine, cyclophosphamide, colchicine, tamoxifen and radiotherapy have been used for treatment of Mesentric Panniculitis. Spontaneous resolution of the illness has also been reported. Surgery is indicated in those patients with compressive or obstructive symptoms. The disease can resolve and recur spontaneously over the years. The best treatment option is unknown.

CONCLUSION: Most cases of epigastric pain are treated with PPI and patients are advised to avoid NSAIDs. Conversely, NSAIDs are used for treatment of mesenteric panniculitis which also presents as epigastric pain.

\section{METASTATIC LUNG CARCINOMA OF UNKNOWN PRIMARY ORIGIN: IS IMMUNOHISTOCHEMISTRY ENOUGH?}

Mario A. Scarpinato ${ }^{1}$; Daniel Roadman ${ }^{2}$; Pakhadi Buddhadev ${ }^{1} .{ }^{1}$ Medical College of Wisconsin, Milwaukee, WI; ${ }^{2}$ Medical College of WIsconsin, Milwaukee, WI. (Control ID \#3185196)

LEARNING OBJECTIVE \#1: Unusual presentation of lung cancer LEARNING OBJECTIVE \#2: Use of immunohistochemistry and genetic testing for lung cancer diagnosis

CASE: A 55-year-old male was admitted for acute respiratory distress. Past medical history included hypertension, hyperlipidemia, schizophrenia, and anxiety. Social history was negative for tobacco or alcohol use. Family history was significant for paternal lung cancer at age 50. A chest radiograph showed a white out of the right hemithorax; a chest tube collected $3,700 \mathrm{~mL}$ of serosanguinous fluid throughout the admission. The pathology was positive for malignancy: immunohistochemistry (IHC) was MOC-31(+), C22(-), C40(-), calretinin(-) and TTF-1(-). Metastatic workup included a $\mathrm{CT}$ chest which identified an anterior mediastinal mass at the right cardiophrenic angle partly invading the suprahepatic inferior vena cava as well as a $3.1 \mathrm{~cm}$ spiculated right upper lobe mass. MRI of the brain identified two small brain lesions at the gray-white junction in each frontal lobe. A PET/CT showed a hypermetabolic anterior mediastinal mass, along with multiple hypermetabolic nodules in the lung and pleural surface. A CT-guided biopsy of the anterior mediastinal mass was positive for metastatic carcinoma, favoring urothelial carcinoma after an IHC staining for GATA-3(+), CK7(+), CK20(+), p40(+), and focal nuclear positivity for TTF-1. A urologic oncologist worked up the suspected urothelial carcinoma with a CT Urogram and cystoscopy without evidence of a primary uroepithelial neoplasm in the genitourinary tract. The patient also had no risk factors for primary urothelial cancer and no urinary symptoms suggesting a urologic primary. The medical oncology team started gemcitabine and cisplatin based on the initial IHC pathology consistent with urothelial carcinoma. However, after just one day of gemcitabine and cisplatin, the patient's genetic testing revealed an EGFR exon 19 deletion, heavily favoring metastatic lung adenocarcinoma. He was switched to osimertinib and has tolerated it well thus far.

IMPACT/DISCUSSION: In diagnosis of metastatic carcinomas of unknown primary origins, the role of IHC helps determine the origin. In this patient, IHC staining of $\mathrm{CK} 7(+) / \mathrm{CK} 20(+)$, GATA-3(+), and p40(+) suggested a urinary bladder origin. In this case, IHC was not enough. Without genomic testing, this patient would still be receiving the treatment for metastatic urothelial carcinoma, which can be used for lung adenocarcinoma but is not the best choice in lung cancers with an EGFR mutation. The definitive diagnosis allowed treatment with osimertinib, targeted chemotherapy, which has shown an additional 6-month life expectancy benefit compared to platinum-based chemotherapy.

CONCLUSION: After initial workup, discussion of risk factors and clinical gestalt should not be ignored. This patient had a family history of lung cancer, a positive clinical picture, and no risk factors for urothelial carcinoma. It took the combination of IHC, consultant services, and genetic testing to make the correct diagnosis.

\section{METFORMIN ASSOCIATED LACTIC ACIDOSIS IN A PATIENT WITH CKD}

Hayley Crossman; Sarah J. Flynn; Dorothy Rimmelin; Shirin Hemmat; Meghna Motiani. University of California, San Francisco, San Francisco, CA. (Control ID \#3186038)

LEARNING OBJECTIVE \#1: Distinguish Type A from Type B lactic acidosis

LEARNING OBJECTIVE \#2: Recognize management of Type B lactic acidosis due to metformin

CASE: An 85-year-old female with a history of HTN, DM, CKD, and CHF presented with abdominal pain, nausea, and vomiting. Her home medications included glipizide, metoprolol, spironolactone, furosemide, aspirin, and atorvastatin. Vital signs were T 33.3 C, HR 93, R 18, BP 63/ $40, \mathrm{SpO} 2100 \%$ on $6 \mathrm{~L}$. Physical exam revealed a well appearing elderly woman in no apparent distress. Rest of exam was unremarkable. Labs were notable for WBC 22.8, Potassium 5.8, Bicarbonate 5, Anion Gap 45, BUN 130 and Creatinine 5.66 (from baseline 1.97). VBG showed $\mathrm{pH}$ 6.9 and pCO2 24, with lactate of 24 . The patient was started on broad spectrum antibiotics and was volume resucitated. She remained hypotensive and norepinephrine, vasopressin, and epinephrine were required for presumed septic shock. Despite this, the patient's lactate uptrended to 26 and she remained profoundly acidotic. A bicarbonate drip was started with no improvement in $\mathrm{pH}$. CVVH was then initiated. Fifteen hours following the initiation of CRRT, the lactate normalized. Vasopressor requirements resolved and CRRT was discontinued. The patient's renal function continued to improve over the ensuing days.

IMPACT/DISCUSSION: Lactate is generated from glucose metabolism via the glycolytic pathway, and lactic acidosis occurs when lactate production exceeds clearance. Most cases of lactic acidosis are due to marked tissue hypoperfusion and subsequent impaired tissue oxygenation (Type A). This can be seen in cases of hypovolemia, heart failure, and sepsis. By contrast, Type B lactic acidosis is characterized by toxin-induced 
impairment of cellular metabolism. In these cases, evidence of systemic hypoperfusion are absent. There are several causes of this, including diabetes, malignancy, alcoholism, HIV, beta-adrenergic agonists, and mitochondrial dysfunction. Our patient had metformin associated lactic acidosis (MALA), a rare clinical entity thought to occur due to decreased gluconeogenesis from alanine, pyruvate, and lactate. The incidence of MALA is estimated as 4.3 cases per 1000,000 patient years. Symptoms, as seen with our patient, are non-specific and include anorexia, nausea/ vomiting, abdominal pain, lethargy, and hypotension. Overdoses of metformin or pre-existing renal insufficiency are at risk factors for developing MALA. Mortality is estimated at $45 \%$. In cases of suspected MALA, metformin should be promptly discontinued. No antidotes to metformin toxicity exist. Supportive care with bicarbonate therapy should be initiated. Extracorporeal removal is considered in those who fail to improve or those in whom $\mathrm{pH}<7.0$ or lactate $>20$. Hemodialysis is preferred to CRRT in MALA, as it promotes rapid clearance of lactic acid. CRRT should be reserved for hemodynamic instability.

CONCLUSION: Metformin associated lactic acidosis is a rare entity with high mortality; management may require extracorporeal removal.

\section{METHEMOGLOBINEMIA \&AMP; MULTI-ORGAN PIGMEN- TATION IN PHENAZOPYRIDINE OVERUSE}

Alicia Cowley $^{2,1}$; David Wei ${ }^{3,1}$. ${ }^{1}$ New York University, New York, NY; ${ }^{2} \mathrm{NYU}$ Langone Tisch Hospital, New York, NY; ${ }^{3} \mathrm{NYC}$ Health + Hospitals/Bellevue, New York, NY. (Control ID \#3160224)

LEARNING OBJECTIVE \#1: Recognize methemoglobinemia \& its sequelae

LEARNING OBJECTIVE \#2: Recall medications that confer risk of iatrogenic methemoglobinemia \& treatment

CASE: An 89-year-old woman was admitted with abdominal discomfort plus five days of dysuria and urinary frequency. Previously, she had seen a urologist for chronic dysuria and was prescribed estrogen cream for vaginal atrophy. On admission, her vital signs were notable for oxygen saturation of $88 \%$, unresponsive to oxygen supplementation. Other clinical findings included jaundice, right upper quadrant and suprapubic tenderness, anemia (hemoglobin $8 \mathrm{~g} / \mathrm{dL}$ ), serum creatinine $2.2 \mathrm{mg} / \mathrm{dL}$, unremarkable liver function testing and ultrasound imaging, plus a urinalysis consistent with a urinary tract infection (UTI). Her chest X-ray was unremarkable and an arterial blood gas (ABG) obtained off of oxygen demonstrated normal partial pressure of oxygen $(\mathrm{PaO} 2)$. Given the discrepancy between oxygen saturation on pulse oximetry and $\mathrm{PaO} 2$, methemoglobinemia was suspected. Co-oximetry revealed oxyhemoglobin $70 \%$, methemoglobin $>21 \%$. The patient denied recent benzocaine, nitrates, or dapsone use, but eventually disclosed taking phenazopyridine for several weeks prior to admission. Intravenous methylene blue was administered, ceftriaxone was given for her UTI, and supportive care was provided until her clinical status returned to baseline.

IMPACT/DISCUSSION: Methemoglobinemia, a condition whereby functional anemia and tissue hypoxia are precipitated by acutely elevated methemoglobin concentration, is often iatrogenic. Nonspecific symptoms plus anemia and acute kidney injury (AKI) can be mistaken for many ailments, highlighting the importance of thorough medication review and having a high index of suspicion when there is a recent use of medications such as oxidizing agents (e.g. topical anesthetics, dapsone), nitrates, phenytoin, or antimalarials. The pathophysiology of acute respiratory distress in methemoglobinemia relates to the reduced erythrocyte oxygen-carrying capacity of functionally impaired hemoglobin. Skin discoloration, hemolytic anemia, and AKI have also been associated with phenazopyridine toxicity. The pathophysiology of AKI is still being investigated but may be multifactorial, including renal tubular epithelial cell injury, heme pigment-induced nephropathy from hemolytic anemia, and hypoxic injury from methemoglobinemia itself.
CONCLUSION: Methemolglobinemia presents with non-specific symptoms (e.g. headache, dizziness, dyspnea, fatigue, mental status changes). When a patient exhibits hypoxia unresponsive to supplemental oxygen, chocolate-brown hued arterial blood, and a discrepancy between pulse oximetry and $\mathrm{ABG}$, methemoglobinemia should be at the top of one's differential. History-taking should include a thorough medication review (including over-the-counter agents) and chemical exposures. Once the diagnosis is confirmed with co-oximetry, methylene blue and supplemental oxygen should be given when methemoglobin level is greater than $20 \%$.

\section{METRONIDAZOLE-INTRODUCED ENCEPHALOPATHY: A RARE BUT POTENTIALLY IRREVERSIBLE NEUROTOXICI- TY OF A COMMONLY USED ANTIBIOTIC}

Tomohiro Matsumoto $^{2}$; Hayato Mitaka ${ }^{1}$; Osamu Hamada ${ }^{3}$; Matthew Harrington ${ }^{4} .{ }^{1}$ Mount Sinai Beth Israel, New York, NY; ${ }^{2}$ Nerima Hikarigaoka Hospital, Nerima-ku, Japan; ${ }^{3}$ Takatsuki General Hospital, Takatsuki, Japan; ${ }^{4}$ Icahn School of Medicine at Mount Sinai, New York, NY. (Control ID \#3169406)

LEARNING OBJECTIVE \#1: - Recognize metronidazole-induced encephalopathy characterized by cognitive and cerebellar dysfunction. Identify the clinical presentation and MRI findings of metronidazoleinduced encephalopathy.

CASE: A 69-year-old man presented to the emergency department with gait disturbance and drowsiness. He was recently hospitalized for cholecystitis complicated by E. coli, K.oxytoca, and C. perfringens liver abscess and bacteremia. He was treated with four weeks of ampicillin-sulbactam and cefmetazole in the hospital followed by levofloxacin $500 \mathrm{mg}$ once daily and metronidazole $500 \mathrm{mg}$ four times daily at discharge, a regimen he had been taking for five weeks prior to presentation. The patient's wife reported four days of unsteady, wide-based gait followed by one day of drowsiness and confusion. On exam, the patient was drowsy and slow in response to verbal commands. Glasgow Coma Scale was E4V4M6. Vital signs were within normal limits. His gait was ataxic and wide-based. Finger-Nose-Finger test showed bilateral dysmetria with intention tremor. Montreal Cognitive Assessment (MoCA) score was 16/30, suggestive of mild cognitive impairment. Complete blood counts, electrolytes, glucose, renal and liver function tests were normal. CT of the abdomen with contrast showed resolution of liver abscess. MRI of the brain showed symmetric, hyperintense FLAIR signals of the cerebellar dentate nuclei, consistent with metronidazoleinduced encephalopathy. He was admitted to the hospital. Metronidazole was discontinued. His cognitive and cerebellar symptoms improved. Repeat MRI on hospital day 8 showed complete resolution of the abnormal findings. At time of discharge on hospital day 23 he had persistent impairment of short-term memory and a slightly wide-based gait. At his follow-up visit one week later, his MoCA score remained below normal at 25/30 but his gait was normal.

IMPACT/DISCUSSION: Encephalopathy is a side effect of metronidazole typically reported among patients receiving prolonged courses of the antibiotic. Clinical manifestations include cerebellar dysfunction (75\%), altered mental status (33\%), and seizure (13\%). MRI shows symmetric enhanced FLAIR signals of the dentate nuclei of the cerebellum, a pathognomonic finding of metronidazole-induced encephalopathy. MRI abnormalities may resolve despite persistence of symptoms, as demonstrated by our patient. The majority of patients experience complete resolution of symptoms after discontinuation of metronidazole, but some patients experience permanent neurologic symptoms. Case reports suggest cognitive symptoms and seizures are more likely to completely resolve than are cerebellar symptoms, however our patient experienced the opposite result.

CONCLUSION: - Encephalopathy is a rare side effect of metronidazole, usually associated with a prolonged course of the antibiotic. - Clinicians utilizing prolonged courses of metronidazole should maintain vigilance for the development cerebellar or cognitive dysfunction in their patients. 
MILIARY TB AND A PARADOXICAL REACTION TO TB TREATMENT IN AN IMMUNOCOMPETENT ADULT

Vikas Gampa; Linda Shipton; Eirini Iliaki. Cambridge Health Alliance, Somerville, MA. (Control ID \#3176542)

LEARNING OBJECTIVE \#1: TB treatment can cause paradoxical reactions in immunocompetent patients

LEARNING OBJECTIVE \#2: Miliary TB can occur in immunocompetent patients

CASE: Mr. D is a $24 \mathrm{y} / \mathrm{o} \mathrm{M}$ with no PMH, who presented with fevers, weight loss, cough, and headache. He was born in Somalia, and had recently traveled to Africa. Physical exam was unremarkable. Initial work-up showed CXR normal, and labs showed mild transaminitis. He was without signs of immunosuppression (HIV negative, CBC unremarkable). Ultrasound of the abdomen showed hypoechoic lesions in the liver; MRI showed innumerable lesions in the liver and spleen. CT chest notable for prominent right hilar lymph nodes. Head CT and LP were unremarkable. Pathology of liver biopsy was unremarkable. A mediastinal lymph node biopsy showed necrotic lymph node and acid-fast bacilli. He was diagnosed with miliary tuberculosis, with concern for resistant TB. He was started on TB treatment, including moxifloxacin given risk for resistant TB; cultures showed a pansensitive organism. One month later, he had new cough and dyspnea; CT scan of the chest showed new cervical and mediastinal lymphadenopathy, and endobronchial lesions. Pathology revealed bronchial mucosa with chronic inflammation, without mycobacterium. The presentation was consistent with a paradoxical reaction to TB treatment. He was treated with prednisone with successful resolution of his symptoms; however, after the completion of 3 month course of steroids, he developed recurrent cough and dyspnea and was diagnosed with recurrent paradoxical reaction with increase in size of lymph nodes and endobronchial lesions, which caused right middle lobe collapse. Steroids were restarted; he also underwent bronchoscopy with lesion debridement with cryoextraction, and recannulization of RML bronchus. Tissue culture was negative for growth of AFB, fungi or other organisms. Pathology showed rare AFB and necrotizing granulomatous inflammation. He was started on an extended course of prednisone and an extended course of TB therapy.

IMPACT/DISCUSSION: Miliary tuberculosis is a rare presentation and occurs most often in immunosuppressed patients. Paradoxical reactions are defined by the radiographic or clinical development of new lesions or pre-existing tuberculous lesions when treated with TB treatment. Paradoxical reactions are rare and some case series have identified immunosuppresion as a potential risk factor for its development. This case represents an unusual occurence in which underlying immunosuppression did not play a significant role in the development of disease.

CONCLUSION: Miliary TB and paradoxical reactions to TB treatment can rarely occur in HIV-negative patients. Paradoxical reactions can result in significant morbidity or mortality. The pathophysiology is unknown; however, patients who have paradoxical TB reactions may have some underlying immunosuppression. Our patient may yet have an underlying immunosuppressive disorder, given the miliary TB and paradoxical reactions.

\section{MIND ALTERING SUBSTANCE: AN ELECTROLYTE STORY}

Jonathan Chang. Tulane University School of Medicine, New Orleans, LA. (Control ID \#3185245)

LEARNING OBJECTIVE \#1: Recognize electrolyte abnormalities as a cause of altered mental status

LEARNING OBJECTIVE \#2: Differentiate the causes of hypercalcemia in malignancy

CASE: A 79-year-old woman with history of metastatic lung adenocarcinoma presented with one day of altered mental status. Vital signs were normal. The patient was somnolent with speech limited to incomprehensible mumbling but aroused to loud voice and followed commands intermittently. At baseline, the patient was fully oriented and functionally independent. Laboratory studies showed serum calcium of $13.3 \mathrm{mg} / \mathrm{dL}$, phosphorus of $6.3 \mathrm{mg} / \mathrm{dL}$, acute kidney injury with creatinine of $2.4 \mathrm{mg} /$ $\mathrm{dL}$, and lactic acid of $4.2 \mathrm{mmol} / \mathrm{L}$. Parathyroid hormone $(\mathrm{PTH})$ was undetectable, 1,25-dihydroxyvitamin D was low, and parathyroid hormone-related peptide (PTHrP) was negative. CT head without contrast was negative for hemorrhage or mass but revealed multiple new osteolytic skull lesions. EEG demonstrated diffuse slowing without epileptiform activity, compatible with toxic-metabolic encephalopathy. Lumbar puncture was unsuccessful, but the patient became fully oriented by the next day and refused a repeat attempt. The hypercalcemia and other abnormalities resolved with aggressive hydration, calcitonin, and pamidronate. Empiric antibiotics for meningitis were discontinued after 24 hours as she exhibited no signs of acute infection and had returned to baseline mental function. She was discharged several days later.

IMPACT/DISCUSSION: Hypercalcemia of malignancy is an important consideration for the general internist as it is by far the leading cause of symptomatic hypercalcemia. This process affects $20-30 \%$ of all cancer patients and portends a worsening prognosis. It is predominately caused by tumor secretion of PTHrP (80\%) or direct bone release from osteolytic metastasis (20\%). In Hodgkin lymphoma, hypercalcemia almost exclusively results from excess 1,25dihydroxyvitamin D. Paraneoplastic PTHrP production, known as humoral hypercalcemia of malignancy, is mostly associated with squamous cell carcinomas and carcinomas of the breast, kidney, bladder, and ovary. PTHrP has significant homology with PTH and drives calcium resorption from the bone and renal tubules as well as urinary phosphorus excretion. Osteolytic metastasis is particularly common in breast carcinomas and multiple myeloma. Bone breakdown is mediated by cytokines that stimulate osteoclast activity, causing concurrent phosphorus elevation. With low PTH, negative PTHrP, and low-to-normal vitamin D levels, clinicians should consider imaging studies and serum/urine protein electrophoresis to evaluate for bony metastases and monoclonal gammopathies.

CONCLUSION: Hypercalcemia of malignancy is the most common cause of symptomatic hypercalcemia. Imaging studies to assess for bony metastasis should be considered with low PTH, negative PTHrP, and lowto-normal vitamin D levels.

\section{MIND THE GAP: AN UNUSUAL CASE OF DYSPNEA IN A RENAL TRANSPLANT PATIENT}

Ann Wang; Samuel Rednor; Sumit Kapoor. Montefiore Medical Center, Bronx, NY. (Control ID \#3186228)

LEARNING OBJECTIVE \#1: Recognize a potentially fatal but intervenable cause of hypoxia in patients taking dapsone.

LEARNING OBJECTIVE \#2: Treat symptomatic methemoglobinemia. CASE: A 51-year-old woman presented with shortness of breath and fatigue. She had a history of lupus nephritis, and two weeks prior to presentation had received a renal transplant and was started on immunosuppressive and prophylactic medications. Physical exam revealed a fatigued and cyanotic-appearing woman with clear breath sounds. Her oxygen saturation on room air was $87 \%$. Labwork was significant for creatinine of $4.8 \mathrm{mg} / \mathrm{dl}$. Non-contrast computed tomography of the chest was negative for infiltrate or edema. A ventilation-perfusion scan was negative for pulmonary embolism. She was placed on high-flow nasal cannula and then BiPAP. However, pulse oximeter continued to read 87$89 \%$. An arterial blood gas (ABG) was then drawn, revealing $\mathrm{pH} 7.49$, pCO2 31mmHg, pO2 $184 \mathrm{mmHg}$, and methemoglobin of $17 \%$. The patient received methylene blue intravenously, with rapid improvement in dyspnea. Of note, she had previously tested normal for G6PD. Within 24 hours, repeat $\mathrm{ABG}$ showed methemoglobin of $9.2 \%$ and pulse oximeter read $97 \%$ on $2 \mathrm{~L} / \mathrm{min}$ nasal cannula. Dapsone was discontinued and atovaquone started for PJP prophylaxis. 
IMPACT/DISCUSSION: Methemoglobin is formed when the ferrous iron in hemoglobin becomes oxidized to ferric iron, which is unable to bind or deliver oxygen. Methemoglobinemia can be congenital or acquired. Clinically, patients present with dyspnea, cyanosis, fatigue, lethargy, and lightheadedness. The "saturation gap" is classic, where pulse oximeter reads $87-88 \%$, and ABG reveals a normal or increased pO2. Symptoms typically are present with methemoglobin levels of $>10 \%$. At methemoglobin levels $>30 \%$, patients may experience respiratory depression, coma, seizures, and death. Dapsone is the most commonly implicated agent in drug-induced methemoglobinemia, although the true incidence of dapsone-induced methemoglobinemia is not well documented. In one study of renal transplant patients at a tertiary care center, over $20 \%$ developed symptomatic methemoglobinemia with a mean of 66 days on dapsone. The incidence can be expected to increase as the number of organ transplant recipients increases. The renal transplant population may be especially vulnerable as dapsone and its metabolites are renally excreted. Patients with methemoglobin levels $>20 \%$, or who are symptomatic at lower levels, should be treated with IV methylene blue. The causative agent should be discontinued. For patients with reduced renal function on dapsone, clinicians may consider periodic monitoring of methemoglobinemia levels, or preemptively using alternative agents for PJP prophylaxis.

CONCLUSION: Dapsone is the most common cause of drug-induced methemoglobinemia. Methemoglobinemia should be suspected in patients with unexplained hypoxia and a saturation gap. Patients with symptomatic methemoglobinemia or methemoglobin levels exceeding $30 \%$ should be treated with the administration of methylene blue

\section{MIND THE GAP: DIABETIC KETOACIDOSIS MASKED BY HEMODIALYSIS}

David Staudt ${ }^{1}$; Deepa R. Nandiwada ${ }^{2} .{ }^{1}$ Hospital of the University of Pennsylvania, Philadelphia, PA; ${ }^{2}$ University of Pennsylvania, Ardmore, PA. (Control ID \#3181591)

LEARNING OBJECTIVE \#1: To recognize the confounding impact of hemodialysis (HD) on the metabolic parameters used for diagnosis of diabetic ketoacidosis (DKA)

LEARNING OBJECTIVE \#2: Critically analyze a differential when there is a discordance between a suspected illness script and diagnostic labs

CASE: A 66 year old woman with a history of type 1 diabetes mellitus (T1DM) complicated by end-stage renal disease presented via ambulance from her dialysis center after 3.5 hours of HD with somnolence. Prior to treatment she had endorsed two days of fatigue and abdominal discomfort. On presentation she was able to confirm that she had missed her insulin for 2 days due to feeling unwell. Vital signs at presentation were unremarkable and her exam was notable for confusion, an ability to answer only simple questions, paradoxical lip smacking, and mild abdominal tenderness. Initial laboratory studies were notable for a glucose of 180 , venous $\mathrm{pH}$ of 7.43 , bicarbonate of $25 \mathrm{mmol} / \mathrm{L}$, anion gap of 24 (from a baseline of 14), a normal lactate, and a urinalysis with $2+$ ketones. Serum beta-hydroxybutyrate was elevated to $5.66 \mathrm{mmol} / \mathrm{L}$ (normal < 0.27). The patient was admitted to the medicine service for altered mental status of unclear etiology. Given her history of missed insulin, abdominal pain, and altered mental status with presentation immediately following dialysis, masked DKA was suspected. With administration of insulin and fluids her abdominal pain, mental status, paradoxical movements, and anion gap improved to her baseline.

IMPACT/DISCUSSION: In this case, our patient presented with clinical symptoms matching the illness script for DKA- missed insulin, abdominal pain, and neuroglycopenic symptoms. However the diagnosis was initially not considered because her laboratory studies did not meet technical criteria for DKA despite her significantly elevated beta-hydroxybutyrate and anion gap. Given her presentation this was likely an effect of dialysis treatment which can normalize glucose levels and mask the metabolic acidosis of DKA by creation of a secondary metabolic alkalosis from bicarbonate- containing dialysate. This phenomenon has been noted in the past. ${ }^{1}$ In fact, dialysis has been used to treat refractory cases of DKA, as it can effectively correct acidosis and improve glycemia., ${ }^{2,3}$ This case is an example of a discrepancy between an illness script (DKA) and laboratory studies likely created by dialysis. In patients with end stage renal disease, clinicians should scrutinize seemingly normal laboratory studies and consider dialysis' modifying effect on diagnostics prior to excluding a diagnosis.

CONCLUSION: -When a discrepancy exists between a clinical presentation and laboratory testing, clinicians need to consider the confounding effect of other factors including dialysis -Clinicians should critically assess their differential of seemingly normal labs in patients who recently received dialysis.

\section{MISUNDERSTOOD AND MISDIAGNOSED: A CASE OF CATA- TONIA IN A WOMAN WITH UNKNOWN MEDICAL HISTORY}

Kathryn Leyens ${ }^{2}$; Jill Allenbaugh ${ }^{1} .{ }^{1}$ Temple University, Philadelphia, PA; ${ }^{2}$ University of Pittsburgh, PITTSBURGH, PA. (Control ID \#3182482)

LEARNING OBJECTIVE \#1: Define the prevalence of catatonia LEARNING OBJECTIVE \#2: Identify the presenting symptoms and treatement of catatonia

CASE: A 35-year-old female with a history of bipolar disorder, depression, and mild mental retardation presented as a transfer from an outside hospital with altered mental status. The patient was minimally interactive, perseverative, and anxious. Limited past medical history was obtained by family members over the phone. Additional medical records were not available as the patient had been living out of state. Her family reported she had returned home after the recent death of her father, and she had been restricting her diet and seemed increasingly depressed. On admission she was hemodynamically stable, with labs remarkable for hypokalemia and UA with ketones. Urine drug screen, chest $\mathrm{x}$-ray, and non- contrasted $\mathrm{CT}$ of her head were unremarkable. After ruling out common toxic, metabolic, and infectious causes for her altered mental status, the focus shifted to her refusal to eat and repetitive hand movements. Catatonia was discussed as a potential cause based on these presenting symptoms and she was given $1 \mathrm{mg}$ of lorazepam. Within ten minutes, she became more alert, interactive, and resumed oral intake. She was subsequently started on lorazepam 1mg BID and was transferred to the psychiatric hospital for further management.

IMPACT/DISCUSSION: This case presented the challenge of managing a patient with unclear past medical history, and inability to participate in her care. The inclusion of catatonia in our differential helped to quickly make an accurate diagnosis and administer the appropriate treatment. Although not commonly included as a differential diagnosis, catatonia is an important consideration. The prevalence of catatonia is reported as high as 38 percent in psychiatric patients. Many of the symptoms of catatonia are non-specific and may be mistaken for other conditions; however, there are certain findings that should prompt the clinician to consider catatonia as a diagnosis. These signs and symptoms include: verbigeration, echolalia/echopraxia, mutism, posturing, perseveration, excitement, and impulsivity. Recognition of these psychomotor symptoms and administration of the appropriate treatment improves the outcome for patients with catatonia. There are various rating scales that can be used to help aid in the identification of catatonia which include and are not limited to the Bush-Francis Catatonia Rating Scale and the Bräunig-Catatonia Rating Scale. If there is suspicion for catatonia, treatment should be considered. Prognosis is good, and lorazepam can induce remission up to $79 \%$ of the time. In the setting of catatonia that is refractory to lorazepam, there are other treatments including amantadine, zolpidem, and electroconvulsive therapy.

CONCLUSION: Catatonia is prevalent and can be present in more than $1 / 3$ of psychiatric patients. Therefore, it should be included in the differential, particularly when a patient has characteristic psychomotor symptoms. 


\section{MIXED BACTERIAL AND FUNGAL INFECTION IN A CASE OF EFFUSIVE CONSTRICTIVE PERICARDITIS IN ESRD}

Satyaki Chakrabarty ${ }^{2}$; Aya Shnawa ${ }^{1}$; Michael Maysky ${ }^{2} .{ }^{1}$ Jordan University of Science and Technology, Irbid, Jordan; ${ }^{2}$ St. Elizabeth's Medical Center, Tufts University School of Medicine, Boston, MA. (Control ID \#3184704)

LEARNING OBJECTIVE \#1: Careful assessment of etiology in pericarditis in patients with end-stage renal disease.

LEARNING OBJECTIVE \#2: Management of effusive constrictive pericarditis due to Candidal infection of pericardium.

CASE: A 79 year old male with a past medical history significant for end stage renal disease and hypertension experienced an episode of near syncope during a hemodialysis session. Vital signs included a body temperature of 98.2 $\mathrm{F}$, blood pressure of $74 / 60 \mathrm{mmHg}$, heart rate of 119 beats $/ \mathrm{min}$, respiratory rate of $24 / \mathrm{min}$ and oxygen saturation of $90 \%$ on room air. Physical examination was notable for muffled heart sounds along with jugular venous distension which increased on inspiration. Initial laboratory findings were significant for: white blood cell count of 16,100/mm3 with $87.0 \%$ neutrophils; BUN, $31 \mathrm{mg}$ / $\mathrm{dL}$; serum creatinine, $5.5 \mathrm{mg} / \mathrm{dL}$; lactic acid, $2 \mathrm{mmol} / \mathrm{L}$; NT pro B-NP, 4380 $\mathrm{pg} / \mathrm{mL}$. There was no evidence of growth on blood cultures. An Echocardiogram showed evidence of pericardial effusion along with diastolic collapse of the right atrium and ventricle, suggesting tamponade physiology. A right heart catheterization confirmed equalization of intracardiac pressures after which $675 \mathrm{~mL}$ of serosanguinous pericardial fluid was removed though pericardiocentesis. Right atrial pressure remained elevated at $28 \mathrm{~mm} \mathrm{Hg}$, confirming an effusive-constrictive physiology. Pericardial fluid demonstrated heavy growth of Candida albicans along with many white blood cells. A further $400 \mathrm{~mL}$ was removed though a pericardial drain. The patient's clinical condition improved significantly and he was started on an oral regimen of Flucanazole and Colchicine, which he continued for 6 weeks. Prior to his follow up, we noted that the pericardial fluid was also growing Mycobacterium avium. At follow up, a repeat Echocardiogram showed complete resolution of the pericardial effusion and we decided to refrain from further medical therapy.

IMPACT/DISCUSSION: Purulent pericarditis caused by Candida species is a rare condition with high mortality rates despite treatment. Our patient was immunocompromised due to his ESRD and Candidal infection of an already uremic pericardium added more insult to injury. What was unusual in this case is the concomitant infection with M. avium. Tuberculosis happens to be a frequent cause of ECP in immunosuppressed patient populations. Our patient's clinical condition resolved with just large volume pericardiocentesis, anti-fungal therapy and antiinflammatory therapy, negating the need for pericardiectomy.

CONCLUSION: To our knowledge, this is the first reported case of ECP caused by mixed bacterial and fungal infection in a patient with ESRD. This argues towards careful assessment of etiology while treating pericarditis in chronic kidney disease. Also, we demonstrate how candidal infection of the pericardium can be successfully treated with oral fluconazole and percutaneous drainage.

\section{MORE THAN DIABETIC NEUROPATHY: A PINS AND NEEDLES CASE OF ANTI-LGI1 AND ANTI-CASPR2 ASSOCI- ATED LIMBIC ENCEPHALITIS}

Sagar Wagle; Simarjeet S. Lamba; Ali Saadi; Elieth Martinez. UCSF Fresno, Fresno, CA. (Control ID \#3183723)

LEARNING OBJECTIVE \#1: Recognize clinical manifestations of anti-LGI1 and anti-CASPR 2 limbic encephalitis.

LEARNING OBJECTIVE \#2: Identify treatment modalities for limbic encephalitis.

CASE: A 78-year-old male with diabetic neuropathy presented with worsening neuropathic pain with stocking-glove pattern. Further history revealed a $30 \mathrm{lb}$ weight loss over 3 months. CT Chest was notable for a $4 \mathrm{~cm}$ thymoma. The thymoma was resected resulting in partial resolution of neuropathic symptoms. Six weeks later, the patient was re-admitted for generalized weakness, confusion, visual hallucinations, and agitation. Vitals were unremarkable and exam was notable for bilateral temporal wasting. He had slurred speech, was oriented only to self, and had poor short-term memory recall. Strength was intact. Workup including MRI brain, EEG, EMG, and lumbar puncture were unremarkable. Serum antibodies were positive for anti-CASPR 2 (contactin-associated protein-like 2) at 1:160 titer, anti-LGI1 (leucine-rich gliomainactivated 1 ) at 1:10 titer, and VGKC (voltage-gated potassium channel) at $257 \mathrm{pmol} / \mathrm{L}$ (normal $<80$ ). The patient received 5 days of methylprednisolone $1 \mathrm{~g}$ daily followed by 5 sessions of plasmapheresis. He had resolution of confusion, hallucinations, and neuropathy.

IMPACT/DISCUSSION: Limbic encephalitis is an autoimmune inflammation of the limbic system. It usually occurs as a paraneoplastic syndrome and can precede a diagnosis of malignancy. Clinical manifestations of anti-LGI1 positive limbic encephalitis include seizures, psychiatric symptoms, neuropathy and memory problems. MRI findings include signal changes in the temporal lobe and hippocampus. Immunotherapy (IVIG, steroids, and plasmapheresis) remains the mainstay of treatment. Most anti-CASPR 2 positive patients with limbic encephalitis are elderly male and thymoma is the most common tumor. Patients with tumor have symptomatic recovery after surgery or chemotherapy, and patients without tumor often have recovery after immunotherapy. With an annual incidence of less than 1 in one million, this case contributes to the literature of autoimmune encephalitis given its broad clinical presentation before a diagnosis is made. While most anti-CASPR 2 patients have full neurological recovery after tumor resection, our patient had transient improvement and subsequent encephalopathy. His symptoms improved only after immunotherapy was started. Currently, there are no set treatment guidelines. Further investigation is required to assess the benefit of sequential use vs concomitant administration of steroids with PLEX. While some providers add other immunotherapies including rituximab and cyclophosphamide, our patient will continue with plasmapheresis in the outpatient setting.

CONCLUSION: Limbic encephalitis presents with memory loss, confusion, seizures, and psychiatric symptoms like personality change, irritability, anxiety, and depression. Its treatment includes immunotherapy and treatment of underlying malignancy (if present).

\section{MULTIPLE ABSCESSES AND RED HERRINGS}

Stephen Sexauer; David O'Dell; Michael P. Smith. University of Nebraska Medical Center, Omaha, NE. (Control ID \#3186340)

LEARNING OBJECTIVE \#1: Develop an evaluation and treatment approach to multiple abscesses

LEARNING OBJECTIVE \#2: Recognize the Streptococcus anginosus group as the potential causative agent for multiple abscesses and common treatment pitfalls

CASE: A 52 year-old man presented with fevers, intractable headaches, photophobia, and SOB that had progressed over the period of one week. He had a history of psoriasis treated with etanercept and who worked as a pig butcher and had a pet squirrel. He was afebrile and hemodynamically stable. There was nuchal rigidity, however, the remainder of the physical exam was unremarkable, including a clear oropharynx. Brain MRI revealed small cerebral abscess and Chest X-ray revealed a lower lobe cavitary lesion. He underwent lumbar puncture that revealed elevated protein, consistent with a viral meningitis. He was treated supportively. He subsequently underwent $\mathrm{CT}$ of his chest to further evaluate the lesion seen on Xray which confirmed the presence of an abscess. He was started on empiric coverage for Nocardiosis with ceftriaxone and his headaches were treated symptomatically. He underwent lung abscess biopsy which was unrevealing and did not grow any organisms. He failed to improve symptomatically after several 
weeks of antibiotic therapy so he subsequently underwent brain abscess biopsy and drainage which grew Streptococcus intermedius. Antibiotics were targeted to the cultured organism based on culture and sensitivity data and he was treated with a prolonged course of TNP-SMX. He underwent oral panoramic xray which revealed multiple periapical abscesses and was referred for outpatient tooth extraction.

IMPACT/DISCUSSION: An abscess is a common problems for the general internist in both the hospital and outpatient setting. Developing a systematic approach to how an abscess should be managed can help expidite appropriate diagnosis and treatment. Commonly abscesses are presumed to be staph aureus or other staph species. Streptococcus intermedius is an aerotolerant anaerobic bacterium that is commensal oral flora but has been implicated in disseminating and forming brain and liver abscesses, especially in immunocompromised hosts. It is a member of the Strep anginosus group that also include Strep anginosis and Strep constellatus. S. anginosis and S. intermedius are more likely to have altered penicillin binding proteins leading to resistance to beta-lactams. Vancomycin is a suitable alternative. It is important to be aware of these treatment implications as this group of organisms are known to causes absceses in areas that are difficult to biopsy including the brain, thoracic cavity, and abdominal cavity. Some members of the $\mathrm{S}$ anginosis group can also produce superantigens leading to necrotizing complications, making prompt recognition even more important

CONCLUSION: General Internists must consider S. intermedius in their diagnostic approach to patients with abscesses and with history of immunomodulators, which are increasingly more common in today's age of targeted biologic therapies

\section{MURINE TYPHUS: A FLEA-TING DIAGNOSIS}

Adam J. Klein; Steven Klemow. Methodist Dallas Medical Center, Dallas, TX. (Control ID \#3184346)

LEARNING OBJECTIVE \#1: Recognize clinical features and risk factors for murine typhus

LEARNING OBJECTIVE \#2: Recognize changing patterns of endemic infections

CASE: 29 year old female with no significant past medical history presented with fever, nausea, vomiting. Patient stated that symptoms started 1 month ago with a prodrome of headache, photophobia and flu like symptoms, which had resolved, but fevers persisted. She had a max temperature of 104 degrees $\mathrm{F}$ with worsening fatigue and lethargy which prompted her presentation to this hospital. On admission, she denied headache, sore throat, chest pain, dyspnea, abdominal pain, diarrhea, dysuria, arthralgias, myalgias, rash, new medications or drug use. She had no known sick contacts and no recent travel/camping and denied any tick or animal bites. She lives in a rural area with her spouse and pet pig. She had a temperature of $102 \mathrm{~F}$, heart rate of 98 , blood pressure $107 / 82$ breathing comfortably on room air. Their was no lymphadenopathy, lung sounds were clear, no cardiac murmur was appreciated, abdomen was soft and non-tender, and no visible rash or wounds. Labwork revealed WBC 12.1, CRP 157, Procalcitonin 4.21, LDH 1,552, ALT 105, AST 103, Alkaline Phosphatase 137. Rapid influenza, acute hepatitis panel, blood cultures, HIV, RPR and T-Spot were negative. Imaging was noncontributory. Anaplasma, ehrlichia, brucella, CMV, EBV, and fungal studies were negative. Rickettsia typhi IgM and IgG antibody by IFA returned at 1:2048 and 1:128 respectively, consistent with a diagnosis of Murine Typhus. Doxycycline was started and her fever defervesced within 48 hours. It was discovered that there was a local R. typhi outbreak in the patient's county. Indoxacarb was provided for the patient's pet pig as possible host. 2 weeks after discharge, symptoms had not returned and repeat IgM antibody was 1:512.

IMPACT/DISCUSSION: Murine typhus is a rare bacterial infection caused by Rickettsia typhi with a worldwide geographic distribution, and in the United States, predominately in Los Angeles County and southern
Texas. Humans are infected by inoculation of infective flea feces in bite wounds. While worldwide, rats are the main host, in the United States, domestic cats and opossums act as the main host with cat fleas acting as the vector. The cat flea is an indiscriminate feeder and commonly parasitizes many animals of similar size. In a review of outbreaks in south Texas, the majority presented with fever, headache, myalgias, fatigue, rash, thrombocytopenia and elevated hepatic transaminases. Overall there has been an upward trend in the number of reported cases in the north Texas region over the last 5 years. While there are many reasons that can be contributing to the changing epidemiology, this has not been formally studied yet.

CONCLUSION: Murine typhus can be challenging to diagnose due to the non-specific presentation. It is important to recognize the clinical signs as the changing epidemiology pattern will impact physicians outside endemic regions more frequently.

\section{MY WATCH SAYS IT'S TIME TO GO TO THE DOCTOR}

John Xie; Sanjin Tunovic. Tulane University School of Medicine, New Orleans, LA. (Control ID \#3184073)

LEARNING OBJECTIVE \#1: Describe the clinical presentation and diagnosis of hyperthyroidism

LEARNING OBJECTIVE \#2: Review recent technology in healthcare assessment and delivery

CASE: A 48-year-old man with recently diagnosed Grave's disease two months prior presented when his Apple watch reported his heart rate was in the $150 \mathrm{~s}$. He endorsed mild anxiety, but had no palpitations, tremor, vision changes, weight loss, nausea, vomiting, or abdominal pain. EKG was consistent with sinus tachycardia with a heart rate in the 160s. Labs returned with TSH suppressed with slightly elevated total T4 as well as free T4. The Burch-Wartofsky Point Scale (BWPS) for Thyrotoxicosis was 30 suggesting impending thyroid storm. He was prescribed methimazole and propranolol with improvement in his symptoms.

IMPACT/DISCUSSION: Thyrotoxicosis, a condition caused by excess circulating thyroid hormone, has an estimated prevalence of $0.1-0.5 \%$. Thyroid hormone can affect any organ in the body, which can make diagnosis challenging. Some presentations can be innocuous, such as anxiety and tachycardia, but can also include heat intolerance, palpitations, weight loss, muscle weakness, and diarrhea. If untreated, thyrotoxicosis can increase the risk for atrial fibrillation, congestive heart failure, osteoporosis, and thromboembolism. The most serious complication is thyroid storm, characterized by altered sensorium, and has a high mortality unless treated promptly. Initial tests to diagnose thyrotoxicosis include a suppressed TSH and elevated total and free T4. The Burch-Wartofsky Point Scale (BWPS) for thyrotoxicosis can also be used to estimate the likelihood of thyroid storm based on clinical presentation. Laboratory data is not included in this scoring system. The advent of new mainstream technology in healthcare has the potential to impact which patients seek medical care. In particular, the recently released Apple Watch Series 4 is the first smartwatch to get FDA clearance for EKG monitoring. This device simulates a one-lead EKG by placing electrodes on the back of the watch and in the digital crown. Thus far, the EKG app can only give two heart rhythms - normal sinus and atrial fibrillation. The Apple Heart Study was started in November 2017 to determine if the Apple Watch can accurately diagnose atrial fibrillation. This study included 588 individuals, half with atrial fibrillation and the other half with normal sinus rhythm. The watch algorithm accurately identified 98.3 percent of people with atrial fibrillation, and correctly identified 99.6 percent of people who did not have atrial fibrillation.

CONCLUSION: Low TSH and elevated thyroid hormones establish a diagnosis of hyperthyroidism. The BWPS can estimate likelihood of thyroid storm based on symptom severity, independent of laboratory results. As wearable device technology continues to advance it will likely have an increasing role in disease detection and monitoring in the outpatient setting. 
MYCOBACTERIUM IMMUNOGENUM INFECTION PRESENTING AS DISSEMINATED CUTANEOUS INFECTION

Michael Yang ${ }^{2}$; Ribka Ayana ${ }^{1} .{ }^{1}$ Washington DC VA Medical Center, Washington DC, DC; ${ }^{2}$ Medstar Georgetown University Hospital, Washington, DC. (Control ID \#3184963)

LEARNING OBJECTIVE \#1: Recognize the clinical features of nontuberculous mycobacteria (NTM) infection

LEARNING OBJECTIVE \#2: Recognize the diagnostic and treatment challenges associated with Mycobacterium Immunogenum (M. immunogenum) infection

CASE: A 46-year-old man presented with a $3 \mathrm{~cm}$ tender, indurated lesion on the left hand five days after injecting IV cocaine into the right arm. He also had discrete, non-tender, non-erythematous nodules on the right forearm and left leg. He was exposed to gardens and fish tanks weeks prior to presentation. He had no fever or leukocytosis. All lesions were surgically drained and cultured, without bacterial growth. He was discharged on Clindamycin but returned two days later with new nonerythematous, rubbery nodules on the right forearm and the left axilla. Infectious disease (ID) was consulted and advised biopsy with AFB and fungal cultures. Mycobacterium chelonae-abscessus group was isolated from the AFB culture. Further gene sequencing at the National Institutes of Health revealed M. immunogenum with resistance to fluoroquinolones, cefoxitin, doxycycline, tobramycin, and TMP-SMX, and intermediate carbapenem sensitivity. The patient received 10 weeks of IV amikacin, meropenem, and azithromycin, followed by 6 week oral course of azithromycin and moxifloxacin.

IMPACT/DISCUSSION: NTM are found in soil, fresh water, and tap water. They include the M. chelonae-abscessus group, M. fortuitum, and M. marinum. M. immunogenum is a NTM first described in 2001 and added to the M. chelonae-abscessus group in 2007. NTM SSTIs have been linked with skin trauma, cosmetic procedures, surgeries, tattoos, piercings, and acupuncture, often in the setting of immunosuppression. The distal extremities are the most common sites of infection. Susceptibility patterns vary by species. M. fortuitum is the most susceptible;M. chelonae-abscessus group is susceptible to aminoglycosides with varying susceptibility to carbapenems, fluoroquinolones, tetracyclines, and linezolid. Widespread penicillin and cephalosporin resistance mitigate their use. Macrolide susceptibility is common, but inducible macrolide resistance exists. Our case adds to the limited number of case reports in literature describing infection with $\mathrm{M}$. immunogenum. M. immunogenum infection can cause substantial morbidity due to clinical under recognition resulting in delayed diagnosis as well as diagnostic challenges as definitive identification requires gene sequencing at a specialized center. Management is further complicated by extensive drug resistance.

CONCLUSION: NTM infection should be considered in all patients in whom bacterial cultures are negative, especially those with distal extremity involvement, immunodeficiency, or the appropriate environmental exposures. M. immunogenum is a difficult-to-treat pathogen. The optimal treatment for disseminated cutaneous M. immunogenum infection remains unknown and is complicated by extensive drug resistance. Management involves AFB culture, ID consultation, where available, and prolonged courses of multiple antibiotics.

\section{MYCOBACTERIUM TUBERCULOSIS IN A PATIENT FROM AN ENDEMIC REGION. WHAT ELSE COULD IT BE?}

Behnam Laderian; Takaaki Kobayashi; Jason H. Barker. University of Iowa Hospitals and Clinics, Iowa City, IA. (Control ID \#3181737)

LEARNING OBJECTIVE \#1: Differential diagnosis in patients suspected to be infected with mycobacterium tuberculosis

LEARNING OBJECTIVE \#2: Recognize the clinical manifestation, diagnosis and treatment for blastomycosis
CASE: A 33 year-old man from Honduras with no significant past medical history arrived for evaluation of refractory pneumonia. Patient's symptoms of dry cough, malaise, night sweats, and fevers mostly occurring at night started a month prior to presentation. Multiple visits to local emergency room resulted in receiving treatment for community-acquired pneumonia. Patient's symptoms continued despite completing appropriate antibiotics. Upon presentation, computer tomography of the chest was obtained with intravenous contrast revealing extensive pneumonia in the right lung with a collapsed right middle lobe bronchus, extensive mediastinal and hilar lymphadenopathy, and numerous pulmonary nodules. The constellation of patient's symptoms, background, and imaging findings prompted testing for pulmonary tuberculosis (TB). Acid fast bacillus stain of sputum, and PCR were negative for TB. Blastomyces urine antigen was elevated at $4.27 \mathrm{ng} /$ $\mathrm{mL}$. Histoplasma urine antigen was also mildly positive at $1.91 \mathrm{ng} / \mathrm{mL}$ due to cross-reaction. Patient was diagnosed with blastomycosis pneumonia and appropriate treatment was initiated.

IMPACT/DISCUSSION: TB should be considered if a patient from an endemic region presents with fevers, night sweats, weight loss, and respiratory symptoms or refractory pneumonia despite adequate treatment with antibiotics. Once TB has been ruled out, consideration must be given to other causes such as viral infection, fungal infection or even malignancy. Blastomycosis is a systemic pyogranulomatous infection that arises after inhalation of conidia of Blastomyces dermatitidis. The fungus lives in the midwestern, south-central, and southeastern states. The clinical manifestations of blastomycosis include asymptomatic infection, acute or chronic pneumonia, and extrapulmonary disease. Symptoms include cough, sputum production, shortness of breath, weight loss, fever, and night sweats. The lungs are the most common site of infection, followed by the skin, bones, and genitourinary system. Chest imaging may show nodules and consolidation with or without cavitation. While culture and cytopathology remains the gold standard for definite diagnosis, the detection of fungal antigen in urine has proven useful. The sensitivity is 89$93 \%$, whereas specificity is $79 \%$ due to cross-reaction to histoplasmosis. The antigen level is also useful for evaluating response to treatment. For patients with mild to moderate disease, itraconazole is recommended, whereas severe disease requires amphotericin B. CONCLUSION: Blastomycosis can mimic other diseases including community-acquired pneumonia, malignancy or TB and is often misdiagnosed due to the variability of presentation and its relative rarity. A high index of suspicion is needed when the diagnosis is uncertain and patients live in or have traveled to areas endemic for blastomycosis.

\section{MYCOPLASMA PNEUMONIAE INFECTION PRESENTING AS STEVEN-JOHNSON SYNDROME-TOXIC EPIDERMAL NECROLYSIS IN AN ADULT}

Ashley Thompson; Minni Shreya Arumugam; Jivan Lamichhane. SUNY Upstate Medical University, Cayuga, NY. (Control ID \#3185854)

LEARNING OBJECTIVE \#1: Recognize that SJS-TEN is a rare dermatologic manifestation of Mycoplasma pneumoniae infection in the adult population

CASE: A 43-year old man with history of smoking and alcohol abuse, and recent upper respiratory illness presented with altered mental status, rash, and severe oropharyngeal bleeding. The patient denied any medication use. Physical exam was notable for maculopapular rash of abdomen with significant desquamation and hemorrhagic bullae of eyelids, nares, oropharynx, extremities, and glans of penis. Vitals were notable for tachycardia, hypertension, and hypothermia. Labs showed leukopenia, thrombocytopenia, mild transaminitis, high-normal lactate, and electrolyte abnormalities. Chest X-ray noted increased interstitial markings. CT chest was remarkable for tree-in-bud appearance and ground glass opacities. M. pneumoniae PCR was positive, which was confirmed with positive IgM serology. Other infectious work up was negative. The patient was started on Azithromycin and Prednisone and transferred to the ICU 
for management of septic shock. Biopsy revealed erythema multiforme, and Stevens-Johnson Syndrome-Toxic Epidermal Necrolysis (SJS-TEN) overlap syndrome was diagnosed based on body surface area involvement. He eventually underwent a tracheostomy and gastric tube placement. Over five weeks, his respiratory status and cutaneous lesions slowly improved, and he was discharged to a rehabilitation facility.

IMPACT/DISCUSSION: Mycoplasma pneumoniae is a well-known cause of mild and self-limited community acquired pneumonia. Extrapulmonary manifestations, however, are reported to occur in $25-33 \%$ of cases, occasionally severe enough to require ICU level of care. While severe rash and mucositis phenomenon are reported to occur in pediatric infections, SJS-TEN is a rarely documented extra-pulmonary manifestation of M. pneumoniae infections in adults. Alternatively, SJS-TEN observed in the adult population is most commonly postulated to be due to drug reactions. The educational objective of this case report is to highlight that dermatologic manifestations as severe as SJS-TEN may occur with M. pneumoniae infection in adults, which, when recognized early and treated appropriately, can lead to significantly better patient outcomes.

CONCLUSION: This case establishes M. pneumoniae as an uncommon etiology of SJS-TEN spectrum in the adult population. Despite several pediatric reports of severe cutaneous manifestations, such as SJS-TEN, associated with $M$. pneumoniae infection, its presentation in adults is not well illustrated in the literature. As such, consideration of this bacterium in the diagnosis and management of the critically ill patient presenting with SJS-TEN can lead to earlier implementation of crucial and necessary treatment, leading to improved patient outcomes.

\section{MYCOTIC ANEURYSM MASQUERADING AS INFERIOR STEMI}

Sherman $\mathrm{Yu}^{2}$; Sudhi Tyagi ${ }^{1}$; Jorge Saucedo ${ }^{2} .{ }^{1}$ Medical College of Wisconsin, Wauwatosa, WI; ${ }^{2}$ Medical College of Wisconsin, Milwaukee, WI. (Control ID \#3175554)

LEARNING OBJECTIVE \#1: Recognize mycotic coronary aneurysms as a complication of infective endocarditis in high risk hemodialysis patients.

CASE: A 60-year old male with ESRD, IV drug abuse and recent massive gastrointestinal bleed was found unresponsive after completing an outpatient hemodialysis session. EMS was dispatched, discovered an inferior STEMI on EKG and transported him to the hospital. On arrival, the patient's MAP was $50 \mathrm{mmHg}$. Inferior STEMI was confirmed. Troponin was elevated at 0.138 . IV fluids, vasopressors and naloxone were administered, normalizing the patient's somnolence, hemodynamics and derangements on EKG. Soon after, the patient's mental status again waned, requiring intubation. Repeat EKG again showed inferior STEMI. He was then taken to the cardiac catheterization lab on vasopressors. His left coronary circulation revealed atherosclerosis without acute plaque rupture. Angiography of the RCA revealed a $2 \mathrm{~cm} \times 3 \mathrm{~cm}$ ovoid aneurysmatic dilatation near the ostium of the artery which was occluded distally and prevented contrast filling of the RCA. STAT TTE revealed a perforated mitral valve and a possible fistula between the left ventricular outflow tract and left atrium. The patient's MAP dropped to $30 \mathrm{mmHg}$ and CPR was initiated. A prompt multidisciplinary conference was held at bedside and the patient was ultimately deemed a poor candidate for further interventions. He died soon after CPR was discontinued due to medical futility. A subsequent review of records from an outside hospital revealed a history of infective endocarditis (IE), severe mitral valve regurgitation and blood cultures positive for Streptococcus pneumoniae six months prior to presentation.

IMPACT/DISCUSSION: This case highlights the extraordinarily high risk of IE in hemodialysis patients with a history of IV drug use and bacteremia. Bloodstream infections are the second most common cause of mortality among ESRD patients. Such patients are 25 times more likely to develop a bacteremia compared to the general population. Furthermore, the severity of illness in ESRD patients is significantly higher, often resulting in prolonged hospital stays. IE is a serious complication of bacteremia, for which dialysis and IV drug use are independent risk factors. Mycotic coronary aneurysms (MCA) are an extremely rare complication of IE, occurring in less than $0.5 \%$ of such cases. Various mechanisms may lead to its development: infarction of the vasa vasorum, immune complex deposition, and bacterial invasion of the vessel wall. The prognosis is grave, characterized by high morbidity and mortality. In this patient who presented with an inferior STEMI, we suspect a MCA located in the RCA compressed the arterial lumen leading to acute myocardial ischemia in the setting of decreased preload after a dialysis session, further exacerbated by severe mitral valve regurgitation.

CONCLUSION: IE may be complicated by MCAs and should be recognized in high-risk populations including hemodialysis patients with IV drug use history and prior bacteremia.

\section{MYOCARDITIS IN UNCONTROLLED ADULT-ONSET ASTHMA}

Lesley Wu; Misa Hyakutake. Mount Sinai Beth Israel, Manhattan, NY. (Control ID \#3161305)

LEARNING OBJECTIVE \#1: Recognize and promptly treat eosinophilic myocarditis

LEARNING OBJECTIVE \#2: Diagnose eosinophilic granulomatosis with polyangiitis in patients with adult-onset asthma

CASE: A 59-year-old Caucasian man with adult-onset asthma and chronic sinusitis presented to ED with dyspnea that awoke him from sleep. He reported fatigue and dyspnea for a year that significantly worsened in the past month with wheezing upon exertion and dry cough refractory to inhaled steroids. Two weeks prior, patient had an exacerbation that responded well to a short course of oral steroids. He denied chest pain, fever, rash, or edema. Three years prior, he was diagnosed with asthma. Eosinophilia of 1500 was noted for a year without identified cause. Patient had allergies to penicillin and aspirin. He was a never smoker. Vitals and physical exam were unremarkable. Laboratory test showed leukocytosis of 14,400 with eosinophil of 4,500 and troponin of 15 . EKG showed loss of R wave in V1-V4. Suspected for myocardial infarction, patient underwent left-heart catheterization, which showed diffuse non-obstructive disease. Transthoracic echocardiogram showed reduced left ventricular ejection fraction (LVEF) of $38 \%$ from $58 \%$ three years prior. Cardiac MRI revealed diffuse myocardial enhancement with edema. Right ventricle biopsy showed inflammatory infiltrates with prominent eosinophils and myocyte damage consistent with eosinophilic myocarditis. Patient met the diagnostic criteria for eosinophilic granulomatosis with polyangiitis, and intravenous steroids were initiated with immediate symptom alleviation and resolution of eosinophilia and troponin elevation. He was discharged on oral steroids with improvement of LVEF to baseline at one-month follow up. Later, ANCA serology returned within normal values.

IMPACT/DISCUSSION: Adult-onset asthma, dyspnea refractory to asthma treatment, and peripheral eosinophilia should prompt investigation of eosinophilic granulomatosis with polyangiitis.

CONCLUSION: Eosinophilic myocarditis (EM) is a rare yet lifethreatening inflammatory complication that accounts for half of the mortality attributable to eosinophilic granulomatosis with polyangiitis (EGPA). The diagnosis of EM requires biopsy to confirm; however, expeditious initiation of systemic glucocorticoids before results of all investigation are available can minimize permanent myocardial damage. Diagnosing EGPA is often difficult because of the sequential nature of clinical manifestations and low sensitivity of laboratory tests. Although ANCA serology is a pathognomonic workup for EGPA, ANCA is only positive in up to $38 \%$ of the patients. In fact, cardiac involvement is observed more in ANCA negative patients. The presence of adult-onset asthma refractory to appropriate asthma treatment warrants workup for masqueraders of asthma including EGPA. Our case supports literature that 
reported 21 out of 22 EGPA patients had asthma diagnosed during adulthood. Adult-onset asthma could be an additional clue with other clinical findings that raise suspicion for EGPA and EM.

\section{NATIVE VALVE ESCHERICHIA COLI ENDOCARDITIS IN A PATIENT WITH NEWLY DIAGNOSED SYSTEMIC LUPUS ERYTHEMATOUS}

Rachel A. Sibley; Michael Rosman; Verity E. Schaye. NYU Langone Medical Center, New York, NY. (Control ID \#3183518)

LEARNING OBJECTIVE \#1: Identify non-HACEK gram-negative endocarditis early in its clinical course.

LEARNING OBJECTIVE \#2: Recognize the morbidity and mortality of Escherichia coli endocarditis.

CASE: A 54 year-old Hispanic man with no known past medical history presented with one month of constitutional symptoms: unintentional weight loss, anorexia, fatigue, and arthralgias. On admission, he was febrile, tachycardic, and breathing comfortably on room air. The exam was otherwise significant for a thin stature with temporal wasting, thrush, a lateral tongue ulcer, a raised non-blanching erythematous macular rash on sun-exposed areas of the extremities, and erythematous papules on the hands. There were no murmurs detected on cardiac auscultation. Initial labs were significant for anemia and leukopenia. A broad differential diagnosis initially included malignancy, rheumatologic disease, and systemic infection. Work-up revealed positive anti-Smith and anti ds-DNA antibodies, $\mathrm{C} 3 / \mathrm{C} 4$ hypocomplementemia, and a pericardial effusion on transthoracic echocardiogram (TTE). He was diagnosed with systemic lupus erythematous (SLE), and started on hydroxychloroquine and steroids with improvement. On hospital day three, blood cultures grew Escherichia coli (E. coli) in four bottles, with an unclear source with aseptic urine and no localizing symptoms. CT scans of the head, chest, abdomen, and pelvis were notable for multiple peripherally located pulmonary airspace opacities concerning for septic emboli. A TTE was negative for vegetation, but given the high clinical suspicion for endocarditis, notwithstanding the rarity of E. coli as a pathologic organism, transesophageal echocardiogram (TEE) was pursued. TEE revealed a mobile echodensity on the aortic valve consistent with vegetation. The patient completed four weeks of ceftriaxone to treat E. coli endocarditis.

IMPACT/DISCUSSION: E. coli bacteremia is common; however, due to decreased adherence of the organism to the endocardium, infective endocarditis from E. coli is rare, accounting for $0.51 \%$ of cases. Risk factors include immunocompromised states. Our patient was leukopenic from SLE. Sources of infection are often gastrointestinal and urinary. However, as in our patient, initial source is unclear in approximately half of cases. Murmur is often absent, and the disease is more common in native valves than prosthetic or degenerative valves. For these reasons, diagnosis is difficult. One study reported at least one month from onset to clinical diagnosis in $90 \%$ of patients with non-HACEK gram-negative endocarditis. However, given its high surgical intervention rate $(42 \%)$, high complication rate (including heart failure and abscess), and high mortality rate of $21 \%$ (drastically higher than the $4 \%$ from HACEK gramnegative endocarditis), clinicians should maintain a high degree of suspicion to make this diagnosis early.

CONCLUSION: E. coli endocarditis is rare, occurs in immunocompromised patients, and is difficult to diagnose. However, given its high morbidity and mortality, timely recognition is critical.

\section{NECROTIZING FASCIITIS: AN UNUSUALLY COMMON PRESENTATION}

Claire Drolen; Emily Sandberg. University of Pennsylvania, Philadelphia,

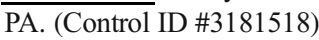

LEARNING OBJECTIVE \#1: Recognize early clinical presentations of necrotizing fasciitis $(\mathrm{NF})$
LEARNING OBJECTIVE \#2: Incorporate tools to assist in diagnosis of early $\mathrm{NF}$

CASE: A 62-year-old man presented after a syncopal episode. He endorsed two months of weight loss, fevers and night sweats. Past medical history included atrial flutter, prostate cancer with recent brachytherapy, and a recent pulmonary embolism (PE) on Rivaroxaban. Physical exam was notable for fever, tachycardia, and bilateral pitting edema to mid-shin. Labs showed elevated NTproBNP; ECG, echocardiogram and telemetry were unrevealing. CT chest revealed a small, acute PE for which he was started on heparin. On hospital day two he developed scrotal swelling and erythema; exam revealed pitting edema without crepitus or tenderness to palpation. Scrotal ultrasound showed cellulitis for which he was started on Vancomycin and Cefepime given continued fevers. On hospital day four he developed worsening fevers, tachycardia, and increased scrotal swelling, pain and discoloration. CT pelvis showed gas throughout the scrotum and perineum. Antibiotics were changed to Vancomycin, PiperacillinTazobactam and Flagyl. He underwent emergent surgical debridement and was found to have a rectoprostatic fistula complicated by Necrotizing Fasciitis (NF). He was discharged one month later and is expected to make a full recovery.

IMPACT/DISCUSSION: NF is a rare, life-threatening infection of the skin, fascia and subcutaneous tissue, with in-hospital mortality as high as $34 \%$. It is typically polymicrobial; E. Coli and Staph species are isolated in most cases. Early recognition and debridement are major determinants of outcome, however diagnosis is often delayed due to nonspecific initial presentation. Classically NF is red, swollen, frankly necrotic, and exquisitely painful. While fulminant cases can present like this, subacute cases are often less obvious with non-specific fever, fatigue and skin changes that can be mistaken for cellulitis or dependent edema. Pathognomonic findings (bullae, crepitus) occur late and in less than $25 \%$ of patients. Localized anesthesia can replace pain if nerve damage has occurred, masking the classic pain out of proportion to exam. Existing risk tools have high diagnostic accuracy and can assist in differentiating NF from other soft tissue infections. Had we used one for our patient, we would have found a $50-75 \%$ risk of NF which may have prompted earlier CT imaging and diagnosis. Diabetes, immunocompromise, trauma and local instrumentation, such as our patient's recent brachytherapy, should serve as red flags. Cross-sectional imaging is the diagnostic study of choice; antibiotics and surgical debridement are definitive treatment.

CONCLUSION: Early, non-fulminant NF can present nonspecifically, without skin necrosis or pain out of proportion to exam. A high index of suspicion and use of diagnostic tools facilitate timely diagnosis of sub-acute NF.

\section{NEVER TOO OLD FOR MYASTHENIA}

Kyle T. Jones; Roshini Pinto- Powell. Dartmouth Medical School, Hanover, NH. (Control ID \#3181205)

LEARNING OBJECTIVE \#1: Recognize the changing incidence of myasthenia gravis

LEARNING OBJECTIVE \#2: Identify cognitive biases that result in delayed diagnosis or misdiagnosis

CASE: A 74-year-old with a history of asthma, 5 weeks post hip arthroplasty, presented to the ED with 2 days of eye-lid droop, dysarthria and shortness of breath. She noted getting winded when speaking. The rest of her review of symptoms was negative. Post-op course had been uneventful, however she began taking aspirin 3 days prior to presentation. FHx was unremarkable. Meds: aspirin, Symbicort and Albuterol. Allergic to sulfa drugs. On Exam: Afebrile, HR 83, BP 148/61, RR 22, SpO2 99\%. Mild tongue swelling, left eyelid droop, mild periorbital edema. Lungs clear, no wheezes. Cranial nerves intact, mild dysarthria, normal strength in all extremities. She was thought to have angioedema and asthma exacerbation due to aspirin allergy. Flexible laryngoscopy showed no laryngeal edema. She improved with epinephrine, Benadryl, and IV solumedrol, and was discharged on a 6-day steroid taper, cetirizine, 
Benadryl, and a follow-up with Allergy. Five days later, the patient came to GIM clinic with worsening symptoms, including bilateral eyelid droop worse as the day progressed, hoarseness, difficulty swallowing, and SOB. Exam showed bilateral ptosis, and difficulty sustaining upward gaze. Neurology was consulted for suspected myasthenic crisis and the patient was admitted and started on IVIG and pyridostigmine. Nerve conduction studies showed $20 \%$ decrement in the right accessory nerve on repetitive stimulation. AChR binding antibody was $5.82 \mathrm{nmol} / \mathrm{L}$ (normal $<=$ $0.02 \mathrm{nmol} / \mathrm{L}$ ). CT chest did not show a thymoma. Her symptoms improved and she was discharged on hospital day 5 .

IMPACT/DISCUSSION: The classic bimodal epidemiology for Myasthenia Gravis is young women and older men. However, as this case illustrates, the incidence of $\mathrm{MG}$ is changing, with an increase in late onset MG (LOMG). Reasons for this changing epidemiology are unclear and may reflect an aging population, improved diagnostics, and environmental factors. LOMG can be difficult to diagnose and requires a high index of suspicion. Studies suggest that ocular MG is more common in LOMG, as seen in this patient. The initial diagnosis in this case demonstrates common cognitive biases that result in misdiagnosis and delayed care. The temporal relationship of symptoms to starting aspirin created a nidus for anchoring bias to an aspirin-related pseudoallergic reaction. Furthermore, the perpetuation of this idea between the treating physicians created diagnostic momentum. On review of the patient's initial symptoms, she had classic signs of ocular and bulbar myasthenia: bilateral ptosis, dysarthria and SOB with speech.

CONCLUSION: The incidence of late onset MG is increasing and should be considered in older patients. Clinicians are subject to cognitive biases that in hindsight are clear. Reviewing cases and exposing these biases can help practitioners become mindful of cognitive errors in an attempt to reduce misdiagnoses.

\section{NEW-ONSET SEIZURES: LOOK FOR A PRECIPITANT, PER- HAPS A DRUG?}

Mohamed Gabr; Radhika Gali; Dharmarajan S. Thiruvinvamalai. Montefiore Medical Center (Wakefield Campus), Bronx, NY. (Control ID \#3183364)

LEARNING OBJECTIVE \#1: Identify the etiology of new-onset seizures

LEARNING OBJECTIVE \#2: Recognize the culprit medications, including SSRIs, that may precipitate seizures

CASE: A 75 year-old man presented after a first-time, witnessed, generalized seizure. Medical history included hypertension and depression; negative for prior strokes and personal or family history of seizures. Only known medications were aspirin, simvastatin and fluoxetine $40 \mathrm{mg}$ daily. $\mathrm{He}$ did not recall symptoms or events that led to the seizure; denied alcohol or drug use. Neurological exam revealed intact motor strength, sensation and cranial nerve functions. His gait was unsteady and the finger-to-nose test was abnormal. Urine and blood toxicology were negative, including ethanol levels. Blood glucose and electrolyte levels were normal, and infectious work-up was negative. CT and MRI of the head revealed chronic microvascular ischemic changes and an old right cerebellar infarct. Transthoracic echocardiogram was normal; carotid ultrasound was negative for stenosis. An electroencephalogram (EEG) did not reveal epileptiform discharges. Fluoxetine was switched to sertraline prior to discharge due to its potential to lower seizure threshold.

IMPACT/DISCUSSION: In the initial evaluation of new-onset seizures, physicians must address personal and family history, history of head trauma, central nervous system (CNS) lesions or infections, childhood seizures, birth injury, and conduct a review of medications. Causes include hypoxia, hypoglycemia, electrolyte abnormalities, infections, drug / alcohol abuse and more. Brain MRI should be performed to rule out structural lesions. EEG helps assess for epileptic seizures, though the likelihood of routine EEG recording epileptiform discharges is $25-50 \%$. Lumbar puncture is done when CNS infection is a possibility. Several medications may lower seizure threshold, including bupropion, tramadol, quinolones, imipenem, penicillins, meperidine, and SSRIs. SSRIs have the ability to induce seizures, especially with chronic use and in higher doses; the exact mechanism is unknown. Fluoxetine, with a long half-life, should be used with caution in the elderly and in those with kidney disease.

CONCLUSION: In our patient's case, his only known medications were aspirin, simvastatin, and fluoxetine $40 \mathrm{mgs} /$ day. Prolonged use of fluoxetine in a high dose was acknowledged as a potential inciting agent for his seizure. Drugs must be a consideration as inducing factors with new onset seizures, warranting meticulous screening of medications.

\section{NIFEDIPINE-INDUCED SHOCK IN 18 WEEK GRAVIDA WITH CONGENITAL AORTIC STENOSIS}

Cullen Soares; Michael Gilson. Brown University, Providence, RI. (Control ID \#3183795)

LEARNING OBJECTIVE \#1: Understand the clinical importance of diagnosing aortic stenosis in pregnancy

CASE: The patient is a 31 year-old G6P2032 at 18 weeks with a history significant for moderate stenosis secondary to a bicuspid aortic valve. Her current twin pregnancy was uncomplicated until 2 weeks prior to admission when she was diagnosed with twin-twin transfusion syndrome (TTTS). On her day of presentation she had an uncomplicated ablation of placental vessels for TTTS. She received two doses of short-acting $20 \mathrm{mg}$ nifedipine over 30 minutes (standard prophylactic tocolytic dose) and developed hypotension (BP 80/40), chest pain, and diffuse ST segment depressions on ECG. She was started on intravenous phenylephrine and transferred to the cardiac care unit. Exam was notable for a harsh mid-peaking systolic murmur radiating to the carotids, elevated venous pressures, and normal perfusion. Phenylephrine was switched to norepinephrine and she received two liters of lactated ringers with calcium gluconate. Bedside echocardiogram showed hyperdynamic ventricular systolic function (Ejection Fraction $78 \%$ ) and moderate aortic stenosis. Lactate was normal, and peak troponin was 4.2. Indomethacin was started for tocolysis. Over 8 hours, blood pressures improved to baseline and she was weaned off pressors. ECG normalized and chest pain resolved. She was discharged to home with follow up with high risk pregnancy clinic and cardiology.

IMPACT/DISCUSSION: Dihydropyridine calcium channel blockers cause peripheral vasodilation, reduced cardiac contractility, and reduced aldosterone production, which together reduce blood pressure. Nifedipine is the preferred tocolytic due to its efficacy in suppressing uterine contractions. It is also the most potent dihydropyridine antihypertensive. Its use in tocolysis is common and generally welltolerated, but studies show it still lowers blood pressure when used for tocolysis. Heart failure and mortality are rare in pregnant women with moderate aortic stenosis (AS). The hemodynamic changes of pregnancy, including increased cardiac output (CO) and decreased systemic vascular resistance (SVR) make this population particularly sensitive to nifedipine. Our patient had compensated for these changes but could not further compensate when stressed by nifedipine due to her stenotic valve. Because obstructive coronary disease is unlikely in this young woman, her ECG changes and troponin elevation can be attributed to global ischemia due to increased myocardial oxygen demand/supply.

CONCLUSION: Nifedipine should be avoided in patients with AS, especially in pregnant women due to their baseline higher $\mathrm{CO}$ and lower SVR. There should be a low threshold to echocardiographically assess pregnant women with exam findings suggesting AS. They should receive alternatives for tocolysis if/when required.

\section{NITROUS OXIDE TOXICITY: NO LAUGHING MATTER}

Nikita Donti; Hiba Sayed; John H. O'Neill. Christiana Care Health Systems, Wilmington, DE. (Control ID \#3178606) 
LEARNING OBJECTIVE \#1: Recognize the clinical features of B12 depletion

LEARNING OBJECTIVE \#2: Recognize the relationship between nitrous oxide poisoning and severe B12 depletion

CASE: A 23-year-old female Chinese university student with a one-year history of inhaled nitrous oxide (N2O) abuse in the form of "whippets" was brought to ER for progressive bilateral lower extremity weakness, dysesthesia, inability to ambulate, altered mentation, and urinary retention. Alcohol intake was infrequent. On exam, she was found to have decreased sensation distal to the ankles, inability to lift her lower extremities off of the bed, inversion of both feet, and $+0 / 5$ dorsiflexion and plantar flexion. No Babinski sign, clonus, or fasciculations were noted. Deep tendon reflexes were absent. Initial labs revealed a macrocytic anemia and B12 level was mildly decreased at $228 \mathrm{pg} / \mathrm{mL}$. She was started on IM cyanocobalamin. A neuropathy workup was negative for HIV, syphilis, lyme disease, and heavy metal poisoning. Glucose, TSH, SPEP, hepatitis antibodies and ANA were normal. Methylmalonic acid (MMA) was normal at $0.21 \mathrm{nmol} / \mathrm{L}$, however homocysteine was markedly elevated at $97.3 \mathrm{mcmol} / \mathrm{L}$. MRI of the spinal cord showed pathologic enhancement of the C1-C6 dorsal columns, concerning for subacute combined degeneration. The patient had gradual clinical improvement of her lower extremity strength and sensation. She was ultimately discharged to a rehabilitation facility.

IMPACT/DISCUSSION: Nitrous oxide poisoning converts B12 from its active monovalent form to an inactive bivalent form, resulting in permanent inactivation of methionine synthase and thus inhibiting the normal production of myelin. Dorsal and lateral column spinal cord demyelination, classically known as subacute combined degeneration (SCD), can result in a severe peripheral neuropathy. Since SCD can also be seen in HIV, copper deficiency, and severe hepatic disease, these causes should be ruled out. Clinical symptoms may include paresthesia, abnormal proprioception, progressive weakness, spasticity, clonus, paraplegia, and urinary and fecal incontinence. Ofnote, the B12 blood level is not indicative of the extent of B12 depletion at the tissue level. Interestingly, the MMA level, which is typically elevated in B12 deficiency, was normal in our patient. For these reasons, this was a clinically difficult diagnosis. Because the relationship between $\mathrm{N} 2 \mathrm{O}$ toxicity and Vitamin B12 depletion can result in grave neurological complications, it is important to recognize these clinical features and begin treatment promptly.

CONCLUSION: Nitrous oxide toxicity leads to inactivation of Vitamin B12, causing subacute combined degeneration of the spinal cord and peripheral neuropathy. The blood level of B12 is not always indicative of the extent of B12 depletion at the tissue level. It is important to recognize the clinical findings associated with Vitamin B12 depletion so that treatment may be started promptly.

\section{NIVOLUMAB INDUCED ADRENAL INSUFFICIENCY (AI): RARE SIDE-EFFECT OF A NEW ANTI-CANCER THERAPY - IMMUNE-CHECKPOINT INHIBITORS}

Maitreyee Rai ${ }^{1}$; Mylene $\mathrm{Go}^{2} .{ }^{1}$ Crozer Chester Medical Center, Brookhaven, PA; ${ }^{2}$ Crozer Chester Medical Center, Upland, PA. (Control ID \#3181657)

LEARNING OBJECTIVE \#1: Recognise \& diagnose a serious sideeffect of the new immune-checkpoint inhibitor (ICPI) drugs

LEARNING OBJECTIVE \#2: Manage a serious side-effect of the ICPIS

CASE: 77 y old male, past medical history of renal cell carcinoma(RCC) status post right nephrectomy, metastatic to lungs, chronic kidney disease, syndrome of inappropriate anti-diuretic hormone, hypertension, presented to the office with fatigue, loss of appetite and dizziness. His blood pressure (BP) was noted $76 / 44 \mathrm{mmHg} \&$ referred to the emergency department(ED). In ED, his BP was $96 / 50 \mathrm{mmHg}$, heart rate $72 /$ minute \& temperature $97.3^{\circ} \mathrm{F}$. He received several boluses of intravenous(IV) fluid, however, BP consistently stayed in $90 \mathrm{~s} / 40$ s. His examinations was significant for orthostatic hypotension. Patient reported being on Nivolumab for metastatic RCC for last 6 months, and the current symptoms started after the last dose given 2 weeks ago. The labs showed low sodium at128 (baseline $133-139 \mathrm{mmol} / \mathrm{L}$ ), creatinine elevated 2.7 (baseline $1.1-1.4 \mathrm{mg} / \mathrm{dL}$ ). In view of the hypotension not responding to fluids, a morning cortisol level was checked to look for possible adrenal insufficiency (AI) due to Nivolumab. It was low $1.3 \mathrm{mcg} / \mathrm{dL}$. Endocrinology was consulted. Cosyntropin stimulation test revealed low basal cortisol $1 \mathrm{mcg} / \mathrm{dL} \&$ only a mild increase to $10.20 \mathrm{mcg} / \mathrm{dL}$ after cosyntropin administration, findings suggestive of AI. To differentiate between primary or secondary cause, adrenocorticotrophic hormone level (ACTH) was checked that was low $<5 \mathrm{pg} / \mathrm{mL}$, favoring a diagnosis of secondary AI, deemed likely due to hypophysitis from Nivolumab. T4 \& TSH were found to be normal. In the meantime, the patient was started on intravenous (IV) hydrocortisone, that improved his hypotension \& acute kidney injury significantly, eventually weaned to oral hydrocortisone. The nivolumab was discontinued and oncology decided to give a nivolumab re-challenge once patient stabilizes.

IMPACT/DISCUSSION: Patient presented with manifestations of AI (fatigue, hypotension, hyponatremia) which is a rare Immune-related Adverse Event (iRAE) occurring in $<1 \%$ of patients on ICPIs. These are nonspecific manifestations and can be easily overlooked if iRAE is not suspected. AI is a life-threatening side-effect of ICPIs that needs to be recognized immediately and managed with IV glucocorticoids. With the increased use of these drugs in the management of advanced cancers, there is an increased number of patients presenting to offices \& EDs with complications due to iRAEs. Therefore, as internists it's imperative to be able to diagnose and intervene at an early stage to prevent serious outcomes that may result in discontinuation of effective anti-cancer therapy.

CONCLUSION: ICPIs like Nivolumab, Pembrolizumab, Ipilimumab are increasingly being used for advanced cancers (melanoma, RCC, nonsmall cell lung cancer). Checkpoint inhibition comes with a wide array of side effects known as irAEs, affecting pulmonary, gastrointestinal, endocrine systems. Early recognition and management can prevent serious outcomes.

\section{NIVOLUMAB INDUCED HYPOPHYSITIS: A RARE CASE REPORT}

Smile Kajal ${ }^{2}$; Ankit Agrawal ${ }^{1}$; Raj Moses ${ }^{3} .{ }^{1}$ Rutgers Robert Wood Johnson Medical School/Saint Peter's University Hospital, New Brunswick, $\mathrm{NJ} ;{ }^{2}$ All India Institute of Medical Sciences, New Delhi, India; ${ }^{3}$ Allegheny General Hospital, Pittsburgh, PA. (Control ID \#3159344)

LEARNING OBJECTIVE \#1: Recognize subtle clinical clues of immunotherpay induced hypophysitits.

CASE: A 84-year old Caucasian female was diagnosed with well differentiated adenocarcinoma of the left upper lobe of lung in 2009. She underwent left upper lobe wedge resection and mesh brachytherapy in November 2009. She was started on systemic chemotherapy with pemetrexed and carboplatin. The treatment response was monitored with CT imaging of chest, abdomen and pelvis. Her CT imaging in January 2012 showed disease recurrence at the wedge resection site. She completed her systemic chemotherapy with pemetrexed and carboplatin in March 2012 and was started on oral erlotinib which was later discontinued in January 2016 due to severe gastrointestinal upset. Nivolumab immunotherapy was initiated in March 2016. The patient had completed 11 cycles of nivolumab by October 2016. At this time, she complained of intense fatigue and excessive sleepiness. Laboratory investigations revealed hypothyroidism and levothyroxine was started. She completed 14 cycles of nivolumab immunotherapy by November 2016 without any symptomatic improvement and now had additional complains of frequent headaches, lightheadedness and nausea. Adrenocorticotropic hormone $(\mathrm{ACTH})$ stimulation test was orderd and the result confirmed 
central adrenal insufficiency. Glucocorticoid therapy was started and significant clinical improvement was noted on follow-up clinic visit.

IMPACT/DISCUSSION: Hypophysitis is an acute or chronic inflammation of pituitary gland. It is a rare condition and the incidence has not been quantified. Immunotherapy associated hypophysitis often presents with headache and anterior hypopituitarism. Anterior hypopituitarism shows a characteristic but atypical pattern of deficiency of ACTH followed by thyroid stimulating hormone (TSH), gonadotrophins and prolactin deficiency or hyperprolactinemia. In a study conducted at MD Anderson Cancer Center in Houston, immunotherapy induced hypophysitis was defined as 1 pituitary hormonal deficiencies (either ACTH or TSH) plus MRI abnormality OR 2 pituitary hormonal deficiencies plus headache or MRI abnormality. Our patient had developed hypothyroidism and central adrenal deficiency. MRI of brain was also obtained which did not show any significant finding but she did report headaches, excessive sleepiness and nausea. Therefore, she was diagnosed with nivolumab induced hypophysitis based on clinical grounds and abnormal hormonal profile. She was started on glucocorticoids to which she responded well symptomatically further supporting our diagnosis.

CONCLUSION: Immunotherapy induced hypophysitis is a rare entity. This is the first case of nivolumab induced hypophysitis in a patient with recurrent non squamous non small cell lung carcinoma. Due to rarity of this condition, a high degree of suspicion and a multimodal approach is required for it's timely diagnosis.

\section{NO ALL HEADACHES ARE MIGRAINES}

Priyanka Majety; Seda Babroudi. Tufts Medical Center, Boston, MA. (Control ID \#3183546)

LEARNING OBJECTIVE \#1: The significance of evaluating red flags in cases of acute headache.

LEARNING OBJECTIVE \#2: Importance of a high clinical index of suspicion for diagnosing cavernous sinus thrombosis.

CASE: A 20-year-old African American female with past history of migraine presented to us with a 9-day history of left-sided throbbing headache associated with nausea, similar in presentation to prior migraine exacerbations but had negligible relief with NSAIDs. She was afebrile with normal complete blood count and was treated with intravenous pain medications including NSAIDs and anti-emetics with only minimal relief. She was diagnosed with "Status migrainosus" that was relieved after multiple IV pain medications, Sumatriptan and Magnesium sulfate and eventually was discharged. Two days later, she re-presented with highgrade fever, blurry vision in her left eye and WBC was elevated to 32,000. She was started on antibiotics for bacterial meningitis \& a CT head showed extensive left sided maxillary, sphenoidal and ethmoidal sinus disease. Lumbar puncture studies were consistent with bacterial meningitis. Cultures from blood and ethmoid sinus grew Streptococcus constellatus sensitive to Ceftriaxone. Upon careful examination, leftsided $6^{\text {th }}$ cranial nerve palsy (limited abduction by Lateral rectus) was noted and she underwent MR Venography that confirmed the diagnosis of cavernous sinus thrombosis with left-sided ophthalmic vein thrombosis, likely in the setting of infection.

IMPACT/DISCUSSION: This is a case of septic cavernous sinus thrombosis (CST) that underscores the importance of a systematic approach and the evaluation of the red flags of headache with detailed neurologic exam in cases of acute headache. The prevalence of migraine in the community is high $(15 \%)$ \& occasionally life-threatening diseases like CST can masquerade as an acute migraine attack. Attention to the danger signs including systemic symptoms (fever, weight loss), abnormal neurologic signs, new onset of headache (especially for age $>50$ ), previous history of headache that has changed in severity and history of trauma is critical, necessitating neuroimaging. Given its location, $6^{\text {th }}$ cranial nerve is the first nerve compressed with increased intracranial pressure. Clinical presentation of CST is highly variable with acute, subacute or chronic onset and can resemble migraine. Infections are responsible only for 6-12\% of cases but are associated with high mortality (30\%) and morbidity (30\%), warranting an early diagnosis. Neuroimaging is valuable in diagnosing CST. The mainstay of treatment is antibiotics, surgical drainage along with anticoagulation, although there is limited data on anticoagulation given the rarity of the condition.

CONCLUSION: Red flags need to be assessed in every case of headache. CST needs to be considered in patients with sinusitis and cranial nerve palsies. Early diagnosis and treatment are essential. Randomized controlled trials are needed to determine the optimal therapy for this condition

\section{NO KIDDING AROUND: A CASE OF CUTANEOUS PARAPOX VIRUS INFECTION}

Michael George; Andrea Carter. University of Pittsburgh Medical Center, Pittsburgh, PA. (Control ID \#3164946)

LEARNING OBJECTIVE \#1: Avoid premature closure in patients with intravenous drug use (IVDU).

LEARNING OBJECTIVE \#2: Recognize the typical presentation of cutaneous zoonotic infections.

CASE: A 23 year-old woman with a history of opioid use disorder presented with one day of right hand swelling and redness. She was otherwise asymptomatic and afebrile. The patient had been abstinent from IVDU for one year, and her urine drug screen was consistent with use of prescribed buprenorphine without any illicit substances. A point-of-care ultrasound was performed in the Emergency Department and due to concern for thrombophlebitis, broad spectrum antibiotics were initiated for presumed septic thrombophlebitis caused by relapsed IVDU. On arrival to the medicine floor, examination of right hand demonstrated two isolated vesicular lesions on the index finger with associated erythema and swelling of the hand. Formal venous dopplers of the right upper extremity were negative for thrombus. Further review of history found that patient had been to a county fair several times over the previous week and had been petting animals including sheep and goats. Dermatology performed a punch biopsy of the ulcer which showed intraepidermal vesicles and a mixed cellular infiltrate without evidence of bacteria or neutrophilic predominant infiltrate seen with typical bacterial cellulitis. Based on the patient's history of animal exposure, biopsy findings, and the typical appearance of the lesion, a presumptive diagnosis of parapox virus was given. Antibiotics were discontinued, and after one day of observation, the patient was discharged home.

IMPACT/DISCUSSION: Parapox (or Orf) virus is a pathogen belonging to the Poxviridae family. It is generally limited to cutaneous infection, although patients may experience fever or lymphangitis and immunocompromised hosts are at risk for disseminated or "giant" pox. The true prevalence of Orf is unknown and is likely limited by limited recognition and incomplete reporting. Transmission occurs from direct contact with a lesion on a vector-typically sheep or goats, and can be facilitated by any damage to the skin barrier. The hallmark of cutaneous infection is a vesicular lesion, often solitary or with 1-2 satellite lesions that progress over weeks to develop a necrotic center and finally dry out to create a papilloma. These lesions typically resolve without treatment within 6 weeks. Confirmatory testing may be performed by examination under electron microscopy or polymerase chain reaction but with limited availability and expense may not be feasible and is unlikely to alter clinical course.

CONCLUSION: This case highlights the distinguishing historical and physical exam features of the cutaneous zoonotic infection parapox virus. This case serves as a reminder of the importance of taking a thorough history and avoiding premature closure, especially in the context of the national opioid epidemic and associated biases clinicians may have when treating patients with opioid use disorder. 
NONSTOP RUNNY NOSE: A CASE OF SPONTANEOUS CSF RHINORRHEA COMPLICATED BY MENINGITIS

Thien Le ${ }^{1,2}$; Carlos Aguirre ${ }^{1,2}$; Brian Pulford ${ }^{1,2}$; Ruta Viktoria Totoraitis ${ }^{1,2}$; Charles W. Graeber ${ }^{1,2}$. ${ }^{1} \mathrm{NCH}$ Healthcare Systems, Naples, FL; ${ }^{2}$ Mayo Clinic School of Medicine and Science, Rochester, MN. (Control ID \#3185109)

LEARNING OBJECTIVE \#1: Managing acute meningitis in the setting of spontaneous cerebrospinal fluid leak

CASE: A 53-year-old Hispanic mother of 5 children with no past medical history presented to our emergency department with headaches. Over the last 10 months she suffered labile headaches, cough and unilateral left sided rhinorrhea. Previously, these headaches were well controlled with acetaminophen and ibuprofen. However, 2 days prior to admission, she experienced subjective fevers, chills, nausea and vomiting. There was no history of foreign travel or head trauma. She went to see an Otolaryngology (ENT) doctor and was diagnosed with allergic rhinitis, treated with steroid nasal spray and recommended for allergy testing. Her headaches were exacerbated with standing and resolved completely when lying flat. She reported no phonophobia, photophobia or vision changes. She was febrile at 102.6F, otherwise vital signs were normal at admission. Laboratory results including a complete blood count, basic metabolic panel and urinalysis were within normal limits. Computerized Tomography (CT) of the head ordered in the emergency department showed intracranial hypertension and a lumbar puncture (LP) was deferred. Empiric antibiotics and antiviral coverage was initiated. Her MRI was negative for intracranial mass lesion and a LP was performed which was consistent with acute meningitis post initiation of antibiotic. A ENT consult was placed for nasal endoscopy which show no visible evidence of CSF leakage. Ultimately, due to the unrelenting nature of her headaches we elected to pursue further investigation of her unilateral nasal fluid leak. Our Neurologist recommended nasal rhinorrhea fluid collection and analysis for Beta2-transferrin which positively identified CSF. Additional imaging with high resolution CT including bone views identified a cribriform plate depression versus questionable fracture with soft tissue extension into the posterior fossa. A consultation with neurosurgery was made and she was transferred for higher level of care with continuation of Ceftriaxone for 2 weeks and neurosurgery to follow.

IMPACT/DISCUSSION: Spontaneous cerebrospinal fluid (CSF) rhinorrhea remains a relatively rare occurrence, incidence of 3-4\% in all cases of CSF leaks (Beckhardt, 1991). Obese females in their $5-6^{\text {th }}$ decades of life are more likely to experience spontaneous CSF nasal leaks (Sayed MSU, Dunn CJ, Alaani A, 2012). Due to the potential complication of meningitis, spontaneous or traumatic CSF leaks requires a keen eye for detection and prompt treatment.

CONCLUSION: Originally postulated by Roman physician Galen during the first century A.D, CSF leak carries substantial risk for meningitis and death (Decherd, 1999). Identification of compromise to the blood brain barrier should be consider when investigating cases of meningitis. Unilateral rhinorrhea with reported changes in volume leak in relation to positional changes should be further investigated with high resolution CT imaging and appriopriate consultation services.

\section{NOT 'JUST A PHLEBITIS': A CASE OF ENDOGENOUS METHICILLIN-RESISTANT STAPHYLOCOCCUS AUREUS ENDOPHTHALMITIS.}

$\underline{\text { Steven Omansky }}^{1}$; Patricia Dharapak ${ }^{2} .{ }^{1}$ Mount Sinai Beth Israel Hospital, New York, NY; ${ }^{2}$ Mount Sinai Beth Israel, New York, NY. (Control ID \#3162820)

LEARNING OBJECTIVE \#1: To identify the common presentations, risk factors, and treatments for bacterial endophthalmitis.

CASE: A 69 year-old female with uncontrolled diabetes mellitus and hypertension was referred from an affiliate eye clinic for further management of suspected endophthalmitis. One week before presentation, she was discharged from an outside hospital where she was admitted for suspected transient ischemic attack. Her family reported that she was febrile prior to discharge, and her peripheral IV site was erythematous and mildly purulent. The IV was removed and she left without medical therapy. Five days later, she developed weakness with worsening visual acuity and pain in the left eye, and after two days of symptoms she visited the eye clinic. She now presents with a fever of $101^{\circ} \mathrm{F}$, left periorbital edema, marked left eye injection, and chemosis but no remaining phlebitis. Labwork was notable for a WBC count of $15 \mathrm{u} / \mathrm{L}$ with neutrophilia. Empiric IV vancomycin and piperacillin-tazobactam were started, and blood cultures later grew methicillin-resistant Staphylococcus aureus (MRSA). Urine culture and chest $\mathrm{x}$-ray were unremarkable, and transesophageal echocardiogram showed no vegetations. Two vitrectomies and several intravitreal antibiotic injections lessened her swelling and pain but did not improve her sight. Her vitreous cultures remained sterile. She completed a 14-day course of IV antibiotics and was discharged with close ophthalmology follow-up.

IMPACT/DISCUSSION: We describe a case of endogenous MRSA endophthalmitis, suspected to be secondary to metastatic seeding from a peripheral IV-associated phlebitis. Endophthalmitis, a bacterial or fungal infection within the eye, is a sight-threatening emergency. Most cases are exogenous as a consequence of trauma, eye surgery, or as an extension of keratitis. Endogenous bacterial cases in the US are uncommon but may result from bacterial seeding of the eye by an endocarditis, urinary tract infections, abdominal abscesses, indwelling catheters, injection drug use, or procedures associated with transient bacteremia. Endogenous endophthlamitis is diagnosed when there are consistent ocular findings in the setting of bacteremia or by positive vitreous or aqueous cultures in patients without direct transmission. Although patients may present with signs of sepsis, less than $20 \%$ present with fever, and many patients present only with eye pain and decreased vision. Treatment includes systemic and intravitreal antibiotics, usually accompanied by vitrectomy. Clinical outcomes, including sight retention, are affected by the timeliness of appropriate antibiotic therapy and the virulence of the pathogen.

CONCLUSION: Acute endogenous bacterial endophthalmitis is a sightthreatening emergency. Given that such patients may have intermittent bacteremia, serial blood cultures and a careful assessment of nosocomial risk are essential adjuncts to optimal ophthalmologic management of those presenting with ocular symptoms in the absence of recent eye trauma or surgery.

\section{NOT FOR HUMAN CONSUMPTION!}

Manojna Konda; Heather Ali; Kinza Ahmed; Susan Beland. University of Arkansas for Medical Sciences, Little Rock, AR. (Control ID \#3186169)

LEARNING OBJECTIVE \#1: Recognize the abuse potential and adverse effects of phenylbutazone, especially in patients with access to livestock

LEARNING OBJECTIVE \#2: Recognize the importance of thorough medication reconciliation

CASE: A 41-year-old previously healthy female presented with 2 weeks duration of nausea, vomiting, generalized weakness. The patient lived on a farm and also reported a tick bite about 1 week prior to the development of her symptoms. Her physical examination was remarkable for dry mucous membranes and tenderness to palpation of RUQ of her abdomen. Laboratory tests revealed pancytopenia with white count of $2.20 \mathrm{~K} / \mathrm{ul}$ with ANC of $1 \mathrm{~K} / \mathrm{ul}, \mathrm{HgB} 10.1 \mathrm{~g} / \mathrm{dl}$, platelets of $48 \mathrm{k} / \mathrm{ul}$. Her BMP was significant for Na $128 \mathrm{mmol} / \mathrm{l}$, Creatinine $1.7 \mathrm{mg} / \mathrm{dl}, \mathrm{CO} 2: 15 \mathrm{mmol} / \mathrm{L}$. LFTs were elevated with AST 617 IU/L, ALT: 211 IU/L, LD: 1039 IU/L, GGT: 54 IU/L, ALP: 81 and Total Bilirubin 0.5. Lactate, procalcitonin, hepatitis, HIV tests were negative. Coagulation panel was within normal limits. Peripheral smear was negative for schistocytes. Blood cultures were obtained and she was started on intravenous fluids and broad spectrum antibiotics including doxycycline to cover for tick borne diseases. She 
was also started on NAC protocol for possible tylenol toxicity. Ultrasound abdomen showed mild splenomegaly and normal examination of the liver. Urine drug screen was negative. Blood cultures and tests for tick borne disease remained negative. The patient later confessed to taking her "horse's bute" i.e. phenylbutazone which was her horse's painkiller for back pain after she sprained her back and was most likely the cause of her presentation. Antibiotics were discontinued. Her blood counts, creatinine and LFTs improved with hydration.

IMPACT/DISCUSSION: Impact: This case illustrates the adverse effects of taking phenylbutazone, including pancytopenia, elevated liver enzymes and kidney injury. It also demonstrates that taking a good history and performing a thorough medication reconciliation can guide the diagnosis. Phenylbutazone, commonly known as "bute", is an NSAID which was originally introduced in the early 1950s as one of the "wonder drugs" to treat rheumatoid arthritis and gout. Eventually in the early 1980s, it was banned from human use due to its severe side effects. However, it continues to be used in veterinary medicine as a pain killer. The most serious adverse effects are related to bone-marrow resulting in pancytopenia and aplastic anemia. Other serious reactions include gastric ulceration with bleeding, hepatitis, renal failure, heart failure, respiratory and/ metabolic acidosis, coma and death. The mainstay of treatment is aggressive supportive care.

CONCLUSION: Commonly called as "Bute", while it is not currently available for human consumption, it is still available for use in livestock. It is important for clinicians to recognize the side effects and also realize the abuse potential in patients with access to livestock.

\section{NOT JUST FOR KIDS: MECKEL'S DIVERTICULUM CAUSING ACUTE LOWER GASTROINTESTINAL BLEEDING IN AN ADULT}

Iris Huang. University of California, San Francisco, San Francisco, CA. (Control ID \#3183679)

LEARNING OBJECTIVE \#1: Recognize Meckel's diverticulum as a cause of acute lower gastrointestinal (GI) bleeding in adults

LEARNING OBJECTIVE \#2: Recognize early predictors of severity in acute lower GI bleeding

CASE: 33 year-old Asian male with medical history of hemorrhoids presented to urgent care with 8 episodes of bloody diarrhea, diffuse abdominal pain and dizziness for one day. He denied fever, vomiting, rectal pain, medication, alcohol or drug use, travel or sick contacts. Physical exam showed an anxious appearance, diffuse abdominal tenderness and blood on rectal exam. He did not have fever, jaundice or hemorrhoids. Sitting blood pressure (BP) was 130/70 and heart rate (HR) was 100; standing BP 86/64 and HR 140. Initial hemoglobin was $13 \mathrm{~g} / \mathrm{dL}$. After referral to the emergency department (ED), he had more bloody diarrhea and hemoglobin decreased to $10 \mathrm{~g} / \mathrm{dL}$ in 6 hours. In the next 12 hours, he was transferred to the Intensive Care Unit and started on massive transfusion protocol and pressors for hypovolemic shock. Upper endoscopy (EGD), colonoscopy and Interventional Radiology angiogram did not reveal a bleeding source. Retrograde double-balloon enteroscopy revealed a diverticulum at the terminal ileum with ulceration consistent with a Meckel's diverticulum (MD). After surgical resection of the MD, his GI bleeding finally stopped. He was discharged home 1 week later.

IMPACT/DISCUSSION: MD is typically associated with GI bleeding in children. About $25-50 \%$ of symptomatic MD cases present under 10 years of age (Park, 2005). Certain clinical features increase the probability of MD in adults: younger age, male sex, lack of risk factors for alternative causes of GI bleeding and no source identified on EGD and colonoscopy. The patient presented to urgent care with several important clinical features: tachycardia, orthostatic hypotension, gross blood on rectal exam and bleeding during the first 4 hours of evaluation. Strate (2003) identified these features along with non-tender abdomen, aspirin use and multiple co-morbidities as early predictors of severity in acute lower GI bleeding.
Multiple features correlated with more severe bleeding. The initial outpatient evaluation of acute GI bleeding assesses hemodynamic stability. His sitting blood pressure of 130/70 appeared misleadingly stable. Measurement of standing vital signs revealed severe orthostatic hypotension (BP $86 / 64$ and HR 140). These high-risk features prompted urgent referral to the ED where severe hypovolemic shock developed within hours.

CONCLUSION: MD is an uncommon cause of acute GI bleeding in adults that should be considered in younger adults who lack risk factors for alternative causes and have normal EGD and colonoscopy. Early predictors of severity in acute lower GI bleeding include tachycardia, orthostatic hypotension, gross blood on rectal exam and bleeding during the first 4 hours of evaluation. Patients with acute GI bleeding may present with normal blood pressure while sitting. Orthostatic blood pressure should also be used to assess hemodynamic stability.

\section{NOT JUST HYPERALGESIA: DIFFUSE PAIN DUE TO MULTI- FOCAL OSTEONECROSIS IN A LUPUS PATIENT}

Callie Plafkin; Tiffany Lin. University of Wisconsin Hospital and Clinics, Madison, WI. (Control ID \#3138587)

LEARNING OBJECTIVE \#1: Recognize osteonecrosis as a common cause of bone pain in lupus patients

LEARNING OBJECTIVE \#2: Recognize systemic lupus erythematosus and corticosteroid use as risk factors for avascular necrosis

CASE: We present a case of a 22-year-old female patient with a history of systemic lupus erythematosus (SLE), complicated by lupus nephritis, antiphospholipid syndrome, Libman-Sacks endocarditis, multifocal cerebral infarcts, pericardial effusion, arthralgias, cytopenias (leukopenia, thrombocytopenia), hypocomplementemia, and positive serologic markers (doublestranded DNA, anti-Smith, and antinuclear antibodies), on chronic corticosteroids and immunosuppressants, who presented with diffuse bone pain. Her primary team was initially concerned for lupus flare versus hyperalgesia or diffuse pain syndrome. The pain was described as affecting 'all of her bones,' more prominent in the lower extremities, and different in character and location (more diffuse) compared to her prior lupus flares. She noted mild knee swelling, but no other swollen joints. Aside from one oral ulcer, she lacked other symptoms suggestive of lupus flare, including rashes, nasal ulcers, alopecia, or hematuria. Her exam was notable for diffuse tenderness, most prominent over the bilateral shins and calves. She had mild bilateral knee effusions without other evidence of synovitis. Labs showed stable blood counts, normal inflammatory markers, normal complement levels that were higher than her prior baseline, normal anti-double-stranded DNA antibody titer, and normal creatine kinase. A diagnosis of tacrolimus pain syndrome was considered, though deemed unlikely when tacrolimus level returned undetectable. Bilateral knee and ankle radiographs were unremarkable. MRI revealed multifocal osteonecrosis involving the left femoral condyles, left patella, left proximal tibial metaphysis, right distal femur and proximal tibia, and bilateral femoral heads, and avascular necrosis was determined to be the cause of the patient's widespread bone pain.

IMPACT/DISCUSSION: Avascular necrosis (AVN) is a common complication affecting SLE patients. Among the most important risk factors for AVN is corticosteroid (CS) use - both duration and total cumulative dose. Some studies suggest concomitant use of immunosuppressants may amplify this risk. Notably, SLE seems to be an independent risk factor for $\mathrm{AVN}$, as AVN occurs more frequently in patients with lupus than in those receiving comparable CS doses for alternate diagnoses. MRI is the diagnostic test of choice. Multifocal AVN should be considered on the differential in lupus patients presenting with widespread bone pain without clear alternate etiology.

CONCLUSION: This case illustrates the importance of maintaining a broad differential for diffuse pain in a lupus patient, beyond merely lupus flare and hyperalgesia, and to further investigate alternate diagnoses when labs and symptomatology are not consistent with a lupus flare. Avascular necrosis should be considered as an etiology of bone pain in the SLE patient. 


\section{NOT THE TYPICAL CAUSE OF AORTIC ANEURYSMS. A RARE CASE OF GIANT CELL ARTERITIS PRESENTING WITH CHEST PAIN}

Sally Salman; Karam Khaddour; Dipesh Ludhwani; Rosemina Patel. Rosalind Franklin University of Medicine and Science- Chicago Medical School, McHenry, IL. (Control ID \#3185522)

LEARNING OBJECTIVE \#1: Recognize giant cell arteritis (GCA) in patients with aortic aneurysms despite the absence of typical GCA symptoms.

LEARNING OBJECTIVE \#2: Conduct more studies to assess the efficacy of screening for aortic involvement in patients with giant cell arteritis.

CASE: A 74-year-old male patient with a known history of hypertension and hypothyroidism presented with atypical chest pain for three days. The patient was a nonsmoker and family history was significant for hypertension and type 2 diabetes mellitus. On physical examination, vitals were significant for mild tachycardia, blood pressure 138/75, oxygen saturation $98 \%$ on ambient air and afebrile. Physical examination was normal. Serial troponins were negative and electrocardiogram showed no ischemic changes. Further evaluation with chest computed tomography (CT) showed large ascending aortic aneurysm measuring $5.7 \mathrm{~cm}$. After medical optimization, the patient underwent surgical repair of the ascending aortic aneurysm. A specimen of the dilated aorta was sent to pathology which showed multinucleated giant cells and associated positive elastin break in the lamina which was consistent with giant cell arteritis. Oral glucocorticoids therapy was recommended. However, the patient declined medical treatment after understanding risks and benefits. During a two year follow up the patient did not have any indication of disease relapse.

IMPACT/DISCUSSION: Giant cell arteritis (GCA) is a granulomatous inflammatory disease affecting individuals older than 50 years. Clinically, it comprises overlapping presentations including cranial arteritis and extracranial GCA (large-vessel GCA). The presentation of GCA in large-vessel is seen in limb claudication, aortic aneurysm, and aortic dissection. Our patient presented with chest pain and did not present with the typical symptoms of GCA; temporal headache, jaw claudication or ophthalmic symptoms. The aorta may be affected in about a third of patients according to one prospective study assessing large vessel involvement. However, aortic aneurysms are very uncommon and appear in only $6 \%$ of patients with large vessel involvement. Retrospective studies have demonstrated a higher prevalence of aortic aneurysm among patients with GCA compared with the general population. Most clinicians recommend steroid treatment as long as the patient is showing disease activity or elevated inflammatory markers. In reviewing the literature, there were no clear guidelines to screen for aortic aneurysms in GCA patients, although studies have shown that aortic aneurysm those patients have increased risk of dissection.

CONCLUSION: Giant cell arteritis is a common cause of vasculitis, however, a rare cause of ascending aortic aneurysm. Giant cell arteritis should be in the differential diagnosis of patients presenting with aortic aneurysms even in the absence of typical GCA symptoms. The need for screening guidelines to look for aortic aneurysms and close follow up should be considered in patients with Giant Cell arteritis.

\section{NOT YOUR TYPICAL HEART ATTACK}

Erica Chung; Omar Saeed. Montefiore Medical Center, Bronx, NY. (Control ID \#3171747)

LEARNING OBJECTIVE \#1: Recognize the clinical features of postpartum spontaneous coronary artery dissection (SCAD)

LEARNING OBJECTIVE \#2: Understand the importance of early recognition of SCAD to reduce mortality

CASE: A 39-year-old woman presented with 1 hour of sudden onset chest pain. She had no cardiovascular $(\mathrm{CV})$ risk factors, no family history of cardiac disease, and denied any drug use. Further history revealed that she had an uncomplicated vaginal delivery 1 month ago. On presentation, she was hemodynamically stable, but troponin T was elevated to $2.39 \mathrm{ng} / \mathrm{mL}$ and ECG revealed ST elevations in the inferior leads. She was taken for urgent coronary catheterization, which showed only mild luminal narrowing of the mid first obtuse marginal. However, given a high clinical suspicion for coronary artery dissection, further intracoronary imaging with optical coherence tomography (OCT) was done, revealing a large intramural hematoma with focal intimal disruption and significant obstruction of the true lumen. This confirmed the diagnosis of spontaneous coronary artery dissection (SCAD), leading to an inferior wall myocardial infarction (MI). She was managed medically with aspirin and metoprolol. Her chest pain soon resolved, troponins trended down, and ST segments normalized. She was discharged on medical therapy and remains stable with preserved cardiac function.

IMPACT/DISCUSSION: SCAD is a rare yet potentially fatal cause of chest pain that accounts for $0.2 \%$ of cases of acute coronary syndrome. In $\mathrm{SCAD}$, an intimal tear creates an intramural hematoma, leading to compression of the true lumen and resultant MI. SCAD mainly occurs in young women with few, if any, of the traditional CV risk factors, and is associated with the post-partum period. The pathogenesis is not fully understood, but may be related to the progesterone-induced alterations in elastic fiber content that weaken the arterial walls, and the increased cardiac demand of pregnancy and stress of labor that further predispose to dissection. Recent studies have also identified specific genetic loci that may be associated with SCAD. In our case, lack of risk factors in this otherwise healthy woman strongly implicated her recent pregnancy as the main precipitating cause of SCAD. Given this clinical suspicion, OCT, with its high resolution and superior ability to visualize intracoronary pathology, was used to confirm the diagnosis. Failure to recognize and diagnose SCAD can lead to an MI, acute heart failure, cardiogenic shock, and death. In fact, the short-term mortality associated with SCAD is about $50 \%$, and reaches $70 \%$ in patients who present with MI. Thus, SCAD presents a unique diagnostic challenge because although uncommon, it can be fatal if missed, and requires accurate history taking and clinical acumen to promptly diagnose and manage these high-risk patients.

CONCLUSION: Acute MIs rarely occur in young patients with no CV risk factors. This case illustrates the need to maintain a high index of suspicion for SCAD in post-partum women presenting with chest pain

\section{NOT YOUR USUAL PALMS-AND-SOLES RASH}

Carolina Gonzalez Lopez ${ }^{1}$; Lindsay Hammons ${ }^{2,}{ }^{1}$. ${ }^{1}$ Mount Sinai Beth Israel, New York, NY; ${ }^{2}$ Mount Sinai Beth Israel, NY, NY. (Control ID \#3186029)

LEARNING OBJECTIVE \#1: To consider disseminated cutaneous varicella zoster virus in an HIV patient with a rash on his palms and soles. CASE: A 46 year-old male with a history of HIV presented with lower back/flank pain and rash. Three days before admission, the patient experienced 8/10 sharp pain in right lower back/flank; the pain was also associated with shooting pain to palpation, with subsequent numbness two days later. At this time, the patient also developed a non-pruritic, nonpainful, diffuse maculopapular rash over his whole body and bruising over his arms. The patient had not been on HAART for over five months due to insurance issues, and had no known history of chickenpox or varicella zoster virus (VZV) vaccinations. On exam, the patient was afebrile. Other vital signs were as follows: BP 114/72 mmHg, HR 109 bpm, RR 20 bpm and $\mathrm{SpO} 297 \%$ on room air. Physical exam was significant for a warm, edematous confluent rash with overlying pustules on his right back/flank following dermatomes T9 through T11 to midback; the area had decreased sensation. Additionally, the patient had a diffuse red maculopapular rash (lesions $1-4 \mathrm{~mm}$ ) over his entire body, including his palms, soles, head and scrotum, with crusting in different stages. Initial labs showed CD4 count 250 with viral load 60,100; CBC 
included WBC $6.9 \mathrm{k} \mathrm{K} / \mu \mathrm{L}, \mathrm{Hb} 14.7 \mathrm{~g} / \mathrm{dL}$ and platelets $13 \mathrm{~K} / \mu \mathrm{L}$. Pertinent positive results included VZV IgM 0.96, VZV IgG >4000, EBV IgG $>600$, CMV IgG $>10$, AST 57 and ALT 78. Pertinent negative results included cryoglobulin and Coxsackie A/B viral titers. The patient was diagnosed with disseminated cutaneous VZV. He was treated with acyclovir for 14 days, dexamethasone and restarted on HAART with substantial improvement of his rash after one week.

IMPACT/DISCUSSION: Varicella zoster virus causes two clinically distinct diseases: varicella and herpes zoster. Varicella is characterized by vesicular lesions on erythematous bases in different stages of healing; the rash spreads first to the trunk then to the extremities, usually sparing palms, soles and genitals. Herpes zoster results from reactivation of the virus leading to painful, usually unilateral vesicular lesions in a dermatomal distribution. HIV patients are at a greater risk of developing herpes zoster than the general population. Additionally, disseminated cutaneous herpes zoster (VZV) is more common in immunocompromised patients, especially in those with HIV and in transplant recipients. Disseminated cutaneous VZV is characterized by a maculopaular rash with $>20$ lesions outside the affected dermatome; this rash also usually spares the palms/ soles. This is the first reported case in the literature of disseminated cutaneous VZV affecting the palms and soles of an HIV patient.

CONCLUSION: VZV infection is common in immunocompromised patients. Therefore, in addition to more common causes-like syphilis and coxsackievirus - it is important to consider VZV infection in an immunocompromised patient who presents with an atypical rash affecting his/her palms and soles.

\section{NOW WATCH ME WHIPPETS: AN ATYPICAL CAUSE OF PE- RIPHERAL NEUROPATHY}

Jason Ng; Angela Arbach. NYU Langone, New York, NY. (Control ID \#3185506)

LEARNING OBJECTIVE \#1: Recognize the clinical \& laboratory presentation of chronic nitrious oxide abuse.

CASE: 51yo F with history of hypothyroidism and anxiety presented to the ED after being brought in from the street by police. Over the course of the past month, she reported mental slowing and relative weakness, numbness and tingling in her hands and feet, incoordination of fine motor movements and gait abnormalities. ROS was otherwise negative. She denied any visual or auditory hallucinations, SI or HI. Past medical, surgical and family history were unremarkable. She lives in Manhattan with her boyfriend, who was apprehended by authorities under similar circumstances. She takes daily synthroid and Valium and reported distant history of alcohol use 1 month ago. VS WNL. She was alert and oriented to self and location but believed the year was 1920 and exhibited word-finding difficulty. Physical exam revealed grossly normal cranial nerve functions without dysarthria, and normal strength, sensation, and bulk muscle tone. She had a negative Romberg exam but exhibited some impairment of proprioception sense in fingers and toes, particularly with downward movement. She was able to stand up and walk unassisted with a wide-based and unsteady gait. BMP, LFTs, coags, TSH, CPK were normal. CBC was remarkable for a leukopenia to 1.7 (ANC 250), Hgb 12.5 with an MCV of 87.8, platelets 166. Iron studies were suggestive of anemia of chronic disease, with LDH 240 and reticulocyte index was 0.9. Vitamin B12 returned low at $183 \mathrm{ng} / \mathrm{L}$ and homocysteine and methylmalonic acid levels were elevated at $191 \mathrm{umol} / \mathrm{L}$ and $0.9 \mathrm{mmol} / \mathrm{L}$ respectively. Utox was positive for benzodiazepines. Many diagnoses were considered including MS, tertiary syphilis, and GBS, but the patient ultimately admitted to abusing up to 8-10 containers of whippets (small pressurized containers containing N2O) daily for the past month. Toxicology and neurology were consulted and her physical exam and lab derangements were thought to be secondary to a functional B12 deficiency, for which she was initiated on IM repletion. An MRI brain and spine was ordered but she ultimately left AMA. She intended on cutting back on whippets use and expressed a desire to "clear her head" on her walk home.

IMPACT/DISCUSSION: Nitrous oxide abuse has recently gained popularity as a recreational party drug due to its availability. When inhaled, it causes euphoria, analgesia within seconds. Chronic use leads to toxicity through the oxidation of cobalt ions in vitamin B12, hence causing inactivation. This causes reduced recycling of homocysteine to methionine, thereby preventing methylation of myelin proteins. Patients present with fatigue, weakness, poor balance and paresthesias and MRI imaging may show posterior column hyperintensities on T2W imaging. High-dose intramuscular B12 replacement is recommended, but recovery may ultimately be slow and incomplete.

CONCLUSION: $\mathrm{N} 2 \mathrm{O}$ is a rare cause of functional B12 deficiency, pernicious anemia and peripheral neuropathy.

\section{NUTCRACKER PHENOMENON MISDIAGNOSED AS LEFT RENAL VEIN THROMBOSIS}

Mohammad Zmaili; Motasem Alkhayyat; Mobin Malik; Deborah Hornacek. Cleveland Clinic Foundation, Cleveland, OH. (Control ID \#3176026)

LEARNING OBJECTIVE \#1: Recognize and diagnose nutcracker phenomenon

LEARNING OBJECTIVE \#2: Recognize the difference between nutcracker phenomenon and nutcracker syndrome

CASE: A 61-year-old African American male with a history of hypertension, hyperlipidemia, and hepatitis $\mathrm{C}$ was incidentally found to have left renal vein thrombosis (RVT) on an abdominal ultrasound (US) during routine surveillance for hepatocellular cancer. The abdominal US appeared to demonstrate a left RVT on greyscale imaging and color power angiography with a recognizable Rouleaux flow. The patient remained asymptomatic. He was admitted and started on heparin infusion. Renal venous duplex US was repeated in a seated, instead of a supine, position with no evidence of RVT on color power angiography and power energy settings. However, there was a dynamic external compression of the vein. We concluded that the initial finding was not a true thrombus but rather a flow artifact due to the compression of the left renal vein as it passes between the aorta and the superior mesenteric artery (SMA), a phenomenon referred to as "Nutcracker phenomenon". Anticoagulation was stopped and the patient was discharged.

IMPACT/DISCUSSION: The patient described above had an incidental finding that was initially interpreted as left RVT. Given the rarity of spontaneous isolated RVT in the absence of nephrotic syndrome or renal malignancy, a repeat study was pursued. Interestingly, reimaging in the seated position alleviated the compression on the left renal vein and revealed no venous thrombosis. Rapid weight loss with reduction of retroperitoneal fat is thought to contribute to this phenomenon by decreasing the aortomesenteric angle (the angle between the aorta and SMA). When extrinsic left renal vein compression leads to the emergence of symptoms, the condition is referred to as nutcracker syndrome. Characteristic symptoms include flank or pelvic pain, hematuria, varicocele, and orthostatic proteinuria. Diagnosis is established based on clinical suspicion by imaging studies with demonstration of reduced aortomesenteric angle and increased left renal vein hilar-toaortomesenteric diameter ratio. Management depends on severity and age of presentation. It ranges from conservative management for mild cases in younger patients, to intravascular or surgical interventions. This patient was asymptomatic with no significant laboratory abnormalities. He was educated on the symptoms of nutcracker syndrome for which he should seek medical attention.

CONCLUSION: Spontaneous isolated RVT is rare unless there is renal malignancy or nephrotic syndrome. The risk increases with inherited 
thrombophilias, hypercoagulability, hypovolemia, and trauma. Nutcracker syndrome is an underdiagnosed condition and should be suspected when clinical presentation suggests left renal venous hypertension. The asymptomatic equivalent of the syndrome is referred to as nutcracker phenomenon which can be managed conservatively.

\section{OBSCURE GI BLEED: GETTING THE "GIST" OF A TRICKY DIAGNOSIS}

Lydia A. Flier. Beth Israel Deaconess Medical Center, Brookline, MA. (Control ID \#3185824)

LEARNING OBJECTIVE \#1: Recognize the difference between occult, overt and obscure gastrointestinal bleeding.

LEARNING OBJECTIVE \#2: Compare and contrast options for deep small bowel imaging to diagnose a source of bleeding.

CASE: The patient is a 74 year old woman with coronary artery disease status-post drug eluting stent to her left anterior descending coronary artery in 1 month prior to initial presentation, atrial fibrillation on Apixaban, remote breast and ovarian cancer, and CKD, who presented with a hemoglobin of 6.2 and concern for GI bleed. She was initially admitted to an affiliated community hospital with a hemoglobin of 5.6 and eventual melena. Upper endoscopy and colonoscopy showed enteritis, superficial ulceration, mucosal petechiae, left-sided diverticular disease, and scant old blood in the colon. Her Apixaban was held and aspirin $81 \mathrm{mg}$ was added to Clopidogrel. The next month, outpatient capsule endoscopy showed antral erythema (suggesting gastritis or GAVE) and diminutive duodenal AVMs. She was admitted weeks later with a $\mathrm{Hgb}$ of 6.3; repeat colonoscopy showed old blood in the left colon, red blood in the right colon, and fresh blood in the terminal ilium without a source. Repeat capsule showed bright red blood 40 minutes into small bowel, but no source. Push enteroscopy did not show a source. She then underwent balloon enteroscopy, which showed an ulcerated mass in the proximal ilium. Biopsy of this mass revealed a gastrointestinal stromal tumor. Imaging was negative for metastatic disease. She underwent a subtotal colectomy and was discharged with outpatient oncology follow up.

IMPACT/DISCUSSION: Approximately 5\% of gastrointestinal bleeding (GIB) qualifies as obscure, or lacking a clear source after upper endoscopy and colonoscopy. Obscure GI bleeds (OGIB) can be occult (chronic) or overt (acute), but usually the source is located between the papilla and the ileocecal valve; consequently, terminology is shifting to call this kind of GI bleed "suspected small bowel bleeding." The source of up to $50 \%$ of OGIB can be found on repeat endoscopy and colonoscopy, but if is not successful, further workup is necessary. This can include capsule endoscopy, push enteroscopy, balloon-assisted enteroscopy (single or double), spiral-assisted enteroscopy, and CT or MR enterography. These modalities have different pros and cons, and hospitals may rely on different modalities based on resources such as advanced radiology capacity, advanced endoscopy, or anesthesiology time. This patient had a GIST as the source of her GI bleed, the treatment of which includes complete surgical resection followed by a course of imatinib.

CONCLUSION: Obscure GI bleeding refers to GI bleeding without a clear source after repeat colonoscopy and endoscopy, and can be overt/ acute or occult/chronic. A systematic approach to occult OGIB may be necessary to diagnose the cause, and includes capsule endoscopy, MR/CT enterography, and push, balloon and/or spiral enteroscopy.

\section{OCCAM'S RAZOR OR HICKAM'S DICTUM: MAKING THE DIAGNOSIS IN COMPLEX CARE}

Caroline Chen; Kristen Kelley; Arielle Sommer; Pourandokht S. Nourbakhsh. University of California - Los Angeles, Los Angeles, CA. (Control ID \#3175641)
LEARNING OBJECTIVE \#1: Recognize atypical features of sinusitis. LEARNING OBJECTIVE \#2: Identify physician, patient, and systems factors that contribute to missed diagnoses.

CASE: A 76 year-old female with Crohn's disease, tobacco-related COPD, obesity, hypertension, and diastolic dysfunction presents to her physician with sinus pain. Antibiotics temporarily improve her symptoms but they return with hearing loss, ear pain, and fatigue. She receives several courses of antibiotics for recurrent sinusitis. During this time, she also reports dyspnea. She is encouraged to comply with her inhalers and is given diuretics for diastolic dysfunction. Nine months later, she presents to the hospital with pre-syncope. Chest x-ray shows bilateral infiltrates and CT of the sinuses shows otomastoiditis for which she is given antibiotics. She is also given diuretics for heart failure exacerbation given intermittent hypoxia. Two weeks later, she presents with 1 day of dizziness and blurred vision. On exam she is lethargic with left anisocoria, right nasolabial fold weakness, and 4/5 strength in the left upper and lower extremities. Imaging reveals an infarct of the thalamus and midbrain. Chest $\mathrm{x}$-ray shows a right lower lobe opacity. She receives antibiotics for pneumonia. Further work-up of her acute stroke is unrevealing. She develops fevers and leukocytosis followed by hypoxemia and renal failure. Active urinary sediment leads to rheumatologic evaluation with positive p-ANCA, c-ANCA, and myeloperoxidase antibodies. Kidney biopsy reveals necrotizing vasculitis with giant cells, consistent with granulomatosis with polyangiitis (GPA). The patient receives steroids, plasmapheresis, and rituximab but unfortunately passes away 17 months after her symptoms began. Physicians involved in her care wondered if her diagnosis could have been made sooner.

IMPACT/DISCUSSION: Annually, 1 in 7 US adults is diagnosed with acute sinusitis while the prevalence of GPA is only 3 per 100,000. Per IDSA Clinical Practice Guidelines, patients who fail first and second line therapies for bacterial sinusitis should be evaluated for non-infectious causes including GPA, a granulomatous small-to-medium vessel vasculitis. As 5 to $12 \%$ of patients with GPA die within the first year and 6 to $13 \%$ develop CNS disease, prompt diagnosis and treatment is essential. In isolation, our patient's problems could be explained: sinusitis in a susceptible host, hearing loss due to sinusitis, dyspnea due to undertreated COPD, hypoxia due to diastolic dysfunction, stroke in an at-risk individual, pneumonia in a patient with aspiration risk, and acute kidney injury due to hypotension from sepsis. Despite Hickam's dictum's tempting explanations, hindsight favors Occam's razor and may have led to earlier diagnosis and treatment for this patient.

CONCLUSION: In complex patients with fragmented care, sumarizing the case to look for a unifying diagnosis by Occam's razor may reveal new insights. When patients fail empiric therapy for acute sinusitis, an alternate diagnosis should be considered.

\section{OCCULT TENSION PNEUMOTHORAX; A LIFE SAVED.}

Ali Azim; Harshit Dua; Allan Tachauer. Weiss Memorial Hospital, Chicago, IL. (Control ID \#3185981)

LEARNING OBJECTIVE \#1: Spontaneous tension pneumothorax is a rare entity and needs high clinical suspicion for prompt diagnosis.

LEARNING OBJECTIVE \#2: Isolated physical or radiological findings are neither sensitive nor specific.

CASE: 56-year-old quadriplegic female was seen in emergency department(ED) for altered mental status(AMS) and abdominal pain. She had history of multiple sclerosis(17years), recurrent urinary tract infections(UTI), dysphagia status post G-tube. Vital signs showed hypotension $(97 / 63 \mathrm{mmHg})$, tachycardia(101/min), hypothermia(95.3F). Physical exam revealed bilateral equal breath sounds with minimal crackles at right lower lung zone, profound sweating and mild epigastric tenderness. Electrocardiogram(EKG) showed sinus tachycardia. Broad spectrum antibiotics and intravenous fluid(IVF) were started. Urinalysis was suggestive of UTI. Labs showed severe hyponatremia $(106 \mathrm{mEq} / \mathrm{L})$ and no 
leukocytosis. Femoral venous catheter was placed for pressor support. Chest X-ray(CXR) was done as a part of sepsis work-up which surprisingly revealed pneumothorax with no mediastinal shift. Given the small pneumothorax reported on CXR, the hypotension was linked to underlying sepsis. Mean arterial pressure did not improve despite multiple pressors and IVF. Follow-up exam of the chest revealed decreased breath sounds on the right side and increased respiratory distress. Immediate needle thoracostomy was performed followed by chest tube placement with gush of air released from the chest cavity. Patient was weaned off of pressors instantly. During her course, she was briefly intubated for worsening AMS. She was successfully treated with broad spectrum antibiotics for UTI and pneumonia and was discharged in stable condition.

IMPACT/DISCUSSION: Tension pneumothorax(TPT) is a clinical diagnosis. Spontaneous tension pneumothorax is not well recognized in spontaneously breathing patients. In these patients, its always the valve mechanism that causes the air to accumulate in pleural cavity. The developing pressure with every breath causes kinking of the great vessels, decreasing cardiac output. All classical signs of TPT are not always present. Mediastinal shift cannot differentiate TPT from simple pneumothorax. Pneumothorax is considered under tension if it results in reversal of respiratory or hemodynamic compromise. Sepsis contributing to hypotension in our case delayed the appropriate management of TPT. Multiple studies of ventilated patients showed increased mortality in undiagnosed or delayed diagnosis of TPT.

CONCLUSION: To our knowledge, none of the previously reported cases of spontaneous tension pneumothorax presented with background sepsis. According to the literature, in a retrospective analysis of $176 \mathrm{ED}$ presentations of pneumothorax, 30 patients deteriorated awaiting chest tube placement with no evidence of mediastinal shift on CXR. Therefore, in patients with developing respiratory distress accompanied with hypotension, tachycardia and pneumothorax, immediate needle thoracostomy and chest tube placement is warranted.

\section{OCULAR SYPHILIS: A DIAGNOSTIC CHALLENGE IN AN IM- MUNOCOMPETENT PATIENT}

Pakhadi H. Buddhadev; Nisreen Mobayed. Medical College of Wisconsin, Milwaukee, WI. (Control ID \#3185115)

LEARNING OBJECTIVE \#1: - Recognizing, diagnosing, and managing ocular syphilis in immunocompetent patients

LEARNING OBJECTIVE \#2: - Distinguishing isolated ocular syphilis from ocular syphilis with concomitant neurosyphilis

CASE: A 75-year-old woman with history significant for stroke presented with 7-months of recurrent uveitis of the right eye. The patient reported right eye photophobia, blurry vision, and throbbing pain refractory to several courses of steroid drops. Social history revealed that patient has not been sexually active for 12 years and has no history of STIs. Exam was only notable for right eye erythema and pain as well as chronic neurological findings secondary to prior stroke. Ophthalmology was consulted and performed optical coherence tomography that revealed cystoid macular edema (CME) in the right eye and normal foveal contour in the left eye. Fluorescein angiography of the right eye confirmed presence of CME without peripheral vasculitis. Slit lamp exam of the right eye demonstrated deep, trace flare with $1+$ cells in the anterior chamber, anterior vitreous cells and haze, and 2+ injection with limbal flush. Visual acuity was 20/40 bilaterally. Further work-up to evaluate for sarcoidosis and infectious etiologies were performed. Work-up revealed a positive treponema pallidum antibody as well as a RPR quantification of 1:16. The remainder of the work-up was negative, including TB and HIV. Evaluation for neurosyphilis, including a MRI of the brain and a LP for VDRL CSF screen, was negative. Patient was admitted for treatment of uveitis syphilitic chorioretinitis, initiating a 14-day course of IV penicillin-G.
IMPACT/DISCUSSION: The CDC has reported increasing prevalence of ocular syphilis; however, few publications document rates of ocular syphilis in immunocompetent patients. Despite the paucity of research evidence, clinicians should consider ocular syphilis as a differential for recurrent uveitis, especially when refractory to standard therapy. This case demonstrates the importance of recognizing and appropriately managing syphilis in an immunocompetent patient. Given the risk of significant complications, including blindness, thorough evaluation and a high degree of clinical suspicion is necessary for the prompt diagnosis and treatment of ocular syphilis. Although the diagnostic criteria of ocular syphilis and neurosyphilis are different, there is a large amount of overlap in presentation. Up to $70 \%$ of patients with ocular syphilis also demonstrate evidence of neurosyphilis on LP. While ocular syphilis and neurosyphilis are treated with the same regimen, it is necessary to evaluate patients for neurosyphilis to mitigate any further neurological complications.

CONCLUSION: - Ocular syphilis may be the only clinical manifestation of syphilis - Clinicians should have a low threshold for performing serologic analysis in patients with uveitis refractory to corticosteroids It is necessary to evaluate for neurosyphilis in patients with ocular syphilis

\section{ODD, BLACK LESIONS - A FATAL CASE OF A GRAVE ILLNESS IN CHRONIC KIDNEY DISEASE}

Varun Shah $^{1}$; Elizabeth Sullivan ${ }^{1}$; Steven G. McKee ${ }^{2} .{ }^{1}$ University of Arkansas for Medical Sciences, Little Rock, AR; ${ }^{2}$ University of Arkansas, Little Rock, AR. (Control ID \#3185168)

LEARNING OBJECTIVE \#1: Contrast early versus delayed timing of skin biopsy in patients with end-stage renal disease (ESRD) and necrotic lesions

LEARNING OBJECTIVE \#2: Contemplate the frequency of enteric ulcers in patients with this rare disease

CASE: A 58-year-old with ESRD secondary to diabetes mellitus, on peritoneal dialysis for one year, presented with fever and painful skin lesions. The initial ulcer appeared behind her left knee six months prior to presentation. Later, similar lesions appeared on both legs culminating in multiple, tender ulcers covered by hard, affixed eschar. The lesions progressed despite five months of standard wound care, antibiotics, and sodium thiosulfate. She did not receive surgical debridement. On admission, she noted fissuring of the eschars with purulent drainage, anorexia, and difficulty with ambulation. She was ill appearing, had wounds as described, and was hypotensive to $95 / 30 \mathrm{mmHg}$. White blood cell count was $15 \mathrm{~K} / \mathrm{uL}$, corrected calcium $9 \mathrm{mg} / \mathrm{dL}$, ionized calcium $1.1 \mathrm{mmol} / \mathrm{L}$, phosphorus $4.8 \mathrm{mg} / \mathrm{dL}$, and parathyroid hormone $78.5 \mathrm{pg} / \mathrm{mL}$. Peritoneal dialysate and blood cultures were negative. Skin biopsy of the wound confirmed calciphylaxis. Wound cultures grew multi-drug resistant Pseudomonas aeruginosa and Achromobacter. She was transitioned to hemodialysis and intravenous sodium thiosulfate. However, she developed severe sepsis and was put on vasopressors nine days after the biopsy resulted. Subsequently, she developed large volume melena from duodenal ulcers that proved recalcitrant to intra-luminal cautery and imageguided arterial embolization. She died 20 days after diagnosis.

IMPACT/DISCUSSION: We argue for early biopsy in patients with ESRD and suspicious lesions. Gastrointestinal (GI) hemorrhage in patients with calciphylaxis is rarely reported and this may be an underappreciated manifestation. Calciphylaxis confers $50 \%$ mortality at one year mainly due to wound infections, and this patient's diagnosis was delayed by six months. Timely diagnosis requires high clinical suspicion and often skin biopsy. Yet prompt recognition is critical since recent evidence suggests early surgical management or hyperbaric oxygen therapy can improve cure rates and survival. Neither treatment was available to our patient due to delay in definitive diagnosis. Calciphylaxis is known to cause cutaneous ulcers but visceral ulcers may be under reported. Literature review uncovered only two other cases of gastrointestinal ulcer and hemorrhage in patients with calciphylaxis, and the sites of these ulcerations were rectal and esophageal, respectively. 
CONCLUSION: Early biopsy and diagnosis of calciphylaxis can reduce time to and preserve the feasibility of curative interventions. Further research is warranted to investigate if anti-acid medications or endoscopic therapies can prevent GI hemorrhage in calciphylaxis patients.

\section{ONE AGEP AT A TIME: A PATIENT WITH A NEW RASH}

Brianna C. Harder; Nicholas F. Maston; Taylor Zuber; Evan Raff. University of North Carolina, Chapel Hill, NC. (Control ID \#3159271)

LEARNING OBJECTIVE \#1: Recognize the presentation of Acute Generalized Exanthematous Pustulosis (AGEP)

LEARNING OBJECTIVE \#2: Identify common medications associated with AGEP.

CASE: A 58-year-old man presented with two weeks of right lower extremity redness and pain. He was diagnosed with cellulitis and received $1 \mathrm{~g}$ intramuscular ceftriaxone followed by a 10-day course of cephalexin. His symptoms did not improve. He received a 10-day course of trimethoprim-sulfamethoxazole and topical mupirocin. Five days later, he developed new pruritic skin lesions and was admitted for further evaluation. His temperature was $37 \mathrm{C}$, blood pressure was 147/71 $\mathrm{mmHg}$, and pulse was $65 \mathrm{bpm}$. Skin demonstrated numerous small, non-follicular papules on an erythematous, edematous background involving the lower extremities, groin, trunk, arms, and face. Significant labs include peripheral white blood cell count of $16.6 \times 109 / \mathrm{L}$ with neutrophilic predominance, eosinophils $0.5 \times 109 / \mathrm{L}$, serum creatinine $1.54 \mathrm{mg} / \mathrm{dL}$ and CRP $25.7 \mathrm{mg} / \mathrm{L}$. Skin biopsy revealed prominent multi-loculated intraepidermal collections of neutrophils in variably-sized pustules, spongiosis, and lympho-eosinophilic infiltrate, overall consistent with acute generalized exanthematous pustulosis (AGEP). Trimethoprimsulfamethoxazole was discontinued. He received clindamycin for his cellulitis and antihistamines, and triamcinolone ointment for his AGEP. At follow-up one week later, his rash had resolved.

IMPACT/DISCUSSION: AGEP has been associated with numerous common medications. Anti-infective agents including sulfonamides (such as sulfamethoxazole), aminopenicillins, quinolones, terbinafine, ketoconazole, fluconazole, and chloroquines have been implicated, as well as nonanti-infective medications, including diltiazem. AGEP is characterized by the acute development of erythema, edema, pruritus or burning, followed by the emergence of numerous non-follicular, sterile pustules, often originating on the face or intertriginous areas and subsequently becoming widespread. AGEP may develop two days to three weeks after drug exposure. Systemic symptoms, including elevated transaminases, renal insufficiency, and respiratory distress, can develop. Pustules may sometimes take on a papular appearance, especially when corticosteroid therapy is employed early in the disease course, and the diagnosis may only be readily apparent on skin biopsy as in this case. Discontinuation of the offending medication, supportive care, and topical corticosteroids are the mainstays of treatment. Spontaneous resolution usually occurs within 2 weeks of drug discontinuation, but topical corticosteroids may help accelerate resolution of the rash. CONCLUSION: The general internist must maintain a high index of suspicion for the development of adverse drug reactions. Recognition of the presentation of AGEP and identification of commonly associated drug classes are critical for prompt discontinuation of the causative drug and resolution of symptoms.

\section{OPHELIA SYNDROME}

Samiullah Arshad. Montefiore Medical Center, Wakefield, Bronx, NY. (Control ID \#3186399)

LEARNING OBJECTIVE \#1: Recognize the paraneoplastic presentation of hodgkin's lymphoma

LEARNING OBJECTIVE \#2: Treat limbic encephalitis due to Hogkin's lymphoma with chemotherapy
CASE: 58 year-old male was admitted for new onset seizures. He had cervical lymphadenopathy (LAD) on exam. Hemoglobin was $8.6 \mathrm{~g} / \mathrm{dl}$ and Erythrocyte sedimentation rate (ESR) was 120 . Computed tomography (CT) chest revealed cervical, thoracic and hilar lymphadenopathy. Patient had another episode of witnessed seizure described as bilateral small amplitude fast frequency tremulous movements of extremities which terminated after patient received lorazepam followed by altered mental status that lasted several days. Further history obtained from the patient's wife revealed that the patient had been having low grade fevers, night sweats and had lost 40 pounds in the past 1 year. Magnetic resonance imaging (MRI) brain without contrast showed subtle asymmetric signal intensity and volume loss involving the left mesial temporal lobe. Electroencephalogram (EEG) revealed positive triphasic waves concerning for encephalopathy, without any epileptiform activity. Lumbar puncture was negative for infection and malignant infiltration. Characteristic Reed Sternberg cells were seen on the cervical lymph node biopsy. Patient was treated with intravenous immunoglobulins (IVIG) for 5 days followed by Adriamycin, bleomycin, vincristine and dacarbazine (ABVD) therapy with patient returning to baseline mental status within 1 week of therapy with no further seizures.

IMPACT/DISCUSSION: Paraneoplastic neurological syndromes (PNSs) are commonly associated with small cell lung cancer, germ cell tumors and breast cancer but rarely associated with Hodgkin's lymphoma. PNSs are caused by immune mechanisms triggered against antigens that are normally present in the nervous system and ectopically expressed by the tumor (onconeural antigens). Paraneoplastic limbic encephalitis (PLE) is a subtype of PNS, characterised by personality changes, irritability, depression, seizures, memory loss and dementia. Generalized or partial complex seizures occur in $50 \%$ of the patients. The most frequent MRI abnormalities in PLE are hyperintensity signals on T2-weighted or fluid attenuation inversion recovery (FLAIR) images involving one or both medial temporal lobes. Early recognition of PNS often leads to the discovery and treatment of the underlying tumor. Limbic encephalitis in patients with Hodgkin's lymphoma is associated with mGluR5 antibod. This is well defined in the literature as Ophelia Syndrome. It is named after the character Ophelia in Shakespeare's drama hamlet, and was first described by Dr. Ian Carr whose daughter, at the age of 15, developed progressive loss of memory, depression, hallucinations, and bizarre behaviour. These symptoms aptly describe the character Ophelia's deluded and obsessional behavior towards Hamlet.

CONCLUSION: Ophelia syndrome is a rare but treatable presentation of Hodgkins lymphoma. Early chemotherapy improves outcomes.

\section{OUT OF SIGHT, OUT OF MIND: DELAYED DIAGNOSIS OF A NECROTIZING SOFT TISSUE INFECTION}

Dustin McCurry; Clark A. Veet; Deborah DiNardo. University of Pittsburgh School of Medicine, Pittsburgh, PA. (Control ID \#3186337)

LEARNING OBJECTIVE \#1: Evaluate the accuracy of the laboratory risk indicator for necrotizing fasciitis (LRINEC) score and review attempts to improve upon its diagnostic value.

LEARNING OBJECTIVE \#2: Recognize how posterior probability error and the two forms of availability bias affect type 1 and type 2 diagnostic reasoning and review strategies to counter these cognitive biases.

CASE: A 54-year-old male with past medical history significant for type 2 diabetes and recurrent stage III squamous cell carcinoma of the rectum presented to oncology clinic for repeat infusion of nivolumab. $\mathrm{He}$ complained of subjective fevers, foul smelling anal drainage, and increased anorectal pain for the past 2 months, worsened over the past week. There were 2 emergency department (ED) visits for the same complaint over the past 2 months. At each visit, his symptoms were attributed to small abscesses caused by obstructing tumor, and he received antibiotics prior to discharge from the ED. In clinic, he was afebrile, HR 123 BP 103/71 RR $1899 \%$ on room air. Exam showed a necrotic 
appearing perirectal mass, with non-purulent discharge. Labs notable for WBC 40,900 Hgb 10.2 sodium 129 BUN 22 creatinine 0.8 and glucose 208. He was diagnosed with a perirectal abscess and triaged to an inpatient oncology service in the evening without further work up and recommendations for surgical oncology evaluation in the morning. Upon arrival, repeat vitals showed $\mathrm{T} 38.1^{\circ} \mathrm{C}$ HR $125 \mathrm{BP} 90 / 5297 \%$ on room air. Compared to his previous exam, he was also noted to have a systolic murmur, tense, leathery induration of the L buttock, and exquisite tenderness with movement of the left hip. There was no crepitus. Additional labs were performed: lactate 3.4 CRP 22.030 and ESR 96. Calculation of a LRINEC score raised suspicion for necrotizing soft tissue infection (NSTI), resulting in urgent imaging, NSTI diagnosis, and surgical intervention.

IMPACT/DISCUSSION: We hypothesize that the patient's history of ED visits led to a type 1 reasoning failure: posterior probability error. In posterior probability error, prior diagnoses unduly influence diagnostic reasoning. In NSTI, where early recognition is challenging but crucial, this case illustrates how adjunctive scoring systems, such as LRINEC or revised forms of LRINEC, can be used to counter biases in type 1 reasoning and prompt type 2 thinking. Additionally, given that NSTI is not a common diagnosis in the outpatient oncology setting, we argue that the non-availability formulation of the availability bias, the "out of sight, out of mind" bias, likely contributed to diagnostic delay. While more reflective and methodical thinking has been shown to mitigate the classic formulation of the availability bias, the non-availability bias can persist despite the use of type 2 reasoning, making this case a diagnostic challenge on multiple levels.

CONCLUSION: A high index of suspicion is required for early diagnosis of NSTI. Cognitive biases affect both type 1 and type 2 diagnostic reasoning.

OUT OF THE FRYING PAN AND INTO THE MRI MACHINE: LONG-TERM PERILS OF CANCER TREATMENT

$\underline{\text { Carlos A. Lopez }}{ }^{1}$; Joanne L. Candela ${ }^{1}$; Nancy A. Kernan ${ }^{1}$; Alexandra Heerdt $^{1}$; Emily S. Tonorezos ${ }^{2} .{ }^{1}$ Memorial Sloan Kettering Cancer Center, New York City, NY; ${ }^{2}$ Memorial Sloan-Kettering Cancer Center, New York City, NY. (Control ID \#3186136)

LEARNING OBJECTIVE \#1: Recognize that female cancer survivors who receive chest radiation therapy (RT) require early breast cancer screening with MRI in addition to mammography.

CASE: A 29 year-old female with a history of acute lymphocytic leukemia (ALL) treated with total body RT, chemotherapy, and a bone marrow transplant (completed treatment at age 21) presented for a routine follow up visit for management of treatment-related late effects. Because she was 8 years post-RT, she underwent her first breast cancer screening via both mammogram and breast MRI. She was generally well, but she reported breast discomfort during breast imaging and when getting an echocardiogram; she denied any other breast symptoms. She was on a combined estrogen-progesterone patch due to being menopausal after her treatment. Exam was notable for an inverted left nipple (unchanged over 6 years) and left breast tenderness without discrete masses but with a heterogenous quality on palpation. There was no axillary lymphadenopathy and there were no skin changes. History and physical were otherwise noncontributory. Though her mammogram was negative, her MRI revealed a $1.2 \mathrm{~cm}$ mass in the left breast and a $0.3 \mathrm{~cm}$ mass medial to the dominant mass suspected to be a satellite lesion. She underwent an ultrasound guided biopsy which revealed an infiltrating duct carcinoma that was $\mathrm{ER}+\mathrm{PR}+$ / HER2- on pathology. Her sentinel lymph node biopsy was positive for micro-metastatic disease. She opted to undergo bilateral mastectomy prior to starting systemic chemotherapy.

IMPACT/DISCUSSION: There were some potential diagnostic pitfalls that arose during our case that were fortunately avoided. First, a mammogram would have been insufficient to detect her lesion; by obtaining a breast MRI, we were able to identify an early malignant lesion that would have been otherwise missed. Secondly, we avoided prematurely ascribing breast pain to a non-malignant etiology: though breast pain makes other diagnoses more likely, her risk factors and the presence of a mass on imaging necessitated an expedient tissue diagnosis. Patients who carry high-risk gene mutations and patients who received chest RT for treatment of childhood cancers are particularly vulnerable to developing breast cancer at an early age. Mammography alone tends to underperform in younger patients because of associated higher breast density, so adding an MRI is recommended in these populations. In patients who have received RT, screening is recommended to begin 8 years after RT or at age 25 , depending on whichever occurs last. Though MRI-based screening can result in more false-positive lesions on initial screen, earlier diagnosis of breast cancer confers a more favorable treatment course.

CONCLUSION: The importance of appropriate and early breast cancer screening in women exposed to chest RT at a young age cannot be overstated. As general providers see more survivors in practice, it is key that they be aware that MRI is recommended in addition to mammography in this population.

\section{OXACILLIN-INDUCED CUTANEOUS LEUKOCYTOCLASTIC VASCULITIS: A CASE REPORT}

Meric Mericliler $^{1,2}$; Aya Shnawa ${ }^{3}$; Dalya AlQaysi ${ }^{1,2}{ }^{2}$; Andrew Moraco ${ }^{1,}$ ${ }^{2}$. ${ }^{1}$ St. Elizabeth's Medical Center, Brighton, MA; ${ }^{2}$ Tufts University School of Medicine, Boston, MA; ${ }^{3}$ Jordan University of Science and Technology, Ramtha, Jordan. (Control ID \#3169457)

LEARNING OBJECTIVE \#1: Drug-induced cutaneous lesions such as those seen with oxacillin-induced cutaneous leukocytoclastic vasculitis, can be mistaken for cutaneous manifestations of endocarditis and other causes of leukocytoclastic vasculitis such as HCV-related cryoglobulinemic vasculitis.

LEARNING OBJECTIVE \#2: A high index of suspicion is imperative for diagnosing drug-related cutaneous manifestations because choosing an alternative therapy is required for complete resolution of vasculitis and completion of infective endocarditis treatment.

CASE: A 32-year-old female with a history of IV drug use presented with fever and malaise. Her blood cultures grew methicillin-susceptible Staphylococcus aureus, and transthoracic echocardiogram showed vegetations on the anterior and posterior leaflets of tricuspid valve. The largest measured $2.7 \times 1.4 \mathrm{~cm}$. The patient was started on IV oxacillin and planned for a six weeks course. On day twelve after the initiation of therapy, skin examination revealed bilateral palpable painless purpuric papules on volar aspect of the hands and dorsal aspect of the feet. Over the next 48 hours, lesions extended up to the anterior knee and inner aspect of thighs and became bullous. Lesions were not associated with abdominal pain, arthralgia, or paresthesia. Investigations revealed weakly positive ANA (1:40) and was negative for p-ANCA and c-ANCA, cryoglobulins, RF, anti-ds DNA antibody. Complement $\mathrm{C} 3$ was low and $\mathrm{C} 4$ was normal. HIV testing was negative. Anti-HCV antibody was positive with a $\mathrm{HCV}$ viral load of $4.16 \times 10^{5} \mathrm{IU} / \mathrm{ml}$. Aspirate from the bullous lesions did not demonstrate any organisms on Gram staining, and culture did not grow any bacteria. Punch biopsies subsequently revealed perivascular neutrophil infiltration with fibrinoid necrosis of small vessels, consistent with leukocytoclastic vasculitis (LCV). Given the temporal relationship between oxacillin administration and development of skin findings, the diagnosis of oxacillinassociated LCV was suspected. Accordingly, vancomycin was started in lieu of oxacillin, resulting in gradual improvement of the lesions.

IMPACT/DISCUSSION: Cutaneous LCV is a rare condition which frequently presents with palpable purpuric nodules of the lower extremities. Medications have been implicated in 10 to $24 \%$ of the cases; sulfa drugs, NSAIDs, and beta-lactams have the most frequent association. Furthermore, cutaneous LCV can also be seen in the setting of infective endocarditis (IE) or HCV infection. In the present case, discontinuation of oxacillin improved the skin lesions suggesting that oxacillin was the likely 
culprit. Thus, we herein present the second reported case of oxacillin induced cutaneous LCV in the literature.

CONCLUSION: Present case illustrates a rare case of oxacillin-induced cutaneous LCV, masquerading the skin manifestations associated with $\mathrm{HCV}$ and IE. A high index of suspicion is imperative for diagnosing drugrelated cutaneous manifestations as discontinuation of the suspected agent is critical for resolution of the vasculitis and cutaneous lesions.

\section{PAIN FROM BUTTOCK TO LEG, BUT IT'S NOT SCIATICA: THE UNMASKING OF EXTRA-NODAL DIFFUSE LARGE B CELL LYMPHOMA IN AN HIV-INFECTED PATIENT}

Jordonna Brown; Swati Vishwanathan; Carol A. Luhrs. SUNY Downstate Medical Center, Brooklyn, NY. (Control ID \#3165797)

LEARNING OBJECTIVE \#1: To recognize unusual manifestations of AIDS-related diffuse large B cell lymphoma (DLBCL) in the post HAART era

LEARNING OBJECTIVE \#2: To diagnose uncommon causes of common lower extremity pain presentations, avoiding anchoring bias

CASE: A 46 year old woman with HTN presented with a dull aching pain in her left hip and buttock for a few months that was initially diagnosed as sciatica. The pain worsened progressively over a 2 week period, radiated down her left thigh with associated paresthesia. Her diagnosis then changed to trochanteric bursitis but the pain became intractable while on appropriate treatment for same so she was admitted to hospital for pain control. Her vitals were normal at presentation. Physical examination revealed an obese woman (BMI 42), with exquisite left hip and gluteal region tenderness, decreased range of motion but no visible or palpable masses. Labs show $\mathrm{Hb} 9$, HCT 29, WBC 5.09, PLT 260, BUN 24, Cr .92, CD4 17, viral load 129178. CT pelvis shows two discrete soft tissue lesions within the left buttock measuring $5.8 \times 4.5 \times 4.8 \mathrm{~cm}$ and $3.6 \times 3.7 \times 4.7 \mathrm{~cm}$. Core biopsy reveals high grade neoplasm, favoring lymphoma, cMYC rearrangements on FISH along with CD20, CD10 and Bc16 positivity and negative MUM on immunohistochemistry, consistent with DLBCL. The patient was discharged home for continued care in oncology and HIV clinics.

IMPACT/DISCUSSION: The advent of HAART has put a dent in the incidence of AIDS-related illnesses such as NHL including DLBCL. Prior to HAART, the risk of AIDS-related NHL maintained an inverse relationship with CD4 counts, the lower the CD4 count, the higher the risk. Post HAART, AIDS-related NHL is seen at higher CD4 counts but vulnerable populations such as African Americans, are disproportionately affected by HIV and are still being diagnosed for the first time with HIV/AIDS when they present with NHL and as such have markedly decreased CD4 counts akin to those seen prior to HAART. Many hospitals have adopted the "Optout" policy for HIV testing where patients are notified and HIV testing gets performed unless the patient declines. This policy has proven useful for diagnosing HIV in individuals who would have otherwise gone undiagnosed as is the case with our patient. DLBCL occurs in two-thirds of AIDS-related NHL cases and usually presents with lymphadenopathy with or without "B" symptoms though immunocompromised individuals tend to present with extra-nodal disease. However, the gluteus muscle is an unusual extra-nodal manifestation of DLBCL. Our patient posed a diagnostic dilemma in that she was morbidly obese which made it difficult to appreciate the gluteal mass on physical exam and her pain characteristics seemed more consistent with sciatica.

CONCLUSION: Despite significant progress in the fight against HIV/ AIDS a subset of high risk individuals continue to present with an AIDSrelated illness such as DLBCL at the time of diagnosis. Maintaining a broad differential even with common presentations is tantamount in preventing anchoring bias.
PAINFUL NECROTIC SKIN ULCERS IN AN END STAGE RENAL DISEASE PATIENT DIAGNOSED AS WARFARIN INDUCED SKIN NECROSIS (WISN) AND CALCIFIC UREMIC ARTERIOLOPATHY (CUA)

Evan R. Gordon-Wollin ${ }^{1}$; Minho Yu ${ }^{1,2}$. ${ }^{1}$ Loma Linda Medical Center, Rancho Cucamonga, CA $;{ }^{2}$ Riverside University Health System, Moreno Valley, CA. (Control ID \#3181023)

LEARNING OBJECTIVE \#1: Identify the features of CUA/ calciphylaxis and WISN in order to treat early.

LEARNING OBJECTIVE \#2: Recognize the risk factors for CUA and WISN in end stage renal disease (ESRD) patients.

CASE: 49-year-old female with a past medical history of ESRD on hemodialysis who was admitted for worsening necrotic skin wounds for one week. She denied fever or chills. Five months prior, she presented with leg ulcers after being on Warfarin for two months, and a punch biopsy confirmed WISN. Warfarin was discontinued, but eventually her wounds progressed and spread, prompting monthly admissions. She underwent one prior surgical debridement, which showed gangrenous necrosis on pathology. Home medications included aspirin, sevelamer, and simvastatin. Surgical history was significant for two failed renal transplants. Her family history was non-contributory. She had a prior 7.5 pack year smoking history, and she denied alcohol or drug use. Her vital signs were stable, and on exam she had multiple necrotic wounds scattered on her bilateral lower extremities and abdomen that had increased in size, number, and severity. Labs were significant for normal white count, calcium and phosphorus within normal limits, and iPTH elevated at $142.7 \mathrm{pg} / \mathrm{mL}$. Prior hypercoagulable workup showed decreased antithrombin III activity at 78\% (ref $88-138 \%$ ). The patient had a second surgical debridement of her wounds, showing calcium deposits, consistent with CUA. She then underwent twenty hyperbaric oxygen sessions, local wound care, and sodium thiosulfate therapy (STS) $25 \mathrm{gm}$ TIW, with significant improvement after two months.

IMPACT/DISCUSSION: Ulcerating wounds among ESRD patients are common given their high prevalence of peripheral arterial disease. Although both conditions are rare, the differential diagnosis should include CUA and WISN when an ulcer is found in an atypical location (i.e. abdomen), does not resolve, the patient is on Warfarin therapy, or has risk factors such as protein $\mathrm{C}$ and $\mathrm{S}$ or antithrombin III deficiency. Warfarin has been found to increase the odds of CUA up to 10-fold, and ESRD decreases the levels of protein S. While WISN typically occurs within the first ten days of initiating Warfarin, as this case and other case reports have shown, it can present much later. Prompt Warfarin discontinuation and reversal greatly improves outcomes. Multiple biopsies and a high clinical suspicion are often necessary to diagnose CUA. Optimal treatment is largely unknown without large randomized controlled studies. However, as seen in our patient, some retrospective reviews have shown that the use of STS led to a $26 \%$ resolution and $47.2 \%$ improvement in CUA wounds (Nigwekar et al. CJASN, 2013). Similarly, An et al. (Nephrology, 2015) showed a 58\% improvement in wounds with HBO therapy among 34 patients.

CONCLUSION: It is vital for a clinician to be aware of the risk factors and presentations of CUA and WISN in ESRD patients, because each diagnosis carries high morbidity and mortality. Early and appropriate treatment has been shown to improve outcomes.

\section{PAINTING THE BIG M}

Navid Berenji; Abdulrahman S. Museedi; Saket Kottewar. UT Health San Antonio, San Antonio, TX. (Control ID \#3185876)

LEARNING OBJECTIVE \#1: Recognize incidence of Multiple Myeloma in young patients with no genetic predisposition

LEARNING OBJECTIVE \#2: Assess occupational risk factors in Multiple Myeloma 
CASE: A 39-year-old Hispanic man presented with four weeks of dry cough. The cough was associated with minimal dyspnea on exertion, low grade intermittent fevers, back pain and pleuritic chest pain related to frequent coughing, and recent unintentional weight loss of 20 pounds over 2 months due to anorexia. Patient denied dyspnea at rest, night sweats, fatigue, recent travel or incarceration, or sick contact. Physical exam was unremarkable except for conjunctival pallor. Labs were significant for Hemoglobin 5.9 g/dl, Hematocrit 19.6\%, MCV 96.6 fl, MCH 29.1 pg, MCHC $30.1 \mathrm{~g} / \mathrm{dl}$, reticulocyte $3.8 \%$, and peripheral smear revealed rouleaux formation. Complete Blood Count with differential, Basic Metabolic panel including serum Calcium and Creatinine, and Urine studies including urine protein were unremarkable on admission. However, patient was found to have a higher total protein to albumin gap of $12.4 \mathrm{~g} / \mathrm{dl}$. Further investigations revealed kappa/lambda ratio of 17 and beta-2 microglobulin of $4.9 \mathrm{mg} / \mathrm{L}$. Serum protein electrophoresis and immunofixation did not demonstrate a monoclonal spike. Skeletal survey revealed diffuse lytic lesions in the axial and appendicular skeleton. We proceeded with Bone marrow biopsy, which revealed $78 \%$ plasma cell population, and fluorescence in-situ hybridization revealed hyperdiploid clone. Patient was started on chemotherapy for Multiple myeloma.

IMPACT/DISCUSSION: Multiple Myeloma (MM) is a disease of older adults with mean age of 68 years old. MM occurs in all races, but the incidence is variable by ethnicity: higher in African Americans, while lower in Mexicans and Japanese. While some cases appear to be familial, there are studies showing an increased incidence of MM with occupational exposure. This is a case of a young patient with non-measurable free light chain only MM that is very rare in patients younger than 40 years of age. Our patient did not have risk factors for MM given his young age, Mexican heritage, and lack of family history. His painting occupation, however, is a concerning risk factor for occupational exposure leading to MM.

CONCLUSION: Few case reports have suggested a higher incidence of MM in patients with Chlorinated solvent exposure, which is commonly found in paint thinners. This relationship is however not well studied. This case serves to make physicians aware to recognize MM in young patients with appropriate clinical suspicion and laboratory findings, while assessing for occupational risk factors.

\section{PARAGLOTTIC FUNGAL ABSCESS IN A PATIENT WITH UN- CONTROLLED DIABETES}

Ayah A. Megahed ${ }^{1}$; Lenar Latypov ${ }^{1}$; Jose Amaya Suarez ${ }^{2} .{ }^{1}$ Lincoln Medical and Mental Health care Centre, New York, NY; ${ }^{2}$ Lincoln Medical and Mental Health Centre, New York, NY. (Control ID \#3165587)

LEARNING OBJECTIVE \#1: To maintain a high clinical index of suspicion for diagnosing paraglottic abscess as a cause of upper airway obstruction, as prompt and urgent treatment is required to prevent potentially lethal outcome

CASE: The patient is a 26 years old female with PMH of DM II, GERD and hyperthyrodism with repeated admissions for DKA with history of multiple intubations. She was admitted due to progressively worsening dyspnea, productive cough, low grade fever, sore throat and hoarseness. Laryngoscopy showing unilateral vocal fold immobility. She was admitted to the medical floor for monitoring of her respiratory status. The patient reported pain in the right side of the neck radiating to the right ear. The pain was not controlled with NSAIDs and the patient was started on opioids. Subsequently, she developed stridor, for which she received prednisone. ENT were consulted. Laryngoscopy was done repeatedly evidencing progressive worsening of vocal cord paralysis, which warranted a CT scan of the neck. CT scan showed right glottic abscess compressing the laryngeal airway. Patient underwent tracheostomy- for worsening stridor- and drainage of the fluid, culture was positive for $\mathrm{C}$. albicans. She was started on IV Fluconazole. Post-operative CT scan revealed multi-loculated mass that required a surgical re-intervention with
Penrose drains, it showed clear purulent discharge. The patient's condition improved, and she was discharged with the tracheostomy, to follow up with ENT as an outpatient.

IMPACT/DISCUSSION: Laryngeal abscess is usually seen in patients with immunosuppression, underlying malignancy, trauma due to airway instrumentation, and external beam radiotherapy. It usually presents as hoarseness, dysphagia, dysphonia, odynophagia, stridor and respiratory distress. The most common isolated organisms are Haemophilus Influenzae, Methicillinresistant Staphylococcus aureus, Streptococcus Pneumoniae, Meningococci, Haemophilus parainfluenza, Kleibsella pneumoniae, Nocardia species and Candida albicans. Treatment decisions should be dictated by the severity of the obstruction of the patient's airway and the size of the abscess. Laryngeal abscess treatments include airway management, surgical drainage of the abscess and IV antibiotics. Airway obstruction can require the immediate establishment of an airway-either by intubation or acute surgical intervention (e.g. a tracheostomy or cricothyroidotomy). Surgical drainage of the abscess is recommended if indicated.

CONCLUSION: Despite being a rare condition, laryngeal abscess should be considered as a differential diagnosis in the presentation of upper airway obstruction in patients with history of poorly controlled diabetes or other medical condition associated with immunosuppression, and history of airway instrumentation.

\section{PARATHYROID CRISIS IN A PATIENT WITH A PARATHY- ROID ADENOMA}

Nicholas Ojile ${ }^{1}$; Georges Elhomsy ${ }^{2} .{ }^{1}$ University of Kansas Wichita, Wichita, KS; ${ }^{2}$ Kansas University-Wichita,, Wichita, KS. (Control ID \#3184259)

LEARNING OBJECTIVE \#1: Manage Parathyroid Crisis

LEARNING OBJECTIVE \#2: Recognize the underlying causes for signficantly elevated parathryoid hormone levels

CASE: A 72 year old female was admitted for hypercalcemia. Family members reported that over the past several days the patient had become more confused, lethargic, and weak; she fell the day of her admission. Initial laboratory evaluation showed serum calcium of $14.7 \mathrm{mg} / \mathrm{dl}$ (normal 8.5-10.1 mg/dl) and phosphorus of $2.5 \mathrm{mg} / \mathrm{dl}$ (normal 2.5-4.9 mg/dl). A serum intact PTH was $1351 \mathrm{pg} / \mathrm{ml}$ (normal 14-85 pg/ml). Her renal function was normal. The patient was volume resuscitated with crystalloid fluids and subsequently treated with intravenous furosemide. She was also given $4 \mathrm{mg}$ of zoledronic acid. A Sestamibi scan was suggestive of a parathyroid adenoma, and her serum calcium normalized prior to parathyroidectomy. A right inferior parathyroid gland mass was resected and was consistent with a parathyroid adenoma on pathology. The first fragment measured 4x 2x $1.3 \mathrm{~cm}$ and had a mass of $3.175 \mathrm{~g}$; the second fragment measured $3 \times 1.4$ x.9 cm with a mass of $1.286 \mathrm{~g}$. Postoperatively, the patient developed hypocalcemia and required calcium supplementation.

IMPACT/DISCUSSION: Parathyroid crisis is a rare complication of primary hyperparathyroidism, occurring in $1.6 \%$ to $6 \%$ of primary hyperparathyroidism cases. Patients usually present with markedly elevated PTH levels and a calcium level greater than $14 \mathrm{mg} / \mathrm{dl}$ with symptoms of hypercalcemia. The laboratory evaluation of parathyroid crisis from an adenoma can be confused with parathyroid malignancy as both can present with markedly elevated PTH levels and should be included in the differential diagnosis. Despite the signficantly elevated laboratory values associated with parathyroid carcinoma, parathyroid adenomas are the usual pathology found. These patients are at high risk for multi-organ decompensation due to the severe hypercalcemia including oliguria, coma, cardiac arrhythmias, or even cardiac arrest if the serum calcium level is not decreased prior to surgery with fluid resuscitation and loop diuretics. The use of bisphosphonates prior to parathyroidectomy as in this case is controversial, as parathyroidectomy is the definitive treatment for parathyroid crisis and bisphosphonates could potentially worsen 
postooperative hypocalcemia. The use of bisphosphonates in parathyroid crisis in the literature is limited to case studies and small case series, which do not suggest, however, an increased risk of postoperative hypocalcemia. CONCLUSION: Parathyroidectomy is the definitive treatment for parathyroid crisis, with fluids and diuretics used as a stabilizing measure. Despite signficantly elevated parathyroid hormone levels being associated with parathyroid carcinoma, parathyroid adenomas are more common.

\section{PAROXYSMAL NOCTURNAL HEMOGLOBINURIA MASQUERADING AS AN ULCERATIVE COLITIS FLARE}

Jared D. Romeo; Joseph M. Rocco. University of Pittsburgh, Pittsburgh, PA. (Control ID \#3185548)

LEARNING OBJECTIVE \#1: Recognize the limits of ulcerative colitis (UC) assessment

LEARNING OBJECTIVE \#2: Highlight an illness script for paroxysmal nocturnal hemoglobinuria $(\mathrm{PNH})$

CASE: A 29-year-old woman with UC on infliximab presented with 1 week of hematochezia and abdominal pain. One month prior, she had right upper quadrant pain with transaminase elevations. CT scan of the abdomen was notable for a heterogenous liver, but she improved with supportive care. Two weeks later, diplopia prompted MRI of the brain which revealed right sigmoid and transverse sinus thrombosis. Warfarin was started but a hypercoagulable workup was negative. One week later, abdominal pain and hematochezia started. The pain was similar but more diffuse than in prior flares. Labs showed Hgb $10.9 \mathrm{~g} / \mathrm{dL}$, platelet 163,000, and bilirubin $1.7 \mathrm{mg} /$ dL. Stool culture, clostridium difficile, and parasite assays were negative. Parenteral steroids for presumed UC flare were initiated. The next day the patient's $\mathrm{Hgb}$ and platelets continued to decrease. Bilirubin was now $2.7 \mathrm{mg} /$ $\mathrm{dL}$, haptoglobin $<30 \mathrm{mg} / \mathrm{dL}$, and LDH 940 suggested hemolysis. A colonoscopy revealed new thrombi within the colonic lamina suggestive of ischemic colitis. Her prior abdominal CT was re-evaluated, and hepatic vein thrombosis was evident. Flow cytometry was performed and revealed decreased expression of CD59 consistent with PNH. The patient was started on eculizumab and at the time of discharge had resolved abdominal pain and stable hemoglobin.

IMPACT/DISCUSSION: Diagnosis of UC is challenging as no set diagnostic criteria exist. Endoscopically, UC presents as continuous erythema, edema, ulceration, and/or bleeding, but up to $15 \%$ of UC cases are indistinguishable from other causes of colitis. Histopathology showing crypt distortion and hypercellularity are consistent with but not specific for UC. Diagnosis is further complicated by lack of correlation between endoscopic and pathologic findings. Thus, clinical correlation is paramount to diagnosing UC and flares. In our patient, slight differences in her symptomatology and unexplained hemolysis suggested an alternative diagnosis for her pain. PNH is characterized by non-immune hemolysis, pancytopenia, and venous thrombosis. The pathophysiology of PNH is an acquired deficiency of glycolipid GPI which anchors CD59 to the surface of RBCs. CD59 typically prevents complement mediated destruction of RBCs. The presentation of Budd-Chiari with dural sinus thrombosis is often seen in $\mathrm{PNH}$, as in our patient, which prompted flow cytometric analysis. Currently, there is no clear mechanism to explain thrombosis in PNH. First line treatment is with eculizumab, but bone marrow transplant is curative. This case highlights the pitfalls of diagnosing abdominal pain in the setting of UC. Physicians should keep in mind that neither endoscopy nor pathology are specific for UC, and alternative diagnoses must always be considered.

CONCLUSION: There is no gold standard for diagnosing UC. PNH should be considered in patients that have atypical venous thrombosis.

\section{PATIENT-DIRECTED CRYOTHERAPY AS A CAUSE OF PE- RIPHERAL NEUROPATHY}

Victoria G. Gillet ${ }^{1}$; David Feldstein ${ }^{2}$; Linda Baier Manwell ${ }^{3}$; Brian Lewis ${ }^{4}$; Katie Miller . ${ }^{1}$ University of Wisconsin, Madison, WI; ${ }^{2}$ University of
Wisconsin School of Medicine and Public Health, Madison, WI; ${ }^{3}$ University of Wisconsin-Madison, Madison, WI; ${ }^{4}$ University of Wisconsin, Middleton, WI. (Control ID \#3185702)

LEARNING OBJECTIVE \#1: Recognize patient-directed cryotherapy as a cause of peripheral nerve damage

LEARNING OBJECTIVE \#2: Explain the importance of giving clear instructions for non-pharmaceutical interventions

CASE: A 53-year-old female consulted urgent care for progressive lower extremity pain of 7 weeks duration. The pain localized to her right medial ankle with associated numbness in her medial arch. She ran 15 miles weekly and did daily bicycling and aerobics. She had tenderness to palpation over the medial talus, no ligamentous laxity, an intact Achilles tendon, intact plantarflexion, and no redness, erythema, or warmth. She was diagnosed with overuse injury and told to decrease her high-impact exercises and "ice as she sees fit". Her pain persisted. She consulted a primary care provider who diagnosed her with tarsal tunnel syndrome and recommended further activity reduction. She then self-referred to podiatry where she received custom orthotics. Her pain continued to progress. Over the next 4 months, she was seen by orthopedic surgery, sports medicine, and primary care without a clear diagnosis or pain alleviation. An MRI of her ankles and feet found no acute nerve or soft tissue injury and her labs were normal including ANA, RF, SPEP, fasting glucose, TSH, CK, B12, BMP, and CBC. Due to worsening pain, an EMG was performed. It revealed multiple absent sensory evoked potentials and motor unit loss in intrinsic foot muscles innervated by the tibial and peroneal nerves. At her next visit, 6 months after initial presentation, she reported icing her lower extremities continuously for two or more hours daily since symptoms began. She was told to stop cryotherapy and monitor symptoms. With the cessation of icing, her pain resolved over 12 months and she returned to her normal activities.

IMPACT/DISCUSSION: Cryotherapy after an acute sports injury is standard practice. It is recommended by professional organizations including the National Athletic Trainer's Association and the American Academy of Orthopedic Surgeons, but there is no high-quality evidence for duration or frequency of cryotherapy. The risk of neuropathy from cryotherapy is rare and experimental studies report that risk increases with treatments longer than 20 minutes. Injuries classically affect single nerves. The most commonly affected nerve is the peroneal, resulting in activitylimiting foot drop. Cryotherapy causing a chronic neuropathy or a peripheral polyneuropathy is less frequently documented. Based on this case, it is now my standard practice to provided written, detailed instructions for safe home cryotherapy to any patient with soft tissue injury.

CONCLUSION: 1. Include patient-directed cryotherapy in your differential diagnosis of peripheral neuropathy especially for physically active patients with normal labs and imaging 2. Make it standard practice to query ways in which patients manage symptoms at home 3 . Instruct patients how to safely use all prescribed therapies including nonpharmacologic techniques

\section{PECTORAL PAIN, A PALPABLE MASS, AND A POOR PROG- NOSIS: CHONDROSARCOMA AS AN ATYPICAL CAUSE OF CHEST PAIN}

Lydia A. Flier; Sydni M. Cole. Beth Israel Deaconess Medical Center, Brookline, MA. (Control ID \#3185781)

LEARNING OBJECTIVE \#1: Recognize features of high-risk chest wall masses in the primary care setting.

LEARNING OBJECTIVE \#2: Diagnose chest wall tumors with the appropriate imaging modality.

CASE: A 72 year old male Jehovah's witness with anxiety, an unspecified learning disability, and remote prostate cancer, presents to his PCP with four weeks of sharp mid-chest pain, increasing in intensity and "incapacitating." Initial evaluation, including ECG, is reassuring, and there was no 
change in management. He re-presents two months later with episodes of chest pain lasting several minutes, having noticed a new $2 \mathrm{~cm}$, nontender, left-sided mass adjacent to the sternum. Chest $\mathrm{x}$-ray and ultrasound at this time are unrevealing. A subsequent CT chest reveals a $9 \mathrm{~cm}$ sternal lesion, and biopsy shows a grade 2 chondrosarcoma of the sternum, which is resected by thoracic surgery. One month post- operatively, he re-presents with a new $2 \mathrm{~cm}$ chest wall mass thought to be a postoperative hematoma, which is imaged and drained. Two weeks later, he re-presents with enlarging left chest wall mass and persistent drainage and is admitted to the thoracic surgery service. MRI shows a $21 \mathrm{~cm}$ mass with lung nodules concerning for metastases, with ongoing chondrosarcoma on pathology. $\mathrm{He}$ is transferred to oncology, where he is initiated on ifosfamide chemotherapy. His course is complicated by ongong bleeding from the surgical site and tumor, as well as agitation and clumsiness thought to be toxicity from ifosfamide. His clinical status does not improve, and he ultimately transitions to palliative-focused care. He passes away 5 months after his initial presentation for chest wall pain.

IMPACT/DISCUSSION: The differential for non-cardiac chest pain is broad, including both musculoskeletal and visceral etiologies. Musculoskeletal chest pain is thought of as either isolated musculoskeletal (e.g. costochondritis), rheumatologic (e.g. spondyloarthritis), or systemic (e.g. neoplastic). Most non-cardiac chest pain is not neoplastic in origin, thus chest wall tumors are rarely considered in this differential. However, chest wall pain is the most common symptom of both benign and malignant chest wall tumors - a diagnosis likely to be missed if a careful examination is not performed. Generally, chest wall imaging should not be pursued for chest pain without a palpable mass. If a mass is palpated on exam, imaging should be done, with $\mathrm{CT}$ chest the imaging modality of choice. $50-80 \%$ of chest wall tumors are malignant; older age and male gender are predictors of malignancy. Chondrosarcomas are the most common malignant chest wall tumors, are not chemosensitive, and require excision with $4 \mathrm{~cm}$ margins. 5-year survival post-resection of chondrosarcomas is $92 \%$, but survival is much lower after local recurrence.

CONCLUSION: Painful palpable chest wall masses, particularly in older men, are highly suspicious for malignancy. CT imaging is the modality of choice to evaluate for chest wall tumors. Survival post-resection of chondrosarcoma is greatly reduced after local recurrence.

\section{PENETRATING CHEST INJURY LEADING TO METHICILLIN- RESISTANT STAPHYLOCOCCUS AUREUS (MRSA) BACTER- EMIA AND MYCOTIC ABDOMINAL AORTIC ANEURYSM IN IMMUNOCOMPROMISED PATIENT}

Sunjay Modi; Dilshan Dhillon; Paul Nona; Daniel S. Moore. Henry Ford Hospital, Detroit, MI. (Control ID \#3170815)

LEARNING OBJECTIVE \#1: Recognize that mycotic aneurysm is a term used to describe any localized dilation of an artery due to destruction of the vessel wall by infection. This aneurysm can result from the infection or a preexisting aneurysm that becomes infected.

LEARNING OBJECTIVE \#2: Recognize that trauma, endocarditis and impaired immunity are common risk factors for infected aneurysms.

CASE: A 52-year-old male with a PMHx of chronic lower back pain s/p laminectomy, presented with a 4 day history of right-sided flank pain, subjective fevers and chills. Additional symptoms included dysuria along with episodic suprapubic pain. Vitals were significant for episodic fever. Physical examination was significant for superficial skin abscesses. He did admit to a recent history of incarceration and reported that he was stabbed in the right upper chest which resulted in a pneumothorax. There was a high index of suspicion for pyelonephritis. UA showed findings consistent with UTI. Initial urine cultures and blood cultures came back positive for MRSA. Due to evidence of persistent bacteremia, IV daptomycin and ceftaroline was initiated. CT chest showed findings consistent with septic emboli in the lungs. Echocardiogram was obtained and subsequent transesophageal echocardiogram which were negative for vegetations. MRI was negative for spinal abscess. HIV-1 quantitative and total CD4+ T cell count were obtained and revealed values 102,587 and 11 , respectively. At this time, the patient began complaining of acute abdominal pain and painful pulsatile abdominal mass was appreciated on physical exam. CT-abdomen revealed a $3.1 \times 2.8 \times 3.5 \mathrm{~cm}$ saccular mycotic aneurysm projecting off the right side of the abdominal aorta. The patient was treated with 6 weeks of daptomycin and placed on dapsone and azithromycin prophylaxis following completion of daptomycin. The patient underwent surgical repair of the aneurysm with a rifampin-soaked Dacron graft and right nephrectomy for an infarcted right kidney.

IMPACT/DISCUSSION: This case highlights that pentrating chest injury in a immunocompromised host led to a MRSA bacteremia causing a mycotic aneurysm. The most commonly involved organisms in mycotic aneurysms include S. aureus, Streptococci and Salmonella. In our case, the patient's risk factors for an infected aneurysm were the stab wound which was a likely inoculation site for MRSA and impaired immunity (HIV+). Clinical findings are a painful, pulsatile mass in the abdomen. CT angiography is the diagnostic test of choice. Empiric antibiotics should include MRSA and gram negative coverage, while definitive treatment is surgical excision of the aneurysm and extensive debridement of infected tissues.

CONCLUSION: - Trauma such as penetrating chest wall injury, endocarditis, and immunocompromised hosts are common risk factors for infected aneurysms - Clinical presentation can include a painful, pulsatile mass - The diagnosis of an infected aneurysm is based upon imaging of the aneurysm and infection is confirmed with blood cultures and/or cultures from aneurysm wall.

\section{PERICARDIAL EFFUSION AS AN INITIAL PRESENTATION OF MYXEDEMA COMA}

Dana Awad; Omar Kousa; Abdallah Qasim; Mohsin Mirza; Emily P. Walker. Creighton University, Omaha, NE. (Control ID \#3186260)

LEARNING OBJECTIVE \#1: Recognize and treat the early manifestation of Myxedema coma that can affect multiple organ systems and cause cardiovascular collapse.

LEARNING OBJECTIVE \#2: Recognize the common cardiovascular changes in Myxedema coma that include cardiomegaly, bradycardia, decreased ventricular contractility, low voltage ECG and rarely, pericardial effusion that can progress to Tamponade.

CASE: A 63-year old white female presented to the ED with worsening dyspnea and cough over the previous two weeks. She has a history of hypothyroidism status post thyroid radio-ablation for Grave's disease greater than 20 years prior. She was poorly compliant with thyroid replacement therapy. On physical examination, she was lethargic and in respiratory distress with low $\mathrm{O} 2$ saturation. Examination of the head revealed facial plethora and enlarged tongue. She had diminished heart sounds and decreased air entry bilaterally on chest auscultation. A a chest x-ray showed enlarged cardiac silhouette and ECG demonstrated low voltage QRS complexes. Echocardiogram was significant for ejection fraction of 50-55\% with circumferential pericardial effusion. She was promptly admitted for stabilization and further work up. On the night following admission, she became more lethargic and unresponsive to stimulus. Temperature was $35 \mathrm{C}$, she developed bradycardia, hypoxia and hypercarbia. Repeat chest $\mathrm{x}$-ray was consistent with acute respiratory distress syndrome (ARDS). She was intubated and remained sedated on a ventilator with vasopressor support and broad-spectrum antibiotic coverage. Initial thyroid function tests resulted with severe hypothyroidism and the diagnosis of myxedema coma was made. She was started on IV levothyroxine daily. Morning serum cortisol on that same day ruled out adrenal insufficiency. On the second day, her heart rate and temperature gradually started to improve. Four days later she became hemodynamically stable and vasopressors were stopped. Thyroxine replacement was continued throughout her hospital stay. She gradually regained 
consciousness, and was successfully extubated on day 10 of hospitalization. She was then switched to oral thyroxine, and discharged to acute rehabilitation a few days later with a plan to follow up shortly with endocrinology.

IMPACT/DISCUSSION: Myxedema coma is an extreme form of hypothyroidism and presents as a life threatening decompensated state presenting most commonly with hypothermia and altered mental status. This case illustrates the importance of early recognition and treatment of myxedema coma, as presentation can sometimes be atypical and clinical manifestations are not restricted to one organ system.

CONCLUSION: Pericardial effusion is relatively rare in the early mild state of hypothyroidism and as prevalent as $80 \%$ when myxedema coma is present. Delay in treatment can be life threatening and it has a high mortality rate of $30-50 \%$.

\section{PERICARDITIS AS A MANIFESTATION OF GRANULOMATO- SIS WITH POLYANGIITIS}

Neeharika R. Ralh; Sourabh Sourabh; Tanaz Salimnia; Daymon Peterson; Shubhkarman Dhillon. Wayne State University, Rochester Hills, MI. (Control ID \#3185241)

LEARNING OBJECTIVE \#1: Granulomatosis with polyangiitis (GPA), formerly known as Wegner's granulomatosis, is an uncommon ANCAassociated vasculitis which is typically known for its sinus, lung and renal involvement. Previously believed to be rare, cardiac manifestations may not be so uncommon. Pericarditis, arteritis, myocarditis, valvulitis and arrhythmias have all been previously recognized in this subset of patients.

CASE: A 48-year-old male with a PMH of GPA with renal involvement presents with acute onset sharp, mid-chest pain associated with positional changes. Workup was significant for diffusely low voltage EKG, elevated troponins in the setting of ESRD with otherwise unremarkable chemistry profile. Echocardiogram and computed tomography of the chest were significant for a moderate to large sized pericardial effusion, $3 \mathrm{~cm}$ in thickness. Hemodynamic instability concerning for cardiac tamponade prompted emergent pericardial window. Pericardial fluid analysis was unremarkable, no exudates, abnormal histology or malignancy noted. Pericardial tissue revealed fibrinous inflammatory changes. Serum

ANCA titers were 1:1280. Treatment with high-dose methylprednisolone and mycophenolate were initiated with immediate relief of symptoms. Patient was diagnosed with pericardial effusion and pericarditis secondary to Wegner's granulomatosis.

IMPACT/DISCUSSION: Cardiac involvement of GPA may occur in up to $6-25 \%$ of patients and up to $44 \%$ in those with known renal involvement. Pericarditis and coronary vasculitis are the most frequent manifestations in up to $50 \%$ of cases.

CONCLUSION: GPA should be considered high on the differential for those with positive history of GPA who present with nonspecific cardiac findings. Complications such as constrictive pericarditis are not uncommon, thus prompt diagnosis and treatment are necessary.

\section{PERIPHERAL NEUROPATHY: DON'T ANCA ON THE DIAGNOSIS}

Staci Saunders; Rahul Gore; Terrance Rodrigues; Elizabeth Targan. Beth Israel Deaconess Medical Center, Jamaica Plain, MA. (Control ID \#3178458)

LEARNING OBJECTIVE \#1: Recognize systemic vasculitis as a cause of peripheral neuropathy

LEARNING OBJECTIVE \#2: Recognize treatment options for severe GPA

CASE: A 66 year-old woman presents with three weeks of acute onset bilateral lower extremity pain and foot weakness; two weeks of dyspnea, non-productive cough, epistaxis; and one week of oliguria and lower extremity edema. Past medical history includes COPD, prior heavy tobacco use, and pre-diabetes. Medications and allergies are noncontributory. Her father is deceased from "kidney failure." Physical exam notable for diffuse rhonchi and crackles, $1+$ bilateral lower extremity edema, $3 / 5$ left dorsiflexion, $4 / 5$ right dorsiflexion, and decreased sensation of bilateral feet. Labs notable for Hgb 7.0 (11.2-15.7), K 5.9 (3.5-5.4), HCO3 13 (22-32), BUN 89 (6-20), and Cr 8.2 (0.4-1.1). Hepatitis C, HIV, ANA, dsDNA, and anti-GBM are negative. Urinalysis shows proteinuria, hematuria, and granular casts. CT chest reveals multifocal pneumonia. The patient is urgently intubated and initiated on dialysis. Bronchoscopy reveals diffuse alveolar hemorrhage ("DAH"). ANCA antibody returns positive with high titers of PR3 antibody, concerning for granulomatosis with polyangiitis. The patient is initiated on methylprednisolone, cyclophosphamide, and plasmapheresis.

IMPACT/DISCUSSION: Granulomatosis with polyangiitis ("GPA") is an autoimmune disease with granulomatous inflammation of the respiratory tract as well as ANCA-associated necrotizing vasculitis of smallmedium sized blood vessels of the lungs and kidneys. This is a case of GPA first presenting with peripheral neuropathy and later evolving to glomerulonephritis and DAH. Peripheral nervous system involvement, such as mononeuritis multiplex, occurs in $15-25 \%$ of GPA patients due to arteritis of the vasa nervorum leading to ischemic neuropathy. As such, patients develop asymmetric and abrupt onset peripheral neuropathy, most commonly of the fibular or tibial nerves. Polyneuropathy is more likely to develop in men, the elderly, or those with renal involvement, large disease burden, or high ANCA titers. Initial treatment of GPA consists of glucocorticoids and either cyclophosphamide or rituximab, as two-year mortality of untreated GPA is $\sim 90 \%$. Both cyclophosphamide and rituximab are highly efficacious in treating GPA. Plasmapheresis is controversial but often favored in those with glomerulonephritis, DAH, or those with concurrent anti-GBM. Given our patient's fulminant clinical course, she received high dose corticosteroids, cyclophosphamide, and five sessions of plasmapheresis.

CONCLUSION: This case demonstrates the importance of recognizing GPA as a systemic disease that can present as peripheral neuropathy, a chief concern often encountered in the primary care setting. Given the potentially devastating consequences of untreated GPA, this should be on the differential for acute onset neuropathy. Initial treatment consists of glucocorticoids and either cyclophosphamide or rituximab; with consideration of plasmapheresis in patients with severe features.

\section{PERSISTENT FATIGUE AS THE SOLE SYMPTOM OF A PO- TENTIALLY FATAL DISEASE}

Nicholas David $^{1}$; Brian Lewis ${ }^{2}$; David Feldstein ${ }^{3}$; Linda Baier ${ }^{4}$; Thomas D. Shiffler ${ }^{3} .{ }^{1}$ University of Wisconsin Hospitals and Clinics, Madison, WI; ${ }^{2}$ University of Wisconsin, Middleton, WI; ${ }^{3}$ University of Wisconsin School of Medicine and Public Health, Madison, WI; ${ }^{4} \mathrm{f}$ Medicine and Public Health, Madison, WI. (Control ID \#3184550)

LEARNING OBJECTIVE \#1: Recognize the ecology of Rocky Mountain Spotted Fever (RMSF).

LEARNING OBJECTIVE \#2: Identify early signs and symptoms of RMSF.

CASE: A 55-year-old man presented to his primary care physician's office in Wisconsin at the end of May with fatigue. He first noticed fatigue a few days ago while driving home to Wisconsin after a recent visit to Maryland, and he needed more coffee than usual to get through the drive. $\mathrm{He}$ reported being bitten by two ticks two weeks ago while working outside in Maryland. The ticks were attached for less than 12 hours. He denied fevers, headaches, myalgias, arthralgias, or rashes. There were four small dots of erythema on his left hip where he was bitten, but the physical exam was otherwise unremarkable. CBC, CMP, and serologies for tick-borne illnesses, including Lyme and RMSF, were all normal. He was counseled about preventing tick exposures and instructed to return if more symptoms 
developed. The patient's fatigue persisted over the next two weeks. Upon follow up with his PCP, he reported no fevers, and his physical exam was unchanged. Repeat testing returned an RMSF IgG titer of 1:256. He was treated successfully for RMSF with seven days of doxycycline.

IMPACT/DISCUSSION: RMSF is a potentially fatal tick-borne illness. It is classically endemic to the southeastern and southcentral United States, where it is transmitted by the American dog tick. Up to $60 \%$ of cases are from five states ( $\mathrm{NC}, \mathrm{OK}, \mathrm{AR}, \mathrm{TN}$, and $\mathrm{MO}$ ), but cases have been reported in all of the lower 48 states except NM. In the northwestern United States, RMSF is spread by the Rocky Mountain wood tick. Although cases have been reported in all months of the year, most occur between May and August. Ticks are both vectors and reservoirs for the disease; thus disease can be transmitted by feeding ticks in all stages of the life cycle. Ticks only need to be attached for 6-10 hours to transmit disease. Most cases become symptomatic 5-7 days after tick exposure, and early symptoms are generally non-specific. They can include fever, headaches, myalgias, and arthralgias. A disseminated rash appears in up to $90 \%$ of patients between the third and fifth days of illness. IgG antibody testing, the gold standard of serological diagnosis, turns positive 7-10 days after the onset of symptoms or 12-17 days after the initial tick bite. The above patient presentation demonstrates the importance of understanding the ecology, signs, and symptoms of RMSF in order to make a timely diagnosis. The case has heightened my clinical suspicion for this deadly disease, even though I don't practice in an endemic area. CONCLUSION: RMSF is characteristically transmitted by the American dog tick in the Southeastern United States in a matter of hours, but geographical distribution and even tick species are variable. Recognizing a concerning symptomatology in the right ecological context is key to making a timely diagnosis.

\section{PERSISTENT HYPERKALEMIA IN CHRONIC KIDNEY DIS- EASE DUE TO TYPE IV RENAL TUBULAR ACIDOSIS}

Brian Chernak $^{1}$; Ryan Bober ${ }^{1}$; Alice Tang ${ }^{2} .{ }^{1}$ NewYork-Presbyterian Weill Cornell Medical Center, New York City, NY; ${ }^{2}$ Weill Cornell Medical College, New York City, NY. (Control ID \#3185758)

LEARNING OBJECTIVE \#1: Develop an approach to diagnosis and management of refractory hyperkalemia

LEARNING OBJECTIVE \#2: Recognize Type IV Renal Tubular Acidosis as a cause for refractory hyperkalemia in chronic kidney disease

CASE: A 71 year old male with diabetes and chronic kidney disease presented with acute on chronic kidney disease following a mechanical fall. His labs were significant for potassium 6.5 and creatinine 2.94; his baseline creatinine was 1.8 . He had a mild metabolic acidosis with a $\mathrm{pH}$ 7.32 , bicarbonate of 23 , and was hyperchloremic to 110 . His Hemoglobin A1c was 8.2. The patient received intravenous furosemide, insulin, and sodium polystyrene, with mild improvement of his potassium. Due to persistent hyperkalemia, he received additional furosemide, insulin, and sodium polystyrene, but his potassium continued to increase. Morning cortisol and aldosterone levels were normal, but plasma free renin was low-normal. Urinary potassium excretion was $43.9 \mathrm{mmol} / \mathrm{L}$. Due to the high suspicion for Type IV Renal Tubular Acidosis (RTA), fludrocortisone $0.1 \mathrm{mg}$ was started with immediate improvement in his potassium from 5.9 to 5.4; it remained within normal levels throughout the remainder of his admission despite minimal change in his creatinine, consistent with a Type IV RTA. He was discharged on fludrocortisone with normal potassium levels.

IMPACT/DISCUSSION: Persistent hyperkalemia is commonly encountered on the general medicine service, especially in patients with chronic kidney disease. Initial evaluation should include assessing for increased potassium intake, pseudohyperkalemia, and medication-related effects. Subsequently, urinary $\mathrm{pH}$, sodium, and potassium can suggest processes which limit potassium excretion, such as type IV RTA, which is characterized by persistent hyperkalemia in the setting of no other identifiable cause. The cause is typically due to hypoaldosteronism, leading to impaired sodium-potassium exchange in the distal tubule, thereby limiting potassium and proton excretion, causing hyperkalemia and hyperchloremic metabolic acidosis. A recent study has found up to $42 \%$ of admitted hyperkalemic patients without ESRD were found to have a Type IV RTA, especially those with underlying renal disease and diabetes. Type IV RTA should be considered in patients with persistent hyperkalemia that is refractory to standard therapies. Low to lownormal plasma renin activity, hyperchloremia, and metabolic acidosis may be suggestive, although lab abnormalities may be minimal. Low urinary potassium excretion can be limited in utility due to a lack of a definitive cutoff value. A mineralocorticoid challenge, which acts in place of aldosterone at receptors in the distal tubule, can facilitate potassium and proton excretion, and identify patients with RTAs.

CONCLUSION: Type IV Renal Tubular Acidosis presents with refractory hyperkalemia but can be difficult to identify in patients with CKD. Diagnosis involves exclusion of other causes of hyperkalemia, lab analysis, and a mineralocorticoid challenge to ensure appropriate diagnosis.

\section{PERSISTENT HYPOGLYCEMIA IN A PATIENT WITH POLYSUBSTANCE USE DISORDER ON HIGH-DOSE METHADONE}

Lurit Bepo. University of California, San Francisco, San Francisco, CA. (Control ID \#3186265)

LEARNING OBJECTIVE \#1: Identify and diagnose etiologies of persistent hypoglycemia

LEARNING OBJECTIVE \#2: Manage hypoglycemia due to suspected sulfonylurea overdose

CASE: A 53 year-old male with methamphetamine, benzodiazepine, and opiate use disorders on methadone, ESRD on hemodialysis, and untreated hepatitis $\mathrm{C}$ was brought in by ambulance for altered mental status and hypoglycemia to 37 after ingesting an unmarked tablet. Oral glucose, intramuscular glucagon, intravenous dextrose, octreotide, and naloxone were administered with mild improvement in his mental status and persistent hypoglycemia to the 40s. Head imaging, lab work, and infectious studies were unrevealing, and the patient was started on a dextrose infusion and admitted to the inpatient medicine service. Due to ongoing low blood glucoses, the dextrose infusion rate was increased, and he was started on octreotide for empiric treatment of a sulfonylurea overdose. Sulfonylurea panel was negative as was work up of insulinoma with normal insulin, proinsulin, and c-peptide levels. High-resolution mass spectrometry revealed metabolites of methadone, antidepressants, and cigarettes. The patient was dialyzed while inpatient, and his methadone dose was decreased from $100 \mathrm{mg}$ to $60 \mathrm{mg}$ daily due to concern for methadone-induced hypoglycemia. He continued to have episodes of hypoglycemia after dextrose was discontinued, but the exact etiology was never identified.

IMPACT/DISCUSSION: The sulfonylurea, glipizide, is similar in appearance to diazepam and is often substituted for the benzodiazepine in illicit drug sales. It is hepatically cleared and ingestion of multiple tablets of extended release glipizide would cause approximately 48-60 hours of hypoglycemia; this is irrespective of impaired renal function as was the case with this patient. Mainstays of management of sulfonylurea overdose are dextrose infusion and IV or IM octreotide administration, the latter which blocks pancreatic insulin release. Though the patient was initially suspected to have an accidental sulfonylurea overdose, his persistent hypoglycemia $>100$ hours after his ingestion raised concerns for alternative etiologies. These included an insulinoma, in which insulin, pro-insulin, and cpeptide levels are notably elevated during episodes of low blood glucose, and methadone-induced hypoglycemia. Previous case reports and observational studies have described glycemic dysregulation with hypoglycemia in the setting of rapid escalation or high 
doses of methadone above $40 \mathrm{mg}$. This patient's case was unusual in that the patient had reportedly been on $100 \mathrm{mg}$ of methadone for 5 years and $90 \mathrm{mg}$ for 10 years prior to that. The patient declined transition to buprenorphine at discharge for management of his opiate use disorder.

CONCLUSION: -Suspect sulfonylurea overdose in patients with a benzodiazepine use disorder who present with persistent hypoglycemia. -Treat suspected sulfonylurea overdose with dextrose infusion and IV or IM octreotide. -High-dose or rapid escalation of methadone can cause hypoglycemia.

\section{PID HIDING IN PLAIN SITE}

Kristen Wilson ${ }^{1}$; Amy H. Farkas ${ }^{1,}{ }^{2}$. ${ }^{1}$ Medical College of Wisconsin, $\overline{\text { Milwaukee, WI }}{ }^{2}$ Milwaukee VA Medical Center, Milwaukee, WI. (Control ID \#3182652)

LEARNING OBJECTIVE \#1: Recognize the importance of diagnosing pelvic inflammatory disease (PID)

LEARNING OBJECTIVE \#2: Understand how clinical biases can impact clinical reasoning

CASE: A 36-year-old female presented to primary care for abdominal pain following ventral hernia repair performed 4 weeks prior. On initial questioning, she reported achy abdominal pain along with difficulty sitting comfortably. She denied any pain at her incision sites, fever or chills, or associated urinary symptoms. Examination of her abdomen revealed healing incisions without tenderness. During the exam, she commented that the pain was more localized to her pelvis. Review of gynecologic history revealed that the patient had one sexual partner, last intercourse was one week prior to surgery, and she denied vaginal discharge. She did note abnormal spotting. Pregnancy test was negative. A pelvic exam revealed purulent discharge from the cervical os with fundal and adnexal tenderness. Cervical swabs were obtained for chlamydia, gonorrhea, and vaginitis panel. She was treated empirically for PID with ceftriaxone IM, oral doxycycline and metronidazole. On one-week follow-up, patient was clinically improved. Cultures were negative.

IMPACT/DISCUSSION: PID is a clinical diagnosis hallmarked by pelvic organ tenderness and inflammation of the lower genital tract which can lead to significant health outcomes. It is often missed by clinicians. While classic presenting symptoms include abrupt onset of severe lower abdominal pain following menses, increasingly women are presenting with less severe symptoms thought to be secondary to a shift in the microbial etiology involving nongonococcal or chlamydial species. Up to $50 \%$ of women with PID will have no evidence of gonorrhea or chlamydia on laboratory testing. As physicians are performing fewer screening pelvic exams they must remember to have a low threshold for performing the bimanual in a woman with an acute abdominal complaint. Too often, clinical bias results in anchoring and premature closure such as in this case where the provider initial anchored on the women's recent abdominal surgery as a cause of her pain only to discovery a benign abdomen on exam. While there are many categories of bias in clinical reasoning, the lead-in thought pattern that precedes them all is Type 1 processing wherein intuitive diagnoses are favored over those obtained via analytical reasoning. It is important for physicians to be aware of clinical bias in their diagnostic reasoning to prevent premature closure and missed diagnoses.

CONCLUSION: 1. PID is a clinical diagnosis that often has subtle findings 2. PID is associated with other organism beyond gonorrhea or chlamydia 3. Awareness of clinical bias is necessary to reduce barriers to identification of disease References Brunham, R. C., Gottlieb, S. L, \& Paavonen, J. (2015). Pelvic inflammatory disease. NEJM, 372(21). Croskerry, P., Mamede, S., \& Singhal, G. (2013). Cognitive debiasing 1: origins of bias and theory of debiasing. BMJ Quality \& Safety, 22(2): ii58-ii64.
PITFALL OF MYCOPLASMA PNEUMONIAE: PNEUMONIA?ENCEPHALITIS?MENINGITIS!

Ryutaro Nagaya; Yoshihiko Kawano; Takamasa Tanaka; Mitsunori Iwase. Toyota memorial hospital, Toyota-city, Japan. (Control ID \#3186269)

LEARNING OBJECTIVE \#1: Recognize that Mycoplasma pneumoniae may lead the aseptic meningitis.

CASE: The patient was a ten-year-old boy without a past medical history. He presented to our emergency room(ER) with a chief complaint of chest pain and nausea. Before visiting ER, he already visited some clinics three times because of mild fever and cough for 5 days but he was treated as just common cold. Chest X ray showed infiltrative shadow bilaterally. It clinically presumed pneumonia and he was admitted to our hospital. On the $2^{\text {nd }}$ day, loop-mediated isothermal amplification(LAMP) was positive, leading to our diagnosis of Mycoplasma pneumonia. Mycoplasma pneumoniae-specific antibody titers was 1:40. Clarithromycin $400 \mathrm{mg} /$ day was administrated. He was discharged without any symptoms on the $5^{\text {th }}$ day,. On the $6^{\text {th }}$ day he went to school, but felt fever, headache and nausea. Because these symptoms continued, he visited ER again. He was febrile and neck stiffness was present without disturbance of consciousness. Brudzinski and Kernig signs were positive. Cerebrospinal fluid (CSF) examination showed no pleocytosis, the protein level was $51 \mathrm{mg} /$ dl. Glucose in CSF was normal $(55 \mathrm{mg} / \mathrm{dl})$ but bacterial meningitis was not ruled out completely. Then cefotaxime and clarithromycin were administrated. On the $9^{\text {th }}$ day, fever sustained and we suspect macrolide-refractory mycoplasma pneumonia and minocycline was administrated. On the $11^{\text {st }}$ day, he responded well and recovered and blood culture test were negative. Mycoplasma pneumoniae-specific antibody titers was 1:360. He eventually discharged in good condition on on the $15^{\text {th }}$ day.

IMPACT/DISCUSSION: Impact When patients with pneumonia caused by Mycoplasma pneumoniae show neurological manifestations, we should consider that the symptoms are caused by Mycoplasma pneumoniae. Discussion Mycoplasma pneumoniae is one of the important causes of upper and lower respiratory tract infections during childhood especially 6 to 14-year-old children. Patients suffering Mycoplasma pneumoniae infection may have varying degrees of neurological complications at a ratio of approximately 6 to $7 \%$. Neurological manifestations include encephalitis, transverse myelitis, acute disseminated encephalomyelitis, Guillain-Barre syndrome, thromboembolic stroke and aseptic meningitis. The time period between the onset of respiratory symptoms and neurological symptoms varies 2 to 14 days. More than $80 \%$ of patients with CNS findings have concomitant respiratory infection. Actually, in this case, aseptic meningitis was evident 6 days after he had symptoms of respiratory tract. Although aseptic meningitis usually do not need any antibiotics, aseptic meningitis caused by Mycoplasma pneumoniae should be administrated some antibiotics clarithromycin and so on

CONCLUSION: We should consider that Mycoplasma pneumoniae cause neurological manifestations including aseptic meningitis after respiratory tract infections.

\section{PLATYPNEA-ORTHODEOXIA SYNDROME: NOT AS SIMPLE AS IT SOUNDS}

Harshit Dua; Ali Azim; Nyi Nyi Kyaw; Allan Tachauer. Weiss Memorial Hospital, Chicago, IL. (Control ID \#3185400)

LEARNING OBJECTIVE \#1: Recognize the clinical features of Platypnea-Orthodeoxia Syndrome (POS).

LEARNING OBJECTIVE \#2: Suspect POS in patients with no apparent causes of hypoxia and perform appropriate diagnostic testing to hasten its identification.

CASE: A 77-year-old female presented to the hospital with shortness of breath. She was found to be tachypneic, anxious and requiring $50 \%$ 
oxygen via ventimask (VM) to maintain saturation of $88 \%$. Chest radiograph was unremarkable, and an arterial blood gas revealed a $\mathrm{PaO} 2$ of $48 \mathrm{mmhg}$ on $100 \%$ oxygen via $\mathrm{VM}$, therefore she was placed on noninvasive ventilation. A ventilation/perfusion scan of the lungs was found to be unremarkable. Further testing ruled out acute coronary syndrome, heart failure and pneumonia. She had no prior history of any lung disease, smoking or other inhaled illicit substances. Her history of work as a mechanical engineer in Ukraine generated a suspicion of interstitial lung disease, leading to a high resolution $\mathrm{CT}$ scan that revealed mild lung base dependent atelectasis. On day 3 of admission, it was coincidentally noticed, after a portable pulse oximeter was left on the patient's finger that, her hypoxia had significantly improved in the supine position. Subsequently, a diagnosis of POS was entertained and a transthoracic echocardiogram (TTE) with bubble study was ordered, which was positive for significant intra-atrial shunting. A trans-esophageal echocardiogram that followed confirmed the size and location of an atrial septal defect. Subsequently she was transferred to the tertiary center for repair, where she underwent successful trans-catheter repair, and was then discharged home in a stable condition as her hypoxia had resolved.

IMPACT/DISCUSSION: This case demonstrates how a rare diagnosis such as POS, which can result in severe hypoxia, can be neglected unless physicians are mindful of it. POS is a condition characterized by dyspnea and hypoxemia in an upright position that resolves when in supine position. The etiology of the condition is believed to a combination of anatomic and functional components. The former includes intracardiac shunting, intrapulmonary shunting, ventilation-perfusion mismatch or a combination of these. Functional components such as cardiac, pulmonary, abdominal and vascular disease further contribute to result in POS. Its pathognomonic finding of orthostatic dyspnea and hypoxia, which improve in the recumbent position, were present in our patient. Diagnosis started with establishment of a relationship between hypoxia and upright position, and further confirmed by echocardiography, although the gold standard for diagnosis remains angiography. The condition is usually only suspected when other causes of hypoxia are ruled out due to its rarity, and in many cases etiology cannot be identified with certainty.

CONCLUSION: The possibility of cure, via an anatomic repair of intracardiac shunt, makes identification of POS crucial. Physician awareness of the disorder, timely diagnosis, and treatment can prevent further morbidity and mortality.

\section{PLOT TWIST IN AN INPATIENT CELLULITIS CASE}

Mohamed Diop; William Turner; Kelsey B. Bryant. Columbia University Medical Center, New York, NY. (Control ID \#3183357)

LEARNING OBJECTIVE \#1: Identify components of a focused physical exam from HPI

LEARNING OBJECTIVE \#2: Diagnose AIDS and CNS Opportunistic Infections

CASE: Male, 55, with no known history presented to the emergency department (ED) with 3 weeks of right lower extremity pain (RLE) and swelling. Progressive pain and difficulty ambulating prompted his presentation. He denied history of trauma; fracture and deep vein thrombosis were ruled out in the ED. He was initially febrile and tachycardic (though remarkably well appearing) and was dosed broad-spectrum antibiotics just prior to hand-off to the hospitalist team. Upon our evaluation, the patient verified his initial history and, and as a casual aside, mentioned he had been experiencing subtle numbness on his right side in addition to double vision. He denied any other neurologic symptoms. Family history was noncontributory. Social history notable for home country of Brazil, sexually active with only wife, no drug use, rare alcohol use, and current smoking. His exam was consistent with RLE cellulitis with warm-totouch-erythema from foot to mid-calf and small punctate areas with purulent drainage. Neurologic exam confirmed sensory deficit over the entire right half of his body. White blood cell count was 6.7. Given systemic signs of infection and inappropriately normal WBC count, an HIV test was sent. While awaiting results, he underwent brain MRI revealing several patchy lesions with rim enhancement in the left corona radiata concerning for toxoplasmosis, lymphoma, or another infectious vs inflammatory process. Lumbar Puncture puncture was quickly performed. At the same time, we received a preliminary (+) HIV test. CSF returned positive for toxoplasma, and lymphoma was ruled out with both central and peripheral flow cytometry. Confirmatory HIV assay returned (+) and CD4 count resulted at 56 confirming a diagnosis of AIDS complicated by CNS toxoplasmosis and cellulitis.

IMPACT/DISCUSSION: Though unrelated to his chief complaint, a thorough neurologic exam was the turning point in a workup for a lifealtering diagnosis. This highlights the importance of a broad differential and systematic approach to physical exam directed by the HPI and, even if the diagnosis seems obvious. Internists are often the first to notice abnormalities leading to specialty involvement, thus maintaining knowledge of things like common CNS lesions is important for workup initiation.

CONCLUSION: This patient did, in fact, have cellulitis, but a systematic approach to the HPI and physical, allowed for the diagnosis of AIDS and an opportunistic infection. Internists should be comfortable with HIV testing and counseling, and familiar with common etiologies of CNS findings to initiate appropriate workup. Througout this patient's admission, our rapport with him grew. This allowed us to disclose his diagnosis in a way that felt comfortable for him, and assist in his transition to outpatient HIV care which he continues to engage in.

\section{PML-IRIS: SEIZE THE OPPORTUNITY}

Julieta Rodriguez; Benison Lau; Deirdre Lewis. Baystate Medical Center, Springfield, MA. (Control ID \#3186673)

\section{LEARNING OBJECTIVE \#1: Define PML-IRIS \\ LEARNING OBJECTIVE \#2: Management of IRIS in the setting of} PML

CASE: A 28-year-old male with perinatal acquired immunodeficiency syndrome (AIDS) prescribed highly active antiretroviral therapy (HAART), complicated by progressive multifocal leukoencephalopathy (PML), recurrent herpes, and Hodgkin's lymphoma in remission presented in status epilepticus. Seizure activity was terminated by levitiracetam and lorazepam soon after onset. Exam was notable for decreased consciousness, asymmetric pupils minimally reactive to light, bradycardia, and asymmetric reflexes with intact muscle tone bilaterally. Labs were remarkable for a normocytic anemia, and a lactate of 3. 4. CT head revealed decreased white matter attenuation, without evidence of mass effect or hemorrhage. He was managed empirically for meningoencephalitis with acyclovir, ceftriaxone, vancomycin, and ampicillin while being monitored on continuous VEEG. Once a full septic workup was negative for an active infection, he was treated with steroids and HAART (CD4 count 22) for a presumed diagnosis of PML- Immune reconstitution inflammatory syndrome (IRIS). Refractory symptoms (continued seizures and unresponsiveness) necessitated initiation of a dexamethasone taper, valproic acid, and mirtazapine with resultant clinical improvement and a repeat CD4 count up to 105 .

IMPACT/DISCUSSION: IRIS refers to an acute inflammatory process that occurs due to rapid regeneration of CD4 cells after initiation of HAART therapy in the setting of an ongoing or preexisting infection. It presents with constitutional symptoms which may be consistent with worsening of the existing infective process, including following the treatment for tuberculosis or leprosy More than $90 \%$ of patients were admitted to the hospital at the time of IRIS diagnosis. Supportive care is generally adequate therapy, however steroids may be required to blunt immune response In the presence of PML, IRIS further lowers the seizure threshold predisposing patients to epileptiform activity as severe as status epilepticus. PML-IRIS onset after HAART treatment can range from 1 week - 26 months with most cases occurring within 4-8 weeks Up to $1 / 3$ rd 
of PML infected HIV patients developed IRIS after starting HAART with $1 / 3$ rd of those developing IRIS requiring steroid therapy. Thus hospitalists should have a high index of suspicion for IRIS and steroid management. While steroids have become the primary treatment, it is important to note that while steroids attenuate immune response it may allow JCV to replicate and lead to initial worsening of neurologic symptoms.

CONCLUSION: PML-IRIS is a rare condition where worsening neurologic symptoms, including seizures, can occur. Steroids may blunt the immune response but may cause a paradoxical worsening of neurologic symptoms initially.

\section{POINT-OF-CARE ULTRASOUND FOR THE DETECTION OF CLINICALLY SIGNIFICANT ELEVATED INTRACRANIAL PRESSURE IN THE PRIMARY CARE CLINIC.}

Kang Zhang; Justin Chen; Greg Heinicke. Internal Medicine Residency Spokane, Spokane, WA. (Control ID \#3167221)

LEARNING OBJECTIVE \#1: Highlight the usefulness of ocular POCUS for evaluation of elevated intracranial pressure (ICP)

CASE: A 32-year-old female presented to the primary care clinic for management of persistent headaches that has worsened in frequency and severity for the past two-years. The headache etiology was multifactorial attributed to migraines, and suspected obstructive sleep apnea. She previously declined polysomnography study. NSAIDs and acetaminophen provided limited relief. The patient was reluctant to try triptan therapy. Previous neuroimaging excluded secondary causes of headaches. Neurological examination revealed no focal deficits. The patient refused direct fundoscopic examination, though previously documented fundoscopic examination did not revealed papilledema. Ocular POCUS performed in clinic demonstrated an increased optic nerve sheath diameter of (ONSD) $5.7 \mathrm{~mm}$ suggestive of elevated ICP. IIH was suspected and lumbar puncture showed an opening pressure measured of $300 \mathrm{mmH}_{2} \mathrm{O}$. A diagnosis of IIH was made based on the modified Dandy criteria. The patient was started on carbonic anhydrase inhibitor and counselled on weight loss. Her daily symptoms improved significantly with initial medical therapy. At 3-month follow up, the patient was headache free.

IMPACT/DISCUSSION: The implications of ocular POCUS to estimate ICP may have large clinical impact in the outpatient setting where headache is common complaint. It is critical for physicians to determine primary and secondary headaches as it dichotomizes the need for potential neuroimaging. The traditional direct fundoscopic exam is an essential skill in general internal medicine. However, a survey of general internists showed the nondilated fundoscopic examination ranked the lowest in self-confidence rating (2.5 on a 5-point Likert scale) compared to 13 other core physical examination maneuvers. Moreover, self-confidence scores of the nondilated fundoscopic examination declined throughout medical training. Ocular POCUS may be an alternative method for evaluating elevated ICP by measuring the ONSD. In the neurocritical care setting, an ONSD $>4.8 \mathrm{~mm}$ had a sensitivity of $98 \%$ and specificity of $94 \%$ and area under the curve (AUC) 0.98 when compared to invasive ICP measurement. Similar results are reported in the emergency room literature using an optimal cut-off value of the ONSD $>5.00 \mathrm{~mm}$. More specifically, in patients proven IIH, the optimal cut-off value for ONSD was $6.3 \mathrm{~mm}$ had a sensitivity, specificity of $94.7 \%$ and $90.9 \%$ respectively. A systematic review and meta-analysis of ocular POCUS showed that depending on pre-test probability, an ONSD $>5 \mathrm{~mm}$ had a positive, negative likelihood ratio of 12.5 , and 0.05 respectively.

CONCLUSION: Depending on the pre-test probability, ocular POCUS (ONSD $>5 \mathrm{~mm}$ ) may be an accurate surrogate marker for the detection of elevated ICP in patients with headache. This non-invasive point-of-care method can have a large clinical impact in the primary care setting.
POST-BABESIOSIS WARM AUTOIMMUNE HEMOLYTIC ANEMIA IN AN ASPLENIC PATIENT

Muhammad Z. Khan ${ }^{1}$; Pragya Shrestha ${ }^{1}$; Daniel Forman ${ }^{2,} 1$. ${ }^{1}$ Reading Hospital - Tower Health, Wyomissing, PA; ${ }^{2}$ Tower Health, Reading, PA. (Control ID \#3155361)

LEARNING OBJECTIVE \#1: Recognize warm autoimmune hemolytic anemia (WAHA) as a complication of Babesiosis

LEARNING OBJECTIVE \#2: Consider screening for WAHA in patients diagnosed with babesia with worsening anemia not responding to anti-parasitic therapy

CASE: An 84-year-old male with history of splenectomy post motor vehicle accident presented with one-month history of fatigue and unintentional weight loss of 20 pounds. He endorsed spending time in woods and cutting trees for the last 10 years. He was afebrile with blood pressure $99 / 57 \mathrm{mmHg}$ with mild tachypnea. Labs were significant for hemoglobin $7.6 \mathrm{mg} / \mathrm{dl}$, LDH $989 \mathrm{IU} / \mathrm{L}$, total bilirubin $2.9 \mathrm{mg} / \mathrm{dl}$ with indirect hyperbilirubinemia. Transaminase and platelets were normal. Blood parasite smear revealed parasitic inclusions morphologically consistent with Babesia. Direct Coombs test was positive with warm autoantibody ( $\operatorname{IgG})$. Titers were negative for Lyme antibody, Ehrlichia \& Anaplasma. After initiation of azithromycin and atovaquone for babesiosis, blood parasitemia load decreased from $4.8 \%$ to $0.2 \%$. However, even with low parasitemia, hemolysis remained persistent with high LDH and further drop in hemoglobin. Immunosuppression was initiated with prednisone $60 \mathrm{mg}$ daily after which a significant improvement in blood counts as well as symptoms was evident.

IMPACT/DISCUSSION: Babesiosis is a tickborne zoonotic disease that is caused by intraerythrocytic protozoa of the genus Babesia with endemic prevalence in the northeastern and midwestern regions of the United States. Presentation can be variable from flu like illness to a lifethreatening state with severe anemia. Older age, asplenia, immunodeficient conditions are risk factors for severe disease process. Post-babesiosis warm autoimmune hemolytic anemia (WAHA) can be a detrimental complication of babesiosis especially in asplenic patients.

CONCLUSION: Screening for WAHA should be considered in cases of worsening anemia despite of appropriate treatment for babesiosis. Postbabesiosis WAHA responds to immunosuppressive treatment rather than antiparasitic treatment.

\section{POST-CHOLECYSTECTOMY BLEEDING COMPLICATION}

Dung Mac; Farzana Harji. University of New Mexico, Albuquerque, NM. (Control ID \#3185648)

LEARNING OBJECTIVE \#1: Recognize cystic artery pseudoaneurysm as a post-cholecystectomy bleeding complication.

CASE: 37 year-old male with history of alcoholic cirrhosis with portal hypertension, peri-esophageal and gastric varices, Mallory-Weiss tear $\mathrm{s} / \mathrm{p}$ clipping, ITP s/p steroids and IVIG therapies, and recent hospitalization for acute cholecystitis s/p laparoscopic cholecystectomy, with subsequent post-op elevated total bilirubin and normal MRCP, re-admitted one week later for spontaneous bacterial peritonitis (sbp), decompensated cirrhosis with new ascites, alcoholic hepatitis, and c. diff colitis. CT showed absent gallbladder with free air in the fossa and no biloma. HIDA scan did not show tracer into the biliary tree. Patient completed sbp treatment, and subsequently placed on prophylaxis antibiotics with worsening of his c. diff colitis and therefore antibiotics were discontinued. 11 days into hospitalization, he acutely decompensated with tachycardia, hypotension and worsening anemia. GI was consulted and EGD and ERCP done emergently did not reveal bleeding source. Over the course of the day, patient's abdomen became more distended. Paracentesis confirmed hemoperitoneum. Arterial phase CT showed arterial bleeding in gallbladder fossa and gastro-hepatic region. Patient was found to have 
pseudoaneurysm of the cystic artery which was successfully embolized by IR. However, patient deteriorated again after successful embolization with re-bleeding that was felt to be due to his underlying liver disease, renal dysfunction, and c. diff colitis with possible disseminated intravascular coagulopathy (DIC). He was placed on CRRT and pressor support. He was not a candidate for TIPS or liver transplant, and was transitioned to comfort care per family request and deceased shortly afterward.

IMPACT/DISCUSSION: Uncontrollable bleeding post lap cholecystectomy is a serious complication with low incidence of $0.1-1.9 \%$ and usually presents intra or post-surgical with acute hemodynamic decline. Uncontrollable bleeding from lap cholecystctomy usually arise from 3 sites, the liver, arterial sources, or port insertion sites. This case is unique in that patient had acute bleeding from cystic artery 3 weeks postprocedure. A recent review identified pseudoaneurysms (PSAs) of the hepatic and/or cystic artery as a rare complication following a laparoscopic cholecystectomy that can occur several weeks after the procedure. In the cases reviewed, trans-arterial embolization had a $94.5 \%$ success rate with a $2.0 \%$ mortality. ${ }^{1}$ Our patient had poor prognosis due to multiple comorbid conditions including underlying liver disease with portal hypertension, renal dysfunction, and possible DIC with c. diff colitis leading to re-bleeding post embolization.

CONCLUSION: Bleeding from pseudoaneurysms is a rare complication from laparoscopic cholecystectomy that can manifest up to a month post procedure.

POST-PARTUM WOMAN WITH WORSENING DYSPNEA: WHEN YOUR EYES DO NOT SEE WHAT YOUR MIND IS NOT LOOKING FOR.

Alison M. Fernandes ${ }^{1}$; Lesly Silva ${ }^{1}$; David H. Lindner ${ }^{2} .{ }^{1} \mathrm{NCH}$ Healthcare system, Naples, FL; ${ }^{2}$ Naples Community Hospital, Naples, FL. (Control ID \#3171914)

LEARNING OBJECTIVE \#1: To illustrate post-partum dyspnea with an underlying congenital heart defect.

LEARNING OBJECTIVE \#2: To characterize a rare disorder in adulthood.

CASE: LS, a 31 y.o (G1P1) without PMH was transferred to the ICU, two days after an uneventful vaginal delivery of a full-term infant, for management of acute hypoxemic respiratory failure. She was noted to have a near-syncopal episode associated with hypoxia. Physical exam revealed clear lungs; $\mathrm{O} 2$ sat $95 \%$ on $3 \mathrm{~L} / \mathrm{min}$ NC. RRR without murmur, and 1-2+ ankle edema. Labs revealed elevated troponins, and D-dimer. Right ventricular hypertrophy on EKG. CT angiogram was negative for pulmonary embolism; however, it alluded to a prominent pulmonary artery $3.86 \mathrm{~cm}$ in diameter. TTE showed LVEF $50 \%$, pulmonary artery systolic pressure $>120 \mathrm{mmHg}$, consistent with severe pulmonary hypertensiongroup I secondary to patent ductus arteriosus (PDA). Over the hospital course, her hypoxemic state worsened, requiring intubation. A right heart catheterization demonstrated pressures in the 100-110 range. It was felt with acute occlusion of the PDA that hemodynamic collapse would occur due to her Eisenmenger's physiology. She would require an assist device as a bridge to transplant, and with her significant neurologic deterioration and subsequent cardiopulmonary arrest, she was not felt to be a candidate for a heart-lung transplant. She ultimately succumbed to death two weeks after initial presentation.

IMPACT/DISCUSSION: This case illustrates a previously undiagnosed PDA in a post-partum woman. The incidence of PDA in full term infants $(0.02-0.04 \%)$ has been rising due to advances in medicine[1]. On average, blood volume increases 50\% during 20-32 weeks of gestation resulting in a high cardiac output[4]. To compensate, the systemic and pulmonary vascular resistance decrease. In those with PAH, pulmonary pressure remains high during this period because the resistance in flow across the PA is already elevated[2]. Recognizing the characteristic continuous flow murmur across the PA is crucial in identification of a PDA. However, there are multiple reports of undiagnosed PDAs in adulthood, as the murmur is seldom detected[1]. Symptomatic PDA's are a common presentation during pregnancy[3]. Kanter et al. reported a 22-week G1P1, who was treated with a transcatheter closure using an Amplatzer ductal occlude device[3]. This device can be used in PDAs up to $12 \mathrm{~mm}$ in diameter. In the case of our patient, the diameter of her PDA was $>3 \mathrm{~cm}$, thereby limiting her options for intervention. Maternal mortality rate is $>50 \%$, while the fetal mortality rate is $\sim 25 \%$, with associated risk of sudden death at 4-6 weeks post-partum in Eisenmenger syndrome[4]. As of yet, there has been no treatment to alleviate this mortality risk. It is therefore been strongly recommended these women terminate their pregnancy early.

CONCLUSION: It is imperative that there be a higher awareness of this issue in primigravida patients, as early detection in this population can decrease adverse outcomes and support proper family planning.

\section{POSTERIOR PLACOID CHORIORETINITIS AS THE ONLY MANIFESTATION OF NEUROSYPHILIS IN HIV NEGATIVE PATIENT}

Mais Abdou ${ }^{1}$; Nooraldin Merza ${ }^{1}$; Asrar Abdou $^{3}$; Hussain Alhamza ${ }^{4}$; Kaylee J. Shepherd ${ }^{2} .{ }^{1}$ TTUHSC, Amarillo, Amarillo, TX; ${ }^{2}$ Texas Tech University Health Sciences Center, Amarillo, TX; ${ }^{3}$ St Joseph Mercy Oakland, Troy, MI; ${ }^{4}$ TTUHSC, Amarillo, TX. (Control ID \#3184557)

LEARNING OBJECTIVE \#1: Recognize the ocular manifestations of systemic diseases. Identify placoid chorioretinitis as an uncommon but distinct ocular manifestation of neurosyphilis.

CASE: A 73 year-old male with medical history of hypertension and bilateral retinal macular degeneration presented to our primary care clinic with worsening blurry vision for about 2 months. There was no headache, weakness, numbness or gait problems. Vitals were stable. Physical exam was unremarkable except for decreased visual acuity bilaterally, which was notably worse when compared to his previous visit. Complete blood counts and basic metabolic panel revealed no significant abnormality. The patient was referred for ophthalmology evaluation. Complete eye exam showed bilateral retinal vasculitis with disseminated choroiditis and posterior placoid chorioretinitis. Syphilis laboratory workup was done and revealed negative RPR but reactive FTA-ABS and TP-PA. Public health database showed a diagnosis of primary syphilis in 1981 that was treated with penicillin. HIV testing was negative. The patient was successfully treated with IV penicillin G for 14 days.

IMPACT/DISCUSSION: Syphilis is a multi-systemic sexually transmitted infection caused by the spirochete Treponema pallidum. Since the introduction of penicillin, the incidence of syphilis decreased dramatically. However, it has been increasing back in the past decade secondary to high rates of human immunodeficiency virus (HIV) co-infection. Ocular syphilis can manifest in many different forms with panuveitis being the most common finding. The diagnosis of ocular syphilis based on ophthalmic findings, is often challenging due to lack of pathognomonic findings and serological confirmation is usually needed. Acute syphilitic posterior placoid chorioretinitis is a rare but characteristic ocular manifestation of syphilis. Once it's present, the patient should be tested for a neurosyphilis and HIV, if positive. Early treatment with intravenous penicillin is usually effective with good visual results.

CONCLUSION: Physicians should be aware of the ocular manifestations of systemic diseases and infections like syphilis. Ocular signs of syphilis may be the only clinical feature of systemic disease. Early recognition and treatment with intravenous penicillin is necessary to prevent complications and vision loss.

\section{POSTERIOR REVERSIBLE ENCEPHALOPATHY SYNDROME (PRES) AS A COMPLICATION OF RED BLOOD CELL TRANSFUSION}

Hayato Mitaka ${ }^{1}$; Leslie L. Seijo ${ }^{1}$; Kenji Motohashi ${ }^{2}$; Mori Nakai ${ }^{2}$; Alfred Burger ${ }^{3}{ }^{1}$ Mount Sinai Beth Israel, New York, NY; ${ }^{2}$ Nerima Hikarigaoka 
Hospital, Tokyo, Japan; ${ }^{3}$ Mount Sinai Beth Israel, Icahn School of Medicine at Mount Sinai, New York, NY. (Control ID \#3185470)

LEARNING OBJECTIVE \#1: - Recognize that PRES can develop after $\mathrm{RBC}$ transfusion - Recognize that rapid correction of chronic anemia is the risk factor for developing PRES

CASE: A 46-year-old woman with iron deficiency anemia presented with headache, right hand weakness, and "difficulty texting and folding clothes," two weeks after being hospitalized for symptomatic anemia with shortness of breath and hemoglobin of $2.9 \mathrm{~g} / \mathrm{dL}$. During her previous admission, she received transfusions of 3 units of red blood cells and was discharged home with a hemoglobin level of $7.5 \mathrm{~g} / \mathrm{dl}$. She now presented to the ED with 3-days of constant, non-localized headache and 1-day history of clumsiness and weakness of the right hand. On exam, her BP was $179 / 78 \mathrm{mmHg}$, and there was numbness, reduced dexterity and apraxia of her right hand. Routine labs were unremarkable and her hemoglobin was $7.5 \mathrm{~g} / \mathrm{dl}$. An MRI of the brain showed multiple subcortical high-intensity lesions on bilateral anterior, parietal, and occipital lobes on T2-FLAIR images. Pregnancy test, hypercoagulable work-up, RF, ANA, and ANCA were negative. The hospital course was complicated by a brief episode of tonic convulsive seizure which self-resolved. Given her neurologic symptoms and radiologic findings, the clinical diagnosis of Posterior Reversible Encephalopathy Syndrome (PRES) secondary to blood transfusion was made. Her headache and neurological symptoms of the right hand gradually improved after starting oral calcium channel blocker. A follow-up MRI 4 weeks after discharge showed resolution of the high-intensity lesions.

IMPACT/DISCUSSION: PRES is a neurologic syndrome characterized by acute onset of headache, altered mental status, seizure, and visual disturbances, with radiographic findings of symmetrical white matter edema in the posterior cerebral hemispheres, particularly in the parietooccipital regions. Rapid correction of anemia with transfusion is a rare cause of PRES with only 10 cases described in literature as of April 2016, according to Cevallos CA and Berman BS. The pathogenesis of PRES after transfusion remains unclear, but it has been proposed that a rapid rise in the hematocrit results in both increased blood viscosity and a loss of hypoxic vasodilation, leading to disordered cerebral autoregulation and endothelial dysfunction. Most of the cases develop from several days to 2 weeks after transfusion, and more likely to occur when the chronic anemia is rapidly corrected. Moderate hypertension as seen in this patient has been described as a complication of PRES. The prognosis is mostly benign and PRES is fully reversible within days to weeks, after removal of the inciting factor (rapid correction of hematocrit in this case) and blood pressure control with vasodilator if hypertensive.

CONCLUSION: Physicians should have a high index of suspicion for this rare but serious condition if a patient with chronic anemia develops neurological symptoms after RBC transfusion, and should avoid rapid correction of chronic anemia without hemodynamic instability.

\section{POTASSIUM CHLORIDE AS TRIPLE THERAPY}

Ammara A. Khan. Creighton University, Omaha, NE. (Control ID \#3185798)

LEARNING OBJECTIVE \#1: 1. Diagnose acute severe metabolic alkalosis

LEARNING OBJECTIVE \#2: Safely treat profound hyponatremia without sodium chloride

CASE: A 48 year old male with a past medical history of chronic pancreatitis, chronic pain and alcoholism presented with a 7 day history of vomiting and diarrhea. At the time of presentation, he was tachycardic with heart rate in $110 \mathrm{~s}$ and hypotensive with blood pressure $90 \mathrm{~s} / 60 \mathrm{~s}$. Physical exam was notable for dry mucosal membranes, tremulousness, tangential thought processes and visual hallucinations. His metabolic panel was significant for multiple abnormalities; AKI with a creatinine of 4 , severe hypochloremia with a chloride of 51 , severe hyponatremia with a sodium of 118 , severe hypokalemia with a potassium of 2.2 , metabolic alkalosis with a bicarbonate of 40 and a coexisting high anion gap metabolic acidosis with an anion gap of 29. ABG showed metabolic alkalosis with partial respiratory compensation. His $\mathrm{pH}$ was 7.6 with a bicarbonate of 56 and $\mathrm{pCO} 2$ of 58 . The patient initially received a liter of lactated ringer's in the ER and his sodium began to overcorrect. His IV fluids were discontinued, and he was placed on a continuous Potassium chloride infusion for the next 24 hours by nephrology. The patient's ketones were metabolized once he received dextrose, and his anion gap began to narrow. With volume repletion and cessation of vomiting, his renal function began to improve as well as his alkalosis. His sodium, as well, appropriately corrected over 48 hours.

IMPACT/DISCUSSION: Our patient developed a pre-renal AKI due to persistent GI losses causing dehydration. Reduced blood flow to the kidneys led to secondary hyperaldosteronism, causing further retention of bicarbonate and excretion of hydrogen, potassium and chloride. Despite the nephron's attempts to retain sodium the increased ADH led to a relative excess of water as compared to sodium leading to a relative hyponatremia. Simultaneously the patient had HAGMA due to lactic acidosis and starvation ketosis. Metabolic alkalosis (MA) is a relatively common, although rarely severe disorder, characterized by elevation in body $\mathrm{pH}$ secondary to elevation of plasma bicarbonate. MA is associated with life threatening complications like arrhythmias due to electrolyte disorders and tissue hypoxia from compensatory respiratory acidosis. MA can lead to hypokalemia, hypernatremia and hypocalcemia. Several studies have shown that mortality with MA increases proportionate to the $\mathrm{pH}$ : $41 \%$ with $\mathrm{pH} 7.55$ to $7.56,47 \%$ with $\mathrm{pH} 7.57$ to $7.59,65 \%$ with $\mathrm{pH} 7.6$ to 7.64 , and $80 \%$ with $\mathrm{pH} 7.65$ to 7.7 . Our patient with a $\mathrm{pH}$ of 7.6 had an expected mortality rate of $65 \%$.

CONCLUSION: In the setting of acute kidney injury, metabolic alkalosis can be severe enough to cause life threatening complications. Secondary hyperaldosteronism due to profound GI fluid loss can complicate acute kidney injury. Metabolic alkalosis, profound hypo-osmolar hyponatremia and hypochloremia can be safely treated with potassium chloride.

\section{PREMATURE CLOSURE IN CLINICAL DECISION MAKING : A CLASSIC PRESENTATION OF LYMPHOMA IS AN UNUSUAL CASE OF DABSKA TUMOUR}

Hoang-long Huynh ${ }^{2}$; Christine Chan ${ }^{2}$; Doris Zavaro ${ }^{1}$; Amarpreet S. Bains ${ }^{2}$; Sheetal Desai-Oghra ${ }^{3}$; Marwa Moussa ${ }^{1} .{ }^{1}$ NYU Langone, Staten Island, NY; ${ }^{2}$ NYU Langone Health, Brooklyn, NY; ${ }^{3}$ NYU, Brooklyn, NY. (Control ID \#3183224)

LEARNING OBJECTIVE \#1: Avoid premature closure when a patient presents with classic lymphoma symptoms. Our case was given a diagnosis of lymphoma by the emergency department and the hematologist oncologist consulted on the case. While the first impression was lympho$\mathrm{ma}$, there is a very rare, locally invasive neoplasm of the lymphatic vascular origin called papillary intralymphatic angioendothelioma (PILA), also referred to as Dabska tumor. Recognize that although PILA commonly presents as a slowly growing, asymptomatic (without B symptoms), violaceous patch or nodule, arising from a preexisting lymphatic or vascular anomaly that occurs typically in young adults, it may also affect the elderly and present with typical B symptoms.

CASE: A 36 year-old otherwise healthy female presented with six days of acute flu-like symptoms, myalgias, persistent fever and chills associated with abdominal bloating and vomiting for three days. Physical exam was notable for temperature of $103 \mathrm{~F}$, sinus tachycardia and mild tenderness at the left upper quadrant on deep palpation. Labs were notable for a normal WBC count, mildly elevated alkaline phosphatase and total bilirubin. The flu swab was negative. All infectious workup including a quantiferon-TB gold test was negative. The Chest CT showed confluent $9 \times 5 \mathrm{~cm}$ anterior mediastinal and $2 \times 2 \mathrm{~cm}$ sub carinal lymphadenopathy extending to the 
aortopulmonary window. On abdominal CT abdomen and pelvis there was marked diffuse, bulky and confluent upper abdominal, mesenteric and retroperitoneal adenopathy. The patient underwent mediastinal video assisted thoracoscopic surgery (VATS) which showed papillary intralymphatic angioendothelioma (PILA). The patient was referred to a vascular anomalies' specialist and was prescribed Sirolimus for prophylaxis immunosuppressant therapy.

IMPACT/DISCUSSION: To our knowledge, less than 40 cases of PILA have been described in literature. Presently, there are diagnostic challenges for pathologists and providers given PILA's rarity and histological complexity to distinguish it from other vascular lesions and lymphoma. We present a new and unusual case of PILA with typical B symptoms and multifocal intra-abdominal lesions. Most case reports describe asymptomatic and external presentations. It is important to rely on final pathology prior to assuming lymphoma is the most likely diagnosis.

CONCLUSION: Premature closure is failure to consider alternative diagnoses after the initial impression is formed. This leads to diagnostic errors that can have substantial effects on our patients lives. Rare diseases are often overlooked and we need to recognize PILA as a differential diagnosis for neoplasms of the lymphatic vascular origin.

\section{PRIMARY CARE OF INDIVIDUALS WITH INTELLECTUAL AND DEVELOPMENTAL DISABILITIES-AN OPPORTUNITY FOR EDUCATION AND ADVOCACY}

Elizabeth "Betty" Kolod ${ }^{1}$; Irene Chern ${ }^{2} .{ }^{1}$ Montefiore Medical Center, Bronx, NY; ${ }^{2}$ Albert Einstein College of Medicine, Bronx, NY. (Control ID \#3185956)

LEARNING OBJECTIVE \#1: Recognize individuals with intellectual and developmental disability as a health disparity population.

LEARNING OBJECTIVE \#2: Explore features of high-quality care for individuals with IDD.

CASE: A 45-year-old woman with a history of depression, obesity, and seizure disorder presents for a routine visit with a new primary care provider (PCP). She has a fractured tooth from a violent encounter with her boyfriend and believes she may have recently had a seizure but is unable to provide more detail. She emigrated from Puerto Rico to the Bronx at age 5 and completed the 10th grade in special education; she is on disability due to chronic pain. She lost of custody of four of her children due to former cocaine use and now cares for her two remaining children and her mother who is illiterate. Prior notes intermittently refer to "underlying cognitive issues" with no formal neuropsychological evaluation. She declines a home visit to evaluate safety and social support and departs with referrals to Neurology and domestic violence services.

IMPACT/DISCUSSION: Intellectual and developmental disabilities (IDD) comprise lifelong limitations of intellectual, physical or emotional development and adaptation. Health disparities are socially-produced differences in burden of illness experienced on a population level; the CDC defines individuals with disabilities as a health disparity population. CDC researchers note a five-fold increase in rate of diabetes and more than two-fold increase in cardiovascular disease among individuals with IDD compared to the general public. This disparity is compounded in people of color with IDD, particularly Hispanic women. Furthermore, individuals with IDD are 1.5 times more likely to be victims of violence and face twice the rate of sexual assault - in one study $49 \%$ of persons with IDD experienced 10 or more sexual assaults. High quality care for patients with IDD requires an understanding of unique health risks and strategies for providing basic preventive care. Patients with IDD are at increased risk for obesity, substance use disorders, seizure disorders, mental illness and violent or sexual victimization. Major barriers to care for patients with IDD include communication challenges, inaccessible office design, and poor physician compensation contributing to lack of access to health professionals trained in IDD. These barriers can be addressed by increasing emphasis on IDD in medical education and public health research, clinical adaptation to patient capabilities, interprofessional coordination, and including caregivers in communication. By utilizing a patient centered approach, PCPs can increase self-advocacy as well as inform physician advocacy for resources to address the disparities faced by this community.

CONCLUSION: Individuals with intellectual and developmental disabilities have worse health outcomes. PCPs can address this health disparity through advocacy and familiarity with best practices in the care of individuals with IDD.

\section{PRIMARY NEUTROPHILIC DERMATOSIS MISDIAGNOSED AS NECROTIZING PORT SITE INFECTION}

Ranish Patel; Shadi Dowlatshahi. Oregon Health and Science University, Portland, OR. (Control ID \#3167602)

LEARNING OBJECTIVE \#1: Recognize clinical features of neutrophilic dermatoses

LEARNING OBJECTIVE \#2: Recognize the significant overlap in clinical presentations of neutrophillic dermatoses and severe infection

CASE: 52-year-old man with myelodysplastic syndrome status post 2 cycles of azacitadine, recent port placement who presented to an outside hospital with painful, erythematous port site. He denied any fevers, chills or sweats. Vitals were notable for tachycardia. Physical exam revealed confluent erythema over the area of the port, extending up to the neck. Labs were significant for WBC $19 \times 10^{\wedge} 3 / \mu \mathrm{L}$. Vancomycin, clindamycin, and piperacillin-tazobactam were started empirically. Surgery was consulted, and the port was removed with copious pus noted at the port site, alongside gas-forming bubbles in the skin. The patient subsequently underwent debridement of the area, with healthy fascial and muscular tissue seen. However, the lesion was noted to continue to expand beyond the surgical margins into a $16 \times 9 \mathrm{~cm}$ necrotic ulcer with a pink-red indurated, and undermined edges. Follow up blood and wound cultures were all negative for pathologic organisms. The patient was transferred to our hospital for further management. Dermatology was consulted, and biopsy was performed. Histopathology demonstrated marked numbers of neutrophils present extending from the surface into the underlying adipose tissue. A diagnosis of pyoderma gangrenosum versus necrotizing Sweet syndrome was made. Antibiotics were discontinued, and the patient was started on a 2-month course of high-dose prednisone and dapsone. The wound displayed marked improvement and healed by secondary intention.

IMPACT/DISCUSSION: This case illustrates the potential for misdiagnosis of necrotizing skin infection in those with primary neutrophilic dermatosis, particularly in patients with compromised immune function. Common distinguishing features include associations with systemic diseases (i.e. hematologic malignancies, inflammatory bowel disease), and sterile, neutrophilic infiltrate on histopathology. Additionally, while not exhibited in all cases, the phenomenon of pathergy is frequently present. In these cases, skin injury is induced or exacerbated at sites of both incidental or iatrogenic trauma, as was seen with our patient. Appropriate diagnosis and management is therefore critical, as aggressive debridement has the potential to cause significant expansion of primary lesions, which in turn warrant further unnecessary resections resulting in significant morbidity.

CONCLUSION: - It is important to consider primary neutrophilic dermatoses in patients with suspected severe skin infections, particularly in patients who are immunocompromised. - Appropriate and timely diagnosis is critical, as delayed management can often result in significant patient morbidity

\section{PRIMARY RENAL LYMPHOMA PRESENTING AS RENAL} CELL CARCINOMA

Joy Tang. Medical College of Wisconsin, Milwaukee, WI. (Control ID \#3179282) 
LEARNING OBJECTIVE \#1: Recognize the increased risk of lymphoma in patients with Crohn's disease and the potential risk with use of TNFinhibitors.

LEARNING OBJECTIVE \#2: Recognize primary renal lymphoma as a differential for a renal mass.

CASE: A 75-year-old male with history of Crohn's disease (CD) on treatment with infliximab for the past 4 years was incidentally found to have an acute kidney injury with creatinine of $2.15 \mathrm{mg} / \mathrm{dL}$. Patient had no complaints. He denied dysuria and hematuria. Vitals and exam were unremarkable. Labs including electrolytes, blood cell counts and urinalysis were unremarkable. A renal ultrasound showed moderate hydronephrosis of the left kidney. CT kidneys then demonstrated a large left retroperitoneal mass measuring $4 \mathrm{~cm} \times 2.5 \mathrm{~cm}$ that was inseparable from the left renal pelvis and enlarged retroperitoneal lymph nodes. Infliximab was stopped due to concern for malignancy. A ureteral stent was placed with improvement in symptoms. Ultrasound of the scrotum was normal. Urine cytology from a diagnostic ureteroscopy was negative for malignancy. Core needle biopsy of the mass then revealed diffuse large B-cell lymphoma. PET scan showed lymphadenopathy above and below the diaphragm. Bone marrow biopsy showed no lymphoma. He established care with hematology/oncology and was started on R-CHOP chemotherapy. He switched treatment to vedolizumab for his CD.

IMPACT/DISCUSSION: Lymphomas are a rare complication of longstanding CD. Most reported cases have occurred in the gastrointestinal tract. Risk factors include advanced age, male gender, use of immunosuppressive therapy, EBV infection and hepatitis C infection. TNF- inhibitors, such as infliximab, are commonly used to treat CD. Recent studies have also reported an increased risk of lymphomas with TNF- inhibitors. The proposed mechanism is by decreasing apoptosis and allowing proliferation of neoplastic cells. Primary renal lymphoma (PRL) is characterized by lymphoma involving the kidney in the absence of primarily extrarenal lymphatic disease. PRL is very rare as the kidney is an extranodal organ and does not have lymphatic tissue. It has a reported incidence of $0.7 \%$ of all extranodal lymphomas and is diagnosed in $<1 \%$ of all kidney lesions. Diagnosis is frequently delayed as it can be difficult to differentiate renal lymphoma from other renal masses. Patients can be asymptomatic, but commonly complain of abdominal or flank pain, weight loss or gross hematuria. The main treatment is chemotherapy using a CHOP regimen, which differs from other renal tumors that may require nephrectomy. It is important for physicians to be aware of PRL and maintain a broad differential in evaluating renal masses as management can change.

CONCLUSION: • Lymphomas are a rare complication of CD. Use of TNF- inhibitors may increase the risk of developing lymphoma. $\bullet$ PRL should be considered when evaluating any renal mass. It can be treated with systemic chemotherapy unlike other renal masses that may require nephrectomy.
PROGRESSIVE RENAL OSTEODYSTROPHY WITH DYSMORPHIC FEATURES (SAGLIKER SYNDROME) ASSOCIATED WITH SUBOPTIMALLY TREATED SECONDARY HYPERPARATHYROIDISM

Naiha Nadeem; Colette Knight. Montefiore Medical Center, Yonkers, NY. (Control ID \#3184955)

LEARNING OBJECTIVE \#1: Recognize the pathophysiology and treatment of renal osteodystrophy

LEARNING OBJECTIVE \#2: Recognize the importance of early intervention in the treatment of secondary hyperparathyroidism (SHPT) to prevent the progression of bone disease

CASE: 40-year-old woman with history of chronic renal disease (unknown etiology) progressing to end-stage renal disease on hemodialysis for seven years and SHPT s/p parathyroidectomy who presented with shortness of breath. She had short stature measuring $\left.150 \mathrm{~cm} \mathrm{(4^{ \prime }} 9^{\prime \prime}\right)$ with large head circumference, facial disfigurement with changes in shape of mandible, teeth and palate. Labs revealed parathyroid hormone (PTH) level $1300 \mathrm{pg} / \mathrm{mL}$, serum calcium $8 \mathrm{mg} / \mathrm{dL}$, serum phosphate $4 \mathrm{mg} / \mathrm{dL}$ and alkaline phosphatase (ALP) $1200 \mathrm{U} / \mathrm{L}$. Radiographic imaging showed diffuse osseous expansion and sclerosis with focal intraosseous lesions consistent with a combination of severe renal osteodystrophy with superimposed brown tumors. The patient also had severe cervical spinal stenosis and extensive thickening of skull. She was treated with calcitriol, phosphorus binders, and calcimimetics. Her clinical presentation was consistent with Sagliker Syndrome.

IMPACT/DISCUSSION: Sagliker syndrome is a rare form of renal osteodystrophy with disfiguring facial changes, associated with overgrowth of facial bone resulting from untreated SHPT associated with renal failure. The syndrome was first described in 2004 and so far 70 cases have been reported in the literature. Inadequate treatment of SHPT causes phosphorus retention, vitamin D deficiency, alteration in the metabolism of calcium and, increase in PTH and ALP level causing skeletal changes, renal rickets, damage to the points of bone growth in children, and subperiosteal changes. Facial disfigurement is caused by alterations of facial bones and tumor-like soft tissue collections. Patients can also develop psychological problems and depression. Renal transplantation prevents progression of the musculoskeletal changes, but established deformities are not reversible. The exact cause is still unknown but genetic mutations were detected in $40 \%$ of patients. Treatment with calcitriol, vitamin $\mathrm{D}$ analogues or calcimimetics is recommended in the early stages of SHPT due to CKD to decrease levels of PTH. In refractory cases, complete parathyroidectomy is suggested.

CONCLUSION: Early monitoring and appropriate treatment of patients with renal insufficiency and secondary hyperparathyroidism can prevent irreversible complications including bone and soft tissue abnormalities. 


\section{PROGRESSIVE VISION LOSS IN A PATIENT WITH ADVANCED MALIGNANCY}

Dame Idossa; Brian A. Costello. Mayo Clinic, Rochester, MN. (Control ID \#3139290)

LEARNING OBJECTIVE \#1: To highlight paraneoplastic syndromes as cause of vision loss in patients with advanced malignancy

CASE: A 49 year-old, previously healthy gentleman presented with daily progressive headaches, low-grade fevers, progressive fatigue, and abdominal pain. A thorough laboratory evaluation solely demonstrated microcytic anemia. As part of the work up for the anemia, an ultrasound of the spleen was obtained and revealed a shadow on the spleen, prompting further studies. A $\mathrm{CT}$ of the abdomen/pelvis revealed a mass on his left kidney with multiple lesions on the liver and extensive retroperitoneal lymphadenopathy, concerning for metastatic disease. He underwent an excisional lymph node biopsy which revealed metastatic papillary renal cell carcinoma with sarcomatoid features. After an evaluation by an oncologist, he ultimately decided not to pursue conventional treatment options. Three months after his initial diagnosis, he presented to his primary care physician with rapidly progressive bilateral loss of vision. Extensive ophthalmologic evaluation revealed melanocyte proliferation of the uvea, diagnostic of bilateral diffuse uveal melanocyte proliferation (BDUMP). This was thought to be a paraneoplastic process arising from his underlying advanced malignancy. He initiated treatment for his visual symptoms with plasmapheresis and IVIG. This transiently improved his vision, but he never regained his baseline visual capabilities. He ultimately developed bilateral retinal detachments and complete vision loss. He continued to defer treatment of his underlying malignancy and developed progression of his underlying metastatic papillary renal cell carcinoma.

IMPACT/DISCUSSION: BDUMP is a rare paraneoplastic ocular syndrome occurring in patients with systemic, often occult, but advanced carcinoma and is the hallmark of poor prognosis. Although rare, BDUMP has consistently resulted in devastating visual consequences. The five cardinal signs of BDUMP described in the literature are: (1) multifocal, faintly visible, round or oval, red, subretinal patches, with (2) associated striking pattern of associated hyperfluorescence during the early phases of angiography; (3) development of multiple, slightly elevated, pigmented and non-pigmented uveal melanocytic tumors, as evidence of diffuse thickening of uveal tract; (4) exudative retinal detachment; and (5) rapid progression of cataract. Ocular symptoms typically precede manifestation of systemic carcinoma by 3-12 months. Thus, an internist would need to have a high index of suspicion, prompt evaluation, and appropriate referral. Treatment options for BDUMP are limited and often unsuccessful. Treatment modalities include: corticosteroids, ocular radiotherapy, plasmapheresis, and treatment of the underlying malignancy.

CONCLUSION: Paraneoplastic syndromes arising from malignancy can present in myriad ways and should always remain on the differential in patient diagnosed with cancer.

PROGRESSIVE VISUAL IMPAIRMENT DUE TO UVEITIS - A RETURN OF SYPHILIS AS THE "GREAT IMITATOR"

Elissa Arnold; Debapria Das; Alexander Barsam; Andrew Jilwan; Kahee Mohammed; Sangeeta Khanna; Stewart Albert. Saint Louis Univeristy, St. Louis, MO. (Control ID \#3186137)

LEARNING OBJECTIVE \#1: Describe the ocular and neurological presentation of Treponema pallidum infection.
CASE: A 47 year old woman presented to ophthalmology for evaluation of painless bilateral vision loss, headaches and ataxia of 1 months duration. She was initially seen for symptoms of right sided vision loss with inability to see faces or read. A brain MRI showed foci in periventricular and subcortical white matter consistent with demyelinating disease. A lumbar puncture (LP) revealed lymphocytic pleocytosis with $26 \mathrm{WBC}$ and was negative for NMDA and Aquaporin-4 antibodies. CSF cultures and cytology examinations were negative. She presented at a later date and was found to have vitritis and papillitis in both eyes. Visual Acuity (VA) was 20/70 on right, 20/100 on left. She had a positive Romberg sign, unequal pupils with diminished response to light and normal accommodation. Repeat MRI showed left optic neuritis. Serologies were negative for HIV, bartonella, chlamydia, gonorrhea, and tuberculosis. FTA-Abs was positive and RPR titer was 1:128. The patient was admitted and started on intravenous penicillin. Within 48 hours, VA improved to 20/25 in the right and 20/30 in the left eye and there was resolution of her ataxia and headaches. Further questioning revealed that she had a maculopapular rash involving her palms five months prior which spontaneously resolved. On discharge (day 4) VA was 20/25 bilaterally.

IMPACT/DISCUSSION: Manifestations of syphilis are broad, warranting its nickname: "The Great Imitator." Syphilis has been increasing in incidence with 9.5 cases per 100,000 in 2017 . $75-90 \%$ of cases are men and there is coinfection with HIV in $50 \%$ of cases. Emerging cases of ocular syphilis prompted advisory from the CDC in 2015, recommending LP in patient with known syphilis and neurologic symptoms. Ocular syphilis may occur in secondary or late syphilis. On average, the diagnosis of ocular syphilis is obtained 2.8 months after onset of symptoms. All structures of the eye may be affected, with uveitis being the most common presentation. It is recommended to perform an LP as if there is a concern for ocular syphilis as $1 / 4$ of cases will have findings consistent with neurosyphilis, as seen in our patient. It is more common for HIV negative neurosyphilis to present with cognitive impairment and ataxia, rather than meningitis, stroke, and vision or hearing loss. Treatment is IV Penicillin G, 3-4 MU every 4 hours for 10-14 days.

CONCLUSION: In an era when sophisticated analyses and therapies have emphasized awareness of HIV, we may have become less suspecting of T. pallidum. Our patient experienced a delayed diagnosis since her initial brain MRI was suggestive of demyelinating disease. This case demonstrates the versatile presentation of syphilis in an unsuspected demographic, the HIV negative female. The diagnosis should be considered with unexplained neurologic or ocular symptoms, as prompt treatment of this potentially fatal condition generally leads to full recovery and successful outcomes.

\section{PSEUDOMONAS PUTIDA CELLULITIS AND BACTEREMIA IN A PATIENT WITH CHRONIC LYMPHOCYTIC LEUKEMIA}

Florina Corpodean $^{2}$; Crystal Xue ${ }^{2}$; Ribka Ayana ${ }^{1}$. ${ }^{1}$ Washington DC VA Medical Center, Washington DC, DC; ${ }^{2}$ George Washington University Hospital, Washington, DC. (Control ID \#3185038)

LEARNING OBJECTIVE \#1: Recognize clinical spectrum of Pseudomonas putida (P. putida) infection

LEARNING OBJECTIVE \#2: Recognize pathogenicity of $P$. putida

CASE: A 73-year-old man with untreated chronic lymphocytic leukemia and lymphedema presented with three weeks of progressive left leg swelling, erythema and pain. On admission, he was febrile and hypotensive. He had diffuse circumferential erythema with warmth and tenderness extending to his upper left leg, along with a large flaccid bulla and multiple three-centimeter lesions with purulent drainage on his posterior calf. He also had a prominent 
left inguinal lymphadenopathy. Laboratory studies revealed leukocytosis to 25 $\mathrm{K} / \mathrm{cmm}$ with lymphocytic predominance. Blood cultures were obtained, and he was started on vancomycin and piperacillin/tazobactam. Patient remained hemodynamically stable but was intermittently febrile with worsening leukocytosis over the subsequent days. CT of the extremity was obtained and revealed edema without fluid collection. On day 4 of hospitalization, his blood cultures grew $P$. putida with intermediate sensitivity to piperacillin/tazobactam and meropenem. His antimicrobial regimen was changed to cefepime and metronidazole with good clinical response.

IMPACT/DISCUSSION: As a gram-negative organism frequently found in the environment, $P$. putida was previously thought to be of low pathogenicity. Although $P$. putida bacteremia and skin and soft tissue infections (SSTI) remain uncommon, it is important for clinicians to be aware of the rise in P. putida-associated nosocomial infections and potential fatality of these infections particularly in immunocompromised patients. $P$. putida is typically found in the natural environment (soil, water, the oropharyngeal flora, and occasionally in the hospital environment). Although most often associated with indwelling catheters and hospital-acquired pneumonias, its spectrum of infection includes urinary tract infections, acute cholecystitis, cholangitis, tonsillitis, thrombophlebitis, septicemia, meningitis and peritonitis. The organism often causes nosocomial infections in those who are immunocompromised and have medical devices. P. putida SSTI and bacteremia are both rare but lethal cases have been described in the literature.

CONCLUSION: Although infections caused by $P$. putida remain uncommon, in the last several decades, it has emerged as an important cause of nosocomial infections particularly in immunocompromised patients. Furthermore, multidrug-resistant and carbapenem-resistant $P$. putida isolates are increasingly being reported in literature. Fatal cases of $P$. putida infection, both soft tissue and bacteremia, have also been reported. It is critical for clinicians to be aware of the potential fatality of nosocomial P. putida infections and initiate early and appropriate antibiotic regimen.

\section{PULMONARY MUCORMYCOSIS IN A PATIENT WITH MYELODYSPLASTIC SYNDROME AND TUBERCULOSIS}

Akane Ariga; Tamaki Kakuwa; Jin Takasaki; Akinari Tsukada; Shuichiro Matsumoto; Eriko Morino; Moe Kato; Toru Igari; Yoshitaka Shida; Shinyu Izumi; Haruhito Sugiyama. National Center for Global Health and Medicine, Tokyo, Japan. (Control ID \#3184821)

LEARNING OBJECTIVE \#1: 1. Consider pulmonary infarction and/or abscess if CT images show a non-aerated, low-density area associated with emboli inside central vessels, or if needle biopsy cannot obtain adequate specimens. 2. Consider a transvascular approach (e.g., right cardiac catheterization) in addition to a percutaneous or transbronchial biopsy, to obtain histological findings that can definitively confirm a mucormycosis diagnosis. 3. Superinfection is always possible, regardless of culture results.

CASE: An 85-year-old man, who had been treated for myelodysplastic syndrome and tuberculosis for several months, presented with subacute fever and worsening left-side chest pain. Contrast-enhanced CT images showed a massive tumor-like consolidation without enhancement, expanding from the left lower lobe. We detected emboli in left descending pulmonary artery. Sputum and abscess cultures were positive for Enterococcus faecium, but no fungal species. Despite intensive treatment, he developed multiple organ failure and died 47 days after hospitalization. Histological examination of the lung autopsy specimen showed invasive mycelium growth by Mucor species in vessels and pulmonary emboli, which did not respond to anticoagulants; it was recognized as the direct cause of death. Mucorales were identified by both real-time polymerase chain reaction and immunohistological analyses of lung tissue.

IMPACT/DISCUSSION: Mucormycosis is a life-threatening infectious disease, seen in immunocompromised patients, such as those with hematological malignancies. As its clinical symptoms and radiological findings vary, and specific biomarkers and culture findings for mucormycosis have not been defined, histological findings are usually necessary to confirm a definitive diagnosis of mucormycosis. Mucormycosis is known to invade vessels, and is associated with embolism or infarction, which may be seen on CT images. Therefore, if CT images show a non-aerated, lowdensity area with emboli inside central vessels, especially in immunocompromised patients, a pulmonary infarction produced by mucormycosis is worth considering. Pulmonary infarction should also be considered when CT images show an abscess-like area, but needle biopsy cannot gather adequate pus-like specimens. A transvascular approach to obtain a histological sample (such as right cardiac catheterization), in addition to a percutaneous or transbronchial biopsy, may help confirm a definitive diagnosis of mucormycosis. Finally, superinfection is always possible, regardless of the culture results, especially when the appropriate antibiotic is inadequate.

CONCLUSION: We encountered a case of pulmonary mucormycosis in a patient with myelodysplastic syndrome and tuberculosis. Mucormycosis should be considered when intensive care according to culture results is not successful, especially for patients in immunosuppression and/or with abnormal CT images. Development of new diagnostic methods for mucormycosis including a transvascular approach is expected.

PUPPY LOVE: A CASE OF CAPNOCYTOPHAGA CAUSING SEPTIC POLYARTHRITIS FAVORING PROSTHETIC JOINTS

Jennifer Rodriguez; Michael G. Simonson; Allison DeKosky. University of Pittsburgh Medical Center, Pittsburgh, PA. (Control ID \#3185891)

LEARNING OBJECTIVE \#1: To diagnose prosthetic joint infection on synovial fluid analysis

LEARNING OBJECTIVE \#2: To recognize Capnocytophaga as a rare etiology for prosthetic septic arthritis

CASE: An 84-year-old otherwise healthy and active female with a history of bilateral total knee and right shoulder replacements presented to the hospital with fever (38.5C), diarrhea, and confusion. Initial chemistries demonstrated a WBC 9x10 $/ \mathrm{L}$, CRP $33 \mathrm{mg} / \mathrm{dL}$, and ESR $46 \mathrm{~mm} / \mathrm{hr}$; however, viral and bacterial infectious work-up, including serologic and radiologic testing, were completely negative. Stool culture was not obtained due to lack of bowel movement. On hospital day 3, she developed acute arthritis with effusion of her left prosthetic knee. Arthrocentesis revealed cloudy synovial fluid with $33,350 \mathrm{cell} / \mathrm{uL}$ without organisms, but repeat blood cultures reported growth of anaerobic gram-negative rods. She underwent surgical washout of the joint and was started empirically on vancomycin and ampicillin-sulbactam. She subsequently developed pain in the right knee and shoulder joints. Arthrocentesis of the newly involved joints revealed similarly elevated cell counts without crystals or organisms and she was taken again for washout. On hospital day 7, blood cultures speciated as Capnocytophaga spp. Notably, her social history revealed she kisses and shares a bed with her dog, who has a disorder of sialorrhea and who was also experiencing diarrhea. Antibiotics were transitioned to a 6-week course of ertapenem prior to discharge and she later presented in improved condition to outpatient clinic. She will subsequently require life-long amoxicillin-clavulanate suppression therapy. 
IMPACT/DISCUSSION: Prosthetic joint infection is a serious clinical entity associated with significant morbidity and mortality. The cutoff value for identifying patients with prosthetic joint infections is lower than in a native joint; a synovial fluid leukocyte differential of $>65 \%$ neutrophils or a leukocyte count of $>2 \times 10^{3} \mathrm{~L}$ is highly predictive of prosthetic joint infection. Capnocytophaga canimorsus is a rarely encountered pathogen in humans but commonly part of canine oral flora. Infection with Capnocytophaga spp. can progress rapidly to sepsis and death if not appropriately diagnosed and treated.

CONCLUSION: Capnocytophaga spp. are capnophilic gram-negative rods that can be difficult to grow under standard microbiology lab conditions. Infection with these organisms has been noted to appear initially 'culture-negative,' without sufficient proinflammatory response, later rapidly progressing to sepsis, arthritis, DIC, and death. Septic arthritis due to Capnocytophaga in the immunocompetent patient without trauma is rare. There has previously been one report of prosthetic joint infection of the hip with Capnocytophaga, however infection in that instance was described as indolent, which is atypical for this organism. To our knowledge, this is the first case reported of acute septic polyarthritis with preferential involvement of prosthetic joints by Capnocytophaga in humans.

\section{PURPURA FULMINANS: A RARE CONDITION WITH A POOR PROGNOSIS}

Munther M Queisi ${ }^{1}$; Sehar Zulfiqar'; Shazia Samanani ${ }^{2}$; Mary Jo Farmer. ${ }^{1}$ University of Massachuetts - Baystate (UMMS), Springfield, MA; ${ }^{2}$ Baystate Medical Center, Springfield, MA. (Control ID \#3186012)

LEARNING OBJECTIVE \#1: Recognize early signs and symptoms of Purpura Fulminans in the acute setting

CASE: A 64-year-old female with a complicated past medical history including ischemic colitis with partial resection, rheumatoid arthritis, methotrexate induced interstitial lung disease, Graves' disease post thyroidectomy, and CKD presented to the hospital with right upper quadrant abdominal pain. While in the ED she became hypoxic and suffered a PEA arrest. After resuscitation, she was admitted to the ICU and work up revealed septic shock from ascending cholangitis with E. coli bacteremia. She underwent ERCP and sphincterotomy and required pressor support for hypotension. Over the next few days she began complaining of pain in her distal extremities and developed necrotic skin lesions over her acral surfaces described as hyperpigmentation with dusky and necrotic appearing fingers and toes as well as bullous ulcerations and surrounding purpura. DIC and heparin-induced-thrombocytopenia were both ruled out as the cause. Vascular Surgery was consulted and advised discontinuing vasopressors, but despite this her lesions rapidly worsened. Shortly after, she was diagnosed with Purpura Fulminans. A few days later despite discontinuation of pressors and aggressive wound care, her necrotic lesions worsened, and she underwent bilateral below the knee amputations.

IMPACT/DISCUSSION: Pupura fulminans (PF) is rare life threatening emergency among critically ill patients, characterized by the appearance of purpuric rash predominantly in the distal extremities, The rash progresses into sharply demarcated, symmetric, blue or purple discoloration that further evolves to black. These then become larger, confluent ecchymotic lesions and later on, haemorrhagic bullae leading to hard eschars of necrotic tissue. This condition occurs due to dermal microthrombosis and disseminated intravascular necrosis of the venules and capillaries, eventually evolving into deep skin necrosis. Multi-organ failure is common secondary to microthrombi in vessels supplying the organs. Management of PF is supportive with adequate hydration, discontinuation of vasoconstrictors and treating the inciting issue. The most common pathogens of sepsis-induced PF are N. meningitides, S. pneumoniae, S. Aureus, and Clostridia species, and if the organism is unknown broadspectrum antibiotics are usually initiated to include coverage of these organisms. Anticoagulation may be started to prevent further necrosis. In severe cases, surgical debridement can be done in situations of skin necrosis, and this has shown to reduce mortality. Despite these interventions $\mathrm{PF}$ is associated with a poor prognosis with mortality exceeding $50 \%$. In those who survive, one-fourth require a quadruple limb amputation. CONCLUSION: The purpose of this abstract is to raise awareness on the importance of recognizing and managing cases of rare purpura fulminans with immediate action as to avoid limb amputations and death in the setting of septic shock.

\section{PYLEPHLEBITIS IN A WOMAN WITH DIVERTICULITIS}

Rachael Stovall; Chongjia Chen. Boston University Medical Center, Boston, MA. (Control ID \#3185999)

LEARNING OBJECTIVE \#1: Recognize the subtle presentation of pylephlebitis

LEARNING OBJECTIVE \#2: Distinguish the risk factors for developing pylephlebitis

CASE: An 86-year-old woman with a history of congestive heart failure, hypertension, chronic kidney disease and diverticulosis presented with three days of fever, anorexia, weakness, nausea and non-bloody diarrhea. She denied vomiting or abdominal pain. She recalls eating a steak and cheese sandwich 24 hours prior to the onset of her symptoms. She denied recent travel, sick contacts, and prior abdominal surgery. She had no alcohol, tobacco or illicit substance use. In the emergency department, she had no flatus or bowel movements. Vitals were notable for a temperature of $100.9 \mathrm{~F}$, blood pressure of 120/56 mm Hg, heart rate of 76 beats per minute, respiratory rate of 16 breaths per minute, and oxygenation of $98 \%$ on ambient air. On examination she had abdominal distention and hypoactive bowel sounds, but no abdominal tenderness. Her laboratory results showed a normal white blood cell count, chronic anemia with a hemoglobin of $9.1 \mathrm{~g} / \mathrm{dL}$, low platelets of 91,000 per microliter of blood (baseline 210,000 per microliter of blood), an elevated creatinine level of $2.82 \mathrm{mg} / \mathrm{dL}$ (baseline $1.92 \mathrm{mg} / \mathrm{dL}$ ) and normal liver function tests. An abdominal radiograph was without evidence of obstruction or colonic dilatation. On hospital day one, her temperature rose to 102 F. A CT abdomen and pelvis showed diverticulitis in the sigmoid colon and a concentric thrombus within the inferior mesenteric vein, superior mesenteric vein and portal vein. Blood cultures grew Bacteriodes fragilis and Eggerthella lenta. She was diagnosed with sigmoid diverticulitis causing pylephlebitis and completed four weeks of antibiotics, ultimately on ceftriaxone. She was bridged on a heparin drip to warfarin and continued warfarin treatment for three months. One year later she is doing well.

IMPACT/DISCUSSION: The patient presented with non-specific symptoms, which is common for pylephlebitis and include fevers, chills, myalgias, weakness, nausea, vomiting, abdominal pain and diarrhea. Physical exam can be benign or include abdominal distention concerning for ileus. Laboratory results may show a leukocytosis and mild transaminitis. The patient's low platelets were atypical and may have been from the thrombus as it resolved with treatment. The most common risk factors for development of pylephlebitis are diverticulitis, appendicitis and 
pancreatitis. Other factors include cholecystitis, inflammatory bowel disease, malignancy, cirrhosis, tobacco use, prior abdominal operations, antiplatelet therapy and steroid use.

CONCLUSION: Patients with pylephlebitis can present with non-specific symptoms including fatigue and nausea and therefore suspicion must remain high in order to secure the diagnosis. Pylephlebitis should be suspected in patients with abdominal sepsis because of its high mortality rate. Triggers for pylephlebitis include diverticulitis, appendicitis, pancreatitis, cholecystitis and ulcerative colitis.

RAPID-ONSET QUADRIPARESIS AND RESPIRATORY FAILURE IN A PATIENT THAT WALKED IN TO THE HOSPITAL Ellen Murray $^{1}$; Juan N. Lessing ${ }^{1}$; Andrew Levy ${ }^{2}$; Miranda E. Merrill ${ }^{1}$. ${ }^{1}$ University of Colorado, Denver, CO; ${ }^{2}$ University of Colorado Denver, Denver, CO. (Control ID \#3184374)

LEARNING OBJECTIVE \#1: Recognize the subtle presentation of SEA and the importance of early recognition

LEARNING OBJECTIVE \#2: Consider SEA as a diagnosis for acute quadriparesis

CASE: A 75-year-old man presented with 1-week of progressive generalized fatigue and weakness. He reported confusion, slurred speech and difficulty standing, dressing and bathing. He denied numbness, tingling, pain, focal weakness, fever, chills, dyspnea, dysuria or diarrhea. Medical history included type II diabetes mellitus, chronic kidney disease, and eczema with frequent skin wounds. Two weeks earlier he had been treated for leg cellulitis. On exam, he had normal vital signs and his cardiopulmonary exam was normal. He had erythema and a shallow ulcer on his left leg. Strength, sensation and reflexes in all extremities were normal. White blood cell count was $10.8 \times 10^{3} / \mu \mathrm{L}$, sodium $126 \mathrm{mg} / \mathrm{dL}$, blood urea nitrogen 83 $\mathrm{mg} / \mathrm{dL}$, creatinine $2.8 \mathrm{mg} / \mathrm{dL}$ and lactate $2.1 \mathrm{mg} / \mathrm{dL}$. Urinalysis was bland. Chest $\mathrm{x}$-ray and CT-head were normal. He received intravenous fluids and cefazolin for cellulitis. Two hours later, he developed dense paresis in bilateral lower-extremities with 1/5 strength and hyporeflexia. Corticosteroids and broad-spectrum antibiotics were administered. While awaiting further neurologic imaging the weakness progressed to his upperextremities and respiratory muscles requiring intubation. MRI demonstrated a spinal epidural abscess (SEA) extending from $\mathrm{C} 1$ to L3 with multi-level spinal cord compression, vertebral osteomyelitis and discitis. Blood cultures and abscess grew MRSA. He developed refractory septic shock and despite aggressive neurosurgical interventions he expired.

IMPACT/DISCUSSION: Extensive SEA is a rare and emergent diagnosis that can lead to quadriparesis and death. Despite being uncommon (2 of 10,000 hospitalizations), the devastating implications of missed or delayed diagnosis of SEA necessitates all clinic and hospital-based internists be aware of this disease. Immune compromised patients, diabetics and intravenous drug users are at risk, however, SEA most frequently occurs from spine instrumentation or bacteremia. Presenting symptoms are subtle: generalized malaise, fevers, back pain, and in some cases, neurologic symptoms. Leukocytosis and elevated ESR are suggestive, however, MRI is diagnostic. Prognosis is poor; $4-20 \%$ of cases result in irreversible paraplegia and 5\% progress to uncontrolled sepsis and death. Management includes broad-spectrum antibiotics and early surgical intervention. Antibiotic coverage should include Staph Aureus (60\%) as well as Strep and gramnegative species. Prompt recognition of this disease and early intervention are keys to its successful treatment.

CONCLUSION: Here we present a case of rapidly progressive paralysis and respiratory failure due to an extensive SEA in a patient presenting with non-specific symptoms. It is critical to include SEA, although rare, in the differential for generalized weakness and back pain in order to prevent a potentially grave outcome.

\section{RARE CASE OF RHEUMATOID ARTHRITIS MEDIATED CAR- DIAC TAMPONADE}

Nikhila Kethireddy ${ }^{1}$; Navya Kuchipudi ${ }^{2} .{ }^{1}$ University of Connecticut, Hartford, CT; ${ }^{2}$ University of Connecticut, Farmington, CT. (Control ID \#3157854)

LEARNING OBJECTIVE \#1: Diffential diagnosis for cardiac tamponade including rheumatoid arthritis.

LEARNING OBJECTIVE \#2: Promptly stablize and treat cardiac tamponade due to rheumatoid arthritis.

CASE: 60 year old male with recent diagnosis of seropositive rheumatoid arthritis presented with sudden onset substernal sharp stabbing chest pain without radiation. He denied dyspnea, lightheadedness, recent upper viral respiratory illness or sick contacts. He was tachycardic to $130-150$ 's and hypotensive to $90 / 70$. Physical exam is pertinent for reduced heart sounds, normal JVP, negative for distended neck veins. White count of 37,500, platelets 528. Troponins peaked at 0.12, elevated ESR 118 and CRP at 1.6 (reference $<0.8$ ). EKG showed sinus tachycardia. Transthoracic echocardiogram showed a pericardial effusion with tamponade physiology. Pericardiocentesis with pigtail catheter placement was performed. Pericardial fluid analysis showed turbid yellow fluid with elevated inflammatory cells (60000 nuclear cells with 90\% polys), high LDH 1188, low glucose at 35. Bacterial gram stain, culture, cytology for malignant cells, adenosine deaminase (ADA) and mycobacterium tuberculosis culture and smear were negative. This was consistent with an inflammatory effusion due to RA. He was started on colchicine, IV solumedrol which was transitioned to oral prednisone. The hydroxychloroquine was restarted and methotrexate was added. Upon follow up, his pericardial effusions subsided upon repeat TTE and he remains asymptomatic.

IMPACT/DISCUSSION: Rheumatoid arthritis is a chronic autoimmune disease with $1 \%$ prevalence among the general population. Incidence of pericardial effusion in individuals with RA on autopsy varies from $11 \%$ to $50 \%$. Silent pericardial effusion is seen more frequently than acute symptomatic pericarditis. Symptomatic rheumatoid pericardial effusions are uncommon and seen in older individuals with long standing RA. RA is one of the least common causes of cardiac tamponade with a prevalence of $0.22 \%$ to $0.5 \%$. There are multiple etiologies for pericardial effusions including viral, bacterial, uremic, neoplastic, tuberculosis, connective tissue diseases, traumatic, medications and idiopathic. Underlying pathophysiology that leads to pericardial effusions in rheumatoid arthritis includes vasculitis via immune complex deposition with inflammatory cell infiltration. Pericardiocentesis with fluid analysis can provide detailed information which can help narrow the diagnosis. In rheumatoid pericardial effusion the $\mathrm{pH}$ is low, high white count, high LDH, very low glucose concentration. In this case, his history of rheumatoid arthritis provided a clue and pericardial fluid analysis being consistent with effusion due to RA further helped establish the etiology.

CONCLUSION: In conclusion, rheumatoid arthritis causing pericardial effusion with cardiac tamponade should be in the differential in individuals who present with acute cardiac chest pain even in those without long standing rheumatoid arthritis.

RARE DIAGNOSIS OF PHEOCHROMOCYTOMA AT AN UNCOMMON AGE OF 96 YEARS

Shiva Shankar Vangimalla; Srinivasa Kasina; Hussam Ammar. Medstar 
Washington Hospital Center, Washington, DC. (Control ID \#3185847)

LEARNING OBJECTIVE \#1: Recognize the clinical presentation of pheochromocytoma in elderly population

LEARNING OBJECTIVE \#2: Recognize that guidelines for surgical interventions are not clear for elderly population

CASE: A 96 year female with a past medical history of coronary artery disease status post percutaneous coronary intervention, heart failure with reduced ejection fraction, hypertension, and hypothyroidism was admitted for acute hypotension and bradycardia. Vital signs on admission were blood pressure 79/42 $\mathrm{mm} \mathrm{Hg}$, heart rate 56 beats per minute, temperature $36.4 \mathrm{C}$. She was confused and drowsy on admission. Initial laboratory work up was notable for hemoglobin $8.9 \mathrm{mg} / \mathrm{dl}$, creatinine $5.07 \mathrm{mg} / \mathrm{dl}$, elevated thyroid stimulating hormone $57.4 \mathrm{uIU} / \mathrm{ml}$, low free thyroxine $0.35 \mathrm{ng} / \mathrm{dl}$. EKG showed sinus bradycardia without any other acute changes. Chest X-ray did not show any acute cardio-pulmonary process. Urine and blood cultures remained with no growth. Vital signs improved with adequate fluid resuscitation. However, over the next few hours, she developed an episode of hypoglycemia (serum glucose $-41 \mathrm{mg} / \mathrm{dl}$ ) and hypothermia (34.2C) that resolved with administration of intravenous (IV) dextrose and brief trial on bair-hugger. Concerning for severe hypothyroidism with impending myxedema coma, she was treated with stress dose IV hydrocortisone and IV levothyroxine. Adrenal insufficiency was ruled out by elevated random serum cortisol of $41.1 \mathrm{mcg} / \mathrm{dl}$. Hospital course was complicated by labile blood pressures, systolic blood pressure ranging from $90 \mathrm{~s}-270$ s and diastolic blood pressure ranging from 40s-130s. Review of her past medical records revealed $4 \times 4 \mathrm{~cm}$ heterogeneous mass arising from left adrenal gland that was incidentally found on a CT scan of abdomen/pelvis in 2016 for hospitalization for nausea and vomiting. Lab work up now revealed elevated plasma metanephrines $6.12 \mathrm{nmol} / \mathrm{L}$, elevated plasma normetanephrines 12.81 $\mathrm{nmol} / \mathrm{L}$, elevated 24 hour-urine fractionated metanephrines $3118 \mathrm{mcg} /$ day, elevated 24 hour-urine normetanephrines $3291 \mathrm{mcg}$ /day confirming the biochemical diagnosis for pheochromocytoma. She was discharged home on low dose alpha blocker (doxazosin) for blood pressure control. Later, patient and her family refused any surgical intervention given her age and other co-morbidities but agreed for optimal medical therapy.

IMPACT/DISCUSSION: Guidelines for surgical management in the elderly are not clear An open discussion about surgical risks/benefits and its impact on overall quality of life is warranted Medical therapy with regular follow up remains a reasonable choice in such patients Our patient of 96 years at the time of diagnosis may be the oldest living individual reported with pheochromocytoma to the best of our knowledge

CONCLUSION: A high index of clinical suspicion is needed to work up pheochromocytoma in elderly given its highly variable clinical presentation Biochemical confirmation should be followed by radiological evaluation for the diagnosis Medical therapy alone might be a reasonable choice in high-risk elderly patients

RARE SUBTYPE AUTOIMMUNE ENCEPHALITIS WITH REFRACTORY NON-CONVULSIVE STATUS EPILEPTICUS: DIAGNOSTIC CHALLENGE

Yasir Loai; Riffat Sabir. Baystate Medical Center, Springfield, MA. (Control ID \#3181725)

LEARNING OBJECTIVE \#1: Recognize atypical presentation of autoimmune encephalitis in patients with refractory seizures

LEARNING OBJECTIVE \#2: Treat suspected autoimmune encephalitis empirically and monitor for interval change

CASE: 33-year-old female with past medical history of depression, anxiety, prior alcohol and cocaine abuse, and newly diagnosed epilepsy admitted for evaluation of recurrent witnessed tonic-clonic seizures despite full adherence to antiepileptic drugs (AED). Routine labs were remarkable for significantly elevated lactate, negative urine toxicology screen, and therapeutic AED level. Computed tomography of head (CT) and magnetic resonance imaging of brain (MRI) showed no abnormal intracranial pathology. Cerebrospinal fluid (CSF) revealed normal glucose and protein levels without presence of cells or oligoclonal bands, with negative viral panel and cultures. Patient course further deteriorated by new onset psychosis, echolalia, blank stares, automatic obedience, catatonia, posturing and purposeless activity. Psychiatry was consulted for evaluation of depressive catatonia, however patient failed to respond to IV lorazepam challenge. Immediate 72-hour Video EEG recorded disorganized slowing with frequent $2 \mathrm{~Hz}$ spike and wave discharges consistent with diffuse epileptiform encephalopathy. With progressive decline in mentation, refractory seizure activity, and failure to respond to AED cocktail, patient was commenced on empiric therapy with high dose IV methylprednisolone and immunoglobulin (IVIG) for presumed autoimmune encephalitis. Within 24 hours patient showed remarkable response with near full resolution of neurological symptoms. Extensive autoimmune and paraneoplastic workup a week later demonstrated elevated P/Q Voltage gated calcium channel (VGCC) type antibodies, thus establishing diagnosis of autoimmune encephalitis.

IMPACT/DISCUSSION: Autoimmune encephalitis (AE) is an antibody mediated inflammation of the brain primarily involving limbic system. AE can be broadly classified into paraneoplastic and non-neoplastic with $\mathrm{P} / \mathrm{Q}$ type VGCC encephalitis being a rare subtype of AE. Clinical presentation can be variable as patients may present with neuropsychiatric or neurological symptoms including seizures, cognitive impairment and autonomic dysfunction. There appears to be no single diagnostic measure that can make this diagnosis in isolation, as small subset of patients will not have any neuroimaging findings despite profound neuropsychiatric symptoms. Therefore, internists need to have high clinical index of suspicion especially in patients not responding to conventional therapy and consider early empiric initiation of corticosteroids and IVIG until disease confirmation by serological testing.

CONCLUSION: Consider diagnosis of AE in patients with neuropsychiatric presentation not improving with AED Initiating empiric treatment with IV steroids and IVIG is warranted for management of presumed autoimmune encephalitis if high clinical index of suspicion

\section{RASH AND LIVER DISEASE IN A PATIENT WITH ROUX-EN-Y GASTRIC BYPASS}

Julie C. Sung $^{1}$; Genevieve Tuveson ${ }^{1}$; Leona Kim-Schluger ${ }^{2}$; Mayce Mansour ${ }^{1} .{ }^{1}$ The Mount Sinai Hospital, New York, NY; ${ }^{2}$ Icahn School of Medicine at Mount Sinai, New York, NY. (Control ID \#3184726)

LEARNING OBJECTIVE \#1: Recognize the clinical features of nutritional deficiencies in patients who have received bariatric surgery

LEARNING OBJECTIVE \#2: Understand patient characteristics and clinical manifestations of zinc deficiency

CASE: A 35-year-old female with history of Roux-en-Y gastric bypass (RYGB) in 2015, alcohol use disorder in remission, and recurrent gallstone/ alcoholic pancreatitis presented with abdominal pain and rash. Patient had normal vitals on presentation. Physical exam remarkable for jaundice and diffuse abdominal pain, but most notable for a palpable, painful purpuric rash over abdomen, lower back, and upper and lower extremities. Noted to have desquamation over dorsum of feet, sparing the palms and soles. Labs notable for AST/ALT 54/26 with Tbili/Dbili 4.4/3.4 with alkaline phosphatase 103. Given elevated liver function tests and rash, patient underwent infectious and 
rheumatologic work-up which was unrevealing. Liver biopsy showed evidence of both biliary fibrosis and alcoholic steatohepatitis, with no evidence of cirrhosis. Urine testing came back positive for alcohol. Skin biopsy of rash showed necrolytic acral erythema. Zinc level was then obtained which showed a low level of 29 . Given otherwise negative work up, rash was likely caused by a zinc deficiency. At discharge, patient was started on zinc supplementation and referred as an outpatient to liver medicine for followup of her alcoholic steatohepatitis and to surgery for cholecystectomy.

IMPACT/DISCUSSION: Gastric bypass surgery, especially RYGB, can put patients at risk for numerous side effects related to changes in absorption. A particularly unique aspect to this case is the purpuric rash. Initial presentation with palpable purpura and new-onset liver disease was concerning for possible vasculitis, however skin biopsy showed necrolytic acral erythema. Necrolytic acral erythema is a rare condition first described among a small cohort of hepatitis C carriers in Egypt in 1996. It is described as a pruritic, hyperkeratotic, and erythematous eruption that affects acral surfaces. However, a few case reports in the literature have described this skin finding in zinc deficiency. Interestingly, the incidence of zinc deficiency occurs almost five times more frequently in RYGB patients than in gastric sleeve patients. RYGB is well-known to be associated with increased risk of iron and B12 deficiencies; however, this case demonstrates other micronutrient deficiencies that should be recognized by practitioners. This is especially critical in complex patients who may have alternative and highly plausible etiologies for possible disease processes.

CONCLUSION: Patients with history of RYGB are at high risk of zinc deficiency. Zinc deficiency can manifest as a purpuric and desquamating rash known as necrolytic acral erythema.

\section{RECTAL PAIN IN A YOUNG MAN}

Rand Dadasovich. University of California, San Francisco, San Francisco, CA. (Control ID \#3183212)

LEARNING OBJECTIVE \#1: Recognize the clinical presentation of lymphogranuloma venereum (LGV)

LEARNING OBJECTIVE \#2: Describe the changing epidemiology of LGV

CASE: A previously healthy 29 year-old man presented to an urban public health clinic with 4 days of dyschezia, bloody rectal discharge, and tenesmus. He reported no fevers, chills, abdominal pain, diarrhea, anal pruritis, rash, arthralgias, or other systemic symptoms. He had no history of hemorrhoids and no family history of IBD or other autoimmune diseases. The patient identified as a gay man and reported unprotected receptive anal sex with a new male partner ten days earlier. His only medication was PrEP, started two years earlier. Vital signs were within normal limits. Examination revealed normal external genitalia, no visible lesions or rash, and no inguinal lymphadenopathy. Anoscopy revealed a thin milky discharge and several small linear non-bloody mucosal ulcerations on the posterior rectal wall. Specimens were collected and sent for testing of chlamydia, gonorrhea, HSV, LGV, syphilis, and HIV. The patient was treated empirically with IM ceftriaxone and a 7-day course of doxycycline. LGV PCR returned positive and the course of doxycycline was extended to 21 days.

IMPACT/DISCUSSION: LGV is caused by 3 serovars of Chlamydia trachomatis that are more invasive than those that cause common chlamydial infections. In the classical presentation, primary LGV infection presents as a small painless genital ulcer at the site of inoculation approximately 3 to 30 days after exposure. Secondary infection appears two to six weeks later as a result of direct extension of the infection to regional lymph nodes, which then leads to lymph node necrosis and abscess formation. In contrast to the classic urogenital presentation, LGV can also produce an anorectal syndrome characterized symptoms of proctocolitis: rectal discharge, anal pain, constipation, fever, and tenesmus. The presentation can be mistaken for IBD and complications include chronic colorectal fistulas and strictures if untreated. LGV was previously considered a tropical disease, endemic among heterosexuals in East and West Africa, India, Southeast Asia, and the Caribbean. There have been increasing reports of LGV since 2003 in temperate climates, including large outbreaks seen in UK and NYC, primarily among MSM. Among 327 cases of LGV identified in the UK from 2004-2006, the major symptoms included rectal discharge (79\%), pain (69\%), rectal bleeding (58\%), tenesmus (29\%), and constipation (25\%). Only four percent of patients had concomitant urogenital LGV. Risk factors included HIV-positivity, history of recent unprotected anal sex, and MSM (Ward et al, 2007).

CONCLUSION: Contrary to the classic urogenital presentation mostly observed in the developing world, LGV can also present as an anorectal syndrome characterized by rectal discharge, anal pain, constipation, fever, and tenesmus. Consider LGV in patients presenting with acute proctocolitis, especially if MSM and reporting recent unprotected anal sex.

\section{REFRACTORY HYPERCALCEMIA AS A KEY TO THE DIAGNO- SIS OF A RARE CASE OF CO-EXISTING HODGKIN'S LYMPHO- MA AND ADULT T-CELL LEUKEMIA/LYMPHOMA.}

Lakshmi Priyanka Mahali; Nikita Fernandes; Colette Knight. Montefiore Medical Center, Bronx, NY. (Control ID \#3184554)

LEARNING OBJECTIVE \#1: Identify that refractory hypercalcemia can be a marker of both disease progression and also development of a new malignancy.

LEARNING OBJECTIVE \#2: Recognize HTLV1-associated ATLL as a cause of relapsing hypercalcemia of malignancy.

CASE: 63 year old Jamaican woman presented with generalized bone pain, headache, nausea and mild abdominal pain. The patient was diagnosed with Hodgkin's lymphoma two years ago and was treated with 6 cycles of ABVD(doxorubicin, bleomycin, vinblastine and dacarbazine) which resulted in marked reduction of adenopathy. Post-treatment Positron Emission Tomography(PET/CT) scan showed resolution of disease. Nearly 1 year after completing chemotherapy, the patient had several admissions for hypercalcemia that was refractory to the usual treatment. During this admission, labs were significant for calcium(Ca): $16 \mathrm{mg} / \mathrm{dl}$, white blood cell count: 26,000 $\mathrm{k} / \mathrm{ul}$ with lymphocytosis, 1,25 hydroxy vitamin $\mathrm{D}$ (calcitriol): $10 \mathrm{pg} / \mathrm{ml}$, parathyroid hormone(PTH): $10 \mathrm{pg} / \mathrm{ml}$, parathyroid hormone-related protein(PTHrP): $32 \mathrm{pg} / \mathrm{ml}$ and alkaline phosphatase(ALP): 328U/1. PET scan showed no increase in adenopathy. The recurrent episodes of hypercalcemia despite no increase in tumor burden raised concern that there was an alternate cause of hypercalcemia. Further evaluation included peripheral blood flow cytometry which was consistent with Adult T-cell Leukemia/Lymphoma (ATLL). Human T Lymphotropic Virus type 1 (HTLV-1) infection was confirmed by western blot.

IMPACT/DISCUSSION: Persistent hypercalcemia that fails to respond to standard therapy may represent worsening of the primary cause or a coexisting disease process. Hypercalcemia of malignancy occurs by several mechanisms, which include tumor secretion of PTHrP, tumor production of 1,25-dihydroxyvitamin $\mathrm{D}$ and local release of cytokines by osteoclasts(OCLs). Increased production of 1,25-dihydroxyvitamin D due to increased expression of 1-alpha hydroxylase is the cause of almost all cases of hypercalcemia in Hodgkin's lymphoma. Hypercalcemia in ATLL is due to marked bone resorption by osteoclasts via receptor activator of nuclear factor kappa- ligand (RANK-L) over-expression, but in some cases can also be 
PTHrP-mediated. In this case the low calcitriol, low PTH and elevated PTHrP is a pattern that is consistent with hypercalcemia due to ATLL. HTLV is endemic in the Caribbean islands and is a major cause of ATLL, either de novo or reactivation after an infection. Case reports have shown reactivation of HTLV after induction chemotherapy for treatment of other cancers. However, it is extremely uncommon for Hodgkin's and ATLL to exist in the same patient as was observed in this case.

CONCLUSION: Alternate diagnosis or co-existing malignancy should be suspected in cases of refractory hypercalcemia, despite optimal therapy. Hypercalcemia of malignancy occurs via different mechanisms, understanding of which helps identify the underlying malignancy.

\section{REFRACTORY ORTHOSTATIC HYPOTENSION, WEAKNESS, \&AMP; PARASTHESIAS IN A VIETNAM VETERAN EXPOSED TO AGENT ORANGE}

Rogelio M. Cruz. NYU, New York City, NY. (Control ID \#3186278)

LEARNING OBJECTIVE \#1: Recognize the clinical manifestations of AL Amyloidosis and its association with Agent Orange

LEARNING OBJECTIVE \#2: Management of refractory orthostatic hypotension

CASE: A 68 year-old man with prior Agent Orange exposure in Vietnam presents with 6 months of progressive orthostatic hypotension $(\mathrm{OH})$, syncope, $60 \mathrm{lb}$ weight loss, and lower extremity weakness, parasthesias, and allodynia. Prior workup found mildly elevated troponin and BNP, but a normal stress test. Midodrine had no improvement in $\mathrm{OH}$, and $\mathrm{BP}$ on admission dropped $138 / 75$ to $67 / 42$. Exam showed lack of pupillary reaction to accommodation, anhidrosis, urinary retention, $3 / 5$ strength in both legs, and decreased sensation below the knees. Routine labs were unremarkable, MRI brain, C/T/L spine was noncontributory, and CT C/A/P showed no malignancy. TTE revealed severe Bi-V hypertrophy and a small pericardial effusion. Further workup showed increased serum lambda ( ) free light chains, and urine immunofixation revealed faint monoclonal free elevation. Cardiac MRI showed LVEF 38\% with diffuse subendocardial gadolinium enhancement suggestive of amyloid cardiomyopathy. An abdominal fat pad biopsy was inconclusive so a Cardiac biopsy was done, confirming AL amyloid (AL) deposition. Bone Marrow biopsy stained positive for amyloid and exhibited a population of lambda-restricted plasma cells $(<10 \%)$ consistent with plasma cell dyscrasia. TTR amyloid genetic testing was negative. EMG \& NCS showed axonal large fiber polyneuropathy favoring amyloid over inflammatory demyelinating neuropathy. Paraneoplastic and autoimmune blood panels were negative. Controlling the $\mathrm{OH}$ was difficult despite a slew of nonpharmacologic interventions such as abdominal binders, and max dose Florinef \& Midodrine. Addition of Droxidopa was required to control symptoms. He was discharged with plans to start chemotherapy.

IMPACT/DISCUSSION: AL amyloidosis is an accumulation of monoclonal Ig light chains into pathologic fibrils that deposit in extracellular spaces and result in tissue dysfunction and cell death, known as proteotoxicity. Our patient showed cardiac involvement as well as autonomic, sensory and motor neuropathy, but manifestations vary depending on location and amount of deposition. Also, while not causal, recognition that the Dept. of Veteran Affairs lists $\mathrm{AL}$ as a disease associated with Agent Orange based on limited suggestive evidence is relevant to this case. At least one study found an odds ratio of 3.02. Pharmocologic management of $\mathrm{OH}$ includes fludrocortisone, which increases sodium and water reabsorption, and midodrine, an alpha-1 agonist, which increases vascular resistance. Droxidopa is an oral synthetic precursor to norepinephrine and while not studied in AL, did show a benefit in $\mathrm{OH}$ in familial amyloid polyneuropathy and was FDA approved in 2014 for $\mathrm{OH}$.
CONCLUSION: The mainstay of treatment for AL is chemotherapy directed at the plasma cell dyscrasia producing the abnormal protein. Immunotherapies targeting the amyloid deposits are currently in development.

\section{RELAPSING POLYCHONDRITIS MASQUERADING AS INFEC- TIOUS CELLULITIS}

Stephanie C. Opusunju; Leigh K. Hunter. Methodist Dallas Medical Center, Dallas, TX. (Control ID \#3162008)

LEARNING OBJECTIVE \#1: 1. Recognize predisposing factors for relapsing polychondritis

LEARNING OBJECTIVE \#2: 2. Distinguish manifestations of relapsing polychondritis from other diagnoses with similar presentations

CASE: A 40-year old African American man presented to the emergency department with three days of facial swelling and tinnitus, was initially diagnosed with facial cellulitis and treated with empirical antibiotics. After two days of minimal improvement, otorhinolaryngology consultation was requested. Physical examination revealed bilateral chondritis with swelling of the nasal tip, extension to periorbital areas and malar eminences, swelling of the cartilaginous portions of ears with sparing of lobules, and signs of conjunctivitis. Diagnosis of Relapsing polychondritis (RP) was established based on McAdam's criteria which requires three or more of the following clinical findings: bilateral auricular chondritis, non-erosive, seronegative inflammatory polyarthritis, nasal chondritis, ocular inflammation (conjunctivitis, keratitis, scleritis/episcleritis, uveitis), respiratory tract chondritis (laryngeal and/or tracheal cartilages), and cochlear and/or vestibular dysfunction (neurosensory hearing loss, tinnitus, and/or vertigo). Of interest, rheumatoid factor and erythrocyte sedimentation rate were also elevated and HIV antibody was positive. The patient declined biopsy but was treated with high dose glucocorticoid therapy resulting in complete resolution of symptoms. The patient was lost to follow up post-discharge.

IMPACT/DISCUSSION: Auricular chondritis, arthritis, nasal chondritis, ocular inflammation, laryngotracheal symptoms, reduced hearing, and vestibular dysfunction are the most common manifestations of RP. Diseases reported in association with RP include systemic vasculitides, rheumatoid arthritis, connective tissue diseases, spondyloarthritis, hematologic diseases, gastrointestinal diseases and other miscellaneous entities such as Sweet's syndrome, retroperitoneal fibrosis, familial Mediterranean fever, sarcoidosis, and immune restoration syndrome. It is imperative that internal medicine physicians recognize the constellation of symptoms of RP in order to avoid diagnostic and treatment delays. Optimal treatment remains an area of active investigation, but immunosuppression is the mainstay.

CONCLUSION: Relapsing polychondritis is a potentially fatal multi-system connective tissue disorder primarily involving cartilaginous structures of ears, eyes, joints, nose, laryngotracheobronchial tree, costal cartilages and proteoglycan rich structures including the heart, kidney, and blood vessels and is characterized by recurrent episodes of inflammation. Because of its rarity, the diagnosis may prove challenging for clinicians resulting in treatment delays and increased morbidity and mortality.

\section{RELIEVING THE PRESSURE A NOVEL APPROACH TO INTES- TINAL PSEUDO-OBSTRUCTION WITH RECTAL FOLEY DE- COMPRESSION.}

Dorjan Pantic; Ceena Chandrabos; Linda Kurian. Hofstra Northwell School of Medicine, Manhasset, NY. (Control ID \#3140347)

LEARNING OBJECTIVE \#1: possible management for intestinal pseudoobstruction with rectal Foley catheter placement 
CASE: We present a novel approach for bowel decompression in Ogilvie syndrome using a rectal Foley catheter. This case series consists of two elderly male bed-bound patients, with recurrent hospital admissions for intestinal pseudo-obstruction with resultant colonic dilation, also known as Ogilvie's syndrome. While this syndrome has been associated with trauma, infections, and abdominal surgeries, it is also frequently seen in patients with prolonged immobility or bed bound state. In such cases, we theorize that decreased abdominal pressure from abdominal muscle laxity allows for increased gaseous distention of the large bowel, resulting in sigmoid colon kinking and subsequent functional outlet obstruction. Both patients presented with severe abdominal distention, and hyperactive bowel sounds. Abdominal CT imaging in both cases showed lack of anatomic obstruction but significant colonic dilation with cecal dilation $>9 \mathrm{~cm}$. Traditionally, these patients are treated with rigid rectal tubes or endoscopic decompression. Our initial patient was initially treated with a rigid rectal tube; however, due to routine patient care, the tube became dislodged resulting in reaccumulation of gaseous distention. Subsequently, a 24 French 3-way straight Foley catheter was placed into the rectum with balloon cuff inflation using $25 \mathrm{ml}$ of sterile water. The catheter was left to drain by gravity with standard Foley catheter tubing and frequent bag emptying. After 24 hours the bowel distention improved and serial abdominal X-rays confirmed bowel decompression. The second case was seen in the emergency department. As before, a Foley catheter was placed into the rectum and connected to catheter tubing and subsequently removed after 6 hours with marked improvement in colonic distention allowing for the patient to be discharged without hospital admission.

IMPACT/DISCUSSION: The use of a Foley catheter to straighten the sigmoid colon enables an outlet for gas and stool. This procedure may be superior to a rectal tube in the ability to remain in place for enough time to successfully decompress colonic distention. Rectal Foley placement does not require advanced training and causes minimal patient discomfort. It can potentially decrease cost of patient care as it does not require advanced procedures or sedation and is cheaper than other methods of decompression. CONCLUSION: Both patients had recurrent pseudo-obstruction potentially resulting from decreased abdominal wall strength due to prolonged immobility allowing for colonic expansion in the setting of a relative decrease in intraabdominal pressure. Both cases subsequently resolved with rectal Foley catheter decompression. Foley catheters are readily available and can be safely placed in patients with large bowel distention due to Ogilvie syndrome. The risk of the procedure is similar to that of a rectal tube and its placement does not require additional expertise.

\section{RENAL CELL CARCINOMA PRESENTING WITH DYSPHAGIA: A CASE REPORT}

Zion Lamm; Andrew Dries; Kevin Humphreys; KEVIN LAMM. Carolinas Medical Center, Charlotte, NC. (Control ID \#3184874)

LEARNING OBJECTIVE \#1: Be able to define and diagnose achalasia and pseudoachalasia.

LEARNING OBJECTIVE \#2: Recognize that rapidly progressive achalasia may be due to a paraneoplastic process

CASE: A 77-year-old female with history of diabetes and vitiligo presented to clinic with progressive dysphagia to solid food and twenty-pound weight loss over six months. Barium esophagram revealed prominent narrowing of the distal esophagus with tertiary contractions. Follow-up with esophageal manometry showed panesophageal pressurization $>30 \mathrm{mmHg}$, suggesting Type II achalasia. A puckered lower esophageal sphincter was noted on esophagogastroduodenoscopy. Subsequent endoscopic ultrasound uncovered a lobulated mass measuring $9 \mathrm{~cm}$ in the lower pole of the left kidney. Given dysphagia and findings of achalasia by manometry thought to be due to paraneoplastic syndrome, 100u of botox was injected into the LES. Patient ultimately underwent left radical nephrectomy for unclassified renal cell carcinoma.

IMPACT/DISCUSSION: Achalasia is a rare motility disorder of the esophagus in which loss of neurons traditionally lead to a slow decline of peristalsis and impaired relaxation of the lower esophageal sphincter (LES). Rapid progression of symptoms including dysphagia and unintentional weight loss suggests pseudoachalasia most often due to a malignancy. Though most cases are due to direct infiltration, primary cancers from lung, prostate, and kidney have been described to create a paraneoplastic syndrome that disrupts esophageal function by anti-neuronal and anti-cholinergic antibodies. We present a case report of a patient diagnosed with achalasia due to a paraneoplastic process from renal cell carcinoma.

CONCLUSION: Rapidly progressing achalasia accompanied by unintentional weight loss is often due to secondary processes. Without controlling for symptoms, $1.5 \%$ of patients diagnosed with achalasia were found to be due to neoplasia. When infiltrative malignancies have been ruled out, imaging of the chest, abdomen, and pelvis should be considered to rule out paraneoplastic syndrome from undiagnosed malignancies.

\section{RETURN OF A FOE OR NEW ADVERSARY?: A CASE OF RECUR- RENT JAUNDICE AND LIVER INJURY IN A 28-YEAR-OLD FE- MALE}

Jesse Solomon ${ }^{1}$; Hugo Kaneku ${ }^{1}$; Anna K. Donovan ${ }^{2} .{ }^{1}$ University of Pittsburgh Medical Center, Pittsburgh, PA; ${ }^{2}$ University of Pittsburgh School of Medicine, Pittsburgh, PA. (Control ID \#3180836)

LEARNING OBJECTIVE \#1: Be familiar with the clinical presentation of relapsing Hepatitis A Virus (HAV)

LEARNING OBJECTIVE \#2: Recognize the limitations of biopsy in distinguishing $\mathrm{HAV}$ infection from autoimmune hepatitis

CASE: A 28-year-old female presented with recurrent jaundice. She was diagnosed with acute HAV infection three months prior and had near complete resolution of her symptoms with supportive care. One month prior to her current presentation, she developed severe fatigue, nausea, vomiting, and jaundice. Her medical history included postural orthostatic tachycardia syndrome, obesity status post gastric sleeve, and anxiety. Family history was noncontributory. Initial physical examination was notable for jaundice and generalized abdominal tenderness without peritoneal signs; exam was negative for Murphy's sign, asterixis, caput medusae, or lower extremity edema. Labs were significant for AST 956 IU/ L, ALT 455 IU/L, total bilirubin 16.5 $\mathrm{mg} / \mathrm{dl}$, albumin $2.5 \mathrm{mg} / \mathrm{dl}$, alkaline phosphatase $88 \mathrm{IU} / \mathrm{L}$, INR 2.4, total IgG 2530, and positive ant-HAV IgM and anti-smooth muscle Ab (ASMA). Abdominal US and CT revealed an unremarkable liver morphology and hepatic vessel flow; however, the liver was noted to be hypoechoic with thickening of the portal venous wall. Differential diagnosis was relapsing HAV vs. autoimmune hepatitis (AIH). A liver biopsy revealed bridging necrosis with a plasmacytic infiltration consistent with a subacute process, such as HAV; however, AIH could not be ruled out. On hospital day 10, the patient became confused and somnolent with worsening coagulopathy. She was transferred to the ICU, where she was treated empirically with high-dose prednisone and plasmapheresis. Family successfully advertised for an altruistic donor on social media resulting in a non-related living donor liver transplant. She was discharged on post-op day 14 with normal mental status and liver function.

IMPACT/DISCUSSION: This case changed our perspective of HAV infection, which is typically self-limiting and without long-term sequelae, and the 
difficulty in distinguishing relapsing HAV from $\mathrm{AIH}$. The diagnostic dilemma is further magnified by the differences in managing the two diagnoses.

CONCLUSION: Up to $10 \%$ of patients may have clinical or biochemical relapsing course of HAV infection, and fulminant hepatitis occurs in just $1 \%$ of cases. Interestingly, HAV infection has been shown to provoke AIH in a minority of patients. Our patient had a recent HAV infection with a concurrently elevated IgG and positive ASMA, raising concern for AIH. Data concordant with relapsing HAV infection include tissue specimen with a subacute pathological process and a lack of response to steroids or plasmapheresis; however, this lack of response may have been an indicator that her liver failure was irreversible. Internists need to consider relapsing HAV and AIH in patients with recent $\mathrm{HAV}$ infection who present with recurrent symptoms.

\section{REVENGE OF A FISH: AN UNUSUAL CAUSE OF PERSISTENT ABDOMINAL PAIN IN A 64 YEAR-OLD FEMALE}

Ryan Burkholder ${ }^{1}$; Hrishikesh Samant ${ }^{2} .{ }^{1}$ Lousiana State University Health Sciences Center, Shreveport, LA; ${ }^{2}$ Lousiana State University, Shreveport, LA. (Control ID \#3184820)

LEARNING OBJECTIVE \#1: Diagnose and treat hepatic abscess due to foreign body implantation

CASE: 64 y.o. female with PMH of hypertension, obesity and GERD presents with 8 days of abdominal pain in right upper quadrant and epigastric region accompanied by nausea, vomiting, fevers and yellow diarrhea. On admission, vital signs were notable for BP 216/90, P 133, RR 18, Temp 102.7. Physical exam showed jaundice but did not appreciate abdominal tenderness or guarding. WBC was $20.2 \mathrm{k}$, total bilirubin 2.4, alkaline phosphatase 114 , AST 62, ALT 59 and albumin 2.6. CT of the abdomen was performed showing a $6.9 \mathrm{~cm}$ lobulated heterogeneously enhancing fluid lesion within the junction of the right and left hepatic lobes, also noting signs of colitis. Interventional radiology performed percutaneous drainage on day of admission. Abscess cultures returned with alpha hemolytic streptococcus. Due to persistent leukocytosis and repeat abdominal CT which showed persistent fluid collection, a drain revision was performed 5 days later, and noticed a $2.1 \mathrm{~cm}$ radiopaque foreign body resembling a fish bone noted at the level of the falciform fissure/ gastro hepatic ligament. Gastroenterology was consulted EGD was done at this time in attempts of retrieval yet there was no visualization of the fishbone from the antrum of the stomach. Surgical team was consulted yet decided against performing retrieval via laparotomy due to clinical improvement in patient. Repeat CT prior to discharge showed subcapsular hepatic collection ( $2 \times 6.5$ $\mathrm{cm}$ ) involving segments $2 / 3$, distal gastric mural thickening and perigastric inflammatory changes. Patient improved clinically, leukocytosis decreased to $8.9 \mathrm{~K}$, and blood cultures remained negative. Patient was discharged on extended course of ceftriaxone and intravenous metronidazole.

IMPACT/DISCUSSION: Gastrointestinal perforations have been reported in less than $1 \%$ of patients with foreign body ingestions with most common sites of perforation of the gut being the stomach and duodenum. Patients have nonspecific complaints including abdominal pain, fever vomiting, weight loss and/ or anorexia as response to infection and abscess formation. History may be unhelpful and patients rarely recall episode of ingestion, and it may be weeks to months prior to presenting complaints. While plain x-rays are only $32 \%$ sensitive in detecting foreign bodies in upper GI tract, CT imaging is preferred technique for diagnosis.

CONCLUSION: Careful correlation of imaging findings and clinical course should be performed in patients with unexplained hepatic abscess formation. As demonstrated in our patient, endoscopy is only helpful when performed early, before migration and mucosal healing of the GI tract. We treated this patient with routine drainage and antibiotics. Our clinical report is similar to previous episodes reported in previous literature, although in our case the decision was made to leave foreign body intact due to clinical improvement.

\section{REVERSAL OF RIGHT VENTRICULAR FAILURE WITH PACE- MAKER LEAD EXTRACTION}

LU CHEN ${ }^{1}$; Uta Guo ${ }^{3}$; PARAG MEHTA ${ }^{2} .{ }^{1}$ NEW YORK PRESBYTERIAN - BROOKLYN METHODIST HOSPITAL, Brooklyn, NY; ${ }^{2}$ NewYorkPresbyterian BMH Hospital, Brooklyn, NY; ${ }^{3}$ SUNRISE GME CONSORTIUM, LAS VEGAS, NV. (Control ID \#3186224)

LEARNING OBJECTIVE \#1: Recognize and treat tricuspid regurgitation (TR) induced right ventricular (RV) failure.

CASE: A 73-year-old female with a medical history of atrial fibrillation (AF), and hypertension presented to the emergency department with gradually worsening dyspnea on exertion, abdominal distension, and lower extremity swelling. For the past year, the patient had decreased exercise tolerance, limited by chest pressure and dyspnea. The chest pressure starts after ambulating one block and is relieved by rest. She was started on furosemide and reported moderate alleviation of her symptoms. In 2008, the patient underwent two unsuccessful ablations for long-standing AF and ultimately received atrioventricular nodal ablation with dual chamber permanent pacemaker (PPM) implantation. On admission, vital signs were stable except for tachypnea at 22 breaths per minute. She had significant jugular venous distension, lower extremity edema up to her thighs, but no crackles on pulmonary exam. Laboratory studies showed elevated brain natriuretic peptide (BNP) at 2980 $\mathrm{pg} / \mathrm{mL}$, hyperbilirubinemia at $1.3 \mathrm{mg} / \mathrm{dL}$, and thrombocytopenia of $55,000 / \mathrm{uL}$. Chest radiography showed cardiomegaly, an implanted PPM, and no pulmonary congestion. Patient was started on high doses of intravenous furosemide. Following successful diuresis, an echocardiogram (TTE) showed left ventricular ejection fraction (LVEF) of 50\%, a severely dilated RV with severe systolic dysfunction, and wide open TR with malcoaptation of the valve leaflets. Right heart catheterization demonstrated elevated right-sided filling pressures without elevation in pulmonary arterial pressure or left-sided filling pressures. Liver biopsy confirmed liver cirrhosis due to congestive hepatopathy. PPM lead-induced severe TR leading to congestive hepatopathy with liver cirrhosis was suspected. Patient underwent laser lead extraction and subsequent implantation of a leadless PPM. Within six months, she reported significant improvement in the quality of life with increased exercise tolerance with no chest pain, shortness of breath or lower extremity edema. Repeat TTE showed moderate RV dilatation with mild dysfunction, and improved tricuspid leaflets coaptation.

IMPACT/DISCUSSION: TR is a recognized complication of transvenous PPM or defibrillator placement as RV lead can impinge the tricuspid leaflet. This case illustrates the importance of a thorough workup for a patient presenting with predominantly right heart failure. Previous experiences with RV lead extraction have shown mixed results on the improvement of RV dysfunction and TR. This can likely be attributed to patient selection. Leadless PPM is a viable option for patients with TR secondary to lead impingement of the tricuspid valve.

CONCLUSION: We present a case of severe TR, and RV failure successfully managed with RV lead extraction. The root of our success likely lies within careful patient selection for lead removal through thorough work-up and excluding alternative causes of TR and RV dysfunction.

\section{REVISITING AN ATYPICAL LYMPHOCYTIC COLITIS: LESSONS FROM A PATIENT WITH CHRONIC DIARRHEA}

$\underline{\text { Harry J. Han }}{ }^{1}$; Adam Tapley ${ }^{1,3}$; Rabih Geha ${ }^{1,2}$. ${ }^{1}$ University of California San 
Francisco, San Francisco, CA; ${ }^{2}$ San Francisco Veterans Affairs Medical Center, San Francisco, CA; ${ }^{3}$ School of Medicine, University of California San Francisco, San Francisco, CA. (Control ID \#3178080)

LEARNING OBJECTIVE \#1: Recognize malignancy as a rare cause for chronic diarrhea

LEARNING OBJECTIVE \#2: Appreciate the challenges in diagnosing intestinal T-cell lymphoma.

CASE: A 76 year-old gentleman with schizophrenia and a 60-pack year tobacco history presented with 10 weeks of profuse diarrhea. Stools were rarely formed, but not oily, greasy, malodorous and without melena or hematochezia. There was associated unintentional weight loss, tenesmus, and nocturnal awakening. Review of systems was otherwise negative. Physical exam was notable for 13-pound weight loss over 3 months and hyperactive bowel sounds. Basic labs were normal as was a CT abdomen/pelvis with contrast. Stool osmolar gap was 130 and stool PCR was positive for Giardia. The patient was treated with a course of metronidazole. Despite antibiotic therapy, the patient had persistent diarrhea and an additional 20-pound weight loss over 2 months. EGD/Colonoscopy showed a diffuse patchy colitis and biopsies were interpreted as diagnostic of lymphocytic colitis. The patient's profound weight loss prompted a reexamination of the colonic biopsies, which revealed a more exuberant lymphocyte response than expected for classic lymphocytic colitis and atypical-appearing intraepithelial lymphocytes (IEL). Further immunohistochemical and genetic studies demonstrated a natural killer (NK) and T-cell mononuclear cell infiltrate in the colonic mucosa with positive T-cell receptor clonality. The patient was diagnosed with monomorphic epitheliotropic intestinal T-cell lymphoma (MEITL).

IMPACT/DISCUSSION: MEITL is an aggressive tumor accounting for less than $5 \%$ of peripheral T-cell lymphomas. Unlike Type I enteropathy-associated T-cell lymphomas (EATL), MEITL (previously known as Type II EATL) is not associated with celiac disease. MEITL is a challenging diagnosis given its rarity, non-specific symptoms, heterogeneous endoscopic appearance, and mixed histopathology. Patients present with non-specific symptoms, including chronic diarrhea, abdominal pain, and weight loss. $50 \%$ of patients are diagnosed after acute abdominal complications (e.g. bowel perforation or obstruction). These tumors are heterogeneous in appearance and can present as normal appearing mucosa, ulcerations, masses, and/or strictures. Histologically, there is an overabundance of mucosal IELs occasionally associated with crypt hyperplasia and villous atrophy. As illustrated in this case, the increased IELs can be mistaken for lymphocytic colitis. Biopsy reanalysis is warranted if the clinical syndrome is inconsistent with microscopic colitis. Median survival for MEITL is $<1$ year and standard of care remains undetermined.

CONCLUSION: MEITL is a rare peripheral T-cell lymphoma that is challenging to diagnose due to non-specific presenting symptoms, heterogeneous macroscopic appearance, and complex histopathology. In patients with chronic diarrhea with associated alarm symptoms (fevers, bleeding, significant weight loss) and lymphocytic colitis, intestinal lymphomas should be considered in the differential diagnosis.

\section{RHABDOMYOLYSIS FOLLOWING SYNTHETIC CANNABINOID USE: "MOJO"}

Hanyuan Shi; Elle Wade; Sarah Cossich. Tulane University School of Medicine, New Orleans, LA. (Control ID \#3184297)

LEARNING OBJECTIVE \#1: Demonstrate the detrimental effects of synthetic cannabinoids.

LEARNING OBJECTIVE \#2: Understand rhabdomyolysis as a lifethreatening side effect of synthetic cannabinoids
CASE: A 22-year old man with a history of depression, schizoaffective disorder, lead exposure, and seizures initially presented to the hospital with altered mental status. Collateral information reported concern for seizure activity with bilateral lower extremity muscle jerking, In the emergency room, the patient was agitated and aggressive. Labs were significant for WBC 14.3, lactate of 2.6, BUN 30, Cr 1.21. Ammonia 89. Liver function tests were elevated with AST 592, ALT 135, normal alkaline phosphatase 87, with normal bilirubin 1. Urine drug screen returned positive for cannabinoid. The patient's initial creatinine kinase (CK) was 49,360. Urinalysis showed $>10.0$ $\mathrm{mg} / \mathrm{dl}$ of blood but $<5 \mathrm{RBCs}$. He was started on $250 \mathrm{ml} / \mathrm{hr}$ of lactated ringers for treatment of rhabdomyolysis. Spot EEG showed no ongoing seizures or status epilepticus. Over the next six days, fluids were continued and his CK trended down to $<5000$. He later reported a use of "K2".

IMPACT/DISCUSSION: Synthetic cannabinoids have been used more frequently as recreational drugs. Common street names include "Spice", "K2", "Yucatan Fire", "Skunk", or "Mojo". Often, users of synthetic marijuana report a more intense high than the natural formulation. Alarmingly, there is an increasing population of teenagers and young adults using these compounds, along with increasing reports of complications including acute kidney injury and nephrotoxicity. The pathogenesis of AKI is unknown but hypothesized that synthetic marijuana often contains non-cannabinoid contaminants that are nephrotoxic. Only a few reports note rhabdomyolysis following synthetic cannabinoid use, including one which details renal failure requiring dialysis. "K2" is a deadly synthetic stimulant that as demonstrated here, can lead to seizures, severe agitation, and acute rhabdomyolysis. This case demonstrates the neurogenic and nephrogenic sequelae of ingestion. Early studies have been released demonstrating significant impairment of behavior and judgment by users; however there has not been many published literature on the consequences of overdose. Liberal regulations of synthetic cannabinoids have allowed for increased availability of these substances. Companies that manufacture "K2" operate in clandestine fashion to avoid detection by the FDA. CONCLUSION: Internists may encounter an increasing number of cases of substance abuse. This case demonstrates the availability and detrimental effects of synthetic cannabinoids.

\section{RHABDOMYOLYSIS: A RARE HERALD SIGN OF HYPERALDOSTERONISM}

Chester J. Kao ${ }^{1}$; Jenny Van Kirk ${ }^{2} .{ }^{1}$ Duke University, Durham, NC; ${ }^{2}$ Duke University Hospital, Durham, NC. (Control ID \#3175921)

LEARNING OBJECTIVE \#1: Recognize hyperaldosteronism as a cause of rhabdomyolysis

LEARNING OBJECTIVE \#2: Diagnose primary hyperaldosteronism

CASE: A 47 year old male with a history of hypertension, chronic hypokalemia, and chronic kidney disease stage III presented with a one week history of non-exertional, atypical chest pain without radiation. His last cardiac catherization was five years prior and was normal. Exam was unremarkable except for hypertension to 160/90. Labs were notable for potassium (K) to 2.9, creatine 2.4, bicarbonate 27, and CK 2100. Acute coronary syndrome (ACS) workup was negative, and EKG demonstrated Q waves in V1 and V3 without ST changes. Medications included carvedilol, amlodipine, losartan, and prior K supplements. Family history was notable for hypertension and hypokalemia. Despite aggressive repletion with over $200 \mathrm{mEq}$ of $\mathrm{K}$ chloride in 48 hours, the patient's potassium remained low at 2.5 to 2.7 . He denied any muscle cramps or hematuria, and his chest pain resolved with normalizing CK. Primary hyperaldosteronism was suspected due to the refractory hypokalemia and hypertension. Thus, losartan was switched to spironolactone $25 \mathrm{mg}$ daily. Aldosterone and renin levels were sent but did not result prior to discharge. 
Due to patient preference, he was discharged with close follow-up. Outpatient endocrinology workup showed elevated plasma aldosterone concentration $(\mathrm{PAC}=27)$ to plasma renin activity $(\mathrm{PRA}=0.7)>15$. Saline infusion test and abdominal computed tomography are pending.

IMPACT/DISCUSSION: Primary aldosteronism (PA) is a rare disease affecting $1 \%$ of hypertensive patients. PA is the most frequent cause of secondary arterial hypertension, and it is often due to aldosterone-producing adenomas or adrenal hyperplasia. PA classically presents with hypertension, hypokalemia, and metabolic alkalosis. Hypokalemia can commonly lead to muscle weakness, but, in rare cases, it can lead to rhabdomyolysis. In the literature, there are only a few cases of rhabdomyolysis as the presenting symptom of PA. In our patient, the rhabdomyolysis was incidentally noted on labwork in the setting of atypical chest pain without muscle weakness or pain. Frank rhabdomyolysis often does not occur unless the serum $\mathrm{K}$ levels are below $2.0 \mathrm{mEq} / \mathrm{L}$. To prevent further progression of symptoms, large amounts of $\mathrm{K}$ supplementation are necessary as well initiation of an anti-aldosterone receptor drug including spironolactone or eplerenone. PAC/PRA > 15 is highly suggestive of PA, but diagnosis should be confirmed with aldosterone suppression with sodium loading or saline infusion test. In addition, imaging is needed for evaluating the cause of PA.

CONCLUSION: Rhabdomyolysis may not present with typical symptoms of muscle weakness or pain, so there should be a high suspicion for PA if rhabdomyolysis occurs in the setting of chronic hypokalemia and medication refractory hypertension. In these cases, initiation of an anti-aldosterone receptor drug can help with supplementation-resistant hypokalemia.

\section{RHINOVIRUS INFECTION A TRIGGER OF SEVERE HYPONATREMIA}

omar kousa; Dana Awad; abdallah qasim; Bailey Waters; Mohsin Mirza. Creighton University, Omaha, NE. (Control ID \#3186065)

LEARNING OBJECTIVE \#1: Recognize the role of rhinovirus in causing hyponatremia.

LEARNING OBJECTIVE \#2: Present hyponatremia as a poor prognostic factor in rhinovirus infection in adults.

CASE: A 52-year-old Caucasian woman with a medical history of hypertension on Lisinopril-Hydrochlorothiazide and anxiety on Sertraline, presented with shortness of breath, productive cough, fatigue, confusion and diarrhea of few days. On physical exam; she was mildly distressed and looked dehydrated. Vital signs were significant for a blood pressure of $100 / 70 \mathrm{mmHg}$, Heart rate of 110 /minute, respiratory rate of 35/minute, a temperature of $100 \mathrm{~F}$ and oxygen (O2) saturation of $85 \%$ requiring 2 Liters (L) of $\mathrm{O} 2$ via nasal cannula. Chest exam revealed diffuse bronchial breathing sounds. Chest X-ray showed consolidation consistent with pneumonia. Metabolic profile was significant for hyponatremia at $101 \mathrm{mmol} / \mathrm{L}$ (normal is $135-145$ ). She was admitted to the intensive care unit as a case of severe sepsis with severe hyponatremia. Further work-up showed low urine sodium at $5 \mathrm{mmol} / \mathrm{L}$, elevated urine osmolality at $736 \mathrm{mOsm} / \mathrm{kg}$, along with low serum osmolality at $230 \mathrm{mOsm} / \mathrm{kg}$ which supported a diagnosis of Syndrome of Inappropriate Anti-Diuretic Hormone Secretion (SIADH) as a cause of her hyponatremia. Renal team was consulted and she was started on hypertonic saline and levofloxacin, in addition to holding all home medications. She was subsequently intubated due to increased work of breathing. Respiratory pathogen screen came positive for Rhinovirus. Her severe hyponatremia was corrected slowly, her mental status improved and she was eventually extubated.

IMPACT/DISCUSSION: The patient had been taking hydrochlorothiazide and sertraline), for many years. sodium six months prior to was normal. We think her severe hyponatremia multifactorial, with Rhinovirus infection being the major contributing factor. Previous studies have shown that respiratory tract infections can induce hyponatremia due to an unknown mechanism, most likely SIADH. When rhinovirus present with hyponatremia, it is associated with a worse course. Multiple previous studies showed the link between rhinovirus and hyponatremia in the pediatric population, but no sufficient data has been reported for the adult population.

CONCLUSION: Rhinovirus can induce severe hyponatremia in high risk patients and low serum sodium at initial presentation is associated with worse clinical outcome in adults. However, larger studies are needed to elucidate the exact mechanism.

\section{RING AROUND THE ENHANCING LESIONS}

Justine Phifer; Andrea Porrovecchio. Montefiore Medical Center, Bronx, NY. (Control ID \#3186330)

LEARNING OBJECTIVE \#1: Identify etiologies of ring enhancing lesions in brain

LEARNING OBJECTIVE \#2: Understand legal and ethical consent process for obtaining HIV testing

CASE: A 60 year old woman from Ghana with unknown HIV status presented with altered mental status of four days and weight loss with trouble ambulating of a few months. On presentation patient was inattentive, disoriented, and had left upper and lower extremity weakness. CT head revealed mass effect in right temporal lobe and right caudate head and a MRI brain revealed 30 predominantly ring enhancing lesions in cerebrum/ cerebellum. Patient was started on dexamethasone given the mass effect of her brain lesions and treated empirically for toxoplasmosis given high clinical suspicion. CSF was eventually positive for toxoplasmosis and HTLV. Although initially unable to provide consent for HIV testing due to her altered mental status, she was eventually able to provide informed consent and was found to have new diagnosis of AIDS with a CD4 count of 29. Given her AIDS status and unusually high number of lesions on MRI, she was thought to potentially have concurrent CNS lymphoma. Brain biopsy was deferred until full malignancy workup had been performed and follow up imaging had been obtained to assess for improvement in lesions with toxoplasmosis treatment. IMPACT/DISCUSSION: The differential for ring enhancing lesions in the brain may be narrowed based on degree of patient's immunosuppression as well as whether lesions cause mass effect. In immunocompetent patients as well as those with CD4 count $>500$, benign and malignant brain tumors and metastases predominate. Moderately immunosuppressed patients or HIV positive patients with CD4 count 200-500 rarely present with focal brain lesions. Severely immunosuppressed patients and those with CD4<200 most likely have an opportunistic infection or AIDS-associated tumors. CNS lesions with mass effect are most often caused by Toxoplasma encephalitis and primary CNS lymphoma in the developed world, and in developing world, tuberculomas. One study of HIV positive persons who underwent brain biopsy for the diagnosis of focal CNS lesions revealed that 6 percent had more than one etiology of a lesion. Laws regarding HIV testing differs amongst states, but in New York, verbal consent must be obtained prior to testing. The law mandates that all patients are offered HIV testing (an "opt-out" program). Certain exceptions do exist, such as for newborns, when testing blood or organs for donation, for instances of occupational exposure, certain cases of indicted sex offenders, etc. While HIV status would have possibly helped narrow the differential in our patient, legal and ethical considerations must be made and under most circumstances, patients have the right to decline testing. CONCLUSION: Toxoplasmosis should be empirically treated if clinical suspicion is high enough, even if HIV status is unknown. In some states, 
HIV testing may only be performed after verbal consent has been obtained, despite clinical scenario

\section{RISING PSA, FALLING FIBRINOGEN: A POTENTIAL PERIL OF METASTATIC PROSTATE CANCER}

Clare Cene-Kush; Sunmin Park; Alfred Shoukry. UPMC, Pittsburgh, PA. (Control ID \#3181524)

LEARNING OBJECTIVE \#1: Recognize disseminated intravascular coagulation (DIC) with excessive fibrinolysis as a potential complication of metastatic prostate cancer

LEARNING OBJECTIVE \#2: Describe the management of acute DIC in the setting of prostate cancer

CASE: A 58-year-old man presented with unintentional 25-lb weight loss and a prostate-specific antigen (PSA) elevation to $602.4 \mathrm{ng} / \mathrm{mL}$. Imaging revealed an enlarged prostate and widespread metastatic osseous disease involving the pelvis, thoracolumbar spine and lower ribs with diagnosis of prostate carcinoma confirmed by iliac lymph node biopsy. He was admitted to our inpatient medicine service for worsening anemia, recurrent epistaxis, spontaneous ecchymosis, hematuria and hemoptysis. Physical exam revealed oozing from bilateral knee abrasions and a large ecchymosis over the right buttock. Laboratories were significant for hemoglobin $6.9 \mathrm{~g} / \mathrm{dL}$, platelets $108 \times 10^{\wedge} 9 / \mathrm{L}$, fibrinogen $70 \mathrm{mg} / \mathrm{dL}$, haptoglobin $<30 \mathrm{mg} / \mathrm{dL}$, lactate dehydrogenase $248 \mathrm{IU} / \mathrm{L}$, D-dimer $>20 \mathrm{ug} / \mathrm{mL}$, positive fibrin degradation products, prothrombin time (PT) 20.2 seconds, partial thromboplastin time (PTT) 45.2 seconds and INR 1.8. His deranged coagulation profile persisted, with fibrinogen levels declining to a nadir of $56 \mathrm{mg} / \mathrm{dL}$ despite supportive transfusions with packed red blood cells and cryoprecipitate, but began to improve six days after initiation of hormonal treatment of his metastatic prostate cancer with bicalutamide and leuprolide. IMPACT/DISCUSSION: This case highlights the need for internists to recognize DIC with excessive fibrinolysis as a potential complication or presenting symptom of prostate cancer. Treatment includes supportive transfusions and urgent initiation of complete androgen blockade or deprivation. In this case, correction of coagulopathy and reduction in spontaneous bleeding occurred within one week of initiation of treatment for his prostate cancer.

CONCLUSION: No single test is diagnostic of DIC; instead it requires recognition of a predisposing clinical scenario combined with characteristic laboratory derangements. Treatment is aimed at reversing the underlying cause. In patients with solid tumors, DIC is usually a chronic, compensated and asymptomatic laboratory phenomenon marked by thrombocytopenia with prolonged PT and PTT. A moderate degree of fibrinolysis is often indicated by an elevated D-dimer, but fibrinogen levels themselves are typically normal. However, a form of acute DIC with prominent fibrinolysis has been described in several case reports as occurring more frequently in prostate cancer, making it unique among the solid tumors associated with this consumptive coagulopathy. It has also been suggested that excessive fibrin degradation portends a poorer prognosis with greater risk for catastrophic bleeding. In this case, our patient's DIC and spontaneous bleeding symptoms began to resolve shortly after initiation of prostate cancer treatment despite a significant degree of fibrinolysis on presentation.

\section{RITUXIMAB IN SYSTEMIC LUPUS ERYTHEMATOSUS FRIEND OR FOE}

Shane Murray $^{1}$; Gustavo Contreras Anez ${ }^{1}$; Ana B Arevalo ${ }^{1}$; Yousaf Ali ${ }^{1,2}$. ${ }^{1}$ Mount Sinai St Luke's - West, New York, NY; ${ }^{2}$ Mount Sinai Hospital, New York, NY. (Control ID \#3185917)
LEARNING OBJECTIVE \#1: Recognize rituximab as a cause of neutropenia in systemic lupus erythematosus

CASE: 47-year-old Female with a past medical history of systemic lupus erythematosus (SLE) presented to the Emergency department with a three-day history chest pain. She described a sharp pain, exacerbated by lying flat associated with chills, muscle ache and sore throat. No rash, joint pain, headache or oral ulcers were noted. Medications included mycophenolate mofetil $2 \mathrm{~g}$ daily, prednisone $1 \mathrm{mg}$ daily and rituximab (RTX) given as a weekly infusion for two weeks every two months. Physical Exam was remarkable for a cardiac friction rub. ECG demonstrated diffuse PR depressions. Initial Laboratory studies revealed troponinemia (peak $2.1 \mathrm{ng} / \mathrm{ml}$ ) and neutropenia with an absolute neutrophil count of zero. Chest radiograph was unremarkable. Treatment was begun with high dose oral corticosteroids and broad-spectrum antibiotics. Initial transthoracic echocardiogram was unrevealing but subsequent cardiac MRI confirmed myopericarditis. Examination of peripheral blood smear noted bands and occasional myelocytes. Laboratory testing for extractable nuclear antigens, including double stranded DNA was negative. Complement levels were normal. Bacterial, viral and fungal testing was unrevealing. Neutrophil counts improved and patient was discharged with a Prednisone taper. Outpatient testing off the drug resulted in normalization of neutrophil counts.

IMPACT/DISCUSSION: RTX has gained popularity in recent years as an off-label therapy for refractory SLE. Studies on the efficacy of the drug have yielded conflicting results and its role as a treatment in SLE remains controversial. Treatment duration is poorly defined and uncertainty exists over the safety and efficacy of redosing. Typically, SLE patients receive between two and four doses as therapy. Retreatment is only considered in those who responded and show signs of disease flare. Here we demonstrate a case of RTX infusions given under a modified protocol at an outside institution which may have contributed to a case of late onset neutropenia (LON). RTX is increasingly recognised as a cause of LON. Recent literature suggests a higher occurrence of LON in SLE patients compared to others treated with the drug. High cumulative doses, as in this case, have also been linked to increased rates of LON. While is difficult to exclude lupus or infection as contributing factors, we felt that RTX was the likely culprit due to the relatively high dose and improvement in neutropenia upon cessation of the drug. Providers should remain cautious when prescribing RTX in SLE patients, record cumulative doses and monitor patients frequently for neutrophil count and signs of infection.

CONCLUSION: Safety and efficacy of RTX in SLE is poorly defined and requires further research. RTX related LON may be more prevalent in SLE patients and high cumulative doses may increase risk. Patients should be monitored closely for neutrophil counts and signs of infection after treatment.

\section{ROLE OF C-INHIBITOR TREATMENT IN GLIOBLASTOMA MULTIFORME WITH REFRACTORY EDEMA}

Gillian R. Naro; Nicholas Noverati; Timothy Craig. Penn State College of Medicine, Hershey, PA. (Control ID \#3165785)

LEARNING OBJECTIVE \#1: Recognize the role of bradykinin in clinical complications of Glioblastoma Multiforme (GBM).

LEARNING OBJECTIVE \#2: Discuss the utility of immune-targeted therapy in glioblastoma associated edema through a clinical vignette.

CASE: A 49-year-old male whose medical history is significant only for GBM diagnosed on 9/26/2016, sought treatment for his cerebral edema and angioedema in $3 / 2018$. His disease progressed significantly despite two craniotomy resections, adjuvant chemotherapy, and radiation. On 1/31/2018 he developed angioedema of the pharynx and intermittent swelling of various 
cutaneous sites that was refractory to antihistamines, as well as progressive cerebral edema that did not respond to high dose corticosteroids. Despite a normal C1q, C4 and C1-inhibitor protein and function, C1-inhibitor treatment was started on 3/12/18 at 20 units/kg IV twice weekly, based on literature suggesting that edema may be bradykinin generated. The patient subsequently reported significant improvement in mental status. An MRI on 5/18/2018 confirmed reduced cerebral edema and midline shift after two months of treatment. He has since been maintained on $\mathrm{C} 1$-inhibitor infusions twice a week.

IMPACT/DISCUSSION: GBM is one of the most common adult primary brain tumors. It has a poor prognosis with 5-year survival after radiation and temozolomide chemotherapy at $9.8 \%$. It is thought that mesenchymal stem cells influence GBM invasiveness through cross-talk pathways that lead to an upregulation of bradykinin receptors. Further, bradykinin is also involved in the formation of vasogenic edema by disrupting the blood-brain barrier. Edema adds to the tumor's mass effect as it invades surrounding parenchyma, ultimately increasing intracranial pressure and contributing to overall morbidity and mortality. Treatment of cerebral edema usually entails corticosteroid therapy; however, if unresponsive, there are few additional medical therapies available. Recent research supports bradykinin's role in both tumor invasion and vasogenic edema, has led to interest in the usefulness of C1-esterase inhibitor as a potential therapy to decrease the amount of bradykinin generated by malignant GBM cells. Our case discusses a patient with advanced GBM who had diffuse edema refractory to steroid therapy, and responded well to recombinant $\mathrm{C} 1$-inhibitor. This success may help inspire future trials to explore the utility of this drug as an addition to the arsenal of available treatment modalities for highly aggressive glioblastomas.

CONCLUSION: •GBM is an aggressive tumor that uses bradykinin pathways to advance its borders and distort the blood-brain barrier to create surrounding edema, which can worsen morbidity and mortality. $\bullet \mathrm{C} 1$-inhibitor works by inhibiting the contact activation system and thus reducing bradykinin levels available to the tumor. •In a patient with vasogenic edema of the $\mathrm{CNS}$ that is refractory to other first-line therapies, $\mathrm{C} 1$-inhibitor may be a safe and effective option.

\section{ROOTING OUT A CURIOUS GARDNER'S RASH}

Justin B. Roy ${ }^{1}$; Melissa Yacur $^{2}$; Ami DeWaters ${ }^{3}$. ${ }^{1}$ Penn State Hershey, Hummelstown, PA; ${ }^{2}$ Hershey Medical Center, Hershey, PA; ${ }^{3}$ Penn State Hershey Medical Center, Hershey, PA. (Control ID \#3185468)

LEARNING OBJECTIVE \#1: Discuss the clinical signs, symptoms and diagnosis of disseminated nocardiosis.

LEARNING OBJECTIVE \#2: Describe the risk factors for nocardiosis.

CASE: A 70 year old man with a history of Hodgkin's lymphoma and Follicular lymphoma treated with chemotherapy one year prior, presented to the hospital due to diffuse subcutaneous nodules, rash and weakness. He had developed an erythematous, mildly painful rash that began on his back six months prior to admission, which progressed to disseminated subcutaneous nodules. He was diagnosed with dermatomyositis and was managed as an outpatient with high intensity glucorticoids. A biopsy was obtained due to concerns for recurrent lymphoma, but returned with only neutrophils. A follow-up PET-CT scan showed innumerable lesions in the lungs, abdomen, diffuse subcutaneous and muscle tissues. A neck lesion was biopsied and cultured, and returned positive for possible actinomyces infection, at which time the patient was referred to the hospital. He had no other pertinent medical, surgical, family or social history apart from the fact that he had been an avid gardener up until six months ago at the onset of his symptoms. On admission, a repeat culture of a biopsy of a nodule in the neck was obtained and sent for additional testing, including nocardia cultures. When his cultures returned positive for nocardia veterana, he was immediately placed on intravenous trimethoprim-sulfamethoxazole. However, his clinical condition continued to deteriorate and he eventually succumbed to his infection one month after the diagnosis of disseminated nocardiosis was made.

IMPACT/DISCUSSION: Nocardiosis is often an elusive diagnosis. The usual hallmarks of infection, such as fever and leukocytosis, can be lacking, particularly when the infection is limited to the skin prior to pulmonary involvement. Subcutaneous, pulmonary, and brain nodules, which have a neutrophil predominance on gram stain, are the most common sign. Cultures (blood/fluid), which require a fourteen day incubation period specifically for nocardia, are necessary for diagnosis. Early diagnosis is critical, since disseminated nocardiosis has mortality rates as high as $64 \%$ without appropriate treatment. Prompt initiation of therapy has been associated with reduction in mortality to $10 \%$. Therefore, clinicians need to maintain a high degree of suspicion to diagnose patients quickly. Identifying risk factors for nocardiosis may help with early diagnosis. Risk factors include immunocompromised states, such as a history of HIV infection, lymphoma, and chronic treatment with high dose glucocorticoids. This patient had both of the later two risk factors. In the presence of subcutaneous nodules, attention to these risk factors may allow for earlier diagnosis and initiation of appropriate management for nocardiosis.

CONCLUSION: Clinicians should be able to describe the risk factors and common presentation of disseminated nocardiosis in order to prevent delays in diagnosis that significantly raise mortality.

\section{ROSAI DORFMAN SYNDROME AS AN UNCOMMON PRESEN- TATION OF SINUS HISTIOCYTOSIS.}

Merza Nooraldin ${ }^{1}$; Assad Mohammedzein ${ }^{2}$; Mazin Saadaldin ${ }^{1}$; Omar Salh ${ }^{1}$; ahmed qasim ${ }^{3}$; Kaylee J. Shepherd ${ }^{4}{ }^{1}$ ttuhsc, Amarillo, TX; ${ }^{2}$ Texas Tech University Health Science Center, Amarillo, TX; ${ }^{3}$ Texas Tech University Health sciences, Amarillo, TX; ${ }^{4}$ Texas Tech University Health Sciences Center, Amarillo, TX. (Control ID \#3184561)

LEARNING OBJECTIVE \#1: 1. Recognize Rosai-Dorfman syndrome as a cause of generalized lymphadenopathy 2 . Learn the available treatment options of this benign rare disease

CASE: A 77-year-old male with medical history of chronic obstructive pulmonary disease on home oxygen, diabetes, hypertension and heart failure with reduced ejection fraction presented with 1 day of worsening shortness of breath, dry cough, orthopnea and lower extremity swelling. Other review of systems unremarkable. Physical exam notable for blood pressure 172/92 mmHg, oxygen saturation $96 \%$ on 2 L nasal cannula, labored breathing, decreased breath sounds in right lung base, bibasilar crackles, pitting edema in lower extremities up to abdomen, and soft and nontender bilateral cervical, axillary, and inguinal lymphadenopathy. Laboratory studies remarkable for eosinophilia 1.1 (0-0.2 10x3/ul), albumin $2.2 \mathrm{~g} / \mathrm{dL}$, LDH $311 \mathrm{UI} / \mathrm{L}$ (87-241), CRP 12.9 mg/l (0-2.9) BNP 1000 pg/ml (0-450), D Dimer 1.8 ng/l (0.190.49 ) with $\mathrm{ABG}$ consistent with acute respiratory acidosis and hypoxemia. Chest $\mathrm{x}$-ray with pulmonary congestion and EKG with rate controlled atrial fibrillation. Transthoracic echocardiogram with preserved ejection fraction, grade 1 diastolic dysfunction, and RVSP $60-70 \mathrm{mmHg}$. CT pulmonary angiogram revealed right lower lobe subsegmental pulmonary embolism, right pleural effusion, and extensive cervical, axillary, and prevascular adenopathy. CT abdomen was obtained which revealed further lymphadenopathy. Pleural fluid analysis was transudative, but had predominant lymphocytes (negative cytology) with reactive mesothelial cells, histiocytes, and lymphocytes. Excisional biopsy of right groin lymph node showed central 
fatty replacement and fibrosis with sinus histiocytosis without significant cortical hyperplasia. There was no evidence of lymphoma or metastatic malignancy.

IMPACT/DISCUSSION: Besides his acute issue for respiratory failure which was multifactorial, our patient had incidental diffuse soft nontender lymphadenopathy with sinus histiocytosis. Sinus histiocytosis itself is a common benign problem often seen as a reaction to infectious or inflammatory process. When accompanied by diffuse bulky lymphadenopathy, which is rare, it's called sinus histiocytosis with massive lymphadenopathy (SHML) or Rosai-Dorfman syndrome. It's often associated with chronic infections or autoimmune disease which our patient had no evidence. Around $40 \%$ of these patient has extranodal manifestation with involvement of the skin, eyes, respiratory tract and CNS. Twenty percent of patients do experience spontaneous resolution hence observation is recommended in most. Alternatively, chemotherapy, radiotherapy or surgery can be considered depending on the extent of the disease.

CONCLUSION: Rosai-Dorfman disease, or SHML, is a disease of benign histiocytic proliferation of unknown etiology. Patients are often asymptomatic, some present with palpable lymphadenopathy, fever or complications from bulky lymph nodes compression.

\section{SACRAL SARCOIDOSIS}

Kenneth M. Fifer. Icahn School of Medicine at Mount Sinai, New York, NY. (Control ID \#3185026)

\section{LEARNING OBJECTIVE \#1: Assess an atypical presentation of low back} pain

LEARNING OBJECTIVE \#2: Recognize extrapulmonary manifestations of sarcoidosis

CASE: A 49 year old man with no significant past medical history presented with several months of progressive bilateral low back pain. The pain was exacerbated by standing for long periods of time at his job. The pain did not radiate to the abdomen or into either lower extremity. He had no fever, cough, shortness of breath, weight loss, or urinary/bowel incontinence. Review of systems was otherwise negative. Physical exam was significant for moderate point tenderness to palpation over the L3-L4 spine. Laboratory studies were significant for a mild anemia (Hgb 12.6) and leukopenia (WBC 4.0), ESR 24, Vitamin D 17. Serum protein electrophoresis was unremarkable. Plain films of the lumbosacral spine demonstrated lytic-like lesions. CT chest/abdomen/ pelvis and MR imaging demonstrated multiple lytic-appearing lesions throughout the lumbar and sacral spine with the appearance of possible metastatic disease or bone marrow replacement process. An enlarged right hilar lymph node was noted. The patient subsequently underwent a CT-guided biopsy which revealed non-necrotizing granulomas. A chest $\mathrm{x}$-ray was unremarkable. Given the back pain, cytopenia and lack of respiratory symptoms, a diagnosis of sarcoidosis of the lumbosacral spine was made.

IMPACT/DISCUSSION: Impact This case demonstrates a valuable lesson in avoiding anchoring bias and evaluating each new piece of clinical data as it is obtained. Each step in the clinical decision making process in this case (physical exam, laboratory testing, imaging, and tissue pathology) had a significant impact on the evolving differential diagnosis. This case adds an unusual presentation of sarcoidosis of the lumbosacral spine to the existing literature. Discussion There are various important extrapulmonary manifestations of sarcoidosis. The musculoskeletal system can be affected in approximately $10 \%$ of patients. The most typical musculoskeletal manifestations include acute and chronic polyarthralgias, periarticular soft tissue swelling, and bone resorption. Less commonly, patients can be afflicted with a progressive diffuse, granulomatous myositis. Bone marrow involvement, however, is relatively uncommon. Case studies as early as 1978 have described sarcoidosis of bone marrow established by histology in the abstract of chest $\mathrm{x}$-ray findings. In one study of 50 patients with sarcoidosis who consented to bone marrow biopsy, 11 were found to have anemia while only 3 of those 11 were found to have bone marrow involvement on biopsy.

CONCLUSION: While extrapulmonary manifestations of sarcoidosis such as musculoskeletal involvement and anemia are relatively common, histological bone marrow involvement is a relatively rare, but important manifestation of systemic sarcoidosis. Providers should consider extrapulmonary involvement in patients with sarcoidosis who present with new, non-pulmonary symptoms

SAVED BY THE SNELL - RECOGNIZING BASILAR SCRAP SYNDROME AS CAUSE OF VERTIGO IN OCCIPITAL CVA Timothy M. Bober $^{1,2}$; Furkan Ertem ${ }^{1,2}$; Kristen M. Livesey ${ }^{2} .{ }^{1}$ UPMC Presbyterian-Shadyside, Pittsburgh, PA; ${ }^{2}$ University of Pittsburgh Medical Center, Pittsburgh, PA. (Control ID \#3163373)

LEARNING OBJECTIVE \#1: Recognize "basilar scrape" syndrome as a cause of vertigo

LEARNING OBJECTIVE \#2: Identify importance of visual acuity and visual field and acuity testing in vertigo evaluation

CASE: This was a 72 year-old female with type II diabetes with right eye blindness secondary to proliferative retinopathy, macular degeneration, rheumatoid arthritis, and migraine presenting with a day of frontotemporal headache, dizziness with vertigo, and worsened left-sided vision. She denied diplopia, slurred speech, eye pain, temporal tenderness, jaw claudication, weakness, numbness, chest pain, or palpitations. Admission vitals were notable for hypertension to 168/90 and heart rate of 68 with regular rhythm. Neurology evaluated the patient and, with a normal Dix-Hallpike and neurological exam, recommended head imaging. Admission exam performed by the medical team showed visual acuity of 20/200 (baseline 20/40) in her left eye and 20/800 (baseline 20/100) in her right eye. Tilting her head to the left to see the Snellen chart, the patient was found to have left homonymous hemianopsia with neglect. Differential included occipital stroke, posterior reversible encephalopathy syndrome, cerebral venous thrombosis, central retinal vein occlusion, and giant cell arteritis. A non-contrasted CT Head was negative. A contrasted MRI showed acute right occipital lobe infarct. Transthoracic echo showed mild atrial enlargement. When stable, patient was discharged and with maintained left sided visual deficit. Holter monitoring revealed normal sinus rhythm with rare ectopy.

IMPACT/DISCUSSION: This case shows the value of comprehensive visual testing in evaluating a patient with vertigo, which can reflect changes in vision, vestibular input, or proprioception. Visual testing localizes lesions and informs further imaging, which is not recommended with dizziness without focal signs. ${ }^{1}$

CONCLUSION: The evaluation of vertigo includes focused history and neurological examination to differentiate peripheral and central causes. ${ }^{1}$ Physicians report "CN II-XII intact" in documentation, but visual testing is often omitted. This exam helped the medical team to provide a more timely diagnosis. This patient's vertigo was attributed to "basilar scrape syndrome" due to transient vertebrobasilar ischemia from an embolus. ${ }^{2}$ Diagnosis warrants evaluation for arrhythmia or cardiomyopathy. ${ }^{3}$ References: 1 . Muncie, H. L., Sirmans, S. M., \& James, E. (2017). Dizziness: Approach to Evaluation and Management. American family physician, 95(3), 154-162. 2. Koroshetz, W. J., \& González, R. G. (2006). Causes of ischemic stroke. In Acute Ischemic Stroke (pp. 27-40). Springer, Berlin, Heidelberg. 3. Kamel, H., \& Healey, J. S. (2017). Cardioembolic stroke. Circulation research, 120(3), 514-526. 
SCRATCHING YOUR HEAD OVER CAT SCRATCH DISEASE Cindy Nguyen; Ashley Busuttil; Chandra Smart. UCLA, Los Angeles, CA. (Control ID \#3177541)

LEARNING OBJECTIVE \#1: Recognize an atypical presentation of Cat Scratch Disease in an elderly patient

CASE: A 71 year old woman presented with fever, fatigue, rigors and confusion. Exam was notable for T 102.7F, 94\% RA, encephalopathy and bibasilar rales. WBC was 9.8. There was no pulmonary consolidation and initial infectious work up was negative. She was empirically treated for presumed atypical pneumonia; however, she continued to have fevers. She had cats at home that do not scratch or bite. She noted a right forearm pustule that was intermittently purulent and crusted over the past two months. Imaging revealed 10-15 hypodense hepatosplenic lesions and abdominal lymphadenopathy. TTE and TEE were normal. A liver biopsy and punch biopsy of the arm pustule were non-diagnostic. Although initial Bartonella serologies and PCR were negative, given the clinical findings of fever, lymphadenopathy, hepatosplenic involvement and cat exposures at home, the patient was treated for presumptive Bartonellosis. She clinically improved and serial serologies (Bartonella IgG) became positive with a titer of 1:256 at 4 weeks. The hepatosplenic lesions and lymphadenopathy decreased in size after four weeks of azithromycin. The positive convalescent serologies and clinical improvement with antibiotics confirmed the diagnosis of Cat Scratch Disease.

IMPACT/DISCUSSION: Cat Scratch Disease commonly presents in children with self-limited regional lymphadenopathy. Elderly patients are more likely to present atypically with endocarditis, encephalitis, and fever of unknown origin. Due to a lower suspicion for Cat Scratch Disease in older patients, the diagnosis is often delayed. A diagnosis is usually made clinically with a history of cat exposure. Serological testing may be performed, however it has poor sensitivity and specificity. IgG titers 1:256 or more suggests active or recent infection. Biopsies are not routinely performed for diagnosis, but the Warthin-Starry stain may reveal B. henselae bacilli. In this case, the patient is an elderly woman with vague symptoms and found to have lymphadenopathy and hepatosplenic lesions. Despite negative serology and biopsy stains, her clinical presentation was consistent with Cat Scratch Disease, and she was empirically treated with antibiotics. The improvement in hepatosplenic lesions and the positive convalescent serologies confirmed the diagnosis of Bartonellosis. Negative serology does not exclude the diagnosis and patients should be treated if there is clinical suspicion. Cat Scratch Disease should be considered in patients with unexplained fevers with known cat exposure, even without a history of scratches or bites. Transmission of Cat Scratch Disease has been observed in individuals with cats with fleas and in younger cats.

CONCLUSION: Elderly patients often present atypically with more generalized and systemic symptoms, but Cat Scratch Disease should be considered if the patient's history includes exposures to cats at home, regardless of history of bites or lack thereof.

\section{SECONDARY ADRENAL INSUFFICIENCY UNMASKED BY IN- FECTIOUS DIARRHEA}

Andrea Pagan; Denise Feradov. University of South Florida, Tampa, FL. (Control ID \#3186554)

LEARNING OBJECTIVE \#1: Clinicians should consider adrenal insufficiency in all acutely ill patients presenting with undifferentiated shock.

LEARNING OBJECTIVE \#2: Adequate treatment of adrenal crisis using stress-dose intravenous steroids shouldn't be delayed for confirmatory testing. CASE: A 22-year-old African American male with no past medical history presented to the ED with altered mental status, fever and diarrhea for one day.
On physical examination he was febrile, hypotensive, tachycardic and oriented by only to person. Initial workup revealed leukocytosis with a left shift, normal BMP, elevated lactic acid of $2.50 \mathrm{mmoll} / \mathrm{L}$, and elevated procalcitonin of 2.23 $\mathrm{ng} / \mathrm{mL}$. Imaging including CXR and CT abdomen with oral contrast were unremarkable. CSF, blood and urine cultures were sterile. Stool GI pathogen panel was positive for Enteroaggregative Escherichia coli. The patient's symptoms improved after antibiotics were started. However, he continued to have episodes of hypotension requiring fluid boluses. Upon further interviewing, the patient reported weight loss, fatigue, and weakness for two years prior to his current presentation. A random cortisol and ACTH stimulation test were ordered, and the results were consistent with secondary adrenal insufficiency. Stress dose steroids were started followed by a taper. MRI of the brain with contrast showed an atrophic pituitary gland without masses. His symptoms improved, and he was discharged on daily hydrocortisone. On outpatient follow-up, repeated testing confirmed the diagnosis of secondary adrenal insufficiency with suppressed ACTH and cortisol levels. The rest of the pituitary workup was normal.

IMPACT/DISCUSSION: Approximately 6,000,000 people in the United States are believed to have undiagnosed adrenal insufficiency, which becomes clinically significant only during times of physiologic stress, as in our patient. Chronic exogenous steroid use is the most common cause of hypothalamicpituitary axis suppression that can result in adrenal insufficiency, although hypopituitarism should also be considered. Secondary adrenal failure is easily missed, since many of the classical symptoms of Addison's disease are absent. Clinicians should be aware that the major impact of a stressor in a patient with corticosteroid insufficiency is on the systemic inflammatory and immune response. When the body is unable to modulate this reaction, it creates the exaggerated response observed in this case and mimics shock. This information is important since patients with undiagnosed and diagnosed adrenal insufficiency and are experiencing an active adrenal crisis episode have a dismal prognosis if left untreated.

CONCLUSION: Secondary adrenal insufficiency occurs because of a disruption in the hypothalamic-pituitary-adrenal axis, causing a myriad of nonspecific symptoms. The hallmark of adrenal crisis is hypotension and shock refractory to fluid resuscitation and vasopressors. This one is a life-threatening condition and treatment shouldn't be delayed for confirmatory testing.

\section{SECONDARY EVAN'S SYNDROME: A RARE PRESENTING MAN- IFESTATION OF SYSTEMIC LUPUS ERYTHEMATOSUS} Ankita Aggarwal ${ }^{1}$; Anubhav Jain ${ }^{1}$; Sarwan Kumar ${ }^{2}{ }^{1}$ Wayne State University, Auburn Hills, MI; ${ }^{2}$ Crittenton Hospital/Wayne state university, Rochester, MI. (Control ID \#3186364)

LEARNING OBJECTIVE \#1: To Diagnose Evans Syndrome(ES) LEARNING OBJECTIVE \#2: To identify systemic lupus erythematosus(SLE) as a cause of secondary Evans Syndrome

CASE: A 24 years old female with previous normal menstrual cycles presented with a history of intermittent spontaneous vaginal bleeding for two months and generalized weakness for six months. No obvious gynecological cause for bleeding was apparent after preliminary workup. A detailed evaluation revealed no history of fever, weight loss, hair loss, oral ulcers, photosensitivity and arthropathic symptoms including morning stiffness. On examination, she had stable vitals, conjunctival pallor with a scleral clot over the right eye and petechial hemorrhages all over the body. Detailed systemic examination was unremarkable. Her routine blood examination detected anemia $(\mathrm{Hb}-$ $4.3 \mathrm{gm} / \mathrm{dl})$, low platelet count $(5 \times 103 / \mu \mathrm{l})$, normal total leukocyte count and corrected reticulocyte count of $7.4 \%$. Peripheral smear (PS) showed microcytic hypochromic anemia with spherocytes. Bone marrow (BM) aspirate was 
cellular with erythroid hyperplasia and megakaryocytes. BT, CT, PT, APTT, ddimer levels were normal. There was unconjugated hyperbilirubinemia with normal transaminases. Direct antiglobulin (Coomb's) test was positive $\{3+\}$. Anti-nuclear antibody (ANA) study was positive (2+, diffuse homogenous pattern) with a tire of $1: 320$, positive dsDNA $(2+)$ with a titer of $95.0 \mathrm{IU} / \mathrm{ml}$ which was suggestive of SLE. Coagulation profile was positive for lupus anticoagulant (LA) and anti-cardiolipin antibodies. She was given three units of whole blood on two consecutive days after admission and eight units of platelets during the hospital stay. She was also started on the treatment of SLE with methylprednisolone $1 \mathrm{gm} /$ day as an infusion for 3 days by intravenous route and was started on tablet prednisolone $1 \mathrm{mg} / \mathrm{kg}$ from the fourth day onwards. Twelve days after hospitalization, she was discharged in a relatively improved condition with a hemoglobin of $9.1 \mathrm{gm} / \mathrm{dl}$ and platelet count of 1.52 x $103 / \mu \mathrm{l}$ on a daily dose of $1 \mathrm{mg} / \mathrm{kg}$ of oral prednisolone.

IMPACT/DISCUSSION: ES was previously considered as a combination of ITP and IHA and /or autoimmune neutropenia in absence of any underlying cause. However, recently the spectrum of illness has broadened to include other systemic diseases, aptly called secondary Evan's syndrome. Though case reports of secondary ES have been reported in literature it is extremely rare to have a patient of SLE presenting as ES. The classical presenting clinical features of SLE like musculoskeletal, constitutional, mucocutaneous, renal and neurologic manifestations were absent with our case making the diagnosis difficult.

CONCLUSION: Our study highlights the need for awareness of the rare entity of secondary ES. A high index of suspicion should be kept by primary care physicians, gynecologists in cases having thrombocytopenia and evidence of hemolysis to look for any underlying secondary etiologies in order to avert a misdiagnosis with attendant consequences in treatment and prognosis.

\section{SECONDARY HYPERTENSION: AN ESSENTIAL DIAGNOSIS}

Samantha J. Allen; Elena Lebduska. University of Colorado, Denver, CO. (Control ID \#3184683)

LEARNING OBJECTIVE \#1: Perform an appropriate workup for newly diagnosed chronic kidney disease

LEARNING OBJECTIVE \#2: Consider a diagnosis of autosomal dominant polycystic kidney disease in patients without a textbook presentation

CASE: A 58-year-old man presented to his new PCP to establish care. He had recently visited a health fair and was told his blood pressure was high. He had not seen a doctor for ten years and had no complaints. He denied any past medical history and was not on any medications, including NSAIDs. He was a current ten pack-year smoker but denied alcohol and drug use. He worked as a landscaper. His mother had lung cancer and his father had COPD; both were heavy smokers. Physical exam was notable for blood pressure 144/94 and BMI 27; exam was otherwise normal. Labs were notable for serum creatinine 2.7 (GFR approximately 30) and hemoglobin of 13.3 with MCV 92; lipid panel was normal. Urinalysis showed moderate protein; spot urine protein:creatinine was 0.2 . EKG was normal. He underwent renal ultrasound, which showed normal bladder and polycystic kidneys, and MRI, with findings consistent with autosomal dominant polycystic kidney disease (ADPKD).

IMPACT/DISCUSSION: Initial workup to evaluate for target organ damage, screen for a limited number of causes of secondary hypertension, and assess cardiovascular risk is indicated at the time of diagnosis of hypertension. This should include serum chemistry, urinalysis, lipid panel, and EKG. A finding of elevated serum creatinine should prompt urinalysis with microscopy as well as serum and urine electrolytes. Renal ultrasound should be considered to assess size, morphology, and symmetry, and rule out obstruction. In this case, obstruction was considered likely given the patient's age-associated risk of
$\mathrm{BPH}$. Obstruction was ruled out with ultrasound, and a surprising and important diagnosis was made. Classically, a diagnosis of ADPKD is made in a patient with a positive family history and is heralded by flank pain and palpable kidneys on exam. However, up to $25 \%$ of patients with the disease have a negative family history, and the majority of patients are asymptomatic. ADPKD is the cause of less than $1 \%$ of cases of secondary hypertension. Conversely, hypertension is the most common clinical manifestation of ADPKD and is often present before renal impairment. Blood pressure management is key in slowing the decline of renal function in ADPKD.

CONCLUSION: All patients should undergo a basic workup at the time of diagnosis of hypertension for risk stratification and screening for secondary hypertension. Renal disease is one of the most common causes of secondary hypertension and should be evaluated with serum and urine labs and ultrasound to assess for obstruction and evaluate kidney size and morphology. ADPKD is often asymptomatic and up to $25 \%$ of patients have a negative family history. Early and effective blood pressure control is key to slowing the decline of renal function in ADPKD.

\section{SEEING THE POSSIBILITIES: DIAGNOSTIC UNCERTAINTY IN BILATERAL OPTIC NEURITIS}

Grace L. Lisius ${ }^{1}$; Andrea Carter ${ }^{2} .{ }^{1}$ University of Pittsburgh, Pittsburgh, PA; ${ }^{2}$ University of Pittsburgh Medical Center, Pittsburgh, PA. (Control ID \#3167499)

LEARNING OBJECTIVE \#1: Describe the typical history, physical exam, and fundoscopic findings of optic neuritis $(\mathrm{ON})$

LEARNING OBJECTIVE \#2: Identify atypical ON findings that should prompt workup for systemic inflammatory and infectious causes

CASE: A 38-year-old healthy male photographer presented to clinic with worsening vision. He had a 1-week history of left eye pain with extraocular movements, 4 days of left peripheral vision distortion with flashes and floaters, and 1 day of diffusely grayed-out left vision. He also had a frontal headache, most severe on awakening, and recent exposure to cats. Exam revealed normal right visual acuity, decreased left visual acuity to finger-counting at 2 feet, and a left afferent pupil defect. Fundoscopic exam revealed bilateral (left greater than right) swelling of the optic nerve. He was admitted. A brain MRI showed bilateral (left greater than right) optic neuropathy without signs of increased intracranial pressure, mass, ischemia, or white matter lesions. Lumbar puncture had normal opening pressure with negative viral and multiple sclerosis (MS) panels. Serologies revealed reactive toxoplasmosis IgM and non-reactive IgG; other infectious and inflammatory markers were negative. Differential diagnosis included primary ON versus ocular toxoplasmosis, and he was treated empirically for both with high dose intravenous corticosteroids and a 6 week course of trimethoprim-sulfamethoxazole, respectively, with complete resolution of headache and vision changes at his 1 month follow up appointment.

IMPACT/DISCUSSION: $\mathrm{ON}$ is an acute inflammatory demyelinating injury to the optic nerve and should be suspected in patients with unilateral painful eye movements and hours-to-days progressive vision loss, classically in a central scotoma. Exam always reveals an afferent pupillary defect on the Swinging Flashlight test, with slower pupil constriction when light is shone in the affected eye if the other eye is unaffected. Fundoscopic exam may reveal papillitis with optic nerve swelling. Intravenous corticosteroids speed the visual recovery in $\mathrm{ON}$ and reduce the 2-year risk of developing MS. Bilateral ON or signs of infection are atypical, and should prompt evaluation of systemic inflammatory and infectious causes. An acute ocular toxoplasmosis infection, considered here due to a reactive $\operatorname{IgM}$ with a non-reactive $\mathrm{IgG}$, was less likely responsible given his lack of chorioretinitis or posterior uveitis. 
Ocular toxoplasmosis usually resolves spontaneously, but atypical presentations, such as our patient with concomitant $\mathrm{ON}$, are an indication to treat with antibiotics.

CONCLUSION: Young adult patients with acute unilateral painful vision loss can be diagnosed clinically with $\mathrm{ON}$, but atypical features such as bilateral findings should prompt workup and treatment of alternative systemic inflammatory or infectious processes, especially given the importance of avoiding consequences in vision-related safety, career, and quality of life.

\section{SEGMENTAL ARTERIAL MEDIOLYSIS (SAM) AS A MIMIC OF SYSTEMIC VASCULITIS}

Bilal Saqi. Cleveland Clinic Foundation, Cleveland, OH. (Control ID \#3186314)

LEARNING OBJECTIVE \#1: To diagnose SAM in a patient being treated primarily as vasculitis resulting in harm

LEARNING OBJECTIVE \#2: To recognize dissections, aneurysms, hemorrhage or ischemia in the celiac, superior mesenteric and other abdominal arteries as features of SAM

CASE: A 44 year old male with history of hypertension and gout who initially presented with chest and epigastric pain. Initial workup was negative for myocardial infarction and pulmonary embolism, but subsequently on worsening of the pain and elevation of white count and liver enzymes, CT angiography was performed and revealed extensive mesenteric thrombosis including hepatic, splenic and gastroduodenal artery occlusion, portal venous air involving the left lobe of the liver and gastric wall pneumatosis. Heparin was started but ensuing CT revealed new splenic and bilateral kidney infarcts. Furthermore he developed flank pain, thrombocytopenia and anemia. Repeat imaging revealed new right perinephric and retroperitoneal hematomas and increased number of bilateral renal infarcts. Heparin was held and initial workup revealed Heparin Induced Thrombocytopenia but confirmatory test was negative. Angiography revealed dissection in the celiac artery but no active bleeding. His course was further complicated by large left main and segmental right lower lobe pulmonary embolism in the setting of new atrial fibrillation, but his echo was negative for vegetation, thrombi or shunt. Treatment with Argatroban was started and extensive hypercoaguable and rheumatological workup including test for Ehlers-Danlos was negative. He was treated primarily as a patient of vasculitis with steroids and cyclophosphamide and discharged with warfarin. He presented again for flank pain and hematuria and was found to have new interval occlusions of the common hepatic artery and evolving bilateral renal and left hepatic infarcts. In light of celiac dissection and diffuse arterial thrombosis, lack of an inflammatory picture, findings on CTA and Angiography, and exclusion of hypercoagulable state, he was diagnosed with SAM. He was treated with Bivalirudin and discharged on Fondaprinux

IMPACT/DISCUSSION: SAM is an arteriopathy characterized by vacuolization and lysis of the arterial smooth muscle cells, arterial gaps, separation, and reparative fibrosis. The pathogenesis of SAM is still unknown. Presentation can range from abdominal pain due to acute intestinal ischemia from thrombosis or dissection to intra-abdominal hemorrhage. Systemic vasculitis, connective tissue diseases, congenital vascular diseases such as Ehlers-Danlos syndrome should always be excluded. Misdiagnosis and treatment directed towards vasculitis can lead to harm such as in our patient. Appropriate therapy with angiography and transcatheter embolization should be performed immediately when indicated

CONCLUSION: In patients presenting with abdominal pain, flank pain, hematuria and arteriopathy, SAM should not be forgotten.
SEIZE THIS PAIN: A CURIOUS CASE OF ACUTE INTERMITTENT PORPHYRIA

Hongyang $\mathrm{Pi}^{1}$; Benjamin Sprague ${ }^{1}$; Akshata Moghe ${ }^{2} .{ }^{1}$ University of Pittsburgh Medical Center, Pittsburgh, PA; ${ }^{2}$ University of Pittsburgh, Pittsburgh, PA. (Control ID \#3185807)

LEARNING OBJECTIVE \#1: Recognize the clinical features of acute intermittent porphyria (AIP)

LEARNING OBJECTIVE \#2: Diagnose and treat an AIP attack

CASE: A 34 yr-old woman with migraines presented with two weeks of severe, intensifying, diffuse abdominal pain. For the past year, she had experienced sporadic abdominal and back pain. Three weeks prior to presentation, she was treated for a urinary tract infection with trimethoprim-sulfamethoxazole. She reported nausea with occasional emesis, without fevers or changes in bowel habits. Family history was notable for functional abdominal pain in her father. She did not use alcohol, tobacco, or illicit drugs. Home medications included acetaminophenhydrocodone and cyclobenzaprine. She was afebrile, with BP of 179/118 $\mathrm{mmHg}$, HR of $144 \mathrm{bpm}$ and RR of 18/min. She was alert with dry mucus membranes. Her abdomen was soft, nondistended, diffusely tender without rigidity or guarding, with reduced bowel sounds. Neurological exam was non-focal. The day after presentation, she had a witnessed generalized tonic-clonic seizure. At that time, her serum sodium level had fallen to $110 \mathrm{mEq} / \mathrm{L}$, from $136 \mathrm{mEq} / \mathrm{L}$ on presentation. Liver function tests were normal. CT of the abdomen was unremarkable. EEG showed mild diffuse encephalopathy. Her urine was reddish-orange but urinalysis showed no RBCs. Differential diagnosis included AIP, cyclobenzaprine toxicity and vasculitis. Urine porphobilinogen (PBG) level returned at $100 \mathrm{mg} / 24 \mathrm{hr}$ (normal < 2.4) clinching the diagnosis of AIP. Daily hematin infusions were initiated with gradual resolution of abdominal pain and normalization of vital signs.

IMPACT/DISCUSSION: AIP is an autosomal dominant metabolic disorder caused by deficiency of the enzyme porphobilinogen deaminase (a.k.a. hydroxymethylbilane synthase) from the heme biosynthesis pathway. Diagnosis of AIP is challenging as i) symptoms can be intermittent \& vague, ii) abdominal pain has a large differential diagnosis, and iii) as a rare disease, AIP is often not considered or appropriate testing is not performed. Presentation of AIP is highly variable, however most attacks begin with abdominal pain and neurological symptoms. Tachycardia and limb paresthesias are common signs of autonomic and peripheral neuropathy, respectively. Hyponatremia is the most common electrolyte abnormality, and can lead to seizures. Urine appears red-brown due to the presence of porphyrins. As in the case of our patient, drugs are common triggers of AIP. Other triggers include dehydration, alcohol, infections, starvation, and hormonal changes of menses or pregnancy. Spot urine PBG is the best diagnostic test, and if elevated, is sufficient to diagnose AIP. Treatment includes daily infusions of hematin in severe attacks, while mild symptoms can be alleviated with carbohydrate loading with IV dextrose.

CONCLUSION: Consider AIP in a patient with abdominal pain, neurological symptoms \& electrolyte abnormalities Spot urine PBG is the preferred diagnostic test Treat with hematin in severe cases, or carbohydrate loading in mild cases

\section{SEPTIC KNEE MONOARTHRITIS DUE TO HISTOPLASMA CAPSULATUM AFTER BONE MARROW TRANSPLANT IN AN APLASTIC ANEMIA PATIENT}

Bhavyaa Bahl; Lourdes Corman. UAB Huntsville Regional Medical Campus, Huntsville, AL. (Control ID \#3186107) 
LEARNING OBJECTIVE \#1: Recognize monoarthritis as a rare presentation of Histoplasmosis

LEARNING OBJECTIVE \#2: Evaluate diagnostic testing for histoplasmosis in an immunocompromised host

CASE: An 18-year-old male from Alabama presented with progressively worsening right knee swelling and pain for four days associated with fever and chills. He had undergone allogeneic bone marrow transplant a year ago as treatment of aplastic anemia for which he had been on an immunosuppressive regimen with prophylactic antimicrobials till two months ago. He was sexually active with his girlfriend and was recently treated for Chlamydia trachomatis urethritis. Magnetic resonance imaging (MRI) knee done five days ago did not reveal any abnormalities. On exam, he was febrile with a $\mathrm{T}_{\max }$ of $102.9^{\circ} \mathrm{F}$. His right knee was inflamed and tender with restricted range of motion. Arthrocentesis done for the knee effusion noted on radiograph revealed grossly purulent synovial aspirate with 19,000 total nucleated cells (90\% neutrophils) along with $1+$ yeast on gram stain. He was empirically started on vancomycin, piperacillin-tazobactam, and fluconazole for septic arthritis. Arthroscopic synovectomy revealed diffuse inflammation of the joint with the involvement of synovium and vessels on pathology. Blood, synovial fluid and biopsy specimens did not reveal bacterial infection on culture and molecular testing. (1,3)-Beta-D-Glucan assay (Fungitell@), T2Candida ${ }^{\circledR}$ and Histoplasma serology resulted as negative. He was switched to micafungin and discharged after being afebrile for 24 hours to complete 6 weeks of treatment for a fungal septic joint. Five days after discharge, Histoplasma polymerase chain reaction (PCR) on the synovial fluid was reported as positive following which he was changed to itraconazole. Two weeks later fungal cultures from blood and synovial fluid resulted as growing mold identified as Histoplasma capsulatum.

IMPACT/DISCUSSION: Histoplasma capsulatum is a dimorphic fungus endemic to the Ohio and Mississippi river valley with primarily asymptomatic pulmonary involvement in immunocompetent hosts. Immunocompromised individuals are particularly at risk for a severe and disseminated disease. Bone and joint infection is very rare and mostly manifests as monoarthritis of the knee due to local inoculation or hematogenous spread. Although tedious, fungal culture is the gold standard of diagnosis with $85 \%$ sensitivity in disseminated disease and was demonstrated in both synovial fluid and blood specimen at four weeks in this case. Earlier diagnosis can be facilitated with PCR testing (100\% specificity) in immunocompromised and disseminated states where Histoplasma antibody testing has a high false negative rate and antigen testing has low sensitivity as seen here.

CONCLUSION: Septic arthritis and tenosynovitis are rare complications of Histoplasmosis that must be suspected in immunocompromised hosts particularly in endemic areas. Molecular testing can aid in the earlier diagnosis of Histoplasmosis even in cases with negative serology.

\section{SEVERE HUNGRY-BONE SYNDROME AFTER RESECTION OF FOLLICULAR PARATHYROID CANCER}

Norman N. Aiad ${ }^{1,2}$; Mat Kladney ${ }^{2,}{ }^{1}$. ${ }^{1}$ NYU Langone Medical Center, New York, NY; ${ }^{2}$ Bellevue Hospital, New York, NY. (Control ID \#3162804)

LEARNING OBJECTIVE \#1: Recognize, manage, and prevent hungrybone syndrome (HBS)

CASE: A 46-year-old male with a history of hypertension presented with urinary hesitancy and frequency for two months. His only other complaint was aching pain in his back and bilateral legs. Physical exam was significant for tenderness of his lower extremities. Labs were significant for creatinine of $3.9 \mathrm{mg} / \mathrm{dL}$, calcium of $12.5 \mathrm{mg} / \mathrm{dL}$, and an alkaline phosphatase of 916 U/L. Parathyroid hormone (PTH) was 4393 pg/mL. Phosphate and 25-hydroxy vitamin D were normal, but 1,25-dihyrdoxy vitamin D was low at $9.1 \mathrm{pg} / \mathrm{mL}$. Renal ultrasound demonstrated bilateral hydronephrosis. CT imaging revealed a left paratracheal soft tissue mass, extensive lytic bone lesions, and multiple renal calculi. Core biopsy of the mass was consistent with follicular parathyroid cancer. The patient received alendronate then underwent parathyroidectomy of the affected gland with post-operative perioral tingling and paresthesias and hypocalcemia with a nadir of $5.5 \mathrm{mg} / \mathrm{dL}$. This patient then required $138 \mathrm{~g}$ of oral calcium carbonate and $118 \mathrm{~g}$ of intravenous calcium gluconate along with calcitriol and cholecalciferol over the course of thirteen days.

IMPACT/DISCUSSION: This case exemplifies the potential severity of HBS. This patient's calcium requirement was $256 \mathrm{~g}$ or an average of $20 \mathrm{~g} /$ day, significantly higher than reported 6 to $12 \mathrm{~g}$ /day that a typical HBS patient requires. HBS is defined as rapid and profound hypocalcemia due to an acute withdrawal of PTH after a parathyroidectomy. This is caused by a reduction of osteoclast activity and an increase in osteoblast activity leading to unopposed deposition of bone and resultant hypocalcemia. Hypophosphatemia and hypomagnesemia may also develop. These electrolyte abnormalities will last for weeks to months, but rarely have been reported to last for over a year. Patients who develop HBS are at risk of arrhythmias, heart failure, and seizures. These problems can be prevented with regular serum testing and repletion of calcium, phosphate, magnesium, and vitamin D. Preoperative bisphosphonates and vitamin D repletion have been shown to decrease the magnitude of hypocalcemia post operatively. Patients at risk of developing HBS include those with older age, higher preoperative serum calcium, alkaline phosphatase, and PTH levels, lower preoperative serum magnesium and albumin, parathyroid mass larger than $5 \mathrm{~cm}^{3}$, and those with evidence of brown tumors or osteitis fibrosa cystica. This patient's impressive calcium requirement may have stemmed from his preoperative kidney disease, vitamin D deficiency, and extensive lytic bone lesions.

CONCLUSION: HBS is a life threatening complication that develops as a result of rapid withdrawal of PTH with subsequent electrolyte disturbances. Some evidence suggests that HBS can be prevented with preoperative administration of bisphosphonates and vitamin D. HBS can be managed with frequent electrolyte monitoring and aggressive repletion.

\section{SEVERE LACTIC ACIDOSIS AND PERSISTENT HYPOGLYCE- MIA AS A UNIQUE PRESENTATION OF BURKITT'S LYMPHO- MA}

Sarah Rawi ${ }^{1}$; Arundati Rao ${ }^{1}$ Bryan F. Stringer ${ }^{2} .{ }^{1}$ University of Connecticut, Hartford, CT; ${ }^{2}$ University of Connecticut, New Birtain, CT. (Control ID \#3185031)

LEARNING OBJECTIVE \#1: Recognize and diagnose a unique presentation of Burkitt's Lymphoma associated with electrolyte abnormalities. LEARNING OBJECTIVE \#2: Identify the Warburg Effect.

CASE: 67-year-old male from Ghana with medical history significant for hepatitis B presented with six months of weakness, fatigue, weight loss, dyspnea, and abdominal distension. On physical exam his vitals were stable, he had a soft, non-tender, distended abdomen, bilateral peripheral pitting edema, and penile ulcers noted. His labs were significant for hemoglobin $7.4 \mathrm{~g} / \mathrm{dL}$, lactic acid $19.5, \mathrm{mmol} / \mathrm{L}$, corrected calcium of 8.6 $\mathrm{mg} / \mathrm{dL}$, glucose of $60 \mathrm{mg} / \mathrm{dL}$, and $\mathrm{LDH}$ of $848 \mathrm{U} / \mathrm{L}$. Abdominal CT showed extensive stranding and abnormal density throughout the retroperitoneum, extraperitoneal, presacral and perirectal spaces. There 
was diffuse anasarca, a stable right adrenal nodule and mild bilateral hydronephrosis. During the first few days of his ICU stay, he exhibited persistent hypoglycemia despite dextrose infusions. C-peptide, insulin and IGF- 1 were all low, supporting a malignant process. Lactic acid peaked at $21 \mathrm{mmol} / \mathrm{L}, \mathrm{LDH}$ peaked at $1105 \mathrm{U} / \mathrm{L}$ and the corrected hypocalcemia trended as low as $7.6 \mathrm{mg} / \mathrm{dL}$. An infectious workup revealed positive testing for HIV, CMV, HSV-1, HSV-2, HSV-6, Syphilis and latent TB. EBV was negative. His HIV viral load was 608k with a CD4 count of 70 . He was started on appropriate therapy. Biopsy of his retroperitoneal mass showed aggressive B cell non-Hodgkin Lymphoma. FISH Studies demonstrated rearrangement of MYC without rearrangement of BCL2 or BCL6, favoring Burkitt Lymphoma. He was started on chemotherapy with CHOP and Rituxan, following which his metabolic derangements normalized.

IMPACT/DISCUSSION: Lactic acidosis and hypoglycemia are rare complications of lymphoma. There have been six cases reported with these as the primary presentation of Burkitt Lymphoma. We present a case of a retroperitoneal lymphoma manifesting as severe lactic acidosis and persistent hypoglycemia. The Warburg Effect rationalizes these derangements, namely, tumor cells favor aerobic glycolysis for their high-energy demand, with resultant lactic acidosis and hypoglycemia. This demonstrates the value of recognition of a constellation of symptoms, signs, and lab abnormalities to identity a life threatening illness.

CONCLUSION: This case vignette increases awareness of and shows the precedence of metabolic derangements such as lactic acidosis and persistent hypoglycemia as initial signs of an underlying Non-Hodgkin's Lymphoma. It demonstrates the value of maintaining a broad differential in patients without a unifying diagnosis.

SEVERE NEUROLOGICAL SIDE EFFECTS OF NOVEL IMMUNE CHECKPOINT INHIBITORS USED IN ONCOLOGY

Joshua M. Lennon ${ }^{1,2}$; Benjamin P. Liu ${ }^{3}$. ${ }^{1}$ Northwestern Memorial Hospital, Evanston, IL; ${ }^{2}$ Ann and Robert H. Lurie Children's Hospital of Chicago, Chicago, IL; ${ }^{3}$ Northwestern Memorial Hospital, Chicago, IL. (Control ID \#3184900)

LEARNING OBJECTIVE \#1: Review the scope of use, and mechanism of action of immune checkpoint inhibitors

LEARNING OBJECTIVE \#2: Recognize severe neurological complications associated with immune checkpoint inhibitors

CASE: A 62 year old male presented to orthopedic surgery on 6/2016 for L elbow pain. During 9/2016 he was found to have a left axillary mass. Biopsy revealed malignant melanoma. In 11/2016 he had L axillary dissection, which was staged at IIIc disease. He completed 4 cycles of ipilumimab from 1/2017-3/2017. During 5/2017, he noted bilateral arm pain, stiffness, and thigh pain for 3 weeks. He was diagnosed with polymyalgia rheumatica, and started on low dose steroids with some improvement. He then complained of $\mathrm{R}$ sided headache and jaw pain, hyponatremia, and word finding difficulties. Differential diagnosis included temporal arteritis, but also side effects from the ipilumimab. Lumbar puncture was performed, noting CSF with 97 WBCs, 90\% lymphocytes, and elevated protein and glucose. Infectious workup was negative. He was started on methylprednisolone $1 \mathrm{~g}$ for 5 days with improvement of symptoms. He was discharged on $60 \mathrm{mg}$ prednisone. He returned to ED 2 days after discharge with sleepiness, confusion, and hyponatremia to 126 . Infectious workup remained negative. He was treated for SIADH, and restarted on methylprednisolone $1 \mathrm{~g}$ x 5 days and IVIG. He was discharged on $80 \mathrm{mg}$ prednisone, and weekly $1 \mathrm{~g}$ IV methylprednisolone. During 7/2017, he was found by police after a motor vehicle accident confused, with delirium. An
MRI brain showed multiple tiny infarctions, Lumbar puncture showed persistent lymphocytic CSF. He was restarted on high dose steroids and IVIG, and discharged on weekly tapering IVIG.

IMPACT/DISCUSSION: Immune checkpoint inhibitors are approved for multiple cancers, and will continue to be more common in oncology therapies. Their method of action involves boosting the immune response to cancer cells, by stopping the inhibitory signals produced by cancer cells to T-cells. Side effects most commonly involve the gastrointestinal system and the skin, but can affect any organ system. The most severe side effects can affect the lungs, and any part of the neurological system. This case is one of three patients that I have cared for and will present regarding severe neurological side effects from immune checkpoint inhibitors. Effects can occur days, or weeks after treatment begins, and the effects can last for long periods of time. Because of the wide variety of possible adverse effects, primary care and hospital medicine physicians need to be educated about the method of action of these medications, the possible side effects of these therapies, and the need to be vigilant to look for possible signs and symptoms in their patients.

CONCLUSION: - Immune checkpoint inhibitors have been approved for and are used to treat many cancers - Severe side effects can be seen in any organ system, including the neurological system - These symptoms can be recurrent and persistent, and start from days to weeks after therapy is started

\section{SGLT-2 INHIBITOR ASSOCIATED EUGLYCEMIC DIABETIC KETOACIDOSIS (DKA)}

Hajira Amir; Sarah Fishman. Lenox Hill Hospital, New York, NY. (Control ID \#3186058)

LEARNING OBJECTIVE \#1: Diagnose euglycemic DKA LEARNING OBJECTIVE \#2: Recognize euglycemic DKA as a complication of SGLT-2 inhibitors

CASE: A 36 year old male with type 1 diabetes mellitus (DM) presented with complaints of chest pain, dyspnea and vomiting. His home medications included glargine 40 units daily, aspart 15 units with meals and ertugliflozin $15 \mathrm{mg}$ daily. On arrival, he was afebrile, normotensive, and tachycardic at $104 \mathrm{bpm}$. He was not in respiratory distress and physical exam was unremarkable. Initial blood work was significant for blood glucose $183 \mathrm{mg} / \mathrm{dl}$ (70-99), lipase $666 \mathrm{U} / \mathrm{L}$ (7-60), trop T $0.15 \mathrm{ng} / \mathrm{ml}$ (0.00-0.01), anion gap $17 \mathrm{mmol} / \mathrm{L}$ (5-17), HCO3 $18 \mathrm{mmol} / \mathrm{L}$ (22-31), $\mathrm{HbA1c} 6.8 \%$ and urine positive for ketones. He was admitted for suspected atypical acute coronary syndrome. Coronary catheterization, echocardiogram, and CT of the chest, abdomen, and pelvis did not reveal any abnormalities to account for the patient's symptoms and blood test results. He was kept NPO and treated with insulin sliding scale with blood glucose values ranging between $160-260 \mathrm{mg} / \mathrm{dl}$. His chest pain and dyspnea worsened over his first two days of admission. Subsequent blood tests revealed worsening acidosis and increasing anion gap. A diagnosis of euglycemia DKA was considered and beta hydroxy butyrate was measured at $11.5 \mathrm{mmol} / \mathrm{L}(<0.4)$ with $\mathrm{pH}$ of 7.1 on venous blood gas. A continuous insulin infusion was initiated and symptoms of dyspnea, nausea, and vomiting slowly improved. The patient was transitioned to subcutaneous insulin with glargine and lispro once ketoacidosis resolved. He was discharged on his pre-admission insulin regimen and advised to discontinue ertugliflozin.

IMPACT/DISCUSSION: DKA is a life-threatening complication of DM that occurs as a result of insulin deficiency, often in association with a precipitating trigger such as infection. It is defined by the presence of 
metabolic acidosis, elevated anion gap and ketosis. Most often, DKA occurs in the setting of hyperglycemia, however some patients have normal blood glucose, a condition termed euglycemic DKA. SGLT-2 inhibitors are associated with an increased incidence of DKA. These medications inhibit glucose uptake in the proximal tubules of the kidney. The resultant glycosuria and reduced blood glucose concentrations contribute to increased glucagon secretion and ketone production as well as decreased insulin production. Furthermore, ketone elimination by the kidney is reduced further promoting ketonemia. In this ketogenic setting, surgical stress, infection and extended fasting can precipitate ketoacidosis. SGLT-2 inhibitor associated euglycemic DKA has been associated with poor B cell functional reserve, longer duration of DM, poor control of DM, lower BMI, and Type 1 DM.

CONCLUSION: DKA is a life threatening complication of DM. SGLT-2 inhibitors have been associated with euglycemic DKA and require treatment with insulin infusion and discontinuation of the medication.

\section{SHE'S GONE BANANAS! A CASE OF ECTOPIC CUSHING'S SYNDROME PRESENTING AS HYPOKALEMIA AND PARA- NOID DELUSIONS}

Christopher C. Marino; Michelle-Linh Nguyen. University of Pittsburgh Medical Center, Pittsburgh, PA. (Control ID \#3184590)

LEARNING OBJECTIVE \#1: Recognize the neuropsychiatric manifestations of ectopic Cushing's syndrome (ECS) and their implications on management.

LEARNING OBJECTIVE \#2: Distinguish the clinical features and management of ECS from Cushing's disease.

CASE: A 67-year-old woman with previously controlled hypertension and recent admission for hypokalemia presented with the urging of her daughter for evaluation of paranoid behavioral changes and hypokalemia refractory to lisinopril and spironolactone. Among several unusual behaviors, she became increasingly reclusive, suspicious of family members, and began donating large sums of money to organizations. On admission, she was found to be hypertensive with plethora, proximal muscle weakness, and peripheral edema. Preceding evaluation for hyperaldosteronism was unremarkable. Plasma cortisol and ACTH levels were markedly elevated. Lowdose and high-dose dexamethasone suppression tests failed to suppress her cortisol. Pituitary MRI and inferior petrosal sinus sampling did not localize a pathologic pituitary ACTH source supporting a diagnosis of ECS. Investigation for the ACTH source with FDG-PET and octreotide scintigraphy failed to identify the ectopic lesion. She ultimately underwent laparoscopic bilateral adrenalectomy and her hypokalemia and neuropsychiatric symptoms resolved.

IMPACT/DISCUSSION: ECS is a rare condition with high morbidity and mortality prompting the need for early recognition and management. Localizing the ectopic source of ACTH is critical as surgical resection is frequently curative and $\mathrm{ACTH}$-secreting tumors are often malignant. Cortisol excess results in a multitude of common disease findings including cardiovascular disease, hypertension, diabetes, myopathy, and osteoporosis, therefore making the diagnosis challenging on initial evaluation. Resistant hypokalemia has been found to be a more prevalent feature of ECS than of Cushing's disease and is associated with a higher severity of hypercortisolism and worse prognosis. Depression, anxiety, and cognitive impairment are the most common neuropsychiatric symptoms, though psychosis and mania can also occur. Neuropsychiatric symptoms generally improve with resolution of hypercortisolism though approximately onethird of patients have been found to have residual symptoms one year in remission. Our case demonstrates that neuropsychiatric manifestations significantly contribute to morbidity and can disrupt the patient's ability to receive appropriate treatment. The preferred treatment of ectopic ACTH syndrome is surgical resection of the ACTH-secreting tumor. However, up to $20 \%$ of tumors are never identified despite thorough evaluation thus necessitating management with bilateral adrenalectomy or adrenal enzyme inhibitors.

CONCLUSION: Early diagnosis of ECS is challenging but critical for timely management and potential cure. Neuropsychiatric symptoms of ECS can disrupt care and make follow up challenging. In these circumstances, bilateral adrenalectomy or adrenal enzyme inhibitor therapy may have to be considered earlier.

\section{SHIFTY MIND AND SHAKEN VOICE}

$\underline{\text { Bishir Clayton }}{ }^{1}$; Ann Goh $^{2}$; Michelle Sweet ${ }^{2} .{ }^{1}$ Rush University, Chicago,

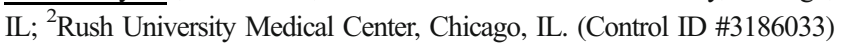

LEARNING OBJECTIVE \#1: West Nile Virus (WNV) can lead to a variety of neurologic symptoms such as dysphasia, parkinsonism, and bulbar symptoms

LEARNING OBJECTIVE \#2: West Nile Virus Encephalitis can have an oscillating presentation which can delay diagnosis

CASE: A 70 year old female with past medical history of diabetes mellitus presented to the emergency department with a 3 day history fever, confusion, and generalized weakness. She had simultaneously developed nausea and anorexia for 2 days that was followed by diarrhea. She was admitted with a diagnosis of viral gastroenteritis, improved with supportive care, and was discharged. Two days after discharge, she began to stare blankly and did not respond to any questions. The fluctuating cognition was accompanied by malaise and persistent fevers. She returned to the hospital and was noted to have oral and abdominal tremulousness. Her labs were significant for leukocytosis with predominant neutrophilia in both serum and CSF, normal CSF glucose, elevated CSF protein, and negative HSV PCR. A CT scan of her brain showed no acute abnormalities. On day 2 of hospitalization, she appeared to be responsive early in the day, but later would not respond to questions or commands. This wavering course continued through hospital day 4 despite labs indicating a down-trending WBC and negative blood cultures. On day 4 of hospitalization her serum and CSF West Nile Virus IgM returned elevated. She continued to improve with supportive care and on day 8 was transferred for acute rehabilitation.

IMPACT/DISCUSSION: WNV can cause a variety of neurologic symptoms in affected individuals that can include combination of parkinsonism, tremors, myoclonus, and encephalitis. Bulbar weakness with symptoms of dysphagia and respiratory failure has been experienced by more than half of individuals. The time it takes from onset of symptoms to development of neurologic disease typically ranges from one day to one week. This patient presents with two notable findings in WNV encephalitis. She experienced bulbar symptoms including dysphasia. While the patient had facial tremors during the course of her illness, the difficulties she displayed appeared to be a challenge of speech production rather than articulation. The fluctuant features of the disease course are also of interest. The initial prodrome that she displayed stereotyped a viral illness with associated confusion and somnolence. The onset of overt encephalitis was unexpected.

CONCLUSION: Awareness of the variable presentations of West Nile Encephalitis may allow clinicians to consider West Nile as a possible etiology of illness in the face of a variety of neurological and/or infectious symptoms that can oscillate in severity. 
SICKLE CELL TRAIT, IMMOBILITY AND A CASE OF SEVERE RHABDOMYOLYSIS

Michael Fattouh; Andrew Hunter; Peter C. Smith. Boston Medical Center, Boston, MA. (Control ID \#3165665)

LEARNING OBJECTIVE \#1: Recognize multiple less commonly cited risk factors for rhabdomyolysis

CASE: $\mathrm{Mr}$. $\mathrm{N}$ is a 40-year-old black male with a chief complaint of "I'm in kidney failure." The patient reported darkened urine and a subjective decrease in urine output. On the day prior, he had used intranasal heroin and woke up 5 hours later leaning on a gate with right shoulder pain. Mr. N has a history of schizoaffective disorder, for which he takes aripiprazole $20 \mathrm{mg}$ daily, and a longstanding history of intranasal heroin use. Exam was notable for tenderness along his right deltoid. Labs were remarkable for a creatine kinase (CK) elevated to $79,946 \mathrm{IU} / \mathrm{L}$ and a creatinine to $6.59 \mathrm{mg} /$ dL. A urine tox screen was positive for only fentanyl. The patient was diagnosed with rhabdomyolysis and acute kidney injury (AKI) and started on IV fluids. The next day, the CK decreased to $41,907 \mathrm{IU} / \mathrm{L}$, however the creatinine increased with an eventual peak three days later to $11.34 \mathrm{mg} / \mathrm{dL}$. When asked how he knew he was in "kidney failure," Mr. N reported a similar presentation 7 months prior: after using heroin he was immobile for multiple hours and was admitted by an outside hospital for rhabdomyolysis with AKI. After accessing the outside records, it was found that his CK was $17,442 \mathrm{IU} / \mathrm{L}$ and his creatinine peaked at $19.83 \mathrm{mg} / \mathrm{dL}$. It was unclear why a patient without a history of primary muscular/neuromuscular pathology and without any clear inciting event had two episodes of such severe rhabdomyolysis. Work up was initiated and a hemoglobin electrophoresis eventually revealed sickle cell trait.

IMPACT/DISCUSSION: Sickle cell trait is recognized as a risk factor for exertional rhabdomyolysis. Further, the use of antipsychotic medications has been reported to increase the risk for exertional rhabdomyolysis in sickle cell trait patients ${ }^{1}$. One small case series suggests heroin itself may increase the severity of rhabdomyolysis ${ }^{2}$. Here we describe a case of rhabdomyolysis and AKI - with CK and creatinine levels seen in patients with crush injury or malignant hyperthermia - secondary to mild muscle compression in an immobile patient with multiple less commonly recognized risk factors: opioid use, antipsychotic use, and sickle cell trait. With an increasing prevalence of opioid use in black patients coupled with higher prevalence of sickle cell in people of African descent, the risk for rhabdomyolysis may be of particular concern in an already vulnerable patient population.

CONCLUSION: - Sickle cell trait increases a patient's risk for exertional rhabdomyolysis - Patients with sickle cell trait may be at an increased risk for severe rhabdomyolysis even without exertion or notable trauma Citations 1. Nelson, D. Alan, et al. "Sickle cell trait, rhabdomyolysis, and mortality among US Army soldiers." New England Journal of Medicine 375.5 (2016): 435-442 2. Kosmadakis, George, et al. "Acute kidney injury due to rhabdomyolysis in narcotic drug users." The International journal of artificial organs 34.7 (2011): 584-588

\section{SIGNIFICANT VISUAL IMPAIRMENT IN AN ALCOHOLIC PA- TIENT}

Tatsuya Fujikawa; Atsushi Taniguchi; Yuka Sogabe. Mitoyo General Hospital, Kanonji, Japan. (Control ID \#3142142)

LEARNING OBJECTIVE \#1: Recognize the clinical features of Wernicke encephalopathy (WE), exhibiting not only typical but nontypical symptoms, such as vision loss
LEARNING OBJECTIVE \#2: Treat with intravenous vitamin B1 when WE is suspected

CASE: A woman in her late 20s, who had depression and alcoholism, complained of vision loss for the past 10 days and reduced dietary intake since the last 3 months. Her clinical history includes alcoholic liver injury and depression. She experienced intermittent appetite loss, nausea, and vomiting 3 months ago, but continued consuming alcohol heavily till 2 months ago. She finally visited the Department of Ophthalmology and General Internal Medicine in our hospital on experiencing vision and appetite loss. On presentation to our hospital, her blood pressure, pulse, respiratory rate, and body temperature were 120/87, 113/min, 18/min, and $97.0^{\circ} \mathrm{F}$, respectively. She had a Glasgow coma scale score of 14 as E4V4M6. Ophthalmologic examination revealed vision loss associated with prominent optic disc edema; physical examination revealed bilateral abduction restriction, gaze-evoked nystagmus, anarthria, and muscle weakness of extremities with Manual Muscle Testing grade 3. FLAIR MRI revealed hyperintense signals in bilateral thalamus, areas around the third ventricle, and the Sylvian aqueduct. Hence, she was diagnosed with WE based on clinical history, physical examination, and MRI findings. Intravenous thiamine replacement therapy was, therefore, started. Metabolic workup, conducted before the thiamine replacement therapy, revealed thiamine deficiency ( $15 \mathrm{ng} / \mathrm{mL}$, reference range 24-66 ng/ $\mathrm{mL}$ ). Thiamine supplementation improved the patient's impaired consciousness and appetite loss within 2-3 days of treatment respectively, abducens nerve paresis within 2 weeks, vision loss over 3 weeks, optic disc edema by 8 weeks, and the nystagmus over several months.

IMPACT/DISCUSSION: WE, an acute neuropsychiatric syndrome, results from thiamine deficiency, mostly due to alcoholism. WE is characterized by a classical triad of symptoms: nystagmus and ophthalmoplegia, mental status changes, and unsteadiness of stance and gait; these conditions were observed in our case. However, optic disc edema, especially associated with vision loss, is rarely reported in WE. MRI can serve as a valuable tool in WE diagnosis; changes in the thalami, mammillary bodies, tectal plate, and periaqueductal area are typically observed. WE prognosis depends on a prompt diagnosis followed by thiamine supplementation at high doses. Early treatment prevents its evolution to Korsakoff syndrome, a chronic form of anterograde and retrograde amnesia, that responds poorly to thiamine.

CONCLUSION: Physicians must highly suspect WE when patients manifest the aforementioned characteristic symptoms. Patients may sometimes visit hospitals with vision loss. If WE is even remotely suspected, thiamine injections should be administered to achieve symptom resolution.

\section{SLOW AND SLEEPY: VALPROIC ACID-INDUCED HYPERAMMONEMIC ENCEPHALOPATHY}

Jake Schlemmer; Ambili Ramachandran. UT Health San Antonio, San Antonio, TX. (Control ID \#3150143)

LEARNING OBJECTIVE \#1: Recognize the clinical features of hyperammonemia.

LEARNING OBJECTIVE \#2: Identify valproic acid as a potential cause of hyperammonemic encephalopathy.

CASE: A 30 year-old man presented with two weeks of progressively worsening fatigue and psychomotor slowing. Other associated symptoms included periodic episodes of confusion, generalized weakness, fine motor deficits, and shuffling gait. The patient's medical history included a traumatic brain injury in 2016 with subdural hematoma status post decompressive hemicraniectomy and cranioplasty, with resulting seizure disorder 
and mood disorder. He had had a recent bone flap excision two months prior that had been complicated by coagulase negative Staphylococcus infection for which he completed 6 weeks of vancomycin. Current medications included brivaracetam, valproic acid, and risperidone. All vital signs were within normal limits. The patient was fully oriented but slightly confused. Strength and sensation were globally intact with normal reflexes and no asterixis. An antalgic, shuffling gait was noted. CT head revealed recent craniectomy changes but was otherwise unremarkable. Electrolytes, cell counts, and liver enzymes were within normal limits. Serum ammonia was 100 micromoles/liter. On further questioning, the patient revealed that he was switched from quetiapine to risperidone a few weeks prior to symptom onset. The patient initially improved with lactulose, levocarnitine, and vitamin B6. Risperidone dose was decreased, and valproic acid was eventually discontinued. At clinic follow-up one month later, patient was improving and eventually reached his baseline at three months after discharge.

IMPACT/DISCUSSION: Altered mental status involves a wide range of etiologies and is a problem commonly encountered by the internist. Determining the etiology of symptoms is key to minimizing morbidity and mortality, especially when the etiology is a patient's own medications. Hyperammonemia can occur in roughly $28 \%$ of patients on valproic acid, even in the absence of liver dysfunction. In patients with elevated ammonia from any cause, presenting symptoms may include gait instability, changes in behavior, slurred speech, excessive sleepiness, and gastrointestinal disturbances. Antipsychotic drugs, including risperidone, have been shown to be an independent risk factor for hyperammonemia in patients on valproic acid, as demonstrated in this case. While treatment may not require immediate cessation of valproic acid, prompt measures to reduce serum ammonia should be taken. Initial treatments may involve lactulose, levocarnitine, antibiotics such as metronidazole, and B vitamins. Continuous renal replacement therapy or hemodialysis may be required in severe cases.

CONCLUSION: Valproic acid-induced hyperammonemia can manifest as acute encephalopathy or other symptoms such as gait instability. Internists should be adept in identifying acute encephalopathy due to medications and medication interactions.

\section{SLOW BUT DEADLY}

Alexandra Valdrighi; Michael P. Smith. University of Nebraska Medical Center, Omaha, NE. (Control ID \#3185928)

LEARNING OBJECTIVE \#1: Develop an approach to risk stratify patients for an aortic dissection

LEARNING OBJECTIVE \#2: Recognize the varied clinical presentations of aortic dissections

CASE: An 87-year-old woman presented with chest pain on inspiration and shortness of breath which came on suddenly. The pain was located on the left side of her chest and in the substernal region, described as a 4/10 "dull ache" and a "pressure sensation." She also reported a mild, nonproductive cough for the past 2-3 days. She denied any previous chest pain episodes and her past medical history included hypertension, left bundle branch block, dementia, and tricuspid regurgitation. She had stable vital signs. She had a known 2/6 murmur along the left sternal border and pain over the xiphoid process with palpation or with deep inspiration. An EKG did not show any changes. Cardiac enzymes, pro-BNP, comprehensive metabolic panel, and complete blood count were all unremarkable. She continued to have a dull aching pain in the same location with a minimal increase in her cardiac enzymes to $0.268 \mathrm{ng} / \mathrm{L}$ but decreased to $0.098 \mathrm{ng} / \mathrm{L}$. She continued to complain of some shortness of breath at rest and general chest discomfort. Vitals continued to remain stable until she spiked a temperature of 101.3 and developed atrial fibrillation with rapid ventricular response. A D-dimer was found to be $4,670 \mathrm{ng} / \mathrm{mL}$. CT angiogram showed abnormal dilation of the aortic root with an aortic dissection. She unfortunately coded and was unable to be resuscitated.

IMPACT/DISCUSSION: Aortic dissections often have varied clinical presentations and thus they can be difficult to diagnose. Subsequently, delays in treatment can lead to fatalities. Since aortic dissections are not encountered frequently, it is important for physicians to consider them in the evaluation of chest pain, especially when it is of sudden onset. The majority of patients describe the pain as "sharp." Neurologic symptoms also occur in approximately $50 \%$ of patients. Some patients may present with painless dissections or more chronic onset of dissections. Other unusual initial presentations include congestive heart failure, stroke, or syncope. Elderly are more likely to present with an atypical presentation which may complicate diagnosis in this age group; yet the mean age of dissection is also 65 . There are twelve history and physical exam findings that have a high sensitivity for detection of an aortic dissection and can be used to risk stratify patients. A D-dimer score of less than $500 \mathrm{ng} / \mathrm{mL}$ can be used as a screening test

CONCLUSION: Although aortic dissections have a relatively low incidence, they have a high mortality rate acutely and it is essential for the general internist to diagnose rapidly.

\section{SMALL CELL LUNG CANCER PRESENTING AS LIMBIC EN- CEPHALITIS.}

Muqsita Nashat ${ }^{1}$; Sheetal Patel ${ }^{2}$; M.Azam Mohiuddin ${ }^{2} .{ }^{1}$ Mercy Nazareth Hospital, Hamilton, NJ; ${ }^{2}$ Mercy Nazareth Hospital, Philadelphia, PA. (Control ID \#3184442)

LEARNING OBJECTIVE \#1: Recognize limbic encephalitis as a paraneoplastic syndrome secondary to malignancy.

CASE: A 58 year old female presented with sudden onset of impaired short term memory of few days duration. Past history includes prediabetes and hyperlipidemia. She is a smoker since age 17 and drinks three glasses of wine every weekend. Her only medication is multivitamin once daily. Physical exam was normal except for a right Hoffman and left extensor plantar reflex and her Folstein score was 25/30. Chest x-ray was normal. MRI brain showed mild small vessel ischemic change without acute intracranial abnormality. She was discharged with a clinical diagnosis of TIA of bilateral posterior cerebral arteries affecting temporal lobes and hippocampus. She was readmitted 48 hours later with new onset seizure and worsening memory. Repeat MRI brain showed hyperintense signal abnormality involving the medial temporal lobes and para hippocampal regions suggestive of infectious or limbic encephalitis. Her CSF showed normal WBC, protein and was negative for JC virus, HSV, Lyme disease and malignant cells. A CT chest showed enlarged right precarinal and paratracheal lymph nodes and biopsy confirmed metastatic small cell neuroendocrine carcinoma. She was discharged home and had outpatient chemotherapy with improvement in memory and remission of cancer.

IMPACT/DISCUSSION: Limbic encephalitis represents a group of autoimmune conditions characterized by inflammation of the limbic system and is commonly associated with paraneoplastic process, infection or autoimmune diseases. Paraneoplastic limbic encephalitis (PLE) can present with acute or subacute onset of mood changes, memory loss, seizure or cognitive dysfunction. The neuropsychiatric symptoms can be present without tumor cell invasion which is caused by an immune response directed to the central nervous proteins which are similar to tumor antigens leading to perivascular 
lymphocyte infiltration and ultimately neuronal cell loss causing these symptoms. Most frequent neoplasms associated with paraneoplastic limbic encephalitis are small cell cancer of the lung, testicular tumors, thymoma, breast cancer and Hodgkin lymphoma. The diagnosis of PLE is generally based on the following four criteria which includes clinical findings mentioned above, analyses of CSF, MRI or EEG, exclusion of other neuro oncologic complications and interval of $<4$ years between onset of neurological symptoms and tumor diagnosis. Furthermore, metabolic encephalopathy, neurotoxic drugs, inflammatory disorders, central nervous system tumors, and neurodegenerative disorders must be excluded. PLE is mostly associated with anti-Hu antibodies and has a positive predictive value of approximately $90 \%$ for small cell cancer of lung. Anti-cancer therapy in combination with immunomodulation is more effective in improving neurological symptoms than immunosuppressive treatment alone.

CONCLUSION: This case illustrates an example where PLE should necessitate further evaluation to rule out malignancy.

\section{SO SLOW, SO FAST}

Sawsan Abulaimoun; Kamelah Abushalha; Peter Silberstein. Creighton University, Omaha, NE. (Control ID \#3185954)

LEARNING OBJECTIVE \#1: Nivolumab is a monoclonal antibody targeted towards programmed cell death-1 protein (PD-1) and works by blocking the interaction between PD-1 and its ligands, PD-L1 and PD-L2, thereby unmasking the tumor cells expressing these ligands attempting to circumvent T-cell mediated cytotoxicity. By increasing the activity of the immune system, immune checkpoint blockade can have inflammatory side effects, which are often termed immune-related adverse events (irAE). Although any organ system can be affected, irAE most commonly involves the gastrointestinal tract, endocrine glands, skin, and liver.

CASE: A 59-year-old male with metastatic renal cell carcinoma (RCC) presents to the ED with anasarca, difficulty breathing (due to tongue swelling), muscle aches, and progressively worsening fatigue over the course of six weeks. He was being treated for RCC with nivolumab and had received a total of 11 doses every other week. Initial workup revealed an elevated creatinine (1.76, baseline 1.2) and thyroid stimulating hormone (TSH) level (149 mIU/L). Creatine kinase (CK) was then measured and found to be critically elevated at 2,029 U/L. He was treated with intravenous (IV) fluids for the rhabdomyolysis with a slow decline in CK levels. Endocrinology was consulted to assist in management, and he was given a loading dose of $200 \mu \mathrm{g}$ IV levothyroxine followed by $100 \mu \mathrm{g}$ the next day. TSH trended down to $71.6 \mathrm{mIU} / \mathrm{L}$ on the day of discharge two days later. Patient continues to receive nivolumab infusions along with levothyroxine. CK and TSH both normalized within two weeks after treatment for hypothyroidism was initiated.

IMPACT/DISCUSSION: Nivolumab is approved for patients with RCC who had received prior anti-angiogenic therapy after showing a statistically significant and clinically meaningful difference in overall survival. In nivolumab monotherapy studies, the median time to onset of immunerelated hypothyroidism or thyroiditis resulting in hypothyroidism was 2.9 months (range: 1 day to 16.6 months). Given the normal baseline thyroid function test and the time frame within which the hypothyroidism developed, it is safe to extrapolate a correlation between this patient's hypothyroidism and nivolumab treatment. The normalization of the CK levels with levothyroxine alone (no glucocorticoids were used) makes it more likely that his rhabdomyolysis was a direct result of severe hypothyroidism, although the possibility of autoimmune-related myositis can't be completely excluded. To our knowledge, this is the first reported case of a PD-1 inhibitor causing hypothyroidism severe enough to induce rhabdomyolysis. A similar presentation was reported with ipilimumab (anti-cytotoxic Tlymphocyte-associated protein 4) and the condition improved with thyroxine therapy.

CONCLUSION: Patients on nivolumab and other PD-1 inhibitors should be monitored and screened regularly for irAE.

\section{SPONTANEOUS CORONARY ARTERY DISSECTION IN A 47- YEAR OLD FEMALE, AN INFREQUENT PRESENTATION OF ACUTE CORONARY SYNDROME}

Michael Gurin. Beth Israel Deaconess, Boston, MA. (Control ID \#3181565)

LEARNING OBJECTIVE \#1: Diagnose acute coronary syndrome in patients without traditional risk factors.

LEARNING OBJECTIVE \#2: Manage and treat spontaneous coronary artery dissection.

CASE: A 47-year old female with past medical history of Hashimoto's thyroiditis resulting in hypothyroidism and irritable bowel syndrome presented to urgent care with indigestion. The prior evening, she felt a lingering sensation of indigestion after preparing a bottle for her baby. She is G5P0 with 3 adopted children, "several" miscarriages, and 1 ectopic pregnancy. She is a former nurse, drinks alcohol occasionally, and does not use tobacco products or illicit drugs. There is no history of premature coronary artery disease or sudden death in her family. Her home medications include levothyroxine, hyoscyamine, and rifaximin. On further evaluation, physical exam did not reveal any abnormalities. ECG showed sinus rhythm with mild $\mathrm{T}$ wave flattening in III and aVF. A troponin level was incidentally checked and was 0.16 . She was admitted to the hospital and treated with heparin, aspirin, atorvastatin, and metoprolol for a presumed NSTEMI. Troponins peaked at 0.22 . Lipid panel revealed total cholesterol 165, triglycerides 78, HDL 95, and LDL 54. She had a coronary angiogram, which showed diffuse narrowing at a small distal left posterolateral branch, consistent with spontaneous coronary artery dissection (SCAD). No obstructive coronary disease was found. Clopidogrel was added to her medication regimen, and she was discharged with cardiology follow-up.

IMPACT/DISCUSSION: The patient was diagnosed with SCAD, a resultant tear within the artery wall that can lead to an intramural hematoma. This case poses the challenge of diagnosing acute coronary syndrome in the absence of obvious cardiac risk factors or significant ECG changes. Her indigestion could have been mistaken for recurrent gastrointestinal symptoms. SCAD is associated with pregnancy, miscarriage, or early postpartum status, as the hormonal effects during these periods lead to weakening of the arterial wall. A suspected recent miscarriage was thought to be the precipitating etiology. Intense Valsalva or weight lifting, hormonal therapy, and emotional stress are other inciting factors. Definitive diagnostic testing is coronary angiography. Many patients with SCAD have underlying connective tissue disorders and fibromuscular dysplasia. Management is typically conservative consisting of antiplatelet therapy, beta-blockers to reduce arterial shear stress, and statins in patients with dyslipidemia. Cardiac rehabilitation has also been shown to be beneficial.

CONCLUSION: 1. Consider SCAD in both young females and males without traditional cardiac risk factors who present with acute coronary syndrome. 2. Management is usually conservative and involves indefinite aspirin, beta-blockers, one year of clopidogrel, and referral to cardiac rehabilitation. 3. Patients diagnosed with SCAD should be evaluated for connective tissue disorders and fibromuscular dysplasia. 
STALLED BY STILL'S - A PUZZLING CASE OF ADULT ONSET STILL'S DISEASE S. MAHARJAN MD, A. OHRI MD, S. FERGUSON MD, M. BHARGAVA MD. HIGHLAND HOSPITAL - ALAMEDA HEALTH SYSTEM, OAKLAND, CALIFORNIA

Suresh Maharjan; Ajay M. Ohri; Monica Bhargava; Sancia Ferguson. Alameda Health System - Highland Hospital, Emeryville, CA. (Control ID \#3183536)

LEARNING OBJECTIVE \#1: Recognize diagnostic complexity of Adult Onset Still's Disease (AOSD). Rule out potential lifethreatening coexisting disorders.

CASE: A 30-year-old healthy, Guatemalan male presented to emergency department with high fevers, headache and neck pain of more than a week. Physical exam was remarkable for a non-pruritic, diffuse, salmon-colored macular rash over his body. Extensive sepsis workup was unrevealing for a clear source of infection. Computer Tomography(CT) head and lumbar puncture were negative. Laboratory data was concerning for elevated transaminases, high C-reactive protein and ferritin of $1731 \mathrm{ng} / \mathrm{ml}$. CT chest and abdomen showed hepatomegaly with extensive lymphadenopathy. Additional laboratory findings of high lactate dehydrogenase, bilirubin, triglycerides and low fibrinogen raised a concern for Hemophagocytic Lymphohistiocytosis(HLH). Hematology was consulted. Bone marrow biopsy ruled out HLH and flow cytometry was negative for malignancies. Multitude of serological tests for autoimmune disorders and viral infections returned negative. Rheumatology was consulted. Based on clinical presentation, workup and patient meeting more than five Yamaguchi criteria, AOSD was suspected. Prednisone was initiated which dramatically improved his symptoms supporting the diagnosis. He was eventually discharged on a tapering steroid regimen with close Rheumatology follow up.

IMPACT/DISCUSSION: George Still first described juvenile Still's disease in 1896 however much remains unknown about the exact etiopathogenesis. Involvement of a complex cytokine network mainly involving macrophages, Tumor Necrosis Factors and Interleukins(IL) is suspected. Several diagnostic criteria have been proposed but so far Yamaguchi criteria(sensitivity of 93\% and specificity of 92\%) has been widely accepted. There's always a possibility of an overlapping/additional clinical entity like HLH, Macrophage Activation Syndrome(MAS) which are thought to be on the same spectrum but can cause life threatening complications. Our patient did not demonstrate hemophagocytosis on bone marrow biopsy, rather, had a rapid clinical improvement in the setting of modest steroids, making these diagnoses unlikely. The goal of treatment is to resolve physical signs/symptoms and laboratory indices of inflammation. Our patient's ferritin decreased to $40 \mathrm{ng} / \mathrm{ml}$ within a week into the treatment, along with clinical improvement. Treatment modalities depend on severity of AOSD ranging from nonsteroidal antiinflammatory drugs to steroids to nonbiologic/biologic Disease Modifying Anti-Rheumatic Drugs.

CONCLUSION: AOSD is a diagnosis of exclusion. The Yamaguchi and Fautrel criteria are both very reliable diagnostic tools. Association with HLH/MAS is rare but not uncommon. Timely diagnosis and treatment is the key to reduce mortality and morbidity which is particularly higher among Asians and patients with disseminated intravascular coagulation. With recent advancement in medical research, hope is high that we might be able to stall Still's disease.
STATIN INDUCED RHABDOMYOLYSIS COMPLICATED BY ACUTE RENAL FAILURE LEADING TO DIALYSIS

Dilshan Dhillon ${ }^{1}$; Paul Nona ${ }^{2}$; Sunjay Modi ${ }^{1}$; Zarina Alam ${ }^{2} .{ }^{1}$ Henry Ford Hospital, Oakland, MI; ${ }^{2}$ Henry Ford Health System, West Bloomfield, MI. (Control ID \#3185617)

LEARNING OBJECTIVE \#1: Recognize life changing consequences of statin induced injury in patients with stage IV CKD LEARNING OBJECTIVE \#2: Identify statin induced muscle and kidney injury

CASE: 56 year old male with presents with generalized malaise, myalgias, tea colored urine and loose stools for 2 days. He denies sick contacts, flu like symptoms, fevers, recent changes in medications, dehydration or recent strenuous exercise. He is a former smoker with 35 pack years, social alcohol consumer and denies illicit drug use. His medications include: aspirin $81 \mathrm{mg}$, atorvastatin $80 \mathrm{mg}$ and metoprolol tartrate $25 \mathrm{mg}$ for coronary artery disease, amlodipine $10 \mathrm{mg}$ for hypertension, lantus 8 units for type 2 diabetes and lasix $60 \mathrm{mg}$ for stage IV chronic kidney disease. On examination he was hemodynamically stable, ill appearing obese gentleman with tenderness in the major muscle groups of his upper and lower extremities. The rest of his exam was unremarkable. Laboratory studies revealed hyperkalemia at $6.2 \mathrm{mEq} / \mathrm{L}$, creatinine $7.70 \mathrm{mg} / \mathrm{dL}$ (baseline around $4 \mathrm{mg} / \mathrm{dL}$ ), calcium $7.8 \mathrm{mg} / \mathrm{dL}$, phosphorus $9.9 \mathrm{mg} / \mathrm{dL}$, his serum ALT was $41 \mathrm{IU} / \mathrm{L}$ and AST was $245 \mathrm{IU} / \mathrm{L}$. Urinalysis was significant for hematuria and proteinuria. Creatine protein kinase (CPK) peaked at 452,312 . Patient was found to be in rhabdomyolysis leading to acute on chronic renal failure. Statin was removed, patient was started on continuous infusion of normal saline and furosemide drip. CPK was down trending however due to worsening hyperkalemia and hyperphosphatemia despite adequate urine output, a tunneled-cuff catheter was placed and patient was initiated on hemodialysis. Electrolytes and CPK normalized however patients renal function did not improve leading to end stage renal disease.

IMPACT/DISCUSSION: Cardiovascular disease is a major cause of morbidity and mortality in the world with the prevalence of cardiovascular diseases doubling by 2020. Statin therapy is the primary pharmacologic therapy to achieve low LDL cholesterol in efforts to improve atherosclerotic cardiovascular disease outcomes in primary and secondary prevention. High doses of statins increase the risk of rhabdomyolysis. The US FDA Adverse Event Reporting System database reports that there are .3-12.5 cases out of 1 million of statin induced rhabdomyolysis. Rhabdomyolysis is syndrome where muscle pain and weakness is caused by muscle tissue breakdown with release of intramuscular contents (enzymes, myoglobin, electrolytes) into the circulation. Myoglobin is cytotoxic to renal tubules and causing tubular cast formation and acute tubular necrosis. Cytokine induced arteriolar vasoconstriction in combination with dehydration results in decreased glomerular filtration rate and eventually acute renal failure.

CONCLUSION: This case report is aimed to highlight the risks of high dose statin therapy and to consider a medication/dose review in the setting of advanced chronic kidney disease to prolong time to dialysis, ultimately improving quality of life.

\section{STAY THE COURSE AND DON'T PUT DOWN ANCHORS}

Nora Kovar; Michael P. Smith. University of Nebraska Medical Center, Omaha, NE. (Control ID \#3186112)

LEARNING OBJECTIVE \#1: Develop an approach to pancytopenia in a patient with human immunodeficiency virus (HIV)

LEARNING OBJECTIVE \#2: Recognize the role of Anchoring bias plays in the diagnosis and management of patients 
CASE: A 45-year-old man presented with unexplained pancytopenia in the absence of any acute symptoms. He endorsed mild headache, right shoulder pain, and bilateral ankle swelling that were chronic. He had a previously identified systolic murmur, decreased range of motion at the right shoulder, and symmetrical swollen ankles. An unintentional 10-pound weight loss in the preceding six months was noted. History included well-controlled HIV infection, on antiretroviral therapy and prophylactic trimethoprimsulfamethoxazole; untreated hepatitis $\mathrm{C}$, including recent ultrasound showing mild splenomegaly; recent hospitalization for bacteremia of unknown source; and aortic stenosis. He admitted to polysubstance abuse with alcohol, methamphetamine, marijuana, and tobacco. Hemoglobin (5.3) and white blood cells (2.9) were decreased from one month prior. Platelets (46) were stable. Differential showed increased nucleated red blood cells. Peripheral smear showed occasional schistocytes. CRP and ferritin were elevated. Other laboratory studies including B12, folate, lactate dehydrogenase, haptoglobin, and testing for parvovirus and herpes simplex virus were negative. Trimethoprimsulfamethoxazole was held, with small improvement in all cell lines. Bone marrow biopsy showed $21 \%$ blasts, myeloperoxidase stain positive, and was diagnostic for acute myeloid leukemia.

IMPACT/DISCUSSION: This patient's complex history highlights many important considerations for this process. Low socioeconomic status and polysubstance abuse increased the risk for nutritional deficits that could impair hematopoiesis. Exposure to toxins such as alcohol can also suppress the bone marrow. Viral infections, including HIV, hepatitis C, Epstein-Barr, and parvovirus can as well. Medications and infiltrative diseases are another potential culprit in cases of bone marrow failure. This patient also had elevated ferritin, which is a diagnostic criterion for hemophagocytic lymphohistiocytosis, and joint pain, which serves as a reminder that rheumatologic disease (e.g., rheumatoid arthritis, systemic lupus erythematosus) can also cause bone marrow failure or hypersplenism. Another important cause of peripheral destruction is disseminated intravascular coagulation. These latter considerations were relevant in this case given the patient's splenic ultrasound and his history of joint pain and bacteremia. In patients with HIV, it can be tempting to attribute new pathology to the virus itself. However, these patients often have complex histories, and HIV carries increased risk for other disorders, as well. In this case, the increased risk of leukemia in HIV was pertinent to the final diagnosis.

CONCLUSION: Pancytopenia is commonly encountered by the general internist. Because of its broad differential, a thorough evaluation is necessary to avoid missed diagnoses.

\section{STEMI IN A YOUNG PATIENT: ROLE OF AN INTERNIST}

Anubhav Jain; Ankita Aggarwal. Wayne State University, Auburn Hills, MI. (Control ID \#3186424)

LEARNING OBJECTIVE \#1: Identify risk factors of coronary artery disease in young patients

CASE: The patient is a 34-year-old male with no past medical history, no known cardiac risk factors and no family history of cardiac disease who presented to the emergency department with acute onset pressure-like chest pain radiating to his back, bilateral shoulders and arms for an hour. In the ED, he continued to have pain, relieved a little with nitro. He was hemodynamically stable. EKG revealed ST elevation in leads V2-V6 suggestive of anterior wall MI. Troponin was negative initially. Cardiology was consulted. He was taken for an emergent left heart catheterization. A left heart catheterization revealed $100 \%$ occlusion of the left anterior descending(LAD). A drugeluting stent was placed in it. He was started on optimal medical therapy including aspirin $81 \mathrm{mg}$ daily, ticagrelor $90 \mathrm{mg}$ Q $12 \mathrm{hrs}$, metoprolol $50 \mathrm{mg}$
Q12 hrs, atorvastatin $80 \mathrm{mg}$ daily. Workup for possible cardiac risk factors revealed dyslipidemia with hypercholesterolemia (Total cholesterol:257), hypertriglyceridemia(TG;772), low HDL(31), prediabetes(HbA1C:5.7), morbid obesity( BMI 35 with complications), metabolic syndrome(Obese, high cholesterol and low HDL) and smoking (3 pack year history). He was advised about lifestyle modification with diet and physical activity and cessation of smoking.

IMPACT/DISCUSSION: The prevalence of coronary artery disease (CAD) in young patients defined as age as less than 45 years is low (1.2\%). The most common risk factors in this population are obesity and a history of smoking, unlike older patients where dyslipidemia and history of diabetes are the most important risk factors. In most young patients, the first manifestation of CAD is STEMI unlike stable angina in older patients.

CONCLUSION: Acute coronary syndrome has a rare incidence in young patients. Since obesity and smoking are the most common cardiovascular risk factors in this population, it is important for us physicians to emphasize the importance of lifestyle modification in these patients to prevent major cardiovascular events. Our case highlights the importance of educating our patients about the risk of developing $\mathrm{CAD}$ and $\mathrm{ACS}$ at a young age with these risk factors even in the absence of family history.

\section{STERNOCLAVICULAR JOINT SEPTIC ARTHRITIS CAUSED BY ESCHERICHIA COLI IN A HEALTHY ADULT WITHOUT RISK FACTORS.}

Liwayway R. Andrade; Saurin Gandhi; Jaison John. The University of Texas Dell Medical School, Austin, TX. (Control ID \#3186306)

LEARNING OBJECTIVE \#1: To recognize the clinical features of sternoclavicular joint septic arthritis for early diagnosis and medical management.

CASE: A 65-year-old woman without significant prior medical history presented to the emergency department with a five-week history of persistent sharp left anterior neck pain radiating to the left shoulder, worse on movement of the left arm. Pain started following a mechanical fall that resulted in a nondisplaced fracture of the right distal fibula, managed non-surgically, but no history of direct trauma to the left neck, shoulder, or arm. She reported fever, chills, nausea and vomiting four-weeks prior attributed to having the flu, but no chest pain, dysphagia, odynophagia, numbness or weakness. Physical exam was significant for asymmetry of the left sternoclavicular joint and mild tenderness to palpation over the area without erythema or edema. Laboratory findings were notable for the absence of leukocytosis and elevation in ESR and CRP. An outpatient MRI showed inflammation of the left sternoclavicular joint. Joint aspirate and blood cultures grew gram-negative rods. Patient was started empirically on cefepime, and infectious disease was consulted. A CT of the abdomen and pelvis was non-revealing for the source of the bacteremia. A transthoracic echocardiogram did not demonstrate vegetation or an embolic source. The source of the infection was never elucidated. Cultures later speciated to pan-sensitive Escherichia coli. Patient was discharged home after seven days and successfully completed a six- week course of antibiotic therapy with ceftriaxone.

IMPACT/DISCUSSION: Infection of the sternoclavicular joint is a rare presentation of septic arthritis occurring in $1 \%$ of the general population. Diagnosis requires a high index of suspicion particularly in healthy adults without risk factors as it typically presents subacutely with minimal symptoms: chest pain localized over the sternoclavicular joint (78\%), shoulder pain (24\%), and neck pain (2\%). Sternoclavicular joint tenderness $(90 \%)$ is the most common and reliable physical finding. Fever, leukocytosis and bacteremia are present in only about half of the cases. Risk factors include history of 
IV drug use, extra-articular infection, diabetes mellitus, trauma, and infected central venous catheter; however, no risk factors were present in $23 \%$ of cases. Methicillin-sensitive Staphylococcus aureus (49\%) is the most common pathogen in both blood and joint aspirate, while Escherichia coli (5\%) is less common. Complications include osteomyelitis, chest wall abscess, and mediastinitis. Soft tissue infection without joint involvement is amenable to medical therapy alone. One study documented a $15 \%$ failure rate for medical therapy alone, while another noted an $83 \%$ failure rate. Joint and bone involvement require surgical debridement or resection.

CONCLUSION: Early diagnosis of sternoclavicular joint septic arthritis will allow for a higher rate of success with medical management alone.

\section{STEROIDS AND SCLERODERMA RENAL CRISIS: NAVIGATING THE UNKNOWN}

Bernard Mendis; Chaya Abelow. Montefiore Medical Center, Albert Einstein College of Medicine, New York, NY. (Control ID \#3184230)

LEARNING OBJECTIVE \#1: Review the diagnostic criteria for systemic sclerosis.

LEARNING OBJECTIVE \#2: Review evidence surrounding steroid use and the risk of scleroderma renal crisis.

CASE: A 70-year-old woman with a past medical history of retinitis pigmentosa and recurrent hospitalizations for hand arthralgias of unknown etiology, on chronic Prednisone and Methotrexate, was referred to the ED by her Rheumatologist after an outpatient BMP revealed a rise from her baseline Creatinine of 0.5 to 2.1 in the setting of new onset hypertension. Prior to presentation, work up for her arthralgias had revealed a positive anti-Scl70 serology. However, given her lack of typical symptoms, she had not yet received a formal diagnosis of systemic sclerosis. On admission, the patient was treated empirically for scleroderma renal crisis with Captopril, and by withholding further prednisone therapy. Initial urine studies were remarkable for hyaline casts, hematuria, and proteinuria. Follow up renal ultrasound revealed bilateral hyperechogenicity prompting a renal biopsy, which revealed thrombotic microangiopathy, secondary renal tubular injury, and interstitial fibrosis consistent with scleroderma renal crisis (SRC). Her creatinine stabilized at 5.3 and the patient was deemed safe with a plan to closely monitor her renal function as an outpatient.

IMPACT/DISCUSSION: Systemic sclerosis (SSc) is characterized by inappropriate deposition of collagen in the skin and internal organs. The pathophysiology is not yet well understood, but has been proposed to be autoimmune, genetic, and environmental in nature. As of yet, there remains no clear consensus on the diagnostic criteria for a preliminary diagnosis of SSc. The Eular Scleroderma Trials and Research Group (EUSTAR) recently proposed a diagnostic approach requiring a combination of: a) Raynaud's Phenomenon, puffy fingers, or ANA, and b) disease specific antibodies, or microvascular changes by capillaroscopy. Initiating therapy would ultimately require a positive HRCT, PFT, or esophageal manometry study. This clinical decision making tool has not yet been validated. The role of steroids in the treatment of systemic sclerosis is controversial. Although the American College of Rheumatology cites corticosteroids (CS) as a treatment option for myalgias in systemic sclerosis, several studies have proposed an association between CS exposure and SRC. A retrospective cohort study published by Teixeira et al observed an OR of 17.4 (95\% CI 2.1-144.0) at 1 month and 24.1(95\% CI 3.0193.8) at 3 months of developing SRC post-CS exposure. This observation has been supported by other literature.

CONCLUSION: -Systemic sclerosis is a diagnosis with important implications regarding choice of therapy, morbidity and mortality. A consensus on early diagnostic criteria has not yet been reached. -Delaying corticosteroid therapy in patients with suspected autoimmune or systemic processes in favor of a more stringent diagnostic work up may be reasonable in select cases.

\section{STEVENS-JOHNSON SYNDROME: POLYPHARMACY GONE WRONG}

Nikhil Kolluri; Justin Brandler; Anjali Bhagra. Mayo Clinic, Rochester, MN. (Control ID \#3183931)

LEARNING OBJECTIVE \#1: Stevens - Johnson syndrome (SJS) is characterized as a severe widespread epidermal necrotizing disorder encompassing $<10 \%$ of the total body surface with the main cause being an idiosyncratic reaction to systemic medications

LEARNING OBJECTIVE \#2: Rapid recognition of SJS is crucial as mortality can be as high as $4-10 \%$ with therapy revolving around swift removal of the offending agent and supportive cares

CASE: A 25-year-old male presented with a 3-day history of painful oral desquamation, odynophagia, diffuse rash, and sloughing lesions on his genitals. Past medical history was significant for mitochondrial encephalomyopathy, lactic acidosis, and stroke-like episodes and seizure disorder. He was recently started on lacosamide (on chronic lamotrigine) for status epilepticus and Bactrim for prostatitis. Physical examination revealed diffuse erythematous maculopapular rash, oral candidiasis, and desquamating ulcers of the lips, oropharynx, and genitals. Labs showed a hemoglobin of $9.8 \mathrm{~g} / \mathrm{dL}$, leukocytes of $6.5 \times 10^{\wedge} 9 \mathrm{cells} / \mathrm{L}$, and lactate of $2.9 \mathrm{mmol} / \mathrm{L}$. A dermatology consult was immediately placed and lesions were biopsied. Workup was negative for blood cultures, HSV, VZV, CMV, Syphilis, Chlamydia, Gonorrhea, Tick borne panel, HIV, and Mycoplasma testing. A fungal culture of his oropharynx revealed candida dubliniensis. Pathology results confirmed Stevens-Johnson syndrome. Ultimately, Bactrim and Lacosamide were thought to be the culprit medications.

IMPACT/DISCUSSION: SJS is characterized as a severe widespread epidermal necrosis causing tissue loss at skin/mucosal surfaces. Diagnosis is based on clinical presentation and biopsy showing full thickness necrosis of the epidermis and keratinocyte apoptosis. The main cause for SJS is an idiosyncratic reaction to systemic medications, with the most common culprits being sulfonamide antibiotics, anticonvulsants (phenytoin, lamotrigine), allopurinol, nevirapine, and NSAIDs. Mycoplasma pneumoniae infection has also been reported to cause SJS. Clinical presentation generally consists of a prodrome of fever and upper respiratory symptoms with progression to ulceration of mucosal areas and painful erythematous vesiculobullous rash. Treatment relies predominantly on quick recognition, prompt withdrawal of the culprit drug, and supportive care with fluids, pain control, and treatment of any superimposed infections (staph aureus and pseudomonas). Adjunctive therapies with corticosteroids, IVIG, and cyclosporine have also shown some promise. Mortality from SJS can range from $4-10 \%$ with disease severity being the main risk factor for mortality. By reporting this classic presentation of SJS, we reinforce the typical presentation and steps for diagnosis and treatment.

CONCLUSION: This case illustrates the enigmatic way SJS can present in the setting of a complex medical history, the importance of rapid identification and management of SJS which may be potentially life threatening, and exemplifies the principle of polypharmacy and its detrimental consequences to patients.

STREPTOCOCCUS PERITONITIS AND TOXIC SHOCK SYNDROME FROM OCCULT TUBO-OVARIAN ABSCESS William R. Mazalewski ${ }^{1}$; John Ebone ${ }^{2}$; Sean Amirzadeh ${ }^{1}{ }^{1}$ University of 
South Florida, Tampa, FL; ${ }^{2}$ Manatee Memorial Hospital, Bradenton, FL. (Control ID \#3185692)

LEARNING OBJECTIVE \#1: Assess severely septic patients by performing a complete, sometimes invasive physical examination for the investigation to find a source of infection.

LEARNING OBJECTIVE \#2: Recognize that group A streptococcal bacteremia and subsequent toxic shock syndrome can arise from tubo-ovarian abscesses.

CASE: A 47-year-old female with a past medical history significant for uterine fibroids presented to the ED with a five-day history of nausea, vomiting, diarrhea, lower abdominal pain, low grade fever, fatigue, and chills. The abdominal pain was non-radiating and described as a sharp pain that was difficult to localize. On physical exam, she was tachycardic with a heart rate of $120 \mathrm{bpm}$, tachypneic at 30/min and afebrile. Abdominal exam was significant for diffuse tenderness with voluntary guarding in the lower quadrants, no rebound. Pelvic examination was within normal limits, as was the rest of the examination. Labs revealed a WBC at $9 \times 10^{\wedge} 3 / \mathrm{uL}$ with bandemia, anemia with hemoglobin of 10.5 , BUN $75 \mathrm{mg} / \mathrm{dL}$, creatinine $6.7 \mathrm{mg} / \mathrm{dL}$, and a lactic acidosis. A CT abdomen and pelvis with contrast revealed trace free fluid in the abdomen with peritoneal enhancement suspicious for peritonitis. A pelvic and transvaginal ultrasound was performed revealing a fibroid uterus without adnexal masses. Blood cultures were positive for group A beta hemolytic streptococcus sp. She was started on broad spectrum antibiotics but subsequently progressed to septic shock, requiring vasopressors, mechanical ventilatory support, as well as continuous renal replacement therapy. On hospital day 3 , a repeat pelvic exam was performed which revealed severe adnexal and cervical motion tenderness with cervical friability. An exploratory laparotomy was performed which showed a ruptured tubo-ovarian abscess. A total abdominal hysterectomy with bilateral salpingo-oophorectomy was performed. Pathology showed necrotizing bilateral fallopian tubes and uterus. Following the surgery, she was maintained on IV antibiotics and was successfully weaned from mechanical ventilation and vasopressor support, ultimately making a full recovery.

IMPACT/DISCUSSION: Tubo-ovarian abscesses (TOA) can result from pelvic inflammatory disease in a pre-menopausal woman. Organisms that are typically implicated are E. coli, Bacteroides sp, and Klebsiella sp. Cases of group A streptococcal (GAS) peritonitis from ruptured TOA have been reported, albeit rare in literature. Toxic shock syndrome is a well described complication of staphylococcal and GAS infections that are often fulminant if not recognized and treated eminently. Presenting symptoms for TOA can be broad, and when caring for a female patient with vague abdominal symptoms, a thorough physical exam can be lifesaving.

CONCLUSION: This case signifies the importance of searching for a cause of septic shock, including repeating invasive portions of a physical examination. It highlights the importance of maintaining vital physical examination skills as an internist to aide in diagnosis when caring for critically ill patients.

\section{STROKE FOLLOWING CARDIOVERSION FOR ATRIAL FIBRIL- LATION DESPITE PATIENT ON ELIQUIS FOR 2 YEARS}

Manishkumar Patel $^{1}$; Mulham Shikh Hamdon ${ }^{2}$; Anubhav Jain ${ }^{1}$; Zain Kulairi ${ }^{1}$. ${ }^{1}$ Wayne State University, Clarkston, MI; ${ }^{2}$ Wayne State University, Shelby TWP, MI. (Control ID \#3185254)

LEARNING OBJECTIVE \#1: Apixaban was approved for the prevention of stroke and systemic embolism in patients with non-valvular atrial fibrillation. This was based on the Apixaban for Reduction in Stroke and Other Thromboembolic Events in Atrial Fibrillation (ARISTOTLE) trial. Despite the use of Apixaban for 2 years of anticoagulation, we describe a patient who suffered an ischemic stroke 24 hours following cardioversion.

CASE: 70-year-old male with history of nonvalvular atrial fibrillation with CHADS-VASc of 2 on apixaban for 2 years, and essential hypertension. Due to symptomatic atrial fibrillation, electrical cardioversion was recommended. The patient was compliant with Eliquis which was confirmed with his wife. A TEE study was not performed prior to the cardioversion as the patient had been anticoagulated for the prior 2 years. The cardioversion attempt was successful and the patient was discharged home on Eliquis in normal sinus rhythm. Approximately 24 hours following the cardioversion, the patient was admitted to our hospital with altered mental status, expressive aphasia and dysarthria, with the remainder of the examination unremarkable. Computed tomography scan showed distal right middle cerebral artery infarct. Computed tomography angiography showed occlusion of the posterior branch of the right middle cerebral artery. Magnetic resonance imaging demonstrated large acute right middle cerebral artery infarct with no shift of the midline structures. Clinically it was determined that he had suffered from a cardioembolic event. Carotid duplex ultrasound showed normal bilateral carotid arteries. Echocardiogram showed ejection fraction $55-60 \%$ with no evidence of left atrial dilation or left atrial appendage.

IMPACT/DISCUSSION: In patients with atrial fibrillation, electrical cardioversion can be used to restore normal sinus rhythm. The risk of cardioversion, however, is that it is associated with an increased occurrence of dislodged thrombi originating from the left atrial appendage resulting in a thromboembolic event. The American Heart Association recommends for patients with atrial fibrillation of unknown length of time or for $>48$ hours therapeutic anticoagulation for at least 3 weeks should be given prior to and 4 weeks after cardioversion. TEE can also be used to rule out thrombi within the heart chambers before cardioversion. Cardioversion data for 577 patients from a phase III trial comparing apixaban with warfarin demonstrated no ischemic strokes or systemic 10 emboli over a 90-day follow up period. Our patient had no evidence of thrombi or echo contrast on TEE prior to apixaban being started, had been compliant with more than 3 weeks of apixaban therapy confirmed with outpatient pharmacy records, and had a low annual risk of stroke. Although no intervention can bring an event rate to zero, an event such as this is concerning.

CONCLUSION: We are reporting a case of failed apixaban therapy to prevent stroke after cardioversion despite this patient being on this medication for 2 years.

\section{STROKE VIS-à-VIS VZV: A CASE OF VZV VASCULOPATHY}

$\underline{\mathrm{Xi} \mathrm{Chu}}{ }^{1}$; Leonidas Palaiodimos ${ }^{1}$; Charles Esenwa ${ }^{2} .{ }^{1}$ Montefiore Medical Center, New York, NY; ${ }^{2}$ Montefiore Medical Center, Bronx, NY. (Control ID \#3182572)

LEARNING OBJECTIVE \#1: Recognize VZV vasculopathy as an etiology of stroke

CASE: A 70-year-old man with diabetes, hypertension, and hyperlipidemia presented with incoherent speech. On exam, he was found to have fluent aphasia with comprehension difficulty, phonemic paraphasias and right homonymous hemianopia. Labs showed HbAlc 7.9\%, LDL 145 mg/dL, ESR/ CRP normal, HIV negative, syphilis TP-PA positive. MRI brain showed acute infarction in the left parietal and occipital lobes, as well as splenium of the corpus callosum and hippocampus, and a chronic left frontal infarct. CTA head/neck showed multifocal areas of moderate to severe intracranial arterial stenoses involving the anterior and posterior circulation, including occlusion in the posterior cerebral artery. Presence of multifocal stroke along with the extensive intracranial stenoses prompted a vasculitis workup. CSF analysis 
did not show pleocytosis, was negative for HSV and VDRL, but showed CSF to serum varicella zoster virus (VZV) IgG ratio of 1:9, consistent with positive CNS VZV infection. VZV DNA was not detected in the CSF by polymerase chain reaction (PCR). The patient was treated with a two-week course of IV acyclovir for VZV.

IMPACT/DISCUSSION: Stroke is frequently encountered by the internist, who is familiar with the classic etiologies of stroke. Less common etiologies, however, include CNS vasculitides that are difficult to diagnose, especially when not associated with systemic presentation. Recent studies illuminate the prevalence of VZV vasculopathy, also termed VZV vasculitis, caused by infection of intracranial and extracranial arteries with resultant granulomatous vasculitis. Previously characterized by large artery stroke following zoster rash in immunocompromised patients, newer studies show large and small artery involvement. Further, a third of patients have no preceding rash and even in those with zoster rash, there is a four-month interval between rash and clinical CNS involvement. ESR/CRP can be normal or high. MRI shows white and grey matter involvement and highly suggestive multifocal greywhite matter junction lesions, while cerebral angiography shows focal narrowing and beading. Given the spectrum of clinical presentations, internists should consider CNS VZV vasculopathy as an etiology of stroke not only in patients with recent zoster, but also in those without systemic findings who have multifocal strokes involving grey-white matter junction and/or multifocal narrowing and beading of cerebral vessels. If suspected, CSF should be obtained and analyzed for VZV DNA by PCR and anti-VZV IgG, with antibody analysis being more sensitive. Treatment with IV acyclovir and steroids for two weeks is recommended by consensus.

CONCLUSION: Stroke associated with VZV vasculopathy can present in otherwise immunocompetent patients without preceding zoster rash. It should be included in the vasculitis workup in patients who present with stroke and areas of narrowing and beading of cerebral vessels, as there is available and effective treatment.

\section{STROKE-LIKE PRESENTATION IN A YOUNG ADULT: PML}

Aiman Zafar ${ }^{1}$; Mukesh Kumar ${ }^{1}$; Marilou Corpuz ${ }^{2} .{ }^{1}$ Montefiore Medical center, Bronx, NY; ${ }^{2}$ Montefiore Medical Center, Bronx, NY. (Control ID \#3185549)

LEARNING OBJECTIVE \#1: To recognize that progressive multifocal leukoencephalopathy (PML) can present like stroke

LEARNING OBJECTIVE \#2: To recognize that PML can be the first manifestation of HIV/AIDS

CASE: A 32 YO man with no prior medical history presented with numbness, tingling and weakness in his left arm, slurred speech and facial muscle weakness for 1 week. His exam showed left sided facial droop, weakness of left upper extremity with decreased sensations and hyperreflexia. MRI brain showed sub-acute to chronic infarcts involving right frontal lobe, centrum semiovale, corona radiata and posterior watershed territory, hence, acute stroke was diagnosed. Extensive work up was done. He was positive for HIV 1 with a CD 4 count of 73 cells/uL. Lumbar puncture was positive for JC polyoma virus PCR (3588 copies $/ \mathrm{ml}$ ). Patient was suspected to have PML although his neuro imaging features were not classic PML findings. Antiretroviral therapy (ART) was initiated. Few weeks later he was readmitted with seizures. A contrast MRI brain performed few months later revealed findings that were more consistent with PML. The follow up MRI brain remained stable and the patient showed clinical improvement in his symptoms.

IMPACT/DISCUSSION: PML is a severe, progressive and fatal demyelinating disease of the central nervous system caused by reactivation of the JC polyomavirus in immunosuppressed individuals. It is now increasingly recognized in patients with HIV and is an AIDS defining illness. It is seen in advanced stages of HIV disease with CD 4 count $<200$ cells/uL and can also herald the manifestation of HIV. Clinical features overlap with stroke and include altered mental status, hemiparesis/monoparesis, gait ataxia, hemianopia and diplopia. Seizures are often seen. On neuroimaging, the lesions are bilateral, asymmetric, multifocal, preferentially involving the periventricular areas and subcortical white matter of frontal/occipital/parietal lobes and do not exhibit mass effect or contrast enhancement. On CT PML lesions appear as hypodense patchy or confluent white matter regions and on MRI brain as areas of increased signal on T2-weighted images and fluid attenuated inversion recovery (FLAIR) sequences. Although brain biopsy is gold standard for making a diagnosis, the presence of JC virus DNA in CSF using PCR and clinical and neuroimaging findings are sufficient to diagnose PML. There is no specific therapy other than improving host's immune response via continuation or initiation of an effective ART.

CONCLUSION: Strokes in patients less than 40 years old are uncommon except for patients with underlying risk factors for atherosclerotic heart disease. Other causes include vasculitis or infections such as syphilis and HIV. Since PML symptoms can overlap with stroke and initial neuro imaging findings can also be misleading, it is crucial to consider HIV testing in a young patient with no known risk factors presenting with stroke like symptoms. High clinical suspicion in the right clinical context is imperative to proper diagnosis.

\section{SUBCUTANEOUS EMPHYSEMA AS A COMPLICATION OF PUL- MONARY ASPERGILLOMA}

Aya Shnawa $^{1}$; Mohammad Almeqdadi ${ }^{2} .{ }^{1}$ Jordan University of Science and Technology, Allston, MA; ${ }^{2}$ St. Elizabeth's Medical Center, Brighton, MA. (Control ID \#3163514)

LEARNING OBJECTIVE \#1: Pulmonary aspergilloma could rarely invade the chest wall without evidence of invasive aspergillosis

CASE: The patient is a 73-year-old male with past medical history of severe chronic obstructive pulmonary disease (COPD), coronary artery disease, 50pack-year smoking and remote left upper non-small cell carcinoma treated with chemoradiation, who presented with one-week history of shortness of breath and hemoptysis. Patient denied any fever, chills, rigors, or night sweats. He had seen his primary care physician who prescribed him a course of antibiotics and a prednisone taper, with no improvement. On exam, he was found to be afebrile, tachycardic, tachypneic, hypoxic ( oxygen saturation of $75 \%$ on 2 liters of nasal cannula), afebrile and normotensive. His lung exam was pertinent for rales and rhonchi bilaterally in all lung fields. Laboratory studies showed leukocytosis of $21.1 \mathrm{k} / \mathrm{uL}$ with $86 \%$ neutrophils, hemoglobin of $7.0 \mathrm{mg} / \mathrm{dL}$, and platelets of $304 \mathrm{k} / \mathrm{uL}$. Beta-1,3-D-Glucan levels of $<31 \mathrm{pg} /$ $\mathrm{mL}$, and galactomannan EIA test index of $<0.5$. Chest $\mathrm{x}$-ray showed a left apical cavitary lesion with increasing surrounding soft tissue loss. A CT of the chest showed a left apical cavity with a lobulated ring of soft tissue and a $4-\mathrm{cm}$ masslike abnormality. He was stabilized and started on antibiotics for presumed pneumonia without improvement. He had an episode of excessive coughing, after which a chest x-ray showed extensive left chest wall subcutaneous emphysema. The cavitary lesion aspirate grew Aspergillus fumigates, he was subsequently started on voriconazole therapy.

IMPACT/DISCUSSION: Aspergillomas are fungal balls arising typically within the frame of a previous pulmonary cavity. Complications may include massive hemoptysis and progression to invasive aspergillosis. Here, we present a case of a pulmonary aspergilloma which was complicated by chest wall subcutaneous emphysema. An aspergilloma consists of Aspergillus 
fungal hyphae, fibrin, and mucus in an established lung parenchymal cavity. It has a predilection to occur in patients with COPD with history of a cavitary lesion due to tuberculosis, sarcoidosis, or necrotizing infections. Rarely does an aspergilloma progress to invasive aspergillosis. Here, we presented a case of an aspergilloma within a previous pulmonary cavity that caused a cavitarysubcutaneous fistula in the setting of excessive cough resulting in massive chest wall subcutaneous emphysema.

CONCLUSION: This shows pulmonary aspergilloma could rarely invade the chest wall without evidence of invasive aspergillosis, and sheds light on subcutaneous emphysema as a potential rare complication of aspergillomas without prior trauma.

SUCCESSFUL PREGNANCY IN A NULLIPAROUS, MULTIGRAVIDA ESRD PATIENT WITH HEMODIALYSIS MARYAM MUBASHIR MD, ALINA WASIM MD SWATI PATHAK MD, ISAIARASI GNANASEKARAN MD

Maryam Mubashir; Alina Wasim. Lincoln Medical center, Bronx, NY. (Control ID \#3185386)

LEARNING OBJECTIVE \#1: Early recognition of pregnancy and initiation of dialysis in ESRD

LEARNING OBJECTIVE \#2: Optimal dialysis modality \& regimen

CASE: 34-year-old female with uncontrolled DM-1 diagnosed at 12, complicated with nephropathy, retinopathy, neuropathy, neurogenic bladder, gastroparesis, on insulin pump. With 2 prior abortions and a stillbirth. Her CKD progressed from stage 4 to stage 5 after the still birth and AV fistula was created. Subsequently she was found to be 8 weeks pregnant on Sonogram. Her BUN was in range of 33-47 mg/dL during first few weeks of her pregnancy. Maintenance hemodialysis was initiated thrice/week for one week (13 hours total). It was subsequently switched to 6 days/week to avoid hemodynamic instability and to maintain steady biochemical profile. She got 122 sessions in total avg. duration 2.5 hrs. Her BP was controlled with MDopa. Glucose was optimized with Insulin pump. Her anemia was managed with IV iron and subsequently with EPO. She received folic acid and Vit D. Predialysis BUN was maintained at $<30 \mathrm{mg} / \mathrm{dL} \& \mathrm{Cr}<4 \mathrm{mg} / \mathrm{dL}$. At 31 weeks patient underwent elective $\mathrm{C}$-section due to polyhydramnios. She delivered a live, healthy $5 \mathrm{lb}$ baby with Apgar score of 8 and 9 at $1 \& 5$ min. Both mother and baby did well during the follow up period.

IMPACT/DISCUSSION: The incidence of pregnancy in women on chronic dialysis is $1-7 \%$ (3). Evidence shows better obstetrical outcomes if women start dialysis after conceiving as compared to getting pregnant while on treatments. Preferred dialysis regimen entails increments in frequency and duration of treatment (5). Shorter and frequent HD was chosen in light of successfully using similar strategy with other cases in past. This resulted in a good maternal and fetal outcome even though CKD had progressed CONCLUSION: No clear superiority of different modalities of dialysis has been established as long as the objective of optimizing the blood chemistry is achieved without drastic changes in fluid shift and balance $(5,8,10)$. Patients require frequent and close monitoring with prompt optimization of maternal $\mathrm{BP}, \mathrm{Hb}$, glucose and chemistries using dialysis along with medications management to ensure successful outcome (3). More over pregnancy outcomes are likely to be more favorable in women who start dialysis after conception (12)

SUGAR, SUGAR HOW YOU GET SO LOW: INSULINOMA, A CASE REPORT

Julia E. Girdler; Sienna Kurland; Alicia Cowley. New York University, New York, NY. (Control ID \#3170152)
LEARNING OBJECTIVE \#1: Recognize the broad differential for hypoglycemia

LEARNING OBJECTIVE \#2: Diagnose an insulinoma

CASE: A 33-year-old male with no significant past medical history was admitted after being found unconscious at his desk with a blood glucose of $28 \mathrm{mg} / \mathrm{dL}$. He reported multiple episodes of confusion, slurred speech, and unsteady gait in the context of fasting that was relieved with meals. He reported almost a decade of high sugar intake - consuming $1.5 \mathrm{~L}$ of soda and multiple candy bars daily - to prevent lightheadedness. He denied medication or drug use. On admission, he was hemodynamically stable with an unremarkable physical exam. Laboratory values were significant for glucose of $156 \mathrm{mg} / \mathrm{dL}$ and then $34 \mathrm{mg} / \mathrm{dL}$ after a 5-hour fast. Further work-up revealed insulin of $21 \mathrm{uIU} / \mathrm{mL}$, c-peptide of $2.4 \mathrm{ng} / \mathrm{mL}$ (RR 0.8- 3.5), B-hydroxybutyric acid $<0.2 \mathrm{mmol} / \mathrm{L}$, hemoglobin Alc of $4.3 \%$, a negative sulfonylurea panel, insulin antibody, toxicology screen, and ethanol level, normal thyroid stimulating hormone, liver function tests, and complete blood count, and borderlinenormal ACTH stimulation test. Magnetic resonance imaging revealed a hyperenhancing mass in the head of the pancreas. He underwent local resection and enucleation of the mass; pathology confirmed an insulinoma.

IMPACT/DISCUSSION: Insulinomas are rare neuroendocrine tumors that classically present with Whipple's Triad: neuroglycopenic symptoms, hypoglycemia, and rapid resolution of symptoms with glucose. Due to their rarity, a broad differential diagnosis for hypoglycemia should be considered first. This includes sepsis, starvation, alcohol-induced hypoglycemia, adrenal insufficiency, hepatic and renal failure. Hyperinsulinemic hypoglycemia narrows the differential to autoimmune hypoglycemia, exogenous insulin injection, secretagogue ingestion, and insulinoma. Anti-insulin antibody and sulfonylurea panel further narrow the differential. Patients with a suspected insulinoma should undergo a supervised fast with serum levels of insulin, c-peptide, and B-hydroxybutyrate drawn once glucose levels reach $<50 \mathrm{mg} / \mathrm{dl}$ for optimal specificity and sensitivity. An insulinoma is suggested by elevated insulin ( 3 $\mathrm{uIU} / \mathrm{mL}$ ) and c-peptide ( $0.6 \mathrm{ng} / \mathrm{mL}$ ) plus low B-hydroxybutyrate ( 2.7 $\mathrm{mmol} / \mathrm{L}$ ). Localization of the tumor is done with computed tomography, magnetic resonance imaging, or endoscopic ultrasound, which has the highest sensitivity and specificity for insulinoma. A selective arterial calcium stimulation test can also aid in localization. The majority of insulinomas are benign and can surgically resected

CONCLUSION: When a patient presents with Whipple's triad, the key to diagnosis is thorough history taking, observation, and diagnostic testing. Essential questions include timing of symptoms, comorbidities, medications, and social history. During a supervised fast, inappropriately elevated levels of insulin and c-peptide with low B-hydroxybutyrate establish the diagnosis of insulinoma. Once imaging confirms the diagnosis, benign solitary insulinomas can be surgically resected.

\section{SUSPECTING SARCOIDOSIS: AN UNUSUAL CAUSE OF HEART FAILURE}

Callie Plafkin. University of Wisconsin Hospital and Clinics, Madison, WI. (Control ID \#3163576)

LEARNING OBJECTIVE \#1: Recognize when to consider infiltrative cardiac disease and pursue further diagnostic work-up

LEARNING OBJECTIVE \#2: Identify the role of various imaging modalities in the diagnosis and management of cardiac sarcoidosis

CASE: A 43-year-old male with a history of hypertension, obstructive sleep apnea, gout, and third-degree heart block status post dual-chamber pacemaker placement was admitted to the cardiology service in 12/2017 for management of new acute decompensated systolic heart failure. Prior to presentation, in 
7/2014, he was found to have bradycardia and complete heart block, for which he received a pacemaker. At that time a transthoracic echocardiogram (TTE) showed normal left ventricular ejection fraction (LVEF) of 60-65\%. Myocardial stress testing in 2014 and 2016 showed no evidence of ischemia. In late 2017, he developed orthopnea, paroxysmal nocturnal dyspnea, leg swelling, and progressive exertional dyspnea precipitated by minimal activity. TTE showed severe dilated cardiomyopathy and biventricular systolic dysfunction with LVEF of $15 \%$. Exam was notable for bibasilar pulmonary rales and bipedal edema. Labs were notable for elevated BNP of $2762 \mathrm{pg} / \mathrm{ml}$ (normal range 0-99). Lyme and HIV serologies were negative. Cardiac MRI (cMRI) demonstrated multiple patchy areas of delayed gadolinium enhancement suggestive of cardiac sarcoidosis. Myocardial FDG PET showed extensive areas of abnormal FDG uptake in both ventricles, consistent with active disease. He lacked other clinical or radiographic evidence of extracardiac sarcoidosis aside from intrathoracic lymphadenopathy on PET. He underwent pacemaker upgrade to cardiac resynchronization therapy defibrillator (CRTD). Signs and symptoms of decompensated heart failure improved with diuresis. Immunosuppressive therapy was initiated with prednisone and mycophenolate, with modest improvement in LVEF (25\% in 10/2018).

IMPACT/DISCUSSION: The majority of patients with sarcoidosis present with lung, skin, or ocular involvement, while only a small proportion (estimated 5\%) have clinically-significant cardiac sarcoidosis (CS). CS has a wide range of presentations and is often diagnosed late. Arrhythmias and noncoronary distribution of wall motion abnormalities on TTE can suggest the diagnosis, but are insensitive and nonspecific. cMRI has a high sensitivity for CS; it can detect edema associated with inflammation, and fibrosis, suggested by late gadolinium enhancement. FDG PET can quantify metabolically active lesions, helping differentiate scar from active disease and permitting monitoring of treatment response.

CONCLUSION: Our patient experienced a three-year delay in diagnosis of CS following presentation with complete heart block, likely due to paucity of extracardiac manifestations, CS rarity, and insensitivity of initial imaging. Had infiltrative disease been considered and evaluated with early cMRI in this young patient with unexplained conduction abnormality and no evidence of ischemia, progression to florid systolic heart failure might have been prevented.

\section{SYMPTOMATIC COPPER DEFICIENCY IN A PATIENT WITH REMOTE GASTRIC BYPASS SURGERY}

Emily C. Atkinson; Morris Jrada; Myint Aye; Charles Okamura. NYU Langone Hospital - Brooklyn, Brooklyn, NY. (Control ID \#3184951)

LEARNING OBJECTIVE \#1: Recognize vitamin deficiencies in a gastric bypass patient

LEARNING OBJECTIVE \#2: Treat copper deficiency

CASE: A 61 year old female with a past medical history of depression, hypothyroidism, dementia, profound insomnia, and remote history of gastric bypass presented with chronic dizziness and orthostatic hypotension with recurrent falls. She had multiple hospital admissions for dizziness, gait instability and falls. Associated symptoms included nausea and vomiting, fatigue, weakness, hypotension, hypoglycemia with low insulin levels, and worsening tingling and numbness in both legs. She denied using zinc containing denture cream or cold tablets. In the $\mathrm{ED}$, her vital signs were stable and blood work was significant for pancytopenia, with a WBC count of 2.2, hemoglobin of 9.4 and platelets of 137 . The levels of many vitamins and minerals were checked given her history of gastric bypass and she was found to have a normal iron level with transferrin saturation of 33\%, normal levels of vitamins B1, B6, B9, B12, and selenium (58) and low levels of copper, ceruloplasmin, zinc, and vitamin D. CT and MRI of the brain were negative for acute pathology but significant for severe left temporal atrophy and overall global volume loss. Following admission, she was given intravenous fluids for persistent hypotension with eventual resolution after increasing her home dose of fludrocortisone and starting her on vitamin supplementation for her noted deficiency. She was discharged on a multivitamin with minerals daily, 50,000 units of Vitamin D weekly, 220mg of zinc sulfate twice daily, and 4mg of copper three times a day with close bariatric specialist follow up.

IMPACT/DISCUSSION: Copper plays an essential role in the synthesis of many proteins and its deficiency can cause a variety different symptoms. Symptomatic copper deficiency is rare but can be seen in patients with a remote history of bariatric surgeries. In these patients, absorption of many minerals, including copper, is disrupted and supplementation may be necessary to prevent deficiency. This patient had been suffering from pancytopenia with a normal iron panel, which can be due to copper deficiency. Copper is a required cofactor in iron oxidation during hematopoiesis and its deficiency therefore results in anemia. Patients with hypocupremia may present with gait abnormalities from myelopathy, as noted in our patient. Additionally, imaging showed cerebral atrophy, possibly related to demyelination, which can be seen in patients with Menkes Disease, a mutation causing defects in copper absorption. Despite having these symptoms for years, her copper levels had not been checked prior, which delayed the diagnosis of copper deficiency.

CONCLUSION: Many of the symptoms of copper deficiency are nonspecific, which may delay diagnosis until after many hospital re-admissions. It is essential to always consider copper deficiency in a patient with chronic neurologic symptoms and anemia with a remote history of gastric bypass surgery.

\section{SYNTHETIC CANNABINOID USE ASSOCIATED WITH ACUTE KIDNEY INJURY}

Nkiru Osude ${ }^{1}$; Anthi Katsouli ${ }^{2}$. ${ }^{1}$ Loyola Medical Center, Crystal Lake, IL; ${ }^{2}$ Loyola University Medical Center, South Barrington, IL. (Control ID \#3184674)

LEARNING OBJECTIVE \#1: Recognize acute tubular necrosis as a complication of synthetic marijuana inhalation.

CASE: A 22 year old with no past medical history but with daily marijuana use initially presented to the outside hospital emergency department (ED) with severe diffuse abdominal pain and intractable nausea and vomiting. Laboratory during his ED admission was notable for a BUN/Cr ratio of 25/1.77. He was discharged home presented to our hospital four days later with similar complaints. On examination his blood pressure was 170/105, heart rate was 75 , respiration rate was 20 with oxygen saturation $100 \%$ on room air. He had a loud mid systolic murmur at the left sternal border associated with a thrill; elevated jugular venous pressure with no peripheral edema. Laboratory was consistent with Cr of 14.7, BUN of 71, HCO3 of 19, and an anion gap of 15. Potassium was 5.1 with phosphorus of 5.7. Urine analysis was positive for large amount of blood, leukocyte esterase, and $>180$ white blood cells. Urine drug screen was positive for cannabinoids. The remaining laboratory tests including HIV, ANA, ANCA, complement levels, hepatitis serologies, extractable nuclear antigen panel were negative. Renal ultrasound demonstrated normal renal size with increased renal echogenicity. CT Abdomen and Pelvis demonstrated extensive perinephric fat surrounding the right kidney. Transthoracic echocardiogram demonstrated normal left ventricular ejection fraction with right ventricular enlargement. No valvular dysfunction was identified. Biopsy of right kidney demonstrated severe acute tubular injury with abundant granular casts. Due to patient's anuria with worsening acidosis dialysis was initiated. He was discharged with close nephrology follow up for daily dialysis 
monitoring. A week later after his discharge he began to make urine and his creatine decreased to 1.31 without need for continuation of dialysis.

IMPACT/DISCUSSION: This case highlights the severity of the current epidemic of synthetic marijuana toxicity, and the need for rapid, reliable testing to allow for in hospital diagnosis of the inciting agent. The patient's initial symptomology and the rapid improvement of symptoms without treatment interventions in the setting of marijuana use makes synthetic marijuana as the causative agent that lead to the patient's ailments.

CONCLUSION: Synthetic marijuana has emerged as a new recreational drug that's toxicities have begun to plague hospitals across the United States. The physiologic effects of synthetic marijuana are similar to that of marijuana, but with more serious toxicities that range from neuropsychiatric disorders, cardiovascular side effects, coagulopathies, to acute tubular necrosis. In 2018 in Illinois alone over 250 cases of suspected synthetic cannabinoid toxicity documented. Synthetic marijuana does not appear on urine drug screen and there is not a quick and reliable screening test that physicians may use to diagnose this intoxication while in the hospital.

\section{SYSTEMIC LUPUS ERYTHEMATOSUS: A PANCYTOPENIC AND PANCREATIC PRESENATION}

Bilawal Ahmed; Shil Shah; Omar Chaudhary; Firas Siddique; Mazhar Mustafa; Byron Okwesili. American University of Antigua, Brooklyn, NY. (Control ID \#3140674)

LEARNING OBJECTIVE \#1: Diagnose Systemic lupus erythematosus with pancreatitis and pancytopenia as initial presentation

CASE: 15 yo $\mathrm{F}$ presented with abdominal pain, nausea, vomiting \& nonbloody diarrhea which began 4 days prior to admission; localizing to RUQ on day 2. Associated symptoms of nonbloody diarrhea \& nonbloody/ nonbilious vomit for 4 days. No contributory PMH or PSH. Denies any drug allergies. General physical examination revealed a fine lacy rash on all 4 extremities. Vitals T 37.9, HR 104, RR 18, BP 99/58. No guarding/rigidity \& no masses/organomegaly appreciated. $\mathrm{CBC}$ revealed profound pancytopenia with a platelet count of $115(150-400 \times 103 / \mathrm{uL})$, hemoglobin $8.5(12-15 \mathrm{~g} / \mathrm{dL})$ and white blood cell 1.5 (4.5-13 x103/uL). Lipase was $489 \mathrm{u} / \mathrm{L}$ and $\mathrm{LDH}$ was 2392 u/L. Other labs, including CMP, lipid panel, CRP \& pregnancy test all within normal limits. Patient admitted with diagnosis of pancreatitis with pancytopenia \& supportive treatment with investigative studies initiated. BM biopsy unequivocal. ID consulted. New laboratory test results indicated positive IgG \& IgM antibodies to Brucella, Rickettsia, Ebstein Barr Virus \& Parvovirus, complicating the diagnosis. Started on doxycycline empirically. Rheumatology consulted most likely etiology to be SLE; await Lupus Panel. ESR was $74 \mathrm{~mm} / \mathrm{hr}$, + antinuclear antibody $1 / 640,+$ antidoublestranded DNA Antibody 1/320, + anti Smith antibody, - rheumatoid factor, C3 $<40 \mathrm{mg} / \mathrm{dL}$, Lipase $2784 \mathrm{u} / \mathrm{L}$, FOBT positive. Following lab results \& clinical course, the diagnosis of Systemic Lupus Erythematosus made on day 4 of admission. Steroids initiated \& status observed closely. Lipase continued to rise to 8,136 $\mathrm{u} / \mathrm{L}$ which prompted an MRCP showing extensive changes of pancreatitis with upper abdominal fluid, no organized collection or dilation of ducts. Also noted was extensive wall edema of the gallbladder with no filling defect. Lipase began trending down on day 2 of steroids. CBC corrected to WNL. Patient reported feeling better \& continued improving.

IMPACT/DISCUSSION: Acute pancreatitis occurs in $2-8 \%$ of patientd in the setting of an active SLE flare [1,2]. The initial presentation of pancreatitis is similar to patients without SLE with an increase in lipase and LDH. The use of IV glucocorticoids or azathioprine has been shown to reduce mortality in SLE associated pancreatitis \& an overall decrease in mortality from $61 \%$ to $20 \%$ [3]. Pathogenesis of SLE leading to acute pancreatitis is not well understood but proposals have identified vascular damage arising from vasculitis \& a concurrent thrombosis as a potential cause [4].

CONCLUSION: The case aims to facilitate diagnosticians in their evaluation of patients presenting with rare manifestations of SLE. SLE should be considered in cases of pancreatitis when common etiologies have been ruled out and symptoms are not residing despite appropriate treatment. In regards to hematologic abnormalities, SLE can cause various cytopenias as an initial presentation; pancytopenia has been reported fewer times in the literature.

\section{TAKAYASU'S ARTERITIS PRESENTING AS VASCULITIC NEU- ROPATHY}

Anna Zemke; Shilpa Sridhar; Xiaomei Meng. The Ohio State University College of Medicine, Columbus, OH. (Control ID \#3183367)

LEARNING OBJECTIVE \#1: Maintain a broad differential when atrial occlusion is demonstrated on imaging, especially if hypercoagulability workup is unremarkable.

LEARNING OBJECTIVE \#2: Recognize that nerve pain could be an insidious presentation of Takayasu's Arteritis, and that neuropathic pain secondary to ischemic nerve damage is possible.

CASE: A 35-year-old female with a history of carpal tunnel syndrome, recent fall, and hypertension presented to the emergency department with chest pain and left-sided neck and shoulder pain with numbness. Physical exam demonstrated asymmetric upper extremity pulses. CT was performed to rule out pulmonary embolism, but demonstrated occlusion of the left subclavian artery. CT angiography showed partial left subclavian occlusion and left brachial artery dissection. MRI confirmed these. Thrombectomy was attempted but no thrombus was retrieved. The brachial artery had extreme intimal thickening and was chronically occluded. Hypercoagulability workup was negative. Rheumatologic workup demonstrated positive dsDNA but negative ANA by IFA. Her age, gender, symptoms, and physical exam are consistent with Takayasu's Arteritis. This patient met 4 out of 6 American College of Rheumatology criteria for TA. Presence of three or more of these criteria has a sensitivity of $90.5 \%$ and specificity of $97.8 \%$ for TA. The patient was discharged with prednisone and aspirin daily.

IMPACT/DISCUSSION: This patient's symptoms of left-sided neck and shoulder pain with left upper extremity numbness can be explained by acute on chronic occlusion of large arteries. Interestingly the patient had a 4 year history of arm weakness and numbness which had been diagnosed as Carpal Tunnel Syndrome. EMG had shown median neuropathies, right ulnar neuropathy, and diminished ulnar sensory amplitudes, consistent with a lengthdependent neuropathy. The patient underwent bilateral carpal tunnel release 1 year prior to presentation, but her symptoms worsened. Her progression makes her initial diagnosis doubtful. It is more likely that her symptoms are attributable to a neuropathy associated with TA. This condition has not been previously reported based on our review. Inflammation of the walls of neural and epineural arteries is the main pathophysiological feature of vasculitic neuropathy. Optic neuropathy has been shown with TA, secondary to acute ischemia of the short posterior ciliary artery. Other specific mononeuropathy has not been shown previously. Unfortunately biopsy was not obtained, but if active inflammation persists her symptoms could be improved with steroid therapy. CONCLUSION: 1. TA is a systemic large-vessel vasculitis of unknown etiology that often affects young women. Here we report a case TA confounded by peripheral neuropathy. 2. Clinicians should have heightened suspicion for TA in young patients with symptoms of limb pain, lightheadedness and unexplained falls. 3. Nerve pain in TA could be secondary to ischemic nerve damage. It is possible for neuropathy to be the initial symptom of an insidious presentation of TA. 
TAKES A LONG TIME TO GET DRESSED WHEN IT'S COLD Jennifer Shiroky $^{1}$; Jarratt Pytell' ${ }^{2}{ }^{1}$ Johns Hopkins Bayview Medical Center, Baltimore, MD; ${ }^{2}$ Johns Hopkins Universtiy, Baltimore, MD. (Control ID \#3167488)

LEARNING OBJECTIVE \#1: Discuss an atypical presentation of Drug Reactions Eosinophilia with Systemic Symptoms syndrome (DRESS)

LEARNING OBJECTIVE \#2: Appreciate cognitive biases that lead to diagnostic delays

CASE: $\mathrm{Mr} \mathrm{V}$ is a 61 yo man with a history of schizophrenia, bipolar disorder, intellectual disability with a speech impediment, who presented with one week of worsening shortness of breath and cough, and one month of diffuse body rash and anasarca. Initial report was one month of dry, peeling skin despite using lotions daily. The rash was accompanied by progressive swelling throughout his body and chills, wearing 4-5 layers of clothing to feel comfortable. He denied recent changes to his home medications, which included lurasidone and aripiprazole for the last year. At presentation, his temperature of $32.5^{\circ} \mathrm{C}$ and his physical exam demonstrated a full body dry desquamating rash with excoriations, diffuse non-pitting edema, diffuse lymphadenopathy, and diminished breath sounds with an expiratory wheeze. Labs were notable for eosinophilia and monocytosis, a normocytic anemia, acute kidney injury, and proteinuria. CT of the chest, abdomen and pelvis revealed diffuse lymphadenopathy and right upper and lower lobe ground glass opacities. He was admitted for evaluation and management of hypothermia with initial concerns for an infectious process, drug reaction, or hematologic malignancy. He was treated empirically for pneumonia without improvement. Dermatology consult initially described skin findings as "dermatitis of neglect" with no evidence of a primary cutaneous process, findings to suggest inflammatory lesions, or drug reactions and declined to do a biopsy. Family collateral reported good personal hygiene and no changes to his lifestyle supporting "dermatitis of neglect." Concern for cutaneous T-cell lymphoma or other lymphoproliferative process prompted an inguinal node resection, which revealed necrotizing granulomatous lymphadenitis with normal lymph node architecture. Blood work revealed an IgG lambda monoclonal gammopathy, normal flow cytometry and T-Cell receptor testing consistent with polyclonality. Repeat dermatology consult resulted in a skin biopsy revealing subacute/chronic spongiotic dermatitis. In the setting of negative infectious and oncologic work up, aripiprazole and lurasidone were discontinued for presumed DRESS syndrome with full resolution of his symptoms.

IMPACT/DISCUSSION: DRESS is characterized by fever, a spectrum of rashes, eosinophilia, and multi-organ damage. $\mathrm{Mr} \mathrm{V}$ experienced diagnostic delay due to an atypical presentation with hypothermia, subacute course of symptoms, and no recent medication changes. This contributed to a variety of diagnostic cognitive biases including the authority bias, attribution effect, and anchoring.

CONCLUSION: This case is a lesson in maintaining a broad differential diagnosis throught the diagnostic and management process, and the importance of encouraging an environment where providers feel comfortable and empowered to question the diagnostic reasoning of colleagues.

\section{TAKING HEPARIN MONITORING TO LEVEL XA}

John Xie. Tulane University School of Medicine, New Orleans, LA. (Control ID \#3184143)

LEARNING OBJECTIVE \#1: Distinguish between the two main methods of monitoring heparin

LEARNING OBJECTIVE \#2: Accurately diagnose heparin resistance using the anti-Xa level
CASE: A 42-year-old woman in the intensive care unit with chronic pancreatitis and bacteremia was found to have a massive pulmonary embolus (PE) and treated with tPA. Heparin drip was then initiated and monitored using anti$\mathrm{Xa}$ levels per hospital protocol. The anti-Xa levels were measured every six hours and returned with values of $0.09 \mathrm{IU} / \mathrm{mL}-0.18 \mathrm{IU} / \mathrm{mL}$ (therapeutic: 0.30 $0.70 \mathrm{IU} / \mathrm{mL}$ ) despite increasing doses of heparin (up to 35,000 IUs/day). Fresh frozen plasma was administered, which transiently increased anti-Xa level to $0.46 \mathrm{IU} / \mathrm{mL}$, but then decreased to $0.17 \mathrm{IU} / \mathrm{mL}$. Treatment was changed to argatrobran and aPTT reached therapeutic levels in less than 24 hours. The ATIII activity level returned low at 26\% (Ref range: $87-130 \%$ ), confirming a diagnosis of heparin resistance due to antithrombin III (ATIII) deficiency.

IMPACT/DISCUSSION: Laboratory monitoring of heparin is traditionally done using the activated partial thromboplastin time (aPTT). The aPTT is a clot-based test that measures the intrinsic and common pathways of the coagulation cascade. Falsely elevated aPTT levels can result from coadministration of warfarin, antiphospholipid antibodies, and factor deficiencies. Falsely depressed aPTT levels can occur with elevated factor VIII and fibrinogen. The anti-Xa assay is a chromogenic assay that directly measures the activity of activated factor X through a spectrophotometer. It is a more specific test for measuring heparin activity than aPTT and is not affected by the other clinical factors mentioned above. Heparin resistance can be considered with a sub-therapeutic response after using 35,000 IUs of heparin per day.[1] The most common cause of heparin resistance is ATIII deficiency but can also be caused by elevated acute phase reactants, particularly factor VIII and fibrinogen. Elevated factor VIII levels are often present in critically ill patients, which will falsely shorten the aPTT level.[2] This makes it difficult to evaluate whether a low aPTT value after heparin administration is due to true resistance (ATIII deficiency) or a pseudo-resistance (elevated factor VIII).[3] Heparin administration can decrease ATIII levels by as much as $30 \%$ due to increased clearance, which can result in falsely low values.[4] Therefore, the anti-Xa level is the most specific test for detecting heparin resistance. In ATIII deficiency, fresh frozen plasma (FFP) challenge will transiently replete the low ATIII level briefly results in a therapeutic anti-Xa. [1] https://www.ncbi. nlm.nih.gov/pubmed/19533051 [2] https:/www.ncbi.nlm.nih.gov/pubmed/ 8778420 [3] https://www.ncbi.nlm.nih.gov/pmc/articles/PMC5998275/ [4] https://www.ncbi.nlm.nih.gov/pmc/articles/PMC5998275/

CONCLUSION: The anti-Xa level is a more specific for diagnosing heparin resistance and can also distinguish between true resistance and pseudoresistance.

\section{TAKOTSUBO CARDIOMYOPATHY ASSOCIATED WITH HYPO- GLYCEMIA}

Soumya Mamidi ${ }^{1}$; Lakshmi Priyanka Mahali ${ }^{2}$; Kristin A. Swedish ${ }^{2}$. ${ }^{1}$ Montefiore medical center, Bronx, NY; ${ }^{2}$ Montefiore Medical Center, Bronx, NY. (Control ID \#3186249)

LEARNING OBJECTIVE \#1: -Recognize hypoglycemia as a potential cause of Takotsubo cardiomyopathy (TCM).

LEARNING OBJECTIVE \#2: -Differentiate TCM from myocardial infarction (MI)

CASE: A 88 year old man with Type II Diabetes Mellitus(T2DM) on insulin and chronic kidney disease (CKD III) was admitted for altered mental status after being found confused on floor. Noted to be hypoglycemic by paramedics with a blood glucose level of $50 \mathrm{mg} / \mathrm{dl}$. Initial labs showed elevated Creatinine kinase(ck) and troponins, Electrocardiogram (EKG) showed non-specific repolarization changes, raising concern for Acute Coronary Syndrome (ACS). Echocardiogram (ECHO) showed severely reduced Ejection Fraction (EF\%) OF $20 \%$ with a large region of apical akinesis, suspicious for TCM. 
Patient's hospital course was complicated by recurrent episodes of hypoglycemia as low as $27 \mathrm{mg} / \mathrm{dl}$. Myocardial perfusion scan obtained on Day 7 of hospitalization showed significant improvement in EF to $45 \%$ and showed only a small area of apical akinesis. Based on above findings, patient was diagnosed with Takotsubo cardiomyopathy associated with hypoglycemia.

IMPACT/DISCUSSION: TCM known as stress induced cardiomyopathy (SICM) is a syndrome characterized by transient regional systolic dysfunction of the left ventricle, mimicking MI. The pathogenesis is not well understood. One of the mechanisms suggest that it may be caused by diffuse catecholamine-induced microvascular spasm or dysfunction, resulting in myocardial stunning or by direct catecholamine-associated myocardial toxicity. Hypoglycemia is thought to be one of the physical stresses triggering catecholamine release. In patients with T2DM and CKD, hypoglycemia can be due to the potentiating effect of insulin in the setting of impaired renal function Hypoglycemia is a relatively common case in emergency department (ED); However, wall motion abnormalities are not usually expected in these patients. Thus, undiagnosed self-limited TCM cases are possible among them. This might warrant an ECHO as not to overlook TCM in the ED TCM can be differentiated from MI by absence of obstructive coronary disease. If coronary disease is found, the diagnosis of stress cardiomyopathy can still be made if the wall motion abnormalities are not in the distribution of the coronary disease. Management of patients with SICM is overall supportive and conservative. The prognosis for SICM is good without management and cardiac function recovers during less than 1 month

CONCLUSION: 1.Careful history taking is important in evaluation of patients presenting with positive cardiac bio-markers. 2.Insulin should be used cautiously in patients with CKD to prevent hypoglycemic episodes, which can lead to severe adverse effects including SCM.

\section{TAKOTSUBO CARDIOMYOPATHY FROM METHAMPHET- AMINE RELATED SEIZURE}

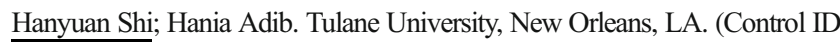
\#3186376)

LEARNING OBJECTIVE \#1: 1. Diagnose seizure related takotsubo cardiomyopathy, and the dangers leading to sudden death

LEARNING OBJECTIVE \#2: 2. Recognize the importance of recognizing cardiac complications such as neurogenic stunned myocardium in the ICU setting

CASE: A 62-year old postmenopausal female with a history of hypertension and COPD initially presented with seizures to an outside hospital. Was loaded with levetirecetam and midazolam but became apneic and was emergently intubated. Drug screen positive for benzodiazepines and mass spectrometry positive for methamphetamine. Initial laboratories significant for leukocytosis (WBC at 16), lactate of 2.1, and troponin of 0.7. EKG showed normal sinus rhythm, 1mm ST elevation in V2, $1.5 \mathrm{~mm}$ ST elevation in V3, T-wave inversions in inferolateral leads. There was concern for acute coronary syndrome so the patient was aspirin loaded (600 mg), clopidogrel loaded (300 $\mathrm{mg}$ ), and started on heparin drip. Transthoracic echocardiogram showed severely decreased ejection fraction $<20 \%$, apical akinesis and ballooning, with only the base of the left ventricle contracting. Troponin peaked at 1.15 and Takotsubo was strongly suspected. When the patient was hemodynamically stable and extubated, a right and left heart catheterization was done for ischemic work-up. RHC showed normal PA pressure (26/8), and wedge (15). LHC showed right dominant coronary system, patent left main, patent LAD and diagonal, as well as patent circumflex and right coronary artery. 24-hour EEG was done by neurology that showed focal periodic epileptiform discharges over the anterior temporal region, focal polymorphic delta activities, and background slowing with no active seizures. An MRI head showed diffuse confluent T2 hyperintensity in the bilateral white matter consistent with Binswanger's Disease. The patient was pre-emptively treated with acyclovir for herpes encephalitis, but discontinued when HSV was negative in the CSF from a lumbar puncture. She recovered well and was discharged uneventfully with levetirecetam.

IMPACT/DISCUSSION: Seizure-related Takotsubo cardiomyopathy can lead to sudden unexpected death in epilepsy (SUDEP), and is frightening underdiagnosed and worked-up in the neurocritical population. Injury to the myocardium can occur due to neurogenic stunning; there is no myocardial infarction (no coronary vessel block) but rising catecholamine levels are believed to be directly toxic to cardiac myocytes as well as vasospasm of the coronary vessels. Here there was transient severe systolic dysfunction after convulsive seizure with completely clean coronaries after the patient stabilized. CONCLUSION: There has been increasing interest in the neurocardiology community in routine monitoring with ECGs and echocardiograms in hospitalized patients with ischemic strokes, hemorrhagic strokes, and epileptic events. Takotsubo's has a reasonably high $8 \%$ mortality rate, and this case elucidates the need importance for controlling the cardiotoxic stressor (seizures) in order to prevent cardiac damage.

\section{TAKOTSUBO CARDIOMYOPATHY UNEXPECTEDLY RE- COVERS IN 24 HOURS}

Kirolus Sourial $^{1,2}{ }^{\text {; }}$ Saif M. Borgan ${ }^{1,2}$; Hiren Patel ${ }^{1}$; George Yazigi ${ }^{1,2}$; Jooby John ${ }^{1,2}$; Ali Ashraf ${ }^{1}$. ${ }^{1}$ University of Central Florida College of Medicine, Orlando, FL; ${ }^{2}$ Central Florida Cardiac \& Vascular Institute at Osceola Regional Medical Center, Kissimmee, FL. (Control ID \#3184263)

LEARNING OBJECTIVE \#1: We describe a case of Takotsubo cardiomyopathy (TTC) presenting as Ventricular Fibrillation (V-fib) cardiac arrest with an acute recovery of left ventricular (LV) systolic function within 24 hours.

CASE: A 71 year old woman with past medical history of CAD with prior CABG was brought to the hospital in V-fib cardiac arrest after an intense argument with her daughter. Return of spontaneous circulation with conversion to normal sinus rhythm was achieved after 15 minutes of effective chest compressions and defibrillator use, followed by amiodarone and norepinephrine drips, along with mechanical ventilation. Emergent coronary angiography revealed widely patent grafts with native vessel disease, unchanged from a prior angiogram several years ago. Transthoracic echocardiogram (TTE) revealed left ventricular ejection fraction (LVEF) of $15 \%$ with severe hypokinesia of the mid and apical segments consistent with TTC. Despite escalation of inotropic support with norepinephrine, dopamine and milrinone drips, hemodynamic and electrical deterioration ensued warranting a percutaneous Left Ventricular Assist Device (pLVAD). A 24-hour interval TTE revealed dramatic improvement of the LVEF to $60 \%$ with resolution of the apical ballooning. At 48 hours from admission the patient was weaned off the pLVAD and inotropic support. Later, she was extubated with excellent hemodynamic parameters, followed by complete recovery.

IMPACT/DISCUSSION: Takotsubo cardiomyopathy (TTC), or Stress cardiomyopathy, is an acute transient worsening of left ventricular (LV) systolic function secondary to catecholamine surge. The diagnosis is based primarily on typical echocardiographic criteria in absence of significant epicardial coronary stenosis. Usual presentation of TTC is chest pain, dyspnea or syncope. The typical recovery of LV function in TTC can be quick and takes about 1-4 weeks, with the fastest recorded recovery within 36 hours of the inciting event. Predictors of delayed recovery include elevated brain natriuretic peptide, elevated LV end diastolic diameter, serious comorbidities and complicated disease course. 
CONCLUSION: In this case, we report an unusual presentation of severe Takotsubo cardiomyopathy as Ventricular Fibrillation cardiac arrest with an ultra-short recovery time of 24 hours. Know a fact like this, can be reassuring for the patient's family that as Takotsubo cardiomyopathy can develop very quickly, it can resolves and heals quickly too with supportive management, even in few hours. Like in our patient, who had full recovery of the ventricular function in less than 24 hours, confirmed by follow up an echocardiogram findings.

\section{TAP IT OUT: A CASE OF SPONTANEOUS BACTERIAL PERITO- NITIS IN CARDIAC ASCITES}

Karen Jong. Emory University, Atlanta, GA. (Control ID \#3184636)

LEARNING OBJECTIVE \#1: Diagnose cardiac ascites and distinguish from other types of ascites.

LEARNING OBJECTIVE \#2: Recognize which patients with cardiac ascites are at higher risk for spontaneous bacterial peritonitis

CASE: 33 year old female with history of cor pulmonale, HIV, Clostridium difficile infection, and ESRD presented with fever, abdominal pain, and distention for five days. She was compliant with antiretroviral therapy for HIV and three times weekly hemodialysis. Exam revealed abdominal distention and diffuse tenderness. CT abdomen demonstrated moderate abdominopelvic ascites but a normal appearing liver. Liver ultrasound was unremarkable. Diagnostic paracentesis revealed ascitic fluid with 10,350 white blood cells with $87 \%$ neutrophils, SAAG of $1.4 \mathrm{~g} / \mathrm{dL}$ and total protein of $4.3 \mathrm{~g} /$ $\mathrm{dL}$. She was diagnosed with ascites due to right-sided heart failure complicated by spontaneous bacterial peritonitis.

IMPACT/DISCUSSION: Congestive hepatopathy is a complication of heart failure, occurring in $15-65 \%$ of patients with significant heart failure. Any condition causing right-sided heart failure will increase central venous hypertension and lead to portal hypertension. Tricuspid regurgitation in particular can lead to severe congestion because right ventricular pressures are transmitted directly into the hepatic veins. Cardiac ascites only accounts for $3 \%$ of ascites as the majority of cases are due to cirrhosis $(81 \%)$ and malignancy (10\%). Cardiac ascites is diagnosed by ascitic fluid with SAAG of $1.1 \mathrm{~g} / \mathrm{dL}$ or greater with an ascitic fluid total protein level of $2.5 \mathrm{~g} / \mathrm{dL}$ or greater. This is in contrast to cirrhotic ascites, which has ascitic total protein level less than $2.5 \mathrm{~g} / \mathrm{dL}$, and also nephrogenic ascites, which has a SAAG of $<1.1 \mathrm{~g} / \mathrm{dL}$. Patients with cirrhosis have known predisposing factors to spontaneous bacterial peritonitis (SBP): extra-intestinal dissemination of microbes from gastrointestinal abnormalities and decreased endogenous antimicrobial activity of cirrhotic ascites fluid. SBP is less common in patients with cardiac ascites, as they do not share these predisposing factors. In this patient, history of $\mathrm{C}$ diff infection may have enabled bacterial gut translocation leading to SBP. This case is a reminder that ascites occurs in conditions other than cirrhosis, and should be evaluated with careful physical exam and/ or bedside ultrasound when patients with heart failure come in with abdominal pain. While less common, spontaneous bacterial peritonitis can arise as a complication of cardiac ascites, and history of intra-abdominal infections should raise concern for SBP in cardiac ascites in the appropriate clinical scenario. There are several case reports in the literature, but more remains to be known about epidemiology, risk factors, and guidelines for diagnosis and treatment of SBP in cardiac ascites.

CONCLUSION: Ascites occurs in conditions other than cirrhosis and metastatic disease. Consider diagnostic paracentesis in patients with abdominal pain and cardiac ascites.
TB OR NOT TB: THAT IS THE QUESTION

Amalia Aldredge ${ }^{1}$; Robin Klein ${ }^{2} .{ }^{1}$ Emory University, Atlanta, GA; ${ }^{2}$ Emory, Atlanta, GA. (Control ID \#3185251)

LEARNING OBJECTIVE \#1: Create a differential for a miliary pattern seen on chest imaging

LEARNING OBJECTIVE \#2: Recognize the diagnostic importance of tissue for in an immunocompromised patient with fever of unknown origin CASE: A 42-year-old Hispanic man presented with three months of fevers, night sweats, weight loss, headache, shortness of breath, productive cough, and abdominal pain. Past medical history was notable for recent diagnoses of AIDS and diabetes mellitus, for which he was taking Bactrim and metformin. He was originally from El Salvador, and had spent significant periods of time all over the United States, mostly in Texas, Colorado, and Georgia. On presentation, he was febrile, tachycardic, and hypotensive. Physical exam was notable for bibasilar crackles and a tender, hyperpigmented papule over the shin. CT Head and MRI Brain were unremarkable. CT Chest showed diffuse small nodules with apical predominance, lymphadenopathy, and bilateral pulmonary effusions. $\mathrm{He}$ underwent lumbar puncture, which revealed mild pleocytosis with lymphocyte predominance, but was otherwise normal. Given the miliary appearance on his chest imaging and his history, he was empirically started on rifabutin, isoniazid, pyrazinamide, ethambutol, and liposomal amphotericin B. AFB sputa, bronchoscopy, and blood cultures were negative. MTB-PCR of the sputum culture was negative. QuantiFERON gold and tuberculin skin test were negative. Histoplasma and Blastomyces antibodies were negative. Coccidioides antibody was positive and antigen was negative. Thoracentesis and transbronchial biopsy were attempted and unsuccessful. He remained febrile and ultimately underwent excisional axillary lymph node biopsy, which showed spherules. His fungal tissue culture was positive for Coccidioides spp. He completed two weeks of amphotericin induction therapy, and was then transitioned to suppressive fluconazole therapy with improvement in his symptoms. Two weeks after discharge, repeat imaging showed improvement of his lung lesions.

IMPACT/DISCUSSION: A miliary pattern on chest imaging is often associated with tuberculosis, although there is a broader differential for this radiographic finding. Miliary nodules represent lymphohematogenous dissemination of a disease process, and are significantly more common in immunocompromised individuals. Generally, the differential includes infection (tuberculosis, fungal, or varicella), metastases (often of the thyroid, kidney, breast, or sarcoma), sarcoidosis, or pneumoconiosis. As in this case, despite a clear source, an underlying diagnosis of the cause of fever of unknown origin is often delayed and may be improved with early consideration of biopsy for a tissue diagnosis.

CONCLUSION: - A miliary pattern on chest imaging can be seen in infection (including tuberculosis, fungal, or varicella), metastases, sarcoidosis, or pneumoconiosis - A tissue biopsy is often essential for diagnosis in an immunocompromised patient with fever of unknown origin

\section{TB OR NOT TB: USE OF ADA IN DIAGNOSING TUBERCU- LOUS VS. LYMPHOMATOUS EFFUSION}

Christian Frese; Priyanka Ballal; Michael Schwartz. Boston Medical Center, Boston, MA. (Control ID \#3178689)

LEARNING OBJECTIVE \#1: Review the differential diagnosis of a pleural effusion in a patient with newly-diagnosed HIV

LEARNING OBJECTIVE \#2: Identify the pitfalls of using ADA to distinguish between tuberculous and malignant effusions 
CASE: A previously healthy 54-year old man who had recently immigrated from Uganda presented with 3 weeks of pleuritic chest pain, dyspnea, and B-symptoms. He denied productive cough, high-risk sexual activity and intravenous drug use. Examination revealed a cachectic man with decreased breath sounds and dullness in the left lung fields. A chest $\mathrm{x}$-ray and subsequent chest CT revealed a large left-sided pleural effusion with right mediastinal shift and hilar lymphadenopathy. He was subsequently found to be HIV positive with a CD4 count of 164 . He underwent a thoracentesis with chest tube insertion and pleural fluid analysis revealed an exudate with lymphocytic predominance, negative cytology and culture, and adenosine deaminase (ADA) of $59.6 \mathrm{U} / \mathrm{L}$ (normal $<9.2 \mathrm{U} / \mathrm{L}$ ). A Quantiferon Gold was positive but all mycobacterial studies including AFB smear, mycobacterial cultures, and tuberculosis PCR of the pleural fluid and induced sputum were negative. Based on the clinical history, positive Quantiferon Gold, and elevated ADA, the leading diagnosis was pleural tuberculosis, but lymphoma could not be definitively excluded. Due to the technical challenges of an excisional biopsy, the decision was made to treat empirically with 4 drug anti-tubercular therapy and monitor the clinical response. While a follow-up CT scan showed resolution of the pleural effusion, there were multiple pleural-based lesions with central cavitation and persistent lymphadenopathy. Due to concern for missed malignancy, a pleural biopsy was obtained which was negative for lymphoma, Kaposi sarcoma, and bacterial, mycobacterial, or fungal infections. Pathology revealed non-caseating granulomatous inflammation. The patient has since completed 8 weeks of four-drug directly observed therapy and is currently on isoniazid and rifabutin with excellent response. He was also started on anti-retroviral therapy and currently has an undetectable viral load.

IMPACT/DISCUSSION: This case shows the complexity of diagnosing extra-pulmonary TB in the absence of positive cultures. Additionally, while ADA is an important tool for diagnosing tuberculosis when cytology and culture are negative, it is important to note that lymphoma can also cause elevated ADA values.

CONCLUSION: Extra-pulmonary TB and lymphoma are both common in immunocompromised patients and can present with similar signs and symptoms. It is important to perform a thorough work-up for such presentations to ensure timely initiation of the appropriate therapy. While tuberculosis almost always causes an elevated ADA, it is important to note that the same can be true for lymphomatous effusions, which in rare cases can exceed $250 \mathrm{U} / \mathrm{L}$. When the diagnosis is equivocal, ADA should be interpreted with caution and a biopsy may be needed to confirm the diagnosis.

\section{TEARING CHEST PAIN IN A PREGNANT WOMAN WHILE WATCHING OLYMPIC GAMES.}

$\underline{\text { Yu Yaegashi }}{ }^{1}$; Mitsunori Iwase ${ }^{1}$; Hiroshi Miyake ${ }^{2} .{ }^{1}$ TOYOTA memorial hospital, Toyota, Japan; ${ }^{2}$ TOYOTA Memorial Hospital, Aichi, Japan. (Control ID \#3182620)

LEARNING OBJECTIVE \#1: Recognize spontaneous coronary artery dissection as a potential cause of chest pain in pregnant women.

LEARNING OBJECTIVE \#2: Recognize clinical features and management of spontaneous coronary artery dissection.

CASE: The patient is a 34 year-old pregnant woman, non-smoker without cardiovascular risk factors. Her pregnancy had been uneventful until one day prior to her due date, when she developed sudden-onset severe "tearing" chest pain while watching the Olympic games on TV. She called emergency medical service and presented to our hospital. Initial electrocardiogram showed anterior ST-segment elevation. Emergent coronary angiography revealed diffuse $90 \%$ stenosis of proximal to mid segments of the left anterior descending coronary artery and total occlusion of the second diagonal branch. Intravascular ultrasound revealed a large intramural hematoma compressing the true lumen, consistent with spontaneous coronary artery dissection (SCAD). Primary percutaneous coronary intervention was performed with balloon angioplasty to left anterior descending artery and the second diagonal branch, resulting in good antegrade coronary flow. We placed intra-aortic balloon pump to provide hemodynamic support during cesarean section, which was successfully performed on the following day. Follow-up coronary angiogram was performed one week later, which showed almost complete resolution of intramural hematoma. Another follow-up coronary angiogram three months later showed complete resolution of intramural hematoma.

IMPACT/DISCUSSION: SCAD is defined as a non-traumatic and noniatrogenic separation of the layers of the coronary arterial wall by which a false lumen is formed. SCAD affects women in more than $90 \%$ of cases and is most prevalent in young women. Pregnancy is one of the key risk factors of SCAD. The large majority of pregnancy-related SCAD occur in the first postpartum month, especially in the first week. Although SCAD is increasingly recognized as an important entity of myocardial infarction in young women, it remains a challenge to promptly diagnose SCAD, especially when presenting without obvious electrocardiographic changes. Prompt recognition and appropriate intervention are essential to achieve favorable maternal and child outcomes. Thus, it is important to have a high index of suspicion for SCAD when encountering a pregnant woman presenting with chest pain. The optimal management of SCAD remains undetermined. Percutaneous coronary intervention for SCAD has a significantly low procedural success rate and high rate of complications. Thus, a conservative approach is preferred. Urgent intervention may still be indicated in cases of ongoing chest pain or persistent ST-elevation.

CONCLUSION: We experienced a young pregnant woman who developed SCAD and was successfully treated with balloon angioplasty, resulting in favorable maternal and child outcomes. It is important to have a high index of suspicion for SCAD in pregnant women presenting with sudden chest pain.

\section{TERRIBLE THORACIC TRANSIT: ACUTE KIDNEY INFARC- TION DUE TO A PARADOXICAL EMBOLUS}

Ye Aung ${ }^{1}$; Holli T. Sadler ${ }^{2} .{ }^{1}$ Dell Medical School at the University of Texas Austin, Austin, TX; ${ }^{2}$ Dell Medical School, Austin, TX. (Control ID \#3183360)

LEARNING OBJECTIVE \#1: Recognize unusual complications of an unprovoked Pulmonary Embolism.

CASE: The patient is a 38 year old African American man with a PMH of cocaine abuse and asthma who presented to the ED due to a witnessed episode of syncope and altered mental status. Upon arrival he was hypoxic, hypotensive and agiated. On physical exam, the Patient had clear lung sounds bilaterally and intact pulses. Severe left flank tenderness was noted. Labs were remarkable for an elevated serum creatinine of $1.3 \mathrm{mg} / \mathrm{dL}$ compared to a baseline of $0.8 \mathrm{mg} / \mathrm{dL}$. Urinalysis revealed mild proteinuria and microscopic hematuria. Urine toxicology was positive for cocaine. Due to concern for pulmonary embolus, computed tomography angiogram of the chest was ordered, which showed massive bilateral PE. The patient was also found to have no perfusion of the Left Kidney. CT head was negative for acute CVA or lesion. Following fluid resuscitation the patient achieved hemodynamic stability and no thrombolysis was indicated. The 
patient was started on heparin drip and his clinical and mental status improved and returned to his baseline within a few hours. Further workup was ordered to determine the cause of left kidney ischemia. Venous ultrasound of the lower extremity showed a large right lower extremity deep vein thrombosis. A nuclear perfusion study showed almost no perfusion to left kidney so function could not be salvaged via endovascular procedure. The patient was discharged on rivoroxaban indefinitely and he was advised to quit cocaine use and smoking. Outpatient hypercoagulability workup is pending.

IMPACT/DISCUSSION: We believe that this patient's acute kidney failure was caused by his large deep vein thrombus in his right lower extremity entering the systemic circulation via his trivial right to left atrial shunt and causing an infarction of the left renal artery. Although the patient's right to left shunt under normal physiologic circumstances is too small to allow a thrombus to pass, we hypothesize that in the context of a massive pulmonary embolism, a temporary increase in pulmonary pressure would cause enlargement of the shunt and allow the passage of a clot. Paradoxical emboli leading to acute unilateral kidney failure is a clinically rare event. This is primarily because there is a requirement for both the existence of a patent cardiac shunt as well as a hyper-coagulable state. We have found one similar case in literature. This case demonstrates the importance of keeping a broad differential and that mild or silent physiologic abnormalities can cause significant morbidity in unique circumstances. (1). R. Schmitt, M. Westhoff-Bleck, H. Haller, A.D. Wagner; Paradoxical renal embolism in a patient with congenital cardiac malformation, QJM: An International Journal of Medicine, Volume 104, Issue 10, 1 October 2011

CONCLUSION: In any patient with risk factors for hypercoagulability such as cocaine use and unexplained kidney injury, paradoxical embolism is a rare yet important possibility that must be considered.

\section{TERRIFIED OF INSULIN: USING A PATIENT-CENTERED, MULTI-DISCIPLINARY APPROACH TO TREAT A CASE OF SE- VERE TYPE 2 DIABETES}

Michael S. Tzeng; Jill Allenbaugh. Temple University, Philadelphia, PA. (Control ID \#3184867)

LEARNING OBJECTIVE \#1: Recognize clinical signs and lab evidence of severe, catabolic diabetes

LEARNING OBJECTIVE \#2: Utilize a patient-centered, multidisciplinary approach to improve patient outcomes in the outpatient setting CASE: A 59 year-old female with type 2 diabetes, hypertension, hyperlipidemia, and obesity presented to the outpatient clinic complaining of worsening fatigue, thirst, urinary frequency, unintentional weight loss, and intermittent abdominal pain. She had lost 15 pounds over six months, and her glucose was $408 \mathrm{mg} / \mathrm{dL}$. Labs revealed a HbA1c of $12.3 \%$ and microalbuminuria, concerning for severe, catabolic diabetes with early evidence of nephropathy. Prior treatment had been complicated by difficulty with lifestyle changes and intolerance of oral diabetic agents. She repeatedly refused subcutaneous insulin, citing a fear of side effects, terror of using needles, and a sentiment that using insulin meant personal failure. After a discussion about alternative therapy, she chose to initiate dulaglutide injections based on its side effect profile and lower frequency of injections. Since she was terrified of needles, she agreed to have her medicine administered by staff during weekly pharmacy clinic visits. Additionally, she was enrolled in diabetic education classes, scheduled for nutritionist visits and encouraged to sign up for a fresh food initiative. Over the next few months, she reported that diabetic education class and nutritionist visits provided her with the knowledge to make healthier food choices. With skills learned from pharmacy clinic, she began closely documenting her glucoses and noticed when certain foods made her sugars spike. Additionally, she began administering her own dulaglutide in clinic and soon graduated to selfadministration at home. With her newly acquired skills and continued support, she eventually reported a resolution in her symptoms. Repeat labs five months later revealed a dramatically improved A1c at goal: $6.4 \%$.

IMPACT/DISCUSSION: In patients with severe diabetes, the failure of lifestyle changes and oral therapy commonly prompts initiation of subcutaneous insulin regimens. However, treatment may be limited by patient preference and poor adherence. This patient was offered an injectable non-insulin medication combined with multi-disciplinary care, diabetic education, and better access to healthy foods, altogether culminating in an A1c at goal and resolution of symptoms.

CONCLUSION: Successful treatment of severe diabetes in the outpatient setting requires not only medical knowledge, but also shared decision making, taking into consideration patient preferences and social determinants of health. Improving patient access to resources and multi-disciplinary care can ultimately prevent the development of hyperglycemic crises or vascular complications.

THAT COUGH SAVED YOUR LIFE: OCULAR MANIFESTATIONS OF ACUTE PROMYELOCYTIC LEUKEMIA

Naomi Habib ${ }^{3}$; suman misra ${ }^{1}$; Santi Karnam ${ }^{1}$; Jaya Raj ${ }^{2}$. ${ }^{1}$ St. Joseph's Hospital and Medical Center, Phoenix, AZ; ${ }^{2}$ St. Joseph's Hospital, Phoenix, AZ; ${ }^{3}$ St Joseph's Hospital and Medical Center/Creighton University Medical School, Phoenix, AZ. (Control ID \#3186545)

LEARNING OBJECTIVE \#1: To identify early signs and symptoms of acute promyelocytic leukemia (APL)

LEARNING OBJECTIVE \#2: To recognize the propensity of APL for causing disseminated intravascular coagulation (DIC) and fatal, systemic hemorrhages

CASE: A 58-year-old woman with a history of an intracranial aneurysm presented to her ophthalmologist after 1 day of bilateral vision loss following 1 month of worsening floaters. She was then sent to our ED for imaging due to concern for Terson syndrome. Her history includes hypertension, a congenital paraclinoid internal carotid artery aneurysm and a bout of severe pneumonia treated one month ago. Her vitals were normal and stable (T:36.7C, HR: 94, RR:16, BP:144/69). Ophthalmologic exam showed bilateral pre-retinal and intra-retinal hemorrhages with heme covering the fovea in the left eye. PERLLA, EOMI. Thre rest of her exam was normal. A CBC showed WBC: 0.5, Hgb: 4.3, Hct: 12.7, Plt: 47. BMP was normal. CT angiogram showed an intact $2 \times 3 \mathrm{~mm}$ paraclinoid ICA aneurysm. MRI of the brain and orbits was grossly negative. A peripheral blood smear showed pancytopenia with $>20 \%$ blasts and a bone marrow biopsy showed virtually $100 \%$ cellular bone marrow with $>70 \%$ blasts. FISH analysis detected the PML-RARalpha translocation which is pathognomonic for APL. She was immediately transfused with pRBCs and started on steroids, ATRA and arsenic. On day 16, she developed dyspnea and leukocytosis. CXR showed evidence of pulmonary edema, and the ATRA was stopped due to concern for retinoic acid/differentiation syndrome. Her leukocyte count normalized and she resumed ATRA therapy with no complications through the rest of her hospital stay. Patient is in remission at 10mo after initial presentation and showed cleared bilateral retinal hemorrhages.

IMPACT/DISCUSSION: The initial presentation of APL is similar to those of patients with other forms of acute myeloid leukemia: including fatigue, easy bruising and epistaxis. However, APL is unique in that it can manifest as hemorrhages and/or DIC in up to $61 \%$ of patients. Early 
initiation of an ATRA-based regimen is necessary to prevent death from hemorrhagic complications. When first described in 1957, no treatment existed, and patients often died due to acute hemorrhage, with a median survival under 7 days. Even now, early mortality remains a concern; the incidence has only decreased from $22.21 \%$ in the 1990 s to $17.5 \%$. In our case, the patient presented with ocular hemorrhage; a known complication of APL and is reported at a rate of $42 \%$ of leukemic ocular findings. However, the incidence of retinal hemorrhage as a solitary finding pointing to the diagnosis of APL is rare, with only 3 cases reported in the literature. We believe that her previous pneumonia, which triggered her severe cough, had increased her propensity for retinal hemorrhage and ultimately led to the diagnosis of APL.

CONCLUSION: Our case demonstrates that maintenance of a high suspicion for APL in the setting of pancytopenia and hemorrhage is paramount, and indeed can help to save a patient's life.

\section{THE ABC'S OF ACUTE VIRAL HEPATITIS IN A HOMELESS MAN WHO INJECTS DRUGS}

Ryan Bonner ${ }^{1}$; Jessica Taylor ${ }^{2} .{ }^{1}$ Boston Medical Center, Boston, MA; ${ }^{2}$ Boston University/Boston Medical Center, Boston, MA. (Control ID \#3162846)

LEARNING OBJECTIVE \#1: Identify the risk for acute hepatitis A among patients experiencing homelessness and/or substance use disorders. LEARNING OBJECTIVE \#2: Offer HAV vaccination to patients with epidemiological risk.

CASE: A 32-year-old man with severe opioid use disorder and homelessness presented to an opioid urgent care clinic. He endorsed receptive syringe sharing. Labs showed mild transaminitis (AST $53 \mathrm{U} / \mathrm{L}$, ALT $84 \mathrm{U} / \mathrm{L}$, total bilirubin $0.3 \mathrm{mg} / \mathrm{dL}$ ) and negative results for $\mathrm{HCV}$ antibody, hepatitis A (HAV) IgG, and hepatitis B (HBV) surface antigen, surface antibody, and core antibody. The patient was started on buprenorphine/naloxone. One week later, he endorsed several days of nausea, vomiting, malaise, and fatigue that began after sustaining a blistering sunburn. Exam showed partial-thickness burns of sun exposed areas, diffuse abdominal tenderness, and no jaundice. The patient was counseled on management of severe sunburn; because of the mild transaminitis, he was also evaluated for acute viral hepatitis. Labs showed a significant rise in AST (2,079 U/L), ALT (2,727 U/L), and total bilirubin (5.2 mg/dL, 4.1 direct). INR was 1.57 . HCV RNA and HBV DNA were not detected, and his HAV IgM antibody returned positive, consistent with acute HAV. The patient was admitted for supportive care. His AST, ALT, and INR down-trended, and he was discharged on hospital day 5 once cleared to return to his shelter by the Department of Public Health. Follow up labs demonstrated further improvement in his liver enzymes and synthetic function.

IMPACT/DISCUSSION: This case highlights the importance of provider awareness of local epidemiological risk and the need for intensified HAV prevention efforts, including vaccination, among people who are homeless and/or inject drugs.

CONCLUSION: People who inject drugs (PWID) are at high risk of viral hepatitis. Testing, prevention, and treatment efforts have focused on HBV and $\mathrm{HCV}$, but recent outbreaks of HAV affecting homeless people and PWID have been described in at least 12 states. Unlike HBV and HCV, which can be transmitted via shared injection, insufflation equipment, or sexual intercourse, HAV is transmitted via the fecal-oral route, and the risk of transmission in communal settings like shelters is high. Nonspecific constitutional or GI symptoms and transaminitis should raise suspicion for acute HAV in individuals affected by substance use disorders or housing insecurity; providers in regions impacted by HAV outbreaks should have a low threshold to test for acute infection with HAV IgM antibody. The management of acute HAV is supportive. Fulminant liver failure is rare in patients without underlying liver disease, but hospitalization and quarantine of patients living in communal settings are often needed. As of November 2 , 2018, 84\% of 141 total outbreak-related cases in Massachusetts have resulted in hospitalization. Overall, PWID should be counseled on strategies to prevent all types of viral hepatitis, including safe injection practices, vaccination for $\mathrm{HAV}$ and $\mathrm{HBV}$, food safety, and hand washing.

\section{THE BLOODY VICE OF BRODIFACOUM AND SPICE}

Alexandra Rezazadeh; Kristi Maso; Marium Khan. Medical College of Wisconsin, Wauwatosa, WI. (Control ID \#3175227)

LEARNING OBJECTIVE \#1: Recognize the bleeding diathesis secondary to brodifacoum-associated synthetic cannabinoid (SCB) use.

LEARNING OBJECTIVE \#2: Collaborate with interdisciplinary hospital and community teams to treat and arrange follow-up for patients with SCBassociated cogulopathy.

CASE: A 23-year-old African American male presented with a 3-day history of right flank pain, hematuria, and emesis. He reported SCB use 3 days prior. Vitals were stable. Exam showed right CVA tenderness. Labs revealed hemoglobin of $11.7 \mathrm{~g} / \mathrm{dl}$, platelets of 105,000, INR of > 10, and urinalysis with $3+$ blood. CT abdomen and pelvis with contrast showed a right retroperitoneal hematoma. He was admitted to the ICU and given IV Vitamin K and continued on oral Vitamin K per Wisconsin Poison Control orders. ICU stay was complicated by expanding hematoma requiring resuscitation with multiple units of fresh frozen plasma, blood transfusions, and a total of $470 \mathrm{mg}$ of Vitamin K over 4 days, after which his INR was 1.6. Vascular surgery was consulted but he required no intervention. Though stable for discharge, due to Vitamin K shortage and his lack of insurance, the regimen cost over $\$ 20,000$. Discharge was delayed one day until pharmacy and social work could secure charity funds. He was given a 30-day supply of Vitamin K for $\$ 5.64$ with an arranged clinic visit 4 days later. He followed up and was put on a Vitamin K taper with a dose decrease of $25 \%$ each week for 4 weeks.

IMPACT/DISCUSSION: SCBs (also called 'Spice,' 'K2') are mind-altering chemicals sprayed on plant material to be smoked or sold as liquids to be vaporized and inhaled. They are chemically related to substances in plantbased marijuana. Their use is preferred due to lower cost and prosecution evasion due to undetectability in drug screens. SCBs have higher binding affinity for cannabinoid receptors than delta-tetrahydrocannabinol, the active component of traditional marijuana. In the spring of 2018, the CDC reported that some SCBs were being laced with brodifacoum, a 'superwarfarin' that binds Vitamin K epoxide reductase more strongly than warfarin, giving it a longer elimination half-life. Use of brodifacoum with SCBs is multifactorial: sources cite intentional addition to prolong SCB effects and unintentional toxicity due to its use as a rodenticide. In Wisconsin in 2018, 67 cases of brodifacoum-laced SCB use were confirmed, including one death. Recent studies describe similar outbreaks. This case reiterates those studies' findings but is unique in highlighting the public health impact. The high cost and shortage of treatment not only hinders discharge but also prevents patients from receiving proper therapy. Interdisciplinary teams were vital to facilitate affordable therapy and follow-up.

CONCLUSION: SCBs are increasing in use due to their affordability and undetectability in drug screens. Recognition of the toxicity requires a high index of suspicion. Failure to diagnose may result in significant adverse outcomes. Proper treatment and follow-up requires strong interdisciplinary cooperation. 
THE CASE OF THE GROWING SNAKE AND THUMPING HEART Iniya Rajendran ${ }^{1}$; Thomas W. Barber ${ }^{2} .{ }^{1}$ Boston Medical Center, Boston, MA; ${ }^{2}$ Boston University School of Medicine, South Boston, MA. (Control ID \#3185845)

LEARNING OBJECTIVE \#1: Recognize the manifestation of high-output heart failure and that an arteriovenous fistula is as a rare but reversible cause. LEARNING OBJECTIVE \#2: Recognize that patient word choices are an important aid in diagnosis.

CASE: Ms N is a 66-year old renal transplant recipient with a history of heart failure with preserved ejection fraction $(\mathrm{HFpEF})$, atrial fibrillation and a patent arteriovenous fistula (AVF) for hemodialysis. She presented to primary care clinic with lower extremity and abdominal swelling despite increasing doses of furosemide. She also reported that her AVF, located in the left forearm, was a "growing snake". Imaging of the abdomen showed "a nodular contour to the liver consistent with cirrhosis" prompting a GI referral and several encounters for therapeutic paracentesis. Ascitic fluid was chylous appearing with a serum albumin/ascites ratio of 1.1. During her clinical course, she constantly reported a "thumping" heart sound which was worsening. New symptoms of fatigue and shortness of breath began to develop. An echocardiogram showed abnormal right ventricular function and morphology and right heart catheterization showed a pulmonary capillary wedge pressure $<15$. Finally, she was diagnosed with pulmonary hypertension and high output heart failure secondary to the large, high flow, AVF. Within three months of ligation of the fistula, she had complete resolution of all symptoms.

IMPACT/DISCUSSION: This is a case of an eloquent historian who developed an uncommon type of HF. Causes of high output HF are broadly categorized as follows (1) increased metabolism leading to an increase in demand for blood and (2) low systemic resistance leading to increased flow into venous circulation such as an AVF. A single center study described the etiologies of high output HF to be obesity (31\%), liver disease (23\%), arteriovenous shunts $(23 \%)$. There is no current evidence for ligation of AVF and is generally maintained in case of return to dialysis and prevention of hemodynamic changes post-transplantation. Decisions should be casebased and in high flow AVFs, ligation is warranted. This case serves as a reminder that an answer to a diagnostic dilemma is often right in front of our us i.e. patients initial report of a growing AFV should be confirmed by examination and Doppler ultrasound. While clinicians appropriately chased a clue (liver findings on the CT scan), it turned out to be the consequence (liver congestion secondary to heart failure) and not the cause. The onomatopoeic word "thump" she repeatedly used through the course is a testament to the patient's subconscious self-awareness of a hardworking heart.

CONCLUSION: An iatrogenic cause of high output heart failure which needs early recognition is the non-removal of enlarging AVFs created for hemodialysis. Active listening and addressing patient concerns may help solve diagnostic dilemmas quicker.

\section{THE CLOT THICKENS: CEREBRAL VEIN THROMBOSIS IN A PATIENT WITH HIV/AIDS FOLLOWING MENINGITIS}

Dylan Atchley; John Hwang. New York Univeristy, New York, NY. (Control ID \#3166582)

LEARNING OBJECTIVE \#1: Recognize the risk factors and clinical features of cerebral vein thrombosis

LEARNING OBJECTIVE \#2: Manage cerebral vein thrombosis and its associated sequalae

CASE: A 26-year-old woman with untreated HIV/AIDS and a CD4 count of 54 presented with three days of fever, confusion, and ear pain, and was found to have pneumococcal meningitis and bacteremia secondary to suppurative otomastoiditis. Myringotomy was performed, and vancomycin, ceftriaxone, and dexamethasone were administered. She improved rapidly and started antiretroviral therapy on hospital day five. However, ten days into her admission, she developed fluctuating sensory loss and weakness of her right side, with sparing of her face. A non-contrast head CT was initially read as normal, but MRI/MRV hours later revealed extensive cerebral vein thrombosis (CVT) involving the superior sagittal and lateral transverse sinuses extending to the internal jugular vein. Tonic-clonic seizures developed shortly after. Heparin, levetiracetam, and phenytoin were started, with resolution of her seizures and neurologic deficits. She was discharged home on enoxaparin and recovered fully.

IMPACT/DISCUSSION: We initially overlooked the possibility of CVT in this patient, exposing several deficits in our illness script for this entity. CVT has a highly variable clinical presentation, usually produces only subtle findings on early unenhanced CT, and can complicate bacterial meningitis even late in its course. Timely diagnosis requires recognition of its risk factors and characteristic findings.

CONCLUSION: A wide variety of underlying disorders predispose to CVT. A systemic prothrombotic state is often present, whether from a genetic thrombophilia or an acquired condition such as malignancy, rheumatologic disease, pregnancy, or oral contraceptive use. Mechanical insults from head trauma, local tumor, or intracranial infection are also common causes; for example, CVT reportedly complicates 7-9\% of cases of pneumococcal meningitis. Perhaps owing to this myriad of causes, the clinical manifestations of CVT are highly variable: headache is most frequent, while seizures, focal neurologic syndromes, and encephalopathy are also commonly observed - but none of these are invariably present. A waxing-waning pattern of stroke-like symptoms that do not conform to a stroke distribution, as seen in this case, is a characteristic presentation. Initial treatment is preferably lowmolecular weight heparin. In the absence of definitive studies, anticoagulation is typically continued for at least six months, but should be individualized based on the patient's thrombotic risk. Warfarin is an acceptable choice, though this patient's cobicistat-containing antiretroviral regimen precluded its use; direct oral anticoagulants, meanwhile, have been insufficiently studied. While we considered the possibility that immune reconstitution may have contributed to this patient's CVT, in the context of severe bacterial infection, early initiation of ART is currently standard of care.

\section{THE CLUE IN THE CULTURE: FUSOBACTERIUM LUNG INFEC- TION LEADING TO DIAGNOSIS OF COLORECTAL ADENO- CARCINOMA}

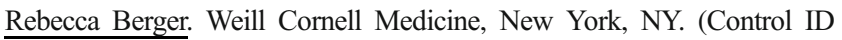
\#3187040)

LEARNING OBJECTIVE \#1: Recognize the association between fusobacterium nucleatum and colorectal cancer

CASE: A 79 year old retired physician presented with 3 weeks of progressive fatigue, dry cough, dyspnea, and dark stools. He had a mass protruding from the right chest wall and decreased breath sounds in the R lung. Hemoglobin was $10.8 \mathrm{~g} / \mathrm{dL}$, ferritin $246 \mathrm{ng} / \mathrm{mL}$, transferrin saturation 9\%. Chest CT showed multiple large peripheral lung opacities, a right infrahilar mass, and a low density rim-enhancing collection invading the right chest wall, concerning for metastatic lung cancer. Fine needle aspiration and core biopsy of the chest wall mass revealed no malignant cells. Bronchoscopy with transbronchial biopsy of the right infrahilar mass also found no evidence of malignancy. The fluid aspirated from the chest wall mass subsequently grew fusobacterium nucleatum. Colonoscopy revealed a $3 \mathrm{~cm}$ infiltrating, ulcerating 
mass in the colon; pathology confirmed colorectal adenocarcinoma. The patient was diagnosed with a fusobacterium lung infection with empyema necessitatis associated with colorectal adenocarcinoma. He was treated with several weeks of ertapenem; repeat CT chest showed near resolution in the intrapulmonary masses and chest wall collection. MRI of the pelvis had no evidence of wall invasion or metastasis. He underwent laparoscopic-assisted anterior resection of the colorectal cancer and ultimately found to have stage IIIB (T3N1cMx) colorectal cancer. He opted to forego adjuvant systemic chemotherapy and is doing well one year after initial presentation with no evidence of recurrence.

IMPACT/DISCUSSION: Fusobacterium nucleatum is a gram-negative obligate anaerobic bacterium that is prevalent in the oral cavity. Several studies in recent years have demonstrated a strong association between fusobacterium nucleatum and human colon adenomas and carcinomas, including some hypotheses that fusobacterium nucleatum promotes the development of colorectal cancer. Existing literature highlights the microbiological overrepresentation of this anaerobe in colorectal adenomas and carcinomas, with no prior reports of clinical fusobacterium infections in patients with colorectal cancer. After the fusobacterium grew in culture, it was on the basis of this literature, with new iron deficiency anemia and dark stools, that the colonoscopy was pursued and the diagnosis of colorectal carcinoma was made. This case represents the first known in the literature in which a clinically evident infection with fusobacterium nucleatum was associated with a concurrent diagnosis of colorectal cancer.

CONCLUSION: When faced with an unexpected or rare finding, in this case an anaerobic lung abscess, availability of the literature can help identify associations that can lead to the ultimate diagnosis.

\section{THE CONFUSING TRUTH ABOUT CEREBRAL AMYLOID ANGIOPATHY}

Shaunak Mulani; Christopher Little; Andres Lopez; Margaret C. Lo. University of Florida College of Medicine, Gainesville, FL. (Control ID \#3186026)

LEARNING OBJECTIVE \#1: Identify the clinical features and classic radiographic findings of CAAI in elderly patients

LEARNING OBJECTIVE \#2: Diagnose and manage CAAI as a reversible cause of dementia

CASE: A 75-year-old female with chronic Bell's palsy presented with newonset worsening visual hallucinations and delusions with behavioral and cognitive changes for 1 month. Vitals and neurologic exam were normal on admission. Psychiatric exam noted visual hallucinations, waxing-waning orientation to situation and inappropriate response to questions. Labs revealed elevated ESR and CRP. Brain CT from a recent hospitalization incidentally found frontal, left occipital and right pontine vasogenic edema. Brain MRI showed hyperintensities in left frontal lobe concerning for inflammatory amyloid angiopathy. Repeat brain MRI at admission showed increasing areas of edema and abnormal FLAIR signal in left parietal lobe with new microhemorrhages in right frontal and parietal lobe, concerning for inflammatory amyloid angiopathy. Lumbar puncture revealed positive oligoclonal bands but no infection or malignancy. Family declined a brain biopsy. Patient was treated for cerebral amyloid angiopathy related inflammation (CAAI) with 5 days of IV solumedrol and a steroid taper. Her symptoms resolved with this regimen. Brain MRI three weeks later showed resolution of the supratentorial edema, improved hyperdensity in frontal lobes, and stable microhemorrhages.

IMPACT/DISCUSSION: Cognitive impairment and dementia are common presentations in the elderly, often attributed to age-related changes. This rare case of CAAI teaches clinicians the risk of pattern recognition and anchoring in the diagnostic evaluation of dementia. CAAI is a rare but known cause of reversible encephalopathy in the elderly and can mimic senile or Alzheimer's dementia. Its prevalence in the elderly is due to deposition of beta-amyloid protein in vessels of the cerebral cortex. Common presenting features are acute/subacute encephalopathy, headache, seizures, and neuropsychiatric signs. Labs reveal elevated ESR and CSF pleocytosis. Risk factors include elderly age and presence of ApoE E4/E4 genotype. Diagnosis is made clinically and by MRI which shows classic findings of multiple microbleeds, confluent white matter hyperintensity, large areas of inflammation, or edema. Stereotactic brain biopsy provides definitive diagnosis but is not required unless MRI is unamenable. Literature reports good response to brief courses of corticosteroid and immunosuppressive agents e.g. cyclophosphamide. As CAAI is an easily reversible disorder, early recognition and prompt diagnosis can prevent further morbidity.

CONCLUSION: CAAI can be diagnosed by clinical presentation and classic MRI findings, thus avoiding the need for invasive brain biopsy. CAAI can present with progressive cognitive decline and neuropsychiatric symptoms in the elderly and can mimic age-related dementia; however, it is easily reversible with immunosuppressive treatment. Prompt recognition and early diagnosis of CAAI are imperative.

\section{THE COST OF COFFEE: RENAL FAILURE IN CENTRAL AMER- ICA}

Emily R. Insetta. Johns Hopkins Bayview Medical Center, Baltimore, MD; Johns Hopkins Community Physicians - Howard County General Hospital, Columbia, MD. (Control ID \#3186401)

LEARNING OBJECTIVE \#1: To increase awareness of Mesoamerican nephropathy.

LEARNING OBJECTIVE \#2: To describe proposed mechanisms of Mesoamerican nephropathy.

CASE: A 29 year old man from Honduras with no medical history presented to the ER with fatigue and dyspnea that began 4 months ago but worsened over 2 weeks. He noted orthopnea and swelling. Labs showed BUN 93, Cr 9.97, potassium 3.1, uric acid 8.2, and 1+ proteinuria. One year prior, BUN was 13 and $\mathrm{Cr}$ was 1.47. He was well appearing, with blood pressure 163/111, jugular venous distention, and ankle edema. He took no medications or substances. During adolescence, he worked harvesting coffee in Honduras. Renal biopsy showed diffuse global glomerulosclerosis and tubulointerstitial scarring. The pathologist diagnosed malignant hypertension versus Mesoamerican nephropathy $(\mathrm{MeN})$. The patient's blood pressure was not severely elevated and he had no history of severe hypertension. His social history and findings of hyperuricemia, hypokalemia, and proteinuria suggested MeN. He was started on renal replacement therapy and discharged on lifelong hemodialysis.

IMPACT/DISCUSSION: MeN is the number one cause of death in sugar cane workers in Nicaragua. It is important for internists to consider this diagnosis in vulnerable populations. From studying sugar farmers in Nicaragua, four features of MeN have emerged: it is specific to Southeast Mexico and Central America, kidney injury is not explained by another cause, young individuals (30-50 years) and men (4:1) are afflicted, and it affects farm workers in hot climates. The primary cause of injury is thought to be recurrent dehydration. This is exacerbated by nephrotoxins, especially NSAIDs, which workers use for musculoskeletal pain. Other proposed factors include toxic agrochemicals, high-fructose drinks, contaminated alcohol products, and local infections such as Leptospirosis. Renin angiotensin activation causes hyperuricemia and hypokalemia. Patients have non-nephrotic proteinuria and pathology shows tubulointerstitial disease and glomerular ischemia. MeN 
progresses to end stage disease within a few years, causing death in regions without dialysis. To provide culturally competent care, the internist must recognize $\mathrm{MeN}$ as an important diagnosis among agricultural workers from afflicted areas. The World Bank has asserted the importance of improving working conditions in these regions. There are no official guidelines, but it may be reasonable to screen for kidney disease and aggressively treat risk factors in high risk patients.

CONCLUSION: Mesoamerican nephropathy causes renal failure in young farm workers from Mexico and Central America. Repetitive bouts of dehydration and nephrotoxic exposures are the proposed causes.

\section{THE CREUTZ OF THE PROBLEM: RAPID CJD DEMENTIA MASKED BY ANXIETY AND INSOMNIA}

Audrey Ready; Ali Shami; Maryam Hasan. Baystate Medical Center, Chicopee, MA. (Control ID \#3186274)

LEARNING OBJECTIVE \#1: Recognize early manifestations of Creutzfeldt-Jakob Disease (CJD) including anxiety and insomnia as presenting symptoms.

LEARNING OBJECTIVE \#2: Discuss diagnostic tools for CJD, including real-time quaking-induced conversion testing (RT-QuIC).

CASE: A 67-year-old female with a history of anxiety and depression came to the clinic with a three-week history of insomnia and increased anxiety. She had returned recently from vacationing in Italy, and the symptoms were ascribed to jet lag. Over the next several weeks she exhibited erratic behavior and confusion and was eventually brought to the emergency department by concerned family. Initial evaluation revealed a middle-aged woman who was alert but very confused, exhibiting echolalia and hyperreflexia in all extremities. Evaluation including blood tests and infectious workup were negative. Magnetic resonance imaging of the brain revealed extensive diffusion abnormalities throughout the cortex of both cerebral hemispheres with the involvement of the caudate head. Video electroencephalogram (EEG) showed periodic lateralized epileptiform discharges in the left temporal region. Her cerebral spinal fluid was negative for viral or bacterial infections. In the context of her rapidly progressive dementia, CSF was also sent to check for the presence of protein 14-3-3 and RT-QuIC. She continued to decline in the hospital, eventually developing mutism; she was ultimately made comfort measures after a multidisciplinary family meeting. She passed away eleven weeks after the presentation of symptoms. Results of her testing returned with greater than $4000 \mathrm{pg} / \mathrm{mL}$ T-tau protein, positive 14-3-3 protein and positive (RT-QuIC) testing.

IMPACT/DISCUSSION: CJD is a fatal, rapidly progressive form of dementia caused by prions. However, the clinical diagnosis is challenging. Psychological disturbances such as anxiety and agitation are noted in $90 \%$ of cases and are often accompanied by hyperreflexia, myoclonus or cerebellar dysfunction. Insomnia itself has only rarely been reported as a presenting symptom in individuals with CJD. Definitive diagnosis requires brain biopsy; therefore other workup is pursued for probable diagnosis. Center for Disease Control (CDC) guidelines dictate that probable diagnosis requires one of the following: EEG with periodic sharp waves; 14-3-3 protein in CSF; or MRI with typical caudate and/or putamen abnormalities. In addition, it requires presence of two out of the following four exam findings: akinetic mutism, myoclonus, pyramidal/extrapyramidal signs, or cerebellar signs. RT-QuIC is a novel test that detects abnormally folded isoforms of prion protein in the CSF and has been shown to exhibit high sensitivity of $92 \%$ and specificity of $100 \%$ for prion disease.

CONCLUSION: This case highlights the difficult diagnosis of CJD which may be masked by other diagnoses such as mood disorders. CJD should be strongly considered in patients with rapid and sudden cognitive decline and novel tests such as RT-QuIC can assist with the diagnosis.

\section{THE DIFFICULT DIFFERENTIATION BETWEEN CAUSES OF INFLUENZA RELATED CARDIOMYOPATHY}

Rohan B. Parikh; Krupa K. Solanki; Muhammad U. Khalid; Ritha Kartan. Western Reserve Health Education, Youngstown, OH. (Control ID \#3146297)

LEARNING OBJECTIVE \#1: The 2017-18 influenza season was a high severity season with an estimated 80,000 flu related deaths. Although mortality in these patients is usually due to respiratory failure, recent studies have implicated influenza in a wide spectrum of cardiovascular diseases. Acute myocardial infarction is a well known complication but myocarditis has been infrequently associated with it. We aim to heighten the awareness of acute viral myocarditis for early recognition and prompt management during seasonal episodes of influenza infection.

CASE: Patient A is an 88-year-old male with known coronary artery disease who presented with dyspnea and productive cough. Lab studies were positive for influenza A, mild kidney injury, and troponin I that trended up to $12.2 \mathrm{ng}$ / $\mathrm{mL}$. ECG showed normal sinus rhythm with few premature ventricular contractions. Echocardiogram showed preserved ejection fraction, grade I diastolic dysfunction, and no wall motion abnormalities. Patient B is an 88year-old male with no significant past medical history who presented with dyspnea. Physical examination was significant for hypoxia and irregular rhythm. ECG showed atrial fibrillation. Lab studies were positive for influenza A, mild kidney injury, and troponin I that trended up to $36.6 \mathrm{ng} / \mathrm{mL}$. Echocardiogram showed ejection fraction of $18 \%$, grade I diastolic dysfunction, and apical akinesis. Coronary catheterization showed severe disease in LMCA, LAD, and RCA.

IMPACT/DISCUSSION: Influenza related myocarditis is due to diffuse inflammation of the myocardium leading to focal myocyte necrosis. Additionally, influenza can trigger myocardial infarction by inducing a hypercoagulable and inflammatory state. Both of these states lead to significant myocardial damage and high troponins leading to cardiomyopathy. While a high degree of suspicion for myocarditis needs to be kept in mind, differentiating between the two is difficult and often requires cardiac catheterization. Recent post-mortem studies have shown that myocarditis is common in fatal cases of influenza and often goes undiagnosed. Patient A was diagnosed with influenza associated myocarditis and was treated conservatively with full recovery. Patient B opted for PCI as opposed to CABG due to his age. He had a complicated hospital course but made a full recovery. This decision making was primarily guided by echocardiographic assessment for wall motion abnormalities in both patients.

CONCLUSION: The pulmonary manifestations in influenza are prominent and may mask cardiac involvement. These cases also highlight the need for guidelines to differentiate ischemic versus non-ischemic cardiomyopathy in the setting of influenza to guide appropriate management.

\section{THE DISEASE YOU KEEP MEANING TO LOOK UP}

Hannah Bernstein; Corey Costantino; Tameka Smith; Celeste Newby. Tulane University School of Medicine, New Orleans, LA. (Control ID \#3184055)

LEARNING OBJECTIVE \#1: Apply the H-score to estimate the probability of HLH

LEARNING OBJECTIVE \#2: Review the current recommended treatment of EBV-associated HLH 
CASE: A 28 year-old man presented with a four-day history of fever, headache, nausea, vomiting, diarrhea, and confusion. He immigrated from Honduras 6 years earlier and had no significant past medical history. He had a temperature of $103.2^{\circ} \mathrm{F}$, pulse of $133 \mathrm{bpm}$, and BP of $126 / 60 \mathrm{mmHg}$. He was alert and oriented but had difficulty answering questions. Physical exam demonstrated hepatosplenomegaly. No nuchal rigidity was present. Lab work revealed platelet count $42 \times 10^{9} / \mathrm{L}$, AST $260 \mathrm{U} / \mathrm{L}$, ALT $139 \mathrm{U} / \mathrm{L}$, triglycerides $991 \mathrm{mg} / \mathrm{dL}$, CRP 196, LDH 2292 U/L, and lipase of 205 U/L. D dimer 6.8 $\mathrm{ng} / \mathrm{mL}$. Empiric treatment for sepsis from possible intra-abdominal infection was initiated. On hospital day one, the patient was found obtunded and required intubation. CT head and MRI brain were did not reveal any acute pathology. CSF had no pleocytosis; CSF bacterial cultures and viral PCR were negative. Lab work revealed worsening transaminase levels and pancytopenia. Further testing revealed a markedly elevated ferritin $(>26,000 \mathrm{ng} / \mathrm{mL})$ and IL-2 (53,000 U/mL). Serum EBV DNA PCR titer was 95,500 cpy/mL. These lab studies and bone marrow biopsy results confirmed a suspected diagnosis of (HLH). Induction chemotherapy per HLH-2004 protocol was initiated. The patient responded to treatment with resolution of encephalopathy, normalization of lab values, and significant improvements of EBV viremia.

IMPACT/DISCUSSION: HLH is an aggressive and potentially lifethreatening immune activation syndrome that is often associated with viral etiologies or malignancy in adult populations. EBV is the most commonly reported viral trigger, with mortality rates ranging 20 to $88 \%$ even with aggressive treatment. The diagnosis of HLH should be considered in adults presenting with multi-organ system failure, cytopenia, acute liver injury, and markedly elevated ferritin levels. The $\mathrm{H}$-score, which includes points for fever, organomegaly, and lab abnormalities, is a risk-stratification tool that can estimate the probability of HLH, allowing for prompt initiation of treatment. The mainstay of HLH treatment is etoposide and dexamethasone, with cyclosporine and intrathecal methotrexate considered in patients with CNS involvement. In EBV-associated HLH the addition of rituximab is associated with lower EBV titers and ferritin level at 1 month. Treatment with anti-virals is also recommended in patients with active viremia, although supporting data is sparse. Hematopoetic stem cell transplantation is considered for patients who are refractory to initial therapy. The patient described herein had excellent initial recovery with cyclosporine induction, etoposide, rituximab, and a course of ganciclovir.

CONCLUSION: HLH is a potentially life-threatening condition that the internist needs to recognize early so appropriate treatment can be initiated. The H-score can be used to risk stratify patients.

\section{THE EYES HAVE IT: RARE MANIFESTATIONS OF ADULT- ONSET IGA VASCULITIS}

Briggs Hoyt $^{2}$; Naomi Habib ${ }^{1}$; Surabhi Uppal ${ }^{3}$; Ishani Shah $^{3}$; Madeline Sing$\mathrm{er}^{1}$; Binh T. Duong ${ }^{4}$. ${ }^{1}$ St Joseph's Hospital and Medical Center/Creighton University Medical School, Phoenix, AZ; ${ }^{2}$ Creighton University School of Medicine, Phoenix, AZ; ${ }^{3}$ St Joseph's Hospital and Medical center, Phoenix, AZ; ${ }^{4}$ St. Joseph Hospital and Medical Center, Phoenix, AZ. (Control ID \#3176629)

LEARNING OBJECTIVE \#1: Identify classic features of IgA Vasculitis in the setting of a complicated medical background.

LEARNING OBJECTIVE \#2: Be able to associate rare manifestations of IgA vasculitis based on disease physiology.

CASE: A 49-year-old man presented with 1 week of chest pain, fever, and a rash. Extensive medical history included replaced congenital bicuspid aortic valve (on warfarin), stroke, pulmonary embolus, and chronic kidney disease.
Exam was significant for bilateral crackles and a hemorrhagic palpable purpuric rash on his legs. Labs showed platelets 105, subtherapeutic INR 1.7, and creatinine 5.46 (baseline 3.5), with urinarlysis significant for microscopic hematuria and proteinuria, and mild pulmonary congestion on chest imaging. He underwent diuresis and hemodialysis for fluid overload due to acute renal failure. On day 4 of admission, he developed sudden left-sided vision loss and was diagnosed with anterior ischemic optic neuropathy (AION). Echocardiogram and MRI were negative for thrombi. On day 5, his rash was noted to extend to his abdomen and chest. Autoimmune and infectious workup was negative; dermatology suspected reactive vasculitis and recommended supportive care. On day 6 , he began experiencing chest pain and went into cardiogenic shock and cardiac arrest. He progressed to multiorgan failure despite intensive therapy, and care was withdrawn. The day after death, skin biopsy with immunofluorescence revealed leukocytoclastic vasculitis and perivascular IgA granules, consistent with IgA vasculitis (IgAV).

IMPACT/DISCUSSION: IgAV is primarily a pediatric disease, with a much rarer in its incidence in adults $(0.1-0.8$ per 100,000$)$, often associated with higher morbidity and mortality. The majority of symptoms are due to formation of IgA complex deposits within end-organs small vessels, with the resultant classic tetrad of palpable purpura, polyarthropathy, gastrointestinal (GI) manifestations and nephritic syndrome. Recognizing IgAV in adults without this classic presentation can be difficult. Our patient's confounding factors, including his previous thrombi and presumed uremic thrombocytopenia, made the diagnosis of IgAV more challenging. Further, in keeping with its effects on small vessels, IgA vasculitis can presents with mononeuropathies, such that monocular vision loss in the presence of a purpuric rash should prompt suspicion of vasculitis. AION is mainly associated with giant cell arteritis, but is a rare complication of other vasculitides as well, with only 2 other IgAV-associated cases described previously. Diagnosis is made on the basis of histopathology. No randomized-controlled trials exist to guide therapy; however experts recommend pulse-dose steroids for rapidlyprogressive renal failure.

CONCLUSION: IgA Vasculitis should be considered when palpable purpura, athralgia, and GI/renal symptoms present concurrently, even in an adult. The sentinel event for a vasculitis can theoretically occur anywhere that vascular occlusion would present acutely.

\section{THE FORGOTTEN PRE-CANCEROUS ITCH: VULVAR LICHEN SCLEROSIS}

Mariella Ntamatungiro. Weill Cornell Medical College-New York Presbyterian Medical Center, New York, NY. (Control ID \#3186124)

LEARNING OBJECTIVE \#1: Review vulvar lichen sclerosis as a differential for recurrent vulvar itching.

LEARNING OBJECTIVE \#2: Recognize vulvar lichen sclerosis as a high risk precursor lesion for vulvar squamous cell carcinoma.

CASE: 41 yo monogamous woman with 2 children presented with recurrent vaginal itching over a period of 4 months. The itching occured intermittently and unpredictably without discharge, dysuria, bleeding, or dyspareunia. Notably she denied new partners, douching, tight clothing, or change in hygiene products. She reported regular menstrual cycles and had her last child 3 years ago. She has had 4 clinic visits over this time frame. She has tested negative for bacterial vaginosis, candidiasis, trichomonas, gonorrhea-chlamydia, HIV, syphilis, and urinary tract infections. Pap with HPV testing was normal. Empiric treatment for candidiasis and bacterial vaginosis with fluconazole and metronidazole did not resolve her itching. On external pelvic exam, pertinent positives included erythema and skin hypo-pigmentation of the labia 
minora with patchy scaly skin. There was an absence of vulvar discharge or tenderness. Speculum exam was unremarkable with a benign cervix and absence of tenderness with bimanual exam. She localized her itching to the hypo-pigmented area on exam. Repeat infectious testing returned negative. Patient was referred to gynecology and dermatology with subsequent pathology confirming lichen sclerosis. She was treated with topical clobetasol ointment.

IMPACT/DISCUSSION: Lichen sclerosis is a rare autoimmune vulvar dermatological pathology that often presents with vulvar pruritus in $90 \%$ of cases. Vulvar pruritus often is severe enough to induce significant scratching resulting in skin scarring, dyspareunia, apareunia, and burning in some women. It causes vulvar skin changes such as hypo-pigmentation, skin thinning and scarring. Vulvar lichen sclerosis prevalence is unknown however it commonly occurs between the ages of 40 to 60 . Under diagnosis is common and are due to many reasons. First, the condition is rare and practitioners often focus on more prevalent vulvar pruritic conditions such as bacterial vaginosis, vulvovaginal candidiasis, and trichomonas especially in sexually active and pre-menopausal women. Second, the vulvar pruritus episodes can be transient in many women leading to inconsistent or delayed treatment with topical corticosteroids. However, recognition matters given $5 \%$ of vulvar lichen sclerosis cases progress to squamous cell carcinoma. Long term therapy with topical corticosteroids does reduce risk of primary vulvar cancer in recent literature. However, only 1 in 4 women with lichen sclerosis are consistently prescribed maintenance therapy. Primary care doctors should refer to vulvar specialists for close monitoring for progression to malignancy and for consistent treatment.

CONCLUSION: Vulvar lichen sclerosis is an under diagnosed and treatable cause of vulvar itching. Vulvar lichen sclerosis progresses to squamous cell carcinoma in $5 \%$ of cases.

\section{THE HAART OF THE MATTER: LIFE THREATENING MEDICA- TION INTERACTIONS IN HIV PATIENTS}

Phillip S. Hamilton. UPMC, Pittsburgh, PA. (Control ID \#3176929)

LEARNING OBJECTIVE \#1: Recognize potential for drug-drug interactions when a medication is discontinued

LEARNING OBJECTIVE \#2: Manage inhaled corticosteroids in patients with HIV on Highly Active Antiretroviral Therapy (HAART)

CASE: A 55-year-old man with a history of HIV, hypertension, and COPD presented with acute onset of hypotension. He had been on the combination antiretroviral Elvitegravir-cobicistat-emtricitabine-tenofovir alafenamide (Genvoya ${ }^{\circledR}$ ) for approximately one year but had discontinued it ten days prior to presentation. His last known CD4 count was $273 \mathrm{c} / \mathrm{uL}$ one month prior. He was compliant with his other medications including HCTZ/lisinopril, inhaled tiotropium and inhaled fluticasone/vilanterol. He presented with a blood pressure of $60 / 40 \mathrm{mmHg}$, a pulse of $130 \mathrm{bpm}$, lactate of $3.7 \mathrm{mmol} / \mathrm{L}$ and $\mathrm{WBC}$ of $9.8 \mathrm{~K} / \mathrm{cmm}$. Due to concern for sepsis he was given IV antibiotics, IV fluids, and IV methylprednisolone and transferred to a higher level of care. On arrival, he had only mild symptoms to report and his blood pressure was 139/87 mmHg. A basic infectious evaluation was unremarkable. Reviewing his medications, it was noted that the cobicistat contained in the Genvoya he had discontinued ten days prior inhibits the metabolism of fluticasone. For several months the patient had noted worsening central obesity and increased prominence of the dorsocervical fat pad consistent with a cushingoid appearance. The sudden removal of the cobicistat resulted in a drop in serum steroid levels in a patient with a suppressed adrenal axis. An ACTH stimulation test was obtained, 0800 cortisol was $1.5 \mathrm{ug} / \mathrm{dL}$ which increased to $16 \mathrm{ug} / \mathrm{dL}$. Endocrine consultants agreed that the transient hypotension was likely related to adrenal suppression from the cobicistat/ fluticasone combination. He was started on hydrocortisone and inhaler changes were made.

IMPACT/DISCUSSION: This is a unique case of a drug-drug interaction leading to a presentation of adrenal insufficiency. The combination of the cobicistat and inhaled fluticasone led to corticosteroid excess and adrenal suppression. The removal of the HAART effectively decreased the level of circulating corticosteroid which resulted in hypotension that improved with steroid administration. The cobicistat in the combination HAART drug is a potent CYP3A inhibitor, whose purpose is to increase or "boost" the levels of elvitegravir via CYP inhibition. In this case the drug-drug interaction became clinically evident with the removal of the HAART. The culprit interacting drug was an inhaler, which can be overlooked as a potential interacting medication. CONCLUSION: Internists must have a heightened suspicion for drug-drug interactions with HIV medications, including inhaled medications which are often overlooked. Among the inhaled corticosteroids, fluticasone has a high likelihood of interaction and should be avoided in patients on a boosted HAART regimen such as Genvoya.

\section{THE HEADACHE WITH A WORLD OF POSSIBLITIES}

Roberto Herrera; Michael P. Smith. University of Nebraska Medical Center, Omaha, NE. (Control ID \#3186091)

LEARNING OBJECTIVE \#1: Identify the differential diagnosis of meningitis to include geographic risk factors.

LEARNING OBJECTIVE \#2: Understand common incidental findings in immigrant populations can mask typical disease presentations.

CASE: A 27 year-old woman presented with one day history of worsening neck pain, stiffness, and headache. She also reported photosensitivity and vomiting. She denied ever being vaccinated for meningitis and immigrated to the United States from Guatemala 3 years ago. She appeared uncomfortable but was afebrile and oriented to person, place, and time. No focal neurologic deficits were observed. She did not have any evidence of rash or petechiae. The neck was stiff and flexion produced pain. A lumbar puncture revealed a CSF glucose of $32 \mathrm{mg} / \mathrm{dL}$ and a CSF protein of $91 \mathrm{mg} / \mathrm{dL}$. CSF cell count with differential revealed 400/cm WBC and $16 \%$ neutrophils. Gram stain of the CSF was negative. A meningitis panel of CSF was negative as well as a West Nile virus IgG and IgM. A head CT showed multiple cysts with nodular mural irregularity and punctate calcifications in and along both cerebral hemispheres consistent with Neurocysticercosis. A Strongyloides antibody IgG was also positive.

IMPACT/DISCUSSION: Headache and neck stiffness are commonly encountered by the internist. As the immigrant population continues to grow in the United States, it is important to identify incidental findings that may mask a typical presentation. It is estimated that 50 million people worldwide have a cysticercosis infection. Cysticercosis is endemic in Central and South America. It is also endemic in India, Asia, and sub-Saharan Africa. Most cases of Neurocysticercosis are found incidentally via radiographic imaging in asymptomatic patients. The most common presentation of symptomatic neurocysticercosis is seizure. Another common presentation is with symptoms of elevated intracranial pressure associated with parenchymal disease. This has an important treatment consideration as any antiparasitic therapy should be held if there is untreated hydrocephalus, high cyst burden, or presence of calcified lesions only. Antiparasitic therapy carries the risk of worsening neurologic symptoms due inflammation around the degenerating cysts. Strongyloides is also endemic in tropical and subtropical regions. It is estimated that the prevalence in subtropical regions may be as high as 25 percent. Typical manifestations of the disease include gastrointestinal symptoms that 
tend to wax and wane. Strongyloidiasis is also associated with cutaneous and pulmonary symptoms that are present chronically.

CONCLUSION: It is important for the general internist to identify common presentations amongst previously uncommon imaging and laboratory findings.

\section{THE HEART OF THE MATTER: A CASE OF CARDIAC AMY-} LOIDOSIS PRESENTING AS SYNCOPE

Curtis Kovaleski ${ }^{1}$; Ami DeWaters ${ }^{2} .{ }^{1}$ Hershey Medical Center, Hummelstown, PA; ${ }^{2}$ Penn State Hershey Medical Center, Hershey, PA. (Control ID \#3186420)

LEARNING OBJECTIVE \#1: Describe the clinical presentation of syncope due to cardiac structural disease.

CASE: A 73-year old woman presented for syncope without palpitations, prodrome or chest pain. On presentation, pulse was 48, blood pressure 108/66, and oxygen saturation $94 \%$. On exam, she was fatigued with no distress, bradycardic with no murmurs, and had one-plus pitting edema. She had an elevated creatinine of three and a brain naturetic peptide of 257. An electrocardiogram showed a junctional rhythm, low voltage, and bradycardia. A transthoracic echocardiogramE was obtained and showed an ejection fraction of $50-55 \%$ and a speckled appearance in the hypertrophied left ventricular wall. With suspicion for infiltrative cardiomyopathy, serum protein electrophoresis was sent and returned positive for monoclonal immunoglobulin. Too unstable for cardiac MRI, she underwent a bone marrow biopsy which showed plasma cell myeloma along with a positive congo red stain and she was diagnosed with cardiac amyloidosis associated with plasma cell myeloma. Chemotherapy was started, however after her first dose; she proceeded into anuric renal failure. After discussion with the patient and family, dialysis was discontinued and she proceeded with hospice care.

IMPACT/DISCUSSION: Syncope has multiple different etiologies, including cardiac arrhythmias, cardiac structural disease, reflex syncope, and neurologic causes. One way to distinguish cardiac etiologies from other etiologies of syncope is the lack of prodrome. In this case, the patient had no warning signs prior to syncope, which was indicative of a cardiac etiology and initiated a cardiac evaluation that revealed cardiac amyloidosis. In cardiac amyloidosis, extracellular abnormal protein deposits in cardiac tissue leading to diastolic and systolic dysfunction and congestive heart failure. Recognizing the symptoms of diastolic heart failure like syncope is key in early diagnosis which is instrumental in a patient's course. Prognosis is dependent on the number and severity of organs involved, with cardiac involvement carrying the worst prognosis. There isa median survival of six months in untreated patients from onset of congestive heart failure. Therefore, prompt evaluation for amyloidosis is necessary. Initial cardiac work up often includes electrocardiogram and echocardiogram. Cardiac MRI can be very helpful to see diffuse subendocardial myocardial enhancement, but definitive diagnosis is best done by cardiac biopsy showing amyloid. However as in the case above, a less invasive bone biopsy with amyloid staining can be done to identify cardiac $\mathrm{AL}$ amyloidosis. From staining, accurate typing of amyloid is possible, allowing tailored treatments necessary for targeting the underlying plasma cell disorder. CONCLUSION: Syncope without a prodrome is indicative of a cardiac etiology. Cardiac amyloidosis can present with syncope, and prompt evaluation for cardiac amyloidosis is necessary to avoid poor outcomes.

\section{THE HUNT THAT TAKES YOUR BREATH AWAY}

syed mohsin saghir ${ }^{1}$; SYED R. SHAH ${ }^{2}$; Arhama A. Malik ${ }^{4}$; SOBIA SYED 3 . ${ }^{1}$ unlv school of medicine, Las Vegas, NV; ${ }^{2}$ UNIVERSITY OF NEVADA LAS VEGAS, Las Vegas, NV; ${ }^{3}$ Ayub Medical College, Las Vegas, NV; ${ }^{4}$ UNLV, Las Vegas, NV. (Control ID \#3186141)
LEARNING OBJECTIVE \#1: Recognize clinical features of Hantavirus pulmonary syndrome (HPS)

\section{LEARNING OBJECTIVE \#2: Manage HPS}

CASE: 64 yr old male with no significant PMH was admitted with $\mathrm{C} / \mathrm{C}$ of high grade fever, myalgias, vomiting and diarrhea. Patient reported a recent history of travel to Cambodia where he worked in areas of rodent exposure and experienced mosquito bites. On presentation he was hemodynamically stable and had a benign physical exam. Pertinent lab findings included a plt count of 48k, Cr 1.3 and PBS showed $1 \%$ parasitemia due to P. vivax. He was started on antimalarials and was admitted for further management. Extensive work up was done to exclude infectious and arthropod borne illnesses. Respiratory biofire, HIV, hepatitis panel and pan cultures were negative. Serology for chikungunya, dengue fever, leptospirosis, RMSF, West Nile and Zika virus was negative. Fungal etiologies were excluded. Patient during hospital course developed respiratory distress with tachypnea, hypoxia and became hypotensive. X-ray chest showed dense bilateral infiltrates. Patient was upgraded to ICU for management of pulmonary edema and was intubated for ventilator support. Echo showed normal left ventricular function. Despite adequate supportive measures patient continued to deteriorate and developed AKI requiring hemodialysis. Repeat PBS showed no organisms. Detection of Hantavirus specific IgM lead to the final diagnosis of HPS. Patienteventually had a tracheostomy and PEG done due to difficulty in weaning off the ventilator and was discharged to an LTAC.

IMPACT/DISCUSSION: The diagnosis of HPS was pursued as patient continued to deteriorate despite getting adequate antimalarial treatment concluding that the malaria was probably a red herring in a traveler returning from an endemic area. HPS is a life threatening rodent borne RNA viral infection. Hantaviruses are divided into New and Old World viruses. New World viruses cause HPS mostly in America. Old World viruses cause hemorrhagic fever with renal syndrome (HFRS) localized to Asia and Europe. Our patient met the clinical picture of HPS although being from Asia where HFRS is more common. Presenting features include fever, myalgias, vomiting, diarrhea and abdominal pain with rapid transition to non-cardiogenic pulmonary edema and shock followed by resolution of respiratory and hemodynamic abnormalities in the next few days. Recovery is associated with prolonged weakness. Lab findings include thrombocytopenia, leukocytosis and an atypical lymphocytosis. Diagnosis is dependent upon detection of Hantavirus IgM and IgGs and PCR on blood or detection of Hantavirus Ag on tissue. No specific treatment is available. Supportive care including mechanical ventilation, vasopressors, inotropes and cautious IV fluids are needed in ICU setting.

CONCLUSION: Renal involvement in HPS is usually mild, but cases have been reported showing severe disease. This case proves that HPS with severe renal failure may not be as uncommon as once thought.

\section{THE IDENTITY OF THE MYSTERY ANASARCA: THE DE- CREASE OF PLATELET COUNTS MAY TELL YOU THE CLUE FOR DIFFERENTIAL DIAGNOSIS.}

$\underline{\text { Ryutaro Nagaya }}^{1}$; Mutsumi Murayama ${ }^{2}$; takehiko takeda ${ }^{1}$; Hidekazu Takahashi $^{2}$; tomokatsu kato ${ }^{1}$; Takamasa Tanaka ${ }^{1}$; Mitsunori Iwase ${ }^{1}{ }^{1}$ Toyota memorial hospital, Toyota-city, Japan; ${ }^{2}$ Toyota memorial hospital, Toyita-city, Japan. (Control ID \#3183744)

LEARNING OBJECTIVE \#1: Identify thrombocytopenia, anasarca, and fever as the triad symptoms in TAFRO syndrome.

LEARNING OBJECTIVE \#2: Understand the importance of prompt diagnosis and early interventions of TAFRO syndrome.

CASE: The patient was a 58-year-old Japanese female without a past medical history. She presented to our emergency department with a chief complaint of 
abdominal pain lasting for 1 week. Her vital signs were within normal range. She had mild diffuse abdominal tenderness without rebound tenderness. Laboratory tests revealed elevation of $\operatorname{WBC}\left(9.7 \times 10^{3} / \mu \mathrm{L}\right), \mathrm{CRP}(20 \mathrm{mg} / \mathrm{dL})$ and ALP(575 U/L). Levels of platelet count $(13.9 \mathrm{~g} / \mathrm{dL})$ and $\mathrm{LDH}(231 \mathrm{U} / \mathrm{L})$ were normal. CT showed moderate ascites without morbid lymphadenopathy or hepatosplenomegaly. She was admitted for presumed peritonitis and started on cefmetazole. On the $3^{\text {rd }}$ day, she had a fever of 39 degrees. Blood and ascitic fluid culture remained negative. Additional laboratory tests for HIV, CMV, hepatitis $\mathrm{B}$ and $\mathrm{C}$ virus, $\mathrm{EBV}$, and tuberculosis were unremarkable. Tumor markers and anti-nuclear antibody were also negative. Upper and lower endoscopies revealed no abnormal pathology. CT showed pleural effusion additionally. On the $9^{\text {th }}$ day, platelet count dropped to $9.2 \times 10^{3} / \mu \mathrm{L}$. We added methylprednisolone and performed a bone marrow biopsy for suspected TAFRO syndrome. we also started anti-DIC therapy. On the $12^{\text {th }}$ day, hemodiafiltration were started but shock vital and decreasing of platelet count sustained. On the $14^{\text {th }}$ day, the results of bone marrow biopsy showed reticulin myelofibrosis, leading to our diagnosis of steroid-refractory TAFRO syndrome. We started tocilizumab as well as ciclosporin. She responded well and recovered, and was eventually discharged in good condition.

IMPACT/DISCUSSION: Impact We should suspect TAFRO syndrome in patients with thrombocytopenia, anasarca, and fever. Discussion TAFRO syndrome was first identified in 2010. Although most cases are reported from Japan, The number of Caucasian patients cases is increasing. It involves a constellation of syndromes:Thrombocytopenia(T), Anasarca(A), Fever(F), Reticulin fibrosis $(\mathrm{R})$, and Organomegaly $(\mathrm{O})$. The first three criteria must be present for the diagnose of this syndrome. Our patient had a normal platelet count at initial presentation, which was atypical. We eventually diagnosed as TAFRO syndrome when a low platelet count became evident. However abdominal pain and elevated ALP levels with normal LDH levels may be also helpful to rule in this syndrome earlier. Most patients are initially treated with corticosteroid, but only $52 \%$ of the patients respond well to it. Some refractory cases require more aggressive therapy such as tocilizumab and ciclosporin. TAFRO syndrome often progresses rapidly but is curable as with our case. Prompt diagnosis and interventions are crucial to improve the outcome of this syndrome.

CONCLUSION: TAFRO syndrome was first reported in Japan and not well known in the US. However we should consider this syndrome as a differential diagnosis in patients with more than 1 criteria of the triad.

\section{THE IMPORTANCE OF FILLING UP THE TANK}

Thomas Rusher; Deepa Bhatnagar. Tulane University School of Medicine, New Orleans, LA. (Control ID \#3185294)

LEARNING OBJECTIVE \#1: Demonstrate the cardiac manifestations of hypophosphatemia.

CASE: A 46 year old woman presented with nausea, vomiting and epigastric pain for 1 day with associated poor oral intake. She had a history of peptic ulcer disease and alcohol abuse. She denied any chest pain, shortness of breath, or palpitations. Exam was significant for epigastric tenderness. Electrocadiogram showed bradycardic sinus rhythm with prolonged QTc to $537 \mathrm{~ms}$ as well as biphasic t-waves. Labwork was significant for severe hypophosphatemia of $0.9 \mathrm{mg} / \mathrm{dL}$. Her potassium was $3.5 \mathrm{mmol} / \mathrm{L}$, and magnesium of $1.8 \mathrm{mg} / \mathrm{dL}$. Her anion gap was calculated to be 18 with positive ketones on urinalysis. The patient received IV ondansetron and promethazine in the emergency department with improvement in her nausea symptoms. Upon transfer to the observation unit, she suddenly became apneic and pulseless. Cardiac monitoring revealed ventricular tachycardia, and later, ventricular fibrillation. The patient was rescucitated with chest compressions and 2 defibrillator shocks.
Emergent coronary angiogram showed no significant coronary disease. Follow-up transthoracic echocardiogram revealed decreased left ventricular function with ejection fraction of 20-25\%. The patient recovered in the hospital with repletion of her phosphorous. Repeat echocardiogram 1 month after dishcarge showed return of normal left ventricular systolic funciton.

IMPACT/DISCUSSION: Phosphate plays an important role in cardiac function due to the formation of adenosine triphosphate (ATP) and red cell 2,3 diphosphoglycerate, which augments the hemoglobin-oxygen dissociation curve. Depletion of ATP can lead to dysfunction in myocardial contractility as well as decreased oxygen delivery to cardiac tissue. The importance of phosphorous to cardiac function is highlighted in studies which have shown an improvement in left ventricular function with correction of severe hypophosphatemia. Hypophosphatemia has also been linked with significant ventricular ectopic activity. Studies have shown low phosphorous is associated with a higher incidence of ventricular arrhythmias in the setting of acute myocardial infarction as well as in sepsis. Hypophosphatemia is caused by a number of mechanisms including decreased dietary intake, intracellular shift, impaired absorption, and increased renal phosphate excretion. In our case, we suspect the patient's low phosphorous was secondary to decreased PO intake. Her initial metabolic derangements were attributed to starvation ketosis and an over the counter diuretic the patient was taking. Additionally, the patient's EKG showed a prolonged QT interval, likely exacerbated by the IV antiemetics she received, ultimately leading to her ventricular tachycardia.

CONCLUSION: Electrolyte repletion and monitoring of QT intervals are common practices in the hospital setting. This case demonstrates the dangers associated with grossly abnormal electrolyte balance as well as the need to monitor therapies to avoid possibly fatal arrhythmias.

\section{THE KEY IS IN THE HISTO-RY: A CASE OF FEVER IN A TRANS- PLANT PATIENT}

Eduardo Mulanovich $^{1}$; Surya Bhatt ${ }^{2} .{ }^{1}$ University of Alabama - Birmingham, Birmingham, AL; ${ }^{2}$ University of Alabama at Birmingham, Birmingham, AL. (Control ID \#3184506)

LEARNING OBJECTIVE \#1: Describe disseminated histoplasmosis as a cause of sepsis in the immunocompromised host

LEARNING OBJECTIVE \#2: Detail the sensitivity of diagnostic tests for histoplasmosis as well as the importance of histological evaluation

CASE: A 55-year-old man from east Texas with a kidney-pancreas transplant 14 years prior on immunosuppression was transferred for 10 days of fever, abdominal pain, and dyspnea. Physical exam was pertinent for fever, tachycardia, tachypnea, hypoxemia, and bilateral diffuse crackles. CT-chest showed diffuse ground glass opacities. He quickly developed worsening dyspnea, altered mentation, and shock requiring intubation and initiation of CRRT and vasopressors. He was started on broad spectrum antibiotic coverage for hospital acquired pneumonia and PJP. Labs showed creatinine $3.2 \mathrm{mg} / \mathrm{dL}$, lactic acid $0.8 \mathrm{mMol} / \mathrm{L}, \mathrm{WBC} 22,000 / \mathrm{cmm}$ (96\% neutrophils), ALT $146 \mathrm{U} / \mathrm{L}$, Alk Phos $785 \mathrm{U} / \mathrm{L}$, ferritin >30,000 ng/mL, and LDH 1,961 U/L. CT-abdomen and CT-head were unremarkable. Cryptococcal and urine legionella antigens (Ag), T2 candida, and blood and urine cultures were negative. BAL aspergillus Ag, legionella Ag, HSV, CMV, aerobic and anaerobic cultures, and AFB were negative. On hospital day 4, peripheral blood smear revealed intracellular yeast and liposomal amphotericin B was started. Urine Histoplasma Ag was positive on day 9. Cultures from BAL and fungal isolator blood were positive for Histoplasma on day 10 and 22 respectively. He deteriorated and died on day 30.

IMPACT/DISCUSSION: Histoplasmosis is an endemic mycosis of the Ohio and Mississippi river valleys that may present as an opportunistic 
infection. Disseminated histoplasmosis occurs mostly in immunocompromised patients. Fever, weight loss, hepatosplenomegaly, lymphadenopathy, and mucocutaneous lesions are common presenting findings. Common labs include pancytopenia, transaminitis, and elevated ferritin and LDH. Chest Xrays can have a miliary pattern. Diagnosis is made by blood or tissue cultures in $65 \%$ of cases, but this may take 2-8 weeks. Histoplasma urine and serum Ag combined have $95 \%$ sensitivity. Rapid diagnosis may be made from buffy coat or peripheral smear of blood in up to $50 \%$ of cases, as in our case. Histologically, Histoplasma capsulatum appear as intracellular round yeast in leukocytes and are associated with disseminated disease and worse prognosis. Standard treatment is liposomal amphotericin B and should be started immediately as the disease is usually fatal within 2-12 weeks.

CONCLUSION: Suspect disseminated histoplasmosis in immunocompromised patients from endemic areas with sepsis, bilateral pulmonary infiltrates, pancytopenia, and elevated LDH and ferritin. Direct examination of blood through smear or buffy coat is a specific test that can provide a rapid diagnosis when positive since Histoplasma Ag, cultures, or tissue biopsy have a long turnaround time. Empiric therapy should be considered in immunocompromised patients with a clinical picture consistent with disseminated Histoplasmosis before laboratory confirmation.

\section{THE LIMITS OF RELIGIOUS AND MEDICAL BELIEF: AN ETH- ICAL LESSON}

Kevin Liu. Beth Israel Mount sinai, New york, NY. (Control ID \#3186411)

LEARNING OBJECTIVE \#1: In the chronic setting, severely anemic levels can be tolerated as long as no signs of organ failure or hemodynamic instability are present.

LEARNING OBJECTIVE \#2: In the chronic setting, severely anemic levels can be tolerated as long as no signs of organ failure or hemodynamic instability are present.

CASE: A 35 year old female with past medical history of CKD stage 5, and intellectual disability presented to the hospital with menorrhagia and sever anemia of a hemoglobin of $2.6 \mathrm{~g} / \mathrm{dL}$ and undetectable reticulocyte count. Patient vital signs did not show signs of hemodynamic instability. On physical exam patient was not showing signs of distress, pale, but otherwise unremarkable exam. The parents had legal guardianship papers, which stated that they were Jehova's Witnesses and refused blood products for their child. The intensive care unit was consulted, and brought the patient into the critical care unit for further observation of decompensation. The parents provided paperwork stating they could withdraw life-sustaining measures: Because their request to withhold blood was on a religious basis, and the patient had an intellectual disability that prevented her from expressing religious belief, the particular life-saving measure of blood transfusion was religious rather than out of futility, blood could not be held from the patient in life-saving circumstances. The patient began showing signs of liver damage through rising liver enzymes, and troponin rose from 0.139 to 0.200 , showing signs of possible organ damage secondary to severe anemia. Given continued hemodynamic stability and no overt signs of organ failure, no transfusion was given at this time. The patient's labs eventually trending towards recovery, and the patient was eventually able to be discharged with gynecology follow-up for the initiation of a contraceptive.

IMPACT/DISCUSSION: Cognitive function tends to decline at around hemoglobin of $5 \mathrm{~g} / \mathrm{dL}$ in the acute setting, in chronic states allowing time for compensation, lower hemoglobin levels can be achieved. The resilience of the human body in these extreme circumstances has been seen in other case reports, mainly of Jehova's Witnesses. Adults with decision-making capacity have the right to refuse life-saving therapy. In this case, the patient is a protected population due to intellectual disability. In the case of HCA Inc v Miller, where life-sustaining treatment was provided for a child despite parents wishes, and ultimately the court ruled in favor of the physicians who helped the child. This case demonstrates that in a population that cannot speak for itself, if there is a life-saving treatment without demonstrated futility, it should be provided.

CONCLUSION: The practice of medicine should blend science and humanity together in order to make the best possible outcome. But, the most important thing is the patient's life and wishes, and if he/she cannot speak for themselves, we must take it upon ourselves as physicians to protect them.

\section{THE MYSTERY COUGH: UNCOMMON CAUSES OF CHRONIC COUGH}

Hassan A. Raza; Adam Hines; Arineh Melkonian; Cindy Sadikot. New York Presbyterian Queens, Dix Hills, NY. (Control ID \#3175692)

LEARNING OBJECTIVE \#1: Recognize the differential diagnosis of a chronic cough, including its uncommon causes

LEARNING OBJECTIVE \#2: Recognize anchoring and availability biases as significant causes of delays in diagnosis

CASE: A 53-year-old man with no smoking history presented to the primary care clinic with persistent productive cough with white sputum and wheezing of 3 months duration. He initially presented to an urgent care center and our $\mathrm{ED}$, was diagnosed with sinusitis and treated with steroids and antibiotics without relief. He had been using an albuterol inhaler with some relief of symptoms. He was suspected to have some form of reactive airway disease, exacerbated by his exposure to dust particles during his work as a mechanic. He was recommended to wear a mask at work, continue albuterol as needed, and to obtain pulmonary function testing when his symptoms resolve. One week later, his symptoms progressed with worsened wheezing and scant hemoptysis. As he had a history of latent tuberculosis (TB) and unresolved symptoms, a computed tomography scan of the lung without contrast was pursued. It showed a linear radiopaque density identified within the bronchus intermedius, extending into the right lower lobe bronchus, consistent with a radiopaque foreign body. He subsequently underwent flexible bronchoscopy and had the foreign body removed. On pathology, it was noted to be consistent with a fishbone. After the procedure, his symptoms completely resolved.

IMPACT/DISCUSSION: Chronic cough affects roughly $12 \%$ of the general population on a daily or weekly basis, ${ }^{1}$ significantly decreasing a patient's quality of life. ${ }^{2}$ Respiratory diseases, including chronic cough, is the most common reason for an adult to visit their primary care physician. ${ }^{3}$ The 3 most common causes of chronic cough include: post-nasal drip syndrome, asthma, and gastroesophageal reflux disease. ${ }^{4}$ Less common causes include: bronchiectasis, lung cancer, and aspiration (food in adults, foreign bodies in children). ${ }^{5}$ Our patient did not recall coughing or choking during any of his meals prior to onset of symptoms. This missing piece of information made this diagnosis more of a challenge. In one study, the most common symptoms of foreign body aspiration were chronic cough, hemoptysis, fever, and dyspnea, and only $7 \%$ of patients presented with choking. ${ }^{6}$ This case also demonstrates anchoring and availability bias, problems of which are common in the primary care setting. ${ }^{7,8}$ His partial response to bronchodilators, and the high prevalence of TB in our community delayed his diagnosis, leading to significant morbidity. We feel this case adds to the medical literature by emphasizing the less common causes of chronic cough.

CONCLUSION: Chronic cough is associated with significant morbidity and decline in quality of life for patients, in which it is imperative for a general internist to maintain broad differential diagnoses. An absence of a history of choking during meal ingestion does not effectively rule out a foreign body aspiration as the etiology for chronic cough. 


\section{THE PERFECT STORM: DISSEMINATED HODGKIN LYMPHO- MA PRESENTING WITH CHOLESTATIC LIVER INJURY AND HEMOPHAGOCYTIC LYMPHOHISTIOCYTOSIS}

Neethu E. Abraham; Nathanael Bailey; Bryan Rea; Kristen M. Livesey. University of Pittsburgh Medical Center, Pittsburgh, PA. (Control ID \#3179470)

LEARNING OBJECTIVE \#1: Recognize hemophagocytic lymphohistiocytosis as a cause of acute cholestatic liver injury

LEARNING OBJECTIVE \#2: Distinguish causes of hemophagocytic lymphohistiocytosis and the impact on management

CASE: A 49-year-old women with irritable bowel syndrome presented with 2 weeks of lethargy, confusion and 3 days of jaundice, abdominal distension and lower extremity edema. Family reported a 40lb weight loss, intermittent fevers and night sweats. Examination was notable for confusion with jaundice, tender hepatosplenomegaly, left axillary lymphadenopathy and anasarca. Labwork was notable for pancytopenia, normal AST/ALT and elevated total bilirubin (17.7 mg/dL), direct bilirubin (13.2 mg/dL), alkaline phosphatase (309 IU/L), ferritin (>1500 ng/mL), triglycerides and LDH. CT of the chest, abdomen and pelvis with contrast was notable for left axillary, right paratracheal and retroperitoneal lymphadenopathy and hepatosplenomegaly with hypodense lesions throughout the liver and spleen. Workup for liver failure included negative hepatitis A, B and C, CMV, HSV and HIV serologies. EBV PCR was 41,000 copies. Left axillary lymph node biopsy revealed Reed-Sternberg cells and EBV positive cells consistent with classic Hodgkin lymphoma (HL). Bone marrow biopsy showed evidence of hemophagocytosis and extensive marrow involvement by HL. Diagnosis of HLH due to classic HL was made and she was treated with etoposide and dexamethasone. She subsequently received Cytoxan, Rituxan and dosereduced Gemcitabine with resolution of her cholestasis.

IMPACT/DISCUSSION: HLH is a rare but fatal disease. This case offers an opportunity to highlight the high degree of suspicion required for diagnosis. Early diagnosis can alter the course and prognosis of the disease if the secondary cause is appropriately addressed. HLH is characterized by excessive inflammation due to a hyper-stimulated but dysregulated immune system. Initial signs and symptoms can mimic common infections, hepatitis or encephalitis, making prompt recognition challenging. In our patient, the cholestasic liver injury made clinical recognition of HLH challenging. While LFT abnormalities are common in cases of HLH, it is a rare cause of cholestasis. The cholestatic injury is due to portal and biliary infiltration by lymphocytes and histiocytes. HLH can occur as a familial or acquired disease; the latter thought to be triggered by infection, malignancy or connective tissue disorder. Development of HLH is rarely associated with $\mathrm{HL}$ and interestingly EBV is more prevalent in these cases. Moreover, HLH extension to the liver by $\mathrm{HL}$ is typically seen in advanced disease.

CONCLUSION: This case demonstrates the need for a high degree of clinical suspicion for the diagnosis of HLH in a patient presenting with fever, cytopenia and cholestasis with an extensive unremarkable infectious work up. The prompt initiation of treatment alters the disease course given the high associated mortality with unrecognized disease.

\section{THE PERICARDIAL EFFUSION WITH A WORLD OF POSSIBIL- ITIES}

Kaiwen Sun; Michael P. Smith. University of Nebraska Medical Center, Omaha, NE. (Control ID \#3185962)

LEARNING OBJECTIVE \#1: Develop an approach to a patient with a pericardial effusion
LEARNING OBJECTIVE \#2: Recognize indications for aggressive treatment of a pericardial effusion

CASE: A 35 year-old man presented for generalized weakness and shortness of breath for two days. The shortness of breath was worse with exertion. He denied chest pain, cough, syncope or edema. He had a history of hypothyroidism, heart failure with preserved ejection fraction, HTN and ESRD. His vitals were unremarkable. He had jugular venous distention $6 \mathrm{~cm}$ above the suprasternal notch, muffled heart sounds, abdominal distention, mild pedal edema, and clear lung fields. He had a CHF peptide of 229, BUN of 90, anion gap of 23, unremarkable troponins and TSH. Chest x-ray remonstrated marked interval increase in cardiac sihouette size compared to a study 2 weeks ago, and TTE was significant for a large circumferential pericardial effusion with tamponade physiology and concentric LVH with ejection fraction of 35-40\%. With a tentative diagnosis of uremic pericardial effusion, $1150 \mathrm{ml}$ of serosanguinous fluid was removed via urgent pericardiocentesis with drain placement. The drain contained $35 \mathrm{ml}$ of fluid and there was resolution of dyspnea and JVD. He received intensified PD with BUN improving to 72 upon discharge. Pericardial fluid demonstrated increased RBC and WBC (91\% PMNs), with negative gram and AFB stains, culture, and cytology. Blood cultures were negative.

IMPACT/DISCUSSION: Confirming the presence of a pericardial effusion requires an echocardiogram, although items from the history and physical, EKG, and chest $\mathrm{x}$-ray can all be suggestive. The general internist, using point of care ultrasound can most often observe a significant pericardial effusion. A formal transthoracic echo can be done to quantify the size of the effusion and to assess for cardiac tamponade. The factors that affect the impact of a pericardial effusion include rate of accumulation, size of effusion, and presence of pericardial scarring or adhesions. Establishing a cause of the effusion is often difficult and is based mostly on additional findings to guide additional evaluation. The course of most effusions tend to be benign but basic laboratory evaluation including a $\mathrm{CBC}, \mathrm{CMP}$, and thyroid studies. ANA and autoimmune evaluation should be undertaken if there are appropriate corresponding clinical factors. Pericardial fluid analysis should be performed in all cases of tamponade physiology, if an infectious or malignant cause is suspected, or if it is a large pericardial effusion. If there is no tamponade, treatment is aimed at treating the underlying cause. Asymptomatic patients with large effusion do not need immediate therapeutic drainage but it should be considered for diagnostic purposes

CONCLUSION: It is essential for the general internist to recognize and appropriately treat pericardial effusions. The approach involves establishing the presence of an effusion, assessing the hemodynamic impact, establishing the cause of the effusion, and then initiating the appropriate treatment.

\section{THE POSITIVE SIDE OF A NEGATIVE}

kohei oguni ${ }^{1}$; Sandra Y. Moody ${ }^{2}$; Makito Yaegashi ${ }^{1} .{ }^{1}$ Kameda Medical Center, Kamogawa, Japan; ${ }^{2}$ Kameda Medica Center \& UCSF/SFVAMC, Kamogawa City, Japan. (Control ID \#3163932)

LEARNING OBJECTIVE \#1: Recognize that a negative inflammatory marker can be a diagnostic clue in a patient with fever.

CASE: A 72-year-old woman with a fever for 10 days was evaluated in the outpatient clinic of our hospital. She had a mild headache, but no other associated symptoms. On examination, she was alert and oriented. Her temperature was $39.5^{\circ} \mathrm{C}$, blood pressure $135 / 75 \mathrm{mmHg}$, heart rate 93 beats per minute, respiratory rate 20 breaths per minute, and oxygen saturation $95 \%$ while breathing ambient air. Her neck was supple, with a normal range of motion. Laboratory testing revealed a white blood cell count of $6300 / \mathrm{mm}^{3}$ ( $73 \%$ neutrophils, $21 \%$ lymphocytes, $6 \%$ monocytes, $0 \%$ eosinophil), serum 
sodium of $131 \mathrm{mEq} / \mathrm{L}$, erythrocyte sedimentation rate of $15 \mathrm{~mm} / \mathrm{h}$, and Creactive protein (CRP) level of $0.05 \mathrm{mg} / \mathrm{dL}$. Blood cultures were negative, and $\mathrm{ACTH}$ stimulation test was normal. CT with contrast of the chest, abdomen, and pelvis did not reveal parenchymal abnormality, lymphadenopathy, or abscess. Given her fever and mild headache with negative CRP, we performed a lumber puncture to rule out meningoencephalitis. Opening pressure was $14 \mathrm{~cm}$ of water. CSF analysis revealed 55 nucleated-cells per cubic millimeter (100\% lymphocytes) and normal protein and glucose levels. The herpes simplex virus (HSV)-PCR was positive. MRI of the brain revealed hyperintensity in the left medial temporal lobe on both T2weighted sequence and FLAIR sequence. HSV encephalitis was diagnosed. After initiation of a three-week course of acyclovir, her fever resolved.

IMPACT/DISCUSSION: There are fewer differential diagnoses to consider in patients with fever and negative inflammatory markers than in patients with positive inflammatory markers. Differential diagnosis in such patients includes early phase of a febrile illness, viral infection, drug fever, systemic lupus erythematosus, hyperthyroidism, thromboses, adrenal insufficiency, meningoencephalitis, alcohol withdrawal syndromes, factitious disorder, malignant hyperthermia, serotonin syndrome and alcohol withdrawal, and others. Viral meningoencephalitis has a good prognosis in general and no specific treatment is required. On the other hand, HSV encephalitis can be a devastating disease with significant morbidity and mortality. Thus, early diagnosis is important. In our patient, euvolemic hyponatremia was noted suggesting the presence of the syndrome of inappropriate antidiuretic hormone secretion, which could be a manifestation of central nervous system disturbance. In evaluating patients with fever and negative inflammatory markers, even when a headache is mild, lumber puncture should be considered to rule out herpes simplex encephalitis, especially in a situation when no alternative diagnosis is made.

CONCLUSION: There are fewer differential diagnoses in patients with fever and negative inflammatory markers than in patients with positive inflammatory markers. In this situation, even when the headache is mild, lumber puncture should be considered to rule out HSV encephalitis.

\section{THE RELENTLESS SORE THROAT, A CASE OF CHRONIC SE- VERE NEUTROPENIA}

Marie Claire Lamb; Jordan Minish; Bradley S. Fletcher. UFL, Gainesville, FL. (Control ID \#3185639)

LEARNING OBJECTIVE \#1: Recognize rare causes of recurrent infections and neutropenia.

CASE: A 63 year old male with a past medical history of malignant melanoma, squamous cell carcinoma of the tonsil, recently diagnosed heart failure with reduced ejection fraction, recently diagnosed pulmonary embolism on warfarin, longstanding hypothyroidism and several false positive syphilis tests presented with a fever of unknown etiology. Patient had had an unrelenting sore throat refractory to several courses of antibiotics and intermittent fevers for the past year. He recently developed diarrhea. On physical exam he had an ulcerated eschar on his right tonsil. He was found to be neutropenic with a white cell count of 1.3 and an absolute neutrophil count of 20. CT scan showed thickening of his right tonsil and splenomegaly of $16 \mathrm{~cm}$. He was started on vancomycin, cefepime and metronidazole with improvement of his symptoms. Further laboratory work-up revealed positive antiphospholipid antibodies, lupus anticoagulant, Epstein Barr Virus IgM, Direct Antiglobulin Test IgG and Clostridium Difficile. Bone marrow biopsy showed an abnormal clonal T-cell population with an expanded T-cell receptor subset consistent with T-cell Large Granulocytic Lymphocytic (LGL) Leukemia. He was started on cyclosporin to suppress his immune system and twice weekly filgrastim to treat his neutropenia. He was discharged on a short course of levofloxacin and close follow-up.

IMPACT/DISCUSSION: LGL Leukemia is a rare lymphoproliferative disorder of the T-cell and Natural Killer cell lineages associated with autoimmunity. There are indolent and aggressive forms. Pathogenesis is hypothesized to be antigen induced monoclonal expansion via Stat3 mutation activation of LGLs. These autoreactive cytotoxic lymphocytes produce cytokines, function as killer cells and avoid apoptosis. Median age of diagnosis is 60 years old and patients present with recurrent infections, oral ulcers, splenomegaly, cytopenias and autoimmunity. This case illuminates the importance of keeping differential diagnoses broad for neutropenia with recurrent infections, as missing one would be catastrophic. It is important to rule out malignancy and full autoimmune work-up is imperative. In this case we saw antiphospholipid syndrome, hypothyroid and possibly related nonischemic cardiomyopathy. Smoldering infection as a cause or consequence of neutropenia must be considered, and a careful history may reveal further associations. LGL leukemia is diagnosed by clinical picture, followed by a blood smear or bone marrow biopsy that demonstrates a clonal LGL population. LGL leukemia that is indolent can be monitored unless it causes severe cytopenias or recurrent infections, in which case we treat. First line is methotrexate, Cyclophosphamide or Cyclosporin as single agents, and G$\mathrm{CSF}$ is added for severe neutropenia.

CONCLUSION: Rare causes of recurrent infections and neutropenia are an important to consider when standard therapies are not working.

\section{THE ROLE OF ECHOCARDIOGRAPHY IN THE WORK-UP OF CHEST PAIN}

Michel Farah; Maya Abdallah. UMMS Baystate Medical Center, Springfield, MA. (Control ID \#3186316)

LEARNING OBJECTIVE \#1: Recognize the importance of thorough history taking in all cases of chest pain, even when the initial diagnosis may seem apparent.

LEARNING OBJECTIVE \#2: Recognize McConnell's sign as an echocardiographic pattern associated with acute pulmonary embolism.

CASE: A 38-year-old obese male with history of insulin-dependent diabetes mellitus presented with a several day history of gradually worsening chest pain and dyspnea. Work-up was notable for an electrocardiogram showing Twave inversions in leads V1-V4 with Q waves in leads III and aVF and elevated cardiac biomarkers. He was treated for acute coronary syndrome and underwent cardiac catheterization which showed chronic occlusion of the left circumflex artery, which could not be stented, but otherwise normal coronary arteries. He continued to experience dyspnea. An echocardiogram was done showing significant right ventricle (RV) dysfunction: severe dilation, reduced systolic function and hypokinesis of the mid-free wall suggestive of McConnell's sign. Acute pulmonary embolus (PE) was suspected. A more detailed history was obtained, with a focus on venous thromboembolic risk factors, and it was discovered that the patient had had prolonged immobilization during travel 2 months prior. Computed tomography angiogram of the chest was done showing bilateral pulmonary emboli in the distal main pulmonary arteries, all lobar arteries and many segmental arteries. He was started on anticoagulation for acute PE.

IMPACT/DISCUSSION: Thorough history taking and avoiding early diagnostic closure are key to appropriate diagnosis and subsequent management of any condition and particularly when life-threatening diseases are being considered. Echocardiogram is an invaluable tool in narrowing the broad differential diagnoses of chest pain. McConnell's sign refers to a distinct echocardiographic pattern of RV dysfunction: regional hypokinesia or 
akinesia of the RV mid-free wall with normal motion at the apex consistent with RV pressure overload. This sign has low sensitivity but high specificity for acute PE.

CONCLUSION: The echocardiographic finding of McConnell's sign is not pathognomonic for acute $\mathrm{PE}$ but should at least prompt clinical suspicion and further work-up if clinically warranted.

\section{THE ROOT OF THE PROBLEM: A CASE OF SEVERE META- BOLIC ALKALOSIS AND HYPOKALEMIA}

Matthew R. Mohorek; Elizabeth R. Jacobs. Medical College of Wisconsin, Milwaukee, WI. (Control ID \#3183266)

LEARNING OBJECTIVE \#1: Recognize glycyrrhizic acid (GZA) toxicity from licorice root as a cause of non-aldosterone mineralocorticoid excess.

LEARNING OBJECTIVE \#2: Review clinical ramifications of nonaldosterone mineralocorticoid excess including secondary hypertension, hypokalemia, and metabolic alkalosis.

CASE: A 73-year-old male presented to outpatient bronchoscopy lab for transbronchial needle aspiration. One month prior, the patient had a CT-chest for lung cancer screen which revealed a right apical spiculated lesion later found to be FDG avid. Upon arrival, the patient was asymptomatic but hypertensive with blood pressure 190/108 $\mathrm{mmHg}$. Work-up was remarkable for potassium $1.6 \mathrm{mmol} / \mathrm{dL}$, bicarbonate $52 \mathrm{mmol} / \mathrm{dL}, \mathrm{pH} 7.655$, and QTc $748 \mathrm{~ms}$. He was admitted to the ICU for management of metabolic derangements. In ICU, history elicited no prescription medications, but the patient was taking a 4,000 mg daily "liver supplement" with active ingredient Glycyrrhiza glabra better known as licorice root. The patient received electrolyte supplementation and was started on spironolactone titrated to $100 \mathrm{mg}$ twice daily. He was hospitalized for five days. Follow-up labs eight days after admission revealed complete resolution of metabolic and ECG derangements. Post-discharge bronchoscopy ultimately revealed stage IIIA non-small cell lung cancer.

IMPACT/DISCUSSION: The patient presented with clinical features suggestive of non-aldosterone mineralocorticoid excess due to glycyrrhizic acid (GZA) toxicity. GZA is a constituent of licorice root (Glycyrrhiza glabra). Its distinctive sweet flavor makes it a popular sweetener for beverages, gum, and tobacco products. It is also used as an herbal supplement for various medical claims. This patient was consuming approximately $800 \mathrm{mg} /$ day of GZA whereas $<100 \mathrm{mg} /$ day is generally considered safe. GZA inhibits 11- -hydroxysteroid dehydrogenase, an enzyme that catalyzes the conversion of cortisone to cortisol. Compared to aldosterone, cortisol has equivalent avidity to the mineralocorticoid receptor, so inhibition leads to a mineralocorticoid excess state. Clinically, this excess produces a constellation of abnormalities including hypertension, hypokalemia, and alkalosis. Unique to this case is the severe metabolic alkalosis present which is likely due to enhanced $\mathrm{H}+$ secretion by intercalated cells in the collecting ducts of the nephron - a direct result of the luminal electronegativity created by the mineralocorticoid receptor mediated $\mathrm{Na}+\mathrm{K}+$ ATPase. Mineralocorticoid antagonists like spironolactone are the cornerstone of treatment.

CONCLUSION: GZA is a constituent of licorice root which remains a popular ingredient in sweetened products and herbal supplements. The latter is of particular interest as recommended doses may contain a dangerous level of the substance, as in this case. GZA can cause a non-aldosterone mineralocorticoid excess state leading to hypertension, hypokalemia, and alkalosis. Treatment of GZA toxicity is centered on mineralocorticoid receptor antagonism, most commonly spironolactone.
THE UNEXPECTED FLIGHT DETOUR WITH FEVER AND AN ABDOMINAL MASS

Jun Chih Wang; George DiSeSa; Daniel Matassa; Joshua Kra. Rutgers New Jersey Medical School, Newark, NJ. (Control ID \#3171095)

LEARNING OBJECTIVE \#1: Recognize the clinical features of lymphoma in patients with HIV

CASE: A 30-year-old Liberian woman with untreated HIV presented with one-day history of generalized weakness and fever that started while flying from Liberia to the United States. She complained of lower abdominal discomfort, weight loss and oligomenorrhea over several months. She denied recent sick contacts or recent infections. She had a fever of 102.7F, pulse 132 bpm, blood pressure 105/64 mmHg. On exam she had a mildly tender lower abdominal mass on palpation, clear lungs and no murmur. Laboratory studies showed WBC 7,000/uL, Hb 4.4 g/dl, platelets 42,000/uL, LDH 878, CD4 243 and a normal chemistry profile. EKG showed sinus tachycardia. Chest xray and UA were unremarkable. CT abdomen/pelvis with contrast revealed a $11 \times 7 \times 9 \mathrm{~cm}$ heterogeneous solid, cystic mass in the pelvis with surrounding hemorrhagic fluid and lymphadenopathy. Despite initial fluid resuscitation, blood transfusion and antibiotics, she continued to be hypotensive and febrile. Due to persistent cytopenia, a bone marrow biopsy was performed but was negative for infectious or neoplastic processes. The pelvic mass was initially thought to represent an incidental finding of a benign mass or a hemorrhagic complex cyst. However, due to minimal improvements with treatments and a non-revealing additional infectious work-up, the patient was eventually taken for a diagnostic laparoscopy and salpingo- oopherectomy. After the operation, the patient's condition stabilized with resolution of hypotension, fevers, and cytopenia. Interestingly, the result of the tissue biopsy of the pelvic mass revealed diffuse large B-cell lymphoma of the ovary.

IMPACT/DISCUSSION: Fever and cytopenia in patients with HIV is often associated with an infectious process; however, autoimmune, malignancy and drug reactions need to be considered. Lymphadenopathy with elevated LDH can be non-specific in this population. In our patient, the association between the septic clinical features and the pelvic mass likely represent a hemorrhagic process with a large tumor burden. Tissue diagnosis remains critical. Lymphoma of the ovary accounts for $0.5 \%$ of all nonHodgkin lymphoma (NHL) and $1.5 \%$ of all ovarian neoplasm. The risk of NHL in patients with untreated HIV is as high as 100-fold; despite the use of anti-retroviral therapy (ART), the risk of NHL remains elevated. HIVrelated primary ovarian lymphoma has only been reported twice in the literature. Despite its rarity, NHL of the ovary should be considered as a differential diagnosis in patients with untreated HIV presenting with fever and an abdominal mass given the potential to alter treatment. The use of adjuvant chemotherapy after resection is not well-studied but may be of benefit to prevent relapse.

CONCLUSION: Ovarian lymphoma is rare but should be considered in patients with HIV presenting with fever and an ovarian mass. Patients with HIV have an increased risk of HIV-related lymphoma, which remains elevated despite the use of ART

\section{THE WOMAN WHO MISTOOK HER HOSPITAL BED FOR A CANOE}

Anna C. Watkins. Brookwood Baptist Health, Hoover, AL. (Control ID \#3183973)

LEARNING OBJECTIVE \#1: Recognize that amyloid B-related angiitis is a rare disorder with diagnostic imaging and histopathological findings 
LEARNING OBJECTIVE \#2: Recognize the common presenting signs and symptoms of amyloid B-related angiitis

CASE: A 72-year-old Caucasian female presented with visual hallucinations, memory loss, lethargy, and intermittent headaches of one month duration. The patient was alert, afebrile, and hypertensive with garbled speech and difficulty following commands, but was able to move all extremities equally; otherwise her physical exam was benign. Labs revealed WBC 8, Na 144, ammonia 14, CRP 5, and ESR 71. CT head showed no intracerebral hemorrhage. MRI brain with contrast showed vasogenic edema in the left temporal lobe with minimal associated enhancement and mild mass effect on the temporal horn of the left lateral ventricle. The patient was started on acyclovir for potential HSV encephalitis. A lumbar puncture revealed normal levels of glucose and protein, and was negative for fungi, AFB, neurosyphilis, Cryptococcus, and HSV-1. Acyclovir was stopped. It was suspected that the patient had a glioma, so the temporal lesion was biopsied and stained positively for amyloid B-related angiitis (ABRA). She was started on $1 \mathrm{~g}$ of methylprednisolone for 7 days then was decreased to $60 \mathrm{mg}$ oral prednisone. Her symptoms improved significantly and she was discharged to inpatient rehab where she continued to improve mentally and physically.

IMPACT/DISCUSSION: ABRA is an autoimmune response to naturally occurring beta-amyloid deposition in the cortical and leptomeningeal vessels of the brain that typically occurs in the 7th decade of life with no gender predilection. Patients most commonly present with cognitive and/or behavioral changes ranging from confusion to hallucinations to coma, but can also present with focal neurological deficits, seizures, and/or headaches. ESR and CRP levels are often elevated, but are nonspecific. LP is non-diagnostic, but aids in ruling out other causes of encephalopathy. MRI distinctively shows leptomeningeal enhancement on T2-weighted images often with microbleeds at cortico-subcortical junctions on susceptibility-weighted images, but tissue biopsy is the gold standard of diagnosis. Initial treatment is high-dose corticosteroids followed by a longer duration of low-dose steroids in addition to immunosuppressive drugs. Mortality of ABRA has been reported to be between 7 and $44 \%$, and a number of patients have neurological deficits after treatment. Therefore, timely diagnosis and aggressive, appropriate treatment are the keys to a successful recovery.

CONCLUSION: ABRA is a relatively rare diagnosis with a mortality of 7 $44 \%$ and with a large number of patients having neurological deficits even after treatment. Therefore, timely diagnosis and aggressive, appropriate treatment are the keys to a successful recovery.

\section{THE WRATH OF HYDROXYCHLOROQUINE: A RECALCI- TRANT CASE OF ACUTE GENERALIZED EXANTHEMATOUS PUSTULOSIS}

Chay Bae; Navya Kuchipudi; Santhanam Lakshminarayanan. University of Connecticut, Farmington, CT. (Control ID \#3183952)

LEARNING OBJECTIVE \#1: Distinguish acute generalized exanthematous pustulosis (AGEP) from other rashes.

LEARNING OBJECTIVE \#2: Recognize the role of cyclosporine in recalcitrant cases of AGEP.

CASE: A 47 year old female with a history of sjogren's syndrome was started on hydroxychloroquine (HCQ) at $5 \mathrm{mg} / \mathrm{kg}$ by her rheumatologist. 4 weeks into HCQ, she developed a rash along her axilla and upper extremity with neutrophilia of $9 \mathrm{k} / \mathrm{uL}$. Despite prednisone and withdrawal of HCQ, her rash spread over 6 days. Ultimately, she was referred to us for a second opinion. On exam, she had mild erosions along her tongue and pin-sized, nonfollicular pustules on an erythematous and edematous base along intertriginous regions, trunk, and extremities. She had neither a Nikolsky sign nor a history of psoriasis. Labs revealed neutrophils of $13 \mathrm{k} / \mathrm{uL}$, ESR of $21 \mathrm{~mm}$, and CRP of $11 \mathrm{mg} / \mathrm{L}$. Although biopsy was histopathologically indeterminant, her findings and the long half-life of HCQ was concerning for a delayed onset of AGEP. She was trialed outpatient on methylprednisolone $1 \mathrm{~g}$ IV (MIV) for 3 days. This halted new lesions and she transitioned to a prednisone taper. She; however, sought an alternate opinion from dermatology. A concern for pustular psoriasis was raised and her prednisone was aggressively tapered. Regrettably, over 48 hours: she manifested new lesions, had an episode of orthostatic syncope, and presented for admission with neutrophils of $18 \mathrm{k} / \mathrm{uL}$, ESR of $58 \mathrm{~mm}$, and CRP of $100 \mathrm{mg} / \mathrm{L}$. Inpatient, she was re-trialed on MIV for 5 days. Despite MIV, she showered lesions in new regions and previous areas of post-pustular desquamation. In this setting, she was re-biopsied, which supported AGEP (corneal pustule and superficial lymphocytic and neutrophilic inflammation). Given the recalcitrance of AGEP, cyclosporine (CSA) was introduced at $4 \mathrm{mg} / \mathrm{kg}$. Within 6 days of CSA, the lesions quiesced and she was discharged. Upon follow up, her CSA was tapered to $1 \mathrm{mg} / \mathrm{kg}$, with concomitant prednisone taper over the next 2 months. At follow up, her rashes had nearly resolved. IMPACT/DISCUSSION: Despite the use of HCQ in rheumatology, AGEP is a rare side effect. AGEP results from a T-cell mediated type IV hypersensitivity reaction. This manifests as a severe pustular reaction typically within days. Due to HCQ's long half-life; however, it often takes weeks to manifest. This makes it difficult to correlate HCQ to AGEP. This is further muddied by differentials, such as pustular psoriasis. In our patient, AGEP was favored by the nature of polymorphous lesions, no prior history of psoriasis, and biopsy findings. Treatment is discontinuation of medication, supportive care, and systemic steroids in refractory cases. Moreover, this case adds to the sparse literature that CSA may have a role in recalcitrant cases due to its $\mathrm{T}$ cell inhibitory effect.

CONCLUSION: This case captures the difficulties of diagnosing HCQ induced AGEP. It also highlights the role of CSA in recalcitrant cases.

\section{THE WRATH OF THE CATH: CARDIAC CATHETERIZATION COMPLICATED BY ACUTE STROKE}

Manan Dhingra $^{1}$; Michael Hagan ${ }^{1}$; Abhishek Thandra ${ }^{2}$; Maryam Gbadamosi ${ }^{2}$. ${ }^{1}$ Creighton University Medical Center, Omaha, NE; ${ }^{2}$ Creighton university, Omaha, NE. (Control ID \#3184705)

LEARNING OBJECTIVE \#1: Recognize that embolic strokes are a known but rare complication of cardiac catheterization.

LEARNING OBJECTIVE \#2: Recognize that preoperative workups are sometimes unnecessary and can have harmful downstream effects.

CASE: An 84-year-old male with history of hypertension, hyperlipidemia, type II diabetes mellitus, coronary artery disease, $7.3 \mathrm{~cm}$ abdominal aortic aneurysm, and cholecystostomy tube in situ underwent coronary catheterization as part of a preoperative evaluation for elective cholecystectomy after a probable single reversible defect in the posterior basilar segment was discovered on a Lexiscan earlier in his preoperative workup. During the procedure, a balloon angioplasty was performed on a stenosis in an existing stent in the distal LAD. Shortly after the procedure, the patient developed diplopia, slurred speech, and left hand and left lower facial numbness. On examination he was found to have internuclear ophthalmoplegia (INO) upon leftward gaze, decreased sensation in the left hand and left lower face, dysmetria on the left, but no focal weakness. He was found to have a NIH stroke scale score of 4. CT head and CTA head/neck showed occlusion in the posterior cerebellar circulation. The patient was deemed a poor candidate for TPA therapy because of the risk of bleeding from his AAA and 
from his cholecystostomy tube. The patient had received heparin and Plavix during the cardiac catheterization procedure and had taken a baby aspirin earlier that morning. A high intensity statin was given after the initial workup was completed. An MRI brain was performed the following day that showed infarcts involving the right paramedian midbrain and cerebellum. A transthoracic echocardiogram with bubble study showed no intracardiac thrombus, vegetations, or any intracardiac shunt. Over the next few days, the decreased sensation the left face and left hand resolved, but the dysarthria, diplopia, and INO persisted. The patient was discharged to a skilled nursing facility for further rehabilitation upon discharge. He will be reassessed as a surgical candidate for cholecystectomy and AAA repair as an outpatient.

IMPACT/DISCUSSION: This case illustrates the complications involved with cardiac catheterization. These complications are rare but need to be considered in patients with multiple risk factors. This case also illustrates that preoperative workups are not always benign. On many occasions, a clinician is able to determine from the clinical picture that a patient is not a good surgical candidate but will go ahead with extensive preoperative workup just to confirm their assessment. These workups not only drain time and money from the healthcare system but can expose the patient to many unintended risks.

CONCLUSION: 1. Embolic strokes are a known but rare complication of cardiac catheterization. 2. Inappropriate preoperative workups not only drain time and money from the healthcare system but can expose the patient to many unintended risks.

\section{THESE CUFFS DON'T LIE: AN ATYPICAL PRESENTATION OF A COMMON CONDITION}

David Hess ${ }^{1}$; Brian Lewis ${ }^{1}$; David Feldstein ${ }^{2}$; Linda Baier ${ }^{2} .{ }^{1}$ University of Wisconsin, Madison, WI; ${ }^{2}$ University of Wisconsin School of Medicine and Public Health, Madison, WI. (Control ID \#3184533)

LEARNING OBJECTIVE \#1: Recognize hypothyroidism as a cause of new or worsening hypertension in the absence of other classic symptoms. LEARNING OBJECTIVE \#2: Appropriately screen patients with a history of radiation therapy to the neck for hypothyroidism.

CASE: A 75-year-old man with a history hypertension and idiopathic cardiomyopathy presented to his primary care physician with elevated blood pressure. His home blood pressure ranged from 150/110 to $170 / 120$ over the past 5 days. He also complained of fatigue and generally feeling unwell. He denied any constipation, cold intolerance, changes in skin or hair, weight changes, headache, visual changes, chest pain, or shortness of breath. His hypertension was previously well controlled with losartan and metoprolol succinate. He was diagnosed with squamous cell carcinoma of the tongue 5 years ago and curatively treated with radiation. Exam was notable for a BP of 182/105. Losartan was increased and a limited workup for secondary causes of hypertension was performed, showing stable renal function and a TSH of $>100$ with free T4 of 0.60 . Levothyroxine was initiated for hypothyroidism. Over 12 weeks, his blood pressure improved and his TSH normalized. He was able to return to his prior anti-hypertensive regimen with good control.

IMPACT/DISCUSSION: Hypothyroidism is commonly managed in primary care. Recognizing its presentation with worsening hypertension is critical. The variety of clinical symptoms of hypothyroidism can be broad, however it is notable that as much as $30 \%$ of people with hypothyroidism have hypertension, particularly diastolic hypertension. For the majority of patients with secondary hypertension due to hypothyroidism, hypertension resolves with appropriate hypothyroidism treatment. This patient was at increased risk of hypothyroidism given his previous neck radiation, but was not being monitored for this. Hypothyroidism is a common adverse effect of neck radiation, with reports of up to $33 \%$ of patients suffering from clinical hypothyroidism. Most develop hypothyroidism within 2 years of radiation, however onset of greater than 5 years after completion of radiation is not uncommon. The National Comprehensive Cancer Network guidelines recommend screening for hypothyroidism with a TSH every 6-12 months indefinitely. In this case, the patient may have avoided the hypertensive urgency if he had been appropriately screened. As a result of this case, I will be more vigilant in evaluating for hypothyroidism in patients with diastolic hypertension. Additionally, now knowing the recommended screening guidelines for patients with neck radiation, I will ensure my patients with this history have at least a yearly evaluation of TSH.

CONCLUSION: - It is important to be vigilant in assessing for hypothyroidism in patients with new onset or worsening diastolic hypertension even in the absence of other classic symptoms. - Patients are at high risk of hypothyroidism after neck radiation and should be screened every 6-12 months.

THIRD TIME'S THE CHARM: DIAGNOSIS OF ADULT ONSET STILL'S DISEASE AFTER REPEAT ADMISSIONS FOR FEVER Katherine Gavinski; Michael Burton. UT Southwestern, Dallas, TX. (Control ID \#3177803)

LEARNING OBJECTIVE \#1: Construct a systematic approach to evaluation of fever of unknown origin (FUO).

LEARNING OBJECTIVE \#2: Recognize diagnostic errors present in complex patient evaluations.

CASE: Mrs. G is a 35-year-old African woman who presented with a multiweek history of fevers, malaise, and rash. She initially developed a pruritic rash to her extremities - treated as an allergic reaction. She was soon hospitalized with fever, weakness, and abdominal pain; workup notable for acute renal injury, leukocytosis, microcytic anemia, thrombocytopenia, coagulopathy, hemolysis, and hypercellular bone marrow; negative infectious workup. She was discharged after IV antibiotics, but soon readmitted with recurrent fevers, disabling knee arthralgias, transaminitis, elevated acute phase reactants, ferritin 56, and nonspecific lymphadenopathy and hepatosplenomegaly. Rickettsia antibody was weakly positive - no history of travel or unusual exposures - so discharged on doxycycline for presumed murine typhus. At home, Mrs. G developed a hypertrophic rash and stopped doxycycline. She was admitted again weeks later for fever, malaise, and maculopapular rash with warm autoimmune hemolytic anemia, acute kidney injury, and ferritin 12,000. Skin biopsy showed persistent, atypical eruption of adult Still's disease. She had rapid clinical improvement with IV steroids.

IMPACT/DISCUSSION: Although adult Still's disease is rare, this case serves as an excellent example of the diagnostic workup for a FUO and common errors in clinical reasoning. FUO workup requires rigorous evaluation for infection, malignancy, and rheumatologic processes, which should be informed by a patient's history and exposures. Despite a conclusive final diagnosis, this case was subject to errors in clinical reasoning. Mrs. G's initial management consisted of testing and treatment for bacterial etiologies of fever. Availability bias makes bacterial infection an accessible diagnostic category, but after lack of response to treatment or positive test, should be abandoned. During her second admission, a positive antibody for Rickessia was found. Despite a lack of exposure and weak antibody titer, confirmation bias and premature closure led to another incorrect diagnosis. The low ferritin led to the cognitive bias of blind obedience - placing undue 
reliance on test results - that caused us to initially dismiss the idea of adult Still's disease. Ultimately, her diagnostic workup required re-framing of her signs and symptoms of disease, repeated testing, and pathologic confirmation to appropriately identify a unifying diagnosis.

CONCLUSION: *Thorough history taking and careful review of patient's records can help elucidate a difficult diagnosis. *If a diagnosis does not fit the clinical information available, renewed effort should be made to identify an alternative diagnosis and avoid errors in reasoning.

\section{THIRD TIMES THE CHARM: A RARE CASE OF RECURRENT PANCREATITIS AND THE IMPORTANCE OF MEDICATION RECONCILIATION}

Rusdeep Mundae; Seth J. Lichtenstein; Ashley Shah. Rush University Medical Center, Chicago, IL. (Control ID \#3186221)

LEARNING OBJECTIVE \#1: Recognize the challenges in identifying drug-induced pancreatitis

LEARNING OBJECTIVE \#2: Perform a thorough history and medication review

CASE: A 57-year-old woman with a past medical history of diabetes mellitus type 2 presented with a two day history of sharp epigastric pain radiating to the back, associated with nausea and vomiting. She denied alcohol, smoking, or drug use. She denied any previous abdominal surgeries. Her vitals on admission were stable. Her exam was notable for epigastric tenderness with deep palpation. Labs showed a serum creatinine $1.2 \mathrm{mg} /$ $\mathrm{dL}$, calcium $9.8 \mathrm{mg} / \mathrm{dL}$, and lipase 2,539 U/L. An abdominal ultrasound was negative for gallstones and a triglyceride level was normal. Upon review of home medications, she was prescribed trimethoprimsulfamethoxazole (TMP/SMX) for a urinary tract infection (UTI) 5 days prior to admission. On further investigation, the patient was noted to have two admissions in 2015 and 2018 for acute pancreatitis, previously thought to be of unknown etiology. However, thorough review revealed that the patient was prescribed TMP/SMX for a UTI prior to each of these admissions. She was treated with fluid resuscitation, pain control, and diet advancement. She improved clinically and was discharged without sequalae after several days. Patient was educated on the etiology of her recurrent pancreatitis and TMP/SMPX was added to the patient's allergy list.

IMPACT/DISCUSSION: Drug induced pancreatitis (DIP) is a rare entity. The true incidence of DIP remains unclear as evidence primarily consists of isolated case reports; however it is estimated to account for approximately $0.1-2 \%$ episodes of acute pancreatitis. The diagnosis of DIP is particularly challenging because it rarely presents with clinical or laboratory evidence that would differentiate it from other causes of acute pancreatitis. DIP is typically a diagnosis of exclusion and is established by assessing the temporal relationship between drug administration and development of acute pancreatitis, or a positive re-challenge with the suspected drug given the lack of other causes. A thorough history, physical, and medication review, is essential in guiding medical decision-making and reducing hospital readmissions for DIP. TMP/SMX is a relatively rare cause of DIP, but there have been several case reports, including one documented report of a re-challenge. This suggests a causal relationship; however, the pathologic mechanism remains unknown. While other sulfonamides have been implicated in causing DIP, it is unclear if the sulfa-moiety of TMP-SMX is in fact the offending agent.

CONCLUSION: DIP is a very rare, but potentially life-threatening diagnosis that is best established by a thorough history and review of a patients past medical history and medication use. Early identification, initiation of appropriate treatment, and withdrawal of the offending agent can aid in preventing worsening disease progression, reducing morbidity, and decreasing hospital readmissions.

THREE'S A CROWD: A CASE OF HEPATITIS B, C, AND D COINFECTION IN A PERSON WHO INJECTS DRUGS

Jill Harrington $^{1}$; Jessica Taylor ${ }^{2}$. ${ }^{1}$ Boston Medical Center, Boston, MA; ${ }^{2}$ Boston Medial Center, Boston, MA. (Control ID \#3185769)

LEARNING OBJECTIVE \#1: Test for hepatitis D in patients with hepatitis B at increased risk for co-infection.

LEARNING OBJECTIVE \#2: Describe the treatment and prognostic implications of viral hepatitis co-infection.

CASE: A 49-year-old man with a history of substance use disorder and injection drug use presented to the Emergency Department (ED) for musculoskeletal back pain. Labs were ordered; because of an $\mathrm{ED}$ initiative to identify cases of hepatitis $\mathrm{C}(\mathrm{HCV})$, an HCV antibody assay was sent and returned reactive with a viral load of $20,774 \mathrm{IU} / \mathrm{mL}$. He was diagnosed with genotype $1 \mathrm{~A} \mathrm{HCV}$, likely chronic, and outpatient follow-up was arranged. At his next visit, the patient reported no symptoms of HCV and was interested in treatment. Additional lab tests and imaging were ordered to stage his liver disease and inform selection of direct acting antiviral therapy (DAA). Hepatitis B virus (HBV) serologies suggested HBV co-infection. Follow-up labs confirmed active, e antigen positive, e antibody negative HBV with a high viral load of $>170,000,000 \mathrm{IU} / \mathrm{mL}$. Because of the patient's history of injection drug use, a hepatitis D virus (HDV) antibody was sent and returned positive. Although the patient's primary care physician manages $\mathrm{HCV}$, this patient was referred to Hepatology for management of possible HBV, HCV, and HDV coinfection. Fortunately, his HDV viral load was undetectable, indicating cleared HDV. Because of the risk of a HBV flare during and after DAA therapy for $\mathrm{HCV}$, the patient was started on tenofovir for his HBV with the plan to start $\mathrm{HCV}$ treatment once an adequate HBV viral load response is achieved.

IMPACT/DISCUSSION: The development of DAAs for HCV has revolutionized treatment and expanded access to high risk populations. Testing for viral co-infection is a crucial component of the HCV pre-treatment evaluation. $\mathrm{HDV}$ is an RNA virus that requires the presence of HBV surface antigen (HbSAg) for replication; patients with HBV/HDV co-infection have higher mortality and a more rapid progression to cirrhosis, which underscores the importance of recognizing HDV. The American Association for the Study of Liver Disease recommends testing for $\mathrm{HDV}$ in patients with a positive $\mathrm{HbSAg}$ at increased risk, including PWID, MSM, people with HIV or HCV coinfection, and people from endemic areas. Studies have estimated that up to one third of HbSAg positive PWID also have chronic HDV. However, HDV testing is not done consistently. When $\mathrm{HBV}$ or HBV/HDV are identified in a patient with chronic $\mathrm{HCV}$, the treatment implications are significant. Due to risk of a HBV flare during or after DAA therapy, patients with HBV coinfection may need to begin HBV treatment or undergo additional monitoring. The treatment approach for patients with $\mathrm{HBV} / \mathrm{HCV} / \mathrm{HDV}$ co-infection is more complex as interferon is the only agent with known HDV activity.

CONCLUSION: Screening for HBV co-infection is an essential part of the $\mathrm{HCV}$ pre-treatment evaluation. Patients with a positive $\mathrm{HbSAg}$ who have a history of injection drug use or other risk factors for HDV, should be screened for HDV.

\section{THYROTOXIC PERIODIC PARALYSIS RESULTING IN ACUTE RESPIRATORY FAILURE}

Charit Taneja $^{1}$; Gorav Sharma ${ }^{1}$; Bryan Blase ${ }^{1}$; Lubna B. Munshi ${ }^{2} .{ }^{1}$ Icahn School of Medicine, Mount Sinai Beth Israel, New York, NY; ${ }^{2}$ The Ohio 
State University, Wexner Medical Center, Columbus, OH. (Control ID \#3139709)

LEARNING OBJECTIVE \#1: Recognize the respiratory complications of thyrotoxic periodic paralysis

LEARNING OBJECTIVE \#2: Suspect impending respiratory failure and need for ICU level of care in these patients

CASE: A 34 year-old Hispanic male with a history of asthma presented to the ER with acute onset of bilateral upper and lower extremity muscle weakness. Two weeks prior to onset, he had a flu-like illness with fever, chills and productive cough, which caused an asthma exacerbation; leading him to use his albuterol inhaler 5-6 times daily along with a course of oral steroids. On admission, he was afebrile and vitals were BP 155/98 mmHg, HR 102 bpm, pulse oximetry $100 \%$ on room air. Physical exam was significant for $2 / 5$ strength in bilateral upper and lower extremities at all joints in all ranges of motion. Laboratory studies revealed potassium $2.1 \mathrm{mmol} / \mathrm{L}$, magnesium 1.3 $\mathrm{mg} / \mathrm{dL}$ and inorganic phosphorus $0.9 \mathrm{mg} / \mathrm{dL}$. Thyrotropin level was $0.1 \mathrm{mIU} /$ $\mathrm{L}$ with a free $\mathrm{T} 4$ of 1.37 and $\mathrm{T} 3$ of 82 . Thyroid autoantibodies were negative. Chest radiograph was unremarkable. Further history was significant for a hospitalization 5 years ago for paralysis in the setting of hypokalemia secondary to a "thyroid problem". Following the resolution of symptoms during this prior episode, he was discharged and subsequently lost to follow-up. Over the next few hours, electrolytes were repleted, yet his muscular weakness worsened rapidly. He became tachycardic and tachypneic with progressive respiratory distress. As a result, the patient was intubated for respiratory support. He was diagnosed with thyrotoxic periodic paralysis and treated with methimazole and propranolol along with concurrent potassium repletion. $\mathrm{He}$ responded appropriately, with full resolution of weakness and normalization of electrolytes. He was discharged with close outpatient endocrinology follow-up.

IMPACT/DISCUSSION: Thyrotoxic periodic paralysis is a subset of hypokalemic periodic paralysis (PP) usually seen in young Asian males with hyperthyroidism, and classically presents with proximal muscular weakness, more prominent in the lower extremities. Later age of onset (usually $>20$ years), findings of hypophosphatemia and hypomagnesemia can be used to distinguish thyrotoxic PP from familial hypokalemic PP. Beta-2 adrenergic bronchodilators and corticosteroids are both known to precipitate attacks by enhancing hypokalemia. Most cases respond rapidly to electrolyte repletion and rarely progress to involve respiratory muscles requiring ventilatory support as seen in our patient.

CONCLUSION: Thyrotoxic periodic paralysis is a rare disorder with potentially grave consequences, including respiratory failure from muscle paralysis and arrhythmias secondary to profound electrolyte disturbances. A high degree of clinical suspicion should always be observed, with patients ideally monitored in the ICU setting and close coordination between intensivists and endocrinologists.

\section{THYROTOXIC PERIODIC PARALYSIS: A RARE MANIFESTA- TION OF HYPERTHYROIDISM}

Alen Sajan $^{1}$; Maushmi Savjani ${ }^{2}$; Rebecca Mazurkiewicz ${ }^{1}$; Samy McFarlane ${ }^{3}$. ${ }^{1}$ Lenox Hill Hospital, New York, NY; ${ }^{2}$ Penn State, Hershey, PA; ${ }^{3}$ SUNY Downstate, Brooklyn, NY. (Control ID \#3186339)

LEARNING OBJECTIVE \#1: Recognize an unusual presentation of a rare complication of thyrotoxicosis associated with IV contrast.

CASE: A 73 year-old Black woman with history of Grave's disease (not adherent to methimazole), atrial fibrillation, hypertension, and diabetes mellitus presented to the Emergency Department with palpitations and dyspnea at rest for a few hours. She had increased frequency of bowel movements for several days, but no fever, chest or abdominal pain, nausea, vomiting, restlessness, diaphoresis, or insomnia. Vital signs were significant for tachycardia at $113 \mathrm{bpm}$, respiratory rate of 26 and oxygen saturation of $98 \%$ on $2 \mathrm{~L} / \mathrm{min}$ nasal cannula. Exam findings included exophthalmos, diffuse thyromegaly with bruit and $2+$ pedal edema. Her potassium level was $3.8 \mathrm{mEq} / \mathrm{L}$. Thyroid function tests (TFTs) were thyroid stimulating hormone (TSH) $<0.008 \mathrm{U} / \mathrm{mL}$, serum total thyroxine (TT4) $>30 \mathrm{ng} / \mathrm{dL}$, and triiodothyronine (T3) uptake $49.82 \%$. She was admitted for thyrotoxicosis with a Burch-Wartofsky Point Scale score of 30, and treatment with methimazole, propranolol and hydrocortisone was initiated. Her course was complicated by acute onset flaccid paralysis after receiving a CT scan with IV radiocontrast to evaluate for possible tracheal obstruction by the goiter. At this time, her neurological exam was remarkable for $0 / 5$ motor strength with hyporeflexia and intact sensation in all extremities and mute plantar reflexes. MRI of the brain and cervical spine showed no acute stroke or transverse myelitis. A diagnosis of Thyrotoxic Periodic Paralysis (TPP) was made, which is known to be associated with hypokalemia.

IMPACT/DISCUSSION: TPP is a well characterized acute onset flaccid paralysis known to occur in the setting of hyperthyroidism and hypokalemia. TTP has been linked to a variety of precipitants from states of physiologic stress to strenuous activity. Thyroid hormone is presumed to play a key role in upregulation of sodium-potassium ATPase activity that drives potassium into the cell, causing hypokalemia and subsequent hyperpolarization of muscle cell membranes leading to inexcitability of skeletal muscles [1]. This case was induced by iodine contrast while the potassium level was within normal limits. There is a similar case reported in 2006, where a patient with hyperthyroidism developed TPP after receiving contrast during cardiac catheterization with potassium level of $3.0 \mathrm{mEq} / \mathrm{L}$ [2]. Our patient was on treatment for thyrotoxicosis for a full week with $50 \%$ decrease in her serum total T4 level $(13.3 \mu \mathrm{g} /$ $\mathrm{dL})$ at the onset of paralysis. Yet she developed TPP within hours of receiving the contrast. The patient regained the majority of her muscle strength without any respiratory complications within 72 hours after continued treatment with methimazole, steroids and propranolol. Restoring euthyroidism generally reverses the paralysis, as in this case [1].

CONCLUSION: Contrast should be avoided or cautiously used in patients with thyrotoxicosis to prevent possible precipitation of TPP.

\section{THYROTOXIC PERIODIC PARALYSIS: A REFLEXIVE DIAGNO-} SIS

$\underline{\text { Samantha J. Allen }}{ }^{1}$; Juan N. Lessing ${ }^{1}$; Mel Anderson ${ }^{1,2}$. ${ }^{1}$ University of $\overline{\text { Colorado, Denver, }} \mathrm{CO} ;{ }^{2}$ Department of Veterans Affairs, Aurora, CO. (Control ID \#3185107)

LEARNING OBJECTIVE \#1: Perform a pertinent exam and obtain appropriate studies on a patient complaining of weakness

LEARNING OBJECTIVE \#2: Recognize thyrotoxic periodic paralysis as a distinct etiology for weakness in a hyperthyroid patient

CASE: A 43-year-old woman presented with one week of weakness, seven kilograms unintentional weight loss, palpitations, and heat intolerance. She had a history of panhypopituitarism following Rathke's cleft cyst resection. Medications included levothyroxine, prednisone, transdermal estrogen, and medroxyprogesterone injections. Her vital signs were notable for tachycardia (HR 105) and weight $80 \mathrm{~kg}$ (87 kg 3 weeks prior). HEENT, chest, lung, and abdominal exams were normal. Bilateral thighs and calves were tender to palpation. Strength was intact in the upper extremities and symmetrically diminished in the lower extremities. She could not rise out of a chair or walk without support. Deep tendon reflexes were diminished in the upper 
extremities and absent in the lower extremities. Neurologic exam was otherwise normal. CBC was normal. ESR was 34 (0-20), CRP was 7.77 (3), and creatine phosphokinase was 190 (19-131). Potassium was 2.8 with an otherwise normal BMP. TSH was undetectable. Free thyroxine was 2.43 (1-1.85). Her musculoskeletal and neurologic exams, along with potassium level and thyroid function tests, pointed toward a diagnosis of thyrotoxic periodic paralysis (TPP). It was noted that her levothyroxine dose was increased 6 weeks prior in response to an elevated thyroid stimulating hormone level on surveillance labs. She now disclosed that at that visit, she had not been forthcoming about inconsistent levothyroxine use; therefore, the dose increase was inappropriate and caused iatrogenic hyperthyroidism complicated by TPP. Symptoms resolved within hours of potassium supplementation. Levothyroxine dose was decreased and follow-up arranged.

IMPACT/DISCUSSION: TPP, like other forms of hypokalemic periodic paralysis, is a membrane channelopathy. One channel that plays a role is Kir 2.6, a potassium channel regulated by thyroid hormone. TPP is characterized by intermittent attacks of proximal muscle weakness and, rarely, respiratory muscle failure. It is more common in men, despite the overall female predominance of thyrotoxicosis. A useful distinguishing finding is deep tendon reflexes, which are absent or diminished in TPP but exaggerated in thyrotoxicosis. Treatment is potassium replacement, management of thyrotoxicosis, and treatment of the underlying thyroid disorder.

CONCLUSION: Weakness is a common symptom encountered by inpatient and outpatient internists. Differential diagnosis is guided by assessing for objective weakness and determining weakness distribution. Thyroid disorders should be considered in the differential diagnosis for weakness. Furthermore, when diminished reflexes, hypokalemia, and thyrotoxicosis are also present, TPP should be considered.

\section{TITLE: A PEDIATRIC RASH IN A GERIATRIC CLINIC}

Jared Schprechman. Naples Community Hospital, Naples, FL. (Control ID \#3184903)

LEARNING OBJECTIVE \#1: Recognize IgA vasculitis in adults LEARNING OBJECTIVE \#2: Distinguish IgA Vasculitis from other vasculitides by history and laboratory analysis

CASE: A 69-year-old non-smoking male with no PMH presented to the clinic with diffuse joint pain and extensive non-pruritic and non-painful rash on his lower extremities that progressed over the past week. He denied any recent travel, over the counter supplements, new medications, occupational exposure to chemicals, or excessive time in the sunlight. ROS was negative. Vitals: BP 128/82, HR 65, and temp 37 degrees Celsius. Physical exam revealed a diffuse erythematous rash with palpable purpura on his legs without ulceration, nodules or livedo reticularis. He had no joint synovitis and a normal neurological exam. Labs showed an increased creatinine of $1.5 \mathrm{mg}$ / $\mathrm{dL}$ from $1.0 \mathrm{mg} / \mathrm{dL}$ baseline, 1+ urine protein on UA, urine protein/creatinine ratio of 1 , suggesting overt proteinuria and no microscopic or gross hematuria. His p-ANCA, c-ANCA, cryoglobulin, ANA, and viral hepatitis antibodies were all negative. Skin biopsy showed neutrophilic predominant small vessel vasculitis. A kidney biopsy revealed mesangial proliferative glomerulonephritis and IM findings revealed granular mesangial positivity for $\operatorname{IgA}, \operatorname{IgM}, \mathrm{C} 3$, fibrinogen and kappa and lambda. These findings were consistent with IgA vasculitis. He was started on lisinopril $5 \mathrm{mg}$ daily and prednisone $30 \mathrm{mg}$ daily, which was tapered over the course of 5 months. His rash resolved within 4 months and his proteinuria resolved within 8 months.

IMPACT/DISCUSSION: IgA vasculitis, once known an Henoch-Schonlein purpura, occurs more than $90 \%$ of the time in children. However, its cardinal symptoms of palpable purpura, arthritis, abdominal pain and renal disease are shared by all age groups. Adults are more likely to have renal disease manifesting in hypertension, nephrotic syndrome and decline in GFR as opposed to isolated proteinuria and hematuria in children. Intussusception is rare in adults. IgA vasculitis may present similarly to other small vessel vasculitides which present with purpura. In the absence of biomarkers including ANCA, one can rule out microscopic polyangiitis (MPA), granulomatosis with polyangiitis (GPA), and Churg-Strauss. A negative ANCA can help make MPA, GPA, and Churg-Strauss less likely. Having negative cryoglobulins, no focal nerve deficits, and no history of hepatitis or connective tissue disease also rules out cryglobulinemia. While medium-vessel vasculitidies such as polyarteritis nodosa may present similarly with renal disease as well, it typically includes sensory and motor deficits as well as skin manifestations such as ulceration, nodules and livedo reticularis.

CONCLUSION: IgA vasculitis can be recognized in adults most often by palpable purpura and worsening renal disease. Key distinguishing factors from other vasculitides include negative ANCA, negative cryoglobulins and a lack of neuro deficits and any other skin findings.

\section{TO B19 AGAIN: TRANSIENT APLASTIC CRISIS IN HEMOGLO- BIN SC DISEASE}

Bridget Azera $^{1,2}$; Odeth Barrett-Campbel1 ${ }^{1,2}$; Andrew A. Chang ${ }^{1,2}$. ${ }^{1} \mathrm{NYC}$ Health + Hospitals/Kings County, New York, NY; ${ }^{2}$ SUNY Downstate Medical Center, Brooklyn, NY. (Control ID \#3185881)

LEARNING OBJECTIVE \#1: To identify and treat Parvovirus B19 transient aplastic crisis (TAC) in patients with Hemoglobin SC disease (HbSC) CASE: A 22-year-old African American woman with HbSC presented with one day of severe lower back and bilateral leg pain, not relieved by ibuprofen. Review of systems was otherwise negative. Initial exam revealed mucosal pallor, scleral icterus, absence of hepatosplenomegaly and diffuse tenderness across the lower back with no focal neurologic deficits. The pain improved with the use of hydromorphone and ketorolac. However, by day 3 of admission, she complained of generalized weakness and dyspnea on exertion. She denied cough, fever, runny nose or sore throat. Labs at that time showed hemoglobin (Hgb) 6.1g/dl (a decline from 9.4g/dl on admission), WBC 7.23 x 109/L, Reticulocyte index was $1.6 \%$, Haptoglobin $<10 \mathrm{mg} / \mathrm{dL}$, Total bilirubin $1.6 \mathrm{mg} / \mathrm{dL}$ and LDH $1049 \mathrm{U} / \mathrm{L}$. No schistocytes were seen on peripheral smear. Parvovirus IgM and IgG antibody titers were elevated. A diagnosis of Parvovirus B19 transient aplastic crisis (TAC) was made, based on decline in Hgb, reticulocytopenia and elevated Parvovirus B19 IgM titers. 2 units of RBCs were given with appropriate increment in Hgb which remained stable over 2 days before she was discharged.

IMPACT/DISCUSSION: Patients with sickle cell anemia (HbSS) are particularly susceptible to Parvovirus B19 infection due to asplenia and can lead to TAC due to the cessation of red blood cell production. Parvovirus B19 accounts for most TAC particularly among children with HbSS disease. In contrast, patients with HbSC disease often have less frequent crises which may delay presentation of Parvovirus B19 TAC to adulthood as in our patient. TAC should be suspected when there is an acute fall in Hgb that is not accompanied by an appreciate bone marrow response in red cell production. Symptoms usually are due to acute on chronic anemia with varying severity but can be life-threatening, highlighting the need for early recognition. Other Parvovirus B19 related complications such as acute chest syndrome, acute splenic or hepatic sequestration and bone marrow necrosis can occur concomitantly with aplastic crisis. Serum IgM testing is recommended for immunocompetent patients and immunocompromised should be tested by viral DNA. Treatment is usually supportive with antipyretics or ibuprofen (if fever is present) and blood 
transfusions if symptomatic. Recurrence of infection is rare due to development of immunity.

CONCLUSION: - HbSC often has a milder clinical course compared to $\mathrm{HbSS}$, which can delay asplenia and its infectious complications. - TAC should be on the considered in HbSC patients and treated aggressively if symptomatic.

\section{TO BELL OR NOT TO BELL: A CASE OF ATYPICAL FACIAL NERVE PALSY}

Niharika Mallepally; Jules Ryan Tabilona; Lee Lu. Baylor College of Medicine, Houston, TX. (Control ID \#3186090)

LEARNING OBJECTIVE \#1: Recognize the gradual onset of symptoms in malignant facial nerve schwannoma (FNS).

LEARNING OBJECTIVE \#2: Review the treatment of malignant FNS. CASE: A 54-year-old female with a history of Bell's palsy presented for an annual well exam. Eighteen months ago, she developed left jaw and facial pain followed by a slow onset of left facial weakness, numbness and inability to close her left eye. These symptoms progressively worsened over 6 months. She was diagnosed with Bell's palsy. Her left facial weakness continued to worsen. She denied hearing loss, tinnitus, vertigo, or other neurological symptoms. Physical exam noted left sided facial paralysis, decreased sensation, and inability to close the left eyelid. Due to the unusual gradual progression of her facial symptoms, an MRI of the orbit was performed and showed diffuse enhancement of the middle ear and mastoid cavities extending through the mastoid foramen. A follow up CT scan of the temporal bone noted a soft tissue mass with aggressive features in the left mastoid cavity with osseous erosion and local extension to the left external auditory canal and middle ear cavity and involvement of the left facial nerve. A biopsy of the mass revealed a malignant tumor with schwannian features. She was diagnosed with malignant FNS and is awaiting surgical excision.

IMPACT/DISCUSSION: Facial nerve schwannoma is a rare tumor arising from schwann cells surrounding the facial nerve. Though comprising less than $1 \%$ of temporal bone tumors, FNS is the most common type of facial nerve tumor. FNS presents with facial nerve paralysis, decreased sensation, tinnitus, hearing loss, pain, and dizziness. Symptom onset usually is slowly progressive over months. On initial presentation, FNS is often misdiagnosed as either vestibular schwannoma or Bell's palsy. Diagnosis is made by MRI imaging showing auditory canal mass with extension to the facial nerve and histopathology. Management can be conservative with serial imaging, and the reported tumor growth is $1.4 \mathrm{~mm}$ annually. Alternatively, stereotactic radiosurgery is a treatment option. Preservation of the facial nerve is a surgical priority, and post-operative improvement in paralysis and paresis is variable. Treatment decisions are based on symptom severity and baseline nerve and hearing function. Though the majority of FNS is benign, $<10 \%$ of all FNS can be malignant, as was the case in our patient. Malignant tumors have aggressive growth and are almost always managed surgically.

CONCLUSION: Unlike the common Bell's palsy's acute presentation, the onset of malignant FNS is gradual; it should be recognized and diagnosed promptly. In a high technological medicine era with less time spent with patients, this case reminds physicians of Sir William Olser's teaching- "Just listen to your patient."

TO CLOT OR TO BLEED: THROMBOSIS AS A FIRST SIGN OF MICROSCOPIC POLYANGIITIS

Kajali Mishra; Sandra Naffouj; Sarah Gorgis. Henry Ford Hospital, Detroit, MI. (Control ID \#3185940)
LEARNING OBJECTIVE \#1: Recognize ANCA-associated vasculitis in a patient with thrombosis

LEARNING OBJECTIVE \#2: Management of diffuse alveolar hemorrhage in the setting of acute thrombosis

CASE: A 59-year-old female with a history of stroke with residual rightsided weakness presented with dyspnea and lower extremity swelling. She was febrile, tachycardic, and required supplemental oxygen (O2). Laboratory studies showed an acute kidney injury (AKI). Lower extremity dopplers revealed bilateral deep vein thrombosis (DVT), and she was started on heparin for DVT and presumed pulmonary embolism (PE). Computed tomography (CT) PE was not performed due to AKI. Chest xray showed bilateral airspace opacities, so antibiotics were started. The patient clinically worsened and developed hemoptysis with increasing $\mathrm{O} 2$ requirements. CT chest showed concern for alveolar hemorrhage. Anticoagulation was stopped and the patient was intubated. Bronchoscopy confirmed diffuse alveolar hemorrhage. Rheumatologic workup showed ESR 111, ANA 1:320 (speckled), and positive MPO (p-ANCA) of 115. Left kidney biopsy revealed focal segmental necrotizing and crescentic glomerulonephritis consistent with ANCA-associated pauci-immune glomerulonephritis, microscopic polyangiitis (MPA). Plasmapheresis and hemodialysis was started due to worsening kidney function. She clinically improved, was extubated, and eventually weaned off oxygen. Induction with Cyclophosphamide and high-dose steroids was begun. Her AKI resolved, no longer requiring dialysis. After a riskbenefit discussion, warfarin was started, and the patient returned home. She was transitioned to maintenance therapy on Azathioprine and remained clinically stable at her three year follow up.

IMPACT/DISCUSSION: We present a case of thrombosis as a first sign of severe microscopic polyangiitis. The most common clinical symptoms of MPA include fatigue, cough, dyspnea, and arthralgias. Although it is a pulmonary-renal syndrome, renal insufficiency is only present in $18 \%$ of patients on presentation. In our patient, a DVT (originally thought to be due to immobilization from stroke) was the first sign of extensive MPA. The accompanying respiratory symptoms were presumed to be due to PE, and therefore a CT scan was not initially performed. The etiology of the hypercoagulable state in such patients is unclear, but circulating antiplasminogen antibodies have been demonstrated in those who develop clots. This case was further complicated by diffuse alveolar hemorrhage, a rare but life-threatening complication of MPA. Immunosuppressive therapy is required in almost all patients with active disease.

CONCLUSION: Patients with ANCA-associated vasculitis have a high risk of thrombosis, but it is rarely the presenting sign. Suspicion in patients with concomitant renal insufficiency and pulmonary symptoms can lead to early diagnosis. Despite being hypercoagulable, anticoagulation may not be the appropriate initial treatment as pulmonary hemorrhage can worsen outcomes.

\section{TO CUT OR NOT TO CUT? A CASE OF ISOLATED PULMONIC VALVE ENDOCARDITIS}

Aravind Seetharaman $^{1}$; Katie Cohen ${ }^{1}$; Sarah Nickoloff ${ }^{1,2}$. ${ }^{1}$ Medical College of Wisconsin, Milwaukee, WI; ${ }^{2}$ Zablocki VA Medical Center, Milwaukee, WI. (Control ID \#3167561)

LEARNING OBJECTIVE \#1: Recognize the clinical manifestations of pulmonary valve endocarditis.

LEARNING OBJECTIVE \#2: Identify high-risk patients who would benefit from cardiovascular surgery evaluation.

CASE: A 70-year-old male with history of hypertension, obstructive sleep apnea, and alcohol abuse was admitted for severe dyspnea, a 30-pound weight loss, and fever. Blood cultures grew Enterococcus faecalis. A new 
heart murmur was auscultated, and transthoracic echocardiogram (TTE) showed a $3.8 \times 0.5 \mathrm{~cm}$ pulmonic valve $(\mathrm{PV})$ vegetation. Confirmatory transesophageal echocardiogram revealed a $4.0 \times 1.0 \mathrm{~cm}$ mobile mass in the RV outflow tract attached to the base of the right cusp of the PV. Treatment with ampicillin and ceftriaxone was initiated. Moderate to severe pulmonary regurgitation and cusp prolapse into the RV outflow tract were seen on cardiac MRI. Cardiothoracic surgery (CTS) was consulted. However, given the patient's stable cardiac function, absence of heart block on EKG, and multiple medical comorbidities, CTS deferred surgical intervention. Despite ongoing treatment with IV antibiotics, the patient had recurrent febrile episodes with hemodynamic instability, and concern for septic embolization was raised. $\mathrm{He}$ was transferred to the affiliate hospital for a second opinion by CTS, who again deferred surgical intervention. Surveillance TTE showed decrease in size of the known mass to $2.7 \times 0.6 \mathrm{~cm}$, a second $1.8 \times 0.3 \mathrm{~cm}$ mass, and severe PV regurgitation with moderate RV enlargement but normal RV systolic function. Repeat TTE two weeks later showed slight enlargement of masses. CTS again evaluated the patient, and continued to recommend medical management, given high risk and uncertain benefit of surgical intervention. He was ultimately discharged home with planned serial TTE re-evaluation.

IMPACT/DISCUSSION: Initial treatment of isolated pulmonic valve endocarditis is usually medical. Our patient's high-risk features such as vegetation size $>20 \mathrm{~mm}$, recurrent pulmonary emboli, severe pulmonary regurgitation and development of new, additional vegetations while on broad-spectrum antibiotics made a compelling case for surgical intervention. However, CTS did not feel that benefit of surgical intervention outweighed risk, and our patient was therefore managed medically.

CONCLUSION: Isolated PV infective endocarditis (IE) is a very rare finding. The rarity of PV IE has been attributed to the lower pressures of the right heart, lower incidence of congenital/acquired valve abnormalities, lower oxygen content of venous blood, and differences in the endothelial lining and vascularization of the valve. There are clearly defined criteria for surgery in left-sided IE. However, guidelines for surgery in right-sided IE are much less clear. Conservative medical management is often recommended, as right-sided heart valve infections are thought to be more responsive to antibiotics and better tolerated than mitral or aortic valve infections. However, further research is needed to elucidate guidelines for surgical management of PV endocarditis.

\section{TO PLEX OR NOT TO PLEX: A CASE OF PULMONARY- LIMITED ANTI-GLOMERULAR BASEMENT MEMBRANE (GBM) DISEASE}

Jeffrey Lee; Justin A. Edward; Emily Waner. University of Colorado, Aurora, CO. (Control ID \#3162616)

LEARNING OBJECTIVE \#1: Recognize that although extremely rare, pulmonary-limited anti-GBM disease can occur in isolation.

LEARNING OBJECTIVE \#2: Identify expert recommended treatment for pulmonary-limited anti-GBM disease.

CASE: A 58-year-old male with a 35-pack-year smoking history and occupational hydrocarbon exposure presented with a 3-day history of submassive hemoptysis and new onset dyspnea. Physical exam was notable for tachycardia, hypoxemia to $72 \%$ on room air, and end-expiratory wheezing bilaterally. Workup was significant for a white blood cell count of $12.1 \times 10^{9} / \mathrm{L}$, negative procalcitonin, normal urinalysis, normal creatinine, and chest $\mathrm{x}$-ray with bilateral hazy opacities. CT angiography was negative for pulmonary embolus and demonstrated extensive bilateral patchy ground-glass opacities. Bronchoalveolar lavage diagnosed diffuse alveolar hemorrhage (DAH) with increasingly hemorrhagic effluent seen on sequential sampling. Bacterial, viral, and fungal cultures were all negative and empiric antibiotics were stopped. Serologic testing resulted in mildly positive anti-GBM antibodies $28 \mathrm{EU} / \mathrm{mL}$ (normal $<20 \mathrm{EU} / \mathrm{mL}$ ) with other autoimmune serologies resulting as negative. The patient improved after a 3-day course of pulse-dose steroids and was started on plasmapheresis (PLEX) for 2 weeks. He was additionally started on induction therapy with oral cyclophosphamide and a prolonged prednisone taper with close pulmonology and rheumatology follow-up.

IMPACT/DISCUSSION: Pulmonary-limited anti-GBM disease is extremely rare and only known via case reports. In reported cases, there is a high prevalence of active smoking and exposure to inhaled agents. ${ }^{2}$ The proposed mechanism of pathogenesis is that inhaled agents damage the non-fenestrated pulmonary capillary endothelium allowing anti-GBM antibodies to reach the alveolar basement membrane. ${ }^{1,2}$ Patients who present with DAH without clinical renal involvement should be screened for anti-GBM disease and treatment should be initiated as soon as possible. Early recognition and treatment of pulmonary-limited anti-GBM disease with immunosuppression and PLEX can prevent progression to renal involvement. ${ }^{2,3}$ Due to the limited cases of pulmonary-limited anti-GBM disease, treatment is based on extrapolation of existing recommendations for pulmonary-renal anti-GBM disease. Historically, there has been an association between anti-GBM antibody titer and disease severity. ${ }^{3,4}$ PLEX is used to expedite the clearance of anti-GBM antibodies, and has been shown to control pulmonary hemorrhage, the leading cause of early death in anti-GBM disease. ${ }^{3,4}$ The combination of PLEX and immunosuppression has improved outcomes as compared to immunosuppression alone. ${ }^{2,3}$ PLEX is a relatively safe procedure with a low risk of serious complications. ${ }^{3,5}$

CONCLUSION: Due its efficacy and safety, PLEX is recommended in patients with pulmonary-limited anti-GBM disease to rapidly control pulmonary hemorrhage and preserve renal function.

\section{TO SNF OR NOT TO SNF? CHALLENGES IN DISCHARGE PLAN- NING FOR PATIENTS WITH A HISTORY OF IVDU REQUIRING LONG COURSES OF ANTIBIOTICS}

Angela O. Suen ${ }^{1}$; Tanya Nikiforova ${ }^{2} .{ }^{1}$ UPMC, Pittsburgh, PA; ${ }^{2}$ University Of Pittsburgh Medical Center, Pittsburgh, PA. (Control ID \#3175932)

LEARNING OBJECTIVE \#1: Identify current evidence to support conservative versus liberal antibiotic management for patients with IVDU history. LEARNING OBJECTIVE \#2: Name risk factors that can predict failure of IV antibiotic therapy in the home setting.

CASE: A 30-year-old woman with a remote history of intravenous drug use (IVDU) presented to the hospital with progressive lower back pain. On admission, she was afebrile and hemodynamically stable. Lab work showed no leukocytosis, CRP 2.0, and ESR 89. Blood cultures grew no bacteria. A basic urine drug screen showed no illicit drugs. Spinal MRI showed T10-11 osteomyelitis. Bone biopsy was negative for bacterial growth. Infectious diseases consultants recommended 6 weeks of IV ampicillin-sulbactam. Given her IVDU history, doctors felt the patient would be at high risk for antibiotic failure and line tampering. Discharge to a skilled nursing facility (SNF) was planned but patient refused, citing barriers around child-care and financial difficulties. Patient decided to leave AMA and take oral antibiotics. She was given a prescription at discharge for oral moxifloxacin $400 \mathrm{mg}$ to complete a 6-week course. She was lost to follow-up.

IMPACT/DISCUSSION: Given concerns for line tampering, recurring drug use, and antibiotic nonadherence, physicians are often wary of discharging patients with a history of substance use to home to complete IV antibiotics. However, there is no conclusive evidence to support this practice. Current 
evidence show low rates of line tampering and high antibiotic completion rates when patients with IVDU history are treated with IV antibiotics at home or at an infusion center. One study found that discharge to home for infusion therapy did not lead to higher rates of line infections, IV drug use recurrence, loss to follow-up, readmission or death when compared to discharge to a skilled nursing facility. However, there are certain patient risk factors associated with a higher failure rate, including active IVDU, unstable housing status, patient unwillingness to comply with therapy, and lack of support systems. Given the absence of conclusive evidence to support our current practice, it is important to consider the patient's risk factors and needs when planning for discharge. This patient was at low-risk for failing home antibiotic therapy; her remote drug use, supportive family, and stable housing are all protective factors. In the future, patients with IVDU history should receive an individualized treatment course that involve a multi-disciplinary team which includes behavioral health, infectious disease, nursing care, and social work.

CONCLUSION: Not all patients with IVDU history necessitate hospital or SNF administration of IV antibiotics. In low risk patients, discharge to home may not lead to higher rates of complications than discharge to SNF. Certain patient risk factors are associated with a higher antibiotic failure rate, including active IVDU, unstable housing, unwillingness to comply with therapy, and lack of support systems.

\section{TRANSIENT ANTI-NMDA RECEPTOR ENCEPHALITIS}

Neil Evans; Amitabh Singh; Cory Cheatham. Saint Louis University, Saint Louis, MO. (Control ID \#3185911)

LEARNING OBJECTIVE \#1: Recognize Anti-NMDA-receptor encephalitis as a frequent cause of encephalitis in young woman

LEARNING OBJECTIVE \#2: Assocaite ovarian teratomas with AntiNMDA-recptor encephalitis

CASE: A 23 year old female with an unremarkable past medical history presents with psychosis. She was recently discharged from a local psychiatric facility for unresolved "Flakka" intoxication. On presentation she was encephalopathic, aggressive, and inappropriate to staff. Initial workup including EEG was unrevealing. MRI of the brain was negative for malignancies and other abnormalities. LP was performed but was negative for common bacterial and viral infectious causes. CT of the abdomen and pelvis was performed demonstrating a right sided ovarian cyst. Pelvic MRI was performed to better characterize the cyst and it had characteristics consistent with a teratoma. A repeat LP demonstrated an elevated oligoclonal bands and 1:20 Anti-NMDAantibody titre. A presumptive diagnosis of NMDA-R encephalitis was made. She was treated with plasmapheresis, IV immunoglobulin and IV solumedrol. She underwent oophorectomy; four days afterwards, she began showing signs of improvement in her cognition and by two weeks afterwards, the patient had returned to her baseline mental status and was discharged home.

IMPACT/DISCUSSION: Anti-NMDA-receptor encephalitis is the leading reversible cause of encephalitis in females under the age of 30. MRI is not sensitive for this condition with $50 \%$ appearing normal. Diagnosis is made by lumbar puncture looking for the antibody. The majority of cases of AntiNMDA-receptor encephalitis have an identifiable ovarian teratoma, which are thought to contribute to the immune-mediated pathogenesis. Treatment protocols emphasize supportive care and teratoma removal. The treatment regimen for anti-NMDA-receptor encephalitis includes high dose IV glucocorticoids, intravenous immune globulin and plasma exchange. In our case the patient received appropriate therapy and had an identifiable teratoma which was excised; leading to her positive outcome.

CONCLUSION: Autoimmune phenomena in the brain are rare and often a diagnostic challenge. It is important to consider anti-NMDA-receptor encephalitis in the evaluation of young females with unknown encephalitis. Exploration of teratomas with ultrasound, CT, or MRI is essential to the treatment as removal improves prognosis.

TRANSIENT TRANSAMINITIS AND HEPATIC GLYCOGENOSIS: A RARE COMPLICATION IN TYPE 2 DIABETES

Daniel Yee ${ }^{1}$; Jack P. Fatica ${ }^{1}$; Bradley Collins ${ }^{2} .{ }^{1}$ Brown University, Providence, RI; ${ }^{2}$ Miriam Hospital, Providence, RI. (Control ID \#3186263)

LEARNING OBJECTIVE \#1: To alert clinicians to a potential cause of transaminitis in poorly controlled diabetics who are given high doses of insulin. CASE: A 33 year-old woman with poorly-controlled type 2 diabetes mellitus (T2DM), left foot osteomyelitis on broad-spectrum antibiotics, hepatic steatosis, and opiate use disorder on methadone was admitted following elopement from a nearby hospital. On admission she was afebrile and hemodynamically stable with labs notable for AST of 67, ALT of 96, glucose of 308 , and no leukocytosis. MRI demonstrated osteomyelitis of the 1st metatarsal head of the left foot, and she was treated with antibiotics and underwent amputation without perioperative complications. Postoperatively, her home insulin regimen was resumed, and she developed fever, abdominal pain, nausea/vomiting, and headache. Labs on post-op day 2 showed AST of 1020, ALT of 299, ALP of 344, GGT of 1097, and LDH >3600. Differential diagnoses included viral or autoimmune hepatitis, hemochromatosis, immunemediated cholestatic disease, and drug-induced liver injury. RUQ ultrasound was suggestive of hepatic steatosis. A viral hepatitis panel was negative while EBV and CMV IgG studies were both positive, with positive EBV PCR studies. Ferritin was significantly elevated to $>16500$, but transferrin saturation was $13 \%$, and ceruloplasmin and hereditary hemochromatosis DNA PCR were negative. Antimitochondrial, anti-smooth muscle, and microsomal antibodies were negative. A toxicology screen was appropriately positive for methadone. There was concern for hemophagocytic lymphocytosis with elevated ferritin and IL-2 elevated to 2838, although the patient declined bone marrow biopsy. Over the following days, she had spontaneous resolution of fevers and abdominal pain with return of transaminases to baseline. Liver biopsy showed glycogenosis of the liver parenchyma and mixed inflammatory infiltrates. In-situ hybridization for EBV RNA was negative.

IMPACT/DISCUSSION: This case illustrates a rare complication of diabetes resulting in transaminitis: hepatic glycogenosis, or hepatic glycogenopathy (HG). HG is more commonly associated with type 1 diabetes, and almost never observed in T2DM (a literature search yielded 3 total case reports). The pathology is thought to be due to fluctuating glucose and insulin levels in poorly controlled diabetics, promoting liver glycogen deposition and inflammation. Clinically, patients present with transaminitis that resolves with improved glycemic control. Definitive diagnosis is via biopsy, as imaging of HG is indistinguishable from non-alcoholic fatty liver disease. In our case, transaminitis was worsened with initial insulin management and likely super-imposed drug injury. This is consistent with Tsujimoto et al., who suggest that large insulin boluses may initially worsen transaminitis in those who do not regularly take insulin.

CONCLUSION: In patients with T2DM and fluctuating transaminases, HG should be considered as a potential etiology, with liver biopsy for definitive diagnosis.

TRAUMATIC INJURY IN AN ELDERLY WOMAN: BIASES AND MISSED OPPORTUNITIES

Khameer Kidia ${ }^{1,2}$; Amy Ship ${ }^{2} .{ }^{1}$ Brigham \& Women's Hospital, Boston, MA; ${ }^{2}$ Atrius Health, Boston, MA. (Control ID \#3185140) 
LEARNING OBJECTIVE \#1: Recognize social determinants of health and cognitive bias in clinical assessment

LEARNING OBJECTIVE \#2: Recognize frailty as a risk factor for complications in the elderly

CASE: An $88 \mathrm{~F}$ fell on the bus, injuring her side and back. She had shoulder, thoracic, and abdominal pain, but didn't seek care until 5 weeks later when CXR and rib xrays were negative. 1 week later, she was seen urgently for pain worse with inspiration. Exam was non-focal. She was sent to pain clinic where she got depomedrol in T8-10 intercostals. Labs later showed: AST 683, ALT 691, Alk Phos 227. The next day, she again walked to the office. She had a homemade cardboard clock to remember her APAP dosing: $1000 \mathrm{mg} \mathrm{q} 8$. She reported living alone and being independent, but that climbing to her 4th-floor walk-up was hard. She had severe pain between her shoulders and in the RUQ, especially with inspiration. Vital signs were normal. She was disheveled and had RUQ tenderness with +Murphy's sign. She was sent to the ED where APAP level = 0, ALT 1586, AST 1298, Alk phos 242, INR 1.2. RUQ US was normal. Work-up showed +Hep A IgM. It was elicited that due to her fixed income she often ate spoiled food from a dumpster. In her 10-day hospitalization, transaminases rose to the 4000s, her INR peaked at 1.4, and she had severe hyponatremia.

IMPACT/DISCUSSION: This case highlights the need to: think about structural reasons for illness, examine biases, and listen carefully. We anchored on the patient's MSK injury and didn't consider structural causes for illness: housing, food, or income. A helpful framework is the structural differential, which "delineates the social, political, and economic factors that may be influencing a patient's health and health care and facilitates responses to the modifiable factors" (Seymour, Griffin et al. 2018). She also lived alone with no social supports. Our rising epidemic of loneliness is increasingly viewed as a health issue, especially in the elderly (Holmen and Furukawa 2002). Addressing loneliness is complex and warrants collective attention. Physicians are not neutral. There were several biases in her care: she was a retired nurse so we assumed high health literacy and financial stability. Her career and diligent cardboard clock, meant we did not formally assess her cognition. There were further clues: she was disheveled. Her constant reassurance about her independence may have been a cry for help. Why did she fall on the bus and sustain such severe injury or mention the stairs to her apartment? This should have clued us into her frailty, which is intimately tied to health outcomes in the elderly (Woo, Goggins et al. 2005).

CONCLUSION: Physician writer Victoria Sweet calls for slowing down the clinical process (2017). This gives doctors time to contextualize a patient and to be systematic. Our case shows the need to look beyond tests. We need to listen to the history and lookout for unspoken signs of distress. We need to slow down.

\section{TREATMENT OF ACQUIRED FACTOR VIII INHIBITOR IN THE} SETTING OF BULLOUS PEMPHIGOID

Meredith Ventura $^{1}$; Bernard Danna ${ }^{1}$; Sara Taveras Alam ${ }^{1,}{ }^{1}$; Perumal Thiagarajan $^{1,}{ }^{1}$; Gustavo Rivero ${ }^{1,}$; Sarvari Yellapragada ${ }^{2,}{ }^{1}$; Madhuri Vasudevan ${ }^{1,1}$. ' ${ }^{1}$ Baylor College of Medicine, Houston, TX; ${ }^{2}$ Michael E Debakey VA Medical Center and Dan L Duncan Cancer Center, Baylor College of Medicine, Houston, TX. (Control ID \#3175632)

LEARNING OBJECTIVE \#1: Recognize and diagnose acquired hemophilia.

LEARNING OBJECTIVE \#2: Treat acquired hemophilia in the setting of bullous pemphigoid.

CASE: A 68-year-old man with bullous pemphigoid (BP) presented to the hospital for ongoing skin blistering and ulceration. Although prednisone was initiated 2 months ago, he continued to develop active lesions. After admission he developed oozing from IV sites and maroon-colored stools. Past medical history included coronary artery disease, hypertension, type II diabetes, and hemorrhoids. No pertinent family or social history. Medications were prednisone and prophylactic lovenox. Physical exam was significant for oozing from IV sites, blood on rectal exam, and blistering and ulceration over the torso, extremities, and oral mucosa but sparing genitals. Labs revealed a prolonged PTT which did not correct with mixing study, low factor VIII activity, and elevated Bethesda titer. Anticardiolipin IgM, anticardiolipin IgG, anti- 2-glycoprotein IgM, and anti- 2-glycoprotein IgG, HIV, hepatitis A, B, and $\mathrm{C}$, and testing for multiple myeloma were negative. Dilute Russell Viper Venom Time ratio was normal. He was diagnosed with acquired hemophilia A (AHA). Prednisone was continued. IVIG and rituximab were started for cotreatment of BP and AHA. He experienced improvement of both skin lesions and bleeding diathesis. Following discharge, he required readmission for staphylococcus epidermidis septic arthritis of the elbow, which was complicated by traumatic tap requiring recombinant Factor VIIa.

IMPACT/DISCUSSION: BP is an autoimmune blistering skin disease driven by autoantibodies against the hemidesmosomes predominantly affecting the elderly. Steroids are first line treatment. IVIG and rituximab benefit patients who fail steroid monotherapy. AHA incidence also increases with age and is associated with an underlying condition in 50\% of cases, the largest being autoimmune diseases. Treatment consists of prednisone and sometimes cyclophosphamide. Although the combination is superior to prednisone alone in inhibitor eradication and disease specific survival, there is no improvement in overall survival. Rituximab is a suggested second line therapy. There is mixed evidence to support IVIG. This case illustrates the typical patient population affected by AHA: an older individual with an autoimmune condition. Co-occurrence of $\mathrm{BP}$ and AHA have been described in the literature. BP typically presents prior to or simultaneously with AHA. There are no guidelines for cotreatment. This case provides a successful example of cotreatment and summarizes treatment modalities used in other co-occurring cases of AHA and BP including steroid monotherapy, rituximab, cyclophosphamide, and azathioprine.

CONCLUSION: AHA incidence increases with age and is associated with autoimmune conditions. Treatment of AHA typically consist of steroids and cyclophosphamide. When cotreating BP and AHA consider the addition of IVIG and rituximab.

\section{TREATMENT RESISTANT HELICOBACTER PYLORI: AN IN- FECTION THAT PROVES DIFFICULT TO STOMACH.}

Erica Bass. UCSF, San Francisco, CA. (Control ID \#3184984)

LEARNING OBJECTIVE \#1: Recognize factors that determine successful eradication of Helicobacter pylori infection.

LEARNING OBJECTIVE \#2: Manage treatment resistant Helicobacter pylori infection.

CASE: Ms. J is a 41 year-old African American woman with chronic Helicobacter pylori (H. pylori) infection refractory to multiple courses of antibiotics who presented to establish care. She was first tested for $\mathrm{H}$. pylori in 2016 when she presented with dyspepsia and was found to have a positive serum immunoglobulin $\mathrm{G}$ (IgG) level. She was treated with clarithromycin triple therapy. Her stool antigen $(\mathrm{Ag})$ remained positive and she was treated with bismuth quadruple therapy. She underwent upper endoscopy with biopsy and pathology revealed chronic active gastritis and H. pylori organisms. Culture was not performed. She received two additional salvage regimens without evidence of eradication. At presentation she endorsed intermittent, mild epigastric pain and reflux following meals. She denied 
dysphagia, odynophagia, nausea, vomiting, and weight loss. She was wellappearing and had no abnormal findings on exam. Her only medication was daily famotidine. She reported strict adherence to past antibiotic regimens. She was seen in gastroenterology clinic and another salvage regimen was prescribed however her stool $\mathrm{Ag}$ remained positive. She was referred to infectious disease who recommended repeat endoscopy with biopsies for H. pylori culture and susceptibility testing. Her isolate was found to be resistant to macrolides.

IMPACT/DISCUSSION: H. pylori infection is prevalent and has been associated with chronic gastritis, peptic ulcer disease, and gastric malignancy. First line treatment is either clarithromycin triple therapy or bismuth quadruple therapy. Confirmation of eradication should be performed in all cases as one in five patients will experience treatment failure after initial therapy. Factors associated with successful eradication include choice of antibiotic regimen, patient adherence to therapy, and sensitivity of the organism to the medications administered. The initial regimen should be selected based on prior antibiotic exposures and allergies. Unfortunately, few data on resistance rates are available to guide antibiotic selection. Current guidelines recommend culture with antibiotic sensitivity testing after two failed treatment attempts. Ms. J underwent five failed treatment attempts over two years before she underwent more definitive testing. Ms. J may have been spared multiple rounds of antibiotics, numerous office visits, and testing with a better understanding of the guidelines for managing treatment resistant $\mathrm{H}$. pylori.

CONCLUSION: Treatment failure after initial therapy and relatively common and occurs in one out of five patients. Treatment selection, patient adherence to treatment, and medication resistance are key factors that predict successful eradication of $\mathrm{H}$. pylori infection. Management for treatment failure for $\mathrm{H}$. pylori infection is salvage therapy and then culture and susceptibility testing to guide antibiotic selection.

\section{TRICKY TO PINPOINT: AN UNUSUAL CAUSE OF CHEST PAIN IN A YOUNG MAN}

Stephen Russell; Patrick Manning; Ricardo Cruz. Boston Medical Center, Boston, MA. (Control ID \#3184995)

LEARNING OBJECTIVE \#1: Review etiology and diagnosis of Thrombotic Thrombocytopenic Purpura (TTP)

LEARNING OBJECTIVE \#2: Recognize and manage cardiac complications of TTP including acute coronary syndromes (ACS)

CASE: A 25 year old male with no past medical history presented with two weeks of progressive dyspnea on exertion and chest pain. The pain was substernal, pressure-like, and improved with rest. He had one day of dark colored urine, abdominal pain and nausea. On exam he was tachycardic, tachypneic, hypertensive, and in moderate distress. Initial labs revealed a platelet count of 13. There was evidence of microangiopathic hemolytic anemia (MAHA) with hemoglobin 7.4, LDH 2388, haptoglobin $<8$, and bilirubin 3.1. Blood smear showed shistocytes. Provisional diagnosis of TTP was made and he was admitted to the medical intensive care unit for therapeutic plasma exchange (TPE). Cardiac evaluation was persued. Initial troponin was 1.64, CK 378. An EKG showed sinus tachycardia. An echocardiogram in the emergency department showed inferolateral and anterolateral hypokinesis with mildly reduced ejection fraction $(\mathrm{EF})$. The troponin peaked at 1.8 and a diagnosis of non-ST-elevation MI (NSTEMI) was made. TTP was confirmed with ADAMTS13 level $<3 \%$ and antibody titer 2.1. He was transfused with packed red blood cells and treated with metoprolol to decrease cardiac demand. Captopril was added for hypertension. The patient stabilized and repeat echocardiogram five days later showed resolution of wall motion abnormalities and an EF of $65 \%$.
IMPACT/DISCUSSION: TTP is an acquired or hereditary hematologic disorder caused by deficiency in ADAMTS13 protease activity leading to platelet aggregation and microvascular thrombi affecting any organ. Patients classically present with MAHA, thrombocytopenia, fever, renal failure, and neurologic findings. Untreated TTP has a high mortality; the therapeutic imperative is urgent plasma exchange. Though cardiac involvement in TTP is considered rare, autopsy series have demonstrated cardiac damage in a significant number of patients. In a single center series myocardial infarction occurred in $21 \%$ of cases. While NSTEMI has been reported, detailed understanding of the clinical course and optimal treatment is unknown. Use of traditional therapeutics such as antiplatelets or anticoagulation is limited by profound thrombocytopenia and bleeding risk. The literature on cardiac imaging in TTP is limited. In case series, few patients had cardiac imaging and fewer had repeat testing after TPE. This patient uniquely had both in hospital and post hospitalization echocardiogram. He had relatively rapid improvement of wall motion abnormalities and there was no evidence of cardiac abnormality on outpatient follow up.

CONCLUSION: Initial evaluation of patients with TTP and cardiac symptoms should include cardiac biomarkers, cardiac imaging, and telemetry. Prompt treatment with TPE is the current best practice for managing cardiac complications of TTP

\section{TWO BROKEN HEARTS}

lingsheng li. Johns Hopkins Bayview Medical Center, Baltimore, MD. (Control ID \#3184687)

LEARNING OBJECTIVE \#1: Recognize the impact of widowhood on health outcomes in older adults

CASE: Mr. M was a 71-year-old retired security agent and primary caregiver for his wife of 49 years. His past medical history was notable for severe coronary artery disease, congestive heart failure, and atrial fibrillation. He was admitted to the cardiac progressive care unit with 2 day history of dyspnea, thought to be in setting of acute decompensated heart failure. On hospital day 3 , he was found to be hypotensive, bradycardic, and went into pulseless electrical activity arrest. Resuscitation attempts were unsuccessful. Four days after Mr. M's death, his wife Mrs. M was admitted to the same service with nausea, vomiting, and elevated troponin. She also had a significant cardiac history with poorly-controlled type 2 diabetes. She underwent a left heart catheterization that showed moderate to severe 2 vessel disease not amenable to revascularization. Per her request, Mrs. M was discharged on hospital day 2 to attend her husband's funeral. Mrs. M subsequently underwent 4 more hospitalizations due to diabetic ketoacidosis. Her family noted a dramatic decline in her emotional and physical health, complicated by ongoing grief, difficulty performing activities of daily living, and managing her insulin regimen. On her last admission, she was intubated for airway protection and found to have profound acidemia, hyperglycemia, acute renal failure, and shock requiring maximum dose of 2 vasopressors. Her children concluded that she would have wanted to pass peacefully and that Mr. M would have supported this decision. Her care was thus transitioned to Do-Not-Resuscitate, Do-Not-Intubate, comfort measures. Mrs. M died 41 days after her husband's death.

IMPACT/DISCUSSION: Older, widowed patients have a $48 \%$ increase in mortality risk in setting of bereavement ${ }^{1,2}$. This phenomenon has been described as the "widowhood effect" ${ }^{3}$. After her husband's death, Mrs. M suffered the loss of her biggest social support, life partner, and caregiver. Their story reflects the profound association between widowhood and adverse health outcomes such as depression, cardiovascular disease, hospitalization, and death. 1. Sullivan AR, Fenelon A. Patterns of widowhood mortality. J 
Gerontol B Psychol Sci Soc Sci 2014; 69(1): 53-62. 2. Patton KK, Glymour MM. In anticipation of grief: Using insights from social epidemiology to improve quality of care. Circulation 2013; 128:2725-2728. 3. Parkes CM, Benjamin B, Fitzgerald RG. Broken heart: A statistical study of increased mortality among widowers. Br Med J 1969; 1(5646):740-3.

CONCLUSION: - The "widowhood effect" is an universal health phenomenon. - There are many risk factors that may contribute to poor quality of life in older adults after the death of a partner. - Healthcare providers must take on an active role in supporting vulnerable individuals during times of grief.

TWO DAY OLD STEMI? WITH LUPUS ANYTHING IS POSSIBLE Ammar Hasnie; Usman Hasnie; James Stanford; Agostino Molteni. UMKC, Kansas City, MO. (Control ID \#3185736)

LEARNING OBJECTIVE \#1: Recognize the varied clinical manifestations of Lupus; more specifically Antiphospholipid Antibody Syndrome CASE: A 37-year-old female with a past medical history of Hypertension, Ischemic Cardiomyopathy ( $\mathrm{EF}=40-45 \%)$, Systemic Lupus Erythematosus, and Antiphospholipid Antibody Syndrome presented to the Emergency Department with complaints of pleuritic chest pain that had been persistent for two days duration which radiated down her left arm. Initial laboratory workup noted a normal BMP \& CBC. Troponin was noted to be elevated at $0.25 \mathrm{ng} / \mathrm{mL}$. Lipid profile ( $\mathrm{LDL}=72$ ) was within normal limits as was $\mathrm{A1C}$ (5. 3). Initial ECG was concerning for ST-elevation MI in the lateral leads(V2-V4) with no bundle branch block. Chest X-ray was unremarkable, however bedside ECHO noted a depressed ejection fraction of $40 \%$ with wall motion abnormality in the distribution of the LAD (left anterior descending). A CT-Angiogram of the chest was performed to rule out aortic dissection. The patient was emergently transferred to the cardiac cath lab for percutaneous coronary intervention with drug-eluting stent placement. Cardiac cath noted a mid-LAD lesion that was $99 \%$ occluded. She tolerated the procedure well and was discharged to home on Ticagrelor and Aspirin.

IMPACT/DISCUSSION: Systemic Lupus Erythematosus is notoriously known for being the "great imitator" due to its ability to affect any organ system. Antiphospholipid Antibody syndrome commonly presents in the setting of Lupus and is associated with the formation of vascular thrombosis. In this case, the patient waited two days before receiving medical attention. A large retrospective analysis showed Antiphospholipid Syndrome was responsible for 100,000 of myocardial infarctions (MIs) in the United States annually, or nearly $4 \%$ of MIs. Given the patient in this case had no obvious traditional risk factors, a delay in diagnosis and treatment could have been fatal given her delay in seeking care. A high index of suspicion of life threatening thrombosis is critical in these patients for the potential for favorable outcome.

CONCLUSION: Antiphospholipid Antibody Syndrome is a systemic autoimmune disease characterized by a hypercoagulable state commonly associated with recurrent pregnancy loss. However, there are other common clinical manifestations ranging from neurological involvement to cardiac involvement. Antiphospholipid antibodies have an ability to induce endothelial activation which can ultimately lead to development of accelerated atherosclerosis. We present a case of an otherwise healthy young female presenting with a myocardial infarction secondary her antiphospholipid antibody syndrome due to plaque rupture in her mid-left anterior descending artery.

\section{TWO DROPS}

Letisha Ferris; Michael P. Smith. University of Nebraska Medical Center, Omaha, NE. (Control ID \#3185746)
LEARNING OBJECTIVE \#1: Identify the three disease phases of Eosinophilic Granulomatosis with Polyangiitis (EGPA)

LEARNING OBJECTIVE \#2: Understand the complications of EGPA CASE: A 53 year-old woman presented with bilateral foot drop. She reported one-month history of progressive clumsiness and a weak hand grip. Symptoms began with a painful rash on her feet. She denied fatigue, myalgia, arthralgia, confusion, dizziness, or loss of bowel or bladder function. She denied recent illness or travel. Past medical history included adultonset allergic sinusitis and asthma refractory to conventional management. Lung imaging with patchy opacities. Multiple sinus surgeries with tissue biopsies noted eosinophilic infiltration. Screening for ANCA twelve years prior was negative. She had no personal or family history of diabetes or neuromuscular diseases. She had never smoked and drank alcohol occasionally. She had weak flexion and extension in her feet and flexion in her wrists. Ankle reflexes were diminished. Sensation to light touch, pinprick, and vibration were diminished in lower extremities. Proprioception was diminished in her great toes. Temperature sense was intact. She exhibited an ataxic gait and was unable to walk on tip-toes or heels. Foot drop was present bilaterally. She had eosinophilia with an absolute count of $14,600 / \mathrm{mL}$ and 65 percent predominance, along with elevated CRP and ESR, trace hematuria, and a positive ANA and p-ANCA. EMG revealed axonal asymmetric sensorimotor neuropathy. Hemoglobin A1c and TSH were unremarkable. Infectious work-up was negative, as were vitamin levels, brain and cervical spine MRI, electrocardiogram, and echocardiogram. She actually met diagnostic criteria for EGPA years prior to presentation

IMPACT/DISCUSSION: EGPA (formerly Churg-Strauss) is a vasculitis of small and medium sized vessels characterized by allergic rhinitis, asthma, and peripheral eosinophilia. It occurs in 18 out of every 1,000,000 people and accounts for 10 percent of vasculitis cases. It is associated with p-ANCA in approximately 50 percent of cases but it is not part of the diagnostic criteria. The diagnostic criteria for EGPA require four of the following to be present: paranasal sinus abnormalities, asthma, non-fixed pulmonary opacities on radiography, greater than 10 percent eosinophils, biopsy revealing extravascular eosinophils, and mononeuropathy or polyneuropathy. EGPA classically presents in three sequential phases. Atopy is representative of the prodromal phase. Peripheral eosinophilia and eosinophilic infiltration of organs, most notably the lungs, is characteristic of the eosinophilic phase. The vasculitic phase commonly manifests as neuropathy, most specifically mononeuritis multiplex, but it may give rise to serious extra-pulmonary complications that account for the majority of mortality in EGPA.

CONCLUSION: It is essential that internists are able to recognize EGPA as early as possible in the disease course to prevent the development of the irreversible vasculitic stage and the associated extra-pulmonary complications.

\section{TYPE 1 OR TYPE 2 DIABETES? A YOUNG, ASYMPTOMATIC MALE WITH A NORMAL BMI, FOUND TO HAVE SEVERE TYPE 2 DIABETES WITH END ORGAN DAMAGE}

Noah Ravenborg; Allison Diamant. UCLA, Los Angeles, CA. (Control ID \#3180727)

LEARNING OBJECTIVE \#1: Recognize that the prevalence of Type 2 Diabetes Mellitus is increasing, including among adults younger than 40

LEARNING OBJECTIVE \#2: Recognize when to consider screening for antibodies associated with Type 1 Diabetes Mellitus in patients who already have a diabetes diagnosis

CASE: An asymptomatic 32-year-old male who had not seen a physician in more than 2 years presented to an outpatient Internal Medicine practice to establish care. History and physical were significant for his social history, 
which revealed a sedentary lifestyle, high consumption of carbohydrates including sugary foods and beverages. His review of systems was negative. Physical exam was notable for an elevated blood pressure of 137/87, BMI of 23, and tinea pedis. Significant Labs: Random glucose 248, Hemoglobin A1C 10.0\%, Non-fasting Total cholesterol 282, Triglycerides 1,075, HDL 29 Repeat tests prior to return visit 1 week later: Hemoglobin A1c 9.7\%, Random glucose 194, Serum ketones: negative, Fasting Total cholesterol 239, Triglycerides 840, HDL 25, Urinalysis: 1+ glucose, Albumin/creatinine ratio: 69.2, Glutamic Acid Decarboxylase Ab: negative, Insulin Ab: negative, IA-2 Ab: negative, C-peptide: $2.2 \mathrm{He}$ was advised to start metformin, long acting insulin, an ACEi, and a statin.

IMPACT/DISCUSSION: This case helps to highlight two important points: Firstly, the importance of being aware of all risk factors associated with DM, especially sedentary lifestyle and dietary composition. Without this historical component, we may not have screened this patient for DM and would have missed the diagnosis and the opportunity to reduce his risk for further complications. He was asymptomatic, with no family history, and a normal BMI, and yet had severe disease and nephropathy. Of note, current USPSTF and $\mathrm{ADA}$ recommendations for screening do not include people in his age category and BMI. We suggest considering screening for diabetes on a broader scale for individuals with significant risk factors, and this case highlights the importance of having a lower threshold to screen. Secondly, when the diagnosis of DM is made in a young adult, when should clinicians consider a further work up for Type 1 Diabetes Mellitus (T1DM)? We want to highlight a screening tool that was developed to identify patients who should receive further screening for antibodies associated with T1DM. This screening tool recommends antibody testing in individuals with at least 2 of the following: age of onset $<50$, symptoms of hyperglycemia, $\mathrm{BMI}<25$, and personal or family history of autoimmune disease.

CONCLUSION: Given the increasing prevalence of diabetes, it is crucial that we screen and diagnose the disease early. Most current guidelines may not be broad enough to diagnose new cases of diabetes, and we should consider having a lower threshold to screen for those at risk.

\section{TYPHOID FEVER IN A NON ENDEMIC URBAN SETTING}

Kateryna Fedorov ${ }^{1}$; Syed Muhammad Ibrahim Rashid ${ }^{2}$; Johanna Daily ${ }^{1}$. ${ }^{1}$ Montefiore Medical Center, Bayside, NY; ${ }^{2}$ Montefiore Medical Center, Bronx, NY. (Control ID \#3182911)

\section{LEARNING OBJECTIVE \#1: Microbiology and epidemiology of Salmo- nella typhi}

LEARNING OBJECTIVE \#2: Signs, symptoms, clinical course, and therapeutic approach to Typhoid fever

CASE: 57-year-old male presented with one week of disabling rigors, fatigue, and myalgias. He denied any sick contacts. No travel outside the United States for himself or his close contacts. His vitals were 130/65mmHg, 79bpm, 102.5F. On exam he had mild abdominal tenderness. WBC was $5.7 \mathrm{k} / \mathrm{ul}$. Blood cultures were drawn. CT abdomen and pelvis showed small lymph nodes, likely reactive. He remained well, defervesced after one dose of Tylenol, and was discharged home. Post discharge he experienced worsening malaise, prostration, rigors, fevers, and anorexia. The patient was called to return to the hospital when blood cultures grew Salmonella typhi. On admission patient's vitals were $101.3 \mathrm{~F}$ and $64 \mathrm{bpm}$, consistent with pulsetemperature dissociation. On exam he was ill appearing, diaphoretic, with mild diffuse abdominal tenderness and had relative leukopenia with WBC of $4.5 \mathrm{k} / \mathrm{ul}$. Stool cultures grew S. typhi. The patient was treated with 3 days of IV Ceftriaxone, followed by 14 days of Cefpodoxime. He reported no recurrent fevers and increasing energy levels one week post-discharge.
IMPACT/DISCUSSION: Typhoid fever is caused by Salmonella typhi, a gram negative flagellated bacillus in the family Enterobacteriaceae. There were 26.9 million cases reported worldwide in 2010; 367 cases in the US in 2015 , with $82 \%$ with history of travel outside US. Remaining $18 \%$ includes those without personal history of travel to typhoid endemic areas. In such cases the transmission of typhoid could occur from an infected traveler to a non-traveler. Even asymptomatic carriers and those recovering from Typhoid fever can act as reservoirs of infection. Transmission of $S$. typhi is typically fecal-oral with humans being the only host. Ingested bacteria penetrate small bowel epithelium and disseminate hematogenously or through lymphatics. Typhoid fever syndrome consists of several stages. Initially, patients have rising fever secondary to bacteremia with a pulse-temperature dissociation followed by higher fevers, abdominal pain, and rose spots. Intestinal perforation, peritonitis, and sepsis can occur in week three. Advanced cases may have neurologic involvement include distorted sleep, psychosis, myalgias, and rigidity. Treatment is based on local sensitivity patterns, usually with fluoroquinolones, third-generation cephalosporins, and azithromycin. Our patient presented with signs strongly suggestive of $S$. typhi infection: pulsetemperature dissociation, relative leukopenia, and reactive abdominal lymphadenopathy. His diagnosis was delayed given negative travel history to typhoid endemic regions. Blood cultures allowed early diagnosis and may have prevented progression to life threatening symptoms.

CONCLUSION: Typhoid fever is uncommon in the US, especially in patients without travel history. In such patients blood cultures are essential to establish the diagnosis prior to disease progression.

\section{TYPICAL PYELONEPHRITIS TRIGGERING ATYPICAL HEMO- LYTIC UREMIC SYNDROME}

Malavika Kapuria; Ahmed Awan; Rajeev Raghavan. Baylor College of Medicine, Houston, TX. (Control ID \#3186967)

LEARNING OBJECTIVE \#1: Identify urinary tract infections (UTI) as a trigger of complement-mediated hemolytic uremic syndrome (HUS)

LEARNING OBJECTIVE \#2: Recognize the challenges related to diagnosing complement-mediated HUS

CASE: A 24-year-old woman with recurrent UTIs and chronic vaginal discharge was admitted for acute pyelonephritis. She had no diarrhea, and urine cultures grew G. vaginalis. Days after initial presentation, she developed worsening anemia and thrombocytopenia, requiring multiple transfusions. Labs revealed Coombs negative, microangiopathic hemolytic anemia (MAHA), nephrotic range proteinuria, and low complement $(\mathrm{C} 3, \mathrm{C} 4)$ levels, suggesting a thrombotic microangiopathy (TMA). Empiric treatment for thrombotic thrombocytopenic purpura (TTP) was initiated with plasma exchange (PLEX) and high-dose steroids, while awaiting ADAMTS13 levels. Thrombocytopenia improved, however there was persistent hemolysis despite continued PLEX. On eliciting additional history, the patient reported that her grandmother had required PLEX after developing acute pancreatitis, and eventually required kidney transplant for unclear reasons. Kidney biopsy confirmed TMA, and ADAMTS13 level was 100\%. Consequently, complement-mediated HUS was diagnosed and Eculizumab was started, after which the hemolysis and renal function improved.

IMPACT/DISCUSSION: Atypical HUS is an umbrella term describing a triad of MAHA, thrombocytopenia and renal failure, in the absence of ADAMTS13 deficiency and Shiga-toxin infection. More recently, it has come to define complement-mediated HUS - a disorder of unregulated complement activation, resulting from the primary deficiency of specific regulatory proteins. Definitive diagnosis involves measuring levels of these deficient proteins; tests which are both hard to obtain and often result too late to defer 
management. Clinically, complement-mediated HUS presents with a HUSlike triad that frequently overlaps with, and is occasionally indistinguishable from other TMAs. Furthermore, its association with infection results in its misdiagnosis as a secondary TMA caused by an infection, as opposed to a hereditary disorder unmasked by the infection. Treating the infectious trigger therefore does not limit the disease process, and missed diagnoses have substantial implications for patient outcomes and family counseling. Our case is particularly interesting as UTIs are seldom reported triggers of complementmediated HUS; and when associated, the UTIs are almost exclusively due to E. coli, and almost never due to G. vaginalis.

CONCLUSION: Complement-mediated HUS is a diagnostic challenge due to its nonspecific clinical presentation and limited complement testing. Delays in recognition however can have devastating patient outcomes, including irreversible end-stage kidney failure. Clinicians must therefore maintain a high index of suspicion: consider infectious triggers and family history, and promptly initiate complement-inhibiting Eculizumab to halt hemolysis and potentially reverse kidney injury.

\section{UNCOMMON BUT LIFE-THREATENING: PERSISTENT EN-} CEPHALOPATHY DUE TO PROLONGED HYPOGLYCEMIA.

Faiza Javed; Charu Ramchandani; Colette Knight. Montefiore Medical center, Wakefield campus, Bronx, NY. (Control ID \#3181453)

LEARNING OBJECTIVE \#1: Recognize that untreated hypoglycemia can result in prolonged neurological sequelae.

LEARNING OBJECTIVE \#2: Assess the role of neuroimaging in diagnosing hypoglycemia-induced encephalopathy

CASE: A 73-year-old woman with type 2 diabetes mellitus and hypertension was brought to the emergency department after being found unresponsive at home. She was last seen in good health 24 hours earlier by her family. Home medications were insulin glargine, repaglinide, and metformin. On examination, vital signs were normal, but glucose finger stick was low at $24 \mathrm{mg} / \mathrm{dL}$. Glasgow coma scale score was $3 / 15$, brain stem reflexes were present, and limbs had a flaccid tone with absent reflexes. She was given Dextrose 50\% and was started on Dextrose 5\% infusion but continued to have hypoglycemic episodes over the next 48 hours. Head CT ruled out stroke or hemorrhage. Brain MRI revealed nonspecific restricted diffusion involving bilateral corona radiata, centrum semiovale, white matter, and splenium of the corpus callosum, concerning for hypoglycemic injury. Infectious etiology and seizures were ruled out. Urine toxicology studies were negative. Hypothyroidism, adrenal insufficiency, and insulin hypersecretion were ruled out. Repeat MRI brain five days after presentation revealed resolution of restricted diffusion in the cerebral white matter; however, the patient did not improve clinically.

IMPACT/DISCUSSION: Severe hypoglycemia can cause encephalopathy. Areas of high energy consumption in the brain, such as cerebral cortex, hippocampus, cerebellum, caudate nucleus, and pallidum, are first to be affected by hypoglycemia. Hypoglycemia causes extensive denaturation, necrosis of neurons, and infiltration of glial cells. Insulin, sulfonylureas, alcohol abuse, renal dysfunction, old age, and infection can all precipitate hypoglycemia. Our patient was taking a combination of insulin, metformin, and repaglinide for many years; infection, drugs, alcohol or renal dysfunction were ruled out. Reversible hypoglycemic encephalopathy has been mentioned in case reports in patients whose hypoglycemic episodes lasted 12-72 hours. In this patient, hypoglycemia was observed for 48 hours after presentation, but it was not known for how long she was hypoglycemic at home. Irreversible, diffuse lesions involving extensive areas of cerebral cortex, hippocampus, and basal ganglia on MRI are associated with worse outcomes. Patients with reversible lesions on MRI were observed to recover either completely or with lingering deficits, such as aphasia. This patient had reversible, restricted diffusion on MRI, but failed to show any clinical improvement after five months.

CONCLUSION: * Early diagnosis and treatment of hypoglycemia can prevent irreversible encephalopathy. * Resolution of MRI findings due to hypoglycemic brain injury may not always predict recovery from encephalopathy.

\section{UNCONSCIOUS AND UNRESPONSIVE: SYNCOPE OR SOME- THING ELSE?}

Jimmy Feeney; Susan Wlodarczyk. UCSF, San Francisco, CA. (Control ID \#3180813)

LEARNING OBJECTIVE \#1: Differentiate syncope from other causes of transient loss of consciousness (TLOC)

LEARNING OBJECTIVE \#2: Identify clinical features that suggest a neurologic cause of TLOC

CASE: A 74 year-old man with a history of eosinophilic granulomatosis with polyangiitis was found slumped over in his chair during a routine rituximab infusion. The patient was unresponsive to sternal rub, did not localize to pain, and had a GCS of 3. His initial vital signs were normal. Labs including glucose, $\mathrm{CBC}$, chemistry panel, Utox, troponin and $\mathrm{ABG}$ were unremarkable. ECG showed sinus bradycardia with HR of 52 . Non-contrast CT of the head was normal. After 30-minutes, the patient abruptly returned to his normal baseline mental status without intervention and was alert, conversant and had a non-focal neurologic exam. He then had a second episode of TLOC, again with spontaneous return to baseline, and was admitted for further evaluation. Given a negative workup for common causes of syncope, including orthostatics, ECG, and TTE, the differential was expanded to include non-syncopal causes of TLOC. A brain MRI/MRA showed multiple punctate infarcts involving the posterior circulation, including the pons, likely from a cardioembolic source given their distribution. The patient was ultimately diagnosed with a posterior circulation TIA as the cause of his episodes of TLOC

IMPACT/DISCUSSION: Syncope, defined as TLOC from global cerebral hypoperfusion, is the most common cause of TLOC, accounting for $6 \%$ of hospitalizations in the US ${ }^{1}$. Current evidence and guidelines recommend avoiding unnecessary brain imaging in the routine diagnostic workup of syncope $^{1}$. However, this case demonstrates the importance of clinically differentiating true syncope from neurologic causes and recognizing when a broader diagnostic workup is indicated. The hypercoagulability that occurs in eGPA increased this patient's likelihood of having a neurovascular cause of $\mathrm{TLOC}^{2}$. Further, the key feature distinguishing this case from true syncope was the prolonged nature of his TLOC with normal vital signs, which is more characteristic of coma. In contrast, syncope is typically brief, self-limited and with rapid recovery. Sudden onset of coma suggests a vascular origin, such as a brainstem stroke involving the reticular activating system. While rare, lesions to the pons are known to cause TLOC and coma. This patient's brain MRI revealed multiple posterior circulation infarcts suggesting that he most likely had a transient basilar artery occlusion. While vertebrobasilar TIA is a rare cause of TLOC, it is crucial that internists recognize when a patient's presentation may point to a neurologic cause that requires a different diagnostic approach compared to true syncope. 1.Mendu 2009, PMID 19636031 2.Aviña 2016, PMID 27213713

CONCLUSION: Clinical features such prolonged duration and complete unresponsiveness distinguish true syncope from neurologic causes of TLOC Vertebrobasilar TIA that causes ischemia in the brainstem, especially the pons, is a rare but important cause of TLOC 
UNCONTROLLED CELL GROWTH: A CASE REPORT OF ACUTE MYELOID LEUKEMIA IN A PATIENT WITH NEUROFIBROMATOSIS TYPE-1

Saboor E. Randhawa ${ }^{1}$; Nagendra Natarajan ${ }^{2}$; Jonathan Gootee ${ }^{1}$; Mohsin Mirza ${ }^{1}{ }^{1}$ Creighton University, Omaha, NE; ${ }^{2}$ Nebraska Cancer Specialists, Omaha, NE. (Control ID \#3186272)

LEARNING OBJECTIVE \#1: Recognize the association of Neurofibromatosis type-1 with Acute Myeloid Leukemia.

LEARNING OBJECTIVE \#2: Recognize the association of Neurofibromatosis type-1 with benign and malignant tumors based on cytogenetics.

CASE: A 67-year-old male presented with severe worsening dyspnea, weakness and unexplained weight loss. His past history was significant for Neurofibromatosis type-1 (NF-1), diabetes mellitus, Barrett's esophagus, and multiple benign lung nodules. He denied fever, chills, night sweats, chest pain or easy bruising. Physical exam was remarkable for numerous raised nodules on his back, arms and face characteristic of NF-1. An extensive diagnostic workup was undertaken. Complete blood count analysis showed hemoglobin of $6.0 \mathrm{~g} / \mathrm{dL}$, platelet count of $29,000 / \mathrm{microL}$, white blood cell count of $42,400 /$ microL with frequent blasts in the peripheral blood. Bone marrow biopsy and aspiration diagnosed acute monoblastic leukemia (a type of Acute Myeloid Leukemia or AML) based on morphology and immunophenotyping. Myeloid mutation panel testing showed alteration in NPM1, TET2, DNMT3A, U2AF1, and cytogenetics/Fluorescent-In-Situ-Hybridization confirmed trisomy 8 . This profile is associated with poor prognosis. He was initiated on intensive remission induction therapy with standard dose cytarabine and idarubicin ( $7+3$ chemotherapy) along with aggressive supportive measures. Remission was achieved, and bone marrow biopsy and aspiration completed on day 14 of treatment revealed hypocellular marrow without evidence of residual AML. His blood counts recovered, with improvement in symptoms.

IMPACT/DISCUSSION: Neurofibromatosis type-1 (NF-1), or Von Recklinghausen disease, is an autosomal dominant genetic disorder with an incidence of approximately 1 in 3000 individuals. NF-1 is due to mutations in the NF1 gene, located at chromosome 17q11.2. These mutations can lead to a number of benign and malignant tumors. The association between hematologic malignancies and germ-line mutations of NF1 has been established in the pediatric setting. In adult population, though not as strong, the association between both leukemia and lymphoma has been described Germline heterozygous alterations of NF1 gene lead to a higher risk to develop juvenile myelomonocytic leukemia and/or AML. More recently, somatic 17q11 deletions encompassing NF1 have been described in many adult myeloid malignancies. In our case, the presence of a malignancy of myeloid lineage supported the association of AML with NF-1, however further studies are needed to establish a direct connection.

CONCLUSION: - Neurofibromatosis type-1 is associated with hematological malignancies like Acute Myeloid Leukemia. - Uncontrolled cell growth due to mutations in NF-1 gene results in both benign and malignant tumors.

\section{UNDER PRESSURE - COMMUNICABLE OR IATROGENIC CAR- DIOMYOPATHY?}

Max Zlatopolsky ${ }^{1,2}$; Vadim Gladwill ${ }^{1}$; Mathar Aldaoud ${ }^{2},{ }^{1}$; Mahmoud Assaad $^{2} .{ }^{1}$ Ascension-Providence, St. Clair Shores, MI; ${ }^{2}$ Ascension-Providence Heart Institute, Southfield, MI. (Control ID \#3180731)

LEARNING OBJECTIVE \#1: Recognize the complexity of stress cardiomyopathy and its major subtypes beyond that of the typical apical (Takotsubo) variant
LEARNING OBJECTIVE \#2: Better understand the more common risk factors for stress cardiomyopathy

CASE: A 65 year old African-American male presented to a tertiary care emergency department with dyspnea and serially positive troponins $(0.21$, 0.24). His past medical history was significant for COPD, hypertension, and tobacco dependence. He had no prior history of acute coronary syndrome (ACS) or relevant family history. Physical examination was essentially unremarkable, however the patient was hypoxic saturating $98 \%$ on $2 \mathrm{~L}$ of oxygen on nasal cannula. Laboratory studies were unrevealing with the exception of the aforementioned elevation in troponin levels. EKG demonstrated normal sinus rhythm without injury pattern. Echocardiography revealed regional hypokinesis of the left ventricular anterior/anterolateral wall with sparing of the apex, along with hypokinesis of the mid/apical right ventricular wall suspicious for bi-ventricular stress cardiomyopathy. A left heart catheterization was performed to evaluate for obstructive plaques and possible stress cardiomyopathy. Non-obstructive CAD was discovered with $<50 \%$ stenosis in the left coronary, right coronary, and left circumflex arteries. Ventriculography confirmed a midventricular stress cardiomyopathy. The patient was initiated on a b-blocker and encouraged to refrain from using his albuterol inhaler and to avoid physical or emotional exertion. Interestingly, further discussion with the patient revealed that his wife experienced stress cardiomyopathy 2 months prior to his presentation. The patient also revealed that due to his dyspnea, he began using his rescue inhaler more frequently (up to 15 times per day) prior to his hospitalization.

IMPACT/DISCUSSION: We believe our case highlights the importance of forming a broad differential in the setting of positive troponins and an anginal equivalent in a patient with risk factors for ACS. It also draws our readers' attention to the complexity of stress cardiomyopathy, and explains that not all stress cardiomyopathy is Takotsubo cardiomyopathy. The current preclinical theory based on animal studies presupposes that the apex contains a higher proportion of adrenergic receptors explaining its ability to be preferentially effected in an a severely stressful situation. And of course, we want to emphasize that COPD is a common but underreported etiology for stress cardiomyopathy that impacts males. Prospective registries identify $90 \%$ of stress cardiomyopathy among women, and the biventricular variant is extremely rare which adds an additional layer of nuance to our case.

CONCLUSION: - Men can develop stress cardiomyopathy despite the overhwleming female predilection - There are multiple variants of stress cardiomyopathy - COPD is a known risk factor for the development of stress cardiomyopathy - Biventricular cardiomyopathy can occur in the absence of cardiogenic shock

\section{UNEXPECTED ASCITES: AN UNCOMMON PRESENTATION FROM COEXISTING COMMON CONDITIONS.}

Shirin Karimi $^{1}$; Ariel Majidi ${ }^{1}$; Priyank Jain ${ }^{2} .{ }^{1}$ Cambridge Health Alliance, Harvard Medical School, Boston, MA; ${ }^{2}$ Cambridge Health Alliance, Cambridge, MA. (Control ID \#3186312)

LEARNING OBJECTIVE \#1: Recognize an uncommon cause of inflammatory ascites

LEARNING OBJECTIVE \#2: Recognize that coexisting common conditions can produce unusual presentations

CASE: A 58 year old man with alcohol use disorder was admitted after being found intoxicated with Listerine. He reported acute onset epigastric pain radiating to the back with progressive difficulty initiating urination and incomplete voiding. His physical exam revealed a markedly distended abdomen with diffuse abdominal tenderness. Basic chemistry showed $\mathrm{K} 7.0$, Anion Gap 29, Cr 7.3, NT-proBNP 789, Lactic Acid 9.5. Additional labs 
showed Lipase 2086, AST 87, ALT 71. Total bilirubin, Alkaline Phosphatase and INR were normal. WBC 23,000 and Platelets 196,000. CT of abdomen and pelvis showed hepatic steatosis, large volume ascites, and distended urinary bladder. A Foley catheter immediately drained $1.5 \mathrm{~L}$ clear urine. $\mathrm{He}$ was treated for pancreatitis and bladder outlet obstruction with resolution of symptoms and normalization of CBC, BMP and lactate within 2 days. Diagnostic paracentesis revealed 2005 nucleated cells in the peritoneal fluid. He was started on cefepime for possible bacterial peritonitis; this was discontinued when bacterial and blood cultures were negative after 48 hours of incubation. Cytology was negative for malignant cells. CT of the abdomen and pelvis with contrast was repeated when pancreatitis and bladder obstruction had resolved. This showed almost complete resolution of ascites and bladder outlet obstruction. The cause of his ascites was attributed to hypervolemia from obstructive renal failure and concurrent inflammation from acute pancreatitis.

IMPACT/DISCUSSION: To our knowledge, this is the first presentation of inflammatory ascites secondary to acute renal failure and acute pancreatitis in the medical literature. While other causes of ascites are more commonly seen as those causes observed in hypervolemia (cirrhosis, congestive heart failure, end-stage renal disease), or inflammation (malignancy or chylous ascites seen in chronic pancreatitis), our literature review did not find any recorded cases of ascites attributed to acute renal failure with concomitant acute pancreatitis. This case educated the team that patients can manifest unusual presentation when different disease states coexist that can have a synergistic pathophysiologic derangement. Neither acute pancreatitis nor acute urinary obstruction causes ascites individually, but in our case, these conditions coexisted and presented with ascites because of hypervolemia and inflammation. In unclear cases of new large volume ascites, one must proceed with thorough investigation through diagnostic paracentesis and CT abdomen/pelvis. However, with concomitant acute renal failure and acute pancreatitis, one can expect significant resolution in ascites with resolution of renal failure and supportive treatment of acute pancreatitis.

CONCLUSION: A new large volume ascites can be observed in cases of acute urinary obstruction renal failure and/or acute pancreatitis.

\section{UNICENTRIC CASTLEMAN DISEASE IN THE SETTING OF HODGKIN LYMPHOMA}

Nishant Munugala; Andrew Rettew. University of Arizona, Banner University Medical Center, Tucson, AZ. (Control ID \#3185258)

LEARNING OBJECTIVE \#1: Evaluate lymphadenopathy in an asymptomatic patient

LEARNING OBJECTIVE \#2: Diagnose Castleman Disease and assess underlying etiology

CASE: A 70-year-old obese male with no significant medical history presented to his primary care provider with progressive, painless left-sided neck swelling of several years duration. Evaluation with computed tomography of the neck revealed a 3-cm lymph node concerning for a neoplastic process. He underwent an excisional biopsy with pathologic findings of angiofollicular lymph node hyperplasia, consistent with Castleman disease of the hyaline vascular subtype. Subsequent PET/CT revealed residual disease confined to the left neck, confirming unicentric disease. Further workup for potential etiologies of Castleman disease was undertaken, including evaluation for HIV, HHV-8, and paraproteinemia, which were all unrevealing. Given the asymptomatic nature of the disease, it was decided to opt for close clinical observation as opposed to additional surgery or systemic therapy. Follow-up PET/CT 6 months later was largely unchanged from prior, continuing to show FDG-avid lymph nodes in the anterior and posterior cervical chains. Given the lack of any significant clinical or radiographic changes, continued observation was pursued. Approximately 1-year later the patient presented for follow-up and noted increased neck swelling in addition to a 35-pound weight loss in the preceding 5 months. Repeat PET/CT revealed increased size and number of FDG-avid lymph nodes in the neck. ENT performed a left neck dissection in an effort to remove all known disease and provide a cure. Pathologic review revealed hyaline vascular Castleman disease intermixed with nodular sclerosing Hodgkin lymphoma. The initial pathology specimen was reviewed, which, in retrospect, did reveal CD30 positive Reed Sternberg cells within the hyaline vascular Castleman disease. Treatment for classical Hodgkin disease was recommended for the patient despite negative post-operative PET/CT given systemic nature of Hodgkin lymphoma.

IMPACT/DISCUSSION: Unicentric Castleman disease is a rare clinical entity, with approximately 2,500 cases diagnosed per year in the United States, that often presents as asymptomatic lymphadenopathy. Pathologic evaluation is of critical importance in diagnosis and thus an excisional biopsy should be pursued. Histologic evaluation reveals one of four subtypes: hyaline vascular, plasma cell, mixed, and plasmablastic. Following diagnosis, workup for an underlying etiology is critical, as Castleman disease is often associated with an underlying disease process, as highlighted by our case. The occurrence of Hodgkin lymphoma concurrently with Castleman disease is exceedingly rare, with few documented cases in the literature.

CONCLUSION: Developing a systematic approach to lymphadenopathy is an essential skill for internists Castleman Disease requires adequate evaluation for an underlying etiology to optimize therapy

\section{UNUSUAL ETIOLOGY OF A USUAL DIAGNOSIS; A CASE OF NON CONVULSIVE STATUS EPILEPTICUS}

Muhammad Hammad; Akhilesh Kumar Thakur; Umair Bajwa. Presence Saint Francis Hospital, Evanston, IL. (Control ID \#3186351)

LEARNING OBJECTIVE \#1: Consider Non-Convulsive Status Epilepticus and Creutzfeldt Jakob Disease as unusual causes of Encephalopathy

LEARNING OBJECTIVE \#2: Recognize the importance of continuous EEG monitoring to diagnose Non-Convulsive Status Epilepticus

CASE: A 68-year-old male presented with altered mental status and an episode of generalized seizure. He had also been more lethargic for the last few months, acting bizarrely and forgetting things. At presentation, he was vitally stable but appeared drowsy. Initial lab work was negative for leukocytosis and electrolyte abnormalities. CXR was concerning for left lower lobe infiltrate. Seizure and encephalopathy was thought to be secondary to underlying infection. CT angiogram of head and neck showed old lacunar infarcts but negative for acute pathology. Spot EEG revealed encephalopathy without any epileptiform activity. MRI brain showed signal alteration on diffusion, $\mathrm{ADC}$, Flair and T2- weighted sequences within the bifrontal and biparietal regions which suggested encephalitis or status epilepticus rather than acute ischemia. CSF analysis was negative for infectious etiology but later came back positive for 14-3-3. Rapidly worsening mental status without seizure like activity raised suspicion for Creutzfeldt Jakob Disease. However, late into the course of his hospital stay, a witnessed seizure raised suspicion for status epilepticus. Continuous EEG monitoring revealed electrographic status epilepticus with sub clinical seizures occurring almost every three minutes. He was treated with anti-epileptics with improvement in his mental status.

IMPACT/DISCUSSION: Encephalopathy in adult population is commonly encountered in clinical practice with underlying cause sometimes difficult to determine. Non-convulsive status epilepticus remains under recognized because the patients do not usually show classical ictal features. This case underscores the importance of continuous EEG monitoring to diagnose 
NCSE as the cause of encephalopathy especially when the etiology remains unclear since the treatment can lead to improved outcomes.

CONCLUSION: Nearly one-half of new-onset seizures occur in individuals over the age of 65 years. Status epilepticus is also not infrequent in older patients. Non convulsive status epilepticus remains a challenging diagnosis as the seizures in older patients are less likely to be convulsive. NCSE can sometimes occur after a single brief convulsive seizure leading to prolonged confusional state that is sometimes mistakenly thought to be postictal. Continuous EEG is therefore needed to make the diagnosis and should be obtained for any patient with altered consciousness of unknown etiology. Detection of 14-3-3 protein in CSF should be considered as an adjunctive rather than absolute test for the diagnosis of prion diseases. The specificity of the test is around 80 percent and can be positive in non-prion diseases as well obscuring the diagnosis. It remains critical to make the right diagnosis. Whereas NCSE is treatable, CJD remains without cure.

\section{UNUSUAL PRESENTATION OF CMV COLITIS IN AN ELDERLY IMMUNOCOMPETENT PATIENT}

Xixi Xu; Muhammad Fazal; Susan Phillips. Boston University School of Medicine, Boston, MA. (Control ID \#3186043)

LEARNING OBJECTIVE \#1: Recognize that CMV colitis should be considered in the differential diagnosis of severe colitis in older immunocompetent patients.

CASE: A 72-year-old male from Cape Verde with an unknown past medical history presented with 2 months of profuse, non-bloody, watery diarrhea that was not responsive to antibiotics. On admission, the patient had diarrhea that occurred up to 8 times per day with poor appetite, no abdominal pain, nausea or vomiting. Physical examination revealed normal abdominal exam. Laboratory studies were pertinent for $\mathrm{WBC} 10.2 \mathrm{~K} / \mathrm{UL}$, potassium of $2.8 \mathrm{mmol} / \mathrm{L}$ and magnesium of $1.1 \mathrm{mg} / \mathrm{dL}$. Inflammatory markers were elevated with CRP of $47.7 \mathrm{mg} / \mathrm{L}$ and ESR of $43 \mathrm{~mm} / \mathrm{Hr}$. Stool culture, stool ova and parasite exam, stool PCR for bacteria, parasite and Microsporidia were negative. Serology testings were negative for HIV and HTLV but positive for IgG of Cytomegalovirus (CMV). Serological viral DNA load was at $9443 \mathrm{IU} / \mathrm{ml}$. A $\mathrm{CT}$ of the abdomen and pelvis showed evidence of pancolitis with dilated transverse colon. Colonoscopy showed severe inflammation in the colon with large well-demarcated patches and the edges were heaped. Biopsy showed diffuse ulceration, granulation tissue, and reparative changes. Immunohistochemistry of the biopsy was positive for CMV. Treatment with ganciclovir was started for 6 days and was transitioned to valganciclovir. The patient was discharged with valganciclovir and loperamide. Four weeks after the initiation of antiviral treatment, he reported significant improvements in symptoms. Repeated Flexible Sigmoidoscopy showed marginal improvements in the colon. Biopsies showed no CMV on immunohistochemistry but the rest of the histological findings were similar to prior biopsies.

IMPACT/DISCUSSION: Gastrointestinal involvement of CMV is usually seen in immunosuppressed patients with common presentations including diarrhea, fever, and abdominal pain. CMV colitis can mimic colitis caused by C.diffile, ischemia, malignant diseases, and inflammatory bowel diseases both clinically and radiologically. Thus, a diagnosis of CMV colitis requires positive IgM titer for CMV, CMV antigen in the blood, positive PCR in blood or urine, and positive immunohistochemistry/histological findings. CMV colitis in immunocompetent hosts is rare. Only 28 cases are reported in a meta-analysis. Most of these patients are over 55 years old, which suggests that the immune system weakens as a consequence of aging and high prevalence of comorbidities. Young and otherwise healthy patients usually do not need antiviral therapy and can recover spontaneously. For older patients with comorbidities, antiviral therapy such as gancyclovir or foscarnet is necessary.

CONCLUSION: After exclusion of common etiologies for severe diarrhea in older patients, CMV colitis should be considered in the immunocompetent patients. Diagnosis of CMV colitis is made with positive serological and histological findings. Gancyclovir or foscarnet should be initiated in older patients once diagnosed.

\section{UTILITY OF FOUR-DIMENSIONAL CORONARY COMPUTED TOMOGRAPHY ANGIOGRAPHY FOR MOBILITY OF A THROM- BUS IN THE ASCENDING AORTA}

Toshiki Kuno $^{1}$; Daisuke Asahara ${ }^{2}$; Kiyoshi Koizumi ${ }^{2}$; Takahito Ito $^{2}$; Yohei Numasawa $^{2}$; Masaki Kodaira ${ }^{2}$; Kazuo Isozumi ${ }^{2}$; Satoru Komatsumoto ${ }^{2}$. ${ }^{1}$ Mount Sinai Beth Israel, New York, NY; ${ }^{2}$ Japanese Red Cross Ashikaga Hospital, Ashikaga, Japan. (Control ID \#3186408)

LEARNING OBJECTIVE \#1: 4DCCTA is an excellent alternative without blind spots to detect and evaluate the mobile thrombus in the ascending aorta. CASE: A 63-year-old man presented with transient left hemiplegia and disturbance of consciousness. He did not have any medical history, but smoked one pack of cigarettes per day. Diffusion weighted magnetic resonance imaging revealed high-intensity signals in the right cortex of the parietal lobe. He again experienced the same symptoms, but improved thereafter. He was diagnosed with acute ischemic stroke, and ascending aortic dissection was suspected because his symptoms fluctuated. However, a thrombus, which was protruding in the ascending aorta, and right renal infarction were detected via contrast-enhanced computed tomography (CT). He was treated with unfractionated heparin to prevent further embolization. Transthoracic echocardiography did not show the thrombus, but four-dimensional coronary computed tomography angiography (4DCCTA) revealed its shape and mobility. Therefore, we strongly considered it to be a mobile thrombus that may have been the cause of ischemic stroke and a risk for further systemic embolization. Preoperative transesophageal echocardiography (TEE) did not reveal a patent foramen ovale, atrial septal defect, or a thrombus in the left atrial appendage. TEE as well as 4DCCTA images showed mobility of the thrombus in the ascending aorta, and no remarkable arteriosclerotic change. The patient underwent a median sternotomy to remove a segment of his ascending aorta, which was replaced with a prosthetic graft material. Pathological examination revealed a thrombus with a ruptured fibrous capsule that was a cholesterol-rich atherosclerotic plaque.

IMPACT/DISCUSSION: Four-dimensional CT is a three-dimensional computed tomography volume containing a moving structure and has previously been utilized in the setting of radiation therapy planning. We performed 4DCCTA to evaluate the movement of a thrombus in the ascending aorta. Since our patient had a further risk of ischemic stroke due to the thrombus in the ascending aorta, we needed to evaluate the state of the thrombus promptly to indicate early surgical intervention. Although TEE is an effective method to evaluate the mobility of the thrombus in the ascending aorta, recent upper gastrointestinal surgery, esophageal varices are contraindications and gastrointestinal perforation, aspiration pneumonia are complications for performing TEE. 4DCCTA could provide more complete evaluations in the aorta without blind spots compared with TEE. If TEE is contraindicated, or not available, 4DCCTA is an excellent alternative to detect and evaluate the mobile thrombus in the aorta.

CONCLUSION: 4DCCTA is an excellent alternative without blind spots to detect and evaluate the mobile thrombus in the ascending aorta. 
UTILIZATION OF HIGH DOSE INSULIN EUGLYCEMIC THERAPY IN INTENTIONAL SOTALOL OVERDOSE

Bryan F. Stringer ${ }^{1}$; Anantha Sriharsha Madgula ${ }^{1}$; Anand Muthu Krishnan ${ }^{1}$; Simrina Sabharwal ${ }^{2}$. ${ }^{1}$ University of Connecticut, Farmington, CT; ${ }^{2}$ University of Connecticut School of Medicine, Hartford, CT. (Control ID \#3185738)

LEARNING OBJECTIVE \#1: Recognize clinical and electrocardiographic findings in sotalol toxicity

LEARNING OBJECTIVE \#2: Manage poison-induced cardiogenic shock with high dose insulin

CASE: Patient is an 83-year old male with past medical history of atrial fibrillation, hypertension, coronary artery disease, anxiety and depression presenting from home after being found unresponsive by family with multiple pill bottles nearby including lorazepam and sotalol. His last seen normal was the previous night. When paramedics arrived, patient was bradycardic to $20 \mathrm{bpm}$, atropine was given and he was transcutaneously paced. Norepinephrine was initiated and upon arrival to the emergency department he was intubated and a transvenous pacer was placed. On admission, vitals included pulse $80 \mathrm{bpm}$ (paced), blood pressure 100/60, respiratory rate 14 . Physical exam revealed an unresponsive male, but no other significant abnormalities. Labs were significant for urine toxicology positive for benzodiazepine, otherwise within normal limits. Computed tomography scan of the brain was negative for acute pathology. Initial electrocardiogram showed junctional bradycardia competing with sinus bradycardia at $30 \mathrm{bpm}$ and QT/QTC of 707/498. Patient was transvenously paced and started on epinephrine and high dose insulin (HDI) for poison-induced cardiogenic shock. He was evaluated by toxicology and nephrology and transferred to intensive care unit where was started on continuous veno-venous hemofiltration. Due to profound hypoglycemia, patient was started on dextrose $25 \%$ infusion. After approximately 48 hours, the patient was able to be weaned off vasopressor support and HDI was discontinued. His transvenous pacer was turned off and he had an intrinsic rate of $50 \mathrm{bmp}$.

IMPACT/DISCUSSION: Beta blockers (BB) are one of the most common causes of poison-induced cardiogenic shock (PCIS). Toxicity typically presents with altered mental status, hypotension and bradycardia. PCIS can be difficult to manage and conventional therapies such as atropine, glucagon, cardiac pacing and vasopressors often offer only a transient response. High dose insulin therapy is an effective therapy in the management of PICS. Its benefit is contributed to its inotropic properties, increased myocardial uptake of carbohydrates and vasodilation improving local microcirculation. To date, there is no randomized clinical trial, however numerous case reports and prospective observational studies have demonstrated benefit of initiating HDI early in patients with PCIS. Electrolyte abnormalities and hypoglycemia are frequently reported adverse effects and require vigilant monitoring. Our patient on HDI required an dextrose (D25) drip to maintain euglycemia.

CONCLUSION: Sotalol toxicity presents like other BB toxicities, with additional EKG changes such as QT prolongation. In patients with PCIS, early HDI can improve hemodynamics. HDI can have adverse effects including hypokalemia and hypoglycemia which require close monitoring. There is a need for randomized clinical trials to attest efficacy of HDI in PCIS.

VAGINAL BLEEDING IN A POST-MENOPAUSAL FEMALE: A CASE REPORT

Laith Numan; Nedaa Husainat; Omar Abughanimeh. University of MissouriKansas City, Kansas City, MO. (Control ID \#3165199)
LEARNING OBJECTIVE \#1: Recognize the rare presentation(s) of sarcoidosis, including vaginal bleeding/uterine mass.

LEARNING OBJECTIVE \#2: Diagnose uterine sarcoidosis after excluding other etiologies with similar histopathology's.

CASE: A 63-year-old African American female with a history of iron deficiency anemia and uterine fibroids, presented to the gynecology clinic complaining of worsening vaginal bleeding. Computed tomography (CT) scan of the abdomen and pelvis showed a large uterus with multiple calcified masses and a dominant calcified mass communicating with the sigmoid colon, and severe bilateral hydroureteronephrosis; which raised concerns of fibroid transformation to leiomyosarcoma. The patient declined uterine biopsy and underwent sigmoidoscopy, which detected a large malignant appearing mass. The mass was obstructing and had necrosis and hard tissue. Biopsy results showed benign colonic mucosa and microcalcification but no evidence of malignancy. Chest CT scan showed bilateral pulmonary nodules, with no evidence of hilar lymph nodes enlargement. Due to concerns of malignancy, a total abdominal hysterectomy, bilateral salpingooophorectomy and sigmoid resection with end colostomy were done. The patient tolerated the procedure well. Histological examination of the specimen demonstrated multiple noncaseating granulomas with no evidence of malignancy. Pathology stains to rule out tuberculosis and fungal infection were negative. Angiotensin-converting enzyme level was elevated. After a multiteam evaluation by Oncology, Pulmonology, and Rheumatology, the patient was believed to have isolated uterine sarcoidosis with invasion to the colon. This is a very unusual way for sarcoidosis to present with only a few documented cases in the literature.

IMPACT/DISCUSSION: Extrapulmonary sarcoidosis is common, however uterine sarcoidosis is extremely rare. Typically, patients presenting with extrapulmonary sarcoidosis will have pulmonary involvement. Isolated extrapulmonary sarcoidosis is very rare. Uterine sarcoidosis is an unusual way for sarcoidosis to present and is challenging to diagnose due to other common etiologies presenting with uterine bleeding. In terms of uterine histopathology: in sarcoidosis, noncaseating granulomas can be found in all the layers of the uterus. Meanwhile, tuberculosis usually spares the myometrium.

CONCLUSION: Uterine sarcoidosis is extremely rare, and the diagnosis can be a challenge. The presentation can be nonspecific, and without pulmonary symptoms, other etiologies of vaginal bleeding should be excluded before confirming the diagnosis. Identification of noncaseating granulomas is the key for the diagnosis, after ruling out other etiology that can present with the same histopathology. Invasion of adjacent organs is possible and will require surgery.

\section{VANISHING BILE DUCTS FROM HODGKIN'S LYMPHOMA}

Neil Evans; Amitabh Singh; Jennifer Schmidt; Kenn Vattathara. Saint Louis University, Saint Louis, MO. (Control ID \#3186108)

LEARNING OBJECTIVE \#1: Recognize vanishing bile duct syndrome (VBDS)

LEARNING OBJECTIVE \#2: Categorize Hodgkin's lymphoma as a cause of VBDS

CASE: A 40-year-old woman with a history of asthma and a cholecystectomy presented with fevers, weight loss, dyspnea, lymphadenopathy, and fatigue. She was prescribed acetaminophen, azithromycin, prednisone, and oseltamivir for presumed flu-induced asthma exacerbation. Four days later, she presented with continued fevers, chills, epigastric pain, and jaundice. Labs showed: aspartate aminotransferase (AST) 225 Units U/L, alanine aminotransferase (ALT) $201 \mathrm{U} / \mathrm{L}$, total bilirubin $11.3 \mathrm{mg} / \mathrm{dL}$, and alkaline 
phosphatase $771 \mathrm{U} / \mathrm{L}$; previously these were normal. Hepatic autoimmune evaluation revealed an anti-nuclear antibody titer of 1:320 (speckled pattern), ANA titer 1:80, antimitochondrial antibody M2 titer 4.1 (positive), F-Actin IgG $10 \mathrm{U}$, viral hepatitis negative, and INR 1.3. Right upper quadrant ultrasound and MRI showed hepatomegaly. Liver biopsy showed bile duct paucity with periportal fibrosis. Steroids and Ursodiol were started for presumed azithromycin induced vanishing bile duct syndrome with improvements in AST/ALT and pruritis, however, bilirubin and alkaline phosphatase continued to uptrend. Patient returned 1 month later with fevers, melena, jaundice and neck swelling. Biopsy of a neck lymph node revealed Hodgkin's lymphoma. She began chemotherapy but progressed to renal failure, liver failure and passed away.

IMPACT/DISCUSSION: Vanishing bile duct syndrome (VBDS) causes progressive destruction of the intrahepatic bile ducts leading to cholestasis. It has several case reports for the condition every year with multiple etiologies found: drug-induced, immunologic, ischemic, infectious, metabolic, and malignant (specifically Hodgkin's lymphoma). Lymphoma remains a commonly reported cause of VBDS. Patients with VBDS associated with lymphoma often present with severe cholestatic hepatitis with autoimmune features. Cholestatic symptoms include: gallstones, hyperlipidemia, malabsorption, xanthelasmas, and fat-soluble vitamin deficiencies. VBDS often manifests 1-6 months after the initial cholestatic episode and is associated with fatigue, anorexia, abdominal pain, pruritis, and weight loss. VBDS has been identified as a paraneoplastic process of Hodgkin's lymphoma (HL). And importantly, HL associated VBDS patients do not recover unless remission of the HL.

CONCLUSION: This case illustrates the importance of avoiding anchoring bias with vanishing bile duct syndrome. Although VBDS is rare and poorly understood, it has a high morbidity and mortality if the underlying cause is not treated. 50\% of Hodgkin's lymphoma patients have liver infiltration and a cholestatic hepatitis with VBDS on biopsy should prompt evaluation of B symptoms and possible work up for lymphoma.

\section{VANITY COSTING THE VEINS}

Ian Cormier; Michael P. Smith. University of Nebraska Medical Center,

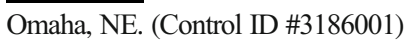

LEARNING OBJECTIVE \#1: Understand the link between off label use of testosterone and risk of hypercoagulability and thrombosis.

LEARNING OBJECTIVE \#2: Recognize the presentation of mesenteric venous thrombosis as a differential for acute abdominal pain.

CASE: A 52 year old man presented with one week of vague abdominal pain exacerbated by oral intake. The patient's medical history was significant for traumatic splenectomy, anabolic-steroid use and hypogonadism. His medications included testosterone, anastrozole, and human chorionic gonadotropin supplements, which were managed by a private men's clinic. On arrival, the patient was afebrile and hypertensive but otherwise hemodynamically stable. Physical exam was significant for diffuse abdominal tenderness to palpation with guarding. Labs were significant for polycythemia and leukocytosis with a normal lactic acid and lipase. Workup was significant for elevated total testosterone, free testosterone and sex hormone globulin. A computed tomography angiogram of the abdomen revealed acute thrombosis of the portal vein and superior mesenteric vein. The patient was kept nil per os, placed on intravenous fluids, and initiated on a heparin infusion. He was gradually reintroduced to oral nutrition and was discharged home on daily enoxaparin injections for anticoagulation. At the time of discharge, his home testosterone boosting supplements were stopped.

IMPACT/DISCUSSION: Mesenteric venous thrombosis is a rare but potentially life threatening cause of abdominal pain. Common causes of mesenteric venous thrombosis include intraabdominal malignancies and inflammatory processes, portal hypertension, acquired and inherited thrombophilias and myeloproliferative disorders. Certain medications and supplements, including testosterone replacement therapy (TRT) and drugs that indirectly increase levels of testosterone may promote a prothrombotic state that can lead to venous thrombosis. TRT causes an acquired prothrombotic state by stimulating erythropoiesis and inducing secondary polycythemia. The link between TRT and VTE, even in men without polycythemia, recently led to the US Food and Drug Administration (FDA) to require manufacturers to place a general warning in the labeling of all approved testosterone products regarding this risk. In the United States, there has been a sharp increase in the number testosterone prescriptions per capita within the last two decades. Many men are initiated on TRT for questionable indications such as sexual dysfunction and decreased energy, yet pathological hypogonadism remains the only indication for TRT. For men initiated on TRT, the Endocrine Society guidelines recommend checking hematocrit at baseline, 3-6 months after starting treatment, and then annually. The Endocrine Society guidelines also suggest stopping therapy if the hematocrit increases to 54 percent.

CONCLUSION: TRT is not a benign therapy and may be associated with life threatening VTE. TRT should be regulated to the standards of other prescribed medications.

\section{VARICELLA ZOSTER VIRUS (VZV) VASCULOPATHY AS A PO- TENTIAL CAUSE OF ALTERED MENTAL STATUS IN AN IMMU- NOCOMPROMISED HOST}

Ryan Handoko; Eric I. Elliott; Sameer Khan; Maricar Malinis. Yale School of Medicine, New Haven, CT. (Control ID \#3162737)

LEARNING OBJECTIVE \#1: Recognize the atypical presentation of varicella zoster virus (VZV) in an immunocompromised host

LEARNING OBJECTIVE \#2: Recognize VZV vasculopathy as an etiology of altered mental status in an immunocompromised host

CASE: This is a 48-year-old male with history of HIV/AIDS (CD4+ count=2 cells $/ \mathrm{mm}^{3}$, HIV RNA=3.66 log copies $/ \mathrm{mL}$ ), nonadherence to antiretroviral therapy, 20-pack year smoking and polysubstance use, who was admitted after being found unresponsive. Diagnostic work-up identified a large right pneumothorax. His hospital course was complicated by polymicrobial pneumonia, MRSA bacteremia, and gastrointestinal bleeding (GIB). Dolutegravir, darunavir, cobicistat, and opportunistic infection prophylaxes were initiated. He was transferred to our institution to treat pneumothorax refractory to pleurodesis with endobronchial valves. On exam, he had temporal wasting, rhonchi over right lung fields, and greasy scaled skin on his face consistent with seborrheic dermatitis. On hospital day 14, he became somnolent with failure to thrive, poor oral intake, and avolition, with no focal neurological deficits. Mirtazapine was given for depression but discontinued for worsening cytopenias. Neurology and psychiatry consultants recommended methylphenidate for CNS stimulation. Contrast-enhanced MRI of the brain revealed hemorrhage in both ganglio-capsular regions and subacute to chronic hemorrhage in the left caudate. Lumbar puncture was performed and CSF revealed 3 $\mathrm{WBC} / \mathrm{mm}^{3}, 41 \mathrm{mg} / \mathrm{dL}$ glucose, $20.1 \mathrm{mg} / \mathrm{dL}$ protein, and $0.8 \mathrm{U} / \mathrm{L}$ adenosine deaminase. Serum TPPA was negative. CSF Cryptococcal antigen, HIV RNA, CMV DNA, JC virus DNA, EBV DNA, HSV DNA, and Toxoplasma DNA were undetected by PCR. However, CSF VZV DNA was detected. CSF VZV IgG was not detected. IV acyclovir was initiated. He subsequently had recurrent GIB and hypoxemia and was transferred to medical step-down. Chest X-ray showed right lung collapse due to mucus plug. His family decided to pursue palliative measures only, and the patient thereafter expired. 
IMPACT/DISCUSSION: VZV vasculopathy occurs in both immunocompetent and immunocompromised hosts. Up to $40 \%$ of patients present without the characteristic rash, and up to $33 \%$ lack CSF pleocytosis. Brain imaging nearly always shows pathology, including ischemic or hemorrhagic lesions involving large or small cerebral arteries. CSF VZV IgG is more sensitive than VZV DNA by PCR, which is only elevated transiently. In our case, CSF VZV $\mathrm{IgG}$ was likely undetected due to hypogammaglobulinemia in a severely immunocompromised host. However, detection of CSF VZV DNA secured the diagnosis. Overall, a comprehensive assessment of changes in mental status in an immunocompromised host must include a work-up for neuroinfectious processes, including VZV vasculopathy.

CONCLUSION: Altered mental status in an immunocompromised patient should include neuro-infectious etiologies such as VZV vasculopathy. Evaluation for VZV vasculopathy includes brain imaging and detection of either VZV IgG (more sensitive) or VZV DNA in CSF (more specific).

\section{VEGETABLES \&GT; VEGETATIONS}

Zhihang Zhang ${ }^{1}$; Alexander Gipsman ${ }^{1}$; Sarah W. Baron ${ }^{2}$; Daniel Sims ${ }^{3}$. ${ }^{1}$ Montefiore Medical Center, New York, NY; ${ }^{2}$ Montefiore, Bronx, NY; ${ }^{3}$ Montefiore Medical Center, BRONX, NY. (Control ID \#3181225)

LEARNING OBJECTIVE \#1: Recognize bilateral infective endocarditis as extremely rare without prior cardiac disease or risk factors.

CASE: A 41-year-old man with past history of uncontrolled T1 Diabetes complicated by toe amputations, presents to the hospital in DKA with a concurrent chronic right foot ulcer with cellulitis. The patient had no history of intravenous drug use. MRI demonstrated osteomyelitis, and blood cultures were positive for methicillin-sensitive Staphylococcus aureus (MSSA). The patient was treated with IV nafcillin, however remained bacteremic for a total of 9 days despite appropriate antibacterial therapy, with 3 days of bacteremia post source control with bone debridement. Transthoracic echocardiography suggested a mobile structure attached to the interventricular septum below the left ventricular outflow tract (LVOT) measuring $0.9 \times 0.3 \mathrm{~cm}$. Transesophageal echocardiography was performed to further characterize the pathology and assess for abscess, and discovered two distinct areas of vegetation, one in the left ventricle attached to the septum and an additional lesion in the right ventricle near the tricuspid annulus measuring $1.5 \mathrm{~cm} \times 1.2 \mathrm{~cm}$ with possible abscess formation. CT imaging of the chest showed septic emboli in the lungs. During the hospital course, he developed a right wrist abscess due to septic emboli, and his foot was debrided for source control. Cardiac MRI for surgical planning confirmed bilateral vegetations. He then underwent removal of the vegetations in the right ventricle with repair of the tricuspid valve, with an uneventful recovery and discharge.

IMPACT/DISCUSSION: Bilateral endocarditis without prior cardiac pathology, prosthetic valve, vascular access, or IV drug use is extremely rare. The majority of infective endocarditis cases involve the left sided system alone, while right sided endocarditis is most associated with venous access infections. One retrospective review of infective endocarditis in patients without IV drug use over 14 years at one center showed that 39 (16\%) were right sided and 204 (84\%) were left sided. Of the right sided endocarditis cases, $36 \%$ had uncorrected congenital heart disease, and another $21 \%$ had central venous catheter access. On literature review of bilateral endocarditis without prior risk factors, case reports of 4 patients total, reported 2 patients with history of aortic valve replacements, and 2 patients without any predisposing condition, 3 of the 4 reported patients had Staphylococcus aureus, which is notably virulent enough to cause disease on previously normal valves. In our case of bilateral infective endocarditis, the only identified risk factor for this rare presentation was a history of uncontrolled diabetes.
CONCLUSION: Endocarditis deserves detailed multimodal imaging to guide management. Bilateral endocarditis without prior cardiac pathology or vascular access is extremely rare, and the authors report this case of bilateral infection with uncontrolled diabetes as the only identified risk factor.

\section{VERTEBRAL OSTEOMYELITIS DUE TO EXTENDED SPEC- TRUM -LACTAMASE (ESBL) PRODUCING ESCHERICHIA COLI FROM A GENITOURINARY SOURCE}

Mais Abdou $^{1}$; Sameer Prakash ${ }^{3}$; Muath Alsharif ${ }^{2}$; Steven Urban ${ }^{1} .{ }^{1}$ TTUHSC, Amarillo, Amarillo, TX; ${ }^{2}$ Texas Tech University Health Science Center, Amarillo, TX; ${ }^{3}$ Texas Tech University Health Sciences Center Amarillo, Amarillo, TX. (Control ID \#3184531)

LEARNING OBJECTIVE \#1: Recognize ESBL-producing gram negative bacilli as an uncommon cause of osteomyelitis especially after a urinary tract infection. Identify the clinical characteristics, diagnostic methods and management of vertebral osteomyelitis.

CASE: A 58-year-old male with past medical history of alcoholism, liver cirrhosis, atrial fibrillation and stage IV squamous cell carcinoma of the hypopharynx, status post radiation and ongoing nivolumab treatment, presented to the hospital with worsening lower back pain and chills for a week. $\mathrm{He}$ also reported nausea, vomiting, and anorexia. Two weeks previously, he had completed a one week course of a carbapenem for ESBL producing E. coli. The patient was afebrile, and physical exam was significant for lower extremities weakness, decreased reflexes and difficulty walking. Laboratory workup revealed normal white cell count, anemia and procalcitonin of 30 . Urinalysis was unremarkable. MRI of lumbar spine showed hydropic degeneration of T12-L1 disc with severe marrow edema and loss of height favoring T12/L1 osteomyelitis/discitis. Blood cultures showed no growth. CT-guided biopsy of T12/L1 disc and the adjacent portion of the T12 vertebral body revealed changes consistent with acute on chronic osteomyelitis, and needle aspiration culture grew ESBL producing $E$ coli. Neurosurgical intervention was not pursued. The patient was started on meropenem with a planned 6 weeks course. However, he was re-admitted to hospital two weeks later with sepsis, and this time blood cultures grew out ESBL producing $E$ coli. The patient's port-a-cath seemed to play a role in persistent infection and therefore was removed. TEE showed no evidence of endocarditis. A short course of gentamicin was added to meropenem. He was then continued on meropenem for the next 4 weeks with subsequent clinical improvement.

IMPACT/DISCUSSION: Vertebral osteomyelitis is a serious bone infection that is classically caused by gram-positive bacteria and less likely gramnegative bacilli. ESBL producing Entrobacteriaceae are rarely reported in the medical literature as the causative agents. The urinary tract is the most frequent reported source of infection. These microorganisms imply a significant challenge in terms of treatment. Carbapenems are the antibiotic class of choice for these infections with no current evidence favoring one carbapenem over another. Most studies favor an antibiotic course of 6-8 weeks of treatment. CONCLUSION: ESBL-producing gram-negative rods should be considered as a causative agent for vertebral osteomyelitis in the setting of recent urinary tract infection. Blood cultures are positive in only $20-59 \%$ of the cases. CT guided biopsy is an important diagnostic method and should not be delayed, as obtaining culture and sensitivity is essential in guiding the treatment.

\section{VISCERAL LEISHMANIASIS AS AN UNEXPECTED CAUSE OF PANCYTOPENIA IN A PATIENT WITH AIDS}

Arie Szeinfeld $^{1}$; Eliran E. Zadikov ${ }^{1}$; Kaitlyn E. Takach ${ }^{1}$; Damian Casadesus ${ }^{2}$. ${ }^{1}$ American University of the Caribbean School of Medicine, Cupecoy, Sint 
Maarten (Dutch part); ${ }^{2}$ Jackson Memorial Hospital, Miami Beach, FL. (Control ID \#3138395)

LEARNING OBJECTIVE \#1: Recognize the importance of having Visceral Leishmaniasis on the differential in HIV-positive patients with pancytopenia.

CASE: A 29 year-old Hispanic male with past medical history of AIDS and multiple-associated co-infections including CNS toxoplasmosis, CMV retinitis, genital HSV, PCP, noncompliance and multiple substance abuse, presented to the ED with 10 days of watery non-bloody diarrhea, abdominal pain, fever, chills, anorexia and dry cough. Patient was admitted for similar symptoms nine months prior. Home medications included Atripla, Azithromycin, Bactrim and Leucovorin. On physical exam, he was awake and alert. Cardiopulmonary examination was normal. The abdomen was tender with hepatosplenomegaly, but non-distended. Cervical lymphadenopathy was palpable. Labs revealed pancytopenia: WBC: $2.3 \times 10^{3} / \mathrm{uL}$; Hgb: $7.8 \mathrm{~g} / \mathrm{dL}$; Hct: 22.8\%; Platelets: 124; LDH: 719. HIV viral load was 12,000 and CD4 count was $1 \mathrm{cell} / \mathrm{uL}$. Chest radiography showed bibasilar patchy opacities suggesting PCP pneumonia and Bactrim was started. Persistent pancytopenia prompted a bone marrow biopsy which demonstrated Donovan bodies consistent with Leishmaniasis. Esophagogastroduodenoscopy and colonoscopy were performed due to persistent diarrhea and biopsy was also consistent with Leishmaniasis. The patient was started on Amphotericin B ( $21 \mathrm{mg} / \mathrm{kg}$ inpatient for 2 weeks and infusions $1 \mathrm{q} /$ week for a total of 6 weeks outpatient) with resolution of the digestive symptoms. Patient followed up in the clinic with improvement of the pancytopenia.

IMPACT/DISCUSSION: Visceral Leishmaniasis (VL) is caused by a parasitic infection characterized by fever, pancytopenia and hepatosplenomegaly with progressive host deterioration. To date, there have been less than six reported cases of HIV with Leishmaniasis co-infection in the United States, thus far all imported. Incidence of VL is increasing in the developed world secondary to climate change, which has extended the natural habitat of the Leishmania vector into the southern United States. This case demonstrates the importance of having VL on the differential in HIV-positive patients with pancytopenia. There is debate regarding adding VL to the list of AIDsdefining opportunistic infections; therefore, a diagnosis of VL should prompt HIV screening. This is especially relevant as the mortality rate in patients with AIDS/Leishmaniasis co-infection is $53.7 \%$ as opposed to $7.5 \%$ in HIVnegative patients.

CONCLUSION: AIDS patients often present with significant hematologic abnormalities such as anemia, lymphocytopenia, thrombocytopenia and various coagulopathies. These irregularities stem from a variety of HIV-associated sources including opportunistic infections, malignancies or as consequences of treatment therapies such as antiretroviral therapy. Physicians should be aware of VL when evaluating an HIV-positive patient with pancytopenia and should consider performing bone marrow biopsy.

\section{VITAMIN B12 DEFICIENCY: AN UNDERAPPRECIATED CAUSE OF HEMOLYTIC ANEMIA}

Hannah Levavi; Rose Yu; Athanasia Vasiliadis. Mount Sinai Hospital, New York, NY. (Control ID \#3186151)

LEARNING OBJECTIVE \#1: Recognize vitamin B12 deficiency as a cause of hemolytic anemia

LEARNING OBJECTIVE \#2: Identify factors that can mask the diagnosis of vitamin B12 deficiency

CASE: A 69 year old woman with beta thalassemia trait presented to her primary care physician with two months of progressive dyspnea. Laboratory evaluation revealed hemoglobin $5.2 \mathrm{~g} / \mathrm{dL}$, mean corpuscular volume (MCV) 94.0 FL and red cell distribution width (RDW) 41\%. Other cell lines were within normal range. The patient was hospitalized for symptomatic anemia. Further evaluation revealed a mild, unconjugated hyperbilirubinemia, with total bilirubin $1.7 \mathrm{mg} / \mathrm{dL}$ and direct bilirubin $0.9 \mathrm{mg} / \mathrm{dL}$, low haptoglobin $<8$ $\mathrm{mg} / \mathrm{dL}$, elevated lactate dehydrogenase $7436 \mathrm{U} / \mathrm{L}$, reticulocytes $2.5 \%$, normal folate level, normal direct coombs, and vitamin B12 $<150 \mathrm{ph} / \mathrm{mL}$. Eventually, intrinsic factor antibodies were identified at $61.0 \mathrm{AU} / \mathrm{mL}$. Two units of packed red blood cells were transfused and vitamin B12 was repleted with serial injections of cyanocobalamin $1000 \mathrm{mcg}$. Oral iron and folate supplementation were also provided in anticipation of accelerated hematopoiesis. In the followup period, the patient had dramatic improvement in anemia.

IMPACT/DISCUSSION: Vitamin B12 is a key cofactor in DNA synthesis. When it is depleted, anemia manifests due to the rapidly dividing nature of hematopoietic precursors. Dissociation between cytoplasmic and nuclear maturation leads to production of large, immature megaloblasts with impaired functionality, which then undergo premature intramedullary cell death. Although the pathogenesis of the hematologic changes from B12 deficiency is well described, the clinically apparent effects of B12 deficiency are not always straightforward. Before clinically evident anemia develops, B12 deficiency causes early red cell morphologic changes. However, despite this, MCV does not correlate with the degree of B12 deficiency, and some studies find that the majority of patients with $\mathrm{B} 12$ deficiency have a normal MCV. The degree of B12 deficiency required before markers of intramedullary hemolysis appear is not known, and vitamin B12 deficiency present with serum bilirubin levels high enough to cause jaundice only rarely. In the current case, our patient had profound intramedullary hemolytic anemia due to B12 deficiency from pernicious anemia, yet her MCV was within the reference range. Confounding her presentation was the fact that pre-existing beta thalassemia trait resulted in two populations of RBCs: one megaloblastic and one microcytic. This fact serves as a reminder to pay close attention to additional red cell indices, such as RDW, which can suggest two simultaneous pathologic mechanisms.

CONCLUSION: The spectrum of manifestations of vitamin B12 deficiency can range from isolated morphologic changes in erythroid cells to overt hemolytic anemia. Despite the classic teaching that megaloblastic anemia results in an elevated $\mathrm{MCV}$, the $\mathrm{MCV}$ is not alone a reliable marker of B12 deficiency, especially in patients with co-occurring microcytosis.

\section{WARBURG AND THE RUNAWAY LACTATE}

Nadine Pardee; John Penner; Brad Sharpe. University of California San Francisco, San Francisco, CA. (Control ID \#3184776)

LEARNING OBJECTIVE \#1: Construct a differential diagnosis for lactic acidosis in the absence of systemic hypoperfusion.

LEARNING OBJECTIVE \#2: Recognize the clinical features of the Warburg effect in the setting of active malignancy.

CASE: A 36 year-old man with HIV and a history of diffuse large B-cell lymphoma (DLBCL) was admitted with three days of severe left wrist pain. He was on emtricitabine, tenofovir, and dolutegravir for his HIV. His DLBCL had been treated with chemotherapy and was in remission based on recent imaging. He was afebrile, tachycardic, and normotensive. He was thin and illappearing, with severe pain in his left wrist without effusion or erythema. He had a white blood cell count of 3.3 cells $/ \mathrm{mm} 3$ and a mild anemia. His lactate was $4.6 \mathrm{mg} / \mathrm{dL}$ with a normal anion gap and creatinine. A computed tomography of the left upper extremity showed focal areas of demineralization of unclear etiology. His blood pressure and heart rate remained unchanged. His lactate rose despite fluid boluses. He was changed to an NRTI-sparing ARV regimen and given empiric high-dose thiamine. He denied metformin, 
salicylate, or albuterol use. His lactate rose to $15.1 \mathrm{mg} / \mathrm{dL}$ and he developed new hypoglycemia that persisted despite D10\% bicarbonate infusion at $500 \mathrm{cc} /$ hour. His lactate then rose to $22.3 \mathrm{mg} / \mathrm{dL}$. Given the severe hyperlactatemia and hypoglycemia in the setting of prior DLCBL, there was a concern for a Warburg effect. A bone marrow biopsy demonstrated recurrent DLBCL. The wrist pain was felt to be secondary to bone marrow infiltration by his lymphoma. He was given palliative chemotherapy and his serum $\mathrm{pH}$, lactate, and glucose levels improved. Unfortunately, on hospital day 25, he passed away.

IMPACT/DISCUSSION: Lactic acidosis is a common problem in the hospital setting. While type A lactic acidosis, a result of tissue hypoperfusion, is the most common, the differential for a type B lactic acidosis, an impairment of cellular metabolism, is less readily encountered. Type B lactic acidosis may be secondary to: 1) Increased metabolic demand (e.g. seizure) 2) Liver disease 3) Renal disease 4) Medications: metformin toxicity, alcohols, propofol, linezolid, NRTIs, or salicylate overdose 5) Thiamine deficiency 6) Increased glycolysis from cocaine ingestion, albuterol, pheochromocytoma or the Warburg effect. The Warburg effect results from an oncogenic switch to increased glycolysis, with avid glucose consumption and consequential lactate production. It is often seen in lymphoproliferative disorders and may or may not present with hypoglycemia. These metabolic derangements can be the initial presenting sign of malignancy and are a poor prognostic indicator. As in our case, the lactic acidosis can worsen with administration of dextrose, supporting the hypothesis that tumor cells aggressively consume glucose and rapidly convert it into lactate.

CONCLUSION: Providers should consider type B lactic acidosis in patients with hyperlactatemia and be aware of the Warburg effect in patients with lymphoproliferative disorders.

\section{WARFARIN - IT'S NOT JUST BLEEDING RISK}

Alexandra Barger; Hannah Oakland. Tulane University School of Medicine, New Orleans, LA. (Control ID \#3184462)

LEARNING OBJECTIVE \#1: Recognize warfarin as a cause of calciphylaxis

LEARNING OBJECTIVE \#2: Identify calciphylaxis as a cause of painful ulcers in patients with no history of ESRD

CASE: A 50-year-old man presented bilateral lower extremity ulcers for 4 months. One week before presentation, ulcers were debrided and then became very painful. Purulent drainage and fever then developed prompting him to seek medical care. He has a history of atrial fibrillation on chronic anticoagulation with warfarin and type 2 diabetes mellitus. He was afebrile with a HR of $114 \mathrm{bpm}$. A $6 \mathrm{~cm}$ wound of the left medial shin with yellow purulent drainage and a $2 \mathrm{~cm}$ RLE medial wound with small amount of purulence were present. WBC was elevated at $14 \times 10^{9} / \mathrm{L}$. Renal function was normal. Arterial duplex ultrasound revealed only mild arterial disease. Pain was controlled and he was discharged on antibiotics. Several weeks later, he returned with uncontrolled pain. A biopsy of the ulcers was performed and returned with focal calcium deposits in subcutaneous fat, with vascular involvement and extensive fat necrosis consistent with calciphylaxis. He was diagnosed with warfarin-induced non-uremic calciphylaxis and was transitioned to apixaban for anticoagulation.

IMPACT/DISCUSSION: Calciphylaxis is a small-vessel disease involving calcification of subcutaneous arterioles and resultant cutaneous necrosis, classically seen in patients with end-stage renal disease (ESRD) but can develop in patients without renal disease. While calciphylaxis in ESRD is thought to be related to hypercalcemia, secondary hyperparathyroidism, or hyperphosphatemia, non-uremic calciphylaxis (NUC) occurs in patients with normal calcium and phosphate levels, and the pathogenesis is not completely understood. Increasingly, warfarin use is being identified as an independent cause of NUC. Calciphylaxis is characterized by medial calcification and intimal fibrosis of arterioles, followed by thrombotic occlusion and ischemia leading to tender, erythematous lesions which can develop into open ulcerations with possible progression to gangrene or sepsis. Calciphylaxis lesions are typically intensely painful, similar to ulcers caused by atherosclerotic peripheral artery disease. One-year mortality rate of those afflicted, regardless of etiology, is approximately fifty percent, with most deaths due to sepsis. The mechanism by which warfarin is thought to cause calciphylaxis is via inhibition of matrix Gla protein, which allows inappropriate calcification of blood vessels. Other risk factors for NUC are hyperparathyroidism, malignancy, alcoholic liver disease, connective tissue disease, female gender, white race, steroid use, diabetes, albumin or blood transfusions, and protein $\mathrm{C}$ or $\mathrm{S}$ deficiency.

CONCLUSION: With a high associated mortality, it is important for the internist to recognize calciphylaxis as a cause of painful ulcers in patients without advanced renal disease. Warfarin has been recognized as a cause of calciphylaxis.

\section{WAS IT THE BEER OR THE BURGERS}

Ian Cormier; Michael P. Smith. University of Nebraska Medical Center,

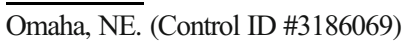

LEARNING OBJECTIVE \#1: Recognize hypertriglyceridemia as a cause of pancreatitis

LEARNING OBJECTIVE \#2: Recognize the effects of hypertryglyceridemia on common labratory evaluations

CASE: A 27-year-old man presented with six hours of severe abdominal pain. The pain was sharp, constant and located across the upper abdomen and radiated to his back. There was associated nausea and vomiting of non-bloody, non-bilious emesis. The patient had consumed a large quantity of alcohol prior to presentation. He had a history of alcohol dependence complicated by withdrawal seizures and frequent episodes of acute pancreatitis requiring hospitalization. Vitals were within normal limits. His BMI was 22. The patient was intoxicated and had marked tenderness to palpation over the left upper quadrant and epigastric area with rebound tenderness. Labs were significant for elevated blood alcohol level, elevated lipase, hyponatremia, hyperkalemia, hypocalcemia, hypermagnesemia and a normal serum osmolality. An ECG revealed no acute abnormalities. Staff from the chemistry lab described the patient's serum as lactescent. A lipid panel revealed triglycerides greater than $5000 \mathrm{mg} / \mathrm{dL}$. Repeat point-of-care-testing of the patient's serum revealed normal electrolyte levels. CT of the abdomen revealed heterogeneous enhancement of the pancreas consistent with acute pancreatitis. The patient was started on intravenous fluids and pain management. He started on an insulin drip with 5\% dextrose and fenofibrate for his hypertriglyceridemia. He was eventually discharged on gemfibrozil.

IMPACT/DISCUSSION: Acute pancreatitis is a commonly encountered diagnosis and is the most common gastrointestinal reason for hospitalization. Approximately $75 \%$ of cases of acute pancreatitis are caused by gallstones or alcohol consumption. In contrast, hypertriglyceridemia only accounts for 2$4 \%$ of acute pancreatitis cases. Though the etiology of acute pancreatitis is usually unambiguous, one must always keep a systematic approach. Pancreatitis in the setting of coexisting hypertriglyceridemia and excessive alcohol consumption is potentially confounding. This case also illustrates the interesting relationship between elevated serum lipids and measured serum electrolytes. The presence of elevated lipids increases the non-aqueous components of the serum with a concomitant decrease in the fraction of serum composed of 
free water. This phenomenon, termed the electrolyte exclusion effect, is common with the use of flame photometry or indirect potentiometry. However, this artifact does not occur with the use of direct potentiometry, as this method directly measures the concentration of electrolytes in the water phase of serum. Point-of-care-testing is more accurate in the setting of severe hypertriglyceridemia as it utilizes direct ion-selective electrodes. This can be used in the setting of hypertriglyceridemia or hyperproteinemia to obtain more precise results.

CONCLUSION: Hypertriglyceridemia is a necessary part of a systematic approach to evaluating patients with pancreatitis

\section{WATERSKIING GONE WRONG: HAMSTRING INJURY FOR THE INTERNIST}

Lily Kornbluth $^{1}$; Carlin Senter ${ }^{2} .{ }^{1}$ UCSF, San Francisco, CA; ${ }^{2}$ University of California San Francisco, San Francisco, CA. (Control ID \#3165778)

LEARNING OBJECTIVE \#1: Determine when imaging is indicated for hamstring injuries

LEARNING OBJECTIVE \#2: Define indications for orthopedic referral for hamstring injuries

CASE: HPI: A 50-year-old healthy gentleman presents to urgent care after a waterskiing accident with posterior right thigh pain. As he tried to stand on water skis, his right leg was thrust in front of him with significant force. He immediately felt a tearing pain down the back of the right thigh and subsequently was unable to bear weight on the right lower extremity. Focused physical exam: Gait: ambulates with crutches, unable to bear weight on the right leg. Inspection: hematoma of the posterior right thigh. Palpation: tenderness of the posterior thigh just distal to the ischial tuberosity. No soft tissue defect. Range of motion: pain with flexion of the right hip. Strength: $4 / 5$ strength on resisted right hip extension and right knee flexion. Strength otherwise 5/5. Sensation: intact.

IMPACT/DISCUSSION: Hamstring injuries are common and may first present to the internist. The most common injury is a mid-muscle strain or tear that is managed with rest and physical therapy. A less common but more concerning injury is a tear of the proximal hamstring tendons. Suspect proximal hamstring tendon tear based on the severity of the mechanism of injury, as in this vignette where the patient's leg was thrust forward with high speed and force. Physical exam in tendon rupture may reveal a hematoma over the posterior thigh accompanied by loss of hamstring muscle function. Palpation is a key exam finding: the more proximal the tenderness of the hamstring (i.e. the closer to the ischial tuberosity), the more concerning for tendon injury. Imaging is indicated if tendon rupture is suspected. Urgent AP pelvis X-ray and hamstring MRI are standard for evaluating for tendon rupture. MSK ultrasound can be a useful alternative to MRI in the acute setting to characterize the injury. Urgent referral to orthopedics is indicated for proximal hamstring tendon rupture as patients with rupture of two or three of the hamstring tendons have better functional outcomes with prompt operative repair. Non-urgent referral to orthopedics is indicated for other hamstring injuries that do not improve with rest and physical therapy.

CONCLUSION: Given the traumatic mechanism of injury for this patient and large posterior thigh hematoma, MRI was ordered to evaluate for hamstring rupture. Unfortunately, the MRI was not covered by the patient's insurance and so was not performed. He was subsequently evaluated by a Primary Care Sports Medicine physician who performed a point-of-care ultrasound. This showed hamstring muscle tear without tendon involvement. He was treated with a 12-week course of physical therapy with gradual improvement.
WE CAN'T AFFORD NOT TO TREAT: THE COSTS OF WITHHOLDING OPIOID USE DISORDER (OUD) TREATMENT DURING INCARCERATION

Samantha Siskind; Karen Hannon; Jessica Taylor. Boston Medical Center, Boston, MA. (Control ID \#3184851)

LEARNING OBJECTIVE \#1: Describe the clinical, human rights, and financial costs of interrupting OUD treatment during incarceration LEARNING OBJECTIVE \#2: Counsel patients on the elevated risk of overdose in the post-incarceration period

CASE: A 64-year-old man with prior incarceration, severe OUD, and cocaine use disorder relapsed with a single use of crack cocaine. A urine drug screen performed by his parole officer returned positive and he was incarcerated for two weeks. His prescribed buprenorphine/naloxone was withheld, as per jail policy, and he experienced severe withdrawal. Shortly after release, he overdosed and was immobile for $>24$ hours. He presented to the emergency department with leg pain and urinary retention. Exam revealed fever, tachycardia, a $10 \times 4 \mathrm{~cm}$ wound on his right thigh, and crackles at his lung bases. Labs showed leukocytosis, acute kidney injury (AKI), and a creatinine kinase of 7,116 U/L. He was admitted for six days with pneumonia, AKI, pressure necrosis, and rhabdomyolysis. Non-healing of his thigh wound led to a second admission. The complications of his overdose resulted in $\$ 25,853$ billed to his state Medicaid program.

IMPACT/DISCUSSION: Criminal justice exposure is common among people with OUD. Although opioid agonist therapy (OAT) reduces relapse and overdose, it is prohibited in many correctional settings due to concern for diversion and safety, logistical barriers, and stigma. Less than one percent of U.S. jails and prisons offer OAT. This patient suffered a feared consequence of OUD in the post-release period: overdose. Due to decreased tolerance after a period of abstinence and the post-release community context, which often includes low social and economic support, exposure to drug use, isolation and hopelessness, the overdose death rate post-release is 120 times that of the general population. A growing body of evidence shows that treating incarcerated individuals with OAT decreases the risk of overdose. Policies denying OAT to inmates have recently been challenged in court as violations of the Eighth Amendment and the Americans with Disabilities Act. Corrections officials have cited cost as a barrier to OAT provision. While the cost of medication, secure storage, and observed administration are important considerations, medication costs at wholesale pricing would likely have been less than $\$ 20 /$ day, contributing to total costs far below the $\$ 25,853$ that resulted in this case.

CONCLUSION: Withholding evidence-based treatment for OUD during periods of incarceration is associated with significant harms. Physicians should counsel patients on the high risk of overdose in the post-release period and discuss strategies to reduce overdose, including OAT and intranasal naloxone. Physicians should also continue to advocate for access to OAT in correctional facilities. Including financial arguments may increase support for this evidence-based treatment.

WE MUST ALWAYS ASK "WHY" — A CASE OF HYPERTRIGLYCERIDEMIA-INDUCED ACUTE PANCREATITIS Abdul Rehman $^{1}$; Manasa Ayyala ${ }^{2} .{ }^{1}$ Rutgers-New Jersey Medical School, Newark, NJ; ${ }^{2}$ Rutgers New Jersey Medical School, Newark, NJ. (Control ID \#3171723)

LEARNING OBJECTIVE \#1: Identify hypertriglyceridemia as a cause of acute pancreatitis 
LEARNING OBJECTIVE \#2: Recognize insulin infusion and apheresis as definitive therapies for hypertriglyceridemia-induced pancreatitis

CASE: A 45-year-old man with a history of hyperlipidemia and gout presented to the emergency room with dizziness for 1 day. He reported having epigastric discomfort and black tarry stools intermittently for 2 weeks. He endorsed taking ibuprofen during this time. Social history was notable for heavy use of alcohol (15-20 drinks of vodka weekly). Physical exam was notable for tachycardia, orthostatic hypotension, and epigastric pain. In addition, generalized pallor and mild scleral icterus were noted. Laboratory investigations revealed anemia (hemoglobin $10.3 \mathrm{~g} / \mathrm{dL}$ ), abnormal liver function tests (alanine aminotransferase $725 \mathrm{U} / \mathrm{L}$, aspartate aminotransferase 763 $\mathrm{U} / \mathrm{L}$ and alkaline phosphatase $419 \mathrm{U} / \mathrm{L})$, elevated lipase (173 U/L) and lactic acidosis (lactate $6.1 \mathrm{mmol} / \mathrm{L}$ ). He was admitted to general medicine for further management. Questioning the etiology of the elevated lipase, the work-up was expanded to evaluate for acute pancreatitis including a fasting lipid panel. Results were notable for a serum triglyceride level of 2,923 mg/dl. Insulin infusion along with dextrose $5 \%$ plus sodium chloride $0.45 \%$ infusion was started and the patient was transferred to the intensive care unit. Apheresis was tentatively planned. The following day, serum triglycerides had improved to $820 \mathrm{mg} / \mathrm{dl}$ and epigastric discomfort had resolved. Subsequently, he underwent esophagogastroduodenoscopy which showed a clean-based gastric ulcer and hypertensive portal gastropathy. Endoscopic ultrasonography of the biliary tree was only notable for biliary sludge but negative for any calculi or mass lesion. His serum triglyceride levels continued to improve and he was subsequently discharged from the hospital.

IMPACT/DISCUSSION: Hypertriglyceridemia is the third most common of acute pancreatitis after alcoholic and biliary pancreatitis. Serum triglycerides undergo hydrolysis by pancreatic lipase to form free fatty acids which induce free radical injury to the pancreatic parenchyma. Generally, acute pancreatitis is attributable to hypertriglyceridemia if the serum triglyceride level is greater than $1,000 \mathrm{mg} / \mathrm{dl}$. In rare cases, serum lipase may be falsely normal. Management of hypertriglyceridemia-induced acute pancreatitis is similar to that for other causes of pancreatitis with the caveat that additional interventions are needed to normalize serum triglyceride levels. Insulin infusion and/or apheresis are two commonly used modalities to normalize serum triglyceride levels.

CONCLUSION: In patients with heavy alcohol use and epigastric pain, pancreatitis due to hypertriglyceridemia must be considered in the differential diagnosis. Insulin infusion and apheresis are the two definitive therapies employed to normalize serum triglyceride levels.

\section{WEIGHT LOSS IN A SALVADORAN WOMAN: THROUGH THE MIGRATION LENS}

Jordana Laks. Boston University School of Medicine, Dorchester, MA. (Control ID \#3186366)

LEARNING OBJECTIVE \#1: Recognize the health consequences of recent migration

LEARNING OBJECTIVE \#2: Assess for trauma in an immigrant patient CASE: A 34-year-old woman from El Salvador presented to a community health center for unintentional weight loss. She reported losing an unknown amount of weight over the past month such that her clothes felt looser. She denied any appetite change, fevers, night sweats, heat intolerance, or gastrointestinal symptoms. When asked about depression symptoms, she endorsed tearfulness and difficulty sleeping since her arrival in the United States three weeks ago. Past medical history and family history were non-contributory. She did not take any medications and denied smoking, alcohol, or drug use. She was currently living with her uncle and searching for work as a tailor. On physical exam, the patient's weight was 134 pounds, down about 10 pounds from her self-reported usual weight, with a normal body mass index of $23 \mathrm{~kg}$ / $\mathrm{m}^{2}$. She had a sad and blunted affect. Basic laboratory tests were unremarkable except for a mild normocytic anemia. At her one-month follow-up appointment, the patient disclosed that she fled her home because of gang violence. She had witnessed gang members kill her brother and niece. She and her daughter walked most of the way on their journey from El Salvador. They had limited access to food, water, and shelter. She was worried about her financial insecurity and distressed by thoughts of family members still in El Salvador. The patient was seen by a mental health provider with trauma expertise. She declined antidepressant pharmacotherapy. She was informed of local resources for free and low-cost food.

IMPACT/DISCUSSION: Refugees in the United States may present to primary care settings with symptoms triggered by migration and trauma. In this case, the patient's unintentional weight loss occurred after her physically arduous journey from El Salvador with poor access to food. This case illustrates the importance of opening a dialogue about the patient's reason for leaving their home country and details of their journey. In recent years, increasing numbers of families from El Salvador, Honduras, and Guatemala are arriving in the U.S. fleeing gang violence and extreme poverty. The World Health Organization has described health harms during migration including deprivation, extreme weather exposure, accidental injuries, and physical and sexual violence. History of trauma is particularly common among Central American migrants. In one study of families arriving at the United StatesMexico border, $83 \%$ of participants cited violence as a reason for migration and $32 \%$ met criteria for post-traumatic stress disorder. Recent immigrants may be hesitant to disclose trauma due to avoidance of painful emotions and/ or fear of political persecution and stigma.

CONCLUSION: Health care providers should ask about prior physical and mental trauma when seeing newly arrived immigrant patients regardless of their chief complaints.

\section{WERNICKE'S ENCEPHALOPATHY FROM POOR NUTRITION- AL INTAKE PRESENTING AS ACUTE ONSET APHASIA}

Saloni Kapoor; Danica Smith; Kristin Berger. University of Pittsburgh Medical Center, Pittsburgh, PA. (Control ID \#3184236)

LEARNING OBJECTIVE \#1: Suspect Wernicke's encephalopathy in elderly patients presenting with acute onset encephalopathy

CASE: Mrs $\mathrm{S}$ is an 82 years old female with history of left parietal lobe meningioma status post resection 1 year back complicated by partial seizures, hypertension, peripheral vascular disease, a stable $4 \mathrm{~mm}$ left PICA aneurysm and an admission 6 months back for post ictal confusion who presented with acute onset aphasia, confusion, and difficulty ambulating. Patient lives and functions independently at baseline and was last seen normal the night before presentation. In the ED, her vitals and labs were unremarkable. NIH stroke score was 17. CT head was negative for acute pathology. CTA Head and neck without major occlusion, no significant change in $4 \mathrm{~mm}$ left PICA aneurysm. MRI brain demonstrated restricted diffusion/enhancement of the left hippocampal cortex. EEG showed left temporal periodic lateralizing epileptic discharges but no seizure activity. TSH, thyroid antibody, HgbAlc were within normal limits. A lumbar puncture demonstrated tube 4 with $2 \mathrm{WBC}, 0 \mathrm{RBC}$, 120 glucose, 68 protein. Subsequently HSV, CMV, VZV, WNV, paraneoplastic panel and anti thyroid antibodies came back negative. Vitamin B1 level was reported as undetectable. She was started on IV Thiamine supplementation. After 3 days she had complete resolution of aphasia. She had reduced her food intake over the past few months as she 
had not been feeling hungry. Her mental status changes were felt to be secondary to Wernicke's encephalopathy (WE) from poor nutrition.

IMPACT/DISCUSSION: Underdiagnosis of Wernicke's has been widely reported based on autopsy results. The triad of encephalopathy, oculomotor dysfunction and gait ataxia at presentation is an exception, rather than the rule. Most patients present only with mental status abnormalities, as with our patient. While chronic alcoholism is certainly the most common underlying pathology, upto $23 \%$ cases have been attributed to other causes including poor nutritional intake. Mammillary body atrophy is the classical sign found on imaging and is very specific for WE. Other MRI findings typical of WE are enhancement of thalamus and periaqueductal gray. In non alcoholic WE atypical MRI findings have been reported and hippocampal enhancement might represent another such case. Untreated, thiamine deficiency can progress to coma. Delayed treatment correlates with increased residual deficits. Given the complete and acute reversibility, early diagnosis is crucial. A high level of suspicion needs to maintained, especially in elderly patients who may not have sufficient nutritional intake in the setting of dementia or deconditioning.

CONCLUSION: 1 . Wernicke's encephalopathy does not usually present with the classic triad of encephalopathy, ataxia and oculomotor abnormalities 2. High suspicion for Vitamin B1 deficiencies in elderly patients presenting with neurological complaints, even without history of alcohol use or malabsorption

\section{WHAT HAPPENED? TRANSFERENCE AND COUNTERTRANS- FERENCE PHENOMENA IN A CLINICAL ENCOUNTER}

Ashwini C. Bapat; Emeric Bojarski. Massachusetts General Hospital, Boston, MA. (Control ID \#3172958)

LEARNING OBJECTIVE \#1: Recognize transference(T) and countertransference(CT)

LEARNING OBJECTIVE \#2: Employ a methodical approach to prevent the harmful consequences of transference phenomena(TP)

CASE: A 54-year-old male with opioid use disorder and lung cancer presents for management of cancer-related pain. His tumors have shrunk with treatment. He reports aching, severe, left-sided chest pain. He continues to exercise. He is on MS Contin $90 \mathrm{mg} 3$ times a day and oxycodone $20 \mathrm{mg}$ every 4 hours as needed, which he takes around the clock regardless of pain level. On exam, he sits and walks comfortably. The clinician suggests opioid down-titration in the setting of a shrinking tumor, meeting functional goals, and concern for oxycodone misuse. The patient responds in anger. T: The patient may feel stigmatized. He has likely been dismissed as a 'junkie' in the past, within the healthcare system or from family due to substance use. He interprets tapering of opioids as a sign the clinician no longer believes his pain reports, leading to reactivation of feelings of helplessness and abandonment. This impacts his willingness to discuss analgesic approaches. CT: The clinician, influenced by prior patients with substance use disorder who diverted or misused opioids, may anticipate another episode of betrayal from this patient with a substance use disorder, reactivating feelings of helplessness and abandonment. To avoid these feelings, the clinician desires to quickly decrease the opioids, severely enforce the terms of the taper, and spend less time with the patient, compromising care. Reflection: Both the patient and clinician act out transferred feelings of helplessness and abandonment. A clinician unaware of TP may fail to appropriately assess pain and discuss management. A clinician attuned to TP can utilize a methodical approach of self-reflection to manage their countertransference, enabling a more effective discussion and plan for pain management.
IMPACT/DISCUSSION: $\mathrm{T}$ is the unconscious redirection of a patient's feelings, attitudes, desires, about a person in their earlier life, onto the clinician, while CT is the unconscious redirection of a clinician's feelings, attitudes, and desires about a person in their earlier life onto the patient. TP is not inherently pathologic, nor does it reflect failure of the clinician or patient. This topic is particularly relevant for internists who frequently care for ill patients with concomitant substance use disorders who evoke intense, often unexamined emotions. Most internists lack the training to recognize and understand this phenomenon, hence transference feelings go unrecognized and can contribute to inappropriate medical care and physician burnout. This case illustrates this phenomenon, highlights the negative impact on patient care, and illustrates how a methodical approach can mitigate the negative consequences

CONCLUSION: Unexamined TP can negatively impact care. A methodical approach to self-reflection can prevent harmful consequences

\section{WHAT HAPPENS ONCE CAN HAPPEN TWICE.}

Naoki Okawa ${ }^{1}$; Makito Yaegashi ${ }^{1}$; Sandra Moody ${ }^{2}{ }^{1}$ Kameda Medical Center, Kamogawa, Japan; ${ }^{2}$ Kameda Medical Center, Kamogawa City, Japan. (Control ID \#3178841)

LEARNING OBJECTIVE \#1: Recognize that necrotizing fasciitis (NF) can recur.

CASE: A 94-year-old man with poorly controlled diabetes mellitus (DM), chronic kidney disease (CKD), and coronary artery bypass surgery presented to our clinic with right upper limb erythema and pain for two days. One day before admission, he noticed erythema and purple discoloration of his right wrist. On physical examination, he appeared ill. His blood pressure was $93 / 57 \mathrm{mmHg}$, temperature $37.6^{\circ} \mathrm{C}$, pulse 86 beats per minute, respiratory rate 18 per minute, and pulse oximetry $98 \%$ in ambient air. There was a large painful dark purple area of his right upper limb, from his wrist to his shoulder. Laboratory studies were remarkable for a white blood cell count of $14,500 / \mu 1$ with $94 \%$ neutrophils, hemoglobin $7.3 \mathrm{~g} / \mathrm{dl}$, blood urea nitrogen $73 \mathrm{mg} / \mathrm{dl}$, and creatinine $2.88 \mathrm{mg} / \mathrm{dl}$. Over the next two hours, the blood pressure fell to $80 / 50 \mathrm{mmHg}$. Intravenous (IV) vancomycin and piperacillin/tazobactam was started empirically with a vasopressor. He was admitted to the intensive care unit and treated with surgical debridement. During the debridement purulent exudate and discoloration of the fascia were observed. Since cultures from blood and tissue revealed the same strain of E. coli sensitive to all antibiotics except ampicillin, antibiotics were de-escalated to cefazolin. In addition, hyperbaric oxygen therapy was initiated. He became stable and finished a 21-day course of IV antibiotic therapy. Twelve days later, he became febrile and tachycardic. Over the next ten hours, his condition deteriorated, and he became comatose. At this presentation, his left elbow and surrounding area were noted to be erythematous with blistering and severe tenderness. Since recurrent NF was suspected, meropenem and a vasopressor were started. His condition deteriorated rapidly and he died within six hours. E. coli with the same sensitivity profile was detected from his blood and tissue. His autopsy confirmed the diagnosis of recurrent NF.

IMPACT/DISCUSSION: NF is categorized by polymicrobial or monomicrobial. E. coli is usually isolated from polymicrobial NF, but has rarely been reported in monomicrobial NF. Monomicrobial NF due to E. coli has also been reported in immunocompromised or diabetic patients; it is rare. In addition, recurrent NF is very rare, and only seven cases has been reported previously. Our patient had several predisposing factors that made him susceptible to NF- poorly controlled DM and CKD. Though it is unclear whether he had a recrudescence of NF from hematogenous seeding 
or a recurrence, we believe his second episode was a recurrence since he remained stable for twelve days after completion of the previous treatment. Recurrent NF should be included in the differential diagnosis in patients with a previous episode. Appropriate antibiotic therapy, early diagnosis and surgical debridement are extremely important for this rare, but deadly disease.

CONCLUSION: Recurrent NF is very rare, but should be excluded in patients with a previous episode of NF.

\section{WHAT LIES BENEATH CATATONIA}

Anna Apostolopoulou; Anita Leon-Jhong. UPMC, Pittsburgh, PA. (Control ID \#3179070)

LEARNING OBJECTIVE \#1: Recognize the signs of malignant catatonia timely

LEARNING OBJECTIVE \#2: Initiate management of malignant catatonia with high doses of benzodiazepines (BZs) in the ICU

CASE: A 23-year-old female with history of autism was transferred from an outside hospital for evaluation of altered mental status. Prior to admission, she had sustained a closed-head injury with negative CT of her head, so a diagnosis of post-concussive syndrome was initially entertained. The patient was at her reported baseline: minimally interactive and not following commands. Her neurologic exam was non-focal; infectious, metabolic/ endocrine workup for AMS was unremarkable. A few days later, she developed waxy flexibility, posturing, staring, and catalepsy. She was evaluated by Neurology and Psychiatry specialists, both suspecting a diagnosis of catatonia. She was challenged with lorazepam but became very sedated, so the plan was to eventually pursue elective ECT. The next morning, she was somnolent and diaphoretic and later became febrile to $39.3 \mathrm{C}$ and tachycardic. Despite repeated negative infectious workup, she was empirically covered with broad-spectrum antibiotics. After 24 hours, and with no sign of improvement, she re-spiked a fever and developed diffuse rigidity. A diagnosis of malignant catatonia was made. Patient was transferred to the ICU for aggressive treatment with escalating doses of lorazepam and emergent ECT.

IMPACT/DISCUSSION: Catatonia is a clinical diagnosis suggested by a constellation of abnormal motor responses such as mutism, stupor, waxy flexibility, posturing, staring, echophenomena. Catatonia can vary in severity from non-malignant to malignant; malignant catatonia can occur as a sequela of non-malignant catatonia, or it can manifest on its own. Malignant catatonia presents with fever, AMS, dysautonomia and rigidity. Given the overlapping features of malignant catatonia with other conditions, such as neuroleptic malignant syndrome and sepsis, a high level of suspicion is required. In this case, key findings were the patient's diffuse muscle rigidity, diaphoresis and tachycardia and her second episode of fever while on antibiotics. Unquestionably, her pre-existing catatonia expedited the diagnosis, which was important given the lethality of malignant catatonia if not diagnosed and managed timely. ECT and escalating doses of BZs are the standards of care for malignant catatonia. Urgent management in an ICU setting is recommended given the autonomic instability and the potential of respiratory compromise with BZs warranting intubation for airway protection. This case gave me insight into the spectrum of catatonia, and given the possible progression of the non-malignant catatonia to the life-threatening malignant catatonia, I realized that a new diagnosis of catatonia should prompt daily surveillance for signs of malignant catatonia.

CONCLUSION: -Monitor patients with new-onset catatonia for signs of fever, dysautonomia and rigidity. -Malignant catatonia warrants immediate transfer to the ICU for escalating doses of BZs.
WHAT THE NECK IS THAT? EVALUATION OF A NECK MASS IN AN IMMUNOCOMPROMISED PATIENT

Shirin Hemmat ${ }^{1}$; Katherine Julian ${ }^{2} .{ }^{1}$ University of California, San Francisco, San Francisco, CA; ${ }^{2}$ UCSF, San Francisco, CA. (Control ID \#3176190)

LEARNING OBJECTIVE \#1: Develop a differential diagnosis for a neck mass in an immunocompromised patient

LEARNING OBJECTIVE \#2: Evaluate and treat drug-induced thrombocytopenia in the outpatient setting

CASE: A 60 year-old woman with a history of renal transplantation presented with a three-week history of enlarging, painful bilateral neck masses. Her past medical history was significant for chronic ureteral reflux resulting in renal transplantation two years prior, maintained on tacrolimus, mycophenolate and prednisone. Her family history was unremarkable. The patient was housed and originally from the Philippines but immigrated to the US more than twenty years ago. Review of systems was negative for fever or chills. Her vital signs were all within normal limits. Her exam was notable for a $3 \mathrm{~cm}$ firm and tender submandibular mass on the left and a $3 \mathrm{~cm}$ firm and tender mandibular mass on the right. The remainder of her exam was unremarkable. Labs were normal. An ultrasound of the neck was concerning for undrained abscesses. The patient was hospitalized and underwent a fine-needle aspiration biopsy which was positive for Mycobacterium Tuberculosis (TB). She was ruled out for active pulmonary TB. She was diagnosed with scrofula (tuberculous lymphadenitis of the cervical lymph nodes) and discharged on rifabutin, isoniazid, pyrazinamide, vitamin B6 and ethambutol (RIPE). Six weeks after initiation of RIPE, the patient platelets decreased from $298 \mathrm{k}$ to $124 \mathrm{k}$ within one week. Her rifabutin was stopped and two weeks later her platelet count returned to $240 \mathrm{k}$.

IMPACT/DISCUSSION: A neck mass likely represents a lymph node, thyroid nodule, congenital or vascular malformation or dermatologic process. Lymph node enlargement can be caused by malignancy, infection or autoimmune diseases. Immunocompromised patients have greater susceptibility to infections. Infections that result in cervical lymphadenopathy include bacterial (Staph Aureus, Bartonella, TB), viral infections (Epstein-Bar Virus, HIV) and endemic fungal infections. Worldwide, it is estimated that TB accounts for almost half of the cases of lymphadenopathy. Scrofula is most often diagnosed in young patients with concurrent HIV (with CD4<300) or who are otherwise immunocompromised. Patients are typically afebrile and present with a firm, chronic, non-tender neck mass. Drug-induced thrombocytopenia in the outpatient setting can be caused by antibiotics (particularly Bactrim and rifabutin), NSAIDs, chemotherapy and H2-blockers. Cessation of the offending medication typically resolves the thrombocytopenia in 2-3 weeks.

CONCLUSION: This case demonstrates evaluation of a neck mass in an immunocompromised patient and the management of rifampin induced thrombocytopenia. It highlights the need to consider TB in the differential of lymphadenopathy, particularly in patients who are originally from countries with high TB prevalence or who have a suppressed immune system.

\section{WHAT YOU "C" MAY NOT BE WHAT YOU EXPECT: A CASE OF SCURVY CAUSING PURPURA}

Rachel Snyder; Deepa R. Nandiwada; Laura Kosseim. University of Pennsylvania, Philadelphia, PA. (Control ID \#3149639)

LEARNING OBJECTIVE \#1: Describe the epidemiology of scurvy in the US

LEARNING OBJECTIVE \#2: Describe the clinical presentation of scurvy 
CASE: A 57 year old woman with history of hypothyroidism, depression and smoking presented with one month of left lower extremity swelling, ecchymosis, purpura and worsening joint pain. Physical exam revealed no teeth and diffuse perifollicular purpura. Her left leg was edematous and tender. She first presented to another hospital and got antibiotics for concern of cellulitis but they were quickly stopped. Abnormal admission labs were hemoglobin 9.4g/ $\mathrm{dL}$ (MCV 91) that dropped to $7.2 \mathrm{~g} / \mathrm{dL}$ after fluids given for orthostasis, sodium $132 \mathrm{mmoL} / \mathrm{L}$ and potassium $3.0 \mathrm{mmoL} / \mathrm{L}$. Rheumatology was consulted for concern of vasculitis. She was empirically started on steroids but showed no improvement so they were stopped. Rheumatology lab panel was unremarkable except Anti-Nuclear Antibody (1:160), CRP (58mg/L) and ESR $(36 \mathrm{~mm} / \mathrm{hr})$. Lower extremity doppler ultrasound showed no deep vein thrombosis. Left lower extremity MRI revealed diffuse edema consistent with nonspecific myositis. Skin biopsy showed subcutaneous lipophages and hemorrhage but no vasculitis. Other work up included Ferritin 160ng/mL, Iron $26 \mathrm{ug} / \mathrm{dL}, \mathrm{TIBC} 142 \mathrm{ug} / \mathrm{dL}, \mathrm{B} 12182 \mathrm{pg} / \mathrm{mL}$, folate $1.3 \mathrm{ng} / \mathrm{dL}$, negative fecal occult blood test. Bone marrow biopsy showed reactive marrow but no malignancy. CT chest/abdomen/pelvis showed no bleeding. She was transferred to our hospital for second opinion of the purpura. Upon further questioning, she endorsed two years of chronic diarrhea that limited her diet to meat and cheese sandwiches and coffee. We reviewed her Vitamin C level after this clinical history was obtained; it was undetectable. Given her dietary history, physical exam and nutrition labs, we diagnosed scurvy. She was discharged home with oral vitamin $\mathrm{C}$.

IMPACT/DISCUSSION: This case highlights the value of a thorough initial history and showcases an often forgotten cause of purpura. The diagnosis was delayed for a month after an extensive workup was done due to an anchoring bias and misconception about the epidemiology of scurvy.

CONCLUSION: Scurvy is a disease of Vitamin C deficiency. It is often thought of as a historic disease or one localized to developing countries. However, age adjusted prevalence is $7.1 \%$ in USA. Risk factors are smoking, restrictive diets, older age, mental illness, alcoholism, low socioeconomic status and malabsorption. Humans must obtain Vitamin $\mathrm{C}$ from the diet. Stores are quickly exhausted after months if not replenished. Vitamin $\mathrm{C}$ is essential for collagen synthesis. Common clinical manifestations are therefore secondary to inadequate collagen leading to impaired blood vessel integrity such as perifollicular hemorrhage, gingivitis, ecchymosis and poor wound healing. Late manifestations are edema, bone pain and even death. Treatment is replenishment of Vitamin C. There is no standardized protocol; one can use $100 \mathrm{mg}$ to $1 \mathrm{~g}$ /daily until stores are repleted. Symptoms improve within a few weeks with complete resolution by about 3 months.

\section{WHEN IT'S NOT BIRDS, THINK HOT TUBS!}

Adam Dossaji ${ }^{1}$; Asad H. Khan ${ }^{2} .{ }^{1}$ Baystate Medical Center, Springfield, MA; ${ }^{2}$ Baystate Medical Centre, Broad Brook, CT. (Control ID \#3186382)

LEARNING OBJECTIVE \#1: Recognize hot tubs as a cause of hypersensitivity pneumonitis

LEARNING OBJECTIVE \#2: Recognize the importance of history in eliciting the cause of hypersensitivity pneumonitis

CASE: A 57 year old female with a past medical history of breast cancer presented with shortness of breath to the emergency department. Two weeks prior, she had a history of dry cough, rhinorrhea, sore throat and malaise which was treated with azithromycin and inhaled steroids followed by AmoxicillinClavulanic Acid as an outpatient. CXR showed bilateral infiltrates suggestive of multifocal pneumonia and patient was started on Vancomycin and Zosyn. On hospital day 2, she developed hypoxia and hypotension requiring high flow nasal cannula and intravenous fluids. Due to concern for lack of clinical improvement, CT Chest was ordered and revealed bilateral ground glass opacities in the setting of positive rhinovirus. A bronchoscopy was performed with bronchalveolar lavage (BAL) and transbronchial biopsy showing interstitial inflammation and Type 2 pneumocyte hyperplasia. Preliminary cultures were negative. Further discussion with patient revealed history of exposure to birdcages at home prompting evaluation for hypersensitivity pneumonitis including hypersensitivity panel which was negative. Patient was started on prednisone with dramatic improvement and she was discharged home. After discharge final BAL cultures grew Mycobacterium Avium and at follow up patient was improved but still endorsing symptoms of coughing and shortness of breath. Continued discussion revealed that patient had a home hot tub which she was repeatedly exposed to in the months prior to presentation. She was advised to strictly avoid this in order to prevent relapse of her pneumonitis. At next follow up patient had complete recovery with repeat CT chest showing complete resolution of bilateral opacities.

IMPACT/DISCUSSION: Hypersensitivity pneumonitis (HP) describes a complex condition in which pulmonary parenchyma has an immune reaction to an inhaled substance, often organic in nature. The most common forms are farmer's lung and bird fanciers lung, but the list of causes is vast. It is often characterized by an initial history of cough, weight loss, crackles and wheezing. Worsening of symptoms after exposure to a specific trigger is a hallmark of this disease. The mainstay of treatment is antigen avoidance, often with complete resolution of symptoms with avoidance of antigen, this makes identifying the cause vital. This case highlights the importance of eliciting a detailed holistic history covering all aspects of the patient's life, in conjunction with the clinician having a low index of suspicion and wide net for causes in mind.

CONCLUSION: Thorough exposure history taking is vital to eliciting cause of HP Complete avoidance of causative exposure is required for total recovery

\section{WHEN PERMETHRIN ISN'T A PERMANENT FIX: CONSIDER- ING IVERMECTIN FOR TREATMENT OF SCABIES IN THE HOMELESS POPULATION}

Alyse Wheelock $^{1}$; Samantha Rawlins-Pilgrim ${ }^{1}$; Jessica Taylor ${ }^{2} .{ }^{1}$ Boston University Medical Center, Boston, MA; ${ }^{2}$ Boston University/Boston Medical Center, Boston, MA. (Control ID \#3183543)

LEARNING OBJECTIVE \#1: Evaluate treatment options for scabies and their relative effectiveness

LEARNING OBJECTIVE \#2: Apply psychosocial considerations to scabies treatment selection

CASE: A 35-year-old woman with a history of AIDS, homelessness, bipolar disorder, and alcohol and cocaine use disorders presented with an intensely pruritic, papular rash. The patient had a recent CD4 count of $173 \mathrm{cells} / \mu \mathrm{L}$ and an undetectable viral load, with intermittent periods of non-adherence to her antiretroviral regimen. She had faced housing insecurity for many years and was presently living on the streets with her partner. She was seen initially in the Emergency Department for a pruritic rash on the lower extremities and abdomen. She was diagnosed with scabies and prescribed $5 \%$ permethrin cream and oral medications to reduce pruritis. She did not apply permethrin according to recommendations. Persistent symptoms prompted another ED visit and a visit to HIV clinic within the next two weeks. In clinic, the rash was thought to be nodular scabies based on appearance and distribution. She was prescribed weight-based ivermectin $(200 \mu \mathrm{g} / \mathrm{kg}$ orally as a single dose and repeated in 2 weeks) given failure of permethrin. She was seen three weeks after her initial ivermectin dose with improvement of the pruritic rash, although the papules were still present. She reported that she did not take her second dose, so a second dose of ivermectin was re-prescribed. When she was next 
seen about ten days later, the lesions were scabbed and her severe pruritis had resolved.

IMPACT/DISCUSSION: Homelessness not only increases one's risk of acquiring scabies infection but also poses important barriers to treatment. This patient presented to medical care four times before achieving resolution of scabies. Consideration of housing status should inform scabies treatment decisions and counseling. Topical permethrin 5\% cream, applied from neck to toe, is typically considered the first-line treatment for scabies. However, applying the cream correctly and washing off 24 hours later may be untenable for patients who have inconsistent access to showers. If feasible, application of permethrin by clinical staff may facilitate correct use. An alternative treatment is systemic ivermectin, dosed $200 \mathrm{ug} / \mathrm{kg}$, using 1-2 doses spaced at least one week apart. Ivermectin treatment has been found to have similar clearance rates to permethrin after two weeks (approximately 67\%) with similar rates of adverse events (Rosumeck et al, 2018). In both cases, environmental measures, such as laundry washing and prophylaxis for close contacts, also affect treatment success.

CONCLUSION: Scabies treatment for homeless patients who do not have regular access to showers and laundry can be complicated. Systemic ivermectin should be considered as a first line option for patients who are unable to complete permethrin treatment.

\section{WHEN POLYARTHRITIS TURNS DANGEROUS, DISABLING, AND EVEN DEADLY}

Megan Winkelman; Jessica Fuller; Nancy Choi. University of California, San Francisco, San Francisco, CA. (Control ID \#3186644)

LEARNING OBJECTIVE \#1: Identify Pancreatitis, Panniculitis, and Polyarthritis (PPP) Syndrome from dermatologic, rheumatologic, orthopedic, and radiologic findings.

CASE: A 46 year old woman with a history of fibromyalgia, psychiatric disease, remote alcohol abuse, and chronic relapsing pancreatitis status post sphincterotomy and pancreatic duct stenting was admitted for acute on chronic pain most severe in her ankles. On hospital day two, multiple erythematous, tender nodules appeared on her legs. Her lipase level on admission was 400 , which soon normalized. Due to ongoing abdominal pain, the GI team performed an ERCP (consistent with chronic pancreatitis), stent removal, and pancreatic sphincteroplasty. During week two, she developed additional skin nodules and worsened pain and swelling in her ankles, wrists, knees, and feet. Rheumatologic work-up was significant only for a mildly elevated ESR/CRP. Pathology from her wrist nodule showed necrosis and saponification of fatty lobules with surrounding neutrophil-rich infiltrates. An ankle and wrist MRI showed early osteonecrosis and joint effusions. Due to concern for joint infection, Orthopedic Surgery performed an ankle synovial biopsy and aspiration, revealing a large amount of yellow-gray fluid with negative cultures. Ultimately, given biopsy and imaging results in the context of her clinical history, she was diagnosed with PPP Syndrome.

IMPACT/DISCUSSION: PPP Syndrome is an uncommon disease (only $2 \%$ to $3 \%$ of patients with pancreatic disease develop cutaneous lesions; those that do rarely go on to have arthritic symptoms) but has a high mortality rate of $24 \%$. Tender, inflammatory lobular nodules are often seen on lower limbs, and may drain oily brown fluid which builds up from the necrosis of adipocytes ${ }^{1}$. The polyarthritis (also described as periarthritis with bone necrosis) is likely caused by systemic activity of pancreatic enzymes leading to microcirculatory disturbances and fat necrosis ${ }^{2}$. Like others with PPP, our patient remains significantly disabled by pain and weight-bearing difficulty, but will continue to receive supportive measures ${ }^{3}$.
CONCLUSION: Early recognition of PPP Syndrome is critical for the hospitalist, who may be the provider best equipped to identify key findings from different consultants and create an integrated diagnosis with a management plan for underlying pancreatic disease as well as the sequelae syndromes. 1. García-Romero, Diana, and Francisco Vanaclocha. "Pancreatic Panniculitis." Dermatologic Clinics, vol. 26, no. 4, Oct. 2008, pp. $465-$ 470., doi:https://doi.org/10.1016/j.det.2008.05.009. 2. Langenhan, Ronny, et al. "Osteomyelitis: A Rare Complication of Pancreatitis and PPPSyndrome." Joint Bone Spine, vol. 83, no. 2, Mar. 2016, pp. 221-224., doi:https://doi.org/10.1016/j.jbspin.2015.05.005. 3. Graham, Paul M, et al. "Panniculitis, Pancreatitis, and Polyarthritis: A Rare Clinical Syndrome." Cutis, vol. 101, no. 1, Jan. 2018, pp. 34-37., doi:https://www.ncbi.nlm.nih. gov/pubmed/29529121.

\section{WHEN STATINS ATTACK: A CASE OF NECROTIZING AUTO- IMMUNE MYOPATHY}

$\underline{\text { Puja Jadav }}{ }^{1}$; Stephanie C. Shieh ${ }^{1,2}$. ${ }^{1}$ Saint Louis University School of Medicine, St. Louis, MO; ${ }^{2}$ VA St. Louis Healthcare System, St. Louis, MO. (Control ID \#3145194)

LEARNING OBJECTIVE \#1: Recognize that SINAM is a rare, but potential side effect of statin use.

LEARNING OBJECTIVE \#2: Identify the main constellation of findings that characterize SINAM.

CASE: Statins are commonly prescribed medications that reduce cardiovascular disease. We present a case of statin-induced necrotizing autoimmune myopathy (SINAM), a recently characterized entity within the well known spectrum of statin myotoxicity. A 58-year-old African American man with a history of cervical spondylosis, diabetes, hyperlipidemia presented with lower extremity swelling and progressive leg weakness following a fall one month prior whereby his legs "gave out". He had been restarted on atorvastatin 1 year prior, which had initially been discontinued for muscle spasms then deemed unrelated. Physical exam was significant for 3+ edema of lower extremities bilaterally to thighs and proximal muscle weakness with strengths of $4 / 5$ in biceps/triceps but $3 / 5$ in hip flexors/extensors. Pertinent workup for his weakness revealed a benign endocrine workup, normal inflammatory markers (ESR 12, CRP 0.26), negative myositis panel, but a positive ANA, greatly elevated muscle markers (Aldolase 66, CK 11,635), and a high titer positivity for Anti-HMG CoA Reductase ( $>200)$. MRI femur showed increased signal within proximal posterior and medial muscle groups indicative of compartmental myositis and skeletal muscle biopsies revealed a necrotizing myopathy with a paucity of chronic inflammation. The patient was started on prednisone 50mg daily, all statins added as allergies, and was discharged with close rheumatology follow up. Given his continued falls despite intense physical therapy, mycophenolate and IVIG were added, with improvement in strength and edema, a downtrend of his CK to 400, and the ability to taper off prednisone.

IMPACT/DISCUSSION: The pathogenesis of SINAM is via the marked up-regulation of the hydroxy-3-methyglutaryl-coenzyme A reductase receptor protein (HMGCR) in regenerating muscle cells of susceptible persons when exposed to statins, which then act as autoantigens to induce antibody production. The anti-HMGCR antibody then triggers necrosis and regeneration of muscle fibers, resulting in severe muscle damage that persists even after the discontinuation of the statin. SINAM is characterized by significant proximal muscle weakness, CK levels over $6000 \mathrm{U} / \mathrm{L}$, evidence of musclecell necrosis on biopsy with minimal inflammatory infiltrates, and the presence of autoantibodies against HMG- CoA reductase as demonstrated in our patient case. Treatment includes discontinuing the statin, with half of 
all patients needing triple therapy consisting of high dose prednisone and two immunosuppressive agents. When recognized and treated, most patients have good outcomes.

CONCLUSION: While inflammatory myopathies are rare, with SINAM having an incidence of 2 out of 100,000 persons on statins, given the large prevalence of statin use, the importance of early recognition and appropriate management of this debilitating condition cannot be under emphasized.

\section{WHEN THE CULTURES DON'T GROW, YOU NEED TB THINK- ING}

Noah Chodos $^{1}$; Zachary Merritt ${ }^{1}$; Luke Andrea ${ }^{1}$; Nikita Agrawal ${ }^{1}$; Amit R. Patel $^{2}$. ${ }^{1}$ Montefiore Medical Center (Moses \& Weiler), New York, NY;

${ }^{2}$ Montefiore Medical Center, New York, NY. (Control ID \#3185554)

LEARNING OBJECTIVE \#1: Describe the work-up of sterile pyuria and when to consider genitourinary tuberculosis

LEARNING OBJECTIVE \#2: Recognize the incidence of TB in a nonimmigrant population

CASE: A 69 year-old man was admitted with 4 months of persistent dysuria after receiving several courses of outpatient antibiotic therapy for a presumed UTI. Serial urinalyses showed sterile pyuria with leukocyte esterase. One month prior to admission, a CT revealed inflammation in his left kidney, ureter, and bladder, and Urology placed a JJ stent which drained purulent urine with negative bacterial culture and no malignant cells. The patient was born and lived in the U.S. and had a recently negative QuantiFERON Gold. Urology was consulted and recommended routine outpatient follow-up. Finally, the team submitted urine for acid fast evaluation which was positive for 1+ AFB on fluorochrome stain. Repeat serum QuantiFERON was positive, and a confirmatory GeneXpert of the patient's urine was positive for Mycobacterium tuberculosis. He was started on appropriate therapy.

IMPACT/DISCUSSION: This patient had a classic presentation of genitourinary tuberculosis despite lacking typical TB risk factors. Extrapulmonary TB is often neglected as a diagnosis in non-immigrant, non-immunocompromised patients without known TB exposure. However, of 9,557 total cases of TB reported in 2015 (most recent CDC data), 3,186 (33\%) were in U.S.-born persons. 1,933 (20\%) of the total TB cases were extrapulmonary, of which 91 were genitourinary. Of note, on further history this patient's only possible risk factor for TB was a brief period of homelessness many years ago. Other causes in the differential diagnosis for sterile pyuria include many of the common sexually transmitted infections (HSV-2, HPV, gonorrhea, chlamydia, syphilis, mycoplasma, trichomoniasis), urinary stones, interstitial cystitis, and bladder neoplasm. When none of these more readily detectable causes are uncovered, TB and fungal infections should be considered, particularly if systemic symptoms are present. This particular patient actually had 6 months of failure to thrive and a new anemia, making TB a more likely suspect. While urine AFB specificity is high, sensitivity is low for both stain (28.5\%) and culture $(37.1 \%)$. There is a more sensitive, alternative test that is not readily available at many U.S. centers. The GeneXpert has a sensitivity of $94.3 \%$ and a specificity of $96.7 \%$ for GU TB and can check for TB DNA in multiple fluid types in less than 2 hours, making it a fast and effective test for ruling out active TB.

CONCLUSION: Persistent dysuria or "recurrent UTI" should prompt additional diagnostic consideration beyond bacterial infection. TB should be considered in individuals with classic presentations even if typical risk factors are lacking. The highly sensitive GeneXpert deserves more use and understanding in the U.S. as a more rapid and appropriate method of ruling out active TB

\section{WHEN THE CURE IS WORSE THAN THE DISEASE}

Jasmine Corona; Louisa Holaday. Montefiore Medical Center, Bronx, NY. (Control ID \#3184555)

LEARNING OBJECTIVE \#1: Recognize amiodarone-induced pulmonary toxicity

LEARNING OBJECTIVE \#2: Monitor for evidence of early pulmonary toxicity in patients on amiodarone

CASE: A 61 year old man with heart failure with reduced ejection fraction with a history of ventricular tachycardia managed with an AICD and amiodarone presented with two weeks of progressive cough with clear sputum and decreased exercise tolerance. Temp 101.7F with oxygen saturation of $83 \%$ with stable BP and HR. Fine crackles noted in all lung fields, with no notable JVD and trace edema to ankles. WBC was 10.8 $\mathrm{k} / \mathrm{uL}$, pro-BNP was elevated but at patient's baseline. Chest xray (CXR) demonstrated bilateral opacities interpreted as multifocal pneumonia. He was treated initially as viral pneumonia versus superimposed bacterial pneumonia, however he did not improve. CT chest was consistent with diffuse pneumonitis and possible amiodarone toxicity, so amiodarone was stopped and systemic corticosteroids begun. However, despite treatment, his respiratory status worsened and he succumbed to the disease. He had not had a CXR or pulmonary function tests (PFTs) in the outpatient setting in more than one year.

IMPACT/DISCUSSION: Heart failure exacerbation and pneumonia are common diagnoses treated by general internists. Amiodarone pulmonary toxicity (APT) has many features common to these diagnoses, and thus is easily misdiagnosed. In 2016, there were nearly 3 million prescriptions written for amiodarone in the United States. The cumulative incidence of APT is estimated at about $5 \%$. It is thus likely that a general internist will encounter APT during their career. However the risk is correlated with daily dosage and cumulative dosage. For those on $400 \mathrm{mg}$ or greater incidence is estimated at up to $15 \%$, while it is only $1.5 \%$ in patients taking less than 400 mg. The mortality for APT is $10 \%$, but for patients with acute APT who develop ARDS it is as high as $50 \%$. The typical presentation of APT is subacute (weeks-months) progressive shortness of breath associated with nonproductive cough, malaise, with or without fever and pleuritic chest pain and hypoxia. It can also present rapidly as ARDS. With symptoms that may mimic heart failure or pneumonia, APT is easy to overlook. APT is most likely to occur in patients with advanced age, high cumulative dose, dosage of more than $400 \mathrm{mg}$ daily, duration of therapy exceeding two months and prior pulmonary disease. Given the severity of illness and challenge of diagnosis, it is most important to monitor for evidence of APT in advance and stop the drug when indicated. Current guidelines recommend baseline PFTs prior to starting the drug and clinical evaluation, chest X-ray, and PFTs every 3-6 months. Additionally, any new respiratory symptoms should lead to urgent clinical evaluation, CXR, and PFTs.

CONCLUSION: Patients on amiodarone should be monitored closely in the outpatient setting for any signs or symptoms of pulmonary toxicity, and APT should be on the differential for any patient presenting with pulmonary symptoms who is taking amiodarone.

WHEN TRUSTING YOUR GUT GOES WRONG: HOW TO AVOID CLINICAL BIASES

Caitlin D. Couper; Edwin Chen; Emad M. Kahlil. UPMC, Pittsburgh, PA. (Control ID \#3180876)

LEARNING OBJECTIVE \#1: Recognize clinical reasoning biases that can often occur with commonly seen admissions 
LEARNING OBJECTIVE \#2: Utilize CT enterography to evaluate small bowel diseases

CASE: 63 year old male with past medical history of hypertension, tobacco use, and chronic back pain on naproxen presenting with painless hematochezia. Physical exam notable for a benign abdomen and rectum without hemorrhoids. Initial vital signs showed systolic pressure in the 90s and tachycardia to the 100 s that rapidly responded to fluid resuscitation. Pertinent labs include hemoglobin of 11.5 (baseline 14). Initial CT A/P showed diverticulosis and a transient distal ileal intussusception felt at the time to be clinically insignificant. He underwent enteroscopy which revealed an unremarkable EGD, but a colonoscopy notable for terminal ileal ulceration with erythematous mucosa. In light of this, a repeat CT with dedicated enterography protocol revealed ileocolic intussusception with nodular enhancement suspicious of a mass. Biopsy of the ulceration revealed a poorly differentiated neuroendocrine tumor. Ultimately, it was felt that the neuroendocrine tumor acted as a pathological leadpoint causing recurrent intussusception that manifested clinically as hematochezia. The patient underwent laparoscopic ileocecectomy with resolution of his symptoms.

IMPACT/DISCUSSION: This case highlights several clinical reasoning biases that can easily occur when presented with common internal medicine chief complaints and serves as a reminder to evaluate data judiciously. This case also introduces $\mathrm{CT}$ enterography as a tool to evaluate and diagnose small bowel diseases.

CONCLUSION: Hematochezia is a common general medicine admission. Etiology in adults is often due to diverticulosis, hemorrhoids, vascular malformations, and colonic malignancies. In this particular case, we committed ascertainment and availability bias by considering only a list of differentials frequently seen on the wards. By anchoring on the diverticular findings of the initial CT and ignoring the intussusception, we committed confirmation bias, which risks premature closure. This case highlights the ease at which one can fall into several clinical reasoning biases in rapid succession. Fortunately, we were able to reevaluate after colonoscopy with CT enterography to identify the pathological lesion, a rare case of intussusception in adults. This serves as a reminder to be judicious when interpreting data. CT enterography is an imaging protocol that can bridge the gap between upper endoscopy and colonoscopy when evaluating gastrointestinal pathologies. Compared to push and capsule endoscopy, enterography is neither limited by reach nor strictures, and allows visualization of extra-luminal areas. It can easily and non-invasively evaluate inflammatory diseases, neoplasms, ischemia, and bleeding in the small bowel. This invaluable diagnostic tool should be in a generalist's repertoire.

\section{WHY AM I STILL SO TIRED?: A CASE OF UNCONTROLLED HYPOTHYROIDISM FROM LACTOSE INTOLERANCE}

Katelin Snow; Robin Klein. Emory University School of Medicine, Atlanta, GA. (Control ID \#3176203)

LEARNING OBJECTIVE \#1: Recognize how malabsorptive syndromes such as lactose intolerance complicate treatment of hypothyroidism.

LEARNING OBJECTIVE \#2: Appreciate lactose intolerance as a potential cause of resistance to levothyroxine treatment in hypothyroidism.

CASE: A 38 year old female with a past medical history of hypothyroidism from Hashimoto's thyroiditis presented to the ER with weakness and lethargy for several months. She also reported constipation, generalized weakness, and dry flaking skin. She was diagnosed with hypothyroidism 10 years prior and takes levothyroxine $100 \mathrm{mcg}$ daily. She takes no other medications and eats a plant based diet as she recently noticed abnormal reactions to certain foods. On exam, she was afebrile with normal vital signs. Her skin was dry and cool, speech was sluggish, and reflexes were decreased. Laboratory workup was remarkable for a TSH of 214. Endocrinology was consulted. Further history revealed she was having bloating and diarrhea with milk products. It was determined that she likely has lactose intolerance. She was discharged on an increased dose of levothyroxine and advised to take her medication with a Lactaid pill. At outpatient follow up 3 months later, her symptoms had resolved and TSH had normalized.

IMPACT/DISCUSSION: Many factors influence the absorption and metabolism of levothyroxine (LT4) including certain foods and many medications. Malabsorptive gastrointestinal disorders, including lactose intolerance, can also inhibit LT4 absorption and complicate treatment in patients with hypothyroidism. Lactose intolerance is a common condition which usually develops in adulthood, causing symptoms such as bloating, flatulence, and diarrhea when lactose is ingested. Most LT4 formulations contain small amounts of lactose. For patients with lactose intolerance, this can inhibit the absorption and metabolism of LT4 into thyroid hormone. In cases of severe lactose intolerance, exposure to the lactose in LT4 can even cause symptoms. In patients not responding appropriately to LT4 therapy, physicians should perform a thorough review of medications, ask how the patient takes the medication, and look for history of or symptoms of malabsorptive disorders. When lactose intolerance is suspected, treatment options include co-administration of LT4 with lactase supplement (such as Lactaid) or switching to a lactose-free LT4 formulation. This should be done along with the removal of lactose containing products from the diet. Typically this allows patients to achieve euthyroidism at normal doses of LT4.

CONCLUSION: Our case illustrates how a malabsorption syndrome may prompt recurrence of symptoms of hypothyroidism. As both lactose intolerance and hypothyroidism are common diseases, physicians need to be cognizant of how the two conditions interact. Lactose intolerance should be considered in patients who appear resistant to LT4 treatment, require increasing LT4 doses or even develop malabsorptive symptoms with LT4 therapy.

\section{WHY CAN'T I BREATHE? A CASE OF PD-L1 INHIBITOR- INDUCED PNEUMONITIS}

Jeffrey Fujimoto; Jennifer Jones. UCLA, Los Angeles, CA. (Control ID \#3179560)

LEARNING OBJECTIVE \#1: Recognize and treat pneumonitis as an adverse side effect of PD-L1 inhibitors

CASE: A 68-year-old man with history of severe COPD and stage IIIB non-small cell-lung cancer (NSCLC) developed acute hypoxemic and hypercapnic respiratory failure while receiving his eighth infusion of durvalumab. Forty minutes into infusion, the patient became short of breath and hypoxic to $80 \%$. He required emergent intubation due to respiratory distress. The etiology of his respiratory symptoms was initially unclear. He lacked infectious signs to suggest pneumonia. His clinical presentation was not consistent with anaphylaxis given absence of other characteristic symptoms such as an edematous oropharynx. He reported compliance with all COPD medications and was without significant wheezing on initial exam though general air movement was poor. The patient was started on empiric antibiotics and pulse dose methylprednisolone. He recovered within 24 hours and was extubated. Antibiotics were de-escalated to azithromycin to complete a 5-day course. CT chest showed bilateral centrilobular groundglass opacities and bronchial wall thickening, consistent with pneumonitis. 
The patient continued methylprednisolone for three days and switched to oral prednisone $40 \mathrm{mg}$ for an additional five days with good clinical recovery.

IMPACT/DISCUSSION: Durvalumab is an immune checkpoint inhibitor that targets programmed death ligand 1 (PD-L1) and has recently been approved for use in unresectable NSCLC. Pneumonitis is a well-known side effect with an incidence of $6.3 \%$ after initiation. Radiographically, the most commonly observed pattern is cryptogenic organizing pneumonia (COP) characterized by multifocal ground glass opacities (65\% of cases) followed by nonspecific interstitial pneumonia ( $15 \%$ of cases). The median time to onset of pneumonitis is 2.6 months with a range of 9 days to 19.2 months. Treatment of PD-L1 associated pneumonitis is based on common terminology criteria for adverse events (CTCAE) guidelines. Treatment of grade 1 (mild) pneumonitis consists of holding immunotherapy and careful clinical observation. Grade 2 (moderate) pneumonitis warrants treatment with oral prednisone and grade 3 or 4 (severe or life-threatening) pneumonitis requires hospitalization and IV methylprednisolone for 5 to 7 days. Prior to initiation of therapy, all patients should undergo high resolution chest CT and be made aware of the symptoms of pneumonitis, especially those with low respiratory reserve, such as our patient. Providers should exercise caution when re-challenging patients after pneumonitis given nearly $25 \%$ may experience recurrence. Patients with confirmed pneumonitis should receive regular $\mathrm{CT}$ imaging of the chest, at least every 1 to 2 weeks in severe cases, until symptoms improve to grade 1 pneumonitis or resolve.

CONCLUSION: Recognizing pneumonitis early with PD-L1 inhibitor therapy can significantly improve mortality Steroids are first-line therapy for symptomatic PD-L1-induced pneumonitis

\section{WHY DO I HAVE CHEST PRESSURE AND LEG PAIN, AGAIN? AN UNUSUAL CASE OF VENOUS THROMBOEMBOLISM} Elise M. Pearson ${ }^{2}$; Joshua H. Jun ${ }^{1}$; Ricardo C. Couso ${ }^{1}$; Rachel Vanderberg ${ }^{1}$. ${ }^{1}$ University of Pittsburgh Medical Center, Pittsburgh, PA; ${ }^{2}$ University of Pittsburgh School of Medicine, Pittsburgh, PA. (Control ID \#3184464)

LEARNING OBJECTIVE \#1: Assess for anatomic risk factors contributing to venous thromboembolism (VTE)

CASE: A 43 year-old female with obesity, unprovoked submassive pulmonary embolism (PE) and extensive deep vein thrombosis (DVT) 23 months prior, now on warfarin, presented to the emergency room with one day of non-radiating substernal chest pain and transient posterior left knee pain. The patient was hemodynamically stable with no oxygen requirement. Her international normalized ratio on presentation was 3.2. On exam of the left lower extremity there was tenderness to palpation of the posterior fossa of the knee, 1+ pitting edema, no calf tenderness, and negative Homan sign. Chest computed tomography (CT) demonstrated a new subsegmental PE in the right lower lobe. Lower extremity duplex ultrasound (US) demonstrated a chronic recanalized DVT of the left femoral and external iliac veins with no evidence of new DVT. Extensive hypercoagulable workup was negative. CT of the abdomen and pelvis with venous phase contrast was obtained to evaluate for May-Thurner syndrome (MTS). CT instead revealed a duplicated left-sided inferior vena cava (IVC) joining the left renal vein, traveling retro-aortically to the right-sided IVC. Given her warfarin failure, the patient was transitioned to enoxaparin and discharged home with hematology, pulmonology, and vascular surgery follow up.

IMPACT/DISCUSSION: Risk for VTE is highest with the presence of one or more elements of Virchow's triad: venous stasis, endothelial injury, and hypercoagulability. Often overlooked in the assessment of VTE are anatomic venous stasis risk factors like varicose veins, MTS and anomalous IVC. Varicose veins affect roughly $23 \%$ of American adults. A recent study demonstrated that patients with varicose veins had higher incidence rates for DVT (hazard ratio (HR) 5.3, [95\% confidence interval (CI), 5.05-5.56]), and PE (HR 1.73, [95\% CI, 1.54-1.94]) compared with the control group. MTS presents with symptoms of pain, swelling, and/or DVTs caused by compression of the common iliac vein by the overlying common iliac artery. Compression occurs most often on the left side, in the 2nd-4th decade of life with a 2:1 female predominance. It is typically diagnosed by duplex US, though CT, magnetic resonance venography or endovascular US may be more sensitive. If DVT is present, therapy involves catheter-directed thrombolysis, stenting, and anticoagulation. Multiple variations of the IVC can occur, related to abnormal persistence or regression of embryonic veins that become the IVC and renal veins. IVC anomalies are typically asymptomatic and found incidentally, however they may be a clinically significant finding in patients with VTE. While data is limited for IVC duplication, definitive treatment for other compressive etiologies of VTE is surgical, thus evaluation by a vascular surgeon should be considered.

CONCLUSION: It is important to consider anatomic risk factors like varicose veins, MTS and anomalous IVCs in patients with VTE as these may impact therapy.

\section{WHY IT IS ESSENTIAL TO THINK TWO MOVES DOWN THE CHESS GAME.}

Chenchen Feng; Alexander Katz; Aimee Sutherland; Jeffrey Wiese. Tulane University School of Medicine, New Orleans, LA. (Control ID \#3184449)

LEARNING OBJECTIVE \#1: Diagnose methemoglobinemia in the setting of acute decompensated heart failure

LEARNING OBJECTIVE \#2: Recognize the association between rasburicase, methemoglobinemia, and G6PD deficiency

CASE: A 64-year-old woman with a history of essential thrombocytosis presented with dyspnea secondary to acute congestive heart failure. She responded well to diuresis and her dyspnea resolved. A peripheral smear revealed thrombocytosis with blasts, raising the concern for acute myeloid leukemia with a blast crisis. A bone marrow biopsy was performed and given the risk for hyper-uricemia secondary to tumor lysis, rasburicase was prescribed. On the second hospital day, she developed refractory hypoxia initially thought to be due to worsening heart failure. This diagnosis was refuted by an arterial blood gas that revealed a $\mathrm{PaO} 2$ of $300 \mathrm{mmHg}$ despite an arterial saturation of $80 \%$ which suggested an abnormal hemoglobin. Methemoglobinemia was suspected based upon recent rasburicase exposure. This was confirmed, methylene blue and ascorbic acid were administered. On the following day, there was an acute drop in hemoglobin which was attributed to hemolytic anemia. G6PD deficiency was confirmed as the cause, likely precipitated by the administration of the methylene blue. Attempts were made to establish an exchange transfusion, but her condition deteriorated resulting in a cardiac arrest and death.

IMPACT/DISCUSSION: Patients with myeloproliferative disorders are commonly encountered by the general internist. While oncologists are primarily responsible for the administration of chemotherapy, it is the general interest who is tasked with the vigilance for and management of common side-effects related to this therapy. Tumor lysis is one of the more common complications, especially for patients with a very high tumor burden. Given the frequency with which these patients are admitted to the 
hospital, the prophylactic administration of agents such as rasburicase and allopurinol is often done without consideration for potential side effects from these medications, Methemoglobinemia occurs in less than one percent of patients who take rasburicase, and the incidence is the same in patients with G6PD deficiency. The treatment for methemoglobinemia is methylene blue and patients with G6PD deficiency are highly susceptible to acute hemolytic anemia in the setting of methylene blue administration. Exchange transfusion is the only alternative when a patient requires methylene blue for methemoglobinemia but is succumbing to hemolytic anemia secondary to G6PD deficiency.

CONCLUSION: There is an FDA warning to screen populations with high incidences of G6PD deficiencies, prior to treatment with methylene blue. The practical reality, however, is that when methemoglobinemia presents, prompt initiation of therapy is required which precludes time for screening. The only way to prevent this scenario is to be aware of the patient's G6PD status prior to beginning methylene blue therapy.

\section{WIDE PULSE PRESSURE IN A TRAUMA PATIENT: HEMOR- RHAGIC VERSUS SEPTIC SHOCK.}

Kenya Iwase $^{1}$; Jintetsu Minami ${ }^{2}$; Munehisa Atsumi ${ }^{2}$; Mitsunori Iwase ${ }^{2}$; hiroki kato ${ }^{2} .{ }^{1}$ TOYOTA memorial hospital, Toyota city, Japan; ${ }^{2}$ Toyota Memorial Hospital, Toyota City, Aichi Pref, Japan. (Control ID \#3183827)

LEARNING OBJECTIVE \#1: Recognize the diagnostic value of pulse pressure to differentiate etiologies of shock.

LEARNING OBJECTIVE \#2: Recognize various causes of wide pulse pressure.

CASE: A 63-year-old man was brought in by ambulance after a single-car rollover traffic accident. His medical history included Crohn's disease for 32 years, long-standing diabetes mellitus and end-stage renal disease on dialysis for 4 years. His blood pressure was $97 / 34 \mathrm{mmHg}$ and heart rate was $121 / \mathrm{min}$. His respiratory rate was $32 / \mathrm{min}$ with normal $\mathrm{SpO} 2$ on room air, and his temperature was $39.9^{\circ} \mathrm{C}$. Physical examination revealed abdominal tenderness and warm extremities. A whole-body CT scan was performed for suspected hemorrhagic shock, which showed neither fracture nor traumatic organ damage. However, there was a "dirty fat" sign around the mesentery, consistent with bowel inflammation. Importantly, history taking revealed persistent fever with shivering and watery stool lasting for 2 weeks prior to the presentation. His immunosuppressant therapy was recently discontinued due to nontuberculous mycobacterial infection. He was admitted for suspected intra-abdominal infection as well as flare-up of Crohn's disease. The patient was treated with meropenem. The initial sets of blood culture were negative. We started administration of 5-ASA for assumed flare-up of Crohn's disease. On the third day, we performed exploratory laparotomy for suspected bowel perforation and abscess formation noted on follow-up CT scan. Although laparotomy showed adhesions of intestine, neither abscess nor perforation was noted. On the fourth day, the second blood cultures grew Candida glabrata. We added Micafungin. However, abdominal pain and bloody stool persisted, which were thought to be due to intestinal ischemia or necrosis secondary to extensive inflammation. Unfortunately, his condition further deteriorated and he passed on the 26th day. By autopsy, dirty ascites retention because of the intestinal inflammation, bleeding and perforation on mucous membranes were detected.

IMPACT/DISCUSSION: The most common etiology of shock in trauma patients is hemorrhagic shock. Most patients with hemorrhagic shock usually have narrow pulse pressure due to peripheral vasoconstriction from intravascular volume depletion and intrinsic catecholamine surge. In contrast, septic shock leads to wide pulse pressure due to profound vasodilation from cytokine surge. In the present case, the observed wide pulse pressure was an important clue indicating distributive shock. Intestinal flora is disturbed in patients with inflammatory bowel disease. Recently, fungal flora dysbiosis are also reported. Importantly, we should keep in mind that the various causes of wide pulse pressure exist. The representative causes include aortic valve insufficiency, severe anemia, hyperthyroidism, and beriberi heart, so on.

CONCLUSION: In trauma patients with wide pulse pressure, we should consider that the cases may have something to do with septic shock.

\section{WITHOUT NAME DAH}

Ian Cormier; Michael P. Smith. University of Nebraska Medical Center, Omaha, NE. (Control ID \#3186078)

LEARNING OBJECTIVE \#1: Develop an approach to diffuse alveolar hemorrhage (DAH)

LEARNING OBJECTIVE \#2: Recognize the presentation of and risk factors for hantavirus

CASE: A 39 year-old woman from Mexico presented with fever and cough for an unspecified duration. Her past medical history was significant for hereditary amyloidosis with cardiac and gastrointestinal involvement. She was febrile and hypotensive. Thrombocytopenia, elevated lactate dehydrogenase, elevated transaminases and a left shift of granulocytes were present. The patient developed acute hypoxic respiratory failure with diffuse pulmonary infiltrates suggestive of adult respiratory distress syndrome (ARDS) and bilateral pleural effusions. CT of the chest revealed severe bilateral pulmonary consolidation and pulmonary edema. She was started on empiric vancomycin, piperacillin-tazobactam, doxycycline and micafungin for atypical pneumonia. Thoracentesis revealed a transudative effusion. The patient's respiratory status worsened despite therapy and a bronchoalveolar lavage (BAL) revealed diffuse alveolar hemorrhage. All cultures and serology were negative. The patient was started on therapy with steroids for suspected pulmonary amyloidosis. The patient's respiratory status gradually improved. Infectious workup all came back negative except for Hantavirus IgM, which was significantly elevated.

IMPACT/DISCUSSION: Hantaviruses are a genus of enveloped viruses within the Bunyaviridae family. The genus is divided into the New World and Old World viruses, with Hantavirus Cardiopulmonary Syndrome caused by the former and Hemorrhagic Fever with Renal Syndrome caused by the latter. In the United States, HCPS is most commonly caused by the Sin Nombre virus (SNV). The majority of reported cases occur in the Southwest, specifically New Mexico, Arizona, and Colorado. All clinically important Hantaviruses are transmitted by aerosolized droplets from urine, feces or saliva of rodents. One of the earliest and most specific laboratory findings in patients with HCPS is thrombocytopenia with a rapid decrease in platelets. A small number of SNV IgM positive / IgG negative samples represent false positive reactivity associated with acute cytomegalovirus or Epstein Barr virus infection. Diffuse alveolar hemorrhage can be divided into infectious and noninfectious causes. Non-infectious causes are the majority of the causes of $\mathrm{DAH}$, however infectious causes must be considered in the appropriate patients due to treatment implications of corticosteroids. DAH secondary to an infectious cause can occur in both immunocompetent and immunocompromised. The most common causes in immunocompetent patients are influenza H1N1, dengue, leptospirosis, malaria, Staphylococcus aureus, and HCPS. The differential for diffuse alveolar hemorrhage must remain broad and should include infectious causes in both immunocompromised and immunocompetent patients. 
CONCLUSION: It is essential that the general internist recognize HCPS in a patient at risk and initiate appropriate evaluation in all parts of the country

\section{YOU BOOZE, YOU LOSE... SENSATION}

Philip Rosenstock. UCLA-Olive View Medical Center, Sylmar, CA. (Control ID \#3183745)

LEARNING OBJECTIVE \#1: Understand the physical exam and EMG differences between alcoholic neuropathy and thiamine deficiency

CASE: A 55 year old Caucasian woman with a history of chronic alcohol use complicated by cirrhosis (MELD Score 8, Child-Pugh Class A) presented to the ED with bilateral lower extremity weakness and frequent falls over 1 to 2 weeks. Patient denied recent sickness, travel, trauma, or symptoms of dysautonomia. She reported drinking two glasses of wine per day but had a history of heavy alcohol use - one liter of vodka daily for many years. The patient denied other substance abuse. On initial presentation, vital signs were stable. Exam was notable for distal bilateral extremity deficits in proprioception and vibratory sensation. She had diminished reflexes, plus numbness and hyperesthesia in a stocking glove distribution. She was unable to ambulate. Motor strength was mildly reduced against resistance in her arms. The patient was alert and oriented to person, place, time, and situation. Labs notable for a WBC 7.3, Hb 10, PLT 279, MCV 95.5, ESR 76, Mg 1.5, and otherwise normal serum electrolyte levels and renal function. Initial management included normal saline, thiamine, multivitamin, and tramadol. Neurology consultation was obtained. The patient's generalized weakness and neuropathic symptoms improved during the fourth day of hospitalization. Vitamin B6, B12, and folate levels were normal. Rheumatologic serologies were normal. Lumbar puncture did not reveal acute viral or bacterial meningitis. West Nile virus antibody titers were negative. CT head was negative for acute and chronic intracranial pathology. MRI of the thoracic and lumbar spine was without myelopathic disease. Electromyogram (EMG) showed evidence of chronic sensory-predominant polyneuropathy with predominantly axonal features in the lower extremities. There was no evidence of acute neuropathy. The diagnosis of chronic sensory axonal polyneuropathy secondary to alcohol use was made.

IMPACT/DISCUSSION: Alcoholic peripheral neuropathy (PN) is a seen in up to $75 \%$ of people with chronic alcohol abuse. It is difficult to diagnose in the initial stages, often mistaken for nutritional deficiencies. In the absence of thiamine deficiency, alcoholic neuropathy can be characterized by slowly progressive symptoms, such as sensory impairment, dysesthesia, and pain. Superficial sensation, frequently nociception, becomes impaired. This is in contrast to both superficial and deep sensory impairments, as well as a motor dominant pattern, seen in thiamine deficiency. Diagnosis can be further validated with an EMG study showing reduced nerve conduction velocities and segmental demyelination. The mainstay of treatment is cessation of alcohol use.

CONCLUSION: - The prevalence of alcoholic peripheral neuropathy is directly correlated with lifetime alcohol consumption more than any nutritional deficiency. - Cessation of alcohol consumption is the only means of reversal.

\section{YOU DON'T DIE FROM NAUSEA AND VOMITING, DO YOU?}

Zhihang Zhang; Emily Baumert; Erin Goss. Montefiore Medical Center, New York, NY. (Control ID \#3181195)

LEARNING OBJECTIVE \#1: Recognize the long-term sequelae of polyarteritis nodosa.
CASE: A 33-year-old male with a past history of polyarteritis nodosa presented to the hospital for nausea and vomiting for 5 days without abdominal pain. He was diagnosed with polyarteritis nodosa (PAN) four years ago and imaging revealed multiple aneurysms in the coronary, renal, splenic, and iliac arteries, as well as thrombosis within multiple mesenteric arteries. Treatment with cyclophosphamide and glucocorticoids followed by azathioprine induced remission. Multiple aneurysms were embolized and he was maintained on systemic anticoagulation. On admission, he denied abdominal pain, but had multiple episodes of non-bloody, non-bilious vomiting often provoked by oral intake, with no hematochezia. His symptoms were originally attributed to gastroenteritis. The patient was afebrile with BP of 110/70 and $\mathrm{HR}$ of 70s, and routine labs were negative for amylase, lipase, WBC count, and the infectious gastroenteritis panel returned negative. During the admission, his vomiting persisted despite conservative treatment. On hospital day 3 , he developed acute epigastric abdominal pain with guarding, but remained normotensive without tachycardia. CT abdomen demonstrated acute rupture of a splenic artery aneurysm with hemoperitoneum. Patient then developed hemorrhagic shock, and despite extensive efforts, was unable to be resuscitated.

IMPACT/DISCUSSION: Polyarteritis nodosa is a systemic necrotizing vasculitis of medium sized vessels. Most cases are idiopathic, though some are associated with Hepatitis $\mathrm{B} / \mathrm{C}$, and hairy cell leukemia. The pathogenesis of PAN is poorly understood, where inflammation leads to thickening of vessel walls that cause luminal narrowing leading to tissue ischemia, as well as weakening of the walls leading to aneurysm formation. 5-year survival rates of PAN are $\sim 80 \%$ with treatment. The French Vasculitis Study Group derived a prognostic tool that identifies risk factors associated with higher mortality including age $>65$, cardiac symptoms, gastrointestinal involvement, and renal insufficiency $(\mathrm{Cr}>1.7 \mathrm{mg} / \mathrm{dL})$. Patients with PAN require regular long-term follow up to monitor disease activity, even after remission. Major causes of death include renal failure and mesenteric, cardiac, or cerebral infarction. Disease recurrence rates are lower compared to other vasculitides but still substantial; $9 \%$ at 1 year and $24 \%$ at 5 years in one study. In addition, complications of PAN can occur when the disease is clinically inactive as the healing of inflamed vessels can narrow lumens leading to ischemia. Follow up with angiography is indicated if there is concern for recurrence, ischemia, or aneurysms that are at risk for expansion or rupture. Ligation or embolization is recommended for any aneurysm presenting with symptoms or $>=2 \mathrm{~cm}$ in diameter. Symptoms of splenic artery aneurysm include nausea, vague abdominal discomfort, or rarely acute rupture (5\%).

CONCLUSION: PAN requires long term follow up for complications and recurrence.

\section{YOU MUST REMEMBER THIS: WHEN A CYST IS NOT JUST A CYST}

Jason Ehrlich; Priyanka Chopra. Northwell, Great Neck, NY. (Control ID \#3186246)

LEARNING OBJECTIVE \#1: Develop the ability to diagnose an often overlooked disorder

LEARNING OBJECTIVE \#2: Identify clinical scenarios in which presentation indicates a need to investigate associated systemic conditions

CASE: Patient is a 57 year old man with polyarthralgias who presents with fairly rapid onset of a left ear mass. The mass began appearing 1 month prior to presentation, and occupied a large aspect of the upper pinna. The mass seemed fluid-filled and produced a throbbing sensation. Upon consultation with Dermatology, the mass was initially regarded as an inflamed epidermoid cyst, and was treated with intralesional corticosteroids. The lesion briefly 
stabilized, but soon resumed its growth. Consultation with Surgery was undertaken. By this point, the soft but tense collection spanned the superior aspect of the upper auricle, obliterating the antihelix. Needle aspiration did not resolve the process. Rapid reaccumulation led to operative evacuation of the mass. Pathology of the excised material demonstrated helical cartilage damage and surrounding exuberant vascularized granulation tissue with histiocytic infiltration, suggestive of chondrodermatitis nodularis helicis.

IMPACT/DISCUSSION: Often the presenting lesion is first regarded as nothing more than a generic cyst in an awkward location. When it grows aggressively, however, it can obliterate underlying tissue and can additionally represent a first sign of an underlying systemic condition. Cartilaginous inflammation is central to the disease process, at times resulting in cartilage necrosis. The etiology may include trauma, frostbite, or repetitive pressure which compromises the local circulation. The pathophysiology may relate to arteriolar narrowing in the perichondrium of the pinna at sites furthest removed from arterial blood supply - the antihelix and the helix - as reported by Upile in 2009. Criber in 2006 found neural hyperplasia present in the lesions, perhaps explaining the notable pain produced even upon light pressure. Two-thirds of patients with the disorder are men, and most commonly it arises in the sixth decade of life, as in our patient. The condition may also be associated, however, with collagen vascular disease, particularly systemic sclerosis, as first identified by Bottomley and Goodfield in 1994, and explored further by Magro in subsequent years. Any case emerging from a patient outside the most typical demographic should be further investigated for links with possible autoimmune disease. Treatment may be undertaken with pressure-relief strategies, intralesional corticosteroids, laser ablation, or surgery. Recurrence may still approach $30 \%$.

CONCLUSION: Chondrodermatitis nodularis helicis is a condition which internists would do well to identify, as its course may be aggressive just as well as it may be indolent. Connecting a patient expeditiously with treatment may reduce the risk of disfigurement. Its association with autoimmune disorders must be recognized, as this benign-appearing cystic mass may lead to discovery of an active systemic condition.

\section{ZOLPIDEM INDUCED SYNDROME OF INAPPROPRIATE ANTIDIURETIC HORMONE}

Ayah A. Megahed ${ }^{1} ;$ Lenar Latypov $^{2} ;$ Sami Rabah $^{1} ;$ Nour Boulad $^{1} .{ }^{1}$ Lincoln Medical and Mental Health care Centre, New York, NY; ${ }^{2}$ Lincoln Medical and Mental Health Center, New York, NY. (Control ID \#3165623)

LEARNING OBJECTIVE \#1: To diagnose hyponatremia secondary to Zolpidem intake, especially in elderly population

CASE: The patient is a 70 year old male with PMH of HTN, DM, CAD s/p CABG, CVA secondary to left vertebral artery dissection, BPH and seizure disorder. He presented with abdominal pain of 3 weeks duration, increasing over the last 3 days. He took 30 pills of Zolpidem over 3 days in an attempt to treat the pain. CT of the abdomen was performed showing calcular gall bladder with chronic atherosclerosis of the coeliac and superior mesenteric trunks. During further evaluation, the patient was found to be severely hyponatremic (Na 116) but asymptomatic. Serum glucose and creatinine were normal. Laboratory work up showed: (Na 116, serum osmolality 242; urine osmolality 400; urine $\mathrm{Na} 64$ ) with normal TSH and cortisol level, consistent with SIADH. The patient has a past medical history of seizure disorder that has been managed with Carbamazepine for many years. Carbamazepine is known to cause SIADH as a side effect, yet he did not have any sodium abnormalities in the past. Therefore, we assumed that this recent hyponatremia was due to ingestion of high dose of Zolpidem. He was initially given NS at a rate of $75 \mathrm{cc} / \mathrm{hr}$ then $150 \mathrm{cc} / \mathrm{hr}$ with a breakthrough seizure in between. The patient was managed with strict fluid restriction and administered $\mathrm{Na}$ tablets with eventual elevation of the Na from 116 to 130 . On final assessment, the patient was asymptomatic without any new complaints. IMPACT/DISCUSSION: Hyponatremia is common among the elderly, and may be caused by physiological changes, pathologic processes or drugs. About half of elderly patients with hyponatremia have features typical of the syndrome of inappropriate antidiuretic hormone secretion (SIADH). The symptoms depend on the degree of hyponatremia and the rapidity with which the syndrome develops and may include anorexia, nausea, vomiting, headache, diarrhea, weakness, lethargy, confusion, convulsions and coma The number of medications that have been reported to cause SIADH is increasing and currently include vasopressin and its analogues, thiazide diuretics, chlorpropamide, carbamazepine, antipsychotics, antidepressants and nonsteroidal anti-inflammatory drugs. Also, few cases of Zolpidem induced SIADH were reported in the literature. Drug-induced SIADH usually is treated by withdrawing the suspect drug and restricting fluid intake

CONCLUSION: It is important to carefully monitor serum sodium in patients receiving Zolpidem. It should be avoided in the elderly population who are more prone to hyponatremia

\section{INNOVATION IN HEALTHCARE DELIVERY (IHD) "THREE STEPS FORWARD, TWO STEPS BACK": A QUEST FOR JOY IN RESIDENT PRACTICE THROUGH AMBULATORY IN- TERPROFESSIONAL TRANSFORMATION}

Jennifer Verbsky $^{1}$; Daniel J. Coletti ${ }^{1}$; Frank Cacace ${ }^{2}$; Nancy A. LaVine ${ }^{3}$; Jason Ehrlich ${ }^{4}$; Nissa Mazzola ${ }^{5}$; Joseph Conigliaro ${ }^{6}$. ${ }^{1}$ Northwell Health, Plainview, NY; ${ }^{2}$ northwell health, Great Neck, NY; ${ }^{3}$ Northwell Health, New Hyde Park, NY; ${ }^{4}$ Northwell, Great Neck, NY; ${ }^{5}$ St. John, Queens, NY; ${ }^{6}$ North Shore LIJ Health System, New Hyde Park, NY. (Control ID \#3185705)

STATEMENT OF PROBLEM OR QUESTION (ONE SENTENCE): Can transformation of a large internal medicine resident ambulatory practice into an interprofessional, teamlet-based structure enhance resident ambulatory experiences and thus promote retention in primary care careers?

OBJECTIVES OF PROGRAM/INTERVENTION (NO MORE THAN THREE OBJECTIVES): 1. Increase frequency of interprofessional interactions in a large resident practice through use of team huddles 2. Improve satisfaction with resident ambulatory training by providing residents with enhanced interprofessional support 3. Augment resident interest in primary care careers

DESCRIPTION OF PROGRAM/INTERVENTION, INCLUDING ORGANIZATIONAL CONTEXT (E.G. INPATIENT VS. OUTPATIENT, PRACTICE OR COMMUNITY CHARACTERISTICS): Our internal medicine residency program has 108 categorical residents rotating through one of 2 ambulatory sites in a $4+1$ block schedule. In one ambulatory site (housing 2 clinics), 10 residents work in the HRSA grant-funded IMPACcT (Improving Patient Access, Care, and Cost through Training) clinic, along with pharmacy, psychology, physician assistant, and medical students in a cohesive interprofessional (IP) team. The IMPACcT clinic has served as our innovation lab for IP practice, where workflows are tested prior to implementation in our other clinics. Based on experiences of the IMPACcT clinic with huddles and co-located staff, we transformed the larger 60 resident clinic to 1) dedicate and co-locate 3 medical assistants (MOAs) alongside residents in the same conference room, 2) group residents, faculty, pharmacy students, and MOAs into smaller "teamlets", and 3) introduce huddles prior to each patient care session.

MEASURES OF SUCCESS (DISCUSS QUALITATIVE AND/OR QUANTITATIVE METRICS WHICH WILL BE USED TO 
EVALUATE PROGRAM/INTERVENTION): Residents complete an anonymous battery of questionnaires at the end of the academic year to evaluate their ambulatory training. The battery includes the IP Socialization and Valuing Scale (ISVS), the Primary Care Attitudes Scale (PCAS), and other questions assessing IP interactions, perceptions of the training site and workflows, and interest in primary care.

FINDINGS TO DATE (IT IS NOT SUFFICIENT TO STATE FINDINGS WILL BE DISCUSSED ): During the 2017-18 assessment period, we observed a significant improvement in the frequency of interactions between residents and pharmacists $(\mathrm{p}=.006)$, MOAs $(\mathrm{p}=.03)$, our health psychologist $(\mathrm{p}=.004)$, and our care management staff $(\mathrm{p}=.004)$. Residents were also more likely to participate in team meetings or huddles $(\mathrm{p}<.001)$. However, we observed no increase in positive attitudes towards primary care careers. Content analysis of open-ended responses suggested that inefficient workflows and an excess of administrative tasks were key reasons for dissatisfaction with the ambulatory experience despite IP transformation.

KEY LESSONS FOR DISSEMINATION (WHAT CAN OTHERS TAKE AWAY FOR IMPLEMENTATION TO THEIR PRACTICE OR COMMUNITY?): We hoped that more regular support from IP team members would lead to a better clinic experience and increased primary care interest. While we found that residents did in fact better appreciate working within an IP team, that alone was not sufficient to improve their overall clinic experience as the volume of administrative tasks was not eased or redistributed. Our next steps will target this feedback and aim to provide additional resident support through task triage strategies, sharing of tasks with MOAs and nurses, and protected task completion time.

\section{A CLINIC RUN COMMUNITY GARDEN THAT SERVES PA- TIENTS LIVING IN FOOD DESERTS \\ Carolyn F. Pedley ${ }^{1}$; Robert Jones ${ }^{4}$; James L. Wofford ${ }^{2}$; Elizabeth Gamble ${ }^{1}$; Peter R. Lichstein ${ }^{3}$; John C. Moskop ${ }^{1}$; Robert L. Rominger ${ }^{4}$; Claudia L. Campos $^{5}$; Ramon Velez ${ }^{4}$; Melanie Martin ${ }^{6}$; Feben Girma ${ }^{7}$; Monica T. Brown $^{4}$; Kirsten Feiereisel ${ }^{8}$; Nancy M. Denizard-Thompson ${ }^{8}$; Deepak Palakshappa $^{3}$; Robert Bloomfield ${ }^{9}{ }^{1}$ Wake Forest, Winston-Salem, NC; ${ }^{2}$ Wake Forest School of Medicine, Winston-Salem, NC; ${ }^{3}$ wake Forest School of Medicine, Winston Salem, NC; ${ }^{4}$ Wake Forest Baptist Health, Winston- Salem, NC; ${ }^{5}$ Wake Forest University, WInston-Salem, NC; ${ }^{6}$ Wake Forest, Greensboro, NC; ${ }^{7}$ Wake Forest Baptist Medical Center, Winston-Salem, NC; ${ }^{8}$ Wake Forest University School of Medicine, Winston-Salem, NC; ${ }^{9}$ Ever- green Internal Medicine, Winston-Salem, NC. (Control ID \#3184420)}

\section{STATEMENT OF PROBLEM OR QUESTION (ONE SENTENCE):}

Many patients who attend an urban, academic affiliated clinic reside in food deserts, lacking in access to fresh fruits and vegetables.

OBJECTIVES OF PROGRAM/INTERVENTION (NO MORE THAN THREE OBJECTIVES): 1 . To establish a community garden next to the clinic to supply patients with fresh fruits and vegetables 2 . To involve patients, medical residents, medical students, physcians, nurses and other clinic members in the operations of the garden 3 . To distruibute the produce to clinic patients and to educate them on the value of eating fresh fruits and vegetables DESCRIPTION OF PROGRAM/INTERVENTION, INCLUDING ORGANIZATIONAL CONTEXT (E.G. INPATIENT VS. OUTPATIENT, PRACTICE OR COMMUNITY CHARACTERISTICS): An abandoned lot next to the medicial clinic located in downtown Winston-Salem was transformed into a community garden which is comprised of 30 raised beds. After consultation with master gardeners and the local agricultural extension agency, volunteers were taught "hands on " techniques of organic gardening. Nearby grade schools and nurseries provided the garden with plants and seeds. Charitable donations were processsed by a church partner and a community development organization provided funds to buy tools and supplies. The medical center donated an irrigation system and the fence surrounding the garden. In 2016 the garden received an award for its community outreach and the award funds were used to purchase a storage building. Volunteers included medical students and residents, clinic physicians, nurses and other personnel, local volunteer groups, garden clubs, scout troops, student service groups, a local temple and church, patients and residents of the neighborhood. Volunteers met at the garden 2-3 hours Saturday mornings March - Nov. to plant, weed, fertilize and harvest. Produce was weighed at each session and distributed to the clinic patients the following week. MEASURES OF SUCCESS (DISCUSS QUALITATIVE AND/OR QUANTITATIVE METRICS WHICH WILL BE USED TO EVALUATE PROGRAM/INTERVENTION): The success of the garden was measured by the following metrics: 1 . Total produce distributed to patients 2 . Numbers of volunteers 3. Numbers of patient/ provider interactions in the distribution of produce in the clinic

FINDINGS TO DATE (IT IS NOT SUFFICIENT TO STATE FINDINGS WILL BE DISCUSSED ): 1. The garden yields of the past three years are as follows: 2016 -1400 pounds 2017- 1500 pounds 2018- 1485 pounds Produce- tomatoes, green beans, squash, okra, kale, lettuce, carrots, beets, cabbage, watermelon, blueberries, strawberries, onions, cucumbers, potatoes, collards, pumpkins, peas, beets, chard, peppers and eggplant. 2. Each growing season had more than 100 volunteers; most volunteered on multiple occasions 3. Assuming that patients received an average of $28 \mathrm{oz}$ bags of produce, there were approximatelyl 3000 opportunites to interact with patients while handing out produce in the clinic each growing season.

KEY LESSONS FOR DISSEMINATION (WHAT CAN OTHERS TAKE AWAY FOR IMPLEMENTATION TO THEIR PRACTICE OR COMMUNITY?): A community garden in association with a medical clinic serves as way to supply much needed fruits and vegetables to an underserved patient population. In addition, volunteers benefit from working together, enjoying "green" physical activity and addressing the common goals of taking care of plants and bringing the produce to their patients. In an informal manner there was exchange of growing techniques, recipes and passing nutritional information on to patients.

\section{A GROUP VISIT MODEL FOR CHRONIC PAIN PATIENTS ON CHRONIC OPIOIDS}

Matthew J. Townsend ${ }^{2}$; Barbara Ogur ${ }^{1,3}$. ${ }^{1}$ Harvard Medical School, Cambridge, MA; ${ }^{2}$ Harvard Medical School, Boston, MA; ${ }^{3}$ Center for Primary Care, Harvard Medical School, Boston, MA. (Control ID \#3180199)

STATEMENT OF PROBLEM OR QUESTION (ONE SENTENCE): New protocols for managing patients with chronic pain on chronic opioid therapy propose tapering off opioids, however clinical experience has shown this to be difficult.

OBJECTIVES OF PROGRAM/INTERVENTION (NO MORE THAN THREE OBJECTIVES): To assess patients on long-term opioids for chronic pain for opioid use disorder (OUD). To assess a program of individualized assessment for OUD and individualized treatment plans coupled with group support. To assist patients to taper opioids or transition to buprenorphine.

DESCRIPTION OF PROGRAM/INTERVENTION, INCLUDING ORGANIZATIONAL CONTEXT (E.G. INPATIENT VS. OUTPATIENT, PRACTICE OR COMMUNITY CHARACTERISTICS): We expanded a program of monthly group visits for chronic pain patients on chronic opioid therapy to include individualized assessments for opioid use disorder and treatment plans based on these assessments. 23 patients 
completed at least 18 months of the program. Group visits consisted of 1 hour of group support and exploration of alternative modalities for controlling pain, mindfulness meditation and principles of cognitive behavioral therapy. Each patient was evaluated for opioid use disorder, using DSM 5 criteria. Patients with criteria for OUD who did not wish to taper were encouraged to switch to buprenorphine therapy. A non-randomized group of chronic pain patients who were not referred to the program was analyzed to compare outcomes.

MEASURES OF SUCCESS (DISCUSS QUALITATIVE AND/OR QUANTITATIVE METRICS WHICH WILL BE USED TO EVALUATE PROGRAM/INTERVENTION): Patients in the pain groups were monitored for depression, anxiety, level of pain, and level of function. Opioid doses and transition to buprenorphine were tracked. Compliance with guidelines for management was monitored and compared with chronic pain patients on opioid not participating in the group.

FINDINGS TO DATE (IT IS NOT SUFFICIENT TO STATE FINDINGS WILL BE DISCUSSED ): 13/23 patients in the chronic pain group met criteria for opioid use disorder ( 9 mild, 3 moderate, 1 severe). 5 of these patients were successfully transitioned to buprenorphine with patients reporting improvement in mental function and levels of anxiety. No comparison group patients transitioned to suboxone. . Identical percentages of patients in group attendees and in the comparison patients tapered off opioids (30\%), decreased their MED (35\%), remained constant ( 17\%), or increased their dose (17\%). All 3 measures of quality care were better delivered in the group setting (provider visit every 3 months, urine drugs screen every 6 months, completion of a controlled substance agreement). Pain group patients' pain, depression, and anxiety scores did not change during group participation. Patients expressed high levels of satisfaction in the group setting, with 14/16 respondents extremely or very satisfied with the groups.

KEY LESSONS FOR DISSEMINATION (WHAT CAN OTHERS TAKE AWAY FOR IMPLEMENTATION TO THEIR PRACTICE OR COMMUNITY?): A group visit format is well received by patients on chronic opioids for chronic pain and simplifies the provision of appropriate processes of care. It facilitates the diagnosis of opioid use disorder and transitioning of appropriate patients to buprenorphine.

\section{A HEALTHCARE SYSTEM'S COORDINATED APPROACH TO PATIENTS ON CHRONIC OPIOIDS}

Joel Sawady $^{3}$, 6 ; Emily Benedetto ${ }^{1}$; Gina Stenhouse ${ }^{2}$; David Roll ${ }^{1}$; Emily Hanson $^{3}$; Aaronson Y. Chew ${ }^{4}$; Randi Sokol ${ }^{5}$; Barbara Ogur ${ }^{6}$; Ellie Grossman ${ }^{1}$; Janice F. Kauffman ${ }^{2} .{ }^{1}$ Cambridge Health Alliance, Somerville, MA; ${ }^{2}$ Cambridge Health Alliance, Cambridge, MA; ${ }^{3}$ Cambridge Health Alliance, Brighton, MA; ${ }^{4}$ Cambridge Health Alliance/Harvard Medical School, Somerville, MA; ${ }^{5}$ Cambridge Health Alliance, Malden, MA; ${ }^{6}$ Harvard Medical School, Cambridge, MA. (Control ID \#3185600)

STATEMENT OF PROBLEM OR QUESTION (ONE SENTENCE): A multidisciplinary, coordinated approach is needed to design and implement treatment programs for patients with chronic pain

OBJECTIVES OF PROGRAM/INTERVENTION (NO MORE THAN THREE OBJECTIVES): 1 . Develop multidisciplinary expertise in the treatment of chronic pain, including long-term opioid therapy, in an academic safety-net healthcare system 2. Design and implement a primary-care-based program that supports providers and patients in safe and evidence-based treatment of chronic pain

DESCRIPTION OF PROGRAM/INTERVENTION, INCLUDING ORGANIZATIONAL CONTEXT (E.G. INPATIENT VS. OUTPATIENT, PRACTICE OR COMMUNITY CHARACTERISTICS): Cambridge Health Alliance (CHA) is a safety-net academic health system with 13 outpatient primary-care practices serving 120,000 patients, with integrated behavioral health $(\mathrm{BH})$ staff at all sites. We brought together a multidisciplinary task force of primary care providers, $\mathrm{BH}$ and addiction specialists, and a clinical pharmacist to focus on developing a chronic pain program. We performed a needs assessment by: $\bullet$ Reviewing evidence-based chronic pain treatment modalities, including their availability in our community $\bullet$ Assessing the extent of opioid prescribing in our system $\bullet$ Speaking with providers and patients about their needs We piloted interventions at several sites, including: • Improved workflows to support compliance with guidelines and regulations $\bullet$ Pharmacist-led medication management $\bullet$ Enhanced mental health supports $\bullet$ Group visits $\bullet$ Multidisciplinary review of complex patients We are now in the process of spreading these interventions across all 13 sites, with attention to educating clinical staff on the biopsychosocial model of chronic pain and leveraging existing integrated BH staff.

MEASURES OF SUCCESS (DISCUSS QUALITATIVE AND/OR QUANTITATIVE METRICS WHICH WILL BE USED TO EVALUATE PROGRAM/INTERVENTION): Each pilot site monitors measures specific to their intervention. System-wide, we monitor rates of utilization of controlled substance agreements, urine drug screens, and brief functional assessments.

FINDINGS TO DATE (IT IS NOT SUFFICIENT TO STATE FINDINGS WILL BE DISCUSSED ): Bringing together a multidisciplinary team has allowed us to deploy a systemwide program, building on primary care's strengths in patient-relationship-building and motivational enhancement, while folding in cognitive-behavioral strategies taken from the psychology world and made feasible for use in primary care. System-wide, we have seen increases in the rate of controlled substance agreements and urine drug screens. Pilot interventions have shown: $\bullet$ Reduction in high-dose opioid prescribing, and in benzodiazepine co-prescribing, in the pilot of workflow supports $\bullet$ Stableto-improving functional status among patients undergoing opioid tapers in the pharmacist-led intervention $\bullet$ High patient satisfaction, and improvement on multiple quality measures, in the group visits $\bullet$ High provider satisfaction with multidisciplinary case reviews

KEY LESSONS FOR DISSEMINATION (WHAT CAN OTHERS TAKE AWAY FOR IMPLEMENTATION TO THEIR PRACTICE OR COMMUNITY?): Creation of a multidisciplinary task force has been instrumental in improving patient care and increasing provider support. Our team's focus on revising processes of care, educating providers, supporting the development of local expertise, and providing new venues of treatment and consultation services has helped shape a more comprehensive, integrated, and evidence-based community of practice around chronic pain management.

A MULTIDISCIPLINARY TEAM APPROACH FOR PHARMACOLOGIC TREATMENT OF ALCOHOL USE DISORDER AT HOSPITAL DISCHARGE

$\underline{\text { Shirin Hemmat }}^{2}$; Joseph Clement ${ }^{1}$; Erica Bass ${ }^{2}$; Rand Dadasovich ${ }^{2}$; Margaret ${\text { Gray }^{2} \text {; Michael Incze }}^{2}$; Nadine Pardee ${ }^{2}$; Michael A. Thomashow ${ }^{2}$; Marlene Martin ${ }^{2}$. ${ }^{1}$ Zuckerberg San Francisco General Hospital, San Francisco, CA; ${ }^{2}$ University of California, San Francisco, San Francisco, CA. (Control ID \#3185647)

STATEMENT OF PROBLEM OR QUESTION (ONE SENTENCE): Alcohol use disorder (AUD) affects up to $12 \%$ of the population and contributes billions of dollars in societal costs, but the use of pharmacotherapy for treatment of AUD is underutilized, particularly at hospital discharge.

OBJECTIVES OF PROGRAM/INTERVENTION (NO MORE THAN THREE OBJECTIVES): Increase the total number of prescriptions for AUD treatment at discharge for patients who are diagnosed with AUD. A 
secondary objective was to educate patients, physicians, nurses and pharmacists about AUD pharmacotherapy.

DESCRIPTION OF PROGRAM/INTERVENTION, INCLUDING ORGANIZATIONAL CONTEXT (E.G. INPATIENT VS. OUTPATIENT, PRACTICE OR COMMUNITY CHARACTERISTICS): At our safety net hospital, $28 \%$ of hospitalized patients have a substance use disorder. AUD accounts for $54 \%$ of these disorders. Furthermore, AUD is among the top 5 diagnoses with the highest 30-day readmission rates (18.9\%) in our hospital. Despite this prevalence, for our inpatient resident-led Internal Medicine teams, only 10 patients per month were receiving prescriptions for treatment of AUD at discharge; reaching less than half of eligible and interested patients already being screened by nurses on the Alcohol and Tobacco Treatment Team (ATTT). The ATTT is an existing nursing-led team that aims to advise physicians on the need for pharmacotherapy for patients with active alcohol and tobacco use disorders. In response to an institution-wide quality improvement incentive program, a team of Internal Medicine residents, nurse leader and a faculty hospitalist partnered with the ATTT to increase the number of patients with AUD who were prescribed medication assisted treatment (MAT) at discharge. We collaborated on an algorithm to expand pharmacologic options available for MAT beyond our institution's previous naltrexoneonly approach. Educational interventions included monthly addiction medicine didactics and widely disseminated educational materials. We also shadowed the ATTT nurses and obtained their input on how to better integrate resident and ATTT workflow. We also provided biweekly updates to housestaff on the number of prescriptions for AUD treatment stratified by medical team to foster healthy competition. Residents will receive a small financial incentive if a prespecified target of 15 patients per month is discharged with a prescription for AUD treatment six out of twelve months of the year.

MEASURES OF SUCCESS (DISCUSS QUALITATIVE AND/OR QUANTITATIVE METRICS WHICH WILL BE USED TO EVALUATE PROGRAM/INTERVENTION): Our primary measure was the number of patients discharged with a prescription for treatment of AUD at hospital discharge.

FINDINGS TO DATE (IT IS NOT SUFFICIENT TO STATE FINDINGS WILL BE DISCUSSED ): Two months after the initiation of our project, the number of patients discharged from our resident-led Internal Medicine service with a prescription for treatment of AUD has increased from average of 10 to 19 patients per month. Findings from subsequent months with be incorporated.

KEY LESSONS FOR DISSEMINATION (WHAT CAN OTHERS TAKE AWAY FOR IMPLEMENTATION TO THEIR PRACTICE OR COMMUNITY?): A multidisciplinary partnership to improve AUD treatment can effectively increase prescribing of pharmacotherapy two months after implementation in a safety-net urban hospital. Key interventions included an educational campaign, working with the ATTT to expand MAT for AUD and incentivizing resident physicians to prescribe medication for treatment of AUD among patients eligible and interested in treatment.

\section{A NEW DISCHARGE INSTRUCTION TEMPLATE: IMPROVING SAFE DISCHARGES ON THE CLEVELAND CLINIC INTERNAL MEDICINE TEACHING SERVICES \\ Megan A. McGervey ${ }^{1}$; Jessica Donato ${ }^{2} .{ }^{1}$ Cleveland Clinic Foundation, Lakewood, OH; ${ }^{2}$ Cleveland Clinic, Beachwood, OH. (Control ID \#3186336)}

\section{STATEMENT OF PROBLEM OR QUESTION (ONE SENTENCE):}

Decreasing hospital readmissions has been a major focus nationally and locally over recent years. Multiple strategies within the discharge process have been show to impact readmission rates with a cumulative effect. The discharge instructions utilize templates and thus provide an opportunity to embed multiple evidence-based components into standard practice. We sought to improve the safety of hospital discharges and reduce readmission rates by developing a novel discharge instruction template on the general medicine teaching services, which hardwires multiple safe discharge practices.

OBJECTIVES OF PROGRAM/INTERVENTION (NO MORE THAN THREE OBJECTIVES): Objectives of the intervention were to identify components of safe discharges that could be improved on teaching services, create a new discharge instruction template that hardwires components with low metrics, and improve safe discharges, patient/physician communication, and reduce readmissions to the hospital.

DESCRIPTION OF PROGRAM/INTERVENTION, INCLUDING ORGANIZATIONAL CONTEXT (E.G. INPATIENT VS. OUTPATIENT, PRACTICE OR COMMUNITY CHARACTERISTICS): A Quality Improvement (QI) project to improve the quality of discharges from the internal medicine teaching services was undertaken starting September 2017 at a large academic medical center. Current state was assessed by reviewing discharges from four teaching service teams and collecting surveys from 48 Internal Medicine senior second and third year residents. Prior to our intervention, residents failed to comply with several components of a safe discharge including confirming the patient's primary care provider (PCP), performing medication reconciliations, follow up information after discharge, and providing patients with customized instructions for their aftercare. A new discharge instruction template was created, requiring residents to confirm the patient's PCP, make follow-up appointments, create customized bulleted instructions, and provide patients with specific anticipatory guidance and instructions on who to contact after discharge.

MEASURES OF SUCCESS (DISCUSS QUALITATIVE AND/OR QUANTITATIVE METRICS WHICH WILL BE USED TO EVALUATE PROGRAM/INTERVENTION): Process measures included PCP confirmation, creation documentation of follow-up appointments, inclusion of customized bulleted instructions and documentation of specific anticipatory guidance in the discharge instructions. Readmission rates are being collected as an outcome measure.

FINDINGS TO DATE (IT IS NOT SUFFICIENT TO STATE FINDINGS WILL BE DISCUSSED ): Forty-one discharges on the general medicine teaching service over one week were studied prior to the intervention. The discharge summary template has been rolled out to four teaching services over the course of this nine-month project with variable compliance from medicine trainees. After the intervention, PCP confirmation increased from $71 \%$ to $100 \%$, follow-up appointments from $20 \%$ to $83 \%$, detailed discharge anticipatory guidance from $<10 \%$ to $100 \%$ and bulleted discharge instructions from $58.8 \%$ to $100 \%$, and detailed discharge anticipatory guidance from $<10 \%$ to $100 \%$.

KEY LESSONS FOR DISSEMINATION (WHAT CAN OTHERS TAKE AWAY FOR IMPLEMENTATION TO THEIR PRACTICE OR COMMUNITY?): By hardwiring several components of a safe discharge into the discharge template that were frequently overlooked by residents previously, we have improved safe discharges showing that these changes can be successfully implemented based on needs at other facilities.

A NEW VISION FOR DIABETIC RETINOPATHY: FACILITATING SCREENINGS USING OPHTHALMOLOGY ECONSULTS Kim Dowdell $^{3,}{ }^{3}$; Samuel Collins ${ }^{1}$; Arjun Dirghangi ${ }^{2}$; Kerry N. Cotter ${ }^{1}$; Ira Helenius ${ }^{3} .{ }^{1}$ Univerisity of Virginia, Charlottesville, VA; ${ }^{2}$ UVA, Charlottesville, VA; ${ }^{3}$ University of Virginia, Charlottesville, VA. (Control ID \#3185134) 
STATEMENT OF PROBLEM OR QUESTION (ONE SENTENCE):

A barrier exists to patient access to diabetic retinopathy screenings, stemming from ophthalmology clinic volume, patient access barriers, and resources in the primary care clinic.

OBJECTIVES OF PROGRAM/INTERVENTION (NO MORE THAN THREE OBJECTIVES): 1 . Provide primary care clinics with the resources to complete diabetic retinopathy screenings (e.g. equipment, software, staff training, and potential reimbursements) 2 . Improve the percentage of eligible diabetic patients being screened for diabetic retinopathy 3 . Improve interprofessional communication and care coordination between primary care and specialty care

DESCRIPTION OF PROGRAM/INTERVENTION, INCLUDING ORGANIZATIONAL CONTEXT (E.G. INPATIENT VS. OUTPATIENT, PRACTICE OR COMMUNITY CHARACTERISTICS): Diabetic retinopathy is the most common cause of blindness in the United States for patients ages 20-74. All patients with diabetes should have a yearly eye exam, but many patients find it difficult to see an ophthalmologist regularly. This is particularly true at our pilot site, University Medical Associates (UMA), which has a high proportion of uninsured, unscreened patients with diabetes. In response to these challenges, the Center for Telehealth and Department of Ophthalmology implemented the Diabetic Retinopathy Screening (DRS) Program. By utilizing the eConsults service, we are implementing the second iteration of this program with new technology and workflow to build a more efficient, sustainable, and scalable screening program. In the U.S, there is a general consensus that the most cost-effective setting for performing retinopathy screenings is at the initial point of care in the PCP clinic. The DRS Program has placed a Remidio camera at UMA, which is the world's first and only smartphone-based, non-mydriatic fundus camera with infrared imaging capabilities. This device comes with an application to facilitate capture, report generation, and storage of the images and is an ideal platform for comprehensive telemedicine enabled eye screening, as it enables images to be synced and archived securely in the cloud for remote review and clinical management

MEASURES OF SUCCESS (DISCUSS QUALITATIVE AND/OR QUANTITATIVE METRICS WHICH WILL BE USED TO EVALUATE PROGRAM/INTERVENTION): -Percentage of eligible diabetics who receive annual diabetic retinopathy screening -Turnaround time of image review and interpretation -Percentage of abnormal findings -Increasing the acuity within referral patterns

FINDINGS TO DATE (IT IS NOT SUFFICIENT TO STATE FINDINGS WILL BE DISCUSSED ): In our first iteration of the DRS Program at UMA, we found the following (plain text of graph due to submission formatting): Year \# of diabetic retinopathy screens $\%$ of diabetic patients screened $20112366.1 \% 201255214.2 \% 2013603$ 15.5\% $201447212.1 \%$ $20152195.6 \% 20162556.5 \% 20173208.2 \% \sim 40 \%$ of screens had abnormal findings and required a follow-up visit to the ophthalmology clinic. KEY LESSONS FOR DISSEMINATION (WHAT CAN OTHERS TAKE AWAY FOR IMPLEMENTATION TO THEIR PRACTICE OR COMMUNITY?): -Consider staffing realities of the primary care clinic -When choosing a device, consider facility limitations -Implement automated scheduling workflow for positive screenings -Ensure that image quality meets the parameters for national standards of diabetic retinopathy screening, -An early primary care champion is critical -Pay careful consideration to outside grant and funding opportunities -Consider emerging capability of artificial intelligence to make diagnoses

A NEW WAY TO USE YOUR ABCS: IMPROVING OUTPATIENT FOLLOW-UP AFTER HOSPITAL DISCHARGE.

Evan Siau; Alejandro Arbelaez; Raghav Aggarwal; Paula Dibo; Rezaul
Hoque; Monica Mauri; Shorabh Sharma; rajan gurunathan. SBH Health System, Norwood, NJ. (Control ID \#3162490)

STATEMENT OF PROBLEM OR QUESTION (ONE SENTENCE): Developing tools to facilitate post-acute care follow-up is a key element in enhancing linkage with primary care services and improving ambulatory outcomes.

OBJECTIVES OF PROGRAM/INTERVENTION (NO MORE THAN THREE OBJECTIVES): To improve effective follow-up after hospital discharge and positively affect linkage with primary care, we set forth to design and implement a scripted educational tool, to standardize the physician approach to scheduling post-hospital discharge care in a userfriendly way.

DESCRIPTION OF PROGRAM/INTERVENTION, INCLUDING ORGANIZATIONAL CONTEXT (E.G. INPATIENT VS. OUTPATIENT, PRACTICE OR COMMUNITY CHARACTERISTICS): A literature review was conducted to identify best practices and focus groups were engaged. Key elements of care were identified, including identifying where patients have established care, where they would like to follow, the timeframe they needed to be seen, and whether access with the type of doctor or service needed was established as priority. Based on literature review, best practice analysis, and focus group inputs, a scripted educational tool was developed. The "ABC"s of Scheduling Post- Discharge Care. AASK: Where is your usual source of care? Where do you want to be seen? Resident Clinic / FQHC / Community Provider / Faculty Practice B- BY WHEN: When does the patient first need to be seen by post-discharge? $<1$ wk $/<2$ wks $/<4$ wks C - COORDINATE: Are the scheduled appointments relevant and coordinated? Is first appointment appropriate (time / place)? Baseline information was reviewed for quality improvement purposes on 88 discharges across 3 selected medical units. 3 PDSA cycles were then conducted over 12 weeks, during which physician champions were engaged, staff were trained using the tool when planning hospital discharge, and selected quality improvement metrics were followed in realtime on the same sample size in order to assess the potential impact of the tool.

MEASURES OF SUCCESS (DISCUSS QUALITATIVE AND/OR QUANTITATIVE METRICS WHICH WILL BE USED TO EVALUATE PROGRAM/INTERVENTION): The \% of patients accurately assigned to their preferred source of care post-hospital discharge. The $\%$ of 'first post-discharge visits' specifically relevant to the patient's hospitalized condition. The $\%$ of no shows for post-hospital discharge visits in our primary resident clinic. The median number of days to first scheduled appointment post-hospital discharge.

FINDINGS TO DATE (IT IS NOT SUFFICIENT TO STATE FINDINGS WILL BE DISCUSSED ): When compared with discharges from the selected units pre-intervention, discharges from the selected units postintervention showed:The $\%$ of patients accurately assigned to their preferred source of care increased from $85 \%$ to $92 \%$. The $\%$ of 'first post-discharge visits' specifically relevant to the patient's hospitalized condition increased from $68 \%$ to $83 \%$. The post-hospital discharge no show-rate in our primary resident clinic decreased from $81 \%$ to $64 \%$. The median number of days to first scheduled appointment decreased from 15 days to 10.5 days.

KEY LESSONS FOR DISSEMINATION (WHAT CAN OTHERS TAKE AWAY FOR IMPLEMENTATION TO THEIR PRACTICE OR COMMUNITY?): Based on our experience, implementation of a scripted educational checklist or tool has significant opportunity to help minimize variability in scheduling of post-hospital discharge visits, enhance access to post-acute care services, and improve patient outcomes within the ambulatory setting. 


\section{A PHARMACIST-DRIVEN POPULATION HEALTH INITIATIVE TO IMPROVE THE QUALITY OF HYPERTENSION MANAGE- MENT IN A GENERAL INTERNAL MEDICINE CLINIC \\ Jennifer Sabatino; Shengyi Mao. The Ohio State University, Columbus, OH. (Control ID \#3186608)}

\section{STATEMENT OF PROBLEM OR QUESTION (ONE SENTENCE):} Globally, hypertension is the number one cause of death and disability which underscores the importance of controlling this modifiable risk factor to reduce cardiovascular mortality in primary care.

OBJECTIVES OF PROGRAM/INTERVENTION (NO MORE THAN THREE OBJECTIVES): To assess utility of pharmacist comanagement of hypertension in improving blood pressure control (defined as blood pressure $<140 / 90$ ) for patients with a diagnosis of hypertension in a primary care clinic.

DESCRIPTION OF PROGRAM/INTERVENTION, INCLUDING ORGANIZATIONAL CONTEXT (E.G. INPATIENT VS. OUTPATIENT, PRACTICE OR COMMUNITY CHARACTERISTICS): A pharmacist flagged charts of patients on the daily clinic schedule with a diagnosis of hypertension whose last office blood pressure was 140/90. Front desk staff notified the pharmacist when flagged patients arrived and clinical staff notified the pharmacist if the flagged patients had a blood pressure that was still $140 / 90$ on rooming. The pharmacist met with these patients real-time to recheck blood pressures, review medications, and assess adherence. If the pharmacist was not able to meet with the patient during their visit, they contacted the physician after the visit to request referral for co-management if appropriate. Alternatively, the physician could contact the pharmacist to initiate referral for co-management.

MEASURES OF SUCCESS (DISCUSS QUALITATIVE AND/OR QUANTITATIVE METRICS WHICH WILL BE USED TO EVALUATE PROGRAM/INTERVENTION): The proportion of adult patients with a diagnosis of hypertension with last office blood pressure $<140 / 90$. We also tracked as a secondary measure, the proportion of patients with uncontrolled hypertension with a defined plan for management following their visit.

FINDINGS TO DATE (IT IS NOT SUFFICIENT TO STATE FINDINGS WILL BE DISCUSSED ): From February 2018 through December 2018, a total of 293 patient visits met the criteria for the new workflow. Of these, 87 patients $(26.7 \%$ ) had a blood pressure 140/90 on rooming or were not checked. The pharmacist was in the office for 49 of the uncontrolled visits (56.3\%). Of these, the pharmacist was notified by clinic staff for seven patients and completed co-management visits for nine patients. Of the 87 uncontrolled patient visits, $70(80.5 \%)$ had hypertension follow-up documented after the visit. Types of follow-up included pharmacist co-management initiated by the pharmacist $(15 / 87$, $17.2 \%)$, pharmacist co-management initiated by the physician $(31 / 87$, $35.6 \%$ ), management by the physician alone (18/87, 20.7\%), monitoring by nursing staff $(4 / 87,4.6 \%)$, or managed by specialist $(2 / 87,0.23 \%)$. During the study period, 42 patients with uncontrolled hypertension at their initial visit had a subsequent visit. Of these, 33 patients $(78.6 \%)$ achieved blood pressures $<140 / 90$. Overall, the percentage of patients with controlled hypertension increased from $58.4 \%$ (380/651) prior to implementation of the new workflow to $70 \%(480 / 681)$ at the end of the study period.

KEY LESSONS FOR DISSEMINATION (WHAT CAN OTHERS TAKE AWAY FOR IMPLEMENTATION TO THEIR PRACTICE OR COMMUNITY?): Establishing a workflow to promote referral for pharmacist co-management of hypertension improved blood pressure control rates compared to usual care.
A PROCESS TO IMPROVE CARDIAC TELEMETRY USE IN A COMMUNITY HOSPITAL

sana chams ${ }^{3}$; jasmeet bal ${ }^{1}$; Ankita Aggarwal ${ }^{1}$; Manishkumar Patel ${ }^{1}$; Gloria J. Hong'; Vesna Tegeltija ${ }^{1}$; Sarwan Kumar ${ }^{2}$. ${ }^{1}$ Wayne State University, Auburn Hills, MI; ${ }^{2}$ Crittenton Hospital/Wayne state university, Rochester, MI; ${ }^{3}$ wayne state university school of medicine, Auburn Hills, MI. (Control ID \#3184688)

STATEMENT OF PROBLEM OR QUESTION (ONE SENTENCE): Inpatient telemetry monitoring is commonly used to identify arrhythmias, QT prolongation, and ischemia. However, it has been noticed that telemetry is being utilized inappropriately which leads to increased health care cost for the patient in addition to alarm fatigue for the health care professionals.

OBJECTIVES OF PROGRAM/INTERVENTION (NO MORE THAN THREE OBJECTIVES): 1 . The primary aim of our project was to explore the reasons for unnecessary telemetry use during hospitalization 2. The secondary aim was to decrease inappropriate telemetry use. DESCRIPTION OF PROGRAM/INTERVENTION, INCLUDING ORGANIZATIONAL CONTEXT (E.G. INPATIENT VS. OUTPATIENT, PRACTICE OR COMMUNITY CHARACTERISTICS): A project team was formed to assess whether telemetry is utilized appropriately amongst patients admitted to our community hospital. The fishbone diagram was used to explore the reasons for inappropriate telemetry use. MEASURES OF SUCCESS (DISCUSS QUALITATIVE AND/OR QUANTITATIVE METRICS WHICH WILL BE USED TO EVALUATE PROGRAM/INTERVENTION): We used the Institute of Healthcare Improvement $[\mathrm{IHI}]$ model to guide this study. PDSA(plan, do, study, act) cycles were used to assess the intervention by comparing pre and post-intervention data.

FINDINGS TO DATE (IT IS NOT SUFFICIENT TO STATE FINDINGS WILL BE DISCUSSED ): PDSA 1: During root cause analysis, a fishbone diagram was utilized to uncover the culprits of inappropriate telemetry use. It was discovered that an order for telemetry can be placed without selecting an indication. We targeted this system problem by creating a list of class I, II and III indications, in line with ACC/AHA guidelines, and integrated it into the EMR(electronic medical records). A post-implementation review revealed no improvement in adherence due to the physician's ability to bypass this checklist. PDSA 2: In the second cycle, a lack of knowledge about indications for telemetry was found as another culprit contributing to unnecessary use. An educational session about indications of telemetry was conducted for internal medicine residents and they were instructed to document it in their note. Post-intervention data revealed that in $30 \%$ of cases, the residents documented an indication for telemetry. Furthermore, chart review revealed that none of the residents discontinued telemetry during the hospitalization despite negative alarms. PDSA 3: A review of 50 charts of patients admitted to the medical floor with telemetry revealed that $60 \%$ of patients had no appropriate indication for telemetry continuation after 72 hours. A pop-up was integrated into the EMR that appears 72 hours after initiation of telemetry guiding physician to either renew telemetry by choosing an indication or to discontinue telemetry if not indicated. A post-implementation review revealed that $34 \%$ of telemetry use was addressed by either renewing telemetry order or by discontinuing it. Only $10 \%$ telemetry was discontinued through the pop-up as physicians were able to bypass it.

KEY LESSONS FOR DISSEMINATION (WHAT CAN OTHERS TAKE AWAY FOR IMPLEMENTATION TO THEIR PRACTICE OR COMMUNITY?): Our project has been successful in bringing attention to overuse of telemetry and physicians are now required to select indications for telemetry when placing an order. However, once an indication is selected, 
the patients continue to remain on the telemetry monitor throughout their hospitalization. Next phase of the project will focus on improving appropriate discontinuation of telemetry.

\section{A STREAMLINED PATHWAY FOR COMMUNITY-ACQUIRED PNEUMONIA WITH RAPID CONVERSION TO ORAL THERAPY IMPROVES HEALTHCARE VALUE \\ Claire Ciarkowski ${ }^{1}$; Emily S. Spivak ${ }^{2}$; Tristan Timbrook ${ }^{4}$; Matthew Sanford ${ }^{3}$; Christy Hopkins ${ }^{5}$; Nathan Hatton ${ }^{1}$; Frank O. Thomas ${ }^{3}$. ${ }^{1}$ University of Utah, Salt Lake, UT; ${ }^{2}$ University of Utah School of Medicine, Salt Lake City, UT; ${ }^{3}$ University of Utah Hospitals and Clinics, Murray, UT; ${ }^{4}$ University of Utah, SALT LAKE CITY, UT; ${ }^{5}$ University of Utah, Salt Lake City, UT. (Control ID \#3171437)}

STATEMENT OF PROBLEM OR QUESTION (ONE SENTENCE): How does the Initiation of a Clinical Care Pathway for Community Acquired Pneumonia (CAP) Effect Antibiotic administration and Hospital Costs? OBJECTIVES OF PROGRAM/INTERVENTION (NO MORE THAN THREE OBJECTIVES): Develop and execute a standardized electronic health record (EHR) "best practice" pathway for the treatment of CAP. Implement a stewardship team to reinforce best practices. Determine how these interventions impact intravenous antibiotic administration, hospital length of stay (LOS), and costs for patients admitted to a medical floor.

DESCRIPTION OF PROGRAM/INTERVENTION, INCLUDING ORGANIZATIONAL CONTEXT (E.G. INPATIENT VS. OUTPATIENT, PRACTICE OR COMMUNITY CHARACTERISTICS): A standardized CAP order set for patients presenting to the emergency department (ED) was developed by a multidisciplinary team. A best practice alert (BPA) is triggered when orders for a chest radiograph and an antibiotic are placed. The BPA asks for confirmation of antibiotic use for pneumonia and if confirmed an order set is provided. The order set offers guidance on appropriate triage, diagnostic testing, risk assessment for drug resistant pathogens and antibiotic selection. Patients admitted to medical floors are given a single intravenous (IV) antibiotic dose, followed by automatic switch to oral antibiotics for a total duration of 5 days. Atypical coverage with azithromycin is discontinued after 24 hours unless Legionella urine antigen returns positive. Pre-implementation training and education was provided to providers, nurses, respiratory therapists and pharmacists. The antimicrobial stewardship team performed prospective audit of patients on medicine floors and provided feedback on appropriate diagnostic testing and antibiotic use for patients with CAP.

MEASURES OF SUCCESS (DISCUSS QUALITATIVE AND/OR QUANTITATIVE METRICS WHICH WILL BE USED TO EVALUATE PROGRAM/INTERVENTION): The primary endpoints were LOS, days of IV antibiotic therapy, and relative mean cost adjusted for CURB65, age and Charlson comorbidity index using interrupted time series analysis. Secondary endpoints were mortality and 30 day readmission.

FINDINGS TO DATE (IT IS NOT SUFFICIENT TO STATE FINDINGS WILL BE DISCUSSED ): The pre-implementation period was $9 / 1 / 2016$ to $8 / 31 / 2017$ and included 409 patients on acute medicine floors and MICU. Implementation date was 9/1/ 2017 followed by a one-month run in period for pathway education. The post-implementation period was $10 / 1 / 2017$ to $8 / 31 / 2018$ and included 457 patients. The preliminary data showed our intervention was associated with a $39 \%$ (95\% confidence interval (CI): $2 \%$ to $62 \%, p=0.04)$ reduction in monthly per visit costs. There was a nonsignificant reduction in both LOS (-1.8 days, $95 \%$ CI: -4.03 to 0.42 , $\mathrm{p}=0.10)$ and IV antibiotic duration $(-0.88$ days, $95 \% \mathrm{CI}:-2.05$ to $0.28, \mathrm{p}=0.18)$. There were no significant changes in 30 -day readmission or mortality. Further analysis will be conducted.

KEY LESSONS FOR DISSEMINATION (WHAT CAN OTHERS TAKE AWAY FOR IMPLEMENTATION TO THEIR PRACTICE OR COMMUNITY?): The implementation of an EHR driven CAP care pathway in the ED supplemented by education and antimicrobial stewardship review appears to improve healthcare value by reducing costs. Our preliminary data suggests our efforts may not have been sustainable, possibly related to new providers and limited stewardship program resources, however further analysis is required.

ADDRESSING LOW RECEIPT OF LIFESAVING MEDICATIONS FOR OPIOID USE DISORDERS IN MINORITY POPULATIONS: IS ENGAGEMENT IN PRIMARY CARE THROUGH PEER RECOVERY COACHING THE ANSWER? Ricardo Cruz ${ }^{1}$; Mayowa T. Sanusi ${ }^{1}$; Rafik Wahbi ${ }^{1}$; Alissa Cruz ${ }^{1}$; Michele Clark $^{3}$; Daniel Hostetler ${ }^{3}$; Molly Higgins-Biddle ${ }^{3}$; Daniel P. Alford ${ }^{2} .{ }^{1}$ Boston Medical Center, Boston, MA; ${ }^{2}$ Boston University School of Medicine and Boston Medical Center, Boston, MA; ${ }^{3}$ JSI, Boston, MA. (Control ID \#3185834)

STATEMENT OF PROBLEM OR QUESTION (ONE SENTENCE): Despite known mortality benefit through receipt of medications for opioid use disorders (MOUD), minority populations (Black \& Latino) are less likely to be engaged and retained in MOUD after completing detox and continue to experience high rates of opioid related mortality.

OBJECTIVES OF PROGRAM/INTERVENTION (NO MORE THAN THREE OBJECTIVES): Peer Recovery Coaches (PRC) deliver interventions to individuals with OUD completing detox assisting them to: 1. Link to primary care and identify, manage and prevent OUD comorbidities such as HIV, hepatitis B and C, and mental illness; 2. Engage in primary care to facilitate receipt of MOUD for a minimum of 6 months; and 3. Receive overdose education and naloxone (OEND).

DESCRIPTION OF PROGRAM/INTERVENTION, INCLUDING ORGANIZATIONAL CONTEXT (E.G. INPATIENT VS. OUTPATIENT, PRACTICE OR COMMUNITY CHARACTERISTICS): Partnering with two addiction treatment programs offering detox services that serve minority populations in Boston, Project RECOVER (Referral, Engagement, Case management, and Overdose preVention Education in Recovery) uses two PRCs who are culturally diverse and in recovery from substance use disorders to support patients through the processes of postdetox linkage, engagement and retention in primary care in order to receive MOUD for up to six months. Through the use of "Recovery Wellness Plans" PRCs prioritize domains of social determinants of health that patients feel are important to their recovery.

MEASURES OF SUCCESS (DISCUSS QUALITATIVE AND/OR QUANTITATIVE METRICS WHICH WILL BE USED TO EVALUATE PROGRAM/INTERVENTION): To determine the degree of linkage, engagement and retention in MOUD and primary care post-detox as a means of decreasing both fatal and non-fatal opioid overdoses, compared to a comparison group from the MA Chapter 55 Opiate Overdose data. FINDINGS TO DATE (IT IS NOT SUFFICIENT TO STATE FINDINGS WILL BE DISCUSSED ): Preliminary findings after three months of implementation are: 52 patients with OUD (54\% male, 54\% were Black and/ or Latino/Hispanic) completing detox were enrolled. Of the enrolled patients $67 \%$ have a history of incarceration, $51 \%$ self-reported Hepatitis C infection, 
$86 \%$ are homeless or with transient housing. Average educational attainment of the participants was high school diploma/GED. The average number of prior overdoses in this group was 7 episodes. Forty-seven patients attempted OUD treatment an average 1.74 times prior to enrollment. Additionally, $46 \%$ had an appointment scheduled for primary care, $71 \%$ have had phone or in person contact with the PRC post-detox, and $75 \%$ have received OEND. Of the 31 patients with one-month data, no overdoses have been reported.

KEY LESSONS FOR DISSEMINATION (WHAT CAN OTHERS TAKE AWAY FOR IMPLEMENTATION TO THEIR PRACTICE OR COMMUNITY?): Using culturally diverse PRCs is a feasible and promising model to link and engage minority patients with OUD to lifesaving medications and primary care and has potential to decrease opioid-related overdose deaths in this population.

\section{ADDRESSING MISSED SCREENING OPPORTUNITIES THROUGH A TEAM HUDDLE IN A RESIDENT PRIMARY CARE PRACTICE \\ Neha S. Paralkar ${ }^{1}$; Yuriy Khanin ${ }^{1}$; Belinda Lee ${ }^{1}$; Nancy A. LaVine ${ }^{2}$; Jennifer Verbsky $^{2}$; Joseph Conigliaro ${ }^{2}$; Eun Ji Kim ${ }^{2} .{ }^{1}$ Northwell Health, Manhasset, NY ${ }^{2}$ Northwell Hofstra School of Medicine, Lake Success, NY. (Control ID \#3144472)}

\section{STATEMENT OF PROBLEM OR QUESTION (ONE SENTENCE):} Can the use of a pre-visit huddle in a resident ambulatory practice improve preventive care screening rates?

OBJECTIVES OF PROGRAM/INTERVENTION (NO MORE THAN THREE OBJECTIVES): 1. Identify eligible patients with missed screening opportunities for hepatitis $\mathrm{C}$, osteoporosis, and colorectal cancer (CRC) during daily pre-visit preparation 2. Improve screening rates for hepatitis $\mathrm{C}$, osteoporosis, and $\mathrm{CRC}$ by discussing individual gaps in care during a team pre-visit huddle

DESCRIPTION OF PROGRAM/INTERVENTION, INCLUDING ORGANIZATIONAL CONTEXT (E.G. INPATIENT VS. OUTPATIENT, PRACTICE OR COMMUNITY CHARACTERISTICS): Our two resident ambulatory practices are located in a large academic medical center and are certified Patient Centered Medical Homes comprised of 108 internal medicine residents rotating on an $n+1$ schedule, nurses, case managers, pharmacists, medical assistants, and other allied health professionals. Pre-visit huddles have been implemented to allow the interprofessional team to prepare for each patient visit. In our intervention, prior to the daily huddle, one medicine resident performed daily chart reviews to identify patients with gaps in care for appropriate screening tests, including screening for hepatitis $\mathrm{C}$, osteoporosis, and CRC. The resident then reviewed those patients with the entire interprofessional team during the huddle to ensure the intervention was addressed during the visit.

MEASURES OF SUCCESS (DISCUSS QUALITATIVE AND/OR QUANTITATIVE METRICS WHICH WILL BE USED TO EVALUATE PROGRAM/INTERVENTION): We evaluated three quality improvement projects utilizing the huddle to identify patients with needed preventive care measures: hepatitis $\mathrm{C}$ screening, osteoporosis screening, and CRC. Screening measures were performed based on current USPSTF guidelines. Pre and post intervention screening rates for eligible patients were measured for each of the projects (Hepatitis C screening, osteoporosis screening, and CRC screening) and the percent change was calculated.

FINDINGS TO DATE (IT IS NOT SUFFICIENT TO STATE FINDINGS WILL BE DISCUSSED ): For eligible patients, the hepatitis C screening rate improved from $66.0 \%$ pre-intervention to $75.8 \%$ postintervention ( $9.8 \%$ increase). The osteoporosis screening rate improved from
$36.3 \%$ pre-intervention to $41.8 \%$ post-intervention (5.5\% increase). The CRC screening rate improved from $36.2 \%$ pre-intervention to $43.2 \%$ postintervention (7.0\% increase).

KEY LESSONS FOR DISSEMINATION (WHAT CAN OTHERS TAKE AWAY FOR IMPLEMENTATION TO THEIR PRACTICE OR COMMUNITY?): Huddles are brief daily interprofessional meetings to improve communication between staff members, enhance accountability and empowerment, and collaborate on plans for improved patient care. Previsit preparation to identify individual gaps in care can be easily incorporated into huddle discussions and can successfully improve screening rates by identifying eligible patients for the entire interprofessional team.

\section{ADDRESSING OBESITY AND MOOD THROUGH A NOVEL PRI- MARY CARE-BASED LIFESTYLE MANAGEMENT PROGRAM IN A SAFETY NET PRACTICE \\ Melissa Mroz. University of Rochester, Rochester, NY. (Control ID \#3184938)}

STATEMENT OF PROBLEM OR QUESTION (ONE SENTENCE): Evidence has shown that lifestyle interventions effectively reduce weight and improve mood; however, barriers to recommending lifestyle interventions include a lack of clinician knowledge and confidence in treating obesity and availability of affordable programs.

OBJECTIVES OF PROGRAM/INTERVENTION (NO MORE THAN THREE OBJECTIVES): Program objectives included: 1 . To provide an affordable, on-site lifestyle management program for primary care patients with obesity 2 . To enhance provider confidence in treating obesity 3 . To improve patient outcomes, as measured by weight reduction and mood improvement

DESCRIPTION OF PROGRAM/INTERVENTION, INCLUDING ORGANIZATIONAL CONTEXT (E.G. INPATIENT VS. OUTPATIENT, PRACTICE OR COMMUNITY CHARACTERISTICS): The Lifestyle Management Group (LMG) intervention was offered to patients with obesity in Strong Internal Medicine (SIM). SIM is a safety net, facultyresident internal medicine practice at the University of Rochester, providing primary care to approximately 12,000 patients. The LMG held bi-weekly sessions over 24 weeks. Each session included a provider visit that focused on goal-setting and comorbidities, followed by group exercise and education. Education topics spanned topics including diet, activity, stress and sleep. Participating staff included providers, nursing, social work and nutrition. The LMG was designed to be revenue neutral provided there were at least 10 patients.

MEASURES OF SUCCESS (DISCUSS QUALITATIVE AND/OR QUANTITATIVE METRICS WHICH WILL BE USED TO EVALUATE PROGRAM/INTERVENTION): Several metrics were used to assess the program's objectives: - $50 \%$ of SIM physicians will refer 1 or more patients - $25 \%$ of referred patients will attend 1 or more sessions and $20 \%$ will attend 2 or more sessions - Weight reduction (\% body weight) and improvements in mood (PHQ-9, GAD-7) for patients attending 2 or more sessions FINDINGS TO DATE (IT IS NOT SUFFICIENT TO STATE FINDINGS WILL BE DISCUSSED ): Initial results are promising. In terms of endorsement, $55 \%$ of physicians referred at least one patient to the LMG. Of 60 total referrals over 6 months, 31 patients were scheduled to attend an LMG session. Of these, 18 attended at least 1 session and 12 attended 2 or more. For patients who attended 2 or more sessions, 9 experienced a decrease in weight, 2 lost $5 \%$ or more of their body weight and 6 showed improvements in mood. The second LMG is ongoing and collecting additional outcome measures, including pain and physical function. 
KEY LESSONS FOR DISSEMINATION (WHAT CAN OTHERS TAKE AWAY FOR IMPLEMENTATION TO THEIR PRACTICE OR COMMUNITY?): Several key lessons warrant highlighting. First, convening a multidisciplinary team was critical to the program's success in light of its duration and scope. Aligning staff interests and skills with sessions maximized program participation and engagement. Support by practice leadership was also needed to block time for staff to facilitate sessions. Second, screening revealed that several patients had food insecurity, limited mobility, and lacked access to safe, outdoor activities. These findings underscore the importance of screening and tailoring activities to patient needs. Last, the engagement of community-based resources and use of curated online exercise videos were vital to meeting patient needs and providing an affordable program. Future work will focus on improving provider skill in motivational interviewing to increase utilization of LMG and community-based resources.

ADDRESSING SOCIAL DETERMINANTS OF HEALTH THROUGH IMPLEMENTATION OF A COMPREHENSIVE SOCIAL NEEDS SCREENING AND REFERRAL PROGRAM

Jane Lindahl $^{2}$; Rachael Odusanya ${ }^{2}$; Omolara T. Uwemedimo ${ }^{1}$; Johanna Martinez $^{2}$; Shari Jardine ${ }^{1}$; Eun Ji Kim ${ }^{2} .{ }^{1}$ Northwell Health, New Hyde Park, NY; ${ }^{2}$ Northwell Hofstra School of Medicine, Lake Success, NY. (Control ID \#3179076)

STATEMENT OF PROBLEM OR QUESTION (ONE SENTENCE): Social determinants of health (SDH) affect more than half of patient health outcomes, but few programs exist to comprehensively screen for SDH and provide resources for addressing unmet needs.

OBJECTIVES OF PROGRAM/INTERVENTION (NO MORE THAN THREE OBJECTIVES): 1) To identify and address unmet social needs of patients at primary care clinics in a large, suburban, academic hospital system. 2) To evaluate whether SDH screening, navigation, and referral can address social needs in vulnerable populations 3) To work towards integrating a standardized, health-system wide program for SDH screening into primary care practices

DESCRIPTION OF PROGRAM/INTERVENTION, INCLUDING ORGANIZATIONAL CONTEXT (E.G. INPATIENT VS. OUTPATIENT, PRACTICE OR COMMUNITY CHARACTERISTICS): OUI health system's SDH Screening Program was established to identify and address adverse SDH in our primary care populations. The program runs at two ambulatory care practices which serve as academic clinical sites for a large teaching hospital serving a primarily low-income, diverse population, many of whom are immigrants. Our program uses a Patient Navigator (PN) model embedded in the clinical workflow to universally screen patients for SDH during well-visits using a screening tool to assess unmet social needs including housing, food, education and utility insecurity, among others. PNs are trained in promoting patient engagement and cultural competency. They conduct SDH screening in the waiting room, provide referrals to connect patients with SDH needs to community resources, and conduct bi-monthly follow-ups to address their needs over the course of 12-weeks.

MEASURES OF SUCCESS (DISCUSS QUALITATIVE AND/OR QUANTITATIVE METRICS WHICH WILL BE USED TO EVALUATE PROGRAM/INTERVENTION): We evaluate the program using the following measures: screening rate, screen completion rate, percent of patients with unmet needs who connect with a PN, percent of patients with unmet needs who are connected to resources, and percent of patients whose needs are resolved within 12 weeks of screening. We examined these measures at an Internal Medicine clinic from July to December 2018.
FINDINGS TO DATE (IT IS NOT SUFFICIENT TO STATE FINDINGS WILL BE DISCUSSED ): 764 patients were screened, of which $326(42 \%)$ screened positive for social needs. Among those with needs, $152(47 \%)$ accepted initial assistance from PNs and 81(25\%) were successfully connected to resources.

KEY LESSONS FOR DISSEMINATION (WHAT CAN OTHERS TAKE AWAY FOR IMPLEMENTATION TO THEIR PRACTICE OR COMMUNITY?): This program model has demonstrated the importance of engaging clinical staff and PNs at each site in order to embed the SDH screening process within the clinical workflow. Further, screening comprehensively for social needs and using a PN model to address those needs through individualized community resource referrals facilitates patient trust and engagement. Prioritizing cultural competency through the recruitment of bilingual navigators, the use of translated screens and interpreter services, and a training program for PNs emphasizing skills for working with diverse, vulnerable populations have also been vital to our success. The program provides streamlined referrals to trusted partners by partnering with local community organizations and establishing a Medical-Legal Partnership allowing for in-house referrals to free legal assistance for patients with health-harming legal needs.

ADDRESSING SOCIAL DETERMINANTS OF HEALTH: CONNECTING DISADVANTAGED PRIMARY CARE PATIENTS TO UNITED WAY COMMUNITY RESOURCE NAVIGATION

Shari Bolen ${ }^{1,}{ }^{2}$; Jonathan Lever ${ }^{3}$; Samantha Smith ${ }^{4}$; David BarShain $^{5}$; Elvira Ordillas ${ }^{6}$; Wanda Ali-Matlock ${ }^{3}$; Joseph Daprano ${ }^{1}$; Matthew Finley ${ }^{7} .{ }^{1}$ MetroHealth/Case Western Reserve University, Cleveland, $\mathrm{OH} ;{ }^{2}$ Case Westerne Reserve University, Cleveland, $\mathrm{OH}$; ${ }^{3}$ Better Health Partnership, Cleveland, $\mathrm{OH} ;{ }^{4} \mathrm{Cuyahoga}$ County Board of Health, Parma, OH; ${ }^{5}$ The MetroHealth System, Cleveland, OH; ${ }^{6}$ MetroHealth Medical Center, Cleveland, OH; ${ }^{7}$ United Way of Greater Cleveland, Cleveland, OH. (Control ID \#3185363)

STATEMENT OF PROBLEM OR QUESTION (ONE SENTENCE): Although disadvantaged patients often have social needs, few programs have implemented and evaluated connecting these patients electronically to a community organization with community resource navigation. We describe early results of a pilot clinic-to-community linkage program addressing social determinants of health for hypertensive adults with uncontrolled blood pressure seen in a safety net clinic.

OBJECTIVES OF PROGRAM/INTERVENTION (NO MORE THAN THREE OBJECTIVES): 1) Determine social determinant of health needs of disadvantaged patients within a safety net clinic; 2) develop a scalable model to address social determinants of health which could be implemented across multiple electronic health record systems and U.S. communities; 3) apply the model to hypertensive adults with uncontrolled blood pressure.

DESCRIPTION OF PROGRAM/INTERVENTION, INCLUDING ORGANIZATIONAL CONTEXT (E.G. INPATIENT VS. OUTPATIENT, PRACTICE OR COMMUNITY CHARACTERISTICS): Hypertensive adult patients with elevated blood pressure (BP $>=140 / 90$ $\mathrm{mmHg}$ ) seen at a safety net clinic in Cleveland, Ohio were referred electronically to United Way 2-1-1 (a non-profit organization which curates a database of $>20,000$ health and social service community resources) with the goal of connecting the patients to health-improving community resources in a 1-month pilot period (9/24/2018 and 10/28/2018). 
MEASURES OF SUCCESS (DISCUSS QUALITATIVE AND/OR QUANTITATIVE METRICS WHICH WILL BE USED TO EVALUATE PROGRAM/INTERVENTION): Our measures of success thus far include: 1) types of needs addressed; and 2) uptake of referrals to community organizations to address identified needs.

FINDINGS TO DATE (IT IS NOT SUFFICIENT TO STATE FINDINGS WILL BE DISCUSSED ): In the 1-month pilot period, 51 hypertensive patients were eligible for referral to United Way 2-1-1 and 25 (49\%) consented to a referral. The two main reasons identified for lack of referral were patient refusal and inconsistencies among staff offering the referrals. Of the 25 referred, 15 patients were reached by the 2-1-1 navigation specialist, 4 patients were still being contacted, and 6 were unreachable. Reached patients reported 67 total needs which fell into 11 distinct categories according to the national 2-1-1 taxonomy: food/meals ( $\mathrm{n}=11$ patients), health care $(\mathrm{n}=11)$, recreation $(n=9)$, utility assistance $(n=4)$, housing $(n=2)$, individual, family, and community support $(n=2)$, income assistance $(n=2)$, information services $(\mathrm{n}=1)$, transportation $(\mathrm{n}=1)$, clothing/personal/household needs $(\mathrm{n}=1)$, and volunteers/donations $(n=1)$. The median number of needs per patient was 4 (range 1-8). A total of 124 referrals to community resources were provided to patients by the navigation specialist to address identified needs, with an average of 8 referrals (SD 4) per patient. Of the 5 patients with follow-up to date, each had either one need resolved or a resolution in progress. These preliminary findings will be updated in May; we anticipate a sample of at least 100 patients, with more extensive follow-up data.

KEY LESSONS FOR DISSEMINATION (WHAT CAN OTHERS TAKE AWAY FOR IMPLEMENTATION TO THEIR PRACTICE OR COMMUNITY?): Patients reported an extensive set of needs with the majority related to food insecurity, health education, physical fitness opportunities, and utility assistance. Successful strategies such as electronic referrals to a national United Way 2-1-1 service could be a model which is scaled up across the U.S. to better address social determinants of health.

\section{ADDRESSING SOCIAL DETERMINANTS OF HEALTH: DEVEL- OPING AND DELIVERING TIMELY, ACTIONABLE AUDIT FEEDBACK REPORTS TO HEALTHCARE TEAMS \\ Harriet Fisher ${ }^{1}$; Jeffrey Wilhite ${ }^{1}$; Lisa Altshuler ${ }^{1}$; Kathleen Hanley ${ }^{1}$; Khemraj Hardowar $^{1}$; Letisha Smith ${ }^{1}$; Sondra Zabar ${ }^{1}$; Isaac Holmes ${ }^{1}$; Andrew B. Wallach $^{1,2}$; Colleen C. Gillespie ${ }^{1}$. ${ }^{1}$ NYU School of Medicine, New York, NY; ${ }^{2}$ Bellevue Hospital, New York, NY. (Control ID \#3185394)}

STATEMENT OF PROBLEM OR QUESTION (ONE SENTENCE): Does actionable feedback on patient safety indicators and responses to disclosed social determinants of health (SDoH) impact clinical behavior? OBJECTIVES OF PROGRAM/INTERVENTION (NO MORE THAN THREE OBJECTIVES): (1) Develop/ disseminate quarterly audit-feedback reports on $\mathrm{SDoH}$ practice behavior, focusing on elicitation of patient information. (2) Enhance our understanding of factors related to disparities in safety/ quality of care. (3) Increase rates of SDoH documentation and referral.

DESCRIPTION OF PROGRAM/INTERVENTION, INCLUDING ORGANIZATIONAL CONTEXT (E.G. INPATIENT VS. OUTPATIENT, PRACTICE OR COMMUNITY CHARACTERISTICS): We sent Unannounced Standardized Patients (USPs) with SDoH-related needs to care teams in two urban, safety-net clinics. Data collected on practice behaviors were used for cycles of audit and feedback on the quality of electronic health record (EHR) documentation, team level information sharing, and appropriate service referral. Reports contained an evolving educational component (e.g. how to recognize, refer, and document SDoH). We disseminated reports to teams (doctors, nurses, physician's assistants, medical assistants, and staff) at routine meetings and via email.

MEASURES OF SUCCESS (DISCUSS QUALITATIVE AND/OR QUANTITATIVE METRICS WHICH WILL BE USED TO EVALUATE PROGRAM/INTERVENTION): Three audit feedback reports have been distributed to date. Survey data was collected at two time points, $2017(n=77)$ and $2018(n=81)$, to assess provider attitude changes and integration of feedback into clinical practice. Measures included change in team knowledge and attitudes towards $\mathrm{SDoH}$, and response to/ documentation of presented SDoH (measured via post-visit checklist and EMR).

FINDINGS TO DATE (IT IS NOT SUFFICIENT TO STATE FINDINGS WILL BE DISCUSSED ): Preliminary data shows no change or improvement in documentation of $\mathrm{SDoH}$ and limited variation between firm-level responses. (1) Only 7\% of providers reported feeling strongly confident in knowing how to make referrals for social needs in 2018; no improvement since 2017. (2) Despite regular report distribution, $58 \%$ of providers reported having received no formalized feedback on responding to SDoH. $24 \%$ reported maybe or not sure. (3) $86 \%$ of 2018 survey participants self-reported having referred a patient to appropriate services when a social need was identified. Our referral data says otherwise, referrals occur for less than $30 \%$ of visits with SDoH-related needs. KEY LESSONS FOR DISSEMINATION (WHAT CAN OTHERS TAKE AWAY FOR IMPLEMENTATION TO THEIR PRACTICE OR COMMUNITY?): Results suggest disconnect between team data and individual reporting: most report they refer but data suggests few do. Deeper integration of reports into team processes, attachment of feedback to curricula, and increased frequency of regular feedback may be needed for accountability. These preliminary results help refine audit feedback methodology but research is needed to understand motivation and systems barriers to referral and documentation. Future research will look at provider attitudes toward referral processes.

\section{AN ACADEMIC CONSORTIUM TO SUPPORT RURAL AMER- ICAN INDIAN HEALTH}

Matthew Tobey; Katrina Armstrong. Massachusetts General Hospital, Boston, MA. (Control ID \#3185835)

STATEMENT OF PROBLEM OR QUESTION (ONE SENTENCE): 5.2 million American Indians and Alaska Native (AIANs) live in the United States, with 3.2 million AIANs belonging to federally recognized tribes. The United States government has a trust relationship to provide healthcare to those 3.2 million AIAN individuals, who experience the lowest life expectancy at birth of any racial or ethnic group. South Dakota is home to nine reservations, including several of the nation's largest reservations by size and population. Despite strong communities and remarkable health innovations, several of these tribes experience the worst health outcomes in the nation. These counties face three of the five lowest life expectancies at birth and the single highest countylevel rates of mortality from diabetes, liver disease, alcohol use and road traffic accidents.

OBJECTIVES OF PROGRAM/INTERVENTION (NO MORE THAN THREE OBJECTIVES): Our seven-year objectives have been to 1) demonstrate sustainability and growth of an academic partnership between a distant large academic health system and a rural American Indian community in South Dakota; and 2) promote and coordinate other academic health center partnerships in the region. 
DESCRIPTION OF PROGRAM/INTERVENTION, INCLUDING ORGANIZATIONAL CONTEXT (E.G. INPATIENT VS. OUTPATIENT, PRACTICE OR COMMUNITY CHARACTERISTICS): In 2012, our academic health center established a partnership with the Indian Health Service (IHS) and the Rosebud Sioux Tribe. In 2016, a rotating team of internists began to provide 365-day-a-year coverage in the IHS center, providing primary care and inpatient care. In 2018, our team hosted an "accelerator workshop" in collaboration with Harvard's Radcliffe Institute of Advance Study. In addition to inviting members of our partnerships, we invited health systems and community leaders from several South Dakota stakeholder organizations and tribes. We also invited leadership from academic health centers, including those in the regional and others with a national reach. Follow-up discussions and partnership building continues.

MEASURES OF SUCCESS (DISCUSS QUALITATIVE AND/OR QUANTITATIVE METRICS WHICH WILL BE USED TO EVALUATE PROGRAM/INTERVENTION): We perform ongoing evaluation of our local partnership with metrics including chronic disease management, health center care quality metrics, care innovations projects, and evaluation of IHS and tribal perspectives. We evaluate the academic consortium on feedback of stakeholders, growth of programs, and ability to effect change in three areas of focus.

FINDINGS TO DATE (IT IS NOT SUFFICIENT TO STATE FINDINGS WILL BE DISCUSSED ): Our local academic partnership has proven sustainable over three years and has helped facilitate the growth of other academic health system partnership efforts in the region. The IHS center has successfully met accreditation standards and met increasingly numbers of national quality metrics. The academic consortium has identified three priority areas: 1 . Health workforce development for local tribes, including youth development 2 . Academic partnerships to improve care systems in rural IHS service units 3 . Health systems transformation to emulate best-practice models from other regions, e.g. Alaska

KEY LESSONS FOR DISSEMINATION (WHAT CAN OTHERS TAKE AWAY FOR IMPLEMENTATION TO THEIR PRACTICE OR COMMUNITY?): We have established partnership pathways by which academic health centers may engage meaningfully with AIAN communities that face health equity challenges.

AN ACADEMIC PARTNERSHIP WITH A RURAL TRIBAL JAIL Matthew Tobey; Rachel E. Simon; Leila H. Borowsky. Massachusetts General Hospital, Boston, MA. (Control ID \#3179136)

\section{STATEMENT OF PROBLEM OR QUESTION (ONE SENTENCE):}

Individuals who experience incarceration carry among the most substantial health risks of any group, including excessive rates of substance use disorders, serious mental illness and chronic medical illness. American Indian and Alaska Native (AI/AN) individuals experience the lowest life expectancy of any ethnic or racial group in the United States, just over 70, with large land-based tribes experiencing worse outcomes yet. The Indian Health Service (IHS) provides services to 2.2 million of the nation's 5.2 million AI/AN individuals, including those incarcerated in tribal jails. Little information exists on the health of incarcerated $\mathrm{AI} / \mathrm{AN}$ individauls, with only five papers in the peer-reviewed literature.

OBJECTIVES OF PROGRAM/INTERVENTION (NO MORE THAN THREE OBJECTIVES): Objectives include: -To improve care access for one nation's largest tribal adult correctional facilities -To provide health education to individuals incarcerated at the tribal jail
DESCRIPTION OF PROGRAM/INTERVENTION, INCLUDING ORGANIZATIONAL CONTEXT (E.G. INPATIENT VS. OUTPATIENT, PRACTICE OR COMMUNITY CHARACTERISTICS): Through a clinical services contract with a rural IHS facility, rotating internists from our academic health center team provide year-round clinical care with the IHS in a rural tribal community. Two programs link this program to the local tribal jail: 1. A once-weekly internal medicine clinic in the jail. 2. A student-led health promotion program at the jail in which a team comprised of a mix of PA, NP and MD students teach one-hour health educational sessions to the jail's women's unit followed by the men's unit. MEASURES OF SUCCESS (DISCUSS QUALITATIVE AND/OR QUANTITATIVE METRICS WHICH WILL BE USED TO EVALUATE PROGRAM/INTERVENTION): Measures - clinical program 1: Stability of the clinic 2: Number of weekly visits 3: Satisfaction of IHS and tribal jail stakeholders 4: Chronic disease outcomes 5: Transitional care postrelease from jail Measures - education program 1 . Sustainability of program and participation of trainees 2: Attendance of individuals who are incarcerated at the jail 3. Satisfaction of participants with the sessions

FINDINGS TO DATE (IT IS NOT SUFFICIENT TO STATE FINDINGS WILL BE DISCUSSED ): Both the clinical and educational programs have proven sustainable over two years. 16 patients are seen during the weekly half-day morning clinic. High levels of satisfaction have been expressed by IHS and tribal jail leadership with the clinic. The tribal jail has repeatedly requested additional weekly clinic sessions. Successful care navigation has led to placements at the tribal alcohol inpatient program, the tribal methamphetamine inpatient program, and our team's hepatitis $\mathrm{C}$ initiative at the IHS. The health promotion curriculum has become the jail's primary health programming for its population. All 60 trainees over the past 18 months have participated. Students often list participation in the sessions as a highlight of the rotation. Intermittent surveys of jail participants have shown high satisfaction and been used to elicit topics for future weeks. 10 women and 15 men participate on a typical week.

KEY LESSONS FOR DISSEMINATION (WHAT CAN OTHERS TAKE AWAY FOR IMPLEMENTATION TO THEIR PRACTICE OR COMMUNITY?): Tribal jails represent an opportunity for the pursuit of health equity and public health agendas. Clinical and nonclinical activities in tribal jails, as described, may strengthen connections between Native health systems and correctional facilities.

\section{AN INTERDISCIPLINARY CLINIC FOR MEDICALLY COM-} PLEX NEW YORKERS WITHOUT HOMES

Yinan $\mathrm{Lan}^{3,}$ 2; Janine Knudsen 3, 1; Ann R. Garment ${ }^{3,2}$; Andrew D. $\overline{\text { Goldstein }^{3}}{ }^{2}$; Jessica Hughes ${ }^{1}$; Anne Marie Young ${ }^{1}$; Megan Hosein ${ }^{3,2}$; Nicole Hosseinipour ${ }^{1}$; Isaac Holmes ${ }^{3}$. ${ }^{1} \mathrm{NYC}$ Health + Hospitals, New York, NY; ${ }^{2}$ Bellevue Hospital Center, New York, NY; ${ }^{3}$ New York University School of Medicine, New York, NY. (Control ID \#3181578)

STATEMENT OF PROBLEM OR QUESTION (ONE SENTENCE): How do we provide effective, dignified primary care for medically complex patients with homelessness in a safety-net health system?

OBJECTIVES OF PROGRAM/INTERVENTION (NO MORE THAN THREE OBJECTIVES): To effectively engage homeless patients with complex barriers to primary care To provide dignified, trauma-informed care focused on patient-oriented care goals while addressing addiction, mental health, and chronic disease To implement an interdisciplinary care team model in a safety-net health care system combining primary care, social work, care coordination, and nursing 
DESCRIPTION OF PROGRAM/INTERVENTION, INCLUDING ORGANIZATIONAL CONTEXT (E.G. INPATIENT VS. OUTPATIENT, PRACTICE OR COMMUNITY CHARACTERISTICS): Unstably housed people with complex chronic disease often receive fragmented care from various emergency departments and inpatient settings, accruing high rates of acute care utilization without improvements in health. Recently, intensive outpatient models have emerged to better manage high need patients. Here we describe our efforts to create a complex care clinic for medically, socially, and behaviorally complex patients with unstable housing at the largest safety-net health system in the

United States. Launched in August 2018, the clinic aims to engage patients in a trusting healthcare environment and break the cycle of disease, addiction, and housing instability. Our team includes four buprenorphinewaivered internal medicine physicians, a social worker, care coordinator, and home care nurse provided by our system's Medicaid Health Home. Patients are referred from the ED, inpatient service, other clinics, street outreach organizations, shelters, and jails. They receive extensive care coordination; on-site addiction, medical, and social services; home nursing visits; and collaboration with shelters and community based organizations. MEASURES OF SUCCESS (DISCUSS QUALITATIVE AND/OR QUANTITATIVE METRICS WHICH WILL BE USED TO EVALUATE PROGRAM/INTERVENTION): A quantitative analysis will be used to determine program impact on clinical outcomes and utilization, patient experience, and provider satisfaction. Both quantitative and qualitative measures will be used to evaluate clinic capacity, services provided, patient engagement, and progress towards patient-oriented care goals.

FINDINGS TO DATE (IT IS NOT SUFFICIENT TO STATE FINDINGS WILL BE DISCUSSED ): From August through December 2018, 156 referrals were given appointments and 83 patients completed at least one appointment. Of those, at least 44 patients (53\%) returned for a second visit. On average patients completed 2.1 visits. We had a $16 \%$ cancellation rate and $38 \%$ no show rate. Patients are mostly male, middle-aged and street or shelter dwelling with common diagnosis of substance use disorder, lower extremity wounds, and hypertension. Our most engaged patients ( $>3$ visits, $\mathrm{n}=15$ ) have seen an average reduction in ED visits by $68 \%$ and inpatient admissions by $58 \%$ within our system compared to pre-clinic intervention. KEY LESSONS FOR DISSEMINATION (WHAT CAN OTHERS TAKE AWAY FOR IMPLEMENTATION TO THEIR PRACTICE OR COMMUNITY?): Relationships have been a core element of patient care, building an interdisciplinary team, and developing referral and collaborative resources internally and in the community. Our focus on a patient-directed care plan, warm hand-offs, continuity of care, and community outreach has also allowed this model to succeed.

\section{BEDSIDE ROUNDS IMPROVE PATIENT SATISFACTION AND CARE TRANSITIONS}

Marwa Moussa $^{1}$; Jennifer Renaud ${ }^{1}$; Charles Okamura ${ }^{1}$; Yvonne Brown ${ }^{1}$; Frank Volpicelli ${ }^{2} .{ }^{1} \mathrm{NYU}$ Langone, Staten Island, NY; ${ }^{2}$ New York University, New York, NY. (Control ID \#3184390)

STATEMENT OF PROBLEM OR QUESTION (ONE SENTENCE): As the lack of a 'face-to-face' interaction between the full team and the patient led to a downtrend in patient experience scores, we were inspired to design a patient centered communication tool that standardizes the multidisciplinary bedside rounds.

OBJECTIVES OF PROGRAM/INTERVENTION (NO MORE THAN THREE OBJECTIVES): 1 . To improve our patients' hospital experience in regards to care transitions and discharge planning by implementing standard bedside rounds that center around the patient's health care needs. 2 . To create a daily scheduled opportunity for the patient to be involved in medical decisions and discharge planning which enhances patients' understanding of their own care plan.

DESCRIPTION OF PROGRAM/INTERVENTION, INCLUDING ORGANIZATIONAL CONTEXT (E.G. INPATIENT VS. OUTPATIENT, PRACTICE OR COMMUNITY CHARACTERISTICS): Our medical-surgical units did not have a standardized approach to ensure clear communication from a patient's multidisciplinary team, comprised of physicians, nurses, case managers and social workers. To this end, we implemented a communication plan based on the acronym "WE CARE" 1) Who was present (who was at bedside in addition to the patient); 2) Everyone on same page ( language and literacy barriers); 3) Connect with patient and family (promote patient-centeredness and compassionate care through eye contact, introducing the full team ); 4) Assessing understanding (explanation of changes to medications, key lab and test results, and postdischarge plans);5) Response from patient and/or caregivers (ensuring understanding); 6) Educate/ empathy/ end of conversation. Centered on the WE CARE model, we gathered all members of the care team and visited each patient at a standardized time every day. The intervention was started on one medical-surgical, unit 5600 on July 2018.

MEASURES OF SUCCESS (DISCUSS QUALITATIVE AND/OR QUANTITATIVE METRICS WHICH WILL BE USED TO EVALUATE PROGRAM/INTERVENTION): HCAPS (Hospital Consumer Assessment of Healthcare Providers and Systems) scores for care transitions and discharge information pre and post intervention will be evaluated for the study group (unit 5600). We will also compare these scores to med/ surg units who did not receive the intervention.

FINDINGS TO DATE (IT IS NOT SUFFICIENT TO STATE FINDINGS WILL BE DISCUSSED ): We compared our pre-intervention 1/1/ 2018-4/31/2018 (approximately 54 surveys) and post-intervention (approximately 42 surveys) scores. In the domain of care transitions, e. g., patient had a good understanding of things patient was responsible for in managing his/her health; patient had a good understanding of purpose of each medication; staff consideration of patient and caregiver preferences postdischarge, there was an increase from $28 \%$ in our top-box (an answer of always) composite HCAHPS score to $58 \%$. Scores for "discharge information delivered" remained high with a top-box response above $85 \%$ both preand post-intervention. In addition, during the post-intervention time, the study group unit had the highest "care transition" and "discharge information" top box responses compared to all control units.

KEY LESSONS FOR DISSEMINATION (WHAT CAN OTHERS TAKE AWAY FOR IMPLEMENTATION TO THEIR PRACTICE OR COMMUNITY?): A focused, structured communication tool WE CARE, implemented as a part of daily standardized multidisciplinary bedside rounds led to an improvement in patient satisfaction scores around care transitions and discharge information delivered.

BEYOND THE WALLS OF THE CLINIC: A TELEMEDICINE PILOT TO ENHANCE CARE DELIVERY IN A RESIDENCY PRACTICE

Zehra Hussain $^{2}$; Gretchen Rickards ${ }^{2}$; Jenna Laughlin²; John Donnelly ${ }^{1}$; $\overline{\text { Sarah Schenck }}{ }^{2}$. ${ }^{1}$ Christiana Care Health System, Newark, DE; ${ }^{2}$ Christiana Care Health System, Wilmington, DE. (Control ID \#3185413)

STATEMENT OF PROBLEM OR QUESTION (ONE SENTENCE): Scheduled telephone visits are an effective, convenient alternative to the traditional face-to-face visit in primary care, but there are not yet established models for incorporating these types of visits into residency clinics. 
OBJECTIVES OF PROGRAM/INTERVENTION (NO MORE THAN THREE OBJECTIVES): To demonstrate the feasibility of adopting telephone visits into a residency clinic and to measure resident utilization of these visits over a two-year period

DESCRIPTION OF PROGRAM/INTERVENTION, INCLUDING ORGANIZATIONAL CONTEXT (E.G. INPATIENT VS. OUTPATIENT, PRACTICE OR COMMUNITY CHARACTERISTICS): A two-year telemedicine pilot was conducted in our urban primary care residency clinic. Nine categorical medicine residents in our 36-resident program participated. Each resident had one hour of dedicated time per week templated into his/her schedule for telephone visits. Openings that went unfilled were converted into acute office visits.

MEASURES OF SUCCESS (DISCUSS QUALITATIVE AND/OR QUANTITATIVE METRICS WHICH WILL BE USED TO EVALUATE PROGRAM/INTERVENTION): We reviewed the number and content of the telephone visits to understand how residents utilized their telephone visit time. We also reviewed whether there was any prescription medication management, testing ordered, and/or referrals placed as a surrogate for the complexity of the visit.

FINDINGS TO DATE (IT IS NOT SUFFICIENT TO STATE FINDINGS WILL BE DISCUSSED ): From October 2016-December 2018, there were 273 telephone visits scheduled, of which 152 (54.7\%) were "completed", meaning the resident reached the patient and documented a note. The remainder of the appointments were categorized as a "no-show", either because the patient could not be reached ( 92 visits) or because there was no documentation about the visit ( 29 visits). The three most common reasons for visits were blood pressure check (74 visits), symptom reevaluation after an office visit (61 visits), and diabetes management (55 visits). Visit types that were most likely to be completed were patient-initiated visits (16 scheduled; $87.5 \%$ completed), symptom reevaluation visits (61 scheduled; $70.5 \%$ completed), and abnormal results review (18 scheduled; $66.7 \%$ completed). Of all completed visits, 48 (31.6\%) included prescription medication management, $12(7.9 \%)$ included ordering of laboratory or imaging testing, and $17(11.2 \%)$ included referrals to other providers.

KEY LESSONS FOR DISSEMINATION (WHAT CAN OTHERS TAKE AWAY FOR IMPLEMENTATION TO THEIR PRACTICE OR COMMUNITY?): Residents used telephone visits for a wide variety of reasons. Over half of the completed visits included prescription medication management, lab/imaging ordering, or referrals, suggesting these visits were relatively complex and may have replaced the need for in-person care. The completion rate for telephone visits was similar to the in-person show rate in our clinic of $60 \%$. We hope to improve this completion rate through resident and patient expectation setting; limiting telephone visits to specific appointment types might also be beneficial. We found inconsistent documentation practices among residents and now reinforce documentation requirements periodically. We did not bill for telephone visits, but we do plan to do so as reimbursement opportunities for telehealth services become available.

BRIDGING THE GAP: A SUCCESSFUL INPATIENT-BASED CONTINUITY AND CARE COORDINATION INTERVENTION FOR HIGH RISK HOSPITAL HIGH UTILIZERS

Kirstin Knox ${ }^{1}$; Jessica Schneider ${ }^{1}$; Heather Charboneau ${ }^{1}$; Todd Hecht ${ }^{2}$. ${ }^{1}$ Hospital of the University of Pennsylvania, Philadelphia, PA; ${ }^{2}$ University of Pennsylvania, Philadelphia, PA. (Control ID \#3183903)

STATEMENT OF PROBLEM OR QUESTION (ONE SENTENCE): Patients who are frequently admitted to the hospital are a highly vulnerable population, facing medical, social, and behavioral health challenges that are compounded by discontinuity between hospital admissions and care settings; this discontinuity leads to unnecessary testing, ineffective/ inconsistent treatment plans, patient/provider frustration, and inability to address the underlying issues that drive repeat hospitalization.

OBJECTIVES OF PROGRAM/INTERVENTION (NO MORE THAN THREE OBJECTIVES): For patients with the highest inpatient utilization on our general medicine service, our objectives were to 1) increase continuity and care coordination across care settings, 2) identify and address the underlying drivers of hospital utilization, and 3) reduce unnecessary health care utilization.

DESCRIPTION OF PROGRAM/INTERVENTION, INCLUDING ORGANIZATIONAL CONTEXT (E.G. INPATIENT VS. OUTPATIENT, PRACTICE OR COMMUNITY CHARACTERISTICS): We designed and implemented a successful multidisciplinary intervention aimed at breaking the cycle of high utilization for the most frequently admitted patients on our service. A continuity team, including a hospitalist physician, nurse, and social worker, is assigned to each patient. With guidance from a multidisciplinary committee, this continuity team partners with the patient, caregivers, and providers to a) develop and implement an individualized longitudinal care plan targeting the biopsychosocial drivers of healthcare utilization, and b) coordinate and streamline care across home, outpatient, emergency, and inpatient settings. To complement this full intervention, we recently developed more targeted behavioral plans that can be rapidly implemented in situations where consistency is essential to patient care and safety. MEASURES OF SUCCESS (DISCUSS QUALITATIVE AND/OR QUANTITATIVE METRICS WHICH WILL BE USED TO EVALUATE PROGRAM/INTERVENTION): Measures of inpatient utilization including hospital admissions, 30-day readmissions, total hospital days, average length of stay, and emergency department visits.

FINDINGS TO DATE (IT IS NOT SUFFICIENT TO STATE FINDINGS WILL BE DISCUSSED ): We have enrolled 47 patients since 2015, and now maintain a 20-25 patient active rolling census. For patients enrolled in our full intervention, six month pre/post analysis demonstrates reduction in admissions, total hospital days, ED visits, and thirty-day readmissions of $52 \%, 40 \%, 43 \%$, and $64 \%$, respectively, with minimal change in LOS ( $n=26$ patients with complete six month follow up). Twelve month pre/post data demonstrate a sustained effect, including reduction in thirty-day readmissions of $58 \%(n=21)$. For patients enrolled in our targeted behavioral plan intervention, six month pre/post analysis demonstrates modest decreases in admissions, LOS, and ED visits (21-28\%), while total hospital days decrease $40 \%$ after plan implementation $(\mathrm{n}=5)$.

KEY LESSONS FOR DISSEMINATION (WHAT CAN OTHERS TAKE AWAY FOR IMPLEMENTATION TO THEIR PRACTICE OR COMMUNITY?): A multidisciplinary and highly individualized approach is essential to developing successful interventions for patients with the highest hospital utilization. The program's greatest strengths lie in our ability to build trust, create consensus among providers, advocate for large scale interventions, and provide continuity and coordination over time and across settings. Investing in a core team to provide extensive care coordination both during and between admissions is key to program success.

BRIDGING THE GAP: THE IMPACT OF AN INPATIENT MEDICAL SUBSTANCE USE GROUP ON KNOWLEDGE GAINS AND OUTPATIENT MAT FOLLOW-UP AMONG OPIOID-USING IV ANTIBIOTIC PATIENTS

Jessica Farrar $^{5}{ }^{6}$; Michelle Gaffaney ${ }^{1,3}$; Tess M. Kilwein ${ }^{1,}$; Sharnay Brown ${ }^{1,4}$. ${ }^{1}$ Denver Health Medical Center, Glendale, CO; ${ }^{2}$ University of Wyoming, Laramie, WY; ${ }^{3}$ University of Colorado Anschutz Medical Center, 
Denver, CO; ${ }^{4}$ University of Colorado School of Medicine, Denver, CO; ${ }^{5}$ Veteran Affairs Salt Lake City Health Care System, Salt Lake City, UT; ${ }^{6}$ University of Utah, Salt Lake City, UT. (Control ID \#3177813)

\section{STATEMENT OF PROBLEM OR QUESTION (ONE SENTENCE):} How do we improve integrated, comprehensive addictions treatment and continuation of care for opioid users inducted on MAT in hospital settings? OBJECTIVES OF PROGRAM/INTERVENTION (NO MORE THAN THREE OBJECTIVES): 1. Improve integrated, comprehensive treatment of addiction in a hospital setting; 2. Increase immediate and longterm outpatient MAT follow-up rates; 3 . Improve continuity of care between inpatient and outpatient settings

DESCRIPTION OF PROGRAM/INTERVENTION, INCLUDING ORGANIZATIONAL CONTEXT (E.G. INPATIENT VS. OUTPATIENT, PRACTICE OR COMMUNITY CHARACTERISTICS): Inpatient medical patients inducted on medication-assisted treatment (MAT) by an addictions consult team at an urban hospital in the Mountain West are invited to participate in a substance use group during their IV antibiotics hospitalization. In addition to their MAT maintenance, patients participate in the weekly, 1-hour group covering topics of psychoeducation, mindfulness, and motivational enhancement to provide more comprehensive treatment during their hospitalization.

MEASURES OF SUCCESS (DISCUSS QUALITATIVE AND/OR QUANTITATIVE METRICS WHICH WILL BE USED TO EVALUATE PROGRAM/INTERVENTION): At both baseline and before discharge, patients are administered a questionnaire assessing knowledge about dual diagnosis conditions (DD) and MAT, outpatient follow-up plans, and intentions to follow-up with MAT after discharge. Immediate (i.e., day after discharge) and long-term (i.e., 6 months after immediate follow-up) outpatient MAT follow-up rates are obtained from chart review and contacting patients/outpatient providers.

FINDINGS TO DATE (IT IS NOT SUFFICIENT TO STATE FINDINGS WILL BE DISCUSSED ): Thus far, $N=11$ patients have participated in the group and discharged from the hospital after completing their IV antibiotics treatment. Discharge questionnaires were not obtained from 2 of these patients. While sample size remains low for reliable data analyses, the remaining 9 patients have demonstrated preliminary significant gains in knowledge about addiction/substance use $(t=2.63, p=.030)$, mental health ( $t=5.12, p=.001)$, co-occurring disorders $(t=2.53, p=.035)$, and mindfulness $(t=4.0, p=.004)$. In addition, all 11 patients were able to identify a follow-up plan at discharge and immediate outpatient MAT followup has been confirmed for 9 of these patients (81.8\%). With ongoing data collection and historical data review, we hypothesize additional significant differences in immediate and long-term (i.e., 6 month) outpatient follow-up rates in comparison to historical patients who did not receive the group intervention.

KEY LESSONS FOR DISSEMINATION (WHAT CAN OTHERS TAKE AWAY FOR IMPLEMENTATION TO THEIR PRACTICE OR COMMUNITY?): Long-term hospitalizations for opioid users completing IV antibiotics are often costly and rarely address the underlying etiology of the admission. In addition, many hospitals struggle with keeping these patients in the hospital, as few initiate MAT, let alone provide addiction treatment during hospitalizations. However, integrated healthcare systems are well positioned to provide brief psychosocial treatments for these patients. Provision of a group intervention may be an effective strategy in increasing relevant knowledge and immediate follow- up to outpatient rates among this population, ultimately improving the continuity of care for patients and saving in healthcare costs.
BRINGING HOME PRIMARY CARE TO EVERYONE: A NURSE PRACTITIONER CONSULT MODEL

Anita B. Leon-Jhong ${ }^{1}$; Susan R. Saxon ${ }^{1}$; Cynthia L. Wilson ${ }^{2}$. ${ }^{1}$ UPMC, Pittsburgh, PA; ${ }^{2}$ UPMC Community Provider Services, Pittsburgh, PA. (Control ID \#3184540)

STATEMENT OF PROBLEM OR QUESTION (ONE SENTENCE): Many PCPs do not have the time, training or inclination to provide home visits, even as their patients may have difficulty travelling to the office.

OBJECTIVES OF PROGRAM/INTERVENTION (NO MORE THAN THREE OBJECTIVES): 1. Provide high-quality medical assessments to frail and homebound patients in collaboration with their PCPs. 2. Increase the program's impact by identifying what types of visits are most effective.

DESCRIPTION OF PROGRAM/INTERVENTION, INCLUDING ORGANIZATIONAL CONTEXT (E.G. INPATIENT VS. OUTPATIENT, PRACTICE OR COMMUNITY CHARACTERISTICS): The UPMC Home Primary Care program is a consult-style service which is staffed by the UPMC Advanced Practice Provider group. This group includes six nurse practitioners (NP) who cover distinct geographic regions of Pittsburgh. When a UPMC PCP identifies a patient who could benefit from a home visit, a referral order is placed and the patient is scheduled with a nurse practitioner within 14 weeks. Common referrals include need for face-to-face (F2F) visit to obtain durable medical equipment (DME) or home health services, management of chronic medical conditions, and evaluation of home safety and support systems. The nurse practitioners have expertise in home-based care, palliative care and community services. The nurse practitioner documents all findings, including physical exam, medication reconciliation and any recommendations, in the electronic medical record. The PCP then decides how to implement changes based on the NP recommendations. Visits are billed through the patient's insurance and patients pay the same co-pay as for a primary care office visit.

MEASURES OF SUCCESS (DISCUSS QUALITATIVE AND/ OR QUANTITATIVE METRICS WHICH WILL BE USED TO EVALUATE PROGRAM/INTERVENTION): The following information is collected via chart review for all visits. Data will be used to better understand the type of patient we are serving in order to target referrals. We also hope to capture the impact of each visit by recording how often measurable tasks are performed. 1. Patient age, sex 2. Date of last PCP visit 3. Hospitalization in last 6 months 4. F2F documented for DME/home health 5. Medication issue identified (patient not taking medication properly, adverse effect) 6. Goals of care discussed, hospice referral Future measures which are not currently being tracked include: 1 . Referral to community service 2 . Recommendation of new medication

FINDINGS TO DATE (IT IS NOT SUFFICIENT TO STATE FINDINGS WILL BE DISCUSSED ): In 2018, 237 home visits were conducted on 157 unique patients. The patients were predominantly female $(61 \%)$ with median age of 79 years. $41 \%$ of patients had been hospitalized in the 6 months prior to the visit. 13 patients were deceased within 1 year of the visit. The median time since last PCP encounter was 140 days, with $17 \%$ of visits occurring on patients who had not seen their PCP in $>1$ year. $29 \%$ of visits included F2F documentation for DME/home health. In $24 \%$ of visits a significant medication issue was identified. Advanced care planning was documented in $8 \%$ of visits and 8 patients were referred to hospice. 
KEY LESSONS FOR DISSEMINATION (WHAT CAN OTHERS TAKE AWAY FOR IMPLEMENTATION TO THEIR PRACTICE OR COMMUNITY?): 1 . The home visit is an ideal way to connect with frail individuals and the nurse practitioner consult model expands access to this service 2. Key services performed during a visit include F2F documenation for DME/home health, identification of medication issues and advanced care planning.

BUILDING A PRIMARY CARE COLLABORATION BETWEEN A COMMUNITY MENTAL HEALTH CENTER AND SAFETY NET MEDICAL CENTER: A QUALITY IMPROVEMENT APPROACH

Bijan Ghaffari ${ }^{1}$; michelle cleeves ${ }^{2} .{ }^{1}$ University of Colorado, Denver, CO; ${ }^{2}$ Denver Health, Denver, CO. (Control ID \#3185551)

\section{STATEMENT OF PROBLEM OR QUESTION (ONE SENTENCE):}

How do we improve access to and engagement in primary care for patients with severe mental illness (SMI)?

OBJECTIVES OF PROGRAM/INTERVENTION (NO MORE THAN THREE OBJECTIVES): 1. Increase enrollment and engagement for patients with SMI in a co-located primary care clinic. 2. Implement referral and communication guidelines between a community mental health center (CMHC) and primary care clinic to optimize access and utilization. DESCRIPTION OF PROGRAM/INTERVENTION, INCLUDING ORGANIZATIONAL CONTEXT (E.G. INPATIENT VS. OUTPATIENT, PRACTICE OR COMMUNITY CHARACTERISTICS): Noting the disparity in mortality and morbidity for patients with SMI compared to the general population, Denver Health and the Mental Health Center of Denver (MHCD) collaborated on a primary care clinic (PCC at MHCD) to improve primary care access for MHCD clients. Co-location within the MHCD clinic reduces barriers such as travel and unfamiliarity for patients needing primary care. Both organizations are safety net care systems serving Denver. 79\% of MHCD's clients reported having no PCP, despite having the PCC at MHCD in the same building. An assessment of access barriers was performed through chart review, reimbursement data, provider surveys, and interviews. The first intervention cycle used reimbursement data to target clients of both organizations that had lapses in primary care and encouraged reengagement. The next intervention includes developing and disseminating training materials for case managers to promote best practices for supporting engagement in primary care.

MEASURES OF SUCCESS (DISCUSS QUALITATIVE AND/OR QUANTITATIVE METRICS WHICH WILL BE USED TO EVALUATE PROGRAM/INTERVENTION): Monthly reports allow us to monitor patients with lapses in care at the PCC at MHCD. The outreach intervention is evaluated through quantitative measures and countermeasures, including patient contact, agreement to schedule, and attendance of appointments. MHCD staff participated in a pre-intervention survey to elicit practices, beliefs, and challenges with primary care engagement. A similar survey will be distributed after the training intervention to evaluate changes in perceptions and practices.

FINDINGS TO DATE (IT IS NOT SUFFICIENT TO STATE FINDINGS WILL BE DISCUSSED ): Survey responses of MHCD staff revealed that a majority believed primary care would benefit their patients, yet there was variability in practices. Identified challenges include lack of knowledge, navigation of healthcare systems, and higher priority given to psychosocial factors. From September to November, 18 PCC at MHCD patients ( $4 \%$ of clinic empanelment) had lapses in primary care longer than 9 months. Direct patient outreach resulted in 7 patients scheduled, and an
$86 \%$ attendance rate. Of 27 emails sent to notify case managers of engagement lapses, 9 patients were scheduled, and $56 \%$ of appointments were attended. A focus group identified best practices that will be compiled into training materials for case managers. In collaboration with MHCD leadership, PCC at MHCD will have formal orientations with new case managers. KEY LESSONS FOR DISSEMINATION (WHAT CAN OTHERS TAKE AWAY FOR IMPLEMENTATION TO THEIR PRACTICE OR COMMUNITY?): This project has illuminated the logistic challenges of an interinstitutional collaboration and the cultural differences between a large medical center and a CMHC. The rapid cycles of QI allow for brief interventions that target the highest need patients while also improving the partnership between organizations.

BUILDING COMMUNITIES AND ALLEVIATING BARRIERS: TRAUMA SENSITIVE YOGA FOR FEMALE IMMIGRANTS IN THE PRIMARY CARE SETTING

Katherine Rizzolo $^{1}$; Sean Lena ${ }^{1}$; Maha Jaber ${ }^{2,}{ }^{1}$; Adriana Eurich ${ }^{2,1}$; Diana Stade $^{2}$; Brian King ${ }^{1}$; Lesley Gordon ${ }^{1} .{ }^{1}$ Maine Medical Center, Portland, ME; ${ }^{2}$ Tufts University School of Medicine, Boston, MA. (Control ID \#3185631)

STATEMENT OF PROBLEM OR QUESTION (ONE SENTENCE): There are significant barriers and limitations to the wellness resources currently available to the immigrant communities in Portland, Maine.

OBJECTIVES OF PROGRAM/INTERVENTION (NO MORE THAN THREE OBJECTIVES): Improve healthcare delivery to immigrants by alleviating barriers to care Improve social isolation of female immigrants through community based programming Integrate community resources for immigrants into the primary care setting

DESCRIPTION OF PROGRAM/INTERVENTION, INCLUDING ORGANIZATIONAL CONTEXT (E.G. INPATIENT VS. OUTPATIENT, PRACTICE OR COMMUNITY CHARACTERISTICS): Maine Medical Center's Internal Medicine Resident Clinic serves a large number of Southern Maine's immigrant population. For many, traditional services such as physical therapy, nutrition, and counseling carry logistical obstacles such as cost, lack of transportation, and unmet childcare needs. Our pilot program is a novel community trauma-sensitive yoga program for immigrant women. The program is low cost (sliding scale) with transportation and childcare included. Women from Iraq, Syria, and Somalia were selected for participation due to their underrepresentation in traditional referral based programs given the aforementioned barriers as well as significant social isolation in the community. A thorough review of community resources indicated this group is underrepresented in nonclinical community programming. A patient focus group of Arabic-speaking women noted the exact issues of social isolation and barriers to attendance noted by providers. Participants were self-referred from our outpatient resident clinic via providers and social workers. Inclusion criteria included women over age 18 and history of immigration.

MEASURES OF SUCCESS (DISCUSS QUALITATIVE AND/OR QUANTITATIVE METRICS WHICH WILL BE USED TO EVALUATE PROGRAM/INTERVENTION): Participants of one or more class completed an anonymous post-intervention survey focused on barriers to class attendance and feedback on how the class affected their pain and quality of life.

FINDINGS TO DATE (IT IS NOT SUFFICIENT TO STATE FINDINGS WILL BE DISCUSSED ): The first session had 12 total participants from Iraq, Djibouti, Afghanistan, and Somalia. $40 \%$ had barriers to attendance including transportation and childcare. $100 \%$ of participants felt 
the class alleviated their physical and/or emotional pain. 100\% reported they would sign up for another class.

KEY LESSONS FOR DISSEMINATION (WHAT CAN OTHERS TAKE AWAY FOR IMPLEMENTATION TO THEIR PRACTICE OR COMMUNITY?): Our immigrant community experiences deep suffering, which encompasses physical and emotional pain from the traumas they endured in their home country, the loss of their homeland, and the many struggles of adapting to a foreign country and culture. Unlike traditional referral-based models, a community-based model offers much more than relief from physical pain. Most participants found a sense of community and improvement in social isolation. In addition, elimination of barriers such as childcare and transportation issues increased participation and enjoyment of the class. This is an opportunity to recognize the benefits of community-based programming in treating patients of significant trauma.

\section{BURNOUT AMONG APPS}

Brianna C. Pearson; Tareq Islam; Ana Everwine. Wake Forest Baptist Health, Winston Salem, NC. (Control ID \#3184549)

\section{STATEMENT OF PROBLEM OR QUESTION (ONE SENTENCE):}

Are APPs (advanced practice provider) burning out and if so, why?

OBJECTIVES OF PROGRAM/INTERVENTION (NO MORE THAN THREE OBJECTIVES): Evaluating the prevalence of APP burnout and how stress, job satisfaction, and workplace factors contribute to burnout.

DESCRIPTION OF PROGRAM/INTERVENTION, INCLUDING ORGANIZATIONAL CONTEXT (E.G. INPATIENT VS. OUTPATIENT, PRACTICE OR COMMUNITY CHARACTERISTICS): AMA's validated 10 question Mini-Z survey was adapted to RedCap and distributed via email to approximately 550 APPs (NP, PA, CRNA, and $\mathrm{CNM}$ ) at Wake Forest Baptist Health (inpatient and outpatient). Information collected include self-reported data on demographic variables (sex, title, setting, specialty, and years in role), subjective feeling of burn out at work and nine work place factors (stress, job satisfaction, work control, environment, teamwork, value alignment with leadership, documentation time, home EMR use, and EMR proficiency) on a Likert scale of 5 (strongly disagree to strongly agree, poor to optimal).

MEASURES OF SUCCESS (DISCUSS QUALITATIVE AND/OR QUANTITATIVE METRICS WHICH WILL BE USED TO EVALUATE PROGRAM/INTERVENTION): Person correlation coefficient was calculated between burnout category and work related factors. Prevalence of different subjective feeling of burnt out at work and work related factors were calculated among overall and stratified by demographic variables. Likelihood ratio was calculated to measure significance of difference. FINDINGS TO DATE (IT IS NOT SUFFICIENT TO STATE FINDINGS WILL BE DISCUSSED ): Total 229 surveys were returned and analyzed. $38.9 \%$ APP feels burnt out at work. This prevalence is higher among NP (44.4\%) compared to PA (33.3\%), but not statistically significant (p-value 0.09). No difference exists by gender, clinical setting (inpatient vs. outpatient). Interestingly APP with $>5$ years of experience $(48.5 \%)$ felt more burnt out than others (0-2 and 3-5 years, $27.1 \%$ and $35.7 \%$ respectively, p-value 0.01 ) and also felt that the care team works poorly together. Self reported work place burn out is highest among APP working in internal medicine (48.2\%) and lowest among Surgery APP (19.4\%) (p-value 0.04). Job satisfaction, stress, control of workload, efficient documentation, hectic work atmosphere, professional values, team work, and EHR efficiency were significantly associated with burn out at work (Pearson co-efficient value of $-0.53,+0.52,-0.53,-0.37,+0.43,-0.40,-0.45,+0.25$ respectively with $\mathrm{p}-$ value $<0.001$ for all). $18.3 \%$ NPs perceive poor control over workload compared to $8.8 \%$ among PAs (p-value 0.03 ). Statistically significant more inpatient APPs feels work atmosphere is hectic and they also spent excessive time in electronic health record (EHR) at home compared to outpatient APP.

KEY LESSONS FOR DISSEMINATION (WHAT CAN OTHERS TAKE AWAY FOR IMPLEMENTATION TO THEIR PRACTICE OR COMMUNITY?): Healthcare has been evolving into a more teambased system in order to help share the burden of a growing patient population which at the same time, demands high quality and timely care. Advanced Practice Providers (APPs) stand at the nucleus of this model and face many of the same obstacles and stress levels as their physician colleagues. However, there is very little research to date on APP burnout. Our survey showed that the prevalence of work place burnout is high among APPs.

CALL ME MAYBE? IMPROVING UTILIZATION OF TELEMEDICINE IN A VA RESIDENT CLINIC

Kaleb Keyserling ${ }^{1}$; Jared Huber ${ }^{1}$; Carol Sprague ${ }^{2,}{ }^{1}$. ${ }^{1}$ Oregon Health \& Science University, Portland, OR; ${ }^{2}$ Portland VAMC, Portland, OR. (Control ID \#3149915)

STATEMENT OF PROBLEM OR QUESTION (ONE SENTENCE): Residents have limited exposure to telemedicine, an important tool in providing care for physically and geographically isolated patients.

OBJECTIVES OF PROGRAM/INTERVENTION (NO MORE THAN THREE OBJECTIVES): 1 . Increase resident exposure to telemedicine, a valuable health care tool. 2. Improve access to care for the veteran population we serve at the Portland VA

DESCRIPTION OF PROGRAM/INTERVENTION, INCLUDING ORGANIZATIONAL CONTEXT (E.G. INPATIENT VS. OUTPATIENT, PRACTICE OR COMMUNITY CHARACTERISTICS): With rapid improvements in technology, telemedicine is becoming common place in modern medical practice, allowing patients quicker and more convenient access to health care and closer follow-up with providers. Residents have little training on utilizing telemedicine. The Portland VA Resident and Faculty Practice offers a unique opportunity for residents to schedule follow-up telephone appointments with patients. Residents were first offered the opportunity to schedule phone appointment at our clinic in 2013. Appointments were originally scheduled in half day blocks, once every three months. A review of the phone clinic data showed that only $18 \%$ of available resident phone appointments were utilized. A resident survey showed opportunity to improve general knowledge on conducting phone visits as well as multiple scheduling issues hindering utilization. Based on these results, we designed and conducted a phone clinic orientation addressing the practical and clinical aspects of the phone visit, including how to schedule and encounter the visit as well as which patients and issues are appropriate for a phone visit. Residents were given a 30 minute protected time slot for phone visits each clinic week and a template was created to facilitate documentation.

MEASURES OF SUCCESS (DISCUSS QUALITATIVE AND/OR QUANTITATIVE METRICS WHICH WILL BE USED TO EVALUATE PROGRAM/INTERVENTION): The number of phone appointments completed per month will be tracked on a run chart as a process measure. Repeat surveys we will be used to assess resident understanding of phone clinic and the scheduling process as our main outcome measures. We will track residents' enthusiasm to keep using phone clinic as a balancing measure. 
FINDINGS TO DATE (IT IS NOT SUFFICIENT TO STATE FINDINGS WILL BE DISCUSSED ): After six months, the number of phone visit encounters increased by $114 \%$ (120 vs 56 appointments) when compared to the same time interval the previous year. Repeat survey results showed residents had improved knowledge of when appointments were scheduled ( $80 \%$ vs $57 \%$ were able to see their schedule in advance). $84 \%$ of residents indicated they plan to utilize the phone clinic going forward. We have identified additional opportunities for improvement. Future plans include working with providers and administrative staff to further streamline the scheduling process, and developing a curriculum to improve comfort with conducting a visit over the phone. Through these changes we hope to continue to increase phone clinic utilization and better serve our patients' healthcare needs.

KEY LESSONS FOR DISSEMINATION (WHAT CAN OTHERS TAKE AWAY FOR IMPLEMENTATION TO THEIR PRACTICE OR COMMUNITY?): Using simple quality improvement methodology, we have dramatically increased resident participation in telephone clinic with continued enthusiasm for the project. Through doing so, we have increased resident exposure to a valuable health care tool that is likely to remain a vital component of primary care going forward.

CAN CONDITION-SPECIFIC EDUCATIONAL VIDEOS ACTIVATE PATIENTS PREPARING FOR DISCHARGE?

Meghan L. Rudder ${ }^{1}$; Theresa Fuller ${ }^{1}$; Nicholas Piniella ${ }^{1}$; Catherine Yoon ${ }^{1}$; Stuart R. Lipsitz ${ }^{2}$; Jeffrey L. Schnipper ${ }^{2}$; Anuj K. Dalal ${ }^{2}$. ${ }^{1}$ Brigham and Women's Hospital, Brookline, MA; ${ }^{2}$ Brigham and Women's Hospital, Boston, MA. (Control ID \#3182281)

STATEMENT OF PROBLEM OR QUESTION (ONE SENTENCE): At discharge, care responsibilities shift from clinician to patient; yet due to rising volume of care transitions, shorter hospital stays, increasing demands on physician time, lack of standardized educational content, and poor patient comprehension, many patients are ill-prepared for this transition.

OBJECTIVES OF PROGRAM/INTERVENTION (NO MORE THAN THREE OBJECTIVES): Activated patients-those with the knowledge, confidence, and skills to manage their health-are more likely to execute a discharge plan. We hypothesized that educational discharge videos may increase patient activation. Our goals were to determine: the acceptability and feasibility of implementing condition-specific educational discharge videos for general medicine inpatients, and whether video education is associated with patient activation.

DESCRIPTION OF PROGRAM/INTERVENTION, INCLUDING ORGANIZATIONAL CONTEXT (E.G. INPATIENT VS. OUTPATIENT, PRACTICE OR COMMUNITY CHARACTERISTICS): We conducted a sub-study of patients enrolled in the Patient-centered Discharge Toolkit (PDTK) study on general medical units at an academic medical center in Boston, MA. Patients were eligible if they had a discharge diagnosis for which one of 19 condition-specific educational videos (Telesofia Medical, Inc) was available. Patients who agreed to participate were invited to view the video on a tablet before discharge. All patients (viewers and non-viewers) were asked to complete a 13-Item Patient Activation Measure survey (PAM-13, Insignia Health) at discharge and during a 30-day follow-up call.

MEASURES OF SUCCESS (DISCUSS QUALITATIVE AND/OR QUANTITATIVE METRICS WHICH WILL BE USED TO EVALUATE PROGRAM/INTERVENTION): We analyzed demographic and clinical data and compared PAM scores at, and 30 days after, discharge as well as 30-day readmission rates between viewers and non-viewers. We also compared patients who viewed the videos to a principle componentmatched cohort of patients from the PDTK study.

FINDINGS TO DATE (IT IS NOT SUFFICIENT TO STATE FINDINGS WILL BE DISCUSSED ): Of 115 patients screened, 45 (39.1\%) were eligible to participate; of the 36 patients who were approached, 18 $(50.0 \%)$ consented to view the video. Viewers were younger than nonviewers (56.2 versus 68.9 years of age, $\mathrm{P}=0.02$ ) and more likely to be male (72.2\% versus $38.9 \%, \mathrm{p}=0.04)$. Viewers had significantly higher mean PAM scores compared to non-viewers ( 75.5 vs $60.2, \mathrm{p}=0.05)$. A greater proportion of viewers than non-viewers had PAM levels $3(66.7 \%$ vs $38.9 \%, \mathrm{p}=0.09)$. At 30 days, viewers had higher PAM scores than nonviewers ( 71.16 vs $58.9, \mathrm{p}=0.32$ ) and fewer readmissions than non- viewers ( $5.6 \%$ vs $11.1 \%, \mathrm{p}=0.55$ ), but these were not statistically significant.

KEY LESSONS FOR DISSEMINATION (WHAT CAN OTHERS TAKE AWAY FOR IMPLEMENTATION TO THEIR PRACTICE OR COMMUNITY?): 1 Many general medicine patients are willing to watch condition-specific educational videos at discharge, but a video specific to the clear, accurate discharge diagnosis must be available. 2 Videos were more acceptable to younger and male patients which may be due to greater comfort with technology, presence of a single discharge diagnosis, or lack of clarity in relevant diagnoses for older patients with co-morbidities. 3 Viewing condition-specific discharge videos may increase patient activation, or more activated patients may elect to view these videos. 4 Future studies should evaluate pre- and post-intervention PAM scores and outcomes for patients randomized to condition-specific videos.

CAN PATIENTS HUMANIZE THEIR ELECTRONIC HEALTH RECORD TO FACILITATE STRONGER CONNECTIONS WITH CARE TEAMS?

Jeana Holt $^{1}$; Rachel Cusatis ${ }^{2}$; Onur Asan ${ }^{3}$; Joni S. Williams ${ }^{2}$; Kathryn E. Flynn $^{2}$; Bradley H. Crotty ${ }^{1} .{ }^{1}$ Medical College of Wisconsin, Milwaukee, WI; ${ }^{2}$ Medical College of wisconsin, Milwaukee, WI; ${ }^{3}$ Stevens Institute of Technology, Hoboken, NJ. (Control ID \#3185284)

\section{STATEMENT OF PROBLEM OR QUESTION (ONE SENTENCE):} Electronic health records (EHR) contain lists of medications, problems, and other healthcare transactions, but the representation of who an individual patient is, their context, and their preferences are poor.

OBJECTIVES OF PROGRAM/INTERVENTION (NO MORE THAN THREE OBJECTIVES): Empower patients to provide their contextual data (needs, values, preferences, and barriers) to their care team through the EHR. Provide tools for care teams to more efficiently develop meaningful connections with patients.

DESCRIPTION OF PROGRAM/INTERVENTION, INCLUDING ORGANIZATIONAL CONTEXT (E.G. INPATIENT VS. OUTPATIENT, PRACTICE OR COMMUNITY CHARACTERISTICS): We implemented a web-based consumer informatics application that creates a patient profile of contextual data using questions derived from communications research. The application was tested in five primary care clinics where patients were invited by email ahead of visits. Patients provide information about "My Self," "My Health," and "My Care" through freetext and checklist entries related to their health, preferences, care, and agenda for their next visit. Data are summarized into a one-screen dashboard that is available for view within the EHR.

MEASURES OF SUCCESS (DISCUSS QUALITATIVE AND/OR QUANTITATIVE METRICS WHICH WILL BE USED TO EVALUATE PROGRAM/INTERVENTION): To assess if patients felt empowered and care teams felt more connected, we conducted a qualitative 
study. We interviewed 21 clinicians (physicians, nurses, assistants, and pharmacists) and conducted 6 patient-user focus groups $(n=26)$ to assess the adoption and use of the application. We used inductive and deductive qualitative analysis drawn from the Consolidated Framework for Implementation Science (CFIR).

FINDINGS TO DATE (IT IS NOT SUFFICIENT TO STATE FINDINGS WILL BE DISCUSSED ): Over 8,000 patients used the application within the first two years at our pilot locations. Regarding facilitators to adoption, clinicians identified the unique information not otherwise captured; enhanced team-based involvement; and alignment of internal motivators to provide patient-centered care. Barriers were concerns with a potential widening of a digital divide and click fatigue of the EHR. The deductive results spanned four domains and seven constructs of CFIR, including Innovation Source and Compatibility, as critical areas that promoted the use of the application. Patients reported the platform was easy to navigate and provided a new avenue to share sensitive topics. Completion of their profile promoted reflection of their health goals, challenges, and priorities, which led to targeted conversations about issues important to them. Patient adoption was enhanced when clinicians endorsed the application and referenced the data during the visit. Regarding effectiveness, inviting patients, summarizing data in an accessible electronic location, aligning with organizational and personal drivers, and focusing attention beyond physicians can facilitate the adoption of patient-contextual data into care.

KEY LESSONS FOR DISSEMINATION (WHAT CAN OTHERS TAKE AWAY FOR IMPLEMENTATION TO THEIR PRACTICE OR COMMUNITY?): We found the purposeful and integrated technology, perceived improvement in team-based efficiencies, and facilitation of patientcentered care were the primary drivers of clinician adoption. Patients identified creating a space for sensitive topics and driving the alignment of patient, and provider goals were important motivators that enhanced the use of the program.

\section{CARE PLUS: FIVE YEARS OF PROVIDING TEAM BASED CARE TO HIGH NEED HIGH COST PATIENTS. \\ Melanie Martin ${ }^{1}$; Feben Girma ${ }^{2}$; Julia Rushing ${ }^{3} .{ }^{1}$ Wake Forest, Greensboro, NC; ${ }^{2}$ Wake Forest Baptist Medical Center, Winston-Salem, NC; ${ }^{3}$ Wake Forest, Winston Salem, NC. (Control ID \#3184094)}

\section{STATEMENT OF PROBLEM OR QUESTION (ONE SENTENCE):}

Multidisciplinary team-based primary care and care coordination can lead to sustained decrease in hospital admissions and ED utilization for high need high cost patients at an academic safety net practice.

OBJECTIVES OF PROGRAM/INTERVENTION (NO MORE THAN THREE OBJECTIVES): Share data of characteristics of high need high cost patients. Describe an effective multidisciplinary care model for these patients and provide data on decreased hospital utilization after enrollment in program. Share lessons learned

DESCRIPTION OF PROGRAM/INTERVENTION, INCLUDING ORGANIZATIONAL CONTEXT (E.G. INPATIENT VS. OUTPATIENT, PRACTICE OR COMMUNITY CHARACTERISTICS): For the past five years, Care Plus has provided team-based primary care and care coordination for high need high cost patients from academic safety net practices. Patients with high admission or ED rates are enrolled in our care model that includes more frequent clinic visits, home visits, intensive outreach, and a focus on both social determinants of health and mental health MEASURES OF SUCCESS (DISCUSS QUALITATIVE AND/OR QUANTITATIVE METRICS WHICH WILL BE USED TO EVALUATE PROGRAM/INTERVENTION): Describe characteristics of high need high cost patients. Outcome of program is measured in terms of rate of admissions and ED utilization of patients in year prior to enrollment as compared to years after enrollment.

FINDINGS TO DATE (IT IS NOT SUFFICIENT TO STATE FINDINGS WILL BE DISCUSSED ): We have enrolled approximately 360 patients in the program. Characteristics of enrolled patients provide compelling evidence of why such a broad team is needed to optimally serve these patients. Our patients are medically complex with an average of five comorbid conditions and twelve medications at the time of enrollment. Additionally, $69 \%$ have mental health diagnoses, $38 \%$ have substance use issues, and $38 \%$ score less than or equal to 21 on the Montreal Cognitive Assessment, indicating moderate cognitive impairment. From a social determinants of health perspective, our patients have a high prevalence of poverty with related food insecurity, difficulty affording medications and safe housing, and transportation challenges. Through a care model that acknowledges and addresses social determinants of health, more frequent visits, and a focus on mental health, we have demonstrated a decrease in ED utilization and hospital admissions compared to pre-enrollment levels that has persisted over years of enrollment. A comprehensive analysis is underway, but preliminary results show that when we compare admissions the year prior to enrollment to the first year of enrollment, admissions decrease by $49.5 \%$. If analysis is limited to patients that remain in our program for at least 12 months, the decrease in the first year in admissions is $39.5 \%$ and in ED visits is $30.2 \%$, compared to patients' historical selves. We also have shown sustained benefit in enrolled patients, with evidence of ED utilization rates over our population decreasing from a median of $0.41 \mathrm{ED}$ visits per month prior to enrollment to $0.22 \mathrm{ED}$ visits per month after enrollment (47\% reduction).

KEY LESSONS FOR DISSEMINATION (WHAT CAN OTHERS TAKE AWAY FOR IMPLEMENTATION TO THEIR PRACTICE OR COMMUNITY?): The challenge of caring for high need high cost patients can be mitigated through multidisciplinary team-based primary care. Improving patient engagement by addressing social determinants of health and mental health are key to solving this challenge.

\section{CHICAGO STREET MEDICINE}

Frederick T. Gibson ${ }^{1}$; Karim G. Khan² ${ }^{2}$ Joshua Smith ${ }^{2}$; Melissa A. Socarras²; Brian Borah ${ }^{2}$; Sarah Messmer ${ }^{2} .{ }^{1}$ University of Illinois College of Medicine, Chicago, IL; ${ }^{2}$ University of Illinois, Chicago, IL. (Control ID \#3185971)

STATEMENT OF PROBLEM OR QUESTION (ONE SENTENCE): In light of inequities in healthcare provision and outcomes for the unsheltered homeless, there is a need for holistic care that will provide seamless integration between outpatient, street-based, and inpatient hospital care.

OBJECTIVES OF PROGRAM/INTERVENTION (NO MORE THAN THREE OBJECTIVES): Create an academic model of street medicine which incorporates students and residents from multiple disciplines in leadership roles. Develop and implement a street medicine system to provide healthcare services and social support to unsheltered homelessness as they transition between traditional avenues of healthcare and street-based care.

DESCRIPTION OF PROGRAM/INTERVENTION, INCLUDING ORGANIZATIONAL CONTEXT (E.G. INPATIENT VS. OUTPATIENT, PRACTICE OR COMMUNITY CHARACTERISTICS): CSM was started by a group of medical students and residents with the goal of improving the well-being of those experiencing unsheltered homelessness in Chicago by performing weekly "street runs" - visiting homeless communities in Chicago to provide medical care, social support, health education, and hygiene essentials. CSM has since expanded to include physicians, 
pharmacy residents, social workers, and occupational therapists. Interventions performed by CSM include wound care, OTC medications, antibiotics, inpatient social support, post-discharge follow-up, suture removal, removal of a PICC line, assistance with housing referrals and documentation, and triage. CSM is uniquely situated to follow and advocate on behalf of patients who are admitted to the hospital, and upon discharge, CSM is able to provide follow-up to a population that was often previously lost to follow-up.

MEASURES OF SUCCESS (DISCUSS QUALITATIVE AND/OR QUANTITATIVE METRICS WHICH WILL BE USED TO EVALUATE PROGRAM/INTERVENTION): Qual: 3 case presentations of CSM patients, 1 presentation of medical student leader development Quant: patients cared for, residents participating, students participating, pharmacists participating, chief complaints, interventions

FINDINGS TO DATE (IT IS NOT SUFFICIENT TO STATE FINDINGS WILL BE DISCUSSED ): A 37yo W who was initially found with rigors and brought to the hospital where she was found to have fungemia, infected pleural effusions and DVTs. Over the course of 1.5 years, a therapeutic relationship has been developed. She has required multiple admissions for SSTIs and fluid overload, but has not left AMA once. We are helping her obtain a new ID. She continues to struggle with addiction but has progressed from contemplation to the preparation stage. A 54yo $\mathrm{M}$ who presented with a chronic arm wound from IVDU. He underwent surgical debridement. CSM continued close wound care upon discharge while also maintaining a link between the patient and social work. He was provided housing via a hospital based housing first initiative. Quant findings: 3 physicians, 13 residents, $40+$ student volunteers, $30+$ street runs, 15 different locations

KEY LESSONS FOR DISSEMINATION (WHAT CAN OTHERS TAKE AWAY FOR IMPLEMENTATION TO THEIR PRACTICE OR COMMUNITY?): Student-fueled academic street medicine is a replicable model that nurtures leadership and the professional development of students and residents. Street medicine has a role in outpatient and inpatient settings of care. Through an interdisciplinary construct, led by medical students, resident physicians, and resident pharmacists, CSM presents an innovative way to advocate for and provide care to the unsheltered homeless everywhere from the tentside to the bedside.

CLINICAL DECISION SUPPORT TO MITIGATE RISKS ASSOCIATED WITH HIGH-DOSE OPIOID USE FOR CHRONIC NONCANCER PAIN

Eboni Price-Haywood; Jeffrey Burton; Todd Burstain. Ochsner Health System, New Orleans, LA. (Control ID \#3184912)

STATEMENT OF PROBLEM OR QUESTION (ONE SENTENCE): Clinical practice guidelines recommend what to do for safe opioid prescribing for chronic non-cancer pain but do not provide guidance on how to implement best practices.

OBJECTIVES OF PROGRAM/INTERVENTION (NO MORE THAN THREE OBJECTIVES): To implement electronic medical record clinical decision support (EMR-CDS) to improve guideline concordant care in ambulatory settings and reduce rates of prescribing high-dose opioids (morphine equivalent daily dose, MEDD) $>90 \mathrm{mg}$.

DESCRIPTION OF PROGRAM/INTERVENTION, INCLUDING ORGANIZATIONAL CONTEXT (E.G. INPATIENT VS. OUTPATIENT, PRACTICE OR COMMUNITY CHARACTERISTICS): In October 2017, Ochsner Health System (Louisiana's largest integrated delivery system) launched EMR-CDS aligned with CDC Practice Guidelines. EMRCDS identifies patients prescribed opioids for the prior 120 days. Providers are prompted to complete the Opioid Risk Tool (ORT) to calculate risk of opioid-aberrant behavior. Patients with at least one of the following conditions are flagged as high-risk: benzodiazepines co-prescriptions; opioid dose $>90$ mg; or diagnosis of substance abuse. The opioid health maintenance tool displays whether patients are up-to-date on best practices. ORT score, MEDD, and hyperlinks to the Louisiana pharmacy drug monitoring program data and pain management agreement are visible in the medication order composer. MEASURES OF SUCCESS (DISCUSS QUALITATIVE AND/OR QUANTITATIVE METRICS WHICH WILL BE USED TO EVALUATE PROGRAM/INTERVENTION): The main outcome is change in percentage of patients with high dose opioid prescriptions.

FINDINGS TO DATE (IT IS NOT SUFFICIENT TO STATE FINDINGS WILL BE DISCUSSED ): The chronic opioid health maintenance tool identified 13,065 patients seen between October 2017 and August 2018 among whom $22 \%$ were prescribed high-dose opioids. Most patients are female $(60 \%)$, white non-Hispanic $(67 \%)$ with a mean age of 57 years. Common pain syndromes include back/neck/knee pain (52\%). Almost $30 \%$ of patients have depression/anxiety while $19 \%$ have a history of substance abuse. Among patients with ORT scores $(n=2040)$ documented, $23 \%$ were medium/high-risk for aberrant behavior. Patients with ORT scores compared to patients without ORT scores have higher rates of pain agreements (38\% vs $8 \%$ ); UDS (26\% vs. $13 \%$ ) and naloxone prescriptions (9\% vs. $1 \%$, all $\mathrm{p}<0.001)$. Overall, while $59 \%$ of patients were screened for depression, $<1 \%$ had PEG-3 (pain) documented. The odds of being prescribed opioids $>90$ MEDD was higher among patients with depression/anxiety (OR [95\% CI]: 1.30 [1.12-1.52] or substance abuse (4.65 [3.99-5.42]); and lower among patients co-prescribed non-opioid pain medications (0.84 [0.73-0.97]) or receiving specialty service $(0.8$ [0.73-0.96]). Among 9,888 patients on chronic opioids before and after launching EMR-CDS, only $6 \%$ had decrease in dosing to $<50$ MEDD.

KEY LESSONS FOR DISSEMINATION (WHAT CAN OTHERS TAKE AWAY FOR IMPLEMENTATION TO THEIR PRACTICE OR COMMUNITY?): EMR-CDS helped providers identify patients who are at risk for opioid misuse and employ mitigation strategies but did not substantially reduce the rate of high-dose opioid prescribing.

\section{CO-PRODUCTION OF VISIT NOTES IN THE ELECTRONIC HEALTH RECORD USING OURNOTES - ONE INSTITUTION'S IMPLEMENTATION}

Stephen K. Liu ${ }^{2}$; Brooke Herndon ${ }^{2}$; Eugene Nelson ${ }^{1}$; Kathryn Sabadosa ${ }^{1}$; Jan Walker ${ }^{4}$; Tom Delbanco ${ }^{3}$. 'Geisel School of Medicine at Dartmouth, Lebanon, $\mathrm{NH} ;{ }^{2}$ Dartmouth-HItchcock, Lebanon, NH; ${ }^{3}$ Beth Israel Deaconess Medical Center, Boston, MA; ${ }^{4}$ BIDMC, Brookline, MA. (Control ID \#3140989)

STATEMENT OF PROBLEM OR QUESTION (ONE SENTENCE): To determine if co-production by patients and providers of visit notes for scheduled office visits is feasible and can improve patient engagement and provider documentation burden at a single site.

OBJECTIVES OF PROGRAM/INTERVENTION (NO MORE THAN THREE OBJECTIVES): Develop a process to implement OurNotes where patients and families can contribute to the writing of their own progress notes before clinic visits by sending recent health history and agenda items before each visit. Assess the program's impact on patient engagement and experience and provider documentation burden from surveys of patients and providers.

DESCRIPTION OF PROGRAM/INTERVENTION, INCLUDING ORGANIZATIONAL CONTEXT (E.G. INPATIENT VS. OUTPATIENT, PRACTICE OR COMMUNITY CHARACTERISTICS): Patient engagement is considered a central tenet of safe, effective, high value care. OpenNotes is an international program that offers patients online access 
to review their visit progress notes. OpenNotes originally was developed at Beth Israel Deaconess Medical Center (BIDMC) and as a next step, the BIDMC team developed a program entitled OurNotes which is a program that invites patients to contribute information online before their office visits, thereby, co-producing their visit notes in the electronic health record. The goal is to have patients complete tasks such as reviewing prior notes, writing an interval health history, and identifying goals for an upcoming visit which could lead to more meaningful patient-doctor interactions, improved patient safety, and decreased documentation burden for doctors. As one of four sites, the General Internal Medicine primary care team at Dartmouth-Hitchcock Medical Center is conducting a pilot test of the OurNotes program using the patient portal through the Epic electronic health record. Patients who participate in the program are sent an invitation a week before a regularly scheduled visit to send an interval history and agenda items before the visit through the secure messaging system. The providers then can review this message and cut and paste the message into the patient's progress note before the visit.

MEASURES OF SUCCESS (DISCUSS QUALITATIVE AND/OR QUANTITATIVE METRICS WHICH WILL BE USED TO EVALUATE PROGRAM/INTERVENTION): We will survey patients and providers about the following outcome measures: patient participation rate, contenof information provided by patients and use of patient information by clinicians during visits.

FINDINGS TO DATE (IT IS NOT SUFFICIENT TO STATE FINDINGS WILL BE DISCUSSED ): While we are in the preliminary stages of implementation, patients that have participated in the OurNotes program find it helpful to send health concerns and agenda items before visits. Providers also find it helpful to know what patients want to address before visits, prioritize agenda items and pend orders before the visit. Visits using OurNotes lead to less time reporting history and transcribing by providers which allows more time for shared decision making and reviewing treatment options.

KEY LESSONS FOR DISSEMINATION (WHAT CAN OTHERS TAKE AWAY FOR IMPLEMENTATION TO THEIR PRACTICE OR COMMUNITY?): Implementation of OurNotes was simple and straightforward using an established patient portal in an electronic health record in a general internal medicine clinic and in selected patients has improved patient experience and engagement and potentially decreases provider documentation burden during office visits.

CREATING AN EMR-BASED DATABASE TO MONITOR ADHERENCE TO THE CDC OPIOID PRESCRIBING GUIDELINE IN PRIMARY CARE PRACTICES

Jaclyn Yamada $^{2}$; Joanna L. Starrels ${ }^{1}$; Anna Flattau ${ }^{1}$; Iby Thomas ${ }^{1}$; Sharon Rikin ${ }^{1}{ }^{1}$ Montefiore - Albert Einstein College of Medicine, Bronx, NY; ${ }^{2}$ Albert Einstein College of Medicine, Bronx, NY. (Control ID \#3185566)

\section{STATEMENT OF PROBLEM OR QUESTION (ONE SENTENCE):}

Primary care practices have a growing need to monitor adherence to guidelines for prescribing opioids for chronic pain, but many have not developed systematic ways to do this using electronic medical record (EMR) data.

OBJECTIVES OF PROGRAM/INTERVENTION (NO MORE THAN THREE OBJECTIVES): To create an EMR-based chronic opioid therapy (COT) database to monitor adherence to the $\mathrm{CDC}$ guideline in primary care settings.

DESCRIPTION OF PROGRAM/INTERVENTION, INCLUDING ORGANIZATIONAL CONTEXT (E.G. INPATIENT VS. OUTPATIENT, PRACTICE OR COMMUNITY CHARACTERISTICS): A team of primary care providers at a large, urban, academic health system in Bronx, NY selected five primary care practices, which share an Epic EMR, to pilot the COT database in 2018. In partnership with a CDC Quality Improvement (QI) Collaborative, the team developed QI metrics corresponding to seven of the CDC Guideline recommendations: regular assessment, evaluation of pain and functional status, avoid concurrent benzodiazepines, discuss risks and benefits, urine drug test, prescription monitoring program (PMP) review, and overdose risk reduction. The COT database and QI metrics were refined based on feasibility of data extraction and feedback from front-line clinicians. MEASURES OF SUCCESS (DISCUSS QUALITATIVE AND/OR QUANTITATIVE METRICS WHICH WILL BE USED TO EVALUATE PROGRAM/INTERVENTION): We judged success as the ability to define the COT population and QI metrics in a way that is reproducible and extractable from the EMR and acceptable to front-line clinicians.

FINDINGS TO DATE (IT IS NOT SUFFICIENT TO STATE FINDINGS WILL BE DISCUSSED ): We identified 408 patients (1.9\% of all patients) that (1) were seen at the clinics within 12 months and (2) were prescribed COT, defined by 4 opioid prescriptions in the last 6 months. Patients were excluded if they received treatment for opioid use disorder. Four of the seven QI metrics were directly extractable from the EMR: regular assessment (office visit $<90$ days), avoid concurrent benzodiazepines (none prescribed in $<6$ months), urine drug test (performed $<12$ months), and overdose risk reduction (naloxone prescribed or dispensed). The other three QI metrics required manual chart review: assessment of pain and functional benefits (PEG scale $<90$ days), discuss risks and benefits (controlled substance agreement $<12$ months), and PMP review (documented verification $<90$ days). The database was shared with front-line clinicians through clinicianand practice-level reports which included the number of patients on COT and completion of QI metrics. Epic EMR enhancements are underway to improve EMR-based capture of the 3 QI metrics that required manual chart review.

KEY LESSONS FOR DISSEMINATION (WHAT CAN OTHERS TAKE AWAY FOR IMPLEMENTATION TO THEIR PRACTICE OR COMMUNITY?): Operationalizing COT guideline recommendations into EMR-based QI metrics requires collaboration and refinement with frontline clinicians, IT analysts, and subject matter experts to understand how primary care practices are documented. EMR-based QI metrics may not fully capture clinical practices. For example, completion of pain and functional assessment was satisfied with a documented PEG scale; however, this may not capture those who met the guideline through another assessment type. This initiative highlighted opportunities for standardization of processes, such as controlled substance agreements. These lessons can help others to create robust EMR-based databases to monitor adherence to COT guidelines.

DECISION SUPPORT AND OFFICE WORKFLOW CHANGES FOR INFLUENZA VACCINATION IN PRIMARY CARE: MEASURED PERFORMANCE AND ACTUALVACCINE ADMINISTRATION

Stephen Persell $^{1,3}$; Ji Young Lee ${ }^{2}$; Nora Lewin ${ }^{2,}{ }^{3}$; Sonali K. Oberoi ${ }^{3}$; EriK Orelind $^{3,1}$; Philip Roemer ${ }^{1,3}$; Michael Schachter ${ }^{3}$; Kathryn Thomas ${ }^{3}$; Banu Yagci ${ }^{3}$. ${ }^{1}$ Northwestern University, Evanston, IL; ${ }^{2}$ Northwestern University, Chicago, IL; ${ }^{3}$ Northwestern Memorial Healthcare, Chicago, IL. (Control ID \#3182720)

STATEMENT OF PROBLEM OR QUESTION (ONE SENTENCE): Influenza vaccination rates are not optimal.

OBJECTIVES OF PROGRAM/INTERVENTION (NO MORE THAN THREE OBJECTIVES): 1) Improve performance on a publically reportable influenza measure, 2) Increase rate of onsite influenza vaccination.

DESCRIPTION OF PROGRAM/INTERVENTION, INCLUDING ORGANIZATIONAL CONTEXT (E.G. INPATIENT VS. 
OUTPATIENT, PRACTICE OR COMMUNITY CHARACTERIS-

TICS): We sought to examine changes in influenza vaccination over three vaccination seasons (2015 to 2018) following decision support improvements and team changes. The setting was 21 adult primary care clinics within a large health system in Chicago, IL and its suburbs. At the start of Season Two, clinical quality leaders and information system experts implemented clinical decision support changes in the electronic health record to facilitate documentation of components related to influenza vaccination (e.g., prior receipt that season, prefers to obtain elsewhere, refusals). Subsequently, they implemented changes to rooming workflow for medical assistants, licensed practical nurses, or registered nurses to promote vaccination at the visit, prepare vaccine orders, document care done elsewhere and record patient refusals. Workflow changes were fully implemented by the start of Season Three.

MEASURES OF SUCCESS (DISCUSS QUALITATIVE AND/OR QUANTITATIVE METRICS WHICH WILL BE USED TO EVALUATE PROGRAM/INTERVENTION): Modified version of performance measure Preventive Care and Screening: Influenza Immunization; proportion of eligible patients receiving onsite influenza vaccination.

FINDINGS TO DATE (IT IS NOT SUFFICIENT TO STATE FINDINGS WILL BE DISCUSSED ): There were approximately 120,000 eligible patients each season and 267 to 298 eligible clinicians. The influenza vaccination performance measure increased substantially each year and was 40.6\% in Season One, 62.5\% in Season Two and 76.4\% in Season Three ( $\mathrm{P}<0.001$ for all changes). Corresponding values for onsite vaccination were $27.7 \%, 28.8 \%$ and $31.5 \%$. The adjusted odds ratio for receiving onsite influenza vaccination in Season Two compared to Season One was 0.94 ( $95 \%$ confidence interval $0.92,0.96$ ). Patients were more likely to receive onsite vaccination in Season Three compared to either of the prior seasons - adjusted odds ratio for Season Three vs. Season Two 1.14 (95\% CI, 1.12, 1.16), adjusted odds ratio for Season Three vs. Season One 1.07 (95\% CI 1.05, 1.09).

KEY LESSONS FOR DISSEMINATION (WHAT CAN OTHERS TAKE AWAY FOR IMPLEMENTATION TO THEIR PRACTICE OR COMMUNITY?): Sequential changes in computerized clinical decision support to make the documentation of elements related to influenza vaccination easier and the addition of new clinical workflows in the patient rooming process were associated with very large improvements in measured performance over two vaccination seasons and with a modest increase in the proportion of patients receiving clinic-administered influenza vaccination. Changes to rooming workflow and the function of care team members appeared to be most important factor for increasing onsite vaccination.

DEMYSTIFYING THE INFLUENZA VACCINE: VIDEO MEDIA IMPROVES VACCINE ACCEPTANCE RATES IN AN UNDERSERVED URBAN RESIDENT-PHYSICIAN PRIMARY CARE CLINIC

Gayatri Malhotra-Gupta $^{3,2}$; Alexandra I. Kreps ${ }^{3,2}$; Lucas Policastro $^{1,2}$; Emmanuel M. Francis $^{1,2}$; Leonel Mendoza ${ }^{1,2}$; Daniel Kashani ${ }^{1,2}$; Andrew A. Chang ${ }^{3,2}$; David L. Stevens ${ }^{4,2}$; Lucas Dreamer ${ }^{3,2}$; Melissa S. Lee ${ }^{1,2} .{ }^{1}$ Kings County Hospital, Brooklyn, NY; ${ }^{2}$ SUNY Downstate Medical Center, Brooklyn, NY; ${ }^{3} \mathrm{NYC}$ Health + Hospitals/Kings County, New York, NY; ${ }^{4} \mathrm{NYC}$ Health + Hospitals/Kings County, Brooklyn, NY. (Control ID \#3186166)

STATEMENT OF PROBLEM OR QUESTION (ONE SENTENCE): Many of our primary care patients are hesitant to accept the influenza vaccine, resulting in a low vaccination rate. Our goal was to increase vaccination from a baseline of $35 \%$ (2017 influenza season) to
50\% during the 2018 influenza season using a resident-made informational video.

OBJECTIVES OF PROGRAM/INTERVENTION (NO MORE THAN THREE OBJECTIVES): - Improve patient health literacy around the influenza vaccine - Identify common reasons for influenza vaccine declination in our patient population - Create and measure the impact of an educational video based on the CDC fact sheet, "No More Excuses: You - Need a Flu Vaccine" on local influenza vaccination rates DESCRIPTION OF PROGRAM/INTERVENTION, INCLUDING ORGANIZATIONAL CONTEXT (E.G. INPATIENT VS. OUTPATIENT, PRACTICE OR COMMUNITY CHARACTERISTICS): Internal Medicine residents at $\mathrm{NYC} \mathrm{H}+\mathrm{H} /$ Kings County produced a 2minute educational video addressing common reasons for flu shot declination. Starring physicians and patient care assistants, the message was delivered by culturally familiar staff. From October to December 2018, patients were asked their reasons for declining influenza vaccination. An educational video was played in the exam room while the resident met with his/her attending preceptor. Subsequently, residents engaged in a shared decision making (SDM) discussion around the risks and benefits of flu vaccination. Patients were then offered the flu vaccine.

MEASURES OF SUCCESS (DISCUSS QUALITATIVE AND/OR QUANTITATIVE METRICS WHICH WILL BE USED TO EVALUATE PROGRAM/INTERVENTION): Vaccine acceptance was recorded both before and after education with a locally produced video and SDM discussion. The intention-to-treat principle was applied in cases where video playback was refused. The primary objective was to measure the conversion rate of initial flu vaccine declination to acceptance following intervention. A secondary objective was to enumerate the frequency of patients' preconceived reasons for flu vaccine refusal.

FINDINGS TO DATE (IT IS NOT SUFFICIENT TO STATE FINDINGS WILL BE DISCUSSED ): The most common reason our patients refused the flu vaccine was, "I don't get the flu." In October, flu vaccine acceptance increased by $16.7 \%$ following the video and SDM intervention; $58.3 \%$ patients received a flu shot. More patients agreed to vaccination in November $(81.25 \%)$ and December (70\%), and 9 out of 23 (39\%) patients who initially declined the flu vaccine changed their mind post-intervention as the flu epidemic hit our community. Consistent use of the educational video and SDM improved uptake of flu vaccination in our population, leading to an increase from a baseline of $35 \%$ in the 2017 season to $72.4 \%$ during our study period.

KEY LESSONS FOR DISSEMINATION (WHAT CAN OTHERS TAKE AWAY FOR IMPLEMENTATION TO THEIR PRACTICE OR COMMUNITY?): After identifying and addressing the prevailing myths of flu vaccination, and locally producing a video to educate patients on the importance of influenza vaccination, the rate of flu vaccination in our patient population increased. This innovative form of patient education using video media during office visit time improved SDM discussions around vaccination. Key components included a tailored, culturally relatable educational platform featuring our patients' healthcare team. We highlight here the importance of using personalized, alternative media as an approach to improving health literacy around preventive health and wellness.

\section{DEVELOPING A DATABASE TO DRIVE AMBULATORY HEALTHCARE INNOVATION}

Lisa Rotenstein $^{1}$; Ashley Shaw ${ }^{2}$; Margaret Krasne $^{2}$; Charles Safran ${ }^{3}$; Sandhya K. Rao ${ }^{4}$ Russell Phillips ${ }^{2}$. ${ }^{1}$ Brigham and Women's Hospital, Boston, MA; ${ }^{2}$ Harvard Medical School, Boston, MA; ${ }^{3}$ Harvard Medical School, Brookline, MA; ${ }^{4}$ Massachusetts General Hospital, Boston, MA. (Control ID \#3185918) 
STATEMENT OF PROBLEM OR QUESTION (ONE SENTENCE):

Mechanisms to share knowledge about ambulatory practice improvement among on-the-ground innovators are lacking, leading to replication and stifling of improvement; we developed and implemented a novel knowledge-sharing platform.

OBJECTIVES OF PROGRAM/INTERVENTION (NO MORE THAN THREE OBJECTIVES): 1) To develop a platform through which physicians can publish and share practical information about improving ambulatory systems of care. 2) To connect clinical innovators conducting similar work across practices to spread innovation. 3) To expose premedical/medical students to principles of ambulatory healthcare improvement.

DESCRIPTION OF PROGRAM/INTERVENTION, INCLUDING ORGANIZATIONAL CONTEXT (E.G. INPATIENT VS. OUTPATIENT, PRACTICE OR COMMUNITY CHARACTERISTICS): $\$ 320$ billion are wasted each year in the US on healthcare systems inefficiency. Although clinicians are constantly improving processes and innovating in how they deliver care, process improvement is often uncompensated and an afterthought. No solution enables on-the-ground clinicians and administrators to share their ambulatory care innovation expertise and to learn from one another. We developed a database for collection of structured information about implementation of healthcare innovations. We subsequently trained student editors to either interview clinicians about implementing new workflows or gather information from written material. Information collected includes a project's impetus, team, budget, challenges, benefits, outcomes, and technology. This information is written in protocol format, stored in a publicly available online database (www.carezooming. com), and can be filtered by setting, technology used, and topic. We facilitated 30-minute phone consultations between experts whose work is featured in our database and clinicians or administrators seeking to implement a new project.

MEASURES OF SUCCESS (DISCUSS QUALITATIVE AND/OR QUANTITATIVE METRICS WHICH WILL BE USED TO EVALUATE PROGRAM/INTERVENTION): 1) The number of protocols featured in our database, their characteristics, and viewership statistics. 2) Quantity of consultations completed and their perceived value. 3) Qualitative information about the student editor experience.

FINDINGS TO DATE (IT IS NOT SUFFICIENT TO STATE FINDINGS WILL BE DISCUSSED ): We have collated 50 implementation protocols led by 55 innovators, spanning innovations in more than 20 cities. They have been viewed by $6000+$ unique individuals across the US. The most commonly read topics were behavioral health integration, Medicare Wellness Visit workflows, and medication assisted treatment for opioid management. Most protocols utilized an EMR and had an MD team member. Budgets ranged from $<\$ 1000$ to $>\$ 1$ million. We have connected 20 innovators working on healthcare improvement through 30minute peer consultations. Users reported the consultations saved them hundreds of dollars and hours of wasted time. Student editors reported enhanced understanding of the quality improvement process and unique exposure to healthcare implementation challenges.

KEY LESSONS FOR DISSEMINATION (WHAT CAN OTHERS TAKE AWAY FOR IMPLEMENTATION TO THEIR PRACTICE OR COMMUNITY?): We demonstrated the feasibility of students collating detailed, structured information about ambulatory care improvement processes. This information has been read by clinicians and administrators across the US and served as the basis for successful peer-to-peer consultations.
DIABETES WEEK: IMPLEMENTATION OF A QUARTERLY DIABETES MANAGEMENT WEEK TO IMPROVE CARE AT A LARGE, ACADEMIC-AFFILIATED VA PRIMARY CARE CLINIC.

Stefanie Deeds $^{2,}{ }^{1}$; Scott Hagan ${ }^{2,}{ }^{1}$. ${ }^{1}$ University of Washington, Seattle, WA; ${ }^{2}$ VA Puget Sound, Seattle, WA. (Control ID \#3186503)

STATEMENT OF PROBLEM OR QUESTION (ONE SENTENCE): The Seattle VA Primary Care clinic was not meeting national quality goals for patients with diabetes, as measured by most recent hemoglobin A1c (HbA1c) 9\% or an HbAlc not done in the past 12 months (HbAlc overdue).

OBJECTIVES OF PROGRAM/INTERVENTION (NO MORE THAN THREE OBJECTIVES): (1) identify patient and PACT barriers and facilitators to care for veterans with diabetes (2) develop interventions to improve reduce the number of patients with $\mathrm{HbA} 1 \mathrm{cs} 9 \%$ and $\mathrm{HbA} 1 \mathrm{cs}$ overdue

DESCRIPTION OF PROGRAM/INTERVENTION, INCLUDING ORGANIZATIONAL CONTEXT (E.G. INPATIENT VS. OUTPATIENT, PRACTICE OR COMMUNITY CHARACTERISTICS): The Seattle VA Primary Care Clinic is a large, academic affiliated clinic with roughly 3900 patients with diabetes, or $23 \%$ of the clinic patient population. Since 2011, the VA has used a medical home model, the Patient Aligned Care Team (PACT), to deliver veteran centric primary care. VA uses two main quality metrics to track diabetes care and targets $<21 \%$ of patients with either an $\mathrm{HbA1c} 9 \%$ or overdue, and $<5 \%$ with an $\mathrm{HbA1c}$ overdue. At baseline, in March 2018 the Seattle VA Primary Care clinic had $13.7 \%$ of patients with diabetes with $\mathrm{HbA1c} 9 \%$ and $10 \%$ with $\mathrm{HbA1c}$ overdue. A multidisciplinary workgroup including PCPs, the clinic director, clinical pharmacists, a PACT $\mathrm{RN}$ and trainees met monthly to review the clinical performance measures, discuss barriers to care, and develop interventions. We conducted a baseline survey and interviews of PACT teams and performed a chart review of demographic factors for patients with $\mathrm{HbA1}$ cs $9 \%$ and/or $\mathrm{HbAlcs}$ overdue to examine patient-level barriers to care. Our primary intervention was a quarterly Diabetes Week during which project leads issued PACT team reports of patients with $\mathrm{HbAlc} 9 \%$ or HbAlc overdue, tips for managing patients with uncontrolled diabetes, and information about obtaining lab work at VA community clinics. A second intervention was a pilot of sending automated lab reminder letters to all patients due for their annual $\mathrm{HbAlc}$.

MEASURES OF SUCCESS (DISCUSS QUALITATIVE AND/OR QUANTITATIVE METRICS WHICH WILL BE USED TO EVALUATE PROGRAM/INTERVENTION): We tracked a monthly run chart of the percentage of diabetes patients with an $\mathrm{HbAlc}$ s $9 \%$ and $\mathrm{HbAlcs}$ overdue.

FINDINGS TO DATE (IT IS NOT SUFFICIENT TO STATE FINDINGS WILL BE DISCUSSED ): From March of 2018 to January 2019, the percentage of patients in our clinic with $\mathrm{HbA} 1 \mathrm{cs}$ overdue and with $\mathrm{HbA1c}$ $9 \%$ declined by $20 \%$ and $5 \%$, respectively. The key finding of staff interviews was that dedicated time for panel management is essential for success. Additionally, a common staff perception was that patient factors were a significant barrier to diabetes care. However, chart review surprisingly showed no significant differences between patients with well controlled and poorly controlled diabetes.

KEY LESSONS FOR DISSEMINATION (WHAT CAN OTHERS TAKE AWAY FOR IMPLEMENTATION TO THEIR PRACTICE OR COMMUNITY?): Our diabetes working group developed a series of interventions to improve diabetes panel management, which are associated with reductions in veterans with diabetes overdue for annual $\mathrm{HbAlc}$ testing and with high A1cs. Forming an interdisciplinary workgroup, collecting staff and patient data on barriers to success, and using registry tools to facilitate 
panel management were important factors to our success. Future directions include enhanced quarterly PACT huddles with clinic leadership and protected time for $\mathrm{RN}$ panel management.

DID YOU POLST TODAY? ADVANCING THE DIRECTIVES ON ADVANCE CARE PLANNING DISCUSSIONS IN RESIDENT CONTINUITY CLINICS.

Karen Jong ${ }^{1}$; Amalia Aldredge ${ }^{1}$; Michael A. Yu ${ }^{2}$; Sarah Koumtouzoua ${ }^{1}$. ${ }^{1}$ Emory University, Atlanta, GA; ${ }^{2}$ Emory University School of Medicine, Atlanta, GA. (Control ID \#3184983)

STATEMENT OF PROBLEM OR QUESTION (ONE SENTENCE): Residents and attendings do not routinely have ACP discussions in primary care clinic with patients $>65$, and most of those patients have never had a documented ACP discussion in the chart.

OBJECTIVES OF PROGRAM/INTERVENTION (NO MORE THAN THREE OBJECTIVES): 1. Identify barriers that prevent provider engagement, accurate documentation and appropriate billing of ACP discussions. 2. Educate providers on engagement in outpatient ACP discussions 3. Increase the percentage of patients who have had ACP discussions in the outpatient setting

DESCRIPTION OF PROGRAM/INTERVENTION, INCLUDING ORGANIZATIONAL CONTEXT (E.G. INPATIENT VS. OUTPATIENT, PRACTICE OR COMMUNITY CHARACTERISTICS): $\mathrm{ACP}$ is not only a key component to resident education and training, but also a critical topic that must routinely be addressed with patients $>65$ years old in order to best respect and advocate for patients wishes when they are not able to speak for themselves. We evaluated resident and attending effectiveness in completion of ACP in the Grady Green Pod Primary Care Clinic, an urban clinic serving a high risk, low resource population. Our intervention will occur in February and March during clinic "lunch-and-learn" sessions. Absent providers will complete an interactive presentation for independent, remote learning. Additional interventions include: creation of patient/ provider reminder cards containing ACP information given at patient triage, printing POLST/AD packets to stock clinic drawers, and distribution of ACP billing guide to attendings.

MEASURES OF SUCCESS (DISCUSS QUALITATIVE AND/OR QUANTITATIVE METRICS WHICH WILL BE USED TO EVALUATE PROGRAM/INTERVENTION): We will measure success qualitatively through a post-intervention survey of providers evaluating their comfort with ACP discussions and knowledge of proper code status ordering, documentation of $\mathrm{AD} / \mathrm{POLST}$, and $\mathrm{ACP}$ billing. A retrospective chart review in April will measure the change in the percentage of patients $>65$ who have had ACP discussions, along with analysis of appropriate documentation, billing and code status ordering.

FINDINGS TO DATE (IT IS NOT SUFFICIENT TO STATE FINDINGS WILL BE DISCUSSED ): A retrospective chart review of 233 established patients $>65$ years of age showed that only 19 (8.2\%) of patients had had ACP discussions documented in our clinic; an additional 13 patients (5.6\%) had discussions inpatient or in other clinics. Over half of these discussions were in patients above the age of 75 . Of the 19 primary care clinic encounters, 8 were with attendings and 11 with residents. Only 2 were billed correctly and only 1 had code status ordered. Fifteen were given AD and/or POLST, but only 2 were uploaded into the chart.

KEY LESSONS FOR DISSEMINATION (WHAT CAN OTHERS TAKE AWAY FOR IMPLEMENTATION TO THEIR PRACTICE OR COMMUNITY?): Increasing incidence of engagement and accurate completion of ACP discussions requires a multifactorial approach; including, but not limited to addressing provider knowledge gaps, making documents accessible, and using tools that remind providers and patients to address ACP. Our generalizable interventions provide a model for resident continuity clinics facing similar patient populations and barriers. Correct documentation, particularly of code status, and billing for ACP directly benefits our patients and financially supports the healthcare system providing their care.

DIRECT REFERRAL INCREASES PULMONARY FUNCTION TEST (PFT) COMPLETION RATES IN A PATIENT CENTERED MEDICAL HOME AND FINDS ALTERNATIVE DIAGNOSIS IN A THIRD OF PATIENTS WITH PREVIOUS DIAGNOSIS OF ASTHMA.

Camellia Banerjee $^{1}$; Dimple Shah ${ }^{1}$; Christina Mai ${ }^{1}$; Travis Halbert ${ }^{1}$; Timothy Wigand $^{1}$; He Qiu ${ }^{1}$; Rebecca Shafer ${ }^{1}$; Sara Tariq ${ }^{1}$; Jacquline Moore ${ }^{1}$; HueiWen Lim ${ }^{1}$; Ankita Sagar ${ }^{2} .{ }^{1}$ Zucker School of Medicine at Northwell/Hofstra, Manhasset, NY; ${ }^{2}$ Hofstra North Shore LIJ, East Meadow, NY. (Control ID \#3184685)

STATEMENT OF PROBLEM OR QUESTION (ONE SENTENCE): Because asthma is often overdiagnosed in primary care, we investigated whether asking patients with a previous diagnosis of asthma to obtain a pulmonary function test via written letter and referral may help our outpatient practice better identify patients with true asthma (or other) diagnosis.

OBJECTIVES OF PROGRAM/INTERVENTION (NO MORE THAN THREE OBJECTIVES): This intervention aimed to increase the amount of pulmonary function testing in our clinic in those diagnosed with asthma, to identify respiratory diseases and confirm asthma based on pulmonary function testing, and to tailor treatment based on new data.

DESCRIPTION OF PROGRAM/INTERVENTION, INCLUDING ORGANIZATIONAL CONTEXT (E.G. INPATIENT VS. OUTPATIENT, PRACTICE OR COMMUNITY CHARACTERISTICS): Our outpatient residency practice patients encompass an array of underserved patients with limited English proficiency, undocumented immigration status, and limited health literacy / ability to navigate the healthcare system, something that was a challenge in our intervention. Patients were drawn from our residency practice based on a diagnosis of asthma in their chart. Patients who had previously undergone pulmonary function testing were excluded. The intervention was a letter to patients explaining the importance of pulmonary function testing (PFT), basis of recommendation for such testing, and referral with directions to make an appointment for PFTs. The letter was sent in both English and Spanish.

MEASURES OF SUCCESS (DISCUSS QUALITATIVE AND/OR QUANTITATIVE METRICS WHICH WILL BE USED TO EVALUATE PROGRAM/INTERVENTION): We evaluated this intervention based on increase in testing after letter was sent. The completed pulmonary function tests were evaluated to confirm the diagnosis and treatments were changed accordingly.

FINDINGS TO DATE (IT IS NOT SUFFICIENT TO STATE FINDINGS WILL BE DISCUSSED ): 330 patients with a diagnosis of asthma were identified. Of those, 32 patients had undergone PFTs prior to the intervention. After the letter was sent, patients were followed for 6 months. In the next 6 months, 34 additional patients had undergone testing. The intervention doubled the number of patients with PFTs in our clinic. Moreover, many of the new PFTs suggested an alternative diagnosis to asthma in $32 \%$ of our patients, which allowed for our providers to discontinue unnecessary medications such as inhalers and prescribe more appropriate treatment. 
KEY LESSONS FOR DISSEMINATION (WHAT CAN OTHERS TAKE AWAY FOR IMPLEMENTATION TO THEIR PRACTICE OR COMMUNITY?): Our study shows that a simple intervention of sending a letter was able to successfully increase the number of PFTs in our residency clinic. Like prior studies, our intervention also showed that many of our patients with a diagnosis of asthma did not meet criteria for asthma by PFTs in $32 \%$ of the population that underwent testing. Those who were identified to have alternative diagnosis were able to be placed on more appropriate treatment, thus reducing both cost and harm in this population.

DISMANTLING A BARRIER TO MINDFUL LAB ORDERING: THE ATTENDING PHYSICIAN

Rachna Rawal $^{1}$; Paul Kunnath ${ }^{2}$; Oluwasayo Adeyemo' ${ }^{1}$ Hala $\mathrm{Saad}^{3}$; Jennifer Schmidt ${ }^{4}$. ${ }^{1}$ Saint Louis University, St. Louis, MO; ${ }^{2}$ Saint Louis University School of Medicine, University City, MO; ${ }^{3}$ Saint Louis University Hospital, Saint Louis, MO; ${ }^{4}$ Saint Louis University, Saint Louis, MO. (Control ID \#3183489)

\section{STATEMENT OF PROBLEM OR QUESTION (ONE SENTENCE):}

Improve resident-attending communication regarding mindful laboratory ordering to create a high-value care culture.

OBJECTIVES OF PROGRAM/INTERVENTION (NO MORE THAN THREE OBJECTIVES): Recognize the attending physician as a barrier to resident mindful laboratory ordering Identify discrepancies between attending/resident perceptions of laboratory ordering communication Understand benefits of educating attending physicians and residents on communication

DESCRIPTION OF PROGRAM/INTERVENTION, INCLUDING ORGANIZATIONAL CONTEXT (E.G. INPATIENT VS. OUTPATIENT, PRACTICE OR COMMUNITY CHARACTERISTICS): Unnecessary laboratory orders are a well-known contributor to healthcare cost. Our residents identified "fear of attending" as a barrier to more mindful lab ordering. This project focused on educating attending physicians on highvalue care and promoting resident-attending ordering discussions.

MEASURES OF SUCCESS (DISCUSS QUALITATIVE AND/OR QUANTITATIVE METRICS WHICH WILL BE USED TO EVALUATE PROGRAM/INTERVENTION): Data was collected via anonymous pre and post-intervention survey (designed by study team). Participants included resident and attending physicians on the inpatient Medicine service. Multiple interventions targeting attending physicians occurred over a 16-week period including presentations at monthly hospitalist meeting (included resident lab-ordering data and resident perceptions of attendings). Electronic requests asked attendings to voluntarily submit a message of support for the project; these were then shared with residents. An attending project champion was identified and emailed attendings on service with reminders to discuss lab ordering with their teams. Surveys assessed sustainability at 6 months and 1 year.

FINDINGS TO DATE (IT IS NOT SUFFICIENT TO STATE FINDINGS WILL BE DISCUSSED ): Initially, 70\% of residents reported worrying about repercussions from attendings if daily labs were not ordered; at 6 months, only $24 \%$ reported this (statistically significant, $\mathrm{p}<0.05$ ). Residents receiving "pushback" from attendings for not ordering daily labs increased from $25 \%$ to $54 \%$ (statistically significant, $\mathrm{p}<0.05$ ), sustained at 6 months. At 6 months, $71 \%$ of interns and $51 \%$ of senior residents reported receiving "pushback" from attendings. At 16 weeks, $62 \%$ of attending physicians reported residents are appropriately mindful of laboratory orders (sustained at 6 and 12 months).
KEY LESSONS FOR DISSEMINATION (WHAT CAN OTHERS TAKE AWAY FOR IMPLEMENTATION TO THEIR PRACTICE OR COMMUNITY?): Our data shows a discrepancy in the perception of mindful ordering discussions between resident and attending physicians. Attendings have a higher perception of attending-initiated discussions and a lower perception of resident-initiated discussion. Interestingly, between 6 and 12-month follow-up, attendings report a decrease in resident-initiated discussions. We hypothesize that this is secondary to a new academic year or may indicate a new barrier. The increased resident-reported discussion of lab ordering has sustained through 6-months. This is further supported by the attendings perceiving the residents as being appropriately mindful of laboratory orders. However, residents have reported receiving more pushback from attendings. This may suggest that attendings are more resistant to mindful lab ordering than they perceive or may reflect increased effort by residents to change lab ordering practices. Despite pushback, fewer residents fear attendings' repercussions.

\section{DISTANCE MAKES THE HEART GROW FONDER}

Hannah Pyle; Ravali R. Inja; Hiral Choksi. St. Louis University, Saint Louis, MO. (Control ID \#3162430)

STATEMENT OF PROBLEM OR QUESTION (ONE SENTENCE): Why do patient's not attend their post-hospital discharge follow-up appointments?

OBJECTIVES OF PROGRAM/INTERVENTION (NO MORE THAN THREE OBJECTIVES): 1. Identify correlations of patient demographics to likelihood of attending an post hospital discharge follow-up appointment 2. Determine opportunities to create interventions to improve show rates

DESCRIPTION OF PROGRAM/INTERVENTION, INCLUDING ORGANIZATIONAL CONTEXT (E.G. INPATIENT VS. OUTPATIENT, PRACTICE OR COMMUNITY CHARACTERISTICS): A retrospective analysis was performed on adult patients admitted to SSM Saint Louis University Hospital, a 356 bed tertiary care facility between February 5 , 2018 and February 28, 2018. Only patients who had follow-up appointments scheduled with Saint Louis University affiliated-physicians were included. Patient charts were accessed through our EMR to acquire demographic and follow-up appointment information. It was sought to examine the effect of multiple available predictors on appointment attendance: distance between clinic and their house, discharge location (home vs facility), length of stay, chronic comorbidities, lead time to appointment, discharge diagnosis, followup service, and number of follow-up appointments

MEASURES OF SUCCESS (DISCUSS QUALITATIVE AND/OR QUANTITATIVE METRICS WHICH WILL BE USED TO EVALUATE PROGRAM/INTERVENTION): Although the dataset contained some repeated measures, a fixed model logistic regression was employed because an exceedingly small intra-class correlation coefficient indicated no violation of independence. The empirically best fit model based on AIC value via stepwise regression was identified.

FINDINGS TO DATE (IT IS NOT SUFFICIENT TO STATE FINDINGS WILL BE DISCUSSED ): The chosen model suggested two significant effects. For every additional mile in distance from the facility, the odds of attending are $2.76 \%$ higher $(p=.0248)$. For every additional day of lead time, the odds of attending decrease by $1.45 \%(\mathrm{p}=.0416)$. The model also suggested two marginally significant effects. Firstly, having heart disease reduces the probability of attendance by $56.18 \%(\mathrm{p}=.0795)$. Secondly, having hyperlipidemia increases the probability of attendance by $262.29 \%$ $(\mathrm{p}=.0577)$. 
KEY LESSONS FOR DISSEMINATION (WHAT CAN OTHERS TAKE AWAY FOR IMPLEMENTATION TO THEIR PRACTICE OR COMMUNITY?): Emphasizing and analyzing the factors that affect patient compliance with follow-up visits is critical in order to provide the best possible care. Once identified, changes can be implemented to increase patient compliance and maximize the impact the healthcare system has on patient care.

\section{E-COMMUNICATION IMPROVES PRIMARY CARE PATIENT ACCESS TO RHEUMATOLOGY}

Zachary Brandon $^{1}$; John P. Shoup ${ }^{1}$; Robert Keenan ${ }^{1}$; Alex H. Cho ${ }^{1}$; Teresa K. Tarrant ${ }^{1}$; Donna Phinney ${ }^{1}$; John Anderson ${ }^{2}$; Elizabeth Malcolm ${ }^{1}$; Kevin Shah ${ }^{1} .{ }^{1}$ Duke University, Durham, NC; ${ }^{2}$ Duke University Health System, Durham, NC. (Control ID \#3186361)

STATEMENT OF PROBLEM OR QUESTION (ONE SENTENCE):

Long wait times, poor referral completion rates, and lack of primary care provider (PCP) and specialist communication combine to prevent appropriate rheumatologic care.

OBJECTIVES OF PROGRAM/INTERVENTION (NO MORE THAN THREE OBJECTIVES): To increase rheumatology referral completion rates and decrease wait times through implementation of $\mathrm{E}$ Communication (or E-Consult). PCP and specialist satisfaction of ECommunication implementation and use were also tracked.

DESCRIPTION OF PROGRAM/INTERVENTION, INCLUDING ORGANIZATIONAL CONTEXT (E.G. INPATIENT VS. OUTPATIENT, PRACTICE OR COMMUNITY CHARACTERISTICS): Three Duke Primary Care (DPC) practices initially participated in the ECommunication program, with expansion to 2 additional practices after 6 and 8 months respectively. In lieu of traditional referrals, primary care providers place an E-Communication request. Each E-Communication is reviewed within $72 \mathrm{hrs}$ by a rheumatologist who provides recommendations for additional workup or treatment. The rheumatologist recommends either continued management by the primary care provider or triaged in-person rheumatologic evaluation.

MEASURES OF SUCCESS (DISCUSS QUALITATIVE AND/OR QUANTITATIVE METRICS WHICH WILL BE USED TO EVALUATE PROGRAM/INTERVENTION): E-Communication completion rates and turnaround time were monitored. Conversion rates of $\mathrm{E}-$ Communication requests to in-person appointments and wait times for these appointments were tracked. E-Communications were compared with traditional referrals from all DPC clinics during the intervention period. Surveys of both primary care and rheumatology participants following the pilot program assessed participant satisfaction.

FINDINGS TO DATE (IT IS NOT SUFFICIENT TO STATE FINDINGS WILL BE DISCUSSED ): Over 12 months, 49 primary care providers submitted 230 E-Communications for 212 individual patients. $58.4 \%$ $(n=124)$ of patients were recommended for in-person evaluation. Of these, $6 \%$ $(n=8)$ were recommended for evaluation within 7 days, $26 \%(n=32)$ within 30 days, and $68.5 \%(\mathrm{n}=85)$ within 90 days or no priority. $86.5 \%(\mathrm{n}=199)$ of $\mathrm{E}-$ Communications were completed within the target $72 \mathrm{hrs}$ (median response 15.5hrs, IQR 4.1hrs-33.5hrs). Compared to DPC patients referred to rheumatology during the same time period, E-Communication patients had shorter wait times (median $31.2 \mathrm{~d}$ vs. $77 \mathrm{~d}$ ). E-Communication patients were significantly more likely to be scheduled $\left(89.5 \%\right.$ vs. $\left.59.3 \%, X^{2}=45.1, p<0.001\right)$ and complete their in person specialty evaluation $\left(78.2 \%\right.$ vs. $40.2 \%, \mathrm{X}^{2}=$ $68.2, \mathrm{p}<0.001)$. We found high levels of PCP satisfaction with ECommunication. When compared to standard referrals, all PCP respondents rated E-Communications as "5- much better" $(79 \%, \mathrm{n}=11)$ or "4- somewhat better" (21\%, n=3) and would recommend E-Communication to a colleague ("strongly agree" $79 \% \mathrm{n}=11$; "somewhat agree" $21 \% \mathrm{n}=3$ ). PCP perception of overall care coordination improved significantly post-implementation (Likert scale 1-2 negative, 3-5 neutral/positive, $\left.X^{2}=19.7, p<0.001\right)$. Rheumatologists had positive experiences as well, with an overall satisfaction mean of 4.2 out of 5 on a Likert scale (IQR 4-5). Clinic level data are pending.

KEY LESSONS FOR DISSEMINATION (WHAT CAN OTHERS TAKE AWAY FOR IMPLEMENTATION TO THEIR PRACTICE OR COMMUNITY?): Through implementation of E-Communication, we improved access and increased visit completion with a high demand specialty. E-Communications were positively received by both PCPs and specialists and perceived care coordination improved.

ECONSULT UTILIZATION AND APPOINTMENT OUTCOMES DIFFER AMONG INTERNAL MEDICINE SPECIALTIES

Sharon Rikin; Eric Epstein; Matthew Berger; Anna Broder; Joann Kwah; Raphael Hulkower; Joseph Deluca; Julia H. Arnsten; Yaron Tomer. Montefiore - Albert Einstein College of Medicine, Bronx, NY. (Control ID \#3174895)

STATEMENT OF PROBLEM OR QUESTION (ONE SENTENCE): Primary care patients frequently experience barriers to timely, patient-centered, and collaborative specialty care.

OBJECTIVES OF PROGRAM/INTERVENTION (NO MORE THAN THREE OBJECTIVES): Improve primary care patient access to specialty expertise by providing a standard tool for primary care physicians (PCPs) and specialists to discuss patient care a) without the need for appointment, b) with triaging of expedited appointment, or c) in preparation for non-expedited appointment. Ensure PCP satisfaction with the intervention.

DESCRIPTION OF PROGRAM/INTERVENTION, INCLUDING ORGANIZATIONAL CONTEXT (E.G. INPATIENT VS. OUTPATIENT, PRACTICE OR COMMUNITY CHARACTERISTICS): An interdisciplinary team based in an urban, academic health system developed the eConsult workflow using the Epic electronic health record. After review of existing eConsult programs, the program was customized: eConsult was an order which PCPs could use as an alternative to routine referrals; one or two eConsultants were designated per specialty and received compensation for each eConsult; questions and responses had a free text format; responses were expected within three days. The program was piloted in three primary care sites and three specialties: endocrinology, gastroenterology, rheumatology. PCPs received on-site training about the program.

MEASURES OF SUCCESS (DISCUSS QUALITATIVE AND/OR QUANTITATIVE METRICS WHICH WILL BE USED TO EVALUATE PROGRAM/INTERVENTION): eConsult program utilization was assessed by the weekly proportion of all specialty referrals (routine referrals + eConsult) initiated through eConsult using the statistical process control $\mathrm{p}$ chart. To evaluate the impact of the program on triaging care, appointment outcomes were assessed using descriptive statistics. These analyses were performed for all specialties combined and stratified by specialty in order to understand demand in anticipation for scaling and sustainability; chi square was used to evaluate associations between specialty and outcomes. PCP satisfaction survey was distributed after three months and evaluated using descriptive statistics.

FINDINGS TO DATE (IT IS NOT SUFFICIENT TO STATE FINDINGS WILL BE DISCUSSED ): Over seven months, a weekly average of $10 \%(n=160)$ of all specialty referral requests were initiated through eConsults with utilization differing by specialty ( $21 \%$ in endocrinology, $3 \%$ in 
gastroenterology, $10 \%$ in rheumatology; $\mathrm{p}<0.001)$. Among all eConsults, $19 \%$ included a recommendation for an expedited appointment and $29 \%$ for a non-expedited appointment also differing by specialty (expedited and nonexpedited: $9 \%$ and $30 \%$ in endocrinology, $33 \%$ and $19 \%$ in gastroenterology, $22 \%$ and $44 \%$ in rheumatology; $\mathrm{p}=0.002)$. The majority of PCPs $(80 \%)$ were very satisfied or somewhat satisfied with eConsults compared to $30 \%$ for routine referrals.

KEY LESSONS FOR DISSEMINATION (WHAT CAN OTHERS TAKE AWAY FOR IMPLEMENTATION TO THEIR PRACTICE OR COMMUNITY?): eConsult utilization and appointment outcomes differ by specialty with the highest use and deferral of appointments in the specialty that relies primarily on laboratory evaluation to guide management (endocrinology) rather than procedures (gastroenterology) or physical exam (rheumatology). eConsults were an effective triaging mechanism for urgent appointments. Outcomes will be used to build capacity for number of eConsultants and for the number of expedited appointments needed per week. PCP feedback will be used to promote the program and to make continuous improvements.

\section{ECONSULTS IMPROVE PRIMARY CARE PATIENT WAIT TIMES TO SPECIALTY CARE}

Ravi Budhan; Joseph Deluca; Eric Epstein; Anna Broder; Raphael Hulkower; Joann Kwah; Sharon Rikin. Montefiore - Albert Einstein College of Medicine, Bronx, NY. (Control ID \#3185225)

\section{STATEMENT OF PROBLEM OR QUESTION (ONE SENTENCE):} Primary care patients face challenges to specialty care including long wait times which are associated with increased no show rates.

OBJECTIVES OF PROGRAM/INTERVENTION (NO MORE THAN THREE OBJECTIVES): To decrease the wait times for specialty care and increase the proportion of completed visits by providing an alternative to routine referrals.

DESCRIPTION OF PROGRAM/INTERVENTION, INCLUDING ORGANIZATIONAL CONTEXT (E.G. INPATIENT VS. OUTPATIENT, PRACTICE OR COMMUNITY CHARACTERISTICS): An eConsult is a standardized form of communication between a primary care physician (PCP) and a specialist within the electronic medical record. From February to July 2018, the eConsult workflow was piloted among three urban, academic primary care practices which included faculty, residents and midlevel providers. The eConsult was developed as an alternative to routine referral orders for Internal Medicine specialties. eConsult orders prompted PCPs to provide a brief summary of the patient along with a clinical question. eConsultants provided recommendations within three days with three potential outcomes: no appointment needed, expedited appointment, or non-expedited appointment. If an expedited appointment was recommended, each specialty was responsible for scheduling the patient, either by overbooking or by utilizing an appointment blocked for this purpose. If a non-expedited appointment was recommended, the same workflow as routine referrals was applied. MEASURES OF SUCCESS (DISCUSS QUALITATIVE AND/OR QUANTITATIVE METRICS WHICH WILL BE USED TO EVALUATE PROGRAM/INTERVENTION): Average wait time to specialty appointment and proportion of completed visits were compared between routine referrals and a) expedited eConsults and b) non-expedited eConsults using student t-test for means and chi-square for categorical outcomes. For routine referrals, a random sample, stratified by specialty and month was evaluated for outcome estimates.

FINDINGS TO DATE (IT IS NOT SUFFICIENT TO STATE FINDINGS WILL BE DISCUSSED ): During the 6 month period, PCPs requested 128 eConsults with an average wait time to initial response of 0.73 days. After the eConsult, $48.4 \%$ of patients did not require an appointment, $20.3 \%$ required expedited appointment, and $31.3 \%$ required nonexpedited appointment. In this same time period, PCPs placed 994 routine referrals and 450 were sampled for estimates. For scheduled visits, the mean wait time was 29 days (SD: 32 days) for eConsult expedited, 78 days (SD: 42 days) for eConsult non-expedited, and 81 days (SD: 59 days) for routine referral (eConsult expedited vs. routine $\mathrm{p}<0.0001$, eConsult non-expedited vs. routine $\mathrm{p}=0.81$ ). The proportion of completed visits was $75 \%$ for eConsult expedited, $46.4 \%$ for eConsult non-expedited, and $50.5 \%$ for routine (eConsult expedited vs. routine $\mathrm{p}=0.02$; eConsult non-expedited vs. routine $\mathrm{p}=0.68)$.

KEY LESSONS FOR DISSEMINATION (WHAT CAN OTHERS TAKE AWAY FOR IMPLEMENTATION TO THEIR PRACTICE OR COMMUNITY?): This eConsult intervention successfully provided a specialist recommendation in $<3$ business days. The majority of eConsults facilitated PCPs management of care without a need for a specialty appointment. eConsults requiring an expedited appointment had decreased wait times and a higher visit completion rate when compared to routine referrals. eConsults requiring non-expedited appointments were not different in appointment outcomes; however, based on specialist recommendations, management could start prior to the appointment.

EFFECTIVENESS OF FECAL IMMUNOCHEMICAL TESTING AS THE PRIMARY SCREENING TOOL FOR COLORECTAL CANCER IN AN AMBULATORY CARE CLINIC FOR THE UNDERINSURED AND UNINSURED

Maurice Marcuard $^{1}$; Edward Oldfield ${ }^{1}$; David Mendel ${ }^{2}$; Sami G. Tahhan ${ }^{1}$; David Johnson ${ }^{2} .{ }^{1}$ EVMS, Norfolk, VA; ${ }^{2}$ EVMS, NORFOLK, VA. (Control ID \#3159189)

STATEMENT OF PROBLEM OR QUESTION (ONE SENTENCE): IS the fecal immunochemical test an effective colorectal cancer screening method in a clinic for the uninsured and underinsured?

OBJECTIVES OF PROGRAM/INTERVENTION (NO MORE THAN THREE OBJECTIVES): 1) To determine the adequacy of the FIT screening program at the ACC and compliance with the core USMSTF quality measures which include: -FIT completion rate to those offered testing of $60 \%$ or greater -Proportion returning FIT that cannot be processed by the laboratory of less than 5\%-Colonoscopy completion rate for those with a positive FIT of $80 \%$ or greater 2) To determine the average time to colonoscopy from positive FIT test 3) To determine the percentage of patients with a negative FIT test who undergo repeat annual FIT testing

DESCRIPTION OF PROGRAM/INTERVENTION, INCLUDING ORGANIZATIONAL CONTEXT (E.G. INPATIENT VS. OUTPATIENT, PRACTICE OR COMMUNITY CHARACTERISTICS): The US MultiSociety Task Force (USMSTF) guidelines for colorectal cancer (CRC) screening recommend either colonoscopy or annual fecal immunochemical testing (FIT) as the $1^{\text {st }}$ tier screening methodologies. As a result, the Sentara Ambulatory Care Center (ACC) started using FIT testing as the preferred screening test. Importantly, the effectiveness of FIT testing is dependent on the quality of the FIT screening program and the ability to follow up positive tests with colonoscopy. This retrospective quality improvement project reviewed all ACC patients ordered for FIT testing during the first year (9/1/2016-8/31/2017) and one year of follow-up time (9/1/2017-8/31/2018). Information was obtained by chart review and included all patients ordered for a FIT test without any exclusion criteria. For patients with negative FIT testing, chart review determined if they were able to complete a follow-up FIT test 
within one year. For patients with positive FIT testing, chart review determined their dates for initial appointment, scheduled and actual colonoscopy dates, and recommended follow up interval.

MEASURES OF SUCCESS (DISCUSS QUALITATIVE AND/OR QUANTITATIVE METRICS WHICH WILL BE USED TO EVALUATE PROGRAM/INTERVENTION): Descriptive statistics was used to characterize the project results to answer the above listed objectives for the project. Continuous outcomes was expressed in mean and standard deviation or median and interquartile range, depending on the distribution. Categorical outcomes was reported as count and frequency.

FINDINGS TO DATE (IT IS NOT SUFFICIENT TO STATE FINDINGS WILL BE DISCUSSED ): A total of 308 patients had a FIT test ordered during the study period ( 26 positive, 230 negatives, and 52 incompletes). The FIT completion rate was $83.1 \%$ (256/308). The colonoscopy completion rate for positive FIT tests was $65.4 \%$ (17/26), and all patients with a positive FIT had a surgery appointment. The average time to colonoscopy was 3.5 months in these patients. For patients with a negative FIT test, only $18 \%$ (38/207) of patients completed a follow-up FIT test within one year. Of completed tests, there were 2 FIT tests $(0.7 \%)$ that could not be processed.

KEY LESSONS FOR DISSEMINATION (WHAT CAN OTHERS TAKE AWAY FOR IMPLEMENTATION TO THEIR PRACTICE OR COMMUNITY?): -We found successful adoption of FIT testing for CRC screening with respect to an initial completion rate of $83.1 \%$-We did not meet colonoscopy goals for positve FIT tests -There was a significant drop-off in the repeat FIT testing with only $18 \%$ of patients completing it. -This highlighted the need for a closely monitored and adjusted system for FIT testing.

\section{EFFECTIVENESS OF NURSE-DRIVEN PROTOCOL FOR BLOOD} PRESSURE MANAGEMENT

Yusuf N. Alalwan; Miriam Jaziri; Alexis C. Haftka-George; Joseph Miller. Henry Ford Hospital, Detroit, MI. (Control ID \#3163465)

STATEMENT OF PROBLEM OR QUESTION (ONE SENTENCE): IS the nurse-driven protocol more effective in controlling blood pressure in patients with hypertension in the outpatient setting than the traditional physician-driven approach?

OBJECTIVES OF PROGRAM/INTERVENTION (NO MORE THAN THREE OBJECTIVES): Improve blood pressure control with dedicated follow-up visits performed by nurses Improve access to health care in traditionally underserved patient populations Utilize a team-based approach in the management of chronic diseases

DESCRIPTION OF PROGRAM/INTERVENTION, INCLUDING ORGANIZATIONAL CONTEXT (E.G. INPATIENT VS. OUTPATIENT, PRACTICE OR COMMUNITY CHARACTERISTICS): The nurse-driven protocol is based on the MUPD campaign (Measure Up Pressure Down) using a team-based approach to improve blood pressure control and counteract clinical inertia in the ambulatory outpatient clinic setting. After patients are referred to the program by their physician, the MUPD nurse will follow up with the patient during cost-free appointments for blood pressure measurements, patient education, and medication titration based on a set protocol. The patients continue in the program until their blood pressures are controlled. The Henry Ford Clinics traditionally treat a disproportionately high number of medically underserved patients from the Detroit City area. The majority of the patients identify as African American.

MEASURES OF SUCCESS (DISCUSS QUALITATIVE AND/OR QUANTITATIVE METRICS WHICH WILL BE USED TO EVALUATE PROGRAM/INTERVENTION): This was a retrospective case- controlled study including all adults ( $>18$ years) with hypertension (SBP $>140$ and/or a DBP $>90$ ) that presented to a Henry Ford internal medicine clinic between 1/2015 and 9/2017 and who participated in the nurse-driven protocol. The control consisted of a matched cohort who only followed up with their physicians. Blood pressures at 6 months and 1 year following enrollment in the nurse-driven HTN program were obtained from electronic medical records. Categorical variables were compared using chi-square tests. Crude and adjusted odds ratios were obtained using generalized estimating equations with a logit link function presence of blood pressure control as the dependent variable.

FINDINGS TO DATE (IT IS NOT SUFFICIENT TO STATE FINDINGS WILL BE DISCUSSED ): The rate of controlled blood pressure (BP) at 6 months is significantly higher in patients who were in the program as compared to those who were not $(61 \%$ versus $45 \%$, $\mathrm{p}<0.001)$. Similarly, the rate of medical management change was significantly higher in patients who were in the program as compared to those who were not $(30 \%$ versus $21 \%$, $\mathrm{p}<0.001$ ). However, for both endpoints, the rates were similar at all other time points. Patients with at least 1 MUPD visit had 1.46 times the odds of having controlled BP in later visits compared to those with no MUPD visits. When controlling for gender, race, baseline systolic $\mathrm{BP}$, and ratio of the medication change, patients with at least one MUPD have 1.48 times the odds of having controlled BP in later visits compared to those with no MUPD visits.

KEY LESSONS FOR DISSEMINATION (WHAT CAN OTHERS TAKE AWAY FOR IMPLEMENTATION TO THEIR PRACTICE OR COMMUNITY?): Nurse-driven clinic visits seem to be more effective in controlling blood pressures in the primary care setting than traditional physician visits. This project furthermore showed that this is also true in outpatient settings with a high proportion of underserved patients with multiple other comorbidities and complex social backgrounds.

EFFECTS OF A COLLABORATIVE FRAMEWORK FOR CHRONIC PAIN MANAGEMENT AT ROSEBUD INDIAN HEALTH SERVICE UNIT

Stephanie Sun; Hannah Wenger. Massachusetts General Hospital, Boston, MA. (Control ID \#3185879)

STATEMENT OF PROBLEM OR QUESTION (ONE SENTENCE): How does the initiation of a committee-based framework affect chronic pain management at Rosebud Indian Health Service (IHS) Unit in rural South Dakota?

OBJECTIVES OF PROGRAM/INTERVENTION (NO MORE THAN THREE OBJECTIVES): The pain committee is intended to: (1) streamline safe opioid prescribing at Rosebud IHS Unit, (2) improve chronic pain management as guided by its outpatient providers, and (3) create a collaborative environment for outpatient providers to discuss chronic pain patients.

DESCRIPTION OF PROGRAM/INTERVENTION, INCLUDING ORGANIZATIONAL CONTEXT (E.G. INPATIENT VS. OUTPATIENT, PRACTICE OR COMMUNITY CHARACTERISTICS): The Rosebud IHS Unit is a rural community-based site on Rosebud Indian Reservation in South Dakota. Its clinical staff launched a pain committee in October 2018 to create a framework for managing patients with chronic pain, including those receiving long-term opioids. Primary care providers first place a pain consult in the electronic record, and then the primary care provider discusses the case and receives recommendations for pain management at the weekly committee meeting, which includes providers and representatives from administration, pharmacy, behavioral health, and other support services in the hospital. 
MEASURES OF SUCCESS (DISCUSS QUALITATIVE AND/OR QUANTITATIVE METRICS WHICH WILL BE USED TO EVALUATE PROGRAM/INTERVENTION): Opioid prescribing will be compared between two major time periods: before and after initiation of the pain committee. We will assess total dispensed morphine milligram equivalents (MMEs) per month and average dispensed MMEs per chronic pain patient per month; we will stratify data based on buprenorphine prescribing. We will also determine the number of pain consults placed per month. PEG scores will be collected for all chronic pain patients, and their scores will be assessed before and after completion of the pain consultation. We will collect qualitative data by surveying providers about the implementation of the pain committee.

FINDINGS TO DATE (IT IS NOT SUFFICIENT TO STATE FINDINGS WILL BE DISCUSSED ): Since initiation of the pain committee in October 2018, total dispensed MMEs/month has been stable while total dispensed MMEs/month excluding buprenorphine has decreased. We do not currently have data to report on patient and provider endpoints.

KEY LESSONS FOR DISSEMINATION (WHAT CAN OTHERS TAKE AWAY FOR IMPLEMENTATION TO THEIR PRACTICE OR COMMUNITY?): This framework for improving the safety and quality of chronic pain management is applicable to other clinical sites in rural and non-rural settings and can be implemented with minimal support staff.

EFFECTS OF A COMMUNITY BASED COLLABORATIVE DIABETES CONTROL AND PREVENTION PROGRAM FOR LOW- INCOME OVERWEIGHT AND OBESE PATIENTS IN A SUBURBAN POPULATION- A PILOT STUDY

Claudia L. Campos ${ }^{1}$; Deepak Palakshappa ${ }^{4}$; Carolyn F. Pedley ${ }^{5}$; Feben Girma $^{3}$; James L. Wofford ${ }^{6}$; Kirsten Feiereisel ${ }^{7}{ }^{1}$; Constance Poe ${ }^{8}$; Melanie Martin ${ }^{2}{ }^{1}$ Wake Forest University, WInston-Salem, NC; ${ }^{2}$ Wake Forest, Greensboro, NC; ${ }^{3}$ Wake Forest Baptist Medical Center, Winston-Salem, NC; ${ }^{4}$ Wake Forest School of Medicine, WINSTON SALEM, NC; ${ }^{5}$ Wake Forest, Winston-Salem, NC; ${ }^{6}$ Wake Forest School of Medicine, WinstonSalem, NC; ${ }^{7}$ Wake Forest University School of Medicine, Winston-Salem, NC; ${ }^{8}$ YWCA, Winston Salem, NC. (Control ID \#3159774)

\section{STATEMENT OF PROBLEM OR QUESTION (ONE SENTENCE):}

With the growing obesity crisis in the US, more adults develop diabetes and its complications. Community organizations and health care systems have begun collaborating to reduce diabetes development and improve glucose control. Linking patients' at higher risk of developing diabetes with community evidence-based behavioral weight loss interventions modeled after the $\mathrm{Na}$ tional Diabetes Prevention Program (DPP) have been successful in decreasing glucose levels and weight in randomized clinical trials settings.

OBJECTIVES OF PROGRAM/INTERVENTION (NO MORE THAN THREE OBJECTIVES): Given this context, the goal of this study was to evaluate the effects of a DPP based program offered by the Winston Salem, NC YWCA, for low income, obese patients with diabetes or pre- diabetes.

DESCRIPTION OF PROGRAM/INTERVENTION, INCLUDING ORGANIZATIONAL CONTEXT (E.G. INPATIENT VS. OUTPATIENT, PRACTICE OR COMMUNITY CHARACTERISTICS): The YWCA enrolled participants from September 2016 through January 2018. Participants were eligible if they had a hemoglobin Alc (HbAlc) 5.7 and a Body Mass Index (BMI) 25. They also had to be English speaking and either have Medicaid insurance or income under $200 \%$ of the Federal Poverty level. A primary care physician from a preselected academic medicine or community practices referred participants to the YWCA program. The program included the following key features: 1) individual case managers or "lifestyle coaches;" 2) frequent contact with participants; 3) a structured, state-of-the-art, 16- session core-curriculum that taught behavioral self-management strategies for weight loss and physical activity; 4) supervised physical activity sessions; 5) a more flexible maintenance intervention, combining group and individual approaches.

MEASURES OF SUCCESS (DISCUSS QUALITATIVE AND/OR QUANTITATIVE METRICS WHICH WILL BE USED TO EVALUATE PROGRAM/INTERVENTION): Primary outcomes were change at 9 months in Mean BMI, HbAlc, and health scores (HRA) as well as percentages of participants who lowered their BMI and HbAlc after the intervention

FINDINGS TO DATE (IT IS NOT SUFFICIENT TO STATE FINDINGS WILL BE DISCUSSED ): As of October 2018, 39 patients completed 9 months or more of the program intervention. Participant characteristics on enrollment were as follows: Race majority AA (36/39), graduated high school (28/39), Mean age 59.5 years (range 46-78), HbAlc 7.8 (range 5.7- 13.4), BMI 41.2 (range 27.3-75), HRA score 56 (range 39-75). At 9 months, participants' Mean Hb Alc decreased to 6.7 (-1.1, $p<0.001)$ and Mean BMI to 40.3 (-0.9, $p=0.007)$. 67\% (26/39) and 82\% (32/39) participants achieved reductions in BMI and in $\mathrm{Hb} A 1 \mathrm{c}$ respectively. Mean HRA increased to $61(+5, p=0.009)$.

KEY LESSONS FOR DISSEMINATION (WHAT CAN OTHERS TAKE AWAY FOR IMPLEMENTATION TO THEIR PRACTICE OR COMMUNITY?): After 9 months of participation, the glucose lowering and health score improvement effect of the collaborative YWCA program for low-income obese patients with prediabetes or diabetes was significant. Its effect on BMI was smaller and not statistically significant. The majority of participants lost weight and lowered their glucose levels. The findings suggest that achieving blood glucose and weight reduction in a real world setting parallel those achieved in the research settings.

EMERGENCY DEPARTMENT USE AMONG PATIENTS WITH MENTAL HEALTH CHIEF COMPLAINTS

Ana Sofia Warner ${ }^{1}$; Trina Chang ${ }^{2}{ }^{1}$; Jason H. Wasfy ${ }^{1}$; Dellara Terry ${ }^{2}$. ${ }^{1}$ Massachusetts General Hospital, Boston, MA; ${ }^{2}$ Partners HealthCare, Boston, MA. (Control ID \#3185791)

STATEMENT OF PROBLEM OR QUESTION (ONE SENTENCE): Can we identify characteristics of frequent emergency department (ED) visitors with mental health comorbidities in order to improve population health-based mental health initiatives and reduce emergency department utilization?

OBJECTIVES OF PROGRAM/INTERVENTION (NO MORE THAN THREE OBJECTIVES): 1. Identify patients who frequently visit the ED who have one or more mental health chief complaints associated with an ED visit during a defined time period. 2. Identify characteristics associated with frequent ED visits, such as insurance status, and enrollment in a care management program. 3 . Use this data to inform existing mental health initiatives to improve ambulatory care and reduce ED utilization.

DESCRIPTION OF PROGRAM/INTERVENTION, INCLUDING ORGANIZATIONAL CONTEXT (E.G. INPATIENT VS. OUTPATIENT, PRACTICE OR COMMUNITY CHARACTERISTICS): Partners Population Health (PHM) is responsible for population health initiatives that span the Partners HealthCare System (PHS) network, including a highrisk care management program and mental health initiatives. Using clinical and claims data, PHM identifies frequent visitors as patients who have 2 or more inpatient and/or emergency department visits in a 11 month period, and uses this data to assess for utilization trends and opportunities for care improvement. 
MEASURES OF SUCCESS (DISCUSS QUALITATIVE AND/OR QUANTITATIVE METRICS WHICH WILL BE USED TO EVALUATE PROGRAM/INTERVENTION): 1. Reduction in the average number of ED visits among frequent visitors with 1 or more mental health discharge diagnoses. 2. Reduction in the average number of patients who present to the ED with a mental health chief complaint and are not admitted. FINDINGS TO DATE (IT IS NOT SUFFICIENT TO STATE FINDINGS WILL BE DISCUSSED ): In August 2018 13,356 patients were identified as frequent ED visitors within the associated 11 month period. 1098 of these patients had at least one ED visit associated with an ICD code that contained one or more of the following diagnoses: anxiety or panic, depression, intentional self-harm, adjustment disorder, alcohol, bipolar or schizoaffective or manic disorder, personality disorder, psychosis or schizophrenia, substance use and suicidal ideation. This subgroup was more likely than their peers without these diagnoses to be male ( $51 \%$ vs. $40 \%$, p $<0.005$ ), have Medicaid ( $29 \%$ vs. $25 \%, \mathrm{p}<0.005)$ or commercial insurance $(43 \%$ vs. $38 \%, \mathrm{p}<0.005)$, and be younger (average age 44 vs. $53, \mathrm{p}<0.005$ ). They were less likely to be in a high-risk care management program (15\% vs. $17 \%, \mathrm{p}<0.05)$. They also had more $E D$ visits on weekends (average 0.72 vs $0.60, p<0.005$ ) and after hours (average 0.77 vs $0.68, \mathrm{p}<0.005$ ) that did not result in a hospital admission. In the 11 month period they had more ED visits than their peers with no mental health chief complaint (average 3.19 vs. $2.28, \mathrm{p}<0.05$ ) but no difference in the number of inpatient stays (average 0.63 vs $0.66, p=0.521$ ).

KEY LESSONS FOR DISSEMINATION (WHAT CAN OTHERS TAKE AWAY FOR IMPLEMENTATION TO THEIR PRACTICE OR COMMUNITY?): Other health systems may be able to similarly assess for differences in ED utilization among patients with mental health chief complaints. In particular, other systems can consider investigating alternatives to ED care for patients with urgent mental health needs, and/or expanding care management programs to include more patients with mental health disorders in order to reduce ED utilization among this population.

ESTABLISHING AN ORTHOPEDICS OBSERVATION UNIT - A NOVEL STRATEGY FOR CONTINUITY OF ORTHOPEDIC COMANAGEMENT

Frances J. Mao ${ }^{2}$; Sonia Siu ${ }^{1}$; Eric L. Hume ${ }^{3}$; Michele Fang ${ }^{1} .{ }^{1}$ University of Pennsylvania, Wynnewood, PA; ${ }^{2}$ University of Pennsylvania, Philadelphia, PA; ${ }^{3}$ Perlman School Medicine, U Pennsylvania, Philadelphia, PA. (Control ID \#3186429)

STATEMENT OF PROBLEM OR QUESTION (ONE SENTENCE): Hospitalists play a key role in orthopedic co-management during the peri- and post-operative period by providing guidance on common internal medicine issues; therefore, we sought to extend hospitalist support after hospital discharge by establishing an orthopedics observation unit for the care of total joint arthroplasty patients.

OBJECTIVES OF PROGRAM/INTERVENTION (NO MORE THAN THREE OBJECTIVES): Objective 1: To develop standard evidence based protocols for common postoperative problems to standardize care and avoid unnecessary tests. Objective 2: To develop an orthopedic observation unit where patients fitting criteria can receive expedited and standard treatment with their orthopedic and comanagement teams. Objective 3: To measure the outcomes of an orthopedic observation unit on length of stay, readmission rates, and complications.

DESCRIPTION OF PROGRAM/INTERVENTION, INCLUDING ORGANIZATIONAL CONTEXT (E.G. INPATIENT VS. OUTPATIENT, PRACTICE OR COMMUNITY CHARACTERISTICS): In October 2017, we established a hospital based orthopedics observation unit that included patients who underwent total knee arthroplasty (TKA), total hip arthroplasty (THA), revision TKA, and revision THA within the past 90 days. We developed evidence-based interdisciplinary protocols in conjunction with orthopedic surgery and emergency medicine for the work-up of fever, shortness of breath, anemia, and joint dislocation. Patients were triaged to the appropriate service (orthopedics observation, orthopedics inpatient, hospitalist observation, or hospitalist inpatient) based on acuity of illness and anticipated length of stay.

MEASURES OF SUCCESS (DISCUSS QUALITATIVE AND/OR QUANTITATIVE METRICS WHICH WILL BE USED TO EVALUATE PROGRAM/INTERVENTION): We will collected data metrics agreed upon by key stakeholders, including length of stay, readmission rate, complication rate, and mortality. Measures of success will focus on the number of patients who were able to be discharged on observation status ( $<48$ hours) without complications or readmissions.

FINDINGS TO DATE (IT IS NOT SUFFICIENT TO STATE FINDINGS WILL BE DISCUSSED ): Preliminary data shows that approximately 17 patients were admitted under orthopedics observation status, of which joint dislocation $(n=5,29.4 \%)$, infection $(n=3,17.6 \%)$ and urinary tract infection or retention $(n=3,17.6 \%)$ were the most common complaints. These patients were able to be discharged to home or skilled nursing facility without increase in complication. Also, traditionally the majority of these patients would have been admitted to general medicine services thereby disrupting continuity of care.

KEY LESSONS FOR DISSEMINATION (WHAT CAN OTHERS TAKE AWAY FOR IMPLEMENTATION TO THEIR PRACTICE OR COMMUNITY?): Orthopedic observation units may decrease readmissions, streamline care, and improve continuity of care for total joint replacement patients. There is no added cost to such a program, and these programs may become more common especially with bundled payments. Close communication with general internists, orthopedics, emergency medicine, and social work is necessary for successful implementation of orthopedic observation units. Further studies will need to be conducted to see if orthopedic observation units will improve outcomes and decrease costs.

EVALUATING THE IMPACT OF A MULTIDISCIPLINARY GASTROINTESTINAL MALIGNANCY CLINIC ON THE DELIVERY OF CARE AT THE DALLAS VA MEDICAL CENTER

Namrah Siddiq ${ }^{1}$; Monicah Gichinga ${ }^{2}$; Sanjai Sabu ${ }^{4}$; Thai H. Pham ${ }^{3}$; David Wang ${ }^{1}$. ${ }^{1}$ University of Texas Southwestern Medical Center, Dallas, TX; ${ }^{2}$ Dallas VA Medical Center, Dallas, TX; ${ }^{3}$ North Texas Dallas Veterans Affairs Medical Center, Dallas, TX; ${ }^{4}$ University of Texas Medical Branch, Galveston, TX. (Control ID \#3180921)

STATEMENT OF PROBLEM OR QUESTION (ONE SENTENCE): Can a biweekly Multidisciplinary Gastrointestinal Malignancy Clinic (MGMC) reduce the time from diagnosis to initiation of definitive treatment in patients with gastrointestinal cancer?

OBJECTIVES OF PROGRAM/INTERVENTION (NO MORE THAN THREE OBJECTIVES): -To reduce the time between oncologic consult and first definitive therapy in patients with gastrointestinal cancer -To reduce the time the between diagnosis and first definitive therapy in patients with gastrointestinal cancer -To improve the efficiency in which patients with gastrointestinal cancer complete their staging following diagnosis

DESCRIPTION OF PROGRAM/INTERVENTION, INCLUDING ORGANIZATIONAL CONTEXT (E.G. INPATIENT VS. OUTPATIENT, PRACTICE OR COMMUNITY CHARACTERISTICS): The outpatient MGMC was established at the Dallas VA in 2016. Prior to the 
MGMC, patients presented to their primary care physicians ( $\mathrm{PCPs}$ ), who once a malignancy was biopsy confirmed would consult an oncologic specialist. Patients requiring multidisciplinary oncologic care would then have up to three separate appointments (medical, surgical, and radiation oncology) scheduled on separate days. With the creation of the MGMC, however, clinicians from all required oncologic specialties evaluate a patient in a single clinic on a given day and a definitive therapy course is planned.

MEASURES OF SUCCESS (DISCUSS QUALITATIVE AND/OR QUANTITATIVE METRICS WHICH WILL BE USED TO EVALUATE PROGRAM/INTERVENTION): Patients seen in the MGMC were matched to patient controls (seen in the prior 2 years before the MGMC was established) by pathologic diagnosis and stage. The primary endpoint was time between the initial oncologic consult and first definitive therapy. Secondary endpoints were time from biopsy to first definitive therapy and time from biopsy to completion of staging. The average times for each endpoint for these two groups were then evaluated statistically.

FINDINGS TO DATE (IT IS NOT SUFFICIENT TO STATE FINDINGS WILL BE DISCUSSED ): 40 patient cases (with 40 available matched controls) were seen in the MGMC from July 2016-June 2018. A statistically significant reduction in the average time between initial oncologic consult to the time of first definitive therapy was found in favor of patients seen in the MGMC (44.3 \pm 20.5 days vs $60.7 \pm 41.4$ days). The average time from biopsy to first definitive therapy was not found to be significantly different between patient groups. The average time from biopsy to completion of staging was significantly reduced in the MGMC group (31.4 \pm 33.1 days vs $53.2 \pm 40.5$ days).

KEY LESSONS FOR DISSEMINATION (WHAT CAN OTHERS TAKE AWAY FOR IMPLEMENTATION TO THEIR PRACTICE OR COMMUNITY?): The establishment of a Multidisciplinary Gastrointestinal Malignancy Clinic allowed cancer patients to meet with various oncology specialists in a single setting and for oncology providers to decide upon an initial consensus treatment plan, resulting in a reduced time between initial consult and first definitive treatment. Staging was also completed more efficiently in patients seen in the multidisciplinary clinic. These results suggest that a multidisciplinary oncology clinic enhances delivery of care in newly diagnosed cancer patients.

\section{EVALUATION OF AN INTERVENTION SUPPORTING GUIDELINE-CONCORDANT, PATIENT-CENTERED CARE FOR PATIENTS ON CHRONIC OPIOIDS}

Joel Sawady $^{2,} 6$; Emily Hanson ${ }^{2}$; Esther Cazeau ${ }^{4}$; KATHERINE Y. COLON ${ }^{5}$; Michael Flores ${ }^{4,3}$; Yanyi Jiang ${ }^{4,3}$; Brian Mullin ${ }^{4,3}$; Ana M. Progovac ${ }^{4,3}$; Lynne A. Seeley ${ }^{1}$; Kathleen Thelen ${ }^{5}$; Benjamin Cook $^{4,3}$. ${ }^{1}$ Cambridge Health Alliane, Cambridge, MA; ${ }^{2}$ Cambridge Health Alliance, Brighton, MA; ${ }^{3} \mathrm{Har}-$ vard Medical School/Health Equity Research Lab, Cambridge, MA; ${ }^{4} \mathrm{Cam}-$ bridge Health Alliance, Cambridge, MA; ${ }^{5}$ Cambridge Health Alliance, Medford, MA; ${ }^{6}$ Harvard Medical School, Cambrige, MA. (Control ID \#3178624)

STATEMENT OF PROBLEM OR QUESTION (ONE SENTENCE): New regulations and guidelines have increased the burden on clinicians caring for patients on long-term opioid therapy (LTOT), compounding the challenge of providing patient-centered care for this vulnerable population OBJECTIVES OF PROGRAM/INTERVENTION (NO MORE THAN THREE OBJECTIVES): 1 . Support clinicians in providing regulation- and guideline-concordant care for patients on LTOT through improved EHR tools and staff support 2. Educate clinicians on best practices for legacy patients already on LTOT 3. Support clinicians in a patient-centered approach to reducing high-risk prescribing
DESCRIPTION OF PROGRAM/INTERVENTION, INCLUDING ORGANIZATIONAL CONTEXT (E.G. INPATIENT VS. OUTPATIENT, PRACTICE OR COMMUNITY CHARACTERISTICS): Cambridge Family Health $(\mathrm{CFH})$ is a primary care practice within the Cambridge Health Alliance (CHA), a safety-net institution caring for a diverse patient population, with a high burden of psychiatric illness, substance use disorders, and chronic pain. LTOT for chronic pain has become more complex due to: 1 . New regulations and evolving best practices, including mandated counseling, prescription monitoring program (PMP) use, controlled substance agreements (CSAs), and urine drug screens (UDSs) 2. Team-based care leading to a need for structured coverage arrangements In response, our multidisciplinary team reviewed relevant regulations, created clinician guidance, and developed workflows to support that guidance. The intervention included: 1 . Regulatory- and guideline-concordant EHR documentation 2. Staffsupported workflows to check the PMP, ensure CSAs are in place, and obtain UDSs 3. Clinician education We hypothesized that educating clinicians and reducing administrative burden would remove barriers to individualized treatment and improve care.

MEASURES OF SUCCESS (DISCUSS QUALITATIVE AND/OR QUANTITATIVE METRICS WHICH WILL BE USED TO EVALUATE PROGRAM/INTERVENTION): Patients on LTOT receiving care at CFH ( $\mathrm{N}=257$; intervention group) were compared with patients on LTOT receiving care at all other CHA clinics ( $\mathrm{N}=1680$; comparison group). We evaluated changes between 2015 and 2018 in: 1. Proportion of patients receiving UDSs 2. Rate of co-prescription of benzodiazepine and opioids 3 . Rate of discontinuation of opioids 4. Proportion of patients on high-dose opioids We conducted a difference-in-differences analysis comparing changes in the intervention group to changes in the control group, adjusting for relevant covariates. Additional analyses were done on a subset of patients on high-dose LTOT (intervention=41, control=212).

FINDINGS TO DATE (IT IS NOT SUFFICIENT TO STATE FINDINGS WILL BE DISCUSSED ): Compared to the comparison group, patients in the $\mathrm{CFH}$ intervention had a significantly greater increase in use of UDSs ( $\mathrm{D}-\mathrm{D}=+8.27 \%$; $\mathrm{p}<.05$ ); a significantly greater decrease in coprescription of benzodiazepines ( $\mathrm{D}-\mathrm{D}=-15.9 \%$; $\mathrm{p}<.05$ ); but no statistically significant change in discontinuation of opioids. Results of D-D analysis were consistent for both unadjusted and adjusted analysis. Controlling for covariates, there was a statistically significant difference in the proportion of patients on high-dose opioids between treatment and comparison groups at the end of the intervention ( $=-.20 \mathrm{p}<.05)$.

KEY LESSONS FOR DISSEMINATION (WHAT CAN OTHERS TAKE AWAY FOR IMPLEMENTATION TO THEIR PRACTICE OR COMMUNITY?): An intervention supporting guideline-concordant care for patients with LTOT shows promise in improving care, particularly for patients on high-dose opioids

EXPANDING ACCESS TO INTRANASAL NALOXONE FOR HIGH RISK PATIENTS UPON HOSPITAL DISCHARGE

Erica Tavares; Rutwij K. Joshi. Massachusetts General Hospital, Boston, MA. (Control ID \#3186346)

STATEMENT OF PROBLEM OR QUESTION (ONE SENTENCE): There is no clearly defined process for providing intranasal naloxone for eligible patients being discharged from their inpatient stay at Massachusetts General Hospital.

OBJECTIVES OF PROGRAM/INTERVENTION (NO MORE THAN THREE OBJECTIVES): A pharmacist driven pilot program was performed on an internal medicine and oncology unit with the objectives of increasing 
hospital provider awareness of high risk candidates and providing access to intranasal naloxone at discharge for these individuals.

DESCRIPTION OF PROGRAM/INTERVENTION, INCLUDING ORGANIZATIONAL CONTEXT (E.G. INPATIENT VS. OUTPATIENT, PRACTICE OR COMMUNITY CHARACTERISTICS): The pharmacist driven protocol on an internal medicine and oncology inpatient unit involved the daily screening of patients meeting criteria for high risk of opioid overdose. Criteria included patients taking $50 \mathrm{mg}$ or greater per day of morphine equivalents, or patients with active or a history of opioid use disorder and those on opioid withdrawal agents. Responding clinicians would then be alerted by the pharmacist of their high-risk patients and naloxone would then be recommended upon discharge. Upon agreement with the responding clinician, the patient would then be seen by the pharmacist, who would counsel them on the signs and symptoms, and risks of opioid overdose. They would then be asked if they would like a prescription for intranasal naloxone upon discharge. If the patient agreed, the pharmacist would write the order and deliver the medication to the patient while still inpatient, to ensure that they left with the medication in hand at discharge. This initiative took place from December 15, 2017 until February 15, 2018.

MEASURES OF SUCCESS (DISCUSS QUALITATIVE AND/OR QUANTITATIVE METRICS WHICH WILL BE USED TO EVALUATE PROGRAM/INTERVENTION): The percentage of eligible patients discharged with intranasal naloxone from this inpatient unit were measured before and after the initiative took place.

FINDINGS TO DATE (IT IS NOT SUFFICIENT TO STATE FINDINGS WILL BE DISCUSSED ): The percentage of eligible patients discharged on intranasal naloxone increased from an average of $13 \%$ to $28 \%$ from December 15, 2017 to January 15, 2018

KEY LESSONS FOR DISSEMINATION (WHAT CAN OTHERS TAKE AWAY FOR IMPLEMENTATION TO THEIR PRACTICE OR COMMUNITY?): This pilot was successful in increasing access to intranasal naloxone to high risk patients upon discharge. The modest percentage increase may be due to limited outpatient pharmacy hours, evening or weekend discharges, and the availability of a pharmacist to counsel patients on naloxone before patients are discharged. Areas for improvement and expansion included a collaborative practice agreement for trained pharmacists in opioid use disorder to prescribe intranasal naloxone upon discharge.

FEASIBILITY OF IMPLEMENTING A 24-HOUR AMBULATORY BLOOD PRESSURE MONITORING (ABPM) TESTING SERVICE INTO PRIMARY CARE

Nadia Liyanage-Don $^{1}$; Nathalie Moise ${ }^{1}$; Kristal Quispe ${ }^{2}$; Jennifer Mizhquiri Barbecho $^{2}$; Hye Gi Shim ${ }^{1}$; Ian M. Kronish ${ }^{1} .{ }^{1}$ Columbia University Medical Center, New York, NY; ${ }^{2}$ Columbia University Medical Center, Brooklyn, NY. (Control ID \#3181226)

STATEMENT OF PROBLEM OR QUESTION (ONE SENTENCE): In 2015 the USPSTF updated their hypertension screening guidelines to recommend that patients with elevated office BP undergo ABPM to confirm the diagnosis of hypertension and exclude white coat hypertension (WCH) (grade A). However, ABPM is infrequently used in primary care due to lack of accessibility, concerns about patients' ability to complete testing, and inadequate reimbursement. We aimed to assess the feasibility and economic viability of an ABPM testing service offered within a primary care network.

OBJECTIVES OF PROGRAM/INTERVENTION (NO MORE THAN THREE OBJECTIVES): 1 . To increase the use of 24-hour ABPM as part of hypertension screening and diagnosis in a primary care network serving a vulnerable patient population. 2. To increase the identification of $\mathrm{WCH}$ in primary care. 3 . To determine the financial sustainability of a 24-hour ABPM testing service with a predominantly publicly insured payor mix.

DESCRIPTION OF PROGRAM/INTERVENTION, INCLUDING ORGANIZATIONAL CONTEXT (E.G. INPATIENT VS. OUTPATIENT, PRACTICE OR COMMUNITY CHARACTERISTICS): ActiveBP is a 24-hour ABPM testing service launched in 2015 at Columbia University Irving Medical Center. Previously, ABPM was only available on campus through referrals to nephrology for patients with kidney disease. ActiveBP offers ABPM testing to patients within the primary care network who have elevated office BP but no diagnosis of hypertension, the only Medicaid/Medicare reimbursable condition. Key features of ActiveBP include an order set within the electronic health record, patient- and provider-friendly ABPM reports, bilingual coordinators for scheduling and device placement, and provider education about ABPM testing availability through presentations and newsletters.

MEASURES OF SUCCESS (DISCUSS QUALITATIVE AND/OR QUANTITATIVE METRICS WHICH WILL BE USED TO EVALUATE PROGRAM/INTERVENTION): BP values from ActiveBP were compared to office BP values from the electronic health record to classify patients as having sustained hypertension (office BP 140/90 mmHg, mean awake BP 135/85 mmHg) or WCH (office BP 140/90 mmHg, mean awake $\mathrm{BP}<135 / 85 \mathrm{mmHg}$ ). Success metrics included the volume of patients referred for ABPM, rates of ABPM completion (defined as 10 awake BP readings), patient and provider satisfaction, and economic viability (revenue versus costs).

FINDINGS TO DATE (IT IS NOT SUFFICIENT TO STATE FINDINGS WILL BE DISCUSSED ): Between September 2015 and June 2018, 436 primary care patients were referred for ABPM, of which 256 initiated testing and $246(95 \%)$ completed testing with sufficient readings. Of patients with sufficient readings, 123 (50\%) were found to have WCH. Patient surveys showed high satisfaction with the testing service. Focus groups to assess provider satisfaction are currently being planned. Economic analysis showed that, including staff time and overhead, cost per test was \$200 whereas Medicaid/Medicare reimbursement per test was $\$ 62$.

KEY LESSONS FOR DISSEMINATION (WHAT CAN OTHERS TAKE AWAY FOR IMPLEMENTATION TO THEIR PRACTICE OR COMMUNITY?): An ABPM testing service can successfully conduct ABPM in primary care patients and can identify $\mathrm{WCH}$ in about half of patients referred. However, with a payor mix comprised of primarily Medicaid/Medicare recipients, reimbursement is insufficient to cover costs. Conducting ABPM testing within the primary care clinic or offering the service to a broader mix of publicly and commercially insured patients may be needed for such testing to be economically viable.

FINANCIAL INCENTIVES FOR MEDICAL ASSISTANTS AS PANEL MANAGERS TO IMPROVE COLORECTAL CANCER SCREENING RATES IN A LARGE, URBAN, ACADEMIC PRIMARY CARE MEDICAL GROUP

$\underline{\text { Laura J. Zimmermann }}{ }^{1}$; Michael Hanak ${ }^{1}$; Tim Reiff'; Aaron Shultz ${ }^{1}$; Daniel Dunham ${ }^{1}{ }^{1}$ Rush University Medical Center, Chicago, IL; ${ }^{2}$ Rush University Medical Center, Oak Park, IL. (Control ID \#3173540)

\section{STATEMENT OF PROBLEM OR QUESTION (ONE SENTENCE):} Cancer screening is becoming increasingly important for financial viability of primary care practices as health systems transition into performance-based payer contracts. As of October $28^{\text {th }}, 2018$, only $65.93 \%$ of eligible primary care patients were screened for colorectal cancer (CRC) in this large, urban, academic medical group (eCQM CMS-130v5). 


\section{OBJECTIVES OF PROGRAM/INTERVENTION (NO MORE THAN} THREE OBJECTIVES): Increase CRC screening rate from $65.93 \%$ to the HEDIS top decile target of $72 \%$ by December 31,2018 , at which time financial incentives for the health system will be determined per payer contracts.

DESCRIPTION OF PROGRAM/INTERVENTION, INCLUDING ORGANIZATIONAL CONTEXT (E.G. INPATIENT VS. OUTPATIENT, PRACTICE OR COMMUNITY CHARACTERISTICS): In addition to evidence-based interventions for improving CRC screening rates (clinical detailing, offering stool-based testing, patient reminder letters and electronic messages, PCP audit-and-feedback, and patient navigation), we offered medical assistants (MAs) in 9 of 13 primary care clinics a financial incentive of $\$ 400$ if their respective clinic achieved a CRC screening rate of $72 \%$. MAs in all 13 clinics were trained to utilize an electronic health record report to call overdue patients to 1) document outside results, 2) transfer patients with existing orders to colonoscopy schedulers, or 3) schedule a visit with the PCP to discuss CRC screening. Setting: 13 urban primary care clinics with 69 PCPs totaling approximately 90,000 patient visits per year and 6,904 patients overdue for CRC screening as of October $28^{\text {th }}, 2018$.

MEASURES OF SUCCESS (DISCUSS QUALITATIVE AND/OR QUANTITATIVE METRICS WHICH WILL BE USED TO EVALUATE PROGRAM/INTERVENTION): We compared CRC screening rates at the provider-, clinic-, and medical-group level on October 28th, 2018 (prior to announcing MA financial incentives), and December $31^{\text {st }}, 2018$. To determine the relative impact of MA financial incentives, we compared the percentage of overdue patients screened and the change in screening rates in the clinics in which MAs were incentivized compared to clinics in which MAs were not incentivized.

FINDINGS TO DATE (IT IS NOT SUFFICIENT TO STATE FINDINGS WILL BE DISCUSSED ): As of December 31st, 2018, these 13 primary care clinics screened an additional 1,532 patients totalling 14,895 of 21,317 eligible patients $(69.87 \%)$, an improvement of $3.94 \%$ in this time period. Sixty of 69 physicians $(86.96 \%$ ) improved (range $-1.34 \%$ to $+13.53 \%$ ). Twelve of the 13 clinics $(92.31 \%$ ) improved (mean 3.12\%, range $-0.17 \%$ to $5.21 \%$ ). A total of 22 MAs received the financial incentive in 5 of 13 clinics. Clinics that incentivized MAs screened a larger percentage of overdue patients (mean $14.18 \%$, range $7.06 \%$ to $20.60 \%$ ) compared to those that did not (mean $5.13 \%$, range $2.38 \%$ to $8.33 \%$ ). Clinics that incentivized MAs also generally achieved greater improvement in CRC screening rates (mean 3.63\%, range $1.59 \%$ to $5.21 \%$ ) compared to those that did not (mean $1.93 \%$, range $-0.17 \%$ to $4.17 \%$ ).

KEY LESSONS FOR DISSEMINATION (WHAT CAN OTHERS TAKE AWAY FOR IMPLEMENTATION TO THEIR PRACTICE OR COMMUNITY?): As reimbursement models evolve to promote valuebased care, health systems will need to utilize healthcare team members to improve quality and avoid PCP burnout. We demonstrated that a large medical group can leverage primary care MAs as panel managers to address care gaps and perform outreach, and the use of modest financial incentives can confer additional improvement in CRC screening rates.

FINDING NEEDLES IN THE EHR HAYSTACK: DESIGN AND EARLY RESULTS FOR A NATURAL LANGUAGE PROCESSING MODEL TO IDENTIFY CLINICAL NOTES RELEVANT TO A PATIENT'S GOALS OF CARE

Azalea Kim $^{1}$; Allison Dunning ${ }^{2}$; Shelley Rusincovitch ${ }^{2}$; Andrew Olson ${ }^{2}$; Julie Childers $^{1}$; Erich Huang ${ }^{2}$; Ursula A. Rogers ${ }^{2}$; Brian Griffith ${ }^{1}$; Lawrence A.

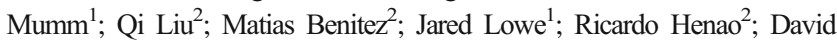
Casarett ${ }^{1}$. Duke University Health System, Durham, NC; ${ }^{2}$ Duke University, Durham, NC. (Control ID \#3184334)
STATEMENT OF PROBLEM OR QUESTION (ONE SENTENCE): Evidence of goals of care (GOC) conversations between patients and providers are scattered throughout the EHR as free text in clinical notes, making it difficult and time-intensive for providers to find.

OBJECTIVES OF PROGRAM/INTERVENTION (NO MORE THAN THREE OBJECTIVES): Describe extent to which GOC-relevant data are scattered throughout the EHR as free text in notes Leverage an integrated clinical and quantitative health data science team to develop a data science model that uses natural language processing (NLP) and machine learning methods to identify notes that contain GOC-relevant data

DESCRIPTION OF PROGRAM/INTERVENTION, INCLUDING ORGANIZATIONAL CONTEXT (E.G. INPATIENT VS. OUTPATIENT, PRACTICE OR COMMUNITY CHARACTERISTICS): We assembled a health data science team with quantitative and clinical members from primary care, hospital medicine, and palliative care experienced in GOC conversations. The data set included $\sim 5.3$ million clinical notes for 97,402 beneficiaries in Duke's Medicare ACO between 2015-2017. Since there's no "gold standard" to define a GOC-relevant note, we drew from palliative care and advance care planning notes and personal experience to create 3 categories to identify GOC-relevant notes: 1 . Surrogate decision makers; 2. Goals and values; 3 . Care preferences. Clinical members identified 51 key phrases within those categories, and quantitative team members wrote them into regular expressions (regex) to search for these phrases.

MEASURES OF SUCCESS (DISCUSS QUALITATIVE AND/ OR QUANTITATIVE METRICS WHICH WILL BE USED TO EVALUATE PROGRAM/INTERVENTION): Use regex to analyze clinical notes and their distribution for GOC-relevant data in an ACO population Develop a consensus-driven standard to identify GOC-relevant notes to train an NLP machine learning algorithm FINDINGS TO DATE (IT IS NOT SUFFICIENT TO STATE FINDINGS WILL BE DISCUSSED ): We evaluated characteristics of regex phrases associated with GOC classifications and their distribution across notes. On average, regex phrases appeared once or twice within the same note, and a single note typically only matched one regex phrase, with maximum 45 matches within a single note. Regex alone are inadequate to identify GOC-relevant notes. Templated notes contributed heavily to this problem. We performed an iterative series of paired clinical note reviews on random samples of notes selected via regex labels that informed our approach to a large scale note adjudication process to train an NLP machine learning algorithm. We then developed a consensusdriven standard to identify notes relevant to real-time GOC discussions. Clinical members are currently labeling $\sim 1,000$ clinical notes as being relevant or not to a real-time GOC discussion, with an additional tag for particularly relevant notes.

KEY LESSONS FOR DISSEMINATION (WHAT CAN OTHERS TAKE AWAY FOR IMPLEMENTATION TO THEIR PRACTICE OR COMMUNITY?): Our analysis confirmed suspicions that GOC data are scattered within a sea of notes within the EHR. From a data science perspective, the lack of a "gold standard" to define GOC relevance is a formidable challenge, but we used our experiences to design a clinically relevant endpoint. Eventually the model will be deployed as a point-of-care tool that displays high probability GOC-relevant notes. Our design and approach could be generalizable to other organizations, and may inform approaches to other nuanced areas of clinical care where data is captured in notes, and a gold standard similarly may not exist. 
FIT VS COLONOSCOPY: A SHARED DECISION MAKING WORKSHOP TO OPTIMIZE COLORECTAL CANCER SCREENING IN AN URBAN UNDERSERVED POPULATION

Denis Yusupov $^{1,2}$; Jordonna Brown ${ }^{1,2}$; Christon Grant ${ }^{1,2}$; Nikita Gupta ${ }^{1,2}$; Gayatri Malhotra-Gupta $^{1,2}$; Michelle Likhtshteyn ${ }^{1,2}$; Melissa S. Lee ${ }^{1,2}$; David L. Stevens ${ }^{1,2}$; Andrew A. Chang ${ }^{1,2}$. ${ }^{1}$ NYC Health + Hospitals/ Kings County, New York, NY; ${ }^{2}$ SUNY Downstate Medical Center, Brooklyn, NY. (Control ID \#3186050)

STATEMENT OF PROBLEM OR QUESTION (ONE SENTENCE): Will a resident run workshop on shared decision making (SDM) improve colorectal cancer (CRC) screening completion rates in an underserved population?

OBJECTIVES OF PROGRAM/INTERVENTION (NO MORE THAN THREE OBJECTIVES): To optimize CRC screening completion of FIT vs colonoscopy through SDM in an urban underserved population. DESCRIPTION OF PROGRAM/INTERVENTION, INCLUDING ORGANIZATIONAL CONTEXT (E.G. INPATIENT VS. OUTPATIENT, PRACTICE OR COMMUNITY CHARACTERISTICS): A retrospective analysis reviewed colon cancer screening rates in average risk patients, as per the U.S. Preventive Services Task Force (USPSTF) guidelines, in an urban underserved resident primary care practice between January and February 2017. The intervention was a resident-run SDM 3-hour workshop using lecture, worksheets, and group practice to teach residents patientcentered CRC screening. A prospective cohort study reviewed charts from August 28th to October 24th, 2018 to assess completion of screening.

MEASURES OF SUCCESS (DISCUSS QUALITATIVE AND/OR QUANTITATIVE METRICS WHICH WILL BE USED TO EVALUATE PROGRAM/INTERVENTION): Overall CRC screening test ordering and completion rates, pre- and post-intervention, were assessed for eligible patients. A subgroup analysis of fecal immunochemical testing (FIT) and colonoscopy completion rates was also performed.

FINDINGS TO DATE (IT IS NOT SUFFICIENT TO STATE FINDINGS WILL BE DISCUSSED ): At baseline, 52 patient charts reviewed 9 (17\%) FIT test ordered, $78 \%$ completion rate $43(82 \%)$ colonoscopies ordered, $26 \%$ completion rate Overall baseline completion rate: $34 \%$ Postintervention: 45 patient charts reviewed 22 (48\%) FIT chosen, 59\% completion rate $23(52 \%)$ colonoscopies chosen, $43.4 \%$ completion rate Overall post-intervention completion rate: $51 \%$ There is a $17 \%$ difference $(\mathrm{p}=0.092,2$ proportion test, trending but not statistically significant) from baseline overall completion rate, however there was statistical significance in patient choice of test after the workshop ( $p=0.0011$ ). KEY LESSONS FOR DISSEMINATION (WHAT CAN OTHERS TAKE AWAY FOR IMPLEMENTATION TO THEIR PRACTICE OR COMMUNITY?): Although CRC is the third most common cancer among men and women and is projected to cause more than 50,000 deaths in 2017 , only $62.6 \%$ of adults 50 years and older were screened (CDC, 2015). Access, insurance/immigration status, education, burden of preparation and ethnicity impact cancer screening. Urban underserved populations are disproportionately affected by these barriers. This study demonstrates that improving SDM between patients and providers can decrease barriers and improve CRC screening. An efficient resident- led curriculum can be easily integrated into a residency schedule. Though the completion rates only trended towards statistical significance, there was a clear difference in tests ordered, reflecting that SDM influences choice of screening method. While SDM improved the show rate for colonoscopy, the no-show rate remained high. Further investigation on patients reasons for not completing their colonoscopy is a possible area for future investigation.
FOOD AS MEDICINE: MEDICAL NUTRITION THERAPY FOR PATIENTS LIVING WITH DIABETES

manjeet bhamra ${ }^{2,1}$; Muhammad afzal Khan ${ }^{2,1}$; Jonathan Francois ${ }^{2,1}$; duarxy R. sainvilien ${ }^{2,1}$; Marlee Ickowicz ${ }^{2}$; Andrew A. Chang ${ }^{2,1}$; Melissa S. Lee ${ }^{2,1}$. ${ }^{1}$ SUNY Downstate, New York, NY; ${ }^{2} \mathrm{NYC}$ Health + Hospitals/Kings County, New York, NY. (Control ID \#3185715)

STATEMENT OF PROBLEM OR QUESTION (ONE SENTENCE): Despite best practice recommendations from the American Diabetes Association, only $20 \%$ of patients living with diabetes at our urban, inner city Internal Medicine resident practice have received medical nutrition therapy (MNT).

OBJECTIVES OF PROGRAM/INTERVENTION (NO MORE THAN THREE OBJECTIVES): To engage the clinical care team in recognizing and referring eligible patients for MNT To increase access to MNT by integrating services into the primary care visit during non-value added time

DESCRIPTION OF PROGRAM/INTERVENTION, INCLUDING ORGANIZATIONAL CONTEXT (E.G. INPATIENT VS. OUTPATIENT, PRACTICE OR COMMUNITY CHARACTERISTICS): The East New York (ENY) Diagnostic and Treatment Center, with a diabetes control rate of $59 \%(\mathrm{HbAlc}<8)$, is the lowest performer in the $\mathrm{NYCH}+\mathrm{H}$ system. Despite having a certified registered dietician on-site, $80 \%$ of patients living with diabetes have not received MNT. Our intervention was designed to address this gap in care. Records of diabetic patients $(\mathrm{HbAlc}>6.5)$ seen in the practice from July to August 2018 were reviewed to identify those who had received MNT within 3 months of their last monitored $\mathrm{HbAlc}$. 30\% eligible patients were referred for MNT. $68 \%$ of those referred completed MNT. A multidisciplinary, collaborative discussion with clinicians, medical assistants, nurses, and our registered dietician confirmed these findings. Using a team-based approach, we sought to engage patients in MNT during their primary care visit. Performance data and clinical guidelines were shared with the clinical team. Medical Assistants (MA) identified patients with diabetes who did not receive MNT within 3 months of their last $\mathrm{HbA1c}$. MA initiated the MNT referral while patients were waiting to see their primary care provider (PCP)

MEASURES OF SUCCESS (DISCUSS QUALITATIVE AND/OR QUANTITATIVE METRICS WHICH WILL BE USED TO EVALUATE PROGRAM/INTERVENTION): To increase the number of MNT referrals and completed MNT visits for diabetic patients by $5 \%$ from a baseline of $30 \%$ and $68 \%$ respectively.

FINDINGS TO DATE (IT IS NOT SUFFICIENT TO STATE FINDINGS WILL BE DISCUSSED ): During the post-intervention study period from October to November 2018, 51\% patients living with diabetes were referred for MNT. MNT completion rate, $68 \%$ prior to the intervention, was $70 \%$ after the intervention, essentially unchanged.

KEY LESSONS FOR DISSEMINATION (WHAT CAN OTHERS TAKE AWAY FOR IMPLEMENTATION TO THEIR PRACTICE OR COMMUNITY?): High rates of uncontrolled diabetes in our patient population necessitate creative team-based clinical interventions to help close gaps in care. Engaging MA in identifying patients living with diabetes who had not received MNT proved an effective way of increasing referrals for diabetes self-management education compared with PCP referral alone. Same-day access to MNT, and a seamless transition from MNT to primary care visits did not impact the percent of completed MNT sessions. Future studies should include the voice of the patient to better understand barriers to integrating MNT into routine care. 
FOOD FOR THOUGHT: CREATING ACCESSIBLE FOOD PHARMACIES FOR AT-RISK POPULATIONS

Divya Venkat ${ }^{1}$; Veli Bakalov ${ }^{1}$; Herman Singh ${ }^{3}$; Courtney Watson ${ }^{2}$; Colleen Ereditario $^{2}$; Bruce Ling ${ }^{2}$; Elizabeth Cuevas ${ }^{2,1}$. ${ }^{1}$ Allegheny General Hospital, Pittsburgh, PA; ${ }^{2}$ Center for Inclusion Health, Pittsburgh, PA; ${ }^{3}$ Allegheny Health Network, Pittsburgh, PA. (Control ID \#3183890)

STATEMENT OF PROBLEM OR QUESTION (ONE SENTENCE): Approximately $47 \%$ of the population in Pittsburgh experiences difficulty accessing food and has the fourth highest rate of obesity in America.

OBJECTIVES OF PROGRAM/INTERVENTION (NO MORE THAN THREE OBJECTIVES): To improve access to healthy foods in low income populations

DESCRIPTION OF PROGRAM/INTERVENTION, INCLUDING ORGANIZATIONAL CONTEXT (E.G. INPATIENT VS. OUTPATIENT, PRACTICE OR COMMUNITY CHARACTERISTICS): The Healthy Food Center was implemented due to a need for food banks for an obese and medically complex population. Through this program, patients receive a referral from a provider and can visit the healthy food center. On-site, a registered dietician tailors each patient's nutrition plan to their comorbid conditions. The food bank provides fresh vegetables, fruits and low sodium goods. Patients are then given 2-3 days worth of food based on family size. From here, patients are given resources to food pantries and boxed food programs. Additionally, patients are given directions on how to shop on a budget and tips on healthy cooking. Patients are also provided with help on completing forms for food assistance programs as needed.

MEASURES OF SUCCESS (DISCUSS QUALITATIVE AND/OR QUANTITATIVE METRICS WHICH WILL BE USED TO EVALUATE PROGRAM/INTERVENTION): Success of this intervention is based on the determination of whether visiting patients had weight loss, change in BMI or $\mathrm{HbAlc}$ level.

FINDINGS TO DATE (IT IS NOT SUFFICIENT TO STATE FINDINGS WILL BE DISCUSSED ): A total of 137 were referred over the period of May 2018 to November 2018. This study included 55 patients with three-month follow up appointment. A majority of patients were females $(98(71.5 \%))$ and $90(65.7 \%)$ patients had households less than 2 people. Our patients were referred from diabetic center (37.2\%), in network PCP (21.9\%) and bariatric center (10.2\%). $66.7 \%$ had diabetes, $49.6 \%$ had hyperlipidemia, $50.3 \%$ hypertension, $8.8 \%$ kidney disease, $8.8 \%$ with heart disease. $22.6 \%$ of the patients followed low sodium diet, $30.7 \%$ met with dietitian and $33.6 \%$ were involved in weight management. Mean BMI at baseline was 36.5 and 35.9 at three month follow up. The majority of patients (40(72.7\%)) had a BMI above 30. Among our patients, 33 (60.0\%) patients had a 1.7 decrease in BMI and $22(40.0 \%)$ patients had 0.7 increase in BMI. Mean weight at baseline was 232.1 pounds and at three months follow up mean weight was 234.6 pounds. Average weight loss was 7.7 pounds and was seen in 30 $(54.5 \%)$ patients, while $25(45.4 \%)$ patients gained mean 3.8 pounds. $\mathrm{Hb} \mathrm{A} 1 \mathrm{c}$ was checked at the first appointment and repeated in 3 months in a total of 27 patients. In 3 months follow up there was no significant $\mathrm{HbAlc}$ change and it was not significantly different with change in weight.

KEY LESSONS FOR DISSEMINATION (WHAT CAN OTHERS TAKE AWAY FOR IMPLEMENTATION TO THEIR PRACTICE OR COMMUNITY?): Our study demonstrates that $60 \%$ of the patients visiting food bank had 1.7 decrease in BMI, and $54.5 \%$ of the patients lost 7.7 pounds. Using a food pharmacy that incorporates a dietician at the recommendation of physicians allows patients to engage in healthy eating while obtaining affordable foods. To further determine our results, follow up would need to be performed at 6 months and one year to evaluate hemoglobin A1C.
GETTING PATIENTS IN THE DOOR: USING MACHINE LEARNING TO INCREASE PATIENT ENGAGEMENT IN RESIDENT CONTINUITY CLINIC

Roma Bhatia; Zachary Lenhart; Patrick Lynch; Dang Tran; Janice A. Cunningham; Gary Fischer; Jaishree Hariharan. University of Pittsburgh Medical Center, Pittsburgh, PA. (Control ID \#3180738)

STATEMENT OF PROBLEM OR QUESTION (ONE SENTENCE): Despite having an established automated phone reminder system in place for patients, in one large academic primary care clinic, the annual no show rate (NSR) averages $27 \%$ for residents' panel patients (RP) versus $12 \%$ for faculty panel patients (FP) in the same clinic.

OBJECTIVES OF PROGRAM/INTERVENTION (NO MORE THAN THREE OBJECTIVES): Continuity clinic is a crucial yet fragmented component of internal medicine training. One major contributing factor is the disproportionate number of missed appointments by RP versus FP. This not only results in poor continuity of care for patients but also decreased learning opportunities for residents. The primary objective of this study is to determine if implementation of a targeted nurse led phone based appointment reminder intervention for patients can reduce the number of missed appointments by RP.

DESCRIPTION OF PROGRAM/INTERVENTION, INCLUDING ORGANIZATIONAL CONTEXT (E.G. INPATIENT VS. OUTPATIENT, PRACTICE OR COMMUNITY CHARACTERISTICS): This urban primary care clinic involves 60 residents who see 3100 patients annually. Residents have one full clinic day weekly for 4 week periods, and see 10-12 patients per full clinic day. Compared to FP who are $71 \%$ White and 23\% Black/Asian, RP are racially heterogenous: $44 \%$ are White and $52 \%$ of are Black/Asian. In addition, RP are more likely to not have insurance at all or have medical assistance compared to FP (44\% vs. $17 \%)$. Usual care for both RP and FP involves an automated phone call appointment reminder that is sent 72 hours prior to their scheduled visit. For the intervention, a machine learning algorithm was utilized to identify RP who were most likely to miss their appointments. This was developed by the health system using 1.7 million office visits with the target of predicting which patient appointments will be missed. RP with at least $20 \%$ chance of missing their appointment were contacted by the nurse 48 hours prior to their scheduled appointment in addition to usual care. Voicemails were left if patients were not reached.

MEASURES OF SUCCESS (DISCUSS QUALITATIVE AND/OR QUANTITATIVE METRICS WHICH WILL BE USED TO EVALUATE PROGRAM/INTERVENTION): The number of patients called, and the outcome of these calls were recorded. The average no show rate, and percent of completed visits was calculated pre and post intervention based on the total number of appointments made and the total number of appointments attended by RP.

FINDINGS TO DATE (IT IS NOT SUFFICIENT TO STATE FINDINGS WILL BE DISCUSSED ): During the 10 weeks of intervention, a total of 346 RP were called and 64\% were reached $(n=222 / 346) .86 \%$ confirmed their appointments ( $n=192 / 222)$ while $14 \%$ either rescheduled or canceled $(n=30 / 226)$ therefore opening slots for other patients. $54 \%$ of patients who confirmed attended their appointment $(n=104 / 192)$. Compared to pre-intervention, the NSR for RP was $22 \%$ (vs 27\%) and the completed patient visits averaged $78 \%$ (vs. $74 \%$ ).

KEY LESSONS FOR DISSEMINATION (WHAT CAN OTHERS TAKE AWAY FOR IMPLEMENTATION TO THEIR PRACTICE OR COMMUNITY?): This intervention decreased the RP NSR by $5 \%$, increased the overall completed visits, and correspondingly increased the number of patients seen in clinic by residents. Compared to FP who may be 
scheduled 1-2 weeks in advance, RP are in flux and may not be scheduled until 72 hours prior to the appointment time. They may also have multiple competing demands that prevent them from coming to clinic, thus requiring reminders much closer to their appointment.

HOW PRIMARY CARE RESIDENTS WORKING WITH PHARMACY TEAMS CAN HELP ADDRESS HEDIS MEASURES WHILE EDUCATING RESIDENT PROVIDERS ON THE IMPORTANCE OF MEDICATION ADHERENCE IN THE AMBULATORY SETTING

Marilyn Chacko; Yi Shan Lee; Morris Jrada; Teresa Attina; Oscar ValderramaTorres; Lisa Anzisi; Mariel Shull; Jennifer Oh; Isaac Dapkins; Neil A. Pasco. NYU Langone Health, New York, NY. (Control ID \#3185116)

STATEMENT OF PROBLEM OR QUESTION (ONE SENTENCE): As healthcare delivery shifts to the value based paradigm how do you educate primary care providers on medication adherence metrics while performing a meaningful educational experience?

OBJECTIVES OF PROGRAM/INTERVENTION (NO MORE THAN THREE OBJECTIVES): 1.Educate Internal Medicine residents in a Primary Care residency program on NCQA HEDIS measures regarding medication adherence metrics 2 . Work with a clinically integrated network (CIN) pharmacy team on identifying patients who have not refilled their medications, and how to engage patient medication adherence

DESCRIPTION OF PROGRAM/INTERVENTION, INCLUDING ORGANIZATIONAL CONTEXT (E.G. INPATIENT VS. OUTPATIENT, PRACTICE OR COMMUNITY CHARACTERISTICS): The Family Health Centers (FHC) at NYU Langone is a network of 8 Federally Qualified Health Centers in Brooklyn New York. Primary care residents, working with the NYU CIN pharmacy team, collaborated on telephonic outreach to engage patients identified by payor contracts as nonadherent on medication refills. After initial training, which included education on how the Proportion of Days Covered (PDC) rate is a quantitative metric used to measure quality of care and scripted exercise on telephonic patient engagement, residents were tasked with identifiying challenges on medication refill as well as intervening when appropriate. At the end of the intervention period a resident focus group was conducted to determine the educational value in this quality initiative.

MEASURES OF SUCCESS (DISCUSS QUALITATIVE AND/OR QUANTITATIVE METRICS WHICH WILL BE USED TO EVALUATE PROGRAM/INTERVENTION): Primary endpoint was increased PDC rates based on payor data for patients who are diagnosed with either having diabetes (non gestational), hypertension or dyslipidemia. A post intervention focus group and semantical content analysis was performed regarding educational value from this exercise.

FINDINGS TO DATE (IT IS NOT SUFFICIENT TO STATE FINDINGS WILL BE DISCUSSED ): In a 6-month period 523 unique patients were engaged with 899 outreaches completed. 1061 barriers were identified. The top 3 patient identified barriers were: patients unaware they had not filled the prescription $(31.05 \%)$, lack of clearly identified reason for non adherence $(20.23 \%)$, and patients did not feel committed to taking the prescribed medication (14.97\%). When comparing PDC rates from the previous year, this intervention saw a $7 \%$ increase in aggregate PDC rates for those who were prescribed medications and having diabetes. Regarding post intervention focus groups with residents, semantic content analysis revealed the highest affinity for positive descriptors in the domains of educational value, need to expand education to resident providers, and continued interest in future quality projects with the pharmacy team.
KEY LESSONS FOR DISSEMINATION (WHAT CAN OTHERS TAKE AWAY FOR IMPLEMENTATION TO THEIR PRACTICE OR COMMUNITY?): Key take home lessons in this intervention is that telephonic pharmacy adherence outreach has a positive impact on maintaining PDC rate compliance, particularly in patients with managed Medicare plans. On educational value, further development is needed in resident curriculum regarding medication adherence and reconciliation in the ambulatory setting. Lastly residents working with pharmacy teams find value in addressing medication adherence barriers and may impact best practices in provider prescribing habits when engaging patients.

HOW TO IMPROVE FOLLOW THROUGH OF CERVICAL CANCER SCREENING RESULTS IN AN INTERNAL MEDICINE RESIDENCY CLINIC

Aparna Sarin. Mount Sinai Hospital, New York, NY. (Control ID \#3186289)

STATEMENT OF PROBLEM OR QUESTION (ONE SENTENCE): Despite the barriers that remain in residency clinics of rotating residents, and the lag between performing and receiving the report of cervical cancer screening tests, how can we improve the rates of follow up on test results, and referrals to gynecology for follow up testing for abnormal results.

OBJECTIVES OF PROGRAM/INTERVENTION (NO MORE THAN THREE OBJECTIVES): 1 . Ensuring that every pap smear done in our women's clinic is tracked manually 2 . Having residents review every abnormal per the American Society for Colposcopy and Cervical Pathology's (ASCCP) guidelines, and to report every result to the patient via EMR, telephone, or letter

DESCRIPTION OF PROGRAM/INTERVENTION, INCLUDING ORGANIZATIONAL CONTEXT (E.G. INPATIENT VS. OUTPATIENT, PRACTICE OR COMMUNITY CHARACTERISTICS): Our internal medicine residency program is in an urban, academic center, and has a robust outpatient women's clinic - a weekly clinic that offers a longitudinal, year long curriculum in women's health to all interns. Our program created a detailed log binder, which is the responsibility of the interns, in which every PAP smear done is logged, and then tracked 1-2 weeks later by the interns on rotation. They check off on the log sheet as well as on EMR what the results were, the plan and how they reached the patient, and refer patients to gynecology is needed, including emailing the appropriate clinic for expedited appointments. This has been in effect for 2 years.

MEASURES OF SUCCESS (DISCUSS QUALITATIVE AND/OR QUANTITATIVE METRICS WHICH WILL BE USED TO EVALUATE PROGRAM/INTERVENTION): We calculated: number of PAP smears performed per calendar year, percentage of normal versus abnormal (abnormal cytology, HPV or insufficient cells requiring repeat PAP smear). We measured how we were able to reach the patient: via letter or EMR messaging (for normal results or those requiring PAP sooner than routine screening but no referral to gyn), telephone call, or certified letter if unable to reach the patient. By manual chart checking, we also will calculate the kind of abnormality, and compare that to standards in literature: percentage of normal vs ASCUS/ LGSIL vs HPV non 16/18 vs HPV 16/18 vs > LGSIL.

FINDINGS TO DATE (IT IS NOT SUFFICIENT TO STATE FINDINGS WILL BE DISCUSSED ): 206 PAP smears performed in 2018. Out of these, 153 were logged as normal (74\%) and 39 as abnormal (19\%). Approximately $10 \%$ PAPs are reported as abnormal and although our number may be elevated falsely due to including those that needed repeat PAP smears due to insufficient cells, it may also be a factor of a more at-risk population. We reached 77 patients $(37 \%)$ by letter or electronic message, 107 were called telephonically (52\%), 3 were called but didn't answer (1.4\%), and 16 were sent 
a certified letter due to abnormal results and patient unable to be reached $(8 \%)$. 2 patients did have any documentation on how they were reached $(0.01 \%)$.

KEY LESSONS FOR DISSEMINATION (WHAT CAN OTHERS TAKE AWAY FOR IMPLEMENTATION TO THEIR PRACTICE OR COMMUNITY?): We were able to perform >200 PAPs per year, of which $19 \%$ were abnormal. We relayed results to $97 \%$ of the our patients via letter (normal or certified) or telephone call. This not only provided improved health care delivery to our vulnerable patients, but also fulfilled the educational mission of our clinic by teaching residents on appropriate follow up for abnormal results.

IMPACT OF "CRITICAL CARE GENERAL INTERNAL MEDICINE (CG-GIM)" OUTSIDE THE INTENSIVE CARE UNIT (ICU) ON THE OTHER DEPARTMENTS IN JAPAN

SATOSHI MATSUNAGA. Aso Iizuka Hospital, lizuka-shi, Japan. (Control ID \#3176260)

STATEMENT OF PROBLEM OR QUESTION (ONE SENTENCE): Critical Care General Internal Medicine (CG-GIM) fills the gap between ICU and Emergency Room(ER), ICU and Internal Medicine, and ICU and the other departments. CG-GIM plays a more significant role than Step-Down hospitalists.

OBJECTIVES OF PROGRAM/INTERVENTION (NO MORE THAN THREE OBJECTIVES): To clarify the role of Critical Care General Internal Medicine (CG-GIM) as a buffer To illustrate the impact of CG-GIM on ICU To describe the impact of CG-GIM on the other departments

DESCRIPTION OF PROGRAM/INTERVENTION, INCLUDING ORGANIZATIONAL CONTEXT (E.G. INPATIENT VS. OUTPATIENT, PRACTICE OR COMMUNITY CHARACTERISTICS): We have created our own team, Critical Care General Internal Medicine (CGGIM), which consists of GIM staff and residents outside of the ICU. In addition to the role for Step-Down hospitalists, CG-GIM directly consults with ER and the other departments all the time.

MEASURES OF SUCCESS (DISCUSS QUALITATIVE AND/OR QUANTITATIVE METRICS WHICH WILL BE USED TO EVALUATE PROGRAM/INTERVENTION): Quantitative metrics FINDINGS TO DATE (IT IS NOT SUFFICIENT TO STATE FINDINGS WILL BE DISCUSSED ): Critical Care General Internal Medicine (CG-GIM) received 363 consultations in one year (from April 1, 2017 to March 31, 2018). CG-GIM received 166 (46\%) consultations from ER, 60 consultations (16\%) from non CG-GIM, 63 consultations (17\%) from ICU, 42 consultations (12\%) from the other Internal-Medicine departments, and 32 consultations $(9 \%)$ from the other departments. On the other hand, ICU received 732 consultations in one year. ICU received 391 consultations (53\%) from ER, 14 consultations (2\%) from CG-GIM, 21 consultations (3\%) from non CG-GIM, 51 consultations (7\%) from the other InternalMedicine departments, and 255 consultations (35\%) from the other departments. CG-GIM accepted 140 consultations (39\%) on the day shift, 95 consultations (26\%) on the semi-night shift, 21 consultations (6\%) on the late night shift, and 107 consultations (29\%) on the weekend shift. In contrast, ICU accepted 218 consultations (30\%) on the day shift, 284 consultations (39\%) on the semi-night shift, 75 consultations $(10 \%)$ on the late night shift, and 155 consultations (21\%) on the weekend shift.

KEY LESSONS FOR DISSEMINATION (WHAT CAN OTHERS TAKE AWAY FOR IMPLEMENTATION TO THEIR PRACTICE OR COMMUNITY?): Critical Care General Internal Medicine (CG-GIM) plays a great role as a buffer. 1.CG-GIM reduces the work burden of ICU, non CG-GIM, other internal-medicine departments, and the other departments.
2.24/7 consultation is a special help on the weekend, the semi-night, and the late-night shift team. 3.CG-GIM enables ER physicians to consult the other departments with less conflict. In addition, CG-GIM provides senior residents with a wonderful opportunity for critical care management and procedures, such as central venous catheter insertion. The number of elderly patients with critical and complex care has dramatically increased in Japan, which requires more sophisticated decision making for CG-GIM.

\section{IMPACT OF MEDICAL SCRIBES ON PHYSICIAN-PATIENT IN- TERACTIONS DURING OUTPATIENT VISITS}

Robert W. Lancey. University of Missouri, Columbia, MO. (Control ID \#3180884)

STATEMENT OF PROBLEM OR QUESTION (ONE SENTENCE): Do medical scribes allow physicians to spend more time interacting with patients during outpatient visits?

OBJECTIVES OF PROGRAM/INTERVENTION (NO MORE THAN THREE OBJECTIVES): Do scribes increase time physicians spend facing patients, reduce time spent facing computer during outpatient visits. Do patients believe their physician spent more time interacting with them with vs without a scribe in their visit

DESCRIPTION OF PROGRAM/INTERVENTION, INCLUDING ORGANIZATIONAL CONTEXT (E.G. INPATIENT VS. OUTPATIENT, PRACTICE OR COMMUNITY CHARACTERISTICS): The widespread introduction of electronic medical records (EMRs) into medical practice has resulted in worsening satisfaction for physicians and patients. Medical scribes are one solution to physician dissatisfaction with EMRs but their ability to impact the time physicians spend interacting with patients versus with the computer in the exam room is uknown. We tested the presence of medical scribes with a prospective observational trial studying interactions between physicians and patients in the presence of computers in exam rooms running Cerner Powerchart in an academic general internal medicine (GIM) outpatient practice at the University of Missouri (MU). Project assistants observed interactions between physicians, patients, exam room computers, and scribes when present. Time data was recorded for several types of interactions in the exam room during outpatient appointments including "physician-patient", "physician-computer", "physician-scribe", and "physical examination". A survey for each patient was administered after physicians exited the rooms. The survey asked patients about their satisfaction with the visit and their perception of their physician's time spent with them versus the computer.

MEASURES OF SUCCESS (DISCUSS QUALITATIVE AND/OR QUANTITATIVE METRICS WHICH WILL BE USED TO EVALUATE PROGRAM/INTERVENTION): Time spent interacting with patients with a scribe present in an outpatient visit compared to time spent interacting with the exam room computer. Patient perception of their visit with a scribe present, including overall visit satisfaction and perception of time physician spent facing them

FINDINGS TO DATE (IT IS NOT SUFFICIENT TO STATE FINDINGS WILL BE DISCUSSED ): With scribes, physicians spent 57\% of their time facing the patient vs. $49 \%$ with no scribe. Physicians spent less time facing EMR with scribes, $27 \%$ vs $38 \%$ without scribes, more time examining the patient with a scribe, $15 \%$ vs $10 \%$ without a scribe. The patient survey showed that patients felt their physician gave them undivided attention significantly more often with a scribe than without, $97 \%$ vs $83 \%$, OR 14.23 ( $\mathrm{p}=0.01$, 95\% CI: 1.52 - 133.42). Physician without a scribe spent too much time looking at the EMR when a medical scribe was not present, $28 \%$ vs 11 with a scribe, and looked at their EMR more than half the time, $22 \%$ vs $5 \%$ with a 
scribe. Patients did not feel that their physicians' interactions with the EMR was a distraction during the visit, $17 \%$ without a scribe vs $16 \%$ with a scribe. KEY LESSONS FOR DISSEMINATION (WHAT CAN OTHERS TAKE AWAY FOR IMPLEMENTATION TO THEIR PRACTICE OR COMMUNITY?): Medical scribes make a difference in the outpatient visit allowing physicians to focus more time on the patient than the EMR. Patients are more satisfied with their outpatient visits when they involve scribes, perceive their physicians spend more time focused on them when a scribe is present.

IMPLEMENTATION AND ENGAGEMENT IN A HOME VISIT PROGRAM DIRECTED TOWARDS PATIENTS AT RISK FOR PREVENTABLE HOSPITALIZATIONS IN A FEDERALLY QUALIFIED HEALTH CENTER (FQHC)

Ramiro Jervis; Neil Pasco; Isaac Dapkins. NYU Langone, New York, NY. (Control ID \#3179156)

STATEMENT OF PROBLEM OR QUESTION (ONE SENTENCE): Can a home visit complex care management program successfully identify and engage high risk patients in a FQHC?

OBJECTIVES OF PROGRAM/INTERVENTION (NO MORE THAN THREE OBJECTIVES): 1 . Identify patients at an FQHC who are at risk for preventable hospitalization 2. Enroll and engage patients in a home visit based complex care management program.

DESCRIPTION OF PROGRAM/INTERVENTION, INCLUDING ORGANIZATIONAL CONTEXT (E.G. INPATIENT VS. OUTPATIENT, PRACTICE OR COMMUNITY CHARACTERISTICS): The Primary Care Plus program ( $\mathrm{PCP}+$ ) is a home visit based program established to address the needs of patients at risk for preventable hospitalizations within the Family Health Centers at NYU Langone. The program staff - a physician, a nurse practitioner, a social worker and 2 community health workers coordinate as a team to identify and address the biopsychosocial needs of high risk patients. A key intervention is the home visit lead by a physician or nurse practitioner to perform the medical assessment, medication reconciliation, and identification of both medical and social impediments to optimal health. The program is not intended to replace the patient's primary care provider, but to function as an addition to the patient's care team, identifying and mitigating risk drivers, and handing off to the primary team and care management resources once the risk drivers have been addressed. Patients are referred into the program by either their primary care doctors or care management. The program is restricted to those patients who have a continuity relationship in the Federally Qualified Health Center, and who are identified as being at risk for a preventable hospitalization. Latitude is given to the referral source in how patients are identified; guidance is given to focus on patients with a history of preventable hospitalizations (as defined by PQI) or patients with advanced disease and potential palliative care needs.

MEASURES OF SUCCESS (DISCUSS QUALITATIVE AND/OR QUANTITATIVE METRICS WHICH WILL BE USED TO EVALUATE PROGRAM/INTERVENTION): The primary measure of success is patient engagement. Patient engagement is defined by both consent to the program and successful home visit by the medical provider. Other outcome metrics are patient characteristics, number of emergency department visits and number of inpatient hospitalizations in the 12 months before program enrollment.

FINDINGS TO DATE (IT IS NOT SUFFICIENT TO STATE FINDINGS WILL BE DISCUSSED ): Since program inception in August 2018 through December 31, 2018, 75 patients have been identified by care management or primary care providers as potential candidates for the program and who met criteria as defined above. Of the 75 patients, $6(8 \%)$ declined the program, and another $10(13.3 \%)$ could not be found. The remaining 59 patients were seen at home and assessed. Total engagement was $78.7 \%$. Patients identified represent a cohort of patients with an average of 2.0 inpatient admissions and 3.2 emergency department visits in the preceding 12 months prior to enrollment.

KEY LESSONS FOR DISSEMINATION (WHAT CAN OTHERS TAKE AWAY FOR IMPLEMENTATION TO THEIR PRACTICE OR COMMUNITY?): Identification of a high-risk patient population in a federally qualified health center and referral into a home visit based care management program is associated with high acceptance and engagement. Future study will determine if patients enrolled in the program have an impact on risk drivers and preventable hospitalizations.

IMPLEMENTATION OF A MODIFIED COLLABORATIVE CARE (CC) MODEL FOR DEPRESSION TREATMENT FOR VULNERABLE POPULATIONS

Colleen McGourty ${ }^{1}$; Brooke Welch ${ }^{2}$; Vanessa Thompson²; Margo Pumar $^{2}$; Lisa Ochoa-Frongia ${ }^{2}$. ${ }^{1}$ University of California San Francisco, San Francisco, CA; ${ }^{2}$ UCSF, San Francisco, CA. (Control ID \#3146811)

STATEMENT OF PROBLEM OR QUESTION (ONE SENTENCE): Significant disparities in mental healthcare exist across the United States: racial and ethnic minorities and those living in poverty have a higher burden of depression and less access to quality mental health services than their white counterparts or those with higher socioeconomic status.

OBJECTIVES OF PROGRAM/INTERVENTION (NO MORE THAN THREE OBJECTIVES): 1. Increase patient access to mental health services for depression treatment through implementation of a modified CC model of team-based depression treatment at a Federally Qualified Health Center (FQHC). 2. Improve depression remission rates for these FQHC primary care patients, where depression prevalence is three times the national rate and only $3-5 \%$ of patients achieve remission compared to $30 \%$ in national studies.

DESCRIPTION OF PROGRAM/INTERVENTION, INCLUDING ORGANIZATIONAL CONTEXT (E.G. INPATIENT VS. OUTPATIENT, PRACTICE OR COMMUNITY CHARACTERISTICS): We piloted our team-based depression treatment program at the Richard Fine People's Clinic (RFPC), an academic internal medicine FQHC that provides primary care to underserved, diverse patients in San Francisco. The team included 3 attending-level primary care physicians, a psychiatrist, a psychologist, nurses, a medical assistant (MA), and a medical student. 14 patients with depression were enrolled based on diagnosis of major depressive disorder and a Patient Health Questionnaire-9 (PHQ-9) score of $>10$. They were offered medication and a referral to an imbedded team of behavioral health clinicians. An MA contacted the patient within a week to verify treatment initiation. Within 2-3 weeks, the patient met with a nurse to confirm medication adherence and to assess medication side effects and titration needs. After 6-8 weeks and again at 3 months, patients were scheduled for clinic visits with a PCP to track symptoms and to determine additional interventions. Progress was tracked in a database and discussed at biweekly team meetings.

MEASURES OF SUCCESS (DISCUSS QUALITATIVE AND/OR QUANTITATIVE METRICS WHICH WILL BE USED TO EVALUATE PROGRAM/INTERVENTION): Fidelity of intervention implementation (percent of patients receiving MA phone calls, nurse visits, and PCP follow-up visits) Patient access to mental health services (rates of medication prescription and patients accessing therapy) Depression remission rates (PHQ-9 scores and qualitative symptom descriptions) 
FINDINGS TO DATE (IT IS NOT SUFFICIENT TO STATE FINDINGS WILL BE DISCUSSED ): Implementation of our CC model was successful in that all patients received a MA phone call to trouble-shoot treatment initiation, and two-thirds of patients attended a visit with a nurse to assess side effects and titration needs. Patients saw an increase in access to mental health treatments: 93\% were prescribed an antidepressant, compared to clinic-wide rates of $27 \%$, and $36 \%$ of pilot patients attended at least one therapy session, compared to only $11 \%$ clinic-wide. This intervention led to improved patient outcomes during this 6-month pilot period. The average PHQ-9 score of patients was 14 upon enrollment, and 9 at the end of the pilot period. $36 \%$ of patients reached full depression remission, compared to clinic-wide remission rates of 3-5\%.

KEY LESSONS FOR DISSEMINATION (WHAT CAN OTHERS TAKE AWAY FOR IMPLEMENTATION TO THEIR PRACTICE OR COMMUNITY?): Team-based management of depression in primary care can be effective for medically underserved patient populations, even when resources do not exist to fund a full CC model.

IMPLEMENTATION OF AN ADAPTED VA MOVE! CURRICULUM COMBINED WITH A MOBILE TEXTING PLATFORM IN GROUP MEDICAL VISITS FOR WEIGHT LOSS AT AN URBAN FEDERALLY QUALIFIED HEALTH CENTER

Elizabeth "Betty" Kolod"1; Viraj V. Patel ${ }^{2}$, ; Sabrina Esbitt'; Shyanne R. Washington ${ }^{3}$; Shwetha Iyer ${ }^{1,}{ }^{1}$. ${ }^{1}$ Montefiore Medical Center, Bronx, NY; ${ }^{2}$ Montefiore Medical Center/Albert Einstein College of Medicine, Bronx, NY; ${ }^{3}$ Lehman College, Bronx, NY. (Control ID \#3186082)

STATEMENT OF PROBLEM OR QUESTION (ONE SENTENCE): Group medical visits supplemented by a mobile text group may be a promising model for primary care-based weight loss intervention, however, feasibility and acceptability are unknown.

OBJECTIVES OF PROGRAM/INTERVENTION (NO MORE THAN THREE OBJECTIVES): 1) Determine the feasibility of an adapted VA MOVE! curriculum in an urban Federally Qualified Health Center (FQHC). 2) Identify characteristics of individuals recruited for group medical visits for weight loss. 3) Use a mobile texting platform to promote patient retention in group medical visits.

DESCRIPTION OF PROGRAM/INTERVENTION, INCLUDING ORGANIZATIONAL CONTEXT (E.G. INPATIENT VS. OUTPATIENT, PRACTICE OR COMMUNITY CHARACTERISTICS): From 9/2018-1/2019, we implemented group medical visits for weight loss at an urban $\mathrm{FQHC}$, with an adapted Managing Obesity for Veterans Everywhere (MOVE!) curriculum. Each of 16 modified session incorporated 1-3 original sessions, and cooking and exercise demonstrations. Additionally, participants joined an encrypted mobile text group. Each session was facilitated by an attending and a resident physician, a psychologist, and a nutrition student. The attending physician met individually with participants during weekly weigh-in for documentation and to bill an individual visit.

MEASURES OF SUCCESS (DISCUSS QUALITATIVE AND/OR QUANTITATIVE METRICS WHICH WILL BE USED TO EVALUATE PROGRAM/INTERVENTION): We assessed rate of recruitment, attendance pattern, and texting platform usage. Additionally, we calculated percent weight change. Finally, we assessed known behavioral mediators of weight loss, pre- and post-intervention.

FINDINGS TO DATE (IT IS NOT SUFFICIENT TO STATE FINDINGS WILL BE DISCUSSED ): Over 1 month, patients self-referred $(n=6)$ or were referred by their provider $(n=42)$. On contact by study coordinator, 22 patients were interested, 6 were not interested or available, and 20 could not be reached. The first 12 interested patients were invited to the group. Ten of the 12 invited participants (83\%) presented. The 10 initial participants had mean age 51.7 years (range 31-66), and mean BMI 45.1 (range 31-57). Seven identified as Black women and 3 as Latina, despite slight clinic predominance of patients identifying as Hispanic over those identifying at Black ( $40 \%$ versus $37 \%$, respectively). Five participants completed $>50 \%$ of the 16 visits. One person attended $31 \%$ of visits, 1 attended monthly for the first 3 months, and 2 participants attended only the first visit due to schedule conflicts. One person was not invited back due to inappropriateness for group visits. Of the five participants completing $50 \%$ of visits, 1 lost $5 \%$ of her total body weight (TBW), 1 lost 1.7\% TBW, 1 halted a pattern of weight gain, 1 maintained her weight, and the final retained participant lost $1 \%$ TBW but then gained 3.7\%. On the text group, the coordinator sent visit reminders and participants communicated attendance plans. Few recipes and exercise routines were shared; participants requested greater emphasis on this function. Participants with intermittent attendance remained active in the group.

KEY LESSONS FOR DISSEMINATION (WHAT CAN OTHERS TAKE AWAY FOR IMPLEMENTATION TO THEIR PRACTICE OR COMMUNITY?): 1) While implementation of group visits for weight loss is feasible, retention remains a challenge. 2) Over-recruitment of participants in group medical visits for weight loss may counteract unforeseen schedule conflicts. 3) The group texting feature was acceptable, though under-utilized in program design.

IMPLEMENTATION OF NALAXONE TRAINING AND DISTRIBUTION FOR HBPC VETERANS ON CHRONIC OPIOID THERAPY IN URBAN AND RURAL SETTINGS

Naomi Karlen. Southeast Louisiana Veterans Health Care System, New Orleans, LA. (Control ID \#3162865)

STATEMENT OF PROBLEM OR QUESTION (ONE SENTENCE): There is a high need for increasing the safety of opioid prescribing in the vulnerable home based population of Veterans in both rural and urban areas. OBJECTIVES OF PROGRAM/INTERVENTION (NO MORE THAN THREE OBJECTIVES): 1) Improve the safety of opioid prescribing to vulnerable urban and rural home based primary care Veteran patients by increasing the amount of completed naloxone training and prescriptions. 2) Educate staff in home based primary care Veteran program on topics including opioid overdose, general nalaxone information, and nalaxone teaching to patients and their friends/family members. 3) Create a sustainable training system for both future new home based primary care staff and patients in order to continue education on opioid overdose and nalaxone.

DESCRIPTION OF PROGRAM/INTERVENTION, INCLUDING ORGANIZATIONAL CONTEXT (E.G. INPATIENT VS. OUTPATIENT, PRACTICE OR COMMUNITY CHARACTERISTICS): The Southeast Louisiana Veterans Health Care System home base primary care program (HBPC) serves approximately 300 home bound Veterans in a 65 mile radius from New Orleans, Slidell, Baton Rouge (rural), and Hammond (rural) clinics. Prior to this quality improvement project, there was no process for the education and prescription of naloxone for HBPC patients at our facility. The project occured as thus: - Facility's Pain Evaluation Program (PEP) pharmacist gave a presentation to HBPC nurses, providers, and pharmacists regarding naloxone training. - A teaching script (along with use of VA created educational videos) and electronic medical record note template were developed for the nurses regarding patient/friend/ 
family education on opioid overdose and use/implementation of nalaxone. HBPC patients actively prescribed opiates were educated one the above by their assigned nurses. Once the education was complete, an intranasal nalaxone kit was mailed to the patient's home (at the time of the project, there was no active patient need for another delivery system).

MEASURES OF SUCCESS (DISCUSS QUALITATIVE AND/OR QUANTITATIVE METRICS WHICH WILL BE USED TO EVALUATE PROGRAM/INTERVENTION): 15 trainings were completed out of 22 eligible patients. Five trainings were unable to be completed due to external factors: opiates weaned off/naloxone no longer indicated (1), patient discharged from program for various reasons prior to training able to be completed (4). Two trainings were not completed by staff despite requests/ reminders. This intervention was successful in increasing the amount of naloxone training and opioid overdose education. This, in turn, increases the safety of our urban and rural home-bound Veterans who are treated with chronic opioid therapy.

FINDINGS TO DATE (IT IS NOT SUFFICIENT TO STATE FINDINGS WILL BE DISCUSSED ): Nalaxone has not been used and/or needed in our home based primary care patient population as of yet. Patients and their family/friends are interested in hearing about opioid overdose and what they can do. Many have already heard overdose and nalaxone in the news given the current opioid epidemic.

KEY LESSONS FOR DISSEMINATION (WHAT CAN OTHERS TAKE AWAY FOR IMPLEMENTATION TO THEIR PRACTICE OR COMMUNITY?): Home bound Veterans do not always have the ability to come to a facility in order to obtain training and education. It is important to develop sustainable programs to "meet patients where they are" in order to best serve their needs.

IMPLEMENTATION OF NURSE DRIVEN CLINICAL DECISION SUPPORT TO IMPROVE PRIMARY CARE MANAGEMENT OF SORE THROAT

$\underline{\text { David Feldstein }}^{1}$; Linda S. Park ${ }^{2}$; Paul Smith ${ }^{3}$; Joseph Palmisano ${ }^{7}$; Rachel Hess $^{4}$; Simon Jones ${ }^{5}$; Sara K. Chokshi ${ }^{6}$; Thomas McGinn ${ }^{8}$; Devin M. Mann ${ }^{6} .{ }^{1}$ University of Wisconsin School of Medicine and Public Health, Madison, WI; ${ }^{2}$ University of Wisconsin Madison, Madison, WI; ${ }^{3}$ University of Wisconsin-Madison, Oregon, WI; ${ }^{4}$ University of Utah, Salt Lake City, UT; ${ }^{5}$ NYU, NY, NY; ${ }^{6}$ NYU School of Medicine, New York, NY; ${ }^{7}$ Boston University, Boston, MA; ${ }^{8}$ Donald and Barbara Zucker School of Medicine at Hofstra/Northwell, Manhasset, NY. (Control ID \#3179759)

STATEMENT OF PROBLEM OR QUESTION (ONE SENTENCE): Underutilization of clinical prediction rules and poor uptake of provideroriented clinical decision support (CDS) has contributed to overuse of antibiotics for sore throat.

OBJECTIVES OF PROGRAM/INTERVENTION (NO MORE THAN THREE OBJECTIVES): 1 . Adapt CDS for registered nurses (RNs) to evaluate and treat patients with sore throat 2. Demonstrate the feasibility of RN visits using CDS to evaluate and treat patients with sore throat

DESCRIPTION OF PROGRAM/INTERVENTION, INCLUDING ORGANIZATIONAL CONTEXT (E.G. INPATIENT VS. OUTPATIENT, PRACTICE OR COMMUNITY CHARACTERISTICS): We performed a 12-week pilot study to evaluate the feasibility of $\mathrm{RN}$ visits using an integrated clinical prediction rule (iCPR) tool to determine patient risk for strep throat and provide appropriate treatment at a family medicine clinic in a Midwest academic healthcare system. iCPR, originally developed for use by primary care physicians (PCPs), includes a risk calculator using
Centor strep throat criteria and ordersets based on patient's risk for strep throat: education for low-risk, testing for intermediate-risk, and testing or antibiotics for high-risk. To adapt the process for RN visits, we developed triage protocols so appropriate patients received nurse visits, very low risk received education and more complex patients received provider visits. No major changes were made to the risk calculator or ordersets. Four RNs, with 2-24 years of experience, received a 10-minute online training session on sore throat evaluation followed by a 45-minute in-person training on physical examination and iCPR use. RNs triaged patients by phone and conducted RN visits using iCPR and following orderset recommendations. RNs could transition to a PCP visit if they were uncomfortable evaluating the patient. MEASURES OF SUCCESS (DISCUSS QUALITATIVE AND/ OR QUANTITATIVE METRICS WHICH WILL BE USED TO EVALUATE PROGRAM/INTERVENTION): Electronic health record data was used to determine the number of nurse visits, frequency of tool use and antibiotic and diagnostic test ordering. RNs completed a self-efficacy survey prior to training and 8-weeks after implementation. At 12 weeks, we interviewed RNs to understand barriers and facilitators to using the tool.

FINDINGS TO DATE (IT IS NOT SUFFICIENT TO STATE FINDINGS WILL BE DISCUSSED ): 162 triage calls for sore throat resulted in $77(48 \%)$ patients with $\mathrm{RN}$-only visits, $45(28 \%)$ with provider visits, $38(23 \%)$ with no visit. Only $2 \mathrm{RN}$ visits $(<3 \%)$ converted to provider visit due to patient complexity. RNs completed the risk calculator for $99 \%$ of visits and followed recommendations in all cases except for ordering antibiotics in 1 high-risk patient with a negative rapid strep. $\mathrm{RN}$ confidence in their ability to evaluate and treat a patient with sore throat was 85 (SD 5.8) (0 cannot do at all; 100 highly certain I can do) prior to training and 97.5 (SD 5.0) at 8-weeks. RNs felt the tool decreased provider visits and strep testing in patients. RN's also felt that the tool increased patient and RN satisfaction.

KEY LESSONS FOR DISSEMINATION (WHAT CAN OTHERS TAKE AWAY FOR IMPLEMENTATION TO THEIR PRACTICE OR COMMUNITY?): This pilot study demonstrates that RNs can use CDS to appropriately triage, evaluate and treat acute low-complexity sore throat patients. Implementation of an $\mathrm{RN}$-driven iCPR tool shows promise to reduce inappropriate antibiotic prescribing and represents a potential model for expanding RN practice using CDS.

IMPLEMENTATION STRATEGIES TO INCREASE USE OF COMMUNITY-BASED DIABETES PREVENTION PROGRAMS BY PRIMARY CARE PATIENTS AT AN URBAN SAFETY-NET HOSPITAL

Jonathan Berz ${ }^{1}$; Lisa M. Quintiliani ${ }^{2}$; Ve Truong ${ }^{1}$; Jennifer Murillo ${ }^{3}$; Karen E. Lasser ${ }^{1} .{ }^{1}$ Boston Medical Center, Boston, MA; ${ }^{2}$ Boston University, Boston, MA; ${ }^{3}$ Boston Medical Center, Boston, MA, MA. (Control ID \#3180276)

STATEMENT OF PROBLEM OR QUESTION (ONE SENTENCE): Primary care practices refer few patients with pre-diabetes to diabetes prevention programs (DPP), and few have systems in place to identify such patients. Little is known about how to implement such systems of care among primary-care practices serving high-risk populations.

OBJECTIVES OF PROGRAM/INTERVENTION (NO MORE THAN THREE OBJECTIVES): 1. Assess the feasibility of implementing a system of identification and referral of patients with prediabetes to a community-based YMCA DPP. 2.Compare effectiveness of 3 implementation strategies to link patients with pre-diabetes to DPPs by comparing frequency of referral and DPP completion 
DESCRIPTION OF PROGRAM/INTERVENTION, INCLUDING ORGANIZATIONAL CONTEXT (E.G. INPATIENT VS. OUTPATIENT, PRACTICE OR COMMUNITY CHARACTERISTICS): Over an 8-month period in 2018, in an urban safety-net internal medicine primary care practice, we identified patients with pre-diabetes based on diagnosis code or most recent Alc within the previous 2 years. We abstracted data from the electronic health record. As the practice is divided into 6 separate suites, we assigned patients in each of 3 suites to one of the following groups: (1) basic patient navigation: navigator called and informed patient about diagnosis of pre-diabetes, assessed interest in a DPP referral, and placed referral if desired by patient; (2) enhanced patient navigation: basic patient navigation plus motivational interviewing to assess and address barriers to DPP attendance; (3) comparison group: Providers in the suite received a 30-minute educational session about benefits of and resources to deliver the DPP.

MEASURES OF SUCCESS (DISCUSS QUALITATIVE AND/OR QUANTITATIVE METRICS WHICH WILL BE USED TO EVALUATE PROGRAM/INTERVENTION): We measured the number of completed phone calls by navigators, call outcomes (defined as spoke with patient or other, voicemail, no answer) and duration of each call in groups 1 and 2. In addition, we assessed preliminary effectiveness of the implementation strategies by comparing frequency of referral and DPP enrollment between groups. We will interview key informants including 2 patient navigators, the DPP program coordinator and a selection of primary care providers to evaluate the acceptability of the implementation strategies and explore barriers to and facilitators of implementation.

FINDINGS TO DATE (IT IS NOT SUFFICIENT TO STATE FINDINGS WILL BE DISCUSSED ): Patient navigators called 209 patients ( $n=171$ in basic patient navigation group and $n=109$ in enhanced patient navigation group). The highest frequency of referral was in the enhanced patient navigation group $(\mathrm{n}=51,47 \%)$, followed by the basic patient navigation group $(n=48,28 \%)$ and the comparison group $(n=0)$. Few patients enrolled in the DPP from either navigation group $(n=4$ in basic patient navigation group and $n=2$ in enhanced patient navigation group). Interview of the DPP coordinator revealed several barriers, including inconvenient class times and location, and limited class capacity.

KEY LESSONS FOR DISSEMINATION (WHAT CAN OTHERS TAKE AWAY FOR IMPLEMENTATION TO THEIR PRACTICE OR COMMUNITY?): A system of proactive outreach was successful in identifying and referring patients with pre-diabetes to a DPP. Identifying and addressing barriers to program enrollment such as scheduling, location and capacity are key for facilitating program enrollment.

IMPLEMENTING A DAILY MEDICINE ROUNDING TOOL TO PROMOTE PATIENT SAFETY AND IMPROVE COMMUNICATION BETWEEN PHYSICIAN AND NURSE DURING HOSPITALIZATION

Marwa Moussa $^{1}$; Lisa Schwartz ${ }^{1}$; Laura Mansfield ${ }^{1}$; Tracey-Ann Knight ${ }^{1}$; Jennifer Renaud $^{3}$; Meg Ferrauiola ${ }^{1}$; Sandra Thompson ${ }^{1}$; Charles Okamura ${ }^{1}$; Frank Volpicelli ${ }^{2} .{ }^{1} \mathrm{NYU}$ Langone, Staten Island, NY; ${ }^{2} \mathrm{New}$ York University, New York, NY; ${ }^{3} \mathrm{NYU}$ Langone Hospital Brooklyn, Brooklyn, NY. (Control ID \#3167999)

\section{STATEMENT OF PROBLEM OR QUESTION (ONE SENTENCE):}

As communication among patient care team members is often dangerously fragmented and effective collaboration becomes essential to provide safe hospital care for patients, we implemented the Daily Medicine Rounding Tool (DMeRT) that improved collaboration between the physician and nurse.
OBJECTIVES OF PROGRAM/INTERVENTION (NO MORE THAN THREE OBJECTIVES): 1 . We aimed to promote a patientcentered, highly reliable rounding tool to reduce hospital adverse events by streamlining real time communication between nurses and physicians. 2 . We hypothesize that this tool will decrease the need for frequent calls throughout the day, ultimately improving team productivity and overall staff satisfaction. DESCRIPTION OF PROGRAM/INTERVENTION, INCLUDING ORGANIZATIONAL CONTEXT (E.G. INPATIENT VS. OUTPATIENT, PRACTICE OR COMMUNITY CHARACTERISTICS): In our institution, the Epic's default patient dashboard columns included patient name, venous thromboemboli (VTE) prophylaxis, Medical Orders for Life Sustaining Treatment (MOLST) completion, glycemic control, and medication reconciliation completion. Expanding upon these prior default columns, we partnered with information technology and nursing to create a customized dashboard that included additional informational columns extracted from the documentation in the charts, to include the administration of intravenous fluids, oxygen supplementation, last bowel movement recorded and high risk medications (anti-coagulants, anti-epileptics, furosemide, opioids, and benzodiazepines). We then trained the physicians and nurses to discuss each patient using the customized DMeRT dashboard during interdisciplinary rounds. The average time spent on the DMeRT is 15 minutes for a total 10 patients. This helps as a reminder and the identification of potential pitfalls and safety concerns. The DMeRT was instituted on a 30 bed medical unit (5500) on June 1, 2018 with iterative improvements to content.

MEASURES OF SUCCESS (DISCUSS QUALITATIVE AND/OR QUANTITATIVE METRICS WHICH WILL BE USED TO EVALUATE PROGRAM/INTERVENTION): We will analyze data pre and post intervention to assess for impact on reducing medication errors during hospitalization, hospital acquired VTE events and improvement in glycemic control. Finally we will track MOLST completion, medication reconciliation compliance, constipation and fluid overload events added to the patient's problem list 48 hours prior to discharge.

FINDINGS TO DATE (IT IS NOT SUFFICIENT TO STATE FINDINGS WILL BE DISCUSSED ): To date, the unit which implemented the intervention had an improvement in the Quality Hyperglycemia Scores (method used to evaluate inpatient glycemic management) from 56 in $4 / 2018$ to 95 in $12 / 2018$. There was an improved MOLST completion from $14 \%$ in $4 / 2018$ to $83 \%$ in $12 / 2018$. A Preliminary survey of 15 nurses on unit 5500 showed that $80 \%$ reported that they rarely need to call house staff within 2 hours of completing the rounding tool and $66 \%$ of nurses were satisfied with the DMeRT.

KEY LESSONS FOR DISSEMINATION (WHAT CAN OTHERS TAKE AWAY FOR IMPLEMENTATION TO THEIR PRACTICE OR COMMUNITY?): During hospitalization, multiple aspects of patient care are overlooked while we focus on the admitting diagnosis, necessary diagnostics and treatments. Medication errors during hospitalization are commonly caused by breakdowns in communication and associated with substantial risk. This is a simple tool that utilizes information technology to efficiently and systematically review standardized aspects of care.

IMPLEMENTING A MAILED FECAL IMMUNOCHEMICAL TESTING PROGRAM FOR COLORECTAL CANCER SCREENING IN FEDERALLY QUALIFIED HEALTH CENTERS Michael Pignone $^{1,}{ }^{1}$; Nicole Kluz ${ }^{1}$; Paul Rathouz'; Eda Baykal-Caglarr; $\overline{\text { Alison Brenner }}{ }^{2}$; Bretta Candelaria ${ }^{1}$; Jessica Trevino'; Jaison John ${ }^{1}$; Daniel S. Reuland ${ }^{2} .{ }^{1}$ Dell Medical School, Austin, TX; ${ }^{2}$ University of North Carolina, Chapel Hill, NC. (Control ID \#3184379) 
STATEMENT OF PROBLEM OR QUESTION (ONE SENTENCE): Colorectal cancer (CRC) screening rates are sub-optimal among patients of Federally Qualified Health Centers (FQHCs), many of whom have low-income and inadequate health insurance. Mailed fecal immunochemical testing (FIT) is an evidence-based method of increasing screening, but has not been widely disseminated.

OBJECTIVES OF PROGRAM/INTERVENTION (NO MORE THAN THREE OBJECTIVES): 1 . To implement a mailed FIT screening program for average-risk adults ages 50-75 who were patients in a network of FQHCs. 2. To measure uptake of mailed screening and positivity rates. 3 . To ensure, through patient navigation, a high rate of completion of followup colonoscopy after positive FIT.

DESCRIPTION OF PROGRAM/INTERVENTION, INCLUDING ORGANIZATIONAL CONTEXT (E.G. INPATIENT VS. OUTPATIENT, PRACTICE OR COMMUNITY CHARACTERISTICS): Age-eligible, average risk patients with no evidence of recent screening who were being seen in a network of FQHCs in Texas were eligible for the program. Eligible patients received a mailing with an introductory letter, return postcard to update screening status, and a single-sample FIT that could be completed and returned by mail. We used reminder text messages and letters to help ensure test completion. Patients with positive tests received support from a bilingual patient navigator to help ensure colonoscopy completion.

MEASURES OF SUCCESS (DISCUSS QUALITATIVE AND/OR QUANTITATIVE METRICS WHICH WILL BE USED TO EVALUATE PROGRAM/INTERVENTION): 1 . Numbers of patients reached 2. Uptake of mailed FIT among eligible patients 3. FIT Positivity rate 4. Proportion of positive FIT with colonoscopy completed within 90 days per medical record review

FINDINGS TO DATE (IT IS NOT SUFFICIENT TO STATE FINDINGS WILL BE DISCUSSED ): We identified 28,306 age-eligible patients and 6,991 (24.7\%) were already up to date based on medical record review. Of the 21,315 not up to date, mean age was 58 and $49.5 \%$ were female; $15.1 \%$ were African-American and $41.0 \%$ Latino. Overall, $68.7 \%$ spoke English preferentially, and 25.8\% Spanish. Less than half $(47.7 \%)$ had commercial or Federal insurance. In Year 1 of the program, we mailed FIT packets to 14,276 patients ( $67.0 \%$ of those not up to date). Of those, $18.4 \%(n=2,633)$ completed FIT and $428(3.0 \%)$ were already up to date with outside screening based on patient report. Of those completing FIT, $142(5.4 \%)$ were positive and 78 have completed a colonoscopy to date (54.9\% completion rate) with another $22.5 \%$ scheduled to be complete within 90 days. Important findings at colonoscopy $(\mathrm{n}=78)$ include 34 (43.6\%) with adenomas, 1 carcinoma in situ, and 2 cancers.

KEY LESSONS FOR DISSEMINATION (WHAT CAN OTHERS TAKE AWAY FOR IMPLEMENTATION TO THEIR PRACTICE OR COMMUNITY?): 1. Mailed FIT is an effective, scalable means of increasing CRC screening in otherwise hard to reach, vulnerable patients in a safety net FQHC system, and should supplement in-office efforts to increase screening. 2. Achieving high rates of follow-up colonoscopy is challenging, even with high-quality patient navigation.

\section{IMPLEMENTING A STUDENT-LED FOOT CARE CLINIC IN AN} OVERNIGHT HOMELESS SHELTER

Aileen L. Doble $^{1}$; Henry C. Ashworth ${ }^{2}$; Kevin G. Sullivan ${ }^{3}$; Marya J. Cohen ${ }^{4}$; Gina R. Kruse ${ }^{5} .{ }^{1}$ MGH Institute of Health Professions, Boston, MA; ${ }^{2}$ Harvard Medical School, Boston, MA; ${ }^{3}$ Boston Healthcare for the Homeless, Boston, MA; ${ }^{4} \mathrm{MGH}$, Boston, MA; ${ }^{5}$ Massachusetts General Hospital, Boston, MA. (Control ID \#3177744)
STATEMENT OF PROBLEM OR QUESTION (ONE SENTENCE): Foot care issues can be severe and debilitating amongst those who experience homelessness; however, more could be known on the distribution of foot disease and prevalence of proper foot care in this vulnerable population. OBJECTIVES OF PROGRAM/INTERVENTION (NO MORE THAN THREE OBJECTIVES): Objective 1: To deliver basic foot care to guests at the Boston Night Center (BNC). Objective 2: To connect BNC guests with foot conditions to primary care services.

DESCRIPTION OF PROGRAM/INTERVENTION, INCLUDING ORGANIZATIONAL CONTEXT (E.G. INPATIENT VS. OUTPATIENT, PRACTICE OR COMMUNITY CHARACTERISTICS): This foot care clinic is an extension of the Crimson Care Collaborative (CCC) at Boston Health Care for the Homeless Program (CCC-BHCHP) clinic site; an interprofessional student-faculty medical clinic that serves homeless guests staying at the BNC, a drop-in overnight shelter, on Monday nights. In order to expand the impact of the clinic and better serve guests at the BNC, a needs assessment was conducted; results clearly pointed to the need for a foot care clinic. CCC volunteers partnered with faculty and leadership at the wellestablished St. Francis House foot clinic to develop a clinical model that involves 1) a comprehensive foot assessment 2) washing of feet in warm soapy water 3) application of preventative medications 4) referral to BHCHP for primary care services if needed.

MEASURES OF SUCCESS (DISCUSS QUALITATIVE AND/OR QUANTITATIVE METRICS WHICH WILL BE USED TO EVALUATE PROGRAM/INTERVENTION): The foot care clinic is evaluated in terms of clinical volume and patients served. The target for clinical volume is to provide foot care to a minimum of four guests each Monday evening. To understand patients served in the foot care clinic, a survey gathering basic demographic data, connection to primary care, and prevalence of foot symptoms and diagnoses is utilized. As this foot care clinic continues, other areas to explore will be guests' perceived importance of foot health and guests' health habits regarding foot hygiene.

FINDINGS TO DATE (IT IS NOT SUFFICIENT TO STATE FINDINGS WILL BE DISCUSSED ): Unofficially, the foot care clinic has received positive feedback from those receiving care and from BNC staff. So far, four CCC volunteers have cared for 21 guests at the foot clinic. The median age of the guests served is 43 years of age. The most prevalent conditions are tinea pedis $(86 \%, \mathrm{n}=21)$, and immersion foot $(29 \%, \mathrm{n}=20)$. Overall, $78 \%$ $(n=18)$ of the guests served were already connected to primary care services, and $22 \%(n=18)$ received referral to primary care services at BHCHP.

KEY LESSONS FOR DISSEMINATION (WHAT CAN OTHERS TAKE AWAY FOR IMPLEMENTATION TO THEIR PRACTICE OR COMMUNITY?): There is value in holding a foot care clinic at a time when other clinics are not open and when homeless persons are not seeking to secure shelter for the evening. This foot care clinic provides a space and time in which guests are able to prioritize their health, which can be difficult during the day when basic needs such as shelter and food often take precedence. Additionally, holding the foot care clinic at the BNC allows us to reach those who may not feel comfortable seeking care in a traditional health-care setting. This foot care clinic also provides a benefit to the CCC volunteers, many of whom have described the experience as transformative, demonstrating how enriching compassionate, hands-on care can be to the student learning experience.

IMPLEMENTING AMBULATORY BLOOD PRESSURE MONITORING INTO AMBULATORY ACADEMIC PRIMARY CARE: A MULTIDISCIPLINARY APPROACH

Jennifer Beach ${ }^{1}$; Stephen P. Juraschek ${ }^{2}$; Anthony Ishak ${ }^{2}$; Kenneth J. Mukamal $^{3} .{ }^{1}$ Beth Israel Deaconess Medical Center, Boston, MA; ${ }^{2}$ BETH 
ISRAEL DEACONESS MEDICAL CENTER, BOSTON, MA; ${ }^{3}$ BIDMC, Brookline, MA. (Control ID \#3184393)

STATEMENT OF PROBLEM OR QUESTION (ONE SENTENCE): Despite expanding recommendations to use $24 \mathrm{hr}$ ambulatory blood pressure monitoring (ABPM) in the diagnosis and management of hypertension (HTN), access to it is limited for many academic practices.

OBJECTIVES OF PROGRAM/INTERVENTION (NO MORE THAN THREE OBJECTIVES): -Create a multidisciplinary outpatient HTN program within a large, urban academic primary care practice to improve HTN management. -Develop a sustainable model of ABPM embedded in primary care for the diagnosis and management of HTN. -Execute an educational curriculum to teach primary care residents to interpret results of ABPM and integrate them into a comprehensive HTN program.

DESCRIPTION OF PROGRAM/INTERVENTION, INCLUDING ORGANIZATIONAL CONTEXT (E.G. INPATIENT VS. OUTPATIENT, PRACTICE OR COMMUNITY CHARACTERISTICS): Healthcare Associates is a large, academic, urban practice in Boston, Massachusetts serving nearly 43,000 patients. We have over 60 faculty physicians, 7 nurse practitioners, 125 residents and 200 other support staff including clinical social workers, medical assistants, LPNs, RNs and phone staff. Embedded within this larger practice, we developed a collaborative multidisciplinary hypertension clinic with a focus on offering $24 \mathrm{hr}$ ambulatory blood pressure monitoring and providing office visits for rapid medication titration and patient education. Our clinic occurs weekly and is staffed with an attending preceptor, a doctorally-trained pharmacist, 1-2 medical residents, and LPN/RN staff. Each clinic session begins with a 30-minute teaching session led by residents, staff, or faculty followed by resident- and pharmacist-staffed office visits.

MEASURES OF SUCCESS (DISCUSS QUALITATIVE AND/OR QUANTITATIVE METRICS WHICH WILL BE USED TO EVALUATE PROGRAM/INTERVENTION): -Number of patients and visits for HTN evaluation/treatment and ABPM -Proportion of patients with medication change -Hypertension-specific teaching modules

FINDINGS TO DATE (IT IS NOT SUFFICIENT TO STATE FINDINGS WILL BE DISCUSSED ): -In 2018, we saw 367 patients overall, 148 for ABPM, and 219 for medication adjustment or follow-up -In patients seen for $\mathrm{ABPM}$, there is an estimated medication change rate of $54 \%$. In those referred to confirm a new diagnosis of HTN, $30 \%$ were found to have white coat hypertension -We have developed 12 new teaching modules on a variety of HTN topics that were selected based on resident needs

KEY LESSONS FOR DISSEMINATION (WHAT CAN OTHERS TAKE AWAY FOR IMPLEMENTATION TO THEIR PRACTICE OR COMMUNITY?): -Challenges that we faced in developing this clinic were myriad and included material costs for monitors and software, personnel time for fitting patients with monitors and uploading the results and educational gaps for providers to learn to interpret results of ABPM testing. -Creating a multidisciplinary team allowed for each provider to work at the top of their licensure -ABPM is a powerful diagnostic and educational tool for managing hypertension, our most common reasons for referral include ruling out white coat hypertension and evaluating resistant/difficult to control hypertension -Training residents to interpret and explain ABPM to patients is a valuable and rewarding addition to their internal medicine training

IMPLEMENTING AN ELECTRONIC SYSTEM TO SCREEN AND ACTIVELY REFER TO COMMUNITY BASED AGENCIES FOR FOOD INSECURITY IN PRIMARY CARE

Amy Smith ${ }^{1}$; Leah Zallman ${ }^{1}$; Kathleen Betts ${ }^{1}$; Lisa Brukilacchio ${ }^{1}$; Fiona McCaughan ${ }^{1}$; Erin McAleer $^{2}$; Noreen Kelly ${ }^{2}$; David Elvin ${ }^{1}$; Lisa M.

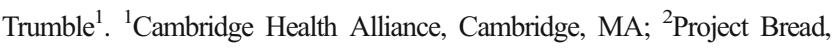
Boston, MA. (Control ID \#3177574)

STATEMENT OF PROBLEM OR QUESTION (ONE SENTENCE): Health related social needs such as food insecurity are prevalent in safety net healthcare settings yet these settings lack feasible systematic ways to actively refer patients to community based agencies (e.g. refer to agencies that will reach out to patients).

OBJECTIVES OF PROGRAM/INTERVENTION (NO MORE THAN THREE OBJECTIVES): 1 . Implement a systematic screening program for food insecurity in a safety net healthcare setting and integrate into the electronic health record 2. Actively and electronically refer patients who screen positive to a community based agency 2 . Examine feasibility of the program

DESCRIPTION OF PROGRAM/INTERVENTION, INCLUDING ORGANIZATIONAL CONTEXT (E.G. INPATIENT VS. OUTPATIENT, PRACTICE OR COMMUNITY CHARACTERISTICS): We implemented a systematic screening program for food insecurity, integrated into the electronic health record, that enables electronic active referral to a community based agency. Patients complete paper forms which are entered into the electronic record by clinic staff. If patients agree that we can share their contact information with a community based agency, our system automatically faxes a referral to a community based agency that addresses food insecurity. We initially piloted this program on paper, then integrated into the electronic medical record and rolled it out to 16 primary care clinics of an integrated safety net health care system. We are currently piloting systems to allow patients to enter their own data via electronic tablets.

MEASURES OF SUCCESS (DISCUSS QUALITATIVE AND/OR QUANTITATIVE METRICS WHICH WILL BE USED TO EVALUATE PROGRAM/INTERVENTION): Ultimately, we aim to reduce the number of health related social needs and improve health outcomes (e.g., quality and costs). In the short, term, we use the following measures: 1. Number of individuals screened for food insecurity and referred to a community based agency 2 . Proportion of patients reached by community based agency 3. Number supplemental nutrition assistance program screenings and community resource referrals (e.g., information provided on resource) by community based agency 4 . Provider and staff feedback on burden and feasibility

FINDINGS TO DATE (IT IS NOT SUFFICIENT TO STATE FINDINGS WILL BE DISCUSSED ): To date, we have screened over 8,000 patients for food insecurity, of whom $25 \%$ screened positive for food insecurity. Our program has resulted in 1,561 referrals to a community based agency. Of these patients, 973 (62\%) were successfully reached by the community based agency and offered SNAP screening. Of these, 428 (44\%) accepted screening for SNAP and $56 \%$ declined because they were already enrolled or were not sure if they were enrolled. The community based agency provided information to patients on 2,001 resources for health related social needs such as food pantries, meal programs and appropriate state agencies. Feedback from providers and staff indicate the program is well received and feasible in a busy primary care setting.

KEY LESSONS FOR DISSEMINATION (WHAT CAN OTHERS TAKE AWAY FOR IMPLEMENTATION TO THEIR PRACTICE OR COMMUNITY?): A program integrated into the electronic medical record that systematically screens for food insecurity and actively refers patients to a community based agency program is feasible in a safety net setting. Such programs can actively connect patients with community based agencies that address health related social needs. 
IMPLEMENTING HUNGER VITAL SIGNS: FOOD INSECURITY SCREENING IN AN URBAN UNDERSERVED RESIDENCY CLINIC

Rene McLeod ${ }^{2,1}$; Lauren V. Wood ${ }^{2,1}$; Iris S. Cheng ${ }^{1,2}$. ${ }^{1}$ University of North Carolina School of Medicine Charlotte Campus/Atrium Health, Charlotte, NC; ${ }^{2}$ Carolinas Medical Center, Charlotte, NC. (Control ID \#3163071)

STATEMENT OF PROBLEM OR QUESTION (ONE SENTENCE): Food insecurity (FI) is associated with a higher incidence of chronic disease including poorly-controlled diabetes and mental heatlh conditions in adults yet it is often overlooked during the office visit; screening for FI in our neediest patients may help us understand root causes of poor health outcomes. OBJECTIVES OF PROGRAM/INTERVENTION (NO MORE THAN THREE OBJECTIVES): 1.To educate residents about the relationship between food insecurity and chronic disease 2 .To screen at least $10 \%$ of our clinic patients for FI using three methods 2 . To refer willing patients who screen positive to a social worker and community food resource

DESCRIPTION OF PROGRAM/INTERVENTION, INCLUDING ORGANIZATIONAL CONTEXT (E.G. INPATIENT VS. OUTPATIENT, PRACTICE OR COMMUNITY CHARACTERISTICS): We initiated screening for FI as a QI project in our underserved urban resident clinic. We utilized two validated USDA questions, the "Hunger Vital Signs" (HVS) to screen patients through 3 PDSA cycles. We first sent an email to our residents to educate them about FI, the screening tools, and screening protocol. Our second PDSA cycle included a medical student who screened patients during an interview. FInally, the patients answered the HVS on a paper screener prior to a medical visit. For all PDSA cycles, residents were alerted when a patient screened positive and addressed FI during the clinic visit and added the ICD-10 code for FI to the chart. Patients who screened positive were offered referral to local food resources and a social worker. MEASURES OF SUCCESS (DISCUSS QUALITATIVE AND/OR QUANTITATIVE METRICS WHICH WILL BE USED TO EVALUATE PROGRAM/INTERVENTION): Only $6 \%$ of resident clinic patients were screened when we informed the residents of our screening directions through e-mail. Our verbal screening effort yielded an $88 \%$ screening rate. Screening by self report on paper yielded a screening rate of $61 \%$. Overall, $61 \%$ of all screened patients over the 3 PDSA cycles screened positive for FI yet only $54 \%$ of these patients were interested in social work assistance, help with SNAP applications or referral to a food pantry.

FINDINGS TO DATE (IT IS NOT SUFFICIENT TO STATE FINDINGS WILL BE DISCUSSED ): We found that food insecurity is a common social factor affecting health in our clinic and many patients were interested in addressing this need but not all. We found that e-mails encouraging providers to screen were not effective and having dedicated staff for inperson screening yielded the highest screening rate but may be hard to sustain. A paper screening tool may be more efficient but literacy and language barriers may pose challenges. We also noted that patients may be more forthcoming with a verbal screening tool compared to a formal paper questionaire. Expanded efforts to screen all of our clinic patients in this underserved resident clinic are planned through validated screening tools embedded in our EHR. We will continue evaluation of this process and its effects on clinic flow, community referral and short and long term health outcomes.

KEY LESSONS FOR DISSEMINATION (WHAT CAN OTHERS TAKE AWAY FOR IMPLEMENTATION TO THEIR PRACTICE OR COMMUNITY?): Verbally screening for FI is an effective way to detect FI. However, not all FI individuals are receptive to help. Clinical flow is minimally impacted and FI can be listed in the problem list as a reminder to readdress this need at future visits. Addressing FI may help improve the management of chronic diseases such as poorly controlled diabetes.
IMPLEMENTING THRIVE, AN EHR-BASED SCREENING AND REFERRAL SYSTEM TO ADDRESS SOCIAL DETERMINANTS OF HEALTH (SDOH) IN AN URBAN SAFETY NET HOSPITAL. Pablo Buitron de la Vega ${ }^{1}$; Stephanie Losi ${ }^{2}$; Linda Sprague Martinez ${ }^{3}$; Allison Bovell-Ammon ${ }^{2}$; Arvin Garg ${ }^{2}$; Thea James ${ }^{4}$; Alana Ewen²; Marna Stack; Heloisa DeCarvalho ${ }^{6}$; Megan Sandel ${ }^{2}$; Rebecca G. Mishuris ${ }^{5}$; Stella Deych ${ }^{2}$; Patrick Pelletier ${ }^{2}$; Nancy R. Kressin ${ }^{7} .{ }^{1}$ Boston Medical Center / Boston University, Dorchester, MA; ${ }^{2}$ Boston Medical Center, Boston, MA; ${ }^{3}$ Boston University School of Social Work, Boston, AL; ${ }^{4}$ Boston Medical Center, Cambridge, MA; ${ }^{5}$ Boston University School of Medicine, Boston, MA; ${ }^{6}$ Boston University School of Medicine, Chelsea, MA; ${ }^{7}$ Dept of Veterans Affairs and Boston University, West Roxbury, MA. (Control ID \#3184950)

\section{STATEMENT OF PROBLEM OR QUESTION (ONE SENTENCE):} Despite the documented impact of social factors on health, few systematic clinical strategies in primary care for addressing SDOH have been employed. OBJECTIVES OF PROGRAM/INTERVENTION (NO MORE THAN THREE OBJECTIVES): We set out to develop and evaluate a systematic clinical strategy for understanding the burden of SDOH by screening patients during primary care visits. As part of this work, we leveraged the EHR by automating patient need related to SDOH using ICD-10 codes which were matched to referral resources and provided to patients in their primary language.

DESCRIPTION OF PROGRAM/INTERVENTION, INCLUDING ORGANIZATIONAL CONTEXT (E.G. INPATIENT VS. OUTPATIENT, PRACTICE OR COMMUNITY CHARACTERISTICS): Boston Medical Center (BMC) is an urban, Safety Net academic center. In transition to an accountable care model for all Medicaid patients, BMC developed THRIVE, a population-based program, adapted from the WECARE model, which aims to improve patient care by identifying and addressing social needs. We secured leadership and IT support to develop a hospital wide EHR process to: (1) screen for SDOH (housing, food, employment, utilities, affording medications, transportation, education and childcare/ older adult care support), (2) capture responses in the form of ICD-10 codes and (3) automate referral guide print outs with information to help address social needs Following a pilot conducted among new patients in general internal medicine (GIM) clinics, we scaled up our intervention to include all patients presenting to 13 ambulatory clinics in GIM, Family Medicine, OBGYN, Infectious Disease and Pediatrics. Moreover, THRIVE is available as part of Oregon Community Health Information Network's (OCHIN) available tools to address $\mathrm{SDOH}$.

MEASURES OF SUCCESS (DISCUSS QUALITATIVE AND/OR QUANTITATIVE METRICS WHICH WILL BE USED TO EVALUATE PROGRAM/INTERVENTION): We measured proportion of patients screened, proportion of signed ICD-10 code orders and proportion of signed orders for $\mathrm{SDOH}$ resource guides. Additionally, proportion of correct entries for a subset of paper screening results transcribed into the EHR and time expended by Medical Assistants (MA) to upload the screener in the EHR. FINDINGS TO DATE (IT IS NOT SUFFICIENT TO STATE FINDINGS WILL BE DISCUSSED ): We found that a hospital wide program to address SDOH was both feasible and acceptable among patients and providers. Between 10/2017 and 12/2018, clinics screened 50,532 unique patients, and $20 \%$ reported at least one $\mathrm{SDOH}$ need. Meanwhile, $12 \%$ of patients requested support with at least one resource. Homelessness / housing insecurity $(10 \%)$ and food insecurity (14\%) were the most prevalent $\mathrm{SDOH}$. We achieved $82 \%$ screening compliance rate across all clinics. $91 \%$ of patients with positive SDOH screenings had ICD-10 code added to visit diagnosis. Of patients requesting resources, $90 \%$ received a resource guide. Among 85 paper screening responses, $75 \%$ were reliably transcribed by MAs into the EHR. 
The time study showed MAs expended an average of 1 minute to enter responses into the EHR.

KEY LESSONS FOR DISSEMINATION (WHAT CAN OTHERS TAKE AWAY FOR IMPLEMENTATION TO THEIR PRACTICE OR COMMUNITY?): We attribute the success of THRIVE to building upon an evidence-based model, aligning efforts with hospital strategic priorities, involving key stakeholders in developing a streamlined standard workflow, leveraging EHR automation, piloting the initial program design before scaling, and incorporating staff feedback at every phase.

IMPLEMENTING TRANSITIONAL CARE MANAGEMENT SERVICE: GETTING PAID FOR COORDINATING SUCCESSFUL TRANSITIONS

Gary Fischer ${ }^{1}$; Janice Cunningham ${ }^{2}$; Zachary Lenhart ${ }^{2}$; Cynthia Arnold ${ }^{3}$; Jaishree Hariharan ${ }^{3} .{ }^{1}$ University of Pittsburgh, Pittsburgh, PA; ${ }^{2}$ University of Pittsburgh Physicians, Pittsburgh, PA; ${ }^{3}$ University of Pittsburgh Medical center, Pittsburgh, PA. (Control ID \#3181550)

\section{STATEMENT OF PROBLEM OR QUESTION (ONE SENTENCE):}

Can the Transitional Care Management (TCM) service, with its operational and documentation requirements, be successfully implemented in a primary care practice?

\section{OBJECTIVES OF PROGRAM/INTERVENTION (NO MORE THAN} THREE OBJECTIVES): 1 . To impement TCM in a large academic general internal medicine (GIM) practice. 2. To perform TCM in $>50 \%$ of targeted patients (pts). 3. To reduce readmissions in the practice population.

DESCRIPTION OF PROGRAM/INTERVENTION, INCLUDING ORGANIZATIONAL CONTEXT (E.G. INPATIENT VS. OUTPATIENT, PRACTICE OR COMMUNITY CHARACTERISTICS): In 2013, Medicare began paying for TCM to improve the care of pts transitioning from a stay in a hospital, rehab, or skilled nursing facility to home and reduce readmissions. TCM covers coordination services over 30 days, but to bill for it, a care team member must contact the pt or caregiver within 2 business days of discharge and the provider must see the pt within 7 or 14 days (depending on the level billed). By 2015 only 7\% of eligible Medicare pts had TCM, even though costs and mortality were lower among TCM pts (JAMA Intern Med. 2018;178(9):1165-71). In 2018 a group involving MDs, operations, clinical IT, revenue cycle IT and compliance developed the infrastructure to provide TCM. We implemented this in an academic GIM practice in Oct 2018. An $\mathrm{RN}$, working from a list of pts discharged the previous day calls targeted pts, ensures that pts' immediate needs are met, and schedules a TCM visit. An APP has dedicated slots to see pts who cannot be seen by their PCP in time. Alerts. templates, and order sets help providers recognize that a visit qualifies for TCM, meet documentation requirements, and bill appropriately.

MEASURES OF SUCCESS (DISCUSS QUALITATIVE AND/OR QUANTITATIVE METRICS WHICH WILL BE USED TO EVALUATE PROGRAM/INTERVENTION): 1. Percent of discharges targeted for TCM. 2. Percent of targeted pts receiving TCM phone call and TCM office visit (by level of service - 99495 (moderate) or 99496 (high)). 3. Marginal increase in revenue generated from TCM. 4. 30 day readmission rate and mortality before and after TCM implementation 5. Qualitative report by TCM $\mathrm{RN}$ on interventions taken during calls.

FINDINGS TO DATE (IT IS NOT SUFFICIENT TO STATE FINDINGS WILL BE DISCUSSED ): From Oct 15 to Dec 31, 373 of 737 (51\%) discharged pts met our outreach criterial. Of these, $264(71 \%)$ had an appt made, 111 (42\%) within 7 days and 112 (42\%) between 8-14 days. $172(64 \%)$ of appts were completed. Some missed apts were due to readmissions. From Oct 15 to Jan 13 (2 wks after Dec 31), 154 TCM services were billed (118 for service 99495 (ave payment/paid claim \$111) and 36 for 99496 (ave payment/paid claim \$146)). If all the TCM pts had had regular E\&M visits during the time, the marginal increase in revenue is $\$ 9,500$ over the time period, likely an underestimate. After sufficient time, changes in readmission and mortality rates will be determined. TCM RN reported cases where post-discharge call resulted in interventions that likely prevented serious harm.

KEY LESSONS FOR DISSEMINATION (WHAT CAN OTHERS TAKE AWAY FOR IMPLEMENTATION TO THEIR PRACTICE OR COMMUNITY?): 1. Coordination between MDs, IT, operations, and compliance facilitate the success of TCM. 2. Implementing TCM can result in timely visits after discharge and increased revenue. 3. Identifying high risk changes (e.g. diuretic doses) during TCM call may prevent readmissions. 4. Increasing pts seen within 7 days will increase revenue and will likely decrease readmissions.

IMPROVING ACCESS TO CARE FOR UNDERINSURED DIABETIC PATIENTS: LESSONS IN COLLABORATION

Stacie Schmidt ${ }^{1}$; Mark Nissley ${ }^{2}$; Yousuf Khalifa ${ }^{1}{ }^{1}$ Emory University, Atlanta, GA; ${ }^{2}$ Intelligent Retinal Imaging Systems (IRIS), Pensacola, FL. (Control ID \#3140871)

STATEMENT OF PROBLEM OR QUESTION (ONE SENTENCE): Ensuring equitable preventive care is delivered to patients with diabetes.

OBJECTIVES OF PROGRAM/INTERVENTION (NO MORE THAN THREE OBJECTIVES): 1. Deliver screening exams with minimal impedance on physician time. 2. Close the gap toward ending preventable blindness among diabetic populations. 3. Provide best practices for engaging health systems, external organizations, and team members in providing health services.

DESCRIPTION OF PROGRAM/INTERVENTION, INCLUDING ORGANIZATIONAL CONTEXT (E.G. INPATIENT VS. OUTPATIENT, PRACTICE OR COMMUNITY CHARACTERISTICS): Diabetes is the leading cause of preventable blindness in the US. Yet, national diabetic retinal screening rates are less than $40 \%$. This is partly related to the fact that annual diabetic eye exams are ordered by the primary care physician, yet patients must schedule eye appointments at times separate from their primary care visits. Our Primary Care Center (PCC) is an academic, safety net, hospital-based clinic that provides visits mostly to uninsured low-literacy patients. Patients in the PCC often attend different appointments between various healthcare specialties. Multiple visits require patients to manipulate their schedules, and secure transportation. We suspect such inefficiencies leads to more scheduling of appointments, increased no-shows, diminished patient satisfaction, and reduced health outcomes. We established diabetic retinal photo services within our PCC that (1) identify patients eligible for annual diabetic retinal screening exams, (2) provide diabetic retinal screening exams with minimal impact to physician workload, and (3) allow images to be reviewed in a timely manner by a trained retinal specialist, with next steps for diagnosis and management clearly delineated. We collaborated with Intelligent Retinal Imaging Systems (IRIS), a company that provides critical access to ophthalmology services for patients. The exam is a critical measure of success in caring for a population; by performing this service, we increased compliance to this metric for our health system. As healthcare shifts from pay for service to pay for performance and quality, we have proven that a simple, collaborative model is capable of closing the gap toward ending preventable blindness. 
MEASURES OF SUCCESS (DISCUSS QUALITATIVE AND/OR QUANTITATIVE METRICS WHICH WILL BE USED TO EVALUATE PROGRAM/INTERVENTION): Monthly reporting metrics enabled us to provide more than 11,000 patients access to diabetic eye exam services, and identify 1800 patients whose vision was saved.

FINDINGS TO DATE (IT IS NOT SUFFICIENT TO STATE FINDINGS WILL BE DISCUSSED ): Results to date include the identification of 4,000 patients with some form of pathology that would not have been known without this service, and the more than 1,800 patients whose vision was saved as a result of this program. We have maintained the percentage of non-readable photos at less than 5\% across the PCC.

KEY LESSONS FOR DISSEMINATION (WHAT CAN OTHERS TAKE AWAY FOR IMPLEMENTATION TO THEIR PRACTICE OR COMMUNITY?): 1) Investment and collaboration with external organizations enhances delivery of preventive services in the primary care center. 2) Ancillary team member involvement improves efficiency of services delivered with minimal impact to the PCP's time and engagement of staff. 3) Integrating services within the PCC provides equitable care to populations who struggle with navigating healthcare systems.

IMPROVING ACCURACY OF INTERNAL MEDICINE RESIDENT PATIENT PANELS IN AN ACADEMIC PRIMARY CARE SETTING

Laura Hallett $^{1}$; Gertrude Manchester ${ }^{1}$; Richard M. Forster ${ }^{2} .{ }^{1}$ University of Massachusetts Medical Center, Worcester, MA; ${ }^{2}$ University of Massachusetts Medical School, Worcester, MA. (Control ID \#3185613)

STATEMENT OF PROBLEM OR QUESTION (ONE SENTENCE): Internal medicine (IM) residents are assigned to be the primary care provider for certain patients in their continuity clinic, but when a patient's PCP is listed incorrectly in the electronic medical record, it can lead to communication delays, lack of continuity for patients, and lower resident satisfaction.

OBJECTIVES OF PROGRAM/INTERVENTION (NO MORE THAN THREE OBJECTIVES): Using an electronic medical record (EMR) displaying pairings of resident PCPs with faculty preceptors, we sought to: 1. Reduce the percentage of resident-owned patients with no coattending listed in the EMR 2. Expand resident patient panels to include previously inaccurate patient/PCP pairings 3 . Initiate a curriculum for residents to work with faculty preceptors on active panel management DESCRIPTION OF PROGRAM/INTERVENTION, INCLUDING ORGANIZATIONAL CONTEXT (E.G. INPATIENT VS. OUTPATIENT, PRACTICE OR COMMUNITY CHARACTERISTICS): TO address inaccuracies in resident patient panels, we met with 20 PGY-2 and PGY-3 residents who had continuity clinic at our residency program's largest primary care site, which serves a diverse, medically complex urban patient population. We created a list of patients currently assigned to each resident and a list of all patients they had seen in their clinic in the previous year. Residents went through both lists to identify patients who were incorrectly assigned and identified corrections to be made. We then addressed these corrections in the EMR and ensured the correct resident PCP and academic PCP were listed for all resident-assigned patients. We created a list of resident patients designated as incorrectly assigned to be distributed to faculty preceptors to ensure accuracy. MEASURES OF SUCCESS (DISCUSS QUALITATIVE AND/OR QUANTITATIVE METRICS WHICH WILL BE USED TO EVALUATE PROGRAM/INTERVENTION): Our metrics to evaluate this intervention were: 1 . Percentage of resident-owned patients with resident PCP and attending preceptor listed correctly in EMR 2. Change in resident panel size post-intervention
FINDINGS TO DATE (IT IS NOT SUFFICIENT TO STATE FINDINGS WILL BE DISCUSSED ): After our intervention, we found: 1. Percentage of resident-owned patients with incorrect resident PCP and faculty pairing listed in EMR decreased from $54 \%$ to $26 \%$ 2. Resident panels expanded by $15 \%$ overall through correct identification of resident-owned patients 3 . Residents identified $21 \%$ of patients assigned to them as incorrectly assigned (i.e. patients they had never seen but were listed as their PCP)

KEY LESSONS FOR DISSEMINATION (WHAT CAN OTHERS TAKE AWAY FOR IMPLEMENTATION TO THEIR PRACTICE OR COMMUNITY?): Conducting a panel review with residents allowed for a significant increase in panel accuracy and expansion of their patient panels. Complex decision-making is required about patients who residents identified as not theirs, and we solicited preceptor input to determine the correct ownership of those patients. We noted anecdotal increased resident satisfaction following this intervention, and hope to collect survey data to further explore resident satisfaction. Correct identification and maintenance of resident patient panels is a labor-intensive process that needs oversight from residents, attendings, nurses, and staff. Our next step is the development of a curriculum focusing on active panel management, in which residents will meet multiple times per year with their faculty preceptors to address panel accuracy and performance on quality metrics.

IMPROVING COLORECTAL CANCER SCREENING RATES WITH ELECTRONIC MEDICAL RECORD INFORMED CARE MANAGEMENT.

Joseph Daprano $^{1,2}{ }^{2}$; David Mansour ${ }^{4}$; David Margolius ${ }^{3}$; James Misak ${ }^{4}$. ${ }^{1}$ MetroHealth Medical Center, Willoughby Hills, $\mathrm{OH} ;{ }^{2}$ Case Western Reserve University, Cleveland, $\mathrm{OH} ;{ }^{3}$ MetroHealth, Cleveland, OH; ${ }^{4}$ MetroHealth Medical Center, Cleveland, OH. (Control ID \#3185864)

STATEMENT OF PROBLEM OR QUESTION (ONE SENTENCE): Colorectal cancer is the third most common cancer diagnosed among men and women and the second leading cause of cancer death in the United States: however, nearly one-third of eligible adults in the United States have never been screened for colorectal cancer.

OBJECTIVES OF PROGRAM/INTERVENTION (NO MORE THAN THREE OBJECTIVES): 1.) Create an EMR based population health process to improve colorectal cancer screening in an urban and suburban outpatient practice setting. 2.) Develop care management processes that can be implemented by any staff in urban and suburban outpatient practice settings. DESCRIPTION OF PROGRAM/INTERVENTION, INCLUDING ORGANIZATIONAL CONTEXT (E.G. INPATIENT VS. OUTPATIENT, PRACTICE OR COMMUNITY CHARACTERISTICS): Patients eligible but not yet screened for CRC were identified with the use of an EMR dashboard. Patient Service Representatives (in the suburban practice) and Community Health Worker students (in the urban practice), were educated on the importance of CRC screening and trained to contact patients in need of CRC screening. Bulk orders for all eligible patients in a practice were created by physicians via EMR. Patient Service Representatives and Community Health Worker students used telephone and in person contact with eligible patients for both the initial and follow up of patients who had not yet completed CRC screening. Contact events were recorded in a paper and eventually EMR file to allow for continued follow-up to completion as needed.

MEASURES OF SUCCESS (DISCUSS QUALITATIVE AND/OR QUANTITATIVE METRICS WHICH WILL BE USED TO EVALUATE PROGRAM/INTERVENTION): 1.) A more than $10 \%$ increased percentage of eligible patients screened for colorectal cancer at each of the two sites. 2.) Uptake of the EMR based Care Management process in each of the two sites. 
FINDINGS TO DATE (IT IS NOT SUFFICIENT TO STATE FINDINGS WILL BE DISCUSSED ): During the thirtteen month period from December 2017 through Dec 2018 an average of 5000 persons were eligible to be screened for colorectal cancer with approximately 4000 of those patients in the suburban practice and 1000 of those patients in the urban practice. The suburban practice achieved a $12 \%$ increase in colorectal cancer screening rising to $73 \%$ of eligible patients screened and the urban practice achieved an $11 \%$ increase in colorectal cancer screening rising to $81 \%$ of eligible patients screened. Although initially only Patient Service Representatives and Community Health Worker students were trained in the process of EMR based care management, Medical Team Assistants and Nurses also began using the process. This uptake by all staff allowed for more consistent follow-up on patients who had been started on the CRC screening pathway but were not completing CRC screening. This persistence in follow up, facilitated by the EMR dashboard, in turn greatly increased CRC screening rates.

KEY LESSONS FOR DISSEMINATION (WHAT CAN OTHERS TAKE AWAY FOR IMPLEMENTATION TO THEIR PRACTICE OR COMMUNITY?): Patients responded to repeated encouragement to complete colorectal cancer screening. A population based, EMR facilitated care management process can quickly and greatly increase colorectal cancer screening in a diversity of practice settings.

IMPROVING ENGAGEMENT IN DEPRESSION TREATMENT AT AN ACADEMIC MEDICAL CENTER THROUGH A TELEPHONEBASED DEPRESSION NAVIGATOR PROGRAM

$\underline{\text { Juliana Macri }}{ }^{1}$; Maria E. Garcia ${ }^{1}$; Hannah Rapp ${ }^{2}$; Maki Aoki ${ }^{1}{ }^{1}$ University of California, San Francisco, San Francisco, CA; ${ }^{2}$ School of Public Health, University of Washington, Seattle, WA. (Control ID \#3184395)

STATEMENT OF PROBLEM OR QUESTION (ONE SENTENCE): Can targeted outreach by a Depression Navigator at an academic primary care office increase rates of depression response/remission by improving receipt and maintenance of evidence-based depression care?

OBJECTIVES OF PROGRAM/INTERVENTION (NO MORE THAN THREE OBJECTIVES): - Outreach to all patients recently prescribed an antidepressant to assess for concerns and adherence - Provide coaching around common reasons for early non-adherence (e.g. side effects, "not working", distrust) - Link patients to behavioral health (therapy/psychiatry) and ensure primary care depression follow-up

DESCRIPTION OF PROGRAM/INTERVENTION, INCLUDING ORGANIZATIONAL CONTEXT (E.G. INPATIENT VS. OUTPATIENT, PRACTICE OR COMMUNITY CHARACTERISTICS): In a diverse, academic general practice, we initiated universal depression screening (using the PHQ-2 followed by the PHQ-9 if positive) in October 2017. A clinic-based Depression Navigator began calling patients with a PHQ-9 >9 who had been newly prescribed an antidepressant. Calls aimed to engage patients in their depression treatment plan by assessing medication adherence and coaching around common reasons for early non-adherence. The Navigator also performed care coordination, linking patients to behavioral health (therapy/ psychiatry) and scheduling 4-6 week follow-up with PCP/primary care team. MEASURES OF SUCCESS (DISCUSS QUALITATIVE AND/OR QUANTITATIVE METRICS WHICH WILL BE USED TO EVALUATE PROGRAM/INTERVENTION): The primary goal of the pilot program was to increase depression response (defined as a decrease in PHQ9 score by $2+$ points) and remission (defined as a $>50 \%$ score decrease or score $<5$ ). To achieve this goal, process measures included: 1) Increased medication adherence at 1 month, 2) Scheduled depression follow-up visit within 4-6 weeks, and 3) Decreased proportion of program-eligible patients who were not contacted or lost to follow up.

FINDINGS TO DATE (IT IS NOT SUFFICIENT TO STATE FINDINGS WILL BE DISCUSSED ): Over the first 4 months of this pilot program, 53 patients with newly prescribed antidepressants were enrolled. Of these, 45 (85\%) patients had at least one contact with the Navigator and $29(55 \%)$ had more than one contact. Among patients with repeat PHQ9s $(\mathrm{n}=26), 11(42 \%)$ achieved response, $6(23 \%)$ achieved remission, and 9 (35\%) had no improvement in PHQ9 score. Medication adherence dropped from $84 \%$ at 1 week $(n=45)$ to $66 \%$ at 1 month $(n=38)$. The Depression Navigator made 19 follow-up appointments for 14 individual patients, though only 6 patients $(11 \%)$ had a repeat PHQ-9 within 6 weeks of anti-depressant initiation. The program's impact was limited primarily by high rates of eligible patients never reached or lost to follow up $(30 \%, \mathrm{n}=16)$ and secondarily by patients declining medications and/or participation $(13 \%, n=7)$.

KEY LESSONS FOR DISSEMINATION (WHAT CAN OTHERS TAKE AWAY FOR IMPLEMENTATION TO THEIR PRACTICE OR COMMUNITY?): Proactive outreach to patients recently initiated on an anti-depressant in their primary care office may better engage a large portion of this vulnerable population in their depression treatment plan. Further implementation success depends on increasing in-office follow up rates, and reducing the proportion of patients never reached and lost to follow up. Systems-level interventions with innovative approaches to patient outreach and improved scheduling access may help resolve these barriers.

IMPROVING LATENT TUBERCULOSIS SCREENING IN A COMBINED INTERNAL MEDICINE AND PEDIATRICS RESIDENCY OUTPATIENT CLINIC AT DENVER HEALTH - A RESIDENT DESIGNED AND IMPLEMENTED QUALITY IMPROVEMENT PROJECT

Timothy P. Newton ${ }^{2}$; Jonathan S. Schultz ${ }^{1}$; Amy Beeson ${ }^{1}$; Joshua M. Gannon $^{1}$; Anne Frank ${ }^{1}$; Julie Venci ${ }^{1} .{ }^{1}$ University of Colorado, Aurora, CO; ${ }^{2}$ University of Colorado School of Medicine, Englewood, CO. (Control ID \#3185338)

STATEMENT OF PROBLEM OR QUESTION (ONE SENTENCE): There is a large proportion of new and established Denver Health (DH) primary care adult and pediatric patients who meet the criteria for screening of latent tuberculosis infection (LBTI) and have never been screened and may be at high risk for development of active TB and possible transmission. OBJECTIVES OF PROGRAM/INTERVENTION (NO MORE THAN THREE OBJECTIVES): We proposed a quality improvement intervention to improve screening at the DH Internal Medicine and Pediatrics (Med-Peds) resident clinic. The objectives were to: 1 . Identify patients who are foreign born and at risk of TB. 2. Discuss screening with those identified, and if positive, treatment options. 3. Perform QFT testing on consenting patients who are at risk.

DESCRIPTION OF PROGRAM/INTERVENTION, INCLUDING ORGANIZATIONAL CONTEXT (E.G. INPATIENT VS. OUTPATIENT, PRACTICE OR COMMUNITY CHARACTERISTICS): In 2016, 64 new cases of active tuberculosis (TB) were reported in Colorado, for a case incidence rate of 1.6 per 100,0000 in Denver. Over half of these cases could have been prevented with appropriate screening and treatment. More than $70 \%$ of patients were born outside of the US; $24 \%$ were from Mexico and the rest from 22 other countries. The Med-Peds residency clinic at DH is located within the Federico F. Peña Southwest Family Health Center in Southwest Denver, a primarily Hispanic and low-income community. MedPeds residents identified that high risk patients, including those who were 
foreign born, were rarely screened for LTBI. An intervention was designed that included an education session, an email reminder to residents and flowchart with LTBI screening criteria posted in the DH resident clinic.

MEASURES OF SUCCESS (DISCUSS QUALITATIVE AND/OR QUANTITATIVE METRICS WHICH WILL BE USED TO EVALUATE PROGRAM/INTERVENTION): The proportion of those who were appropriately screened out of all who met the inclusion criteria at clinic visits were compared 12 months before and after the intervention period with a chisquared test. Also a run chart was created to show changes overtime. All analyses were conducted in SAS 9.4.

FINDINGS TO DATE (IT IS NOT SUFFICIENT TO STATE FINDINGS WILL BE DISCUSSED ): A total of 405 patients met the inclusion criteria from May $1^{\text {st }} 2016$ to April $30^{\text {st }} 2017$, prior to the intervention. The mean age was 30 years (SD 24) and primarily female (55\%). Only 16 (4\%) of the 405 patients were appropriately screened with a QFT test. After the intervention, from June $1^{\text {st }} 2017$ to May $31^{\text {st }} 2018$, a total of 619 patients met the inclusion criteria, with a mean age of 33 years (SD 25) and primarily female $(56 \%)$. An increase to $67(11 \%)$ of the 619 patients were appropriately screened $(\mathrm{p}<0.001)$. After the intervention, Med-Peds residents were nearly 3 times as likely to screen patients appropriately for LTBI (RR 2.74, 95\% CI (1.61-4.66)). Among those, 9 of the 83 (11\%) had a positive QFT, had a chest X-ray performed and were referred for LTBI treatment.

KEY LESSONS FOR DISSEMINATION (WHAT CAN OTHERS TAKE AWAY FOR IMPLEMENTATION TO THEIR PRACTICE OR COMMUNITY?): - Resident led QI initiatives can be effective in primary care clinics - Primary care clinics can effectively initate LTBI screening programs

IMPROVING MENTAL HEALTH CARE APPOINTMENT SHOWRATES AT AN URBAN PRIMARY CARE TRAINING SITE

Olufunke Adeusi ${ }^{2}$; Tamara Goldberg ${ }^{1}$. ${ }^{1}$ Mount Sinai, New York, NY; ${ }^{2}$ Mount Sinai St. Lukes's and Mount Sinai West, New York, NY. (Control ID \#3180926)

STATEMENT OF PROBLEM OR QUESTION (ONE SENTENCE): Does the method of communication between care providers and on-site behavioral health integration professionals (BHI) at time of referral impact mental health appointment show-rates among an underserved patient population?

OBJECTIVES OF PROGRAM/INTERVENTION (NO MORE THAN THREE OBJECTIVES): To improve patient show-rates for initial mental health appointments. To assess residents' familiarity with the collaborative care model and on-site BHI services.

DESCRIPTION OF PROGRAM/INTERVENTION, INCLUDING ORGANIZATIONAL CONTEXT (E.G. INPATIENT VS. OUTPATIENT, PRACTICE OR COMMUNITY CHARACTERISTICS): This project was implemented at a Federally Qualified Health Center in New York City that serves as the primary care practice site for our internal medicine residents. We reviewed data over a three-month period regarding method of BHI referral (EMR order versus warm-handoff) and mental health appointment show-rates. Resident providers were surveyed regarding knowledge of the collaborative care model and satisfaction with their degree of engagement with BHI professionals. Next, we implemented a collaborative intervention to improve resident engagement with BHI services including: presence of a BHI team member at preclinic huddles and dissemination of a resource sheet describing the collaborative care model and how to conduct a real-time, warm handoff with BHI staff. Finally, post-intervention referral data and show-rate data were obtained, and will continue to be collected through the Spring of 2019.

MEASURES OF SUCCESS (DISCUSS QUALITATIVE AND/OR QUANTITATIVE METRICS WHICH WILL BE USED TO EVALUATE PROGRAM/INTERVENTION): Metrics include BHI referral data and mental health show-rate data, pre and post intervention. FINDINGS TO DATE (IT IS NOT SUFFICIENT TO STATE FINDINGS WILL BE DISCUSSED ): Baseline show-rates to a first mental health appointment were $23.8 \%(25 / 105)$ overall. Show rates were $20.7 \%$ (19/92) with EMR referral only, and 46.2\% (6/13) for EMR referral with a warm handoff. Post-intervention patient show-rates were $15.1 \%$ (14/ 86) for EMR referrals only, and 50\% (9/18) for EMR referrals with a warm handoff. 19 of 40 residents completed the baseline survey. Only $42 \%$ felt somewhat confident about their knowledge of the collaborative care model and $61 \%$ felt satisfied with their degree of engagement with BHI.

KEY LESSONS FOR DISSEMINATION (WHAT CAN OTHERS TAKE AWAY FOR IMPLEMENTATION TO THEIR PRACTICE OR COMMUNITY?): Despite on-site BHI services and overall provider satisfaction with BHI engagement, the vast majority of BHI referrals at our urban underserved clinic lacked a warm-handoff between providers, with overall show-rates less than $25 \%$ for initial mental health appointments. However, show-rates more than doubled when a warm-handoff between the primary care provider and $\mathrm{BHI}$ professional was performed rather than EMR alone. This suggests that open collaboration can lead to improved show-rates and patient engagement. Visibility of BHI staff at morning huddles and educational pamphlets for residents may help improve resident knowledge gaps and increase the motivation for warm-handoffs.

IMPROVING MONOFILAMENT TESTING IN UNCONTROLLED DIABETIC PATIENTS IN AN OUTPATIENT COMMUNITY CLINIC - A RESIDENT LED INITIATIVE

Priya Jaisinghani; Payal Parikh. Robert Wood Johnson, Edison, NJ. (Control ID \#3179963)

STATEMENT OF PROBLEM OR QUESTION (ONE SENTENCE): How to improve annual monofilament exams in diabetic patients in an underserved community health clinic

OBJECTIVES OF PROGRAM/INTERVENTION (NO MORE THAN THREE OBJECTIVES): To improve the rates of annual monofilament exam as well as resident documentation from the baseline of $44 \%$ to $70 \%$ for the cohort of diabetic patients at Eric B. Chandler Health Clinic (EBCHC)

DESCRIPTION OF PROGRAM/INTERVENTION, INCLUDING ORGANIZATIONAL CONTEXT (E.G. INPATIENT VS. OUTPATIENT, PRACTICE OR COMMUNITY CHARACTERISTICS): We identified barriers to performing annual monofilament exams by creating an in-depth root cause analysis. We charted our possible interventions on the pay-off priority matrix and enacted them in a multitude of PDSA cycles. Our goal was to ensure that a greater percentage of patients at EBCHC with uncontrolled diabetes were receiving monofilament exams during their patient encounters. Our interventions included a multidisciplinary approach involving environmental factors such as supplies, systemic factors such as the electronic medical record (EMR), provider factors such as education, communication factors, and patient factors such as increasing awareness on preventative medicine. First, it was ensured that all patient exam rooms had a full supply of monofilaments. Then, providers were educated regarding the location of the monofilaments in an exam room and the importance of regular monofilament exams in patients with diabetes. All resident 
physicians were further educated on the proper location to document diabetic foot exams in the EMR. We also included the MA and RNs in our process improvement by encouraging them to ask every patient who was getting an A1c checked to remove their shoes and socks in preparation for the annual visit. Informational posters were created and placed in exams rooms in English and Spanish requesting patients to remove their shoes and socks if they have diabetes so that their resident physician could perform the monofilament exam.

MEASURES OF SUCCESS (DISCUSS QUALITATIVE AND/OR QUANTITATIVE METRICS WHICH WILL BE USED TO EVALUATE PROGRAM/INTERVENTION): Pre-intervention data was obtained from the EMR which showed that out of 1046 diabetic patients, $44 \%$ of patients had monofilament testing within the past 1 year.

FINDINGS TO DATE (IT IS NOT SUFFICIENT TO STATE FIND-

INGS WILL BE DISCUSSED ): For the cohort of diabetic patients seen at EBCHC between October 1, 2017 to December 31,2017, 66\% of patients had a visit with documentation and completion of annual monofilament exam compared to $44 \%$ prior to interventions.

KEY LESSONS FOR DISSEMINATION (WHAT CAN OTHERS TAKE AWAY FOR IMPLEMENTATION TO THEIR PRACTICE OR COMMUNITY?): Patients with diabetes are 15 to 25 times more likely to lose part of a leg than people without the condition (International Diabetes Federation (IDF)). Throughout the world, up to $70 \%$ of leg amputations are performed in people with diabetes (IDF). Data from 2001 shows diabetic foot care costed $\$ 10.9$ billion and is continuously increasing (Raghav). One study on diabetic foot examinations in the clinic setting found that 844 (41.4\%) patients with diabetes reports that their healthcare provider checked their feet for sores during the past year (Al Sayah). With the high risk of amputation in diabetics and increasing healthcare costs, there is room for improvement in implementing easy screening techniques such as the monofilament exam in outpatient clinics which have high impact on patient outcome and healthcare cost.

IMPROVING NALOXONE COPRESCRIBING FOR PATIENTS ON HIGH DOSE OPIOIDS AT HOSPITAL DISCHARGE

Rebecca Richardson; Polina Kukhareva; Kensaku Kawamoto; Christopher Walls; Phillip B. Warner; Jacob McKenna; Kencee K. Graves. University of Utah, Salt Lake City, UT. (Control ID \#3145408)

\section{STATEMENT OF PROBLEM OR QUESTION (ONE SENTENCE):}

Taking high doses of opioids places patients at high risk of accidental opioid overdose, and the Centers of Disease Control and Prevention (CDC) has recommended co-prescription of an opioid reversal agent to these patients; however, there was no standardized process to ensure this was done at hospital discharge.

OBJECTIVES OF PROGRAM/INTERVENTION (NO MORE THAN THREE OBJECTIVES): The objectives of this study were to first understand the current state of opioid and naloxone co-prescribing on hospital discharge based on CDC guidelines; and second, to increase the number of naloxone prescriptions at the time of discharge from the University of Utah Hospital by $20 \%$ for patients on morphine milligram equivalents (MME) of 100 or greater within the 6 months following the implementation of an electronic alert through the hospital electronic medical record (EMR). DESCRIPTION OF PROGRAM/INTERVENTION, INCLUDING ORGANIZATIONAL CONTEXT (E.G. INPATIENT VS. OUTPATIENT, PRACTICE OR COMMUNITY CHARACTERISTICS): Our study was set at a single academic medical center and is focused on a single group of general internal medicine hospitalist physicians and advanced practice clinicians (APCs) caring for adult patients in an inpatient setting. Prior to the intervention, discharge data from the University of Utah Internal Medicine Hospitalist group in August 2017 was analyzed to assess opioid and naloxone co-prescriptions at the time of discharge. Data query indicated that there were 71 patients who were discharged on an opioid (oxycodone, hydrocodone (including combination with acetaminophen) and hydromorphone). A chart review of a small subset of 23 patients showed that $8(35 \%)$ were discharged on greater than $100 \mathrm{MME}$ and 14 out of 23 were discharged on greater than $50 \mathrm{MME}$. Zero patients were discharged with a co-prescription of naloxone. Therefore, an intervention was created to identify patients on high doses of opiates that would put them at risk for accidental overdose. A best practice alert (BPA) was built into our Epic EMR via the discharge order set to calculate the MME a patient is prescribed at discharge, then alert the provider if a patient is prescribed more than $100 \mathrm{MME}$, and lastly, prompt the clinician to coprescribe naloxone prior to hospital discharge. The BPA was introduced to a pilot group of physicians and APCs in September 2018.

MEASURES OF SUCCESS (DISCUSS QUALITATIVE AND/OR QUANTITATIVE METRICS WHICH WILL BE USED TO EVALUATE PROGRAM/INTERVENTION): Using discharge data collected from the EMR, we compared the number of patients discharged on opiates greater than $100 \mathrm{MME}$ and the percentage of those patients discharged with naloxone before and after the BPA was implemented. FINDINGS TO DATE (IT IS NOT SUFFICIENT TO STATE FINDINGS WILL BE DISCUSSED ): Data collected from September to November 2018 showed that the BPA fired for 8 patients. Naloxone was co-prescribed for 5 out of the 8 patients (about 62\%).

KEY LESSONS FOR DISSEMINATION (WHAT CAN OTHERS TAKE AWAY FOR IMPLEMENTATION TO THEIR PRACTICE OR COMMUNITY?): Our intervention of implementing an electronic alert prompting providers to co-prescribe naloxone significantly increased the percentage of naloxone prescriptions for patients on high dose narcotics. As we have shown improvement with our pilot, we have now launched the BPA for all internal medicine units at our hospital. This is an adaptable strategy for other institutions using Epic as their electronic medical record.

IMPROVING PATIENT ENROLLMENT IN A PATIENT-WEB PORTAL THROUGH A MULTI-TIERED INTERVENTION

Eugene J. Lucas ${ }^{2}$; Carlos A. Lopez ${ }^{1}$; Srineil Nizambad³ ${ }^{3}$ Ankita Sagar ${ }^{2}$. ${ }^{1}$ Memorial Sloan Kettering Cancer Center, New York City, NY; ${ }^{2}$ Zucker School of Medicine at Hofstra/Northwell, Brooklyn, NY; ${ }^{3}$ State University of New York at Stony Brook, Stony Brook, NY. (Control ID \#3186320)

STATEMENT OF PROBLEM OR QUESTION (ONE SENTENCE): In a National Committee for Quality Assurance PatientCentered Medical Home Level III site that caters to an at-risk patient population, the proportion of patients signed up for access to their health care data via an online patient portal was found to be below the desired goal and highlighted the need for an improvement-oriented strategy.

OBJECTIVES OF PROGRAM/INTERVENTION (NO MORE THAN THREE OBJECTIVES): The objective was to increase the proportion of enrolled patients from a baseline of $18.5 \%$ of the clinic population to $25 \%$. We employed a 3-phase intervention over the course of 9 months and also sought to compare the various success rates of each of the 3 methods. 
DESCRIPTION OF PROGRAM/INTERVENTION, INCLUDING ORGANIZATIONAL CONTEXT (E.G. INPATIENT VS. OUTPATIENT, PRACTICE OR COMMUNITY CHARACTERISTICS): Baseline enrollment data for the cohort of patients being followed by participating residents was obtained via electronic health record query for the period of January 2017 to September 2017; subsequent enrollment data was obtained at the conclusion of each of the three intervention phases. During phase-1 (from October 2017 to December 2017), participating residents incorporated a discussion of the portal with patients and provided an instructional handout during clinic visits. During phase-2 (from January 2018 to March 2018), a volunteer was introduced into routine clinic visits to provide portal education and assistance with portal enrollment to patients. During phase-3, e-mail invitations with instructions on how to enroll in the portal were automatically sent to patients at the time of their registration for their visits.

MEASURES OF SUCCESS (DISCUSS QUALITATIVE AND/OR QUANTITATIVE METRICS WHICH WILL BE USED TO EVALUATE PROGRAM/INTERVENTION): The primary metric to evaluate success was the overall percentage of patients signed up for access to the online patient portal. A secondary metric was the sign-up success rate for each of the 3 separate approaches.

FINDINGS TO DATE (IT IS NOT SUFFICIENT TO STATE FINDINGS WILL BE DISCUSSED ): From January 2017 to September 2017 - the period before our intervention - a total of 535 patients were seen by the participating internal medicine clinic residents, of which $99(18.5 \%)$ were already enrolled in the portal. After phase- 1 of the intervention, 58 of $303(19.1 \%)$ patients seen by participating residents were enrolled in the patient portal. At the conclusion of phase-2, 54 of 228 (23.7\%) patients seen by participating residents were enrolled in the patient portal. Furthermore, 47 patients of 228 patients completed their visit with the volunteer, and of these 47 patients, 21 patients enrolled into the portal, giving a yield of $44.6 \%$ from the volunteer intervention alone. At the end of phase- 3,22 of $100(22 \%)$ patients seen by participating residents were enrolled in the patient portal.

KEY LESSONS FOR DISSEMINATION (WHAT CAN OTHERS TAKE AWAY FOR IMPLEMENTATION TO THEIR PRACTICE OR COMMUNITY?): While our study demonstrated modest overall success rates for combining the various methods of improving patient portal sign up rates, a more important outcome was the much greater success rate of the volunteer-based method. In the setting of an at-risk patient population, efforts to dramatically increase patient portal enrollment may need to consider having a staff member or members devoted to assisting with the sign-up process and usage of online patient portals.

\section{IMPROVING PPI PRESCRIBING PRACTICES IN AN ACADEMIC SAFETY-NET PRIMARY CARE CLINIC: QUALITY IMPROVE- MENT PROJECT}

Naren S. Nallapeta ${ }^{1}$; Smita Y. Bakhai ${ }^{2}{ }^{1}$ University at Buffalo, Buffalo, NY; ${ }^{2}$ SUNY at BUffalo, Williamsville, NY. (Control ID \#3173031)

\section{STATEMENT OF PROBLEM OR QUESTION (ONE SENTENCE):}

Proton Pump Inhibitors (PPI) overuse in both inpatient and outpatient settings have increased drastically over the last 2 decades. Overuse of PPIs continues despite evidence based guidelines and prolonged PPI use carries serious adverse effects.

OBJECTIVES OF PROGRAM/INTERVENTION (NO MORE THAN THREE OBJECTIVES): The aim of this quality improvement (QI) is to reduce the percentage of patients inappropriately prescribed PPIs between the ages of $40-75$ years from a baseline rate of $80 \%$ to less than $60 \%$ within 1 year in hospital based internal medicine clinic (IMC).
DESCRIPTION OF PROGRAM/INTERVENTION, INCLUDING ORGANIZATIONAL CONTEXT (E.G. INPATIENT VS. OUTPATIENT, PRACTICE OR COMMUNITY CHARACTERISTICS): We used the Plan-Do-Study-Act (PDSA) model and performed a root cause analysis to identify barriers. The major barriers included system, lack of electronic medical record (EMR) alerts, gaps in knowledge in physician and the patient, and fear of discontinuation of the PPI. Multidisciplinary QI team included nursing and ancillary staff, residents, attending physicians and social worker from IMC, staff from Gastroenterology (GI ) Information technology (IT) department and patients. Outcome measure included reducing rates of patients inappropriately prescribed PPIs. Process measures included the percentage of patients on PPI who have their Gastroesophageal reflux disease assessed during the clinic including assessment for alarm symptoms and the percentage of patients who are appropriately referred to GI clinic for an esophagogastroduodenoscopy (EGD). Balancing measures included increase in patient wait times in the clinic and increase in poor access to EGD. QI team performed four PDSA cycles from January 2018 to October 2018. The first PDSA cycle consisted of creation of EMR templates with goals of redesigning of nursing work flow to alert physicians to review chronic PPI use and reminding physicians to evaluate alarm symptoms; and to improve medical documentation for continuation of chronic PPI use. Other PDSA cycles included education to the physicians, nursing staff and patients. Physician education consisted of PowerPoint Presentation with small group discussion about evidence based guidelines for treatment of GERD. Nursing staff and physicians were also trained on a new EHR template and workflow for prescription of PPI. Electronic patient registry was created in collaboration with IT department

MEASURES OF SUCCESS (DISCUSS QUALITATIVE AND/OR QUANTITATIVE METRICS WHICH WILL BE USED TO EVALUATE PROGRAM/INTERVENTION): Data analysis was performed by monthly run chart. The percentage of patients' inappropriately on PPI's were reduced from $80 \%$ in January 2018 to $67.7 \%$ by October 2018 .

FINDINGS TO DATE (IT IS NOT SUFFICIENT TO STATE FINDINGS WILL BE DISCUSSED ): We achieved $51.1 \%$ for patients on long term PPI use that completed EGD from the baseline of $<40 \%$. During project period, we referred $83.87 \%$ of patients on chronic PPI use to GI clinic from $72.9 \%$ in Jan 2018.

KEY LESSONS FOR DISSEMINATION (WHAT CAN OTHERS TAKE AWAY FOR IMPLEMENTATION TO THEIR PRACTICE OR COMMUNITY?): Lack of automated medical decision support tool was identified as the biggest barrier. Optimization of EHR and education to QI team members were crucial for the success of this QI.

IMPROVING REFERRALS FOR PENICILLIN ALLERGY TESTING: A PRIMARY CARE STRATEGY FOR PENICILLIN ALLERGY DELABELING

Dustin Fowler; Samuel O. Schumann. Medical University of South Carolina, Charleston, SC. (Control ID \#3186231)

STATEMENT OF PROBLEM OR QUESTION (ONE SENTENCE): Despite approximately $90 \%$ of patients who report a penicillin allergy having penicillin tolerance, less than $0.1 \%$ of penicillin allergy reporters undergo appropriate confirmatory testing, lending to increases in unnecessary broadspectrum antibiotics use, the rates of certain drug-resistant infections and medication costs.

OBJECTIVES OF PROGRAM/INTERVENTION (NO MORE THAN THREE OBJECTIVES): 1. Identify patients who report a penicillin allergy and are candidates for penicillin allergy testing, through a 
standardized screening protocol 2. Refer identified patients for penicillin allergy testing, through a streamlined workflow

DESCRIPTION OF PROGRAM/INTERVENTION, INCLUDING ORGANIZATIONAL CONTEXT (E.G. INPATIENT VS. OUTPATIENT, PRACTICE OR COMMUNITY CHARACTERISTICS): Within our academic internal medicine residents' clinic, we screened all patients presenting for an appointment over the course of one month for a history of penicillin drug reactions and interest in penicillin allergy testing. Excluding patients who reported tolerating multiple, subsequent episodes of penicillin exposure, we offered referral for penicillin allergy testing to interested patients. Additionally, written educational material was provided to patients. Prior to project initiation, we partnered with colleagues in the division of allergy / immunology to ensure appropriate referral workflow and availability of testing. Internal medicine residents also received academic detailing on the impact of penicillin allergy testing and the quality improvement project.

MEASURES OF SUCCESS (DISCUSS QUALITATIVE AND/OR QUANTITATIVE METRICS WHICH WILL BE USED TO EVALUATE PROGRAM/INTERVENTION): Measures of success include the response rate of screened patients and the proportion of candidates for penicillin allergy testing who received a referral for testing.

FINDINGS TO DATE (IT IS NOT SUFFICIENT TO STATE FINDINGS WILL BE DISCUSSED ): 412 out of 800 outpatients completed screening over one month, providing a response rate of $52 \%$. According to chart documentation the prevalence of penicillin allergy among our outpatients is $4 \%$, however $17 \%$ (71) screened patients reported a history of penicillin reaction. $10 \%$ (7) of patients who reported a history of penicillin reaction also reported continued penicillin use without consequence. $35 \%$ (22) of patients reporting history of penicillin reaction were interested in penicillin skin testing. 36\% (8) of interested patients were referred for testing. KEY LESSONS FOR DISSEMINATION (WHAT CAN OTHERS TAKE AWAY FOR IMPLEMENTATION TO THEIR PRACTICE OR COMMUNITY?): Implementation of a standardized screening protocol, can quickly identify appropriate candidates who for penicillin allergy testing, in line with American Academy of Allergy, Asthma, and Immunology and Infectious Diseases Society of America recommendations. However, less than half of patients reporting interest in penicillin allergy testing received a referral for testing. Further data is needed to determine the drivers of penicillin allergy testing referral. Additionally, it is unclear why a larger percentage of patients reported a history of penicillin reaction compared to chart documentation of allergy.

IMPROVING SAFE PRESCRIBING OF OPIOIDS FOR HOSPITALIZED OLDER ADULTS WITHIN AN ELECTRONIC HEALTH RECORD (EHR) BASED PRESCRIBING CONTEXT

Kathleen Drago; Jackie Sharpe; Bryanna De Lima; Abdulaziz S. Alhomod; Elizabeth Eckstrom. Oregon Health \& Science University, Portland, OR. (Control ID \#3183324)

STATEMENT OF PROBLEM OR QUESTION (ONE SENTENCE): Hospitalized older adults are at risk of being prescribed medications at inappropriate doses and medication-related adverse events are a common hazard in acute care, driven in part by provider reliance on age-independent dose defaults within EHR medication orders.

OBJECTIVES OF PROGRAM/INTERVENTION (NO MORE THAN THREE OBJECTIVES): We developed an EHR-based, agetriggered set of alternate dose defaults to enhance thoughtful, age- appropriate prescribing without significant alterations to normal prescriber workflows.
DESCRIPTION OF PROGRAM/INTERVENTION, INCLUDING ORGANIZATIONAL CONTEXT (E.G. INPATIENT VS. OUTPATIENT, PRACTICE OR COMMUNITY CHARACTERISTICS): The Geriatric Prescribing Context (GPC) is an Epic-based set of alternate dose defaults triggered for patients 75 and over. It offers pre-set age-sensitive dose and regimen defaults for 74 medications compiled by a geriatrician and pharmacist team using evidence-based tools. GPC went live in Epic at our Level 1 tertiary care academic medical center in July 2017 and is active only for inpatients, not Emergency or outpatients. Development \& implementation by the core team took approximately 50 hours. There was no formal training of prescribers.

MEASURES OF SUCCESS (DISCUSS QUALITATIVE AND/OR QUANTITATIVE METRICS WHICH WILL BE USED TO EVALUATE PROGRAM/INTERVENTION): This analysis compared average dose, total daily dose and doses per day for oxycodone, oral \& IV hydromorphone for 6 months before and after GPC went live in July 2017. The data were retrieved from the EHR database retrospectively; including patients 75 and older with an inpatient encounter within the study time frame and who received at least one dose of any of the three medications of interest as a standing order, not from an order set. Unpaired T-tests assuming the unequal variance were performed via STATA 15 to determine statistical significance.

FINDINGS TO DATE (IT IS NOT SUFFICIENT TO STATE FINDINGS WILL BE DISCUSSED ): Data from pre and post-GPC implementation revealed the following changes: Oxycodone Pre $(n=1646)$ Post $(\mathrm{n}=1874)$ Average Dose $6.34 \mathrm{mg} 5.92 \mathrm{mg} \mathrm{p}=0.008$ Doses per Day $2.712 .89 \mathrm{p}=0.003$ Total Daily Dose $19.72 \mathrm{mg} 19.19 \mathrm{mg} \mathrm{p}=$ 0.51 Oral Hydromorphone Pre $(n=162)$ Post $(n=260)$ Average Dose $2.88 \mathrm{mg} 1.8 \mathrm{mg} \mathrm{p}<0.05$ Doses per Day $2.993 .07 \mathrm{p}=0.65$ Total Daily Dose $9.92 \mathrm{mg} 5.84 \mathrm{mg} \mathrm{p}=0.0001 \mathrm{IV}$ Hydromorphone Pre ( $\mathrm{n}=$ 714) Post $(\mathrm{n}=644)$ Average Dose $0.42 \mathrm{mg} 0.38 \mathrm{mg} \mathrm{p}=0.02$ Doses per Day $2.742 .55 \mathrm{p}=0.1$ Total Daily Dose $1.24 \mathrm{mg} 1.13 \mathrm{mg} \mathrm{p}=0.17$ KEY LESSONS FOR DISSEMINATION (WHAT CAN OTHERS TAKE AWAY FOR IMPLEMENTATION TO THEIR PRACTICE OR COMMUNITY?): These results suggest that an EHR-based set of alternate dose defaults for a high risk population of older adults may be effective at aligning prescribing practice with populationspecific recommendations with relatively little time or cost investments. This simple and potentially effective intervention in resourcelimited environments may minimize risk to hospitalized older adults.

IMPROVING TREATMENT OF PREDIABETES WITH METFORMIN IN AN INTERNAL MEDICINE OUTPATIENT CLINIC

Kelly Koehn ${ }^{1}$; Natalie Cohen ${ }^{2}$; Ankit R. Parikh ${ }^{1}$; Gudbjorg Jonsdottir $^{1}$; Melissa B. Ludgate ${ }^{4}$; Heidi Ahmed ${ }^{3}$; Nanmeng Yu ${ }^{1}$; Robert Szalewski ${ }^{1}$; Hashim Chaudhry ${ }^{4}$; Rebecca S. Davis ${ }^{1}$; Krista M. Johnson ${ }^{4}$. ${ }^{1}$ University of Iowa Hospitals and Clinics, North Liberty, IA; ${ }^{2}$ University of Iowa Hospital and Clinics, North Liberty, IA; ${ }^{3}$ The University of Iowa Hospitals and Clinics, Iowa City, IA; ${ }^{4}$ University of Iowa, Iowa City, IA. (Control ID \#3184097)

STATEMENT OF PROBLEM OR QUESTION (ONE SENTENCE): The American Diabetes Association (ADA) recommends metformin in addition to lifestyle modifications in patients with prediabetes $<60$ years old to prevent progression to type II diabetes; however, a recent study found only $3.7 \%$ of eligible patients with prediabetes were prescribed metformin. 
OBJECTIVES OF PROGRAM/INTERVENTION (NO MORE THAN THREE OBJECTIVES): Our aim was to increase metformin use in eligible patients $<60$ years old with prediabetes to $15 \%$ from November 2017 to April 2018.

DESCRIPTION OF PROGRAM/INTERVENTION, INCLUDING ORGANIZATIONAL CONTEXT (E.G. INPATIENT VS. OUTPATIENT, PRACTICE OR COMMUNITY CHARACTERISTICS): This quality improvement project was implemented at an outpatient IM clinic associated with University of Iowa Hospitals and Clinics. Participants included both IM residents ( $N=37)$ and IM faculty $(\mathrm{N}=19)$ who had their primary care clinics at this site. Our interventions consisted of teaching sessions to other IM residents including a brief didactic on the impact of prediabetes and current ADA guidelines. Residents viewed a video clip explaining how metformin works, as well as how to prescribe and titrate the medication. We also placed bright pink placards with information regarding the diagnosis and treatment of prediabetes on computers in the outpatient clinic workstations as a visual reminder to consider prescribing metformin for prediabetes if appropriate.

MEASURES OF SUCCESS (DISCUSS QUALITATIVE AND/OR QUANTITATIVE METRICS WHICH WILL BE USED TO EVALUATE PROGRAM/INTERVENTION): We searched our electronic health record database for all patients aged 18-60 seen in our clinic from October 1, 2016 to October 1, 2017 that met criteria for prediabetes as defined by A1c level between 5.7-6.4, and who did not have a diagnosis of type 1 or type 2 diabetes mellitus in their problem list $(\mathrm{N}=410)$. The same method was used for selection of patients post-intervention from November 1, 2017 to February 1, 2018 ( $N=229$ ). A two proportional $\mathrm{Z}$ test was used to compare the proportion of patients with prediabetes who had metformin listed on their medication list before and after the intervention.

FINDINGS TO DATE (IT IS NOT SUFFICIENT TO STATE FINDINGS WILL BE DISCUSSED ): There were no significant differences between the pre- and post-intervention groups in gender, median age, BMI and mean hemoglobin A1c. The total percentage of patients with prediabetes prescribed metformin increased from $8.3 \%$ pre-intervention to $13.5 \%$ postintervention $(\mathrm{p}=0.048)$. In a separate analysis for patients with a BMI greater than 35 , metformin prescriptions increased from $11.4 \%$ pre-intervention to $16.5 \%$ post-intervention, although this result did not reach statistical significance $(\mathrm{p}=0.330)$

KEY LESSONS FOR DISSEMINATION (WHAT CAN OTHERS TAKE AWAY FOR IMPLEMENTATION TO THEIR PRACTICE OR COMMUNITY?): Although we did not reach our aim of $15 \%$, we did observe a significant increase in the percentage of patients $<60$ years old with prediabetes treated with metformin at our IM clinic. By increasing the number of patients treated for prediabetes, we hope to decrease the rate of progression to diabetes. It is also possible that raising provider awareness of prediabetes will lead to improved screening and improved early interventions. Strengths of our study include a large pre- and post- sample size and the ease of obtaining Alc levels from the electronic health record. Also, our interventions were easy to implement and could be adopted by other clinics and/or institutions.

INCREASING PREP IMPLEMENTATION IN HIGH-RISK PATIENTS SEEN BY RESIDENTS IN A PRIMARY CARE CLINIC

Heather Viola; Devorah Edelman; Surksha Sirichand; Dipal R. Patel. Mount Sinai St. Luke's-West, New York, NY. (Control ID \#3174348)

STATEMENT OF PROBLEM OR QUESTION (ONE SENTENCE): The CDC estimates that approximately 1.2 million U.S adults may benefit from HIV pre-exposure prophylaxis (PrEP), a preventive measure that has been shown to significantly reduce HIV transmission among at-risk populations, but only a minority are using; PrEP initiation should largely originate from primary care providers (PCP's) however their knowledge of PrEP initiation guidelines and comfort in prescribing PrEP remains low.

OBJECTIVES OF PROGRAM/INTERVENTION (NO MORE THAN THREE OBJECTIVES): The objectives of this resident-driven QI project were to 1) improve resident knowledge and awareness of PrEP, 2) identify high-risk individuals seen in residency clinic, and 3) refer them to a designated PrEP counselor to ultimately increase PrEP implementation rates.

DESCRIPTION OF PROGRAM/INTERVENTION, INCLUDING ORGANIZATIONAL CONTEXT (E.G. INPATIENT VS. OUTPATIENT, PRACTICE OR COMMUNITY CHARACTERISTICS): Initially, a 3-year retrospective chart review was performed at a federally qualified urban community clinic to identify patients seen who were likely to benefit from a referral for PrEP initiation. Subsequently, an educational intervention, including a comprehensive lecture on the CDC clinical practice guidelines, was carried out over a 6 -week period. The action phase entailed identifying patients that would benefit from PrEP at routine health visits, using the NYC-DOHMH sponsored HPLUS screening form. If the patient was identified as high risk, the provider discussed PrEP. Residents were provided with a simple PrEP initiation algorithm. Those patients amenable were then referred to a PrEP counselor and obtained initial lab testing. A follow-up provider visit was then scheduled to initiate Truvada. MEASURES OF SUCCESS (DISCUSS QUALITATIVE AND/OR QUANTITATIVE METRICS WHICH WILL BE USED TO EVALUATE PROGRAM/INTERVENTION): The educational intervention was meant to assess and improve resident knowledge and comfort level with offering PrEP by using pre and post intervention resident surveys. During the action phase, the initiation of PrEP in high-risk patients over a 6-month period was documented in one HIPAA compliant, password secured Excel file. The initial pre-intervention retrospective chart review was then used as a comparison to this data to assess an improvement in implementation rates.

FINDINGS TO DATE (IT IS NOT SUFFICIENT TO STATE FINDINGS WILL BE DISCUSSED ): Compared to pre survey, residents surveyed post intervention $(n=20)$ demonstrated expanded knowledge of proper candidates for PrEP (59\% vs. 90\%) and mandatory initial testing (34\% vs. 95\%). 90\% felt more comfortable discussing PrEP with their patients. After initiating the action phase, 6 individuals in the first 6 months were referred to begin PrEP, representing a 6-fold increase from the 1 patient who was referred in the 6 months prior including the educational phase. This is also in stark contrast to the 3 years prior where at least 117 high-risk patients were seen and none were referred to begin PrEP. Subsequently, all 7 patients referred to the PrEP counselor were initiated on Truvada. To date, each of the patients are still taking PrEP without adverse effect and have negative followup HIV testing.

KEY LESSONS FOR DISSEMINATION (WHAT CAN OTHERS TAKE AWAY FOR IMPLEMENTATION TO THEIR PRACTICE OR COMMUNITY?): This QI initiative reveals that a vast majority of our high-risk population is not being offered PrEP. Our easily reproducible multimodal intervention increased PrEP referral and implementation rates.

INNOVATIONS IN TEAM-BASED PRIMARY CARE: PHYSICIAN ASSISTANT AND INTERNAL MEDICINE RESIDENT PANEL COMANAGEMENT

Alev Atalay ${ }^{1,}{ }^{3}$; Christina M. Meade ${ }^{1}$; Caroline Cupelo ${ }^{2}$; Michele Elms $^{2}$; Teresa Zhang ${ }^{2}$; Richard S. Gitomer ${ }^{1}$; Bonnie Southworth ${ }^{2}$. ${ }^{1}$ Brigham and Women's Hospital, Jamaica Plain, MA; ${ }^{2}$ Brigham and Women's Hospital, Boston, MA; ${ }^{3}$ Harvard Medical School, Boston, MA. (Control ID \#3182838) 
STATEMENT OF PROBLEM OR QUESTION (ONE SENTENCE): Resident primary care physicians (rPCPs) struggle to provide continuity and complete inter-visit tasks while on inpatient rotations, thus we hypothesize that a primary care practice-sharing model between rPCPs and Physician Assistants (PAs) will improve patient outcomes.

OBJECTIVES OF PROGRAM/INTERVENTION (NO MORE THAN THREE OBJECTIVES): Attending physicians (aPCPs) have superior clinical outcomes and no-show rates as compared to rPCPs. Incorporation of PAs on surgical resident teams improves patient outcomes and quality of care. Through a primary care practice-sharing model between rPCPs and PA, we aim to: 1 . Improve clinical outcomes for patients of rPCPs, 2. Improve clinic engagement by reducing no-show rates for rPCPs.

DESCRIPTION OF PROGRAM/INTERVENTION, INCLUDING ORGANIZATIONAL CONTEXT (E.G. INPATIENT VS. OUTPATIENT, PRACTICE OR COMMUNITY CHARACTERISTICS): Resident/PA co-management will occur at the Phyllis Jen Center for Primary Care (PJC), a large, academic internal medicine practice at Brigham and Women's Hospital, Boston, One team will serve as the intervention group and the PA will co-manage rPCP panels [ $\sim 800$ patients] while rPCPs are on inpatient rotations. Two other teams will serve as controls without comanagement. The PA addresses clinical concerns, refills prescriptions, and sees patients for urgent complaints, chronic disease management, hospital discharge follow up, wellness visits, and participates in population health management. To ensure that ambulatory educational needs are met, rPCPs perform all the above during ambulatory rotations. New workflows were created to facilitate team co-management. Based on the performance measures these workflows have and will require frequent iteration.

MEASURES OF SUCCESS (DISCUSS QUALITATIVE AND/ OR QUANTITATIVE METRICS WHICH WILL BE USED TO EVALUATE PROGRAM/INTERVENTION): We will measure (1) appropriateness of care metrics for diabetes, hypertension, and cardiovascular disease and age-appropriate cancer screening rates; (2) no-show rates. We compare these metrics for rPCPs and aPCPs to drive the improvement of the co-management care team operation.

FINDINGS TO DATE (IT IS NOT SUFFICIENT TO STATE FINDINGS WILL BE DISCUSSED ): Baseline data reveals poorer outcomes for rPCPs as compared to aPCPs. Across control and intervention groups, rPCP baseline rates were similar, thus rPCPs are compared as a group to aPCPs. Patients of aPCPs met appropriateness of care metrics more frequently than $\mathrm{rPCPs}$, with diabetes at A1c goal (76.4\% vs. $62.1 \%)$, hypertension at blood pressure goal $(81.4 \%$ vs. $80.1 \%)$ and hyperlipidemia at goal $(87.0 \%$ vs. $85.5 \%)$. Patients of aPCPs had higher rates of cancer screening than rPCPs (mammogram $84.3 \%$ vs. $78.0 \%$, pap smear $79.7 \%$ vs. $68.7 \%$, colorectal cancer screening $76.8 \%$ vs. $64.3 \%$ ). No- show rate for rPCPs (17.7\%) was significantly higher than aPCPs (13.2\%).

KEY LESSONS FOR DISSEMINATION (WHAT CAN OTHERS TAKE AWAY FOR IMPLEMENTATION TO THEIR PRACTICE OR COMMUNITY?): Once optimized, the intent is to expand to all rPCPs at PJC and will serve as a model for other large academic practices to improve the quality of care provided to patients with rPCPs. Additionally, rPCPs uniformly felt that panel sharing is highly appealing, given the challenges of performing effective management while on inpatient rotations. We thus hope this model will improve the ambulatory educational experience.
INNOVATIVE WAY TO CREATE A PHYSICIAN SUPERVISED WEIGHT LOSS PROGRAM IN A PRIMARY CARE PROVIDER OFFICE

Varalakshmi Niranjan. University of Connecticut, West Hartford, CT. (Control ID \#3138992)

STATEMENT OF PROBLEM OR QUESTION (ONE SENTENCE): There is limited time available in a primary care provider schedule to offer effective weight loss counselling to obese patients.

OBJECTIVES OF PROGRAM/INTERVENTION (NO MORE THAN THREE OBJECTIVES): To create an effective insurance based physician supervised weight loss program in a primary care provider office setting. To assess the average number of months it takes to achieve effective weight loss of $5 \%$ or more of body weight in a physician supervised weight loss program. To assess the number of counselling office visits it takes to achieve effective weight loss of $5 \%$ or more of the body weight.

DESCRIPTION OF PROGRAM/INTERVENTION, INCLUDING ORGANIZATIONAL CONTEXT (E.G. INPATIENT VS. OUTPATIENT, PRACTICE OR COMMUNITY CHARACTERISTICS): A physician supervised weight loss program was designed for a primary care provider office in a private practice setting. The program was made available for all overweight and obese patients to enroll voluntarily. The program included bi weekly outpatient office visits with the provider. Patients were provided with nutritional counselling, meal plans, exercise ideas, and behavioral therapy at each visit by the provider. Patients were provided with tools to track food intake and exercise with electronic apps and or paper journal. The patient's medical insurances were billed for these office visits with no extra cost from the patient.

MEASURES OF SUCCESS (DISCUSS QUALITATIVE AND/OR QUANTITATIVE METRICS WHICH WILL BE USED TO EVALUATE PROGRAM/INTERVENTION): The number of voluntary enrollment by the patients and dropouts were tracked annually. The demographics of the patients who enrolled in a physician supervised weight loss program were analyzed. The initial weight and monthly weights were monitored. The average time it took for a patient to lose $5 \%$ or more of body weight was determined with respect to each class of obesity. The relationship between number of visits and weight loss achieved were tracked.

FINDINGS TO DATE (IT IS NOT SUFFICIENT TO STATE FINDINGS WILL BE DISCUSSED ): A total of 126 patients were enrolled between September 2016 and August 2018. There was a 20.63\% dropout noted at the end of the two year mark. The program enrolled $84.1 \%$ females and $15.9 \%$ males. A total of 1111.8 pounds were lost during the two year period. The average time it took for a patient to lose $5 \%$ or more of body weight was 2.97 months. The subgroup analysis showed obesity class 2 and class 3 patients took lesser time to achieve this weight loss in comparison to class 1 and overweight individuals. A higher percentage of class 2 and class 3 obese patients achieved 5\% or more weight loss than class 1 and overweight patients. Patients who had more than 12 visits 1 lost $7.8 \%$ of their body weight. Patients with fewer than 12 visits only lost $3.6 \%$ of their body weight.

KEY LESSONS FOR DISSEMINATION (WHAT CAN OTHERS TAKE AWAY FOR IMPLEMENTATION TO THEIR PRACTICE OR COMMUNITY?): Physician supervised weight loss program can be designed with minimal cost investment. Effective weight loss can be achieved by counselling patients more frequently. The primary care provider schedule can be modified easily to accommodate these obese patients biweekly. The longitudinal trust with the primary care provider ensures higher follow up rates and minimal dropout rates in this program. 
INPATIENT ADDICTION MEDICINE CONSULTATIONS AT THE URBAN/RURAL FRONTIER: IMPROVING QUALITY OF CARE AND LINKAGE TO OUTPATIENT SERVICES FOR PATIENTS WITH SUBSTANCE USE DISORDER IN CENTRAL KENTUCKY Devin Oller ${ }^{1}$; Laura Fanucchi ${ }^{2} .{ }^{1}$ University of Kentucky, Lexington, KY; ${ }^{2}$ University of Kentucky College of Medicine, Lexington, KY. (Control ID \#3181470)

STATEMENT OF PROBLEM OR QUESTION (ONE SENTENCE): Does an addiction medicine consult service facilitate opioid use disorder(OUD) medication initiation and linkage to outpatient services in an academic medical center serving both urban and rural areas?

OBJECTIVES OF PROGRAM/INTERVENTION (NO MORE THAN THREE OBJECTIVES): Provide timely consultations for individuals with substance use disorders(SUD) admitted to hospital medicine teams Secure linkage to outpatient treatment for consult patients

DESCRIPTION OF PROGRAM/INTERVENTION, INCLUDING ORGANIZATIONAL CONTEXT (E.G. INPATIENT VS. OUTPATIENT, PRACTICE OR COMMUNITY CHARACTERISTICS): The Addiction Consultation and Education Service(ACES) began 10/4/ 18 at the University of Kentucky Hospital, a 945-bed tertiary referral academic medical center with more than 3000 opioid-comorbid encounters in 2018. ACES was available on weekdays to 16 inpatient hospital medicine teams. The team was comprised of several physicians and an APRN. A patient navigator assisted with prior authorizations and outpatient linkage. ACES referred to UK First Bridge Clinic for office-based opioid treatment as well as community programs when possible.

MEASURES OF SUCCESS (DISCUSS QUALITATIVE AND/OR QUANTITATIVE METRICS WHICH WILL BE USED TO EVALUATE PROGRAM/INTERVENTION): Patient demographics, SUD diagnoses, insurance, and comorbidities will be collected, as well as key process metrics including: time-to-consultation and medication initiation, length of stay(LOS), discharge against medical advice(AMA), and details of linkage to outpatient services (type, travel distance, and time-to-follow-up)

FINDINGS TO DATE (IT IS NOT SUFFICIENT TO STATE FINDINGS WILL BE DISCUSSED ): ACES was staffed by a single physician seeing an average 1.7 new consults/day. Of the 91 patients seen by ACES over the first 3 months of the service, 73 of consults met DSM- 5 criteria for OUD or OUD plus an additional SUD, 10 for stimulant use disorder, and 8 for alcohol use disorder. 58\% of patients lived in rural counties (Rural-Urban Continuum Codes 4-9). Average LOS was 19.5 days; consult orders were entered an average of 5.8 days after admission. Medication treatment for SUD was initiated in the majority of ACES patients, with medications started an average of 0.9 days after consultation. Among OUD patients, $71 \%$ underwent buprenorphine/naloxone (BUP/NX) induction, $9 \%$ were started on methadone, and $20 \%$ did not receive medication. Of patients started on BUP/NX $58 \%$ were linked to the UK First Bridge Clinic.

KEY LESSONS FOR DISSEMINATION (WHAT CAN OTHERS TAKE AWAY FOR IMPLEMENTATION TO THEIR PRACTICE OR COMMUNITY?): Establishing a bridge clinic prior to the implementation of an inpatient consult service is critical. ACES relied heavily on the UK First Bridge clinic for patients started on BUP/NX - waiting lists and costs made linkage to closer clinics difficult. Compared to other addiction medicine consult services, a greater percentage of patients seen by ACES resided in rural counties. ${ }^{1,2}$ To address transportation barriers, we are evaluating options for initiating depot buprenorphine for ACES patients. While ACES plans to increase staffing, our pilot demonstrates that services with minimal staff can increase the number of inpatients receiving medications for OUD. 1.Priest et al. Role of the Hospital in the $21{ }^{\text {st }}$ Century opioid overdose epidemic: the addiction medicine consult service JAM Dec 2018 2.Trowbridge et al. Addiction consultation services:Linking hospitalized patients to outpatient addiction treatment $J$ Subst Abuse Treat.2017;79:1-5

INTEGRATING BEHAVIORAL HEALTH INTO PRIMARY CARE: MAKING A PATIENT WHOLE

Divya Venkat ${ }^{1}$; Herman Singh ${ }^{2}$; Bruce Ling ${ }^{2}$; Courtney Watson ${ }^{2}$; Maggie Feinstein ${ }^{2}$; Elizabeth Cuevas ${ }^{2} .{ }^{1}$ Allegheny General Hospital, Pittsburgh, PA; ${ }^{2}$ Allegheny Health Network, Pittsburgh, PA. (Control ID \#3183916)

STATEMENT OF PROBLEM OR QUESTION (ONE SENTENCE): Patients with significant mental health diagnoses have a shorter life span, often in the setting of poorly managed and underrecognized co-existing medical illnesses.

OBJECTIVES OF PROGRAM/INTERVENTION (NO MORE THAN THREE OBJECTIVES): To evaluate the utility of the integration of behavioral health into the primary care setting.

DESCRIPTION OF PROGRAM/INTERVENTION, INCLUDING ORGANIZATIONAL CONTEXT (E.G. INPATIENT VS. OUTPATIENT, PRACTICE OR COMMUNITY CHARACTERISTICS): The setting of this study occurs at two clinics affiliated with a residency program. One clinic meets the needs of a low-income neighborhood with a variety of age ranges while the other serves an upper middle class neighborhood with a predominantly middle-aged population. Behavioral health specialists were integrated into these clinics in July 2015 and January 2017 respectively. Medical assistants first speak with the patients and obtain a PHQ-2. Based on this value, residents will perform a PHQ-9 and address the initial issues of behavioral health. Based on this, the behavioral health specialist will speak with the patient and recommend treatment strategies or further assessment by a psychiatrist. Residents will then begin medications in the clinic or speak directly with the psychiatry outpatient clinic.

MEASURES OF SUCCESS (DISCUSS QUALITATIVE AND/OR QUANTITATIVE METRICS WHICH WILL BE USED TO EVALUATE PROGRAM/INTERVENTION): In this quality improvement project, behavioral health specialists were integrated into the above mentioned residency clinics. Data was collected on the following: (1) number of visits made to the behavioral health specialist, (2) the frequency of clinic visits in which depression screening with the Patient Health Questionnaire (PHQ) occurred, and (3) the number of referrals made to outpatient psychiatry. FINDINGS TO DATE (IT IS NOT SUFFICIENT TO STATE FINDINGS WILL BE DISCUSSED ): The primary analysis is the assessment of the number of referrals to outpatient psychiatry prior to the integration of the behavioral health specialist compared to the post-integration period as evaluated in monthly time periods. At the residency clinic located in the low income neighborhood, there was a decrease in the number of referrals in the post-integration period (a low of $n=6$ in the assessed month) when compared to the pre-integration period $(n=32)$. In the upper middle class neighborhood clinic, there were 33 referrals to psychiatry during the month in which the behavioral health specialist was integrated. At this clinic, there has been a gradual decline in the number of referrals to a low of 13. After institution of BHI, a target of $80 \%$ of all patients were screened for PHQ-9. Furthermore, there have been over 140 visits per month consistently to each behavioral health specialist.

KEY LESSONS FOR DISSEMINATION (WHAT CAN OTHERS TAKE AWAY FOR IMPLEMENTATION TO THEIR PRACTICE OR COMMUNITY?): Integrating behavioral health specialists within primary clinic gives patients a well-rounded care approach. Patients who cannot obtain adequate mental health care are able to reach out to a behavioral health 
specialist while primary care physicians can continue to address other chronic medical conditions. Primary care physicians are then able to more adequately assess the impact of behavioral health concerns on a patient's chronic conditions. Further work needs to be done to assess the impact of this on chronic conditions, such as management of diabetes mellitus and hypertension.

INTEGRATING GROUP THERAPY FOR BEHAVIORAL HEALTH INTO A PRIMARY CARE PROGRAM FOR PATIENTS WITH COMPLEX MEDICAL AND SOCIAL NEEDS: A PILOT.

Venkatesan R. Krishnamoorthi; Nicole Gier; Emily Perish; David Meltzer. University of Chicago, Chicago, IL. (Control ID \#3186262)

\section{STATEMENT OF PROBLEM OR QUESTION (ONE SENTENCE):} Individuals with complex medical and social needs often have behavioral health $(\mathrm{BH})$ problems that impair their ability for self-care, but rates of referrals for and attendance at BH specialists' appointments are low.

OBJECTIVES OF PROGRAM/INTERVENTION (NO MORE THAN THREE OBJECTIVES): 1 . Develop a group therapy approach to $\mathrm{BH}$ integrated into and led by members of the primary care clinic. 2 . Improve the rate of $\mathrm{BH}$ care through attendance at group therapy sessions, over conventional referrals to BH specialists outside of the clinic.

DESCRIPTION OF PROGRAM/INTERVENTION, INCLUDING ORGANIZATIONAL CONTEXT (E.G. INPATIENT VS. OUTPATIENT, PRACTICE OR COMMUNITY CHARACTERISTICS): The Comprehensive Care Program (CCP) at the University of Chicago provides continuity care for Medicare patients at high risk for hospitalization in both outpatient and inpatient settings. The team includes a licensed clinical social worker with training in BH care. Multi-disciplinary rounds occur daily, allowing referral patients for $\mathrm{BH}$ care via warm hand-offs. Noting the common challenges among CCP patients in attending appointments, a $\mathrm{BH}$ group therapy progam was created due to several advantages over individual therapy: a safe space for those not comfortable with psychotherapy; support from peers with similar health and social problems; more efficiency for providers. Patients who expressed interest were enrolled and scheduled into a 5 week group therapy module. Participants are encouraged to establish personal goals and are taught skills of mindfulness, emotion regulation, distress tolerance, interpersonal effectiveness, and self-compassion. In order to accommodate barriers to attendance (e.g. illness, transportation) participants are allowed to drop in to the group throughout the year.

MEASURES OF SUCCESS (DISCUSS QUALITATIVE AND/OR QUANTITATIVE METRICS WHICH WILL BE USED TO EVALUATE PROGRAM/INTERVENTION): We compared the attendance rate of enrollees in the CCP group therapy program to the same enrollees' referral rate and attendance rate to $\mathrm{BH}$ specialists' appointments outside of the $\mathrm{CCP}$ program (psychiatry or psychology at Univ of Chicago or other institutions). We performed a retrospective chart review of those enrollees to investigate 1) who attended at least one $\mathrm{CCP}$ group session, 2) who had been referred to a $\mathrm{BH}$ specialist in the prior 12 months, and 3) who had attended at least one $\mathrm{BH}$ specialist appointment in the prior 12 months.

FINDINGS TO DATE (IT IS NOT SUFFICIENT TO STATE FINDINGS WILL BE DISCUSSED ): Twelve patients expressed interest and were scheduled into a 5-week group therapy program. Ten were female, the average age was 61, all were African-American. Of these, six (50\%) attended at least one group session. Of the twelve, six had been previously referred to a BH specialist outside of CCP in the prior 12 months, but only two (33\%) had attended an appointment. Of the six who attended a group session, only two had been previously referred for outside $\mathrm{BH}$ care (33\%), and only one attended their appointment (17\%). Among the six who enrolled in CCP group therapy but did not attend, four had been previously referred but only one actually attended an outside BH specialist (17\%).

KEY LESSONS FOR DISSEMINATION (WHAT CAN OTHERS TAKE AWAY FOR IMPLEMENTATION TO THEIR PRACTICE OR COMMUNITY?): Integrating a group therapy approach to $\mathrm{BH}$ care into a program for high-risk patients with high rates of $\mathrm{BH}$ problems may improve attendance for at least their first experience with $\mathrm{BH}$ care.

INTEGRATING PREDICTIVE ANALYTIC RISK TOOLS FOR IMPROVEMENT IN UTILIZATION OF CARE MANAGEMENT RESOURCES IN THE PRIMARY CARE SETTING

Bethany D. Corbin ${ }^{1}$; Shalom Schlagman ${ }^{1}$; Alice Baker ${ }^{1}$; Enrico Caiola ${ }^{1}$; Angela K. Nagel ${ }^{2}$; Amanda M. Ramos ${ }^{1}$; Robert J. Fortuna ${ }^{1}$. ${ }^{1}$ University of Rochester, Rochester, NY; ${ }^{2}$ St John Fisher College, Rochester, NY. (Control ID \#3185814)

STATEMENT OF PROBLEM OR QUESTION (ONE SENTENCE): The average primary care physician cares for about 1,700 patients, but has limited access to finite care management resources, leading to a necessary area for improvement on how to evaluate risk within large populations, and identifying potential tools and processes to efficiently guide the allocation of care management resources to most appropriately meet patients' needs.

OBJECTIVES OF PROGRAM/INTERVENTION (NO MORE THAN THREE OBJECTIVES): 1 . Implement a system for identifying patients for whom care management may improve clinical outcomes, using predictive analytic tools in addition to clinical judgment 2. Monitor the average aggregate risk score for patients under care management 3 . Decrease the number of ED visits and hospitalizations for patients under care management

DESCRIPTION OF PROGRAM/INTERVENTION, INCLUDING ORGANIZATIONAL CONTEXT (E.G. INPATIENT VS. OUTPATIENT, PRACTICE OR COMMUNITY CHARACTERISTICS): In our outpatient residency practice, we implemented an operational process and predictive analytic tool to identify patients at risk for ED visits or hospitalization, for whom early intervention with care management may reduce this overall risk. This predictive analytic model was developed by Epic over 7 years using data from 345,000 patients. The model identified 19 variables that were predictive of a patient's risk for ED visits or hospitalization, and provides a score between 0 to 100 that correlates to the risk of ED visit or hospitalization within the next year. Validation of the tool yielded an average $\mathrm{C}$ statistic of 0.71 . In conjunction with this analytic tool, the operational process engages clinical teams to assess the potential of care management to impact the outcome (impactability). This impactability aspect includes consideration of a patient's medical factors (e.g. health conditions, adherence) and social determinants (e.g. health literacy, housing, transportation).

MEASURES OF SUCCESS (DISCUSS QUALITATIVE AND/OR QUANTITATIVE METRICS WHICH WILL BE USED TO EVALUATE PROGRAM/INTERVENTION): 1. Process measures include number of patients enrolled in care management, risk score of enrolled patients, and improved monitoring parameters of medical condition (e.g. A1c, BP control) 2. Outcome measures include increasing the average risk score of patients under care management, and decreasing the number of managed patient $\mathrm{ED}$ visits and hospitalizations

FINDINGS TO DATE (IT IS NOT SUFFICIENT TO STATE FINDINGS WILL BE DISCUSSED ): Currently, 134 patients are enrolled with care management. The baseline average risk score of patients enrolled in November, 2018 was $33.4 \%$. More than half of enrolled patients had a score less than $30 \%$. Over the first 2 months of this intervention, the average risk 
score of patients enrolled in care management has increased to $40.4 \%$, reflecting an increase risk of the changing cohort of patients. Further analyses will be completed after additional data are collected.

KEY LESSONS FOR DISSEMINATION (WHAT CAN OTHERS TAKE AWAY FOR IMPLEMENTATION TO THEIR PRACTICE OR COMMUNITY?): An operational process guided by predictive analytics and clinical judgement can help direct the allocation of care management resources within a residency practice. In the first 2 months of this intervention, the patient population under care management evolved to include higher risk individuals. With further implementation of this system, we hope to increase the average risk score for patients under care management to $60 \%$ by June 2019

INTER-PROFESSIONAL INTEGRATION IMPROVES THE PRIMARY CARE TO PHYSICAL THERAPY REFERRAL PROCESS Sharen E. McKay ${ }^{1}$; Frank D. Buono ${ }^{2}$; Jennifer Walker ${ }^{3}$; Carly Croteau ${ }^{4}$; Destiny Printz ${ }^{5}$ Rebecca Brienza ${ }^{6} .{ }^{1}$ Yale University School of Medicine, West Haven, CT; ${ }^{2}$ Yale School of Medicine, New Haven, CT; ${ }^{3}$ Veterans Hospital West Haven CT, Derby, CT; ${ }^{4}$ US Department of Veteran Affairs, West Haven, CT; ${ }^{5}$ Veterans Affairs Connecticut Healthcare System, West Haven, CT; ${ }^{6}$ VA Connecticut Healthcare System, West Haven, CT. (Control ID \#3176647)

STATEMENT OF PROBLEM OR QUESTION (ONE SENTENCE): Musculoskeletal (MSK) pain has been estimated to comprise $20-25 \%$ of patient complaints in the primary care (PC) setting resulting in many referrals to physical therapy (PT); however, the referral system can include long wait times and discontinuation of care necessitating new delivery methods.

OBJECTIVES OF PROGRAM/INTERVENTION (NO MORE THAN THREE OBJECTIVES): The objective of the program is to improve PT delivery by integrating physical therapists in an interdisciplinary patient-centered, team-based care medical home $(\mathrm{PCMH})$ model in a VA ambulatory care training clinic.

DESCRIPTION OF PROGRAM/INTERVENTION, INCLUDING ORGANIZATIONAL CONTEXT (E.G. INPATIENT VS. OUTPATIENT, PRACTICE OR COMMUNITY CHARACTERISTICS):

The VA Health Administration's Centers of Excellence in Primary Care Education (COE) are interprofessional post-graduate training programs in primary care that integrate providers of different professions in a $\mathrm{PCMH}$ model. There are COEs at seven VA sites nationally and all include physician, nurse practitioner, health psychology, and pharmacy trainees and faculty. The West Haven, CT COE has additionally integrated physical therapy faculty who participate in co-precepting, enabling direct "warm handoffs" from a PC provider to a PT when patients arrive with MSK complaints.

MEASURES OF SUCCESS (DISCUSS QUALITATIVE AND/OR QUANTITATIVE METRICS WHICH WILL BE USED TO EVALUATE PROGRAM/INTERVENTION): To evaluate the efficacy of this model we tracked the numbers of PT referrals, the number of completed referrals, the numbers of discontinued/cancelled (D/C) appointments, and the wait time between referral and encounter. The listed PT referral parameters were monitored for 3 years (FY2015-2017) and were compared between the COE training clinic and two other PC training clinics at the same VA site (Firm A and Firm B.) where PT referrals are done in the traditional manner. FINDINGS TO DATE (IT IS NOT SUFFICIENT TO STATE FINDINGS WILL BE DISCUSSED ): Over three years there was a $64 \%$ increase in referrals for COE, an increasing trend of $35 \%$ in Clinic A, and decreasing trend of $-0.8 \%$ in Clinic B. The COE showed a steady decline in D/C PT referrals and steady increase in completed PT encounters. The other Firms showed diffrent patterns of change in completed and $\mathrm{D} / \mathrm{C}$ visits over the same time. The average wait time for a PT appointment was considerably shorter in COE than either of the other Firms beginning in FY2015 (mean=18.1 days COE vs.45.2 days Firm Avs. 28.7 days Firm B) and the COE trend continued downward in FY2016 and 2017. Patients gave high ratings to seeing a physical therapist, receiving a home exercise program, and/or receiving equipment such as a cane, walker, rollator, or TENs unit on the same day as their PC appointment.

KEY LESSONS FOR DISSEMINATION (WHAT CAN OTHERS TAKE AWAY FOR IMPLEMENTATION TO THEIR PRACTICE OR COMMUNITY?): PT Integration in a PC setting increases overall PT referral, enhances completion of PT referrals and reduces wait times for appointments. We can only speculate on the mechanisms for these improvements but they may include enhanced awareness and knowledge of MSK complaints by PC providers who regularly interact with PTs, improved diagnosis of MSK issues by co-precepting and warm-handoffs, and a "jump-starting" effect on PT made possible by preliminary interventions made at the time of the warm-handoff where PT providers can recommend home exercise programs and provide equipment.

INTERDISCIPLINARY COMMUNICATION PROJECT: USING GROUP LEVEL ASSESSMENT TO GUIDE HEALTHCARE SYSTEM IMPROVEMENT

Ashley Jenkins ${ }^{1,}{ }^{2}$; Andrew Arken ${ }^{3}$; Jennifer Jasmine E. Arfaa ${ }^{3}$; Danielle L. Clark $^{1}$; Katherine Clark ${ }^{1}$; Emily Dobbs ${ }^{1}$; Betina Lahbabi ${ }^{3}$; Brian May ${ }^{1,2}$; Sriyutha Reddy ${ }^{1}$; Sana Rockwell ${ }^{3}$; Jamie Tu ${ }^{3}$; Danielle Weber ${ }^{1}$, ; Katherine Clarke-Myers ${ }^{2} .{ }^{1}$ University of Cincinnati Medical Center, Cincinnati, $\mathrm{OH}$; ${ }^{2}$ Cincinnati Children's Hospital Medical Center, Cincinnati, OH; ${ }^{3} \mathrm{UCHealth}$, Cincinnati, OH. (Control ID \#3182299)

STATEMENT OF PROBLEM OR QUESTION (ONE SENTENCE): Communication failures amongst interdisciplinary internal medicine (IM) teams in the inpatient setting inhibit mutual understanding of care plans, increase the potential for medical error, and contribute to patient dissatisfaction.

OBJECTIVES OF PROGRAM/INTERVENTION (NO MORE THAN THREE OBJECTIVES): 1 . Conduct a Group Level Assessment (GLA) to assess facilitators and barriers of effective communication within interdisciplinary inpatient IM teams 2 . Use results from GLA to identify and prioritize development of future interventions to facilitate communication DESCRIPTION OF PROGRAM/INTERVENTION, INCLUDING ORGANIZATIONAL CONTEXT (E.G. INPATIENT VS. OUTPATIENT, PRACTICE OR COMMUNITY CHARACTERISTICS): Efforts to enhance interdisciplinary collaborative teamwork within inpatient IM teams highlighted a critical need to address concerns related to communication among IM teams. We used GLA, a participatory qualitative method, to examine current barriers and facilitators of effective communication. GLA follows a structured process designed to engage diverse groups of stakeholders in the inquiry process. Our recruited stakeholders were IM faculty, residents, nurses and ancillary staff, patients/families, and care managers who work or have experienced care on inpatient IM teams at an 800-bed tertiary academic hospital. Participants attended disciplinespecific GLA sessions. During the sessions, participants first responded individually to open-ended prompts addressing interdisciplinary communication (e.g. Ideal methods of communication include). Following generation of individual responses, participants worked in small groups to synthesize the qualitative data, identify key themes, and reach consensus on priority improvement areas. 
MEASURES OF SUCCESS (DISCUSS QUALITATIVE AND/OR QUANTITATIVE METRICS WHICH WILL BE USED TO EVALUATE PROGRAM/INTERVENTION): Key features of success when using a participatory approach are enhanced participant involvement, production of meaningful results, confirmation of mutual values, and increased commitment to future action. We successfully recruited at least 15 participants per stakeholder group (total $\mathrm{n}=104)$ ): patients and families (18\%); IM faculty (22\%), IM residents (29\%); nurses and other ancillary staff (e.g. unit coordinator, 15\%); and care managers (16\%). Future success measures include validating thematic analysis with stakeholders and utilizing data to drive improvement work in the inpatient setting.

FINDINGS TO DATE (IT IS NOT SUFFICIENT TO STATE FINDINGS WILL BE DISCUSSED ): Stakeholders identified several themes related to interdisciplinary communication: 1) patient and family inclusion; 2) shared mental models and expectations; 3 ) execution of communication (modalities); 4) access to essential information; 5) cultural and behavioral components of an optimal environment; 6) role of communication in patient outcomes. Further evaluation will look specifically for overlap and divergence of themes and subthemes.

KEY LESSONS FOR DISSEMINATION (WHAT CAN OTHERS TAKE AWAY FOR IMPLEMENTATION TO THEIR PRACTICE OR COMMUNITY?): Understanding current state of effective interdisciplinary communication will allow the design of contextually-relevant interventions to improve patient care and outcomes on inpatient IM teams. Demonstrating feasibility of using a structured participatory approach in the inpatient setting shows that this method can be employed and adapted at other institutions to facilitate interdisciplinary collaboration and engagement.

INTERPROFESSIONAL MANAGEMENT OF OBSTRUCTIVE LUNG DISEASE IN AN URBAN PENNSYLVANIA FREE CLINIC FOR THE UNINSURED

Christopher C. Marino ${ }^{1}$; Theo Pham $^{2}$; Sharon E. Connor ${ }^{2}$; Mary Herbert ${ }^{1}$; Thuy Bui ${ }^{1}$; Faraaz A. Shah ${ }^{1} .{ }^{1}$ University of Pittsburgh Medical Center, Pittsburgh, PA; ${ }^{2}$ University of Pittsburgh, Pittsburgh, PA. (Control ID \#3163115)

STATEMENT OF PROBLEM OR QUESTION (ONE SENTENCE): Low-income uninsured patients face numerous barriers to optimal management of obstructive lung disease and have a higher prevalence of asthma and chronic obstructive pulmonary disease (COPD).

OBJECTIVES OF PROGRAM/INTERVENTION (NO MORE THAN THREE OBJECTIVES): The Birmingham Free Clinic (BFC) aims to provide access to high-quality care and timely diagnosis for uninsured patients with obstructive lung disease in an interprofessional free clinic setting. DESCRIPTION OF PROGRAM/INTERVENTION, INCLUDING ORGANIZATIONAL CONTEXT (E.G. INPATIENT VS. OUTPATIENT, PRACTICE OR COMMUNITY CHARACTERISTICS): Comprehensive pulmonary care at BFC includes a smoking cessation program, pharmacist-driven medication therapy management clinic, medication assistance programs, and a specialty pulmonary clinic with on-site spirometry offered every three months. This project identified patients with poorlycontrolled obstructive lung disease and explored associated contributing factors.

MEASURES OF SUCCESS (DISCUSS QUALITATIVE AND/OR QUANTITATIVE METRICS WHICH WILL BE USED TO EVALUATE PROGRAM/INTERVENTION): A retrospective chart review was performed for all patients seen at the clinic with a diagnosis of asthma or COPD from January 2016 to June 2018. Cases were identified using ICD-10-
CM diagnostic codes. A case report form was applied to abstract data on demographics, tobacco use, spirometry, medications, emergency department visits, and hospitalizations. Patients were classified as uncontrolled if chart review revealed: (1) daily albuterol inhaler use, (2) nighttime awakening, (3) functional limitations, or (4) emergency department visits or hospitalizations due to obstructive lung disease within the past year.

FINDINGS TO DATE (IT IS NOT SUFFICIENT TO STATE FINDINGS WILL BE DISCUSSED ): A total of 106 adults (age $45 \pm 15$ years, $51 \%$ female) presented with obstructive lung disease over the 30-month period (asthma 75\%, COPD 21\%, combined asthma-COPD 4\%). Seventy (66\%) had no documented spirometry testing, demonstrating spirometry underutilization and reliance on clinical assessment for diagnosis. Forty-six (43\%) reported ongoing tobacco use and nineteen $(18 \%)$ reported former tobacco use. Twenty-nine (27\%) had uncontrolled disease. Factors associated with poor control included active tobacco use $(\mathrm{p}=0.04)$, older age $(\mathrm{p}=0.03)$, male sex $(\mathrm{p}=0.01)$, and a diagnosis of COPD or combined COPD-asthma $(\mathrm{p}<0.01)$. Race and body mass index did not significantly differ between controlled and uncontrolled groups. Suboptimal control of obstructive lung disease resulted in 58 emergency department visits and 15 hospitalizations. A minority of patients (27\%) continued to follow regularly at the clinic into 2018.

KEY LESSONS FOR DISSEMINATION (WHAT CAN OTHERS TAKE AWAY FOR IMPLEMENTATION TO THEIR PRACTICE OR COMMUNITY?): Management of obstructive lung disease in an uninsured population presents unique challenges as patients are at high risk of fragmented care and have limited access to diagnostic spirometry and appropriate therapy. This quality improvement study identified targets to optimize care in a free clinic setting including enhanced inter-visit follow up, pharmacist-assisted management, improved access to on-site spirometry, increased tobacco cessation efforts, and incorporation of validated assessment tools in asthma and COPD care.

IT TAKES A VILLAGE-AMBULATORY INTERDISCIPLINARY ROUNDS TO ADDRESS HIGH COST-HIGH NEED PATIENTS William Carroll; Sun Yoo. UCLA, Los Angeles, CA. (Control ID \#3185880)

STATEMENT OF PROBLEM OR QUESTION (ONE SENTENCE): High cost-high need patients require intensive care coordination to address medical and social complexity, but traditional primary care models do not provide an efficient structure for interdisciplinary discussion to craft comprehensive care plans that address drivers of utilization.

OBJECTIVES OF PROGRAM/INTERVENTION (NO MORE THAN THREE OBJECTIVES): Perform a root cause analysis to determine underlying drivers of inpatient hospital admissions and emergency room visits. Provide a venue for interdisciplinary development of individualized complex chronic care management plans for high risk patients in order to decrease preventable hospital admissions and 30-day readmissions. Stratification of specifc tasks to appropriate team member who will serve as the point of contact between the medical team, community stakeholders and the patient to minimize redundancy and allow members maximize clinical licenses.

DESCRIPTION OF PROGRAM/INTERVENTION, INCLUDING ORGANIZATIONAL CONTEXT (E.G. INPATIENT VS. OUTPATIENT, PRACTICE OR COMMUNITY CHARACTERISTICS): An interdisciplinary team consisting of 4 internal medicine extensivist physicians, 1 nurse practitioner, 1 registered nurse $(\mathrm{RN}), 1$ clinical pharmacist, 1 comprehensive care coordinator (CCC), 2 patient service representatives (PSR), 1 hemodialysis coordinator, and 1 social worker meet weekly to evaluate previously identified cases to evaluate pertinent social determinants of health to stabilize chronic disease management in patients identified as a high utilizer. 
Inclusion criteria includes 2 or more inpatient hospitalizations within a sixmonth period or 3 or more emergency room visits in a six-month period. Various community stakeholders are invited to participate in patient discussion including home health agencies, hemodialysis center representatives and behavioral health representatives. During discussion, interventions are stratified to various members of the interdisciplinary team.

MEASURES OF SUCCESS (DISCUSS QUALITATIVE AND/OR QUANTITATIVE METRICS WHICH WILL BE USED TO EVALUATE PROGRAM/INTERVENTION): Number of individualized care plans created and qualitative evaluation of the effectiveness of interdisciplinary rounds for patients in a 12-week period. 30-day hospital readmissions following creation of care plan in interdisciplinary rounds.

FINDINGS TO DATE (IT IS NOT SUFFICIENT TO STATE FINDINGS WILL BE DISCUSSED ): 75 patients were discussed in 12 conference sessions with creation of 75 individual care plans. Patients discussed were evaluated for subsequent interventions in care coordination tasked to appropriate team members. $61.3 \%$ of patients required $\mathrm{RN}$ clinical follow-up, $54.7 \%$ of patients required CCC intervention, $32 \%$ of patients required PSR administrative intervention, $12 \%$ of patients required social work intervention and $8 \%$ of patients required hemodialysis coordination intervention. $36 \%$ of patients discussed were readmitted within 30 days of discussion.

KEY LESSONS FOR DISSEMINATION (WHAT CAN OTHERS TAKE AWAY FOR IMPLEMENTATION TO THEIR PRACTICE OR COMMUNITY?): Chronic care management for high utilizer patient population requires the input of interdisciplinary team in order to address the social determinants of health that impact chronic underlying diseases. Interdisciplinary review of high need-high cost patient populations identifies both clinical and non-clinical interventions to chronic disease management to address social determinants of health, symptom control and subspecialty care.

LOW-BARRIER INITIATION OF BUPRENORPHINE FOR TREATMENT OF OPIATE USE DISORDER AT HOMELESS ENCAMPMENTS: MEETING PEOPLE WHERE THEY ARE AT

Michael Incze ${ }^{1}$; Soraya Azari ${ }^{2}$; Deborah Borne ${ }^{3}$; Rebecca PfeiferRosenblum ${ }^{3} .{ }^{1}$ Univeristy of California, San Francisco, San Francisco, CA; ${ }^{2}$ University of California, San Francisco, San Francisco, CA; ${ }^{3}$ San Francisco Department of Public Health, San Francisco, CA. (Control ID \#3160049)

\section{STATEMENT OF PROBLEM OR QUESTION (ONE SENTENCE):}

Though access to medication assisted treatment for opioid use disorder is widely available in San Francisco, barriers such as transportation, stigma and lack of social supports prevent a significant number of high risk individuals from receiving treatment.

OBJECTIVES OF PROGRAM/INTERVENTION (NO MORE THAN THREE OBJECTIVES): Our intervention was to offer low-barrier, sameday initiation of medication assisted treatment (MAT) with buprenorphinenaloxone with prompt connection to care at monthly pop-up health fairs located at a rotating group of homeless encampments in San Francisco.

DESCRIPTION OF PROGRAM/INTERVENTION, INCLUDING ORGANIZATIONAL CONTEXT (E.G. INPATIENT VS. OUTPATIENT, PRACTICE OR COMMUNITY CHARACTERISTICS): A multidisciplinary group of health care providers and community non-profits organize monthly health fairs at a rotating group of homeless encampments around San Francisco. Participants were routinely screened by medical providers for opioid use disorder and assessed for readiness to start MAT. For those who desired to start treatment with buprenorphine-naloxone, California's state PDMP was checked onsite using mobile devices, and a 2-3 day prescription was called in at the prescribing provider's discretion to a partnering pharmacy with expertise in harm reduction and MAT. No utox was required for the initial prescription. Follow up was arranged at one of three local walk-in treatment centers based on proximity and patient preference. The state PDMP and the SFDPH electronic health record were used to monitor follow-up MEASURES OF SUCCESS (DISCUSS QUALITATIVE AND/OR QUANTITATIVE METRICS WHICH WILL BE USED TO EVALUATE PROGRAM/INTERVENTION): Our primary measure was the percentage of patients who filled a prescription and attended at least one follow up visit. Secondary measures included the percentage of patients who remained in treatment at six months, the total buprenorphine-naloxone prescriptions at each event, and qualititative feedback from trainees.

FINDINGS TO DATE (IT IS NOT SUFFICIENT TO STATE FINDINGS WILL BE DISCUSSED ): After 12 months, 23* people received new prescriptions for buprenorphine-naloxone on-site, of which $37 \%$ attended at least one follow-up visit. $14 \%$ were still in treatment at six months. $45 \%$ of patients prescribed buprenorphine-naloxone never picked up a prescription or had a documented follow up visit in the electronic health record. The total number of buprenorphine-naloxone prescriptions at each event has increased. Qualitative feedback from trainees was positive. *Please note that we have new data for the past six months that will be incorporated into the presentation, with a total number of bupe starts now at $\sim 60$.

KEY LESSONS FOR DISSEMINATION (WHAT CAN OTHERS TAKE AWAY FOR IMPLEMENTATION TO THEIR PRACTICE OR COMMUNITY?): Low-barrier, on-site initiation of MAT of opiate use disorder was effective in connecting a substantial number of people from a marginalized and hard-to-reach population with a high prevalence of opiate use disorder to treatment. As San Francisco invests in resources to address the opiate epidemic, street-based outreach will remain an important mode of connecting with people who inject drugs that may not utilize existing infrastructure for accessing treatment because of stigma and significant psychosocial barriers. Next steps include improving attendance at follow up visits and retention in treatment by developing more intensive patient navigation resources at future events.

\section{MAKING PRIMARY CARE NEIGHBORHOOD CARE}

Elizabeth Jacobs $^{2,}{ }^{1}$; William M. Tierney ${ }^{2,1}$; Brandon Allport ${ }^{1,2}$; Alexandra Garcia $^{2,1} .{ }^{1}$ University of Texas at Austin, Austin, TX; ${ }^{2}$ Dell Medical School, University of Texas at Austin, Carmel, IN. (Control ID \#3181837)

\section{STATEMENT OF PROBLEM OR QUESTION (ONE SENTENCE):} Primary care providers increasingly recognize that zip-code is a more important determinant of health than a person's genetic code.

\section{OBJECTIVES OF PROGRAM/INTERVENTION (NO MORE THAN} THREE OBJECTIVES): 1) To understand and address the medical and social determinants of health in a geographic neighborhood in Austin, TX. 2) To develop an innovative primary care delivery program that addresses the medical and social needs of patients.

DESCRIPTION OF PROGRAM/INTERVENTION, INCLUDING ORGANIZATIONAL CONTEXT (E.G. INPATIENT VS. OUTPATIENT, PRACTICE OR COMMUNITY CHARACTERISTICS): We have developed and are in the early stages of implementing Dell Med School's Neighborhood Health Initiative. Its 3 main components are: (1) a partnership with a Federally Qualified Health Center; (2) partnerships with communitybased organizations in our target neighborhood; and (3) the household level health needs assessments (HoLA). Using data from HoLA and our community and FQHC partner, we have developed a plan to connect families to primary care and social services to address barriers to health identified by residents. 
MEASURES OF SUCCESS (DISCUSS QUALITATIVE AND/OR QUANTITATIVE METRICS WHICH WILL BE USED TO EVALUATE PROGRAM/INTERVENTION): We will assess success by (1) establishment of trusting, productive relationships with our $\mathrm{FQHC}$, community partners and neighborhood residents; (2) increasing numbers of neighborhood residents engaged with primary care; (3) number of residents with whom the community health worker has established a relationship and the number of referrals to services they have made; and (4) the well-being and health of residents served.

FINDINGS TO DATE (IT IS NOT SUFFICIENT TO STATE FINDINGS WILL BE DISCUSSED ): To date we have established successful community partnerships and a clinical partnership in the neighborhood, including co-hiring a medicine-pediatrics physician. We collected data from over 100 diverse households: $64 \%$ identified as Latino and 25\% Black, 55\% selfreported as immigrant, and most were uninsured (23\%), received Medicaid (21\%), or covered by a gap coverage provided by the local Health District (33\%). Only $65 \%$ were engaged with primary care and $23 \%$ did not access any medical services in the past year. Residents faced a number of challenges: A third had difficulty meeting expenses every month, $42 \%$ worried about not having enough food, almost $20 \%$ said their health was diminished by concerns about immigration and deportation, $10 \%$ were affected by racism and discrimination, and $10 \%$ by crime, grief, and violence. Top requests for services were for dental services (32\%), vision care, mental health, assistance with paying for medications, accessing health care, and housing (about 25\% each), and legal services $(10 \%)$.

KEY LESSONS FOR DISSEMINATION (WHAT CAN OTHERS TAKE AWAY FOR IMPLEMENTATION TO THEIR PRACTICE OR COMMUNITY?): Thinking outside the box to improve the health of a community requires addressing both social and medical needs, partnering with community social and medical service providers, and listening to and being open to residents' needs. All of this takes time and a willingness to partner across sectors and organizational cultures.

MEDICAL ASSISTANT PROFESSIONALISM PROGRAM BRINGS EDUCATION, TRAINING, COMMUNITY AND CAREER SATISFACTION TO AN INDISPENSABLE WORKFORCE IN PRIMARY CARE

Rachel Haft ${ }^{1}$; Karen Victor ${ }^{1}$; Meredith Hobson ${ }^{1}$; Beth A. Lown ${ }^{1}$; Andrew Curran $^{1}$; John D'Angelo ${ }^{3}$; Diane Shapiro ${ }^{3}$; Valerie E. Stone ${ }^{2} .{ }^{1}$ Mount Auburn Hospital, Cambridge, MA; ${ }^{2}$ Mount Auburn Hosp, Harvard Medical School, Cambridge, MA; ${ }^{3}$ Mount Auburn Cambridge Independent Practitioners Association, Brighton, MA. (Control ID \#3185887)

STATEMENT OF PROBLEM OR QUESTION (ONE SENTENCE): The Medical Assistant (MA) profession has entered an era of expanding competencies and expectations, yet MA education is not customized to primary care practice, standards do not exist to ensure that postgraduate education and training parallel advances in clinical care, and healthcare organizations miss an opportunity to improve MA satisfaction and retention through the dignity of educational investment and community-building among this workforce.

OBJECTIVES OF PROGRAM/INTERVENTION (NO MORE THAN THREE OBJECTIVES): We built a Medical Assistant Professionalism Program to deliver education and training of high value to our primary care MAs and to evaluate their satisfaction with the program's first year.

DESCRIPTION OF PROGRAM/INTERVENTION, INCLUDING ORGANIZATIONAL CONTEXT (E.G. INPATIENT VS. OUTPATIENT, PRACTICE OR COMMUNITY CHARACTERISTICS): Our
11 primary care clinics employ approximately 40 MAs. We convened focus groups to ascertain what motivated the MAs in their work, tally their selfidentified strengths and ideas, and formulate a high-value curriculum. Sessions were moderated without supervisors or providers present, to promote frank discussion. We then divided our workforce into three cohorts to stagger clinic staffing and bring MAs from different clinics together for cross-fertilization. Each cohort attended four 1.5 hour classes, held at 3-month intervals. Curricula included advanced blood pressure measurement, cancer screening, EMR workflows, nuances of glucose testing, PlanDoStudyAct (PDSA) training for the primary care setting, and Service Excellence topics aimed at patient experience.

MEASURES OF SUCCESS (DISCUSS QUALITATIVE AND/OR QUANTITATIVE METRICS WHICH WILL BE USED TO EVALUATE PROGRAM/INTERVENTION): The majority of MAs, individually and in teams, designed and implemented PDSA projects to address issues of personal interest and reported back to their respective cohorts. Also, participants were surveyed at each session to assess satisfaction with programming and its potential impact on their work.

FINDINGS TO DATE (IT IS NOT SUFFICIENT TO STATE FINDINGS WILL BE DISCUSSED ): Several MA-designed PDSA exercises yielded positive results in primary care delivery. One project effected $20 \%$ improvement in fecal immunochemical test completion. Another PDSA reconfigured the demographic data collection process as a more patientcentric experience, elevating participation to $100 \%$. MAs reported feeling that programming had been designed specifically with them in mind (89.5\%); they considered the covered topics clinically relevant to their work (94.4\%) and reported that the sessions would change how they care for patients $(82.8 \%)$. They positively rated the quality of presentations on Service Excellence (94.5\%) and all other topics (96.6\%). This is the program's second year.

KEY LESSONS FOR DISSEMINATION (WHAT CAN OTHERS TAKE AWAY FOR IMPLEMENTATION TO THEIR PRACTICE OR COMMUNITY?): A Medical Assistant Professionalism Program offers a venue for primary care MAs to continue their medical education and build community, resulting in a high level of satisfaction for this workforce and with significant potential benefits for their contributions to primary care practice. Healthcare systems that deliver primary care services could leverage such programming to meet their obligations of MA recruitment, competency advancement, and patient and provider satisfaction. Furthermore, we believe this program can contribute to improved MA retention; data analysis is underway.

MILE HIGH PROBLEMS: IMPROVING TURNAROUND TIME FOR NEW HOME OXYGEN ORDERS

Cody Dashiell-Earp ${ }^{1}$; Edward Collins ${ }^{1}$; Carmen L. Lewis ${ }^{2} .{ }^{1}$ University of Colorado, Denver, CO; ${ }^{2}$ Univesrity of Colorado, Denver, CO. (Control ID \#3184873)

STATEMENT OF PROBLEM OR QUESTION (ONE SENTENCE): Although providers and staff in our practice spent at least one hour on paperwork for every new oxygen order, the average time to fulfillment was 12.7 days, leading to poor health outcomes for patients and significant frustration.

OBJECTIVES OF PROGRAM/INTERVENTION (NO MORE THAN THREE OBJECTIVES): Reduce the average time to fulfillment of an oxygen order by $50 \%$. Reduce the amount of time spent by physicians completing paperwork for new oxygen orders. Improve patient, provider and staff satisfaction with the oxygen ordering process. 
DESCRIPTION OF PROGRAM/INTERVENTION, INCLUDING ORGANIZATIONAL CONTEXT (E.G. INPATIENT VS. OUTPATIENT, PRACTICE OR COMMUNITY CHARACTERISTICS): Due to our location in Denver, Colorado, our outpatient internal medicine practice has an unusually high burden of new oxygen orders, with an average of 20 new orders per month. Since most Medicare patients seen in our office are contracted with just one oxygen vendor, there is little incentive for this vendor to improve customer service. The 18 providers in our office and the medical assistants (MAs) and nurses (RNs) that support them each had a different process for initiating an oxygen order, variable familiarity with the requirements for such orders, and no standard way of following up with vendors to ensure the order was fulfilled. To solve this problem, two MAs were designated as oxygen specialists and given $0.2 \mathrm{FTE}$ to work on this task. They met in person twice a week with a representative from our primary oxygen vendor to review the status of oxygen orders and once a month with practice leadership to review performance data.

MEASURES OF SUCCESS (DISCUSS QUALITATIVE AND/OR QUANTITATIVE METRICS WHICH WILL BE USED TO EVALUATE PROGRAM/INTERVENTION): Our primary outcome was the number of days between the first oxygen order and the delivery of oxygen to the patient's home. We also performed qualitative interviews to assess the satisfaction of our physicians, the MAs who worked on the project, and the MAs who were not involved in the project.

FINDINGS TO DATE (IT IS NOT SUFFICIENT TO STATE FINDINGS WILL BE DISCUSSED ): The average time to fulfillment of a new oxygen order dropped from 12.7 to 5.4 days and has been sustained for 2 months. This means that for every hour our MAs work in this capacity, they reduce the time our patients wait for oxygen by 2.2 days. Qualitative interviews with providers and staff have revealed that both the MAs who became our oxygen specialists and the MAs who no longer need to spend time on oxygen orders are highly satisfied with this new workflow. Provider satisfaction has increased slightly, however there continue to be frustrating delays for oxygen order renewals and CPAP orders, which were not the focus of this project.

KEY LESSONS FOR DISSEMINATION (WHAT CAN OTHERS TAKE AWAY FOR IMPLEMENTATION TO THEIR PRACTICE OR COMMUNITY?): The oxygen ordering process is too complex to be managed efficiently on a case by case basis by all members of the primary care team. Creating an oxygen specialist for a practice can dramatically reduce the time it takes to fulfill a new oxygen order and the paperwork burden on providers and staff. In a non-competitive oxygen marketplace, exchanging performance data and best practices with the primary oxygen vendor improves accountability and turnaround time.

MYTHBUSTERS! IMPROVING INFLUENZA VACCINATION RATES IN AN URBAN UNDERSERVED RESIDENT PRACTICE USING TARGETED EDUCATIONAL INTERVENTION

Helen Cha ${ }^{1,2} ;$ Filip Oleszak $^{1,2} ;$ Ahmed Awwad ${ }^{1,2} ;$ Hira Chaudhary ${ }^{1,2} ;$ Lucas $\overline{\text { Dreamer }^{1}}{ }^{2}$; Melissa S. Lee ${ }^{1,2}$; Andrew A. Chang ${ }^{1,2}$; David L. Stevens ${ }^{1,2}$. ${ }^{1} \mathrm{NYC}$ Health + Hospitals/Kings County, New York, NY; ${ }^{2}$ SUNY Downstate Medical Center, Brooklyn, NY. (Control ID \#3171379)

STATEMENT OF PROBLEM OR QUESTION (ONE SENTENCE): Will influenza (flu) vaccination rates improve by targeting patient concerns and providing standardized educational information?

OBJECTIVES OF PROGRAM/INTERVENTION (NO MORE THAN THREE OBJECTIVES): To increase flu shot administration rates by $10 \%$ from our practice's usual care $(67 \%)$ over two months (OctoberDecember 2018)
DESCRIPTION OF PROGRAM/INTERVENTION, INCLUDING ORGANIZATIONAL CONTEXT (E.G. INPATIENT VS. OUTPATIENT, PRACTICE OR COMMUNITY CHARACTERISTICS): The resident primary care practice serves a predominantly Afro-Caribbean urban underserved population in Brooklyn, NY. All patients were offered the flu vaccine. Those that declined were engaged in conversation based on the CDC "No More Excuses: You Need a Flu Vaccine" fact sheet. Most common categories of declination reasons were: - The flu vaccine can give me the flu. - I do not need to get the flu vaccine every season. - I can wait to get vaccinated. The flu vaccine is not $100 \%$ effective/protective. Residents were educated on the most common reasons for declination and targeted educational interventions based off the fact sheet addressed each patient's specific concern. The number of patients who converted to accepting vaccination was recorded. Patients with reasons other than the above were excluded from the study.

MEASURES OF SUCCESS (DISCUSS QUALITATIVE AND/OR QUANTITATIVE METRICS WHICH WILL BE USED TO EVALUATE PROGRAM/INTERVENTION): The percentage of patients who converted to accepting the flu vaccine following the intervention.

FINDINGS TO DATE (IT IS NOT SUFFICIENT TO STATE FINDINGS WILL BE DISCUSSED ): The flu vaccine was offered to 71 patients, 37 patients (52\%) accepted, 34 patients (48\%) initially declined. Of these 34 patients, 23 (68\%) patients converted to accepting flu vaccination following the targeted educational intervention. In total, 60 out of 71 patients $(84 \%)$ ultimately accepted flu vaccine administration. Our intervention had an absolute improvement of flu shot acceptance of $17 \%$ over usual care $67 \%$ in our practice the previous year). 34 patients were surveyed on reasons for declination, with the following results: 15 patients $(44.1 \%)$ "The flu vaccine can give you the flu." 9 patients $(26.4 \%)$ "The flu vaccine is not $100 \%$ effective/ protective." 6 patients (17.6\%) "I can wait to get vaccinated." 4 patients $(11.7 \%)$ "I do not need to get the flu vaccine every season." 6 patients (17.6\%) "Other reasons." These patients were excluded from the study.

KEY LESSONS FOR DISSEMINATION (WHAT CAN OTHERS TAKE AWAY FOR IMPLEMENTATION TO THEIR PRACTICE OR COMMUNITY?): Patients decline influenza vaccination for a few common reasons as mentioned above. By identifying and addressing each patient's particular concern, and by using a focused standardized educational intervention, physicians can improve vaccination rates.

NICE TO NOT MEET YOU! ELIMINATING BARRIERS TO PRIMARY CARE FOR HOUSE STAFF THROUGH NO-VISIT ESTABLISHMENT

Dan Henderson; J. B. Crocker; Emily Shipp. Massachusetts General Hospital,

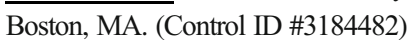

STATEMENT OF PROBLEM OR QUESTION (ONE SENTENCE): For residents and fellows seeking primary care, long wait times for new patients, tight clinical schedules, and the universal requirement of an initial visit pose significant barriers to establishing care.

OBJECTIVES OF PROGRAM/INTERVENTION (NO MORE THAN THREE OBJECTIVES): 1 . Create a new pathway to safely establish primary care without an initial visit. 2. Decrease delays in obtaining primary care for house staff. 3 . Increase the proportion of internal medicine residents with a primary care physician.

DESCRIPTION OF PROGRAM/INTERVENTION, INCLUDING ORGANIZATIONAL CONTEXT (E.G. INPATIENT VS. OUTPATIENT, PRACTICE OR COMMUNITY CHARACTERISTICS): Our office is a hospital-affiliated primary care practice with a special mission to provide high-value care to hospital employees and their family members. 
Many of our patients are residents and fellows, who carry a significant burden of stress-related symptoms and conditions, as well as reduced access to primary and preventive care due to competing demands. Given the pressures on residents and the critical roles they play in hospital operations, we sought to bridge the gap between the care residents need and the care they get. Across our institution, the average wait for a new patient visit was 44 days, and so many house staff are unable to establish care without taking a vacation. Since they tend to be medically well and health savvy, we sought to use a questionnaire to triage need for visit, and create a pathway to non-visit empanelment. We designed the questionnaire to elicit a variety of needs, including routine prevention, physical or behavioral symptoms, and sexual health. We also built in mechanisms for patients to request routine and recommended testing without needing to be seen. We intended to make the program available to all housestaff, but were limited by logistical issues to the internal medicine residents and fellows. We announced the program as part of intern orientation and through department communications.

MEASURES OF SUCCESS (DISCUSS QUALITATIVE AND/OR QUANTITATIVE METRICS WHICH WILL BE USED TO EVALUATE PROGRAM/INTERVENTION): 1. Number of residents establishing care in the first six months of the pilot, compared to the previous year. 2. Median time in residency before establishing care, compared to those establishing in the previous year. 3. Proportion of patients who establish care without a visit.

FINDINGS TO DATE (IT IS NOT SUFFICIENT TO STATE FINDINGS WILL BE DISCUSSED ): The program was implemented June 21st, and so we have only recently passed the six month time point. As such, the analysis is ongoing at the time of submission. Initial results show that 36 interns established care in that time period, though this likely underestimates the total, as it reflects only those actively counted by one PCP. We are currently analyzing appointment and panel data against the roster of residents. The questionnaire appeared to function well, and correctly identified a handful of patients with significant medical or behavioral health issues, based on historical vital signs or standard questionnaires.

KEY LESSONS FOR DISSEMINATION (WHAT CAN OTHERS TAKE AWAY FOR IMPLEMENTATION TO THEIR PRACTICE OR COMMUNITY?): A well-designed questionnaire, applied to the right population, may be a safe alternative or supplement to the standard establishing visit. We intend to continue the program and explore expanding the questionnaire to other populations.

OD IN THE ED: A TEAM-BASED APPROACH TO THE OPIOID EPIDEMIC

Divya Venkat ${ }^{1}$; James Bruce ${ }^{1}$; Bruce Ling ${ }^{2}$; Courtney Watson ${ }^{1} .{ }^{1}$ Allegheny General Hospital, Pittsburgh, PA; ${ }^{2}$ Allegheny Health Network, Pittsburgh, PA. (Control ID \#3183904)

STATEMENT OF PROBLEM OR QUESTION (ONE SENTENCE): Opioid overdose is one of the leading causes of death in the United States. OBJECTIVES OF PROGRAM/INTERVENTION (NO MORE THAN THREE OBJECTIVES): To evaluate the initiation of buprenorphine in the emergency department to patients who present following an opioid overdose and high risk opioid use.

DESCRIPTION OF PROGRAM/INTERVENTION, INCLUDING ORGANIZATIONAL CONTEXT (E.G. INPATIENT VS. OUTPATIENT, PRACTICE OR COMMUNITY CHARACTERISTICS): This healthcare innovation occurs in a community based hospital in an urban part of Pittsburgh. This hospital serves a significant number of low-income patients with a have a history of illicit opioid use. Due to the hospital's location, its emergency department treats a large number of opioid overdoses and high risk patients. Within the hospital, the Addiction Medicine team collaborates with the Emergency Department on an Opioid Use Disorder Initiative to treat those with opioid use disorder. Through this initiative, patients who are seen in the emergency department and are diagnosed with opioid use disorder are offered a dose of buprenorphine to transition patients to opioid replacement therapy. This dose is given either by the Emergency Department providers or through a referral to the Addiction Medicine team. Following this dose of buprenorphine, a warm hand off is provided to the Medication Assisted Treatment (MAT) clinic via the emergency medicine physician or through the Addiction Medicine team for the transition of patients to outpatient drug recovery services.

MEASURES OF SUCCESS (DISCUSS QUALITATIVE AND/OR QUANTITATIVE METRICS WHICH WILL BE USED TO EVALUATE PROGRAM/INTERVENTION): Success of this intervention is measured in the amount of patients receive this intervention and subsequently follow up in MAT clinic.

FINDINGS TO DATE (IT IS NOT SUFFICIENT TO STATE FINDINGS WILL BE DISCUSSED ): This program was initiated in May 2018 with the study period consisting of May 2018 to November 2018. There were 24 patients who received buprenorphine after meeting applicable clinical criteria. As a result of this initiative, there was a median of 2.5 patients per month who received buprenorphine. The Addiction Medicine team and ED providers ordered buprenorphine with similar frequency. During this time, 12 out of 24 patients were referred to the Addiction Medicine team's MAT clinic. Seven of these patients were seen at the MAT clinic and three remain active. An additional three patients were seen at external MAT clinics and 6 out of the 24 patients were referred to a drug and alcohol treatment center. Twelve patients had repeated ER visits, half of which were opioid related.

KEY LESSONS FOR DISSEMINATION (WHAT CAN OTHERS TAKE AWAY FOR IMPLEMENTATION TO THEIR PRACTICE OR COMMUNITY?): This program shows the potential importance of initiating buprenorphine to patients with opioid use disorder while in the emergency room. While this was a small sample, a substantial amount of patients followed up with a MAT clinic or were connected to a drug and alcohol treatment center. By intervening with patients who have opioid use disorder while they are engaged in a healthcare setting such as the ED, buprenorphine can be initiated within the appropriate timeframe and patients can be transitioned to continued care in the outpatient setting. Collaborative efforts such as this between the emergency department and experts in addiction medicine may help facilitate the care of patients with opioid use disorder.

ON THE FRONTLINES OF THE SILENT EPIDEMIC: FINDINGS FROM A MULTIYEAR PROGRAM TO EXTEND COMMUNITYBASED HEPATITIS C SCREENING AND TREATMENT IN LOS ANGELES'S SKID ROW

Trista M. Benitez; Shannon Fernando. Los Angeles Christian Health Centers, Los Angeles, CA. (Control ID \#3184368)

STATEMENT OF PROBLEM OR QUESTION (ONE SENTENCE): An inequitable prevalence of hepatitis C (HCV) exists within Los Angeles's Skid Row, yet screening, linkage, and treatment uptake remains marginal.

OBJECTIVES OF PROGRAM/INTERVENTION (NO MORE THAN THREE OBJECTIVES): Project HEAL (Hepatitis C Engagement and Accessible Linkage) will reduce inequities in $\mathrm{HCV}$ screening and treatment through the following objectives: 1) The proportion of screened HCV+ participants who are linked to care, i.e. return for an evaluation to discuss lab results and receive initial $\mathrm{HCV}$ care, will increase $13 \%$ from $76 \%$ to $86 \%$ 
2) Organizational capacity to cure hepatitis $C$ will be expanded through training of four new $\mathrm{HCV}$ treaters, primary care providers who prescribe direct acting antivirals (DAAs) 3) Treatment outcomes of homeless participants on DAAs will be similar to those observed in traditional cohort studies DESCRIPTION OF PROGRAM/INTERVENTION, INCLUDING ORGANIZATIONAL CONTEXT (E.G. INPATIENT VS. OUTPATIENT, PRACTICE OR COMMUNITY CHARACTERISTICS): An estimated quarter of Los Angeles's Skid Row population is $\mathrm{HCV}+$, though many are unaware of their diagnosis or how to access treatment. Project HEAL addressed this inequity through routine HCV screening at two sites directly serving Skid Row - an FQHC and a clinic co-located in a shelter. Project HEAL employed a care coordination model to accomplish its primary objective of enhancing linkage to care. A dedicated care coordinator received alerts of all $\mathrm{HCV}+$ antibody cases in real- time via EMR integration with an internal database. This allowed the care coordinator to track and engage $\mathrm{HCV}+$ patients via phone and in-person at their visits. The care coordinator served as an advocate by appealing health plan denials, pursuing treatment for the uninsured, and ensuring timeliness of medication delivery.

MEASURES OF SUCCESS (DISCUSS QUALITATIVE AND/OR QUANTITATIVE METRICS WHICH WILL BE USED TO EVALUATE PROGRAM/INTERVENTION): Project HEAL measured its progress by the number of $\mathrm{HCV}$ tests conducted, unique $\mathrm{HCV}+$ patients identified through testing, and the proportion who were linked to care. Project HEAL monitored each participant's progress in the HCV care cascade, notably those who commenced treatment, completed therapy, and achieved sustained virologic response at 12-weeks post-treatment (SVR-12).

FINDINGS TO DATE (IT IS NOT SUFFICIENT TO STATE FINDINGS WILL BE DISCUSSED ): From 10/2016 to 11/2018, 6,760 HCV antibody tests were performed and a total of $408 \mathrm{HCV}+$ patients were confirmed. The proportion of linked participants increased $13 \%$ from $76 \%$ (187/246) in year one to $86 \%$ (140/162) in year two. From 3/2017 to 7/2018, $28 \mathrm{HCV}+$ participants had commenced treatment with DAAs, $79 \%$ of which experienced homelessness (22/28). At time of analysis, the treatment completion rate of participants experiencing homelessness was $95 \%$ (21/22), 86\% had obtained SVR-12 (18/21) with three participants lost to follow-up.

KEY LESSONS FOR DISSEMINATION (WHAT CAN OTHERS TAKE AWAY FOR IMPLEMENTATION TO THEIR PRACTICE OR COMMUNITY?): - Co-location of screening and linkage to care in nontraditional settings, such as homeless shelters, is essential to confront inequities in HCV screening and treatment. - EMR integration and real-time alert of HCV+ cases enhances prompt outreach and successful linkage to care - Care coordination facilitates progress through the HCV care cascade by building trust and rapport with participants - A primary care program with an established HCV Champion can mobilize organizational capacity to treat $\mathrm{HCV}$

ONLINE ASSESSMENTS OF MEDICATION ADHERENCE AND RISKS FOR INADEQUATE ADHERENCE TO CRITICAL MEDICATIONS IN AMBULATORY POPULATIONS

Marina Arvanitis ${ }^{1}$; Amy Moore ${ }^{1}$; Scott Hur ${ }^{1}$; Laura M. Curtis ${ }^{1}$; Daniela Ladner ${ }^{2}$; Michael S. Wolf ${ }^{1} .{ }^{1}$ Northwestern University, Chicago, IL; ${ }^{2}$ Northwetsern University, Chicago, IL. (Control ID \#3186400)

STATEMENT OF PROBLEM OR QUESTION (ONE SENTENCE): Among patients receiving critical mediation regimens, such as transplant recipients, there is a high rate of inadequate medication adherence, yet few strategies exist for assessing and addressing risk for inadequate adherence.
OBJECTIVES OF PROGRAM/INTERVENTION (NO MORE THAN THREE OBJECTIVES): 1. Leverage existing clinic technology and resources to routinely assess patients' adherence to critical medication regimens, and risk factors of inadequate adherence 2. Create clinic workflows to utilize survey data and intervene in real-time, in order to prevent complications related to inadequate adherence

DESCRIPTION OF PROGRAM/INTERVENTION, INCLUDING ORGANIZATIONAL CONTEXT (E.G. INPATIENT VS. OUTPATIENT, PRACTICE OR COMMUNITY CHARACTERISTICS): In two academic transplant clinics, we have implemented a medication adherence monitoring strategy - TAKE IT - which includes 1) routine, online selfreported assessments of patient adherence to medications and suspected psychological and social comorbidities that affect medication adherence; 2) direct alert notifications of the results via the electronic health record to transplant nurses; 3) quarterly adherence reports to monitor immunosuppression drug levels and summarize adherence trends; 4) deployment of adherence support tools tailored to specific adherence concerns. The online, self-reported 21-item assessment is comprised of novel questions specific to transplant patients, and the following: the Basel Assessment of Adherence Immunosuppression Scale (BAASIS), the 2-item Patient Health Questionnaire (PHQ-2), the 4-item Consumer Health Activation Index (CHAI), the Woloshin et al. scale of Perceived Adequacy of Tangible Social Support, and the cost-item of the ASK-12 Adherence Barrier Survey. This assessment is completed completely online via a secure patient portal, and takes under 5 minutes to complete.

MEASURES OF SUCCESS (DISCUSS QUALITATIVE AND/OR QUANTITATIVE METRICS WHICH WILL BE USED TO EVALUATE PROGRAM/INTERVENTION): 1. Completion of the online, self-report assessment 2. Identification of risk for inadequate adherence 3. Response by clinic staff to assessment results

FINDINGS TO DATE (IT IS NOT SUFFICIENT TO STATE FINDINGS WILL BE DISCUSSED ): Among 61 patients randomized to receive the TAKE IT intervention, $75 \%$ completed the initial online selfreport adherence assessment. Over the first three months, $74 \%$ of participants completed at least two assessments. In all, $51 \%$ were considered at risk of inadequate adherence. Among all identified adherence concerns, $31 \%$ were classified as cognitive (e.g. forgetfulness), $25 \%$ psychological (e.g. depression), $17 \%$ medical, $4 \%$ regimen-related (e.g. side effects), $15 \%$ social, and $8 \%$ economic. In nearly all patients with reported concerns, transplant center staff responded and resolved the issue. KEY LESSONS FOR DISSEMINATION (WHAT CAN OTHERS TAKE AWAY FOR IMPLEMENTATION TO THEIR PRACTICE OR COMMUNITY?): Assessments of medication adherence and potential root causes of inadequate adherence, including psychological and social comorbidities, can be addressed via brief, routine online survey via a patient portal. Such regular assessments have the potential to identify problems or potential problems with adherence between clinic visits, and before adverse health outcomes develop. Future work will focus on deploying adherence support tools tailored to specific adherence barriers, and determining how to integrate these tools into existing clinic workflows without burdening clinical staff.

OUTCOMES OF A MOBILE-BASED BEHAVIORAL ECONOMIC INTERVENTION TO IMPROVE ADHERENCE AMONG HIGHNEED, HIGH-COST PATIENTS

Maria Duenas ${ }^{1}$; Sire Sow ${ }^{2}$; Yixuan $\mathrm{Ma}^{4}$; Mike Fuccillo ${ }^{4}$; Ania Wajnberg ${ }^{3}$. ${ }^{1}$ Mount Sinai Hospital, New York, NY; ${ }^{2}$ Mount Sinai, New York, NY; ${ }^{3}$ Icahn School of Medicine at Mount Sinai, New York, NY; ${ }^{4}$ Wellth, New York, NY. (Control ID \#3185826) 
STATEMENT OF PROBLEM OR QUESTION (ONE SENTENCE):

High-need, high-cost patients suffer from multiple chronic medical conditions and struggle to adhere to medications and engage in self-management behaviors. Effective tools and strategies are needed to engage and activate this patient population.

OBJECTIVES OF PROGRAM/INTERVENTION (NO MORE THAN THREE OBJECTIVES): Improve adherence to medication and self-management behaviors Improve glycemic control in diabetic patients Achieve high patient satisfaction rates

DESCRIPTION OF PROGRAM/INTERVENTION, INCLUDING ORGANIZATIONAL CONTEXT (E.G. INPATIENT VS. OUTPATIENT, PRACTICE OR COMMUNITY CHARACTERISTICS): Wellth is a New York-based digital health company that has developed a mobile application rooted in behavioral economic principles to help patients with chronic conditions improve their adherence to medication and self-management behaviors. Wellth's app prompts patients with daily reminders and financial incentives to motivate long-term habit formation through daily reinforcement. Patients use the app to upload photos to track their adherence to required behaviors. For this program, $\$ 75$ in rewards was offered in increments of $\$ 15$ paid out every two weeks as long as patients were fully adherent. For missed tasks, a deduction of up to $\$ 2$ was made. The patients participating in this pilot are part of the PEAK Health Clinic at Mount Sinai Hospital, an outpatient clinic that offers intensive primary care services and complex care management to a high-need, high-cost, multimorbid, mixed Medicaid/Medicare patient population. Patients with suspected or known nonadherence were referred for enrollment by their primary care provider. Patients were ineligible if their device was incompatible with the Wellth software. Providers received weekly reports summarizing adherence rates and glucose and weight measurements. Hypo or hyperglycemia, significant weight gain, or sustained nonadherence triggered alerts to the patient's care team.

MEASURES OF SUCCESS (DISCUSS QUALITATIVE AND/OR QUANTITATIVE METRICS WHICH WILL BE USED TO EVALUATE PROGRAM/INTERVENTION): Percent adherence to medication, glucose-monitoring and weight check-in. Change in A1c pre and post intervention in diabetic patients. Change in weight pre and post intervention in CHF patients. Patient satisfaction with the intervention.

FINDINGS TO DATE (IT IS NOT SUFFICIENT TO STATE FINDINGS WILL BE DISCUSSED ): We successfully enrolled 56 patients, overall they demonstrated $84 \%$ adherence to medications, $87 \%$ adherence to glucose-monitoring and $95 \%$ adherence to daily weight check-ins. Among patients with Type 1 or Type 2 Diabetes, there was an average decrease in hemoglobin A1c of $0.5 \%$. Patients enrolled in Wellth for weight check-ins due to a history of congestive heart failure demonstrated an average decrease in weight of $3.7 \mathrm{lbs}$. Patient surveys have revealed a net promoter score of $94 \%$, indicating a high level of patient satisfaction with Wellth.

KEY LESSONS FOR DISSEMINATION (WHAT CAN OTHERS TAKE AWAY FOR IMPLEMENTATION TO THEIR PRACTICE OR COMMUNITY?): High-need, highcost patients with multiple chronic medical conditions and suboptimal adherence can be engaged to improve adherence to medication and self-management behaviors through the use of a mobilebased intervention that incentivizes patients using behavioral economic principles. Adherence rates were above $80 \%$ for all three target behaviors, indicating a high success rate among a generally difficult to engage patient population.
PA-HOSPITALIST TASK FORCE: USING APPRECIATIVE INQUIRY TO DRIVE POSITIVE CHANGE WITHIN A HOSPITAL MEDICINE GROUP

Sydney Katz ${ }^{1}$; Alice Tang ${ }^{1}$; Rachel Aubuchon ${ }^{2}$; Vanessa Oliveira ${ }^{2}$; Stephanie J. Tang ${ }^{1}{ }^{1}$ Weill Cornell Medical College, New York, NY; ${ }^{2}$ New York Presbyterian, New York, NY. (Control ID \#3167531)

STATEMENT OF PROBLEM OR QUESTION (ONE SENTENCE): Burnout and provider attrition is pervasive in hospital medicine and negatively impacts physicians (MDs), physician assistants (PAs), and patient care. OBJECTIVES OF PROGRAM/INTERVENTION (NO MORE THAN THREE OBJECTIVES): A collaborative working group, the "PA-Hospitalist Task Force," was created to formulate strategies to: 1 . Provide excellent patient care 2. Improve hospitalist and PA job satisfaction and retention 3. Improve hospitalist and PA professional development

DESCRIPTION OF PROGRAM/INTERVENTION, INCLUDING ORGANIZATIONAL CONTEXT (E.G. INPATIENT VS. OUTPATIENT, PRACTICE OR COMMUNITY CHARACTERISTICS): A PA-Hospitalist Task Force comprised of three PAs and three hospitalist physicians was created at an affiliate hospital of a major urban academic center. The Task Force used an appreciative inquiry (AI) approach, through surveys and focus groups, to elicit the strengths of the group and harness them to achieve shared goals (defined above). To discover the best of "what is," MDs and PAs were prompted to reflect upon the strengths and behaviors of colleagues in an open-ended survey. To dream "what could be," participants were asked in focus groups to envision their ideal work environment. To design "what should be," Task Force members analyzed survey responses and focus group field notes for themes that were used to develop a set of Best Practice Guidelines. To "create what will be," guidelines were distributed to the group for implementation and a series of skills development workshops ensured successful implementation of best practices.

MEASURES OF SUCCESS (DISCUSS QUALITATIVE AND/OR QUANTITATIVE METRICS WHICH WILL BE USED TO EVALUATE PROGRAM/INTERVENTION): Qualitative data was collected via two surveys that evaluated the impact of the program on patient care, job satisfaction and professional development, motivation and meaning in work, and decision to continue employment at the institution.

FINDINGS TO DATE (IT IS NOT SUFFICIENT TO STATE FINDINGS WILL BE DISCUSSED ): A survey was sent out six months after the Best Practice Guidelines were disseminated. 17 MDs (74\%) and 11 PAs (55\%) responded. Participants noted that the new standardized handoff format improved patient care by facilitating efficient and accurate transitions of care and medication reconciliation. PA-led rounds fostered critical thinking, teaching, and improved communication between providers. PAs noted enhanced professional development through increased autonomy in clinical decisionmaking and communication with patients and providers. PAs and MDs noted a closer partnership in patient care and increased professional development with role optimization. PAs and MDs found enhanced meaning and motivation in work when surrounded by colleagues who drive positive change. Future areas of study include impact on patient care (HCAHP scores, length of stay), efficiency (PA clock-out times), job satisfaction (length of employment), and professional development (scholarly output).

KEY LESSONS FOR DISSEMINATION (WHAT CAN OTHERS TAKE AWAY FOR IMPLEMENTATION TO THEIR PRACTICE OR COMMUNITY?): Appreciative inquiry is a powerful tool that can be used within the healthcare setting to change culture and drive positive change. A collaborative approach can be used to optimize hospital medicine PA and MD clinical care delivery, meaning in work, and professional development, and can be translated to other fields. 
PATIENT CARE OUTCOMES FROM A RESIDENT SUBOXONE CLINIC

Melanie Woodward ${ }^{1}$; Deepa R. Nandiwada ${ }^{1}$; Marc Shalaby ${ }^{2}$; Benjamin Larson ${ }^{3} .{ }^{1}$ University of Pennsylvania, Philadelphia, PA; ${ }^{2}$ Perelman School of Medicine at the University of Pennsylvania, Philadelphia, PA; ${ }^{3}$ Main Line Health, Philadelphia, PA. (Control ID \#3181600)

STATEMENT OF PROBLEM OR QUESTION (ONE SENTENCE): Incorporating suboxone treatment into the resident clinic provides unique challenges of frequent handoffs, varying provider input into care decisions, and fragmented continuity in the patient-doctor relationship which may affect patient outcomes.

OBJECTIVES OF PROGRAM/INTERVENTION (NO MORE THAN THREE OBJECTIVES): The intent of the program is to treat opiate use disorder in an educational environment with treatment outcomes equivalent to those of primary care based programs.

DESCRIPTION OF PROGRAM/INTERVENTION, INCLUDING ORGANIZATIONAL CONTEXT (E.G. INPATIENT VS. OUTPATIENT, PRACTICE OR COMMUNITY CHARACTERISTICS): The START (Solutions To Addiction Recovery and Treatment) Clinic was embedded in a resident-based practice in late 2017 with four rotating attendings which attend on a 6 week rotation and residents which rotate in 1-2 week rotations. The half day clinic occurs weekly based on referrals from across the healthcare system with the goal of providing primary care and suboxone services. Policies are in place to ensure appropriate prescription and urine drug screen monitoring, address relapses, refer to mental health care, and screen for chronic illnesses. However, there is variation in how these policies are carried out amongst providers which may ultimately affect patient success.

MEASURES OF SUCCESS (DISCUSS QUALITATIVE AND/OR QUANTITATIVE METRICS WHICH WILL BE USED TO EVALUATE PROGRAM/INTERVENTION): We reviewed patient records of those seen in the START program to assess demographics, types of substance abuse, referral modality, and retention with appropriate urine drug screens. The number of relapses and attention to primary care treatment was secondarily measured. Given the unique nature of embedding the program within a resident clinic, special attention was paid to whether these measures were comparable to non-resident outcomes and whether there was unexpected variation in these parameters as a result of breaks in provider continuity.

FINDINGS TO DATE (IT IS NOT SUFFICIENT TO STATE FINDINGS WILL BE DISCUSSED ): In the first 14 months of the program, 39 patients were successfully inducted. 3 failed home inductions and did not return. Of those succesffuly inducted, $59 \%$ remain active in the program with average retention of 21.9 weeks and $35.8 \%$ retention at 24 weeks. $41 \%$ of patients experienced a relapse with an average of 4 appropriate urine drug screens prior to relapses. Variable responses to relapse included signing recommitment contracts, returning to weekly office visits, requiring mental health treatment, engaging partners/family members in care, and retaining wrappers. $88 \%$ of patients had routine HIV, HCV screening with 9 patients referred for initial HCV or HIV treatment. Patients received care from an average of 6 residents and 3 attendings.

KEY LESSONS FOR DISSEMINATION (WHAT CAN OTHERS TAKE AWAY FOR IMPLEMENTATION TO THEIR PRACTICE OR COMMUNITY?): The resident-based clinic with referral from a health system presents unique issues created by involvement of multiple providers which may lead to decreased rates of retention when compared to other primary care based programs that refer from within their own practice. However, the resident-embedded model has the capacity to increase the number of patients served by suboxone exponentially, caring for patients immediately and training waivered providers of the future. It will continue to grow in success with time and experience and should be considered for expansion to other residency programs.

PATIENT EXPERIENCE AND CULTURALLY COMPETENT CARE FROM THE PERSPECTIVE OF AFRICAN AMERICANS/ BLACKS IN DENVER, COLORADO

Beza Jobira $^{1,}{ }^{3}$; Terri Richardson ${ }^{3}$; Virginia Visconti ${ }^{2}$; Cerise Hunt ${ }^{2}, 3$. ${ }^{1}$ University of Colorado Denver, Denver, CO; ${ }^{2}$ Colorado School of Public Health, Denver, CO; ${ }^{3}$ Colorado Black Health Collaborative, Denver, CO. (Control ID \#3164479)

\section{STATEMENT OF PROBLEM OR QUESTION (ONE SENTENCE):}

African Americans/Blacks (Blacks) have higher rates of morbidity and mortality than other races and ethnicities, and a lack of culturally competent healthcare hinders effective healthcare delivery and increases the likelihood of poor clinical outcomes

OBJECTIVES OF PROGRAM/INTERVENTION (NO MORE THAN THREE OBJECTIVES): 1) Assess Black patients' experience of culturally competent care in outpatient primary care through facilitated focus group discussions 2) Conduct qualitative analysis of themes related to cultural competency 3) Disseminate patients' experience and perception of culturally competent care to healthcare providers

DESCRIPTION OF PROGRAM/INTERVENTION, INCLUDING ORGANIZATIONAL CONTEXT (E.G. INPATIENT VS. OUTPATIENT, PRACTICE OR COMMUNITY CHARACTERISTICS): This study used focus groups to understand patient experiences and perceptions of healthcare providers' cultural competency. Blacks that receive healthcare from three different primary care clinics settings- HMO, federally qualified health center, and center for uninsured patients - were recruited from the Denver Metropolitan area. A flyer was distributed for participation in community centers and an email outreach was done through a community organization. A total of 28 patients participated in one of the three focus groups.

MEASURES OF SUCCESS (DISCUSS QUALITATIVE AND/OR QUANTITATIVE METRICS WHICH WILL BE USED TO EVALUATE PROGRAM/INTERVENTION): All focus groups were facilitated by a trained research assistant using a semi-structured protocol, and were recorded and transcribed verbatim. Framework Analysis was used to analyze and identify convergent themes.

FINDINGS TO DATE (IT IS NOT SUFFICIENT TO STATE FINDINGS WILL BE DISCUSSED ): Patient experiences were influenced by the dynamic interaction between the healthcare system, clinic environment, and healthcare providers. At the system level, patient experiences were shaped cost, time spent with providers, and providers' cultural competence training. At the clinic level, location and workforce diversity affect patient experiences. At the healthcare provider level, patients highly value the quality of the patient-provider interaction. This interaction was not only influenced by the providers' quality of engagement, cultural competency and communication skills, but also by the patients' ability to advocate for their healthcare.

KEY LESSONS FOR DISSEMINATION (WHAT CAN OTHERS TAKE AWAY FOR IMPLEMENTATION TO THEIR PRACTICE OR COMMUNITY?): Regular diversity and inclusion training can support the enrichment of healthcare staff and providers' cultural competency. Integrating culturally competent care appropriate to patients' cultural backgrounds is imperative in improving patient satisfaction and engagement in healthcare. 
PAVING A ROAD TO RECOVERY: THREE-PILLARED APPROACH TO IMPROVING DISCHARGE REFERRAL PARTNERSHIPS FOR PATIENTS WITH SUBSTANCE USE DISORDER AND HOUSING INSTABILITY

Nicholas Iverson; Nancy Choi; James Hardy; Meher Singh; Jermaine Blakley; Sarah Imershein; Catherine Lau. UCSF, San Francisco, CA. (Control ID \#3177486)

STATEMENT OF PROBLEM OR QUESTION (ONE SENTENCE): In caring for patients with homelessness and substance use disorder (SUD), how can a health system optimize new discharge referral partnerships with community-based organizations (CBO)?

OBJECTIVES OF PROGRAM/INTERVENTION (NO MORE THAN THREE OBJECTIVES): 1) Improve provider education of CBO partner services. 2) Improve multidisciplinary team (MDT) workflow and satisfaction around discharge of complex patients to $\mathrm{CBO}$ partners. 3) Measure the return on investment (ROI) for a health system paying for extended outpatient services for hospitalized patients with SUD and homelessness.

DESCRIPTION OF PROGRAM/INTERVENTION, INCLUDING ORGANIZATIONAL CONTEXT (E.G. INPATIENT VS. OUTPATIENT, PRACTICE OR COMMUNITY CHARACTERISTICS): We describe three components of a partnership between an urban academic hospital's inpatient medicine service and a discharge referral partner Salvation Army Harbor Light (SAHL), a CBO that provides acute detoxification services and opportunity for long term residential treatment. One component is a needs assessment to examine provider understanding of the $\mathrm{CBO}$, followed by targeted education and visits with former patients. A second component improves direct communication between inpatient providers and $\mathrm{CBO}$ managers for safe transition of care and examines social worker (SW) satisfaction around discharges. A final component calculates the value equation for the health system to pay for a patient's stay at SAHL up to 30 days, while analyzing pre- and post- referral ED visits, length of stay, and 30-day readmission rates.

MEASURES OF SUCCESS (DISCUSS QUALITATIVE AND/OR QUANTITATIVE METRICS WHICH WILL BE USED TO EVALUATE PROGRAM/INTERVENTION): 1) Provider knowledge of services at the CBO. 2) SW satisfaction with providers regarding communication during referrals. 3) ROI to the health system by decreased ED visits, LOS, and 30-day readmissions.

FINDINGS TO DATE (IT IS NOT SUFFICIENT TO STATE FINDINGS WILL BE DISCUSSED ): Component 1: Provider survey ( $\mathrm{n}=33$ ) demonstrated that $54 \%$ of providers were not sure or were incorrect about key features of SAHL, and $67 \%$ did not feel confident discussing the benefits of SAHL with their patients. Component 2: SW satisfaction survey $(n=3)$ revealed that having providers talk with patients about the $\mathrm{CBO}$ prior to referral often made it more difficult to later discuss disposition with patients and requested a change in workflow. An open line of communication between providers and the $\mathrm{CBO}$ was established for complex patients, facilitated by SW discussion with the CBO. Component 3: From July 2017 to July 2018, 101 patients have been discharged to the SAHL. Readmission rates decreased from $38 \%$ to $19 \%$ and number of inpatient days decreased by 1.9 , resulting in a direct cost savings of $\$ 5,400$ per patient.

KEY LESSONS FOR DISSEMINATION (WHAT CAN OTHERS TAKE AWAY FOR IMPLEMENTATION TO THEIR PRACTICE OR COMMUNITY?): 1) Many inpatient providers do not understand the intricacies of $\mathrm{CBO}$ partner services to help patients make informed discharge decisions. Education and improvement of discharge workflow for the entire MDT are key to improving referrals and MDT satisfaction. 2) Safe discharges of more complex patients with homelessness and SUD can be coordinated by leadership establishing a direct line of provider-CBO communication. 3) Calculating value to the health system of investment in outpatient services for inpatients with homelessness and SUD demonstrated that these services can be cost-beneficial and improve patient outcomes.

\section{PEP IT UP: CATALYZING PRACTICE IMPROVEMENT}

Anuradha Phadke ${ }^{1}$; Jake Mickelsen ${ }^{2}$; Megan Mahoney ${ }^{3} .{ }^{1}$ Stanford University School if Medicine, Palo Alto, CA; ${ }^{2}$ Stanford Healthcare, Palo Alto, CA; ${ }^{3}$ Stanford University, Palo Alto, CA. (Control ID \#3185190)

STATEMENT OF PROBLEM OR QUESTION (ONE SENTENCE): How do we create an environment that supports front line teams' engagement in continuous quality improvement?

OBJECTIVES OF PROGRAM/INTERVENTION (NO MORE THAN THREE OBJECTIVES): 1) Support front line individuals and teams in conducting rapid-cycle quality improvement projects 2) Grow improvement capability by educating participants in quality improvement fundamentals 3) Align improvement efforts with division strategic objectives while also addressing front line needs

DESCRIPTION OF PROGRAM/INTERVENTION, INCLUDING ORGANIZATIONAL CONTEXT (E.G. INPATIENT VS. OUTPATIENT, PRACTICE OR COMMUNITY CHARACTERISTICS): Primary Care Performance Enhancement Program (PC-PEP) is a structured quality improvement program within the primary care division at our academic medical center. Its aim is to provide accountability and support for an individual or small team working in one of 13 academic medical center primary care clinics to perform a quality improvement project. Projects are scoped to be completed in less than 8 weeks. Key elements of program structure are as follows: 1) A project is identified in one of two ways: a) a participant with an improvement idea seeks approval to participate from clinic leadership or b) clinic leadership identifies an area of strategic importance for which they solicit a volunteer to lead a project 2) Participants receive a problem-solving guide and coach to assist them with their project 3) Participants present their projects in progress at a thirty-minute weekly teleconference check-in. Check-ins serve as an opportunity for participants to ask questions and engage in brief teaching about quality improvement 4) At the close of projects, participants submit completed project materials, including benefits capture information, to the program team 5) Program and clinic leadership celebrate participants at a quarterly graduation.

MEASURES OF SUCCESS (DISCUSS QUALITATIVE AND/OR QUANTITATIVE METRICS WHICH WILL BE USED TO EVALUATE PROGRAM/INTERVENTION): Quantitative measures of program success include: 1) benefits such as annualized time savings, financial saving, and care-delivery improvement 2) percentage of primary care staff and faculty engaged in a PC-PEP project 3) enhanced participant comfort with quality improvement as demonstrated by a pre-post survey. Qualitative measures of program success include participant comments on project closing questionnaire documents.

FINDINGS TO DATE (IT IS NOT SUFFICIENT TO STATE FINDINGS WILL BE DISCUSSED ): Over the 11 months since this program's inception, PC-PEP has incubated 24 projects across 12 primary care clinics. Over 30 medical assistants and 10 physicians have participated. Project topics have ranged from operations topics such as improving the process of fax management to population health topics such as improving tobacco screening and counseling. Annualized improvements include 6,600 hours in time savings for staff and physicians, approximately $\$ 72,000$ in annualized 
financial benefits for clinics, and improved care delivery for 8200 patients. Comments from project participants as noted on project closing documents include improved understanding of quality improvement approaches as well as enhanced participant self-efficacy at problem solving.

KEY LESSONS FOR DISSEMINATION (WHAT CAN OTHERS TAKE AWAY FOR IMPLEMENTATION TO THEIR PRACTICE OR COMMUNITY?): Institutions can catalyze front line problem solving by creating a structure of supportive leadership, trained coaches, and regular check-ins.

PICC-ING AWAY THE WASTE: A MULTIDISCLIPINARY APPROACH TO VASCULAR ACCESS IN PATIENTS WITH CHRONIC KIDNEY DISEASE

June Kampangkaew; Nathan C. Nowalk; Maulin Shah; Charlie Lan. Baylor College of Medicine, Houston, TX. (Control ID \#3186219)

STATEMENT OF PROBLEM OR QUESTION (ONE SENTENCE): At the Michael E DeBakey VA Medical Center, the current process for obtaining renal approval for placement of Peripherally Inserted Central Catheters (PICCs) in patients with Chronic Kidney Disease (CKD) 3-5 is complicated by work task redundancy and delays in PICC placement.

OBJECTIVES OF PROGRAM/INTERVENTION (NO MORE THAN THREE OBJECTIVES): To review PICC approval patterns in patients with CKD 3-5 at MEDVAMC To reduce redundant work in the current PICC approval process to improve provider experience To reduce delays in PICC placement in patients with CKD 3-5

DESCRIPTION OF PROGRAM/INTERVENTION, INCLUDING ORGANIZATIONAL CONTEXT (E.G. INPATIENT VS. OUTPATIENT, PRACTICE OR COMMUNITY CHARACTERISTICS): Michigan Appropriateness Guide for Intravenous Catheters (MAGIC) was developed in response to the increasing use of peripherally inserted central catheters (PICCs) to help physicians choose the appropriate catheter for the indication. In June 2017, the Michael E Debakey Veteran Affairs Medical Center (MEDVAMC) implemented MAGIC through a new vascular access team (VAT). The responsibilities of VAT include teaching nursing staff proper PICC maintenance and independently determining the appropriateness of type of catheter placed when consulted for access. This latter role ultimately reduced the number of PICCs placed. In patients with Chronic Kidney Disease (CKD) 3-5 who may be a candidate for dialysis in the future, there is particular concern that PICCs can damage large veins and thus make them unusable for dialysis access in the future. At the MEDVAMC, before a PICC line can be placed for all patients with CKD 3-5, the current process requires: an order for PICC, VAT confirming appropriate indication, and renal consultation for documented approval in electronic medical record. We used a multidisciplinary team approach (renal, medicine, VAT) to evaluate the current process using quality improvement (QI) tools to improve work flow, reduce redundancy, and to decrease time to PICC placement.

MEASURES OF SUCCESS (DISCUSS QUALITATIVE AND/OR QUANTITATIVE METRICS WHICH WILL BE USED TO EVALUATE PROGRAM/INTERVENTION): Number of hours to place PICC Number of tasks required by team requesting PICC Surveys assessing provider burnout

FINDINGS TO DATE (IT IS NOT SUFFICIENT TO STATE FINDINGS WILL BE DISCUSSED ): Between September 2017 and October 2018, MEDVAMC cared for 23 patients who had an indication for PICC with CKD 3-5. Seventy-eight percent $(n=18)$ were age $>65$ years old. Indications for PICC included $57.5 \%(n=13)$ for vasopressors or inotropes, $13.0 \%(n=3)$ for venous access, $17.4 \%(\mathrm{n}=4)$ for long-term intravenous (IV) antibiotics,
$4.3 \%(\mathrm{n}=1)$ for chemotherapy. One year all-cause mortality rates were $60.9 \%$ $(n=14)$. After consultation with renal, $100 \%(n=23)$ of PICCs were approved. To date, no patients from our cohort are undergoing evaluation for dialysis access.

KEY LESSONS FOR DISSEMINATION (WHAT CAN OTHERS TAKE AWAY FOR IMPLEMENTATION TO THEIR PRACTICE OR COMMUNITY?): Although there are likely gaps in physician knowledge and practice for PICCs, the VAT has successfully modified requests for PICCs to midline or ultrasound-guided IV catheters. This autonomy has decreased the total number of PICCs placed in all patients, including patients with CKD 3-5. Post-implementation process analyses can identify other areas of potential waste, which can be later reduced by a multi-disciplinary intervention.

PREDICTING RISK OF CLINICAL DETERIORATION: A CLOSER LOOK AT RESDIENT HANDOFFS AND RAPID RESPONSE

Khaled Sanber; Rachael Jacobson; Sarah Tuthill; Bhavika Kaul; Vagish Hemmige; Elizabeth Guy; Chirayu Shah; Stephen B. Greenberg. Baylor College of Medicine, Houston, TX. (Control ID \#3187119)

STATEMENT OF PROBLEM OR QUESTION (ONE SENTENCE): A key component of the I-PASS handoff includes assigning each patient an illness severity, which is categorized into three groups in order of increasing severity: "stable", "watcher", "unstable" (renamed as "star" in this study). To our knowledge, the utility of these categories as a means of predicting clinical deterioration has not been evaluated.

OBJECTIVES OF PROGRAM/INTERVENTION (NO MORE THAN THREE OBJECTIVES): The main objective of this study was to evaluate whether the illness severity category was predictive of overnight clinical deterioration, defined as a rapid response team (RRT) activation, after resident handoff.

DESCRIPTION OF PROGRAM/INTERVENTION, INCLUDING ORGANIZATIONAL CONTEXT (E.G. INPATIENT VS. OUTPATIENT, PRACTICE OR COMMUNITY CHARACTERISTICS): This study is a retrospective chart review of in-patients at Ben Taub Hospital (Houston, Texas) who were under the care of internal medicine teaching teams during a 12-month period (April 2016 through March 2017). Patients who had an RRT activation between 6PM and 7AM (after resident hand-off and during the care of a cross-covering night team) were analyzed. The data were collected by accessing a previously developed list of rapid response events documented by rapid response nurses. Resident-generated I-PASS Excel handoff sheets and the electronic medical records for all patients with RRTs during the 12-month study period were retrospectively reviewed. Prior to starting residency, BCM residents received training on performing an effective handoff using the I-PASS bundle including patient classification based on perceived clinical stability.

MEASURES OF SUCCESS (DISCUSS QUALITATIVE AND/OR QUANTITATIVE METRICS WHICH WILL BE USED TO EVALUATE PROGRAM/INTERVENTION): A total of 5,176 patients met inclusion criteria. For these patients, 28,235 handoffs and 98 RRTs were analyzed. Of the 98 RRTs, 5 (5\%) patients were labeled as "star", 35 (36\%) as "watcher", and $58(59 \%)$ as "stable". When analyzing all patient handoffs, $377(1.3 \%)$ were categorized as "star", 5,309 (19\%) as "watcher", and 22,549 $(80 \%)$ as "stable". When compared to patients listed as "stable" during handoff, patients listed as "watcher" had an odds ratio of 2.6 (95\% CI 1.7$3.9, \mathrm{p}<0.001)$ and patients listed as star had an odds ratio of 5.2 (95\% CI 2.1$13.1, \mathrm{p}<0.001)$ of an overnight RRT activation. Overall hospital mortality for patients with an RRT was $28 \%$. 
FINDINGS TO DATE (IT IS NOT SUFFICIENT TO STATE FINDINGS WILL BE DISCUSSED ): A modified I-PASS handoff bundle was effective in predicting which patients on an internal medicine teaching service were at risk of clinical deterioration after patient handoff. This helps the night cross-covering team focus care on high-risk patients who are more likely to require closer monitoring and intervention. We have instituted new interventions, including modifications to the I-PASS handoff bundle, that are aimed at enhancing the ability of residents to identify high-risk patients and improving patient outcomes. This will be followed by another round of data collection and analysis.

KEY LESSONS FOR DISSEMINATION (WHAT CAN OTHERS TAKE AWAY FOR IMPLEMENTATION TO THEIR PRACTICE OR COMMUNITY?): A modified I-PASS handoff bundle was effective in predicting which patients on an internal medicine teaching service were at risk of clinical deterioration after patient handoff. This helps the night crosscovering team focus care on high-risk patients who are more likely to require closer monitoring and intervention.

PRESCRIPTION REPORTING WITH IMMEDIATE MEDICATION UTILIZATION MAPPING (PRIMUM) IMPLEMENTATION : DO NO HARM

Animita Saha ${ }^{1}$; Rachel B. Seymour ${ }^{2} .{ }^{1}$ Atrium Health, Charlotte, NC; ${ }^{2}$ atrium Health, Charlotte, NC. (Control ID \#3175511)

STATEMENT OF PROBLEM OR QUESTION (ONE SENTENCE): An important step to reduce the number of people who are associated with prescription medication abuse, misuse and diversion is identification of at risk patients prior to narcotics prescription.

OBJECTIVES OF PROGRAM/INTERVENTION (NO MORE THAN THREE OBJECTIVES): To assess the changes in prescribers' behavior when presented with immediate feedback through an electronic alert system based on searchable objective data on EMR on potential misuse of prescription narcotics in acute and chronic pain patients..

DESCRIPTION OF PROGRAM/INTERVENTION, INCLUDING ORGANIZATIONAL CONTEXT (E.G. INPATIENT VS. OUTPATIENT, PRACTICE OR COMMUNITY CHARACTERISTICS): The study was conducted at Atrium Health, Charlotte, NC. We used PRIMUM, which is a computerized alert system to identify patients at high risk for misuse, abuse, and diversion of prescription narcotics and to provide critical information to the prescriber at the point of care to help clinical decision making. Evidence-based triggers were built in to EMR to fire within when a prescription for a narcotic was initiated. The following indications prompted the alert to the prescriber: 1. Current prescription of a narcotic with $>50 \%$ remaining expected; 2 . $2+$ visits to $\mathrm{ED}$ or Urgent Care with onsite treatment for narcotics (excluded visits led to admission) within past 30 days; 3. $3+$ prescriptions for narcotics within past 30 days and 4. Previous presentation for overdose; 5. Positive screen for alcohol, cocaine and marijuana MEASURES OF SUCCESS (DISCUSS QUALITATIVE AND/OR QUANTITATIVE METRICS WHICH WILL BE USED TO EVALUATE PROGRAM/INTERVENTION): Baseline data were collected for one month by running the alert algorithm silently (no alert) followed by five months of live data when the prescribers received the alerts. Following outcomes were measured: 1 . Number of alerts; 2 . Number of cancellations of narcotic prescriptions following alert; and 3. Overrides of the alert system. FINDINGS TO DATE (IT IS NOT SUFFICIENT TO STATE FINDINGS WILL BE DISCUSSED ): During the test period, our system had $5,133,278$ encounters. For opioid $(n=282,870)$, the alert was triggered in $71,498(25.3 \%)$ cases, of which $13.7 \%$ were temporarily cancelled, however, ultimately $5.4 \%(\mathrm{n}=3,860)$ were resulted in no prescription. For BZD $(n=96,999)$, the alert fired in $21,810(22.5 \%)$ cases, of which $23.4 \%$ were initially cancelled $(n=5,104)$ and $13.1 \%$ resulted in no prescription $(n=2,857)$. Altogether, cancellations resulted a reduction of 6,717 prescriptions. Rates of cancellation and no prescription significantly differed by specialty $(\mathrm{p}<0.0001)$. Cancellation for opioid prescription was most when patients received more than 3 prescriptions over last 30 days $(\mathrm{p}<0.0001)$ and for BZD, it was early refill request $(\mathrm{p}<0.0001)$.

KEY LESSONS FOR DISSEMINATION (WHAT CAN OTHERS TAKE AWAY FOR IMPLEMENTATION TO THEIR PRACTICE OR COMMUNITY?): The PRIMUM-generated alert system presented an opportunity to utilize EMR to provide targeted clinical-decision support for appropriate prescribing. This platform laid the groundwork for further clinical decision support, which included evidence-based guidelines for acute and chronic pain management, including risk assessment prior to prescribing and providing a streamlined method to drug monitoring programs. There was a high rate of cancellation still a huge number of patients with risk factors received a prescription. Although these may have been clinically indicated this system presents an opportunity to train providers as many alerts were ignored.

PRIMARY CARE \&AMP; COMMUNITY HEALTH WORKER BASED QUALITY IMPROVEMENT PROGRAM IMPROVES HYPERTENSION OUTCOMES IN URBAN INDIAN SLUMS

Turja Chakrabarti'; Debolina Pramanik ${ }^{2}$; Arpan Pramanik ${ }^{2}$; Karthik Man$\mathrm{na}^{3}$; Sourav Mondal ${ }^{3}$; Paul O'Rourke ${ }^{4}$. ${ }^{1}$ John Hopkins University School of Medicine, Baltimore, MD; ${ }^{2}$ Medical College Kolkata, Kolkata, India; ${ }^{3}$ Pratit International, Kolkata, India; ${ }^{4}$ Johns Hopkins University, Baltimore, MD. (Control ID \#3185843)

STATEMENT OF PROBLEM OR QUESTION (ONE SENTENCE): Hypertension management is especially challenging in the context of extreme poverty found in Urban Indian Slums.

OBJECTIVES OF PROGRAM/INTERVENTION (NO MORE THAN THREE OBJECTIVES): The purpose of this Quality Improvement (QI) program serves to build a multidisciplinary effort to manage hypertension in multiple slum communities. The program aims to combine evidence-based pharmacotherapy with counselling on lifestyle modifications to bring patients living in extreme poverty to meet the American College of Cardiology (ACC) defined blood pressure goal < 130/80 $\mathrm{mmHg}$.

DESCRIPTION OF PROGRAM/INTERVENTION, INCLUDING ORGANIZATIONAL CONTEXT (E.G. INPATIENT VS. OUTPATIENT, PRACTICE OR COMMUNITY CHARACTERISTICS): A mobile health clinic that provides routine primary care in three underserved slum areas in Kolkata, India was selected for a QI program for hypertension management. The population of these slum communities live in extreme poverty with an average per capita daily income of $\$ 1.05$ US. A team comprised of 2 Primary Care Physicians (PCP) and 5 Community Health Workers (CHW) participated in this QI program which involved 59 patients. PCPs and CHWs underwent a workshop on hypertension guidelines and specialized counseling for patients with hypertension. PCPs treated hypertension in clinic with 4 available drug classes (Thiazide diuretics, ACE Inhibitors, ARBs and CCBs) while also providing initial guidance and counseling on lifestyle modifications. Patients were routinely followed up in clinic at 1 to 2 month intervals. CHWs visited hypertensive patients in their homes on a weekly basis to measure blood pressure, teach medication management and counsel on dietary and lifestyle modification. CHWs also reported back to the PCP from the patient's home on abnormal blood 
pressure readings, and if deemed necessary, medication changes were made in real time prior to clinic visits.

MEASURES OF SUCCESS (DISCUSS QUALITATIVE AND/OR QUANTITATIVE METRICS WHICH WILL BE USED TO EVALUATE PROGRAM/INTERVENTION): Key outcome measures being studied include changes in blood pressure, changes in weight, adherence to medications, adoption of a low sodium diet $(<2 \mathrm{~g} /$ day $)$, cardiovascular exercise ( $>30 \mathrm{mins} /$ day), tobacco use status, and alcohol use status. A checklist survey is completed by CHWs at each home visit recording these outcomes. FINDINGS TO DATE (IT IS NOT SUFFICIENT TO STATE FINDINGS WILL BE DISCUSSED ): 59 patients with blood pressures > 130/ 80 were selected to be part of this quality improvement program. Average age of participants was 52.6 years $(\min =19$, median $=42.5 \max =80)$. The group comprised of $74.6 \%$ females and $25.4 \%$ males. Average blood pressure on enrollment was $\mathrm{SBP}=148.81 \mathrm{mmHg}$ and $\mathrm{DBP}=84.6 \mathrm{mmHg}$. At 6 months, average blood pressure was $\mathrm{SBP}=126.78 \mathrm{mmHg}$ and $\mathrm{DBP}=$ $81.19 \mathrm{mmHg}$. Mean reduction in systolic blood pressure was $22.03 \mathrm{mmHg}$. At the end of 6 months, $50.8 \%$ of patients within the quality improvement program had blood pressures at ACC goal of $<130 / 80$.

KEY LESSONS FOR DISSEMINATION (WHAT CAN OTHERS TAKE AWAY FOR IMPLEMENTATION TO THEIR PRACTICE OR COMMUNITY?): In the context of extreme poverty, this multidisciplinary community based approach led to a mean reduction in SBP of $22.03 \mathrm{mmHg}$ and led to $50.8 \%$ of patients to be within the ACC blood pressure goal. These findings should encourage development of similar programs in resource deprived settings globally.

PROMOTING HIGH-VALUE PRACTICE BY STANDARDIZING COMMUNICATION BETWEEN THE HOSPITALIST AND PRIMARY CARE PROVIDER DURING HOSPITALIZATION

Marwa Moussa $^{1}$; Carrie Mahowald ${ }^{1}$; Charles Okamura ${ }^{1}$; Olena ksovreli ${ }^{1}$; Myint $\mathrm{Aye}^{2}$; Himali Weerahandi ${ }^{1} .{ }^{1}$ NYU Langone, Staten Island, NY; ${ }^{2}$ NYU Langone Hospitals- Brooklyn, Brooklyn, NY. (Control ID \#3167861)

\section{STATEMENT OF PROBLEM OR QUESTION (ONE SENTENCE):} The increasing complexity of admitted patients, shorter hospital stays and post-acute care adverse events demand a more sophisticated and effective coordination of care between hospitalists and Primary care providers (PCPs). OBJECTIVES OF PROGRAM/INTERVENTION (NO MORE THAN THREE OBJECTIVES): 1 . Standardizing communication between Hospitalist and PCP during hospitalization will lower the rate of readmission due to lack of PCP follow up and post-acute care adverse events. 2. Implementing this practice into our daily workflow will improve PCP satisfaction and increase referrals to our institution.

DESCRIPTION OF PROGRAM/INTERVENTION, INCLUDING ORGANIZATIONAL CONTEXT (E.G. INPATIENT VS. OUTPATIENT, PRACTICE OR COMMUNITY CHARACTERISTICS): We reviewed a root-cause survey of 30 day readmissions between 1/2018$4 / 2018$ as well as readmission rates for each of our hospitalists. We surveyed our PCPs' satisfaction with communication experiences with our hospitalist group. Finally, we conducted a semi-structured interview of the hospitalist with the lowest readmission (8\% vs 12\% average for other hospitalists) and highest PCP satisfaction rates, Dr. A, to develop best practices for closed loop communication. Based on this data, we designed a protocol and piloted on 5/1/2018, where the hospitalist contacts the PCP via phone call on admission and delivers a discharge narrative to the PCP via our EMR's routing capability. We used a trackable smart phrase to document the communication. For the prospective phase, we will operationalize these best practices in a study group, Family Health Center PCPs. A control group (community PCPs) will receive usual practice.

MEASURES OF SUCCESS (DISCUSS QUALITATIVE AND/OR QUANTITATIVE METRICS WHICH WILL BE USED TO EVALUATE PROGRAM/INTERVENTION): We will compare readmission rates between the study group and control group, monitoring the proportion and absolute number of readmissions attributed to no PCP follow up or medication errors. Follow up satisfaction surveys will be sent to the PCPs 6 months after our revised communication practice. Finally, we will monitor the hospitalists' compliance with the smart phrase.

FINDINGS TO DATE (IT IS NOT SUFFICIENT TO STATE FINDINGS WILL BE DISCUSSED ): A review of our institution's 30 day readmissions between 1/2018-4/2018 found that $19 \%$ were attributed to lack of PCP/outpatient provider follow-up. Surveys of our community PCPs showed $70 \%$ reported being contacted by the hospitalist group in less than $25 \%$ of the time. Results from Dr. A's interview revealed that after her encounter with the patient, she calls the patient's PCP highlighting the admitting diagnosis, significant events, pertinent labs, imaging and medications. Dr. A then delivers a discharge narrative to the PCP on the day of discharge highlighting any medication changes, incidental findings and follow up. On a random audit of 100 charts between 5/1/2018-10/30/2018 our preliminary data show that there was $88 \%$ compliance with using the smart phrase by the hospitalists.

KEY LESSONS FOR DISSEMINATION (WHAT CAN OTHERS TAKE AWAY FOR IMPLEMENTATION TO THEIR PRACTICE OR COMMUNITY?): Using a "positive deviance" approach, we identified best practices for hospitalist-PCP closed loop communication to develop an intervention to improve this aspect of care. If we are successful in reducing readmission rates and improving PCP satisfaction, we will expand to all of our PCPs and ultimately expand to other services to implement this program as best practice.

PROTON PUMP INHIBITOR STEWARDSHIP: DEPRESCRIBING PROTON PUMP INHIBITORS IN A PRIMARY CARE CLINIC Cecilia Y. Cai ${ }^{1}$; Chiemeziem Eke ${ }^{1}$; Niharika Mallepally ${ }^{1}$; David Szafron ${ }^{1}$; Andrew S. Brown ${ }^{1}$; Derek Lin ${ }^{1}$; Jerry Wong ${ }^{2}$; Erica Lescinskas ${ }^{1}$; Lee Lu ${ }^{1}$. ${ }^{1}$ Baylor College of Medicine, Houston, TX; ${ }^{2}$ Harris Health System, Houston, TX. (Control ID \#3159428)

\section{STATEMENT OF PROBLEM OR QUESTION (ONE SENTENCE):}

The proton pump inhibitors (PPIs) are commonly used for treatment of upper gastrointestinal disorders; however, the rise in PPI prescriptions has led to increased prevalence of adverse drug effects and increased cost of therapy. OBJECTIVES OF PROGRAM/INTERVENTION (NO MORE THAN THREE OBJECTIVES): 1. Identify rate of inappropriate PPI prescriptions at a resident-run primary care clinic. 2. Implement a PPI taper order set. 3. Educate residents on appropriate indications for PPI and reduce the rate of inappropriate PPI prescriptions.

DESCRIPTION OF PROGRAM/INTERVENTION, INCLUDING ORGANIZATIONAL CONTEXT (E.G. INPATIENT VS. OUTPATIENT, PRACTICE OR COMMUNITY CHARACTERISTICS): We conducted an observational chart review of PPI prescriptions over a 14-month period from January 2017 to August 2018 at an Internal Medicine resident-run primary care clinic. Medical records of 315 patients prescribed PPIs were reviewed for specific indications and duration of therapy. Intervention entailed implementation of a four-week PPI taper order set to the clinic electronic health record, in addition to provider education through flyers and emails that highlighted PPI indications and consequences of long- 
term use. During analysis of prescribed PPIs, usage was categorized as appropriate or inappropriate based on criteria set by Gastroenterology clinical guidelines.

MEASURES OF SUCCESS (DISCUSS QUALITATIVE AND/OR QUANTITATIVE METRICS WHICH WILL BE USED TO EVALUATE PROGRAM/INTERVENTION): We analyzed the number of PPI prescriptions per month, indications for prescriptions, and the rate of inappropriate prescriptions per month.

FINDINGS TO DATE (IT IS NOT SUFFICIENT TO STATE FINDINGS WILL BE DISCUSSED ): 70 patient charts were analyzed over six months pre-intervention, and 245 charts were analyzed over eight months post-intervention. Monthly number of prescriptions fluctuated; however, the rate of inappropriate PPI prescriptions has steadily declined since beginning of intervention. Pre-intervention, a substantial number of PPIs were inappropriately prescribed (77\%). Of these prescriptions, a majority (67\%) was for gastroesophageal reflux disease (GERD), followed by nonsteroidal antiinflammatory drug (NSAID) use (9\%), and unspecified dyspepsia (7\%). The most common mistake in GERD and dyspepsia prescriptions was providing a several month supply and refills without close interval followup to assess treatment efficacy. Preexisting NSAID use as documented reason for PPI prescription for ulcer prophylaxis was commonly inappropriate due to young patient age with low bleeding risk. Post-intervention, inappropriate PPI prescriptions decreased to $53 \%$, of which $61 \%$ was attributed to GERD, $5 \%$ to NSAID use, and $8 \%$ to dyspepsia.

KEY LESSONS FOR DISSEMINATION (WHAT CAN OTHERS TAKE AWAY FOR IMPLEMENTATION TO THEIR PRACTICE OR COMMUNITY?): Many resident physicians lack adequate knowledge on indications for long-term PPI use and unintentionally over-prescribe. With a structured deprescribing tool and enhanced awareness, residents were able to reduce inappropriate prescriptions significantly. Primary care providers can use a similar intervention at their practice to reduce unnecessary PPI prescriptions.

PROVIDER BARRIERS TO LINKING PATIENTS WITH COMMUNITY HEALTH COACHES AT AN URBAN CLINIC

Kun Chen ${ }^{1}$; Tamara Goldberg ${ }^{2} .{ }^{1}$ Mt Sinai St Luke's/West, New York, NY; ${ }^{2}$ Mount Sinai St. Lukes West, New York, NY. (Control ID \#3185823)

\section{STATEMENT OF PROBLEM OR QUESTION (ONE SENTENCE):}

Though clinical-community partnerships have increasingly emerged to address social determinant barriers for patients, best practices to engage providers and patients with such services are not well studied.

OBJECTIVES OF PROGRAM/INTERVENTION (NO MORE THAN THREE OBJECTIVES): To understand provider-perceived barriers in engaging patients with community health coach services. To increase referrals to community health coaching services.

DESCRIPTION OF PROGRAM/INTERVENTION, INCLUDING ORGANIZATIONAL CONTEXT (E.G. INPATIENT VS. OUTPATIENT, PRACTICE OR COMMUNITY CHARACTERISTICS): The Ryan Adair Community Health Center is a Federally Qualified Health Center (FQHC) in Manhattan that serves as a training site for our internal medicine residents who rotate through the clinic in quartiles every two weeks. The center recently partnered with a local community-based organization (CBO) which provides free community health coaching services to patients with common chronic conditions. A resident physician-champion was selected to spend time with the CBO and focus on ways to create awareness of this service among resident providers. To increase referrals, the physician-champion disseminated information about the $\mathrm{CBO}$ services and referral instructions via email reminders, postings in exam rooms, and intermittent verbal announcements during pre-clinic huddles. We then tracked referrals to the $\mathrm{CBO}$ over a six month period and surveyed residents about their awareness of the services offered and referral process.

MEASURES OF SUCCESS (DISCUSS QUALITATIVE AND/OR QUANTITATIVE METRICS WHICH WILL BE USED TO EVALUATE PROGRAM/INTERVENTION): The number of referrals generated by providers to the community-based organization was tracked over a six month period. Provider-perceived barriers were assessed via survey administration.

FINDINGS TO DATE (IT IS NOT SUFFICIENT TO STATE FINDINGS WILL BE DISCUSSED ): Over the course of a six-month period, five referrals were generated from our clinic to the CBO. 69\% (22 out of 32) of resident providers completed the survey. $45 \%$ of respondents $(n=10)$ were unaware of the $\mathrm{CBO}$ and services offered, $55 \%(\mathrm{n}=12)$ did not know the criteria for a referral, and $25 \%(n=5)$ reported making referrals to the CBO. Providers noted that the most common reasons for patient refusal of services when offered was patient preference to try to manage their disease on their own and perceived time commitment.

KEY LESSONS FOR DISSEMINATION (WHAT CAN OTHERS TAKE AWAY FOR IMPLEMENTATION TO THEIR PRACTICE OR COMMUNITY?): The data above highlights that provider unawareness is a key barrier in connecting patients to community-based resources. Neither email reminders nor postings in clinic were high-yield contributors in generating referrals, as evidenced by the number of resident providers who were either unaware of the $\mathrm{CBO}$ or did not know the criteria for a referral. Key next steps include engaging other members of the clinic team in advocating for the $\mathrm{CBO}$ within the daily work flow, ensuring consistency of daily reminders at all huddles, and addressing reasons for patient refusal when a referral is offered.

QUALITY IMPROVEMENT IN HOSPITAL AT HOME: AN INTERDISCIPLINARY AND RAPID IMPROVEMENT APPROACH FOR A NOVEL MODEL OF CARE

Pamela Saenger; Claire Davenport; Elisse Catalan; Sara Lubetsky; Diana Motti; Katherine Ornstein; Linda DeCherrie. Icahn School of Medicine at Mount Sinai, New York, NY. (Control ID \#3185411)

STATEMENT OF PROBLEM OR QUESTION (ONE SENTENCE): In implementing an innovative home-based care model, Hospitalization at Home $(\mathrm{HaH})$ programs face the dual challenge of ensuring high quality patient care as well as seamless coordination within the interdisciplinary team and with contracted service vendors (CSVs), who perform integral functions.

OBJECTIVES OF PROGRAM/INTERVENTION (NO MORE THAN THREE OBJECTIVES): 1. Develop an intuitive adverse event and incident reporting tool 2. Engage interdisciplinary team members in identifying and addressing key systems issues 3. Track our progress and report findings

DESCRIPTION OF PROGRAM/INTERVENTION, INCLUDING ORGANIZATIONAL CONTEXT (E.G. INPATIENT VS. OUTPATIENT, PRACTICE OR COMMUNITY CHARACTERISTICS): $\mathrm{HaH}$ provides hospital-level care to patients in their homes. Our team provides care alongside CSVs who assist with nursing, labs, imaging, durable medical equipment, medication and oxygen delivery, and patient transportation. After discharging patients from the acute hospital episode, we follow them clinically for a short "plus" period. We developed a customized Web-based Incident Reporting System (WIRS) for the team to report both 
adverse clinical events as well as communication lapses, barriers to efficiency, and deviations from protocols. A Quality Improvement (QI) team member reviewed each incident report (IR), put an action plan in motion, and then reviewed it during a weekly QI workgroup; action plans included one or more of the following: plan-do-study-act (PDSA) cycle, team huddle, case review, Root Cause Analysis (RCA), or change in protocol. Summaries and longitudinal trends were presented to the larger team monthly.

MEASURES OF SUCCESS (DISCUSS QUALITATIVE AND/OR QUANTITATIVE METRICS WHICH WILL BE USED TO EVALUATE PROGRAM/INTERVENTION): All team members were oriented to the goals of incident reporting, the culture of non-blame, and trained how to access and submit a report. The QI workgroup met weekly to review new IRs and new or previously developed action plans. We presented qualitative and quantitative summaries of our work monthly to the larger team.

FINDINGS TO DATE (IT IS NOT SUFFICIENT TO STATE FINDINGS WILL BE DISCUSSED ): 194 IRs were submitted between 9/2015 and 12/2018. IRs per HaH episode increased 10-fold after we switched from email or hard copy reports to a web-based platform (2.42 vs 0.23$)$. While MDs submitted the largest share (49\%), $20 \%$ were submitted by NPs or RNs, and $17 \%$ were submitted by other staff. $27 \%$ of events occurred during the "plus" period. IRs were most often related to CSV or communication issues (37\% and 26\%, respectively). Clinical events (e.g. falls) were less common (5.7\%). Despite an increased patient census, IRs related to communication lapses, DME delivery, patient transportation, and infusions declined from July to December 2018.

KEY LESSONS FOR DISSEMINATION (WHAT CAN OTHERS TAKE AWAY FOR IMPLEMENTATION TO THEIR PRACTICE OR COMMUNITY?): Our WIRS is the cornerstone of our program QI, allowing our team to investigate adverse clinical outcomes and CSV issues alike, identify underlying systems issues, and act quickly to address them. Other $\mathrm{HaH}$ programs and any care model that leverages CSV services may benefit from implementing a customized WIRS with near real time reviews by a core QI team who are empowered to rapidly implement changes. Interdisciplinary collaboration and regular reporting to stakeholders solidify buy-in and continued participation.

RAMP (REAL TIME ANALYTICS MONITORING PLATFORM): USING AND DISPLAYING PREDICTIVE MODELING AND CARE COORDINATION FOR PATIENT CARE MANAGEMENT

William R. Clay; John Voss; John E. Ainsworth; Joel M. Schectman; Marissa McKay; Andrew Barros; Megan Pierce; Valentina Baljak. University of Virginia, Charlottesville, VA. (Control ID \#3180640)

STATEMENT OF PROBLEM OR QUESTION (ONE SENTENCE): There is a broad need to improve the efficiency of patient evaluation, acuity status assessment and care resource allocation in the inpatient setting. OBJECTIVES OF PROGRAM/INTERVENTION (NO MORE THAN THREE OBJECTIVES): 1. Provide physicians and nurses with new real-time patient care decision-making tools 2. Overcome inherent EMR limitations for developing, testing, and deploying complex predictive models DESCRIPTION OF PROGRAM/INTERVENTION, INCLUDING ORGANIZATIONAL CONTEXT (E.G. INPATIENT VS. OUTPATIENT, PRACTICE OR COMMUNITY CHARACTERISTICS): The Real-time Analytics and Modeling Platform (RAMP) was developed as a tool for aggregating and displaying trends in real-time inpatient acuity and for deploying complex predictive models. RAMP extracts current patient information from the electronic medical record (EMR) and physiologic monitoring interfaces, uses this information as inputs to predictive models, and displays the information to a monitor or mobile device organized by patient care team, unit or floor. The system is not restricted by model type, quantity, or complexity. Data visualization can be built into RAMP and/or RAMP can write data back to the EMR. Our current implementation of RAMP displays a map of a floor or unit with colored patient icons representing patient status. When patients of interest are scattered across geographic units, patient scores and icons can be viewed in list format. Individual patients can be selected by mouse click or touch screen to show current patient data and 48 hour trends. MEASURES OF SUCCESS (DISCUSS QUALITATIVE AND/OR QUANTITATIVE METRICS WHICH WILL BE USED TO EVALUATE PROGRAM/INTERVENTION): RAMP value will be measured by reduced mortality, reduced length of stay, and the count of new patient interventions including new antibiotics, fluid boluses, imaging orders, nursing staff changes or ICU transfer. Additional benefits to be measured include improved care team efficiency and communication and fewer 'false-alarm' rapid response alerts.

FINDINGS TO DATE (IT IS NOT SUFFICIENT TO STATE FINDINGS WILL BE DISCUSSED ): A pilot test is underway on a 27 bed medical nursing unit using a 40 " wall mounted monitor at the center of the unit passively displaying the NEWS (National Early Warning System) score for all unit patients. Nurses alert MDs when they identify 1) nursing concern re: illness course or 2) a NEWS score that meets or exceeds the designated acuity threshold. In the first month of a planned 6 month trial, $50 \%$ of the alerts (12 of 24) have been triggered by a high RAMP NEWS score with an overall fire rate of 0.77 alerts/day. There was also a $31 \%$ increase in patient interventions (new antibiotics, imaging or fluids) attributable to high RAMP NEWS triggers. The small sample precludes formal statistical testing of the outcomes identified as measures of success, but it is clear to date that the system is influencing care team behavior. Testing of active alerting is also planned.

KEY LESSONS FOR DISSEMINATION (WHAT CAN OTHERS TAKE AWAY FOR IMPLEMENTATION TO THEIR PRACTICE OR COMMUNITY?): Unit-based standard work supported by passive display of early warning scores may improve delivery of patient care. Input from clinicians and analytics staff is essential for successful development of such a software tool. Predictive models and the interventions that utilize them require input and buy-in from the end-users in order to change the complex processes associated with hospital standard work.

RESIDENT LED INITIATIVE TO IMPROVE SCREENING FOR SOCIAL DETERMINENTS OF HEALTH IN AN OUTPATIENT CLINIC

Robert J. DeGrazia $^{1}$; Nicole Laslett ${ }^{1}$; Ronak Patel ${ }^{1}$; Anthony Tramontozzi ${ }^{1}$; Zehra Hussain ${ }^{2} .{ }^{1}$ Christiana Care Health System, Aberdeen, MD; ${ }^{2}$ Christiana Care, Chadds Ford, PA. (Control ID \#3153502)

STATEMENT OF PROBLEM OR QUESTION (ONE SENTENCE): There is no standarized approach to screening for $\mathrm{SDoH}$ in our resident outpatient clinic

OBJECTIVES OF PROGRAM/INTERVENTION (NO MORE THAN THREE OBJECTIVES): Our quality improvement initiative aimed to develop a standardized approach to screen patients for SDoH in the primary care setting, create a streamlined referral system for patient resources and to quantify the degree to which these factors affect our clinic population.

DESCRIPTION OF PROGRAM/INTERVENTION, INCLUDING ORGANIZATIONAL CONTEXT (E.G. INPATIENT VS. OUTPATIENT, PRACTICE OR COMMUNITY CHARACTERISTICS): Wilmington Adult Medicine clinic at Christiana Care Health System in Wilmington, DE serves an underserved population. To address the lack of standardized 
screening for SDoH, we developed a questionnaire based on a validated survey guidelines. This was developed into a EMR "quicktext" that could be used to screen patients. The first PDSA cycle residents implemented this questionnaire into their workflow. The second PDSA cycle involved front desk staff screening patients at check-in. The third cycle shifted to medical assistants screening patients.

MEASURES OF SUCCESS (DISCUSS QUALITATIVE AND/OR QUANTITATIVE METRICS WHICH WILL BE USED TO EVALUATE PROGRAM/INTERVENTION): Measures of success included the number of patients screened and user acceptability to workflow.

FINDINGS TO DATE (IT IS NOT SUFFICIENT TO STATE FINDINGS WILL BE DISCUSSED ): Baseline data was obtained through chart review. $\sim 10 \%$ of each resident's patients were manually reviewed for documented SDoH. Out of $\sim 100$ charts reviewed, 33 included at least one documented SDoH and none had any systematic screening performed. In the first PDSA cycle, 86 patients were screened using the quicktext. Of those screened, 25 (29\%) screened positive for at least $1 \mathrm{SDoH}$. This led to 13 new referrals to social work. 1 month after initiation, residents were surveyed and revealed that none continued to use the quicktext after implementation. We next worked with clinic staff to have the front desk screen patients, however 2 weeks into this cycle, only 1 patient had been screened with the major barrier being "time constraints." For our next cycle, we attempted to have medical assistants(MA's) screen patients during the rooming process. After 2 weeks, 33 patients were screened. MA's also cited "lack of time" and "disruption of workflow" as the primary barriers to screening all patients.

KEY LESSONS FOR DISSEMINATION (WHAT CAN OTHERS TAKE AWAY FOR IMPLEMENTATION TO THEIR PRACTICE OR COMMUNITY?): Among several barriers faced while working on this initiative, the most significant was our office changing EMRs part way through our initiative, and we were unable to track our data in the EMR, instead having to track the number of patients screened manually. Despite this, we gathered useful information to help inform next steps, including factors that influence whether residents and office staff are able to integrate screening into their workflows. We learned that fine-tuning the workflow in our office on a small scale was extremely valuable prior broader implementation. Next, we will work on integrating the screening questionnaire directly into our new EMR to address barriers. Lastly, depite these barriers, we still screened 120 patients and identified significant unmet needs in our patient population. We learned that this screening strategy is not only valuable for patients, but also, creates valuable data on the needs of the population to be used to allocate resources.

RESOURCE INTENSIVE CARE TRANSITIONS PROGRAM IMPACT ON 90-DAY HOSPITAL READMISSIONS

Shreya P. Trivedi ${ }^{1}$; Emma Trawick ${ }^{1}$; Jillian Diuguid-Gerber ${ }^{2}$; Ian Fagan ${ }^{1}$; Mack Lipkin ${ }^{3}$; Mark D. Schwartz ${ }^{1}{ }^{1}$ NYU School of Medicine, New York, NY; ${ }^{2}$ New York University, New York, NY; ${ }^{3}$ New York University School of Medicine, New York, NY. (Control ID \#3179204)

STATEMENT OF PROBLEM OR QUESTION (ONE SENTENCE): IS enrollment in a Care Transitions Program (CTP) associated with decrease in 90-day hospital admissions compared to standard discharge care for high utilizers in a large safety-net city hospital?

OBJECTIVES OF PROGRAM/INTERVENTION (NO MORE THAN THREE OBJECTIVES): 1 . Implement a team to follow high-utilizers for 30 days post-discharge; 2 . Assess patient needs with a home visit or other faceto-face visit and coordinate care based on needs, and 3. Decrease hospital admissions of high utilizers
DESCRIPTION OF PROGRAM/INTERVENTION, INCLUDING ORGANIZATIONAL CONTEXT (E.G. INPATIENT VS. OUTPATIENT, PRACTICE OR COMMUNITY CHARACTERISTICS): In a large safety-net hospital, high utilizers were Medicaid-insured or uninsured patients who had four or more admissions in the prior year. Inpatient medical teams made referrals to CTP. In CTP, a multidisciplinary team of a social worker, nurse, community liaison and, at times, a medicine resident followed the patient for 30 days post-discharge. Interventions included medication reconciliation, weekly phone calls, and either a home visit or face-to-face visit at a preferred location for the patient. Eligible high utilizers not referred to CTP received standard discharge follow-up and served as controls in this quasiexperimental study.

MEASURES OF SUCCESS (DISCUSS QUALITATIVE AND/OR QUANTITATIVE METRICS WHICH WILL BE USED TO EVALUATE PROGRAM/INTERVENTION): We identified all admissions of high-utilizers eligible for CTP enrollment between 4/1/17 and 9/30/17. Patients were categorized as enrolled in CTP, with or without a home or face-toface visit, or not enrolled in CTP. Changes in 90-day admission rates before and after the eligible admission were compared in the CTP and control groups, with the eligible admission included in the pre-intervention period. Subgroup comparisons were also performed. The decrease in 90-day admission rates across groups were compared using ANOVA.

FINDINGS TO DATE (IT IS NOT SUFFICIENT TO STATE FINDINGS WILL BE DISCUSSED ): Of the 358 admissions of eligible highutilizers, $31 \%$ (112) were enrolled in CTP. Of those in CTP, $54 \%$ had either a home visit (28) or a face-to-face visit (32). Within control patients, 90-day admission rates fell from 2.90 to 1.26 , a decrease of $50.6 \%$ (95\% CI $39.0 \%$ $62.3 \%$ ). Within CTP patients, admission rates fell from 2.53 to 1.02 , a decrease of $54.6 \%$ (95\% CI $41.0 \%-67.6 \%$ ). The difference in 90-day admission reductions between CTP and control groups, 3.7\% (95\% CI -22.8\%-30.2\%), was not significant. Per-protocol analysis of the CTP subgroups who had either a home visit or a face-to-face visit showed decreases in 90-day admissions of $68.7 \%$ (95\% CI $51.2 \%-86.2 \%$ ) and $64.0 \%$ (95\% CI $46.9 \%-81.1 \%$ ) respectively. The differences in 90-day admission reductions between CTP patients with home or face-to-face visit vs. controls, $18 \%$ (95\% CI - $13 \%-49 \%, \mathrm{p}=0.59$ ) and $13.3 \%$ (95\% CI $-17.8 \%-44.5 \%, \mathrm{p}=0.84)$ respectively, were not significant. KEY LESSONS FOR DISSEMINATION (WHAT CAN OTHERS TAKE AWAY FOR IMPLEMENTATION TO THEIR PRACTICE OR COMMUNITY?): The trend towards greater decrease in 90-day admissions for high utilizers that had a home or face-to-face visit suggests that assessing high utilizers in their community environment may improve care for these patients. Given the small sample size and non-randomized allocation, interpretation of the results is preliminary, and subsequent, randomized, adequately powered study with criteria-based high-utilizer referral to these highintensity resources is warranted.

\section{REVISITING PRIMARY CARE: A QUALITY IMPROVEMENT STUDY}

Ben Long; Samuel O. Schumann; Jingwen Zhang. Medical University of South Carolina, Charleston, SC. (Control ID \#3184910)

STATEMENT OF PROBLEM OR QUESTION (ONE SENTENCE): Why do established patients of a practice choose to utilize emergency department (ED) services over ambulatory services?

OBJECTIVES OF PROGRAM/INTERVENTION (NO MORE THAN THREE OBJECTIVES): 1. Assess patient reasoning for utilizing emergency services rather than ambulatory services. 2. Identify future targets for interventions to reduce non-emergent utilization of (ED) services. 
DESCRIPTION OF PROGRAM/INTERVENTION, INCLUDING ORGANIZATIONAL CONTEXT (E.G. INPATIENT VS. OUTPATIENT, PRACTICE OR COMMUNITY CHARACTERISTICS): During April 2018, all patients presenting for an office visit at an academic internal medicine clinic were screened at registration for ED visits within the last 30 days. Patients who screened positive were asked to complete a survey querying all applicable reasons for ED presentation. Questions were modified from prior published surveys on the reasons for ED presentation. Survey responses were consolidated into groups based on response themes and then linked at the patient level to the ED visit. The majority of prior published work on this subject has occurred in the ED instead of in ambulatory clinics, where clinic resources can be leveraged to keep non-emergent visits out of the ED.

MEASURES OF SUCCESS (DISCUSS QUALITATIVE AND/OR QUANTITATIVE METRICS WHICH WILL BE USED TO EVALUATE PROGRAM/INTERVENTION): Measures of success include quantifying patient reasons for visiting ED and correlating patient reasons with severity of illness.

FINDINGS TO DATE (IT IS NOT SUFFICIENT TO STATE FINDINGS WILL BE DISCUSSED ): 200 surveys were delivered with a response rate of $56 \% .73 \%$ of patients cited "problem severity" as a reason for seeking care in the ED. $45 \%$ of patients contacted their PCP's office prior to seeking care in the ED. 13\% could not wait for an answer, $15 \%$ could not wait for the appointment, and 27\% were sent by their PCP's office or another MD. $19 \%$ of patients sought care after hours, $9 \%$ perceived the need for testing only available in the ED, and $10 \%$ cited superior quality of care in the ED. $2 \%$ stated transportation was a barrier to accessing primary care and a reason for $\mathrm{ED}$ visit. Of the patients seen in the ED, $30 \%$ reported a subsequent hospital admission. Patient responses were then linked to electronic health record data and stratified by the Emergency Severity Index (ESI), in which lower score represents more severe illness. In our respondents, there were no patients with an ESI of 1 or $5.100 \%$ of patients with an ESI of 2 reported severity as a reason for ED visit and $69 \%$ of these patients were admitted. $77.3 \%$ of patients with an ESI of 3 reported severity as a reason for ED visit and 34\% of these patients were admitted. $60 \%$ of patients with an ESI of 4 reported severity as a reason for ED visit and $20 \%$ of these patients were admitted.

KEY LESSONS FOR DISSEMINATION (WHAT CAN OTHERS TAKE AWAY FOR IMPLEMENTATION TO THEIR PRACTICE OR COMMUNITY?): Reducing unnecessary ED visits is on the forefront of utilization and continuity initiatives. Our findings suggest that improving patient education of reasons for ED visits and of resources (testing and treatments) available in ambulatory clinics could reduce the number of ambulatory care sensitive conditions treated in the $\mathrm{ED}$, allowing for cost savings and improved patient satisfaction. Other opportunities such as expansion of telehealth, expansion of case management, and establishment of ambulatory clinics within EDs should be explored.

SERIOUS ILLNESS CONVERSATIONS: HELPING CARE TEAMS TAKE THE LEAP

Deborah Swiderski. Montefiore Medical Center, Bronx, NY. (Control ID \#3184183)

STATEMENT OF PROBLEM OR QUESTION (ONE SENTENCE): Despite robust evidence of the benefits of goals of care conversations with patients who have late-stage chronic illness, and widespread agreement among both patients and physicians that such discussions are beneficial these conversations are usually infrequent, late, and limited.

OBJECTIVES OF PROGRAM/INTERVENTION (NO MORE THAN THREE OBJECTIVES): To train and support care teams in 2 urban academic health centers to have goals of care conversations with patients in the late stages of chronic illness using the Serious Illness Conversation Guide DESCRIPTION OF PROGRAM/INTERVENTION, INCLUDING ORGANIZATIONAL CONTEXT (E.G. INPATIENT VS. OUTPATIENT, PRACTICE OR COMMUNITY CHARACTERISTICS): The Serious Illness Conversation Guide, a tool developed by Harvard's Ariadne Labs, has shown great promise in helping physicians and care teams to have goals of care conversations with patients in the late stages of chronic illness. Such conversations are typically difficult for both physician and patient and often never take place until the patient is terminally ill and no real choices remain. This project is designed to assess the use of the Guide in academic health centers serving an urban underserved community. In this implementation phase the project was introduced in small group meetings and in larger presentations such as Grand Rounds. Physicians, nurses, and social workers at 2 academic neighborhood health centers were trained in the use of the Guide by project leaders who attended training at Ariadne Labs. A leadership group meets monthly to assess progress and address problems. Site champions have assumed active roles in helping trained providers use the Guide with appropriate patients. Documentation and coding and billing emerged as important issues and are being addressed.

MEASURES OF SUCCESS (DISCUSS QUALITATIVE AND/OR QUANTITATIVE METRICS WHICH WILL BE USED TO EVALUATE PROGRAM/INTERVENTION): Training began in September 2018. Our goal was to train at least 5 providers at each site over a 3 month period and for each of those providers to have 1 conversation by the end of December. FINDINGS TO DATE (IT IS NOT SUFFICIENT TO STATE FINDINGS WILL BE DISCUSSED ): The implementation phase of this project has been remarkably successful: $15 \mathrm{MDs}, 4 \mathrm{RNs}$, and 4 SWs completed the 2 hour training in the use of the Guide over a 3 month period. Flexible workflows were developed by each site to promote the use of the Guide. 7 providers completed 14 conversations by the end of the 4 months from the beginning of training. A protocol for documentation of these visits in the EMR was established. Working groups were formed at the departmental level to address long term solutions for EMR documentation and coding and billing. Informal feedback from providers has been strongly positive.

KEY LESSONS FOR DISSEMINATION (WHAT CAN OTHERS TAKE AWAY FOR IMPLEMENTATION TO THEIR PRACTICE OR COMMUNITY?): Several factors contributed to the success of the project thus far. Project leadership by a physician champion with expertise in the field of provider-patient communciation, and modest salary support for the project leader facilitated implementation. Strong support from both administrative and clinical leadership was critical. And it is likely that part of this acceptance stems from the fact that the project addresses physicians' desire to provide personalized, respectful care for their seriously ill patients.

SHARING THE BURDEN OF DISEASE: MULTI-DISCIPLINARY SHARED MEDICAL APPOINTMENTS FOR OSTEOARTHRITIS Katie Miller ${ }^{1}$; Linda Baier ${ }^{3}$; David Rabago ${ }^{2} .{ }^{1}$ University of Wisconsin, Madison, WI; ${ }^{2}$ University of Wisconsin School of Medicine and Public Health, Madison, WI; ${ }^{3} \mathrm{f}$ Medicine and Public Health, Madison, WI. (Control ID \#3170064)

STATEMENT OF PROBLEM OR QUESTION (ONE SENTENCE): Knee and hip osteoarthritis (OA) is a disabling condition affecting over $50 \%$ of US adults over 65 . Patients often do not receive guideline-recommended care. OBJECTIVES OF PROGRAM/INTERVENTION (NO MORE THAN THREE OBJECTIVES): The University of Wisconsin Knee and Hip Comprehensive Non-Surgical Management Clinic uses shared medical visits 
(SMV) with multi-disciplinary providers to manage patients with knee or hip OA. The long-term clinic goals are to decrease the gap between recommended and actual OA care and to improve self-reported and objectively assessed outcomes in these patients.

DESCRIPTION OF PROGRAM/INTERVENTION, INCLUDING ORGANIZATIONAL CONTEXT (E.G. INPATIENT VS. OUTPATIENT, PRACTICE OR COMMUNITY CHARACTERISTICS): We recently implemented an innovative SMV program. Patients commit to attending six 90-minute appointments (plus optional 30-minute exercise session) twice monthly for 12 weeks. SMV care is provided by a general internist, physical therapist, dietitian, and health psychologist. During each visit, patients are given an opportunity to share their progress and challenges, learn about managing OA in an interactive format, and set treatment goals.

MEASURES OF SUCCESS (DISCUSS QUALITATIVE AND/OR QUANTITATIVE METRICS WHICH WILL BE USED TO EVALUATE PROGRAM/INTERVENTION): At the first and last SMV, we assess: Quality of life: Veterans Rand 12-Item Health Survey (VR-12) physical and mental subscales Objectively-assessed function: Timed Up and Go (TUG) Test, 30 second Chair Stand Test Weight loss Satisfaction, knowledge, and confidence: (0-10 Likert scales).

FINDINGS TO DATE (IT IS NOT SUFFICIENT TO STATE FINDINGS WILL BE DISCUSSED ): From Fall 2017 to Summer 2018, 27 patients (mean age 59.6 $\pm 8.4,25$ female) attended an average of 4.6 of the 6 visits. All were obese at baseline; $19(70.4 \%)$ had a BMI over $40 \mathrm{~kg} / \mathrm{m}^{2}$. Sixteen patients $(59.2 \%)$ had 5 or more significant co-morbid conditions and the mean VR-12 Physical Health Score was low ( $26.60 \pm 8.35$ points). At $30-56$ weeks post, $6(22.2 \%)$ patients had a weight loss of $10 \%$ or more while 4 (14.8\%) lost 5-10\% of baseline weight. Changes in the VR-12 were small; the Cohen's d effect size for physical and mental subscales was 0.22 and 0.24 respectively. Improvements were seen for the Chair Stand Test (2.2 rises) and TUG time (5.3 seconds). Patients valued the SMV program and would recommend it to a friend $(9.16 \pm 0.80,10$-point scale). They indicated increased confidence in managing osteoarthritis ( $7.8 \pm 1.61$ points) and reported a high level of knowledge gained ( $8.6 \pm 1.76$ points).

KEY LESSONS FOR DISSEMINATION (WHAT CAN OTHERS TAKE AWAY FOR IMPLEMENTATION TO THEIR PRACTICE OR COMMUNITY?): These early data suggest that SMVs have the potential to improve quality of life and function, and facilitate weight loss, in patients with hip and knee OA. SMVs were well-liked and improved patient knowledge and confidence regarding OA and its management. Additional planned assessments include more formal tracking of SMV- associated changes, comparison to other management strategies, and determination of OA guideline adherence

SMALL STATE, BIG CHANGE: A 5-YEAR DESCRIPTION OF VERMONT'S PRIMARY CARE TRANSFORMATION TO EMBRACE POPULATION HEALTH

Jennifer Gilwee $^{1}$; Halle G. Sobel ${ }^{2} .{ }^{1}$ University of Vermont, Shelburne, VT; ${ }^{2}$ University of Vermont Medical Center, Burlington, VT. (Control ID \#3153492)

STATEMENT OF PROBLEM OR QUESTION (ONE SENTENCE): How do you transform the delivery of care across primary care disciplines in an academic medical center to positively affect outcomes for an attributed population?

OBJECTIVES OF PROGRAM/INTERVENTION (NO MORE THAN THREE OBJECTIVES): - Describe the standardization of care to a population of patients across primary care specialties - Share successful practice of administrative/leadership coordination among three primary care specialties at our organization. - Summarize positive impacts of the transforming primary care process on patient outcomes

DESCRIPTION OF PROGRAM/INTERVENTION, INCLUDING ORGANIZATIONAL CONTEXT (E.G. INPATIENT VS. OUTPATIENT, PRACTICE OR COMMUNITY CHARACTERISTICS): The University of Vermont Medical Center consists of 12 ambulatory clinics which span three primary care specialties (Internal medicine, Family medicine, Pediatric medicine) and care for $\sim 80,000$ empaneled patients. There are $\sim 160,000$ visits per year which cover acute care, chronic disease management and preventive healthcare. Five years ago we realized the need to transform our care delivery to efficiently and effectively meet the comprehensive needs of our patients and providers. This led to the implementation of standardized previsit protocols, daily huddles and weekly care coordination meetings all designed to optimize the care delivered to patients, ensuring their preventative as well as chronic disease (including chronic opiate use) needs are met. We instituted a specific administrative structure including physician and staff supervisor representatives from each clinic site. The operational deployment and review of protocols was done by multidisciplinary groups (including MDs, RNs, medical assistants and clerical staff) to foster empowerment and adoption.

MEASURES OF SUCCESS (DISCUSS QUALITATIVE AND/OR QUANTITATIVE METRICS WHICH WILL BE USED TO EVALUATE PROGRAM/INTERVENTION): Over the course of our transformation we have had the implementation of several protocols spanning all aspects of the patient-health system interaction including pre-visit planning, rooming, medication refill, post-hospitalization follow up and specific condition RNdirected treatment protocols. As a result we have demonstrated success in measures that cut across multiple ages and conditions. These include preventive health measures including increased immunization rates and screening for hepatitis C. We demonstrated success in achieving advanced care planning documentation on our population. Most recently, we have demonstrated success in increasing the number of opiate informed consent and prescription agreements on file for those patients who are on chronic opiates.

FINDINGS TO DATE (IT IS NOT SUFFICIENT TO STATE FINDINGS WILL BE DISCUSSED ): Over a 3 year span we noted a $4 \%$ increase in overall immunization rate for our entire population. Our hepatitis $\mathrm{C}$ screening rates went up from $66 \%$ to $71 \%$ over a 5 year timeframe. Our advance care planning documentation rates rose from $30 \%$ to $39 \%$ over a 1 year timeframe. Our percent of patients with opioid informed consent and prescription agreement has gone up to $>85 \%$

KEY LESSONS FOR DISSEMINATION (WHAT CAN OTHERS TAKE AWAY FOR IMPLEMENTATION TO THEIR PRACTICE OR COMMUNITY?): -Must have leadership support and administrative champions (MD, supervisors) -Imperative to empower all members of the team to function at the top of their job description -Implementation and adoption can be hard without clear communication of why things are changing. Cannot feel "top down'

STANDARDIZED HANDOFF EDUCATION FOR INTERNAL MEDICINE RESIDENTS: AN INNOVATIVE EDUCATION AND PATIENT SAFETY INITIATIVE

Morgan R. Moore ${ }^{1}$; Steven G. McKee $2 .{ }^{1}$ University of Arkansas for Medical Sciences, Little Rock, AR; ${ }^{2}$ University of Arkansas, Little Rock, AR. (Control ID \#3184433)

STATEMENT OF PROBLEM OR QUESTION (ONE SENTENCE): Internal medicine residents received no formal education on standardized 
patient handoff, which led to wide variations in the quality of handoffs and posed a risk to patients' safety.

OBJECTIVES OF PROGRAM/INTERVENTION (NO MORE THAN THREE OBJECTIVES): The primary objective was to teach residents the IPASS model for handoff. Additionally, an electronic medical record (EMR)based tool was provided to residents. The final goal was to assess residents' perceptions of the training, the tool, and compliance with the I-PASS format. DESCRIPTION OF PROGRAM/INTERVENTION, INCLUDING ORGANIZATIONAL CONTEXT (E.G. INPATIENT VS. OUTPATIENT, PRACTICE OR COMMUNITY CHARACTERISTICS): Residents participated in a one-hour. resident-led session that included didactics and case-based practice. There were 60 internal medicine (IM) and 18 medicine-pediatrics (MP) residents. A template macro was disseminated to the residents through the electronic medical record (EMR) to standardize written handoff.

MEASURES OF SUCCESS (DISCUSS QUALITATIVE AND/OR QUANTITATIVE METRICS WHICH WILL BE USED TO EVALUATE PROGRAM/INTERVENTION): Before the sessions, residents were surveyed to assess baseline knowledge and practices. Post-intervention surveys were sent immediately and again three months following the intervention. Survey data is based on residents who responded to the surveys and is not inclusive of all residents who completed the training. The $\mathrm{n}$ value represents total number of respondents and includes residents in IM post-graduate years (PGYs) 1-3 and MP PGYs 1-4.

FINDINGS TO DATE (IT IS NOT SUFFICIENT TO STATE FINDINGS WILL BE DISCUSSED ): Only 18\% of IM and MP residents ( $\mathrm{n}=22$ ), reported any previous training on standardized handoff. Immediately postintervention, $92 \%$ of respondents $(n=39)$, rated the training as valuable. At three months post-intervention, $94 \%$ of residents $(n=31)$ selected that the EMR macro improved patient safety and streamlined written handoff creation. The EMR tool enabled $80 \%$ of residents $(n=31)$ to quickly create a high-quality, IPASS format written handoff. However, after three months, only $32 \%(n=31)$ of residents reported that contingency planning was always included in the written handoff.

KEY LESSONS FOR DISSEMINATION (WHAT CAN OTHERS TAKE AWAY FOR IMPLEMENTATION TO THEIR PRACTICE OR COMMUNITY?): The education sessions were well received yet results reveal that residents omit important components of the I-PASS model after only three months. Dissemination of an EMR-based macro proved simple and effective. As we move forward, we plan to further assess uptake of the tool and the I-PASS format through random chart review of handoffs and to improve upon the training based on feedback from participants. Clearly there is room for increased adherence to standardized handoff and we hope to define the ideal timing and format of further trainings.

\section{STANDARDIZING FRAGILITY HIP FRACTURE CARE USING AN} ELECTRONIC HEALTH RECORD APPROACH

$\underline{\text { Kencee K. Graves }}^{1}$; Polina Kukhareva ${ }^{1}$; Kensaku Kawamoto ${ }^{2}$; Logan A. Horne $^{1}$; David Rothberg ${ }^{1}$; Jacob McKenna ${ }^{1}$; Steven M. Johnson ${ }^{1}$; Jeffrey Young ${ }^{1}$; Mark A. Supiano ${ }^{1} .{ }^{1}$ University of Utah, Salt Lake City, UT; ${ }^{2}$ University of Utah, Sandy, UT. (Control ID \#3163409)

STATEMENT OF PROBLEM OR QUESTION (ONE SENTENCE): Fragility hip fractures are a devastating injury to elderly patients, resulting in significant morbidity, mortality and cost; however, in-hospital care for these patients can be variable.

OBJECTIVES OF PROGRAM/INTERVENTION (NO MORE THAN THREE OBJECTIVES): Our first objective was to standardize the care for patients with fragility fracture as much as possible. Our second objective was to provide feedback to providers on the quality of their hip fracture care as defined by pre-determined metrics. Finally, we aimed to provide notification to specific providers when a patient is admitted to the hospital.

DESCRIPTION OF PROGRAM/INTERVENTION, INCLUDING ORGANIZATIONAL CONTEXT (E.G. INPATIENT VS. OUTPATIENT, PRACTICE OR COMMUNITY CHARACTERISTICS): First, we implemented a forced function hip fracture order set containing evidencebased interventions, such as treatment for osteoporosis, anticoagulation to prevent venous thromboembolism, and early physical therapy. Second, we implemented an electronic score card to provide quarterly feedback to providers, including orthopedics and internal medicine; and third, we designed an electronic alert to targeted providers notifying them of patients admitted with fragility hip fracture to allow for data analysis and follow up for these patients. This program was for patients older than 65 years of age admitted with hip fracture to a single academic medical center.

MEASURES OF SUCCESS (DISCUSS QUALITATIVE AND/OR QUANTITATIVE METRICS WHICH WILL BE USED TO EVALUATE PROGRAM/INTERVENTION): To evaluate the effects our program, we compared adherence to components of the order set, readmissions, mortality, length of stay and total direct cost.

FINDINGS TO DATE (IT IS NOT SUFFICIENT TO STATE FINDINGS WILL BE DISCUSSED ): For statistical analysis, we compared a preintervention cohort of 39 patients to two post-intervention cohorts. Postintervention cohort 1 includes 115 patients who received care with the order set and whose providers received score card feedback. Post-intervention cohort 2 included 50 patients who received the interventions in cohort 1 and, in addition, whose providers received an electronic alert of their admission. Our primary outcome was defined as receiving all components of the order set, considered to constitute "perfect care." The percentage of patients receiving "perfect care" increased from $15.4 \%$ for the pre-intervention cohort to $33.6 \%$ for post-intervention cohort 1 and to $45.1 \%$ for post-intervention cohort 2 $(\mathrm{p}=0.012$ ). Of our individual components, use of osteoporosis medications increased from $69.2 \%$ pre-intervention to more than $88 \%$ post-interventions $(\mathrm{p}=0.006)$. However, there were no significant differences seen in mortality, 30-day readmissions, or total direct cost.

KEY LESSONS FOR DISSEMINATION (WHAT CAN OTHERS TAKE AWAY FOR IMPLEMENTATION TO THEIR PRACTICE OR COMMUNITY?): Implementation of a hip fracture care improvement program with the goal of standardizing care is feasible in a single academic medical center. However, this program did not improve the outcomes of mortality, length of stay or readmission in our cohort. Future studies with larger sample size will be needed to determine if better adherence to perfect care will be associated with improved mortality, length of stay or readmission. Outcomes such as delirium incidence, mobility, or return to home may be more sensitive to assess the benefits of "perfect care." While this method may be reproducible, the benefit of dissemination is unclear.

STOP THE MADNESS: A TALE OF TWO DE-IMPLEMENTATION INITIATIVES TO REDUCE EHR BURDEN IN PRIMARY CARE

$\mathrm{Ling} \mathrm{Chu}^{1}$; Ethan Halm${ }^{2}$; Duwayne L. Willett ${ }^{3} .{ }^{1}$ UT Southwestern, Dallas, TX; ${ }^{2}$ Univ of TX Southwestern Med Ctr, Dallas, TX; ${ }^{3}$ University of Texas Southwestern Medical Center, Dallas, TX. (Control ID \#3179325)

STATEMENT OF PROBLEM OR QUESTION (ONE SENTENCE): Frustration with the burden of using EHRs is a major driver of physician burnout, can selective de-implementation decrease EHR burden? 
OBJECTIVES OF PROGRAM/INTERVENTION (NO MORE THAN THREE OBJECTIVES): 1. Reduce primary care providers (PCPs) EHR burden by identifying opportunities to reduce the amount of low value added information to be reviewed and eliminate unnecessary keystrokes. 2. Showcase success of two practical, high impact deimplementation initiatives that reduced EHR inbox message volume in PCPs inbox and eliminated most second password authentications.

DESCRIPTION OF PROGRAM/INTERVENTION, INCLUDING ORGANIZATIONAL CONTEXT (E.G. INPATIENT VS. OUTPATIENT, PRACTICE OR COMMUNITY CHARACTERISTICS): Our first initiative focused on ways to reduce the exploding volume of Epic EHR inbox messages among PCPs. Analysis of 3 years of inbox messages received by PCPs in GIM and Family Medicine (FM) found that "CC Chart" messages were the most common $(332 \mathrm{~K})$ and more frequent than Results (256K), Patient Calls (234K), and any other type. PCPs were receiving 3 times as many "CC Chart" messages in their inbox compared to specialty physicians. PCPs validated that almost none of these CC Charts were helpful. The EHR was programmed to automatically send a copy of every specialty outpatient encounter to the PCP. This programming decision was made over 10 years ago and then propagated to over 50 clinic-specific settings over the years (including routine dental and ophthalmology visits). Consensus was reached among physician leadership and frontline PCPs to de-implement this automatic messaging and use only intentional CC Chart routing. Our second initiative sought to eliminate the need for $2^{\text {nd }}$ password authentication for medication ordering. Our EHR build required providers to enter their password to approve nearly all types of orders (not just controlled substance orders) even though they were already logged in. After garnering operational and IT leadership support, the EHR team located and removed the automated programming point for automatic $\mathrm{CC}$ Charts and turned off the requirement for $2^{\text {nd }}$ password entry for non-controlled medication ordering.

MEASURES OF SUCCESS (DISCUSS QUALITATIVE AND/OR QUANTITATIVE METRICS WHICH WILL BE USED TO EVALUATE PROGRAM/INTERVENTION): Reduction in number of CC Chart messages and $2^{\text {nd }}$ password entries

FINDINGS TO DATE (IT IS NOT SUFFICIENT TO STATE FINDINGS WILL BE DISCUSSED ): Prior to the intervention, the number of CC Chart messages received by GIM PCPs was growing steadily, exceeding 9,500 per month. After "DCing the CC", the number of messages dropped nearly in half, to under 5,000 messages per month. Among FM PCPs, CC Chart messages dropped from 2,500 to 1,000 per month. Specialty departments served as controls and saw no decrease in their CC Chart messages. In our second initiative, eliminating the need for 2nd password entry for noncontrolled medication orders saved 692,465 password entries among 1,917 clinicians over a 7.5 month period in our outpatient setting, 1,961,674 in our hospitals for a total of 2,654,139 entries saved.

KEY LESSONS FOR DISSEMINATION (WHAT CAN OTHERS TAKE AWAY FOR IMPLEMENTATION TO THEIR PRACTICE OR COMMUNITY?): Identifying common EHR tasks that are low value added or redundant and eliminating them can reduce EHR burden. While the natural history of EHR adoption is to add more and more features and requirements, more attention is needed to periodically review and deimplement unnecessary or dated ones.

SUSTAINABLE IMPROVEMENT OF COLORECTAL CANCER SCREENING RATES THROUGH A RESIDENT LED INITIATIVE Daniel Jonas ${ }^{1}$; Matt Welzenbach ${ }^{1,1}$; Patrick Ryan ${ }^{2} .{ }^{1}$ University of Colorado, Denver, CO; ${ }^{2}$ Denver Health and Hospital Authority, Denver, CO. (Control ID \#3183608)
STATEMENT OF PROBLEM OR QUESTION (ONE SENTENCE): How can we increase our colorectal cancer screening rates in a sustainable, automated method at a Federally Qualified Community Health Center? OBJECTIVES OF PROGRAM/INTERVENTION (NO MORE THAN THREE OBJECTIVES): At the Westside Adult Internal Medicine Clinic in Denver, Colorado our colorectal cancer screening rates were below a hospital wide goal. We aimed to increase our colorectal cancer screening rate from $54 \%$ to a hospital wide goal of $65 \%$ using a resident led quality improvement project by focusing on increasing screening through FIT card testing.

DESCRIPTION OF PROGRAM/INTERVENTION, INCLUDING ORGANIZATIONAL CONTEXT (E.G. INPATIENT VS. OUTPATIENT, PRACTICE OR COMMUNITY CHARACTERISTICS): This was a resident led quality improvement project at the Westside Adult Primary Care clinic, an outpatient clinic affiliated with Denver Health in Denver, Colorado. We first underwent a root cause analysis to identify reasons why our screening rates were low. We then underwent three plan-do-study-act cycles. First we had medical assistants pend FIT orders for provider on patients who were due for colorectal cancer screening. Our second cycle implemented a patient handout in exam rooms to cut back on time spent discussing screening options. And finally, we developed an automated system through our electronic medical record to identify patients prescribed FITs and who had not returned them. We then sent reminder letters to these patients to return their FIT kits.

MEASURES OF SUCCESS (DISCUSS QUALITATIVE AND/OR QUANTITATIVE METRICS WHICH WILL BE USED TO EVALUATE PROGRAM/INTERVENTION): The quantitative metric used to measure success were electronic medical record system reports that calculate the percent of eligible adults who have received colorectal cancer screening which we did before and after initiation of the new screening protocol. Additionally we broke this data down to individual physicians in the clinic and provided them with this data monthly. A qualitative metric used to measure success was ongoing adoption of the new screening protocol in the clinic after completion of the project and adoption by other clinics as well.

FINDINGS TO DATE (IT IS NOT SUFFICIENT TO STATE FINDINGS WILL BE DISCUSSED ): Over an academic year from June 2017 to May 2018 we increased our clinic wide screening rate from $54 \%$ to $63 \%$. The process developed empowered medical assistants to chart review and pend FIT orders as well as created an automated reminder letter to return kits. This has continued to be utilized in the clinic and has been adopted by other clinics in the Denver Health Hospital System with similar success.

KEY LESSONS FOR DISSEMINATION (WHAT CAN OTHERS TAKE AWAY FOR IMPLEMENTATION TO THEIR PRACTICE OR COMMUNITY?): Successful resident-led quality improvement projects are those that can be become automated with minimal time commitment and have buy-in from clinic leadership and hospital administration leadership. Annual FIT testing with medical assistant pended orders for providers and automated reminder letters to return FIT kits is a successful approach to increase colorectal cancer screening rates.

SUSTAINING MINDFUL LABORATORY ORDERING PRACTICES: A CULTURE CHANGE

$\underline{\text { Rachna Rawal }}^{1}$; Ara Vartanyan ${ }^{2}$; Paul Kunnath ${ }^{3}$; Oluwasayo Adeyemo'; Hala Saad ${ }^{4}$; Alex Lane ${ }^{1}$; Jennifer Schmidt ${ }^{5}$. ${ }^{1}$ Saint Louis University, St. Louis, MO; ${ }^{2}$ Saint Louis University School of Medicine, St. Louis, MO; ${ }^{3}$ Saint Louis University School of Medicine, University City, MO; ${ }^{4}$ Saint Louis University Hospital, Saint Louis, MO; ${ }^{5}$ Saint Louis University, Saint Louis, MO. (Control ID \#3183457) 
STATEMENT OF PROBLEM OR QUESTION (ONE SENTENCE):

How to sustain a high-value care mindful lab ordering culture in a medicine residency program?

OBJECTIVES OF PROGRAM/INTERVENTION (NO MORE THAN THREE OBJECTIVES): Recognize barriers to mindful lab ordering Engage in multi-faceted interventions to promote mindful lab ordering

DESCRIPTION OF PROGRAM/INTERVENTION, INCLUDING ORGANIZATIONAL CONTEXT (E.G. INPATIENT VS. OUTPATIENT, PRACTICE OR COMMUNITY CHARACTERISTICS):

The United States is in a health quality crisis; we must transform to a high-value, low-cost healthcare culture. To achieve such a culture, trainees must learn high-value care habits. We developed a 95-week longitudinal study to promote mindful laboratory habits in an Internal Medicine residency program.

MEASURES OF SUCCESS (DISCUSS QUALITATIVE AND/OR QUANTITATIVE METRICS WHICH WILL BE USED TO EVALUATE PROGRAM/INTERVENTION): Subjects included residents and attending physicians on the general medicine inpatient service. The primary aim was to decrease total laboratory orders (BMP, CMP, $\mathrm{CBC}$ with and without differentials) by $15 \%$ within one academic year and to sustain these changes. Total duration was 95 weeks (control-15 weeks, interventions - 80 weeks, divided into 5 16-week phases); each was cumulative. Phase 1: resident education, phase 2: EMR (Epic) changes, phase 3 : interactive cases, phase 4 : attending physician education, phase 5: night-float resident education and additional EMR changes. Aggregate order data was obtained from the EMR and culture change was assessed with pre/post surveys. Primary outcomes were total labs ordered, the type of laboratory panel ordered, resident attitudes of mindful laboratory ordering and money saved. Secondary outcomes included length of stay, 30-day readmission rate, case-mix index and venipuncture utilization.

FINDINGS TO DATE (IT IS NOT SUFFICIENT TO STATE FINDINGS WILL BE DISCUSSED ): Survey data showed increased resident perception of mindful lab ordering from $40 \%$ (32 weeks) to $85 \%$ ( 80 weeks $)(\mathrm{p}<0.05)$. Additionally, residents reported team discussion of laboratory ordering increased from $25 \%$ ( 0 weeks) to $85 \%$ $(\mathrm{p}<0.05)$. EMR lab frequency utilization data showed a $50 \%$ decrease in use of "daily" frequency and a $50 \%$ increase of "one-time" ordering option. By week 45 , there was a $20 \%$ decrease in total labs ordered/ week; this was sustained through week 95 . Both BMP and $\mathrm{CBC}$ without differential increased while $\mathrm{CMP}$ and $\mathrm{CBC}$ with differential decreased per a thousand patient days. Hepatic panels, a balancing measure, did not significantly change. Secondary outcomes demonstrated a downtrend in 30-day readmission rate (1.18 to 0.92) and length-of-stay (LOS) (1.02 to 1.0$)$ and uptrend incase-mix index (CMI) (1.37 to 1.83). The percentage of patients on medicine service undergoing venipuncture also decreased.

KEY LESSONS FOR DISSEMINATION (WHAT CAN OTHERS TAKE AWAY FOR IMPLEMENTATION TO THEIR PRACTICE OR COMMUNITY?): We believe we have created and sustained a cultural change toward more mindful laboratory ordering. Residents not only perceive that they are more mindful, they have actually decreased the number of labs ordered. While we cannot attribute the downtrend in LOS and readmissions to our study, we can state that decreasing labs did NOT result in an increased LOS or readmission rate. We also know that our decrease in laboratory orders is not secondary to lower acuity patients. Additionally, in the setting of higher acuity patients we have seen a decrease in the percentage of patients undergoing venipuncture.
TEAM-BASED PROCESS FOR MEDICATION RECONCILIATION \&AMP; REVIEW IN AMBULATORY CARE

Celia Lu ${ }^{1,2}$; Christine Chim ${ }^{1,2}$; Danielle Ezzo ${ }^{1,2}$; Nissa Mazzola ${ }^{1,2}$; Rachel $\overline{\text { S. Kashan }}{ }^{3}$; Daniel J. Coletti ${ }^{2}$. ${ }^{1}$ St. John's University College of Pharmacy \& Health Sciences, Lake Success, NY; ${ }^{2}$ Northwell Health, Great Neck, NY; ${ }^{3}$ Yeshiva University, Ferkauf Graduate School of Psychology, New York, NY. (Control ID \#3185199)

STATEMENT OF PROBLEM OR QUESTION (ONE SENTENCE): Discrepancies between the medications the patient is taking and those listed in the electronic medical record (EMR) can lead to medication errors and patient harm.

OBJECTIVES OF PROGRAM/INTERVENTION (NO MORE THAN THREE OBJECTIVES): Develop, implement, and evaluate an interprofessional (IP) team-based medication reconciliation process, with the objectives of reducing medication discrepancies and medication errors.

DESCRIPTION OF PROGRAM/INTERVENTION, INCLUDING ORGANIZATIONAL CONTEXT (E.G. INPATIENT VS. OUTPATIENT, PRACTICE OR COMMUNITY CHARACTERISTICS): IMPACcT (Improving Patient Access, Care, and Cost through Training) is an internal medicine training practice where residents collaborate with medical students and trainees from psychology, physician assistant, and pharmacy programs. Our pharmacy-led IP medication review process consisted of four steps: "scrubbing" the chart, huddle, interview, and reconciliation. First, a pharmacy student identifies high-risk patients who need a medication review. The student then presents identified patient medication needs during the huddle and identifies team partners who can assist. Third, the student obtains a medication history with the patient and identifies discrepancies, while the partnering student focuses on adherence. During reconciliation, the team discusses identified discrepancies, updates the patient's medication list in the EMR, and communicates the plan to the patient.

MEASURES OF SUCCESS (DISCUSS QUALITATIVE AND/OR QUANTITATIVE METRICS WHICH WILL BE USED TO EVALUATE PROGRAM/INTERVENTION): A coding system was utilized to classify medication-related problems and to record changes made to the treatment plan. We examined the frequency and types of discrepancies identified and the percentage of successfully updated medication lists.

FINDINGS TO DATE (IT IS NOT SUFFICIENT TO STATE FINDINGS WILL BE DISCUSSED ): A Plan-Do-Study-Act cycle was conducted examining 164 medication reviews. We used prior practice data to benchmark a $20 \%$ improvement in the number of identified medication discrepancies that were correctly adjusted in the patient's medical record. We identified 1 discrepancy in the vast majority of patients $(n=133 ; 81.1 \%)$ and found an average of 3.06 discrepancies per discrepant record (range $=1$ 10). A total of 95 patients $(58.5 \%)$ had 1 discrepancy involving a daily prescription medication taken for a chronic condition. The most frequently identified medications included central nervous system agents $(\mathrm{n}=38)$, cardiac medications $(n=31)$, gastrointestinal drugs $(n=26)$, prescribed analgesics $(n=21)$, and hypoglycemic agents $(n=18)$. The medication review team recommended a chart update to address 326 of the 407 identified discrepancies. Changes were successfully made to address 244 of these 326 discrepancies (74.8\%). Of the charts for which medication reviews were completed, $68.9 \%$ were free of discrepancies by the end of the visit.

KEY LESSONS FOR DISSEMINATION (WHAT CAN OTHERS TAKE AWAY FOR IMPLEMENTATION TO THEIR PRACTICE OR COMMUNITY?): A structured IP medication review process encouraged identification and discussion of discrepancies, which improved accuracy of the medication lists. However, workflow barriers hindered successful 
medication updates in some patients even after medication reconciliation was performed.

THE EAST BALTIMORE FARM TO CLINIC VEGETABLE INITIATIVE: A PILOT PROGRAM TO DISTRIBUTE VEGETABLES TO PATIENTS WITH CHRONIC DISEASE IN AN URBAN CLINIC Theresa R. Aguilar ${ }^{1}$; Amelia Cover ${ }^{2}$; Laura Harding-Fukushima ${ }^{3}$; Katherine Rediger $^{2,3}$; Donald R. Miles ${ }^{1,}{ }^{3}$. ${ }^{1}$ Johns Hopkins University School of Medicine, Baltimore, MD; ${ }^{2}$ Johns Hopkins University School of Nursing, Baltimore, MD; ${ }^{3}$ Johns Hopkins Community Physicians, Baltimore, MD. (Control ID \#3184233)

STATEMENT OF PROBLEM OR QUESTION (ONE SENTENCE): Inadequate intake of healthy foods is associated with chronic disease, and East Baltimore Medical Center (EBMC) is located in a neighborhood where one third of residents lack access to fruits and vegetables.

OBJECTIVES OF PROGRAM/INTERVENTION (NO MORE THAN THREE OBJECTIVES): Establish a partnership between an urban clinic and a local farm to provide fresh vegetables to underserved patients Assess the feasibility of a Community Supported Agriculture (CSA) model to distribute fresh vegetables to adult patients at a clinic in an under-resourced neighborhood Engage the EBMC patients in consumption of increased amounts of vegetables and healthy diet educational initiatives

DESCRIPTION OF PROGRAM/INTERVENTION, INCLUDING ORGANIZATIONAL CONTEXT (E.G. INPATIENT VS. OUTPATIENT, PRACTICE OR COMMUNITY CHARACTERISTICS): EBMC, an outpatient office of Johns Hopkins, is in the Greenmount East neighborhood, which is predominantly black (96.6\%) and $33.8 \%$ live in poverty. Heart disease is the leading cause of death. Primary food sources are corner stores, which have limited fresh vegetables, making it difficult to eat a healthy diet to reduce cardiovascular disease risk or contributing risk factors, like diabetes and obesity. The initiative was developed by health care professionals at EBMC and staff at Karma Farm of Monkton, Maryland. Fresh vegetable bags were distributed to patients with obesity, diabetes, or hypertension at EBMC weekly for 13 weeks during Summer 2018. Three monthly cooking classes were held.

MEASURES OF SUCCESS (DISCUSS QUALITATIVE AND/OR QUANTITATIVE METRICS WHICH WILL BE USED TO EVALUATE PROGRAM/INTERVENTION): Participants were surveyed for demographics, insurance, use of federal food assistance, and food insecurity, using a sensitive and specific 2 -item screen. Those returning were surveyed for satisfaction to measure engagement. EBMC staff were surveyed to assess perception of patient food insecurity, program awareness, and likelihood of referring patients to a long-term program. Descriptive statistics and a Chi-square Test of Independence were performed with R. A focus group was conducted for qualitative feedback. In a one hour session, 7 participants were asked open-ended questions about program satisfaction and suggestions for future initiatives.

FINDINGS TO DATE (IT IS NOT SUFFICIENT TO STATE FINDINGS WILL BE DISCUSSED ): 541 CSA bags were provided to 421 participants. $75.5 \%$ were females. $44.9 \%$ had Medicaid insurance. $47.7 \%$ had federal nutrition assistance. $63 \%$ has food insecurity. Those with federal nutrition assistance were more likely to report food insecurity. $10 \%$ returned to pick up a bag. Those returning scored average satisfaction at 9.4 out of $10.95 \%$ of those returning would want a weekly bag. Every cooking class participant would attend again. $95.7 \%$ of staff would refer patients to a long-term program. Focus group responses highlighted appreciation of vegetables, benefits of cooking classes, financial barriers to healthy eating, and desire to learn about nutrition.

KEY LESSONS FOR DISSEMINATION (WHAT CAN OTHERS TAKE AWAY FOR IMPLEMENTATION TO THEIR PRACTICE OR COMMUNITY?): Participants rated vegetable bags and cooking classes highly, corroborated by focus group qualitative data. We believe vegetable distribution and cooking classes are valuable for clinics with vulnerable patients. While the amount of vegetables provided was not enough to affect food insecurity, we believe this program fostered health behavior change among participants.

THE EFFECT OF A FRACTURE LIAISON SERVICE FOLLOWING HIP FRACTURE ON A VA POPULATION.

Sean Bradley $^{1,2}{ }^{2}$; Sarah Hall ${ }^{2}$; Yarbrough Peter ${ }^{2} .{ }^{1}$ University of Utah, Salt lake city, UT; ${ }^{2}$ George E Whalen VA Hospital, Salt Lake City, UT. (Control ID \#3184397)

STATEMENT OF PROBLEM OR QUESTION (ONE SENTENCE): Determine if a Fracture Liaison Service (FLS) improves treatment of osteoporosis following first time hip fractures in a single VA population. OBJECTIVES OF PROGRAM/INTERVENTION (NO MORE THAN THREE OBJECTIVES): The objective of the intervention was: 1. Idenfity patients with hip fractures during hospitalization with a new diagnosis of osteoporosis. 2. Start appropriate medical therapy for newly diagnosed osteoporosis following hip fracture.

DESCRIPTION OF PROGRAM/INTERVENTION, INCLUDING ORGANIZATIONAL CONTEXT (E.G. INPATIENT VS. OUTPATIENT, PRACTICE OR COMMUNITY CHARACTERISTICS): A Fracture Liason Service (FLS) is composed of a team of licensed pracitcal nurses and an endocrinologist at our VA hospital. Inpatient nurses identifing high risk patients for osteoporosis on the wards with signs of fragility fractures, ie hip fracture by reviewing inpatient team list and referring new hip fractures to endocrinology via e-consult for appropriate management either during or post hospitalization. We performed a retrospective chart review. ICD-9 and ICD-10 codes for hip fracture and osteoporosis were queried for the fiscal year 2015 and 2016 at a single VA site (SLCVA) resulting in a total of 61 first-time hip fractures to be reviewed (31 in 2015 and 30 in 2016). Primary and secondary outcomes for each year were compared using a Chi-squared test.

MEASURES OF SUCCESS (DISCUSS QUALITATIVE AND/OR QUANTITATIVE METRICS WHICH WILL BE USED TO EVALUATE PROGRAM/INTERVENTION): Primary outcomes: 1. Referral to endocrine bone health via E-consult. 2. DEXA scan within 6 months of hip fracture 3. Bisphosphonate/denosumab initiation within 6 months of hip fracture. Secondary outcomes: 1. Vitamin D labs drawn within 6 months of hip fracture 2. Vitamin D/Calcium supplementation within 6 months of hip fracture 3. 1 year mortality following hip fracture

FINDINGS TO DATE (IT IS NOT SUFFICIENT TO STATE FINDINGS WILL BE DISCUSSED ): There was a significant difference in increased endocrine bone referral $(p=0.03785)$, medication use $(\mathrm{p}=0.000003)$, vitamin $\mathrm{D}$ level drawn $(\mathrm{p}=0.023119)$, and vitamin D supplementation ( $\mathrm{p}=0.022705)$ following the implementation of a fracture liaison service prior to the fiscal year of 2016. There was a trend towards significance in increased DEXA screening $(p=0.058125)$. There was no significance noted between calcium supplementation $(p=0.822565)$ or mortality $(p=0.912117)$ Interestingly, the one-year mortality rate for the two years was noted to be $42.62 \%$. 
KEY LESSONS FOR DISSEMINATION (WHAT CAN OTHERS TAKE AWAY FOR IMPLEMENTATION TO THEIR PRACTICE OR COMMUNITY?): The use of a fracture liaison service can capture patients with osteoporosis in an effective way to deliver appropriate work-up and treatment following hip fracture

THE EFFECTS OF TELEHEALTH ON THE FREQUENCY OF PRIMARY CARE CLINIC VISITS

Morgan H. Randall ${ }^{1}$; Jingwen Zhang ${ }^{2}$; Elizabeth B. Kirkland ${ }^{2} .{ }^{1}$ Medical University of South Carolina, Charleston, SC; ${ }^{2}$ MUSC, Charleston, SC. (Control ID \#3185783)

STATEMENT OF PROBLEM OR QUESTION (ONE SENTENCE): Does patient participation in remote patient monitoring affect the frequency of primary care clinic visits?

OBJECTIVES OF PROGRAM/INTERVENTION (NO MORE THAN THREE OBJECTIVES): 1) Compare the frequency of primary care visits before and after enrollment in a remote patient monitoring program. 2) Compare the frequency of primary care visits between patients participating in remote patient monitoring to those not participating.

DESCRIPTION OF PROGRAM/INTERVENTION, INCLUDING ORGANIZATIONAL CONTEXT (E.G. INPATIENT VS. OUTPATIENT, PRACTICE OR COMMUNITY CHARACTERISTICS):

Telehealth has the potential to mitigate barriers to care and affect social determinants of health by providing access to medical professionals without requiring a patient's physical presence in a medical office. Our institution implemented a remote monitoring program targeting patients with uncontrolled diabetes and hypertension. Specifically, patients with a hemoglobin Alc greater than eight and with diabetes and hypertension managed exclusively by our primary care clinic are eligible to participate in a program utilizing a 2-in-1 automated device with glucose and blood pressure monitoring capability. Recorded values are transmitted directly via cell tower to a secure web server for review by healthcare providers. Based on this data, adjustments in medication regimens were made by physicians within the primary care clinic on a regular basis. This study compared visit frequency among patients who participated in the telehealth program and patients who were eligible but did not participate. While outcomes data are being assessed and will be reported elsewhere, we also aimed to discern whether enrollment in this program has altered the average number of clinic appointments for these patients before and after enrollment. The first available enrollment class offered after identification was used as the intervention date for the unenrolled cohort. MEASURES OF SUCCESS (DISCUSS QUALITATIVE AND/OR QUANTITATIVE METRICS WHICH WILL BE USED TO EVALUATE PROGRAM/INTERVENTION): Success in this study was defined as a decrease in the average number of clinic visits for participating patients compared to the same period of time prior to enrollment or compared to eligible but non-participating patients.

FINDINGS TO DATE (IT IS NOT SUFFICIENT TO STATE FINDINGS WILL BE DISCUSSED ): A total of 55 telehealth participants and 80 eligible but not enrolled patients have completed 9 months follow up. Participation in telehealth did not decrease the average number of primary care clinic visits when comparing visit frequency pre- and post-enrollment for the participant group ( 3.9 vs 3.6, p=0.51). Furthermore, there was no difference in visit frequency between patients participating in telehealth compared to those who were not participating (3.6 vs 3.9, p 0.54).

KEY LESSONS FOR DISSEMINATION (WHAT CAN OTHERS TAKE AWAY FOR IMPLEMENTATION TO THEIR PRACTICE OR COMMUNITY?): Telehealth is a tool that has the potential to help overcome barriers to care. Regardless of the effect of telehealth on the clinical outcomes for diabetes and hypertension, this study revealed no significant decrease in primary care clinic visits for patients participating in a telehealth program. This suggests that telehealth acts as a supplement rather than a replacement for primary care. As additional clinical data emerges and the follow up period is extended, the relationship between healthcare utilization and telehealth should be re-examined.

THE PRIMARY CARE PANEL RETENTION TOOL: A QUALITY IMPROVEMENT PROJECT TO REDUCE LOSS TO FOLLOW-UP IN A VA PRIMARY CARE CLINIC

Andrew T. Harris; Catherine Hoover; Mariel Zaun; Brendan L. Cmolik; Corinna D. Falck-Ytter. Louis Stokes Cleveland VA Medical Center, University Heights, OH. (Control ID \#3186691)

\section{STATEMENT OF PROBLEM OR QUESTION (ONE SENTENCE):} Can primary care panel retention be improved by a tool that identifies patients who are lost to follow-up?

OBJECTIVES OF PROGRAM/INTERVENTION (NO MORE THAN THREE OBJECTIVES): 1. Retain paneled patients by ensuring they have an encounter within the past 12 months and a future appointment or recall reminder 2. Promote a team-based approach to panel management across a large integrated healthcare system.

DESCRIPTION OF PROGRAM/INTERVENTION, INCLUDING ORGANIZATIONAL CONTEXT (E.G. INPATIENT VS. OUTPATIENT, PRACTICE OR COMMUNITY CHARACTERISTICS): The Primary Care Panel Retention Tool was implemented at the VA Northeast Ohio Healthcare System, the nation's $3^{\text {rd }}$ largest 1a high-complexity facility with 14 primary care locations and 143 Patient Aligned Care Teams (PACTs) serving over 90,000 veterans. The Primary Care Panel Retention Tool is a web based application that: 1 . Sorts patients by facility, Patient Aligned Care Team (PACT) and primary care provider (PCP) 2. Filters by last encounter with PACT team ( $<10 \mathrm{mo}, 10-12 \mathrm{mo}, 12-24 \mathrm{mo}$, no encounter) 3. Identifies patients lost to follow-up (ie, no future appointment, return to clinic order or recall) 4. Triages patients based on likelihood of adverse outcomes using the Care Assessment Needs (CAN) We pilot tested the tool in one primary care PACT and modified the tool based on feedback. Following pilot testing, we decided to address the following key contributing factors as part of our intervention: 1. Develop a tool to identify lost to follow-up patients - the Primary Care Panel Retention Tool 2. Standardize the outreach process for veterans that are lost to follow up 3. Identify best practices for difficult to reach veterans 4 . Standardize policy on how often veterans should be seen ideally once per year 5. Develop a toolkit for handling common scenarios such as death, incarceration, moved, etc. 6. Standardize processes for depaneling inactive patients Following multiple PDSA cycles, the Primary Care Panel Retention Tool was implemented across all primary care PACTs by September 2018. Each PACT was coached on use of the tool in their weekly team huddles. Outreach was targeted to patients not seen in the past 10-24 months.

MEASURES OF SUCCESS (DISCUSS QUALITATIVE AND/OR QUANTITATIVE METRICS WHICH WILL BE USED TO EVALUATE PROGRAM/INTERVENTION): 1 . Percentage of primary care patients not seen in the past year 2. Percentage of primary care patients with no scheduled follow-up (appointment, recall reminder or return to clinic order) FINDINGS TO DATE (IT IS NOT SUFFICIENT TO STATE FINDINGS WILL BE DISCUSSED ): Since starting data collection in December 2017, the number of primary care patients not seen within the past year has reduced from $10,039(10.5 \%)$ to $6,127(6.5 \%)$. The number of primary care 
patients without scheduled follow-up has decreased from 21,704 (22.6\%) to $16,061(17.1 \%)$.

KEY LESSONS FOR DISSEMINATION (WHAT CAN OTHERS TAKE AWAY FOR IMPLEMENTATION TO THEIR PRACTICE OR COMMUNITY?): 1. Primary Care teams can be empowered to control their own access 2 . Repeated small tests/pilots allowed us to identify problems early and customize 3 . Regular feedback from end users and customizability of the tool enhanced spread 4. Innovation and QI can work together. We developed an innovative solution, but it was addressing a recognized gap in care. The innovation was designed to address root causes. 5. Site visits and attending team huddle helped increased visibility and buy-in among team members.

THE SCOPE OF THE PROBLEM: A NOVEL MULTIDISCIPLINARY APPROACH TO REDUCE INPATIENT ENDOSCOPY DELAYS

Cameron Locke ${ }^{1}$; Molly A. Kantor ${ }^{1}$; Najwa El-Nachef ${ }^{1}$; Kendall Beck ${ }^{1}$; Sajan Patel $^{1}$; Helge Eilers ${ }^{1}$; Ryan David ${ }^{2}$; Scott Pasternak ${ }^{1}$; Bertina Herman ${ }^{2}$; Daniel Roseland ${ }^{1}$; Sara Lewin ${ }^{1} .{ }^{1}$ UCSF, San Francisco, CA; ${ }^{2}$ UCSF Health, San Francisco, CA. (Control ID \#3184690)

STATEMENT OF PROBLEM OR QUESTION (ONE SENTENCE): Endoscopies are commonly performed in the inpatient setting, and due to organizational complexity are prone to delays, which can lead to frustration amongst care providers and patients, patient discomfort from prolonged nil per os time, and excess costs from lengthened hospitalizations.

OBJECTIVES OF PROGRAM/INTERVENTION (NO MORE THAN THREE OBJECTIVES): 1 . Identify and characterize etiologies of delay to inpatient endoscopy 2. Reduce avoidable inpatient endoscopy delays to $<5 \%$ by optimizing workflow, scheduling, and staffing.

DESCRIPTION OF PROGRAM/INTERVENTION, INCLUDING ORGANIZATIONAL CONTEXT (E.G. INPATIENT VS. OUTPATIENT, PRACTICE OR COMMUNITY CHARACTERISTICS): A survey of hospitalists at our large, urban university medical center identified endoscopy as a common contributor to delays in patient care. Representatives from gastroenterology, anesthesiology, hospital medicine, inpatient nursing, and endoscopy nursing were engaged to create a multidisciplinary team to evaluate and intervene upon these delays. Root-cause analysis demonstrated multifactorial causes of endoscopy delays, and baseline data showed that $16.4 \%$ of inpatient endoscopies were delayed due to reasons characterized as avoidable, with an average length of delay of 1.22 days. The identified highest impact contributors to avoidable reasons for delay were endoscopy nursing availability, anesthesia provider availability, and volume of competing scheduled outpatient procedures. Three interventions to address these common causes of delay were implemented. First, dedicated anesthesiologist availability for inpatient endoscopy cases was provided on prespecified weekdays. Second, the endoscopy nursing staff instituted later and extended shifts to accommodate more procedures. Third, the on-call gastroenterologist reduced the number of daily scheduled outpatient endoscopic procedures, allowing for increased endoscopy suite allocation to inpatient procedures. The proposed interventions underwent several plan-do-study-act cycles to identify optimal implementation, with a target goal of 5\% delay rate.

MEASURES OF SUCCESS (DISCUSS QUALITATIVE AND/OR QUANTITATIVE METRICS WHICH WILL BE USED TO EVALUATE PROGRAM/INTERVENTION): Following intervention implementation, number of total endoscopies and number of delayed endoscopies, with subgroup analysis of cause of delay, were tracked for a 10 month period.
FINDINGS TO DATE (IT IS NOT SUFFICIENT TO STATE FINDINGS WILL BE DISCUSSED ): Scheduling conflicts, provider availability, and failure of adequate bowel preparation were found to be the most common causes of delay. Following implementation of the above interventions, the average rate of avoidable endoscopy delays fell to from $16.4 \%$ to $3.5 \%$ while average endoscopies per month remained constant.

KEY LESSONS FOR DISSEMINATION (WHAT CAN OTHERS TAKE AWAY FOR IMPLEMENTATION TO THEIR PRACTICE OR COMMUNITY?): Successful completion of inpatient endoscopies frequently requires close coordination among nurses, hospitalists, gastroenterologists, and anesthesiologists. Given the complexity of this multidisciplinary involvement, as well as variability in patient factors like clinical stability and presentation, endoscopies are prone to delays. However, multidisciplinary examination and optimization of nursing and anesthesiology availability coupled with optimal endoscopy suite utilization can significantly reduce inpatient endoscopy delays, independent of patient factors.

\section{TOBACCO CESSATION: BEYOND THE AFTER-VISIT SUMMARY \\ Richard Altman ${ }^{2}$; Hillary Chrastil ${ }^{2}$; Lauren Drake ${ }^{2}$; Carmen L. Lewis ${ }^{1}$. ${ }^{1}$ University of Colorado, Denver, CO; ${ }^{2}$ University of Colorado, Aurora, CO. (Control ID \#3178346)}

STATEMENT OF PROBLEM OR QUESTION (ONE SENTENCE): Tobacco cessation is one of the most high value interventions available with brief intervention by clinicians increasing cessation by 1-3\%; however, tobacco interventions are underutilized in ambulatory settings with national data showing only $20 \%$ of current tobacco users had tobacco cessation documented during visits with clinicians.

OBJECTIVES OF PROGRAM/INTERVENTION (NO MORE THAN THREE OBJECTIVES): 1 . Implement clinical architecture to maximize the opportunity for tobacco cessation interventions in the clinic setting. 2. Evaluate the implementation strategy.

DESCRIPTION OF PROGRAM/INTERVENTION, INCLUDING ORGANIZATIONAL CONTEXT (E.G. INPATIENT VS. OUTPATIENT, PRACTICE OR COMMUNITY CHARACTERISTICS): The intervention was first implemented at an academic General Internal Medicine practice in Denver (Practice A). The intervention was then implemented at additional academic practices, one Family Medicine practice and one of combined Family Medicine and General Internal Medicine practice (Practices B and C). Each practice received a Tobacco Implementation Package (TIP) that included baseline data, electronic medical record (EMR) tools, training tools, and report access. EMR tools included MA and clinician workflows in which the MA collects current tobacco use information and the clinician receives a prompt with support for documenting brief tobacco counseling, an assessment, and plan. Training tools included a presentation and EMR tip sheets. Report access included audit and feedback and care management tools.

MEASURES OF SUCCESS (DISCUSS QUALITATIVE AND/OR QUANTITATIVE METRICS WHICH WILL BE USED TO EVALUATE PROGRAM/INTERVENTION): 1. Proportion of visits where 1) tobacco status was reviewed by the MA and 2) MA asked if the patient was ready to quit (RTQ). 2. Proportion of visits among tobacco users with clinician documentation about tobacco in the assessment and plan. 3. Practice use of new reports.

FINDINGS TO DATE (IT IS NOT SUFFICIENT TO STATE FINDINGS WILL BE DISCUSSED ): At Practice A, baseline data show that tobacco status was reviewed at $91 \%$ of visits and RTQ was asked $46 \%$ of the 
time. At Practices B and $\mathrm{C}$, baseline rates for reviewing tobacco status were $94 \%$ and $96 \%$ and RTQ was asked $20 \%$ and $21 \%$ of the time. After TIP, Practice A reviewed tobacco status $94 \%$ of the time and asked RTQ $75 \%$ of the time, Practice B reviewed tobacco status $92 \%$ of the time and asked RTQ $32 \%$ of the time, and Practice C reviewed tobacco status $99 \%$ of the time and asked RTQ $32 \%$ of the time. After TIP, Practice A clinician documentation trended upward over 12 months ( 40 to $51 \%$ ) as did Practice B over 5 months (10 to $25 \%$ ), Practice $\mathrm{C}$ demonstrated a downward trend over 3 months (26\% to $19 \%$ ) On average, Practice A accessed the reports eight times per month, Practice $\mathrm{B}$ accessed the reports four times per month, and Practice $\mathrm{C}$ did not access the reports.

KEY LESSONS FOR DISSEMINATION (WHAT CAN OTHERS TAKE AWAY FOR IMPLEMENTATION TO THEIR PRACTICE OR COMMUNITY?): MA performance of reviewing current tobacco status was high and remained high. TIP improved performance of MAs asking current tobacco users if they are ready to quit. Clinician documentation is trending up at two practices and trending down at one, who has had the least amount of time with the TIP. During this implementation period, the practices who accessed the reports saw improvement in their clinician documentation. This project is on-going so will include an additional practice site and data at the time of presentation.

UNCOVERING BARRIERS TO PRESCRIBING POSTDISCHARGE NICOTINE REPLACEMENT THERAPY FOR HOSPITALIZED SMOKERS

$\underline{\text { John Landefeld }}^{1,2}{ }^{2}$; Scott Goldberg ${ }^{1,}{ }^{2}$; Joseph Clement ${ }^{1,2}$; Maya Vijayaraghavan ${ }^{1,2}$. ${ }^{1}$ ucsf, San Francisco, CA; ${ }^{2}$ Zuckerberg San Francisco General Hospital, San Francisco, CA. (Control ID \#3184785)

STATEMENT OF PROBLEM OR QUESTION (ONE SENTENCE): Despite guidelines recommending that all hospitalized smokers receive tobacco-cessation counseling and nicotine replacement therapy (NRT) during hospitalization, low-income patients who have high rates of smoking and who are frequently cared for in public hospitals often do not receive this treatment. OBJECTIVES OF PROGRAM/INTERVENTION (NO MORE THAN THREE OBJECTIVES): Our hospital implemented an Alcohol and Tobacco Treatment Team (ATTT), comprised of licensed vocational nurses overseen by a Clinical Nurse Specialist to deliver tobacco and alcohol cessation interventions to hospitalized patients upon admission. The focus of this abstract is on tobacco cessation interventions.

DESCRIPTION OF PROGRAM/INTERVENTION, INCLUDING ORGANIZATIONAL CONTEXT (E.G. INPATIENT VS. OUTPATIENT, PRACTICE OR COMMUNITY CHARACTERISTICS): The ATTT is an inpatient service that provides at least one-time, in-person tobacco cessation counseling, outpatient referrals for cessation, and medication recommendations during the inpatient stay and upon discharge to all smokers on admission.

MEASURES OF SUCCESS (DISCUSS QUALITATIVE AND/OR QUANTITATIVE METRICS WHICH WILL BE USED TO EVALUATE PROGRAM/INTERVENTION): While the ATTP achieved near complete success in reaching all hospitalized smokers for counseling, the rates of receiving discharge NRT was substantially lower. We examined characteristics associated with lower rates of being prescribed NRT on discharge. Our analysis included 1577 hospitalized smokers, seen for a single visit by the ATTT in 2017. We extracted the following data from the electronic health record in order to examine factors associated with receiving discharge NRT: patient demographics, insurance status (Medicaid, Medicare, private, uninsured), English proficiency status (English-speaking vs non-English-speaking), and service type (Medicine, Cardiology, Family Practice, Neurology, and Surgery/Surgical subspecialties). We also conducted six in-depth, semi-structured interviews of Internal Medicine housestaff.

FINDINGS TO DATE (IT IS NOT SUFFICIENT TO STATE FINDINGS WILL BE DISCUSSED ): Of the patients, $75 \%$ were male, $90 \%$ were English-speaking, and $93 \%$ were covered by public health insurance (i.e. Medicaid or Medicare). Whereas $26.2 \%$ of patients received NRT on discharge, $56.2 \%$ refused NRT, and $17.2 \%$ were recommended to receive NRT but were not prescribed. Younger patients and non-English-speaking patients were more likely to be recommended to receive NRT but not receive a prescription $(p<0.03)$. Patients on the medicine and family practice services were more likely to receive discharge NRT than patients on other services. In interviews, housestaff identified several barriers to prescribing NRT: 1) ATTT recommendations are often communicated via text page later in the day when the primary medical team is off; and 2) ATTT recommendations are not documented in the same area of the EHR used for inpatient care.

KEY LESSONS FOR DISSEMINATION (WHAT CAN OTHERS TAKE AWAY FOR IMPLEMENTATION TO THEIR PRACTICE OR COMMUNITY?): A single-enterprise EHR may enable improved communication around cessation care between the ATTT and primary MDs, and could facilitate automatic precribing of NRT for eligible and interested patients at discharge. Additional quantitative and qualitative analyses are necessary to understand the reasons behind the lower screening and prescribing rates of discharge NRT for non-English-speaking patients as well as patients on nonmedicine services.

\section{USE OF ASSESSMENTS AND FACILITATION TO REDESIGN AN INTEGRATED HEALTH SYSTEM}

Stella Safo; Brooke Gogel. Icahn School of Medicine Mount Sinai, New York, NY. (Control ID \#3174062)

STATEMENT OF PROBLEM OR QUESTION (ONE SENTENCE): Redesigning an urban primary care network to deliver value-based care OBJECTIVES OF PROGRAM/INTERVENTION (NO MORE THAN THREE OBJECTIVES): To review an approach to large scale primary care redesign To evaluate how an assessment tool and facilitation impacts primary care redesign

DESCRIPTION OF PROGRAM/INTERVENTION, INCLUDING ORGANIZATIONAL CONTEXT (E.G. INPATIENT VS. OUTPATIENT, PRACTICE OR COMMUNITY CHARACTERISTICS): Mount Sinai Health System (MSHS) in New York is an integrated health system aiming to become a population health management center. As part of these efforts, 47 primary care practices are undergoing comprehensive assessment and implementing improvements around technology, quality improvement (QI) infrastructure, and operational workflows. Practice facilitators, individuals trained in QI and project management, are paired with practices to guide them through redesign initiatives. The Redesign Team developed an assessment tool to determine the amount of practice facilitation needed for each practice. The tool evaluated physical space, technology efficacy, population health management capabilities, accessibility, culture of transformation, staffing/workflows, and patient population. Assessments were administered over visits at the first 8 sites through a series of observations and key informant interviews with practice leadership and staff. Facilitators were then assigned to practices based on the results.

MEASURES OF SUCCESS (DISCUSS QUALITATIVE AND/OR QUANTITATIVE METRICS WHICH WILL BE USED TO EVALUATE PROGRAM/INTERVENTION): Each practice is evaluated by metrics defined broadly by workflow optimization, quality improvement, and technology. Measures of success include evaluating staff satisfaction, teaching 
Lean QI to practice leadership, increasing same day access, and implementing daily team huddles and standardized workflows around care transitions.

FINDINGS TO DATE (IT IS NOT SUFFICIENT TO STATE FINDINGS WILL BE DISCUSSED ): Results varied across each category. For example, 4 practices showed weakness in population health metrics as measured by their lack of proactive patient empanelment, resulting in episodic care. Within staffing metrics, 3 practices had limited ancillary staff, meaning resources like behavioral health and pharmacy were either unavailable or only remotely available. Certain challenges were experienced by all 8 practices, including after hours care alternatives, limited workflow standardization, staffing vacancies, inadequate EHR training, minimal usage of population health tools, and inconsistencies in QI engagement. Based on these findings, practices were assigned a facilitator. 1 practice which was struggling with population health and leadership received a combination of facilitation and intensive regional leadership oversight. 5 practices with moderate rating received embedded facilitation. 2 practices demonstrated strength across all categories and thus did not receive continuous facilitation.

KEY LESSONS FOR DISSEMINATION (WHAT CAN OTHERS TAKE AWAY FOR IMPLEMENTATION TO THEIR PRACTICE OR COMMUNITY?): This case represents an important effort by an integrated urban health system to become a population health manager. Our experience at MSHS suggests that redesign can be rolled out at scale if a health system is willing to invest in certain resources; in our case, we designed an easy-to-administer assessment tool which then allowed us to allocate practice support (in the form of facilitators) to the appropriate practices.

\section{USING ACGME MILESTONES TO IMPROVES DIABETES MAN- AGEMENT}

Emily D. Fondahn; Melvin Blanchard. Washington University, St Louis, MO. (Control ID \#3164099)

\section{STATEMENT OF PROBLEM OR QUESTION (ONE SENTENCE):} Does linking practice-based milestones to completion of diabetes foot exam improve performance?

OBJECTIVES OF PROGRAM/INTERVENTION (NO MORE THAN THREE OBJECTIVES): Improve percent of diabetic patients with up-todate foot exam Implement standard method to assess residents on PBL1 and PBL2 milestones

DESCRIPTION OF PROGRAM/INTERVENTION, INCLUDING ORGANIZATIONAL CONTEXT (E.G. INPATIENT VS. OUTPATIENT, PRACTICE OR COMMUNITY CHARACTERISTICS): Residents received an email twice per year with their individualized diabetes quality metrics, along with overall clinic performance on the quality metrics for the preceding 6 months. The residents completed a chart audit to determine the number of diabetic patients having an up-to-date foot exam performed and documented. Patients were excluded from the chart audit if the primary care physician was incorrect, had only been seen once in the clinic or followed for less than a year, did not have diabetes, or had a lower extremity amputation. The residents emailed their results to the Associate Program Director (EF). The results from the diabetes foot exam chart audit were linked to assessment on the following subcompentencies: Monitor practice with a goal for improvement (PBLI 1) and Learns and improves via performance audit (PBLI 2).

MEASURES OF SUCCESS (DISCUSS QUALITATIVE AND/OR QUANTITATIVE METRICS WHICH WILL BE USED TO EVALUATE PROGRAM/INTERVENTION): The goals were to achieve at least $80 \%$ of diabetic patients with an up-to-date foot exam and have $100 \%$ of residents complete the chart audit.
FINDINGS TO DATE (IT IS NOT SUFFICIENT TO STATE FINDINGS WILL BE DISCUSSED ): The percent of residents who submitted their diabetes data each year was very high (96-100\%). The overall foot exam rate for the clinic increased from $56 \%$ to $72 \%$. The percent of patients with diabetic foot exams improved each year of residency, with the largest improvement between first and second year (PGY1- 56\%; PGY2-70\%; PGY3-72\%). There were no meaningful, sustained changes in other diabetes metrics, include $\mathrm{HbAlc}$ control, blood pressure control and screening for nephropathy.

KEY LESSONS FOR DISSEMINATION (WHAT CAN OTHERS TAKE AWAY FOR IMPLEMENTATION TO THEIR PRACTICE OR COMMUNITY?): Developing a standard method to assess PBLI milestones across a large residency program can be challenging. Having clear expectations and a unified goal for the clinic were critical for implementation of this project and buy-in from the residents and attendings.

USING TELEHEALTH TO IMPROVE PATIENTS' POSTDISCHARGE ACCESS TO PCPS, FOLLOWING DISCHARGE FROM THE HOSPITAL.

Priyanka Agarwal $^{1}$; Tannaz Safari-Vejin ${ }^{3}$; Nathaniel Gleason ${ }^{2}$; Yumiko AbeJones ${ }^{1}$; Michelle Mourad ${ }^{1} .{ }^{1}$ University of California, San Francisco, San Francisco, CA; ${ }^{2} \mathrm{UC}$ San Francisco, Nathaniel Gleason, CA; ${ }^{3}$ Lincoln Memorial University, Harrogate, TN. (Control ID \#3185300)

STATEMENT OF PROBLEM OR QUESTION (ONE SENTENCE): The transition home from the hospital is a high-risk time for patients, and prompt follow up with a patient's PCP is important. Due to primary care and patient access constraints, only $38 \%$ of patients with a PCP discharged home from hospital medicine see their PCP within 2 weeks of discharge, at our institution.

OBJECTIVES OF PROGRAM/INTERVENTION (NO MORE THAN THREE OBJECTIVES): The goal of the Post-Discharge Video Visit Program was to increase patient access to PCPs in the post discharge period, and to have $100 \%$ of patients discharged home from the hospital medicine service seen by their PCP within 2 weeks of discharge. To do so, we piloted a program in which patients discharged home from hospital medicine were offered a Video Visit (VV) with their PCP.

DESCRIPTION OF PROGRAM/INTERVENTION, INCLUDING ORGANIZATIONAL CONTEXT (E.G. INPATIENT VS. OUTPATIENT, PRACTICE OR COMMUNITY CHARACTERISTICS): The program was implemented as follows: The telehealth discharge coordinator (TDC) ran a custom Epic Workbench report to identify eligible patients on the hospital medicine service (patients being discharged home, with a UCSF PCP). The TDC approached eligible patients to offer a VV with their PCP; in-person visits were preferentially booked as possible. PCPs of interested patients were emailed asking if they were amenable to conducting a VV. VVs were then conducted using the Zoom platform.

MEASURES OF SUCCESS (DISCUSS QUALITATIVE AND/OR QUANTITATIVE METRICS WHICH WILL BE USED TO EVALUATE PROGRAM/INTERVENTION): The following metrics were collected: a) Volumes of patients discharged home from the hospital medicine service, b) Volumes of patients offered and/or scheduled for a VV with their PCP d) Volumes of patients discharged home who were able to see their PCP in person or via telehealth, and e) Volumes of PCPs who participated. Qualitative reasons for declining VVs were also collected.

FINDINGS TO DATE (IT IS NOT SUFFICIENT TO STATE FINDINGS WILL BE DISCUSSED ): The program was conducted from JulyDecember 2017, over a period of 64 days. During this period 1236 patients 
were discharged from the hospital medicine service. 649 patients were discharged home and 171 of these patients had a UCSF PCP, representing the patient population eligible for the Program. 125 patients were approached by the TDC, and of those 56 patients (45\% of those screened), expressed interest in a post discharge VV with their PCP. Of these 56 patients, 14 had VVs scheduled, with $11 \mathrm{VVs}$ completed (or $6 \%$ of the eligible patient population). 69 screened patients declined VVs due to a preference for an in-person visit ( $\mathrm{n}=36$ patients), lack of access to VV technology ( $\mathrm{n}=14$ patients), lack of comfort in conducting a VV ( $\mathrm{n}=9$ patients), or for other reasons ( $\mathrm{n}=10$ patients). 36 PCPs were approached to conduct a VV for their patient; 20 PCPs were amenable, and 10 PCPs participated in the program. 16 PCPs declined to participate due to lack of availability ( $n=5$ PCPs), a preference for an in-person visit ( $\mathrm{n}=7$ PCPs), or a lack of comfort in conducting VVs ( $n=4$ PCPs).

KEY LESSONS FOR DISSEMINATION (WHAT CAN OTHERS TAKE AWAY FOR IMPLEMENTATION TO THEIR PRACTICE OR COMMUNITY?): Patients do have an interest in engaging in care using Telehealth. However, the post-discharge period may not be the ideal setting to encourage telehealth adoption based on our program's low adoption rate. In a specific patient population, post discharge VVs may be a way to increase access to PCPs following hospital discharge.

UTILIZATION OF PATIENT NAVIGATORS: AN INNOVATIVE METHOD FOR CONNECTING PATIENTS WITH COMMUNITY RESOURCES WITHIN THE SOCIAL HEALTH ALLIANCE TO PROMOTE HEALTH EQUITY (S.H.A.P.E) PROGRAM.

Rachael Odusanya $^{2}$; Jane Lindahl ${ }^{2}$; Johanna Martinez ${ }^{3}$; Omolara T. Uwemedimo $^{1}$; Shari Jardine ${ }^{1}$; Eun Ji Kim ${ }^{4}{ }^{1}$ Northwell Health, New Hyde Park, NY; ${ }^{2}$ Zucker School of Medicine at Hofstra/Northwell, Lake Success, NY; ${ }^{3}$ Northwell Health, Lake Success, NY; ${ }^{4}$ Northwell Hofstra School of Medicine, Lake Success, NY. (Control ID \#3183353)

STATEMENT OF PROBLEM OR QUESTION (ONE SENTENCE): Many programs have adopted social determinants of health (SDH) screening and referral processes, however, there is a need to provide support and ensure that patients are utilizing referrals.

OBJECTIVES OF PROGRAM/INTERVENTION (NO MORE THAN THREE OBJECTIVES): 1) To recruit student interns as Patient Navigators (PNs) to act as social support staff in order to address SDH and follow-up with patients continuously for 12 weeks. 2) To equip PNs with motivational interviewing, cultural competency, and resource navigation skills that allow for patient centered follow-up. 3) To examine PNs impact on patient satisfaction and resource utilization.

DESCRIPTION OF PROGRAM/INTERVENTION, INCLUDING ORGANIZATIONAL CONTEXT (E.G. INPATIENT VS. OUTPATIENT, PRACTICE OR COMMUNITY CHARACTERISTICS): Our health system's Social Needs Screening Program was established as a way to identify and address adverse SDH in Internal Medicine and Pediatric patient populations. The program includes undergraduate students interested in healthcare, health policy, and social work as PNs. They screen patients for unmet social needs and help them navigate community resources. PNs completed a week-long training on SDH content, the screening process, motivational interviewing, cultural competency, community health organizations, and a medical legal partnership. They also precept case managers and physicians to get clinical experiences. The training prepares $\mathrm{PNs}$ for in-depth patient interaction during the screening and 12 week follow-up process, where patients are followed bi-weeky via telephone, e-mail or in-person.
MEASURES OF SUCCESS (DISCUSS QUALITATIVE AND/OR QUANTITATIVE METRICS WHICH WILL BE USED TO EVALUATE PROGRAM/INTERVENTION): We evaluate the effectiveness of PN social support in the clinical setting by examining; questions centered around patient experience with program as well as evaluation surveys on training and overall PN experience, percent of patients with unmet needs who are connected to resources, and percent of patients who successfully utilize resources within 12 weeks,

FINDINGS TO DATE (IT IS NOT SUFFICIENT TO STATE FINDINGS WILL BE DISCUSSED ): In 2018, of the 326 patients who screened positive for social needs, $152(47 \%)$ accepted PN assistance. Of those, $81(53 \%)$ were connected to resources. To date, 11/16 (69\%) who have completed the navigation process have successfully utilized the resources provided. When asked "How helpful did you find this experience," $73 \%(n=8 / 11)$ of patients found the process helpful or very helpful. A survey of current PNs demonstrated that $88 \%(n=14 / 16)$ have a better understanding of SDHs than before participating in program, and $69 \%$ $(n=11 / 16)$ believed that the week of training prepared them for their role.

KEY LESSONS FOR DISSEMINATION (WHAT CAN OTHERS TAKE AWAY FOR IMPLEMENTATION TO THEIR PRACTICE OR COMMUNITY?): The use of PNs to provide dedicated screening and referrals to address resource needs in a primary care setting allows for a patient-centered approach to addressing SDH through "warm hand-offs" to community health resources, personalized follow-up and navigation services. Integration of the PN's into clinical sites has alleviated the burden on other clinical staff and providers, who often may not have time to address $\mathrm{SDH}$ with their patients. Additionally, having bilingual Navigators, who have been trained in cultural competency and motivational interviewing allows for an added level of comfort and trust to promote patient engagement in the program.

VA VIRTUAL PRIMARY CARE PROVIDERS REDUCE OPIOID USE AND INCREASE OPIOID SAFETY IN RURAL VA CLINICS. Anna Abramson ${ }^{1}$; Henry Crevensten ${ }^{2} .{ }^{1}$ UCSF/Veterans Affairs, Mill Valley, CA; ${ }^{2}$ University of California, San Francisco, San Francisco, CA. (Control ID \#3186111)

STATEMENT OF PROBLEM OR QUESTION (ONE SENTENCE): Rural communities have the highest rates of opioid prescriptions and opioid-related deaths, yet lack consistent access to primary care. OBJECTIVES OF PROGRAM/INTERVENTION (NO MORE THAN THREE OBJECTIVES): 1 . Assess the opioid prescription metrics in rural community clinics affiliated with the San Francisco Veterans Affairs Health Care System before and after initiation of a virtual primary care program. 2. Improve opioid prescription safety through continuous patient panel management.

DESCRIPTION OF PROGRAM/INTERVENTION, INCLUDING ORGANIZATIONAL CONTEXT (E.G. INPATIENT VS. OUTPATIENT, PRACTICE OR COMMUNITY CHARACTERISTICS): Rural clinics in socioeconomically disadvantaged populations are experiencing staff shortages and rely on locums and gap provider coverage. Patients with chronic pain require continuity of care for successful pain and opioid management. In 2017, San Francisco Veteran's Affairs Medical Center implemented a virtual primary care team known as "Teleprimary Care" to increase continuous primary care access by leveraging video teleconferencing modalities. Teleprimary Care providers (TelePCPs) were integrated into existing multidisciplinary teams to cover one or more panels among three chronically understaffed rural clinics. This review focuses on 
the largest regional rural clinic ("Clinic A") to demonstrate the trends seen in all 3 clinics. Clinic A has 2600 patients on 5 patient panels. A single inperson primary care provider had been at the clinic for several years prior to the study period and maintained their existing panel during the 12 months studied. Three TelePCPs shared 2 patient panels of which one TelePCP served as clinic medical director. The other 2 panels had 7 locums and 9 gap coverage providers over 12 months.

MEASURES OF SUCCESS (DISCUSS QUALITATIVE AND/OR QUANTITATIVE METRICS WHICH WILL BE USED TO EVALUATE PROGRAM/INTERVENTION): Decrease in number of patients receiving greater than 90 MEDD and co-prescription of opioid and benzodiazepines.

FINDINGS TO DATE (IT IS NOT SUFFICIENT TO STATE FINDINGS WILL BE DISCUSSED ): Nationally in the VA in 2018, the percentage of patients with greater than 90 morphine equivalent daily doses (MEDD) decreased from 1.13 to $0.86 \%$. Regionally at the SFVA, $>90$ MEDD scripts decreased from 1.5 to $1.2 \%$. The Rural Clinic A panel covered by the single, in-person continuous PCP, $>90$ MEDD scripts decreased from 0.97 to $0.61 \%$. Rural Clinic A panels covered by TelePCPs $>90$ MEDD scripts decreased from 4-7\% to $1.2-1.8 \%$ over 12 months. Eleven months after implementation, of the 27 patients on $>90$ MEDD written in 1 month: 4 prescriptions (15\%) were written by PCP ( 1 inperson, 1 virtual) of 925 paneled patients; 17 prescriptions (63\%) by gap providers (3 locums, 1 virtual) of 973 paneled patients; and 6 prescriptions (22\%) written by medical director for his panel and unassigned patients. After integration of the virtual PCP teams to Clinic A, the number of patients with co-prescription of benzodiazepines and opioids decreased from $2.2 \%$ to $1.3 \%$, compared to VA national level of $1.14 \%$ to $0.86 \%$ and SFVA level of $1.25 \%$ to $1.03 \%$. These trends were also observed at the other 2 rural clinics that were assigned TelePCPs.

KEY LESSONS FOR DISSEMINATION (WHAT CAN OTHERS TAKE AWAY FOR IMPLEMENTATION TO THEIR PRACTICE OR COMMUNITY?): Adequate pain assessment and opioid management requires continuity of primary care to patients in rural communities. Consider virtual primary care providers for management of chronic pain patients.

VIDEO VISITS IN PRIMARY CARE: A FEASIBLE AND CONVENIENT ADDITION TO TRADITIONAL CARE

$\underline{\text { Kathryn L. Hughes }}{ }^{1}$; Susan B. Glick ${ }^{2}$; Anthony J. Perry ${ }^{2}$; Marisa Truesdell ${ }^{2}$; Amanda Tosto ${ }^{2}$; Lisa Ravindra ${ }^{2}$. ${ }^{1}$ Rush University Medical Center, Highland Park, IL; ${ }^{2}$ Rush University Medical Center, Chicago, IL. (Control ID \#3163533)

STATEMENT OF PROBLEM OR QUESTION (ONE SENTENCE): Can the addition of video visits enhance a fee for service practice environment?

OBJECTIVES OF PROGRAM/INTERVENTION (NO MORE THAN THREE OBJECTIVES): Explore patient interest in video visits for low acuity convenient care Maintain continuity of care for patients with non-urgent medical conditions Evaluate the feasibility and acceptability of video visits in primary care

DESCRIPTION OF PROGRAM/INTERVENTION, INCLUDING ORGANIZATIONAL CONTEXT (E.G. INPATIENT VS. OUTPATIENT, PRACTICE OR COMMUNITY CHARACTERISTICS): We initiated a video visit (VV) program for established primary care (PC) patients at a large urban academic medical center. A committee of clinicians, information technologists, and administrators met twice weekly for 3 months to develop workflows, acquire hardware, train staff, and set goals. Patients were notified of VVs via email and flyers. We used an established video platform in the electronic medical record. Physicians used a tablet in an exam room and a headset with a microphone. Patients accessed VVs via their online portal using smartphones or computers with a camera. 9 medical assistants and 3 nurses were trained in VV execution. VVs were piloted by 2 physicians for 23 acute, non-urgent conditions, including urinary tract infection, cough, contraception, and follow up at physician's discretion. After a free 6-week pilot, patients paid \$49 per visit. Patients received a 5-question post-visit survey regarding convenience, satisfaction, need for additional care, alternative care options, and comparison to an inperson visit.

MEASURES OF SUCCESS (DISCUSS QUALITATIVE AND/OR QUANTITATIVE METRICS WHICH WILL BE USED TO EVALUATE PROGRAM/INTERVENTION): Success was assessed via number of VVs completed, number of patients utilizing the program, net promoter score (NPS), relative value units (RVU), and a post-visit patient survey (PVS).

FINDINGS TO DATE (IT IS NOT SUFFICIENT TO STATE FINDINGS WILL BE DISCUSSED ): During the pilot, 2 physicians completed $8 \mathrm{VVs}$ in the course of a normal workday with 8 patients (7 women, 1 man). One 19-year-old man rescheduled due to lack of internet access. VVs were scheduled with the patient's PC physician. Mean age was $39.2 \pm 17.7$ years. Maximum VV time was 15 minutes. Conditions addressed included anxiety, acute sinusitis, travel advice, contraception, urinary tract infection, low back pain and follow up (herpes labialis and hydrocele). Each visit earned 0.97 RVUs. 6 patients completed PVS. All (6/6) completed visits without needing further care and reported the visit was easy to complete. Were VVs unavailable, 2/6 respondents would have seen a doctor in person, 2/6 would have visited a retail clinic, and 1/6 would have visited another medical center. All (6/6) rated VV quality equal to that of an inperson visit. NPS was $60 \%(n=5)$. Key challenges were privacy, internet connection, and protocols for refunds and rescheduling. Physician concerns included continuity of care, reimbursement, and inability to examine patients.

KEY LESSONS FOR DISSEMINATION (WHAT CAN OTHERS TAKE AWAY FOR IMPLEMENTATION TO THEIR PRACTICE OR COMMUNITY?): Incorporating VVs in PC is both feasible and acceptable to patients. Were VVs unavailable, half of our patients would have sought care outside of our organization. For our VVs, the NPS, a measure of customer loyalty, was comparable to that of Apple laptops $(63 \%)$, iPhones $(60 \%)$, and Netflix $(62 \%)$. Implementing a VV program requires attention to privacy, connectivity, and protocols for rescheduling.

\section{WANT 100\% SEPSIS BUNDLE COMPLIANCE?}

Ali Al-Hilli ${ }^{1}$; Michaela Wilsmann ${ }^{1}$; Joanna Pluta ${ }^{2}$; Jonathan A. Schiller ${ }^{1}$. ${ }^{1}$ Marshfield Medical Center, Marshfield, WI; ${ }^{2}$ Marshfield Clinic, Marshfield, WI. (Control ID \#3171799)

STATEMENT OF PROBLEM OR QUESTION (ONE SENTENCE): Early recognition and treatment of sepsis continue to be a major local, national and global challenge.

OBJECTIVES OF PROGRAM/INTERVENTION (NO MORE THAN THREE OBJECTIVES): 1. Identify factors that contribute to delays in early recognition and treatment of sepsis 2 . Implement interventions to improve compliance with the recommended sepsis care bundle 3 . Measure outcomes as percentage of care bundle compliance and mortality 
DESCRIPTION OF PROGRAM/INTERVENTION, INCLUDING ORGANIZATIONAL CONTEXT (E.G. INPATIENT VS. OUTPATIENT, PRACTICE OR COMMUNITY CHARACTERISTICS): Marshfield Medical Center is a 500-bed tertiary hospital in north central Wisconsin. We formed the Sepsis Committee in October 2015, at the time when CMS (Centers for Medicare \& Medicaid Services) introduced the new SEP-1 core measure for adult patients with severe sepsis and septic shock. Arguably, this is the most challenging core measure ever introduced by CMS MEASURES OF SUCCESS (DISCUSS QUALITATIVE AND/OR QUANTITATIVE METRICS WHICH WILL BE USED TO EVALUATE PROGRAM/INTERVENTION): We obtained all adult severe sepsis and septic shock diagnoses through our coding and billing departments. We analyzed each patient case for the following metrics: Received within three hours: - Sepsis lactate (will repeat in $3 \mathrm{hrs}$ if $>2$ ) - Broad spectrum antibiotic Blood cultures drawn prior to antibiotics AND if SBP $<90$ or lactate $>4$ : Initiated within three hours: - Resuscitation with $30 \mathrm{ml} / \mathrm{kg}$ crystalloid fluids (may base on ideal body weight [IBW] if morbidly obese and fluids are ordered based on IBW. Documentation must be clear.) AND received within six hours: - Repeat lactate level measurement only if initial lactate level is elevated over 2.0 - Vasopressors for persistent hypotension - MD focused sepsis exam-documented All cases were brought for discussion during our monthly CME-approved meetings. For cases where the core measure was not adhered to, follow up was delegated to the appropriate team member.

FINDINGS TO DATE (IT IS NOT SUFFICIENT TO STATE FINDINGS WILL BE DISCUSSED ): The latest national average compliance rate with the SEP-1 core measure was $50 \%$, our running average to date was $77 \%$ and our latest audit showed that we have now reached $100 \%$ compliance with the SEP-1 core measure.

KEY LESSONS FOR DISSEMINATION (WHAT CAN OTHERS TAKE AWAY FOR IMPLEMENTATION TO THEIR PRACTICE OR COMMUNITY?): Excellent sepsis care requires a multidisciplinary and system-based approach to streamline the workflow. We accomplished this by involving the appropriate representatives and leaders in our meetings to help us facilitate the required changes. We automated our laboratory system to order repeat lactate within 3 hours, without any provider having to place a separate order. We created a sepsis order set to guide providers towards meeting all the care bundle components. We invested in a cloud-based software and modified it to serve us as an early warning system, triggering alerts when a combination of parameters is suggestive of sepsis. We provided education via email, Grand Rounds, departmental meetings, resident lectures, posters, and pocket cards. We created online learning modules for different members of the healthcare team to cater to their scope of duties. Furthermore, we ventured beyond the hospital's walls to spread sepsis education. We gave talks to Emergency Medical Crews, we presented at a regional nursing conference and we provided education to all the local nursing home staff.

WELLNESS FOR PRIMARY CARE PROVIDERS: THE IMPACT OF WELLNESS COMMITTEE INTERVENTIONS ON PROVIDER SATISFACTION

Stephanie Duarte ${ }^{1}$; Gabi Schiller ${ }^{2}$; Kira Y. Xie ${ }^{3}$; Lauren Peccoralo ${ }^{4} .{ }^{1}$ Mount Sinai Hospital, New York, NY; ${ }^{2}$ Mount Sinai Health System, New York, NY; ${ }^{3}$ Icahn School of Medicine at Mount Sinai, New York, NY; ${ }^{4}$ Mount Sinai School of Medicine, New York, NY. (Control ID \#3185490)

STATEMENT OF PROBLEM OR QUESTION (ONE SENTENCE): Burnout rates in primary care providers (PCP) can be as high as 50\% which can lead to mental illness, decreased morale, decreased productivity, decreased retention, and lower patient satisfaction and quality of care.
OBJECTIVES OF PROGRAM/INTERVENTION (NO MORE THAN THREE OBJECTIVES): To determine if implementing a number of wellbeing, work flow, and staffing interventions has improved faculty physician (MD) and nurse practitioner (NP) satisfaction and reduced burnout in an urban academic hospital based clinic.

DESCRIPTION OF PROGRAM/INTERVENTION, INCLUDING ORGANIZATIONAL CONTEXT (E.G. INPATIENT VS. OUTPATIENT, PRACTICE OR COMMUNITY CHARACTERISTICS): A wellness committee comprised of 7 members convened individually and with leadership on a monthly basis over the course of 4 months to create a preintervention survey for the faculty physician and NP providers at an academic primary care clinic. Based on the results of the survey, interventions were created and instituted including hiring 3 administrative assistants, addition of IT equipment to work space, scheduling changes to evenly distribute evening clinic sessions, as well as annual bonus letters and biannual wellness grand rounds. A 1 year follow up survey was administered, mirroring the pre-survey (modified Mini-Z, 16 questions, quantitative and qualitative). The survey responses were compared to assess effectiveness of the implemented interventions.

MEASURES OF SUCCESS (DISCUSS QUALITATIVE AND/OR QUANTITATIVE METRICS WHICH WILL BE USED TO EVALUATE PROGRAM/INTERVENTION): Primary outcomes are level of provider burnout and satisfaction with current job. Secondary outcomes include sense of control, teamwork, stress, and desire to leave the practice. The quantitative data of the pre- and post-survey was compared using the Chi Squared test (nonparametric). Likert scales were dichotomized for the analysis. FINDINGS TO DATE (IT IS NOT SUFFICIENT TO STATE FINDINGS WILL BE DISCUSSED ): Overall, 28 PCPs responded to the presurvey (response rate 70\%) and 29 PCPs to the post-survey (response rate $73 \%$ ). Results showed a nonsignificant decrease in provider burnout from $43 \%$ to $24 \%$, p 0.134 , after the implemented interventions. There was no change in provider satisfaction with their job. Level of job-related stress significantly decreased after wellness interventions, $64 \%$ to $35 \%, \mathrm{p}<0.05$. Meaning in work significantly increased from $37 \%$ to $93 \%, \mathrm{p}<0.001$. There also seemed to be a nonsignificant decrease in desire to leave practice and increase in the perceived amount of "time to provide care."

KEY LESSONS FOR DISSEMINATION (WHAT CAN OTHERS TAKE AWAY FOR IMPLEMENTATION TO THEIR PRACTICE OR COMMUNITY?): While the primary endpoints did not achieve statistical significance, there was a trend toward decreased burnout and significantly decreased job-related stress and increased meaning in work after the interventions. Our practice wellness committee partnered with leadership to implement various measures to promote wellness for providers. The impact assessment is limited by moderate level of participation in survey, as well as survey anonymity preventing paired analysis. Future steps will be to assess the impact of further interventions and a paired analysis, using codes to protect confidentiality.

YOU GET WHAT YOU PAY FOR: AN ANALYSIS OF PAY FOR PERFORMANCE RESULTS IN A LARGE MULTI-SITE ACADEMIC PRIMARY CARE PRACTICE

Benjamin L. Ranard ${ }^{1}$; John Anderson ${ }^{2,1}$; Kevin Shah ${ }^{1,2}$. ${ }^{1}$ Duke University School of Medicine, Durham, NC; ${ }^{2}$ Duke University Health System, Durham, NC. (Control ID \#3170979)

STATEMENT OF PROBLEM OR QUESTION (ONE SENTENCE): Does pay for performance improve statin prescribing by primary care providers (PCPs) for diabetic patients? 
OBJECTIVES OF PROGRAM/INTERVENTION (NO MORE THAN

THREE OBJECTIVES): To increase guideline-directed medical care of diabetic patients by providing a financial incentive to PCPs.

DESCRIPTION OF PROGRAM/INTERVENTION, INCLUDING ORGANIZATIONAL CONTEXT (E.G. INPATIENT VS. OUTPATIENT, PRACTICE OR COMMUNITY CHARACTERISTICS): Duke Primary Care (DPC) is an entity within the Duke University Health System in North Carolina that consists of over 30 clinics in 8 counties, with over 240 PCPs and 730,000 annual patient encounters. DPC has utilized a bundled Diabetes Care metric as a quality incentive for several years. PCPs received a $\$ 2,500$ bonus if at least $25 \%$ of their diabetic patients met all 5 quality metrics: 1) statin prescription in patients ages 40-75 without statin allergies, 2) HgbA1c < 8\%, 3) BP < 140/90 (age 18-59) or <150/90 (age 6075), 4) non-smoker, and 5) aspirin prescription for patients with 10 year ASCVD risk $>10 \%$. The analysis included only PCPs and patients who were present throughout the incentive period of July 2016 - July 2017, PCPs with at least 20 diabetic patients and patients aged 40-75.

MEASURES OF SUCCESS (DISCUSS QUALITATIVE AND/OR QUANTITATIVE METRICS WHICH WILL BE USED TO EVALUATE PROGRAM/INTERVENTION): A 10\% absolute increase in statin prescriptions without an increase in statin allergies recorded in the medical record.

FINDINGS TO DATE (IT IS NOT SUFFICIENT TO STATE FINDINGS WILL BE DISCUSSED ): There were 134 PCPs and 15,652 patients in the analysis. PCPs varied substantially in prescribing statins and recording statin allergies. Statins were prescribed at baseline by PCPs at a median of $69 \%$ (range: $41-91 \%$, IQR: $62-75 \%$ ). Statin allergies at baseline by PCP were median of $6.3 \%$ (range: $0-19 \%$, IQR 3.4-8.9\%). After the incentive period, there was a $7 \%$ relative increase in prescribed statins (550 patients) and a $40 \%$ relative increase in statin allergies recorded (455 patients). The median change in statin prescriptions by PCPs was 4\% (IQR $0.9-7.7 \%$ ). The median change in statin allergies was $2.2 \%$ (IQR $0.78 \%-3.4 \%$ ); however, there were four positive outliers (range: $8.8-17 \%$ ). A total of 107 (80\%) PCPs had an increase in statin prescription or statin allergies, and $91(68 \%)$ had an increase in both statin prescriptions and statin allergies.

KEY LESSONS FOR DISSEMINATION (WHAT CAN OTHERS TAKE AWAY FOR IMPLEMENTATION TO THEIR PRACTICE OR COMMUNITY?): Pay for performance financial incentives impact provider behavior, but it can be challenging to design incentives that improve patient health, fairly compensate providers, and avoid adverse behaviors. In this analysis, there was variation in response to the financial incentive. By allowing PCPs to exclude patients with allergies from their statin prescription metric, providers had two ways to meet the metric - either prescribe a statin or mark a patient as having a statin allergy in the medical record. Providers responded to this incentive by prescribing more statins as well as documenting more statin allergies. While the former was the expected response, the latter may be an undesired outcome with potential negative consequences for patients.

INNOVATION IN MEDICAL EDUCATION (IME)

"ARE YOUR LEARNERS READY TO HOLD THE ADMISSION PAGER?": BUILDING A RESIDENT CURRICULUM FOR TRIAGE AND DISPOSITION DECISION-MAKING - PART 2 AN ASYNCHRONOUS ONLINE COURSE ON HOSPITAL TRIAGE AND SYSTEMS-BASED PRACTICE

David Schmit $^{1,2}$; Emily S. Wang ${ }^{1,2}$; Sadie Trammell Velasquez ${ }^{1,2}$; Sherwin $\mathrm{Hsu}^{3}$; Christopher J. Smith ${ }^{4}$; Tabatha Matthias ${ }^{4}$; Raj Sehgal ${ }^{1,2}$. ${ }^{1}$ University of Texas Health at San Antonio, San Antonio, TX; ${ }^{2}$ South Texas Veterans Health
Care System, San Antonio, TX; ${ }^{3}$ Olive View - UCLA Medical Center, Sylmar, CA; ${ }^{4}$ University of Nebraska Medical Center, Omaha, NE. (Control ID \#3184562)

NEEDS AND OBJECTIVES: In the context of Internal Medicine (IM), triage refers to activities related to determining the most appropriate disposition and management plans for patients. When triaging, residents must use effective inter-professional communication, coordinate care in complex healthcare systems, and make appropriate evidence-based decisions. Because of duty hour restrictions, many triaging duties have transitioned from resident learners to faculty thus creating a gap in triage skills and knowledge. In the first part of this project, we developed an e-module on inter-professional communication, which is currently available on the Society of Hospital Medicine's (SHM) online learning portal. The focus of the second part in this series, through a 2017 UT Kenneth Shine Academy Health Science Education small grant, is an asynchronous online course/e-module on systems-based practice (SBP)/ healthcare systems. 1. Define triage and the role of an internist as a triagist 2. Demonstrate understanding of how each of the following competencies apply to the triagist role: Inter-professional communication, SBP/healthcare systems, and evidence-based clinical decision making 3. Development of an asynchronous online course for IM resident learners focusing on SBP/healthcare systems

SETTING AND PARTICIPANTS: Through collaboration between 3 academic medical centers, the curriculum on SBP/healthcare systems includes a narrated e-module with closed captioned videos, faculty teaching guide, and pre-/post-knowledge assessments, all of which are freely available through SHM's online learning portal. This curriculum is intended to be utilized by IM residents and facilitated by faculty.

DESCRIPTION: With the lack of a formal curriculum on triaging, our group sought to create an innovative curriculum that IM faculty can easily implement in their home institutions. We identified 3 core competencies of triaging: interprofessional communication, SBP, and evidence-based decision making. This e-module focuses on understanding SBP/healthcare systems with the corresponding teaching guide providing guidance on incorporating the e-module into local curriculums.

EVALUATION: 1. Utilization of the e-module (geographical distribution and number of times accessed) 2. Assess improvement in residents' SBP knowledge through quizzes embedded in the e-module 3. Pre-/post-surveys from resident learners and faculty

DISCUSSION / REFLECTION / LESSONS LEARNED: With the increasing need to deliver healthcare to the right patients at the right time with the right resources, our IM residents need further education and training on triaging to achieve this goal. We hope our e-module will meet the needs of IM residents and faculty. As the project nears completion, our group appreciates the time commitment and dedication required to develop a formal online curriculum and would like to pass our insights to future educators.

ONLINE RESOURCE URL (OPTIONAL): Part 1 on Interprofessional communication: https:/www.shmlearningportal.org/ content/teaching-guide-building-resident-curriculum Part 2 on SBP: Will be available for the 2019 SGIM Annual Meeting

"CHOOSE AND TEACH YOUR OWN ADVENTURE": A RESIDENT-CREATED CASE-BASED APPROACH TO PRIMARY CARE CLERKSHIP DIDACTICS

Josephine Cool ${ }^{1}$; Brian Eiss ${ }^{2} .{ }^{1} \mathrm{New}$ York Presbyterian-Weill Cornell, New York, NY; ${ }^{2}$ Weill Cornell Medicine, New York, NY. (Control ID \#3183060) 
NEEDS AND OBJECTIVES: The objectives of this project were a. to develop an educational session that filled a curricular gap utilizing an interactive "choose your own adventure" approach, b. to assess baseline and new knowledge in several core gastroenterology/hepatology topics using a small group case-based format and pre- and post-tests, c. to facilitate the development of teaching skills in student learners by asking them to use teach-back to educate their peers and d. to assess whether teach-back peer education improves performance on relevant post-test questions.

SETTING AND PARTICIPANTS: This is a one hour session set in a PBL classroom that recurs each clerkship. The participants are medical students rotating through the primary care clerkship. The facilitators are one resident and one faculty member.

DESCRIPTION: The students first complete a pre-test that covers topics from each case. They are then divided into two groups, each of which works through a different case. Each case uses the online presentation tool "Prezi", and the students are prompted to make decisions that will take the case in different directions. At each branching point, they are prompted to look through guidelines, relevant clinical images and evidence-based links that are embedded within the presentation. Although the order of slides varies based on the students' choices, they will always review all content included. Once the case ends, the facilitator helps each student formulate a take home point to present to the students who worked through the other case. Finally, the students reunite and teach each other, and then complete the post-test. At the end they receive a .pdf document with key information to use as a study guide.

EVALUATION: At the beginning of each session, students take a brief pre-test using NBME examination-style vignette-based questions that reflect gastroenterology/hepatology content. The students take the same test at the end of the session, allowing for a comparison of the effects of more direct learning with peer education. Students evaluate the educational value of this session as part of standard post-clerkship evaluations.

DISCUSSION / REFLECTION / LESSONS LEARNED: Feedback for this new session has been positive; students describe higher engagement through the interactive case portion, and they find the teach-back portion to be the most useful. In this last part the role of the facilitator to help students formulate and refine succinct, high yield teaching points is more important than we expected; their eagerness and engagement during the teach-back session led to time constraints, leading us to create a time script for the session. This project has also allowed us to involve senior internal medicine residents in the design and facilitating of the sessions. In this case, a senior resident was able to create an innovative session, recruit assistant chief residents to help facilitate the sessions, thus fostering collaborative educational efforts and stimulating more interest in medical education career paths for both residents and students.

ONLINE RESOURCE URL (OPTIONAL): www.prezi.com IMPROVING RESIDENTS SKILLS AND COMFORT LEVEL USING EHR IN OUTPATIENT CONTINUITY CLINIC

Adrianna Wegrecki; Mehrshid Kiazand. UPMC Mercy, Pittsburgh, PA. (Control ID \#3185407)

NEEDS AND OBJECTIVES: The Electronic Health Record (EHR) is an important advancement in health care. However, it may create challenges for trainees. Most residents in our program do not have prior exposure to the specific EHR system used in our continuity clinic (EPIC). A different system is used in the inpatient setting. This adds another learning curve for new interns. Efficiently using EHR in the outpatient setting may be more challenging due to the limited visit time and insufficient training as opposed to inpatient rotations. This negative experience may be one of the factors determining if a trainee pursues an outpatient medicine career. We designed Supervised Peer Teaching Sessions aimed at helping residents improve their EHR skills.

SETTING AND PARTICIPANTS: Participants were $1^{\text {st }}, 2^{\text {nd }}$ and $3^{\text {rd }}$-year Internal Medicine Residents during their 4-week ambulatory rotation at an academic outpatient clinic.

DESCRIPTION: Medicine residents worked individually or in pairs and presented a specific skill related to EPIC at a weekly didactic session. The topics for presentations were suggested by a faculty champion and reflected the most commonly used features of EPIC needed to efficiently document a visit or update the chart. A pre- and post-session 8-question survey was sent to the residents between January and June 2018. The questions were designed to assess their level of comfort using EPIC in general and its specific functions using a 4-point rating scale. Resident cohort responses were compared using chi-square test.

EVALUATION: The survey was sent to 25 residents and we received 17 pre- and 15 post-rotation responses. The most significant skill improvements were being able to help others with their EPIC questions $(\mathrm{p}=0.01$ ), updating medication list $(\mathrm{p}=0.026)$ and health maintenance section $(\mathrm{p}=0.024)$. Skills that were not covered during the didactic sessions did not achieve significant improvement in post-survey, i.e. using Smartphrases $(p=0.75)$ or Smartsets $(p=0.065)$. Skills that the residents were comfortable with in pre-survey only marginally improved in post-survey, i.e. updating problem lists $(\mathrm{p}=0.62)$. Comments submitted by residents indicated that most of them appreciated and enjoyed the sessions and felt they were helpful.

DISCUSSION / REFLECTION / LESSONS LEARNED: This Peer Teaching Session offered an effective way to improve medicine residents' comfort level and set of skills needed to efficiently utilize an outpatient EHR. The sessions were brief and enjoyable and did not add much additional burden to the ambulatory rotation. The limitations of this survey were small sample size, misperceptions about own skills in the pre-session survey and short sampling time. Based on this study we implemented this session as a permanent part of the didactic curriculum during the ambulatory rotation and we will continue assess its utility and effectiveness.

\section{REFRAMING THE MEDICAL HISTORY: APPLYING PSYCHIATRY'S FOUR PERSPECTIVES TO THE PRIMARY CARE PATIENT}

Patrick Hemming. Duke University School of Medicine, Durham, NC. (Control ID \#3142526)

NEEDS AND OBJECTIVES: The medical history lays the groundwork for all other endeavors of patient care. Current trends in medicine (such as short visit length and the electronic health record) may distract learners from asking about patients' circumstances, priorities and unique needs. To aid learners in individualizing the medical history, the Four Perspectives (Disease, Behavior, Dimensions, Life Story) can be adapted from psychiatric education to offer a unique teaching framework. The goal of this curriculum is that learners will be able to describe and apply all four perspectives in the context of a primary care patient encounter. Such a curriculum will be successful if learners view the information gained as valuable to their patient care and decision-making.

SETTING AND PARTICIPANTS: During the pilot curriculum, each internal medicine resident participated separately in a half-day clinic session with one-on-one precepting. Each resident chose 3-4 patients they identified as having a known or suspected mental health diagnosis and each was 
scheduled for a 1-hour appointment. Immediately prior to the session, the preceptor shared a didactic with the resident regarding the Four Perspectives, and prompted the resident to incorporate the Perspectives into each patient visit.

DESCRIPTION: Each perspective covers a different aspect of the patient's history. The Disease Perspective encourages the provider to synthesize objective and subjective information into a physical or mental diagnosis; this Perspective is incomplete without the other three. The Behavior Perspective identifies what actions the patient is taking that impact her disorder; examples include substance abuse and addictions. The Dimensions Perspective challenges the learner to think about the personal characteristics of the patient, and may include health literacy, level of perceived trust, and the provider's own interpersonal chemistry with the patient. Finally, the Lifestory Perspective expands the concept of "social history" and asks the learner to gain an understanding of the patients' background.

EVALUATION: Thirteen residents each participated in a session. In postsession surveys, $83 \%$ found that it was useful for their patient, and many commented that they understood their patients better. Only $58 \%$ felt that they had met applied the Perspectives, although in a chart review by the preceptor of their patient notes, $67 \%$ incorporated the Perspectives. Thirtythree percent of residents reported a high positive impact on their patient care skills.

DISCUSSION / REFLECTION / LESSONS LEARNED: Teaching and observing medical history taking is a time-intensive process; importantly, this teaching can allow learners better understanding of their patients. This pilot suggests that learners may benefit from the following: further adaptation of the Four Perspectives for the non-psychiatric general medical setting, and a longitudinal method of instruction.

\section{A CLINICAL LEARNING EXPERIENCE FOR RESIDENTS IN THE MANAGEMENT OF CHRONIC PAIN AND OPIOIDS}

Christine Soran ${ }^{1}$; Soraya Azari ${ }^{2} .{ }^{1}$ University of California San Francisco, San Francisco, CA; ${ }^{2}$ University of California, San Francisco, San Francisco, CA. (Control ID \#3187218)

NEEDS AND OBJECTIVES: In the era of the opioid epidemic and tightening regulations on prescribing opioid medications, resident physicians are increasingly uncomfortable with the management of chronic opioid therapy (COT). Additionally, attending practice patterns differ dramatically leaving residents with uncertainty. We sought to create a structured clinical learning experience for primary care residents on a patientcentered approach to the management of chronic pain and opioids.

SETTING AND PARTICIPANTS: HOPE clinic is a multi-disciplinary, once weekly clinic based in an academic primary care clinic based at the county hospital, Zuckerberg San Francisco General (ZSFG). The team includes pharmacy, quality improvement, and physicians with addiction medicine training. The primary care clinic has 24 primary care residents and 23 categorical from the UCSF Internal Medicine residency program.

DESCRIPTION: Primary care residents in their second or third year rotate through the clinic twice per year. Each session has a dedicated curricular topic on chronic pain management. Residents see patients alongside the physician supervisor with the focus of the visit on management of chronic pain and opioids and motivational interviewing communication skills. Additionally, we discuss a plan of care for all resident primary care patients on COT and residents present patients with complex chronic pain or substance use issues. Residents were given a survey to assess confidence with managing pain and COT before and after each clinic session on a 5 point Likert-like scale $(1=$ not confident through $5=$ very confident $)$.
Residents were also asked about stress and enjoyment in working with patients with chronic pain and substance use $(1=$ completely disagree, $5=$ completely agree).

EVALUATION: A total of 14 primary care residents rotated through HOPE clinic one time in the first 6 months of the academic year. Prior to attending HOPE clinic, residents expressed feeling low confience in the all assessed domains but reported increase in confidence after the session. The change in confidence level was: - conducting a comprehensive pain assessment (average score pre $=2.8$ and post $=3.9$ ) - safely prescribing opioids (pre $=2.5$ and post $=3.4$ ) - recognizing and managing concerning behaviors in patients on COT (pre $=2.5$ and post $=3.5$ ) - creating a treatment plan for chronic pain ( pre $=2.8$ and post $=3.2$ ) - identifying substance use disorder in patients on COT (pre $=2.1$ and post $=3.4$ ) - treating substance use disorders in primary care (pre $=2.3$ and post $=3.3$ ) Residents report feeling a great deal of stress working with patients with chronic pain and substance use with slight improvement by one-half point on a Likert scale after the session. All residents agreed they enjoyed the clinical learning experience. DISCUSSION / REFLECTION / LESSONS LEARNED: Resident physicians face stress and uncertainty when managing chronic pain and opioid therapy. A clinical learning experience with guided mentorship can improve resident confidence and decrease stress when working on these complex issues.

\section{A DIVERSITY CURRICULUM DESIGNED TO FIT A PROVIDER'S BUSY SCHEDULE.}

Chirag R. Patel; Marwan Mohammad; Taru Saigal; Mahrous Abo Hassan; Keiko Smith. The Ohio State University Wexner Medical Center, Columbus, OH. (Control ID \#3182913)

NEEDS AND OBJECTIVES: In response to feedback received from our hospitalized patients about opportunities for improved cultural and religious sensitivity, we were determined to help our faculty promote and sustain culturally and linguistically appropriate services, and effectively interact with individuals from diverse backgrounds. The purpose of this project was to improve provider cultural competency.

SETTING AND PARTICIPANTS: Setting: Large, tertiary care, academic medical center. Participants: Hospital Medicine physician faculty

DESCRIPTION: As the U.S. population becomes more diverse, Hospital staff need to be better educated to care for patients of varying cultural and social backgrounds. More than half of all U.S. babies born today are people of color, and by 2050 our nation will have no clear racial or ethnic majority ${ }^{1}$. As such, the authors created and enacted a diversity curriculum within the division of hospital medicine designed to educate faculty and help minimize cultural barriers that may exist between them and the diverse patient population they serve.

EVALUATION: Using a questionnaire, a baseline assessment was conducted of staff's perception of their cultural competency. The findings validated the authors' hypothesis that a diversity curriculum was needed. The curriculum was developed and involved a multifaceted and asynchronous approach. A monthly lecture series was created with each one dedicated to improving provider familiarity and knowledge of frequently encountered ethnic, religious, cultural, sexual orientation and gender identity groups from our community. Additionally, monthly newsletters which highlighted and provided background on numerous cultural holidays and religious observances were circulated via email and posted in staff work rooms. All staff who attended a lecture were surveyed monthly to obtain a longitudinal assessment of their knowledge and comfort level taking care of culturally diverse patients. 
DISCUSSION / REFLECTION / LESSONS LEARNED: Prior to implementation of the diversity curriculum, the average physician selfassessment scores for "knowledge about cultural issues" and "comfort level in taking care of patients from diverse background" were 1.5 and 2 respectively on a 0-5 rating scale with 5 being the highest score. Over the course of nine months, 190 survey responses were received. Within six months of curriculum implementation, the average scores had increased to 4.5 out of 5 for both "knowledge" and "comfort". The subsequent three months demonstrated sustained high scoring. Based on the results, the diversity curriculum was felt to be highly impactful and successful in improving staff confidence in caring for a diverse patient population. As the curriculum progresses and evolves, we will be measuring if improved provider comfort and knowledge levels will have a downstream impact on care quality and health disparities.

\section{A FOCUS ON INTERPROFESSIONAL TEAMS: HIGHLIGHTING THE NURSING ROLE IN RESIDENT EDUCATION}

Jennifer Ohtola ${ }^{1}$; Dianna Copley ${ }^{1}$; Kimberly Kalo ${ }^{1}$; Jessica Donato ${ }^{2} .{ }^{1}$ Cleveland Clinic, Cleveland, $\mathrm{OH} ;{ }^{2}$ Cleveland Clinic, Beachwood, OH. (Control ID \#3185818)

NEEDS AND OBJECTIVES: Success in systems based practice includes knowledge of the interprofessional team, the roles of each member and qualities of effective teams. Internal medicine trainees frequently work within interprofessional teams in multiple clinical environments but rarely receive formal education on the scope of practice and tasks of their nursing colleagues. We sought to develop a didactic session focused on knowledge delivery related to nursing scope of practice and attitudinal change related to communication and mutual respect between residents and nursing.

SETTING AND PARTICIPANTS: A one-hour didactic session was implemented at our large internal medicine training program with 43 residents in attendance (12 PGY3, 15 PGY2, 16 PGY1). The session was led by a PGY2 medicine resident and a group of five med-surg nursing staff.

DESCRIPTION: The session started with a resident-led discussion of interprofessional team structure and features of effective teams, followed by a nursing-led review of nursing education and orientation, workflow and tasks, and effective communication strategies. This included discussion of patient assignments, medication passes, nursing management of physician orders, and paging standards. A nursing assistant (NA) reviewed the scope of practice, tasks and training for NAs. The session concluded with a review of "what we wish residents knew" for both nurses and NAs. This was followed by time allotted for audience questions to the panel of 4 nurses and a NA. Residents were provided with a handout highlighting electronic medical record (EMR) communication pitfalls and orders to optimize effective communication with nurses regarding medications, lines, and drains.

EVALUATION: Residents completed pre- and post-session surveys assessing knowledge of nursing and NA scope of practice and attitude related to communication with nursing with a 1-4 point Likert scale ( 1 = strongly disagree, $4=$ strongly agree). Thirty pre-session and 27 post-session surveys were completed. The average level of trainee-reported knowledge of scope of practice for nurses and NAs improved from 2.6 to 3.3 and 2.4 to 3.3, respectively. Knowledge of EMR orders for communication improved from 2.7 to 3.4, and knowledge of differences between EMR layouts of physicians and nurses improved from 2 to 3.1. Self-reported comfort with communication style with nursing improved from 3 to 3.3. The mean rating of the session was 3.6, with 1 being poor and 4 being excellent.

DISCUSSION / REFLECTION / LESSONS LEARNED: We describe a novel interprofessional educational session focused on enhancing trainee knowledge of nursing scope of practice and improving trainee-nursing relationships and communication. We showed an improvement in traineereported knowledge of nursing practice and attitude regarding communication with nursing. We also showed an increase in trainee knowledge of EMR orders available for communication and differences in EMR functionality between physicians and nurses. The session was well-received and well-rated.

\section{A HEALTH EQUITY AND DISPARITIES CURRICULUM WHICH DOES NOT REDACT HISTORICAL AND POLITICAL ROOTS Yalda Shahram. UCSF, San Francisco, CA. (Control ID \#3176650)}

NEEDS AND OBJECTIVES: Treating all patients equally does not eliminate health disparities; providers ignoring their own cultural background and biases when delivering health care to patients does not eliminate health disparities; equal access to health care does not eliminate health disparities; these and other misconceptions exist among health care providers. We present an innovative curriculum to address the key gap in current health equity and disparities curricula: the avoidance of historical and political roots of inequity. Our objectives are to break misconceptions about health disparities and to provide tools for practicing equitable care.

SETTING AND PARTICIPANTS: 24 participants ranging in specialty from anesthesia to radiology, from 2 years of practice to over 20. Attending physicians have a choice when selecting half-day workshops for faculty development and all participants had selected this seminar.

DESCRIPTION: It is inadequate to discuss poverty without oppression, race without racism, income inequality without capitalism, gender inequality without sexism... Avoidance of the roots contributing to inequity in health care presents to the learner a redacted curriculum that will always lead to a perpetuation of inequity, only with the new guise of awareness. This curriculum about health equity and disparities is presented in an interactive style including polling, small group discussions, case reviews and presentations, and debriefing activities. The curriculum content is presented in Table 1.

EVALUATION: Post-intervention survey showed participants ranking their understanding of health disparities from average/good to good/great. True/False statements in the survey were used to demonstrate misconceptions, for example, "the Tuskegee experiments were conducted to treat adult African Americans suffering from syphilis" showed $66.7 \%$ correct pre-seminar compared to $100 \%$ post-seminar. Confidence was also measured after each statement in the survey and for the example given, confidence increased from 4.2 to 4.9 on a 5 -point scale.

DISCUSSION / REFLECTION / LESSONS LEARNED: Addressing health care disparities begins with awareness. In this curriculum, interactive learning by small group discussions is the primary mode of distributing the knowledge about the historical and political roots of inequities. The topics covered can elicit an emotional response from participants and it is imperative that a safe space is established prior to opening discussions of sensitive topics, such as privilege or bias. Limiting the number of participants to 24 and having small group activities helped to establish a safe space. After an awareness is reached, action is practiced by the method of unlearning. Participants are introduced to the method of unlearning and encouraged to continue practicing using this tool.

ONLINE RESOURCE URL (OPTIONAL): Table 1: https://docs.google. com/document/d/1HHnIusk2CuRpY5jBO6C2F9zYUizBnGSjyADOVP qENZM/edit?usp=sharing

\section{A HOTSPOTTING CURRICULUM TO ENHANCE EDUCATION ON COMPLEX AMBULATORY PATIENT CARE}

Zehra Hussain; Michaela Seigo; Tabassum Salam; Gretchen Rickards. Christiana Care Health System, Wilmington, DE. (Control ID \#3181816) 
NEEDS AND OBJECTIVES: A small number of individuals with complex health and social needs drive much of the total cost of care in the US health system today. This group of patients, referred to as "high-utilizers," often receive fragmented care that poorly addresses their chronic physical and psychosocial issues. Healthcare hotspotting involves the strategic use of data to identify high-utilizer patients, understand their underlying issues, allocate resources, and design interventions to tailor care. We introduced a novel hotspotting curriculum to enhance population health training in our ambulatory resident clinic.

SETTING AND PARTICIPANTS: Wilmington Adult Medicine is an urban primary care resident clinic. Thirty-six residents in our categorical internal medicine program participated in this curriculum.

DESCRIPTION: Using data from our accountable care organization (ACO) as well as ER utilization reports, each resident was matched with a highutilizer patient on their continuity panel. At the beginning of the academic year, a didactic session was presented that introduced basic concepts of population health management and high-value care. Each resident was then provided the name of the high-utilizer patient he/she had been assigned to follow for the year. The primary tasks were to review chart and cost of care data for the patient, to identify barriers to the patient's care, and to design and implement interventions to address the patient's unique needs. The final deliverable was a structured written reflection about the exercise.

EVALUATION: Data included the residents' reflections as well as pre- and post-intervention patient cost data from our ACO database. Twenty-two of the 36 residents (61\% of participants) submitted reflection papers. Three primary themes emerged from the reflections: (1) social determinants are key; (2) early intervention is critical; and (3) resources exist to help. With regard to ACO cost data, 12 of the 36 patients were excluded from the analysis due to missing data. The mean annual pre-intervention cost per patient was $\$ 20,111.54$ (range $\$ 6.87-\$ 117,937.49$ ) and the mean post-intervention cost for the same group was $\$ 23,121.65$ (range $\$ 363.61-\$ 177,544.74 ; p=0.78$ ).

DISCUSSION / REFLECTION / LESSONS LEARNED: Through this curriculum, residents applied hotspotting techniques to address the health of our clinic's sickest and most vulnerable patients. Although we did not achieve cost reduction, our hope is that the exercise resulted in a deeper understanding of the costs of care, the social determinants of health, and the value of interprofessional and community resources in complex care. The curriculum was well received by residents, who described greater confidence in caring for their most medically and socially challenging patients post-intervention.

\section{A LONGITUDINAL RESILIENCE CURRICULUM FOR MEDICAL STUDENTS DURING CLERKSHIPS}

Jennifer Houpy $^{1}$; Sonia P. Oyola ${ }^{1}$; Amber-Nicole Bird ${ }^{2}$; Oana Tomescu ${ }^{2}$; Amber Pincavage ${ }^{1}$. ${ }^{1}$ University of Chicago, Chicago, IL; ${ }^{2}$ University of Pennsylvania, Philadelphia, PA. (Control ID \#3186319)

NEEDS AND OBJECTIVES: Fostering resilience is a promising way to mitigate negative effects of difficult experiences and prevent burnout. In our needs assessment of third year medical students, mean resilience was lower than in a general population sample but higher in students who felt able to cope with difficult clinical events and who felt comfortable discussing stress and burnout with peers. We aimed to create a curriculum to help cultivate resilience in medical students during their clerkship year.

SETTING AND PARTICIPANTS: Our curriculum included three skillbuilding workshops interspersed during the clerkship year of seventy-four third year medical students at the University of Chicago.

DESCRIPTION: Three skill-building workshops focused on difficult team interactions, challenging patient interactions, and disappointments and setbacks. These themes were frequently identified clinical stressors based on our needs assessment. Each session encouraged students to engage in facilitated reflection on their personal experiences with the topic and theoretical cases. Cases were based upon experiences from prior clerkship reflection sessions. After group discussion, skill-building exercises were introduced and used to stimulate further discussion.

EVALUATION: The curriculum was assessed based upon surveys of student satisfaction and Connor Davidson Resilience Scales (CD-RISC 10, range 040 ), as well as focus groups. Seventy-four medical students were surveyed at the end of their third year with a $72 \%$ response rate (53/74). Compared to third year students in 2016 at the end of the third year prior to the implementation of the curriculum ( $\mathrm{n}=57)$, the post curriculum students' CD-RISC 10 resilience scores improved (pre-mean $26.91 \pm 6.47$ vs. post-mean 29.59 \pm 5.33 ; $\mathrm{p}=0.019$ ). More than half of students agreed or strongly agreed that the sessions had been an open forum for reflection $(55 \%, 29 / 53)$ and allowed them to connect with peers $(51 \%, 27 / 53)$. Fifty-one percent $(27 / 53)$ thought having time to discuss these topics was valuable. Themes in students' written comments and focus groups included connecting with peers and feeling less alone. Some students commented holding sessions with clerkship directors present was not ideal for them, and others felt they had other outlets for these discussions and sessions were not necessary. Many students appreciated that attention was paid to their wellness during clerkships.

DISCUSSION / REFLECTION / LESSONS LEARNED: This curriculum targeted clinical stressors highlighted by medical students as a springboard to teach resilience skills. A challenge was constructing a setting conducive to comfortable reflection for all learners, and not all students found these sessions necessary. The curriculum was valuable for half of students, and it is possible resilience was improved.

\section{A MEDICINE CLERKSHIP CURRICULUM TO PROMOTE PA- TIENT ADVOCACY}

Nancy Choi; Sneha S. Daya; Cindy Lai. UCSF, San Francisco, CA. (Control ID \#3166711)

NEEDS AND OBJECTIVES: Patient advocacy is taught in the pre-clinical medical school years as a core concept of the medical profession, yet clerkship students are rarely taught practical skills on how to translate this concept to direct patient care. We developed an advocacy curriculum teaching students to: 1) elicit how social determinants of health ( $\mathrm{SDOH}$ ) impact care of patients, 2) develop concrete skills to reduce healthcare barriers, and 3) reflect and debrief on their advocacy experience.

SETTING AND PARTICIPANTS: During the six blocks of the academic year (January-December 2018), internal medicine clerkship students ( $n=66)$ at a large, urban academic medical center were required to participate.

DESCRIPTION: The curriculum consisted of four components spanning a two-month clerkship: 1) Faculty-facilitated, interactive workshop that included the following: "Advocacy Toolkit" (handout summarizing practical tools and resources); case studies on how to apply advocacy skills during hospitalization and transitions of care; "best practices" discussion with a social worker (SW) and case manager (CM); and time to practice how to discuss advocacy concepts in team rounds. Students utilized these to practice their advocacy skills during their clerkship, and there was concurrent faculty development in this. 2) Post-discharge patient phone call to assess success of the discharge transition. 3) Written and oral reflection to medical team discussing patient advocacy. 4) Debriefing workshop at the end, discussing strategies to continue advocacy.

EVALUATION: Students ( $\mathrm{n}=66$ ) completed surveys pre- and postcurriculum regarding advocacy. Pre-curriculum, $87 \%$ of students agreed that 
advocacy education in clerkships was important to their professional development, yet only $26 \%$ agreed that they had received formal education on this. Pre-curriculum, $35 \%$ of students agreed that they felt confident in their skills to advocate for patients in the inpatient setting; this increased to $100 \%$ postcurriculum. $93 \%$ of students agreed this curriculum helped them work closely with patients to ensure they received equitable care. Qualitative comments about what students mostly valued included themes related to advocacy education (e.g., "The ability to teach all of us that these are tangible factors that can radically change one's outcome when dc'd from the hospital") and the SW/CM interactive components of this curriculum.

DISCUSSION / REFLECTION / LESSONS LEARNED: We hope to prepare all students to advocate for better patient health outcomes and health equity. Our survey results indicate our curriculum improved students' confidence and skills in patient advocacy topics. Their assignment and debriefing sessions allowed critical time for meaningful reflection. Student feedback has been critical to the evolution of the curriculum. Faculty development surrounding patient advocacy education has been instrumental in the implementation of this project, and we encourage other institutions to incorporate patient advocacy skills education into their clerkship curricula.

\section{A MULTIFACETED APPROACH TO INCREASE TRAINEE SCHOLARLY ACTIVITY}

Shradha A. Kulkarni. Baylor College of Medicine, Houston, TX. (Control ID \#3187004)

NEEDS AND OBJECTIVES: Due to the clinical rigors of residency, time to pursue extracurricular scholarly activity can be limited for residents. Additionally, mentorship and support for research endeavors is also variable among residency programs. At Baylor College of Medicine (BCM), the Accreditation Council for Graduate Medical Education (ACGME) annual program evaluation survey identified scholarly activity as an area in which to potentially improve. To directly address this need, a task force was created and developed the following multifaceted innovation.

SETTING AND PARTICIPANTS: This innovation involved medical students on the internal medicine core clerkship and residents in the internal medicine residency program at Baylor College of Medicine, and took place as an after-hours, voluntary session on campus.

DESCRIPTION: First, to increase awareness regarding opportunities for scholarly activity, an extensive list of local, regional, and national academic conferences was compiled and distributed to all residents and medical students. The list was organized by specialty and included submission deadlines, conference locations, and dates. Next, a workshop was held in which faculty members provided general tips and suggestions for writing an abstract to submit to a conference. The final portion of this innovation was the poster workshop, which was held three times during the year. The workshop began with a brief introduction with tips for creating effective poster and oral presentations. Subsequently, medical students and residents practiced presenting rough drafts of their posters to a mock judging panel comprised of faculty members, several of whom have served on abstract review committees or judging panels for conferences.

EVALUATION: The number of abstract submissions and acceptances to academic conferences were tracked before and after the innovation was created.

DISCUSSION / REFLECTION / LESSONS LEARNED: After the above interventions began in 2017, abstract submissions and acceptances to academic conferences have increased. For the Texas chapter of the American College of Physicians annual meeting (TxACP), the total number of posters/ oral presentations by students and residents in 2015 and 2016 were 15 and 19, respectively. After development of this innovation, the number increased in 2017 and 2018 to 25 and 26, respectively. This innovation has both increased awareness and scholarly activity output by $50 \%$ by trainees at BCM. The most striking impact has been on medical students, as the number of student presentations at TxACP has increased over 300\% from 6 in 2015 to 20 in 2018. Residents played an integral role as most student presentations included at least one resident as a co-author.

\section{A NOVEL CONTRACEPTION COUNSELING AND SHARED DE- CISION MAKING CURRICULUM: IMPACT ON RESIDENT KNOWLEDGE AND ATTITUDES}

Rebeca Ortiz Worthington $^{1}$; Amber Pincavage ${ }^{1}$; Julie Oyler ${ }^{2}$; Jen Rusiecki ${ }^{1}$. ${ }^{1}$ University of Chicago, Chicago, IL; ${ }^{2}$ University of Chicago Medical Center, Chicago, IL. (Control ID \#3185024)

NEEDS AND OBJECTIVES: After completion of this curriculum residents will be able to: 1.Discuss a patients reprodcutive goals. 2.Engage a patient in a Shared Decision Making (SDM) conversation including counsel a patient on contraception options including discussing efficacy. 3.Identify contraindication and common side effects of these options. 4.Explore a patient's values and concerns surrounding contraception.

SETTING AND PARTICIPANTS: IM and Med/Peds PGY-2-4 residents at a single institution participated in the curriculum. This included a 1-hour large group, interactive teaching session with the use of an original educational video. The initial needs assessment was distributed a year prior to the curriculum and the post survey was performed immediately following the curriculum. Clinical faculty preceptors were also included in the initial needs assessment.

DESCRIPTION: The 2014 CDC guideline states that PCPs should provide contraceptive counseling (CC) and prescribe the selected method. IM and $\mathrm{Med} / \mathrm{Peds}$ physicians are responsible for providing care to medically complex women of reproductive age, yet past studies have shown that inadequate knowledge is a barrier to CC. The CHOICE study demonstrated an increase in long-acting reversible contraception (LARC) prescribing with a CC interventions highlighting that counseling is an important factor in reproductive planning. SDM is a method of patient-centered communication that has been shown to promote contraception adherence. We initiated an innovative curriculum to teach residents $\mathrm{CC}$ through the framework of SDM.

EVALUATION: 38 pre and 58 post-curriculum residents (48\% and $73 \%$ ) completed surveys. Residents reported improvement in comfort with CC overall (mean pre- 3.21 , post- $3.58, \mathrm{p}<0.001$, scale $1=$ "I need close supervision", and 5="I can teach this skill"). The greatest improvements were seen in comfort discussing efficacy, patient beliefs and negotiating a decision. $66 \%$ reported comfort with counseling on Nexplanon after the curriculum (37\% pre). Gains were seen in most of the questions focused on medical knowledge (IUDs and PID, 95\% correct post versus 59\% pre). Residents found the video to be a helpful tool ( $90 \%$ felt the video helped with discussing contraception options). Residents and faculty both under recognized teratogenic risk (13\% faculty and $11 \%$ residents correctly identify teratogens unchanged with curriculum). The faculty survey revealed a gap in contraceptive knowledge and comfort with teaching these topics. $22 \%$ felt they had not received adequate training. Only $17 \%$ of faculty reported comfort teaching this skillset. Though $78 \%$ of faculty feel it is very important that residents learn about contraception.

DISCUSSION / REFLECTION / LESSONS LEARNED: Prior to this curriculum, residents felt that $\mathrm{CC}$ was important, but lacked knowledge and training. This curriculum successfully addresses gaps in comfort and knowledge with CC including the use of LARC. Residents feel that the video 
component of the curriculum was beneficial. Future iterations of the curriculum will need to highlight teratogenic risk.

\section{A NOVEL UNIVERSITY-WIDE PATIENT-CENTERED INTER- PROFESSIONAL 1ST YEAR CURRICULUM}

Christopher Bruti ${ }^{1}$; Jan Odiaga ${ }^{2}$; Joanne M. Miller ${ }^{2}$; Gabriella Cs-Szabo ${ }^{2}$; Mary Jo Guglielmo ${ }^{2}$; Steven Taylor ${ }^{2}$; Theresa Gierlowski ${ }^{2} .{ }^{1}$ Rush University Medical Center, Chicago, IL; ${ }^{2}$ Rush University, Chicago, IL. (Control ID \#3181212)

NEEDS AND OBJECTIVES: Highly effective patient-centered interprofessional health care teams are one of the best ways to meet the IHI's proposed quadruple aim of healthcare (improved care, population health, cost effectiveness, and joy of work). Rush University created a novel $1^{\text {st }}$ year University-wide Interprofessional Patient-Centered (IPPC) curriculum to give students the foundation needed to lead and succeed in this environment.

SETTING AND PARTICIPANTS: Rush inaugurated the IPPC curriculum in the 2016-17 academic year with 376 students from 11 degree programs. Based on feedback from students and faculty, the course was redesigned and expanded in 2017-18 to include 698 students from 18 different academic programs including another university. The course involves 6 live team-based experiential workshops with online precourse work over an academic year. Students stay in the same interprofessional team with at least 4 different professionals. The course is pass/fail with the expectation of spending approximately 20 hours of work over the year.

DESCRIPTION: The course objectives are for students to acquire the 4 Interprofessional Education Collaborative (IPEC) competencies (roles/responsibilities, interprofessional-communication, teams/ teamwork, values/ethics) utilizing a patient-centered approach. Highlights of the workshops include the Community Health Mentor $(\mathrm{CHM})$ where teams work with a community member with a chronic disease. They learn from their CHM about living with a chronic disease, work with them to create a health care goal, and learn about their community. They are tasked with following the CHM longitudinally and creating a community advocacy proposal to improve the CHM's community's health outcomes. Team skills are evaluated at the end of the course through a Team Objective Structured Clinical Exam (TOSCE), where the student teams work with and counsel a standardized patient about a health problem.

EVALUATION: Quantitative and qualitative data were obtained following each workshop. Students developed competency in all four IPEC domains. They valued the interprofessional team approach to their health mentor, assessing health situations and providing whole-person care.

DISCUSSION / REFLECTION / LESSONS LEARNED: Implementation of an IPE curriculum spanning 2 universities, 5 colleges, 18 programs, and 698 students was full of challenges and lessons learned. Most important to the success of the curriculum was building an interprofessional leadership team. This team continued to meet weekly for the past 4 years to build support, design and iterate the curriculum, and to resolve logistical issues. The first version of the curriculum could not maintain team consistency due to scheduling conflicts and thus relied on online team-based modules, which were poorly received and ineffective. This was overcome by implementation of a university common calendar with Wednesday afternoons reserved for IPE. Since IPE successfully aligned with the mission, vision, and strategy of Rush, the program gained high-level attention and financial support.

A PILOT CLUSTER RANDOMIZED, CONTROLLED TRIAL OF A CLERKSHIP-BASED VISUAL ARTS CURRICULUM TO COMBAT BIAS AND PROMOTE WELL-BEING

Stephanie J. Hoffman ${ }^{1}$; Garth W. Strohbehnn ${ }^{2}$, ; Molly Tokaz ${ }^{1}$; Ruth Slavin ${ }^{3,4}$; Suzanne Winter ${ }^{1}$; Martha Quinn ${ }^{1}$; David Ratz ${ }^{5}$; Sanjay Saint ${ }^{6,}{ }^{2}$; Vineet Chopra ${ }^{1,2}$; Nathan Houchens ${ }^{2,}{ }^{1}$; Joel Howel1 ${ }^{7,}{ }^{4}$. ${ }^{1}$ University of Michigan, Ann Arbor, MI; ${ }^{2}$ Veterans Affairs Medical Center, Ann Arbor, MI; ${ }^{3}$ University of Michigan Museum of Art, Ann Arbor, MI; ${ }^{4}$ Medical Arts Program, Ann Arbor, MI; ${ }^{5}$ Veterans Affairs, Ann Arbor, MI; ${ }^{6}$ University of Michigan Health System, Ann Arbor, MI; ${ }^{7}$ U Michigan, Ann Arbor, MI. (Control ID \#3138376)

NEEDS AND OBJECTIVES: Retrospective analyses have shown associations between arts exposure and protective personality traits (e.g., empathy, tolerance of ambiguity), suggesting that facilitating arts exposure may help learners gain these traits. Unfortunately, most visual arts-based curricular interventions lack rigorous assessment and are rarely performed in the clinical setting. We conducted a pilot randomized, controlled trial to assess impacts of incorporating a visual arts curriculum into a clinical clerkship.

SETTING AND PARTICIPANTS: Study participants were University of Michigan (UM) medical students on VA Ann Arbor Healthcare System (VA) inpatient internal medicine teams. Museum arts (MA) interventions occurred at the UM Museum of Art whereas hospital arts (HA) interventions and case-based conferences (CBC) were at the VA.

DESCRIPTION: After participants provided written informed consent and completed a baseline survey, we randomized teams to three weekly one-hour sessions: MA $(n=14)$, HA $(n=14)$, or CBC $(n=15)$. Developed by an expert arts educator, MA and HA used active, guided discussion of artwork to explore themes of compassion, suffering, and ambiguity. The HA curriculum was identical to MA except for the use of digital representations of artwork and facilitation by non-experts. CBC served as contact time and environmental control arm.

EVALUATION: Psychometric surveys were repeated after curriculum completion, using validated scales of empathy, mindfulness, tolerance of ambiguity, and grit. We also compared NBME shelf exam performance. No statistically significant results were identified within quantitative outcome measures, including shelf exam performance between the arms. Focus groups with formal qualitative analysis, however, suggested that the arts curriculum provided a fulfilling escape from clinical medicine, increased cognizance of implicit bias, and promoted differentiation between observation and interpretation.

DISCUSSION / REFLECTION / LESSONS LEARNED: In our pilot study, students were willing to participate in art-based interventions while on clinical duties. No statistically significant quantitative results emerged, but absence of difference in NBME shelf exam scores was reassuring. Focus group discussions identified benefits of the arts intervention not otherwise captured by quantitative measures. Despite being shelf exam neutral, students felt stress while away from clinical duties. Study strengths include randomization of an unbiased study sample and use of outcome 
measures relevant to curricular stakeholders. Inclusion of the HA group also supports the feasibility of implementing arts interventions at other centers.

\section{A PILOT OFFICE-BASED OPIOID TREATMENT CLINIC IN AN INTERNAL MEDICINE RESIDENT CONTINUITY PRACTICE: PROVIDER, STAFF, AND PATIENT OUT- COMES.}

Jarratt Pytell ${ }^{1}$; Michael Brady ${ }^{1}$; Megan Buresh ${ }^{1,2}$; Ryan Graddy ${ }^{1} .{ }^{1}$ Johns Hopkins Bayview Medical Center, Baltimore, MD; ${ }^{2}$ Johns Hopkins Bloomberg School of Public Health,, Baltimore, MD. (Control ID \#3182464)

NEEDS AND OBJECTIVES: The integration of substance use disorder (SUD) care and competencies in graduate medical education training is needed. Previous research shows improvements in provider knowledge, attitudes, and practices after exposure to SUD care. Few studies report outcomes for patients with SUD in resident physician continuity practices. We describe a pilot integrating office-based opioid treatment (OBOT) with buprenorphine in an internal medicine resident continuity practice. We report survey results of resident and staff knowledge and attitudes of OBOT and patient outcomes.

SETTING AND PARTICIPANTS: We surveyed all internal medicine residents $(\mathrm{N}=48)$ and staff $(\mathrm{N}=22)$. A retrospective chart review was completed for all patients seen $(\mathrm{N}=12)$.

DESCRIPTION: The OBOT clinic operated within the GIM practice for 1.5 hours weekly during a clinic session and was staffed by 2 attendings and 3 residents with prior OBOT experience who alternated coverage. Two support staff coordinated appointments and collect urine toxicology screens. Patients with opioid use disorder (OUD) were identified by their primary care provider, referred to the OBOT clinic, and an individualized treatment plan was created. All care, including buprenorphine prescriptions and counseling, was provided by the residents and attendings. Surveys of provider and staff knowledge and attitudes of OBOT were administered at baseline and 4 months into the pilot. We analyzed survey results using Fisher's exact test. A retrospective chart review of the first 15-months obtained patient characteristics and treatment outcomes including retention and measured good clinical response. A good clinical response was defined as being illicit opioid negative and buprenorphine positive for $>50 \%$ of time in treatment.

EVALUATION: Survey response rates were $83 \%$ for residents and $100 \%$ for staff. No statistically significant differences were observed for any survey item between baseline and 4 months. Most residents (87\%) reported caring for patients with OUD, yet only $27 \%$ felt comfortable providing care and $30 \%$ believed OBOT was ineffective without on-site drug counseling. Among staff, $53 \%$ felt primary care practices were inappropriate places to treat OUD. Results from the retrospective chart review showed the average age was 55 years (range 30-78 years) and all patients were white. Most patients were male $(75 \%)$ and referred from residents $(75 \%)$. Seven patients (58\%) were retained in care at the end of the study period (range of 9-15 months); 8 patients (75\%) demonstrated a good clinical response. Of those not retained, 3 patients transferred, 1 self-tapered, and 1 was lost to follow up. DISCUSSION / REFLECTION / LESSONS LEARNED: OBOT can be successfully integrated into existing resident continuity practices utilizing existing staff and resources with positive patient outcomes. We did not observe changes towards acceptance in provider and staff attitudes of OBOT likely due to the small scope of the OBOT pilot clinic. It is possible that more direct and frequent exposure to OUD care would lead to increased acceptance.

\section{A PILOT USAGE OF POSITIVE DEVIANCE TO ENHANCE TEACHING OF THE 3RD-YEAR-INTERNAL MEDICINE CLERK- SHIP \\ Ali S. Jahansooz. KUSM-W, Wichita, KS. (Control ID \#3181522)}

NEEDS AND OBJECTIVES: To enhance the quality of teaching on our 3rd Year Internal Medicine clerkship, we designed a positive-deviance (PD) intervention to identify unique teaching tactics used by our most effective faculty teachers and disseminate their unique tactics to the remaining faculty. SETTING AND PARTICIPANTS: Area hospitals Academic hospitalists DESCRIPTION: Modeled after Google's Project Oxygen, we identified the hospitalist faculty rated the highest for "overall effectiveness" using the endof-3rd-year faculty evaluations. We surveyed our hospitalist faculty to query the teaching tactics they perceive are effective or unique. We created a Table of Tactics with one row listing tactics reported by our positive deviance and the other row listing tactics by other faculty members. We created a faculty development session that used the Table of Tactics to prompt a discussion of the most effective teaching strategies identified by our positive deviants. The Faculty was given recommendations excerpted from Palmer and Blake on creating a safe learning environment for successful peer learning.

EVALUATION: $80 \%(12 / 15)$ of hospitalist faculty responded to the survey of tactics and all positive deviants responded to the survey. Some of the responses, including from PDs, were general and did not offer specific tactics. $75 \%$ (3/4) positive deviants considered creating a safe learning environment most effective while 3 of 7 non-deviants considered safe learning environment most effective; thus, the development session focused on this discussing implementation of this tactic. We received 5 post-session surveys with four positive responses.

DISCUSSION / REFLECTION / LESSONS LEARNED: Most faculty members received the PD intervention positively. Lessons learned from this pilot are 1) use a plot of faculty assessments by students overlaying a binomial distribution to improve selecting the PDs 2) improve our survey of tactics by priming the faculty with a framework of teaching tactics from the literature, but not stating best practices within the framework in order to encourage honest reporting by the faculty, 3) improve our introduction of the session to reduce threat and perception of criticism of non-deviants, 4) expand comments in the discussion by creating ad hoc scenarios for role play.

\section{ONLINE RESOURCE URL (OPTIONAL): N/A A RESIDENT-CENTERED WELLNESS CURRICULUM} Amanda Ray ${ }^{1}$; Yukiko Kunitomo ${ }^{1}$; Brita Roy ${ }^{2}$; Katherine A. Gielissen ${ }^{1}$. ${ }^{1}$ Yale-New Haven Hospital, New Haven, CT; ${ }^{2}$ Yale University School of Medicine, New Haven, CT. (Control ID \#3186061)

NEEDS AND OBJECTIVES: Studies show high burnout rates among medical residents. Many US programs have developed interventions to minimize burnout but most only target interns despite evidence of high levels of burnout among upper-level residents. Nearly all curricula are led by faculty or Chief Residents, however, a preliminary survey of internal medicine (IM) residents at Yale New Haven Hospital (YNHH) revealed that many residents find the presence of faculty or chiefs detracts from effective and open exploration of burnout and wellness. We describe a resident-designed, resident-led wellness curriculum for IM trainees of all levels.

SETTING AND PARTICIPANTS: The curriculum was implemented in the 2018-2019 academic year's ambulatory didactics provided for YNHH's Traditional IM residents, occurring on a rotating basis throughout the year. 
DESCRIPTION: After performing a survey-based needs assessment of residents, we developed six, hour-long sessions covering topics on burnout, wellness, and resilience. Each session includes 1) introductory exercises, 2) open discussion, and 3) teaching of specific coping tools. Designated resident "Wellness Leaders" (peer-elected upper-level residents) facilitate the sessions.

EVALUATION: We collected resident baseline scores of burnout, wellbeing, and self-efficacy in Spring 2018 using the Oldenburg Inventory (OBI), Short Flourishing Scale (SFS), and General Self-Efficacy Scale (GSE), respectively. Residents will complete a post-intervention survey in Spring 2019. All residents receive post-session surveys utilizing Likert scales and narrative comments to obtain feedback on curriculum content and format and to evaluate the subjective usefulness of the sessions and their activities.

DISCUSSION / REFLECTION / LESSONS LEARNED: At baseline, $77 \%$ scored high on the OBI overall $(n=62)$ with $90 \%$ and $63 \%$ scoring high on the exhaustion and disengagement subscales, respectively. $33 \%$ scored low-to-moderate $(<45)$ on the SFS $(n=59)$, and $17 \%$ scored low-tomoderate $(<30)$ on the GSE $(n=59)$. Although we await post-intervention data to quantify curriculum impacts on these indices, $85 \%$ of respondents on average report leaving sessions with increased coping tools $(n=72)$. Common themes from feedback surveys include the benefits of having space to discover shared experiences across all years and learning specific coping strategies from peers for the demands of residency. Challenges raised are how to keep sessions relevant to third-year residents and the difficulty of sharing personal challenges in a group setting.

\section{A ROBUST FACULTY DEVELOPMENT PROGRAM FOR MEDI- CAL EDUCATORS: A DECADE OF EXPERIENCE}

Sarah B. Merriam $^{1,}{ }^{2}$; Rachel Vanderberg ${ }^{3}$; Melissa McNeil ${ }^{1}$; Tanya Nikiforova ${ }^{4}$; Carla Spagnoletti ${ }^{5}$. ${ }^{1}$ University of Pittsburgh Medical Center, Pittsburgh, PA; ${ }^{2}$ VA Pittsburgh Healthcare System, Pittsburgh, PA; ${ }^{3}$ UPMC, Pittsburgh, PA; ${ }^{4}$ University Of Pittsburgh Medical Center, Pittsburgh, PA; ${ }^{5}$ University of Pittsburgh, Pittsburgh, PA. (Control ID \#3185591)

NEEDS AND OBJECTIVES: Faculty development programs (FDPs) foster learning communities and enhance professional identity formation for medical educators. Recent work has highlighted the need to consider competency-based frameworks when designing FDPs, to both identify deficiencies in existing curricula and to drive skill development across clinical practice, teaching, and scholarship domains. We aim to outline the components of a novel FDP in the context of established medical educator competencies, describe its outcomes to date, and identify steps for implementation and sustainment of similar programming.

SETTING AND PARTICIPANTS: The FDP consists of 4 one-hour-long conferences held weekly since 2007 at the University of Pittsburgh SOM, open to all health sciences faculty, fellows, and others interested in medical education. Evaluation data is available for faculty attendees and presenters in four consecutive academic years from 2014 to 2018.

DESCRIPTION: The overarching goal of the FDP is to support the advancement of educator faculty, promote curricular innovation and teaching excellence, enhance educational scholarship, and cultivate a community of educators. The FDP includes four rotating conferences: Academy of Master Educators Seminar Series, Medical Education Research Conference, Medical Education Journal Club, and Medical Education Research Methods and Innovative Design Conference.

EVALUATION: We outlined the relationship of these FDP conferences to established medical educator competencies by consensus. We also evaluated 1) presenter and attendee rank and department, 2) average conference attendance over 4 academic years (2014-2018) and 3) impact of this program on faculty presenters.

DISCUSSION / REFLECTION / LESSONS LEARNED: Collectively, this well-attended (average \# of attendees per conference $=20$ ) internallydriven FDP meets all established competencies for educator faculty. Presenters and attendees were diverse in terms of academic rank and represented 12 clinical departments, 7 basic science departments, dental medicine and physical therapy. Of research "works in progress" from 2014-2017, 14 peer-reviewed fellow and faculty publications resulted, with an additional 5 manuscripts in revision or under review. Of those projects presented during the past academic year (2017-2018), 2 have gone on to peer-reviewed publication and 4 others are currently in revision or under review. We report data from the most recent academic year separately, given the expected lag time prior to publication. Because presenters and attendees are volunteers, the FDP's 10-year track record is evidence of faculty satisfaction with the programming. We have identified key factors for implementation and sustainment of similar FDPs at other institutions: establish a clearly defined mission statement that meets the needs of the target audience and engenders engagement and ownership, identify champion(s) to lead the effort, capitalize on local talent and expertise, and secure institutional backing.

A TEACHER, A LEARNER AND A SET OF OBJECTIVES. SAME PLAYERS, DIFFERENT GAME. INNOVATING MEDICAL EDUCATION THROUGH CHANGING FOCUS.

$\underline{\text { Shahnur Saiyad }}^{1}$; Jennifer Velasco ${ }^{1}$; Amar R. Chadaga ${ }^{2}$; Armand Krikorian ${ }^{3}{ }^{1}$ UIC/Advocate Christ Medical Center, Oak Lawn, IL; ${ }^{2}$ Advocate Christ Medical Center, Oak Lawn, IL; ${ }^{3}$ University of Illinois at Chicago/ Christ Medical Center, Oak Lawn, IL. (Control ID \#3186073)

NEEDS AND OBJECTIVES: As residents, we have the responsibility to teach medical students beyond the books during their wards rotation. A Dedicated Teaching Resident (DTR) has the ability to influence medical students' learning. - To provide a framework for the DTR to enhance students' learning experience; explore his/her own style of teaching, practice creativity and flexibility in approaching medical topics; practice leadership and mentorship. - To facilitate active learning; provide opportunities to research and learn how to learn effectively. - To practice consistent teaching amongst the inpatient teams, with set objectives.

SETTING AND PARTICIPANTS: An ACGME-accredited Internal Medicine Program in a tertiary care center An inpatient team consists of a DTR with students on their internal medicine rotation.

DESCRIPTION: 3 components of this initiative: 1) DTR, 2) dedicated teaching curriculum, 3) implementation of curriculum via exploration of various teaching strategies Every month, each team has a DTR who is responsible for the medical education of 2-3 students. Each day, the DTRs have the flexibility to teach any topic the students are interested in based on their teaching style and the students' learning styles. For each topic, the DTR and students are encouraged to find a patient with the specific diagnosis and learn through incorporating the history and physical exam, clinical presentation, labs, differential diagnosis and management in their learning.

EVALUATION: - Qualitative survey for the students, assessing knowledge checks and overall learning experience - Weekly DTR meetings for formative feedback with the program director and development of his/her teaching portfolio

DISCUSSION / REFLECTION / LESSONS LEARNED: The curriculum provides structure and specific objectives, thus serving as a focused framework for the DTR for the concrete delivery of medical topics. In 
addition, strategic guidance from the faculty allows the DTR to explore their own style of teaching, identify roadblocks to knowledge, and sharpen their leadership and mentorship skills. It is not enough to just work on facts or recite guidelines. Becoming a good clinician requires a solid foundation of medicine, but equally requires the application of this knowledge in the context of a patient. Hence, the actual process of learning is integral to becoming a good clinician. Through this initiative, we hope to facilitate learning for the students, and help our educators realize their own potential. We are still in the initial phases of our project and hope to achieve feedback through the surveys and weekly meetings in the coming months.

ONLINE RESOURCE URL (OPTIONAL): https://rive.google.com/ file/d/1nig_BADE7t0GI_mh3maAygmySvRZDBRa/view?usp=sharing

\section{A TELEHEALTH CURRICULUM FOR INTERPROFESSIONAL TRAINEES AND STAFF}

Janeen E. Smith ${ }^{1,}{ }^{2}$; Bridget C. OBrien ${ }^{3}$; Maya H. Dulay ${ }^{4}$; Leonie Heyworth $^{5}$; Omar Aljundi ${ }^{1}$; Rebecca L. Shunk ${ }^{6}$; Christopher Sha ${ }^{1,2}$; William B. Smith ${ }^{2}$; Amy D. Lu ${ }^{7}$. ${ }^{1}$ San Francisco VA Health Care System, San Francisco, CA; ${ }^{2}$ University of California, San Francisco, San Francisco, CA; ${ }^{3}$ Univ of California, San Francisco, San Rafael, CA; ${ }^{4}$ UCSF/VA, San Francisco, CA; ${ }^{5}$ VA San Diego Healthcare System, La Jolla, CA; ${ }^{6}$ UCSF, San Francisco, CA; ${ }^{7}$ San Francisco VA Medical Center, San Francisco, CA. (Control ID \#3174741)

NEEDS AND OBJECTIVES: Access to medical care for rural and underserved patients is adversely affected by distance associated time and cost burdens. Telehealth has expanded access to care for many patients, allowing them to see their providers from the convenience of their own homes. However, telehealth home visits are underutilized because providers lack the confidence and skills necessary to conduct such visits. To address this training gap, we designed a curriculum to teach trainees and staff to perform safe video visits, employ virtual physical exam techniques, utilize peripheral devices and develop new care pathways.

SETTING AND PARTICIPANTS: The Department of Veterans Affairs introduced VA Video Connect (VVC) in July of 2017. VVC connects Veterans to their care team via a secure online platform. Residents, students and staff from medicine, nursing, pharmacy and psychology participate in the curriculum.

DESCRIPTION: The curriculum consists of 3 hour-long sessions delivered over 6 months. The first session covers equipment, technology, safety, contingency planning, documentation and completion of national VA VVC training requirements to perform video visits. Learners take turns role playing providers and Veterans to learn the functions of the virtual medical room. In the second session learners use VA issued tablets and peripheral devices and practice a virtual physical exam in simulated cases. The third session focuses on appropriate health concerns for video visits and integration into clinic workflow.

EVALUATION: Thirteen trainees and staff participated in the first session. Their average self-assessed ability to do a video visit, rated from $1=$ poor to $5=$ excellent, increased from $1.9( \pm 1.3)$ pre-session to $3.5( \pm 1.1)$ postsession. Participants rated their likelihood of incorporating video visits into practice as a result of the session $4.7( \pm 0.75)$, on average. As implementation continues, we will track the percentage of learners who conduct at least one video visit within 6 months and conduct focus groups to identify what content and educational strategies are most and least effective.

DISCUSSION / REFLECTION / LESSONS LEARNED: As early adopters of VVC, we recognize the barriers socioeconomic status and limited facility with telecommunication technologies pose to Veterans, so we added education on use of VA provided technology and devices into the curriculum. By including real-time practice, learners experience some of the technology challenges encountered in real patient visits. In contrast to most telehealth orientations, our transformative curriculum moves beyond a tour of technology to address integration of technology into routine patient encounters.

\section{ABSTRACT TITLE: FACILITATING REFLECTION ON THE PA- TIENT DISCHARGE PROCESS: AN EDUCATIONAL INTER- VENTION AT THE VA \\ Matthew H. Zegarek ${ }^{1}$; Seonaid F. Hay ${ }^{2}$; Rebecca Brienza ${ }^{3}$. ${ }^{1}$ Yale University School of Medicine, West Haven, CT; ${ }^{2}$ West Haven VA Hospital, Madison, CT; ${ }^{3}$ VA Connecticut Healthcare System, West Haven, CT. (Control ID \#3178921)}

NEEDS AND OBJECTIVES: Adverse events occur frequently after patients are discharged from the hospital. Successfully orchestrating care transitions is a core milestone for internal medicine residents as defined by the ACGME and ABIM; however, focused education on discharge planning is rare. A 2009 survey showed that only $16 \%$ of internal medicine residencies have formal curricula on the topic. Reflection on practice is also a key competency of physicians. The ACGME outlines the needs for residents to investigate, evaluate, and improve their practice. Prior interventions to increase resident reflection on patient discharges have either not been based in the patient care that the learner directly participated in or have required significant investment from institutions and the learner. We designed an interactive session to foster reflection on the post-discharge course of patients that learners directly cared for using easily accessible data at the VA. Our goal is to design an educational program to engage learners in reflection on the discharge process in order to help increase their skills in managing care transitions.

SETTING AND PARTICIPANTS: The intervention consisted of a onehour educational session at the end of a two-week inpatient internal medicine rotation for residents and students.

DESCRIPTION: Emphasis was placed on reviewing cases that resulted in readmissions or "near misses," using data from the electronic medical record at the VA. The facilitator guided the participants in reflection by reviewing salient themes that arose from the case reviews and assisted participants in identifying practices that they could adopt to prevent future errors or near misses in patient discharges. The participants were asked to complete a survey immediately before and after the educational session to assess its effect.

EVALUATION: After participating, learners had more agreement with the statement "following up on patients improves my understanding of how health systems function." More learners commented on the importance of communication with other providers and with patients in preventing adverse events after discharge after the session. Learners indicated that the session helped them to reflect on the process of discharging patients and helped them create specific goals to change their process of discharging patients. Further statistical analysis is underway.

DISCUSSION / REFLECTION / LESSONS LEARNED: A major goal of medical training is to prepare students and residents to independently and safely design discharge plans. This educational intervention was unique in that it leveraged the electronic medical record of the VA to facilitate learner reflection. The survey results indicate that learners found the session helpful in reflecting on their practice in discharging patients and increased their appreciation of the impact of health systems. Learners reported increased appreciation for the role of communication during the discharge process. 
ADDRESSING RESIDENT-IDENTIFIED BARRIERS TO ATTENDING NOON CONFERENCE

David M. Hayes ${ }^{1}$; Garrett Rampon ${ }^{1}$; Rachna Rawal ${ }^{1}$; Fred R. Buckhold ${ }^{2}$. ${ }^{1}$ Saint Louis University, St. Louis, MO; ${ }^{2}$ Saint Louis University School of Medicine, St. Louis, MO. (Control ID \#3179394)

NEEDS AND OBJECTIVES: 1. To identify barriers to resident attendance to noon-conference educational sessions 2 . To improve the quality of noon-conference 3 . To engage attending physicians in noon-conferene sessions relating to medical decision making

SETTING AND PARTICIPANTS: Internal Medicine Residency programs utilize noon conference as a key opportunity for formal education for residents. In response to low attendance at our noon conferences, we distributed a survey to our residents assessing conference quality and composition as well as barriers to attendance and implemented changes based on the results of the survey.

DESCRIPTION: Main barriers included patient care obligations (24\% of respondents), answering pages (22\%), and rounds continuing near or through the beginning of conference (17\%). $69 \%$ of residents reported attending conference less than $60 \%$ of the time. $61 \%$ felt guilty for not attending. $86 \%$ of residents agreed they would benefit more from conferences if attendings handled their pagers. Comments indicated that faculty attendance and participation at conferences would make the sessions more educationally robust. Interactive conferences and conferences that focused on medical decision-making were more popular than standard didactics.

EVALUATION: In response to survey results, we developed a twopronged approach to 1) revise and improve our curriculum and 2) address the barriers to attendance. We changed the format of case reports to focus on a medical decision-making teaching point rather than the diagnosis of a rare disease. We introduced a new "Case Series" conference in which senior residents present a challenging case specifically to a panel of faculty. We revived journal club with a new format including pre-session discussion questions and interactive small group discussions. We added a new "High Value Care" conference to introduce educational opportunities pertaining to optimizing resource utilization. We moved much of the core curriculum to a new "Afternoon School" series - a small group based interactive learning series designed to replace standard didactics on high yield topics. We started tracking attendance using a QR code scanned from mobile devices. We revised faculty expectations to include completing rounds thirty minutes prior to conference and strongly encouraged them to attend conferences and hold their residents' pagers. We scheduled educational conferences in the scheduling software to reinforce attendance expectations and empower residents to show attendings their scheduled obligations.

DISCUSSION / REFLECTION / LESSONS LEARNED: Postintervention survey data is pending. Preliminary data demonstrate clearly higher conference attendance with increased faculty and resident engagement. Residents responded positively to faculty participation in the medical decision-making conferences.

\section{ADVOCACY 101: A CURRICULUM TO ENGAGE PHYSICIAN TRAINEES IN HEALTHCARE ADVOCACY AND ANALYSIS OF ITS IMPACT}

Andrea S. Christopher ${ }^{1,2}{ }^{2}$; Magni Hamso ${ }^{3,}{ }^{1}$. ${ }^{1}$ Boise Veterans Affairs Medical Center, Boise, ID; ${ }^{2}$ University of Washington School of Medicine, Seattle, WA; ${ }^{3}$ University of Washington Boise Internal Medicine Residency, Boise, ID. (Control ID \#3187147)
NEEDS AND OBJECTIVES: Over 90\% of physicians believe community participation and collective advocacy are very important. Professional organizations highlight training in advocacy skills as a means to improve population health. Movements such as \#whitecoatsforblacklives demonstrate trainee interest in advocacy. However, few opportunities exist for physician trainees to learn advocacy skills. We present our Advocacy 101 curriculum, which seeks to empower physician trainees from multiple community training programs in Boise, Idaho with the knowledge and skills to engage in healthcare advocacy. This curriculum is novel due to the inclusion of both undergraduate and graduate level physician trainees from multiple specialties. Advocacy 101 objectives are: 1. Appreciate the physician's role as advocate for patients in the healthcare system, both locally and nationally 2 . Gain concrete skills in advocacy and leadership including speaking with legislators, writing and publishing op eds, and conducting research to inform advocacy 3 . Practice advocacy during the Idaho state legislative session.

SETTING AND PARTICIPANTS: We invite participation in Advocacy 101 from University of Washington (UW) medical students, Family Medicine Residency of Idaho residents, UW Boise Internal Medicine residents, and UW Psychiatry Idaho Advanced Track residents. About 20 trainees attend at least one session during each delivery year.

DESCRIPTION: Through an elective series of six 1.5 hour seminars, trainees learn about the history of health reform; discuss the role of physicians as advocates; identify local issues in the state legislature that affect them and their patients; practice writing op-eds and interacting with the media; learn how to most effectively interact with legislators; and learn how to use research to inform advocacy. The curriculum runs concurrent with the Idaho legislative session from January to March, in order to maximize exposure to active policy issues.

EVALUATION: We conduct a pre and post survey of Advocacy 101 participants, which started during the pilot of the curriculum in 2017. Using a 5 point Likert scale, participants reflect on their knowledge of national and local health policy, confidence in various advocacy skills, and personal views about the role of advocacy in their careers. At the course conclusion, trainees showed the most growth in their access to a community of physicians interested in advocacy, knowledge of local health policy issues, experience engaging in advocacy and confidence in speaking with a legislator.

DISCUSSION / REFLECTION / LESSONS LEARNED: Preliminary data analysis shows that as a result of this course, physician trainees reported more knowledge, skills and experience engaging in advocacy. We additionally identify benefits from exposing trainees from different levels of training and specialties to this content together, including collaboration between trainees and faculty from medical education programs across the region and growing the population of physician advocates for rural and medically underserved populations in Idaho.

ADVOCATING FOR CHANGE: A DESCRIPTION OF AN ADVOCACY CURRICULUM FOR INTERNAL MEDICINE RESIDENTS Benjamin A. Howell $^{1,2}$; Lacey R. Whitmire ${ }^{3}$; Sanjeet Baidwan ${ }^{2}$; Ross B. Kristal $^{2}$; Stephen J. Huot ${ }^{2}$; Tracy Rabin ${ }^{2}$; Julie R. Rosenbaum ${ }^{2} .{ }^{1}$ Yale School of Medicine, New Haven, CT; ${ }^{2}$ Yale University School of Medicine, New Haven, CT; ${ }^{3}$ Iora Primary Care, Hartford, CT. (Control ID \#3183987)

NEEDS AND OBJECTIVES: Advocating for the promotion of societal health is a core professional activity. We propose that formal training of physicians in the knowledge, skills, and attitudes of effective advocacy 
should be incorporated into graduate medical education. There are few published models of how to teach public advocacy skills to physicians-intraining. We developed a curriculum that exposes residents to a conceptual framework for physician advocacy and builds skills through workshops that are anchored in a topic selected by each resident to enhance engagement.

SETTING AND PARTICIPANTS: Participants are senior residents in a university-based internal medicine program located in an urban mediumsized city. The curriculum is compulsory, contain 12 hours of content, and is delivered in five sessions over a 4-week ambulatory rotation to groups of 4-6 residents. Two faculty members and one chief resident teach the course. DESCRIPTION: Session 1. Core Concepts: Trainees discuss the ethics of advocacy and how advocacy may fit into their own careers. A framework for developing an advocacy campaign is reviewed and used to evaluate a local advocacy project. Trainees then identify an advocacy topic which they will use as a vehicle to learn skills during future sessions. Session 2. Persuasive Communication: Residents learn techniques for creating public narratives, review examples of persuasive communication, and the elevator pitch. The residents then have a conversation with a local community organizer on local advocacy and coalition building. Session 3. The Power of Narrative: Residents learn the skills of op-ed writing to develop their topics into written calls to action. Session 4. Legislative Advocacy: State and federal legislative processes are reviewed and a primer on techniques for communicating with legislators and their aides is presented. Session 5 . Advocacy in Action: Residents research state bills to learn how other advocacy topics have fared in the legislature. Then, residents are joined by a local policymaker for a conversation about health advocacy and the residents' topics of interest. It concludes with the residents delivering their elevator pitches and receiving feedback.

EVALUATION: To date, 48 residents have completed the curriculum. In course evaluations residents most appreciated the skill-building components and the inclusion of non-physician experts. Most residents stated that they would be more likely to engage in advocacy following exposure to the curriculum. Long-term impact of the curriculum will be evaluated through survey of graduates that are five years' post-residency to assess involvement in advocacy and lasting impact of the curriculum on knowledge, skills, and attitudes regarding physician advocacy.

DISCUSSION / REFLECTION / LESSONS LEARNED: Our curriculum on physician advocacy has been well-received. Key success factors include: using a topic of personal interest to each resident as the vehicle for learning advocacy skills, inclusion of non-physician advocates and policymakers, and emphasizing practical skills.

\section{AMBULATORY HANDOFFS: A PRIMARY CARE INTERVEN- TION TO IMPROVE THE END-OF-YEAR TRANSITION TO NEW RESIDENTS FOR HIGH RISK PATIENTS}

Lesley E. King ${ }^{1}$; Maryann K. Overland ${ }^{2}$; Christopher $\mathrm{Wong}^{2}$; Ginger A. Evans ${ }^{3} .{ }^{1}$ VA Puget Sound/University of Washington, Seattle, WA; ${ }^{2}$ University of Washington, Seattle, WA; ${ }^{3}$ VA Puget Sounds HCS Seattle, Seattle, WA. (Control ID \#3183982)

NEEDS AND OBJECTIVES: Best practices have been established for safe patient handoffs in the inpatient setting. The outpatient setting is inherently different, with a single transition often serving as the only means of transmitting multiple years of relevant health information. This is particularly pronounced in resident continuity clinics when departing senior residents handoff to incoming interns. While some seminal work has been accomplished in different systems (Pincavage et al, 2012, 2013), in most residency programs there is little to no signout between residents, and few innovations have focused on transitions to incoming interns. Our objective was to improve the end-of-year ambulatory care transition process by improving communication between departing and incoming residents.

SETTING AND PARTICIPANTS: Internal medicine continuity clinic residents affiliated with an academic internal medicine residency program. We surveyed 164 residents at 8 unique continuity clinic sites. 2 clinical sites participated in a structured signout process with 44 out of 63 potential pairs of departing-incoming residents performing an in-person signout.

DESCRIPTION: We developed an intervention that was implemented at 2 of the 8 continuity clinic sites within our academic system. Our intervention was multi-pronged: a system for departing senior residents to identify patients most at risk of adverse events during their transition in residents, chart documentation of relevant and outstanding issues, and the opportunity to give an in-person signout (during a structured event) to the incoming intern.

EVALUATION: Residents across all 8 clinic sites (intervention and nonintervention) were surveyed on a 5-point scale about the handoff process and asked openended questions on their experiences. Residents who participated in the signout event had increased satisfaction of how their highrisk patients were handed off (3.5 for residents who received signout, compared with 2.9 for residents who had not received signout). Interns received an additional survey and uniformly reported the event as "very helpful" and reported increased confidence in their ability to care for their patients ( 4.9 out of $5, \mathrm{n}=10$ ). Aggregate results for all surveyed in 2018 showed that those who had received signout had higher transition satisfaction scores compared with those who did not (3.8 compared with 3.1). Those who received in-person signout were much more likely to prefer that method ( $81 \%$ compared with $56 \%$ among those who did not receive signout).

DISCUSSION / REFLECTION / LESSONS LEARNED: While the best practices for ambulatory transitions during the handoff between residents remains to be defined, our intervention shows that an in-person "warm handoff" between departing and incoming residents is feasible, desired, and may increase resident satisfaction. We are continuing to work on improvements to the process including identifying ways to incorporate the multidisciplinary team. Our initial work focused on analysis of the resident experience - next steps should include patient satisfaction and outcomes.

\section{AN 'EPIC' IMPROVEMENT IN CARE COORDINATION: IMPLEMENTING A SKILLS LOG TO INCREASE RESIDENT PROFICIENCY IN NEWLY ADOPTED EMR}

Rebecca A. Griffith; Anushka Chadha; Afrasiab Khan; David Kuo. Atlantic Health/ Morristown Medical Center, Morristown, NJ. (Control ID \#3185849)

NEEDS AND OBJECTIVES: Recent data from the SGIM Medical Residency Clinic Directors Interest Group survey found that $97 \%$ of Internal Medicine residency programs use an electronic medical record (EMR) system, $66 \%$ of which have a shared single EMR with the hospital, such as EPIC, the current industry leader. EPIC is a powerful platform that holds promise in streamlining workflow but falls short when physicians do not utilize all the functions. This often stems from suboptimal training and can preclude the delivery of comprehensive patient care. Our objective is to investigate a new teaching strategy to increase resident competency in using essential EPIC skills, termed the "3D" approach: demonstration, direct observation, and documentation. 
SETTING AND PARTICIPANTS: Our study was conducted at a large regional teaching hospital in New Jersey that recently adopted EPIC in both ambulatory and inpatient settings. Participants included 52 Internal Medicine residents (15 PGY-1, 9 preliminary, 15 PGY-2, and 13 PGY-3) supervised by Internal Medicine and Clinical Pharmacy teaching faculty DESCRIPTION: This was a 6 month longitudinal study. Program faculty identified 6 functions in EPIC considered essential for the coordination of patient care but underutilized based on previous year's experiences. Trainees completed a survey to self-report their confidence level for writing a result note and result letter, completing a phone encounter, writing a letter to a patient, posting an out-of-office notification, and writing a remind-me note. Residents were provided a one-hour training session on how to accomplish these skills. For the next six months, when trainees performed these tasks in their outpatient clinic, they were required to demonstrate their proficiency for a faculty member and record it in a central skills log. All residents were surveyed again six months later to self-report their confidence level.

EVALUATION: 42 residents completed the initial survey and as of this time 28 residents completed the post survey. Results were stratified by PGY level and interpreted using weighted averages. Confidence levels for completing a phone encounter, posting an out-of-office notification, and posting a remind-me note, increased in at least two of the PGY levels; meanwhile, writing a result note, result letter and letter to a patient, decreased in at least two of the PGY levels. The greatest improvement in confidence level was seen in the PGY-2 class. To date, 59\% of the residents completed the EPIC skills $\log$.

DISCUSSION / REFLECTION / LESSONS LEARNED: Using our novel "3D" approach, our residents reported increased confidence in performing more than $80 \%$ of essential EPIC skills. Direct observation of residents utilizing EPIC functions provided opportunity for evaluation as well as feedback and instruction. Due to the anonymous nature of the postsurvey, we were unable to tell which respondents had actually completed the skills $\log$ thus we were unable to determine if there was a correlation between log completion and confidence level.

\section{APPLYING DESIGN THINKING PRINCIPLES TO AN ETHICAL IMPERATIVE: LIVER TRANSPLANTATION}

Amanda Simone ${ }^{1}$; Lauren Block ${ }^{2}$; David Marcus ${ }^{3} .{ }^{1}$ Northwell Health, Manhasset, NY; ${ }^{2}$ Northwell Health, Lake Success, NY; ${ }^{3}$ Northwell Health, New Hyde Park, NY. (Control ID \#3184936)

NEEDS AND OBJECTIVES: Design thinking (DT) has been used in business to generate practical ideas to solve problems, but has only recently been applied as a tool in medical education. Here, it has been described as a tool for curricular design and problem solving, but there are no published instances of it being used in a medical ethics context. We used the DT model to redesign a session on the ethics of liver transplantation at one medical school. DT was chosen to allow students to draw on their own experiences in proposing solutions to a real-world problem within the lens of ethics and professionalism.

SETTING AND PARTICIPANTS: Content was delivered to 100 first year medical students in a 150-minute session as part of their ethics curriculum.

DESCRIPTION: The session began as a group discussion on challenges of organ donation in the US and the issue of developing a fair system to allocate limited resources. Students transitioned to groups of 5 to begin the design thinking challenge. Groups were asked to consider alternative solutions to improve patient wait times and fairness of organ distribution and the ethical impacts of changing the current model. Facilitators led students through DT steps of empathizing on the problem, defining the team's point of view, ideating on potential solutions and ideas, prototyping a specific idea, and testing the idea through oral presentation. The session ended with presentations by the teams on their proposals, with questions and answers by peers and faculty.

EVALUATION: The curriculum was evaluated with a mixed methods design, with confidential pre-and post-surveys including quantitative and qualitative items. Unpaired t-tests and chi-square tests compared survey data, and thematic analysis was used on qualitative items. After the session, students reported an increased understanding of the current liver transplant allocation system $(\mathrm{p}<0.01)$ and an increased appreciation of shortcomings of the current organ allocation system in the US $(p-<0.01)$. Before the session, $75.3 \%$ of students never had any practical experience with DT, highlighting the novel nature of this teaching modality in medical education. After the session, $73.8 \%$ of students felt that DT could be used to approach complex health system problems.

DISCUSSION / REFLECTION / LESSONS LEARNED: Design thinking is a tool for generating and exploring ideas from multiple stakeholders to solve problems. We used these principles to encourage medical students to engage with the ethical problems inherent to organ donation. Students displayed improved knowledge and attitudes towards organ donation and design thinking. These results indicate that design thinking is a novel, effective and useful learning framework to use when addressing ethical problems in healthcare.

\section{ASSESSING BURDEN OF CHRONIC DISEASE AMONG THE UNINSURED: AN INTERDISCIPLINARY COMMUNITY OUT- REACH}

Sayeef Mirza $^{1}$; Shams Rahman ${ }^{2}$; Noura Ayoubi ${ }^{1}$; Laurie Woodard ${ }^{1}$; Rahul Mhaskar ${ }^{1} .{ }^{1}$ University of South Florida, Tampa, FL; ${ }^{2}$ Bethune Cookman, Daytona Beach, FL. (Control ID \#3186402)

NEEDS AND OBJECTIVES: 1. Define specific socioeconomic factors that impact chronic disease morbidity and mortality among the uninsured frequenting free clinics. 2. Integrate the concepts of interprofessional community outreach, health services research, student mentorship, with biostatistics/epidemiology didactics as a part of medical education curricula. SETTING AND PARTICIPANTS: A retrospective chart review was conducted to collect data on chronic diseases from electronic and paperbased medical records at 9 free clinics in the Tampa Bay area. Charts containing clinic visits from January 2016 to December 2017 were included and analyzed. Redcap was used to organize a comprehensive chart review. DESCRIPTION: There are over 1,200 free clinics managing uninsured and under-insured patients throughout the United States. Only about $10 \%$ of these clinics are affiliated with a medical school where students are involved with operational management. However, data is rarely recorded systematically that would facilitate statistical analysis and outcomes research. Assessing the burden of chronic disease (ABCD) may be an elementary step in assessing the health of a largely 'undocumented' population. A multidisciplinary effort can unite different professions in order to study, analyze and report data about uninsured patients.

EVALUATION: From January 1, 2016 to December 31, 2017, a total of 8857 patients were managed at 9 free clinics. After omitting missing variables, 5074 (58\%) were women, 3408 (54\%) were Hispanic, and 2019 (46\%) were unemployed. In terms of follow-up, 4207 (48\%) were seen more than once, and 2613 (30\%) were seen more than twice. The mean number of visits was 2.51 . Follow-up visits were most common among patients with the 
following documented conditions: hypertension $(1350,63 \%)$, diabetes $(840$, $65 \%)$, hyperlipidemia $(890,73 \%)$, depression $(529,67 \%)$, anxiety $(462$, $68 \%$ ), arthritis $(344,63 \%)$, asthma $(336,66 \%)$, anemia $(260,73 \%)$, coronary artery disease $(130,59 \%)$, cancer $(147,71 \%)$, COPD (101, 64\%), CVA (102, $71 \%)$, MI $(55,63 \%)$. The average CCI for all patients was 0.84 with the CCI being 0.64 for patients seen only once, and 1.06 for patients seen for 2 or more visits. Further univariable analyses including chi-square and correlations are reported.

DISCUSSION / REFLECTION / LESSONS LEARNED: Uninsured patients with multiple comorbidities and elevated mortality risk continue to follow up with free clinic providers. Future public health programs should target the common diseases experienced by uninsured patients. Future studies are needed to study effective management of these multiple comorbidities in the free clinic setting. By describing the clinical characteristics of uninsured patients, we can paint a more accurate picture of the state of American health care as we struggle to pay for the price of poverty.

\section{ASSESSMENT AND MANAGEMENT OF SOCIAL DETERMI- NANTS OF HEALTH IN THE PRIMARY CARE SETTING, AN INTERNAL MEDICINE RESIDENT CURRICULUM}

$\underline{\text { Mary McAllister }}{ }^{1}$; Mark Troyer ${ }^{2}$; Lisa Kearns ${ }^{1} .{ }^{1}$ Ohio State University, Columbus, $\mathrm{OH} ;{ }^{2}$ Ohio State University Wexner Medical Center, Columbus, OH. (Control ID \#3186222)

NEEDS AND OBJECTIVES: Clinicians may see the effects of social determinants on their patients' health but often lack knowledge or confidence in addressing SDH in clinical practice. Medical training has traditionally emphasized the biomedical model over social and environmental factors, leaving physicians with few tools to address SDH. Course objectives: Learners will be able to recognize socially determined barriers contributing to health disparity in at-risk populations. Learners will apply concepts of interdisciplinary care to address barriers to health care. Learners will show consideration for societal determinants of health in care plans in the clinic.

SETTING AND PARTICIPANTS: This course takes place in an ambulatory noon lecture setting. Participants are primary care track residents in the Ohio State University Internal Medicine Residency Program.

DESCRIPTION: We have developed an interactive course to increase knowledge of social determinants of health (SDH) and increase confidence in addressing SDH in the ambulatory setting. Topics addressed include economic stability, language \& health literacy, built environment, food security, addressing SDH for individual health, and community-specific resources to optimize patient outcomes. Eight 45-minute sessions are held across two months, consisting of brief didactic teaching followed by interactive activities. These include role play, attitude/bias discussion, vignette or article analysis. Learners complete virtual neighborhood visits of zip codes served by their own clinic by researching SDH metrics (ie income, crime, language) and reviewing evidence tying each metric to health outcomes and disparities. Discussions such as "what can we do?" include pharmacists, social workers, and nurses sharing available clinic resources for addressing SDH with patients.

EVALUATION: To evaluate this curriculum, we are utilizing a pre/post survey for participants. We will measure improvements in knowledge of SDH using multiple choice questions and assess improvements in attitudinal and behavioral/skills outcomes using a 5-point scale that relies on learner self-assessment of confidence in recognizing and managing healthcare barriers. Each survey question is cross-referenced to a learning objective identified in sessions 1-8. The course is currently in progress.
DISCUSSION / REFLECTION / LESSONS LEARNED: This course is a resident-driven initiative to improve awareness of societal determinants of heath. Pre-course surveys indicate an awareness of SDH but overall lack in knowledge of how this directly impacts patient care. While our course is still ongoing, we have had anecdotal feedback on the direct applicability of content to patient care. Moreover, resident participants are actively brainstorming and creating additional initiatives to address these concerns specific to our patient population. We are encouraged that by formally introducing this new curriculum, it has resulted in self-directed learning outside of the specific lecture content.

\section{BLOCK OF ADDICTION MEDICINE (BAM!): AN INTENSIVE RESIDENT CURRICULUM IMPROVES COMFORT WITH SUB- STANCE USE DISORDERS}

Hadas Reich $^{1}$; Kathleen Hanley ${ }^{1}$; Lisa Altshuler ${ }^{2} .{ }^{1}$ NYU, New York, NY; ${ }^{2}$ NYU School of Medicine, New York, NY. (Control ID \#3181811)

NEEDS AND OBJECTIVES: There is an increasing need for resident education on substance use disorders (SUDs). The purpose of our curriculum was to improve residents' knowledge, skills, and attitudes on treating patients with SUDs.

SETTING AND PARTICIPANTS: First and second year residents from NYU's Primary Care, Internal Medicine program participated in the Block of Addiction Medicine (BAM!) curriculum. Clinical settings included buprenorphine/methadone clinics and outpatient treatment programs in a large, urban safety net hospital system.

DESCRIPTION: BAM! is an intensive two week curriculum focused on SUDs. To improve residents' knowledge, we included didactic sessions on substances, including alcohol, opiates, and tobacco. Sessions covered epidemiology, biology, and treatment, including pharmacologic options, with all residents receiving buprenorphine prescribing waiver training. BAM! was delivered by an interdisciplinary faculty that included addiction medicine specialists, department of health officials, and general practitioners, nurses, and social workers who have worked extensively with patients with SUDs. Workshops built skills including screening, brief interventions, and referral to treatment (SBIRT) and motivational interviewing. Residents attended buprenorphine/methadone clinics, outpatient treatment programs, and 12-step (AA/NA) meetings. Residents shared lunch in a non-clinical setting with patients in recovery to understand their perspectives on living with addiction.

EVALUATION: Residents' attitudes and self-perceived efficacy in treating SUDs were surveyed. Pre and post data was obtained on 15 of 16 participants. Using the medical condition regard scale (MCRS), an 11 item questionnaire on biases/emotions/expectations for treating patients with SUD, we found a statistically significant improvement in the composite score, from 44.46 to 47.0 ( $\mathrm{p}=0.026$ ). Of 15 residents, 11 reported improved ability to effectively screen for SUD, 10 reported improved comfort in screening patients for SUD, 12 reported improved knowledge in using medically assisted treatment (MAT), and 14 reported improved ability to effectively treat patients with MAT (all $\mathrm{p}<.001$ in Wilcoxon signed rank test). Qualitative feedback showed residents felt this curriculum was an essential part of their education; one participant commented: "this is a course that should be offered to every medical care provider."

DISCUSSION / REFLECTION / LESSONS LEARNED: BAM! included a varied curriculum delivered by inter-professional faculty. Residents reported improved comfort in treating patients with SUDs and demonstrated a significant improvement on the MCRS in their already positive attitudes towards treating this patient population. Qualitative feedback indicated that 
residents enjoyed BAM! and found it important to their training. Given the increasing need for providers who are able to effectively treat SUDs, courses such as BAM! are an effective and essential part of residency. Further studies are needed to assess if the changes in residents' attitudes persist and whether we influenced practice.

\section{BUILDING A NEEDS-BASED EBM CURRICULUM WITHIN AM- BULATORY BLOCK EDUCATION}

Melissa Mroz; Thomas Carroll. University of Rochester, Rochester, NY. (Control ID \#3185609)

NEEDS AND OBJECTIVES: The learning and practice of evidence based medicine (EBM) is a critical component to internal medicine residency education. Despite dedicated sessions to support EBM skill development, traditional journal club formats have failed to engage learners and foster conceptual understanding. Results of an internal needs assessment identified several areas to target including residents' understanding of probability, interpreting test results, and developing answerable clinical questions using the literature. Given that residents reported feeling disinterested in the current EBM curriculum, we aimed to deliver content in way that actively engaged resident learners and addressed knowledge gaps.

SETTING AND PARTICIPANTS: Seventy-two categorical internal medicine residents at the University of Rochester participated in a revised EBM curriculum delivered during dedicated ambulatory educational half-days. Ambulatory education is protected time from clinical duties held weekly during two-week ambulatory blocks. All residents have at least six blocks of ambulatory medicine per year.

DESCRIPTION: The revised EBM curriculum consisted of six one-hour sessions over one year. The sessions incorporated interactive lecture utilizing audience response and problem-based learning to apply EBM concepts to clinical settings. Sessions topics included: 1) recognition of overconfidence and understanding probability; 2) test interpretation; 3) study design; 4) asking a clinical question; 5) abstract review; and 6) feedback and skills assessment.

EVALUATION: Sixty-eight residents completed the Fresno test of EBM competence at the end of the year-long curriculum and had a mean score of 124 - a value between novice (95.6) and expert (147.5) categories. Resident evaluation further showed that the EBM curriculum was well received, with an overall course rating of 7.8 (SD 1.8) on a Likert scale ranging from 1 to 10 (best). Resident comments supported the numeric score: "EBM this year has been much better than the last 2 years. It was focused on the basic, relevant things we need to know"; "I have enjoyed the back to the basics nature as opposed to reading articles and trying to apply topics we don't truly understand."

DISCUSSION / REFLECTION / LESSONS LEARNED: Resident physicians begin residency with varying familiarity of EBM concepts. Although traditional models of journal club help to facilitate a critical review of the literature, residents may still lack an appropriate foundation to apply EBM skills. Further, many residents were unaware of knowledge gaps. Thus, highlighting learner needs served to emphasize the value of the curriculum. The first session purposely challenged learners' understanding of EBM while remaining well received. Linking EBM concepts to meaningful clinical examples improved resident engagement. Overall our approach was highly rated by resident learners. Future work will expand EBM across a three-year curriculum to reinforce key concepts and further develop critical appraisal skills.

\section{BUILDING A THREE-YEAR HIGH VALUE CARE CURRIC- ULUM}

Jessica Donato ${ }^{1}$; Richard Cartabuke ${ }^{2} .{ }^{1}$ Cleveland Clinic, $\overline{\text { Beachwood, } \mathrm{OH} ;}{ }^{2}$ Cleveland Clinic, Painesville, OH. (Control ID \#3185087)

NEEDS AND OBJECTIVES: The current economic burden imposed by healthcare is not sustainable. Medical educators have been tasked with not only educating trainees about costcontainment but also shaping their clinical practice with regards to high value care (HVC). Various high value curricular structures have been described but few focus on a longitudinal curriculum spanning the duration of residency training. We sought to develop a three-year HVC curriculum.

SETTING AND PARTICIPANTS: A monthly HVC conference targeting all post-graduate years (1-3) was implemented at a large internal medicine training program starting in the fall of 2017.

DESCRIPTION: Ten one-hour conferences are delivered per academic year from August through May. Each conference covers an inpatient or ambulatory topic including syncope, acute kidney injury, low back pain, and cellulitis. A three-year curriculum has been developed with 30 different topics, such that trainees are not exposed to the same conference throughout their training, and at least one conference per year is dedicated to popular HVC topics such as "things we do for no reason" and "imaging wisely." Each conference includes an interactive small group discussion and faculty-led review of evidence-based management strategies. Each conference also incorporates a discussion of benefits, harm, charges for our institution and value. The conference concludes with take home points for high-value strategies and a 1-page handout to summarize key points.

EVALUATION: Fourteen conferences have been implemented. Attendance has sustained at 43-81 trainees per conference. A survey was administered in March $2018(\mathrm{~N}=55)$ and revealed knowledge acquisition: $88.9 \%$ of residents correctly identified the definition of value, $70.6 \%$ correctly identified the high value strategy for managing an upper gastrointestinal bleed, and $83.7 \%$ correctly identified the high value strategy for a suspected pulmonary embolism. $100 \%$ of trainees noted the principles discussed were relevant to their daily clinical practice. $98 \%$ of respondents noted they reflect on their ordering practices and gain new perspective after the conference, and $96 \%$ report changes in ordering practices because of the HVC conference. An annual survey is planned to maintain ongoing assessment of this curriculum.

DISCUSSION / REFLECTION / LESSONS LEARNED: Many HVC curricula have been described but few have been established as a three-year longitudinal curriculum. We describe the development of a successful HVC conference with sustained attendance, knowledge acquisition and trainee-reported attitudinal and behavior change. The longitudinal nature of this monthly conference helps to maintain interest and focus on cost-conscious, high value care throughout the three-year training program. Utilization of different topics over three years also avoids redundancy and 
allows the curriculum to target all trainee levels concurrently. The conference focuses on fundamental medical topics to maintain relevance and provides evidence-based discussions to provide readily applicable clinical teaching points.

BUILDING A TOOLKIT FOR MEDICAL STUDENTS: ADDRESSING MICROAGGRESSIONS \&AMP; DISCRIMINATION ON THE WARDS

Raquel Sandoval3; Titilayo Afolabi3; Jordan Said3; Avik Chatterjee2; Daniele D. Olveczky1. 1Beth Israel Deaconess Medical Center, Boston, MA; 2Harvard Medical School, Cambridge, MA; 3Harvard Medical School, Boston, MA. (Control ID \#3187230)

NEEDS AND OBJECTIVES: Multiple studies suggest that physicians' implicit biases can affect the care that they provide to their patients ${ }^{1}$,

${ }^{2}$. This, in addition to inequalities in the distribution of social determinants of health in society, is thought to contribute to stubbornly persistent health disparities between minority and non-minority populations. These implicit biases often manifest as microaggressions. There has been a deepening understanding of the harmful impact of micro-aggressions on the clinical relationship between patients and health care professionals $<$ font size $=" 1 ">$ SETTING AND PARTICIPANTS: Setting: Harvard Medical Participants: First year students prior to the start of clinical rotations.

DESCRIPTION: A two-hour workshop for rising first year medical and dental students who had completed their first fifteen months of undergraduate medical education and were going to transition to clinical responsibilities was developed. The workshop consisted of (1) a thirty-minute interactive large-group didactic session via PowerPoint presentation that introduced micro-aggressions, discrimination, and frameworks for addressing these issues in the clinical setting, and (2) a seventy-five-minute small-group session during which students worked through two cases by applying the presented frameworks and role-playing scenarios.

EVALUATION: 121 and 105 respondents completed the pre and post workshop surveys respectively. An analysis of 81 pairs of pre- and postworkshop surveys was performed. Pre-workshop, $77.7 \%$ had encountered some form of a micro-aggression during a clinical experience at least once. Students were also asked to self-assess their challenges responding to micro-aggressions in the following competencies: "Fear of retribution," "Difficulty recognizing," "Not sure what to say or do," "Lack of allies," and "Lack of familiarity with institutional support." Self-assessment strength was checked against a micro-aggressions definition and identification task in the post-survey.

DISCUSSION / REFLECTION / LESSONS LEARNED: Discussion: Our results demonstrated that the workshop broadly improved self-assessed participant competencies for recognizing and combating micro-aggressions in a clinical setting. Post-workshop, perceived challenges to addressing micro-aggressions and episodes of discrimination on the wards decreased for all of the barriers and sub-groups except "Fear of retribution," which remained a barrier for all respondents post-workshop. Conclusion: The prevalence of micro-aggressions in the clinical environment remains significant. A two-hour workshop resulted in a significant decrease in perceived challenges in addressing micro-aggressions.

BUILDING CAMARADERIE, COMMUNITY AND MENTORSHIP: FOSTERING PROFESSIONAL DEVELOPMENT THROUGH RESIDENT-LED WOMEN IN INTERNAL MEDICINE PEER GROUP (WIIM)

Anna Buteau ${ }^{1}$; Anupama M. Kapadia ${ }^{2}$; Meera Bhakta ${ }^{1}$; Liwayway R.
Andrade $^{1}$; Holli T. Sadler ${ }^{1}$; Anne Cioletti ${ }^{3} .{ }^{1}$ Dell Medical School at UT Austin, Austin, TX; ${ }^{2}$ Dell Medical School at the University of Texas at Austin, Austin, TX; ${ }^{3}$ University of Texas Dell Medical School, Austin, TX. (Control ID \#3185740)

NEEDS AND OBJECTIVES: As women enter the field of medicine in increasing numbers, literature shows they have decreased quality of life and blunted career advancement. One solution is to amplify mentorship and sponsorship for women by women. Due to limited numbers of mentors and sponsors, it is difficult for female learners to receive individualized guidance. Objectives for WiIM: 1. Enhance opportunities for peer mentoring amongst female residents 2 . Amplify mentorship and sponsorship by internal medicine female faculty

SETTING AND PARTICIPANTS: This informal curriculum is for all female residents and faculty. Each event was hosted at a member's home.

DESCRIPTION: Eager for camaraderie, a group of female residents united to create an informal peer group focused on women in internal medicine, coined WiIM. Initially, only residents attended, but the group rapidly identified the added value of including faculty. The monthly gatherings provide an opportunity to socialize, network and to discuss relevant readings and topics, including advocacy, mentorship and resident-based concerns. Crowdsourcing topics from the group, we have discussed challenges unique to women, including preserving balance yet advancing in the hierarchical worlds of medicine and academia. Faculty shared their experiences negotiating contracts and salaries as well as ongoing challenges as women in medicine.

EVALUATION: Initially, feedback was informal and anecdotal. Attendance has been our strongest measure of success, as $64 \%$ percent of women residents and $64 \%$ of women faculty have attended at least 1 out of 5 meetings. While data on participation preferences, motivation for attendance, and benefits of attending is still being collected, we continue to collect comments that illustrate the level of interest: "after 2 hours at one of these sessions, it is the same high I get after going to a conference for a couple of days!", "loved the collaborative aspect and networking" and "I absolutely loved these events- I was able to get to know my attendings on a very personal level and identified with their continued struggles as women physicians in medicine."

DISCUSSION / REFLECTION / LESSONS LEARNED: Though research regarding career fulfillment among women in medicine is limited, there is an increasing demand to create an environment where women can thrive and to ensure that they are given opportunities for career advancement. Our WiIM group is unique in that a resident-driven solution to an unfulfilled need now amplifies mentorship and sponsorship by faculty. The growth of WiIM speaks for itself. Female residents from other programs within our institution and visiting medical students have enthusiastically asked to be included in our meetings. Next steps for the organization are to establish a framework to pass on management of the group year over year, to invite more women leaders to our group, and to explore expanding across all residencies. Maintaining an intimate environment will be a challenge.

\section{CHALLENGES IN RESIDENT CLINIC EMPANELMENT: INVES- TIGATING AND INTERVENING UPON THE PANEL TRANSFER PROCESS}

Lydia A. Flier; Sarah Knapp; Mariana Gonzalez; Rebecca Glassman. Beth Israel Deaconess Medical Center, Boston, MA. (Control ID \#3163550)

NEEDS AND OBJECTIVES: (1) To assess the annual panel transfer process between graduating residents and incoming interns at a primary care 
clinic. (2) To propose and enact improvements to the panel transfer process. (3) To assess the improvement in the panel transfer process following interventions.

SETTING AND PARTICIPANTS: A fully-integrated resident primary care practice within an academic primary care center at a tertiary care hospital. Approximately 30 residents graduate each year and transfer their panels to new interns.

DESCRIPTION: Empanelment refers to patients being linked with individual primary care providers and care teams in a way that is recognized by the patient, providers, and practice. This is essential to functional primary care, and in particular - as part of the continued shift towards Accountable Care Organizations - practices need to be able to identify a given physician's panel. Resident practices have frequent, predictable turnover, as every year outgoing residents pass along a panel of patients to new interns. Unfortunately, there are no "best practices" for panel transfer. This is a high-risk period for patients, especially those with multiple co-morbidities. We assessed the existing panel transfer process between residents who graduated in 2016 and incoming interns that year. We reviewed a 50\% random sample, 15 resident panels with a total of 1439 patients who were transferred. A chart review identified whether the patients were seen over 18 months following transfer and by whom. We selected 18 months to provide a generous margin of error to capture annual visits in addition to chronic disease follow-up. Based on our findings, we enacted several changes of various scale to address gaps in the panel transfer process and to improve empanelment.

EVALUATION: We found that $41.6 \%$ of patients were not seen by our institution's primary care providers over this period time. On further assessment, the majority were under 40 years old and did not have chronic diseases. However, we found that one third of patients (248) were due for preventative health screening, and that 59 patients with a chronic disease were lost to follow up. There was no apparent difference in attrition based on gender, race/ethnicity, or language.

DISCUSSION / REFLECTION / LESSONS LEARNED: We enacted multiple changes to improve the panel transfer process. We initiated a personalized letter written by the outgoing physician informing patients of their new provider. We provided dedicated time for graduating senior residents and incoming interns to discuss their patients. We also created a "return to care" order in our electronic medical records that allows for outgoing residents to indicate when patients should return for care, helping the practice to provide outreach for scheduling and better track patients who are lost to follow up. These changes have been in place for six months and we are just beginning to collect data on the utility and efficacy of the changes.

\section{CHOOSE YOUR OWN ADVENTURE IN INTERPROFESSIONAL EDUCATION}

Madelyn Alvarez $^{1}$; Christine J. Kolehmainen ${ }^{2}$; Jessica M. Baier ${ }^{1}$; Gladys Roman-Rosado $^{1}$; Ronda Tippie ${ }^{1}$; Jo Ellen Uptegraw ${ }^{1}$; Heather O'Hearn ${ }^{1}$; Alishea Serwe-Behnke ${ }^{1}$; Bennett Vogelman ${ }^{3}$; Molly Carnes ${ }^{4}$; Robert Holland ${ }^{1}$. ${ }^{1}$ William S Middleton Memorial VA Hospital, Madison, WI; ${ }^{2}$ University of Wisconsin, Madison, WI; ${ }^{3}$ University of Wisconsin, Madison, Madison, WI; ${ }^{4}$ University of Wisconsin-Madison, Madison, WI. (Control ID \#3184223)

NEEDS AND OBJECTIVES: The ACGME requires training in interprofessional (IP) models of care. The University of Wisconsin Internal Medicine (IM) Residency Program developed an interprofessional education (IPE) curriculum using gaming to help learners advance their IPE foundational and applied knowledge. The primary objective is for residents to describe the importance of the four domains of IPE as defined by the Interprofessional Education Collaborative (ethics/values, roles/ responsibilities, communication, and teamwork) and practice application of this understanding using a game.

SETTING AND PARTICIPANTS: Our pilot curriculum includes 32 IM residents with a continuity clinic at the affiliate Veteran's Hospital. They will participate in the training during their four-week primary care rotation block.

DESCRIPTION: We created a blended curriculum with online and faceto-face activities. The online portion includes a novel choose-your-ownadventure (CYOA) game, didactic PowerPoints and videos. The in-person activities include facilitated discussions, interviewing and shadowing a team member for perspective taking and leading an IP team meeting. We chose to develop a CYOA style game that creates an individualized story for each learner and a memorable and enjoyable experience. The game provides the resident the flexibility of asynchronous education and allows them to interact with a virtual patient and IP team in a realistic way in that their choices have consequences. The resident's personalized avatar fosters emotional buy-in for these outcomes. The adventure follows a resident through clinical scenarios and highlights the importance of an IP approach to achieve a "good ending" to the story. In development, we engaged an IP team, including physicians, nurses, clerical staff, pharmacists, and social workers to enhance IP learning, make the storyline cohesive and choices realistic. UW-Madison's technology team used the software "Storyline" to make the game and add visual appeal.

EVALUATION: The residents will complete the Interprofessional Collaborative Competency Attainment Survey as a pre and post self-evaluation and write a post-course reflection. An IP team member will use the individual Teamwork Observation and Feedback Tool to evaluate teamwork, shared decision making and leadership.

DISCUSSION / REFLECTION / LESSONS LEARNED: We have learned that developing a CYOA game to promote IPE is challenging but has many advantages. CYOA gaming offers asynchronous methods of learning that maintain realistic, interprofessional interactions to enhance IPE applied knowledge in a fun and stimulating way. Intentionally constructing a strategy for each storyline, specifying the scope of the story at the beginning of the project, and being explicit about the educational objective for each decision tier can help to offset development challenges. Collaborative development of the game with an interprofessional team was critical to optimize the learning value of the game and include rich educational elements into the story from informal interactions, which are typically unobserved.

CLINICALREASONING.ORG: IMPROVING ACCESS AND ENGAGEMENT WITH CLINICAL REASONING TEACHING MATERIALS THROUGH WEBSITE REDESIGN

Gregory M. Ow ${ }^{1}$; Gabrielle Berger ${ }^{2}$; Juan N. Lessing ${ }^{3}$; Denise M. Connor ${ }^{4}$; Rabih Geha ${ }^{4}$; reza manesh ${ }^{5}$; Jeff Kohlwes ${ }^{4}$; Geoffrey V. Stetson ${ }^{4} .{ }^{1}$ University of California, San Francisco, San Francisco, CA; ${ }^{2}$ University of Washington, Seattle, WA; ${ }^{3}$ University of Colorado, Denver, CO; ${ }^{4}$ University of California, San Francisco and San Francisco VA Medical Center, San Francisco, CA; ${ }^{5}$ Johns Hopkins University, Baltimore, MD. (Control ID \#3143133)

NEEDS AND OBJECTIVES: The Exercises in Clinical Reasoning (ECR) series in the Journal of General Internal Medicine (JGIM) is a unique and growing series of 30 case-based problem-solving manuscripts, designed 
to teach the foundations of clinical reasoning to both educators and students. For selected cases, the ECR team has produced case presentations and instructor guides. These novel and freely downloadable materials are available on the JGIM website. However, site analytics reveal that: although these materials are frequently accessed ( $~ 8100$ users in 2017), users do not remain engaged with the material ( $\sim 90 \%$ of visitors visit only one page in the website, i.e. the bounce rate $=\sim 90 \%$ ). The ECR editorial team has hypothesized that the lack of engagement is due to poor website design. 1 . The goal of this project is to create a new, streamlined, and easy-to-use ECR website to promote free access to case-based clinical reasoning materials. 2 . The improved design will be evaluated for the hypothesized increased user engagement. The specific target is to reduce the bounce rate from $\sim 90 \%$ to $70 \%$, measured over the course of 6 months (September 2018 to March 2019). 3. The design will also be evaluated with the validated LIDA assessment tool from Minervation, a tool which provides a granular quantitative scoring of a website's usability, accessibility, and reliability.

SETTING AND PARTICIPANTS: ClinicalReasoning.org is accessible to educators and students worldwide.

DESCRIPTION: An updated ECR website was designed using the foundational principles of visual design - proximity, alignment, repetition, and contrast. In summary, these principles respectively state that: (1) logically related elements should be placed close together, (2) elements should be purposefully positioned, (3) style conventions and patterns should be consistent throughout, and (4) use large (rather than small) visual differences in color, typography, and size. In addition to visual improvements, the redesign also included simplification of website navigation. ClinicalReasoning.org went live on September 2018, and provides free access to the ECR teaching materials. EVALUATION: Design evaluation utilized the LIDA tool, which evealed an improvement in usability from 29 to 48 on a 54 point scale. Data from Sept. to Dec. 2018, when compared to data from Jan. to Dec. of 2017, reveals a large and statistically significant improvement in bounce rate from $87.70 \%$ ( $\mathrm{n}=$ 8100 views) to $47.49 \%$ ( $n=1625$ views), $p<0.0001$ using N-1 chi-squared. DISCUSSION / REFLECTION / LESSONS LEARNED: The freedom of web resources has increased accessibility of education materials. Anyone in the world with any level of training can access ECR resources to learn, practice, and teach clinical reasoning. However, the freedom of web resources also creates an unstructured environment, where user interaction can suffer because of poor or inconsistent design. Incorporation of visual design principles and a user-centric approach to education material greatly increases immediate visual appeal and long-term user engagement.

ONLINE RESOURCE URL (OPTIONAL): ClinicalReasoning.org

CREATING A LEADERSHIP CURRICULUM FOR WOMEN IN MEDICINE: WOMEN IN LEADERSHIP DEVELOPMENT (WILD) Bridget Keenan; Anne G. Montgomery; Anjali Thakkar; Catherine Burke; Haley Crossman; Shirin Hemmat; Arielle Klepper; Lekshmi Santhosh. UCSF, San Francisco, CA. (Control ID \#3180516)

NEEDS AND OBJECTIVES: Though women account for nearly half of US medical students and residents, they are underrepresented at higher levels in academic medicine. A lack of structured leadership skills curricula targeting women trainees contributes to this gap. The Women in Leadership Development (WILD) curriculum was therefore developed within the Department of Medicine at the University of California, San Francisco (UCSF) with the goal of building community and providing leadership training for women trainees hoping to launch their careers in academic medicine.

SETTING AND PARTICIPANTS: WILD serves women residents and fellows at UCSF. During the first two years of programming (2017 and
2018), a total of 77 trainees within the Department of Medicine attended a daylong WILD symposium. Based on the Symposium's success, a yearly longitudinal curriculum was implemented at the GME level for the 2018-2019 academic year, serving women trainees across all specialties.

DESCRIPTION: The WILD Symposium is a daylong course with the objectives of skill-building, mentorship, and networking. Skill sessions focus on public speaking, conflict resolution, microaggressions, and advocacy, with presenters from both inside and outside of academic medicine. This year, we have expanded WILD to include monthly skill-building sessions for women trainees across specialties ("WILD: Series") as well as quarterly events for trainees within the Department of Medicine focused on networking and community building ("WILD: IM").

EVALUATION: Prior to implementation of the WILD Symposium, we conducted a needs assessment to survey women residents about their interest in a leadership course. 93\% had some level of interest. Topic areas of interest included negotiation, CV development, self-promotion, communication, networking, work-life balance, and the art of "saying no." Of 77 participants surveyed after the event, over $90 \%$ agreed that participation in the symposium was a useful experience and would strongly recommend the session to other trainees. The public speaking session received the highest reviews.

DISCUSSION / REFLECTION / LESSONS LEARNED: While challenges remain for women trainees in academic medicine, leadership training represents one possible solution to address gender disparities. The strong positive response to the WILD Symposium reinforced the need for improved leadership training among women residents and fellows at UCSF.

\section{CREATING CLINICIAN EDUCATORS: ASSESSMENT OF A RESIDENT-AS-TEACHER ELECTIVE IN GRADUATE MEDICAL EDUCATION.}

Geoffrey V. Stetson ${ }^{1}$; Jessica Beaman ${ }^{1}$; Adeena Khan ${ }^{2}$; Bradley A. Sharpe ${ }^{2}$. ${ }^{1}$ University of California, San Francisco, San Francisco, CA; ${ }^{2}$ UCSF, San Francisco, CA. (Control ID \#3183696)

NEEDS AND OBJECTIVES: Residents are expected to assume many roles: clinician, team leader, and teacher. At UCSF, there are brief formal didactics focused on teaching but no longitudinal experience for internal medicine residents to gain the skills necessary to be a successful teacher. In Spring of 2015, a needs assessment using focus groups revealed uniform desire for a primer in foundational clinical teaching skills. Key experts and stakeholders gathered to determine objectives for a month-long elective: understand best practices and demonstrate improvement in the areas of outpatient precepting, delivering feedback, whiteboard presentations, public speaking, entrusting learners, and teaching at the bedside.

SETTING AND PARTICIPANTS: Participants were third-year internal medicine residents at UCSF. The elective accommodates one learner per month. The course is spread across the three principal learning sites of our university. In our first two years we worked with learners during 22 of the available 24 month-long sessions.

DESCRIPTION: The elective consists of 11 unique and tailored half-day activities which build foundational concepts that support the learning objectives. Opportunities for implementation of skills are provided, followed by targeted feedback, and an opportunity to demonstrate improvement. Each activity or exercise has an accompanying faculty mentor, pre-reading, and reflection activity. Assessment of the elective was done using a Likert-style retrospective pre/post self-assessment method (Skeff 1992) to analyze changes in confidence level related to each of the teaching skills addressed in the curriculum and satisfaction with the elective experience (Kirkpatrick Levels 1\&2). 20/22 learners completed the end-of-rotation evaluation. 
EVALUATION: Data from the surveys compared pre-elective to postelective using chi-squared statistics. Results showed a significant increase in confidence $(\mathrm{p}<0.05)$ in learners' ability to deliver an effective whiteboard talk $(\mathrm{p}=0.018)$, and in their upcoming transition to a post-residency role $(\mathrm{p}=0.025)$. Two other areas approached significance $(\mathrm{p}<0.10)$ : giving effective feedback to a learner $(\mathrm{p}=0.082)$, and teaching at the bedside $(\mathrm{p}=0.10) .95 \%$ of learners reported they would either recommend or strongly recommend the elective to a classmate.

DISCUSSION / REFLECTION / LESSONS LEARNED: This curriculum provides a unique opportunity for residents to invest time in learning evidence-based strategies and build a foundation for becoming a medical educator. Our goal was to create an elective that would fit within the current scheduling structure of our residency but allow for an individualized approach to building specific skills. Both quantitative data and narrative responses show that we have developed an effective, flexible, and learner-centered course.

\section{CRITICAL CARE CURRICULUM FOR INTERNAL MEDICINE INTERNS TRANSITIONING TO SUPERVISING RESIDENTS IN THE MEDICAL INTENSIVE CARE UNIT- A TARGETED NEEDS ASSESSMENT}

Dimple Shah; Karen Friedman; Gulrukh Zaidi. Donald and Barbara Zucker School of Medicine at Hofstra/Northwell, Manhasset, NY. (Control ID \#3184858)

NEEDS AND OBJECTIVES: To conduct a medical education needs Assessment of internal medicine (IM) residents and critical care faculty (CCF) to determine if there is a gap in critical care knowledge in residents transitioning from internship into second year residency.

SETTING AND PARTICIPANTS: Categorical IM residents (PGY 1,2,3) and CCF at Northwell Health.

DESCRIPTION: We surveyed the categorical IM residents and CCF to determine 1) the perceived level of preparedness of rising second year IM residents to take on the role of a supervising resident in the medical intensive care unit (MICU) 2) whether a critical care curriculum would be beneficial for rising second year IM residents, and 3) which subject areas in a critical care curriculum would be most valuable for residents. Surveys were conducted on a 5 point Likert Scale (strongly disagree, disagree, neither agree/disagree, agree or strongly agree) to determine preparedness and necessity of the curriculum, and a ranking system to determine the topics of greatest importance (1-8).

EVALUATION: 42 IM Residents (41.5\%) and $16 \mathrm{CCF}$ (59.2\%) filled out the survey. For senior resident preparedness in MICU, $41 \%$ of IM residents and $70 \%$ of CCF felt the rising second year IM residents were not adequately prepared. $90 \%$ of IM residents and $100 \%$ of CCF agreed that a critical care curriculum would be beneficial prior to IM residents taking on the role of senior resident. Both IM residents and CCF ranked (on scale from 1-8, 8 being most important) shock and vasopressor management (average score IM: 6.83, CCF: 6.44) as the most important topic to cover, followed by acute respiratory failure (IM: 6.49; CCF 6.06), ventilator management (IM: 5.55, CCF: 4.06) and acid base disorders (IM: 5.3 ; CCF 4.5).

DISCUSSION / REFLECTION / LESSONS LEARNED: The results of our study show both IM residents and CCF felt there is a gap in critical care education for residents transitioning from internship to taking on the role of a supervising senior resident. Key areas of focus for a critical care curriculum were identified via our survey study. Based on our needs assessment, we will develop a curriculum aimed to prepare rising second year residents to take on the role of a supervising resident. The pilot curriculum with provide high yield didactic and interactive videos on the topics of shock and vasopressor management, acid/base disorders and acute respiratory failure. IM residents will be tested on practical knowledge in the form of a pre and post multiple choice test and OSCE.

\section{DESIGNING A MEDICAL-LEGAL COLLABORATION FOR MED- ICINE RESIDENTS}

Jo Henderson-Frost $^{1}$; Aisha James ${ }^{1}$; Nancy Lorenz ${ }^{2}$; Hannah Tanabe ${ }^{2}$; Julian Mitton ${ }^{1} .{ }^{1}$ Massachusetts General Hospital, Newton, MA; ${ }^{2}$ Greater Boston Legal Services, Boston, MA. (Control ID \#3185764)

NEEDS AND OBJECTIVES: Health concerns, from disability to mental illness, impact the legal considerations of clients, while social determinants of health, from housing insecurity to criminal records, impact health outcomes. Despite this interdependence, few partnerships exist between the medical and legal professions, and training opportunities in this space are rare. To address this need, we designed a participatory, community-based, medical-legal collaboration (MLC) for medicine residents.

SETTING AND PARTICIPANTS: The Massachusetts General Hospital (MGH) Department of Medicine partnered with the Greater Boston Legal Services (GBLS) to create the MLC. Eight sessions took place over the 20172018 pilot year, and consisted of case presentations and discussion in an interdisciplinary seminar format. Residents participated in the MLC during a physician advocacy elective.

DESCRIPTION: The sessions were led by two MGH faculty, one resident, and two GBLS attorneys. In preparation for the seminar, residents were asked to identify a patient with a legal question impacting their health. Consent was obtained and background information collected to prepare a de-identified case presentation. During the seminar, attorneys provided advice and counsel, and offered resources to help address legal concerns. In turn, the GBLS attorneys prepared questions from de-identified client medical charts, or asked general medical questions relevant to case arguments. The resident reviewed the charts and presented educational tutorials during the seminar to address these questions. In the final step, learned information was relayed back to the patient and care team, and client and legal team respectively.

EVALUATION: After each MLC, the residents' experience was reviewed. Additionally, all participants were asked to complete an anonymous survey regarding the utility of the session and the main learning points. Results are currently under review.

DISCUSSION / REFLECTION / LESSONS LEARNED: During the MLC, participants had the opportunity to probe to the depth of the issues facing their patients and clients and share relevant, case-based expertise. The information from the legal advice frequently resulted in actionable items that impacted a patient's care. Moreover, the information from the medical advice impacted legal cases and led to client success. We anticipate participants will value the educational component of the collaboration. We expect to identify challenges in relaying salient information from the MLC to patients.

\section{DEVELOPING AN INTEGRATED CASE CONFERENCE IN A HOMELESS VETERANS CLINIC}

Michael Soh ${ }^{1}$; Brianna J. Cowan ${ }^{3}$; Kerri Schutz ${ }^{2}{ }^{1}$ Greater Los Angeles VA, Los Angeles, CA; ${ }^{2}$ West Los Angeles VA MC, Los Angeles, CA; ${ }^{3}$ UCLA, Los Angeles, CA. (Control ID \#3186191)

NEEDS AND OBJECTIVES: Research has shown that because homeless Veterans generally access care in a haphazard fashion (Tsai et al., 2017), lack consistent follow-up, and typically do not have established access to a phone or mailing address, it is imperative that a space be created for team members to prepare for these issues and provide intervention, consultation, and 
collaboration (Lie, et al., 2016). In our clinic, we implemented an integrated case conference (ICC) that was accessible to all parties and identified specific actions items for improved quality and delivery of care.

SETTING AND PARTICIPANTS: This interprofessional (IP) practice/ training site is in a large VA clinic for homeless Veterans ( $\sim 3,000$ patients). The clinic provides primary and mental health care and social work services within a patient-centered medical home (PCMH). As of January 2019, over 30 trainees representing specialties including internal medicine, advanced nursing, pharmacy, psychiatry, and psychology are currently in clinic.

DESCRIPTION: The goals of our ICC were to discuss urgent needs, identify opportunities for collaboration, and create an integrated treatment plan. That said, cases were selected by the trainee based on urgency and opportunity for IP collaboration. Prior to, presenters conducted a chart review, prepared a brief summary, and shared potential IP questions with their team. During the ICC, meeting roles were assigned, questions were discussed, and action items were identified.

EVALUATION: The aims of the conference were to recognize needs for IP collaboration, perform IP roles, educate others about their professional expertise, and incorporate the expertise of other professions into their practice. As such, over the course of one training year, we deployed two evaluation tools - a weekly instrument that examined each case presentation and its impact and satisfaction, and a 6- and 12-month instrument that explored changes in trainee attitudes and behaviors related to IP collaboration, professional expertise integration, and IP role confidence. In total, weekly data from 21 ICCs was collected, with approximately non-unique 250 trainees and attending participants, and 20 trainees completed the 6- and 12-month instruments.

DISCUSSION / REFLECTION / LESSONS LEARNED: Evaluation data from our trainees indicates that the ICC led to statistically significant increases in translation of knowledge from other professions into a treatment plan, ability to formulate and recommend a collaborative and coordinated care plan for their patients, and confidence in working collaboratively with primary care and/or mental health providers. Additionally, nearly $75 \%$ of trainees reported being "very satisfied" with the ICCs they participated in. Over the past year, we've learned that our ICCs benefit from a consistent structure (day, time, location), advanced notice of case-based questions, and selecting patients that are at least semi- engaged with the clinic, among many others.

\section{DEVELOPING EQUITY REPORTS FOR RESIDENTS TRAINING IN PRIMARY CARE}

$\underline{\text { Muriel Jean-Jacques }}{ }^{1}$; Andrew J. Cooper ${ }^{2}$; Lauren A. Gard ${ }^{2}$; Mita S. Goel ${ }^{2}$; Paul Ravenna $^{2}$; Aashish Didwania ${ }^{3}$; Matthew J. O'Brien ${ }^{2}$; Stephen Persell ${ }^{2}$. ${ }^{1}$ Northwestern University Feinberg School of Medicine, Chicago, IL; ${ }^{2}$ Northwestern University, Chicago, IL; ${ }^{3}$ Northwestern Medicine, Chicago, IL. (Control ID \#3185578)

NEEDS AND OBJECTIVES: The ACGME has concluded that there is a substantive deficiency in preparing residents to identify and address health disparities. In particular, most clinical learning environments lack a formal approach for identifying disparities in quality. Barriers to systematically examining disparities include inconsistent documentation of race/ethnicity and limited capture of sociodemographic variables. Resident-specific challenges include accurate patient attribution and smaller patient panels. Our goal was to develop a methodology for systematically providing feedback about disparities in ambulatory care quality to residents.

SETTING AND PARTICIPANTS: Equity reports were developed for internal and family medicine residents training in two urban GME programs within a single healthcare system.

DESCRIPTION: Five quality measures were included in the equity reports: breast and colon cancer screening, blood pressure and diabetes control, and statin therapy. Each measure was stratified by race/ethnicity, insurance, preferred language, and whether or not the patient lives in a Census block group with a high level of poverty. We tested 3 methods for attributing patients to residents and examined 3 levels of granularity for the equity reports: individual resident panel; panel of all patients attributed to residents; and all patients in the practice. For each level, we determined the number of measures with sufficient numbers of patients to make meaningful comparisons by subgroup in order to identify the appropriate level of granularity for the equity reports.

EVALUATION: The attribution rule that assigned patients to the physician who had seen them for the greatest proportion of encounters performed best on measures of resident panel size and continuity, while attribution based on listed PCP peformed worst. It was not possible to create equity reports at the individual resident panel level as no measures had sufficient numbers of patients to allow for reliable comparisons. Most measures had sufficient patients when all resident panels were combined, and all measures had sufficient patients when all patients in the practice were included. Disparities were identified for at least one subgroup for 4 measures.

DISCUSSION / REFLECTION / LESSONS LEARNED: We identified several lessons in our effort to develop health equity reports for residents training in primary care that may be applicable to others. 1. Even in a healthcare system that, like most, has not yet implemented the routine assessment of disparities as part of their quality tracking protocols, it was feasible to create equity reports that included a range of sociodemographic variables. 2 . Geocoding allowed us to include a measure of socioeconomic position. 3 . Relatively straightforward methods could be applied to attribute patients to resident physicians. 4. While residents did not have large enough patient panels to create equity reports at the individual resident panel level, it was possible to create equity reports when all resident panels were combined or at the practice level.

DEVELOPING MEDICAL STUDENT LEADERSHIP, TEAM-
WORK, AND COMMUNITY ENGAGEMENT THROUGH IMPLE-
MENTATION OF A NOVEL SERVICE-LEARNING CURRICULUM
IN THE DENVER HEALTH LONGITUDINAL INTEGRATED
CLERKSHIP (DH-LIC)
Catherine Ard ${ }^{1}$; Jennifer Adams ${ }^{2}$; Margaret Tomcho ${ }^{2} .{ }^{1}$ University of Colora$\frac{\text { Catherine Ard }}{1}{ }^{1}$; Jennifer Adams ${ }^{2}$; Margaret Tomcho ${ }^{2} .{ }^{1}$ University of Cor, CO; ${ }^{2}$ Denver Health, Denver, CO. (Control ID \#3179970)

NEEDS AND OBJECTIVES: It is estimated that the Social Determinants of Health $(\mathrm{SDoH})$ contribute to $80 \%$ of a patient's health outcomes, yet most medical students receive little to no training on addressing them; instead focusing on a biomedical model of disease. In a needs assessment of DHLIC students, $66 \%$ of students reported low confidence in working with communities to find solutions to their self-identified problems related to $\mathrm{SDoH}$. Based on these results, an innovative service learning curriculum was developed with the objectives to increase medical student teamwork, community engagement, and understanding of $\mathrm{SDoH}$.

SETTING AND PARTICIPANTS: The University of Colorado School of Medicine's DH-LIC is a year-long clerkship in which 10 medical students complete all core clerkships in a longitudinal, integrated experience at Denver's safety net hospital.

DESCRIPTION: This innovation consists of a multimodal curriculum woven into the DH-LIC, including didactic sessions focused on $\mathrm{SDoH}$, community windshield surveys and case investigations, team dynamics workshops, written reflections with group discussion, and the development of a longitudinal service project in collaboration with community partners. 
EVALUATION: Pre-curricular surveys assessed student values and attitudes surrounding leadership, community engagement, altruism, and confidence in addressing SDoH of their patients. Results showed students highly value community involvement, physician leadership, and altruism, with mixed results in confidence and experience in addressing SDoH. Post-curricular surveys will be administered to students with results to be presented at the conference. Additionally, community partners will be surveyed about impact of service projects and student characteristics and interactions with community members.

DISCUSSION / REFLECTION / LESSONS LEARNED: A service learning curriculum proved to be a powerful tool to introduce students to experiential learning around community engagement and organizing, teamwork, leadership, and the influence of SDoH on individual and community health outcomes. Students reflected on the roles of physicians in communities and felt empowered by their partnerships with community members. Workshops with facilitated reflection focused on team dynamics and leadership led to powerful discussions among students and pushed students to expand their roles outside of comfort zones leading to personal and professional growth.

\section{DEVELOPMENT AND IMPLEMENTATION OF A PRE-CLINICAL LGBTQ+ HEALTH ELECTIVE}

Uriel Kim ${ }^{2}$; Eric Chen ${ }^{2}$; Megan McNamara ${ }^{1}$. ${ }^{1}$ Louis Stokes Cleveland VAMC, Cleveland, $\mathrm{OH} ;{ }^{2}$ Case Western Reserve University School of Medicine, Cleveland, OH. (Control ID \#3186565)

NEEDS AND OBJECTIVES: Despite the documented healthcare barriers and disparities of LGBTQ+ individuals, there is often little medical school curricular emphasis on the topic. The main objective of the LGBTQ+ Health Elective is to provide learners with a 360-degree, "whole person" perspective of LGBTQ+ health through multidisciplinary lectures and shadowing experiences.

SETTING AND PARTICIPANTS: Fourteen second year medical students from Case Western Reserve University School of Medicine (CWRU-SOM) participated in the elective.

DESCRIPTION: This zero-credit, pre-clinical elective met for three hours every other week over the course of a semester. Key strengths of the elective include the implementation of national curricular guidelines from the AAMC, multi-disciplinary lectures, multi-disciplinary and clinical shadowing experiences. The lectures were delivered by physicians, social workers, sociologists, and other allied health professionals, and covered topics including the Social Determinants of LGBTQ+ Health, Gender Affirmation Surgery, Spirituality, and the Sociology of Sexuality. Students were required to complete three shadowing experiences, each four hours long. Students shadowed either a social worker or psychologist at least once. The shadowing experiences occurred at LGBTQ-focused Clinics at the Louis Stokes Cleveland VA Medical Center and the Cleveland Clinic Foundation.

EVALUATION: For the program evaluation, we asked learners to complete a "minute paper," a validated quality improvement instrument, after each lecture. We also asked learners to quantitively assess their clinical shadowing experiences and their preceptors. For the student evaluation, we administered a validated, LGBTQ+ health-specific pre/post survey consisting of 16 likertscale questions. Additionally, at the conclusion of the elective, students completed a brief, 55-word, reflective practice exercise.

DISCUSSION / REFLECTION / LESSONS LEARNED: To our knowledge, this elective is the among the most comprehensive pre-clinical curricula for LGBTQ+ Health. The results of the pre/post survey demonstrate improvement in domains of "knowledge," "attitudes," and "experiences." The average standardized mean differences between the pre and post samples $(\mathrm{N}=13)$ for the 5 knowledge questions, 6 attitudes questions, and 5 experience questions were $0.23,0.37$, and 0.19 , respectively. Secondary benefits of the elective include elevating the importance of LGBTQ+ Health in the medical curriculum and the opportunity to knit together LGBTQ+ providers and allies at CWRU-SOM's various clinical affiliates.

\section{DEVELOPMENT AND INITIAL EVALUATION OF COMMUNITY HEALTH CURRICULUM IN AN INTERNAL MEDICINE RESI- DENCY PROGRAM: YEAR ONE}

Rachael W. Hayes ${ }^{1}$; Jennifer Adams ${ }^{2}$; Lisa Altshuler ${ }^{2}$; John Martin ${ }^{1} .{ }^{1} \mathrm{NYU}$ Langone Brooklyn, New York, NY; ${ }^{2}$ NYU School of Medicine, New York, NY. (Control ID \#3175501)

NEEDS AND OBJECTIVES: In the changing landscape of healthcare, physicians must be adaptive, visionary and evidence-based in their approach to care. Medical education must be adjusted to allow learners to gain skills that prepare them to function effectively in this new paradigm. In order to meet these needs, we developed a community based curriculum with emphasis on transitions of care, population health and innovation of care.

SETTING AND PARTICIPANTS: Curriculum was developed as part of a new NYU Internal Medicine Residency Community Health Track, housed at NYU Langone Hospital- Brooklyn, a community based, academic teaching hospital. Ambulatory training is based at the Family Health Centers at NYU Langone, a network of FQHCs. Both the hospital and FQHCs serve a vulnerable, diverse community in south Brooklyn. To date we have recruited one class of 10 interns. As of July 2020 we anticipate having a full track consisting of 30 residents.

DESCRIPTION: Our first year curriculum aims to develop a framework for thinking about community health introducing key concepts such as population-based care, novel delivery of care, and interdisciplinary collaboration. Early in their training, residents completed a community assessment using observational data, interviews and census track data. These assessments paired with collaboration with CHW and community organizations gave them first hand exposure to our area's specific challenges and gaps in care. The residents began to develop skills in home care by working with an interdisciplinary team of doctors, nurses and CHWs. Additionally, they participated in a transitions of care workshop, examining their own hospital patients who had been readmitted and identifying best practices for hospital discharge.

EVALUATION: A multi-method evaluation plan is essential as we evaluate and strengthen the curriculum. Qualitative feedback is gathered at regular intervals throughout the year along with surveys of trainees. Initial results suggest that curricula is well-received by residents. Aggregated longitudinal educational data including resident self-report, $360^{\circ}$ evaluations and performance-based assessment, (OSCEs, USP visits) will contribute to program evaluation. The most important outcome will be how these trainees practice once they have graduated. We plan to use postgraduate surveys to judge the impact of the curriculum.

DISCUSSION / REFLECTION / LESSONS LEARNED: We adapted curriculum to focus on our particular community and created innovative programs to improve the population's health. Essential to these accomplishments was our partnership with learners and our reliance on their feedback to guide curriculum development. Allowing trainees to explore their interests has lead to visionary projects. We have learned that by being flexible and adapting to the learners' needs and interests we can serve our community in deeper ways than we had initially anticipated. However, structural limitations of the clinic coupled with institutional changes resulted in a slower time frame for clinical adaptations. 
DEVELOPMENT AND PILOT TESTING OF A TOOL TO IMPROVE GENERIC ORAL CONTRACEPTIVE PILL PRESCRIBING

Gena Lenti ${ }^{1}$; Hannah Wilson ${ }^{3}$; Jen Rusiecki ${ }^{1}$; Vineet M. Arora ${ }^{2} .{ }^{1}$ University of Chicago, Chicago, IL; ${ }^{2}$ University of Chicago Medical Center, Chicago, IL; ${ }^{3}$ University of Chicago Pritzker School of Medicine, Chicago, IL. (Control ID \#3185697)

NEEDS AND OBJECTIVES: Brand and generic oral contraceptives (OCPs) are therapeutically equivalent, but generics still tend to be under-prescribed. In order to asses barriers to generic OCP prescription, a needs assessment was conducted using focus groups and surveys completed by Primary Care Providers and Nurse Practitioners at American College of Physicians Internal Medicine and American Association of Nurse Practitioners Annual Meetings. Focus groups identified confusion about generic naming as a barrier to prescription. One participant stated, "If you're going to generically prescribe an oral contraceptive...you're going to have to go in there and be savvy enough to be able to ask for that drug based on the generic drug equivalents that are in it." Based on focus group and survey findings, our objective was to develop and test a clinical tool to facilitate generic OCP prescription.

SETTING AND PARTICIPANTS: Following development, our tool was disseminated among residents, attendings, and APN's in the University of Chicago Primary Care Group (PCG), and focus groups and surveys were used to explore usefulness and gather feedback on the tool.

DESCRIPTION: Our tool consisted of a pocket card designed to clarify OCP nomenclature. The front of the card included benefits of generic medications to be communicated to patients as well as a description of how to use the card. The back of the card comprised of a table of specific types of branded OCPs and their generic equivalents, matched based on estrogen (dose) and progesterone (type and dose) components. The medication types listed in the table were drawn from the University of Chicago PCG most frequently prescribed OCPs, and the card was reviewed by University of Chicago pharmacists and primary care leaders.

EVALUATION: Surveys completed before dissemination of the pocket card indicated $63 \%(n=38)$ of participants found the naming of OCPs to be confusing, $74 \%$ reported they do not discuss switching to generic OCPs, and $58 \%$ were not confident prescribing OCPs without a tool. Provider knowledge of generic OCPs was low, indicated by the fact that $85 \%$ and $50 \%$ were able to correctly identify Yaz and Ortho-cyclen as brand OCPs, yet only $45 \%$ and $29 \%$ were able to identify Sprintec and TriNessa as generic. Following dissemination, $100 \%$ agreed that the pocket card was understandable, and $89 \%$ said the pocket card would be useful in clinical practice. Feedback for improvement of the tool included presence of drug prices, identification of common "go-to" varieties, development of an OCP algorithm, and creation of an online version.

DISCUSSION / REFLECTION / LESSONS LEARNED: Nomenclature of generic OCPs is confusing and this creates a barrier to generic prescription. An educational tool such as ours may help to fill this knowledge gap among OCP prescribers. In the future, we would like to develop a post-survey for participants to complete after integrating the card into their clinical workflow and to conduct a similar assessment with other physicians who prescribe OCPs, such as obstetrician-gynecologists.
ONLINE RESOURCE URL (OPTIONAL): https://bit.ly/ 2VZWZ4P

DEVELOPMENT AND TESTING OF A WEB MODULE TO IMPROVE GENERIC PRESCRIBING OF ORAL CONTRACEPTIVES AMONG INTERNAL MEDICINE PHYSICIANS Gena Lenti $^{1}$; Jeanne M. Farnan ${ }^{1}$; Arlene Weissman ${ }^{3}$; Michelle L. Cook ${ }^{4}$; Marilyn Stebbins ${ }^{8}$; Christopher Moriates ${ }^{5}$; Shalini S. Lynch ${ }^{8}$; Anita Samarth $^{9}$; James X. Zhang ${ }^{6}$; September Wallingford ${ }^{7}$; Neel Shah ${ }^{10}$; Samantha Ngooi ${ }^{1}$; David Meltzer ${ }^{1}$; Vineet M. Arora ${ }^{2} .{ }^{1}$ University of Chicago, Chicago, IL; ${ }^{2}$ University of Chicago Medical Center, Chicago, IL; ${ }^{3}$ American College of Physicians, Philadelphia, PA; ${ }^{4}$ American Assocation of Nurse Practitioners, Austin, TX; ${ }^{5}$ Dell Medical School at The University of Texas, Austin, Austin, TX; ${ }^{6}$ The University of Chicago, Chicago, IL; ${ }^{7}$ Costs of Care, Quincy, MA; ${ }^{8}$ University of California San Francisco, San Francisco, CA; ${ }^{9}$ Clinovations Government and Health, Washington D.C., DC; ${ }^{10}$ Harvard Medical School, Boston, MA. (Control ID \#3185575)

NEEDS AND OBJECTIVES: Use of generic oral contraceptives (OCPs) can improve medication adherence and reduce healthcare costs for patients, yet skepticism of generic drugs remains a barrier to generic OCP prescription. Generic skepticism, defined as lack of agreement that generic drugs are as effective as, as safe as, or do not cause more adverse events than brand counterparts, persists among clinicians in many fields and is associated with less willingness to discuss and prescribe generic drugs. While several strategies to reduce generic skepticism and increase generic prescribing have been attempted, none have targeted use of OCPs specifically. An educational web module was developed to reduce generic skepticism related to OCPs, improve knowledge of generic drugs, and increase willingness to discuss and prescribe generic OCPs among physicians.

SETTING AND PARTICIPANTS: A baseline survey asessing generic skepticism and prescription of generic drugs was disseminated via email to an ACP research panel. Eligibility criteria selected for physicians specializing in internal medicine and those who spend greater than $50 \%$ of time in patient care. The module was sent to physicians who completed this baseline survey.

DESCRIPTION: Kern's six step approach to curriculum development was used to develop a generic OCP web-module. A primary needs assessment was completed using focus groups at American College of Physicians (ACP) Annual meetings and a survey targeting baseline generic skepticism. Insights gained from needs assessments were used to build an educational web module discussing benefits to generic OCP prescription. Post-module evaluation assessed learner reaction, knowledge, intention to discuss and prescribe generic OCPs, and changes in generic skepticism.

EVALUATION: The module had a response rate of 56\% ( $n=208 / 369)$. Individuals defined as generic skeptics at baseline were significantly less likely to complete our module compared to non-skeptics (responders $9.6 \%$ vs. non-responders $16.8 \%, \mathrm{p}=0.04)$. The majority $(85 \%, \mathrm{n}=17 / 20)$ of baseline skeptics were converted to non-skeptics $(\mathrm{p}<0.01)$ following completion of the module. Compared to non-skeptics, post-module generic skeptics reported less willingness to discuss (skeptic 33.3\% vs. nonskeptic $71.5 \%, \mathrm{p}<0.01$ ), but not less willingness to prescribe generic OCPs (skeptic $53.3 \%$ vs. non-skeptic $67.9 \%$, p=0.25). Non-white physicians and international medical graduates (IMG) were more likely to be generic skeptics at baseline (non-white $86.9 \%$ vs. white $69.9 \%, \mathrm{p}=0.01$, 
IMG $13.0 \%$ vs. USMG $5.0 \%$ vs. unknown $18.2 \%, \mathrm{p}=0.03$ ) but also more likely to report intention to prescribe generic OCPs as a result of the module (non-white $78.7 \%$ vs. white $57.3 \%$, p $<0.01$, IMG $76.1 \%$ vs. USMG $50.3 \%$ vs. unknown $77.3 \%$, $\mathrm{p}=0.03$ ).

DISCUSSION / REFLECTION / LESSONS LEARNED: Our study demonstrated an effective way to promote generic medications and educate providers using a brief web module disseminated on a national scale. Future interventions such as ours should target groups with low knowledge of generic drugs as well as those with high generic skepticism.

DEVELOPMENT OF A MEDICAL STUDENT CLINICAL ELECTIVE FOCUSING ON HEALTH EQUITY: "EXPLORING SOCIAL DETERMINANTS OF HEALTH AT AN URBAN COMMUNITY HOSPITAL." A LOOK AT IMPACTS ON STUDENT ATTITUDES AND READINESS TO SERVE A DIVERSE PATIENT POPULATION.

Amanda Ramsdell. Weill Cornell Medical College, New York, NY. (Control ID \#3164992)

NEEDS AND OBJECTIVES: The importance of health equity is gaining awareness throughout every level of the healthcare system. Medical providers bear witness to some of the most severe downstream effects of health inequity in a community. Serving our patients involves taking a closer look at the root cause of disease and health disparities long before a patient enters the hospital. How we address these upstream factors in our clinical practice is evolving, and the question of how we explicitly teach our approach to medical students in the clinical setting needs to be explored. Our goal is to allow medical students the time to focus on and explore these issues through both lectures and real-time clinical encounters. In doing so, we hope to reinforce the importance of social determinants of health in their future practice of medicine.

SETTING AND PARTICIPANTS: Students qualify to take this elective after having completed the core clinical clerkships in internal medicine, pediatrics and family medicine, which typically occurs during their third or fourth year. The clinical portion of the elective involves patients admitted to the Hospitalist service, referred by Hospitalist providers.

DESCRIPTION: The core experience of the course centers around student interviews of patients at the bedside, exploring the patients' experience and discussing prior history and root causes of disease and wellness. Working backwards from this knowledge, the students and preceptor explore upstream factors of observed downstream outcomes. Elective team then presents information to the primary inpatient team, and brainstorms current actionable interventions. Topics of didactics include relevant areas related to housing, immigration, implicit bias involving race and incarceration which are common areas of concern for many of our patients. Learning is reinforced by targeted field visits to housing/ homelessness advocacy groups, methadone clinics and community centers. Continued discussions extend to our patients' political determinants of health and areas of opportunity for advocacy as physicians.

EVALUATION: IRB approval pending for upcoming students for qualitative research project, details to be determined.

DISCUSSION / REFLECTION / LESSONS LEARNED: While Health Equity is often addressed in the pre-clinical years explicitly, modeling addressing these important factors into real-time clinical work remains largely implicit and dependent on the attending of service for most medical students. Explicitly speaking about health equity in the clinical setting is often lacking at many levels of medical training (i.e. medical students, Physician Assistants, and Attendings.) During our pilot elective, students appreciated being able to directly focus on these factors and felt they added value to the primary clinical team. The preceptor and medical team on service also benefited from greater explicit communication about social determinants of health and considering broader systems based interventions that may be of benefit to their patients in the longer term.

\section{DEVELOPMENT OF AN EVALUATION FRAMEWORK FOR COMMUNITY HEALTH WORKERS IN A MEDICAID ACO}

$\underline{\text { Salina Bakshi }}^{2,}{ }^{3}$; Kayt Kitt ${ }^{4}$; Sarah $\mathrm{Oo}^{5}$; Danelle Marable ${ }^{5}$; Maryann Vienneau ${ }^{4}$; Amy O. Flaster ${ }^{1,4}$. ${ }^{1}$ Brigham and Women's Hospital, Brookline, MA; ${ }^{2}$ Massachusetts General Hospital, Boston, MA; ${ }^{3}$ Harvard Medical School, Boston, MA; ${ }^{4}$ Partners Healthcare, Boston, MA; ${ }^{5}$ Massachusetts General Hospital, Chelsea, MA. (Control ID \#3186004)

NEEDS AND OBJECTIVES: 1) To learn how to leverage and implement a community health worker (CHW) model to assist in managing patients' social determinants of health (SDOH) needs. 2) To develop key performance indicators to ensure the operational success and to support the evaluation of a CHW model. 3) To encourage the continued use of CHWs as a resource supporting the success of a Medicaid Accountable Care Organization model.

SETTING AND PARTICIPANTS: This study was an observational quality assessment completed by Partners Healthcare, a health system located in Boston, MA. This assessment was conducted at two academic medical centers, specifically Massachusetts General Hospital and Brigham and Women's Hospital, both in Boston, MA. The participants included CHWs hired by both institutions and the Partners Community Health Worker Executive Committee.

DESCRIPTION: One of the great imperatives in patient education is to develop superior methods of educating patients on their condition and to better empower patients to improve their health. Community Health Workers (CHWs) are a potential solution to this challenge. CHWs are public health workers who can help provide and augment the care received by patients. Their roles include, but are not limited to, patient outreach and education, cultural mediation, providing direct patient services, and patient advocacy. CHWs have previously been demonstrated to reduce barriers to healthcare while simultaneously decreasing medical spending and unneeded healthcare utilization. However, there is no consensus framework detailing the key operational metrics for the work done by CHWs, and the metrics organizations should employ in evaluating their own CHW programs.

EVALUATION: Herein, we propose a series of metrics that seek to critically and comprehensively assess CHW performance. These metrics include (1) CHW workflow (2) average interactions per patient per month (3) methods of contact with patients (face-to-face, telephone, etc.) and (4) type and number of contacts per CHW per month. Furthermore, we plan to tie these metrics to relevant clinical outcomes, including ED visits, primary care physician (PCP) visits, primary care no-show rate, and total medical expenditure.

DISCUSSION / REFLECTION / LESSONS LEARNED: These metrics have been internally piloted and validated. Collectively, we feel the outlined framework of metrics can provide a structure for operational oversight of CHWs, as well as criteria for evaluating the impact of a CHW within an ACO setting. They are generalizable and may be readily repurposed for implementation within other health systems. 
DISABILITY AND ABLEISM IN MEDICINE: A CURRICULUM FOR FIRST-YEAR MEDICAL STUDENTS

Hannah M. Borowsky ${ }^{1}$; Leora Morinis ${ }^{1}$; Megha Garg ${ }^{2,}{ }^{1}$. ${ }^{1}$ University of California San Francisco, San Francisco, CA; ${ }^{2}$ San Francisco VA Medical Center, University of California San Francisco, San Francisco, CA. (Control ID \#3186092)

NEEDS AND OBJECTIVES: By the end of this session, students will: 1. Explain possible influences of disability on patient health and clinical encounters 2. Compare and contrast the medical and social models of disability 3. Discuss the meaning and manifestations of ableism 4. Recognize the ways in which ableism contributes to health and health care disparities 5 . Brainstorm solutions through which medical communities can address ableist norms and practices 6 . Reflect on their relationship to disability and how it could impact their future clinical practice

SETTING AND PARTICIPANTS: We designed and implemented a 2hour course for all UCSF first-year medical students, divided into groups of 12 , with one faculty-facilitator. The session is now a permanent part of the UCSF Bridges Health and the Individual block, a three week course dedicated to social and behavioral foundational sciences.

DESCRIPTION: Founded on the belief that medical education can provoke personal, professional, and systemic change, we developed a "Disability and Ableism in Medicine" curriculum to offer medical students a new lens through which to understand themselves and their future patients. Our small group session was designed to equip students with tools to care for patients with disabilities and to identify ableism - a worldview that values autonomy, and perceives disabilities as deficits to be ideally overcome. We included four key activities: (a) norm-setting; (b) student-led group presentations on: health and healthcare disparities, disability history and policy, disability and intersectionality, and disabilities amongst medical professionals; (c) brainstorm of interventions for overcoming healthcare barriers and biases; (d) privilege awareness exercise. The course materials acknowledged that members of the UCSF community have disabilities and that medical school has historically been a difficult environment in which to be open about them.

EVALUATION: The session's mean value was 4.58 on a 5-point scale ( $\mathrm{n}=45$ students). A pre- and post-session paired-sample t-test $(\mathrm{n}=17)$ indicated significant increases in students' self-reported understanding of ableism ( $p=0.002$ ) and confidence in assessing barriers to care for patients with disabilities $(\mathrm{p}<0.001)$. Faculty facilitators reported that the session provoked powerful student-centered learning, leadership, and widespread participation. DISCUSSION / REFLECTION / LESSONS LEARNED: Our curriculum on disability and ableism introduces a crucial topic often absent from medical training. By drawing on input from disability scholars, community members, and advocates in the design of this curriculum, students encountered perspectives not always included in medical education. These perspectives prompted students to connect individual experience to structural determinants, spurring new thinking about medicine and advocacy. Being secondyear medical students at the time we developed the curriculum also greatly influenced the way we structured our course, illustrating the ways in which near-peer curriculum development can uniquely address certain educational needs.

DOCUMENTATION OF LEARNING OUTCOMES TO GRANT MAINTENANCE OF CERTIFICATION CREDIT FOR PARTICIPATION IN MORBIDITY AND MORTALITY CONFERENCE

Kelly A. Kieffer ${ }^{1,2}$; Harley Friedman ${ }^{1,2}$. ' Dartmouth-Hitchcock Medical Center, Lebanon, $\mathrm{NH} ;{ }^{2}$ Geisel School of Medicine at Dartmouth, Lebanon, NH. (Control ID \#3180179)
NEEDS AND OBJECTIVES: Morbidity and Mortality Conference (M\&M) can provide a positive environment for discussion of unexpected negative outcomes and medical error, and can incorporate learning related to the clinical content of a case, the diagnostic reasoning process, and strategies to promote patient safety. Continuing Medical Education (CME) providers offering credit for M\&M can also offer Maintenance of Certification (MOC) credit through a 2015 collaboration of the American Board of Internal Medicine (ABIM) and the Accreditation Council of Continuing Medical Education (ACCME). Granting MOC requires a formative assessment process including feedback, which can be challenging in the context of a large group interactive format.

SETTING AND PARTICIPANTS: Internists enrolled in ABIM MOC who participate in the Department of Medicine Morbidity, Mortality and Improvement conference at Dartmouth-Hitchcock Medical Center in Lebanon, New Hampshire.

DESCRIPTION: We developed MOCME, an assessment process incorporating a web-based tool that uses only open source resources. The tool enables physicians to document learning outcomes, receive feedback, and earn MOC credit. Users log in after each M\&M conference, complete an evaluation, and reflect on what they learned and how it will change their practice. The conference facilitator reviews all entries, assesses MOC eligibility, and enters a summary of key learning points that is emailed to participants. A report of MOC eligible physicians is routed to the institutional CME office for submission to the ACCME.

EVALUATION: In 15 months, 518 of 525 MOC requests by 53 physicians were approved; 7 were declined due to insufficient quality of reflective statements regarding participants' learning. A median of 3 points were approved per physician. $84 \%$ of respondents to an anonymous survey felt that the system is easy to use and $86 \%$ reported being satisfied with it. $65 \%$ agreed that the engaging with MOCME reinforced their learning from M\&M. Most respondents take an average of 5 minutes or fewer to complete the process. MOCME added minimal administrative burden for the facilitator.

DISCUSSION / REFLECTION / LESSONS LEARNED: MOCME is a user-friendly system that meets ABIM's guidelines for granting $\mathrm{MOC}$ for CME. The system could be adapted for other institutions and other types of Regularly Scheduled Series. Further study is needed to identify factors that influence participants to utilize the system and to measure long term learning outcomes and impact on practice. The content of MOCME participants' selfidentified learning outcomes provides valuable insight to the facilitator that will inform improvements in the structure and content of the conference to better meet participants' needs.

\section{DOES A PERSONAL EXPERIENCE INFLUENCE RECOMMEN- DATIONS BY PRECLINICAL MEDICAL STUDENTS? EFFECTS OF A VIRTUAL DEMENTIA SIMULATION}

Arati Kelekar ${ }^{1}$; Nelia Afonso ${ }^{2} .{ }^{1}$ Oakland University William Beaumont School of Medicine, Huntington Woods, MI; ${ }^{2}$ Oakland University William Beaumont school of Medicine, Rochester, MI. (Control ID \#3164035)

NEEDS AND OBJECTIVES: When faced with clinical decisions, learners with limited experience tend to be linear thinkers and focus mainly on single problems. The objective of this project was to evaluate the influence of a personal experience by preclinical students in a simulated setting on: - the recognition of the multidimensional nature of clinical problems - identification of potential solutions and - provision of patient centered care.

SETTING AND PARTICIPANTS: The Virtual Dementia Tour (VDT)® is a simulation-based sensitivity training program for health care providers to the plight of patients with dementia. This project received IRB approval. 
DESCRIPTION: Prior to the VDT® experience preclinical students documented a management plan for an 86-year-old with dementia presenting with uncontrolled hypertension. Following the 8-minute VDT® simulation, students submitted a reflection on their experience and were allowed to modify their original plan. Qualitative research methods using grounded theory were used to gain insights from student comments on recognition of the multidimensional nature of the problem and the effect on their treatment plans.

EVALUATION: Of the 117 students who experienced the VDT®, 101 completed the assignment. Pre VDT® Prior to the experience $60 \%$ of students focused on pharmacologic management, $28 \%$ addressed care-giver issues. VDT® reflections Common themes and sample statements identified in the reflections included: Emotional/physical discomfort "The biggest challenge was hearing/interpreting/communicating with others. The sensory overload is something that I will never forget" "It's scary. Even the temporary experience was unbearable..." "Feeling rushed by others, feeling abandoned, a large sense of frustration, ... I was unable to make sense of what was around me" Experience of challenges faced by patient Dementia is much more than just memory loss "... gave the unique insight as far as how a person with dementia experiences problems" Empathy I could understand better if someone with dementia reacted with anger or frustration or exhaustion knowing that they deal with this every day" Modification of communication behaviors I would take extra time to address their frustrations. Give more time and support. Check for understanding. Provide written and verbal instructions. Insight I always isolated dementia from other sensory modalities of the body. I did not understand the extent of emotional changes the patient goes through on a day to day basis. Post VDT A majority of students amended their original plans to include various non-pharmacologic strategies such as caregiver assistance (33\%), assistive devices and environmental modifications (33\%).

DISCUSSION / REFLECTION / LESSONS LEARNED: The VDT® simulation resulted in increased empathy and insight into the struggles faced by patients with dementia as evidenced by the reflections noted by the preclinical students. Additionally, it resulted in a modification of their management plans to include consideration of home environment and assistance requirements.

\section{DON'T WAIT, ESCALATE!: IMPROVING RESIDENT PER- CEIVED ESCALATION BARRIERS THROUGH A COMPREHEN- SIVE CURRICULUM}

Stefanie Reiff $^{1}$; Lisa Altshuler ${ }^{2}$; Lisa Schwartz'; Marwa Moussa ${ }^{1} .{ }^{1} \mathrm{NYU}$ Langone Hospital - Brooklyn, Brooklyn, NY; ${ }^{2}$ NYU Medical Center, New York, NY. (Control ID \#3142840)

NEEDS AND OBJECTIVES: Residents often fail to escalate care due to uncertainty resulting in delays of care and possible harm. Multiple studies have identified trainee self-reported barriers to escalation, but none have evaluated the impact of a multi-faceted curriculum aimed to reduce perceived escalation barriers. Our objective was to identify, address, and improve residents' perceived barriers to escalation.

SETTING AND PARTICIPANTS: This study was conducted at an urban, academic medical center within the Internal Medicine residency program over one year.

DESCRIPTION: A baseline Likert-scale survey categorized residents' perceived escalation issues. A four-lecture curriculum about common causes of patient deterioration and an objective structured clinical examination (OSCE) were created to address the found issues. In the OSCE PGY1 residents first entered the room with the option to escalate to a PGY2 or a PGY3 acting as the rapid response team (RRT) leader with an attending physician creating pushback/intimidation throughout. Debrief focused on both knowledge and collapsing hierarchies. A retrospective pre-post Likert-scale survey evaluated for change in resident attitudes after the interventions in three areas: Communication Skills, Awareness/proper knowledge base of the problem, and Selfassertiveness/handling intimidation from superiors.

EVALUATION: A total of 54/77 of IM residents completed the baseline survey. Only the PGY1,2 received intervention, and 34/54 completed the prepost survey. Baseline survey results Identified barriers included feeling intimidated when escalating (33\% rated this as at least a fairly common problem), feeling pushback when escalating (31\%), worrying others will view them negatively $(10 \%)$, gaps in knowledge (12\%)/awareness (32\%), and misunderstanding severity of the problem (11\%). Retrospective Pre-Post Results Paired t-tests were conducted on pre and post summary scores. All postintervention summary scores rose compared to pre scores, and the Awareness scale approached significance $(\mathrm{p}=.08)$. The seven most targeted questions were examined using Wilcoxin Sign tests. Three questions showed statistically significant improvement: improved frequency of being told information needing escalation $(p=0.004)$, less feelings of self-blame $(p=0.035)$, less limitation of autonomy with mandatory RRTs $(p=0.009)$. The other four questions including comfort with, worries about repercussions for, feeling intimidated about, and viewing self negatively if needing to escalate showed change in the positive direction without reaching statistical significance.

DISCUSSION / REFLECTION / LESSONS LEARNED: This study demonstrates the implementation of a year-long curriculum and OSCE can lead to significant change in resident attitudes about perceived escalation barriers. It is likely this study was hindered by a small sample size due to the number of near-significant findings. Future studies are needed involving larger numbers of residents and looking at changes in RRT instances and outcomes to determine if clinical change accompanies the found perceptual change.

\section{EDUCATING HEALTHCARE PROFESSIONALS ON THE EF- FECTS OF INCARCERATION ON PATIENTS}

Rachael Williams; Marya J. Cohen; Matthew Tobey. MGH, Boston, MA. (Control ID \#3184019)

NEEDS AND OBJECTIVES: The U.S. incarcerates a greater proportion of its population than any other nation at a rate of 698 per $100,000{ }^{1}$ Those involved in the criminal-legal system have a higher burden of chronic disease and mental illness compared to the general population., During the first two weeks following release from incarceration, the mortality rate of this population is 12.7 times that of the general population, with a 129 times increased risk for drug overdose. ${ }^{4}$ Formerly incarcerated patients (FIPs) have unique barriers to healthcare including missed appointments, insurance lapses and difficulty obtaining prescriptions upon release. Healthcare providers often lack training on how to care for FIPs. One study found that $42 \%$ of formerly incarcerated men experienced discrimination by healthcare workers. It is important for healthcare providers to be equipped to address the unique needs of FIPs in a sensitive manner.

SETTING AND PARTICIPANTS: This work is anchored in partnership between Massachusetts General Hospital's Crimson Care CollaborativeNashua Street Jail (CCC-NSJ) and the Suffolk County Sheriff's Department (SCSD). CCC-NSJ is a student-faculty clinic located in a local jail where faculty from MGH teach health professions students in the correctional setting. Through a grant from the Massachusetts Medical Society Charitable Foundation, we have utilized our network of MGH faculty, CCC-NSJ volunteers, and SCSD staff to establish curricula for community clinics to improve the quality of care provided to FIPs. 
DESCRIPTION: We have created two 1-hour educational sessions for healthcare professionals on the effects of incarceration. One session targets clinicians and the other staff. Topics include history of U.S. incarceration rates, recommended language use, opportunities to screen for incarceration, an overview of the health risks of incarceration and how to best practice trauma-informed care for this population.

EVALUATION: Surveys evaluating each session were distributed to providers following each of the first two sessions. Twenty-two responses were recorded. On a 5-point Likert scale, the mean session rating was 4.8/ 5 and the mean rating for the session's content was $4.8 / 5$.

DISCUSSION / REFLECTION / LESSONS LEARNED: We received positive feedback from participants. Many respondents commented that this topic was new and necessary. One person noted, "I've never heard this information presented in the health center before." Respondents also mentioned that more time devoted to trauma-informed care would be helpful. Our team noted the conversations with physicians and staff differed. Some clinicians had theoretical knowledge of the topic, but staff had more lived experience. Staff members were able to relate multiple anecdotes of family members who had experienced health risks related to incarceration. Despite personal experience, staff described lack of professional empowerment to speak with patients on this matter. As we continue to develop teaching sessions, we will increase the length of sessions to allow more time to focus on trauma-informed care.

EFFECT OF GENDER ON ATTENDING PHYSICIAN'S FEEDBACK TO INTERNAL MEDICINE RESIDENTS: A QUANTITATIVE AND QUALITATIVE STUDY

Ana Sofia Warner; Nneka Ufere; Kerri Palamara. Massachusetts General Hospital, Boston, MA. (Control ID \#3185760)

NEEDS AND OBJECTIVES: Residency is an important period of professional learning and growth for physicians-in-training. The Accreditation Council for Graduate Medical Education (ACGME) adopted the Milestones-based Next Accreditation System in 2013, which relies heavily on faculty evaluations of residents. While evaluations are an important aspect of resident professional development, the inherently subjective nature of these evaluation leaves room for implicit bias. To our knowledge, no contemporary data exists assessing for gender differences in faculty evaluations of internal medicine trainees using the ACGME milestones-based evaluations. We aim to use qualitative and quantitative methods to evaluate the impact of gender on internal medicine trainees' performance evaluations. SETTING AND PARTICIPANTS: Data for this longitudinal, retrospective project will be collected from Massachusetts General Hospital internal medicine resident evaluations from the 2016-2017 academic year. This study is part of a larger multi-site study involving six internal medicine residency programs across the U.S.

DESCRIPTION: We will be collecting data from attending evaluation forms of internal medicine residents using the ACGME milestones-based evaluations. Each evaluation consists of written comments and quantitative scoring of residents across six clinical competency domains: patient care, medical knowledge, system-based practice, practice-based learning and improvement professionalism and interpersonal and communication skills. EVALUATION: Our investigation involves analysis of both the quantitative and qualitative evaluation data. For the quantitative data, we will convert the rankings into numerical scores. For the qualitative analysis, we will examine the written comments by attendings of trainees.

DISCUSSION / REFLECTION / LESSONS LEARNED: At the MGH site, we have 1070 resident ( 68 female, 82 male) evaluations that were completed by faculty ( 62 female, 99 male) during the 2016-2017 academic year. For our quantitative analysis, we will convert scores on the ACGME milestone-based performance scales to numerical scores for each trainee. We aim to compare the mean scores for each milestone domain between male and female trainees using t-tests and estimate associations of mean scores for each milestone domain with attending-trainee gender pairings using linear mixed effect models. For our qualitative analysis, we will use a multistage, multianalysis procedure for coding and analyzing the written feedback in the evaluations of trainees to categorize the content of these evaluations into groups according to broader issues or themes.

\section{EMPOWERING RESIDENTS TO EXPLORE BARRIERS TO WELL-BEING: CREATION OF A WELL-BEING ELECTIVE}

Chloe Ciccariello ${ }^{1}$; Larissa Thomas ${ }^{2}$. ${ }^{1}$ University of California San Francisco, San Francisco, CA; ${ }^{2}$ University of California, San Francisco at Zuckerberg San Francisco General, San Francisco, CA. (Control ID \#3179519)

NEEDS AND OBJECTIVES: The drivers of physician well-being are complex and involve cultural, organizational, and individual components. To address this, we created a Well-being Elective, the objective of which is to allow residents to explore cultural and structural drivers of physician wellbeing, as well as possible interventions

SETTING AND PARTICIPANTS: At the UCSF Internal Medicine Residency, we have had a Well-being Committee comprised of resident physicians who meet monthly to design interventions to improve the resident experience, but resident ability to work on these projects has been limited by the lack of protected time. Through advocacy within the Internal Medicine Residency, we were able to create a Well-being elective that is available to third year internal medicine residents on their ambulatory rotations.

DESCRIPTION: The residents spend 2-3 half days per week on the elective over one month; time is split three ways. The main focus of the elective is completion of a deep-dive project that is focused on a cultural or systems aspect of physician well-being, aligned with the commitments of the Charter on Physician Well-being. They also prepare a well-being case conference, focused on an emotionally challenging patient care experience and its impact on the care team. Finally, the residents participate in mentorship and skill-building activities happening at the medical center. EVALUATION: There was widespread interest in the elective; 9 residents will complete the elective in 2018-19. To date, residents have completed projects across a breadth of organizational well-being priorities. Residents prepare reports summarizing their findings, which are presented at program leadership meetings, and two-thirds of residents have successfully submitted their projects as innovation abstracts to scholarly meetings. Long-term follow-up is planned to assess programmatic changes implemented as a result of these projects.

DISCUSSION / REFLECTION / LESSONS LEARNED: Residents are interested in well-being as a field of study, and providing protected time for residents to study this places it as equally important to other academic endeavors. Residents have much to offer programs, if given the time and resources to study drivers of well-being.

ENHANCING CLINICAL SKILLS DEVELOPMENT THROUGH A LONGITUDINAL CROSS-CLERKSHIP CLINICAL SKILLS EXAM Kristy Y. Kosub ${ }^{1}$; Kevin Schindler ${ }^{1}$; Diane Ferguson ${ }^{2} .{ }^{1}$ UT Health San Antonio, San Antonio, TX; ${ }^{2}$ UTHSCSA, San Antonio, TX. (Control ID \#3185531) 
NEEDS AND OBJECTIVES: Assessment of clerkship students' clinical skills is frequently based upon faculty/resident evaluations. Direct observation is challenging ${ }^{1}$ and evaluations are frequently unreliable ${ }^{2,3}$. We designed a longitudinal objective structured clinical examination series, the Crossclerkship Clinical Skills Exam (C3SE) to improve observation/feedback. We hypothesized that multiple formative skills evaluations would augment early intervention with struggling learners and Step 2CS preparation. The C3SE replaced our traditional senior year Clinical Skills Exam (CSE).

SETTING AND PARTICIPANTS: Exams are administered to all students in core clerkship year.

DESCRIPTION: Multiple clerkship directors, evaluation specialists, clinical skills center directors and standardized patient (SP) educators reviewed the school of medicine and clerkship competencies and created milestones for each C3SE. Balancing the number of competencies for evaluation and student/ skills center time, we developed three exams with four SP encounters each. Cases had diversity of patient ages, genders, and medical conditions to assess competencies for interpersonal skills, interviewing, physical examination, note documentation, and clinical reasoning. We developed a remediation exam for students who did not meet milestones. Assessment tools for each case were designed. SPs were trained for role portrayal and assessment tool accuracy. Student surveys were administered after each exam.

EVALUATION: We successfully implemented the C3SE during AY17-18. Post-C3SE surveys revealed students felt confident in improvement in historytaking, physical examination, clinical reasoning, note writing, and communication skills (average response: $\mathrm{C} 3 \mathrm{SE} 1=3.1, \mathrm{C} 3 \mathrm{SE} 2=3.0$, and $\mathrm{C} 3 \mathrm{SE} 3=3.1$ on 4-point Likert scale). Results did not decline throughout the year despite increasing case complexity. Periodic assessments allowed faculty to identify struggling students ( $n=20$ of 219).

DISCUSSION / REFLECTION / LESSONS LEARNED: Periodic structured observation and feedback may provide sustained clinical skills development and focused Step 2CS preparation. Score comparison from cases used in previous CSE was surprisingly equivalent no matter when cases appeared on C3SE schedule. Replacement of CSE with C3SE assessments allowed students to enroll in Step 2CS earlier than past years and more effective use of the skills center. We are closely monitoring Step 2CS results for effect on pass rates. Development of a complex examination requires significant resources, so support from clerkship personnel and UME Deans is vital to successful implementation.

ONLINE RESOURCE URL (OPTIONAL): [1]Carline JD, Paauw DS, Thiede KW, et al. Factors affecting the reliability of ratings of students' clinical skills in a medicine clerkship. J Gen Intern Med. 1992;7(5):506-10. 2 Plymale MA, French J, Donnelly MB, et al. Variation in faculty evaluations of clerkship students attributable to surgical service. J Surg Educ. 2010;67(3):179-83. ${ }^{3}$ Howley LD, Wilson WG. Direct observation of students during clerkship rotations: A multiyear descriptive study. Acad Med. 2004;79:276-280

ENHANCING TRAINEE EDUCATION AND PRACTICE THROUGH COMMUNITY PARTNERSHIPS: BUILDING AN INTERDISCIPLINARY, INTERPROFESSIONAL COMMUNITY HEALTH TRACK

Mariecel Pilapil ${ }^{1}$; Johanna Martinez ${ }^{2}$; Lauren Block²; Daniel J. Coletti ${ }^{2}$; Barbara Keber ${ }^{6}$; Celia $\mathrm{Lu}^{3}$; Julie DiGregorio ${ }^{4}$; Alice Fornari ${ }^{5}$. ${ }^{1}$ Zucker School of Medicine at Hofstra/Northwell, New Hyde Park, NY; ${ }^{2}$ Northwell Health, Lake Success, NY; ${ }^{3}$ St. John's University College of Pharmacy \& Health Sciences, Lake Success, NY; ${ }^{4}$ Northwell Health, Huntington Station, NY; ${ }^{5}$ Northwell Health, Great Neck, NY; ${ }^{6}$ Zucker School of Medicine at Hofstra/ Northwell, Hempstead, NY. (Control ID \#3181815)
NEEDS AND OBJECTIVES: Achieving health equity is critical to medical training, particularly in the primary care specialties of internal medicine (IM), family medicine (FM), and pediatrics. The Institute for Healthcare Improvement proposes addressing social determinants of health (SDH) and developing community partnerships as two key components to achieving health equity. However, training in these skills is lacking in medical residency and nurse practitioner (NP) curricula. We developed an interdisciplinary, interprofessional track that provides a longitudinal community health experience for trainees. SETTING AND PARTICIPANTS: Faculty from IM, FM, and pediatrics planned and launched our health system's first multidisciplinary community health track in July 2018. The initial cohort for the 2018-2019 year included 8 trainees: 2 pediatric residents, 2 IM residents, 2 FM residents, and 2 NP students.

DESCRIPTION: In 2016, through a professional development grant, faculty in IM, FM, and pediatrics identified the need for improved community health training among residents. The ENHANCE track (ENgaging in Health Advocacy through Neighborhood Collaboratives and Education) was developed with the vision of developing trainee skills in population health and community engagement. The goals are to teach trainees how to: (1) Evaluate SDH and address their interplay with illness, (2) Partner with community-based organizations (CBOs), (3) Develop, implement, and evaluate a longitudinal, community-based project that addresses a population health need evident from their CBO partnership, and (4) Work collaboratively with professionals across disciplines. These goals are met through workshops, online webinars, CBO visits, and a collaborative health-related project. Mentoring and communication between trainees and faculty also occurs through group chats using the social media platform What's App.

EVALUATION: The ENHANCE track evaluation plan includes: postworkshop reflective evaluations from trainees and faculty, analysis of resident comments about their CBO visits during What's App conversations, focus groups on trainee experiences of the track, and surveys to $\mathrm{CBO}$ partners. Long term evaluation will be an assessment and dissemination of their community based scholarly project. To date, trainee reflections suggest an increased understanding of the barriers patients face, evidence of SDH, and the risks of implicit biases.

DISCUSSION / REFLECTION / LESSONS LEARNED: Development of an interdisciplinary community health track for residents and NP students is needed and feasible. Trainees can integrate classroom skills, apply them through service learning experiences, and incorporate lessons learned into their own clinical practice. Establishing trust between trainees and their $\mathrm{CBO}$ partners can be difficult due to trainee schedules, resulting in spaced visits to the CBOs. This demonstrates a challenge of community-based work. In addition, trainees report a perceived tension between their roles as traditional volunteers and as medical professionals able to provide expertise.

EPAS TO COMPETENCIES: A ONE DAY SUB-INTERNSHIP PREPARATORY EXPERIENCE TO BRIDGE THE GAP

Irsk J. Anderson ${ }^{1}$; Jeanne M. Farnan ${ }^{2}$; Diane Altkorn ${ }^{2} .{ }^{1}$ University of Chicago Hospitals, Chicago, IL; ${ }^{2}$ University of Chicago, Chicago, IL. (Control ID \#3177909)

NEEDS AND OBJECTIVES: In early 2018, the AAIM Sub-internship Task Force published its revised Internal Medicine Sub-internship Core Curriculum 2.0 in direct response to an IM residency program director survey. Program directors identified four core skills expected of new interns: recognizing sick vs. non-sick patients, knowing when to ask for assistance, managing time wisely, and communicating effectively within healthcare teams. Only the first and fourth skills align the AAMC EPAs with ACGME Sub- 
competencies. Given the short duration of sub-internship experience as well as competing responsibilities, it is unlikely sub-interns will be exposed to much of the proposed core curriculum. In response, we created a one-day Subinternship Preparatory Course to cover core curricular material as well as to address topics that are often not formally covered during the clinical years.

SETTING AND PARTICIPANTS: All third-year medical students were invited to enroll and attend the one-day preparatory course approximately one month prior to starting their fourth year clinical work. All students who attended the Prep Day received course credit.

DESCRIPTION: A one-day preparatory experience was designed to address topics not often formally addressed in M4 clinical training, specifically those based on student feedback and literature review that align AAMC EPAs with ACGME Sub-competencies. These included hand-off communication, opioids and pain management, antibiotic stewardship, high-value care and intravenous access. We invited local experts to deliver interactive talks on all five topics. Students were asked to assess their preparedness in each of the topic areas before and after the interactive discussions, as well as evaluate the Preparatory Course experience overall.

EVALUATION: Eighty percent (70/88) of the third-year medical students attended the course. 84\% (59/70) of students completed the pre-course survey while $94 \%$ (66/70) completed the post-course survey. The two-sample Wilcoxon rank-sum (Mann-Whitney) test was used to compare students' responses to 10 items questions (2 per topic) before and after Prep Day. Students perceived preparedness improved significantly after the Prep Day experience in 9 of 10 topics ( $\mathrm{P}$ 0.0000). The only non-significant improvement was locating the University of Chicago antibiotic stewardship antibiogram and treatment pathways.

DISCUSSION / REFLECTION / LESSONS LEARNED: The clerkship years are not necessarily preparing students for advanced communication skills or safe, effective care around high-risk medications. A one-day boot camp experience improved perceived preparedness significantly in all but one category. We are currently assessing late-term (6 months) knowledge and preparedness and hope to incorporate a direct patient care observation experience for one or more of the covered topics following the 2019 Prep Day Course.

\section{EQUAL MEDICINE: A NOVEL CAREER DEVELOPMENT PRO- GRAM FOR WOMEN PHYSICIANS IN AN INTERNAL MEDICINE RESIDENCY PROGRAM}

Aditi Ramakrishnan ${ }^{2}$; Nicole Hadeed ${ }^{1}$; Jennifer R. Lukela ${ }^{1}{ }^{1}$ University of Michigan Medical School, Ann Arbor, MI; ${ }^{2}$ The Ohio State University, Columbus, OH. (Control ID \#3185742)

NEEDS AND OBJECTIVES: 1. Identify the career development needs of women residents in Internal Medicine (IM). 2. Develop a curriculum to address gaps in traditional IM residency education, empower women residents, and foster gender equity.

SETTING AND PARTICIPANTS: We conducted a survey to understand the unique career development needs of women residents in our IM training program. We evaluated resident perception of the importance of various career advancement topics, preferred educational formats for content delivery, and perception of access to mentorship and female faculty role models. All women IM and Medicine-Pediatrics residents were invited to complete the needs assessment. Based on survey results, a pilot curriculum was developed. Post-workshop evaluations were distributed to all participating residents.

DESCRIPTION: The pilot curriculum consisted of three workshops open to all women residents in our training program. Sessions focused on the topics ranked most highly in the needs assessment: negotiation strategies (19\%), leadership skills (16\%), and strategies for career advancement (15\%). Bold and Brave featured a panel with women leaders in academic and privatepractice medicine with small and large group discussions. Art of Negotiation focused on negotiation and career advancement strategies. The final workshop, Perfecting your Pitch, was an interactive session which featured strategies to combat imposter syndrome and become comfortable with selfpromotion for career advancement. EVALUATION: Needs assessment survey response rate was $63 \%$. These results were used to develop the pilot curriculum themes and format. Both quantitative and qualitative analysis was applied to post-workshop evaluations. Following Bold and Brave, 92\% participants agreed/strongly agreed that they had a better understanding of how to develop leadership skills. Qualitative coding revealed that hearing "personal stories and experiences" of women physician leaders was most formative for resident attendees. All residents (100\%) who attended the Art of Negotiation workshop agreed/strongly agreed that they had a better understanding of how to develop skills in negotiation following the session. Residents again highlighted personal stories and "hearing from real women" as most valuable. After Perfecting your Pitch, $89 \%$ of participants strongly agreed that the session was useful and covered relevant topics. $100 \%$ trainees agreed/strongly agreed that the session was conducive to increasing selfconfidence.

DISCUSSION / REFLECTION / LESSONS LEARNED: Our survey revealed gaps in the traditional IM residency curriculum, particularly for women trainees. This educational initiative sought to bridge those gaps and empower women residents with key skills and knowledge to advance their careers and foster equity in medicine. Based on post-workshop assessments, this curriculum achieved these goals and provided additional opportunities for women residents to identify women faculty mentors. This curriculum has created a novel, impactful educational space for women residents in our residency program.

\section{EVALUATING RESIDENT OBSERVED STRUCTURED CLINICAL EXAMINATION (OSCE) PERFORMANCE WITH A MILESTONE- BASED SCORING TOOL}

Molly A. Kantor ${ }^{1}$; Brian Kwan ${ }^{2,3}$; Talya Bordin-Wosk ${ }^{2,4}$; Nivedita Ghosh ${ }^{2,3}$; Blaine Lovetro $^{2}, 3$; Meghan Sebasky ${ }^{2,}{ }^{4}$; Rebecca Sell ${ }^{2,}$; ; Stacy T. Charat ${ }^{2,3}$. ${ }^{1}$ University of California, San Francisco, San Fransisco, CA; ${ }^{2}$ University of California, San Diego, Del Mar, CA; ${ }^{3}$ VA San Diego Healthcare, San Diego, CA; ${ }^{4}$ University of California, San Diego, San Diego, CA. (Control ID \#3184477)

NEEDS AND OBJECTIVES: Internal medicine (IM) residency programs currently evaluate resident performance based on six Accreditation Council for Graduate Medical Education (ACGME) domains of clinical competency that encompass 22 milestones representing core competencies of becoming a physician. Recognizing that these domains can also be evaluated during Observed Structured Clinical Examination (OSCEs), we sought to create an OSCE scoring tool that reflected these milestones. Objectives: 1. Develop a novel evaluation tool for the OSCE based on competency milestones 2. Assess ease of use and consistency of a milestone-based OSCE evaluation tool SETTING AND PARTICIPANTS: In 2016 and 2017, all PGY-1 IM residents (interns) completed an OSCE during the first month of residency. Interns rotated through four 15-minute scenarios: evaluation of a patient with chest pain, disclosure of medical error (two distinct scenarios), and delivery of feedback to a learner with knowledge deficits. Participants received immediate feedback from faculty and peer observers. The encounters were videotaped. DESCRIPTION: We developed a structured scoring tool based on ACGME milestones and adapted to each clinical scenario, scored on a five-point Likert 
scale. This tool was intended to mirror the global assessment of performance in the clinical setting. A scoring guide for each case was collectively developed. Faculty were trained in the use of the scoring instrument using practice exercises. Faculty then watched the videotaped encounters and completed the scoring tool, with one primary faculty member scoring each clinical scenario and a fifth faculty member scoring a random sample from each scenario to verify interrater reliability.

EVALUATION: Over two years, 98 IM interns participated in the OSCE, yielding 196 clinical scenarios (two per intern). Faculty evaluated 189 unique OSCE encounters (96.4\%) and were successfully able to use the scoring tool. Qualitative evaluation of faculty experience with the tool revealed that it could be easily completed during OSCE observation. Interrater reliability is currently being evaluated.

DISCUSSION / REFLECTION / LESSONS LEARNED: A milestonebased OSCE scoring tool is feasible to develop, easy to use, and is an innovative method of evaluating an OSCE. Scoring guides helped faculty understand the application of a milestone-based scoring system for each OSCE scenario. Future analysis will include examining correlations between results from the OSCE scoring tool and other measures of resident performance.

\section{EVALUATING RESIDENTS' IMPRESSION OF PRECEPTORSHIP BY USING NEAR- PEER TEACHING TECHNIQUE}

Mehrshid Kiazand. UPMC Mercy, Pittsburgh, PA. (Control ID \#3185067)

NEEDS AND OBJECTIVES: The Accreditation Council for Graduate Medical Education Internal Medicine Residency Review Committee (RRCIM) requires that training programs address resident teaching skills as a part of the practice-based learning competency. This need seems to be more challenging in the outpatient setting considering the limited visit time and rapid turnover. This lack of experience might be a major factor in determining whether a trainee pursues an academic career. We designed a Near- Peer Teaching curriculum to introduce trainees to principals of clinical teaching in outpatient setting and evaluate their opinion about taking a preceptor role as a future career.

SETTING AND PARTICIPANTS: Participants were third year (PGY3) Internal Medicine Residents at an academic outpatient clinic. We implemented a curriculum emphasizing the five microskills of the one minute preceptor teaching model during the 4-week Ambulatory Rotation. A total of 15 residents have participated in this curriculum from June 2018 to January 2019. DESCRIPTION: Senior residents were scheduled for a 4-hour session during Ambulatory Rotation. They were able to have a one-on-one teaching session with a trained faculty to discuss teaching techniques in outpatient setting and review different scenarios. PGY3s were asked to use a 4-hour protected time session to precept first year trainees on a weekly basis. Both third and first year residents were observed by a faculty preceptor and received immediate feedback after the session.

EVALUATION: A survey questionnaire was sent to all PGY3s at the end of the rotation. It included 13 questions to evaluate residents' opinion and satisfaction about this experience on a 5-point scale. Residents cohort responses compared using chi-square test. Most residents had no previous experience with a preceptorship curriculum (73\%). Prior to this exposure, $46.5 \%$ of residents felt precepting is easy or very easy and just $13 \%$ planned to pursue an academic career in outpatient setting. After participating in this curriculum, the majority stated they may or most definitely will plan to work as a preceptor $(88.8 \%, \mathrm{P}=0.004)$ although just 2 out of 15 felt it was easy or very easy to work as a preceptor $(\mathrm{P}=0.03)$. Most residents agreed the visit time would be longer if preceptors see patients with all trainees (73.3\%). About $73.3 \%$ of responders believed this session changed their opinion about preceptorship in an outpatient setting.

DISCUSSION / REFLECTION / LESSONS LEARNED: Understanding the clinical skills and the job responsibilities of outpatient preceptors is not clear for most residents. This curriculum allowed PGY3s the opportunity to practice teaching skills in outpatient setting in a supervised environment. Also, the curriculum was successful in enhancing the trainees' interest in pursuing a teaching career in outpatient setting. The trainees' interest, the teaching opportunities, and the time efficient curriculum indicates the Near-Peer teaching technique's potential in any residency program.

EVALUATING THE ROLE OF A FORMAL CXR CURRICULUM ON HOUSESTAFF EDUCATION

Angela O. Suen ${ }^{1}$; Rachel Wojcik ${ }^{1}$; Michael G. Simonson ${ }^{1}$; Nicholas $\mathrm{Vu}^{3}$; Andrew Klobuka ${ }^{3}$; Richard H. Zou ${ }^{1}$; Jared Chiarchiaro ${ }^{2}$. ${ }^{1}$ UPMC, Pittsburgh, PA; ${ }^{2}$ University of Pittsburgh, Pittsburgh, PA; ${ }^{3}$ University of Pittsburgh Medical Center, Pittsburgh, PA. (Control ID \#3186009)

NEEDS AND OBJECTIVES: Internal Medicine residents cite varying comfort levels with interpretation of chest radiographs (CXR) during clinical training. Being able to independently review CXRs is an important, but often overlooked, skill especially as formal interpretation may not be immediately available. The current gold standard for learning how to interpret CXRs is through clinical rotations, MKSAP, and Radiology Rounds. To the best of our knowledge, a standardized educational imaging curriculum tailored to Internal Medicine residents does not exist. In conjunction with the Department of Radiology, we created a prospective case-based chest radiograph curriculum aimed to increase clinical competency and comfort with image interpretation.

SETTING AND PARTICIPANTS: Internal Medicine interns $(n=75)$ at the University of Pittsburgh Medical Center were randomized in 1:1 fashion into control $(n=37)$ and intervention arms $(n=38)$. A pre-curriculum quiz and survey were administered to all interns during their orientation. The final assessment will be conducted at an end-of-year retreat.

DESCRIPTION: The initial survey given during orientation focused on knowledge of and comfort in interpreting CXRs. Those randomized into the intervention arm were asked to anonymously participate voluntarily. Cases were sent out on a monthly basis with plans for a total of twelve months. Each of the 12 cases include a brief patient case, de-identified CXR, and a clinical-based multiple-choice question, with the focus on common clinical scenarios. Answers were accompanied by systematic image interpretation and case summaries. Those in the control arm participated in CXR interpretation as usual with clinical exposure and self-directed learning. At the conclusion of the study, all interns will participate in a post-curriculum quiz and survey to reassess knowledge, attitudes, and feedback. Educational exemption was obtained by the local Institutional Review Board (PRO17070620).

EVALUATION: Seventy-two (96\%) interns completed the precurriculum quiz with a mean score of $75 \%$. Through the first 4 cases of the prospective curriculum, there is a $37 \%$ response rate among interns in the intervention arm, with a mean score of $63 \%$. The January 2019 case had a $13 \%(n=5)$ response rate with a mean score of $100 \%$. 
DISCUSSION / REFLECTION / LESSONS LEARNED: This is the first prospective randomized study of Internal Medicine residents evaluating the role of a standardized chest radiograph curriculum on education, knowledge, and clinical competency. While there is demonstrated interest in the curriculum, we have encountered limitations in content delivery. As established in other small studies, we saw that the voluntary aspect of this intervention resulted in relatively few responses that continued to decrease over time. It seems that there are limitations in deploying a voluntary intervention via email, including internal motivation, email fatigue, and prioritization. Because of the relatively few responses at present, it may be difficult to assess for efficacy by the end of the 12-month study.

EVALUATION OF A HUMANITIES CURRICULUM TO PROMOTE HIGHER LEVEL CLINICAL REASONING AT INTERNAL MEDICINE MORNING REPORT: RESULTS OF A QUALITATIVE ANALYSIS

Grace Prince; Rimma Osipov; Anthony Mazzella; Paul Chelminski. University of North Carolina, Chapel Hill, NC. (Control ID \#3177717)

NEEDS AND OBJECTIVES: 1. Develop a humanities-based curriculum using art media (visual, music, and literature) to promote higher level clinical reasoning at Internal Medicine morning report. 2. Solicit interactive, structured observation and analysis of art media using Visual Thinking Strategy (VTS) [Katz, Khoshbin, 2014]. 3. Apply techniques of qualitative analysis to session recordings to demonstrate how participants apply and develop clinical reasoning skills through engagement with humanities media.

SETTING AND PARTICIPANTS: In medical education, the humanities are used to teach communication, promote wellness, and elucidate the social context of care. We piloted a curriculum to expand these roles, using the humanities to catalyze higher level clinical reasoning. Drawing on accepted theories of clinical reasoning, this project adapts the casebased morning report format to analysis of a "case" from the humanities. The pilot spanned four sessions and included medical students, residents and faculty.

DESCRIPTION: With a facilitator, the audience engaged collaboratively in observation and interpretation of a work of art, music, or literature using VTS

EVALUATION: Each session was recorded and transcribed. Transcripts were analyzed using ATLAS.ti. Six codes, derived from the work of Judith Bowen, were assigned to responses: data acquisition, problem representation, contextualization, hypothesis generation, pattern recognition, and illness scripts. Coding was validated for reliability. Analysis to date reveals that clinicians apply analytic techniques used in the clinical setting to art. Within a single session, we saw progression from rudimentary data acquisition (15 occ) to higher level hypothesis generation (16 occ), contextualization (16 occ), and application of illness scripts (9 occ). Of 43 coded responses, we found 16 instances in which codes cooccurred within a statement, indicating that participants' clinical reasoning was not compartmentalized. Rather, clinicians iteratively blended and refined the processes of clinical reasoning, thus evincing Dual Processing Theory. This analysis suggests that clinical reasoning is more analogous to the epistemology of the humanities than often perceived and demonstrates a role for humanistic media in developing clinical reasoning.

DISCUSSION / REFLECTION / LESSONS LEARNED: The humanities offer a dynamic platform for cultivating observation and contextualization. Though humanities courses are well established for medical students, this project integrates the humanities into a clinical learning environment for learners at all levels. Linking the respective frameworks of medical and humanities education is feasible and productive despite the time constraints within a busy residency program.

EVALUATION OF THE EFFECT OF INTRODUCTION OF A CLINICAL REASONING CURRICULUM IN A CLINICAL SKILLS COURSE FOR NOVICE MEDICAL STUDENTS.

Arati Kelekar ${ }^{1}$; Nelia Afonso ${ }^{2} .{ }^{1}$ Oakland University William Beaumont School of Medicine, Huntington Woods, MI; ${ }^{2}$ Oakland University William Beaumont School of Medicine, Sterling Heights, MI. (Control ID \#3167094)

NEEDS AND OBJECTIVES: Clinical reasoning (CR) is often not explictly taught to novice medical students. A significant proportion of students graduate with suboptimal diagnostic reasoning abilities. Preclerkship clinical skills (CS) courses are an ideal venue to teach the $\mathrm{CR}$ process as they encourage students to integrate their basic science knowledge with patient centered communication skills. The goal of our study was to evaluate the impact of a clinical reasoning curriculum on the accuracy of relevant symptom query and generation of appropriate differential diagnoses by second year (M2) medical students assessed through an end of semester objective structured clinical examination (OSCE).

SETTING AND PARTICIPANTS: The study was approved by the institutional IRB. M2 students participating in the clinical skills course of winter 2018 formed the study group. These students received 3 new clinical reasoning sessions (total 6 hours). Whole case approach, serial cue approach, self explantion of pathophysiological mechanisms and comparison of closely related diagnoses were strategies used to teach clinical reasoning. Students from the previous year who had not received the curricular intervention formed the comparison group.

DESCRIPTION: The students interviewed a standardized patient (SP) presenting with a chief complaint of palpitations and dizziness and were asked to document a comprehensive history. They were also asked to list their top 3 differential diagnoses. In order to generate the items and value for scoring the case, 16 experienced faculty clinicians were asked to determine the importance of symptoms and historical elements in the presentation and required to generate a prioritized differential diagnosis. Students who consented (101 in CR group and 107 in non CR group) had their OSCE writeups graded. They were given 1 point for each significant symptom queried and 1 point for identifying each high priority differential diagnoses. EVALUATION: Students in the CR group were more likely to query relevant symptoms than those in the non CR group but the results were not statistically significant (3.74 vs 3.34). For the high priority differential diagnoses, out of a maximum score of 3, students in the CR group achieved 1.98 while students in the non CR group scored 1.64, a result that was statistically significant $(\mathrm{p}=0.0002)$.

DISCUSSION / REFLECTION / LESSONS LEARNED: Students who underwent the curriculum were more likely to identify symptoms of organ systems not anatomically related to the organ system of the chief complaint. For the differential diagnosis, hyperthyroidism and anxiety (pathophysiolocally distant from the chest) were more likely to be identified by the curricular group and diagnoses like pulmonary embolism and myocardial infarction were more often noted by the non curricular group. The top 5 differential diagnosis generated by students who underwent the CR curriculum mirrored those entertained by the clinicians. It is our impression that our curriculum which emphasized multi- organ system based differential diagnosis contributed to this occurrence. 
EXPLORING PRIMARY CARE TRAINING CHARACTERISTICS OF UNITED STATES PRIMARY CARE INTERNAL MEDICINE TRAINING PROGRAMS

Samantha Alonso. Emory, Atlanta, GA. (Control ID \#3185247)

NEEDS AND OBJECTIVES: There is a need for primary care physicians in the United States coupled with deficiencies in ambulatory training for resident physicians. To address this, specialized Primary Care Internal Medicine (PC IM) resident training programs or tracts provide focused training in primary care and ambulatory medicine. We surveyed leaders of specialized PC IM programs with the aim of collectively assessing how PC IM programs provide training and education in primary care.

SETTING AND PARTICIPANTS: We conducted a cross sectional survey of leaders of U.S. Primary Care Internal Medicine training programs or tracks identified via search of National Resident Matching Program (NRMP), the Fellowship and Residency Electronic Interactive Database (FREIDA), and program websites.

DESCRIPTION: Please see above in Setting and Participants

EVALUATION: Forty-five of the 70 programs identified as PC IM training programs or tracks completed the survey (64\% response rate). On average, PC IM programs report PC residents engage in 22.8 weeks of training in the ambulatory setting per year and 69.4 continuity clinic sessions per year. Majority report PC residents engage in more weeks of ambulatory training and continuity clinic sessions than their categorical IM peers, specifically PC residents engage in an additional 10.1 weeks of ambulatory training per year and 20.8 additional continuity clinic sessions per year than their categorical IM peers. Majority, 77\%, offered multiple continuity clinic sites which included university clinics, community nonprofit clinics, public hospital based clinics, Veterans Administration-based clinics, private practice clinics, and Health Department or other government clinics. Number of patient visits per continuity clinic session varied by resident level of training (PGY1 3.5 visits; PGY2 4.9 visits; PGY3 5.7 visits). Standard ambulatory clinical rotations varied across programs with majority offering rotations in geriatrics, dermatology, orthopedics, and HIV medicine.

DISCUSSION / REFLECTION / LESSONS LEARNED: PC IM training programs aim to address the nation's shortage of primary care physicians by providing focused training in Primary Care. Our survey demonstrates that PC IM programs offer more ambulatory training time and continuity clinic exposure than traditional categorical IM training programs. Given their mission, more training in this setting is expected however raises the question of what level of ambulatory training should be considered standard for PC IM programs beyond what is required for ambulatory training per the Accreditation Counseling for Graduate Medical Education. PC IM programs should lean into their role as standard bearers in Primary Care training including setting expectations for PC IM training programs in terms of primary care training and education.

FAMILY FEUD MEETS SCRIPT CONCORDANCE: USING GAMIFICATION TO TEACH CLINICAL REASONING TO PRECLERKSHIP MEDICAL STUDENTS

Thilan P. Wijesekera. Yale School of Medicine, New Haven, CT. (Control ID \#3185772)

NEEDS AND OBJECTIVES: Prioritizing a differential diagnosis- a key step in clinical reasoning- is a challenging skill to develop, ranking last in self-reported comfort in a survey of Yale School of Medicine (YSM) rising clerkship students. As part of the YSM Clinical Reasoning Course, this educational innovation was created to: -Prepare 2nd-year medical students to prioritize a differential diagnosis for high-yield chief concerns during clerkships -Develop an engaging and versatile teaching modality grounded in clinical reasoning pedagogy

SETTING AND PARTICIPANTS: -Setting: As part of a larger student orientation prior to beginning clerkships, workshops took place for 70 minutes in a small conference room with a laptop connected to a large monitor to display educational content. -Participants: There were a total of 120 students and 10 faculty. Each pair of faculty (one attending and one 4thyear student) facilitated three back-to-back sessions with eight sets of 2ndyear students (two teams of four).

DESCRIPTION: -Preparation: Clerkship directors provided a list of the 10 most high-yield chief concerns for their respective rotations, which were given to students two weeks in advance. Case scenarios were created for six chief concerns (e.g., syncope, abdominal pain). These were provided to the faculty, whose answers were collected and weighted by frequency to create a consensus differential diagnosis, similar to a script concordance test. Points were assigned based on how often that diagnosis was selected. These values were uploaded into a PowerPoint formatted with the sound and visual effects of the game show "Family Feud". -During: For each round, both teams read each clinical scenario, took turns sharing their differential, explained their reasoning, and received points based on how high the diagnosis was on the faculty differential. Faculty ended each round with a 5-minute discussion demonstrating their own clinical reasoning on the case. The team wins who has the most points at the end of the six rounds.

EVALUATION: On a scale of 1-5 (i.e., poor, fair, good, very good, and excellent), students rated the workshop a 4.68 , significantly higher than any of the eight clinical reasoning workshops over the past four years (range 3.27-4.25). One particularly representative student comment was: "Family Feud was INCREDIBLE! I wish we used this more during the preclinical curriculum... Creating comprehensive differentials is something I think we are all struggling with, and both the format and the clinical guidance from the student and attending present were so helpful and engaging. It was a really fun environment to make mistakes and learn."

DISCUSSION / REFLECTION / LESSONS LEARNED: Prioritizing a differential diagnosis is challenging, a sentiment shared before and after the session from our students. Our "Family Feud" game showed significant promise as an educational tool for teaching it by employing gamification to engage and motivate students while giving them the opportunity to learn through targeted pre-reading, discussion with colleagues, and faculty role modeling.

FEEDBACK ON A TRANSITION TO ATTENDING ELECTIVE: A NOVEL HEALTH SYSTEMS SCIENCE CURRICULUM EMPOWERING GRADUATING INTERNAL MEDICINE RESIDENTS FOR INDEPENDENT PRACTICE

$\underline{\text { Andrew Orr }}^{1}$; Kendal Williams ${ }^{1}$; Nadia L. Bennett ${ }^{2}$; Kamini Hogan ${ }^{1}$; Deepa R. Nandiwada ${ }^{2}$. ${ }^{1}$ Perelman University School of Medicine, Philadelphia, PA; ${ }^{2}$ University of Pennsylvania, Philadelphia, PA. (Control ID \#3167780)

NEEDS AND OBJECTIVES: The transition from residency to independent practice as a hospitalist is a challenging time in one's career. Residency prepares learners with much of the necessary clinical knowledge; however, exposure to autonomous decision-making, leadership training for interprofessional teams, and practical elements of attending daily work flow remain limited. Despite the increasing popularity of hospitalist medicine, only half of large academic Internal Medicine residency programs currently offer 
hospitalist-based rotations to ease this transition (Ludwin et al. Journal of Hospital Medicine, 2018). Further, of the rotations offered, the majority focus on quality improvement/high value care or enhancing clinical skills. These rotations generally do not have a targeted curriculum focused on higher level health systems attending skills Curricular Objectives: Discover challenges and opportunities of working as the attending physician on interdisciplinary teams Provide an opportunity for residents to oversee direct patient care and conduct independent rounds with an advanced practice provider (APP) Develop practice patterns and leadership skills for independent practice in conjunction with an APP

SETTING AND PARTICIPANTS: This study involves Internal Medicine PGY-3s who have participated in the "Transition to Attending" Elective, APPs at Pennsylvania Presbyterian Medical Center, and current hospitalist faculty members within the University of Pennsylvania Health System.

DESCRIPTION: We developed a novel, two-week "Transition to Attending" elective in which third-year Internal Medicine residents assume the responsibility of an attending hospitalist, lead a team of APPs, and receive personalized training on high-yield elements of independent clinical practice (teaching on rounds, hospital systems, billing/documentation, Point of Care Ultrasound, leadership skills, and interprofessional best practices) from expert clinical educators. Residents work with an APP providing direct patient care while the attending of records sees patients separately and provides higher level feedback on systems based skills.

EVALUATION: From November 2017 to date, 7 residents completed the 2 week rotation and associated post-rotation survey. Two of the study authors analyzed this qualitative data to identify themes regarding the rotation's strengths, areas for improvement, and resident perception of preparedness for independent practice.

DISCUSSION / REFLECTION / LESSONS LEARNED: We found that this elective filled a gap in providing residents a true clinical experience working with APPs on higher level skills with deliberate feedback focusing on the transition to becoming an attending hospitalist. Residents identified that they did not realize how independent APPs are clinically and spent much of their time developing shared patient work flows. Our total number of participants is small but overall feedback shows that this has been a high yield rotation with an appropriate balance of patient care and acquisition of practical knowledge.

\section{FEEDBACK ON GENERAL MEDICINE ROTATIONS: RECON- CILING TWO PERSPECTIVES}

Conor Walsh; Rupal Shastri; Manya Gupta. Rush University Medical Center, Chicago, IL. (Control ID \#3175725)

NEEDS AND OBJECTIVES: The objective of our study was to unveil and understand differences in perceived frequency and quality of feedback between hospitalists and residents with the ultimate goal of improving the system and culture of effective feedback on general medical floors.

SETTING AND PARTICIPANTS: Our surveys were for residents who had completed at least 1 month of general medicine wards and hospitalists on teaching services.

DESCRIPTION: During October 2018, 114 internal medicine residents/ preliminary medicine interns and 50 hospitalists were surveyed. The surveys included 14-16 similar questions for residents and hospitalists regarding feedback.

EVALUATION: Forty seven out of 114 residents responded to our survey while 26/50 hospitalists completed the survey. There was broad agreement of what constituted effective feedback with $>55 \%$ of both groups agreeing that it should be specific, timely, and include both positive and negative feedback. Of note, only $10.6 \%$ of residents thought that avoiding criticism was an important component of feedback. Regarding the frequency of feedback, $84.6 \%$ of hospitalists reported giving end of rotation feedback $75-100 \%$ of the time whereas $44.7 \%$ of residents reported receiving it that often. Regarding mid-rotation feedback, $57.7 \%$ of hospitalists reported giving it at least $50-100 \%$ of the time whereas only $32.6 \%$ of residents reported receiving it that often. A total of $37 \%$ of residents reported receiving mid-rotation feedback $0-25 \%$ of the time. Regarding feedback in general other than at the end of the rotation, $57.7 \%$ of hospitalists reported giving feedback either daily or every few days compared to $8.5 \%$ of residents reporting receiving feedback that often. On average, hospitalists rated themselves as 6.6/10 while residents rated the feedback as 6.1/10. Overall, $44.7 \%$ of residents reported being told "keep up the good work" or to "continue reading" without additional specific feedback. Regarding challenges to giving feedback, $76.8 \%$ of hospitalists reported time constraints, $42.3 \%$ reported difficulty with negative feedback, and $38.5 \%$ reported lack of enough direct observation of residents.

DISCUSSION / REFLECTION / LESSONS LEARNED: Both residents and hospitalists agree that feedback is important and that a more robust culture of feedback is desired. Our project shows that there are large discrepancies between the perceived frequency of giving/receiving feedback between hospitalists and residents. Of particular interest, residents want more specific and negative feedback even as hospitalists acknowledged their own discomfort with giving constructive criticism. Other challenges cited by hospitalists include time constraints and not enough direct observation of residents. Improving the culture of feedback at any institution will require a deliberate and collaborative effort between residents and attendings. By reconciling both perspectives, this project serves as a starting point for our institution towards achieving that goal.

\section{FILLING THE GAP: A GERIATRICS AND PALLIATIVE CARE LEADERSHIP NEEDS ASSESSMENT}

Gabi Schiller $^{2}$; Ayla Pelleg ${ }^{1}$; Helen M. Fernandez ${ }^{1}$; Elizabeth Lindenberger ${ }^{1}{ }^{1}$ Mount Sinai School of Medicine, New York, NY; ${ }^{2}$ Mount Sinai Hospital, New York, NY. (Control ID \#3169844)

NEEDS AND OBJECTIVES: By 2030, those over the age of 85 will double to about 10 million. Despite this demographic imperative, there is a significant shortage in geriatricians and palliative care specialists. To best utilize this scarce, specialty resource, we propose the development of an elite workforce of future geriatrics and palliative care leaders who will lead health systems to best meet the needs of a vulnerable, aging population. Currently, there are no leadership curricula developed for Geriatric and Palliative Care fellows. Therefore, a needs assessment was conducted as an initial step to help establish a future leadership fellowship track within Geriatrics and Palliative Care fellowship programs.

SETTING AND PARTICIPANTS: Thirty-three key informants were interviewed, including national experts in the field of Geriatrics and/or Palliative care as well as former fellows who have graduated from Mount Sinai's Geriatric and Palliative Care Fellowship program. In total, 19 experts and 14 former fellows were interviewed. These interviews were conducted via video and/or phone conference over six months. Subsequent participants were recruited via the "snowball method," i.e. asking key informants to suggest other leaders in the field.

DESCRIPTION: Key informants $(\mathrm{N}=33)$ participated in qualitative interviews that ranged from 15-60 minutes. Interview questions were formulated by both a created taskforce for this needs assessment and based on 
previously distributed surveys to former Mount Sinai Geriatric and Palliative Care fellows. All interviews were audio-recorded and transcribed.

EVALUATION: A qualitative thematic analysis of all interviews was completed. Each interview was coded by two researchers to improve inter-rater reliability using the coding software NVivo. A coding dictionary, broken down by skills and knowledge categories, was created using both inductive and deductive reasoning.

DISCUSSION / REFLECTION / LESSONS LEARNED: Recruited participants saw the added value of a healthcare leadership track. Interviewees emphasized the need for fellows to have practical, diverse experiences during training to help prepare them for future leadership positions. Knowledge deficits emphasized were healthcare finance literacy and healthcare policy. Important skills a junior faculty should acquire included: negotiation, mentorship, and networking. Working in interdisciplinary teams and across professions was seen as a strength among Geriatricians and Palliative Care providers.

\section{FLOURISHING IN THE CLERKSHIP YEAR: A LONGITUDINAL CURRICULUM INTRODUCING SKILLS TO REDUCE BURN- OUT AND FOSTER RESILIENCE IN MEDICAL STUDENTS}

Rachel H. Kon ${ }^{1}$; Tabor E. Flickinger ${ }^{1}$; John Schorling ${ }^{1}$; Natalie May ${ }^{1}$; Justine E. Owens ${ }^{1}$; Madaline Harrison ${ }^{2}$; Margaret L. Plews-Ogan ${ }^{1} .{ }^{1}$ University of Virginia, Charlottesville, VA; ${ }^{2}$ University of Virginia School of Medicine, Charlottesville, VA. (Control ID \#3185156)

NEEDS AND OBJECTIVES: Burnout occurs in physicians at all levels of training and leads to poor patient satisfaction and physician wellbeing. At our institution, burnout rates in medical students are highest during the clerkship year. We developed a curriculum to foster resilience and wisdom with the objective of mitigating burnout.

SETTING AND PARTICIPANTS: We recruited students prior to starting the clerkship year for an optional program of 12 monthly sessions throughout the year. Students were randomized to an intervention group receiving instruction in resilience techniques or a control group. Of 139 eligible students, 29 chose to participate and were randomized. 27 students attended the first session and consented to be part of the study. 3 later dropped out. DESCRIPTION: Students in the program met monthly for two hours in control or intervention small groups with the same two faculty members. All sessions included debriefing on clerkship events and the intervention group also had instruction in specific techniques in four areas: reflective practice, mindfulness, appreciative practices, and narrative medicine. Each area was addressed three times during the year to reinforce skill acquisition.

EVALUATION: In pre- and post-clerkship qualitative surveys, themes were identified related to students' desires for shared experiences, peer support, mentorship, and reflection. Intervention students rated techniques in narrative medicine, mindfulness, appreciative practice, and reflective practice as high or very high value. More students in the intervention than the control group rated these aspects of the curriculum as high or very high value: having a place to process my clerkship experience (92\% vs $70 \%$ ), helping me manage stress and difficult emotions ( $77 \%$ vs $60 \%) .92 \%$ of intervention students would recommend the sessions to others. Students completed pre and post questionnaires including the 22- item Maslach Burnout Inventory (MBI), the 10-item Connor Davidson Resilience Scale (CD-RISC), and the 39-item 3-D Wisdom Scale. There were significant differences at follow-up in overall wisdom (3.90 vs 3.60, $\mathrm{p}=0.026$ ), but not in MBI or CD-RISC scores between the two groups.

DISCUSSION / REFLECTION / LESSONS LEARNED: Students in the intervention recognized high value in the techniques taught in this curriculum and both groups valued having this setting to process their clerkship experiences. Students were able to give examples of how they used these skills in their clinical work. The positive feedback from participants is shaping an expansion of this program to all our students. A larger study is still needed to see if this training can reduce burnout and improve resilience.

\section{FOCUSED POCUS: HOW CAN WE TEACH ULTRASOUND IN INTERNAL MEDICINE RESIDENCY?}

Laura Horton; Stephanie R. Eizember; Rafael Paez; Sanjay M. Salgado; Lindsey Wu. Brigham and Women's Hospital, Boston, MA. (Control ID \#3184993)

NEEDS AND OBJECTIVES: Diagnostic point-of-care ultrasound (POCUS) is an ACGME requirement in emergency medicine and critical care training programs, and the American College of Physicians has formally acknowledged the important role of POCUS in internal medicine (IM). However, because few IM faculty use POCUS in their own practice, the optimal method to teach POCUS to IM residents is unclear. The immersive workshops often used for faculty training are not a feasible option for training large groups of residents. Our aim was to design and evaluate an easily-learned and high-yield POCUS curriculum that could be delivered to residents longitudinally during a clinical rotation.

SETTING AND PARTICIPANTS: Given the demands of residency, we designed a POCUS curriculum that could be taught in four one-hour sessions, delivered weekly during residents' month-long inpatient rotations. Each session was led by faculty trained in the curriculum and consisted of three to five resident learners.

DESCRIPTION: We intentionally limited the curricular content based on the teaching time allocated. We therefore focused solely on select exams in volume assessment, chosen out of over 15 exams taught in most IM POCUS courses because they are well-studied, technically straightforward, and valuable on general inpatient medicine. The four sessions included: 1) knobology/jugular venous pressure, 2) inferior vena cava, 3) pulmonary (Blines, pleural effusions), and 4) review. The first three sessions consisted of didactics followed by directed POCUS on pre-identified and consented hospitalized patients, and the final session was spent on skill development entirely at the bedside. We piloted the POCUS curriculum on a single general medicine team over three month-long rotations. At the end of each month, all residents on general medicine (including those on the intervention team and those on control/non-intervention teams) were surveyed.

EVALUATION: Compared to the control group, residents who rotated on intervention teams reported greater POCUS comfort (1.9 versus 0.6 on a scale of $0=$ not comfortable to $4=$ extremely comfortable), frequency of use ( 2.7 versus 0.5 on a scale of $0=$ never to $4=$ daily), and knowledge $(66 \%$ versus $42 \%$ of questions correct on an assessement of factual knowledge, i.e. ability to identify key pathology on still ultrasound images).

DISCUSSION / REFLECTION / LESSONS LEARNED: POCUS is increasingly recognized as an effective tool in IM, but the time required to reach proficiency in its vast applications is a barrier to training residents. By choosing focused content, we successfully delivered a longitudinal curriculum within the timeframe of a clinical resident rotation. Using real-time patient applications of POCUS allowed both for learner skill development and garnered buy-in on the value of POCUS in the clinical setting, maximizing key principles of adult learning theory. By increasing portable ultrasound equipment and training a small group of core faculty, we hope to expand this pilot so that it becomes integrated into all inpatient general medicine rotations. 
FREQUENCY OF ETHICAL ISSUES ON A GENERAL INTERNAL MEDICINE TEACHING SERVICE AT AN URBAN, TERTIARY CARE CENTER

Matthew W. McCarthy. Weill Cornell Medicine, Irvington, NY. (Control ID \#3182900)

NEEDS AND OBJECTIVES: Little is known about the daily ethical conflicts encountered by hospitalists on the General Internal Medicine (GIM) service that do not prompt a formal clinical ethics consultation. These issues may cause significant moral distress and are an underrecognized educational opportunity. We sought to characterize the epidemiology of ethical issues arising on a GIM teaching service during an academic year and compare them to those seen by the ethics consultation service.

SETTING AND PARTICIPANTS: We describe the frequencies of ethical issues identified during daily rounds on GIM teaching services at a metropolitan, tertiary-care, teaching hospital. Data was collected from September 2017 through May 2018 by two attending hospitalists from the ethics committee who were embedded on GIM rounds and was compared to all ethics consults at the institution during 2017.

DESCRIPTION: 270 patients were evaluated and 113 ethical issues were identified in 77 of those patients (28. 5\%) on the GIM service. These issues most frequently involved discussions about goals of care, treatment refusals, decision-making capacity, discharge planning, cardiopulmonary resuscitation status, and pain management. Only 5 formal consults were brought to the Hospital Ethics Committee for these 270 patients $(p<0.001)$. When compared to the cases seen by the ethics service, hospitalists and trainees on the GIM service dealt significantly more frequently with problems around clarifying goals of care $(\mathrm{p}<0.01)$, whereas clinical ethicists dealt more frequently with problems involving surrogate decision-making $(p<0.05)$.

EVALUATION: The results of this study have been used to create an undergraduate seminar in medical ethics at the Macaualay Honors College at the City University of New York (CUNY). The course is now in its second year and has expanded to cover ethical issues encountered in GIM, psychiatry, social work, nursing, and other subspecialities.

DISCUSSION / REFLECTION / LESSONS LEARNED: Our data is the first prospective description of ethical issues arising on academic GIM teaching services. These scenarios present an unrealized opportunity and is an important step in the development of a targeted ethics curriculum for hospitalists. This has already begun with the creation of an undergraduate curriculum and will soon expand to graduate medical education.

ONLINE RESOURCE URL (OPTIONAL): https://macaulay.cuny.edu/ academics/upper-level-courses/spring-2019-courses/issues-in-medicalethics/

\section{GAMING FOR THE WIN IN RESIDENT EDUCATION: GAMING TO ENHANCE RESIDENT LEARNING}

Allie Dakroub $^{1,2}$; Diane L. Levine ${ }^{3} .{ }^{1}$ University of Pittsburgh Medical Center, Pittsburgh, PA; ${ }^{2}$ Wayne State University/Detroit Medical Center, Detroit, MI; ${ }^{3}$ Wayne State University, Detroit, MI. (Control ID \#3186285)

NEEDS AND OBJECTIVES: In response to declining attendance and engagement, we sought to revitalize our resident board review. We designed more active educational sessions through the implementation of gaming. We hypothesized that a game-based board-review curriculum would improve attendance and enhance residents' learning experience.

SETTING AND PARTICIPANTS: Our residency program is at an academic, urban health care system with approximately 105 IM residents.
Board review is held weekly and learning games were implemented for 1 hour, weekly.

DESCRIPTION: We developed several learning games that provided a team-based competitive experience for resident board review. These games were implemented during weekly board review sessions for 20 weeks throughout the 2017-2018 academic year. The residency is divided into 5 cohorts, each comprising about 21 residents distributed evenly by PGY level. Each cohort competed in these learning games, and the competition concluded with a 4-hour "Cohort Cup Olympics" grand finale. The cohort with the highest overall score was treated to dinner and their team name was engraved on a trophy, the "Cohort Cup". Pre- and post-curriculum electronic surveys comprising 5-point Likert-type items were used to assess resident perceptions of the academic half-day experience and satisfaction with the "Cohort Cup". Attendance was also tracked.

EVALUATION: A pre- and post-intervention survey comprising 5-point Likert-type items was used to gauge resident perceptions and satisfaction of the novel game-based board review. 84 residents completed the pre-survey, and 74 completed the post-survey. Additionally, resident attendance was tracked and improved from an average of 12 residents per session for the didactic board-review to 25 residents per session for the game-based board review. [survey item, pre-intervention average, post-intervention average] (Survey Likert Scale: 1 - strongly disagree, 2-strongly agree, 3-neither agree nor disagree, 4-agree, 5-strongly agree). I am engaged in the academic halfday as they are currently designed, 2.74, 3.8 The current structure of the academic half-day contributes to my education, 2.68, 3.95 I prefer games and active learning as opposed to standard lecture based didactics for my learning, 3.77, 4.32 I prefer the "Cohort Cup" activity as opposed to standard lecture based didactics for my learning, n/a, 4.24 I feel that I learn more from the "Cohort Cup" activity than I do from standard lecture based didactics. n/a, 4.03 I prefer to have the "Cohort Cup" remain a part of my didactics. n/a, 4.42 I feel that the "Cohort Cup" activity has helped me bond with others in my residency. n/a, 4.36

DISCUSSION / REFLECTION / LESSONS LEARNED: Implementation of an educational strategy that incorporates the fun of games and the motivation of competition resulted in increased resident attendance and engagement in our residency program's board review. The "Cohort Cup" model could be adapted for other curricular content or for board review curricula in other programs looking for innovative ways to incorporate active learning.

\section{GRIT AMONG FIRST-YEAR INTERNAL MEDICINE RESI- DENTS}

Andrew J. Klein ${ }^{1}$; Scott D. Rothenberger ${ }^{2}$; Kathryn Berlacher $^{3}$; Thomas Grau ${ }^{1} .{ }^{1}$ University of Pittsburgh School of Medicine, Pittsburgh, PA; ${ }^{2}$ University of Pittsburgh, Pittsburgh, PA; ${ }^{3}$ University of Pittsburgh Medical Center, Pittsburgh, PA. (Control ID \#3186326)

NEEDS AND OBJECTIVES: Grit is a characteristic, defined as passion and perseverance for long-term goals, that is associated with success and avoidance of burnout in a number of fields, but whether this is a relevant construct among physicians has not been well established. We aimed to evaluate Grit among our first-year internal medicine residents in relation to burnout. Our hypothesis is that July Grit level may help predict those who may be at highest risk of developing burnout.

SETTING AND PARTICIPANTS: We recruited 75 postgraduate year 1 (PGY1) residents in Internal Medicine at a major academic hospital program. DESCRIPTION: We administered the well-validated short Grit survey and Maslach Burnout Inventory in July and December of 2018 to all PGY1 
residents in Internal Medicine. An additional background survey was administered in December to collect information on potential confounders, including age, gender identification, race, ethnicity, marital status, social supports, career goals, current rotation, work hours, daily exercise, and level of financial debt. Survey responses from the December administrations are still being collected. Levels of Grit and burnout in July and December were compared using paired Students t-tests with a 2 -sided p-value $<0.05$ considered statistically significant. Additional analysis to control for confounders and elucidate any association between Grit and burnout using linear regression is planned. EVALUATION: Among 75 PGY1 residents recruited, 49 have completed both the initial and 6-month surveys at this time. Two measures of burnout significantly increased, including both emotional exhaustion $(\mathrm{p}<0.001)$ and cynicism $(\mathrm{p}<0.001)$, while personal efficacy scores were unchanged $(\mathrm{p}=$ $0.56)$. Grit scores did not change $(\mathrm{p}=0.32)$. Additional analysis to determine the relationship between Grit and burnout in this population is pending.

DISCUSSION / REFLECTION / LESSONS LEARNED: Our current analysis is unsurprising. PGY1 residents are at high risk of developing burnout, particularly in the first 6 months of residency. Similarly, the short Grit scale was developed to determine one's trait-level Grit and its ability to evaluate changes in Grit over time has not been assessed. Additional analysis is needed to determine if Grit is a helpful construct in this population.

\section{HEALING PRESENCE: AN ELECTIVE ICU CURRICULUM FOR MEDICAL STUDENTS BASED IN THE CLINICAL PASTORAL EDUCATION TRAINING MODEL}

Jeremy Baruch ${ }^{2}$; Kristin M. Collier ${ }^{1}$. ${ }^{1}$ University of Michigan, Bloomfield Hills, MI; ${ }^{2}$ University of Michigan, Ann Arbor, MI. (Control ID \#3171991)

NEEDS AND OBJECTIVES: Medicine is best practiced interprofessionally. We have much to learn from our partners in spiritual care with regards to reflection, formation and meaning in medicine. The "ICU-Chaplaincy Healing Presence" is an elective experience for medical students on their adult ICU rotations which complements their ICU education through exposure to the art and skills of hospital chaplaincy. The learning objectives of this program include: 1 . Providing students with a framework with which to process emotions that arise in caring for acutely ill and dying patients. 2 . Reflecting on the interpersonal dynamics of patient care. 3. Developing patient care skills based in Clinical Pastoral Education. 4. Exploring how students' spirituality shapes their approach to patient care and how they cope with the challenges of training.

SETTING AND PARTICIPANTS: The elective is open to senior medical students during adult ICU rotations at Michigan Medicine. The chaplains are members of Michigan Medicine's Department of Spiritual Care.

DESCRIPTION: To achieve these objectives, this elective provides students with the opportunity to round on ICU patients with chaplains, develop chaplaincy skills and engage in four structured, small group experiences facilitated by a chaplain. The first session involves discussion and reflection on such questions as "What is spirituality?" "How does your spirituality inform your patient care?" "What thoughts/feeling often arise in patients and their families during times of illness?" Sessions 2 and 3 involve "verbatims" where students transcribe a conversation they had with a patient during one of their chaplain supervised encounters. The transcript is then read to the group, and with feedback, the student reflects on the questions asked, themes that arose, and missed opportunities. Session 4 is a wrap up session where students are asked to reflect upon how their perspectives may have changed as a result of the elective and what insights were gained.

EVALUATION: Students are asked to fill out pre- and post- elective questions (N=17) Post Participation Survey Results: Pre-participation, 53\% of students thought it was important or very important to consider how their values can be actualized in the hospital; which increased to $94 \%$ post participation. $88 \%$ thought that finding personal meaning in taking care of patients was important or very important prior to participation, compared with $100 \%$ post participation. Pre-participation, $54 \%$ thought that responding to patients' spiritual concerns was important or very important, compared with 94\% post-participation.

DISCUSSION / REFLECTION / LESSONS LEARNED: From narrative feedback provided by the students, we learned that students who participated in the elective reclaimed a sense of meaning and purpose in their training. In addition, students perceived therapeutic value as they were able to process the ICU experience in a supportive group led by a chaplain.

HEALTH IN THE NEIGHBORHOOD CONTEXT: A SOCIAL DETERMINANTS OF HEALTH (SDH) FOCUSED HOME VISIT CURRICULUM FOR INTERNAL MEDICINE RESIDENTS

Iman Hassan $^{1}$; Etsemaye P. Agonafer ${ }^{2}$; Kwonho Jeong ${ }^{3}$; Scott D. Rothenberger $^{3}$; Thuy Bui ${ }^{3}$; Carla L. Spagnoletti ${ }^{1}$; Maggie K. Benson ${ }^{1}$. ${ }^{1}$ University of Pittsburgh Medical Center, Pittsburgh, PA; ${ }^{2}$ UCLA, Los Angeles, CA; ${ }^{3}$ University of Pittsburgh, Pittsburgh, PA. (Control ID \#3186242)

NEEDS AND OBJECTIVES: To enhance residents' awareness of SDH by providing a framework for understanding the connection between patients' health with their social and neighborhood context. Curriculum goals are to use home visits to increase: 1) recognition of the link between neighborhoodlevel SDH and health; 2) familiarity with local neighborhoods and tools for identifying community resources; and 3) rates of asking about and addressing SDH with clinic patients.

SETTING AND PARTICIPANTS: All $462^{\text {nd }}$ year internal medicine (IM) residents participated in the curriculum during a 4-week ambulatory rotation. A total of $48 \mathrm{IM}$ residents who did not receive the curriculum were used as historical controls.

DESCRIPTION: The curriculum began with a 60-min didactic, smallgroup introduction on how neighborhoods impact health and a demonstration of electronic tools to identify community characteristics and resources. Residents selected one of their clinic patients. They then conducted a guided virtual assessment of their selected patient's neighborhood, followed by a $1 / 2$ day SDH-focused home visit and exploration of the patient's neighborhood. Residents participated in a 60-min debriefing session.

EVALUATION: Pre and post intervention surveys assessed attitudes and self-reported behavior on a 5-point Likert scale. After the curriculum, residents were more likely to agree that, "It is important for me to be knowledgeable about the neighborhoods in which my patients live" (4.2 pre vs. 4.4 post, $\mathrm{p}<0.01$ ) and were more comfortable asking patients about their SDH (3.7 pre vs. 4.1 post, $\mathrm{p}<0.01$ ). They reported asking more frequently about SDH (3.2 pre vs. 3.5 post, $\mathrm{p}<0.01$ ) and addressing SDH (3.1 pre vs. 3.4 post, $\mathrm{p}<0.01$ ). Specifically, residents asked more about neighborhoods in which patients live, access to healthy food, housing, transportation barriers, and health insurance (all $\mathrm{p}<0.02$ ). They more frequently addressed access to healthy food, housing, and transportation barriers (all $\mathrm{p}<0.05$ ). Residents also considered a patient's neighborhood more frequently when developing a plan of care (2.7 pre vs. 3.0 post, $\mathrm{p}=0.02$ ) and used online tools to find resources for patients ( $7 \%$ pre vs. $64 \%$ post, $\mathrm{p}<0.01$ ). Compared to controls, those who underwent the curriculum more frequently asked about the neighborhood where their patients live ( $\mathrm{p}=0.03)$, asked about and addressed access to healthy food (both $\mathrm{p}<0.01)$, and used online tools to identify resources $(\mathrm{p}<0.01)$. 
DISCUSSION / REFLECTION / LESSONS LEARNED: This home visit curriculum increased overall attitudes and self-reported behaviors regarding SDH. Chart review to assess rates of health record tool use to address SDH and 6-month survey data to assess curricular durability currently underway.

\section{HEALTH SYSTEMS SCIENCE IN THE CLERKSHIP: APPLICA- TION TO PATIENT CARE}

Ana M. Yepes-Rios ${ }^{1}$; Mark Mayer ${ }^{1}$; Neil Mehta ${ }^{1}$; Aaron Hamilton ${ }^{2}$; Colleen Colbert $^{2}$; Beth Bierer ${ }^{2}$. ${ }^{1}$ Cleveland Clinic Lerner College of Medicine, Cleveland, $\mathrm{OH} ;{ }^{2} \mathrm{Cleveland}$ Clinic, Cleveland, OH. (Control ID \#3178315)

NEEDS AND OBJECTIVES: Education in Health Systems Science (HSS) has emerged as the third pillar of medical education to complement basic and clinical sciences and address the demands of evolving healthcare environments. In the Cleveland Clinic Longitudinal Clerkship (CCLC), an innovative HSS curriculum challenges medical students to reflect on the care of patients in the context of the healthcare system and the populations they serve. The objectives are to 1 . Demonstrate an awareness of their roles in an interprofessional, collaborative healthcare team to improve healthcare outcomes; 2. Demonstrate the application of core didactic objectives to the care of patients via a blog reflection.

SETTING AND PARTICIPANTS: Participants are a cohort of 75 thirdyear medical students in the CCLC. Students come from Case Western Reserve University's School of Medicine, including university and college (Cleveland Clinic Lerner College of Medicine) programs. Internal Medicine residents review the blogs

DESCRIPTION: Building upon a foundation implemented in the preclerkship, the HSS curriculum spans the academic year with eight, 1-2-hour, interactive learning sessions on the topics of Patient Safety, Quality Improvement, Transitions of Care, Social Determinants of Health, Inter-professional Education, Population Health and High Value Care. After each session, objectives and guiding questions prompt students to blog a reflection on the application of the session to patient care in the clerkship. Blogs are posted in a Google + private community that is further subdivided into 8 small groups to facilitate interactive discussions. Each group is actively monitored by 2 Internal Medicine residents who offer near-peer teaching and feedback, and by the course director.

EVALUATION: Students are assessed on their ability to transfer learning from the classroom session to their clinical experiences (experiential learning). To date students consistently reflect on their role in the healthcare team, and residents have offered feedback and support with near-peer experiences. Each session is systematically evaluated by students. To date, students report the sessions meet course objectives and engages them at an appropriate level of training.

DISCUSSION / REFLECTION / LESSONS LEARNED: The HSS thread in the clerkship and associated blogs meets the objectives of incorporating HSS education into the curriculum, with an associated authentic learning experience. Resident reviews of student blogs have created a safe, invaluable near-peer learning experience for both residents and students, offering a continuum of education in HSS.

\section{HERPES, HYPERTENSION, AND HEALTH SYSTEMS: DEVELOP- ING AND DELIVERING A HEALTH EDUCATION PROGRAM AT A COUNTY JAIL}

Daniel Cole ${ }^{1}$; Cameron Bright ${ }^{2}$; Wendy Miller ${ }^{1}$; Saida Yassin ${ }^{1}$; Jessica Sjoholm ${ }^{1}$; Rachel S. Silva ${ }^{1}{ }^{1}$ Hennepin Healthcare, Minneapolis, MN; ${ }^{2}$ University of Minnesota, Minneapolis, MN. (Control ID \#3182862)
NEEDS AND OBJECTIVES: Provide health education to justice-involved individuals Increase resident exposure to health disparities in justice-involved populations. Foster teaching and curriculum development skills for residents SETTING AND PARTICIPANTS: Four internal medicine residents and one Masters of Public Health student developed a curriculum and led health education classes at the Hennepin County Adult Detention Center (ADC) in Minneapolis, Minnesota. Bimonthly meetings were held in a classrooms in the ADC, typically facilitated by 1 to 2 participants and attended by 10 to 18 male attendees per session.

DESCRIPTION: Participants contributed to the development of a health education curriculum which included topics such as diabetes and sexually transmitted diseases. Hour-long programming was lecture-based with an emphasis on class participation and group discussions related to the chosen topics. Participants and class attendees completed post-participation surveys which were used to evaluate the pilot program.

EVALUATION: Participants were surveyed regarding their experiences teaching in the program. They strongly agreed that teaching improved their community education skills and impacted their perspective on incarceration with average ratings of 91 and 89 out of 100, respectively. Major themes learned included communication skills for a classroom, knowledge base and misconceptions about health topics among individuals currently incarcerated, and appreciation for the daily life of incarcerated individuals. Class attendees were also given a survey regarding the classes with 238 surveys collected over the first 6 months of the program. Attendees overwhelmingly marked that they found the class helpful. On a 5-point Likert Scale measuring how much attendees felt they knew before and after the class regarding the topic, there was a mean increase of 1.12 points $(\mathrm{p}=<.01)$.

DISCUSSION / REFLECTION / LESSONS LEARNED: Research has shown that justice-involved individuals have higher rates of chronic health conditions, substance use disorders, and mental illness than the general population. Yet very few residency programs offer opportunities for trainees to gain intentional exposure to justice-involved populations. The ADC health education program provided an opportunity for a team of trainees to gain experience working with the local jail population while simultaneously providing a valuable service to the justice-involved individuals. Feedback from trainees indicates that the experience not only improved their understanding of the health needs and behaviors of the justice-involved individuals, but that they improved their ability to provide general health education. In addition, justiceinvolved participant feedback suggests that the material presented was not only informative but impactful with many indicating they would make a behavioral change as a result of what they learned. Based on the overall positive feedback from both groups, plans are underway to expand the ADC health education program to cover additional topics and to include medical students as learner/educators.

\section{HIGH VALUE CARE AT YOUR FINGERTIPS: DEVELOPMENT OF AN IPHONE APP}

Jessica Donato $^{1}$; Courtney A. Covert ${ }^{2} .{ }^{1}$ Cleveland Clinic, Beachwood, OH; ${ }^{2}$ Cleveland Clinic, Cleveland, OH. (Control ID \#3184023)

NEEDS AND OBJECTIVES: A monthly high value care (HVC) conference was implemented at our large internal medicine training program. Each conference includes a review of evidence and guidelines related to the clinical topic for the given conference and a 1-page handout with key take-home points related to high value management strategies and key evidence. Trainees within our program sought an electronic resource for easy access to curricular materials from each HVC conference. We sought to develop a high value care iPhone application to make high value care curricular materials and other 
electronic high value care resources readily available to our training program. SETTING AND PARTICIPANTS: A monthly, high value care conference targeting all post-graduate years (1-3) was implemented at our large internal medicine training program starting in the fall of 2017 with 43-81 trainees in attendance at each conference.

DESCRIPTION: An iPhone application was developed in December 2018. The application includes access to PowerPoint slides and 1-page handouts from each high value care conference. The application also includes links to six key HVC websites including Choosing Wisely, Costs of Care, and the American College of Radiology Appropriateness Criteria. The application also includes a "Question of the Week" in which users can complete a MKSAP question under the domain of high value care.

EVALUATION: The application will be released to our training program in the coming months through the Apple App Store. We plan to measure the number of downloads and assess the utility and impact of the application in our annual survey evaluating the high value care conference.

DISCUSSION / REFLECTION / LESSONS LEARNED: Medical education in the current state must adapt to millennial learners. Our high value care conference includes high-yield PowerPoint slides which summarize evidence and guidelines, in addition to a 1-page handout. In order to make these curricular resources readily available to our trainees, we developed an iPhone application. This electronic tool is convenient, user-friendly and includes other HVC resources and educational tools such as a question of the week. We believe this will enhance trainee implementation of high-value care practices through more readily available resources and evidence to support high-value management strategies taught in our HVC conference.

HIGH VALUE CARE MORNING REPORT: A TEAM-BASED, INTERDISCIPLINARY CONFERENCE TO TEACH COSTEFFECTIVE CARE

June Kampangkaew ${ }^{2,}{ }^{4}$; Nathan C. Nowalk ${ }^{2,}{ }^{4}$; Diana Stewart ${ }^{3}$; Prathit $\overline{\text { Kulkarni }^{2,}{ }^{4} \text {; Michelle Sibille }}{ }^{1,2} .{ }^{1} \mathrm{MD}$ Anderson Cancer Center, Houston, TX; ${ }^{2}$ Baylor College of Medicine, Houston, TX; ${ }^{3}$ Baylor College of Medicine/Michael E. DeBakey VA Medical Center, Houston, TX; ${ }^{4}$ Michael E. DeBakey VA Medical Center, Houston, TX. (Control ID \#3185335)

NEEDS AND OBJECTIVES: Health care spending continues to rise with billions of dollars wasted annually, which has inspired a high value care (HVC) movement to curb expenses while improving patient outcomes. We aimed to implement a team-based, interdisciplinary morning report (MR) conference emphasizing high value, evidence-based work-up in order to educate physicians on the tools and resources of HVC practice. 1. Improve knowledge in HVC principles. 2. Improve confidence in utilizing HVC resources. 3. Provide a forum for interdisciplinary collaboration.

SETTING AND PARTICIPANTS: We utilized the existing MR case-based conference series to address the need for HVC training in our curriculum. Participants included Baylor College of Medicine residents and medical students rotating on inpatient internal medicine rotations.

DESCRIPTION: The conference follows the typical MR format, which includes presentation of the history and physical of an actual case. During preparation, the resident is encouraged to work with a content-expert faculty mentor as well as a HVC mentor who provides guidance on HVC resources. Each hour-long conference begins with the resident presenting the case. The audience is then divided into teams of different learner levels to discuss the differential diagnosis, what diagnostics they would order for evaluation, and an estimation of the prices for each test ordered. After the teams discuss their proposed evaluation and management, the presenter discusses the actual evaluation and management, as well as the ideal evidence-based HVC evaluation for the case and the estimated prices of these services.

EVALUATION: From January 2018 to December 2018, 24 conferences were held with approximately 300 total attendees. Seventy attendees were surveyed before and after implementation of the HVC morning report curriculum. Surveys addressed knowledge, skills, and attitudes for HVC using a 5-point Likert scale ( 1 = strongly disagree or very unlikely, $5=$ strongly agree or very likely). Interns showed lowest likelihood to "consider cost when discussing management" prior to the HVC morning report, while attendings showed highest likelihood to consider cost. The most agreed upon statement, before and after the MR, was the statement: "Physicians have a responsibility to patients to consider cost when providing treatment." Following conference attendance, all items surveyed showed an increase towards "strongly agree."

DISCUSSION / REFLECTION / LESSONS LEARNED: We have now adapted this conference to three different hospital settings in our residency program which demonstrates generalizability for replication. Our conference was expanded to MD Anderson Cancer Center, where the presenter emphasized a HVC approach to palliative care. In this way, the series is adept at underscoring the specific qualities of practice settings to create a unique and enriching conference.

\section{HIGH-VALUE CARE EDUCATION FOR THIRD-YEAR MEDI- CAL STUDENTS}

Rachna Rawal ${ }^{1}$; Paul Kunnath ${ }^{2}$; Jennifer Schmidt ${ }^{3}$. ${ }^{1}$ Saint Louis University, $\overline{\text { St. Louis, MO; }}{ }^{2}$ Saint Louis University School of Medicine, University City, MO; ${ }^{3}$ Saint Louis University, Saint Louis, MO. (Control ID \#3183527)

NEEDS AND OBJECTIVES: Recognize medical student need for highvalue care education Identify improvements in medical student selfawareness of high-value care Identify barriers for medical students practicing high-value care

SETTING AND PARTICIPANTS: Literature shows that habits established during training follow physicians through their career. Therefore, providing education to medical students will enable them to recognize and apply highvalue care, establishing it as a practice pattern early in their careers. Current state assessment surveys revealed $80 \%$ of our institutions' incoming third year medical students were not familiar with the term "high-value care". Additionally, $100 \%$ of students identified no prior high-value care education. Our data confirmed what is known in literature - medical students get little exposure to high value care concepts. This presented an opportunity to develop a high-value care curriculum for students.

DESCRIPTION: Participants are third year medical students rotating on inpatient Internal Medicine core clerkship. Data is collected via pre and postintervention survey. Objectives include defining high-value care, distinguishing between low and high-value care, and applying evidencebased guidelines to patient care. Interventions include two sessions: an introduction to high-value care during clerkship orientation and a casebased, interactive session mid-way through the clerkship. The material is designed to show students how to consider the risks, benefits and costs of diagnostic testing to make informed diagnostic decisions.

EVALUATION: When students defined high-value care in the pre-survey as a free response, $75 \%$ of the free responses included "costs", "waste", or "quality" suggesting familiarity with high-value care concepts but lack of more nuanced meanings. Students who felt comfortable utilizing evidencebased medicine for decision making increased from 59\% to $91 \%$ (statistically significant, $\mathrm{p}<0.05$ ). Post-intervention, $89 \%$ of students felt comfortable discussing testing indications with their team, statistically significant increase 
from $48 \%$ pre-intervention. $83 \%$ of students felt comfortable choosing between a $\mathrm{CBC}$ with differential versus without compared to $10 \%$ preintervention $(p<0.05)$. Additionally, $93 \%$ of students felt comfortable choosing between a BMP and CMP compared to $19 \%$ pre-intervention $(\mathrm{p}<0.05)$. The students identified a lack of knowledge and not having a voice as barriers to engaging in high-value care discussions.

DISCUSSION / REFLECTION / LESSONS LEARNED: Our data reveals that while students may be familiar with high-value care, they require additional training on its application. After two didactic sessions, students had improved attitudes and application of evidence based guidelines and increased engagement in high-value care discussions with their teams. However, additional initiatives are needed to reduce self-identified hierarchy barriers.

IMAGING WISELY: AN INTRODUCTION TO THE ACR APPROPRIATENESS CRITERIA AND ANALYSIS OF ITS IMPACT ON INTERNAL MEDICINE RESIDENTS

Mike K. Cheng ${ }^{1}$; Andrea L. Magee ${ }^{2}$; Carina W. Yang ${ }^{1}$; Joyce W. Tang ${ }^{1}$. ${ }^{1}$ University of Chicago, Chicago, IL; ${ }^{2}$ The University of Chicago, Chicago, IL. (Control ID \#3178183)

NEEDS AND OBJECTIVES: Inappropriate radiological exam ordering contributes significantly to healthcare waste. The American College of Radiology's (ACR) Appropriateness Criteria (AC) were designed to inform radiological exam ordering practices, but many internists are unfamiliar with them. To promote high-value ordering practices and use of this evidencebased resource, our team of internists and radiologists developed a curriculum introducing medicine residents to the ACR AC.

SETTING AND PARTICIPANTS: We offered our Imaging Wisely curriculum to University of Chicago internal medicine interns from 2017-2018. To date, 23 completed the curriculum.

DESCRIPTION: Our curriculum included: (1) discussion of medical risks of radiological exam overuse and sample exam charges; (2) introduction to the ACR AC website and app; (3) application of the AC to case vignettes through Radiology-TEACHES ${ }^{\mathrm{TM}}$ software; (4) group discussions about the vignettes; and (5) a radiologist-led session about principles of high-value exam ordering. EVALUATION: We used pre- and post-intervention surveys to assess for change in knowledge (through open-book testing with RadiologyTEACHES ${ }^{\mathrm{TM}}$ vignettes) and attitudes related to high-value exam ordering. Follow-up surveys 3-11 months post-intervention assessed for durability of change. We used McNemar's tests and paired t-tests for analysis.

DISCUSSION / REFLECTION / LESSONS LEARNED: N=23 completed the initial surveys; $n=18$ completed the follow-up survey. Preintervention, $35 \%$ of residents were unaware of the ACR AC; only $25 \%$ reported it among their top 3 resources guiding ordering decisions. Postintervention, knowledge scores for appropriate exam ordering increased from $59 \%$ correct answers (SD 0.16) to $89 \%$ (SD 0.14); $\mathrm{p}=0.0001$. The ACR AC were more often referenced post-intervention (17\% of residents pre vs. $74 \%$ post; $\mathrm{p}=0.0003$ ). Post-intervention, more residents felt comfortable with their knowledge of exam costs (4\% pre vs. $74 \%$ post) and discussing costs with patients ( $9 \%$ pre vs. $61 \%$ post), and more residents valued the ACR AC $(65 \%$ pre vs. $96 \%$ post); p-values all $<0.05$. All residents found the curriculum helpful and $91 \%$ planned to change their ordering practices. At 3-11 months post-intervention, $78 \%$ referenced the $\mathrm{AC}$ at least monthly; $67 \%$ reported changing ordering practices due to the curriculum. Knowledge scores remained significantly improved. This curriculum was well-received and produced durable change in knowledge and attitudes towards high-value radiological exam ordering and use of the ACR AC. We believe a factor of its success was the engagement of residents with the utilization of different learning styles and reinforcement of concepts through various formats (didactic sessions, group discussion, problem-based learning). Also, it promoted ongoing, self-driven learning as it equipped learners with a resource that they can continually reference. Given residents' reported sustained change in ordering practices, the curriculum shows strong potential to influence downstream ordering practices. It is currently being piloted with faculty hospitalists.

\section{IMPLEMENTATION OF A SOCIAL DETERMINANTS OF HEALTH CURRICULUM FOR INTERNAL MEDICINE RESI- DENTS AT THE UNIVERSITY OF ROCHESTER MEDICAL CEN- TER}

Mahala Schlagman; Clare Park; Carlos Irwin Oronce. University of Rochester Medical Center, Rochester, NY. (Control ID \#3170695)

NEEDS AND OBJECTIVES: The ACGME requires residency programs to provide training that includes "an understanding of health care disparities." Despite this mandate, few formal curricula exist to teach residents about health disparities. Our goal was to develop a social determinants of health (SDH) curriculum to teach internal medicine (IM) residents at the University of Rochester Medical Center (URMC) about the patient population they serve and provide an introduction to the social services that are available to help facilitate health. Once established, we plan to make our curriculum available to other residency programs locally and nationwide.

SETTING AND PARTICIPANTS: Rochester, NY has the most individuals living below half the federal poverty level of any mid-sized city in America. The URMC is home to the largest IM resident practice in the city, which serves 73\% Medicaid or dual-eligible individuals.

DESCRIPTION: We developed a curriculum to educate residents about SDH. Modules include: introduction to SDH and impact on health in the city of Rochester, poverty, introduction to health policy, transportation, structural racism, SDH screening, and medical legal partnerships. The program was implemented within the existing ambulatory education sessions as part of the residency program requirements.

EVALUATION: $81 \%$ (56 of 69) of residents completed a survey following the first three sessions. Responses were tallied using average Likert scales and word-association pattern development. Of residents surveyed, 96.4\% reported an increase in their comfort with an underserved patient population. In free-response answers, $24.6 \%$ reported an increase in understanding or empathy for their patients, $33.3 \%$ indicated they would be more likely to consider patients' out-of-pocket costs, and $21.7 \%$ reported an increased awareness of available resources. Residents also felt increased awareness of how costs affect patient decision-making and the social factors that affect access to care. An additional survey will be administered after curriculum completion.

DISCUSSION / REFLECTION / LESSONS LEARNED: The SDH curriculum increased resident empathy for our underserved patients, helping many of them commit to patient care that considers individuals within their social context. Development of the program required identification of a faculty champion and buy in from program leadership to prioritize these topics within the already robust IM curriculum. Our results provide important preliminary data to guide curriculum advancement.

IMPLEMENTATION OF A TRAUMA INFORMED CARE PRE CLINIC CONFERENCE CURRICULUM FOR RESIDENTS IN A COUNTY HOSPITAL

Giulia Leggett; Alejandro Díaz; Katherine Dang. Highland Hospital, Oakland, CA. (Control ID \#3154900) 
NEEDS AND OBJECTIVES: Adverse childhood experiences (ACE) and trauma impact roughly $61.7 \%$ of adults in California with likely increased proportions within the safety net network. We identified that the current Pre Clinic Conference curriculum did not address trauma identification and the concept of trauma informed care. Given the fact that residents working in our safety net primary care clinic have daily contact with survivors of trauma, the aim and objective of this project was to evaluate and implement a new Pre Clinic Conference Curriculum which would focus on trauma informed care. SETTING AND PARTICIPANTS: This 20 minute educational didactic lecture was implemented during regularly scheduled pre clinic conferences for Resident physicians between 4/2018 until 06/2018 in the outpatient clinic. A pre and post lecture questionnaire was emailed out to all eligible participants including PGY1, 2 and 3 requesting voluntary participation to assess baseline knowledge, effectiveness of the curriculum and obtain additional feedback.

DESCRIPTION: The educational curriculum was developed using a case based format followed by questions. The preceptor version included answers and additional probing questions to implement discussion. Our educational module highlighted two cases of patients with histories of ACE and various forms of trauma. Questions included illustrated the objective learning points with such examples: What is trauma? Is it appropriate to screen for trauma in a primary care setting? What is trauma informed care?

EVALUATION: A pre intervention questionnaire was administered asking questions with a three point likert scale to identify baseline knowledge and attitudes around trauma informed care. Some questions included: How familiar are you with Trauma Informed Care? How comfortable are you discussing with your patient their personal history of physical, emotional and sexual abuse? How familiar are you with the clinical and scientific findings of the Adverse Childhood Experiences (ACEs) Study? An identical post intervention questionnaire was administered to assess for any difference in knowledge or attitudes. A mixed effects model was used to calculate the difference in mean total scores and showed a significant change between PGY3 and the aggregate.

DISCUSSION / REFLECTION / LESSONS LEARNED: We found that the Trauma Informed Care Pre Clinic Conference module our group developed was an effective teaching tool which was well received by learners. Total difference between pre and post survey scores was noted to have a $p$ value of $<0.001$ indicating significant change in knowledge and attitudes. Notably, our faculty found the module to be a fast, effective, and easy way to implement teaching on the critically important subject of trauma informed care. In summary, this project illustrates the recognition of developing a Trauma Informed Care curriculum within our county system and the success of implementation through a Pre Clinic

Conference module.

\section{IMPLEMENTATION OF AN ONLINE CASE-BASED CLINICAL REASONING CURRICULUM}

Michelle Fleshner ${ }^{1}$; Ben Smith ${ }^{2}$; Joseph M. Rocco ${ }^{3}$; Thomas Painter ${ }^{3}$. ${ }^{1}$ UPMC, Pittsburgh, PA; ${ }^{2}$ UPMC Presbyterian Shadyside, Pittsburgh, PA, PA; ${ }^{3}$ University of Pittsburgh, Pittsburgh, PA. (Control ID \#3185469)

NEEDS AND OBJECTIVES: 1 . To improve availability of standardized clinical reasoning education for learners of all levels. 2. To create a case-based clinical reasoning curriculum distributed online to promote learner engagement. 3. To determine the best means for curriculum distribution both internal to and external to our institution.

SETTING AND PARTICIPANTS: This project was initiated by the University of Pittsburgh Department of Medicine's (UPMC) Clinical Reasoning Group. The curriculum was initially geared towards UPMC Internal Medicine residents and later distributed on social media to interested learners of different levels.

DESCRIPTION: The curriculum was based on learning objectives chosen by the Clinical Reasoning Group, which are in line with those promoted by the Society to Improve Diagnosis in Medicine. Cases were selected to exemplify potential biases that could lead to diagnostic error. Each case was organized into sections separated by multiple-choice questions that prompted learners to prioritize their differential diagnosis and work up decisions. The online survey platform Qualtrics was utilized and cases are distributed monthly via email to residents and faculty at UPMC and also via Twitter for open participation and discussion. Each case takes 5-6 minutes to complete. Data collected includes number of persons completing each case and means of accessing the case.

EVALUATION: Three cases were distributed via email to Internal Medicine Residents, Faculty, and medical students at UPMC over three months. The cases were also distributed on Twitter by an educational account. The number of participants for cases one, two, and three were 152, 183, and 246, respectively. Participants were surveyed at the end of the case to determine how they accessed the cases and to provide feedback. The proportion of participants accessing the case from Twitter increased each month. Participants indicated that they would appreciate more review of evidence-based testing, disease pathology, and mechanistic learning within the cases.

DISCUSSION / REFLECTION / LESSONS LEARNED: An online survey platform is an effective way to distribute clinical reasoning cases for educational purposes. The number of participants has steadily increased since initiation and feedback has overall been positive. In general, participants appreciate the accessibility of cases on smartphones, their brevity, and the interactive nature of the cases. Both email and social media have been effective platforms for distribution, but social media may have the ability to distribute educational materials more widely.

ONLINE RESOURCE URL (OPTIONAL): https://docs.google.com/document/d/11Xuk4fqk55QFLVXs6GLgc5kmb7VhdLuy0YDOQVGw-QY/edit?usp=sharing

IMPLEMENTATION OF TELEPHONE VISITS IN A RESIDENCY CLINIC: A STRATEGY TO BUILD TELEHEALTH SKILLS, IMPROVE PATIENT OWNERSHIP, AND ENHANCE CONTINUITY

Jenna Laughlin $^{1}$; Zehra Hussain ${ }^{1}$; Gretchen Rickards ${ }^{1}$; Sarah Schenck ${ }^{1}$; John Donnelly ${ }^{2}$. ${ }^{1}$ Christiana Care Health System, Wilmington, DE; ${ }^{2}$ Christiana Care Health System, Newark, DE. (Control ID \#3185420)

NEEDS AND OBJECTIVES: As primary care moves towards patientcentered care models, alternatives to the traditional in-person clinic visit are becoming increasingly utilized. Scheduled telephone visits are a convenient, effective method to deliver care. We piloted a telephone visit initiative in our residency clinic to develop telehealth skills in our trainees. We hoped that this novel visit type would also increase patient ownership and provider-patient continuity.

SETTING AND PARTICIPANTS: Since October 2016, nine internal medicine residents from our 36-resident categorical medicine program have had dedicated openings for telephone visits built in to their outpatient clinic schedule.

DESCRIPTION: Each resident had one hour of dedicated time per week templated into their clinic schedule for telephone visits. Depending on level of training, up to three telephone visits could be conducted during this protected time. Openings that went unfilled were converted into acute office visits. With regard to supervision, PGY1 residents received direct attending supervision for all visits. PGY2s were required to discuss all cases with the preceptor, and PGY3s discussed cases only as needed. All notes were routed to and co-signed 
by the preceptor, who provided immediate guidance and feedback to residents. EVALUATION: Measures included resident surveys as well as continuity data obtained through chart reviews. Seven residents were surveyed about their experience providing telephone visits; 2 of the original 9 participants were excluded because they had left the institution. The response rate was $86 \%$ (6/7 residents surveyed). All respondents reported that these visits increased their patient ownership, allowed them to build rapport with patients, and that the level of supervision they received was appropriate. With regard to continuity, out of the 273 telephone visits scheduled, 179 (65.6\%) were with the patient's PCP and 71 (26.0\%) were within the PCP's firm.

DISCUSSION / REFLECTION / LESSONS LEARNED: We encountered several scheduling and supervision challenges during our pilot. Unused phone visits were initially all automatically converted to acute visits, which was overwhelming for PGY1 residents. We have thus adjusted this scheduling practice. Because inappropriate patient bookings occurred when staff had scheduling access, we now limit scheduling of telephone visits to the provider only. We found supervision to be easiest when all resident telephone visits were scheduled at the same time. We did not bill for telephone visits, although residents did learn about current and upcoming opportunities for reimbursement.

\section{IMPROVING EASE OF ACCESS TO CLINICAL INFORMATION WITH A HOSPITAL-SPECIFIC INTERNAL MEDICINE ANDROID MOBILE APPLICATION}

Matthew Gerling. University of New Mexico, Albuquerque, NM. (Control ID $\# 3172469)$

NEEDS AND OBJECTIVES: Recognize that the majority of Resident Physicians in the United States are from the Millennial Generation, 92\% of whom carry a smart phone Recognize the opportunity that smart phones provide for demand-based access to important hospital system-specific clinical and administrative information

SETTING AND PARTICIPANTS: Setting: A single large academic medical institution consisting of an academic/community hospital and a Veterans Affairs hospital Participants: Internal Medicine residents and rotating medical students

DESCRIPTION: In September 2018, I developed an Android application called "VA Night Call" for Internal Medicine housestaff and students and made it available for free download on the Google Play store. Information provided in the application was intended to be specifically for the interns' night call shift. With the success of the VA Night Call app, I created a programspecific Internal Medicine Android app published on Google Play on November $30^{\text {th }}, 2018$. It includes hospital-specific information divided by subspecialty service as well as rotation-specific information. The app has current information under the Wards Handbook, Resident Wellness, VA Night Call, Infectious Diseases, and Palliative Medicine categories and may be accessed for free at https://play.google.com/store/apps/details?id=com. UNMIMResources.unmimresources

EVALUATION: Before downloading the VA Night Call app, surveys were administered to interns during their orientation. The same questionnaire was then administered at the end of the rotation, after they had downloaded and used the app. Interns reported being more confident in their medical decision making with the use of the app. Additionally, senior residents reported multiple cases where they have seen interns using the New Mexico VA Night Call application while on their inpatient medicine rotation. As of December 29th, there are 15 active installations of the app on unique devices. Our residency program has 39 interns, indicating a $38 \%$ participation rate. Currently, I am working to reprogram the app into Swift so that it can be published in the
Apple Store and made available for download by interns and residents who have iPhones. Interest in the app has spread beyond the Internal Medicine department, as several faculty members from other departments have expressed their interest in retooling its code for use in the development of apps for their respective residency programs.

DISCUSSION / REFLECTION / LESSONS LEARNED: As of December 29th, there are 15 active installations of the app on unique devices. Our residency program has 39 interns, indicating a 38\% participation rate in use of the app. Currently, I am working to reprogram the app into Swift so that it can be published in the Apple Store and made available for download by interns and residents who have iPhones. Interest in the app has spread beyond the Internal Medicine department, as several faculty members from other departments have expressed their interest in retooling its code for use in the development of apps for their respective residency programs.

\section{IMPROVING HIGH-RISK PATIENT HANDOFFS IN A RESIDENT PRIMARY CARE CLINIC}

Emily Fessler. Hospital of the University of Pennsylvania, Philadelphia, PA. (Control ID \#3183613)

NEEDS AND OBJECTIVES: The majority of Internal Medicine (IM) residency programs do not have a formal outpatient handoff process, though every 3 years graduating residents must transition their primary care panel to a new provider. In our resident clinic, current practice is for senior residents to select high risk patients in the spring using clinical judgment, and provide a warm handoff to an assigned junior resident. There is no protocol in place to guide patient selection, the content or format of the handoff, or to ensure that patients establish care with the receiving resident. This study will characterize patients deemed high risk by graduating residents and assess follow up surrounding the year-end transition to develop a handoff system that will reduce lapses in care.

SETTING AND PARTICIPANTS: High risk patients in a resident primary care clinic.

DESCRIPTION: Charts reviewed for 54 patients selected as high risk by 2017 graduating IM residents. Data collected re: comorbidities, healthcare visits in the year prior to handoff, and follow up after handoff. Handoff protocol developed to address identified gaps in care.

EVALUATION: High risk patients varied in age (median 64, 26-93) and had an average of 3 significant comorbidities; $50 \%$ carried a psychiatric diagnosis. Patients also varied in healthcare utilization in the year prior to handoff, with 119 clinic visits (median 4), 0-11 ED visits, and 0-7 admissions. 44\% had a transitions of care note in the medical record. At 1 year, only half of patients had seen the resident who received their high risk sign-out. 20\% established care with a different clinic provider, and the remainder were lost to care or left the clinic. The median time between last primary care (PCP) visit prior to yearend transition and first PCP visit post-transition was 7.7 months (1.9-26.4). Many were seen for another visit type (e.g. CRNP) in the interim.

DISCUSSION / REFLECTION / LESSONS LEARNED: Based on this data, the following clinic handoff will be implemented (1) Patient selection: Many of these patients would not be identified based on standard quality metrics. Residents will continue using clinical judgement to identify their highest risk patients. (2) Format: 50\% of high risk patients were never seen by the resident who received their handoff, and many saw other practitioners before a new PCP. Handoff must therefore be available in the medical record. "High risk patient - see $\mathrm{dd} / \mathrm{mm} / \mathrm{yy}$ transitions note" will be added to the problem list. (3) Content: A handoff note template will include reason for selection, medical summary/pending tasks, and psychosocial context. (4) Tracking: Residents will create an Epic list of high risk patients early in senior 
year. List will be used to collate pending tasks and ensure that follow up is scheduled prior to handoff. Graduating residents will be paired 1:1 with a junior resident and share patient list. Junior resident will then monitor to ensure patients seen by a new PCP. This should reduce lost information, and decrease time between last pre-transition PCP visit and first new PCP visit.

\section{IMPROVING HYPERTENSION IN AFRICAN AMERICANS: IM- PLEMENTATION OF A QI CLERKSHIP ADDRESSING HEALTH EQUITY}

Christopher Bautista ${ }^{1}$; Christopher $\mathrm{Cai}^{2}$; Carmen $\mathrm{Lee}^{2}$; Jordie Martin ${ }^{2}$; Isabel Ostrer $^{2}$; Noemi Plaza ${ }^{2}$; Ethan Zaccagnino ${ }^{2}$. ${ }^{1}$ University of California, San Francisco, San Francisco, CA; ${ }^{2}$ University of California, San Francisco School of Medicine, San Francisco, CA. (Control ID \#3163556)

NEEDS AND OBJECTIVES: Medical schools are incorporating health systems science into curricula, including quality improvement (QI), population health, and social determinants of health. While most medical schools have curricula that address health disparities, the intensity and quality of curricula vary. QI and health disparities competencies are naturally aligned, providing an opportunity for innovative curricular implementation.

SETTING AND PARTICIPANTS: At the University of California, San Francisco, all pre-clinical students participate in a longitudinal "clinical microsystems clerkship" focused on QI. Six pre-clinical medical students were assigned to a small group, guided by a faculty "coach" in a primary care clinic (the clinical microsystem) in San Francisco. Students met in the microsystem for one afternoon weekly over 13 months.

DESCRIPTION: The QI project chosen by the students in consultation with faculty coach addressed the disparity in hypertension control rates in African American patients compared to non-African American patients in the primary care clinic. Students were tasked to complete a "systems improvement template" (A3). Following this template, the students clarified the problem, and performed background, current state, and gap analyses, using local data and inter-professional interviews. They designed and implemented a telephone patient outreach intervention to identify gaps and facilitate patient follow up. Students completed 2 PDSA cycles.

EVALUATION: Educational impact: All 6 students successfully passed the QIKAT administered to all students; completed, designed, and implemented a QI intervention; and completed the systems improvement template. Work was disseminated as a poster in a QI symposium and recognized as one of the highest impact QI projects in the health system. The students gave an oral presentation at QI symposium. Clinical Impact: Telephone outreach protocol was developed and used to guide future outreach efforts. About 70 patients were included in the intervention, with $30 \%$ improvement in hypertension control in this group at the end of the study period.

DISCUSSION / REFLECTION / LESSONS LEARNED: Pre-clinical medical students learned and practiced QI by addressing a health disparity in hypertension control for African American patients. By choosing this gap, students learned about issues around health equity, including access to care, diversity and inclusion, and racial inequity. Pre-clinical students participated in direct patient care, reinforcing communication and doctoring skills. Their work had an observable and significant impact in improving the health of patients and addressing health inequity.

\section{IMPROVING INTERN EFFICIENCY THROUGH LAPTOP BASED ROUNDING IN FLOW}

Heather Balch; Casey Gradick. University of Utah School of Medicine, Salt Lake City, UT. (Control ID \#3180323)
NEEDS AND OBJECTIVES: The most common method used by residents to round on hospitalized patients involves batched work-flow (i.e. reviewing all patients in the computer, then rounding on all patients, then placing orders, then doing notes). This creates redundancy, which can reduce residents' efficiency, and lead to duty hour violations, decreased resident satisfaction, and delayed completion of patient care activities. To address this we implemented a novel work-flow intervention aimed to improve resident efficiency. SETTING AND PARTICIPANTS: The study took place on the inpatient general medicine teams at the University of Utah. These teams are comprised of an attending, a senior resident, and 2-3 interns. The work-flow intervention was implemented on 1 team for 3 months (13 interns), and 2 other teams were controls (21 interns).

DESCRIPTION: Residents on the intervention team were provided a recommended work flow (see URL), and their own laptop to allow them to round "in-flow" (i.e. doing all the work for one patient before moving on to the next). The interns pre-rounded with the laptops and then presented from them, which decreased the amount of written information. Orders and notes were then completed during rounds.

EVALUATION: There were 3 parts to the evaluation: a time in motion (TIM) study, subjective survey data, and EMR data (formal analysis underway) on progress note completion, order, and discharge times. The TIM study included 14 intern days, 7 each of the control and intervention work-flows. Once averaged, the interns on the intervention team (IT) spent 30 more minutes per day on direct patient care, 30 minutes less on computer time, and 15 more minutes on education, compared to those on a control team (CT). $38 \%(5 / 13)$ of interns on the IT and $48 \%$ (10/21) of interns on the CT responded to the post-intervention survey. Full analysis of this data is underway, but raw data shows the following differences: $80 \%$ vs $30 \%$ thought that orders discussed on rounds were always placed before $1230 ; 80 \%$ vs $40 \%$, said that all, or most, of their progress notes were done before $1230 ; 60 \%$ vs $10 \%$, said they were always able to leave the hospital by 7PM. Additionally, respondents from the IT compared to the CT felt better prepared on rounds, thought that they knew their patients better, and wrote less info down before rounds.

DISCUSSION / REFLECTION / LESSONS LEARNED: Since the implementation of the EMR, the majority of interns' work is now done on the computer, but the structure of rounding and pre-rounding has changed little. Our preliminary survey data show that increasing access to reliable, fast, and mobile workstations, in addition to encouraging a more in-flow style of rounding, led to decreased redundancy, improved resident efficiency with progress notes, order entry, and leaving the hospital in a timely manner. Our time-in-motion study suggests that the intervention may also have increased time for direct patient care, decreased overall time on the computer, and given more time for education, though this is likely not statistically significant.

ONLINE RESOURCE URL (OPTIONAL): https://goo.gl/ SDBWN1

\section{IMPROVING MEDICAL STUDENT BEHAVIOR AND SKILLS TOWARDS ADDRESSING SOCIAL DETERMINANTS OF HEALTH (SDOH)}

Haeyeon Hong ${ }^{2}$; Daniel Sylvester ${ }^{2}$; Stephanie Losi ${ }^{3}$; Marna Stack ${ }^{4}$; Mara Eyllon ${ }^{2}$; Arvin Garg ${ }^{3}$; Nancy R. Kressin ${ }^{5}$; Priya S. Garg ${ }^{2}$; Jodi Abbott $^{2}$; Lindsay B. Demers ${ }^{2}$; Thea James ${ }^{6}$; Pablo Buitron de la Vega $^{1}$. ${ }^{1}$ Boston Medical Center / Boston University, Dorchester, $\overline{\mathrm{MA}}{ }^{2}$ Boston University, Boston, MA; ${ }^{3}$ Boston Medical Center, Boston, MA; ${ }^{4}$ Boston University School of Medicine, Boston, MA; ${ }^{5}$ Dept of Veterans Affairs and Boston University, West Roxbury, MA; ${ }^{6}$ Boston Medical Center, Cambridge, MA. (Control ID \#3172896) 
NEEDS AND OBJECTIVES: Addressing the SDOH for patients is critical to providing high-quality care. SDOH are usually taught as immutable realities rather than actionable circumstances that should be addressed with specific tools. As a result, medical students are underprepared to address the SDOH. To address this gap, we developed a quality improvement initiative for third-year medical students (MS3s) at Boston University School of Medicine with the goal of improving their behaviors and skills towards addressing SDOH.

SETTING AND PARTICIPANTS: MS3s rotating through Internal Medicine 8 week inpatient clerkships at Boston Medical Center (BMC). An SDOH screening and referral model called THRIVE, adapted from the WECARE model, has been implemented at BMC primary care clinics. THRIVE is unfamiliar to emerging medical students and has not previously been used in inpatient settings.

DESCRIPTION: Starting in July 2018 MS3s screened and referred patients for eight SDOH domains and then followed-up with their progress through phone calls. During orientation, MS3s learned how to use the THRIVE screening tool and the THRIVE Directory (BMC's web-based repository of resources to help patients with social needs). MS3s followedup by phone 1-2 weeks after each patients' hospital stay to determine if they were able to receive support for identified SDOH needs.

EVALUATION: We held huddles with MS3s at the end of their rotation to explore feasibility and acceptability of the experience. Additionally, we used a pre and post survey to examine the impact of this activity on MS3's behavior and skills towards addressing SDOH. During huddles, the majority of MS3s mentioned having no trouble with the screening and referral process. Barriers included difficulty documenting responses in the Electronic Health Record, lack of formal training on calling patients to follow up about connecting to resources, and lack of experience collaborating with social workers. We performed a series of sign tests to compare students' behavior and perception of skills before and after participating in THRIVE. Twentyfour MS3s completed the pre and post survey. Overall, THRIVE had a significant positive impact on enabling MS3s to ask patients about social needs ( $\mathrm{p}<.001$ ). THRIVE also significantly impacted students' confidence in providing resources to help patients with their social needs $(\mathrm{p}<.001)$. Although it was not statistically significant, there is a trend showing that participation in THRIVE improved students' confidence in asking patients about their social needs $(p<1)$. There was no statistically significant change in MS3s perceptions regarding the importance and their role in addressing SDOH.

DISCUSSION / REFLECTION / LESSONS LEARNED: The initiative significantly improved students' asking patients about their social needs and confidence providing resources to help mitigate these needs. We learned that students need further education in understanding how to discuss addressing patients' social needs. These skills should be learned and simulated prior to starting their clerkship rotations.

\section{IMPROVING RESIDENT POPULATION HEALTH EDUCATION AND CARE THROUGH TEAM-BASED QUALITY IMPROVE- MENT}

John P. Shoup $^{1}$; Azalea Kim² ${ }^{2}$ Benjamin L. Ranard ${ }^{3}$; Jonathan Wilson ${ }^{3}$; Jane Pendergast $^{3}$; Joel C. Boggan ${ }^{4}{ }^{1}$ Duke University School of Medicine, Durham, NC; ${ }^{2}$ Duke Health, Durham, NC; ${ }^{3}$ Duke University, Durham, NC; ${ }^{4}$ Duke University Health System, Durham, NC. (Control ID \#3186388)

NEEDS AND OBJECTIVES: There is an urgent need to equip physicians with the knowledge and skills to manage population health to succeed in a landscape shifting to value-based-care contracts. We sought to improve the preparedness of residents to provide high-value, population-focused care through a combination of didactic and practice-based learning.

SETTING AND PARTICIPANTS: Internal Medicine residents at a large, quaternary-care academic medical center and an associated resident continuity clinic.

DESCRIPTION: We surveyed Internal Medicine, Med-Peds, and MedPsych residents to assess confidence in providing population-based healthcare. Specifically, residents were asked to assess their confidence in defining population health, identifying gaps in patient care, creating a strategy to address gaps without in-person visits, managing a shared patient panel, leading an interdisciplinary team, and utilizing population health strategies following training. We developed and incorporated 5 hours of population-health didactics into the academic curriculum to address these topics. Practice-based learning will be facilitated through a shared patient panel of a "practice partnerships" (PP) of an intern, second, and third year resident. We created an EPIC-based dashboard to report PP performance on population health metrics and identify patients with gaps in care. Each partnership selected one of three metrics to target: colorectal cancer (CRC) screening, statin prescription in diabetics, or patients with $\mathrm{A} 1 \mathrm{C}>9$. Participants had one administrative half-day session exclusively focused on improving their target metric. All PPs received quarterly control-charts of longitudinal performance.

EVALUATION: $75 \%(\mathrm{n}=133)$ of residents completed the initial survey. Less than $25 \%$ of surveyed residents reported confidence on most population health topics. Residents reported most confidence in identifying patients who were not up-to-date on routine care $(40.6 \%, \mathrm{n}=54)$, but only $13.5 \%$ (n $=18$ ) reported confidence in creating a plan to address gaps in care. Only a reported race of "Other" was associated with higher odds of confidence in creating a care plan as compared to white respondents (OR 3.44, 95\% CI 1.03-11.46) after adjusting for measured characteristics. There was no significant impact of sex, PGY year, anticipated future practice, or having an additional advanced degree. Among PPs, 9 selected statin prescription in diabetics, 10 CRC screening, and 1 uncontrolled diabetes. PP's received quarterly updates in the form of p-charts. Follow-up surveys together with metric performance at one year will be collected.

DISCUSSION / REFLECTION / LESSONS LEARNED: Surveyed internal medicine residents reported a lack of confidence in all population health related topics, and are not confident creating a care plan to address gaps in care.

\section{IMPROVING WEIGHT MANAGEMENT SKILLS IN TRAINEES} Noor Khan. UPMC Mercy, Moon Township, PA. (Control ID \#3184901)

NEEDS AND OBJECTIVES: Overweight and Obesity affect $69 \%$ of the US adult population and contribute greatly to many other conditions. Obesity is a disease that often goes unaddressed in primary care visits. Barely a quarter of physicians report feeling adequately trained in weight management and fewer than $30 \%$ of medical schools meet the minimum hours of nutrition education recommended by the National Research Council. We developed a curriculum that introduces trainees to principles and practice of weight management and improves their confidence in addressing this condition.

SETTING AND PARTICIPANTS: Participants were third year (PGY3) Internal Medicine Residents at an outpatient clinic. We implemented a curriculum emphasizing didactics, small group discussions and patient care during the 4-week ambulatory rotation. A total of 22 residents have participated in this curriculum from November 2017-December 2018.

DESCRIPTION: Patients with Obesity were scheduled for weight 
management visits during a half-day session during the PGY3 ambulatory rotation. Residents were asked to review material on evaluating obesity and to take an Implicit Association Test (Project Implicit) to increase awareness of subconscious bias prior to the session. Patients were evaluated, counseled and managed by a team consisting of the resident and a trained (American Board of Obesity Medicine certified) faculty member. Counseling skills, motivational interviewing and patient centered decision making was modeled by the faculty. At the end of the patient care session, small group discussions reinforced lessons learnt. Weight management specific didactic sessions included a 30-minute Pre-Clinic conference during the Ambulatory Rotation for all residents and the addition of 2 noon conferences to the annual schedule. Residents were encouraged to schedule weight management visits for patients on their continuity panel throughout the year.

EVALUATION: Participating PGY3 residents rated their comfort level with managing obesity before and after the half-day session and evaluated other aspects on a 5-point scale. The mean score for the overall experience was 4.7. The mean self-reported comfort with weight management was 2.4 before the session, improving to 4 post-session ( $p$ value of $<0.0001$, Wilcoxon signed rank test).

DISCUSSION / REFLECTION / LESSONS LEARNED: This successfully implemented curriculum demonstrated how dedicated weight management visits along with a few didactic sessions can substantially improve resident confidence. Similar interventions can be implemented at other programs interested in addressing this knowledge and training deficit in trainees without committing too much valuable residency time. There is a need to train more faculty members in weight management. Residents should be encouraged to treat patients with obesity in an evidence based proactive manner throughout their training to solidify weight management skills. We need to assess whether residents will continue to apply lessons learned after completing this curriculum.

\section{INCREASING CONNECTION AND ENGAGEMENT: A STUDY OF PEER-LED REFLECTION GROUPS FOR FELLOWS.}

Arabella L. Simpkin $^{1,2}{ }^{2}$; Susan Hata ${ }^{1,2}$; Merranda Logan ${ }^{1,2}$; Katrina Armstrong ${ }^{1,2}$. ${ }^{1}$ Massachusetts General Hospital, Boston, MA; ${ }^{2}$ Harvard Medical School, Boston, MA. (Control ID \#3185763)

NEEDS AND OBJECTIVES: Burnout appears to be an accelerating phenomenon, with implications for physician wellbeing, patient safety, and the quality of healthcare delivery. While many studies and interventions been directed at medical students, residents, and faculty to better understand what impacts burnout, subspecialty fellows have been largely untargeted, despite being a vulnerable population, critical to the future of patient care. Fellowship training presents unique challenges, often coinciding with geographic uprooting, increased responsibility, pressure to enter the job market, and loss of the team structure that can define residency. To tackle the goals of reducing burnout, increasing engagement, inclusion and belonging, we have created a program that creates small communities of fellows, who will gather to reflect on meaningful and challenging aspects of their work, and support one another. SETTING AND PARTICIPANTS: Sub-specialty fellows at Massachusetts General Hospital from the Department of Medicine. Peer support groups will convene for one hour, every 2 weeks, in a centrally located room on the main campus.

DESCRIPTION: Innovative program with 8-10 interdisciplinary fellows gathering on a two-weekly basis for a self-facilitated peer-support group with a provided discussion guide.

EVALUATION: All participants complete surveys at the beginning and end of the study to measure: work engagement; empowerment at work; resilience; reaction to uncertainty; and burnout. Baseline surveys show 32\% of fellows meet criteria for burnout.

DISCUSSION / REFLECTION / LESSONS LEARNED: We have experienced challenges in fellows being able to attend the lunchtime sessions, despite positive feedback about the endeavor. Fellows have a set of unique demands that ironically puts them at huge risk of burnout whilst impeding their ability to attend structured wellness sessions: unpredictable and changing patient volumes make regular sessions on a standardized day an impossibility; consults are increasingly complex; voicing concerns about overwhelming workloads can feel unwise given fellows are often looking for faculty jobs in the institution. Recommendations to overcome these challenges include: connecting fellows to mentors and coaches outside their divisions; flexibility in timing and composition of peer-support groups; connecting fellows from different sub-specialties and years to nurture vertical associations.

\section{INCREASING HEALTH PROFESSION STUDENTS' INTERPRO- FESSIONAL COMPETENCIES: THE INTERPROFESSIONAL DEDICATED EDUCATION UNIT (IPDEU)}

$\underline{\text { Rachel Jantea }}^{1}$; Victoria Hornyak ${ }^{1}$; Cassandra Leighton ${ }^{1}$; Teresa Pacella ${ }^{1}$; Rosemary Hoffmann $^{1}$; Sandra Engberg ${ }^{1}$; Subashan Perera ${ }^{1}$; Susan Meyer ${ }^{1}$, ${ }^{1}$; Benjamin Reynolds ${ }^{1}$; Debra K. Weiner ${ }^{1}$; David M. Elnicki²; Alton E. James ${ }^{1,}{ }^{1}$; Rollin M. Wright ${ }^{1}$. ${ }^{1}$ University of Pittsburgh, Pittsburgh, PA; ${ }^{2}$ University of Pittsburgh, Wexford, PA. (Control ID \#3177640)

NEEDS AND OBJECTIVES: Many health professionals work in interprofessional (IP) teams to care for older adults with complex needs. Interprofessional education (IPE) is now required for health profession students, but the best educational model is unknown. Further, clinical preceptors are often untrained in IPE themselves, creating heterogeneity in students' IPE experiences. To address these needs, we designed and evaluated an Interprofessional Dedicated Education Unit (IPDEU) acute care IPE experience for health profession students.

SETTING AND PARTICIPANTS: We designated a neurotrauma hospital unit (older population with complex needs) the IPDEU at our large academic medical center. Unit nurse, occupational therapy (OT), physical therapy (PT), and speech therapy (ST) providers were trained as IPE instructors in JulyAugust 2018. Learners were University of Pittsburgh students from different health professions ( 3 audiology, 13 nursing, 8 OT, 9 PT, 9 ST, 8 physician assistant) who rotated on the IPDEU in fall 2018.

DESCRIPTION: We trained IPE instructors in both IP practice skills and IPE-specific instructor skills. Instructors completed 2 hours of web-based skill modules and 4 hours of skill practice with simulation-trained actors. Students from different health professions were paired. Each pair spent 2 half-day sessions on the IPDEU within a one-week period (1 with a nurse IPE instructor \& 1 with a therapy IPE instructor). During IPDEU sessions, students actively observed their IPE instructors during usual patient care activities. Active observation explicitly focused on IP aspects of care (not clinical skills) and was facilitated through instructor techniques such as IPE-directed questions and thinking-out-loud. A 30-minute group debrief concluded each session.

EVALUATION: Students self-rated their IP competencies using the Interprofessional Collaborative Competency Attainment Scale (ICCAS) before/ after the IPDEU experience. Pre/Post composite ICCAS scores were analyzed by independent t-tests. Pre/Post individual item Likert responses were dichotomized and analyzed by $X^{2}$. Fifty students completed the experience. Twentyseven (54\%) reported prior formal IPE. Composite ICCAS scores improved post-IPDEU (65.3 vs $81.5, p<0.0001)$. Pre-IPDEU, few students rated individual competencies highly ( $<70 \%$ rated themselves Very Good or Excellent on any item). Post-IPDEU, all item competency ratings improved significantly 
( $p$-values ranged 0.02 to $<0.0001$ ). Areas of greatest improvement were IP team utilization, communication, roles, and providing constructive team feedback. Sample size limited subset analyses.

DISCUSSION / REFLECTION / LESSONS LEARNED: Despite most students reporting prior IPE exposure, few IP competencies were rated highly at baseline. All competency ratings improved significantly post-IPDEU, suggesting the experience improved confidence in knowledge, skills, or both. Next steps will assess long-term competency retention, evaluate differences between professions and levels of pre-existing clinical experience, and explore thematic content of student debriefs.

\section{INCREASING RESIDENT COMPETENCE AND CONFIDENCE IN RESUSCITATION LEADERSHIP}

Nathan T. Douthit ${ }^{1}$; Christine Mcbride ${ }^{2,}{ }^{1}$; Erin Townsley ${ }^{1}$. ${ }^{1}$ Brookwood Baptist Health, Birmingham, AL; ${ }^{2}$ Virginia College Of Medicine, Auburn, AL. (Control ID \#3185223)

NEEDS AND OBJECTIVES: Internal Medicine (IM) residents are experiencing fewer cardiopulmonary resuscitations. Most are intimidated by leading a resuscitation. Many IM residents go to careers in hospital medicine, where competence and confidence in resuscitations are essential qualities. SETTING AND PARTICIPANTS: This intervention took place in a community hospital residency training program. Included participants were all categorical internal medicine residents from the 2017/2018 and 2018/2019 academic years. $(n=39)$

DESCRIPTION: 12 months of records were reviewed in order to determine the outcomes of resident-led resuscitations compared to other resuscitations led by hospitalist and intensivist. Variables measured were ROSC, survival to discharge (sDC), time of event, and location of event. Surveys assessing resident confidence were distributed before and after an intervention. The intervention consisted of a "Code Conference" which took place 8 times in the academic year. It featured a simulation led by an intern and a high-yield resuscitation topic lecture. Staff from the intensivist, nursing, and pharmacy services were all present and provided immediate feedback after the simulation. Outcome data was tracked during the intervention and compared to the pre-intervention data. Data was analyzed using SAS 9.4. EVALUATION: On a 0-5 Likert scale, PGY-2 residents in 2018 compared to PGY-2 residents in 2017 did not show an improvement in confidence following orders during a resuscitation (averages of 4.75 pre and 4.375 post). In contrast they did show an increase in confidence in leading a resuscitation ( 2.5 pre and 4.125 post) and teaching others how to lead a resuscitation (2.5 pre and 3.125 post). In the post intervention survey, residents gave the intervention an importance rating of 4.5 on a 0-5 scale. Resident ROSC improved (63.27\% compared to $79.17 \%$; $\mathrm{p}=0.10)$ and $\mathrm{sDC}$ were stable $(16.33 \%$ compared to $16.67 \% \mathrm{p}=0.90)$ despite decreases in the hospital wide rates between the two years of ROSC (72.85\% pre and $68.28 \%$ post) and SDC (21.19\% pre and $17.24 \%$ post).

DISCUSSION / REFLECTION / LESSONS LEARNED: Limitations included the small sample size and inability to quantify resuscitation complexity. Despite these limitations, there was an improvement in resident competence with a positive trend. Despite the decrease in ROSC and SDC in the hospital, residents had more success in ROSC and stable rates of sDC. Residents confidence increased and they viewed the intervention as extremely important to their IM training. These code conferences are a low-cost multidisciplinary intervention that can be used to improve resident confidence and competence in leading resuscitations, an important part of internal medicine education.

\section{INNOVATION IN INPATIENT HIV EDUCATION: A FLEXIBLE FLIPPED CURRICULUM}

Mollie B. Tucker; Merceditas Villanueva. Yale School of Medicine, New Haven, CT. (Control ID \#3178593)

NEEDS AND OBJECTIVES: The Inpatient Infectious Disease (ID) Rotation provides an opportunity for residents to learn about HIV management. Previously, teaching on the rotation was not standardized, partly due to constraints placed by intensive inpatient clinical needs. In the flipped classroom (FC) model, learners build a knowledge foundation outside of the classroom, while classroom time is devoted to knowledge application. We adopted a modification of the FC model, a flexible flipped curriculum (FFC), to provide residents with a standardized HIV curriculum with additional flexible topics, geared towards adult learners.

SETTING AND PARTICIPANTS: This curriculum was implemented on the Inpatient ID Service at Yale New Haven Hospital. The service consists of two teams, each team with an attending, resident, intern, and medical students. Participants rotate in 2- or 4-week blocks. All team members participated in the curriculum, and residents and interns were surveyed about their educational experience.

DESCRIPTION: The web-based curriculum consisted of 3 core modules and 8 optional modules. Each module contained "out-of-classroom" (OC) and "in-classroom" (IC) materials. OC materials included videos made by core ID faculty and links to articles from the AIDS Education and Training Center (AETC) National HIV curriculum. IC materials included relevant questions from the AETC question bank and links to cases from the New England Journal of Medicine. Teams were asked to complete all core modules and to choose optional modules based on relevance to active cases. Residents reviewed OC materials on their own, then engaged in IC question-based and case-based sessions led by their attendings.

EVALUATION: Implementation began in July 2018. Residents received pre- and post-tests designed to assess utilization and perceived effectiveness of the curriculum. In the first 6 months, 39 residents participated. Of those, $66.7 \%$ completed the pre-test and $53.8 \%$ completed the post-test. There was variation in utilization of modules. Overall, $28.5 \%$ preferred the FFC model, $19 \%$ preferred the classic teaching model, and $52.3 \%$ had no preference.

DISCUSSION / REFLECTION / LESSONS LEARNED: Residents generally appreciated the availability of a standardized curriculum. However, there remained variability in its utilization. Those who used the curriculum enjoyed the opportunity to apply knowledge with expert guidance. More widespread utilization was hampered by the ongoing challenge of finding time when all team members were available to hold IC sessions. Improving implementation of the curriculum, with attention to variable schedules of team members, is still needed.

\section{INNOVATIONS IN CLERKSHIP ASSESSMENT: THE EARLY EX- PERIENCES AT DELL MEDICAL SCHOOL}

Sherine Salib $^{1}$; Stephanie Corliss ${ }^{2}$; Kimberly Brown ${ }^{2}$; Valli Annamalai ${ }^{2}$; $\overline{\text { Beth Nelson }}{ }^{2}$. Dell Medical School, University of Texas at Austin, Austin, TX; ${ }^{2}$ Dell Medical School, Austin, TX. (Control ID \#3163280)

NEEDS AND OBJECTIVES: A fairly ubiquitous challenge in the core clerkships is that of observing and assessing specific desirable medical student behaviors and skills in the clinical setting. Clarity in how those behaviors and skills are observed and assessed is an important and yet often elusive task, particularly with some of the less traditionally assessed characteristics that we seek to cultivate in our students, such as Clinical Curiosity, Initiative, Problem-Solving and Safety and Value considerations. 
SETTING AND PARTICIPANTS: Dell Medical School has a robust 4 year clinical skills, leadership and value-in-healthcare curriculum. These skills are explicitly taught and assessed throughout the 4 years of medical school, including the core clerkships.

DESCRIPTION: Dell Medical School incorporates several longitudinal themes into its mission, including clinical skills, leadership and value and safety in healthcare. Creating an environment within the core clerkships where these competencies can be applied is challenging, both for students to demonstrate, and for faculty to recognize and assess. We have implemented several strategies and tools to assess those competencies in the clerkships. These tools align with the competencies outlined in the clerkship clinical performance assessment form, which includes competencies such as leadership, initiative, value and safety considerations, amongst others.

EVALUATION: At the end of the MS1 year, students rated themselves on their level of attainment of each DMS core competency. During the MS2 Clerkship, faculty assess students using the Clinical Performance Assessment Form. 1212 forms were completed for 50 students during clerkship rotations 1-3 of the 2017-2018 academic year. On the student self assessments, students assessed themselves the highest in the areas of "Emotional Intelligence \& Situational Awareness", "Continuous Personal Improvement" and "Interprofessional Care". On the clinical performance assessment forms completed by faculty \& residents, "Performance exceeds expectations" was selected in the highest percentages for Clinical Curiosity (40\%), Emotional Intelligence and Situational Awareness (29\%), Continuous Personal Improvement (25\%) and Patient Safety (24\%). Several items stood out as being difficult for faculty to assess, with "Not enough information to evaluate" being selected in responses for Patient Safety (35\%), Problem Solving \& Innovation in Healthcare (34\%), and Healthcare System Context (31\%) being the most challenging. Our data indicate that, with these tools, along with clear grading rubrics and ongoing faculty development, faculty are able to reliably and confidently assess most competencies, although some challenges remain.

DISCUSSION / REFLECTION / LESSONS LEARNED: Creating experiences to practice specific skills in the clinical setting, and implementing tools to observe and assess those behaviors and skills is imperative, but not always easy. This is particularly challenging with subcompetencies such as initiative, clinical curiosity, problem-solving and value and safety considerations.

\section{INNOVATIONS IN POPULATION HEALTH MANAGEMENT: IM- PROVING CERVICAL CANCER SCREENING RATES AMONG INTERNAL MEDICINE RESIDENT PATIENT PANELS}

Phillip Yun ${ }^{1}$; James Doolin ${ }^{1}$; Jeanne Gosselin ${ }^{2}$; Rebecca Glassman ${ }^{2} .{ }^{1}$ Beth Israel Deaconess Medical Center, Cambridge, MA; ${ }^{2}$ Beth Israel Deaconess Medical Center, Boston, MA. (Control ID \#3178425)

NEEDS AND OBJECTIVES: In June 2017, we identified a significant disparity in the cervical cancer screening rates among patients seen by internal medicine residents and faculty attendings, $66 \%$ and $80 \%$, respectively, at Beth Israel Deaconess Medical Center's ("BIDMC") ambulatory clinic, Healthcare Associates ("HCA"). Our objective was to improve cervical cancer screening rates among patients seen by BIDMC's internal medicine residents. The first phase of our quality improvement project included a needs assessment and the development of our curricular intervention. Here, we report on the second phase of our project: preliminary outcomes from the first 12 months of our curricular intervention.

SETTING AND PARTICIPANTS: Our intervention was conducted within the internal medicine residency program at BIDMC's main primary care clinic, HCA, in Boston, Massachusetts. We delivered our curricular intervention to $2^{\text {nd }}$ and $3^{\text {rd }}$ year residents during their ambulatory blocks in six-week cycles from November 2017-January 2019.

DESCRIPTION: After surveying residents on their understanding of cervical cancer screening, we designed a curricular intervention targeting perceived barriers. The curriculum was co-created by residents and attendings. The primary care track residents led the weekly sessions. The content included increasing awareness of ancillary support staff at HCA and proper navigation of population health tools and documentation. The intervention also included guided panel management time alongside HCA's outreach team to help identify and reach out to patients in need of pap smears.

EVALUATION: In order to evaluate the impact of the intervention, we focused on the residents who were in their 2nd year in June 2017. We tracked changes in the cervical cancer screening rates among their patients into September 2018. From June 2017 to September 2018, the cervical cancer screening rate for this group of residents increased from $63 \%$ to $70 \%$. In addition, we administered a post intervention survey to $2^{\text {nd }}$ and $3^{\text {rd }}$ year residents beginning in December 2018. Preliminary results included responses from eighteen $3^{\text {rd }}$ residents and seventeen $2^{\text {nd }}$ year residents. When asked if they were more comfortable with population health after attending the curricular intervention, 33/35 (94\%) agreed. When asked if they would recommend the curricular intervention to other internal medicine residency programs, 32/35 (91\%) agreed. When asked if they were more comfortable with cervical cancer screening after attending the curricular intervention, 18/ $35(51 \%)$ agreed.

DISCUSSION / REFLECTION / LESSONS LEARNED: We believe the increase in cervical cancer screening rates among resident patients from June 2017 to September 2018 is largely due to our curricular intervention, as no other large-scale intervention or practice change at HCA occurred during this time period. Our post intervention survey also supports this hypothesis as a majority of residents responded positively to our intervention.

\section{INSULIN PRESCRIBING WORKSHOP: A BRIDGE BETWEEN} PHARMACY AND PRIMARY CARE

David Kuo ${ }^{1}$; Anthony J. Longo ${ }^{4}$; Olesya Taylor ${ }^{2,3}$; Christine Lam $^{3,}$; Rebecca A. Griffith ${ }^{2} .{ }^{1}$ Atlantic Health System, Morristown, NJ; ${ }^{2}$ Atlantic Health/ Morristown Medical Center, Morristown, NJ; ${ }^{3}$ Fairleigh Dickinson University, Bedminster, NJ; ${ }^{4}$ Atlantic Health, Little Falls, NJ. (Control ID \#3176518)

NEEDS AND OBJECTIVES: Internal Medicine residents and medical students often have a strong conceptual knowledge about prescribing insulin but are often uncomfortable with real life skills required in managing insulin. Such skills include using proper insulin injection technique, proper glucometer technique, and being able to convert between different forms of insulin. Since the practical aspects of these skills are not routinely taught in medical school or even residency, we implemented a workshop to teach these skills to our medical students and residents.

SETTING AND PARTICIPANTS: Morristown Medical Center Internal Medicine Residency is a moderate sized program with 15 categorical residents each year and a clinical training site for three medical schools. The target audience for the workshop consisted of the residents and medical students currently rotating at the hospital. The workshop faculty consisted of ambulatory Internal Medicine faculty and an academic clinical pharmacy department which also has pharmacy residents and students from two schools. The onehour workshop took place in lieu of a morning report session.

DESCRIPTION: The workshop consisted of three stations each led by either a primary care or pharmacy faculty member, with senior residents or 
pharmacy students as junior instructors. The stations were: (1) how to use a glucometer, (2) how to use an insulin pen, and (3) how to calculate and write mixed insulin prescriptions. Each session lasted 14 minutes in duration. Equipment such as insulin pens, GLP-1 devices, glucometers and testing strips and lancets and skin models were provided by the clinical pharmacy department.

EVALUATION: The program was evaluated with a brief survey of the participants' self-reported confidence performing the skills taught during the workshop. The four-point scale ranged from "not at all confident" to "very confident." 27 residents participated in the workshop, with 17 completing the pre- and post-test survey. Of those completing the survey, 94\% reported at least a one-point increase in their confidence in calculating a 30-day supply of insulin pens or vials; $88 \%$ reported at least a one-point increase in converting mixed insulin to a basal-bolus regimen and teaching patients how to use a glucometer; $76 \%$ reported at least a one-point increase in confidence level using an insulin pen.

DISCUSSION / REFLECTION / LESSONS LEARNED: Over $80 \%$ of diabetes patients are managed by primary care physicians, many without the support of an endocrinologist. In the absence of subspecialty support, most primary care physicians are comfortable prescribing oral diabetes drugs, but practical aspects of managing insulin and glucose monitoring are often relegated to nurses or pharmacists. In this workshop, residents and students learned common and fundamental insulin management skills which will help them better care for their diabetic patients.

INTEGRATED SONOGRAPHIC COMPETENCY AT NYU (ISCAN): PROGRAM DESCRIPTION AND EARLY EVALUATION Michael Janjigian ${ }^{1}$; Anne Dembitzer ${ }^{2}{ }^{1}$; Caroline Srisarajivakul-Klein ${ }^{1}$; Khemraj Hardowar ${ }^{1}$; Penelope Lusk ${ }^{1}$; Sondra Zabar ${ }^{1}$; Harald Sauthoff ${ }^{1}$. ${ }^{1}$ NYU Langone Health, New York, NY; ${ }^{2}$ VA NY Harbor Healthcare System, New York, NY. (Control ID \#3168682)

NEEDS AND OBJECTIVES: Point-of-care ultrasound (POCUS), when integrated with a physical examination, increases accuracy of diagnosis and decreases procedural complications. However, most hospitalists have not been trained to use this new technology. We developed a year-long curriculum, the Integrated Sonographic Competency at NYU (I-ScaN), to train hospitalists in POCUS.

SETTING AND PARTICIPANTS: Twenty-three hospitalists from across the 4 hospitals affiliated with NYU Langone Health participated. Sixteen of the participants (72\%) reported prior ultrasound training, with a range of 2-80 hours (median $=4$ hours); 3 reported more than 5 hours of prior training. Three reported active clinical use of POCUS though none of them had more than 5 hours of prior training. The group averaged 4.5 years of clinical practice (range $=1-13$ years).

DESCRIPTION: The program began with an intensive 2-day course consisting of lectures and hands-on training on human models covering views of the heart, lungs/pleura, abdomen, and leg vasculature. We developed the remainder of the year-long program with the goal of helping participants retain and improve upon the skills acquired during the initial course. Our program included hands-on teaching sessions held at each institution by local experts, presentations at monthly conferences with the course director (HS), and online feedback on uploaded ultrasound images. To facilitate this final item, each participant was given access to portable ultrasound devices with the ability to upload ultrasound clips to a HIPAA-compliant website. Participants provided a clinical interpretation and assessment of image quality for each of their clips. The clips were then reviewed by an expert who provided feedback on both of these domains. Participants could then use these clips to create personal portfolios in accordance with national standards set by the Society of Hospital Medicine.

EVALUATION: Participants rated the 2-day intensive program as very useful and satisfaction with the individual components of the program ranged from useful to very useful. Participants reported statistically significant increases in their confidence in acquiring images, interpreting images, and performing a clinical evaluation using POCUS for all domains except in performing paracentesis. Knowledge scores increased from a baseline of $58 \%$ to $83 \%$; $\mathrm{p}<0.001$. At 6 months into the program, 2 hospitalists had uploaded $>400$ clips each and 7 had uploaded fewer than 20 clips each.

DISCUSSION / REFLECTION / LESSONS LEARNED: I-ScaN is a highly rated and effective program to train hospitalists in core POCUS competencies. The 2-day intensive program significantly improves confidence and knowledge. Monitoring of progress and scanning activity was difficult because few hospitalists uploaded clips regularly. Our focus now is on identifying barriers to using POCUS for new trainees. Future analysis will include frequency of scanning, retention of knowledge and skill, and generation of learning curves for each view.

\section{INTEGRATING A NOVEL HIGH-VALUE CARE CURRICULUM FOR INTERNAL MEDICINE RESIDENTS}

Rachna Rawal $^{1}$; Paul Kunnath ${ }^{2}$; Fred R. Buckhold ${ }^{2}$; Jennifer Schmidt ${ }^{3}$. ${ }^{1}$ Saint Louis University, St. Louis, MO; ${ }^{2}$ Saint Louis University School of Medicine, University City, MO; ${ }^{3}$ Saint Louis University, Saint Louis, MO. (Control ID \#3183505)

NEEDS AND OBJECTIVES: Understand internal medicine resident need for high-value care education Identify components of a high-value care curriculum for internal medicine residents Recognize residents improved self-awareness of high-value care

SETTING AND PARTICIPANTS: The healthcare crisis in the United States is prompting a transformation toward a high-value, cost-conscious culture. Physicians develop their ordering practices early in their training, therefore directing high-value care education at residents will have the greatest impact. A needs assessment demonstrated that only $8 \%$ of our residents could provide an adequate free-response explanation of high-value care. Only $18 \%$ of residents participated in a prior high-value care initiative. This need prompted development of a high-value curriculum.

DESCRIPTION: We designed and implemented a high-value care curriculum directed at Internal Medicine residents. It included six noon didactic sessions, three afternoon school interactive case sessions, and high-value rapid-fire cases during monthly floor orientation. Objectives included defining high-value care, applying evidence-based medicine, "how-to" develop a highvalue care initiative, cognitive biases, integrating pathology consults, and applying American College of Radiology (ACR) Appropriateness Criteria. Residents completed pre and quarterly post surveys consisting of Likert scale, multiple choice and free responses questions.

EVALUATION: Pre-intervention data showed $85 \%$ of residents identified the definition of high-value care in a multiple-choice question. $8 \%$ and $52 \%$ of residents distinguished the definitions of "price" and "cost", respectively. $14 \%$ reported familiarity with the ACR criteria and $24 \%$ felt comfortable applying those guidelines. After the first didactic session, the number of residents who felt "very comfortable" recognizing hospital overuse increased from $27 \%$ to $52 \%$ (statistically signifncant, $\mathrm{p}<0.05$ ). $96 \%$ felt encouraged to participate in a high-value care initiative in the future. Additional data is pending.

DISCUSSION / REFLECTION / LESSONS LEARNED: While the majority of residents could choose the definition of high-value care from a 
list, they could not generate a free-response definition. This discrepancy highlights that while residents have some basic knowledge of high-value care, they lack the skills to apply and generalize it. Residents were unfamiliar with radiology guidelines.

\section{INTEGRATING SCIENTIFIC RESEARCH INTO MEDICAL EDU- CATION THROUGH A COMPREHENSIVE SCIENCE SCHOLARS ELECTIVE COURSE}

Yasmin Kamal; Marek Svoboda; Roshini Pinto- Powell. Dartmouth Medical School, Hanover, NH. (Control ID \#3185873)

NEEDS AND OBJECTIVES: One of the goals of evidence-based medical education is to familiarize future physicians with the scientific method in order for them to comprehend scholarly literature, and communicate an evidenced based plan of care to their patients. However, many medical students lack the skills necessary to participate in scholarly research. We describe the implementation of a comprehensive elective course designed to equip interested students with these skills through a series of workshops, one-on-one mentorship, and pursuit of a summer research experience. The program aims to provide medical students with the knowledge and skills required to incorporate research into their medical training and contribute to their synthesis and increased use of evidence-based medicine as future physicians.

SETTING AND PARTICIPANTS: Course participants are first-year medical students at the Dartmouth Geisel School of Medicine.

DESCRIPTION: This year-long course consists of four main parts: scientific mentorship meetings, small group workshops, final research project, and a summer research experience. Each M1 student is assigned a mentor - MD$\mathrm{PhD}, \mathrm{PhD}$, or senior MD student, based on their research interests. Mentors help students navigate the research environment at Dartmouth and obtain summer research positions. The instructional portion of the course comprises a series of ten workshops taught by the program coordinators (MD-PhD students) and Dartmouth faculty. At course completion, all participating medical student write a research aims page to effectively communicate the goals of their research project when they apply to local as well as national competitive external research funding opportunities for a first year summer research experience.

EVALUATION: Nine students participated in the first year of the elective (2017-2018). All students secured a research position for the summer following their first year of medical school and four of the nine students obtained nationally competitive funding. $78 \%$ of the students indicated that participation in the elective significantly helped them find and secure these positions and $88 \%$ said they learned valuable research skills not taught in the medical curriculum.

DISCUSSION / REFLECTION / LESSONS LEARNED: M1 participation in a well-designed research elective has the potential to provide immediate value in the acquisition of a summer research position and to stimulate a lifelong interest in scientific inquiry.

ONLINE RESOURCE URL (OPTIONAL): https://geiselmed.dartmouth. edu/mdphd/links/science/

\section{INTEGRATING TRAINEES TO TELEHEALTH INFRASTRUTURE IN PRIMARY CARE IN THE DEPARTMENT OF VETERANS AF- FAIRS}

Sarai Ambert-Pompey $^{1}$; Leoni Heyworth ${ }^{3}$; Janeen E. Smith ${ }^{2}$; Ijeoma KeneEwulu ${ }^{4} .{ }^{1}$ VA, Boise, ID; ${ }^{2}$ San Francisco VA Health Care System, San Francisco, CA; ${ }^{3}$ VA, San Diego, CA; ${ }^{4} \mathrm{VA}$, Atlanta, GA. (Control ID \#3186353)
NEEDS AND OBJECTIVES: The next generation of healthcare professionals must become competent in delivering clinical services remotely. Nationally, however, little standardized telehealth education exists in training programs. The Department of Veterans Affairs (VA) is working to provide a formalized model of telehealth education for its more than 120,000 trainees. The VA has already delivered over 12 million telehealth encounters and has developed extensive educational materials for its clinicians. Now the VA is using its clinician telehealth curriculum and telehealth best practices to design a telehealth education infrastructure for all healthcare trainees who rotate through primary care in its more than 150 facilities. The intervention to provide national standardized telehealth education for trainees is: 1) Using existing VA Telehealth Training Curricula for clinicians and making it available to all trainees; 2) Developing an inventory of telehealth trained clinicians and pairing them with trainees; 3 ) Cataloguing best practices in telehealth training at VA facilities; 4) Addressing policy obstacles

SETTING AND PARTICIPANTS: This intervention focuses on the $120,000+$ healthcare professional trainees that rotate through over $150 \mathrm{VA}$ healthcare facilities throughout the nation. The development of standardized national telehealth education began with an assessment of the scope of telehealth training and found $69 \%$ of all medical centers were providing individualized telehealth training opportunities for trainees.

DESCRIPTION: Graduate, undergraduate and associated health trainees are receiving telehealth didactics, participating in live interactive telehealth simulation sessions, and work-based learning across healthcare facilities and to the patient via mobile devices. Clinicians from different states met once a month via video conferencing technology to discuss different components of the curriculum to adjust to the local resources and time constraint from other competing educational opportunities. The curriculum addresses common and interprofessional components in addition to individual profession-directed components that are within the scope of license.

EVALUATION: Evaluation of the development of a national standardized telehealth education model for trainees will be measured quantitatively and qualitatively. The numbers and expansion of didactic web-based trainings completed by trainees are tracked along with their simulation sessions. Each facility will provide data on the numbers and types of telehealth trainee opportunities they are offering. Telehealth satisfaction surveys for providers and patients will be expanded and accreditation body competencies evaluations.

DISCUSSION / REFLECTION / LESSONS LEARNED: Many of the lessons learns across the different sites were shared and new challenges particularly challenges in common were tackled amongst the group as a whole. We noted there were small things in one site that work well other sites incorporated those like simulations, participation in established team virtual meetings and huddles.

INTERPROFESSIONAL QUALITY IMPROVEMENT PROJECTS: USING QI PRINCIPLES TO DEVELOP A ROBUST QI CURRICULUM

William G. Weppner ${ }^{1}$; Rick Tivis ${ }^{2}$; Sarah Naidoo ${ }^{2}$; Lisa Inouye ${ }^{1}$; Michael F. $\mathrm{Krug}^{1}$; India C. King ${ }^{3} .{ }^{1}$ University of Washington, Boise, ID; ${ }^{2}$ Boise VA Medical Center, Boise, ID; ${ }^{3}$ Family Medicine Residency of Idaho, Boise, ID. (Control ID \#3184460)

NEEDS AND OBJECTIVES: The goal of this project was to apply QI principles to improve a QI curriculum for interprofessional trainees.

SETTING AND PARTICIPANTS: The Boise VAMC serves as the host to a Center of Excellence in Primary Care Education, as well as multiple graduate health care training programs from internal medicine, psychology, pharmacy 
and nursing. Trainees specifically engaged in the QI curriculum include nurse practitioner residents $\left(1^{\text {st }}\right.$ year), nursing students (final year and VALOR students), PGY-2 pharmacy residents (ambulatory care and mental health), psychology trainees (interns and postdoctoral candidates), and internal medicine residents (PGY-2).

DESCRIPTION: The "interprofessional Quality Improvement Projects" (iQIP) curriculum consists of an initial half-day didactic session to introduce QI concepts. This employs a "Zombie Attack" theme with hand-on work for aims statements, pareto charts, process mapping and gamified run charts, among other activities. This is followed by a mentored workplace learning experience for the rest of the year, where trainees rank their interest in a proposed project relevant to the health care system. Each team is composed of at least 3 members, from at least 2 disciplines, with at least 1 faculty mentor. Trainee teams are required to present 2 work in progress presentations, followed by a final presentation at grand rounds for the entire facility.

EVALUATION: The training has been continually evaluated and adapted using an informal PDSA cycle framework by curriculum leads. We employed pre-post evaluation using the Quality Assessment Instrument (QIKAT) (Lawrence et al JGME, Mar 2011, p41-48). This project examiness the changes in the program since 2014 and how these changes effected differences in pre-post measures on the QIKAT. In 2014-2015 there was very little change between the pre-post with none of the pre-post change rising the statistical or clinical significance $(\mathrm{P}>.18)$. This initiated changes emphaszing more targeted learning objectives, and intentional restructuring of the workshop and project components, resulting in significant improvement across the next two academic years in four of the nine measures (P .05). Major modifications were implemented prior to the 2017 academic year to further streamline and focus the process. These changes resulted in significant changes in learning as measured by the QIKAT on five of the nine $(\mathrm{P}<.05)$ with two others approaching significance $(\mathrm{P}=.06)$.

DISCUSSION / REFLECTION / LESSONS LEARNED: Curriculum development \& implementation is a process, and should be subject to ongoing evaluation with a feedback and improvement mechanism. Cyclic evaluation leads to improvement and assures that the educational intervention remains relevant and related to desired educational goals. Evaluation should not be limited to a single measure of changes in perceived knowledge, but should include measures of the quality of the projects produced.

ONLINE RESOURCE URL (OPTIONAL): <a href="http://boisevacoe. org">http://boisevacoe.org

\section{IT TAKES A VILLAGE: AN INTERDISCIPLINARY APPROACH TO INTEGRATING WOMEN'S HEALTH INTO RESIDENCY EDU- CATION}

Janet Henrich $^{1}$; Luz S. Vasquez ${ }^{1}$; Tracy Rabin ${ }^{2}$; jacqueline satchell ${ }^{1}$; Mukta Dhond ${ }^{1}$; susan maya ${ }^{1} .{ }^{1}$ Yale University, New Haven, CT; ${ }^{2}$ Yale University School of Medicine, New Haven, CT. (Control ID \#3180068)

NEEDS AND OBJECTIVES: In response to identified gaps in residency education in women's health (WH), we developed and implemented a series of ongoing WH education modules. The goals are to: develop an interdisciplinary approach to $\mathrm{WH}$ education by partnering with other disciplines and members of the community; introduce topics at the interface of medicine with other fields, or not covered elsewhere in the curriculum; and create a learning environment that fosters the development of a cadre of interdisciplinary WH educators.

SETTING AND PARTICIPANTS: Modules are directed at all 209 residents from the Yale Traditional, Primary Care and Med-Peds Residency Programs and presented according to a rotating schedule in half-day sessions,
8 times a year. PGY1 through PGY4 residents are exposed to all modules over 4 years in protected educational time during ambulatory block rotations. A core group of general medicine faculty are the primary teachers. Partner faculty include community panelists (transgender community leaders) and representatives from Family Planning, Urogynecology, Radiology and Endocrinology. DESCRIPTION: Four discrete modules supplement the WH content in residents' core ambulatory curriculum and online WH resources. Each module is thematic using interactive teaching methods (practicums/debates/cases/ videos/community panels). Residents are assigned key readings before each module. Senior residents serve as debate and discussion leaders. Modules include: From contraception to menopause: an evidence-based approach to caring for women across the reproductive life span (menopausal symptoms, contraception options, pelvic and breast exam practicum). Clinical controversies in women's health: using best evidence to partner with patients around shared clinical decision-making (breast cancer screening, preconceptual care, abnormal uterine bleeding). Clinical guideline updates (osteoporosis and cervical cancer screening and management). From anatomy to identity (the difficult pelvic exam and trauma focused care, urinary incontinence, transgender identity).

EVALUATION: Residents complete an anonymous pre and post selfassessment of their mastery of each topic (on a continuous scale of I know nothing about this subject to I can teach this module). Mastery increases uniformly from the lower to the upper end of the continuum with varying degree, depending on the topic. The modules are highly rated by residents compared with other education sessions.

DISCUSSION / REFLECTION / LESSONS LEARNED: The WH modules allow us to enhance the education of all residents in areas under their purview but in which they have little mastery. Many areas cross disciplines, and we structured the modules to highlight and model the central role of general medicine faculty in managing these topics with the appropriate use of consultants. Involvement of community members is a powerful way to make residents aware of disparities in care and engage them in advocacy. Residents, faculty and community members are all teachers and learners in this model adding to a growing cadre of WH educators.

\section{JOINING FORCES: A NEW COURSE BRIDGING STUDENTS' PASSION FOR SOCIAL JUSTICE WITH RUSH'S EQUITY MIS- SION}

Ritika Dhawan ${ }^{2}$; Coralie Pardo ${ }^{2}$; Paul Kent² ${ }^{2}$ Laura Deon $^{2}$; David Ansell ${ }^{1,2}$; Danesha Lewis ${ }^{2}$; Elizabeth S. Davis ${ }^{1,2}$. ${ }^{1}$ Rush University Medical Center, Chicago, IL; ${ }^{2}$ Rush Medical College, Chicago, IL. (Control ID \#3186133)

NEEDS AND OBJECTIVES: We started an elective for first year medical students, the Health Equity and Social Justice Leadership elective, to connect students passionate about social justice to Rush University Medical Center's health equity strategy. Learning objectives: Identify social and structural factors that affect the health of a community Use data to identify an actionable community or population health problem. Learn how to partner with community organizations Design an evaluation plan for a community health intervention

SETTING AND PARTICIPANTS: A Rush Medical College elective for 20 first year medical students.

DESCRIPTION: The Health Equity and Social Justice Leadership elective was started in the 2017/18 academic year. The fall session includes ten sessions covering key domains of social medicine. Teaching methods include site visits, speakers with lived experience, interactive sessions, and key readings. In the spring semester, the students break into groups, each of which partner with a community organization. The groups use community 
engagement, data analysis, and quality improvement tools in their work on the projects. Each group is mentored by one of the faculty members. For the 2018/19 academic year, we incorporated feedback from students who took the course the first year. This included expanding site visits and mentoring, adding a course coordinator, and using an application system instead of a lottery for selecting students.

EVALUATION: Students were evaluated using an anonymous survey. Questions reflected learning objectives, clarity of expectations, and feedback on class sessions. For the 2018/19 academic year, we also added a pre-course questionnaire exploring student interests and baseline knowledge. For the first year of the elective, there were 20 slots in the elective. Sixty one students ranked this elective, with 34 students ranking it first on their elective list. For the second year of the elective we required short applications and 40 students applied to the elective. For the 2017/18 academic year, all students who took the course said they would recommend the course to incoming students. We received feedback that the course could be better organized and that there was not sufficient mentoring. Overall, students were positive about the class with comments about how this course was the highlight of their year and was inspiring to them. Of the four projects started during the course, one has become a student led service learning program for all students at Rush. Two others are actively ongoing even though the class ended during the last academic year.

DISCUSSION / REFLECTION / LESSONS LEARNED: Health equity is an interest and priority for medical students. Through this course, we were able to connect students to community leaders in meaningful ways. We learned the value of soliciting feedback from students often and changing the class based on that feedback. Student leadership has been key to this course from its inception. We learned that it was important to have sufficient faculty and course coordinator time to run the course effectively.

\section{LEARNERS WHO STRUGGLE IN MEDICAL EDUCATION: COM- MON PRESENTATIONS}

Melis G. Celdirr ; Alexander Steeves-Fuentes ${ }^{4}$; Antoinette Schoenthaler ${ }^{3}$; Paul B. Yellin ${ }^{4,5}$; Mack Lipkin ${ }^{2} .{ }^{1}$ Mayo Clinic, Rochester, MN; ${ }^{2}$ New York University School of Medicine, New York, NY; ${ }^{3}$ NYU School of Medicine, New York, NY; ${ }^{4}$ New York University School of Medicine, New York City, NY; ${ }^{5}$ The Yellin Center for Mind, Brain, and Education, New York City, NY. (Control ID \#3185900)

NEEDS AND OBJECTIVES: It is common for students to struggle in the challenging academic and social environments of medical education. We posit that some who struggle are not simply the edge of a Gaussian distribution of success but present definable syndromes which are recognizable, testable, and remediable. In this first heuristic look at our hypothesis, we aimed to identify prominent learning profiles and early signs of struggle among medical students with poor academic performance and measurable factors that correlate with their struggles.

SETTING AND PARTICIPANTS: Ninety medical students with academic struggles were referred to a learning assessment center between 2006 and 2018. Reports of the assessment process were constructed to provide remediation strategies for students and educators.

DESCRIPTION: We evaluated reports of the comprehensive neurodevelopmental evaluations. Reports documented referral concerns, students' self-assessments of their competencies, academic history, neuropsychological evaluations and interviews in the assessment process to establish learning profiles of strengths and challenges of each student and recommended remediation strategies. We applied grounded theory methods to identify common patterns in students' comprehensive evaluations.
EVALUATION: Students who displayed signs of struggle earlier in medical school commonly presented after poor performance on standardized assessments $(63 \%, 50 / 80)$. They had historically excelled in academic areas rewarding intuitive ways of learning and chosen academic degrees that played to their strengths. In medical school, rote memorization and passive study methods such as transcribing lectures, without active information processing led to inefficient learning, requiring more time for their studies. They lacked strategies to plan their studies and exams, regulate their attention, filter and systematically store information of salient details. Students experienced high rates of anxiety and depression $(41 \%, 37 / 90)$ and some received psycho/ pharmacotherapy $(27 \%, 24 / 90)$. Social isolation and feelings of inadequacy further exacerbated their struggles.

DISCUSSION / REFLECTION / LESSONS LEARNED: Lack of learning and test-taking strategies appropriate for the unique and standardized medical school curriculum, combined with psychosocial stress in a competitive learning environment, expose challenges which may have been unnoticed by students in prior academic pursuits. Their patterns of presentation might alert instructors and students to seek evaluation and assistance early, receive guidance and remediation of specific learning challenges and avoid distressing, diminished academic performance.

\section{LEAVE NO DISCHARGE BEHIND: A TRANSITIONS OF CARE PILOT PROJECT}

Neha Jindal; Seagram M. Villagomez. New York University, New York, NY. (Control ID \#3183535)

NEEDS AND OBJECTIVES: Transitioning patients safely from the inpatient to the outpatient setting is crucial to patient outcomes and readmission rates, but residents receive little formal training to manage this. Transitions are fraught with issues, including poor communication of discharge instructions and a lack of timely follow-up with the primary care provider (PCP). In addition to the impact on patient outcomes, unsuccessful transitions have been shown to evoke strong negative reactions in student learners. We aim to improve resident education and confidence in managing transitions of care through the implementation of a Transitions of Care (TC) Team at the NY campus of the VA New York

Harbor Healthcare System (NYVA NYHHS).

SETTING AND PARTICIPANTS: Our study is taking place at NYVA NYHHS. The TC Team consists of the discharging hospitalist, NYU Internal Medicine residents, and a nurse practitioner. The patients are those who are admitted with a diagnosis of CHF or COPD.

DESCRIPTION: This pilot study sets up a TC Team involving medical residents to provide experiential learning in safe and successful conversion to the outpatient setting. Just prior to the discharge of a patient, an inpatient resident caring for the patient contacts the TC Team to provide a warm handoff. The patient is then scheduled for a follow-up home visit within 1 week. During the home visit, the patient is examined and assessed by the TC Team, medications are reviewed and follow-up instructions are discussed. Prior to completing the home visit, the TC Team resident contacts the discharging hospitalist who provides precepting and education. The TC team resident then communicates with the patient's PCP.

EVALUATION: We are studying the impact on readmissions and resident confidence in discharge planning and follow-up. The quantitative outcome measure is pre / post intervention thirty-day readmission rates for CHF and COPD. Qualitative outcomes collected through survey data include resident confidence in the discharge process: the ability to provide clear follow-up discharge planning, conduct a post-hospital discharge follow-up visit, perform accurate medication reconciliation, and reduce medical errors after discharge 
from the hospital. We are also looking at resident satisfaction with educational experience within the TC Team model.

DISCUSSION / REFLECTION / LESSONS LEARNED: Discharge from the hospital is one of the most complex and vulnerable points in a patient's care. Currently, there is wide variation in resident confidence and guidance during transition to outpatient care. The TC team has created an opportunity for residents to see early outcomes of discharge, particularly how seemingly small details can have great impact on the patient's ability to manage their disease independently. Frequently patients need additional intervention and counseling at the home visit. Residents report satisfaction in care and heightened connection to patients when they see them in the hospital and again in the home setting. Most importantly, they feel this experience strengthens their discharge skills.

\section{LESSONS LEARNED IN CLERKSHIP INNOVATION: A QUALITA- TIVE STUDY OF THE DESIGN AND IMPLEMENTATION OF LONGITUDINAL INTEGRATED CLERKSHIPS}

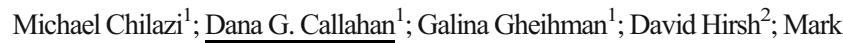
A. Schuster ${ }^{3}$. Harvard Medical School, Boston, MA; ${ }^{2}$ Harvard Medical School, Cambridge, MA; ${ }^{3}$ Kaiser Permanente School of Medicine, Pasadena, CA. (Control ID \#3184593)

NEEDS AND OBJECTIVES: Longitudinal Integrated Clerkships (LICs) offer an opportunity to advance health equity through medical education reform. LIC students participate in the comprehensive care of patients over time, maintain continuing learning relationships with preceptors, and meet clinical competencies across disciplines simultaneously. Compared to traditional block rotations, LICs are more likely to cultivate patient-centeredness, promote work in health advocacy, and address community needs. Despite the growing number of LICs nationally and internationally, the current literature lacks an empirical review of best practices in LIC implementation. Our study aims to fill this gap in order to provide a resource to medical educators and institutional leadership contemplating, implementing, or expanding LICs.

SETTING AND PARTICIPANTS: We interviewed LIC leaders (clerkship directors, discipline directors, administrative coordinators) across the United States, Canada, and the United Kingdom.

DESCRIPTION: Using convenience sampling, we conducted in-depth, semi-structured interviews with leaders of established LIC programs to explore experiences related to designing and implementing LICs.

EVALUATION: We interviewed 29 participants representing 20 LICs. We performed qualitative thematic analysis, which identified 4 key challenges in LIC implementation and possible solutions: (1) Administration: facilitating continuity between learners and patients requires administrative support and technological solutions; (2) Buy-in: outcomes data and shared professional values promote engagement of key stakeholders; (3) Comparability: rigorous program evaluation enables demonstration of comparable or improved outcomes for learners and patients; and (4) Growth: LICs face structural and cognitive barriers to expansion. Our analysis also highlighted that LICs meet key goals for modern undergraduate medical education by: (1) Providing meaningful continuity with patients and providers; (2) Integrating curricula related to community engagement and systems improvement; and (3) Addressing workforce needs of underserved communities.

DISCUSSION / REFLECTION / LESSONS LEARNED: Program leaders experienced similar challenges during the design and implementation of LICs, highlighting future opportunities for research towards shared solutions. Rogers' theory of "Diffusion of Innovation" provides a framework for organizing these challenges and identifying LIC features that promote successful adoption and spread.
LET'S TALK ABOUT THE FUTURE: RESIDENT COMFORT AND EXPERIENCE WITH OUTPATIENT ADVANCE CARE PLANNING

Kaylan Christianer. Columbia University Medical Center, New York, NY. (Control ID \#3181317)

NEEDS AND OBJECTIVES: Advance care planning (ACP) allows patients to communicate their goals for future care and appoint surrogate decision makers. Holding these discussions early in the outpatient setting allows for comprehensive planning. Prior studies have identified several barriers in accomplishing outpatient ACP, notably lack of time and provider training. However, in 2016 the Centers for Medicare and Medicaid Services added payment codes to reimburse for ACP conversations, incentivizing their integration into routine primary care. While many residency programs have implemented curricula to improve residents' comfort delivering bad news and discussing goals of care with inpatients, these skills do not necessarily translate to the outpatient setting. This project seeks to assess resident experiences facilitating outpatient ACP which can guide development of future curricula.

SETTING AND PARTICIPANTS: Second- and third-year Internal Medicine residents on a 2-week ambulatory rotation at NewYork-Presbyterian Columbia resident clinic serving a largely Spanish-speaking Medicaid patient population.

DESCRIPTION: Participants were invited to complete a survey assessing their comfort facilitating care planning, as well as their familiarity with common documents. They were also asked to quantify their prior experience facilitating ACP among their outpatient panel, and to identify motivations and barriers to initiating these discussions.

EVALUATION: Preliminary results from 17 participants (100\% response rate) reveal residents are significantly more comfortable addressing care planning with inpatients as compared to outpatients (mean 4.2 vs. 2.9 out of $5, \mathrm{p}<0.001)$. Participants had variable comfort with common documents including Out-of-Hospital DNR/DNI (mean 3.9 out of 5), NY State Health Care Proxy (mean 3.4), MOLST/POLST (mean 3.4), and Living Will (mean 2.4). A majority (76\%) of residents report discussing ACP with less than $10 \%$ of their total outpatient panel, and only $17 \%$ of residents report discussing ACP with at least half of their "very old or seriously ill" outpatients. Common barriers identified included lack of time (82\%), lack of access to forms (41\%), inability to implement (29\%), lack of training or experience $(24 \%)$ and language barriers (24\%).

DISCUSSION / REFLECTION / LESSONS LEARNED: This assessment reveals an important discordance in residents' ability to conduct care planning in the outpatient as compared to inpatient setting, as well as low rates of addressing ACP among resident panels.

LIFELONG LEARNING FOR FACULTY: THE CREATION OF A CLINICAL RESEARCH USING SECONDAY DATA COURSE AT AN ACADEMIC CENTER

Halle G. Sobel ${ }^{1}$; Abigail Crocker ${ }^{2}$; Charles D. MacLean ${ }^{2}$; Benjamin Littenberg ${ }^{2} .{ }^{1}$ University of Vermont Medical Center, Burlington, VT; ${ }^{2}$ University of Vermont, Burlington, VT. (Control ID \#3181461)

NEEDS AND OBJECTIVES: 1. Deans and department chairs at academic medical centers want to increase academic productivity at their institutions. A faculty development course in designing and implementing clinical research can help achieve this goal. 2. Faculty at academic institutions are surrounded by secondary data through electronic medical records (EMRs) and can benefit by learning how to use this data for scholarly research. 3. Clinicians who better understand and perform clinical research can promote improved quality and 
safety at their institution and beyond. EMR data is often under-utilized and learning about such data can promote both scholarly work and improved clinical care.

SETTING AND PARTICIPANTS: Clinical faculty members at The University of Vermont Medical Center were offered an opportunity to enroll in a 10-month faculty development course led by a team of 2 physician researchers, and 3 epidemiologists/ entitled Introduction to Clinical Research Using Secondary Data. Participants from Medicine, Surgery, Family Medicine, Pediatrics, Psychiatry, Dermatology, Radiology, Emergency Medicine, Pathology and Hospital medicine completed the course over the first three years, 2015-2018, in three cohorts of 15-19 particpants.

DESCRIPTION: The course content focused on an analysis of secondary data rather than primary data collection because of timely data availability and to promote use of data from our EMR. The curriulum included the topics of developing a reserach question, searching the medical literature, navigating the IRB process, understanding the data extraction process and communicating with data analysts, selected topics in statistics, and how to summarize and present findings in abstract and manuscript format. Each participant was asked to develop a research question and were paired with both a faculty researcher (one of the course teachers) as well as a statistician.

EVALUATION: Each year we completed pre and post student course evaluations. Students rated the experience very positively with $91 \%$ noting that the time commitment was "just right" and $100 \%$ agreeing or strongly agreeing that they would recommend the course to a colleague. Student confidence in defining a research question increased significantly in a prepost evaluation with over $84 \%$ reporting being "confident or very confident" at the end of the course, an increase of $25 \%$ from the baseline ( $p<0.01$ ).

DISCUSSION / REFLECTION / LESSONS LEARNED: A clinical reserch course led by experience faculty for faculty without experience in clinical research is a successful strategy to increase the output of research, QI and patient safety projects at an academic institution. By coordinating institutional resources to be part of a 10-month curriculum, faculty can better learn both the process of clinical research and what resources are available at their institution. The teaching of faculty can help clinicians better understand their practice, be more engagment and better understand how to use secondary EMR data.

\section{LONGITUDINAL ASSESSMENT OF PERSONAL AND PROFES- SIONAL DEVELOPMENT COMPETENCIES AT THE PRITZKER SCHOOL OF MEDICINE}

Hannah D. Caldwell $^{1}$; Christopher Mattson ${ }^{3}$; James N. Woodruff ${ }^{1,2}$; Wei Wei

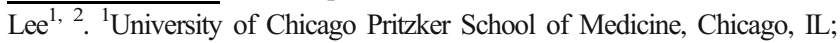
${ }^{2}$ University of Chicago, Chicago, IL; ${ }^{3}$ University of Chicago Medicine Comer Children's Hospital, Chicago, IL. (Control ID \#3175503)

NEEDS AND OBJECTIVES: While the Association of American Medical Colleges named Personal and Professional Development (PPD) a foundational competency for medical students in 2013, few studies have examined how best to teach and assess PPD skills. Our study aims to 1) analyze results of a biannual PPD self-assessment survey administered to the Pritzker School of Medicine Class of 2018 and 2) use results to identify areas for future interventions.

SETTING AND PARTICIPANTS: Following a literature review, we developed the Personal and Professional Development Skills Assessment Survey to assess PPD proficiency and attitudes. The optional survey was emailed to the student body every six months, Fall 2014 to Spring 2018. The analyzed cohort were students who matriculated Fall 2014 as well as those joining this cohort following time off.
DESCRIPTION: The 46-item survey consisted of 44 five-point Likert-scale items assessing personal $(n=15$, i.e. coping skills), professional $(n=15$, i.e. teamwork), and academic ( $\mathrm{n}=9$, i.e. study skills) development, as well as attitudes ( $\mathrm{n}=5$, i.e. reflection is important to my personal development) and 2 open-ended questions. (i.e. list three personal development goals). The Likertscale responses were dichotomized with proficient defined as 4. Descriptive statistics were used to summarize the findings over eight timepoints (S1-S8) and compare preclinical (S1-S4) and clinical (S5-S8) responses.

EVALUATION: A total of 93 students completed at least one survey. Response rates ranged from 38\% at S6 (Spring 2017, second half of third year clerkships) to $91 \%$ at S2 (Spring 2015, second half of first year). Within each domain (Personal, Professional, Academic), scores were reported as the percentage of total items rated as proficient. The Personal Domain score significantly declined from S2 to S3 $(79 \%$ to $72 \%, \mathrm{p}<0.01)$ at the transition from first to second year. The lowest Personal Domain score of $72 \%$ occurred at the start of second and third year (S3 and S5) and peaked at $84 \%$ at the end of fourth year (S8). The Professional Domain score increased significantly between the start of clerkship year to the half-way point (S5 and S6, 83\% to $89 \%, \mathrm{p}<0.01)$. The Professional Domain score was lowest throughout first year (79\%, S1 and S2) and highest throughout fourth year (92\%, S7 and S8). The Academic Domain score showed no statistically significant changes between any consecutive time points. When comparing PPD proficiency in preclinical (S1-S4) and clinical (S5-S8) phases, there were significant increases in Professional $(79.7 \%$ to $86.4 \%, \mathrm{p}<0.01)$ and Academic $(76.1 \%$ to $81.4 \%$, $\mathrm{p}<0.01)$ Domains. The Personal Domain did not differ significantly $(76.3 \%$ to $76.0 \%)$.

DISCUSSION / REFLECTION / LESSONS LEARNED: Our study used a longitudinal self-assessment to measure students' PPD skill attainment. While we found that personal development skills were lowest during critical transitional periods, these same transition points may offer opportunities for professional and academic growth.

\section{LONGITUDINAL RESIDENT COACHING IN THE AMBULATO- RY SETTING: A NOVEL INTERVENTION TO IMPROVE CLINI- CAL SKILLS}

Ryan Graddy; Stasia Reynolds; Scott Wright. jhusom, Baltimore, MD. (Control ID \#3180277)

NEEDS AND OBJECTIVES: Direct observation of residents' clinical practice is essential for accurately assessing their skills and to facilitate specific feedback. Opportunities to do this are infrequent, especially in busy outpatient teaching practices. We aimed to assess the effects of a longitudinal direct observation and coaching intervention on residents' clinical performance in an academic GIM practice.

SETTING AND PARTICIPANTS: The Johns Hopkins Bayview GIM Practice is a mixed faculty/resident practice. Although faculty preceptors are encouraged to oversee residents in the presence of their patients, direct observation of complete patient visits is rare.

DESCRIPTION: All 16 interns in a single IM residency class were randomized to the coaching (intervention) or control group at the start of the 2017-18 academic year. Two faculty members with extensive coaching experience performed 3 complete visit observations over the course of internship year for the 8 trainees in the coaching arm. Faculty used a 23 item "yes/no" behavioral checklist, based on tenets of clinical excellence, during the direct observation to focus their surveillance and to facilitate targeted feedback and coaching to residents after each visit. At the end of the academic year, 15/16 interns had a complete patient visit in the practice videotaped. Each video was viewed by 2 senior generalist clinician-educators blinded to the interns' 
intervention or control group assignment. These evaluators scored each encounter using the same behavioral checklist and assigned each resident a letter grade (A-F) as an overall evaluation. The proportion of each behavior performed was assessed with Chi-square test and differences in the 2 groups' overall performance of behaviors on the checklist was assessed with student ttest.

EVALUATION: Coached interns completed an average of 21/23 behaviors as graded by expert observers, while uncoached interns completed 18 $(\mathrm{p}=0.046$ ). The average overall grade for coached interns was $\mathrm{B}+$ and for uncoached interns it was B-. While no individual checklist items were performed statistically significantly more often by either group (all $\mathrm{p}>0.05$ ), 11 of the 23 behaviors were more regularly executed among those who had been coached.

DISCUSSION / REFLECTION / LESSONS LEARNED: Episodic longitudinal direct observation and coaching by experienced preceptors was associated with improved clinical performance in real patient encounters among IM interns. Previous work has shown that this type of coaching is both appreciated by learners and useful for identifying blind spots in residents' self-assessment. Longitudinal direct observation and coaching, while timeintensive, is valuable for residents' clinical growth and development.

\section{MAKING THE VOICES OF FEMALE TRAINEES HEARD: YEAR DATA}

Lisa Rotenstein $^{1}$; Abeba Leveille ${ }^{1}$; Rebecca Berman ${ }^{2}$; Joel T. Katz ${ }^{1}$; Maria Yialamas ${ }^{1}{ }^{1}$ Brigham and Women's Hospital, Boston, MA; ${ }^{2}$ University of California, San Francisco, San Francisco, CA. (Control ID \#3185810)

NEEDS AND OBJECTIVES: While there is increasing awareness of gender-based discrepancies at a faculty level, there is less understanding of how discrepancies apply to trainees. After discovering a potential discrepancy in residency-wide, ad-hoc recognition (via email "shout-outs") of publications for male versus female trainees by program leadership as well as a perceived differential willingness of male trainees to share publishing accomplishments with residency leadership, we developed and implemented a systematic process to identify and publicize resident publications. We analyzed the year 1 data to determine any differences in publication number of female vs. male residents.

SETTING AND PARTICIPANTS: This project was conducted in the Internal Medicine Residency Program at Brigham and Women's Hospital, an academic medical center affiliated with Harvard Medical School.

DESCRIPTION: In January 2018, we adopted a process by which our residency administrative support personnel systematically search for resident publications each month via a structured query of PubMed and our hospital's compilation of lay press articles by affiliates. We also developed an online submission form for residents to notify us of publications. Our program directors then email results to the entire residency monthly.

EVALUATION: We assessed the number of publications identified as authored by male vs. female authors and the distribution of publication type (journal article vs. lay article) by gender in the one year after our program's implementation. We compared this to the gender distribution of publication shout-outs in the six months prior to our program's initiation. Between January and December 2018, there was no difference in the proportion of publications authored by males versus females $(\mathrm{n}=86(47.3 \%)$ by females, $\mathrm{n}=96(52.7 \%)$ by males, $\mathrm{p}=0.51) .10$ of $24(41.7 \%)$ lay press articles and 76 of $158(48.1 \%)$ of journal articles were published by female authors; there was no significant difference in article type by gender. In contrast, only $1 / 6(16.7 \%)$ of email shout-outs in the six months prior to program implementation were for female publications.
DISCUSSION / REFLECTION / LESSONS LEARNED: We demonstrated that systematic search and dissemination of trainee publications can eliminate gender-based disparities in recognition of academic accomplishment. Through the process of implementing this program, we identified that differential self-promotion habits start early in training and are more pronounced in female trainees. The presence of a program leadership committed to advancement of female physicians and willing to engage constructive improvement was key to this program's success.

MASSACHUSETTS GENERAL HOSPITAL WOMEN IN MEDICINE TRAINEE'S COUNCIL: AN INNOVATIVE MODEL FOR FEMALE TRAINEE SUPPORT, ADVOCACY AND PROFESSIONAL DEVELOPMENT

Ana Sofia Warner; Emily Lau; Nneka Ufere; Varsha Tanguturi; Katrina Armstrong; Nancy A. Rigotti; Doreen DeFaria Yeh. Massachusetts General Hospital, Boston, MA. (Control ID \#3185708)

NEEDS AND OBJECTIVES: The Massachusetts General Hospital (MGH) is the largest hospital in New England, and is recognized as a pioneer in medical innovation, healthcare delivery, and workforce education and development. There are 386 residents and fellows within the Department of Medicine, $44.8 \%$ of whom are female. The MGH supports the professional advancement of women physicians through initiatives including the Office for Women's Careers and dedicated funding for early career female physician researchers. In response to a recognition that similar resources were lacking for trainees, the MGH Women in Medicine Trainee's Council (WIMTC) was developed in 2016 with the goal of supporting the personal and professional development of women trainees at MGH within the Department of Medicine.

SETTING AND PARTICIPANTS: The WIMTC consists of a core group of 30 trainees and 20 faculty advisors. The council is made up of five working groups focused on career development and mentorship, advocacy, recruitment, research and work-life balance. The council meets monthly to discuss goals, progress and future programming.

DESCRIPTION: The council is funded through the Department of Medicine with an annual budget of approximately $\$ 5000$. To-date the council has sponsored dinners with notable female physicians, travel grants to fund childcare for trainees travelling to conferences, negotiation and micro-aggressions workshops, mock fellowship interviews, and networking events with faculty. The council has successfully lobbied for improvement in hospital lactation spaces and is working to improve access to childcare services for trainees.

EVALUATION: This coming year the WIMTC plans to begin evaluating our initiatives and workshops to assess the impact that these sessions have on trainees preparedness and to identify areas for improvement.

DISCUSSION / REFLECTION / LESSONS LEARNED: Since its implementation in 2016, the WIMTC has become a sustainable model for female trainee professional development at MGH. The WIMTC has improved female trainees' access to important professional development activities such as networking, negotiations training and mock interviews. Additionally, the council provides a mechanism for fellows and residents to collaborate on initiatives that cross departmental lines. These types of hospital-wide initiatives provide a leadership opportunity for the trainees involved in the council. Perhaps most sustainable is the sense of community that the Council has created among women trainees and involved faculty. 


\section{MASTERING CO-MANAGEMENT: A CURRICULUM FOR HOSPITALISTS}

Aron Mednick $^{1}$; Anne Dembitzer ${ }^{2}$; Adin Nelson ${ }^{4}$; Shreya P. Trivedi ${ }^{3}$; Anand Viswanathan ${ }^{1} .{ }^{1} \mathrm{NYU}$ Langone Health, New York, NY; ${ }^{2} \mathrm{NYU}$ School of Medicine, New York, NY; ${ }^{3}$ NYU, New York, NY; ${ }^{4}$ Rutgers New Jersey Medical School, Newark, NJ. (Control ID \#3184475)

NEEDS AND OBJECTIVES: The U.S. surgical population is becoming increasingly medically complex, increasing the risk of post-operative complications. Surgeons have traditionally consulted internists and subspecialists for medical management during inpatient admissions, but this has failed to decrease complications or healthcare costs. To address this issue hospitalists have taken on the role of co-managing patients admitted to surgical services. However, internal medicine residencies don't adequately prepare trainees for this role. Safe and effective modern medical care requires training in medical co-management (MCM), yet there exists no robust theory- and evidence-based curricula to teach these competencies. We designed a curriculum to fill this need.

SETTING AND PARTICIPANTS: Thirteen hospitalists with 0-7 years' clinical experience in an academic medical center in New York, NY. Hospitalists spend $50 \%$ of their clinical time on an MCM service covering general surgery, vascular surgery, neurosurgery, general neurology/ epilepsy, and orthopedic surgery.

DESCRIPTION: We developed a yearlong curriculum based on the Society for Hospital Medicine's guidelines on creating MCM teams and other published frameworks. We applied evidence-based learning theories including: Adult Learning Theory, Cognitivism, Constructivism, and Ericsson's Theory of Expertise. We developed a conceptual framework incorporating key stakeholders in MCM (medical attending, surgical attending, PCP, mid-levels, and patients/families) and 6 core content topics (Roles \& Responsibilities, Communication Strategies, Trust \& Respect, Common Complications, Transitions of Care, and Deliberate Practice). The curriculum includes two parts: a week-long intensive orientation with Objective Structured Clinical Examinations (OSCEs) and workshops in the main content areas, and a series of monthly continuing professional development (CPD) sessions to facilitate deliberate practice and improve skills needed to manage niche patients on surgical services. These CPD skills are based upon needs identified by the hospitalists and surgical teams.

EVALUATION: Effectiveness will be measured at the program and hospitalist level. The program will be assessed by patient quality metrics (eg, LOS and readmission rates) and satisfaction by the surgical teams yearly. We will evaluate the impact on hospitalists using retrospective prepost surveys to measure changes in clinical knowledge, confidence and engagement. Assessments will be collected using Qualtrics ${ }^{\circledR}$ survey software.

DISCUSSION / REFLECTION / LESSONS LEARNED: In the early stages of this curriculum, $79 \%$ of participants found the CPD sessions "very or extremely helpful." Participants have shown increasing engagement in the curriculum as evidenced by proposals of future session topics and attendance. Data collection is ongoing.

\section{MAXIMIZE THE X+Y: LONGITUDINAL AMBULATORY THREADS}

Daniella A. Zipkin; Dinushika Mohottige; Lynsey Michnowicz; Aimee K. Zaas. Duke University, Durham, NC. (Control ID \#3185054)
NEEDS AND OBJECTIVES: The Accreditation Committee for Graduate Medical Education requires residency training programs (RTPs) to minimize tension between inpatient and outpatient responsibilities. However, RTPs struggle to balance these arenas while ensuring breadth and depth in internal medicine training. Many programs now use "X+Y" scheduling patterns to balance inpatient and ambulatory time. The Duke Internal Medicine Residency transitioned to a "4+2" schedule in 2016. In the fall of 2017 , we collectively engaged residents and faculty across all divisions in a review of the ambulatory education mission. Faculty and residents desired continuity in the ambulatory learning environment in order to achieve learning goals. Residents also requested a formal curriculum bridging clinical experience with didactics. In response, we developed the Ambulatory Threads model described here, allowing residents to experience continuity in the clinic environment in every subspecialty of medicine over the course of two years.

SETTING AND PARTICIPANTS: First and second year Duke internal medicine residents were included in the intervention, which was implemented in July 2018 and leveraged our broad base of faculty in all subspecialties.

DESCRIPTION: The Ambulatory Threads consist of weekly templates made up of grouped clinical sub-specialties such that each template contains 1-2 sessions with each subspecialty, along with continuity clinics, academic half day, and self-care half days. Templates remain consistent across all of the ambulatory blocks in a six-month period, allowing for continuity with sub-specialty faculty throughout that time. Residents migrate to a new thread every six months. Intern threads include "nephrology-rheumatology-endocrine" and "gastroenterology-infectious diseases", allowing early exposure in these fields which do not have inpatient ward services at Duke. The second year threads are "pulmonary- oncology", and "cardiology-hematology". Through a collaborative effort with education leads in all of the divisions, the academic half day content is matched to the clinical thread. In the academic half day teaching, all faculty are encouraged to utilize innovative educational strategies that optimize interactive, engaging learning techniques while meeting their subspecialty's core educational objectives.

EVALUATION: Of the 162 rotation evaluations sent out between July-October 2018, 122 were completed, for a response rate of $75 \%$. Feedback has been tremendously positive, noting an enriching learning environment enhanced by a strong faculty commitment to teaching. Constructive feedback regarding errors in the complex schedule and geographic locations of clinics have been addressed in revised templates.

DISCUSSION / REFLECTION / LESSONS LEARNED: Beyond simply implementing a 4+2 system with regularly spaced ambulatory time, linking together those ambulatory blocks with longitudinal exposure to multiple subspecialties has substantially improved the ambulatory educational experience at Duke. Faculty and residents have commended the improved learning environment.

MEDICAL STUDENT ATTITUDES TOWARDS INTERDISCIPLINARY INTERACTIONS WITH PHARMACY STUDENTS: A STUDENT-RUN FREE CLINIC PERSPECTIVE

Mark N. Alshak ${ }^{2}$; Jason Harris²; Alexandra H. Miller²; Pamela Charney ${ }^{1}$. ${ }^{1}$ Weill Cornell Medical College, New York City, NY; ${ }^{2}$ Weill Cornell Medicine, New York, NY. (Control ID \#3186032) 
NEEDS AND OBJECTIVES: The Weill Cornell Community Clinic (WCCC) has recently expanded early interprofessional exposure (IPE) for medical students by integrating pharmacy students and their supervising pharmacists into clinic practice. We decided to explore the impact of this program on the knowledge and attitudes of clinical medical students towards IPE while they rotated in WCCC for six weeks as part of their required Ambulatory Medicine clerkship.

SETTING AND PARTICIPANTS: The Weill Cornell Community Clinic (WCCC) is a medical student developed and run comphrensive primary care clinic that cares for uninsured New Yorkers with Medical, Women and Mental Health clinics. Within the Medicine clinic both clinical and preclinical medical students with attending Internal Medicine physicians provide care. Social workers and social work students have been integrated for years. Additionally, nutritionists and a Nurse Practioner educator have volunteered. More recently a Pharmacy school approached about providing on-site pharmacist evaluations within our medical practice.

DESCRIPTION: Using the pre-validated Student Perceptions of Physician-Pharmacist Interprofessional Clinical Education Instrument (SPICE-R2), surveys were distributed to third year medical students at the beginning and end of their 6-week rotation within the clinic. Attitudes towards interprofessional exposure were assessed on a 1-5 scale (1=strongly disagree, $5=$ strongly agree). Interprofessional exposure was provided in clinic where pharmacy students worked alongside medical students to provide medication reconciliation and counseling. Pre- and postintervention data was analyzed using 1-way ANOVA across categories and two-tailed paired t-tests within three categories based on SPICE-R2 designations: interprofessional teamwork and team-based practice, roles/ responsibilities for collaborative practice, and patient outcomes from collaborative practice. Twenty medical students were assessed.

EVALUATION: Overall SPICE-R2 score improved from 4.04 to 4.35 (7.67\% increase) $(\mathrm{p}=0.01)$. The biggest change in category score was observed in the overall roles and responsibilities section, which increased from 3.27 to 3.91 ( $19.5 \%$ increase) $(\mathrm{p}=0.0001)$ with the understanding of the course taken by and training requirements of other health professionals showing the largest area of improvement of any question with an improvement in score of $34 \%(\mathrm{p}=0.0058)$. Overall teamwork scores increased 4.5 to 4.7 (4.7\% increase) ( $\mathrm{p}=0.0026)$. Overall outcome scores did increase, although not significantly $(\mathrm{p}=0.62)$.

DISCUSSION / REFLECTION / LESSONS LEARNED: Early exposure for medical students to pharmacy students demonstrated improved teamwork and a better understanding of the roles, responsibilities, trainings, and courses required by other professions. Interprofessional exposure in medical school student run free clinics provides an excellent opportunity to foster interprofessional growth and collaboration.

\section{MEETING PATIENTS WHERE THEY ARE: ENHANCING COM- PETENCE IN ADDRESSING SOCIAL BARRIERS TO CARE THROUGH A MEDICAL STUDENT PATIENT NAVIGATOR PROGRAM}

Priscilla Wang ${ }^{1}$; Lorenzo R. Sewanan ${ }^{2}$; Eamon Duffy ${ }^{3}$; Matthew Meizlish ${ }^{2}$; Juliana Berk-Krauss ${ }^{4}$; Sophia Gamez ${ }^{2}$; Emily L. Coleman ${ }^{2}$; Pinar OraySchrom ${ }^{2}$. ${ }^{1}$ Brigham and Women's Hospital, Brookline, MA; ${ }^{2}$ Yale University, New Haven, CT; ${ }^{3}$ Johns Hopkins, Baltimore, MD; ${ }^{4}$ Mount Sinai, New York, NY. (Control ID \#3186291)

NEEDS AND OBJECTIVES: Research consistently demonstrates that health outcomes are determined largely by what happens outside of the walls of hospitals and clinics. Yet medical student training predominantly occurs in health care settings and focuses on addressing biological mechanisms of health. To be effective future clinicians, medical students must understand the context in which patients live and make health-related decisions. The objective of this medical student-initiated patient navigator program (PNP) was to utilize a service-learning model to provide medical students with the knowledge, skills and experience needed to help patients overcome social barriers to health.

SETTING AND PARTICIPANTS: Teams in New Haven, CT were formed, each with 2 Yale School of Medicine medical students (1 MS1, 1 MS4+) working with a preceptor (chief resident / attending) supervising multiple teams. In 2016-2017, 8 teams participated; in 2017-2018 the program expanded to 16 teams across 4 sites ( 2 primary care centers, a diabetes clinic, the Connecticut Mental Health Center).

DESCRIPTION: Recruitment of high-need patients (identified by a care provider) occurs in July and August, with one patient assigned to each team. Teams perform a health / social needs-related intake and form health goals with patients via shared decision making. From September to May, medical student patient navigators (PN) contact patients weekly and help them navigate the health care system and address socioeconomic barriers to health goals, via home visits, attending medical appointments, and other means. This service component is coupled with didactic sessions twice a month teaching means of addressing social determinants of health (e.g. food insecurity, motivational interviewing) and group solicitation of ideas to advance patient care.

EVALUATION: Pre- and post-intervention surveys were conducted assessing knowledge and skills relating to addressing social determinants of health, such as comfort level conducting a home visit and understanding how to obtain transportation to an appointment. Paired analysis demonstrated statistically significant improvement in the majority of areas assessed. PN rated the program very highly and clinicians reported improvements in patient care outcomes (e.g. decreased HgbA1C).

DISCUSSION / REFLECTION / LESSONS LEARNED: Patient navigation is a powerful means of teaching medical students critical skills for addressing social determinants of health. It enables students to regularly apply learning in context, while serving their community and increasing patient contact.

\section{MIND THE GAP: ENHANCING RESIDENT-DELIVERED FOR- MATIVE FEEDBACK}

Malavika Kapuria ${ }^{1}$; Shira A. Sachs ${ }^{2,}{ }^{1}$. ${ }^{1}$ Baylor College of Medicine, Houston, TX; ${ }^{2}$ Weill Cornell Medicine, New York, NY. (Control ID \#3185682)

NEEDS AND OBJECTIVES: Delivering feedback is challenging for clinicians at all levels. Residents face unique barriers to providing feedback given their limited training in this area and their near-peer status to fellow trainees. This has a significant impact on medical students, who rely on feedback from residents to further their professional development. Objectives: (1) Design an intervention to coach residents in providing effective feedback (2) Assess the impact of this intervention on the frequency and quality of resident-delivered feedback

SETTING AND PARTICIPANTS: Over a six-month period, 48 upperlevel residents and 96 medical students rotating on Internal Medicine wards at the Michael E. DeBakey VA Hospital were enrolled in the study.

DESCRIPTION: We designed a tool with a four-step model for giving feedback. The steps were as follows: setting (identifying a time and venue for feedback), assessment (soliciting the learner's self-assessment before providing feedback based on direct observation), action plan (assisting the learner in 
identifying steps for performance improvement), and summary (making arrangements for follow-up). Residents were randomized to receive one-onone coaching in using the feedback tool compared to standard preparation.

EVALUATION: Residents in the intervention and control groups completed pre-post surveys assessing self-competence in delivering feedback. Medical students who worked with residents enrolled in the study completed postsurveys assessing the frequency and quality of resident feedback. Trainees who completed assigned surveys, 33 of 48 eligible residents and 35 of 96 eligible medical students, were included in the final data analysis. Regarding the resident surveys, there was no significant difference in the reported frequency of feedback between intervention and control groups. There was a significant difference in self-rated competency setting expectations for feedback, with $83 \%$ in the intervention group reporting being "quite competent" or "very competent" in this domain compared to $47 \%$ in the control group $(\mathrm{p}=0.018)$. While there was no statistically significant difference in selfrated competency in providing formative feedback, there was a trend towards improvement with $76 \%$ in the intervention group reporting being "quite competent" or "very competent" in this domain compared to $57 \%$ in the control group. Regarding the student surveys, there was no significant difference in the reported frequency or quality of feedback between residents in the intervention or control groups.

DISCUSSION / REFLECTION / LESSONS LEARNED: The goal of our intervention was to improve formative feedback provided by residents to medical students. The intervention required a brief orientation session. Residents using the feedback tool reported higher rates of self-competency with regards to setting expectations around feedback, with a trend towards higher rates of self-competency in delivering feedback. Our study was limited by the small sample size of residents, and low survey response rates by medical students.

\section{MINI-GRAND ROUNDS: A CME SERIES FOR BUSY CLINICIANS} Lisa Kearns ${ }^{1}$; Sarah Jonaus ${ }^{1}$; Cynthia G. Kreger ${ }^{1}$; Neeraj H. Tayal ${ }^{2} .{ }^{1}$ Ohio State University, Columbus, $\mathrm{OH} ;{ }^{2}$ The Ohio State University Wexner Medical Center, Columbus, OH. (Control ID \#3186380)

NEEDS AND OBJECTIVES: As physicians graduate from residency and enter into clinical practice, they are faced with a deluge of new clinical questions. Similarly, those physicians who have been in practice for many years often times find it difficult to remain abreast with new updates or guidelines. While formal education series such as department wide Grand Rounds assist with continuing education, not all sessions are geared toward the busy practicing generalist. Other perceived barriers to continuing education include learning time, infrastructure as many of our physicians practice in different locations, and opportunity. Our objective is to create a sustainable continuing medical education series pertinent to busy clinicians.

SETTING AND PARTICIPANTS: The Ohio State University Wexner Medical Center Division of General Internal Medicine consists of 57 physicians and nurse practitioners who work in seven distinct office locations throughout Franklin County.

DESCRIPTION: The live conference is held at main practice location with Webex conferencing available to all clinic sites. These sessions are held during the lunch hour twice monthly and are approximately 30 minutes during. Each session answers a pertinent clinical question applicable to the practicing general internist. Sessions are delivered by general internal medicine faculty and are peer-reviewed. CME is awarded to those who attend live either in person or via Webex. Sessions are recorded for future viewing for faculty who cannot attend.
EVALUATION: Evaluations are sent to general medicine faculty immediately following each presentation. This provides opportunity for faculty presenters to receive feedback on their teaching. In addition, evaluations are used to track attendance and to receive suggestions for future topics. This is an ongoing project and data are currently being collected.

DISCUSSION / REFLECTION / LESSONS LEARNED: We have had preliminary success with implementing a succinct, general internist-focused CME series for a large group practice scattered at different locations. Not only does this series serve to keep faculty current without sacrificing their busy clinical practices, it also acts as a vehicle for peer-reviewed teaching to help with teaching portfolio development. Preliminary data collection suggest faculty find this series beneficial.

\section{MISTREATMENT BY PATIENTS: A PILOT CURRICULUM BASED ON RESIDENT EXPERIENCES}

Sally Namboodiri ${ }^{1}$; Timica Campbell ${ }^{2}$; Carolyn $\mathrm{Chan}^{3} .{ }^{1}$ Cleveland VAMC, Cleveland Heights, $\mathrm{OH} ;{ }^{2}$ Louis Stokes VA Medical Center, Cleveland, $\mathrm{OH}$; ${ }^{3}$ Metrohealth Medical Center, Cleveland, OH. (Control ID \#3138493)

NEEDS AND OBJECTIVES: Mistreatment of physicians by patients is a problem that contributes to burnout. Few educational curricula address this issue for physicians in training. This pilot project sought to characterize resident mistreatment by patients at a Veterans Affairs (VA) and an affiliated university (non-VA) hospital and develop a novel curriculum that provides tools to handle mistreatment.

SETTING AND PARTICIPANTS: One 60-minute session was held as a noon conference for PGY 1-3 internal medicine (IM) residents in two settings: VA and non-VA. VA attendees were $\mathrm{n}=10$ (male $=5$, female $=$ 5). Non-VA attendees were $n=16($ male $=12$, female $=4)$. Two IM physician educators were facilitators.

DESCRIPTION: Curriculum development was informed by VA residents' experiences with mistreatment. VA residents $(n=20)$ were asked about the prevalence, type, perpetrators and effects of mistreatment; based on this data, we developed an interactive, case-based curriculum related to mistreatment by patients with the following learning objectives: 1.Define resident mistreatment 2.Review the prevalence and forms of mistreatment 3.Discuss approaches to mistreatment by patients as a victim and bystander. Cases included unwanted sexual behavior, racial discrimination, and gender harassment. At the VA, large group discussions required residents to demonstrate use of tools from the literature to approach cases. Due to some attendees' reticence to speak out in this format, the non-VA residents were divided into small groups for case studies, which enhanced open discussions. We developed minute papers for pre- and post-sessions; Likert scales were used to determine mistreatment frequency and comfort level with response to mistreatment.

EVALUATION: Mistreatment frequency differed between settings: $70 \%$ of VA residents experienced mistreatment frequently or often and $30 \%$ experienced it sometimes, while $33 \%$ of non-VA residents experienced it sometimes or often and $67 \%$ only rarely or never. Pre-session, $70 \%$ of VA residents felt uncomfortable or very uncomfortable with their response to mistreatment while only $0-1 \%$ of non-VA residents felt uncomfortable or very uncomfortable. Post-session, $90 \%$ of residents at both sites felt comfortable or very comfortable handling mistreatment. Residents at both sites were very satisfied with the quality and effectiveness of the presentations. The most helpful aspects were tools reviewed in the session for VA residents and the small group discussions for non-VA residents.

DISCUSSION / REFLECTION / LESSONS LEARNED: Results suggest that IM residents were more likely to experience mistreatment by patients in VA compared to non-VA settings. This could be due to a growing mistrust 
of the VA by veterans or the disinhibitory effect of increasingly racist and sexist politics on the largely male veteran population. However, a lack of gender balance in the non-VA group may have led to lower responses. The high satisfaction levels post-session in both settings suggest that the curriculum was beneficial. Limitations included small sample sizes and lack of gender balance.

\section{MODIFIED PROBLEM-BASED LEARNING: AN INNOVATIVE AP- PROACH TO IMPROVE RESIDENCY EDUCATION, CRITICAL REASONING, AND COLLABORATION}

Megan A. McGervey ${ }^{1}$; Susan C. Scott ${ }^{1}$; Jamal H. Mahar ${ }^{1}$; Mohammad G. Mohmand $^{1}$; Carlos Isada ${ }^{2}$; Abby Spencer ${ }^{3} .{ }^{1}$ Cleveland Clinic Foundation, Lakewood, OH; ${ }^{2}$ Cleveland Clinic, Cleveland, OH; ${ }^{3}$ Cleveland Clinic, Chagrin Falls, OH. (Control ID \#3186277)

NEEDS AND OBJECTIVES: Adult learners require different teaching modalities that are not employed in residency programs. Large residency programs rely heavily on large group didactics for the delivery of educational material. However, retention rates following lectures has been shown to be very low. Problem-based learning (PBL) is a student-centered approach where students work in groups to solve a problem using an active and iterative process to identify what students know and, more importantly, what they don't know. While it has used in undergraduate medical education for many years, there are scarce reports of it being used in internal medicine residency programs as a longitudinal and primary curriculum. Our objective was to create a residency curriculum using adult learning theory that engages learners and allows for better knowledge retention.

SETTING AND PARTICIPANTS: This innovation takes place at a large academic medical center that hosts 181 internal medicine residents. All second and third year residents take part in the mPBL sessions that occur during an academic half day within protected learning time during the longitudinal clinic week using the $4+1$ block scheduling model.

DESCRIPTION: In this innovative Modified PBL structure, the focus lies on self-directed learning and discussion of discrete learning objectives (LOs) derived from clinical cases in the presence of a multidisciplinary team of experts. Our mPBL consists of two sessions that allow learners to become familiarized with the cases and set discrete learning objectives within small groups. The second session the following day revolves around discussion of LOs with active facilitation to ensure case based learning with input from specialists. The learning is led by residents, while faculty facilitation is centered on more concrete understanding of medical concepts as well as correct interpretation of information and literature. The small group discussions allow learners to make the transition from knowledge gathering to analysis, application and practical utility of knowledge with the ultimate goal of having the learner apply the assimilated knowledge into clinical practice.

EVALUATION: Twenty-nine surveys were collected from second year residents indicating that $>75 \%$ of participants "agreed" or "strongly agreed" that PBL had supplemented their education. Residents overwhelmingly enjoyed the content expert and the interactive case-based learning approach. Survey comments indicated that knowledge retention increased and that residents were made aware of knowledge gaps during discussion with their peers. Focus groups and survey data are currently being collected for this academic year.

DISCUSSION / REFLECTION / LESSONS LEARNED: Based on survey data, we modified to provide learners with cases well before sessions, adding suggested LOs, choosing high-yield cases spanning different disciplines, using multi-case formats with 4 cases per session, employing discrete learning objectives for the content expert discussion, and increasing the use of handouts and online learning materials.
NARRATIVE ASSESSMENT PROTOCOL (NAP) FOR MEDICAL STUDENTS IN A LONGITUDINAL INTEGRATED CLERKSHIP

Michael McShane ${ }^{1,2}$; Britta Thompson ${ }^{1}$; Daniel R. Wolpaw ${ }^{1} .{ }^{1}$ Penn State College of Medicine, Hershey, PA; ${ }^{2}$ Mount Nittany Medical Center, State College, PA. (Control ID \#3184883)

NEEDS AND OBJECTIVES: With the goal of achieving standardization and objectivity in assessments, educators have relied on methods that deconstruct observed behaviors into likert scale numbers. These values often fail to offer either meaningful feedback or the promised "objectivity". This has led to increased emphasis on narrative assessments, which in GME have demonstrated to reliably identify learners at risk, and provide authentic feedback. However, it has been challenging to get clinical faculty to do more than complete scales and write short, unhelpful narratives such as "good job" or "read more". Here, we describe our efforts to overcome these barriers through a narrative assessment protocol (NAP) that features faculty semi-scripted narrative prompts, student goal setting, calibration discussions, and faculty supported performance improvement plans.

SETTING AND PARTICIPANTS: Penn State College of Medicine recently launched a four-year curriculum track for 12 students based in State College, Pennsylvania, featuring a longitudinal integrated clerkship (LIC) in the second year. 4 months into this first clerkship iteration, we implemented the NAP.

DESCRIPTION: To construct the semi-scripted narrative prompts, we asked clerkship directors to identify feedback opportunities (FOs) in their disciplines. These discipline specific FOs were combined with two prompts to be completed in reference to the FO: (1) "I really liked the way you..." and (2) "One thing that you could to do take your performance to the next level is to..." Faculty were surveyed by email. Next each student filled out 1 FO and compared it to faculty statement. Then students met with the LIC director in a "Calibration Meeting", where this information was reviewed to recalibrate student and faculty observations about clerkship performance, and create an across clerkship goal. In a collaborative meeting, clerkship directors discussed how to best help the student fulfill their goal within each clerkship. To better understand the quality of the NAP, qualitative analysis of faculty narrative statements, student goals and student-specific performance improvement plan was performed.

EVALUATION: Response rate for faculty narrative statements was $77 \%$. Narrative statements were primarily behaviorally based with limited judgmental or non-specific feedback. Initially, student goals were about improving presentation and clinical reasoning. The "Calibration Meeting" helped to improve these goals and the subsequent clerkship director's discussion was characterized by supportive conversation and helpful recommendations.

DISCUSSION / REFLECTION / LESSONS LEARNED: Using a sequence of faculty-identified feedback opportunities, semi-scripted narrative prompts, calibrated SMART goals, and collaborative clerkship director input, we were able to create a meaningful combination of narrative feedback and refined goals for LIC students. We feel that this approach to capturing narrative faculty feedback and supporting student learning and professional growth in the clerkships is promising.

ONE STEP FORWARD AND NO STEPS BACK! NBME SUBJECT EXAM SCORES AND TRANSITION TO AN INTEGRATED CLINICAL CURRICULUM

Laura Zakowski; Kirstin A. Nackers; Raquel Tartar; Eileen Cowan; Katharina Stewart; Sarah E. Ahrens; David Tillman; Laura Jacques; Shobhina G. Chheda. University of Wisconsin School of Medicine and Public Health, Madison, WI. (Control ID \#3170282) 
NEEDS AND OBJECTIVES: Recent AAMC data shows that two-thirds of medical schools are currently undergoing or planning substantial curriculum changes. During periods of curricular change, it is desirable to track assessment outcomes to inform leadership of possible negative impacts to students during the transition. We used NBME subject exam scores and pass rates to study how moving from a traditional clerkship model to integrated clinical blocks affected student acquisition of medical knowledge.

SETTING AND PARTICIPANTS: The Legacy Curriculum at University of Wisconsin School of Medicine and Public Health followed the standard medical school educational model, including traditional, department-based, clinical clerkships that students entered after 2 years of preclinical basic science curriculum. The school transitioned to the ForWard Curriculum in 2016 and this cohort of students entered new integrated clinical experiences in January 2018 following 18 months of integrated preclinical basic science curriculum. We integrated our Neurology and Psychiatry clinical experiences with Internal Medicine in a new course entitled Acute Care. Family Medicine and Ambulatory Internal Medicine are integrated in Chronic and Preventative Care. OBGYN and Pediatric experiences are integrated in Care Across the Life Cycle. Surgery, Anesthesia, and other procedural specialties are integrated in Surgical and Procedural Care. Finally, we integrated clinically oriented basic science curriculum with the clinical experiences.

DESCRIPTION: We compared NBME subject exam scores and passing rates between the final cohort of Legacy M3 students and first cohort of ForWard students for Adult Ambulatory Medicine, Medicine, Neurology, OBGYN, Pediatrics, Psychiatry, and Surgery. Institutional passing thresholds were not changed.

EVALUATION: NBME scores and passing rates to date for the ForWard Curriculum cohort demonstrate no statistically significant differences from those of the Legacy cohort ( $>0.05$ ). We do not find substantive negative effects to student acquisition of medical knowledge during the transition period to the integrated curriculum.

DISCUSSION / REFLECTION / LESSONS LEARNED: Medical schools undergoing substantial curricular transformation can be reassured that integration of specialties and reduction of preclinical curricular time does not affect NBME subject exam scores. We will continue to collect data comparing the ForWard and Legacy curriculum students, including NBME Step 1 score comparison, to provide more information about how our integration affects acquisition of medical knowledge.

OPIOID CRISIS: DIVERSIFYING MEDICAL SCHOOL CURRICULA IN RESPONSE TO THE CRIES OF OUR COMMUNITIES Sandeep Kapoor ${ }^{1,2}$; Melissa Pawelczak ${ }^{2}$; Bruce Goldman ${ }^{3,}$; Laura Harrison $^{1}$; Lauren Block ${ }^{1,}{ }^{2}$; Joseph Conigliaro ${ }^{1,}{ }^{2}$; R. Ellen Pearlman ${ }^{2}$. ${ }^{1}$ Northwell Health, New Hyde Park, NY; ${ }^{2}$ Zucker School of Medicine at Hofstra/Northwell, Hempstead, NY; ${ }^{3}$ Northwell Health-Zucker Hillside Hospital, Glen Oaks, NY. (Control ID \#3185869)

NEEDS AND OBJECTIVES: We are amidst of a large-scale epidemic that impacts our patient population and communities with no sense of discrimination or short-term relief. The misuse and abuse of alcohol, illicit and prescribed opioids, has motivated important dialogue throughout the Nation, our State, our homes, our workplaces, our health system and medical school. Transparently inventorying health professional training, it is apparent that little time is dedicated to develop basic competencies, yielding discomfort, lack of training and experience, manifesting as barriers to addressing substance use. SETTING AND PARTICIPANTS: The newly designed curriculum was delivered to third year (MS3) students in an allopathic medical school during an inter-clerkship themed week.
DESCRIPTION: We developed and incorporated an 11-hour "opioid epidemic" themed inter-clerkship week, part of a larger 4-year longitudinal "Addressing Substance Use Curriculum", within an allopathic medical school. The themed week included three first-person perspective panel discussions (1. patient in recovery; 2 . families dealing with addiction/loss; 3 . healthcare professionals in recovery), and six dynamic skills-based workshops (1. opioid overdose prevention/Naloxone rescue; 2. judicious prescribing guidelines; 3. alternatives to pain mgmt.; 4. assessment/diagnosis of opioid use disorder; 5. medications for addiciton treatment (MAT); 6. landscape of addiction treatment services). To cap off the week, a team-based interactive session was held to encourage learners to formulate better processes using quality improvement science/tools. With over 55 faculty/facilitators representing 25 clinical and non-clinical departments, the interprofessional interdisciplinary approach we were able to execute served to reinforce the reality that the current opioid crisis spans every discipline and aspect of healthcare.

EVALUATION: Learners: pre-/post- $(n=86 / 82)$ short-answer quizzes, and qualitative/quantitative surveys consisting of Likert scaled questions and open ended questions to assess knowledge, attitudes, and perceived skills. Quantitative survey data was analyzed using t-tests. Faculty/facilitator: post-session surveys to comment on quality and percieved effectiveness, and overall general feedback.

DISCUSSION / REFLECTION / LESSONS LEARNED: Learner post-: 95\% Know 2+ options for MAT (Pre: 27\%); 95\% Know 2+ DSM criteria for Opioid Use Disorder (Pre: 53\%); $91 \%$ Know 3+ signs of an overdose (Pre: $65 \%)$ Learner: "Issues and problems facing the community were raised (opioid crisis) but we weren't left with a cliff hanger, we were brought through the process of diagnosing, recognizing, understanding the patient, and treatment." Faculty: "I was quite impressed and genuinely moved. The quality of the students was outstanding, and their commitment to the greater good was evident in their thoughtful participation. It was obviously a milestone in med school education, and you should be very proud."

OUR "RIGHT TO HEALTH": SELF-GUIDED EXPERIENTIAL LEARNING IMPACTS INTERNAL MEDICINE RESIDENTS ATTITUDES AND BELIEFS ABOUT OF SOCIAL DETERMINANTS OF HEALTH IN AN UNDERSERVED PRIMARY CARE SETTING.

Stacie Schmidt. Emory University, Atlanta, GA. (Control ID \#3184907)

NEEDS AND OBJECTIVES: 1 . Introduce learners to social determinants of health through experiential activities highlighting disparities in the healthcare experience. 2. Create opportunities for learner self-reflection about their knowledge and attitudes regarding social determinants of health. 3. Inspire learners, in a self-directed manner, to learn about local community resources that address social determinants of health.

SETTING AND PARTICIPANTS: Our Primary Care Center (PCC) is an academic, safety net, hospital-based clinic accommodating 70,000 visits a year to uninsured low-literacy patients. It serves as the main continuity clinic site for more than 130 Internal Medicine residents. Many disparities exist in managing chronic disease among poorer individuals, including decreased access to healthy foods, homelessness, low health literacy, and difficulty navigating large hospital systems. A survey of resident physicians found significant self-identified gaps in preparedness to provide cross-cultural care; education of residents is needed to promote consideration of patients' social and cultural barriers in managing disease and navigating the healthcare system.

DESCRIPTION: We designed an ambulatory experiential module to help Internal Medicine residents at an urban safety-net institution better understand how social constructs hinder the patients' health in the primary care setting. 
During their ambulatory month, second-year residents attend an interactive one-hour introductory session on social determinants of health, taught by a faculty member implementing group discussion and social media. Experiential activities are then chosen by learners from a list of options, and carried out in small groups of residents during a half day of protected time. Reflective writing exercises at the end of the experience elicit resident attitudes and lessons learned from their experience.

EVALUATION: 39 second year residents participated in the module. $41 \%$ of residents submitted reflective statements about their experience. Reflective responses suggest (1) enhanced appreciation for social determinants of health (2) empowerment to advocate for patient resources (3) appreciation for systems-level factors that impact social determinants of health (4) demonstrated ability to learn about community resources in a self-directed manner.

DISCUSSION / REFLECTION / LESSONS LEARNED: A short, experience-based module can impact resident attitudes about social determinants and improve their advocacy around providing enhanced patient resources. We believe our module to be effective; while a resident response rate of $41 \%$ for submitting written reflective statements may seem low at the outset, we believe this to be a high response rate in the context of a busy clinical environment and minimial faculty oversight, thereby demonstrating a high degree of engagement in the module.

ONLINE RESOURCE URL (OPTIONAL): https://doi.org/10.15766/ mep_2374-8265.10647

\section{OUTPATIENT HANDOFF MATTERS, TOO! AN INTERVENTION FOR GRADUATING PGY-3 PANEL PATIENTS AND EFFECT ON RESIDENT PERCEPTION IN A SINGLE LARGE ACADEMIC PROGRAM SETTING}

Vanessa Scowden. University of Michigan, Brighton, MI. (Control ID \#3178376)

NEEDS AND OBJECTIVES: Much attention has been paid to transition of care upon hospital discharge, although outpatient transition of care is an area perceived by many of lesser importance. Graduating residents in primary care settings experience this cycle predictably, and a paucity of evidence exists to help guide programs in best practice. Goals were to establish handoff process and standardize documentation and improve resident satisfaction with close of clinic as well as perception of importance of ambulatory handoff.

SETTING AND PARTICIPANTS: Single continuity clinic site for large academic internal medicine residency.

DESCRIPTION: Graduating PGY-3 resident PCPs and their continuity attending at a single academic continuity site (resident $n=5$ ) were selected to review their panels. Patients subjectively identified as "higher risk" were then discussed individually to identify who was best served to undergo intervention- options were to transition to an established resident (rising PGY-2) or an attending panel, or to complete a formal transition of care process. Patients identified for formal transition of care process were identified for in-office return visit prior to resident graduating. This visit note was standardized, designed to remain in the medical record. To evaluate the impact on the residents, an anonymous retrospective pre-then-post survey was completed to assess perception and sense of value for handoff in the ambulatory setting with $100 \%$ of surveys returned $(n=5)$. There were four questions designed to evaluate perception of importance for both inpatient and ambulatory handoff, and if the resident felt current curriculum provided adequate instruction for best practice in both settings.

EVALUATION: There were a total of 331 patients identified as having a PGY-3 as PCP and individually reviewed approximately 6 months prior to resident graduation. Of these patients, $39(11.8 \%)$ were determined to be medically or socially complex enough to be best served by intervention process. Regarding value for inpatient handoff, all PGY-3 agreed or strongly agreed that there is value. They also all agreed or strongly agreed that their current curriculum provides instruction for best practice. This study did not show any change in level of response after our intervention regarding inpatient handoff attitude or provided instruction. All respondents did also agree or strongly agree that there is value in ambulatory handoff, although 4 of the 5 disagreed or strongly disagreed that there was adequate instruction prior to this intervention regarding handoff in the ambulatory setting. After the intervention, 4 of the 5 did improve on ambulatory question metrics, ranging from one to three levels of improvement.

DISCUSSION / REFLECTION / LESSONS LEARNED: This study was created to address a need for improved formal handoff in an ambulatory setting during resident transition. This was formalized in recommendations both for review process, individual patient discussion as well as standardizing documentation.

\section{PC TEACH: DEMONSTRATION OF A NOVEL PEER TEACHING TEAM MODEL IN RESIDENT PRIMARY CARE CLINIC}

Katherine L. Chen; Mina Ma; Antonio M. Pessegueiro. UCLA, Los Angeles,

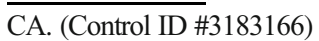

NEEDS AND OBJECTIVES: Whereas residents regularly teach and mentor each other on the wards, they rarely collaborate with their peers in ambulatory practice. Continuity clinic represents a missed opportunity to use peer teaching to boost primary care skills and attitudes. The PC Teach pilot program aimed to assess the feasibility and impact of a novel outpatient teaching team model.

SETTING AND PARTICIPANTS: 5 residents, 6 interns, and 4 attendings in an internal medicine residency program participated in the pilot.

DESCRIPTION: One resident was paired with one intern in each PC Teach session. In the first 6 months, the resident directly observed the intern's clinic visits and helped the intern prepare for the presentation to the attending. In the second 6 months, the intern saw each patient independently and presented to the resident, who acted as preceptor; the attending supervised the teaching and then confirmed the plan. At the end of each session, participants used worksheets to guide self-assessment and feedback on the intern's clinical skills and the resident's teaching.

EVALUATION: Participants completed anonymous surveys pre- and postintervention. The study was not powered to demonstrate statistical significance, but several trends were observed. After the intervention, interns and residents reported that they enjoyed working together in PC Teach. Interns also conveyed more positive attitudes toward primary care after PC Teach. Meanwhile, residents reported increased confidence as outpatient teachers and greater interest in pursuing primary care careers. After PC Teach, attendings were less confident in their ability to gauge residents' outpatient teaching abilities and less confident that graduating residents were ready to be outpatient preceptors. All participants agreed that future trainees would benefit from PC Teach. Trainees agreed that the impact on patient care was somewhat positive, and attendings reported that it was neutral.

DISCUSSION / REFLECTION / LESSONS LEARNED: The PC Teach pilot study demonstrated that an outpatient teaching team model was feasible and well received by trainees. Although any of the observed trends could reflect random error, attendings' decreased confidence in residents' outpatient teaching skills might imply that PC Teach gave faculty a novel glimpse of residents' teaching abilities, which previously they had overestimated. More rigorous evaluation of $\mathrm{PC}$ Teach will be possible as the program grows. Now in our second year of PC Teach, we have tripled the number of participants 
and applied lessons learned from the pilot study, including a more robust orientation, continuity in resident-intern pairings, and improved worksheets for skills-based feedback and reflection.

\section{PERCEPTION VERSUS REALITY IN USE OF TEACH-BACK BE- FORE AND AFTER A ONE-HOUR SKILLS TRAINING}

Kimberly C. Bates ${ }^{1}$; Michelle Ogrodnick ${ }^{2}$; Iris Feinberg ${ }^{2}$; Robert Hendrick ${ }^{2}$; Kevin Johnson ${ }^{1}$. ${ }^{1}$ Gwinnett Medical Center, Lawrenceville, GA; ${ }^{2}$ Georgia State University, Atlanta, GA. (Control ID \#3163132)

NEEDS AND OBJECTIVES: Physicians ask patients if they understand their diagnosis or instructions during clinic visits; patients often say yes. However, many patients leave with little idea of their medication and discharge instructions. Teach-Back (TB) is a health-literate technique that allows physicians to confirm patient understanding during clinic visits. The purpose of this pilot study was to evaluate perception vs actual use of TB by medical residents in primary care clinics; if the observed rate of TB was discordant with perception, we examined if a TB skills training intervention had any impact on use of TB. We were also interested in language used during TB and its relationship to patient demographics or health literacy level.

SETTING AND PARTICIPANTS: Participants were Family Medicine and Internal Medicine residents who provide patient care in two clinics in suburban Atlanta, Georgia. Residents were recruited at a regularly scheduled graduate medical education meeting. Residents were told that we would audiotape ten of their patient clinic visits- 5 before and 5 after a skills training intervention. Patients were recruited in waiting areas of the clinics prior to their encounter. Patients were over 18 and spoke English.

DESCRIPTION: Residents completed the "Always Use Teach-Back Confidence and Conviction Scale". Clinic visits were audiotaped and scored for TB. A one-hour TB skills training took place after the first five audiotaped visits. A second round of visits were audiotaped after the training. Content analysis was performed on TB language. Demographics and health literacy level were collected on patients.

EVALUATION: n/a

DISCUSSION / REFLECTION / LESSONS LEARNED: Despite high level of perception of TB $(60 \%)$, TB was only used $2.5 \%$ of the time. Postintervention, TB use increased to $53 \%$. Findings may be critical to improving physician communication: a single one-hour intervention dramatically increased use of TB from 2 to 41 instances. Residents used more patientcentered and collaborative TB language after the training intervention. There is a range of how residents perform TB with no relationship to patient demographics or health literacy level.

\section{PERFORMANCE AND CONFIDENCE OF MEDICAL RESIDENTS IN LEADING A RAPID RESPONSE TEAM: A QUALITY IM- PROVEMENT PROJECT}

Maythawee Bintvihok $^{1}$; Esilida Sula Karreci ${ }^{1}$; Bertrand Jaber ${ }^{1}$; Andrew Moraco $^{2} .{ }^{1}$ St.Elizabeth's Medical Center, Brighton, MA; ${ }^{2}$ St. Elizabeth's Medical Center, Brighton, MA. (Control ID \#3186993)

NEEDS AND OBJECTIVES: A rapid response team (RRT) is a team of providers that is summoned to the bedside to immediately assess and treat a patient demonstrating signs of imminent clinical deterioration, with the goal of preventing intensive care unit transfer, cardiac arrest, or death. RRT activation is associated with reduced in-hospital cardiopulmonary arrest and mortality. We reviewed the total number of RRTs activated at SEMC in 2016 and established a rate of 15.6 per 1,000 admissions, lower than rates of 20-40 reported in published literature. Prior studies have shown that RRT training can improve team confidence and ability.

SETTING AND PARTICIPANTS: We conducted a survey of 54 medical residents (63\% response rate) to examine the level of comfort with activating and leading a RRT. Amongst PGY-1 residents, $6 \%$ had previously activated a RRT, only $12.5 \%$ felt comfortable leading a RRT, $81 \%$ never had formal RRT practice, and $25 \%$ were reluctant to call a RRT due to concerns with ineptitude. Amongst PGY-2 residents, 58\% had ever activated a RRT, 66\% felt comfortable leading a RRT, 25\% never had formal practice, and $60 \%$ felt the need for orientation on how to lead a RRT. The survey results demonstrated that the concerns with ineptitude amongst residents in conducting a RRT were secondary to lack of training in common urgent scenarios. The goal of this quality improvement project was to improve resident performance and confidence in leading a RRT through education and training.

DESCRIPTION: We provided a series of 9 didactic sessions, conducted mainly by a staff neurologist, a cardiology fellow, and a pulmonary and critical care fellow (in March and May 2018). Each session included simulation-based training in common urgent scenarios that lead to RRT activation. We also provided a pocket information card with a brief algorithm for each scenario and a pocket sized list of hospital phone extensions to aid in reaching certain services during a rapid response.

EVALUATION: To assess the impact of our intervention, we conducted a post-implementation resident survey. Amongst PGY-1 residents, 93\% had activated a RRT, which improved from $6 \%$ in the pre-intervention period. $64 \%$ felt comfortable leading a RRT, which significantly improved from $12.5 \%$ in the pre-intervention period $(\mathrm{P}=0.001)$. Amongst $\mathrm{PGY}-2$ residents, $78 \%$ reported activating a RRT, which improved from $58 \% .100 \%$ felt more comfortable leading a RRT, an improvement from $66 \%$ in the pre-intervention period but did not reach statistical significance $(\mathrm{P}=0.157)$. The RRT activation rate increased from 15.6 to 16.7 per 1,000 admissions following our intervention.

DISCUSSION / REFLECTION / LESSONS LEARNED: Formal instruction on RRT through didactic sessions and simulation-based training can potentially improve knowledge and confidence of medical residents in leading RRTs. This can lead to increased awareness of RRT activation to prevent intensive care unit transfer, cardiac arrest, and death. Future efforts will focus on coordinating with the Code Committee to implement mock RRT scenarios.

\section{PGY-1 TO PGY-2 TRANSITION: LEADING RAPID RESPONSES}

Emily Cetrone $^{1}$; Hallie Rozansky ${ }^{1}$; Marina Zambrotta ${ }^{1}$; Subha Ramani ${ }^{2}$; Matt Difrancesco $^{1}$; Tomas Cordova ${ }^{1} .{ }^{1}$ Brigham and Women's Hospital, Brookline, MA; ${ }^{2}$ Brigham and Women's Hospital, Harvard Medical School, Boston, MA. (Control ID \#3178333)

NEEDS AND OBJECTIVES: When PGY-1 residents transition to PGY-2 year, they function as supervisors of junior trainees, teachers, and team leaders with scant training in these skills. One of their most challenging roles is leading management of clinically unstable patients in "rapid response" scenarios ("rapids"). In our new curriculum, PGY-1s will run simulated rapids and receive feedback on their performance. The curriculum will also help program leadership identify gaps for further training. Our curricular objectives are that residents will: 1) Demonstrate increased confidence in running rapids 2) Exhibit awareness of the roles of the various participating staff 3) Gain knowledge of criteria that trigger rapids 4) Be open to receiving feedback on performance 5) Demonstrate effective leadership and communication skills SETTING AND PARTICIPANTS: In 2018, residents in the Scholars in Medical Education pathway implemented the first "Intern to Junior Transition Retreat" at our institution. The retreat included a didactic session on running 
rapids and codes. Resident evaluation of the retreat indicated that further training in rapid responses was needed, and over one-third of respondents requested hands-on simulation training. The new curriculum will take place toward the end of intern year at our simulation center, each intern will have the opportunity to lead a simulated rapid, followed by feedback and debriefing. DESCRIPTION: - Pre-simulation: Didactic teaching on clinical parameters for calling a rapid and the personnel who arrive. Emphasis will be placed on leadership and communication skills. - Simulation: Each intern will lead one scenario and participate in one scenario as a nurse. Both scenarios will use the same chief complaint with two different underlying causes and clinical trajectories. - Post-simulation: We will conclude with a debriefing guided by checklists outlining key leadership skills.

EVALUATION: Program evaluation will include: 1) A questionnaire to assess residents' self-reported confidence in running rapids before, immediately after and six months after simulation training. Results will be compared to data from the 2018 Transition Curriculum, where interns received didactic teaching only. 2) Achievement of specific leadership and communication endpoints during each scenario. This information will guide debriefing and identify areas for future curricular revisions. 3) Audio-recording of debriefing sessions and thematic analysis to identify areas for future training.

DISCUSSION / REFLECTION / LESSONS LEARNED: The planning team learned the following key lessons: needs assessment is key to successful planning of educational content and strategies and curriculum implementation; scholarly approach to curriculum design is important; and getting buy-in from key stakeholders, such as chief residents and the program director, is critical to success.

POLICY AND ADVOCACY FOR CLINICIANS: TEACHING SYSTEMS THINKING TO INTERNAL MEDICINE RESIDENTS Jennifer B. Cowart ${ }^{1}$; Michael Maniaci ${ }^{2}$; Jaimie Grega ${ }^{2}$; Michele D. Lewis ${ }^{2}$. ${ }^{1}$ Mayo Clinic, Jacksonville, FL; ${ }^{2}$ Mayo Clinic in Florida, Jacksonville, FL. (Control ID \#3153766)

NEEDS AND OBJECTIVES: 1. Increase practical knowledge of the US healthcare system and the impact of healthcare policy on the daily work of internists. 2. Improve awareness of and comfort with physician advocacy. 3. Provide education in the ACGME Core Competency of Systems-Based Practice.

SETTING AND PARTICIPANTS: Year-long, monthly morning report series for residents in an academic internal medicine program.

DESCRIPTION: We created a curriculum in healthcare policy and physician advocacy for internal medicine residents, delivered in half-hour lectures during morning report. The course began in the 2018-2019 academic year. A precourse survey was administered to all internal medicine residents in our program prior to the first lecture, and a post-course survey will be administered in June 2019.

EVALUATION: The pre-course survey identified gaps in self-reported knowledge of the healthcare system: low levels of ability to describe process of federal healthcare policy making, staying up to date on current healthcare policy news, familiarity with US healthcare payers, or comfort contacting elected officials about issues. Residents agreed that advocacy was important to the work of physicians, but most reported having never participated in an advocacy effort. Most residents agreed that voting was important and $90 \%$ reported having voted before.

DISCUSSION / REFLECTION / LESSONS LEARNED: Discussion: With current events showing continued changes in healthcare delivery and medical education, we identified a growing need for physicians to enter the policy and advocacy space. This course was designed to address gaps in knowledge and awareness in these domains. Pre-course survey data confirmed these information gaps, while showing that residents agree that advocacy is an important part of their role as physicians. The curriculum is being administered currently as a series of monthly lectures, and will also include encouraging residents to participate in physician advocacy activities at the state or federal level with internal medicine professional societies, including "Hill Day". This type of curriculum can be implemented in a variety of settings, not exclusive to internal medicine or to postgraduate medical education. Reflections/Lessons learned: During the first year of curriculum development, a traditional lecture model is being used. The time constraints of the 30-minute morning report model limit the time for meaningful discussion around the topics, which are often new to the learners. Future iterations may employ other modalities, to potentially include a "flipped classroom". Written or video materials could be delivered to the learners in advance, so that lecture time may be spent discussing and interacting with the content. This material shares some commonality with patient safety and quality improvement, in terms of the systemsbased practice focus, and some policy material could be incorporated into a quality-focused morbidity and mortality conference or into other lectures on purely clinical topics.

\section{PREPARING FUTURE PHYSICIANS TO CARE FOR PATIENTS WITH OPIOID USE DISORDER - BUPRENORPHINE WAIVER TRAINING COURSE DURING MEDICAL SCHOOL}

Irvin Lien ${ }^{1}$; Jody S. Chou ${ }^{4}$; Diane L. Levine ${ }^{2}$; Eva Waineo ${ }^{3} .{ }^{1}$ Wayne State University School of Medicine, Detroit, MI; ${ }^{2}$ Wayne State University, Detroit, MI; ${ }^{3}$ Wayne State University school of medicine, Detroit, MI; ${ }^{4}$ Wayne State University, Okemos, MI. (Control ID \#3184545)

NEEDS AND OBJECTIVES: The opioid epidemic in the US has become a major issue in healthcare and is destructive to individuals, families, and communities. Despite the decrease in opioid prescriptions, an unintended consequence is increased use of illicit or dangerous opioids such as heroin and fentanyl. In 2017, there was an estimated 72,306 drug overdose related deaths and the Emergency Departments (ED) nationally saw a $30 \%$ increase in opioid related overdoses. Innovative programs can help ensure patients are offered optimal treatment options. Most primary care physicians self-report they lack the skills to identify and appropriately treat substance abuse disorders (SUDs). Studies have suggested that the best solution is to improve medical school curricula, which translates to better educated future physicians. Unfortunately, most curricula do not provide adequate education to identify and treat SUDs in the medical practice. Buprenorphine is a partial $\mu$-receptor agonist that is utilized to treat opioid addiction. To prescribe buprenorphine, an 8-hour Medication Assisted Treatment (MAT) training must be completed. Only 808 of the 28,206 active physicians $(<3 \%)$ in the state of Michigan are registered to prescribe buprenorphine. We developed an innovative approach to provide students with the skills to understand how to prescribe buprenorphine and build confidence to medically manage opioid use disorders in the future. By completing the training and obtaining their permanent license, students will have completed all requirements to prescribe buprenorphine as a physician. SETTING AND PARTICIPANTS: Third year medical students on their internal medicine clerkship are offered the MAT training during their 8-week rotation.

DESCRIPTION: The MAT training is offered online by the American Osteopathic Academy of Addiction Medicine twice a month and is free of charge. Training consists of a $4.25-\mathrm{hr}$ webinar and $3.75-\mathrm{hr}$ online module. Students who complete the training can choose to take one day off their clerkship. A pre- and post-experience survey is completed to assess how the training has impacted perspectives on patients with SUDs. 
EVALUATION: Preliminary data suggests medical students do feel there is a lack of SUDs education in the curricula. However students have a strong interest in learning how to prescribe and utilize buprenorphine in their medical practice. After completing the training, medical students report that they have a better foundation on when buprenorphine can be prescribed and how to recognize SUDs.

DISCUSSION / REFLECTION / LESSONS LEARNED: Medical students recognize opioid use disorders is an important issue within communities and want to help their future patients. This training has importance to all students regardless of their interested specialty. Offering the MAT training can supplement the school's curricula and is generalizable to use by other medical schools.

PRIMARY CARE BEHAVIORAL HEALTH INTEGRATION: USE OF A PSYCHOLOGIST IN RESIDENT DIRECT OBSERVATION Lisa N. Kransdorf; Stephanie R. Young; Jessica M. Bernacki; Colin L. Robinson; Mina Ma; Alice Kuo. UCLA, Los Angeles, CA. (Control ID \#3184754)

NEEDS AND OBJECTIVES: Structured direct observation is an important component of resident training to ensure achievement of core competencies. For residents who will eventually practice in primary care, mastering these core competencies as they pertain to behavioral health is especially important. Recognizing this need, a growing number of residency programs are incorporating integrated behavioral health clinicians (IBHCs) in clinical training sites. A study of residents in training programs by Hemmings et al. that currently incorporate IBHCs reported an increase in interpersonal communication skills awareness and a positive impact on patient care. We are not aware of existing literature, however, regarding the use of IBHCs in the direct evaluation of residents. As noted in a study of family medicine residents (Martin et al.), though trainees may understand the concepts behind behavioral health competencies, they may not have insight into their own knowledge and skill deficits without direct instructor feedback. IBHCs bring a unique perspective in the assessment of residents in behavioral health-related core competencies. At our institution, we will be using IBHCs in the assessment of residents in structured direct observation. We plan to compare resident core competencies in behavioral health in trainees who experience structured direct observation by both IBHCs and medical attendings compared to trainees exposed to direct observation by only medical attendings.

SETTING AND PARTICIPANTS: The study setting is a large academic medical center in a densely populated urban area. The study population will be Internal Medicine residents and Combined Internal Medicine-Pediatrics residents at UCLA. Location of the study will be their continuity clinic site. The behavioral health integration at these sites will be provided by doctoral level psychologists (PsyD and PhD).

DESCRIPTION: This will be a cross-sectional study of Internal Medicine residents in the UCLA- Olive View Primary Care track and Combined Internal Medicine-Pediatrics residents (IHBC + medical attending direct observation) compared to categorical UCLA Internal Medicine residents (medical attending-only direct observation).

EVALUATION: UCLA has a structured direct resident observation program with a templated evaluation form assessing specific core competencies for each resident. We will compare core competency scores with specific attention to interpersonal communication, professionalism and management of mood disorders between study groups over a 12-month academic year.

DISCUSSION / REFLECTION / LESSONS LEARNED: We hypothesize that the feedback provided by IBHCs in the structured direct observation of residents will result in improved core competencies with regard to behavioral health. This specific area of core competencies is of special importance as the behavioral health care in our country becomes increasingly fragmented; our primary-care oriented graduates will need to be especially effective and engaged behavioral health advocates for their patients.

PRIMARY CARE PROFESSIONS PERCEPTIONS: A CURRICULUM TO CORRECT STEREOTYPES AND CLARIFY TEAM ROLES AMONG TRAINEES OF MULTIPLE HEALTH PROFESSIONS

Anna Golob ${ }^{1,2}$; Linda Pyke ${ }^{4}$; Mary-Catherine J. Kane ${ }^{3}$; Cassandra Song ${ }^{5}$; Diane Smith ${ }^{4}$; Joyce E. Wipf ${ }^{6} .{ }^{1}$ VA Puget Sound Healthcare System, Seattle, WA; ${ }^{2}$ University of Washington, Seattle, WA; ${ }^{3}$ VA Puget Sound, Seattle, WA; ${ }^{4}$ Seattle VA Puget Sound Health Care System, Seattle, WA; ${ }^{5}$ VA Puget Sound Health Care System, Seattle, WA; ${ }^{6}$ VA Puget Sound, University of Washington, Edmonds, WA. (Control ID \#3185610)

NEEDS AND OBJECTIVES: The Seattle VA Center of Excellence in Primary Care Education (CoEPCE) aims to transform primary care education out of professional silos and train physicians and other health professionals to be highly competent in providing team-based collaborative care. Even with shared $\mathrm{CoE}$ educational and clinical training experiences, we find our trainees may still have limited knowledge and misperceptions about skills and roles of their colleagues. Here we describe our curriculum, adapted from Case Western Reserve University's "Interprofessional Exercise," aimed at exploring biases and enhancing understanding of other professions' roles and expertise.

SETTING AND PARTICIPANTS: Our "Professions Perceptions" seminars are held in primary care once yearly when all participating $\mathrm{CoE}$ trainees $(n=\sim 35 /$ year) are $>3$ months into their respective graduate training program. We include all $\mathrm{CoE}$ trainees and faculty based in primary care, including internal medicine MDs, nursing (NPs and RNs), psychology, pharmacy, social work, and psychiatry.

DESCRIPTION: In the seminars, trainees and faculty gather in a conference room with several large posters on the wall. Each poster has the name of one profession with two questions: 1) What are three things you think of when you hear this profession name? and 2) What are this profession's skills and roles on the primary care team? Trainees visit each profession's poster and write their perceptions. Next, trainees and faculty of each profession gather by their profession's poster and review/reflect on the perceptions recorded. Each profession then shares with the large group whether they feel perceptions and roles of their profession were accurately captured; corrects misperceptions; and reviews their team roles.

EVALUATION: We tracked perceptions poster data for the past two academic years and recorded faculty observations. For some professions, there was near consensus on descriptors: for example, social workers as "magicians" and RNs as "heroes." For other professions, there was a broad range of descriptors and more role confusion; for example, differences and commonalities between MD and NP primary care providers; and psychologists and psychiatrists. Our faculty observe that over time, trainees have increasing sophistication of understanding and appreciation of their interprofessional colleagues.

DISCUSSION / REFLECTION / LESSONS LEARNED: This curriculum provides a safe, intentional environment for trainees to share their perceptions and confirm or correct perceptions by others. Lessons learned include: 1) timing is crucial to maximize trainee participation and comfort: previous sessions held at orientation were less successful given trainees were still learning about their own professional roles and were less comfortable discussing biases; 2) this seminar builds on CoE trainees' shared training experience to better inform perceptions, and shows increasing knowledge of 
others' expertise and roles; 3 ) misperceptions remain, particularly for professions with "overlapping roles" versus unique contributions.

PRIMARY CARE QUALITY IMPROVEMENT TEACHING FOR INTERNAL MEDICINE RESIDENTS TO IMPROVE CARE GAPS kristin huang; Daniel Chandler; Deborah Blazey-Martin; Stacy Schwartz; Julie Tishler. Tufts Medical Center, Newton, MA. (Control ID \#3185119)

NEEDS AND OBJECTIVES: Multiple care gaps between resident and attending patients exist in our academic primary care practice, including in colon cancer screening and HTN control. We sought to use quality improvement (QI) teaching and practice as one tool to decrease these disparities and improve health outcomes for residents' primary care patients. A systematic review of the literature has shown a paucity of data regarding effectiveness of residents' participation in QI efforts, particularly concerning patient outcomes (Patow et al). We sought to design an intervention that would improve both resident education around basic QI concepts and clinical outcomes. We hypothesized that this intervention would improve residents' familiarity with their patient panel as well as their comfort level with QI skills. We also hypothesized that this intervention would improve the residents' rates of colon cancer screening and HTN control.

SETTING AND PARTICIPANTS: Our internal medicine residency program includes 74 residents, all of whom have continuity clinic at a single academic primary care practice on the main teaching hospital campus. Residents' average panel size is 96 patients.

DESCRIPTION: Over the semester, junior and senior residents had one 3week primary care rotation during which they participated in a 2-hour QI workshop and received individualized data on their panels regarding colon cancer screening and HTN control. They designed aim statements and plando-study-act (PDSA) cycles, and then implemented their PDSA cycles during their rotation. They were surveyed before and after this rotation regarding their attitudes and knowledge about QI.

EVALUATION: On a 5-point Likert scale, residents' comfort level with managing their panel rose from $3.85(n=55)$ before the QI training to 3.92 $(n=39)$ after it. Residents' comfort level with crafting an aim statement rose from 3.51 to 4.26; their comfort with designing and implementing a PDSA cycle rose from 2.80 to 3.92 . Residents' interest in making QI a part of their career also rose from 3.35 to 3.56 . Only $62 \%$ of residents had performed an intervention based on their panel report in the past, and this rose to $85 \% .62 \%$ of residents knew the rate of HTN and colon cancer screening in their patient panels before the training, and this rose to $97 \%$ after. Only $45 \%$ of residents had performed a PDSA cycle prior to the QI training, and this rose to $77 \%$ after. Residents' baseline rate of colon cancer screening in January 2018 was $34 \%$, which rose to $43 \%$ by July 2018 . Residents' baseline HTN control rate was 65\% in January 2018, and this stayed constant through July 2018.

DISCUSSION / REFLECTION / LESSONS LEARNED: A quality improvement workshop followed by implementation of individual, selfdesigned PDSA cycles focused on colon cancer screening and HTN control improved both residents' comfort with QI skills and colon cancer screening rates among resident patients.

PRIMER COURSE DURING RESIDENCY FOR IN-FLIGHT MEDICAL EMERGENCIES (PRIME): A SINGLE-CENTER RANDOMIZED CONTROLLED TRIAL

Yazan Daaboul; D. Alexander Morrison; Anupama Kumar; Joseph Rencic; Laura K. Snydman. Tufts Medical Center, Boston, MA. (Control ID \#3176018)
NEEDS AND OBJECTIVES: Management of in-flight medical emergency remains absent from medical curricula among residents and is an unmet need. The PRIME study will be the first randomized controlled trial that will test the hypothesis that a multidisciplinary primer course, including video series and role playing, for in-flight emergencies will improve the fund of theoretical knowledge and confidence level among first-year residents when managing inflight medical emergencies.

SETTING AND PARTICIPANTS: First-year resident physicians at Tufts Medical Center in Boston, MA are eligible to participate.

DESCRIPTION: First-year residents will be randomly assigned to receive either 1) an educational primer course including video series and role playing for in-flight medical emergencies [intervention arm] or 2) no educational material [control arm]. The videos and role playing content will include the approach to commonly encountered emergencies, as well as aviation physiology, medico-legal considerations, familiarity of medical kits, triaging techniques, and documentation. The course will not include pediatric or obstetric emergencies.

EVALUATION: The primary endpoint of the trial is pre-specified and is defined as the mean difference in the score of the MCQ test between the intervention vs. control arm. The 20-question multiple choice question (MCQ) assessment will evaluate subjects' fund of knowledge regarding relevant clinical pearls. In addition, a retrospective pre/post survey will evaluate learners' attitudes and comfort pre-exposure to the course (all subjects) vs. their comfort post-exposure (only intervention arm).

DISCUSSION / REFLECTION / LESSONS LEARNED: The results of this trial will be available April, 2019 by the time of presentation at SGIM. The required sample size is $\mathrm{N}=24$, which was estimated based on the two-means Student's t-test to provide a mean difference of $20 \%$ in the primary outcome. The intervention of video series and role playing, as opposed to traditional teaching, was chosen to allow replicating the course elsewhere and minimize the effect of confounding, such as teacher techniques. If the differences between the study groups are statistically significant, this trial will be the first to provide a scientifically reliable, standardized means to help physicians address commonly encountered in-flight medical emergencies.

PROSPECTIVE VALIDATION OF HIV-ASSIST, AN ONLINE EDUCATIONAL TOOL FOR HIV TREATMENT DECISION SUPPORT Nicky J. Mehtani'; Manoj V. Maddali ${ }^{2}$; Jonathan Z. Li ${ }^{3}$; Maunank Shah ${ }^{1}$. ${ }^{1}$ Johns Hopkins University School of Medicine, Baltimore, MD; ${ }^{2}$ University of California San Francisco, San Francisco, CA; ${ }^{3}$ Brigham \& Women's Hospital, Boston, MA. (Control ID \#3177776)

NEEDS AND OBJECTIVES: The transformation of HIV into a chronic disease has increased demand for providers proficient in HIV management, yet the supply of such providers is declining. This is in part due to a perpetually evolving landscape of HIV with continual changes in clinical guidelines and an expanding repertoire of antiretroviral (ARV) drugs. Guidelines advocate for an individualized approach to ARV selection, but synthesizing the various factors pertinent to optimal regimen selection is complex and time-consuming. There is critical need for innovations that promote increased involvement of primary care physicians in HIV care and integrate efficient learning of HIV management principles into clinical practice.

SETTING AND PARTICIPANTS: Multi-criteria design analysis was used to construct HIV-ASSIST - a free, online interactive tool to inform clinical decision-making for ARV selection. Development included construction of a multi-attribute utility function drawing upon existing literature, guidelines, expert opinion, and focus groups to refine algorithms. A prospective survey of experienced HIV providers from Johns Hopkins, Brigham \& Women's, 
Massachusetts General Hospital, and UCSF was conducted to evaluate concordance of HIV-ASSIST with expert prescribing practices.

DESCRIPTION: An initial literature review identified 11 key attributes pertinent to ARV selection, including viral load, mutations, tropism, HLAB5701 status, CD4 count, comorbidities, comedications, treatment history, and factors related to adherence. The HIV-ASSIST online interface allows user input of these attributes, across which the algorithm measures and ranks all potential ARV regimens, which are presented to users, along with dosing guidelines, drug interactions, clinical trial data, and an explanation of all decision-making steps to provide tailored educational support while simultaneously improving clinical care.

EVALUATION: Ten patient case scenarios of varying degrees of complexity were distributed to $15 \mathrm{HIV}$ clinicians across multiple institutions, who were asked to recommend a specific ARV regimen for each case. Among 4 cases of ARV-naïve patients with and without comedications and comorbidities there was $100 \%$ concordance between provider free responses and the top 5 HIV-ASSIST ranked outputs when guideline-contradictory responses were excluded; among 3 cases of virally-suppressed ARVexperienced patients there was $93 \%$ concordance; and among 3 cases of viremic ARV-experienced patients there was $84 \%$ concordance. Concordance was under $93 \%$ for only two cases, and for each the 15 providers suggested 9 discrete ARV regimens - demonstrating heterogeneity in clinical practice.

DISCUSSION / REFLECTION / LESSONS LEARNED: HIV-ASSIST demonstrated a high degree of concordance with ARV selection by experienced HIV providers. Despite substantial variation in provider prescribing practices for ARV-experienced patients, the results suggest that HIVASSIST is a robust resource to aid clinical decision-making and educational support in primary care settings.

ONLINE RESOURCE URL (OPTIONAL): www.hivassist.com Username: SGIM Password: SGIM

QUALITATIVE ASSESSMENT OF PRIMARY CARE EDUCATION IN AN INTERPROFESSIONAL TEAM: THEMES FROM THE IMPROVING PATIENT ACCESS, CARE, AND COST THROUGH TRAINING (IMPACCT) EXPERIENCE

$\underline{\text { Don-Andre Jackson }}{ }^{1}$; Lyndonna M. Marrast ${ }^{2}$; Alice Fornari ${ }^{1} .{ }^{1}$ Northwell Health, Great Neck, NY; ${ }^{2}$ Zucker School of Medicine at Hofstra/Northwell, Kew Gardens, NY. (Control ID \#3178273)

NEEDS AND OBJECTIVES: This IP clinical training program requires integration of skills contributed by learners from across various disciplines: psychology, medicine, pharmacy and physicians assistant (PA). Now in the third year since its inception, we sought to identify the elements of this IP team that make the huddle and precepting experience effective. We anticipate this data will help design faculty development training material to improve IP participation of learners and faculty.

SETTING AND PARTICIPANTS: This is a qualitative study employing semi-structured interviews with 14 IP healthcare team members(4 pharmacists, 9 medicine attendings, 1 psychologist) to elicit experiences of conducting huddles, precepting in an interprofessional team, and educating learners of various disciplines in this model of care. Themes were identified using an iterative content analysis approach of the interviews, post transcription.

DESCRIPTION: Northwell Health has a federally funded grant from HRSA to develop and implement a longitudinal clinical program entitled Improving Patient Access, Care, and Cost through Training (IMPACcT). The goal is to provide interprofessional(IP) trainees with a mentored primary care experience integrating education and clinical skills focused on patientcentered care, quality improvement and population health in an IP teambased clinical setting. Teams interact via patient interactions, precepting and huddles. Huddles are prepared pre-visit discussions to assess the unique needs of every patient prior to each clinical session.

EVALUATION: As our population ages and medical comorbidities become more complex, there is an increasing need for IP collaborative approach to patient centered care. This analysis has demonstrated several important themes:(1) Huddles as effective tools organize patient care in an IP setting, (2) Precepting in this model of care increases interprofessional communication and camaraderie. Further interviews from all IP team members are needed to develop these initial themes into faculty development content. We plan to use this as part of our continuous quality improvement effort for IMPACcT clinic.

DISCUSSION / REFLECTION / LESSONS LEARNED: Eight interviews were conducted with the IP attendings who precept the IP trainees. Participants described huddles as being resident driven, prone to tangents, more effective with defined leadership. Most important is that successful huddle leaders can be team members other than physicians. Additionally, participation of pharmacy and PA learners improved when they were assigned specific tasks. Study participants reported that this model of care had unique challenges and rewards when precepting. Common themes about the precepting experience were (1) increased need to balance work flow and efficiency while educating multiple learners, (2) deeper connections with other professionals, and (3) increased reliance on other IP clinicians on the team.

RED EYE ROUNDS: KNOWLEDGE IN THE CLOAK OF DARKNESS

Kimberly Indovina $^{1,}{ }^{2}$; Chi Zheng ${ }^{1,}$ 2; Noelle Northcutt ${ }^{1,}{ }^{2}$; Anna M. Munoa ${ }^{1,2}$. ${ }^{1}$ Denver Health, Denver, CO; ${ }^{2}$ University of Colorado School of Medicine, Denver, CO. (Control ID \#3185975)

NEEDS AND OBJECTIVES: At our hospital, there has historically been little interaction between residents and hospitalists during the night shift. Residents reported inadequate communication with hospitalists overnight about patient flow and triage, and identified lack of faculty-led overnight education as a programmatic weakness. In designing Red Eye Rounds (RER), our goals were 1) to increase nighttime communication between residents and hospitalists, and 2) to add structured teaching to nighttime workflow using a high-yield, sustainable curriculum.

SETTING AND PARTICIPANTS: Hospitalists and internal medicine residents working night shifts at an urban academic safety-net hospital.

DESCRIPTION: RER is a meeting of night shift hospitalists and residents that occurs every night at midnight and lasts about 15 minutes. It provides a structured framework to troubleshoot patient care or flow issues and to discuss a clinical pearl. To help our faculty facilitate RER with minimal preparation, we created a curriculum of teaching resources consisting of: 1) high-yield board review questions using MKSAP and other resources, 2) brief summaries of key practice-changing articles, and 3) a guide to clinical reasoning for common cross-cover calls. We spent 6 months developing RER prior to its launch. We first surveyed residents to understand their preferences for nighttime teaching, then developed the RER curriculum, then piloted RER and made small changes based on initial feedback. We officially integrated RER into overnight workflow in fall 2017.

EVALUATION: To evaluate the impact of RER, we gather monthly attendance data and participant feedback. Tracking attendance has increased accountability and promoted RER as standard practice. While in the first 
month RER occurred only $48 \%$ of nights, frequency of attendance has increased steadily, and has consistently been above our goal of $90 \%$ since month 7. Participant feedback has been overwhelmingly positive. Hospitalists commented, "It's a really good thing. It's changing the dynamic between us and the residents literally overnight!" and, "There is more of a sense that the residents should feel free to call us anytime they have questions or concerns." The chief resident noted, "The residents have really enjoyed the opportunity to ask faculty clinical questions and discuss admission flow. It's been the most successful project implemented this year."

DISCUSSION / REFLECTION / LESSONS LEARNED: We've found that RER improves resident-attending nighttime communication and encourages frequent dialogue, increases hospitalist awareness of unstable patients and logistic problems, facilitates high-yield learning, and boosts overall night shift satisfaction for both residents and hospitalists. We are now partnering with colleagues at our affiliated teaching sites (university and VA hospitals) to expand RER and standardize the curriculum across sites. Our framework could easily be adapted to other programs, and we hope that structured nighttime communication and education will become a national standard among internal medicine residencies.

RESIDENT INITIATIVE FOR AN ULTRASOUND CURRICULUM Raymond Lam; Artin Galoosian; Ingeborg Schafhalter-Zoppoth; Iris Ma. California Pacific Medical Center, San Francisco, CA. (Control ID \#3184673)

NEEDS AND OBJECTIVES: Internal medicine (IM) physicians are increasingly using point of care ultrasound (POCUS) to aid in bedside diagnostics. However, ultrasound education in IM residency programs is lacking. Only $25 \%$ of surveyed IM residency programs offer formal ultrasound training. We evaluate a resident-driven ultrasound curriculum to address this educational need.

SETTING AND PARTICIPANTS: IM residents and medical students at the California Pacific Medical Center participated in the ultrasound curriculum between October 2017 and May 2018. Two Sonosite X-Porte and two handheld GE Healthcare V-Scan ultrasound machines were used in the study. Motivated residents volunteered to develop workshops under the guidance of fellows and attending physicians with expertise in bedside ultrasound. These residents led weekly one-hour ultrasound rounds consisting of brief didactics followed by hands-on mentored scanning of inpatient volunteers. The curriculum included core topics in cardiac, pulmonary, hepatobiliary, venous, and renal ultrasound.

DESCRIPTION: Baseline knowledge of trainees was assessed anonymously through a written evaluation administered before and after each session. Each evaluation included the basics of ultrasound physics, scanning technique, and interpretation of clinically relevant ultrasound images. Residents were anonymously evaluated on a 10-point confidence scale at the start and end of the program. Qualitative feedback was also solicited. Our primary outcome was the change in performance in the written evaluation score assessing ultrasound knowledge, and a secondary outcome was the change in resident confidence level.

EVALUATION: A total of 56 residents and 9 medical students completed the ultrasound knowledge assessment. Their average score increased from $55.2 \%$ (standard deviation [SD] 27.4) before teaching to $83.6 \%$ (SD 18.5) after the teaching, with a significant increase of $28.5 \%(\mathrm{p}<0.001$, paired $\mathrm{t}$ test). There were 69 residents who completed the confidence assessment. Their average confidence score increased from 2.6 (SD 1.7) at the beginning of the year to 4.2 (SD 2.2) at the end of the year, with an increase in average confidence levels by $16 \%$ ( $p<0.001$, two-sample $\mathrm{t}$ test). The qualitative feedback included a desire to keep attendance optional and to maintain the hands-on format of scanning patient volunteers. Trainees also expressed a desire to facilitate attendance by creating protected time for the ultrasound sessions.

DISCUSSION / REFLECTION / LESSONS LEARNED: A residentdriven ultrasound initiative was an effective educational model in IM residency as shown by a significant improvement in knowledge scores and confidence ratings globally across all training levels. Future directions for this program include integration with inpatient medicine rounds or morning report, studying the effect of POCUS on patient satisfaction, and testing residents for diagnostic accuracy.

\section{RESIDENT REPRESENTATION ON HOSPITAL COMMITTEES: A MODEL FOR ADVOCACY, QUALITY IMPROVEMENT AND PROFESSIONAL GROWTH}

Nathan C. Nowalk ${ }^{1}$; June Kampangkaew ${ }^{1}$; Diana Stewart ${ }^{2}$; Erica Lescinskas $^{1}$; Charlie Lan ${ }^{1} .{ }^{1}$ Baylor College of Medicine, Houston, TX; ${ }^{2}$ Baylor College of Medicine/Michael E. DeBakey VA Medical Center, Houston, TX. (Control ID \#3186257)

NEEDS AND OBJECTIVES: Needs: Resident representation on hospital and institutional committees allows residents to learn how the leaders of their institution approach systems-based issues and implement change. We have developed a framework within our institution to increase resident representation at hospital committees to facilitate resident advocacy, involvement in quality improvement, and resident empowerment. Objectives: To provide residents opportunities to participate in quality improvement initiatives through committees To develop a sustainable model for meaningful resident participation on committees

SETTING AND PARTICIPANTS: Hospital committees were selected from four hospitals in the Texas Medical Center. Participants included all $2^{\text {nd }}$ year residents from Baylor College of Medicine's Internal Medicine Residency $(n=51)$.

DESCRIPTION: There is a universal expectation for physicians to serve on institutional committees after their training. However, no similar expectation exists for the resident in training. Only recently did the Clinical Learning Environment Review (CLER) suggest training programs involve residents in committee participation to integrate trainees into quality improvement. When hospital committees involve residents, there is a mutual benefit between hospital leadership and the trainee. Thus, we aimed to develop a sustainable framework for ensuring residents have the opportunity to serve on hospital committees in an impactful way. Seventeen committees were identified among the VA, county hospital, private hospital and large oncology center. A group of 3 to 4 PGY-2 residents were assigned to one hospital committee with a committee administrator as a point of contact. Examples of some of these committees include: Code Blue committee, Quality Review, Infection Prevention, Pharmacy and Therapeutics and Antibiotic Stewardship. A monthly electronic survey was used to confirm attendance, receive feedback from residents, and demonstrate meaningful impact.

EVALUATION: Early qualitative data suggests our initiative is succeeding in involving residents in hospital processes. A Patient Flow Committee member has reported that the residents have been "very helpful in providing valuable insight into the process map for admissions from the emergency room." Other residents have standardized code blue notes while serving on the CPR committee. We continue to collect monthly surveys to monitor compliance, resident contributions, and obstacles encountered while serving on these committees. 
DISCUSSION / REFLECTION / LESSONS LEARNED: We recognized an untapped potential in having residents participate on hospital committees. At a teaching hospital, residents offer a unique perspective as they intersect with multiple disciplines and work at the front line of patient care. Thus, for a hospital initiative to achieve the fullest success, the buy-in of the resident stakeholder is paramount. For the resident, committees offer an opportunity to practice leadership, network with specialists in their desired field, and contribute to hospital projects.

\section{RESIDENT RESPONSE TO A SPECIALTY CLINIC SESSION FOR PATIENTS WITH CHRONIC PAIN ON OPIOIDS}

Serena L. Roth ${ }^{1}$; Laila Khalid ${ }^{1}$; Aaron Burkenroad ${ }^{3}$; Joanna L. Starrels ${ }^{2}$. ${ }^{1}$ Montefiore Medical Center, Bronx, NY; ${ }^{2}$ Albert Einstein College of Medicine \& Montefiore Medical Center, Bronx, NY; ${ }^{3}$ University of California, Los Angeles, Los Angeles, CA. (Control ID \#3184404)

NEEDS AND OBJECTIVES: Internal medicine (IM) residents have low confidence and satisfaction treating patients with chronic pain on chronic opioid therapy (COT). To address the dual aims of training residents about judicious opioid prescribing and providing guideline-adherent patient care, we developed a weekly resident teaching practice session for patients on COT, the Power Over Pain (POP) Clinic. We report on resident survey responses after completion of the 2-year curriculum.

SETTING AND PARTICIPANTS: POP Clinic is a weekly session at a large urban IM teaching practice in Bronx, NY that cares for 150 patients on COT. IM residents rotate through POP Clinic 8 times during their PGY2\&3 years.

DESCRIPTION: Since 9/2016, all patients on COT must be seen in POP Clinic at least yearly. Residents in POP Clinic see 1-3 patients per session in hour-long visits precepted by two IM attendings with expertise in chronic pain and opioids. Residents receive 30-minute didactic lectures before each session on topics including opioid safety and efficacy, multimodal pain care, opioid use disorder, and interpreting urine drug tests. Precepting focuses on summarizing the risks and benefits of opioid therapy, optimizing medications and non-pharmacologic treatments, counseling patients, and naloxone provision.

EVALUATION: Residents completed an online survey prior to their first POP session during PGY2 (baseline) and at the end of PGY3 (post-POP). The survey assessed confidence and reward in managing patients on COT and experiences in the POP Clinic on a 5-point Likert scale from "strongly disagree" to "strongly agree." Paired t-tests were used to compare confidence and reward in the baseline and post-POP periods. Of 23 residents who received the full POP curriculum between 2016-2018, 21 (91\%) completed both surveys and were included in the analysis. In the post-POP survey, residents agreed that POP Clinic improved their confidence in managing patients with chronic pain, their ability to perform comprehensive pain assessments and identify opioid use disorder, and their confidence in prescribing nonopioid pain medication (median response 4 for all items). Resident confidence in prescribing opioids significantly decreased from baseline (mean 3.8) to post-POP (mean 2.7, p<0.001). There was no significant difference in resident enjoyment in managing patients with chronic pain from baseline (mean 2.6) to post-POP (mean 2.3, p=0.23).

DISCUSSION / REFLECTION / LESSONS LEARNED: IM residents reported positive impact of POP Clinic on their confidence managing chronic pain and COT. However, confidence in opioid prescribing decreased and reward in managing patients with chronic pain was unchanged. The lack of improvement in confidence in opioid prescribing may reflect increased awareness of the challenges and risks associated with prescribing opioids and may suggest a need to revise the curriculum. Even with improved skills, it is challenging to increase resident enjoyment of managing patients with chronic pain.

\section{RESIDENT-LED EHR TRAINING IN AN ACADEMIC AMBULA- TORY CLINIC}

Elizabeth Tran ${ }^{1}$; Jacob Murray ${ }^{1}$; Bernadette Miller ${ }^{2}$; Carmen Vesbianu'; Kristin Rodriguez ${ }^{2}$. ${ }^{1}$ The University of Oklahoma, Tulsa, OK; ${ }^{2}$ University of Oklahoma School of Community Medicine, Tulsa, OK. (Control ID \#3178579)

NEEDS AND OBJECTIVES: Despite the widespread use of electronic health records (EHR), medical trainees continue to struggle with effectively navigating these systems. Evidence shows that the usual classroom training is insufficient for new EHR users, yet we found no studies that have evaluated or disseminated the effectiveness of alternative training methods. Consequently, we developed a comprehensive, "hands-on" training course for the incoming PGY1 residents with the aim of improving the competency and efficiency of their use with our EHR system.

SETTING AND PARTICIPANTS: Participants included all PGY1 Internal Medicine residents $(\mathrm{n}=12)$. The training sessions were led by PGY2 and PGY3 residents $(n=18)$. Two faculty members administered pre- and postEHR skill assessments independent from the training sessions.

DESCRIPTION: Each year, incoming interns participate in a 4-hour classroom EHR training taught by the on-campus EHR specialist during their orientation week. For this project, we administered an assessment to evaluate the interns' understanding and utilization of basic functions of Centricity EHR after the conventional training was completed. From these results, we identified common problem areas and deficiencies that required correction. We designed a comprehensive resident-led training that included placing orders, e-prescribing medications, reviewing labs and test results, etc. The training was delivered during the first week of +1 ambulatory block and consisted of a one-hour noon session from Monday to Thursday for a total of four sessions. The interns completed a post-assessment at the conclusion of the training.

EVALUATION: Twelve PGY-1 residents were evaluated using a 13-item workflow skills assessment tool. The assessment required the intern to demonstrate each function/skill in real-time with each skill graded based on the ability to complete tasks independently, with assistance, or needing additional training. Before the training, $27.9 \%$ of interns were able to perform basic EHR tasks independently. After the training, $90.5 \%$ of participants achieved competency. The overall difference between pre- and posttests was statistically significant $(\mathrm{p}<.001)$.

DISCUSSION / REFLECTION / LESSONS LEARNED: While EHR systems are becoming a necessity in all clinics, training, specifically for residents, remains subpar. We demonstrated that conventional EHR training is insufficient and that resident-based training has a significant impact on the effective utilization of EHR in every day clinical situations.

\section{RESIDENT-LED REVIEW SESSION FOR THE INTERNAL MED- ICINE CORE CLERKSHIP}

Shradha A. Kulkarni. Baylor College of Medicine, Houston, TX. (Control ID \#3187019)

NEEDS AND OBJECTIVES: Every year, over 20,000 students matriculate to medical schools in the United States. Every one of these students are required to complete the core clerkships including Internal Medicine (IM). 
The National Board of Medical Examiners (NBME) subject examinations remain a staple of undergraduate medical education (UME) core clerkships in North America due to their established standardized and objective assessment of medical knowledge. While there are widely accepted review materials such as NBME self-assessments, question banks, and Step up to Medicine and the Board Review Series texts, no standardized oral shelf exam reviews are recognized among medical students. At Baylor College of Medicine, feedback from students on the IM clerkship revealed a need for improved review content for the NBME exam. The following innovation was developed to address this need.

SETTING AND PARTICIPANTS: This innovation involved medical students on the IM clerkship at Baylor College of Medicine, and took place as an after-hours, voluntary session on campus.

DESCRIPTION: A voluntary, resident-led review was held during the middle of each IM clerkship for an 18-month period. The review was 2 hours in duration, led by a senior IM resident, and structured as a contentbased PowerPoint (with intermittent audience participation questions) covering all major organ systems. Additionally, tools and strategies for studying were provided to the students.

EVALUATION: Anonymous surveys were distributed before and after the session to examine the effect on the students' perceived level of preparedness. The surveys consisted of qualitative questions with a 5-point Likert scale (with 5 being the most positive).

DISCUSSION / REFLECTION / LESSONS LEARNED: Six review sessions were held and over $80 \%$ of clerkship students attended the sessions. Results showed a statistically significant increase (using a t-test) in perceived knowledge of the content and structure of the exam (2.6 to 4.0), confidence studying for the exam (3.7 to 4.2), and overall preparedness for the exam (3.2 to 3.5). The overall rating for the session had a mean of 4.8. This innovation increased medical student confidence and perceived preparedness for the NBME IM exam. The high attendance rate and positive reviews likely indicate effectiveness and potential for continued success. Given the standardized content and structure of the NBME exam, this shelf review session is likely applicable to any clerkship at any medical school that administers the NBME exam.

\section{RESIDENTS AS TEACHERS IN MEDICAL STUDENT CLINICAL SKILLS COURSE EFFECTS ON BEDSIDE TEACHING}

Hector H. Gonzalez ${ }^{1}$; Lisa C. Martinez ${ }^{2} .{ }^{1}$ FAU Charles E. Schmidt College of Medicine, Delray Beach, FL; ${ }^{2}$ Florida Atlantic University, Boca Raton, FL. (Control ID \#3184633)

NEEDS AND OBJECTIVES: There has been a decrease in bedside teaching in both undergraduate and graduate medical education. Our objective was to implement a clinical skills curriculum where internal medicine residents served as teachers for first year medical students. We wish to explore the influence of faculty development in residents as teachers by analyzing bedside teaching following participation in clinical skills course sessions. SETTING AND PARTICIPANTS: We implemented the curriculum during first semester clinical skills course at our medical school's simulation center. The study population consisted of 10 PGY-2 or PGY-3 residents randomly selected based on their clinic schedules. Sixty residents who did not participate in the teaching sessions were used as controls during the survey. The course consisted of bedside clinical skills teaching with first year students incorporating history taking, and whole organ system physical examination.

DESCRIPTION: Residents were assigned afternoon teaching sessions at the simulation center, participating on average in 3-4 sessions. On assigned days residents attended faculty development at $12: 15$ focusing on properly performing clinical skills and effectively teach them. Teaching followed this from 1:20-4pm with medical students. This course focused on teaching communication, physical exam techniques, professionalism, and clinical reasoning. Residents observed students, providing verbal coaching and feedback. To assure resident teaching was equivalent to using faculty, residents were assessed by student evaluation of residents and end of course OSCE of students.

EVALUATION: An anonymous survey link was sent via email to all residents for completion. This study was approved by the institutional IRB. The survey evaluated resident's previous teaching experience, interest in teaching and personal comfort with bedside teaching. Residents participating were asked about their perceived improvement in clinical skills teaching and comfort with bedside education following completion of the course.

DISCUSSION / REFLECTION / LESSONS LEARNED: This study sought to be more than just an adaptation to local needs. An important lesson was the important correlation between the symbiotic benefit of medical student and resident teaching. We sought to determine whether involvement in first year medical student clinical skills teaching increases resident comfort in teaching at bedside. We have created a formalized curriculum where the needs of satisfying course objectives can be accomplished by our residents, while providing the courage to lead their clinical teams at bedside.

\section{RESTRUCTURING AMBULATORY CURRICULUM}

Jasmine Omar; Danielle L. Heidemann; Alexis C. Haftka. Henry Ford Health System, Detroit, MI. (Control ID \#3186315)

NEEDS AND OBJECTIVES: During the 2017-2018 academic year, the curriculum for ambulatory medicine consisted mainly of discussion of clinical trials from scholarly journals. Results of both the mid-year and end of the year survey administered to residents showed dissatisfaction with the educational session, due to emphasis on clinical trials and lack of discussion regarding medical management of common outpatient pathology. This prompted restructuring of the curriculum with the goal of improving resident satisfaction, providing more management guidelines, and better preparing residents for board exams.

SETTING AND PARTICIPANTS: Ambulatory education in our institution takes place prior to our residents' traditional half-day continuity clinic. Small groups of up to 10 residents and 4 faculty participate in a "flipped classroom" format.

DESCRIPTION: In the 2018-2019 academic year, our ambulatory curriculum underwent several key changes. First, educational sessions were shifted to focus more on guidelines and medical management of outpatient conditions. Second, each month a subspecialty topic was assigned that paralleled our inpatient educational noon conference. In this format, the outpatient curriculum built on concepts and topics addressed in our inpatient curriculum. Third, we utilized our in-training results to help optimize topic selection to help residents focus on areas where scores tend to be lower. At the end of each month we had a resident-lead board review session to review key points and answer questions as a group.

EVALUATION: Mid -year surveys identical to the previous academic year were used to assess the resident and preceptor response to changes in the curriculum. $77 \%$ of residents rated the curriculum as good or excellent, a $30 \%$ increase from the year prior. $23 \%$ of residents rated it as fair/poor, a $30 \%$ decrease from the year prior. When asked about board review content, $56 \%$ of residents felt there were enough board review questions, a $38 \%$ increase from the year prior. Preceptors also felt their residents were more engaged during the sessions then they had been previously. Direct resident feedback has been 
positive, including comments that they appreciate the synchrony of the inpatient and outpatient curriculum

DISCUSSION / REFLECTION / LESSONS LEARNED: Resident satisfaction with the ambulatory curriculum has improved with the recent curriculum changes, leading to increased engagement in our educational sessions. Residents prefer broad discussions about clinic management rather than discussing clinical trials. By organizing these discussions to supplement the inpatient curriculum, residents felt they had a more structured educational experience. Board review questions help to reinforce the monthly topic while simultaneously preparing the residents for the ABIM. Choosing topics that are relevant and enjoyable to the targeted learners help enrich the ambulatory educational experience through increased participation and learning opportunities.

\section{RETHINKING THE MEDICINE SUB-INTERNSHIP: TARGETING CRITICAL SKILLS THROUGH ACTIVE LEARNING AND MEN- TORSHIP}

Alice Tang ${ }^{1}$; Sydney Katz ${ }^{2}$; Stephanie J. Tang ${ }^{3} .{ }^{1}$ Weill Cornell Medical College, New York, NY; ${ }^{2}$ Weill Cornell, Brooklyn, NY; ${ }^{3}$ Weill Cornell Medicine | NewYork-Presbyterian Hospital, Long Island City, NY. (Control ID \#3184749)

NEEDS AND OBJECTIVES: While sub-internship rotations are typically viewed as a capstone experience prior to internship, most lack a formal curriculum. Students are often expected to become competent in essential clinical skills by passively observatng others; however, clinical teachers are varied in their skills and observation may not be the most effective means for learning these skills. Furthermore, students often do not receive sufficient observation-based formative feedback. To address these needs, we created a new sub-internship rotation with key objectives to: (1) Hone essential clinical skills and entrustable professional activities (EPAs) through interactive workshops, (2) Stimulate weekly self- reflection, feedback, and goal-setting, and (3) Provide frequent high quality feedback on core clinical skills.

SETTING AND PARTICIPANTS: Fifteen fourth year medical students completed a novel medicine sub-internship rotation at an affiliate hospital of a major urban academic medical center between July 2017 and September 2018.

DESCRIPTION: Our four-week sub-internship rotation was designed to foster students' mastery of the EPAs through integrating direct faculty observation, collaborative feedback, and a clinical skills-based "Foundations" curriculum. High quality real-time feedback was facilitated by the team structure of one attending, one PA, and one sub-intern. Self-reflection, feedback, and goal-setting were facilitated by weekly meetings with faculty reviewing a collaborative feedback form. Clinical skills were developed through interactive workshops that targeted essential skills necessary for residency.

EVALUATION: All students completed an anonymous course evaluation comprised of free response and Likert-type items. Mean confidence in starting internship improved significantly after completing the rotation (from 2.2 to 4.3 on a 5-point scale). Students rated autonomy, coaching and mentorship, observation and feedback, and culturally sensitive care highly (mean ratings ranging 4.9 to 5 on a 5 -point scale).

DISCUSSION / REFLECTION / LESSONS LEARNED: Our innovative sub-internship rotation highlights the benefits of direct observation and mentorship, collaborative feedback, and a skills-based curriculum. Our rotation demonstrates the value of a 1:1 attending to learner ratio. Future directions include expanding the curriculum and the collaborative feedback form across all other medicine sub-internships sites. Our preliminary data is limited by the small sample size. We acknowledge the challenges in maintaining quality assurance as we expand this rotation to other sites. We recognize how crucial faculty development will be to ensure students receive the same highquality coaching and mentorship across sites.

\section{RISKY BUSINESS: ON THE ROAD TO ELIMINATION OF HCV THROUGH IMPROVED RISK ASSESSMENT}

Dawn Fishbein ${ }^{2}$; Shari Sawney²; Stephen Fernandez ${ }^{2}$; Emily Paku²; Sara Miller ${ }^{1} .{ }^{1}$ Med-IQ, Baltimore, MD; ${ }^{2}$ MedStar Health Reasearch Institute, Washington, DC. (Control ID \#3185129)

NEEDS AND OBJECTIVES: Hepatitis C virus infection (HCV) represents a major public health threat that continues to grow, largely due to the increased use of injection drugs. A focus on patients at high risk for $\mathrm{HCV}$ is a critical part of the public health effort to identify, cure, and eliminate this disease. The rise in HCV rates demonstrates a continued need for risk-based testing and requires a more informed and educated clinician compared to birth cohort testing. This educational initiative was employed to improve riskbased HCV testing and linkage to care.

SETTING AND PARTICIPANTS: We implemented an iterative and multifaceted HCV quality improvement initiative beginning in February 2018 that included multiple educational opportunities and continuous sharing of data regarding clinical practice patterns. This initiative included clinicians in 8 primary care and/or women's health clinics across the MedStar Health, a large regional healthcare system in Maryland and Washington, DC.

DESCRIPTION: Education was delivered live in-person, online, and through short evidence-based text publications distributed on a bimonthly basis. Data evaluating baseline practice and changes included survey evaluation of perceptions, attitudes, and barriers and quantitative assessment of practice patterns from March 2018 through the intervention period as documented in the EHR. Changes in practice patterns before and after the intervention were compared with a chisquared test; a $Z$ test was used to calculate the odds ratio across groups. EVALUATION: In the baseline provider survey, $64.2 \%$ of clinicians $(\mathrm{n}=53)$ ranked their ability to identify candidates for HCV testing based on risk as a 3 or 4 on a 4 -point Likert scale ( $4=$ highest); $58.5 \%$ ranked their ability to elicit an accurate social history to evaluate behavioral risks as a 3 or 4 . Among clinicians who had been in the same location for 3 or more years $(n=42), 28.6 \%$ reported increased use of injection drugs among their patients. EHR data demonstrate low levels of risk evaluations as well as $\mathrm{HCV}$ testing for those with documented high risk among both the intervention and non-intervention groups at baseline. Improvement in the proportion of evaluations were observed in the intervention group (18.6\% to $20.5 \%$ ) as compared to the non-intervention group (8.5\% to $8.78 \%$ ) (OR: 1.094 [CI 1.025-1.166]). When high-risk behaviors were documented, $\mathrm{HCV}$ testing improved in both groups $(21.8 \%$ to $24.1 \%$ intervention and $22.01 \%$ to $24.6 \%$ non-intervention groups), the improvements were not statistically significantly better among the intervention group (OR .983; CL .715-1.351).

DISCUSSION / REFLECTION / LESSONS LEARNED: Improving clinical practice within contemporary overtaxed healthcare settings requires well-designed, continuous, and iterative interventions which include both education and data sharing. Even in the context of such initiatives, improvements are frequently slow. If HCV elimination is to become a reality, particularly in a climate of increased risks associated with the ongoing opioid epidemic, initiatives such as this one are critical. 
SGIM LEADERSHIP IN HEALTH POLICY CURRICULUM INITIATIVE

Molly A. Fisher ${ }^{1}$; Sarah G. Candler ${ }^{2}$; Susan Lane ${ }^{4}$; Colin Robinson ${ }^{3}$; Latonya Riddle-Jones ${ }^{5}$; Michi Yukawa ${ }^{6}$. ${ }^{1}$ University of Pittsburgh Medical Center, Pittsburgh, PA; ${ }^{2}$ Baylor College of Medicine, Houston, TX; ${ }^{3}$ UCLA, Los Angeles, CA; ${ }^{4}$ Stony Brook Medicine, Stony Brook, NY; ${ }^{5}$ Wayne State University School of Medicine, Farmington Hills, MI; ${ }^{6}$ University of California San Francisco, San Francisco, CA. (Control ID \#3185098)

NEEDS AND OBJECTIVES: 1 . Conduct a Delphi survey of health policy and education experts to determine which health policy topics to teach to internal medicine residents 2. Create four one-hour lessons that focus on Medicare, Medicaid, social determinants of health, and private insurance and the healthcare marketplace 3. Implement and evaluate a pilot curriculum at participating institutions 4 . Disseminate this curriculum to medical training programs to adapt to their home institutions SETTING AND PARTICIPANTS: This curriculum is to be taught to internal medicine and med-peds residents. We have designed the curriculum so that it can be taught in either small group or large lecture settings. There are four separate one-hour lessons that can be taught independently or as a complete curriculum.

DESCRIPTION: Several major healthcare organizations have stated the importance of educating physicians to learn about health policy. However, there are no current guidelines on what information should be taught to physicians or trainees, and how this content should be delivered. Based on a Delphi survey of health policy experts conducted by the authors, four major topics were identified as essential components of a health policy curriculum for internal medicine residents: Medicare, Medicaid, social determinants of health, and private insurance and the healthcare marketplace. Members of the SGIM Leadership in Health Policy (LEAHP) fellowship worked to create four one-hour lessons based on these topics. Each lesson consists of a didactic component and a group activity. The authors of this curriculum plan to pilot the curriculum at their home institutions during the 2019-2020 academic year. Once the pilot year is complete, a standardized evaluation tool that assesses attitudes and knowledge will help to inform the authors on how to alter the curriculum prior to dissemination. Once edited, we intend to disseminate this curriculum through the Society for General Internal Medicine Health Policy Committee. Future plans include teaching the curriculum at the medical student level and creating additional modules to teach if more curricular time is available.

EVALUATION: We developed a standardized evaluation tool that will assess the knowledge and attitudes of the residents participating in the program. It is a pre-survey and post-survey that we will administer shortly after completing the program.

DISCUSSION / REFLECTION / LESSONS LEARNED: Creating this curriculum has been a large multi-institutional effort with faculty members from a wide variety of backgrounds. Through the pilot project, we intend to find areas of improvement in the way the content is delivered as well as adapting the activities as needed.

SHOW ME THE MONEY! RESULTS FROM A PILOT FINANCIAL WELLNESS PROGRAM FOR INTERNAL MEDICINE RESIDENTS

Patricia Ng; Rachel Wong. Stony Brook University School of Medicine, Port Jefferson Station, NY. (Control ID \#3160604)
NEEDS AND OBJECTIVES: Financial wellness has become a topic of interest, with a 2012 study showing that higher educational debt is associated with lower quality of life and increased burnout rates. Our initial needs assessment identified several resident profiles with varying financial capabilities and concern for personal debt. Our aims were to pilot and assess the efficacy of a tailored financial wellness program in improving the finance planning behaviors of internal medicine residents.

SETTING AND PARTICIPANTS: Our pilot program was offered to 84 categorical and 10 prelimiary internal medicine residents at a universitybased program in Stony Brook, NY during the 2017-2018 academic year.

DESCRIPTION: Our pilot program was created by faculty champions and an institution hired financial advisor. The program included 90-minute interactive workshops and a mandatory mid-year financial wellness checkin with program directors. Workshops were given during intern orientation to capture new interns and in the evening to target seniors. Program topics included loan repayment, budgeting, retirement planning, and income protection. During mid-year check-ins, program directors inquired about financial stress during overall wellness screening and ensured that all residents were aware of local financial resources. Residents also received educational handouts and simplified financial planning to-do lists created by faculty developers.

EVALUATION: Residents completed surveys immediately before and after the workshop, and a follow-up survey at the end of the year. Surveys included modified questions from the FINRA National Financial Capability Survey and feedback about the intervention. Fifty-three (56.4\%) residents completed the pre-workshop survey, 49 (52.1\%) completed the postworkshop survey, and 40 (42.6\%) completed the follow-up survey. Majority $(97.9 \%$ ) rated the workshops "Good/Excellent" and all requested additional didactics. At the end of the year, residents reported improvements in several positive financial planning behaviors. For debt management, more residents reported creating a student loan repayment plan $(43.4 \%$ vs $58.8 \%)$ and consolidating to the public service loan forgiveness program $(29.4 \%$ vs $74.1 \%$ ). For savings and retirement, there was a statistically significant increase in residents being aware about their employers' retirement plans (73.6\% vs $94.2 \%, \mathrm{p}=0.0113$ ) and contributing regularly to retirement $(11.3 \%$ vs $35.3 \%$, $\mathrm{p}=0.0214$ ). Lastly for catastrophic planning, there was a statistically significant increase in residents having rainy day funds $(30.8 \%$ vs $65.0 \%, \mathrm{p}=0.0007)$.

DISCUSSION / REFLECTION / LESSONS LEARNED: Our results suggest that a tailored financial wellness program may improve financial planning behaviors of internal medicine residents. When planning a financial wellness program, interventions should include local financial experts, simplified to-do lists, and multiple didactic sessions. We plan assess for generalizability of our program by implementing similar financial wellness programs at 2 other internal medicine residency programs.

\section{SMARTPHONE DEVICE FOR OPHTHALMOSCOPY SIMULA- TION TRAINING IN UNDERGRADUATE MEDICAL EDUCA- TION.}

Patricia Nelson ${ }^{2}$; xin lu $^{2}$; Marah Hamdan²; Akaanksh Shetty ${ }^{2}$; Jessica R. Hungate $^{2}$; Thwe $\mathrm{Htay}^{1}{ }^{1}$ Texas Tech University HSC El Paso, El Paso, TX; ${ }^{2}$ Texas Tech University Health Sciences Center, El Paso, TX. (Control ID \#3184781)

NEEDS AND OBJECTIVES: The objective of this study is to measure the effectiveness of using a reproducible smartphone imaging for ophthalmology simulation training in enhancing learners' confidence and skill technique in imaging the retina. 
SETTING AND PARTICIPANTS: The setting of this study involved surveying 96 second year medical students new to the study of observing the eye during clinical skill training.

DESCRIPTION: The students were asked to compare the proficiency and ease of use of a traditional direct ophthalmoscope versus a new smartphone enabled imaging device. Each student was given pre-recorded instructional videos and directions prior to the use of both devices in order to ensure proper understanding of observing methods. The students were given pretraining surveys prior to the instructions on how to use the Ophthalmoscope and the imaging device, as well as a similar post training survey. The survey measured confidence on a 5 point scale. 88 responses were included in the study analysis.

EVALUATION: There was a low pre-survey confidence in imaging the retina, including obtaining the red reflex. "Not all confident" was ranked $42 \%$ for using a direct ophthalmoscope, $56.8 \%$ for identifying the red reflex, $58 \%$ for focusing on the optic nerve head, $63.6 \%$ for focusing on the fovea, $48.9 \%$ for focusing on an artery or vein, $63.6 \%$ for apertures and filters, and $35.2 \%$ for differentiating healthy vs. pathologic retina. Post training surveys showed a significant improvement in confidence. "Not at all confident" was ranked $0 \%$ for using the direct ophthalmoscope, $2.3 \%$ for identifying the red reflex, $0 \%$ for focusing on the optic nerve head, $4 \%$ for focusing on the fovea, $1.1 \%$ for focusing on an artery or vein, $57 \%$ for apertures and filters, and $2.3 \%$ for differentiating healthy vs. Pathologic retina.

DISCUSSION / REFLECTION / LESSONS LEARNED: Ophthalmoscopy allows for the non-invasive evaluation of patients eyes microvasculature, permitting direct detection of end organ pathology. Given its importance, it is a difficult skill for medical students to learn. Medical students often have a few contact hours of training on the direct ophthalmoscope throughout the undergraduate medical education. In addition, our questionnaire showed that 82 students $(92.3 \%)$ did not own a direct ophthalmoscope, which limits the opportunity of further independent skill development. This study shows that simulation training in ophthalmology appears to be an effective method in increasing learners confidence in skill technique and identifying retinal structures. Additionally, $47 \%$ of students preferred the smartphone imaging device to the direct ophthalmoscope The imaging device is 3-D printed, inexpensive, and allows for direct retinal photography that could be used to educate the patient. This indicates that this method could be used in undergraduate medical education in conjunction with the traditional ophthalmoscope.

\section{SOCIAL DETERMINANTS AND HOME VISITS}

Achilia Morrow $^{1}$; Amy Baldwin ${ }^{2}$; Laurel B. Murrow ${ }^{3}$; Suzanne H. Lester ${ }^{1}$. ${ }^{1}$ AU/UGA Medical Partnership, Athens, GA; ${ }^{2}$ AU / UGA Medical Partnership, Athens, GA; ${ }^{3}$ Mercy Health Center, Athens, GA. (Control ID \#3163251)

NEEDS AND OBJECTIVES: Social determinants play a major role in health and many medical schools have introduced curricula to teach these principles. However, early on, students may not connect the material to actual patient care. This year patient care was introduced into the award-winning community health course at the AU/UGA Medical Partnership to facilitate student understanding of the role of social determinants in patient management.

SETTING AND PARTICIPANTS: The community health course is a service-learning course that occurs during the first year of medical school. Students are placed in groups of 8-9, assigned 2 faculty preceptors (one clinical, one non-clinical), and then paired with a community organization. Our community partner, Mercy Health Center, is a free clinic serving uninsured patients in Athens-Clarke and surrounding counties.
DESCRIPTION: Students conduct home visits for selected patients. Visits include taking a history inclusive of the social determinants of health, developing a plan with guidance from their preceptors, and adding a note about the encounter to the patient's chart. Templates and prompts were provided to help students gather this information. Students completed reflective writing and presented "exemplar" patients to the entire class.

EVALUATION: Workshops and reflections have revealed that students have identified factors beyond patient control, have broadened their view of "who uninsured patients are," recognized the role of family/social support, and appreciated patient difficulties navigating the health care system. Surveys conducted at the beginning and end of the course will be used to formally gauge changes in student perspectives.

DISCUSSION / REFLECTION / LESSONS LEARNED: In addition to allowing students to identify social determinants that affect their patients' health, these visits have also provided early learning opportunities for the management of chronic diseases, including depression, role of specialty referrals, and how fragmentation of care can affect health. Additionally, promotion of a team approach has led to the investigation of legal assistance, transportation solutions, and assistance from community agencies. These early experiences will allow students to continue to recognize and incorporate these factors into patient care as they progress through their careers.

\section{SPANISH FOR MEDICAL PROFESSIONALS (INTERMEDIATE LEVEL). \\ Josune N. Iglesias. Rush University, Chicago, IL. (Control ID \#3185744)}

NEEDS AND OBJECTIVES: Objectives: 1-Communicate in Spanish with Spanish speaking patients at an intermediate level. 2- Students will be able to communicate in simple Spanish using mainly the present tense wiht the 3rd person "usted" and different expressions to indicate past and future actions. They should be able to utilize specific medical terms learned in class. 3- Students should be able to communicate with Spanish speaking patients and take a chief complain, history of present illness, past medical history, medication list and allergies.

SETTING AND PARTICIPANTS: The elective course runs throughout the M1 year (first year) in small groups (10-16 students) with an intermediate level of knolwdge of Spanish. Two hour per small group session and one hour online work per week of classes. Taught with lectures and in small group activity by a native spanish speaker faculty. The course is organized with emphasis to integration of topics covered in the new organ based curriculum.

DESCRIPTION: This course addresses the needs of medical students with intermediate experience in Spanish. Students participate in language tasks through listening, reading, writing, and conversation. Classes will be taught in Spanish in order to immerse students in the target language. This medical Spanish Course consists of: a. Formal instruction:There are in- class activities, such as role-playing and history taking. Students also expand their Spanish vocabulary with an emphasis on medical terminology. I- Introduction to the patient. II- Chief complaints III- History of Present Illness IVChest pain V- Shortness of breath and Past medical history VI- End Stage Renal Disease and Past medical history VII- amenorrhea and Urinary Tract Infection VIII- Mid term exam IX-Rash and Itching/ Medication list XAngioedema and medication list XI- New patient and allergies XII- Upper respiratory tract infection XIII- Ear pain XIV- Headache XV- Abdominal pain XVI- Final Exam/ Oral test b. Extracurricular activities and independent study via internet resources This course also includes a series of cultural extracurricular activities and Spanish language websites for students to practice Spanish independently outside of the classroom. By visiting a 
hispanic neighborhood near our instution and watching movie sessions, students will be able to engage in Spanish learning activities.

EVALUATION: I. Daily class participation (4 x 10 points each) $40 \%$ II. Attendance (10points) 10\% III. Quizzes (100 points) 10\% IV. Midterm exam (100 points) $10 \%$ V. Oral exam (100 points) $15 \%$ VI. Final exam (150 points) $15 \%$

DISCUSSION / REFLECTION / LESSONS LEARNED: This is the first Intermediate Medical Spanish course in our instuition driven by the need to learn spanish and communicate with our community in their native language. The eagerness of our medical students and leadership to learn medical Spanish has been instrumental. This course will improve by practicing history taking with Standarized Spanish speakers patients

STEMMING THE RED TIDE: DESIGN OF AN INEXPENSIVE MANNEQUIN TO TEACH TEAMS TO MANAGE MASSIVE VARICEAL BLEEDING.

Jorge Lara-Gutierrez; Erika L. Hoffman; Mary Ellen Elias. VA Pittsburgh, Pittsburgh, PA. (Control ID \#3182929)

NEEDS AND OBJECTIVES: Variceal hemorrhage is an example of the type of medical emergency that requires efficient care provided by a rapid response team. Teams must simultaneously ensure hemodynamic stability, airway protection, prevention and treatment of complications, and management of the bleeding. Balloon tamponade is an option for temporarily stopping bleeding from esophageal or gastric bleed while definitive treatment is being arranged. Simulation can be used to teach nursing staff to gather the appropriate equipment, to maintain the device after insertion, and to set up the traction and weights needed. It can also be used to help teach clinicians how to insert the tube and to inflate the balloons in the correct order to decrease potential complications. This type of simulation was felt to be essential for employee development to increase comfort with the equipment. We were not able to find a mannequin that possessed the desired capabilities. Our objective was to create a mannequin to facilitate team-based training and to provide the opportunity to practice responding to this scenario in a safe learning environment.

SETTING AND PARTICIPANTS: Training will take place in a simulated emergency department at the VA Pittsburgh Healthcare System. A scenario was created in which a patient with cirrhosis presents with massive hematemesis. Participants will be attending physicians, fellows, residents, and nurses from the emergency department and the intensive care unit.

DESCRIPTION: To address the need for a specialized mannequin a multidisciplinary planning team was formed. Participants included critical care \& ER attending, a simulation fellow, dentists, speech pathologists, and a biomedical engineer. The team identified that the simulator needed to allow faculty to teach teams to intubate the patient \& to insert and inflate tamponade tubes in the correct order during active bleeding. An old Laerdal mannequin was modified.

EVALUATION: A checklist has been created for each type of learner. Physicians will be assessed on treatment priorities in this clinical scenario including airway management, and balloon tamponade insertion. Nurses will be assessed on the ability to gather the correct supplies needed for insertion and to secure and maintain the balloon tamponade tube. They will learn safe set up of the over the bed traction and how to use the massive transfusion pump.

DISCUSSION / REFLECTION / LESSONS LEARNED: To create the level of fidelity deemed important by a multidisciplinary team, a full body mannequin was built utilizing an old Laerdal mannequin, a rebuilt upper airway and GI tract, a water pump, and a soda bottle. We can simulate massive upper GI bleeding. The mannequin can be intubated, and a balloon tamponade tube can be inserted \& inflated. The trainer can be replicated and at the cost of less than 200.00 USD. A team-based employee development curriculum has been created to help improve comfort with handling massive upper GI bleeding which is a low-frequency high-risk event. We are training our initial team and collecting data.

\section{STICKING TO THE SCRIPT: USING ILLNESS SCRIPT GRIDS TO SCAFFOLD LEARNING FOR PRE-CLERKSHIP MEDICAL STUDENTS}

McKinsey M. Pillsbury $^{1}$; Sirisha Narayana ${ }^{1,1}$; Susannah Cornes ${ }^{1,}{ }^{1}$; Denise M. Connor ${ }^{1,2}$. ${ }^{1}$ University of California, San Francisco, San Francisco, CA; ${ }^{2}$ University of California, San Francisco and San Francisco VA Medical Center, San Francisco, CA. (Control ID \#3181351)

NEEDS AND OBJECTIVES: Pre-clerkship medical students lack the clinical insights needed to structure their growing knowledge in ways that will optimize clinical reasoning. We sought to: $\bullet$ Establish the feasibility of using illness script grids to reinforce and structure early medical students' clinical knowledge $\bullet$ Determine pre-clerkship students' acceptance of illness script grids as a way to crystallize small group learning $\bullet$ Explore the feasibility of using freely available mobile software to create illness script libraries to encourage use in clerkships

SETTING AND PARTICIPANTS: All pre-clerkship UCSF medical students participate in a longitudinal diagnostic reasoning curriculum. In first year, reasoning workshops introduce illness scripts and compare/contrast (vertical) learning. In second year, students take a deep dive into clinical reasoning through a capstone course, the Diagnostic Reasoning (DR) Block. DESCRIPTION: Students review an online module describing the illness script grid format and create grids for clinical syndromes discussed in reasoning workshops as wrap-up work. Key and differentiating features of diagnoses relevant to a clinical syndrome (e.g. acute chest pain) are entered in an illness script format (predisposing factors, clinical features, time course, pathophysiology). Model grids are released to students, who also receive personalized feedback on one grid. Students use free, mobile-based software (e.g. GoogleDocs) to construct grids with an illness script template. This system (ScriptSmart) enables students to construct an illness script library organized around core clinical problems. Students can access, modify, and share this library in multiple settings, and can enrich scripts during clerkships.

EVALUATION: Students complete a survey after the DR Block, including questions evaluating their impression of illness script grids. We will analyze responses to determine acceptability and feasibility of using grids to structure learning. An IRB proposal is underway to enable us to disseminate this data, and we anticipate including this analysis if accepted at SGIM. Future work will include study of clerkship students to assess the impact of early exposure to illness script grids, and optimization of ScriptSmart using human-centered design.

DISCUSSION / REFLECTION / LESSONS LEARNED: In medical schools across the United States, students are entering clinical settings earlier in training, requiring new approaches to support early integration of clinical and classroom-based knowledge. Solidifying learning using compare/ contrast illness script grids has the potential to help students build mental frameworks that support their clinical reasoning. ScriptSmart is a practical way to scaffold clinical knowledge and reinforce clinical reasoning concepts. The use of freely available software to create a personalized, searchable, and modifiable mobile illness script library represents a novel way to model knowledge acquisition and encourage integration during clerkships. 
ONLINE RESOURCE URL (OPTIONAL): ScriptSmart module: https:// ucsf.box.com/s/z2gga2iuuwpu1183sooly6krtmjp3ggj

\section{STRETCHING BEYOND CLERKSHIPS: A LONGITUDINAL} POST-CLERKSHIP COURSE IN SOCIAL MEDICINE

Michelle Cleeves ${ }^{1}$; Margaret $\mathrm{Tomcho}^{2}$; Jennifer Adams ${ }^{2} .{ }^{1}$ Denver Health and Hospital Authority, Denver, CO; ${ }^{2}$ Denver Health, Denver, CO. (Control ID \#3183888)

NEEDS AND OBJECTIVES: The University of Colorado Denver Health Longitudinal Integrated Clerkship (DH-LIC) is based at an urban safety-net hospital. Nationally, there is variability in how post-clerkship students and medical schools structure learning, ${ }^{1}$ and debate over best utilization of this time. A needs assessment of DH-LIC students $(n=12)$ demonstrated high interest in a post-clerkship elective. Based on these results, the Longitudinal Social Medicine Elective (LSME) was implemented and encompasses a mentored project and a course in social medicine. Primary goals were to allow students to continue mentorship relationships with faculty, engage in mentored scholarly work, and further develop knowledge and skills in social medicine. SETTING AND PARTICIPANTS: Students in the DH-LIC were eligible to enroll in the LSME during their fourth year. Currently, the elective is in its second year and has enrolled nine students over two years (from16 eligible LIC students).

DESCRIPTION: Students identify a capstone project in the umbrella areas of medical education, community engagement, or quality improvement (QI). The course also includes a week-long intensive course in social medicine encompassing topics such as trauma-informed care, implicit bias, correctional care, and health care for the homeless, as well as service learning and reflective writing. The capstone of the course is student presentations of project work to peers, faculty and health system and community leaders. Illustrative examples of projects include: utilization of palliative care in the SICU, a qualitative study of impact of LIC students on patient satisfaction, development of a radiology curriculum for LIC students, implementation of a service learning curriculum for LIC students, and a collaborative QI project to improve primary care engagement for patients with serious mental illness.

EVALUATION: Students rated their overall satisfaction with the course as high to very high (100\%) and all students felt this elective filled an important gap in their education. $100 \%$ reported their interest in practicing at Denver Health after residency was high or very high at completion of the longitudinal immersive course. To assess long-term impact on career focus on underserved care, scholarly work and advocacy, graduates of the program will be surveyed annually.

DISCUSSION / REFLECTION / LESSONS LEARNED: Strengths of this innovation include meaningful projects that serve the Denver Health community and allow students to produce scholarly work. Student participation strengthened student commitment to the underserved and understanding of social medicine.

\section{STUDENT-DIRECTED COMMUNITY ENGAGEMENT PRO- JECTS AS A LEARNING LABORATORY FOR PHYSICIAN LEAD- ERSHIP - A SIX-YEAR EXPERIENCE}

$\underline{\text { Brent C. Williams }}^{1}$; Joy C. Williams ${ }^{1}$; Jason Bell ${ }^{1}$; Katherine L. Hughey ${ }^{1}$; Patricia Mullan ${ }^{1}$; Andrew J. Haig ${ }^{2} .{ }^{1}$ University of Michigan, Ann Arbor, MI;

${ }^{2}$ The University of Michigan, Ann Arbor, MI. (Control ID \#3185312)

NEEDS AND OBJECTIVES: Two challenges facing medical schools in fostering students as leaders in community-engaged healthcare are: a) few leadership curricula include content based on medical students' perceived needs, and b) true collaborations between students and community-based organizations (CBOs) (as opposed to service-learning) are difficult due to student turnover and full curricula. Our objective was to design a communitybased 'learning laboratory' to promote leadership skills in academiccommunity collaboration through reflection-on-action.

SETTING AND PARTICIPANTS: Second year medical students in an elective co-curriculum in health disparities at University of Michigan Medical School from 2012 to 2017.

DESCRIPTION: Each year, teams of 4-6 students were tasked to engage with a CBO of their choice over 5-7 months to complete a 'Mini Field Project'. Initially the assignment was kept small, requiring only structured interviews or site visits. Later, the task was expanded to require collaborating with a $\mathrm{CBO}$ to design and complete a project resulting in a 'deliverable' to the CBO. At the mid- and endpoints of the assignment teams wrote structured reflective narratives on physician leadership and organizational collaboration. After completion teams presented their projects to each other using persuasive, creative formats. Over the six years, 174 students formed 45 teams to engage with 38 CBOs. 15 projects consisted of interviews only; 5 were site visits; and 25 were projects, 21 of which were completed.

EVALUATION: In early years, students' observations on physicians as leaders included traditional features of leadership (e.g., strategic vision) along with non-traditional domains (e.g., professional identity development, worklife balance). In later years prompts related to the non-traditional topics were included in the reflective narrative reports. Annual student feedback on the value of the Mini Field Projects was highly positive. Feedback on work burden for this extracurricular activity informed the size and scope of projects over time. A one-time survey of CBO experience (Year 2) was highly positive.

DISCUSSION / REFLECTION / LESSONS LEARNED: Mini Field Projects are now a standard component of the health disparities cocurriculum, and have been highlighted by the medical school as exemplary community engagement by our students. Our next goals are to continue projects at individual sites over several years, and involve students in other health professions schools to promote interprofessional education in community settings.

SUCCESSFUL IMPLEMENTATION OF A REFLECTIVE PRACTICE CURRICULUM IN AN INTERNAL MEDICINE RESIDENCY TRAINING PROGRAM

Julie M. Chen ${ }^{2}$; Joseph Maciuba ${ }^{1}$; Joan B. Ritter ${ }^{1}$; Sarah Ordway ${ }^{1}{ }^{1}$ Walter Reed National Military Medical Center, Bethesda, MD; ${ }^{2}$ Kaiser Permanente Mid-Atlantic States, Rockville, MD. (Control ID \#3167936)

NEEDS AND OBJECTIVES: Redesign of internal medicine programs has led to the transformation of the ambulatory experience to block scheduling systems. However, block schedules decrease continuity between learners and teachers with consequent challenges in developing longitudinal relationships that contribute to professional identity formation. Additionally, burnout during residency has led to an increased need for a forum to discuss factors that lead to burnout and its mitigation. This presentation outlines a novel approach using reflective practice to cultivate professional identity formation and promote continuity for faculty and trainees. It provides one platform to foster resilience, joy and meaning in medicine through an innovative curriculum.

SETTING AND PARTICIPANTS: The participants were 75 internal medicine residents and 30 faculty. Novel reflective practice tools were used successfully in a large internal medicine residency. There were 15 reflective practice groups, each consisting of 5 trainees/2 faculty facilitators. The reflective practice curriculum included readings, videos and TED talks, artwork and 
different scenarios that promote reflection/discussion of pertinent topics (burnout, caregiver fatigue, etc.).

DESCRIPTION: It is imperative to address professional identity development and resiliency during residency training. However, the challenges to implementation are ever-increasing with productivity demands on clinicianeducators. Over one academic year in this IM residency, reflective practice groups held 2-hour sessions every 10 weeks during duty hours. Sessions were mandatory, and residents had no clinical responsibilities during this time. Reflective practice groups followed a curriculum for one year, using several tools for creating a safe environment that fostered camaraderie and dedicated time to introspection and shared reflections between faculty and trainees.

EVALUATION: Residents and faculty surveys on the effectiveness of the reflective practice sessions were administered at the 12- and 20-month mark after implementation of the curriculum. The response rate was $60 \%$ overall. Results include the perceived value of sessions (quantitative ratings and qualitative feedback), and topics that the residents found to be most beneficial or would like added. Data includes ratings of attitudes regarding the helpfulness of this curriculum.

DISCUSSION / REFLECTION / LESSONS LEARNED: Reflective practice helped guide residents and physicians in this residency program to become more aware of their personal context, biases and reactions while addressing significant topics such as providing equitable care. It also addressed personal feelings of physician burnout and fatigue, as well as how to continue fostering joy and meaning in medicine. Data after one year revealed that medicine residents surveyed rated compassion fatigue, resilience, mistakes and guilt/shame as the most important topics, and the majority would choose to continue the reflective practice curriculum.

ONLINE RESOURCE URL (OPTIONAL): http://reflectivepractice.net/ institution/walter-reed-internal-medicine-residency/

\section{SURVEY OF LEADERS OF U.S. PRIMARY CARE INTERNAL MEDICINE TRAINING PROGRAMS: EXPLORING GRADUATE CAREER PURSUITS}

Robin Klein ${ }^{2}$; CAITLIN ANDERSON ${ }^{1}$; Samantha Alonso ${ }^{2}$; Nour Chams ${ }^{2}$; Anu Kurl ${ }^{1}$; Katryna Lim ${ }^{3}$; Caitlin Taylor ${ }^{2}$; Akanksha Vaidya ${ }^{3}$; Stacy Higgins ${ }^{3}$. ${ }^{1}$ Emory University School of Medicine, Atlanta, GA; ${ }^{2}$ Emory, Atlanta, GA; ${ }^{3}$ Emory University, Atlanta, GA. (Control ID \#3185275)

NEEDS AND OBJECTIVES: There is a critical shortage of primary care physicians in the United States. To address this need, specialized Primary Care Internal Medicine (PC IM) residency programs provide dedicated training in ambulatory medicine. Literature assessing the ultimate career pursuits of PC IM graduates is limited. We aimed to explore the career choices of PC IM graduates and to identify program features that were associated with more graduates remaining in General Internal Medicine (GIM).

SETTING AND PARTICIPANTS: We conducted a cross sectional survey of Program Leaders of PC IM training programs or tracks in the United States. DESCRIPTION: We identified PC IM programs via the National Resident Matching Program, the American Medical Association's Fellowship and Residency Electronic Interactive Database, and individual program websites. Forty-five of the 70 programs identified as PC IM training programs or tracks completed the survey (64\% response rate).

EVALUATION: Program leaders report that $55.8 \%$ of graduates within the last 5 years pursued PC or GIM careers, 23.1\% pursued subspecialty fellowship training (i.e. cardiology) and $10.3 \%$ pursued primary-care oriented fellowship (i.e. geriatrics). Amount of training time in ambulatory settings, but not the number of continuity clinic sessions, was mildly associated with PC IM graduate career choices. Less ambulatory time was associated with more graduates pursuing subspecialty fellowship training (correlation coefficient $-0.38, \mathrm{p}$ value 0.02 ). There was no significant association between careers in $\mathrm{PC}$ or GIM and program size, age of program, number of continuity clinic sessions, number of continuity clinic sites, number of patient visits per continuity clinic session, breadth of subspecialty ambulatory rotations required or widely available, or breadth of topics included in PC curriculum.

DISCUSSION / REFLECTION / LESSONS LEARNED: Dedicated PC IM programs serve a critical role in addressing the shortage of primary care physicians in the U.S. We found that more than half of PC IM residents choose careers in PC or GIM. Less ambulatory time, but not less continuity clinic exposure, was associated with more graduates pursuing subspecialty fellowship training. This suggests that increasing ambulatory exposure across subspecialty clinics may be more effective at increasing the PC workforce than increasing time in continuity clinic. Future research could identify specific features of ambulatory exposure and of continuity clinics that are associated with more graduates pursuing careers in GIM.

TEACH THE TEACHER MODEL: AN INNOVATIVE METHOD FOR DEVELOPING A POINT OF CARE ULTRASOUND CURRICULUM WITHIN A LARGE ACADEMIC INSTITUTION

Christopher T. Kelly $^{1}$; Felix J. Nau ${ }^{1}$; Casey Glass ${ }^{2}$; Chaudry N. Majeed ${ }^{1}$. ${ }^{1}$ Wake Forest, Winston Salem, NC; ${ }^{2}$ Wake Forest, Winston-Salem, NC. (Control ID \#3183781)

NEEDS AND OBJECTIVES: Point-of-Care Ultrasound (POCUS) enhances the physical exam and is emerging as a necessary skill in modern medicine. Designing an Internal Medicine (IM) resident POCUS curriculum is difficult with limited faculty who are trained and comfortable using ultrasound. Our objective is to circumvent this obstacle and promote a cultural shift towards embracing bedside ultrasound by creating a "teach the teacher model" as the foundation of our IM POCUS curriculum.

SETTING AND PARTICIPANTS: In July 2018, 105 IM residents were in need of ultrasound training and only 1 inpatient POCUS trained IM faculty was available for teaching. 25 upper level residents received early structured POCUS training from July through September to be "POCUS champions." They went on to be peer instructors alongside faculty during workshops and in the clinical setting the remainder of the year.

DESCRIPTION: Training begins with POCUS lectures on the lung, heart, and IVC followed by hands on workshops with standardized patients. POCUS champions participate in gel rounds, where they perform 25 lung, heart, and IVC exams on real patients under trained faculty supervision. They must demonstrate proficiency before training other residents. In the multiplier phase, POCUS champions become mentors for 4-5 untrained residents and are allowed to supervise exams. All untrained residents are expected to complete 25 supervised lung, heart, and IVC exams by the end of the academic year. The rising PGY2s and PGY3s with the most interest and skill are selected to be the new POCUS

champions the subsequent year. The current curriculum focuses only on cardiac and pulmonary imaging. It will expand to cover other organ systems and POCUS education will be further augmented through additional training methods.

EVALUATION: A survey prior to implementing the curriculum showed that over $70 \%$ of residents felt uncomfortable or very uncomfortable performing POCUS exams. 21 of the initial 25 residents received their POCUS champion teaching privileges at the faculty's discretion. The curriculum is still in its early stages so compiled data is limited. We plan to evaluate the curriculum and resident POCUS proficiency in the following ways: 1) annual surveys to assess resident comfort; 2) tracking exams to measure POCUS use; 3) remote 
image assessment to evaluate quality; 4) tests to assess knowledge, image interpretation, and clinical application.

DISCUSSION / REFLECTION / LESSONS LEARNED: POCUS is not meant to replace radiology or cardiology expertise, but using POCUS as an extension to the physical exam is becoming an essential skill in general medicine. Our "teach the teacher" model overcomes the obstacle of having limited faculty who are comfortable using and teaching ultrasound by distributing the responsibility for POCUS education to the stakeholders themselves. Upper level residents perpetuate a continuous cycle to teach POCUS and interns have the opportunity to learn while simultaneously taking care of their hospitalized patients. This helps produce a cultural shift towards using POCUS within IM.

\section{TEACHING DIGITAL PROFESSIONALISM AND ASSESSING PHYSICIAN BEHAVIOR CHANGE}

Katherine Wrenn; Kelly L. Graham. Beth Israel Deaconess Medical Center, Boston, MA. (Control ID \#3181078)

NEEDS AND OBJECTIVES: Social media use has rapidly grown and is high among medical trainees. Their use raises privacy issues and concerns about boundaries in the patient-physician relationship. There is an emerging body of literature about social media use among medical trainees and physicians, leading the AMA, ACP, and FSMB to publish guidelines on appropriate use. However, there is minimal evidence or guidance about how best to teach medical trainees about digital professionalism. Therefore, we aim to (1) develop a novel curriculum for IM residents on digital professionalism, and (2) assess whether participation in the curriculum leads to changes in attitudes, behavior, and social media use among this population.

SETTING AND PARTICIPANTS: All residents in the IM Residency Program at Beth Israel Deaconess Medical Center were invited to participate in our study, which took place during their ambulatory medicine curriculum. While attending the didactic session was required, participation in the study was voluntary.

DESCRIPTION: The case-based curriculum is delivered in a 90-minute small group session. Residents discuss cases related to patient and physician privacy, the patient-doctor relationship, and professionalism. During discussion, we present guidelines on digital professionalism and social media use, along with data on unprofessional behavior among medical trainees and its implications. Prior to participating, residents complete a pre-survey, which includes demographic information, confounding variables such as previous exposure to digital professionalism training, an assessment of specific behaviors relating to digital professionalism, and attitudes about these behaviors. Following the session, residents complete a post-survey. This presents the same checklists of attitudes and behaviors, and asks whether they are likely to change their behavior after participating in the curriculum. Two months later, residents receive a second post-survey, asking them to identify whether or not they changed these specific behaviors. The BIDMC IRB deemed the study exempt.

EVALUATION: Data collection is ongoing. We will compare responses from pre- and post-surveys to determine if residents' attitudes about social media use and digital professionalism change after participating in the curriculum, and how likely residents feel they are to change behaviors after participating. By incorporating a delayed post-survey, this will also be a durability study, to assess whether residents indeed changed their behaviors. We will conduct analysis of attitude and behavior change pre/post using McNemar's test for paired data.

DISCUSSION / REFLECTION / LESSONS LEARNED: With a growing number of studies citing unprofessional behavior among medical trainees, there is a need for enhanced training in digital professionalism. We developed and implemented a novel, case-based curriculum for residents to help meet this need. Initial feedback has been positive, and we will perform an analysis to determine if residents' attitudes and behavior change after participating in the curriculum.

\section{TEACHING HAND OFFS TO JUNIOR MEDICAL STUDENTS: IMPACT OF AN EDUCATIONAL INTERVENTION}

Diane L. Levine; Omar M. Saaed. Wayne State University, Detroit, MI. (Control ID \#3185930)

NEEDS AND OBJECTIVES: Handoff is the process of transferring medical information from one healthcare provider to another and is crucial for safe patient care. A survey of Medicine Program Directors found an important skill for incoming interns was the ability to provide a prioritized and organized written hand off and conduct an organized verbal hand off. This provides impetus to educate medical students and provide opportunities to practice and develop handover skills. Although studies on handovers have been published (Acad Med.2009;84,1775-87), few focus on students. We delivered educational training tomedical students to prepare them to handover independently.

SETTING AND PARTICIPANTS: Students on the Medicine clerkship viewed material about handovers including the importance of sign-out, consequences of inadequate sign-out, content of oral and written sign-out, and use of I-PASS. They completed a formal written sign-out using IPASS, observed resident's handovers, and completed surveys about their perceptions of the safety of handovers and how prepared they felt to provide a handover in the future. Student written sign-outs were evaluated using a scoring system we developed; 2 points were assigned for each component of IPASS (0-missing/inadequate/non-actionable; 1-unnecessary, nonspecific information, limited detail, hard to follow; 2- appropriate detail, specific information, actionable, easy to follow, for a potential score of 8 .

DESCRIPTION: 123 students rotated on the clerkship. Sign-outs were submitted by $123 ; 2$ were illegible; 121 were analyzed. $19 \%$ scored $8,5 \%$ scored 7, $17 \%$ scored 6, 35\% scored 5, $11 \%$ scored 4, 7\% scored 3, and $2 \%$ scored 2 . Analyzing only by Action List (AL) and Situation Awareness (SA) resulted in $23 \%$ scoring 4, 61\% scoring 2, 7\% scoring 1 and $6 \%$ scoring 0 . Students who scored 2 on AL all scored 2 on SA. $93 \%$ of students who scored 2 AS scored 2 on AL. Average sign-out scores were calculated by rotation block. Students on the first 8 week block scored 5.2, 5.4 on the second block and 5.8 on block 3.117 completed the signout observation survey; 108 completed sign-in survey. Approximately one third observed handovers that had elements that decreased safety (distractions, interruptions). More than half felt prepared or very prepared to provide a handover. Approximately $40 \%$ felt somewhat prepared, 3\% did not feel prepared.

EVALUATION: Introducing students to hand overs during a clinical clerkship is feasible, however, students require additional practice to develop competency in this skill.

DISCUSSION / REFLECTION / LESSONS LEARNED: An educational intervention to teach junior students to handover and perform signouts demonstrated students were able to complete sign-outs using IPASS. Although most felt prepared to perform sign-outs only a quarter included crucial information necessary for safe handovers. A single educational intervention was sufficient to train students to perform sign-outs but was not sufficient to ensure competency for all students. Performance increased with each block possibly due to clinical experience. 
TEACHING PRACTICAL RESPONSES TO SEXUAL HARASSMENT IN MEDICINE: REFLECTIONS FROM A NATIONAL WORKSHOP

Emmanuelle B. Yecies ${ }^{1,2}$; Amy H. Farkas ${ }^{3}$; Melissa McNeil ${ }^{4} .{ }^{1}$ University of Pittsburgh School of Medicine, Pittsburgh, PA; ${ }^{2}$ VA Pittsburgh Healthcare System, Pittsburgh, PA; ${ }^{3}$ Medical College of Wiscponsin, Milwaukee, WI; ${ }^{4}$ University of Pittsburgh Medical Center, Pittsburgh, PA. (Control ID \#3185065)

NEEDS AND OBJECTIVES: In the wake of the \#MeToo movement, it is imperative that the medical field address sexual harassment rapidly to improve the work environment of our physicians and trainees. Little has been done to provide guidance and training on appropriate responses when experiencing or witnessing harassment, leaving many physicians feeling powerless and unsure of how to proceed when such instances do occur. Compounding the difficulty, harassment in medicine comes from a multitude of sources, with patients complicating the traditional colleague hierarchical paradigm. We aimed to develop a workshop providing a repertoire of responses to incidents of experienced and witnessed sexual harassment that participants would find useful and applicable to academic Internal Medicine settings.

SETTING AND PARTICIPANTS: The workshop was presented at the Association of Program Directors of Internal Medicine (APDIM) national meeting in Orlando, Florida in October 2018. Approximately 50 participants were in attendance from across the country.

DESCRIPTION: We began the workshop with a review of the current data on sexual harassment in medicine. We then reviewed guiding principles and provided a handout of approximately 40 sample responses, organized by situation and source. Examples of responses included "I prefer to be addressed as...." and "Let's keep things professional." In small groups, participants had the opportunity to develop skills and practice responses to different scenarios including experienced and witnessed instances of sexual harassment, from both patients and colleagues. Evaluations were submitted through the standardized APDIM system.

EVALUATION: Twenty-eight evaluations were returned. For "relevance of content to your job," "quality of speaker as a content leader," and "overall satisfaction with the session," participants rated the workshop 4.76, 4.68, and 4.75, respectively (1-5 Likert scale, average for conference 4.40). Free-text comments included: "This was a great session! Being able to walk out with scripts for a variety of situations [as] both a target and observer made the entire conference worthwhile!!! Thank you" and "Challenging, yet clearly and well presented. I have tangible practical takeaways that I am planning to start implementing with my residents. Thank you for doing this!" The content has been requested for dissemination by three institutions.

DISCUSSION / REFLECTION / LESSONS LEARNED: The workshop was very well received by all in attendance and promoted lively discussion. Multiple participants expressed the desire to implement these educational methods at their institutions, and formal dissemination is already in process. Continued efforts are needed to incorporate these communication drills into the standard residency curriculum, explore their applicability to other settings, and evaluate their impact on real-life communication skills and confidence.

\section{TEACHING SAFE AND EFFECTIVE OPIOID PRESCRIBING TO IM CLERKSHIP STUDENTS}

Mim Ari; Amber Pincavage. University of Chicago, Chicago, IL. (Control ID \#3184966)

NEEDS AND OBJECTIVES: Managing acute and chronic pain with opioids should optimally address the morbidity and mortality associated with the opioid epidemic. A 2016 AAMC statement highlighted that for teaching on this topic to be most effective, experiences must be "reinforced throughout the continuum of medical education." Our objective was to update our current IM clerkship curriculum on pain management with evidence-based principles of safe and effective opioid prescribing.

SETTING AND PARTICIPANTS: Participants were IM clerkship students at the University of Chicago. The workshop was part of their required didactic experience.

DESCRIPTION: A 1-hour case-based workshop was created to discuss concepts that guide chronic opioid prescribing, recognize red flags for opioid misuse, learn how to screen for opioid use disorder, and manage acute pain appropriately. A pre-post survey was administered to assess the impact of the workshop on experiences, knowledge and confidence caring for patients on opioids during the IM clerkship.

EVALUATION: Forty-three students have participated in our workshop and completed pre-post surveys (100\% response rate). During the IM clerkship, $81 \%$ and $75 \%$ of students care for patients on acute and chronic opioids respectively once per week or more. Seventy-nine percent cared for patients with opioid use disorder once per month or more. Despite frequent exposure, the majority of students were not familiar with guidelines for prescribing chronic opioids (79\%), and not confident in determining appropriate acute pain regimens $(90 \%)$ and identifying patient at risk for opioid use disorder $(60 \%)$. On 5-point Likert scales of familiarity and confidence, completion of the curriculum was associated with increases in familiarity with guidelines ( 2.0 pre, 3.8 post, $p<0.0001$ ), confidence in determining acute pain regimens (1.6 pre, 3.4 post, $\mathrm{p}<0.0001$ ) and identifying patients at risk ( 2.4 pre, 3.8 post, $\mathrm{p}<0.0001$ ). Performance on 3 knowledge questions improved from $46 \%$, $11 \%$ and $60 \%$ correct to $90 \%$ ( $\mathrm{p}<0.001), 100 \%(\mathrm{p}<0.001)$ and $90 \%$ correct $(\mathrm{p}=0.006)$. Ninety-five percent felt the workshop was helpful in practicing effective opioid prescribing and $98 \%$ reported gaining new knowledge DISCUSSION / REFLECTION / LESSONS LEARNED: Implementing a workshop for third year medicine clerkship students focused on safe and effective opioid prescribing improved students' knowledge, familiarity and confidence. This limited and feasible curricular innovation shows promise, although more in-depth curricula throughout the continuum will be critical to address the opioid epidemic.

TEAM-BASED LEARNING: AN AMBULATORY CURRICULUM TO INSTILL COMPETITION, FUN, AND SELF-DIRECTED LEARNING INTO INTERNAL MEDICINE RESIDENCY EDUCATION

Siew May Wang; Marianne Reeves; Kelsey Black; Atsuko Yamahiro. Metrohealth System, Cleveland, OH. (Control ID \#3183934)

NEEDS AND OBJECTIVES: Traditional lecture-based didactics are commonly used for educational conferences in internal medicine (IM) residency programs but remain a passive form of learning. Team-based learning (TBL) is commonly used in undergraduate medical education, as it is interactive and it promotes self-directed learning. We created a modified TBL curriculum at our residency program to foster active resident learning and to augment resident knowledge in common ambulatory topics.

SETTING AND PARTICIPANTS: All residents $(n=68)$ participated in the TBL ambulatory curriculum at the MetroHealth System IM residency program. The residents follow a "4+1" schedule. During their fifth ambulatory week, residents have an educational half-day. Residents attended a two-hour TBL session during their educational half day. There were up to 18 residents per session. The TBL session occurred every week, and 10 topics rotated throughout the year. 
DESCRIPTION: Each TBL session reviewed a common ambulatory topic. One week prior to each TBL session, residents received a relevant article to read based on the topic of the week (known in TBL as pre-class preparation). At the start of each TBL session, each resident completed a timed-quiz related to the topic, similar in format to the ABIM exam (individual readiness assurance test). The residents were then assigned to a team, to discuss the same questions to reach consensus answers (readiness assurance test). Following this, the facilitator led an inter-team discussion of the questions. Finally, a clinical vignette based on the topic was presented with questions for team discussion. Points were given to teams for each correct answer. Each session had a total number of points possible, and points were tracked for the all teams throughout the academic year. The point system is a modification from traditional TBL, as grades were not instituted.

EVALUATION: We administered anonymous surveys prior to the TBL sessions to all resident participants, measuring resident perceptions of lecture-based versus TBL-based teaching methods. Three months after the implementation of the TBL curriculum, we conducted post-TBL surveys to the same participants to gauge resident completion of pre-class reading assignments and their perception of TBL.

DISCUSSION / REFLECTION / LESSONS LEARNED: Most residents agreed or strongly agreed (93\% to $98 \%$ ) that TBL was well-suited to their learning style, felt engaged during the TBL sessions, and believed that TBL improved their clinical decision-making skills. The majority of residents $(96 \%$ to $98 \%$ ) also reported that this method nurtured positive interactions between peers. This study showed that most residents $(87 \%)$ completed the pre-lecture reading most or all of the time, with the most common reason of self-directed reading as "being a valuable team player." Our data demonstrates that TBL is an effective teaching format to improve resident engagement in didactic sessions, team work, and motivation for self-directed learning.

\section{THE ADVOCACY AND COMMUNITY HEALTH CHIEF RESI- DENT: A NOVEL POSITION TO ENHANCE COMMUNITY EN- GAGEMENT}

$\underline{\text { Benjamin A. Howell }}^{1}$; Sanjeet Baidwan ${ }^{1}$; Lacey R. Whitmire ${ }^{2}$; Ross B. Kristal $^{1}$; Julie R. Rosenbaum ${ }^{3}$; Stephen J. Huot ${ }^{3}$; Tracy Rabin ${ }^{3}$. ${ }^{1}$ Yale School of Medicine, New Haven, CT; ${ }^{2}$ Iora Primary Care, New Haven, CT; ${ }^{3}$ Yale University School of Medicine, New Haven, CT. (Control ID \#3185657)

NEEDS AND OBJECTIVES: Effective community engagement enables physicians to collaborate with populations to improve health. To address these barriers to in residency training to learn about community engagement we created a chief resident position geared towards advocacy and community engagement and function as nidus for curricular and community-based experiential innovations. In 2015, we designed a novel position, Chief Resident for Advocacy and Community Health. The objectives for this chief resident are to: collaborate with the community to better understand local priorities, develop an integrated curriculum for physician advocacy and social justice, serve as a mentor to residents interested in advocacy and community engagement projects.

SETTING AND PARTICIPANTS: The setting is an academic medical center-based internal medicine-primary care residency program located in a mid-size northeast city. The local community is made up of a majority racial/ ethnic minorities with $\sim 25 \%$ living below the poverty line. The majority of patients served by the resident practice have Medicaid.

DESCRIPTION: The novel chief resident position, an addition to the existing chief resident group, was created with funding provided by the sponsoring institution. Primary roles include working with faculty to develop and co-lead a three-year, mandatory, longitudinal community engagement and advocacy curriculum, develop a conference series on social justice topics, and function as a liaison between housestaff and the community. The chief resident also collaborates with non-profit organizations, and actively fosters relationships with community through regular attendance at community meetings. In addition, the chief resident shares clinical, administrative and teaching responsibilities with the other chief residents. The chief resident also delivers a grand rounds to the Department of Medicine on an advocacy or community engagement topic. The chief resident also leads a committee of residents to facilitate and mentor residents in self-directed advocacy projects such as collaboration with local farm programs around health promotion and local providers of diabetes prevention programs. At the end of the 2018-2019 academic year, four individuals will have served in this role.

EVALUATION: The creation of this position has resulted in an expansion of curricular offerings on community engagement and advocacy, including specific knowledge of community-based resources. The chief residents' collaboration with faculty supporting this role has also enhanced the continuity and depth of relationships with community organizations despite the transient nature of residency training.

DISCUSSION / REFLECTION / LESSONS LEARNED: Our residency program has successfully integrated a Chief Resident in Advocacy and Community Health. Through this role, the residency has fostered a deeper understanding of the community which has helped to inform and guide care in the clinic. The position has also provided additional support for residents interested in advocacy, community engagement and social justice.

\section{THE ART OF BALANCE: CARING FOR PHYSICIAN AND PA- TIENT}

Mukta Panda ${ }^{2}$; Paul Chelminski ${ }^{1}$; Angela Dittmar ${ }^{3} .{ }^{1}$ University of North Carolina at Chapel HIll, Chapel HIll, NC; ${ }^{2}$ University of Tennessee, Chattanooga, TN; ${ }^{3}$ UTC, Chattanooga, TN. (Control ID \#3182902)

NEEDS AND OBJECTIVES: Medical students and residents often recite the Hippocratic Oath (or its equivalent) at their white coat ceremonies. Oaths enjoin physicians to care for patients with sincerity, empathy and kindness. Some have suggested that these same values should apply to the physician's self-care as well. That is, repurposing the Hippocratic Oath to emphasize a reciprocal interaction benefiting the servant and the served. Given the alerming prevalence of physician burnout, there is a growing need for resilience training in medical education. Medical education often includes texts in the form of case studies as a way to develop ethical reasoning and diagnostic skills. Building resilience requires similar explicit training. Our work showcases the Hippocratic Oath as a text repurposed to enhance professional wellbeing and undergird ethical and compassionate practice.

SETTING AND PARTICIPANTS: We will share our curriculum, The Art of Balance: Caring for Physician and Patient, which is designed to build physician resiliency by emphasizing teamwork, reflection and empathy as catalysts for overcoming physician burnout.

DESCRIPTION: The Art of Balance: Caring for Physician and Patient was developed in partnership among The University of Tennessee, Hunter Art Museum Southeast Center for Art Education. There is practical emphasis on developing strategies for overcoming physician burnout. Sessions are comprised of two to three main sections: group engagement and discussion with a work of art; a transitional activity involving individual museum exploration or small group activity; reflective collaboration on a creative prompt. This presentation unpacks a sample session and how the program supports varying needs related to physician burnout.

EVALUATION: This program has served over 150 learners. Each session has a theme but remains organic and responsive to learner needs as they 
emerge. Some learners have returned frequently and many remark that their appreciation surpassed their expectations. Qualitative evaluations will be shared.

DISCUSSION / REFLECTION / LESSONS LEARNED: Time to interpret through guided questions has been a success factor of the richness of conversation during discussion. The significance of the relationship between interpreting art and emotional intelligence and been documented. In the Art of Balance, individuals develop and refine their emotional intelligence. Students find the experirnce de-isolating, realizing they are not alone in their professional struggles. De-isolation results from conversations catalyzed by observations and reflections on art. Individual interpretations are woven together to become a shared tapestry of thoughts and feelings. This shared construction of meaning lead to empathy. Emergent expression and dialogue is a critical tool in combatting burnout. Loss of autonomy and agency are frequently cited contributors to burnout. Emergent discovery promotes autonomy and agency in a social profession such as medicine.

THE CSI (CLINICAL SCHOLARS IN INFORMATICS): A NOVEL INTERNAL MEDICINE (IM) RESIDENT-DRIVEN APPLIED INFORMATICS PATHWAY

Ajay Dharod $^{1,1}$; Hal H. Atkinson ${ }^{2,1}$; Gary Rosenthal ${ }^{2} .{ }^{1}$ Wake Forest School of Medicine, Winston Salem, NC; ${ }^{2}$ Wake Forest School of Medicine, Winston-Salem, NC. (Control ID \#3183877)

NEEDS AND OBJECTIVES: In the changing landscape of healthcare, applied informatics shapes clinical workflows and decision making. It is important to train physicians leaders in applied informatics allowing for novel locally-relevant workflows targeted to improve healthcare effectiveness, efficiency, equity, and patient engagement.

SETTING AND PARTICIPANTS: A single academic medical center, IM residency program. All PGY1 IM residents are eligible. Each year, two PGY1 residents are selected for the pathway to begin their PGY2 year.

DESCRIPTION: The CSI is a two-year applied educational and mentorship pathway for IM residents to develop skills in applied informatics. At residency completion, CSI residents are prepared to tackle most health-system challenges with additional comprehensive understanding of informatics (CDS, workflow, change management, IT systems, EHR, database structures, SQL, regulatory issues, Security/Privacy, etc.). The pathway translates a resident derived project (addressing system pain points) into reality. The presenter will elucidate details of the CSI curriculum, timeline considerations, resources required, budgetary considerations and journal club structure in the presentation (please see online resource URL).

EVALUATION: Of our inaugural class of CSI residents, both completed EHR certification, IRB protocol, workflow analysis, intervention design, baseline data analysis and intervention implementation. One CSI project has been presented at a national meeting. Projects range in scope from predictive analytics to clinical decision support to alert systems to data visualization (please see online resource URL for project details). Of our second class of CSI residents, both are in the design phase. Additionally, we received the following message excerpt from a CSI resident: "I am blown away by the enthusiasm fellowship programs have shown learning about CSI. They are excited about the skill set of a potential future fellow and are all amazed by the structure of CSI. When I applied for CSI, I had no idea the training received would be this valuable to my career, both now and long-term. Interviewers have been very impressed by the project that we have implemented. Informatics has dominated the conversation in almost all of my interviews."

DISCUSSION / REFLECTION / LESSONS LEARNED: One resident plans to continue informatics-related work in the Hem-Onc and one resident plans to join general IM faculty to pursue the practice pathway clinical informatics board certification. CSI has been cited as successful by residents, mentoring faculty, and residency/departmental leadership. We continue to evaluate the impact of CSI projects and draft and disseminate CSI residentdriven innovations. CSI is overall low-cost with $\$ 20,000$ in direct costs. Personnel considerations include collaboration with ITS and faculty time. CSI can be leveraged as a residency resident recruitment tool.

ONLINE RESOURCE URL (OPTIONAL): https://school.wakehealth. edu/Education-and-Training/Residencies-and-Fellowships/Internal-Medicine-Residency/Curriculum-Overview/Clinical-Scholars-in-Informatics-Pathway

\section{THE DENVER HEALTH LONGITUDINAL INTEGRATED CLERKSHIP (DH-LIC): AN INNOVATION FOSTERING PATIENT-CENTERED ADVOCATES FOR SOCIAL JUSTICE AND HEALTH EQUITY}

Jennifer Adams $^{1,2}$; Vishnu Kulasekaran ${ }^{1}$; Gretchen Guiton ${ }^{2}$; Kristina Oatis ${ }^{2}$; jennifer gong ${ }^{2} .{ }^{1}$ Denver Health, Denver, CO; ${ }^{2}$ University of Colorado, Aurora, CO. (Control ID \#3183687)

NEEDS AND OBJECTIVES: The DH-LIC was established in 2014 to pilot this model at the University of Colorado School of Medicine (CUSOM) and demonstrate feasibility and comparability to rotation based clerkships (RBC). The DH-LIC aimed to increase observation and feedback, create opportunities for meaningful contribution to patient care, and maintain patient-centered values in students. The curriculum focused on social justice and health equity, reinforced by locating this program at an urban safety net hospital with a fully integrated community health system.

SETTING AND PARTICIPANTS: The DH-LIC selects 10 students each year based on their commitment to underserved patients, career aspirations related to health equity, and leadership potential. Students spend one year at DH completing all core clerkships in an LIC model.

DESCRIPTION: LICs share foundational elements of 1) comprehensive care of patients over time, 2) continuous learning relationships with preceptors, and 3) meeting core clinical competencies across multiple disciplines simultaneously. DH-LIC students attain all core clinical competencies expected of CUSOM students and academic achievement has met or exceeded RBC students.

EVALUATION: The DH-LIC has remained the highest rated course at the CUSOM since its inception. Students rate their value as team members, experience with faculty, and ability to impact patient care especially high. Focus groups reveal themes related to the importance of longitudinal relationships with patients, mentorship and role modeling from preceptors, and sustained idealism and commitment to serving vulnerable patients. Postgraduate surveys examine career paths and durability of desire to work with the underserved. $91.3 \%$ of DH-LIC graduates report high/very high commitment to working with the underserved and $43.5 \%$ plan careers in primary care. DISCUSSION / REFLECTION / LESSONS LEARNED: Focus groups have led to a deeper understanding of how the DH-LIC model and its curricular focus on social medicine influence attitudes towards caring for vulnerable patients, students' professional identity, and the career paths of student participants. Students are not selected based on an interest in primary care (PC), but chose PC fields disproportionately due to role modeling and ability to follow patients longitudinally. Students who did not choose PC careers or who describe themselves as less likely to work with the underserved described how the DH-LIC experience led them to better understand the care coordination role of primary care and recognize the importance of advocating for disadvantaged patients. 
ONLINE RESOURCE URL (OPTIONAL): http://medschool.ucdenver. edu/dhlic

\section{THE GIMBOREE EXPERIENCE: STRENGTHENING PRIMARY} CARE CAREER INTEREST AND COMMUNITY IN RESIDENCY Deborah G. Freeland ${ }^{2}$; Paul O'Rourke ${ }^{1} .{ }^{1}$ Johns Hopkins University, Baltimore, MD; ${ }^{2}$ Johns Hopkins University School of Medicine, Baltimore, MD. (Control ID \#3185885)

NEEDS AND OBJECTIVES: There is a shortage of primary care physicians in the United States, and traditionally internal medicine residency training has focused on inpatient care. To address this growing need, residencies must find ways to emphasize the importance of primary care and support residents who are interested in the fields of general internal medicine (GIM) and geriatrics. We developed a monthly primary care community night for residents and faculty called "GIMboree" with the goal of increasing resident sense of community and interest in primary care careers through a tripartite model: 1) exposure to GIM and geriatrics role models; 2) strengthening medical knowledge related to outpatient medicine through journal club; and 3) fostering a community in which residents feel supported in their outpatient interests.

SETTING AND PARTICIPANTS: We invited Johns Hopkins Bayview Medical Center primary care track residents and GIM/geriatrics faculty to attend voluntary GIMboree events held at either a faculty member's or resident's home. In the first 6 months of implementation (July to December 2018), attendance has ranged from 4 to 8 residents (of 18 total residents) and 2 to 5 faculty per evening.

DESCRIPTION: GIMboree meetings are held monthly and last about 2 hours. The structure includes dinner, primary literature journal article discussion, a faculty member's career story, and time for reflection about GIM clinic. Articles focus on outpatient medicine and were published within the last year. A resident volunteer leads the group through analysis of the evidence, and faculty members volunteer to share their story with prompting questions. Clinic reflection allows residents to obtain feedback about an encounter or to discuss joys and challenges specific to outpatient medicine.

EVALUATION: At each meetings' end, residents and faculty were surveyed with Likert scale and open-ended reflection questions. The short-answer responses were assessed for common themes across meetings and data collection is still ongoing.

DISCUSSION / REFLECTION / LESSONS LEARNED: Preliminary results reveal three main themes from the open-ended reflection questions: excitement about a career in primary care medicine ("Love that you can have a long-term relationship with patients and fulfill a role that no one else is able to for the patient"), growing sense of community ("Having the faculty share joys and difficulties made the community feel tighter"), and goals for selfimprovement ("I will be more brave about discussing risks with patients and building a network of colleagues").

THE HIDDEN CURRICULUM: A RESIDENT-LED PILOT PROGRAM TO INCREASE CONFIDENCE AND EXPERTISE IN THE TRANSITION TO CLINICAL PRACTICE

Leah B. Kosyakovsky; Michael Ruiz; Jonah Himelfarb. University of Toronto, Toronto, ON, Canada. (Control ID \#3185668)

NEEDS AND OBJECTIVES: The entrance into clinical clerkship is a challenging and intimidating transition. Given the gap between the goals of the preclinical curriculum and the practical skills necessary to begin patient care, this transition presents a challenge unique to this stage of training. In order to facilitate this new phase of training, we created a resident-led pilot initiative focused on developing the areas often under-represented in the preclinical curriculum. These topics included documentation, medical communication, and applied clinical reasoning.

SETTING AND PARTICIPANTS: This longitudinal program was run as a combination of large and small-group sessions over the course of the academic year. 96 second-year medical students participated in our first session.

DESCRIPTION: Our pilot session involved an interactive case focused on the process of an initial patient assessment and admission. Students completed a pre-survey regarding their confidence in different clinical domains. Students rated their confidence in performing several skills on a scale from 1 (not confident at all) to 5 (very confident). After the session, students completed a similar post-session survey re-assessing their confidence in the taught skills.

EVALUATION: The pre-program survey, completed by 68 students, demonstrated that students had low perceived confidence in several critical skills. In particular, students reported the lowest scores in admitting an inpatient (1.73/5), creating a management plan (1.95/5), and assessing patients in the Emergency Department (2.09/5). After the session, 25 students responded to the post-session survey. $100 \%$ of these students reported the session was helpful or very helpful. $96 \%$ felt more comfortable documenting an inpatient consultation, 84\% felt more comfortable performing an admission, and 96\% reported an improvement in their confidence in beginning clerkship.

DISCUSSION / REFLECTION / LESSONS LEARNED: Our study indicates that pre-clerkship students have low confidence in performing several of the critical skills needed in clinical practice. Our program was designed to surmount these gaps through practical, small-group sessions focused on the development of these key competencies. This pilot session demonstrated an improvement in students' confidence in performing and documenting inpatient admissions, as well as allaying fears regarding the transition to clerkship. Further quantitative and qualitative data will be collected throughout future sessions as we continue to shape and refine this preparatory program for clinical practice.

THE HOOFBEATS / COREIM SERIES: PODCASTING AS A PROMISING MEDIUM TO EXPLORE THE LANGUAGE OF CLINICAL PROBLEM-SOLVING.

John I. Hwang $^{4}{ }^{4}$; Shira A. Sachs ${ }^{5}$; Amy Ou ${ }^{3}$; Shreya P. Trivedi ${ }^{4}$; Martin Fried $^{6}$; Neil Shapiro ${ }^{2,4}$; Cindy Fang ${ }^{4}$. ${ }^{1}$ Bellevue Hospital Center, New York, NY; ${ }^{2}$ Dept. Of Veterans Affairs, New York, NY; ${ }^{3}$ NYU School of Medicine, New York, NY; ${ }^{4}$ New York University, New York, NY; ${ }^{5}$ Weill Cornell Medicine, New York, NY; ${ }^{6}$ Ohio State University Wexner Medical Center, Columbus, OH. (Control ID \#3183711)

NEEDS AND OBJECTIVES: Superior clinical reasoning is a hallmark of the expert clinician. However, to a passive listener at a traditional case conference, the process by which this expert arrives at a diagnosis may not be obvious. Discussion often focuses on medical knowledge, rather than on the methods the expert uses to reach a conclusion. Experts vary in their ability to describe how they approach a case, and it is debatable whether narratives produced in this manner are in fact reliable representations of their mental processes. Teaching clinical reasoning can thus be challenging even for experienced faculty. In response, we designed a podcast series using actual cases to explore the conscious and unconscious habits and strategies used by physicians. Our intention was to immerse listeners in the language of clinical problem solving in an accessible and interactive way.

SETTING AND PARTICIPANTS: Hoofbeats is one of several series that comprise the CoreIM podcast. It is targeted at trainees and medical professionals across all specialties within internal medicine. 
DESCRIPTION: Hoofbeats listeners play the role of the clinician, as a difficult case is presented in stepwise fashion with pauses to allow revision of an impression or differential. Listeners hear a discussant work through the case, accompanied by commentary from the show's hosts, who interpret and elaborate on the discussant's reasoning behaviors. Clinical pearls, useful schema, and biases are highlighted. To reinforce the challenge, a visual representation of the case is sent out via social media. In some episodes users can solve an interactive version of the case on the online database HumanDx; the resulting collective differential is then explored within the episode.

EVALUATION: From March to December 2018 we released six Hoofbeats episodes, with over 64,000 total downloads by listeners across 170 countries. In our descriptive analysis of feedback, we found that listeners valued the explicit analysis of the problem-solving behavior of experienced clinicians. Interactive elements successfully engaged our listeners, who actively attempted cases and reflected on their reasoning. Through role modeling from the hosts, listeners reported greater confidence articulating and teaching clinical reasoning concepts. Interestingly, commentary on particularly challenging points in a difficult case led our listeners to appreciate the honesty and humility of our hosts and experts, who frequently recognized their own shortcomings.

DISCUSSION / REFLECTION / LESSONS LEARNED: Decades of research into clinical problem-solving have produced a terminology now familiar to many trainees and medical professionals, such as the practice of "problem representation" or the concept of the "availability heuristic." Many of these abstract concepts originated in behavioral science and artificial intelligence programming, and do not lend themselves naturally to book or lecturebased learning. However, our experience with Hoofbeats suggests there is significant interest among learners in the practical application of these constructs.

THE HUMAN DIAGNOSIS PROJECT: AN OBJECTIVE, REALTIME, AND SCALABLE WAY TO MEASURE CLINICAL REASONING

Marc Rabner; Seiji Hayashi. The Human Diagnosis Project, Washington, DC. (Control ID \#3184948)

NEEDS AND OBJECTIVES: Medical errors are the third leading cause of death. Roughly 40,000-80,000 of these deaths stem from errors in diagnosis, and nearly every American will experience a diagnostic error in their lifetime. Despite this magnitude, efforts to reduce medical errors have typically focused on medical treatment and not diagnosis. The largest contributor to diagnostic error is failures of clinical reasoning. Despite the emphasis on the importance of diagnosis and clinical reasoning in medical education today, current approaches to assess and improve clinical reasoning skills are resource intensive and/or subjective, which limit their impact and reach.

SETTING AND PARTICIPANTS: The Human Diagnosis Project (Human $\mathrm{Dx}$ ) is an online tool for measuring and training clinicians' diagnostic reasoning. Basic access to Human Dx is free and accessible to anyone via a mobile app for iPhone and Android, and on the web at www.humandx.org. Over 15,000 medical professionals and trainees have solved Human Dx's interactive clinical case simulations nearly 250,000 times. Participants are from over 500 institutions, in 80 countries, and from 40 specialties. About $45 \%$ are trained in internal medicine. Approximately 30\% are attending physicians, 30\% are graduate medical trainees, and the remainder are medical students and other health professionals (e. g., NPs, PAs, RNs).

DESCRIPTION: Human Dx has over 3,500 brief, interactive case simulations. Each case is designed to be highly engaging to the practicing physician in order to ensure critical thinking about each case. Cases include clinical information based on a real patient encounter (de-identified) and promote the evidence-based clinical decision-making found in real patient care. Learners can solve the case simulations in less than 5 minutes and enter their responses in free-text, instead of multiple choice, mimicking real-world practice. Learners' responses are automatically scored by the Human Dx system in real-time - allowing for immediate feedback on learner diagnostic performance.

EVALUATION: A cohort study published in JAMA Open investigated the performance of 1,728 physicians and trainees in over 11,000 Human Dx case simulations. Three measures of Clinical Quotient (CQ), a metric Human Dx uses to estimate clinical competency, were used to assess physicians' diagnostic performance: accuracy, efficiency, and a combined score called the Diagnostic Acumen Precision Performance. The study found that medical professionals with more experience and training demonstrated higher CQ scores on Human Dx case simulations.

DISCUSSION / REFLECTION / LESSONS LEARNED: The Human Diagnosis Project introduces an objective and scalable way to measure diagnostic reasoning. Physicians and trainees anywhere can now use an open, online system to quickly and easily assess and develop their clinical reasoning skills.

ONLINE RESOURCE URL (OPTIONAL): www.humandx.org

\section{THE HUMANISM POCKET TOOL: FINDING THE JOY IN TREATING COMPLEX PATIENTS}

Michael Soh ${ }^{1}$; Andrew Shaner ${ }^{1}$; Lillian Gelberg ${ }^{2}$; Margi Stuber ${ }^{1}$; Kristin Kopelson ${ }^{3}$; Carole Warde ${ }^{4}$. ${ }^{1}$ Greater Los Angeles VA, Los Angeles, CA; ${ }^{2}$ UCLA, Los Angeles, CA; ${ }^{3} \mathrm{VA}$, Los Angeles, CA; ${ }^{4}$ Greater Los Angeles VA Health System, North Hills, CA. (Control ID \#3186183)

NEEDS AND OBJECTIVES: Your brain is equipped with automatic, emotional responses biased to protect you from people who might be dangerous, infectious, or time-consuming. These emotions can sneak up on you and replace compassion with fear, disgust, or anger. Existing practices to counteract these emotions can be time and labor intensive and challenging to implement. The Humanism Pocket Tool (HPT) emphasizes simple yet explicit strategies and techniques designed for providers to promote patient-centeredness by counteracting reflexive, dehumanizing responses to patients with challenging behaviors.

SETTING AND PARTICIPANTS: Our IP practice/training site is in a large VA clinic for homeless Veterans ( $\sim 3,000$ patients). The clinic provides primary care, mental health, and social work services within a patient-centered medical home. To date, we have over 30 trainees and a dozen faculty from five different professions in primary and mental health care.

DESCRIPTION: The HPT pulls from the realms of appreciative inquiry, storytelling, active listening, and mindfulness and integrates intra/interpersonal techniques into common clinical interactions. Via our HPT, clinicians and trainees learn to (1) coach themselves; (2) be warm; (3) listen actively; (4) condense each patient's personal story into a highly compact form, termed a vivid vignette; (5) use the Vivid Vignette to identify the patient in progress notes and in conversations with colleagues, so as to inspire and coordinate care; (6) appreciate differing professional perspectives on the patient; and (7) know their team members as people. Each technique builds upon each other to create and enrich the patient's story in order to prioritize their quality of care. EVALUATION: The HPT is still a work in progress and we intend to learn more about how different teams teach and practice and the various obstacles they run into during patient care. To date, we have over 15 collaborators across the country that have begun piloting the HPT at their respective sites and we've begun a process of reaching out to these providers to learn more about how they utilize the HPT and what can be done to improve it. 
DISCUSSION / REFLECTION / LESSONS LEARNED: The HPT has been a product of, and catalyst for, strong collaboration and interdisciplinarity. The vivid vignette, a strategy designed to illuminate both the person and their goals, rather than just their problems - has become a fundamental part of case presentations. Additionally, the explicit nature of the HPT has put humanism at the forefront of how our trainees interact with their patients and colleagues and how our clinic culture has defined itself.

\section{THE HYPOTHESIS-DRIVEN PHYSICAL EXAM FOR COMMON INPATIENT PRESENTING SYMPTOMS}

Allison Kanakis ${ }^{1}$; Deborah DiNardo ${ }^{2}$; Eliana Bonifacino ${ }^{3}$; Melissa McNeil ${ }^{1}$. ${ }^{1}$ University of Pittsburgh Medical Center, Pittsburgh, PA; ${ }^{2}$ University of Pittsburgh School of Medicine, Pittsburgh, PA; ${ }^{3}$ UPMC, Pittsburgh, PA. (Control ID \#3185516)

NEEDS AND OBJECTIVES: The declining emphasis on the physical exam in medical education and in clinical practice has been widely recognized. More recently, there has been a renewed interest in physical diagnosis as an essential component medical decision-making. As opposed to the complete "head-totoe" physical exam, the hypothesis-driven physical exam (HDPE) involves predicting and recognizing specific physical exam findings to refine differential diagnoses. However, there is a lack of consensus regarding the essential elements in a HDPE across a spectrum of disease processes and learner levels. We therefore aimed to develop a rubric for assessing the medical student HDPE for common inpatient presenting symptoms.

SETTING AND PARTICIPANTS: We reviewed 248 admission notes submitted by 67 medical students for documentation of the chief complaints. The admission notes were gathered from students rotating on their third year internal medicine clerkship at the University of Pittsburgh Medical Center who submit notes for grading to a faculty facilitator.

DESCRIPTION: We selected the 3 most common chief complaints as presented in the students' admission notes. The 3 most common chief complaints were shortness of breath (32 patients), abdominal pain (23 patients), and chest pain (21 patients). These 3 chief complaints comprised 76 (31\%) of submitted admission notes. For each of these 3 selected symptoms, four clinical reasoning experts generated a list of the most common associated diagnoses and the relevant diagnostic physical exam maneuvers based on clinical experience and literature review.

EVALUATION: We created a survey to administer to a group of clinical reasoning and physical diagnosis experts. In the first part of the survey, participants are provided with potential physical exam maneuvers for each symptom and are asked to rank how important each maneuver is to be performed on initial assessment using a Likert scale. In the second part of the survey, participants are asked to identify whether or not an exam maneuver should be performed only if a specific diagnosis is being considered using a dichotomous scale. We plan to conduct a two-round Delphi to establish a consensus list of essential physical exam maneuvers for the HDPE for the 3 selected symptoms. Based on literature review and expert group consensus as described, we will consolidate the list into physical exam maneuvers that (1) should always be performed for a particular presenting symptom and (2) should be done in a hypothesis-driven manner if a specific diagnosis is being considered.

DISCUSSION / REFLECTION / LESSONS LEARNED: By developing a rubric for common inpatient presenting symptoms, the teaching and evaluation of the inpatient HDPE in medical students can be standardized. This tool has the potential to help students learn the pertinence of physical exam findings and enhance their clinical reasoning skills.
THE I-TOPC CASE-BASED CURRICULUM: A NOVEL APPROACH TO TEACHING PRIMARY CARE TOPICS TO INTERPROFESSIONAL LEARNERS.

Timica Campbell ${ }^{1}$; Megan McNamara ${ }^{2}$; Andrew T. Harris ${ }^{3}$; Elizabeth Paint$\mathrm{er}^{4}$; Kristina Pascuzzi ${ }^{4}$; Pamela Lynch ${ }^{4}$; Mary A. Dolansky ${ }^{5,4}$. ${ }^{1}$ Louis Stokes VA Medical Center, Cleveland, OH; ${ }^{2}$ Louis Stokes Cleveland VAMC, Cleveland, OH; ${ }^{3}$ Louis Stokes Cleveland VA Medical Center, University Heights, $\mathrm{OH} ;{ }^{4}$ Louis Stokes Cleveland VA Medical Center, Cleveland, $\mathrm{OH}$; ${ }^{5}$ Case Western Reserve University, Cleveland, OH. (Control ID \#3185511)

NEEDS AND OBJECTIVES: Traditional teaching of primary care concepts usually takes a physician-centered approach to diagnosis and management. With the advent of the Patient Centered Medical Home, we recognize the need for teaching cases that model the teambased approach that ideally happens in workplace learning. Currently, there is a paucity of ambulatory curricula that address interprofessional primary care management. Therefore, the objective of the teaching innovation was to develop and implement modules that engage learners in the collaborative nature of primary care.

SETTING AND PARTICIPANTS: The Cleveland VA Medical Center is one of five original Centers of Excellence in Primary Care Education (COE-PCE) established in 2010 with funding from the Veterans Health Administration's Office of Academic Affiliations. The curriculum is an innovative interprofessional program that focuses on training future health care professionals to manage chronic condition. The program includes Medical (MD) residents, Nurse Practitioner (NP) residents, Pharmacy residents, Psychology residents, Social Work and NP students.

DESCRIPTION: A core group of interprofessional faculty from medicine, advanced practice nursing, pharmacy, behavioral health and social work developed and implemented a case-based curriculum that emphasized a team-based approach to primary care topics. In the development of this Interprofessional Transforming Out-patient Care (i-TOPC) case-based curriculum, we used an agreed upon framework and an iterative process to create modules that included interprofessional learning objectives, a content specific case, guiding questions, and a facilitator guide. Examples of topics covered included fibromyalgia, headaches, and dizziness. A team of interprofessional learners were assigned to facilitate the session. Prior to the conference, learners were coached on techniques of presenting to an interprofessional group. Following each case conference, learners were provided with real-time feedback from the core group faculty. At the end of each session all learners were provided with a copy of the facilitator guide.

EVALUATION: After each conference, audience members completed an online evaluation. The evaluation consisted of a combination of Likert-scale questions and short answer questions that rated presentation style, delivery, and the interprofessional nature of the presentation. The average overall rating for the sessions was 4.69 on a 5point scale. Examples of some of the short answer feedback include: "Fantastic! Love the new i-TOPC format." "No better way [to present information]!"

DISCUSSION / REFLECTION / LESSONS LEARNED: Over the course of the development and implementation of i-TOPC cases, we learned that maintaining a small, core group of faculty members was key. After identifying the core group, we solidified into an efficient and committed team. We also recognized the utility of interprofessional faculty members attending case presentations to facilitate discussion, gauge case effectiveness and for future case improvement. 
THE INPATIENT ACADEMIC HALF DAY; THE WHYS AND HOWS IN A LARGE MULTI-SITE PROGRAM THAT USES AN X+Y SCHEDULING SYSTEM

Jasmyn Miller $^{1}$; Roberto O. Diaz Del Carpio ${ }^{2}$; Scott Gruarin ${ }^{3}$; Regina Makdissi ${ }^{4} .{ }^{1}$ Jacobs School of Medicine and Biomedical Sciences at University at Buffalo, Niagara Falls, NY; ${ }^{2}$ University at Buffalo, Buffalo, NY; ${ }^{3}$ Jacobs School of Medicine and Biomedical Sciences, Buffalo, NY; ${ }^{4}$ State University of New York at Buffalo, Buffalo, NY. (Control ID \#3185127)

NEEDS AND OBJECTIVES: Delivering high quality conferences at 3 sites is challenging given the lack of standardization, difficulty finding speakers, and video broadcasted conferences felt impersonal and passive. Daily clinical work prevents active trainee participation with the lack of true protected time for meaningful education. Objectives: 1 . To increase the quality of inpatient educational teaching sessions. 2. Provide the trainees with high quality, evidence-based content to use during their clinical rotations within a protected educational time. 3. To enhance camaraderie and teamwork amongst all trainees. 4. To increase trainee satisfaction with their educational activities. Create a venue where trainees are accountable for their active learning and can interact with their core faculty in an encouraging, judgment free environment.

SETTING AND PARTICIPANTS: A large multi-site (3 major teaching hospitals) with 4+1 scheduling.

DESCRIPTION: Sessions are topic guided: We based the objectives on the Society of Hospital Medicine Curriculum. One separate half day for the residents and interns: 1st Hour: Case discussion with questions to be researched and presented by the trainees next conference day 2nd Hour: Topic review with board-review questions at the end to test for retention. 3rd Hour: Evidence-based medicine presentation by trainees.

EVALUATION: We surveyed 23 Academic Hospitalists prior to implementation, $50 \%$ rated the noontime conferences as average or below average, $50 \%$ did not know. Trainee surveys by ACGME and our Wellness Committee showed overall poor satisfaction with their education and poor professional satisfaction with noontime conference. Focus group discussions identified the lack of protected time, constant interruptions for calls from the nursing staff, and the lack of active learning as major limitations to their education. This redesign is part of a trainee-led initiative supported by the Back to Bedside Grant awarded by the ACGME. After implementation, we administered ABIM style quizzes and brief evaluations of the conferences quarterly. We also administered a follow up wellness survey in June 2018. Thirty six trainees completed these questions with an overwhelmingly positive response to the new academic half day. Close to $90 \%$ of trainees looked forward to the academic half day with the remaining percent neutral. Over $85 \%$ agreed that the new half day was better than the previous noontime conferences. A high majority of trainees believed the topics were tailored to their education level/needs, were satisfied with the topics and length of presentation. A high majority also disagreed that having team members leave for the academic half day negatively affected patient care.

DISCUSSION / REFLECTION / LESSONS LEARNED: Major changes require anticipation, culture change and buy-in from all parties. It requires careful planning to find the balance between patient care and education where neither is compromised. Reaching out to other programs to learn from them was an important part of our planning.
THE MASTER CLINICIAN'S ELECTIVE: INTEGRATING EVIDENCE-BASED PHYSICAL EXAMINATION (EBPE) AND POINT OF CARE ULTRASONOGRAPHY (POCUS) IN MODERN CLINICAL MEDICINE

Maria G. Frank $^{1}$; Cason Pierce ${ }^{2}$; Noelle Northcutt ${ }^{3}$; joseph W. keach ${ }^{4}$; Gerard Salame ${ }^{1}$; Rebecca Allyn ${ }^{5} .{ }^{1}$ Denver Health Hospital Authority, Denver, CO; ${ }^{2}$ Denver Health, Denver, CO; ${ }^{3}$ Denver Health, Aurora, CO; ${ }^{4}$ Denver Health Medical Center, University of Colorado School of Medicine, Denver, CO; ${ }^{5}$ Denver Health Medical Center, Denver, CO. (Control ID \#3186385)

NEEDS AND OBJECTIVES: The program aims to create a reproducible elective that combines physical exam and bedside ultrasound as a method of improving resident skills in these techniques. The objectives for learners include: understand the utility and evidence behind physical exam maneuvers, exhibit the ability to execute an EBPE, confirm or challenge clinical diagnosis by skillfully performing POCUS and utilize POCUS to increase the success and safety of medical bedside procedures.

SETTING AND PARTICIPANTS: Our program is offered as an elective rotation at an academic, safety-net hospital in Denver, CO. The course is open to up to six senior Internal Medicine residents per year. Since inception, 9 residents have participated and over 20 are currently on the waitlist for the elective this year.

DESCRIPTION: The two-week curriculum has been offered since 2016 and combines teaching EBPE skills and POCUS. Six faculty members (four for physical exam and two for POCUS) from the division of Hospital Medicine teach the course. Faculty rely upon published literature to determine the highest yield exam maneuvers and bedside ultrasound techniques for establishing commonly encountered diagnoses. Additionally the limited diagnostic value of certain commonly performed exam maneuvers is highlighted. Teaching is done in interactive, small group sessions. To ensure integration, each instructional day provides morning physical exam teaching and afternoon ultrasound training sessions which are focused on a common theme. Enrollment is capped to optimize the trainee to attending ratio.

EVALUATION: Residents perform a practical pre-test and a post-test under direct observation by course faculty. Each resident is measured on the ability to perform pre-determined EBPE maneuvers and POCUS skills. Thus far, in the pre-test, participants have correctly performed an average of $40 \%$ of expected physical exam maneuvers and $32 \%$ of expected POCUS skills. At the conclusion of the elective, all participants have been able to effectively demonstrate $100 \%$ of the highest yield physical exam and ultrasound maneuvers.

DISCUSSION / REFLECTION / LESSONS LEARNED: In the era of high value care, we believe it is imperative for all practitioners to master EBPE and POCUS. Trained faculty and ultrasound resources are limited. While our elective is a collaboration of 4 exam-focused and 2 POCUS providers, it could be run with as few as 1 of each with enrollment limited to four participants. Challenges encountered include faculty availability due to competing clinical duties, accessibility of bedside ultrasound technology and faculty with a level of expertise to teach bedside ultrasound applications.

THE OTHER SBP - TEACHING RESIDENTS FUNDAMENTALS OF NAVIGATING COMPLEX CLINIC AND COMMUNITY SYSTEMS VIA A SYSTEMS BASED PRACTICE CURRICULUM

Arash Nafisi; Sahar Soleymani. Olive View - UCLA Medical Center, Encino, CA. (Control ID \#3184765)

NEEDS AND OBJECTIVES: Medical education in residency programs has often consisted of curriculum that primarily emphasizes traditional medical knowledge, such as the medical treatment of diabetes and obesity. However, 
the changing nature of medicine has required physicians to become increasingly familiar with coordinating patient care within larger health care systems. Thus, it is more important than ever that programs highlight Systems Based Practice as a core competency to emphasize an interdisciplinary approach to chronic disease management and to teach residents how to effectively navigate clinic and community resources for treatment chronic disease and addressing underlying social determinants that impact patient health.

SETTING AND PARTICIPANTS: We developed an outpatient Systems Based Practice (SBP) curriculum for interns in our internal medicine residency program at Olive View - UCLA Medical Center, a safety-net hospital in Los Angeles that cares for vulnerable, underserved patients.

DESCRIPTION: Our yearlong curriculum begins during orientation, where the concept of SBP is defined, key members of the Patient-Centered Medical Home team are introduced and their roles are reviewed. Subsequent sessions occur at the start of each outpatient block for all 10 blocks during intern year. The curriculum is presented in small groups by clinic faculty and includes topics such as inbox management, clinic/community resources and Quality Improvement. Sessions are interactive and often include guest presenters such as social workers (SW), community health workers (CHW) and dietitians.

EVALUATION: To evaluate the effectiveness of this curriculum, we developed a survey that was administered to interns before and after our intervention. We found that at the end of the intervention, interns subjectively reported significantly increased confidence in their ability to navigate system complexities ( $p$ 0.01) and utilize both clinic and community resources to help meet patient needs ( $p<0.001$ ). Residents were also objectively found to navigate clinic and community systems significantly better, correctly identifying resources for alcohol use disorders ( $\mathrm{p}<0.001)$, food and transportation insecurity (p 0.001), behavioral health ( $\mathrm{p}$ 0.002) and referrals to community health workers $(\mathrm{p}<0.001)$.

DISCUSSION / REFLECTION / LESSONS LEARNED: We have developed an SBP curriculum to emphasize the importance of effectively navigating clinic and community resources for treatment of prevalent chronic diseases. After our intervention, interns subjectively reported increased confidence in navigating the system and objectively demonstrated significant gains in understanding and utilizing clinic and community resources to provide optimal care for patients. We also found that interns were more engaged when guest speakers were present. Though we do not have a comparison group, we believe this format has been effective in highlighting this core competency; we plan to continue adapting our SBP curriculum to engage our learners and teach them how to advocate for patients in our community.

\section{THE ROLE OF NARRATIVE MEDICINE AS A MEANS OF RE- FLECTION AMONG INTERNAL MEDICINE INTERNS}

Louisa W. Whitesides; Meredith McAdams; Sheila Quinn; Farah Hussain. Hospital of the University of Pennsylvania, Philadelphia, PA. (Control ID \#3184568)

NEEDS AND OBJECTIVES: Narrative Medicine (NM) is an activity shown to have positive effects on the emotional and professional development of healthcare professionals and has even been shown to decrease burnout. Yet, few sustainable curricula have been developed for residency-level trainees. This study integrates a longitudinal NM curriculum during intern year of an internal medicine residency program with aims to explore its role in fostering empathy and social connectedness among interns. Objective 1: to determine the feasibility and acceptability of incorporating a pilot NM curriculum into existing learning modules for internal medicine (IM) interns. Objective 2: to explore the role of NM in the development of empathy and social connectedness among IM interns
SETTING AND PARTICIPANTS: Each intern participated in a total of 3 one-hour NM sessions embedded in protected didactic time during outpatient blocks over 6 months. Sessions included approximately 12-15 IM interns and were facilitated by a project leader. Fourty-four total interns participated in the curriculum.

DESCRIPTION: Each session focused on 2-3 different pieces of media selected by the investigators (prose, poetry, visual art, or video clips). Interns were provided with the excerpts one at a time. Each excerpt was read or viewed together and followed by a group discussion of salient themes. Interns spent 5-7 minutes writing in response to a prompt. Participants then shared their writing in small groups. Each session focused on a different theme or perspective, for example, the transition from student to intern; characteristics of a "good" doctor; the Inner Critic vs Inner Coach.

EVALUATION: Participants completed an evaluation form after each session. Semi-structured in-depth Interviews are underway to study NM's role in empathy and social connectedness among interns.

DISCUSSION / REFLECTION / LESSONS LEARNED: This curriculum was easily integrated into the intern didactic plan, demonstrating feasibility. Forty-four interns rated the overall quality of the sessions with a mean score of 4.4/5 on a Likert scale of (1) not at all useful to (5) extremely useful. Interestingly, writing was considered to be the least useful component of the session (3.9/5), while group discussion was considered to be the most useful $(4.5 / 5)$. Although this is a relatively small sample in a single setting, preliminary findings suggest that protected time for reflection and discussion among peers is an effective method of processing the challenging experiences in medical training. Reflective writing may also be useful, but preliminarily appears to be a less favorable method. We aim to further understand this concept in the qualitative portion of our study, which is currently underway.

\section{THE TOPSY-TURVY AND WONDERFUL WORLD OF FLIPPED CLASSROOMS. UTILIZING FLIPPED CLASSROOMS IN THE IN- TERNAL MEDICINE CLERKSHIP AS A CURRICULAR INTE- GRATION TOOL.}

Sherine Salib ${ }^{1}$; Beth Nelson ${ }^{2} .{ }^{1}$ Dell Medical School, University of Texas at Austin, Austin, TX; ${ }^{2}$ Dell Medical School at UT Austin, Austin, TX. (Control ID \#3168241)

NEEDS AND OBJECTIVES: Curricular integration of pre-clerkship and clinical material can be challenging. We review one tool that we have found to be effective in facilitating this.

SETTING AND PARTICIPANTS: At Dell Medical School, students follow a non-traditional 4 year curriculum, entering the clerkship year in the second year (MS2) after an accelerated one year pre-clerkship (MS1) curriculum. The MS1 curriculum actively uses problem based learning and interactive large group learning. Students take the USMLE step 1 exam at the end of the second year with an average of five weeks of dedicated study time.

DESCRIPTION: All core clerkships were charged by the Curriculum Committee to incorporate active learning techniques into the clerkship didactics. During our 8-week Internal Medicine Clerkship, we utilize an interactive, case-based "flipped classroom" model to both highlight high-yield clinical curricular components, as well as vertically integrate material from the preclerkship and clinical years. Clinician-educators, several of whom have experience teaching in both settings, help lead these sessions. To make the flipped classrooms effective, students review pre-class material, including the systems-related items from the pre-clerkship year. Additionally, faculty who facilitate these classrooms incorporate questions and cases that help students integrate learning from the pre-clerkship and longitudinal courses such as interprofessional education, population health, and social determinates of health. 
EVALUATION: Our data indicates very good student evaluations of these sessions. For the academic year 2017-2018, the flipped classroom sessions received an overall score of 4.7 out of 5.0 , compared to a score of 3.9 out of 5.0 from the prior year when a more traditional didactic set-up was utilized. Students' mean equated percent correct score on the Medicine NBME exam was 74.9, which is within one point of the national mean, further supporting that there is certainly no harm in the flipped classroom model. Additionally, our early experience with curricular integration, of which these flipped classrooms are just one tool amongst many others utilized in other courses, has resulted in robust step 1 scores. Every member of the 2017-2018 student cohort earned a passing score on the USMLE Step 1 exam, with a mean score of 244 .

DISCUSSION / REFLECTION / LESSONS LEARNED: Our data support better student outcomes in both satisfaction and board exam performance, demonstrating that our students have excelled in this curricular model. Embedding an intentional integration model to align learning goals from the preclerkship and clinical years, in the form of flipped classrooms, is one tool which may help achieve this. Traditional faculty believe they must instruct students in everything they need to learn, whereas in the flipped classroom there is an assumption of trusting our learners to come prepared, taking responsibility for their learning. Ongoing faculty development, as well as robust communication between the various courses are imperative for the success of this model.

\section{TRAINING HEALTH PROFESSIONALS TO BE INSTRUCTORS} OF INTERPROFESSIONAL PRACTICE: A NOVEL APPROACH

Rachel Jantea; Victoria Hornyak; Cassandra Leighton; Teresa Pacella; Rosemary Hoffmann; Sandra Engberg; Susan Meyer; Subashan Perera; Benjamin Reynolds; David M. Elnicki; Debra K. Weiner; Alton E. James; Rollin M. Wright. University of Pittsburgh, Pittsburgh, PA. (Control ID \#3175044)

NEEDS AND OBJECTIVES: Interprofessional (IP) collaborative practice is essential for older adults with complex medical problems and improves health outcomes. Interprofessional education (IPE) is required for students across the health professions, but many preceptors remain untrained in IP practice and instruction. After formal needs assessment, we designed an integrated IP practice + IPE instructor skills training to prepare preceptors to teach IPE competencies to health profession students in the clinical setting.

SETTING AND PARTICIPANTS: Ten health professionals (5 nurse, 2 physical therapy, 2 occupational therapy, 1 speech therapy) at a major academic medical center neurotrauma unit [designated the Interprofessional Dedicated Education Unit (IPDEU)] were trained as IPE instructors in JulAug 2018.

DESCRIPTION: IPE Instructor training focused on IP practice skills and IPE-specific instructor skills. IP practice training included 1) a 1-hour webbased skills module based on the 2016 Interprofessional Education Collaborative Core Competencies, and 2) a 2-hour simulated physician colleague interaction for IP skill practice. IPE-specific instructor training included 1) a 1hour web-based module emphasizing skills to facilitate active IPE-directed observation by students, and 2) a 2-hour simulated IP student interaction for instructor skill practice.

EVALUATION: We measured pre-post change in IP practice and instructor knowledge (multiple-choice test), IP attitudes [Interprofessional Collaborative Competency Attainment Scale (ICCAS) \& Assessment for Collaborative Environments (ACE15)], and IP practice/instructor skills (simulation skill checklists). Composite scores for each measure were analyzed using paired t-tests. Nine participants had complete data. ICCAS \& ACE15 scores increased after training (diff 7.8, $p=0.011 ; 12.2, p=0.049$, respectively). Both IP practice and IP instructor skill use increased after training, with a greater increase in instructor skills (diff $1.86, p=0.0626 ; 2.99, p=0.026$, respectively). Knowledge test scores were unchanged.

DISCUSSION / REFLECTION / LESSONS LEARNED: All measures improved after training, with the exception of knowledge test scores, which were unchanged. Improvement in IPE instructor skills suggests IPE-specific teaching is a new skill that improves with training. Baseline self-rated IP competencies (ICCAS) were higher than was reflected in baseline skill use, indicating a discrepancy between confidence and competence. Both self-rated IP competencies and skill use improved with training. The training was feasible for this small sample. Next steps include evaluation of student outcomes and implementation on a larger scale.

\section{TRAINING MEDICAL RESIDENTS TO BE PHYSICIAN ADVO- CATES IN IMMIGRATION DETENTION HEALTH}

Chanelle Diaz ${ }^{1}$; Tanuja Devaraj ${ }^{1}$; Robyn Winsor ${ }^{1}$; Nathaniel Kratz ${ }^{1}$; Lauren Quijano ${ }^{2}$; Shruthi Rajashekara ${ }^{3}$; Matthew Anderson ${ }^{1} .{ }^{1}$ Montefiore Medical Center, Bronx, NY; ${ }^{2}$ New York Lawyers for Public Interest, New York, NY; ${ }^{3}$ Baylor College of Medicine, Houston, TX. (Control ID \#3184927)

NEEDS AND OBJECTIVES: Every day U.S. Immigration and Customs Enforcement (ICE) detains over 40,000 people in detention facilities where substandard medical care has resulted in poor health outcomes and in-custody deaths. Medical providers can play an important role in advocating for the release of detained immigrants by partnering with lawyers to document health inequities in detention facilities. Medical residents developed a pilot community-based track focused on immigration detention health advocacy. The objectives of this track were to: 1 . Train residents in medical advocacy on behalf of individuals who need medical attention and are at risk of deportation. 2. Expose residents to the realities of mass deportation and its impact on community health. 3. Empower residents to use their voices in public spaces to advocate for immigrant rights.

SETTING AND PARTICIPANTS: Third-year medical residents in a primary care residency program in Bronx, NY. In-person medical assessments took place at local detention facilities under the supervision of an attending physician. Visits to detention facilities were facilitated through an existing immigration-focused community-medical-legal partnership.

DESCRIPTION: Four residents participated in the pilot track, consisting of approximately six half-day sessions. Residents were required to complete a training workshop that provided knowledge and skills in the medical evaluation of individuals in immigration detention, standards of care in detention, unique health needs and barriers to care, and writing affidavits. Residents were expected to complete 1-2 evaluations, either through review of medical records alone or in-person assessment depending on the nature of the request made to us by our legal partners. Based on these evaluations, residents wrote medical affidavits that lawyers used to advocate for the individual's release or access to needed medical care. Residents also met with community-based legal and advocacy organizations that work with individuals at risk of deportation. Completion of an additional advocacy project was optional.

EVALUATION: There are 10 third-year primary care residents; 4 listed the immigration detention health track as their first choice out of 4 options. Four listed it as their second choice. Participating residents completed a 2-hour training workshop. A total of 9 medical evaluations were completed (1-3 per resident). Residents visited 3 community-based organizations (75\% attendance), and rotated at a community-based clinic that serves undocumented minors and families, including families who were separated at the border. One resident published an opinion editorial on medical neglect in immigration detention as an advocacy project. 
DISCUSSION / REFLECTION / LESSONS LEARNED: We report the successful launch of a residency track to develop physician advocates with skills in immigration detention health. Residents gained an in-depth understanding of mass deportation as a public health issue, as well as the impact of immigration status on the mental and physical health of individuals.

\section{TRAINING PROVIDERS FOR THE FUTURE: INTERDISCIPLIN- ARY CARE OF CHRONIC PAIN AND OPIOID MANAGEMENT IN THE AGE OF TELEHEALTH}

Rachel Wong; Patricia Ng; Tracey Spinnato; Kimberly Noel; Erin Dainer; Mark Lerman; Amit Kaushal; Alice Fernan. Stony Brook University School of Medicine, Stony Brook, NY. (Control ID \#3160786)

NEEDS AND OBJECTIVES: Patients with chronic pain, who have a high rate of anxiety and depression, are challenging to manage in primary care. Given the complex biopsychosocial nature of chronic pain, we need to train physicians to provide interdisciplinary care using tools such as telehealth to reduce barriers to care. Our aim is to create and implement an interdisciplinary, longitudinal simulation which integrates team-based telehealth care of complex patients with chronic pain. We will develop metrics for evaluation of residents in synchronous and asynchronous communication skills with patients and relevant specialties. We also aim to study the effect of this interdisciplinary telehealth simulation on the knowledge, attitudes and self-reported behaviors of residents in caring for patients with complex chronic pain.

SETTING AND PARTICIPANTS: The training will be offered to 56 PGY1 and PGY3 Internal Medicine residents at a university-based program in Stony Brook, NY during the 2018-19 academic year.

DESCRIPTION: We developed a longitudinal, 5-week simulation with 1) a virtual tele-visit Observed Standardized Clinical Evaluation (OSCE) with a patient with chronic pain, opioid use and comorbid psychiatric disease, 2) post-visit asynchronous care of the patient using electronic messaging between nursing, social work and subspecialty consultants to manage clinical deterioration and 3) a virtual, interdisciplinary tele-conference to discuss patient care and decision-making.

EVALUATION: Resident knowledge and attitudes regarding telehealth will be measured using pre- and post-simulation surveys. Resident skills will be evaluated through assessment of 1) standardized patient checklists during televisit patient encounters, 2) post-visit written electronic consultation and communication between residents with specialists and patients 3) a post teleconference message from the resident to the patient that summarizes and conveys tele-conference recommendations for management.

DISCUSSION / REFLECTION / LESSONS LEARNED: The educational simulation will be implemented from February to April 2019, with plan for data analysis and reporting in May 2019.

\section{TRAINING THE FUTURE GENERATION OF PRIMARY CARE}

Nautam Ganatra. Michael E DeBakey VA Medical Center, Houston, TX. (Control ID \#3186099)

NEEDS AND OBJECTIVES: The estimated shortage of primary care physicians will be between $8,700-43,100$ by 2030 . Primary care residency positions are increasing less rapidly than other positions. Lower reimbursements are often cited as the reason for shortage of primary care physicians. ACGME requires 130 half days of continuity clinic during 3 years of residency, which is less than half a day a week. Majority of training during the residency is focused towards the hospital medicine, and subspecialties. Residents' continuity with their patients and preceptors is challenging concept.
It can improve the educational experience in ambulatory care and may lead to more trainees to choose primary care as their career choices.

SETTING AND PARTICIPANTS: Presented here are the findings form the academic primary care practice at the New Orleans VA. The practice comprised of four academic internists, who supervised more than 80 residents across all PGY levels, form Tulane University School of Medicine. They were dedicated to the teaching and precepting 8 half days week, and 2 half days week they were provided with administrative time, with 3300 veterans assigned. Nonacademic providers had combined 9400 veterans. They were scheduled for 9 half days of direct patient care, and one half a day of administrative time.

DESCRIPTION: Continuity rate for the residents with their patients in academic clinic were compared to the national average. Diabetes and Hypertension outcomes were compared to the non-academic veterans' panel at the New Orleans VA. This comparison was made during the first four months of second half of the academic year.

EVALUATION: During the year 2017, from January to April, continuity with residents was between 56-59\% at our site, while national average during the same time for residents' continuity was between $43-45 \%$. HTN : BP > 150/ $90 \mathrm{~mm}$ of Hg; (goal < 15\%) [Jan 2017- April 2017] - (Lower The Better) EPRP review The \% of patients who had inadequate $\mathrm{BP}$ control were higher in resident clinic at $19.4 \%$, while non academic teams had $17.5 \%$ of patient with poor BP control. Chi square $=0.0213, p$ value $=0.999$ Diabetes: $\%$ Alc $>9 \mathrm{Or}$ Not Done: [Jan 2017-April 2017] - (Lower The Better) - EPRP Review The \% of poorly controlled DM were better in academic continuity team, $21.30 \%$; compared to non-academic teams combined, at New Orleans VA were 23.30\%. $z$ score $=3.2944, p$ value $=0.001$ Diabetes: $\%$ DM $-B P<140 / 90$, goal $75 \%$ or more [aggregate: Jan 2017- April 2017] - EPRP Review BP control in patients with DM, academic continuity team were at $76.70 \%$, compared to non-academic teams combined at New Orleans VA were $74.90 \% . z$ score $=6.9783, p$ value $=0.00001$

DISCUSSION / REFLECTION / LESSONS LEARNED: This observational data shows better performance of academic clinic in Diabetes care. Hypertension control were better in non academic teams in diabetic patients. In non diabetics, there were better trends in non academic teams. Despite having better than average continuity experience for the residents, the quality metrics outcomes are inconsistent in academic primary care settings.

\section{TRUST ME, I'M AN INTERNAL MEDICINE DOCTOR - A COL- LABORATIVE PROCESS TO DEVELOP SPECIALTY-SPECIFIC EPAS}

Shanu Gupta $^{1}$; Kevin E. O'Brien ${ }^{1}$; Kellee Oller ${ }^{1}$; Robert Ledford ${ }^{1}$; Cuc Mai ${ }^{2}$; Deborah DeWaay ${ }^{1}$. ${ }^{1}$ University of South Florida, St Petersburg, FL; ${ }^{2}$ USF, Tampa, FL. (Control ID \#3184954)

NEEDS AND OBJECTIVES: Since the early 2000s, the medical education community has developed competency-based education to identify the professional roles of a physician in training. Measuring competency requires several steps: identification of tasks, implementation of curricular activities to teach these tasks, and development of measurement tools to determine competence. The AAMC has packaged tasks into generalizable EPAs. Little is known about what evidence GME programs would need to agree with a UME program's decision of entrustment. At our institution, we sought to foster a closer alliance between UME and GME in developing EPAs that deliver an entrusted professional ready to take on specialtyspecific tasks at the start of Internal Medicine residency. Our objectives were to: 1. Identify Internal Medicine specific Entrustable Professional Activities (EPA) 2. Develop a process for implementing, assessing and evaluating an innovative EPA-based curriculum 
SETTING AND PARTICIPANTS: The primary stakeholders in the development process were Internal Medicine Clerkship Directors, the Acting Internship Director, the residency Program Directors and the Chair of Internal Medicine.

DESCRIPTION: Ten percent (10\%) faculty effort was procured to develop EPAs. Department chairs committed to invest in upstream efforts because $20 \%$ of our medical students matriculate into our residencies. Currently, $4^{\text {th }}$ year learners participate in a specialty-specific curriculum. The internal medicine faculty champion worked in partnership with department, clerkship and residency program leadership to describe specialty-specific EPAs using the AMEE EPA guide. Each IM EPA was mapped to AAMC EPAs and core competencies. Assessments for entrustment were interwoven into 4th year track requirements.

EVALUATION: The first year goal is to assign the EPA lead, establish joint meetings with clerkship and program directors, and identifying specialty specific UME EPAs for competency assessment. The 3-5 year goal is to develop a specialty specific EPA program that would be cited on the students' MSPE.

DISCUSSION / REFLECTION / LESSONS LEARNED: We are challenging the idea that all graduating medical students should be general practitioners. This EPA-based curriculum is specialty specific - a strength and a weakness. We gained stakeholder buy-in as $20 \%$ of graduates remain at our institution. For our learners the link between medical school and their specific experience on July 1 is clearer. Although this pilot represents a small cohort, a focused skill set improves feasibility. Institution specificity may reduce generalizability. The collaborative process of developing EPAs opens a channel for true educational continuity. Moving entrustment from a theoretical model to a pragmatic curricular blueprint is a paradigm shift for most clinician educators. We anticipate the need for faculty training in establishing a frame of reference for entrustment in Internal Medicine.

\section{TWITTER REPORT: A NOVEL APPROACH FOR TEACHING CLINICAL REASONING ON SOCIAL MEDIA}

Casey N. McQuade ${ }^{1}$; Michael G. Simonson ${ }^{1}$; Kristen A. Ehrenberger ${ }^{1,}$; Amar Kohli ${ }^{2} .{ }^{1}$ University of Pittsburgh Medical Center, Pittsburgh, PA; ${ }^{2}$ UPMC, Mars, PA. (Control ID \#3178107)

NEEDS AND OBJECTIVES: Social media is increasingly popular for disseminating educational material to busy trainees. Resident report-style learning is particularly valuable, but cases take 30-60 minutes to conduct. We adapted report-style, case-based teaching into an asynchronous format using the Twitter social media platform.

SETTING AND PARTICIPANTS: We developed a Twitter profile, @ MedEdPGH, starting in February 2018 to disseminate educational material. Unconditional email invitations were sent to Internal Medicine residents $(n=200)$ and faculty $(n=165)$ at our institution to encourage participation. The profile was viewable by any Twitter user, including those not at our institution.

DESCRIPTION: Each Friday, the authors posted a brief clinical vignette followed by the question, "What else do you want to know?" to the @MedEdPGH profile. Twitter users responded to the post asking for additional details, and the authors replied in real-time. Participants were asked to explain their clinical reasoning, when applicable, and the authors emphasized clinical reasoning pearls in reply. The authors moderated the discussion so all pertinent aspects of the case were explored, including eliciting summary statements and a differential diagnosis. After the correct answer was elucidated, the case was concluded with relevant teaching points. Online engagement was assessed using Twitter Analytics. Resident and faculty attitudes after 6 months were assessed at our institution using a post-intervention survey instrument.

EVALUATION: Over the 6-month intervention period, the 11 Twitter Report cases were viewed 21,845 times and were engaged by users 1,773 times. A total of 86 Twitter profiles spanning 22 U.S. states and 6 countries contributed to Twitter Report discussions. Participants from all training levels were present, including faculty. The most common clinical reasoning topics discussed were cognitive biases, test characteristics, and diagnostic frameworks. Moderators required 30-90 minutes of total screen-time spaced over a 24-48 hour period to lead each discussion. Of 86 trainees who responded to the post-intervention survey, 22 (15 residents, 7 faculty) reported interacting with Twitter Report. 15 of 22 respondents at least somewhat agreed that participation in Twitter Report increased their clinical reasoning skills. 16 of 22 at least somewhat agreed that Twitter Report increased their clinical knowledge.

DISCUSSION / REFLECTION / LESSONS LEARNED: Twitter Report is effective for engaging learners of all levels in a clinical reasoning discussion on social media. It reached a global audience using a case-based format. Participants reported favorable effects on their clinical reasoning and knowledge. The intervention required time and effort similar to inperson report-style teaching, but the asynchronous format allowed time commitments to be distributed favorably throughout the day for busy trainees and educators. Future iterations will leverage the multimedia capabilities of social media to further illustrate clinical reasoning teaching points. ONLINE RESOURCE URL (OPTIONAL): https://twitter.com/ MedEdPGH

\section{UCONN IMMIGRATION RIGHTS INITIATIVIVE: THE DEVEL- OPMENT OF A STUDENT RUN ASYLUM CLINIC AND AN AV- ENUE FOR REFUGEE ADVOCACY}

susan levine. University of Connecticut, Farmington, CT. (Control ID \#3175910)

NEEDS AND OBJECTIVES: There are over 68 million individuals displaced globally due to human rights abuses. Of these 262,000 applied for asylum in the United States in 2016. As the caps for refugee resettlement continue to diminish and the criteria for asylum status become more restrictive, it is increasingly important that physicians become trained to advocate for this vulnerable population. An individual's liklihood of successfully attaining asylum increases from 38 to $89 \%$ when an affidavit encorporates a trained medical or psychiatric forensic evaluation. A student run asylum clinic is a way to provide critical medical testimony, to teach trainees essential clinical skills for recognizing signs of abuse, and to advocate for vulnerable populations.

SETTING AND PARTICIPANTS: The UConn Immigration Rights initiative is the advocacy arm of a 3 part entity, UConn Immigrant Health. UConn Immigrant Health also includes a faculty run refugee and Immigrant Health clinic as well as a medical student and resident curriculum in refugee health. The UConn Immigration Rights Initiative is a student run asylum clinic wherin medical student trained by Physicians for Human Rights (PHR) perform probono forensic evaluations for attorneys representing clients seeking asylum. In addition to providing forensic affidavits, students work directly with community partners to advocate on a wide variety of immigrant and refugee issues. Students apply the advocacy skills they learn within their medical school community.

DESCRIPTION: The asylum clinic is a collaboration between UConn Law school, PHR and UConn Health. Medical students and supervising faculty attend PHR training which reviews: legal aspects of asylum, 
conduction of an evaluation for physical and psychological symptoms of torture in order to correlate consistency between symptoms and abuse allegations, as well as drafting of an affidavit based on the Instanbul protocol. Student follow cases through to their ajudication, attending case hearings. Student leadership roles in the clinic include executive director, director of education, director of outreach, director of asylum services and board of advisors. Student meetings review pending and upcoming cases, plan community events and provide educational updates in a mini journal club format.

EVALUATION: Asylum affidavit drafts are submitted to PHR for review and then revised with the attorney. Ultimately the success of this clinic will be determined by case records. To date our 2 nd case is awaiting hearing. Our first case resulted in asylum being granted.

DISCUSSION / REFLECTION / LESSONS LEARNED: UConn is one of a small number of medical schools in the country with a student run asylum clnic. What is particularly unique is the integration of this clinic with the educaion and clincal care components of UConn Immigrant health. Thru this integration students learn first hand the power of advocacy and contextualize learning to care for a particularly vulnerable population.

UNTANGLING INPATIENT CARE FROM POLICING: TRAINING TO ADDRESS CARING FOR HOSPITALIZED PATIENTS IN CUSTODY

Jo Henderson-Frost; John Weems; Rachel Simon. Massachusetts General Hospital, Newton, MA. (Control ID \#3186318)

NEEDS AND OBJECTIVES: Patients who are unable to fully communicate his or her clinical or social history due to fear of legal repercussions, stigmatization or threats may receive inferior medical care, despite best intentions of providers. Typical medical education includes discussion of HIPAA and patient privacy, but not necessarily clinical examination of patients in custody and maintenance of patient confidentiality in the presence of correctional officers. We have observed challenges providing confidential medical care in the presence of the correctional officers. We aim to develop and disseminate a training module to educate staff on clinical care and the rights of detained patients.

SETTING AND PARTICIPANTS: To best formulate the training, we are evaluating baseline knowledge, attitudes and practices of resident physicians in internal medicine and emergency medicine at Massachusetts General Hospital. We chose this population because physicians in the emergency room and the internal medicine wards are the most likely to care for hospitalized patients in custody.

DESCRIPTION: In an online survey, participants will be asked about their knowledge on the privacy rights of hospitalized patients in custody by the Department of Corrections; their attitudes toward caring for patients in custody; and their observations of comparative clinical practice in the care of patients in and not in custody. The survey results will be used to develop a training to be offered to all internal medicine and emergency medicine residents physicians.

EVALUATION: In the preliminary phase to design the survey, we asked a smaller group of residents to describe their interactions with correctional officers. In these anecdotes, officers were sometimes asked to step outside of the patient's room and on other occasions remain present during the clinical encounter. With this survey, we will evaluate the knowledge of providers, e.g. does HIPAA applies equally to all patients, to design the content of the training module. We will also evaluate attitudes to address fears and concerns, and experience to design a training that addresses current practices in our hospital. An evaluation of the training module will be subsequently designed with a comparison of pre- and post- knowledge, attitudes and practices.

DISCUSSION / REFLECTION / LESSONS LEARNED: To our knowledge, there are no published studies that evaluate providers' knowledge, attitudes towards and preparation to care for hospitalized patients in custody. This survey evaluates if and how care of patients in custody differs from those not in custody from the perspective of the provider. The survey results will directly inform design of our training module. Participation in this study may also provide space for residents to reflect on their care for patients in custody, particularly if and how the presence of law enforcement personnel in the patient room affects the care they provide.

\section{USE OF SIMULATION TO IMPROVE RESIDENT CONFIDENCE DURING RESPONSE TO IN-HOSPITAL CARDIAC ARREST}

Razia Jayman-Aristide ${ }^{1}$; Sadia Arshad ${ }^{1}$; craig hertz $^{2}$; Erica Beauplan ${ }^{1}$. ${ }^{1}$ Southside Hospital, Bay Shore, NY; ${ }^{2}$ Northwell Health, Lake Success, NY. (Control ID \#3184913)

NEEDS AND OBJECTIVES: In-hospital cardiac arrests (IHCA) occur about 200,000 times per year in the United States with an 18-20\% survival rate. While resident physicians are expected to competently lead an IHCA code team, restrictions on residency duty hours decreases exposure to IHCAs. Research suggests that simulation improves resident confidence, communication and quality of care during IHCAs through deliberate practice in a supportive learning environment. The objective of this study is to evaluate the effect of IHCA simulations on residents' perceived confidence in assuming critical code team roles as well as their perception of the effectiveness of the multidisciplinary code team.

SETTING AND PARTICIPANTS: Six IHCA simulations were conducted over a four-month period on medical floors from January to April 2018. Family medicine residents responded as they would during actual codes as a part of their inpatient service curriculum. Faculty physicians observed the event, giving immediate feedback via a debriefing. Anonymous confidence surveys were sent to residents prior to and after the intervention. Respondents to Simulations included: On Call Residents, Nurses, \& Respiratory Therapists

DESCRIPTION: Simulations were developed in one of two ways - from ACLS (Advanced cardiac life suffort) protocol scenarios or from true occurences within the hospital. ACLS protocols such as identifying ventricular tachycardia vs. ventricular fibrillation, identifying PEA (pulseless electrical activity), and various other arrhythmias were the focus of a portion of the simulations. 'True occurences' within the hospital were used as reflective teaching during simulations.

EVALUATION: Surveys were created using Qualtrics software. A link to surveys was sent to the residents to complete over two-weeks. Pre- and postintervention data were calculated by Qualtrics. PGY-1s felt most comfortable in their "assumed" role as a compressor. Only 25\% of PGY-3 respondents felt comfortable as a team leader. The remaining $75 \%$ felt somewhat comfortable. Approximately $56 \%$ of the PGY-2 respondents felt uncomfortable in the role of a team leader, whereas $33 \%$ felt somewhat comfortable. Post-intervention, across all years of training, overall comfort in every role increased. PGY-1 and PGY-3 residents felt more comfort as team leader, ventilator and medication administrator. PGY-1 residents felt no difference as a defibrillator. PGY-2's noted less overall comfort as the team leader, medication administrator or defibrillator with an unchanged comfort level as the ventilator.

DISCUSSION / REFLECTION / LESSONS LEARNED: Simulation can help address residents' perceived lack of confidence, comfort and 
knowledge in IHCA responses. In this study, it was noted that residents do not feel comfortable assuming the role as team leader. This poses a significant implication in an inpatient setting where residents are in fact expected to be able to lead code blue scenarios. This lack of comfort in assuming the role of a leader can ultimately affect performance during real-time scenarios

\section{USING A GROUP OBSERVED STANDARDIZED CLINICAL EX- PERIENCE (GOSE) TO TEACH MOTIVATIONAL INTERVIEWING}

Barbara Porter $^{1}$; Kelly J. Crotty ${ }^{2}$; Sarah J. Moore ${ }^{3}$; Joanna Dognin ${ }^{4}$; Margaret Horlick ${ }^{4} .{ }^{1} \mathrm{NYU}$ SOM, New York, NY; ${ }^{2}$ New York University, NY, NY; ${ }^{3}$ NYU School of Medicine, New York, NY; ${ }^{4}$ NY Harbor VA Healthcare Center, Brooklyn, NY. (Control ID \#3185304)

NEEDS AND OBJECTIVES: Didactic training in motivation interviewing (MI) lacks efficacy, because opportunities to practice skills while being directly observed are rare. The goal of our educational innovation was to train interns in the advanced communication skills of motivation interviewing through a group observed standardized clinical experience (GOSCE). Our learning objectives were as follows: After an experiential learning session on MI, interns will be able to: 1 . Identify opportunities to use MI with patients 2 . Recognize "change talk" from a patient as an opportunity to use MI techniques 3. Use MI techniques when discussing behavior change with a patient

SETTING AND PARTICIPANTS: 46 internal medicine interns in an academic internal medicine residency program.

DESCRIPTION: Each session began with a 20 minute review of MI for behavior change given by a psychologist trained in Motivational Interviewing. Then, interns participated in a 3 station, one-hour long Group Observed Standardized Clinical Exercise (GOSCE). Interns worked in teams of 3 , and for each station, one of the 3 interns was the active physician, while the other 2 observed the encounter. Each intern had an opportunity to be the active physician for a case. Each case was observed by one or two faculty members, one of whom was a psychologist trained in MI. After a 10 minute interaction with the standardized patient, the active physician received feedback on their MI skills and debriefed the encounter with the faculty and their peers. After the 3 cases, the session concluded with a group debrief and summary of the experience. Interns completed a retrospective pre/post survey to assess the impact of the session.

EVALUATION: Residents reported statistically significant improvement in all domains, including confidence with identifying opportunities to use MI, comfort using reflective and summary statements during MI, and likelihood of using motivational interviewing in future patient encounters. Qualitative comments after the session reflect that residents developed an appreciation for silence as a tool during MI, felt comfortable with tools such as decisional balance, and recognized the value of patient centeredness during MI.

DISCUSSION / REFLECTION / LESSONS LEARNED: Our interprofessional educational team (psychologists and internists) provided different perspective for both learners and our internal medicine faculty. Our residents appreciated practicing skills and receiving feedback in real time. This academic year (one year after the intern GOSCE), these same learners will participate in an OSCE that includes a case requiring motivational internviewing skills, and we will evaluate the durability of motivation interviewing skills taught during this session. We are interested in expanding opportunities to use GOSCE as a low stakes skills practice and development tool.
USING OBJECTIVE STRUCTURED TEACHING ENCOUNTERS (OSTES) TO PREPARE RESIDENTS FOR CLINICAL TEACHING ON THE WARDS

Jacob H. Johnson ${ }^{1}$; Madeleine Matthiesen ${ }^{2}$, ; Zoë Gottlieb ${ }^{2}$; Caitlin N. Li ; $\overline{\text { Arielle Medford }}{ }^{2}$; Nilay Patel ${ }^{2}$; Anna K. Rubin ${ }^{2}$; Ariel Frey-Vogel ${ }^{2}$; Kerri Palamara ${ }^{2}$. ${ }^{1}$ Brigham and Women's Hospital, Boston, MA; ${ }^{2}$ Massachusetts General Hospital, Boston, MA. (Control ID \#3183724)

NEEDS AND OBJECTIVES: Education of peers, students, and health professionals is an important role for residents during their graduate medical training. However, formal methods to improve trainees' clinical teaching are lacking. An Objective Structured Teaching Encounter (OSTE) is a simulated scenario in which participants teach scripted standardized trainees (actors). We previously developed a realistic and accurate 4-case OSTE for summative assessment of resident clinical teaching. This project's objective is to adapt the OSTEs for formative assessment by adding in structured debriefs after each case with the goal of improving clinical teaching.

SETTING AND PARTICIPANTS: Medicine and pediatric residents who lead clinical teams were invited to participate. Multiple sessions were offered in 2017-2018.

DESCRIPTION: The OSTE curriculum contains four cases: Teaching on Rounds, Teaching at the Bedside, Giving a Chalk Talk, and Giving Feedback on an HPI. Cases have up to 3 actors to simulate clinical teaching. The clinical content was adapted based on specialty. A 1-page debrief guide was developed for each case based on a literature review and iteratively updated based on expert opinion. Faculty and actors received 15 minutes of training. For each case, the resident read the prompt and then taught for 8 minutes followed by a 10 minute debrief with the faculty observer and actor(s). The 4-case session lasted 80 minutes.

EVALUATION: Forty-four residents participated, 21 in medicine and 23 in pediatrics; 22 as resident teachers and 32 as actors (10 as both). 12 faculty participated as debriefers. Teachers, faculty, and actors completed surveys with a 97\% response rate. All residents who participated as a teacher would recommend the session. On a 5-point Likert scale, with higher score indicating more agreement, all residents agreed or strongly agreed they felt more prepared to teach on the wards (mean 4.48), and all felt the debrief was helpful (mean 4.61). Teaching take-aways included, "Asking more probing questions to fully assess knowledge" and "Pause longer for answers." Residents cited "Hearing immediate feedback with people we know well" and "Great feedback from actors" as the most effective portion of the OSTE. 97\% of actors agreed or strongly agreed that they learned new teaching skills (mean 4.64) and remarked, "liked participating in the debrief - great way to learn from juniors/attendings/fellow actors" and "Helpful to see the different learners' approaches." All actors felt prepared to portray their part. All faculty, teachers, and actors agreed or strongly agreed that the cases were reflective of actual teaching scenarios. One faculty member noted, "[this case] is realistic and can be taken in so many directions that it captures many of the elements which cause learners and teachers angst."

DISCUSSION / REFLECTION / LESSONS LEARNED: Our 80-minute 4-station OSTE with faculty and actor-led debrief was well-received, feasible and an efficient way to train clinical teachers. Nearly all residents who participated, regardless of role, reported improving their teaching.

USING THE MEDIAN NERVE AS A MODEL FOR INTRODUCING BASIC PRINCIPLES OF POINT OF CARE ULTRASOUND IN INTERNAL MEDICINE RESIDENT EDUCATION

$\underline{\text { Rita Poon }}{ }^{1}$; Christopher T. Kelly ${ }^{1}$; Claudia L. Campos ${ }^{2} .{ }^{1}$ Wake Forest University School of Medicine, Winston Salem, NC; ${ }^{2}$ Wake Forest University, WInston-Salem, NC. (Control ID \#3167769) 
NEEDS AND OBJECTIVES: The structure and content of POCUS curricula in outpatient internal medicine (IM) have not been standardized and are understudied. We conducted a novel hands-on model for an introduction to ambulatory POCUS education during IM residency training. Ultrasound (US) evaluation of the median nerve to diagnose carpal tunnel syndrome may be a promising alternative to nerve conduction studies (LR 5.87). The educational objectives for the session were $1 /$ to successfully locate and measure the median nerve at the wrist 2/ demonstrate principles of US knobology.

SETTING AND PARTICIPANTS: 18 Internal Medicine residents (PG1-3) at Wake Forest Baptist Medical Center in Winston-Salem, NC participated in an introductory POCUS workshop in a classroom environment during noon conference

DESCRIPTION: A 30-minute hands-on POCUS workshop was conducted. Overview of basic US concepts and the median nerve anatomy at the wrist was reviewed in a brief introductory PowerPoint presentation. Residents were then divided into 4 small groups (4-5 participants) with 1 facilitator and 1 POCUS machine in each group. Only the main instructor was proficient in identifying the median nerve. Residents took turns finding the median nerve on each other, while adjusting the gain and depth to obtain an ideal image for measurement.

EVALUATION: The facilitator in each group observed residents using the US and assessed their ability to identify the median nerve, acquire an adequate image, and perform measurements. Residents also completed a survey at the end of the session. By the end of the workshop, 18/18 residents were able to visualize and measure the median nerve. All 18 residents were able to successfully choose the correct transducer, and adjust focus, depth, and gain. All residents either agreed or strongly agreed that this workshop was effective in acquiring basic US principles and gain a better understanding of knobology. DISCUSSION / REFLECTION / LESSONS LEARNED: Using the median nerve at the wrist is an easy and effective hands-on model to teach basic US principles. Following the recommendations from the Consensus on a Canadian Internal Medicine US Curriculum (2014), examination of the median nerve 1 / was selected on basis of clinical or educational need, 2/was feasible (both cognitive and technical components of the curriculum could be reasonably taught and learned in a competency-based manner while minimizing potential risks to patients); 3/was evidence-based. The exam is quick and eliminates the need for standardized patients or simulation models. Instructors do not need prior training in neuro-US.

\section{UTILIZATION OF QUICK RESPONSE FEEDBACK TOOL TO IDENTIFY EARLY STRUGGLING INTERNS AND PROMOTE SU- PERVISING RESIDENT COACHING}

Rita Poon; Adam R. Schertz; Jessica Hollingsworth; George Waits; Andrew Dothard; Hal H. Atkinson; Donna Williams. Wake Forest School of Medicine, Winston-Salem, NC. (Control ID \#3179018)

NEEDS AND OBJECTIVES: Early identification of struggling interns during internal medicine (IM) residency training is difficult, yet critical. Late identification limits opportunities for early intervention and remediation. Formal evaluations are limited by the length and relative rarity of administration. Supervising residents (SR) are an untapped resource for identifying and assisting struggling interns in real time. Here, we describe the implementation of a tool administered weekly utilizing Quick Response (QR) codes for SR evaluation of interns. This tool was adapted from Loyola University IM Residency Program. We aimed to identify struggling interns, and promote SR coaching in areas of concern.

SETTING AND PARTICIPANTS: The QR feedback tool (QR-FT) was administered weekly by chief residents (CR) to SRs rotating on ward services from July 2018 through January 2019. The survey included 11 specific questions about the following areas of intern performance: efficiency, organization, communication, professionalism, and mental health. Evaluees consist of 45 interns, (33 categorical, 12 preliminary). Non-IM interns were excluded. Evaluators consist of senior IM residents who answered "yes" or "no" to each question in the survey where "yes" always indicated a concern.

DESCRIPTION: SR accessed the survey on Google Forms by using their smartphones to scan a QR code brought to the workroom by the CR weekly. The SR spent $\sim 5$ minute per intern on the following tasks: 1) complete tool, 2) explain concerns, 3) receive feedback on how to coach. The CR followed up on each intern's progress next week with the same survey. If an intern had 2 or more concerns for 2 or more consecutive weeks, they were identified as struggling, and the CR began coaching that intern.

EVALUATION: 13 of 45 interns were evaluated each week via the QR-FT yielding a total of 194 responses. 41 interns were evaluated total, with a mean of 4 evaluations each. Concerns were raised on $63.4 \%$ of interns $(n=26)$. Feedback was not provided in 28 of 66 responses (44\%) when a concern was identified. The most common concern related to efficiency: "Has the intern stayed past $6 \mathrm{pm}$ to finish notes?" ( $46.3 \%, \mathrm{n}=19$ interns), and concern was resolved with a mean time of 2.29 weeks. 9 interns were identified as struggling learners via implementation of this tool, as compared to 0 interns identified by the formal evaluations.

DISCUSSION / REFLECTION / LESSONS LEARNED: The QR-FT identified struggling interns, and showed that SR provided feedback half of the time when a concern was identified. These findings indicate that the tool is feasible, timely, and effective in identifying early concerns and represents a platform to improve coaching of struggling interns. Future directions include measuring SR and intern attitudes toward this tool and ensuring SR followthrough on suggested coaching.

\section{VALUE-ADDED MEDICAL EDUCATION THROUGH STUDENT DOCUMENTATION: ADOPTING THE CMS RULE CHANGE IN THE MEDICINE SUB-INTERNSHIP}

Avni Bavishi3; Jennifer Bierman3; Kathryn Hufmeyer1; Celia O'Brien3; Michael Angarone2; Heather Heiman3. 1Northwestern, Chicago, IL; 2Northwestern university Feinberg School of Medicine, Chicago, IL; 3Northwestern University Feinberg School of Medicine, Chicago, IL. (Control ID \#3183130)

NEEDS AND OBJECTIVES: Medical students desire meaningful clinical roles, and educators have advocated "value-added" medical education, in which health systems benefit from the presence of learners. In 2018, The Center for Medicare and Medicaid Services (CMS) revised rules to allow students, with appropriate supervision, to document billable notes for patient encounters. We aimed to determine whether students' perception of their notes' value and of their supervisors' engagement and feedback would change after implementation of the rules.

SETTING AND PARTICIPANTS: Our primary teaching hospital adopted the revised CMS rules in July 2018. In December 2018, we surveyed 48 fourth-year students who completed their medicine sub-internship during the two months before (group 1) and two months after (group 2) the rules were adopted.

DESCRIPTION: Leaders from education, compliance, legal, and information systems developed a hospital-wide policy and workflow permitting billable student documentation of supervised encounters. Students were advised about the change and the intended workflow. Faculty and residents were asked to apply the new policy to medicine sub-intern notes and were provided language for addending student notes. 
EVALUATION: Surveys included 6 structured-response items rated on a Likert scale (1=never, 5=always) and one open-ended item. Forty-five of 48 students (93\%) responded; we evaluated the 35 students placed at the primary teaching hospital. Group 2 sub-interns $(n=18)$ reported that their notes more often served as the team note for their patient (mean 3.9 vs 1.9, $\mathrm{p}<.001$ ), were valuable to the work of the team (mean 3.9 vs $2.7, \mathrm{p}=.01$ ), and were read by attendings (mean 4.0 vs. 3.2, p=.02) when compared with group 1 sub-interns $(\mathrm{n}=17)$. Group 2 sub-interns also reported more frequent resident note review and more feedback from residents and attendings; these changes were not statistically significant. Group 2 sub-interns whose notes usually/always served as the team note $(n=11)$ reported significant increases in all outcomes except attending feedback compared with group 1 sub-interns $(n=12)$ whose notes were rarely/never used as the team note.

DISCUSSION / REFLECTION / LESSONS LEARNED: After implementation of the CMS documentation rule change in the sub-internship, students reported significantly higher perceived value of their notes. We demonstrated trends toward improved feedback, though they did not reach significance in the primary analysis of this small cohort. Our findings suggest that permitting students to take on this clinical role may enhance learning of documentation, a critical entrustable professional activity.

\section{WELLNESS WEDNESDAYS AND MORE: BUILDLING A WELL- NESS CURRICULUM THAT FOSTERS WELL-BEING AND COM- BATS BURNOUT}

Megan A. McGervey1; Susan Vehar1; Cory Chevalier1; Jennifer Ramsey1; Abby Spencer2. 1Cleveland Clinic Foundation, Lakewood, OH; 2Cleveland Clinic, Chagrin Falls, OH. (Control ID \#3181399)

NEEDS AND OBJECTIVES: In an era where physicians experience high rates of depression, burnout, and suicide, it is imperative that training programs support residents using an interdisciplinary approach to foster connection, build coping strategies, and enhance resilience as we work towards systemic change that supports resident well-being. Our objectives were to create change that fosters well-being through leadership roles and scheduling changes, creating learning objectives to educate residents on components of well-being and recognizing burnout, and providing resources to promote a culture of wellness.

SETTING AND PARTICIPANTS: This innovation takes place at a large tertiary care academic medical center that hosts 181 internal medicine residents. All residents receive protected learning time for the wellness curriculum during the longitudinal clinic week using the $4+1$ block scheduling model and during the lecture series delivered at noon conference.

DESCRIPTION: The inftructure for our wellness curriculum was built with the appointment of a Director of Wellness and Well-being and schedule restructure using the $4+1$ model in order to promote work-life balance that includes protected time for sessions that focus on caring for the caregiver. During this Y week model, residents receive protected learning time with a focus on forming relationships, recognizing burnout, and addresses issues specific to physicians in today's age. The curriculum uses a multi-faceted approach targeting the different domains of physical, emotional, social, and professional wellbeing. This has included access to free gym memberships, resident PCP appointments, development of community building activities, weekly "Wellness Wednesday" walk round activities, sessions on nutrition, social media support, resident-led wellness interest groups, and increased awareness and normalization of free caregiver therapy and hospital resources utilizing holistic medicine. It includes resident-led "Wellness Groups" featuring opportunities for food-based gatherings, joint runs, cooking classes, movie nights, camping trips, and plans for a talent show this upcoming spring.
EVALUATION: Surveys were sent to 181 residents indicating that Wellness Walk Rounds and Wellness Wednesday emails have been overwhelmingly popular and are extremely effective means for distribution of upcoming events and resources that promote well-being. Prior to the intervention, few residents were aware of the "Caring for Caregivers" free therapy sessions or free gym memberships. Wellness groups have been created and have grown in size and expanded to 10 different domains. Thus far, metrics for recognition or prevention of burnout have been less effective.

DISCUSSION / REFLECTION / LESSONS LEARNED: Our current initiatives have opened the door to conversation and the potential for collaboration with other residency programs who are interested in this directorship. We are currently creating a tool to assess for burnout during advisor meetings that can be used to focus on residents particularly at risk.

\section{WHAT CAN I DO ABOUT IT?: A PRACTICE-ORIENTED ADVO- CACY CURRICULUM FOR INTERNAL MEDICINE RESIDENTS} Priscilla Wang; Ayrenne Adams; Zoe Tseng. Brigham and Women's Hospital, Brookline, MA. (Control ID \#3186381)

NEEDS AND OBJECTIVES: The critical role of social determinants in affecting health has been well-documented. However, the majority of internal medicine residency training focuses on teaching biologic disease interventions, as opposed to addressing social determinants of health. Brigham and Women's internal medicine residents reported via survey interest in advocating for patients, but hesitancy to do so due to limited experience and lack of awareness of how clinicians can create systemslevel change. The primary objective of our curriculum is to enable internal medicine trainees to graduate residency equipped with foundational knowledge and skills necessary to advocate for patients in all settings, whether in the clinic or a legislator's office. Secondary objectives include teaching self-advocacy as a means of advancing health equity, and increasing resident interest in participating in advocacy during residency and after training.

SETTING AND PARTICIPANTS: Primary care PGY-1 and PGY-2 cohorts at Brigham and Women's Hospital (15 individuals)

DESCRIPTION: We designed a peer-led advocacy curriculum grounded in adult learning theory with active participation. Scheduled during ambulatory blocks, each session features (1) a brief didactic component, (2) interactive breakouts and (3) a group activity to build an advocacy toolkit session-by-session (e.g. targeted electronic medical record "SmartPhrases"). Sessions include: (1) Structural competency as framework to address health inequity (2) Legislative advocacy (3) Self- and patient-advocacy in clinical settings (4) "Hill Day": residents interface with state legislators

EVALUATION: The PGY-2 cohort completed a pre-survey prior to the curriculum, followed by post-session feedback forms. Pre and postresponses were matched and compared using anonymous personal identifiers. The pre-survey revealed that $85 \%$ of participants did not feel that they possessed concrete skills to use for advocacy and $85 \%$ were not comfortable in engaging in legislative advocacy. Themes in post-survey written and verbal feedback included appreciation of interactive sessions and the focus on practical applications, and clear recognition of skillbuilding during sessions. Post-curriculum survey data and PGY-1 data will similarly be analyzed following completion of scheduled sessions.

DISCUSSION / REFLECTION / LESSONS LEARNED: Our curriculum has preliminarily demonstrated that a small number of concise, practice-based sessions can increase resident comfort with engaging in advocacy and likelihood of participating in advocacy in the future. 
WHAT CAN PHYSICIANS DO?: BUILDING AN ADVOCACY CURRICULUM FOR FIRST-YEAR MEDICAL STUDENTS Elizabeth P. Griffiths ${ }^{1}$; Arianne Teherani ${ }^{1}$; Megha Garg ${ }^{2} .{ }^{1} \mathrm{UCSF}$, San Francisco, CA; ${ }^{2}$ San Francisco VA Medical Center, University of California San Francisco, San Francisco, CA. (Control ID \#3181665)

NEEDS AND OBJECTIVES: The role of physicians as advocates is increasingly recognized. However, most medical students still receive little to no training on advocacy skills. Prior exit surveys of University of California, San Francisco (UCSF) medical students indicate that students feel unprepared to engage in advocacy activities. Our objective was to develop and evaluate a novel advocacy curriculum for first-year medical students.

SETTING AND PARTICIPANTS: In 2016, UCSF School of Medicine launched a course in which first-year students explore the social and behavioral sciences. Our new advocacy curriculum was delivered to all first-year medical students beginning in 2016-17, then modified for the 2017-18 year. DESCRIPTION: The new small group session on physician advocacy in 2016-17 included an advocacy debate and opinion writing practice. Based on student feedback, in 2017-18 the session was revised to include a written reflection and discussion of physicians' roles as advocates rather than a debate, and a legislative advocacy role play was added. Discussion included a focus on the level at which students plan to be involved as advocates - on behalf of individual patients, to improve the health system in which they work, and/or for policy change at the county, state, or national level.

EVALUATION: In the 2017-18 year, quantitative data was collected via a matched pre- and post-curriculum survey. Forty-five students (32\%) completed both surveys. We found no significant change in students' views on the importance of advocacy (4.36 vs 4.36 on a 5 point Likert scale, $\mathrm{p}=1.00$ ) or their likelihood of advocacy as future practicing physicians ( $4.22 \mathrm{vs} 4.24, \mathrm{p}=$ $0.74)$. We did find a significant increase in their likelihood of advocacy as medical students ( $4.00 \mathrm{vs} 4.20, \mathrm{p}=0.02)$ and their confidence in their ability to advocate ( 3.58 vs $3.89, \mathrm{p}=0.01)$. We also found that $87 \%$ of students plan to be involved in advocacy beyond the individual patient level (i.e. health system level or beyond) and 58\% plan to be involved in advocacy beyond the health system level (i.e. county, state, or national level).

DISCUSSION / REFLECTION / LESSONS LEARNED: A two-hour small group session on physician advocacy showed significant increases in students' likelihood of involvement in advocacy and their confidence in their ability to advocate. This data is limited by response rate and proximity to the session. We plan to complete qualitative analysis of students' reflections on physician advocacy and repeat surveys as students near completion of medical school to better understand students' views on advocacy, as well as how these views change over the course of training.

WITH HER: WOMEN'S HEALTH EDUCATION FOR INTERNAL MEDICINE RESIDENTS. FIRST YEAR RESULTS FOR AN INNOVATIVE 3-YEAR LONGITUDINAL CURRICULUM

Patricia $\mathrm{Ng}^{1}$; Kimberly A. Kranz ${ }^{2} .{ }^{1}$ Stony Brook University School of Medicine, Port Jefferson Station, NY; ${ }^{2}$ Stony Brook University School of Medicine, Stony Brook, NY. (Control ID \#3160590)

NEEDS AND OBJECTIVES: Recent studies surveying Internal Medicine Program Directors identified women's health to be an essential education topic, but there is limited data about structured women's health curricula for programs who use $\mathrm{X}+\mathrm{Y}$ block schedules. Our program's needs assessment revealed that the majority of our residents were unsatisfied with our current women's health curricula and were not confident in managing core women's health topics. Our study aims to develop and assess a longitudinal women's health curriculum that includes interactive workshops using the "Jigsaw Method" (a cooperative learning strategy where peers are responsible for teaching specific content in teams) and a women's preventive health clinic (WPHC) to improve Internal Medicine residents' knowledge, confidence, and skills in addressing core women's health topics.

SETTING AND PARTICIPANTS: Our curriculum was delivered to 84 categorical residents at a university-based Internal Medicine program in Stony Brook, NY. We implemented only the workshop portion of our curriculum during the first year.

DESCRIPTION: We delivered a 2-hour weekly "jigsaw" workshop over 5 ambulatory blocks, which focused on menopause, osteoporosis, urinary incontinence, and abnormal uterine bleeding. In the Jigsaw Method, residents divide into teams of 4 , their "home base group," and each member is assigned to become an "expert" in 1 of the 4 workshop topics. To become "experts," teams split up to join peers who were given the same assignment and these groups will review readings together. Eventually residents return to their home base group to peer teach their topic and apply their collective expertise to solve patient cases. To review answers, teams compete in a gameshow quiz which allowed facilitators to reinforce main teaching points.

EVALUATION: Participants completed a pre-test and post-test immediately before and after the workshop, and a follow-up survey at the end of the academic year. Surveys included knowledge and confidence questions, and feedback questions about the curriculum. Seventy-four residents $(88.1 \%)$ completed the pre-test, $62(73.8 \%)$ completed the post-test and $53(63.1 \%)$ completed the follow-up survey. At post-test, mean knowledge scores increased from $51.3 \%$ to $69.2 \%(\mathrm{p}<0.0001)$ and the majority (94.6\%) were "very satisfied/satisfied" with the workshop. End of the year results showed less improvement in knowledge scores but there was significant improvement in the confidence of managing menopause, hormone replacement therapy, and urinary incontinence $(\mathrm{p}<0.05)$.

DISCUSSION / REFLECTION / LESSONS LEARNED: Our preliminary results suggest that workshops using the Jigsaw Method may be an effective technique to increase Internal Medicine residents' knowledge and confidence in women's health, but repetition is needed to enhance retention. We plan to continue to develop and assess this longitudinal curriculum and implement the WPHC.

ONLINE RESOURCE URL (OPTIONAL): www.jigsaw.org; www. kahoot.com

\section{WOMEN IN MEDICINE MENTORING AND EMPOWERMENT TRAINING PROGRAM: A NEEDS ASSESSMENT AND INTER- VENTION IN PROGRESS}

Amanda Simone ${ }^{1}$; Melissa Simone ${ }^{2}$; Lauren Block ${ }^{3}$; Nancy A. LaVine ${ }^{4}$. ${ }^{1}$ Northwell Health, Flushing, NY; ${ }^{2}$ University of Minnesota, Minneapolis, MN; ${ }^{3}$ Northwell Health, Lake Success, NY; ${ }^{4}$ Northwell Health, New Hyde Park, NY. (Control ID \#3184965)

NEEDS AND OBJECTIVES: Women face unique challenges throughout their careers as physicians, including disparities in salary and leadership positions. Interventions to address these issues have been made at the faculty level, however little has been done to address the same challenges for female residents. This is concerning, as many decisions determining career trajectory take place during residency. Thus, we conducted a needs assessment on gender in the workplace to establish a mentoring and empowerment training program to support female residents.

SETTING AND PARTICIPANTS: A needs assessment survey was distributed amongst female internal medicine residents within the Northwell 
Health System. There residents were also the participants in our focus group and ongoing training program.

DESCRIPTION: A targeted needs assessment, a combination of 3 validated surveys on workplace/gender culture, burnout and empowerment was sent to female residents. Surveys were sent to 60 female residents, and to date we have a $30 \%$ response rate. Preliminary results show that $69 \%$ of respondents felt that women had fewer opportunities for professional development at work than men, $69 \%$ felt that men receive more organizational support than women, and $87 \%$ stated they would like to receive more support in the workplace. Data collection still is ongoing. These early results reveal an opportunity to provide support and professional development to our female trainees. A focus group of five female trainees identified a need for a "safespace" to discuss issues specific to being female residents. Participants identified desired topics, including negotiation, leading an effective team, work-life integration, public speaking, self-advocacy and addressing microaggressions and gender bias in the workplace. Based on this preliminary data, we developed a 6-session mentoring and empowerment training program for female internal medicine residents. Each session includes a learnercentered workshop on a topic from the focus group, and time for mentoring. Sessions are held at the hospital after working hours for ease of attendance. EVALUATION: Three workshops have been held, with attendance ranging from 5-15 participants. Pre- and post-workshop surveys focused on perceived knowledge and comfort with topics covered will be given at each session, the results of which are forthcoming. The needs assessment survey will be repeated at the end of the year to reassess participant's sense of support and opportunity for career advancement.

DISCUSSION / REFLECTION / LESSONS LEARNED: Our needs assessment shows a need amongst female residents for a place to receive support and career training. Our program provides a learner-centered, innovative way to provide this. Variability in attendance is attributed to variable resident schedules, and we plan to optimize schedules to enhance attendance going forward.

YES WE CAN! A SELF-MANAGEMENT CURRICULUM TEACHING PATIENT SELF-EFFICACY CAN IMPACT PATIENT OUTCOMES IN A RESOURCE POOR, UNDERSERVED ACADEMIC PRIMARY CARE SETTING.

Stacie Schmidt ${ }^{1}$; Erica Sheline ${ }^{2} .{ }^{1}$ Emory University, Atlanta, GA; ${ }^{2}$ Emory University School of Medicine, Atlanta, GA. (Control ID \#3183009)

NEEDS AND OBJECTIVES: 1 . Outline an experiential curriculum for medical students to teach patient self-management skills 2. Enhance learner confidence and satisfaction in helping patients set behavioral health goals. 3 . Demonstrate that student-administered health coaching can improve patient clinical and behavioral health outcomes.

SETTING AND PARTICIPANTS: Our academic, safety net, hospitalbased clinic provides care to uninsured patients with multiple chronic illnesses. To address barriers that underserved patients face towards adopting healthier nutrition habits, our clinic provides weekly Healthy Living Group Classes. These interactive nutrition and behavior based classes are taught by medical student volunteers who also spend class time facilitating patient goal setting.

DESCRIPTION: The Healthy Living class curriculum is continually modified by patient feedback and has been reviewed by nutritionists. Students review the class syllabus outlining topics and educational discussion points for each session. Volunteer students also work with patients via telephone to set, assess, and reframe (if necessary) self-management goals every 1-2 weeks.
EVALUATION: In 2017-2018, more than thirty medical students voluntarily delivered health coaching to patients via weekly group classes or individualized telephone health coaching. Likert-scale survey results show high learner satisfaction; reflections submitted by student volunteers disclose impactful experiences and a deep understanding of barriers patients face in accessing health care. The tele-health coaching pilot (mean duration 4.6 months) conducted by phone involved 27 patients, with 4 patients dropping out. Changes in patient behaviors (goal attainment, medication adherence, and show rates to appointments) as well as patient systolic blood pressures improved during that time period. Preliminary data from 26 patients who attended more than one Healthy Living class between October 2017-September 2018 showed that $73 \%$ of patients lost weight. Of those who lost weight, 10 patients (52\%) lost over $5 \mathrm{lbs}$ each.

DISCUSSION / REFLECTION / LESSONS LEARNED: Experiencebased models may improve medical student confidence in teaching patient self-management skills. Such interventions demonstrate positive clinical outcomes and improved health behaviors for small samples of patients. Our intervention has several limitations including small sample size, no control group, and selection bias as students participate voluntarily.

\section{YOU CAN DO IT: USING A MI CURRICULUM TO ENHANCE STUDENT LEARNING, ENGAGE PATIENTS, AND PROMOTE HEALTHY BEHAVIORS}

Chavon Onumah; Mihir Patel; Matthew Mintz; Namarik Alenezy. George Washington University, Washington, DC. (Control ID \#3184287)

NEEDS AND OBJECTIVES: Describe the fundamental principles, steps, and techniques of Motivational Interviewing. Demonstrate core skills of Motivational Interviewing. Apply Motivational Interviewing skills for health promotion in the clinical setting.

SETTING AND PARTICIPANTS: The curricular initiative was implemented as a core requirement for all clinical clerkship students at a single medical school $(\mathrm{N}=155)$.

DESCRIPTION: Using Kolb's Experiential Learning Cycle as a Model, a 4-part MI curriculum, "Promoting Healthy Behaviors," was implemented in our longitudinal primary care clerkship to enhance learning in motivational interviewing. The curriculum includes self-directed learning, small-group exercises, standardized patient practice, AND a real-life clinical intervention (medication adherence, lifestyle modification, or substance cessation) with patient follow-up and reflection.

EVALUATION: Students completed anonymous surveys regarding their learning experience with the project, their respective patient's goal progress at the time of follow-up, and feedback on the project. Data was analyzed via quantitative and qualitative methods. Three faculty members coded qualitative responses independently through open coding. Disagreement was resolved by discussion and data recoded by a final coding scheme. The authors discussed the coded data to arrive at a final set of themes.

DISCUSSION / REFLECTION / LESSONS LEARNED: Rich reflections were collected through the student evaluations and the following themes across the knowledge, skills, and attitude learning domains emerged: Meeting patients "where they are at" on the Stages of Change scale is key in promoting change. Behavioral change is difficult and takes considerable support and follow-up. Incremental changes, tapping into internal motivation, and addressing barriers aid in success. Motivational interviewing is an impactful way to promote healthy behaviors and important skill to learn. $78 \%$ of patients reported progress towards their selfdirected health behavioral change goal established in the student-led MI encounter at 4 to 6 weeks post-encounter. Over half of students agreed that 
the project helped them learn to effectively counsel patients using a patientcentered approach. Lessons learned: Patient follow-up after the initial MI encounter enhanced learnign and contributed greatly to the transformative process but was also a barrier for some students Students appreciate earlier exposure and expanded opportunities to utilize and receive feedback on MI skills. Conclusion: The Promoting Healthy Behaviors project is an effective method of enhancing multi-dimensional medical student learning in motivational interviewing and engaging patients in behavior change and health promotion. A clinical intervention with follow-up helped students appreciate their potential impact, recognize the difficulties patients experience in moving towards behavioral change, and provided "immediate applicability" for skills learned through the in-class and standardized patient exercises. 